\title{
$42^{\text {nd }}$ EASD Annual Meeting of the European Association for the Study of Diabetes
}

\author{
Copenhagen, Denmark, 14-17 September 2006
}

\author{
Abstracts \\ Index of Oral Sessions \\ OP 1 Novel treatments for diabetes \\ OP 2 Nephropathy - clinical \\ OP 3 Immunopathogenesis of type 1 diabetes - from \\ mice to men \\ OP 4 TCF7L2 - a novel gene for type 2 diabetes \\ OP 5 Beta cell differentiation and neogenesis \\ OP 6 Insulin and nutrient signaling pathways \\ OP 7 Novel therapies - type 2 diabetes \\ OP 8 Predictors and markers of cardiovascular disease \\ and mortality \\ OP 9 Retinopathy \\ OP 10 Novel genes in type 2 diabetes \\ OP 11 Molecular components of the insulin secretion \\ pathway \\ OP 12 Animal models of type 2 diabetes and insulin \\ resistance \\ OP 13 Progression and mortality in type 2 diabetes \\ OP 14 Central control of metabolism \\ OP 15 Inflammation and arterial modelling \\ OP 16 Epidemiology of CVD in type 2 diabetes \\ OP 17 Mechanisms of beta cell dysfunction and death \\ OP 18 Diabetes in pregnancy \\ OP 19 Lessons from clinical studies \\ OP 20 Glucose metabolism and the brain \\ OP 21 Inflammatory mediators in type 2 diabetes \\ OP 22 Mechanisms of prevention of endothelial damage \\ OP 23 Experimental genetics: regulation of insulin \\ secretion \\ OP 24 Fetal programming \\ OP 25 Insulin therapy \\ OP 26 Wound healing - diabetic foot \\ OP 27 Genetics of type 1 diabetes \\ OP 28 Nephropathy - experimental \\ OP 29 Incretin hormone action - animal studies \\ OP 30 Diabetes in childhood \\ OP 31 Inhaled insulin \\ OP 32 Neuropathy \\ OP 33 Mitochondrial dysfunction and insulin resistance \\ OP 34 Molecular mechanisms of obesity \\ OP 35 Second messengers and oscillatory behaviour \\ in beta cell function \\ OP 36 New therapies - pre-clinical \\ OP 37 Hypertension \\ OP 38 Postprandial glucose regulation \\ OP 39 Optimising clinical diabetes care \\ OP 40 Coronary endothelial funcion \\ OP 41 Prediction and prevention of type 1 diabetes \\ OP 42 Pancreas and islet transplantation \\ OP 43 Continuous glucose monitoring \\ OP 44 Transcriptional regulation of beta cell function \\ OP 45 Retinopathy - clinical \\ OP 46 Modulation of inflammatory markers in diabetes \\ OP 47 Highlights in genetics and prediction of diabetes \\ OP 48 Clinical immunology

\section{Index of Poster Sessions} \\ PS 1 Epidemiology of type 1 diabetes I \\ PS 2 Epidemiology of type 1 diabetes II \\ PS 3 Genetics of type 1 diabetes \\ PS 4 The grey zone between type 1 diabetes and type 2 \\ diabetes - LADA \\ PS 5 The spectrum of diabetic disorders \\ PS 6 Incidence and prevalence of type 2 diabetes \\ in different ethnic populations \\ PS 7 Prediction and prevention of type 2 diabetes and \\ its complications \\ PS 8 Type 2 diabetes genes \\ PS 9 PPAR and adiponectin genes \\ PS 10 Obesity genes \\ PS 11 Genetics of insulin resistance and cardiovascular \\ disease \\ PS 12 Cardiovascular risk factors \\ PS 13 "Metabolic syndrome" \\ PS 14 Obesity, abdominal obesity and insulin resistance \\ PS 15 Treatment, metabolic control and outcome \\ in diabetes \\ PS 16 Acute and chronic complications in diabetes
}


PS 17 Cytokines, chemokines and beta cell death

PS 18 Beta cell dysfunction and death in models of type 2 diabetes

PS 19 Beta cell damage and apoptosis

PS 20 Pancreas and islet transplantation

PS 21 Immunopathogenesis of type 1 diabetes

PS 22 Immunology and prediction of type 1 diabetes

PS 23 Alpha and delta cell function

PS 24 Developmental biology of the endocrine pancreas

PS 25 Transcriptional regulation in beta cells

PS 26 Intracellular mediators of insulin secretion

PS 27 Mediators of insulin secretion

PS 28 Novel approaches to restore beta cell mass

PS 29 Novel signaling molecules in beta cell function and dysfunction

PS 30 Lipid metabolism

PS 31 Cardiac metabolism

PS 32 Brain metabolism

PS 33 Mitochondria

PS 34 Effects of glitazones on insulin sensitivity

PS 35 Pathophysiology, metabolism

PS 36 Other hormones I

PS 37 Other hormones II

PS 38 Glucose transport

PS 39 Insulin action - human studies

PS 40 Insulin signaling

PS 41 Insulin action

PS 42 Insulin secretion I

PS 43 Insulin secretion II

PS 44 Human liver metabolism

PS 45 Liver metabolism - animal models

PS 46 Regulation of beta cell function by incretin hormones

PS 47 Metabolic effects of incretin hormones I

PS 48 Metabolic effects of incretin hormones II

PS 49 Inflammation and insulin resistance

PS 50 Metabolic effects of adipocytokines

PS 51 Adipokines - in vitro studies

PS 52 Adipose tissue metabolism

PS 53 Inflammation and adipose tissue

PS 54 Prediction of risk in insulin resistance and diabetes

PS 55 Markers of insulin resistance and metabolic syndrome

PS 56 Metabolic syndrome and lipid lowering in type 2 diabetes

PS 57 Life style interventions in metabolic syndrome and obesity

PS 58 Mechanisms of obesity and weight loss

PS 59 Metabolic effects of exercise

PS 60 GLP-I analogues and incretin effect

PS 61 DPP-4 inhibitors

PS 62 Oral agents: new drugs and new combinations
PS 63 Thiazolidinediones in combination therapy

PS 64 Diabetes in the "real world"

PS 65 Liver disease and transplantation-induced diabetes

PS 66 Hypoglycaemia

PS 67 Diet and natural remedies

PS 68 Novel therapies (pre-clinical)

PS 69 Psychological complications of diabetes

PS 70 Psychiatric complications of diabetes

PS 71 Education in diabetes

PS 72 Social and economic implications of diabetes treatment

PS 73 Quality of care

PS 74 Diabetes management in outpatient care

PS 75 Diabetes in childhood

PS 76 Gestational diabetes

PS 77 Type 1 diabetes in pregnancy

PS 78 Diagnosis and treatment of diabetes in pregnancy

PS 79 Continuous glucose monitoring

PS 80 Inpatient glycaemia / diabetes management

PS 81 Continuous insulin infusion - subcutaneous or intraperitoneal

PS 82 Insulin treatment modalities

PS 83 Insulin trials in type 2 diabetes

PS 84 Insulin trials in type 1 or type 2 diabetes

PS 85 Inhaled insulin

PS 86 Pharmacodynamics of new insulin

PS 87 Genetics of complications

PS 88 Cardiovascular disease - clinical interventions

PS 89 Hypertension

PS 90 Nephropathy - clinical interventions

PS 91 Nephropathy - clinical

PS 92 Erythropoietin and nephropathy

PS 93 Nephropathy - experimental

PS 94 Retinopathy

PS 95 Somatic neuropathy

PS 96 Somatic neuropathy - pathogenesis and treatment

PS 97 Autonomic neuropathy - cardiovascular

PS 98 Autonomic neuropathy - gastrointestinal

PS 99 Diabetic foot

PS 100 Glycation and advanced glycation endproducts

PS 101 Endothelium, oxidative stress and inflammation

PS 102 Pancreas, liver and metabolic syndrome

PS 103 Glitazones: mechanisms, effects and costs

PS 104 Coronary artery disease and assessment of cardiac function

PS 105 Predictors of morbidity and mortality

PS 106 Diabetic cardiomyopathy

PS 107 Peripheral endothelial function

PS 108 Arterial stiffness and remodelling

PS 109 Peripheral arterial disease

PS 110 Erythrocytes, clotting and thrombosis

PS 111 Lipid metabolism and lipid-lowering therapy

PS 112 Brain, bone and skin in diabetes 


\section{OP 1}

\section{Novel treatments for diabetes}

0001

Effects of exenatide compared with twice-daily biphasic insulin aspart in patients with type 2 diabetes using metformin and a sulphonylurea

M. A. Nauck ${ }^{1}$, S. Duran ${ }^{2}$, D. Kim ${ }^{3}$, D. Johns ${ }^{4}$, A. Festa ${ }^{4}$, M. Trautmann ${ }^{4}$;

${ }^{1}$ Clinical Diabetology, Diabeteszentrum Bad Lauterberg, Germany, ${ }^{2}$ Cátedra de Endocrinología, Hospital de Valme, Seville, Spain, ${ }^{3}$ Amylin Pharmaceuticals, Inc., San Diego, United States, ${ }^{4}$ Lilly Research Laboratories, Eli Lilly and Company, Indianapolis, United States.

Background and Aims: In patients with type 2 diabetes (T2D) inadequately treated with oral antidiabetic agents (OADs), studies have shown that exenatide treatment significantly improves glycaemic control with the added benefit of weight reduction. Alternative treatment options include insulin with a formulation containing both intermediate and rapid-acting components. We conducted a 1-y trial to compare the safety and efficacy of exenatide and biphasic insulin aspart 30/70 in patients inadequately treated with metformin (MET) and a sulphonylurea (SU).

Materials and Methods: 66 sites in 13 countries participated in this open-label trial. Intention-to-treat patients with T2D (mean \pm SD: age $58.7 \pm 9.0$ y, $\mathrm{HbA}_{1 \mathrm{c}} 8.6 \pm 1.0 \%$, BMI $30.4 \pm 4.1 \mathrm{~kg} / \mathrm{m}^{2}$, body weight $84.4 \pm 15.7 \mathrm{~kg}$ ) were randomised to exenatide ( $\mathrm{n}=253 ; 5 \mu \mathrm{g}$ BID for $4 \mathrm{wks}, 10 \mu \mathrm{g}$ BID thereafter) or biphasic insulin aspart $(\mathrm{n}=248$; BID doses individually titrated), adjunctive to pre-existing SU/MET treatment. The primary endpoint in this noninferiority trial was change in $\mathrm{HbA}_{1 \mathrm{c}}$ at $\mathrm{Wk}$ 52. The noninferiority margin for the difference between treatments was $0.4 \%$.

Results: The mean dose of biphasic insulin aspart increased from $15.7 \mathrm{U} / \mathrm{d}$ ( Wk 2) to $24.4 \mathrm{U} / \mathrm{d}$ (Wk 52). Both treatments resulted in reductions in $\mathrm{HbA}_{1 \mathrm{c}}$ at endpoint (mean \pm SEM: exenatide $-1.04 \pm 0.07 \%$, biphasic insulin aspart $-0.89 \pm 0.06 \%$; difference $-0.15 \%[95 \% \mathrm{CI}-0.32$ to $+0.01 \%]$ ). A greater proportion of exenatide-treated patients achieved target $\mathrm{HbA}_{1 \mathrm{c}} \leq 6.5 \%$ (exenatide $18 \%$, biphasic insulin aspart 9\%; between-group $p=.002)$ and target $\mathrm{HbA}_{1 \mathrm{c}} \leq 7 \%$ (exenatide $32 \%$, biphasic insulin aspart 24\%; between-group $\mathrm{p}=.078$ ). Exenatide-treated patients experienced a steady decline in body weight $(-2.5 \pm 0.2 \mathrm{~kg}$, $\mathrm{p}<0.001$ ), while those receiving biphasic insulin aspart gained weight $(+2.9 \pm 0.2 \mathrm{~kg}, \mathrm{p}<0.001)$, for a difference between treatments of $-5.4 \mathrm{~kg}(95 \% \mathrm{CI}-5.9$ to $-5.0 \mathrm{~kg}$, $\mathrm{p}<0.001)$. Both treatments reduced fasting serum glucose (exenatide $-1.8 \pm 0.2 \mathrm{mmol} / \mathrm{L}, \mathrm{p}<0.001$; biphasic insulin aspart $-1.6 \pm 0.2 \mathrm{mmol} / \mathrm{L}, \mathrm{p}<0.001)$. Between-group analyses revealed greater reductions in 2-h postbreakfast and 2-h postdinner glucose concentrations in the exenatide group, while biphasic insulin aspart predominantly reduced premeal glucose. Nausea (33\% incidence, $3.5 \%$ withdrawal rate) and vomiting (15\% incidence, $1.6 \%$ withdrawal rate), mostly mild/moderate intensity, were the most common adverse events reported by exenatide-treated patients. Hypoglycaemia rates were not significantly different between treatment groups (exenatide $4.7 \pm 0.7$ events/patient-y, biphasic insulin aspart $5.6 \pm 0.7$ events/patient-y). No severe hypoglycaemia was reported.

Conclusion: Exenatide treatment resulted in overall glycaemic control similar to conventional insulin treatment with biphasic insulin aspart, but without the inconvenience of ongoing titration. Nausea and vomiting were not a significant cause of patient discontinuation from the study. Exenatide reduced fasting glucose, provided better postprandial glucose control, and was associated with weight reduction, making it a potential alternative to biphasic insulin aspart for the treatment of T2D not adequately treated with OADs.

Supported by Eli Lilly and Company and Amylin Pharmaceuticals, Inc.

0002

Safety and effects of a once-weekly, long-acting release formulation of exenatide over 15 weeks in patients with type 2 diabetes

D. Maggs ${ }^{1}$, L. MacConell ${ }^{1}$, D. Zhuang ${ }^{1}$, C. Schnabel ${ }^{1}$, K. Taylor ${ }^{1}$, M. Trautmann ${ }^{2}$, D. Kim ${ }^{1}$;

${ }^{1}$ Amylin Pharmaceuticals, Inc., San Diego, United States, ${ }^{2}$ Lilly Forschung GmbH, Hamburg, Germany.

Background and Aims: Exenatide is an incretin mimetic that improves glycaemic control and reduces body weight when administered twice daily (BID) by subcutaneous (SC) injection in patients with type 2 diabetes treated with metformin and/or a sulphonylurea. This randomized, double-blind, placebo-controlled study assessed the safety, pharmacokinetics and pharmacodynamics of a long-acting release (LAR) formulation of exenatide in 45 patients $(40 \%$ female, age $53 \pm 11$ y, BMI $36 \pm 6 \mathrm{~kg} / \mathrm{m}^{2}$, WT $106 \pm 20 \mathrm{~kg}$, $\mathrm{HbA}_{1 \mathrm{c}} 8.5 \pm 1.2 \%$, FPG $10 \pm 2 \mathrm{mmol} / \mathrm{L}$, diabetes duration $5 \pm 4$ y, mean \pm SD) with type 2 diabetes treated with metformin (MET) or diet and exercise (DE).

Materials and Methods: Once-weekly SC doses of either 0.8 or $2.0 \mathrm{mg}$ exenatide LAR, or placebo (PBO) were administered for $15 \mathrm{wks}$, following a 3-day lead-in period during which patients received either $5 \mu \mathrm{g}$ exenatide or PBO BID by SC injection. 
Results: Dose-related increases in plasma exenatide concentrations were observed, reaching steady-state levels between Wks 6-7. Exenatide LAR 0.8 and $2.0 \mathrm{mg}$ treatment resulted in reductions from baseline in $\mathrm{HbA}_{1 \mathrm{c}}$ $(\%)$ of $-1.4 \pm 0.3$ and $-1.7 \pm 0.3($ mean $\pm \mathrm{SE})$, and in $\mathrm{FPG}$ $(\mathrm{mmol} / \mathrm{L})$ of $-2.37 \pm 0.9$ and $-2.17 \pm 0.5$ compared to corresponding increases of $+0.4 \pm 0.3$ and $+1.0 \pm 0.6$ with $\mathrm{PBO}$ at Wk 15. With exenatide, the proportion of patients achieving an $\mathrm{HbA}_{1 \mathrm{c}} \leq 7 \%$ was $33 \%$ and $86 \%$ with 0.8 and $2.0 \mathrm{mg}$ LAR and $0 \%$ with PBO. Mean reductions from baseline in body weight $(\mathrm{kg})$ were $-0.03 \pm 0.7$ and $-3.81 \pm 1.5$ in the 0.8 and $2.0 \mathrm{mg}$ exenatide LAR arms versus $-0.04 \pm 0.7$ with $\mathrm{PBO}$. The most frequent adverse event during LAR treatment was mild nausea (LAR 0.8, $2.0 \mathrm{mg}$ : 19\%, 27\%; PBO: 15\%) and no severe hypoglycaemia was observed. No adverse events leading to early withdrawal were reported for the LAR treatment arms.

Conclusion: Exenatide administered weekly as an LAR formulation over $15 \mathrm{wks}$ was well-tolerated, improved glycaemic control, and reduced body weight in a doserelated manner in patients with type 2 diabetes.

\section{3}

Liraglutide treatment, blood pressure and biomarkers of cardiovascular risk in patients with type 2 diabetes: 14 weeks monotherapy study

J.-P. Courrèges ${ }^{1}$, M. Zdravkovic ${ }^{1}$, T. Le-Thi ${ }^{2}$,

T. Krarup ${ }^{3}$, O. Schmitz ${ }^{4}$, T. Vilsbøll ${ }^{3}$, R. Verhoeven ${ }^{5}$, I. Buganova ${ }^{6}$, S. Madsbad ${ }^{7}$;

${ }^{1}$ Service de Médecine A, Centre Hospitalier de Narbonne, France, ${ }^{2}$ Novo Nordisk A/S, Bagsværd, Denmark, ${ }^{3}$ Internal Medicine (F), Gentofte Hospital, Hellerup, Denmark, ${ }^{4}$ Internal Medicine (M), Aarhus University Hospital, Denmark, ${ }^{5}$ Poli Interne Geneeskunde, Gelre Ziekenhuizen, Apeldoorn, The Netherlands, ${ }^{6}$ Diabetologická Ambulancia, Žilina, Slovakia, ${ }^{7}$ Endocrinology, Hvidovre University Hospital, Denmark.

Background and Aims: Liraglutide, a once-daily GLP-1 analogue, has been shown to reduce blood glucose and weight in patients with type 2 diabetes. In addition to glucose-lowering effect, the impact of treatment on blood pressure, lipids, adiponectin, leptin, hsCRP, IL-6, TNF- $\alpha$, PAI-1 and BNP was evaluated.

Materials and Methods: 165 type 2 diabetic patients, drug-naive or on single OAD (washed-out for 4 weeks) were randomised $1: 1: 1: 1$ to receive $0.65,1.25$ or $1.90 \mathrm{mg}$ of liraglutide as monotherapy or placebo.

Results: After 14 weeks, significant reduction in SBP vs placebo was observed in liraglutide groups: -7.37 (0.65 mg), $-5.21(1.25 \mathrm{mg})$ and $-7.91 \mathrm{mmHg}(1.90 \mathrm{mg})$
( $\mathrm{p}<0.05$ for all). Change in DBP $(-1.46,-0.59$ and $-2.45 \mathrm{mmHg}$ ) did not reach statistical significance. Reduction in triglycerides was seen in liraglutide-treated patients when compared to placebo, adjusted differences were $-19 \%$ (0.65 mg, $\mathrm{p}=0.03),-15 \%(1.25 \mathrm{mg}, \mathrm{p}=0.09)$ and $-22 \%$ (1.90 mg, $\mathrm{p}=0.01)$. There were no clinically meaningful and consistent differences in total cholesterol, HDL, LDL, ApoB, IL-6, TNF- $\alpha$, leptin and adiponectin levels between liraglutide groups and placebo (a significantly greater reduction in LDL was seen for placebo vs active dose groups, but this was not confirmed for ApoB where difference was only observed in the $1.25 \mathrm{mg}$ dose group). We saw marked effect of liraglutide on PAI-1 levels of $-14 \%(p=0.29),-29 \%(p=0.02)$ and $-25 \%(p=0.05)$ for $0.65,1.25$ and $1.90 \mathrm{mg}$ liraglutide vs. placebo, respectively. Also, a dose-dependent decrease of BNP levels: $-26 \%$ $(p=0.1),-30 \%(p=0.05)$ and $-38 \%(p=0.01)$ vs. placebo for the respective liraglutide groups. Observed dose-dependent reduction in CRP versus placebo $(-3 \%,-12 \%$ and $-20 \%)$ did not reach statistical significance.

Conclusion: Liraglutide treatment was associated with a decrease in blood pressure, triglycerides, PAI-1 and BNP concentrations, but the observed effects must be confirmed in longer-term studies and need to be further explored.

Supported by Novo Nordisk A/S

0004

Fourteen weeks of liraglutide therapy in subjects with type 2 diabetes significantly improves first phase insulin secretion and maximal beta-cell secretory capacity S. Madsbad ${ }^{1}$, B. Brock ${ }^{2}$, H. Perrild ${ }^{3}$, H. H. Lervang ${ }^{4}$, K. Kølendorf ${ }^{5}$, T. Krarup ${ }^{6}$, O. Schmitz ${ }^{2}$, T. Le-Thi ${ }^{7}$, M. Zdravkovic ${ }^{7}$, T. Vilsbø11 ${ }^{6}$;

${ }^{1}$ Endocrinology, Hvidovre University Hospital, ${ }^{2}$ Internal Medicine (M), Aarhus University Hospital, ${ }^{3}$ Internal Medicine, Bispebjerg University Hospital, ${ }^{4}$ Endocrinology, Aarhus University Hospital, Aalborg, ${ }^{5}$ Internal Medicine, Roskilde County Hospital, Køge ${ }^{6}$ Internal Medicine, Gentofte Hospital, Hellerup, ${ }^{7}$ Novo Nordisk A/S, Bagsværd, Denmark,

Background and Aims: Liraglutide is a once-daily s.c. GLP-1 analogue under development for the treatment of type 2 diabetes. The data presented here is from a sub-study to a main study including 165 subjects with type 2 diabetes with the aim of assessing beta cell related parameters

Materials and Methods: Subjects were randomized $(1: 1: 1: 1)$ to four treatment arms (placebo, 0.65, 1.25 and $1.90 \mathrm{mg} /$ day of liraglutide). Prior to study drug administration and after 14 weeks subjects underwent both an insulin modified frequently sampled iv glucose tolerance test 
(FSIGTT) and a hyperglycaemic clamp (20 mM) combined with and i.v. arginine stimulation.

Results: Out of 39 subjects randomized to the sub-study 28 subjects completed the 14 weeks treatment period. In addition a group of 12 matched healthy subjects (BMI, gender and age) underwent the same experimental procedures (FSIGT and clamp) without being treated with liraglutide. The two highest dosages of liraglutide significantly increased the maximal beta cell secretory capacity compared to placebo, by $6.27 \mathrm{pM}(95 \%$ CI $[2.92 ; 9.63])$ $114 \%$ and $7.17 \mathrm{pM}(95 \%$ CI $[3.32 ; 11.01]) \quad 97 \%$ for the $1.25 \mathrm{mg}$ and $1.90 \mathrm{mg}$ doses, respectively $(\mathrm{p}<0.05)$. The same dosages increased the first phase insulin secretion significantly by $11.0 \mathrm{pM} * \mathrm{~h}(95 \%$ CI $[6.6 ; 15.4]) \quad 124 \%$ $(1.25 \mathrm{mg})$, and $9.5 \mathrm{pM} * \mathrm{~h}(95 \%$ CI $[3.5 ; 15.5]) \quad 107 \%$ $(1.90 \mathrm{mg})(\mathrm{p}<0.05)$. Second phase insulin secretion increased significantly at the $1.25 \mathrm{mg}$ dose level, but did not reach statistical significance at the highest dose level ( $\mathrm{p}=0.17$ for the $1.9 \mathrm{mg}$ dose group, and $\mathrm{p}<0.01$ for the $1.25 \mathrm{mg}$ dose group). No treatment related effect on glucose effectiveness or insulin sensitivity as assessed by the minimal model was seen. For all parameters, except 2nd phase insulin secretion, there was a significantly greater response in healthy subjects in comparison to subjects treated with liraglutide.

Conclusion: Once daily treatment with liraglutide markedly improves beta cell function in subjects with type 2 diabetes.

Supported by Novo Nordisk A/S

\section{5}

Sitagliptin monotherapy improved glycaemic control in patients with type 2 diabetes

P. Aschner ${ }^{1}$, M. Kipnes ${ }^{2}$, J. Lunceford ${ }^{3}$, M. Sanchez ${ }^{3}$, C. $\mathrm{Mickel}^{3}$, M. J. Davies ${ }^{3}$, D. Williams-Herman ${ }^{3}$;

${ }^{1}$ Department of Science, Colombian Diabetes Association, Bogota, Colombia, ${ }^{2}$ Clinical Research, Diabetes \& Glandular Disease Research Associates, San Antonio, United States, ${ }^{3}$ Merck \& Co. Inc., Rahway, United States.

Background and Aims: In response to a meal, glucagonlike peptide-1 and glucose-dependent insulinotropic peptide are released and effect several different processes important for glucose control including stimulating glucose-dependent insulin release, suppressing glucagon release, and delaying gastric emptying. These incretins are, however, rapidly degraded by the enzyme, dipeptidyl peptidase-4 (DPP-4). DPP-4 inhibitors are a novel class of oral antihyperglycemic agents currently in development for the treatment of type 2 diabetes. The aims of this pivotal Phase III study were to examine the efficacy and safety of once-daily sitagliptin, a DPP-4 inhibitor, in patients with inadequately controlled type 2 diabetes $\left(\mathrm{HbA}_{1 \mathrm{c}} \geq 7.0 \%\right.$ and $\left.\leq 10.0 \%\right)$.

Methods: This was a Phase III, 24-week, randomized, double-blind, placebo-controlled study. After a drug washout period (for those patients on an antihyperglycemic agent) and a 2-wk single-blind placebo run-in period, 741 patients (ages 18 to 75 years) were randomized to 1 of 3 treatments: placebo, sitagliptin $100 \mathrm{mg}$ q.d., or sitagliptin $200 \mathrm{mg}$ q.d.

Results: For the entire randomized cohort, average baseline $\mathrm{HbA}_{1 \mathrm{c}}$ and $\mathrm{FPG}$ were $8.0 \%$ and $9.6 \mathrm{mmol} / \mathrm{L}$, respectively. After 24 weeks, sitagliptin $100 \mathrm{mg}$ and $200 \mathrm{mg}$ produced significant $(\mathrm{p}<0.001)$ placebo-adjusted reductions in $\mathrm{HbA}_{1 \mathrm{c}}$ $(-0.79 \%$ and $-0.94 \%$, respectively) and fasting plasma glucose (FPG: -1.0 and $-1.2 \mathrm{mmol} / \mathrm{L}$, respectively). Patients with higher baseline $\mathrm{HbA}_{1 \mathrm{c}}(\geq 9 \%)$ had greater reductions in placebo-adjusted $\mathrm{HbA}_{1 \mathrm{c}}$ with sitagliptin $(-1.52 \%$ for $100 \mathrm{mg}$ and $-1.50 \%$ for $200 \mathrm{mg})$ than those with baseline $\mathrm{HbA}_{1 \mathrm{c}}<8 \%(-0.57 \%$ and $-0.65 \%)$ or $\geq 8 \%$ to $8.9 \%(-0.80 \%$ and $-1.13 \%)$. In a meal tolerance test, sitagliptin $100 \mathrm{mg}$ and $200 \mathrm{mg}$ significantly decreased 2-hr post-meal glucose (PPG) compared with placebo (placeboadjusted PPG: -2.6 and $-3.0 \mathrm{mmol} / \mathrm{L}$, respectively). Postmeal insulin and C-peptide AUC, ratio of insulin AUC to glucose AUC, HOMA- $\beta$, and proinsulin/insulin ratio were significantly improved with sitagliptin relative to placebo, suggesting improvements in beta cell function. There was no increased incidence of hypoglycemia or gastrointestinal adverse events with sitagliptin compared with placebo. Minimal reductions from baseline in body weight were observed with sitagliptin $100 \mathrm{mg}(-0.2 \mathrm{~kg})$ and $200 \mathrm{mg}$ $(-0.1 \mathrm{~kg})$; body weight was significantly $(\mathrm{p}<0.01)$ reduced with placebo $(-1.1 \mathrm{~kg})$ compared to sitagliptin.

Conclusions: In this 24 -week study, sitagliptin monotherapy improved glycemic control in the fasting and postprandial states, improved measures of beta-cell function and was well tolerated in patients with type 2 diabetes.

Supported by Merck \& Co. Inc.

0006

Efficacy and safety of sitagliptin added to ongoing metformin therapy in type 2 diabetes patients who were inadequately controlled on metformin alone

G. Meininger ${ }^{1}$, B. Charbonnel ${ }^{2}$, A. Karasik ${ }^{3}$, J. Liu ${ }^{1}$, M. $\mathrm{Wu}^{1}$, A. Meehan ${ }^{1}$;

${ }^{1}$ Merck \& Co. Inc., Rahway, United States, ${ }^{2}$ Hotel Dieu Hospital Centre de Recherche, Centre Hospitalier Universitiaire de Nantes, France, ${ }^{3}$ Endocrinology Institute, Chaim Sheba Medical Center, Tel Hashomer, Israel.

Background and Aims: Sitagliptin, a selective dipeptidyl peptidase-4 (DPP-4) inhibitor, is in development for the 
treatment of type 2 diabetes. Sitagliptin acts by blocking DPP-4-mediated inactivation of active incretin hormones that are released into the circulation following ingestion of a meal. The incretins regulate blood glucose through a number of glucose-dependent mechanisms, including increased insulin release, suppressed glucagon release, and delayed gastric emptying. This Phase III study assessed the efficacy and safety of sitagliptin added to ongoing metformin therapy in patients with type 2 diabetes who were inadequately controlled ( $\mathrm{HbA}_{1 \mathrm{c}} \geq 7 \%$ and $\leq 10 \%$ ) on a maximally effective dose of metformin alone.

Material and Methods: After a screening diet/exercise runin, a metformin dose titration/stabilization period, and a 2week, single-blind, placebo run-in, 701 patients ages 19 to 78 years (mean $\mathrm{HbA}_{1 \mathrm{c}}: 8.0 \%$ ) receiving ongoing metformin $(\geq 1500 \mathrm{mg} /$ day) were randomized to receive the addition of placebo or sitagliptin $100 \mathrm{mg}$ q.d. in a 1:2 ratio for 24 weeks. Patients not meeting predefined glycemic thresholds were provided rescue therapy (pioglitazone) until the end of the study. The efficacy analysis was based on an allpatients-treated population using an ANCOVA and excluded data obtained after glycemic rescue.

Results: After 24 weeks, the addition of sitagliptin to ongoing metformin therapy led to a significant $(\mathrm{p}<0.001)$ mean placebo-subtracted reduction from baseline in $\mathrm{HbA}_{1 \mathrm{c}}$ $(-0.65 \%)$. Treatment with sitagliptin also led to significant $(\mathrm{p}<0.001)$ mean placebo-subtracted reductions from baseline at Week 24 in fasting plasma glucose $(-1.4 \mathrm{mmol} / \mathrm{L})$ and 2-hour post-meal plasma glucose $(-2.8 \mathrm{mmol} / \mathrm{L})$. Fasting insulin and C-peptide, post-meal insulin and Cpeptide AUCs, post-meal insulin/glucose AUC ratio, and QUICKI, were all significantly increased with sitagliptin relative to placebo. HOMA- $\beta$, an index of insulin secretion, was also significantly increased with sitagliptin compared to placebo. In addition, there was a significant decrease in the fasting proinsulin/insulin ratio with sitagliptin relative to placebo, reflecting improved $\beta$-cell function. A significantly smaller proportion of patients in the sitagliptin group required glycemic rescue therapy compared to the placebo group. Additionally, the time to initiation of rescue therapy was significantly delayed in the sitagliptin group compared to the placebo group. Sitagliptin was generally well tolerated, with no increased risk of hypoglycemia or gastrointestinal adverse events compared to placebo. The mean change from baseline in body weight at Week 24 was not significantly different between sitagliptin $(-0.7 \mathrm{~kg})$ and placebo $(-0.6 \mathrm{~kg})$.

Conclusion: In this 24-week study, the addition of sitagliptin $100 \mathrm{mg}$ q.d. to ongoing metformin therapy was well tolerated and provided effective and sustained improvement in $\mathrm{HbA}_{1 \mathrm{c}}, \mathrm{FBG}$, and post-meal glucose, as well as significant improvements in indices of insulin secretion and $\beta$-cell function, in patients with type 2 diabetes who had inadequate glycemic control on a maximally effective dose of metformin alone.

Supported by Merck \& Co. Inc.

\section{OP 2}

\section{Nephropathy - clinical}

\section{7}

Mild renal impairment and cardiovascular outcomes in type 2 diabetes: results from the FIELD study

P. L. Drury on behalf of the FIELD Study Investigators NHMRC Clinical Trials Centre, Sydney, Australia.

Background and Aims: Several studies in non-diabetic patients have shown that even mild degrees of renal impairment predict worse cardiovascular outcomes. We examined this hypothesis in a large cohort of patients with type 2 diabetes using estimated glomerular filtration rates.

Materials and Methods: The FIELD study is a randomised double-blind controlled trial of co-micronised fenofibrate $200 \mathrm{mg}$ daily versus placebo in 9795 patients with type 2 diabetes and mild dyslipidaemia over a median follow-up of 5 years in 63 centres in Australia, New Zealand and Finland. Exclusion criteria included a basal serum creatinine of $\geq 130 \mu \mathrm{mol} / \mathrm{L}$. We studied only the placebo cohort $(\mathrm{n}=4900$, mean age $62,63 \%$ male) to exclude effects of fenofibrate on plasma creatinine and outcomes. Estimated glomerular filtration rate (eGFR) was calculated by Cockroft-Gault $(\mathrm{C}-\mathrm{G})$ and Modification of Diet in Renal Disease method (MDRD)

Results: Total cardiovascular (CV) event rate were calculated according to estimated GFR in bands of $<60,60-90$ and $>=90 \mathrm{ml} / \mathrm{min} / 1.73 \mathrm{~m}^{2}$ or $\mathrm{ml} / \mathrm{min}$ respectively. Patients with $\mathrm{C}-\mathrm{G}$ eGFR $<60$ showed a $\mathrm{CV}$ event rate 1.98 fold that of those with eGFR $>=90$ while those with eGFR of $60-90$ showed a 1.33 fold excess $(p<0.0001$ for both). Parallel analyses with MDRD eGFR gave corresponding hazard ratios of 3.57 and 1.75 respectively ( $<<0.0001$ for both). These effects persisted after correction for systolic and diastolic blood pressure (BP) or for mean BP: ratios 1.78 and 1.27 for $\mathrm{C}-\mathrm{G} ; 3.23$ and 1.75 for MDRD (all $\mathrm{p}<0.0001$ ). The CV event hazard ratios were greater than those seen for macroalbuminuria and microalbuminuria compared with normoalbumninuria, and identified a small group of patients with an extremelyhigh rate of one or more $\mathrm{CV}$ events over 5 years.

Conclusion: We conclude that relatively minor degrees of renal impairment are associated with a worse cardiovascular prognosis and that eGFR, especially by the MDRD method, may be a useful marker for this. It may be simpler, more 
consistent and less modified by treatment than BP or degree of proteinuria, and thus allow more accurate risk assessment and improved targeting of cardioprotective therapy.

\section{8}

Serum prorenin, renin genotype and glycated haemoglobin are predictors of persistent microalbuminuria in type 1 diabetes

P. Hovind ${ }^{1,2}$, W. Hop ${ }^{3}$, J. A. M. Janssen ${ }^{3}$, L. Tarnow ${ }^{1}$, H.-H. Parving ${ }^{1,4}$, J. Deinum ${ }^{3}$;

${ }^{1}$ Steno Diabetes Center, Gentofte, Denmark, ${ }^{2}$ Department of Clinical Physiology and Nuclear Medicine, University of Copenhagen, Rigshospitalet, Denmark, ${ }^{3}$ Department of Endocrinology, Erasmus Medical Centre, Rotterdam, The Netherlands, ${ }^{4}$ Faculty of Health Science, University of Aarhus, Denmark.

Background and Aims: The renin angiotensin aldosterone system (RAAS) is implicated in the development of diabetic nephropathy (DN). The most striking abnormality is an increase in prorenin, the enzymatically inactive precursor of renin. Previously we observed in two separate cross-sectional cohort studies with varying duration of diabetes that increased prorenin and $\mathrm{HbA}_{1 \mathrm{c}}$ predict the development of microalbuminuria, and that renin genotype for a biallelic polymorphism may weakly influence risk of DN. The aim of the present study is to study prorenin, $\mathrm{HbA}_{1 \mathrm{c}}$ and renin genotype in a prospective follow-up study of an inception cohort of 277 patients with newly diagnosed type 1 diabetes.

Materials and Methods: An inception cohort of 277 patients with newly diagnosed type 1 diabetes, consecutively admitted to the Steno Diabetes Center between 1 September 1979 and 31 August 1984. Development of persistent microalbuminuria was the main outcome measure. Serum prorenin, $\mathrm{HbA}_{1 \mathrm{c}}$ and renin genotype were evaluated as predictors for this outcome.

Results: During a median follow-up of 18.0 years (range 1.0-21.5 years), 79 of 277 patients developed persistent microalbuminuria. At baseline sex, median albumin excretion rate, and systolic and diastolic blood pressure differed between subjects that eventually developed microalbuminuria and those that did not (respectively 70 vs. 55\% male, $\mathrm{p}=0.02,11$ vs. $8 \mathrm{mg} / 24 \mathrm{~h}, \mathrm{p}=0.002$, systolic blood pressure 128 vs. $122 \mathrm{~mm} \mathrm{Hg}$ and diastolic blood pressure 80 vs. $76 \mathrm{~mm} \mathrm{Hg}, \mathrm{p}=0.004$ for both). Univariate Cox regression analysis with persistent microalbuminuria as dependent variable showed prorenin, $\mathrm{HbA}_{1 \mathrm{c}}$, mean arterial blood pressure (MABP) (all as time-dependent variables), and sex to be significant covariates. Renin genotype data were available for 223 subjects. In multivariate Cox regression analysis MABP and sex lost significance. In the final model $\mathrm{HbA}_{1 \mathrm{c}}$, serum prorenin and renin genotype (collapsed to absence or presence of one or two copies of the most frequent allele) were significant covariates $(p=0.001,0.008$ and 0.026 respectively). An even stronger covariate than absolute prorenin level was prorenin increase from baseline prorenin (measured in the 1 st or 2 nd year of diabetes), $\beta=3.004, p=0.001$ ). No interaction was observed between prorenin level and renin genotype. A prognostic index, indicating risk of microalbuminuria, can be calculated from the formula: $1.11 *$ renin genotype $(0$ or 1$)+2.344 * \log$ [prorenin] $+0.435 * \mathrm{HbA}_{1 \mathrm{c}}$.

Conclusion: An increase in serum prorenin and $\mathrm{HbA}_{1 \mathrm{c}}$ predicts persistent microalbuminuria, the herald of diabetic nephropathy. Renin genotype appears to modify risk as well, but this does not occur through increased prorenin level. From a clinical point of view our findings allow identification of normoalbuminuric subjects at high risk and should lead to a study that assesses the benefits of starting treatment (e.g. blockade of the RAAS) in such patients.

Supported by the Danish Diabetes Association

0009

Plasma $\alpha$-defensin is associated with diabetic nephropathy and mortality in type 1 diabetic patients

G. Joseph ${ }^{1}$, L. Tarnow ${ }^{2}$, T. K. Hansen ${ }^{1}$, H.-H. Parving ${ }^{2}$, A. Flyvbjerg ${ }^{1}$, J. Frystyk ${ }^{1}$;

${ }^{1}$ Medical Research Laboratories and Medical Department M (Endocrinology \& Metabolism), Aarhus University Hospital, ${ }^{2}$ Steno Diabetes Center, Gentofte, Denmark.

Background and Aims: $\alpha$-defensins are antimicrobial peptides of the innate immune system. They are secreted by neutrophils during phagocytosis. In addition, $\alpha$-defensins are suggested to be involved in atherosclerosis by stimulating binding of lipoproteins and LDL cholesterol to endothelial cells in the blood vessels. By binding to tissueplasminogen activator, $\alpha$-defensins inhibit fibrinolysis, causing thrombotic occlusions. Finally, $\alpha$-defensins may participate in angiogenesis through binding to integrin molecules. To elucidate a potential role of $\alpha$-defensin in micro- and macrovascular complications, we investigated the association between plasma levels of $\alpha$-defensin and the presence of diabetic nephropathy as well as cardiovascular disease (CVD) in type 1 diabetic patients.

Materials and Methods: A total of 391 patients with long lasting type 1 diabetes were recruited through the outpatient clinic at Steno Diabetes Center, Gentofte. Approximately half of the study population $(n=199)$ had diabetic nephropathy, the rest $(n=192)$ had normoalbuminuria. After an overnight fast, venous blood was drawn from an 
antecubital vein and plasma stored at $-80^{\circ} \mathrm{C}$. Occurrence of CVD and ischemic heart failure were evaluated. Blood pressure, se-creatinine, $\mathrm{BMI}, \mathrm{HbA}_{1 \mathrm{C}}$, cholesterol levels and other clinical parameters were measured. To study the association between plasma $\alpha$-defensin, the development of vascular complications and mortality, we prospectively followed the study cohort for more than 10 years. Plasma $\alpha$-defensin was measured using a validated in-house radioimmunoassay (RIA).

Results: The median plasma $\alpha$-defensin concentration at baseline was $258 \mu \mathrm{g} / \mathrm{L}$ (IQR 205-321 $\mu \mathrm{g} / \mathrm{L}$ ). Patients with nephropathy had significantly higher levels of plasma $\alpha$ defensin compared to those with normoalbuminuria: $305 \mu \mathrm{g} / \mathrm{L}$ (IQR 182-263) vs. $223 \mu \mathrm{g} / \mathrm{L}$ (IQR 205-321), $P<0.0001$. This difference remained significant after adjustment for diabetes duration, HbAlc, BMI, blood pressure, UAE and GFR; nephropathy vs. normoalbuminuria: $302 \mu \mathrm{g} / \mathrm{L}(95 \% \mathrm{CI} 287-317)$ vs. $250 \mu \mathrm{g} / \mathrm{L}(95 \% \mathrm{CI}$ 235-266), $P<0.0001$.

During follow-up 76 patients died, hereof 32 from CVD. Patients who died had significantly higher levels of $\alpha$ defensin than survivors: $335 \mu \mathrm{g} / \mathrm{L}$ (IQR 269-394) vs. $245 \mu \mathrm{g} / \mathrm{L}$ (IQR 200-301), $P<0.0001$. From ROC curve analysis, an $\alpha$-defensin level of $310 \mu \mathrm{g} / \mathrm{L}$ was found to be the best predictive cut-off level (sensitivity 0.61 , specificity 0.81 , area under the ROC curve $0.74, P<0.0001$ ). All-cause mortality in patients with plasma $\alpha$-defensin levels $>310 \mu \mathrm{g} / \mathrm{L}$ was $43 \%$ (46 deaths among 107 patients) vs. $11 \%(30 / 282)$ among patients with $\alpha$-defensin $<310 \mu \mathrm{g} / \mathrm{L}$, hazard ratio $4.9(95 \%$ CI 3.1-7.7), $P<0.0001$. Of note, a plasma $\alpha$-defensin level $>310 \mu \mathrm{g} / \mathrm{L}$ remained an independent predictor of mortality, even after adjustment for nephropathy status at baseline and for the possible confounding effects of age, sex, $\mathrm{HgbA}_{1 \mathrm{c}}$, plasma creatinine, BMI, total cholesterol and systolic blood pressure (hazard ratio $2.0,95 \%$ CI 1.1-3.6, $P<0.05$ ).

Conclusion: Our data indicate that plasma $\alpha$-defensin may be related to the development of diabetic nephropathy. Further, our findings suggest that measurements of plasma levels of $\alpha$-defensin may be used as a prognostic factor for nephropathy and mortality in type 1 diabetic patients. Supported by the Danish National Board of Health

\section{0}

Polymorphisms in the adiponectin gene are associated with the incidence of renal event in type 2 diabetic subjects with a 4-year follow-up

R. Jaziri ${ }^{1}$, N. Bellili ${ }^{1}$, I. Porchay ${ }^{1}$, F. Pean ${ }^{1}$, R. Aubert ${ }^{1}$, G. Chatellier ${ }^{2}$, M. Lievre ${ }^{3}$, J. Menard ${ }^{4}$, M. Marre ${ }^{1,5}$,

F. Fumeron ${ }^{1}$;
${ }^{1}$ Inserm U695, Inserm, Paris, ${ }^{2}$ Département d'Informatique Médicale, Hôpital Européen Georges Pompidou, Paris, ${ }^{3}$ Service de Pharmacologie Clinique, Université Claude Bernard, Lyon, ${ }^{4}$ Laboratoire de Santé Publique et Informatique Médicale, Université Paris, ${ }^{5}$ Service d'Endocrinologie, Diabétologie, Nutrition, Hôpital Bichat, Paris, France.

Background and Aims: Adiponectin concentration is decreased in Type 2 diabetic (T2D) subjects, and subjects with coronary artery disease. However the concentration of this adipokine is increased in subjects with renal disease. Our objective was to evaluate the association between three adiponectin gene polymorphisms (SNP), $-11391 \mathrm{G}>\mathrm{A},+45$ $\mathrm{T}>\mathrm{G}$ and $+276 \mathrm{G}>\mathrm{T}$, and the prospective risk of renal event in a cohort of T2D subjects: the Diabhycar study

Materials and Methods: Participants were 3,123 French T2D with high vascular risk from the DIABHYCAR Study (non-insulin-dependent DIABetes HYpertension microalbuminuria or proteinuria, CArdiovascular events, and Ramipril). The participants were older than 50 years and had urinary albumin excretion $\geq 20 \mathrm{mg} / \mathrm{l}$. The renal events incident cases $(n=75)$ were defined as creatininemia doubling and/or end stage renal disease. The subjects were genotyped by using the TaqMan ${ }^{\circledR}$ allelic discrimination and the Molecular Beacons ${ }^{\circledR}$. We achieved survival analysis with multiple adjustment by using the Cox regression analysis.

Results: The -11391 AA genotype was associated with higher baseline urinary albumin excretion $(194.0 \mathrm{mg} / \mathrm{l}$ in AA vs $97.4 \mathrm{mg} / \mathrm{l}$ in $\mathrm{G}$ carriers, $\mathrm{p}=0.002$ ). In this cohort, the risk of incident renal event was higher in $-11391 \mathrm{~A}$ carriers (HR 1.89 [1.11-3.20], $\mathrm{p}=0.019$, adjusted for baseline values of HbA1c, HDL-cholesterol, log [creatinine], log [CRP] and $\log$ [urinary albumin excretion]), and higher in $+45 \mathrm{G}$ carriers (HR 2.18 [1.31-3.60], $\mathrm{p}=0.003$ ). The highest risk was found for the combination of both alleles $(+45 \mathrm{G} /$ $-11391 \mathrm{~A}$ vs $+45 \mathrm{~T} /-11391 \mathrm{G}$ : OR 3.61) indicating that the effects of the two polymorphisms were cumulative. Furthermore, the $+45 \mathrm{~T}>\mathrm{G}$ and $-11391 \mathrm{G}>\mathrm{A}$ SNPs were associated with the 4-year increase in creatinine levels (respectively $p=0.001$ and $p=0.043$ ). The $+276 \mathrm{G}>\mathrm{T}$ SNP was associated neither with the incidence of renal events nor with variations in serum creatinine levels.

Conclusion: Our results show that adiponectin genetic polymorphisms are predictive for the incidence of renal complications in type 2 diabetic patients. Our data support a causal role of the adiponectin in the incidence of renal disease. 
Pharmacogenetic association of the ACE/ID polymorphism on renal outcome and death in relation to losartan treatment in patients with type 2 diabetes and nephropathy H.-H. Parving ${ }^{1}$, D. de Zeeuw ${ }^{2}$, M. Cooper ${ }^{3}$, N. Liu ${ }^{4}$, J. Lunceford ${ }^{4}$, S. Shahinfar ${ }^{4}$, P. Wong ${ }^{4}$, P. Lyle ${ }^{4}$, B. Brenner;

${ }^{1}$ Steno Diabetes Center, Gentofte, Denmark, ${ }^{2}$ Department of Clinical Pharmacology, University of Groningen, The Netherlands, ${ }^{3}$ Department of Medicine, University of Melbourne, Australia, ${ }^{4}$ Merck Research Laboratories, West Point, United States, ${ }^{5}$ Renal Division, Brigham \& Women's Hospital, Boston, United States.

Background and Aims: The RENAAL study showed that losartan treatment significantly reduced renal outcomes in patients with type 2 diabetes and nephropathy. We investigated the whether an insertion (I)/deletion (D) polymorphism in the angiotensin-I-converting enzyme (ACE) gene predicts renal outcomes and death and influences with the effect of losartan in the RENAAL study. Materials and Methods: A total of 1435 (95\%) out of 1513 patients enrolled in this randomized, double blind study comparing losartan with placebo on top of conventional blood pressure lowering therapy were available for this investigation. The primary outcome was the composite of doubling of baseline creatinine concentration, end stage renal disease (ESRD) or death. This endpoint was analyzed using a stratified COX regression model with terms including treatment, geographic region, ACE/ID genotype and treatment-by-genotype interaction.

Results: The composite endpoint was lower in II vs. ID and $\mathrm{DD}, 17.5 \%$ and $38.1 \%, \mathrm{P}=0.029$. The individual components of this endpoint showed the same trend. The adjusted risk reduction for losartan versus placebo for the composite endpoints was 5.8\% (95\% CI $-23.3,28.0), 17.6 \%$ (3.8, $29.4)$ and $27.9 \%(7.0,44.1)$ for II, ID and DD respectively. A significant interaction between losartan treatment and ACE/ID genotypes was demonstrated for the risk reduction for ESRD of $3.1 \%, 30.5 \%$ and $50.4 \%$ for II, ID and DD respectively $(\mathrm{P}=0.032)$. A similar trend was demonstrated for the other endpoints.

Conclusion: Proteinuric Type 2 diabetic patients with the $\mathrm{D}$ allele of the ACE/ID polymorphism have an unfavorable renal prognosis which can be mitigated and even improved by losartan.

Supported by Merck
Improved survival of patients with diabetes and endstage renal disease in Denmark

V. R. Sørensen ${ }^{1}$, E. Mathiesen ${ }^{2}$, J. Heaf ${ }^{3}$,

B. Feldt-Rasmussen ${ }^{1}$;

${ }^{1}$ Department of Nephrology, Rigshospitalet, Copenhagen,

${ }^{2}$ Department of Endocrinology, Rigshospitalet, Copenhagen,

${ }^{3}$ Department of Nephrology, Herlev Hospital, Copenhagen, Denmark.

Background and Aim: Treatment in patients with diabetic nephropathy has improved during the last 15 years. Whether life expectancy of diabetic patients with end-stage renal disease (ESRD) has improved is unknown. The aim of our study was to investigate the survival of diabetic patients with ESRD in Denmark in the time period from 19902004, and to evaluate the effect of type of diabetes, transplantation, age and calendar time.

Materials and Methods: Data were obtained from The Danish National Registry; Report on Dialysis and Transplantation, where all patients initiating active treatment for ESRD has been registered and followed up since 1990. ESRD is defined as need of chronic dialysis or renal transplantation. The patients were stratified into 3 groups according to the 5-year period during which renal replacement therapy was initialized. Log-rank test was used to compare survival rates and cox-regression to estimate proportional hazards.

Results: A total of 8618 patients (1123 (13\%) with type 1 diabetes (T1DM), 719 (8\%) with type 2 diabetes (T2DM) and 6776 (79\%) without diabetes (non-DM)) initiated renal replacement therapy during the study period. Mean age (years)(SD) was: T1DM: 50(14), T2DM: 66(9), non-DM: 58(17). The overall patient survival rate is shown in figure 1. The survival of T1DM patients has approached that of non-DM patients and was superior to patients with T2DM. Receiving a renal transplantation was the most important predictor of survival with $80 \%$ lower mortality risk (hazard ratio $(\mathrm{HR})=0.2095 \% \mathrm{CI}: 0.18-0.22)$ compared to patients on dialysis. Patients with DM (T1 and T2) had a 56\% increased mortality risk $(\mathrm{HR}=1.56 \mathrm{CI} 1.46-1.68)$ compared to non-DM patients, whereas the HR for age was 1.4 (CI: 1.36-1.43) per decade of increasing age. Survival improved during the study period with a $21 \%$ lower mortality risk $(\mathrm{HR}=0.79 \mathrm{CI}$ : $0.73-86)$ in patients with DM (T1 and T2) and a $15 \%$ lower mortality risk in non-DM patients $(\mathrm{HR}=0.87 \mathrm{CI}$ : 0.83-0.91) per 5 calendar years. The percentage of patients who received a transplantation was: T1DM: 27\%, T2DM: 5\% and non-DM: 26\%. The survival of transplanted patients with DM (T1 and T2) compared to non-DM patients at one year was: $95 \%$ vs. 93\%, 5 years: 
$80 \%$ vs. $85 \%$ and 10 years: $52 \%$ vs. $71 \%$. There was no difference in graft survival between the two groups.

Conclusion: The survival of diabetic patients with ESRD has markedly improved during the past 15 years in Denmark. The overall survival of T1DM patients has approached that of non-DM patients and the percentage of patients who were receiving a renal transplantation was similar among T1DM and non-DM patients. The 5-year survival after renal transplantation in DM (T1 and $\mathrm{T} 2$ ) patients was $80 \%$ and comparable to the survival in patients without diabetes.

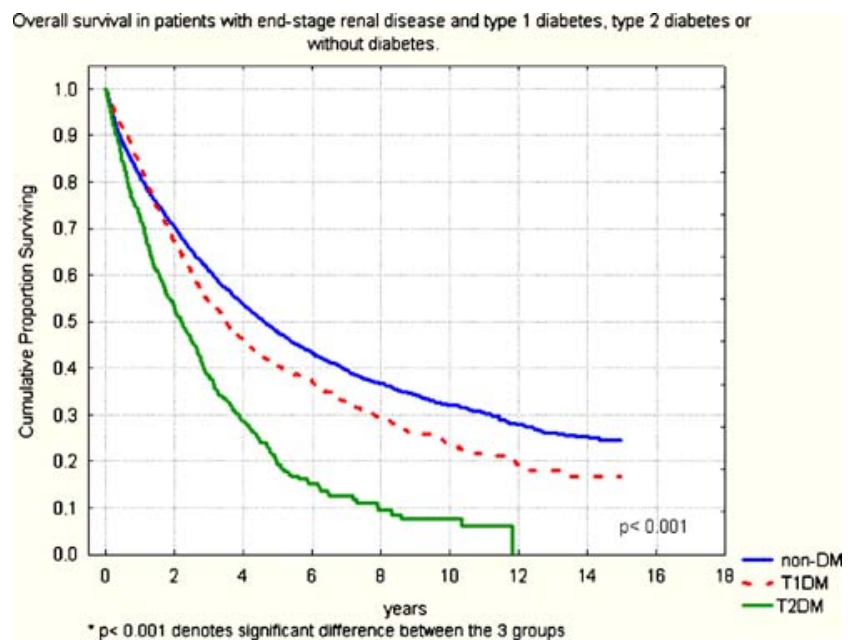

Supported by Nyreforeningen, Diabetesforeningen

\section{OP 3}

\section{Immunopathogenesis of type 1 diabetes - from mice to men}

\section{3}

\section{Peripherin is the major neuroendocrine autoantigen targeted by islet-infiltrating B-lymphocytes in NOD mice}

M. C. Puertas, J. Carrillo, X. Pastor, R. M. Ampudia, A. Alba, R. Planas, M. Vives-Pi, J. Verdaguer;

Laboratory of Immunobiology for Research and Diagnosis (LIRAD-BST), Institut d'Investigacio Germans Trias i Pujol, Badalona, Spain.

Background and Aims: B-lymphocytes migrate to the pancreatic islets during T1D development. Our current appreciation of the antigenic repertoire of autoreactive B-cells in T1D almost exclusively stems from studies of the antigenic specificity of circulating islet-reactive autoantibodies and from analyses of the antigenic specificity of peripheral B-cell hybridomas from human blood or rodent spleen. In order to characterize the antigenic specificity of islet-infiltrating Blymphocytes during the progression of the disease, we generated hybridoma cell lines of these infiltrating B-cells rom NOD and (NODxNOR)F1 mice. We found that a prevalent number of hybridomas $(55,8 \%)$ produced MoAbs restricted to peripheral nervous system (PNS) elements. The aim of the present study has been to characterize the molecular nature recognized by these MoAbs.

Materials and Methods: On neuroblastoma and insulinoma cell lines, MoAbs anti-PNS displayed a reticular intracellular staining. Then we pursued a fractioned protein extraction protocol from these cell lines, western blot immunodetection on protein extracts, isolation and trypsin digestion of the spot recognized by MoAbs, and subsequent mass-spectrometry analysis. An intermediate filament network-defective cell line was transfected with plasmids encoding the peripherin isoform Per56, Per58 or Per61. Specificity of MoAbs for every isoform was assessed by Western blot analysis.

Results: Interestingly, our studies revealed that peripherin was the autoantigen recognized by all the MoAbs (21/21). Peripherin is an intermediate filament protein expressed in PNS as well as in pancreatic beta cells. Three peripherin isoforms generated by alternative splicing have been described. Our studies indicate that all MoAbs target Per58 and Per 61 isoforms, whereas only one (1/21) binds Per56 isoform as well. Since Per56 isoform differs from Per58 and Per61 in the C-terminal aminoacidic sequence, the results strongly suggest that the epitope recognized by most of MoAbs is found at the carboxiterminal end of the Per58 and Per 61 isoforms.

Conclusions: Peripherin is the major neuroendocrine autoantigen targeted by islet-infiltrating B-lymphocytes. The dominant(s) epitope(s) of peripherin recognized by islet-infiltrating B-lymphocytes maps at the carboxi-terminal region of the protein.

Supported by Fondo de Investigaciones Sanitarias (FIS 03/ 0775) and Fundación Ferrer para la Investigación

\section{4}

\section{A unique $\mathrm{CD204}^{+}$subpopulation of dendritic cells in} NOD mice

K. Takahashi ${ }^{1}$, J. Satoh ${ }^{2}$, Y. Oka ${ }^{1}$;

${ }^{1}$ Division of Molecular Metabolism and Diabetes, Tohoku University, Sendai, ${ }^{2}$ Department of Diabetes and Metabolism, Iwate Medical University, Morioka, Japan.

Background and Aims: Dendritic cells (DC) are professional antigen presenting cells, and central to the immune system. DC display defective function and phenotype in human type 1 diabetes, as well as in NOD mice and BB rats, 
and possibly play crucial roles in the pathogenesis of this disease. To characterise molecular changes in $\mathrm{CD} 11 \mathrm{c}^{+}$bone marrow (BM)-derived DC from NOD, we recently compared transcript profiles of these cells with those from normal NON mice, and then found that BMDC from NOD express significantly higher level of CD204 (macrophage scavenger receptor type A) than those from NON. Interestingly in monkeys, CD204 on DC reportedly mediates a unique capacity to capture antigens from live cells, a process referred to as nibbling. Given that CD204 intervenes in this process irrespective of race, strong expression of CD204 on DC from NOD possibly leads to active antigen uptake from autologous cells, and then to the predisposition to autoimmune diseases. Focusing on this point, we here investigated the phenotype and function of CD204 ${ }^{+}$BM and splenic DC from NOD, and the role of this molecule in nibbling.

Materials and Methods: Bone marrow cells from 6-weekold female mice were cultivated in the presence of GM-CSF and IL- 4 over 6 days. CD11 ${ }^{+}$DC were sorted by magnetic beads-conjugated anti-CD11c antibodies (MACSTM). BM and splenic DC were phenotyped by a flow cytometer. Syngeneic mixed lymphocyte reaction was performed coculturing CD204 ${ }^{+}$or CD204 ${ }^{-}$DC sorted with FACSAria ${ }^{\mathrm{TM}}$ and syngeneic $\mathrm{CD}^{+} \mathrm{T}$ cells, and then measured with the MTT assay. CD11 ${ }^{+}$BMDC were dual labeled with the fluorescent dyes $\mathrm{DiO}$ and $\mathrm{DiD}$, and nibbling was evaluated by monitoring the trafficking of plasma membrane between labeled and unlabeled cells.

Results: BMDC from 6-week-old female NOD displayed 7-8 times higher mean fluorescence intensity of CD204, than normal strains (NOD $65.7 \pm 3.4, \mathrm{NON} 8.2 \pm 0.34, \mathrm{C} 3 \mathrm{H} /$ $\mathrm{HeN} 8.0 \pm 0.55$, Balb/c 9.0 $\pm 0.49, \mathrm{p}<0.05$, Mann-Whitney U test). Among splenocytes, the proportion of $\mathrm{CD} 204^{+}$ CD11 $\mathrm{c}^{+}$DC from 6-week-old female NOD was significantly higher than normal strains (NOD $0.65 \pm 0.09 \%$, NON $0.10 \pm 0.02 \%, \mathrm{C} 3 \mathrm{H} / \mathrm{HeN} 0.10 \pm 0.02 \%$, Balb/c $0.04 \pm 0.03 \%$, $\mathrm{p}<0.05$ ). The phenotype of $\mathrm{CD} 204^{+} \mathrm{CD} 11 \mathrm{c}^{+} \mathrm{BM}$ and splenic DC was $\mathrm{CD} 4{ }^{10 \mathrm{w}} \mathrm{CD} 8^{-} \mathrm{CD} 11 \mathrm{~b}^{10 \mathrm{w}} \mathrm{CD} 16 /$ $32^{+} \mathrm{CD} 19^{\text {low }} \mathrm{CD} 80^{\text {high }} \mathrm{CD} 86^{\text {high }} \mathrm{MHC}$ class $\mathrm{I}^{+}$class $\mathrm{II}^{+} \mathrm{F} 4 /$ $80^{+} \mathrm{Gr}-1^{\text {low }}$. CD204 ${ }^{+} \mathrm{CD} 11 \mathrm{c}^{+} \mathrm{DC}$ showed equivalent capacity to stimulate syngeneic $\mathrm{CD}^{+} \mathrm{T}$ cells to $\mathrm{CD} 204^{-}$ CD11c ${ }^{+}$DC (Splenic DC, OD $0.10 \pm 0.03$ vs $0.10 \pm 0.10$; BMDC, OD $0.26 \pm 0.02$ vs $0.25 \pm 0.01$, stimulator: responder $=1: 100$, NS). Unlabeled BMDC from NOD acquired fluorescently dual labeled membrane lipid from other cells more rapidly than those from NON (Figure). The trafficking of plasma membrane was blocked with polyG, one of CD204 ligands.

Conclusion: We propose that CD204 highly expressed in a unique subpopulation of DC from NOD possibly leads to active uptake of self-antigens, and then to the predisposition to autoimmune diseases in NOD. The effect by genetic disruption of CD204 on NOD is now under investigation.

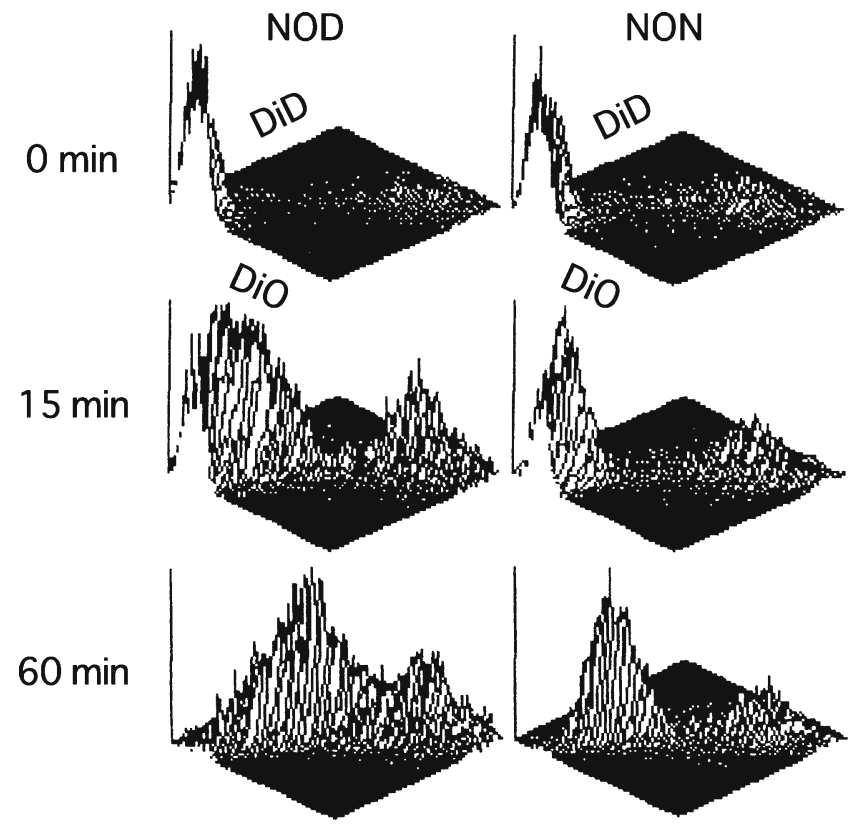

Figure Flow cytometric analysis of plasma membrane trafficking between BMDC dual labeled with DiO and DiD and those unlabeled.

Supported by the Grant-in-Aid for Scientific Research organised by the Japan Society for the Promotion of Research \#17590911

0015

Unaltered development of insulitis and diabetes in vitamin $D$ receptor KO NOD mice

C. Gysemans, E. van Etten, L. Overbergh, A. Giulietti,

G. Eelen, R. Bouillon, A. Verstuyf, C. Mathieu;

Experimentele Geneeskunde, LEGENDO, Leuven, Belgium.

Background and Aims: 1,25-dihydroxyvitamin $D_{3}$ or active vitamin D prevents type 1 diabetes in animal models, modulates dendritic cell differentiation and induces regulatory $\mathrm{T}$ cells. On the other hand, vitamin $\mathrm{D}$ deficiency increases the incidence of type 1 diabetes in geneticallypredisposed individuals and results in impaired maturation of bone marrow-derived macrophages. This steroid hormone, which is also produced in macrophages and dendritic cells, acts through specific binding to a member of the nuclear receptor family, the vitamin D receptor (VDR). The aim of the present study was to evaluate the effect of eliminating VDR in non-obese diabetic (NOD) mice on disease pathogenesis. 
Materials and Methods: This was done by introducing the VDR-null mutation onto the NOD genetic background, by backcrossing $\mathrm{VDR}^{-1-}$ mice $(\mathrm{C} 57 \mathrm{BL} / 6)$ into the NOD strain for 14 generations.

Results: VDR-deficient NOD mice (on regular diet containing $1 \%$ calcium, $1.25 \%$ phosphorus and $2500 \mathrm{IU} / \mathrm{kg}$ vitamin D) had typical features of vitamin D-dependent rickets type II, including hypocalcaemia, osteomalacia, and alopecia. $\mathrm{VDR}^{-1-}$ NOD mice had growth retardation as reflected by decreased body weight during development. Despite these characteristics, lack of VDR did not affect the development of islet cells and the general morphology of islets in NOD mice as compared to wild-type mice, in contrast to previous observations in NOD mice lacking the ligand, vitamin D. Moreover, VDR-deficient NOD mice developed insulitis similar to VDR-sufficient NOD mice. Development of diabetes by the age of 250 days in the N10 generation was identical between VDR-deficient and sufficient NOD mice, in final outcome (in females $70 \%$ vs $80 \%$, NS; in males $40 \%$ vs $40 \%$, NS) as well as in kinetics (onset of diabetes; in females $142 \pm 18$ vs $139 \pm 13$ days, NS; in males $163 \pm 7$ vs $166 \pm 10$ days, NS). The cumulative diabetes incidence in mutant females remained unchanged after increasing the number of backcrosses to N14 (69\% vs $70 \%$ in wild type females), as was the case in males $(30 \%$ vs $38 \%$ in wild type males, NS). As expected, no major immune defects between $\mathrm{VDR}^{-/-}$and $\mathrm{VDR}^{+/+} \mathrm{NOD}$ mice were observed at 100 days of age. Lymphocyte subset composition in different immune organs was normal, apart from a profound decrease in immature $\mathrm{CD} 4^{-} \mathrm{CD} 8^{-}$and regulatory $\mathrm{CD} 4{ }^{+} \mathrm{CD} 25^{+} \mathrm{T}$ cells. Splenocyte proliferation was normal after aCD3 stimulation, but a lower response to alloantigens was observed in a mixed lymphocyte reaction. No differences in phagocytic capacity of macrophages and neutrophils were observed between the groups.

Conclusion: These results suggest that VDR is not critically involved in islet formation and that VDR expression is not an absolute requirement for development of autoimmune diabetes in the NOD mouse.

\section{6}

Characterisation of the $T$-cell repertoire in the LEW.1AR1-iddm rat

T. Arndt ${ }^{1,2}$, H. Weiss ${ }^{3}$, M. Elsner ${ }^{1}$, A. Jörns ${ }^{4}$, S. Lenzen ${ }^{1}$, H. J. Hedrich ${ }^{2}$, D. Wedekind ${ }^{2}$;

${ }^{1}$ Hannover Medical School, Institute of Clinical Biochemistry,

${ }^{2}$ Hannover Medical School, Institute of Laboratory Animal Science, ${ }^{3}$ University of Rostock, Institute of Medical Biochemistry and Molecular Biology, ${ }^{4}$ Hannover Medical School, Centre of Anatomy, Germany.
Background and Aims: The LEW.1AR1-iddm rat is an animal model of human Type 1 diabetes mellitus (T1DM) which arose through a spontaneous mutation in the diabetes resistant inbred strain LEW.1AR1. Recently, flow cytometry of peripheral blood lymphocytes (PBL) indicated that the T-cell content of LEW.1AR1-iddm rats is altered as compared to the background strain LEW.1AR1. The aim of this study was to characterise the lymphocyte repertoire within the PBLs of the diabetes susceptible LEW.1AR1$i d d m$ strain and its diabetes resistant control strain LEW.1AR1 during the first twelve weeks of life.

Materials and Methods: Blood of the two congenic LEW strains, LEW.1AR1-iddm (diabetic) and LEW.1AR1 (diabetes resistent) was taken every five days between 30 120 days of age. PBLs were differentiated by flow cytometry using a set of monoclonal antibodies (OX-38 FITC, OX-8 FITC, OX-8 PE, G4.18 PE, R73 FITC, OX-33 FITC, NKR 10/78 FITC, all BD; ED1 FITC, Serotec).

Results: The LEW.1AR1 strain was characterised by an age-dependent composition of lymphocyte subpopulations within the PBLs. In contrast, the course of the lymphocyte composition within the PBLs of the LEW.1AR1-iddm rats during the first twelve weeks of life was almost unique for each single animal. The alterations of the T-cell subpopulations in the course of life of the LEW.1AR1-iddm rats lead to a strong variation of the $\mathrm{CD} 4^{+} / \mathrm{CD}^{+}$T-cell ratio, varying between 0.9 and 2.2 with a mean value of 1.52 $( \pm 0,06)$. The $\mathrm{CD}^{+} / \mathrm{CD}^{+} \mathrm{T}$-cell ratio in PBLs of the background strain LEW.1AR1 was much more stable with a mean values of $2.1( \pm 0,02)$.

Conclusion: The mutation of the LEW.1AR1-iddm rat has an effect on the lymphocyte composition of the PBLs at variance from the coisogenic background strain LEW.1AR1. Moreover, it can be postulated that these alterations lead to T1DM development in $60 \%$ of the LEW.1AR1-iddm colony. Interestingly, recent investigations in diabetic patients also indicate that alterations in the lymphocyte repertoire may play a role in the pathogenesis of human T1DM. Perspectively, the identification of the functional role of the variant T-cell content may help to understand the mechanisms of autoimmunity in T1DM.

0017

The $70 \mathrm{kDa}$ heat shock protein DnaK targets the same B-chain epitope of human insulin as autoimmune T-cells V. Burkart ${ }^{1}$, R. K. Siegenthaler ${ }^{2}$, K. Vandenbroeck ${ }^{3}$, I. Alloza ${ }^{3}$, W. Fingberg ${ }^{1}$, P. Christen ${ }^{2}$, H. Kolb ${ }^{1}$;

${ }^{1}$ German Diabetes Clinic, German DiabetesCenter, Düsseldorf, ${ }^{2}$ Biochemical Institute, University of Zurich, Switzerland, ${ }^{3}$ McClay Research Center for Pharmaceutical Sciences, Queeńs University, Belfast, United Kingdom. 
Background and Aims: Autologous insulin represents a dominant antigen in the development of immune reactivity against pancreatic beta cells. The hormone is a target of autoantibodies and of cell-mediated immune processes including insulin-reactive T-cells, which emerge in the prediabetic phase in type 1 diabetes and in animal models of the human disease. Recent studies have shown that heat shock proteins (Hsp) have a high capacity to stimulate the proinflammatory activity of innate immune cells, such as macrophages. In addition, due to their chaperone activity, Hsp can induce peptide-specific immune responses by efficient transfer of peptides to MHC molecules for antigen presentation. In the present study we investigated the potential interaction of insulin with $\mathrm{Hsp} 70$ as a critical initial event in the selection of beta cell-specific (auto-) antigenic peptides.

Materials and Methods: The specificity of Hsp-insulin interactions was studied by analysing the binding of DnaK, an Hsp70 homolog of Escherichia coli, to preproinsulinderived 13-mer peptides and to (pro-)insulin variants.

Results: First, we studied the binding of DnaK to immobilized 13-mer peptides spanning the entire 110 amino acid (aa) sequence of preproinsulin. Four clusters of the preproinsulin peptides exhibited strongly increased DnaK retention in a range similar to those of control peptides with well-documented high affinities to DnaK. Each of the four functional preproinsulin domains contained one DnaK binding region: the signal peptide (aa S7-23), the A-chain (aa A6-21), the B-chain (aa B11-25) and the C-peptide (aa C15-31). The binding strength of DnaK to the immobilized peptides was assessed by determining the kinetics of DnaK release from the peptides during fractionated electro-elution. In this approach peptide B11-23 exhibited the strongest binding to DnaK of all 13mer peptides within the proinsulin sequence. In addition, strong binding of DnaK to the signal peptide was observed. The apparent dissociation equilibrium constants $(\mathrm{Kd})$ of the complexes of DnaK and selected 13-mer insulin peptides, proinsulin and insulin were determined by a competition binding assay using a fluorescence-labeled peptide with high affinity to DnaK. The B-chain region B11-23 exhibited high affinity for ADP-liganded DnaK, the substrate sequestering form of the chaperone ( $\mathrm{Ki}$ $2.2 \pm 0.4 \mu \mathrm{M})$. This region had previously been identified as dominant T-cell epitope and includes the hydrophobic core motif Lys-Tyr-Lys, which also represents a binding motif for MHC. However, a Tyr to Ala exchange did not affect the affinity of the peptide to DnaK (Ki $1.8 \pm 0.3 \mu \mathrm{M}$ ). The physiologically active insulin heterodimer shows extremely low DnaK affinity (Ki 67.8 $\pm 21.0 \mu \mathrm{M}$ ) while an only weakly aggregating proinsulin variant exhibited substantial binding ( $\mathrm{Ki} 11.3 \pm 7.8 \mu \mathrm{M})$.
Conclusion: Our observations demonstrate a high affinity of Hsp70 to distinct proinsulin-peptides with a preference for the B-chain peptide B11-23. We conclude that the initial preselection and chaperoning of sets of potentially (auto-)antigenic peptides by Hsp might link innate with adaptive immunity and thus play a central role in the induction of immune reactivity directed against autologous antigens such as insulin.

0018

Reduced expression of regulatory $T$-cell associated transcription factor Foxp3 in cord blood $\mathrm{T}$ lymphocytes from newborn infants at genetic risk of type 1 diabetes K. Luopajärvi ${ }^{1}$, J. Honkanen ${ }^{2}$, J. Ilonen ${ }^{3,4}$,

H. K. Åkerblom ${ }^{1}$, O. Vaarala ${ }^{2}$ on behalf of the TRIGR Study Group

${ }^{1}$ Hospital for Children and Adolescents, University of Helsinki, ${ }^{2}$ Laboratory for Immunobiology, Department of Viral Diseases and Immunology, National Public Health Institute, Helsinki, ${ }^{3}$ Department of Clinical Microbiology, University of Kuopio, ${ }^{4}$ Immunogenetics Laboratory, University of Turku, Finland.

Background and Aims: In type 1 diabetes the function of the regulatory $\mathrm{T}$ cells has been reported to be deficient. The aim of this study was to characterize the in vitro expression of regulatory T-cell related transcription factor Foxp3 in cord blood (CB) lymphocytes from newborn infants carrying the HLA risk haplotypes for type 1 diabetes.

Materials and Methods: CB EDTA samples were randomly received from newborn infants taking part in the Trial to Reduce IDDM in Genetically at Risk (TRIGR) project with a first-degree relative (mother, father, or full-sibling) with type 1 diabetes or the Finnish Dietary Intervention Trial for Prevention of type 1 diabetes (FINDIA) project with no affected family member. We included 16 infants carrying DR4-DQ8 (DQB1*0302) haplotype, 12 infants with DR3DQ2 (HLA DQA1*0501-DQB1*02) haplotype, and 36 newborn infants who did not have either DR4-DQ8 or DR3-DQ2 haplotype. CB mononuclear cells were stimulated in type 1 (IL-12, anti-IL-4), or type 2 cytokine (IL-4, anti-IL12) environment for 6 days. Expression of transcription factor Foxp3 was determined by real time quantitative RT-PCR.

Results: After culture of $\mathrm{CB}$ mononuclear cells in type 2 cytokine environment the expression of Foxp3 differed between the groups with various HLA genotypes $(p=0.020$, Kruskal-Wallis test). The relative level of Foxp3 mRNA was lower in newborn infants carrying DR4-DQ8 haplotype compared to subjects with DR3-DQ2 haplotype (median 11.1 vs 18.50; p=0.011, Mann-Whitney U-test) and newborn infants without DR4-DQ8 or DR3-DQ2 
haplotypes (median 11.1 vs $18.10 ; \mathrm{p}=0.013$ ). No difference was seen between groups grown in type 1 cytokine environment.

Conclusion: The up-regulation of Foxp3 is a prerequisite for the function of regulatory T-cells, and the poor activation of Foxp3 in the infants with HLA DR4-DQ8 may explain their susceptibility to autoimmunity later in their life.

\section{OP 4}

\section{TCF7L2 - a novel gene for type 2 diabetes}

\section{9}

Large scale case-control and family-based analyses of TCF $7 L 2$ variants in $>6000 \mathrm{UK}$ subjects demonstrates an almost two-fold difference in relative risk between homozygote classes

C. J. Groves ${ }^{1}$, E. Zeggini ${ }^{1,2}$, J. Minton ${ }^{3}$, T. M. Frayling ${ }^{3}$, M. N. Weedon ${ }^{3}$, N. W. Rayner ${ }^{1,2}$, G. A. Hitman ${ }^{4}$,

M. Walker ${ }^{5}$, S. Wiltshire ${ }^{2}$, A. T. Hattersley ${ }^{3}$,

M. I. McCarthy ${ }^{1,2}$;

${ }^{1}$ Oxford Centre for Diabtes Endocrinology and Metabolism, University of Oxford, ${ }^{2}$ Wellcome Trust Centre for Human Genetics, University of Oxford, ${ }^{3}$ Department of Diabetes Research \& Vascular Medicine, Peninsula Medical School, Exeter, ${ }^{4}$ Department of Diabetes \& Metabolic Medicine, Barts and the London, Queen Mary School of Medicine and Dentistry, London ${ }^{5}$ Department of Medicine, School of Medicine, Newcastle-upon-Tyne, United Kingdom.

Background and Aims: Evidence has recently emerged relating common variation in the gene TCF7L2 (encoding transcription factor 7 like 2) to type 2 diabetes susceptibility in subjects of Northern European origin. The generalizability of these findings and the mechanisms involved are, as yet, unknown.

Materials and Methods: We typed four TCF7L2 SNPs (the two most associated in the previous study [rs12255372 \& rs7903146] plus the two best correlated proxies available from Phase II Hapmap [rs4506565 \& rs12243326]), and performed a case-control analysis in 2158 UK T2D subjects and 2574 controls, supplemented with family-based association analysis in 388 parent-offspring trios. Genotyping was performed using a fluorescence-based competitive allele-specific PCR method (KASPar) with overall call rates for each SNP exceeding 95\%. All SNPs performed well against stringent quality control criteria. There was no evidence of departure from Hardy-Weinberg equilibrium (all $\mathrm{P}>0.05$ in controls), two Mendelian inconsistencies in
963 families and a discrepancy rate on duplicate genotyping of $2 / 2416$ (ie $0.04 \%$ error).

Results: Case-control analysis demonstrated powerful association with T2D at all four SNPs, with the strongest effect at rs27903146 (allele-wise Relative Risk 1.36 95\% CI 1.24-1.48) $\left(P=1.3 \times 10^{-11}\right)$. Data were consistent with a multiplicative model. Compared to baseline, the genotype relative risk of diabetes in individuals carrying two copies of the risk allele was 1.90 (95\% CI $1.54-2.33)$ $\left(P=3.6 \times 10^{-10}\right)$. Independent evidence for association at all loci was provided by the family-based analyses (eg. TDT at rs 4506565 showed $62 \%$ transmission, $P=7 \times 10^{-5}$ ) with no parent of origin effects evident. TCF $2 L 2$ genotypes associated with T2D were more frequent in cases selected for positive family history and early onset (rs4506565 $P=0.02$ ), and the population attributable risk (based on the least selected cases) was estimated at $16 \%$. No aetiological mutations were detected when all exons were re-sequenced in 48 subjects with permanent early-onset diabetes. The global evidence for association, derived by combining casecontrol and family-based analyses, exceeded genome-wide significance $\left(\mathrm{P}=4.4 \times 10^{-14}\right)$.

Conclusion: Our results provide overwhelming support and replication of the previously published association between TCF7L2 variation and type 2 diabetes demonstrated in Icelandic, Danish and US cohorts. Indeed, TCF $7 L 2$ variation confers the greatest T2D-susceptibility effect yet demonstrated. The effect size and allele frequency of this variant augurs well for ongoing efforts to identify additional diabetes susceptibility genes through genome wide approaches.

0020

TCF7L2 gene strongly associates with type 2 diabetes, with lower age of onset and BMI in the French population, and its adipose expression is impaired in diabetes

S. Cauchi ${ }^{1}$, D. Meyre ${ }^{1}$, H. Choquet ${ }^{1}$, C. Samson ${ }^{1}$, C. Polychronakos ${ }^{2}$, B. Balkau ${ }^{3}$, R. Scharfmann ${ }^{4}$, G. Frühbeck ${ }^{5}$, G. Charpentier ${ }^{6}$, R. Sladek ${ }^{2}$, P. Froguel ${ }^{1}$; ${ }^{1}$ Genomics and Molecular Physiology of Metabolic Diseases, CNRS, Lille, France, ${ }^{2}$ Division of Pediatric Endocrinology, McGill University Health Center, Montréal, Canada, ${ }^{3}$ Cardiovascular and Epidemiological Epidemiology, INSERM U258-UFR69, Villejuif, France, ${ }^{4}$ Necker Hospital, INSERM E363, Paris, France, ${ }^{5}$ Laboratory of Metabolic Research, Clinica Universitaria de Navarra, Pamplona, Spain, ${ }^{6}$ Corbeil Hospital, CH Gilles de Corbeil, Corbeil Essonnes, France. 
Background and Aims: Recently, non-coding variants in TCF7L2, a transcription factor in the Wnt signaling pathway, were strongly associated with increased risk of type 2 diabetes (T2D) in samples from Iceland, Denmark and the US. We analyzed the two most associated variants in the French population.

Materials and Methods: Case-control study was carried out with 2,421 obese and lean T2D patients and in 926 control individuals (BMI $<27 \mathrm{~kg} / \mathrm{m}^{2}$ and fasting glycemia $<5.6 \mathrm{mmol} / \mathrm{l}$ ) from a French population. High-throughput genotyping of rs7903146 and rs12255372 variants was performed by Taqman (Applied Biosystem). $\mathrm{Chi}^{2}$ test and $\mathrm{P}$ values were empirically computed with the CLUMP program for analyses. A multivariate linear regression model taking into account age and sex was performed for BMI parameters. The same analysis was done with BMI adjustment for diabetes onset parameters. Affymetrix microarrays and MTC panels were used to assess the TCF7L2 expression in different tissues.

Results: We confirmed the previously-showed association of TCF7L2 variants (rs7903146 and rs12255372) with risk of $\mathrm{T} 2 \mathrm{D}$ in French population (respectively $\mathrm{OR}=1.69, \mathrm{P}=1.7$ $10-19$ and $\mathrm{OR}=1.64, \mathrm{P}=8.210-17$ ). Due to strong linkage disequilibrium between the two variants (delta $=0.86$ ), we decided to only analyse the most associated with T2D (rs7903146). We found a strong OR increase $(\mathrm{OR}=1.93$, $\mathrm{P}=1.310-24$ ) in T2D non-obese subgroup (BMI $<30 \mathrm{~kg}$ / $\mathrm{m}^{2}, 926$ control individuals, $1265 \mathrm{~T} 2 \mathrm{D}$ patients). Contrary to the previously published data, we found that the at-risk allele is associated with lower BMI $(\mathrm{P}=2.8 \quad 10-4)$. In addition, there was a 2.4 year decrease of the age at diabetes onset with the at-risk allele $(\mathrm{P}=3.7$ 10-5). In human tissues, TCF7L2 expression was found to be quite ubiquitous although poorly expressed in the skeletal muscle. Its expression pattern looks like following NGN3 expression during the early steps of rat foetal $\beta$-cells differentiation. In subcutaneous and omental adipose tissues, the TCF7L2 expression is impaired in glucoseintolerant and diabetic obese subjects.

Conclusion: In French population, we found a very strong association between two previously studied TCF7L2 variants and $\mathrm{T} 2 \mathrm{D}$, especially in non-obese subjects. These variants are also associated with early diabetes onset and a lower BMI. In humans, TCF7L2 expression is ubiquitous and is impaired in the diabetes state. TCF7L2 is particularly expressed at early steps of $\beta$-cells differentiation, suggesting a physiological role in several key organs for glucose homeostasis.
0021

Common variants in the TCF $7 L 2$ and PPARG genes predict future type 2 diabetes in 7,000 persons followed for 22 years from the Malmö preventive project (MPP) V. Lyssenko ${ }^{1}$, G. Berglund ${ }^{2}$, L. Groop ${ }^{1}$, P. Nilsson ${ }^{2}$;

${ }^{1}$ Department of Clinical Sciences/Diabetes \& Endocrinology, Lund University, Malmö, ${ }^{2}$ Department of Medicine, Malmö University Hospital, Lund University, Malmö, Sweden.

Background and Aims: Type 2 diabetes (T2D) is considered a complex polygenic disorder where common genetic variants interact with environmental factors to unmask the disease. Common variants in a number of genes have been associated with type 2 diabetes in cross-sectional studies. The question whether this information could be used to identify individuals at risk of developing future diabetes has been addressed in some studies, but the number of subjects converting to diabetes has been limited and the follow-up time relatively short. The MPP offers sufficient power to test this hypothesis as 30,000 individuals have been followed for a median of 22 years. Thus, we tested the ability of common variants in TCF7L2 (rs12255372; rs7903146), PPARG (P12A), ENPP1 (K121Q), CAPN10 (SNP43), IRS1 (972C > T), UCP2 ( $-866 \mathrm{G}>\mathrm{A}$ ), PTPN1 (rs1044498), PGC1 (G482S) to predict overt T2D in 7,061 individuals with DNA and follow-up data available thus far. Altogether 22,000 of the original participants will be restudied.

Materials and Methods: 4392 men and 2669 women (age $46 \pm 6$ yrs, BMI $24 \pm 3.4 \mathrm{~kg} / \mathrm{m}^{2} ; 24.5 \%$ with IFG/IGT) were included. Genotyping was performed using the IPLEX system adopted to mass spectroscopy on a Sequenom platform. It was not possible to multiplex all potential candidate genes in the same setting, so other candidate genes will be added in the future, e.g. KCNJ11.

Results: In total, 1422 (20.1\%) persons converted to T2D; of them $1072(24.4 \%)$ men with a mean 25-year and 350 (13.1\%) women with a mean 18-year follow-up period. In Cox regression analyses adjusted for age, sex and BMI, the PPARG PP-genotype (Hazard ratio 1.20, 95\%CI 1.05-1.38, $P=0.0045)$ and the T-allele of both TCF7L2 $(1.30,1.17-$ 1.45, $P=1.8 \times 10^{-6}$ and $1.42,1.28-1.58, P<10^{-10}$, for rs 12255372 and rs 7903146 , respectively) increased the risk of future T2D. Furthermore, in the multivariate analyses TCF7L2 (rs7903146) and PPARG (P12A) (1.42, 1.27-1.57, $\mathrm{P}<10 \times^{-10}$ and $\left.1.23,1.09-1.40, \mathrm{P}=0.00058\right)$ were independent predictors of T2D. None of the other common variants in ENPP1, CAPN10, IRS1, UCP2, PTPN1 and PGC1 genes were associated with increased risk of T2D.

Conclusion: These data from a large prospective study with 22 years follow up clearly demonstrate that common variants in the TCF7L2 and PPARG genes predict future T2D and can be considered true T2D susceptibility genes. 
0022

The transcription factor 7-like 2 gene (TCF 7L2) is strongly associated with type 2 diabetes in the Botnia Study

M. Sjögren ${ }^{1}$, V. Lyssenko ${ }^{1}$, P. Almgren ${ }^{1}$, M. Svensson ${ }^{1}$, T. Tuomi ${ }^{2,3}$, B. Isomaa ${ }^{4,5}$, L. Groop ${ }^{1}$, M. Orho-Melander ${ }^{1}$ on behalf of the Botnia Study Group ${ }^{1}$;

${ }^{1}$ Clinical Sciences, Diabetes \& Endocrinology, Lund University, Malmö, Sweden, ${ }^{2}$ Department of Medicine, Division of Diabetology, Helsinki University Hospital, Finland, ${ }^{3}$ Institute of Genetics, Folkhälsan Research Center, Helsinki, Finland, ${ }^{4}$ Folkhälsan Genetic Institute, Folkhälsan Research Center and Research Program for Molecular Medicine, Helsinki University, Finland, ${ }^{5}$ Malmska Municipal Health Care Center and Hospital, Folkhälsan Ostanlid, Jakobstad, Finland.

Background and Aims: Genetic complexity of type 2 diabetes (T2D) is demonstrated by the fact that so far no susceptibility gene has confidently been associated with the disease in all studied populations. Recently, single nucleotide polymorphisms (SNPs) in TCF7L2 were found to be strongly associated with T2D in Icelandic and two other populations. Our aim was to investigate the putative role of TCF7L2 in the Botnia study.

Materials and Methods: Two SNPs in TCF7L2 (rs7903146, rs12255372) were genotyped in 716 controls (m/f 346/370, age 54 $\pm 11 \mathrm{y}$, BMI $\left.25.9 \pm 3.7 \mathrm{~kg} / \mathrm{m}^{2}\right), 751 \mathrm{~T} 2 \mathrm{D}$ patients $\left(\mathrm{m} / \mathrm{f} 399 / 352\right.$, age $60 \pm 10 \mathrm{y}$, BMI $\left.28.9 \pm 4.8 \mathrm{~kg} / \mathrm{m}^{2}\right)$ and 2293 non-diabetic individuals participating a prospective follow-up (1051 men/1242 women, age $45 \pm 14 \mathrm{yrs,}$ BMI $25 \pm 4 \mathrm{~kg} / \mathrm{m}^{2}$ ). All individuals originate from the Botnia region in Western Finland and genotyping was performed by allelic discrimination on ABI 7900. During a mean follow-up period of 6 years 132 individuals developed T2D.

Results: The earlier described "at risk" T-alleles (minor alleles) of both SNPs were more frequent in T2D patients compared to controls (rs7903146: 41.0 vs. $31.0 \%$, $\mathrm{p}=0.000097$; rs12255372: 39.0 vs. $29.9 \%, \mathrm{p}=0.00027$ ). The T-allele carriers had increased risk of T2D as compared to homozygous major allele carriers (rs7903146: OR 1.54 [1.24-1.92], $\mathrm{p}=0.00010$, rs12255372: OR 1.50 [1.20-1.86], $\mathrm{p}=0.00028)$. Carriers of the at-risk alleles of both SNPs had an even higher risk of T2D (OR 2.38 [1.86-3.05], $\mathrm{p}<0.000001)$. In the non-diabetic subjects participating in the Botnia prospective study, the T-allele carriers of rs7903146 had higher fasting- $(5.60 \pm 0.58$ vs. $5.54 \pm 0.58 \mathrm{mmol} / \mathrm{l}, \mathrm{p}=0.031)$ and $2 \mathrm{~h}$-plasma-glucose $(6.45 \pm 1.6$ vs. $6.20 \pm 1.6 \mathrm{mmol} / \mathrm{l}, \mathrm{p}=0.00028)$ as well as lower insulinogenic index $(4.91 \pm 3.9$ vs. $5.36 \pm 4.4, \mathrm{p}=0.022)$ and disposition index $(4.35 \pm 3.3$ vs. $4.75 \pm 3.8, p=0.016)$ at baseline. Moreover, the homozygous rs7903146 TT-genotype carriers were leaner compared to the other genotype carriers ( 24.2 vs. $\left.25.7 \mathrm{~kg} / \mathrm{m}^{2}, \mathrm{p}=0.0052\right)$. Finally, already at baseline the individuals with IGT or IFG had a higher frequency of the risk genotypes (rs7903146: 39.6 vs. $34.1 \%, \mathrm{P}=0.012$; rs12255372: 36.1 vs. $31.1 \%, \mathrm{P}=0.018$ ) and the T-allele of rs7903146 was associated with increased risk of developing T2D among individuals with IFG at baseline (2.64 [1.09-6.42], $\mathrm{p}=0.032$ ).

Conclusion: Variants in TCF7L2 are strongly associated with $\mathrm{T} 2 \mathrm{D}$ and predict morbidity to T2D in the Botnia study. Our results point at a role for TCF7L2 in regulation of insulin secretion and glucose tolerance.

\section{3}

Refinement of the association between the $T C F 7 L 2$ gene and type 2 diabetes in a West African population

S. F. A. Grant ${ }^{1}$, G. Thorleifsson ${ }^{1}$, A. Helgason ${ }^{1}$, A. Adeyemo ${ }^{2}$, Y. Chen ${ }^{2}$, G. Chen ${ }^{2}$, R. Benediktsson ${ }^{3,4}$, M. Faruque ${ }^{2}$, A. Doumatey ${ }^{2}$, J. Zhou ${ }^{2}$, U. Thorsteinsdottir ${ }^{1}$, J. R. Gulcher ${ }^{1}$, A. Kong ${ }^{1}$, C. Rotimi ${ }^{2}$, K. Stefansson ${ }^{1}$;

${ }^{1}$ Population Genomics, deCODE Genetics, Reykjavik, Iceland, ${ }^{2}$ National Human Genome Center, Howard University, Washington, United States, ${ }^{3}$ Landspitali, National University Hospital, Reykjavik, Iceland, ${ }^{4}$ Hjartavernd, Icelandic Heart Association, Reykjavik, Iceland.

We recently described association between the Transcription Factor 7-like 2 gene (TCF7L2; formerly TCF4) and type 2 diabetes (T2D) with a population attributable risk (PAR) of $21 \%$. We reported the association of three markers, the composite allele $\mathrm{X}$ of microsatellite DG10S478 and the T alleles of SNPs rs12255372 and rs7903146, based on an initial discovery in Iceland and subsequent replication in Danish and European American cohorts. The strength of association of these highly correlated markers in Caucasian populations was comparable so we recommended that all three should be genotyped in any replication effort.

In an attempt to replicate and refine this association, we genotyped these markers in a more genetically diverse West African cohort, consisting of 618 T2D patients and 434 controls derived from the Africa America Diabetes Mellitus Study. Following adjustment for relatedness and ethnic origin, we replicated the association of allele $\mathrm{T}$ of rs7903146 with T2D (Relative Risk=1.45 [1.20,1.76], $P=7.3 \times 10^{-5}, \mathrm{PAR}=22.2 \%$ ); however, the other two markers were not significantly associated.

The West African cohort has therefore enabled us to define allele T of rs7903146 as either the risk variant itself or the closest correlate of a still unidentified risk variant. This 
refinement was possible because the T allele of rs7903146 occurs almost exclusively on chromosomes carrying both allele X of DG10S478 and allele T of rs12255372 in populations with European ancestry, while this is not the case in the West African cohort, where it occurs with both alleles of DG10S478 and rs12255372. This is consistent with the observation that $\mathrm{T}$ is the ancestral allele of rs7903146, whereas allele X of DG10S478 and allele T of rs 12255372 are both derived relative to the chimpanzee reference sequence. More generally, this finding is also consistent with the expectation that non-African populations tend to carry only a subset of the variants present in African populations.

\section{4}

A KLF9 promoter variant that is predicted to affect TCF7L2 binding is associated with type 2 diabetes

B. Neve ${ }^{1,2}$, R. Gutiérrez-Aguilar ${ }^{1}$, Y. Benmezroua ${ }^{1}$, E. Vaillant ${ }^{1}$, C. Dina ${ }^{1}$, B. Balkau ${ }^{3}$, M. Marre ${ }^{4}$,

G. Charpentier ${ }^{5}$, P. Froguel ${ }^{1,2}$;

${ }^{1}$ ICnrs8090, Institute of Biology, Pasteur Institute, Lille, France, ${ }^{2}$ Genomic Medicine, Imperial College, London, United Kingdom, ${ }^{3}$ Xavier Bichat Faculty of Medicine, Paris, France, ${ }^{4}$ Inserm u258-ifr69, Université Paris XI, Villejuif, France, ${ }^{5}$ Department of Diabetes, Sud Francilien Hospital, Corbeil-Essonnes, France.

Background and Aims: We investigated contribution of Kruppel-Like Zinc Finger (KLF) transcription factor family genes to type 2 diabetes (T2D), since they regulate genes involved in cell proliferation and differentiation in several different tissues (including pancreatic beta-cells). In particular, KLF2, 5, 6, 7 and 15 were shown to regulate expression of genes involved adipose differentiation.

Materials and Methods: We genotyped 51 SNPs of 12 KLFs (minor allele frequency $>5 \%$ ) in a first case-control set of 365 T2D French Caucasian subjects with at least one affected first-degree relative $(196 / 169 \mathrm{M} / \mathrm{F}$, age at diagnosis $45 \pm 11$ years) and 363 normoglycaemic subjects $(136 / 227$ $\mathrm{M} / \mathrm{F}$, age at examination $59 \pm 13$ years) using Taqman (ABI), SNPlex (ABI) or Lightyper technology (Roche). SNPs showing a trend for association were analyzed in a second set consisting of $750 \mathrm{~T} 2 \mathrm{D}$ subjects $(455 / 295 \mathrm{M} / \mathrm{F}$, age at diagnosis $48 \pm 10$ years) and 741 normoglycaemic subjects (294/447 M/F, age at examination $53 \pm 6$ years).

Results: In the first set, 13 SNPs showed a trend for association with T2D and these were further analyzed in the second set. A KLF7 intron 3 variant associated with T2D $(\mathrm{P}<0.01)$. Moreover, a KLF9 promoter variant showed a trend towards $\mathrm{T} 2 \mathrm{D}$ association in both sets $(\mathrm{P}=0.03)$, which needs to be replicated in additional samples. In-silico analysis (Genomatix) of this promoter variant predicted that either TCF1 or TCF7L2 transcription factor binding is lost when the variant allele is present. We are now analysing TCF1/TCF7L2 binding to this variant by gelshift experiments and chromatin-immunoprecipitation.

Conclusion: Besides the previously reported T2D association of KLF11, our study suggests that KLF7 (confirming earlier findings in the Japanese population) and KLF9 may be associated to T2D. No contribution of other KLFtranscription factor variants was observed in the French population. The potential association of the KLF9 promoter variant with T2D suggests that KLF9 is a target of TCF7L2 and may be involved in the signaling pathway of this strong T2D susceptibility gene.

Supported by a Grant of the European Regional Development Fund (FEDER) and Region Nord-Pas de Calais (ARCir) to BN and PF

\section{OP 5}

\section{Beta cell differentiation and neogenesis}

\section{5}

Function of the transcription factor RFX3, a novel link between cilia and development of the endocrine pancreas A. Ait-Lounis ${ }^{1}$, E. Barras ${ }^{1}$, D. Baas ${ }^{2}$, C. Bénadiba ${ }^{2}$, P. Meda ${ }^{3}$, B. Durand ${ }^{2}$, W. Reith ${ }^{1}$;

${ }^{1}$ Pathology and Immunology, University of Geneva Medical School, Switzerland, ${ }^{2}$ Centre de Génétique Moléculaire et Cellulaire, Université Claude Bernard, Lyon, France, ${ }^{3}$ Cell Physiology and Metabolism, University of Geneva Medical School, Switzerland.

Background and Aims: In mammals there are five members of the RFX family of transcription factors. While RFX5 plays a well-defined role in the immune system, the functions of RFX1-RFX4 remain largely unknown. We recently found that RFX3 plays a key role in controlling the expression of genes required for the formation and function of cilia. As consequence of defects in nodal cilia, RFX3 deficient mice exhibit frequent left-right asymmetry defects leading to a high rate of embryonic lethality and situs inversus in surviving adults.

Results: We now show that RFX3 is also expressed specifically in the islets of Langerhans and that, in the absence of RFX3, islet morphogenesis is severely perturbed. The rare RFX3-deficient mice that survive to adulthood exhibit a reduced tolerance to glucose injection, a marked reduction in the size of pancreatic islets, reduced insulin production and defective glucose-stimulated insulin secretion. These abnormalities result from a developmental 
defect leading to an alteration in the cellular composition of the islets during embryogenesis. By embryonic day 19, the islets in RFX3-deficient mice contain considerably less insulin-producing $\beta$ cells, glucagon-producing $\alpha$ cells and ghrelin-producing cells, whereas there is a marked increase in the number of pancreatic peptide (PP)-producing cells.

Conclusion: This skewed cell composition of the islets suggests that RFX3 is implicated in one or more cell fate decisions in the developing endocrine pancreas. Finally, we have examined expression in the pancreas of known or suspected RFX3 target genes coding for cilia related proteins. We find that expression of two of these genes, D2lic and $\operatorname{Tg} 737$ is significantly reduced in the pancreas, thereby providing a link between cilia and the regulation of pancreatic islet cell development.

Supported by Roche, ALFEDIAM, the Sir Jules Thorn Charitable Trusts

\section{6}

Regulation of Pax6 and Pax6(5a) in pancreatic beta cells G. Wolf, A. Karkour, U. Henrion, R. Walther; Department of Medical Biochemistry and Molecular Biology, University of Greifswald, Germany.

Background and Aims: The transcription factor Pax6 is an important regulator of alpha- and beta cell function. These cell types produce and release the hormones glucagon and insulin, which are reciprocally regulated in response to glucose. In alpha cells Pax6 is important for activation as well as for insulin dependent inhibition of the glucagon synthesis. The influence of this transcription factor in beta cells on insulin gene expression is discussed controversially. Also the importance of alternative splicing and the function of the resulting Pax6(5a) in islet cells is not understood. We investigated the regulation of both isoforms in pancreatic beta cells to analyse whether these factors are involved in the regulation of insulin biosynthesis.

Materials and Methods: Gel shifts and chromatin immunoprecipitations were done to identify DNA binding sites of Pax6 within the rat insulin gene II promoter. After treatment of Insulinoma cells with different concentrations of glucose or $\mathrm{H}_{2} \mathrm{O}_{2}$, alteration of the intracellular localisation was visualised by fluorescence microscopy. The mRNA amount of Pax6(5a) was detected by RT-PCR. The effect of the exon 5a on interaction with BETA2 and on Pax6 regulated insulin gene expression was analysed using GST capture assay and the Dual Luciferase Assay system respectively. Furthermore in vitro kinase assays were performed.

Results: Pax6 is able to bind to insulin promoter ciselements not similar to PISCES. We observed an augment nuclear localisation of EGFP-Pax6 and EGFP-Pax6(5a) in
INS-1 cells under low glucose concentrations and during oxidative stress. The isoform Pax6(5a) is not able to interact with BETA2 and to suppress insulin gene expression to the same extent as Pax6. Furthermore we demonstrate that Pax6 and Pax6(5a) are phosphorylated by CaMK and CK2. Conclusion: At low concentrations of glucose and enhanced amounts of $\mathrm{H}_{2} \mathrm{O}_{2}$ Pax6 acts as a negative regulator of insulin gene expression. Further Pax6 activity is modulated via alternative splicing and phosphorylation by CaMK and CK2.

0027

P27 regulates the transition of beta cells from quiescence to proliferation

S. K. Georgia ${ }^{1}$, A. Bhushan ${ }^{2}$;

${ }^{1}$ Molecular Biology IDP, University of California, Los Angeles, ${ }^{2}$ Department of Medicine, Division of Endocrinology, University of California, Los Angeles, United States.

Background and Aims: Diabetes results from an inadequate mass of functional beta cells. Such inadequacy could result from loss of beta cells due to an immune assault or the inability to compensate for insulin resistance. Thus, mechanisms that regulate the number of beta cells will be key to understanding both the pathogenesis of diabetes and for developing therapies. In this study we show that cell cycle regulator, p27 plays a crucial role in establishing the number of beta cells formed before birth.

Methods: Expression patterns of islet transcription factors and islet hormones were analyzed by fluorescent immunohistochemistry on wildtype and p27-/- littermate mice, aged E10.5 to adulthood. Beta cell mass was measured by multiplying the insulin/area ratio (quantified by insulin area divided by total tissue area) by the mass of the pancreas. Plasma levels were measured, and interperitoneal glucose tolerance and insulin tolerance tests were performed on p 27-/ - and WT mice aged 10-12 weeks, after 6-16 hour fasts. The statistical significance of differences was measured by unpaired student t-test.

Results: We show that p27 accumulates in terminally differentiated beta cells during embryogenesis. Disabling p27 allows terminally differentiated beta cells that are normally quiescent during embryogenesis to reenter the cell cycle and proliferate. As a consequence, excess beta cells are generated in the p27-/- mice, doubling their beta cell mass at birth. The early postnatal expansion of beta cell mass was unaffected in $\mathrm{p} 27-/-$ mice, indicating that the main function of p27 is to maintain the quiescent state of newly differentiated beta cells generated during embryogenesis. The expanded beta cell mass 
was accompanied by increased insulin secretion, however, the p27-/- mice were glucose intolerant as these mice were insulin insensitive. To assess the role of p27 to affect regeneration of beta cells in models of diabetes, p27-/- mice were injected with streptozocin. In contrast to control mice that displayed elevated blood glucose levels, p27-/- mice showed decreased susceptibility to develop streptozotocin-induced diabetes. Furthermore, beta cells retained the ability to reenter the cell cycle at a far greater frequency in p27-/- mice after develop streptozotocin-induced diabetes compared to wild-type littermates.

Conclusion :This data indicates that p27 is a key regulator in establishing beta cell mass and an important target for facilitating beta cell regeneration in therapies for diabetes.

Supported by Kirschstein National Research Service Award GM07185, NIH R01 DK-6876, Larry HIllblom Foundation, JDRF

\section{8}

Extracellular matrix increases rat but not human beta cell proliferation

G. Parnaud ${ }^{1}$, D. Bosco ${ }^{2}$, T. Berney ${ }^{2}$, P. A. Halban ${ }^{1}$;

${ }^{1}$ Department of Genetic Medicine and Development, University Medical Center, Geneva, ${ }^{2}$ Cell Isolation and Transplantation Center, Department of Surgery, University Hospital, Geneva, Switzerland.

Background and Aims: Inducing proliferation of beta cells in vitro is attractive for both transplantation and biological studies. The aim of the study was to investigate a possible role of extracellular matrix $(\mathrm{ECM})$ in inducing cell proliferation of rat and human beta cells.

Materials and Methods: Autofluorescence FACS-sorted rat pancreatic beta cells were plated on poly-L-lysine (PLL) or on matrix produced by $804 \mathrm{G}$ rat bladder carcinoma cell line (804G-ECM) in continuous presence of BrdU. FACS purification of human beta cells involved exclusion of ductal cells labelled with anti CA19-9 and dead cells stained with 7-aminoactinomycin $\mathrm{D}$, with beta cells sorted by virtue of their high zinc content using Newport Green, yielding a population with $90 \%$ beta cells. Human beta cells were plated either on lawns of $\mathrm{NH}_{4} \mathrm{OH}$-lysed HTB9 human bladder carcinoma cells or on $804 \mathrm{G}-\mathrm{ECM}$ or plastic in presence of BrdU during the last five days. Cell spreading was monitored microscopically, and insulin secretion measured after static incubation $(1 \mathrm{~h}$ at $2.2 \mathrm{mM}$ glucose $+1 \mathrm{~h}$ at $22.2 \mathrm{mM}$ glucose) by radioimmunoassay. Cell replication was determined using BrdU incorporation and beta cells identified by insulin immunofluoresence. Data are mean \pm SEM of at least 3 independent experiments.

Results: As previously shown, rat beta cells spread on 804G-ECM but not on PLL. Rat beta cells proliferated on PLL and 804G-ECM with a significant beneficial effect of the matrix at all time points. After 2 days, percentage of BrdU positive beta cells was $1.8 \pm 0.4$ vs. $4.6 \pm 0.5$ ( $\mathrm{P}=0.006)$ on PLL vs. 804G-ECM respectively. After 5 days of continuous BrdU labelling, these values were $25.18 \% \pm$ 1.85 vs. $41.5 \% \pm 3.6(\mathrm{P}=0.007)$. The results for the purified human beta cells were strikingly different. After one or two weeks of culture most of the human beta cells were spread on lysed HTB9 lawns but not on PLL or on 804G-ECM. However, little if any beta-cell replication was observed under any condition $(\mathrm{n}=6)$. The method used for sorting human beta cells is not in cause because sorted human beta cells responded well to glucose (stimulation index high vs. low glucose $=6.1 \pm 0.9$ ) and no apoptosis was observed in these preparations. Furthermore rat beta cells purified with the same Newport Green sorting method used for human cells, can proliferate and show similar stimulation by $804 \mathrm{G}-$ ECM. Intact human islets were also plated on lysed HTB9 lawns or on plastic. There were BrdU positive cells in islets; however, after confocal analysis, we observed that BrdU positive cells were almost always insulin negative with $<1 \mathrm{BrdU}$ insulin double positive cell/islet.

Conclusion: It is concluded that rat beta cells can proliferate in vitro and this is increased significantly by ECM. By contrast, sorted human beta cells do not replicate to any significant extent in vitro regardless of the substratum. Our data do not exclude, however, that division of human beta cells is accompanied by rapid loss of insulin. Supported by the JDRF program for regeneration of beta cell function

0029

Expansion of mesenchymal cells from human pancreatic islets of Langerhans and their "in vitro" differentiation in insulin secreting cells

R. Gallo ${ }^{1}$, V. Tellone ${ }^{1}$, G. Bernardi ${ }^{1}$, R. Lupi ${ }^{2}$, M. Masini ${ }^{2}$, P. Marchetti ${ }^{2}$, F. Dotta ${ }^{1}$;

${ }^{1}$ Internal Medicine and Endocrine Sciences, University of Siena, ${ }^{2}$ Department of Endocrinology and Metabolism, University of Pisa, Italy.

Background and Aims: "In vitro" development of cells able to produce and secrete insulin in a physiological manner is crucial to produce a proper cell-therapy as a cure for type I diabetes. Aims of our work have been the development of cell culture strategies for expansion of proliferating cells with potential beta-cell differentiation 
capacity obtained from human pancreatic islets and the characterization of such processes.

Material and Methods: Freshly, highly purified human pancreatic islets from 6 normal cadaveric multi-organ donors were cultured in $100 \mathrm{~mm}$ tissue-dishes in presence of RPMI 1640 medium containing 10\% FCS (growth medium-GM) at $37^{\circ} \mathrm{C}$ in $5 \% \mathrm{CO}_{2}$ to obtain a proliferating cell population which was expanded. To induce beta-cell differentiation, the proliferating cells were plated in serum-free RPMI supplemented with BSA, transferrin, insulin and sodium selenite as a differentiation medium (DM). Morphological and molecular characterization of proliferating and differentiating cells was performed by electron-microscopy, FACS, immunofluorescence and Real-Time-PCR

Results: The human islet preparations, with no sign of dissociation, were seeded in GM and, after 48 hours, a proliferating cell population migrated out of adherent islets. These cells were harvested with trypsin every 8-10 days and after 7 months, approximately $10^{10}$ cells were obtained from each original islet seeded, before showing evident signs of senescence. The expression of CD90, CD73, CD13, CD44, CD49e, CD49b, Nestin, aSmooth-MuscleActin, Slug e Snaill analyzed by FACS, immunofluorescence and Real-Time-PCR indicated a mesenchymal phenotype of our proliferating cells. Such mesenchymallike cells, when cultured in DM, generated "islets-like" aggregates within 10-20 days. Electron microscopy analysis of such aggregates showed mesenchymal-like cells in the outer layers surrounding a "core" of cells with a well developed Golgi apparatus characterized by enlarged cisternes, and beta-cell like secretory granules. Immunofluorescence analysis showed the presence of C-peptide in these cellular aggregates and, accordingly, in vitro insulin secretion in the presence of $3.3 \mathrm{mM}$ glucose was detected by RIA. Real-Time-PCR analysis, showed a significant induction of gene expression of insulin, glucagon, somatostatin, Ngn3, NeuroD1, Isl1, Nkx2-2, Nkx6-1, IRS1 and synaptophysin, confirming an acquired endocrine pancreatic phenotype of our aggregate.

Conclusion: We obtained from human pancreatic islets a proliferating mesenchymal-like cell population which was expanded and induced to re-differentiate into insulinsecreting cells.

Supported by the Ministry of Health
0030

Pancreatic islet-cell differentiation from adult hepatic stem cells directed in vivo by $\mathrm{Ad} / \mathrm{AAV}$ hybrid vectormediated expression of NGN3, Beta2, and RIPE3b1 Y. Song ${ }^{1}$, E. Lee ${ }^{2}$, C. Ahn ${ }^{2}$, P. Yashar ${ }^{3}$, L. E. Pfaff ${ }^{3}$, L. J. Jameson ${ }^{3}$;

${ }^{1}$ Endocrinology, NHIC Hospital, Goyang-si, Republic of Korea, ${ }^{2}$ Endocrinology, Yonsei University, Seoul, Republic of Korea, ${ }^{3}$ Endocrinology, Metabolism and Molecular Medicine, Northwestern University, Chicago, United States.

Background and Aims: Recent work showed that adenovirus - mediated ectopic expression of PDX-1 induced differentiation of insulin producing cells in mouse liver. PDX-1 also activated exocrine genes in these cells, resulting in destruction of insulin producing cells. Using BETA2 (NeuroD), downstream factor of PDX-1, specific differentiation of endocrine islet - cells was demonstrated. We hypothesized that simultaneous expression of multiple transcription factors involved in islet development may enhance the differentiation of stem cells.

Materials and Methods: Expression cassettes carrying rat PDX-1 (852 bps), mouse BETA2 (1,074 bps), NGN3 (645 bps), and/or RIP3b1 (1084 bp) with IRES were inserted into Adenovirus/adeno-associated virus (Ad/AAV) hybrid vectors, in which the AAV integration machinery was incorporated into adenoviral vectors. Male CD1 mice were injected with $2 \times 10^{9} \mathrm{PFUs}$ of $\mathrm{Ad} / \mathrm{AAV}$ hybrid vectors through the tail vein after induction of diabetes by intraperitoneal administration of streptozotocin. Daily blood glucose levels were monitored and intraperitoneal glucose tolerance test were performed in mice showing normalization of blood glucose level.

Results: Hyperglycemia in diabetic mice was ameliorated after injection of Ad/AAV hybrid vectors carrying bicistronic constructs. Intraperitoneal glucose tolerance test showed near normal responses. We detected the activation of insulin and other islet-specific transcripts, including glucagon, somatostatin, islet-amyloid peptide, and PDX-1. Immunocytochemistry confirmed the presences insulin and glucagon producing cells at the hepatic periportal area.

Conclusion: Our results suggest that directed differentiation of adult stem cells by the delivery of cell-lineage specific transcription factors may be a useful strategy for the treatment of hormonal insufficiency. 


\section{OP 6}

\section{Insulin and nutrient signaling pathways}

0031

Identification of TXNIP as a marker and regulator of glucose homeostasis in humans

H. Parikh ${ }^{1}$, E. Carlsson ${ }^{1}$, W. A. Chutkow ${ }^{2}$, L. Johansson ${ }^{1}$, R. Saxena ${ }^{3}$, A. Krook ${ }^{4}$, M. Björnholm ${ }^{5}$, J. Zierath ${ }^{5}$, M. Ridderstråle ${ }^{1}$, D. Altshuler ${ }^{3}$, R. T. Lee ${ }^{2}$, A. Vaag ${ }^{1}$, L. C. Groop ${ }^{1}$, V. K. Mootha ${ }^{6}$;

${ }^{1}$ Department of Clinical Sciences/Diabetes \& Endocrinology, Lund University, Malmö, Sweden, ${ }^{2}$ Cardiovascular Division, Brigham and Women's Hospital, Cambridge, United States, ${ }^{3}$ Center for Human Genetic Research, Massachusetts General Hospital, Boston, United States, ${ }^{4}$ Department of Physiology and Pharmacology, Section Integrative Physiology, Karolinska Institutet, Stockholm, Sweden, ${ }^{5}$ Department of Molecular Medicine and Surgical Sciences, Section Integrative Physiology, Karolinska Institutet, Stockholm, Sweden, ${ }^{6}$ Department of Systems Biology, Harvard Medical School, Boston, United States.

Background and Aims: To identify novel pathways regulated by insulin in skeletal muscle using genome-wide expression profiling in human skeletal muscle.

Materials and Methods: We performed gene expression profiling in muscle biopsies taken before and after a euglycemic, hyperinsulinemic clamp using oligonucleotide (Affymetrix HGU133A GeneChip ${ }^{\circledR}$ ) microarrays in 6 nondiabetic individuals. For replication we performed real time-PCR of genes significantly influenced by insulin in 96 young non-diabetic humans. Functional studies included studies of effect of insulin and glucose in human adipoctyes, overexpression (lentiviral transduction) and siRNA gene silencing in 3T3-L1 cells and human skeletal muscle cells.

Results: Three genes were consistently influenced by insulin in all protocols $G 0 S 2$, was strongly induced by insulin $\left(1.65 \pm 0.17\right.$ vs. $\left.6.01 \pm 0.62, P<1 \times 10^{-16}\right)$, while TXNIP (thioredoxin interacting protein) $(0.18 \pm 0.02$ vs. $\left.0.06 \pm 0.01, P<1 \times 10^{-14}\right)$ and BCL6 $(0.27 \pm 0.02$ vs. $0.22 \pm 0.03, P<1 \times 10^{-5}$ ) were strongly repressed by insulin. TXNIP expression was also consistently elevated in individuals with T2DM as compare to NGT (1558.5 141.24 vs. $973.71 \pm 122.42, P=0.01 \& 14211.93 \pm 1241.44$ vs. $10181.72 \pm 673.09, P=0.02$ ) in two previously published microarray datasets of human muscle. Moreover, TXNIP expression is inversely correlated with insulin-stimulated glucose metabolism in NGT or IGT subjects $(\mathrm{N}=25$, $r=-0.67, P<0.001)$, but lost in the patients with T2DM $(\mathrm{N}=18, r=-0.21, P=0.41)$. While insulin suppressed, glu- cose upregulated TXNIP expression by $>1.5$-fold $(P<0.05)$ at $25 \mathrm{mM}$ glucose concentration in a human adipocytes cell line. A two-fold forced overexpression of TXNIP using lentiviral transduction results in both diminished basal $(-59 \%, P<0.02)$ and insulin stimulated $(-28 \%, P<0.005)$ glucose uptake while forced reduction of TXNIP expression by siRNA gene-silencing achieves the opposite effect and enhances both basal $(+157 \%, P<0.02 \&+48 \%, P<0.01)$ and insulin stimulated $(+61 \%, P<0.001 \&+49 \%, P<0.01)$ glucose uptake in 3T3-L1 cell line and human skeletal muscle cells respectively.

Conclusion: TXNIP represents a novel pathway regulated by glucose and insulin and could play a central role in the pathogenesis of metabolic defects leading to human T2DM.

\section{2}

siRNA based gene silencing reveals specialized roles of IRS-1 and IRS-2 in glucose and lipid metabolism in human skeletal muscle

K. Bouzakri ${ }^{1}$, A. Zachrisson ${ }^{1}$, L. Al-Khalili ${ }^{1}$,

B. B. Zhang ${ }^{2}$, H. A. Koistinen ${ }^{3}$, A. Krook ${ }^{1}$, J. R. Zierath ${ }^{1}$; ${ }^{1}$ Molecular Medicine and Surgery, Karolinska Institutet, Stockholm, Sweden, ${ }^{2}$ Merck Research Laboratories, Rahway, United States, ${ }^{3}$ Division of Cardiology, Department of Medicine, Helsinki University Central Hospital and Biomedicum, Finland.

Background and Aims: Insulin receptor substrates (IRS) 1 and 2 control metabolic and mitogenic responses and participate in insulin action in human skeletal muscle. Defects in IRS-1 or IRS-2 tyrosine phosphorylation contribute to insulin resistance, impaired glucose tolerance and Type 2 diabetes. We determined the importance and relative contribution of IRS-1 and IRS-2 to diverse, insulinmediated metabolic responses in human skeletal muscle.

Materials and Methods: siRNA-mediated specific gene silencing was used to suppress IRS-1 and IRS-2 in primary human skeletal muscles cells to determine whether there are specialized or redundant roles of these insulin signal transducers on insulin-mediated glucose and lipid metabolism. siRNA was transfected into $80 \%$ confluent cells and differentiation was induced the following day.

Results: In myoblasts, depletion of IRS-1 impaired cell growth and differentiation. Therefore, the effect of IRS ablation was determined in myotubes. IRS-1 silencing completely abolished insulin-mediated phosphorylation of Akt at $\mathrm{Ser}^{473}$, AS160, and FoxO1/FoxO4 and partly (50\%) decreased Akt $\mathrm{Thr}^{308}$ phosphorylation. Conversely, IRS-2 suppression was without effect on phosphorylation of $\mathrm{Akt}$ at $\mathrm{Ser}^{473}$, AS160 and, FoxO1/FoxO4, but dramatically decreased Akt Thr ${ }^{308}$ phosphorylation. The effect of siRNA 
silencing of IRS-1 or IRS-2, was determined on glucose uptake and glycogen synthesis in differentiated myotubes. In control cells (untransfected cells or cultured cells transfected with a scrambled siRNA construct), insulin increased glucose uptake 2 -fold $(\mathrm{P}<0.01)$. Insulin action on glucose uptake was prevented in IRS-1 depleted myotubes and preserved in IRS-2 depleted myotubes. Insulin increased glucose incorporation into glycogen 2.2-fold in control cells $(\mathrm{P}<0.01)$. IRS-1 silencing inhibited insulin action on glycogen synthesis $(\mathrm{p}<0.001)$, whereas IRS-2 silencing had no effect. In control cells, insulin increased palmitate uptake $(2$-fold; $\mathrm{P}<0.01)$ and decreased betaoxidation (2.8-fold; $\mathrm{P}<0.01)$. IRS-2 suppression increased basal palmitate uptake, whereas IRS-1 depletion was without effect. Insulin action on palmitate uptake was unaltered by IRS-1 silencing and reduced by IRS-2 silencing. Basal beta-oxidation was decreased $220 \%$ $(\mathrm{P}<0.02)$ in IRS-1 depleted cells. Insulin action on betaoxidation was preserved in IRS-1 depleted cells, whereas IRS-2 depletion completely abolished insulin action.

Conclusion: We provide evidence for specificity in insulin signaling responses governing myotube formation and glucose/lipid metabolism. Insulin signaling pathways involving IRS-1 are necessary for myotube formation, glucose uptake, and phosphorylation of Akt and AS160 whereas IRS-2 is dispensable. In contrast, insulin signaling pathways involving IRS-2 are necessary for lipid uptake and metabolism.

Supported by the Commission of the European Communities

\section{3}

siRNA based gene silencing reveals specialised roles for Akt1 and Akt2 on glucose and lipid metabolism in human skeletal muscle

A. K. Zachrisson ${ }^{1}$, K. Bouzakri ${ }^{1}$, L. Al-Khalili ${ }^{1}$, B. B. Zhang ${ }^{2}$, H. A. Koistinen ${ }^{3}$, A. Krook ${ }^{4}$, J. R. Zierath ${ }^{1}$; ${ }^{1}$ Molecular Medicine and Surgery, Karolinska Institutet, Stockholm, Sweden, ${ }^{2}$ Metabolic Disorders-Diabetes, Merck Research Laboratories, Rahway, United States, ${ }^{3}$ Division of Cardiology, Department of Medicine, Helsinki University Central Hospital and Biomedicum, Finland, ${ }^{4}$ Physiology and Pharmacology, Karolinska Institutet, Stockholm, Sweden.

Background and Aims: Insulin-mediated activation of PI3-kinase and Akt has been implicated in glucose and lipid metabolism. The complexity of the pathway is magnified by existence of multiple isoforms of these signaling transducers. Genetic studies in knockout mice have shown
Akt1 is involved in growth and Akt2 is involved in maintaining normal glucose homeostasis. The objective of this study was to determine the importance and relative contribution of Akt1 and Akt2 to diverse, insulin-mediated metabolic responses in human skeletal muscle.

Materials and Methods: We have used siRNA-mediated specific gene silencing to suppress Akt1 and Akt2 in primary human skeletal muscle cells to determine whether there are specialized or redundant roles of these targets in insulin-mediated signal transduction, as well as in glucose and lipid metabolism. Transfection was performed on day 3 of differentiation.

Results: Depletion of Akt2 in myoblasts impaired cell growth and differentiation, whereas Akt1 was without effect. Therefore, the effect of Akt isoform silencing was studied in myotubes. Akt2 silencing abolished insulinmediated phosphorylation of Akt at $\mathrm{Ser}^{473}, \mathrm{AS} 160$, and FoxO1/FoxO4 and partly decreased $(33 \%$; $\mathrm{P}<0.001)$ Akt $\mathrm{Thr}^{308}$ phosphorylation. Conversely, Akt1 suppression was without effect on phosphorylation of Akt at $\mathrm{Ser}^{473}$, AS160 and, FoxO1/FoxO4, but decreased $(55 \% ; \mathrm{P}<0.01)$ Akt $\mathrm{Thr}^{308}$ phosphorylation. The effect of siRNA silencing of Akt1 and Akt2, was determined on glucose uptake and glycogen synthesis 4 days after transfection. In control cells insulin increased glucose uptake 1.8 -fold $(\mathrm{P}<0.01)$. This insulin-mediated increase was prevented in Akt2 depleted cells $(\mathrm{P}<0.05)$, but preserved in cells depleted of Akt1. Insulin increased glucose incorporation into glycogen 3.2fold in control cells $(\mathrm{P}<0.001)$. Akt 2 ablation inhibited insulin action on glycogen synthesis $(\mathrm{P}<0.01)$, whereas Akt1 silencing was without affect. In control cells, insulin increased palmitate uptake 2.6-fold $(\mathrm{P}<0.01)$ and decreased beta-oxidation 3.5-fold $(\mathrm{P}<0.001)$. Akt1 suppression increased basal palmitate uptake 1.6-fold $(\mathrm{P}<0.05)$, whereas Akt2 depletion was without effect. Insulin action on palmitate uptake was increased 2-fold by Akt2 silencing $(\mathrm{P}<0.05)$ and unaltered by Akt1 silencing. Basal betaoxidation was increased 1.8 -fold $(\mathrm{P}<0.001)$ in Akt1 depleted cells and decreased 0.3 -fold $(\mathrm{P}<0.05)$ in Akt2 depleted cells. Insulin action on beta-oxidation was preserved in Akt2 depleted cells.

Conclusion: Using siRNA-mediated gene silencing, we provide that insulin signaling pathways involving Akt2 are required for myotube formation, glucose uptake, and phosphorylation of Akt and AS160. In contrast, insulin signaling pathways involving Akt1 are necessary for lipid uptake and metabolism. Akt2 can partly compensate for Akt1 to mediate lipid metabolism.

Supported by the Commission of the European Communities 


\section{4}

Reduced phosphorylation of proline-rich PKB/Akt substrate PRAS40 under conditions of high-fat diet induced insulin resistance

E. B. M. Nascimento ${ }^{1}$, M. Fodor ${ }^{2}$, R. Vlasblom ${ }^{3}$, B. Baan ${ }^{1}$, J. A. Maassen ${ }^{1}$, M. Diamant ${ }^{4}$, D. M. Ouwens ${ }^{1}$; ${ }^{1}$ Molecular Cell Biology, Leiden University Medical Center, ${ }^{2}$ Anatomy and Embryology/Central Animal Facility, Leiden University Medical Center, ${ }^{3}$ Laboratory of Physiology, ${ }^{4}$ Endocrinology/Diabetes Center, VU Medical Center, Amsterdam, The Netherlands.

Background and Aims: The number of people diagnosed with type 2 diabetes mellitus (T2DM) is rising rapidly. Insulin has its action via the insulin receptor, which results in the activation of downstream factors phosphatidylinositol 3'-kinase (PI3K) and its substrate protein kinase B (PKB/ Akt). An early clinical hallmark of T2DM is insulin resistance. This condition is associated with decreased activation of $\mathrm{PI} 3 \mathrm{~K}$ and $\mathrm{PKB} / \mathrm{Akt}$. Activation of $\mathrm{PKB} / \mathrm{Akt}$ has been implicated in different cellular processes eg. glucose uptake, cell survival and stress signaling, however the downstream protein substrates regulating these responses are poorly characterized. In the current study, novel endogenous substrates for PKB/Akt were characterized in different insulin target tissues under normal conditions and under conditions of insulin resistance.

Materials and Methods: To this end, adult male Wistar rats were put on a high-fat diet (HFD) or low-fat diet (LFD) for 7 weeks. $30 \mathrm{~min}$ prior to sacrifice, animals received insulin or saline via i.p. injection.

Results: In all examined tissues (cardiac tissue, hepatic tissue, skeletal muscle) of LFD-fed rats, insulin strongly induced the phosphorylation of a $\sim 30-\mathrm{kDa}$ (p30) and a $\sim 40$ $\mathrm{kDa}(\mathrm{p} 40)$ protein. Only in skeletal muscle and cardiac muscle, phosphorylation of a $\sim 160-\mathrm{kDa}$ (p160) was observed. p30 was identified as ribosomal protein S6 and p160 as AS160, which are involved in the regulation of protein synthesis and GLUT4 mediated glucose uptake respectively. p40 was identified as proline-rich $\mathrm{PKB} / \mathrm{Akt}$ substrate $40 \mathrm{kDa}$ (PRAS40). PRAS40 has been implicated in protection of neural cells from apoptosis, however its role in insulin action remains elusive.

In vivo phosphorylation of PRAS40 on Thr246 after insulin stimulation was observed in adipose tissue, cardiac muscle, skeletal muscle and hepatic tissue. In vitro experiments confirmed PI3K dependent signaling with the use of PI3K inhibitor wortmannin. Immunohistochemical staining showed nuclear localization of phosphorylated PRAS40 under physiological conditions in LFD-fed animals. In vitro immunofluorescent staining also displayed nuclear localization of phosphorylated PRAS40. Insulin stimulation of cultured cells increased nuclear localization of phosphorylated PRAS40, next to detection of a small amount of phosphorylated PRAS40 in the cytoplasm. Finally in HFDinduced insulin resistant animals, phosphorylation of PRAS40 was significantly reduced compared to LFD-fed animals in all examined tissues. The reduction of phosphorylated PRAS40 in HFD-fed animals was also accompanied with reduced phosphorylation of PKB/Akt.

Conclusion: PRAS40 has been identified as a novel target of in vivo insulin action. Phosphorylation of PRAS40 is increased by insulin in different insulin target tissues (skeletal muscle, cardiac muscle, liver, adipose tissue). HFD-induced insulin resistance induced results in reduced PRAS40 phosphorylation. Future experiments are planned to identify the role of PRAS40 in insulin action.

Supported by Dutch Diabetes Research

\section{5}

Insulin differentially affects glucose metabolism in human endothelial- and smooth muscle cells

M. Artwohl ${ }^{1}$, B. Brunmair ${ }^{1}$, C. Fürnsinn ${ }^{1}$, T. Hölzenbein ${ }^{2}$, G. Rainer ${ }^{3}$, A. Freudenthaler ${ }^{1}$, N. Huttary ${ }^{4}$, E. M. Porod ${ }^{1}$, S. M. Baumgartner-Parzer ${ }^{1}$;

${ }^{1}$ Department of Internal Medicine III, Division of Endocrinology and Metabolism, ${ }^{2}$ Department of Surgery, Division of Vascular Surgery, ${ }^{3}$ Department of Ophthalmology, ${ }^{4}$ Clinical Institute of Pathology, Medical University of Vienna, Austria.

Background and Aims: Diabetes mellitus and insulin resistance are characterized by vascular endothelial cell dysfunction. The molecular mechanisms linking endothelial glucose metabolism with diabetes-associated vascular disease and insulin resistance have, however, not been fully elucidated. So far, available data are controversial and are primarily restricted to animal endothelial cells. Therefore, this study aimed at analysis of the effects of insulin on glucose metabolism of human micro- and macrovascular endothelial cells and human smooth muscle cells.

Materials and Methods: Human umbilical vein(HUVECs), adult vein- (HAVECs), aortal- (HAECs), and retinal (HRECs) endothelial cells as well as smooth muscle cells (HSMCs) were incubated with insulin $(0.3 \mathrm{nmol} / \mathrm{l}$ and $1 \mu \mathrm{mol} / \mathrm{l})$ or $\mathrm{LiCl}(5$ and $10 \mathrm{mmol} / \mathrm{l})$ and evaluated for glucose transport $\left({ }^{3} \mathrm{H}-2\right.$-deoxy-glucose accumulation), glycogen synthesis (net glucose incorporation into glycogen), glycogen content (quantification of glucose from glycogen), proliferation $\left({ }^{3} \mathrm{H}\right.$-thymidine incorporation), and protein expression (Western blots). Most experiments were performed in medium containing $5.5 \mathrm{mmol} / 1$ glucose, some in the presence of $30 \mathrm{mmol} / 1$ glucose. 
Results: In HUVECs $(\mathrm{n}=6)$, insulin increased $(\mathrm{p}<0.05)$ proliferation $(+63 \pm 13 \%)$, endothelial NO synthase expression $(+61 \pm 23 \%)$ and Akt (protein kinase B) phosphorylation $(+101 \pm 38 \%)$, the latter, however, to a lesser extent than in HSMCs $(+1093 \pm 500 \%)$. Insulin stimulated glycogen synthesis in HSMCs from $1866 \pm 358$ to $3248 \pm 702 \mathrm{fmol} /$ cell/hour $(\mathrm{p}<0.05)$, but had no effect on glucose transport, glycogen synthesis, and glycogen content in the different endothelial cell species. Nevertheless, the rates of glycogen synthesis in endothelial cells were increased $(p<0.05)$ by $i$ ) $\mathrm{LiCl}$, an inhibitor of glycogen synthase kinase-3 $\beta$ (up to $+105 \pm 25 \%)$, ii) overnight glucose starvation $(+556 \pm 38 \%)$, and iii) incubation $(48 \mathrm{~h})$ with $30 \mathrm{mmol} / \mathrm{l}$ glucose $(+66 \pm 10 \%)$. But even in HAECs $(n=8)$, preincubated with high glucose, insulin neither stimulated glucose transport (339 \pm 49 versus $337 \pm 36 \mathrm{cpm}\left[{ }^{3} \mathrm{H}-2 \mathrm{~d}\right.$-glucose $] / 10^{6}$ cells/ 10 minutes, n.s.) nor glycogen synthesis $(694 \pm 98$ versus $707 \pm 71 \mathrm{fmol} /$ cell/hour, n.s.).

Conclusion: Insulin increased endothelial proliferation and endothelial NO-synthase expression and activated Akt, a key regulator of glycogen metabolism. Incubation with $\mathrm{LiCl}$ (an inhibitor of glycogen synthase kinase-3 $\beta$ ), overnight glucose starvation, and preincubation with high glucose stimulated glycogen synthesis in endothelial cells. Exposure to insulin, however, had no effect on glucose transport, glycogen synthesis, and glycogen content of human micro- and macrovascular endothelial cells. A recently described high glucose-induced de novo insulin sensitization of HAECs could not be confirmed in our study. In contrast to smooth muscle cells, insulin, apparently, has no major effects on human endothelial glucose metabolism, independent of the cells' vascular origin (micro- versus macrovascular).

\section{6}

Amino acids inhibit glucose oxidation independently of the mTOR/p70S6-kinase pathway in isolated rat skeletal muscle

K. Stadlbauer, B. Brunmair, Z. Szöcs, M. Krebs, W. Waldhäusl, C. Fürnsinn;

Dept. Med. III, Div. Endocrinol. Metab., Medical University of Vienna, Austria.

Background and Aims: Mammalian target of rapamycin (mTOR) and its downstream target p70S6-kinase (p70S6K) have been proposed to be implicated in the development of insulin resistance. This relates to the finding that activation of the mTOR/p70S6K pathway by amino acids causes serine/threonine phosphorylation of insulin receptor substrate-1, which disrupts downstream signaling events and eventually impairs glucose utilisation. The present study challenges this concept by showing that excess supply of amino acids affects glucose metabolism of isolated skeletal muscle predominantly via an other mechanism.

Materials and Methods: Isolated specimens of skeletal muscle from healthy male Sprague-Dawley rats were incubated for $2 \mathrm{~h}$ with $2.5 \%, 5 \%$, or $10 \%$ (vol/vol) of an amino acid mixture (Aminoplasmal-10\% ${ }^{\circledR}$ AP). Where indicated, 20 or $40 \mathrm{nmol} / 1$ rapamycin, a specific inhibitor of the mTOR/p70S6K pathway, was added. During the last hour of the experiment, rates of glucose oxidation and glycogen synthesis were measured in the absence or presence of $10 \mathrm{nmol} / 1$ insulin. The activity of p70S6K is given as $\%$ of the total kinase being in the phosphorylated (=active) state as detected by Western Blot analysis.

Results: In line with previous reports, the activity of p70S6K in isolated skeletal muscle decreased in response to rapamycin (\% of kinase in phosphorylated state: control, $95 \pm 3 ; 20 \mathrm{nmol} / 1 \mathrm{rapamycin}: 67 \pm 8 ; 40 \mathrm{nmol} / 1 \mathrm{rapamycin}$ : $60 \pm 8 ; \mathrm{p}<0.01$ each vs. control) and increased in response to amino acids (\% of kinase in phosphorylated state: control, $53 \pm 4 ; 2.5 \%$ AP, $64 \pm 3, \mathrm{p}<0.05 ; 5 \% \mathrm{AP}, 69 \pm 3, \mathrm{p}<0.002 ; 10 \%$ AP, $76 \pm 5 ; \mathrm{p}=0.056$, ns). Amino acid-induced activation of p70S6K was accompanied by a distinct dose-dependent reduction of insulin-stimulated glucose oxidation $(\mu \mathrm{mol}$ glucose converted into $\mathrm{CO}_{2}{ }^{*} \mathrm{~g}^{-1} * \mathrm{~h}^{-1}$ : control, 3.6 \pm 0.2 ; $2.5 \% \mathrm{AP}: 2.1 \pm 0.2 ; 5 \% \mathrm{AP}: 1.4 \pm 0.1 ; \quad 10 \% \mathrm{AP}: 0.8 \pm 0.1$; $\mathrm{p}<0.001$ each vs control). However, our findings argued against insulin desensitisation via the mTOR/p70S6K pathway being responsible for the observed impairment of glucose oxidation, (i) because rapamycin failed to counteract the effect of amino acids on glucose oxidation

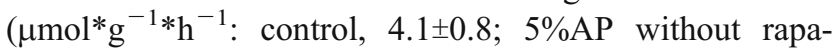
mycin: $1.5 \pm 0.2, \mathrm{p}<0.01 ; 5 \% \mathrm{AP}$ with $20 \mathrm{nmol} / 1$ rapamycin, $1.6 \pm 0.2,5 \% \mathrm{AP}$ with $40 \mathrm{nmol} / 1$ rapamycin, $1.7 \pm 0.1$, ns for effect of rapamycin), (ii) because amino acids impaired also basal, i.e. insulin independent, glucose oxidation ( $\mu \mathrm{mol}^{*} \mathrm{~g}^{-1} * \mathrm{~h}^{-1}$ : control, $2.9 \pm 0.3 ; 2.5 \% \mathrm{AP}: 1.4 \pm 0.2 ; 10 \%$ AP: $0.6 \pm 0.1 ; \mathrm{p}<0.005$ each vs control), and (iii) because insulin-stimulated glycogen synthesis, which is known to be severely blunted in insulin resistant muscle, was not affected by amino acids ( $\mu \mathrm{mol}$ glucose incorporated into glycogen ${ }^{*} \mathrm{~g}^{-1} \mathrm{~h}^{-1}$ : control, $4.2 \pm 0.3 ; 2.5 \% \mathrm{AP}: 4.4 \pm 0.3 ; 5 \%$ AP: $4.4 \pm 0.3 ; 10 \%$ AP: $3.7 \pm 0.3$; ns vs control).

Conclusion: High concentrations of amino acids distinctly inhibit glucose oxidation in isolated native skeletal muscle, which can be attributed neither to insulin desensitisation nor to activation of the $\mathrm{mTOR} / \mathrm{p} 70 \mathrm{~S} 6 \mathrm{~K}$ pathway. We speculate that the effects of excess amino acids on cellular glucose metabolism could relate to substrate competition on the level of the tricarboxylic acid cycle. 


\section{OP 7}

\section{Novel therapies - type 2 diabetes}

0037

Initial safety, tolerability and glucose lowering of CS917, a novel fructose 1,6-bisphosphatase (FBPase) inhibitor, in subjects with type 2 diabetes

S. R. Bruce, J. Walker, K. Feins, B. Tao, J. Triscari; Sankyo Pharma Development, Edison, United States.

Background and Aims: CS-917 is the prodrug of a potent and specific inhibitor of the enzyme, fructose 1,6-bisphosphatase (FBPase), being developed as a potential therapy for type 2 diabetes. This rationally designed agent is activated preferentially in the hepatocyte where it acts to suppress the augmented gluconeogenesis that contributes to fasting and post-prandial hyperglycemia in these patients.

Materials and Methods: Thirty-nine subjects (30 male, 9 female) with type 2 diabetes, mean age 52-62 years, with mean fasting glucose $205-249 \mathrm{mg} / \mathrm{dL}$, were randomized to receive one of four doses of CS-917 $(50,100,200$ or $400 \mathrm{mg}$ ) or placebo, orally, once daily in the morning, for 14 days, in sequential escalating dose panels. Evaluations included assessments of safety and tolerability. Measurements of pharmacokinetic and pharmacodynamic parameters were collected during 24-hour blood sampling periods and areas under the curve for relevant parameters were calculated on Days $-1,+1$ and +14 . An additional cohort was added that received CS-917 $400 \mathrm{mg}(\mathrm{N}=10)$ or placebo $(\mathrm{N}=4)$ QHS, evaluated for safety and tolerability.

Results: CS-917 was well tolerated in subjects with type 2 diabetes over 14 days of dosing. There were no serious adverse events. No hypoglycemia occurred. One subject (400 mg QHS) withdrew due to rash. Two subjects(400 mg QHS and $200 \mathrm{mg}$ QAM) withdrew consent. No subjects reported vomiting with QAM dosing. In the $400 \mathrm{mg}$ QHS group one subject reported nausea and one subject experienced vomiting on Day 1. Both episodes resolved and both subjects continued and completed the 14 day course.

CS-917 was rapidly absorbed, extensively metabolised and rapidly cleared from plasma in patients with type 2 diabetes. Maximum concentrations of the active metabolite, R-125338, were reached between 2 to 3 hours after both single and multiple doses. Mean exposure and Cmax for the parent and active metabolite were approximately linear over the range of 50-200 $\mathrm{mg}$.

CS-917 treatment resulted in statistically significant lowering of post-dose fasting plasma glucose (delta $\mathrm{AUC}_{\text {Glucose }}(0-6$ hours)), compared to placebo. Two subjects in the $400 \mathrm{mg}$ morning-dose group had isolated asymptomatic elevated post-prandial lactic acid levels $>4.5 \mathrm{mmol} / \mathrm{L}$. Lactic acid profiles were not different compared to placebo for doses of 50 and $100 \mathrm{mg}$ but $\mathrm{AUC}_{\text {lactic acid }}(0-6$ hours $)$ was increased versus placebo in the $400 \mathrm{mg}$ group.

Conclusion: CS-917 was generally safe and well tolerated during 14 days of treatment in subjects with type 2 diabetes. The incidence of gastrointestinal side effects was low but dose related nausea and vomiting occured at $400 \mathrm{mg}$. An initial glucose lowering effect was demonstrated with no occurence of hypoglycemia. Mean lactic acid concentrations remained within normal limits but were increased versus placebo at the highest dose of $400 \mathrm{mg}$. Further exploration of the potential efficacy of CS-917 as a novel treatment for type 2 diabetes is warranted.

Supported by Sankyo Pharma Development

\section{8}

Twelve-week efficacy and tolerability of sitagliptin, a Dipeptidyl Peptidase-4 (DPP-4) inhibitor, in Japanese patients with type 2 diabetes

K. Nonaka ${ }^{1}$, T. Kakikawa ${ }^{1}$, A. Sato ${ }^{1}$, K. Okuyama ${ }^{1}$, G. Fujimoto ${ }^{1}$, N. Hayashi ${ }^{1}$, H. Suzuki ${ }^{1}$, Y. Hirayama ${ }^{1}$, P. P. Stein ${ }^{2}$;

${ }^{1}$ Clinical Development Institute, Banyu Pharmaceutical CO., LTD., Tokyo, Japan, ${ }^{2}$ Merck \& Co., Inc., Rahway, United States.

Background and Aims: Sitagliptin (MK-0431) is an orally active, potent, selective DPP-4 inhibhitor in development for the treatment of type 2 diabetes. This randomized, double-blind, placebo-controlled, parallel group study evaluated the efficacy and tolerability of sitagliptin in Japanese patients with type 2 diabetes.

Materials and Methods: Patients with type 2 diabetes either not on an antihyperglycemic agent or on an antihyperglycemic agent in monotherapy (discontinued at entry into the run-in period), entered an 8 week diet and exercise run-in period. Patients with an A1C of 6.5 to $10.0 \%$ after the run-in period were eligible to be randomized to sitagliptin $100 \mathrm{mg}$ q.e. or placebo for a 12-week double-blind treatment period. The efficacy analysis was based on a modified intention-to-treat population with last observation carried forward.

Results: Patients ( $\mathrm{N}=151)$, ages 27-69 years, were randomized $1: 1$ to sitagliptin $(\mathrm{n}=75)$ or to placebo $(\mathrm{n}=76)$. Mean baseline A1C was 7.5\% in the sitagliptin and $7.7 \%$ in the placebo group. At week 12, A1C decreased by $0.65 \%$ in the sitagliptin group compared with an increase of $0.41 \%$ in the placebo group, for a between-treatment group difference in A1C of $1.05 \%$ (95\% CI: $1.27,0.84 \%$; $\mathrm{p}<0.001)$. In an analysis of patients achieving A1C goal, 
$58.1 \%$ of patients on sitagliptin achieved an A1C of $<7 \%$ compared with $14.5 \%$ on placebo. Fasting plasma glucose decreased by $22.5 \mathrm{mg} / \mathrm{dL}$ with sitagliptin and increased by $9.4 \mathrm{mg} / \mathrm{dL}$ with placebo after 12 weeks, for a between treatment difference of $31.9 \mathrm{mg} / \mathrm{dL} \quad(95 \%$ CI: 39.7 , $24.1 \mathrm{mg} / \mathrm{dL} ; \mathrm{p}<0.001)$. $\beta$ cell function as assessed by HOMA- $\beta$ index was significantly improved with sitagliptin group compared to placebo group. In a subset patients that underwent a meal tolerance test at baseline and at Week 12, 2-hour postprandial glucose decreased by $69.2 \mathrm{mg} / \mathrm{dL}$ in the sitagliptin group compared with an increase of $11.7 \mathrm{mg} / \mathrm{dL}$ on placebo group. 2-hour insulin and C-peptide were also increased after treatment with sitagliptin group versus placebo group. Treatment with sitagliptin was well tolerated with no reported adverse experiences of hypoglycemia. The overall incidence of adverse experiences reported was similar between treatment groups. Although body weight was slightly reduced in the sitagliptin group after 12 weeks $(-0.1 \mathrm{~kg})$, the reduction in body weight was significantly $(\mathrm{p}=0.003)$ greater in teh placebo group $(-0.7 \mathrm{~kg})$.

Conclusion: In this study, sitagliptin $100 \mathrm{mg}$ q.d. in monotherapy was efficacious and well tolerated in Japanese patients with type 2 diabetes, who no reports of hypoglycemia.

0039

Addition of Sitagliptin to pioglitazone improved glycaemic control with neutral weight effect over 24 weeks in inadequately controlled type 2 diabetes

J. Rosenstock ${ }^{1}$, R. Brazg ${ }^{2}$, P. J. Andryuk ${ }^{3}$,

C. McCrary Sisk ${ }^{3}$, K. Lu $^{3}$, P. Stein ${ }^{3}$;

${ }^{1}$ Clinical Research, Dallas Diabetes and Endocrine Center, Dallas, ${ }^{2}$ Clinical Research, Rainier Clinical Research Center, Inc., Renton, ${ }^{3}$ Merck \& Co. Inc., Rahway, United States.

Background and Aims: Thiazolidinediones are increasingly used in type 2 diabetes (T2DM) but may not provide sufficient glycemic control in monotherapy. This 24-wk, randomized, double-blind, placebo (PBO)-controlled study assessed the efficacy and safety of adding once-daily sitagliptin (SIT), a DPP-4 inhibitor, to patients with T2DM treated with pioglitazone (PIO) monotherapy.

Materials and Methods: After a diet/exercise run-in and an 8-14 wk dose-stable period on PIO (duration according to previous therapy) and a 2 -wk single-blind $\mathrm{PBO}$ run-in period, patients with $\mathrm{HbA}_{1 \mathrm{c}} \geq 7 \%-\leq 10 \%$ were randomized (1:1) to addition of PBO or SIT $100 \mathrm{mg}$ q.d. to ongoing PIO. Mean baseline characteristics of randomized patients $(\mathrm{N}=353)$ including age $56 \mathrm{yrs}$ (range 24-87 yrs); diabetes duration $6.1 \mathrm{yrs} ; \mathrm{HbA}_{1 \mathrm{c}} 8.0 \%$; fasting plasma glucose
(FPG) $9.2 \mathrm{mmol} / \mathrm{L}$; and BMI $31.5 \mathrm{~kg} / \mathrm{m}^{2}$ were similar between groups. Efficacy analysis was based on an all patients treated using ANCOVA.

Results: After 24-wks, addition of SIT to PIO produced significant $\mathrm{PBO}-$ subtracted reductions in $\mathrm{HbA}_{1 \mathrm{c}}(-0.70 \%$, $\mathrm{p}<0.001)$ and FPG $(-0.98 \mathrm{mmol} / \mathrm{L}, \mathrm{p}<0.001)$. SIT effects on glycemic control were maintained over the 24 -wk study. Endpoint $\mathrm{HbA}_{1 \mathrm{c}}$ was $7.2 \%$ and $7.8 \%$ for SIT and $\mathrm{PBO}$ and $\%$ of patients reaching target $\mathrm{HbA}_{1 \mathrm{c}}<7 \%$ was $45 \%$ and $23 \%$ $(\mathrm{p}<0.001)$, respectively. Proinsulin levels and proinsulin/ insulin ratio were significantly reduced with SIT compared with PBO, suggesting improvements in $\beta$-cell function. Sitagliptin was well tolerated with no increased risk of hypoglycemia compared with $\mathrm{PBO}$; a slightly higher incidence of abdominal pain was observed with SIT, with no significant differences in other GI AEs compared to PBO. Mean body weight change was not different between SIT and PBO when added to PIO.

Conclusion: In patients with T2DM with inadequate glycemic control on PIO, the addition of sitagliptin $100 \mathrm{mg}$ once daily led to significant lowering of $\mathrm{HbA}_{1 \mathrm{c}}$ and FPG while improving some measures of $\beta$-cell function without additional weight gain and with good tolerance over a 24-wk period.

Supported by Merck \& Co. Inc.

0040

The effect of the dipeptidyl peptidase IV inhibitor LAF237 on gastrointestinal function and glucose metabolism in type 2 diabetes

A. Vella ${ }^{1}$, G. Bock ${ }^{1}$, P. D. Giesler ${ }^{1}$, D. B. Burton ${ }^{2}$, R. A. Rizza ${ }^{1}$, M. Camilleri ${ }^{2}$;

${ }^{1}$ Endocrinology \& Metabolism, ${ }^{2}$ Gastroenterology \& Hepatology, Mayo Clinic College of Medicine, Rochester, United States.

Background and Aims: Incretin-based therapy is a useful adjunct for the treatment of type 2 diabetes. Administration of glucagon-like peptide-1 (GLP1) or GLP1 receptor (GLP1R) agonists has been shown to delay gastric emptying. Chronic administration of GLP1R agonists leads to weight loss. However, the effect of compounds that raise endogenous GLP1 concentrations by inhibition of dipeptidyl peptidase IV (DPP-IV) on gastrointestinal function is unknown. In addition, the relative contribution of DPP-IV induced changes in gastric function (if any), insulin secretion and/or glucagon suppression to improved glycemic control in people with type 2 diabetes is unknown.

Materials and Methods: To address these questions, we studied 14 subjects with type 2 diabetes who had no history of pre-existing gastrointestinal dysfunction. Using a double 
blind, placebo-controlled crossover design, subjects were given LAF237 (50 mg bid) or placebo for 10-days in random order separated by a three-week washout. On day 9 of each treatment period, subjects ate a meal consisting of $50 \mathrm{~g}$ of bacon, 2 scrambled eggs labeled with ${ }^{99 \mathrm{~m}} \mathrm{Tc}$-Sulfur colloid and $75 \mathrm{~g}$ of Jell-O labeled with $\left[1-{ }^{13} \mathrm{C}\right]$-glucose. $\left[6-{ }^{3} \mathrm{H}\right]$ glucose also was infused intravenously to enable measurement of the systemic rate of appearance of the ingested glucose. Gastric accommodation was measured on a separate study day (day 7) using single photo emission computed tomography (SPECT) and intravenous ${ }^{99 \mathrm{~m}} \mathrm{Tc}-$ sodium pertechnetate.

Results: As expected, treatment with LAF237 reduced postprandial glucose concentrations (1.048 \pm 0.0105 vs. $0.941 \pm 0.0095 \mathrm{~mol} / \mathrm{L}$ per $6 \mathrm{~h}, P=0.018)$ and suppressed glucagon $(29695 \pm 1452$ vs. $26975 \pm 1135 \mathrm{ng} / \mathrm{L}$ per $6 \mathrm{~h}$, $P=0.03)$. C-peptide concentrations did not differ between study days ( $746 \pm 92$ vs. $697 \pm 60 \mathrm{nmol} / \mathrm{L}, P=0.35$ ). LAF237 did not alter gastric emptying, gastric accommodation, or the systemic rate of appearance of the ingested glucose (table 1) Conclusion: LAF237 does not alter gastric emptying, gastric accommodation or the rate of entry of ingested glucose into the systemic circulation in humans. Therefore, in contrast to GLP1R agonists, DPP-IV inhibitors do not lower postprandial glucose concentrations by altering the rate of nutrient absorption.

Data are shown as mean + SEM

\begin{tabular}{|c|c|c|c|c|c|c|}
\hline \multirow{2}{*}{$\begin{array}{l}\text { Meal Ra } \\
\mu \mathrm{mol} / \mathrm{kg} \\
\text { per } 6 \mathrm{~h}\end{array}$} & \multicolumn{3}{|c|}{ Gastric Emptying } & \multicolumn{3}{|c|}{ Gastric Accommodation } \\
\hline & $\begin{array}{l}{ }^{9}{ }^{99 \mathrm{~m}} \mathrm{Tc} \\
\text { counts } \\
\text { retained }\end{array}$ & $\begin{array}{l}\mathrm{T}_{\text {lag }} \\
\text { (mins) }\end{array}$ & $\begin{array}{l}\mathrm{T}_{50} \\
\text { (mins) }\end{array}$ & $\begin{array}{l}\text { Fasting } \\
\text { Volume } \\
(\mathrm{mL})\end{array}$ & $\begin{array}{l}\text { Initial } \\
(0 \mathrm{~min}) \\
\text { Fed } \\
\text { Volume } \\
(\mathrm{mL})\end{array}$ & $\begin{array}{l}15 \text { minute } \\
\text { Fed } \\
\text { Volume } \\
(\mathrm{mL})\end{array}$ \\
\hline
\end{tabular}

$\begin{array}{llllllll}\text { LAF237 } & 8974 \pm 918 & 0.17 \pm 0.05 & 35 \pm 4 & 145 \pm 7 & 248.2 \pm 20.8 & 731.0 \pm 30.5 & 746.2 \pm 27.9\end{array}$

$\begin{array}{lllllllll}\text { Placebo } & 8902 \pm 525 & 0.15 \pm 0.05 & 40 \pm 6 & 143 \pm 7 & 247.3 \pm 18.9 & 747.0 \pm 23.9 & 771.8 \pm 25.9\end{array}$

\begin{tabular}{llllllll}
$P$ & 0.91 & 0.36 & 0.46 & 0.79 & 0.98 & 0.65 & 0.54 \\
\hline
\end{tabular}

Supported by Novartis, who provided LAF237 and salary support

0041

Vildagliptin suppresses Endogenous Glucose Production (EGP) and increases beta cell function after single dose administration in type 2 diabetic (T2D) patients M. Baig ${ }^{1}$, B. Balas ${ }^{1}$, C. Watson ${ }^{2}$, B. E. Dunning ${ }^{3}$, M. Ligueros-Saylan ${ }^{4}$, Y.-L. He ${ }^{5}$, K. Cusi ${ }^{1}$, J. E. Foley ${ }^{4}$, R. A. DeFronzo ${ }^{1}$;
${ }^{1}$ Diabetes, UTHSCSA, San Antonio, ${ }^{2}$ Novartis Institutes of Biomedical Research, Cambridge, ${ }^{3}$ Novartis, Princeton, ${ }^{4}$ Novartis Pharmaceuticals, East Hanover, ${ }^{5}$ Novartis Institutes of Biomedical Research, Cambridge, United States.

Background and Aims: Vildagliptin is a selective DPP-4 inhibitor that augments meal-stimulated levels of biologically active GLP-1 levels. Chronic vildagliptin treatment decreases postprandial glucose levels and reduces $\mathrm{HbA}_{1 \mathrm{c}}$ in type 2 diabetes (T2D). However, little is known about the mechanisms by which vildagliptin promotes reduction in fasting plasma glucose (FPG) concentration.

Materials and Methods: To examine the mechanisms via which vildagliptin improves glucose tolerance and reduces FPG in T2D.

Results: 16 T2D (age $=48 \pm 12$ y; $\mathrm{BMI}=34.4 \pm 6.7 ; \mathrm{HbA}_{1 \mathrm{c}}=$ $9 \pm 1.2 \%$ ) participated in a randomized, double-blind, placebo (PBO)-controlled, trial. On separate days patients received $100 \mathrm{mg}$ vildagliptin or placebo at 5:30PM followed $30 \mathrm{~min}$ later by 6 -h meal tolerance test (MTT) performed with double tracer technique $\left(3-{ }^{3} \mathrm{H}\right.$-glucose iv and $1-{ }^{14} \mathrm{C}$-glucose incorporated in meal) to assess hepatic and peripheral glucose metabolism. Following vildagliptin, suppression of endogenous glucose production (EGP) during 6-h MTT was greater than with $\mathrm{PBO}(1.02 \pm 0.06$ vs $0.74 \pm 0.06 \mathrm{mg} / \mathrm{kg} \cdot \mathrm{min}$, $\mathrm{p}=0.004$ ) while rate of total glucose disposal (TGD) was similar. During 6-h MTT performed with vildagliptin, insulin secretion rate (ISR)-calculated by deconvolution of Cpeptide plasma concentration-increased by $21 \%(\mathrm{p}=0.003$ vs PBO) despite significant reduction in mean plasma glucose $(213 \pm 4$ vs $230 \pm 4 \mathrm{mg} / \mathrm{dl}, \mathrm{p}=0.006)$; consequently, the insulinogeneic index $(\Delta \mathrm{ISR} \div \Delta$ plasma glucose) increased by $29 \%(p=0.01)$. Suppression of mean plasma glucagon during MTT was 5 times greater with vildagliptin $(p<0.02)$. From 6PM to 8AM EGP was significantly reduced $(0.8 \pm 0.06$ vs $0.5 \pm 0.06 \mathrm{mg} / \mathrm{kg} \bullet \mathrm{min}, \mathrm{p}<0.003)$ in vildagliptin vs $\mathrm{PBO}$ and the decline in EGP was positively correlated in the treated $\operatorname{arm}(\mathrm{r}=0.55, \mathrm{p}<0.03)$ with the $14 \mathrm{mg} / \mathrm{dl}$ decrease in FPG observed at 8AM.

Conclusion: During a MTT, vildagliptin augments insulin secretion and inhibits glucagon release, leading to enhanced suppression of EGP. During the entire overnight period (6PM-8AM), a single dose of vildagliptin reduced FPG by enhancing suppression of EGP.

Supported by Novartis Pharmaceuticals 


\section{2}

Sustained efficacy and tolerability of vildagliptin monotherapy during one-year treatment of drug-naive patients with type 2 diabetes

B. Goeke ${ }^{1}$, A. Schweizer ${ }^{2}$, A. Couturier ${ }^{2}$, A. LeBeaut ${ }^{2}$, S. Dejager ${ }^{2}$;

${ }^{1}$ Medicine, Ludwig Maximilian University, Munich, Germany,

${ }^{2}$ Novartis, Basel, Switzerland.

Background and Aims: Vildagliptin is a potent and selective DPP-4 inhibitor that improves glycemic control by increasing pancreatic islet responsiveness to glucose. The purpose of the present study was to assess the efficacy and tolerability of vildagliptin in comparison to metformin in drug-naïve patients (pts) with type 2 diabetes (T2DM).

Methods: This was a 52-wk, multicenter, randomized, double-blind, active-controlled, parallel-group study of vildagliptin $50 \mathrm{mg}$ bid $(\mathrm{n}=526)$ and metformin, titrated to $1000 \mathrm{mg}$ bid $(\mathrm{n}=254)$ in drug-naïve pts with T2DM. The primary endpoint was the between-group difference and the associated $95 \%$ confidence interval in the adjusted mean change $(\mathrm{AM} \Delta)$ in $\mathrm{HbA}_{1 \mathrm{c}}$ from baseline (BL) to $\mathrm{Wk} 52$ or study endpoint.

Results: The demographic and disease characteristics of the two groups were well-balanced at BL. In the randomized population, mean age $=53.1 \mathrm{y}, \mathrm{BMI}=32.4 \mathrm{~kg} / \mathrm{m}^{2}$, $\mathrm{HbA}_{1 \mathrm{c}}=8.7 \%$ and disease duration=2.4 y. As shown below, both vildagliptin $50 \mathrm{mg}$ bid and metformin $1000 \mathrm{mg}$ bid rapidly decreased $\mathrm{HbA}_{1 \mathrm{c}}$ and this improvement was sustained throughout 52-wk treatment. The AM $\Delta$ from BL to endpoint was $-1.0 \pm 0.1 \%$ in pts receiving vildagliptin and $-1.4 \pm 0.1 \%$ in those receiving metformin. The study did not establish non-inferiority of vildagliptin $100 \mathrm{mg}$ daily to metformin $2000 \mathrm{mg}$ daily in these drug-naïve patients.

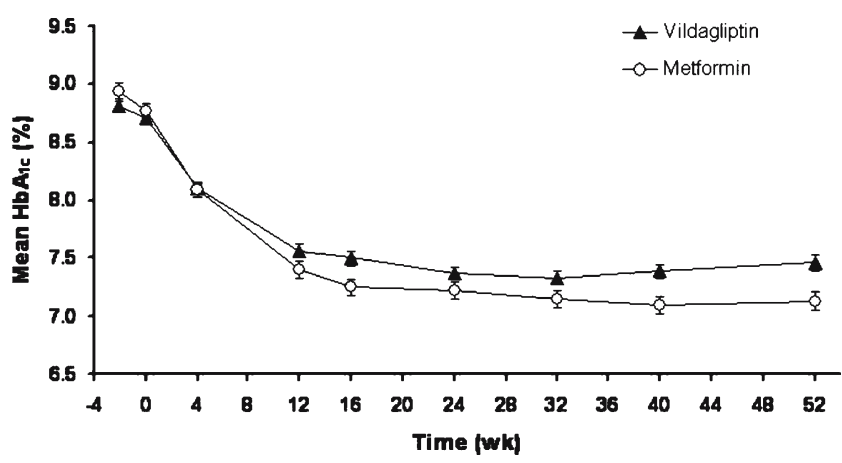

However, the percentage of pts reporting side effects was $70.1 \%$ in the vildagliptin group and $75.4 \%$ in the metformin group, and the incidence of gastrointestinal (GI) side effects was significantly less in patients pts receiving vildagliptin $(21.8 \%)$ vs those receiving metformin $(43.7 \%, \mathrm{P}<0.001)$. Diarrhea was reported by $6.0 \%$ vs $26.2 \%$ of pts treated with vildagliptin vs metformin; nausea, by $3.3 \%$ vs $10.3 \%$; abdominal pain, by 2.3 vs $7.1 \%$; dyspepsia, by 1.2 vs $4.8 \%$; vomiting, by 2.1 vs $4.4 \%$ and flatulence, by 1.0 vs $4.0 \%$. Further, a smaller percentage of pts in the vildagliptin group (4.2\%) discontinued due to an adverse event than in the metformin group (7.1\%). A mild hypoglycemic episode was reported by 3 vildagliptin-treated pts $(0.6 \%)$ and by one metformin-treated pt $(0.4 \%)$. Vildagliptin did not cause weight gain (AM $\Delta$ body weight $=0.3 \pm 0.2 \mathrm{~kg}$ ) despite the improvement in metabolic control.

Conclusions: 1) a clinically meaningful reduction in $\mathrm{HbA}_{1 \mathrm{c}}$ is sustained throughout one-year treatment with vildagliptin $50 \mathrm{mg}$ bid, 2) vildagliptin is well-tolerated and associated with fewer GI side effects than metformin.

Supported by Novartis

\section{OP 8}

\section{Predictors and markers of cardiovascular disease and mortality}

\section{3}

Predictors of mortality in type 1 diabetes - a 20 year prosepective study

P. Rossing ${ }^{1}$, H.-H. Parving ${ }^{1,2}$;

${ }^{1}$ Steno Diabetes Center, Gentofte, Denmark, ${ }^{2}$ Faculty of Health Science, Aarhus University, Denmark.

Background and Aims: To evaluate the prognostic significance of microalbuminuria and overt diabetic nephropathy and other putative risk factors for all-cause mortality in type 1 diabetic patients in a 20 year prospective observational follow up study.

Materials and Methods: All adult type 1 diabetic patients, duration of diabetes $\geq 5$ years, attending our clinic were identified in 1984 ( $n=939)$; At baseline 593 patients had normal urinary albumin excretion $(\leq 30 \mathrm{mg} / 24 \mathrm{~h}), 181 \mathrm{had}$ persistent microalbuminuria ( $>30$ and $<300 \mathrm{mg} / 24 \mathrm{~h}$ ), and 165 had overt nephropathy ( $\geq 300 \mathrm{mg} / 24 \mathrm{~h}$ ).

Results: After 20 years of follow up $34 \%$ of patients with normoalbuminuria, $47 \%$ of patients with microalbuminuria ( $p<0.001$ vs. normoalbuminuria) and $62 \%$ of patients with overt nephropathy at baseline died $(p<0.001)$. A stepwise multiple Cox regression analysis revealed the following predictors of all-cause mortality: male sex 1.84 (1.45 to 2.34) (relative risk ( $95 \%$ confidence interval)), age (years) 1.05 (1.04 to 1.06), smoking 1.67 (1.31 to 2.12), social class 4 (compared to class 5) 0.57 (0.44 to 0.75$), \log _{10}$ urinary albumin excretion rate 1.50 (1.29 to 1.74$)$, Cockroft-Gault estimated creatinine clearance $(\mathrm{ml} / \mathrm{min})$ 0.99 (0.986 to 0.995$)$ and haemoglobin $\mathrm{A}_{1 \mathrm{c}}(\%) 1.7$ (1.01 
to 1.14$)$, systolic blood pressure $(\mathrm{mm} \mathrm{Hg}) 1.01$ (1.01 to 1.02), vibration perception threshold $(\mathrm{mV}) 1.01$ (1.009 to 1.02). Height, presence of retinopathy, pulse pressure, diastolic blood pressure, and duration of diabetes were not included in the model. Median survival time after onset of overt diabetic nephropathy was 13.9 (11.8 to 17.2) years.

Conclusion: Abnormally elevated urinary albumin excretion and other potentially modifiable risk factors such as hypertension, smoking, poor glycaemic control and social class predicts increased mortality in type 1 diabetic patients.

\section{4}

High levels of advanced glycation end products predict increased cardiovascular disease mortality in women, but not in men with type 2 diabetes. A population-based 18-year follow-up study

B. K. Kilhovd ${ }^{1,2}$, A. Juutilainen ${ }^{3,4}$, S. Lehto ${ }^{3,4}$,

T. Rönnemaa ${ }^{5}$, P. A. Torjesen ${ }^{6,2}$, K. F. Hanssen ${ }^{1,2}$, M. Laakso ${ }^{3,4}$;

${ }^{1}$ Department of Medicine, Aker University Hospital HF, Oslo, Norway, ${ }^{2}$ Faculty of Medicine, University of Oslo, Norway, ${ }^{3}$ Faculty of Medicine, University of Kuopio, Finland, ${ }^{4}$ Department of Medicine, Kuopio University Hospital, Finland, ${ }^{5}$ Department of Medicine, University of Turku, Finland, ${ }^{6}$ Hormone Laboratory, Aker University Hospital HF, Oslo, Norway.

Background: Advanced glycation end products (AGEs), short- and long-term modification products of glycation or glycoxidation of proteins and lipids, have been linked to the development of premature atherosclerosis in non-diabetic and diabetic subjects. We have recently reported that high serum levels of AGEs predict increased coronary heart disease (CHD) mortality in nondiabetic women, but not in nondiabetic men. The present study investigated whether cardiovascular disease mortality (CVD) was predicted by increased serum levels of AGEs in patients with type 2 diabetes in a population based study.

Methods and Results: Serum levels of AGEs were measured with a competitive immunoassay utilizing a polyclonal antiAGE antibody and time delayed fluorescence (DELFIA), in samples obtained at baseline examination of a random sample of 874 individuals (488 men and 386 women) with diabetes aged 45 to 64 years living in Kuopio, East Finland or Turku, West Finland in 1982 to 1984 . After 18 years of follow-up, allcause mortality, CVD, and CHD mortality were registered on the basis of death certificates. Multivariate Cox regression model showed a significant association of serum AGEs with all-cause ( $\mathrm{P}=0.004)$, and CVD mortality $(\mathrm{P}=0.042)$ in women, but not in men. Fasting serum AGEs in the highest quartile was an independent risk factor for all-cause (hazards ratio [HR],
$1.61 ; 95 \% \mathrm{CI}, 1.22$ to $2.12 ; \mathrm{P}=0.001$ ), and CVD mortality (HR $1.42 ; 95 \%$ CI 1.01 to $2.00 ; \mathrm{P}=0.046$ ) in women, even after adjusting for confounding factors including highly sensitive $\mathrm{C}$ reactive protein (Table). Non-CVD deaths were mainly due to diabetes or cancer.

Conclusions: The present study is the first to show that serum levels of AGEs predict total and CVD mortality in women with type 2 diabetes.

\begin{tabular}{|c|c|c|c|c|}
\hline \multirow[b]{2}{*}{$p$-value } & \multicolumn{2}{|l|}{ Model 1} & \multicolumn{2}{|l|}{ Model 2} \\
\hline & $\operatorname{HR}(95 \% \mathrm{CI})$ & $p$-value & $\operatorname{HR}(95 \% \mathrm{CI})$ & $p$-value \\
\hline \multicolumn{5}{|c|}{ Total mortality } \\
\hline All & $1.14(0.95-1.36)$ & 0.160 & $1.18(0.98-1.43)$ & 0.077 \\
\hline Men & $0.85(0.66-1.09)$ & 0.197 & $0.89(0.69-1.16)$ & 0.399 \\
\hline Women & $1.61(1.24-2.11)$ & $<0.001$ & $1.61(1.22-2.12)$ & 0.001 \\
\hline \multicolumn{5}{|c|}{ Non-CVD mortality } \\
\hline All & $1.05(0.76-1.45)$ & 0.757 & $1.16(0.83-1.63)$ & 0.382 \\
\hline Men & $0.66(0.41-1.07)$ & 0.091 & $0.71(0.42-1.19)$ & 0.189 \\
\hline Women & $1.99(1.27-3.13)$ & 0.003 & $2.11(1.32-3.37)$ & 0.002 \\
\hline \multicolumn{5}{|c|}{ CVD mortality } \\
\hline All & $1.18(0.95-1.46)$ & 0.141 & $1.19(0.95-1.49)$ & 0.129 \\
\hline Men & $0.94(0.70-1.26)$ & 0.678 & $0.98(0.72-1.32)$ & 0.876 \\
\hline Women & $1.45(1.04-2.02)$ & 0.027 & $1.42(1.01-2.00)$ & 0.046 \\
\hline \multicolumn{5}{|c|}{ CHD mortality } \\
\hline All & $1.13(0.87-1.45)$ & 0.361 & $1.14(0.87-1.49)$ & 0.332 \\
\hline Men & $0.85(0.61-1.19)$ & 0.352 & $0.87(0.62-1.24)$ & 0.449 \\
\hline Women & $1.48(0.99-2.22)$ & 0.055 & $1.51(1.00-2.29)$ & 0.052 \\
\hline
\end{tabular}

High serum levels (the gender-specific highest quartile range vs. the three lowest quartile ranges: cutpoints $\geq 10.3 \mathrm{mU} / 1$ in diabetic men and $9.6 \geq \mathrm{mU} / 1$ in diabetic women) of AGEs as a risk factor for total, non-CVD, CVD and CHD mortality in diabetic subjects. Adjusted in model 1: for age, area of residence, and area of residence (in all), total cholesterol, current smoking, hypertension, body mass index, HDL cholesterol, triglycerides, and postmenopausal status (in women); in model 2: as in model 1 and additionally for CRP.

Supported by the Norwegian Foundation for Health and Rehabilitation, Aker Diabetes Research Fund

0045

Skin autofluorescence is a better predictor for mortality than HbA1c in well-controlled type 2 diabetes patients A. J. Smit ${ }^{1}$, H. L. Lutgers ${ }^{2}$, W. J. Sluiter ${ }^{1}$, T. P. Links ${ }^{1}$, R. G. Graaff ${ }^{3}$, H. J. G. Bilo ${ }^{2}$;

${ }^{1}$ Department of Medicine, University Medical Center Groningen, ${ }^{2}$ Diabetes Outpatient Clinic, Isala Clinics, Zwolle, ${ }^{3}$ Department of Biomedical Engineering, University Medical Center Groningen, The Netherlands. 
Background and Aims: In DCCT substudies in type 1 diabetes skin Advanced Glycation End products (AGEs) predicted diabetic complications better than $\mathrm{HbAlc}$. Type 2 diabetes mellitus is also associated with increased formation of AGEs. Some AGEs encompass a characteristic fluorescence pattern, which can be non-invasively measured as skin autofluorescence (AF). We have previously demonstrated that skin $\mathrm{AF}$ is elevated in type 2 diabetes mellitus, and strongly related to, and also predictive of chronic complications. This study aims to assess whether skin AF predicts life expectancy of T2DM-patients.

Materials and Methods: In 2001, 973 primary care T2DMpatients, participating in a shared-care project, were included. This group (mean age $66 \mathrm{yrs}$ ) was well-controlled (mean HbA1c 7.0\%) and had a relatively short diabetes duration (median 4.2 yrs), 19\% were current smokers. Skin AF was assessed at that time, and is calculated by dividing the mean value of the emitted light intensity per $\mathrm{nm}$ between 420 $600 \mathrm{~nm}$ by the mean value of the excitation light intensity per $\mathrm{nm}$ between $300-420 \mathrm{~nm}$, using a prototype of the current AGE reader.Vital status was assessed at January 2005. Life expectancy was assessed by transforming survival time to standardised survival time (SST) allowing adjustment for the baseline mortality rate of the general population.

Results: During a median follow-up of 3.2 years, 86 patients died (44 from cardiovascular disease). All cause and cardiovascular mortality were increased in the non-smoking group, with a relative risk (RR) of 2.4 (CI 1.3-3.9; $\mathrm{p}<0.005$ ) and $2.7(1,2-6.5 ; p=0.02)$, respectively. This was not found in the smokers $(19 \%)$. In a Cox regression analysis smoking, the presence of peripheral vascular disease and skin AF were the only 3 remaining factors for mortality or lifetime expectancy. HbAlc (actual, or of the previous year), blood pressure and lipid parameters were not.

Conclusions: This study shows that skin AF is a strong and independent predictor of mortality and life expectancy in a non-smoking and well-supported T2DM population, and performs better as predictor than conventional measures like $\mathrm{HbAlc}$, and blood pressure. This makes it a promising tool for rapid, non-invasive cardiovascular risk assessment in type 2 diabetes mellitus.

Supported by Diabetes Fonds Nederland

\section{6}

High level Impaired Fasting Glucose (IFG) (110 $126 \mathrm{mg} / \mathrm{dl})$, but not low level IFG $(100-110 \mathrm{mg} / \mathrm{dl})$ is associated with increased mortality and heart failure after MI

B. Vergès ${ }^{1}$, M. Zeller ${ }^{2}$, G. Dentan ${ }^{3}$, J. Beer ${ }^{2}$, Y. Laurent ${ }^{4}$, L. Janin-Manificat ${ }^{5}$, H. Makki ${ }^{6}$, Y. Cottin ${ }^{2}$;
${ }^{1}$ Endocrinology-Diabetology, ${ }^{2}$ Cardiology, Hôpital du Bocage, Dijon, ${ }^{3}$ Cardiology, Clinique de Fontaine, Fontaine les Dijon, ${ }^{4}$ Cardiology, Hôpital de Semur, ${ }^{5}$ Cardiology, Hôpital de Beaune, France, ${ }^{6}$ Cardiology, Hôpital de Chatillon, France.

Background and Aims: In a previous study, we have shown that patients hospitalized for acute MI, with IFG, according to the previous ADA criteria (110-126 mg/dl) had an overall worse outcome characterized by a significant higher risk of developing heart failure during their hospital stay. Since 2004, ADA has defined new criteria for IFG (fasting blood glucose between 100 and $126 \mathrm{mg} / \mathrm{dl}$ ), because of evidence for increased cardiovascular risk in individuals with fasting glycemia above $100 \mathrm{mg} / \mathrm{dl}$. However, so far the short-term prognosis after MI according to the new ADA criteria for IFG, and more particularly for the lower IFG level $(100-110 \mathrm{mg} / \mathrm{dl})$ has not been evaluated. This prompted us to study in-hospital outcomes according to the new criteria for IFG, using a large French regional MI registry collecting data from patients hospitalized for acute MI.

Materials and Methods: We studied 2353 patients with acute MI. The diagnosis of fasting glucose abnormality was performed using the mean of the fasting glycemias obtained at day 4 and 5 after admission. Patients were classified as diabetics (D) (known diabetes or $\mathrm{FG} \geq 126 \mathrm{mg} / \mathrm{dl}$ ), high IFG $(110 \leq \mathrm{FG}<126 \mathrm{mg} / \mathrm{dl})$, low IFG $(100 \leq \mathrm{FG}<110 \mathrm{mg} / \mathrm{dl})$ and normal fasting glycemia (NFG) (FG $<100 \mathrm{mg} / \mathrm{dl}$ ).

Results: Among the 2353 patients, 968 (41.1\%) were D, $262(11.1 \%)$ were high IFG, $332(14.1 \%)$ were low IFG and $791(33.7 \%)$ were NFG. As compared to NFG individuals, in-hospital cardiovascular mortality was increased in high IFG subjects $(5.3 \%$ vs $1.8 \%, \mathrm{p}=0.002)$ but not in low IFG subjects ( $1 \%$ vs $1.8 \%)$. As compared to NFG individuals, in-hospital heart failure was increased in high IFG subjects $(42 \%$ vs $20 \%, \mathrm{p}<0.0001)$ but not in low IFG subjects $(21 \%$ vs $20 \%)$. In multivariate analysis,after adjusment for age, gender, history of hypertension, anterior infaction, Killip class or LVEF, heart rate and blood pressure at admission, use of reperfusion therapy, high IFG, but not low IFG, was an independent factor for inhospital mortality (OR: 2.33 [1.55-3.47], $\mathrm{p}=0.03$ ) and for heart failure (OR: 1.70 [1.36-2.07], $\mathrm{p}=0.01$ ).

Conclusion: The high level of the new ADA criteria for IFG $(110-126 \mathrm{mg} / \mathrm{dl})$ is an independent factor for in-hospital CV mortality and heart failure after MI, when the low level of the new ADA criteria for IFG $(100-110 \mathrm{mg} / \mathrm{dl})$ is not. 
NT-proBNP and mortality in type 1 diabetes

L. Grimmeshave ${ }^{1}$, U. Pedersen-Bjergaard ${ }^{2}$, C. Kistorp ${ }^{3}$, J. Faber ${ }^{2}$

${ }^{1}$ Dept. Internal Medicine I, Hilleroed Hospital, ${ }^{2}$ Dept. of Endocrinology, Herlev University Hospital, Copenhagen, ${ }^{3}$ Dept. of Cardiology and Endocrinology, Frederiksberg University Hospital, Copenhagen, Denmark.

Background and Aims: N-terminal pro brain natriuretic peptide (NT-proBNP) is a well-established prognostic biomarker in patients with heart failure and acute coronary syndrome. Recent data suggest that NTproBNP may be an independent predictor of mortality in patients with diabetic nephropathy. We assessed whether NT-proBNP is a risk marker of death in patients with type 1 diabetes both with and without nephropathy.

Materials and Methods: Prospective cohort study in type 1 diabetes. Micro- and macro vascular complications were recorded at baseline, and NT-proBNP was measured in all patients. Five-year follow-up with recording of all-cause mortality.

Results: A total of 285 patients were included (median age 44, range 19-84 years, median duration of diabetes 19 years, range 3-56 years at baseline). Nineteen percent had hypertension, 7\% had known cardiovascular disease (2\% former stroke and 5\% former myocardial infarction/angina), $9 \%$ had diabetic nephropathy and $18 \%$ had microalbuminuria. The five-year all-cause mortality rate was $6.0 \%$.

At stepwise multivariate analysis, female gender $(p<0.0005)$, high age $(p<0.0005)$, presence of nephropathy $(p<0.05)$, peripheral neuropathy $(p<0.01)$ and former myocardial infarction/angina $(\mathrm{p}=0.01)$ were positively associated with elevated NT-proBNP at baseline.

All-cause mortality was significantly associated with high levels of NT-proBNP $(p<0.001)$. After exclusion of subjects with known cardiovascular disease, hypertension and diabetic nephropathy $(\mathrm{n}=195)$ high NTproBNP levels were still significantly associated $(\mathrm{p}<0.0005)$ with death.

Conclusion: Elevated NT-proBNP is associated with presence of microvascular complications as nephropathy and neuropathy. The present data suggest, that NTproBNP is a novel strong risk marker of all-cause mortality in type 1 diabetic patients with and without overt nephropathy and independent of present cardiovascular disease.
Insulin-like growth factor binding protein-1 predicts cardiovascular morbidity and mortality in patients with acute myocardial infarction and type 2 diabetes. A report from the DIGAMI 2 trial

M. Wallander ${ }^{1}$, K. Malmberg ${ }^{1}$, A. Norhammar ${ }^{1}$, J. Ohrvik ${ }^{1}$, L. Ryden ${ }^{1}$, K. Brismar ${ }^{2}$ on behalf of the DIGAMI 2 study group

${ }^{1}$ Dep. of Medicine, Cardiology Unit, Karolinska Institutet, Stockholm, ${ }^{2}$ Dep. of Molecular Medicine and Surgery, Endocrine and Diabetes Unit, Karolinska Institutet, Stockholm, Sweden.

Background and Aims: Although not a consistent finding there are indications that high levels of insulin-like growth factor binding protein-1 (IGFBP-1) may be related to cardiovascular $(\mathrm{CV})$ mortality and morbidity. The aim of this study was to test the hypothesis that high levels of IGFBP-1 predict future CV mortality and morbidity in patients with acute myocardial infarction (AMI) and type 2 diabetes (T2DM).

Materials and Methods: The DIGAMI 2 trial recruited 1253 patients with T2DM and suspected AMI of which 575 patients (men 67\%), the present material, were enrolled in a biochemical program with repeated blood sampling. Primary and secondary endpoints were CV mortality and a composite of $\mathrm{CV}$ events (CV death, reinfarction or stroke). Biochemical and clinical factors at admission where analyzed by a multiple Cox proportional hazard regression analysis. The results are presented as HR and $95 \%$ CI.

Results: During a median follow-up of 2.2 years, 102 out of the 575 patients $(18 \%)$ died from CV reasons while $175(30 \%)$ suffered from a CV event. Age ( +5 years; 1.6 , $1.4-1.8 ; \mathrm{p}<0.001)$, Ln S-IGFBP-1 (1.5, 1.2-1.9; $\mathrm{p}<0.001)$ and Ln S-Creatinine $(2.0,1.2-3.5 ; \mathrm{p}=0.013)$ remained independent predictors of $\mathrm{CV}$ mortality. The model predicting major $\mathrm{CV}$ events contained the same variables and in addition previous congestive heart failure $(1.5,1.1-2.1 ; \mathrm{p}=0.025)$. Variables that were eliminated in best subset analyses were B-glucose, HbA1c, S-Potassium, S-cholesterol, S-triglycerides, BMI, gender, duration of diabetes, smoking, previous AMI and U-albumin. Figure is presenting Kaplan-Meier curves of $\mathrm{CV}$ mortality statified for IGFBP-1 tertiles $(\mathrm{p}<0.001)$.

Conclusion: High levels of IGFBP-1 in the early phase of an AMI indicate increased risk for $\mathrm{CV}$ mortality and morbidity in patients with T2DM. 


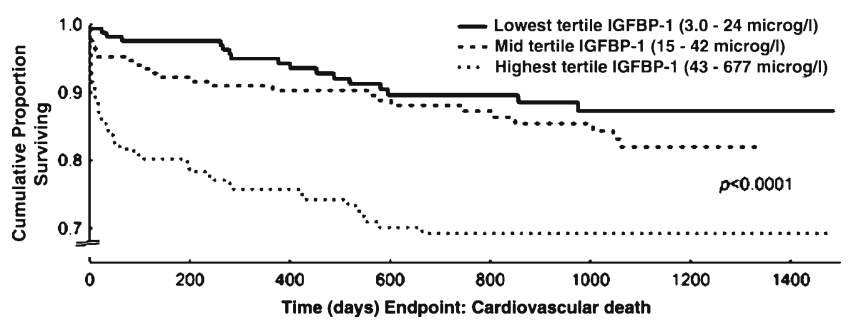

OP 9

\section{Retinopathy}

0049

Early treatment with combined antisense oligonucleotides against ECM components prevents vascular BM thickening and lesions in diabetic rat retinas

S. Roy, S. Roy, T. Oshitari, C. Allain, M. Yee, N. Tane; Medicine and Ophthalmology, Boston University School of Medicine, United States.

Background and Aims: Thickening of capillary basement membrane (BM) has been widely observed in diabetic humans and animals, and is the most prominent histological abnormality in the diabetic retina. Studies have shown that diabetes increases BM gene expression in retinal vascular cells and that the excess synthesis of BM components contributes, at least in part, to the development of thickened vascular BMs. Our findings suggest that combined antisense oligos administered soon after diabetes inhibit abnormal synthesis of extracellular matrix (ECM) components and reduce pericyte loss, acellular capillaries, and vascular leakage in the diabetic retina. In this study we determined whether combined antisense oligos approach is effective if administered after 3 months of diabetes in preventing vascular lesions associated with diabetic retinopathy.

Materials and Methods: Streptozotocin-induced diabetic rats were randomly assigned to receive either combined AS-oligos (fibronectin (FN), collagen IV (Col), and laminin (LM) oligos) or random (Ran) oligos. After 3 months of diabetes, $3 \mathrm{uM}$ combined AS oligos or Ran oligos were administered monthly for 3 months by intravitreal injection. At the end of 6 month period, the rats were sacrificed, glycohemoglobin level measured, retinas isolated, retinal trypsin digest (RTD) prepared and Western Blot (WB) analysis performed for FN, Col, and LM protein expression. Retinal vascular BM thickening was assessed by electron microscopy, and pericyte loss/acellular capillaries were determined from RTD preparations stained with hematoxylin and PAS.

Results: WB analysis showed retinal FN, Col, and LM level was upregulated in the diabetic rats compared to those of non-diabetic rats $(228 \pm 25 \%, 178 \pm 21 \%, 155 \pm 18 \%$ of control, respectively, $\mathrm{p}<0.05)$. The eyes injected with combined antisense oligos showed reduced protein expression for the three components $(131 \pm 11 \%$ of control, $136 \pm 28 \%$ of control, $114 \pm 17 \%$ of control, respectively, $\mathrm{p}<0.05)$. Retinal capillary $\mathrm{BM}$ thickness measured in diabetic rats showed a significant increase compared to those of non-diabetic control rats $(148 \pm 19 \mathrm{~nm}$ vs. $91 \pm 15 \mathrm{~nm}, \mathrm{p}<0.01)$. The BM width in retinal capillaries of diabetic rats injected with combined antisense oligos showed a significant reduction compared to those of untreated diabetic rat eyes $(109 \pm 21$ vs. $148 \pm 19 \mathrm{~nm}$, $\mathrm{p}<0.02)$. Diabetic rats treated with Ran oligos showed no difference in the retinal capillary width. The number of pericyte loss and acellular capillaries was significantly increased in retinas of diabetic rats compared with nondiabetic control rats $(160 \%$ of control, $\mathrm{p}<0.03$, and $240 \%$ of control, $\mathrm{p}<0.02$, respectively). Diabetic rats treated with combined AS oligos exhibited a significant decrease in the number of pericyte loss and acellular capillaries compared to those of untreated diabetic rats $(160 \%$ vs. $110 \%$ of control, $\mathrm{p}<0.01$, and $240 \%$ vs. $160 \%$ of control, $\mathrm{p}<0.01$, respectively. The number of pericyte loss and acellular vessels of diabetic rats that received random oligos were not different from those of untreated diabetic rats. Glycohemoglobin levels were elevated in all diabetic rats compared to those of control rats $(1.7 \pm 0.3$ fold $)$.

Conclusion: Early treatment with combined (FN, Col, LM) antisense oligos may provide a beneficial effect in reducing BM thickening and vascular lesions associated with diabetic retinopathy.

Supported by National Institutes of Health

0050

\section{WITHDRAWN}

0051

Downregulation of high glucose-induced fibronectin overexpression modulates integrin expression in microvascular endothelial cells

Y. Tane ${ }^{1,2}$, N. Tane ${ }^{1,2}$, S. Roy ${ }^{2}$;

${ }^{1}$ Ophthalmology, Shimane University Faculty of Medicine, Shimane, Japan, ${ }^{2}$ Medicine and Ophthalmology, Boston University School of Medicine, United States.

Background and Aims: Vascular basement membrane thickening and retinal vascular cell loss are two prominent lesions associated with diabetic retinopathy. While increased synthesis of extracellular matrix (ECM) compo- 
nents has been shown to contribute to the development of basement membrane thickening, the underlying cause of retinal vascular cell loss in diabetic retinopathy remains unclear. Studies have shown that high glucose and diabetes increase integrin expression, including fibronectin (FN) specific integrin receptor alpha 5 beta 1 , and may compromise interactions between cells and their basement membranes. Having downregulated fibronectin overexpression with antisense oligonucleotides targeted to fibronectin transcript, in this study, we have determined whether direct modulation of fibronectin expression alters expression of integrin alpha 5 subunit.

Materials and Methods: To determine whether modulation of FN expression affects alpha 5 subunit expression, rat microvascular endothelial cells (RMECs) were grown in high glucose condition for 9 days and transfected with 0.2 uM FN-AS oligos. After 3 days post-transfection, cells were harvested and protein analyzed by Western blot for $\mathrm{FN}$ and alpha 5 subunit expression. In parallel, cells plated on cover slips and similarly transfected with FN-AS oligos, FN and alpha 5 immunoreactivity were examined under fluorescence microscopy. Cells transfected with random oligos were used as control in Western blot analysis and experiments using immunofluorescence microscopy.

Results: Western blot analysis showed increased alpha 5 expression in cells grown in $\mathrm{HG}$ condition compared to cells grown in $\mathrm{N}$ medium $(233 \pm 23 \%$ of control, $\mathrm{p}<0.01)$. In cells transfected with AS-FN oligos, alpha 5 subunit expression was significantly reduced $(161 \pm 30 \%$ of control, $\mathrm{p}<0.01)$ compared to cells grown in HG condition. The cells grown in HG medium and transfected with random oligos showed no effect in either alpha 5 subunit or FN expression. Immunofluorescence microscopy indicated increased integrin alpha 5 immunostaining in RMECs grown in $\mathrm{HG}$ medium compared to cells grown in $\mathrm{N}$ medium $(140 \pm 11 \%$ of control, $\mathrm{p}<0.01)$. The alpha 5 subunit expression was reduced $(101 \pm 10 \%$ of control, $\mathrm{p}<0.05)$ in cells transfected with AS-FN oligos compared to cells grown in HG condition. The cells grown in HG medium and transfected with random oligos showed no effect. In both analyses, strong correlations were observed between FN expression and integrin expression.

Conclusion: The findings indicate a direct link between FN and integrin expression in microvascular endothelial cells. While downregulation of FN overexpression may prevent basement membrane thickening, it is possible that it may also prevent integrin overexpression associated with endothelial dysfunction in diabetes.

Supported by National Eye Institute, NIH, American Diabetes Association, Massachusetts Lions Eye Research Foundation, Inc., and Research to Prevent Blindness, Inc.
0052

Tight blood pressure control prevents early inflammatory events in diabetic retina

K. C. Silva, C. C. Pinto, S. K. Biswas,

J. B. Lopes de Faria, J. M. Lopes de Faria;

Faculty of Medical Sciences, State University of Campinas, Brazil.

Background and Aims: Hypertension is the main secondary factor associated with diabetic retinopathy (DR). The mechanisms by which hypertension increase the risk for DR are poorly understood. The aims of this study were to investigate the effect of diabetes and hypertension on early inflammatory phenomena in the retina of hypertensive and diabetic rats and to test the effects of blood pressure control on retinal inflammation.

Materials and Methods: Four week old spontaneously hypertensive rats (SHR) and their normotensive counterpart Wystar Kyoto (WKY) rats were rendered diabetic by intravenous injection of streptozotocin. Control groups received only vehicle (Wc and $\mathrm{Sc}$ ). Diabetic SHR rats $(\mathrm{Sd})$ were randomized for no antihypertensive treatment, for treatment with losartan $(\mathrm{Sd}+\mathrm{Los})$, or triple therapy $(\mathrm{Sd}+\mathrm{Tri})$. After 20 days, rats were sacrificed and the retinas were collected.

Results: The number of immunohistochemically detected ED1/microglial positive cells $(13.4 \pm 2.8$ vs $25.0 \pm 0.07$, $\mathrm{p}<0.0001)$ and the expression of ICAM- $1(0.7 \pm 0.1$ vs $1.6 \pm 0.6$ arbitrary units, $\mathrm{p}<0.004)$ in the retina were significantly higher in diabetic SHR than in the control. The NF-kB p65 levels were higher in SHR compared with WKY groups $(0.4 \pm 0.2$ vs $1.6 \pm 0.6$ arbitrary units, $p<0.005)$ and its increment in diabetic SHR was not significant. These abnormalities were not observed in diabetic WKY rats. The control of hypertension in SHR, either with triple therapy or losartan, reestablished the microglial status $(4.5 \pm 1.6$ and $5.0 \pm 1.0$ vs $6.6 \pm 2.0$ for $\mathrm{Sd}+$ tri and $\mathrm{Sd}+$ los vs Wc, $p=0.1)$, ICAM- 1 retinal levels $(0.5 \pm 0.2$ and $0.4 \pm 0.2$ vs $0.3 \pm 0.1$ arbitrary units for $\mathrm{Sd}+\mathrm{Tri}$ and $\mathrm{Sd}+\mathrm{Los}$ vs $\mathrm{Wc}$, $\mathrm{p}=0.1)$, and NF-kB p65 levels $(0.7 \pm 0.1$ and $0.5 \pm 0.06$ vs $0.4 \pm 0.3$ arbitrary units for $\mathrm{Sd}+\mathrm{Tri}$ and $\mathrm{Sd}+$ Los vs $\mathrm{Wc}$, $\mathrm{p}=0.1)$ to WKY levels.

Conclusion: The concomitance of diabetes and hypertension leads to exuberant inflammatory response in the retina and the tight control of blood pressure was able to abrogate these abnormalities. Inflammatory abnormalities may be involved in the mechanism by which hypertension exacerbates retinopathy in patients with diabetes.

Supported by FAPESP, CNPq 
0053

Down-regulation of the anti-angiogenic factor PEDF by insulin, rosiglitazone and VEGF: implications for neovascularization in poorly controlled diabetes

B. Wang ${ }^{1}$, P. Atherton ${ }^{2}$, G. Manning ${ }^{1}$, R. Donnelly ${ }^{1}$;

${ }^{1}$ Division of Vascular, ${ }^{2}$ Division of Clinical Physiology, The University of Nottingham, Derby, United Kingdom.

Background and Aims: Pigment Epithelium Derived Factor (PEDF) is an important anti-angiogenic protein associated inversely with diabetes-related microvascular complications, especially retinopathy, but very little is known about the metabolic and pharmacological regulation of PEDF expression in vascular cells.

Materials and Methods: Human umbilical smooth muscle cells were cultured to confluence, then starved and quiesced in low-serum medium for $24 \mathrm{~h}$. Cells were then exposed to VEGF $10 \mathrm{ng} / \mathrm{mL}$, insulin $400 \mathrm{nM}$, rosiglitazone $100 \mu \mathrm{M}$, and D-glucose 10-20 $\mu \mathrm{M}$ for $24 \mathrm{~h}$. Following cell lysis, RNA was extracted for real time RT-PCR in Bio-Rad iCycle PCR programmes.

Results: In comparison with untreated cells, three experimental interventions produced significant decreases in PEDF mRNA expression relative to the housekeeping gene B2M: e.g VEGF 0.672-fold reduction in expression $(n=4$, $\mathrm{p}=0.018)$, rosiglitazone 0.598 -fold $(\mathrm{n}=4, \mathrm{p}<0.01)$ and insulin 0.123 -fold $(n=4, p<0.01)$. In contrast, D-glucose had no effect (1.08-fold expression; $n=14, p=0.574$ ).

Conclusion: This is the first study to show that rosiglitazone, VEGF and especially insulin down-regulate the expression of an important molecule, PEDF, which inhibits key steps in angiogenesis.

\section{4}

The apoptotic response of retinal pericytes after fluctuation from high to low glucose: a comparison between bovine and human models

E. Berrone, E. Beltramo, S. Tarallo, M. Porta;

Department of Internal Medicine, University of Turin, Italy.

Background and Aims: Loss of pericytes is one of the earliest events in diabetic retinopathy, due to mechanisms that have not yet been elucidated. The pathophysiological role of pericytes has been investigated, so far, using primary bovine or rat cells in in vitro studies. However, some observations suggest that human and bovine pericytes may behave differently in experimental conditions designed to mimick the diabetic milieu. This study aimed at verifying whether pulsed exposure to high glucose may have different effects on human (HRP) and bovine retinal pericyte (BRP) apoptosis.

Materials and Methods: BRP and HRP were grown in physiological $(5.6 \mathrm{mmol} / \mathrm{L})$ or high $(28 \mathrm{mmol} / \mathrm{L})$ glucose for 7 days, and then exposed to glucose $5.6 \mathrm{mmol} / \mathrm{L}$ for another 24, 48 or $72 \mathrm{~h}$. DNA fragmentation was evaluated by ELISA, as a marker of early apoptosis. In parallel, the expression of two genes involved in apoptosis, bax and p53, was determined by RT-PCR. Results are expressed as percentages of BPR grown in physiological glucose.

Results: Apoptosis rate in physiological glucose in HRP was $9.3 \%$ than in BRP $(p=0.000)$. Seven-day exposure to high glucose increased apoptosis in BRP (190.5 $\pm 77.0 \%$, $\mathrm{p}=0.050)$, but not in HRP $(16.6 \pm 22.7 \%)$. However, when BRP kept in high glucose were returned to $5.6 \mathrm{mmol} / \mathrm{L}$, apoptosis reverted within $24 \mathrm{hrs}$ to the levels measured in BRP that had always been in physiological glucose. On the contrary, in HRP kept for 7 days in high glucose, apoptosis increased $48(\mathrm{p}=0.010)$ and $72 \mathrm{hrs}(\mathrm{p}=0.010)$ after reexposure to $5.6 \mathrm{mmol} / \mathrm{L}$.

bax expression increased and decreased concurrently with DNA fragmentation in BRP and HRP, with a clear overexpression in HRP within $24 \mathrm{~h}$ of returning to physiological glucose. p53 mRNA was unchanged in both cell types, regardless of glucose exposure.

Conclusion: Human pericytes appear to be less prone to apoptosis induced by persistently high glucose than bovine cells. However, while BRP recover when brought back to a physiological milieu, HRP appear to be more vulnerable to downwardly fluctuating glucose levels. These data reinforce the hypothesis that daily fluctuations in blood glucose play a major role in the development of diabetic retinopathy.

\section{OP 10}

\section{Novel genes in type 2 diabetes}

\section{5}

$\mathrm{N}$-myc downstream-regulated gene 2 is a novel type 2 diabetes candidate gene

M. J. Prior ${ }^{1}$, C. Swinton ${ }^{1}$, R. Fahey ${ }^{1}$, K. Carey ${ }^{2}$,

D. Cameron-Smith ${ }^{2}$, K. Walder ${ }^{1}$, G. R. Collier ${ }^{3}$,

V. C. Foletta ${ }^{1}$;

${ }^{1}$ Metabolic Research Unit, Deakin University, Geelong,

${ }^{2}$ School of Exercise and Nutrition Sciences, Deakin University, Burwood, Melbourne, ${ }^{3}$ ChemGenex

Pharmaceuticals, Geelong, Australia.

Background and Aims: Skeletal muscle is the major site for insulin-mediated glucose uptake and it is therefore a therapeutic target to reduce blood glucose levels in insulin 
resistant and diabetic individuals. In Psammomys obesus ( $P$. obesus), a polygenic rodent model of obesity and type 2 diabetes, we used microarray analysis to identify genes in the red gastrocnemius muscle that responded to exercise training. We identified a number of exercise-responsive genes, including N-myc down-regulating gene 2 (NDRG2). In both rodents and humans, NDRG2 is predominantly expressed in the brain and skeletal muscle. In skeletal muscle NDRG2 is a substrate of AKT/PKB, SGK1, RSK, and $\mathrm{p} 70 \mathrm{~S} 6 \mathrm{~K}$ but its function is not known. To further characterise NDRG2 and its possible role in diabetes, NDRG2 gene expression was analysed in normal and insulin resistant P.obesus and human individuals and in differentiating myoblasts. In addition, a yeast two hybrid screen of a human skeletal muscle cDNA library was performed using NDRG2 as bait to identify novel interacting protein partners of NDRG2.

Materials and Methods: Normal glucose tolerant (NGT) and impaired glucose tolerant (IGT) P.obesus ( $\mathrm{n}=8$ for each) were fasted for $24 \mathrm{~h}$. NGT $(\mathrm{n}=8)$ and IGT $(\mathrm{n}=20)$ human subjects were fasted for 8 hours. NDRG2 mRNA levels were quantitated by real time PCR and statistical analysis was performed using independent samples t-test. For the yeast two hybrid screen, full length NDRG2 cDNA along with a human skeletal muscle cDNA library was transformed into MaV203 yeast cells. The HIS3, URA3 and lacZ reporter genes were utilised as selectable markers of protein interaction. Protein interactions were confirmed following co-immunoprecipitation studies of overexpressed NDRG2, Arfaptin 2 and Protein interacting with C kinase 1 (PICK1) in HEK293 cells.

Results: NDRG2 gene expression was increased significantly in skeletal muscle from human IGT subjects $(\mathrm{P}=0.005)$ and from IGT P.obesus $(\mathrm{P}=0.036)$ compared to NGT controls. In differentiating L6 myotubes, NDRG2 gene expression was up-regulated 13 fold. The yeast two hybrid screen identified 9 clones that strongly interacted with NDRG2. Eight clones were identified as Arfaptin 2, a protein known to interact with RAC and ADP-ribosylation factors (ARFs) GTPases, molecules that are linked to the control of the cytoskeleton and membrane trafficking, respectively. The remaining clone was found to be PICK1, a protein that binds lipids and regulates protein traffic. Both Arfaptin 2 and PICK1 contain an arfaptin homology domain indicative of proteins involved in intracellular transport. These interactions were confirmed by co-immunoprecipitation analyses in HEK293 cells.

Conclusion: Here, we have identified NDRG2 as a novel interacting partner of PICK1 and Arfaptin 2 revealing for the first time a potential role for this molecule. Since NDRG2 is a substrate of kinases integral to insulin signaling and found differentially expressed in IGT $P$. obesus animals and human individuals, together these data suggest an involvement for NDRG2 in protein trafficking and in the development of type 2 diabetes.

Supported by ChemGenex Pharmaceuticals and Deakin University

0056

The H566H polymorphism of the lamin $\mathrm{A} / \mathrm{C}$ gene (LMNA) on chromosome 1q associates with type 2 diabetes and elevated fasting glycaemia in studies of 7,098 Danish whites

L. Wegner ${ }^{1}$, N. Grarup ${ }^{1}$, L. Hansen ${ }^{1,2}$,

K. Borch-Johnsen ${ }^{1,3}$, T. Jørgensen ${ }^{3}$, A. Vaag ${ }^{1}$, T. Hansen ${ }^{1}$, O. Pedersen ${ }^{1}$;

${ }^{1}$ Steno Diabetes Center, Niels Steensens Vej 2, Gentofte, ${ }^{2}$ Novo Nordisk A/S, Bagsværd, ${ }^{3}$ Research Centre for Prevention and Health, Glostrup University Hospital, Glostrup, Denmark.

Background and Aims: Evidence for linkage of type 2 diabetes to a region on chromosome 1q21-q24 has been provided in several populations. Among genes in this region is $L M N A$ encoding nuclear lamin $\mathrm{A}$ and $\mathrm{C}$ proteins which serve as nuclear lamina intermediate-filament proteins in nearly all somatic cells. Rare mutations in $L M N A$ cause several distinct monogenic syndromes including different subtypes of muscular dystrophy and familial partial lipodystrophy; the latter characterized by altered fat distribution, insulin resistance, and type 2 diabetes. A common synonymous variant $(\mathrm{H} 566 \mathrm{H})$ in LMNA has previously been associated with features of the metabolic syndrome. Thus, the aim of the present study was to examine the $\mathrm{H} 566 \mathrm{H}$ variant in relation to type 2 diabetes and associated quantitative metabolic traits in Danish whites.

Materials and Methods: The LMNA $\mathrm{H} 566 \mathrm{H}$ variant (c. $1908 \mathrm{C}>\mathrm{T}$, rs4641) was genotyped in the populationbased Inter99 cohort $(n=5,754)$ and in a group of 1,324 type 2 diabetic patients using mass-spectrometry of PCR products.

Results: The minor T-allele was more prevalent among 1,324 type 2 diabetic patients than in 4,386 subjects with normal glucose tolerance (27.0\% [95\% CI: $25.3-28.7 \%$ ] vs. 24.5\% [23.6-25.4\%]; odds ratio 1.17 [1.03-1.26]; $p=0.01$ ). Adjusting for the effect of age, gender, and BMI strengthened the association (additive model: $p=0.003$ ). Furthermore, a study of quantitative traits among 5,395 population-based sampled, middle-aged people without known diabetes revealed that individuals carrying the minor T-allele had higher fasting plasma glucose concentration (CC: mean $\pm \mathrm{SD}$ $5.52 \pm 0.75 \mathrm{mmol} / \mathrm{l}, \mathrm{CT}: 5.56 \pm 0.87$, TT: $5.60 \pm 1.0$; additive model: $p=0.008)$. No association with obesity or measures of insulin resistance was observed. 
Conclusion: The minor T-allele of the synonymous $\mathrm{H} 566 \mathrm{H}$ variant in $L M N A$ associates with type 2 diabetes and elevated fasting glycaemia in the general population. A possible mechanism of altered protein function is through a change in lamin $\mathrm{A}$ to $\mathrm{C}$ ratio since the nucleotide substitution occurs at the site of alternative mRNA splicing in exon 10 .

\section{7}

The genetic variation in the gene (TNMD) is associated with impaired glucose metabolism and conversion to type 2 diabetes in men of the Finnish Diabetes Prevention Study

A.-M. Tolppanen ${ }^{1}$, L. Pulkkinen ${ }^{1}$, M. Kolehmainen ${ }^{1}$, T. Salopuro ${ }^{1}$, U. Schwab ${ }^{1}$, J. Tuomilehto ${ }^{2}$, M. Uusitupa ${ }^{1}$; ${ }^{1}$ Department of Public Health and Clinical Nutrition, Division of Clinical Nutrition, University of Kuopio, ${ }^{2}$ Department of Epidemiology and Health Promotion, Diabetes and Genetic Epidemiology Unit, National Public Health Institute, Helsinki, Finland.

Background and Aims: We recently demonstrated that long-term weight reduction changes the gene expression profile of adipose tissue in overweight individuals with impaired glucose tolerance. One of the responding genes was the $\mathrm{X}$-chromosomal tenomodulin (TNMD), whose expression was decreased by weight reduction. Tenomodulin is a transmembrane protein expressed mainly in extracellular matrix of tendons, ligaments and eyes. In this study our aim was to study genetic associations between TNMD SNPs, glucose metabolism and the risk for T2DM in the Finnish Diabetes Prevention Study (DPS).

Methods: The DPS is a longitudinal intervention study consistiong of 522 obese or overweight subjects with impaired glucose tolerance (IGT). The seven SNPs from two haploblocks of the TNMD gene were selected for association studies on the basis of HapMap data. Genotyping was performed by Taqman allelic discrimination method. The linkage disequilibrium statistics were analyzed by Haploview software. Associations of SNPs with glucose and insulin levels were analyzed by general linear model using univariate analysis and the associations of SNPs with the progression of IGT to T2DM by Cox regression using SPSS 11.5 software.

Results: Genotypic effects of TNMD gene SNPs were gender-specific. The two SNPs, rs2073163 and rs1155794 belonging to the same haploblock were associated with 2hour glucose levels in men $(p=0.038$ and 0.011$)$. Furthermore, the same two markers and an additional marker, rs2073162 associated with the conversion of IGT to T2DM in men. The genotypes associating with elevated 2-hour glucose values were approximately two times more likely to develop
T2DM during follow-up. The hazard ratios were 2.192 $(p=0.025)$ for $r 22073162,2.191 \quad(p=0.027)$ for $\mathrm{rs} 2073163$ and $1.998(p=0.054)$ for rs1155974, with the model adjusted for BMI, WHR, fasting plasma glucose and intervention.

Conclusion: These results suggest that SNPs of the TNMD gene mediate gender-dependent effects on insulin and glucose metabolism and on conversion of IGT to T2DM in men. Furthermore, this study provides evidence that extracellular matrix components may play a role in the development of T2DM via modulating glucose metabolism. Supported by the Finnish Academy (211497)

\section{8}

The TT genotype of the subtype 2 bradykinin receptor (BDKRB2) is associated with a high risk for type 2 diabetes (T2D) in the general population: the DESIR study

R. Roussel ${ }^{1,2}$, N. Bellili' ${ }^{2}$, F. Fumeron ${ }^{2}$, D. Meyre ${ }^{3}$, M. Fysekidis ${ }^{2,1}$, G. Velho ${ }^{2}$, S. Hadjadj ${ }^{4}$, J. Tichet ${ }^{5}$,

M. Vaxillaire ${ }^{3}$, F. Alhenc-Gelas ${ }^{6}$, B. Balkau ${ }^{7}$, P. Froguel ${ }^{3}$, M. Marre ${ }^{1,2}$;

${ }^{1}$ Diabetology, Bichat Hospital, APHP, Paris, ${ }^{2}$ U695, INSERM, Paris, France, ${ }^{3} \mathrm{Cnrs}$ umr 8090, Département de Génétique Humaine, Lille, ${ }^{4}$ Diabetology, CHU,

Poitiers, ${ }^{5}$ IRSA, Institut Régional pour la Santé, La Riche, ${ }^{6}$ U652, INSERM, Paris, ${ }^{7}$ U258, INSERM, Villejuif, France.

Background and Aims: The Kallikrein-Kinin system may affect glucose metabolism through an insulin facilitating action, as supported by experimental data and the prevention of diabetes by ACE inhibitors in cardiovascular trials. The bradykinin metabolism and effectiveness are conditioned by subtype 2 receptor (BDKRB2), whose C-58T polymorphism was reported to affect blood pressure, cardiovascular risk and diabetic nephropathy. We tested this polymorphism on risk for T2D in a large cohort from the French general population.

Materials and Methods: Subjects took part in DESIR (Epidemiologic Data on the Insulin Resistance Syndrome), a cohort of 2576 men and 2636 women from the middleaged general population who were clinically and biologically evaluated at inclusion and at 3,6 and 9-year visits. We genotyped them for the C-58T BDKRB2 promoter polymorphism. We studied the association of this polymorphism with the prevalence of diabetes at inclusion and its incidence during the 9-year follow-up. We used a logistic model for the incidence of diabetes taking into account the BMI, age, fasting glycemia at baseline, waist circumference and gender. Also, we tested the impact of the genotypes on insulin resistance. 
Results: The $\mathrm{C}$ and $\mathrm{T}$ allelic frequencies were 57 and $43 \%$ respectively. The distribution of genotypes was in HardyWeinberg equilibrium. The genotype frequencies differed according to the presence or not of diabetes at baseline (Table, Prevalence: $p=0.002$ ) and the incidence of new cases of diabetes during the follow-up (Table, Incidence: $\mathrm{p}=0.01$ ), consistent with a recessive effect of the $\mathrm{T}$ allele.

\begin{tabular}{lllll}
\hline $\begin{array}{l}\text { Prevalence of } \\
\text { diabetes } \\
\text { at baseline }\end{array}$ & $\mathbf{C C}$ & $\mathbf{C T}$ & $\mathbf{T T}$ & Total \\
No Diabetes & 1592 & 2373 & 868 & 4833 \\
& $(97.3 \%)$ & $(97.9 \%)$ & $(95.3 \%)$ & $(100 \%)$ \\
Diabetes & 45 & 50 & 43 & 138 \\
& $(2.7 \%)$ & $(2.1 \%)$ & $(4.7 \%)$ & $(100 \%)$ \\
$\begin{array}{l}\text { Total } \\
\text { Incidence }\end{array}$ & $\mathbf{C C}$ & 2423 & 911 & 4971 \\
$\begin{array}{l}\text { of Diabetes } \\
\text { during follow-up }\end{array}$ & $\mathbf{C T}$ & $\mathbf{T T}$ & \\
$\begin{array}{l}\text { No new-onset } \\
\text { diabetes }\end{array}$ & 1158 & 1694 & 621 & 3473 \\
$\begin{array}{l}\text { New-onset } \\
\text { diabetes }\end{array}$ & $(95.7 \%)$ & $(95.5 \%)$ & $(93.0 \%)$ & $(100 \%)$ \\
Total & $(4.3 \%)$ & $(4.5 \%)$ & $(7.0 \%)$ & $(100 \%)$ \\
\hline
\end{tabular}

The odds ratio of new-onset diabetes associated with the presence of the TT genotype was 1.77 (95\% CI: 1.11-2.80) after adjustment. The population risk for incident T2D attributable to the BDKRB2 TT genotype was 10,3\% (95\% CI $2,0-18,0 \%$ ). There was no difference in HOMA-IR according to genotypes.

Conclusions: These data suggest that the BDKRB2 C-58T polymorphism strongly affects the risk for T2D in the general population. This effect is not likely to be mediated by increased insulin resistance.

Supported by ALFEDIAM

\section{9}

Variants in the GPR40 gene and association with type 2 diabetes

M. Kalis ${ }^{1}$, P. Levéen ${ }^{2}$, V. Lyssenko ${ }^{3}$, P. Almgren ${ }^{3}$, B. Olde ${ }^{4}$, C. Owman ${ }^{4}$, L. Groop ${ }^{3}$, C. M. Cilio ${ }^{1}$;

${ }^{1}$ Dept. of Clinical Sciences/Cellular Autoimmunity Unit, Lund University, Malmö, ${ }^{2}$ Dept. of Clinical Sciences/ Division of Paediatrics, Lund University, Lund, ${ }^{3}$ Dept. of Clinical Sciences/Diabetes and Endocrinology, Lund University, Malmö, ${ }^{4}$ Dept. of Clinical Sciences/Division of Molecular Neurobiology, Lund University, Malmö, Sweden.

Background and Aims: The G protein-coupled receptor GPR40 is the first gene product identified to act as an extracellular membrane receptor for FFAs. The receptor is expressed mainly in pancreatic $\beta$-cells but also in the human brain. In $\beta$-cells in vitro, the receptor is activated by medium to long chain FFAs as well as by thiazolidinediones resulting in elevated $\mathrm{Ca}^{2+}$ concentrations and promotion of insulin secretion. These properties suggest that GPR40 could be a mediator of lipotoxicity and a potential candidate gene for Type 2 diabetes (T2D). We therefore studied whether variations in the GPR 40 locus are associated with T2D by re-sequencing the GPR 40 gene and genotyping single nucleotide polymorphisms (SNPs).

Materials and Methods: GPR40 region was sequenced in 96 subjects to identify SNP polymorphisms and then SNP genotyping was used in 1929 patients with T2D and 754 healthy control subjects.

Results: This study suggests an effect of one polymorphism, located in a putative regulatory region of the GPR40 gene, on the risk of developing T2D $(\mathrm{p}=0.01)$. Furthermore, we observed an association with insulin secretion $(\mathrm{p}=0.01)$ and postprandial suppression of postprandial FFA levels $(p=0.003)$ in a sample of 1011 healthy individuals from Botnia and the rest of Finland suggesting a role for GPR40 in the regulation of the lipotoxic effect of FFAs in humans. Conclusion: In conclusion, variation in the GPR40 gene is associated with T2D, $\beta$-cell function and postprandial FFA levels.

Supported by Universitetssjukhuset MAS stiftelser och gåvor, Diabetes Program at Lund University, Albert Påhlsson's Foundation, Swedish Research Council, Sigrid Juselius Foundation, Folkhalsan Research Foundation and Swedish Diabetes Research Foundation.

\section{0}

Evidence that the $L A R S 2$ gene represents a novel type 2 diabetes mellitus gene

E. Reiling ${ }^{1}$, J. M. Dekker ${ }^{2}$, T. Hansen ${ }^{3}$, M. N. Weedon ${ }^{4}$, A. T. Hattersley ${ }^{4}$, T. M. Frayling ${ }^{4}$, E. C. van Hove ${ }^{1}$, G. Nijpels ${ }^{2}$, G. A. Hitman ${ }^{5}$, M. Walker ${ }^{6}$, M. I. McCarthy ${ }^{7}$, O. Pedersen ${ }^{3}$, R. J. Heine ${ }^{2}$, J. A. Maassen ${ }^{1,2}$, L. M. 't Hart', ${ }^{1}$ Molecular Cell Biology, Leiden University Medical Center, The Netherlands, ${ }^{2}$ EMGO Institute, VUMC, Amsterdam, The Netherlands, ${ }^{3}$ Steno Diabetes Centre, Gentofte, Denmark, ${ }^{4}$ Molecular Genetics, Peninsula Medical School, Exeter, United Kingdom, ${ }^{5}$ Centre for Diabetes and Metabolic Medicine, Bart's and The London Queen Mary's School of Medicine and Dentistry, London, ${ }^{6}$ School of Clinical Medical Sciences, University of Newcastle, ${ }^{7}$ Division of Medicine, Oxford Centre for Diabetes, United Kingdom. 
Background and Aims: It is known that mitochondrial dysfunction can lead to an increased risk for type2 diabetes mellitus (T2DM). LARS2 encodes for the mitochondrial leucyl tRNA synthetase (LeuRS), which is essential for mitochondrial protein synthesis. Previously we have shown that an H324Q polymorphism associates with an enhanced risk for T2DM in populations from the Netherlands and Denmark. In this study we performed a replication study and reconstruct the LARS2 LD structure for a haplotype based analysis.

Materials and Methods: LARS2 SNPs were genotyped using Taqman SNP genotyping assays. Enzyme activity and mischarging rate were determined using recombinant enzymes. siRNA was performed using Rat-I and INS-1E cells.

Results: In this study the large UK Warren2 cohort was genotyped for the H324Q variant (OR 1.14 (95\% CI 0.95 1.36), $\mathrm{p}=0.16, \mathrm{n}=7063)$. In a meta analysis including almost 15000 subjects from five independent studies the overall OR was 1.23 (95\% CI 1.06-1.41, $\mathrm{p}=0.004, \mathrm{n}=14899)$. We have also reconstructed LARS2 haplotypes in 1521 subjects from the Netherlands and Denmark. Eleven haplotype tagging SNPs were selected for further study based on data from the Hapmap database. We examined the LD structure and from this set five tagging SNPs were selected, which can discriminate between the most frequent haplotypes in our pilot study ( $n=354, f>2 \%$ ). This set of SNPs was used to reconstruct the haplotypes of the larger cohort. None of the 7 common haplotypes observed were significantly associated with T2DM $(\mathrm{P}>0.05)$. When we added the H324Q variant to our analysis a single haplotype carrying the $\mathrm{H} 324 \mathrm{Q}$ risk allele associated with T2DM susceptibility $(\mathrm{P}<0.05)$. Until now it is not clear via which pathogenic mechanism the $\mathrm{H} 324 \mathrm{Q}$ variant enhances T2DM susceptibility. The enzyme activity of both wild type and mutant LeuRS was measured in vitro, but no significant differences were observed. Furthermore the mischarging rate was measured. Although not significant, the mischarging rate of the mutant enzyme was increased which will induce a higher error rate in mitochondrial protein synthesis. Because LeuRS is indispensable for mitochondrial protein synthesis we have tested whether or not mitochondrial protein synthesis is affected in fibroblasts of heterozygous H324Q carriers, but we found no significant differences. Preliminary siRNA data suggest that knockdown of LeuRS results, as expected, in a decreased function of mtDNA encoded proteins confirming the importance of correct tRNA synthetase activity for mitochondrial function.

Conclusion: In conclusion we have shown that the LARS2 gene represents a T2DM susceptibility gene in several Caucasian populations. Additional risk or protecting haplotypes were not identified. Further research is needed to elucidate the exact pathogenic mechanism.

Supported by ZonMW

\section{OP 11}

\section{Molecular components of the insulin secretion pathway}

0061

Knockdown of the newly identified mitochondrial glutamate carrier GC1 and effects on glucose stimulated insulin secretion in INS-1E beta-cells

M. Casimir, B. Rubi, P. Maechler;

Department of Cell Physiology and Metabolism, University of Geneva, Switzerland.

Background and Aims: The mitochondrial glutamate carrier 1 (GC1), transporting glutamate with $\mathrm{H}+$ or in exchange to $\mathrm{OH}$ - through inner mitochondrial membrane, was previously cloned from human brain cDNA and showed high levels of expression in the whole pancreas. According to the role that glutamate might play in betacells as an additive factor in glucose stimulated insulin secretion, it was of interest to determine: i) putative expression of GC1 in insulin secreting cells, ii) in case of expression the intracellular localization of $\mathrm{GC} 1$, iii) whether knockdown of GC1 expression would affect glucose stimulated insulin secretion. Specifically, we cloned GC1 from the rat insulinoma INS-1E cells, generated an antibody against $\mathrm{GC} 1$, and investigated $\mathrm{GC} 1$ function with respect to insulin secretion by use of siRNA approach to specifically down-regulate GC1 in INS-1E beta-cells.

Materials and Methods: We first identified the rat GC1 ortholog in INS-1E beta-cells, which exhibits 95\% similarity with its human counterpart. Next, we identified siRNA construct down-regulating $\mathrm{GC} 1$ by about $70 \%$. This construct was cloned in a recombinant adenovirus and its down-regulation was assessed by immunoblotting using an antibody raised against $\mathrm{GC} 1$ selected sequence. The most efficient titer, i.e. $0.06 \mu \mathrm{l}$ per well of 24 -well plates, was used to transduce INS-1E cells for one hour and cells were subsequently analysed four days post-infection. As a control, an empty adenovirus construct was used. Following this transduction protocol, we measured several parameters, including mitochondrial membrane potential (rhodamine-123 fluorescence), cytosolic ATP changes (luciferase bioluminescence), cellular calcium levels (Fura-2 fluorescence). Insulin secretory responses (analysed by radioimmunoassay) to stimulatory glucose concentrations were tested with and without addition of cell permeant methylated glutamate.

Results: Immunofluorescence studies on INS-1E cells revealed expression and mitochondrial localization of GC1. Four days after infection with anti-GC1 siRNA adenovirus, down-regulation of $\mathrm{GC} 1$ expression was 
observed by immunoblotting using the GC1 specific antiserum. In parallel experiment, the decrease in GC1 expression correlated with significant effects on insulin secretion. In control cells, elevation of glucose from basal $2.5 \mathrm{mM}$ to $15 \mathrm{mM}$ stimulated insulin secretion 3-fold. In cells transduced with the anti-GC1 siRNA construct, we observed inhibition of glucose evoked insulin secretion by $31 \%(\mathrm{p}<0.01)$. The depletion of $\mathrm{GC} 1$, which promoted a decrease in insulin secretion, was partially restored upon provision of cell permeant methyl-glutamate. Parameters such as mitochondrial membrane potential, cytosolic ATP, and intracellular calcium levels, were not affected in cells treated with anti-GC1 siRNA construct compared to cells transduced with the empty construct. Cellular glutamate measurements are ongoing to determine changes in glutamate levels inside and outside of the mitochondrion, in order to provide insight into the directionality of glutamate transport across the mitochondrial membrane.

Conclusion: Insulin secreting cells express the newly identified glutamate carrier GC1. Knockdown experiments demonstrate that this mitochondrial carrier is necessary for the full development of the secretory response to glucose. Future investigations should delineate the specific role of $\mathrm{GC} 1$ in insulin release.

Supported by SwissNF and European Foundation for the Study of Diabetes

\section{2}

Presence and functionl importance of Kv1.1 channels in mouse islets: evidence from mice with truncated Kv1.1

A. Björklund ${ }^{1}, \mathrm{Z}_{\mathrm{Ma}}{ }^{1}, \mathrm{C}$. Lavebratt ${ }^{1}$, M. Almgren ${ }^{1}$, N. Portwood ${ }^{1}$, S. Falkmer'

${ }^{1}$ Molecular Medicine and Surgery, Karolinska Institutet, Stockholm, ${ }^{2}$ Laboratory of Pathology and Clinical Cytology, Ryhov Hospital, Jönköping, Sweden.

Background and Aims: Voltage-gated potassium channels ( $\mathrm{Kv}$ channels) are involved in repolarization. In pancreatic beta cells blocking of Kv2.1 channels leads to increase in intracellular calcium and enhanced glucose-induced insulin secretion. However, the presence and functional importance of Kv1.1 channels in beta cells is presently unclear.

The megencephaly mouse BALB/cByJ-Kv1.1 mceph/mceph (called $\mathrm{mceph} / \mathrm{mceph}$ ) displays neurological abnormalities, which are coupled to an 11 base-pair deletion in the gene coding for Kv1.1. The predicted MCEPH protein is truncated at amino acid 230 out of 495 .

The aims of the present study were 1) to test for presence of Kv1.1 expression in pancreatic islets of normal mice and to verify abnormal expression in beta cells of mceph mice.
2) to test for functional importance of Kv1.1 on insulin secretion.

Materials and Methods: Mutant and wild type mice (BALB/cByJ) were used for experiments at the age of 2.53 months. Isolated islets of Langerhans were used for RT-PCR and histopathological analysis. Insulin release was measured in batch-type incubations and perifusion experiments.

Results: Weight, blood-glucose and histological staining of pancreata by hematoxylin/eosin did not differ between mutant and wild type mice. By RT-PCR we documented for the first time, expression of the Kv1.1 gene in normal mouse islets and its presence, in mutated form, in islets from the $\mathrm{mceph} / \mathrm{mceph}$ mouse. Incremental glucose-induced insulin release was increased in mutant vs. wild type islets ( $70 \pm 10$ vs. $51 \pm 7.4 \mu \mathrm{U} /$ islet/h, $\mathrm{p}<0.03, \mathrm{n}=11)$ in batchtype incubations. The Kv1.1 channel specific blocker dendrotoxin-k increased insulin secretion in wild type islets but had no effect in mutant islets tested both in batch type incubations and in a perifusion system $(43 \%$ increase in wild type vs. $12 \%$ decrease in mutant islets, $\mathrm{p}<0.05, \mathrm{n}=7$ ).

Conclusion: Kv1.1 channels are present and of functional importance in mouse beta cells.

Supported by the Medical Research Council, Sweden

0063

$K_{\text {ATP }}$ channel independent coupling between insulin, membrane potential and $\left[\mathrm{Ca}^{2+}\right]_{c}$ in mouse pancreatic $\beta$-cells

M. Düfer ${ }^{1}$, D. Haspel ${ }^{1}$, P. Krippeit-Drews ${ }^{1}$, L. Aguilar-Bryan ${ }^{2}$, J. Bryan ${ }^{3}$, G. Drews ${ }^{1}$;

${ }^{1}$ Pharmazeutisches Institut, Universität Tübingen, Germany, ${ }^{2}$ Department of Medicine, ${ }^{3}$ Department of Molecular and Cellular Biology, Baylor College of Medicine, Houston, United States.

Background and Aims: In pancreatic $\beta$-cells from wildtype (WT) mice hyperpolarization of the plasma membrane by insulin is usually ascribed to the opening of $\mathrm{K}_{\mathrm{ATP}}$ channels. However, insulin alters the oscillations of membrane potential in $\mathrm{K}_{\mathrm{ATP}}$ channel null, Sur1KO mice thus we investigated $\mathrm{K}_{\mathrm{ATP}}$ channel independent mechanisms.

Materials and Methods: Membrane potential $\left(\mathrm{V}_{\mathrm{m}}\right)$ recordings were performed with intracellular microelectrodes or in the perforated-patch configuration; $\left[\mathrm{Ca}^{2+}\right]_{\mathrm{c}}$ and $\left[\mathrm{Na}^{+}\right]_{\mathrm{c}}$ were determined using fluorescence techniques.

Results: In Sur1KO $\beta$-cells 200-400 nM insulin hyperpolarised $\mathrm{V}_{\mathrm{m}}$ by $-4 \pm 1 \mathrm{mV}(\mathrm{n}=4)$. An initial hyperpolarization of $\mathrm{V}_{\mathrm{m}}$ was also induced by elevation of glucose from 0.5 to $15 \mathrm{mM}(-8 \pm 1 \mathrm{mV} ; \mathrm{n}=6)$. This effect coincided with a transient reduction of $\left[\mathrm{Ca}^{2+}\right]_{\mathrm{c}}(236 \pm 52$ to $33 \pm 7 \mathrm{nM}$, 
$\mathrm{P}<0.01 ; \mathrm{n}=7$ ) that was inhibited by the mitochondrial uncoupler FCCP $(0.5 \mu \mathrm{M}, \mathrm{n}=6)$. The transient drop of $\left[\mathrm{Ca}^{2+}\right]_{\mathrm{c}}$ was not due to $\mathrm{Ca}^{2+}$ sequestration by the ER and persisted in the presence of the SERCA inhibitor cyclopiazonic acid $(10 \mu \mathrm{M}, \mathrm{n}=7)$. Thus, the decrease in $\left[\mathrm{Ca}^{2+}\right]_{\mathrm{c}}$ may be caused by $\mathrm{Na} / \mathrm{Ca}$ exchange. In WT $\beta$-cells glucose stimulation also resulted in a SERCA-independent transient reduction of $\left[\mathrm{Ca}^{2+}\right]_{\mathrm{c}}$ when $\mathrm{K}_{\mathrm{ATP}}$ channels were blocked by $1 \mathrm{mM}$ tolbutamide $(278 \pm 28$ to $51 \pm 10 \mathrm{nM}, \mathrm{P}<0.001 ; \mathrm{n}=4)$. Interruption of insulin signaling by the tyrosine kinase inhibitor genistein $(n=3)$ or the PI3 kinase blocker wortmannin $(n=7)$ attenuated the $\mathrm{Ca}^{2+}$ lowering effect of glucose in Sur1KO $\beta$-cells significantly. The result suggested an autocrine effect of insulin. Since insulin is known to activate the $\mathrm{Na} / \mathrm{K}$ ATPase we tested whether glucose stimulation reduces $\left[\mathrm{Na}^{+}\right]_{\mathrm{c}}$. Increasing glucose from 0.5 to $15 \mathrm{mM}$ decreased $\left[\mathrm{Na}^{+}\right]_{\mathrm{c}}$ by $49 \pm 4$ fluorescence units $(\mathrm{n}=3)$ potentially reflecting increased $\mathrm{Na} / \mathrm{K}$ ATPase activity. The hyperpolarizing effect of glucose and the transient drop in $\left[\mathrm{Ca}^{2+}\right]_{\mathrm{c}}$ were completely suppressed by ouabain, a $\mathrm{Na} / \mathrm{K}$ ATPase blocker ( $1 \mathrm{mM}, \mathrm{n}=3)$.

Conclusion: In Sur1KO and in tolbutamide-treated WT $\beta$-cells an increase in glucose concentration lowers $\left[\mathrm{Ca}^{2+}\right]_{\mathrm{c}}$ by activation of hyperpolarizing ionic currents. This effect is diminished by interrupting the insulin signaling and by ouabain, consistent with a glucose-induced hyperpolarization of $\mathrm{V}_{\mathrm{m}}$ mediated through an autocrine inhibitory effect of insulin on $\mathrm{Na} / \mathrm{K}$ ATPase activity.

Supported by DFG Du425/1-1

\section{4}

Snapin, a collectrin binding protein, regulates insulin secretion

K. Fukui ${ }^{1}$, Y. Sato ${ }^{2}$, I. Shimomura ${ }^{1}$, K. Yamagata ${ }^{1}$;

${ }^{1}$ Department of Metabolic Medicine, ${ }^{2}$ Department of Medicine and Pathophysiology, Graduate School of Medicine, Osaka University, Japan.

Background and Aims: Mutations in the hepatocyte nuclear factor-1 $\alpha$ (HNF-1 $\alpha)$ gene cause a form of maturity onset diabetes of the young (MODY3) that is characterized by impaired insulin secretion. We have recently reported that collectrin, a novel target of HNF-1 $\alpha$ in pancreatic $\beta$-cells, controls insulin exocytosis by interacting with snapin. Although snapin has been suggested to be involved in neurotransmitter secretion, the precise role of snapin in insulin secretion remains unclear. The present study is aimed to investigate the effects of snapin in insulin secretion.

Materials and Methods: Expression of snapin was examined by Northern blotting and immunohistochemistry.
Gene expression of snapin in mouse islets was studied by quantitative RT-PCR. To study the effect of snapin on insulin secretion, the expression of snapin in INS-1 cells was suppressed by siRNA and glucose stimulated insulin secretion was examined. Functional interaction betseen snapin and collectrin was explored by transiently cotransfecting INS-1 cells with snapin and/or collectrin expression vector together with human GH expression vector. GST pull-down assay was performed to study the interaction of snapin and SNARE proteins.

Results: Immunohistochemical analysis revealed that snapin was expressed by pancreatic $\beta$-cells, but was not detected in exocrine cells. Snapin gene expression in the islets was not changed by feeding. Insulin secreton stimulated by $22 \mathrm{mM}$ glucose was significantly inhibited $(73.1 \%$ of control, $\mathrm{p}<0.05)$ in INS-1 cells by suppression of snapin (63.0\% of control). GH secretion assay revealed that while overexpression of snapin alone did not increase hGH secretion, co-transfection of collectrin and snapin significantly increased hGH secretion than transfection of collectrin alone $(162.5 \pm 7.1 \%$ vs $139.3 \pm 11.1 \%$, percent to control, $\mathrm{p}<0.05)$. GST pull-down study revealed that snapin bound to SNAP25 as well as collectrin.

Conclusion: These data suggest that snapin regulates insulin secretion by interacting with collectrin.

\section{5}

Stimulation of exocytosis by the miR-375 target protein myotrophin in insulin-secreting cells

A. Wendt, A. Lindqvist, L. Eliasson;

Endocrinology, Clinical Sciences, Malmö, Sweden.

Introduction and Aims: Micro-RNAs (miRNAs) are short stretches of non-coding RNA, which negatively regulates specific target mRNAs. We have earlier described (Poy et al 2004, Nature 432:226-230) the finding of a miRNA, miR-375, which is expressed in pancreatic $\beta$-cells. In that study, it was shown that the exocytotic response, measured using the patch clamp technique in combination with capacitance measurements, was markedly reduced in single $\beta$-cells overexpressing miR-375. This effect was specific to $\beta$-cells as no change in the exocytotic response from $\alpha$-cells was detected. To elucidate the target mRNA for miR-375, bioinformatic studies was performed. They revealed a series of potential target genes, of which myotrophin (Mtpn) was the most interesting. Myotrophin is a cytoplasmic protein expressed at high levels in the pancreatic $\beta$-cells. The aim of this study is to investigate by which meachanisms myotrophin regulates insulin secretion. 
Material and Methods: Silencing RNA (siRNA) aimed towards myotrophin (NM_008098) was designed by Ambion (UK) and cloned into pRNAT-H1.1/Shuttle (Genscript, USA). Exocytosis was monitored as increases in membrane capacitance $\left(\Delta \mathrm{C}_{\mathrm{m}}\right)$ using the standard whole-cell configuration of the patch-clamp technique. The cells were transfected with the appropriate construct $48 \mathrm{~h}$ prior to experiments. Individual insulin-containing LDCVs were visualized using TIRF (total internal reflection of fluorescence)-microscope.

Results: To investigate the effects of myotrophin on insulin secretion, siRNA directed against myotrophin (siMtpn) was introduced into single insulin-secreting cells. Exocytosis was evoked by a train of ten 500-ms depolarizations from -70 to $0 \mathrm{mV}$. In the siMtpn treated cells the exocytotic response averaged $93 \pm 21 \mathrm{fF}(\mathrm{n}=8)$ while in the control cells (treated with a scrambled non homologous sequence) the same response averaged $177 \pm 32 \mathrm{fF}(\mathrm{n}=12 ; \mathrm{P}<0.05)$. Further analysis of the exocytotic response revealed that the average capacitance increase in the initial depolarization amounted to $25 \pm 5 \mathrm{fF}$ and $59 \pm 13 \mathrm{fF}(\mathrm{P}<0.01)$ in the siMtpn treated cells and control cells, respectively. Similarly, the capacitance increase during the last nine depolarizations was reduced from $118 \pm 31 \mathrm{fF}$ under control conditions to $68 \pm 19 \mathrm{fF}$ in the presence of siMtpn. To investigate if Mtpn is involved in the docking and/ or priming of the insulin-containing vesicles, the timedependent reduction of docked vesicles upon glucose-stimulation was evaluated using TIRF-microscopy. The insulin vesicles were labelled with IAPP-emerald. The preliminary results indicate that the relative number of docked granules 5 minutes after glucose-stimulation $(15 \mathrm{mM})$ was reduced $67 \pm 7 \%(n=4)$ in cells transfected with siMtpn. This is to be compared with the control situation where the corresponding reduction amounted to only $40 \pm 10 \%(\mathrm{n}=3, \mathrm{P}<0.05)$.

Conclusions: Exocytosis as well as number of docked vesicles during glucose stimulation is reduced in cells treated with siMtpn suggesting a decreased ability in the treated cells do dock and/or prime the vesicles. Accordingly, we hypothesise that myotrophin stimulates the docking/ priming of insulin-containing LDCVs.

Supported by ESFD/Novo Nordisk Program in Diabetes Research

\section{6}

Expression of $\mathrm{GABA}_{\mathrm{A}}$ receptor $\mathrm{Cl}^{-}$channels and glucose-dependent GABA release in human pancreatic islets

M. Braun, R. Ramracheya, P. E. MacDonald,

C. J. Partridge, A. Clark, P. Rorsman;

Oxford Centre for Diabetes, Endocrinology and Metabo-

lism, Oxford University, United Kingdom.
Background and Aims: Human pancreatic $\beta$-cells contain high concentrations of the neurotransmitter $\gamma$-aminobutyric acid (GABA). Previous work on rodent islets has shown that GABA is secreted from $\beta$-cells by regulated exocytosis and may act as a paracrine and autocrine regulator of hormone secretion. We now studied the expression of GABA receptors and GABA release in human pancreatic islets.

Materials and Methods: Patch-clamp experiments were performed on dispersed human pancreatic islet cells. To detect expression of endogenous $\mathrm{GABA}_{\mathrm{A}}$ receptors, GABA (1 $\mathrm{mM}$ ) was rapidly applied to the surface of single cells using an oocyte injection system. After patch-clamping, the cell type was determined by immunocytochemistry. For studying GABA secretion from single $\beta$-cells or cell clusters, (auto-) synapses were created by infecting cells with adenoviral vectors encoding the $\mathrm{GABA}_{\mathrm{A}}$ receptor $\alpha_{1}$ and $\beta_{1}$ subunit, respectively. Exocytosis of GABA-containing vesicles was detected as transient inward currents sensitive to the $\mathrm{GABA}_{\mathrm{A}}$ receptor antagonists bicuculline $(100 \mu \mathrm{M})$ or SR95531 $(10 \mu \mathrm{M})$.

Results: Expression of $\mathrm{GABA}_{\mathrm{A}}$ receptor $\mathrm{Cl}^{-}$channels was detected in $\beta$-, $\alpha$ - and $\delta$-cells. The current density was higher in $\delta$-cells $(207 \pm 126 \mathrm{pA} / \mathrm{pF}$, receptors detected in 4 out of 4 cells) than in $\beta$-cells $(12 \pm 4 \mathrm{pA} / \mathrm{pF}, 9$ out of 18 cells $)$ and $\alpha$-cells ( $3 \mathrm{pA} / \mathrm{pF}, \mathrm{n}=1$ ). The currents could be blocked by SR95531 $(50 \mu \mathrm{M})$. Exocytotic release of GABA was observed in single $\beta$-cells infused with buffer containing $3 \mu \mathrm{M}$ free $\mathrm{Ca}^{2+}$ ( $\mathrm{n}=7$ from 3 donors). With EGTA $(50 \mu \mathrm{M})$ containing intracellular solution, GABA release was triggered by voltage-clamp depolarisations from -70 to $0 \mathrm{mV}$ $(n=3)$. GABA exocytosis was also studied in islet cell clusters. The patch-clamped cell was held at $-70 \mathrm{mV}$ and infused with solution containing $10 \mathrm{mM}$ EGTA, thus serving as a "sniffer" cell for release from neighbouring cells. Vesicular release of GABA was rarely observed at $5 \mathrm{mM}$ glucose in the extracellular medium $(0.5 \pm 0.3$ events/ min) and was strongly stimulated by increasing the glucose concentration to $10 \mathrm{mM}(39 \pm 14$ events $/ \mathrm{min} 2 \mathrm{~min}$ after wash-in, $\mathrm{p}<0.05, \mathrm{n}=4)$. At $4 \mathrm{mM}$ glucose, exocytosis of GABA was triggered by addition of the $\mathrm{K}_{\mathrm{ATP}}$ channel antagonist tolbutamide (100 $\mu \mathrm{M} ; \mathrm{n}=2)$.

Conclusion: We show here that human $\beta$-cells secrete GABA by regulated exocytosis. We demonstrate, for the first time in primary $\beta$-cells, that GABA exocytosis is stimulated by glucose. Expression of functional $\mathrm{GABA}_{\mathrm{A}}$ receptor $\mathrm{Cl}^{-}$channels was found in the 3 major islet cell types. These findings constitute the basis for a putative function of GABA as a paracrine and/or autocrine transmitter in human islets.

Supported by The Wellcome Trust and Diabetes UK 


\section{OP 12}

\section{Animal models of type 2 diabetes mellitus and insulin resistance}

0067

Cardiac overexpression of adipose differentiationrelated protein (ADRP) leads to myocardial steatosis M. Ueno ${ }^{1}$, J. Suzuki ${ }^{1}$, M. Handa ${ }^{1}$, M. Kasen ${ }^{1}$, Y. Zenimaru ${ }^{1}$, S. Takahashi ${ }^{1}$, S. Ikuyama ${ }^{2}$, T. Koizumi ${ }^{3}$, F. B. Kraemer ${ }^{4}$, I. Miyamori ${ }^{1}$;

${ }^{1}$ Medicine, University of Fukui, Japan, ${ }^{2}$ The Medical Institute of Bioregulation, University of Kyushu, Oita, Japan, ${ }^{3}$ Laboratory Animal Resources, University of Fukui, Japan ${ }^{4}$ Medicine, Stanford University, United States.

Background and Aims: Diabetic cardiomyopathy is characterized by intracellular accumulation of triglyceride (TG) droplets that leads to cellular apoptosis and eventually cardiac dysfunction. The adipose differentiation-related protein (ADRP) is a lipid droplet-associated molecule expressed in many tissues including heart, although its patho-physiological function remains uncertain. We have found that cardiac ADRP expression is upregulated in concert with cardiac TG accumulation upon fasting, while this physiological regulation is impaired in diabetes. To explore the function of cardiac ADRP, we have generated transgenic mice with heart-specific overexpression of ADRP.

Materials and Methods: The cDNA of mouse ADRP and enhanced green fluorescent protein was subcloned under a promoter of myosin heavy-chain- $\alpha$. The founders were backcrossed with C56BL/6 for 6 to 7 generations and heterozygous transgenic (Tg) mice and their Wildtype (Wt) littermates were studied. The expression of ADRP was analyzed by Western blotting and cardiac lipid content was measured enzymatically. Histological studies were performed using light and electron microscopy. To study cardiac uptake of fatty acids (FA), glucose and very lowdensity lipoprotein (VLDL), ${ }^{123}$ I-beta-methyl iodophenyl pentadecanoic acid (BMIPP), ${ }^{14} \mathrm{C}$-deoxy-D-glucose (DG) and ${ }^{125}$ I-rabbit VLDL were injected into animals. Cardiac gene expressions were analyzed by quantitative RT-PCR (qRT-PCR).

Results: We have established $2 \mathrm{Tg}$ lines, AD17 and AD19, that showed a high level of heart-specific ADRP expression on Western blotting. Light microscopy with oil red-O staining showed that $\mathrm{Tg}$ mice had many visible lipid droplets in cardiomyocytes. Electron microscopy revealed these lipid droplets as bubbly chains around clusters of mitochondria. Cardiac TG and FA contents were 8 times and $40 \%$ higher, respectively, in fed $\mathrm{Tg}$ mice compared to
Wt mice. Interestingly, $24 \mathrm{~h}$-fasted $\mathrm{Tg}$ mice showed $80 \%$ lower ADRP mRNA expression and 40\% lower TG content compared with fed Tg mice, whereas Wt mice had higher ADRP expression and TG content with fasting than feeding. Cardiac ${ }^{14} \mathrm{C}-\mathrm{DG}$ uptake was markedly reduced with fasting compared to fed condition in both genotypes, while no significant difference were seen between $\mathrm{Tg}$ and Wt. Cardiac uptake of ${ }^{123}$ I-BMIPP and ${ }^{125}$ I-VLDL were also similar in $\mathrm{Tg}$ compared with $\mathrm{Wt}$ mice in any conditions. qRT-PCR showed that expression of carnitinepalmitoyle transferase-1 (CPT-1) and uncoupling protein-2 (UCP-2) were significantly upregulated in Wt mice upon fasting, but this induction was blunted in $\mathrm{Tg}$ mice.

Conclusion: Cardiac overexpression of ADRP is sufficient to induce intracellular accumulation of TG droplets that histologically mimics diabetic cardiomyopathy. Although cardiac uptake of glucose, FA and VLDL were not altered, the blunted induction of CPT-1 and UCP-2 in Tg mice suggets a potential function of ADRP in controlling FA metabolism in the heart. Thus, cardiac ADRP could be a target to modulate diabetic cardiomyopathy.

Supported by a grant from the Ministry of Education, Culture, Sports, Science and Technology

\section{8}

In vivo lipoprotein lipase-mediated triglyceride metabolism in the human heart

J. M. Miles ${ }^{1}$, R. H. Nelson ${ }^{1}$, A. Prasad ${ }^{2}$, A. Lerman ${ }^{2}$;

${ }^{1}$ Endocrine Research Unit, ${ }^{2}$ Cardiovascular Diseases, Mayo Clinic, Rochester, United States.

Background and Aims: Animal studies have shown that circulating triglycerides are a major source of energy for the myocardium, and that excess triglyceride availability may lead to myocardial steatosis and cardiac dysfunction. Although the human heart is known to contain lipoprotein lipase (LPL), virtually no information is available regarding the possible uptake of circulating triglycerides by this tissue in humans.

Materials and Methods: We studied postabsorptive nondiabetic adults $(\mathrm{N}=6)$ during diagnostic coronary angiography. $\left[1-{ }^{14} \mathrm{C}\right]$ oleate and a lipid emulsion labeled with $\left[{ }^{3} \mathrm{H}\right]$ triolein were infused in tracer amounts to steady-state concentrations. Paired arterial and coronary sinus samples were taken every $4 \mathrm{~min}$ for $20 \mathrm{~min}$. Systemic and myocardial kinetics, including the production of $\left[{ }^{3} \mathrm{H}\right]$ oleate generated by the action of LPL on the infused triglyceride tracer, were determined. Fractional spillover of LPL-generated fatty acids was calculated as the rate of appearance $\left(\mathrm{R}_{\mathrm{a}}\right)$ of $\left[{ }^{3} \mathrm{H}\right]$ oleate $\div$ the disappearance rate of the $\left[{ }^{3} \mathrm{H}\right]$ triglyceride tracer, both for whole body and for myocardium. 
Results: The coronary simus hematocrit was higher than arterial $(36.5 \pm 1.3 \%$ vs $35.9 \pm 1.4 \%, \mathrm{P}<0.03)$. Because of this, small corrections for hemoconcentration were made for plasma FFA and triglyceride concentrations in individual subjects. Plasma total free fatty acid concentrations were lower in the coronary sinus than in the artery $(507 \pm 119$ vs $653 \pm 106 \mu \mathrm{mol} / \mathrm{L}, \mathrm{P}<0.001) .\left[{ }^{14} \mathrm{C}\right]$ oleate specific activity was lower in the coronary sinus than in the artery $(1.20 \pm 0.17$ vs $1.34 \pm 0.18 \mathrm{dpm} / \mathrm{nmol}, \mathrm{P}<0.05)$, whereas there was no difference in $\left[{ }^{3} \mathrm{H}\right]$ oleate specific activity ( $4.85 \pm 0.97$ vs $4.61 \pm 0.96 \mathrm{dpm} / \mathrm{nmol}, \mathrm{P}=\mathrm{NS})$. Systemic oleate $\mathrm{R}_{\mathrm{a}}$ was $2.7 \pm 0.6 \mu \mathrm{mol} \bullet \mathrm{kg}^{-1} \cdot \mathrm{min}^{-1}$. Myocardial production of oleate was $0.5 \pm 0.1 \%$ of systemic $R_{a}$. The fractional myocardial extraction of radiolabeled oleate was higher than that of radiolabeled triglyceride $(34 \pm 5 \%$ vs $11 \pm 3 \%, \mathrm{P}<0.001)$. The contribution of the heart to whole body disappearance of free fatty acids and triglycerides was $3.1 \pm 1.1 \%$ and $2.1 \pm 0.7 \%$, respectively. Systemic fractional spillover of LPL-generated fatty acids was higher than myocardial ( $49 \pm 7 \%$ vs $20 \pm 3 \%, \mathrm{P}<0.02)$.

Conclusion: These results indicate that in humans, myocardial LPL acts on circulating lipoproteins, resulting in uptake of fatty acids from triglycerides. Systemically, the heart is a minor site of uptake for both free fatty acids and triglycerides. It is also a minor source of systemic FFA $R_{a}$. Myocardial uptake of LPL-generated triglyceride fatty acids is relatively efficient compared with whole body uptake. Additional study is needed to determine whether myocardial triglyceride uptake is increased in individuals with hypertriglyceridemia, and whether it increases after ingestion of meals containing fat.

Supported by NIH RO1 HL67933

\section{9}

Skeletal muscle mitochondrial function and intramyocellular lipids in the diabetic Zucker rat: baseline data of a longitudinal study using in vivo ${ }^{31} \mathrm{P}$ and ${ }^{1} \mathrm{H} \mathrm{MR}$ spectroscopy

H. M. M. De Feyter ${ }^{1}$, S. J. Majoor ${ }^{1}$, M. K. Hesselink ${ }^{2}$, P. Schrauwen ${ }^{3}$, J. J. Prompers ${ }^{1}$, K. Nicolay ${ }^{1}$;

${ }^{1}$ Biomedical NMR, Eindhoven University of Technology,

${ }^{2}$ Department of Movement Sciences, ${ }^{3}$ Department of Human Biology, Maastricht University, The Netherlands.

Background and Aims: Various mechanisms have been proposed to explain the relation between mitochondrial dysfunction and insulin resistance, often involving a role for intramyocellular lipids (IMCL). Data supporting these different hypotheses are derived from cross-sectional studies, making it difficult to determine whether mitochondrial dysfunction is either a cause or a consequence of insulin resistance. Thus, we investigate both mitochondrial function and IMCL levels using a longitudinal study design in the growing diabetic ZDF rat, a rodent model with progressive loss of insulin sensitivity during maturation resulting in overt diabetes in adulthood.

Materials and Methods: ZDF (fa/fa) $(n=10)$ and control $(\mathrm{fa} /+)(\mathrm{n}=11)$ rats are observed during the development of type 2 diabetes. At distinct time points during the pathogenesis (pre-diabetic: 6 weeks, diabetic: 12 weeks and long-term diabetic: 18 weeks of age) mitochondrial function and IMCL are measured using non-invasive ${ }^{31} \mathrm{P}$ MR spectroscopy (MRS) and ${ }^{1} \mathrm{H}$ MRS, respectively. Mitochondrial function was measured in tibialis anterior muscle (TA) as post-exercise phosphocreatine (PCr) recovery time constant $\left(\tau_{\mathrm{PCr}}\right)$ using ${ }^{31} \mathrm{P}$ MRS after in vivo electrical stimulation. ${ }^{1} \mathrm{H}$ MRS is used to measure IMCL levels in the white (ventral) and red (dorsal) TA.

Results: Baseline data at 6 weeks of age are shown in table 1. Fasting plasma glucose levels and in vivo mitochondrial oxidative capacity $\left(\tau_{\mathrm{PCr}}\right)$ was similar in fa/fa and fa/+rats. The fa/fa rats had a higher body mass and higher food intake compared to the fa/tanimals (all $\mathrm{p}<0.001$ ). The in vivo ${ }^{1} \mathrm{H}$ MRS measurements clearly showed higher IMCL levels in both the white and red TA (all $\mathrm{p}<0.001$ for $\mathrm{fa} / \mathrm{fa}$ vs. fa/ + ).

Conclusion: At 6 weeks of age, the fa/fa rats are hyperphagic, have a higher body weight and also IMCL content was already higher in fa/fa rats. Plasma glucose levels in the $\mathrm{fa} / \mathrm{fa}$ rats were still normal. Interestingly, despite obviously higher IMCL levels, the fa/fa rats did not show an impaired in vivo skeletal muscle mitochondrial function since $\tau_{\mathrm{PCr}}$ was the same as in fa/+animals. Overall, these results suggest that in the pre-diabetic stage, fa/fa animals do not have an impaired skeletal muscle mitochondrial function. Mitochondrial function and IMCL content will be monitored during the pathogenesis of type 2 diabetes and data at 12 and 18 weeks of age will be shown.

\begin{tabular}{|c|c|c|c|c|c|c|}
\hline & $\begin{array}{l}\text { Body } \\
\text { weight } \\
\text { (gr) }\end{array}$ & $\begin{array}{l}\text { Food } \\
\text { intake } \\
\text { (gr) }\end{array}$ & $\begin{array}{l}\text { Fasting } \\
\text { glucose } \\
(\mathrm{mM})\end{array}$ & $\begin{array}{l}\text { IMCL } \\
\text { white } \\
\text { TA } \\
(\% \text { of } \\
\mathrm{H}_{2} \mathrm{O} \\
\text { signal) }\end{array}$ & $\begin{array}{l}\text { IMCL } \\
\text { red TA } \\
(\% \text { of } \\
\mathrm{H} 2 \mathrm{O} \\
\text { signal) }\end{array}$ & $\begin{array}{l}\tau \mathrm{p}_{\mathrm{Cr}} \\
(\mathrm{s})\end{array}$ \\
\hline $\begin{array}{l}\mathrm{fa} / \mathrm{fa} \\
(\mathrm{n}=10)\end{array}$ & $193.6 \pm 1.9 *$ & $22.7 \pm 1.0 *$ & $5.3 \pm 0.3$ & $\begin{array}{l}0.46 \pm \\
0.03 *\end{array}$ & $\begin{array}{l}0.80 \pm \\
0.04 *\end{array}$ & $45.0 \pm 4.4$ \\
\hline $\begin{array}{l}\mathrm{fa} /+ \\
(\mathrm{n}=11)\end{array}$ & $147.2 \pm 3.3$ & $13.8 \pm 0.2$ & $4.7 \pm 0.4$ & $\begin{array}{l}0.16 \pm \\
0.02\end{array}$ & $\begin{array}{l}0.34 \pm \\
0.01\end{array}$ & $45.6 \pm 3.0$ \\
\hline
\end{tabular}

Typical parameters at 6 weeks of age. $\mathrm{IMCL}=$ intramyocellular lipids, $\mathrm{TA}=$ tibialis anterior muscle, $\tau \mathrm{p}_{\mathrm{Cr}}=$ phosphocreatine recovery time constant, ${ }^{*}=\mathrm{p}>0.001$ vs. $\mathrm{Fa} /+$ 
0070

Tg972 mice show a global impairment of glucose homeostasis and insulin action

M. L. Hribal ${ }^{1}$, M. Federici ${ }^{2}$, F. Tornei ${ }^{2}$, R. Amoruso ${ }^{2}$, A. Pujol ${ }^{3}$, R. Menghini ${ }^{2}$, R. Lauro ${ }^{2}$, F. Bosch ${ }^{3}$, G. Sesti ${ }^{1}$; ${ }^{1}$ Exp. and Clinical Medicine, University of Catanzaro Magna Graecia, Italy, ${ }^{2}$ Internal Medicine, University of Rome Tor Vergata, Italy, ${ }^{3}$ Biochemistry and Molecular Biology, University of Barcelona, Bellaterra, Spain.

Background and Aims: Several association studies have suggested that the G972 variant of the IRS-1 gene increase the risk for diabetes, obesity and atherosclerosis. In the last decade, we have addressed the functional significance of G972R variant in in vitro models, showing that the presence of this variant results in an impaired association of IRS-1 with the p85 subunit of PI3K , impaired glucose metabolism at the level of skeletal muscle and impaired insulin secretion and survival in pancreatic beta-cells. More recently we have genearated a transgenic mouse model overexpressing G972R IRS-1 under the control of the CMV promoter ( $\mathrm{Tg} 972)$ to address the impact of this variant on insulin resistance pathogenesis in vivo. Preliminary data showed that $\operatorname{Tg} 972$ mice develop age-related hyperglycemia and impaired glucose tolerance. On the basis of these preliminary findings, we proceeded to investigate the contribution of different insulin-sensitive tissues to the worsening of glucose metabolism in these mice.

Materials and Methods: We performed glucose tolerance tests incorporating the tracer ${ }^{3} \mathrm{H}$-2-deoxyglucose to evaluate tissue specific glucose utilization and western blot assays to assess insulin signaling pathway activation in the different tissues. To specifically assess insulin secretion in these mice we purified pancreatic islets from Tg972 mice and their WT littermates and evaluated insulin content and insulin release after glucose stimulation from the isolated islets.

Results: We demonstrated that glucose uptake was markedly impaired in both muscle and adipose tissue from Tg972 compared to WT littermates $(\mathrm{p}<0.05)$ and glycogen incorporation was significantly reduced in liver from $\operatorname{Tg} 972$ $(p<0.01)$. These changes were paralleled by a reduced activation of the insulin signaling pathway; in fact, despite an increased IRS-1 expression and total pTyr phosphorylation in all the three tissues, we observed a decreased IRS-1 associated PI3K activity and Akt phosphorylation $(p<0.001)$. Furthermore it has recently been demonstrated that the introduction of the G972 variant in a recombinant peptide comprising amino acids 925-1008 from IRS-1 caused a reduction of Tyr phosphorylation on two specific residues Tyr941 and Tyr989, which are known binding sites for the p85alpha regulatory subunit. Using tissue lysates from $\operatorname{Tg} 972$ mice we were able to demonstrated for the first time in vivo a $70 \%$ reduction of Tyr ${ }^{989}$ phosphorylation $(p<0.001)$. To examine whether the mutation under study affects insulin secretion, we purified islets from $\mathrm{Tg} 972$ mice and WT littermates, and incubated them in the presence of increasing glucose concentration. Glucose-induced insulin secretion was significantly decreased in $\mathrm{Tg} 972$ islets at all the concentrations tested $(\mathrm{p}<0.01)$.

Conclusion: Tg972 show a global impairment of insulin signaling and action in all the key target tissues, thus reproducing the alterations observed in insulin resistance and glucose intolerance states and therefore representing an excellent model to study these conditions.

Supported by European Community FP6 EUGENE2 to G. S. and Italian Ministry of Education PRIN-COFIN 2005 to R.L

0071

Adenosine $A_{2 B}$ receptor knock-out mice display an improved metabolic phenotype

J. L. Treadway ${ }^{1}$, R. Sacca ${ }^{1}$, B. K. Jones ${ }^{2}$;

${ }^{1}$ Pfizer Inc, Groton, ${ }^{2}$ Xenogen Biosciences, Xenogen Corp, Cranbury, United States.

Background and Aims: The adenosine $A_{2 B}$ receptor is a G-protein coupled receptor expressed in liver, muscle, and other tissues. Several reports suggest that $\mathrm{A}_{2 \mathrm{~B}}$ receptor antagonists could be used for diabetes treatment by supressing hepatic glucose output and increasing insulinmediated glucose uptake in skeletal muscle, resulting in improved glycemic control. To further evaluate this concept, and to determine other consequences of $\mathrm{A}_{2 \mathrm{~B}}$ receptor antagonism, we characterized $\mathrm{A}_{2 \mathrm{~B}}$ knock-out mice. Materials and Methods: Adenosine $\mathrm{A}_{2 \mathrm{~B}}$ knockout mice $(50 \% 129 \mathrm{ola}+50 \% \mathrm{C} 57 \mathrm{BL} / 6 \mathrm{~N})$ were obtained from Deltagen and crossed with $\mathrm{C} 57 \mathrm{BL} / 6 \mathrm{~N}$ mice to a hybrid background of $80-95 \%$. Het $\mathrm{x}$ Het matings produced the homozygous $-/-(\mathrm{KO})$ male and female mice used for the study. Age and sex-matched littermates served as wild-type (WT) controls. Animals were studied at 11-28 weeks of age ( $\mathrm{n}=4-25 /$ group). The phenotype analysis consisted of $>40$ bioassays designed to evaluate effects on major physiologic systems (e.g. neurologic, cardiovascular, metabolic, endocrine, immune, inflammatory, behavioral, musculoskeletal, urologic, and gastrointestinal) and metabolic response to a 7 week high fat diet (HFD, males only).

Results: There were several significant $(\mathrm{p}<0.05)$ findings for the adenosine $A_{2 B}$ receptor $\mathrm{KO}$ mice relative to $\mathrm{WT}$ controls. 
A) Standard diet: Body Composition-Female KO mice demonstrated a marked reduction in fat mass $(60 \%$, $\mathrm{p}<0.05)$, resulting in a $40 \%(\mathrm{p}<0.05)$ reduction in $\%$ body fat and $25 \%(p<0.05)$ increase in $\%$ lean mass compared to WT. Female KO mice also demonstrated increased $(\mathrm{p}<0.05)$ relative mass of the tibialis, EDL, gastrocnemius, and soleus muscles. Tissue Glycogen-Muscle glycogen concentration tended to increase in female KO mice $(\mathrm{p}=0.09)$, while liver glycogen was markedly reduced $(87 \%, \mathrm{p}<0.05)$ compared to WT. Energy Expenditure-Male KO mice showed a $20-25 \%(\mathrm{p}<0.05)$ increase in $\mathrm{O}_{2}$ consumption, $\mathrm{CO}_{2}$ consumption, heat production and respiratory exchange ratio compared to WT.

B) High-Fat Diet: Body Composition-Male KO mice responded to the HFD with significantly $(\mathrm{p}<0.05)$ lower percent total body fat, and increased lean mass, percent lean mass, and a trend towards a decrease in fat mass compared to WT. Plasma Chemistries and Hormones-No differences in serum insulin levels were observed between male $\mathrm{KO}$ and WT mice. The KO mice had slightly $(p=0.09)$ decreased adiponectin levels compared to WT before, but not after, the HFD. Glucose Tolerance (GTT) - No difference was observed prior to the HFD, but there was a trend for reduced glucose excursion $(22 \%, \mathrm{p}=0.052)$ during the GTT in the HFD KO mice compared to WT.

C) Other Parameters: No differences between KO and WT mice were observed for all other tests performed, except for inconsistent changes in bone endpoints, Elevated Maze test, RBCs and Phospholipid B, and Sebum Production.

Conclusions: $A_{2 B}$ receptor $\mathrm{KO}$ mice demonstrated a positive metabolic phenotype through reduced adiposity and increased lean body mass. GTT results suggested improved glucose tolerance in mice fed a HFD. There were also positive changes in energy expenditure, muscle mass, and tissue glycogen levels suggesting anti-obesity/anabolic effects. Further, there were no major adverse effects of the $\mathrm{KO}$ phenotype. Overall, these results suggest that reduction or antagonism of the adenosine $\mathrm{A}_{2 \mathrm{~B}}$ receptor could be useful for the treatment of several metabolic disorders, including obesity, frailty, and type 2 diabetes.

\section{2}

\section{Cardiac energy metabolism in hormone-sensitive lipase} knockout mice

J. Suzuki ${ }^{1}$, M. Ueno ${ }^{1}$, Y. Zenimaru ${ }^{1}$, M. Kasen ${ }^{1}$,

M. Handa ${ }^{1}$, S. Takahashi ${ }^{1}$, J. Osuga ${ }^{2}$, S. Ishibashi ${ }^{3}$, F. B. Kraemer ${ }^{4}$, I. Miyamori ${ }^{1}$;

${ }^{1}$ Medicine, University of Fukui, Japan, ${ }^{2}$ Endocrinology and Metabolism, University of Tokyo, Japan, ${ }^{3}$ Endocrinology and Metabolism, Jichi Medical University, Tochigi, Japan, ${ }^{4}$ Medicine, Stanford University, United States.
Background and Aims: Hormone-sensitive lipase (HSL) regulates intracellular lipolysis in many tissues including adipose, pancreatic beta cells and heart. HSL-knockout (KO) mice have been created and impaired adipocyte lipolysis was reported. Since free fatty acids (FA) are the major energy source for heart, and energy balance between glucose and fatty acids dramatically changes depending on nutritional condition, we asked how disruption of HSL affects cardiac energy metabolism. To clarify the impact of HSL-KO in cardiac metabolism, KO mice were fasted and refed, and the cardiac phenotypes investigated.

Materials and Methods: HSL-KO mice in C56BL/6 background aged 24 to 32 wks were studied. Homozygous KO mice and wildtype (Wt) littermates were fasted for $24 \mathrm{~h}$ and then refed for 3-6 h prior to the experiments. Plasma lipids, glucose and insulin concentration, and cardiac lipid contents were measured. Cardiac lipase activities were measured using radioactive substrates. Histological studies were performed by electron microscopy. To study cardiac uptake of FA, glucose and very low-density lipoprotein (VLDL), ${ }^{123}$ I-beta-methyl iodophenyl pentadecanoic acid (BMIPP), ${ }^{14}$ C-deoxy-D-glucose and rabbit VLDL whose tyrosine was labeled with ${ }^{125} \mathrm{I}$, were injected into animals. Cardiac gene expressions were analyzed by quantitative RT-PCR.

Results: KO mice showed 30\% higher plasma glucose and 9-times higher plasma insulin levels compared to Wt mice, indicating insulin resistance. Plasma FA concentration in Wt mice was elevated 2-fold with fasting, while this increase was not observed in KO mice. BMIPP uptake showed that cardiac FA influx paralleled plasma FA level; thus, KO mice had lower FA influx during fasting. Accordingly, upregulation of a FA-responsive gene, mitochondrial HMG CoA-synthase, was markedly blunted in $\mathrm{KO}$ mice in fasted and refed conditions. Although cardiac glucose uptake was similar between $\mathrm{KO}$ and $\mathrm{Wt}$ mice, cardiac VLDL uptake was 2-fold higher in $\mathrm{KO}$ than $\mathrm{Wt}$ mice during fasting, accompanied by a 2-fold increased cardiac lipoprotein lipase (LPL) activity. This LPL activation was not diminished by heparin perfusion, suggesting that LPL is located inside or on the surface of cardiomyocytes. Neutral cholesteryl esterase activity in $\mathrm{KO}$ heart was undetectable, while LPL and non-LPL triglyceride (TG) lipase activities were similar to Wt heart in fed condition. However, non-LPL TG lipase activity in KO heart was 40\% lower and TG droplets accumulated during refeeding. The residual TG lipase in $\mathrm{KO}$ heart might be adipose TG lipase (ATGL) since its mRNA was expressed in heart and was upregulated 3 -fold by fasting and refeeding in both $\mathrm{KO}$ and Wt.

Conclusion: Systemic disruption of HSL impairs FA release from adipose tissue upon fasting, and diminishes FA flux into cardiomyocytes. As compensation, cardiac 
LPL is activated to facilitate VLDL-uptake presumably via lipoprotein receptors. HSL-KO affects activity of cardiac non-LPL TG lipases, including ATGL, and leads to aberrant lipid accumulation in cardiomyocytes.

Supported by the Japanese Ministry of Education, Culture, Sports, Science and Technology

\section{OP 13}

\section{Progression and mortality in type 2 diabetes}

\section{3}

The age at diagnosis predicts change in $\mathrm{HbA}_{1 \mathrm{c}}$ over time in patients with newly diagnosed type 2 diabetes

T. Elgzyri, P. Almgren, M. Eliasson, H. Parikh,

E. Lindholm, K.-F. Eriksson, L. Groop;

Clinical Sciences/Diabetes\&Endocrinology, Lund University, Malmö, Sweden.

Background and Aims: Type 2 diabetes (T2D) is characterized by an initial decline followed by a progressive rise in blood glucose and HbAlc over time, which until now has been independent of mode of therapy. Although the underlying mechanisms are not fully understood, it has partially been ascribed to deterioration of $\beta$-cell function. The aim of this study was to identify non-genetic predictors of this deterioration with a future goal to also include genetic factors.

Materials and Methods: 1209 T2D patients from the local diabetes registry in southern Sweden were followed with repeated $\mathrm{HbAlc}$ measurements during 7 years after diagnosis. Fasting plasma glucose, fasting C-peptide and subsequently homeostasis model assessment of percent $\beta$-cell function (HOMA-B\%) and HOMA of insulin resistence index (HOMA-IR) were measured at diagnosis in 585 of these patients. The relation between age at diagnosis, BMI at diagnosis, sex, HOMA-B\% (estimated from $\mathrm{C}$-peptide), HOMA-IR and ethnic background and changes in HbAlc during the follow up period adjusted for age, sex and BMI at diagnosis were studied using a generalized estimating equation procedure.

Results: HbA1c improved at one year of diagnosis from $7.6 \pm 2.18$ to $6.3 \pm 1.4 \%(\mathrm{P}=0.0001)$. Thereafter, HbA1c showed a progressive rise from $6.3 \pm 1.4$ to $7.0 \pm 1.5 \%$ $(\mathrm{P}<0.000)$ during subsequent 6 years. The mean time to initiation of insulin therapy was 2.5 years. After 7 years $47 \%$ were on insulin therapy. In patients aged $<50$ years at diagnosis there was a steeper increase in $\mathrm{HbAlc}$ than in those with age at diagnosis $\geq 50$ years ( $\mathrm{HR}-0.10$; CI $-0.16-0.04, \mathrm{P}=0.001)$. HOMA-B\% was inversely associated with $\mathrm{HbAlc}$ values during the whole period (HR -0.004 ; CI $-0.007-0.002, \mathrm{P}<0.000$ ). No relationship was observed between the rise in HbAlc over time and HOMA-IR, sex, BMI at diagnosis, or ethnicity (Scandinavian vs. non-Scandinavian origin).

Conclusion: We observed the expected rise in $\mathrm{HbAlc}$ over time in patients with newly diagnosed T2D. Early age at diagnosis $(<50$ years) was the strongest predictor of this secular increase in $\mathrm{HbA1c}$ concentrations and related to impaired $\beta$-cell function. This provides a basis to test whether genetic factors can modify these relationships.

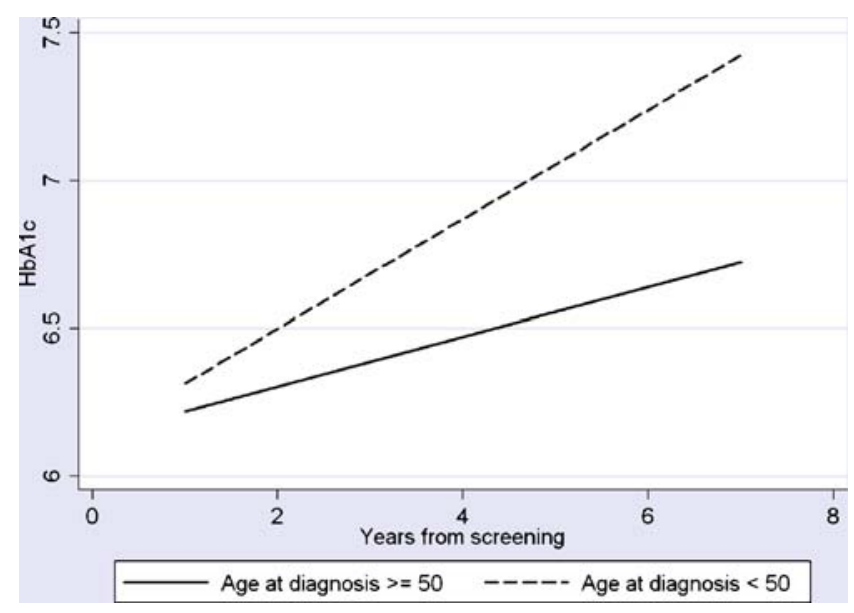

0074

Early intensive insulin therapy on newly diagnosed type 2 diabetic patients: a long term follow-up study

W. Xu, Y. B. Li, J. Liu, W. P. Deng, J. P. Weng;

Department of Endocrinology, the First Affiliated Hospital of Sun Yat-Sen University, Guangzhou, China.

Background and Aims: In our previous study, we demonstrated that 2-week intensive insulin therapy with CSII could induce long-term glycemic control in newly diagnosed type 2 diabetic patients with severe hyperglycemia. Nearly one-half of the patients can maintain euglycemia on diet only for more than 12 months. 61 patients in the study had been followed for more than 24 months. We report here a study of these patients to analyze the followup data and assess the variables predictive of long-term near-normoglycemic remission.

Materials and Methods: 61 patients in our previous study (newly diagnosed type 2 diabetic patients with severe hyperglycemia who were treated with 2-week CSII and then on diet) had been followed for more than 24 months. Intravenous glucose tolerance tests (IVGTT) were performed. Insulin and C-peptide during IVGTT, FPG, glycosylated hemoglobin $\mathrm{A}_{1 \mathrm{C}}\left(\mathrm{GHbA}_{1 \mathrm{C}}\right)$ and lipid profiles were measured. Group means were compared with Student's t test. A Kaplan-Meier survival analysis and 
Cox hazard proportion analysis were used to determine the time to hyperglycemic relapse and variables predictive of long-term near-normoglycemic remission. The patients' initial data before and after CSII (including insulin and Cpeptide during IVGTT, FPG, 2-h postbreakfast plasma glucose (PPG), $\mathrm{GHbA}_{1 \mathrm{c}}$ and lipid profiles) were used for the Cox hazard proportion analysis.

Results: All of the patients (36 men and 25 women) with the initial FPG $14.3 \pm 4.8 \mathrm{mmol} / \mathrm{L}$ had been followed for more than 24 months. A total of 26 (18 men and 8 women) still maintained near-normoglycemic control without medication. 4 of these 26 patients had been in near-normoglycemic remission for more than 50 months. There were no differences in age, body mass index (BMI), GHbA $1 \mathrm{C}, \mathrm{FPG}$, $\mathrm{PBG}$, insulin response, insulin resistance and lipid profiles before and after CSII between the patients still in remission (remission group) and those relapsed (relapse group). In this visit, the patients' final BMI were similar (remission vs relapse: $25.18 \pm 4.19$ vs $24.50 \pm 3.76 \mathrm{~kg} / \mathrm{m}^{2}$ ). FPG of the patients in remission was significantly lower than that of the patients relapsed $(5.88 \pm 0.88$ vs $8.69 \pm 3.36 \mathrm{mmol} / \mathrm{L})$, so as $\mathrm{GHbA}_{1 \mathrm{c}}(6.07 \pm 0.70 \%$ vs $7.53 \pm 1.72 \%)$. And the acute insulin response of the remission group was markedly higher than that of the relapse group $(1607.46 \pm 1221.85 \mathrm{vs}$ $409.27 \pm 721.19 \mathrm{pmol} / \mathrm{l} / \mathrm{min})$. A Kaplan-Meier survival analysis gave a median time of 17 months (95\%CI $11.67-$ 22.33) to relapse. And the Cox models showed that the duration of near-normoglycemic remission was not significantly related to age, sex, FPG, BMI, insulin response, insulin resistance and weight change. The data suggested that the baseline LDL-cholesterol and PPG measured after CSII (on the following day at least $15 \mathrm{~h}$ after insulin cessation) were the risk factors affected the duration of near-nomoglycemic remission (odd ratio 2.471, 95\% CI $1.282-4.760$ and $1.410,95 \%$ CI $1.136-1.752$, respectively) Conclusion: 26 of 61 newly diagnosed type 2 diabetic patients who were treated with 2 -week CSII and then on diet had maintained near-normoglycemic remission without medication for more than 24 months. The median time to relapse was 17 months. The baseline LDL-cholesterol and PPG measured after CSII were the risk factors affected the duration of near-nomoglycemic remission.

Supported by NCET 2004-08-07 (Jianping Weng) and Guangdong natural science fundation 2005 (Liyan Bing)

\section{5}

Rosiglitazone combined with insulin therapy improves islet $\beta$ cell function and increases serum GLP-1 level in type 2 diabetic patients

H. Wu, N. W. Tong, L. Cao;
Department of Endocrinology, West China Hospital, Chengdu, China.

Background and Aims: To investigate the effects of rosiglitazone(RSG) combined with insulin therapy on islet $\beta$ cell function, especially on early phase of insulin secretion, and on serum glucagon-like peptide-1(GLP-1) level in type 2 diabetic patients (T2DP) who need to use insulin therapy to control blood glucose.

Materials and Methods: T2DP who need to use insulin therapy were selected. According to age, sex, diabetic duration, glycosylated hemoglobin $\mathrm{A}_{1 \mathrm{C}}\left(\mathrm{HbA}_{1 \mathrm{C}}\right)$, matchedcontrol study (1:1) was used. One was study group and the other was control group. The study group injected insulin and combined with RSG (4 mg/day) orally, the control group only used insulin. The therapy lasted for 3 months. $100 \mathrm{~g}$ carbohydrate (steamed bread) challenge of C-peptide (CP) releasing test was performed to evaluate $\beta$ cell function. Other indices such as serum GLP-1(0 min, $30 \mathrm{~min}, 120 \mathrm{~min}), \mathrm{HbA}_{1 \mathrm{C}}$, tumor necrosis factor(TNF) $-\alpha$, urinary ratio of albumin to creatinine $(\mathrm{UA} / \mathrm{Cr})$, free fatty acid(FFA), triglycerides (TG), high density lipoprotein cholesterol(HDL-ch) and low density lipoprotein cholesterol(LDL-ch) were detected.

Results: Thirty patients were included in the study and 28 patients finished follow-up. At the end, in the study group $(n=14)$ serum levels of TG decreased[(1.98 \pm 1.20 vs $1.61 \pm 0.72) \mathrm{mmol} / \mathrm{L}, \mathrm{p}=0.049]$, HDL-ch increased $[(1.15 \pm$ 0.23 vs $1.36 \pm 0.28) \mathrm{mmol} / \mathrm{L}, \mathrm{p}<0.001]$, FFA decreased $[(663.14 \pm 88.18$ vs $499.48 \pm 94.36) \mu \mathrm{mmol} / \mathrm{L}, \mathrm{p}<0.001]$ significantly; In the control group $(n=14)$ these indices were not changed significantly. The diastolic pressure in the study group decreased [(77.14 \pm 12.45 vs $74.50 \pm 5.92) \mathrm{mmHg}, \mathrm{p}=0.008]$, the blood pressure of control group did not have significant changes. Indices of early phase insulin secretion(EPIS) in the study group were improved significantly [CP30 min $(0.63 \pm 0.23$ vs $0.84 \pm 0.33) \mathrm{mmol} / \mathrm{L}, \mathrm{p}=0.002, \mathrm{AUCcp} 30$ $(15.51 \pm 6.05$ vs $19.85 \pm 7.19)$ mmol.min. $\mathrm{L}^{-1}, \mathrm{p}=0.000, \Delta \mathrm{CP} 30 /$ $\Delta \mathrm{G} 30(0.07 \pm 0.05$ vs $0.14 \pm 0.10, \mathrm{p}=0.02)]$. Other indices of $\beta$ cell function were also improved. Some indices of GLP-1 in the study group were increased significantly [GLP-130 $\mathrm{min}$ $(24.45 \pm 1.23$ vs $25.60 \pm 1.13) \mathrm{pmol} / \mathrm{L}, \mathrm{p}=0.000, \mathrm{AUCglp} 30$ $(637.82 \pm 53.60$ vs $659.68 \pm 50.25)$ pmol.min. $\mathrm{L}^{-1}, \mathrm{p}=0.000$, AUCglp120(2601.43 \pm 184.41 vs 2686.29 \pm 171.07$)$ pmol.min. $\left.\mathrm{L}^{-1}, \mathrm{p}=0.000\right]$. TNF $-\alpha[(7.92 \pm 1.60$ vs $7.12 \pm 1.00) \mathrm{fmol} / \mathrm{L}$, $\mathrm{p}=0.032]$ and ratio of $\mathrm{UA} / \mathrm{Cr}[(42.59 \pm 27.43$ vs $35.81 \pm 25.66)$ $\mu \mathrm{g} / \mathrm{mg}, \mathrm{p}=0.047]$ in the study group decreased significantly, and these indices in the control group were not changed significantly.

Conclusion: Rosiglitazone may improve islet $\beta$ cell function including EPIS in T2DP who need insulin therapy, the protective effects of RSG on $\beta$ cell might relate to the 
increase of GLP-1 secretion partly except for the decrease of IR.

Supported by the Ethics Committee of West China Hospital

0076

Triple oral therapy with pioglitazone decreases the requirement for insulin therapy: a 42-week, doubleblind, randomised, placebo-controlled study

J.-F. Blickle ${ }^{1}$, G. Charpentier ${ }^{2}$, S. Halimi ${ }^{3}$ on behalf of the Study F-PIO-100 Investigators;

${ }^{1}$ Medicin Interne, Diabete, Mal Metabolisme, Hôpitaux Universitaires Strasbourg, ${ }^{2}$ Service de Diabetologie, Centre Hospitalier Sud Francilien, Corbeil-Essonnes, ${ }^{3}$ Diabetology Nutrition, Centre Hospitalier Universitaire de Grenoble, France.

Background and Aims: According to current guidelines in France, insulin should be started in patients taking dual oral antidiabetic therapy who fail to achieve $\mathrm{HbA}_{1 \mathrm{c}}<8 \%$. In the PROactive study, pioglitazone (PIO), a thiazolidinedione, has been shown to delay the need for permanent insulin use. There is, however, at present no clear evidence that adding PIO to failing metformin (MET) and sulphonylurea (SU) dual therapy postpones the need for exogenous insulin (INS). We evaluated the effect on the requirement for INS therapy of adding PIO to MET/SU dual therapy in inadequately controlled INS-naïve patients with Type 2 diabetes.

Materials and Methods: This prospective, double-blind, placebo (PBO)-controlled study, performed in 57 French centres, randomised 299 patients with Type 2 diabetes previously treated with MET $(\geq 1700 \mathrm{mg} /$ day $)$ and SU (maximal dose) for more than 3 months and $\mathrm{HbA}_{1 \mathrm{c}}$ between $7.0 \%$ and $9.5 \%$. After a 4 -week run-in, patients received either PIO $30 \mathrm{mg}$ once daily or PBO. After 3 months, if $\mathrm{HbA}_{1 \mathrm{c}}>6.5 \%$, PIO or $\mathrm{PBO}$ was up-titrated to $45 \mathrm{mg}$ for the remaining 4 months. Endpoints included the proportion of patients 1) requiring INS therapy at study end $\left(\mathrm{HbA}_{1 \mathrm{c}}>8 \%\right)$ and 2) for whom the investigator prescribed INS at study end.

Results: Overall, 289 patients (mean age $60 \pm 9$ years, mean duration Type 2 diabetes $12 \pm 8$ years) had at least 3 months' study treatment. At study end, $92 \%$ of the PIO group received $45 \mathrm{mg}$ once daily and $82 \%$ of the PIO group versus $42 \%$ of the $\mathrm{PBO}$ group had $\mathrm{HbA}_{1 \mathrm{c}} \leq 8 \%$.

At study end, one patient in the PIO group was prescribed INS in addition to oral therapy versus 11 patients in PBO group: nine in addition to oral therapy and two transferred to INS alone. After the study, the majority of investigators preferred to continue triple oral therapy than to prescribe INS. Triple oral therapy was prescribed to $67.5 \%$ of the PIO patients versus $58.5 \%$ of $\mathrm{PBO}$ group, dual therapy $30.0 \%$ versus $38.8 \%$ and monotherapy $0.7 \%$ versus $1.4 \%$.

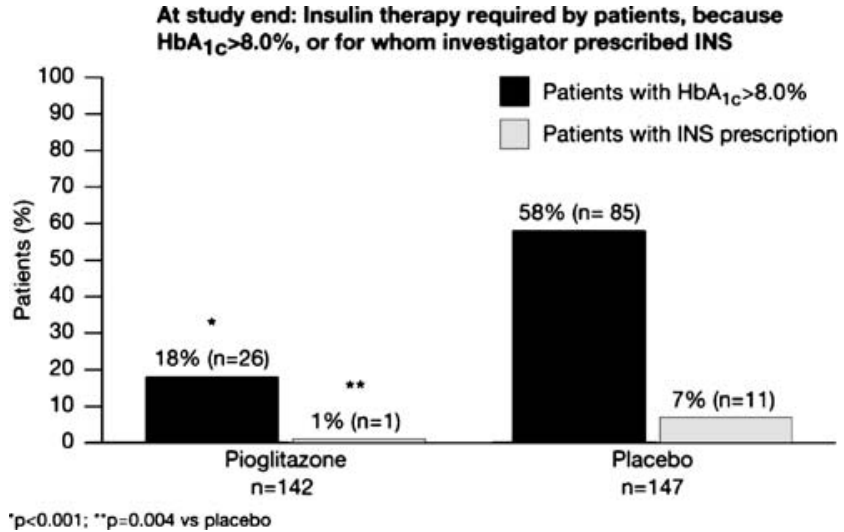

Conclusion: The addition of PIO to MET and SU significantly reduced the requirement for INS, with significantly more patients on PIO than PBO achieving $\mathrm{HbA}_{1 \mathrm{c}} \leq 8.0 \%$ after 7 months' treatment. Of interest was the disparity between clinical practice and clinical guidelines for the introduction of INS therapy in patients requiring tighter glycaemic control.

Supported by Laboratoires Takeda, France

0077

Lifestyle changes according to general guidelines on inflammation in subjects with impaired glucose tolerance: the SLIM project

C. Roumen ${ }^{1}$, E. Corpeleijn ${ }^{1}$, E. J. M. Feskens ${ }^{2}$, W. H. M. Saris ${ }^{1}$, E. E. Blaak ${ }^{1}$;

${ }^{1}$ Human Biology, Maastricht University, ${ }^{2}$ Division of Human Nutrition, Wageningen University,

The Netherlands.

Background and Aims: To evaluate the effect of changes in C-reactive protein (CRP) and Interleukin 6 (IL-6) on changes in $2 \mathrm{~h}$ glucose levels (after an OGTT) and insulin sensitivity (as assessed by HOMA-IR) in subjects with impaired glucose tolerance (IGT), who participate in a lifestyle intervention program.

Materials and Methods: We studied 104 IGT subjects, intervention group; $\mathrm{n}=51$ (INT) and control group; $\mathrm{n}=53$ $(\mathrm{CON})$ at baseline and after 1 year of lifestyle intervention. Subjects underwent measurements of body composition, body fat distribution, glucose tolerance (OGTT), and maximal aerobic capacity (VO2 max). The intervention program included dietary advice and a physical activity program, based on general public health recommendations. 
Results: One year lifestyle intervention improved VO2 max and insulin sensitivity and reduced saturated fat intake $(-2.34 \pm 3.00 \mathrm{En} \%$ in INT versus $-0.52 \pm 3.34 \mathrm{En} \%$ in CON, $\mathrm{p}=0.004)$, reduced body weight $(-2.67 \pm 3.57 \mathrm{~kg}$ in INT versus $-0.53 \pm 3.86 \mathrm{~kg}$ in $\mathrm{CON}, \mathrm{p}=0.004)$ and improved glucose tolerance $(-0.74 \pm 1.79 \mathrm{mM}$ in INT versus $+0.36 \pm 2.17 \mathrm{mM}$ in CON, $\mathrm{p}=0.006$ ). No differences were observed in the change of CRP and IL- 6 between INT and CON, $p=0.545$ and $p=0.559$, respectively. Also, no associations were seen between changes in CRP and IL-6 and changes in HOMA-IR. However, one-year changes in CRP $(-0.07 \pm 6.36 \mathrm{mg} / \mathrm{l})$ and IL-6 $(-0.30 \pm 2.05 \mu \mathrm{g} / \mathrm{l})$ were positively associated with change in $2 \mathrm{~h}$ glucose levels $(-0.18 \pm 2.06 \mathrm{mM})$, adjusted $\mathrm{R}$ square $7.8 \%, \mathrm{p}=0.007$ and adjusted $\mathrm{R}$ square $8.4 \%, \mathrm{p}=0.005$, even after correction for changes in saturated fat intake, body weight and VO2 max change.

Conclusion: Lifestyle intervention improves glucose tolerance and insulin sensitivity in impaired glucose tolerant subjects, indicating that it may be effective in the prevention of type 2 diabetes mellitus. Changes in CRP and IL-6 were positively associated with changes in $2 \mathrm{~h}$ glucose levels, independent of lifestyle factors like change in saturated fat intake, weight loss and change in $\mathrm{VO} 2$ max. Therefore, changes in CRP and IL-6 seem important independent mediators of changes in glucose tolerance and may be considered as 'nontraditional' risk factors for the development of the impaired glucose tolerant state and type 2 diabetes mellitus.

Supported by the Dutch Diabetes Foundation, the Netherlands Organisation for Health Research and Development and Netherlands Organization for Scientific Research

\section{8}

Three-year mortality in diabetic patients treated with different combinations if insulin secretagogues and metformin

E. Mannucci, M. Monami, C. Lamanna, C. M. Desideri, G. Masotti, N. Marchionni;

Critical Care Medicine and Surgery, Unit of Gerontology University of Florence, Italy.

Background and Aims: Several studies have shown an increase of mortality in diabetic patients treated with combinations of sulphonylureas and biguanides. Comparisons between different insulin secretagogues in combination with metformin with respect to all-cause mortality have not been reported so far.

Materials and Methods: An observational cohort study was performed on a consecutive series of 2002 outpatients with type 2 diabetes mellitus. Of these patients, 696
(34.8\%) were receiving combinations of insulin secretagogues and biguanides at enrolment. Three-year mortality was assessed through search in the City of Florence Registry Office.

Results: During follow-up, 295 deaths were recorded. Among patients on combined secretagogue and biguanide treatment, glibenclamide was associated with a significantly higher yearly mortality $(8.7 \%)$ than repaglinide $(3.1 \%$; $\mathrm{p}=0.002)$, gliclazide $(2.1 \% ; \mathrm{p}=0.001)$ and glimepiride $(0.4 \% ; \mathrm{p}<0.0001)$. After adjusting for potential confounders (including age, duration of diabetes, BMI, lipid profile, HbA1c, insulin treatment, metformin doses, Charlson comorbidity score), mortality remained significantly higher in patients treated with combinations of glibenclamide and metformin when compared to those treated with different insulin secretagogues (OR with 95\% CI: 2.09 [1.07;4.11]). Conclusion: In the present study, sulphonylureas with greater selectivity for beta cell receptors, such as glimepiride and gliclazide were associated with a lower mortality, when used in combination with metformin, in comparison with glibenclamide. Safety of such combinations deserves further investigation.

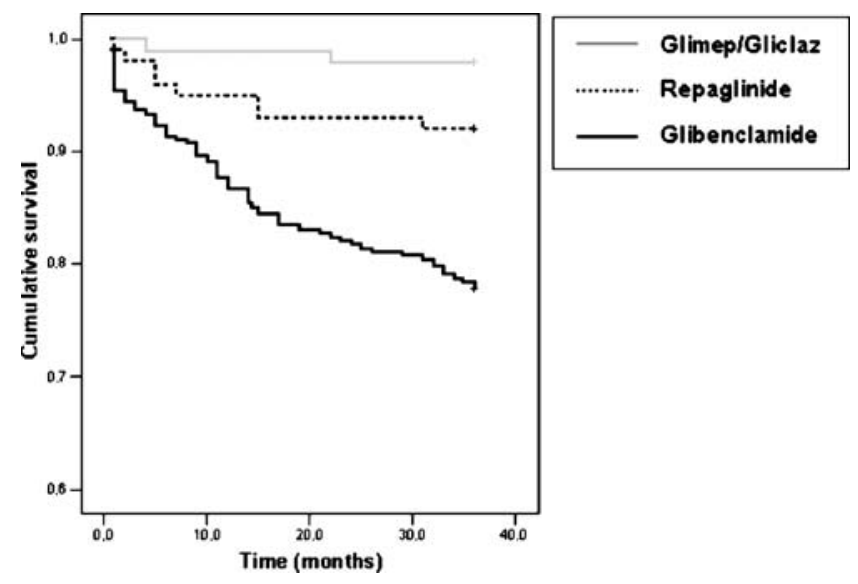

\section{OP 14}

\section{Central control of metabolism}

0079

Mice lacking inhibitory leptin receptor signals are lean with normal endocrine function

M. Björnholm ${ }^{1}$, H. Münzberg ${ }^{2}$, R. L. Leshan ${ }^{2}$, S. H. Bates ${ }^{2}$, J. C. Jones ${ }^{2}$, C. Bjørbæk ${ }^{3}$, M. G. Myers Jr ${ }^{2}$; ${ }^{1}$ Dept of Molecular Medicine and Surgery, Karolinska Institutet, Stockholm, Sweden, ${ }^{2}$ Dept of Medicine, University of Michigan, Ann Arbor, United States, ${ }^{3}$ Dept of Medicine, Beth Israel Deaconess Medical Center, Boston, United States. 
Background and Aims: The adipocyte-derived hormone, leptin, activates the long form leptin receptor (LRb) in regions of the brain to signal the status of body energy stores and regulate food intake, energy expenditure and neuroendocrine function. Lack of leptin or LRb results in obesity, infertility and growth retardation; the failure of elevated leptin levels to promote weight loss in common forms of obesity suggests that leptin-induced leptin resistance may promote obesity. LRb mediates intracellular signals via four pathways: (1) $\mathrm{Tyr}_{1138}$-mediated activation of STAT3, (2) Tyr $_{985}$-mediated association with SHP-2 and SOCS3, (3) $\mathrm{Tyr}_{1077}$-mediated phosphorylation of STAT5 and (4) via numerous tyrosine phosphorylation sites on the associated Jak2 molecule. We have previously shown that $\mathrm{Tyr}_{1138} / \mathrm{STAT} 3$ is required for leptin regulation of energy homeostasis, but not growth or reproduction in mice. We have now examined the role of $\mathrm{Tyr}_{985}$ in LRb action in vivo.

Material and Methods: We have generated a line of genetically engineered mice in which the gene for LRb (lepr) is replaced by a mutant allele $\left(l e p r^{l 985}\right)$ encoding a site-directed substitution mutation of $\mathrm{Tyr}_{985}$. We investigated parameters such as body weight, food intake, leptin sensitivity, fertility and growth in these animals.

Results: Compared to wild-type mice, animals homozygous $(l / l)$ for $l e p r^{1985}$ display decreased weight and adiposity, with commensurately decreased levels of circulating leptin. In spite of these decreased leptin levels, 1/1 mice displayed increased hypothalamic LRb/STAT3 signaling and consumed less food than wild-type animals when fed a high fat diet. Furthermore, the $l / l$ mice show increased sensitivity to exogenous leptin, as they demonstrated a greater reduction of body weight and food intake in response to leptin injections than did control animals. No impairments in growth or reproductive function were observed in $1 / 1$ animals.

Conclusion: LRb signals to growth and reproduction are not mediated by $\mathrm{Tyr}_{985}$ or $\mathrm{Tyr}_{1138}$, suggesting a role for LRb $\mathrm{Tyr}_{1077}$ or Jak2 mediated signals in these actions of leptin. The finding that $\mathrm{Tyr}_{985}$-mediated signals decrease feeding and fat mass while not altering the growth and reproductive axes in mice suggests that leptin activates physiologically important autoinhibitory signals via LRb $\mathrm{Tyr}_{985}$. These autoinhibitory signals act to attenuate leptin action and thus promote increased feeding and adiposity in vivo and are hence potential mediators of leptin resistance in obesity.

Supported by NIH DK57768, DK56731 and grants from ADA and Swedish Society for Medical Research.
0080

Central leptin infusion impairs insulin-stimulated GLUT4 translocation to plasma membrane in white adipose tissue in rats

A. Andres ${ }^{1}$, E. Bonzon-Kulichenko ${ }^{1}$,

T. Fernandez-Agullo ${ }^{2}$, R. Serrano ${ }^{1}$, C. Martinez ${ }^{1}$, P. Blanco ${ }^{1}$, M. Ros ${ }^{2}$, N. Gallardo ${ }^{1}$;

${ }^{1}$ Bioquimica, Universidad de Castilla-La Mancha, Ciudad Real, ${ }^{2}$ Facultad Ciencias de la Salud, Universidad Rey Juan Carlos, Alcorcon (Madrid), Spain.

Background and Aims: Leptin modulates glucose homeostasis by acting as an insulin-sensitizing factor in most insulin target tissues. Nevertheless, insulin-dependent glucose uptake in white adipose tissue decreases after in vivo treatment with leptin. In the present work, we have analyzed the effects of central leptin on insulin-stimulated GLUT4 translocation in rat WAT.

Materials and Methods: 3-months-old Wistar rats were used throughout the study. Central leptin infusion was performed using osmotic pumps (Alzet) implanted in the right lateral cerebral ventricle, with a releasing rate of $1 \mu 1 / \mathrm{h}$ with rat leptin $(0.2 \mu \mathrm{g} /$ day $)$ or saline. During this time, rats were allowed free access to food and water. After 7 days the rats were subjected, under anesthesia, to an intravenous injection of insulin (10 U/kg body weight) or saline for $30 \mathrm{~min}$ and killed by decapitation. The epididymal WAT was rapidly dissected out, flash frozen in liquid nitrogen and stored at $-70^{\circ} \mathrm{C}$ until use. Total RNA was isolated from EWAT and the mRNA expression of IR isoforms, IRS-1, and GLUT4 was studied by semiquantitative RTPCR. IRS-1, Akt and GLUT4 protein content, and PY-IRS1 and PS-Akt in different subcellular fractions of EWAT was analyzed by Western blotting.

Results: Central leptin infusion during 7 days reduce the mRNA expression of IR-B, IRS-1 and GLUT4 in the EWAT, without affecting the IR-A isoform. In addition, this treatment increased the basal phosphorylation levels on tyrosine and serine of both cytosolic IRS-1 and Akt, respectively. Nevertheless, central leptin infusion impaired the insulin-stimulated IRS-1 tyrosine phosphorylation and translocation to the cytosol, as well as Akt activation and GLUT4 translocation to the plasma membrane.

Conclusion: The observed effects of central leptin on the insulin signaling pathway in WAT may contribute to explain the reported decrease in insulin-stimulated glucose uptake in this tissue in leptin treated rats.

Supported by Grants BFI2002-04030-C03-01 from Ministerio de Ciencia y Tecnología, Spain and PAC-02-004 from Junta de Comunidades de Castilla-La Mancha, Spain. 
0081

Central insulin response in Wistar rats: effects of ageing and food restriction

M. G. San Frutos ${ }^{1}$, T. Fernández-Agulló ${ }^{1}$, M. L. Gavete ${ }^{1}$, A. Andrés ${ }^{2}$, J. M. Carrascosa ${ }^{3}$, M. Ros ${ }^{1}$; ${ }^{1}$ Facultad de Ciencias de la Salud, Universidad Rey Juan Carlos, Alcorcón, ${ }^{2}$ Facultad de Ciencias Químicas, Universidad de Castilla-La Mancha, Ciudad Real, ${ }^{3}$ Centro de Biología Molecular, UAM-CSIC, Cantoblanco, Spain.

Background and Aims: There is now compelling evidence supporting the role of insulin as a feed-back or adiposity signal, acting at central level. In the present report, we have studied the effects of ageing on central insulin action in Wistar rats. In spite of normal fasting serum levels of both, insulin and glucose, these animals present peripheral insulin resistance and central leptin resistance. Thus, we have explored whether or not these animals may also present alterations of the central insulin response. The fact that central leptin resistance associated with ageing in these rats can be ameliorated by food restriction also led us to investigate the effects of food restriction on central insulin action in these animals.

Materials and Methods: 3 and 24 months Wistar albino male rats were used in these experiments. The effects of central insulin administration on body weight and food intake was performed using osmotic pumps (Alzet) connected to the lateral ventricle, with a releasing rate of $1 \mu \mathrm{l} / \mathrm{h}$ with humaninsulin (10 and $100 \mathrm{mU} /$ day) or saline during 7 days. During this time, rats were allowed free access to food and water and body weight and food intake was measured. Central insulin signal transduction was studied after a single dose of insulin $(10 \mathrm{mU})$ or vehicle into the lateral ventricle. $15 \mathrm{~min}$ after the treatment, rats were sacrificed and the hypothalamus was dissected, homogenated and used to check IR and GSK3 phosphorilation by imnmunoprecipitation and western blot analysis. The expression of IR also was studied by quantitative RTPCR and western blot in rat hypothalamus of young, old and old food restricted rats.

Food restriction. 20 to 21 months-old rats were placed in individual cages and were fed daily with an amount of chow equivalent to 75 to $80 \%$ of the normal food intake until they reached a reduction of about $25 \%$ of their body weight at the beginning of the experiment. Rats under this food restriction protocol during 3 month reached adiposity index similar to those of young rats.

Results: Insulin infusion during 7 days brought about a decrease in both body weight and food intake in a dose dependent manner in both young and old rats. Nevertheless, this effect was less evident in old rats and significance was only reached in young rats. IR and GSK3 phosphorylation was also decreased in old rats (43.7 and $28.78 \%$, respectively, $\mathrm{P}<0.001)$ compared to young ones. No significant differences were appreciated in the expression of IR in rat hypothalamus with ageing. Food restriction increased central insulin responsiveness (body weight and food intake) in old rats as well as signal transduction.

Conclusion: The data presented herein demonstrate that aged rats present a lower response to central insulin. This can be explained by the impairment of the insulin signal transduction pathway. These alterations may contribute to the state of insulin resistance that takes place during ageing. The fact that food restriction improves central insulin responsiveness points to the role that adiposity may play in the development of insulin resistance associated with ageing.

Supported by Grants from Universidad Rey Juan Carlos and from Ministerio de Ciencia y Tecnología, Spain. We thank E. Oliveros and J. A. Mass for their technical assistance.

\section{2}

Hypothalamic NPY during elderly and chronic food restriction: effect of central insulin

T. Fernandez- Agullo ${ }^{1}$, M. G. San Frutos ${ }^{1}$, M. L. Gavete ${ }^{1}$, C. $\operatorname{Arribas}^{2}$, J. M. Carrascosa ${ }^{3}$, M. Ros ${ }^{1}$;

${ }^{1}$ Facultad de Ciencias de la Salud, Universidad Rey Juan Carlos, Alcorcon (Madrid), ${ }^{2}$ Facultad de Medio Ambiente, Universidad Castilla-La Mancha, Toledo, ${ }^{3}$ Centro de Biologia Molecular, UAM-CSIC, Canto Blanco (Madrid), Spain.

Background and Aims: Male Wistar rats develop central leptin resistance during ageing which is ameliorated by chronic food restriction. Arcuate NPY serves as one of the downstream mediators of leptin's actions and arcuate NPY neurons are also a target for insulin action. In the present study we have analized the expression of NPY in the arcuate nucleus in a model of chronic food restriction in aging and the effect of central infusion of insulin. As NPY actions in the hypothalamus are both pre and postsynaptic, we also analized the expression on Y5 and Y2 NPY receptors.

Materials and Methods: 3 and 24 months Wistar albino male rats were used in these experiments. The effects of central insulin administration was performed using osmotic pumps (Alzet) connected to the lateral ventricle, with a releasing rate of $1 \mu \mathrm{l} / \mathrm{h}$ with human-insulin $(10$ and $100 \mathrm{mU} /$ day) or saline during 7 days. During this time, rats were allowed free access to food and water. The expression of 
NPY, $Y_{5}$ and $Y_{2}$ NPY receptors was studied by quantitative RT-PCR of rat hypothalamus of young, old and old food restricted rats. NPY expression in arcuate nucleus was also analized by immunohistochemistry and in situ hybridization using digoxigenin labeled riboprobes.

Food restriction. 20 to 21 months-old rats were placed in individual cages and were fed daily with an amount of chow equivalent to 75 to $80 \%$ of the normal food intake until they reached a reduction of about $25 \%$ of their body weight at the beginning of the experiment. Rats under this food restriction protocol during 3 month reached adiposity index similar to those of young rats.

Results: Aging is associated with a decrease in hypothalamic NPY expression analized by RT-PCR and correlated with less number of arcuate NPY cells analized by in situ hybridization histochemistry and also less inmunostaining for NPY. Chronic caloric restriction increases the expression of NPY in hypothalamus by RT-PCR and also by in situ hybridization in arcuate nucleus. Central infusion of insulin during 7 days reduce the expression of NPY in hypothalamus in a dose dependent manner in young but not in old rats. Caloric restriccion has no effect in the modulation of NPY by insulin in old rats. NPY Y 5 receptor expression decrease in old rats and food restriction increase its expression but at lower levels than in young animals. In young animals insulin infusion produce an upregulation of NPY $\mathrm{Y}_{5}$ receptor and has the opposite effect in old annimals which is not changed by caloric restriction. NPY $\mathrm{Y}_{2}$ receptor expression pressents the opossite changes than NPY Y ${ }_{5}$ receptor during elderly and there is no changes during caloric restriction. Central infussion of insulin induce a decrease in the expression of NPY $\mathrm{Y}_{2}$ in young and old rats without variation in food restricted animals.

Conclusion: Low levels of NPY arcuate expression in old animals cannot explain the same food intake in young than in old animals. The different response in young and old rats (also in food restricted annimals) in NPY expression after treatment with central insuline and the different regulation of pre and postsynaptic NPY receptors point to a central insuline resistance during aging. Supported by Grants from Universidad Rey Juan Carlos and from Ministerio de Ciencia y Tecnología, Spain. We thank E. Oliveros and J. A. Mass for their technical assistance.

\section{3}

Brain infusion of low dose glucosamine impairs glucose handling during ivGTT

M. Osundiji, J. Shaw, C.-Y. Yueh, M. L. Evans; Dept of Medicine, University of Cambridge, United Kingdom.
Background and Aims: Hypothalamic sensing of glucose/ nutrition may be important in the integrated control of homeostatic metabolic processes such as appetite and hepatic glucose output. In order to sense changes in glucose, specialised neurones may use mechanisms used by pancreatic beta-cells such as the low affinity hexokinase, glucokinase (GK). We hypothesised that brain delivery of the GK inhibitor, glucosamine (GSN) would reduce brain sensing of glucose and reduce glucose handling during a glucose challenge.

Materials and Methods: We performed 3 sequential studies to look at the effects of brain delivery of GSN on glucose tolerance.

(1) We examined pooled Sprague Dawley rat hypothalamic preparations. At a glucose concentration of $3 \mathrm{mM}$, GK represented approximately $5 \%$ of total hypothalamic glucose phosphorylation and was selectively inhibited by $1 \mathrm{mM} \mathrm{GSN}$ (reduced to $52+7 \%$ activity compared with $100+1 \%$ for nonGK phosphorylation).

(2) We then studied overnight-fasted chronically catheterised (vein and brain ICV catheters) adult male rats. In pilot studies, a 30 minute ICV infusion of $75 \mathrm{nmol} / \mathrm{min}$ GSN resulted in an approximate $1 \mathrm{mM}$ rise in hypothalamic hexosamine levels compared with vehicle $(1.4+0.4$ vs $0.2+$ $0.1 \mathrm{mM}, \mathrm{p}<0.001)$ with no changes in levels in other brain areas or blood.

(3) Using this dose of GSN, we then examined the effects of 90 minute ICV infusion of GSN $(n=7)$ or vehicle $(n=7)$ on glucose and insulin responses to an intravenous glucose tolerance test (ivGTT- $0.5 \mathrm{~g} / \mathrm{kg}$ ) in chronically catheterised Sprague Dawley rats.

Results: Following ICV GSN, rats displayed impaired handling of glucose during the first minutes of ivGTT, with a significantly higher glucose peak $(18.5+0.2 v s 15.8+0.9 \mathrm{mM}$, $\mathrm{p}=0.02)$ and $\mathrm{AUC}_{0-10 \min }(86+2$ vs $66+7 \mathrm{mM} . \mathrm{min}, \mathrm{p}=0.02)$. Despite these higher circulating glucose levels, insulin levels tended to be lower in ICV GSN rats (peak $3.6+0.5$ vs $4.3+$ $0.6 \mathrm{ug} / \mathrm{l}, \mathrm{p}=\mathrm{NS}$ ).

Conclusion: Inhibition of hypothalamic GK reduced the efficiency of disposal of a glucose load supporting a role for brain GK-mediated glucose-sensing in the integrated responses to hyperglycaemia. The trend for a lower insulin response indicates that this may be mediated, at least in part, by a reduction in first phase insulin release suggesting a contribution from brain glucose-sensing in the integrated control of glucose-stimulated insulin release in vivo. 


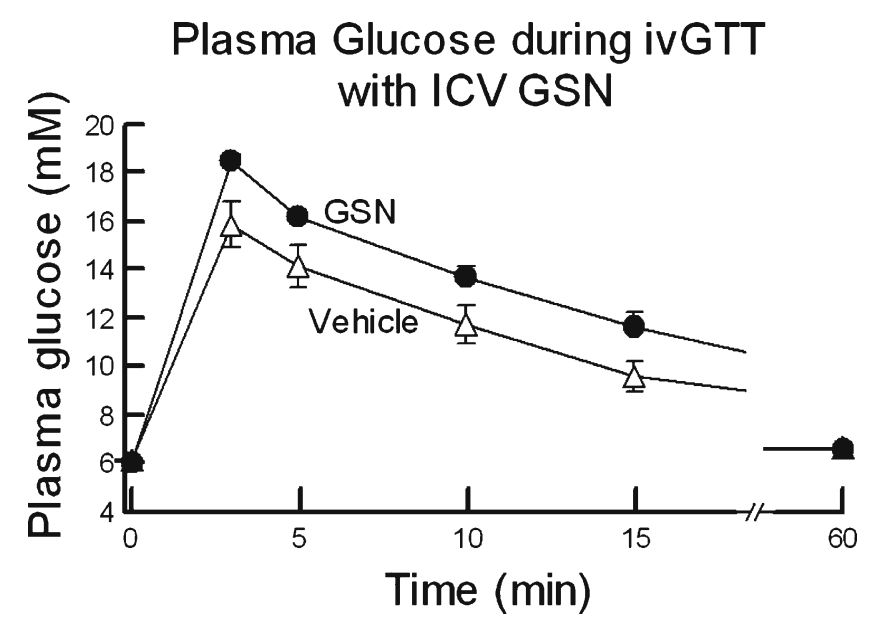

Supported by JDRF, Diabetes Research Wellness Foundation

0084

Central lipids overload increases NOS activity and modulate peripheral insulin secretion and action in response to glucose

N. Marsollier, A. Lacombe, C. Magnan,

C. Cruciani-Guglielmacci;

Laboratoire de Physiopathologie de la Nutrition, CNRS UMR 7059, Paris, France.

Background and Aims: We have previously shown that rats infused with lipids for $24 \mathrm{~h}$ towards brain displayed a hepatic insulin resistance and an increased GIIS (Glucose Induced Insulin Secretion), both due to changes in autonomic nervous activities. Some data showed that deleterious effects of fatty acids (lipotoxicity) implicate inflammatory Nitric Oxide (NO) pathway. So we aimed to test whether central NO could be involved in the early dysfunctions of glucose homeostasis induced by lipids.

Materials and Methods: Two groups of rats were infused during 24 hours into carotid artery towards brain, control group received saline with heparin (C), and the other group a lipids emulsion (Intralipid ${ }^{\circledR}$ ) with heparin (IL). We measured hypothalamic Nitric Oxide Synthases (NOS) activity and mRNA expression for inducible NOS (iNOS, $\mathrm{Ca}^{2+}$ independent), and endothelial/neural NOS (eNOS, nNOS) in both group.

Then we treated rats with an acute injection of competitive NOS inhibitor $N_{G}$-Monomethyl-L-Arginine Monoacetate (L-NMMA) into carotid artery, just before the Intra-Venous Glucose Tolerance Test (IVGTT). We also injected LNMMA during euglycemic-hyperinsulinemic clamps to test the effects of central NO production on glucose kinetics.
Results: The lipid infusion did not lead to any modifications of plasma triglycerides and free fatty acids. Food intake and body weight remained similar between groups. eNOS, nNOS and iNOS mRNA expression was unchanged in the hypothalamus of IL rats, whereas iNOS activity was significantly increased $(+56 \%, \mathrm{p}<0,05)$, as well as eNOS and nNOS activities $(+90 \%, \mathrm{p}<0,05)$. L-NMMA treatment had no effect on glycaemia time courses during IVGTT and no effect on insulinaemia time course of $\mathrm{C}$ rats, whereas the increased GIIS characteristic of IL rats was normalized after L-NMMA injection. The clamps study showed a hepatic insulin resistance in IL rats, which is abolished following L-NMMA treatment.

Conclusion: These results suggest that NO pathway is involved in the central effect of lipid overload: the increase activity of NOS is probably responsible, at least in part, of the hepatic insulin resistance and the concomitant increase in insulin secretion in response to glucose. Indeed we shown that central NOS blockade restore normal GIIS and hepatic insulin sensitivity in IL group. We propose that induction of NO pathway could be at the crossroad of inflammatory process, lipotoxicity and autonomic control of glucose homeostasis and energy balance.

\section{OP 15}

\section{Inflammation and arterial modelling}

0085

Benfotiamine reduces the postprandial increase in serum inflammatory markers induced by dietary AGE (Advanced Glycation Endproducts) in patients with type 2 diabetes mellitus (T2DM)

M. Negrean ${ }^{1}$, A. Stirban ${ }^{1}$, B. Stratmann ${ }^{1}$, T. Gawlowski ${ }^{1}$, C. Götting ${ }^{2}$, K. Kleesiek ${ }^{2}$, T. Koschinsky ${ }^{3}$, D. Tschöpe ${ }^{1}$;

${ }^{1}$ Diabetes Clinic, Heart-and Diabetes Center NRW, Bad Oeynhausen, ${ }^{2}$ Institute for Laboratory and Transfusion Medicine, Heart-and Diabetes Center NRW, Bad Oeynhausen, ${ }^{3}$ Diabetes Centre, Heinrich Heine University, Düsseldorf, Germany.

Background and Aims: In patients with T2DM, an AGErich diet can induce after 2-6 weeks persistent increases in inflammatory mediators (CRP, TNF $\alpha$ ). Benfotiamine (BT), the liposoluble derivative of vitamin $\mathrm{B} 1$, has the capacity to inhibit AGE formation in vitro and in vivo. The acute effects of dietary AGE on inflammatory markers and their modulation by BT have not been studied until now.

Materials and Methods: Therefore, 19 T2DM inpatients (age: $55.4 \pm 2.2$ years, $\mathrm{HbA1c}: 8.8 \pm 0.5 \%$, without acute cardiovascular events within the previous 6 months) 
received a standard diabetes diet for 9 days. On day 4 and 6, the acute effects of a high AGE or a low AGE meal (HAGE vs. LAGE, 15.100 vs. $2.750 \mathrm{kU}$ AGE) on serum inflammatory markers (IL-6, IL-8) were studied in a randomized, cross-over, investigator-blinded design. In a subgroup of 11 patients the HAGE meal was repeated on day 9 after a 3-day pre-treatment with BT (Milgamma $^{\circledR}$, Wörwag, Germany): $3 \times 350 \mathrm{mg} / \mathrm{d}$ on day 7 and $8,1.050 \mathrm{mg}$ on day $9,1 \mathrm{~h}$ prior to the meal. Inflammatory markers (determined by ELISA, ImmuLite 2000, DPC Biermann, Bad Nauheim, Germany) were assessed after an overnight fast, prior and 2, 4 and $6 \mathrm{~h}$ after HAGE or LAGE. Both meals had the same ingredients (580 kcal, $54 \mathrm{~g}$ protein, $17 \mathrm{~g}$ lipids, $48 \mathrm{~g}$ carbohydrates) and differed only by the cooking conditions (temperature and time).

Results: IL- 6 levels increased after HAGE by $100 \%$, from $2.9 \pm 0.6 \mathrm{pg} / \mathrm{ml}$ at baseline to $3.9 \pm 1.1(2 \mathrm{~h}), 3.4 \pm 0.6(4 \mathrm{~h})$ and $5.8 \pm 1.2 * \dagger(6 \mathrm{~h})(* \mathrm{p}<0.05$ vs. baseline, $\dagger \mathrm{p}<0.05$ vs. LAGE), and changed transiently and non-significantly after LAGE, from $3.5 \pm 0.8 \mathrm{pg} / \mathrm{ml}$ at baseline to $2.9 \pm 0.4(2 \mathrm{~h}), 3.1 \pm 0.4$ $(4 \mathrm{~h})$ and $4.0 \pm 0.6(6 \mathrm{~h})(\mathrm{p}=\mathrm{ns})$. BT pre-treatment could not prevent this increase, but reduced its extent to $69,5 \%$ : from $2.2 \pm 0.1 \mathrm{pg} / \mathrm{ml}$ at baseline to $2.2 \pm 0.1(2 \mathrm{~h}), 2.3 \pm 0.2(4 \mathrm{~h})$ and $2.9 \pm 0.3 *(6 \mathrm{~h})(* \mathrm{p}<0.05$ vs. baseline $)$.

IL-8 levels changed after HAGE from $9.5 \pm 0.9 \mathrm{pg} / \mathrm{ml}$ at baseline to $10.6 \pm 1.6(2 \mathrm{~h}), 10.1 \pm 1.5(4 \mathrm{~h})$ and $10.4 \pm 1.6(6 \mathrm{~h})$ and after LAGE from $10.4 \pm 1.5 \mathrm{pg} / \mathrm{ml}$ at baseline to $8.9 \pm 0.9$ $(2 \mathrm{~h}), 8.0 \pm 0.7(4 \mathrm{~h})$ and $8.3 \pm 0.7(6 \mathrm{~h})(\mathrm{p}=\mathrm{ns}$ vs. baseline for all). Pre-treatment with benfotiamine before the repeated HAGE meal led to a persistent and significant decrease in IL-8 levels: from $9.7 \pm 0.8 \mathrm{pg} / \mathrm{ml}$ at baseline to $9.2 \pm 0.9(2 \mathrm{~h})$, $8.8 \pm 0.8^{*}(4 \mathrm{~h})$ and $8.6 \pm 0.7^{*}(6 \mathrm{~h})(\mathrm{p}<0.05$ vs. baseline $)$.

Conclusion: Even a single real-life AGE-rich meal induces an acute increase in serum inflammatory markers (IL-6, IL8) in T2DM, while a LAGE meal with identical ingredients does not. Pre-treatment with benfotiamine partly prevents the increase of IL- 6 and induces a persistent decrease of IL8 . The benefits of long-term supplementation with benfotiamine remain to be established.

\section{6}

Inflammation in epicardial adipose tissue: comparison of inflammatory mechanisms for production of adipocytokines in human epicardial and gluteo-femoral adipose tissue in CABG patients

A. R. Baker ${ }^{1}$, A. Ranasinghe ${ }^{2}$, N. Howell ${ }^{2}$, D. Pagano ${ }^{2}$, R. Bonser ${ }^{2}$, S. Kumar ${ }^{1}$, P. G. McTernan ${ }^{1}$;

${ }^{1}$ Unit for Diabetes and Metabolism, University of Warwick, Coventry, ${ }^{2}$ Department of Cardiothoracic Surgery, University of Birmingham, United Kingdom.
Background and Aims: Studies have established that adipose tissue distribution has significant impact on disease with central abdominal fat increasing both type 2 diabetes (T2DM) and cardiovascular disease (CVD) risk compared with gluteo-femoral fat. Whilst the relative pathogenic risk of abdominal and gluteo-femoral adipose tissue are apparent, few studies to date have investigated site specific nature of human epicardial adipose tissue compared with gluteo-femoral fat. Our recent studies have implicated epicardial adipose tissue (AT) as an important source of pro-inflammatory cytokines which may have important impact on myocardial function due to its proximity to the heart. Therefore the aim of this study using matched paired epicardial adipose tissue (AT) and gluteo-femoral (thigh) AT from patients undergoing coronary artery bypass grafting (CABG) patients was to examine (1) the key mediators of the innate immune pathway toll like receptors, TLR 2 and TLR 4 (2) the inflammatory signaling molecules nuclear factor kappa B and c-jun kinase (JNK) key mediators of adipocytokine production (3) and the mRNA expression of CD68 as a marker of macrophages investigating the role of macrophages in epicardial adipose tissue. Materials and Methods: Epicardial and gluteo-femoral adipose tissue was obtained from subjects undergoing coronary artery bypass grafting $(n=16, A g e=66.7 \pm 6.7$, $\mathrm{BMI}=28.4 \pm 3.6$ ). Components of the NFkB pathway were analysed at a protein level by Western Blotting. Gene expression was analysed by quantitative RT-PCR using the ABI 7500 Real-Time PCR system.

Results: Gene expression data in the epicardial adipose tissue showed strong correlations between CD-68 with both TLR-2 and 4 (TLR-2 $\mathrm{r}=0.94, \mathrm{p}<0.001$; TLR-4 $\mathrm{r}=0.95$, $\mathrm{p}<0.001)$ and also TNF $\alpha(r=0.81, \mathrm{p}<0.001)$. Protein expression data of NFKB and the IKK complex showed epicardial AT had significantly higher NFкB, IKK- $\gamma$, IKK- $\beta$ compared with thigh AT (NFKB: Epicardial $2.83 \pm(\mathrm{SEM}) 0.37$ vs Thigh: $1.69 \pm 0.37 \mathrm{p}=0.06$, IKK $\beta$ Epicardial $3.59 \pm 0.25$ vs Thigh $1.50 \pm 0.33 \mathrm{p}=0.01$, IKK $\gamma$ Epicardial $2.22 \pm 0.10$ vs Thigh $1.02 \pm 0.03 \mathrm{p}=0.001$ ). In addition JNK protein expression data indicated a trend towards upregulation in epicardial AT compared with thigh (JNK: Epicardial $3.15 \pm 0.57$ vs Thigh $1.35 \pm 0.21 \mathrm{p}=\mathrm{NS}, \mathrm{n}=4$ ).

Conclusion: In summary these studies highlight that epicardial adipose tissue has increased NFкB, and IKK $\beta$ an important determinant in inflammation activation compared with matched thigh tissue. We identified a close positive correlation between both TLR-2 and-4 affirms there known interaction in inflammation. Further our mRNA expression data of CD68 also implicates macrophage infiltration in this increased inflammatory state. As such increased levels of NFKB pathway observed in the epicardial depot may arise through activated macrophages in conjunction with the adipocytes, this is supported by the 
strong correlations of mRNA expression of the NFкB target gene and TNF- $\alpha$ with CD-68. In conclusion, these studies highlight epicardial AT as a source of inflammatory cytokines mediated through $\mathrm{NF} \kappa \mathrm{B}$ and components of key inflammatory pathways and further highlights the role of the macrophage in inflammation within this tissue.

Supported by Dunhill Medical Trust

\section{7}

Plasma adiponectin levels are inversely associated with carotid intima media thickness and markers of insulin resistance in middle-aged men

P. M. Nilsson ${ }^{1}$, G. Engström ${ }^{2}$, B. Hedblad ${ }^{2}$, J. Frystyk $^{3}$, M. Persson ${ }^{4}$, G. Berglund ${ }^{1}$, J. Flyvbjerg ${ }^{3}$;

${ }^{1}$ Medicine, Department of Clinical Sciences Medicine, Malmö, Sweden, ${ }^{2}$ Epidemiology, Department of Clinical Sciences Medicine, Malmö, Sweden, ${ }^{3}$ Medical Research Laboratories, Clinical Institute and Medical Department M (Diabetes and Endocrinology), Aarhus University Hospital, Denmark, ${ }^{4}$ Clinical Research Unit Medicine, Department of Internal Medicine, Malmö, Sweden.

Background and Aims: Circulating plasma adiponectin is a peptide marker for insulin sensitivity and glucose metabolism, derived from fat cells. It is still largely unknown if adiponectin is also an independent marker for early atherosclerosis or not.

Materials and Methods: We measured plasma adiponectin levels in a sample of 384 middle-aged men and 524 middleaged women, as a subgroup of the Malmö Diet CancerCardiovascular Branch (MDC-CVB; n=6103) study. The subjects were sampled stratified for degree of insulin sensitivity as evaluated by the HOMA-IR index. Clinical examination (height, weight, waist and hip circumference, blood pressure (BP)) and fasting blood sampling (lipids, haemoglobin $\mathrm{A}_{1 \mathrm{c}}\left(\mathrm{Hb}_{1 \mathrm{c}}\right)$ ) was carried out in all subjects. An ultrasound measurement of the right common carotid artery intima media thickness (IMT) was made by use of Accuson device. Plasma adiponectin was measured by a novel in-house (Aarhus) time-resolved immunofluorometric assay (TR-IFMA).

Results: When the distribution of adiponectin was stratified into sex-specific quartiles (Q1-Q4), men in Q4 differed from Q1 in higher mean age and HDL-cholesterol, but lower diastolic BP (DBP), $\mathrm{HbA}_{1 \mathrm{c}}$, HOMA-index, BMI, and prevalence of diabetes. Women in adiponectin Q4 compared to Q1 had higher HDL-cholesterol, but lower $\mathrm{HbA}_{1 \mathrm{c}}$, HOMA-index, BMI, waist-hip ratio and prevalence of diabetes. Mean IMT for men was significantly lower $(\mathrm{p}=0.03)$ in adiponectin Q4 as compared to Q1 when adjusted for age, waist circumference, smoking, HDLcholesterol, and DBP in multiple regression analyses. When adding $\mathrm{HbA}_{1 \mathrm{c}}$ to the model, the association was no longer significant $(p=0.08)$. For women no difference in IMT was noticed across adiponectin quartiles.

Conclusion: Plasma adiponectin is a marker of glucose metabolism and obesity and shows an inverse age-adjusted association with carotid ultrasound IMT in men, but not in women. This association is is attenuated after adjustment for metabolic risk factors.

Supported by the Heart- and Lung Foundation of Sweden, the Danish Medical Research Council, the Danish Diabetes Association, the Novo Nordisk Foundation, and Clinical Institute, Aarhus University, Aarhus, Denmark

\section{8}

Undiagnosed dysglycaemia and inflammation in cardiovascular disease

O. Johansen ${ }^{1,2}$, K. I. Birkeland ${ }^{3,4}$, E. Brustad ${ }^{1}$, E. Aaser ${ }^{1}$, A.-K. Lindahl ${ }^{5}$, R. Midha ${ }^{6}$, T. Ueland ${ }^{7}$, P. Aukrust ${ }^{7}$, L. Gullestad ${ }^{8}$;

${ }^{1}$ Medical Department, Asker and Baerum Hospital, Rud, ${ }^{2}$ Novo Nordisk Scandinavia AS, Rud, ${ }^{3}$ Department of Endocrinology, Aker University Hospital, Oslo, ${ }^{4}$ Faculty Division Aker University of Oslo, ${ }^{5}$ Department of Surgery, Asker and Baerum Hospital, Rud, ${ }^{6}$ Department of Clinical Chemistry, Asker and Baerum Hospital, Rud, ${ }^{7}$ Resarch Institue for Internal Medicine, Rikshospitalet University Hospital, Oslo, ${ }^{8}$ Department of Cardiology, Rikshospitalet University Hospital, Oslo, Norway.

Background and Aims: Cardiovascular (CV) disease is associated with increased levels of glucose and enhanced inflammation, but the prevalence of dysglycaemia in $\mathrm{CV}$ diseases is not fully known. We examined the prevalence of unknown dysglycaemia and its association with inflammation in Caucasian patients with ischemic vascular complications (coronary artery disease [CAD], cerebrovascular disease [CVD], peripheral artery disease [PAD]).

Materials and Methods: This case control study involved 149 patients (mean age 68 years) hospitalised for CAD $(n=51)$, PAD $(n=47)$ or CVD $(n=51)$ and 59 control subjects (CTR, mean age 66 years) free from CV-disease. Anthropometric measures were similar among all groups. The prevalence of dysglycaemia according to WHO/ADA criteria (impaired fasting glycaemia, impaired glucose tolerance or diabetes mellitus) was assessed by a $75 \mathrm{~g}$ oral glucose tolerance test (OGTT). Inflammatory parameters were analysed in fasting serum samples. 
Results: Dysglycaemia was found in $49 \%, 55 \%$ and $57 \%$ (Fig 1) among patients with CAD, CVD and PAD, respectively, all significantly higher than among controls $(29 \%)$. The odds ratio for being dysglycaemic were 1.7 (95\% CI: 1.04-2.77), 1.9 (1.19-3.06) and 2.0 (1.25-3.19) for $\mathrm{CAD}, \mathrm{CVD}$ and $\mathrm{PAD}$ respectively. Inflammatory markers (the total leukocyte count, sTNF-R1, CRP) were elevated in patient groups (Table 1) while the opposite was true for the anti-inflammatory cytokine TGF- $\beta 1$ and IGF-BP3 in patients with CAD. Inflammatory markers tended to parallel increasing blood glucose levels in all groups (not shown).

Conclusion: Undiagnosed dysglycaemia was common in patients with ischemic $\mathrm{CV}$ manifestations regardless of vascular bed involved. Inflammation was enhanced in patients with CV-complications and associated in a dosage-related-manner to glucose levels.

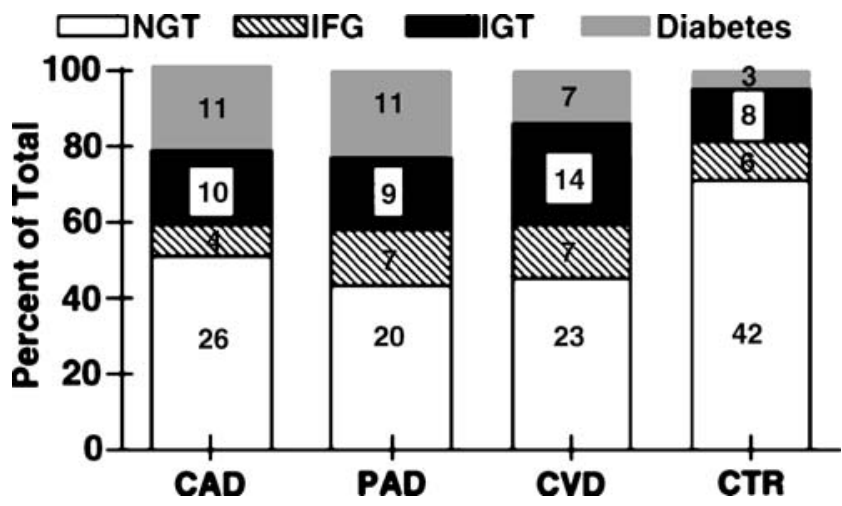

Table 1. Inflammatory markers of the different groups and controls.

\begin{tabular}{lllll}
\hline & CAD & PAD & CVD & CTR \\
\hline $\begin{array}{l}\text { Total leukocyte } \\
\text { count }\left(10^{9} / 1\right)\end{array}$ & $6.9 \pm 2.0^{* *}$ & $7.2 \pm 1.8^{* * *}$ & $6.8 \pm 1.9 * *$ & $5.9 \pm 1.4$ \\
CRP $(\mathrm{mg} / \mathrm{dl})$ & 3.7 & $4.8^{*}$ & 3.7 & 2.8 \\
$(95 \% \mathrm{CI})$ & $(2.2-5.2)$ & $(3.3-6.4)$ & $(2.3-5.1)$ & $(1.8-3.7)$ \\
sTNF-R1 $(\mathrm{pg} / \mathrm{ml})$ & $999 \pm 493^{*}$ & $1036 \pm 376^{* *}$ & $965 \pm 539$ & $850 \pm 283$ \\
TGF-B1 $(\mathrm{ng} / \mathrm{ml})$ & $5.4 \pm 2.9^{* *}$ & $6.5 \pm 3.4$ & $6.6 \pm 3.8$ & $7.4 \pm 3.6$ \\
IGF-1 $(\mathrm{ng} / \mathrm{ml})$ & $62.9 \pm 24.3$ & $78.7 \pm 28.6$ & $72.4 \pm 26.0$ & $70.5 \pm 29.5$ \\
IGFBP3 $(\mu \mathrm{g} / \mathrm{ml})$ & $1.5 \pm 0.4^{*}$ & $1.5 \pm 0.4$ & $1.6 \pm 0.4$ & $1.6 \pm 0.4$ \\
\hline
\end{tabular}

Data are mean \pm SD unless otherwise stated. ${ }^{*} p<0.05$, $* * \mathrm{p}<0.01$ and $* * * \mathrm{p}<0.001$ versus CTR. CAD, coronary artery disease; PAD, peripheral artery disease; CVD, cerebrovascular disease; CTR, controls; CRP, C-reactive protein; sTNF-RI, soluble tumor necrosis factor receptor type I; TGF, transforming growth factor; IGF, insulin-like growth factor, IGFBP, IGF binding protein.

Supported by Eastern Norway Regional Health Authority
0089

Additive anti-inflammatory effect of pioglitazone to simvastatin in non-diabetic cardiovascular disease, the role of insulin resistance

M. Hanefeld ${ }^{1}$, N. Marx ${ }^{2}$, A. Pfuetzner ${ }^{3}$, J. Mueller ${ }^{4}$,

G. Luebben ${ }^{5}$, E. Karagiannis ${ }^{5}$, U. Stier ${ }^{1}$, T. Forst ${ }^{3}$;

${ }^{1}$ Endocrinology and Metabolism, Center for Clinical Studies, Dresden, ${ }^{2}$ Department of Cardiology, University of Ulm, ${ }^{3}$ Institute for Clinical Research and Development, Mainz, ${ }^{4}$ Statistics, Acromion, Frechen, ${ }^{5}$ Takeda Pharma, Aachen, Germany.

Background and Aims: Low-grade inflammation (LGI) is a pathogenic factor for atherosclerosis. High sensitivity CRP (hs-CRP), matrix metalloproteinase 9 (MMP9), and plaminogen activator inhibitor (PAI-1) are markers of LGI, associated with cardiovascular disease (CVD). Statins and PPAR $\gamma$ antagonists (i.e. pioglitazone) lower inflammatory markers and reduce CVD in type 2 diabetes. We aimed to test the safety and efficacy of the combination of pioglitazone and simvastatin versus each individual drug in non-diabetic subjects with CVD and high hs-CRP.

Materials and Methods: In a prospective, double blinded, double dummy, three arm parallel trial 125 subjects were randomly assigned to receive simvastatin or pioglitazone plus placebo or both drugs. Follow-up was 12 weeks. We tested the changes in hs-CRP by analysis of covariance (ANCOVA) with fixed effect factors for treatment group and baseline hs-CRP as covariate. The correlation between changes in hs-CRP and insulin resistance (HOMA) was calculated with Spearmans rank correlation test.

Results: At baseline there were no significant differences in the groups. Pioglitazone and simvastatin significantly reduced hs-CRP $(3.64 \pm 2.42$ to $2.48 \pm 1.77 \mathrm{mg} / \mathrm{L}$ and $3.26 \pm 2.02$ to $2.81 \pm 2.11 \mathrm{mg} / \mathrm{L}$ ); but the combined treatment had an additive effect (hs-CRP from $3.49 \pm 1.97$ to $2.06 \pm 1.42 \mathrm{mg} / \mathrm{L}, \mathrm{p}<0.0103)$. HOMA decreased in both groups on pioglitazone and the correlation between the changes in insulin sensitivity and in hs-CRP was significant $(r=0.43 ; p<0.05)$. No serious adverse events occurred in any group. Additionally PAI-1 decreased significantly only in the pioglitazone groups and MMP 9 in the 3 groups

Conclusion: Pioglitazone, by reducing insulin resistance, has additive antiinflammatory effects to Simvastatin in nondiabetic subjects with CVD and high hs-CRP.

Supported by a grant from Takeda 
0090

Divergent relationship between soluble TNF-alpha receptor 1 and 2 , insulin resistance and endothelial function

A. Castro ${ }^{1}$, E. Esteve ${ }^{2}$, A. López-Bermejo ${ }^{2}$, J. Vendrell ${ }^{3}$, W. Ricart ${ }^{2}$, J. Fernández-Real ${ }^{2}$;

${ }^{1}$ Internal Medicine, ${ }^{2}$ Endocrinology, Hospital of Girona,

${ }^{3}$ Endocrinology, Hospital of Tarragona, Spain.

Background and Aims: Tumor necrosis factor- $\alpha$ (TNF- $\alpha$ ) is a pro-inflammatory cytokine that is implicated in the pathogenesis of endothelium dysfunction and insulin resistance. Serum concentrations of soluble TNF- $\alpha$ receptor 1 and 2 (sTNFR1 and sTNFR2) are thought to reflect TNF- $\alpha$ action. We aimed to evaluate whether circulating sTNFR1 and 2 contribute to explain the complex effects of TNF- $\alpha$ on endothelial function independent of insulin resistance.

Materials and Methods: Circulating sTNFR1 and sTNFR2 concentrations were measured by EASIA. Insulin sensitivity (minimal model) and brachial vascular reactivity (high resolution external ultrasound) were assessed in 100 consecutive nondiabetic Caucasian men (70 with normal and 30 with impaired glucose tolerance).

Results: In subjects with normal glucose tolerance, serum sTNFR1 was an independent contributor to the variance of endothelium-dependent vasodilatation after adjusting for age, BMI, smoking status, systolic and diastolic blood pressure and insulin sensitivity $(p=$ 0.002). In fact, circulating sTNFR1 was positively associated with endothelium-dependent vasodilatation in these subjects $(\mathrm{r}=0.291, \mathrm{p}=0.02)$. In contrast, serum sTNFR2 concentration was negatively associated with endothelium-dependent vasodilatation in all subjects as a whole (trend $\mathrm{r}=-0.190, \mathrm{p}=0.058$ ), and the latter association was especially significant in subjects with impaired glucose tolerance $(\mathrm{r}=-0.366, \mathrm{p}=0.047)$. No significant associations were observed between endothelium-independent vasodilatation and serum sTNFR1 or sTNFR2 concentration.

Conclusion: TNF- $\alpha$ receptor shedding (circulating sTNFR1 and sTNFR2) could help to explain the complex effects of TNF- $\alpha$ on endothelial function.

Supported by Redes G03/28

\section{OP 16}

\section{Epidemiology of CVD in type 2 diabetes}

0091

Use of sulphonylureas and mortality after myocardial infarction in diabetic patients: a Danish nationwide population-based study

H. Thisted ${ }^{1,2}$, J. Jacobsen ${ }^{1}$, R. W. Thomsen ${ }^{1}$,

S. P. Johnsen ${ }^{1,3}$, J. Rungby ${ }^{2,4}$;

${ }^{1}$ Department of Clinical Epidemiology, Aarhus University Hospital, ${ }^{2}$ Department of Pharmacology, University of Aarhus, ${ }^{3}$ Center of Cardiovascular Research, Aalborg Hospital, ${ }^{4}$ Department of Endocrinology C, Aarhus University Hospital, Denmark.

Background and Aims: Sulphonylureas (SUs) can block the ATP-sensitive potassium channels in the heart, thereby inhibiting the myoprotective effects of ischemic preconditioning (IP). However, in experimental studies, only glibenclamide inhibit IP, whereas newer SUs (gliclazide and glimepiride) do not abolish this protection. The clinical implications of these pharmacological differences are unclear as data from clinical studies are sparse, but in a preliminary regional Danish study, we found a lower risk of myocardial infarction (MI) with gliclazide and glimepiride when compared to other SUs and a trend towards a lower 30-day mortality among users of gliclazide compared to users of other SUs.

Material and Methods: We examined the mortality among users of "new" once-daily SUs (gliclazide and glimepiride) and "old" SUs (glibenclamide, glipizide and tolbutamide) in a nationwide population-based follow-up study in Denmark. We identified 54,835 patients with a first-time hospitalization for MI in Denmark in the period 1996-2002 using data from the Danish National Hospital Registry (DNHR). Among MI patients, data on diabetes and type of antidiabetic treatment were obtained from the DNHR and a national prescription database. Mortality was determined through the Civil Registration System. We estimated 30-day mortality rate ratios (MRRs) associated with the use of different SUs before MI adjusted for a range of prognostic factors including duration of diabetes, use of cardiovascular drugs and level of comorbidity, using Cox regression.

Results: Among MI patients, we identified a total of 6,644 patients with diabetes. Treatment with "new" SUs before 
MI was associated with a decreased 30-day mortality both when compared to no pharmacotherapy for diabetes (see table below) and when compared to treatment with "old" SUs (adjusted MRR $=0.75,95 \%$ CI: 0.61-0.93, $\mathrm{p}=0.009$ ). The apparent advantage in 30-day mortality of treatment with "new" SUs was also evident during the entire followup period (median follow-up time 1.68 years).

30-day MRRs according to antidiabetic treatment

Antidiabetic medication Number Deaths Mortality Adjusted MRR $(95 \% \mathrm{CI})$

\begin{tabular}{lllll}
\hline No pharmacotherapy & 1,636 & 375 & $22.9 \%$ & 1.00 (reference) \\
"New" sulphonylureas & 579 & 110 & $19.0 \%$ & $0.81(0.65-1.01)$ \\
-Glimepiride & 465 & 90 & $19.4 \%$ & $0.84(0.66-1.06)$ \\
-Gliclazide & 115 & 20 & $17.4 \%$ & $0.75(0.47-1.17)$ \\
"Old" sulphonylureas & 1,526 & 395 & $25.9 \%$ & $1.08(0.93-1.27)$ \\
-Glibenclamide & 776 & 193 & $24.9 \%$ & $1.08(0.90-1.30)$ \\
-Glipizide & 378 & 108 & $28.6 \%$ & $1.15(0.92-1.43)$ \\
-Tolbutamide & 387 & 101 & $26.1 \%$ & $1.10(0.87-1.38)$ \\
\hline
\end{tabular}

Conclusion: Our findings support the hypothesis that "new" SUs (gliclazide and glimepiride) have cardioprotective effects that may translate into improved survival after MI. Supported by the Danish Diabetes Association and Servier

0092

High risk of cardiovascular mortality in subjects with impaired fasting glucose is mostly explained by those who convert to diabetes

J. M. Rijkelijkhuizen ${ }^{1}$, G. Nijpels ${ }^{1,2}$, R. J. Heine ${ }^{1,3}$, L. M. Bouter ${ }^{1}$, C. D. A. Stehouwer ${ }^{1,4}$, J. M. Dekker $^{1}$; ${ }^{1}$ EMGO Institute, ${ }^{2}$ Department of General Practice, ${ }^{3}$ Department of Endocrinology, VU University Medical Center, Amsterdam, ${ }^{4}$ Department of Internal Medicine, Academic Hospital Maastricht, The Netherlands.

Background and Aims: Impaired glucose regulation is considered a high-risk state for type 2 diabetes and cardiovascular disease (CVD). The American Diabetes Association (ADA) introduced diagnostic criteria for impaired fasting glucose (IFG) in 1997. In 2003, the ADA lowered the threshold for the definition of impaired fasting glucose (IFG) in order to increase the sensitivity of identifying subjects who will convert to diabetes within 10 years. However, the consequences of lowering this cut point of IFG for CVD risk were not considered. Our aim was to assess the impact of lowering the IFG cut point on the associated risk of CVD mortality and to evaluate whether this risk is explained by subjects who later convert to type 2 diabetes.
Materials and Methods: The risk of CVD mortality for subjects with impaired fasting glucose was studied in a population-based cohort, the Hoorn Study. A 75-g oral glucose tolerance test (OGTT) was administered at baseline (1989) and at the follow-up examination in $1996(\mathrm{n}=1428)$. Subjects were classified at baseline according to criteria of the ADA of 1997 (IFG if fasting plasma glucose (FPG) 6.1$7.0 \mathrm{mmol} / \mathrm{l}$ ) and of 2003 (IFG if FPG 5.6-7.0 mmol/l). For the subjects who were classified as IFG at baseline, it was determined whether they had converted to diabetes or not by classifying them again in 1996. For CVD mortality in the period from 1996 until January 1st 2005, hazard ratios (HRs) and $95 \%$ confidence intervals (CIs) were obtained from Cox proportional hazards models (adjusted for age and sex).

Results: For the ADA-1997 criteria but not for the ADA2003 criteria, IFG-subjects had a significantly higher CVD mortality risk than normal fasting glucose (NFG)-subjects (Table 1). For both sets of criteria, subjects who converted from IFG to diabetes had a more than two-fold risk at CVD mortality (ADA-2003: 2.14 (1.12-4.10) and ADA-1997: 2.47 (1.17-5.19), Table 1) than subjects who were classified as NFG. Subjects who remained IFG or had converted to NFG had only slightly (and not significantly) higher CVD mortality risks than subjects with NFG (ADA2003: 1.15 (0.69-1.93) and ADA-1997: 1.50(0.72-3.15)).

Conclusion: The lower cut point for IFG (ADA-2003 criteria) results in a category of IFG which no longer represents a high-risk state of CVD. Furthermore, we showed that the association between IFG and the risk at CVD mortality is mostly explained by the conversion to diabetes.

Table 1. Age- and sex-adjusted hazard ratios of CVD mortality for glucose tolerance categories

\begin{tabular}{|c|c|c|c|c|c|}
\hline \multicolumn{3}{|c|}{ ADA-1997 } & \multicolumn{3}{|c|}{ ADA-2003 } \\
\hline $\mathrm{N}$ & $\begin{array}{l}\text { CVD } \\
\text { mortality } \\
\text { (n) }\end{array}$ & $\operatorname{HR}(95 \% \mathrm{CI})$ & $\mathrm{N}$ & $\begin{array}{l}\text { CVD } \\
\text { mortality } \\
\text { (n) }\end{array}$ & $\mathrm{HR}(95 \% \mathrm{CI})$ \\
\hline 1217 & 758 & 1 & 878 & 39 & 1 \\
\hline 149 & 16 & $\begin{array}{l}1.87 \\
(1.07-3.25)\end{array}$ & 488 & 35 & $\begin{array}{l}1.37 \\
(0.87-2.16)\end{array}$ \\
\hline 87 & 8 & $\begin{array}{l}1.50 \\
(0.72-3.15)\end{array}$ & 387 & 723 & $\begin{array}{l}1.15 \\
(0.69-1.93)\end{array}$ \\
\hline 62 & 8 & $\begin{array}{l}2.47 \\
(1.17-5.19)\end{array}$ & 101 & 12 & $\begin{array}{l}2.14 \\
(1.12-4.10)\end{array}$ \\
\hline 62 & 7 & $\begin{array}{l}1.72 \\
(0.78-3.80)\end{array}$ & 62 & 7 & $\begin{array}{l}1.78 \\
(0.79-4.00)\end{array}$ \\
\hline
\end{tabular}

$\mathrm{N} / \mathrm{n}$ : number of cases, NFG: normal fasting glucose, IFG: impaired fasting glucose, IFG $\rightarrow$ IFG/NFG: subjects classified as IFG at baseline and as IFG or NFG at follow-up, IFG $\rightarrow$ DM: subjects classified as IFG at baseline and as DM at follow-up, DM: diabetes mellitus type 2 


\section{3}

Physical inactivity and all-cause mortality in Australians with 'undiagnosed' impaired glucose metabolism. The AUSDIAB Study

D. W. Dunstan ${ }^{1}$, E. L. M. Barr ${ }^{1}$, D. J. Magliano ${ }^{1}$, J. Salmon ${ }^{2}$, N. Owen ${ }^{3}$, D. Jolley ${ }^{4}$, J. E. Shaw ${ }^{1}$,

P. Z. Zimmet' ${ }^{1}$;

${ }^{1}$ Epidemiology, International Diabetes Institute, Melbourne,

${ }^{2}$ School of Exercise and Nutrition Sciences, Deakin University, Melbourne, ${ }^{3}$ Cancer Prevention Research Unit, The University of Queensland, Brisbane, ${ }^{4}$ Monash Institute of Health Services Research, Monash University, Melbourne, Australia.

Background and Aims: Physical inactivity has been associated with increased all-cause mortality in the general population and in people with type 2 diabetes. However, little is known about the association of physical inactivity among people with 'undiagnosed' impaired glucose metabolism. The aim of this study was to examine the association between physical inactivity and all-cause mortality in Australian adults with impaired glucose metabolism.

Materials and Methods: The Australian Diabetes, Obesity and Lifestyle Study (AusDiab) is a nationally representative population-based survey of adults aged $\geq 25$ years. Baseline measurements were obtained between 1999 and 2000. Based on an OGTT, 2,471 individuals (1,238 men, 1,233 women) were identified as having impaired glucose metabolism (impaired fasting glycemia [IFG], impaired glucose tolerance [IGT], or undiagnosed type 2 diabetes) at baseline. Selfreported leisure-time physical activity status (physically inactive ( 0 mins/week) or physically active) was determined using interviewer-administered questionnaires. Potential confounders measured at baseline included: education, smoking status, diseases present at baseline (prior cardiovascular disease, hypertension, dyslipidaemia), waist circumference and television viewing time. Vital status was ascertained in 2005 from the National Death Index.

Results: Over a median follow-up of 5.2 years there were 131 deaths in those identified as having impaired glucose metabolism at baseline. Collectively, in those with impaired glucose metabolism, the age- and sex-adjusted odds ratio (OR) of all-cause mortality associated with being physically inactive was 2.10 (95\% CI 1.40-3.15), which remained significant after adjustment for confounders - OR: 1.95 (95\% CI 1.28-2.95). Among those with IGT and undiagnosed type 2 diabetes the odds ratios (adjusted for all confounders) of all-cause mortality associated with physical inactivity were $2.26(95 \%$ CI $1.25-4.1)$ and $2.56(95 \%$ CI 1.12-5.86) respectively. There was no association observed between all-cause mortality and physical inactivity in those with IFG - OR: 1.68 (95\% CI 0.53-5.31).
Conclusion: These findings show that physically inactive adults with 'undiagnosed' impaired glucose metabolism have more than a two-fold increased risk of all-cause mortality over 5 years. These results further reinforce the importance of promoting physical activity in people with impaired glucose metabolism.

0094

Are patients with type 2 diabetes more likely to have diagnosed atherosclerosis? A large US epidemiologic study

L. Glass ${ }^{1}$, P. $\operatorname{Sun}^{1}$, R. Wang ${ }^{2}$;

${ }^{1}$ Lilly Research Laboratories, Indianapolis, ${ }^{2}$ Quantum Research Group, Austin, United States.

Background \& Aim: Over last 2 decades, advances in cell and molecular biology have improved our understanding on the impact of atherosclerosis among patients with type 2 diabetes (T2D). However, up to now, no large US epidemiologic study has been conducted to compare atherosclerosis prevalence between patients with T2DM and patients without diabetes (NDM). Our study aims to fill this information gap through comparing age and gender specific atherosclerosis prevalence rates between patients with T2D and patients without diabetes (NDM) in a US primary care setting.

Methods: A retrospective cohort study was conducted based on a large national electronic medical records database that covered 4.2 million unique Americans in 2005. In the study, we reviewed medical records of 231,492 patients with T2D and a random sample of NDM patients $(1,219,047)$. Based on our medical record review, we estimated the age and gender specific atherosclerosis prevalence rates for individual gender and age groups, as well as relative risks (RR) and odds ratios (OR) of having atherosclerosis between T2D and NDM patients.

Results: Table 1 presents age and gender specific atherosclerosis prevalence rates for T2D and NDM patients. On average, the atherosclerosis prevalence rates were $1.6 \%$ for patients with T2D, but $0.7 \%$ for NDM. The age and gender specific relative risks (or odds ratios) of having atherosclerosis between patients with T2D and those without ranged from 1.3 through 5.0 for female and from 1.1 through 2.3 for male with a possible inverse age trend.

Conclusions: Patients with T2D, on average, were twice likely to have atherosclerosis than patients without diabetes. The odds ratios of having atherosclerosis tend to be higher among females and among younger age groups. These results suggest the necessity of preventing, diagnosing and treating atherosclerosis aggressively among subjects with T2D. 
Table 1: Age and Gender Specific Prevalence, Relative Risks and Odds Ratios of Atherosclerosis

\begin{tabular}{clllcc}
\hline $\begin{array}{c}\text { Age } \\
\text { Group } \\
\text { (years) }\end{array}$ & Both & T2DM & NDM & $\begin{array}{c}\text { Relative } \\
\text { Risk* }\end{array}$ & $\begin{array}{c}\text { Odds } \\
\text { Ratios* }\end{array}$ \\
\hline Female & & & & \\
$20-29$ & $0.1 \%$ & $0.2 \%$ & $0.0 \%$ & 5.0 & 5.0 \\
$30-39$ & $0.1 \%$ & $0.2 \%$ & $0.1 \%$ & 4.7 & 4.7 \\
$40-49$ & $0.2 \%$ & $0.2 \%$ & $0.2 \%$ & 1.3 & 1.3 \\
$50-59$ & $0.4 \%$ & $0.7 \%$ & $0.4 \%$ & 1.9 & 1.9 \\
$60-69$ & $1.0 \%$ & $1.4 \%$ & $0.8 \%$ & 1.7 & 1.7 \\
$70-79$ & $1.8 \%$ & $2.4 \%$ & $1.6 \%$ & 1.6 & 1.6 \\
$80+$ & $2.1 \%$ & $2.9 \%$ & $1.9 \%$ & 1.6 & 1.6 \\
All Ages & $0.8 \%$ & $1.6 \%$ & $0.7 \%$ & 2.3 & 2.3 \\
& Male & & & & \\
$20-29$ & $0.0 \%$ & $0.0 \%$ & $0.0 \%$ & - & - \\
$30-39$ & $0.1 \%$ & $0.2 \%$ & $0.1 \%$ & 2.3 & 2.3 \\
$40-49$ & $0.2 \%$ & $0.3 \%$ & $0.2 \%$ & 1.1 & 1.1 \\
$50-59 ;$ & $0.6 \%$ & $0.8 \%$ & $0.5 \%$ & 1.7 & 1.7 \\
$60-69$ & $1.2 \%$ & $1.5 \%$ & $1.1 \%$ & 1.4 & 1.4 \\
$70-79$ & $2.0 \%$ & $2.6 \%$ & $1.7 \%$ & 1.5 & 1.5 \\
$80+$ & $2.2 \%$ & $3.0 \%$ & $2.0 \%$ & 1.5 & 1.5 \\
All Ages & $0.9 \%$ & $1.6 \%$ & $0.8 \%$ & 2.1 & 2.1 \\
\hline Note: & & & & & \\
\hline
\end{tabular}

Note: $\mathrm{p}<0.01$ for all relative risks and odds ratios.

Supported by: Unrestricted fund from Eli Lilly and Company

\section{5}

Population screening for type 2 diabetes and associated cardiovascular risk factors reduces mortality

S. J. Griffin ${ }^{1}$, M. Rahman ${ }^{2}$, R. W. Jakes ${ }^{1}$, M. F. Yuyun ${ }^{3}$, A. Niggebrugge ${ }^{4}$, S. H. Hennings ${ }^{1}$, D. R. R. Williams ${ }^{5}$, N. J. Wareham ${ }^{1}$;

${ }^{1}$ Epidemiology Unit, Medical Research Council, Cambridge, ${ }^{2}$ Department of Public Health and Primary Care, University of Cambridge, ${ }^{3}$ Department of Medicine, Addenbrooke's Hospital NHS Trust, Cambridge, ${ }^{4}$ Eastern Region Public Health Observatory, Institute of Public Health, Cambridge, ${ }^{5}$ Centre for Health Improvement through Research and Evaluation, Swansea University School of Medicine, United Kingdom.

Background and Aims: Uncertainty remains over whether screening the general population for type 2 diabetes and associated cardiovascular risk factors will lead to overall health improvement. Prospective randomised trials of such screening programmes with mortality endpoints that might address this question will be expensive and take many years to complete. Material and Methods: A parallel group population-based cohort study was undertaken in Ely, Cambridgeshire. In 19901706 people were randomly selected from a popula- tion of 4933 individuals aged 40 to 65 years, without known diabetes, and were invited for five-yearly screening for diabetes using the oral glucose tolerance test (OGTT). Results including values for cholesterol, triglyceride, glycosylated haemoglobin and blood pressure were forwarded to general practitioners without treatment recommendations. Mortality follow-up lasted 14.3 years.

Results: $68 \%$ of those invited attended the first round of screening, attendance was lowest among men $(\mathrm{p}=0.035)$ and those with greater material deprivation (Townsend index $)(\mathrm{p}=0.005) .108$ participants had positive OGTTs during the three rounds of screening. A total of 581 participants died during follow-up, 245 cancer-related and 197 cardiovascular. Diabetes was recorded on 41 death certificates. After adjusting for age, gender and deprivation, invitation to screening was associated with a $20 \%$ reduction in overall mortality (hazard ratio: $0.80,95 \% \mathrm{CI}: 0.67$ to 0.95). Compared to those not invited the hazard ratio for mortality was lower for those taking up the offer of screening $(0.59,95 \% \mathrm{CI}$ : 0.47 to 0.73$)$ and higher for those invited but not attending (1.28, 95\% CI: 1.02 to 1.60$)$.

Conclusion: Invitation to a screening programme for diabetes including assessment of cardiovascular risk factors appears to significantly reduce overall population mortality, although many at high risk did not attend. It is not possible to ascertain the mechanism underlying this observation from this retrospective study. Earlier detection of diabetes may partly account for these findings, but they may also be due to changes in patient and practitioner behaviour associated with the screening programme. We await the findings from prospective studies before advocating population screening.

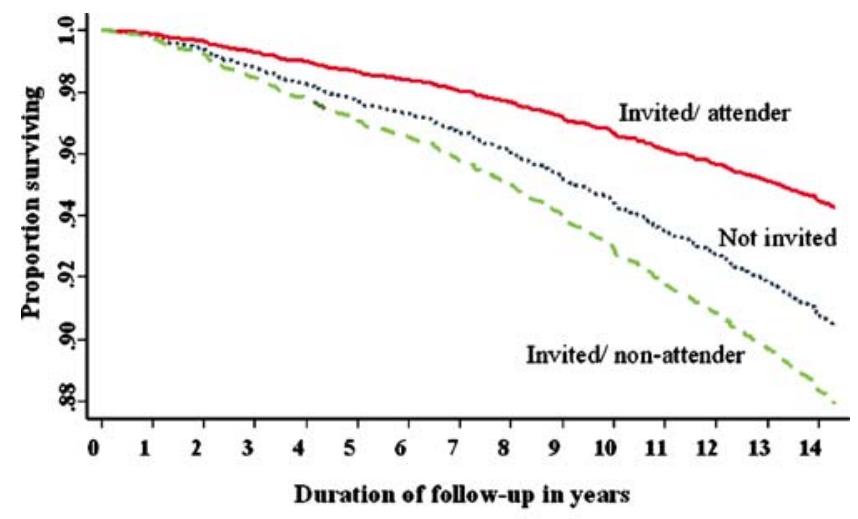

Kaplan-Meier survival curves according to whether individuals were invited to screening and whether invited individuals attended screening (adjusted for age, gender and Townsend Index of deprivation)

Supported by the Medical Research Council, NHS R\&D 


\section{6}

Insufficient cardiovascular risk factor control following myocardial infarction in patients with type 2 diabetes: a register linkage study from the Swedish national diabetes register (NDR)

S. Gudbjörnsdottir ${ }^{1}$, J. Cederholm ${ }^{2}$, K. Eeg-Olofsson ${ }^{1}$, B. Eliasson ${ }^{1}$, P. M. Nilsson ${ }^{3}$;

${ }^{1}$ Department of Medicine, Lundberg Laboratory for Diabetes Research, Göteborg, ${ }^{2}$ Department of Public Health and Caring Sciences, Uppsala University, ${ }^{3}$ Division of Medicine, Lund University, Malmö, Sweden.

Background and Aims: Cardiovascular risk factor control following myocardial infarction in patients with type 2 diabetes (DM2) is essential. The aim of this study was to examine the adherence to recent treatment guidelines in clinical practice.

Material and Methods: Patients with DM2 registered in the NDR $(n=41475)$ were analysed for comparisons of cardiovascular risk factors between patients with and without previous myocardial infarction (MI), based on national register linkage analyses for hospital care (2003). The epidemiological definition of DM2 was: (i) therapy with diet alone or oral hypoglycaemic agents (OHAs), (ii) insulin in monotherapy or combined with OHAs in patients with an age $>39$ years at the onset of diabetes.

Results: The mean age of the patients with a previous myocardial infarction $(\mathrm{n}=4215 ; 32 \%$ women) were 72 years. The mean duration of diabetes in these patients was 11 years and the mean follow-up time after the MI was 9 years. The achievement of treatment goals of cardiovascular risk factors were $90 \%$ for non-smoking status, $65 \%$ for nonobesity, $64 \%$ for total cholesterol (TC) $<5.0 \mathrm{mmol} / \mathrm{L}, 56 \%$ for both $\mathrm{HbAlc}<7.3 \%$ and triglycerides $<2.0 \mathrm{mmol} / \mathrm{L}$, and $34 \%$ for blood pressure (BP) $<130 / 85 \mathrm{mmHg}$. These frequencies, compared to patients without previous MI $(\mathrm{n}=32498)$, were considerably higher concerning BP and $\mathrm{TC}$, and somewhat higher regarding $\mathrm{HbAlc}$, non-obesity and non-smoking.

Treatment with antihypertensive- (34\% vs. $19 \%)$ and lipidlowering drugs ( $70 \%$ vs. $58 \%$ ) and aspirin ( 80 vs $33 \%$ ) were considerably higher in patients with a previous MI. The DM2 patients with a previous MI who had reached the treatment targets, were more oftenly treated with diet $(88-75 \%)$ and OHAs $(62-60 \%)$, but not with insulin, for the achieved HbA1c level, than the DM2 patients without a previous MI. Patients with a longer follow-up period after the MI than 9 years had, compared to patients with a shorter follow-up period, a tendency to higher achievement of the HbAlc target in 2003, but lower achievement of the TC level.

A logistic regression analysis of a subgroup of 33586 DM2 patients (with complete data for all variables in 2003) showed that a previous MI (dependent variable) was independently associated with hyperlipidaemia (untreated with $\mathrm{TC}>5.0 \mathrm{mmol} / \mathrm{L}$ or triglycerides $>1.7 \mathrm{mmol} / \mathrm{L}$, or treated), odds ratio (OR) $2.89(\mathrm{p}<0.001)$, and with hypertension (untreated with $\mathrm{BP}>140 / 90 \mathrm{mmHg}$, or treat ed), OR $2.49(\mathrm{p}<0.001)$. The association was less evident for $\mathrm{HbAlc}>8.3 \%$, OR $1.24(\mathrm{p}<0.001)$, and with smoking, OR $1.13(\mathrm{p}<0.05)$.

Conclusion: Although risk factor treatment seems to be intensified in type 2 diabetic patients, who have suffered a MI, the degree of fulfilment of the treatment goals is still insufficient in this high-risk group of patients.

\section{OP 17}

\section{Mechanisms of beta cell dysfunction and death}

0097

Pdx-1-driven differentiation-dependent sensitivity to interleukin-1 toxicity in insulin-producing INS1 $\alpha \beta$ cells is related to enhanced $J_{N K}$ and $N F \kappa B$ activity and a distinct gene expression profile

M. Tonnesen ${ }^{1}$, P. Hagedorn ${ }^{2}$, M. Kruhøffer ${ }^{3}$, F. Pociot ${ }^{1}$, T. Mandrup-Poulsen ${ }^{1}$;

${ }^{1}$ Steno Diabetes Center, Gentofte, ${ }^{2}$ Biosystems Department, Riso National Laboratory, Roskilde, ${ }^{3}$ Department of Clinical Biochemistry, Aarhus University Hospital, Denmark.

Background and Aims: Pancreatic $\beta$-cells are more sensitive to several toxins (e.g. streptozotocin, alloxan, and cytokines) than the other endocrine cell types in the islets of Langerhans. We have previously shown that $\beta$-cell acquisition of sensitivity to interleukin (IL)-1 induced apoptosis is differentiation-dependent and associated with pancreatic duodenal homeobox factor-1 (Pdx-1) expression. Pdx-1 is an important transcription factor regulating pancreatic development and islet cell function, but the molecular mechanisms by which Pdx-1 leads to enhanced cytokine-sensitivity are currently unknown. Rationale: Cytokines secreted by infiltrating inflammatory cells have been implicated in 1) islet allograft rejection, 2) disease recurrence in isografts and 3) non-specific inflammation caused by transplantation trauma even when using islet microencapsulation. It is therefore important to understand the molecular actions of cytokines on the $\beta$-cell as well as differentiation-dependent steps causing the particular sensitivity of pancreatic $\beta$-cells to inflammatory cytokines compared to non- $\beta$-cells. Aims: (1) To compare IL-1-induced apoptosis and signaling mechanisms in INS-1 $\alpha \beta \beta$ cells $+/-$ Pdx-1 overexpression. (2) To clarify gene expression profiling and transcription 
factor networks of differentiation-aquired $\beta$-cell-sensitivity to cytokines providing a molecular fingerprint of inflammatory sensitivity.

Materials and Methods: Doxycycline-inducible Pdx-1 expression in INS-1r $\alpha \beta$ was used as a model of $\beta$-cell maturation. IL-1-induced cell death was determined by TUNEL, cleavage of caspase-3 and ELISA detection of histone-DNA complexes in the cytoplasm. Activation of MAPKs was determined by Western blot analysis using phosphospecific antibodies and nuclear translocation of NF $\kappa$ B was determined by electric mobility shift assay (EMSA) and Luciferase reporter assay. Affymetrix microarray analysis was performed to investigate the molecular mechanisms of enhanced IL-1 sensitivity.

Results: Pdx-1 over-expressing INS-1 $\mathrm{r} \alpha \beta$ cells were more sensitive $(310 \%, \mathrm{p}<0,05)$ to the apoptotic effect of IL- 1 as determined by TUNEL, cytoplasmic histone-DNA complexes and a distinct IL-1-induced cleavage of caspase-3. IL-1-induced JNK activity determined by phosphorylation was markedly enhanced and prolonged in $\mathrm{Pdx}-1$ overexpressing INS-1r $\alpha \beta$ cells. Furthermore, IL-1-induced ІкB degradation was increased correlating with augmented $\mathrm{NF} \kappa \mathrm{B}$ binding to DNA $\kappa \mathrm{B}$ sites and $\mathrm{a}>2$ fold increase in $\mathrm{NF} \kappa \mathrm{B}$ transcriptional activity in Pdx-1 overexpressing cells. These observations were associated with complete separation of the expression of gene clusters in response to IL-1 depending upon Pdx-1 overexpression, indicating that Pdxexpression distinctly modifies IL-1 induced signaling pathways and transcription factor networks.

Conclusion: Our data suggest that the final $\beta$-cell maturation accompanied by increased IL-1 sensitivity depend upon the expression of genes regulated by Pdx-1 leading to enhanced and prolonged JNK and NFK $\dot{\phi}$ activity.

Supported by JDRF center for beta cell therapy and diabetes and the European Union

\section{8}

Molecular pathways underlying Toll-like receptor 3 (TLR3)-induced beta cell apoptosis

J. Rasschaert ${ }^{1}$, Z. Dogusan ${ }^{1}$, D. Flamez ${ }^{1}$,

L. Alexopoulou ${ }^{2}$, C. Mathieu ${ }^{3}$, D. Eizirik ${ }^{1}$;

${ }^{1}$ Laboratory of Experimental Medicine, Université Libre de Bruxelles, Belgium, ${ }^{2}$ Laboratory of TLRs and Innate Immunity, Université de la Méditerranée, Marseille, France, ${ }^{3}$ Laboratory of Experimental Medicine and Endocrinology, Katholieke Universiteit van Leuven, Belgium.

Background and Aims: The mechanisms by which viruses, or viral products such as double-stranded RNA (dsRNA), affect beta cell function and survival and trigger autoimmunity in type 1 diabetes remain unknown. We have shown that dsRNA, in association with IFN- $\gamma$, triggers beta cell apoptosis. By microarray analysis of human islets and FACS-purified rat beta cells we observed that infection by the Coxsackievirus B5 or treatment with dsRNA+IFN- $\gamma$ upregulates the expression of type $\mathrm{I}$ interferons and other genes contributing for islet inflammation and beta cell death. Several of these viral/dsRNA-induced genes are potentially regulated by the Toll-like receptor 3 (TLR3), which is expressed and apparently functional in beta cells. We presently investigated the pro-apoptotic signaling pathway downstream of TLR3 in beta cells.

Materials and Methods: FACS-purified rat beta cells and dispersed islet cells from wild type (wt) or TLR $3^{-1-}$ mice were cultured for 3 to 5 days, with or without synthetic dsRNA (PIC; $1-100 \mu \mathrm{g} / \mathrm{ml}$; transfected or not with lipofectamine), IFN- $\gamma(500 \mathrm{U} / \mathrm{ml})$ and/or IL-1 $\beta(50 \mathrm{U} / \mathrm{ml})$. Viability was determined by the nuclear dyes HO 342 and propidium iodide, while gene expression was determined by real-time RT-PCR. Activation of NF-кB and IRF-3 was studied respectively by immunocytochemistry and Western blot using an antibody specific for the phosphorylated form of IRF-3.

Results: Exposure of dispersed islet cells from wt mice for 5 days to PIC $(100 \mu \mathrm{g} / \mathrm{ml})+\mathrm{IFN}-\gamma$, but not to PIC alone, increased apoptosis ( $47 \%$ vs $21 \%$ in control wt cells; $p<0.005$; $\mathrm{n}=5$ ). On the other hand, islet cells from TLR ${ }^{-/-}$mice were completely resistant to dsRNA+IFN- $\gamma$-induced apoptosis $\left(18 \%\right.$ vs $17 \%$ in control TLR $3^{-1-}$ cells; $\left.n=5\right)$. PIC induced NF-kB and IRF-3 activation in wt but not in $\mathrm{TLR}^{-/-}$islet cells. These data suggest a key role for TLR3 in beta cell apoptosis triggered by exogenous dsRNA. During viral infection beta cells are exposed to both extra- and intracellular dsRNA, and introduction of foreign dsRNA (PIC; $1 \mu \mathrm{g} / \mathrm{ml}$ ) by transfection into islet cells induced similar degree of apoptosis in wt and TLR3 $3^{-1-}$ islet cells. This suggests that TLR-3-independent mechanisms are involved in the intracellular recognition of dsRNA. Exogenously added or transfected dsRNA up-regulated TLR3 and PKR mRNAs to a similar extent and did not affect iNOS expression in rat beta cells. Internal dsRNA, however, induced a 50-100 fold higher up-regulation of IFN- $\alpha$ and IFN- $\beta$ mRNAs. We have previously shown that dsRNA+IFN- $\beta$ induces beta cell apoptosis.

Conclusions: The TLR3 plays a crucial role in exogenous dsRNA-induced NF- $\mathrm{BB}$ and IRF-3 activation and dsRNA+ IFN- $\gamma$-induced apoptosis. Introduction of foreign dsRNA into pancreatic beta cells triggers apoptosis by a TLR3- and NO-independent manner. Increased production of type I interferons probably contributes for this effect.

Supported by the EFSD/JDRF/Novo Nordisk Type 1 Diabetes Research Programme 


\section{9}

Transgenic overexpression of hormone-sensitive lipase in immortalised mouse $\beta$-cells rescues the cells from lipotoxicity

C. Klint ${ }^{1}$, R. N. Kulkarni ${ }^{2}$, C. Holm ${ }^{1}$, M. Sorhede Winzell ${ }^{3}$;

${ }^{1}$ Dept. of Experimental Medical Science, Lund University, ${ }^{2}$ Dept. of Medicine, Joslin Diabetes Center, Boston, United States, ${ }^{3}$ Dept. of Clinical Sciences, Lund University, Sweden.

Background and Aims: Lipid perturbation and accumulation of triglycerides in pancreatic $\beta$-cells are associated with impaired insulin secretion. Hormone-sensitive lipase (HSL) is expressed and active in $\beta$-cells and may be involved in regulating the turn-over of the triglyceride pool. The aim of the study was to create a stable mouse pancreatic $\beta$-cell line overexpressing HSL to evaluate the ability of these $\beta$ cells to retain GSIS after exposure to fatty acids in the presence of low and high glucose levels.

Materials and Methods: A mouse strain carrying an HSL transgene under the control of the insulin promoter was crossed with a mouse strain developing spontaneous insulinomas (RIP SV40). Tumour cells from offspring containing the transgene were used to establish a $\beta$ cell line with a stable overexpression of HSL (tgHSL- $\beta$ ) together with a control cell line that did not carry the transgene (wt- $\beta$ ).

The two cell lines were exposed to glucose or glucose in combination with palmitate for $72 \mathrm{~h}$, after which glucose responsiveness was assessed by measuring insulin release during $60 \mathrm{~min}$ in response to $1.0,11.1$ or $16.7 \mathrm{mM}$ glucose. Viable count was assessed after $72 \mathrm{~h}$ using a commercially available MTS assay. Palmitate oxidation and triglyceride (TG) content in cells were analysed after $72 \mathrm{~h}$ exposure to $0.5 \mathrm{mM}$ palmitate in 3.3 or $16.7 \mathrm{mM}$ glucose.

Results: The number of viable cells was increased in tgHSL- $\beta$ compared to wt- $\beta$ cells when cultivated for $72 \mathrm{~h}$ in $16.7 \mathrm{mM}$ glucose $(191 \%$ viable tgHSL- $\beta$ cells and $150 \%$ viable wt $\beta$ cells). Exposure of the $\beta$ cell lines to $0.25 \mathrm{mM}$ palmitate and $16.7 \mathrm{mM}$ glucose for $72 \mathrm{~h}$ decreased wt $\beta$ cell viable count to $65 \%$, whereas tgHSL- $\beta$ retained the same number of viable cells $(116 \%)$ as with $3.3 \mathrm{mM}$ glucose.

TG content in tgHSL- $\beta$ and wt $\beta$ cells was equal after $72 \mathrm{~h}$ exposure to $3.3 \mathrm{mM}$ and $16.7 \mathrm{mM}$ glucose. Exposure for $72 \mathrm{~h}$ to $0.5 \mathrm{mM}$ palmitate and 3.3 or $16.7 \mathrm{mM}$ glucose the TG content was increased to $50 / 56 \mu \mathrm{g} \mathrm{TG} / \mathrm{mg}$ protein in wt $\beta$ cells but remained 19/16 $\mu \mathrm{g} \mathrm{TG} / \mathrm{mg}$ protein in tgHSL- $\beta$ cells. Palmitate oxidation in either of the cell lines was not increased by incubation for $72 \mathrm{~h}$ with palmitate disregarding glucose level.
Insulin content was increased to $205 \%$ in tgHSL- $\beta$ compared to in wt- $\beta$ cells when cultured in $3.3 \mathrm{mM}$ glucose. After exposure to $0.5 \mathrm{mM}$ palmitate, the insulin content in the two cell lines was found to be equal ( 0.23 and $0.25 \mu \mathrm{g}$ insulin/mg protein respectively).

At acute exposure to glucose and GLP-1, carbacholin or forskolin insulin secretion was improved in tgHSL- $\beta$ compared to the wt $\beta$ cell line at glucose concentrations over $8.3 \mathrm{mM}$. Acute exposure to palmitate lead to higher insulin secretion in wt $\beta$ than in tgHSL- $\beta$ cells at low but not at higher glucose levels. Chronic $(72 \mathrm{~h}$ ) exposure to $0.5 \mathrm{mM}$ palmitate at high glucose concentration $(16.7 \mathrm{mM})$ resulted in intact glucose responsiveness in tgHSL- $\beta$ cells although insulin secretion was increased compared to tgHSL- $\beta$ cells without palmitate. However, the wt- $\beta$ cell line displayed blunted glucose responsiveness after $72 \mathrm{~h}$ of palmitate exposure, also with an overall increase in insulin secretion.

Conclusion: The $\beta$ cell line overexpressing HSL displays resistance to conditions of high glucose as well as high palmitate levels compared to the wildtype $\beta$ cell line, manifested both as a higher maintained proliferation rate and a retained GSIS function after long time exposure to high levels of glucose and palmitate.

\section{0}

Unfolded protein response in fatty acid treated pancreatic $\beta$-cells

H. Ortsäter, K. Thörn, E. Sargsyan, P. Bergsten; Medical Cell Biology, Uppsala University, Sweden.

Background and Aims: The unfolded protein response (UPR) is a physiological reaction elicited by conditions of endoplasmic reticulum (ER) -stress such as accumulation of unfolded, misfolded or excessive amounts of proteins in the ER. The UPR coordinates attenuation of general protein synthesis, increased capacity of protein folding through upregulation chaperones and enhancement of protein degradation with the aim to relieve the cell from the ER-stress. Failure to cope with ER-stress can trigger apoptotic cell death. When either primary $\beta$-cells or insulin-producing cell lines are cultured with free fatty acids for long time periods apoptosis is increased. Recently it was found that palmitate and oleate increased mRNA levels of some ER-stress markers indicating that free fatty acids can induce ERstress. The aim of the present study was to investigate the activity and timing of the UPR pathways in response to fatty acid treatment.

Materials and Methods: The insulin-producing cell lines INS-1E and MIN6 were exposed for 0,2,4,8,12 and 24 hours to palmitate $(0.5 \mathrm{mM})$, oleate $(0.5 \mathrm{mM})$ or palmitate 
in combination with diazoxide $(250 \mu \mathrm{M})$. In addition, cells were exposed to thapsigargin $(300 \mathrm{nM})$, an ER-stress inducer. After exposure, the cells were lysed and key proteins of the PERK and ATF6 signaling pathways of UPR were measured by western blotting.

Results: To analyse the activity in the PERK pathway we measured phosphorylation of eukaryotic initiation factor $2 \alpha$ (eIF2 $\alpha$ ) and the cellular levels of the apoptotic protein CHOP. In INS-1E cells palmitate caused a 2.5 -fold increase of eIF $2 \alpha$ phosphorylation already after 2 hours of exposure. The degree of phosphorylation continued to rise over the study period reaching a 10 -fold increase after 24 hours. In MIN6 cells palmitate triggered a kinetically similar response although the maximal induction of eIF $2 \alpha$ phosphorylation was only 2 -fold in these cells. Oleate did not induce phosphorylation of eIF $2 \alpha$ in either INS-1E or MIN6 cells. The degree of eIF $2 \alpha$ phosphorylation in cells cultured in the presence of palmitate was reduced by $40-60 \%$ when diazoxide was included in the culture medium. Thapsigargin induced a prompt (after 2 hours) and sustained elevation of eIF $2 \alpha$ phosphorylation in both INS-1E and MIN6 cells. Palmitate also increased CHOP levels but at a later $(6 \mathrm{~h})$ stage than eIF $2 \alpha$ phosphorylation. In INS-1E and MIN6 cells the cellular level of CHOP protein began to rise after 8 hours and after 24 hours a more than 4-fold increase was observed. The induction of CHOP protein expression by palmitate was counteracted by inclusion of diazoxide in the culture medium. No rise in CHOP was observed in cells exposed to oleate. Elevated levels of CHOP were also evident in thapsigargin treated cells. To analyse the activity in the ATF6 pathway we measured the cellular levels of the chaperone proteins GRP78/BiP, PDI and PPI-B. None of the chaperone proteins demonstrated elevated levels in cells exposed to either palmitate, oleate or thapisgargin.

Conclusion: Palmitate and thapsigargin, but not oleate, preferentially activate the PERK pathway of the UPR. Since phosphorylation of eIF $2 \alpha$ negatively regulates protein synthesis our results (sustained elevation of eIF $2 \alpha$ phosphorylation) could provide an explanation for the inability of pancreatic $\beta$-cells to biosynthetically compensate enhanced basal insulin secretion caused by fatty acids. This in turn would lead to a reduced insulin content.

\section{1}

Beta cell damage in nutritionally-induced T2DM in the gerbil Psammomys obesus (sand rat) is not cytokine mediated

A. Jörns ${ }^{1}$, O. Bock ${ }^{2}$, K. Rath ${ }^{1}$, S. Lenzen ${ }^{1}$;

${ }^{1}$ Institute of Clinical Biochemistry, ${ }^{2}$ Institute of Pathology, Hannover Medical School, Germany.
Background and Aims: Psammomys obesus is a well established animal model for type 2 diabetes mellitus (T2DM) developing hyperglycaemia, initially hyperinsulinaemia and later hypoinsulinaemia accompanied by insulin resistance when placed for one to three weeks on a high energy (HE) diet. Recently it has been proposed that cytokines may be involved also in the aetiopathology of T2DM. Therefore a morphological and biochemical analysis using either isolated pancreatic islets or fixed pancreatic sections during the time course of islet destruction was performed to evaluate changes in the cytokine and enzyme expression in pancreatic beta cells in the time interval from one to three weeks on HE diet in comparison to the control situation.

Materials and Methods: The gene expression of the proinflammatory cytokines IL- $1 \beta$, IFN- $\gamma$ and TNF- $\alpha$ and of the IL-1 $\beta$ induced enzyme iNOS was analyzed using gerbil specific primers by quantitative real time RT-PCR and by in situ RT-PCR, whereas for insulin and the enzyme caspase 3 expression analysis rat primers were used because these gerbil gene sequences are unknown. To detect the protein of these cytokines and enzymes antibodies were used recognizing human, rat and mouse specific protein sequences because gerbil specific antibodies are not commercially available.

Results: The studies on gene expression by in situ and $q$ RT-PCR showed no mRNA expression for IFN- $\gamma$, TNF- $\alpha$ and iNOS as well as caspase 3 during diabetes development in the sand rat at different stages of islet destruction. In comparison to pancreas draining lymph nodes a more than 10 -fold lower gene expression of IL-1 $\beta$ was observed in isolated control islets, which was not induced by hyperglycemia for 7 or 21 days during diabetes development. Pancreatic beta cells both in the normoglycaemic as well as hyperglycaemic situation remained immunohistochemically unstained for all proinflammatory cytokines, iNOS and caspase 3 whereas the insulin staining on the protein and the gene expression level decreased stepwise from day 7 to day 21 on HE diet. The small numbers of immune cells, only macrophages and neutrophils, showed also no gene and protein expression for proinflammatory cytokines and iNOS in pancreatic islets of normoglycaemic and hyperglycaemic animals. All proinflammatory cytokines were detectable on the gene and protein level in the lymphoid tissue of both the pancreas draining lymph nodes and the spleen in the control and the diabetic situation.

Conclusion: Thus it can be concluded that the mechanisms leading to beta cell damage during the development of hyperglycaemia in the sand rat on HE diet were not mediated by induction of proinflammatory cytokines and a resulting upregulation of iNOS induced by IL-1 $\beta$. Caspase 3 as the first executor caspase leading to apoptotic cell death remained undetectable confirming that the intra- 
cytoplasmic vacuoles appearing in beta cells on HE diet are a sign of a necrotic cell damage is this T2DM animal model.

0102

Bcl-2 acutely regulates $\beta$-cell $\mathrm{Ca}^{2+}$ signaling and apoptosis via $\mathrm{IP}_{3} \mathrm{R}$ - and RyR-mediated mechanisms

D. S. Luciani, Y. Bychkivska, T. B. Kalynyak,

T. M. Underhill, A. V. Sampaio, J. D. Johnson;

Diabetes Research Group, Department of Cellular and Physiological Sciences, University of British Columbia, Vancouver, Canada.

Background and Aims: Members of the Bcl-2 family of proteins are well known regulators of $\beta$-cell death, and the diabetic state is associated with an increased ratio of proapoptotic to anti-apoptotic members in the pancreatic islet. However, to date little is known about the acute physiological functions played by endogenously expressed antiapoptotic Bcl-2 proteins. The aim of the present study was to examine the moment-to-moment role of $\mathrm{Bcl}-2$ in the regulation of $\beta$-cell function and survival, with emphasis on its putative functional interaction with intracellular $\mathrm{Ca}^{2+}$ release channels and $\beta$-cell $\mathrm{Ca}^{2+}$ homeostasis.

Materials and Methods: We used the cell-permeable small molecule Bcl-2 inhibitors HA14-1 and Bcl-2 inhibitor (Bcl2i) to acutely inhibit anti-apoptotic Bcl-2 in primary mouse islet cells and MIN6 cells. The effect of various doses of the inhibitors was examined alone or in combination with modulators of cellular $\mathrm{Ca}^{2+}$ homeostasis. Cell death was quantified in real-time over a period of up to 48 hours by monitoring cellular propidium Iodide (PI) incorporation using an automated Cellomics KineticScan ${ }^{\mathrm{TM}}$ imaging system. The involvement of cell death pathways was investigated by Western blotting, and the results quantified by band densitometry. Caspase-3 activation was also monitored by real-time microscopy as the loss of FRET between cyan- and orange-emitting fluorescent proteins linked by a peptide containing the 'DEVD' caspase-3 cleavage sequence. Changes in cytosolic $\mathrm{Ca}^{2+}$ were recorded using fura- 2 .

Results: Inhibition of endogenously expressed Bcl-2 proteins, by HA14-1 or Bcl-2i, induced caspase-3 activation and cell death of MIN6 and primary $\beta$-cells. The onset of $\beta$-cell death was rapid in response to $20 \mu \mathrm{M} \mathrm{Bcl-2i}$, with PI incorporation and caspase- 3 activation occurring between 2 and 5 hours after treatment. Cell death induced by Bcl-2i and HA14-1 was not associated with activation of the ER stress, as indicated by normal levels of CHOP/ GADD153 expression after 24 hours exposure to $20 \mu \mathrm{M}$ and $100 \mu \mathrm{M}$ of the inhibitors. In primary $\beta$-cells both Bcl-2i and HA14-1 stimulated rapid cytosolic $\mathrm{Ca}^{2+}$ fluctuations, followed by a slow rise to sustained supraphysiological
$\mathrm{Ca}^{2+}$ levels, and eventually loss of plasma membrane integrity. The first phase of Bcl-2i-induced fluctuations was rapidly reversible at all doses tested. Cell death was significantly reduced after pre-treatment with $50 \mu \mathrm{M}$ BAPTA or inhibition of intracellular $\mathrm{IP}_{3} \mathrm{R}$ and $\mathrm{RyR} \mathrm{Ca}^{2+}$ release channels with $1 \mu \mathrm{M}$ xestospongin $\mathrm{C}$ and $100 \mu \mathrm{M}$ ryanodine, respectively. Moreover, xestospongin $\mathrm{C}$ and ryanodine both lowered the amount of cleaved caspase- 3 detected at 2 hours by approximately $50 \%$.

Conclusion: Acute inhibition of Bcl-2 rapidly activates caspase- 3 and evokes $\mathrm{Ca}^{2+}$-regulated $\beta$-cell death. Moreover, our findings provide evidence for a moment-tomoment role for endogenous Bcl- 2 in regulation of $\beta$-cell calcium homeostasis. Specifically, inhibitor-induced caspase-3 activation and apoptosis depends on flux through $\mathrm{IP}_{3}$ and ryanodine receptors. Together, these results suggest that $\mathrm{Bcl}-2$ regulates $\beta$-cell ER $\mathrm{Ca}^{2+}$ homeostasis and exerts its anti-apoptotic effect in the $\beta$-cell by controlling $\mathrm{Ca}^{2+}$ mediated signaling between ER and mitochondria.

Supported by CIHR, CDA, JDRF, MSFHR and CFI

\section{OP 18}

\section{Diabetes in pregnancy}

\section{3}

Following in Mom's footsteps? Preliminary assessment of the health and lifestyle status of teenage girls born to gestational diabetic women

G. M. Egeland ${ }^{1}$, S. J. Meltzer ${ }^{2}$, M. Nudi ${ }^{3}$;

${ }^{1}$ Human Nutrition, ${ }^{2}$ Medicine, ${ }^{3}$ MUHC, McGill University, Montreal, Canada.

Background and Aims: Gestational Diabetes (GDM) appears to increase the risk of obesity, type 2 Diabetes Mellitus (DM) and the metabolic syndrome in women and their offspring. This study seeks to determine health and lifestyle risk factors that might make daughters of GDM women more vulnerable to diabetes and its associated conditions.

Study Design: This is a longitudinal follow-up of a casecontrol cohort of women that delivered a baby girl between 1989 and 1991 at the Royal Victoria Hospital. Consenting mother-daughter pairs were assessed for anthropometric, behavioral (diet, physical activity) and metabolic (glucose intolerance, insulin resistance, dyslipidemia) and other clinical and novel indicators.

Results: A double entered and edited database of 103 (48 case and 55 control) mother-daughter pairs was available for preliminary analyses. Characteristics of case (GDM) and control (Non-GDM) moms and daughters are presented in Table 1. To date, no daughters of cases or controls have 
type $2 \mathrm{DM}$, impaired fasting glucose (IFG) or impaired glucose tolerance (IGT). HOMA IR was significantly elevated in daughters of women that either had GDM or glucose intolerance during their pregnancy, however the difference was not significant in linear regression analyses controlling for daughters' waist circumference. Case daughters, as a group, were non-obese but tended to have a higher body mass index (BMI) and were less active (in MET scores). As for the mothers, fasting glucose and $2 \mathrm{hr}$ OGTT results indicate that $17(37 \%)$ cases are now diabetic, and that an additional $6(13 \%)$ either have IFG, IGT or both IFG and IGT. In contrast, 2 (3.6\%) control mothers have diabetes, 7 (12.7\%) have IFG and one (1.8\%) has both IGT and IFG. As expected, women that were glucose intolerant during their pregnancy were significantly heavier, had a worse lipid profile, were found to be more insulin resistant and tended to be less active than women with a history of glucose tolerant pregnancies (Table 1). In the available data,WC measures were highly correlated between mothers and daughters $(r=0.39, \mathrm{p}<0.0001)$, whether they were cases or controls. Mother's GDM case status was an important and statistically significant determinant of daughters' waist circumference ( $p$-values $<0.01)$. The saturated fat intake and leisure time physical activity in MET scores of the mothers was positively and significantly correlated with their daughter's saturated fat intake as a percent of total energy $(r=0.42, \mathrm{p}<0.0001)$ and daughter's MET scores $(r=0.24, \mathrm{p}=0.017)$.

Table 1- Characteristics of Case (GDM) and Control (NonGDM)

\begin{tabular}{|c|c|c|c|c|c|c|}
\hline \multirow[b]{2}{*}{ Characteristics } & \multicolumn{3}{|c|}{ DAUGHTERS } & \multicolumn{3}{|c|}{ MOTHERS } \\
\hline & GDM & $\begin{array}{l}\text { Non- } \\
\text { GDM }\end{array}$ & p-value & GDM & $\begin{array}{l}\text { Non- } \\
\text { GDM }\end{array}$ & p-value \\
\hline Age (years) & $14.6 \pm 0.7$ & $15.1 \pm 0.6$ & $<0.001$ & $46.9 \pm 4.0$ & $46.8 \pm 4.8$ & N.S. \\
\hline BMI (kg/m2) & $23.5 \pm 4.6$ & $22.1 \pm 3.3$ & 0.07 & $29.4 \pm 6.2$ & $26.4 \pm 4.5$ & $<0.01$ \\
\hline Waist & $79.9 \pm 10.8$ & $74.9 \pm 8.3$ & $<0.01$ & $95.6 \pm 14.1$ & $87.8 \pm 12.3$ & $<0.01$ \\
\hline \multicolumn{7}{|l|}{$\begin{array}{l}\text { Circumference } \\
(\mathrm{cm})\end{array}$} \\
\hline Body Fat $\%$ & $30.6 \pm 6.7$ & $28.8 \pm 7.0$ & N.S. & $37.9 \pm 7.2$ & $34.3 \pm 7.3$ & $<0.01$ \\
\hline $\begin{array}{l}\text { HDL } \\
\text { Cholesterol }\end{array}$ & $1.2 \pm 0.3$ & $1.2 \pm 0.2$ & N.S. & $1.2 \pm 0.3$ & $1.5 \pm 0.4$ & $<0.01$ \\
\hline $\begin{array}{l}\text { Total } \\
\text { Cholesterol } \\
(\mathrm{mmol} / \mathrm{L})\end{array}$ & $4.0 \pm 0.6$ & $4.3 \pm 0.7$ & 0.07 & $5.4 \pm 1.0$ & $5.3 \pm 1.3$ & N.S. \\
\hline Triglycerides & $0.7 \pm 0.3$ & $0.7 \pm 0.4$ & N.S. & $1.5 \pm 1.2$ & $1.1 \pm 0.9$ & 0.05 \\
\hline HOMA-IR & $2.2 \pm 1.3$ & $1.7 \pm 0.7$ & $<0.01$ & $3.4 \pm 3.6$ & $1.5 \pm 1.0$ & $<0.001$ \\
\hline MET (hrs/week) & $60.6 \pm 37.1$ & $74.2 \pm 49.2$ & 0.09 & $18.0 \pm 22.7$ & $26.1 \pm 22.1$ & 0.07 \\
\hline $\begin{array}{l}\text { Saturated } \\
\text { Fat Intake } \\
\text { (\% total energy) }\end{array}$ & $11.1 \pm 2.8$ & $11.2 \pm 2.1$ & N.S. & $11.2 \pm 2.8$ & $11.3 \pm 2.5$ & N.S. \\
\hline
\end{tabular}

Conclusion: Although teenage girls of GDM cases in our population appeared to be healthier than similar offspring of other study populations, maternal GDM was an important determinant of daughters' BMI, central fat patterning and insulin resistance levels. Shared lifestyle factors such as low physical activity levels may be aggravating risks of diabetes in offspring of pregnancies affected by GDM.

\section{4}

Insulin sensitivity and beta cell function in pregnancy M. Dalfrà ${ }^{1}$, E. Parretti ${ }^{2}$, G. Pacini ${ }^{3}$, A. Mari ${ }^{3}$, R. Cioni ${ }^{2}$, C. Marzari ${ }^{4}$, M. Masin ${ }^{1}$, G. Scarselli ${ }^{2}$, D. Fedele ${ }^{1}$, G. Mello ${ }^{2}$, A. Lapolla ${ }^{1}$;

${ }^{1}$ Medical and Surgical Sciences, University of Padova, ${ }^{2}$ Gynecology Perinatology and Human Reproduction, University of Firenze, ${ }^{3}$ Metabolic Modeling Unit, National Research Council, Padova, ${ }^{4}$ Aging Branch Institute of Neuroscience, National Research Council, Padova, Italy.

Background and Aims: Uncomplicated pregnancy is characterized by insulin resistance and increased insulin secretion. We retain of interest to evaluate insulin sensitivity and secretion in pregnant women with normal glucose tolerance and gestational diabetes, in the early and late period of pregnancy by utilizing as fasting indices of insulin sensitivity $\mathrm{IS}_{\mathrm{QUICKI}}$ and as OGTT index of insulin action OGIS as insulin secretion the disposition index (DI) Materials and Methods: 922 pregnant women among which 829 with normal glucose tolerance (NPW) (mean age $31 \pm 4$ years, mean BMI 22.5 \pm 3 ) and 93 GDM patients (mean age $33 \pm 4$ years, mean BMI $23 \pm 3$ ) were evaluated in early (16-20 g.w.)and in late pregnancy (26-30 g. w). Plasma glucose and insulinemia levels were evaluated in fasting state and after 1 and 2 hours og OGTT (75 gr glucose). As for sensitivity index we calculated IS-QUICKI and OGIS. Insulin secretion was evaluated with the area under the insulin concentration curve (AUC), beta cell function: with the ratio of the insulin AUC to glucose AUC for the whole test period (insulinogenic index).The parameter that describes this relationship is the disposition index (DI), calculated as OGIS times the insulinogenic index. DI quantifies the ability of the beta cell to compensate for reduced insulin sensitivity by increasing its secretion.

Results: In early stage GDM women with respect to NPW had higher levels of fasting plasma glucose (FPG $74 \pm 7 \mathrm{mg} /$ dl vs $78 \pm 8 \mathrm{mg} / \mathrm{dl} ; \mathrm{p}<0,001$ ) and comparable insulin (FPI $6.4 \pm 6.7 \mu \mathrm{U} / \mathrm{ml}$ vs $6.2 \pm 4.0)$; AUC under the respective curves was significantly $(\mathrm{p}<0.00001)$ elevated in GDM (AUC glucose $12.1 \pm 2.2$ vs $15.1 \pm 2.9$ and AUC insulin 
$4.7 \pm 2.3$ vs $5.9 \pm 2.9$ respectively)QUICKI $(0,40 \pm 0.06$ vs $0.39 \pm 0.06)$ are not different, while periferal insulin sensitivity OGIS was significantly lower in $\operatorname{GDM}(468 \pm 68$ vs $427 \pm 58 ; \mathrm{p}<0.001)$.insulin secretion in basal condition and dynamic B-cell function were similar in both groups.In Late pregnancy GDM women show higher FPG( $<<0.0004)$, not different FPI,dynamic AUC higher significantly and both liver and periferal insulin sensitivity lower (QUICKI $0.39 \pm 0.06$ vs $0.37 \pm 0.04$; $<<0.05$; OGIS $432 \pm 60$ vs $396 \pm 57$; $\mathrm{p}<0.0001)$ No difference was detected in B-cell function. During pregnancy, from early to late period, in both group FPG, FPI increased as well as AUC,QUICKI and OGIS instead were reduced.B-cell function increased in NPW $(17 \%, \mathrm{p}<0.00001)$ but less in $\operatorname{GDM}(13 \%, \mathrm{p} 0.03)$. Disposition index ,not different in early period, show in late pregnancy an overcompensation in NPW, lower in GDM (p0.02) showing an inability of GDM women B-cell to relase more hormone to augment glucose disposal.

Conclusion: Our data, on a large number of cases, show that insulin sensitivity decreases in pregnancy, but in early pregnancy only OGIS already predicts the deterioration in metabolic status that GDM encounter later on .Beta cell function is reduced and the DI is less able than betac cell function to respond to physiological insulin resistance in GDM.

\section{5}

Increased levels of circulating adhesion molecules (ICAM, VCAM) in women with previous gestational diabetes

T. Prikoszovich ${ }^{1}$, O. Wagner ${ }^{2}$, W. Kollmann ${ }^{1}$, G. Pacini ${ }^{3}$, A. Tura ${ }^{3}$, C. Winzer ${ }^{1}$, A. Luger ${ }^{1}$, A. Kautzky-Willer ${ }^{1}$;

${ }^{1}$ Department of Internal Medicine III, Division of Endocrinology and Metabolism, ${ }^{2}$ Department of Medical and Chemical Laboratory Diagnostics, Medical University of Vienna, Austria, ${ }^{3}$ Institute of Biomedical Engineering, CNR, Padova, Italy.

Background and Aim: Gestational Diabetes (GD) can serve as a model of type 2 diabetes (T2DM). Women with prior GD often feature insulin resistance and are at increased risk of premature atherosclerosis. Therefore we investigated in women with a history of GD in comparison to women with normal glucose tolerance during and after pregnancy (controls) surrogate parameters of inflammation and endothelial dysfunction.

Material and Methods: 119 women with previous GD (Age: $33.76 \pm 0.73$ years, BMI: $27.65 \pm 0.51 \mathrm{~kg} / \mathrm{m}^{2}$ ) and 41 control women (Age: $31.55 \pm 0.88$ years, BMI: $\left.25.01 \pm 0.89 \mathrm{~kg} / \mathrm{m}^{2}\right)$ underwent an oral $(75 \mathrm{~g}$; OGTT) and an intravenous (FSIGT) glucose tolerance test 14-16 weeks after delivery. The women with prior GD were reinvestigated by an OGTT after one year. At both visits no woman had any sign of infection and plasma concentrations of the circulating adhesion molecules vascular cell adhesion molecule (VCAM), intercellular adhesion molecule-1 (ICAM-1) and E-selectin (ELAM), plasma endothelin and leptin as well as inflammatory markers (sCRP, IL-6, fibrinogen, PAI-1) were measured. Insulin sensitivity $\left(\mathrm{S}_{\mathrm{I}}\right)$ was calculated from the insulin modified FSIGTs.

Results: At baseline women with a history of GD showed higher concentrations of ICAMs $(298.5 \pm 7.36 \mathrm{ng} / \mathrm{ml}$; $\mathrm{p}<0.0001)$ and VCAMs $(611.8 \pm 18.71 \mathrm{ng} / \mathrm{ml} ; \mathrm{p}<0.003)$ but not E-Selectin or endothelin compared to the control women (ICAM: $238.0 \pm 8.67 \mathrm{ng} / \mathrm{ml}$, VCAM: $502.3 \pm 26.31 \mathrm{ng} / \mathrm{ml}$, respectively). In addition sCRP $(\mathrm{p}<0.05)$ and PAI-1 $(\mathrm{p}=0.01)$ were higher and $\mathrm{S}_{\mathrm{I}}$ $(p<0.001)$ was lower in patients with prior GD than in the control women. $\mathrm{S}_{\mathrm{I}}$ was inversely related with ELAM $(\mathrm{r}=-0.21 ; \mathrm{p}=0.01), \operatorname{VCAM}(\mathrm{r}=-0.23 ; \mathrm{p}<0.01)$ and ICAM-1 $(\mathrm{r}=-0.22 ; \mathrm{p}<0.01)$ as well as with sCRP $(\mathrm{r}=-0.52 ; \mathrm{p}<0.0001)$, IL-6 $(\mathrm{r}=-0.25 ; \mathrm{p}=0.01)$, and fibrinogen $(\mathrm{r}=-0.24 ; \mathrm{p}=0.003)$. Furthermore ICAM $(\mathrm{r}=0.22$; $\mathrm{p}<0.01)$ and VCAM $(\mathrm{r}=0.18 ; \mathrm{p}=0.02)$ were associated with sCRP, while ICAM and ELAM correlated with fibrinogen concentrations. All adhesion molecules also related to plasma leptin $(\mathrm{r}=0.17 ; \mathrm{p}<0.02)$ and $\mathrm{BMI}(\mathrm{r}=0.18 ; \mathrm{p}<0.01)$. At one year follow up ELAM $(\mathrm{p}<0.0007)$ decreased in women with prior GD, while ICAM-1 and VCAM did not change significantly. Furthermore plasma leptin $(p<0.02)$ increased, but all other markers of inflammation or endothelial dysfunction and also BMI remained unchanged at the follow-up visit.

Conclusion: Women with a history of GD are characterised by higher plasma levels of adhesion molecules which relate to insulin resistance, inflammatory parameters and plasma leptin and the degree of obesity. Whether these persistingly increased levels of ICAMs and VCAMs can predict development of a premature atherosclerosis in women with a history of GD remains to be determined.

\section{6}

Intensive management of pregestational diabetes mellitus in pregnancy: Comparison of outcomes of type 2 diabetes with type 1 diabetes

B. T. Kinsley, R. G. R. Firth, M. M. Byrne, R. Al-Agha, A. Khamis, S. Murray;

Dublin Diabetes in Pregnancy Centre, Mater Misericordiae University Hospital, Ireland.

Background: The incidence of T2DM in the reproductive years is increasing. Previous data suggests that outcomes in 
T2DM in pregnancy are poor. There is little information on the impact of intensive clinical management on pregnancy outcomes in T2DM.

Materials and Methods: We present data on 67 women with T2DM in pregnancy and 372 women with T1DM who attended at the Combined Diabetes/Obstetrical Clinics in three university maternity hospitals in Dublin, Ireland from 1995-2005. Diabetes care at each clinic was delivered by the same endocrinologists (RF, BTK, MMB). All women were treated intensively with insulin from time of booking at clinic. Management and glycaemic goals were identical for T1DM and T2DM groups.

Results:

\begin{tabular}{llll}
\hline & T2DM (n=67) & T1DM (n=372) & P value \\
\hline Age (yrs) & $340 \pm 5$ & $30 \pm 5$ & $<0.001$ \\
DM Duration (yrs) & $3.3 \pm 2$ & $13.0 \pm 8$ & $<0.001$ \\
Baseline Wt (kg) & $88 \pm 17$ & $72 \pm 12$ & $<0.001$ \\
Booking Gest (Wks) & $9.8 \pm 5$ & $8.4 \pm 5$ & $<0.05$ \\
Hba1c \%-Booking & $7.3 \pm 1.6$ & $7.7 \pm 1.5$ & 0.06 \\
13 weeks & $6.2 \pm 1.0$ & $6.6 \pm 1.0$ & 0.01 \\
26 weeks & $5.4 \pm 0.8$ & $6.6 \pm 0.8$ & $<0.001$ \\
Term (>36 weeks) & $5.9 \pm 0.9$ & $6.4 \pm 0.3$ & $<0.001$ \\
Birth Weight (Kg) & $3.4 \pm 0.8$ & $3.6 \pm 0.7$ & 0.01 \\
\hline
\end{tabular}

First trimester miscarriage rates were similar in T2DM (13/ 67) $19 \%$ and T1DM (70/372) $18 \%$. Perinatal mortality rate in the T2DM group was 55/1000 (3/54) vs 36/1000 (11/ 302) total births in the T1DM group. Major congenital malformation rate (CMR) was $93 / 1000(5 / 54)$ vs $37 / 1000$ (14/302) total births for T1DM. Lethal CMR was 55/1000 in T2DM vs 10/1000 in T1DM. All 3 lethal CMR in T2DM were congenital cardiac defects.

Conclusion: Compared to women with T1DM in pregnancy, women with T2DM are older, heavier, have shorter DM duration, book later to a specialist clinic, achieve better glycaemic control and have lower infant birth weights. However T2DM in pregnancy is associated with higher rates of major congenital malformations, especially congenital cardiac defects. Women of reproductive age with T2DM should be identified for intensive therapy and education prepregnancy in an attempt to improve outcomes.

\section{7}

Interaction between maternal type 1 diabetes and HLA DR4 increases fetal growth

M. Hummel ${ }^{1}$, S. Marienfeld ${ }^{1}$, M. Huppmann ${ }^{1}$, A. Knopff ${ }^{1}$, M. Voigt ${ }^{2}$, E. Bonifacio ${ }^{3}$, A.-G. Ziegler ${ }^{1}$;
${ }^{1}$ Diabetes Research Institute, Munich, Germany, ${ }^{2}$ Paediatric Department, EMA-University Greifswald, Germany, ${ }^{3}$ Istituto Scientifico San Raffaele, Milan, Italy.

Background and Aims: Maternal diabetes and genetic factors affect intrauterine growth. The aim of this study was to determine the interaction between maternal diabetes, birth weight and type 1 diabetes susceptibility genes.

Materials and Methods: Birth weight and gestational age were obtained in newborn children of mothers with type 1 diabetes $(\mathrm{n}=1191)$, fathers or siblings with type 1 diabetes $(n=917)$, and mothers with gestational diabetes $(n=619)$. Birth weight, weight and height at age 1,2 and 5 years were obtained from pediatric records and expressed as percentiles of national reference data corrected for age and sex. All 2108 children with mother, father or sibling with T1DM had HLA DR typing, and 1120 of these children also had INS VNTR typing. Results: The median birth weight in children of mothers with T1DM was at the 69th percentile (IQR, 36th-91th percentile; $\mathrm{P}<0.0001$ ) and in offspring of mothers with GDM was at the 64th percentile (IQR, 35th-90th percentile; $\mathrm{P}<0.0001$ ) of the normative population. Fetal growth was influenced by strong interactions between maternal type 1 diabetes, sub-optimal metabolic control and the presence of the HLA DR4 allele in the child. Birth weight increased with moderately elevated $\mathrm{HbAlc}(5.5 \%-6.5 \%$ : median, 74th percentile; IQR, 47th-91st percentile; P6.5\% (median, 86th percentile; IQR, 65th to 96th percentile, $\mathrm{P}<0.0001$ ). In children of mothers with type 1 diabetes, birth weight was significantly increased if children had the HLA DR4 allele (75th vs 63rd percentile; $\mathrm{P}<0.0001$ ). No differences were observed with other HLA alleles. HLA DR4 did not influence birth weight in children of non-diabetic mothers. Multivariate analysis indicated that HLA-DR4 in the child $(\mathrm{P}=0.001)$ contributed to birth weight independently from maternal HbAlc during pregnancy $(\mathrm{P}<0.0001)$, and parity status (P6.5\% during pregnancy had the highest proportion of overweight children ( $23 \%$ at age 5 years).

Conclusion: A diabetic intrauterine environment interacts with gene(s) marked by the type 1 diabetes susceptibility HLA DR4 allele to increase fetal growth.

0108

Preeclampsia - a predictor of diabetic nephropathy?

D. Gordin ${ }^{1,2}$, C. Forsblom ${ }^{1,2}$, V. Hiilesmaa ${ }^{3}$, J. Fagerudd ${ }^{1,2}$, M. Rönnback ${ }^{1,2}$, R. Kaaja $^{3}$, K. Teramo ${ }^{3}$, P.-H. Groop ${ }^{1,2}$;

${ }^{1}$ Folkhälsan Research Center, Institute of Genetics, Helsinki, ${ }^{2}$ Department of Medicine, Division of Nephrology, Helsinki, ${ }^{3}$ Depatment of Obstetrics and Gynaecology, Helsinki University Hospital, Finland. 
Background and Aims: A preeclamptic pregnancy is associated with a substantially increased risk of cardiovascular disease later in life in non-diabetic women. It is also well known that type 1 diabetes increases the risk of preeclampsia (PE) and pregnancy-induced hypertension (PIH). However, it is not known whether PE or PIH increase the risk of diabetic nephropathy (DN) later in life. The aim was to study whether $\mathrm{PE}$ and $\mathrm{PIH}$ are predictors of $\mathrm{DN}$ in type 1 diabetic patients in a prospective cross-sectional setting.

Materials and Methods: A total of 203 type 1 diabetic women, who were pregnant during the time period 1988 1996 and followed at the Department of Obstetrics and Gynecology in Helsinki, were re-studied after a median of 10.6 years within the nationwide, multi-center Finnish Diabetic Nephropathy Study (FinnDiane). Patients with proteinuria in the two first trimesters (Whites class F) or prepregnancy hypertension were excluded from the nephropathy analyses. So far, we have been able to verify (at least three recent urine collections) the renal status of 180 patients. Further characterization is ongoing. Incident $\mathrm{DN}$ was defined as microalbuminuria, macroalbuminuria or end stage renal disease (ESRD).

$\mathrm{PIH}$ was defined as an increase in the diastolic blood pressure of $=15 \mathrm{mmHg}$ or the final level $=90 \mathrm{mmHg}$ after 20 weeks of gestation. If proteinuria $(=0.3 \mathrm{~g} / 24 \mathrm{~h})$ was detected after 20 weeks, the patient fulfilled the criteria for PE. At the FinnDiane visit, data on micro- and macrovascular complications as well as phenotypic characteristics were collected. SPSS 12.0.1 for Windows was used to analyze categorical variables with the $\chi^{2}$-test and continuous variables with Student's $t$-test. A logistic regression analysis with DN as the dependent variable was performed. Results: Out of 180 patients 43 had had PE, $32 \mathrm{PIH}$ and 105 a non-hypertensive pregnancy. A total of 38 patients had developed DN (2 ESRD, 9 macroalbuminuria, 27 microalbuminuria). Patients with prior PE had more often DN (DN vs normal albumin excretion rate (AER); 41.9\% vs $8.9 \%, P<0.001)$. Patients with a history of PIH did not have more $\mathrm{DN}(10.3 \%$ vs $8.9 \% ; P=0.81)$. Coronary heart disease was more prevalent in patients with a history of $\mathrm{PE}$ (12.2\% vs. 2.2, $P=0.03)$ In the logistic regression analysis $\mathrm{PE}(\mathrm{P}<0.001)$ and $\mathrm{HbA}_{1 \mathrm{c}}(\mathrm{P}<0.001)$ were independently associated with incident DN in a model that further included follow-up time, BMI, smoking, diabetes duration and age.

Conclusion: These preliminary data suggest that preeclampsia (PE), but not pregnancy induced hypertension (PIH), may be considered a predictor of incident diabetic nephropathy. PE predicts also CHD in type 1 diabetic patients. Supported by Finska Läkaresällskapet, Nylands Nation

\section{OP 19}

\section{Lessons from clinical studies}

\section{9}

The effects of long-term pioglitazone therapy on lipid profiles in high-risk type 2 diabetes patients: results from PROactive

R. Spanheimer ${ }^{1}$, M. $\operatorname{Tan}^{2}$, J. Yates ${ }^{3}$

on behalf of the PROactive Investigators;

${ }^{1}$ Takeda Pharmaceuticals North America, Inc., Lincolnshire, ${ }^{2}$ Eli Lilly and Company, Indianapolis, ${ }^{3}$ Takeda Global Research and Development Center, Lincolnshire, United States.

Background and Aims: Dyslipidemia associated with type 2 diabetes is characterized by elevated triglyceride (TG) levels and decreased high-density lipoprotein cholesterol (HDL-C) levels. Pioglitazone (PIO) has shown benefits in lipid metabolism in patients with type 2 diabetes, in addition to its glucose-lowering effects. Subanalyses of patients in PROactive have assessed the lipid-altering effects in four cohorts: PIO vs placebo (PBO), PIO vs PBO with or without insulin at baseline, PIO when added to metformin (MET) or sulfonylurea (SU) monotherapy, and PIO added to MET+SU combination therapy.

Materials and Methods: PROactive was a randomized, double-blind, placebo-controlled cardiovascular outcome study in which 5,238 patients received randomized treatment of either PIO or matching PBO, in addition to other blood-glucose and cardiovascular medications. Doses of PIO were titrated from $15 \mathrm{mg}$ to $30 \mathrm{mg}$ at the first month visit, and to $45 \mathrm{mg}$ at the second month visit, where it was maintained unless there were tolerability concerns. Mean follow up was 34.5 months and mean exposure to study medication was 30 months.

Results: There were statistically significantly greater mean decreases in TG levels and mean increases in HDL-C levels in the PIO group compared with other treatments in all four cohorts of patients. Despite a significant increase in LDL-C with PIO, the LDL-C/ HDL-C ratio was significantly improved compared to PBO for those receiving and not receiving insulin at baseline. Similar improvements in LDL-C/HDL-C ratios were seen between the PIO vs $\mathrm{PIO}+\mathrm{MET}$ and/or $\mathrm{SU}$ treatment groups. 
Changes from baseline in lipid parameters. PIO vs PBO and PIO with or without insulin at baseline

\begin{tabular}{|c|c|c|c|c|c|c|}
\hline \multirow{2}{*}{$\begin{array}{l}\text { Mean \% } \\
\text { change } \\
\text { from } \\
\text { baseline }\end{array}$} & \multirow{2}{*}{$\begin{array}{l}\mathrm{PIO} \\
(\mathrm{N}=2605)\end{array}$} & \multirow[b]{2}{*}{$\begin{array}{l}\mathrm{PBO} \\
(\mathrm{N}=2633)\end{array}$} & \multicolumn{2}{|c|}{$\begin{array}{l}\text { Receiving any } \\
\text { combination } \\
\text { of insulin }\end{array}$} & \multicolumn{2}{|c|}{$\begin{array}{l}\text { Not receiving } \\
\text { insulin }\end{array}$} \\
\hline & & & $\begin{array}{l}\mathrm{PIO} \\
(\mathrm{N}=864)\end{array}$ & $\begin{array}{l}\mathrm{PBO} \\
(\mathrm{N}=896)\end{array}$ & $\begin{array}{l}\mathrm{PIO} \\
(\mathrm{N}=1741)\end{array}$ & $\begin{array}{l}\mathrm{PBO} \\
(\mathrm{N}=1737)\end{array}$ \\
\hline $\begin{array}{l}\mathrm{TG} \\
(\mathrm{mmol} / \\
\mathrm{L})\end{array}$ & -1.7 & +12.5 & -2.21 & +14.11 & -1.41 & +11.7 \\
\hline $\begin{array}{l}\text { HDL-C } \\
(\mathrm{mmol} / \\
\mathrm{L})\end{array}$ & +21.2 & +11.3 & +20.5 & +10.1 & +21.6 & +11.9 \\
\hline $\begin{array}{l}\text { LDL-C } \\
(\mathrm{mmol} / \\
\mathrm{L})\end{array}$ & +12.1 & +8.4 & +15.1 & +12.1 & +10.6 & +6.5 \\
\hline $\begin{array}{l}\text { LDL-C/ } \\
\text { HDL-C }\end{array}$ & -4.39 & -0.04 & -0.95 & +4.66 & -6.1 & -2.5 \\
\hline
\end{tabular}

Changes from baseline in lipid parameters: PIO+MET or SU and PIO+MET+SU

\begin{tabular}{|c|c|c|c|c|c|c|}
\hline $\begin{array}{l}\text { Mean \% } \\
\text { change } \\
\text { from } \\
\text { baseline }\end{array}$ & $\begin{array}{l}\mathrm{PIO}+ \\
\mathrm{MET} \\
(\mathrm{N}=253)\end{array}$ & $\begin{array}{l}\mathrm{PBO}+ \\
\mathrm{MET} \\
(\mathrm{N}=261)\end{array}$ & $\begin{array}{l}\mathrm{PIO}+ \\
\mathrm{SU} \\
(\mathrm{N}=508)\end{array}$ & $\begin{array}{l}\mathrm{PBO}+ \\
\mathrm{SU} \\
(\mathrm{N}=493)\end{array}$ & $\begin{array}{l}\mathrm{PIO}+ \\
\mathrm{MET}+ \\
\mathrm{SU} \\
(\mathrm{N}=654)\end{array}$ & $\begin{array}{l}\mathrm{PBO}+ \\
\mathrm{MET}+ \\
\mathrm{SU} \\
(\mathrm{N}=660)\end{array}$ \\
\hline $\begin{array}{l}\text { TG } \\
(\mathrm{mmol} / \mathrm{L})\end{array}$ & -2.60 & +14.94 & -1.09 & +11.70 & -1.1 & +8.3 \\
\hline $\begin{array}{l}\text { HDL-C } \\
(\mathrm{mmol} / \mathrm{L})\end{array}$ & +20.62 & +10.37 & +21.82 & +12.67 & +21.2 & +12.6 \\
\hline $\begin{array}{l}\text { LDL-C } \\
(\mathrm{mmol} / \mathrm{L})\end{array}$ & +12.60 & +10.03 & +9.83 & +4.76 & +12.3 & +6.1 \\
\hline $\begin{array}{l}\text { LDL-C/ } \\
\text { HDL-C }\end{array}$ & -2.67 & +1.30 & -6.86 & -5.31 & -4.4 & -2.6 \\
\hline
\end{tabular}

Conclusion: Improvements in lipid parameters were observed in the PIO group on top of the known benefits of the concomitant glucose-lowering and lipid-altering medications taken in all of the treatment groups.

\section{0}

Effect of simvastatin and/or pioglitazone on adiponectin, proinsulin and glucose metabolism in non-diabetic patients at cardiovascular risk - the PIOSTAT Study

B. Wilhelm ${ }^{1}$, T. Forst ${ }^{1,2}$, G. Lübben ${ }^{3}$, A. Pfützner ${ }^{1}$, C. Koehler ${ }^{4}$, M. Hanefeld ${ }^{4}$, E. Karagiannis ${ }^{3}$;
${ }^{1}$ Institute for Clinical Research and Development, Mainz, ${ }^{2}$ Endocrinology, University Mainz, Germany, ${ }^{3}$ Takeda Pharma, Aachen, ${ }^{4} \mathrm{GWT}$, Dresden, Germany.

Background and Aims: Insulin resistance and impaired postprandial glucose metabolism are well known confounders in cardiovascular disease. We investigated the effect of acute intervention with Pioglitazone in comparison to and in combination with Simvastatin on insulin resistance, plasma adiponectin levels, and the postprandial time course of plasma glucose, insulin and proinsulin concentrations in a non-diabetic cardiovascular risk population.

Materials and Methods: 125 non-diabetic patients with increased cardiovascular risk, defined as hypertension and/ or a previously sustained cardiovascular event (47 male, 78 female; age $58.6 \pm 6.3$ years; BMI $30.8 \pm 3.2 \mathrm{~kg} / \mathrm{m}^{2}$; mean \pm SD) were included in our three arm, double blinded, double dummy - parallel study. Patients were randomised to $45 \mathrm{mg}$ Pioglitazone and Plazebo (PIO), $45 \mathrm{mg}$ Pioglitazone and $20 \mathrm{mg}$ Simvastatin (PIO/SIM), or Plazebo and $20 \mathrm{mg}$ Simvastatin (SIM). At baseline and after 3 months, fasting blood samples were taken for the measurement of glucose, insulin and adiponectin concentrations and the HOMAs Score was calculated. In addition, an oral glucose load (OGTT) with the measurement of glucose, insulin and proinsulin intact at 0,30 and 120 minutes was performed.

Results: The HOMAs Score declined from $3.27 \pm 2.21$ to $2.40 \pm 0.97(\mathrm{p}=0.003)$ during PIO treatment and from $3.85 \pm 2.19$ to $2.81 \pm 1.15(\mathrm{p}=0.0003)$ during treatment with $\mathrm{PIO} / \mathrm{SIM}$. Inversely, adiponectin plasma levels increased from $14.0 \pm 8.2$ to $27.6 \pm 14.5 \mu \mathrm{g} / \mathrm{mL}(\mathrm{p}<0.0001)$ during PIO and from $11.7 \pm 10.0$ to $26.7 \pm 15.7 \mu \mathrm{g} / \mathrm{mL}(\mathrm{p}<0.0001)$ during $\mathrm{PIO} / \mathrm{SIM}$ treatment. A slight decrease in adiponectin levels from $15.5 \pm 12.7$ to $11.6 \pm 7.0 \mu \mathrm{g} / \mathrm{mL}(\mathrm{p}<0.05)$ could be observed during SIM treatment. An inverse correlation was found between the change in $\mathrm{HOMA}_{\mathrm{S}}$ Score and plasma adiponectin levels $(\mathrm{r}=-0.31, \mathrm{p}=0.0004)$. In addition, treatment with $\mathrm{PIO}$ and $\mathrm{PIO} / \mathrm{SIM}$ resulted in an overall improvement in glucose and insulin levels during OGTT $(\mathrm{p}<0.05$, respectively). As shown in table 1 , treatment with $\mathrm{PIO}$ and PIO/SIM significantly reduced postprandial glucose and proinsulin levels. Significant improvements in total cholesterol, HDL and LDL levels could be observed during PIO/SIM and SIM treatment ( $\mathrm{p}<0.05$ respectively), but not during single treatment with PIO.

Conclusion: Despite every single drug evolves beneficial effects on distinct cardiovascular risk parameters, only the combined treatment with both study drugs was sufficient to 
provide an overall improvement in fasting and postprandial cardiovascular risk predictors in a non-diabetic population at cardiovascular risk.

Table 1: Glucose and Proinsulin Levels during OGTT (mean $\pm \mathrm{SD} ; * \mathrm{p}<0.05$ vs baseline)

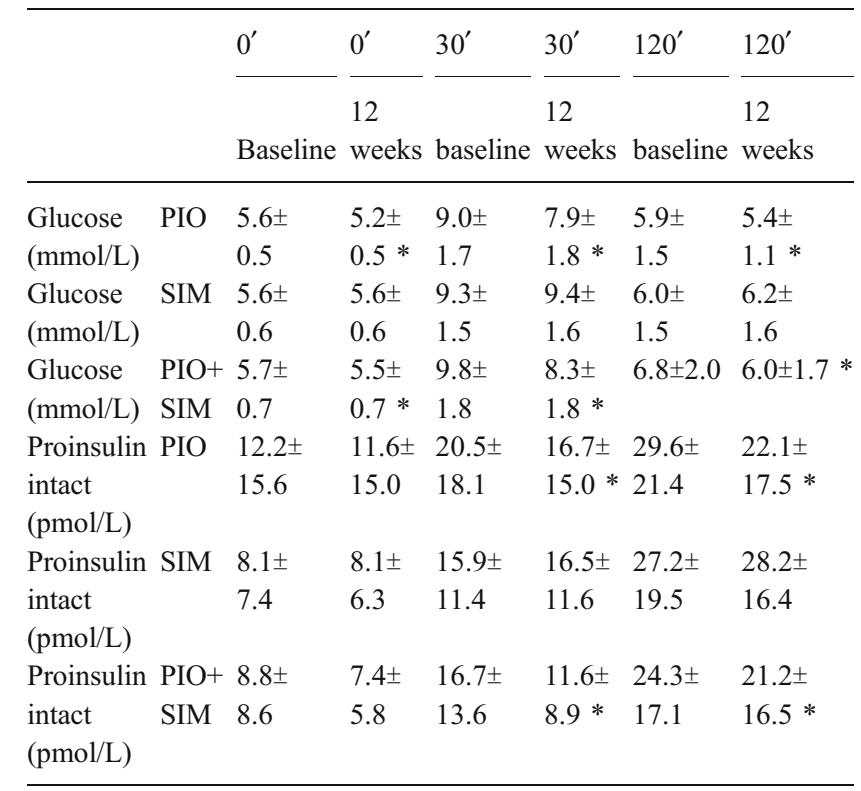

\section{Supported by TAKEDA}

\section{1}

Cardiovascular risk modification after combined intervention of rosiglitazone and exercise in patients with type 2 diabetes mellitus and metabolic syndrome N. Kadoglou ${ }^{1}$, F. Iliadis ${ }^{1}$, N. Angelopoulou ${ }^{2}$, C. Liapis ${ }^{3}$, D. Perrea ${ }^{4}$, M. Alevizos ${ }^{1}$;

${ }^{1}$ Propedeutic Department of Medicine, AHEPA Hospital, Aristotle University, Thessaloniki, ${ }^{2}$ Physical Education and Sports Science, Aristotle University, Thessaloniki, ${ }^{3}$ Vascular Surgery, University of Athens, ${ }^{4}$ Laboratory of Experimental Surgery and Surgical Research, University of Athens, Greece.

Background and Aims: Growing evidence indicates that rosiglitazone (ROSI) and exercise confer beneficial effects on numerous cardiovascular $(\mathrm{CV})$ risk factors. The aim of this study was to determine the $\mathrm{CV}$ effects of combined intervention of ROSI and exercise training program in patients with type 2 diabetes mellitus (NIDDM) and metabolic syndrome (MS).
Materials and Methods: A total of 100 individuals with NIDDM and MS, aged 44-70 years, without micro-/macrovascular complications entered this 6month study. All the participants were overweight $\left(\mathrm{BMI}>27 \mathrm{kgr} / \mathrm{m}^{2}\right)$, with inadequate glycemic control (HbAlc $>7 \%)$ under stable anti-diabetic regimen with $1700 \mathrm{mgr}$ metformin and 180 mgr gliclazide daily. At baseline and at the end of the study all the patients performed a graded exercise test and blood samples were drawn to determine biochemical parameters. The patients were randomly assigned to the following groups: A) (ROSI+EX, N=25) Patients received $4 \mathrm{mgr} / \mathrm{d}$ of ROSI as added-on therapy and performed a supervised EX program (4 times/week) for 6 months. B) (ROSI, N=25) ROSI (4 mgr/d) was administered additionally for 6 months. C) (EX, $\mathrm{N}=25$ ) Diabetics participated only in EX program similar to group A. D) (Control, $\mathrm{N}=25$ ) The rest of participants served as control group. We used one-way ANOVA and post-hoc Tukey test for the comparison between groups.

Results: At the end of the study we observed a significant reduction of $\mathrm{HbAlc}$ and fasting plasma glucose, respectively, in group: A $(-1.67 \pm 0.87 \%$ and $-43.58 \pm 27.02 \mathrm{mg} /$ $\mathrm{dl}), \mathrm{B}(-0.96 \pm 0.4 \%$ and $-39.96 \pm 26.58 \mathrm{mg} / \mathrm{dl}), \mathrm{C}$ $(-0.51 \pm 0.21 \%$ and $-20.15 \pm 19.14 \mathrm{mg} / \mathrm{dl})$, while glycemic control deteriorated in group D $(+0.34 \pm 0.06 \%$, $10.32 \pm 3.63 \mathrm{mg} / \mathrm{dl})$. With the exception of control group systolic/diastolic blood pressure was reduced considerably in group A $(-13.08 \pm 11.28 /-7.31 \pm 7.53 \mathrm{mmHg})$, group B $(-7.32 \pm 7.64 /-3.93 \pm 4.16 \mathrm{mmHg})$ and group $\mathrm{C}$ $(-7.86 \pm 9.69 /-2.9 \pm 4.33 \mathrm{mmHg})$. Exercise program contributed to significant reduction of cholesterol and LDL $(p<0.05)$ in group A and C. The latter lipid parameters remained unaltered in groups B and D. In the following table the additive effects of ROSI and EX in HDL and hsCRP are easily recognized. Concerning insulin resistance, triglycerides and $\mathrm{VO} 2 \mathrm{max}$ the combined intervention revealed the synergistic effects of ROSI and EX in group A. Finally the performance of systemic exercise seemed to counteract the weight gain of ROSI treatment.

\begin{tabular}{|c|c|c|c|c|}
\hline $\begin{array}{l}\text { Change of } \\
\text { variables }\end{array}$ & $\mathbf{A}$ & $\mathbf{B}$ & $\mathrm{C}$ & D \\
\hline Weight (kgr) & $0.09 \pm 0.18^{*}$ & $2.52 \pm 1.27 *$ & $-1.59 \pm 0.71 *$ & $0.61 \pm 0.24$ \\
\hline $\begin{array}{l}\text { hsCRP } \\
(\mathrm{mg} / \mathrm{dl})\end{array}$ & $-0.29 \pm 0.031 *$ & $-0.17 \pm 0.02 *$ & $-0.15 \pm 0.01 *$ & $0.043 \pm 0.01$ \\
\hline $\begin{array}{l}\text { Insulin } \\
(\mathrm{mU} / \mathrm{lt})\end{array}$ & $-5.33 \pm 3.21 *$ & $-2.7 \pm 1.58^{*}$ & $-2.16 \pm 1.1 * \#$ & $1.25 \pm 0.33$ \\
\hline HOMA & $-4.06 \pm 1.91 * \#$ & $-1.99 \pm 1.06^{*}$ & $-1.59 \pm 0.65 *$ & $0.96 \pm 0.42$ \\
\hline $\begin{array}{l}\text { HDL (mg/ } \\
\text { dl) }\end{array}$ & $11.08 \pm 4.87 * \#$ & $3.86 \pm 2.04 *$ & $6.59 \pm 4.97 *$ & $-2.61 \pm 1.38$ \\
\hline $\begin{array}{l}\text { VO2max } \\
(\mathrm{ml} / \mathrm{kgr} / \mathrm{min})\end{array}$ & $7,98 \pm 1,44 * \#$ & $2,08 \pm 0,39 *$ & $4,32 \pm 2,14^{*}$ & $-1,26 \pm 0,75$ \\
\hline $\begin{array}{l}\text { Triglycerides } \\
(\mathrm{mg} / \mathrm{dl})\end{array}$ & $-32.39 \pm 3.20 *$ & $-7.33 \pm 3.83$ & $-19.43 \pm 3.63 *$ & $16.61 \pm 4.94$ \\
\hline
\end{tabular}


Conclusion: The combined therapy with ROSI and exercise resulted in multiple beneficial effects on $\mathrm{CV}$ risk profile in patients with NIDDM and MS. These findings indicate that ROSI in conjunction with physical activity exert a greater cardioprotective role in high risk patients. Supported by Project "Pythagoras I" and Propondis Foundation

\section{2}

An analysis from PROactive on the effects of pioglitazone on major adverse cardiovascular events (MACE) and myocardial infarction

R. Wilcox on behalf of the PROactive Investigators; Department of Cardiovascular Medicine, Queen's Medical Centre, Nottingham, United Kingdom.

Background and Aims: Composite endpoints of cardiovascular events are commonly used to compare treatments in large cardiovascular outcome studies and are referred to as major adverse cardiovascular events (MACE). Patients with type 2 diabetes are at a higher risk of macrovascular events than the general population. In this analysis from PROactive, we evaluated the effects of pioglitazone on the prespecified endpoints of fatal/non-fatal myocardial infarction (MI; excluding silent MI) and a MACE1 composite of cardiovascular death, non-fatal MI (excluding silent MI), or non-fatal stroke; and on a post-hoc MACE2 composite of all-cause mortality, non-fatal MI (excluding silent MI), nonfatal stroke, or acute coronary syndrome in high-risk patients with type 2 diabetes.

Materials and Methods: PROactive was a double-blind, placebo-controlled cardiovascular outcome study in which 5,238 patients were randomised to pioglitazone or placebo, in addition to their existing glucose-lowering and cardiovascular medication. Patients started with $15 \mathrm{mg}$ /day of pioglitazone, titrated to maximum tolerated doses in $15 \mathrm{mg}$ increments to a maximum of $45 \mathrm{mg}$. Mean follow-up was 34.5 months.

Results: A Cox proportional hazards model showed statistically significant risk reductions with pioglitazone compared with placebo for the prespecified endpoints of MI $(23 \%$; HR $=0.77 ; 95 \% \mathrm{CI}=0.60,1.00 ; \mathrm{P}=0.046)$ and MACE1 $(18 \%, \mathrm{HR}=0.82 ; 95 \% \mathrm{CI}=0.70,0.97 ; \mathrm{P}=0.020)$, and the post hoc MACE2 endpoint $(17 \%$; $\mathrm{HR}=0.83 ; 95 \% \mathrm{CI}=0.72,0.96$; $\mathrm{P}=0.010)$. At study end, 339 (13.0\%) patients in the pioglitazone group had a first event that contributed to the MACE2 endpoint, compared with 409 (15.5\%) in the placebo group and $108(4.1 \%)$ pioglitazone patients and 140 (5.3\%) placebo patients had an MI (excluding silent MI). A similar result occurred when non-cardiovascular deaths were excluded from the MACE2 endpoint ( $\mathrm{HR}=0.80 ; 95 \% \mathrm{CI}=0.69,0.94$; $\mathrm{P}=0.005$ ).

Time to fatal/nonfatal MI (excluding silent MI) and time to the MACE2 composite endpoint

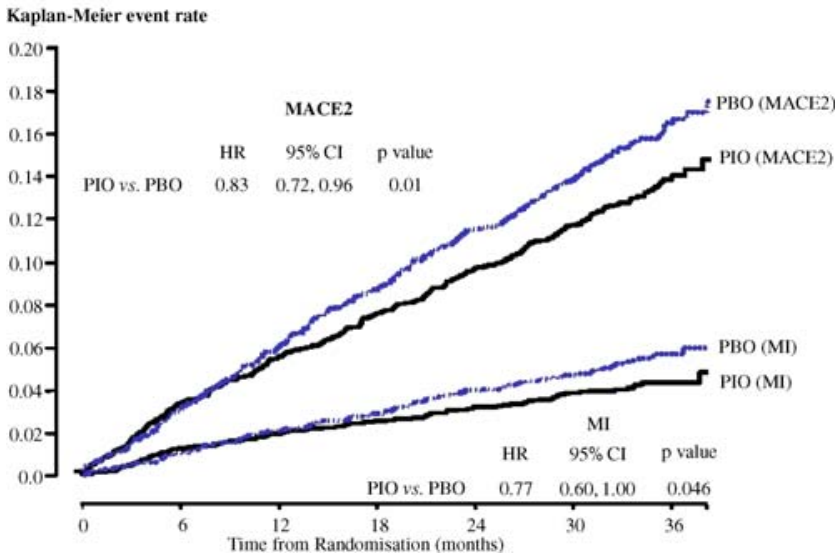

Conclusion: Pioglitazone treatment resulted in a $23 \%$ clinically relevant and statistically significant risk reduction in the prespecified time to first fatal/non-fatal MI (excluding silent MI) and nearly a $20 \%$ reduction in time to MACE1 compared with placebo in this population of patients with advanced type 2 diabetes at high risk for cardiovascular events.

0113

Long-term treatment with pioglitazone improves markers of liver function in patients with type 2 diabetes: results from PROactive

M. Diamant, R. Schindhelm, R. J. Heine on behalf of the PROactive Investigators;

Department of Endocrinology, VA University Medical Center, Amsterdam, The Netherlands.

Background and Aims: Due to hepatotoxicity concerns, liver function monitoring is recommended in patients receiving thiazolidinedione treatment. Pioglitazone was administered to 5,238 high-risk patients with type 2 diabetes and macrovascular disease in PROactive, a cardiovascular outcome study. We examined the effects of pioglitazone on liver safety in this patient population.

Materials and Methods: PROactive was a double-blind, placebo-controlled study in 5,238 patients with type 2 diabetes 
and macrovascular disease randomised to pioglitazone or matching placebo in addition to existing glucose-lowering and cardiovascular medication, including anti-hypertensive, antiplatelet, and lipid-modifying drugs. The starting dose of pioglitazone $(15 \mathrm{mg})$ was titrated to a maximum tolerated dose of $45 \mathrm{mg}$. Mean follow-up was 34.5 months. Laboratory assessments for liver function (alanine aminotransferase [ALT], aspartate aminotransferase [AST], and alkaline phosphatase $[\mathrm{AP}]$ ) were performed at every visit during the first year and every 6 months thereafter.

Results: The results show that there was a general shift toward normalisation of ALT and AST values in the pioglitazone group from baseline to the final visit compared to no change or an increase in values in the placebo group. At study entry, similar proportions of pioglitazone and placebo patients had ALT (9.7\% and $10.7 \%)$, AST $(6.1 \%$ and $6.9 \%)$, or AP $(6.7 \%$ and $5.8 \%$ ) values above the upper limit of normal (>ULN), respectively. In the pioglitazone group at final visit, the occurrence of elevated ALT decreased to $5.9 \%$, whereas there was a small increase in the placebo group $(11.9 \%$ at final visit; $\mathrm{P}<0.0001$ between groups). More placebo than pioglitazone patients also had elevated levels of AST (7.3\% versus $4.5 \%$; $\mathrm{P}=0.0001$ ), respectively. There was a small decrease in the proportion of patients with AP values $>$ ULN in the pioglitazone group (to $5.9 \%$ ) and a small increase in the placebo group (to 6.6\%; $\mathrm{P}=\mathrm{NS}$ ). In the pioglitazone group, mean ALT decreased from $28.3 \mathrm{IU} / \mathrm{L}$ at baseline to $26.2 \mathrm{IU} / \mathrm{L}$ at the final visit, whereas there was a small mean increase $(2.0 \mathrm{IU} / \mathrm{L})$ in the placebo group ( $\mathrm{P}<0.0001$ between groups).

Patients with at least one ALT, AST, or AP $23 \times$ ULN at end of the trial

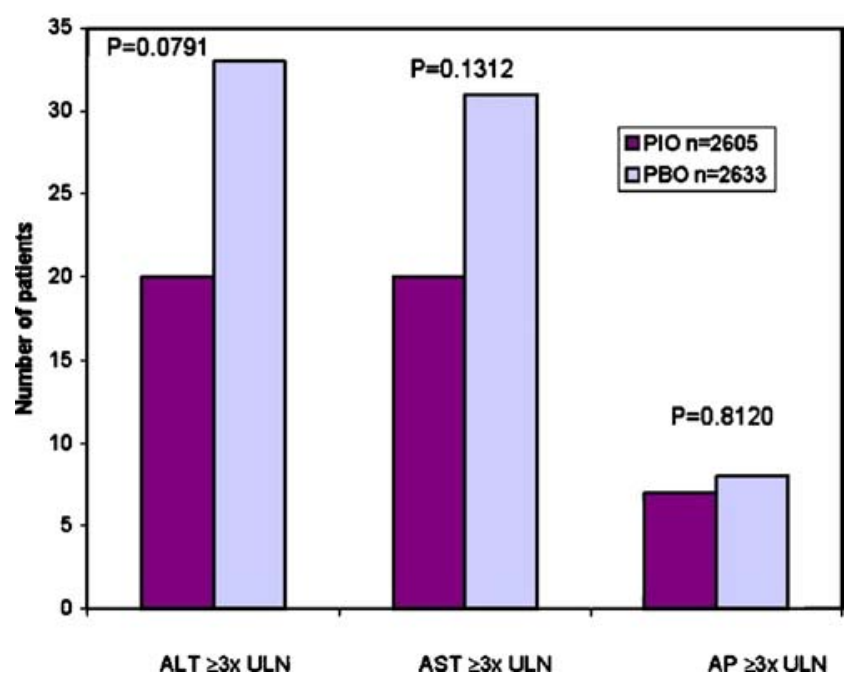

Conclusion: Results show that long-term pioglitazone treatment was associated with an improved hepatic laboratory profile compared with placebo in this high-risk patient population with type 2 diabetes. Because ALT is a marker of the amount of liver fat related to liver insulin resistance, these data suggest that treatment with pioglitazone is associated with a lowering of liver fat content.

\section{4}

Evaluation of ezetimibe/simvastatin versus rosuvastatin in hypercholesterolaemic patients with type 2 diabetes or metabolic syndrome

A. L. Catapano ${ }^{1}$, C. M. Ballantyne ${ }^{2}$, M. H. Davidson ${ }^{3}$, W. E. Brady ${ }^{4}$, R. A. Gazzara ${ }^{4}$, J. E. Tomassini ${ }^{4}$,

A. M. Tershakovec ${ }^{4}$;

${ }^{1}$ Department of Pharmacological Sciences, University of Milan, Italy, ${ }^{2}$ Section of Atherosclerosis, Baylor College of Medicine, Houston, ${ }^{3}$ Department of Internal Medicine, Rush University School of Medicine, Chicago, ${ }^{4}$ Merck \& Co., Inc., West Point, United States.

Background and Aims: Type 2 diabetes mellitus (DM) is considered a coronary heart disease (CHD) risk equivalent. DM and metabolic syndrome (MS) share elements of an atherogenic dyslipidemia characterized by high levels of triglycerides (TG), low levels of high-density lipoprotein cholesterol (HDL-C), and small, dense low-density lipoprotein (LDL) particles. This post-hoc analysis compared the efficacy of ezetimibe/simvastatin (E/S) vs. rosuvastatin (R) in patients with DM, MS without DM, or neither disorder.

Material and Methods: This was a multicenter, doubleblind, randomized, parallel-group, 6-week study in which patients were randomized equally to 1 of 6 treatment arms: E/ $\mathrm{S} 10 / 20,10 / 40$, or $10 / 80 \mathrm{mg}$, or R 10 , R 20 , or R $40 \mathrm{mg}$. In this post-hoc analysis, the efficacy of E/S was compared with $\mathrm{R}$ at the usual start doses, E/S 10/20 mg vs. R $10 \mathrm{mg}$; alternate start doses, E/S 10/40 vs. R 20; maximum doses, E/S 10/80 vs. R 40, and averaged across all doses, All E/S vs. All R.

Results: Of 2959 patients randomized, 2855 patients were included in the modified intent-to-treat population (E/ $\mathrm{S}=1428, \mathrm{R}=1427)$, of which 367 (12.9\%) had DM, 811 (28.6\%) had MS without DM, and 1661 (58.5\%) had neither disorder. Sixteen patients, who did not have DM, could not be placed in a category because they were missing other data that precluded their MS classification. Efficacy results are shown below for LDL-C, HDL-C, and TG, averaged across all doses (All E/S vs. All R). 


\begin{tabular}{|c|c|c|c|c|c|c|}
\hline \multirow{2}{*}{$\begin{array}{l}\text { Parameter } \\
\mathrm{Mg} / \mathrm{dL}\end{array}$} & \multicolumn{2}{|l|}{$\mathrm{DM}$} & \multicolumn{2}{|c|}{ MS without DM } & \multicolumn{2}{|l|}{ Neither } \\
\hline & $\begin{array}{l}E / S \\
(n=186)\end{array}$ & $\begin{array}{l}\mathrm{R} \\
(\mathrm{n}=181)\end{array}$ & $\begin{array}{l}E / S \\
(n=395)\end{array}$ & $\begin{array}{l}R \\
(n=416)\end{array}$ & $\begin{array}{l}E / S \\
(n=837)\end{array}$ & $\begin{array}{l}\mathrm{R} \\
(\mathrm{n}=824)\end{array}$ \\
\hline
\end{tabular}

\begin{tabular}{|c|c|c|c|c|c|c|}
\hline \multicolumn{7}{|l|}{ LDL-C } \\
\hline Baseline $\dagger$ & 170.0 & 169.8 & 174.2 & 172.9 & 173.0 & 173.8 \\
\hline \multirow[t]{2}{*}{$\%$ change } & -58.5 & -54.2 & -55.0 & -51.8 & -55.6 & -51.0 \\
\hline & $(0.9)$ & $(0.9)$ & $(0.6)$ & $(0.6)$ & $(0.4)$ & $(0.4)$ \\
\hline Tx Diff§** & \multicolumn{2}{|c|}{$-4.3(-6.7,-1.9)$} & \multicolumn{2}{|c|}{$-3.2(-4.8,-1.6)$} & \multicolumn{2}{|c|}{$-4.6(-5.7,-3.4)$} \\
\hline \multicolumn{7}{|l|}{ HDL-C } \\
\hline Baseline $\dagger$ & 46.6 & 47.0 & 44.4 & 44.2 & 53.9 & 53.8 \\
\hline$\%$ change $\ddagger$ & $7.0(0.8)$ & $6.3(0.8)$ & $9.4(0.5)$ & $8.9(0.5)$ & $6.9(0.4)$ & $7.3(0.4)$ \\
\hline Tx Diff & \multicolumn{2}{|c|}{$0.7(-1.4,2.9)$} & \multicolumn{2}{|c|}{$0.5(-0.9,1.9)$} & \multicolumn{2}{|c|}{$-0.4(-1.4,0.6)$} \\
\hline \multicolumn{7}{|l|}{$\mathrm{TG}$} \\
\hline Baselinett & 172.8 & 185.0 & 210.0 & 203.8 & 129.5 & 132.5 \\
\hline$\%$ change $\dagger \dagger$ & -28.2 & -26.7 & -31.6 & -29.2 & -23.4 & -21.5 \\
\hline Tx Diff:t*** & \multicolumn{2}{|c|}{$-3.0(-6.7,0.7)$} & \multicolumn{2}{|c|}{$-2.7(-5.2,-0.4)$} & \multicolumn{2}{|c|}{$-2.8(-4.7,-0.8)$} \\
\hline
\end{tabular}

$\dagger$ Means; †LS Means (SE); §LS Means (95\% CI); $\dagger$ Median; + Hedian $(95 \% \mathrm{CI}) ; * * \mathrm{p}<0.001$ for treatment effect, treatment by subgroup interaction NS.

Conclusion: DM and MS patients treated with ezetimibe/ simvastatin showed greater improvements in LDL-C and TG, with similar improvements in HDL-C, compared with those treated with rosuvastatin. Results were similar for the individual dose comparisons. These findings were consistent with the main study results. Thus, the dual cholesterollowering mechanisms of ezetimibe/simvastatin provided additional lipid-modifying benefits compared to rosuvastatin monotherapy in patients with DM and MS without DM. Supported by Merck/Schering Plough Pharmaceuticals

\section{OP 20}

\section{Glucose metabolism and the brain}

\section{5}

Prominent decrease of hippocampal neurogenesis in a spontaneous model of type 1 diabetes, the nonobese diabetic mice (NOD): a probable link with diabetes associated-depression?

F. E. Saravia ${ }^{1,2}$, J. Beauquis ${ }^{1}$, J. Coulaud ${ }^{3}$, P. Roig ${ }^{1}$, F. Homo-Delarche ${ }^{3}$, A. De Nicola ${ }^{1,2}$;

${ }^{1}$ Neuroendocrine Biochemistry, Institute of Biology and Experimental Medicine, Buenos Aires, Argentina, ${ }^{2}$ Faculty of Medicine, University of Buenos Aires, Argentina, ${ }^{3}$ CNRS 7059, Universite Paris, France.

Background and Aims: The production of new neurons (neurogenesis) in the adult brain is restricted to two brain areas, the subventricular zone and the dentate gyrus (DG) of the hippocampus. In the DG, this plastic event is associated to specific types of learning and is especially modulated by hormones, environmental factors (stress, exercise) and aging. Defective neurogenesis is intimately linked to inflammation, affective-related disorders and depression. In human diabetes, degenerative and functional disorders of the central nervous system, including a high incidence of depression, are common findings. We recently reported that DG neurogenesis is reduced in streptozotocin (STZ)-treated mice, a pharmacological model of T1D. Here, we wanted to explore DG neurogenesis before and after diabetes onset in the NOD mouse, a well described spontaneous T1D model.

Materials and Methods: DG cell proliferation and survival were studied as a function of age in prediabetic and diabetic NOD and control C57BL/6 and BALB/C female mice, using different protocols of 5-bromo-2'-deoxyuridine (BrdU) incorporation, and cell phenotype analysis by immunocytochemical co-detection for glial (GFAP) or neuronal (Neu-N) markers and confocal microscopy.

Results: In the first protocol, cell proliferation was assessed in the DG of 4, 8 and 12-week-old control strains and NOD mice (the 12-week-old NOD mice were overtly diabetic, whereas the 4- and 8-week-old groups were pre-diabetic). Levels of BrdU positive cells in NOD DG were, regardless of age, constantly and significantly lower than in controls, showing that hippocampal alterations are present far before diabetes onset in NOD mice. The second protocol was designed to study survival of the newly-generated cells: 15week-old animals were sacrificed 21 days after BrdU injection. Here, in addition to controls, two subsets of NOD mice were considered: non diabetic (noDb) and diabetic $(\mathrm{Db})$. Cell survival in NOD DG was significantly lower than C57BL/6, where 599.4 $\pm 78.9 \mathrm{BrdU}+$ cells were found per DG while the values corresponding to NOD Db

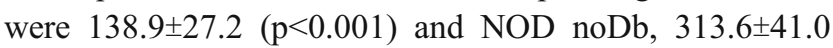
$(p<0.01)$. Moreover, the presence of diabetes strongly aggravated cell survival in DG $(\mathrm{p}<0.05)$. However, confocal analysis of cell phenotypes determined that the proportion of neurons in these groups was not altered by the diabetic state.

Conclusion: Like in the pharmacological (STZ) T1D model, hippocampal neurogenesis is strongly diminished in the spontaneous T1D model of the NOD mouse. Notably, in the STZ model, we previously showed that antidepressant treatment (fluoxetine) induced complete recovering of proliferation of cells potentially becoming neurons, an effect that might result from improvement of hypothalamo-pituitary-adrenal axis that is known to be altered in rodent and human diabetes. Here, in addition, we showed that hippocampal DG cell proliferation defect takes place during the prediabetic stage in the NOD mouse. Whether this early alteration might result, in this autoim- 
mune strain, from HPA axis alterations and/or ongoing brain inflammatory process remains to be investigated.

Supported by Inserm-Conicet International Agreement, University of Buenos Aires Grant.

\section{6}

\section{Hippocampal neuronal death and gliosis during} development of streptozotocin diabetes mellitus

M. A. Orlovsky, O. M. Tsupykov, G. G. Skibo;

Cytology, Bogomoletz Institute of Physiology, Kiev, Ukraine.

Background and Aims: Diabetes mellitus is characterized by hypothalamo-pituitary-adrenal (HPA) axis hyperactivity, shown both in experimental and clinical studies. However, mechanisms of this phenomenon remains still unclear. It is suggested that diabetes-induced hippocampal damage can produce morphological substrate for diminished glucocordicoid negative feedback regulation. The present investigation was objected to study expression of pro- and anti-apoptotic factors and glial reaction in hippocampus at different terms of experimental diabetes.

Materials and Methods: Diabetes was induced by a single intraperitoneal streptozotocin administration $(45 \mathrm{mg} / \mathrm{kg})$ in adult female Wistar rats during diestrus. Animals were killed 3, 7, 14, 21 and 35 days after. Hippocampal expression of bcl2, inducible nitric oxide synthase (iNOS), neuronal enolase (Neu, specific neuronal marker) GFAP (astroglial marker) and BrdU were studied using indirect immunofluorescence. Additionally, blood corticosterone, insulin and glucose level were measured using ELISA and standard biochemical techniques.

Results: Diabetes development in all terms was characterized by progressive hypoinsulinemia and hyperglycemia; the blood costicosterone concentration was maximally increased at day 14. Starting from day 21 diabetes led to progressive hippocampal neuronal loss at all areas and at day 35 the maximum decrease $(-43,0 \pm 5,2 \%)$ in number of neuronal cells was observed in CA3 area. This was associated with prominent astrogliosis: both number of astroglial cells in GFAP expression level was increased already at day 14 and later gliosis was further enhanced till the end of the experiments (35 days). iNOS expression was found in hippocampus prior to neuronal loss already at day 3 totally disappearing after the first week of diabetes development.

Conclusion: Thus, it can be concluded, that diabetes development is associated with progressive neuronal degeneration and gliosis in hippocampus. This process is tightly linked to the elevated blood corticosterone levels.

Supported by INTAS foundation, young scientists postdoctoral fellowship grant
0117

Early vascular risk factors impair brain metabolism in healthy men

O. Heikkilä ${ }^{1}$, N. Lundbom ${ }^{2}$, M. Timonen ${ }^{2}$, P.-H. Groop ${ }^{1}$, S. Heikkinen $^{3}$, S. Mäkimattila ${ }^{1}$;

${ }^{1}$ Institute of Genetics, Folkhälsan Research Center, Helsinki, ${ }^{2}$ Helsinki Medical Imaging Center, University of Helsinki, ${ }^{3}$ Laboratory of Organic Chemistry, University of Helsinki, Finland.

Background and Aims: Silent brain infarctions and cognitive decline are common findings in type 2 diabetes (T2D). Typical location for a silent brain infarction is the thalamus with its long penetrating end arteries. It is an important relay station for many neural loops that regulate the initiation and termination of planning, working memory, and attention. We hypothesized that early vascular risk factors may derange thalamic metabolism, which predispose brain tissue to later damage.

Materials and Methods: Eighteen healthy non-smoking men (22-45 yrs) without any medication and with normal brain MRI were studied. Nine subjects had a first degree relative with $\mathrm{T} 2 \mathrm{D}$ and 3-4 of the following vascular risk factors: waist $\geq 94 \mathrm{~cm}, \quad \mathrm{fS}-\mathrm{Gluc} \geq 5.6 \mathrm{mmol} / 1, \quad \mathrm{BP} \geq 130$ / $85 \mathrm{mmHg}, \mathrm{S}-\mathrm{Tg} \geq 1.7 \mathrm{mmol} / \mathrm{l}$, and $\mathrm{S}-\mathrm{HDL}<1.03 \mathrm{mmol} / \mathrm{l}$. The controls had no family history of $\mathrm{T} 2 \mathrm{D}$ and $0-1$ vascular risk factors. The groups were matched for age but differed $(p<0.01)$ in weight $(94 \pm 13$ vs. $77 \pm 10 \mathrm{~kg})$, waist $(104 \pm 5$ vs. $78 \pm 8 \mathrm{~cm})$, systolic BP $(141 \pm 11$ vs. $126 \pm$ $8 \mathrm{mmHg})$, diastolic BP $(88 \pm 4$ vs. $76 \pm 8 \mathrm{mmHg})$, and fSGluc (5.6 \pm 0.7 vs. $4.7 \pm 0.3 \mathrm{mmol} / \mathrm{l})$. There was no difference in S-Chol (4.8 \pm 0.9 vs. $4.4 \pm 1.0 \mathrm{mmol} / \mathrm{l}), \mathrm{S}-\mathrm{HDL}(1.4 \pm 0.2$ vs. $1.4 \pm 0.3 \mathrm{mmol} / \mathrm{l}), \mathrm{S}-\mathrm{LDL}(2.6 \pm 0.7$ vs. $2.5 \pm 1.0 \mathrm{mmol} / \mathrm{l})$ or $\mathrm{S}-$ $\operatorname{Tg}(1.7 \pm 1.2$ vs. $0.9 \pm 0.3 \mathrm{mmol} / \mathrm{l})$ between the groups. The risk-factor group underwent a 75-g OGTT: 2 h S-Gluc was $6.7 \pm 1.7 \mathrm{mmol} / 1$.

Single-voxel short echo time (TE $30 \mathrm{~ms}$ ) 1D-spectra were collected after 12 hours fast in the thalamus. Brain metabolites creatine ( $\mathrm{Cr}$; energy metabolite), N-acetyl aspartate (NAA; a neuronal marker), choline and myoinositol (Cho and m-Ins; membrane markers), glucose (Gluc), glutamine and glutamate (Glx; neurotransmitters), and tissue water $\left(\mathrm{H}_{2} \mathrm{O}\right)$ were evaluated by integration of the characteristic spectral regions. ${ }^{1} \mathrm{H}$ MRS studies were performed with a clinical 1.5 T MR-system using the PRESS method. Metabolites were referred to the unsuppressed tissue water signal.

Results: In the risk-factor group, $\mathrm{Cr} / \mathrm{H}_{2} \mathrm{O}$ was $14 \%$ higher in the thalamus (Table). Intensity of $\mathrm{H}_{2} \mathrm{O}$ signal was similar in the groups $\left(2.5 \pm 0.3\right.$ vs. $2.6 \pm 0.1 \cdot 10^{8}$ a.u. $)$. 


\begin{tabular}{lllllll}
\hline $10^{-4}$ & $\mathrm{Cr} / \mathrm{H}_{2} 0$ & $\mathrm{NAA} / \mathrm{H}_{2} 0$ & $\mathrm{Cho} / \mathrm{H}_{2} 0$ & $\mathrm{~m}-\mathrm{Ins} / \mathrm{H}_{2} 0$ & $\mathrm{Gluc} / \mathrm{H}_{2} 0$ & $\mathrm{Glx} / \mathrm{H}_{2} 0$ \\
Subjects & $2.4 \pm 0.3$ & $4.4 \pm 0.3$ & $1.9 \pm 0.2$ & $1.6 \pm 0.3$ & $0.3 \pm 0.1$ & $1.6 \pm 0.2$ \\
Controls & $2.1 \pm 0.2$ & $4.2 \pm 0.2$ & $1.8 \pm 0.2$ & $1.5 \pm 0.3$ & $0.3 \pm 0.1$ & $1.5 \pm 0.1$ \\
$p$ & 0.019 & 0.058 & $N S$ & $N S$ & $N S$ & $N S$ \\
\hline
\end{tabular}

Cr levels correlated positively with OGTT $2 \mathrm{~h}$ S-Gluc concentration $(r=0.75, p<0.05)$

Conclusions: This data suggests that the $\mathrm{Cr}$ level in thalamus is increased in healthy men with early vascular risk factors. Increased $\mathrm{Cr}$ may indicate enhanced glial metabolism, activated possibly by alterations in glucose availability to neurons.

\section{8}

Diabetes status and fasting plasma glucose are independent determinants of cognitive status in people at high risk for cardiovascular disease

T. Cukierman-Yaffe ${ }^{1}$, H. C. Gerstein ${ }^{1}$, C. Anderson ${ }^{2}$,

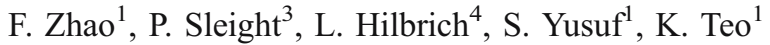
on behalf of the ONTARGET/TRANSCEND Investigators; ${ }^{1}$ Population Health Research Institute, McMaster University, Hamilton, Canada, ${ }^{2}$ Royal Prince Alfred Hospital, Sydney, Australia, ${ }^{3}$ John Radcliffe, Oxford, United Kingdom, ${ }^{4}$ Boehringer-Ingelheim Pharma KG, Ingelheim Am Rhein, Germany.

Background and Aims: Dementia and cognitive dysfunction (predementia) are a growing problem worldwide. Recent evidence suggests that elevated levels of glucose are an independent determinant of cognitive dysfunction. The objective of this study was to assess the cross-sectional relationship between measures of glycemia and cognitive status.

Materials and Methods: The ONgoing Telmisartan Alone and in combination with Ramipril Global Endpoint Trial (ON TARGET) $(\mathrm{N}=25,620)$ trial and the concurrent Telmisartan Randomized Assessment Study in ACE intolerant Subjects with Cardiovascular Disease (TRANSCEND) $(\mathrm{N}=5926)$ trials are two long term parallel international multi-centre randomized trials assessing the cardiovascular effects of an angiotensin receptor blocker (ARB), telmisartan and an angiotensin converting enzyme (ACE) inhibitor, ramipril in high risk individuals. Baseline data from both trials provide a unique opportunity to analyze the cross-sectional relationship between measures of glycemia and cognitive status. The Mini Mental State Examination (MMSE) was used to measure cognitive function. The relationship between diabetes status, fasting plasma glucose (FPG) and MMSE score was assessed; a $B$ coefficient as well as a $p$ value was reported. The $p$ value indicates the statistical significance. The beta coefficient for diabetes status is the mean difference in MMSE score between those with and without diabetes. For FPG the B coefficient describes the mean difference in MMSE score for every $1 \mathrm{mmol} / \mathrm{L}$ difference in $\mathrm{FPG}$ value.

Results: In people with diabetes the mean MMSE score was 0.4 units lower than in people without diabetes $(\mathrm{P}<0.0001)$. In all participants, a $1 \mathrm{mmol} / \mathrm{L}$ higher $\mathrm{FPG}$ value was associated with a MMSE score that was 0.06 units lower $(\mathrm{P}<0.0001)$. The association persisted even after adjustment for several cardiovascular risk factors.

Conclusion: Dysglycemia is an independent determinant of cognitive function in people at high risk for cardiovascular events. Prospective studies of this relationship can discern the temporal relationship between these variables and clinical trials may reveal whether or not glucose lowering can affect the progression of cognitive decline in this population.

\section{The association between FPG levels and MMSE score after adjusting for baseline characteristics}

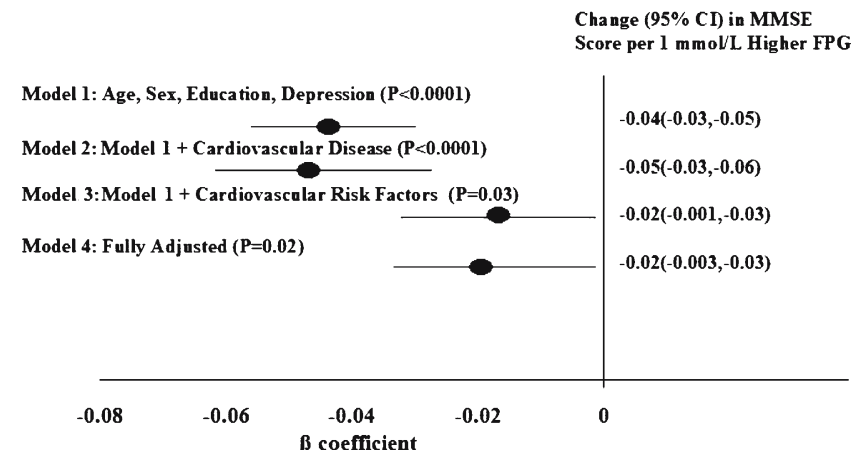

0119

Brainstem auditory evoked potentials in patients with diabetes mellitus

N. Gherdeva ${ }^{1}$, L. Savchuk ${ }^{2}$, B. Mankovsky ${ }^{1}$;

${ }^{1}$ Diabetology, Center for Endocrinology, Kiev, ${ }^{2}$ Institute of Otolaryngology, Kiev, Ukraine.

Background and Aims: The evaluation of brainstem auditory evoked potentials provides the possibility to detect subclinical cerebral damage. Therefore, the aim of our study was to investigate the parameters of short-latency brainstem auditory evoked potentials in patients with type 1 and type 2 diabetes mellitus without clinical signs of cerebral or cochlear damage.

Materials and Methods: One hundred and five patients with diabetes mellitus were examined including 43 patients with type 1 and 62 with type 2 diabetes. Patients were divided into three age groups. The first group consisted of patients 1739 years old ( 30 persons), mean age $26.1+1.3$ years, BMI $23.6+0.6 \mathrm{~kg} / \mathrm{m}^{2}, \mathrm{HbAlc}-8.9+0.3 \%$. The second group 
included middle age patients - 40-59 years old (44 persons), mean age $50.2+0.8$ years, BMI $-29.5+0.7 \mathrm{~kg} /$ $\mathrm{m}^{2}$, HbAlc $-8.8+0.3 \%$. The third group consisted of elderly patients $-60-79$ years ( 31 persons), mean age $65.7+$ 0.7 years, BMI $-29.8+0.7 \mathrm{~kg} / \mathrm{m}^{2}$, HbAlc $-9.0+0.3 \%$. Control group included 32 persons without history of diabetes mellitus who were also divided into three respective age-matched groups. Statistical analysis was performed by Student's test.

Results: We found the significant prolongation of the $\mathrm{V}$ wave latency time of auditory brainstem evoked potentials and the $\mathrm{I}-\mathrm{V}$ interwave interval in diabetic patients in all age groups compared to age-matched controls. The latency of $\mathrm{V}$ wave in the first age group patients with diabetes was 5.71+ $0.04 \mathrm{~ms}$ compared to $5.58+0.02 \mathrm{~ms}$ in the control group; in the second age group $-5.79+0.02 \mathrm{~ms}$ and $5.61+0.02 \mathrm{~ms}$; in the third age group $-5.86+0.03 \mathrm{~ms}$ and $5.62+0.03 \mathrm{~ms}$, respectively, $\mathrm{p}<0.05$ for all comparisons. Interwave interval $\mathrm{I}-\mathrm{V}$ in the first age group of diabetic patients was $4.10+$ $0.02 \mathrm{~ms}$ and $3.88+0.03 \mathrm{~ms}$ in the control group; in the second age group $-4.10+0.02 \mathrm{~ms}$ and $3.93+0.03 \mathrm{~ms}$; in the third age group $-4.19+0.08 \mathrm{~ms}$ and $3.95+0.02 \mathrm{~ms}$, in diabetic and control subjects, respectively, $\mathrm{p}<0.05$.

Conclusion: The revealed prolongation of latency time of auditory brainstem evoked potentials could reflect the subclinical cerebral and cochlear impairments in patients with type 1 and type 2 diabetes mellitus.

\section{0}

Pioglitazone in patients with or without a history of stroke: results from PROactive

\section{J. Dormandy ${ }^{1}$, M.-G. Bousser ${ }^{2}$, R. Wilcox ${ }^{3}$ on behalf}

of the PROactive Investigators;

${ }^{1}$ Department of Clinical Vascular Research, St. George's Hospital, London, United Kingdom, ${ }^{2}$ Hospital Lariboisière, Hospital Lariboisière, Paris, France, ${ }^{3}$ Department of Cardiovascular Medicine, Queen's Medical Centre, Nottingham, United Kingdom.

Background and Aims: The risk of stroke is markedly higher in patients with diabetes than in those without. The most effective strategies to prevent stroke among people with diabetes include antihypertensive, antiplatelet, and lipidaltering therapy; however, benefit has not been demonstrated with any glucose-lowering drug. No data have been published on the potential benefit of the use of thiazolidinediones in preventing primary and secondary stroke (all stroke) in patients with type 2 diabetes. We conducted a prespecified analysis from PROactive of the risk of stroke and other cardiovascular outcomes in patients with type 2 diabetes with and without prior stroke ( $>6$ months before randomisation).
Materials and Methods: The prospective, double-blind PROactive trial randomised 5,238 patients with type 2 diabetes and increased risk of cardiovascular disease to pioglitazone (force-titrated to $45 \mathrm{mg}$ ) or placebo, in addition to current medication for diabetes and cardiovascular disease. Cardiovascular endpoint events were independently adjudicated.

Results: In the patients with previous stroke, there were statistically significant benefits of pioglitazone on the endpoints of 1 . first fatal or non-fatal stroke and 2 . cardiovascular death, MI (excl. silent MI), or stroke. There was a trend of benefit with pioglitazone for the primary and the principal secondary endpoints. Thirteen patients in the pioglitazone group $(2.7 \%)$ and 16 in the placebo group $(3.2 \%)$ had a transient ischaemic attack. There was no effect of pioglitazone on subsequent strokes in the patients without prior stroke.

\begin{tabular}{|c|c|c|c|c|c|c|}
\hline \multirow[t]{2}{*}{ Endpoints } & \multicolumn{3}{|c|}{$\begin{array}{l}\text { Prior Stroke } \\
\text { n events (\%) }\end{array}$} & \multicolumn{3}{|c|}{$\begin{array}{l}\text { No Prior Stroke } \\
\mathrm{n} \text { events }(\%)\end{array}$} \\
\hline & $\begin{array}{l}\mathrm{PIO} \\
\mathrm{N}=486\end{array}$ & $\begin{array}{l}\mathrm{PBO} \\
\mathrm{N}=498\end{array}$ & $\begin{array}{l}\text { HR } \\
(95 \% \mathrm{CI})\end{array}$ & $\begin{array}{l}\mathrm{PIO} \\
\mathrm{N}=2119\end{array}$ & $\begin{array}{l}\mathrm{PBO} \\
\mathrm{N}=2135\end{array}$ & $\begin{array}{l}\mathrm{HR} \\
(95 \% \mathrm{CI})\end{array}$ \\
\hline All-stroke & $\begin{array}{l}27 \\
(5.6)\end{array}$ & $\begin{array}{l}51 \\
(10.2)\end{array}$ & $\begin{array}{l}0.53 \\
(0.34,0.85)\end{array}$ & $\begin{array}{l}59 \\
(2.8)\end{array}$ & $\begin{array}{l}56 \\
(2.6)\end{array}$ & $\begin{array}{l}1.06 \\
(0.73,1.52)\end{array}$ \\
\hline $\begin{array}{l}\text { Cardiovascular } \\
\text { death, MI } \\
\text { or stroke }^{1}\end{array}$ & $\begin{array}{l}63 \\
(13.0)\end{array}$ & $\begin{array}{l}88 \\
(17.7)\end{array}$ & $\begin{array}{l}0.72 \\
(0.52,1.00)\end{array}$ & $\begin{array}{l}194 \\
(9.2)\end{array}$ & $\begin{array}{l}225 \\
(10.5)\end{array}$ & $\begin{array}{l}0.86 \\
(0.71,1.04)\end{array}$ \\
\hline Primary $^{2}$ & $\begin{array}{l}98 \\
(20.2)\end{array}$ & $\begin{array}{l}126 \\
(25.3)\end{array}$ & $\begin{array}{l}0.78 \\
(0.60,1.02)\end{array}$ & $\begin{array}{l}416 \\
(19.6)\end{array}$ & $\begin{array}{l}446 \\
(20.09)\end{array}$ & $\begin{array}{l}0.94 \\
(0.82,1.07)\end{array}$ \\
\hline $\begin{array}{l}\text { Principal } \\
\text { secondary }\end{array}$ & $\begin{array}{l}76 \\
(15.6)\end{array}$ & $\begin{array}{l}98 \\
(19.7)\end{array}$ & $\begin{array}{l}0.78 \\
(0.58,1.06)\end{array}$ & $\begin{array}{l}225 \\
(10.6)\end{array}$ & $\begin{array}{l}260 \\
(12.2)\end{array}$ & $\begin{array}{l}0.86 \\
(0.72,1.03)\end{array}$ \\
\hline $\begin{array}{l}\text { All-cause } \\
\text { death }\end{array}$ & $\begin{array}{l}46 \\
(9.5)\end{array}$ & $\begin{array}{l}49 \\
(9.8)\end{array}$ & $\begin{array}{l}0.96 \\
(0.64,1.44)\end{array}$ & $\begin{array}{l}131 \\
(6.2)\end{array}$ & $\begin{array}{l}137 \\
(6.4)\end{array}$ & $\begin{array}{l}0.96 \\
(0.75,1.22)\end{array}$ \\
\hline
\end{tabular}

1. Excluding silent MI.

2. All-cause death, non-fatal MI (incl. silent MI), acute coronary syndrome, cardiac intervention including CABG or PCI, stroke, major leg amputation, or bypass surgery or revascularisation in the leg.

3. All-cause death, non-fatal MI (excl. silent MI), or stroke

Conclusion: PROactive was a large outcome study that examined the effects of pioglitazone on macrovascular outcomes and included stroke prevention in its composite endpoint. In a pre-specified subgroup analysis from this largescale, prospective study, pioglitazone appeared to significantly reduce the risk of recurrent stroke in high-risk patients with type 2 diabetes and previous stroke. The addition of pioglitazone to other standard therapies used in stroke prevention appears to improve outcomes. This may be due to pioglitazone's other non-hypoglycemic effects such as its action on diabetic dyslipidemia and pleiotropic mediators. 


\section{OP 21}

\section{Inflammatory mediators in type 2 diabetes}

0121

\section{Sub-clinical inflammation in T2DM: the role of Toll like receptors in the activation of $\mathrm{NF} \kappa \mathrm{B}$ and cJun Kinase in the innate immune pathway in human abdominal isolated adipocytes and explants}

S. J. Creely, F. M. Fisher, A. L. Harte, P. G. McTernan,

S. Kumar;

Diabetes and Metabolism, University of Warwick Medical School, Coventry, United Kingdom.

Background and Aims: Type 2 diabetes (T2DM) may result from a combination of obesity mediated hyperinsulinaemia/insulin resistance (IR) and sub-clinical inflammation. Our hypothesis is that hyperinsulinaemia/IR enhances absorption of endotoxin, which may have an effect on adipose tissue to elicit an inflammatory response. Our studies have shown circulating lipopolysaccharide (LPS), is significantly higher in T2DM than in BMI and aged matched controls $(\mathrm{p}<0.001, \mathrm{n}=25)$, and is reduced by treatment with the insulin senstizer, rosiglitazone, in T2DM patients $(\mathrm{p}<0.05)$. In addition, toll-like receptors (TLR) 2 and 4, which are LPS receptors, are noted to increase with obesity and T2DM in human adipose tissue (AT). Therefore we investigated (1) the role of LPS in mediating the innate immune pathway in human AT (2) the resulting effect on adipocytokines release (3) the effect of blockade of inflammatory signaling molecules nuclear factor kappa B (NFKB, blocking agent SN50) and c-jun kinase (JNK) inhibitor (SP600125) on adipocytokine release (4) and the influence of explants versus isolated adipocytes in their inflammatory response.

Methods and Materials: Abdominal subcutaneous $(\mathrm{AbdSc})$ adipose tissue explants and isolated AbdSc adipocytes were obtained from patients undergoing elective liposuction surgery (age 35.5 \pm 10.2 yrs; BMI $27.2 \pm 6.1 \mathrm{~kg} /$ $\mathrm{m} 2 ; \mathrm{n}=18)$. AbdSc adipocytes and explants were treated with and without LPS $(10 \mathrm{ng} / \mathrm{mL})$ and with or without $\mathrm{NF} \kappa \mathrm{B}$ or JNK blocker $(\mathrm{n}=8)$. Following treatment conditioned media was analysed for adiponectin, IL-6 and TNF$\alpha$. Protein expression of TLR 2 and 4 was also assessed $(\mathrm{n}=10)$.

Results: TLR-2 protein expression increased two-fold $(p<0.05)$ compared with control in response to LPS treatment. TLR-4 protein expression remained unchanged. LPS treated AbdSc isolated adipocytes and explants both showed a significant increase in TNF- $\alpha$ and IL-6 response (IL-6, Control: $2700.0 \pm 40 \mathrm{pg} / \mathrm{mL}$ vs LPS: $4800 \pm 30 \mathrm{pg} / \mathrm{mL}$; $\mathrm{p}<0.001$; TNF- $\alpha$, Control: $1.0 \pm 0.83 \mathrm{pg} / \mathrm{mL}$ vs LPS:
$32.8 \pm 6.23 \mathrm{pg} / \mathrm{mL} ; \mathrm{p}<0.0001$ ) and explants (IL-6, Control: $3300 \pm 90 \mathrm{pg} / \mathrm{mL}$ vs LPS: $1030 \pm 130 \mathrm{pg} / \mathrm{mL}$; $\mathrm{p}<0.001$; TNF$\alpha$, Control: $1.4 \pm 0.3 \mathrm{pg} / \mathrm{mL}$ vs $68.7 \pm 23.9 \mathrm{pg} / \mathrm{mL} ; \mathrm{p}<0.05$ ). Adiponectin levels were unchanged in Abd Sc adipocytes but significantly reduced in explants $(p<0.05)$. NFкB blockade reduced the levels of IL-6 in Abd Sc adipocytes (Control: $2.7 \pm 0.5 \mathrm{ng} / \mathrm{mL}$ vs Blocker: $2.1 \pm 0.4 \mathrm{ng} / \mathrm{mL}$; $\mathrm{p}<0.001$ ) whilst there was no significant change in TNF- $\alpha$ or adiponectin levels. In AbdSc explants however there were significant reductions in both TNF- $\alpha$ and adiponectin (TNF$\alpha$, Control: $1.4 \pm 0.3 \mathrm{pg} / \mathrm{mL}$ vs Blocker: $1.2 \pm 0.2 \mathrm{pg} / \mathrm{mL}$; $\mathrm{p}<0.05$ : Adiponectin, Control: $0.47 \pm 0.07 \mu \mathrm{g} / \mathrm{mL}$ vs Blocker: $0.32 \pm 0.05 \mu \mathrm{g} / \mathrm{mL} ; \mathrm{p}<0.01$ ) with a trend towards reduction in IL-6 $(3.3 \pm 0.9 \mathrm{ng} / \mathrm{mL}$ vs $2.4 \pm 0.7 \mathrm{ng} / \mathrm{mL} ; \mathrm{p}=0.069)$. JNK blockade appeared to have more effect on adipocytokines reduction in explants than Abd Sc adipocytes.

Conclusions: In summary our studies indicate that LPS increases TLR 2 in isolated adipocytes, elicites an inflammatory response and reduces adiponectin secretion and that blockade of NFKB and or JNK pathways reduces cytokine release in human AT. These findings may be important for targeting new therapies to reduce inflammation. Thus LPS action in AT may represent a factor in initiating inflammation and therefore insulin resistance, and represent an important mechanism for pathogenesis of type 2 diabetes.

Supported by Dunhill Medical Trust, Welcome Trust

0122

An LPS/CD14 mechanism is responsible for the onset of high-fat diet-induced obesity and insulin resistance

R. G. Burcelin ${ }^{1}$, M. A. Iglesias ${ }^{1}$, M. Poggi ${ }^{2}$, J. Amar ${ }^{3}$, C. Knauf ${ }^{1}$, B. Cousin ${ }^{1}$, L. Casteilla ${ }^{1}$, J. Ferrières ${ }^{3}$, M. C. Alessis ${ }^{4}$;

${ }^{1}$ Functional Genomics, CNRS, Toulouse, ${ }^{2}$ Thombosis and Metabolism, Inserm, Marseille, ${ }^{3}$ Cardiologie, Hopital Rangueil, Toulouse, ${ }^{4}$ Thombosis and Metabolism, Inserm, Toulouse, France.

Background and Aims: A recent ongoing mechanistic hypothesis for high-fat diet (HFD) induced-insulin resistance and obesity is that inflammation would trigger cytokine release impairing energy homeostasis and adipose tissue plasticity. However, no HFD-linked molecular mechanism has been proposed for the triggering of inflammation. Among natural inflammatory reagents, the bacterial endotoxin (LPS) is a potent candidate because it triggers inflammatory cytokines secretion by macrophages upon binding to its membrane receptor, it is ubiquitously distributed in the environment, and it is transported in the blood by triglyceride-enriched lipoproteins. 
Materials, Methods and Results: To demonstrate this hypothesis we show that a fat enriched-diet increases fasted endotoxemia in healthy by $100 \%$ humans and by $40 \%$ in mice. To determine how elevated endotoxemia contributes to the development of obesity and diabetes, we challenged CD14 knockout mice with a HFD. Such mice were protected against LPS-induced cytokine secretion (IL6, IL1b, PAI-1). After 24 weeks of HFD, the degree of HFD-induced obesity and liver steatosis was reduced by $40 \%$. To avoid the confounding effect of excessive body weight on insulin resistance we studied insulin sensitivity by an euglycaemic clamp after only 5 weeks of HFD, when diabetes is present but obesity undetectable. Under these conditions, insulin resistance was blunted in CD14 mutant mice. Furthermore, fasted hyperglycemia and glucose intolerance were totally prevented. These observations are likely due to tissue rather than circulating cell types, as mice transplanted with CD14-depleted hematopoietic cells retained LPS-induced insulin resistance.

Conclusion: Our data demonstrate that LPS is a potent inflammatory factor that during high-fat feeding triggers increased cytokine secretion, obesity and insulin resistance. Strategies aimed at lowering LPS in food and/or plasma may delay the development of metabolic diseases and obesity in humans.

\section{3}

Monocyte chemoattractant protein-1 in obesity and type 2 diabetes. Relationship with insulin-resistance and proinflammatory cytokines

M. R. Chacón ${ }^{1}$, J. Fernández-Real ${ }^{2}$, C. Richart ${ }^{1}$, A. Megia ${ }^{1}$, J. Gómez ${ }^{3}$, M. Miranda ${ }^{1}$, E. Caubet ${ }^{4}$,

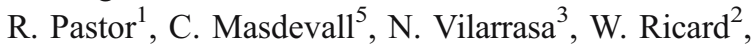
J. Vendrell ${ }^{1}$;

${ }^{1}$ Research Unit, Hospital Universitari Joan XXIII de Tarragona, ${ }^{2}$ Endocrinology and Diabetes Unit, University Hospital of Girona. "Dr. Josep Trueta", ${ }^{3}$ Endocrinology and Diabetes Unit, Hospital of Bellvitge, Barcelona, ${ }^{4}$ Surgery Service, Hospital "St Pau i StaTecla", Tarragona, ${ }^{5}$ Surgery Service, Hospital of Bellvitge, Barcelona, Spain.

Background and Aims: Monocyte chemoattractant protein (MCP-1) mRNA is elevated in the adipose tissue of genetically obese mice remaining responsive to exogenous insulin both in mice and in insulin-resistant 3T3L-1 adipocytes. It has been shown that MCP-1 may be released from human preadipocytes as well as from isolated mature adipocytes, with an increased secretion and expression in human obesity. In humans, the correlation between circulating MCP-1 (cMCP-1) and its local adipose tissue secretion has shown discordant results.
We studied the relationship between cMCP-1 plasma levels and insulin-resistance in a large group of healthy Caucasian male to whom $\mathrm{S}_{\mathrm{i}}$ test was performed. Additionally, we aimed to investigate MCP-1 adipose tissue gene expression in relation with cMCP-1 plasma protein levels in a representative group of non-diabetic and Type 2 diabetic subjects with different degrees of obesity

Materials and Methods: cMCP- 1 were measured in $n=116$ consecutive healthy male subjects to whom insulin sensitivity $\left(S_{\mathrm{i}}\right)$ test was performed. Circulating levels of sCD14, sTNFR2, sIL-6 and adiponectin were also measured in this population. Subcutaneous adipose tissue samples were obtained from $\mathrm{n}=107$ non-diabetic and Type 2 diabetic subjects with different degrees of obesity. Real Time PCR was used to measure gene expression of $M C P-1, C D 68$, $T N F-\alpha$ and its receptor TNFR2. cMCP-1 plasma levels were also measured.

Results: In the $S_{\mathrm{i}}$ study no independent effect of cMCP-1 levels on insulin sensitivity was observed. In the expression study, in non-diabetic subjects, MCP-1 mRNA had a positive correlation with BMI $(\mathrm{r}=0.407, p=0.003)$, TNF- $\alpha$ mRNA $(\mathrm{r}=0.419, p=0.002)$ and TNFR2 mRNA $(\mathrm{r}=0.410$, $p=0.003$ ). In these subjects cMCP-1 were found to correlate with WHR $(\mathrm{r}=0.322, p=0.048)$. In patients with Type 2 diabetes, MCP-1 mRNA was up regulated compared to non-diabetic subjects. TNF- $\alpha$ mRNA was found to independently contribute to MCP-1 mRNA expression. In this group, CD68 mRNA was found to correlate with BMI $(\mathrm{r}=0.455, p=0.001)$.

Conclusions: cMCP-1 is not associated with insulin sensitivity in apparently healthy men. TNF- $\alpha$ is the inflammatory cytokine associated to MCP-1 expression in subcutaneous adipose tissue of non-diabetic and Type 2 diabetic subjects, suggesting a local inflammatory role of MCP-1 in this tissue with little or very weak contribution into circulating levels.

Supported by by grants from: FIS (04/0377) of the Instituto de Salud Carlos III, Red de Centros RCMN (C03/08) and Red de Grupos (G03/212 and G03/028), Madrid, Spain.

\section{4}

The in vitro effects of $\mathrm{JNK}$ of $\mathrm{NF}-\kappa \mathrm{B}$ inhibitor on resistin secretion from isolated human abdominal subcutaneous adipocytes

C. M. Kusminski, S. J. Creely, N. F. da Silva, A. L. Harte, S. Kumar, P. G. McTernan;

Unit of Diabetes and Metabolism, University of Warwick, Coventry, United Kingdom.

Background and Aims: Obesity-associated low-grade inflammation is one of the foremost contributory factor in 
the pathogenesis of type 2 diabetes mellitus (T2DM). Resistin has recently been implicated as a pro-inflammatory cytokine in obesity-related type 2 diabetes. The aims of this study were to characterise a role for resistin in sub-clinical inflammation, specifically the innate immune signaling pathway in human abdominal subcutaneous (AbdSc) adipose tissue (AT). Therefore, for this study we investigated (1) whether resistin could act as a positive mediator of cytokine production in isolated abdominal subcutaneous (Abd Sc) adipocytes; (2) the acute in-vitro effects of human recombinant $(\mathrm{h}-\mathrm{r})$ resistin on the expression of components of the innate immune pathway, specifically the nuclear factor-kappa B (NF-KB) and c-Jun N-terminal kinase (JNK) signaling pathways; (3) determine whether a regulatory feedback mechanism exists to reduce resistin secretion through use of JNK and NF- $\mathrm{KB}$ inhibitors, in isolated adipocytes and matched Abd Sc explants.

Materials and Methods: Isolated human Abd Sc adipocytes were treated with h-r resistin $(10-50 \mathrm{ng} / \mathrm{mL}), \mathrm{NF}-\kappa \mathrm{B}$ inhibitor $(50 \mu \mathrm{g} / \mathrm{mL})$ or $J \mathrm{NK}$ inhibitor $(10 \mu \mathrm{M} / \mathrm{mL})$. The response of components of the innate immune pathway to $\mathrm{h}-\mathrm{r}$ resistin was determined by Western blot. Additionally, the level of resistin secretion from isolated adipocytes and IL- 6 and TNF- $\alpha$ was examined by ELISA.

Results: Both TNF- $\alpha$ and IL- 6 secretion were increased in response to $\mathrm{h}-\mathrm{r}$ resistin (TNF- $\alpha, \mathrm{p}<0.001$; IL-6, $\mathrm{p}<0.001$ ). Our findings also demonstrated that $\mathrm{h}-\mathrm{r}$ resistin upregulated components of the NF-кB pathway. Protein expression of MyD88 (activated upon Toll-like receptor stimulation; $\mathrm{n}=4$, $\mathrm{p}<0.01)$ and NF- $\mathrm{kB}(\mathrm{n}=4, \mathrm{p}<0.05)$ were increased in response to $\mathrm{h}-\mathrm{r}$ resistin. Furthermore, when examining the IKK complex, whilst there was no effect on IKK- $\gamma$, IKK- $\beta$ was significantly upregulated by $\mathrm{h}-\mathrm{r}$ resistin treatment $(n=6, p<0.01)$. Similarly, $h-r$ resistin significantly upregulated mediators of the insulin signaling pathway, in particular, phosphospecific JNK-1 and JNK-2 protein expression was increased in response to $\mathrm{h}-\mathrm{r}$ resistin treatment (JNK-1: $\mathrm{n}=6, \mathrm{p}<0.05 ;$ JNK-2: $\mathrm{n}=6, \mathrm{p}<0.001)$. Finally, AbdSc adipocytes treated with either NF- $\mathrm{BB}$ or $J \mathrm{NK}$ inhibitor significantly reduced the level of resistin

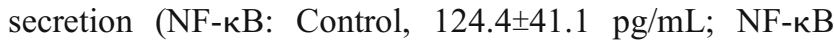
inhibitor, $85.5 \pm 24.8 \mathrm{pg} / \mathrm{mL} ; \mathrm{n}=8, \mathrm{p}<0.01$; JNK: Control, $101.5 \pm 19.2 \mathrm{pg} / \mathrm{mL}$; JNK inhibitor, $77.0 \pm 11.4 \mathrm{pg} / \mathrm{mL} ; \mathrm{n}=6$, $\mathrm{p}<0.01)$. However, whilst AT explants treated with NF-кB blocker reduced resistin secretion significantly (Control, $1222.9 \pm 195.4 \mathrm{pg} / \mathrm{mL}$; NF- $\mathrm{kB}$ inhibitor, $913.0 \pm 147.4 \mathrm{pg} /$ $\mathrm{mL} ; \mathrm{n}=8, \mathrm{p}<0.05$ ), no significant effect on resistin secretion from adipocytes was observed when inhibiting JNK $(n=6)$. Conclusions: These findings suggest resistin could participate in more than one mechanism for the upregulation of pro-inflammatory cytokine release from isolated adipocytes, potentially via the integration of NF- $\mathrm{KB}$ and JNK signaling pathways. Furthermore, this study emphasises potential crosstalk between insulin signaling and inflammatory pathways. Collectively, this study emphasises potential crosstalk between insulin signaling and inflammatory pathways with these metabolic functions being dysregulated in obesity and type 2 diabetes.

Supported by Diabetes UK

\section{5}

Tace $^{+/-}$mice are protected from obesity and type 2 diabetes

M. Federici, M. Serino, R. Menghini, M. Hribal, D. Lauro, P. Sbraccia, R. Lauro;

Dept. of Internal Medicine, University of Tor Vergata, Rome, Italy.

Background and Aims: Soluble TNF-alpha is believed to contribute to insulin resistance in prediabetes and type 2 diabetes. We reported that an increased activity of TNFalpha Converting Enzyme (TACE) increases soluble TNFalpha levels in Insulin Receptor null mice shifting the phenotype from normal glucose homeostasis to hyperinsulinemia/hyperglycemia. Moreover, increased Tace activity has been recently linked to development of atherosclerosis and chronic kidney disease in murine models.

Methods and Materials: To gain insights the role of TACE in insulin resistance related diseases we fed Tace $+/-$ and WT littermates with a High-Fat Diet (HFD) for 16 weeks, studying glucose and lipid metabolism at the beginning and at the end of the study for each genotype.

Results: Growth curves showed that Tace $+/-$ mice gained less weight compared with WT littermates $(\mathrm{p}<0.01$, ANOVA-2). Interestingly, WT mice tend to develop hyperinsulinemia and have higher glucose levels compared to Tace $+/-$ mice $(\mathrm{p}<0.01$, ANOVA-1). IPGTT and ITT confirmed the protective effect of Tace haploinsufficiency against diet-induced insulin resistance. Wild-type mice showed abnormal glucose and insulin tolerance compared with Tace $^{+/-}$mice $(\mathrm{p}<0.01$, ANOVA-2). HFD WT mice showed increased fat pads compared with HFD Tace ${ }^{+/-}$ mice ( $<<0.01$, ANOVA-1). In HFD WT mice we observed as expected that the increase in WAT weight was associated with increased adipocyte cell size and decreased cell density. By contrast, in Tace ${ }^{+-}$mice HFD did not cause a significant change in both cell density and size compared with mice fed a normal chow. Next, we inquired whether the protective phenotype determined by Tace inhibition under a HFD is explained by reduction of food intake or improvement in peripheral fat oxidation or both. Food intake was slightly but not significantly reduced in HFD $\mathrm{Tace}^{+/-}$mice compared with WT littermates. NEFA did not 
increase and Adiponectin did not decrease in HFD Tace ${ }^{+/-}$ compared to HFD WT mice. mRNA analysis revealed that Tace+/- compared with WT mice express significantly higher levels of Adiponectin and PPARgamma and lower levels of ccl-2/MCP-1 in WAT and increased UCP-1 expression in the skeletal muscle. Consistently, in $\mathrm{db} / \mathrm{db}$ mice treatment with a Tace inhibitor, TAPI-1, with daily administration for 1 week led to improvement of fed glucose and insulin levels, total cholesterol and triglycerides levels ( $p<0.05$ for each value compared to basal value, $\mathrm{t}$-Test).

Conclusions: TACE inhibition in animal models, through genetic or pharmacological approach, significantly reduced the detrimental effect of overnutrition and obesity, regulating adipose tissue remodeling and gene expression in both WAT and muscle. Altogether these data favor the hypothesis that TACE inhibition may play a role in protecting from obesity, insulin resistance and dyslipidemia.

\section{6}

Role of oxidative stress and inflammation in lipid-induced B-cell dysfunction

A. I. Oprescu ${ }^{1}$, G. F. Lewis ${ }^{2}$, I. G. Fantus ${ }^{2}$, M. B. Wheeler ${ }^{2}$, A. Giacca ${ }^{2}$;

${ }^{1}$ Institute of Medical Science, ${ }^{2}$ Physiology, University of Toronto, Canada.

Background and Aims: An important mechanism in pathogenesis of type 2 diabetes is elevation of plasma free fatty acids (FFA) which induce insulin resistance and may impair $\beta$-cell function and mass. In our previous studies the antioxidant taurine (TAU) prevented the impairment in $\beta$-cell function induced by prolonged exposure to FFA. TAU is a sulfur containing amino acid which may have other effects in addition to its antioxidant action. In support to taurine's action as an antioxidant, another antioxidant, N-Acetylcysteine (NAC), proved to be equally effective to TAU in preventing the FFA-induced impairment in glucose stimulated insulin secretion (GSIS); however, since NAC can be converted to TAU, it cannot be excluded that also the effect of NAC is independent of its antioxidant properties. Our current objective was to investigate the role of oxidative stress in the FFA-induced impairment in $\beta$-cell function using an antioxidant unrelated to TAU or NAC, TEMPOL (TPO, SOD mimetic), and to address potential downstream effects of oxidative stress involving inflammation.

Materials and Methods: Oleate $\left(1.3 \mu \mathrm{mol} \cdot \mathrm{min}^{-1}\right)$ was infused iv for $48 \mathrm{~h}$ with or without the antioxidant TPO $\left(2.41 \mu \mathrm{mol} \cdot \mathrm{kg}^{-1} \mathrm{~min}^{-1}\right)$ to elevate plasma FFA levels 2 fold in Wistar rats. After the $48 \mathrm{~h}$ infusion islets were isolated to evaluate GSIS ex vivo and to measure reactive oxygen species (ROS) by the dichlorofluorescein method. The same studies of $48 \mathrm{~h}$ oleate infusion or $48 \mathrm{~h}$ olive oilt+ heparin infusion were also performed with or without the anti-inflammatory salicylate.

Results: GSIS in isolated islets was impaired by oleate (OLE: $1.009 \pm 0.102 \mathrm{pmol} /$ islet $/ \mathrm{h}, \mathrm{n}=13, \mathrm{p}<0.01$ vs control: $1.618 \pm 0.224 \mathrm{pmol} / \mathrm{islet} / \mathrm{h}, \mathrm{n}=12$, at $22 \mathrm{mM}$ ) but was restored by TPO (OLE+TPO: $1.421 \pm 0.200 \mathrm{pmol} / \mathrm{islet} / \mathrm{h}$ at $22 \mathrm{mM}$, $\mathrm{n}=7, \mathrm{p}=\mathrm{NS}$ vs control) while TPO alone had no effect. Furthermore TPO abolished ( $p=\mathrm{NS}$ OLE + TPO vs control) the increase in ROS induced by OLE in islets $(\mathrm{p}<0.01)$. Our laboratory has shown that the increase in ROS induced by lipids activates the IKK $\beta / N F k B$ inflammatory pathway in liver, inducing hepatic insulin resistance, which led us to our next objective, to determine whether IKK $\beta$ is also involved in lipid-induced decrease in GSIS. Oleate or an emulsion of $20 \%$ olive oil+heparin $\left(40 \mathrm{U} \cdot \mathrm{ml}^{-1}\right)$ was infused as mentioned above, for $48 \mathrm{~h}$ in Wistar rats \pm the IKK $\beta$ inhibitor salicylate $\left(0.117 \mathrm{mg} \cdot \mathrm{kg}^{-1} \mathrm{~min}^{-1}\right.$, the dose that inhibited IKK $\beta$ and prevented lipid-induced insulin resistance). After $48 \mathrm{~h}$ infusion plasma FFA were elevated 2 fold with either oleate or olive oil. GSIS in isolated islets was impaired by olive oil or oleate (olive oil: $0.996 \pm 0.132 \mathrm{pmol} / \mathrm{islet} / \mathrm{h}$ at $22 \mathrm{mM}, \mathrm{n}=12$; oleate: $1.033 \pm 0.097 \mathrm{pmol} / \mathrm{islet} / \mathrm{h}$ at $22 \mathrm{mM}, \mathrm{n}=14$, both $\mathrm{p}<0.01$ vs. control: $1.542 \pm 0.159 \mathrm{pmol} /$ islet/h at $22 \mathrm{mM}$, $\mathrm{n}=12$ ) but was restored by salicylate (olive oil+ salicylate: $1.754 \pm 0.367 \mathrm{pmol} / \mathrm{islet} / \mathrm{h}$ at $22 \mathrm{mM}, \mathrm{n}=6$; oleate + salicylate: $1.737 \pm 0.311 \mathrm{pmol} / \mathrm{islet} / \mathrm{h}$ at $22 \mathrm{mM}, \mathrm{n}=8$, both $\mathrm{p}=\mathrm{NS}$ vs control) while salicylate alone had no effect (salicylate: $1.524 \pm 0.218 \mathrm{pmol} /$ islet/ $\mathrm{h}$ at $22 \mathrm{mM}, \mathrm{n}=10, \mathrm{p}=\mathrm{NS}$ vs. control). Conclusion: Our results suggest a potential role for both oxidative stress and activation of inflammatory pathways involving IKK $\beta$ in lipid-induced $\beta$-cell dysfunction. Supported by CIHR, BBDC-Novo Nordisk, IMS

\section{OP 22}

\section{Mechanisms of prevention of endothelial damage}

\section{7}

Activation of 5'AMP-activated protein kinase induces NO-dependent vasodilation and uncovers insulinmediated vasodilatation in muscle resistance arteries

E. C. Eringa ${ }^{1}$, C. D. A. Stehouwer ${ }^{2}$, I. Korstjens ${ }^{1}$, G. P. van Nieuw Amerongen ${ }^{1}$, R. J. Musters ${ }^{1}$, P. Sipkema ${ }^{1}$; ${ }^{1}$ Laboratory for Physiology, VU University Medical Centre, Amsterdam, ${ }^{2}$ Internal Medicine, Academic Hospital Maastricht, The Netherlands. 
Background and Aims: Exercise enhances blood flow and insulin-mediated glucose uptake in muscle, but the underlying mechanisms are unknown. 5'-AMP-activated protein kinase (AMPK), which regulates whole-body insulin sensitivity, has been proposed to mediate these effects. Interaction of AMPK with insulin-mediated vasoreactivity in muscle may increase blood flow as well as insulinmediated glucose uptake in muscle. Aim: To study the effects of AMPK activation on vascular tone, insulinmediated vasoreactivity and insulin-mediated cell signaling in skeletal muscle resistance arteries.

Methods/Results: To study effects of AMPK activation, resistance arteries from the rat cremaster muscle and human microvascular endothelial cells (MVEC) were stimulated in vitro with the AMPK agonist 5-amino-4-imidazolecarboxamide riboside (AICAR; $2 \mathrm{mM}$ ). In MVEC and muscle resistance arteries AICAR enhanced Thr 172 phosphorylation of AMPK, Ser 72 phosphorylation of Acetyl CoA Carboxylase (ACC) and Ser 1177 phosphorylation of eNOS. 3D fluorescence microscopy showed localization of phosphorylated AMPK in the plasma membrane and in the nucleus (figure 1). In muscle resistance arteries, AICAR induced a marked vasodilatation of resistance arteries $(18+5$ percent diameter change, $\mathrm{P}=0.01$ ) that was abolished by inhibition of AMPK activity with Compound $\mathrm{C}$ (to $3+3$ percent) or inhibition of NO synthesis with N-G-nitro-Larginine (L-NA; to $-9+3$ percent). To study effects of AMPK activation on insulin-mediated vasoreactivity of skeletal muscle resistance arteries, vasoreactivity to insulin (4-3400 microU/ml) was studied in the absence and presence of AICAR. In the absence of AICAR, insulin did not induce vasodilatation $(0+3$ percent diameter change at $272 \mathrm{microU} / \mathrm{ml}$ ). In contrast, insulin induced vasodilatation in the presence of AICAR $(20+5$ percent at 272 microU $/ \mathrm{ml}, \mathrm{P}<0.05$ vs. insulin). This vasodilatation was abolished by pretreatment with L-NA (to $3+1$ percent). In contrast, insulin induced dose-dependent vasoconstriction in the absence of AICAR $(-26+2$ percent at 272 microU/ $\mathrm{ml}$ ), showing that AICAR inhibits vasoconstrictor effects of insulin. Western blot analysis showed that this inhibition was accompanied by an inhibition of insulin-mediated activation extracellular-regulated kinase 1/2 (ERK1/2).

Conclusion: Activation of AMPK induces NO-mediated vasodilatation of skeletal muscle resistance arteries and uncovers insulin-induced, NO-dependent vasodilatation. This interaction of AMPK with insulin-mediated vasoreactivity is achieved by inhibition of insulin's ERK1/2dependent vasoconstrictor effects and potentiation of insulin's ERK1/2-independent vasodilator effects. These data provide a new mechanism by which exercise improves endothelial function in muscle.

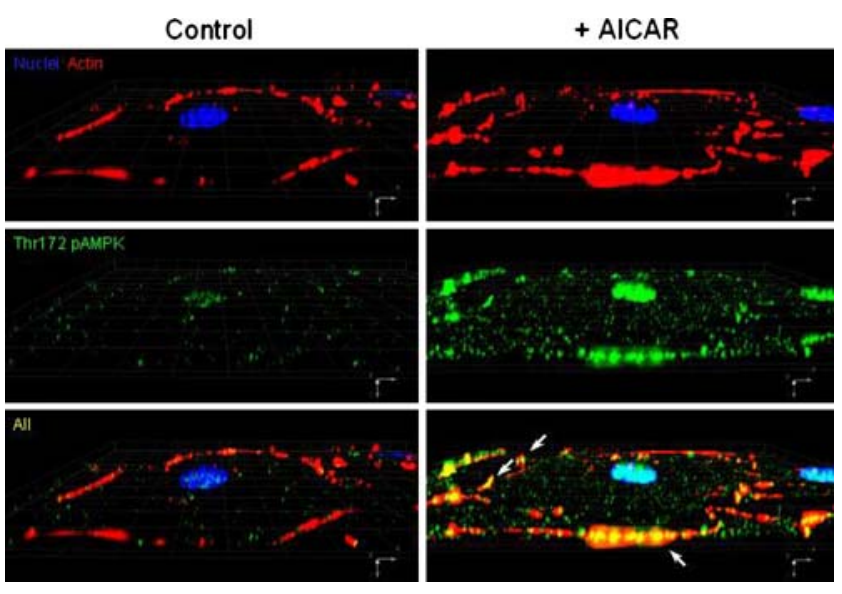

Fig 1. Intracellular localization of activated AMPK in microvascular endothelial cells

Supported by Dutch Diabetes Foundation

0128

Peroxisome-proliferator activated receptor- $\delta$ (PPAR $\delta$ ) mediates high glucose-induced downregulation of glucose transport in vascular endothelial cells

S. Sasson, E. Alpert, Y. Sin-Malia;

Dept. of Pharmacology, Hebrew University School of Medicine, Jerusalem, Israel.

Background and Aims: Increased influx of glucose to vascular endothelial cells (VEC) is closely associated with the development of cellular dysfunction and contributes to the development of macrovascular disease in diabetic patients. Yet, VEC protect their intracellular environment against such effects by reducing the rate of glucose transport when exposed to high glucose levels. It has been shown that eicosanoids, and in particular, 12-hydroxyeicosatetraenoic acid (12-HETE, the product of 12-lipoxygenase), whose production is increased in VEC exposed to high glucose, participate in this protective mechanism. Several eicosanoids are known ligands to PPAR isoforms. The present study was performed to determine whether members of the PPAR family were involved in the downregulation of glucose transporter-1 (GLUT1) expression and its plasma membrane abundance in VEC maintained at high glucose conditions.

Materials and Methods: Primary bovine aortic endothelial cell cultures were incubated at 5.5 or $25.0 \mathrm{mmol} / 1$ glucose up to $48 \mathrm{~h}$, in the absence or presence of PPAR $\alpha,-\gamma$ and $-\delta$ agonists. The capacity of these agonists to mimic the effect of high glucose levels and reduce the rate of hexose uptake, GLUT-1 expression and plasma membrane localization in 
VEC was then determined using the $\left[{ }^{3} \mathrm{H}\right]-2$-deoxy-Dglucose uptake assay, cell-surface biotinylation and Western blot analysis.

Results: VEC, exposed to the high glucose level, reduced the rate of hexose uptake, GLUT-1 content and plasma membrane abundance in VEC by $40-60 \%$, in comparison with cultures that were maintained at $5.5 \mathrm{mmol} / 1$ glucose. The PPAR $\alpha$ agonist WY-14,643 and the PPAR $\gamma$ agonist troglitazone did not reduce the rate of hexose transport into the cells. In fact, both agonists further increased the rate of transport, indicating that they possessed the opposite effect of high glucose. However, the selective PPAR $\delta$ agonist GW501516 fully mimicked the effect of high glucose and reduced the rate of hexose transport in a dose- and a timedependent manner in VEC exposed to $5.5 \mathrm{mmol} / 1$ glucose. Moreover, this effect resulted from a similar decrease of total cell content of GLUT-1 and its plasma membrane localization. We then asked whether 12-HETE might be a specific ligand for PPAR $\delta$, which mediated the downregulatory response. We exposed VEC, maintained at $25.0 \mathrm{mmol} / \mathrm{l}$ glucose, to baicalein (a selective inhibitor of 12-lipoxygenae) in the absence or presence of GW501516. In the former case baicalein prevented high glucosedependent downregulation of hexose transport. However, in the latter case GW501516 reversed this action of baicalein and the rate of hexose transport remained low. Thus, the synthetic PPAR $\delta$ agonist GW501516 substituted for 12-HETE, whose prudction was blocked.

Conclusion: This study shows that hyperglycemia-induced downregulation of hexose transport and GLUT-1 expression in VEC requires the formation of an active 12-HETEPPAR $\delta$ complex. This complex may dimerize with its cognate RXR (retinoid X receptor) to regulate the expression of specific genes involved in this regulatory mechanism. Our model is particularly attractive in view of recent studies that show a PPAR $\delta$-dependent increase of peripheral glucose utilization in various animal models. These reports and our present findings indicate that PPAR $\delta$ might be a potential target for drug development, aimed at reducing hyperglycemia-induced VEC dysfunction and macrovascular disease in diabetes.

Supported by the Israel Ministry of Health

\section{9}

Visfatin expresses in the vascular endothelial cells and inhibits apoptosis induced by high glucose

W.-B. Xu, L. Yu, X.-L. Fu, M. Luo;

Study Group of Diabetic Complications, Shanghai Institute of Endocrine and Metabolic Diseases, China.
Background and Aims: Diabetes mellitus causes various cardiovascular complications. Hyperglycemia induces apoptosis in endothelial cells, but the precise molecular mechanisms are not yet well understood. Visfatin, also known as pre-B cell colony-enhancing factor (PBEF), was identified as a novel adipocytokine exerting insulin-mimetic effects in various insulin-sensitive tissues. Previous work has shown that PBEF is expressed in many tissues and play an antiapoptotic role in neutrophil stimulated by inflammatory stimuli. This study was to investigate the effect of high glucose on expression of visfatin in vascular endothelial cells and the role of visfatin on apoptosis induced by high glucose.

Materials and Methods: Human umbilical vein endothelial cells (HUVECs), were incubated in high glucose $(25 \mathrm{mmol} / \mathrm{L})$ for different time intervals. Visfatin mRNA and protein expression were measured by quantitative realtime reverse transcription-polymerase chain reaction and western blotting, respectively. To view the effect of visfatin on cell apoptosis induced by high glucose, HUVECs were incubated in high glucose $(25 \mathrm{mmol} / \mathrm{L})$ in the presence or absence of different doses of human recombinant visfatin with or without a special antibody to visfatin for the indicated time. For detection of apoptosis Annexin V/ propidium iodide (PI) staining was used, and the enzymic activity of caspase-3, which is the final effector caspase in cytosol, was determined by colorimetric protease assay as well.

Results: Visfatin is expressed in endothelial cells. Compared with normoglycemia, exposure to hyperglycemia, but not mannitol, changed its expression at both mRNA and protein levels which exhibited a temporal increase reaching maximal levels about 4(for mRNA) or 8(for protein) hours after high glucose incubation followed by durative decrease $(P<0.05, n=6)$. Recombinant visfatin $(20-100 \mathrm{ng} / \mathrm{ml})$ dosedependently inhibited apoptosis in endothelial cells induced by high glucose $(p<0.05, n=6)$, which was specific as antivisfatin antibody(1:500) completely neutralized this effect $(P<0.01, n=3)$. Visfatin pretreatment also resulted in reduced activation of caspase- 3 reflected in reduced levels of in vitro enzymatic activity $(P<0.05, n=4)$.

Conclusion: Hyperglycemia could regulate visfatin expression in endothelial cells and extraneous visfatin significantly inhibited hyperglycemia-induced apoptosis in endothelial cells through reducing caspase-3 activation. These results suggest that visfatin may be a potential therapeutic target for preventing cardiovascular complications of diabetes. Supported by the Major State Basic Research Development Program of the People's Republic of China and the National Nature Science foundation of China 


\section{0}

Inhibition of glycogen synthase Kinase 3 beta (GSK3B) prevents palmitate-induced human umbilical vein endothelial cells (HUVEC) apoptosis

H.-J. Jang ${ }^{1}$, S.-E. Choi ${ }^{1}$, S.-H. Jung ${ }^{2}$, S.-M. Ahn ${ }^{2}$, Y.-K. Kim ${ }^{2}$, Y.-E. Jo ${ }^{2}$, Y.-J. Choi ${ }^{2}$, H.-J. Kim², D.-J. Kim², Y. Kang ${ }^{1}$, K.-W. Lee ${ }^{2}$;

${ }^{1}$ Institutes for Medical Sciences, ${ }^{2}$ Endocrinology and Metabolism, Ajou University School of Medicine, Suwon, Republic of Korea.

Background and Aims: Dyslipidemia is a risk factor for diabetes-accelerated atherosclerosis. Free fatty acid such as palmitate have been reported to induce failure of endothelial cells function and to increase endothelial cells apoptosis. GSK $3 \beta$ as a serin/threonine kinase, plays a central role in neuronal cells death. However, the role of GSK $3 \beta$ on the death of endothelial cells is unclear. In this study, we investigated whether GSK3 $\beta$ plays any role in palmitateinduced HUVEC apoptosis

Materials and Methods: Palmitate induced HUVEC death was measured by DNA ladder, DAPI and MTT assay. To investigated the mechanism of HUVEC death by palmitate, we used several inhibitors and adenovirus of kinase death and constitutively active form of GSK (GSK-S9A: 9th serine switched to alanine).

Results: Incubation of HUVEC with palmitate significantly increased dephosphorylation of GSK3 $\beta$ at 9th serine and also increased apoptotic features such as activation of caspases 3 and 9 , release of cytochrome $\mathrm{c}$ and poly-ADP ribose polymerase (PARP) cleavage. Blocking GSK3 $\beta$ activity with selective inhibitor (TDZD-8, lithium, SB216763) significantly prevented activation of palmitate-induced caspase 3 and 9 , PARP cleavage and HUVEC apoptosis. To further assess the role of GSK $3 \beta$ in palmitate-induced apoptosis, HUVEC were infected with adenovirus of kinase-inactive GSK $3 \beta$ and these cells resisted palmitate-induced apoptosis.

Conclusion: These results therefore suggest that the prevention of palmitate induced endothelial cells apoptosis through GSK3 $\beta$ and GSK3 $\beta$ represents a novel target for therapeutic agents.

Supported by the "GRRC" Project of Gyeonggi Provincial Government, Republic of Korea

0131

Rosiglitazone reverses the alterations of capillary permeability but does not improve nerve function in Zucker Diabetic Fatty rats

A. Behar, F. Cohen-Boulakia, K. Tarhzaoui, G. Léger,

R. Lestrade, P. Valensi;
Laboratory of Nutrition, Metabolic Diseases and Cardiovascular Prevention, Paris-Nord University, Bobigny, France.

Background and Aim: An increase in capillary permeability is a common disorder in diabetes and may play a role in the pathophysiology of peripheral neuropathy. Several data suggest that glitazones exert beneficial effects on endothelial function but may induce edema. The aim of the present study was to test the effect of rosiglitazone on the capillary filtration of albumin (CFA) and peripheral nerve function in ZDF rats.

Materials and Methods: Fifteen ZDF rats were treated by rosiglitazone from 2 to 6 months of age, and were compared to 15 untreated $\mathrm{ZDF}$ rats and 15 lean (fa/?) controls. CFA and lymphatic function were evaluated by a noninvasive isotopic test using technetium-labelled albumin at 2 and 6 months. Sensory and motor nerve conduction velocities were measured at 6 months.

Results: At 2 months CFA was significantly higher in ZDF rats than in lean controls $(\mathrm{p}<0.04)$. At 6 months, as compared to lean controls, CFA was markedly higher in untreated ZDF rats $(p<0.001)$ but very similar in rosiglitazone-treated rats. Lymphatic function was similar in ZDF rats and controls at 2 months but significantly impaired in ZDF rats at 6 months, whatever treated or not treated $(\mathrm{p}<0.001)$. Motor and sensory nerve conduction velocities were significantly impaired at 6 months in both treated and untreated ZDF rats as compared with controls.

Conclusion: The present data show that in ZDF rats, 1) rosiglitazone prevents the increase in capillary permeability but not the impairment in lymphatic function, which suggests that alteration of lymphatic function might be involved in induction of edema during rosiglitazone treatment ; 2) rosiglitazone does not prevent the impairment in nerve function.

Supported by GlaxoSmithKline-Ltd

0132

Insulin resistance and postprandial endothelial dysfunction: Does attenuation of postprandial hyperglycaemia make a difference?

A. Major-Pedersen ${ }^{1}$, N. Ihlemann ${ }^{1}$, B. Kveiborg ${ }^{1}$,

B. Christiansen ${ }^{1}$, T. S. Hermann ${ }^{1}$, H. Dominguez ${ }^{1}$,

D. B. Nielsen ${ }^{1}$, C. R. Madsen ${ }^{2}$, O. L. Svendsen ${ }^{3}$,

L. Køber ${ }^{4}$, C. Torp-Pedersen ${ }^{1}$;

${ }^{1}$ Cardiology, Bispebjerg Hospital, Copenhagen, Denmark,

${ }^{2}$ Cardiology, Joslin Diabetes Center, Boston, United States,

${ }^{3}$ Internal Medicine I, Bispebjerg Hospital, Copenhagen, Denmark, ${ }^{4}$ Cardiology, Rigshospitalet, Copenhagen, Denmark. 
Background and Aims: Postprandial hyperglycemia is an independent risk factor for cardiovascular disease, even before the development of overt diabetes. We aimed to determine if attenuation/normalization of postprandial hyperglycemia (with nateglinide administration - an oral antidiabetic of ultra-rapid action that stimulates postprandial first-phase insulin secretion) could diminish endothelial dysfunction, one of the earliest markers of atherosclerosis.

Materials and Methods: We assessed post-oral glucose load endothelial function in 56 insulin-resistant subjects with impaired glucose tolerance (IGT) and with the following baseline characteristics: (mean values \pm SEM) Homeostasis Model Assessment (HOMA) 6.41 \pm 0.7 , body mass index (BMI) $34.4 \pm 5.68 \mathrm{~kg} / \mathrm{m}$, Oral Glucose Tolerance Test (OGTT): $0 \mathrm{~min} 6.2 \pm 1.0 \mathrm{mmol} / 1$ and $120 \mathrm{~min}$. $11.9 \pm 2.7 \mathrm{mmo} / \mathrm{l}, \mathrm{HbA1C} 6.4 \pm 0.82 \%$, waist circumference $115.8 \pm 20.5 \mathrm{~cm}$, age $57.5 \pm 7.3$ years. All subjects were randomized to either intervention or time control group. In addition, subjects in the intervention group received an individually adjusted dose of nateglinide on a second day (Day 2), $10 \mathrm{~min}$. before oral glucose load. Furthermore, we examined post-oral glucose load endothelial function in 9 healthy, insulin-

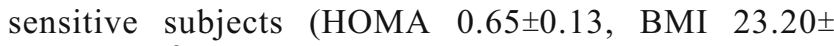
$2.13 \mathrm{~kg} / \mathrm{m}^{2}$; OGTT $0 \mathrm{~min} 4.12 \pm 0.36$ and $120 \mathrm{~min}$. $5.58 \pm 0.95 \mathrm{mmol} / \mathrm{l}$, waist circumference $80.77 \pm 2.93$, age $41.11 \pm 3.02$ ). Flow-mediated dilation (FMD) technique was used to measure endothelial function.

Results: The following postprandial Delta FMD values (fasting FMD value subtracted from postprandial FMD value) were seen in intervention and control groups respectively (mean value \pm SEM) : $(-3.42 \pm 1.67 \%) \mathrm{p}=0.04$ and $(-2.20 \pm 1.19 \%) \mathrm{p}=0.07$. The Delta postprandial FMD value in the healthy group was $4.80 \pm 1.41, \mathrm{p}=0.009$. On Day 2 , when nateglinide was administered $10 \mathrm{~min}$. prior to glucose-load in the intervention group, Delta FMD values increased to $2.32 \pm 1.45 \%, \mathrm{p}=0.02$.

Conclusion: Endothelial function in insulin resistant individuals deteriorates after an oral glucose load (which represents a standard meal). Attenuation of post load hyperglycemia not only nullifies this negative effect, but also enhances endothelial function to the extent that it mimicks post glucose-load endothelial function in healthy. These findings could substantially impact the prevention of cardiovascular disease in insulin resistant individuals with pre-diabetes or early type 2 diabetes.

Supported by the Danish Diabetes Association, Bayer, Novartis

\section{OP 23}

\section{Experimental genetics: regulation of insulin secretion}

\section{3}

Distinct diabetes susceptibility loci impair insulin exocytosis and glucose metabolism in pancreatic beta cells A. Rosengren, C. Granhall, E. Renström, H. Luthman; Dep. Clinical Sciences Malmoe, Lund University, Sweden.

Background and Aims: Genetic factors are important in the development of type 2 diabetes. Coupling of candidate genes to cellular functional defects in type 2 diabetes is facilitated by studies of inbred animal models with inherited susceptibility to the disease. The Niddm1i locus, a major glucose-regulating locus in the diabetic GK rat, has previously been coupled to defect insulin secretion in pancreatic $\beta$-cells. Interestingly, this locus corresponds to a diabetes susceptibility locus on human chromosome 10q24-26. Here, to further fine-map the Niddm1i locus, we have generated a series of subcongenic strains harbouring different parts of the Niddml and investigated their insulin release properties.

Materials and Methods: Transfer of GK alleles onto the genome of F344 rats by repeated backcrossing established the homozygous congenic strain F344.GK-Niddm 1i (NIDDM1I) and the subcongenic strains were established in a similar way by backcrossing NIDDM1I onto F344 rats. In vivo hormone release, islet morphology, pancreatic RNA and protein content measurements, capacitance measurements, mitochondrial potential measurements, and ATP/ ADP assays were applied to the congenic strains.

Results: All congenic strains displayed normal insulin content and $\beta$-cell mass. Glucose-evoked insulin secretion, as studied in batch-incubated islets, was $61 \%$ lower in the NIDDM1I strain (with GK-derived genome in the entire $16 \mathrm{Mb}$ Niddm 1 i-region) compared to control rats $(\mathrm{p}<0.001)$. The insulin secretion in N1IREC1, harbouring $4 \mathrm{Mb}$ GK genome proximal in Niddmli, was suppressed by $54 \%$ $(\mathrm{p}<0.001)$, and N1I12, harbouring $8.7 \mathrm{Mb}$ GK genome distal in Niddm1i (no overlap with N1IREC1), showed a $47 \%$ reduction in insulin secretion upon glucose stimulation $(\mathrm{p}<0.001)$. Both NIDDM1 and N1I12 showed a 50\% reduced insulin release $(\mathrm{p}<0.05)$ in response to depolarization with high potassium, whereas the response of N1IREC1 to high potassium was intact. In excellent agreement with the islet insulin release measurements, single $\beta$-cell exocytosis was reduced by $45 \%$ in NIDDM1 
$(\mathrm{p}<0,05)$ and by $49 \%$ in N1I12. Interestingly, the N1IREC 1 strain showed a $35-50 \%$ enhanced exocytotic capacity compared to NIDDM1I $(\mathrm{p}<0.05)$ and N1I12 $(\mathrm{p}<0.01)$.

Since the increase in ATP/ADP ratio is fundamental to initiation of $\beta$ cell electrical activity and insulin secretion, we also examined the cytosolic adenine nucleotide levels. N1IREC1 islets showed a markedly lower ATP/ADP ratio $(30-40 \%, \mathrm{p}<0.01)$ compared to control, NIDDM1 and N1I12. Furthermore, N1IREC1 demonstrated an increased mitochondrial hyperpolarization compared to the other strains upon glucose addition $(\mathrm{p}<0.05)$.

Conclusion: 1. A $3 \mathrm{Mb}$ locus located proximally in Niddml encodes for defective glucose metabolism in the $\beta$-cells.

2. A $4.5 \mathrm{Mb}$ locus in the distal part of Niddm 1 generates a more profound defect in insulin exocytosis.

3. A few highly interesting candidate genes exist in both loci.

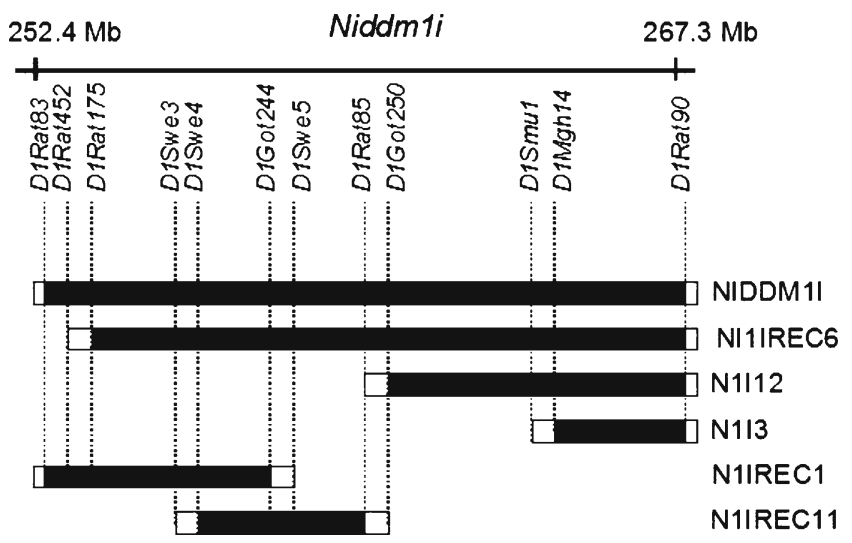

Supported by the European Foundation for the Study of Diabetes

0134

Global analysis of gene expression profiles reveals both shared and distinct roles of $\mathrm{Hnf} 4 \alpha$

S. F. Boj, J.-M. Servitja, N. Pozo, I. Papatheodorou,

J. Ferrer;

Genomic Programming of Beta Cells, IDIBAPS, Barcelona, Spain.

Background and Aims: Mutations in HNF4 $\alpha$ and HNF $1 \alpha$ (also known as TCF1) transcription factor genes cause forms of MODY diabetes that are closely related based on clinical or other phenotypic criteria. This suggests that the two genes may interact functionally in beta cells. It is known that $\mathrm{Hnfl} \alpha$ is a regulator of $\mathrm{Hnf} 4 \alpha$ in pancreatic islets, and human genetic evidence suggests that $\mathrm{Hnf} 4 \alpha$ may regulate Hnfl $\alpha$. However, the precise manner in which these two transcriptional factors interact in beta cell transcriptional regulatory networks is still unknown.

Materials and Methods: Pancreas-specific Hnf4 $\alpha$ deficient mice $(\mathrm{pHnf} 4 \alpha \mathrm{KO})$ were generated by crossing Pdx1-Cre with $\mathrm{Hnf} 4 \alpha$-loxP mice. Greater than $85 \%$ inactivation was verified by real-time PCR of Hnf4 $\alpha$ mRNA in isolated islets. Expression profiles of cultured islets from control, pHNF $4 \alpha \mathrm{KO}$, and Hnfl $\alpha \mathrm{KO}$ mice were analyzed with Affymetrix chips. Statistical analysis of the data was carried out with SAM, and genes exhibiting differential regulation at a $5 \%$ false discovery rate were selected.

Results: The absence of Hnf $4 \alpha$ in mouse pancreas did not result in diabetes or impaired intraperitoneal glucose tolerance. Blood glucose in fed mice was mildly but significantly decreased. Despite this lack of severe phenotypic consequences, expression analysis of $\mathrm{pHnf} 4 \alpha \mathrm{KO}$ islets revealed significantly increased or reduced regulation of $7 \%$ of islet genes. This regulatory role of $\mathrm{Hnf} 4 \alpha$ affected several key functional categories. Interestingly, while many genes were uniquely regulated in either $\mathrm{pHNF} 4 \alpha \mathrm{KO}$ or Hnfl $\alpha$-deficient islets, nearly $30 \%$ of genes exhibiting more than 2 -fold reduction in pHNF $4 \alpha \mathrm{KO}$ were also down regulated $>2$-fold in Hnfl $\alpha$-deficient islets. This overlap varied among different functional categories, with some gene classes exhibiting shared regulation in $100 \%$ of genes. These results could not be explained solely by the fact that $\mathrm{Hnfl} \alpha$ regulates $\mathrm{Hnf} 4 \alpha$, suggesting the existence of complex regulatory interactions between these genes.

Conclusion: These results provide new insights to understand the role of $\mathrm{Hnf} 4 \alpha$ in pancreatic islets. They also demonstrate a shared gene expression signature of $\mathrm{Hnf} 4 \alpha$ and Hnfl $\alpha$ deficient-islets, in keeping with the common phenotype during the diabetic stage of MODY1 and MODY3. This framework will allow us to gain an in depth understanding of a key regulatory interaction between two genes involved in human diabetes.

0135

Mice with conditional beta-cell knockout of glutamate dehydrogenase exhibit severe insulin secretion impairment S. Carobbio ${ }^{1}$, B. Rubi ${ }^{1}$, M. Bloksgaard-Nielsen ${ }^{2}$, A. Gjinovci ${ }^{1}$, P. L. Herrera ${ }^{3}$, W. Reith ${ }^{4}$, S. Mandrup ${ }^{2}$, P. Maechler ${ }^{1}$;

${ }^{1}$ Department of Cell Physiology and Metabolism, University of Geneva, Switzerland, ${ }^{2}$ Department of Biochemistry and Molecular Biology, University of Southern Denmark, Odense, Denmark, ${ }^{3}$ Department of Genetic Medicine and Development, University of Geneva, Switzerland, ${ }^{4}$ Department of Pathology and Immunology, University of Geneva, Switzerland. 
Background and Aims: Glutamate dehydrogenase (GDH) is a mitochondrial enzyme that can reversibly form glutamate from the TCA cycle intermediate alpha-ketoglutarate using $\mathrm{NAD}(\mathrm{P}) \mathrm{H}$ as coenzyme. The importance of GDH for the control of insulin secretion is well recognized, although its specific role is still debated. Accordingly, betacell specific abrogation of GDH in a mouse model would allow unprecedented investigations of this critical pathway. Materials and Methods: We recently generated transgenic mice with tissue specific conditional abrogation of GDH using the lox/P-flp-recombinase strategy. The targeting vector carried Glud-1 exon 7, comprising the NADH binding site essential for GDH activity, flanked by two lox/P sites. The construct was introduced into mouse ES cells by electroporation. Selected recombinant ES clones were screened by PCR and Southern blot to check for the occurrence of homologous recombination before proceeding to mouse blastocyst injection. The founder mice were crossed to obtain F1 mice carrying a mutant Glud-1 genomic locus containing the exon 7 flanked by lox/P sites and the neoR-cassette. Next step was the excision of the neoR-cassette by crossing the F1 with Flp deleter mice. The mice generated this way were crossed with mice expressing the Cre recombinase under the control of an insulin promoter (RIP), resulting in the knockout of GDH exon 7, specifically in beta-cells, thereby leading to the abrogation of GDH activity.

Results: Immunohistochemistry on pancreas sections revealed disorganized islets in homozygous GDH beta-cell specific knockout mice. Specifically, alpha-cells were present in the core of the islet, normally composed essentially of beta-cells. GDH beta-cell knockout mice did not show any difference in body weight compared to wild type mice. Interestingly, homozygous mice appeared to be normoglycaemic despite markedly reduced blood insulin levels at the fasting state ( 7 weeks: $-56 \%$; 12 weeks: $-61 \%$ ). Glucose tolerance during an ipGTT was also normal, again accompanied by marked reduction in plasma insulin levels $15 \mathrm{~min}$ after glucose injection (7 weeks: $-45 \%$; 12 weeks: $-47 \%$ ). In situ pancreatic perfusions confirmed that knockout mice exhibited severe impairment of glucose stimulated insulin release compared to control mice. The secretory responses were reduced both during the first phase $(-51 \%, \mathrm{p}<0.005)$ and the second phase $(-48 \%$, $\mathrm{p}<0.05)$. Ongoing studies investigate the susceptibility of these mice to diet-induced obesity and diabetes. Preliminary results showed that homozygous knockout mice challenged during 15 weeks with a high fat diet did not become obese compared to control mice. They were also able to maintain normoglycaemia as opposed to hyperglycaemic controls.
Conclusion: Based on our recently generated beta-cell specific GDH null mice, present data show the importance of GDH in the control of insulin secretion. The results also indicate unexpected adaptative capacity of the organism to maintain normoglycaemia despite severe beta-cell dysfunction.

Supported by SwissNF \& European Foundation for the Study of Diabetes

\section{6}

GPR40 knock-out mice do not develop obesity induced glucose intolerance

I. Treinies ${ }^{1}$, C. Shrake ${ }^{2}$, A. Koester ${ }^{2}$;

${ }^{1}$ Lilly Forschung GmbH, Hamburg, Germany, ${ }^{2}$ Eli Lilly \& Co, Indianapolis, United States.

Background and Aims: GPR40 is a G-protein coupled receptor that is highly expressed in pancreatic beta cells and is activated by medium and long-chain free fatty acids (FFA). Acute FFA-induced insulin secretion has been shown to be dependent on GPR40, however, it is unclear whether GPR40 is also involved in mediating detrimental effects of chronic fatty acid exposure on beta-cell function. In order to elucidate the role of GPR40 to chronic exposure of elevated levels of FFA, we characterized GPR40 knockout mice fed a high-fat diet and investigated their islet function in vitro.

Materials and Methods: Male GPR40 knock-out mice $(n=6)$ and wild type littermates $(n=6)$ were fed a high-fat diet (Research Diets D12492) for 15 weeks. Bodyweight and food consumption were monitored weekly, fasted insulin and glucose monthly. An oral glucose tolerance test (OGTT) was performed after 15 weeks on the diet. In vitro insulin secretion was measured from batches of 3 islets isolated by collagenase treatment.

Results: After 15 weeks of high-fat feeding male GPR40 knock-out mice showed a significant lower glucose excursion combined with a trend of lower insulin response during OGTT compared to their control littermates. GPR40 knock-out mice had lower fasted insulin $(p=0.04)$ but not glucose levels in comparison to control animals. In vitro characterization of GPR40 knock-out islets isolated from the mice after the feeding experiment showed that glucose induced insulin secretion was comparable to wild type islets but acute fatty acid-enhanced insulin secretion was abolished. In order to investigate if GPR40 is involved in longterm fatty acid induced impairment of beta cell function, glucose stimulated insulin secretion was analysed after incubation of GPR40 knock-out and wild type islets for 
$48 \mathrm{~h}$ with either $400 \mu \mathrm{M}$ palmitic acid or oleic acid. Exposure of wild type islets to either fatty acid resulted as expected in a reduction of glucose-induced insulin secretion. However, in contrast to a previous report GPR40 knock-out islets were not protected from long-term inhibitory effects of fatty acids.

Conclusion: Our in vivo data on GPR40 knock-out mice suggest that loss of GPR40 leads to decreased hyperinsulinemia, which results in increased insulin sensitivity. The data also shows that in vitro GPR40 mediates only acute effects of FFA on insulin secretion but is most likely not involved in FFA induced islet lipotoxicity.

\section{7}

\section{Sterol regulatory element binding protein1 (SREBP1) is required for the induction by glucose of $\beta$-cell genes involved in glucose sensing}

F. Diraison ${ }^{1}$, M. A. Ravier ${ }^{1}$, H. Shimano ${ }^{2}$, G. A. Rutter ${ }^{1}$; ${ }^{1}$ Department of Biochemistry, Henry Wellcome Signaling Laboratories, Bristol, United Kingdom, ${ }^{2}$ Department of Internal Medecine, University of Tsukuba, Tsusuka, Japan.

Background and Aims: Culture of mouse islets at elevated glucose concentrations leads to the enhanced secretion of insulin at both low and high glucose concentrations. In a recent study using islets from control mice or mice deleted for the sterol regulatory element binding protein 1 (SREBP1) gene, we found that the latter transcription factor, a "master" controler of lipogenesis, was required for this response. Here, we have examined the changes in the expression of $\beta$-cell specific genes associated with insulin hypersecretion and the role of SREBP1 in regulating the expression of each one.

Materials and Methods: Islets from 12-16 week old SREBP $1^{-/-}$, SREBP1 $1^{+-}$, or wild-type C57BL/6 mice were isolated by collagenase digestion and handpicking. After isolation, islets were incubated for $16 \mathrm{~h}$ at $11 \mathrm{mmol} /$ 1 glucose, and then cultured for $96 \mathrm{~h}$ at 8 or $30 \mathrm{mmol} /$ 1 glucose before measuring glucose-stimulated insulin secretion and gene expression. Secreted insulin was measured after static incubation at the indicated glucose concentration for $30 \mathrm{~min}$., by radioimmunoassay. SREBP1c, fatty acid synthetase (FAS), acetyl-CoA carboxylase-1 (ACC1), glucose transporter 2 (Glut2), glucokinase (Gk), sulfonylurea receptor 1 (SUR1), potassium inward rectifier 6.2 (Kir6.2), pancreatic-duodenal homeobox factor-1 (Pdx1), Nkx6.1, and neurogenin3 (Ngn3) mRNA levels were determined after RNA extraction in Trizol (Sigma) and quantitative real-time RT-PCR (Taqman ${ }^{\mathrm{TM}}$ ) and were normalised to cyclophilin A levels which were not different between groups. Data from three mice per condition are quoted as means \pm S.E.M. Statistical comparision was by ANOVA and Newman Keuls test.

Results: Cultured at $8 \mathrm{mmol} / 1$ glucose, islets from SREBP $1^{-/-}$ mice displayed decreased levels of FAS $(63 \%, \mathrm{p}<0.05)$ and $\mathrm{Gk}$ $(41 \%, \mathrm{p}<0.05)$ mRNAs, but increased Ngn3 mRNA $(2.19 \pm 0.35$-fold, $\mathrm{p}<0.05)$ compared to wild-type littermates. Islets from wild-type mice chronically ( $96 \mathrm{~h}$ ) exposed to elevated (30 versus $8 \mathrm{mmol} / \mathrm{l}$ ) glucose concentration displayed a 5-10-fold increase in both basal and glucose (16 versus $3 \mathrm{mmol} / \mathrm{l}$ )-stimulated insulin release. This change was associated with increased levels of mRNAs encoding SREBP-1c ( $1.37 \pm 0.13$-fold, $\mathrm{p}<0.05)$, FAS $(2.01 \pm 0.35$-fold, $\mathrm{p}<0.05)$, ACC1 (2.65 \pm 0.45 -fold, $\mathrm{p}<0.05)$, Glut2 (2.05 \pm 0.37 -fold, $\mathrm{p}<0.05)$, Gk (2.46 \pm 0.82 -fold, $\mathrm{p}<0.05)$, Pdx1 (3.58 \pm 0.60 -fold, $\mathrm{p}<0.05)$, SUR1 (3.18 \pm 0.63 -fold, $\mathrm{p}<0.05)$, Kir 6.2 (2.99 \pm 0.50 -fold, $\mathrm{p}<0.05), \mathrm{Ngn} 3(2.33 \pm 0.14$-fold, $\mathrm{p}<0.001)$ and $\mathrm{Nkx}$ 6.1 (3.65 \pm 0.81 -fold, $\mathrm{p}<0.05$ ). With the exception of FAS $\mathrm{mRNA}$ which was still induced by culture at $30 \mathrm{mmol} / \mathrm{l}$ glucose in islets from SREBP1 ${ }^{-/+}$mice, the up-regulation by high glucose of each of the above genes was completely abolished in islets from mice deleted for one or both SREBP1 allele.

Conclusions: These results demonstrate a requirement for SREBP1 in the hypersecretion of insulin resulting from chronic exposure to high glucose concentrations. In addition to the requirement for SREBP-1 in the induction of lipogenic genes, SREBP1 is also, unexpectedly, necessary for the up-regulation of genes directly involved in the control of the $\beta$-cell transcriptome (Pdx1) and in glucose sensing (Glut2, Gk, Kir6.2, SUR1). Induction of SREBP-1c may therefore be involved in the adaptive insulin hypersecretion observed in hyperglycemic states such as insulin resistance and pregnancy.

Supported by the Medical Research Council, Diabetes UK

0138

Beta-cell function is improved in RIP-SOCS-3 transgenic mice fed normal and high-fat diets M. Sörhede Winzell ${ }^{1}$, H. Frobøse ${ }^{2}$, S. Groth Rønn ${ }^{2}$, N. Billestrup ${ }^{2}$, T. Mandrup-Poulsen ${ }^{2}$, B. Ahrén ${ }^{1}$;

${ }^{1}$ Clinical Sciences, Medicine, Lund University, Sweden, ${ }^{2}$ Steno Diabetes Center, Gentofte, Denmark.

Background and Aim: Suppressor of cytokine signaling 3 (SOCS-3) regulates pancreatic $\beta$-cell mass and proliferation. SOCS-3 is induced by leptin and inhibits leptinmediated JAK/STAT signaling in $\beta$-cells. Leptin has been demonstrated to inhibit insulin secretion and insulin gene 
expression. Dysregulation of the adipoinsular axis may contribute to hyperinsulinemia and insulin resistance. High-fat diet (HFD) results in obesity and insulin resistance and glucose intolerance due to insufficient islet compensation to increased insulin demands, and leptin levels are elevated in parallel with increased body weight $(\mathrm{BW})$. The aim of this study was to investigate in vivo and ex vivo effects of overexpression of SOCS-3 on $\beta$-cell function in normal mice and in mice with HFDinduced insulin resistance.

Material and Methods: Transgenic mice (tg) with $\beta$-cell specific overexpression of SOCS-3 (SOCS3-RIP-Tg) were generated. At 8 weeks of age, female wildtype (wt) and SOCS-3 tg mice were fed HFD (58\% fat by energy) for 12 weeks. Control wt and tg mice were fed a normal diet (ND) with $11 \%$ fat. Islet function was studied in vivo by intravenous glucose tolerance test (IVGTT) and intravenous arginine challenge. Islets were isolated and glucose-stimulated insulin secretion (GSIS) was measured. Western blots confirmed the overexpression of SOCS-3 in tg islets

Results: SOCS-3 expression was induced in islets from wt mice fed the HF-diet. BW gain was similar in SOCS-3 tg mice and in wt mice in both diet groups. There was no difference in fasting plasma glucose while insulin levels were slightly reduced in SOCS-3 tg fed HFD. Tg mice on ND had improved insulin response to glucose in the IVGTT ( 1 min peak value: $2250 \pm 340$ vs. $1460 \pm 190 \mathrm{pM}, \mathrm{P}<0.05$ ), which resulted in improved glucose elimination $\left(\mathrm{K}_{\mathrm{G}}\right.$ : $2.6 \pm 2.1$ vs. $1.3 \pm 0.2 \% / \mathrm{min}, \mathrm{P}=0.002)$. Glucose tolerance was similar in the HFD-fed wt and tg mice. Insulin secretion in vitro was augmented 4.6 fold with $11.1 \mathrm{mM}$ glucose compared to $2.8 \mathrm{mM}$ glucose in both wt and $\mathrm{tg}$ islets from ND-fed mice ( $\mathrm{P}<0.01$ in both groups). GSIS was impaired in wt HF-diet fed mice while the response was restored in SOCS-3 tg islets $(1.3 \pm 0.3$ fold increase in HFDwt vs. $3.2 \pm 0.7$ fold increase in HFDtg, $\mathrm{P}<0.05)$. The islet response to intravenous arginine was similar in wt and tg mice on both diets.

Conclusions: SOCS-3 improved $\beta$-cell function in vivo and ex vivo in SOCS-3-RIP-tg mice fed ND, and restored HFD-induced impaired GSIS ex vivo. We hypothesise that the in vivo increased response to glucose and improved glucose elimination observed in SOCS3 tg mice on ND is masked in SOCS-3 tg mice on HFD, since HF-feeding in itself stimulates islet expression of SOCS3. We suggest that increased expression of SOCS-3 in islets is involved in the islet adaptation in high-fat diet induced glucose intolerance.

\section{OP 24}

\section{Fetal programming}

\section{9}

Compromised beta-cell development and reduced glucokinase expression in weanling offspring from dams maintained on a high fat diet during gestation

M. E. Cerf, K. Williams, C. Chapman, J. Louw; Diabetes, Medical Research Council, Tygerberg, South Africa.

Background and Aims: A high fat diet (HFD) is known to adversely affect beta-cell development and function evident by reduced beta-cell mass, hyperglycemia and hyperinsulinemia. Glucokinase $(\mathrm{GK})$ is the key glycolytic enzyme involved in glucose stimulated insulin secretion from betacells. An insult in utero is believed to programme progeny metabolically predisposing them to develop Type 2 diabetes in adulthood. Our previous data have shown that dams fed a HFD during gestation produced weanlings that were hyperglycemic and hypoinsulinemic. The aim of the present study was to investigate whether the HFD also affected beta- and alpha-cell development. In addition, we sought to establish whether the HFD altered expression of GK.

Materials and Methods: Weanling offspring were obtained from dams maintained on a HFD $(2.06 \mathrm{kcal} / \mathrm{g}$ : $40 \%$ of mainly saturated fat as energy, $14 \%$ protein and $46 \%$ carbohydrate) for either the first (HF1), or second (HF2), or third (HF3) week of gestation, or throughout (HF-G) gestation, followed by a standard laboratory diet $(2.60 \mathrm{kcal} / \mathrm{g}$ : $10 \%$ fat, $15 \%$ protein and $75 \%$ carbohydrate) for the duration of lactation. At postnatal day 21, pancreata were excised and either snapfrozen for quantitative PCR (of GK mRNA expression) or processed for immunocytochemical examination and image analysis (to determine beta- and alpha-cell volume, number and size and the levels of GK immunoreactivity).

Results: HF1, HF2 and HF3 weanlings displayed a reduction in beta-cell volume, number and size. In addition, the alpha-cell sizes were also smaller in these weanlings. However, the alpha-cell volume and number was not affected by the HFD. Relative quantification revealed a decrease in GK mRNA expression in HF1, HF2 and HF3 weanlings. This was supported by reduced immunoreactivity for GK in these weanlings. In contrast, HF-G weanlings had no significant differences in beta-cell volume, number or size. Alpha-cell development also appeared to be normal with no changes also detected in either GK mRNA expression or immunoreactivity.

Conclusion: Maintenance of dams on a HFD, for any single week of gestation, has a deleterious effect on the 
beta-cell development of their weanling offspring. In contrast, offspring from dams fed a HFD throughout gestation showed no significant changes in islet cell development. Switching the maternal diet during gestation appears to programme weanling progeny inducing diabetogenic effects characterized by compromised beta-cell development and reduced GK expression.

\section{0}

Effects of maternal obesity on metabolic syndrome in the offspring

A. C. M. Heinsbroek, G. van Dijk;

Animal Physiology, Unit Neuroendocrinology, University of Groningen, Haren, The Netherlands.

Background and Aims: Epidemiological studies have shown that offspring born to women that have been starved during pregnancy in the Second World War in the Netherlands, often had a low birth-weight and higher risk of developing metabolic syndrome (diabetes, obesity, high blood-pressure). This suggests that there are prenatal environmental factors involved in development of the metabolic syndrome.

Not only starvation, but also maternal obesity may be important for the predisposition to the metabolic syndrome in the offspring. In ethnic groups with a high incidence of diabetes and obesity, like the Pima indians, children from diabetic mothers have a higher risk of becoming diabetic compared with children from non-diabetic mothers. This effect is much stronger with maternal diabetes compared with paternal diabetes, suggesting a non-genetic mode of transfer to the offspring.In this project, the effects of maternal obesity on the development of the metabolic syndrome in the offspring were investigated in rats.

Materials and Methods: Maternal obesity was induced by i3vt infusion of the melanocortin 34 antagonist SHU9119 during the last two weeks of pregnancy and the first two weeks of lactation. Male offspring rats from these pregnancies were used at the age of 3-4 months to assess the risk of metabolic syndrome.

Results: Body weight was increased in offspring from SHU mothers, starting from 68 days of age. Food intake was not changed, but water intake was increased in SHU offspring. Respirometric measurements showed increased RQ and a trend to decreased energy expenditure in the dark phase. This was associated to decreased locomotor activity in the dark phase. Oral glucose tolerance tests showed an increase in basal glucose, with no difference in insulin levels, suggesting glucose intolerance in SHU offspring.

Conclusion: We conclude that maternal obesity increases the risk of the metabolic syndrome in the offspring in a non-genetic way. This is mainly due to changes in fat and glucose metabolism, and decreased physical activity, resulting in high body weight and glucose intolerance.

Supported by a career development grant from the dutch Diabetes Fonds to Dr. G. van Dijk on aetiology of the insulin resistance syndrome.

0141

Intrauterine growth retardation leads to the functional change of insulin secretion in the newborn rat

Q. X. Yuan ${ }^{1}$, C. Liu ${ }^{1}$, W. De ${ }^{2}$;

${ }^{1}$ Department of Endocrinology, The First Affiliated Hospital of Nanjing Medical University, ${ }^{2}$ Department of Biochemistry and Molecular Biology, Nanjing Medical University, China.

Background and Aims: Embryonic pancreatic development had a close relation to adult diseases. Intrauterine growth retardation (IUGR) is a common complication of pregnancy and a risk factor for both perinatal disease and disorders of later life, especially impaired glucose tolerance and diabetes. The aim of this study is to investigate the functional change of insulin secretion in IUGR newborn rat and explore the mechanism of reduced blood insulin level. Materials and Methods: SD pregnant rats were divided into two groups: control group and intrauterine energy restriction group. The animal model of IUGR in rats was made by $50 \%$ calorie restriction in pregnant rats from gestational day 15 until term. Pancreata of control and IUGR newborn rats were dissected respectively. After abstraction of totle RNA, RTPCR was used to study the expression of genes related to insulin synthesis and exocytosis. Intraperitoneal glucose tolerance tests(IPGTT) and ELISA were done to detect the function of pancreatic islet.

Results: Birth weight and pancreas mass of IUGR newborn rats were significantly lower than those of controls. Although no significant differences were observed in genes expression of insulin and PDX-1, the expressions of genes related to insulin exocytosis such as munc13-1, vamp-2, syntaxin1a, rab3a were reduced markedly in IUGR newborn rats. The blood insulin level and insulin secretion response to glucose challenge were also reduced simultaneously in IUGR newborn rats compared with normal newborn rats. 
Conclusion: IUGR can induce changes in glucose homeostasis in newborn rats. The main cause of this abnormality in the process might be the reduced function of insulin exocytosis. Supported by Jiangsu Province government

\section{2}

Diminished beta-cell neogenesis and proliferation contribute to reduce beta-cell mass in neonatal undernourished rats

C. Alvarez ${ }^{1}$, L. de Miguel ${ }^{1}$, E. Fernández ${ }^{1}$, P. Serradas ${ }^{2}$, M. A. Martín ${ }^{3}$, F. Escrivá ${ }^{1}$;

${ }^{1}$ Bioquímica y Biología Molecular II, Universidad Complutense, Madrid, Spain, ${ }^{2}$ Lab. Physiopathol. Nutr. CNRS UMR 7059, Université Paris, France, ${ }^{3}$ Metabolismo y Nutrición, CSIC, Madrid, Spain.

Background and Aims: We have previously shown that Wistar fetuses from protein caloric undernourished pregnant rats $(35 \%$ of control diet during the last week of pregnancy) at 21 days post coitum (dpc) exhibit increased beta-cell mass. This alteration can be related to the stimulation of beta-cell proliferation induced by locally increased IGF-1 in pancreas. On the contrary, continuation of maternal food restriction caused reduced beta-cell mass in undernourished (U) neonates at 4 days of life, representing only $58 \%$ of control values. This effect persists until adult age. During pancreas development, new beta-cells can be formed either by replication of pre-existing differentiated beta-cells or by neogenesis (differentiation from stem-cells) and the loss of beta-cells can occur by apoptosis. The aim of this study was to investigate whether a condition of undernutrition affects both beta cell proliferation and differentiation in $U$ rats on postnatal day 4 and the implication of the IGF system, highly responsive to nutritional status, in these processes. To this end we have evaluated 1) replication and differentiation of beta-cells in pancreas, 2) serum IGF-1 and -2 levels and 3) IGF-1 and -2 protein content in liver and pancreas.

Materials and Methods: Food restricted pregnant rats received $35 \%$ of control diet since the last week of gestation. Spontaneous beta-cell regeneration and beta-cell replication were evaluated by immunocytochemistry and morphometry. Serum concentrations of IGF-1 and IGF-2 were measured by radioimmunoassay and radioreceptor assay respectively. The protein content of IGF-1 and -2 was analyzed by Western blot in pancreas and liver.

Results: The results show that both beta cell replication and beta cell neogenesis were markedly decreased in $U$ rats at
4 days of life. Serum IGF-2 levels as well as liver and pancreatic protein content were found increased in $U$ rats, whereas the serum concentration and abundance of IGF-1 protein in pancreas and liver were decreased. All values were compared to those of controls.

Conclusion: Our data suggest that in U rats at 4 days of life 1) the decreased beta-cell mass found could be related to the inhibition of both beta cell proliferation and beta cell neogenesis; in which, 2) low serum IGF-1 levels together with a reduced pancreatic IGF-1 protein content could play a crucial role, and 3 ) in contrast to other animal models in which a reduced pancreatic expression of IGF-2 was related to a decreased beta-cell mass and to an enhancement of apoptosis, the enhanced pancreatic IGF-2 levels in the present $\mathrm{U}$ model could let us assume that malnutrition does not affect apoptosis in our conditions. Therefore, understanding differences in the relationship between the IGF system and the process of beta-cell growth may lead to therapeutic and preventive interventions in diabetic patients with previous episodes of undernutrition.

Supported by Ministerio de Ciencia y Tecnología, Spain

\section{3}

The effects of undernutrition on beta-cell development require a functional glucocorticoid receptor in pancreatic precursor cells

B. Breant ${ }^{1}$, C. Dupuis ${ }^{2}$, D. Zenaty ${ }^{2}$, B. Blondeau ${ }^{2}$, E. Gesina ${ }^{2}$, A. Milet ${ }^{3}$, F. Tronche ${ }^{3}$;

${ }^{1}$ Unit 671, Inserm, Paris, ${ }^{2}$ Unit 690, Inserm, Paris, ${ }^{3}$ UMR7148, CNRS, Paris, France.

Background and Aims: Low birth weight is strongly predictive of hypertension, cardiovascular diseases, obesity, insulin resistance and diabetes. The hypothesis of a primary defect in beta-cell development was initially studied in a rat model of maternal General Food Restriction (GFR), leading to impaired glucose tolerance at adult age. GFR caused maternal and foetal glucocorticoid (GC) elevation, which, in turn, caused a reduction in beta-cell mass in the foetus. Conversely, low GC levels were associated with increased beta-cell mass when nutrient supply is normal. In line with this, the selective inactivation of the glucocorticoid receptor (GR) gene in pancreatic precursor cells (GR-Pdx-Cre mice) was associated with a 2-fold increased beta-cell mass in adult mice while its inactivation in insulin-expressing cells (GR-RIP-Cre mice) had no consequences.

The aim of the present study were 1) to determine if the effects observed on the mutants were postnatal or antenatal, 
2) to investigate if all the effects of undernutrition on betacell development involve the glucocorticoid receptor gene activation and 3) to decipher the cellular mechanisms involved, using the GR mutant mouse models.

Materials and Methods: GR-lox/lox female mice crossed with GR-lox/lox-Pdx-Cre male mice and carrying half GRlox/lox-Pdx-Cre and half GR-lox/lox foetuses were either nourished ad libitum (AL) or given 50\% food restriction (GFR group) from E11.5 until E18.5. A similar protocol was conducted with the GR-RIP-Cre mouse line. Maternal food intake and body weight were monitored daily. At E18.5, the dams were i.p. injected with $50 \mathrm{mg} / \mathrm{kg} \mathrm{BrdU}$ and the foetuses were excised $2 \mathrm{~h}$ later, weighed and the pancreas were dissected. Beta-cell fraction was analysed by morphometrical measurements after immunohistochemistry for insulin. Double immunohistochemistry for insulin and $\mathrm{BrdU}$ was used to measure beta-cell proliferation rate.

Results: As pregnancy progresses AL dams gained weight regularly while the body weight from GFR dams remained stable. No differences in body weight were observed between normally nourished GR-lox/lox, GR-Pdx-Cre or GR-RIP-Cre foetuses. Irrespective of the genotype, foetal weight was decreased $33 \%$ in GFR compared with AL foetuses $(0.70 \pm 0.13 \mathrm{~g}$ vs $1.04 \pm 0.17 \mathrm{~g}, \mathrm{p}<0.001)$.

When comparing AL foetuses, the inactivation of the GR in precursor cells (GR-Pdx-Cre) led to a 2 -fold increased betacell fraction $(\mathrm{p}<0.01)$ and beta-cell proliferation rate $(p<0.05)$ compared with GR-lox/lox foetuses, while GR inactivation in beta cells (GR-RIP-Cre) had no consequences. General Food Restriction decreased beta-cell fraction in GR-lox/lox and GR-RIP-Cre, compared to their corresponding AL-fed foetuses, while GFR-GR-Pdx-Cre and AL-GR-Pdx-Cre foetuses showed similarly elevated beta-cell fractions.

Conclusion: The absence of GC signaling in pancreatic cells favours beta-cell mass expansion and proliferation in the foetus, suggesting that GC negatively regulate these process antenatally. The cellular targets for physiological or excess circulating GC concentrations are Pdx-1-expressing precursor cells. Our results strongly suggest that all the effects of undernutrition on beta-cell development involve a functional Glucocorticoid Receptor in pancreatic precursor cells.

Supported by INSERM, CNRS, EU-Food-CT-2005-007036 and Fondation pour la Recherche Médicale

\section{4}

Non-genetic association between birth weight and insulin resistance in twins

L. Grunnet, A. Vaag, P. Poulsen;

Steno Diabetes Center, Gentofte, Denmark
Background and Aims: Several studies have show an association between low birth weight and increased risk of impaired glucose tolerance, insulin resistance and type 2 diabetes later in life. Whether this association is due to a common genotype resulting in both low birth weight and metabolic abnormalities or a result of a prenatal environmental insult is still unknown. Twin studies represent a tool in investigating whether these associations between birth weight (BW) and measures of glucose metabolism are of genetic or non-genetic origin.

Materials and Methods: An oral glucose tolerance test (OGTT) was performed in a population-based cohort of twins including 142 same-sex monozygotic (MZ) and 214 dizygotic (DZ) twin pairs aged 55-73 years. Information on BW was obtained from original midwife records. Intra-twin-pair correlations (correlations between within-twin-pair differences) and multiple regression analyses with within-twin-pair differences with adjustment for BMI were performed.

Results: We found a non-genetic negative association between BW and fasting insulin, HOMA-IR and index for whole body insulin sensitivity illustrated by significant intra-twin-pair correlations among MZ twins (fasting insulin: $\mathrm{r}=-0.27 \mathrm{p}=0.001$, HOMA-IR: $\mathrm{r}=-0.26 \mathrm{p}=0.002$ and whole body insulin sensitivity index: $r=0.23 \mathrm{p}=0.007$ ). The equivalent correlations in DZ twins were non-significant. Furthermore, a non-genetic negative association was found between BW and fasting plasma glucose data in MZ twins $(\mathrm{r}=-0.19 \mathrm{p}=0.023)$. The association between $120 \mathrm{~min}$ post OGTT plasma glucose levels and BW was significant in both $\mathrm{MZ}$ and DZ twins (MZ: $\mathrm{r}=-0.27 \mathrm{p}=0.001$, DZ: $\mathrm{r}=-0.19 \mathrm{p}=0.004)$.

After adjustment for BMI the association between BW and HOMA-IR, fasting plasma glucose and 120 min plasma glucose, respectively, remained significant. A $100 \mathrm{~g}$ decrease in BW increased HOMA-IR with $3 \%$ and $120 \mathrm{~min}$ plasma glucose with $2.5 \%$ in $\mathrm{MZ}$ twins and $\mathrm{DZ}$ twins, while fasting plasma glucose increased with $1.4 \%$ in $\mathrm{MZ}$ twins. The impact of BW on fasting insulin and whole body insulin sensitivity index, however, were not significant after adjustment for BMI.

Conclusion: We demonstrate a non-genetic negative association between BW and insulin resistance supporting an impact of the fetal environment on insulin resistance in elderly twins, independent of current body size. In addition, glucose intolerance is associated with BW, although a genetic association can not be excluded.

Supported by the Danish Diabetes Foundation, the Danish National Research Council and Sygekassernes Helsefond 


\section{OP 25}

\section{Insulin therapy}

0145

Equivalence of basal insulin glargine versus prandial insulin lispro for glucose control in type 2 diabetes patients on oral agents - results of the APOLLO study R. G. Bretzel ${ }^{1}$, U. Nuber ${ }^{1}$, K. El-Haschimi ${ }^{2}$, T. Linn ${ }^{1}$ on behalf of the APOLLO Study Group;

${ }^{1}$ Third Medical Department and Policlinic, University Hospital Giessen, ${ }^{2}$ Sanofi-Aventis, Berlin, Germany.

Background and Aims: One option to control blood glucose (BG), to delay or prevent complications associated with type 2 diabetes (T2DM), is combination therapy with oral antidiabetic agents (OADs) +insulin. However, there is debate as to whether it is more beneficial to treat patients by targeting postprandial glucose (PPG) levels with a short-acting insulin, or to target fasting $\mathrm{BG}$ (FBG) levels with a basal insulin+OADs.

Materials and Methods: We present the results of $a$ 44week, parallel, open, randomized, multinational study showing the first direct comparison of the efficacy and safety of an $O A D$ regimen+either once-daily insulin glargine $(\mathrm{n}=174)$ or mealtime insulin lispro $(\mathrm{n}=174)$ in T2DM patients failing oral treatment (APOLLO).

Results: $\mathrm{HbA}_{1 \mathrm{c}}$ significantly improved, reaching target levels $<7 \%$ in both groups (Table). Equivalence was established for glargine vs lispro, with the confidence interval $(\mathrm{CI})$ of the mean $\mathrm{HbA}_{1 \mathrm{c}}$ decrease within the preestablished $0.4 \%$ limit for non-inferiority $(0.157 ; 95 \%$ $\mathrm{CI}=0.35-0.05$ [per protocol set]). Glargine provided significantly better control of FBG $(\mathrm{p}<0.0001)$ and nocturnal BG ( $p=0.0017$; Table); lispro gave better PPG control, particularly after lunch and dinner (both $\mathrm{p}<0.0001$ ). The mean number of overall hypoglycaemic events was significantly lower with glargine vs lispro (5.4 vs 24.4 events/patient-year). Daily insulin doses at endpoint did not differ with glargine vs lispro $(42.1 \pm 25.9$ vs $45.1 \pm 25.6 \mathrm{IU})$.

Conclusion: A regimen using the long-acting insulin analogue glargine plus OADs to target FBG offers glucose control equivalent to that with the short-acting insulin analogue lispro plus OADs to target PPG in T2DM. However, the glargine regimen was associated with a reduced risk of hypoglycaemia, fewer insulin injections and less self-monitoring of BG compared with lispro. Thus, the glargine regimen may improve patient treatment satisfaction and compliance compared with the lispro regimen.

\begin{tabular}{llllll}
\hline Mean \pm SD & \multicolumn{2}{l}{ Glargine } & & & \multicolumn{2}{l}{ Lispro } \\
\cline { 2 - 3 } & Baseline & Endpoint & & Baseline & Endpoint \\
\hline $\mathrm{HbA}_{1 \mathrm{c}}(\%)$ & $8.71 \pm 0.95$ & $6.96 \pm 0.67$ & & $8.64 \pm 0.95$ & $6.77 \pm 0.83$ \\
$\mathrm{FBG}(\mathrm{mmol} / \mathrm{L})$ & $10.3 \pm 2.0$ & $6.2 \pm 1.5$ & & $9.9 \pm 2.7$ & $8.0 \pm 1.9$ \\
$(\mathrm{mg} / \mathrm{dL})$ & $(186 \pm 36)$ & $(111 \pm 27)$ & & $(179 \pm 41)$ & $(145 \pm 34)$ \\
Nocturnal BG & $9.8 \pm 2.4$ & $6.5 \pm 2.2$ & & $9.8 \pm 2.9$ & $7.2 \pm 1.8$ \\
$(\mathrm{mmol} / \mathrm{L})(\mathrm{mg} / \mathrm{dL})$ & $(177 \pm 44)$ & $(118 \pm 39)$ & & $(177 \pm 53)$ & $(129 \pm 33)$ \\
\hline
\end{tabular}

Supported by sanofi-aventis

0146

Insulin detemir and insulin glargine: similar time-action profiles in subjects with type 2 diabetes

T. Heise ${ }^{1}$, O. Klein ${ }^{1}$, J. Lynge ${ }^{2}$, L. Endahl ${ }^{2}$, B. Damholt ${ }^{2}$, L. Nosek ${ }^{1}$;

${ }^{1}$ Profil Institut für Stoffwechselforschung $\mathrm{GmbH}$, Neuss, Germany, ${ }^{2}$ Novo Nordisk A/S, Bagsvaerd, Denmark.

Background and Aims: This is the first head-to-head comparison of the pharmacodynamic (PD) and pharmacokinetic (PK) properties of insulin detemir (IDet) and insulin glargine (IGlar) in subjects with type 2 diabetes (T2D).

Materials and Methods: Twenty-seven insulin-treated male subjects with T2D (BMI $30.8 \pm 2.6 \mathrm{~kg} / \mathrm{m}^{2}$ (mean \pm SD), HbAlc $7.6 \pm 1.1 \%$ ) were enrolled in this randomised, double-blind, parallel trial and received $0.4,0.8$, and $1.4 \mathrm{U} / \mathrm{kg}$ of IDet or IGlar under glucose clamp conditions (target blood glucose (BG) $90 \mathrm{mg} / \mathrm{dL}$, iv insulin infusion (rate $0.3 \mathrm{mU} / \mathrm{kg} / \mathrm{min} 3 \mathrm{~h}-0.5 \mathrm{~h}$ before dosing), clamp duration $24 \mathrm{~h}$ or until $\mathrm{BG}>200 \mathrm{mg} / \mathrm{dL}$ ). Assessment of endogenous glucose production (EGP) and peripheral glucose uptake (PGU) were made using the isotope dilution technique $\left(6,6-{ }^{2} \mathrm{H}_{2}-\right.$ Glucose $)$.

Results: The mean glucose infusion rate (GIR) profiles for IDet and IGlar were similar in shape/flatness (figure) and showed increasing effect with increasing dose. The doseresponse relationship (log-effect versus log-dose) was similar for the insulin preparations in intercept and slope (p-values: slope $=0.23$, intercept $=0.84)$. Within-subject variability was lower for IDet than for IGlar (p-values: withinsubject variability $<0.0001$, between-subject variability $=0.38$ ). The duration of action (time from dosing to GIR $<0.5 \mathrm{mg} / \mathrm{kg} / \mathrm{min}$ ) increased with rising doses for both IDet and IGlar without major difference between the two preparations for the clinically relevant doses 0.4 and $0.8 \mathrm{U} / \mathrm{kg}$ (IDet 719 $\pm 512,1007 \pm 408$; IGlar: 613 \pm 443 , $1162 \pm 413 \mathrm{~min}$ ) and was $>24 \mathrm{~h}$ in most subjects with the highest dose ( $1328 \pm 154$ vs. $1440 \pm 0 \mathrm{~min})$. IDet and IGlar led to comparable, dose-dependent suppression of EGP 
(Area over EGP curve; IDet: $295 \pm 344,610 \pm 563$, $800 \pm 429 \mathrm{mg} / \mathrm{kg}$, IGlar: $211 \pm 265,519 \pm 427,1034 \pm 721 \mathrm{mg} /$ $\mathrm{kg}$, NS for IDet vs. IGlar) and stimulation of PGU (Area under PGU curve; IDet: $393 \pm 324,817 \pm 584,1257 \pm 893 \mathrm{mg} /$ $\mathrm{kg}$, IGlar: $415 \pm 447,721 \pm 1007,1706 \pm 1485 \mathrm{mg} / \mathrm{kg}$, NS for IDet vs. IGlar).

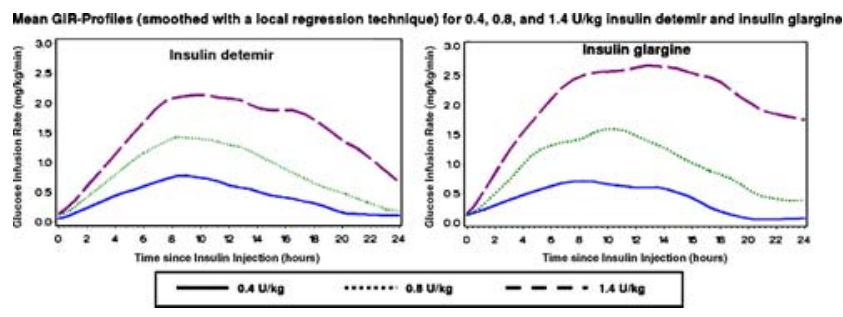

Conclusion: This first head-to-head comparison of the pharmacodynamic properties of IDet and IGlar in subjects with type 2 diabetes confirmed lower within-subject variability for IDet and showed no substantial differences in the shape of the GIR-profiles, duration of action and ability to stimulate glucose uptake and suppress endogenous glucose production.

Supported by a research grant from Novo Nordisk A/S

\section{7}

No correlation between weight gain and number of hypoglycaemic events in patients with type 2 diabetes treated with insulin detemir as compared to NPH insulin T. Derezinski ${ }^{1}$, M. Davies ${ }^{2}$, H. Kim ${ }^{3}$, P. Clauson ${ }^{4}$;

${ }^{1}$ Out-patient Clinic, Eskulap Gniewkowo, Poland, ${ }^{2}$

Department of Metabolic Medicine, University Hospitals of Leicester, United Kingdom, ${ }^{3}$ Novo Nordisk Asia Pacific Pte, Singapore, ${ }^{4}$ Novo Nordisk, Bagsvaerd, Denmark.

Background and Aims: Insulin detemir (detemir), a new long-acting insulin analogue, has been shown to improve glycaemic control in people with Type 1 and Type 2 diabetes when used as part of a basal-bolus regimen or as add-on to oral anti-diabetic drugs (OADs). Addition of basal insulin to OADs is a common regimen for people inadequately controlled on oral agens, but weight gain usually occurs when insulin treatment is initiated. The aim of this exploratory analysis was to investigate if any possible correlation existed between weight gain and risk of hypoglycaemia with use of detemir.

Materials and Methods: The analysis was based on a 26week, randomised, multi-centre, open-label, parallel group trial, in which glycaemic control, risk of hypoglycaemia and weight development were compared between detemir and NPH insulin (NPH). A total of 475 insulin-naïve Type
2 diabetes patients (detemir: 237, NPH: 238) treated with one or two OADs added basal insulin morning and evening to their current oral treatment. Baseline $\mathrm{HbA}_{1 \mathrm{c}}$ was $8.6 \%$ (detemir) and 8.5\% (NPH), respectively.

Results: Both groups achieved excellent metabolic control $\left(\mathrm{HbA}_{1 \mathrm{c}}\right.$ detemir: 6.6\%, NPH: 6.5\%, NS). Weight gain was less with detemir compared to NPH (1.2 vs. $2.8 \mathrm{~kg}$, $\mathrm{p}<0.001)$ and the overall risk of hypoglycaemia was $47 \%$ lower with detemir $(\mathrm{p}<0.001)$. No significant relationship between hypoglycaemia and weight gain was seen with detemir $(\mathrm{p}=0.2)$, while a statistically significant correlation was found for NPH ( $p=0.003)$. At an equal level of $\mathrm{HbA}_{1 \mathrm{c}}$, detemir results in less weight gain than NPH. This may be caused by fewer hypoglycaemic events, which could result in less defensive snacking with detemir.

Conclusion: The results of this analysis contribute to our understanding of some of the underlying mechanisms of insulin associated weight differences between detemir and NPH.

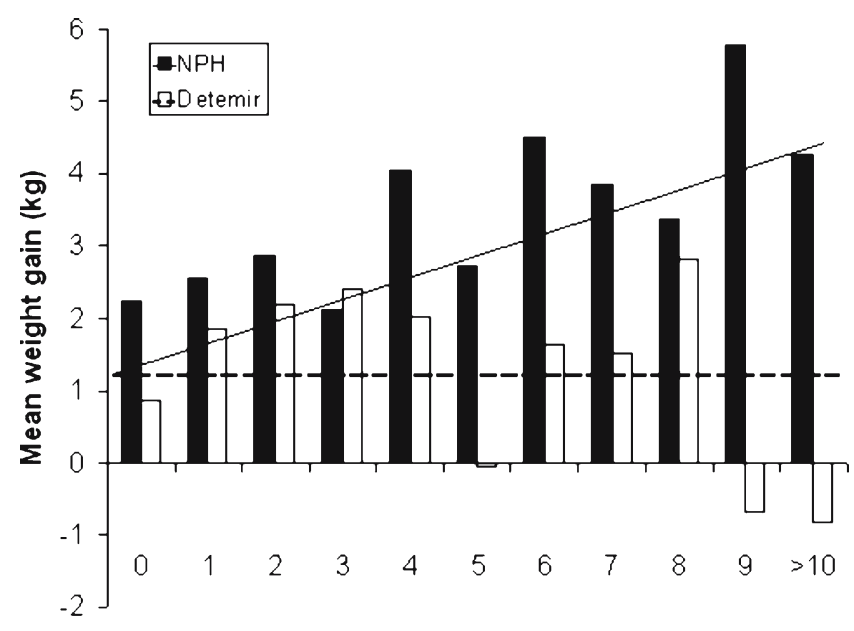

Hypoglycaemic events per patient year

Supported by Novo Nordisk A/S

0148

Insulin glulisine: faster onset of absorption and action than insulin lispro in lean to obese subjects

H. Spitzer ${ }^{1}$, T. Heise ${ }^{1}$, L. Heinemann ${ }^{1}$, L. Nosek ${ }^{1}$,

A. D. Frick ${ }^{2}$, R. H. A. Becker' ${ }^{2}$;

${ }^{1}$ Profil Institute for Metabolic Research $\mathrm{GmbH}$, Neuss, ${ }^{2}$ sanofi-aventis Deutschland $\mathrm{GmbH}$, Frankfurt/Main, Germany.

Background and Aims: This randomized, double-blind, cross-over study compared the pharmacodynamic (PD) and pharmacokinetic (PK) properties of the rapid-acting insulin analogues insulin glulisine (GLU) and insulin lispro (LIS) in lean to obese subjects. 
Materials and Methods: Eighty healthy subjects were stratified into 4 body mass index (BMI) classes $(<25$, $25 \geq 30,30>35$ and $\geq 35 \mathrm{~kg} / \mathrm{m}^{2}$ ) and received single injections of GLU and LIS $(0.2 \mathrm{U} / \mathrm{kg}$ and $0.4 \mathrm{U} / \mathrm{kg})$ in a randomized order on 4 study days under euglycaemic glucose clamp conditions. Glucose infusion rate (GIR) and insulin (INS) concentration were assessed for 10 hours post-dose. Areas under the curve (AUC) from individual INS and GIR profiles were calculated from untransformed data with the trapezoidal rule. All PD and PK data (after a natural logtransformation) were analysed using an analysis of variance model to allow the estimation of least square means and to test differences between treatments.

Results: Early metabolic action (GIR-AUC ${ }_{0-1 \mathrm{~h}}$ ) was significantly greater with GLU than with LIS in the total population and in a number of BMI groups $(p<0.05$, see Table) while the total metabolic effect was not significantly different between treatment groups. Correspondingly, onset of action (GIR- $\mathrm{t}_{10 \%}$ ) was faster with GLU than with LIS. This is in agreement with a consistently faster early INS exposure (INS- $\mathrm{t}_{10 \%}$ ) with GLU compared with LIS at either dose.

Conclusion: These findings in lean to obese subjects support previous observations of a slightly faster BMIindependent onset of action of GLU in obese to morbidly obese subjects. Because of its faster onset of action, GLU may offer improved postprandial glucose control.

\begin{tabular}{|c|c|c|c|}
\hline & $\begin{array}{l}\text { BMI } \\
\left(\mathrm{kg} / \mathrm{m}^{2}\right)\end{array}$ & $\begin{array}{l}0.2 \mathrm{U} / \mathrm{kg} \\
\text { GLU - LIS }\end{array}$ & $\begin{array}{l}0.4 \mathrm{U} / \mathrm{kg} \\
\text { GLU - LIS }\end{array}$ \\
\hline \multirow{5}{*}{$\begin{array}{l}\text { GIR-AUC } \mathbf{0}_{\mathbf{0} \mathbf{~ h}} \\
(\mathrm{mg} / \mathrm{kg})\end{array}$} & Total & $19.1(6.6 ; 31.7)^{*}$ & $45.6(33.1 ; 58.2)^{*}$ \\
\hline & $<25$ & $18.2(-6.8 ; 43.3)$ & $78.6(53.5 ; 103.7)^{*}$ \\
\hline & $25 \geq 30$ & $28.9(3.8 ; 54.0)^{*}$ & $33.0(7.9 ; 58.1)^{*}$ \\
\hline & $30>35$ & $10.6(-14.5 ; 35.7)$ & $54.0(28.8 ; 79.0)^{*}$ \\
\hline & $\geq 35$ & $18.7(-6.4 ; 43.8)$ & $17.1(-8.0 ; 42.2)$ \\
\hline \multirow{5}{*}{$\begin{array}{l}\text { GIR-t }_{\mathbf{1 0}} \% \\
(\mathrm{~min})\end{array}$} & Total & $-4.5(-8.0 ;-1.0)^{*}$ & $-4.0(-6.5 ;-1.0)^{*}$ \\
\hline & $<25$ & $-3.5(-9.5 ; 2.0)$ & $-6.0(-9.0 ;-2.5)^{*}$ \\
\hline & $25 \geq 30$ & $-5.0(-13.0 ; 0.0)^{*}$ & $-4.3(-10.0 ; 1.5)$ \\
\hline & $30>35$ & $-2.5(-11.5 ; 9.5)$ & $-5.3(-13.0 ; 1.0)$ \\
\hline & $\geq 35$ & $-5.0(-18.0 ; 9.0)$ & $1.5(-6.5 ; 10.0)$ \\
\hline \multirow{5}{*}{$\begin{array}{l}\text { INS-t } \mathbf{1}_{\mathbf{1 0}} \% \\
(\mathrm{~min})\end{array}$} & Total & $-5.4(-7.0 ;-3.8)^{*}$ & $-5.0(-6.8 ;-3.3)^{*}$ \\
\hline & $<25$ & $-4.3(-7.0 ;-1.3)^{*}$ & $-6.4(-8.5 ;-3.8)^{*}$ \\
\hline & $25 \geq 30$ & $-4.6(-10.9 ;-2.6)^{*}$ & $-8.8(-12.3 ;-5.2)^{*}$ \\
\hline & $30>35$ & $-5.4(-8.7 ;-1.3)^{*}$ & $-3.0(-6.9 ;-0.2)^{*}$ \\
\hline & $\geq 35$ & $-7.0(-10.6 ;-2.7)^{*}$ & $-0.9(-6.1 ; 4.0)$ \\
\hline
\end{tabular}

Values are point estimates with $95 \%$ confidence limits of differences between treatments; $* \mathrm{p}<0.05$ versus corresponding dose and BMI group for LIS

Supported by sanofi-aventis

\section{9}

Sexual activity in diabetic patients treated by continuous subcutaneous insulin therapy

J.-P. Riveline ${ }^{1}$, F.-X. Jollois ${ }^{2}$, N. Messaoudi ${ }^{1}$, F. Lagarde ${ }^{3}$, D. Charitanski ${ }^{4}$, B. Lormeau ${ }^{5}$, A. Benoit ${ }^{1}$, S. Pichard $^{6}$, E. Requeda $^{1}$, A. Deburge $^{7}$, E. Dresco $^{8}$, M. Lejeune ${ }^{1}$, A. Ciofu ${ }^{3}$, O. Dupuy ${ }^{9}$, G. Charpentier ${ }^{1}$ on behalf of the GPSF Group;

${ }^{1}$ Service de Diabétologie Endocrinologie, Hopital Sud Francilien, Corbeil Essonnes, ${ }^{2}$ CRIP 5, Université Rene Descartes, Paris, ${ }^{3}$ Service de Diabétologie Endocrinologie, Centre Hospitalier, Montargis, ${ }^{4}$ Service de Diabétologie, Centre Hospitalier Victor Jousselin, Dreux, ${ }^{5}$ Service de Diabétologie, Hopital Jean Vercier, Bondy, ${ }^{6}$ Service de Diabétologie Endocrinologie, Centre Hospitalier, Etampes, ${ }^{7}$ Service de Diabétologie Endocrinologie, Centre Hospitalier, Ferolles Atilly, ${ }^{8}$ Service de Diabétologie Endocrinologie, Centre Hospitalier, Dourdan, ${ }^{9}$ Service de Diabétologie Endocrinologie, HIA Begin, Saint Mandé, France.

Background and Aims: Concerns about interference with sexual activity could be an obstacle to initiating pump therapy in type 1 diabetic patients. The aim of this study was to evaluate the level of sexual activity reported by diabetic patients treated with insulin pump therapy (CSII).

Materials and Methods: Between July 2005 and December 2005, patients filled out a questionnaire concerning their demographic data, diabetes history, pump treatment history, metabolic control, the inconvenient/convenient from pump and catheter (via visual analogic scales) and information on sexual activity.

Results: 336 diabetic patients treated with CSII since $4.2+$ / -5.9 yrs, age $43+/-15.9$ yrs, women 53\%, diabetes duration $19+/-11$ yrs, single $20 \%$, were included in a cohort by 42 diabetologist in 15 hospital center or private diabetologic center. Pump indications were : bad metabolic control (HbA1c>8\%) :42\%, unstability:34\%, pregnancy: $13 \%$, injections intolerance: $6 \%$, life style :2\%, insulin resistance $: 3 \%$. HbAlc was $7.8+/-1.26 \%$, with $2.45+/$ -2.27 mild hypoG/week. Complications: retinopathy: $45.2 \%$, nephropathy: $17.8 \%$, neuropathy: $31.8 \%$, macroangiopathy: $9.9 \%$. Sexual activity frequency was: never: $29.9 \%,<1 /$ month: $12.3 \%,>1 /$ month and $<1 /$ week: $18.2 \%$, $>1 /$ week: $39.6 \%$. These frequencies were correlated with age $(p<0.0001)$, co-habitation $(p<0.01)$, good metabolic control $(p<0.05)$, absence of complications (no nephropathy $(\mathrm{p}<0.05)$, no neuropathy $(\mathrm{p}<0.05)$, and no macroangiopathy $(\mathrm{p}<0.01)$ ), but not with "dislike/like" pump or catheter. Multivariate analysis showed that only age and cohabitation were correlated with sexual activity frequency $(p<0.0001$ and 0.0003 respectively) but not with diabetes duration or complications. To the question: "Does the pump 
have an influence on your sexual activity?" the answer was: no: $89.4 \%$, yes: $10.6 \%$. In multivariate analysis, this influence was correlated with $\mathrm{HbAlc}(\mathrm{p}<0.05)$, the fact to report a discomfort with the pump $(\mathrm{p}<0.05)$, the number of mild hypoglycaemia $(p<0.01)$. Finally, to the question: "Do you disconnect the catheter during sexual activity?" the answer was: never: $22.3 \%$, rarely: $11.6 \%$, sometime: $18.2 \%$, always: $47.9 \%$. Multivariate analysis showed that men and younger people disconnect the pump more often $(p<0.05)$. This was also the case for patient with bad metabolic control $(\mathrm{p}<0.05)$ and those who reported inconvenient by the pump. This disconnexion was less frequent in patient with high pump duration $(\mathrm{p}<0.01)$.

Conclusion: Sexual activity frequency seems not affected by pump therapy or diabetes but is correlated with age and co-habitation. The use of the pump (i.e. disconnection) during sexual activity is different according the gender, age and pump duration.

Supported by Novo Nordisk A/S

\section{0}

Ten year-experience with implanted insulin pumps documents safe and effective control in type 1 diabetic patients with initial poor prognosis

P. Schaepelynck Belicar ${ }^{1}$, E. Renard ${ }^{2}$, C. Fermon ${ }^{3}$, S. Rudoni ${ }^{4}$, J.-P. Riveline ${ }^{5}$, B. Catargi ${ }^{6}$, B. Guerci ${ }^{7}$, L. Millot ${ }^{8}$, J.-F. Martin ${ }^{9}$, A. Sola ${ }^{10}$, L. Dufaitre ${ }^{1}$, L. Rocher ${ }^{1}$ on behalf of the EVADIAC Study Group, France;

${ }^{1}$ Endocrinology and Diabetes, University Hospital, Marseille, ${ }^{2}$ Endocrinology and Diabetes, University Hospital, Montpellier, ${ }^{3}$ Endocrinology and Diabetes, University Hospital, Lille, ${ }^{4}$ Endocrinology and Diabetes, University Hospital, Dijon, ${ }^{5}$ Endocrinology and Diabetes, General Hospital, Corbeil, ${ }^{6}$ Endocrinology and Diabetes, University Hospital, Bordeaux, ${ }^{7}$ Endocrinology and Diabetes, University Hospital, Nancy, ${ }^{8}$ Endocrinology and Diabetes, University Hospital, Saint-Etienne, ${ }^{9}$ Endocrinology and Diabetes, General Hospital, Le Mans, ${ }^{10}$ Endocrinology and Diabetes, University Hospital, Paris, France.

Background and Aims: Early studies with implanted insulin pumps (IP) from 1989 to 1993 proved treatment feasibility, safety and effectiveness on glucose control in type 1 diabetes mellitus. After poor physical stability of infused U400 insulin, that occurred in 1993-1994 and resulted in recurrent underdelivery incidents, has been improved, the EVADIAC group resumed implantations of IP from 1995 to assess long-term safety and effectiveness of this therapy. The goal of the present study was to investigate the benefit- risk ratio of IP therapy on a long-term basis.
Materials and Methods: Current cumulated data concern 137 type 1 diabetic patients (58 males, 79 females) who started with IP therapy between 1995 and 2005. At implantation time (T0), mean age was 42.3 (range:19-63) yrs and diabetes duration 22.3 (2-52) yrs. Status of diabetic complications included neuropathy in $30.4 \%$ of patients, nephropathy in $23.4 \%$, retinopathy in $60.4 \%$, cardiovascular disease in $15 \%$. Prior to implantation, insulin treatment regimen was MDI in $17.2 \%$ of patients and CSII in $82.8 \%$. Motivations for pump implantation included: poor blood glucose stability (43.1\%), recurrent hypoglycaemia (either severe or more than four non severe per week) (33.1\%), poor metabolic control based upon HbAlc levels $(10 \%)$, SC insulin resistance (4.6\%), others $(9.2 \%)$.

Results: Reported mean duration of treatment with IP was 46.4 months (6-124) with most of the patients currently continuing this therapy. HbAlc levels significantly decreased from $\mathrm{T} 0$ to $\mathrm{T} 1$ (one year later) (in 128 patients) i.e. 7.91 to $7.69 \%$ ( $\mathrm{p}<0.003$ ), T0 to T2 (two years later) (in 102 patients) $\mathrm{i}$. e. 7.92 to $7.69 \%$ ( $\mathrm{p}=0.023$ ), T0 to T4 (in 46 patients) i.e. 8 to $7.69 \%(\mathrm{p}=0.04)$ and from T0 to T5 (in 34 patients) i.e. 8 to $7.6 \%(\mathrm{p}=0.03$ ). Meanwhile, no significant variation of body weight and complication status assessed on a yearly basis occurred. No correlation was identified between body weight and $\mathrm{HbA} 1 \mathrm{c}$ levels. Seventeen premature pump removals were motivated by blocked pumps $(\mathrm{n}=5)$, premature low battery power $(n=4)$, pump-pocket complications $(n=5)$, recurrent catheter encapsulations $(\mathrm{n}=3)$.

Conclusions: This unique and large-scale experience supports a positive benefit upon risk ratio for IP therapy in poorly controlled type 1 diabetes mellitus in spite of an intensive SC insulin treatment regimen, mostly associated with advanced complications. From these data, IP should deserve a renewed interest as a mean to improve metabolic status and impaired prognosis of these patients, when more widely available.

\section{OP 26}

\section{Wound healing — diabetic foot}

0151

Novel PKC based therapeutic overcoming diabetes related wound healing impairment

M. Meir ${ }^{1}$, I. Solomonik ${ }^{1}$, E. Brener ${ }^{1}$, R. Mandil ${ }^{1}$, M. Ben-Hamo ${ }^{1}$, O. $\mathrm{Nov}^{2}$, E. Braude ${ }^{3}$, T. Tennenbaum ${ }^{2}$, L. Braiman-Wiksman ${ }^{1}$;

${ }^{1}$ HealOr Ltd., Ness Ziona, ${ }^{2}$ Life Sciences, Bar-Ilan University, Ramat Gan, ${ }^{3} \mathrm{HealOr}$ Ltd., Ness Ziona, Israel. 
Background and Aims: Diabetes involves skin related complications including chronic untreatable wounds which lead, in many cases, to lower extremity amputations. Protein kinase C (PKC), a family of serine-threonine kinases is known to be involved in skin physiology and diabetes pathology as well as in regulation of glucose homeostasis. In this study we introduce an innovative PKC based formulation for the treatment of diabetes ulcers, composed of a novel PKC activating peptide and a classical PKC inhibiting peptide (HO/03/03).

Materials and Methods: Wound healing studies were set in two model systems: STZ (175 mg/kg) injected C57BL mice which were sustained until blood glucose levels were $>450 \mathrm{mg} / \mathrm{dl}$ for at least 2 days (diabetes type 1 model) and Psammomys Obesus (diabetes type 2 model); biopsies were collected 7-9 days post wounding. Morphological analysis of healing progression was performed during the experiment followed by a novel quantitative immunohistochemical assessment strategy carried out by utilizing specific epidermal and dermal markers: keratin 14 (K14) staining (epidermal regeneration), keratin 1 (K1) (epidermal differentiation), keratin 6 (K6) (epidermal migration), proliferating cell nuclear antigen (PCNA) (cell proliferation and granulation tissue formation) and Masson trichrome staining for collagen fiber distribution. Dermal contraction and remodeling were assessed by relative wound size and hair follicles regeneration. Organization of the hypodermis and severity of inflammation were assessed utilizing hematoxylin-eosin (H\&E) and myeloperoxidase.

Results: Our results demonstrate a uniform wound healing impairment in both diabetic animal models including all parameters tested: epidermal closure $67 \%$ vs. $100 \%$ in diabetics and non diabetic wounds respectively; a complete absence of hypodermis layer in diabetics vs. $100 \%$ in controls; severe inflammation in $83 \%$ vs. 33\%; granulation tissue formation $50 \%$ vs. $100 \%$ in controls. In addition, abnormal angiogenesis in the wound gap was distinctive of diabetic wounds ( $83 \%$ vs. 0$)$. These results clearly demonstrate that the diabetic state is consistent with wound healing impairment. Topical treatment with HO/03/03 dramatically induced wound healing and accelerated critical healing parameters when compared to control diabetes non treated wounds including: epidermal closure (71\% vs. $17 \%$ ), epidermal differentiation (33\% vs. $0 \%$ ) and dermal contraction (28\% vs. $0 \%$ ). In addition, HO/03/03 significantly reduced the inflammatory response characteristic of impaired diabetic wounds.Further examination of non healing wounds in larger animal models, such as dogs and horses, showed that treatment with $\mathrm{HO} / 03 / 03$, accelerated wound healing similarly to diabetic animal wounds. A clinical trial protocol using this formulation for the treatment of diabetic ulcers was approved by the Israeli Ministry of Health. Preliminary human case studies of chronic non healing wounds revealed positive results in facilitating wound closure when treated with $\mathrm{HO} / 03 / 03$.

Conclusion: These results demonstrate that specific PKC modulation is imperative to wound healing processes and can overcome the pathology of impaired wound healing as it appears in diabetes. Moreover, we demonstrated the therapeutic ability of a novel PKC based formulation ( $\mathrm{HO} /$ 03/03) in impaired wound healing in various models.

0152

Negative pressure wound therapy in complex diabetic foot wounds: costs, resource utilisation and outcomes J. Apelqvist ${ }^{1}$, D. G. Armstrong ${ }^{2}$, L. A. Lavery ${ }^{3}$, A. J. M. Boulton ${ }^{4}$;

${ }^{1}$ Medicine, Division of Endocrinology, University of Malmo, Sweden, ${ }^{2}$ Scholl's Center for Lower Extremity Ambulatory Research (CLEAR), Rosalind Franklin University of Medicine and Science, North Chicago, United States, ${ }^{3}$ Surgery, Texas A\&M University College of Medicine, Temple, United States, ${ }^{4}$ Medicine, University of Manchester, United Kingdom.

Background and Aims: To report on health economic and resource utilization aspects of negative pressure wound therapy (NPWT) compared to standard wound therapy (SWT) based on results of a randomized clinical trial.

Methods: 162 patients were enrolled into a sixteen-week, eighteen-center randomized clinical trial. Inclusion criteria consisted of partial foot amputation wounds up to the transmetatarsal level and evidence of adequate perfusion. Patients randomized to NPWT $(n=77)$ received therapy with dressing changes every 48 hours. Control patients $(n=85)$ received SWT according to consensus guidelines. NPWT was delivered through the Vacuum Assisted Closure (V.A.C.) Therapy System. Wounds were treated until healing or completion of the active treatment period.

Results: NPWT was superior to SWT in both proportion $(p=0.04)$ and rate $(p=0.005)$ of healing. The median time to healing in the NPWT group was 8 weeks compared to 11 weeks for SWT. More reamputation $(10.6 \%$ vs. $2.6 \%$ and all high level amputations (5.9\% vs. $0 \%$ ) occurred in the SWT group compared to the control group $(\mathrm{p}=0.06)$. Total skilled nursing hours dedicated to the wound was 3-fold higher in the SWT group (86.5+/- 76.6 vs. $27.6+/-16.1$, $\mathrm{p}<0.01)$. Independent of healing status, the number of dressing changes per 24-hour period was lower in the NPWT group $(0.5+/-0.2$ vs. $1.4+/-0.6, \mathrm{p}<0.01)$ compared to SWT. 61\% fewer dressing changes (603 vs. 1555) were performed by physicians in the NPWT group compared to SWT. This trend was consistent for other health care providers and family members $(p=0.03)$. Estimated hospital- 
ization and procedure-related costs for reamputation alone (excluding prostheses, disability and rehabilitation) were lower in NPWT $(\$ 79,913$ vs. $\$ \$ 362,840$ USD).

Conclusion: Treatment with NPWT resulted in a higher proportion of wounds that healed at an improved rate, while at the same time resulting in less resource utilization than SWT. Because of the reduced number of interactions required with the wound, weekly reductions in utilization and direct cost may be independent of healing status.

Supported by KCI

\section{3}

Thrombin-activated platelets enhance healing of ulcerative skin lesion by inducing IGF-1 release by skin fibroblasts

F. Giacco ${ }^{1}$, G. Perruolo ${ }^{1}$, F. Oriente ${ }^{1}$, G. Fratellanza ${ }^{1}$,

E. D’Agostino ${ }^{1}$, A. Cassese ${ }^{1}$, A. P. M. Barbagallo ${ }^{1}$,

F. Paturzo ${ }^{1}$, G. A. Raciti ${ }^{1}$, C. Romano ${ }^{1}$, C. Miele ${ }^{1,2}$,

F. Beguinot ${ }^{1,2}$, P. Formisano ${ }^{1}$;

${ }^{1}$ Dipartimento di Biologia e Patologia Cellulare e Molecolare, University of Naples, ${ }^{2}$ Ieos, CNR, Naples, Italy.

Background and Aims: Diabetic foot is a common and hard to treat complication of diabetes accompanied by a strong impairment of life quality. Platelet components have found successful clinical utilization to initiate or to accelerate tissue repair in this pathology. However, the molecular pathways by which platelet factors contribute to tissue regeneration have not been fully elucidated. We have studied the effect of thrombin-activated platelets (TAPs) on cell growth in vivo and in cultured cell systems.

Materials and Methods: Shoulder skin fibroblasts were obtained by punch biopsy and were grown at $37 \mathrm{C}$ in DMEM supplemented with $10 \%$ fetal calf serum in a $5 \% \mathrm{CO}_{2}-95 \%$ air humidified atmosphere. Cultures were used for experimental procedures between the 8 th and 15 th passage. Platelets'rich plasma (PRP) was obtained from healthy donor volunteers (for in vitro experiments) and from diabetic patients undergoing autologous TAPs treatment for ulcers of the lower extremities. For TAPs preparations, thrombin $(1: 10 \mathrm{vol} / \mathrm{vol})$ and calcium gluconate $(1: 10 \mathrm{vol} / \mathrm{vol})$ were added to PRP for $5 \mathrm{~min}$ at room temperature. For the treatment of the patients, the volumes were dictated by the size of the lesions and the applications were repeated twice a week. For in vitro experiments, $1 \mathrm{~cm}^{2}$ aliquots of PG were applied to cells plated in $100 \mathrm{~mm}$ diameter dishes.

Results: Application of TAPs to ulcerative skin lesions of diabetic patients induced local activation of ERK1/2 and Akt/ PKB. Moreover, when applied to cultured human skin fibroblasts, obtained both from healthy donors and diabetic patients, TAPs promoted cell growth, DNA synthesis and activated PDGF and IGF-1 receptor tyrosine kinases. PDGF was released by TAPs and rapidly achieved a plateau. At variance, the release of IGF-1 was mainly provided by the TAPs-stimulated fibroblasts and progressively increased up to $48 \mathrm{~h}$. The PDGF-R blocker Ag1296 reduced the activation of $\mathrm{Akt} / \mathrm{PKB}$ and, at a lesser extent, of ERK1/2. Conversely, inhibition of IGF-1 signaling by Ag1024 and expression of a dominant negative IGF-1R mutant selectively reduced the stimulation of ERK1/2 by TAPs and fibroblast-released factors, with minor changes of Akt/PKB activity.

Conclusion: Here we show that TAPs represent an important source of growth factors to induce the proliferation of human fibroblasts, in vivo as well as in cultured systems. Platelet factors promote fibroblast growth by acutely activating Akt/PKB and ERK1/2. Sustained activation of ERK1/2, however, requires autocrine production of IGF-1 by TAPs-stimulated fibroblasts.

\section{4}

Use of foam dressing for lower-extremity necrotising fasciitis in type 2 diabetic patients

A. Kumar Varma ${ }^{1}$, N. Papanas ${ }^{2}$, A. Bal ${ }^{1}$, H. Kumar ${ }^{1}$, K. Rajesh ${ }^{1}$, M. N. Sandhya ${ }^{1}$, E. Maltezos ${ }^{2}$;

${ }^{1}$ Department of Endocrinology, Diabetes and Podiatric Surgery, Amrita Institute of Medical Sciences and Research Center, Kochi, Kerala, India, ${ }^{2}$ Second Department of Internal Medicine, Democritus University of Thrace, Alexandroupolis, Greece.

Background and Aims: Extensive necrotising fasciitis requiring major debridement belongs to the most severe diabetic foot complications. Foam dressings retain moisture, absorb exudates, facilitate removal of slough, and thereby promote wound healing. The aim of this study was to investigate whether foam dressings are superior to conventional dressings in type 2 diabetic patients with lower-extremity necrotising fasciitis.

Materials and Methods: This study included 48 type 2 patients (28 men, 20 women) with a mean age of $65 \pm 10$ years and a mean diabetes duration of $9 \pm 5$ years. The study was conducted between 1st January and 31st July 2005. All patients suffered from lower-extremity necrotising fasciitis and had already undergone debridement. Patients were randomly assigned to the study group and the control group. In the study group $(\mathrm{N}=24)$, foam of $10 \mathrm{~mm} \times 10 \mathrm{~cm} \times 30 \mathrm{~cm}$ and shore hardness of 10 was used, without topical antibiotics or desloughing ointments. In the control group $(\mathrm{N}=24)$, patients were dressed with conventional techniques, using antibiotic and hydrocolloid ointments. Dressings were changed on a daily basis and bedside debridement was performed as required in both groups. Patients were offloaded on the operated limb in 
both groups. When sufficient granulation tissue was evident and the ulceration exceeded $5 \mathrm{~cm}$ in diameter, split skin grafting (s.s.g) was employed. Time to heal was regarded as the number of days from 1 st debridement until s.s.g. In patients who were not skin-grafted, time to heal was taken as the number of days from 1 st debridement until complete healing or until 31st July 2005.

Results: Successful granulation to enable s.s.g was accomplished in 19 patients $(79.2 \%)$ in the study group vs. 11 patients $(45.8 \%)$ in the control group $(p=0.037)$, denoting better granulation in the study group. Failure to heal completely was observed in 7 patients $(29.2 \%)$ in the control group, while all patients in the study group healed completely by the end of the study $(p=0.009)$. The mean time to heal was $22.5+15.4$ days in the foam group, while it was $48.6+21.0$ days in the control group $(\mathrm{p}<0.0001)$.

Conclusions: Application of foam dressing is associated with higher healing rates and a significantly shorter time to heal as compared to treatment with conventional dressings in type 2 diabetic patients with lower-extremity necrotising fasciitis. These encouraging results warrant further investigation to address the issue of the potential reduction in hospital stay, treatment costs and morbidity.

\section{5}

Accelerated cutaneous wound healing of streptozotocin diabetic mice via non-viral VEGF ${ }^{165}$ gene therapy using biocompatible super-coiled minicircle plasmid

J. H. Park ${ }^{1}$, C. S. Yoon ${ }^{1}$, H. S. Chung ${ }^{1}$, M. J. Kwon ${ }^{1}$, S. H. Lee ${ }^{1}$, M. K. Kim²;

${ }^{1}$ Division of Endocrinology \& Metabolism, Department of Internal Medicine, Molecular Therapy Lab, Paik Memorial Insitute for Clinical Research, Pusan Paik Hospital, College of Medicine, Inje University, Busan, ${ }^{2}$ Department of Internal Medicine, Maryknoll General Hospital, Busan, Republic of Korea

Background and Aims: Chronic un-healing foot wound is a serious problem in the complicated diabetic patients. To accelerate the healing of diabetic cutaneous wounds, various kinds of growth factors have been employed with the limited success. The short half life of the administered growth factors in the hostile wound beds might have limited wide-spread clinical usage. To overcome this, growth factor gene therapy might be an attractive alternative rather than the direct application of factors onto the wound beds. However, possible biological risks of foreign DNA integration into the chromosome and the immunologic side effects of viral gene carrier have made it very difficult to be used in the actual human treatment. To develop the more safe and effective non-viral growth factor gene therapy techniques, we performed this study.

Materials and Methods: We cloned super-coiled minicircle plasmid containing human $\mathrm{VEGF}^{165}$ cDNA devoid of hazardous bacterially originated sequences including $\mathrm{CpG}$ motifs which could stimulate the host immune responses. We injected mincircle pMINI-beta-VEGF165 via non-viral gene delivery techniques into the margin of cutaneous wound of streptozotocin diabetic C57BL/6J mice.

Results: Administration of pMINI-beta-VEGF ${ }^{165}$ showed much accelerated wound healing as compared with the control groups without the any adverse events. Increased neovascularizations in the wound were confirmed by the quantitative and qualitative laser Doppler flowmetry and the immunohistochemical staining of the various vascular markers. The VEGF ${ }^{165}$ expression decreased again to the basal level shortly after the healing of cutaneous wounds.

Conclusion: These data might suggest that the non-viral VEGF165 minicircle plasmid gene therapy can be effectively used without the harmful side effects of viral gene therapy for the treatment of chronic un-healing cutaneous wounds of diabetic patients.

Supported by a Grant from Inje University 2005

0156

Long-term effects of intranasal calcitonin on healing times and recurrence of acute Charcot foot: a randomized controlled trial

R. Bem, A. Jirkovska, V. Fejfarova, J. Skibova;

Diabetes Centre, Institute for Clinical and Experimental Medicine, Prague, Czech Republic.

Background and Aims: Charcot foot represents a late complication of diabetes and can lead to progressive and worsening disruption of the bone architecture of the foot. The aim of this study was to evaluate the effectiveness of intra-nasal calcitonin on clinical features (healing times and recurrence of acute Charcot foot attack) in a randomized controlled trial.

Materials and Methods: Thirty-six patients with acute Charcot foot treated in our foot clinic during a 16-month period were randomized to receive intranasal calcitonin 200 IU/day together with a calcium supplementation $1000 \mathrm{mg} /$ day [study group $(\mathrm{n}=18)$ ] or on calcium supplementation of $1000 \mathrm{mg} /$ day in monotherapy [control group $(n=18)]$. All patients also had standard treatment of the Charcot foot, including off-loading by removable contact cast or cast walkers, with no significant difference between groups in the type of used off-loading device. All subjects were instructed to bear weight on their casted extremity using assistive devices (crutches). Healing was defined as 
the cessation of off-loading. Until March 2006, 31 (sixteen in the study group and fifteen patients in controls), who were primary healed and finished a one-year follow-up period after primary healing, were assessed in our preliminary study. Healing time was defined as the number of days from the initial off-loading device application until the cessation of the casting. The decision of definitive offloading cessation was made on the basis of clinical signs (foot without oedema and redness with skin temperature difference $<2^{\circ} \mathrm{C}$ during 6 weeks period of temporary intermittent off-loading) and a favourable radiological and isotopic examination of affected foot. The recurrence was defined as new signs of acute Charcot foot requiring new intensive off-loading and it was assessed during one- year period since cessation of casting.

Results: The mid-foot was the most commonly affected site [13 (81\%) in the study group, $13(86 \%)$ in controls; NS]. There were a significant shorter healing times in the study group in comparison with controls [mean 159.8 days $(95 \%$ CI 137.6-182.1) vs. 195.2 days (95\% CI 166.8-223.7); $\mathrm{p}<0.05]$. Three in the study group $(18.7 \%)$ and six $(40 \%)$ subjects in controls had acute recurrences of Charcot foot within the first year of follow-up after the initial casting period, but no significant difference was seen between groups. Seven patients in each group were ulcerated $(43.7 \%$ vs. $46.6 \%$; NS) and two patients underwent minor amputation in both groups during the study period $(12.5 \%$ vs. $13.3 \%$; NS), no major amputation was required.

Conclusion: This study suggests that patients with acute Charcot foot may have a clinically apparent benefit from the calcitonin treatment, which shortens healing times of the Charcot foot. The tendency of the lowering the recurrence rate of acute Charcot foot attack is promising, but a longer follow-up period is necessary.

\section{OP 27}

\section{Genetics of type 1 diabetes}

\section{7}

Genetic interaction in type 1 diabetes susceptibility supported by data mining and protein interaction analyses

R. Bergholdt ${ }^{1}$, Z. M. Størling ${ }^{2}$, K. Lage ${ }^{2}$, E. O. Karlberg ${ }^{2}$, P. Í. Ólason ${ }^{2}$, M. Aalund ${ }^{3}$, J. Nerup ${ }^{1,4}$, S. Brunak ${ }^{2}$, F. Pociot ${ }^{1,4}$;

${ }^{1}$ Steno Diabetes Center, Gentofte, Denmark, ${ }^{2}$ Center for Biological Sequence Analysis, Technical University of Denmark, Lyngby, Denmark, ${ }^{3}$ Neurotech A/S, Copenhagen, Denmark, ${ }^{4}$ Institute for Clinical Science, University of Lund, Sweden.
Background and Aims: Complex traits like type 1 diabetes (T1D) are influenced by multiple genes presumed to interact with each other to confer disease susceptibility and/or protection. The aim of the present study was to use analytical methods of data mining and decision trees to evaluate genetic interaction in T1D pathogenesis. For evaluation of functionality of observed genetic interactions, protein interaction networks were generated.

Material and Methods: T1D genome scan data (IBDstatus) on 1321 affected sib pairs, obtained via the Type 1 Diabetes Genetics Consortium, was used. Decision tree learning (SAS Enterprise Miner) was used to detect combinations (rules) of microsatellite markers splitting data into cleaner subgroups of T1D vs. Non-T1D. Proteins encoded by $10 \mathrm{Mb}$ on each site of microsatellite markers included in the rules were used as input proteins, and queried by a newly developed protein interaction network analysis scheme, for interactions at the protein level. Resulting protein interaction networks were visualized by Cytoscape v.2.0.

Results: Evidence for genetic interaction between several loci was observed. Rules of two markers providing more information than any of the markers themselves were obtained $(\mathrm{P}=<0.001$ to 0.04$)$. For further evaluation of these predicted genetic interactions we queried the loci for physical interactions with each other. By generation of protein interaction networks, we demonstrated evidence for interactions at the protein level for several of the most significant genetic interactions predicted by data mining studies, e.g. between the HLA-region and a region on chromosome 19 (TNFA - D19S591), as well as between HLA and chromosome 21 (TNFA - D21S270), and between regions on chromosome 17 and 21 (D17S798 - D21S167). The molecular function and biological processes of several of the proteins in the high-confidence networks supports oxidative stress, signal transduction, regulation of transcription and apoptosis to be important in T1D pathogenesis.

Conclusion: Implication for functionality was obtained for several of the observed genetic interactions conferring susceptibility to T1D. This may be the most extended suggestion of genetic epistasis in a multifactorial disease as T1D, strengthened significantly by the functional evidence for the interactions. This support the concept of genes encoding proteins, which function within complex networks to carry out specific biological processes, and supports that genetic interaction is important in T1D pathogenesis.

The Type 1 Diabetes Genetics Consortium (www.t1dgc.org) kindly provided access to their genome scan data. 


\section{8}

Differential expression of $H L A-D Q A 1$ and $-D Q B 1$ alleles associated with susceptibility to and protection from autoimmune type 1 diabetes

A. C. Britten, K. Jones, A. N. Dixon, S. D. Rees,

C. H. Mijovic, A. H. Barnett, M. A. Kelly;

Medicine, University of Birmingham, United Kingdom.

Background and Aims: The risk of developing type 1 diabetes is influenced by HLA-DQ alleles; $D Q A 1^{*} 0301$, $D Q A 1 * 0501, D Q B 1 * 0201$ and $D Q B 1^{*} 0302$ predispose to the disease while $D Q A 1 * 0102$ and $D Q B 1 * 0602$ confer protection. This effect may be related to the ability of the encoded molecules to present diabetogenic antigens to autoreactive $\mathrm{T}$ cells. The three-dimensional structure of the DQ molecules is an important determinant of this function, but the activation of an autoimmune response may also be influenced by the density of these molecules on the surface of antigen presenting cells. Disease risk may therefore be determined by the relative levels of expression of predisposing and protective/neutral alleles. To explore this possibility, we investigated the relative levels of mRNA transcripts from pairs of DQA1 and DQB1 alleles in peripheral blood lymphocytes (PBLs) from DQ-heterozygous individuals with and without type 1 diabetes. We also assessed the promoter activity of the alleles associated with the disease. Our aim was to determine whether a) different alleles are expressed at different levels, b) these differences are attributable to differences in promoter activity, c) the relative level of expression of an allele is related to its influence on disease risk and d) the ratio of expression of protective and predisposing alleles correlates with disease risk.

Materials and Methods: The relative abundance of mRNA transcripts from pairs of DQA1 and DQB1 alleles was determined in 59 DQ-heterozygous individuals (29 with type 1 diabetes and 30 healthy controls) using RT-PCR followed by allele-specific restriction digestion. The proximal promoter and polymorphic upstream regulatory regions of the predisposing and protective DQ alleles were cloned into the pGL3 Basic vector. The constructs were introduced into three DQ homozygous B lymphocyte cell lines using electroporation and promoter activity was measured using the Dual-Glo luciferase assay.

Results: The $D Q A 1^{*} 0301$ and $D Q B 1^{*} 06$ alleles were generally over-expressed at the mRNA level compared to other alleles. In contrast, mRNA for the $D Q B 1 * 02$ allele was generally less abundant than other DQB1 transcripts There was no consistent pattern of expression for $D Q A 1^{*} 0102$ and $D Q A 1^{*} 0501$. The ratio of expression of predisposing/protective alleles did not differ between the diabetic and control subjects. The results of the promoter study were concordant with those from the mRNA analysis.
The $D Q A 1 * 0301$ promoter showed higher activity compared with $D Q A 1^{*} 0102$ and $D Q A 1^{*} 0501$; no consistent pattern was observed for the latter two alleles. The $D Q B 1 * 0602$ promoter showed greater activity compared with $D Q B 1^{*} 0201$ and $D Q B 1 * 0302$.

Conclusion: This is the first study to demonstrate differential allelic expression at both the DQA1 and DQB1 loci in PBLs. Our data suggest that the relative abundance of mRNA transcripts for different alleles may be partly determined by differences in promoter activity. No simple correlation was found between the level of expression of specific DQ alleles and their influence on the risk of diabetes. The unbalanced expression of DQ alleles may influence the stoichiometry of the DQ $\alpha \beta$ heterodimers expressed on the surface of antigen presenting cells. This could have important implications for the pathogenesis of autoimmune disease and requires further study at the protein level.

Supported by Eli Lilly

0159

Major histocompatibility complex, class II, transactivator, MHC2TA, is associated with type 1 diabetes susceptibility S. Ahmed ${ }^{1}$, A. Gyllenberg ${ }^{1}$, M. Janer ${ }^{2}$, E. A. Rutledge ${ }^{3}$, L. Padyukov ${ }^{4}$, J. Graham ${ }^{5}$, F. Piehl ${ }^{6}$, T. Olsson ${ }^{6}$, C. Törn ${ }^{7}$, M. Landin-Olsson ${ }^{7}, \AA$. Lernmark $^{3}$, I. Kockum ${ }^{8}$;

${ }^{1}$ Molecular Med and Surgery, Karolinska Institutet, Stockholm, Sweden, ${ }^{2}$ Biology, Systems Biology, Seattle, United States, ${ }^{3}$ Dept of Medicine, University of Washington, Seattle, United States, ${ }^{4}$ Molecular Med., Karolinska Institutet, Stockholm, Sweden, ${ }^{5}$ Dept of Statistical and Actuarial Sciences, Simon Fraser University, Burnaby, Canada, ${ }^{6} \mathrm{Clin}$. Neurosciences, Karolinska Institutet, Stockholm, Sweden, ${ }^{7}$ Dept of Medicine, University Hospital, Lund, Sweden, ${ }^{8}$ Dept of Medicince, Karolinska Institutet, Stockholm, Sweden.

Background and Aims: The major histocompatibility complex (MHCII) is thought to be the major susceptibility factor for type 1 diabetes (T1DM). Other non-HLA genetic factors are also shown to contribute to T1DM susceptibility. The MHC2TA (16p13) gene codes for the non DNA binding transcriptional co-activator CIITA. This protein is transcribed from four different MHC2TA promoters (PIPIV).The CIITA protein is crucial for MHC II transcription and it is therefore thought that the MHC2TA gene is the main controlling factor of MHC II expression. The rs3087456 marker located in the PIII region of the MHC2TA gene, has been reported to be involved in the susceptibility of Multiple Sclerosis (MS), Rheumatoid Arthritis (RA) and Myocardial Infarction. We have previously observed linkage in the region of MHC2TA to 
T1DM. Thus the aim of our study was to test the hypothesis that MHC2TA is a T1DM susceptibility gene.

Material and Methods: Genotyping was carried out using the DASH method and the Taqman system. Genotypes from 28 Single Nucleotide Polymorphisms (SNP) in 373 unrelated Caucasian controls were used to define haplotype blocks and tagging SNPs in the HapBlock program. In total 20 of these SNPs were genotyped in a Swedish case-control study (586 T1DM patients and 836 controls). The age at onset of the patients varied between 15 and 34 years of age. Test of association were calculated using the cocaphase command in the Unphased computer program. Logistic regression analysis was performed using the proc logistic command in the SAS package.

Results: The HapBlock program revealed 5 blocks in the MHC2TA gene extending from before PI to the 3UTR region. The blocks were tagged by 1 to 3 SNPs each, comprising a total of 12 SNPs. These SNPs were analysed in the Swedish case-control study to show that SNPs in the first two haplotype blocks in MHC2TA were associated with T1DM $(p<0.006$ and $p<0.04$ respectively). An additional 8 SNPs were typed in these two blocks (see figure 1). The rs3087456 marker, in PIII, which was associated to MS, RA and MI was also associated with T1DM $(p<0.04)$, however a stronger association is observed for rs11074932, between PI and PIII, $p<0.003$. The association of MHC2TA remained significant in a logistic regression analysis including HLA DRB1, DQA1 and DQB1 as well as INS VNTR. The presence of $\mathrm{T}$ at rs11074932 was associated with T1DM $(\mathrm{OR}=2.48,95 \% \mathrm{CI}$ $1.25-4.92, \mathrm{p}=0.003)$.

Conclusion: We conclude that the rs 11074932 SNP located between PI and PIII is a significant susceptibility marker for T1DM. However, since no markers located in PI could be typed and due to the close proximity of rs11074932 to PI it is suggested that sequencing of PI should be done in order to fully understand which marker "truly" is involved in the susceptibility of T1DM. Taken together these data, we propose that the MHC2TA gene contributes to the susceptibility of T1DM.

Supported by JDRF

0160

Association of FOXP3 polymorphisms with GAD65 autoantibodies in type 1 diabetes

B. C. Holm ${ }^{1}$, E. Lindholm ${ }^{2}$, E. Bakhtadze ${ }^{2}$, C.-D. Agardh ${ }^{2}$, C. M. Cilio ${ }^{2,3}$;

${ }^{1}$ Dept. of Clinical Sciences, Lund University, Malmö, Sweden, ${ }^{2}$ Dept. of Clinical Sciences, ${ }^{3}$ Dept. of Pediatrics, Lund University, Malmö University Hospital, Sweden.
Background and Aims: Although increasing efforts have been made to identify susceptibility genes, only a few candidate genes besides the MHC have clearly been associated with type 1 diabetes (T1D) development. The human FOXP3 is a forkhead transcription factor, encoded by a gene on chromosome Xp11.23, which is important for the development and function of regulatory $\mathrm{T}$ cells. The aim of this study was to define if variants in the FOXP3 gene are associated with T1D development.

Materials and Methods: We screened single nucleotide polymorphisms (SNPs) within the regulatory $\mathrm{T}$ cell specific gene FOXP3 among 803 T1D patients and compared the allele frequencies with 208 healthy individuals. Importantly, we also correlated the SNPs with various clinical parameters in the T1D patient group, including levels of GAD65 autoantibody (GADA), HbA1c, fasting plasma glucose, fasting plasma C-peptide, body mass index (BMI) and HLA genotypes.

Results: Three of four SNPs were in linkage disequilibrium (LD), and one of these was thoroughly analyzed. Male T1D patients with the minor A allele of FOXP3 rs 2232365 (2365) displayed lower frequencies of GADA $(\mathrm{p}=0.004)$ and lower GADA titers $(\mathrm{p}=0.003)$ compared to those with the $G$ allele. When only male T1D patients with short disease duration ( 0 to 3 years) were considered, patients with the $\mathrm{G}$ allele had higher frequencies of undetectable $\mathrm{C}$ peptide $(30.3 \%)$ than those with the A allele $(4.2 \%$; $\mathrm{p}=0.02$ ). When patients were stratified for HLA genotypes a significant difference in the frequency of GADA positive individuals and GADA titers in the male T1D group with risk HLA-DQB1 genotypes was observed. Logistic regression analyses among all T1D males demonstrated that GADA were associated with shorter disease duration, older age, older age at onset of diabetes, HLA-DQB1 risk genotypes and with the FOXP3 $2365 \mathrm{G}$ allele $(\mathrm{p}=0.0001)$.

Conclusion: The minor A allele in the FOXP3 2365 SNP seems to be a protective allele in T1D development and therefore this novel finding suggests a role of FOXP 3 in the pathogenesis of T1D.

Supported by JDRF grant (CMC) and JDRF postdoctoral fellowship (BCH)

0161

TIM-3 and CTLA-4 polymorphisms in German type 1 diabetes families: two genes associated with T-lymphocyte regulation

E. Ramos-Lopez, W. Bartsch, P. Brück, H. Kahles, M. Penna-Martinez, J. Herwig, K. Badenhoop; Endocrinology, University Frankfurt am Main, Germany. 
Background: The balance between Th1 and Th2 cells is critical in the pathogenesis of type 1 diabetes. It has been previously reported that the family of T-cell immunoglobulin domain and mucin domain (TIM) proteins are expressed on the T cells. TIM-3 is present in Th1 cells and appears to play an essential role in Th1-mediated immune responses and in macrophage activation. While the cytotoxic $\mathrm{T}$ lymphocyte antigen-4 (CTLA-4) is a negative regulator of activated Tcells, TIM-3 downregulates the T-lymphocyte response at the effector level. Furthermore TIM-3 deficient mice develop a more severe form of experimental autoimmune diabetes. Therefore we investigated the role of TIM-3 gene polymorphisms in German type 1 diabetes families.

Materials and Methods: German type 1 diabetes families [191, (573 subjects)] were genotyped for the rs10515746 and rs1036199 polymorphisms using real time PCR, while for the rs10053538 by restriction fragment length polymorphism. Genotype-, haplotype-frequencies and linkage disequilibrium (LD) were estimated using Unphased version 2.403. A corrected $P(P \mathrm{c})<0.05$ was considered significant.

Results: No differences were observed in the transmission and not transmission frequencies of the TIM-3 polymorphisms $\left(P_{\mathrm{rs} 10515746}=0.6548, P_{\mathrm{rs} 1036199}=0.7114\right.$, $P_{\mathrm{rs} 10053538}=0.2214$, respectively). Allele frequencies range from 5 to $20 \%$.

We correlated the two CTLA-4 polymorphisms with the strongest type 1 diabetes association (CT_60 and MH30) with TIM-3. In the presence of TIM-3-rs10053538C the relative risk to type 1 diabetes - stratified for the polymorphisms CTLA-4 MH30 or CT_60 - is higher than if these CTLA-4 SNPs were considered alone: odds ratios rise from 1.83 to 1.93 and from 1.57 to 1.64 , respectively, but this difference is not significant. Thus, the allele combinations rs10053538C-MH30G $(P=0.0001)$ and rs10053538CCT_60G $(P=0.0011)$ were more frequently transmitted, while the allele combinations rs10053538C-MH30C and rs10053538C-CT_60A were less often transmitted to offspring ( $P=0.0002$ and $P=0.0041)$.

Conclusion: A combined transmission of TIM-3 and CTLA-4 variants on risk haplotypes enhances the genetic susceptibility to type 1 diabetes but the TIM-3 contribution is marginal.

\section{2}

Temporal trends in HLA genotype distribution in 1986-2005 suggest altered risk for type 1 diabetes

S. Lindehammer ${ }^{1}$, A. Nilsson ${ }^{1}$, S. Lindehammer ${ }^{1}$,

K. Larsson ${ }^{1}$, E. Örtqvist ${ }^{2}$, A. Carlsson ${ }^{3}$, E. Cederwall ${ }^{4}$, S. Ivarsson ${ }^{1}$, B. A. Jönsson ${ }^{1}$, H. Larsson ${ }^{1}$, B. Lindberg ${ }^{1}$, K. Lynch ${ }^{1}$, J. Neiderud ${ }^{5}$, S. Sjöblad ${ }^{3}$, A. Lernmark ${ }^{1}$;
${ }^{1}$ University Hospital MAS, Lund University, Malmö,

${ }^{2}$ Temporal Trends in HLA Genotype die, Karolinska Institute, Malmö, ${ }^{3}$ University Hospital, Lund University, ${ }^{4}$ Department of Clinical Sciences, Ängelholm Hospital, ${ }^{5}$ Department of Pediatrics, Helsingborgshospital, Helsingborg, Sweden.

Background: It has been suggested that the frequency of certain HLA genotypes among new onset type 1 diabetes patients may show a temporal change. The relative risk of certain HLA genotypes are gaining while others are losing in risk. Such changes may influence the triggering events, the age at clinical onset and may perhaps explain the trend that children tend to develop the disease at a younger age. Aims: Our aims were to compare the frequency of HLA genotypes in new onset type 1 diabetes patients diagnosed at 1-18 years of age in 1986-1987 $(n=430), 1996-2000$ $(n=342)$ and 2003-2005 $(n=171)$. We tested the hypothesis that the distribution of the HLA genotype frequencies changes over time.

Methods: Consecutively diagnosed Swedish type 1 diabetes patients were typed for HLA using PCR amplification and allele-specific probes for $\mathrm{DQ} \mathrm{A} 1^{*}$ and $\mathrm{B} 1^{*}$ alleles. Buffy coats or Dried Blood Spots were used as the source of DNA. The case-control study of all patients developing type 1 diabetes in 1986-87 was the baseline reference study available at http://stat-db.stat.sfu.ca/magenta/. The 19962000 cohort were ascertained in Ská¥ne and the 2003-2005 group from Stockholm.

Results: The most common T1D HLA genotype DQA1*B1*: 0501-0201/0301-0302 changed from 36\% (153/430) in $1986-87$ to $37 \%(127 / 342)$ in $1996-2000$ and to $19 \%(33 /$ $171)$ in $2003-2005$. The decrease in the high-risk heterozygous genotype may be mirrored by an increase in the homozygous 0301-0302/0301-0302 genotype: 8\% in 1986$87,10 \%$ in $1996-2000$ and $12 \%$ in $2003-2005$. There was also an increase in the frequency of the 0301-0302/01020604 genotype: $2 \%$ in $1986-87 ; 2 \%$ in $1996-2000$ and $4 \%$ in 2003-2005. The 0501-0201/0501-0201 genotype also increased: $2 \%$ in $1986-87 ; 7 \%$ in $1996-2000$ and $5 \%$ in 2003-2005. All changes over time were significant $(\mathrm{p}<0.05)$. Conclusion: This study in Swedish type 1 diabetes patients diagnosed at 1-18 years of age support the notion that there is a temporal change in HLA risk.

Supported by Swedish Research Council, Swedish Diabetes Association, Swedish Childhood Diabetes Fund, UMAS Funds, Skane County Council, National Institutes of Health 


\section{OP 28}

\section{Nephropathy - experimental}

0163

Podocyte inducible angiopoietin-2 overexpression results in albuminuria and glomerulosclerosis in transgenic mice

L. Gnudi ${ }^{1}$, B. Davis ${ }^{1}$, A. Dei Cas ${ }^{1}$, K. E. White ${ }^{2}$, R. Bilous ${ }^{2}$, G. Viberti ${ }^{1}$;

${ }^{1}$ Department of Diabetes and Endocrinology, Cardiovascular Division, King's College London, ${ }^{2}$ Department of Diabetes and Metabolism, University of Newcastle, United Kingdom.

Background and Aims: The development of nephropathy in diabetes is associated with glomerular upregulation of angiopoietin-2 (Ang2), a critical growth factor implicated in angiogenesis and vascular integrity. The specific role of this growth factor in renal dysfunction and pathology is unknown. Materials and Methods: We generated transgenic animals with glomerular specific overexpression of Ang2 and $\beta$ galactosidase cDNA using the Tet-On regulatable inducible system. We utilised the podocyte-specific promoter podocin driving the expression of the reverse tetracycline transactivator. To activate the transgene, Doxycycline $(2 \mathrm{mg} /$ $\mathrm{mL}$ ) was administered with water. We studied the following groups of mice for up to 10 weeks: a) transgenic (TG) with doxycycline (DOX), b) TG without DOX, c) controls (C) with DOX and d) $\mathrm{C}$ without DOX. Kidney cortex was collected and prepared for subsequent analysis for $\beta$-galactosidase staining, western immunoblotting, immunohistochemistry, and electron microscopy studies. 24-hour albuminuria was determined with ELISA.

Results: Podocyte positive $\beta$-galactosidase staining was present only in TG-DOX treated animals, indicating absence of leaky transgene activity. Western immunoblotting and immunogold studies showed a 3-4 fold increase $(p<0.05)$ in Ang2 protein overexpression only in the DOX treated TG mice. Albuminuria was increased significantly by $2-3$ fold in the TG-DOX treated mice $(\mathrm{p}<0.05)$ as early as 5 weeks after treatment with DOX. At 10 weeks a significant increase in glomerular extracellular matrix deposition was observed (PAS staining) $(\mathrm{p}<0.05)$.

The expression of vascular endothelial growth factor was reduced in TG DOX treated animals when compared to controls $(\mathrm{p}<0.05)$.

Conclusion: We have generated a transgenic mouse model that allows podocyte-specific inducible Ang2 overexpression. This resulted in induction of albuminuria and excess extracellular matrix accumulation suggesting a role of Ang2 in renal pathology.

Supported by EFSD/Servier Research Programme

0164

TGFß1-mediated activation of protein kinase B/Akt is important for epithelial-mesenchymal transition in diabetic nephropathy

D. P. Brazil, J. J. Kattla, C. Godson;

UCD School of Medicine and Medical Science, UCD Conway Institute, Dublin, Ireland.

Background and Aims: The cellular signaling events driving the pathogenesis of diabetic nephropathy are complex and not fully elucidated. Transforming growth factor-beta 1 (TGF-beta1) is a primary mediator of cellular damage during $\mathrm{DN}$, and its upregulation is implicated in epithelial-mesenchymal transition (EMT) associated with $\mathrm{DN}$-associated renal fibrosis. Protein kinase $\mathrm{B}(\mathrm{PKB} / \mathrm{Akt})$ is a multi-functional regulator of multiple key cellular processes, and some of its downstream targets have been implicated in the onset and progression of EMT in renal fibrosis. Importantly, the regulation of Snail, a transcription factor that represses the expression of e-cadherin during renal fibrosis has been shown to be regulated by GSK3beta, a downstream target of PKB/Akt.

Materials and Methods: In vitro cell culture models of epithelial to mesenchymal transition were used by treated kidney epithelial cells with TGFbeta and/or EGF. CElls were analysed using Western blotting or immunfluoresence. Results: To investigate the relevance of PKB/Akt activation downstream of TGFbeta1 in tubulointerstitial fibrosis, kidney epithelial cells (NRK-52E and HK2 cells) were exposed to TGFbeta1 for various periods. Phosphorylation of $\mathrm{PKB} / \mathrm{Akt}$ was evident in cells exposed to TGFbetal for $60 \mathrm{~min}$. Activation of downstream targets of PKB/Akt such as GSK3beta and p27Kip1 was also detected. PI3K inhibitors such as LY294002 and wortmannin reduced this TGFbeta1-mediated activation. TGFbeta1 treatment of epithelial cells induces epithelial-mesenchymal transition as judged by decreased levels of epithelial markers ecadherin and ZO-1, and increased levels of mesenchymal markers such as vimentin and alpha smooth muscle actin. Inhibition of PKB/Akt using LY294002 significantly reduced the ability of TGFbetal to induce EMT in these cells, suggesting that $\mathrm{PKB} /$ Akt activity is involved in this response.

To examine the role of $\mathrm{PKB} / \mathrm{Akt}$ activation in $\mathrm{DN}$ in vivo, we analyzed $\mathrm{PKB} / \mathrm{Akt}$ activation in kidney sections from both control Wistar and type 2 diabetic Goto-Kakizaki rats (GK). GK rats displayed mild to moderate diabetic 
nephropathy at 9-10 months of age. Elevated levels of phosphorylated $\mathrm{PKB} / \mathrm{Akt}$ and its downstream target pGSK3beta were observed in the kidneys of diabetic, but not control Wistar rats, with pronounced staining in the kidney tubules.

Conclusion: These data suggest that TGFbetal mediated $\mathrm{PKB} /$ Akt signaling plays an important role in the fibrotic response triggered by the extracellular milieu in diabetic nephropathy.

Supported by Health Research Board Ireland and Science Foundation Ireland

\section{5}

Cholecystokinin plays protective roles against progression of diabetic nephropathy through anti-inflammatory effects

M. Sasaki, K. Shikata, S. Nishishita, C. Sato, S. Ohga, S. Okada, H. Makino;

Department of Medicine and Clinical Science, Okayama University Graduate School of Medicine, Dentistry and Pharmaceutical Sciences, Japan.

Background and Aims: We have shown that intercellular adhesion molecule-1(ICAM-1) is upregulated and mediates macrophage infiltration into diabetic kidney. ICAM-1 deficiency protects against renal injuries after induction of diabetes suggesting that inflammatory process is involved in the pathogenesis of diabetic nephropathy. DNA microarray analyses using diabetic ICAM-1 knockout (KO) mice suggested that cholecystokinin (CCK) is one of the key molecule for inflammatory process in the progression of diabetic nephropathy. CCK is a peptide with various functions including regulation of inflammatory reaction, gastrointestinal homeostasis, and neurotransmittion. Two types of $\mathrm{CCK}$ receptors, types $\mathrm{A}$ and $\mathrm{B}$, have been identified. Both type receptors are expressed in macrophage and kidney. The aim of this study is to clarify the role of CCK in the pathogenesis of diabetic nephropathy using $5 / 6$ nephrectomy which is the model of non-immune glomerular sclerosis.

Materials and Methods: 1) We performed DNA microarray analysis using the kidneys from diabetic and nondiabetic ICAM-1 KO (ID and IND) and wild type mice (WD and WND). We also used 5/6 nephrectomized and sham-operated ICAM-1 KO (IN and IS) and wild type mice (WN and WS). 2) We performed 5/6 nephrectomy or sham operation on CCK-A receptor KO (AN), CCK-B receptor $\mathrm{KO}(\mathrm{BN})$, CCK-A and $\mathrm{B}$ receptor double $\mathrm{KO}(\mathrm{ABN})$ mice and wild type mice. We evaluated renal function, blood pressure and renal pathology over a period 3 month. Glomerular size and mesangial matrix area were measured by morphometry using NIH Image. DNA microarray analysis (GeneChip ${ }^{\mathrm{TM}}$,Affimetrix) was performed with RNA from the kidney of $A B N$ and WN. 3) Cultured macrophages (PMA-treated THP-1 cells) were treated with sulfated CCK octapeptide (CCK-8s), control peptide and vehicle for $24 \mathrm{~h}$. Expression of TNF- $\alpha$, IFN- $\gamma$, IL-1 $\beta$ and MCP-1 in the 3 groups of macrophages was measured by quantitative real time RT-PCR.

Results: 1) Signal intensity of CCK was increased in WD or IN compared with WND or WS, and decreased in ID or IN compared with WD or WN. CCK was detected in renal tubules by immunoperoxidase staining. 2) Blood pressure was not different in the 8 groups. Creatinine clearance (Ccr) was lower in $\mathrm{ABN}$ than in $\mathrm{WN}$. The glomerular size was significantly larger in $\mathrm{ABN}$ than $\mathrm{WN}(\mathrm{p}<0.05)$. Mesangial matrix area was significantly greater in $\mathrm{ABN}$ than in the $\mathrm{WN}(\mathrm{p}<0.05)$. AN and $\mathrm{BN}$ had no significant difference from $\mathrm{WN}$ in $\mathrm{Ccr}$ and morphometry. DNA microarray indicated that 93 genes were upregulated and 101 genes were downregulated in $\mathrm{ABN}$ as compared with WN. These genes were classified into functional Gene Ontology categories. The analysis indicated that the category of the most significantly upregulated genes was "immune response" including inflammatory related genes $(p<0.001)$. 3) Cultured macrophages treated with CCK-8s suppressed mRNA expression of TNF- $\alpha$ as compared with control peptide and vehicle.

Conclusions: CCK mRNA expression was increased in diabetic and 5/6 nephrectomized kidney. Deficiency of both CCK-A and B receptors accelerated renal injuries as compared with wild type mice. Inflammatory related gene expression was upregulated in the kidney of $\mathrm{ABN}$ as compared with WN. CCK-8s inhibited the production of inflammatory cytokines from cultured macrophages. CCK may play protective roles against progression of diabetic nephropathy through anti-inflammatory effects.

Supported by Grants-in-Aid for Scientific Research from the Ministry of Education, Science, Culture, Sports and Technology of Japan

0166

Proinsulin C-peptide constricts afferent glomerular arterioles in diabetic mice. A potential reno-protective mechanism of action

L. Nordquist, E.-Y. Lai, M. Sjöquist, A. E. G. Persson; Dept. of Medical Cell Biology, Div. of Integrative Physiology, Uppsala University, Sweden. 
Background and Aims: The initial phase of diabetes is commonly associated with glomerular hyperfiltration filtration, and this has been postulated to be involved in the progression of diabetic nephropathy. Several studies have reported reno-protective effects of C-peptide in both diabetic subjects and in animal models. Furthermore, C-peptide has been shown to decrease the diabetes-induced hyperfiltration in insulin-dependent diabetic subjects. In this study, we investigated the vascular reactivity to C-peptide in isolated afferent arterioles from control and alloxan-induced diabetic C57/B1 mice.

Materials and Methods: Diabetes was induced by an intravenous injection of alloxan $(75 \mathrm{mg} / \mathrm{kg}$ bw) two weeks prior to the experiment. Age-matched normoglycemic mice served as control. Afferent arterioles, with intact glomerulus, were isolated and fixed in a confocal microscope. The arterioles were perfused with DMEM, and thereafter with DMEM containing C-peptide (1 nM). All results were compared to that of vehicle and a scrambled peptide of identical size as C-peptide, but with no known physiological function.

Results: The blood glucose level was elevated in the alloxan-treated mice $(18.2 \pm 0.6$ vs. control $6.4 \pm 0.2 \mathrm{mmol} / 1)$. C-peptide reduced the diameter of the afferent arterioles from diabetic mice (final reduction $-27 \pm 8 \% ; n=7$ ) within 10 minutes, whereas arterioles from control animals first showed a modest response to C-peptide after 30 minutes $(-4 \pm 1 ; n=7)$. The vehicle or the scrambled peptide did not have any effect in either group.

Conclusion: Our findings show that C-peptide has a pronounced constricting effect on afferent arterioles isolated from diabetic mice, whereas it has only a modest and delayed effect on arterioles originating from normoglycemic control mice. This suggests that the mechanism, by which C-peptide reduces the diabetes-induced glomerular hyperfiltration, is to constrict the afferent arteriole, thereby reducing the glomerular filtration pressure. Thus, new treatment strategies taking into account the beneficial effects of C-peptide could potentially reduce the prevalence and incidence of diabetic nephropathy.

\section{7}

The role of the angiotensin II type 2 receptor subtype in the development and progression of end-organ injury in experimental diabetes

A. Koitka, K. A. M. Jandeleit-Dahm, P. Koh, C. Tikellis, M. E. Cooper, T. J. Allen;

Diabetes and Metabolism Division, Baker Heart Research Institute, Melbourne, Australia.
Background: Although many of the renal and cardiac actions of angiotensin II (Ang II) are mediated via the type 1 angiotensin (AT1) receptor subtype, there is evidence that under pathophysiological conditions, the type 2 (AT2) receptor subtype may also be an important regulator of the response to injury. However, the status and role of the AT2 receptor in the diabetic context has not been fully characterised.

Aims: The aims of this study were firstly to assess the renal and cardiac expression of the AT2 receptor subtype (with concomitant measurement of AT1 receptor expression) in long term diabetes and secondly to evaluate the effect of an AT1 receptor antagonist on AT2 receptor expression.

Methods: Diabetes was induced by injection of streptozotocin (55 mg.kg-1) in 6-week-old C57Bl6 and Apo E deficient (a model of accelerated renal and vascular complications) mice and further randomised to no treatment or to the AT1 receptor antagonist, irbesartan (10 mg.kg-1) for 20 weeks ( $n=6-8 /$ group). The status of the AT2 receptor was assessed by real time RT-PCR, immunohistochemistry and in vitro autoradiography.

Results: The induction of diabetes was associated with increased expression of the AT2 receptor at sites of endorgan injury, including the kidney (control, $1.0 \pm 0.2$ vs diabetic C57, 6.2 $\pm 1.8, \mathrm{p}<0.05$ ) and the heart (control, $1.0 \pm 0.4$ vs diabetic C57, 18.6 $\pm 4.9, \mathrm{p}<0.01)$. A similar pattern was seen in diabetic apo $\mathrm{E}$ deficient mice with an approximately 7 and 25 fold increase in AT2 receptor expression in the kidney and heart respectively. Treatment with the AT1 receptor antagonist, which attenuates endorgan injury at all sites in these 2 models, ameliorated the diabetes associated increase in AT2 receptor expression (renal, diabetic $\mathrm{C} 57+$ irbesartan, $1.2 \pm 0.8, \mathrm{p}<0.05$ vs untreated diabetes). A similar reduction in AT2 receptor expression was also seen in both the kidney and heart in diabetic apoE deficient mice treated with irbesartan. In the same organs, the gene expression of the AT1 receptor was reduced or unchanged by diabetes and was in general upregulated $>100 \%$ by irbesartan treatment.

Conclusions: There was increased cardiac and renal expression of the AT2 receptor subtype in experimental diabetes. Whether this represents a protective and/ or pathogenic response in diabetes remains to be established by further studies in either AT2 deficient mice or mice treated with an AT2 receptor antagonist. Audrey Koitka was supported by ALFEDIAM. 


\section{8}

Tight control of arterial pressure and not inhibition of renin-angiotensin system determines prevention of glomerular hypertrophy and nephrin loss in experimental diabetes

J. B. Lopes de Faria, R. B. Amazonas, R. A. Sanita; Internal Medicine, University of Campinas, Brazil.

Background and Aims: A recent systematic review and meta-analysis on the effect of inhibitors of the reninangiotensin system in diabetic renal disease have concluded that: "additional renoprotective actions of these substances beyond lowering blood pressure remain unproven" (Casas et al. Lancet 2005;336:2026). The present study tested the hypothesis that in spontaneously hypertensive rats (SHR) with streptozotocin-induced diabetes mellitus (DM) strict control of arterial pressure with or without inhibition of the renin-angiotensin system can prevent the development of glomerular hypertrophy and the reduction in renal expression of nephrin.

Materials and Methods: DM was induced by injecting streptozotocin in 4-week-old, prehypertensive, SHR and the rats were randomized for no treatment, or for treatment with captopril, losartan or triple therapy (hydrochlorothiazide, reserpine and hydralazine) for 20 days.

Results: Plasma glucose levels in treated and non-treated diabetic rats were consistently and significantly elevated $(>20 \mathrm{mmol} / \mathrm{l}, \mathrm{p}<0.0001)$ when compared to control SHR (about $8 \mathrm{mmol} / \mathrm{l}$ ). The increase in systolic blood pressure was equally prevented by captopril $(115 \pm 16 \mathrm{mmHg})$, losartan $(108 \pm 12)$, and triple therapy $(113 \pm 9)$. Albuminuria was higher in diabetic rats $[400(111-501) \mu \mathrm{g} / 24 \mathrm{~h}]$ and similarly reduced $(\mathrm{p}<0.05)$ by captopril $[210(68-449)]$, losartan [190 (70-543)], and triple therapy [171 (62-381)]. Glomerular expression of nephrin, estimated by Western blot and immunofluorescence, was reduced in diabetic SHR in comparison with controls and the antihypertensive treatment prevented the reduction in glomerular expression of nephrin. Glomerular size was higher $(\mathrm{p}<0.005)$ in diabetic SHR $(27,300 \pm 2130 \mu \mathrm{m} 2)$ compared with nondiabetic SHR $(23,800 \pm 307)$. The antihypertensive therapy with captopril $(23,900 \pm 175)$, losartan $(23,800 \pm 120)$, and triple therapy $(23,400 \pm 210)$ prevented the glomerular enlargement in diabetic SHR. Glomerular expression of fibronectin and TGF- $\beta$ IIR were increased in diabetic SHR and they were normalized by treatment with the antihypertensive drugs.

Conclusion: In diabetic SHR rats strict control of arterial pressure with or without inhibition of the renin-angiotensin system can prevent the development of glomerular hypertrophy and reduction in renal expression of nephrin.

Supported by FAPESP, CNPq

\section{OP 29}

\section{Incretin hormone action - animal studies}

\section{9}

The classic incretin receptors for GLP-1 and GIP are essential for the sustained glucoregulatory actions of vildagliptin in mice

G. Flock ${ }^{1}$, J. L. Zhang ${ }^{2}$, A. Duttaroy ${ }^{2}$, D. J. Drucker ${ }^{1}$; ${ }^{1}$ BBDC, Medicine, University of Toronto, Canada, ${ }^{2}$ Novartis Institutes for Biomedical Research, Cambridge, United States.

Background and Aims: DPP-4 inhibitors (DPP-4i) lower blood glucose and $\mathrm{HbA1c}$ in experimental models and in clinical therapy of human subjects with type 2 diabetes, however the mechanism of action through which these agents improve glucose homeostasis remains incompletely understood. Although GLP-1 and GIP represent the predominant substrates mediating glucose reduction following acute DPP-4 inhibition, whether sustained inhibition of DPP-4 activity exerts anti-diabetic actions via recruitment of additional glucoregulatory substrates such as GRP or PACAP remains uncertain. To determine whether additional substrates contribute to the antidiabetic actions of DPP-4i, we examined the efficacy of LAF-237 (Vildagliptin) in high fat (HF)-fed wildtype (WT) or dual incretin receptor knockout (DIRKO) mice over 8 weeks.

Results: Vildagliptin had no significant effect on food intake, energy expenditure, body composition, body weight gain or insulin sensitivity, as assessed by ITT, in WT or DIRKO mice. Although Vildagliptin had no effect on fasting glucose, glycemic excursion following OGTT was significantly reduced in WT but not in DIRKO mice after 7 weeks of drug treatment. Pancreatic insulin content was comparable in mice with or without 8 weeks of Vildagliptin therapy. Vildagliptin increased levels of glucose-stimulated plasma insulin, acutely decreased levels of plasma glucagon, and reduced levels of cholesterol in WT but not in DIRKO mice. Furthermore, Vildagliptin therapy was associated with reduced hepatic expression of genes important for cholesterol synthesis. As GIP exerts complex actions on fat tissues, we analyzed adipokine expression in different fat depots. Although Vildagliptin had no effect on levels of epididymal or inguinal fat pad adipokine mRNA transcripts for adiponectin, leptin, resistin, TNF-alpha, IL- 
1beta, and IL-6 WT mice, PAI-1 mRNA transcripts were increased in WT yet significantly decreased in epididymal fat pads from DIRKO mice following 8 weeks of Vildagliptin therapy

Conclusion: These findings illustrate that 8 weeks of continuous DPP-4 inhibition improves glucose homeostasis and parameters of cholesterol synthesis in WT but not in DIRKO mice. Hence classic incretin receptors represent the dominant molecular mechanisms for transducing the chronic anti-diabetic actions mediated by DPP-4 inhibition in vivo. Supported in part by a grant from Novartis Inc

\section{0}

CJC-1134, an exendin-4-albumin GLP-1R agonist potently activates GLP-1R-dependent actions controlling glucose homeostasis

L. L. Baggio, D. J. Drucker;

BBDC, Medicine, University of Toronto, Canada.

Background and Aims: GLP-1 lowers blood glucose via multiple actions however the therapeutic potential of native GLP-1 is limited by its short plasma half-life, due to rapid inactivation by DPP-4 and renal clearance. We have now examined the biological actions of CJC-1134, a conjugate of exendin-4 covalently linked, ex vivo, to human serum albumin, to determine if much larger peptide-albumin hybrid molecules can exert similar actions to those exhibited by native exendin-4.

Materials and Methods: The acute actions of CJC-1134 were examined in both wild-type C57BL/6 and GLP-1 receptor $-/-(\mathrm{GLP}-1 \mathrm{R}-/-)$ mice. In longer-term studies, C57BL/6 mice were maintained on a high fat diet (HFD; $45 \%$ kcal fat) for an initial 4 weeks followed by an additional 4 week period of high fat feeding in the presence of ip injections of (i) twice daily saline, (ii) twice daily exendin-4 (Ex-4; $24 \mathrm{nmol} / \mathrm{kg}$ ), (iii) once daily human serum albumin (HSA; $100 \mathrm{nmol} / \mathrm{kg}$ ), or (iv) once daily CJC-1134 (100 nmol/kg).

Results: CJC-1134 dose-dependently lowered the glycemic excursion in response to either oral or ip glucose challenge following acute administration in mice. Although larger GLP-1R agonists may not penetrate the CNS to the same extent as smaller peptides like GLP-1 or exendin-4, both CJC-1134 and exendin-4 produced comparable inhibition of gastric emptying (PBS: $70.7 \pm 3.9 \%$ vs. Ex-4: $11.4 \pm 6.6 \%$, $P<0.001$; HSA: $75.7 \pm 5.8 \%$ vs. CJC-1134: $23.3 \pm 4.0$, $P<0.001)$. CJC-1134 also reduced 24-hr food intake, and activated c-fos expression in the murine CNS in wild-type mice. Furthermore, consistent with the GLP-1R-dependent specificity of CJC-1134 action, CJC-1134 did not lower blood glucose, inhibit gastric emptying or activate CNS c-fos expression in GLP-1R-/- mice. Chronic administration of exendin-4 or CJC-1134 significantly reduced body weight (PBS: $33.9 \pm 0.9$ g vs. Ex-4: $28.8 \pm 0.5 \mathrm{~g}$, $P<0.001$; HSA: $33.7 \pm 0.7$ g vs. CJC-1134: $27.8 \pm 0.5 \mathrm{~g}$, $P<0.001$ ), epididymal fat pad weight (PBS: $1.68 \pm 0.16 \mathrm{~g}$ vs. Ex-4: $0.68 \pm 0.07 \mathrm{~g}, P<0.001$; HSA: $1.42 \pm 0.18 \mathrm{~g}$ vs. CJC1134: $0.61 \pm 0.06 \mathrm{~g}, P<0.001$ ), and total fat mass (PBS: $10.5 \pm 0.7$ g vs. Ex-4: $4.7 \pm 0.7 \mathrm{~g}, P<0.05$; HSA: $6.9 \pm 1.1 \mathrm{~g}$ vs. CJC-1134: $2.4 \pm 0.4 \mathrm{~g}, P<0.05)$ and increased total pancreas weight (PBS: $0.28 \pm 0.01 \mathrm{~g}$ vs. Ex-4: $0.33 \pm 0.01 \mathrm{~g}, P<0.01$; HSA: $0.30 \pm 0.01 \mathrm{~g}$ vs. CJC-1134: $0.38 \pm 0.01 \mathrm{~g}, P<0.001)$. CJC-1134 also significantly decreased HbA1c levels (HSA: $3.99 \pm 0.12 \%$ vs. CJC-1134: $3.58 \pm 0.05 \%, P<0.01)$. Moreover, both exendin-4 and CJC-1134 reduced the glucose excursion in response to ip or oral glucose challenge performed after 3 or 4 weeks of drug treatment, respectively. Plasma cholesterol was reduced in CJC-1134-treated mice (HSA: 145.9 $\pm 8.6 \mathrm{mg} / \mathrm{dl}$ vs. CJC-1134: $120.9 \pm 5.5 \mathrm{mg} /$ dl, $P<0.05$ ), and hepatic triglyceride content was significantly lower in mice treated with either exendin-4 or CJC1134 (PBS: $7.6 \pm 1.2 \mathrm{mg} / \mathrm{g}$ vs. Ex-4: $5.5 \pm 0.5 \mathrm{mg} / \mathrm{g}, P<0.05$; HSA: $6.0 \pm 0.7 \mathrm{mg} / \mathrm{g}$ vs. CJC-1134: $3.4 \pm 0.4 \mathrm{mg} / \mathrm{g}, P<0.01)$. The effects of once-daily CJC-1134 were greater than those observed for twice-daily exendin-4 for the majority of GLP1R-dependent endpoints examined

Conclusion: These studies illustrate that the larger exendin4:albumin conjugate CJC1134 can elicit the same profile of GLP-1R-dependent biological actions, both in the periphery and CNS, relative to that obtained with smaller GLP-1R agonists such as exendin-4. The extended pharmacokinetic profile of albumin-based therapeutics in human subjects raises the possibility that $\mathrm{CJC}-1134$ may be suitable for once weekly dosing for the treatment of type 2 diabetes. Supported in part by a Travel grant from Conjuchem Inc

0171

Early administration of the GIP receptor antagonist $\left(\right.$ Pro $\left.^{3}\right)$ GIP counters development of hyperglycaemia, glucose intolerance and insulin resistance in genetic obesity

P. R. Flatt, N. Irwin, P. L. McClean, B. D. Green,

V. A. Gault, C. J. Bailey, F. P. M. O'Harte;

School of Biomedical Sciences, University of Ulster, Coleraine, United Kingdom.

Background and Aims: Gastric inhibitory polypeptide (GIP) is a gastrointestinal hormone secreted in response to feeding that stimulates insulin secretion and regulates carbohydrate and lipid metabolism. The actions of GIP are blocked by $\left(\right.$ Pro $\left.^{3}\right)$ GIP, a well-characterised stable and specific GIP receptor antagonist. This study utilised (Pro ${ }^{3}$ GIP to inves- 
tigate the effects of longer-term (60-day) GIP receptor ablation on development of spontaneous obesity and diabetes in young obese $(o b / o b)$ mice.

Materials and Methods: $\left(\mathrm{Pro}^{3}\right)$ GIP was synthesised using solid-phase Fmoc peptide chemistry and structure was confirmed by matrix assisted laser desorption ionisation mass spectrometry. Obese $(o b / o b)$ mice (5-7 weeks) were divided into two groups $(\mathrm{n}=8)$ that received, once daily i.p. injections $(17: 00 \mathrm{~h})$ over a 60 -day period, of either saline vehicle $(0.9 \%, \mathrm{w} / \mathrm{v}, \mathrm{NaCl})$ or $\left(\mathrm{Pro}^{3}\right) \mathrm{GIP}(25 \mathrm{nmol} / \mathrm{kg}$ body weight). Food intake and body weight were recorded five days before and daily throughout the study period. Plasma glucose and insulin concentrations were measured at 4 7 day intervals. At the end of the treatment period (day 60), glucose tolerance $(18 \mathrm{mmol} / \mathrm{kg}$ glucose i.p.), insulin sensitivity (50 U insulin/kg bw), glycated haemoglobin $\left(\% \mathrm{HbA}_{1 \mathrm{c}}\right)$ and plasma lipidse were assessed.

Results: Weight gain was suppressed in $\left(\mathrm{Pro}^{3}\right)$ GIP treated mice, but body weights and food intake did not significantly differ between groups at 60-days. However, non-fasting plasma glucose levels were significantly decreased in mice receiving $\left(\mathrm{Pro}^{3}\right) \mathrm{GIP}$ treatment from day $13(10.8 \pm 0.5 \mathrm{mmol} /$ 1 vs. $12.9 \pm 0.7 \mathrm{mmol} / 1$ for control; $\mathrm{P}<0.05$ ), did not rise thereafter and remained significantly lower throughout the entire study ( $\mathrm{P}<0.05$ to $\mathrm{P}<0.001)$. Circulating plasma insulin levels were also significantly reduced in $\left(\mathrm{Pro}^{3}\right)$ GIP treated mice, being $45.9 \pm 4.5 \mathrm{ng} / \mathrm{ml} v s .73 .1 \pm 8.9 \mathrm{ng} / \mathrm{ml}$ for control $(\mathrm{P}<0.05)$ on day 44. Following i.p. glucose administration on day 60 , the overall plasma glucose excursion was significantly $(1.9$-fold; $\mathrm{P}<0.001)$ lowered in $\left(\mathrm{Pro}^{3}\right)$ GIP treated mice (712.8 $\pm 73.8 \mathrm{mmol} / \mathrm{l} . \mathrm{min}$ vs. $1339.0 \pm 64.4 \mathrm{mmol} / \mathrm{l} . \mathrm{min}$ for control). Plasma insulin concentrations were also significantly diminished 15 min post glucose injection $(37 \%$; $\mathrm{P}<0.05)$, which was reflected by a significantly 2.1 -fold reduced $(\mathrm{P}<0.05)$ overall insulinotropic response in $\left(\mathrm{Pro}^{3}\right)$ GIP treated mice. Exogenous insulin injection caused a significantly greater fall of glucose concentrations in $\left(\mathrm{Pro}^{3}\right)$ GIP treated $o b /$ $o b$ mice $(105.9 \pm 17.5 \mathrm{mmol} / \mathrm{l} . \mathrm{min}$ vs. $145.5 \pm 32.0 \mathrm{mmol} / \mathrm{l} . \mathrm{min}$ for control; $\mathrm{P}<0.05$ ) indicative of substantial alleviation of insulin resistance. In keeping with improved glycaemic control, $\left(\mathrm{Pro}^{3}\right)$ GIP treated mice had significantly $(\mathrm{P}<0.05)$ decreased $\% \mathrm{HbA}_{1 \mathrm{c}}(4.5 \pm 0.4 \%)$ levels compared to controls $(6.0 \pm 0.3 \%)$. Assessment of plasma lipids revealed that 60 -day $\left(\mathrm{Pro}^{3}\right)$ GIP treated mice had significantly lower triglyceride $(122.3 \pm 9.6 \mathrm{mg} / \mathrm{dl} v s .150 .1 \pm 4.9 \mathrm{mg} / \mathrm{dl}$ for control; $\mathrm{P}<0.05)$ and LDL-cholesterol concentrations (1.5-fold; $\mathrm{P}<0.05)$ compared to controls.

Conclusion: Once daily administration of $\left(\mathrm{Pro}^{3}\right) \mathrm{GIP}$ for 60 days improved metabolic regulation and countered development of diabetes in $o b / o b$ mice. This illustrates the potential of $\left(\mathrm{Pro}^{3}\right)$ GIP for treatment of obesity-related type 2 diabetes, acting through beta cell sparing and the alleviation of insulin resistance.
0172

Long-acting analogues of GIP with significantly improved antihyperglycaemic and insulinotropic properties: N-terminal modification versus mid-chain acylation

N. Irwin ${ }^{1}$, G. C. Clarke ${ }^{1}$, V. A. Gault ${ }^{1}$, B. D. Green ${ }^{1}$, P. Harriott ${ }^{2}$, F. P. M. O'Harte ${ }^{1}$, P. R. Flatt ${ }^{1}$;

${ }^{1}$ School of Biomedical Sciences, University of Ulster, ${ }^{2}$ School of Biology and Biochemistry, Queens University Belfast, United Kingdom.

Background and Aims: Glucose dependent insulinotropic polypeptide (GIP) is an insulinotropic gastrointestinal hormone with therapeutic potential for type 2 diabetes. However, metabolic instability and rapid renal clearance limit the development of GIP-based therapies. Numerous GIP analogues have now been generated exhibiting DPP IV resistance and extended bioactive profiles. The present study reports a direct evaluation of the long-term antidiabetic actions of three such GIP molecules, $N$-AcGIP, GIP(Lys $\left.{ }^{37} \mathrm{PAL}\right)$ and $N$-AcGIP $\left(\mathrm{Lys}^{37} \mathrm{PAL}\right)$ in obese diabetic $(o b / o b)$ mice.

Materials and Methods: GIP-related peptides were synthesised using solid-phase Fmoc peptide chemistry and structure confirmed by matrix assisted laser desorption ionisation mass spectrometry. Obese-diabetic $(o b / o b)$ mice $(12-15$ weeks) were divided into four groups $(n=8)$ that received, once daily i.p. injections (17:00 h) over a 14-day period, of either saline vehicle $(0.9 \%, \mathrm{w} / \mathrm{v}, \mathrm{NaCl}), \mathrm{N}$-AcGIP, GIP(Lys ${ }^{37} \mathrm{PAL}$ ) or $N$-AcGIP(Lys ${ }^{37} \mathrm{PAL}$ ) (all at $25 \mathrm{nmol} / \mathrm{kg}$ body weight). Food intake and body weight were recorded five days before and daily throughout the 14-day study period. Plasma glucose and insulin concentrations were measured at 3-4 day intervals. At the end of the treatment period, circulating glucagon, glucose tolerance $(18 \mathrm{mmol} / \mathrm{kg}$ glucose), insulin sensitivity (50 $\mathrm{U}$ insulin/kg bodyweight), response to GIP (25 nmol/kg body weight), pancreatic insulin content and islet morphology were assessed.

Results: Body weight, food intake, circulating glucagon and insulin did not significantly differ $(\mathrm{P}>0.05)$ between groups during the study. However, on day 14 of treatment, non-fasting plasma glucose levels were significantly $(\mathrm{P}<0.05)$ reduced in all GIP analogue treated groups compared to control (12.0$12.8 \pm 1.2 \mathrm{mmol} / 1$ vs. $15.0 \pm 0.6 \mathrm{mmol} / \mathrm{l} . \mathrm{min}$ for control). Following i.p. glucose administration, the overall plasma glucose excursion was significantly lowered in the 14-day $\mathrm{N}$ AcGIP (1.3-fold; $\mathrm{P}<0.01)$, GIP(Lys $\left.{ }^{37} \mathrm{PAL}\right)(1.2$-fold; $\mathrm{P}<0.05)$ and $N$-AcGIP(Lys $\left.{ }^{37} \mathrm{PAL}\right)(1.2$-fold; $\mathrm{P}<0.05)$ treated mice compared to saline control. In harmony with this, all three GIP analogues significantly enhanced glucose-induced insulin release $(\mathrm{P}<0.05)$. Exogenous insulin injection caused a significantly greater fall of glucose concentrations in $N$-AcGIP, GIP(Lys $\left.{ }^{37} \mathrm{PAL}\right)$ and $N$-AcGIP(Lys $\left.{ }^{37} \mathrm{PAL}\right)$ treated $o b / o b$ mice 
compared to control (118.0-126.6 $\pm 13.8 \mathrm{mmol} / \mathrm{l} . \mathrm{min} v s$. $174 \pm 14.4 \mathrm{mmol} / \mathrm{l}$.min for control; $\mathrm{P}<0.05$ in all cases). The metabolic and insulin secretory responses to native GIP were also significantly $(\mathrm{P}<0.05-\mathrm{P}<0.01)$ enhanced in all 14-day analogue treated mice compared to control, revealing no evidence of GIP-receptor desensitization. These effects were accompanied by a significant increase in pancreatic islet area (0.029-0.031 $\pm 0.0038 \mathrm{~mm}^{2}$ vs. $0.021 \pm 0.0027 \mathrm{~mm}^{2}$ for control; $\mathrm{P}<0.01$ in all cases) and islet number (1.4-1.6 fold; $\mathrm{P}<0.05-\mathrm{P}<0.01)$ in all GIP analogue groups.

Conclusion: These data demonstrate the therapeutic potential of once daily injection of enzyme resistant GIP analogues and suggest that $N$-AcGIP is equally as effective as related palmitate derivatised analogues of GIP, GIP (Lys ${ }^{37} \mathrm{PAL}$ ) and $N$-AcGIP(Lys $\left.{ }^{37} \mathrm{PAL}\right)$.

\section{3}

Liraglutide, a long-acting GLP-1 analogue, expands the $\beta$-cell mass of human pancreatic cells implanted in immunodeficient diabetic mice and corrects their diabetes: gastrin potentiates liraglutide's effects

W. L. Suarez-Pinzon ${ }^{1}$, J. R. T. Lakey ${ }^{2}$, C. F. Gotfredsen ${ }^{3}$, T. B. Bodvarsdottir ${ }^{3}$, L. Bjerre Knudsen ${ }^{3}$, J. S. Petersen ${ }^{3}$, A. E. Karlsen ${ }^{3}$, A. Rabinovitch ${ }^{1}$;

${ }^{1}$ Medicine, ${ }^{2}$ Surgery, University of Alberta, Edmonton, Canada, ${ }^{3}$ NovoNordisk, Maaloev, Denmark.

Background and Aims: Pancreatic islet transplantation is a viable treatment for type 1 diabetes, but is limited by human donor tissue availability. In this study we investigated whether the $\beta$-cell mass in human pancreatic cell preparations with low endocrine cell purity could be expanded by treating the cells with liraglutide, a long-acting glucagon-like peptide-1 (GLP-1) analog and gastrin, peptides reported to induce $\beta$-cell neogenesis in diabetic animal models.

Materials and Methods: Islets were isolated from pancreases of adult human brain-dead organ donors, dissociated and $5 \times 10^{6}$ cells $(7 \pm 2 \%$ insulin $+\beta$ cells, $37 \pm 7 \% \quad \mathrm{CK}$ $7^{+}+$CK $19+$ duct cells, and $11 \pm 2 \%$ amylase+acinar cells) were implanted under the left renal capsule of nonobese diabeticsevere combined immune deficiency (NOD-scid) mice made diabetic (blood glucose 20-25 mmol/l) with streptozotocin. The mice were injected daily with vehicle, a low dose of liraglutide $100 \mathrm{ug} / \mathrm{kg} /$ day, gastrin $300 \mathrm{ug} / \mathrm{kg} /$ day, and liraglutide+gastrin. In the first study, blood glucose (BG) was measured weekly, and after 5 weeks the human pancreatic cell grafts were harvested and dissociated and their cell compositions were analyzed by immunocytochemistry. In the second study, BG was measured weekly, and after 4 weeks plasma human C-peptide was measured by RIA ; then the left kidneys with the human pancreatic cell grafts were removed and insulin contents were measured by ELISA, and the mice were monitored daily for return of hyperglycemia.

Results: After 5 weeks, BG rose to $27.1 \pm 1.6 \mathrm{mmol} / \mathrm{l}$ in vehicle - treated mice $(n=9)$, and decreased to $18.7 \pm 1.8 \mathrm{mmol} /$ 1 in gastrin-treated mice $(n=12) ; 11.8 \pm 1.9 \mathrm{mmol} / \mathrm{l}$ in liraglutide-treated mice $(\mathrm{n}=10)$ and $7.9 \pm 0.9 \mathrm{mmol} / 1$ in liraglutide+gastrin treated mice. BG was not decreased in sham-implanted mice receiving any treatment. $\beta$-cells in the human pancreatic cell implants increased from $2.38 \pm$ $0.45 \times 10^{5}$ cells to $3.99 \pm 0.79 \times 10^{5}$ cells in vehicle-treated mice, $7.72 \pm 1.23 \times 10^{5}$ cells in gastrin-treated mice, $11.65 \pm 0.96 \times 10^{5}$ cells in liraglu tide-treated mice, and $16.10 \pm 2.12 \times 10^{5}$ cells in liraglutide+gastrin-treated mice. Insulin content in the human pancreatic cell implants in the mice decreased from $126 \pm 17 \mathrm{ng}$ to $14 \pm 1 \mathrm{ng}$ after 5 weeks in vehicle-treated mice, but increased to $288 \pm 52 \mathrm{ng}$ in gastrin-treated mice, $459 \pm 123 \mathrm{ng}$ in liraglutide-treated mice and $1105 \pm 257$ in liraglutide+gastrin-treated mice. Correction of hyperglycemia by the peptides correlated with the insulin content in the human pancreatic cell implants $(\mathrm{r}=$ -0.978) and with the plasma human C-peptide level ( $\mathrm{r}=$ $-0.862)$ in the mice. Diabetes $(B G \geq 20 \mathrm{mmol} / \mathrm{l})$ recurred within a few days after removal of the implants.

Conclusion: These results show that a low dose of liraglutide expands the $\beta$-cell mass of adult human pancreatic cell preparations of low endocrine cell purity after implantation in immunodeficient diabetic mice. The increase in $\beta$-cells is 5-fold and sufficient to correct hyperglycemia in the diabetic mice. Gastrin potentiates these effects of liraglutide.

Supported by Novo Nordisk

0174

Differential effects of DPP-4 inhibition on incretin hormone levels in drug-naive and metformin-treated patients with type 2 diabetes

B. E. Dunning ${ }^{1}$, M. Ligueros-Saylan ${ }^{2}$, D. A. D'Alessio ${ }^{3}$, B. Balas ${ }^{4}$, D. E. Kelley ${ }^{5}$, C. F. Deacon ${ }^{6}$, J. J. Holst ${ }^{6}$, J. E. Foley ${ }^{2}$;

${ }^{1}$ PharmaWrite, Princeton, ${ }^{2}$ Novartis, East Hanover, ${ }^{3}$ Endocrinology, University of Cincinnati, ${ }^{4}$ Diabetes, Univ of Texas Health Science Center, San Antonio, ${ }^{5}$ Medicine, University of Pittsburgh, United States, ${ }^{6}$ Panum Institute, University of Copenhagen, Denmark

Background and Aims: Vildagliptin is an inhibitor of DPP4, the enzyme that degrades the incretin hormones GLP-1 and GIP. Vildagliptin lowers fasting (FPG) and postprandial (PP) glucose in patients (pts) with type 2 diabetes (T2DM) via incretin hormone-mediated improvements in islet function. Because metformin (Met) has been suggested to increase GLP-1 release, we sought to determine if the effect of 
vildagliptin on plasma levels of intact (active) GLP-1 and GIP was greater in patients (pts) with T2DM receiving concomitant Met than in pts receiving vildagliptin alone.

Methods: Three randomized, double-blind, placebo (PBO)controlled studies were performed and plasma levels of active GLP-1 and GIP were measured by ELISA and RIA, respectively, using N-terminally directed antisera. In Study 1, GLP-1 and GIP were measured in overnight-fasted pts before (Wk 0) and after 12-wk treatment with vildagliptin $50 \mathrm{mg}$ bid. In Study 2, PP GLP-1 and GIP were measured after a single $100 \mathrm{mg}$ dose of vildagliptin or PBO given before a meal. In Study 3, fasting and PP GLP-1 and GIP were measured after 6 -wk treatment with vildagliptin $50 \mathrm{mg}$ bid or PBO. Each study included pts continuing a stable Met regimen (Met) and pts receiving vildagliptin alone (No Met).

Results: As shown below, in Study 1, vildagliptin significantly increased fasting levels of active GLP-1 in the Met group, but did not affect fasting GIP. In the No Met group, fasting GIP increased, but fasting GLP-1 did not. In Study 2, vildagliptin increased the 2 -h mean PP levels (AUC $\div$ time) of active GLP-1 and GIP in both subgroups, but PP GIP was higher in the No Met vs the Met subgroup. In Study 3 vildagliptin significantly increased fasting levels of active GLP-1 only in the Met subgroup, but it did not significantly affect fasting GIP in either subgroup. In Study 3, vildagliptin increased PP levels of the active forms of both GLP-1 and GIP, but the increase in PP GLP-1 was significantly greater in the Met than in the No Met subgroup.

\begin{tabular}{|c|c|c|c|c|}
\hline & \multicolumn{2}{|c|}{ Active GLP-1 (pM) } & \multicolumn{2}{|c|}{ Active GIP (pM) } \\
\hline & $\begin{array}{l}\text { No Met } \\
(n=7)\end{array}$ & $\begin{array}{l}\text { Met } \\
(n=12)\end{array}$ & $\begin{array}{l}\text { No Met } \\
(n=7)\end{array}$ & $\begin{array}{l}\text { Met } \\
(n=12)\end{array}$ \\
\hline \multicolumn{5}{|l|}{ Study 1} \\
\hline Wk 0, Fasting & $5.7 \pm 2.2$ & $2.3 \pm 0.1$ & $16 \pm 2$ & $17 \pm 2$ \\
\hline \multirow{2}{*}{$\begin{array}{l}\text { Wk } 12 \text {, Fasting } \\
\text { Study } 2\end{array}$} & $7.3 \pm 3.4$ & $12.5 \pm 4.9^{\mathrm{a}}$ & $25 \pm 5^{\mathrm{a}}$ & $19 \pm 3$ \\
\hline & $\begin{array}{l}\text { No Met } \\
(n=4)\end{array}$ & $\begin{array}{l}\text { Met } \\
(n=8)\end{array}$ & $\begin{array}{l}\text { No Met } \\
(n=4)\end{array}$ & $\begin{array}{l}\text { Met } \\
(n=8)\end{array}$ \\
\hline $\begin{array}{l}\mathrm{PBO}, 2-\mathrm{h} \\
\text { mean PP }\end{array}$ & $3.3 \pm 0.8$ & $5.2 \pm 0.8$ & $42.4 \pm 7.8$ & $36.1 \pm 4.2$ \\
\hline \multirow{3}{*}{$\begin{array}{l}\text { Vildagliptin, } \\
\text { 2-h mean PP } \\
\text { Study } 3\end{array}$} & $7.4 \pm 0.9^{\mathrm{b}}$ & $10.8 \pm 1.4^{\mathrm{b}}$ & $79.0 \pm 7.0^{\mathrm{b}}$ & $56.1 \pm 3.0^{\mathrm{b}, \mathrm{c}}$ \\
\hline & & & & \\
\hline & $\begin{array}{l}\text { No Met } \\
(n=5)\end{array}$ & $\begin{array}{l}\text { Met } \\
(n=12)\end{array}$ & $\begin{array}{l}\text { No Met } \\
(n=5)\end{array}$ & $\begin{array}{l}\text { Met } \\
(n=12)\end{array}$ \\
\hline PBO, Fasting & $2.8 \pm 0.8$ & $3.4 \pm 1.0$ & $2.4 \pm 0.2$ & $11.3 \pm 1.5$ \\
\hline Vildagliptin, Fasting & $4.1 \pm 1.4$ & $11.3 \pm 2.9^{\mathrm{b}}$ & $7.8 \pm 3.0$ & $13.5 \pm 3.0$ \\
\hline PBO, 2-h mean PP & $5.0 \pm 1.5$ & $8.6 \pm 2.4$ & $14.2 \pm 3.5$ & $23.5 \pm 3.1$ \\
\hline $\begin{array}{l}\text { Vildagliptin, } \\
\text { 2-h mean PP }\end{array}$ & $9.7 \pm 1.7^{b}$ & $22.7 \pm 3.1^{\mathrm{b}, \mathrm{c}}$ & $34.7 \pm 6.9^{\mathrm{b}}$ & $40.0 \pm 3.6^{\mathrm{b}}$ \\
\hline
\end{tabular}

${ }^{\mathrm{a}} \mathrm{P}<0.05$ vs Wk $0 ;{ }^{\mathrm{b}} \mathrm{P}<0.05$ vs $\mathrm{PBO} ;{ }^{\mathrm{c}} \mathrm{P}<0.05$ vs No Met Values below limit of quantification (LOQ) set equal to LOQ
Conclusions: DPP-4 inhibition with vildagliptin has a larger effect on active GLP-1 in pts receiving concomitant Met than in pts receiving no concomitant antidiabetic agent. In pts receiving no Met there is a more pronounced effect on active GIP. Although both GLP-1 and GIP enhance insulin release, GIP stimulates, but GLP-1 suppresses glucagon release. Thus DPP-4 inhibition may also have differential effects on $\alpha$ - and $\beta$-cell function in drug-naïve vs Met-treated pts. The more robust effect on GLP-1 (vs GIP) in Met-treated pts may underlie the particularly pronounced efficacy of vildagliptin to reduce both FPG and $\mathrm{HbA}_{1 \mathrm{c}}$ in Met-treated pts.

Supported by Novartis

\section{OP 30}

\section{Diabetes in childhood}

\section{5}

\section{Alarming continued increase of overweight at onset of insulin-treated diabetes in both African-American and Caucasian children \\ I. M. Libman ${ }^{1}$, P. Balfour ${ }^{1}$, S. Brooke ${ }^{2}$, R. E. LaPorte ${ }^{3}$, D. J. Becker' ${ }^{1}$; \\ ${ }^{1}$ Pediatric Endocrinology and Diabetes, Children's Hospital of Pittsburgh, ${ }^{2}$ School of Medicine, Drexel University, Philadelphia, ${ }^{3}$ Graduate School of Public Health, Universi- ty of Pittsburgh, United States.}

Background and Aims: Insulin-treated diabetes in childhood was thought to be a disease of wasting, not overweight. We have previously reported that the prevalence of being overweight more than tripled from 19791989 (period I) to $1990-1998$ (period II) at onset of islet autoantibody positive childhood insulin-treated diabetes the concept of "double" diabetes. The current study evaluates whether this increase has continued in the period 1999-2002 (period III).

Materials and Methods: All Black children $<19$ years of age, diagnosed with diabetes and treated with insulin at onset, admitted to Children's Hospital of Pittsburgh from January 1st 1979 to December 31st 2002 were matched with White children by sex, age at onset and year of diagnosis. Data were obtained from retrospective review of medical records and $\beta$-cell antibodies measured at onset. Overweight was defined as body mass index $\geq 85$ th percentile for age and sex.

Results: Complete data were available for 252 children out of 332 (76\%). Overall, the prevalence of being overweight in all 252 insulin treated subjects more than tripled in 24 years of observation, $12.6 \%$ in period I to $36.8 \%$ in period II, to $43.9 \%$ in period III ( $\mathrm{p}=0.0005)$. In period II, 
the effect was greatest in Black females $<11$ years $(0 \%$ to $50 \%$ to $25 \%$ in periods I II and III respectively, $\mathrm{p}=0.04$ ). In period III, the biggest increase was seen in those previously less affected, i.e. younger White males $(0 \%$ to $9.1 \%$ to $37.5 \%$ in periods I, II and III respectively, $\mathrm{p}=0.05$ ). The prevalence by race, sex and age at onset is seen in the table below.

\begin{tabular}{|c|c|c|c|c|c|c|}
\hline & \multicolumn{3}{|c|}{$<11$ years $(\mathrm{n}=108)$} & \multicolumn{3}{|c|}{$>=11$ years $(n=144)$} \\
\hline & Period I & Period II & Period III & Period I & Period II & Period III \\
\hline $\begin{array}{l}\text { Whites } \\
(\mathrm{n}=124)\end{array}$ & 5.0 & 4.8 & $25^{*}$ & 0 & 25.8 & $33.3 * *$ \\
\hline $\begin{array}{l}\text { Blacks } \\
(\mathrm{n}=128)\end{array}$ & 5.9 & 31.8 & $41.8^{*}$ & 38.9 & 68.4 & $67.7^{*}$ \\
\hline $\begin{array}{l}\text { Males } \\
(\mathrm{n}=117)\end{array}$ & 5.2 & 9.5 & $44.4 * * *$ & 29.4 & 59.2 & 50 \\
\hline $\begin{array}{l}\text { Females } \\
(\mathrm{n}=135)\end{array}$ & 5.5 & 25 & 12.5 & 11.7 & 42.8 & $50 * *$ \\
\hline
\end{tabular}

${ }^{*} \mathrm{p}=0.08 ; * * \mathrm{p}<0.05 ; * * * \mathrm{p}<0.005$ comparing the three periods by age

There was no significant difference in terms of frequency of ketoacidosis between the three periods $(36.5 \%$ vs $37 \%$ vs $31.2 \%$ respectively, $\mathrm{p}=0.71$ ). The frequency of addition of metformin to the treatment regimen with insulin increased from period I (2.8\%) to period II and III (14.4\% and $11.9 \%$ respectively, $\mathrm{p}=0.04)$.

Conclusion: The phenotype of children with insulin-treated diabetes at onset is changing. Almost half of the children with insulin-treated diabetes are now overweight despite frequent onset weight loss. The recent rise is greatest in younger white males. It is yet to be determined whether this weight excess is related to the continued increase in type 1 diabetes incidence or if it affects rates of complications. Lifestyle intervention studies in high risk populations (e.g. first degree relatives) are needed to assess potential beneficial effects in prevention/delay of the onset of the disease or its comorbidities.

Supported by the Cochrane-Weber Fund

\section{6}

An attempt of pancreatic islet beta-cells prevention after clinical diagnosis of type 1 diabetes mellitus

L. Szewczyk, Y. Azab, A. Bury, R. Piekarski;

Pediatric Endocrinology and Neurology, Medical University of Lublin, Poland.

Backgroud: In recent years studies focused on prevention of type 1 diabetes mellitus (DM) and possibility of beta-cell protection at the clinical diagnosis of DM. Some recent studies have demonstrated beneficial role of vit. D3 analogue in human beta-cell activity maintenance at the early stages of DM.

Material and Methods: The study was performed in 63 children and adolescents ( 32 female and 31 male) aged 4 16 years who received insulin and vit D3 analogue (alfacalcidol - 1alpha-OH-vitamin D3) in daily dose $0.25 \mu$ g. 37 peers (17 female and 20 male) with type $1 \mathrm{DM}$ who received only insulin therapy without vit. D3 administration were the control group. After one year therapy we compared the level of HbA1c, body mass, daily insulin dose in both groups.

Results: There are no differencies in mean body mass and in metabolic control of diabetes reported by mean $\mathrm{HbAlc}$ level at the onset of the DB and after one year observation of these patients among studied groups. However, daily insulin dose in group of patients who received vit D3 therapy after one year observation were at the similar level compared to the early stage of DM $(0.36 \mathrm{u} / \mathrm{kg}-0.37 \mathrm{u} / \mathrm{kg})$. On the contrary, insuline requirement in the group of patients without vit D3 treatment significantly increased after one year observation (from $0.37 \mathrm{u} / \mathrm{kg}$ to $0.63 \mathrm{u} / \mathrm{kg}$ ).

Conclusion: In group of children treated alfacalcidol insulin requirement after one year therapy with vit D3 analogue sustained at the level found at the early stage of DM. Children without vit D3 administration had twofold incerase of insulin requirement after one year observation from clinical diagnosis. Our observations suggest, that alfacalcidol could play a role in prevention of further beta-cells destruction.

\section{7}

Post-prandial glucose excursions following three methods of bolus insulin administration in adolescent girls with type 1 diabetes

A. Kernell, A. Lindholm-Olinder, J. Runefors;

Clinical Research and Education, Karolinska Institute, Stockholm, Sweden.

Objectives: To determine if one method of short-acting insulin bolus administration is superior to other methods in managing a meal with the same content of calories and carbohydrates but with different content of fat.

Methods: Thirteen girls aged 13-19 years with type 1 diabetes agreed to consume two different lunch meals on 6 occasions with one week apart. The meals were exactly identical with the same content of calories and carbohydrates but with 30 or $36 \%$ fat. They received the same dose of bolus insulin on each of the 6 occasions as a single dose immediately prior to the meal as a single bolus $(\mathrm{N})$, a dual- 
wave $(60 \%$ as a single bolus and $40 \%$ as square wave over $1 \mathrm{~h}, \mathrm{DF})$ or as a square wave over $1 \mathrm{~h}(\mathrm{~F})$. The plasma-glucose levels were followed with CGMS every fifth minute between $30 \mathrm{~min}$ before and until $3 \mathrm{~h}$ after the meal.

Results: The maximal change of plasma-glucose occurred after $110 \mathrm{~min}(2,7 \mathrm{mmol}), 85 \mathrm{~min}(2,6 \mathrm{mmol})$ and 55 $(3,8 \mathrm{mmol}) \mathrm{min}$ after the $30 \%$ fat meal with the N, DF and $\mathrm{F}$ bolus administration. The maximal change of plasmaglucose after the $36 \%$ fat meal with the N, DF and F bolus after $75 \mathrm{~min}(2,6 \mathrm{mmol}), 45 \mathrm{~min}(1,8 \mathrm{mmol})$ and $65 \mathrm{~min}$ $(2,8 \mathrm{mmol})$ The area under the curve between start and 180 min was lowest after the DF bolus for both types of meals $(\mathrm{p}<0,001)$.

Conclusions: The dual wave method for bolus administration seams to be the most effective method for a recommended type of main-meal or a "pizza"-type of meal. Supported by Barndiabetesfonden, Stiftelsen Samariten

\section{8}

Contribution of basal insulin on daily dose is related to c-peptide secretions in diabetes children treated with continuous subcutaneous insulin infusion

A. Szypowska, E. Pankowska, M. Lipka;

Department of Pediatric Diabetology and Birth Defects, Medical University of Warsaw, Poland.

Background and Aims: Continuous subcutaneous insulin infusion has become an increasingly used option in type 1 diabetes children management. This advanced technology provides the most precise insulin administration and also allows to keep the records of delivered insulin doses over the period of last 12 weeks. The analysis of registered data gives an opportunity to review obligatory algorithms of insulin adjustment in intensive therapy.

The aim was to investigate the insulin requirement, basal insulin contribution on total insulin daily dose (TIDD) and analysis key determinant factors for basal to bolus proportion in TIDD.

Materials and Methods: 78 (35 female/43 male) well controlled type 1 diabetes children were included in the study; mean age $10,19 \pm 4,19$ y $(1,05-17,91)$, mean diabetes

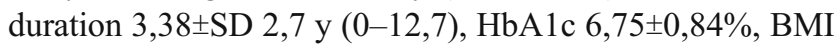
$17,93 \pm \mathrm{SD} 2,78 \mathrm{~kg} / \mathrm{m} 2$. During hospital visits pumps memory data was downloaded; clinical and biochemical: fasting c-peptide, GlyHbA1c were assessed. Patients with DKA (ketoacidosis), SH(severe hypoglycemia) or infection diseases during last 3 months were excluded.

Results: The average requirement for insulin was $0,75 \pm 0,21 \mathrm{U} / \mathrm{kg} / \mathrm{d}$, and it was not significantly different in age groups: it was comparable in prepubertal and pubertal children $(0,71 \pm 0,17$ vs $0,79 \pm 0,24 \mathrm{U} / \mathrm{kg} / \mathrm{d})$ but basal contribution was statistically lower in prepubertal patients than in pubertal $(22,35 \pm 8,76$ vs $31,23 \pm 14,82 \% \mathrm{p}<0,05)$. Analysis of factors of basal contribution on TIDD shows significant correlation with age $(\mathrm{r}=0,33 \mathrm{p}=0,05)$, diabetes durations $(\mathrm{r}=0,54, \mathrm{p}<0,0001)$ and BMI $(\mathrm{r}=0,325 \mathrm{p}<0,0037)$ but inversely correlated with C-peptide $(\mathrm{r}=-0,349 \mathrm{p}=0,0017)$. However C-peptide did not significantly correlated with TIDD.

Conclusion: The basal to bolus insulin is below $50 \%$ of TIDD in children with type 1 diabetes. The basal contribution in growing-up population should be differentiated by the age, diabetes duration and BMI. Remission phase related to residual secretion of insulin by beta cell, clinically is expressed by contribution of basal insulin on TIDD but not by insulin amounts per $\mathrm{kg}$ of body mass per day.

0179

Risk for hospitalisation in diabetic children and adolescents and its association with social status

R. W. Holl ${ }^{1}$, J. Rosenbauer ${ }^{2}$, K. Strassburger ${ }^{2}$, M. Grabert ${ }^{1}$, G. Giani ${ }^{2}$, A. Icks ${ }^{2}$;

${ }^{1}$ Epidemiology, University of Ulm, ${ }^{2}$ Epidemiology, University of Düsseldorf, Germany.

Background and Aims: Hospitalization in type 1 diabetic children and adolescents is high, despite of many efforts to reduce inpatient care in chronic diseases. Children from socially deprived families seem to have a disproportionately high risk to be hospitalized. However, data regarding the association between hospitalization risk and social status are limited, and results are conflicting. Thus, aim of our study was to evaluate the association between post-onset hospitalization and the social situation in a large cohort of newly diagnosed type 1 diabetic children and adolescents, based on data of a nationwide established documentation program (DPV).

Materials and Methods: From 62 pediatric departments, which document social status, all children and adolescents $<20$ years of age with diabetes onset in 2002 to 2005 and with at least one in- or outpatient contact and documentation of the social status, were included $(n=1,127,55 \%$ male, age at onset (SD) 8.5 (4.2) years, mean follow up 1.2 years (SD 0.9, range 0.2-3.7 years)). We evaluated the association between four social parameters (parental school education and professional training, family situation, nationality) and the risk for post-onset hospital admission as well as number of hospital days. We estimated relative risks with $95 \%$ confidence intervals (RR, CI), adjusting for 
age and sex. To take the cluster effect of centres into account, we used random effects models.

Results: $42 \%$ of the study subjects were hospitalized at least once during the follow up period. $50 \%$ of hospital admissions were due to education. Incidence of hospital admissions was $0.47(0.43-0.50)$ per person-year (py), hospital days 2.78 (2.70-2.86) per py. Hospital admissions and hospital days were significantly higher in children from single parent families (RR 1.57; 95\% CI 1.31-1.89 and $1.63 ; 1.51-1.76)$ and from lower school and professionally educated parents (RR's between 1.45 and 2.34, all $\mathrm{p}<0.05$ ). In children from non-German and German families, the differences in hospital days were smaller (RR 1.25; 1.15 1.35 ), and there was no significant difference with respect to hospital admissions (RR 1.15; 0.94-1.39).

Conclusion: As expected, hospitalization was higher in diabetic children and adolescents from lower educated parents and incomplete families, indicators for low social status. Interestingly, there were less differences between German and non-German children. This finding may be explained by different patterns of health care seeking, maybe by lower participation in structured diabetes education in non-German families. The observations need to be confirmed and explained. Supported by German Research Foundation

\section{0}

A comparison of diabetes-related quality of life between adolescents with type 1 diabetes and their parents

D. C. A. Taylor ${ }^{1}$, J. Zhang ${ }^{1}$, L. Menditto ${ }^{2}$, D. Thompson ${ }^{1}$; ${ }^{1}$ Innovus Research Inc., Medford, ${ }^{2}$ sanofi-aventis U.S., Bridgewater, United States.

Backround and Aims: As part of a randomized 24-week clinical trial in which insulin glargine was compared to twicedaily intermediate-acting insulin, diabetes-related quality of life (QOL) among adolescents with type 1 diabetes (T1DM) and their parents was evaluated at baseline.

Materials and Methods: The inclusion criteria for the study were males and females aged 9 to 17 years, Tanner stage $\geq 2$, with a diagnosis of T1DM for at least 1 year that was treated only with insulin. QOL was measured using the Youth and Caregiver modified versions of the Diabetes Quality-of-Life Measure, a self-administered questionnaire in which subjects responded to questions within 3 domains (diabetes impact [DI], diabetes-related worries [DW], and life satisfaction [LS]) using a 5-point Likert scale where higher scores represent poorer QOL. We limited our analysis to domain items that were similar in both the youth and parent questionnaires in order to facilitate direct comparisons. The data were analyzed using mixed-effects models with domain score as the dependent variable and youths' gender, respon- dent (parent or youth), study site, and gender-by-respondent interaction as independent variables.

Results: A total of 175 adolescents ( 80 male, 95 female) were enrolled with a mean baseline A1C of $7.9 \%$.

The female parent was the respondent in $86 \%$ of the surveys. Adjusted mean scores at baseline were higher (worse) for parents than children in the DI, DW, and LS domains, these differences were more pronounced when the child was male (Table).

Adjusted Mean Domain Scores by Gender and Respondent

\begin{tabular}{lccl}
\hline Domain/Child's Gender & Parents & Youths & $P$ Value \\
DI & & & \\
-Overall & 23.2 & 20.6 & $<0.0001$ \\
-Female & 23.0 & 21.4 & .0216 \\
-Male & 23.5 & 19.7 & $<0.0001$ \\
DW & & & \\
-Overall & 22.7 & 15.9 & $<0.0001$ \\
-Female & 23.0 & 16.9 & $<0.0001$ \\
-Male & 22.5 & 14.8 & $<0.0001$ \\
LS & & & \\
-Overall & 27.6 & 26.9 & .2306 \\
-Female & 27.4 & 28.0 & .4341 \\
-Male & 27.9 & 25.7 & .0199 \\
\hline
\end{tabular}

Conclusion: We conclude that T1DM has a greater perceived effect on the QOL of parents than on the children themselves in terms of diabetes impact and diabetes-related worries, the parent-child difference being greater when the child is male.

Supported by sanofi-aventis U.S.

\section{OP 31}

\section{Inhaled insulin}

\section{1}

Superior post prandial glucose control in patients with type 1 diabetes when using prandial technosphere insulin compared to NovoLog

A. H. Boss ${ }^{1}$, S. H. Evans ${ }^{1}$, H. Ren ${ }^{1}$, R. Harris ${ }^{1}$, I. Firsov ${ }^{2}$, P. Richardson ${ }^{1}$;

${ }^{1}$ MannKind Corporation, Danbury, United States, ${ }^{2}$ ClinStar Europe LLC, Moscow, Russian Federation.

Background and Aims: A major challenge in basalprandial insulin therapy is to achieve appropriate synchronization between meal absorption and prandial insulin activity. Technosphere insulin (TI) has an action profile with a faster onset and shorter duration of action than seen with existing insulin therapy, and might be ideally suited 
for prandial insulin substitution. This study evaluated postprandial glucose excursions following a standard liquid meal and overall glycemic control in a randomized controlled study of patients with type 1 diabetes.

Materials and Methods: 110 patients with type 1 diabetes (41 male and 69 female) already using a basal-prandial regimen were randomized to receiving $\mathrm{TI}(\mathrm{N}=54)$ or insulin aspart, a rapid acting insulin analogue (RAA), $(\mathrm{N}=56)$ at meal times together with insulin glargine as basal insulin. After a transfer period, patients where followed for 3 month during which individual adjustments of therapy was performed, and standardized meal test (Boost Plus) were conducted at 0,8 and 12 weeks of therapy.

Results: The study population (all Caucasian) had a mean age of 34 years, and a BMI of $24.4 \mathrm{~kg} / \mathrm{m}^{2}$, with no statistical difference between groups. Similar reductions inHbA1c where seen in both groups. The reduction from baseline was $0.83(1.11)$ (mean $(\mathrm{SD}) ;(\mathrm{p}<0.001)$ in the TI group and $0.99(1.07) ;(p<0.001)$ in the group receiving sc RAA, with no statistical difference between groups; $(p=0.458)$. At the same time, body weight decreased by $0.41(2.22) \mathrm{kg}$ in the TI group, while it increased by 0.89 (1.92) $\mathrm{kg}$ in the group receiving SC insulin. The difference in weight change between the groups was statistically significant $(\mathrm{p}=0.0016)$. An improvement in post-prandial blood glucose excursions was observed in subjects receiving TI as compared to RAA. Maximal post-prandial excursion at visit 10 was $0.92 \mathrm{mmol} / \mathrm{L}$ for TI vs. $3.0 \mathrm{mmol} / \mathrm{L}$ for RAA. The total post-prandial glucose elevation (AUCgluc) was $96.7 \mathrm{mmol} / \mathrm{L}^{*} \mathrm{~min}$ for TI and $400.6 \mathrm{mmol} / \mathrm{L}^{*} \min$ for RAA. No changes in lung function was seen after 3 months treatment, (change in FEV1 was $-0.0641(0.189)$ for TI and $-0.072(0.193)$ for RAA $(\mathrm{p}=0.82$; n.s. $)$, and for DLco -1.62 (3.29) and -1.094 (3.08) ( $\mathrm{p}=0.39$; n.s.) respectively.

Conclusion: Used in a basal-prandial regimen, post prandial excursions where significantly less when using TI compared to RAA. TI provided similar improvement in glycemic control (expressed as change from baseline HbAlc) to RAA, without an increase in body weight. No adverse effect was seen on pulmonary function.

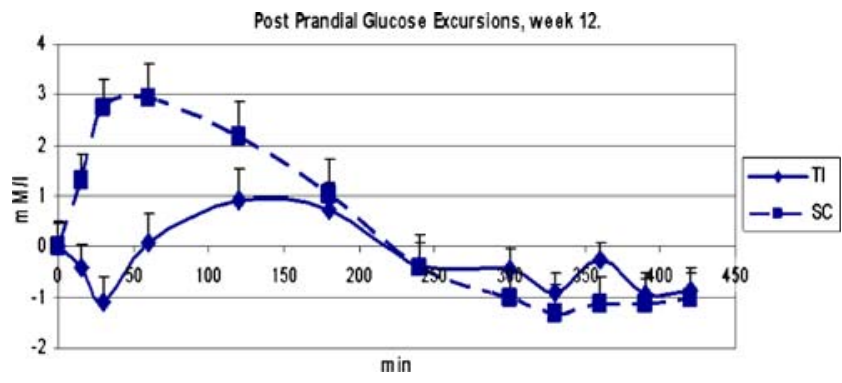

Supported by MannKind Corporation
0182

Impact of exercise on absorption of inhaled insulin A. H. Petersen ${ }^{1,2}$, G. Köhler ${ }^{1}$, S. Korsatko ${ }^{1}$, A. Wutte ${ }^{1}$, M. Wonisch ${ }^{1}$, A. Mautner ${ }^{1}$, B. B. Rönn ${ }^{3}$, P. Clauson ${ }^{3}$, T. Laursen ${ }^{2}$, P. Wollmer ${ }^{4}$, T. Pieber ${ }^{1}$;

${ }^{1}$ Department of Internal Medicine, Medical University Graz, Austria, ${ }^{2}$ Department of Pharmacology, University of Aarhus, Denmark, ${ }^{3}$ Novo Nordisk A/S, Bagsvaerd, Denmark, ${ }^{4}$ Department of Clinical Sciences, Lund University, Malmö, Sweden.

Background and Aims: Inhaled insulin is a new alternative to injection of insulin. Little is however known about the effect of exercise on the absorption of inhaled insulin. Thus, the purpose of this trial was to investigate the effect of moderate exercise on the absorption of inhaled human insulin in administered by a commercial nebuliser.

Materials and Methods: This single-center, open-labeled, three-period cross-over trial included a total 12 nonsmoking healthy subjects $(28.6 \pm 4.9$ years (mean \pm SD), BMI $23.2 \pm 2.7 \mathrm{~kg} / \mathrm{m}^{2}$ ). On three different dosing days, in randomised order, the subjects received a dose of $3.5 \mathrm{mg}$ fast-acting inhaled human insulin administered by a commercial nebuliser (MedicAidpro-JAEGER APS) followed by either 1) No Exercise, 2) $30 \mathrm{~min}$ moderate exercise starting immediately after dosing (Exercise $\left.{ }_{0}\right)$, or 3 ) $30 \mathrm{~min}$ moderate exercise starting $30 \mathrm{~min}$ after dosing (Exercise En ). The study was carried out as a 10 hour euglycemic glucose clamp (90 mg/dL (5.0 mM)).

Results: Area under the serum insulin curve $\left(\mathrm{AUC}_{\mathrm{ins}}\right)$ from 0 to 120 minutes after start of exercise was $16 \%$ increased for Exercise $_{0}$ (Ratio[95\%CI] for $\mathrm{AUC}_{\mathrm{ins}(0-120 \mathrm{~min})}: 1.16$ $[1.04 ; 1.30], p=0.01)$ and $20 \%$ increased for Exercise 30 $\left(\mathrm{AUC}_{\text {ins }(30-150 \mathrm{~min})}: 1.20[1.05 ; 1.36], \mathrm{p}<0.01\right)$, both compared to No Exercise. The effect of exercise on the overall insulin absorption during the first 6 hours $\left(\mathrm{AUC}_{\mathrm{ins}(0-360 \mathrm{~min})}\right)$, and the complete 10 hours $\left(\mathrm{AUC}_{\mathrm{ins}(0-600 \mathrm{~min})}\right)$ after dosing was not statistically significant. The maximum insulin concentration $\left(\mathrm{C}_{\max }\right)$ was increased by $32 \%$ for Exercise E $_{0}$ and $35 \%$ for Exercise $_{30}$ (both $\mathrm{p}<0.01$ ) compared to No Exercise, while the time to $\mathrm{C}_{\max }\left(\mathrm{t}_{\max }\right)$ was $31 \mathrm{~min}$ faster for Exercise $_{0}(\mathrm{p}<0.01)$, but not significantly different after Exercise $_{30}$ compared to No Exercise. The appearance of insulin in plasma was faster after exercise compared to No Exercise, with an initial rate of increase (best fitted line from baseline to $\left.t_{\max }\right) 150 \%$ larger for Exercise $e_{0}(p<0.01)$ and $72 \%$ larger for Exercise Ex $_{30}(p=0.02)$. There was no effect of exercise on the terminal elimination rate constant.

The integrated glucose infusion rate $\left(\mathrm{AUC}_{\mathrm{GIR}}\right)$ for 2 hours after start of exercise increased by $95 \%$ for Exercise $_{0}$ and $123 \%$ for Exercise $_{30}$ (both $\mathrm{p}<0.01$ ) compared to No Exercise, whereas $\mathrm{AUC}_{\mathrm{GIR}}$ for the first 6 hours, and 
complete 10 hours after exercise was not statistically significant different. Compared to No Exercise, the maximal GIR $\left(\mathrm{GIR}_{\max }\right)$ was increased by $59 \%$ for Exercise $_{0}$ $(p=0.02)$ and $93 \%$ for Exercise Ex $_{30}(p<0.01)$, and occurred $66 \mathrm{~min}$ faster for Exercise $_{0}(\mathrm{p}<0.01)$ and $38 \mathrm{~min}$ faster for Exercise $_{30}(p=0.02)$.No safety issues or adverse events related to the inhaled human insulin were observed.

Conclusion: Thirty minutes of moderate exercise immediately or 30 minutes after dosing of inhaled human insulin leads to increased absorption, with higher $\mathrm{C}_{\max }$ over the first 2 hours after start of exercise, and a shorter $t_{\max }$ for exercise immediately after dosing. These data indicate that patients using inhaled insulin should expect an increased absorption of insulin in connection with exercise shortly after inhaling insulin, but not in the overall absorption.

Supported by grants from the Ministry of Science, Technology and Innovation, Denmark, and Novo Nordisk A/S, Denmark

\section{3}

Effects of albuterol and fluticasone on Inhaled Human Insulin (Exubera ${ }^{\circledR}$ ) pharmacokinetics in subjects with mild-to-moderate asthma

J. G. Teeter, R. Foutaine, A. Milton, G. Wei, T. Checchio, M. Stolar, D. A. Fryburg;

Pfizer Inc, Research and Development, Groton, United States.

Background and Aims: Inhaled asthma treatments and inhaled human insulin (INH; Exubera ${ }^{\circledR}$ (insulin human [rDNA origin]) Inhalation Powder) share a common route of administration, therefore it is important to characterize the potential effects of concomitant administration. This study assessed the effects of prior administration of albuterol (ALB, a short-acting $\beta$-agonist) and fluticasone (FLU, an inhaled corticosteroid) on INH pharmacokinetics. Materials and Methods: This open-label, crossover study randomized 67 nondiabetic subjects with asthma (mean unmedicated forced expiratory volume $\left[\mathrm{FEV}_{1}\right] \geq 80 \%-100 \%$ [mild] or $\geq 50 \%-<80 \%$ [moderate] of predicted values) to INH (3 mg) alone, ALB (180 $\mu \mathrm{g})+\mathrm{INH}(3 \mathrm{mg}) 30$ minutes later, INH $3 \mathrm{mg}$ then ALB $180 \mu \mathrm{g} 10$ minutes later, or FLU $(440 \mu \mathrm{g})+\mathrm{INH}(3 \mathrm{mg}) 30$ minutes later. Healthy nonasthmatic volunteers received INH (3 mg).

Results: Prior ALB administration increased mean maximum insulin concentration $\left(\mathrm{C}_{\max }\right)$ and area under the curve $\left(\mathrm{AUC}_{0-360}\right.$ ) by $\sim 25 \%$ to $35 \%$ in patients with mild asthma and by $\sim 45 \%$ to $50 \%$ in patients with moderate asthma relative to INH administration without ALB (Table 1). The increased INH bioavailability following ALB administration resulted in mean $\mathrm{AUC}$ and $\mathrm{C}_{\max }$ values that approximated those in healthy subjects without asthma (data not shown). Prior FLU administration had no significant effect on INH pharmacokinetics; point estimates of ratios for AUC and $\mathrm{C}_{\max }$ ranged from $94.3 \%$ to $98.8 \%$ and all $95 \%$ CIs included $100 \%$ (Table 2). In asthmatic subjects, there were no remarkable differences in frequency or severity of adverse events between treatment groups.

Conclusion: In the absence of a bronchodilator, mild-tomoderate asthma was associated with reduced pulmonary absorption of INH. Administration of a bronchodilator 30 minutes before INH in subjects with mild-to-moderate asthma resulted in $\mathrm{INH}$ bioavailability that approximated bioavailability in nonasthmatic subjects. Therefore, to avoid undue variability in exposure, it is recommended that the timing of such administration to be consistent. These results suggest INH and fluticasone can be administered concomitantly without impacting insulin levels.

Table 1

\begin{tabular}{|c|c|c|c|c|c|c|}
\hline & \multicolumn{3}{|c|}{ Moderate asthma } & \multicolumn{3}{|c|}{ Mild asthma } \\
\hline & $\mathrm{ALB}+\mathrm{INH}^{*}$ & $\begin{array}{l}\text { INH } \\
\text { Alone* }\end{array}$ & $\begin{array}{l}\text { Ratio (\%) } \\
(95 \% \mathrm{CI})\end{array}$ & $\begin{array}{l}\text { ALB } \\
+\mathrm{INH}^{*}\end{array}$ & INH Alone* & $\begin{array}{l}\text { Ratio (\%) } \\
(95 \% \mathrm{CI})\end{array}$ \\
\hline $\begin{array}{l}\text { Insulin } \\
\mathrm{AUC}_{0-360} \\
(\mu \mathrm{U} \cdot \mathrm{min} / \mathrm{mL})\end{array}$ & 2941 & 1953 & $\begin{array}{l}150.6 \\
(119.8, \\
89.4)\end{array}$ & 5052 & 4056 & $\begin{array}{l}124.6 \\
(106.2, \\
146.2)\end{array}$ \\
\hline $\begin{array}{l}\text { Insulin } \\
C_{\max } \\
(\mu \mathrm{U} / \mathrm{mL})\end{array}$ & 29.6 & 20.1 & $\begin{array}{l}147.0 \\
(120.2, \\
179.8)\end{array}$ & 46.8 & 34.6 & $\begin{array}{l}135.2 \\
(115.1, \\
158.7)\end{array}$ \\
\hline
\end{tabular}

*Adjusted geometric means. $\mathrm{CI}=$ confidence interval.

Table 2

\begin{tabular}{|c|c|c|c|c|c|c|}
\hline & \multicolumn{3}{|c|}{ Moderate Asthma } & \multicolumn{3}{|c|}{ Mild Asthma } \\
\hline & $\begin{array}{l}\text { FLU } \\
+\mathrm{INH}^{*}\end{array}$ & $\begin{array}{l}\text { INH } \\
\text { Alone* }\end{array}$ & $\begin{array}{l}\text { Ratio }(\%) \\
(95 \% \text { CI })\end{array}$ & $\begin{array}{l}\text { FLU } \\
+\mathrm{INH}^{*}\end{array}$ & $\begin{array}{l}\text { INH } \\
\text { Alone* }\end{array}$ & $\begin{array}{l}\text { Ratio }(\%) \\
(95 \% \mathrm{CI})\end{array}$ \\
\hline $\begin{array}{l}\text { Insulin } \\
\text { AUC }_{0-360} \\
(\mu U . m i n / m L)\end{array}$ & 1843 & 1953 & $\begin{array}{l}94.3 \\
(74.9- \\
118.8)\end{array}$ & 4009 & 4056 & $\begin{array}{l}98.8 \\
(83.9- \\
116.4)\end{array}$ \\
\hline $\begin{array}{l}\text { Insulin } \\
\mathrm{C}_{\max } \\
(\mu \mathrm{U} / \mathrm{mL})\end{array}$ & 19.1 & 20.1 & $\begin{array}{l}94.8 \\
(77.4- \\
116.1)\end{array}$ & 34.2 & 34.6 & $\begin{array}{l}98.8 \\
(83.8- \\
116.4)\end{array}$ \\
\hline
\end{tabular}

*Adjusted geometric means

Supported by: Pfizer Inc 


\section{4}

Human insulin inhalation powder (HIIP) in subjects with chronic obstructive pulmonary disease (COPD): pharmacokinetic (PK) and glucodynamic (GD) responses K. Rave ${ }^{1}$, A. de la Peña ${ }^{2}$, F. S. Tibaldi ${ }^{3}$, L. Zhang ${ }^{2}$, B. L. Silverman ${ }^{4}$, L. Heinemann ${ }^{1}$, M. Hausmann ${ }^{1}$, D. B. Muchmore;

${ }^{1}$ Profil Institut fur Stoffwechselforschung, Neuss, Germany, ${ }^{2}$ Eli Lilly and Company, Indianapolis, United States, ${ }^{3}$ Eli Lilly and Company, Mont-Saint-Guibert, Belgium, ${ }^{4}$ Alkermes Incorporated, Cambridge, United States.

Background and Aims: Only limited information is available on the PK and GD effects of inhaled insulin in patients with COPD. This open-label, randomized, 3period crossover trial compared PK and GD responses in healthy subjects and in subjects with COPD after administration of HIIP (Lilly/Alkermes inhaled insulin system) versus administration of subcutaneous (SC) insulin lispro.

Materials and Methods: Nonsmoking subjects, 15 healthy (age $38 \pm 13$ years) and 30 with moderate COPD (IIA or IIB per Global Initiative for Chronic Obstructive Lung Disease criteria), 15 each with chronic bronchitis ( $53 \pm 9$ years) and emphysaema ( $58 \pm 6$ years) received 2 single doses of HIIP (5.2 $\mathrm{mg}$ each) and 1 dose of SC insulin lispro (12 U). PK parameters were assessed by analyzing the serum insulin concentration data and GD parameters were derived from the euglycaemic glucose clamp data. Safety was assessed by laboratory pulmonary function tests (PFTs) before and after each clamp and by spirometry performed 4 times during clamps.

Results: HIIP and insulin lispro were absorbed rapidly in all 3 subject groups (median $t_{\max } 45-60 \mathrm{~min}$ ). Total insulin exposure $\left(\mathrm{AUC}_{\left(0-\mathrm{t}^{\prime}\right)}\right)$ and metabolic effect $\left(\mathrm{GIR}_{\mathrm{tot}}\right)$ after $\mathrm{SC}$ insulin lispro were comparable in all 3 groups. Insulin exposure $\left(\mathrm{AUC}_{\left(0-t^{\prime}\right)}\right)$ after HIIP administration was reduced by $22 \%(\mathrm{p}=0.13)$ in subjects with emphysaema and by $44 \%$ $(p<0.001)$ in those with chronic bronchitis as compared to healthy subjects. Accordingly, GIR tot after HIIP was reduced in subjects with emphysema $(33 \%, \mathrm{p}<0.01)$ and chronic bronchitis $(40 \%, \mathrm{p}<0.01)$. Intra-subject variability for HIIP exposure, based on $\mathrm{PK} \mathrm{AUC}_{\left(0-t^{\prime}\right)}$ was comparable between healthy subjects and those with emphysaema (29 and $28 \% \mathrm{CV}$ ), but was greater in subjects with chronic bronchitis $(52 \% \mathrm{CV})$. No statistically significant differences between the pre- and post-clamp PFTs were observed. During clamps, FEV1 and FVC declined modestly in both COPD groups, with no difference between HIIP and SC insulin lispro treatments.
Conclusion: The Lilly/Alkermes HIIP system was well tolerated by COPD subjects, showing similar time-exposure and time-action profiles but reduced overall exposure and effect compared to healthy subjects. Long-term safety evaluation of HIIP in patients with COPD and diabetes is warranted.

Supported by Eli Lilly and Company

\section{5}

Technosphere insulin does not affect pulmonary function in a 6 month study of patients with type 2 diabetes R. A. Baughman ${ }^{1}$, S. H. Evans ${ }^{1}$, A. H. Boss ${ }^{1}$, I. Firsov ${ }^{2}$, R. Harris ${ }^{1}$, H. Ren ${ }^{1}$, P. C. Richardson ${ }^{1}$;

${ }^{1}$ MannKind Corporation, Danbury, United States, ${ }^{2}$ ClinStar, Moscow, Russian Federation.

Background and Aims: The prospect of replacing prandial insulin injections with insulin inhalation is attractive to many physicians and patients. However, changes in pulmonary function have been reported with other formulations of pulmonary insulin, and this study was undertaken to evaluate the safety of Technosphere insulin (TI) in this respect.

Materials and Methods: 306 patients with type 2 diabetes with $\mathrm{HbA} 1 \mathrm{c}$ levels of $7-11.5 \%$ were enrolled in a randomized, controlled, 6 month study. Prior to enrolling, patients had been treated with sc insulin for at least 3 month. At entry, patients initiated treatment with insulin glargine. Following a 3 week titration period, patients were randomized to either TI or insulin aspart at meal times. Pulmonary function testing, including FEV1, FVC and FEF $25-75 \%$, was conducted at entry, randomization and at 2 and 6 month. All pulmonary laboratories were uniformly trained and monitored and procedures were standardized according to American Thoracic Society recommendations.

Results: The study population $(\mathrm{n}=306)$ had a mean age of 58 years, and a BMI of $30.8 \mathrm{~kg} / \mathrm{m}^{2}$, with no statistical difference between groups. The TI group consisted of 148 subjects (39 male/109 female) and the control group of 158 subjects ( 33 male/125 female). The results of pulmonary function testing are listed in the table. For all parameters, the differences were not statistically significant. Pulmonary function is susceptible to changes in age and weight. When a correction for these parameters was applied, (using the algorithm of A Miller et al), no numeric change from baseline was observed. 
Pulmonary Function Measurements

\begin{tabular}{lllllll}
\hline L mean & FEV1 & FEV1 & FVC & FVC & FEF25- & FEF25- \\
(SD) & TI & SC & TI & SC & $75 \%$ TI & $75 \%$ SC \\
Week -4 & 2.559 & 2.501 & 3.282 & 3.237 & 2.362 & 2.273 \\
& $(0.71)$ & $(0.54)$ & $(0.88)$ & $(0.70)$ & $(1.00)$ & $(0.88)$ \\
Week 0 & 2.540 & 2.476 & 3.266 & 3.196 & 2.330 & 2.281 \\
& $(0.71)$ & $(0.53)$ & $(0.88)$ & $(0.67)$ & $(1.0)$ & $(0.93)$ \\
Week 8 & 2.545 & 2.471 & 3.287 & 3.198 & 2.276 & 2.257 \\
& $(0.74)$ & $(0.53)$ & $(0.89)$ & $(0.67)$ & $(1.04)$ & $(0.94)$ \\
Week 12 & 2.542 & 2.433 & 3.29 & 3.152 & 2.298 & 2.190 \\
& $(0.76)$ & $(0.53)$ & $(0.93)$ & $(0.64)$ & $(1.6)$ & $(0.92)$ \\
\hline
\end{tabular}

Conclusion: There was no change pulmonary function after 6 month treatment with TI compared with treatment with sc insulin.

Supported by MannKind Corporation

0186

Sustained efficacy and tolerability of inhaled human insulin $\left(\right.$ Exuber $\left.^{\circledR}\right)$. Therapy over 2 years: patients with type 1 diabetes

J. Skyler ${ }^{1}$, L. Jovanovic ${ }^{2}$, S. Klioze ${ }^{3}$, J. Reis ${ }^{3}$, W. Duggan ${ }^{3}$;

${ }^{1}$ Division of Endocrinology, Diabetes \& Metabolism, University of Miami Miller School of Medicine, ${ }^{2}$ Sansum Diabetes Research Institute, Santa Barbara, ${ }^{3}$ Pfizer Inc, Groton, United States.

Background and Aims: The availability of inhaled human insulin (INH; Exuber ${ }^{\circledR}$ (insulin human [rDNA origin]) Inhalation Powder) may increase patient acceptance of insulin, improve glycemic control, and reduce the burden of diabetes. Two previous 12- and 24-week studies of INH versus subcutaneous (SC) insulin in patients with type 1 diabetes mellitus (T1DM) showed comparable efficacy and small treatment group differences in lung function (measured using routine pulmonary function testing [PFT]), but long-term studies are critical to better characterize pulmonary safety. The aim of this study was to investigate the long-term (2-year) efficacy and safety profile of INH therapy in patients with T1DM.

Materials and Methods: An open-label, parallel-group, multicenter study where patients (between 18 and 65 years of age) were randomized to INH $(\mathrm{n}=291)$ or $\mathrm{SC}$ insulin $(\mathrm{n}=291)$. The primary end point was pulmonary function, as measured using highly standardized PFT. Secondary end points included glycosylated hemoglobin $\left(\mathrm{HbA}_{1 \mathrm{c}}\right)$, hypoglycemia, fasting plasma glucose (FPG), and body weight. Results: Treatment group differences in changes from baseline lung function (forced expiratory volume in 1 second $\left[\mathrm{FEV}_{1}\right]$ and carbon monoxide diffusing capacity
$\left.\left[\mathrm{DL}_{\mathrm{co}}\right]\right)$ were small $(<1 \%$ and $<2 \%$ of mean baseline values, respectively), occurred early (Month 3 data: $-0.021 \mathrm{~L}$ [90\% confidence interval $[\mathrm{CI}],-0.041,-0.002]$ for $\mathrm{FEV}_{1}$ and $-0.687 \mathrm{~mL} / \mathrm{min} / \mathrm{mm} \mathrm{Hg}[-0.969,-0.406]$ for $\mathrm{DL}_{\mathrm{co}}$ ), and remained stable with no progression for up to 2 years $(-0.023[-0.044,-0.002]$ and $-0.439[-0.732,-0.145]$, for $\mathrm{FEV}_{1}$ and $\mathrm{DL}_{\mathrm{co}}$, respectively). Glycemic control was maintained in both groups (change from baseline to Month 24 in $\mathrm{HbA}_{1 \mathrm{c}}$ was $0.1 \%$ and $-0.2 \%$ for INH and SC groups, respectively). The incidence of hypoglycemic events was similar in both groups (4.0 vs 3.8 events/subject-month, for $\mathrm{INH}$ and SC, respectively), but the incidence of severe hypoglycemic events was lower with INH (2.8 events/100 subject-month) versus SC (4.1 events/100 subject-month). After 24 months, INH resulted in greater reductions in FPG compared with SC insulin (from 9.5 to 8.7 , vs 9.3 to $9.6 \mathrm{mmol} / \mathrm{L}$, [170.1 to 156.8 , vs 166.9 to $173.5 \mathrm{mg} / \mathrm{dL}$, respectively), and less body weight gain (mean $0.8 \pm 4.2 \mathrm{~kg}$ vs $2.0 \pm 4.6 \mathrm{~kg}$ ). Adverse events were generally comparable with the exception of cough in the INH group, which was mild in most patients, nonproductive, and closely associated with dosing.

Conclusion: This 2-year study shows that INH is well tolerated and provides comparable glycemic control to SC insulin in adult patients with type 1 diabetes.

Supported by Pfizer Inc

\section{OP 32}

\section{Neuropathy}

\section{7}

Influence of blood glucose oscillations, measured by continuous glucose monitoring, on QTe interval and sympatho-vagal balance, during the day-life of type 1 diabetic patients

E. Cicconetti, D. Bracaglia, V. Santini, C. Verri, G. Cervoni, G. Ciampittiello, S. Gambardella, S. Frontoni; Dept. of Internal Medicine, Diabetes Center, Rome, Italy.

Background and Aims: Several recent studies have described a relationship between QTc prolongation and increased mortality in type 1 diabetic patients. Both hypoglycaemia and hyperglycaemia have shown to acutely increase QTc duration and stimulate sympathetic activation. However, only few, preliminary data are available on the role of blood glucose modifications on cardiac repolarization and sympatho-vagal balance, under physiological condition. Aim of the present study was to investigate the influence of blood glucose oscillations, during the day-life 
of type 1 diabetic patients, on QTc duration and on sympatho-vagal balance.

Materials and Methods: 22 type 1 diabetic patients (11 males and 11 females) were studied. Patients were in good metabolic control, mean age 34.4+9.9 years; exclusion criteria were hypertension, medication known to modify QT and diabetic complications. A 24-h electrocardiogram was recorded in each patient, with automatic QTc and R-R measurement, for the evaluation of sympatho-vagal balance (LF/HF ratio). Simultaneously, interstitial glucose concentrations were measured via a subcutaneous abdominal catheter using a continuous glucose monitoring system. Mean hourly values of blood glucose were divided into quintiles: I. $<60$; II. $>60<100$; III. $>100<140$; IV. $>140<190$; V. $>190 \mathrm{mg} / \mathrm{dl}$.

Results: A significant increase of QTc interval was observed in the V quintile vs. II and III $(436+19.4$ vs. $423.4+22.5$ and $425.4+24.6 \mathrm{~ms}$, respectively, $\mathrm{p}<0.001)$, with a significant correlation between blood glucose and QTc in the V quintile, thus suggesting a role of hyperglycemia on QTc lengthening. LF/HF ratio was significantly increased in the I vs. the II and the III quintile $(2.2+1.5$ vs. $2.7+1.7$ and vs. $2.8+1.9$, respectively, $\mathrm{p}<0.03$ ) and in the $\mathrm{V}$ vs. the II and the III quintile $(\mathrm{p}<0.04)$; in the $\mathrm{V}$ quintile, $\mathrm{LF} / \mathrm{HF}$ significantly correlated with QTc $(\mathrm{p}=0.005)$.

Conclusion: In conclusion, hyperglycemia seems to represent the most important factor in influencing QTc interval increase and sympathetic activation in well controlled, type 1 diabetic patients, without diabetic complications.

\section{8}

Preservation of thalamic neuronal function may be a prerequisite for pain perception in diabetic neuropathy R. A. Gandhi ${ }^{1}$, D. Selvarajah ${ }^{1}$, I. Wilkinson ${ }^{2}$, C. Emery ${ }^{1}$, P. J. Shaw ${ }^{3}$, P. D. Griffiths ${ }^{2}$, S. Tesfaye ${ }^{1}$;

${ }^{1}$ Diabetes Research Unit, Royal Hallamshire Hospital, Sheffield, ${ }^{2}$ Academic Unit of Radiology, University of Sheffield, ${ }^{3}$ Academic Unit of Neurology, University of Sheffield, United Kingdom.

Background: Pain is the most distressing symptom of diabetic neuropathy (DN). Despite extensive research, the pathogenesis of pain in DN remains poorly understood. As a result, treatment of painful DN is frequently unsatisfactory. The thalamus, which is the gateway to the somtosensory cortex, may have a role in the pathogenesis and persistence of painful DN.

Methods: 23 subjects with diabetes ( 7 No-DN, 8 painless $\mathrm{DN}$ and 8 painful DN) and 6 healthy controls (HC) underwent detailed clinical and neurophysiological assessments (Dyck's staging criteria; NIS(LL)+7). Proton magnetic resonance spectroscopy of the right postero-lateral thalamic nucleus was performed to measure established markers (N-acetyl [NA] compounds) of neuronal function using long echo time (LTE) and neuronal integrity using short echo time (STE) sequences.

Results: At LTE (ANOVA $\mathrm{p}=0.003$ ), subjects with painless $\mathrm{DN}$ (mean=1.56 $[\mathrm{SD}=0.18]$ ) had lower NA compared to painful DN $(1.93[0.23] \mathrm{p}<0.001)$. There was no significant difference between painful DN and the other groups (vs No$\mathrm{DN}$ (1.78[0.15], $\mathrm{p}=0.11$; vs HC (1.74[0.11], $\mathrm{p}=0.052)$. There were no significant differences in STE between the groups. Conclusions: As NA is largely confined to neuronal cell bodies and axons, the significant difference between painless and painful DN is suggestive of a higher degree of thalamic dysfunction in painless DN and preserved thalamic sensory neuronal function in the latter. The lack of a difference in STE between the groups suggests no neuronal loss. Preservation of thalamic neuronal function may therefore be a prerequisite for the perception of pain. Supported by Diabetes UK

\section{9}

Effects of venlafaxine and carbamazepine for painful peripheral diabetic neuropathy: a randomized controlled double-blind and double-dummy multicenter clinical trial

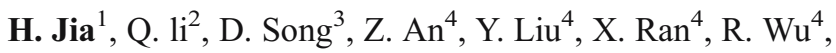
H. Tian ${ }^{4}$;

${ }^{1}$ Laboratory of Endocrinology and Metabolism, West China Hospital of Sichuan University, Chengdu, ${ }^{2}$ Department of Endocrinology, the First Affiliated Hospital of Chongqing University of Medical Science, Chongqing, ${ }^{3}$ Department of Diabetes Mellitus, Kunming Medical College, Kunming, ${ }^{4}$ Department of Endocrinology, West China Hospital of Sichuan University, Chengdu, China.

Background and Aims: Diabetic neuropathies are chronic complications of diabetes mellitus. Venlafaxine is a unique antidepressant with dual mechanisms of action. Several studies suggested that venlafaxine may benefit the patients with severe painful diabetic neuropathy. Venlafaxine extended release appeared effictive and safe in relieving painful diabetic neuropathy.Carbamazepine is an iminostilbene derivative chemically related to the tricyclic antidepressants. The studies showed that it can improve the symptoms of painful diabetic neuropathy.

Aim:To evaluate the safety and efficacy of venlafaxine and carbamazepine on painful peripheral diabetic neuropathy.

Materials and Methods: This clinical trial was a randomized, parallel-group, double-blind, double-dummy study. 132 Patients (venlafaxine group $(\mathrm{n}=66)$ and carbamazepine group $(\mathrm{n}=66)$ ) with painful peripheral diabetic neuropathy 
were recruited from 3 clinical centers. Venlafaxine group took venlafaxine $25 \mathrm{mg}$ plus one dummy carbamazepine tablet twice times a day and carbamazepine group took carbamazepine $0.1 \mathrm{~g}$ plus one dummy venlafaxine tablet twice times a day for 2 weeks. The primary efficacy parameter was a numeric pain intensity scale and the secondary was to assess quality of life.

Results: 119 patients have completed the trial. Venlafaxine was superior to carbamazepine in improving mean pain intensity score at $5,7,10$ and 14 days by PP analysis $(\mathrm{p}=0.0229, \mathrm{p}=0.0256, \mathrm{p}=0.0028$ and $\mathrm{p}=0.0005$ respectively). The effects of venlafaxine on improvement of the totle scores of quality of life were better than that of carbamazepine at 10 and 14 days $(\mathrm{p}=0.0183$ and $\mathrm{p}=0.0037$ respectively). Sleep interference and mood were improved by venlafaxine and carbamazepine, and the efficacy of venlafaxine was superior to that of carbamazepine. The common venlafaxine's adverse events included mild gastrointestinal discomfort, dizziness and somnolence. The frequency of adverse events in venlafaxine group was about $43.9 \%$ (4 patients withdrew for adverse events) and in carbamazepine group about $25.76 \%$ (2 patients withdrew for adverse events) $(\mathrm{p}=0.0558)$.

Conclusion: Venlafaxine and carbamazepine are efficacious in the treatment of painful diabetic neuropathy, venlafaxine is superior to carbamazepine in improving pain and quality of life. Both two drugs are safe and well tolerated.

\section{0}

Safety and efficacy of CNS 5161, a new neuronal glutamate receptor antagonist (NMDA) in the treatment of painful peripheral neuropathy

T. Forst ${ }^{1,2}$, T. W. Smith ${ }^{3}$, K. Schütte ${ }^{4}$, P. Marcus ${ }^{5}$, A. Pfützner ${ }^{1}$;

${ }^{1}$ Institute for Clinical Research and Development, Mainz, Germany, ${ }^{2}$ Endocrinology, University Mainz, Germany, ${ }^{3}$ Development, CeNeS, Cambridge, United Kingdom, ${ }^{4}$ Ergomed, Frankfurt, Germany, ${ }^{5}$ Medical Consulting, Marcus Ridley Ass., Devon, United Kingdom.

Background and Aims: Treatment of chronic pain syndromes associated with peripheral nerve injury is often a challenge. NMDA antagonists attenuate the frequencydependent potentiation of dorsal horn nociceptive neurons and have been found to exert beneficial pain relief in patients with peripheral neuropathies.

Materials and Methods: This study was a double blind, dose escalating, crossover study evaluating the safety and efficacy of the new NMDA antagonist CNS 5161 compared to placebo. Forty patients with chronic pain syndromes; post herpetic pain, diabetic neuropathy, post traumatic nerve injury or chronic regional pain syndrome I or II; (23 male, 17 female, age $55.2 \pm 11.5$ years, weight $81.9 \pm 17.9 \mathrm{~kg}(\operatorname{mean} \pm \mathrm{SD}))$ were recruited. Patients were treated with one of four different dosages of CNS 5161 $(125,250,500$ and $750 \mu \mathrm{g})$. The patients received the study medication as a bolus intravenous infusion of $30 \%$ over 10 minutes and as a subsequent infusion of $70 \%$ over 5 hours 50 minutes. The incidence, severity and relationship to study drug of all adverse events were determined. Blood pressure, heart rate, ECG and clinical laboratory values were assessed. Pain relief was measured by visual analogue scales (VAS) and ordinal verbal pain scores at baseline and at 2, 6, 12 and 24 hours after study drug administration.

Results: Infusion with CNS 5161 at all dosages appeared to be associated with an increase in mean systolic blood pressure and to a lesser extent in diastolic blood pressure. Moderate blood pressure elevations were observed in $8.3 \%$ of patients receiving $250 \mu \mathrm{g}$ and in $50 \%$ of patients receiving $500 \mu \mathrm{g}$ CNS 5161, compared with $15.4 \%$ during placebo treatment. Only two patients were recruited for treatment with $750 \mu \mathrm{g}$ CNS 5161 due to the blood pressure effects with increasing dosage. The most commonly occurring nervous system disorder was headache, which was found more often during placebo than during CNS 5161 treatment. Blurred vision was experienced by $16.7 \%$ receiving $250 \mu \mathrm{g}$ and by $33.3 \%$ receiving $500 \mu \mathrm{g}$ CNS 5161 , but not during placebo treatment. Post treatment VAS revealed a tendency for better pain improvement after $500 \mu \mathrm{g}$ CNS 5161 compared to placebo $(1.16 \pm 2.15$ vs. $0.23 \pm 1.87 ; \mathrm{p}=0.11)$. No difference in the verbal pain scores could be observed between CNS 5161 and placebo treatment. The mean improvement in VAS was considerable higher in patients with diabetic neuropathy compared with all other nerve disorders: $1.7,2.5$ and $2.3 \mathrm{~cm}$ at 2,6 and 12 hours respectively. In addition, the effect of CNS 5161 on verbal pain intensity and verbal pain relief seemed to be stronger in diabetic patients than in the other nerve disorders.

Conclusion: The new NMDA antagonist CNS 5161 was shown to be sufficiently well tolerated when used for treatment of neuropathic pain, with the most common side effects being a moderate increase in blood pressure and mild visual disturbances. Although this study was not powered for efficacy evaluation, treatment with CNS 5161 appeared to exert analgesic effects, especially in patients with diabetic neuropathy. Further sufficiently powered studies will investigate the clinical effects of CNS 5161 in this special patient population.

Supported by $\mathrm{CeNeS}$ 


\section{1}

Transcutaneous electrical nerve stimulation (TENS) in diabetic neuropathy

S. Kalra, B. Kalra, G. Nanda;

Endocrinology, Bharti Hospital, Karnal, India

Background and Aims: Painful neuropathy is a common, and difficult to manage, complication of diabetes. There is a need for effective, efficient and safe methods of pain relief in these patients. This paper reviews the effect of TENS on different symptoms of painful neuropathy in patients of diabetes mellitus.

Materials and Methods: A single blind, randomized, prospective single-centre study was planned to compare TENS with oxcarbamazepine in the management of painful diabetic neuropathy. 180 adult diabetic patients receiving five sittings of TENS on daily or alternate day basis were compared with 125 age-matched, disease-matched patients who were given daily oxcarbamazepine and five sittings with sham electrodes.

Results: Pain scores reduced from $4.40 \pm 0.54$ to $3.60 \pm 0.54$ in the oxcarbamazepine group while they fell from $4.40 \pm 0.54$ to $1.60 \pm 0.54$ in the TENS group $(\mathrm{p}<0.05)$. This difference was maintained after 3 week, even though the TENS sittings had stopped. Best reductions in pain score were achieved in subjects with burning (3.28 \pm 0.64$)$ and lancinating (3.12 \pm 0.64$)$. Least improvement was seen in allodynia $(2.15 \pm 0.35)$ and restless legs syndrome (2.16 \pm 0.56$)$

A significant improvement was seen in health distress and disease intrusion scores in the TENS group

Conclusion: This study demonstrates the beneficial effects of low dose TENS in patients with painful diabetic neuropathy

\section{2}

Gene transfer of an engineered transcription factor promoting expression of VEGF-A protects against experimental diabetic neuropathy

S. A. Price ${ }^{1}$, B. Duran-Jimenez ${ }^{1}$, Y. Liang ${ }^{2}$, L. Zhang ${ }^{2}$, E. J. Rebar ${ }^{2}$, C. C. Case $^{2}$, P. Gregory ${ }^{2}$, T. J. Martin' ${ }^{2}$, D. $\mathrm{Ando}^{2}, \mathrm{~K}$. $\mathrm{Spratt}^{2}$, D. R. Tomlinson ${ }^{1}$;

${ }^{1}$ Faculty of Life Sciences, University of Manchester, United Kingdom, ${ }^{2}$ Sangamo Biosciences Inc., Richmond, United States.

Background and Aims: We investigated whether gene transfer of an engineered zinc finger protein transcription factor (ZFP TF) designed to upregulate expression of the endogenous vascular endothelial growth factor (VEGF)-A gene could protect against experimental diabetic neuropathy (DN). ZFP TF-driven activation of the endogenous gene results in expression of all of the VEGF-A isoforms.

Materials and Methods: Rats were made diabetic with streptozotocin (55 mg/kg i.p.) after an overnight fast and group-housed with food and water ad libitum. They were then maintained for 8-week protocols at the end of which motor and sensory nerve conduction velocities (MNCV, SNCV) were measured in both hind-limbs under isoflurane anaesthesia. Different groups were given intramuscular injections of a plasmid encoding the ZFP TF in the left hind-limb only; the right limb was not injected. Three diabetic groups were given this treatment at 2 or 4 or 6 weeks after induction of diabetes; another diabetic group was given this treatment at all these time points and a final diabetic group was injected at the three time point with a placebo plasmid that did not encode the ZFP TF (shaminjected group). A non-diabetic group formed the controls. Results: The controls and sham injected group showed similar NCVs in both limbs, with the sham-injected diabetic rats showing falls of $19.1 \%(\mathrm{MNCV})$ and $21.4 \%$ (SNCV; both $\mathrm{p}<0.001)$ compared to controls. The diabetic rats injected 3 times with active plasmid showed reductions in $\mathrm{NCV}$ in the non-injected (right) limb of $17.0 \%$ (MNCV) and $25.0 \%$ (SNCV), with no significant reductions in NCV on the injected side and a marked statistically significant difference between the two sides ( $p=0.011$ for MNCV and 0.002 for SNCV). The diabetic group injected with active plasmid at week 6 only ( 2 weeks before NCV measurement) showed similar data to the group receiving three injections (left-right comparisons were $\mathrm{p}=0.066$ for $\mathrm{MNCV}$ and 0.005 for SNCV). There was a residual effect in the group injected at week 4 , but the group injected at week 2 showed data similar to the sham-injected animals.

Conclusion: These data suggest that VEGF-A-activating ZFP TFs may ultimately be of clinical utility in the treatment of diabetic neuropathy.

Supported by Diabetes UK, Sangamo Bioscinces Inc.

\section{OP 33}

\section{Mitochondrial dysfunction and insulin resistance}

\section{3}

Mitochondrial respiratory function is decreased in skeletal muscles of patients with type 2 diabetes

M. Mogensen ${ }^{1}$, K. Sahlin ${ }^{1}$, M. Fernström², D. Glintborg ${ }^{3}$, H. Beck-Nielsen ${ }^{3}$, K. Højlund ${ }^{3}$; 
${ }^{1}$ Institute of Sports Science and Clinical Biomechanics, University of Southern Denmark, Odense, Denmark, ${ }^{2}$ Department of Physiology and Pharmacology, Karolinska Institute, Stockholm, Sweden, ${ }^{3}$ Diabetes Research Centre, Department of Endocrinology, Odense University Hospital, Denmark.

Aim: This study was undertaken to investigate whether decreased oxidative capacity in type 2 diabetic (T2D) patients is caused by decreased mitochondrial volume or impaired mitochondrial functionality.

Methods: Skeletal muscle biopsies obtained from 10 obese T2D patients and 8 obese control subjects were used for assessment of 3-hydroxy-Acyl-CoA-dehydrogenase (HAD) and citrate synthase (CS) activity, uncoupling protein 3 (UCP3) content, fiber type distribution and respiration in isolated mitochondria using different substrates. To account for mitochondrial volume measures of respiration were corrected for CS activity.

Results: T2D patients had a significant lower state 3 respiration compared to obese controls using pyruvate+ malate but not when using palmitoyl-L-carnitine+malate. Furthermore, maximal activity of the electron transport chain was lower in T2D patients. No differences in CS or $\mathrm{HAD}$, or overall respiration per mg muscle protein were observed between T2D patients and obese controls. T2D patients had a higher percentage of type $2 \mathrm{~b}$ fibers whereas no differences in percentage type 1 fibers or UCP3 content were observed. Correlation analysis in whole population $(n=18)$ revealed a significant negative correlation between state 3 using pyruvate+malate and HbA1c. State 4 respiration (regardless of substrate) was positively correlated to percentage of type $2 \mathrm{~b}$ fibers. Furthermore, percentage type $2 \mathrm{~b}$ fibers correlated positively with several markers of insulin resistance.

Conclusion: In conclusion we provide evidence for a functional impairment in mitochondrial respiration and increased amount of type $2 \mathrm{~b}$ fibers in skeletal muscle of patients with T2D. These alterations may be important factors contributing to the progression from obesity to type 2 diabetes.

Supported by Statens Sundhedsvidenskabelige Forskningsråd

\section{4}

Muscle mitochondrial dysfunction with age-impact of body composition and insulin sensitivity

H. Karakelides, M. Bigelow, K. R. Short, J. Kahl, K. Nair; Endocrinology, Diabetes, Metabolism and Nutrition, Mayo Clinic College of Medicine, Rochester, United States.
Background and Aims: Muscle mitochondrial dysfunction has been reported in aging, type 2 diabetes mellitus, obesity and other insulin resistant states. We sought to determine whether muscle mitochondrial dysfunction in aging is related to change in body composition, insulin resistance or age per se.

Materials and Methods: We studied 12 each of elderly lean $\left(\mathrm{EL}, \mathrm{BMI}=22.6 \pm 0.6 \mathrm{~kg} / \mathrm{m}^{2}\right)$, elderly obese (EO, $\mathrm{BMI}=30.0 \pm 0.6$ ), young lean ( $\mathrm{YL}, \mathrm{BMI}=23.0 \pm 0.5)$ and young obese $(\mathrm{YO}, \mathrm{BMI}=30.9 \pm 0.6)$ who have similar leisure time activity levels and fat free mass. Hyperinsulinemic (1.5 mU/kg FFM/min) euglycemic clamp to assess insulin sensitivity was performed. Freshly isolated mitochondria (mt) from needle vastus lateralis muscle biopsy samples was used to measure ATP production based on luciferase reaction.

Results: Elderly people irrespective of their differences in BMI had lower $\mathrm{VO}_{2}$ peak $(32.42 \pm 1.26 \mathrm{ml} / \mathrm{kgFFM} / \mathrm{min})$ than in younger people $(47.58 \pm 0.94 \mathrm{ml} / \mathrm{kgFFM} / \mathrm{min}$, $\mathrm{p}<0.0001)$. mtATP production using pyruvate, palmitoylL-carnitine, ketoglutarate and malate (PPKM) was higher $(5.51 \pm 0.2 \mu \mathrm{mol} / \mathrm{min} / \mathrm{g}$ tissue) in younger people than in older people $(3.97 \pm 0.33, \mathrm{p}=0.0003)$ and there were no differences between obese and lean groups. Glucose infusion rate (GIR) was higher in lean people $(54.28 \pm 2.41 \mathrm{mmol} / \mathrm{kgFFM} / \mathrm{min})$ than in obese group $(42.7 \pm 2.46, \mathrm{p}=0.002)$ to maintain similar glucose levels at steady state $(\mathrm{lean}-90.9 \pm 0.06 \mathrm{mg} / \mathrm{dL}$ vs obese $-90.9 \pm 0.04$, $\mathrm{p}=\mathrm{NS}$ ). There was no effect of age on GIR. Insulin sensitivity had no effect on mtATP production or vice versa.

Conclusion: In conclusion, obesity is the major determinant of insulin sensitivity, while age and mtATP production show no significant effect. However, the decline in muscle mitochondrial ATP production with age is unrelated to the differences in obesity and insulin sensitivity.

Supported by NIH R01 DK 41973

0195

Impaired in vivo mitochondrial function but similar intramyocellular lipid content in patients with type 2 diabetes mellitus compared to BMI-matched controls

V. B. Schrauwen-Hinderling ${ }^{1}$, M. E. Kooi ${ }^{1}$, M. Mensink ${ }^{2}$, M. K. C. Hesselink ${ }^{3}$, J. M. A. van Engelshoven ${ }^{1}$, P. Schrauwen ${ }^{2}$;

${ }^{1}$ Radiology, University Hospital Maastricht, ${ }^{2}$ Human Biology, Maastricht University, ${ }^{3}$ Movement Sciences, Maastricht University, The Netherlands. 
Background and Aims: Mitochondrial dysfunction and increased IMCL content have both been implicated in the development of type 2 diabetes mellitus (T2DM), but the relative contribution of these two factors in the aetiology of diabetes is unknown. As obesity is an independent determinant of IMCL content, we here examined mitochondrial function and IMCL content in overweight type 2 diabetic patients and BMI matched controls.

Materials and Methods: Twelve patients with T2DM and nine BMI matched normoglycemic controls were investigated (age (y): 61.8 \pm 4.4 and 56.1 $\pm 6.8, \mathrm{p}<0.05$; BMI $\left(\mathrm{kg} / \mathrm{m}^{2}\right): 29.4 \pm 3.3$ and $29.3 \pm 2.7, \quad \mathrm{p}=\mathrm{n} . \mathrm{s}$.; $\mathrm{VO}_{2} \max$ $\left(\mathrm{ml}^{*} \min ^{-1} * \mathrm{~kg}^{-1}\right): \quad 30.6 \pm 5.8$ and $34.6 \pm 4.8, \mathrm{p}=\mathrm{n} . \mathrm{s}$., in T2DM and controls, respectively (mean \pm stdev)). Mitochondrial function was determined by measuring $\mathrm{PCr}$ recovery half-time immediately after exercise with 31-phosphorus magnetic resonance spectroscopy $\left({ }^{31} \mathrm{P}-\mathrm{MRS}\right)$. IMCL content was determined by proton magnetic resonance spectroscopic imaging ( $\left.{ }^{1} \mathrm{H}-\mathrm{MRSI}\right)$ and insulin sensitivity was assessed with a hyperinsulinemic euglycemic clamp.

Results: The half-time of $\mathrm{PCr}$ recovery was $45 \%$ longer in T2DM patients $(27.3 \pm 3.5 \mathrm{~s}$ versus $18.7 \pm 0.9 \mathrm{~s}$, $)$, while IMCL content was similar as in controls $(1.37 \pm 0.30 \%$ vs. $1.25 \pm 0.22 \%$ of the water resonance, $\mathrm{p}=\mathrm{n} . \mathrm{s}$.). Insulin sensitivity was lower in T2DM patients $(26.0 \pm 2.2$ and $18.9 \pm 2.3 \mu \mathrm{mol}^{*} \min ^{-1} * \mathrm{~kg}^{-1}$, (all mean \pm SEM), $\mathrm{p}<0.05$ ). The recovery half-time of $\mathrm{PCr}$ correlated positively with plasma glucose $\left(\mathrm{R}^{2}=0.42, \mathrm{p}<0.01\right)$ and $\mathrm{HbA1c}\left(\mathrm{R}^{2}=0.48\right.$, $\mathrm{p}<0.05)$ in diabetic patients.

Conclusion: The finding that in vivo mitochondrial function is decreased in T2DM patients while IMCL content is similar as in controls suggests that a low mitochondrial function is a more important determinant than a high IMCL content in the aetiology of T2DM. The correlation of plasma glucose concentration as well as HbAlc with $\mathrm{PCr}$ recovery half-time reveals that the impairment of in vivo mitochondrial function is related to the degree of the disease. Our results are in line with the hypothesis that decreased mitochondrial function is a predisposition to develop type 2 diabetes mellitus.

\section{6}

In vivo mitochondrial function in young men born with low birth weight, as assessed by ${ }^{31} \mathrm{P}$-NMR spectroscopy after energy depleting exercise

C. Brøns ${ }^{1}$, C. B. Jensen ${ }^{1}$, H. Storgaard ${ }^{1}$, A. V. Astrup ${ }^{2}$, B. Quistorff ${ }^{3}$, A. Vaag ${ }^{1}$;

${ }^{1}$ Steno Diabetes Center, Gentofte, ${ }^{2}$ Department of Human Nutrition, The Royal Veterinary and Agricultural University, Frederiksberg, ${ }^{3}$ Department of Medical Biochemistry and Genetics, University of Copenhagen, Denmark.
Background and Aims: Low birth weight (LBW) is associated with increased risk of insulin resistance and development of type 2 diabetes (T2D) later in life. The underlying molecular mechanism(s) remain largely undetermined. Recent magnetic resonance spectroscopy studies in humans suggest, that even subtle defects in mitochondrial function may play a role in the pathogenesis of (muscle) insulin resistance in aging and T2D. Mitochondrial dysfunction in the liver and pancreatic beta cells has also recently been linked with impaired insulin secretion in growth retarded rats, indicating a general intrauterine programming mechanism. The aim of this study was to determine whether LBW in humans is associated with impaired mitochondrial function in skeletal muscle tissue after energy depleting exercise.

Materials and Methods: We evaluated in vivo mitochondrial function in the forearm flexor muscle by ${ }^{31}$ phosphorus nuclear magnetic resonance spectroscopy $\left({ }^{31} \mathrm{P}\right.$ NMR) in 18 24-year old healthy lean men with LBW (BW $\leq 10$ th percentile) and 16 matched normal birth weight (NBW) (50th $\leq \mathrm{BW} \leq 90$ th percentile) controls. There was no difference between the groups with regards to BMI, W/H, fat mass and lean mass; however fasting b-glucose was higher in LBW subjects $4.59 \pm 0.43$ vs. $4.99 \pm 0.48 \mathrm{mmol} / \mathrm{L}$ $(P=0.003)$. A standard exercise protocol was performed with 4 successive maximal voluntary contractions each lasting $30 \mathrm{sec}$ interspersed by a $60 \mathrm{sec}$ recovery period. The ${ }^{31} \mathrm{P}$ NMR technique provides non-invasive real-time estimates of resting state parameters of energy metabolism as well as mitochondrial aerobic capacity.

Results: In the forearm flexor muscle, the recovery of $\mathrm{PCr}$ (doubling time) after exercise was increased by $35 \%$ in the LBW group, $57 \pm 19$ vs. $76 \pm 28 \mathrm{sec}$. $(P=0.05)$. There were no other differences between the NBW and LBW group with regards to resting $\mathrm{PCr}$ or $\mathrm{PCr}$ levels after exercise, $\mathrm{pH}$ at rest and after exercise or in the calculated Vmax for mitochondrial ATP synthesis. Insulin resistance as determined by using the euglycemic hyperinsulinemic clamp technique was not correlated with recovery of $\mathrm{PCr}$ or other measures of in vivo mitochondrial function.

Conclusion: The $\mathrm{PCr}$ recovery kinetics was altered in 24year old healthy men with low birth weight, suggesting that the mitochondrial function is affected in this group. These data could imply that the altered mitochondrial function could possibly forego the development of an impaired glucose metabolism. Importantly, as these changes occur in young, lean non-diabetic subjects, we speculate that mitochondrial dysfunction may play a primary role in the pathogenesis of muscle insulin resistance and T2D associated with low birth weight.

Supported by European Foundation for the Study of Diabetes, Diabetesforeningen, Aase og Ejnar Danielsens Fond 
0197

Mitochondrial dysfunction is not due to impaired transmembrane glucose transport in type 2 diabetes J. Szendroedi ${ }^{1}$, A. I. Schmid ${ }^{2}$, M. Chmelik ${ }^{2}$, C. Toth ${ }^{1}$, A. Brehm ${ }^{3}$, P. Nowotny ${ }^{1}$, W. Waldhäusl ${ }^{1}$, M. Roden ${ }^{3}$;

${ }^{1}$ Division of Endocrinology and Metabolism, Medical University of Vienna, ${ }^{2}$ MR Centre of Excellence, Medical University of Vienna, ${ }^{3}$. Medical Department, Hanusch Hospital, Vienna, Austria.

Background: Type 2 diabetic patients (T2DM) and their relatives exhibit defective energy metabolism. However, factors determining mitochondrial dysfunction are yet unclear. We measured mitochondrial function noninvasively from ATP synthetic flux (fATP) and analyzed relationships with insulin resistance and transmembrane myocellular glucose transport in humans.

Materials and Methods: Well controlled T2DM $(n=10$; age:59 $\pm 6 \mathrm{a}$, nondiabetic older (CONo: $\mathrm{n}=10 ; 57 \pm 6 \mathrm{a}$ ) and younger humans (CONy: $n=11 ; 27 \pm 2 a ; p<10^{-6}$ vs T2DM and $\mathrm{CONo}$ ) were studied. Muscle glucose-6-phosphate (G6P), inorganic phosphate (Pi) and fATP were measured before (bfATP) and during hyperinsulinemic-euglycemic clamp (ifATP) using ${ }^{31} \mathrm{P}$ magnetic resonance spectroscopy (MRS). Four T2DM underwent a further hyperinsulinemichyperglycemic clamp. Insulin sensitivity was assessed from steady state glucose infusion rate (M). Intramyocellular lipid contents (IMCL) were quantified with ${ }^{1} \mathrm{H}$ MRS.

Results: $\mathrm{M}$ differed between T2DM, CONo and CONy $\left(5.4 \pm 2,8.3 \pm 1,12.2 \pm 3 \mathrm{mg} \cdot \mathrm{kg}^{-1} \cdot \mathrm{min}^{-1} ; \mathrm{p}<0.05\right)$, whereas IMCL were similar in all groups. BfATP was $\sim 37 \%$ lower in $\mathrm{T} 2 \mathrm{DM}$ than in CONy $\left(8.6 \pm 2\right.$, vs $11.8 \pm 3 \mu \mathrm{mol} . \mathrm{g}^{-1}$. $\left.\min ^{-1} ; \mathrm{p}<0.05\right)$. Insulin increased fATP by $26 \%$ and $9 \%$ only in CONy and CONo $(\mathrm{p}<0.05)$. Fasting G6P and Pi were comparable and increased by $\sim 50 \%$ and $\sim 17 \%$ during the clamp in all study groups (G6P: T2DM: $224 \pm 97$, CONo: $225 \pm 45$, CONy: $221 \pm 72$; $<<0.05$ vs baseline; Pi: T2DM: $3.3 \pm 0.4$, CONo: $3.6 \pm 0.5$, CONy: $3.2 \pm 0.3$; $<<0.005$ vs baseline). BfATP and ifATP related positively to $\mathrm{M}$ $(R \geq 0.40, \mathrm{p}<0.05)$ and negatively to basal plasma free fatty acids $(R \geq-0.45, \mathrm{p}<0.05)$. During hyperglycemic clamps, $\mathrm{M}$ was $\sim 70 \%$ ( $8.1 \pm 2$ vs $4.8 \pm 1 \mathrm{mg} . \mathrm{kg}^{-1}$ body weight. $\mathrm{min}^{-1}$, $P<0.05)$ and G6P was $\sim 100 \%$ higher than during normoglycemic clamps $\left(290 \pm 1\right.$ vs. $145 \pm 5 \mu$ g.kg muscle ${ }^{-1}$, $\mathrm{p}=0.01$ ). BfATP and ifATP did not differ between normoglycemic and hyperglycemic clamps (bfATP: $9.0 \pm 2$ vs 9.4 \pm 2 , ifATP: $9.2 \pm 4$ vs $10.3 \pm 2 \mu \mathrm{mol} . \mathrm{g}^{-1} \cdot \mathrm{min}^{-1}$ ).

Conclusion: Muscle ATP synthesis reflects insulin sensitive glucose disposal and decreases with increased lipid availability. Hyperglycemia increases glucose transport but fails to improve the impairment of ATP synthesis in well- controlled T2DM indicating the presence of a mitochondrial defect independent of substrate flux.

Supported by Austrian Science Foundation (FWF P15656)

0198

Genetic, epigenetic and environmental factors influence oxidative phosphorylation genes and metabolism in human muscle

C. A. Ling $^{1}$, P. Poulsen ${ }^{2}$, S. Simonsson ${ }^{3}$, J. Holmkvist ${ }^{1}$, P. Almgren ${ }^{1}$, P. Hagert ${ }^{1}$, E. Nilsson ${ }^{2}$, A. G. Mabey ${ }^{1}$,

A. Vaag $^{2}$, L. Groop ${ }^{1}$;

${ }^{1}$ Department of Clinical Sciences, Lund University, Malmö, Sweden, ${ }^{2}$ Steno Diabetes Center, Gentofte, Denmark, ${ }^{3}$ Institute of Biomedicine, The Sahlgrenska Academy, Göteborg, Sweden.

Background and Aims: A set of genes involved in oxidative phosphorylation (OXPHOS) show reduced expression in muscle of patients with type 2 diabetes, but it remains unknown whether this is an inherited or acquired defect. Here we investigated whether genetic, epigenetic and non-genetic factors influence the expression of 4 OXPHOS genes, NDUFB6 (complex 1), UQCRB (complex 3), COX7A1 (complex 4) and ATP5O (complex 5) in human muscle.

Materials and Methods: Muscle biopsies were taken from young ( $\mathrm{n}=86$, age $28 \pm 0.2$ years) and elderly $(\mathrm{n}=68$, age $62.4 \pm 0.2$ years) monozygotic (MZ) and dizygotic (DZ) healthy twins before and after a hyperinsulinemic euglycemic clamp.

Results: The mRNA expression of NDUFB6, UQCRB, COX7A1 and ATP5O was reduced by $15 \%-40 \%(\mathrm{P}<0.007)$ in muscle biopsies from elderly compared with young twins. When a classical twin approach and a biometric model were used, we found that both genetic and environmental factors influence the expression of these four OXPHOS genes in muscle. Regression analysis were then performed to test if any of the following non-genetic factors: muscle PGC- $1 \alpha$ and PGC- $1 \beta$ expression, birth weight, age, sex, percentage body fat, trunk-leg fat ratio and $\mathrm{VO}_{2}$ max, influence muscle OXPHOS expression. Indeed, NDUFB6 expression was positively related to PGC-1 $\alpha$ levels $(P<0.0001)$, trunk-leg fat ratio $(P<0.05)$ and $\mathrm{VO}_{2} \max (P<0.007)$. UQCRB expression was positively related to PGC-1 $\alpha(P<0.0001)$, percentage body fat $(P<0.02)$ and $\mathrm{VO}_{2} \max (P<0.001)$. COX7A1 expression was positively related to PGC- $1 \alpha(P<0.0001)$, PGC- $1 \beta$ levels $(P<0.02)$ and percentage body fat $(P<0.04)$, and related to age $(P<0.0001)$ and sex $(P<0.0001)$. ATP5O expression was positively related to PGC-1 $\alpha \quad(P<0.0001)$ and related to age $(P<0.0001)$ and sex $(P<0.003)$. We then 
used regression analysis to test if any of the four OXPHOS genes influence muscle metabolism, and NDUFB6 was the only OXPHOS gene associated with insulin-stimulated glucose -uptake $(\mathrm{P}<0.05)$, -oxidation $(\mathrm{P}<0.008)$ and -storage $(\mathrm{P}<0.05)$ as well as lipid oxidation $(\mathrm{P}<0.03)$. We therefore focused our genetic and epigenetic analysis on NDUFB6 and we identified a polymorphism $(\mathrm{A} / \mathrm{G})$ in the promoter-region of the NDUFB6 gene, creating a possible transcription factor binding site and a DNA methylation site (a CG-dinucleotide). Interestingly, elderly carriers of the G/G genotype had reduced NDUFB6 expression compared with carriers of $\mathrm{A} / \mathrm{A}$ and $\mathrm{A} / \mathrm{G}$ genotypes $(\mathrm{G} / \mathrm{G} 0.24 \pm 0.03$ vs. $\mathrm{A} / \mathrm{G} \quad 0.33 \pm 0.02$ and $\mathrm{A} / \mathrm{A} \quad 0.36 \pm 0.02 ; \mathrm{P}<0.05)$ and this polymorphism influenced insulin- stimulated glucose disposal (G/G 264 \pm 28, A/G $233 \pm 14$ vs. A/A 294 $\pm 13 ; \mathrm{P}<0.05$ ), nonoxidative glucose metabolism (G/G 146 $\pm 27, \mathrm{~A} / \mathrm{G}$ $128 \pm 13$ vs. $\mathrm{A} / \mathrm{A} 187 \pm 13 ; \mathrm{P}<0.05)$ in muscle and $\mathrm{VO}_{2} \max$ (G/G 24 \pm 2, A/G $24 \pm 1$ vs. A/A 29 $\pm 1 ; \mathrm{P}<0.05)$. Finally, this DNA methylation site was only methylated in elderly and in none of the young twins carrying the $G / G$ genotype and the degree of DNA methylation correlated negatively to muscle NDUFB6 expression $(r=-0.61 ; P<0.05)$.

Conclusion: Our data show that OXPHOS expression in human muscle is influenced by genetic, epigenetic and nongenetic factors (e.g. age), thereby providing an example how genes and environment may interact to increase agedependent susceptibility to type 2 diabetes.

Supported by Swegene

\section{OP 34}

\section{Molecular mechanisms of obesity}

\section{9}

Obese are resistant to the catabolic, but not to the cognitive effects of central nervous insulin W. Kern ${ }^{1}$, C. Benedict ${ }^{2}$, B. Perras ${ }^{1}$, H. L. Fehm ${ }^{1}$, J. Born ${ }^{2}$, M. Hallschmid ${ }^{2}$;

${ }^{1}$ Medical Clinic I, ${ }^{2}$ Dept. of Neuroendocrinology, Medical University, Luebeck, Germany.

Background and Aims: Insulin acts in the central nervous system to reduce food intake and body weight and is considered a major adiposity signal. Intranasal insulin administration increases cerebrospinal fluid concentrations of the peptide without absorption into the blood stream. In a previous study, eight weeks of intranasal insulin administration induced loss of body weight and body fat and improved declarative memory in lean men. The present experiments aimed to generalize these findings to obese subjects.
Materials and Methods: Insulin and placebo, respectively, were intranasally administered 4 times a day (amounting to $160 \mathrm{IU} / \mathrm{d}$ ) over a period of 8 weeks to two groups of 15 obese men each in a double-blind manner (body mass index, mean \pm SEM: insulin: $32.24 \pm 1.21$; placebo: $33.13 \pm 1.50 \mathrm{~kg} / \mathrm{m}^{2}$ ). Body weight and body composition as well as declarative memory (recall of word lists) were evaluated during baseline and at the end of the treatment period.

Results: Contrasting with the catabolic effects in normalweight men, insulin treatment did not induce any significant reduction of body weight and body fat in the obese subjects. However, in accordance with the effects in normal-weight men, declarative memory was improved (remembered words, placebo: $1.13 \pm 0.38$; insulin: $2.87 \pm$ $0.56, \mathrm{p}<0.05)$ and hypothalamic-pituitary-adrenal axis activity in the morning was reduced in obese subjects (ACTH, placebo: $25.65 \pm 3.62$; insulin: $20.11 \pm 3.27 \mathrm{pg} / \mathrm{ml}$, $\mathrm{p}<0.01$; cortisol, placebo: $13.03 \pm 1.42$; insulin: $9.55 \pm$ $0.55 \mu \mathrm{g} / \mathrm{dl}, \mathrm{p}<0.04)$.

Conclusion: Our results indicate that intranasal insulin is functionally active in obese men, inducing an improvement in declarative memory, but fails to affect the neuronal networks critically involved in body weight regulation. They confirm for the first time in humans that obesity is associated with diminished central nervous sensitivity to the adiposity signal insulin. This defect likely contributes to the persistence of obesity in spite of elevated levels of circulating insulin in these patients.

Supported by Deutsche Forschungsgemeinschaft

0200

Adiponectin and resistin in human cerebrospinal fluid and disturbance of the blood brain barrier with obesity K. Kos ${ }^{1}$, P. G. McTernan ${ }^{1}$, A. L. Harte ${ }^{1}$, S. James ${ }^{2}$, D. Snead ${ }^{2}$, G. Chaldakov ${ }^{3}$, A. Tonchev ${ }^{3}$, B. Hoggart ${ }^{4}$, P. 0'Hare ${ }^{1}$, S. Kumar';

${ }^{1}$ Department of Diabetes and Metabolism, Clinical Sciences, Coventry, United Kingdom, ${ }^{2}$ Department of Pathology, Clinical Sciences, Coventry, United Kingdom, ${ }^{3}$ Division of Cell Biology, Medical University, Varna, Bulgaria, ${ }^{4}$ Department of Anaesthesia, Birmingham Heartlands and Solihull NHS Trust, Solihull, United Kingdom.

Background and Aims: The adipocytokines adiponectin and resistin are both implicated in the pathogenesis of obesity mediated diabetes but central action in humans is unknown. In contrast, the adipocytokine leptin has been extensively studied in regards of its central appetite regulating pathways, with leptin resistance identified in obesity. The aims of these studies were 1) to examine serum 
and CSF levels of adiponectin and resistin and their relationship with age, gender, body mass index (BMI), insulin resistance (IR) and parameters of inflammation 2) to compare their $\mathrm{CSF} /$ serum ratio with leptin and 3) to establish the presence of adiponectin receptors (AdipoR1 \& R2) in the human hypothalamus.

Materials and Methods: Serum and CSF samples were analysed from 39 consenting non-diabetic Caucasian subjects undergoing elective surgery with spinal anaesthesia. In total, 20 men (Age: $69.8 \pm(\mathrm{SD}) 8.6$ years; BMI: 29.4(SD) \pm $3.4 \mathrm{~kg} / \mathrm{m}^{2}$ ) and 19 women: (Age: $69.4 \pm 4.3$ years, BMI: $27.3 \pm 4.8 \mathrm{~kg} / \mathrm{m}^{2}$ ) were analysed for adiponectin, resistin, leptin, insulin and high sensitivity (hs) CRP levels. Further, human brain slices were analysed for ACRP 1 and 2 by immunohistochemistry. The HOMA index was used as a measure of IR.

Results: The mean total CSF adiponectin level was $16.4 \pm 6.25 \mathrm{pg} / \mathrm{ml}$. Serum and CSF adiponectin levels correlated positively with serum levels $(\mathrm{r}=0.34 ; \mathrm{p}=0.047)$. Serum and CSF adiponectin levels showed a positive correlation with age $(\mathrm{CSF} r=0.36, \mathrm{p}=0.035)$. Serum adiponectin levels were significantly higher in females than in males $(\mathrm{p}=0.015)$, but CSF levels were not gender. Serum resistin levels correlated with age $(\mathrm{r}=0.45, \mathrm{p}=0.006)$, but not in CSF levels (mean $0.2 \pm 0.13 \mathrm{ng} / \mathrm{ml}$ ). Serum adiponectin but not resistin levels showed an inverse correlation with HOMA index (serum Adiponectin $\mathrm{r}=-0.36, \mathrm{p}=0.036$ ), whilst no pattern was observed for CSF levels. CSF Adiponectin and resistin appeared unrelated to hsCRP.

In males, serum and CSF adiponectin showed a significant, negative correlation with BMI, which remained significant even when age was adjusted for (serum: $r=-0.569, p=0.01$, $\mathrm{n}=17$; CSF: $\mathrm{r}=-0.62, \mathrm{p}=0.01, \mathrm{n}=14)$. The $\mathrm{CSF} / \mathrm{Serum}$ ratio was inversely correlated with BMI in women $(\mathrm{r}=-0.61$, $\mathrm{p}=0.02$ ) whilst a similar correlation was found for leptin $(\mathrm{r}=-0.7, \mathrm{p}<0.01)$. Furthermore, adiponectin receptors were identified in the human hypothalamus.

Conclusion: The study demonstrated that both adiponectin and resistin are detectable in human CSF and CSF adiponectin levels are age and BMI dependant. Both adipokines appear to enter CSF by a mechanism yet to be determined -either transported into the CSF from serum or produced within brain. The presence of adiponectin in the CSF suggests a potential regulatory role in energy homeostasis, which may influence body fat mass in humans. As with leptin, there is a gender dependent decrease in the adiponectin serum/CSF ratio with BMI. This suggests alteration of transport into the CSF for adiponectin in obesity is unrelated to insulin resistance and inflammation. These findings implicate a role for these adipokines in energy homeostasis and the cause of changes in obesity.
0201

Lifestyle intervention downregulates the expression of genes linked to extracellular matrix (ECM) and cell cycle in adipose tissue in subjects with metabolic syndrome - the GENOBIN study

M. Kolehmainen ${ }^{1}$, U. Schwab ${ }^{2}$, T. Salopuro ${ }^{2}$, J. Kekäläinen ${ }^{2}$, D. E. Laaksonen ${ }^{3}$, L. Pulkkinen ${ }^{2}$, P. Kallio ${ }^{1}$, R. Kouki ${ }^{4}$, V. Lindi ${ }^{2}$, U. Mager ${ }^{2}$, N. Siitonen ${ }^{2}$, L. Niskanen ${ }^{3}$, H. Gylling ${ }^{2}$, R. Rauramaa ${ }^{4}$, M. Uusitupa ${ }^{2}$;

${ }^{1}$ Food and Health Research Centre, University of Kuopio, ${ }^{2}$ Department of Clinical Nutrition, University of Kuopio, ${ }^{3}$ Department of Medicine, Kuopio University Hospital, ${ }^{4}$ Kuopio Research Institute of Exercise Medicine, University of Kuopio, Finland.

Background and Aims: Lifestyle modification has been proven to be effective in the prevention of type 2 diabetes, although the underlying molecular mechanisms are not well known. The aim of the present study was to examine the effect of a lifestyle intervention on gene expression in adipose tissue and to find out genes and gene clusters contributing to the development of type 2 diabetes.

Materials and Methods: Seventy-five overweight to obese subjects (BMI 28-40 $\mathrm{kg} / \mathrm{m}^{2}$ ) with impaired glucose tolerance, aged $60 \pm 7$ years $($ mean $\pm \mathrm{SD})$ were randomized either to weight reduction (WR) $(\mathrm{N}=28)$, aerobic training $(\mathrm{N}=15)$, resistance training $(\mathrm{N}=14)$ or control $(\mathrm{N}=18)$ groups according to gender, age and BMI. Oral and intravenous glucose tolerance tests, fasting blood samples, anthropometric measurements, and subcutaneous adipose tissue (AT) biopsies were performed before and after the 33-week intervention. Gene expression using microchip technology was studied in AT samples in 9 subjects from the WR and 10 from control group that was confirmed by real time PCR in the whole population. The study was approved by the Ethics Committee of the Kuopio University Hospital and was performed in accordance with the standards of the Helsinki Declaration.

Results: The subjects in WR group decreased body weight $(p<0.0001)$, BMI $(p<0.0001)$, waist circumference $(p<0.0001)$ and lean body mass $(p<0.005)$. No significant changes were seen in other groups. The WR group was divided according to success in weight reduction: $\geq 5 \%$ (Group A, N=11) and $<5 \%$ (Group B, N=17) of their initial weight. Fasting serum insulin $(\mathrm{p}=0.027)$ and fasting plasma glucose $(p=0.057)$ concentrations decreased during the intervention in Group A. The insulin sensitivity index increased more in Group A than in Group B ( $p=0.037)$. Downregulation in AT gene expression was evident in Group A (of 105 genes with changes in gene expression $82 \%$ were downregulated, $\mathrm{p}<0.01$ ), whereas an opposite effect was seen in the control group (of 62 genes with 
changes in gene expression $82 \%$ were upregulated, $\mathrm{p}<0.01)$. The changes in gene expression were modest, with fold changes ranging from 0.67 to 1.68 in Group A and from 0.63 to 1.33 in the control group. Gene Ontology (GO) clusters for biological processes were assessed for enrichment of genes in each cluster with $\mathrm{p}<0.001$. Altogether seven GO biological process clusters were found in 105 genes linked to ECM, cell cycle and proliferation in Group A.

Conclusion: Long-term weight reduction resulted in significant overall downregulation in gene expression in AT, especially in genes linked to ECM and cell cycle. This suggests that ECM might have a role in the development of obesity and insulin resistance. Downregulation of genes linked to the cell cycle suggests adaptation also at the molecular level after intensive weight loss.

Supported by the Academy of, the Ministry of Education in, the Finnish Foundation for Diabetes Research, and the Sigrid Juselius Foundation

\section{2}

Obesity, exercise and variants in the adioponutrin gene: a population based study

L. E. Johansson ${ }^{1}$, C. A. Larsson ${ }^{2}$, U. Lindblad ${ }^{2,3}$,

L. Råstam ${ }^{2}$, M. Ridderstråle ${ }^{1}$;

${ }^{1}$ Department of Clinical Sciences Malmö, Clinical Obesity, Lund University, Malmo, ${ }^{2}$ Department of Clinical Sciences Malmö, Community Medicine, Lund University, Malmo, ${ }^{3}$ Skaraborg Institute, Skövde, Sweden.

Background and Aims: We have recently shown that obese subjects have an increased adipose adiponutrin $(A D P N)$ mRNA expression and that variants in the gene show association with obesity. Carriers of the associated alleles had a lesser increase in adipose mRNA expression but also an increased basal lipolysis. Functional studies show that ADPN has both lipase and transacylase activity i.e. working both in a lipogenic and a lipolytic way. Additionally, the $A D P N$ mRNA expression is widely regulated by nutritional status. A high carbohydrates diet increases the mRNA expression in adipose tissue. There seem to be an insulin dependent effect on the increased expression seen by glucose in human adipocytes suggesting insulin as an important regulator. The aim of the study was to further investigate the association between $A D P N$ and obesity and the possible influence of exercise.

Materials and Methods: The coding SNP rs738409 and the intronic SNP rs2072907 in the $A D P N$ gene were genotyped using TaqMan assays on the ABI 7900HT (Applied Biosystems) in a population-based material from Vara, Sweden $(\mathrm{n}[\mathrm{m} / \mathrm{f}]=904 / 905$, age $46[58-56]$ years, BMI
$26.0[23.7-29.3] \mathrm{kg} / \mathrm{m}^{2}$ ). Information about physical activity and anthropometrical measures were available.

Results: Both SNPs differed significantly in allele frequency where the common allele was over-represented in the obese subjects $\left(P_{\mathrm{rs} 738409}=0.012, P_{\mathrm{rs} 2072907}=0.046\right)$. There was significantly more carriers of the homozygous rs738409 common allele among the obese compared to non-obese $[P=0.030]$. Homozygous carriers of rs 738409 common allele had an increased risk of obesity, OR 2.26 $(1.11-4.59)[P=0.024]$. The effect was stronger in subjects above 50 years of age, OR 4.82(1.12-20.69) $[P=0.035]$. Homozygous carriers of the common allele had an increased risk of developing obesity when the level of physical activity was low, OR 2.16(1.00-4.67) $[P=0.049]$. There was no significant increased risk of developing obesity when carrying the risk allele when the level of physical activity was high $[P=0.22]$. There was no significant effect of rs2072907 although the trends were similar to those of rs738409. Grouping carriers of the rs2072907 variant allele resulted in a borderline significant higher risk of obesity if you are a homozygous carrier of the common allele, OR 1.28(0.99-1.65) $[P=0.056]$.

Conclusion: This study confirms the previous association between the $A D P N$ SNPs and obesity. Homozygous carriers of the common allele have an increased risk of obesity but an increased physical activity might lower this risk. It still remains to be clarified if this is an actual effect of the workout itself or if it is due to concomitant dietary habits. The functionality of the coding SNP rs738409 may be of importance for future studies.

0203

Single nucleotide polymorphisms in the Krüppel-like factor $7(K L F 7)$ gene are involved in the pathogenesis of obesity

D. P. Jensen ${ }^{1}$, K. L. Andersen ${ }^{1}$, H. Eiberg ${ }^{2}$, K. Borch-Johnsen ${ }^{1,3}$, T. Jørgensen ${ }^{3}$, S. Maeda ${ }^{4}$, Y. Nakamura ${ }^{4}$, T. Hansen ${ }^{1}$, O. Pedersen ${ }^{1}$;

${ }^{1}$ Steno Diabetes Center, Gentofte, Denmark, ${ }^{2}$ Department of Medical Biochemistry and Genetics, University of Copenhagen, Denmark, ${ }^{3}$ Research Centre for Prevention and Health, Glostrup University Hospital, Denmark, ${ }^{4}$ SNP Research Center, RIKEN, Yokohama, Japan.

Background and Aims: The $K L F 7$ gene encodes Krüppellike factor (KLF) 7, which is a member of the KLF family of transcription factors, initially shown to play important roles in cellular development and differentiation, and recently reported to be involved in adipogenesis. Several single nucleotide polymorphisms (SNPs) have been identified in the KLF7 gene, of which some have been associated 
with type 2 diabetes in a Japanese population; however, a possible association of KLF7 SNPs with obesity has not been investigated. The aim of this study was to examine $K L F 7$ SNPs in relation to obesity and associated metabolic quantitative traits in Danish whites. Two intronic SNPs (rs1263608 and rs1263627) were initially investigated, whilst studies of interactions with other SNPs in the KLF7 gene are ongoing.

Materials and Methods: Studies were performed in the population-based Inter99 cohort of middle-aged people ( $n=5,570 ; 2,803$ men, 2,767 women). 1,182 of these subjects $(21 \%)$ were obese $\left(B M I \geq 30 \mathrm{~kg} / \mathrm{m}^{2}\right)$. The two SNPs were genotyped using the PCR-Invader assay.

Results: The KLF7 variants rs1263608 and rs1263627 were associated with obesity in case-control studies. The minor allele frequency of rs1263608 was higher among obese subjects (BMI $\geq$ above $30 \mathrm{~kg} / \mathrm{m}^{2}$ ) than among control subjects $\left(\mathrm{BMI}<30 \mathrm{~kg} / \mathrm{m}^{2}\right)(39.8 \%$ [95\% CI: 37.6-42.0\%] vs. $37.2 \%$ [36.2-38.1\%], $\mathrm{p}=0.03$, odds ratio 1.17 [1.01$1.36]$ when carrying the minor allele); this was also the case for $\operatorname{rs} 1263627(28.8 \%$ [26.8-30.9\%] vs. 25.9\% [25.0$26.8 \%$ ], $\mathrm{p}=0.009$, odds ratio $1.22[1.06-1.41]$ when carrying the minor allele). Further evidence of an association with obesity was found in an analysis of quantitative traits, where subjects carrying the minor alleles of either rs1263608 or rs1263627 had a higher BMI than wild type subjects $(p=0.006$ and $p=0.003$ for the two SNPs, respectively). Finally, all carriers of the rs 1263608 variant had statistically significant elevated levels of serum insulin $30 \mathrm{~min}$ post-OGTT $(\mathrm{p}=0.03)$, and the incremental area under the curve for circulating levels of glucose, insulin, and C-peptide during a 2-h OGTT was also elevated in these subjects ( $p=0.04,0.02$, and 0.04 , respectively).

Conclusion: In a large study sample of Danish whites we identified an association between SNP rs1263608 and rs1263627 in KLF7 and obesity. Studies of interactions with other SNPs in the gene are ongoing.

\section{4}

Variants in the hepatic lipase gene ( $L I P C)$ associate with obesity and elevated fasting plsama HDL-cholesterol levels in a population based sample of 5,850 middle-aged people

C. H. Andreasen ${ }^{1}$, M. K. Andersen ${ }^{1}$, N. Grarup ${ }^{1}$,

A. Albrechtsen ${ }^{1}$, K. Borch-Johnsen ${ }^{1,2}$, T. Jørgensen ${ }^{2}$,

T. Hansen ${ }^{1}$, O. Pedersen ${ }^{1}$;

${ }^{1}$ Steno Diabetes Center, Gentofte, ${ }^{2}$ Research Centre for Prevention and Health, Glostrup University Hospital, Denmark.

Background and Aims: High density lipoprotein (HDL) is involved in the transport of cholesterol from peripheral tissues to the liver, where it is metabolised. Hepatic lipase (HL) is a liver-specific lipolytic enzyme, catalyzing the hydrolysis of triglycerides and phospholipids in HDL particles. Promoter variants of the gene encoding HL (LIPC) have been associated with lower HL activity. Thus, the aim of the study was to elucidate if variants in $L I P C$ associate with obesity and related quantitative metabolic traits. We also hypothesized that lifestyle factors modify the effect of genotype.

Materials and Methods: We genotyped the LIPC $250 \mathrm{G}>\mathrm{A}$ promoter (rs2070895) and the Ser215Asn (rs6083) polymorphisms in 5,850 individuals from a population-based cohort (Inter99) using mass-spectrometry of PCR products and TaqMan allelic discrimination.

Results: We observed no linkage disequilibrium between the two variants $\left(D^{\prime}=0.05 ; r^{2}=0.001\right)$. The Ser215Asn variant associated with obesity $\left(\mathrm{BMI} \geq 30 \mathrm{~kg} / \mathrm{m}^{2}\right)$ in a casecontrol study of 2,585 lean and 966 obese individuals (minor allele frequency 36.7\% [95\% CI: 35.4-38.0\%] vs. $39.7 \%$ [37.5-41.9\%]; odds ratio 1.14 [1.02-1.27]; $p=0.019$ ), and with elevated BMI in a study of quantitative traits (additive model: $p=0.016$ ). The $-250 \mathrm{G}>\mathrm{A}$ polymorphism showed no association with obesity, yet carriers of the A allele (A allele frequency 22.6\% [95\% CI: $21.8-$ 23.4\%]) had increased fasting plasma HDL-cholesterol levels compared to subjects with the GG genotype (GG: $1.42 \pm 0.39 \mathrm{mmol} / \mathrm{L}$, GA: $1.46 \pm 0.41 \mathrm{mmol} / \mathrm{L}$, AA: $1.53 \pm 0.44 \mathrm{mmol} / \mathrm{L}$; additive model: $p=7 * 10^{-10}$ ). Moreover, we observed a significant interaction of smoking habits with the $-250 \mathrm{G}>\mathrm{A}$ variant, indicating a greater effect of smoking on lowering the HDL-cholesterol levels in subjects with the GG or GA genotype compared to subjects with the AA genotype (GG/GA: $-0.11 \mathrm{mmol} / \mathrm{L}$ vs. AA: $0.01 \mathrm{mmol} / \mathrm{L}$; recessive model: $p=0.01)$.

Conclusion: The LIPC 215Asn allele associates with obesity and the $-250 \mathrm{G}>\mathrm{A}$ polymorphism associates with plasma HDL-cholesterol levels influenced by interaction of smoking status. This study underlines that variation in LIPC modulates risk factors of cardiovascular disease.

\section{OP 35}

\section{Second messengers and oscillatory behaviour in beta cell function}

0205

Glucokinase and PFK-2/FBPase-2 interact to fulfil crucial roles in glucose induced insulin secretion in pancreatic beta cells and in glucose disposal in liver S. Langer ${ }^{1}$, S. Baltrusch ${ }^{1}$, W. E. Smith ${ }^{2}$, D. A. Okar ${ }^{2}$; 
${ }^{1}$ Institute of Clinical Biochemistry, Hannover Medical School, Germany, ${ }^{2}$ Veterans Administration, Medical Center, Minneapolis, United States.

Background and Aims: Glucokinase (GK) plays a pivotal role in the process of glucose recognition in pancreatic beta cells and in glucose metabolism in liver. The bifunctional enzyme 6-phosphofructo-2-kinase/fructose-2,6-bisphosphatase (PFK-2/FBPase-2) is a key regulator in liver metabolism. Pancreatic beta cells express the PFK-2/FBPase-2 brain isoform. In previous experiments we elucidated the binding between both proteins. Interestingly, PFK-2/ FBPase-2 activates GK in vivo irrespective of the isoform. Therefore, it was the aim of this study to elucidate the underlying molecular mechanisms of the GK-PFK-2/ FBPase-2 complex formation.

Materials and Methods: Interaction between recombinant GK protein and recombinant PFK-2/FBPase-2 or FBPase-2 protein was analyzed with in vitro pull-down assays, fluorescence spectroscopy and steady state kinetics. Intracellular binding of the enzymes were analyzed in GK and PFK-2/FBPase-2 overexpressing RINm5F cells by immunohistochemistry.

Results: Incubation with recombinant liver PFK-2/FBPase-2 for $1 \mathrm{~h}$ in vitro resulted in a significant 3 fold increase in GK enzyme activity. In a Ni-NTA affinity assay recombinant Histag GK was able to pull-down AlexaFluor-594 labeled FBPase-2 and addition of unlabeled FBPase-2 confirmed that the binding is specific and reversible. In vitro titration of AlexaFluor-594 labeled PFK-2/FBPase-2 with GK labeled by AlexaFluor-488, while following the FRET signal between the fluorescence dyes, suggests a 1:1 stoichiometric GK-PFK-2/FBPase-2 complex. In insulin-producing RINm5F cells GK and PFK-2/FBPase-2 immunofluorescence was detectable in the cytoplasm. Quantitative analyses revealed a significant 3 fold increase of colocalization between both proteins in cells cultured at $10 \mathrm{mmol} /$ 1 glucose in comparison to cells cultured at $3 \mathrm{mmol} /$ 1 glucose.

Conclusion: Glucose promotes the GK-PFK-2/FBPase-2 interaction. This indicates a glucose-dependent stabilization of a specific GK conformation by PFK-2/FBPase-2 and a major regulatory principle of the GK-PFK-2/FBPase-2 complex in glucose sensing cell types. In beta cells activation of the glucose sensor GK stimulates glucoseinduced insulin secretion. In liver GK stabilization mediates a coordinated upregulation of the glucose disposal. Our results may open the perspective for a therapeutic targeting of this interaction in type 2 diabetes therapy.

Supported by VA Merit Award to DAO
0206

Synchronous oscillations of cAMP and $\mathrm{Ca}^{2+}$ in hormone-stimulated insulin-secreting $\beta$-cells

O. Dyachok, Y. Isakov, J. Sågetorp, E. Gylfe,

A. Tengholm;

Department of Medical Cell Biology, Uppsala University, Sweden.

Background and Aims: $\mathrm{Ca}^{2+}$ signaling has been extensively characterized and found to involve oscillations important for the temporal control of insulin secretion. In pancreatic $\beta$-cells, cAMP affects the cytoplasmic $\mathrm{Ca}^{2+}$ concentration $\left(\left[\mathrm{Ca}^{2+}\right]_{\mathrm{i}}\right)$ and amplifies $\mathrm{Ca}^{2+}$-dependent insulin release. The hormones glucagon and glucagon-like peptide-1 (GLP-1), strongly amplify nutrient-stimulated insulin secretion by raising cAMP and modulating $\mathrm{Ca}^{2+}$ signaling. We therefore studied the kinetics of hormone-evoked cAMP and $\mathrm{Ca}^{2+}$ signaling in insulin-secreting $\beta$-cells.

Materials and Methods: We developed a fluorescent biosensor that reports the cytoplasmic cAMP concentration beneath the plasma membrane $\left([\mathrm{cAMP}]_{\mathrm{i}}\right)$. Insulin-secreting INS-1 cells were transfected with a truncated form of the protein kinase A (PKA) regulatory subunit fused with CFP and targeted to the plasma membrane. Co-expressed YFPtagged PKA catalytic subunit also localized to the membrane under basal conditions, but dissociated upon elevation of $[\mathrm{cAMP}]_{\mathrm{i}}$. This translocation was detected as an increase of the CFP/YFP fluorescence ratio. Simultaneous recording of $[\mathrm{cAMP}]_{\mathrm{i}}$ and $\left[\mathrm{Ca}^{2+}\right]_{\mathrm{i}}$ was performed with evanescent wave microscopy and the fluorescent indicator fura-red.

Results: An increase in $[\mathrm{cAMP}]_{\mathrm{i}}$ evoked by IBMX inhibition of phosphodiesterases or forskolin stimulation of adenylyl cyclases resulted in rapid and pronounced rise of the CFP/YFP fluorescence ratio. Stimulation of INS-1 cells with $0.3-10 \mathrm{nM}$ glucagon or GLP-1 evoked pronounced oscillations of $[\mathrm{cAMP}]_{\mathrm{i}}$. These $[\mathrm{cAMP}]_{\mathrm{i}}$ oscillations disappeared upon removal of extracellular $\mathrm{Ca}^{2+}$ and this effect was readily reversible. Simultaneous recording of $[\mathrm{cAMP}]_{\mathrm{i}}$ and $\left[\mathrm{Ca}^{2+}\right]_{\mathrm{i}}$ from GLP-1 stimulated cells revealed that the initial $[\mathrm{cAMP}]_{\mathrm{i}}$ elevation was followed by rise of $\left[\mathrm{Ca}^{2+}\right]_{\mathrm{i}}$, and that subsequent $[\mathrm{cAMP}]_{\mathrm{i}}$ oscillations were synchronized with oscillations of $\left[\mathrm{Ca}^{2+}\right]_{\mathrm{i}}$.

Conclusion: These data demonstrate receptor-triggered oscillations of cAMP synchronized with those of $\mathrm{Ca}^{2+}$. The interplay between cAMP and $\mathrm{Ca}^{2+}$ should increase the signal information content improving low-level signal detection and further contribute to the specificity of the downstream cellular responses like ion channel activity, insulin secretion, $\beta$-cell survival and proliferation. 
The temporal pattern of cAMP signals determines protein kinase $\mathbf{A}$ activity range in insulin-secreting cells A. Tengholm, J. Sågetorp, O. Dyachok;

Department of Medical Cell Biology, Uppsala University, Sweden.

Background and Aims: Stimulation of insulin-secreting cells with glucagon or glucagon-like peptide-1 (GLP-1) has been found to cause oscillations of the cAMP concentration beneath the plasma membrane ([cAMP]). To investigate whether [cAMP] oscillations can contribute to selective regulation of downstream cellular targets we studied the effect of different time-courses of [cAMP] signals on the generation of cytoplasmic $\mathrm{Ca}^{2+}$ signals and on the nuclear translocation of the protein kinase A (PKA) catalytic subunit in individual INS-1 $\beta$-cells.

Materials and Methods: [cAMP] was measured with a novel fluorescent translocation biosensor and ratiometric evanescent wave microscopy. The cytoplasmic $\mathrm{Ca}^{2+}$ concentration $\left(\left[\mathrm{Ca}^{2+}\right]_{\mathrm{i}}\right)$ was measured with ratiometric epifluo rescence imaging and the fura- 2 indicator. Analysis of PKA nuclear translocation was performed with epifluorescence imaging and FlAsH-labelling of tetracysteinetagged PKA-C $\alpha$ subunit.

Results: Stimulation of INS-1 cells with GLP-1 induced dose-dependent increases of [cAMP] with oscillations in $>80 \%$ of the cells at $0.3-1 \mathrm{nM}$, whereras higher concentrations of the hormone most frequently elicited stable [cAMP] elevations $(>60 \%)$. Inhibition of phosphodiesterases with $100 \mu \mathrm{M}$ IBMX resulted in a prompt rise $\left(\mathrm{t}_{1 / 2}=23 \pm 2 \mathrm{~s}\right)$ of [cAMP], consistent with a high basal rate of cAMP formation. The IBMX-induced elevation of [cAMP] lasted throughout the stimulation period but rapidly returned to basal levels upon removal of the stimulus $\left(\mathrm{t}_{1 / 2}=14 \pm 3\right)$. Pulsatile application of IBMX ( $1 \mathrm{~min}$ stimulation, 3 min wash) generated oscillations of [cAMP] mimicking those observed after hormone stimulation. Both stable and pulsatile application of IBMX induced pronounced $\left[\mathrm{Ca}^{2+}\right]_{\mathrm{i}}$ spiking and in the case of $[\mathrm{cAMP}]$ oscillations the $\left[\mathrm{Ca}^{2+}\right]_{\mathrm{i}}$ spikes were grouped and correlated with the periods of elevated [cAMP]. In contrast, only prolonged stable [cAMP] elevation induced nuclear entry of FlAsH-labelled PKA-C $\alpha$ subunit.

Conclusion: These data indicate that cAMP oscillations can contribute to selective regulation of downstream targets by restricting the spatial redistribution of PKA.
Oscillations of PIP3 in insulin-secreting cells evoked by glucose- and insulin-induced co-activation of PI3-kinase O. Idevall Hagren, A. Tengholm;

Medical Cell Biology, Uppsala University, Sweden.

Background and Aims: Glucose-induced insulin release from pancreatic beta-cells is associated with autocrine activation of insulin receptors on the beta-cell surface, which leads to stimulation of PI3-kinase and formation of the lipid messenger phosphatidylinositol-3,4,5-trisphosphate (PIP3). PIP3 regulates many important cellular functions, including gene transcription, cell survival and proliferation as well as insulin secretion. The present study aimed at investigating the time-course of PIP3 signals evoked by glucose in individual beta-cells.

Materials and Methods: Insulin-secreting MIN6 cells were transfected with the pleckstrin homology domain from Akt fused to GFP (GFP-AktPH). This biosensor resides in the cytoplasm under basal conditions but redistributes to the plasma membrane upon generation of PIP3. The translocation was detected with evanescent wave microscopy and the cytoplasmic $\mathrm{Ca} 2+$ concentration $([\mathrm{Ca} 2+] \mathrm{i})$ was simultaneously measured with the fluorescent $\mathrm{Ca} 2+$ indicator fura red.

Results: MIN6 cells responded to $100 \mathrm{nM}$ insulin with GFPAktPH translocation resulting in a $30 \pm 3 \%(n=42)$ increase of plasma membrane fluorescence. Depolarization with $30 \mathrm{mM}$ $\mathrm{KCl}$ caused rapid elevation of $[\mathrm{Ca} 2+]$, which was paralleled by a $28 \pm 3 \% \quad(n=21)$ increase of membrane GFP-AktPH fluorescence. Removal of extracellular $\mathrm{Ca} 2$ + prevented both the depolarization-induced $[\mathrm{Ca} 2+] \mathrm{i}$ increase and the GFP-AktPH translocation, whereas inhibition of insulin secretion with $5 \mu \mathrm{M}$ epinephrine abolished the $\mathrm{KCl}$ induced GFP-AktPH translocation without affecting the [Ca2 $+]$ i response. Elevation of the glucose concentration from $3 \mathrm{mM}$ to $20 \mathrm{mM}$ resulted in an initial lowering of $[\mathrm{Ca} 2+] \mathrm{i}$ followed within 3 minutes by a rapid rise, that was associated with an increase of GFP-AktPH membrane fluorescence $(48 \pm 3 \%, \mathrm{n}=58)$. After $5-10 \mathrm{~min}$, there was regular oscillations of membrane GFP-AktPH fluorescence $(0.22 \pm 0.015 \min -1, n=28)$. Each oscillation was preceded by a distinct elevation of $[\mathrm{Ca} 2+] \mathrm{i}$. In the presence of $250 \mu \mathrm{M}$ of the hyperpolarizing agent diazoxide, glucose did not induce significant GFP-AktPH translocation by its own, but markedly enhanced the response to $100 \mathrm{nM}$ exogenous insulin ( $48 \pm 5 \%$ vs. $28 \pm 2, \mathrm{P}<0.001, \mathrm{n}=42$ ).

Conclusion: It is concluded that glucose and insulin synergistically activate PI3-kinase in insulin-secreting cells and that oscillations of PIP3 occur as a result of pulsatile insulin release. 
0209

Plasma membrane PIP $_{2}$ concentration is dynamically regulated by $\mathrm{Ca}^{2+}$ and the $A T P / A D P$ ratio in insulin secreting cells

S. Thore, J. Sågetorp, A. Tengholm;

Department of Medical Cell Biology, Uppsala University, Sweden.

Background and Aims: Phosphatidylinositol 4,5-bisphosphate $\left(\mathrm{PIP}_{2}\right)$ is important for a variety of cellular processes by serving as precursor for second messengers and by regulating ion channels, the cytoskeleton, and vesicle traffic in many types of cells, including insulin-secreting $\beta$-cells. The present study aimed at investigating how the plasma membrane concentration of $\mathrm{PIP}_{2}$ is regulated by glucose, intracellular $\mathrm{Ca}^{2+}$, ATP and by different phosphoinositide kinases in individual insulin-secreting cells.

Materials and Methods: Plasma membrane PIP2 concentration was recorded in insulin-secreting MIN6-cells with evanescent wave microscopy and the $\mathrm{PIP}_{2}$-binding GFPtagged PH-domain from PLC $\delta$. In some experiments, the cytoplasmic $\mathrm{Ca}^{2+}$ concentration $\left(\left[\mathrm{Ca}^{2+}\right]_{\mathrm{i}}\right)$ was simultaneously measured using the fluorescent indicator fura red.

Results: Elevation of the glucose concentration from 3 to $11 \mathrm{mM}$ evoked anti-synchronous oscillations of $\left[\mathrm{PIP}_{2}\right]$ and cytoplasmic $\mathrm{Ca}^{2+}$ concentration, consistent with phospholipase $\mathrm{C}$ being periodically activated by the voltage-dependent $\mathrm{Ca}^{2+}$. influx. In $\alpha$-toxin-permeabilized cells, $0.1-1 \mathrm{mM}$ ATP induced dose-dependent increases of $\left[\mathrm{PIP}_{2}\right]$ counteracted by the ADP-analogue adenosine-5'-O-2-thiodiphosphate. There was rapid turnover of $\mathrm{PIP}_{2}$ in the membrane with $\mathrm{t}_{1 / 2} 16 \mathrm{~s}$. RT-PCR analysis identified transcripts of 10 different phosphoinositide-kinase isoforms. Adenosine-sensitive type-II- and wortmannin-sensitive type-III PI4-kinases were equally important for ATP-dependent $\mathrm{PIP}_{2}$-formation. Moreover, $\mathrm{PIP}_{2}$ synthesis was selectively enhanced by overexpression of PIP5-kinase I $\beta$, but not by the $\alpha$ - or $\gamma$-isoforms. Conclusion: We conclude that plasma membrane $\mathrm{PIP}_{2}$ is dynamically regulated by intracellular $\mathrm{Ca}^{2+}$ and the ATP/ ADP ratio in insulin-secreting cells. The rapid turnover allows generation of second messengers with maintenance of the $\mathrm{PIP}_{2}$ levels required for sustained exocytosis of insulin secretory granules.

\section{0}

Unmasking glucose-induced insulin secretion in mouse islets lacking $\mathbf{K}_{\mathrm{ATP}}$ channels

A. Szollosi, M. Nenquin, J.-C. Henquin;

Unit of Endocrinology and Metabolism, University of

Louvain Faculty of Medicine, Brussels, Belgium.
Background and Aims: A current model ascribes glucose stimulation of insulin secretion (GSIS) to the cooperation of a $\mathrm{K}_{\text {ATP }}$ channel-dependent triggering pathway and a $\mathrm{K}_{\text {ATP }}$ channel-independent amplifying pathway. Closure of $\mathrm{K}_{\text {АTP }}$ channels leads to $\beta$-cell depolarization, increased $\mathrm{Ca}^{2+}$ influx and rise of cytosolic $\mathrm{Ca}^{2+}\left(\left[\mathrm{Ca}^{2+}\right]_{\mathrm{c}}\right)$ that triggers secretion. The amplifying pathway does not produce a further rise of $\left[\mathrm{Ca}^{2+}\right]_{\mathrm{c}}$, but is thought to augment $\mathrm{Ca}^{2+}$ action via still unidentified mechanisms. However, several groups using islets from sulfonylurea receptor 1 knockout (Sur1KO) mice failed to measure significant GSIS in these islets whose $\beta$-cells lack $K_{\text {ATP }}$ channels. Our aim was to address this issue that casts doubt on the validity of the model of $\beta$-cell function.

Materials and Methods: Islets were isolated from adult control and Sur1KO mice (provided by J. Bryan, Houston), and tested rapidly after isolation $(2 \mathrm{~h}$ preincubation in Krebs medium with $10 \mathrm{mmol} / \mathrm{l}$ glucose - G10) or after overnight culture in RPMI medium with G10. The islets were perifused for measurement of IS or $\left[\mathrm{Ca}^{2+}\right]_{\mathrm{c}}$ or incubated with $\mathrm{U}-{ }^{14} \mathrm{C}$ glucose for measurement of glucose oxidation.

Results: Compared with control islets, freshly isolated Sur1KO islets had elevated basal $(\mathrm{G} 1)\left[\mathrm{Ca}^{2+}\right]_{\mathrm{c}}$ and IS rate that were both corrected by preventing $\mathrm{Ca}^{2+}$ influx. Despite the lack of $\mathrm{K}_{\mathrm{ATP}}$ channels, they responded to G15 by a transient drop in $\left[\mathrm{Ca}^{2+}\right]_{\mathrm{c}}$ followed by an increase which, however, remained lower than glucose-stimulated $\left[\mathrm{Ca}^{2+}\right]_{\mathrm{c}}$ in control islets. The absolute rate of GSIS was $\sim 3$-fold lower in Sur1KO than control islets, with only a poor increase above baseline (G15: G1=1.2-fold in Sur1KO vs $>20$-fold in controls). These differences could not be explained by differences in insulin content, alterations of glucose oxidation or poorer synchronization of $\left[\mathrm{Ca}^{2+}\right]_{\mathrm{c}}$ oscillations in islets. Overnight culture of Sur1KO islets slightly augmented basal IS (G1) and considerably improved their secretory response. Stimulation with G15 now induced a 4-fold increase in IS above baseline, reaching higher absolute values than in cultured control islets. Importantly, overnight culture did not produce similar effects in control islets, whose GSIS was barely different from that of fresh islets. This major impact of culture on GSIS in Sur1KO islets did not require serum but was markedly attenuated when the RPMI medium contained only G5 instead of G10. It was not attributable to changes in the rate of glucose oxidation, but could at least partly be explained by higher $\left[\mathrm{Ca}^{2+}\right]_{\mathrm{c}}$ levels in the islets. Testing the amplifying pathway during $\mathrm{KCl}$-depolarization of fresh and cultured Sur1KO islets did not evidence significant differences, indicating that this pathway is functional immediately after isolation provided $\left[\mathrm{Ca}^{2+}\right]_{\mathrm{c}}$ is sufficiently increased.

Conclusion: Overnight culture of Sur1KO islets unmasks the ability of glucose to increase IS in $\beta$-cells lacking $\mathrm{K}_{\text {АTP }}$ channels. The poor efficacy of glucose in fresh Sur1KO islets (probably reflecting the in vivo situation) is attributed 
to a weak triggering signal $\left(\left[\mathrm{Ca}^{2+}\right]_{\mathrm{c}}\right.$ is low compared to Gstimulated control islets) and a poor efficacy of the amplifying signals (because their action requires higher $\left.\left[\mathrm{Ca}^{2+}\right]_{\mathrm{c}}\right)$. Altogether, these data help explaining controversies in the literature and support models implicating two pathways in the control of GSIS.

\section{OP 36}

\section{New therapies - pre-clinical}

\section{1}

Comparative effects of CS-917, a fructose 1,6-bisphosphatase inhibitor, and metformin

A. Okuno, T. Yoshida, J. Tanaka, K. Takahashi, S. Kanda, R. Nakashima, J. Ogawa, Y. Hagisawa;

Sankyo Co. Ltd., Tokyo, Japan.

Background and Aims: CS-917 (MB06322) is a prodrug of an inhibitor of fructose 1,6-bisphosphatase, a ratelimiting enzyme of gluconeogenesis (GNG). CS-917 decreases plasma glucose in cynomolgus monkeys and various rodent models of type 2 diabetes (T2DM) by inhibition of GNG. The mechanism of a biguanide, metformin (Met), has not been elucidated in detail although a predominant effect in patients with T2DM is reported to be inhibition of hepatic glucose production through reduced GNG. In the present study, we compared the glucoselowering mechanisms and effects between CS-917 and Met. Materials and Methods: 1) Blood glucose kinetics was analyzed using the Steele equation. CS-917 $(7.5 \mathrm{mg} / \mathrm{kg})$ or Met $(300 \mathrm{mg} / \mathrm{kg})$ was administered orally to overnightfasted Goto-Kakizaki (GK) rats (insulin deficient model), followed by intravenous injection of ${ }^{3} \mathrm{H}$-glucose.

2) Glucose-lowering effects in fasted and non-fasted GK rats were evaluated after oral administration of CS-917 $(15 \mathrm{mg} / \mathrm{kg})$ or Met $(300 \mathrm{mg} / \mathrm{kg})$. The AUC of plasma glucose for $8 \mathrm{~h}$ was calculated.

3) In vivo efficacy was then evaluated by $\mathrm{AM} / \mathrm{PM}$ combination treatment regimens in Zucker diabetic fatty rats (insulin resistant model). AM and PM doses were followed by 8 h-daylong fasting and nocturnal ad lib feeding, respectively. CS-917 (150 mg/kg) and Met $(150 \mathrm{mg} / \mathrm{kg})$ were orally administered AM or PM in one of the AM/PM combination treatment regimens. Glycohemoglobin levels were measured after 6-week treatment.

Results: 1) CS-917 decreased the appearance of blood glucose, but not the disappearance of blood glucose. In contrast, Met increased the appearance and disappearance of blood glucose (see Table 1).
2) CS-917 significantly decreased the AUC of plasma glucose in the fasted state and Met decreased that in the fed state (see Table 1).

3) The regimen of dosing CS-917 AM with Met PM decreased glycohemoglobin levels more than its reverse regimen (AM/PM regimen, glycohemoglobin level (\%, meanSE): control, 9.6 \pm 0.4 ; CS-917/Met, 7.5 $\pm 0.5^{*} \dagger$; Met/CS-917, $8.4 \pm 0.4^{*}\left({ }^{*} P<0.05\right.$ vs. control, ${ }^{\dagger} P<0.05$ vs. Met/CS-917)).

Table 1 Glucose kinetics and effect on AUC of plasma glucose levels in GK rats

\begin{tabular}{|c|c|c|c|c|c|c|}
\hline \multirow[t]{2}{*}{ Treatment } & \multicolumn{3}{|c|}{ Glucose rate (mg/dL/min) } & \multicolumn{3}{|c|}{$\begin{array}{l}\text { AUC of plasma glucose } \\
(\mathrm{mg} / \mathrm{dLxh})\end{array}$} \\
\hline & $\mathrm{N}$ & Appearance & Disappearance & $\mathrm{N}$ & Fasted state & Fed state \\
\hline Control & 11 & $1.69 \pm 0.47$ & $1.26 \pm 0.87$ & 6 & $\begin{array}{l}1758.5 \pm \\
162.3\end{array}$ & $\begin{array}{l}2472.6 \pm \\
149.4\end{array}$ \\
\hline CS-917 & 11 & $0.43 \pm 0.16^{*}$ & $1.15 \pm 0.30$ & 6 & $\begin{array}{l}1060.7 \pm \\
13.2^{*}\end{array}$ & $\begin{array}{l}2284.8 \pm \\
89.0\end{array}$ \\
\hline Met & 12 & $2.85 \pm 0.18^{*}$ & $3.45 \pm 0.28^{*}$ & 6 & $\begin{array}{l}1593.0 \pm \\
61.0\end{array}$ & $\begin{array}{l}2015.6 \pm \\
98.5^{*}\end{array}$ \\
\hline
\end{tabular}

Mean \pm SE; $* P<0.05$ vs. control

Conclusion: CS-917 decreased glucose appearance consistent with GNG inhibition. In contrast, Met increased glucose disappearance. The glucose-lowering effect of CS-917 was observed in the fasted state where GNG is predominant. In contrast, that of Met was observed in the feeding state. The repeated bid regimen of CS-917 before fasting with Met before feeding represented better reduction in glycohemoglobin than the reverse dosing regimen. These results suggested that CS-917 and Met have different glucose-lowering mechanisms and that their efficacies are partly dependent on the timing of administration and/or on the feeding state. CS-917 is the first in a new class of oral antidiabetic agents and provides a new therapeutic strategy for the treatment of T2DM.

\section{2}

Evaluation of SVT002149, a novel PPAR $\gamma$ modulator with in vivo antiadipogenic properties

D. Balsa, J. Cabellos, O. Rey, C. Serra, A. Enrich, T. Pellicer, S. De Davalillo, C. Lagunas, C. Salcedo, A. G. Fernández; Preclinical R\&D, SALVAT, Esplugues de Llobregat, Barcelona, Spain.

Background and Aims: PPAR $\gamma$ agonists confer anti-diabetic effects in animals and humans. Nevertheless, they are associated with significant adverse effects, including weight gain, edema, mild anemia, risk for congestive heart failure and tumour formation, which limits their clinical use. An alternative approach is the recent discovery of ligands with 
partial agonist activity, which offer the promise of retained efficacy and potentially reduced toxicity. The aim of this work is to describe the in vitro profile of SVT002149, a PPAR $\gamma$ modulator and its effects in a rodent model of obesity. Material and Methods: - Binding assay: [3H]-rosiglitazone (RSG) and $[3 \mathrm{H}] \mathrm{GW}-2433$ were used as radioligands for GST-PPAR $\gamma$, and GST-PPAR $\alpha /$ PPAR $\delta$, respectively. Bound radioligand was separated by affinity chromatography.

- Trans-activation assay: COS-7 cells were transfected with pFACMV-hPPAR $\gamma$ LBD/GAL4, pUAS $(5 \times)$-tk-luciferase and pRLrenilla. After $48 \mathrm{~h}$ exposure to compounds, luciferase activity was determined.

- 3T3-L1 preadipocytes differentiation: After reaching the confluence, cells were treated with RSG and insulin for 9 days, and stained with Oil Red $O$.

- Molecular Docking: Ligands were docked by means of Induced Fit Docking (Schrödinger LLC).

- Diet Induced-Obese (DIO) Mice: Male C57BL/6J mice were used. LF control was fed a low fat diet (5\% kcal) and the HF control a high-fat diet $(45 \% \mathrm{kcal})$. Animals were treated with the compound incorporated into the diet. Body weight and food intake were routinely determined for 10 weeks. Plasma and hematology parameters were tested and the necropsy evaluated. The study was performed in accordance with the institutional ethical guidelines.

Results: SVT002149, a compound structurally distinct from glitazones, is a selective PPAR $\gamma$ ligand $(\mathrm{Ki}=470 \mathrm{nM}$, compared to $\mathrm{RSG}, \mathrm{Ki}=230 \mathrm{nM}$ ). This ligand shows partial agonism in cell-based trans-activation of PPAR $\gamma$. Even more, it inhibits the trans-activation induced by RSG in a concentration-dependent manner. In comparison with RSG, SVT002149 has a reduced adipogenic activity in 3T3-L1 cells. Induced Fit Docking studies reveal that both molecules interact with PPAR $\gamma$ throught a distinct binding mode: whereas RSG is packed between $\alpha$-helix 4 and $\beta$-sheet 1, SVT002149 binds to a hydrophobic pocket formed by $\alpha$-helixes 4,7 and 8 , and also to the pocket formed by $\alpha$ helixes $4,6, \beta$-sheet 1 , and loop between $\alpha$ helixes 2 and 3 . These biochemical properties may provide a mechanistic basis for its distinct pharmacological profile: oral treatment of DIO mice with SVT002149 (1-10 mg/kg) is well tolerated, showing an opposite profile to that of RSG: SVT002149 markedly dose-dependent reduced body weight $(72 \%)$, adiposity index $(82 \%)$ and hyperinsulinemia $(87 \%)$. Although neither tryglyceride nor cholesterol accumulation is observed in liver, plasma lipidic profile is significantly improved. Heart weight, plasma biochemical and hematological markers were not affected.

Conclusions: Together, the in vitro and the in vivo data support that SVT002149 appears to modulate PPAR $\gamma$ response, showing an improvement in insulin- and lipidlowering efficacy, without eliciting the major side-effects characteristic of full agonists. These results reinforce the concept that selective modulators are attractive drug candidates that may offer an improved therapeutic profile over existing PPAR agonists.

\section{3}

Supplementation with polyphenolic complex from red grapes rehabilitates lipid profile and ameliorates insulin resistance in Golden Syrian hamsters with experimental metabolic syndrome

N. S. Krasova ${ }^{1}$, A. L. Zagayko ${ }^{2}$, L. N. Voronina ${ }^{2}$, V. V. Poltorak ${ }^{1}$, A. I. Gladkih ${ }^{1}$;

${ }^{1}$ Pathophysiology Laboratory, Institute of Endocrine Pathology Problems, Kharkiv, ${ }^{2}$ Department of Biological Chemistry, National University of Pharmacy, Kharkiv, Ukraine.

Background and Aims: Polyphenolic extract from "Cabernet" grapes (named Enoant, contained approx. 20 g polyphenols per 1) has numerous activities, such as antimutagenic, immunopotentiating, cholesterol-lowering, hypotensive and antioxidant effects. We and others have reported that Golden Syrian hamsters can be induced to develop atherosclerosis and metabolic syndrome in a manner that closely mimics human pathology. The aim of the present study was to characterize therapeutic (hypolipidaemic) effect of Enoant in animal model of metabolic syndrome.

Materials and Methods: 30 female 1-mo-old Golden Syrian hamsters were randomized into 3 groups (10 animals each). A control group was submitted to a standard diet. Two groups were submitted for 9 weeks to a diet containing $29 \%$ vegetable- and animal oils and fructose $(2 \mathrm{~g} / 100 \mathrm{~g}$ body weight/day) - metabolic syndrome groups (MS). After than MS groups received Enoant $(0.05 \mathrm{ml} / 100 \mathrm{~g}$ body weight, per os, MS+E group) or placebo for the last 14 days. Fasting blood samples were used for glycaemia, plasma insulin, total lipids (TL) and cholesterol, triglycerides (TG), NEFA, LDL + VLDL and $\alpha$-tocopherol $(\alpha-\mathrm{T})$ levels determinations. TL, $\alpha-T$, ascorbic acid levels and glucose-6-phosphate-dehydrogenase (G6PDH) activity were measured in liver by spectrophotometrically. Homeostasis Model Assessment (HOMA) was used to estimate insulin resistance (IR). Statistical analyses were carried out using SPSS and Microsoft Excel. Results are expressed as mean \pm SEM.

Results: IR, hyperinsulinaemia and dyslipidaemia (hypertriglyceridaemia and atherogenic lipid profile) were developed in hamsters fed a half unsaturated fat and fructoseenriched diet compared to controls. Two weeks' oral administration of Enoant to the MS hamsters resulted in marked reduction of serum insulin (by $54 \%, \mathrm{p}<0.001$ ), HOMA-IR $(8.03 \pm 0.73$ vs $19.43 \pm 2.39, \quad \mathrm{p}<0.001)$, TL ( $3.54 \pm 0.16$ vs $4.52 \pm 0.17 \mathrm{mg} / \mathrm{ml}, \mathrm{p}<0.001)$, TG $(91.2 \pm 2.3$ vs $108.4 \pm 4.9 \mathrm{mg} / \mathrm{dl}, \mathrm{p}<0.01)$ and NEFA $(0.95 \pm 0.02$ vs 
$1.17 \pm 0.06 \mathrm{mmol} / \mathrm{l}, \mathrm{p}<0.01$ ) levels in comparison with $\mathrm{MS}$ +placebo. This was associated with suppression of G6PDH activity $(4.62 \pm 0.07$ vs $4.96 \pm 0.11 \mathrm{nmol} \mathrm{NADPH} /$ min $\mathrm{mg}$ protein in $\mathrm{MS}, \mathrm{p}<0.05)$ and reduction of $\mathrm{TL}$ $(111.53 \pm 4.08$ vs $140.75 \pm 9.15 \mathrm{mg} / \mathrm{g}$ in $\mathrm{MS}, \mathrm{p}<0.01)$ in liver. Serum and liver $\alpha-\mathrm{T}$ contents were also elevated (by 47 and $20.5 \%$, respectively, $\mathrm{p}<0.01$ ) in $\mathrm{MS}+\mathrm{E}$ group.

Conclusion: These results show that the abnormal lipid metabolism associated with insulin resistance might be improved in the Golden Syrian hamsters with experimental metabolic syndrome by the oral administration with polyphenolic extract from red grapes. This suggests the potentiality of the Enoant as a novel agent for the treatment of the metabolic syndrome and lipid abnormalities.

\section{4}

Long-term treatment with GLP-1 analogue liraglutide reduces body weight and improves glucose tolerance in diet-induced obese rats more efficaciously than conventional doses of sibutramine and rimonabant

K. Lykkegaard ${ }^{1}$, L. Bjerre Knudsen ${ }^{2}$, G. Hansen ${ }^{1}$, P. J. Larsen ${ }^{1}$;

${ }^{1}$ Rheoscience A/S, Roedovre, ${ }^{2}$ NovoNordisk A/S, Bagsvaerd, Denmark.

Background and Aims: The GLP-1 analog liraglutide is currently in late stage clinical development for a once daily treatment of people with type 2 diabetes (T2DM). Beyond improvement of glycaemic control, chronic administration of GLP-1 agonists confer reduction of body weight.

Materials and Methods: We have compared weight reducing efficacy of liraglutide (200 and $300 \mathrm{microg} / \mathrm{kg}$ BID) with conventional doses of two oral weight control agents rimonabant $(10 \mathrm{mg} / \mathrm{kg}$ QD) or sibutramine $(5 \mathrm{mg} / \mathrm{kg})$. Adult male diet-induced obese Sprague Dawley (DIO) rats with impaired glucose tolerance were dosed for 28 days.

Results: Liraglutide treatment significantly reduced cumulated food intake (both after 14 and 28 days) and significantly more so than sibutramine and rimonabant. Resulting loss in body weight was twice as large for liraglutide compared to sibutramine and rimonabant. Liraglutide and sibutramine significantly decreased all examined body fat depots (mesenterial, perirenal, epididymal, inguinal), whilst rimonabant only decreased inguinal and epididymal depots. Compared to vehicle, fasting plasma glucose levels and $\mathrm{HbA1c}$ levels were unaffected in all treatment groups at the final day of treatment. Plasma triglyceride levels decreased in all treatment groups. On the final day of dosing, an oral glucose tolerance test was performed. Liraglutide was the only treatment which significantly improved glucose tolerance in DIO rats, whereas rimonabant treated animals displayed impaired glucose toler- ance. Hovever, all treatments significantly lowered oral glucose induced insulin secretion with sibutramine being less efficacious than liraglutide and rimonabant.

Conclusion: Based on these data it is concluded that liraglutide is a promising weight loss agent having more potent effect on body weight than both rimonabant and sibutramine.

Liraglutide treatment reduces adiposity

\begin{tabular}{|c|c|c|c|c|c|}
\hline $\begin{array}{l}\text { Day } 28 \\
\text { data }\end{array}$ & $\begin{array}{l}\text { Body } \\
\text { weight } \\
\text { gain }(\%)\end{array}$ & $\begin{array}{l}\text { s.c. } \\
\text { inguinal } \\
(\mathrm{g})\end{array}$ & $\begin{array}{l}\text { Epididymal } \\
\text { (g) }\end{array}$ & $\begin{array}{l}\text { Mesenterial } \\
(\mathrm{g})\end{array}$ & $\begin{array}{l}\text { Perirenal } \\
\text { (g) }\end{array}$ \\
\hline & $105.3 \pm 0.9$ & $11.4 \pm 0.6$ & $12.8 \pm 0.8$ & $12.5 \pm 0.9$ & $25.1 \pm 1.0$ \\
\hline Liraglutide & $\begin{array}{l}90.4 \pm \\
0.8^{\mathrm{abc}}\end{array}$ & $\begin{array}{l}7.0 \pm \\
0.6^{\mathrm{ac}}\end{array}$ & $9.4 \pm 0.8^{\mathrm{a}}$ & $6.8 \pm 0.4^{\mathrm{a}}$ & $\begin{array}{l}14.9 \pm \\
1.2^{\mathrm{abc}}\end{array}$ \\
\hline Sibutrami & $96.6 \pm 1.0^{a}$ & $8.2 \pm 0.5^{a}$ & $10.6 \pm 0.7^{\mathrm{a}}$ & $8.5 \pm 0.7^{\mathrm{a}}$ & $18.9 \pm 1.2^{\mathrm{a}}$ \\
\hline Rimonabant & $96.1 \pm 0.7^{a}$ & $9.7 \pm 1.0$ & $10.6 \pm 0.7^{\mathrm{a}}$ & $8.64 \pm 0.8^{a}$ & $18.1 \pm 1.3^{\mathrm{a}}$ \\
\hline
\end{tabular}

${ }^{\mathrm{a}} \mathrm{p}<0.05$ vs. vehicle, ${ }^{\mathrm{b}} \mathrm{p}<0.05$ vs. sibutramine, ${ }^{\mathrm{c}} \mathrm{p}<0.05$ vs. rimonabant

0215

Abnormal feeding behavior and ghrelin secretion in mice lacking glial cell line-derived factor family receptor GFRo2

A. Huotari ${ }^{1}$, J. Rossi ${ }^{2}$, V. Võikar ${ }^{2}$, K.-H. Herzig ${ }^{1,3}$, M. S. Airaksinen ${ }^{2}$;

${ }^{1}$ Dept of Biotechnology and Molecular Medicine, University of Kuopio, ${ }^{2}$ Neuroscience Center, University of Helsinki, ${ }^{3}$ Department of Internal Medicine, University of Kuopio, Finland.

Background and Aims: Neurturin signaling via GDNFfamily receptor GFR $\alpha 2$ is required for development and target innervation for subsets of parasympathetic and enteric neurons. Knockout of the receptor gene (Gfra2-/-) (ko) in mice leads to postnatal growth impairment by an unknown mechanism. Ko mice have deficient innervations, impaired function of exocrine and endocrine pancreas and postnatal growth retardation. In ko mice, the growth deficit ( $\sim 50 \%$ compared to wild type mice littermates (wt)) is most pronounced shortly after weaning, but they regain growth during the following months, yet remain app. $\sim 20 \%$ smaller than their wt littermates.

Materials and Methods: In this study, we analyzed in detail the feeding behavior and energy expenditure using an automated monitoring system with indirect open circuit calorimeter, food and water consumption recorded in parallel with metabolic measurements in intervals of 15 min and activity measurement by an array of infrared beams. Ghrelin and leptin plasma levels were analyzed by commercially available RIAs. 
Results: Total food intake and locomotor activity did not differ between genotypes. However, in ko mice, the duration of individual meals was $\sim 50 \%$ longer, meal sizes were reduced by $\sim 40 \%$, and the number of feeding bouts during dark period was increased by $\sim 40 \%$ compared to wt mice. In addition, the average time between individual meals during the night phase was significantly shorter in GFR $\alpha 2-k o$ mice (ko $12 \mathrm{~min}$ vs. wt $20 \mathrm{~min}$ ) suggesting decreased satiety. The biochemical analysis demonstrated equal glycogen levels in the livers. Determination of peptide hormones involved in food intake regulation we found significantly increased plasma ghrelin levels in both fasted $(\sim+35 \%)$ and in fed $(\sim+51 \%)$ ko mice compared to their wt control littermates. Feeding reduced ghrelin levels by $45 \%$ in the ko mice and $50 \%$ in the wt littermates. Plasma leptin levels were reduced by $40 \%$ (from 10,2 in wt to $6,1 \mathrm{ng} / \mathrm{ml}$ in ko mice) in the fed state.

Conclusion: Our data suggest that elevated ghrelin levels promotes the increase in meal frequency, possibly compensating for the lack of food induced satiety in adult GFR $\alpha 2-k o$ mice. Previously, it has been shown that ghrelin secretion is mostly of gastric origin and under cholinergic control. Since our ko mice have deficient peripheral cholinergic innervations, this control seems to be overridden in our mice suggesting that high ghrelin levels are a superior physiological adaptation mechanism in order to increase food intake as indicated by the increased feeding bouts. Analysis of young animals is ongoing to study the possible development of compensatory modifications in feeding behaviour and energy expenditure.

Supported in part by the Academy of Finland

\section{6}

\section{Leptin mediates sodium tungstate antiobesity effects}

I. Canals, S. Moreno, M. Amigó, R. Gomis;

Endocrinology and Nutrition Unit., Hospital Clinic Universitari, Institut d'Investigacions Biomèdiques August Pi i Sunyer (IDIBAPS). Faculty of Medicine, University of Barcelona, Spain.

Background and Aims: Oral administration of sodium tungstate in diet-induced obese rats reduces body weight gain and adiposity, without altering food intake. Moreover, it improves the lipid profile and insulin resistance. These effects are due, at least partially, to an increase in energy expenditure and in lipid oxidation. A reduction in blood leptin levels was observed in treated obese animals. Since leptin is a key regulator of energy homeostasis, this finding suggests that leptin could play a major role in the tungstate effect. The aim of this study is to confirm the implication of leptin on the tungstate antiobesity effects.

Materials and Methods: 12 weeks old leptin deficient ob/ ob mice and their respective controls $(\mathrm{ob} /+)$ were divided in two groups: one was treated with tungstate $(2 \mathrm{~g} / \mathrm{l}$ in the drinking water) for 30 days $(T)$, and the other was left untreated $(U T)$. The body weight $(B W)$ and food intake $(F I)$ were measured daily. The oxygen consumption was determined by indirect calorimetry in untreated animals after one tungstate dose $(60 \mathrm{mg} / \mathrm{Kg})$ administered by gavage. $500 \mathrm{mg}$ epididimal adipose tissue (epWAT) from $\mathrm{ob} /+$ mice were divided in four explants and transplanted into ob/ob mice scapular and lumbar subcutaneous zone. The animals were divided in two groups: untreated ( $t x U T$ ) and tungstate treated ( $2 \mathrm{~g} / 1$ for four weeks, $t x T)$. An additional group of ob/ob mice transplanted with ob/ob adipose tissue was used as a leptin deficiency control $(t x C N)$. At the end of the treatment, fat transplants were removed and the treatment was carried out for an additional two weeks.

Results: Tungstate treatment significantly reduces BW gain (3.23 \pm 0.33 vs. $0.7 \pm 0.86 \mathrm{~g} B W$ gain $U T$ vs. $T$; $p$-value

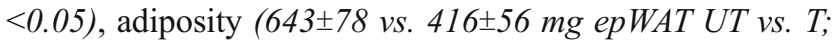
p-value $<0.05)$ and FI (105.2 \pm 6.6 vs. 87.9 $\pm 6.7 \mathrm{~g}$ chow/ 30 days UT vs. T; p-value <0.05), and increases oxygen consumption in ob/+mice $\left(100.0 \pm 6.6\right.$ vs. $116.8 \pm 3.4 \% \mathrm{O}_{2}$ consumption UT vs. T; p-value <0.05). However, none of these parameters are significantly changed in treated ob/ob mice $(6.67 \pm 0.46$ vs. $6.11 \pm 0.56 \mathrm{~g} \mathrm{BW}$ gain, $3430 \pm 180 \mathrm{vs}$. $3450 \pm 150 \mathrm{mg}$ epWAT, $149 \pm 19$ vs. $134 \pm 13 \mathrm{~g}$ chow $/ 30$ days and $100.0 \pm 8.0$ vs. $87.2 \pm 2.9 \% \mathrm{O}_{2}$ consumption UT vs. T; $p$ value NS in all measurements). Ob/ob mice transplanted with epWAT from ob/+have detectable blood leptin levels and show a decrease in body weight gain, compared to transplanted control mice $(-2.32 \pm 010 \mathrm{vs}$. $4.23 \pm 0.02 \mathrm{~g} \mathrm{BW}$ gain txUT vs. txCN; p-value $<0.05)$. In addition, food intake is also reduced in these animals $(137.6 \pm 9.8 \mathrm{vs} .158 .8 \pm 9.8 \mathrm{~g}$ chow/4 weeks txUT vs. txT; p-value <0.05), and oxygen consumption is increased $\left(1153 \pm 24 \mathrm{vs} .1072 \pm 20 \mathrm{ml} \mathrm{O} / \mathrm{h} / \mathrm{Kg}^{0.75}\right.$ txUT vs. txT; p-value <0.05). These effects are amplified in tungstate treated transplanted mice $(-8.10 \pm 0.05 \mathrm{~g}$ BW gain, $113.9 \pm 5.8 \mathrm{~g}$ chow $/ 4$ weeks and $1280 \pm 15.3 \mathrm{ml} \mathrm{O} / \mathrm{h} / \mathrm{Kg}^{0.75}$ txT; $p$-value $<0.05$ vs. $t x U T)$, indicating that leptin presence is necessary for tungstate to have antiobesity effects. Moreover, morphological differences in brown adipose tissue were observed in transplanted untreated ob/ob mice, which are more accentuated in treated animals. Finally, the removal of transplanted adipose tissue from the treated mice immediately halts tungstate antiobesity effects. These animals show an increase in food intake which leads to raise in body weight. 
Conclusions: Tungstate needs leptin presence to increase energy expenditure and to reduce food intake, leading to an antiobesity effect.

Supported by Redes C03/08, G03/212 from the Ministerio de Sanidad y Consumo (Spain)

\section{OP 37}

\section{Hypertension}

\section{7}

\begin{abstract}
Aliskiren, alone and in combination with ramipril, significantly lowers blood pressure and plasma renin activity in patients with diabetes and hypertension, regardless of the degree of glycaemic control

D. Tschoepe ${ }^{1}$, A. Taylor ${ }^{2}$, C. Kilo ${ }^{3}$, G. Ibram $^{4}$, H. Fang ${ }^{4}$, A. Satlin ${ }^{4}$, M. F. Prescott

${ }^{1}$ Diabetes Center, Ruhr-Universitat Bochum, Bad Oeynhausen, Germany, ${ }^{2}$ Department of Medicine, Baylor College of Medicine, Houston, ${ }^{3}$ Kilo Diabetes and Vascular Research Foundation, Washington University School of Medicine, St Louis, ${ }^{4}$ Cardiovascular and Metabolism, Novartis Pharmaceuticals Corporation, East Hanover, United States.
\end{abstract}

Background and Aims: Hypertension, a common comorbidity in patients with diabetes, greatly increases the risk of cardiovascular and renal disease. While glycaemic control is the primary objective in diabetes management, morbidity and mortality are also reduced by effective blood pressure (BP) control. We investigated antihypertensive efficacy of the novel renin inhibitor aliskiren and the ACE inhibitor ramipril, alone and in combination, in patients with different degrees of glycaemic control (indicated by glycosylated haemoglobin [HbA1c] levels). Plasma renin activity (PRA; capacity for generation of angiotensin), was measured in a subset of patients from each $\mathrm{HbAlc}$ subgroup to determine treatment effects on renin system activation.

Materials and Methods: Patients with type 1 or 2 diabetes and mean sitting diastolic BP (MSDBP) 95-109 $\mathrm{mmHg}$ were randomized to double-blind aliskiren $150 \mathrm{mg}(\mathrm{n}=282)$, ramipril $5 \mathrm{mg}(\mathrm{n}=278)$ or aliskiren/ramipril $150 / 5 \mathrm{mg}$ $(n=277)$ once daily for 4 weeks, followed by forced titration to aliskiren $300 \mathrm{mg}$, ramipril $10 \mathrm{mg}$ or aliskiren/ramipril $300 / 10 \mathrm{mg}$ for a further 4 weeks. Reductions in MSDBP and mean sitting systolic BP (MSSBP) were assessed in patient subgroups with baseline $\mathrm{HbAlc}<$ and $\geq$ median
( $\mathrm{n}=400$ and 426, respectively, of whom 121 and 107, respectively, had PRA measured).

Results: Median $\mathrm{HbAlc}$ at baseline was $7.0 \%$, which is also the goal for glycaemic control recommended by the American Diabetes Association. Mean HbAlc levels in the two subgroups were $6.2 \%$ (range $4.6-6.9 \%$ ) and $8.2 \%$ (7.0-13.8\%). Baseline MSSBP and MSDBP were comparable in the two subgroups. Table 1 shows $\mathrm{BP}$ reductions at endpoint. Both subgroups showed similar patterns of BP lowering to the overall population; combination therapy was generally superior to ramipril monotherapy. From geometric mean baseline values of 0.45 and $0.51 \mathrm{ng} / \mathrm{mL} / \mathrm{h}$ in patients with $\mathrm{HbA} 1 \mathrm{c}<$ and $\geq 7 \%$, respectively, aliskiren reduced PRA by $62 \%$ and $75 \%$, respectively, as monotherapy, and by $46 \%$ and $51 \%$ when combined with ramipril. Ramipril monotherapy increased PRA by $92 \%$ and $118 \%$, respectively.

Conclusion: Aliskiren monotherapy provides effective BP lowering and renin system suppression. When combined with ramipril, aliskiren provides additional BP lowering and neutralizes ramipril-induced increases in PRA. These effects are independent of the degree of glycaemic control at baseline. Aliskiren may offer the potential of enhanced end-organ protection in patients with hypertension and diabetes, including those with poor glycaemic control at greatest risk of cardiovascular and renal disease.

Least squares mean change from baseline ( $\mathrm{mmHg}$ )

\begin{tabular}{lllll}
\hline & & Aliskiren & Ramipril & Combination \\
Overall population & MSSBP & $-14.6^{*}$ & -11.9 & $-16.5^{* * *}$ \\
$\mathrm{n}=826$ & MSDBP & -11.3 & -10.7 & $-12.7 \dagger^{* *}$ \\
$\mathrm{HbA} 1 \mathrm{c}<7.0 \%$ & MSSBP & -14.9 & -11.8 & $-15.6^{*}$ \\
$\mathrm{n}=400$ & MSDBP & -11.3 & -10.8 & $-13.3 \dagger^{*}$ \\
$\mathrm{HbA} 1 \mathrm{c} \geq 7.0 \%$ & MSSBP & -14.9 & -12.1 & $-17.8^{* * *}$ \\
$\mathrm{n}=426$ & MSDBP & -11.5 & -10.8 & -12.4 \\
\hline
\end{tabular}

${ }^{*} \mathrm{p} \leq 0.05,{ }^{* *} \mathrm{p} \leq 0.01,{ }^{* * *} \mathrm{p} \leq 0.001$ vs ramipril; $\dagger \mathrm{p} \leq 0.05$ vs aliskiren

Supported by Novartis Pharma $A G$

0218

The Anglo-Scandinavian Cardiac Outcomes Trial: Blood Pressure-Lowering Limb (ASCOT-BPLA): effects in patients with type 2 diabetes J. Östergren ${ }^{1}$, P. Sever ${ }^{2}$, N. Poulter ${ }^{2}$, B. Dahlöf ${ }^{3}$, H. Wedel ${ }^{4}$, G. Beevers ${ }^{5}$, M. Caulfield ${ }^{6}$, R. Collins ${ }^{7}$, S. Kjeldsen ${ }^{8}$, A. Kristinsson ${ }^{9}$, G. McInnes ${ }^{10}$, J. Mehlsen ${ }^{11}$, M. Nieminen ${ }^{12}$, E. ÓBrien ${ }^{13}$; 
${ }^{1}$ Dept of Medicine, Karolinska University Hospital, Stockholm, Sweden, ${ }^{2}$ Imperial College, London, United Kingdom, ${ }^{3}$ Östra, Sahlgrenska University Hospital, Göteborg, Sweden, ${ }^{4}$ Nordic School of Public Health, Göteborg, Sweden, ${ }^{5}$ City Hospital, Birmingham, United Kingdom, ${ }^{6}$ Queen Marys School of Medicine, Barts, London, United Kingdom, ${ }^{7}$ Radcliffe Infirmary, Oxford, United Kingdom, ${ }^{8}$ Ullevål Hospital, Oslo, Norway, ${ }^{9}$ University Hospital, Reykjavik, Iceland, ${ }^{10}$ University of Glasgow, United Kingdom, ${ }^{11} \mathrm{H}$ S Fredriksberg Hospital, Denmark, ${ }^{12}$ University Central Hospital, Helsinki, Finland, ${ }^{13}$ Beaumont Hospital, Dublin, Ireland.

Background and Aims: Diabetes (DM) and hypertension (HT) are both important risk factors for cardio-vascular disease. Despite this very few large trials have been specifically oriented towards patients with DM and HT. The Anglo-Scandinavian Cardiac Outcomes Trial (ASCOT) compared two antihypertensive treatment strategies for the prevention of coronary heart disease (CHD) in more than 19,000 hypertensive patients who had no history of CHD. This report is an analysis of the large subpopulation with DM in this study.

Materials and Methods: Patients were between 40 and 79 years with either untreated HT, systolic blood pressure (SBP) of $160 \mathrm{mmHg}$ or more, and/or diastolic (DBP) of $100 \mathrm{mmHg}$ or more, or treated HT with SBP of $140 \mathrm{mmHg}$ or more, and/or DBP $90 \mathrm{mmHg}$ or more. For those with type II DM at least two additional risk factors were required. Patients were randomised to an amlodipine \pm perindopril or atenolol \pm thiazide regimen with possible addition of doxazosin and other antihypertensives in both arms. Therapy was titrated to achieve a target blood pressure $<130 / 80 \mathrm{mmHg}$.

Results: The trial was terminated early on recommendation of the safety committee due to benefit in mortality and stroke for the amlodipine based regimen. In the 5137 patients with DM the amlodipine based treatment significantly lowered the incidence of combined major cardio-vascular events including coronary events, strokes and interventional procedures as compared to the atenolol based regimen (hazard ratio 0.86 , CI $0.76-0.98),(\mathrm{p}=0.026)$. Fatal and non-fatal stroke was $25 \%$ lower $(\mathrm{p}=0.017)$, peripheral arterial disease $48 \%$ lower $(\mathrm{p}=0.004)$ and non-coronary revascularisation procedures $57 \%$ lower $(p<0.001)$ in the amlodipine group. For the other endpoints included in the composite endpoint differences were moderate and non-significant. Non-fatal myocardial infarction was reduced by $11 \%$ (n s). The absolute risk reduction in major cardio-vascular events by amlodipine \pm perindopril was very similar in the non-diabetic $(2.6 \%)$ and diabetic (2.4\%) subgroups in ASCOT. Blood pressure (BP) was slightly $(1-3 \mathrm{~mm} \mathrm{Hg}$ ) more reduced by treatment based on amlodipine. By the end of the study in those allocated to amlodipine BP was 136/75 $\mathrm{mm} \mathrm{Hg}$ and in those allocated to atenolol $137 / 76 \mathrm{~mm} \mathrm{Hg}$.

Conclusion: In the diabetic subgroup in ASCOT, treatment with a regimen based on amlodipine \pm perindopril as compared to atenolol \pm thiazide reduced the incidence of first total cardiovascular events and procedures by $14 \%$. The finding of this analyses is well in line with the overall finding of a $16 \%$ risk reduction of first total cardiovascular events and procedures by treatment based on amlodipine \pm perindopril in the overall population of ASCOT.

Supported by Pfizer

0219

CYP2C9 polymorphism modifies blood pressure lowering response to losartan in type 1 diabetic nephropathy M. Lajer ${ }^{1}$, L. Tarnow ${ }^{1}$, S. Andersen ${ }^{1}$, H.-H. Parving ${ }^{1,2}$; ${ }^{1}$ Steno Diabetes Center, Gentofte, Denmark, ${ }^{2}$ Faculty of Health Science, University of Aarhus, Denmark.

Background and Aims: CYP2C9 of the cytochrome P450 superfamily catalyzes the metabolism of the antihypertensive treatment (AHT), losartan, to its active metabolite E3174. Two functional polymorphisms within the CYP2C9 gene result in the expression of three variants, CYP2C $9 * 1$, *2 (Arg144Cys) and *3 (Ile359Leu) encoding enzymes with declining catalytic activity. We investigated whether the CYP2C9 genotype predicts blood pressure (BP) and albuminuria lowering response and preservation of GFR during long-term treatment with losartan.

Materials and Methods: The CYP2C9 alleles were determined in 60 hypertensive type 1 diabetic patients with overt diabetic nephropathy. After a 4 week wash-out period, the patients received losartan (100 $\mathrm{mg}$ o.d.) for a mean follow-up of 36 months. Additional AHT was prescribed after 4 months if BP was $>135 / 85 \mathrm{mmHg}$. At baseline, after 2 and 4 months, and every 6 months thereafter, 24-h BP, albuminuria, and glomerular filtration rate (GFR) were measured. The CYP2C9 genotypes were determined by TaqMan technique. Results: The CYP2C9*1, *2 and *3 allele frequencies were $78 \%, 12 \%$, and $10 \%$, respectively. At baseline, 24-h BP, albuminuria, and GFR did not differ between the group of genotypes carrying the $* 3$ allele $(\mathrm{n}=12$, moderate metabolizers) compared to the non- $* 3$ allele carriers $(n=48$, high metabolizers). After 4 months the non-*3 carriers had obtained a significantly greater reduction in systolic 24-h BP than *3-carriers (mean (SE) $\mathrm{mmHg}$ ) -15 (16) and 5 (17), respectively $(p=0.001)$ and a tendency to a greater reduction in plasma aldosterone levels (geometric mean $(95 \% \mathrm{CI}) \%)-24$ $(-4$ to -41$)$ and $22(-50$ to 195$)(p=0.13)$, respectively.

After addition of amlodipine and diuretics in 2 out of 3 patients in both groups, systolic 24-h BP was significantly 
lowered in patients carrying the $* 3$ allele, $p=0.004$, whereas it remained unchanged in non-*3 allele carrying patients. During long-term treatment, albuminuria $(p=0.9)$, or rate of decline in GFR (median (range)) $3.3(-0.1$ to 8.0$) \mathrm{ml} / \mathrm{min} /$ year and $3.0(-1.6$ to 16.0$)(\mathrm{p}=0.4)$ declined similarly in *3 allele carriers and non-*3-carriers, respectively.

Conclusion: The CYP2C $9 * 3$ polymorphism is associated with attenuated BP response to losartan treatment. However, by adding other AHT a similar progression of diabetic nephropathy was obtained in Type 1 diabetic patients with or without the CYP2C $9 * 3$ allele.

\section{0}

Anti-hypertensive effect of device-guided paced breathing in diabetics: a randomized controlled study

M. H. Schein ${ }^{1}$, A. Alter ${ }^{2}$, S. Levine ${ }^{3}$, T. Baevsky ${ }^{3}$, A. Nessing ${ }^{3}$, B. Gavish ${ }^{2}$;

${ }^{1}$ Family Medicine, Hadassah, Hebrew University Medical Center, Jerusalem, ${ }^{2}$ Clinical Dept., InterCure, Lod, ${ }^{3}$ Family Medicine, Clalit Health Services, Rehovot, Israel.

Background and Aims: Hypertension is a common comorbidity of diabetes. Slow breathing has been shown to reduce sympathetic activity and blood pressure (BP) in hypertensives. The objective of this study was to assess the efficacy of device-guided breathing in lowering blood pressure in diabetics with uncontrolled BP.

Materials and Methods: A randomized controlled study, carried out in urban family practice clinics in Israel. Noninsulin dependent diabetics with uncontrolled BP $(>130 /$ $80 \mathrm{mmHg}$ ), receiving anti-hypertensive therapy or unmedicated, were enrolled. The intervention group used a device (RESPeRATE, InterCure Ltd, Israel), which interactively guides the user towards slow and regular breathing by synchronizing breathing voluntarily to musical tones, for 15 minutes daily at home for an 8 -week period. Compliance and performance were monitored and analyzed from data automatically stored by the device during the treatment period. The control group continued with their regular treatment. The main outcome measure was the BP change, measured in the clinics at baseline, after 4 weeks and at termination at 8 weeks.

Results: Baseline characteristics of 60 patients (30 treatment and 30 controls), $36 \mathrm{M} / 24 \mathrm{~F}$ were: age $63 \pm 8$ years (mean+SD); BMI $30 \pm 3 \mathrm{~kg} / \mathrm{m} 2$; BP148 $\pm 11 / 81 \pm 9 \mathrm{mmHg}$. The difference in BP change (mean+SE) between treatment and control groups was significant for systolic BP $-9.5 \pm 1.9$ vs. $+2.1 \pm 2.4 \mathrm{mmHg}, \mathrm{p}<0.0005$ for comparison between groups. Reduction of pulse pressure was significantly higher in the treatment group compared to control $\mathrm{p}=0.0007$. Reduction of systolic BP correlated with the total time spent in slow breathing (less than 10 breaths per minute) during the 8 weeks of treatment, $\mathrm{p}=0.01$.

BP value at start and end of study (mean+SE, $\mathrm{mmHg}$ )

\begin{tabular}{|c|c|c|c|c|c|c|c|}
\hline & START & END & $p$ value & START & END & $\begin{array}{l}p \\
\text { value }\end{array}$ & $\begin{array}{l}p \text { value } \\
\text { by } \\
\text { group }\end{array}$ \\
\hline & \multicolumn{3}{|c|}{ Treatment } & \multicolumn{4}{|l|}{ Control } \\
\hline Systolic & $149.4 \pm$ & $139.9 \pm$ & 0.00002 & $146.2 \pm$ & $148.3 \pm$ & 0.38 & 0.0002 \\
\hline BP & 2.1 & 2.6 & & 1.8 & 3.0 & & \\
\hline Diastolic & $82.1 \pm$ & $78.6 \pm$ & 0.01 & $80.6 \pm$ & $79.1 \pm$ & 0.33 & 0.15 \\
\hline BP & 1.8 & 2.2 & & 1.4 & 1.1 & & \\
\hline Pulse & $67.3 \pm$ & $61.4 \pm$ & 0.01 & $65.6 \pm$ & $69.2 \pm 3$ & 0.14 & 0.0007 \\
\hline Pressure & 2.5 & 2.5 & & 2.1 & & & \\
\hline
\end{tabular}

Conclusion: Self-treatment with device-guided breathing at home for 2 months by non-insulin dependent diabetic patients was associated with a significant reduction in office systolic BP and pulse pressure.

\section{OP 38}

\section{Postprandial glucose regulation}

\section{1}

Is postprandial $2 \mathrm{~h}$ glucose level a real determinant for glucose intolerance in people at risk for type 2 diabetes? RISK study results-I

T. Yilmaz ${ }^{1}$, M. Sargin ${ }^{2}$, B. Olmez ${ }^{3}$, S. Karadeniz ${ }^{4}$, B. Oguzhan ${ }^{5}$ on behalf of the RISK Study Group, Turkey; ${ }^{1}$ Endocrinology and Metabolism Dept., Istanbul University, ${ }^{2}$ Endocrinology and Metabolism Dept., Kartal Educ. and Res. Hosp., Istanbul, ${ }^{3}$ Novartis Pharma, Istanbul, ${ }^{4}$ Hospitals, Group Florence Nightingale, Istanbul, ${ }^{5}$ Diabetes Centers, Turkish Diabetes Foundation, Istanbul, Turkey.

Background and Aims: To compare the efficacy of impaired fasting glycemia (IFG), impaired glucose tolerance at 1.h (IGT1) and impaired glucose tolerance at 2.h (IGT2) for the determination of glucose intolerance in population at risk for type 2 diabetes according to the ADA criteria.

Materials and Methods: The multicentric RISK study is population based and cross-sectional. It comprises 13.209 subjects (F/M: 1.75, mean chr. age: $52.95 \pm 11.5$ years) randomly selected from 191 centres in Turkey. Inclusion criteria for the study were to be at a chr. age of $\geq 45$ years or have risk factors for type 2 diabetes according to ADA criteria regardless of age. Family history of diabetes and for female subjects' history of gestational diabetes (GDM) 
were interrogated. The examination included 75 -g oral glucose tolerance test (OGTT), measurement of body mass index (BMI), blood pressure and lipid profile. Fasting blood glucose between $100-125 \mathrm{mg} / \mathrm{dl}$ and $1 . \mathrm{h} / 2 . \mathrm{h}$ postprandial glucose levels between 140-199 mg/dl was considered as IFG, IGT1 and IGT2, respectively.

Results: IFG was diagnosed in $30.5 \%$ of the subjects. The OGTT revealed IGT1 in $41.4 \%$, IGT2 in $23.9 \%$, combined glucose intolerance (CGI: IFG+IGT) in $10 \%$ and diabetes $(\mathrm{DM})$ in $3.3 \%$ of the subjects. Mean age was $53.68 \pm$ 11.1 yrs. in IFG, $53.20 \pm 11.6$ yrs. in IGT1, $53.8 \pm 11.2$ yrs. in IGT, $54.12 \pm 10.7$ yrs. in CGI and $54.74 \pm 10.8$ yrs. in DM group. Female/Male ratio was 1.89 in IFG, 1.98 in IGT1, 1.84 in IGT, 1.88 in CGI and 1.89 in DM group. Ratio of positive family history of diabetes was $55.7 \%$ in IFG, $49.7 \%$ in IGT1, $56.9 \%$ in IGT, $58.6 \%$ in CGI and $67.2 \%$ in DM group. The prevalence of subjects with BMI $>25 \mathrm{~kg} / \mathrm{m}^{2}$ was $84.2 \%$ in IFG, $84.8 \%$ in IGT1, $87.3 \%$ in IGT, $87.1 \%$ in CGI and $91.7 \%$ in DM group. Hypertension prevalence was $50.4 \%$ in IFG, $46.5 \%$ in IGT1, $53.9 \%$ in IGT, $57.4 \%$ in CGI and $64.3 \%$ in DM group.

Conclusion: In the evaluation of people at risk for type 2 diabetes according to ADA criteria, the rate of IGT according to WHO criteria was higher in IGT1(41.4\%) than IGT2 $(23.9 \%)$. If only $2 . \mathrm{h}$ glucose level was considered, $17.5 \%$ of people at risk of type 2 diabetes were missed, and with IFG $10.9 \%$. These results show that fasting and 2.h glucose levels are not solely suggestive of glucose intolerance and evaluation of also the glucose values at the $1 . \mathrm{h}$ should be opened to discussion.

Supported by Novartis Pharma

0222

Effect of adjunctive mealtime pramlintide therapy on treatment satisfaction mediated primarily via attenuation of postprandial glucose excursions

J. Crean ${ }^{1}$, N. Nanayakkara ${ }^{1}$, R. Rubin ${ }^{2}$;

${ }^{1}$ Amylin Pharmaceuticals, Inc., San Diego, ${ }^{2}$ Medicine \& Pediatrics, Johns Hopkins Univ., Baltimore, United States.

Background and Aims: In this study, subcutaneous (SC) pramlintide or placebo injections administered adjunctively with mealtime insulin in a cohort of intensively treated patients with type 1 diabetes (baseline $\mathrm{A} 1 \mathrm{C}=8.1 \%$ ) resulted in similar $\mathrm{A} 1 \mathrm{C}$ reductions $(-0.4 \mathrm{vs} .-0.5 \%, \mathrm{p}=0.6)$ after 29 wks of double-blind therapy. However, in pramlintidetreated patients, prandial insulin use decreased and the increase in basal insulin use was less than for placebo- treated patients (wk29\% from baseline: prandial $-20 \mathrm{vs.}$ $+2 \%, \mathrm{p}<0.01$; basal+6 vs. $+19 \%, \mathrm{p}=0.08$ ). Pramlintidetreated patients experienced superior postprandial glucose (PPG) control (average PPG: $151 \mathrm{vs} .172 \mathrm{mg} / \mathrm{dl}, \mathrm{p}<0.01$ ) and lost rather than gained weight (wk29 $\Delta$ from baseline: -1.5 vs. $+1.3 \mathrm{~kg}, \mathrm{p}<0.01$ ). Responses on a 14 -item studyspecific treatment satisfaction (TS) survey completed under double-blind conditions also indicated pramlintide-treated patients were significantly more satisfied with the effects of study medication compared to PBO-treated controls. Given overall glucose control (A1C) was similar between the treatment groups, it was of interest to understand the relative contribution of other clinical endpoints in determining patients' reported treatment satisfaction. This post-hoc analysis evaluated the unique influence of 4variables - including average PPG, A1C, weight and insulin use - on the aforementioned survey responses.

Materials and Methods: Intensively treated (CSII or MDI) patients with type 1 diabetes received double-blind SC mealtime injections of pramlintide $(n=130)$ or PBO $(n=136)$ for 29 wks. Mean baseline characteristics including A1C (8.1\%), BMI $\left(28 \mathrm{~kg} / \mathrm{m}^{2}\right)$, duration diabetes ( $\left.20 \mathrm{yrs}\right)$, and age $(41 \mathrm{yrs})$ were similar across the groups. A 14-item TS survey was completed at wk29 under double-blind conditions. Items were rated on a 6-point Likert scale. Regression analysis was used to evaluate the independent associations of average PPG and wk-29 changes in weight, A1C, and total daily insulin dose with variation in TS. Analyses were performed for the combined study population and separately within each study group.

Results: After controlling for other clinical endpoints, average PPG was the strongest predictor of most (10/14) of the TS survey items for the combined study population, with lower (improved) PPG associated with higher TS. These relationships were stronger when the regression analysis was performed separately for the pramlintide-treated group and strongest for the following items: study medication made me feel better overall $(\mathrm{F}=16.0, \mathrm{p}<0.01)$, outweighed the extra injections $(\mathrm{F}=15.4, \mathrm{p}<0.01)$, increased flexibility in eating $(\mathrm{F}=12.9, \mathrm{p}<0.01)$, made $\mathrm{BG}$ control more predictable $(\mathrm{F}=12.5, \mathrm{p}<0.01)$ and provided more benefits than taking insulin alone $(\mathrm{F}=11.8, \mathrm{p}<0.01)$; all partial correlation coefficients were $\geq-0.35$. In contrast, average PPG held little predictive utility within the $\mathrm{PBO}$ group.

Conclusion: Pramlintide-treated patients scored significantly higher than PBO-treated patients on the majority of items listed on a treatment satisfaction survey. In this study, the effect of pramlintide on attenuating PPG excursions was the primary factor differentiating the groups across survey items. 
0223

Exenatide delays gastric emptying and reduces postprandial glucose in type 2 diabetes

H. Linnebjerg ${ }^{1}$, S. Park ${ }^{2}$, P. Kothare ${ }^{2}$, M. Trautmann ${ }^{3}$, K. Mace ${ }^{2}$, M. Fineman ${ }^{4}$, I. Wilding ${ }^{5}$, M. Nauck ${ }^{6}$, M. Horowitz ${ }^{7}$;

${ }^{1}$ Eli Lilly and Company, Windlesham, United Kingdom,

${ }^{2}$ Eli Lilly and Company, Indianapolis, United States, ${ }^{3}$ Eli Lilly and Company, Hamburg, Germany, ${ }^{4}$ Amylin Pharmaceuticals, Inc., San Diego, United States, ${ }^{5}$ Pharmaceutical Profiles, Ltd, Nottingham, United Kingdom, ${ }^{6}$ Dept of Medicine, Ruhr University, Bochum, Germany, ${ }^{7}$ Dept of Medicine, Universiy of Adelaide, Australia.

Background and Aims: Exenatide mimics several glucoselowering actions of glucagon-like protein-1 (GLP-1). The effect of exenatide in type 2 diabetes may in part reflect delay of gastric emptying (GE). Exenatide slows acetaminophen absorption in healthy subjects and in patients with type 2 diabetes. This is the first study to directly quantify the effect of exenatide on GE by scintigraphy.

Materials and Methods: Seventeen patients with type 2 diabetes participated in a randomised, 3-period, singleblind, crossover study (16 males, 1 female; age, 39-69 yrs; BMI, 22.7-34.6 kg/m²; HbAlc, 7.0-10.0\%; treated with oral agents). Each patient was randomised to one of three treatment sequences containing all three treatments (PBO, $5 \mu \mathrm{g}$ exenatide BID, and $10 \mu \mathrm{g}$ exenatide BID). In the first 4 days of a 5-day period, patients received either $5 \mu \mathrm{g}$ exenatide or placebo (PBO) subcutaneously (SC) BID, and patients in the $10 \mu \mathrm{g}$ group received exenatide $5 \mu \mathrm{g}$ SC BID for 2 days followed by $10 \mu \mathrm{g}$ SC BID on days 3 and 4 . Individual treatment periods were separated by a washout interval of at least 2.5 days. In all treatment groups, patients consumed a $450-\mathrm{kcal}$ breakfast that included $99 \mathrm{mTc}-$ labeled eggs and 111In-labeled water immediately after the single morning dose on Day 5. Plasma exenatide, serum glucose and insulin, perceptions of appetite, and GE time were assessed for 6 hrs post-dose.

Results: Exenatide significantly delayed GE and reduced increases in postprandial glucose in a dose-dependent manner compared with PBO (see table below). Based on Visual Analogue Scales, exenatide had no significant effect on hunger, satiety, or fullness but significantly reduced prospective food consumption after the test meal compared with PBO (5 $\mu \mathrm{g}$, $\mathrm{P}=0.03 ; 10 \mu \mathrm{g}, \mathrm{P}=0.07)$. The most frequent adverse event was mild-to-moderate nausea; no hypoglycemia occurred.

Conclusion: In patients with type 2 diabetes, exenatide treatment significantly delayed GE of solids and liquids and was associated with a significant reduction in postprandial glucose excursions.

\begin{tabular}{|c|c|c|c|c|}
\hline & $\begin{array}{l}\text { GE solids } \\
\text { (T50, mins) }\end{array}$ & $\begin{array}{l}\text { GE liquids } \\
\text { (T50, mins) }\end{array}$ & $\begin{array}{l}\text { Glucose*, } \\
\mathrm{C}_{\max } \\
(\mathrm{mmol} / \mathrm{L})\end{array}$ & $\begin{array}{l}\text { Glucose*, } \\
\text { AUC } \\
(\mathrm{mmol} \times \mathrm{h} / \mathrm{L})\end{array}$ \\
\hline PBO & 59.6 & 33.9 & 13.3 & 56.8 \\
\hline $\begin{array}{l}\text { Exenatide, } \\
5 \mu \mathrm{g}\end{array}$ & $111.3^{* *}$ & $87.0^{* *}$ & $10.9 * *$ & $47.7 * *$ \\
\hline $\begin{array}{l}\text { Exenatide, } \\
10 \mu \mathrm{g}\end{array}$ & $169.4 * *$ & $114.2 * *$ & $9.7 * *$ & $41.6^{* *}$ \\
\hline
\end{tabular}

Least Squares Geometric Means shown; *Mean premeal glucose concentrations (mmol/L): $\mathrm{PBO}, 10.3 ; 5 \mu \mathrm{g}$ exenatide, 8.9; and $10 \mu \mathrm{g}$ exenatide, 9.6. Premeal values adjusted for in statistical analysis. T50: half-emptying time. $* * \mathrm{P}<0.01$ vs. $\mathrm{PBO}$.

Supported by Eli Lilly and Company and Amylin Pharmaceuticals, Inc.

0224

Long-term treatment with exenatide improved postprandial glycaemic control and was associated with a shift from small to large HDL and LDL particles

C. Kapitza ${ }^{1}$, M. A. Nauck ${ }^{2}$, D. Kim ${ }^{3}$, M. Trautmann ${ }^{4}$, D. Johns ${ }^{4}$, A. Festa ${ }^{4}$;

${ }^{1}$ Profil Institut fuer Stoffwechselforschung, Neuss, Germany, ${ }^{2}$ Diabeteszentrum Bad Lauterberg, Germany, ${ }^{3}$ Amylin Pharmaceuticals, Inc., San Diego, United States, ${ }^{4}$ Eli Lilly and Company, Indianapolis, United States.

Background and Aims: Dyslipidemia in patients with type 2 diabetes is characterised by abnormalities in lipid concentration, lipoprotein size, and subclass composition. Recent studies have suggested that postprandial hyperglycemia is associated with vascular disease. The aim of this 52wk study was to explore the effects of long-term exenatide treatment on dyslipidemia and postprandial glycaemia.

Materials and Methods: 52 patients participated in a test meal study which was part of a 1-year, open-label, phase 3 clinical trial comparing exenatide and biphasic insulin aspart $30 / 70(n=505)$. Patients with type 2 diabetes inadequately treated with sulphonylurea and metformin were randomised to exenatide $(n=30)$ or biphasic insulin aspart $(n=22)$. For test meal data, we calculated postprandial glucose incremental $\mathrm{AUC}_{0-4 \mathrm{~h}}$ at baseline and Wk 52. Nontraditional cardiovascular (CV) markers, including precursor brain natriuretic protein, oxidized LDL-C, high-sensitivity C-reactive protein (hsCRP), fibrinogen, and apolipoproteins $\mathrm{A} 1$ and $\mathrm{B}$, were assessed during fasting. Lipoprotein size and subclass composition were assessed using nuclear magnetic resonance technology. Results:Both exenatide and biphasic insulin aspart treatment resulted in $\mathrm{HbA}_{1 \mathrm{c}}$ reductions (mean $\pm \mathrm{SEM}$ : 
$-1.4 \pm 0.2 \%$ and $-0.8 \pm 0.2 \%$, respectively). Postprandial glucose responses were similar at baseline (incremental $\mathrm{AUC}_{0-4 \mathrm{~h}}$ : exenatide $4.4 \pm 1.1 \mathrm{mmol}-\mathrm{h} / \mathrm{L}$, biphasic insulin aspart $5.2 \pm 1.4 \mathrm{mmol}-\mathrm{h} / \mathrm{L}$ ). Both treatments significantly reduced postprandial glucose incremental $\mathrm{AUC}_{0-4} \mathrm{~h}$ from baseline (exenatide $-6.5 \pm 1.2 \mathrm{mmol}-\mathrm{h} / \mathrm{L}, \mathrm{p}<0.001$; biphasic insulin aspart $-5.4 \pm 1.6 \mathrm{mmol}-\mathrm{h} / \mathrm{L}, \mathrm{p}=.002)$. Body weight changes were $-4.8 \pm 0.8 \mathrm{~kg}(\mathrm{p}<0.001)$ for exenatide and $+0.8 \pm 0.8 \mathrm{~kg}(\mathrm{p}=.33)$ for biphasic insulin aspart. Exenatidetreated patients demonstrated a statistically significant reduction in fibrinogen from baseline (exenatide $-0.45 \pm 0.22 \mathrm{~g} / \mathrm{L}$, $\mathrm{p}=.045$; biphasic insulin aspart $-0.24 \pm 0.24, \mathrm{p}=\mathrm{NS}$ ). No other statistically significant changes were observed at $\mathrm{Wk} 52$ for the nontraditional CV markers; however, a trend toward reduction of hsCRP was observed (exenatide $-0.62 \pm$ $0.33 \mathrm{mg} / \mathrm{L}, \mathrm{p}=.07$; biphasic insulin aspart $-0.06 \pm 0.40 \mathrm{mg} / \mathrm{L}$, $\mathrm{p}=.87$ ). Baseline to endpoint changes in lipoprotein particle size and concentration are shown in the table.

Conclusion: Exenatide and biphasic insulin aspart improved postprandial glycaemic control. Exenatide treatment was associated with reduced fibrinogen levels and a shift from small to large LDL and HDL particles. These findings suggest an improved cardiovascular risk profile after longterm treatment with exenatide.

Table: Lipoprotein Particle Size and Concentration

\begin{tabular}{|c|c|c|c|}
\hline & $\begin{array}{l}\text { Exenatide Change } \\
\text { (mean, SEM) }\end{array}$ & $\begin{array}{l}\text { Insulin Change } \\
\text { (mean, SEM) }\end{array}$ & $\begin{array}{l}\text { Between-Group } \\
\text { Difference }\end{array}$ \\
\hline LDL Particle & $+0.33(0.10)$ & $+0.11(0.12)$ & $\mathrm{p}=.144$ \\
\hline Size $(\mathrm{nm})$ & $p=.001$ & $\mathrm{p}=.356$ & \\
\hline HDL Particle & $+0.11(0.04)$ & $+0.04(0.05)$ & $\mathrm{p}=.318$ \\
\hline Size (nm) & $p=.013$ & $\mathrm{p}=.391$ & \\
\hline VLDL Particle & $-3.2(1.9)$ & $-1.6(2.2)$ & $\mathrm{p}=.590$ \\
\hline Size (nm) & $\mathrm{p}=.097$ & $\mathrm{p}=.482$ & \\
\hline $\begin{array}{l}\text { Total LDL } \\
(\mathrm{nmol} / \mathrm{L})\end{array}$ & $\begin{array}{l}-87.3(62.2) \\
p=.169\end{array}$ & $\begin{array}{l}+5.0(74.6) \\
\mathrm{p}=.947\end{array}$ & $\mathrm{p}=.348$ \\
\hline $\begin{array}{l}\text { Large LDL } \\
(\mathrm{nmol} / \mathrm{L})\end{array}$ & $\begin{array}{l}+68.4(27.3) \\
\mathbf{p}=. \mathbf{0 1 7}\end{array}$ & $\begin{array}{l}+37.7(32.8) \\
\mathrm{p}=.258\end{array}$ & $\mathrm{p}=.479$ \\
\hline $\begin{array}{l}\text { Small LDL } \\
(\mathrm{nmol} / \mathrm{L})\end{array}$ & $\begin{array}{l}-146.4(67.7) \\
\mathbf{p}=. \mathbf{0 3 7}\end{array}$ & $\begin{array}{l}-25.8(81.2) \\
p=.753\end{array}$ & $\mathrm{p}=.262$ \\
\hline $\begin{array}{l}\text { Very Small } \\
\text { LDL }(\mathrm{nmol} / \mathrm{L})\end{array}$ & $\begin{array}{l}-126.8(53.9) \\
\mathbf{p}=. \mathbf{0 2 4}\end{array}$ & $\begin{array}{l}-17.0(64.6) \\
p=.794\end{array}$ & $\mathrm{p}=.201$ \\
\hline $\begin{array}{l}\text { Total HDL } \\
(\mu \mathrm{mol} / \mathrm{L})\end{array}$ & $\begin{array}{l}+0.33(0.79) \\
\mathrm{p}=.678\end{array}$ & $\begin{array}{l}+2.5(0.9) \\
\mathbf{p}=. \mathbf{0 1 2}\end{array}$ & $\mathrm{p}=.088$ \\
\hline $\begin{array}{l}\text { Large HDL } \\
(\mu \mathrm{mol} / \mathrm{L})\end{array}$ & $\begin{array}{l}+0.69(0.33) \\
\mathbf{p}=.042\end{array}$ & $\begin{array}{l}+1.0(0.4) \\
\mathbf{p}=.014\end{array}$ & $\mathrm{p}=.528$ \\
\hline $\begin{array}{l}\text { Total VLDL } \\
(\mathrm{nmol} / \mathrm{L})\end{array}$ & $\begin{array}{l}-9.3(5.5) \\
\mathrm{p}=.097\end{array}$ & $\begin{array}{l}+12.9(6.6) \\
p=.056\end{array}$ & $p=.014$ \\
\hline $\begin{array}{l}\text { Large VLDL } \\
(\mathrm{nmol} / \mathrm{L})\end{array}$ & $\begin{array}{l}-2.5(1.6) \\
p=.124\end{array}$ & $\begin{array}{l}+0.13(1.9) \\
\mathrm{p}=.945\end{array}$ & $\mathrm{p}=.296$ \\
\hline
\end{tabular}

Supported by Eli Lilly and Company and Amylin Pharmaceuticals, Inc

\section{OP 39}

\section{Optimising clinical diabetes care}

\section{5}

Costs of self-measurement of blood glucose (SMBG) regarding morbidity and mortality in type 2 diabetes in a reality of care setting (The ROSSO study No. 6)

K. Neeser ${ }^{1}$, C. Weber ${ }^{1}$, H. Wenzel ${ }^{2}$, B. Schneider ${ }^{3}$;

${ }^{1}$ Medical Affairs, Institute for Medical Informatics and Biostatistics, Basel, Switzerland, ${ }^{2} \mathrm{~S}-\mathrm{IPH}$, University of Bielefeld, Germany, ${ }^{3}$ Institute of Biometrics, Medical University Hanover, Germany.

Background: A recently published large retrospective multi-centre study (RoSSO; Martin et al. Diabetologia 2006) showed in a German population that self measurement of blood glucose (SMBG) in patients with type 2 diabetes had a positive long-term outcome on hard endpoints. The aim of our analysis was to compare the costs of diabetes in the two study groups (patients with and without SMBG).

Methods: The original study groups (patients with and without $\mathrm{SMBG}$ ) are in some points not comparable, because (e.g.) one group was in average 3.5 years older than the other. If adjustment for these differences was done by Cox regression in the original publication, adjustment for the present analysis had to be done by matching pairs based on age, gender, smoking status and blood glucose at diagnosis. In total 813 matched pairs (1626 patients) have been eligible for analysis. A further segmentation on the antidiabetic medication revealed 971 patients with oral antidiabetic drugs only ("OAD only") and 372 patients with oral antidiabetic drugs plus insulin ("OAD plus insulin"). Our assessment is based on costs for 18 diabetes related complications (including surgical interventions), follow-upcosts for these complications, costs for outpatient physician services, cost of antidiabetic and additional pharmaceutical treatment and costs for strips and lancets for patients performing SMBG using a German cost parameter set reflecting the situation in Germany in the year 2005.

Results: The following diagram summarizes the cost of type 2 diabetes and SMBG for patients treated with "OAD only" and patients with "OAD plus insulin". Use of SMBG in type 2 diabetic patients with "OAD only" leads to a nonsignificant cost saving of EUR 214 per patients and year, whereas a combined therapy "OAD plus insulin" causes significant cost savings of EUR 1'727 per patient and year. 


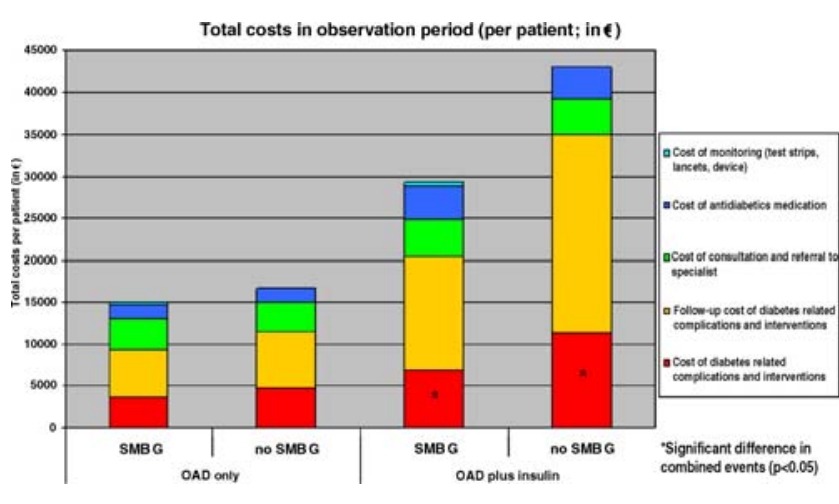

Conclusion: Use of SMBG in type 2 diabetic patients may reduce complication related costs, whereby the costs for self-monitoring (test strips, devices) are compensated.

Supported by an unrestricted grant of Roche Diagnostics Germany

0226

The implementation of a diabetes care system: a prospective observational 8-years follow-up study

L. M. C. Welschen ${ }^{1,2}$, S. D. M. Bot ${ }^{1,2}$, J. M. Dekker ${ }^{1}$, W. A. B. Stalman ${ }^{1,2}$, G. Nijpels ${ }^{1,2}$;

${ }^{1}$ EMGO Institute, ${ }^{2}$ Department of General Practice, VU University Medical Center, Amsterdam, The Netherlands.

Background and Aims: In 1997, an extensive diabetes management programme, 'the Diabetes Care System (DCS)', was implemented in West-Friesland, The Netherlands. General practitioners (GPs) were invited to refer all their patients with diabetes to this care system, in addition to their care. The objective of the DCS was to provide the co-ordination of the sub-regional care including benchmarking of the main treatment outcomes, and to give feedback to the GPs. Additionally, to provide annual medical examinations for patients, and extensive education by diabetes nurses and dieticians in order to improve patient empowerment. At the start in 1997, 65 patients were included in the system. After a sharp increase of patients included during the first two years $(\mathrm{n}=803$ in 1998 and $n=1,330$ in 1999), the average number of new patients every year was between 300 and 400 . The aim of this study was to describe the changes in clinical characteristics of the patients after their entry into the system.

Material and Methods: A prospective observational cohort study of patients with diabetes who were included in the DCS was performed. From 1998 till 2005, data were collected every year on $\mathrm{HbA}_{1 \mathrm{c}}$, weight, blood pressure, fasting blood glucose, total and HDL-cholesterol, triglycer- ides, medication and smoking status. To evaluate individual patients characteristics following entry into the care system, a study cohort was defined. In this cohort, for every individual patient, the measurements in their first year of entry into the system were set at $\mathrm{T}=1$. Follow-up measurements were defined as $\mathrm{T}=2$, for the second year, to $\mathrm{T}=8$, for the eight year after entry into the system.

Results: The defined study cohort started with 4,933 patients at $\mathrm{T}=1$. Mean age of the patients was 62 years ( $\mathrm{SD} \pm 12$ years) and $51 \%$ was male. Body mass index was $29.8 \mathrm{~kg} / \mathrm{m}^{2}$ and did not change during follow-up. Glycaemic control showed an initial sharp decrease, followed by stabilization during follow-up. The use of insulin therapy and diabetes duration increased. Total cholesterol and triglycerides decreased during the 8 years of follow-up whereas HDL-cholesterol increased. Systolic blood pressure increased, but diastolic blood pressure decreased. The percentage of smoking patients decreased from $21 \%$ at $\mathrm{T}=1$ to $15 \%$ at $\mathrm{T}=8$.

Changes in clinical characteristics during a prospective observational 8-years follow-up study

\begin{tabular}{|c|c|c|c|c|c|c|c|c|}
\hline & $\mathrm{T} 1$ & $\mathrm{~T} 2$ & $\mathrm{~T} 3$ & $\mathrm{~T} 4$ & T5 & T6 & $\mathrm{T} 7$ & $\mathrm{~T} 8$ \\
\hline $\begin{array}{l}\text { Number } \\
\text { of patients }\end{array}$ & 4,933 & 3,709 & 3,179 & 2,539 & 1,974 & 1,526 & 1,049 & 461 \\
\hline $\begin{array}{l}\text { Diabetes } \\
\text { duration } \\
\text { (years) }\end{array}$ & $\begin{array}{l}4.6 \\
(6.1)\end{array}$ & $\begin{array}{l}5.5 \\
(5.7)\end{array}$ & $\begin{array}{l}6.7 \\
(5.7)\end{array}$ & $\begin{array}{l}7.7 \\
(5.6)\end{array}$ & $\begin{array}{l}8.8 \\
(5.5)\end{array}$ & $\begin{array}{l}10.1 \\
(5.8)\end{array}$ & $\begin{array}{l}11.5 \\
(5.8)\end{array}$ & $\begin{array}{l}13.4 \\
(5.7)\end{array}$ \\
\hline $\begin{array}{l}\mathrm{HbA}_{1 \mathrm{c}} \\
(\%)\end{array}$ & $\begin{array}{l}7.7 \\
(1.8)\end{array}$ & $\begin{array}{l}7.0 \\
(1.2)\end{array}$ & $\begin{array}{l}7.1 \\
(1.2)\end{array}$ & $\begin{array}{l}7.2 \\
(1.2)\end{array}$ & $\begin{array}{l}7.1 \\
(1.2)\end{array}$ & $\begin{array}{l}7.2 \\
(1.2)\end{array}$ & $\begin{array}{l}7.1 \\
(1.1)\end{array}$ & $\begin{array}{l}7.1 \\
(1.1)\end{array}$ \\
\hline $\begin{array}{l}\text { Systolic BP } \\
(\mathrm{mmHg})\end{array}$ & $\begin{array}{l}143.3 \\
(21.8)\end{array}$ & $\begin{array}{l}141.6 \\
(21.7)\end{array}$ & $\begin{array}{l}142.4 \\
(21.6)\end{array}$ & $\begin{array}{l}144.1 \\
(21.7)\end{array}$ & $\begin{array}{l}147.3 \\
(21.5)\end{array}$ & $\begin{array}{l}149.4 \\
(22.2)\end{array}$ & $\begin{array}{l}151.3 \\
(21.7)\end{array}$ & $\begin{array}{l}151.6 \\
(21.3)\end{array}$ \\
\hline Diastolic & 82.6 & 81.3 & 80.9 & 80.5 & 79.7 & 78.6 & 77.9 & 76.4 \\
\hline BP (mmHg) & (11.3) & (11.1) & (11.3) & $(10.8)$ & (10.9) & $(10.5)$ & $(10.0)$ & $(10.3)$ \\
\hline $\begin{array}{l}\text { Fasting } \\
\text { blood } \\
\text { glucose } \\
(\mathrm{mmol} / \mathrm{l})\end{array}$ & $\begin{array}{l}8.9 \\
(3.5)\end{array}$ & $\begin{array}{l}8.2 \\
(2.2)\end{array}$ & $\begin{array}{l}8.3 \\
(3.0)\end{array}$ & $\begin{array}{l}8.3 \\
(2.5)\end{array}$ & $\begin{array}{l}8.2 \\
(2.9)\end{array}$ & $\begin{array}{l}8.3 \\
(2.2)\end{array}$ & $\begin{array}{l}8.2 \\
(2.1)\end{array}$ & $\begin{array}{l}8.2 \\
(2.3)\end{array}$ \\
\hline $\begin{array}{l}\text { Total } \\
\text { cholesterol } \\
(\mathrm{mmol} / \mathrm{l})\end{array}$ & $\begin{array}{l}5.4 \\
(1.1)\end{array}$ & $\begin{array}{l}5.3 \\
(1.1)\end{array}$ & $\begin{array}{l}5.2 \\
(1.1)\end{array}$ & $\begin{array}{l}5.2 \\
(1.1)\end{array}$ & $\begin{array}{l}5.1 \\
(1.0)\end{array}$ & $\begin{array}{l}5.1 \\
(1.1)\end{array}$ & $\begin{array}{l}4.9 \\
(1.0)\end{array}$ & $\begin{array}{l}4.8 \\
(1.0)\end{array}$ \\
\hline $\begin{array}{l}\text { HDL- } \\
\text { cholesterol } \\
(\mathrm{mmol} / \mathrm{l})\end{array}$ & $\begin{array}{l}1.2 \\
(0.4)\end{array}$ & $\begin{array}{l}1.3 \\
(0.4)\end{array}$ & $\begin{array}{l}1.3 \\
(0.3)\end{array}$ & $\begin{array}{l}1.3 \\
(0.4)\end{array}$ & $\begin{array}{l}1.3 \\
(0.4)\end{array}$ & $\begin{array}{l}1.3 \\
(0.4)\end{array}$ & $\begin{array}{l}1.3 \\
(0.4)\end{array}$ & $\begin{array}{l}1.4 \\
(0.4)\end{array}$ \\
\hline $\begin{array}{l}\text { Triglycerides } \\
(\mathrm{mmol} / \mathrm{l})\end{array}$ & $\begin{array}{l}2.1 \\
(2.0)\end{array}$ & $\begin{array}{l}1.9 \\
(1.2)\end{array}$ & $\begin{array}{l}1.9 \\
(1.2)\end{array}$ & $\begin{array}{l}1.9 \\
(1.1)\end{array}$ & $\begin{array}{l}1.8 \\
(1.0)\end{array}$ & $\begin{array}{l}1.8 \\
(1.1)\end{array}$ & $\begin{array}{l}1.8 \\
(1.1)\end{array}$ & $\begin{array}{l}1.8 \\
(1.0)\end{array}$ \\
\hline $\begin{array}{l}\text { Patients on } \\
\text { insulin (\%) }\end{array}$ & 12 & 17 & 20 & 24 & 28 & 32 & 35 & 41 \\
\hline
\end{tabular}

Data are means (standard deviation) or \%

Conclusion: The Diabetes Care System was successful in improving and stabilizing the clinical characteristics for individual patients, despite the longer mean diabetes duration. This implies that a disease management system 
may be a major step in the improvement of the care of patients with diabetes.

0227

Cost-effectiveness of an intensified multi-factorial intervention vs. conventional treatment in microalbuminuric patients with type 2 diabetes at a high CVD risk: results from the Steno-2 study

P. Gaede ${ }^{1}$, H.-H. Parving ${ }^{1}$, A. J. Palmer ${ }^{2}$, O. Pedersen ${ }^{1}$; ${ }^{1}$ Steno Diabetes Center, Copenhagen, Denmark, ${ }^{2}$ Health Economics and Outcomes Research, CORE - A Unit of IMS, Basel, Switzerland.

Background and Aims: The Steno-2 study was a longterm, randomised, open, parallel trial that compared an intensified, multitargeted intervention versus conventional multifactorial treatment in patients with type 2 diabetes and microalbuminuria. Patients received conventional treatment in accordance with national guidelines, or an intensified, integrated treatment targeting glycaemia, lipid and blood pressure abnormalities, microalbuminuria and platelets. The primary composite endpoint was cardiovascular disease (CVD) i.e. non-fatal MI, non-fatal stroke, PCI, coronary artery bypass grafting, revascularisation of the leg, amputation and CVD death. After 7.8 yrs, patients in the intensive arm had a $53 \%(95 \%$ CI: $27-76)$ lower relative risk of CVD. The aim of the current study was to assess the cost-effectiveness of intensive versus conventional therapy as applied in the Steno 2 study from the perspective of a Danish reimbursement authority.

Materials and Methods: A mathematical simulation Markov model was developed which incorporated annual CVD events, heart failure-related hospital admissions, dialysis and total mortality rates that were capture during the Steno 2 study. Danish-specific event- and intensive/ conventional intervention direct medical costs were extracted from Danish cost databases by local experts. An 8 -year analysis (approximating the with-in trial period), as well as longer-term projections were made. Outcomes that were calculated included: quality-adjusted life expectancy (in quality-adjusted life years [QALYs]), mean total costs per patient, and incremental costs per QALY gained. Future costs and QALYs were discounted in line with current international guidelines ( $3 \%$ annually) to account for time preferences for money and good health. Extensive sensitivity analyses were performed to assess the robustness of the findings.

Results: Intensive intervention led to substantial improvements in QALY versus conventional intervention. The higher costs of implementing the intensive therapy were partially offset by reduced costs of complications. Projec- tions over longer time horizons led to greater improvements in QALYs and smaller differences in costs between the treatment arms. Sensitivity analysis revealed that if the intensified therapy could be implemented as effectively in a primary care setting, it would be cost saving compared to conventional therapy.

Conclusion: A multifactorial intensified intervention is highly cost-effective compared to conventional therapy in a Danish setting by current international standards. If the intensified intervention can be implemented as effectively in a primary care setting, substantial cost savings to the Danish healthcare system would be anticipated.

Summary of Economic Outcomes for Intensive versus Conventional Therapy

\begin{tabular}{|c|c|c|c|c|c|c|}
\hline \multicolumn{7}{|c|}{ Time Horizon } \\
\hline & $\begin{array}{l}\text { 8-Years } \\
\text { (approximating } \\
\text { with-in trial } \\
\text { period) }\end{array}$ & $\begin{array}{l}\text { 8-Years } \\
\text { (approximating } \\
\text { with-in trial } \\
\text { period) }\end{array}$ & $\begin{array}{l}\text { 10- } \\
\text { Years }\end{array}$ & $\begin{array}{l}10- \\
\text { Years }\end{array}$ & $\begin{array}{l}30- \\
\text { Years }\end{array}$ & $\begin{array}{l}30- \\
\text { Years }\end{array}$ \\
\hline & $\begin{array}{l}\text { Costs } \\
\text { (Danish } \\
\text { Kroner) }\end{array}$ & $\begin{array}{l}\text { QALY } \\
\text { (Years) }\end{array}$ & $\begin{array}{l}\text { Costs } \\
\text { (Danish } \\
\text { Kroner) }\end{array}$ & $\begin{array}{l}\text { QALY } \\
\text { (Years) }\end{array}$ & $\begin{array}{l}\text { Costs } \\
\text { (Danish } \\
\text { Kroner) }\end{array}$ & $\begin{array}{l}\text { QALY } \\
\text { (Years) }\end{array}$ \\
\hline Intensive & 161,059 & 5.50 & 191,794 & 6.54 & 339,170 & 10.72 \\
\hline Conventional & 127,610 & 5.34 & 160,531 & 6.27 & 308,420 & 9.41 \\
\hline Difference & 33,449 & 0.16 & 31,263 & 0.28 & 30,750 & 1.31 \\
\hline $\begin{array}{l}\text { Costs/QALY } \\
\text { gained }\end{array}$ & 211,702 & - & 113,271 & - & 23,366 & - \\
\hline $\begin{array}{l}\text { Costs/QALY } \\
\text { gained }(€)\end{array}$ & 28,377 & - & 15,183 & - & 3,132 & - \\
\hline
\end{tabular}

Supported by: Supported by Novo Nordisk A/S, Denmark

0228

The influence of an intensive treatment and teaching programme on the progression of diabetes late complications in type 1 diabetic patients in Moscow (15-year results)

A. Y. Mayorov ${ }^{1}$, G. R. Galstyan ${ }^{1}$, O. M. Dvoynishnikova ${ }^{1}$, M. B. Antsiferov ${ }^{2}$, E. G. Bessmertnaya ${ }^{1}$, T. M. Milenkaya ${ }^{1}$, I. I. Dedov';

${ }^{1}$ Institute of Diabetes, National Research Centre for Endocrinology, Moscow, ${ }^{2}$ Department of Diabetes, Moscow City Centre for Endocrinology, Russian Federation.

Background and Aims: Patient education and self-monitoring is regarded as the basis for a reduction of diabetesrelated acute and late complications, improvement of diabetes care and saving of health care cost. The aim of 
the study was to evaluate metabolic control and progression of late diabetes complications after structured programme for intensive treatment and teaching of Type 1 diabetic patients (DTTP) during long-term follow-up.

Materials and Methods: 65 Type 1 diabetic patients (28 male, 37 female) were re-examined after DTTP during follow-up period of 15 yrs. The mean age at the end of study was 43,6 6 8,6 (30-62) yrs; mean diabetes duration $25,8 \pm 7,4$ (15-46) yrs. Patients were divided two subgroups (SG I, SG II). SG I ( $\mathrm{n}=24)$ was followed-up in study center, these visits included elements of individual education (review and adjustment regimens of insulin therapy, improvement knowledge and skills, discussion urgent problems and etc.). Patients of SG II $(n=41)$ were treated in system of public health without intensive follow-up. All results of this study were compared with data before DTTP. To evaluate progression of diabetic retinopathy fundus photos were taken before DTTP and during follow-up period. For detection of diabetic nephropathy we measured albumin excretion rate and glomerular filtration rate.

Results: There was significant improvement of $\mathrm{HbA} 1 \mathrm{c} 1 \mathrm{yr}$ after DTTP (from $9,5 \pm 0,9 \%$ to $7,5 \pm 1,0 \%, \mathrm{p}<0,001$ ), SG I did not differ from SG II. HbAlc increased to $8,4 \pm 1,7 \%$ $(\mathrm{p}<0,005$ compared initial level) 7 yrs after and to $8,9 \pm 1,8 \%$ $(p<0,01) 15$ yrs after DTTP. HbA1c was significantly lower in SG I compared with SG II: $7,7 \pm 1,1 \%$ vs $8,8 \pm 1,8 \%$ $(p<0,001)$ in 7 yrs and $7,9 \pm 1,1 \%$ vs $9,3 \pm 1,9 \%(p<0,001)$ in 15 yrs. There was significance difference in blood glucose self-monitoring frequency between subgroups 15 yrs after DTTP: $26,1 \pm 17,7$ in SG I vs $10,3 \pm 9,5$ per week in SG II $(p<0,001) .40 \%$ patients had diabetic retinopathy before DTTP, $72 \%$ and $100 \%$ patients had such late complication 7 and $15 \mathrm{yrs}$ after. The prevalence of proliferative retinopathy during follow-up increased from $1,5 \%$ before to $12,3 \% 7 \mathrm{yrs}$ and $20,0 \% 15 \mathrm{yrs}$ after DTTP, but there was significant difference between SG I and SG II in 15 yrs: $8,3 \%$ vs $26,8 \% \quad(p<0,05)$. The prevalence of diabetic nephropathy did not increased. $20 \%$ patients had microalbuminuria initially, $7,7 \%$ - in $7 \mathrm{yrs}, 15,4 \%$ - in $15 \mathrm{yrs}$ $(p>0,05)$. Proteinuria was found in $4,6 \%$ patients before DTTP, in $12,3 \% 7 \mathrm{yrs}$ after, in $9,2 \% 15 \mathrm{yrs}$ after $(\mathrm{p}>0,05)$. The prevalence of all stages of nephropathy was similar in both subgroups.

Conclusion: Type 1 diabetic patients had significantly better level of glycemic control during 15 yrs follow-up compared with baseline before DTTP. Also this study has demonstrated deterioration of metabolic control during long-term follow-up after DTTP. But intensive follow-up after DTTP can help to achieve better HbAlc level compared with routine diabetes care in public health system. This study has shown effective- ness of DTTP and long-term intensive follow-up in respect of late diabetes complications.

\section{OP 40}

\section{Coronary endothelial function}

\section{9}

\section{Coronary vasoreactivity is not altered in young uncom-} plicated type 1 diabetes

B. Capaldo, M. Galderisi, A. A. Turco, A. D'Errico, G. Nosso, S. Turco, O. de Divitiis, G. Riccardi;

Department of Clinical and Experimental Medicine, University Federico II Naples, Italy.

Background and Aims: Abnormalities of coronary vasoreactivity have been reported in diabetic patients even in the absence of coronary artery disease. How early these abnormalities occur in Type 1 diabetes is still unsettled.

Materials and Methods: We evaluated coronary microcirculation function in thirty-one Type 1 diabetic patients (D) (age $31 \pm 8 \mathrm{y}$, BMI $24 \pm 3 \mathrm{~kg} / \mathrm{m} 2$, duration of diabetes $11 \pm 8 \mathrm{y}$, HbAlc $7.9 \pm 1.0 \%$ ) and in 11 normal subjects $(\mathrm{N}$ ) (age $32 \pm 7 \mathrm{y}$, BMI $23 \pm 2 \mathrm{Kg} / \mathrm{m} 2$ ). The two groups were similar for blood pressure and lipid profile, and no subject had a positive family history of coronary heart disease. Diabetic patients were free of macrovascular complications, had a normal exercise treadmill test and only few of them had initial microvascular complications $(6 / 31$ showed signs of background retinopathy and 7/31 microalbuminuria). Blood flow velocity in the left anterior descending coronary artery was measured at rest and after dipyridamole $(0,56 \mathrm{mg} / \mathrm{kg}$ for $4 \mathrm{~min}$ followed by $0,26 \mathrm{mg} / \mathrm{Kg}$ for $2 \mathrm{~min}$ ) using transthoracic color-guided pulsed Doppler echocardiography. Coronary flow reserve (CFR) was defined as the ratio of dipyridamole-induced coronary peak flow velocity and resting flow velocity. CFR assessment was performed in the fasting state before administration of the morning shortacting insulin dose.

Results: Both systolic and diastolic blood pressure were similar in D $(119 \pm 10 / 74 \pm 8 \mathrm{mmHg})$ and $\mathrm{C}(121 \pm 24 /$ $78 \pm 8 \mathrm{mmHg}$ ) and remained substantially unchanged during dipyridamole infusion. Heart rate at rest was $72 \pm 12$ in D and $73 \pm 12$ beats $/ \mathrm{min}$ in $\mathrm{N}$ and increased by $30-40 \%$ after dipyridamole in both groups. Coronary flow velocity was $23 \pm 0.7$ in D and $24 \pm 0.5 \mathrm{~cm} / \mathrm{sec}$ in $\mathrm{N}$ at rest and increased to $69 \pm 1.6 \mathrm{~cm} / \mathrm{sec}$ and $77 \pm 1.7 \mathrm{~cm} / \mathrm{sec}$ in $\mathrm{D}$ and $\mathrm{N}$, respectively ( $\mathrm{p}=$ n.s.) CFR was similar in the two groups $(3.15 \pm 0.7$ and $3.20 \pm 0.7$ in D and N). No correlation was found between 
CFR and duration of the disease or degree of glycemic control. All indexes of both diastolic and systolic function were similar in D and $\mathrm{N}$.

Conclusion: Coronary vasoreactivity and myocardial function are well preserved in young uncomplicated Type 1 diabetic patients. This observation suggests that in the absence of vardiovascular risk factors, coronary vasoreactivity impairs later on in the course of type 1 diabetes.

\section{0}

Coronary endothelial dysfunction and arterial stiffness, but not microalbuminuria, are of poor cardiovascular prognosis in diabetics without other cardiovascular risk factor

I. Pham ${ }^{1}$, E. Cosson ${ }^{2}$, A. Nitenberg ${ }^{1}$, M. Brahimi ${ }^{2}$, J.-R. Attali ${ }^{2}$, P. Valensi ${ }^{2}$;

${ }^{1}$ Functional Investigations Department, ${ }^{2}$ EndocrinologyDiabetology-Nutrition, Jean Verdier Hospital, Bondy, France.

Background and Aims: An association between peripheral endothelium dysfunction, microalbuminuria (MA) and arterial stiffness has been suggested. MA has been shown to be a powerful predictor of cardiovascular events (CVE) in diabetic patients. The aim of the study was to evaluate the relation between coronary endothelium dysfunction (CED), MA and arterial stiffness and their respective prognostic value in patients with type 2 diabetes (T2D) but no other major $\mathrm{CV}$ risk factor.

Patients and Methods: A total of 84 patients with T2D for $9.3 \pm 2.7$ years, with atypical cardiac symptoms and with positive or equivocal ECG stress test or myocardial scintigraphy but angiographically normal coronary arteries were included. They were aged $<65 \mathrm{yrs}$, normotensive, non smokers, non obese, and had LDL cholesterol $<3.7 \mathrm{mmol} / 1$. CED was evaluated according to the diameter changes of the left anterior descending coronary artery $(\triangle \mathrm{DC})$ after a cold pressor testing during a quantitative coronary angiography. Arterial stiffness was estimated by the brachial pulse pressure. The patients were followed up for CVE (cardiac death, myocardial infarction, stable or unstable angina, coronary artery revascularization, stroke).

Results: A CED, defined as a decrease in coronary artery diameter $>6 \%$, was present in $62(74 \%)$ patients. MA was present in 25 patients and urinary albumin excretion rate correlated with $\triangle \mathrm{DC}(\mathrm{r}=-0.39, \mathrm{p}=0.003)$ and pulse pressure $(\mathrm{r}=0.244, \mathrm{p}<0.05)$. A CVE occurred in 19 patients, after $45 \pm 19$ months of follow-up. The predictive factors for CVE were CED ( $29 \%$ vs $4.5 \%, \mathrm{p}<0.01)$, higher systolic blood pressure $(p<0.05)$ and pulse pressure $(p<0.05)$. The risk associated with CED was unmodified by the presence of MA (CED- MA-: $5.6 \%$ of CVE, CED- MA+: $0 \%$, CED+MA-: $31.7 \%$, CED+MA+: $23.8 \%$ ).

Conclusions: These data suggest that 1 ) CED and arterial stiffness are predictive of early CVE in the T2D population without other CV risk factor ; 2) MA is a marker of CED and arterial stiffness. 3) CED and arterial stiffness might account for the increased CV risk linked to MA.

\section{1}

Pioglitazone improves insulin sensitivity and endothelial function in coronary artery disease patients with unknown impaired glucose tolerance

S. Rizza, M. Cardellini, A. Savo, F. Clementi, O. Porzio, F. Romeo, R. Lauro, M. Federici;

Dept. of Internal Medicine, University of Tor Vergata, Rome, Italy.

Objective: Diabetes is often undiagnosed and untreated in patients suffering from coronary heart disease (CHD). However, a medical history characterized by concurrence of previous Acute Myocardial Infarction (AMI) and Type 2 diabetes (T2DM) is known to identify patients at the highest risk for cardiovascular mortality, due to recurrent AMI events. Moreover, activation of inflammatory pathways often is present in patients with unknown diabetes and in CHD patients with tendency to recurrent events. The aims of this study were 1) to evaluate the prevalence of glucose metabolism disturbances in CHD patients; 2) to correlate the glucose metabolism status with inflammatory markers; and 3) to identify in a pilot study a reasonable therapeutic approach acting to dampen both impaired glucose metabolism and inflammation.

Methods: To evaluate the prevalence of glucose metabolism disturbances in CHD patients, we performed an oral glucose tolerance test (OGTT) in a large group of patients with a previous MI and without known T2DM, no differences in secondary prevention treatments. In all patients we also evaluate inflammatory markers (hsCRP, TNF-alpha, CD40L and adiponectin). Then, we tested the short-term effect of pioglitazone $(n=12)$ compared to placebo $(n=11)$ on inflammation state, insulin sensitivity (euglycemic hyperinsulinemic clamp, IS) in a sub-group of patients affected by impaired glucose tolerance (IGT) after the OGTT.

Results: In 185 non T2DM CHD patients all matched for age, sex, waist, BMI, smoking and pharmacological treatments, upon OGTT, we observed that $21 \%$ patients were affected by T2DM, $38 \%$ by IGT, and only $41 \%$ were 
normoglycemic (NGT). Among the inflammation markers (compared with univariate ANOVA) we observed that only TNF-alpha was significantly higher in both IGT and T2DM compared to NGT patients $(\mathrm{p}<0.01)$. Interestingly, adiponectin was higher in NGT compared to the other two groups $(p<0.05)$. In overall patients, a stepwise logistic regression analysis showed that TNF-alpha was the main predictor for defects of glucose tolerance $(p<0.01)$. Next, 23 patients from the IGT group were randomized to receive pioglitazone (30 mg/die, 12 patients) or placebo (11 patients) for 12 weeks. At the end of treatment we observed that pioglitazone significantly reduced fasting insulin (but not fasting glucose) as well as TNF-alpha levels, when compared to the placebo group. Remarkably, we observed that Pioglitazone increased adiponectin and IS, assessed by a hyperinsulinemic euglycemic Clamp compared to the placebo group ( $\mathrm{p}<0.05$ for all).

Conclusions: Our study suggests that an unchecked inflammatory state characterized by increased TNF-alpha and reduced adiponectin levels in patients with a prior AMI is associated to disturbances of glucose metabolism. Pioglitazone can impede progression to diabetes and reduce inflammatory status. Since previous studies separately observed that increased TNF-alpha and undiagnosed diabetes in CHD patients were associated to higher mortality due to recurrent coronary events and/or heart failure, our study suggest that all CHD subjects must be evaluated through OGTT to adequately treat IGT and T2DM.

\section{2}

Exendin-4 rapidly activates eNOS and protects human coronary artery endothelial cells against glucolipoapoptosis Ö. Tutuncu ${ }^{1}$, D. Nathanson ${ }^{2}, \AA$. Sjöholm ${ }^{2}$, T. Nyström², Q. Zhang';

${ }^{1}$ South Hospital, Research Center, Karolinska Institute, Stockholm, ${ }^{2}$ Internal Medicine, Karolinska Institute, Stockholm, Sweden.

Background and Aims: Endothelial dysfunction plays an important role in macrovascular complications in diabetes. We recently showed that the glucagon-like peptide-1 receptor is expressed in human coronary artery endothelial cells (HCAEC) and that GLP-1 improves endothelial dysfunction in type 2 diabetic patients with coronary artery disease. The aim of the present study was to investigate the role of the stable GLP-1 receptor agonist exendin-4 on neogenesis and apoptosis under normal and hyperglucolipi- demic conditions (simulating the diabetic milieu) in HCAEC cells in vitro. Exendin-4 was approved for clinical use in the U.S. in 2005 against type 2 diabetes.

Materials and Methods: HCAEC cells were cultured in the endothelial growth medium in the presence of $5 \mathrm{mM}$ glucose. Phosphorylation and expression of endothelial nitric oxide synthase (eNOS) were examined by Western blotting using anti-phospho-eNOS and anti-eNOS antibodies, respectively. $\beta$-actin examined in same blots acted as loading control. The concentration of NO in the culture medium was determined by the Saltzman assay. Results were normalized by subtracting the NO content in the wells with medium alone in the incubation. ${ }^{3} \mathrm{H}$-thymidine incorporation was assayed in 96well plates as a measure of DNA synthesis, and caspase-3 activity was evaluated using ELISA kit as a measure of apoptosis. DNA synthesis and apoptosis were examined after 48 h-incubation with the reagents. Cell death was assessed using Trypan-blue inclusion.

Results: Incubation of HCAEC cells with exendin-4 from 1 to $10 \mathrm{nM}$ for $10 \mathrm{~min}$ resulted in a dose-dependent increase of eNOS phosphorylation to an extent comparable with that of GLP-1 and acetylcholine. Continuous treatment of the cells with exendin- 4 for $48 \mathrm{~h}$ resulted in increased expression of eNOS protein. Similar to the acute effects of the peptide, long-term treatment with exendin-4 also enhanced the phosphorylation state of the enzyme even when normalized for eNOS expression. Incubating cells in $30 \mathrm{mM}$ glucose resulted in decreased NO production, an effect normalized by co-incubation with exendin-4. In contrast, palmitate-mediated NO production was not affected by this treatment. Exendin-4 promoted HCAEC DNA synthesis after $48 \mathrm{~h}$ incubation in $5 \mathrm{mM}$ glucose, and neogenesis was confirmed by an increased cell number. Incubation of the cells with high glucose or palmitate provoked apoptosis and cell death, both of which were completely prevented by exendin- 4 .

Conclusions: Our study reveals salutary effects of exendin4 on cell regeneration and protection from glucolipoapoptosis in human coronary artery endothelial cells, These novel findings add yet other beneficial properties of exendin-4, increasing its clinical utility in type 2 diabetic patients in whom endothelial dysfunction is a salient feature that adversely affect their survival. Elucidation of the mechanisms underlying these beneficial vascular effects of exendin-4 may be exploited when intervening pharmacologically against vascular dysfunction in diabetes and may form the basis of improved incretin enhancers that hold the potential to prevent vascular lesions in diabetic patients. 


\section{OP 41}

\section{Prediction and prevention of type 1 diabetes}

0233

Serum metabolite patterns between birth and development of autoantibodies and overt type 1 diabetes: application of large-scale metabolomics to the Type 1 Diabetes Prediction and Prevention study (DIPP)

M. Oresic ${ }^{1}$, T. Seppänen-Laakso ${ }^{1}$, S. Simell ${ }^{2}$, T. Suortti ${ }^{1}$, V. Parikka ${ }^{2}$, K. Nänto-Salonen ${ }^{2}$, J. Lähde ${ }^{3}$, M. Sysi-Aho ${ }^{1}$, A. Reinikainen ${ }^{4}$, T. Simell ${ }^{2}$, J. Ilonen ${ }^{5}$, H. Hyöty ${ }^{6}$, M. Knip ${ }^{3}$, R. Veijola ${ }^{7}$, O. Simell ${ }^{2}$;

${ }^{1}$ Quantitative Biology and Bioinformatics, VTT Technical Research Centre of Finland, Espoo, ${ }^{2}$ Department of Pediatrics, University of Turku, ${ }^{3}$ Department of Pediatrics, University of Tampere, ${ }^{4}$ Molecular Immunology, Turku Centre for Biotechnology, ${ }^{5}$ Department of Virology, University of Turku, ${ }^{6}$ Department of Virology, University of Tampere, ${ }^{7}$ Department of Pediatrics, University of Oulu, Finland.

Background and Aims: To explore etiopathogenesis and prevention possibilities of Type 1 diabetes (T1D), over 8000 children carrying HLA-conferred genetic risk for T1D have been followed since birth at 3- to 12-month intervals for development of T1D-related autoantibodies (ICA, IAA, GADA and IA-2A) and overt T1D in Type 1 Diabetes Prediction and Prevention Study (DIPP), launched in Finland in 1994. We continue screening of over 10,000 newborn babies annually for HLA-conferred T1D risk to enroll those at risk in the study. As changes in groups of metabolites may be descriptive of systemic responses to genetic or environmental exposures, they may function as a powerful tool for characterization of complex phenotypes and as biomarkers for specific physiological responses. We thus hypothesized that extended serum metabolite patterns might differ between children remaining autoantibody negative (controls) and children who later develop autoantibodies and progress to T1D (cases), and that the changes might reflect etiopathogenetic events that by far precede development of autoimmunity. Thus, our aim was to elucidate these early events leading to autoimmunity and overt T1D by analyzing our 11.5-year collection of longitudinal serum sample series from the DIPP case and control children.

Materials and Methods: DIPP serum samples were stored at $-70^{\circ} \mathrm{C}$. The metabolite profiles of the sample series collected from 46 cases (progressed to T1D) were compared with the series collected from 59 controls (remained autoantibody negative) matched for time of birth, gender, genetic T1D risk, and city of birth (total 1234 samples). Ultra Performance Liquid Chromatography coupled to high resolution mass spectrometry (MS) and gas chromatography coupled to mass spectrometry were used. Data were processed with MZmine software, and metabolites identified using in-house databanks and tandem MS.

Results: Metabolite profiles and identified key metabolites separate the cases from the controls much before autoantibodies emerge. The changes reflect alterations in gut permeability, antioxidant capacity and inflammation.

Conclusion: Our data suggest that early metabolic insult(s) may lead to alterations in the immune system making it autoimmunity-prone. The pre-seroconversion changes support the trigger-booster hypothesis claiming that the processes leading to T1D are triggered by an exogenous factor driven by one or several environmental determinants. Our data further suggest that advanced high-throughput metabolomics methods may markedly antedate and improve accuracy of defining which children will later progress to autoimmunity and T1D.

Supported by Tekes FinnWell Program, Diabetes Research Foundation International

\section{4}

Elevated IL-4 levels at birth may predict type 1 diabetes S. Eising ${ }^{1,2}$, K. Skogstrand ${ }^{2,3}$, B. Carstensen ${ }^{1}$, A. Nilsson ${ }^{4}$, A. Lernmark ${ }^{4}$, B. Nørgaard-Pedersen ${ }^{2}$, D. M. Hougaard ${ }^{2}$, F. Pociot ${ }^{1,4}$, J. Nerup ${ }^{1,4}$;

${ }^{1}$ Steno Diabetes Center, Gentofte, Denmark, ${ }^{2}$ Clinical Biochemistry, Statens Serum Institut, Copenhagen, Denmark, ${ }^{3}$ Department of Epidemiology and Social Medicine, NANEA, Aarhus, Denmark, ${ }^{4}$ Clinical Science, CRC, Lund University, Malmø, Sweden.

Background: Reliable prediction is a prerequisite for developing and testing prevention strategies for type 1 diabetes (T1D). Beta cell destruction in T1D pathogenesis is thought to be mediated by an immune response against the beta cells involving pro-inflammatory $\mathrm{T}$ helper cell type 1(Th1) cytokines (IL-1b, IFNg, TNFa) from islet-infiltrating $\mathrm{T}$ cells, Natural Killer (NK) cells and macrophages, whereas anti-inflammatory Th2 cytokines such as IL-4 and IL-10 may protect against T1D. At time of birth, predominantly Th2 immune responses are present in healthy individuals. Th1 levels are increasing during childhood. It is still unknown if cytokine levels differ in newborns who later in life develop T1D as compared to non-diabetic controls. 
Aims: To determine levels of cytokines and other inflammatory markers at time of birth in a large unselected population-based case-control population of newborns using Dried Blood Spots (DBS) at day 5 and test the hypothesis that levels of inflammatory markers at birth predict T1D.

Materials: DBS of 2086 Danish T1D patients from the birth cohorts of 1981-2002, and 2 controls per patient were selected. Patient and control samples were matched by place and date of birth. The samples were stored for 324 years at $-25^{\circ}$ at the Statens Serum Institut, Copenhagen, Denmark.

Methods: Cytokines (IL-1b, IFNg, TNFa, TGFb, IL-4, IL6, IL-8, IL-10, IL-12), MBL, CrP, TREM-1, Adiponectin and Leptin were measured by use of the flowmetric Lumine ${ }^{\circledR}$ xMAP technology. Samples were analyzed simultaneously for all markers. Analysis has been performed and evaluated in the first 2780 samples. Cases and matched controls were run together. GAD65 (GADA) and IA-2 (IA-2A) autoantibodies in combination was measured by standard radiobinding assay on DBS eluates. Conditional logistic regression was used to estimate hazard ratios of diabetes occurrence from the matched case control data.

Results: Proportional Hazard Regression model in patients compared to their matched controls showed a Hazard Ratio (HR) of 1.108 (confidence interval (CI) 1.034-1.186, $\mathrm{p}<0.0035)$ and $1.402(\mathrm{CI} 1.033-1.901, \mathrm{p}<0.03$ ) for every increase with $10 \mathrm{pg} / \mathrm{ml}$ of IL-4 and for every 10 -fold increase of IL-4 $(=\log 10)$, respectively. HR for possibly interacting cytokines was 1.136 (CI 1.024-1.261, $\mathrm{p}<0.017$ ) and 1.299 (CI $1.044-1.616, \mathrm{p}<0.019$ ) for $\log 10$ (IL-4*IL$10)$ and $\log 10$ (IL-4*IL-12), respectively. No differences were detected between cases and controls for any of the other inflammatory markers. In cases positive for GADA, IA-2A, or both, HR for $\log 10$ (IL-4) was 5.475 (CI 2.841$10.55, \mathrm{p}<0.0001$ ), compared to HR=1.366 (CI 1.001-1.863, $\mathrm{p}<0.049$ ) for $\log 10$ (IL-4) in patients compared to controls negative for GADA and IA-2A.

Conclusion: Elevated IL-4 levels at the time of birth may be a prediction marker of T1D diagnosed before 24 years of age, particularly in individuals positive for islet autoantibodies against GAD65, IA-2, or both.

Supported by Danish Medical Research Council, Danish Diabetes Association, P.Carl Petersen Found, EFSD/Pfizer Research Partnership, E.+A.Danielsens Found, Danish Medical Research Found, The Swedish Research Council, Danish Study Group for Childhood Diabetes
0235

Islet antibodies at birth predict development of type 1 diabetes

A. Nilsson ${ }^{1}$, S. Eising ${ }^{2,3}$, D. M. Hougaard ${ }^{3}$, B. NørgaardPedersen $^{3}$, F. Pociot ${ }^{2,1}$, J. Nerup ${ }^{2,1}$, A. Lernmark ${ }^{1}$;

${ }^{1}$ Clinical Science, CRC, Lund University, Malmø, Sweden, ${ }^{2}$ Steno Diabetes Center, Gentofte, Denmark, ${ }^{3}$ Clinical Biochemistry, Statens Serum Institut, Copenhagen, Denmark.

Background: It is still unknown if diabetes-related autoantibodies at the time of birth impact the development of type 1 diabetes (T1D). A reason for current controversies is the lack of sufficiently large investigations at time of birth of subjects who later developed T1D. Despite considerable efforts current prospective studies ascertain only a limited number of new patients per year. Other complicating issues affecting statistical power include transplacental transfer of autoantibodies and their disappearance during the first year of life, and the fact that studies such as BABY DIAB focus on children with first degree relatives with T1D, despite only $15 \%$ of new onset patients belong to this category. Population-based investigations would therefore seem to be a requisite to establish whether islet autoantibodies at birth predict T1D.

Aims: To determine GAD65 and IA-2 autoantibodies at time of birth in a large unselected population-based casecontrol population of newborns using Dried Blood Spot (DBS) collected 5 days after birth. We test the hypothesis that islet autantibodies at birth predict T1D.

Materials: DBS of 2028 Danish T1D patients from the birth cohorts of 1981-2002, and 2 controls per patient were selected. Patient and control samples were matched by place and date of birth. The samples were stored for 324 years at $-25^{\circ}$ at the Statens Serum Institut, Copenhagen, Denmark. Gender, age of gestation, birth weight (BW), birth length (BL), age at clinical diagnosis of T1D, and mothers' potential diabetes diagnoses were known.

Methods: A radiobinding assay was performed on 3.2 diameter DBS punch-out samples, simultaneously detecting autoantibodies to GAD65, IA-2, or both. Autoantibody positive samples (by $99 \%$ percentile) are re-analyzed for IA-2 autoantibodies alone to distinguish the possible role of GAD65 from IA-2 autoantibodies.

Results: In the case group, 70/2028 (3.45\%) were autoantibody positive compared to $21 / 4021(0.52 \%)$ in the control group $(p<0.0001)$. The $99.5 \%$ percentile in the control population was used as a cut-off point. Proportional Hazard Regression Model in cases compared to their 
matched controls showed a Hazard Ratio of 2.54 (confidence limits 2.002-3.242) for every 10-fold increase in relative units (an expression of autoantibody levels) $(p<0.0001)$. Antibody positive compared to negative patients did not differ with respect to age at diabetes diagnosis, birth weight, birth length, or gestational age (determined by regression analysis). Only 2/70 (2.86\%) autoantibody positive cases were born to mothers with known diabetes (T1D). A total of 88/1958 (4.49\%) autoantibody negative cases were born to mothers known to have any form for diabetes $(1.74-2.24 \%$ T1D $(0.5 \%$ not specified), $1.63 \% \mathrm{~T} 2 \mathrm{D}, 0.7 \%$ gestational diabetes).

Conclusion: Detection of islet autoantibodies against GAD65 and IA-2 at the time of birth is a significant prediction marker of T1D diagnosed before 24 years of age. Supported by Danish Medical Research Council, Danish Diabetes Assoc., P.C.Petersen Foundation, EFSD/Pfizer Research Partnership, Danielsens Foundation, Danish Medical Research Foundation, the Swedish Research Council, National Institutes of Health (DK26190), DSBD

0236

The Swedish GAD-vaccination Trial: outcomes of a phase II safety and efficacy trial with Diamyd ${ }^{\text {TM }}$ for preservation of beta cell function in children with T1D J. Ludvigsson ${ }^{1}$, R. Casas ${ }^{1}$, O. Vaarala ${ }^{1}$, G. Forsander ${ }^{2}$, S. A. Ivarsson ${ }^{3}$, C. Johansson ${ }^{4}$, A. Lindh ${ }^{5}$, N. O. Nilsson ${ }^{6}$, J. Åman ${ }^{7}$, E. Örtqvist ${ }^{8}$, J. A. Robertson ${ }^{9}$;

${ }^{1}$ Div. of Pediatrics and Diabetes Research Centre, Dept. of Molecular and Clinical Medicine, Linköping University, ${ }^{2}$ Dept. of Pediatrics, Queen Silvia Pediatric Hospital, Göteborg, ${ }^{3}$ Div. of Pediatrics, University Hospital MAS, Malmö, ${ }^{4}$ Dept. of Pediatrics, Ryhovs Hospital, Jönköping, ${ }^{5}$ Dept. of Pediatrics, Central Hospital, Borås, ${ }^{6}$ Dept. of Pediatrics, Central Hospital, Halmstad, ${ }^{7}$ Dept. of Pediatrics, University Hospital, Örebro, ${ }^{8}$ Astrid Lindgren Pediatric Hospital, Karolinska Institute, Stockholm, ${ }^{9}$ Research \& Development, Diamyd Medical AB, Stockholm, Sweden.

Background and Aims: Numerous studies suggest Glutamic Acid Decarboxylase (GAD65) is a major autoantigen in type 1 diabetes. Evidence has recently been reported for the preservation of beta cell function by vaccination with alum-formulated rhGAD65 (DiamydTM) in adults with slowly progressive autoimmune diabetes (LADA). To investigate if GAD-treatment is safe and can preserve beta cell function in patients with Type 1 diabetes (T1D) which is considered as a more aggressive form of autoimmune diabetes than in LADA - a phase II randomised, double blind, and placebo controlled trial has been conducted in $70 \mathrm{~T} 1 \mathrm{D}$ patients.
Materials and Methods: The study was approved by The Regulatory Authorities in Uppsala, Sweden and by the Ethic's Committee in Linkoping, Sweden. The study was carried out at eight centers in Sweden. Informed consent was granted by patients and their parents.

118 patients between $10-18$ years of age and diagnosed with T1D within 18 months were screened before 70 patients were found to meet the two main inclusion criteria: GADA titer $>23 \mathrm{WHO} \mathrm{U} / \mathrm{mL}$ and a residual fasting Cpeptide level of at least $0.1 \mathrm{nmol} / \mathrm{l}$. Patients were random ised into 2 groups with 35 patients receiving $20 \mathrm{ug}$ DiamydTM at day 1 and 28, and 35 patients assigned to the placebo arm receiving the same formulation without rhGAD65. Patients revisited at 3,6,9 and 15 months at which point metabolic and immunologic parameters were measured. C-peptide levels before and after Sustacal challenge were measured as a surrogate for beta cell function.

Patients are stratified for several parameters including HLA, age, gender, disease duration, GADA, HbA1C and C-peptide levels at inclusion.

Results: One patient developed a serious adverse event after having received one of the two intended injections, and did not receive the second injection. The treatment code was broken showing that the patient had been allocated to the placebo group. No other Serious Adverse Reactions have been reported. Clinical outcomes regarding safety and efficacy, together with the results from analyses of impact of treatment on parameters reflecting immunologic and beta cell function, will be reported from this first clinical trial with DiamydTM in patients with recent-onset T1D.

Conclusion: Treatment of children with recent onset T1D with 20 ug DiamydTM seems safe and feasible. Impact of treatment on parameters reflecting immunologic and beta cell function will be reported.

Supported by Swedish Child Diabetes Foundation (Barndiabetesfonden)

\section{OP 42}

\section{Pancreas and islet transplantation}

0237

Islet-graft response to transplantation injury includes up-regulation of protective as well as apoptotic genes

S. Rodriguez-Mulero, M. Montolio, E. Estilles, N. Téllez, M. Nacher, J. Soler, E. Montanya;

Lab. Diabetes and Experimental Endocrinology, IDIBELL, Hospital Universitari Bellvitge, University of Barcelona, Spain. 
Background and Aims: Around $60 \%$ of pancreatic islet tissue is lost within the first days after transplantation. Islet damage is multifactorial and includes an early non-specific inflammatory response that takes place before rejection or autoimmunity recurrence. We have recently shown that the expression of IL- $1 \beta$, IL- 6 , IL-10 and TNF- $\alpha$ genes is increased early after islet transplantation, suggesting their contribution to the initial graft loss. Here, we investigated the molecular mechanisms involved in beta cell damage and survival after pancreatic islet transplantation. We studied the expression of a set of selected genes involved in apoptosis (bcl-2, bax, bad, bid and C/EBP homologous protein $[\mathrm{CHOP}]$ ), cytokine defense, (suppressor of cytokine signaling [socs]-1 and socs-3) or free radical protection (heme oxygenase-1 [HO-1], $\mathrm{Cu} / \mathrm{Zn}$-superoxide dismutase [SOD], Mn-sod and hsp70). Since hyperglycemia has deleterious effects on islet transplantation outcome, we studied its effect on the expression of these genes.

Materials and Methods: 500 islets were syngeneically transplanted under the kidney capsule of normoglycemic or streptozotocin-induced diabetic Lewis rats. Gene expression was analyzed by quantitative real-time RT-PCR in grafts harvested 1, 3 and 7 days after transplantation, and in freshly isolated islets.

Results: The expression of protective genes socs-1, socs-3, HO-1 and Mn-sod as well as that of pro-apoptotic genes bid and CHOP was maximally increased one day after transplantation, and in most cases it remained increased, although at a lower level, seven days later. In contrast, the expression of bcl-2, bax, bad, $\mathrm{Cu}$-sod and hsp70 genes was unchanged. No differences were found between grafts transplanted to normoglycemic and hyperglycemic recipients. SOCS-1 and SOCS-3 negatively regulate cytokine signaling, and HO-1 expression increases in beta cells exposed to TNF- $\alpha$ or IL-1 $\beta$ contributing to the anti-inflammatory response of islets. Altogether, the increased expression of SOCS-1, SOCS-3 and HO-1 supports the involvement of pro-inflammatory cytokines in the early inflammatory response in islet grafts. The expression of bid may be related to anoikis and to the extrinsic apoptotic pathway, linking membrane death signaling to mitochondria. The expression of mitochondrial antioxidant gene $\mathrm{Mn}$-sod, but not that of cytosolic $\mathrm{Cu}$-sod, was increased suggesting a role of mitochondrial free radicals in islet damage. The increased expression of CHOP gene points to the occurrence of severe ER-stress, which has been recently linked to beta cell death.

Conclusion: Our results suggest an important role of inflammatory cytokines and free radical formation in islet graft damage early after transplantation. Anoikis can also be relevant as suggested by the increased bid expression. ERstress and mitochondrial dysfunction are early events in islet graft dysfunction. In contrast, hyperglycemia may not be a major islet insult in the immediate post-transplantation period. Supported by the Juvenile Diabetes Research Foundation International

0238

Metabolic effect of sirolimus versus mycophenolate mofetil on pancreatic graft function - a prospective randomised study

T. Havrdova, F. Saudek, P. Boucek, K. Lipar,

R. Koznarova, T. Jedinakova, J. Skibova;

Diabetes Center, Inst. Clin. Exp. Medicine, Prague, Czech Republic.

Background: Metabolic effects of immunosuppressive agents are of great importance in pancreas or islet transplantation. The aim of our study was to compare the glucose metabolism in Type 1 diabetic recipients of kidney and pancreatic grafts on tacrolimus-based immunosuppression in conjunction with sirolimus (RAPA) or mycophenolate mofetil (MMF) in a prospective randomised study.

Methods: The investigation was performed in 26 insulinindependent rejection-free patients after simultaneous pancreas and kidney transplantation (with systemic venous drainage of pancreatic graft) at 2 occasions: at $0.5 \pm 0.2$ [mean $\pm \mathrm{SD}$ ] month post-transplant (with steroid dose $12.1 \pm 4.0 \mathrm{mg} /$ day) and subsequently at $24.2 \pm 10.7$ months (steroid-free). All recipients had a good function of the kidney graft (serum creatinine level $114.0 \pm 26.1 \mu \mathrm{mol} / \mathrm{L}$ ). Fasting glycemia, insulin and C-peptide levels, $\mathrm{HbA}_{1 \mathrm{c}}$, standard intravenous glucose tolerance test (IVGTT) with coefficient of glucose assimilation $\left(\mathrm{K}_{\mathrm{G}}\right)$ calculation were assessed in both groups. Insulin sensitivity was evaluated by the homeostasis model assessment (HOMA-IR). Areas under the insulin/C-peptide curves during the IVGTT (AUC-IRI, AUC-CP) were used as the parameters of insulin/C-peptide secretion.

Results: The RAPA and MMF groups did not differ in age, post-transplant period and steroid daily dose. The results (with differences between 1st and 2nd examinations in single groups) are shown in the table. However, we did not find any significant difference between the groups in main parameters. The only difference between RAPA and MMF groups was lower stimulated C-peptide level in the first mentioned one (Examination 2: IVGTT - Minute 6: $1.3 \pm 0.54$ vs. $1.9 \pm 0.78 \mathrm{pmol} / \mathrm{mL}, \mathrm{p}<0.05$ ). Trough levels of tacrolimus (in therapeutic range) had no significant impact on any of examined parameters. The response of IVGTT $\left(\mathrm{K}_{\mathrm{G}}\right)$ and HOMA-IR of whole study group significantly improved 
between the exams $(1.03 \pm 0.43$ vs. $1.4 \pm 0.51, \mathrm{p}<0.01$ and $3.49 \pm 2.39$ vs. $2.47 \pm 1.77, \mathrm{p}<0.05$, respectively).

Conclusion: Glucose tolerance measured with the use of IVGTT significantly improved due to the increased insulin sensitivity. The stimulated insulin production was not changed during our study. Parameters of glucose metabolism were similar in patients treated with sirolimus and mycophenolate mofetil.

\begin{tabular}{|c|c|c|c|c|}
\hline & \multicolumn{2}{|c|}{ RAPA group ( $\mathrm{n}=13$ ) } & \multicolumn{2}{|c|}{ MMF group $(n=13)$} \\
\hline & $\begin{array}{l}\text { Examination } \\
1\end{array}$ & $\begin{array}{l}\text { Examination } \\
2\end{array}$ & $\begin{array}{l}\text { Examination } \\
1\end{array}$ & $\begin{array}{l}\text { Examination } \\
2\end{array}$ \\
\hline BMI & $22.9 \pm 2.3$ & $\begin{array}{l}24.6 \pm 2.5 \\
(p=0.05)\end{array}$ & $23.1 \pm 3.9$ & $\begin{array}{l}24.1 \pm 3.7 \\
\text { NS }\end{array}$ \\
\hline HbA1c (\%) & $6.4 \pm 1.0$ & $\begin{array}{l}5.6 \pm 0.49 \\
(p<0.05)\end{array}$ & $5.8 \pm 0.5$ & $\begin{array}{l}5.5 \pm 0.49 \\
\text { NS }\end{array}$ \\
\hline $\begin{array}{l}\text { Fasting } \\
\text { glycemia } \\
(\mathrm{mmol} / \mathrm{L})\end{array}$ & $5.2 \pm 0.72$ & $\begin{array}{l}4.7 \pm 0.51 \\
(p<0.05)\end{array}$ & $5.4 \pm 0.82$ & $\begin{array}{l}4.68 \pm 0.89 \\
(p=0.01)\end{array}$ \\
\hline $\begin{array}{l}\mathrm{K}_{\mathrm{G}}(\% / \\
\min .)\end{array}$ & $1.03 \pm 0.52$ & $\begin{array}{l}1.26 \pm 0.39 \\
\mathrm{NS}\end{array}$ & $1.02 \pm 0.34$ & $\begin{array}{l}1.55 \pm 0.59 \\
(p=0.01)\end{array}$ \\
\hline $\begin{array}{l}\text { AUC-IRI } \\
(\mathrm{mIU} / \mathrm{L} / \\
60 \mathrm{~min} .)\end{array}$ & $1853 \pm 1042$ & $\begin{array}{l}1944 \pm 950 \\
\text { NS }\end{array}$ & $2555 \pm 1055$ & $\begin{array}{l}2564 \pm 1353 \\
\text { NS }\end{array}$ \\
\hline $\begin{array}{l}\text { AUC-CP } \\
\text { (pmol/mL/ } \\
60 \mathrm{~min} .)\end{array}$ & $140.4 \pm 65.2$ & $\begin{array}{l}90.7 \pm 31 \\
(p<0.01)\end{array}$ & $151.8 \pm 43.1$ & $\begin{array}{l}110.5 \pm 29.6 \\
(p<0.01)\end{array}$ \\
\hline HOMA-IR & $2.88 \pm 1.87$ & $\begin{array}{l}2.3 \pm 1.14 \\
\text { NS }\end{array}$ & $4.11 \pm 2.76$ & $\begin{array}{l}2.65 \pm 2.27 \\
\mathrm{NS}\end{array}$ \\
\hline
\end{tabular}

Supported by: IKEM (MZO 00023001)

0239

Which to prefer: Simultaneous islet-kidney or pancreaskidney transplantation in type 1 diabetes mellitus: A six year single center follow-up

P. A. Gerber ${ }^{1}$, V. Pavlicek ${ }^{1}$, R. Zuellig ${ }^{1}$, T. Pfammatter ${ }^{2}$, R. Wuethrich ${ }^{3}$, M. Weber ${ }^{4}$, G. A. Spinas ${ }^{1}$, N. Demartines ${ }^{4}$, R. Lehmann ${ }^{1}$;

${ }^{1}$ Internal Medicine, Endocrinology and Diabetes,

${ }^{2}$ Radiology, Diagnostic Radiology, ${ }^{3}$ Internal Medicine, Nephrology, ${ }^{4}$ Surgery, Transplantation Surgery, University Hospital Zurich, Switzerland.

Background and Aims: Steroid-free immunosuppression has given hope that islet transplantation alone (ITA) is developing into an alternative to pancreas transplantation in patients with type 1 diabetes mellitus. We compared glycemic control and renal function in recipients of simultaneous islet-kidney (SIK) with recipients of pancreas-kidney (SPK) transplants in our hospital.

Materials and Methods: Glycated hemoglobin, need for exogenous insulin and renal function (creatinine/creatinine clearance) were compared in 13 patients with SIK with a mean of 2.2 islet transplantations and 25 patients with SPK transplanted during the same time period (January 2000 December 2004).

Results: The mean follow-up was 40.5 (12-67) months in both groups. Adverse outcomes: One primary non-function (PNF) of the kidney (retransplanted), one PNF of islets, and 2 incidents of minor liver bleedings in the SIK versus $44 \%$ relaparotomies in the SPK group. The mean HbAlc during follow-up in the SIK group as compared to the SPK group was $6.4 \pm 0.9$ (SD) $\%$ vs. $5.9 \pm 0.5 \%(\mathrm{p}=0.01)$ (pretransplant: $8.4 \pm 1.8 \%$ vs. $8.7 \pm 1.9 \%$ ). 5 out of 7 patients ( $71 \%$ ) with $\geq 2$ islet transplantations were initially off insulin (currently 2 ) as compared to all 25 patients in the SPK group (currently 24). The higher creatinine and lower creatinine clearance in the SIK group $\left(177 \pm 130 \mu \mathrm{mol} / 1\right.$ and $\left.45.5 \pm 20.9 \mathrm{ml} / \mathrm{min} / 1.73 \mathrm{~m}^{2}\right)$ as compared to $109 \pm 30 \mu \mathrm{mol} / 1$ and $65.0 \pm 14.9 \mathrm{ml} / \mathrm{min} /$ $1.73 \mathrm{~m}^{2}(\mathrm{p}<0.01)$ in the SPK group is most likely due to the 15 years higher donor and 13 years higher recipient age.

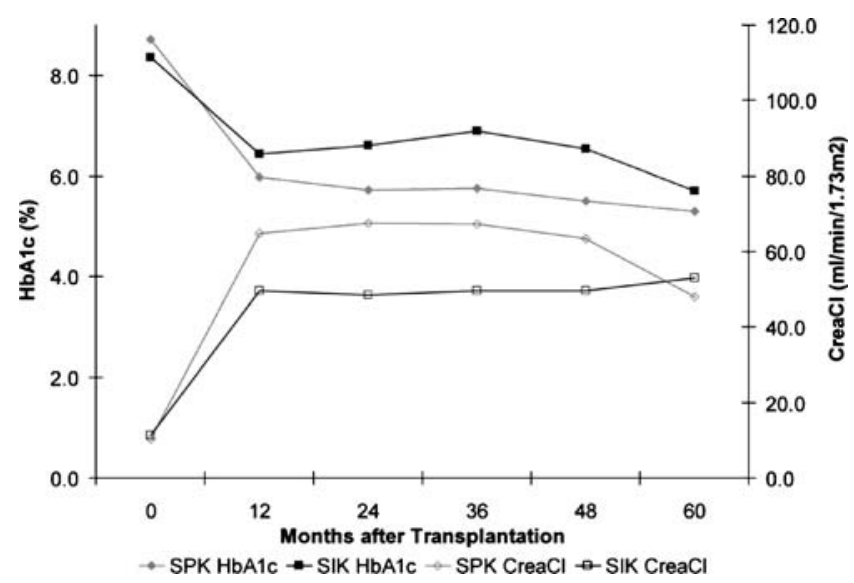

Figure 1. $\mathrm{HbA}_{1 \mathrm{c}}$ and Creatinine Clearance of the SPK and SIK group over 60 months

Conclusion: This series, using about the same number of pancreata for transplantation for SIK or SPK with a mean follow-up of over 3 years indicates that SPK achieves a much higher insulin independence at the cost of more surgical complications, whereas glycemic control is only slightly different in both groups. Residual insulin production combined with optimal insulin therapy seems to be sufficient for maintaining near normal glucose control which is the primary objective of islet transplantation in our institution and not insulin independence alone. 
0240

Pancreas transplant alone (PTA) in type 1 diabetic patients (T1DM): a 3-year follow-up

A. Coppelli, R. Giannarelli, U. Boggi, F. Vistoli,

M. Del Chiaro, A. Tedeschi, A. Piaggesi, F. Mosca,

S. Del Prato, P. Marchetti;

Endocrinology and Metabolism, University of Pisa, Italy.

Background and Aims: Whereas combined pancreas and kidney transplantation is a recommendable procedure in uremic T1DM, the role of PTA in T1DM without advanced kidney disease is still debated. We report our single centre experience on PTA.

Materials and Methods: Between April 2001 and December 2005, 63 T1DM (M/F: 32/31; age: $37 \pm 8$ yrs; body mass index: $24 \pm 3 \mathrm{~kg} / \mathrm{m}^{2}$; duration of diabetes: $23 \pm 10 \mathrm{yrs}$ ) received a PTA, with portal (49 patients, $77.8 \%$ ) or systemic (14 patients, $22.2 \%$ ) endocrine drainage. Indications were hyperlability of diabetes in 9 cases (14.3\%), and progressive chronic diabetes complications in the remaining 54 cases $(85.7 \%)$. Immunosuppression consisted of anti-IL2 receptor antibodies for induction, and MMF, tacrolimus and low dose steroid for maintainance.

Results: Patient survival was $98.2 \%$ at 3 years post-transplant, and graft survival (insulin-independence) was 89.8, 87.2 and $79.8 \%$ at 1,2 , and 3 years, with graft losses due to immunological reasons. Successful PTA determined sustained normoglycemia (at 1,2 and 3 years fasting plasma glucose was $86 \pm 11,86 \pm 12$ and $88 \pm 11 \mathrm{mg} / \mathrm{dl}$; Alc was $5.3 \pm 0.4$, $5.3 \pm 0.4$ and $5.4 \pm 0.4 \%$ ), with fasting C-peptide levels of $2.8 \pm 1.6,2.7 \pm 1.4$ and $2.9 \pm 1.5 \mathrm{ng} / \mathrm{ml}$. Several cardiovascular risk factors (blood pressure, total and LDL-cholesterol, fibrinogen) improved significantly after PTA, and daily proteinuria decreased from $1.3 \pm 2.3$ to $0.4 \pm 0.8 \mathrm{~g} \quad(\mathrm{p}<0.01)$. No new case of diabetic retinopathy (DR) occurred, whereas $82 \%$ of patients with DR showed improvements or stabilization; however, $14 \%$ of subjects with advanced DR had deterioration of retinal status. Neuropathy parameters improved significantly in all tested PTA recipients. The observed improvements in PTA were statistically significant when compared with data from non-transplanted, matched T1DM. No cancer disease occurred in PTA recipients.

Conclusion: In conclusion, PTA in T1DM determines prolonged insulin-independence in the majority of patients, with a favourable (although not yet optimal) benefits/risks ratio; most appropriate timing for the procedure and effects on life expectancy remain major open issue.

\section{OP 43}

\section{Continuous glucose monitoring}

0241

Association between nocturnal hyperglycaemia and severe sleep-disordered breathing in type 2 diabetic patients: a study based on continuous glucose monitoring M. Pallayova ${ }^{1}$, V. Donicova ${ }^{2}$, V. Donic ${ }^{1}$;

${ }^{1}$ Department of Physiology and Sleep Laboratory, Faculty of Medicine, PJ Safarik University, Kosice, ${ }^{2}$ Department of Internal Medicine and Diabetology, Outpatient Clinic, Kosice, Slovakia.

Background and Aims: It has been previously shown that sleep-disordered breathing (SDB) is pathophysiologically related to impaired glucose homeostasis. The aim of this study was to evaluate changes in interstitial glucose levels monitored by continuous glucose monitoring system (CGMS, Medtronic MiniMed) in selected type 2 diabetic patients with or without SDB (verified by polysomnography) and study the possible relationships between nocturnal hyperglycemia and sleep apnea.

Materials and Methods: 30 patients with type 2 diabetes and near-normal blood sugar levels on oral hypoglycemic therapy (22 men, 8 women; mean age \pm SD: $49,23 \pm 4,81$ years, $\mathrm{HbA1c/HPLC}$ method $6,72 \pm 0,73 \%$, duration of diabetes $2,7 \pm 1,44$ years, BMI $32,46 \pm 7,41$ ) were monitored using the CGMS for several days, in conjunction with self-monitoring of blood glucose at least 4 times/day. Patients were divided into two groups according to previous history of SDB. Subjects with severe SDB were referred to sleep laboratory for parallel polysomnographic evaluation of disturbed sleep. We evaluated and compared the CGMS data between both groups. The primary endpoint was the area under the interstitial glucose concentration curve AUC $>_{7,8}(0-480 \mathrm{~min})$ for a 8 -hour period of night $(10 \mathrm{PM}-$ $6 \mathrm{AM})$. The secondary parameters assessed were: nocturnal and daytime CGMS-glucose values, apnea-hypopnea index (AHI), oxygen saturation parameters, HbAlc. Results were presented as mean \pm SD. Statistical analyses included correlation, mean absolute difference (MAD) and Student's t-test.

Results: Patients were each monitored with CGMS for an average of $98,67 \pm 26,24 \mathrm{~h}(\mathrm{r}=0,86 \pm 0,09 ; \mathrm{MAD}=14,37 \pm$ $3,5 \%$ ). The parallel polysomnographic recording in the SDB-group ( $\mathrm{n}=8$; HbA1c 7,36 $\pm 0,45 \%$; AHI 57,64 $\pm 9,64 / \mathrm{h}$; oxygen saturation $82,5 \pm 5,58 \%$; minimal oxygen saturation $49,13 \pm 10,19 \%)$ revealed frequent episodes of sleep apnea/ hypopnea with severe oxygen desaturation followed by a significant rise in blood glucose of up to $12,3 \mathrm{mmol} / \mathrm{l}$. The nocturnal increment in blood glucose expressed as 
AUC $>_{7,8}(0-480 \mathrm{~min})$ was significantly lower for non-SDB group ( $\mathrm{n}=22$; HbA1c $6,49 \pm 0,68 \%$; AHI $<10 / \mathrm{h}$ ) as compared to group of patients with severe $\operatorname{SDB}(0,2 \pm 0,1 \mathrm{mmol} / \mathrm{l}$.night vs. $1,11 \pm 0,2 \mathrm{mmol} / \mathrm{l}$.night; $\mathrm{p}<0,01)$ and strongly correlated with severe oxygen desaturation $(r=0,72, p<0,01)$. CGMS showed mean nocturnal glucose val ues significantly higher in SDB group $(8,24 \pm 0,99 \mathrm{mmol} / 1$ vs. $6,15 \pm 1,16 \mathrm{mmol} / \mathrm{l}$; $\mathrm{p}<0,001)$. The peak of nocturnal glucose levels tended to appear within 1 hour post hypoxia. Mean duration of posthypoxic hyperglycemia was 48,2 $\pm 9,79$ min. Morning fasting glucose level was significantly higher in SDB group $(8,01 \pm 0,19 \mathrm{mmol} / 1$ vs. $6,6 \pm 0,4 \mathrm{mmol} / 1 ; \mathrm{p}<0,05)$. No significant difference in other daytime CGMS-glucose levels was observed between both groups.

Conclusion: The results suggested that sleep apnea may have an immediate elevating effect on nocturnal blood glucose even in patients with near-normal blood sugar level on oral hypoglycemic therapy, but without adequate treatment of severe SDB. These findings indicated that satisfactory treatment of sleep apnea should be considered as an important part of the complex management of diabetic patients with severe SDB in order to prevent postapnoeic nocturnal hyperglycemia and to maintain long-term tight glycemic control.

0242

CGMS reveals critical point in glycaemic excursions requiring therapeutic intervention in people with type 2 diabetics with near-normoglycaemia on long acting sulfonylurea

V. Donicova ${ }^{1}$, M. Pallayova ${ }^{2}$, V. Donic ${ }^{2}$;

${ }^{1}$ Department of Internal Medicine and Diabetology, Outpatient Clinic, Kosice, ${ }^{2}$ Department of Physiology and Sleep Laboratory, Faculty of Medicine, PJ Safarik University, Kosice, Slovakia.

Background and Aims: The greatest postprandial glucose excursion in type 2 diabetes occurs after breakfast. The aim of this study was to examine plasma glucose profiles of people with type 2 diabetes with near-normoglycaemia, using continuous glucose monitoring (CGMS, Medtronic MiniMed) to ascertain the frequency of post-breakfast hyperglycaemia and whether this could be modified by carbohydrate intake.

Materials and Methods:30 people with type 2 diabetes on long-acting sulfonylurea monotherapy (14 men, 16 women; mean age \pm SD: $57.53 \pm 6.63$ years, $\mathrm{HbA1c} / \mathrm{HPLC}$ method $6.68 \pm 0.76 \%$, BMI 29.13 \pm 3.26 ) were divided into two groups according to amount of carbohydrates eaten in breakfast ( $\leq 15 \mathrm{~g} ; \mathrm{n}=15$ and $\geq 30 \mathrm{~g} ; \mathrm{n}=15$ ). CGMS was applied for several days during normal activity along with self-monitoring of blood glucose at least 4 times/day. Patients recorded: times of meals, exercise, and hypoglycaemic drug administration. The primary endpoint was the area under the interstitial glucose concentration curve AUC $>_{7,8(0-120 \mathrm{~min})}$ for breakfast. The secondary parameters assessed were mean CGMS-glucose values for breakfast, lunch and dinner. Results were presented as mean \pm SD. Statistical analyses included correlation, mean absolute difference (MAD) and Student's t-test.

Results: All subjects were each monitored with CGMS for an average of $113.63 \pm 22.85 \mathrm{~h}(\mathrm{r}=0.82 \pm 0.07$; $\mathrm{MAD}=12.82 \pm$ $2.5 \%)$. AUC $>_{7,8}(0-120 \mathrm{~min})$ in group with $\leq 15$ g carbohydrates in breakfast was significantly lower than in the group of $\geq 30 \mathrm{~g}$ carbohydrates $(0.4 \pm 0.3 \mathrm{mmol} / 1 . \mathrm{h}$ vs. $4.34 \pm 1.88 \mathrm{mmol} / \mathrm{l} . \mathrm{h} ; \mathrm{p}<0,001)$. AUC $>_{7,8} \quad(0-120 \mathrm{~min})$ for breakfast strongly correlated with the amount of breakfast carbohydrates $(\mathrm{r}=0.82)$. The mean CGMS-glucose values were: fasting, $7.3 \pm 2.2 \mathrm{mmol} / 1 ; 1.5 \mathrm{~h}$ post-breakfast, $11.6 \pm 2.27 \mathrm{mmol} / \mathrm{l}$; pre-lunch, $6.3 \pm 2.59 \mathrm{mmol} / \mathrm{l}$; $1.5 \mathrm{~h}$ postlunch, $8.03 \pm 2.89 \mathrm{mmol} / \mathrm{l}$; pre-dinner, $6.4 \pm 2.1 \mathrm{mmol} / \mathrm{l} ; 1.5 \mathrm{~h}$ post-dinner, $8.1 \pm 2.3 \mathrm{mmol} / 1$. Despite the comparable carbohydrates intake during the day, the highest peak of post-meal hyperglycemia occurred after breakfast.

Conclusion: The CGMS results demonstrated that postbreakfast hyperglycemia appears to be most important time for glycaemic control even in patients with near-normoglycaemia. From the results of this study, $15 \mathrm{G}$ of carbohydrate appears to be the maximum amount in breakfast to prevent an excessive postprandial hyperglycaemic excursions in most people with 2 diabetes.

\section{3}

Glycaemic control improvement with the weekly use of the Guardian $^{\text {RT }}$ together with a wireless assistant for asynchronic remote advice in pump treated type 1 diabetic patients

M. Rigla ${ }^{1}$, M. Hernando ${ }^{2}$, E. J. Gómez ${ }^{2}$, E. Brugués ${ }^{1}$, V. Torralba ${ }^{2}$, G. García-Sáez ${ }^{2}$, R. Cabezas ${ }^{1}$, A. de Leiva ${ }^{1}$; ${ }^{1}$ Endocrinology, Hospital de Sant Pau, Barcelona, ${ }^{2}$ Bioengineering and Telemedicine, Universidad Politécnica, Madrid, Spain.

Background and Aims: The s.c. continuous glucose monitoring system Guardian ${ }^{\mathrm{RT}}$ allows the patient to have real time access to the interstitial glucose data. The PARIS_Diabtel system provides physicians and patients of 24 hours telemonitoring and telecare services based on web 
applications and a wireless personal assistant (PA). We sought to evaluate, in terms of metabolic control, the use of the Guardian ${ }^{\mathrm{RT}}$ in addition to the PARIS_Diabtel system in a randomized cross-over clinical.

Materials and Methods: Ten type 1 diabetic patients (5 women, age 40.6 (21-62), diabetes duration 14.7 (3-52) years) treated with insulin pumps for more than a year, were included. Along the study, patients used the PARIS_Diabtel system for glucose and insulin data transmission to the diabetes center, analysis by the physician who advised modifications of the basal rate and boli. During the 4-weeks intervention phase, patients used 3 days a week the Guardian $^{\mathrm{RT}}$. At the end of the control phase a 3-day glucose monitoring (MiniMed CGMS).

Results: After the intervention phase, HbAlc decreased significantly $(8.1 \pm 1.1$ vs. $7.3 \pm 0.8 ; \mathrm{p}=0.01)$ while no significant changes were observed during the control phase. Data regarding the use of the system (mean number of daily glucose values and boli sent by patients, PA functionalities: data visualization, data download from the insulin pump and synchronization with the Telemedicine Server as well data of the therapy changes advised and performed by patients will be presented. We are now analysing the results of the s.c. glucose monitoring performed in both study phases using the poincare plot described by Kovatchev B. et al.

Conclusion: The Guardian ${ }^{\mathrm{RT}}$ together with the PARIS Diabtel system, a telemedicine system that includes a wireless personal assistant for remote treatment advising, improves glycemic control in pump treated type 1 diabetic patients

Supported by FIS 04/2372

\section{4}

Improvement in glucose excursions using a seven-day continuous glucose sensor: managing the extremes L. Jovanovic ${ }^{1}$, S. K. Garg ${ }^{2}$;

${ }^{1}$ Research, Sansum Diabetes Research Institute, Santa Barbara, ${ }^{2}$ Barbara Davis Center for Childhood Diabetes, University of Colorado at Denver and Aurora, United States.

Background and Aims: Hypoglycaemia and wide glucose excursions continue to be major obstacles to achieving target $\mathrm{HbAlc}$ values and the associated reductions in longterm complications (and economic costs) in people with insulin-treated diabetes. In this study we evaluated the accuracy, safety, and clinical effectiveness of a continuous glucose-sensing device.
Materials and Methods: Baseline $\mathrm{HbAlc}$ was measured within 30 days of enrollment of 86 patients with insulinrequiring diabetes who participated in this trial. Patients inserted and wore a transcutaneous glucose sensor (STS System, DexCom, Inc.) for 21 days in three 7-day periods during normal activities. Sensors were BLINDED during period 1. During periods 2 and 3 (DISPLAY), glucose values, trend graphs, and high/low alerts and alarms were provided.

Results: Of 6357 paired sensor-SMBG points prospectively analyzed, mean absolute relative difference was $15.7 \%$. Patients with $\mathrm{HbAlc}>9 \%$ spent $94.6 \%$ (median) more time in euglycaemia $(81-140 \mathrm{mg} / \mathrm{dL})$ during the unblinded period without an increase in hypoglycaemia (Table 1A). Patients with $\mathrm{HbA} 1 \mathrm{c} \leq 7 \%$ maintained euglycaemia as in the blinded state but spent $46.4 \%$ (median) less time below $55 \mathrm{mg} / \mathrm{dL}$ during DISPLAY use (Table 1B).

Conclusion: We conclude that 7-day use of the STS System was safe, accurate, and stable. Moreover, patients with poorly controlled diabetes can use the added information from a continuous sensor to significantly improve glycaemic profiles without increased risk of hypoglycaemia. The well-controlled patient can also maintain their glucose control while using a continuous sensor, but have a reduced incidence of hypoglycaemia.

Patients with A1c $<7 \%$

\begin{tabular}{lll}
\hline $\begin{array}{l}\text { Glucose Range } \\
(\mathbf{m g} / \mathbf{d L})\end{array}$ & $\begin{array}{l}\text { Median Percent Change } \\
\text { in Time Spent } \\
\text { (BLINDED vs. DISPLAY) }\end{array}$ & p-value \\
\hline$<55$ & $-\mathbf{4 6 . 4 \%}$ & $\mathbf{0 . 0 3 1 6}$ \\
$55-80$ & $-14.5 \%$ & 0.1046 \\
$81-140$ & $18.8 \%$ & 0.1705 \\
$141-240$ & $-8.5 \%$ & 0.6099 \\
$241-400$ & $-14.2 \%$ & 0.7243 \\
\hline
\end{tabular}

Patients with A1c $>9 \%$

\begin{tabular}{lcc}
\hline $\begin{array}{l}\text { Glucose Range } \\
(\mathbf{m g} / \mathbf{d L})\end{array}$ & $\begin{array}{l}\text { Median Percent Change } \\
\text { in Time Spent } \\
\text { (BLINDED vs DISPLAY) }\end{array}$ & p-value \\
\hline$<55$ & $-31.1 \%$ & 0.4238 \\
$55-80$ & $27.4 \%$ & 0.4697 \\
$81-140$ & $\mathbf{9 4 . 6 \%}$ & $\mathbf{0 . 0 0 6 8}$ \\
$141-240$ & $0.8 \%$ & 0.4697 \\
$241-400$ & $-36.4 \%$ & 0.6772 \\
\hline
\end{tabular}

Supported by DexCom, Inc. 


\section{OP 44}

\section{Transcriptional regulation of beta cell function}

0245

Construction of a pancreatic beta-cell specific gene atlas B. Kutlu ${ }^{1}$, D. Burdick ${ }^{1}$, E. Helton ${ }^{1}$, P. Pirot ${ }^{2}$, D. Eizirik ${ }^{2}$, N. Welsh ${ }^{3}$, N. Goodman';

${ }^{1}$ JDRF Center for Bioinformatics, Institute for Systems Biology, Seattle, United States, ${ }^{2}$ Laboratory of Experimental Medicine, Universite Libre de Bruxelles, Belgium, ${ }^{3}$ Department of Medical Cell Biology, Uppsala University, Sweden.

Background: Understanding and exploring the basal gene expression in a given tissue provides new insights in the functioning of genes under normal and disease conditions. Genes that are specifically or highly expressed in pancreatic islets are candidates for biomarkers which can potentially serve as an indicator of physiological or disease processes in diabetes.

Material and Methods: Massively Parallel Signature Sequencing (MPSS) is a sequencing-based technology to detect and quantify the expression of almost all known genes by counting the number of short sequence signatures. To explore the transcriptome of human pancreatic islets and prepare a comprehensive inventory of insulin-producing cell gene expression, we performed MPSS analysis of two human untreated pancreatic islet samples. A total of $1,051,000$ and 1,313,239 signatures were sequenced for samples 1 and 2 , respectively. The abundance of each signature was represented as transcripts per million (tpm). With the aim to determine genes that are restricted to $\beta$ cells, gene expression profiles of FACS-purified primary $\beta$ cells and INS cell lines were detected by Affymetrix Rat230 2 chip. The secreted and transmembrane proteins were predicted using SecretomeP 3.0 and TMHMM 2.0, respectively.

Results: After filtering the bad quality signatures with hits to multiple Unigene clusters and applying a stringent cut-off threshold of 10 tpm, we identified 3869 shared unique genes in both samples and 6939 genes in one or both human pancreatic islet samples. MPSS method is particularly sensitive in determining low abundance transcripts that microarray platforms fail to detect. Comparison of MPPS results with published microarray datasets performed with untreated human pancreatic islets identified 1036 genes that could be detected by MPSS analysis alone. The majority (736) of these genes were low abundant transcripts with counts lower than $30 \mathrm{tpm}$. We subsequently compared islet MPSS results to a published MPSS dataset sampling a collection of 31 normal human tissues. This analysis revealed
848 unique genes that are either specific to or highly enriched in human islets. This list contains 114 putative transmembrane proteins of which 44 are highly expressed in primary $\beta$ cells. Similarly, the list of islet-enriched genes contains 228 classic (signal peptide-triggered) or non-classical (lacking a signal peptide) secreted proteins that are also expressed strongly in primary $\beta$ cells. In order to complement our MPSS data, we collected and analyzed almost all published microarray gene expression studies from endocrine and exocrine pancreas from different species. We have constructed a database of pancreas gene expression atlas and will make it publicly available via, T1DBase (http://t1dbase.org), a T1DM-specific bioinformatics resource.

Conclusion: Our pancreas gene atlas provides interesting insights on the gene expression landscape of pancreatic $\beta$ cells, and is a valuable source for detailed information on the gene expression distribution in beta cells and pancreatic islets. Moreover, the list of pancreatic islet enriched genes contains proteins that are secreted or reside in plasma membrane and have the potential to serve as $\beta$ cell specific markers.

Supported by the Juvenile Diabetes Research Foundation

0246

Global analysis of the transcriptional role of $\mathrm{HNF}-1 \alpha$ in pancreatic islets

J.-M. Servitja ${ }^{1}$, M. Garrido ${ }^{1}$, M. Pignatelli ${ }^{2}$, M. A. Maestro ${ }^{1}$, R. Guigo ${ }^{2}$, J. Ferrer ${ }^{1}$;

${ }^{1}$ Hospital Clinic de Barcelona-Endocrinology, IDIBAPS, ${ }^{2}$ Grup de Recerca en Informatica Biomedica, IMIM-UPFCRG, Barcelona, Spain.

Background and Aims: The most common form of monogenic diabetes (MODY 3) is caused by mutations in the gene encoding HNF-1 $\alpha$ (also known as TCF1). Lack of HNF-1 $\alpha$ has a dramatic effect on beta-cell function, which indicates that HNF-1 $\alpha$ targets play key functional roles in the beta cell. Despite knowledge of several genes that are regulated by HNF-1 $\alpha$ in mouse islets, a complete picture of the role of HNF-1 $\alpha$ in beta cells remains elusive. We have combined genome-wide expression profiling and transcription factor binding to address the role of HNF-1 $\alpha$ as a regulator of a beta-cell transcriptional programme.

Materials and Methods: Expression profiles of cultured islets from control and Hnfl $\alpha-/-$ mice were analyzed with Affymetrix chips. Statistical analysis of the data was carried out with SAM, and genes exhibiting differential regulation at a $5 \%$ false discovery rate were selected. HNF-1 $\alpha$ binding to targets genes was analyzed by chromatin immunoprecipitation combined with promoter microarrays from the Beta Cell Biology Consortium and custom arrays produced 
by Agilent. High affinity, evolutionary conserved HNF1 consensus cis elements were identified after aligning the mouse, human, dog, and chicken genomic sequences.

Results: HNF- $1 \alpha$ plays an unexpectedly wide-ranging role in regulating gene expression in the pancreatic islets, with significantly increased or reduced expression of over $15 \%$ of active genes. To distinguish between direct and indirect effects of HNF-1 $\alpha$, we carried out a computational analysis of evolutionary conserved HNF1 sites in the mouse genome and chromatin immunoprecipitation/microarray analysis of HNF-1 $\alpha$-bound chromatin. This revealed that HNF-1 $\alpha$ primarily acts as an essential activator in a substantial number of genes, but is unlikely to carry out direct repressor functions in islets. It also showed that HNF-1a directly regulates a disporportionate number of genes regulating specific biological processes, most notably genes encoding secreted regulatory proteases, transcriptional regulators, and regulators of amino acid and glucose metabolism. Interestingly, this study also uncovered an altered expression of genes involved in important signaling events for the beta cell. Genes that exhibit full dependence on HNF-1 $\alpha$ were more likely to be direct targets. In contrast, indirect targets are functionally pleiotropic and exhibit only moderate dependence on HNF-1 $\alpha$.

Conclusion: These findings provide a framework to understand the structure of a key beta-cell genetic programme, and suggest unanticipated biological insights into the role of the MODY3 gene in the beta cell.

Supported by GEN2001-14478-C05-02 (MCyT, Spain) and JDRF 1-2002-21

\section{7}

SREBP-1c antagonises PDX1 functions through the direct interaction and suppresses PDX1 expression M. Amemiya-Kudo ${ }^{1}$, J. Oka ${ }^{1}$, N. Yahagi ${ }^{2}$, J.-I. Osuga ${ }^{2}$, N. Yamada ${ }^{3}$, T. Murase $^{1}$, H. Shimano ${ }^{3}$;

${ }^{1}$ Laboratory of Lipid Research, Okinaka Memorial Institute for Medical Research, Tokyo, ${ }^{2}$ Department of Diabetes and Metabolic Diseases, University of Tokyo, ${ }^{3}$ Department of Internal Medicine, University of Tsukuba, Tsukuba Ibaraki, Japan.

Background and Aims: Overexpression of SREBP-1c in $\beta$-cells caused impaired glucose-stimulated insulin secretion associated with diminished PDX1 expression in vitro and in vivo, suggesting that a potential role of SREBP-1c in the lipotoxicity could be mediated through direct suppression of PDX1. The aim of this study is to clarify the molecular mechanism of PDX1 repression by SREBP-1c.
Materials and Methods: Promoter analysis for a mouse PDX1 gene $(2.7 \mathrm{~kb})$ was performed in $\beta$-cells (HIT) and non- $\beta$-cells (HepG2) with a luciferase reporter gene system. The direct interaction of PDX1 and SREBP-1c was estimated by GST pull-down and EMSA assays. Gene expression was investigated by Northern blot analysis in $\beta$-cells (INS1) after adenovirus-mediated overexpression of SREBP-1c and GFP as a control.

Results: In luciferase assays, the promoter activity of the mouse PDX1 gene was not affected by co-expression of nuclear SREBP-1c in HepG2 cells, but strongly suppressed in HIT cells. PDX1 itself activated the promoter, but SREBP-1c completely antagonized PDX1 feedforwardloop activation in both cells. To investigate the inhibitory mechanism by SREBP-1c, a series of deleted PDX1 promoter activities by SREBP-1c were examined. The cis element for SREBP-1c was not identified on the promoter. Next, protein-protein interaction was examined by GST pull-down assay. SREBP-1c directly interacted with PDX1. The PDX1 cis element was previously reported in the distal enhancer region (Area I). EMSA with radiolabeled probe of Area I demonstrated that SREBP-1c dose-dependently reduced PDX-1 binding ability to the Area I, whereas SREBP-1c did not bind to this region, excluding the possibility that SREBP-1c might suppress PDX1 promoter through their direct binding. Adenovirus mediated SREBP1c overexpression in INS1 cells suppressed both PDX1 and insulin mRNA levels.

Conclusion: These results demonstrate that SREBP-1c antagonizes PDX1 functions through protein-protein interaction and reduced PDX1 expression potentially leading to defects in insulin secretion and $\beta$-cell mass as a potential role of SREBP-1c in $\beta$-cell lipotoxicity.

Supported by Grants-in-aid from the Ministry of Science, Education, Culture, and Technology of Japan

0248

PTB1 binds to the 5'-UTR of mRNAs encoding proteins of the insulin secretory granules

K.-P. Knoch ${ }^{1}$, H. Schneider ${ }^{1}$, F. Ehehalt ${ }^{2}$, C. Wegbrod ${ }^{1}$, A. Altkrüger ${ }^{1}$, M. Solimena ${ }^{1}$;

${ }^{1}$ Experimental Diabetology, ${ }^{2}$ Department of Visceral-, Thoracic- and Vascular Surgery, Medical Faculty "Carl Gustav Carus", Dresden, Germany.

Background and Aims: The biosynthesis of insulin secretory granules (SG) is regulated by glucose. Previously we have shown that in rat islets and cultured insulinoma INS-1 cells glucose and glucagon-like peptide 1 (GLP-1) 
rapidly increase the expression of various SG proteins through the activation of a posttranscriptional pathway involving polypyrimidine tract-binding protein (PTB1). Upon its glucose-stimulated nucleocytoplasmic translocation, PTB1 binds to the $3^{\prime}$-untranslated regions (UTR) of SG mRNAs, including ICA512, PC1/3 and PC2, thereby leading to their stabilization and increased translation. Especially in view of its role in translation, we investigated here whether PTB1 can also directly bind to the $5^{\prime}$-UTR of ICA512, PC1/3 and PC2 mRNAs, and whether such binding is regulated by glucose.

Material and Methods: The 5'-UTR of rat ICA512 was extended beyond its previously known boundaries by 5'-RACE. The 5'-UTRs of rat ICA512, PC1/3 and PC2 were biotinylated in vitro and incubated with cytosolic extracts from INS-1 cells non-stimulated ( $0 \mathrm{mM}$ glucose $)$ or stimulated ( $25 \mathrm{mM}$ glucose) for $2 \mathrm{hrs}$. After RNA pulldown with streptavidin-magnetobeads, binding of PTB1 was analysed by immunoblot. The same 5'-UTRs have been cloned upstream of the firefly luciferase cDNA in the pGL3 vector (Promega) and co-transfected with renilla luciferase in INS-1 cells to assess the ability of these $5^{\prime}$-UTRs to enhance luciferase activity in response to glucose.

Results: Bioinformatic analyses indicated the presence of a canonic consensus sequence for PTB1 binding (-CYYYY CYYYYYG-) in the $5^{\prime}-$ UTRs of PC1/3 and PC2, but not in the $5^{\prime}$-UTR of rat ICA512. The latter only includes a short pyrimidine-rich sequence. Nevertheless we found that PTB1 binds also to the 5'-UTR of ICA512. The recovery of PTB1 was $\sim 3$ fold higher when each of the three biotinylated 5 -UTRs was incubated with cytosolic extracts from glucose stimulated INS-1 cells. Inclusion of the PC2 5'-UTR enhanced firefly luciferase activity by $\sim 15$ fold in resting INS-1 cells and $\sim 56$ fold upon glucose stimulation compared to pGL3 alone. These increments were abolished by mutation of the PTB1 binding site in the PC2 $5^{\prime}$-UTR. The impact of the ICA512 and PC1 $5^{\prime}$-UTRs on luciferase expression is currently under investigation.

Conclusions: Our studies provide evidence that in response to glucose stimulation PTB1 promotes not only the stability but also the translation of several mRNAs encoding SG proteins by binding to their $5^{\prime}$-UTRs. These findings are consistent with the ability of PTB1 to enhance the translation of viral mRNAs by binding to motifs in their 5'-UTRs.

Supported by GDRF research grant

\section{OP 45}

\section{Retinopathy - clinical}

\section{9}

Effect of ruboxistaurin on diabetic macular edema and visual loss: integrated analysis of the PKC-DRS and PKC-DRS2

P. M. Dodson ${ }^{1}$, L. P. Aiello ${ }^{2}$, L. Vignati ${ }^{3}$, M. J. Sheetz ${ }^{3}$, X. Zhi ${ }^{3}$, A. Girach ${ }^{4}$, M. D. Davis ${ }^{5}$, R. C. Milton ${ }^{6}$;

${ }^{1}$ Diabetes Centre, Birmingham Heartlands Hospital, Birmingham, United Kingdom, ${ }^{2}$ Department of Ophthalmology, Joslin Diabetes Center, Harvard University Medical School, Boston, United States, ${ }^{3}$ Eli Lilly and Company, Indianapolis, United States, ${ }^{4}$ Eli Lilly and Company, Windlesham, United Kingdom, ${ }^{5}$ Department of Ophthalmology and Visual Sciences, University of Wisconsin, Madison, United States, ${ }^{6}$ AREDS Coordinating Center, EMMES Corporation, Rockville, United States.

Background and Aims: Persons with advanced nonproliferative diabetic retinopathy (NPDR) are at increased risk of vision loss. The PKC-DRS, a multidose trial in 252 patients, evaluated whether ruboxistaurin (RBX), an oral protein kinase $C \beta$ inhibitor, slowed progression of NPDR to proliferative diabetic retinopathy. No effect was seen for this outcome, but RBX did reduce the 3-year occurrence of sustained moderate visual loss (SMVL; a $\geq 15$-letter loss on the ETDRS chart sustained for the last 6 months of study participation). The PKC-DRS2 evaluated these outcomes in 685 patients at the dose with greatest effect in the PKCDRS. We report data for placebo $(n=401)$ and $32 \mathrm{mg} / \mathrm{d}$ RBX ( $n=412)$ groups combined from both trials.

Material and Methods: Both the PKC-DRS and PKCDRS2 were 3-year, multi-center, randomized, placebocontrolled, double-masked, phase 3 trials. Patients had a best-corrected ETDRS visual acuity (VA) $\geq 45$ letters (approximately $20 / 125$ Snellen $=6 / 40=0.16$, or better), moderately severe to very severe NPDR (ETDRS retinopathy level $\geq 47 \mathrm{~A} / 47 \mathrm{~B}$ and $\leq 53 \mathrm{E}$ ), and no prior panretinal photocoagulation in at least one eye. There were no restrictions on the presence of diabetic macular edema (DME) or prior focal/grid photocoagulation at baseline.

Results: For the combined population, mean $\mathrm{HbAlc}$ was $8.2 \%$ and mean systolic and diastolic blood pressures were 138 and $78 \mathrm{~mm} \mathrm{Hg}$ at baseline. SMVL occurred in 10.2\% of placebo vs. $6.1 \%$ of RBX patients $(41 \%$ risk reduction, $\mathrm{P}=0.011$ ). A significant between-group difference in mean VA change from baseline was observed starting at 
12 months of therapy, and continued through the duration of the studies. $\mathrm{A} \geq 15$-letter gain of VA occurred in $2.4 \%$ of placebo vs. $4.7 \%$ of $\mathrm{RBX}$ eyes $(\mathrm{P}=0.021)$, and $\mathrm{a} \geq 15$-letter loss of VA occurred in $11.4 \%$ of placebo vs. $7.4 \%$ of RBX eyes $(\mathrm{P}=0.012)$. Of placebo and $\mathrm{RBX}$ eyes with clinically significant macular edema (CSME) $>100 \mu \mathrm{m}$ from the center of the macula at baseline, $66.9 \%$ vs. $50.0 \%$, respectively, progressed to having CSME $\leq 100 \mu \mathrm{m}$ from the center of the macula ( $\mathrm{P}=0.002)$.

Conclusion: Combined analyses of these two similarly designed trials demonstrated that, compared with placebo, RBX reduced the occurrence of vision loss, reduced the progression of DME to the center of the macula, and increased the chance of VA improvement in patients with moderately severe to very severe NPDR.

Supported by Lilly Research Laboratories

0250

Proteomic analysis of human vitreous fluid by DIGE: a new strategy for identifying potential candidates in the pathogenesis of proliferative diabetic retinopathy

M. García-Ramírez ${ }^{1}$, F. Canals ${ }^{2}$, C. Hernández ${ }^{1}$, N. Colomé ${ }^{2}$, C. Ferrer $^{2}$, E. Carrasco ${ }^{1}$, J. García-Arumí ${ }^{3}$, R. Simó ${ }^{1}$;

${ }^{1}$ Diabetes Research Unit, ${ }^{2}$ Proteomics Laboratory, Institut de Recerca Hospital Universitari Vall d'Hebron, Barcelona, ${ }^{3}$ Ophthalmology Department, Hospital Universitari Vall d'Hebron, Barcelona, Spain.

Background and Aims: Vitreous fluid analysis has been used as a surrogate of the metabolic events that are taking place in the retina in vivo. However, the volume of vitreous fluid obtained after vitrectomy is about $1 \mathrm{ml}$ and, therefore, only a few peptides can be analyzed simultaneously. The recent development of proteome analysis has made it feasible to compare protein profiles in body fluids with only a small sample and, therefore it could be a useful tool in exploring new candidates potentially involved in the pathogenesis of diabetic retinopathy. Fluorescence-based Difference Gel Electrophoresis (DIGE), a method that permit us to perform an accurate quantitative comparison, has not been previously used in the proteomic analysis of the vitreous fluid in the setting of diabetic eye disease. The aim of the study was to compare for the first time the protein profiles of human vitreous from diabetic patients with proliferative diabetic retinopathy (PDR) with those obtained from non-diabetic patients with idiopathic macular holes (MH) by using DIGE strategy.

Materials and Methods: Vitreous humors from type 1 diabetic patients with PDR $(\mathrm{n}=8)$ and from non-diabetic patients with $\mathrm{MH}(\mathrm{n}=10)$ closely matched by age were included. Vitreous hemorrhage was ruled out by measuring intravitreal hemoglobin. DIGE strategy was used to carry out the comparative proteomic analysis. In order to improve the coverage of the proteins present in the vitreous we have used an affinity chromatographic separation to deplete the highest abundant proteins (ie. IgG and albumin) before their electrophoretic separation. Differentially expressed proteins (abundance ratio $>1.4$ fold; $\mathrm{p}<0.05$ ) were identified by mass spectrometric analysis.

Results: 8 proteins were highly expressed in PDR patients in comparison with non diabetic subjects (Zinc- $\alpha$-2-glycoprotein -ZAG-, Apo A1, Apo H, Fibrinogen A, and the following complement factors: C3, C4b, C9 and Factor B). By contrast, we found 3 proteins underexpressed in PDR subjects (Pigment epithelial derived factor -PEDF-, Interstitial retinol-binding protein -IRBP-, and Inter-alpha tripsin inhibitor heavy chain -ITIH2-). There was no overlap in the vitreous levels of the above-mentioned proteins between PDR patients and non-diabetic controls. The different expression of ZAG, C3, Factor B, PEDF and IRBP was further confirmed by Western blot.

Conclusion: Proteomic analysis by DIGE, which permits an accurate quantitative comparison, was useful in identifying new potential candidates involved in the pathogenesis of PDR.

Ssupported by grants from Novo Nordisk Pharma S.A., the Instituto de Salud Carlos III and the Ministerio de Ciencia y Tecnología

0251

Deficit of somatostatin expression in diabetic human retina: an early event in the pathogenesis of diabetic retinopathy

E. Carrasco ${ }^{1}$, C. Hernández ${ }^{1}$, A. Miralles ${ }^{2}$, J. Mesa $^{1}$, J. Farrés ${ }^{3}$, R. Simó ${ }^{1}$;

${ }^{1}$ Diabetes Research Unit, Institut de Recerca Hospital Universitari Vall d'Hebron, Barcelona, ${ }^{2}$ Tissue Bank and Cell Therapy Center, Hospital Universitari Vall d'Hebron, Barcelona, ${ }^{3}$ Biochemistry Department, Universitat Autònoma de Barcelona, Spain.

Background and Aims: Somatostatin (SST) and its receptors are found in the neuroretina of various species, including humans, and there is growing experimental evidence that in the retina SST acts as both a neuromodulator and antiangiogenic factor. In fact, we have previously shown that SST exists in abundant quantities in the human vitreous fluid, thus supporting the concept that it is essential in retinal homeostais. In addition, lower intravitreous SST levels were found in patients with proliferative diabetic retinopathy than in nondiabetic subjects. 
Although vitreous fluid analysis can be considered as a surrogate of the events that are taking place in the retina, studies addressed to confirm the deficit of SST production by the retina of diabetic patients have not been previously reported. The aim of the present study is to compare mRNA SST in the retina from diabetic and nondiabetic donors. In addition, the differential SST expression between neuroretina and retinal pigment epithelium (RPE) was also explored for the first time.

Materials and Methods: Human postmortem eyes $(n=16)$ were obtained from 4 diabetic donors without retinopathy and 4 non diabetic donors age-matched. The time elapsed from death to eye enucleation was $3.8 \pm 1.4 \mathrm{~h}$. SST mRNA expression was determined by semi-quantitative RT-PCR. To evaluate the quantitative expression of the SST mRNA a Quantitative RT-PCR was performed using specific primers (TaqMan ${ }^{\circledR}$ pre-made gene expression assay Hs00174949m1, Applied Biosystems) in an ABI Prism 7000 Sequence Detection System (PE Applied Biosystems).

Results: A significant higher expression of SST mRNA in the RPE than in the neuroretina in retinas from nondiabetic donors was detected $(0.98 \pm 0.02$ vs. $0.38 \pm 0.24 ; \mathrm{p}<0.001)$. In retinas from diabetic donors, SST mRNA expression was also higher in the RPE than in the neuroretina $(0.44 \pm 0.30$ vs. 0.11 $\pm 0.09 ; \mathrm{p}=0.018$ ). Finally, SST mRNA levels were lower in both RPE and neuroretina from diabetic donors in comparison with non-diabetic donors $(0.44 \pm 0.30 \mathrm{vs}$. $0.98 \pm 0.02 ; \mathrm{p}=0.001$ and $0.11 \pm 0.09$ vs. $0.38 \pm 0.24$; $\mathrm{p}=0.018$, respectively).

Conclusion: 1) Apart from neuroretina, RPE is a significant contributor to intraocular SST production. 2) A significant deficit of SST mRNA expression were found in both neuroretina and RPE from diabetic donors. 3) Since diabetic donors were free of clinically detectable microvascular changes, SST deficit can be considered as an early event in the pathogenesis of diabetic retinopathy. Finally, our results suggest that intravitreous SST delivery would be considered a more rational approach than systemic administration to prevent or arrest diabetic retinopathy.

Supported by grants from Novartis Pharma S.A., Novo Nordisk Pharma S.A, the Instituto de Salud Carlos III and the Ministerio de Ciencia y Tecnología

\section{2}

Erythropoietin is expressed in human retina and it is highly elevated in the vitreous fluid of patients with diabetic macular edema

R. Simó ${ }^{1}$, M. García-Ramírez ${ }^{1}$, A. Fonollosa ${ }^{2}$, M. Higuera ${ }^{1}$, R. Catalán ${ }^{3}$, A. Miralles ${ }^{4}$, J. García-Arumí ${ }^{2}$, C. Hernández ${ }^{1}$;
${ }^{1}$ Diabetes Research Unit, Institut de Recerca Hospital Universitari Vall d'Hebron, Barcelona, ${ }^{2}$ Ophthalmology Department, ${ }^{3}$ Biochemistry Department, ${ }^{4}$ Tissue Bank and Cell Therapy Center, Hospital Universitari Vall d'Hebron, Barcelona, Spain.

Background and Aims: Erythropoietin (Epo) has been recently found increased in the vitreous fluid from ischemic retinal diseases such as proliferative diabetic retinopathy (PDR). The aims of the present study were: 1) To measure Epo levels in the vitreous fluid from patients with diabetic macular edema (DME), a condition in which the ischemia is not a predominant event. 2) To compare Epo mRNA expression between human retinas from non-diabetic and diabetic donors without retinopathy.

Materials and Methods: Vitreous from 12 type 2 diabetic patients with DME without significant retinal ischemia and 12 PDR patients were prospectively analysed. Vitreous from 10 non-diabetic patients with macular holes served as the control group. Epo was assessed by RIA (mU/ml). Epo m-RNA expression was measured by quantitative real-time RT-PCR analysis in the retina from 8 non-diabetic and 8 age-matched diabetic donors without diabetic retinopathy.

Results: Intravitreal Epo concentration was higher in both PDR and DME patients than in non-diabetic controls (PDR vs. controls: 302 [117-1850] vs. 30 [10-75], $\mathrm{p}<0.01$; DME vs. controls: 430 [41-3000] vs. 30 [10-75], $\mathrm{p}<0.01)$. Higher intravitreous Epo concentration was detected in subjects with DME in comparison with PDR patients, but the difference was not statistically significant either in absolute terms or after adjusting for intravitreal proteins $(430 \mathrm{mU} / \mathrm{ml}$ [41-3000] vs. $302 \mathrm{mU} / \mathrm{ml}$ [117-850]; $\mathrm{p}=0.21$ and $105 \mathrm{mU} /$ $\mathrm{mg}$ [13-967] vs. $65 \mathrm{mU} / \mathrm{mg}$ [32-1027]; $\mathrm{p}=0.53$, respectively). Epo mRNA expression was detected in the human retina and it was higher in the retina from diabetic than from non-diabetic donors. In addition, higher expression of Epo mRNA was detected in retinal pigment epithelium than in the neuroretina in both, diabetic donors and non-diabetic donors.

Conclusion: Intravitreous Epo concentration is strikingly elevated in patients with DME without significant retinal ischemia. In addition, Epo is expressed in the human retina and it is upregulated in diabetic donors even without retinopathy. These findings strongly suggest that other factors apart from ischemia are involved in the overexpression of Epo in diabetic retinopathy.

Supported by grants from Novo Nordisk Pharma S.A., the Instituto de Salud Carlos III) and the Ministerio de Ciencia y Tecnología 


\section{OP 46}

\section{Modulation of inflammatory markers in diabetes}

0253

Treatment with interleukin-1 receptor antagonist improves glycaemic control and $\beta$-cell function in patients with type 2 diabetes mellitus

M. Y. Donath ${ }^{1}$, M. Faulenbach ${ }^{1}$, C. M. Larsen ${ }^{2}$, A. Vaag ${ }^{2,3}$, T. Mandrup-Poulsen ${ }^{2,4}$;

${ }^{1}$ Endocrinology \& Diabetes, Internal Medicine, Zurich, Switzerland, ${ }^{2}$ Steno, Diabetes Center, Gentofte, Denmark, ${ }^{3}$ University of Lund, Sweden, ${ }^{4}$ Department of Molecular Medicine, Karolinska Institute, Stockholm, Sweden

Background and Aims: High concentrations of glucose induce $\beta$-cell production of interleukin-1(IL-1) $\beta$ leading to impaired $\beta$-cell function and apoptosis in isolated human islets. Islet production of natural IL-1 receptor antagonist (IRAP) is reduced in Type 2 diabetes (T2D). Therefore, the balance of islet derived IL-1 and IRAP may play a crucial role in progressive $\beta$-cell failure in T2D. Aim: To investigate if IRAP improved glycemia and $\beta$-cell function in patients with T2D.

Materials and Methods: In an investigator-initiated, randomised, placebo-controlled, double-masked two-centre clinical trial, 70 out-patients with T2D (age 60.1 yrs, 27\% female, diabetes duration 11 yrs, baseline BMI 31.4, HbAlc 8.4\%, basal C-peptide 1061 pM (ambient plglucose $10.3 \mathrm{mM}$ ), $2 \%$ on diet, $47 \%$ on oral agents (OA), $51 \%$ on $\mathrm{OA}+$ insulin or insulin alone) were randomized (sequence and code concealment by County Pharmacy Zürich) to treatment with $100 \mathrm{mg}$ sc of rhIRAP (Kineret, Amgen, prepared and labelled by County Pharmacy Zürich) once daily or placebo (P) for $13 \mathrm{w}$ and followed up for 39 w. Diet and anti-diabetic therapy were unchanged during IRAP treatment. At baseline and $13 \mathrm{w}$ an OGTT followed by $0.3 \mathrm{~g} / \mathrm{kg}$ glucose, $0.5 \mathrm{mg}$ glucagon and $5 \mathrm{~g}$ arginine iv (IV) and in 36 random patients hyperinsulinemic-euglycemic clamps were performed after overnight fast, no exercise and no anti-diabetic medication. $\Delta \mathrm{HbA} 1 \mathrm{c}$ was $\mathbf{1}^{\circ}$ end-point and $\Delta$ - $\beta$-cell function and -insulin sensitivity $2^{\circ}$ end-points between baseline and $13 \mathrm{w}$. HbAlc was measured (DCCT/ IFCC standards) with exchange between the two centers, Cpeptide and insulin by ELISA at SDC and data analysed as per-protocol. 42 patients were required to obtain $80 \%$ power to detect a change in Hbalc of $0,5 \%$ (one-sided significance of $\alpha=0.05$ ).

Results: HbA1c (but not $\mathrm{Hb}$ ) and $120 \mathrm{~min}$ pl-glucose in OGTT decreased in IRAP- and increased in placebo-treated patients $(-0.46 \pm 0.2$ vs $0.08 \pm 0.12 \%, p=0.011 ;-0.6 \pm 0.5$ vs
$1.0 \pm 0.8 \mathrm{mM}, \mathrm{p}=0.04$, respectively). $\Delta \mathrm{C}$-peptide (total AUC (AUCt) during OGTT) improved by IRAP- but deteriorated by placebo-treatment $(10.5 \pm 6.8$ vs $-8.2 \pm 7.8 \mathrm{nMxmin}$, $\mathrm{p}=0.038$ ), as did the AUCt during OGTT+IV test $(\mathrm{p}=0.041)$. IV $\triangle \mathrm{AUCt}$-insulin was also improved in IRAP treated patients $(1.2 \pm 0.5$ vs $-0.2 \pm 0.5 \mathrm{nMxmin}, \mathrm{p}=0.031)$. IRAP did not alter BMI or insulin sensitivity, but reduced C-reactive peptide $(-4.4 \pm 1.6$ vs $0.3 \pm 0.3 \mathrm{mg} / 1, \mathrm{p}<0.001)$, and was well-tolerated, apart from self-limiting local injection reactions in 10 patients.

Conclusion: Blocking IL-1 action for 13 weeks improves glycemic control and $\beta$-cell function, but does not alter BMI or insulin sensitivity in poorly controlled T2D patients. This study provides proof-of-principle that IL-1 is an important mediator of impaired glycemia in T2D by affecting $\beta$-cell function.

0254

Effect of adjunctive pramlintide treatment on hsCRP and adiponectin levels in intensively treated patients with type 1 diabetes

C. Lush, J. Frias, N. Nanayakkara, T. Darsow, D. Maggs, D. Kendall;

Amylin Pharmaceuticals, Inc., San Diego, United States.

Background and Aims: Elevated plasma concentrations of high-sensitivity C-reactive protein (hsCRP) and decreased concentrations of adiponectin (ADPN) have been related to risk of cardiovascular disease (CVD) in diabetes. Higher concentrations of hsCRP were observed in intensively treated patients who gained weight in the Diabetes Control and Complications Trial. Low plasma adiponectin concentrations were associated with progression of atherosclerosis in the Coronary Artery Calcification in Type 1 Diabetes study. The aim of the present study was to assess the effects of pramlintide therapy on these markers in intensively treated patients with type 1 diabetes.

Materials and Methods: We performed a post-hoc analysis of a randomized, double-blind study in 187 subjects with type 1 diabetes on intensive insulin therapy. Subjects were treated with either adjunctive pramlintide (30 or $60 \mu \mathrm{g}$ with major meals, $n=84)$ or placebo $(n=103)$, with both groups targeting similar glycemic goals. Plasma samples were assayed for both hsCRP and ADPN at baseline and Week 25. Baseline characteristics (age $42 \pm 14$ vs. $42 \pm 12 \mathrm{y}$, disease duration $20 \pm 12$ vs. $21 \pm 12 \mathrm{y}$, body weight $81.0 \pm 17.8$ vs. $80.6 \pm 16.8 \mathrm{~kg}$, mean \pm SD) for pramlintide vs. placebo subjects were well balanced, as were metabolic measures (A1C $8.0 \pm 0.8$ vs. $8.1 \pm 0.9 \%$, hsCRP $2.0 \pm 1.9$ vs. $1.7 \pm 1.7 \mathrm{mg} / \mathrm{L}, \mathrm{ADPN} 12.9 \pm 7.6$ vs. $14.4 \pm 9.5 \mu \mathrm{g} / \mathrm{mL})$. 
Results: At 25 weeks, pramlintide improved postprandial glucose control ( $152 \pm 4$ vs. placebo $174 \pm 4 \mathrm{mg} / \mathrm{dL}, \mathrm{p}<0.001$, mean \pm SE), reduced body weight $(-0.93 \pm 0.4$ vs. placebo $+1.38 \pm 0.3 \mathrm{~kg}, \mathrm{p}<0.001$ ), decreased mealtime insulin dose $(-22 \pm 5$ vs. placebo $+4 \pm 4 \%, \mathrm{p}<0.001)$, and resulted in similar reductions in $\mathrm{A} 1 \mathrm{C}(-0.4 \pm 0.1$ vs. placebo $-0.6 \pm 0.1 \%$ ) compared with placebo. Placebo subjects had a significant increase in hsCRP compared with pramlintide subjects $(+0.66 \pm 0.2$ vs. $-0.06 \pm 0.2 \mathrm{mg} / \mathrm{L}, \mathrm{p}<0.01)$ while ADPN was significantly increased in pramlintide compared with placebo subjects $(+0.69 \pm 0.5$ vs. $-0.43 \pm 0.3 \mu \mathrm{g} / \mathrm{mL}$, $\mathrm{p}<0.05$ ) at Week 25. After 25 weeks of therapy, a significantly larger proportion of placebo subjects shifted to hsCRP levels indicative of higher relative risk (hsCRP $>3.0 \mathrm{mg} / \mathrm{L}$ ) compared with pramlintide subjects after 25 weeks of therapy ( 19 vs. $5 \%, \mathrm{p}<0.01)$. Multiple regression analysis, adjusting for baseline hsCRP, treatment, and changes from baseline in $\mathrm{A} 1 \mathrm{C}$ and body weight revealed that each of these factors was significantly associated with Week 25 hsCRP. Similar modeling indicated a significant relationship between change in body weight and Week 25 ADPN.

Conclusion: Adjunctive pramlintide in patients with type 1 diabetes intensively treated with insulin therapy lead to an increased concentration of ADPN and an attenuated increase in hsCRP when compared with patients treated with insulin alone, even at similar levels of glycemia. Longer-term studies are warranted to further investigate the clinical relevance of these findings to cardiovascular risk in type 1 diabetes.

\section{5}

Metabolic and anti-inflammatory effects of pioglitazone (PIO) lead to histological improvement in patients with non-alcoholic steatohepatitis (NASH)

B. Balas ${ }^{1}$, R. Belfort ${ }^{1}$, A. Gastaldelli ${ }^{1}$, S. Harrison ${ }^{2}$, J. Finch ${ }^{1}$, C. Darland ${ }^{1}$, S. Schenker ${ }^{3}$, K. Cusi ${ }^{1}$;

${ }^{1}$ Diabetes, UTHSCSA, San Antonio, ${ }^{2}$ Gastroenterology, Brooke Army Medical Center, San Antonio, ${ }^{3}$ Gastroenterology, UTHSCSA, San Antonio, United States.

Background and Aims: NASH is a chronic liver condition associated with steatosis, necroinflammation (+/-fibrosis), insulin resistance and glucose intolerance. We have shown that pioglitazone treatment in NASH has beneficial effects on liver histology, although the contributions of improved glucose metabolism and reduction in inflammation remain unclear.

Materials and Methods: We studied 48 IGT/T2DM biopsy-proven patients with NASH (age: $51 \pm 2$, BMI: $33 \pm 1 \mathrm{~kg} / \mathrm{m}^{2}$, FPG: $116 \pm 6 \mathrm{mg} / \mathrm{dl}$ ) and 12 normal controls
(CON) matched for age, gender and ethnicity. Patients received a hypocaloric diet and were randomized (doubleblind) to PIO (45 mg/d) or placebo (Pbo) for 6 months. Before and after treatment patients had a liver biopsy and a double-tracer OGTT $\left(3-{ }^{3} \mathrm{H}\right.$ glucose infusion $/{ }^{14} \mathrm{C}$ in oral glucose load) to assess glucose tolerance/clearance and hepatic/peripheral glucose metabolism. In addition to metabolic parameters, we measured in plasma the following inflammatory markers: hsCRP, interleukin-6 (IL-6), tumor necrosis factor (TNF)- $\alpha$, tumor growth factor (TGF) $\beta$ and vascular- and intracellular adhesion molecules (VCAM/ ICAM).

Results: Pbo reduced only AST/ALT $(\mathrm{p}<0.02)$ and hepatic inflammation $(\mathrm{p}<0.03)$. Compared to CON, pts with NASH had hepatic insulin resistance (fasting hepatic insulin sensitivity index $[\mathrm{HISI}]=100 /[\mathrm{EGP} \times \mathrm{FPI}] ; 5.0 \pm 1.4$ vs. $19.3 \pm 2.2$ ) and reduced glucose clearance during the OGTT $(2.17 \pm 0.2$ vs. $3.15 \pm 0.3 \mathrm{mg} / \mathrm{min}$, both $\mathrm{p}<0.01)$. PIO lowered $\mathrm{HbA}_{1 \mathrm{c}}(-0.8 \%)$ and ALT/AST to normal, improved HISI by $\sim 60 \%$ and increased adiponectin $\sim 2.5$ fold (all $\mathrm{p}<0.0001 ; \mathrm{p}<0.01$ vs. Pbo). Steatosis and necroinflammation decreased by $\sim 50 \%$ (both $\mathrm{p}<0.0001, \mathrm{p}<0.01$ vs. Pbo). PIO significantly increased glucose clearance $(2.2 \pm 0.2$ vs. $2.5 \pm 0.2 \mathrm{mg} / \mathrm{min}, \mathrm{p}<0.01 ; \mathrm{p}<0.0001$ vs. Pbo). We calculated an index of peripheral insulin sensitivity from the OGTT ( $\mathrm{Rd} / \mathrm{I})$, by dividing glucose disposal rate (largely muscle) by the plasma insulin. PIO enhanced $\mathrm{Rd} / \mathrm{I}(6.8 \pm 0.7$ vs. $10.0 \pm 0.9 \mathrm{mg} / \mathrm{kg} \mathrm{min} \div \mu \mathrm{U} / \mathrm{ml}, \mathrm{p}<0.01)$ but was unchanged by Pbo. Compared to CON, hsCRP was $\sim 3$-fold higher in NASH patients, while IL- 6 , TNF- $\alpha$, VCAM and ICAM were all $40-50 \%$ higher (all $\mathrm{p}<0.02-0.001$ vs. CON). PIO significantly reduced hsCRP $(6.1 \pm 1.0$ to $3.1 \pm 0.8 \mathrm{mg} / \mathrm{L}$, $\mathrm{p}<0.04), \mathrm{TNF}-\alpha(2.2 \pm 0.1$ vs. $2.0 \pm 0.1 \mathrm{pg} / \mathrm{ml}, \mathrm{p}<0.01)$, TGF$\beta(31 \pm 2$ vs. $26 \pm 2 \mathrm{ng} / \mathrm{ml}, \mathrm{p}<0.05), \operatorname{VCAM}(523 \pm 32$ vs. $480 \pm 32 \mathrm{ng} / \mathrm{ml}, \mathrm{p}<0.01)$ and ICAM $(315 \pm 17$ vs. $268 \pm 10 \mathrm{ng} /$ $\mathrm{ml}, \mathrm{p}=0.001)$. In the PIO arm, steatosis inversely correlated with both basal $(\mathrm{r}=-0.41)$ and OGTT $(\mathrm{r}=-0.51)$ improvement in $\mathrm{Rd} / \mathrm{I}$ (both $\mathrm{p}<0.01$ ).

Conclusion: PIO improves both liver and muscle insulin sensitivity in patients with NASH. These metabolic effects of PIO may be important both for the histological response seen in patients with NASH as well as for the reduction of the pro-inflammatory state associated with this condition. Supported by Takeda Pharmaceuticals

0256

Metabolic and histologic improvement by pioglitazone treatment in NASH increases plasma adiponectin levels and reduces visceral and liver fat in patients with NASH A. Gastaldelli ${ }^{1}$, J. Hardies ${ }^{2}$, R. Belfort ${ }^{1}$, S. Harrison ${ }^{3}$, B. Balas ${ }^{1}$, K. Brown ${ }^{4}$, S. Schenker ${ }^{4}$, K. Cusi ${ }^{1}$; 
${ }^{1}$ Diabetes, ${ }^{2}$ Research Imaging Center, UTHSCSA, San Antonio, ${ }^{3}$ Gastroenterology, Brooke Army Medical Center, San Antonio, ${ }^{4}$ Gastroenterology, UTHSCSA, San Antonio, United States.

Background and Aims: We have previously shown that pioglitazone (PIO) treatment in non-alcoholic steatohepatitis (NASH) improves glucose/lipid metabolism, insulin sensitivity, steatosis and leads to marked histologic improvement. However, the relationship between these beneficial metabolic/histologic effects and changes in visceral/ liver fat content have not been studied before.

Materials and Methods: We present data on 34 IGT/T2DM biopsy-proven pts with NASH (age: $51 \pm 2$, BMI: $32 \pm 1 \mathrm{~kg} / \mathrm{m}^{2}$, FPG: $118 \pm 5 \mathrm{mg} / \mathrm{dl}$ ) and 8 normal controls (CON) matched for age, gender and ethnicity. Pts received a hypocaloric diet and were randomized (double-blind) to PIO $(45 \mathrm{mg} / \mathrm{d})$ or placebo (Pbo) for 6 months. Before and after treatment pts underwent the following studies: 1) liver biopsy; 2) doubletracer 75 gram OGTT $\left(3-{ }^{3} \mathrm{H}\right.$ glucose infusion $/{ }^{14} \mathrm{C}$ in oral glucose load) to assess glucose tolerance/clearance and hepatic/peripheral glucose metabolism; 3) measurements of liver fat content by magnetic resonance spectroscopy (MRS) and of visceral (VF)/subcutaneous (SC) fat by magnetic resonance imaging (MRI).

Results: Patients with NASH had increased liver fat by MRS $(21 \pm 2 \%$ vs. $10 \pm 2 \%, \mathrm{p}<0.04)$ and a $\sim 2.5$-fold increase in VF ( $p=0.01)$ compared to CON. Treatment with PIO for 6 months led to a significant improvement in basal (fasting) hepatic insulin sensitivity, glucose tolerance and glucose clearance during the OGTT, and marked histologic improvement (all $\mathrm{p}<0.01-0.001$ vs. Pbo). Plasma FFA levels decreased by $\sim 30 \%$ with PIO while plasma adiponectin levels increased $\sim 3$-fold ( $\mathrm{p}<0.001$ vs. Pbo) in association with a $\sim 50 \%$ reduction of liver fat and $\sim 30 \%$ decrease in VF (both $\mathrm{p}<0.01$ vs. Pbo, which was unchanged). In the PIO group, reduction in both liver fat content by MRS and VF were associated with an improvement in glucose clearance $(r=0.44$ and $r=0.55$, respectively, both $\mathrm{p}<0.01)$. There was a strong correlation between the increase in plasma adiponectin concentration and the decrease in liver fat by MRS ( $\mathrm{r}=-0.57, \mathrm{p}<0.001)$, VF $(\mathrm{r}=-0.46, \mathrm{p}<0.01)$, as well as enhanced hepatic insulin sensitivity $(\mathrm{r}=0.37$, $\mathrm{p}<0.01)$, glucose clearance $(\mathrm{r}=0.44, \mathrm{p}<0.004)$ and decreased mean glycemia $(r=-0.34, p<0.03)$ during the OGTT. The decrease in liver fat by MRS and VF with PIO occurred despite a mild increase in body weight $(\mathrm{PIO}=+2.7$ vs. $\mathrm{Pbo}-$ $0.4 \mathrm{~kg}, \mathrm{p}<0.02$ ). The improvement in the total histologic score of steatosis, necroinflammation and fibrosis was directly associated with decrease in visceral fat $(r=0.48$, $\mathrm{p}=0.01)$ and inversely with an increase in plasma adiponectin concentration $(r=-0.43, p<0.006)$.

Conclusion: In pts with IGT/T2DM and NASH, the improvement in metabolic/histologic parameters by PIO, and in particular the reduction of liver fat content and VF, have a good correlation with the increase in plasma adiponectin levels. This suggests that changes in plasma adiponectin concentration may play an important role in mediating the beneficial effects of PIO in patients with NASH.

Supported by Takeda Pharmaceuticals

\section{OP 47}

\section{Highlights in genetics and prediction of diabetes}

0257

Association of KLF11 with insulin resistance is mediated via STAT3 binding to KLF11 variant $-1659 \mathrm{G}>\mathrm{C}$ R. Gutiérrez-Aguilar ${ }^{1}$, Y. H. Hamid ${ }^{2}$, E. Vaillant ${ }^{1}$, Y. Benmezroua ${ }^{1}$, T. Jørgensen ${ }^{3}$, K. Borch-Johnsen ${ }^{3,2}$, T. Hansen ${ }^{2}$, P. Froguel ${ }^{4,1}$, O. Pedersen ${ }^{5,2}$, B. Neve ${ }^{1}$;

${ }^{1}$ Cnrs umr8090, Institut de Biologie, Lille, France, ${ }^{2}$ Steno Diabetes Center and Hagedorn Research Institute, Gentofte, Denmark, ${ }^{3}$ Research Centre for Prevention and Health, Glostrup University Hospital, Glostrup, Denmark, ${ }^{4}$ Genome Centre and Genomic Medicine, Imperial College, London, United Kingdom, ${ }^{5}$ Faculty of Health Science, University of Aarhus, Denmark.

Background and Aims: Recently, it has been shown that the TGF- $\beta$ inducible transcription factor, KLF11, affects transcriptional regulation of the insulin gene. Genetic variants of the KLF11 gene are associated to type 2 diabetes (T2D), including the Q62R and promoter - 1659 $\mathrm{G}>\mathrm{C}$ (p-SNP) that are in complete LD. We aimed to study the T2D-association of KLF11 variants by genotyping a general Danish population and analyzing transcriptional regulation of KLF11 affected by p-SNP. This SNP is situated in a putative RBP-JK or STAT3 binding site $((\mathrm{G}>\mathrm{C})$ TGGGAA). The transition of G- to $\mathrm{C}$ - allele of $\mathrm{p}$-SNP is predicted to result in a loss of transcription factors binding. RBP-JK plays an important role in the Notch signaling pathway that enhances differentiation of the endocrine pancreatic beta-cells. On the other hand, STAT3 is necessary for regulation of gluconeogenesis in hepatocytes. 
Materials and Methods:We genotyped Q62R in 4,443 normal glucose tolerant (NGT), 491 impaired fasting glucose, 679 impaired glucose tolerance, 251 newly identified T2D and 118 subjects with history of T2D (INTER99 cohort) and analysed association with glucose related-traits. We analyzed nuclear proteins binding onto the p-SNP sequence by gelshift experiments (EMSA). We cloned the KLF11 promoter region containing wild type or mutant $\mathrm{p}-\mathrm{SNP}$ in a pGL3-luciferase construct and cotransfected them with pCMV-RL, whereafter relative luciferase activity was measured.

Results: In NGT subjects the 62R-allele was a significantly associated with higher fasting serum insulin levels, higher fasting serum C-peptide levels and increased HOMA insulin resistance indexes $(\mathrm{P}=0.00004, \mathrm{P}=0.006$ and $\mathrm{P}=0.00002$, respectively). We showed that a protein complex binds onto the wild type p-SNP sequence and that it is absent on the mutant sequence. Our results proof that STAT3 is present in this complex. The analysis of KLF11 promoter activity showed an inhibition of the G-allele compared to the C- allele, suggesting that p-SNP affects KLF11 expression levels.

Conclusion: KLF11 is associated to insulin resistance in the Danish population. The fact that p-SNP alters STAT3 binding and repression of the KLF11 promoter, suggests that KLF11 regulation plays an important role in liver glucose sensibility, which could explain the association of KLF11 with insulin resistance.

Supported by the European Regional Development Fund (FEDER) and Region Nord-Pas de Calais (ARCir) to PF and BN. Grant from Consejo Nacional De Ciencia $Y$ Tecnologia (CONACYT) and Societe Francaise D'exportation Des Ressources Educatives (SFERE) to RGA

\section{8}

Modulation of genetic susceptibility by behavioral factors and glucose regulation status in the general population exemplified by the impact of the $A P O A 5$ -1131T $>C$ variant on serum lipids

G. Andersen ${ }^{1}$, T. Spars $\varnothing^{1}$, A. Albrechtsen ${ }^{1}$, S. I. Castella ${ }^{1}$, C. Glümer ${ }^{1,2}$, K. Borch-Johnsen ${ }^{1,2}$, T. Jørgensen ${ }^{2}$,

T. Hansen ${ }^{1}$, O. Pedersen ${ }^{1}$;

${ }^{1}$ Steno Diabetes Center, Gentofte, ${ }^{2}$ Research Centre for Prevention and Health, Glostrup University Hospital, Denmark.

Background and Aims: Previous studies suggest that the C-allele of the APOA5 $-1131 \mathrm{~T}>\mathrm{C}$ polymorphism confers an increase in serum triglyceride concentrations. The aim of the present study was at the population level to estimate the effect of the APOA5 $-1131 \mathrm{~T}>\mathrm{C}$ polymorphism on fasting serum lipids and to elucidate the potential modulating impact of health behavior and glucose regulation status.

Materials and Methods: The APOA5 - 1131T $>\mathrm{C}$ polymorphism (rs662799) was genotyped in a population-based sample (Inter99) of 5,873 treatment-naïve Danes $(2,929$ men, 2,944 women; age $46 \pm 8$ years; BMI $26.3 \pm 4.6 \mathrm{~kg} / \mathrm{m}^{2}$ ) using a chip-based matrix-assisted laser desorption/ionization time-of-flight mass spectrometry of PCR-generated primer extension products.

Results: The APOA5 $-1131 \mathrm{~T}>\mathrm{C}$ polymorphism was associated with dyslipidaemia (defined according to WHO) in a case-control study of 1,439 patients with dyslipidaemia and 4,432 normolipidaemic subjects, $P=4 \cdot 10^{-6}$ for minor allele frequency $(8.2 \%$ [7.2-9.2\%] vs. $5.7 \%[5.2-6.2 \%]$ ) and $P=1 \cdot 10^{-5}$ for genotype distribution. Correspondingly, in the total population of 5,873 participants the $\mathrm{C}$-allele was associated with increased fasting serum levels of triglyceride $\left(P=1 \cdot 10^{-15}\right)$ and total cholesterol $(P=0.0003)$ and decreased fasting serum HDL-cholesterol $(P=0.004)$. Significant epistasis on fasting serum triglyceride concentrations was observed between the APOA5 - 1131T $>\mathrm{C}$ polymorphism and smoking $(P=0.0003)$, daily alcohol intake $(P=0.005)$, and dietary monounsaturated fatty acid intake $\left(P=8 \cdot 10^{-6}\right)$. The effect of the polymorphism on fasting serum triglyceride was more profound in people with impaired glucose regulation than for glucose-tolerant subjects $\left(P=5 \cdot 10^{-9}\right)$.

Conclusion: At the level of the general population of middle-aged people the common C-allele of the APOA5 $1131 \mathrm{~T}>\mathrm{C}$ polymorphism greatly influences the fasting serum lipid levels. Importantly, the impact of this gene variant on fasting serum triglycerides is strongly modulated by behavioral factors. Similarly, the APOA5 variant effect is much more profound in subjects with hyperglycaemia.

0259

Predictive value of diabetes-associated autoantibodies among children with HLA-conferred disease susceptibility recruited from the general population

H. T. A. Siljander ${ }^{1,2}$, S. Simell ${ }^{3}$, A. Hekkala ${ }^{4}$, J. Lähde ${ }^{2}$,

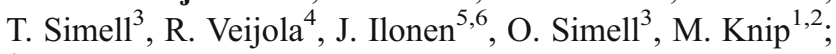
${ }^{1}$ Hospital for Children and Adolescents, University of Helsinki, ${ }^{2}$ Dept. of Paediatrics, Tampere University Hospital, Finland, ${ }^{3}$ Dept. of Paediatrics, University of Turku, ${ }^{4}$ Dept. of Paediatrics, University of Oulu, ${ }^{5}$ Dept. of Clinical Microbiology, University of Kuopio, ${ }^{6}$ Immunogenetics Laboratory, University of Turku, Finland.

Background and Aims: There is a shortage of data on the predictive value of diabetes-associated autoantibodies in the general population, from which about $90 \%$ of new cases 
affected by type 1 diabetes (T1D) are derived. We aimed at assessing the predictive characteristics of ICA in combination with IAA, GADA, and/or IA-2A among children with HLA-conferred disease susceptibility recruited from general population.

Materials and Methods: We observed from birth 7412 participants (3513 boys; 47.4\%) in the Finnish Type 1 Diabetes Prediction and Prevention study (DIPP) for progression to T1D for an average of 5.6 years $(0.9$ 10.4 years). All subjects carried HLA genotypes conferring increased T1D risk. Blood samples were taken with an interval of 3-12 months throughout the follow-up period. ICA were used as the primary screening tool. If a subject tested positive for ICA, IAA, GADA and IA-2A were measured from all samples available from that individual. Subjects were categorised according to their autoantibody profile in such a way that each child could belong to only one category. Persistent antibody positivity was defined as positive results in at least two consecutive samples including the last sample available. ICA were detected with conventional immunofluorescence, and the other autoantibodies were analysed with specific radiobinding assays.

Results: 763 children $(10.3 \%)$ tested positive for ICA at least once, 437 of whom (5.9\%) were positive for ICA only, $100(1.3 \%)$ for two antibodies, $72(1.0 \%)$ for three antibodies and $154(2.1 \%)$ for all four autoantibodies. Persistent isolated ICA positivity was seen in 287 (3.9\%) children, while $75(1.0 \%)$ subjects were persistently positive for two antibodies, $82(1.1 \%)$ for three antibodies and $74(1.0 \%)$ for all four antibodies. $97(1.3 \%)$ children presented with clinical T1D at a mean age of 3.9 years (0.9-9.2 years). The highest disease sensitivity [52.6\% $\left.\left(\mathrm{CI}_{95} 42.3-62.8 \%\right)\right]$ was observed for positivity for all four antibodies, while persistent positivity for the combination of ICA+IAA+IA-2A resulted in the highest specificity $\left[100 \%\left(\mathrm{CI}_{95} 99.9-100 \%\right)\right]$ and positive predictive value [62.5\% $\left.\left(\mathrm{CI}_{95} 24.5-91.5 \%\right)\right]$. In the survival analyses the highest progression rate was seen in those persistently positive for ICA+IAA+IA-2A, among whom all children observed for more than 7 years $(n=5)$ presented with T1D. Among the children persistently positive for ICA+IAA all the affected children (7/15) were diagnosed before the age of 2 years.

Conclusion: It is possible to identify a cohort of children from the general population with as high a disease risk as that seen among siblings positive for multiple autoantibodies by screening genetically susceptible children for autoantibodies. The high-risk cohort is small, and the disease sensitivity of high-risk antibody markers remains below $55 \%$. However, based on autoantibody markers one can sort out a subgroup of children with rapid progression to T1D and another group in which almost all children appear to progress to overt T1D before the age of 8 years. Supported by Special Public Grants for Medical Research at Tampere, Oulu and Turku University Hospitals; the Academy of Finland; the Juvenile Diabetes Research Foundation International; the Novo Nordisk Foundation; European Union Biomed 2

\section{0}

Functional impact of the vitamin D receptor gene Fok I polymorphism on immune cells

E. Van Etten ${ }^{1}$, A. Giulietti ${ }^{1}$, L. Verlinden ${ }^{1}$, E. RamosLopez $^{2}$, L. Overbergh ${ }^{1}$, A. Verstuyf ${ }^{1}$, K. Badenhoop ${ }^{2}$, C. Mathieu;

${ }^{1}$ LEGENDO, Katholieke Universiteit Leuven (KUL), Belgium, ${ }^{2}$ Division of Endocrinology, Diabetes and Metabolism, University Hospital Frankfurt am Main, Germany.

Background and Aims: The active form of vitamin D, 1,25-Dihydroxyvitamin $\mathrm{D}_{3}\left(1,25(\mathrm{OH})_{2} \mathrm{D}_{3}\right)$, has potent immunomodulatory properties and is able to prevent autoimmune diabetes in NOD mice. These effects of 1,25 $(\mathrm{OH})_{2} \mathrm{D}_{3}$ are mediated through binding with the vitamin $\mathrm{D}$ receptor (VDR). Several polymorphisms of the human VDR gene have been identified of which the FokI polymorphism results in a VDR protein with a different structure, a long $f$-VDR or a 3 amino acids shorter $F$-VDR. Multiple studies have tried to correlate this VDR FokI polymorphism with the genetic predisposition to type 1 diabetes but failed to reveal a solid association or a functional explanation for the observed correlation. The aim of the present study was to investigate the functional consequences of the FokI polymorphism in immune cells.

Materials and Methods: Transfection experiments with a luciferase reporter construct under control of different promoter sequences were performed in the presence of the long $f$-VDR or short $F$-VDR. Functional experiments were performed on monocytes and lymphocytes isolated from human subjects with a homogenous long $f f$ or short $F F$ VDR-genotype. IL-12p40 and p35 mRNA expression levels were determined by quantitative real time RT-PCR in IFN- $\gamma$ stimulated monocytes. Proliferation of PHA-stimulated lymphocytes was assessed by thymidine incorporation.

Results: In the transfection experiments, the presence of the shorter $F$-VDR resulted in higher NFкB- and NFATdriven transcription as well as higher transcription driven by the endogenous promoter of IL-12p40. No differential effects were observed for AP-1-driven transcription or transactivation of the classical mouse osteopontin DR3type vitamin D responsive element. Concordantly, in monocytes from subjects with a homogenous short $F F$ 
VDR-genotype, expression of IL-12p40 and p35 after in vitro IFN- $\gamma$ stimulation was higher than in monocytes expressing the long ff VDR-genotype. Additionally, lymphocytes with a short $F F$ VDR-genotype proliferated stronger in response to phytohemagglutinin.

Effects of the VDR FokI polymorphism on immune cell behavior

\begin{tabular}{lll}
\hline & long ff genotype & short FF genotype \\
\hline p40 & $1.1 \pm 0.5$ & $3.8 \pm 0.7(\mathrm{p}<0.05)$ \\
p35 & $8.5 \pm 3.2$ & $62.8 \pm 18.4(\mathrm{p}<0.05)$ \\
proliferation (SI) & $26.6 \pm 12.6$ & $70.1 \pm 13.6(\mathrm{p}=0.05)$ \\
\hline
\end{tabular}

Conclusion: In his study we have demonstrated clearly that different FokI genotypes interact differently with transcription factors that are important in the immune system. The observed differences between the FokI genotypes at transcriptional level were reflected in a different immune behavior of monocytes and lymphocytes. Together these data provide the first functional evidence that the VDR FokI polymorphism affects immune cell behavior, with a more active immune system for the short $F$-VDR, thus possibly playing a role in autoimmune diseases.

Supported by grants from the Katholieke Universiteit Leuven (KUL), the Flemish Research Foundation (Fonds voor Wetenschappelijk Onderzoek (FWO)) and the Juvenile Diabetes Research Foundation (JDRF).

\section{OP 48}

\section{Clinical immunology}

\section{1}

Ii-key/MHC class II epitope hybrid technology and ELISPOT assay in type 1 diabetes

M. Vadacca $^{1}$, G. Valorani ${ }^{2}$, R. E. Humphreys ${ }^{3}$, N. Kallinteris ${ }^{3}$, L. Valente ${ }^{1}$, P. Pozzilli ${ }^{1}$, I. Group ${ }^{4}$;

${ }^{1}$ Endocrinologia, Università Campus Bio-Medico, Roma, Italy, ${ }^{2}$ Diabetes and Metabolic Medicine, Queen Mary University of London, United Kingdom, ${ }^{3}$ Biotechnology, Antigen Express USA, Worcester, United States, ${ }^{4}$ Endocrinologia, MultiCentre, Roma, Italy.

Background and Aims: Type 1 diabetes (T1D) is the result of the immunomediated destruction of insulin producing $\beta$ cells in the islets of Langherans. This immune response involves both cellular and humoral changes that persist over a prolonged period up to diagnosis. Autontibodies have become a useful marker for the prediction of the disease. Less is known on the specificity of cellular responsiveness and on T-cell profiling in T1D. MHC class II epitopes are normally bound to MHC class II molecules in a post-Golgi, antigenic peptide-binding compartment of antigen presenting $\mathrm{T}$ cells. The shortest sequence (Ii-key peptide) (LRKM) of the Ii protein acts at an allosteric site on MHC class II molecules to facilitate charging and presentation of peptides into the antigenic binding site, entirely circumventing the normal process of antigen phagocytosis, processing and intracellular charging onto MHC class II molecules. This offers us an efficient way to augment the binding of epitopes to the antigenic peptide-binding groove, thus enhancing the immunogenecity of epitopes. These observations have been supported by studies in cancer and infectious diseases. The main objective of this study was to investigate the cellular immune response in T1D by characterizing the cytokines secreted by peripheral blood mononuclear cells (PBMCs) in response to the exposure to Ii-key/MHC class II insulin epitope hybrids.

Materials and Methods: Sequences of the human insulin were obtained from Genebank. Predicted DRB1*0301 and DRB $1 * 0401$ epitopes were identified by application of the Rammensee SYFPEITH program (http://syfpeithi.bmi-hei delberg.com/Scripts/MHCServer.dll/EpPredict.htm). Five peptide series (15 hybrids and five epitope-controls) were analyzed. The study was carried out in 31 patients (20 M) affected by T1D, and in 10 normal subjects. Diagnosis was defined according ADA classification. PBMCs were prepared from heparinised blood using standard Ficoll Hypaque separation. ELISPOT assays were performed with BD Pharmingen set for IFN- $\gamma$ according to the manufacturer's instructions. Digitalized images of spots were analyzed with an Eli.Analyse 4.0 software.

Results: All assays detected significant cytokine secretion in response to the polyclonal stimulators PMA/Ionomycin. Six out 31 patients $(19.4 \%)$ demonstrated a positive response to one or more peptides. Control subjects $(4 / 10$ subjects were DR3 or DR4) showed no detectable response to stimulation with Ii-key hybrids or peptides. Tested peptides were recognized only by DR3/DR4 0302+ve diabetic patients.

Conclusion: The Ii-key hybrid technique is a new strategy for augmenting the potency of MHC class-II restricted epitopes resulting in a profound increase in $\mathrm{T}$ cell stimulation. Our study has demonstrated that HLA is crucial for the response to Ii-Key hybrids and this can help to better understand epitope specificity in T1D. Since extensive studies have confirmed the role of ELISPOT assay to detect early antigen-directed activation of lymphocyte subpopulations in T1D, the combined use of the keyhybrids technique and the ELISPOT assay assures a more selective and sensitive cytokine detection in response to 
antigen stimulation and could advance our understanding of the cell mediated immune response in T1D.

Supported by Antigen Express USA/University Campus Bio-Medico

\section{2}

Dietary eicosapentaenoic acid (EPA) and docosahexaenoic acid (DHA) intake is associated with decreased risk of islet autoimmunity in children at risk for type 1 diabetes J. M. Norris ${ }^{1}$, X. Yin ${ }^{1}$, K. Barriga ${ }^{1}$, M. M. Lamb ${ }^{1}$, H. L. Brady ${ }^{1}$, H. Orton ${ }^{1}$, M. Hoffman ${ }^{1}$, M. Rewers ${ }^{2}$; ${ }^{1}$ Preventive Medicine and Biometrics, UCDHSC, Denver, ${ }^{2}$ The Barbara Davis Center for Childhood Diabetes, UCDHSC, Denver, United States.

Background and Aims: Studies have suggested that fish oil supplementation in infancy may protect against type 1 diabetes, but little is known about the role of fish oil after infancy on risk of type 1 diabetes or its precursors. We examined whether childhood intake of EPA and DHA, the omega-3 fatty acids found in fish oil, is associated with the development of islet autoimmunity (IA) in children in the Diabetes Autoimmunity Study in the Young (DAISY). Material and Methods: DAISY is following children who either possess a higher risk HLA genotype or are a first degree relative of someone with type 1 diabetes, for development of IA, which is defined as being positive for insulin, glutamic acid decarboxylase or IA-2 autoantibodies on at least two consecutive occasions and still positive or diabetic at last follow-up. Data on omega-3 and omega-6 fatty acid intake were collected annually starting at the age of 1 , using the Willett food frequency questionnaire. To validate the reported data on fatty acid intake, we measured erythrocyte membrane fatty acid levels in 314 children, and found a strong statistically significant association between reported EPA and DHA intake and erythrocyte membrane EPA and DHA levels $(p<0.0001)$. Of the 1474 children with complete dietary data, 57 children developed IA at a mean age of 4.8 years, and 1417 children were IA negative after a mean follow-up of 5.5 years. Cox proportional hazards models were used, and hazard ratios (HR) and 95\% confidence intervals (CI) were calculated based on a standard deviation difference in nutrient intake. We examined four fatty acid intake variables: 1) total omega-3 fatty acids, 2)EPA\&DHA, a summed intake of the two omega-3 fatty acids found in fish oil, 3) total omega- 6 fatty acid intake, and 4) arachidonic acid, an omega-6 fatty acid.

Results: Adjusting for HLA DR3/4 status, family history of type 1 diabetes, maternal age, caloric intake, and age at introduction of cereals in the infant diet, EPA\&DHA intake was inversely associated (HR: 0.35, CI: 0.16-0.78), and arachidonic acid intake was directly associated (HR: 1.83, CI: 1.12-3.00) with risk of IA. In separate models, total omega- 6 fatty acid intake was not associated with risk of IA; and total omega-3 fatty acid intake was marginally inversely associated with IA.

Conclusion: We found that childhood intake of EPA and DHA, the omega-3 fatty acids found in fish oil, may lower risk of developing islet autoimmunity in children at increased risk for diabetes.

Supported by NIH R01 DK32493 and DK49654

0263

Screening for celiac disease in childhood diabetes: is it enough to screen at the time of clinical diagnosis of diabetes?

K. A. M. Larsson;

Dept of Pediatrics, Dept of Pediatrics, Kristianstad, Sweden.

Background and Aims: The prevalence of Celiac Disease (CD) in type 1 Diabetes Mellitus (T1DM) is known from several studies and varies from 2.3 to $10 \%$. CD is often asymptomatic or atypical in children with T1DM. Few children have CD before T1DM is diagnosed. Longitudinal studies are also needed to investigate the development of CD in T1DM patients.

Aims: To investigate the prevalence of $\mathrm{CD}$ in a cohort of children and adolescents at the onset of T1DM and the occurence of $\mathrm{CD}$ during a six year follow up.

Materials and Methods: A total of 300 patients with T1DM were prospectively studied for $\mathrm{CD}$. The study group was a cohort of children and youths under the age of 19 year at six clinical centers for pediatric diabetes in Scania (Skåne), Sweden. The children were diagnosed with diabetes between Januari 1, 1996 and December 31, 2000. We performed repeated serological analyses from the onset of diabetes and annually thereafter during six years. The IgA-antiendomysium antibody (EMA) test was selected as the screening test and patients with a positive test were considered appropiate for a jejunal biopsy. Two patients had been diagnosed with CD before the onset of their diabetes. Results: The prevalence of biopsy proven $\mathrm{CD}$ at the time of clinical diagnosis of T1DM was 4\% (12/300). During the follow up a total of 17 patients developed $\mathrm{CD}$ as follows: 10 after one year, 5 after two years, 1 after three years and 1 after five years. The cumulativ frequency of $\mathrm{CD}$ therefore represented 29/300 (9,7\%), all confirmed by intestinal biopsies. At diagnosis of diabetes in patients with $\mathrm{CD}$ the mean age was 8,8 year and in patients without CD 9,9 year. The prevalence of CD in patients with T1DM is approximately 20 times higher than in the general population. It was noted that while $12 / 29$ had $\mathrm{CD}$ at the time of clinical 
onset of T1DM the remaining group of 17 T1DM children developed $\mathrm{CD}$ without clinical symtoms.

Conclusion: Our study confirmes a high prevalence of biopsy proven $\mathrm{CD}$ in children and youths with T1DM with the overall prevalence of $9,7 \%$. We suggest that children with T1DM should be screened for CD annully, at least for three years with a possible reduction in test thereafter unless the patient show symptoms.

Supported by the Children Diabetes Foundation

\section{4}

Autoantibodies against cytokeratin 19 in patients with type 1 diabetes mellitus

E. Di Cesare ${ }^{1}$, M. Previti ${ }^{1}$, F. Lombardo ${ }^{2}$, N. Mazzù ${ }^{11}$, A. Mirto ${ }^{1}$, D. Cucinotta ${ }^{1}$;

${ }^{1}$ Department of Internal Medicine, University of Messina,

${ }^{2}$ Institute of Paediatrics, University of Messina, Italy.

Background and Aims: Autoantibodies against antigens expressed in exocrine pancreatic cells, such as carbonic anhydrase II and lactoferrin, have been reported in patients with type 1 diabetes mellitus. Citokeratin 19 (CK19) is an antigen expressed in both pancreatic and biliary ductal cells. Autoantibodies against CK19 (anti-CK19) have been found in patients with autoimmune hepatitis. In this study we verify whether CK19 represents a target antigen for autoimmunity in type 1 diabetes.

Materials and Methods: We evaluated the presence of anti-CK19 in sera from 62 patients $(39 \mathrm{M} / 23 \mathrm{~F}$; age $1-$ 19 years) with duration of type 1 diabetes mellitus $<3$ months and from 30 healthy controls $(13 \mathrm{M} / 14 \mathrm{~F}$; age 3-18 years). Serum levels of autoantibodies anti-CK19 of the IgG class were measured by ELISA and the mean OD +2 SD of controls was considered the upper limit of normal range. Results were expressed as median and range.

Results: Diabetic patients showed anti-CK19 levels higher $(0.430$, range $0.210-1.690 ; p<0.0001)$ than control subjects $(0.300$, range $0.140-0.510)$. Values above the normal range were found in $23 / 63(37 \%)$ type 1 diabetic patients and in $1 / 30(3.3 \%)$ healthy controls $(\mathrm{p}<0.001)$.

Conclusion: Our results on the presence of anti-CK19 in sera of type 1 diabetic patients represent another evidence of autoimmunity against exocrine pancreatic antigens in type 1 diabetes mellitus.

\section{PS 1}

\section{Epidemiology of type 1 diabetes I}

\section{5}

Further clues to the aetiology of type 1 diabetes: spatial clustering amongst 0-29 year olds in Yorkshire, UK

R. G. Feltbower ${ }^{1}$, R. J. Q. McNally ${ }^{2}$, L. Parker ${ }^{2}$, H. J. Bodansky ${ }^{3}$, F. M. Campbell ${ }^{4}$, P. A. McKinney ${ }^{1}$; ${ }^{1}$ Paediatric Epidemiology Group, University of Leeds, ${ }^{2}$ School of Clinical Medical Sciences (Child Health), University of Newcastle Upon Tyne, ${ }^{3}$ Diabetes Centre, The General Infirmary at Leeds, Paediatrics, St. James's University Hospital, Leeds, United Kingdom.

Background and Aims: The aetiology of type 1 diabetes in children and young people has a strong environmental component. The presence of geographical or spatial clustering would suggest localised excesses of relevant environmental exposures. We studied spatial clustering amongst children and young adults aged $0-29$ years using population-based data from a specialist register in Yorkshire, UK.

Material and Methods Two data sets of children and young people diagnosed with type 1 diabetes while living in Yorkshire were analyzed: (1) cases aged 0-14 years diagnosed between 1978-2002; (2) cases aged 15-29 years diagnosed between 1991-2002. The Potthoff-Whittinghill method was used to test for spatial clustering.

Results 3019 cases of type 1 diabetes aged $0-14$ years and 989 cases of type 1 diabetes aged 15-29 years were analysed. There was statistically significant spatial clustering for 0 14 year olds diagnosed during the period 1978-1985 only ( $P=0.009)$, mainly involving younger children $(P=0.01$ for $0-4$ year olds and $P=0.005$ for $5-9$ year olds). There was also significant clustering for $15-29$ year olds $(P=0.003$ and $P<0.001$ for cases diagnosed during the periods 1991-1995 and 1996-2005, respectively), especially involving 2024 year olds $(P=0.002$ and $P=0.01$ for cases diagnosed during the periods 1991-1995 and 1996-2005, respectively). Conclusion The present study is the first to analyse spatial clustering of type 1 diabetes amongst older teenagers and young adults. The findings show that geographical clustering of cases is present and differs by age and time period. This pattern suggests that environmental factors associated with persistent localised exposure may influence the risk of developing type 1 diabetes in different age groups during different time periods.

Supported by Department of Health 


\section{6}

\section{WITHDRAWN}

0267

Continued increasing trend of type 1 diabetes incidence in childhood in North Rhine-Westphalia, Germany J. Rosenbauer ${ }^{1}$, K. Straßburger ${ }^{1}$, A. Icks ${ }^{1}$, U. Krause ${ }^{2}$, R. W. Holl ${ }^{2}$, G. Giani ${ }^{1}$;

${ }^{1}$ Institute for Biometrics and Epidemiology, German Diabetes Research Institute, Leibniz Institute at Duesseldorf University, ${ }^{2}$ Department of Epidemiology, University of Ulm, Germany.

Background and Aims: A large risk population and a sufficiently long observation period are important preconditions for valid estimation of Type 1 diabetes incidence in childhood and its temporal trend. Aim of the present study was to estimate incidence and time trend of Type 1 diabetes in children 0-14 years of age in the large risk population of the German federal state North Rhine-Westphalia (NRW) during 1996-2003.

Materials and Methods: NRW has the largest population among all German federal states comprising about one quarter of all children in the age group 0-14 years in Germany. During the study period the average risk population was 2.91 millions children. The North Rhine-Wesphalian diabetes incidence register ascertains newly diagnosed cases of type 1 diabetes by means of three data sources: the prospective hospital-based active surveillance system ESPED, annual inquiries among paediatric, internal, and general medical practices, and the computer-based documentation system DPV founded for quality control and scientific research in paediatric diabetes care. Completeness of ascertainment was estimated by the capture-recapture-method using log-linear modelling. Point and interval estimates (95\%-CI) of incidence rates (per 100,000 person-years) were based on Poisson distribution. Age-and sex-standardized rates were estimated by the direct method using equal weights. Poisson regression analysis was applied to assess time trends. Results: During 1996-2003 a total of 4,261 newly diagnosed diabetic children aged $0-14$ years $(2,227$ boys, 2,034 girls) were registered. Ascertainment was estimated to be $96.8 \%$ (95\%-CI: $96.4 \%-97.2 \%)$ complete. The overall age- and sex-standardized incidence rate was 18.1 (17.6-18.7). The age-standardized incidence among boys $(18.5,17.7-19.3)$ was slightly higher than among girls $(17.8,17.0-18.5, \mathrm{p}=0.188)$. Incidence depended significantly on age $(\mathrm{p}<0.001)$. Age-specific estimates for age groups $0-4,5-9$, and $10-14$ years were 12.5 (11.7-12.4), 19.8 (18.8-20.8), and 22.0 (21.0-23.1), respectively.
Annual incidence rates ranged between 15.4 in 1998 and 21.7 in 2003 . The average annual incidence increase was estimated as $3.9 \%(2.5 \%-5.3 \%)$. There was no significant difference in incidence trend among boys and girls (annual increase: $4.3 \%$ vs. $3.4 \%, p=0.526)$. But incidence trend varied significantly between age-groups $(\mathrm{p}=0.012)$, with the steepest increase in youngest children. Annual increases for the age groups $0-4,5-9$, and $10-14$ years were $7.7 \%$, $3.8 \%$, and $2.1 \%$, respectively.

Conclusion: These data based on a large risk population and high case ascertainment confirm that the incidence of type 1 diabetes in childhood is steadily increasing in Germany. The annual increase was slightly higher than the average increase in Europe. In accordance with other European data the steepest rise was observed among youngest children. Based on the observed incidence rate, there are annually about 2,500 children newly diagnosed with type 1 diabetes in Germany, what underlines the public health importance of childhood diabetes care. Further research is needed to identify causes of the continuous rise of diabetes incidence.

Supported by German Ministry of Health, Ministry of Innovation, Science, Research and Technology of the State of North Rhine-Westphalia

0268

The parallel increase of type 1 diabetes incidence in two different regions of Poland: highly industralised Upper Silesia and agricultural North-Eastern regions of Podlasie and Warmia-Mazury

A. Kretowski ${ }^{1,2}$, P. Jarosz-Chobot ${ }^{3}$, J. Peczynska ${ }^{4}$, E. Bandurska-Stankiewicz ${ }^{5}$, J. Rutkowska ${ }^{5}$, M. Urban ${ }^{4}$, M. Rewers ${ }^{2}$;

${ }^{1}$ Department of Endocrinology Diabetology and Internal Medicine, Medical University of Bialystok, Poland, ${ }^{2}$ Barbara Davis Center, University of Colorado, Aurora, United States, ${ }^{3}$ Department of Pediatrics, Endocrinology and Diabetes, Silesian Medical University, Gliwice, Poland, ${ }^{4}$ 2nd Department of Pediatrics, Medical University of Bialystok, Poland, ${ }^{5}$ Department of Endocrinology and Diabetology, General District Hospital, Olsztyn, Poland.

Background and Aims:The incidence of childhood type 1diabetes in Poland has been increasing, at least since the early 1970 's (3.5/100.000 in $1970-1981$ vs. $6.6 / 100.000$ in 1982-1985). A number of subsequent studies from several regions of Poland have suggested a further increase, but the reasons remain unknown.

Material and Methods: The aim of the present study was to evaluate if the increase of T1D incidence could be related to the 
environmental factors. Upper Silesia is the most industrialized, urbanized and polluted part of Poland, in contrast to the Podlasie and Warmia-Mazury regions - forested and agricultural. In both regions data concerning new cases of T1D among children aged $0-14$ years were collected prospectively between 1989 and 2003 as a part of EURODIAB program.

Results and Conclusions: The most recent data shows that in both regions the incidence of T1D has reached levels typical to Western Europe (14.5/100.000 in Upper Silesia and 18.8/100.000 in North-Eastern Poland). The average annual incidence increases since 1989 in Upper Silesia $(8.4 \% / y)$ and North-Eastern Poland $(7.0 \% / y)$ suggest that the epidemic of T1D has more features of a secular trend than year-to-year outbreaks. In addition, the factor(s) driving the epidemic are apparently independent of the environmental pollution and currently unabating in Poland.

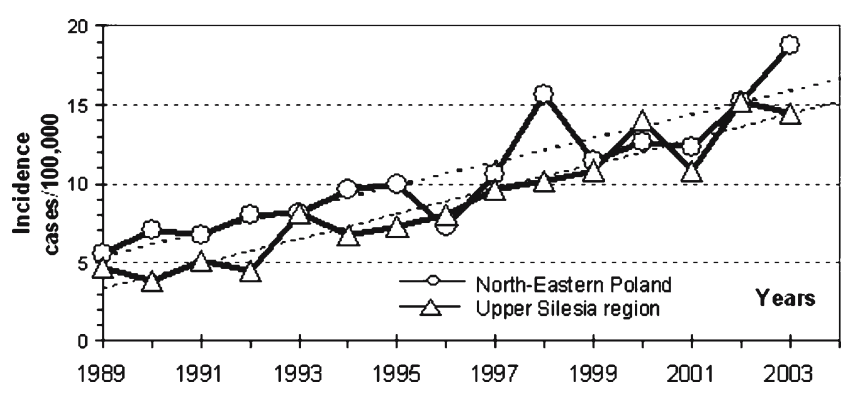

0269

Environmental risk factors affecting high incidence rate of type 1 diabetes in north-eastern Poland

E. Bandurska-Stankiewicz, J. Rutkowska;

Endocrinology and Diabetology, Olsztyn General District Hospital, Poland.

Background and Aims: In north-eastern Poland, Warmia and Mazury Region (W\&M Region) an increase in the incidence rate (IR) of type 1 diabetes (T1DM) was observed in the years 1994-2003. The annual IR increase in the age group 0-29 was $4.6 \%$, in this $9.8 \%$ in the age group $0-14$. The aim of the study was to assess the influence of selected environmental risk factors on the T1DM IR.

Materials and Methods: The prospective register was carried on in W\&M region according to EURODIAB methods. Three hundred thirty-one patients aged 0-29 were registered in the years 1994-2003. Perinatal, alimentary, infectious, socio-economic, climatic and pollution risk factors were assessed. The data obtained were compared with the data characteristic for the general Polish population. Results: Type 1 diabetes IR was closely associated with urban area residence compared to rural area; i.e. $12.7 / 100$
000 (95\%CI:10.4-15.0) and 8.8/100 000 (95\%CI:6.7-10.9) $(p<0.05)$, respectively, but only for children aged $0-14$ years. The place of residence was not a risk factor for the older age groups. The age of mothers over 25 , and especially over 35 years, was significantly associated with increased risk of T1DM $(\mathrm{p}<0.05)$. Diabetes was diagnosed more often in children born from the second and the following deliveries compared to the first one $(\mathrm{p}<0.05)$. The duration of full breast-feeding shorter than 3 months and early exposure to cow's milk was associated with higher IR of T1DM in our region $(p<0.05)$. Seasonality was reflected in higher IR of T1DM in autumn and winter than in summer; i.e. 1.2/ 100,000 vs $0.2 / 100,000(\mathrm{p}<0.0)$, respectively. The secondary and higher education level of mothers of patients with T1DM proved high risk factors $(\mathrm{p}<0.05)$. No connection was found between birth weight, caesarean delivery, viral infections (mumps, roseola, chicken-pox), vaccinations, water composition and pollution after the Chernobyl accident and the incidence of T1DM in our region.

Conclusion: Among the studied risk factors for T1DM the significant ones were:

-place of residence

-age of mother

-number of deliveries

-full breast feeding period shorter than 3 months

-seasonality of incidence

-hygienisation of life resulting from mother's education level

0270

New data regarding the influence of gender and ethnicity on the incidence of childhood type 1 diabetes mellitus in Romania

V. Serban ${ }^{1}$, A. Vlad ${ }^{1}$, L. Dumbrava ${ }^{2}$, I. Velea ${ }^{3}$;

${ }^{1}$ Diabetes Clinic, University of Medicine and Pharmacy, Timisoara, ${ }^{2}$ Pediatric Department, Districtual Hospital, Oradea, ${ }^{3}$ Second Pediatric Clinic, University of Medicine and Pharmacy, Timisoara, Romania.

Background and Aims. Romania is a country having a low/intermediate incidence of type 1 diabetes mellitus (T1DM) in children. As in other countries, the number of new cases of T1DM is getting higher in Romania, too. The purpose of this work was to assess the time trends in the incidence of childhood T1DM and to reveal the influences determined by gender and ethnicity.

Material and Methods. Data were obtained from two sources: 1. Romanian Childhood Diabetes Registry, developed since 1995 by ONROCAD (Romanian acronym for "Romanian National Organization for the Protection of Children and Adolescents with Diabetes"); 2. Medical records from Clinical Center "Cristian Serban" Buzias, 
where children with T1DM from Romania are treated. Till year 2001, only data from children aged $0-14$ years were obtained. Starting year 2002, ONROCAD collected data from age group $0-18$ years, too

Results. Table 1 presents the incidence of T1DM in children from Romania, between years 1996 and 2005.

\section{Table 1}

\begin{tabular}{|c|c|c|c|c|c|c|c|c|c|c|c|}
\hline \multicolumn{2}{|l|}{ Incidence* } & 1996 & 1997 & 1998 & 1999 & 2000 & 2001 & 12002 & 2003 & 2004 & 2005 \\
\hline \multicolumn{2}{|c|}{ General (0-14 years) } & 3.91 & 4.02 & 4.10 & 4.97 & 5.05 & 5.66 & 4.88 & 5.47 & 5.36 & 6.30 \\
\hline Gender & G & 3.96 & 4.68 & 4.34 & 5.23 & 4.94 & 5.04 & 4.34 & 5.41 & 4.93 & 6.21 \\
\hline (0-14 years) & B & 3.88 & 3.39 & 3.72 & 4.56 & 5.15 & 6.15 & 5.34 & 5.52 & 5.78 & 6.39 \\
\hline Ethnicity & $\mathbf{R}$ & - & - & 3.90 & 4.57 & 4.82 & 5.53 & 4.64 & 5.23 & 5.38 & 6.30 \\
\hline (0-14 years) & H & - & - & 7.02 & 8.98 & 7.88 & 7.19 & 5.91 & 11.63 & 9.77 & 8.84 \\
\hline General (0-18 & years) & - & - & - & - & - & - & 5.51 & 5.67 & 5.93 & 6.12 \\
\hline Gender & $\mathbf{G}$ & - & - & - & - & - & - & 4.71 & 5.31 & 5.23 & 5.87 \\
\hline (0-18 years) & B & - & - & - & - & - & - & 6.28 & 6.01 & 6.66 & 6.36 \\
\hline Ethnicity & $\mathbf{R}$ & - & - & - & - & - & - & 5.34 & 5.62 & 5.93 & 6.13 \\
\hline (0-18 years) & $\mathbf{H}$ & - & - & - & - & - & - & 8.27 & 9.31 & 10.69 & 8.27 \\
\hline
\end{tabular}

Legend: *=no. of cases $/ 100,000 ; \mathrm{G}=$ girls; $\mathrm{B}=$ boys; $\mathrm{R}=$ Romanians; $\mathrm{H}=$ Hungarians.

Some remarks can be made: 1 . The general incidence in age group 0-14 years increased with 61\% between 1996 and 2005. A plateau was registered between years 2001 and 2004. 2. Incidence in age group $0-18$ years increased from 2002 to 2005 , especially in girls. 3. The incidence was higher in girls between 1996 and1999 and in boys thereafter. 4. The incidence in Romanians was lower than in Hungarians (the main ethnic minority); environmental factors being the same, the only explanation is given by genetic differences.

Conclusions. The incidence in T1DM in Romania showed an increasing trend over the past 10 years. The girls were more frequent affected in the first half of the decade and the boys thereafter. Children from the Hungarian minority have a higher incidence than those from the Romanian majority, due to their genetic background.

\section{PS 2}

\section{Epidemiology of type 1 diabetes II}

\section{1}

\section{Factors affecting Body Mass Index from onset of diabetes to age 18}

J. Svensson ${ }^{1}$, N. Birkebæk ${ }^{2}$, K. Kristensen ${ }^{2}$ on behalf of the Danish Diabetes Register (DSBD), Glostrup, Denmark, ${ }^{1}$ Pediatric Department Glostrup, DSBD, Glostrup, ${ }^{2}$ Pediatric Department Skejby, DSBD, Aarhus, Denmark,
Background and Aims: The Danish Childhood Diabetes Register was founded in 1996. It is a nation-wide register collecting data from all Danish paediatric diabetes centres treating type-1-diabetic patients aged $0-15$ years. Weight and height is measured at onset of the disease and yearly thereafter. The aim of the present study was to describe the factors affecting body mass index (BMI) from onset to age 18 . Materials and Methods: Weight and height is measured by health professionals at each centre. Weight is measured in kilogram, height in $\mathrm{m}$. Weight and height measured less than 30 day after onset were excluded. BMI was calculated as weight $(\mathbf{k g}) /$ height $(\mathbf{m})^{2}$ In the model BMI was entered as sds-score. Standard deviation score (SDS) was defined as (observed - expected)/standard deviation for age and sex using a Danish reference material. General linear modelling was used to estimate the association between the different risk factors and the BMI sds.

Results: There are 2906 children in the register, and 13310 year reports with height and weight data. There was a significant increase in BMI sds with length of diabetes $(p<0.01)$ and age of onset $(p<0.01)$. There was a significant interaction between diabetes duration and sex. Girls tended to start on a lower level, but had a more steep increase in BMI. The BMI sds were the same for boy and girls after approximately two years of diabetes, thereafter the girls tended to have a higher BMI sds. The BMI sds differed between centres. There were a significant association between BMI sds and $\mathrm{HbAlc}$ and centre. There were no association with year of onset.

Conclusion: BMI sds differed between centres and was associated with diabetes duration, sex, HbAlc and age at onset.

0272

Breastfeeding among mothers with type 1 diabetes (T1D) in a primary diabetes prevention trial

S. Bärlund ${ }^{1}$, D. Cuthbertson ${ }^{2}$, A. M. Nucci ${ }^{3}$, J. P. Krischer ${ }^{2}$, M. Knip ${ }^{4}$, H. K. Åkerblom ${ }^{4}$,

S. M. Virtanen ${ }^{1,5}$ TRIGR Study Group;

${ }^{1}$ Department of Health Promotion and Chronic Disease Prevention, Public Health Institute, Helsinki, Finland, ${ }^{2}$ Pediatrics Epidemiology Center, University of South Florida, Tampa, United States, ${ }^{3}$ Nutrition Support and Intestinal Care Center, Children's Hospital of Pittsburgh, United States, ${ }^{4}$ Hospital for Children and Adolescents, University of Helsinki, Finland, ${ }^{5}$ Tampere School of Public Health, University of Tampere, Finland.

Background and Aims: Difficulties in initiating and maintaining breastfeeding among mothers with T1D have previously been reported. The aim of this study was to 
compare breastfeeding among mothers with and without T1D participating in the Trial to Reduce IDDM in the Genetically at Risk (TRIGR).

Materials and Methods: The present analysis included 696 mothers with and 690 mothers without T1D, whose infants were at least 9 months old by the end of February 2006. Information on breastfeeding was acquired from the family by dietary interviews conducted at 2 weeks and at 1 , 2, 3, 4, 5, 6 and 9 months of age of the infant.

Results: During the first 2 weeks of life $89 \%$ of the mothers with T1D breastfed their infants versus $93 \%$ of the mothers without T1D. At 3, 6 and 9 months the proportions of infants being breastfed were significantly different $(\mathrm{p}<0.001)$ with 65,50 and $38 \%$ among the mothers with T1D and 82, 71 and 53\% among the other mothers, respectively. Median duration of exclusive breastfeeding was 0.1 weeks and 1.4 weeks among mothers with and without T1D, respectively $(\mathrm{p}<0.001)$.

Conclusion: The proportion of mothers with or without T1D who initially breastfed their infants did not differ significantly. However, the duration of both exclusive and total breastfeeding was shorter among the mothers with T1D.

Supported by NICHD, NIDDK, NIH (HD040364, HD042444), CIHR, JDRF, EC (QLK1-2002-00372), EFSD, Academy of Finland, DFN, Finnish Diabetes Research Foundation, Mead Johnson Nutritionals.

\section{3}

Seasonal variation of newborns with cord blood islet autoantibodies

K. Lynch, B. Lernmark, J. Merlo, A. Nilsson, G. Hansson, H. Rastkhani, C. Cilio, S. Ivarsson, A. Lernmark;

Clinical Sciences, Lund University, Malmö, Sweden.

Background and Aims: Genetic factors and unknown environmental factors are important in the development of islet autoimmunity and Type1 Diabetes (TID). The aim of this study was to test whether autoantibodies against GAD65, IA-2 and insulin, which predict type 1 diabetes, in the cord blood of newborns born to non-diabetic mothers may mark a triggering event during pregnancy.

Materials and Methods: HLA-DQ genotypes were determined in dried blood spots taken at birth from more than 35,000 children in the Diabetes Prediction in Skåne (DiPiS) study. Autoantibodies to glutamic acid decarboxylase $(\mathrm{GAD} 65 \mathrm{Ab})$, islet associated antigen-2 (IA-2Ab), and insulin (IAA) were determined in standard radioligand binding assays. Information about the pregnancy was obtain at birth by the midwife and from a questionnaire administered to the mother when the child was two months of age. Newborns of mothers reporting they had diabetes (T1D or T2D) or gestational diabetes at birth were excluded from this study.

Results: A total of $1.6 \%$ (GAD65Ab, 0.58\%; IA- $2 \mathrm{Ab}$ $0.06 \%$; IAA, $1.0 \%$ ) newborns had high levels of islet autoantibodies in the cord blood. Cord blood serum levels of GAD65Ab $(\mathrm{r}=0.96, \mathrm{p}<0.001)$ and IA- $2 \mathrm{Ab}(\mathrm{r}=0.90$, $\mathrm{p}<0.001$ ) correlated strongly with levels in the mother at the time of delivery. HLA-DQ2/8 conferring the highest genetic risk of T1D was associated with GAD65Ab (OR 1.98 (95\%CI was $1.13-3.49), \mathrm{p}=0.017)$, IA-2Ab (OR 4.86 $(95 \% \mathrm{CI}$ was $1.42-16.57), \mathrm{p}=0.011)$ as well as IAA (OR $2.20(95 \%$ CI was $1.45-3.34), p<0.0005)$. The prevalence of newborns with islet autoantibodies was increased in the third yearly quarter (July, August and September in four consecutive years): GAD65Ab: OR 1.37 (95\% CI was $1.01-$ 1.85), $\mathrm{p}=0.046)$; IA-2Ab OR $2.09(95 \% \mathrm{CI}$ was $0.86-5.10)$, $\mathrm{p}=0.10$; IAA, OR $2.02(95 \%$ CI was $1.63-2.52), \mathrm{p}<0.0001)$ compared to the rest of the year. The prevalence of reported gastroenteritis $(p<0.0001)$ or fever $(p<0.0001)$ during pregnancy also showed a monthly seasonal variation, with a consistent lag time correlation with prevalence of autoantibodies that was strongest at 6-7 months prior to birth $(\mathrm{p}<0.001)$. Among children born in the third quarter there was a positive association between islet autoantibodies in the newborn and reported gastroenteritis (OR 1.80 (1.04-3.11), $\mathrm{p}=0.037)$ in the mother. The HLA and environmental associations with cord blood autoantibodies were independently significant.

Conclusion: Autoantibodies detected in cord blood showed consistent seasonal variation over four years. The increased frequency of islet autoantibodies was associated with genetic and environmental association that occurs primarily through the mother. The data suggest that environmental exposure during early pregnancy may trigger islet autoimmunity which may have consequences not only for the risk of the mother to develop type 1 diabetes during or after pregnancy but also for the offspring.

Supported by Lund University

0274

Increased relative birthweight in children with diabetes high risk HLA may be enhanced by infections during pregnancy

H. E. Larsson ${ }^{1}$, K. Lynch ${ }^{2}$, B. Lernmark ${ }^{2}$, A. Nilsson ${ }^{2}$, H. Rastkhani ${ }^{2}$, G. Hansson ${ }^{1}$, A. Lernmark ${ }^{2}$, S.-A. Ivarsson ${ }^{1}$ and the DiPiS study group;

${ }^{1}$ Department of Clinical Sciences - Pediatrics, ${ }^{2}$ Department of Clinical Sciences, Lund University, Malmö, Sweden. 
Background and Aims: It has been reported that children born with HLA conferring high risk for type 1 diabetes (T1D) have an increased high relative birthweight (HrBW). The underlying mechanisms for the HrBW is not understood, but gestational infections, islet autoantibodies, or both, are thought to contribute to T1D risk. The aim of this study was to test following hypotheses: 1) Mothers reporting infections during pregnancy give birth to children with HrBW; 2) Infections during pregnancy affect the association between HLA and HrBW; 3) The reduction in $\mathrm{HrBW}$ by islet autoantibodies as shown previously is explained by infections during pregnancy.

Material and Methods: HLA-genotypes were determined in dried blood spots of cord blood from children in the population-based Diabetes Prediction in Skane (DiPiS) study. Children born preterm (<37 weeks of gestation) and to mothers with diabetes or gestational diabetes were excluded. Standard radioligand binding assays were used to determine GAD65Ab, IA-2Ab and IAA. Birthweight (BW) was adjusted for gestational age and divided into quartiles. Upper quartile was defined as HrBW. Two months after delivery, the mother completed questionnaires on gastroenteritis, fever or both during pregnancy.

Results: A total of $19.3 \%$ (3832/19 756) mothers reported infections during pregnancy. $10.7 \%$ reported gastroenteritis with or without fever and 3.7\% fever without gastroenteritis. Islet autoantibody negative children born to mothers reporting infections during pregnancy had an increased risk for $\mathrm{HrBW}$, with the highest risk with fever and/or gastroenteritis across several trimesters (OR 1.51 (95\% CI was 1.19-1.91), $\mathrm{p}<0.001$ ). This effect on HrBW was aggravated in children born with the HLA-DQ2/8 or DQ8/X T1D high risk genotypes (OR 2.31 $(1.11-4.82), p=0.025)$ and in the T1D low risk genotype HLADQB1*0603 (OR 2.32 (1.14 4.72), p=0.021). Autoantibodies present in cord blood modified the effect of infections by decreasing the risk of $\mathrm{HrBW}$ with fever and/or gastroenteritis in one trimester (OR $0.32(0.17-0.60), \mathrm{p}<0.0005)$ or several trimesters (OR $0.10(0.01-0.74), \mathrm{p}=0.024)$.

Conclusion: This study revealed 1) that children have an increased HrBW when born to mothers reporting infections with fever, gastroenteritis, or both, during pregnancy; 2) that the previously reported association between T1D high risk HLA with $\mathrm{HrBW}$ is aggravated in children born to mothers reporting infections during pregnancy and 3) that islet autoantibodies in cord blood decreases $\mathrm{HrBW}$ if mothers reports infections during pregnancy. These data suggests an interaction between HLA, gestational infections, islet autoantibodies and fetal growth.

Supported by the Swedish Research Council (72X-14064), Skane County Council, Swedish Childhood Diabetes Fund, Swedish Diabetes Association, UMAS Funds, the American Diabetes Association Gregg Award and the National Institutes of Health (DK026190)
0275

Enterovirus but not adenovirus infections are associated with autoantibody defined risk in children from the general population and in children at onset of the disease

M. Schlosser ${ }^{1}$, V. Moya-Suri ${ }^{2}$, H. Kenk ${ }^{3}$, W. Kerner ${ }^{4}$, R. Walther ${ }^{1}$, R. Mentel ${ }^{2}$;

${ }^{1}$ Department of Medical Biochemistry and Molecular Biology,, ${ }^{2}$ Friedrich Loeffler Institute of Medical Microbiology, University Greifswald, Germany, ${ }^{3}$ Institute of Pathophysiology, University Greifswald, Karlsburg, Germany, ${ }^{4}$ Clinic for Diabetes and Metabolic Diseases, Klinikum Karlsburg, Germany.

Background and Aims: Environmental factors as virus infections have been associated with the manifestation of type 1 diabetes (T1DM) in several but not all studies performed so far. As shown for autoantibody (AAb) positive first degree relatives of patients, the risk to develop T1DM is also increased in probands from the general population if multiple AAbs against different beta-cell antigens occur. The aim was to study if enterovirus (EV) and adenovirus (ADV) infections are associated with the risk to develop T1DM in AAb positive children from the general population and in children at onset.

Materials and Methods: EV-RNA and ADV-DNA sequences were detected by conventional RT-PCR as well as by two-step TaqMan RT-PCR in fifty healthy schoolchildren without heredity of T1DM recruited from the Karlsburg type 1 diabetes risk study and differentiated according to their AAb profile in the first or second followup serum sample into three risk groups: 29 probands at high risk (HR; $>1 \mathrm{AAb}), 10$ at moderate risk (MR; $1 \mathrm{AAb}$ at high titer) and 11 at low risk (LR; $1 \mathrm{AAb}$ at low titer). Furthermore, 47 children with newly diagnosed T1DM (median diabetes duration 5 days) and $50 \mathrm{AAb}$ negative control children were involved.

Results: The overall prevalence of EV-RNA amounted to $20 \%(10 / 50)$ in AAb positive children and 36\% (17/47) in children at onset and was significantly increased comparison to controls $(4 \% ; 2 / 50 ; \mathrm{p}=0.03$ and $\mathrm{p}<0.001$, respectively). However, if the prevalence of EV-RNA is analyzed according to $\mathrm{AAb}$ defined risk, only children at HR have significantly increased frequencies of virus infections in comparison to controls: $24.1 \%(7 / 29, \mathrm{p}=0.02)$ in RT-PCR and $28.6 \%(8 / 28, p=0.002)$ in two-step RT-PCR. The prevalence of EV-RNA in children at MR or LR did not differ from those of controls. Characterization of the EV amplicons by direct sequencing revealed homology of $66.7 \%(8 / 12)$ with coxsackievirus B group (B4: $n=5, B 2$ : $n=2$, and B6: $n=1)$ in the HR group and of $50 \%(10 / 20)$ with coxsackievirus B4 in probands at onset. During the 
follow-up of the study, 12 children at HR (four tested positive for EV-RNA) and one child at MR have progressed to clinical T1DM so far. For ADV no data supporting an association with T1DM have been found; ADV-DNA was detected in $14 \%(7 / 50)$ samples of $\mathrm{AAb}$ positive children, in $2.1 \%(1 / 47)$ samples at onset children and in $4 \%(2 / 50)$ of controls. There was no significant seasonality of sampling in any of the groups investigated. However, EVRNA was found significant frequently in 55\% (11/20) of children at onset in autumn and winter (September to February) in comparison to $22.2 \%(6 / 27)$ of children at onset in spring and summer (March to August; $p=0.046$ ).

Conclusion: Our data demonstrate that EV infections but not ADV infections are a risk factor for T1DM and are associated with the induction of beta-cell autoimmunity before the onset of the disease. Especially in genetically susceptible probands at high risk identified by combined $\mathrm{AAb}$ screening from the general population, EV infections seems to accelerate and/or initiate the beta-cell destruction.

Supported by Else Kroener Fresenius Foundation, German Diabetes Foundation

0276

Activity of constitutive, antiviral enzyme ( $2^{\prime} 5^{\prime}$-oligoadenylate synthetase) is significantly correlated with GADA levels in T1D families

V. Bonnevie-Nielsen ${ }^{1}$, Å. Lernmark ${ }^{2}$, D.-J. Zheng ${ }^{1}$, T. Daniels ${ }^{2}$, S. Lu ${ }^{3}$, L. Bekris ${ }^{2}$, L. Field ${ }^{3}$;

${ }^{1}$ Pathology and Laboratory Medicine, University of British Columbia, Vancouver, Canada, ${ }^{2}$ Department of Medicine, University of Washington, Seattle, United States, ${ }^{3}$ Medical Genetics, University of British Columbia, Vancouver, Canada.

Background and Aims: During the pathogenesis of Type 1 diabetes (T1D), the processes that initiate damage to the $\beta$ cells in the islets of Langerhans remain unknown. Increasing evidence indicates that infection with pancreotropic viruses could be one of the first events that initiates the pathogenic course, ultimately leading to eradication of the $\beta$ ells. In our human studies of the innate, antiviral immune defense in T1D, we have found that activity of the antiviral, IFN- $\alpha$ inducible enzyme, 2'5'-oligoadenylate synthetase $\left(2^{\prime} 5^{\prime} \mathrm{AS}\right)$ is significantly and persistently increased in T1D patients. We have demonstrated that $2^{\prime} 5^{\prime} \mathrm{AS}$ activity is under strong genetic control by specific genotypes in the OAS1 ( $\left.2^{\prime} 5^{\prime} \mathrm{AS}\right)$ gene, implicating a possible role for $2^{\prime} 5^{\prime} \mathrm{AS}$ in the pathogenesis of T1D. The total, constitutive $2^{\prime} 5^{\prime} \mathrm{AS}$ activity would result in induction of apoptosis and reduction of the $\beta$ cell mass. GADA (glutamic acid decarboxylase autoanti- bodies) is a widely used marker for identifying subjects at risk of developing T1D. The appearance of GAD epitopes reflects a process where $\beta$ cells are dysfunctional or are undergoing apoptosis. Whether these processes are caused by latent virus infections or $\beta$ cell specific intrinsic factors are not well understood. We propose that constitutive $2^{\prime} 5^{\prime} \mathrm{AS}$ activity could be a contributing factor in $\beta$ cell killing. The aim of the study is to determine a possible association between constitutively expressed $2^{\prime} 5^{\prime} \mathrm{AS}$ enzyme activity and the levels of GADA in individuals with T1D.

Materials and Methods: The study group was Danish nuclear families. In these families there were 82 diabetic and 82 healthy siblings. GADA levels were determined according to international standards. Cutoff level chosen for autoantibody detection was $70 \mathrm{U} / \mathrm{ml}$. Three extreme GADA values, 1-2 orders of magnitude higher than mean values, were excluded from calculations. Activity of $2^{\prime} 5^{\prime} \mathrm{AS}$ was analyzed in a radiometric assay, in which $2^{\prime} 5^{\prime} \mathrm{AS}$ catalyzes the conversion of ATP to $2^{\prime} 5^{\prime}$ - oligoadenylates of different chain-lenghts.

Statistical testing was done by correlation and regression analyses.

Results: In siblings with T1D, 49\% (40/82) were positive for GADA. In healthy siblings, 7.3\% (6/82) were positive for GADA. Totally, 46 individuals were found positive for GADA. Of these, six individuals were not having T1D. When the levels of GADA in the included individuals $(n=43)$ were tested against $2^{\prime} 5^{\prime}$ AS activity levels, we found a significant correlation, $r=0.43, p=0.004$. These results indicate a high degree of co-variation between the two parameters.

Conclusion: Previously we have shown that activity of the antiviral enzyme $2^{\prime} 5^{\prime} \mathrm{AS}$ is under strong genetic control, a finding that is of interest in studies of pathogenesis of T1D because $2^{\prime} 5^{\prime} \mathrm{AS}$ activity induces apoptosis. Functionally, the enzyme plays two roles, induction of a constitutive, "physiological" apoptosis in $\beta$ cells and/or induction of very high levels of apoptosis when the enzyme becomes activated during virus infections. In both cases $\beta$ cells will die to different degrees. It is suggested that a genetically controlled $2^{\prime} 5^{\prime}$ AS activity might facilitate $\beta$ cell killing and lead to generation of GADA in predisposed individuals. Supported by JDRF, NIH and Children's Hospital Foundation, Vancouver

0277

Serum adiponectin and the development of microangiopathy in type 1 diabetes: An inception cohort Study

K. K. Andersen ${ }^{1}$, J. Frystyk ${ }^{1}$, P. Hovind ${ }^{2}$, L. Tarnow ${ }^{2}$, H.-H. Parving ${ }^{2,3}$, A. Flyvbjerg ${ }^{1,3}$; 
${ }^{1}$ Clinical Institute and Medical Research Laboratories M, Medical Research Laboratories, Århus, Denmark, ${ }^{2}$ Steno Diabetes Center, Gentofte, Denmark, ${ }^{3}$ Faculty of Health Science, University of Århus, Denmark.

Background and Aims: Low circulating adiponectin (ADPN) levels have been associated with an increased risk of cardiovascular disease and retinopathy in patients with type 2 diabetes. In contrast, several studies have observed elevated serum ADPN levels in patients with type 1 diabetes. Furthermore, a recent cross-sectional study from our group showed even higher serum ADPN levels in type 1 diabetic patients with microvascular complications as compared to matched counterparts without complications. Accordingly, we wanted to investigate the potential predictive value of serum ADPN for the development of microvascular complications in type 1 diabetes.

Materials and Methods: A cohort $(n=270)$ of newly diagnosed type 1 diabetic patients was followed for 18.0 years (median; range 1.0-21.8 years). Baseline levels of ADPN were measured in all participants after 3 years of diabetes. A second ADPN measurement was performed in 206 patients and took place either when they developed a microvascular complication or when the diabetes duration exceeded 15 years. Serum ADPN was measured by a validated in-house sandwich assay.

Results: Baseline serum ADPN levels did not differ between those patients who later developed micro- or macroalbuminuria and those who did not develop diabetic nephropathy $(\mathrm{P}=0.5)$. Similarly, there were no differences in baseline levels of ADPN between patients who developed diabetic retinopathy (simplex as well as proliferative) and those who did not $(\mathrm{P}=0.3)$. When patients were stratified according to baseline serum ADPN levels above or below the median and analyzed for the time to develop simplex retinopathy, there was a tendency towards a protective role of high ADPN levels $(\mathrm{P}=0.05)$.

Conclusion: Despite of previously reported increased levels of serum ADPN levels in type 1 diabetic patients with microvascular complications, we found no significant predictive value of serum ADPN in an inception cohort study comprising 270 type 1 diabetic patients with an median follow-up of 18 years.

Supported by the Danish Diabetes Association, the Poul and Erne Sehested Hansen Foundation, the Aase and Ejnar Danielsen Foundation, and the Per S. Henriksen Foundation

\section{PS 3}

\section{Genetics of type 1 diabetes}

0278

T1DBase: Integration of public and private genetics, genomics and functional data in type 1 diabetes

N. Goodman ${ }^{1}$, E. C. Adlem ${ }^{2}$, J. Allen ${ }^{2}$, D. B. Burdick ${ }^{1}$, O. S. Burren ${ }^{2}$, V. M. Cassen ${ }^{1}$, C. C. Cavnor ${ }^{1}$,

G. E. Dolman ${ }^{2}$, K. F. Friery ${ }^{1}$, B. C. Healy ${ }^{2}$, E. M. Hulbert ${ }^{1}$, S. A. Killcoyne ${ }^{1}$, B. Kutlu ${ }^{1}$, J. Mazzarelli ${ }^{3}$, B. Rajagopalan ${ }^{4}$, H. Schuilenburg ${ }^{2}$, N.M. Walker ${ }^{2}$, C.J. Stoeckert ${ }^{3}$, J.C. Mychaleckyj ${ }^{4}$, J. A. Todd ${ }^{2}$, L. J. Smink ${ }^{2}$;

${ }^{1}$ Juvenile Diabetes Research Foundation Center for Bioinformatics, Institute for Systems Biology, Seattle, United States, ${ }^{2}$ Juvenile Diabetes Research Foundation/Wellcome Trust Diabetes and Inflammation Laboratory, University of Cambridge, United Kingdom, ${ }^{3}$ Center for Bioinformatics, University of Pennsylvania, United States, ${ }^{4}$ Wake Forest University School of Medicine, Winston-Salem, United States.

Background \& Aims: T1DBase (http://T1DBase.org) is a website and database that supports the type 1 diabetes (T1D) research community. T1DBase is a collaboration between the JDRF Center for Bioinformatics at ISB and the JDRF/Wellcome Trust (WT) Diabetes and Inflammation Laboratory (DIL) at the University of Cambridge. The Beta Cell Gene Expression Bank at T1DBase is the result of collaboration with D. Eizirik (Laboratory for Experimental Medicine, Free University of Brussels). We are also functionally interfacing T1DBase with EPConDB (Beta Cell Biology Consortium). Our aim is to integrate T1D specific and general biological data to create an overview of ongoing research and to provide informatics and genetics tools to T1D researchers in order to promote collaboration by allowing researchers to compare data and share results. Methods: T1DBase focuses on the molecular genetics and biology of T1D susceptibility and pathogenesis. T1DBase contains data from human, mouse and rat. Datasets include: annotated genomes, genetically identified T1D susceptibility regions and genes, genotypes from the recently published Quad-Scan and other studies, descriptions of NOD mouse congenic strains (with L. Wicker, JDRF/WT DIL), tissue-specific gene expression, microarray data from published experiments, protein interactions from public databases, and biological pathways from KEGG and BioCarta. Data for a given gene is presented on a single page, using well-defined orthology. Tools include the GBrowse genome browser, site-wide search, Connect-theDots for connecting gene identifiers, Cytoscape for biological networks, and a microarray viewer. 
Over the last year, we continued to develop new tools, integrate new data sources, and seek new collaborations. T1DBase includes gene models from Ensembl, EPConDB, CCDS and DIL and provides consensus models. The Beta Cell Gene Atlas provides beta cell gene expression under different conditions. Using this data, we have compiled a list of genes that are expressed in the beta cell under basal conditions. The Beta Cell Gene Expression Bank contains over 750 beta cell-specific gene annotations and curated beta cell-specific pathways.

Marker data from dbSNP and HapMap, including genotypes, has been added and is available through a BioMartstyle filter. The HapMap and dbSNP data has been extracted and imported into GLIDER (Genetics Laboratory Interactive Database and Experimental Repostory). GLIDER provides a generic data store for all genotype data and can be easily expanded to contain additional datasets.

My T1DBase allows users to create folders of genes, markers or other features of interest. These collections can be used as a starting point for tools and to save state for future visits.

In the T1DBase Gene Dossier, a list of genes is scored across a number of T1D specific datasets, including pathways and interaction networks.

Conclusions: T1DBase has approximately 500 visits per day (a visit is a continuous session by a single user and generally involves many page hits), indicating that the system is widely employed. We aim to extend its utility to all T1D research areas from DNA sequence to disease mechanism.

Supported by JDRF, Wellcome Trust

0279

Better Diabetes Diagnostic (BDD): A country-wide registry of incident patients suggests an altered HLA genotype distribution from 1986-87

A. Carlsson ${ }^{1}$, L. Blom ${ }^{2}$, G. Forsander ${ }^{3}$, S. A. Ivarsson ${ }^{2}$, A.-K. Karlsson ${ }^{3}$, A. Kernell ${ }^{4}$, A. Lernmark ${ }^{2}$, B. Lindblad ${ }^{3}$, J. Ludvigsson ${ }^{5}$, C. Marcus ${ }^{6}$, A. Nilsson ${ }^{2}$, H. Rastkhani' ${ }^{2}$,

I. Zachrisson ${ }^{6}$ on behalf of BDD study group.

${ }^{1}$ Department of Pediatrics, Institution of Clinical Science Lund University Hospital, Lund, ${ }^{2}$ Department of Pediatrics, Institution of Clinical Science Malmö University Hospital, Malmö, ${ }^{3}$ Department of Pediatrics, Institution of Clinical Science The Sahlgrenska Academy at Göteborg University Hospital, ${ }^{4}$ Department of Clinical Research and Education, Karolinska University Hospital, Stockholm, ${ }^{5}$ Div of Pediatrics and Diabetes Research Centre, Faculty of Health Sciences, Linköping University, ${ }^{6}$ Department of Pediatrics, Karolinska University Hospital, Stockholm, Sweden.
Background: HLA genotypes among new onset type 1 diabetes patients is changing overtime and that there is an increased frequency of type 2 diabetes among teenagers. Sweden has the second highest incidence rate in the world and the age at onset has been shown to decrease.

Aim. The aim of the present study was to test the hypothesis that HLA typing at the time of diagnosis would better diabetes diagnosis and improve classification and thereby treatment.

Materials and Methods: The case-control study of all patients developing type 1 diabetes in 1986-87 $(n=430)$ was the baseline reference study available at http://stat-db. stat.sfu.ca/magenta/. The Better Diabetes Diagnosis (BDD) project was initiated in May 2005. Almost all 42 Pediatric clinics in Sweden participate by preparing Dried Blood Spots (DBS) of blood samples taken on the day of clinical diagnosis. HLA DQA1-B1 genotypes were determined by PCR on the DBS using allele specific probes detected by time-resolved fluorescence.

Results: A total of 491 incident patients have been analyzed about nine months into the BDD study. The most common HLA DQA1-B1 genotype, DQB1*0501-0201/ $0301-0302$, which was $36 \%$ in $1986-87$ had decreased in frequency to $27 \%(130 / 491)$ in 2005-2006. The DQ A1*B1* genotype 0301-0302/0301-0302 had increased from $8 \%$ to $28 \%$. Other significant changes included the $0301-$ 0302/0102-0604 genotype which showed a modest increased from $4 \%$ to $6 \%(31 / 491)$. The homozygous genotype 0501-0201/0501-0201 increased from 3\% in $1986-87$ to $11 \%(56 / 491)$. DQB1*0602 was not present in any child 1968-87 but in BDD 6 0602-positive children have developed diabetes.

Conclusion: It is concluded that there is a temporal change in HLA genotype distribution. The increased frequency of the DQB $1 * 0201 / 0201$ genotype may explain an increasing prevalence of celiac disease at the time of clinical diagnosis of autoimmune diabetes. We speculate that the change in HLA distribution may reflect adaptation of triggering infectious agent(s) to specific HLA.

Supported by the Swedish Child Diabetes Foundation and the BDD Children Diabetes Clinics in Sweden

0280

HLA-DQ alleles in children and adolescents with type 1 diabetes: temporal changes

J. A. Willis ${ }^{1}$, R. S. Scott ${ }^{1}$, B. A. Darlow ${ }^{2}$, J. W. Nesbit ${ }^{1}$, J. F. McRae ${ }^{1}$, A. C. Johnstone ${ }^{1}$, C. M. Frampton ${ }^{3}$;

${ }^{1}$ Lipid \& Diabetes Research Group, Christchurch Hospital, ${ }^{2}$ Department of Paediatrics, Christchurch School of Medicine and Health Sciences, ${ }^{3}$ Department of Medicine, Christchurch School of Medicine and Health Sciences, New Zealand. 
Background and Aims: The incidence of type 1 diabetes presenting in individuals aged 0-19 years in Canterbury (NZ) has increased significantly with time over the last three decades. Ascertainment of all cases is complete for the years 1970 to the present day. Reduction in the prevalence of high-risk HLA genotypes in patients with type 1 diabetes has been reported, against increasing incidence of the disease, suggesting that environmental factors are contributing to increasing disease penetrance. This research investigated the frequencies of HLA-DQ genotypes in individuals with type 1 diabetes diagnosed prior to 20 years of age.

Materials and Methods: All new cases of diabetes aged 019 years in the study area were either admitted to the regional hospital or attended acute intervention outpatient clinics at the same institution. Blood samples have been taken at diagnosis for determination of antibodies against islet antigens and for characterisation of HLA-DQ alleles in all consenting individuals diagnosed since 1990. HLA-DQ $\alpha$ and $\beta$ alleles were characterised by PCR-RFLP using group specific primers. The distribution of alleles conferring susceptibility for type 1 diabetes was compared across incidence years

Results: HLA-DQ $\alpha$ and $\beta$ genotypes are available for 252 of the 344 cases diagnosed between 1990 and 2004. The proportion of cases expressing one or two of the HLA-DQ $\alpha$ susceptibility alleles, 0301 and 0501 , was $37 \%$ and $59 \%$ respectively. The proportion of cases presenting with two copies of the HLA-DQ $\alpha$ susceptibility alleles did not change over time. The HLA-DQ $\beta$ susceptibility alleles, 0201 and 0302 , were present in $32 \%$ and $50 \%$ of cases respectively. The proportion of cases carrying two HLA-DQ $\beta$ susceptibility alleles decreased over the study period $(\mathrm{p}<0.01)$.

Conclusion: The prevalence of HLA-DQ $\beta$ susceptibility alleles in type 1 diabetes cases diagnosed before 20 years of age has reduced over time reflecting a shift in the relative importance of environmental factors over genetic factors in the development of type 1 diabetes.

Supported by Health Research Council of New Zealand, Lottery Health Research, RG Bell Charitable Trust

\section{1}

DNA typing in Russian patients with type 1 diabetes mellitus

D. Avzaletdinova ${ }^{1}$, Z. Balhiyarova ${ }^{1,2}$, T. Morugova ${ }^{1}$, L. I. Samigullina ${ }^{1}$, O. Mustafina ${ }^{2}$;

${ }^{1}$ Endocrinology Department, Bashkir State Medical University, Ufa, ${ }^{2}$ Institute of Biochemistry and Genetics, Ufa Research Center, Russian Academy of Sciences, Ufa, Russian Federation.
Background and Aims: Type 1 diabetes mellitus (T1DM) is caused by the interplay of genetic and environmental factors. Human leukocyte antigen system (HLA) has been demonstrated to contribute to T1DM genetic susceptibility. Tumor necrosis factor $\alpha(\mathrm{TNF} \alpha)$ and interleukin $1 \beta$ (IL-1 $\beta$ ) activate inducible NO-synthase in $\beta$-cells causing their destruction. 1,25-Dihydroxivitamin $\mathrm{D}_{3}$ inhibits lymphocyte activation and affects cytokine and immunoglobulin production preventing the development of autoimmune diabetes in NOD mice. Thus, the genes, encoding TNF $\alpha$, IL-1 $\beta$ and vitamin $\mathrm{D}$ receptor can be supposed as candidate genes for T1DM. We aimed to analyze the relationship between $H L A-D R B 1$ and $H L A-D Q B 1$ genes, $-308 G / A$ polymorphism of TNF $\alpha$ gene $(T N F A),-511 T / C$ polymorphism of IL-1 $\beta$ gene $(I L 1 B)$, FokI polymorphism of vitamin D receptor gene $(V D R)$ and susceptibility to T1DM in Russians of Bashkortostan.

Materials and Methods: We PCR-analyzed DNA of 118 Russian T1DM patients and 254 Russian controls living in Bashkortostan. Two-tailed test of Fisher was used for statistical analysis. Bonferroni correction for multiple comparisons was applied during HLA polymorphism investigation.

Results: The HLA-DRB1 (investigated in 99 T1DM patients and 100 controls) and $H L A-D Q B 1$ (analyzed in 100 T1DM patients and 100 controls) genomic typing revealed increased frequencies of $D R B 1 * 04(33.3 \%$ vs. $11.0 \%, \mathrm{P}=0.006), D R B 1 * 17(23.7 \%$ vs. $9.0 \%, \mathrm{P}=0.006)$, $D R B 1 * 04 * 17(23.2 \%$ vs. $3.0 \%, \mathrm{P}=0.006), D Q B 1 * 0201 /$ *0501 (43.0\% vs. $14.0 \%, \mathrm{P}=0.001)$ in $\mathrm{T} 1 \mathrm{DM}$ patients compared to controls. The ratio of the $D R B 1 * 11(4.0 \%$ vs. $15.0 \%, \mathrm{P}=0.006), D R B 1 * 15(0.0 \%$ vs. $8.0 \%, \mathrm{P}=0.006)$, $D Q B 1 * 0301(12.5 \%$ vs. $25.0 \%, \mathrm{P}=0.020), D Q B 1 * 0602-08$ $(0.5 \%$ vs. $12.0 \%, \mathrm{P}<0.001)$ were lower in $\mathrm{T} 1 \mathrm{DM}$ patients than in controls. Concerning $-308 G / A$ polymorphism of TNFA gene (researched in 116 T1DM patients and 226 controls), we couldn't find any differences of the genotype and allele frequencies between T1DM patients and healthy people. The distribution of the $I L 1 B$ gene $-511 T / C$ polymorphism $T T(25.9 \%$ vs. $10.1 \%, \mathrm{P}=0.001), T C$ $(68.5 \%$ vs. $46.8 \%, \mathrm{P}<0.001)$ and $C C(5.6 \%$ vs. $43.1 \%$, $\mathrm{P}<0.001$ ) genotypes (examined in 109 T1DM patients and 216 controls) was different among controls and T1DM patients. The typing of FokI polymorphism of VDR gene (carried out in 118 T1DM patients and 214 controls) revealed that the genotype $f f$ is more frequent in controls than in patients $(17.8 \%$ vs. $8.5 \%, \mathrm{P}=0.023)$.

Conclusion: Thus, the predisposing markers of T1DM in Russians of Bashkortostan are $H L A$ alleles $D R B 1 * 04$ $(\mathrm{OR}=4.05, \mathrm{CI}=2.28-6.89), D R B 1^{*} 17(\mathrm{OR}=3.15, \mathrm{CI}=1.75-$ 5.65), HLA genotypes $D R B 1 * 04 / * 17(\mathrm{OR}=9.79, \mathrm{CI}=2.83-$ $33.81), D Q B 1 * 0201 / * 0501(\mathrm{OR}=3.94, \mathrm{CI}=2.00-7.80)$, genotype $C C$ of $I L 1 B$ gene $-511 T / C$ polymorphism 
$(\mathrm{OR}=8.36, \mathrm{CI}=3.10-22.60)$. The protective genetic markers are $H L A$ alleles $D R B 1 * 11(\mathrm{OR}=0.24, \mathrm{CI}=0.11-0.53)$, $D R B 1 * 15 \quad(\mathrm{OR}=0.03, \mathrm{CI}=0.00-0.47), \quad D Q B 1 * 0301$ $(\mathrm{OR}=0.43, \mathrm{CI}=0.25-0.73), D Q B 1 * 0602-08 \quad(\mathrm{OR}=0.04$, $\mathrm{CI}=0.01-0.33)$, genotypes $T T(\mathrm{OR}=0.32, \mathrm{CI}=0.16-0.34)$ and $T C(\mathrm{OR}=0.40, \mathrm{CI}=0.25-0.47)$ of $I L 1 B$ gene $-511 T / C$ polymorphism, genotype $f f(\mathrm{OR}=0.43, \mathrm{CI}=0.21-0.90)$ of VDR gene FokI polymorphism.

Supported by Republic programm "Diabetes mellitus"

\section{2}

Analysis of genetic factors predisposing to type 1 diabetes in children under 5 years of age

A. Skorka ${ }^{1}$, E. Pankowska ${ }^{1}$, A. Szypowska ${ }^{1}$, M. Choim ${ }^{1}$, G. Kostrzewa ${ }^{1}$, M. Szalecki ${ }^{2}$, L. Korniszewski ${ }^{1}$, R. Ploski ${ }^{3}$; ${ }^{1}$ Department of Diabetology, Neonatology and Birth defects, Medical University of Warsaw, ${ }^{2}$ Department of Diabetology and Endocrinology, Children Hospital of Kielce, ${ }^{3}$ Department of Medical Genetics and Department of Forensic Medicine, Medical University of Warsaw, Poland.

Background and Aims: Type 1 diabetes is a multifactorial disease. The genetic contribution is not straightforward and involves many loci. The strongest association is with HLA region and the results of multilocus analysis provided evidence of between 12 and 20 other susceptibility loci. Recently it has been claimed that the incidence of type 1 diabetes has increased in many countries including Poland. The highest increase occurred in children under 5 years of age. There are data suggesting that the type 1 diabetes in very young children may have different genetic background. The aim of the study was to compare the frequencies of several genetic factors in two groups of children: diagnosed under 5 years of age and the group of older children.

Materials and Methods: The study group consisted of 357 children with type 1 diabetes aged from 0.33 to 18.2 (mean age 8.34, SD 4.61). In that group there were 99 children diagnosed under 5 years of age (mean age 2.68, SD 1.24). We analyzed the following markers: DRB1 alleles, CTLA-4 gene polymorphisms (+49A/G, CT60, MH30), six polymorphisms of Il-10 gene $(592 \mathrm{C} / \mathrm{A}, 819 \mathrm{C} / \mathrm{T}, 1082 \mathrm{G} / \mathrm{A}$, 2763 C/A, 2849 G/A, 3575 T/A), polymorphism 1085 C/T of PTPN22 gene, polymorphism -23 HphI of INS gene, polymorphism -1260 C/A of CYP27B1 gene, polymorphism rs $1544410 \mathrm{~A} / \mathrm{G}$ of VDR gene and polymorphism rs 10774671 A/G of OAS1 gene. Two types of methods were used to analyze polymorphisms either PCR-RFLP or SnapShot minisequencing method.
Results: There were no statistically significant differences in frequencies of studied polymorphisms between children with diabetes diagnosed under 5 years of aged and those diagnosed over 5 years of age. For CTLA-4 gene polymorphism $+49 \mathrm{~A} / \mathrm{G} \mathrm{p}=0.92, \mathrm{OR}=0.96$, for CT60 $\mathrm{p}=0.60, \mathrm{OR}=0.87$, for $\mathrm{MH} 30 \mathrm{p}=0.67, \mathrm{OR}=0.89$, for haplotypes of Il-10 polymorphisms $\mathrm{p}=0.41$, for polymorphism $1085 \mathrm{C} / \mathrm{T}$ of PTPN22 gene $\mathrm{p}=0.58, \mathrm{OR}=1.14$, for polymorphism $-23 \mathrm{HphI}$ of INS gene $\mathrm{p}=0.31$, $\mathrm{OR}=0.75$, for polymorphism $-1260 \mathrm{C} / \mathrm{A}$ of CYP27B1 gene $\mathrm{p}=0.73$, $\mathrm{OR}=1.08$, polymorphism rs $1544410 \mathrm{~A} / \mathrm{G}$ of VDR gene $\mathrm{p}=0.59, \mathrm{OR}=1.12$ and polymorphism rs $10774671 \mathrm{~A} / \mathrm{G}$ of OAS1 gene $\mathrm{p}=0.29, \mathrm{OR}=1.22$.

Conclusion: The youngest children with type 1 diabetes do not differ genetically from children diagnosed at later age. It seems probable that the increasing incidence of type 1 diabetes reported recently in children under 5 years of age is due to some environmental factors that operate early in prenatal period or early infancy.

This study was supported by the State Committee for Scientific Research

\section{3}

Identification of novel type 1 diabetes susceptibility loci in the Faroese population, a genetic isolate

R. L. Nolsøe ${ }^{1}$, R. Bergholdt ${ }^{1}$, M. Deckert ${ }^{1}$, J. Róin ${ }^{2}$, S. F. Olsen ${ }^{3}$, J. A. Kelly ${ }^{4}$, F. Pociot ${ }^{1}$, T. Mandrup-Poulsen ${ }^{1}$; ${ }^{1}$ Steno Diabetes Center, Gentofte, Denmark, ${ }^{2}$ Internal Medicine, Landssygehuset, Tórshavn, Faeroe Islands, ${ }^{3}$ Department of Epidemiology, Aarhus University, Denmark, ${ }^{4}$ Department of Medicine, Oklahoma Medical Research Foundation, Oklahoma City, United States.

Background: Recent genome wide scans identify the MHC complex as the major susceptibility region for Type 1 diabetes. Non-MHC genes typically had lod scores below 3 and have minor contributions to Type 1 diabetes genetic susceptibility. As increasing numbers of Type 1 diabetic families are required for identification of non-MHC susceptibility genes the identification of these may be confounded by pooling of genetically heterogeneous populations. In genetic isolates patients with genetic diseases are likely to share extended haplotypes inherited from a few common founders. The Faroese Islands were populated at about 900 AD with a founder population of mainly Norwegians and British of a few thousand people. The size of this population remained stable at approximately 4000 inhabitants until around 1800. Since then the population has increased to its present level of 45.000 inhabitants. Thus this genetic isolate is almost ideal for genetic studies due to a probable strong founder effect. We hypothesize that 
Faroese Type 1 diabetic patients share extended segments of DNA around disease genes allowing genome wide scans with fewer markers to identify novel susceptibility genes.

Aims: 1. To collect, clinically characterize and genotype with approximately 400 genetic markers the Faroese Type 1 diabetic families. 2. To identify novel Type 1 diabetes susceptibility genes.

Materials and Methods: We have succeeded in obtaining clinical information and DNA from $94 \%$ of the 110 Type 1 diabetic Faroese families, including 104 Type 1 diabetic probands and 294 relatives. We have performed a genome wide scan with 400 markers equal to a marker density of $10 \mathrm{cM}$. Based on genealogical evidence we have analyzed 21 families, of which 11 have a common founder and are unrelated in 6 generations.

Results: We have identified 6 chromosomal regions, with evidence for association to T1D, of which 5 were not previously reported. Using highly polymorphic markers in the HLA region we have shown that Faroese Type 1 diabetic patients do have genetic association with the MHC region and that this association does not differ between the 21 unrelated families and the remaining Type 1 diabetic families. Conclusion: We have performed the first genome wide scan in the majority of Type 1 diabetic families from the Faroese genetic isolate. Five new genetic regions linked to Type 1 diabetes have been identified.

Supported by Danish Diabetes Association

\section{4}

INS VNTR polymorphism in young onset diabetes subjects of Bangladesh

Z. Hassan ${ }^{1}$, M. I. Hawa ${ }^{2}$, M. O. Faruque ${ }^{3}$, K. B. Biswas ${ }^{1}$, K. Azad ${ }^{4}$, A. K. Azad Khan ${ }^{5}$, L. Ali ${ }^{3}$, R. D. G. Leslie', G. A. Hitman';

${ }^{1}$ Physiology and Molecular Biology, BIRDEM, Dhaka, Bangladesh, ${ }^{2}$ Centre for Diabetes and Molecular Medicine, Bart's and The London Queen Mary's School of Medicine and Dentistry, London, United Kingdom, ${ }^{3}$ Biochemistry and Cell Biology, BIRDEM, Dhaka, Bangladesh, ${ }^{4}$ Dept of Pediatrics, BIRDEM, Dhaka, Bangladesh, ${ }^{5}$ Dept of Gastroenterology, BIRDEM, Dhaka, Bangladesh.

Background and Aims: A remarkable heterogeneity in clinical and biochemical presentation creates a special problem in the characterization and classification of young diabetic patients in Bangladesh. In an attempt to characterize these patients we have studied INS VNTR polymorphism in young diabetic patients in relation to their insulin secretory capacity and autoantibody status.

Materials and Methods: A group of 372 young nonketotic diabetes mellitus (YDM) subjects (diabetes diagnosed under 30 years of age) and 332 healthy controls were studied. INS VNTR T/A polymorphism was analyzed by PCR RFLP using endonuclease $H p h 1$ and serum C-peptide was measured by ELISA. Insulin secretory capacity (HOMAB) was calculated by Homeostatic Model Assessment. GAD antibody was determined by radioimmunoprecipitation method.

Results: Mean ( \pm SD) BMI in the controls was $19.7 \pm 3.2$ and YDM subjects $18.3 \pm 4.9(\mathrm{p}<0.001)$. Genotype frequencies of INS VNTR T/A polymorphism did not show any significant difference between Controls and YDM (homozygous wild 0.753 vs 0.712 ; heterozygous variant 0.229 vs 0.259 and homozygous variant 0.018 vs 0.030$)$. HOMAB (\%, median-range), of the YDM subjects [1.02 (0.11-2.11)] was significantly lower compared to the controls [1.94 (1.57-2.34), $(\mathrm{p}<0.001)]$. In the controls HOMAB with wild genotype [1.94 (1.57-2.34)] did not show any difference with variant genotype [1.96 (1.68-2.32)], but in YDM group those with wild TT genotype [0.96 (0.11-2.17)] had significantly lower value compared to those with variant (TA and AA) genotype [0.1.13 $(0.15-1.89),(p=0.019)]$. GAD antibody was positive in $3.2 \%$ of the controls and $22.6 \%$ of YDM subjects. GAD antibody positive YDM cases had significantly lower HOMAB [0.67 (0.11-0.84)] compared to the negative cases $[1.12(0.11-2.17)$, $(p<0.001)]$. The proportion of wild genotype was significantly higher in antibody positive cases $(83 \%)$ compared to negative cases $(69 \%, \mathrm{p}<0.018)$.

Conclusion: The association of INS VNTR wild TT genotype with GAD antibody positivity and lower insulin secretion suggest that young diabetic subjects in Bangladesh include a subgroup of typical type 1 diabetic patients with atypical clinical presentation and residual insulin secretory capacity.

The study was supported by grants from International Program in the Chemical Sciences (IPICS) and Diabetic Association of Bangladesh (DAB)

\section{5}

HLA haplotypes and insulin gene VNTR polymorphism in relation to markers of beta cell autoimmunity in children from the general population

H. Holmberg ${ }^{1}$, O. Vaarala ${ }^{1,2}$, J. Ilonen ${ }^{3}$, J. Ludvigsson ${ }^{1}$ on behalf of the ABIS study group,

${ }^{1}$ Department of Molecular and Clinical Medicine, Division of Pediatrics and Diabetes Research Centre, Faculty of Health Sciences, Linköpings Universitet, Sweden, ${ }^{2}$ Department of Viral Diseases and Immunology, Laboratory for Immunobiology, National Public Health Institute, Helsinki, Finland, ${ }^{3}$ Department of Virology, University of Turku, Finland. 
Background and Aims: The emergence of beta-cell autoantibodies and their predictive value for type 1 diabetes (T1D) have been mostly studied in follow-up cohorts of children with a genetic predisposition for T1D. The present knowledge of the effects of HLA and insulin gene (INS) VNTR genotypes on the development of beta-cell autoimmunity may not be relevant in a genetically unselected population. Our aim was to retrieve information on early of beta-cell autoimmunity, and the association with HLA risk haplotypes and INS VNTR polymorphism in children from the general population.

Materials and Methods: The current study was part of a prospective population-based follow-up study of all infants born between 1st October 1997 and 1st October 1999 in Southeast Sweden (the ABIS study; All Babies in Southeast Sweden). We analysed autoantibodies to insulin (IAA), glutamic acid decarboxylase (GADA) and the tyrosine phosphatase like IA-2 (IA-2A) by radiobinding assays in sera taken from 714 children at 5-6 years of age. HLA genotyping was done using panels of sequence specific oligonucleotide probes, which were hybridized with amplified polymorphic regions of DQB1, DQA1 and DRB1 genes. A similar method was used for detection of polymorphism in the insulin gene at the $-23 H p h I \mathrm{~A} / \mathrm{T}$ restriction site.

Results: We found that children from the general population with the risk associated haplotype DR4-DQ8 had an increased frequency of GADA above the 99th percentile at $5-6$ years of age $(p=0.005)$. The number of children positive for GADA and/or IAA above the 98th percentile tended to be lower in children with (DR15)-DQB1*0602 $(p=0.056)$. The frequency of children positive for IAA, as well as for GADA or IA-2A, was similar in children with INS-23 A/A, A/T or T/T genotype.

Conclusion: The beta-cell autoantibody GADA is associated with the HLA DR4-DQ8 in our study population, but the impact of other disease associated risk genotypes on the development of autoantibodies seems to be small in a general population. These results suggest that the risk of beta-cell autoimmunity in a general population is not strongly associated with the risk genes of T1D other than the HLA DR4-DQ8 haplotype indicating that environmental factors are important determinants in the induction of beta-cell autoimmunity. The risk genes of T1D may rather determine the development from beta-cell autoimmunity to the destruction of beta-cells and clinical disease.

Supported by JDRF-Wallenberg foundations, The Swedish Medical Research Council (MFR; Vetenskapsrådet, K99-72X-11242-05A), Swedish Child Diabetes Foundation (Barndiabetesfonden), The Jerring Foundation (Jerringfonden)
0286

Progression to type 1 diabetes and autoantibody positivity in relation to HLA risk genotypes in children participating in the ABIS study

C. Gullstrand ${ }^{1}$, J. Wahlberg ${ }^{1}$, J. Ilonen ${ }^{2,3}$, O. Vaarala ${ }^{1}$, J. Ludvigsson ${ }^{1}$;

${ }^{1}$ Department of Molecular and Clinical Medicine, Division of Pediatrics, Linköping University, Sweden, ${ }^{2}$ Department of Clinical Microbiology, University of Kuopio, Finland, ${ }^{3}$ Immunogenetics Laboratory, University of Turku, Finland.

Background and Aims: The incidence of childhood type 1 diabetes (T1D) has increased during the last decades, especially in children younger than 5 years of age. Autoantibodies against beta-cell antigens together with HLA risk genotypes can be used as predictive markers for development of T1D. The aim of this study was to describe the frequency of T1D and autoantibodies in children from the ABIS study and to investigate the relationship between autoantibodies and HLA risk and protective genotypes.

Materials and Methods: Autoantibodies against GAD (GADA) and IA-2 (IA-2A) were investigated at 1, 2.5 and 5 years of age in 2227 children, who participated in a prospective population-based follow-up study; the ABIS study (All Babies in Southeast Sweden). The frequency of children progressing to T1D was studied in all 17055 children from the ABIS study. GADA and IA-2A were determined with immunoprecipitation.

Results: T1D: By the age of 6-7 years 31 of total 17055 $(0.18 \%)$ children in the ABIS study had developed T1D. $73 \%$ $(16 / 22)$ had GADA and/or IA-2A before onset of the disease. HLA genotypes were analysed in 18 children and all had at least one susceptibility associated haplotype ( $p=0.0035$ compared to autoantibody negative children) (table 1). Permanent autoantibodies: 16 children (0.7\%) showed permanent GADA and/or IA-2A at both 2.5 and 5 years of age and 4 of them were also positive at 1 year of age. 9 of 15 children with permanent autoantibodies and studied for HLA genotypes were positive for HLA risk haplotypes $(60 \%) \quad(p=0.094$ compared to autoantibody negative children), 3 of them together with a protective allele, and 6 had protective genotypes (40\%). Transient autoantibodies: 374 children (17\%) were positive for GADA and/or IA-2A at 1 and/or 2.5 years of age, but negative later. $29 \%$ of 363 children with transient autoantibodies and studied for HLA genotype had risk genotype without protective alleles, $17 \%$ had risk and protective alleles, and $41 \%$ had protective haplotypes, similar to figures seen in autoantibody negative children.

Conclusion: Most of the children with T1D developed GADA and/or IA-2A before diagnosis, indicating an ongoing autoimmune process. Permanent autoantibodies are rare in the ABIS population, while transient autoanti- 
bodies occur more frequently. Induction of autoantibodies, especially transient occurrence, seems not to be controlled by HLA risk genotypes but rather by environmental factors. However, HLA risk genotypes play an important role for development of manifest T1D.

Table 1. HLA genotypes in relation to T1D and autoantibodies.

\begin{tabular}{lllll}
\hline & Risk (S/S, S/N) & $\begin{array}{l}\text { Protective } \\
(\mathrm{P} / \mathrm{P}, \mathrm{P} / \mathrm{N})\end{array}$ & $\begin{array}{l}\text { Risk and protective } \\
(\mathrm{S} / \mathrm{P})\end{array}$ & $\begin{array}{l}\text { Neutral } \\
(\mathrm{N} / \mathrm{N})\end{array}$ \\
\hline $\begin{array}{l}\text { Type 1 } \\
\text { diabetes (18) }\end{array}$ & $16(89 \%)$ & 0 & $2(11 \%)$ & 0 \\
$\begin{array}{l}\text { Permanent } \\
\text { ab's (15) }\end{array}$ & $6(40 \%)$ & $6(40 \%)$ & $3(20 \%)$ & 0 \\
$\begin{array}{l}\text { Transient } \\
\text { ab's (363) }\end{array}$ & $104(29 \%)$ & $151(41 \%)$ & $62(17 \%)$ & $46(13 \%)$ \\
$\begin{array}{l}\text { Negative } \\
\text { ab's (357) }\end{array}$ & $98(28 \%)$ & $126(35 \%)$ & $78(22 \%)$ & $55(15 \%)$ \\
\hline
\end{tabular}

$\mathrm{S}=$ susceptible, $\mathrm{N}=$ neutral, $\mathrm{P}=$ protective haplotype

Supported by the Swedish Research Council

0287

Promoter activity of the gene encoding repair enzyme protein carboxyl methyltransferase (PCMT1) is enhanced by exposure to INS-1e cells to cytokines

A. M. Wägner ${ }^{1}$, P. Cloos ${ }^{2}$, J. Nerup ${ }^{1}$, F. Pociot ${ }^{1}$;

${ }^{1}$ Steno Diabetes Center, Gentofte, ${ }^{2}$ Biotech Research Innovation Centre, Symbion Science Park, Copenhagen, Denmark.

Background and Aims: PCMT is an enzyme which recognises and repairs posttranslational isoaspartyl-formation in proteins. An increase in its expression is associated with a reduction in apoptosis in rat astroglial cells. The gene encoding this enzyme in humans, PCMT1 is located on chromosome $6 \mathrm{q} 24-25$, in a region previously linked to type 1 diabetes (IDDM5). A variant in the promoter region of PCMT1 $(-509 \mathrm{C}>\mathrm{G})$ which is in complete linkage disequilibrium with a functional variant in exon 5 $(358 \mathrm{G}>\mathrm{A})$, was transmitted to $46 \%$ of the affected siblings in a sample of 248 Danish families with type 1 diabetes, and to $53 \%$ of the non-affected ( $\mathrm{p}=0.099$ for transmission to affected vs. non-affected). The aim of this study is to assess the response of PCMT1 promoter activity to cytokine exposure in a murine insulin-producing cell line, as well as the potential functional consequences of the promoter variant previously described.

Materials and Methods: Seven promoter constructs were cloned upstream of the firefly luciferase (luc) reporter gene, comprising 1817-201 nucleotides upstream of PCMT1 exon
1. All constructs were verified by sequencing and restriction enzyme cleavage. INS-1e cells (split numbers 65-89) were co-transfected with each reporter construct and renillaluciferase (pRL-TK vector, internal control, Promega) using a cationic lysosome based method (SuperFect, Qiagen), in the absence or presence of IL-1, IFN $\gamma$ or both. Luciferase activity was measured using an Orion micro-plate luminometer. Ratio of firefly/renilla luciferase activity was calculated for each construct and treatment. Five experiments were performed, with each condition in duplicate. Each treatment and promoter construct was compared with the rest (ANOVA, Tukey's post-hoc correction).

Results: Exposure to cytokines significantly increased promoter activity for all the constructs tested (firefly/renilla luciferase ratio $11.6 \pm 7.0$ for unstimulated vs. $18.6 \pm 5.6$ for IL1 vs. $16.4 \pm 5.3$ for IFN $\gamma$ and $20.1 \pm 6.8$ for the cytokine mix, $p \leq 0.003$ for all cytokines vs. no treatment). The shorter construct showed significantly lower promoter activity $(10.0 \pm 3.4, \mathrm{p} \leq 0.05)$ than the rest (except for the 2 longest). There was no difference in the promoter activity of the constructs adjacent to the previously described variant $(18.7 \pm 7.3$ vs. $21.2 \pm 8.0, \mathrm{p}=0.92)$.

Conclusion: PCMT1 promoter activity is enhanced in INS1e cells by exposure to cytokines. The previously described variant in the PCMT1 promoter region $(-509$ $\mathrm{C}>\mathrm{G})$ doesn't seem to have functional consequences on promoter activity.

\section{PS 4}

\section{The grey zone between type 1 diabetes and type 2 diabetes - LADA}

0288

Identification of two subtypes of adult-onset autoimmune diabetes (the NIRAD Study)

R. Buzzetti ${ }^{1}$, M. Locatelli ${ }^{2}$, A. Giaccari ${ }^{3}$, A. Petrone ${ }^{1}$, S. Di Pietro ${ }^{4}$, C. Suraci ${ }^{5}$, M. Capizzi ${ }^{1}$, C. Tiberti ${ }^{1}$, M. Arpi ${ }^{6}$, F. Dotta ${ }^{7}$, E. Bosi on behalf of NIRAD Study Group $^{8}$;

${ }^{1}$ Clinical Science, "La Sapienza" University, Rome, ${ }^{2}$ Scientific Institute, Bambino Gesù Hospital, Rome, ${ }^{3}$ Endocrinology, Catholic University, Rome, ${ }^{4}$ Ircss INRCA, Diabetes, Rome, Italy, ${ }^{5}$ Diabetes, S. Pertini Hospital, Rome, ${ }^{6}$ Endocrinology, Garibaldi Hospital, University of Catania, ${ }^{7}$ Endocrine and Met. Sciences, University of Siena, ${ }^{8}$ San Raffaele Hospital, Vita-Salute University, Milan, Italy.

Background and Aims: Autoimmune diabetes developing in adults is a clinically heterogeneous disorder, commonly identified by the pathogenetic marker glutamate decarbox- 
ylase autoantibodies (GADA). In the present study we aimed to define heterogeneity of adult onset autoimmune diabetes based on characterization of GADA.

Materials and Methods: Patients enrolled in the NIRAD (Non Insulin Requiring Autoimmune Diabetes) Study, a nationwide survey aiming to assess prevalence and characteristics of adult onset autoimmune diabetes in Italy, have been screened for GADA and antibodies to IA-2 (IA-2A) and further characterized for GADA titre, antibodies to thyroid peroxidase (TPO) and HLA DRB1-DQB1 alleles.

Results: Of 4250 patients, enrolled between February 2001 and June 2004 in 83 diabetes centres in continental Italy, Sicily and Sardinia, $4.5 \%$ had either GADA and/or IA-2A. Of these, 189 (4.4\%) had GADA, 39 (0.91\%) had IA-2A and $37(0.87 \%)$ had both. Analysis of GADA titres was independent from diabetes duration and showed a bimodal distribution which identified two subgroups of autoimmune patients, with high and low GADA titre, characterized by distinctive clinical and genetic features: compared with classical type 2 diabetes, high GADA patients showed significantly higher fasting glucose, $\mathrm{HbAlc}$, prevalence of IA-2A, TPO antibodies, and frequency of DRB $1 * 03$ DQB1*0201 and lower age of diabetes onset, Body Mass Index, waist circumference, total cholesterol, triglycerides, uric acid and prevalence of metabolic syndrome (all $\mathrm{p}<0.001$ ); patients with low GADA titre showed intermediate values, but still significantly different from type 2 diabetes for all these measures (all $\mathrm{p}<0.03$ vs type 2), except for total cholesterol, triglycerides and prevalence of metabolic syndrome which were similar to type 2 diabetes. Frequency of DRB $1 * 04-\mathrm{DQB} 1 * 0302$ was comparable in all autoimmune patients and higher than in type 2 diabetes $(\mathrm{p}<0.001)$.

Conclusion: GADA titration allows a dissection of heterogeneity within adult onset autoimmune diabetes, with the identification of two subgroups of patients having distinctive clinical and genetic features.

Support from FoRiSID (Research Foundation of the Italian Society of Diabetes) thanks to an unrestricted grant from Novo Nordisk Italy

\section{9}

Insulin resistance precipitates autoimmune diabetes in the adult (LADA) without affecting parameters of the immune process. Results from the Nord-Trøndelag Study

S. Carlsson ${ }^{1,2}$, K. Midthjell ${ }^{3}$, V. Grill ${ }^{4}$;
${ }^{1}$ Department of Epidemiology, Institute of Environmental Medicine, Stockholm, Sweden, ${ }^{2}$ Division of Epidemiology, Stockholm Centre of Public Health, Stockholm, Sweden, ${ }^{3}$ HUNT Research Center, Institute of Community Medicine, Trondheim, Norway, ${ }^{4}$ Institute of Cancer Research and Molecular Medicine, Norwegian University of Science and Technology, Trondheim, Norway.

Background and Aims: The prevalence of latent autoimmune diabetes in the adult (LADA) is high, yet the risk factors for this type of diabetes are poorly characterized. The aim of this study was to investigate the influence of age, body mass index and physical activity on the 11-year incidence of LADA. For comparison, we also investigated these factors in relation to the incidence of Type 2 and Type 1 diabetes.

Materials and Methods: The study population consisted of 38,800 men and women of the Nord-Trndelag health study (HUNT). This is a prospective study where inhabitants of the county of Nord-Trndelag on two occasions (1984-86 and 1995-97) participated in a health examination and filled out a questionnaire on lifestyle factors. Patients classified as LADA had antibodies against glutamic acid decarboxylase (GAD) and were insulin independent at diagnosis.

Results: The results indicated that body mass index was strongly associated with LADA incidence. BMI $\geq 28 \mathrm{~kg} / \mathrm{m}^{2}$ at baseline was associated with a relative risk (RR) of 10.1 for LADA, $95 \%$ confidence interval $(\mathrm{CI})=5.1-19.7, \mathrm{n}=81$. The association was similar for type 2 diabetes $(R R=10.8$, $95 \% \mathrm{CI}=8.6-13.6, \mathrm{n}=834$ ) but was not found for type 1 diabetes $(\mathrm{RR}=0.55,95 \% \mathrm{CI}=0.12-2.55, \mathrm{n}=24)$. Similarly, age was as important for the risk of LADA as for type 2 diabetes. Age $\geq 60$ years compared to age $<40$ was associated with a relative risk of $5.6(95 \% \mathrm{CI}=2.4-13.4)$ for LADA and $6.8(95 \% \mathrm{CI}=5.1-9.1)$ for type 2 diabetes. In contrast, there was no clear association between age and type 1 diabetes $(\mathrm{RR}=0.86,95 \%=\mathrm{CI} 0.25-2.99)$. Subjects with high physical activity had a more than $50 \%$ reduced risk of LADA and Type 2 diabetes compared to sedentary subjects $(\mathrm{RR}=0.32,95 \% \quad \mathrm{CI}=0.12-0.85 \quad(\mathrm{LADA})$ and $\mathrm{RR}=0.49,95 \% \mathrm{CI}=0.37-0.66$ (Type 2 diabetes)). These results persisted after adjustment for smoking (previously shown to be protective for LADA and Type 1 diabetes, Diabetologia 47, 1953, 2004). Baseline and follow-up BMI correlated strongly with fasting C-peptide in LADA and Type 2 diabetes but not with titres of anti-GAD.

Conclusion: We conclude that environmental and agerelated insulin resistance precipitate diabetes in autoimmune-reactive individuals to the same extent as in Type 2 diabetes by increasing demands on beta-cell function but seemingly without affecting the immune process. 


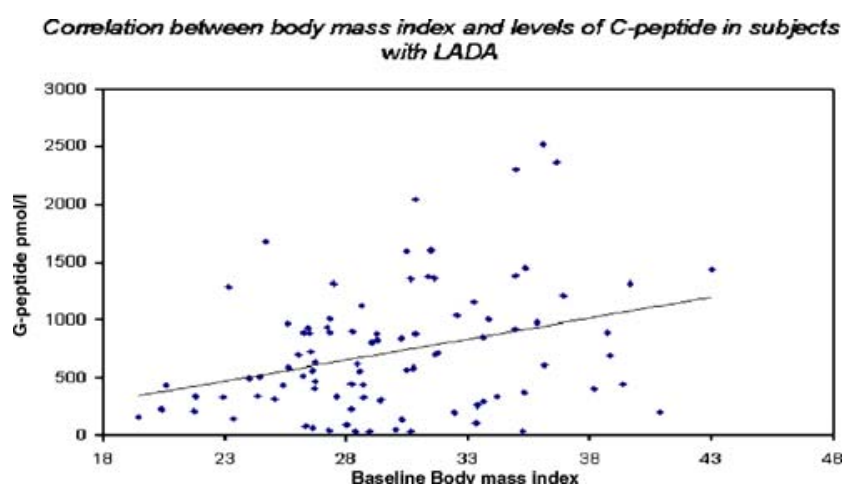

Supported by the Nord-Trøndelag Health Study is a collaboration between HUNT Research Centre, Faculty of Medicine, Norwegian University of Science and Technology (NTNU), Verdal, the Norwegian Institute of Public Health, and the Nord-Trøndelag County Council. GlaxoSmithKline Norway and the Norwegian Diabetes Association also supported the diabetes part of the HUNT surveys.

0290

Action LADA, a multinational European project in latent autoimmune diabetes in adults (LADA)

M. I. Hawa, T. O. Ola, A. Gigante, J. Teng, R. D. G. Leslie on behalf of Action LADA consortium,

Department of Diabetes \& Metabolic Medicine, St Bartholomews and The London Hospital Queen Mary University of London, United Kingdom.

Background: LADA is defined by key features including adult age at diagnosis, the presence of diabetes associated autoantibodies and a delay in the need for insulin therapy. LADA represents about $5-10 \%$ of newly diagnosed noninsulin requiring diabetes patients. ACTION LADA aims to define the prevalence of LADA in 9 European countries including England, Northern Ireland, Denmark, Spain, Finland, Germany, France, Austria, and Italy. Entry criteria includes diagnosis of diabetes between 30 and 70 years, duration of less than 5 years, non-insulin requirement for the first 6 months of diagnosis. Accurate estimates of prevalence have important implications both for the correct classification of diabetes and potential for developing early intervention strategies.

Patients and Methods: We tested GADA from samples collected from participating centers in the Action LADA consortium using an IDS validated Radio-immuno-precipitation assay. GADA was standardized to IDS workshop and positivity defined as antibody concentration $\geq 25 \mathrm{WHO}$ units.
Results: Of 5652 LADA samples identified according to the selection criteria, GADA were detected in 551 samples $(10.6 \%)$. We analysed a single centre data from England according to GADA levels. Of 575 samples tested 416 were GAD negative, mean age 49 years (30-69). GADA was detected in $14.4 \%(83 / 575)$. Age at diagnosis was significantly lower in those who were GADA positive compared to GAD negative patients $(43 \mathrm{Vs} 49)(\mathrm{p}<0.0002)$ however gender did not distinguish those with or without GADA antibodies. GADA positive patients were identified in $32 \%$ of the Insulin treated group in comparison to the non insulin treated group $(7 \%)(p<0.001)$. Further analysis of these variables according to GADA levels showed a significant tendency for those with higher GADA levels to progress to insulin therapy.

Conclusion: We conclude that LADA is prevalent throughout Europe. In England GADA was associated with younger age at diagnosis and progression to insulin treatment. Preliminary evidence suggests heterogeneity within LADA according to GADA levels Supported by the European Commission \& develogen

0291

Adult-onset autoimmune diabetes: identification of a new marker for the detection of anti-tyrosine phosphatase 2 (IA-2) antibodies (The NIRAD Study)

C. Tiberti $^{1}$, C. Giordano ${ }^{2}$, M. Locatelli ${ }^{3}$, E. Bosi $^{4}$, G. Bottazzo ${ }^{5}$, R. Buzzetti ${ }^{1}$, A. Galluzzo ${ }^{6}$, A. Falorni ${ }^{7}$, F. Dotta on behalf of NIRAD Study Group ${ }^{8}$;

${ }^{1}$ Clinical Science, "La Sapienza" University, Rome, ${ }^{2}$ Scientific Institute, Bambino Gesù Hospital, Rome, ${ }^{3}$ San Raffaele Hospital, Vita Salute University, Milan, ${ }^{4}$ Scientific Directorate, Bambino Gesù Hospital, Rome, ${ }^{5}$ Department of Endocrinology, University of Palermo, ${ }^{6}$ Internal Medicine, University of Perugia, ${ }^{7}$ Endocrine and Met. Sciences, University of Siena, Italy.

Background and Aims: The presence of glutamic acid decarboxylase (GADA) and protein tyrosine phospatase IA-2 (IA-2A) in type 2 diabetic (T2DM) sera identifies those subjects that initially do not require insulin treatment but that are at high risk to develop future insulin dependency. Our aim was to identify, in Caucasian type 1 diabetes (T1DM) and adult-onset diabetes patients, the antigenic domains target of anti-IA-2 humoral autoimmunity, evaluating if age at disease diagnosis may influence the IA-2 immunoreactivity.

Materials and Methods: Adult-onset autoimmune diabetic patients were recruited from 4250 consecutive cases of T2DM enrolled in the NIRAD (Non Insulin Requiring Autoimmune Diabetes) study, a nationwide survey aiming 
to assess prevalence and characteristics of adult onset autoimmune diabetes in Italy. The sera from $n=88$ T1DM (all at disease diagnosis T1DMa: $\mathrm{n}=44$, mean age 7.1 \pm 2.8 ; T1DMb: $n=44$ mean age 29.6 \pm 5.1 yrs) and from $n=125$ adult-onset autoimmune diabetes patients, referred as NIRAD patients (NIRADa: $\mathrm{n}=31$ mean age 44,2 \pm 13.8 within 6 months from diagnosis; NIRADb: $\mathrm{n}=94$, mean age 52.6 \pm 12.6 ), with a disease duration of from 6 to 60 months) were analyzed. The reactivity of 5 IA-2 constructs [IA-2PTP(687-979), IA-2(761-964), IA-2(256760), IA-2JM(609-631) e IA-2IC(605-979)] were tested using a radioimmunoprecipitation assay.

Results: T1DMa children had IA-2Abs mainly directed against the $\mathrm{C}$-terminal portion of the protein, with significantly higher $\mathrm{Ab}$ frequencies for IA-2PTP(687-979) [T1DMa $57,5 \%$ vs T1DMb $15,9 \% \quad(\mathrm{p}<0.0001)$ and vs NIRADa $25,8 \%(\mathrm{p}=0,03)]$ and for IA-2(761-964) $[45 \%$ vs $19,4 \%(\mathrm{p}=0,042)]$ with respect to adult TIDMb and NIRADa subjects. T1DMb and NIRADa sera reacted predominantly with the middle and juxtmembrane portion of the protein, with significantly higher IA-2JM(609-631) $\mathrm{Ab}$ frequencies with respect to T1DMa patients [T1DMb $27,3 \%$ and NIRADa $19,4 \%$ vs T1DMa $2,5 \%, p=0.002$ and $\mathrm{p}=0,04$ respectively]. No difference was found among the IA-2 construct reactivity relative to the different groups of adult diabetic patients (T1DMb, NIRADa e NIRADb). IA2IC(605-979), actually considered the most sensitive construct for IA-2Ab detection, reacted with $65 \%$ of the $\mathrm{T} 1 \mathrm{DMa}, 45.5 \%$ of the T1DMb and only with the $24.8 \%$ of NIRAD sera, detecting in this latter group of patients about $30 \%$ less positivity with respect to IA-2(256-760) $(35,2 \%)$.

Conclusion: The results of this study indicate that a) the specificity of the IA-2 humoral immune response in diabetic patients is age-dependent b) the loss of IA-2 C-terminal immunoreactivity in adult diabetic patients results in a lower diagnostic sensitivity of the IA-2IC(605-979) fragment c) IA-2(256-760), an IA-2 construct lacking the C-terminal portion of the protein, may represent a new marker with high sensitivity for the study of the anti-IA-2 humoral immunoreactivity in adult-onset autoimmune diabetes.

Foundation of the Italian Society of Diabetes and Novo Nordisk Italy

\section{2}

The Protein Tyrosine Phosphatase Non receptor (PTPN22) is associated with high GAD antibody titre in adult-onset autoimmune diabetes (The NIRAD Study)

A. Petrone ${ }^{1}$, M. Capizzi ${ }^{1}$, A. Giaccari ${ }^{2}$, E. Bosi ${ }^{3}$, D. Cucinotta ${ }^{4}$, E. Cossu ${ }^{5}$, C. Suraci ${ }^{6}$, A. Falorni ${ }^{7}$,

P. Pozzilli ${ }^{8}$, R. Buzzetti ${ }^{1}$ on behalf of the NIRAD Study Group,
${ }^{1}$ Clinical Science, "La Sapienza" University, Rome, ${ }^{2}$ Endocrinology, Catholic University, Rome, Italy, ${ }^{3}$ San Raffaele Hospital, Vita-Salute University, Milan, ${ }^{4}$ Internal Med., University of Messina, ${ }^{5}$ Center of Diabetology, University of Cagliari, Cagliari, Italy, ${ }^{6}$ Diabetes, S. Pertini Hospital, Rome, ${ }^{7}$ Internal Med., University of Perugia, ${ }^{8}$ Endocrinology, Campus Biomedico, Rome, Italy.

Background and Aims: The Protein Tyrosine Phosphatase Non-receptor type 22 (PTPN22) gene encodes for lymphoid-specific phosphatase known as LYP, a powerful inhibitor of T-cell activation. Recently, many studies showed an association between the $\mathrm{C} 1858 \mathrm{~T}$ polymorphism (Arg620Trp) of the PTPN22 gene and type 1 diabetes (T1DM) and other autoimmune diseases. The aim of the present study was to evaluate whether the missense C1858T SNP of PTPN22 confers susceptibility to adult-onset autoimmune diabetes also referred to as Latent Autoimmune Diabetes in Adults (LADA) or Non Insulin Requiring Autoimmune Diabetes (NIRAD).

Materials and Methods: The study was performed in $\mathrm{n}=191$ GADA positive patients recruited from 4250 consecutive cases of type 2 diabetes patients (T2DM) enrolled in the NIRAD (Non Insulin Requiring Autoimmune Diabetes) study. This NIRAD study is a nationwide survey aiming to assess prevalence and characteristics of adult-onset autoimmune diabetes in Italy. The bimodal distribution of GAD titres in antibody positive T2DM patients, allowed us to divide antibody positive $(\mathrm{Ab}+)$ patients into two subgroups, namely low $(\mathrm{LAb}+) \mathrm{n}=94$ and high $(\mathrm{HAb}+) \mathrm{n}=97 \mathrm{GAD}$ antibody titre positive patients. The analysis was also performed on $n=382$ T2DM patients (matched for sex, BMI age of recruitment and duration of disease), and defined as antibody-negative (Ab-), $\mathrm{n}=558$ T1DM and $n=235$ controls. The C1858T SNP was genotyped using the fluorogenic $5^{\prime}$ nuclease assay application of the ABI PRISM 7900 HT Sequence Detection System. Statistical analysis was performed using SPSS statistical software, version 13. The genotype frequencies of the investigated SNP were in agreement with HardyWeinberg equilibrium. Genotype and allelic distributions of the C1858T polymorphism were compared using the $x^{2}$ test.

Results: The frequency of the $1858 \mathrm{~T}$ carriers of the PTPN22 gene was significantly increased in High $\mathrm{Ab}$ $+(16.2 \%)$ compared to $\mathrm{Ab}-(7 \%) \quad(\mathrm{p}=0.01)$, and even compared to Low $\mathrm{Ab}+(5.5 \%)(\mathrm{p}=0.02)$. Low $\mathrm{Ab}+, \mathrm{Ab}-$ patients and controls showed similar genotype and allelic distributions. Moreover, the PTPN22 1858T allele frequency was $8.8 \%$ in Low $\mathrm{Ab}+$ and $3.8 \%$ in $\mathrm{Ab}-(\mathrm{p}=0.01)$. The $1858 \mathrm{~T}$ phenotype confers an $\mathrm{OR}=2.6$

Conclusion: In conclusion in GADA positive T2DM patients the C1858T polymorphism of PTPN22 gene is 
associated with a high titre of GADA antibody rather than with the presence of autoimmunity "per se".

Support from FoRiSID (Research Foundation of the Italian Society of Diabetes) thanks to an unrestricted grant from Novo Nordisk Italy

\section{3}

\section{Plasma adiponectin in latent autoimmune diabetes of adults (LADA)}

E. Hatziagelaki, K. Kantartzis, M. Panagiotidou,

I. Garofallakis, T. Economopoulos, S. A. Raptis;

2nd Dpt. of Internal Medicine, Research Institute and Diabetes Center, Athens University, Greece.

Background and Aims: The role of adiponectin as an insulin-sensitizing protein in both subjects with type 2 diabetes and non-diabetics is widely accepted. Plasma adiponectin levels are known to show an inverse correlation with body mass index (BMI) in healthy individuals and to be decreased in patients with type 2 diabetes. In type 1 diabetes, plasma adiponectin concentrations were found to be higher than in healthy subjects, independently of BMI and diabetes control. Mechanisms for and the physiologic relevance of this finding are still unknown. The role of adiponectin in the Latent Autoimmune Diabetes of Adulthood (LADA), which shows characteristics of both type 1 (progressive autoimmune-mediated destruction of $\beta$-cells) and type 2 diabetes (insulin resistance), has not been investigated yet.

Materials and Methods: In a cross-sectional setting, we measured fasting adiponectin levels and explored their relationships to $\beta$-cell function and common metabolic parameters, as BMI and insulin resistance (estimated by homeostasis model assessment - HOMA-IR) in patients with LADA $(n=33)$, with type 1 diabetes $(n=41)$ and in healthy controls $(n=72)$. Patients with LADA were identified by their age at diagnosis of diabetes ( $>40$ years), a type 2 diabetes phenotype and the presence of GAD 65 and IA-2 antibodies.

Results: Patients with LADA had lower, compared to patients with type 1 diabetes, plasma adiponectin levels, adjusted for gender, age and BMI $(\mathrm{p}=0.006)$, and comparable levels to healthy controls. Inclusion of C-peptide levels in the model resulted in a highly significant inverse association of adiponectin levels with C-peptide in patients with type 1 diabetes $(p=0.003)$ and LADA $(p=0.002)$, but not in healthy controls $(\mathrm{p}=0.68)$. Even adjusted for $\mathrm{C}$ peptide, patients with type 1 diabetes had higher adiponectin levels than patients with LADA $(p=0.03)$. In patients with LADA, adiponectin was significantly associated with female sex (adjusted for age and BMI $\mathrm{p}=0.04$ ) and negatively with BMI (adjusted for sex and age $p=0.02$ ) and HOMA-IR (adjusted $\mathrm{p}=0.03$ ), whereas in patients with type 1 diabetes only with female sex $(\mathrm{p}=0.02)$.

Conclusion: Our results suggest that hypoinsulinemia is a major determinant of adiponectin levels in type 1 diabetes and LADA. However, in the latter, adiponectin levels are also affected by adiposity and inversely correlated with insulin resistance, suggesting a physiologic role similar to healthy subjects.

0294

Alteration of peripheral blood lymphocyte subsets in autoimmune diabetes of the adults (ADA) prior of insulin deficiency

C. Åkesson ${ }^{1}, \AA$ Å. Lethagen ${ }^{2}$, J. Arvastsson ${ }^{1}$, A.. Lernmark ${ }^{3}$, C.-D. Agardh ${ }^{2}$, C. M. Cilio ${ }^{1}$;

${ }^{1}$ Dept. of Clinical Sciences, Cellular Autoimmunity Unit, Malmö, ${ }^{2}$ Dept. of Clinical Sciences, Diabetes and Metabolism Unit, Malmö, ${ }^{3}$ Dept. of Clinical Sciences, Diabetes and Celiac Unit, Malmö, Sweden.

Background/Rational: About $10 \%$ of the patients diagnosed with T2DM, have detectable serum levels of autoantibodies against GAD65. These patients usually progress to insulin dependency within a few years and are classified as Latent Autoimmune Diabetes in Adults (LADA) or as suggested more recently Autoimmune Diabetes of the Adult (ADA). Specific changes in lymphocyte subsets, like a decrease in the frequency and function of peripheral blood NK cells as well as $\mathrm{CD} 4+\mathrm{CD} 25+$ regulatory $\mathrm{T}$ cells, have been observed in newly diagnosed T1DM and in pre-diabetic high-risk individuals. However, peripheral blood lymphocytes in ADA patients have been poorly investigated.

Aim: The aim of this study was to determine the frequency of major peripheral lymphocyte subsets in pre insulindependent ADA patients.

Methods: The frequency of peripheral blood lymphocyte subsets was determined in 44 ADA patients and 29 controls using four colour flow cytometry. All patients had autoantibodies against GAD65 in their blood but none of them were insulin-dependent. At the time of blood withdraw all patients were metabolically compensated.

Results: No differences were observed regarding the frequency of total T, CD4+, CD8+and B cells. A specific decrease of NK cell proportion was found in ADA patients. Moreover the frequency of total $\mathrm{CD} 4+\mathrm{CD} 25+\mathrm{T}$ cells was reduced in ADA patients.

Conclusions: These observations highlight a defect in the generation and frequency of NK and CD4+CD25+ lymphocytes in ADA patients. Recent evidences have 
demonstrated a regulatory function of both these lymphocyte subsets. Thus, we suggest that these specific immunological alterations may contribute to the development of autoimmune diabetes by affecting important mechanisms of peripheral tolerance. Moreover, these data are in line with observations obtained in T1DM suggesting that similar immunological dysfunctions may contribute in both ADA and T1DM.

\section{5}

The functional polymorphism $(1858 \mathrm{C} / \mathrm{T})$ in the PTPN22 gene is associate with late autoimmune diabetes in adults - LADA.

M. Szelachowska, B. Szepietowska, A. Okruszko, N. Wawrusiewicz-Kurylonek, M. Gorska, A. Kretowski; Department of Endocrinology, Diabetology and Internal Medicine, Medical University, Bialystok, Poland.

Background and Aims: The functional polymorphism $(1858 \mathrm{C} / \mathrm{T})$ of gene encoding protein tyrosine phosphatase N22 (PTPN22) has been recently found to be associated with a higher risk of type 1 diabetes in several populations (including the Polish population). However, it is little known about the role of this gene polymorphism in patients with late autoimmune diabetes in adults (LADA). The aim of our study was to assess the association of the PTPN22 gene polymorphism 1858C/T and HLA DRB1, DQB1, DQA1 with the predisposition to LADA in the Polish population.

Materials and Methods: The study was performed in the group consisting of 175 individuals with classic clinical type 1 diabetes, 80 patients with LADA (age of diagnosis > 35 yrs., with anti-islet antibodies and low $\mathrm{C}$ peptide levels) and the control group comprising of 151 healthy non-related subjects. The GADA, IA2A and IAA antibodies were measured with RIA. HLA typing of DRB1, DQB1, DQA1 alleles was performed by PCR-SSP method. The presence of different variants of the PTPN22 gene polymorphisms was estimated using the DNA sequencing method.

Results: Patients with late onset autoimmune diabetes had a lower frequency of DRB $1 * 03 \quad(37.0 \%$ vs. $75.0 \%$, $\mathrm{p}<0.0001), \mathrm{DRB} 1 * 04(45.3 \%$ vs. $85.3 \%, \mathrm{p}<0.0001)$ and DQB $1 * 02$ alleles $(54.2 \%$ vs. $80.6 \%, \mathrm{p}=0.0007)$ in comparison to those with type 1 diabetes, but still had the higher frequencies of these alleles in comparison to the controls. In contrast, the higher frequency of $1858 \mathrm{C} / \mathrm{T}$ polymorphism of PTPN22 gene have been shown in subjects with LADA in comparison to those with type 1 diabetes and healthy controls (1858T allele respectively: $26.8 \%$ vs. $17.4 \%$, $\mathrm{p}=0.013$, and vs. $13,2 \% \mathrm{p}=0,002$ ).
Conclusion: Our results shows, that similarly to type 1 diabetes, the HLA genes and non-HLA regions (PTPN22) play a role in the LADA genetic susceptibility, however different combinations of the risk alleles seems to influence the time of clinical onset of the disease.

0296

Association of IRS-2 Gly1057Asp IRS-1 Ala513Pro polymorphism with insulin resistance in patients with latent autoimmune diabetes in adults (LADA)

\section{V. Kononenko;}

Newly Diagnosted Diabetes Mellitus, Russian Endocrinology Research Centre, Moscow, Russian Federation.

Background and Aims: Presence of autoantibodies to GAD and/or ICA in patients diagnosed with type 2 diabetes (T2DM) is main diagnostic criteria of LADA. However results of some clinical study demonstrated that LADA comprises a heterogeneous population of diabetic patients, some of them may have insulin resistance.

The aim of the study was to evaluate the insulin sensitivity (IS) in patients with LADA during the first 5-years of disease and to investigate the association of IRS-2 Gly1057Asp and IRS-1 Ala513Pro polymorphism with LADA. It's important to understand the pathogenesis of this form of diabetes and for identification therapeutic approach.

Materials and Methods: We investigated 43 patients with latent autoimmune diabetes in adults (age 48,7 $\pm 9,5$ years, BMI $26,7 \pm 4,3 \mathrm{~kg} / \mathrm{m}$ ). The diagnosis of LADA was confirmed by the presence of autoantibodies (GAD65, ICA, IAA) in diabetes patients with initial diagnosis of T2DM, who didn't require insulin treatment for at least six months after the diagnosis. Control group consisted of 31 healthy volunteers (age 39,7 $\pm 9,8$ years old, BMI $22,9 \pm 3,6 \mathrm{~kg} / \mathrm{m}$.).IS was measured as Matsuda index (ISI) in 26 patients with LADA, by results of OGTT, and as CARO-index and HOMA-index. Data are given as means \pm SD. IRS-2 Gly1057Asp and IRS-1 Ala513Pro polymorphism was studied in 43 patients with LADA and 31 healthy controls. Results: During OGTT patients with LADA had three types of insulin responses: hypersecretory, moderate and hyposecretory $53,7 \%$ patients with hypersecretory and moderate types had high insulin resistance (ISI 1,8 $8 \pm 0,3$ and $2,8 \pm 1,3$ ). $46,3 \%$ LADA patients did not have stimulated insulin secretion and have high insulin sensitivity (ISI $-8,6 \pm 3,0$ ). LADA was positively associated with the marker Gly1057Asp gene IRS-2. Distribution of alleles and genotypes frequencies of marker Gly1057Asp gene IRS-2 was differently in LADA patient and control group. The presence of the 1057Asp allele was significant higher in LADA patients $(13,9 \%$ and $1,6 \%$, respectively, $\mathrm{p}<0,05 \mathrm{OR}=4,64(1,46-14,6)$. The pres- 
ence of the genotype Asp/Gly in codon 1057 of IRS-2 in LADA patients was also significant higher than in control subjects $(18,6 \%$ and $3,2 \%$, respectively, $\mathrm{p}<0,05 \mathrm{OR}=4,14$ $(1,01-16,8)$. (1,6\% and 3,2\%). In LADA group 1057Asp allele was associated with lower BMI and waist-to-hip ratio and 8 from 10 LADA patients with 1057Asp allele needs of insulin treatment during 5 years after the diagnosis. Patients with 1057 Asp allele had the same IS as patients with $G l y / G l y$ genotype. We didn't find association between LADA and IRS-1 Ala513Pro polymorphism.

Conclusion: Our results demonstrated the association of IRS-2 Gly1057Asp polymorphism with latent autoimmune diabetes in adults. 1057Asp allele may influence over early insulin dependency.

\section{7}

Development of latent autoimmune diabetes of adulthood in patients, infected by hepatotropic viruses

L. Y. Hamnueva ${ }^{1}$, I. A. Bondar ${ }^{2}$, I. V. Malov ${ }^{1}$,

A. V. Davydova ${ }^{1}$, L. S. Andreeva ${ }^{1}$;

${ }^{1}$ Endocrinology, State Medical University, Irkutsk, ${ }^{2}$ Endocrinology, State Medical Academy, Novosibirsk, Russian Federation.

Background and Aims: To study the influence of hepatotropic viruses (HBV and HCV) on development of autoimmune processes to pancreatic beta-cells and it's secretory function in patients with diabetes mellitus (DM). Materials and Methods: 173 patients with type 2 DM (41 male, 132 female, middle age $57.5 \pm 0.8$ year, average diabetes duration $8.55 \pm 0.53$ years) were surveyed. Patients were distributed into five groups: first group $(n=7)$ diabetic patients with HBV infection in replicative phase; second $(\mathrm{n}=68)-\mathrm{HBV}$ infected in nonreplicative phase; third $(n=23)-\mathrm{HCV}$ infected in replicative phase; fourth $(n=13)-$ HCV infected in nonreplicative phase; fifth $(n=62)$ - noninfected type 2 diabetic patients. Markers of a viral hepatites B and C, glutamate decarboxilase autoantibodies (GADA), islet-cells cytoplasm autoantibodies (ICA) and basal C-peptide level were studied by immune-enzyme assay, viral DNA and RNA - by polymerase chain reaction. Results: GADA was revealed more often in $\mathrm{HBV}$ and $\mathrm{HCV}$ infected type $2 \mathrm{DM}$ patients in comparison with noninfected: in group $1-57.1 \%, \mathrm{p}=0.007$; in $2-36.7 \%, \mathrm{p}=0.003$; in $3-$ $34.8 \%, \mathrm{p}=0.003$; in $4-53.9 \%, \mathrm{p}=0.01$ vs. group $5-9.7 \%$ (chisquare test). ICA in infected patients also met more often, than in noninfected: in group $1-28.6 \%, \mathrm{p}=0.019$; in 2 $29.4 \%, \mathrm{p}<0.001$; in $3-17.4 \%, \mathrm{p}=0.026$, in $4-15.4 \%, \mathrm{p}=0.15$ vs group $5-1.6 \%$ (chi-square test). GADA and ICA revealing frequency was not depend on a virus replication. In $13.5 \%$ HBV- and HCV-infected patients both kinds of autoanti- bodies were determined. In noninfected patients simultaneously ICA and GADA wasn't revealed. In HBV and HCV infected, GADA and/or ICA-positive diabetic patients ( $\mathrm{n}=18$, patients with $0 \mathrm{pmol} / \mathrm{l} \mathrm{C}$-peptide level were excluded) the Cpeptide median was essentially lower, than in GADA and/or ICA-negative diabetic patients $(\mathrm{n}=10)(0.84 \mathrm{vs} 1.49 \mathrm{pmol} /$ 1 respectively; $p=0.027$, Mann-Whitney $U$ test). The survival analysis (Kaplan-Meier product limit method with use of Cox F-test) has been lead before an outcome (decrease Cpeptide) depending on duration DM. It is revealed, that in $\mathrm{HBV}$ - and HCV-infected type 2 diabetic group a share of persons, which are not having C-peptide level decrease, was less, than in noninfected; the difference made from 8 up to $13 \%(\mathrm{~F}(158.66)=1.59, \mathrm{p}=0.016)$.

Conclusion: In type 2 diabetic $\mathrm{HBV}$ and $\mathrm{HCV}$ infected patients autoantibodies to pancreatic beta-cells were found out statistically significantly more often, than in noninfected DM patients. In HBV and $\mathrm{HCV}$ infected patients with type $2 \mathrm{DM}$, with increase in duration of disease, decrease of insulin secretion faster progressed than in noninfected. One of the pathogenetic mechanisms of insulin secretion decrease can be autoimmune aggression directed to pancreatic beta-cells, initiated by HBV- and HCV-infection. In $36 \%$ of $\mathrm{HBV}$ - and $\mathrm{HCV}$-infected diabetic patients were found out both autoantibodies to beta-cells and down level of insulin secretion that testifies to development in them of latent autoimmune diabetes of adulthood.

\section{PS 5}

\section{The spectrum of diabetic disorders}

\section{8}

Permanent neonatal diabetes could be much more frequent than estimated: Slovak incidence and successful transfer to sulphonylureas

J. Stanik ${ }^{1,2}$, D. Gasperikova ${ }^{1}$, L. Barak ${ }^{2}$, M. Paskova ${ }^{3}$, J. Javorkova ${ }^{4}$, M. Ciljakova ${ }^{4}$, P. Hlava ${ }^{5}$, S. Flanagan ${ }^{6}$, J. Michalek ${ }^{7}$, S. Ellard ${ }^{6}$, E. Pearson ${ }^{6,8}$, A. T. Hattersley ${ }^{6}$, I. Klimes ${ }^{1}$;

${ }^{1}$ DIABGENE, Inst. Exp. Endocrinology, Bratislava, Slovakia, ${ }^{2}$ First Dept. of Pediatrics, Comenius University, Bratislava, Slovakia, ${ }^{3}$ Second Dept. of Pediatrics, Safarik University, Kosice, Slovakia, ${ }^{4}$ Dept. of Pediatrics, Jessenius Faculty of Comenius University, Martin, Slovakia, ${ }^{5}$ Diabetes Registry, Inst. of Medical Statistics, Bratislava, Slovakia, ${ }^{6}$ Inst. of Biomedical and Clinical Science, Peninsula Medical School, Exeter, United Kingdom, ${ }^{7}$ Pediatric Dept., National Inst. Diabetes and Endocrinology, Lubochna, Slovakia, ${ }^{8}$ Ninewells Hospital, Medical School, Dundee, United Kingdom. 
Background and Aims: Permanent neonatal diabetes (PND) has been recently intensively investigated across Europe. Nonetheless, the information on frequency of PND cases has been based on non-population based collections only. Thus the aim of this study was to identify the incidence for PND in Slovakia and to transfer these patients to sulphonylureas (SU).

Materials and Methods: We have searched for PND patients in the Slovak National Diabetes Registry (SNDR), which includes all children with diabetes since 1981. In the insulin treated patiens who matched the clinical criteria for PND, the KCNJ11 gene was sequenced and the KCNJ11 mutation carriers were invited for transfer to SU.

Results: Based on information from SNDR, eight patients with diabetes onset under 6 months were identified. With a mean of 71,805 live births per year since 1981, the PND incidence in Slovakia is approximately one case in 215,000 live births. One of the 8 PND identified had died and one has the Wolcott-Rallison syndrome. In four of the remaining six patients, 3 different KCNJ11 mutations were found (i.e. R201H, and two novel mutations: H46Y and L164P). Three patients (two R201H and H46Y carriers) were transferred from insulin to SU. Before therapy change, the subjects had an insulin requirement of $0.88 \mathrm{U} / \mathrm{kg} /$ day (0.66-1.00), HbA1c 10.0\% (9.3-11.0) and C-peptide concentrations $0.06 \mathrm{nmol} / \mathrm{ml}(0.04-0.11)$. All subject responded well to $\mathrm{SU}$ and insulin was stopped. After 1 month off insulin, the HbAlc decreased to $6.7 \%$ (5.9-7.3) and the C-peptide concentrations increased to $0.73 \mathrm{nmol} / \mathrm{ml}$ (0.61-0.79). Insulin and C-peptide secretion response to oral glucose (in OGTT) was higher than to intravenouse glucose. The L164P carrier suffers from active chronic hepatitis $\mathrm{C}$, and so far has not been suitable for the treatment change.

Conclusion: The PND frequency in Slovakia is approximately one case to 215,000 live births, which is much higher than previous international estimations ( 1 in 800,000 live births). We have identified $4 \mathrm{KCNJ} 11$ mutation cariers and 3 of them suscessfully tranferred to SU, dramatically improving their diabetes control and quality of life.

This work was supported in part by research grants MZ.2005/15-NEDU-01, SP 51/0280800/028 0802-2003 and $A P V V-51-014205$.

\section{9}

Kir6.2 mutation search in patients with PND from a Polish population. Successful transfer to sulphonylurea therapy in children and adult subjects

M. T. Malecki ${ }^{1}$, W. Mlynarski ${ }^{2}$, T. Klupa ${ }^{1}$, A. Gach ${ }^{2}$, J. Nazim ${ }^{3}$, J. Skupien ${ }^{1}$, A. Noczynska ${ }^{4}$, J. Sieradzki ${ }^{1}$;
${ }^{1}$ Department of Metabolic Diseases, Jagiellonian University, Medical College, Krakow, ${ }^{2}$ Clinic of Pediatrics, Institute of Pediatrics, Medical University, Lodz, ${ }^{3}$ Department of Paediatric Endocrinology, Polish-American Children's Hospital, Jagiellonian University, Medical College, Krakow, ${ }^{4}$ Department of Endocrinology for Children and Adolescents, University of Wroclaw, Faculty of Medicine, Wroclaw, Poland.

Introduction: Activating mutations in the KCJN11 gene encoding Kir6.2 have been described in patients with permanent neonatal diabetes (PND). Aim: To examine the contribution of $K C J N 11$ mutations to PND in patients from Poland.

Material and Methods: To dissect the genetic background of PND in Poland, the Nationwide Registry was established. So far, 32 subjects were included, all diagnosed below 6 months (range 1-26 weeks). This constitutes one of the world's largest national PND collections. Until now, we sequenced the KCJN11 gene in 25 insulin treated, diabetic patients (age at exam: 1-50 years).

Results: We identified eleven patients with heterozygous missense diabetes causing mutations. Five subjects carried the R201H mutation, three had the V59M substitution, one patient was a carrier of the R50Q, the H46L, and the K170N mutations, respectively. There was evidence of clinical heterogeneity: the age of diagnosis varied from 2 to 26 weeks; and insulin requirements varied from 0.25 to $1 \mathrm{U} / \mathrm{kg}$. During euglycemic hyperinsulinemic clamp in three adult $\mathrm{R} 201 \mathrm{H}$ mutation carriers, we found various degrees of insulin resistance. So far, ten patients, seven children and three adults, (range 5-50 years) were transferred to sulphonylurea therapy (SU). In five cases with $\mathrm{R} 201 \mathrm{H}$ mutation we used controlled-release glipizide GITS (gastrointestinal therapeutic system). One carrier of R50Q mutation was well controlled on $1 \mathrm{mg}$ of glimepiride. In four children with evidence of developmental delay (V59M and H46L mutations) glibenclamide was successfully used. HbA1c improved in all patients. To our knowledge, a 50 year old man transferred by us to glipizide is the oldest human with the Kir6.2 mutation in whom SU was used. We were not able to fully transfer a 20 year old carrier of $\mathrm{K} 170 \mathrm{~N}$ mutation who is currently on combined glibenclamide/insulin regimen.

Conclusions: We summarize that Kir6.2 mutations are a common cause of PND in a Polish population. The results showed evidence of clinical heterogeneity among Kir6.2 mutation carriers. We report successful transfer of paediatric and adult patients from insulin to various SU (sustainedrelease glipizide, glimepiride, and glibenclamide).

Supported by the Ministry of Scientific Research and Information GrantTechnology Grant No. 2 POE 13629. 
0300

Catalytic instability of the glucokinase MODY-2 mutants V62M and G72R in pancreatic beta cells

C. Arden ${ }^{1}$, A. L. Gloyn ${ }^{2}$, J. A. M. Shaw ${ }^{1}$,

F. M. Matschinsky ${ }^{3}$, L. Agius ${ }^{1}$;

${ }^{1}$ SCMS - Diabetes, University of Newcastle Upon Tyne, Newcastle Upon Tyne, United Kingdom, ${ }^{2}$ Diabetes Research Laboratories, University of Oxford, United Kingdom, ${ }^{3}$ Diabetes Research Center, University of Pennsylvania School of Medicine, Philadelphia, United States.

Background and Aims: The glucokinase (GK) mutations V62M and G72R demonstrate mildly activating kinetics and are thermally stable compared with wildtype GK but paradoxically cause Maturity Onset Diabetes of the Young Type 2 (MODY-2). Lack of responsiveness to a pharmacological activator and the liver regulatory protein GKRP has been reported. However, neither abnormality can explain the defect in insulin secretion in MODY-2. Therefore, the aim of the present study was to use a cell biology approach to determine the mechanism by which these mutants compromise GK function in pancreatic beta-cells.

Materials and Methods: To investigate cellular stability, expression and subcellular location of the mutant GK, stable pancreatic beta-cell MIN6 cell lines expressing GFPtagged wildtype, V62M and G72R GK were generated.

Results: As proteosomal inhibition revealed increased turnover of GFP-tagged GK in the stable cell lines, kinetic and cellular analysis was performed during proteosomal suppression. Kinetic analysis of the stable cell lines confirmed the mildly activating kinetics of the mutant enzymes within the pancreatic beta cell. GFP-GKwildtype demonstrated similar sigmoidal kinetics to untransfected MIN6 cells $\left(\mathrm{S}_{0.5}=9.0 \pm 1.0 \mathrm{mM}\right.$ vs $\left.9.1 \pm 1.3 \mathrm{mM}\right)$, whilst GFP-V62M and GFP-G72R demonstrated a decreased $\mathrm{S}_{0.5}$ for glucose $(5.2 \pm 0.4 \mathrm{mM}$ and $4.6 \pm 1.0 \mathrm{mM}$, respectively). GFP-GKwildtype demonstrated activation by a pharmacological GK activator $\left(\mathrm{S}_{0.5}=1.9 \pm 0.2 \mathrm{mM}\right)$ whereas GFPV62M and GFP-G72R did not respond to the GK activator. Similarly, GFP-GKwildtype demonstrated appropriate interaction with liver GKRP whilst neither mutant protein demonstrated interaction with GKRP, either in hepatocytes or following adenoviral expression in the stable beta-cell lines. Cellular stability analysis revealed a decreased GK activity/immunoreactivity and GK activity/mRNA ratio for GFP-V62M and GFP-G72R compared to GFP-GKwildtype (2-4fold), both in the absence and presence of proteosomal suppression, indicating catalytic instability. Protein stability, determined by the immunoreactivity/mRNA ratio, was similar for GFP-GKwildtype, GFP-V62M and GFP-G72R. Conclusion: The GK mutants V62M and G72R demonstrate cellular catalytic instability in pancreatic beta-cells and are not responsive to a pharmacological GK activator or liver GKRP. The catalytic instability of V62M and G72R may explain the clinical MODY-2 phenotype.

Supported by Diabetes UK

0301

The $-30 \mathrm{G}>\mathrm{A}$ polymorphism in the glucokinase gene predicts the effect of a lifestyle intervention on insulin secretion in a population at risk for type 2 diabetes

K. Kantartzis, F. Machicao, A. Fritsche, B. Gallwitz, S. Schäfer, O. Tschritter, N. Stefan, H.-U. Häring; Department of Internal Medicine, Division of Endocrinology, Metabolism and Pathobiochemistry, University of Tübingen, Germany.

Background and Aims: Glucokinase is considered to play a key role in glucose sensing and regulation of insulin secretion. The impact of the $-30 \mathrm{G}>\mathrm{A}$ polymorphism in the $\beta$-cell specific promoter region of the glucokinase gene on insulin secretion has been investigated in cross-sectional studies yielding inconsistent results. We investigated the long-term effect of the polymorphism on insulin secretion in the setting of a lifestyle intervention program to prevent type 2 diabetes.

Materials and Methods: The effect of the $-30 \mathrm{G}>\mathrm{A}$ polymorphism on insulin secretory function in our population was determined in 116 non-diabetic subjects by means of a hyperglycemic clamp using different secretagogues. During a lifestyle intervention with diet and increase in physical activity we investigated whether this polymorphism predicted the change in glucose-stimulated insulin secretion as assessed by first phase insulin secretion and 30 minute C-peptide plasma levels during an oral glucose tolerance test.

Results: The $-30 \mathrm{~A}$ allele was associated with lower first $(\mathrm{p}=0.03)$ and second phase $(\mathrm{p}=0.02)$ of glucose-stimulated insulin secretion, and with lower second phase of GLP-1 stimulated insulin secretion $(p=0.007)$ independently of age, gender and insulin sensitivity. During the lifestyle intervention in subjects with impaired glucose tolerance, there was an increase in insulin secretory function in homozygote carriers of the $-30 \mathrm{G}$ allele, while there was a decrease in carriers of the $-30 \mathrm{~A}$ allele, resulting in a significant difference in the change in insulin secretion between genotypes (first phase: $p=0.009 ; 30$ min C-peptide: $\mathrm{p}=0.01$ ). In subjects with normal glucose tolerance no difference in the change in insulin secretion was found between genotype groups.

Conclusion: In German population at risk for developing type 2 diabetes, carriers of the $-30 \mathrm{~A}$ allele in the glucokinase gene have lower insulin secretory capacity than $\mathrm{G} / \mathrm{G}$ homozygotes. Intervention for preventing diabetes in these subjects should 
be carried out as early as they maintain normal glucose tolerance, because, once impairment of insulin secretion is established, it continues to decline, even when a lifestyle modification program is implemented.

\section{2}

The impact of HNF1及 (R276X) mutation on insulin secretion and morphology of kidney

K. Fujimoto ${ }^{1,2}$, T. Sasaki ${ }^{1,2}$, N. Masami ${ }^{1,2}$, Y. Hiki ${ }^{2}$, N. Nakai ${ }^{1}$, T. Ohashi ${ }^{2}$, N. Tajima ${ }^{1}$;

${ }^{1}$ Diabetes, Metabolism and Endocrinology, Jikei University School of Medicine, Tokyo, Japan, ${ }^{2}$ Department of Gene Therapy, Institute of DNA Medicine, Jikei University School of Medicine, Tokyo, Japan.

Background and Aims: HNF1 $\beta$ is a transcription factor that expresses in pancreatic $\beta$ cells and has an important role in the development and maintenance of islet cell function. A definite evidence for its significance in islet function is phenotypic expression of MODY 5 patients with the HNF1 $\beta$ gene mutation, but the mechanism for the development of diabetes and polycystic kidney disease remains unknown. Here we report observations of a case with HNF1 $\beta$ (R276X) mutation, a second case in the world. The aim of this study is to examine the clinical phenotype, histology of the kidney and affectedness to insulin secretion.

Methods: Genomic DNA was extracted from peripheral blood sample, followed by direct sequencing. Imaging studies of kidneys, consisting of ultrasonography and computed tomography, and renal biopsy specimens were reviewed by a nephrologists and a renal pathologist. To clarify the physiological relevance of HNF1 $\beta$ mutation in vitro, we monitored insulin secretion indirectly by measuring human $\mathrm{C}$-peptide secretion by transfecting HNF1 $\beta$ mutant cDNA with human preproinsulin cDNA into insulin secreting cell lines.

Results: The patient is a 31 year-old male who was diagnosed as an insulin-requiring diabetes at age 27 . It was unlikely that he had type 1 diabetes because basal insulin secretion is relatively low but his urine C-peptide excretion still remained detectable by $36.1 \mu \mathrm{g} /$ day, isletassociated autoantibodies including anti-GAD, anti-IA2 and anti-ICA antibodies were all negative, and also he had no susceptible HLA allele to type 1 diabetes. In the family history, his father had developed renal failure with mild diabetes but had already past. By clinical examination, size of his left kidney was small ( $85 \mathrm{~mm}$ in the height) accompanied by cortical atrophy, and right kidney had been extensively occupied by multiple cysts. The creatinine clearance was reduced as low as $54 \mathrm{ml} / \mathrm{min}$ though he had no detectable proteinuria, suggesting that the renal damage is not based on common diabetic nephropathy. Electron microscopic examination of left kidney whose sample was obtained by needle biopsy revealed presence of focally enlarged glomeruli, but no evidence of typical diabetic nephropathy. Direct sequence analysis of genomic DNA has revealed that his one allele of HNF1 $\beta$ has $\mathrm{C}$ to $\mathrm{T}$ point mutation that leads to truncated protein in exon 4 . In order to assess its functional relevance, we assayed C-peptide concentration by transfecting human preproinsulin gene to hamster insulinoma (HIT) cell line. When simultaneously transfecting the mutant HNF1 $\beta$ gene with insulin gene, excess expression of R276X-HNF1 $\beta$ gene did not change concentration of human $\mathrm{C}$-peptide in culture media, suggesting that the mutation has little influence on basal insulin secretion as a dominant negative manner.

Conclusion: We have identified HNF1 $\beta$ mutation HNF1 $\beta$ (R276X) with small kidney and multiple renal cysts for the second time. This is the first report that showing imaging studies of kidneys of HNF1 $\beta$ mutation. Additional studies are needed to further clarify the relevance between HNF1 $\beta$ mutation and damped insulin secretion.

Grant-in-Aid of Ministry of Science, Education and Culture

\section{3}

Familial persistent hyperinsulinaemic hypoglycaemia of infancy due to a novel glucokinase (GCK) activating mutation G68V

A. L. Gloyn ${ }^{1}$, G. Lahr ${ }^{2}$, S. Boehler ${ }^{3}$, M. Lindner ${ }^{4}$, M. Wabitsch ${ }^{2}$;

${ }^{1}$ Diabetes Research Laboratories, University of Oxford, United Kingdom, ${ }^{2}$ Pediatric Endocrinology, Department of Pediatrics, University of Ulm, Germany, ${ }^{3}$ Aventis Pharma, Bad Soden, Germany, ${ }^{4}$ Department of Pediatrics, University of Heidelberg, Germany.

Background \& Aims: Persistent hyperinsulinaemic hypoglycaemia of infancy (PHHI) is a rare heterogenous disorder. Heterozygous activating mutations in the glucokinase (GCK) gene have been described in a small number $(n=6)$ of cases. Patients with $G C K$ activating mutations have regulated insulin secretion and can usually be treated with diazoxide. We identified a 7 year old female proband with a history of hypoglycaemia from infancy. The proband's mother, maternal-grandmother and 2 siblings were also affected. Clinical investigations in the proband demonstrated that hypoglycaemia (fpg 2.3-3.5 mmol/L with corresponding insulin values of $50-110 \mathrm{pmol} / \mathrm{L}$ ) was the only abnormal finding. The patient is successfully treated with diazoxide and a biopsy revealed that pancreatic histology is normal. Given this clinical phenotype we hypothesised that the proband had an activating mutation in the $G C K$ gene. 
Methods: The entire coding region and exon-intron boundaries of the human islet $G C K$ gene were sequenced in the proband and her family. Functional analysis of purified wild-type (WT) and mutant G68V recombinant glutathionyl S-transferase fusion proteins were performed spectrophotometrically using an NADP coupled assay.

Results: We have identified a novel $G C K$ mutation (exon 2, GGC $>$ GTC, G68V) which co-segregates in the family with hypoglycaemia. This mutation is not present in 200 normal chromosomes. Structural modelling localised the mutated residue to the allosteric activator site. Kinetic analysis revealed that G68V-GCK is 22 times as active as WT- GCK with an increased affinity for glucose (glucose $\mathrm{S}_{0.5} 1.70 \pm 0.18$ for $\mathrm{G} 68 \mathrm{~V}-\mathrm{GCK}$ vs $7.25 \pm 0.34 \mathrm{mmol} / \mathrm{L}$ for WT-GCK), a decreased ATP $\mathrm{K}_{\mathrm{m}}(0.27 \pm 0.06$ for G68VGCK vs $0.53 \pm 0.03 \mathrm{mmol} / \mathrm{L}$ for WT-GCK), the maximal specific activity $\left(\mathrm{K}_{\mathrm{cat}}\right)$ and Hill number were unchanged. Mathematical modelling predicted a threshold for glucose stimulated insulin (GSIR) release of $1.45 \mathrm{mmol} / \mathrm{L}$.

Conclusion: We have identified a novel activating mutation in the $G C K$ gene in a family with PHHI. Our results increase the number of reported cases of GCK-PHHI and add to our knowledge of the GCK activator site which is an important target for therapeutic agents.

Supported by Diabetes UK

\section{4}

The role of a family history of type $\mathbf{2}$ diabetes in patients with type 1 diabetes (the FinnDiane Study)

L. M. Thorn ${ }^{1,2}$, C. M. Forsblom ${ }^{1,2}$, P.-H. Groop ${ }^{1,2}$;

${ }^{1}$ Folkhälsan Institute of Genetics, Folkhälsan Research Center, Helsinki, ${ }^{2}$ Department of Medicine, Division of Nephrology, Helsinki University Central Hospital, Helsinki, Finland.

Background and Aims: Non-diabetic offspring of type 2 diabetic patients (T2D) are insulin resistant and show components of the metabolic syndrome. We have recently reported a $39 \%$ prevalence of the metabolic syndrome in Finnish type 1 diabetic patients. In the present study, the aim was to study the association between family history of T2D and clinical characteristics, as well as presence of diabetic late complications in patients with type 1 diabetes. Materials and Methods: Of 1860 type 1 diabetic patients from the cross-sectional Finnish Diabetic Nephropathy (FinnDiane) Study, 620 with and 1240 without a family history of T2D were matched for age and sex. Family history of T2D was based on the information provided by the type 1 diabetic proband using a standardized questionnaire (sensitivity $89 \%$, specificity $98 \%$ ).

Results: The mean age of the probands was $43.5 \pm 0.2$ and $50 \%$ were male. The table below shows characteristics of patients with (FH T2D+) and without (FH T2D-) a family history of T2D. BMI=body-mass index, eGDR=estimated glucose disposal rate.

\begin{tabular}{|c|c|c|c|}
\hline & $\begin{array}{l}\text { FH T2D+ } \\
n=620\end{array}$ & $\begin{array}{l}\text { FH T2D- } \\
n=1240\end{array}$ & $P$ value \\
\hline $\begin{array}{l}\text { Age at onset of } \\
\text { diabetes (yrs) }\end{array}$ & $17.2 \pm 0.4$ & $16.1 \pm 0.3$ & 0.008 \\
\hline BMI $\left(\mathrm{kg} / \mathrm{m}^{2}\right)$ & $25.7 \pm 0.2$ & $25.0 \pm 0.1$ & $<0.001$ \\
\hline Wiast cicumference $(\mathrm{cm})$ & $88.0 \pm 0.5$ & $86.1 \pm 0.3$ & 0.002 \\
\hline $\mathrm{HbA}_{1 \mathrm{c}}(\%)$ & $8.4 \pm 0.1$ & $8.3 \pm 0.1$ & 0.020 \\
\hline eGDR $\left(\mathrm{mg}^{*} \mathrm{~kg}^{-1} * \mathrm{~min}^{-1}\right)$ & $5.8 \pm 0.1$ & $6.1 \pm 0.1$ & 0.023 \\
\hline Insulin dose (IU/kg) & $0.68 \pm 0.01$ & $0.65 \pm 0.01$ & 0.005 \\
\hline Triglycerides $(\mathrm{mmol} / \mathrm{l})$ & $1.36 \pm 0.04$ & $1.20 \pm 0.02$ & $<0.001$ \\
\hline HDL Cholesterol (mmol/l) & $1.37 \pm 0.02$ & $1.38 \pm 0.01$ & 0.478 \\
\hline $\begin{array}{l}\text { Systolic blood pressure } \\
(\mathrm{mmHg})\end{array}$ & $138 \pm 1$ & $137 \pm 1$ & 0.195 \\
\hline $\begin{array}{l}\text { Diastolic blood pressure } \\
(\mathrm{mmHg})\end{array}$ & $80 \pm 1$ & $80 \pm 1$ & 0.570 \\
\hline
\end{tabular}

In a logistic regression analysis, an independent association with family history of T2D was found for age at onset of diabetes, BMI, insulin dose, and triglycerides. Family history of T2D was associated with the metabolic syndrome (NCEP criteria; $44 \%$ vs. $38 \%$, p=0.014, IDF definition; $43 \%$ vs. $38 \%, p=0.031$ ), but not with coronary heart disease (9.9\% vs. $7.4 \%)$, cardiovascular hard end-points (13\% vs. $12 \%)$, diabetic nephropathy ( $25 \%$ vs. $25 \%)$, retinal laser treatment ( $44 \%$ vs. $44 \%)$, or antihypertensive medication (49\% vs. $49 \%$ ). Maternal history of T2D was associated with larger waist circumference, lower eGDR, higher triglycerides, $\mathrm{HbA}_{1 \mathrm{c}}$, insulin dose, $\mathrm{BMI}$, and later onset of diabetes, as well as coronary heart disease, cardiovascular hard end-points, and the metabolic syndrome (NCEP and IDF). Paternal history of T2D was associated with higher triglycerides, insulin dose, and BMI.

Conclusion: A family history of T2D is associated with some, but not all, components of the metabolic syndrome, and also with a later onset of diabetes. In these still rather young type 1 diabetic patients, a family history of T2D was not associated with diabetic late complications.

0305

HLA-DQB1 genotypes predict autoimmune diabetes development and $\beta$ cell failure in adult (40-59 years old) diabetic patients

E. Bakhtadze, E. Lindholm, L. Groop, G. Sundkvist; 
Department of Clinical Sciences, Lund University, Malmö, Sweden.

Background and Aims: Common allelic variants at the HLA class II loci account for the major genetic risk for type 1 diabetes among children and young adults. However, HLA predisposition to autoimmune diabetes development among adult diabetic patients is not well established. We assessed HLA-DQB1 genotypes, islet antibodies (GADA) and fasting plasma C-peptide (F-P-C-peptide) among 1339 Swedish adult (40-59 years old) diabetic patients from a diabetes registry in Southern Sweden. Our aim was to relate HLA-DQB1 genotypes to GADA and $\beta$-cell function ( $F-$ $\mathrm{P}-\mathrm{C}$-peptide) in adult diabetic patients.

Materials and Methods: Age at onset of diabetes was between 40-59 years in registered patients. F-P-C-peptide concentration was measured in duplicate by a radioimmunoassay: normal range was $0.25-0.75 \mathrm{nmol} / \mathrm{l}$. GADA were measured by radioimmunoprecipitation method: cut-off limit for positivity was 5 RU. HLA-DQB1 genotyping was performed using hybridisation assay.

Results: Among the 1339 patients, risk HLA-DQB1 genotypes were found in 283 (21\%), GADA in 170 (13\%) and low F-P-C-peptide $(<0.25 \mathrm{nmol} / \mathrm{l})$ in $172(13 \%)$. Patients with GADA had significantly $(\mathrm{p}<0.0001)$ lower median BMI $(25$ vs. $29 \mathrm{~kg} / \mathrm{m}^{2}$ ) and F-P-C-peptide (0.3 vs. $0.9 \mathrm{nmol} / \mathrm{l}$ ) than those without GADA. Compared with GADA-, GADA +was associated with female gender (49\% vs. $35 \%$; $\mathrm{p}=0.0001)$ and insulin treatment $(60 \%$ vs. $23 \%$; $<<0.0001)$. HLA-DQB1 $* 0302 / \mathrm{X}$ were increased in diabetic patients compared with non-diabetic controls ( $10 \%$ vs. $4.2 \%$; $\mathrm{p}=0.0049$ ). After stratification for GADA presence, compared with GADA-, GADA+had a fivefold increased risk of having HLA-DQB $1 * 02 / * 0302(27 \%$ vs. $6.7 \%$; $\mathrm{p}<0.0001)$, significantly $(\mathrm{p}<0.0001)$ lower $(<0.25 \mathrm{nmol} / \mathrm{l})$ F-P-C-peptide (6.6\% vs. $48 \%)$ and lower $\left(<25 \mathrm{~kg} / \mathrm{m}^{2}\right)$ BMI (20\% vs. 50\%). In multinominal regression analysis, HLADQB1 *02/*0302, low F-P-C-peptide $(<0.25 \mathrm{nmol} / \mathrm{l})$, low BMI $\left(<25 \mathrm{~kg} / \mathrm{m}^{2}\right)$ and female gender remained independently significant in association with GADA. Evaluation of F-PC-peptide concentrations separately in GADA+and GADA - groups related to diabetes duration and HLADQB1 genotypes showed that, low F-P-C-peptide significantly $(<0.0001)$ correlated with a long duration of diabetes, both in GADA $+(\mathrm{r}=-0.445)$ and $\mathrm{GADA}-(\mathrm{r}=-0.179)$. Moreover, amongst GADA+, low F-P-C-peptide concentrations were significantly associated with HLA-DQB1 *02/ *0302 ( $\mathrm{p}=0.039)$, whereas amongst GADA - , low F-P-Cpeptide concentrations were significantly associated with HLA-DQB $1 * 0302 / \mathrm{X} \quad(\mathrm{p}=0.001)$ and $* 0302 / * 0604$ $(p=0.033)$. After stratification of F-P-C-peptide concentra- tions into $(<0.25 \mathrm{nmol} / \mathrm{l})$ and $(>0.25 \mathrm{nmol} / \mathrm{l})$, multinominal regression showed that GADA $+(\mathrm{p}<0.0001)$, HLA-DQB1 $* 0302 / X(p=0.018), * 0302 / * 0604(p=0.006)$, low $(<25 \mathrm{~kg} /$ $\left.\mathrm{m}^{2}\right)$ BMI $(\mathrm{p}<0.0001)$ and a long duration of diabetes $(\mathrm{p}<0.0001)$ were independently associated with low $(<0.25 \mathrm{nmol} / \mathrm{l}) \mathrm{F}-\mathrm{P}-\mathrm{C}$-peptide concentrations.

Conclusions:Although decrement of F-P-C-peptide concentrations is associated with a long duration of diabetes, risk HLA-DQB1 genotypes independently predict autoimmune diabetes development and $\beta$-cell failure in 40-59 years old diabetic patients: ${ }^{*} 02 / * 0302$ is associated with GADA + , but GADA+independently of HLA-DQB1 risk, contributes to $\beta$-cell failure, whereas among GADA - HLA-DQB1*0302/ $\mathrm{X}$ and $* 0302 / * 0604$ contribute to $\beta$-cell failure. Hence, our study suggests that risk HLA-DQB1 genotypes contribute to $\beta$-cell failure in patients without GADA.

Supported by Skane County Council Research and Development Foundation

\section{6}

Epitope specificity of autoantibodies directed to the Nterminus of glutamate decarboxylase (GAD65) correlate with beta cell functional reserve in patients with ketosis prone diabetes mellitus

T. Hall ${ }^{1}$, R. Nalini ${ }^{2}$, M. Maldonado ${ }^{3}$, G. Garza ${ }^{4}$, D. Iyer $^{2}$, A. Balasubramanyam ${ }^{2}$, C. S. Hampe ${ }^{5}$;

${ }^{1}$ Department of Medicine, University of Washington, Seattle,

${ }^{2}$ Translational Metabolism Unit, Baylor College of Medicine, Houston, ${ }^{3}$ Medicine, Bristol-Myers-Squibb Co., Princeton, ${ }^{4}$ Endocrine Service, Ben Taub General Hospital, Houston,

${ }^{5}$ Medicine, University of Washington, Seattle, United States.

Background and Aims: Diabetic ketoacidosis (DKA) has traditionally been viewed as a complication of type 1 diabetes, but several recent studies have shown that patients presenting with DKA are heterogeneous in regard to the type of diabetes. We have prospectively characterized these heterogeneous forms of ketosis-prone diabetes mellitus (KPDM) into four subgroups, based on the presence or absence of beta cell autoimmunity $(\mathrm{A}+$ or $\mathrm{A}-$ ) and beta cell functional reserve $(\mathrm{B}$ +or $\mathrm{B}-$ ). The $\mathrm{A}+\mathrm{B}-\mathrm{KPDM}$ subgroup comprises patients with classic, autoimmune type 1 diabetes (complete beta cell loss), whereas the $\mathrm{A}+\mathrm{B}+\mathrm{KPDM}$ subgroup, despite possessing the same autoantibodies as $\mathrm{A}+\mathrm{B}-$ patients, have only partial beta cell loss and a distinct clinical phenotype. We hypothesized that the autoimmune reaction differs between these two KPDM subgroups. We analyzed the epitope specificity of autoantibodies directed against the $65 \mathrm{kDa}$ isoform of glutamic acid decarboxylase (GAD65) as a reflection of the underlying islet cell autoimmunity. 
Material and Methods: We analyzed the sera of GAD65Abpositive KPDM patients $(n=36)$ for their epitope recognition using recombinant Fabs specific to six different GAD65 epitopes. Binding to the different epitopes was correlated with measures of beta cell function ( $\mathrm{HOMA} \% \mathrm{~B}$, Fasting C-peptide (FCP)), age, gender and other clinical parameters.

Results: The sera's binding to a GAD65 N-terminal epitope correlated with longitudinally assessed beta cell functional reserve. Binding to the $\mathrm{N}$-terminal epitope correlated with FCP $(\mathrm{R}=0.56, \mathrm{p}=0.0008)$ at the initial sampling and with both HOMA\%B and FCP 12 months later $(\mathrm{R}=0.4, \mathrm{p}=0.03, \mathrm{R}=0.54, \mathrm{p}=0.007$, respectively $)$. In the two phenotypic subgroups of GAD65Ab-positive patients, binding to the $\mathrm{N}$-terminal epitope was significantly stronger in $\mathrm{A}+\mathrm{B}+($ median binding $31 \%$ ) than in $\mathrm{A}+\mathrm{B}-$ patients (median binding 12\%) $(\mathrm{p}=0.001)$.

Conclusion: Binding to a N-terminal epitope of GAD65 is correlated with preserved beta cell functional reserve in GAD65Ab-positive patients with KPDM, and therefore associated with the $\mathrm{A}+\mathrm{B}+$ phenotype. Whether GAD65Ab specific to the $\mathrm{N}$-terminal epitope can be used as markers for a slower or more latent form of beta cell destruction or may even be involved in this more benign clinical course, remains to be investigated.

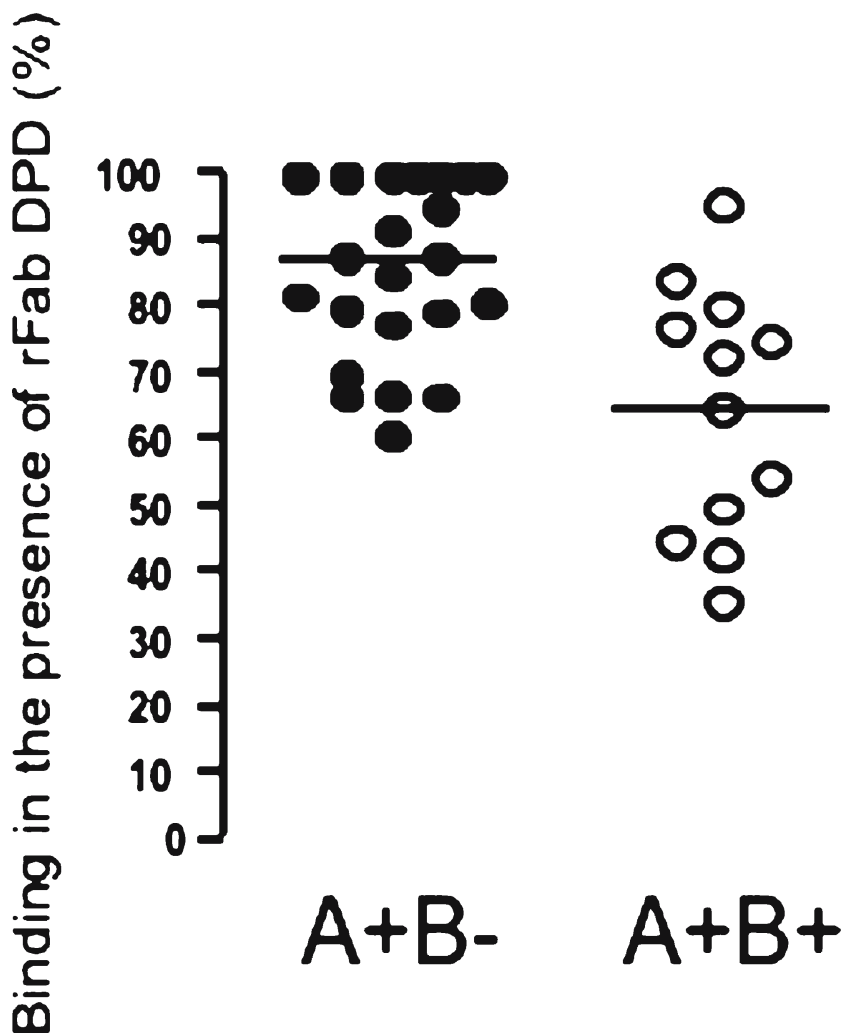

Supported by American Diabetes Association
0307

Autoimmunity and prevention of type 2 diabetes: the Diabetes Prevention Program (DPP)

D. Dabelea, Y. Ma, W. C. Knowler, S. Marcovina,

C. Saudek, R. Arakaki, N. H. White, S. Kahn,

S. M. Haffner, T. Orchard, R. Goldberg, R. F. Hamman;

George Washington University, The Diabetes Prevention Program, Rockville, United States.

Limited data exist on the prevalence and predictive role of diabetes-related autoantibodies (DRAA) among adults at risk for DM. Moreover, it is unknown whether preventive interventions are equally effective in subjects with and without DRAA. The DPP measured baseline GAD65 and IA-2 antibodies in 3,050 IGT subjects aged 25-84, randomized to intensive lifestyle (ILS; $\mathrm{N}=1015$ ), metformin (MET; $\mathrm{N}=1013$ ) and placebo (PLB; $\mathrm{N}=1022$ ) and followed 3.2 years. DRAA positive (DRAA+) was either GAD65 index $\geq 0.085$ or IA-2 index $\geq 0.017$. Cox models assessed the effect of DRAA on DM risk, accounting for the potential interaction with treatment assignment, and adjusted for age, sex, race/ethnicity, baseline $\mathrm{BMI}, \mathrm{HbAlc}$, insulin resistance and beta-cell function. A total of 367 subjects $(12 \%)$ had DRAA+: $9.5 \%$ of non-Hispanic whites, 9.7\% of Hispanics, $16.0 \%$ of African-Americans, $14.1 \%$ of Asians and $29.6 \%$ of American Indians. Race/ethnic differences were mostly due to IA-2. Crude DM incidence by DRAA and DPP intervention is shown in the figure. Overall, DRAA did not predict DM [Hazard ratio, $\mathrm{HR}=1.18(95 \% \mathrm{CI}=0.96-1.46)]$. However, DRAA+ persons in the PLB group had $40 \%$ higher $\mathrm{DM}$ risk $(\mathrm{CI}=3-91 \%)$ than DRAA negative. The increased risk in the PLB group was largely conveyed by IA-2 alone $[$ HR=1.50 (1.0-2.4)]. The effects of ILS or MET (vs. PLB) on DM prevention were similar regardless of DRAA $(p=0.3$ for interaction). In conclusion, among people with IGT, DRAA predict faster progression to DM in untreated persons, and ILS and MET are equally effective in subjects with and without autoimmunity.

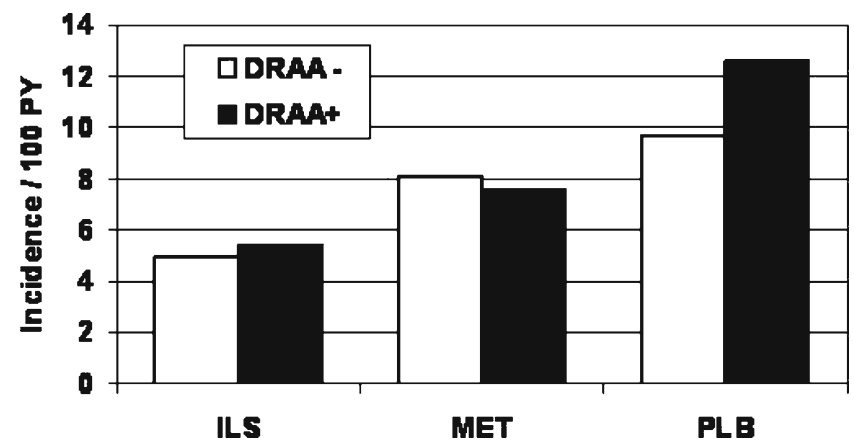




\section{PS 6}

\section{Incidence and prevalence of type 2 diabetes in different ethnic populations}

\section{8}

\section{Incidence of type 2 diabetes in Pima Indian and Mexican populations}

A. Jimenez-Corona ${ }^{1}$, R. Lopez-Ridaura ${ }^{1}$, M. E. Pavkov ${ }^{2}$, C. Gonzalez-Villalpando ${ }^{1}$, R. G. Nelson ${ }^{2}$;

${ }^{1}$ Direccion de Enfermedades Cronicas, Instituto Nacional de Salud Publica, Cuernavaca, Mexico, ${ }^{2}$ Epidemiology and Clinical Research Branch, National Institute of Diabetes and Digestive and Kidney Diseases, Phoenix, United States.

Background and Aim: Pima Indians from Arizona have the greatest incidence of type 2 diabetes in the world, while in Mexico type 2 diabetes prevalence has increased over the last decades. The aim of this study was to compare the adjusted incidence of type 2 diabetes in two longitudinal studies from these populations with differences in genetic susceptibility and in the prevalence of risk factors.

Methods: A population based follow up study began in 1989 in low income neighborhoods of Mexico City with 1460 subjects (592 men and 868 women) free of diabetes and aged 35 to 64 years at baseline. Follow up visits, including fasting and 2 hours plasma glucose, were performed on average every 3 years up to 2000. A study in Pima Indians started in 1965 with biannual follow up visits up to 2004, similar to the ones in the Mexico City cohort. From this study we included 1,466 subjects (615 men and 851 women) with their first non diabetic examination between ages 35 to 65 years. Incidence rate (IR) was calculated as the number of persons who developed diabetes divided by the person-years of followup and expressed per 1,000 person-years and standardized to the 1998 World population aged 35 to 65 years. The risk period extended up to the examination at which diabetes was diagnosed or to the date of the last examination within the study period in each cohort. Diabetes was diagnosed by the WHO criteria (fasting plasma glucose $>7.8 \mathrm{mmol} / \mathrm{l}$ or 2 hour post load plasma glucose $>11.1 \mathrm{mmol} / \mathrm{l})$.

Results: At baseline, Pima Indians had worse risk profile than Mexican participants, especially for mean BMI (33.3 vs $28.0 \mathrm{~kg} / \mathrm{m} 2$ ), fasting plasma glucose (97.6 vs $84.7 \mathrm{mg} /$ $\mathrm{dL})$ and 2 hour post load plasma glucose (120.1 vs. $104.72 \mathrm{mg} / \mathrm{dL}$ ). During a median follow-up of 5.6 years (range 1.0 to 9.5 years), 161 Mexican subjects developed diabetes. Age-adjusted IR were 18.5 cases/1000 pyrs $(95 \%$ CI 13.9-23.13) in men and 19.2 (95\% CI 15.2-23.1) in women. For Pima Indians, during a median follow-up of 12.6 years (range $0.05-39.2$ years) 607 of them developed diabetes. Age-adjusted IR were 41.6 cases $/ 1000$ pyrs $(95 \%$ CI 36.0-47.3) in men and 53.6 (95\% CI 48.2-58.9) in women. The sex-adjusted IR stratified by age categories are presented in table 1. Given the differences on risk factor at baseline, we selected a subset from the Mexican cohort with high risk to develop diabetes defined as fasting plasma glucose $>100 \mathrm{mg} / \mathrm{dL}$ at baseline. The age and sex adjusted IR on this high risk group of Mexicans was 41.3 cases $/ 1000$ pyrs (95\% CI $34.2-48.4)$ similar to the IR in the general Pima Indian population.

Sex-adjusted IR (per 1,000/year) of diabetes in Mexicans and Pima Indians by age category

\begin{tabular}{lllll}
\hline & \multicolumn{2}{l}{ Mexicans } & \multicolumn{2}{l}{ Pima Indians } \\
\hline Age (years) & IR & $95 \%$ CI & IR & $95 \%$ CI \\
$35-44$ & 14.3 & $9.8-18.9$ & 41.6 & $36.0-47.3$ \\
$45-54$ & 24.7 & $19.1-30.4$ & 54.4 & $47.4-61.3$ \\
$55-64$ & 19.4 & $13.7-25.2$ & 49.5 & $40.1-58.9$ \\
Age-sex-adjusted & 18.9 & $15.8-21.8$ & 47.6 & $62.9-88.8$ \\
\hline
\end{tabular}

Conclusions: The IR of diabetes in Pima Indians is more than 2 times higher than that in Mexican subjects. However this difference does not hold once we select a high risk subset of the Mexican population, suggesting that the original difference is partially explained by differences in baseline risk factors.

0309

Increased prevalence of diabetes in an industrial area of southern Poland

A. Wittek ${ }^{1}$, B. Sokalski ${ }^{2}$, W. Grzeszczak ${ }^{1}$, K. Strojek ${ }^{1}$;

${ }^{1}$ Department of Internal Diseases Diabetology

and Nephrology, Silesian Medical Academy, Zabrze, ${ }^{2}$ Outpatient Clinic for Diabetics, Ruda Slaska, Poland.

Background and Aims: Prevalence of diabetes is growing up and global data shows the increasing burden. No realistic data on epidemiology of diabetes from Poland, for the last 20 years, is available. Aim: Estimation of the prevalence of diabetes and cardiovascular risk factors in industrial area of Southern Poland in adult population.

Materials and Methods: We randomly selected and invited for screening the group of 782 subjects from the voters' list consisting of 23442 inhabitants of suburb Halemba, town Ruda Slaska. The attendance rate was $56 \%$ but $85 \%$ in the group of age $>50$ yrs. OGTT $75 \mathrm{~g}$, anthropometric measurements, cholesterol (total, HDL, LDL), triglicerides were measured. Adjusted prevalenceestimated error using fraction index were calculated. 
Results: The overall prevalence of diabetes (DM) was $8.06 \pm 0.90 \%$ (male $7.15 \pm 1.25$; female $8.94 \pm 1.30$ ) including $5.56 \pm 0.77 \%$ of known DM and $2.52 \pm 0.56 \%$ of newly diagnosed DM. Respective figures for male were: known $-5.56 \pm 1.13 \%$, new $-1.59 \pm 0.64 \%$; and for female were: known $-5.56 \pm 1.06 \%$ and new $-3.37 \pm 0.89 \%$. Impaired glucose tolerance (both IGT and IFG) was found additionally in $8.44 \pm 0.93 \%$ (male $6.09 \pm 1.17 \%$; female $10.70 \pm 1.41 \%$. After adding registered cases of diabetes in children and number of inhabitants at the age $<18 \mathrm{yrs}$ the prevalence of diabetes in overall population was calculated as $6.54 \pm 0.73 \%$ ( $4.59 \pm 0.62$ known; $2.05 \pm 0.44 \%$ new $)$ and impaired glucose tolerance as $6.85 \pm 0.68$. The prevalence of metabolic syndrom (IDF criteria) was $34.1 \%$ in the group studied including $21.8 \%$ in normal glucose tolerant subjects; $76.5 \%$ in diabetics; $54.4 \%$ in IFG/IGT group.

Conclusion: Prevalence of diabetes in Southern Poland is higher than estimated indirectly but the percentage of undiagnosed diabetes is reduced. The prevalence of cardiovascular risk factors (metabolic syndrom) is similar to other caucasian populations.

Unrestricted financial support from Novo Nordisk and Servier.

\section{0}

Diabetes among immigrants from Turkey and Lebanon compared to Danes. Prevalence, treatment, screening for complications and cardiovascular outcome

J. K. Kristensen ${ }^{1}$, T. Lauritzen ${ }^{1}$, J. F. Bak ${ }^{2}$;

${ }^{1}$ Institute of Public Health, Department of General Medicine, University of Aarhus, Denmark, ${ }^{2}$ Department of Endocrinology and Internal Medicine C, Aarhus University Hospital, Denmark.

Background and Aims: Population survey data have shown globally increased prevalence rates of diabetes. For unknown reasons Europeans (Caucasians) may have a lower progression rate of Type 2 diabetes than patients from other parts of the world. By use of national and regional registries, this study aimed to identify trends in diabetes prevalence, treatment, care and cardiovascular complications among Lebanese (L) and Turkish (T) immigrants compared to native Danes (DK).

Materials and Methods: Patients with diabetes, their country of origin, laboratory measurements, medical treatment, frequency of examinations by ophthalmologists and cardiovascular complications were identified from The National Health Service Registry, The Regional Laboratory Database, The Danish National Hospital Registry and the Danish Civil Registration System. Patients were classified as Type 2 diabetics if they were treated with diet alone, with oral anti-diabetic agents or, irrespective of treatment, if they were over 40 years of age at the time of diagnosis (results from a questionnaire). Data were collected from 2000 through 2003 in a background population of 649,177 citizens in Aarhus County, corresponding to $12 \%$ of the total Danish population.

Results: Age-standardised prevalences of Type 2 diabetes were $18.9 \%(\mathrm{~L}, \mathrm{~N}=85) ; 19.8 \%(\mathrm{~T}, \mathrm{~N}=152)$ and $3.6 \%(\mathrm{DK}$, $\mathrm{N}=8885$ ). In the period of 2000-2003 a considerable variation in the quality of glycaemic control both within and between ethnic groups was found. Thus, in 2003 $\mathrm{HbA} 1 \mathrm{c}$ was measured at least once in $84 \%$ (DK), $87 \%$ (L) and $83 \%(\mathrm{~T})$ of the patients. Furthermore, $72 \%$ (DK), 53\% (L), and 51\% (T) had an HbAlc $<8 \%(\mathrm{p}<0.05)$. In 2003, a large proportion of Danes were in non-pharmacological treatment: $40 \%$ (DK), 29\% (T), and 32\% (L) (DK vs. T, $\mathrm{p}<0.05$ ), while immigrants were more likely to be treated with oral antidiabetic agents: $42 \%(\mathrm{DK}), 53 \%$ (T), $53 \%(\mathrm{~L})$; $(p<0.05)$. There were no differences in the proportions of patients treated with insulin: 18\% (DK), 19\% (T), and 15\% (T) $(\mathrm{p}=\mathrm{NS})$. During 2000-2003 increasing proportions of patients with total cholesterol $<5.0 \mathrm{mmol} / 1$ were found in all groups: $37-42 \%$ (L), $41-48 \%(\mathrm{~T})$, and $34-47 \%$ (DK) $(\mathrm{p}=\mathrm{NS})$. This corresponds with an increase in the use of lipid-lowering treatment: $17-27 \%(\mathrm{~L}), 13-32 \%(\mathrm{~T})$, and $18-38 \%$ (DK) (DK and $\mathrm{L} ; \mathrm{p}<0.05$ ). In 39\% (DK), 35\% $(\mathrm{L})$, and $37 \%(\mathrm{~T})$ of the patients no examination for retinopathy was registered during the period of 2000 2003. A cardiovascular diagnosis was registered in $27 \%$ (DK), $32 \%(\mathrm{~L})$ and $33 \%(\mathrm{~T})$ of the patients ( $\mathrm{p}=\mathrm{NS}$ ).

Conclusion: Compared to native Danes, Lebanese and Turkish immigrants had a much higher prevalence of Type 2 diabetes and worse glycaemic regulation. The routine monitoring of diabetes care for native Danes, Lebanese or Turkish immigrants was comparable. Still, there is room for improvement in complication screening programmes. Despite differences in glycaemic control, cardiovascular complications were equivalent in the three groups.

0311

High prevalence of undiagnosed type 2 diabetes, other types of impaired glucose regulation and associated cardiovascular risk factors among working population of Chelyabinsk

I. A. Vaychulis ${ }^{1}$, I. I. Shaposhnik ${ }^{1}$, T. N. Vaychulis ${ }^{2}$;

${ }^{1}$ Internal Medicine Department, Chelyabinsk State Medical Academy, Chelyabinsk, ${ }^{2}$ Endocrinology Department, Chelyabinsk Municipal Hospital No.1, Chelyabinsk, Russian Federation.

Background and Aims: It is known that great proportion of patients (pts) with type 2 diabetes (DM 2) remains 
undiagnosed for several yrs resulting in high prevalence of cardiovascular (CV) complications at the time of diagnosis. The aim of the study was to estimate the prevalence of DM 2, impaired fasting glucose (IFG), impaired glucose tolerance (IGT) and associated CV risk factors among working population of Chelyabinsk aged 40 yrs and older.

Materials and Methods: Chelyabinsk is a big industrial city with the total population of 1095053 and population aged $40 \mathrm{yrs}$ and older of 493963 (197533 males/296430 females). We examined a total of $4238(1661 \mathrm{~m} / 2577 \mathrm{f})$ randomly selected workers of 15 enterprises and institutions of different types, located in all 7 administrative districts of the city, aged $40-70$, mean age \pm SD $52.0 \pm 6.8$ yrs. Fasting capillary glucose (FCG) was analysed with a glucometer Accu-Chek Active (Roche Diagnostics) and in people with FCG 5.6-6.0 standard oral glucose tolerance test was performed. Weight, height, waist circumference (WC), systolic (SBP) and diastolic blood pressure (DBP) were measured and body mass index (BMI) was calculated. Prevalence data are shown as mean $\% \pm 95 \%$ CI. General linear univariate model with age-adjustment was used to compare prevalence rates.

Results: According to WHO (1999) criteria the prevalence of isolated IFG and combined IFG/IGT was 7.9 [7.1-8.7] and $1.8[1.4-2.2] \%$, respectively. The estimated prevalence of previously and newly diagnosed DM2 was $2.1[1.6-2.5]$ and $5.9[5.2-6.6] \%$, respectively. The estimated number needed to screen (NNTS) to identify one person with previously unknown dysglycaemia was 6.4 [6.0-6.9].

Age-adjusted prevalence of obesity $\left(\mathrm{BMI} \geq 30 \mathrm{~kg} / \mathrm{m}^{2}\right)$, abdominal obesity (WC>88 $\mathrm{cm}$ in women, $\mathrm{WC}>102 \mathrm{~cm}$ in men) and arterial hypertension ( $\mathrm{SBP} \geq 140$ and/or $\mathrm{DBP} \geq 90 \mathrm{~mm} \mathrm{Hg}$ ) was significantly higher in all groups with abnormal glucose level compared to normoglycaemic individuals $(p<0.001)$ but no significant difference $(p>0.05)$ was found when compared isolated IFG, combined IFG/ IGT and newly diagnosed DM2 groups with previously diagnosed DM2 pts (table 1).

Conclusion: Screening among working population aged $40 \mathrm{yrs}$ and older is an efficient method to identify dysglycaemic subjects. The prevalence of undiagnosed DM2 among working residents of Chelyabinsk is almost 3 times higher than the prevalence of registered DM 2. Any type of impaired glucose regulation was associated with higher prevalence of CV risk factors. Subjects with undiagnosed DM 2 and intermediate glucose abnormalities demonstrated $\mathrm{CV}$ risk profile similar to pts with previously diagnosed DM2.
Table 1. Age-adjusted prevalence of $\mathrm{CV}$ risk factors according to the categories of dysglycaemia in the study population.

\begin{tabular}{llllll}
\hline $\begin{array}{l}\text { Risk } \\
\text { factor }\end{array}$ & $\begin{array}{l}\text { Normal } \\
\text { FCG }\end{array}$ & IFG & $\begin{array}{l}\text { IFG } \\
+ \text { IGT }\end{array}$ & $\begin{array}{l}\text { Previously } \\
\text { diagnosed } \\
\text { DM2 }\end{array}$ & $\begin{array}{l}\text { Undiagnosed } \\
\text { DM2 }\end{array}$ \\
n & 3491 & 332 & 77 & 88 & 250 \\
Obesity & $27.8 \%$ & $40.2 \%$ & $51.6 \%$ & $54.5 \%$ & $57.1 \%$ \\
& {$[26.3-$} & {$[34.9-$} & {$[40.2-$} & {$[43.9-$} & {$[51.0-63.3]^{* \S}$} \\
& $29.2]$ & $45.5]^{*}$ & $63.0]^{*}$ & $65.1]^{*}$ & \\
Abdominal & $25.9 \%$ & $45.5 \%$ & $54.4 \%$ & $56.9 \%$ & $56.9 \%$ \\
obesity & {$[24.5-$} & {$[40.1-$} & {$[43.0-$} & {$[46.3-$} & {$[50.7-63.0]^{* \#}$} \\
& $27.4]$ & $50.9]^{*}$ & $65.8]^{*}$ & $67.4]^{*}$ & \\
Arterial & $28.5 \%$ & $41.9 \%$ & $41.7 \%$ & $44.9 \%$ & $53.5 \%$ \\
hypertension & {$[27.0-$} & {$[36.6-$} & {$[30.4-$} & {$[34.3-$} & {$[47.3-59.7]^{* \#}$} \\
& $30.0]$ & $47.2]^{*}$ & $52.9]^{*}$ & $55.5]^{*}$ & \\
\hline
\end{tabular}

* $\mathrm{p}<0,001$ compared to normal $\mathrm{FCG}$

$\S \mathrm{p}<0.001$ compared to IFG

$\# \mathrm{p}<0.01$ compared to IFG

Supported by Servier

0312

Gender differences in the prevalence of impaired fasting glucose (IFG) in healthy Thai

K. Athirakul ${ }^{1}$, S. Anuras ${ }^{1}$, W. Lausoontornsiri ${ }^{2}$;

${ }^{1}$ Medicine, Bumrungrad International Hospital, Bangkok,

${ }^{2}$ Medicine, The Ministry of Public Health of Thailand, Bangkok, Thailand.

Background and Aims: A substantial number of patients are unaware of their glycemic status and have already developed chronic complications at the time of the diagnosis of hyperglycemia. These undiagnosed patients can only be discovered by fasting plasma glucose (FG) measurement. Individuals with IFG are predisposed for DM and metabolic syndrome. Screening these individuals is essential for early identification and management of DM. However, the prevalence of IFG by age and gender using the recent ADA classification in healthy Thai is not known.

Materials and Methods: We retrospectively reviewed FGs in Thai in one tertiary care medical center from 1/2002-12/ 2004. FG was determined by glucose oxidase method. Individuals were categorized to 8 age groups at 5 -year interval, beginning from 25 to $>60$ years old (y). The agespecific FG was calculated to represent the average FG of each age group. The population estimated was based on Thailand 2002 census (41.4 million people aged $\geq 25$ y). Data were presented as mean \pm SD.

Results: There were 14837 patients, most were middle class and resided in metropolitan area. None is known to 
have prior history of hyperglycemia. Forty six percent were male. The mean age was $43 \pm 11(25-84)$ y for male and was $42 \pm 11(25-84)$ y for female. FG increased with age in both genders with the predicted incremental rates of 0.67 and $0.62 \mathrm{mg} / \mathrm{dl} /$ year for male and female respectively $(\mathrm{r}=0.30$ for male and $\mathrm{r}=0.36$ for female, $\mathrm{p}<0.001$ each). As a group, FG was elevated in male compared with female $(101 \pm 25 \mathrm{mg} / \mathrm{dl}$ vs. $93 \pm 19 \mathrm{mg} / \mathrm{dl}, \mathrm{P}<0.001)$. In addition, the age-specific FG was also higher in male (ANCOVA, $\mathrm{P}<0.01$ ). For Thai aged $\geq 25 \mathrm{y}$, the overall estimated prevalence was $22.2 \%$ for IFG ( 8.3 million people) and $4.4 \%$ for undiagnosed DM (1.8 million people). As with FG, the undiagnosed hyperglycemia (IFG and undiagnosed $\mathrm{DM})$ were more common in older individuals in both genders ( $\mathrm{r}=0.98, \mathrm{p}<0.001$, each, figure 1$)$. When adjusted for age, the undiagnosed hyperglycemia was again more prevalent in male than female (ANCOVA, $\mathrm{P}<0.01$ ).

Conclusion: Our study has observed the influence of age and gender on plasma glucose and the prevalence of hyperglycemia in Thai. The epidemic of the undiagnosed hyperglycemia is an important public health concern. This information is essential for establishing a screening program to control and prevent DM and its long term complications.

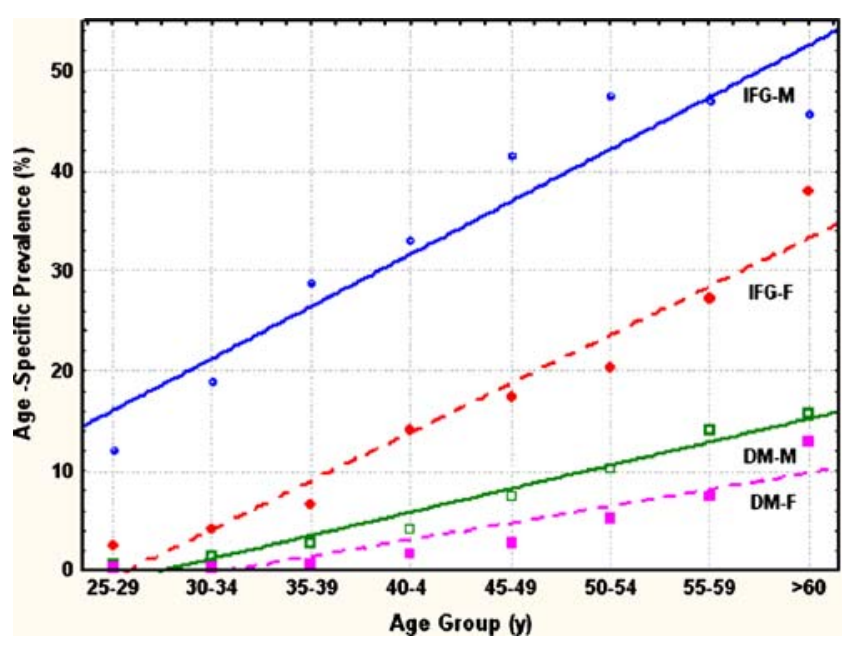

0313

Prevalence of diabetes, impaired fasting glucose and obesity in Iran

M. M. Gooya ${ }^{1}$, A. Esteghamati ${ }^{2}$, A. Delavari ${ }^{1}$, S. Alikhani ${ }^{1}$, A. Moghisi ${ }^{1}$, A. Yarahmadi ${ }^{1}$, F. Alaedini ${ }^{1}$, M. Abbasi ${ }^{2}$, S. Mahdavi ${ }^{1}$, F. Azizi ${ }^{3}$;

${ }^{1}$ CDC, Ministry of Health, Tehran, ${ }^{2}$ Tehran University of Medical Sciences, Endocrinology and Metabolism Research Center, Tehran, ${ }^{3}$ Shahid Beheshti Medical University, Endocrine Research Center, Tehran, Islamic Republic of Iran
Background and Aims: Obesity and diabetes currently threaten the health, well-being and economic welfare of virtually every country in the world. The purpose of this study was to estimate the prevalence of diabetes (DM), impaired fasting glucose (IFG) and obesity and the association of DM\& IFG with risk factors in the Iranian population.

Materials and Methods: The Iranian National Health and Nutrition Surveillance system 2004- 2005 was a nationally representative survey with multistage cluster sampling design. Data from a comprehensive questionnaire, a physical examination, and blood tests were obtained from 52519 Iranian adults in urban and rural areas (25244 men and 27275 women) aged $25-65$ years.

Results: The age-adjusted prevalence of diabetes in this population was $5.9 \%$, and the age-adjusted prevalence of previously diagnosed diabetes was $2.7 \%$. Overall, these results indicate that $5.6 \%$ of Iranian men and $6.1 \%$ of Iranian women have diabetes. The age-adjusted prevalence of IFG was $15.4 \%$, using the new American Diabetes Association criteria. The overall prevalence of overweight (BMI 25-29.9), obesity (BMI>30) and morbid obesity (BMI $>35)$ were $28.6 \%, 10.8 \%$ and $3.4 \%$ respectively. The prevalence of obesity was significantly higher in women than men $(14 \%$ versus $7.4 \% \mathrm{P}<0.001)$. Diabetes and IFG prevalence increased with age and peaked in the oldest agegroup. Diabetes was found to be associated with age, BMI, waist circumference, smoking, blood pressure, cholesterol, exercise, education, high caloric diet and a family history of diabetes.

Conclusion: This study shows that diabetes, IFG and obesity are common in Iran, and about one-half of diabetes cases remain undiagnosed. These results emphasize the need to develop an urgent public program to improve the detection, prevention, and treatment of diabetes.

Supported by $\mathrm{MOH}$

0314

Phenotypic characteristics among young adult-onset diabetic subjects in Sri Lanka (A South Asian population) - Sri Lanka Young Diabetes Study (SLYDS)

D. R. Matthews ${ }^{1}$, P. Katulanda ${ }^{1,2}$, M. M. McCarthy ${ }^{1}$, J. G. Mahesh', M. H. R. Sheriff; ;

${ }^{1}$ Oxford Centre for Diabetes Endocrinology and Metabolism, University of Oxford, United Kingdom, ${ }^{2}$ Dept of Clinical Medicine, University of Colombo, Sri Lanka.

Background and Aims: Diabetes mellitus is rapidly becoming a public health problem among South Asians. Clinical phenotypes among young adults are not well characterised. We aimed to characterise the different clinical 
phenotypes among young adult onset diabetic subjects with a view to identifying phenotypes that need different clinical management and primary preventive strategies.

Materials and Methods: Cross sectional descriptive study. Ethical approval obtained from the Ethical Review Committee of the Faculty of Medicine, Univrsity of Colombo. Diabetic subjects who were diagnosed between 16 to 40 years of age were recruited from free government clinics and from fee levying private sector clinics. Data collection conducted at the Diabetes research Unit of the University of Colombo in Sri Lanka. Detailed clinical and metabolic assessments were undertaken using a prevalidated case record form by trained medical graduates according to standard methods. Lipid analysis done using Hitachi 704 analyser and HBA1c in BIO-RAD HPLC analyser. Statistical analysis was performed using SPSS version 13.

Results: Total numbers of subjects 1007, mean age 36.62 years (SD 5.82), mean age at diagnosis 31.87 years (SD 5.62), females 57.8\%, and mean diabetes duration 4.81 years (SD 4.18). Overall, $16.4 \%$ of subjects are treated with insulin. The percentage that was treated with insulin from the onset or due to primary failure of oral therapy is $12.4 \%$. The mean body mass index is $24.64 \mathrm{~kg} / \mathrm{m}^{2}$. According to the cut-off values for South Asians $(23 \mathrm{~kg} /$ $\mathrm{m}^{2}$ ), $64.8 \%$ are overweight or obese. Central obesity is significantly higher among females; males $43.5 \%$ and females $80.4 \%(\mathrm{p}<0.001)$. Acanthosis Nigricans is present in $20.3 \%$ of subjects. In this sample $73 \%$ has positive first degree family history and $9.6 \%$ has family history among three consecutive generations. Among females who had been pregnant, $22.32 \%$ gives a history of gestational diabetes mellitus. Incidence of diagnosed Polycystic Ovarian Disease is $0.87 \%$. The mean HBA1c is $8 \%$ (SD 2). HDL Cholesterol is lower in $66.9 \%$ and Triglycerides high among $36.7 \%$ of subjects. The LDL is elevated in $74.8 \%$ and $94.2 \%$ have some lipid abnormality.

Conclusion: In this sample Type 2 diabetes is the predominant clinical phenotype. The prevalence of overweight and obesity is high. Females are more centrally obese than males and a high incidence of gestational diabetes is reported among the parous females. Most subjects had abnormal lipid profiles. Insulin resistance, islet cell antibodies and markers for monogenic diabetes are being analysed to refine further the aetiological diagnosis. Prospective follow up is being undertaken to define the natural history among different phenotypes.

Supported by Oxford Centre for Diabetes Endocrinology and Metabolism

\section{5}

Population-based incidence of type 2 diabetes in Asturias, Spain

S. Valdés ${ }^{1}$, P. Botas ${ }^{2}$, E. Delgado ${ }^{3}$, F. Díaz-Cadórniga ${ }^{3}$, F. Álvarez

${ }^{1}$ Endocrinology and Nutrition, Hospital Universitario Carlos Haya, Málaga, ${ }^{2}$ Endocrinology, Hospital San Agustín, Avilés, ${ }^{3}$ Endocrinology and Nutrition, Hospital Universitario Central de Asturias, Oviedo, Spain, ${ }^{4}$ Clinical Biochemistry, Hospital Universitario Central de Asturias, Oviedo, Spain.

Background and Aims: Type 2 diabetes (DM) is reaching epidemic proportions worldwide and for prevention purposes, identification of persons at high risk is mandatory. The aim of the present study was to define the incidence of type 2 diabetes in a Caucasian low-risk population in the north of Spain and its relationship with impaired fasting glucose (IFG), impaired glucose tolerance (IGT) and other risk factors.

Materials and Methods: The "Asturias Study" is a prospective population-based survey of diabetes and cardiovascular risk factors. The baseline examination was carried out during 1998-1999 when 1034 individuals aged 30-75 years were randomly selected to determine the prevalence of DM and prediabetes in the region of Asturias (north of Spain). The results showed that $11.3 \%$ of all participants had diabetes, $12.4 \%$ ITG and $7.6 \%$ IFG.

In 2004-2005, these same subjects were invited for a followup examination. 42 of them had died and 19 had moved out of Asturias. Of the remaining 973, 700 participated (72\%). For the present study we include only the 630 individuals without diabetes at baseline.Glucose was measured in venous blood both fasting (FPG) and $2 \mathrm{~h}$ after 75 gr oral glucose tolerance test (2hPG). We used WHO 1999 criteria to classify glucose metabolism at both baseline and follow-up: IFG:FPG 110-125 mg/dl, IGT: 2hPG 140-199 mg/dl, DM: FPG $\geq 126 \mathrm{mg} / \mathrm{dl}$ and/or $2 \mathrm{hPG} \geq 200 \mathrm{mg} / \mathrm{dl}$.

Results: The study population includes 630 individuals aged 30-75 years at baseline and with an average follow-up of 6.3 years. During follow-up, 44 of them developed diabetes $(7 \%)$. The incidence of diabetes adjusted to age and sex structure of Asturias is 10.8 cases per 1000 persons/years. Cumulative incidence of diabetes in prediabetic states are $36.5 \%$ for IFG, $23,9 \%$ for IGT and $60 \%$ for individuals with combined IFG-IGT. In a multivariate logistic regression analysis, in addition to IFG and IGT, hypertrigliceridemia ( $p$ $0.000)$ and diastolic hypertension (0.002) were also independently associated with development of DM. 
Conclusions: Our study shows in this population of the north of Spain an incidence rate of type 2 diabetes of 10.8 cases per 1000 persons/years. IFG as defined by the WHO 1999 was the strongest predictor of diabetes. The risk was extremely high for people with both IFG and IGT. Supported by grants from the Consejeria de Sanidad del Principado de Asturias and Pfizer

\section{6}

Exocrine and endocrine pancreatic function in type 2 diabetes patients of Bangladesh

S. Sattar ${ }^{1}$, C. Hanck ${ }^{2}$, Z. Hassan ${ }^{1}$, U. Keller ${ }^{3}$, D. Whitcomb ${ }^{4}$, L. Ali ${ }^{5}$, A. K. Azad Khan ${ }^{6}$, N. Gyr ${ }^{7}$;

${ }^{1}$ Dept of Physiology and Molecular Biology, BIRDEM, Dhaka, Bangladesh, ${ }^{2}$ Dept of Gastroenterology, University Hospital, Basel, Switzerland, ${ }^{3}$ Dept of Endocrinology and Metabolism, University Hospital, Basel, Switzerland, ${ }^{4}$ Centre for Genomic Sciences, University of Pittsburgh, Pittsburgh, United States, ${ }^{5}$ Dept of Biochemistry and Cell Biology, BIRDEM, Dhaka, Bangladesh, ${ }^{6}$ Dept of Gastroenterology, BIRDEM, Dhaka, Bangladesh, ${ }^{7}$ Dept of Internal Medicine, University Hospital, Basel, Switzerland.

Background and Aims: Exocrine pancreatic dysfunction in type 2 diabetes is still an unsettled issue. Since SPINK1 gene N34S variant has been associated with pancreatopathy we aimed to analyze the gene variant and evaluate the exocrine and endocrine functional status in a cohort of typical type 2 diabetic patients in comparison to tropical calcific pancreatitis patients with and without diabetes.

Materials and Methods: Age-matched type 2 diabetic patients $(n=49)$, tropical calcific pancreatitis without diabetes (TCP, $n=35)$ and with diabetes [Fibrocalculus calculus pancreatic diabetes $(\mathrm{FCPD}), \mathrm{n}=83$ )] were studied. Exocrine function was evaluated by measurement of fecal elastase 1 (by ELISA) and endocrine function by measurement of insulin (by RIA) following IV agrinine stimulation test. SPINK1 gene N34S variant was analyzed by direct DNA sequencing. Student's unpaired-' $t$ ', Mann-Whitney and Chi-square tests were performed where applicable.

Results: Mean $( \pm \mathrm{SD}) \mathrm{BMI}$ of the T2D $(23.8 \pm 4.7)$ subjects was significantly higher compared to TCP $(19.5 \pm 3.1$, $\mathrm{p}<0.001)$ and FCPD $(18.6 \pm 3.1, \mathrm{p}<0.001)$ subjects. Fecal elastase1 was severely $(<100 \mu \mathrm{g} / \mathrm{g}$ of stool) reduced in $58 \%$ of T2D subjects compared to $81 \%(\mathrm{p}=0.082)$ of TCP and 95\% ( $\mathrm{p}=0.001)$ of FCPD subjects. SPINK1 gene N34S variant was positive in $8.8 \%$ of T2D, $37 \%$ TCP $(\mathrm{p}=0.011)$ and $33 \%$ FCPD $(\mathrm{p}=0.011)$ subjects. SPINK1 gene N34S variant positivity was present in subjects with severely reduced Fecal elastase 1 of $15 \%$ of T2D, $18 \%$ TCP and $39 \%$ FCPD subjects. In all three groups SPINK1 N34S variant positive and negative cases did not show any difference regarding Fecal elastase1. Insulin levels (median, U/l) at basal and on arginine stimulation state (at 15, 30, 45 and $60 \mathrm{~min}$ ) in T2D (basal- 24.4, stimulated- 41.7, 42.3, 34.3 and 21.9) subjects was significantly higher compared to TCP (basal- 13.0, stimulated- 21.6, 23.4, 18.7 and 14.0) and FCPD (basal- 15.9, stimulated- 20.7, 19.5, 22.5 and 15.1) subjects. Between SPINK1 gene variant positive and negative cases insulin levels at basal and stimulated state did not show any statistical difference.

Conclusion: The data suggest that i) a substantial proportion of type 2 diabetic patients in Bangladesh have exocrine pancreatic dysfunction; ii) in T2D the exocrine dysfunction and insulin secretory capacity is not associated with SPINK1 gene variant.

The study was supported by grant from IPICS and DAB

\section{PS 7}

\section{Prediction and prevention of type 2 diabetes and its complications}

0317

Do fasting serum insulin and proinsulin levels in prediabetic individuals identified in a high-risk screening programme predict progression to diabetes. The ADDITION study (DK)

S. S. Rasmussen ${ }^{1}$, C. Glümer ${ }^{1}$, A. Sandbaek ${ }^{2}$, O. Pedersen ${ }^{1}$, T. Jørgensen ${ }^{3}$, T. Lauritzen ${ }^{2}$,

K. Borch-Johnsen ${ }^{1}$;

${ }^{1}$ Steno Diabetes Center, Steno Diabetes Center, Gentofte, ${ }^{2}$ Department of General Practice, Institute of Public Health, Aarhus, ${ }^{3}$ Research Centre for Prevention and Health, University Hospital of Glostrup, Denmark.

Background and Aims: Persons identified with pre-diabetes (IFG and IGT) in a high-risk screening programme are at a particularly high risk of developing diabetes. Fasting serum proinsulin is a determinant for progression in populationbased studies. We wanted to compare the level of fasting serum insulin in pre-diabetic individuals identified in a highrisk screening programme with that in individuals identified by mass-screening. Secondly, we wanted to examine whether fasting serum insulin and proinsulin or proinsulin/insulin ratio are predictors for progression to diabetes after one year in high-risk screened pre-diabetic individuals. 
Materials and Methods: A high-risk screening programme, the ADDITION study Denmark, identified 699 individuals with IFG and IGT (WHO1999 definition), in whom there were one-year follow-up data. The Inter99 study, a massscreening study in Denmark, identified 1,637 individuals with IFG and IGT. Baseline fasting serum insulin levels in these populations were compared. Poisson regression was used in the ADDITION population to estimate rate ratios of progression to diabetes between levels of fasting serum insulin, serum proinsulin and serum proinsulin/insulin ratio. Secondly, the model was adjusted for screening variables (age, sex, family history of diabetes, gestational diabetes, known hypertension, BMI, physical activity, random blood glucose, HbA1c), glucose tolerance status, and systolic blood pressure. Serum insulin was determined by ELISA with a narrow specificity excluding $\operatorname{Des}(31,32)$ - and intact proinsulin and serum proinsulin was determined by ELISA with a broad specificity including 4 proinsulin conversion intermediates (65-99\%) together with intact proinsulin (100\%).

Results: Fasting serum insulin levels in the ADDITION and the Inter99 populations were (median) 50.0 and $45.0 \mathrm{pmol} / 1$, respectively $(\mathrm{p}<0.0001)$. In the ADDITION study, the rate ratios of progression from pre-diabetes to diabetes associated with a two-fold increase were: insulin $1.13(\mathrm{p}=0.09)$, proinsulin $1.30(\mathrm{p}<0.001)$ and proinsulin/ insulin ratio $1.52(\mathrm{p}<0.001)$. After adjustment, these associations diminished and became insignificant: insulin 1.00 [95\% CI:0.84-1.18] $(\mathrm{p}=0.98)$, proinsulin 1.09 [0.921.30] $(p=0.34)$, proinsulin/insulin ratio 1.26 [0.95-1.67] $(p=0.10)$. Glucose tolerance status was not significantly associated with progression.

Conclusion: Pre-diabetic individuals in high-risk screening programmes are more insulin resistant as estimated from fasting serum insulin levels than those in classical surveys. In our high-risk screened population, fasting serum insulin and proinsulin or proinsulin/insulin ratio did not predict progression to diabetes when adjusted for study design, glucose tolerance status and systolic blood pressure.

This fully investigator initiated, designed and controlled study received funding from a number of public as well as private sources.

\section{8}

Carbohydrate and fiber intake and incidence of type 2 diabetes in the EPIC-Potsdam Study

M. B. Schulze, M. Schulz, C. Heidemann,

A. Schienkiewitz, K. Hoffmann, H. Boeing;

Department of Epidemiology, German Institute of Human

Nutrition Potsdam-Rehbruecke, Nuthetal, Germany.
Background and Aims: Although some prospective studies have evaluated whether intake of carbohydrates is associated with risk for type 2 diabetes, it remains unclear what long-term effects substituting protein at the expense of carbohydrates, common with popular low-carbohydrate protein-rich diets, may have with regard to diabetes risk. Our objective was to evaluate carbohydrate intake in predicting type 2 diabetes using substitution models for fat and protein and to evaluate whether fiber intake is associated with risk.

Materials and Methods: We conducted a prospective cohort study of 9,711 men and 15,402 women aged 3565 years and free of diabetes who were followed for incident type 2 diabetes. Dietary intake of macronutrients and fiber were estimated with a validated food-frequency questionnaire. We estimated the relative risk (RR) using Cox proportional hazards analysis. Because there were no significant interactions with sex, we pooled men and women in all analyses.

Results: During 176,780 person-years of follow-up we observed 849 incident cases of physician confirmed type 2 diabetes. There was no significant association between carbohydrate intake and diabetes risk in models substituting for total fat. After adjustment for age, BMI, waist circumference, potential lifestyle confounders, soluble fiber, insoluble fiber, alcohol, and magnesium intake, the relative risk for substituting 5\% of energy intake from total fat with carbohydrates was 1.03 (95\% KI: 0.95-1.11). In contrast, substituting carbohydrates for protein significantly reduced diabetes risk (RR for a 5\% energy substitution: $0.74 ; 95 \%$ CI: $0.63-0.88)$. This association was similar across strata of carbohydrate intake $(<\mathrm{vs} .>=45 \%$ energy) and obesity (BMI $<$ vs. $>=30$ ). A non-significant inverse association with diabetes risk was observed for total fiber intake (RR for extreme quintiles of energy adjusted intake: 0.81 [95\% CI: 0.62-1.06]). However, higher cereal fiber intake was significantly associated with a reduced diabetes risk independent of other fiber sources (RR for extreme quintiles of energy adjusted intake: 0.70 [95\% CI: $0.55-$ $0.89])$, while fruit fiber $(0.87$ [0.68-1.10]) and vegetable fiber (0.95 [0.76-1.20]) were not significantly associated. Among carbohydrate-rich foods, whole-grain bread intake was associated with reduced risk of developing diabetes. The RR for the highest quintile compared to the lowest was 0.79 (95\% CI: 0.64-0.99).

Conclusion: Substituting carbohydrates for fat appears not to be related to diabetes risk. However, a higher protein intake at the expense of carbohydrates may increase diabetes risk suggesting potential harmful effects of popular low-carbohydrate protein-rich diets. Cereal fiber and whole-grain bread intake may reduce diabetes risk.

Supported by Federal Ministry of Science, Germany, European Union, German Cancer Aid, European Community 


\section{9}

Is measurement of $\mathrm{HbA}_{1 \mathrm{c}}$ alone appropriate as an initial screening test for undiagnosed diabetes?

T. Nakagami $^{1}$, M. Tominaga ${ }^{2}$, R. Nishimura ${ }^{3}$, N. Yoshiike ${ }^{4}$, N. Tajima ${ }^{3}$;

${ }^{1}$ Diabetes Center, Tokyo Women's Medical University, ${ }^{2}$ Department of Laboratory Medicine, Yamagata University School of Medicine, ${ }^{3}$ Division of Diabetes, Metabolism and Endocrinology, Department of Internal Medicine, Jikei University School of Medicine, Tokyo, ${ }^{4}$ Department of the National Nutrition Survey in Japan, National Institute of Health and Nutrition, Tokyo, Japan.

Background and Aims: Glycated hemoglobin A1c (HbA1c) is currently used to estimate number of people with diabetes (DM) in the Japan National Diabetes Survey (JNDS). In the JNDS, the possible DM was defined as people with $\mathrm{HbA} 1 \mathrm{c} \geq 6.1 \%$ or under treatment of $\mathrm{DM}$ and the probable DM was defined as those with HbA1c 5.6$6.0 \%$ in people without treatment of DM. According to the JNDS in 2002, the total number of people having probable and possible DM was estimated as 7.4 million and 8.8 million in the whole population over 20 years old. The aim of this study is to assess the efficacy of HbA1c as an initial screening test for undiagnosed DM, by using populationbased data from the Funagata study in 2000-2002.

Subjects and Methods: 1,904 Funagata-town inhabitants aged 35-89 years old were analysed. HbA1c was measured together with plasma glucose during an oral glucose tolerance test and DM was diagnosed according to the 1999-WHO criteria. The area under the receiver operating characteristic (ROC) curve predicting undiagnosed DM was compared between $\mathrm{HbAlc}$ and fasting plasma glucose. Screening properties for HbA1c predicting undiagnosed DM was examined, with respect to sensitivity, specificity, predictive values positive (PPV) and negative (NPV), and the proportion of people above the cut-off point. Moreover, impact of sex, age, and BMI on the ROC curve of HbAlc for undiagnosed DM was examined.

Results: In the Funagata study, the prevalence of previous DM, undiagnosed DM, and Impaired Glucose Regulation according to the WHO criteria were: $5.5 \%, 5.8 \%$, and $18.8 \%$ in the population. The area under the ROC curve for undiagnosed DM was similar between HbA1c (0.849 [95\% $\mathrm{Cl}: 0.814-0.895])$ and fasting plasma glucose $(0.899$ [0.864 0.933]). The area under the ROC curve of HbAlc for undiagnosed DM had a trend to increase with increase of BMI and decrease of age, with statistical insignificance. $\mathrm{HbA} 1 \mathrm{c}$ of $5.6 \%$ gave a sensitivity of $54.9 \%$, a specificity of $94.9 \%$, a PPV of $41.5 \%$, a NPV of $97.0 \%$, and the proportion of people above the cut-off point of $8.2 \%$. At HbAlc of $5.3 \%$ where maximizing sensitivity plus specificity, the corresponding values were: $76.6 \%, 81.9 \%, 21.7 \%, 98.2 \%$, and $21.7 \%$. The PPV, 1-NPV, and the proportion of people above the cut-off level increased with increase of HbAlc. The current cut-off point of 5.6\% was lower than the points where maximizing sensitivity plus specificity predicting retinopathy in the future among Japanese general population (HbAlc: $5.7 \%$ ) and the treatment target of HbAlc recommended by the guideline in Japan (HbAlc: 5.8\%).

Conclusions: HbA1c seems to be a useful initial screening test for undiagnosed DM and the current cut-off point of $5.6 \%$ may be appropriate, with respect to screening properties for undiagnosed DM, and prediction and prevention of microvascular complications in Japan.

The analysis of this study was supported by the grant from the Japanese Ministry of Health, Welfare and Labour.

0320

Childhood predictors of young onset type 2 diabetes mellitus

P. W. Franks ${ }^{1,2}$, R. L. Hanson ${ }^{1}$, A. M. Infante ${ }^{1}$, W. C. Knowler ${ }^{1}$, H. C. Looker ${ }^{1}$;

${ }^{1}$ National Institutes of Diabetes \& Digestive \& Kidney Diseases, National Institutes of Health, Phoenix, United States, ${ }^{2}$ Dept. Public Health \& Clinical Medicine, Umeå University Hospital, Sweden.

Background: Optimal strategies for preventing young onset type 2 diabetes mellitus (T2D) require the identification of the disease's early-life modifiable risk factors. We aimed to do this using longitudinal data in 1,604 American Indian children and adolescents who were nondiabetic at baseline (5-19 yrs; 5.7\% developed T2D during follow-up). Methods: For T2D prediction, we derived an optimallyweighted continuously distributed standardized multivariate score (zMS) comprised of commonly measured metabolic risk factors (i.e., fasting and $2 \mathrm{hr}$ glucose, $\mathrm{HbA}_{1 \mathrm{c}}, \mathrm{BMI}$, waist circumference, fasting insulin, HDL-C, triglycerides, and systolic and diastolic blood pressures), and compared the predictive power for each feature against zMS using ROC analyses.

Results: In separate Cox proportional hazard models, adjusted for age, sex, and fraction Indian heritage, associations with incident T2D were observed for all metabolic risk factors. Stepwise proportional hazards regression models selected fasting glucose, $2 \mathrm{hr}$ glucose, HDL-C, and waist as independent predictors of $\mathrm{T} 2 \mathrm{D}$, but individually these were weaker predictors than zMS $(\mathrm{p}<0.01)$. However, a parsimonious summary score constructed from only these variables had similar predictive power as zMS (ROC AUC: 0.77 vs. $0.78 ; \mathrm{p}=0.10)$. Although intrauterine exposure to diabetes or parental history of early onset T2D substantially increased a 
child's absolute risk of developing T2D, the magnitude of the $\mathrm{T} 2 \mathrm{D}$ risk relationships for $\mathrm{zMS}$ and the parsimonious score were no stronger than in children without these familial risk factors.

Conclusions: It may be possible to accurately predict young onset T2D using simple and non-invasive measures of fasting glucose, $2 \mathrm{hr}$ glucose, HDL-C, and waist. The predictors of T2D differ in younger and older children possibly because of changes in metabolism that occur during puberty. These data may prove informative for strategies that seek to target the early-life risk factors for T2D. They also point to the features of a childhood-specific metabolic syndrome score that are relevant for $\mathrm{T} 2 \mathrm{D}$ prediction.

Table. Pair-wise comparisons of the full model (zMS) with each parsimonious model derived from a step-wise regression procedure.

\begin{tabular}{|c|c|c|c|c|}
\hline & Model & $\mathbf{A U C}$ & (SE) & p-value \\
\hline \multirow[t]{2}{*}{ 5-9 yr olds } & 1. Full model (zMS) & 0.90 & $(0.02)$ & - \\
\hline & 2. Waist & 0.88 & $(0.03)$ & 0.17 \\
\hline \multirow[t]{2}{*}{ 10-14 yr old } & 1. Full model (zMS) & 0.82 & $(0.03)$ & - \\
\hline & 2. BMI, $\mathrm{HbA}_{1 \mathrm{c}}, 2 \mathrm{hr}$ gluc & 0.79 & $(0.03)$ & 0.06 \\
\hline \multirow[t]{2}{*}{ 15-19 yr olds } & 1. Full model (zMS) & 0.76 & $(0.03)$ & - \\
\hline & 2. Waist, $\mathrm{HbA}_{1 \mathrm{c}}, 2 \mathrm{hr}$ gluc & 0.74 & $(0.03)$ & 0.04 \\
\hline \multirow[t]{2}{*}{ 5-19 yr olds } & 1. Full model (zMS) & 0.78 & $(0.02)$ & - \\
\hline & $\begin{array}{l}\text { 2. Fast gluc, } 2 \text { hr gluc, BMI, } \\
\text { HDL-C }\end{array}$ & 0.77 & $(0.02)$ & 0.10 \\
\hline
\end{tabular}

P-value is for pair-wise comparison of Full model with Partial model. All models include terms for age, sex and fraction Indian heritage. BMI=body mass index; Fast gluc=fasting glucose; $2 \mathrm{hr}$ gluc=2 hr hour glucose; $\mathrm{HDL}=$ high density lipoprotein cholesterol. Predictor variables were standardised (z-score) within one year age strata.

Parsimoniously predictive subsets of the variables (shown above as model 2) were identified by a backward stepwise procedure. All predictor variables were included in the initial model and the weakest predictor was sequentially eliminated until all of the remaining variables were retained at $\mathrm{p}<0.05$; (age, sex and heritage were not made available to the stepwise procedure and are, thus, forcibly included in all models). To assess the extent that this submodel captured the information contained in the fully optimized model containing all variables, the ROC AUCs for these two models were compared (difference between models indicated by $\mathrm{p}$-values in table).

This study was conducted under the NIDDK intramural research program
0321

\section{Smoking and the risk of type 2 diabetes mellitus}

L. Radzeviciene, R. Ostrauskas;

Institute of Endocrinology, Kaunas University of Medicine, Lithuania.

Background and Aims: The aim of study was to determine the association between smoking and the risk of type 2 diabetes mellitus.

Materials and Methods: A case-control study included 234 cases with newly confirmed diagnose of type 2 diabetes mellitus and 468 controls who were free of the disease in 2001. Cases and controls were matched by gender and age ( +5 yr.). Information on age, gender, body mass index, family history on diabetes, education level, years of total education, occupation status, nutrition habits, alcohol consumption, cigarette smoking, physical activity and stress was collected from questionnaire. Conditional logistic regression was used to compute the odds ratio (OR), 95\% confidence intervals (CI) and $\mathrm{p}$ for trend. The ORs were adjusted for potentials confounder such as: family history on diabetes, body mass index, speed of eating, portion of food, using of salty and fatty food, physical activity early in life, exercise, years of total education, and stress that were related to type 2 diabetes mellitus in univariate regression model and alcohol drinking, that were associated with the disease.

Results: After adjustment for possible confounders increased risk of type 2 diabetes mellitus determined for current smokers $(\mathrm{OR}=2.25 ; 95 \% \mathrm{CI} 1.03-4.92)$, duration of smoking $(\mathrm{OR}=2.43 ; 95 \%$ CI 1.14-5.20) for 20-39 yrs, compared with never-smokers. There were no statistically significant associations between risks of type 2 diabetes mellitus, and number of cigarettes smoked a day, number of pack-years and time after quit up smoking.

Conclusion: Our data suggest that smoking is a significant risk factor of type 2 diabetes mellitus.

0322

Microalbuminuria predicts diabetes mellitus in men from the general population

J.-M. Halimi ${ }^{1}$, F. Bonnet ${ }^{2}$, B. Balkau ${ }^{3}$, J. Tichet ${ }^{4}$, M. Marre ${ }^{5}$;

${ }^{1}$ Nephrology, Tours University, France, ${ }^{2}$ Endocrinology, Lyon University, ${ }^{3}$ INSERM, INSERM U258, Villejuif, ${ }^{4}$ IRSA, IRSA, La Riche, France, ${ }^{5}$ Service de Diabétologie, Hôpital Bichat, Paris, France.

Background: Microalbuminuria (Malb) is associated to metabolic syndrome, hypertension and many other cardio- 
vascular risks. Whether elevated Malb constitutes a risk for diabetes mellitus is presently unknown.

Objectives: We prospectively assessed the predictive value of Malb for the development of diabetes mellitus in men and women selected from the general population.

Subjects and Methods: 3252 non diabetic subjects (aged 30 to 64 years) were included in the D.E.S.I.R. Study. At baseline, the subjects had a complete medical and biological checkup. After a 9-year follow-up, new cases of diabetes mellitus were recorded.

Results: The 9-year incidence of diabetes mellitus was $4.6 \%$; it occurred more frequently in men $(6.8 \%)$ than in women $(2.0 \%)$. Diabetes mellitus was more frequent in microalbuminuric than in normoalbuminuric subjects (RR: 2.00 [1.29-3.10], $\mathrm{P}=0.0020)$. The risk associated to microalbuminuria was greater in men (2.11 [1.29-3.44], $\mathrm{P}=0.0030$ ); however, it did not reach the significance threshold in women (1.44 [0.50-4.19], $\mathrm{P}=0.4986)$. The risk of diabetes mellitus associated with microalbuminuria in men was unchanged despite multiple adjustments.

Conclusions: Microalbuminuria is a strong, independent risk for diabetes mellitus in men.

Supported by INSERM - CNAMTS grants

\section{3}

Clinical and cost-effectiveness of primary prevention of type 2 diabetes in a routine health care setting: Model based on the KORA Survey 2000

A. Icks ${ }^{1}$, W. Rathmann ${ }^{1}$, B. Haastert ${ }^{1}$, A. Gandjour ${ }^{2}$, R. Holle ${ }^{3}$, J. John ${ }^{3}$, G. Giani ${ }^{1}$;

${ }^{1}$ Institute for Biometrics and Epidemiology, German Diabetes Center, Düsseldorf, Germany, ${ }^{2}$ Institute of Health Economics and Clinical Epidemiology, Cologne University, Köln, Germany, ${ }^{3}$ Institute of Health Economics and Health Care Management, GSF National Research Centre for Environment and Health, Neuherberg, Germany.

Background and Aims: Lifestyle modification and Metformin have been demonstrated to prevent progression from prediabetic status to type 2 diabetes (Diabetes Prevention Program (DPP)). Several studies have analyzed costs and cost effectiveness of type 2 diabetes prevention, and found both medication and lifestyle modification to be cost effective. However, so far, no analysis considered an implementation in a routine health care setting. In particular, a high participation of patients in the prevention interventions was assumed, which might be unrealistic in clinical practice. The aim of our study was to evaluate the clinical and cost-effectiveness of metformin and lifestyle intervention to prevent type 2 diabetes in an elderly high risk population, which was identified by a targeted screening, in a routine health care setting, using population-based data (KORA-Survey in Augsburg, Germany, whole population about 600,000).

Material and Methods: We performed a decision analytic model with a time horizon three years. Interventions were staff education, targeted screening, and lifestyle modification or metformin in subjects aged 60-74 years with BMI $>=24$ and prediabetic status (target population, about 72,500), according to the Diabetes Prevention Program trial. Main outcome measures were cases of type 2 diabetes prevented or delayed, costs (Euro), incremental costs per additionally avoided case of diabetes (incremental costeffectiveness ratios, ICER's), all from third party payers' and societal perspectives.

Results: Under model assumptions, 14,908 subjects of the target population would develop diabetes without intervention. 184 and 42 cases would be avoided with lifestyle and metformin intervention, respectively. Costs were Euro 856,507 and Euro 4,961,340 for lifestyle modification and Euro 797,539 and Euro 1,335,204 for metformin from statutory health insurance' and societal perspective. Up to $5 \%$ of costs were due to staff education, and up to $36 \%$ to screening. Lifestyle was more cost effective than metformin. ICERs for lifestyle versus no intervention were Euro 4,664 and Euro 27,015 per case prevented from the statutory health insurance' and societal perspective.

Conclusions: Total costs and costs per diabetic case avoided were high, and staff education and screening had a considerable impact. Considering low participation in a routine health care setting, with both strategies, there was only a small number of diabetic cases that would be prevented or delayed. Before implementing a program, more effort should be undertaken to improve patient participation to achieve more clinical and cost effectiveness in type 2 diabetes prevention in clinical practice.

\section{4}

Feasibility and uptake of screening for type 2 diabetes in primary care

L. A. Sargeant ${ }^{1}$, A. Kinmonth ${ }^{2}$, N. J. Wareham ${ }^{3}$, S. J. Griffin ${ }^{1}$;

${ }^{1}$ Prevention Group, MRC Epidemiology Unit, Cambridge, United Kingdom, ${ }^{2}$ Department of Public Health and Primary Care, University of Cambridge, United Kingdom, ${ }^{3}$ Director, MRC Epidemiology Unit, Cambridge, United Kingdom.

Background and Aims: Uncertainty remains over whether screening for type 2 diabetes is cost-effective. Screening can only be cost-effective if there is sufficient uptake. Identification of those with poor uptake can facilitate appropriate targeting of screening programmes. The 
ADDITION Study (Cambridge) is a randomised trial of a programme of screening and intensive multifactorial intervention for type 2 diabetes in primary care in the east of England. This investigation aimed to determine the factors influencing screening uptake.

Materials and Methods: A validated risk score based on age, sex, body mass index (BMI) and prescribed steroid and antihypertensive medication was calculated from information held in general practice notes for people aged 40-69 years without known diabetes. Those with a risk score above a cutoff equivalent to the top $25 \%$ of the population distribution were invited to attend an appointment at their practice for a random capillary blood glucose (RBG) test (HemoCue) and $\mathrm{HbA}_{1 \mathrm{c}}$ test (Biorad). Participants with an RBG of $\geq 11.1 \mathrm{mmol} / 1$ were invited for a standard $75 \mathrm{~g}$ oral glucose tolerance test (OGTT). Those with a RBG of 5.5-11.1 mmol/ 1 were invited to return for a fasting capillary blood glucose (FBG) test. Those with a FBG of $\geq 6.1 \mathrm{mmol} / 1$, or a FBG of 5.5-6.1 mmol/1 and an $\mathrm{HbA}_{1 \mathrm{c}}$ of $\geq 6.1 \%$, were invited for an OGTT. By the end of June 2005, 33 general practices had completed the screening process and were included in these analyses. There were 76,574 patients between the ages of 40 and 69 years in these practices representing $33.5 \%$ of the overall practice population. The prevalence of known diabetes in this age category was $3.9 \%$ with a range of 2.1 to $6.1 \%$. The proportion of 40-69 year olds without a calculated risk score was $27.2 \%$ (range -10.3 to $67.3 \%$ ). The mean (SD) BMI was $26.5(5.0) \mathrm{kg} / \mathrm{m}^{2}$.

Results: Of the 17814 with a high risk score and invited for an RBG test $77 \%$ attended. Screening uptake increased to $93 \%$ for the subsequent two stages (FBG testing and diagnostic OGTT). A total of 4930 were eligible for FBG and 846 for OGTT. There were 503 screen-detected cases of diabetes (3.7\% of those attending the RBG test). Screening uptake for the RBG test was significantly higher among older people, women and those with a high BMI and a high risk score ( $\mathrm{p}$-value $<0.001$ for all comparisons) while age $(p<0.001)$ and $R B G$ result $(p=0.009)$ were associated with uptake of the FBG test. The practice level factors investigated (practice size, prevalence of known diabetes, urban-rural location, and proportion with missing data on risk score variables) were not consistently associated with screening uptake.

Conclusion: Targeted RBG screening of individuals assessed as high risk by data routinely available in general practice is feasible and acceptable. Uptake of screening was highest amongst those individuals at highest risk by virtue of age and BMI, but could not be predicted by practice level characteristics. Different strategies may be required to increase uptake of screening among younger men, should screening be shown to be beneficial.

Supported by MRC and Wellcome Trust
0325

Basic characteristics and treatment regimens in people with type 2 diabetes in the international diabetes management practices study (IDMPS)

N. Hancu ${ }^{1}$, S.-H. Baik ${ }^{2}$, S. Ferreira ${ }^{3}$, J.-J. Gagliardino ${ }^{4}$, J. Chan ${ }^{5}$, P. Aschner ${ }^{6}$, A. Ramachandran ${ }^{7}$, S. Twigg ${ }^{8}$, H. Ilkova ${ }^{9}$;

${ }^{1}$ Diabetes Center and Clinic, Iuliu Hatieganu University of Medicine, Cluj-Napoca, Romania, ${ }^{2}$ Department of Internal Medicine, Korea University Guro Hospital, Seoul, Republic of Korea, ${ }^{3}$ Department of Preventive Medicine, Federal University of Sao Paulo, Brazil, ${ }^{4}$ Faculty of Medical Sciences, National University of La Plata, Argentina, ${ }^{5}$ Department of Medicine and Therapeutics, Prince of Wales Hospital, Shatin, Hong Kong, ${ }^{6}$ Endocrinology Unit, Unidad Médica de diagnóstico, Bogotá, Colombia, ${ }^{7}$ Diabetes Research Centre, M.V. Hospital for Diabetes, Chennai, India, ${ }^{8}$ Department of Medicine, University of Sydney, Australia, ${ }^{9}$ Department of Internal Medicine, Istanbul University Cerrahpasa Medical Faculty, Turkey.

Background and Aims: The IDMPS is a multinational observational study that aims to gather data that reflect current care practices of people with diabetes in different areas of the world and to evaluate the compliance with international care guidelines.

Materials and Methods: Data are being collected from type 2 diabetic patients ( $\geq 18$ years) in 1-year cycles (a 2week cross-sectional period followed by a 9-month longitudinal period) for 5 years. Data from the first crosssectional period are currently available from 7543 people (48\% men) in 11 countries in Eastern Europe, Asia, and Latin America.

Results: The mean age was $58 \pm 11$ years, mean BMI $27 \pm 5 \mathrm{~kg}$ / $\mathrm{m}^{2}$ (men: $26 \pm 4 \mathrm{~kg} / \mathrm{m}^{2}$, women: $27 \pm 5 \mathrm{~kg} / \mathrm{m}^{2}$ ) and mean disease duration 8 years. Mean $\mathrm{HbA}_{1 \mathrm{c}}$ was $7.7 \pm 1.75 \%$ and $38 \%$ of people had an $\mathrm{HbA}_{1 \mathrm{c}}<7 \%$. Among people with $\mathrm{HbA}_{1 \mathrm{c}}<7 \%$, 4\% were treated with diet and exercise only, $77 \%$ with oral antihyperglyemic agents (OHAs) alone and $19 \%$ with insulin \pm OHAs. A regimen of diet and exercise was reported in $3 \%$ of people (mean $\mathrm{HbA}_{1 \mathrm{c}}$ of $6.9 \pm 1.67 \%$ ). OHAs alone were used by $67 \%$ of people (mean $\mathrm{HbA}_{1 \mathrm{c}}$ of $7.4 \pm 1.60 \%$ ). Insulin \pm OHAs were used by $16 \%$ of people (average insulin dose $36 \mathrm{IU}$, mean $\mathrm{HbA}_{1 \mathrm{c}}$ of $8.3 \pm 1.89 \%$ ). Mean FBG (laboratory) was $152 \pm 60 \mathrm{mg} / \mathrm{dL}$. Mean systolic/ diastolic blood pressure was $135 / 81 \mathrm{mmHg}$. Target treatment values were obtained for LDL $(<100 \mathrm{mg} / \mathrm{dL})$ in $34 \%$ of people, HDL ( $>40 \mathrm{mg} / \mathrm{dL})$ in $64 \%$ of people, and triglycerides $(<150 \mathrm{mg} / \mathrm{dL})$ in $48 \%$ of people.

Conclusion: As can be seen, despite the evidence provided by the UKPDS and the recommendations of the ADA, the majority of people did not achieve the recommended 
treatment goals for $\mathrm{HbA}_{1 \mathrm{c}}$ and $\mathrm{FBG}$. It is thus apparent that more efficient guidance and education be made available to improve treatment outcomes in people with type 2 diabetes world wide.

Supported by Sanofi-Aventis

0326

\section{WITHDRAWN}

\section{PS 8}

\section{Type 2 diabetes genes}

0327

The combined impact of replicated hyperglycaemia susceptibility alleles on fasting and stimulated glycaemia in the general population: a study of 5,603 middle-aged treatment-naïve subjects

C. S. Rose ${ }^{1}$, A. Albrechtsen ${ }^{1}$, G. Andersen ${ }^{1}$, B. Carstensen ${ }^{1}$, K. Borch-Johnsen ${ }^{1,2}$, T. Jørgensen ${ }^{2}$, S. Maeda ${ }^{3}$, Y. Nakamura ${ }^{3}$, T. Hansen ${ }^{1}$, O. Pedersen ${ }^{1}$;

${ }^{1}$ Steno Diabetes Center, Denmark, ${ }^{2}$ Research Centre for Prevention and Health, Glostrup University Hospital, Denmark, ${ }^{3}$ SNP Research Center, RIKEN, Kanagawa 2300045, Yokohama, Japan.

Background and Aims: Previous population studies have below the threshold for diabetes shown a graded relationship between fasting and postprandial plasma glucose concentrations and the subsequent incidence of cardiovascular morbidity and mortality. Insights into the genetic determinants of this relationship may therefore be of relevance in identifying subgroups of the general population at high risk and in targeting genotype-based intervention against glycaemia induced morbidity. Four polymorphisms in $G C K(-30 \mathrm{G}>\mathrm{A}, \mathrm{rs} 1799884), H N F 4 A$ (rs1884614), KCNJ11 (Glu23Lys, rs5219), and TFAP2B (IVS1+774G $>\mathrm{T}$ ), respectively, that are reported to associate with hyperglycaemia were selected for this study. The objective was at the population level to elucidate the combined effect of these risk alleles on fasting and postOGTT plasma glucose levels.

Materials and Methods: The four polymorphisms were genotyped in the population-based Inter99 cohort of 5,603 middle-aged individuals $(4,238$ subjects with normal glucose tolerance, 478 subjects with impaired fasting glycaemia, 649 subjects with impaired glucose tolerance, and 238 screen-detected and treatment-naïve type 2 diabetic patients). The same polymorphisms were genotyped in a case-control study of 1,023 type 2 diabetic patients and 4,338 glucose-tolerant subjects. For each of the four polymorphisms the allele that was reported to contribute to hyperglycaemia was defined as the risk allele. We assumed that each risk allele has an additive effect and that the effect of all risk alleles is the same. A general linear model and a binary logistic regression analysis were used to estimate the effect of the risk alleles on glycaemic levels and prevalence of type 2 diabetes, respectively.

Results: Among 5,603 middle-aged treatment-naïve Inter99 participants a relationship between the additive effect of risk alleles and increased fasting plasma glucose levels was observed with an effect size of $0.059(\mathrm{mmol} / \mathrm{l})$ per risk allele $(p<0.0001)$. This same relationship was observed for $30 \mathrm{~min}$. and $120 \mathrm{~min}$. post-OGTT plasma glucose levels with effect sizes of $0.117(\mathrm{mmol} / \mathrm{l})(p<0.0001)$ and 0.135 $(\mathrm{mmol} / \mathrm{l})(p<0.0001)$, respectively. Interestingly, in a study of 1,023 type 2 diabetic patients and 4,338 glucose-tolerant subjects the risk of having type 2 diabetes increased significantly as a function of the number of risk alleles with an odds ratio of 1.15 [95\% CI $1.08-1.22]$ per risk allele $(p<0.0001)$.

Conclusion: Combining susceptibility alleles which individually have been reported to associate with hyperglycaemia may be one important way to identify subjects in the general population at increased risk of developing premature cardiovascular morbidity and mortality.

\section{8}

DUSP12 polymorphisms interact with the GCK $-30 \mathrm{G}>\mathrm{A}$ variant to increase fasting glycaemia in a large-scale population-based sample of middle-aged whites

T. Sparsø ${ }^{1}$, G. Andersen ${ }^{1}$, L. Hansen ${ }^{2}$, S. C. Elbein ${ }^{3}$, K. Borch-Johnsen ${ }^{1,4}$, T. Jørgensen ${ }^{4}$, T. Hansen ${ }^{1}$, O. Pedersen ${ }^{1}$;

${ }^{1}$ Steno Diabetes Center, Gentofte, Denmark, ${ }^{2}$ Development Projects, Novo Nordisk, Bagsværd, Denmark, ${ }^{3}$ Medical Service, Central Arkansas Veterans Healthcare System, Little Rock, United States, ${ }^{4}$ Research Centre for Prevention and Health, Glostrup University Hospital, Denmark.

Background and Aims: DUSP12 is a dual-specificity phosphatase identified using a two-hybrid system assay as a protein capable of interacting with glucokinase. DUSP12 dephosphorylates glucokinase in vitro and in a dosedependent manner accelerates its activity and hence may play an important role in glucose homeostasis. DUSP12 is located on chromosome 1q21-q22, a region showing linkage to type 2 diabetes. We hypothesised that variation 
in DUSP12 associates with hyperglycaemia - either alone or in combination with a common $-30 \mathrm{G}>\mathrm{A}$ polymorphism in the gene encoding glucokinase (GCK).

Materials and Methods: The DUSP12 rs1503814, rs1027702, rs1063178, and rs3820449 polymorphisms were genotyped in a population-based sample (Inter99) of 5,862 Danish whites (2,902 men, 2,960 women, age $46 \pm 8$ years BMI $26.3 \pm 4.6 \mathrm{~kg} / \mathrm{m}^{2}$ (mean $\left.\pm \mathrm{SD}\right)$ ). All subjects underwent a standard $75 \mathrm{~g}$ OGTT.

Results: Applying a general linear model no association with fasting or post-OGTT plasma glucose levels was observed when analysing the DUSP12 rs1503814 (minor allele frequency $(\mathrm{MAF})=0.261), \operatorname{rs} 1027702(\mathrm{MAF}=0.371)$, rs1063178 (MAF=0.330), and rs3820449 (MAF=0.257) polymorphisms. Some degree of linkage disequilibrium was observed between the four DUSP12 variants (pair-wise $r^{2}$ in the range of 0.4-0.8) and the most common haplotype had a frequency of 0.62 ; however, no association was observed with plasma glucose levels. Interestingly, an interaction was observed between the DUSP12 variants and the $G C K-30 \mathrm{G}>\mathrm{A}$ polymorphism on fasting plasma glucose: $p=0.04$ (rs1027702) TT/AA $6.3 \pm 0.6 \mathrm{mmol} / \mathrm{l}$, $p=0.02(\mathrm{rs} 3820449)$ TT/AA $6.3 \pm 0.8 \mathrm{mmol} / \mathrm{l}$, and $p=0.02$ (rs1063178) TT/AA $6.4 \pm 0.6 \mathrm{mmol} / \mathrm{l}$, whereas the remaining genotype combinations had fasting plasma glucose levels of $5.5 \pm 0.02 \mathrm{mmol} / 1$.

Conclusion: DUSP12 variation does not per se play a major role in the pathogenesis of hyperglycaemia in the general population of middle-aged whites. However, variants in DUSP12 may in combination with the $G C K$ $-30 \mathrm{G}>\mathrm{A}$ polymorphism contribute to the inter-individual variation in the fasting plasma glucose level.

\section{9}

Polymorphisms of the $\operatorname{Reg} 1 \alpha$ gene are associated with early onset type 2 diabetes in the Korean population B.-K. Koo ${ }^{1}$, Y. Cho ${ }^{1}$, S. Lim ${ }^{1}$, J. Kim ${ }^{1}$, M. Moon ${ }^{1}$, S. Choi ${ }^{1}$, H. Shin ${ }^{2}$, H. Jang ${ }^{1}$, S. Kim ${ }^{1}$, H. Lee ${ }^{1}$, K. Park ${ }^{1}$; ${ }^{1}$ Internal Medicine, Seoul National University College of Medicine, Seoul, ${ }^{2}$ Department of Genetic Epidemiology, SNP Genetics, Inc., Seoul, Republic of Korea.

Background and Aims: The Reg gene has been reported to be expressed in regenerating islets and Reg1 protein to be up-regulated early in the progression of type 2 diabetes in mice. As human Reg1 $\alpha$ is homologous with murine Reg1, we investigated whether common variants in the Reg1a gene are associated with type 2 diabetes or with its related phenotypes in the Korean population.

Materials and Methods: We sequenced the $\operatorname{Reg} 1 \alpha$ gene to identify common polymorphisms using 24 Korean DNA samples. Of 11 polymorphisms of the $\operatorname{Reg} 1 \alpha$ gene found, five common ones (g.-385T $>$ C, g. $-36 \mathrm{~T}>\mathrm{G}$, g. $209 \mathrm{G}>\mathrm{T}$, g. $1385 \mathrm{C}>\mathrm{G}$, and g. $2199 \mathrm{G}>\mathrm{A}$ ) were genotyped in 770 type 2 diabetic patients and 643 nondiabetic subjects. In addition, the influence of the polymorphism on the promoter activity was investigated by electrophoretic mobility shift assays (EMSA).

Results: No polymorphism was found to be associated with the risk of type 2 diabetes. However, g.-385C and g.2199A lowered the risk of early-onset type 2 diabetes, which was defined as the first quartile in terms of the age at diagnosis $(\mathrm{OR}=0.750$ [0.594-0.946] with $P=0.015$ and 0.737 [0.5850.929] with $P=0.023$, respectively) and the haplotype consisting of g. $-385 \mathrm{C}$ and g.2199A decreased the risk of early-onset diabetes (sex-adjusted $\mathrm{OR}=0.648$ [0.4690.894], $P=0.008$ in the dominant model). In addition, EMSA showed that NeuroD and upstream stimulating factor 1 (USF 1) specifically bound to the promoter region g.-385C of human Reg1 $\alpha$ gene.

\section{Conclusion:}

Polymorphisms in the Reg1 $\alpha$ gene were not found to be associated with overall susceptibility to type 2 diabetes, but were associated with early-onset type 2 diabetes in the Korean population possibly by modifying transcription activity.

Supported by Korean Health $21 R \&$ D Project, Ministry of Health \& Welfare, Republic of Korea

0330

The HNF-4A P2 promotor and risk of future type 2 diabetes

J. Holmkvist ${ }^{1}$, V. Lyssenko ${ }^{1}$, P. Almgren ${ }^{1}$, P. Nilsson ${ }^{2}$, L. Groop ${ }^{1}$;

${ }^{1}$ Dept. of Clinical Sciences - Diabetes \& Endocrinology, Lund University, Malmö, ${ }^{2}$ Dept. of Medicine, Lund University, Malmö, Sweden.

Background and Aims: Mutations in the transcription factor Hepatocyte Nuclear Factor-4 alpha (HNF-4 $\alpha$, TCF14) gene is known to cause monogenic diabetes, Maturity Onset Diabetes of the Young type 1 (MODY1). $H N F-4 \alpha$ is located on chromosome 20q12-q13, a region that has shown suggestive linkage to type 2 diabetes (T2D), and an important regulator in a network of transcription factors in the liver and pancreas. Variation in the $H N F-4 \alpha$, $\beta$-cell specific, P2 promoter have recently shown association to T2D. We hypothesized that variation in the P2promoter will increase future risk of T2D.

Materials and Methods: Individuals in the Botnia Prospective ( $\mathrm{n}=2293$; 132 T2D converters during mean 6-year follow-up) and in the Malmö Preventive Project (MPP; $\mathrm{n}=4873$; $491 \mathrm{~T} 2 \mathrm{D}$ converters during mean 20-year follow- 
up) cohorts were genotyped for three single nucleotide polymorphisms (SNPs) (rs4810424 [G/C], rs1884614 [C/T] and rs2144908 [G/A]) using the Taqman Allelic Discrimination technique on an Applied Biosystems 7900HT. Cohorts were analysed using multiple regression analysis at basal and last visits. The Cox proportional hazard model, applying a recessive genetic model, was used to predict future T2D. Analyses were adjusted for BMI, gender, age at visit and within family correlation when necessary.

Results: Genotype frequencies were in the same range as previously reported with minor allele frequencies at $18 \%$ and in Hardy-Weinberg equilibrium. The three SNPs were in linkage disequilibrium $\left(\mathrm{D}^{\prime}>0.87, \mathrm{r} 2>0.75\right)$. Two of the three SNPs in the Botnia prospective cohort were associated with increased risk of T2D; rs4810424, $\mathrm{HR}=1.9(95 \%$ CI 1.0-3.5; $\mathrm{p}=0.04)$ and $\mathrm{rs} 1884614, \mathrm{HR}=1.9(1.0-3.4, \mathrm{p}=0.04)$. In the MPP cohort the rs 1884614 and the rs 2144908 SNPs showed increased risk of future T2D; HR=1.8 (1.2-2.7, $\mathrm{p}=0.005)$ and $\mathrm{HR}=1.8(1.2-2.7, \mathrm{p}=0.004)$.

These differences were not associated with significant phenotypic differences (fasting plasma glucose, $2 \mathrm{~h}$ glucose, fasting insulin, $2 \mathrm{~h}$ insulin, HOMA-IR, HOMA-beta, insulinogenic index or disposition index) neither at the base line visit nor at the last visit.

Conclusion: Genetic variation in the $H N F-4 \alpha \mathrm{P} 2$ promoter significantly increases risk of future $\mathrm{T} 2 \mathrm{D}$ as determined by two large prospective cohorts from Finland and Sweden.

\section{1}

Common variation in the $L M N A$ gene increases susceptibility to type 2 diabetes

K. R. Owen ${ }^{1}$, C. J. Groves ${ }^{1}$, E. Zeggini ${ }^{2}$, G. Hitman ${ }^{3}$, M. Walker ${ }^{4}$, T. M. Frayling ${ }^{5}$, A. T. Hattersley,

M. I. McCarthy ${ }^{1}$;

${ }^{1}$ Diabetes Research Laboratories, University of Oxford,

${ }^{2}$ Genetics of Diabetes, Wellcome Trust Centre for Human Genetics, Oxford, ${ }^{3}$ Diabetes and Metabolic Medicine, University of London, ${ }^{4}$ Department of Medicine, University of Newcastle, ${ }^{5}$ Diabetes Research and Vascular Medicine, Peninsula Medical School, Exeter, United Kingdom.

Background and Aims: $L M N A$ is a positional and biological candidate gene for type 2 diabetes (T2D). The gene is located in the replicated area of T2D linkage on chromosome 1q, while mutations in $L M N A$ cause a form of monogenic insulin resistance and diabetes (familial partial lipodystrophy). We hypothesised that, as in other monogenic causes of diabetes - such as those due to mutations in PPARG, HNF4A and KCNJ11- common variation in LMNA might predispose to multifactorial T2D.
Materials and Methods: We analysed 6 tagging SNPS (rs12063564, rs6661281, rs955383, rs693671, rs505058 and rs4641) in cases of T2D $(n=2158)$ and non-diabetic controls $(n=2574)$, all of UK origin. SNPs were selected from HapMap and previously published data, capturing $>90 \%$ of common variation in the selected region at an $\mathrm{r}^{2}$ of 0.8 .

Results: Association with diabetes was seen with the rare allele for 3 SNPs which was strongest using a recessive model: rs 12063564 ( $5^{\prime}$ upstream, MAF 0.17) OR $1.83(95 \%$ CI 1.23-2.7), $\mathrm{p}=0.002$; rs6661281 ( $5^{\prime}$ upstream, MAF 0.41) OR 1.19 (1.02-1.40), $\mathrm{p}=0.03$; rs505058 (synonymous SNP exon 7, MAF 0.06) OR 3.68 (1.3-12.95), $\mathrm{p}=0.01$.

Haplotype analysis did not reveal any additional association compared to the single point analysis.

In the diabetic cases no association was seen between any SNP and age at diagnosis of diabetes, BMI or waist-hip ratio.

Conclusion: In this first large case-control study, our results suggest that common variation in the LMNA gene affects susceptibility to T2D. This finding requires confirmation in further cohorts but is consistent with other examples of overlap between genes involved in both monogenic causation and multifactorial susceptibility.

Supported by Diabetes UK

0332

Does the Val262Leu polymorphism in the mitochondial rhomboid protease $P S A R L$ predispose to type 2 diabetes in South Asians?

A. N. Dixon ${ }^{1,2}$, A. C. Britten ${ }^{1}$, K. Jones ${ }^{1}$, S. Bellary ${ }^{1,2}$, S. Mughal ${ }^{2}$, S. Kumar ${ }^{3}$, J. P. O'Hare ${ }^{3}$, A. H. Barnett ${ }^{1,2}$, M. A. Kelly ${ }^{1}$;

${ }^{1}$ Department of Medicine, University of Birmingham, ${ }^{2}$ Department of Diabetes, Heart of England NHS Trust, Birmingham, ${ }^{3}$ Warwick Medical School, University of Warwick, Coventry, United Kingdom.

Background and Aims: The genes encoding mitochondrial proteins involved in beta-oxidation of fatty acids are potential susceptibility genes for type 2 diabetes. One such candidate is the gene encoding the mitochondrial intramembrane protease presenilins-associated rhomboid-like protein (PSARL). PSARL is located on chromosome $3 \mathrm{q} 27$ in a region with substantial evidence for linkage with type 2 diabetes and metabolic syndrome phenotypes. Expression of the PSARL gene in human skeletal muscle is associated with insulin sensitivity and mitochondrial oxidative capacity. The common $\mathrm{G} \rightarrow \mathrm{C}$ SNP in exon 7 of the PSARL gene results in a change from valine to leucine at amino acid 262 in the encoded protein. A study of a population of northern European ancestry demonstrated that individuals homozygous for the $G$ allele had higher plasma insulin concen- 
trations compared with individuals with the $\mathrm{GC}$ or $\mathrm{CC}$ genotypes. The association of the PSARL genotype with type 2 diabetes was not explored, however. We present data from a pilot study which aimed to determine whether the $\mathrm{G} \rightarrow \mathrm{C}$ SNP of the PSARL gene is associated with type 2 diabetes in a distinct South Asian ethnic group.

Materials and Methods: Genotyping was performed in subjects recruited to the United Kingdom Asian Diabetes Study. All the individuals were from an ethnically homogeneous population, originating from north Pakistan and Azad Kashmir. This population is known to have a high prevalence of insulin resistance.

Results: 631 patients with type 2 diabetes and 250 ethnically-matched normoglycaemic control subjects were genotyped. The frequency of the less common $\mathrm{C}$ allele was 0.449 in the diabetic group compared to 0.452 in the control group $(\mathrm{p}=\mathrm{NS})$. There was no significant difference in the genotype frequencies between the groups. In subjects above the age of 50 years, however, the GG genotype was significantly more frequent in the control population compared to the diabetic group $(\mathrm{p}<0.01)$.

Conclusion: This pilot study had $81 \%$ power to detect a disease association with an odds ratio of 1.35 or above. The data suggest, therefore, that the $\mathrm{G} \rightarrow \mathrm{C}$ SNP in exon 7 of the PSARL gene is unlikely to be a major susceptibility determinant for type 2 diabetes, although we cannot exclude a minor role. The GG genotype of the PSARL gene may be protective against type 2 diabetes in older individuals, although this requires confirmation in a larger data set. Further studies investigating the effect of this polymorphism on beta-cell function and the development of insulin resistance are required.

Supported by Pfizer, Aventis UK, Servier Laboratories UK, Merck Sharp \& Dohme/Schering-Plough, Takeda UK, Boehringer Ingleheim, Eli Lilly, Merck Pharma, NovoNordisk, Bristol Myers Squibb, Sanofi-Synthelabo, Roche and Sankyo UK

\section{3}

Adenylyl Cyclase 3 (AC3) and the genetic susceptibility to type 2 diabetes in Swedish Caucasians

S. Nordman ${ }^{1}$, A. Abulaiti ${ }^{1}$, K. Humphreys ${ }^{2}$, C.-G. Östenson ${ }^{1}$, S. Efendic ${ }^{1}$, H. F. Gu ${ }^{1}$;

${ }^{1}$ Department of Molecular Medicine and Surgery, Karolinska Institute, ${ }^{2}$ Department of Medical Epidemiology and Biostatistics, Karolinska Institute, Stockholm, Sweden.

Background and Aims: Goto-Kakizaki (GK) rat is an animal model of non-obese type 2 diabetes (T2D). A previous study from our laboratory demonstrated that the adenylyl cyclase 3 (AC3) gene is over-expressed in the pancreatic islets of the GK rat due to two point mutations in the promoter region. In the present study, we explore whether AC3 genetic variation confers susceptibility to T2D in men.

Materials and Methods: We first screened for mutation in the promoter region and then studied the single nucleotide polymorphisms (SNPs) covering the whole genomic region of the human AC3 gene. Genotyping experiments for 15 valid SNPs, including a novel identified variant $-17 \mathrm{~A} / \mathrm{T}$ in the promoter, were performed in 294 patients with T2D, 319 subjects with impaired glucose tolerance (IGT) and 317 non-diabetic healthy individuals. All patients and subjects studied were Swedes.

Results: The single marker association analysis indicated that four SNPs, including rs2033655, rs1968482, rs934611 and rs 1044040 were significantly associated with T2D. Further quantitative trait analysis related to SNP rs934611 revealed that T2D patients with GG genotype as compared with those carrying GA and AA genotypes had increased BMI $(30.2 \pm 5.1$ vs $28.3 \pm 5.6, \mathrm{P}=0.032)$ and WHR $(0.96 \pm 0.08$ vs $0.90 \pm 0.05, \mathrm{P}=0.041)$.

Conclusion: We demonstrate that $\mathrm{AC} 3$ genetic variations confer the susceptibility to the development of T2D among Swedish Caucasians.

Supported by Novo Nordic Consortium, Swedish Research Council, Vetenskapligt arbete inom diabetologi foundation, Loo and Hans Osterman foundation and Swedish Diabetes Association

\section{4}

Gene-gene interaction between the type 2 deiodinase gene (DIO2) Thr92Ala variant and the $\beta 2$ adrenergic receptor gene (ADRB2) Arg16Gly variant is associated with elevated glycaemia in the general population M. K. Andersen ${ }^{1}$, C. H. Andreasen ${ }^{1}$, N. Grarup ${ }^{1}$, A. Albrechtsen ${ }^{1}$, K. Borch-Johnsen ${ }^{1,2}$, T. Jørgensen ${ }^{2}$, J. Auwerx ${ }^{3,4}$, T. Hansen ${ }^{1}$, O. Pedersen ${ }^{1}$;

${ }^{1}$ Steno Diabetes Center, Gentofte, Denmark, ${ }^{2}$ Research Centre for Prevention and Health, University Hospital, Glostrup, Denmark, ${ }^{3}$ Institut de Génétique et Biologie Moléculaire et Cellulaire, CNRS/INSERM/ULP, Illkirch, France, ${ }^{4}$ Institut Clinique de la Souris, Génopole Strasbourg, Illkirch, France.

Background and Aims: The $\mathrm{DIO} 2$ gene encodes the type 2 deiodinase (D2), which is activated by the $\beta$-adrenergic pathway. D2 catalyzes the conversion of thyroxine $\left(\mathrm{T}_{4}\right)$ to the active form of thyroid hormone $\left(\mathrm{T}_{3}\right)$. $\mathrm{T}_{3}$ is an important factor in energy metabolism by its ability to stimulate the expression of genes involved in thermogenesis and regulation of glucose metabolism in skeletal muscle and adipose 
tissue. The Thr92Ala polymorphism (rs225014) in DIO2 has previously been associated with insulin resistance in women and diabetic patients. The aim of this study was to investigate the possible association between the DIO2 Thr92Ala polymorphism and type 2 diabetes (T2D), obesity, and related metabolic quantitative traits including measures of insulin resistance in 7,348 middle-aged Danish whites. We also hypothesized that variation in $\beta$-adrenergic receptor genes contributes to association with metabolic traits via interactions with $\mathrm{DIO}$.

Materials and Methods: The DIO2 Thr92Ala polymorphism was genotyped in the large population-based Inter99 cohort of 5,942 subjects and in a group of 1,406 type 2 diabetic patients.

Results: No significant association between either T2D or obesity and the DIO2 Thr92Ala polymorphism was found. Neither, did we find association between the genotype and obesity- and T2D related quantitative metabolic traits, including fasting and post-OGTT levels of plasma glucose and serum insulin, and measures of obesity. However, we did observe a significant gene-gene interaction of the Thr92Ala DIO2 variant and the Arg16Gly variant in the gene encoding the $\beta 2$ adrenergic receptor (ADRB2) on fasting plasma glucose in a double recessive model (Ala92/ Arg16 double homozygous $(n=100)$ : mean \pm SE $5.8 \mathrm{mmol} /$ $1 \pm 0.08$ vs. all other genotypes: $5.5 \pm 0.01$; epistatic model $p=0.004$, global model $p=0.005$ ). A possible mechanism for this interaction is through $\beta$-adrenergic receptor-D2thyroid hormone stimulation of GLUT4 expression.

Conclusion: Middle-aged subjects homozygous for both the DIO2 Ala-allele and the ADRB2 Arg-allele have elevated fasting plasma glucose level. Further large-scale studies are needed to substantiate this finding.

\section{5}

ATF6, a key regulator of the unfolded protein response, is associated with type 2 diabetes

S. J. R. Meex ${ }^{1}$, M. M. J. van Greevenbroek ${ }^{1}$,

T. A. Ayoubi ${ }^{2}$, R. Vlietinck ${ }^{2}$, V. M. M. Vermeulen ${ }^{1}$,

E. Feskens ${ }^{3}$, C. G. Schalkwijk ${ }^{1}$, C. D. A. Stehouwer ${ }^{1}$,

C. J. H. van der Kallen ${ }^{1}$, T. W. A. de Bruin ${ }^{4}$;

${ }^{1}$ Internal Medicine, Maastricht University, The Netherlands, ${ }^{2}$ Populationgenetics, Maastricht University, The Netherlands, ${ }^{3}$ Division of Human Nutrition, Wageningen University, Wageningen, The Netherlands, ${ }^{4}$ Translational Medicine \& Genetics, GlaxoSmithKline, Research Triangle Park, United States.

Background and Aims: Activating Transcription Factor 6 (ATF6) is critical for initiation and full activation of the unfolded protein response. We investigated ATF6 for its association with type 2 diabetes in the Dutch population. An association between variations in ATF6 and type 2 diabetes was recently reported in Pima Indians, but additional studies in more outbred study samples are strongly required to evaluate its significance.

Materials and Methods: Two independent Dutch cohorts, comprising in total 949 subjects, were used to investigate the genetic association between polymorphisms in the ATF6-gene and type 2 diabetes. Four SNPs with a high potential to affect the ATF6 protein levels/activity were genotyped in 152 subjects from the general population and analysed for their association with glucose, insulin and HOMA-index: SNP $-116 \mathrm{C} / \mathrm{T}$ in the promotor, an $\mathrm{A} / \mathrm{G}$ Met67Val substitution in exon 3, a C/G Pro145Ala substitution in exon 5, and $+124 \mathrm{G} / \mathrm{A}$ in the 3 'untranslated region of the ATF6 gene. Significantly associatiated polymorphisms in the first cohort were further investigated for their contribution to type 2 diabetes in a second Dutch casecontrol cohort $(\mathrm{N}=797)$, that consisted of 210 type 2 diabetes patients, 208 IFG/IGT subjects and 379 normoglycaemic controls

Results: In subjects from the general population, the $+124 \mathrm{G}$-allele was associated with increased glucose levels $(\mathrm{p}=0.02)$, and HOMA-index $(\mathrm{p}=0.05)$. Furthermore, in the Dutch case-control cohort, the $+124 \mathrm{G}$-allele was more prevalent in subjects with IFG, IGT or type 2 diabetes, than in healthy individuals ( $49 \%$ versus $40 \%$, OR $=1.4,95 \%$ confidence interval $1.09-1.91, \mathrm{p}=0.011)$. This effect remained significant after adjustment for age, sex and body mass index $(\mathrm{p}=0.016)$.

Conclusion: These results confirm the recently reported association of ATF6 with type 2 diabetes in Pima Indians, and highlight the potential importance of ATF6 for conferring genetic susceptibility to type 2 diabetes in more outbred populations.

Collection of the type 2 diabetes patients was supported by grants of the Netherlands Organisation for Scientific Research (940-35-034) and the Dutch Diabetes Foundation (DFN98.901).

0336

Gene polymorphisms of antioxidant enzymes in diabetes mellitus

M. Flekac, J. Skrha, J. Hilgertova, Z. Lacinova; 3rd Department of Internal Medicine, Charles University, Prague, Czech Republic.

Background and Aims: Reactive oxygen species generated by hyperglycemia modify structure and function of lipids, proteins and other molecules taking part in chronic vascular changes in diabetes. Low activity of scavenger 
enzymes has been observed in diabetic patients and their protective role in oxidative stress may be deteriorated. This study was undertaken to investigate the association between polymorphisms of selected genes of antioxidant enzymes and diabetic angiopathy.

Materials and Methods: Total of 220 patients with Type 2 diabetes (T2DM), 66 with Type 1 diabetes (T1DM) and control group of 110 nondiabetic healthy subjects unrelated to diabetic patients were examined. Microangiopathy was present in 159 patients, 35 subjects had macrovascular complications and 92 patients were free of any complications. Venous blood samples were drawn after an overnight fast. Paraoxonase (PON1) and superoxide dismutase (SOD) activities were determined spectrophotometrically. Genomic DNA was obtained by automated isolator. Polymorphisms were estimated by PCR-RFLP.

Results: The allele frequencies of PON1, SOD1, SOD2 and CAT are shown in Tab.1. Significant diferences between T1DM,T2DM and control persons $(p<0,01)$ were found in coding region of PON1 and SOD2 genes (in table expressed by bold letters) but not in SOD1 and CAT genes. Serum PON1 activity was significantly decreased in T1DM $(110 \pm 68 \mathrm{nmol} / \mathrm{ml} / \mathrm{min})$ and T2DM subjects $(118 \pm 69 \mathrm{nmol} /$ $\mathrm{ml} / \mathrm{min})$ compared to the control persons $(203 \pm 58 \mathrm{nmol} / \mathrm{ml} /$ min). L55M and Q192R polymorphisms effected PON1 activity. Serum PON1 activity was higher in LL than in MM genotype $(p<0,05)$ and similarly the presence of $R R$ genotype was associated with higher PON1 activity than of QQ genotype $(p<0,05)$ in both Types of diabetes. No associations were observed between SOD gene polymorphisms and SOD activity. Better diabetes control was found in patients with $\mathrm{LL}(\mathrm{HbAlc}=7,4 \pm 0,2)$ than with $\mathrm{MM}$ $(\mathrm{HbA} 1 \mathrm{c}=7,6 \pm 0,1 ; \mathrm{p}<0,05)$ genotype of PON1. Significantly different allele frequencies were found in diabetic patients with macroangiopathy $(\mathrm{M}=0,59, \mathrm{R}=0,11)$ than in those without $(\mathrm{M}=0,44, \mathrm{R}=0,18 ; \mathrm{p}<0,01)$ but no difference was associated with/without microangiopathy.

Tab1. The Allele frequencies.

\begin{tabular}{|c|c|c|c|c|c|c|c|}
\hline & PON1 & PON1 & PON1 & PON1 & SOD1 & SOD2 & CAT \\
\hline & L55M & Q192R & $-107 \mathrm{C} / \mathrm{T}$ & $-907 \mathrm{G} / \mathrm{C}$ & $35 \mathrm{~A} / \mathrm{C}$ & Ala-9Val & $-262 \mathrm{~T} / \mathrm{A}$ \\
\hline \multirow[t]{2}{*}{ T1DM } & $\mathrm{L}=\mathbf{0 , 5 2 1}$ & $\mathrm{L}=\mathbf{0}, \mathbf{8 1 3}$ & $\mathrm{C}=0,515$ & $\mathrm{G}=0,550$ & $\mathrm{~A}=0,90$ & $A=\mathbf{0 , 4 8 0}$ & $\mathrm{T}=0,57$ \\
\hline & $M=0,479$ & $M=\mathbf{0 , 1 8 7}$ & $\mathrm{T}=0,485$ & $\mathrm{C}=0,450$ & $\mathrm{C}=0,10$ & $V=\mathbf{0 , 5 2 0}$ & $\mathrm{A}=0,43$ \\
\hline \multirow[t]{2}{*}{ T2DM } & $\mathrm{L}=\mathbf{0 , 5 8 3}$ & $Q=0,849$ & $\mathrm{C}=0,520$ & $\mathrm{G}=0,553$ & $\mathrm{~A}=0,91$ & $A=0,475$ & $\mathrm{~T}=0,58$ \\
\hline & $M=0,417$ & $\mathrm{R}=\mathbf{0 , 1 5 1}$ & $\mathrm{T}=0,480$ & $\mathrm{C}=0,447$ & $\mathrm{C}=0,09$ & $V=\mathbf{0 , 5 2 5}$ & $\mathrm{A}=0,42$ \\
\hline \multirow[t]{2}{*}{ Controls } & $\mathrm{L}=\mathbf{0 , 6 9 0}$ & $Q=0,543$ & $\mathrm{C}=0,555$ & $\mathrm{G}=0,590$ & $\mathrm{~A}=0,92$ & $\mathrm{~A}=\mathbf{0 , 3 0 0}$ & $\mathrm{T}=0,57$ \\
\hline & $M=0,310$ & $\mathrm{R}=\mathbf{0 , 4 5 7}$ & $\mathrm{T}=0,445$ & $\mathrm{C}=0,410$ & $\mathrm{C}=0,08$ & $\mathrm{C}=\mathbf{0 , 7 0 0}$ & $\mathrm{A}=0,43$ \\
\hline
\end{tabular}

Conclusion: The results of our study demonstrate that oxidative stress in diabetes mellitus can be accelerated nor only due to increased production of reactive oxygen species by hyperglycemia but also by reduced ability of antioxidant defense caused at least partly by genetic polymorphisms of some scavenger enzymes. Relationship was found between PON1 polymorphisms and macroangiopathy in diabetic patients.

Study was supported by research project MSM0021620807

\section{PS 9}

\section{PPAR and adiponectin genes}

\section{$\mathbf{0 3 3 7}$}

Genetic and non-genetic regulation of PPAR $\mathrm{mRNA}$ expression in human skeletal muscle

E. Nilsson ${ }^{1}$, P. Poulsen ${ }^{1}$, M. Sjögren ${ }^{2}$, A. Vaag ${ }^{1}$, L. Groop ${ }^{2}$; ${ }^{1}$ Steno Diabetes Center, Gentofte, Denmark, ${ }^{2}$ Department of Clinical Sciences, Diabetes \& Endocrinology, Malmö, Sweden.

Background and Aims: Peroxisome proliferator activated receptors (PPARs) are nuclear receptors regulating the expression of genes involved in lipid and glucose metabolism in a subtype- and tissue-specific manner. There are three different PPAR isoforms named $\alpha, \delta$ and $\gamma$. In mice, PPAR $\delta$ seems to be implicated in the regulation of fatty acid burning capacities of skeletal muscle and adipose tissue by controlling the expression of genes involved in $\beta$ oxidation and energy uncoupling. Our aim was to study the influence of age and insulin on PPAR $\delta$ mRNA expression in human skeletal muscle, and investigate whether PPAR $\delta$ mRNA expression is an inherited trait.

Materials and Methods: Muscle biopsies were obtained before and after hyperinsulinemic euglycemic clamps from 102 young (age $28 \pm 2$ years, 62 monozygotic and 40 dizygotic) and 74 elderly twins (age $62 \pm 2$ years, 36 monozygotic and 38 dizygotic). Body composition was determined by dual-energy x-ray absorptiometry. PPAR $\delta$ mRNA levels were quantified by real-time PCR using the ABI PRISM 7900 Sequence Detection System. Cyclophilin $A$ was used as an endogenous control. Genetic modelling to estimate the degree of genetic vs. environmental influence on PPAR $\delta$ mRNA expression was performed separately in the two age groups using standard Mx scripts. Six SNPs were chosen for genotyping after analyzing HapMap data using the Tagger program. The PPAR $\delta$ SNPs (rs7744392, rs9470001, rs2267665, rs2076169, rs2076168 and rs1053046) were genotyped using allelic discrimination in the ABI PRISM 7900 Sequence Detection System.

Results: Young twins had significantly increased PPAR mRNA levels compared to elderly twins both in the basal state $(2.5 \pm 0.18$ vs. $1.3 \pm 0.08, P<0.000001)$ and after the 2 hour hyperinsulinemic euglycemic clamp $(2.6 \pm 0.30$ vs. 
$1.3 \pm 0.08, P<0.000001)$. The insulin clamp had no significant effect on PPAR $\delta$ mRNA levels neither in all individuals $(2.0 \pm 0.12$ vs. $2.1 \pm 0.19, P=0.4)$ nor in the young $(P=0.4)$ or elderly twins $(P=0.8)$ when analyzed separately. Biometric models were calculated for basal PPAR $\delta$ mRNA expression to estimate the degree of genetic vs. environmental influence. In both young $\left(\mathrm{a}^{2}\right.$ [additive genetic] $=0.59$, $\mathrm{e}^{2}$ [unique environment] $\left.=0.41\right)$ and elderly twins $\left(\mathrm{a}^{2}=0.63\right.$, $\mathrm{e}^{2}=0.37$ ) there was a major genetic component influencing basal PPAR $\delta$ mRNA levels. Young carriers of the rare allele at SNP rs 1053046 displayed a trend towards higher PPAR $\delta$ mRNA levels compared to non-carriers in the basal state $(2.9 \pm 0.40$ vs. $2.4 \pm 0.19, P=0.08)$ and significantly higher levels after the hyperinsulinemic euglycemic clamp ( $4.8 \pm 2.0$ vs $2.4 \pm 0.28, P=0.02$ ). No such difference was observed among the elderly twins $(0.9 \pm 0.17$ vs. $1.3 \pm 0.08$, $P=0.14$ in the basal state and $0.9 \pm 0.07$ vs. $1.3 \pm 0.07, P=0.39$ after the insulin clamp). No genotype-specific differences in PPAR $\delta$ mRNA expression were observed for any of the other polymorphisms. Regression analysis revealed that age $(P<0.0001)$, central adiposity $(P=0.004)$ and birth weight $(P=0.03)$ were the best predictors of basal PPAR $\delta$ mRNA expression. Age $(P=0.002)$ was the factor that best explained PPAR $\delta$ mRNA levels after insulin clamp.

Conclusion: We provide evidence that mRNA expression of PPAR $\delta$ in human skeletal muscle is reduced by age and under genetic control.

\section{8}

Studies of association of the PPAR $\alpha$ Leu162Val polymorphism in relation to obesity,dyslipidaemia, type 2 diabetes, and related quantitative traits

M. S. Hussain ${ }^{1}$, T. Sparsø ${ }^{1}$, G. Andersen ${ }^{1}$, C. Glümer ${ }^{1,2}$, K. Borch-Johnsen ${ }^{1,2}$, T. Jørgensen ${ }^{2}$, T. Hansen ${ }^{1}$,

O. Pedersen ${ }^{1}$;

${ }^{1}$ Steno Diabetes Center, Gentofte, ${ }^{2}$ Research Centre for Prevention and Health, Glostrup University Hospital, Denmark.

Background and Aims: Peroxisome proliferator-activated receptor- $\alpha$ (PPAR $\alpha)$ is a nuclear receptor capable of regulating proteins involved in lipid metabolism and glucose homeostasis in the liver and in skeletal muscle. PPAR $\alpha$ regulates the expression of genes involved in peroxisomal and mitochondrial $\beta$-oxidation pathways, fatty acid uptake, and the catabolism of triglycerides. Previous studies of the Leu162Val polymorphism in PPAR $\alpha$ have indicated association with obesity, type 2 diabetes, and dyslipidaemia, although the results have been somewhat discrepant.
Materials and Methods: The Leu162Val polymorphism was genotyped in a population-based sample (Inter99) of 6,174 Danish Caucasians (2,955 men, 2,986 women, age $46 \pm 8$ years (mean \pm SD), BMI $26.3 \pm 4.6 \mathrm{~kg} / \mathrm{m}^{2}$ ) and 1,037 type 2 diabetic patients sampled at Steno Diabetes Center $(625$ men, 412 women, age $58.9 \pm 11$ years, BMI $\left.29.4 \pm 5.1 \mathrm{~kg} / \mathrm{m}^{2}\right)$. All participants of Inter99 underwent a standard $75 \mathrm{~g}$ oral glucose tolerance test. Genotyping was performed using chip-based matrix-assisted laser desorption/ionisation timeof-flight (MALDI-TOF) mass spectrometry.

Results: Using a general linear model with correction for sex, age, and BMI we found no association between the Leu162Val polymorphism and obesity-related quantitative traits (BMI, fasting serum triglyceride, fasting serum total and HDL cholesterol, and waist-to-hip ratio). In a casecontrol study no significant differences in allele frequency or genotype distribution between 1,383 type 2 diabetic patients and 4,401 healthy control subjects were observed. In addition, the Leu162Val polymorphism was not associated with dyslipidaemia in a case-control study comprising 1,399 dyslipidaemic subjects and 4,399 normolipidaemic subjects. Furthermore, we were not able to show any interaction between the Leu162Val polymorphism and SNPs in other PPAR isotypes (PPAR $\delta$, PPAR $\gamma$ ) on serum triglyceride levels. Interestingly, for the Val162Val genotype an increase in fasting serum triglyceride concentration was observed (Leu/Leu+Leu/Val $n=5,777,1.33 \pm$ $1.35 \mathrm{mmol} / 1$ vs. Val $/ \mathrm{Val} n=17,2.22 \pm 2.4 \mathrm{mmol} / 1, p=0.007$ ). Conclusion: The PPAR $\alpha$ Leu162Val polymorphism is not a major contributor in the pathogenesis of obesity, type 2 diabetes, dyslipidaemia, or related quantitative traits in a Danish Caucasian population-based cohort.

\section{9}

Common polymorphisms of the PPAR- $\gamma 2$, PGC- $1 \alpha$ and adiponectin genes interact in their effects on the conversion from impaired glucose tolerance to type 2 diabetes: the STOP-NIDDM trial

L. Andrulionyte ${ }^{1}$, J. Zacharova ${ }^{1}$, J.-L. Chiasson ${ }^{2}$, M. Laakso ;

${ }^{1}$ Department of Medicine, University of Kuopio, Finland, ${ }^{2}$ Department of Medicine, University of Montreal, Research Centre, Centre Hospitalier de l'Université de Montréal, Hôtel-Dieu, Quebec, Canada.

Background and Aims: Peroxisome proliferator-activated receptor (PPAR) $\gamma$ is a nuclear hormone receptor that plays a role in adipocyte differentiation, particularly when coactivated by PPAR- $\gamma$ coactivator $1 \alpha$ (PGC- $1 \alpha)$, and modulates the production and release of adipose-derived factors including adiponectin, leptin, plasminogen activator inhib- 
itor 1 (PAI-1), tumor necrosis factor $\alpha$ (TNF- $\alpha$ ), and interleukin 6 (IL-6). The Pro12Ala polymorphism of the PPAR- $\gamma 2$ gene (PPARG2) was the first genetic variant identified consistently associated with the risk of type 2 diabetes. Single nucleotide polymorphisms (SNPs) of the PGC-1 $\alpha$ gene $(P G C 1 A)$, and genes regulating adipokines have also been associated with diabetes. The aim of the present study was to investigate whether or not there is an interaction between SNPs of PPARG2, PGC1A and SNPs of genes encoding different adipokines in their effects on the conversion from impaired glucose tolerance to type 2 diabetes in subjects from the Study To Prevent Non Insulin Depending Diabetes Mellitus (STOP-NIDDM).

Materials and Methods: The STOP-NIDDM trial evaluated the effect of acarbose compared to placebo on the prevention of type 2 diabetes. The following SNPs were genotyped in 768 DNA samples available: Pro12Ala of PPARG2, Gly482Ser of PGC1A, SNPs +45 in exon 2 and +276 in intron 2 of the adiponectin gene, G-308A in the promoter of the TNF- $\alpha$ gene, $\mathrm{C}-174 \mathrm{G}$ in the promoter of the IL-6 gene, pentanucleotide insertion/deletion polymorphism of the leptin receptor gene and $-6754 \mathrm{G} / 5 \mathrm{G}$ polymorphism in the promoter of the PAI-1 gene.

Results: The Pro12Ala of PPARG2 and Gly482Ser of the $P G C 1 A$ had a significant interaction with the haplotype of the two SNPs of the adiponectin gene with respect to the conversion to diabetes $(P$ values for the interaction 0.005 and 0.036 , respectively). Subjects carrying the three risk genotypes of SNP+45 and SNP+276 of the adiponectin gene and the Pro12Ala polymorphism of PPARG2 had a 2.82-fold higher risk for the development of diabetes $(95 \%$ confidence interval (CI) $1.28-6.23, P=0.010)$ compared to those who did not carry these risk alleles. In the placebo group, carriers of the previously mentioned risk genotypes had a 5.71-fold increase in the risk of diabetes $(95 \% \mathrm{CI}$ 1.57-20.78; $P=0.008)$. The presence of the three risk genotypes of the Gly482Ser polymorphism of $P G C 1 A$ and both SNPs of the adiponectin gene resulted in 3.42-fold increase in the risk of diabetes (95\% CI 1.44-8.14, $P=0.005$ ) compared to subjects who did not have the risk genotypes. In the placebo group the corresponding odds ratio (OR) was 13.26 (95\% CI 2.65-66.28, $P=0.002)$. The ORs for the conversion to diabetes associated with the four risk alleles (SNPs of PPARG2, PGC1A and the adiponectin gene) was not higher than that of the three risk alleles reported above. $P$ values for the interaction between the polymorphisms of PPARG2, PGC-1A and SNPs of TNF- $\alpha$, IL-6, leptin receptor and PAI-1genes with respect to the conversion to type 2 diabetes were not statistically significant, and the effect of the combined genotypes on the risk of diabetes was not increased beyond that of individual SNPs.
Conclusion: Our results suggest that SNPs in the PPARG2, $P G C-1 A$ and adiponectin genes may interact in their effects on the risk for type 2 diabetes.

The STOP-NIDDM trial and DNA analyses were funded by an unrestricted grant from Bayer AG to Markku Laakso.

\section{0}

The protective effect on the risk of type 2 diabetes given by the PPAR $\gamma 2$ Ala12 variant is heterogeneous across different populations

O. Ludovico ${ }^{1}$, F. Pellegrini ${ }^{2}$, R. Di Paola ${ }^{1}$, A. Minenna ${ }^{1}$, S. Mastroianno ${ }^{1}$, M. Cardellini ${ }^{3}$, M. Marini ${ }^{3}$, F. Andreozzi $^{4}$, O. Vaccaro ${ }^{5}$, G. Sesti ${ }^{3,4}$, V. Trischitta ${ }^{1,6}$;

${ }^{1}$ Endocrinology, IRCCS Casa Sollievo della Sofferenza, San Giovanni Rotondo, ${ }^{2}$ Clinical Pharmacology and Epidemiology, Consorzio Mario Negri Sud, S. Maria Imbaro, ${ }^{3}$ Internal Medicine, University of Tor Vergata, Roma, ${ }^{4}$ Experimental and Clinical Medicine, University "Magna Graecia", Catanzaro, ${ }^{5}$ Clinical and Experimental Medicine, University Federico II, Napoli, ${ }^{6}$ Clinical Sciences, Univeristy La Sapienza, Roma, Italy.

The P12A SNP of PPAR $\gamma 2$ gene has been studied for association with type 2 diabetes (T2D) with controversial results, thus suggesting variability in its contribution to disease risk. To deeper investigate this issue, a metaanalysis of all 39 published association studies (i.e. PubMed search using "PPAR $\gamma$ and diabetes", "genetics of type 2 diabetes" and "candidate gene" as key-words) and two unpublished Italian studies for a total of 41,296 individuals was performed.

In the 2 Italian studies, Caucasians from Gargano $(n=1183)$ or Calabria $(n=1196)$ were recruited. The 2 studies were approved by local Ethical committees. The P12A SNP was screened by dideoxy single-base extension of an unlabeled primer (ABI PRISM SNaPshot Multiplex Kit 4323151).

Risks are expressed As OR and 95\% CI. Between-studies heterogeneity was assessed with Cochran's Q test. Random effects meta-analysis and meta-regression were used to estimate overall ORs and to explore heterogeneity. Permutation resampling p-values were calculated to address the risk of spurious significant results. All the analyses were performed using SAS (Release 9.1, Cary, NC, USA, 2002-2003).

In all 41 studies the P12A SNP was in Hardy-Weinberg equilibrium. In random-effects meta-analysis of data from all 41,296 individuals, A12 carriers showed a 19\% risk reduction of $\mathrm{T} 2 \mathrm{D}(\mathrm{OR}=0.81,95 \%$ C.I. $=0.75-0.88)$; this effect was heterogeneous across all studies $(\mathrm{p}=0.004)$. Data were then stratified according to the 3 regions where studies had been conducted: Asia, Europe and North-America. The 
risk reduction given by the $\mathrm{A} 12$ variant was significantly different (ORs heterogeneity $\mathrm{p}=0.05$ ), being 35\% among Asians and 15\% among both Europeans and North Americans. Risk reduction in Asia was different from that in Europe $(\mathrm{p}=0.02)$ but not from that in North America $(p=0.09)$, very likely because of the small size of the latter sample. When data from Europe were stratified according to a North to South gradient, the risk reduction of T2D in Ala12 carriers was progressively reduced (test for trend in the ORs $\mathrm{p}=0.02)$ from North $(25 \%, \mathrm{p}<0.0001)$ to Centre $(10 \%, \mathrm{p}=0.04)$ and South $(0 \%, \mathrm{p}=0.94)$ Europe. The same trend was significant even when assessed via permutation resampling $(\mathrm{p}=0.041)$. Our meta-analysis on more than 41,000 individuals shows that the $\mathrm{A} 12$ variant confers a reduced risk of T2D. This effect is not homogeneous, being greater in Asians than in other populations, and among Caucasians from Europe, being stronger in Northern, lower and barely significant in Central and null in Southern Europe. To the best of our knowledge this is the first study which clearly indicates heterogeneity of the genetic risk of T2D and stress, therefore, the need of great caution when trying to interpret and reconcile data obtained in populations from different geographical regions and/or of different ethnicity.

Supported by Telethon Grant GGP02423

\section{1}

Haplotype structure and association testing of the peroxisome proliferator-activated receptor $\delta P P A R D$ with insulin resistance in a population-based sample 6,125 middle-aged subjects

N. Grarup ${ }^{1}$, J. Ek ${ }^{1,2}$, A. Albrechtsen ${ }^{1}$, G. Andersen ${ }^{1}$, K. Borch-Johnsen ${ }^{1,3}$, T. Jørgensen ${ }^{3}$, T. Hansen ${ }^{1}$, O. Pedersen ${ }^{1}$; ${ }^{1}$ Steno Diabetes Center, Gentofte, Denmark, ${ }^{2}$ Kennedy Institute, National Eye Clinic, Glostrup, Denmark, ${ }^{3}$ Research Centre for Prevention and Health, Glostrup University Hospital, Denmark.

Background and Aims: Studies in animals reveal that peroxisome proliferator-activated receptor $\delta$ (PPAR $\delta$ ) regulates glucose metabolism and insulin sensitivity both in the liver and skeletal muscles. Moreover, PPAR $\delta$ enhances fatty acid catabolism and in skeletal muscle PPAR $\delta$ additionally augments physical endurance, increases the proportion of slow twitch type I muscle fibres and increases oxidative metabolism thereby averting obesity. Thus, we hypothesized that variation in PPARD associates with insulin resistance and this impact is accentuated by interaction with known variants in PPAR and PPARGCl genes reported to increase the risk of insulin resistant traits. Materials and Methods: We studied variation in the coding region of PPARD in 64 type 2 diabetic patients and 24 healthy subjects selected for high or low physical fitness. Based upon the results of this variant detection and information derived from the HapMap data resource common variants and haplotype tagging single nucleotide polymorphisms (SNPs) selected using the Tagger approach were genotyped in the population-based Inter99 cohort of Danish whites $(n=6,125)$ using TaqMan allelic discrimination.

Results: Fifteen nucleotide variants were identified; four with an estimated minor allele frequency above $5 \%$. A total of twelve SNPs including haplotype tagging variants were genotyped. In the population-based Inter99 sample we studied quantitative metabolic traits and observed a significant association of rs2076167 and haplotypes including this variant and rs6902123 with measures of insulin resistance (fasting serum insulin, $p<0.001$; HOMA insulin resistance, $p=0.001$; and post-OGTT incremental area under the insulin curve, $p=0.03$ ) when evaluating subjects with treatment-naïve impaired glucose regulation (IFG, IGT, or screen-detected type 2 diabetes, $n=1,393)$. When examining subjects with normal glucose tolerance $(n=4,346)$ this effect was not evident. In addition, we tested for a potential epistatic effect of SNPs in PPARD and common variants in the PPARG, PPARA, PPARGC1A, and PPARGC1B genes on measures of insulin resistance. No epistatic effects were, however, identified.

Conclusion: Exhaustive evaluation of variation in PPARD reveals that SNPs and haplotypes of PPARD associate with measures of insulin resistance in subjects with treatmentnaïve impaired glucose regulation. These results suggest that variation in PPARD may influence insulin resistance at a critical and vulnerable time point in the pathogenesis of type 2 diabetes at which subjects are further strained by environmental and genetic risk modulators.

\section{2}

Association between the $\mathrm{C}-11377 \mathrm{G}$ promoter variant of the $A C D C$ gene and carotid intima thickness in healthy subjects

M. Walker ${ }^{1}$ et al., S. Patel ${ }^{1}$ et al.; A. Flyvbjerg ${ }^{2}$, M. Kozàkova ${ }^{3}$, J. Frystyk ${ }^{2}$, I. M. Ibrahim ${ }^{1}$ and the RISC investigators,

${ }^{1}$ School of Clinical Medical Science, Diabetes, University of Newcastle, United Kingdom, ${ }^{2}$ Medical Research Laboratories, Clinical Institute and Medical Department (Diabetes \& Endocrinology), Aarhus University Hospital, Denmark, ${ }^{3}$ Department of Internal Medicine, University of Pisa, Italy.

Background and Aims: The $A C D C$ gene encodes the protein adiponectin and lower adiponectin levels have been reported in patients with type 2 diabetes and coronary artery 
disease. Common variants in the promoter region of the $A C D C$ gene have been associated with adiponectin levels and type 2 diabetes. The purpose of this study was to examine the relationships between common variants in the $A C D C$ promoter region and carotid intima-media thickness, a marker of cardiovascular disease, in a cohort of healthy subjects participating in the RISC study (Relationship between Insulin Sensitivity and Cardiovascular disease study).

Materials and Methods: Healthy subjects aged 30-60 yrs were recruited at 19 centres in 14 European countries. B-mode ultrasound images of the common carotid artery were recorded by a single operator at each centre, and intima media thickness (cIMT) was measured in digitized end-diastolic frames in the central laboratory by a single reader. SNPs A11426G, G-11391A and C-11377G were genotyped using the TaqMan system (Applied Biosystems, Warrington, UK). Haplotypes were inferred using the PHASE program. Fasting adiponectin levels were normalised by log transformation and the geometric means are presented.

Results: The study cohort consists of 1325 subjects (597 men and 728 women) aged $43.8 \pm 8.3$ yrs (mean \pm SD), with a mean BMI of $25.5 \pm 4.1 \mathrm{~kg} / \mathrm{m}^{2}$. All SNPs were in Hardy-Weinberg equilibrium and common (minor allele frequency $>0.05$ ). Of the 3 SNPs, only C-11377G was significantly associated with cIMT. Carriers of the $\mathrm{G}$ allele had significantly greater cIMT values compared to $\mathrm{C}$ allele homozygotes (mean $\pm \mathrm{SE}$, $608 \pm 3.9$ vs. $596 \pm 3.3 \mu \mathrm{m}, \mathrm{p}=0.017$ ). This difference remained after adjusting for age, sex, recruitment centre and BMI $(p=0.02)$, and after inclusion of fasting adiponectin levels to these covariates $(p=0.025)$. The haplotype pair (diplotype) containing the alleles $-11426 \mathrm{~A},-11391 \mathrm{G}$ and $-11377 \mathrm{G}$ was significantly associated with higher cIMT values compared to all other diplotypes $(610 \pm 4.8$ vs. $595 \pm 3.4 \mu \mathrm{m}, \mathrm{p}=0.006)$. This difference remained significant after accounting for the same factors and covariates.

Conclusion: Variation at SNP C-11377G in the $A C D C$ gene promoter is associated with increased cIMT in healthy subjects, and is independent of circulating adiponectin levels. RISC is supported by the European Union and AstraZeneca

\section{3}

Studies of polymorphisms of the adiponectin encoding gene $A D I P O Q$ show an association of a $-11391 \mathrm{G}$ allele with insulin resistance in the general middle-aged population of whites

Y. H. Hamid ${ }^{1}$, S. A. Urhammer ${ }^{1}$, B. Carstensen ${ }^{1}$, A. Albrechtsen ${ }^{1}$, G. Andersen ${ }^{1}$, K. Borch-Johnsen ${ }^{1}$, T. Jørgensen ${ }^{2}$, T. Hansen ${ }^{1}$, O. Pedersen ${ }^{1}$;

${ }^{1}$ Steno Diabetes Center, Gentofte, ${ }^{2}$ Research Center for Prevention and Health, Glostrup University Hospital, Denmark.
Background and Aims: The $A D I P O Q$ gene at chromosome 3q27 encoding adiponectin, which plays an important role in glucose and lipid metabolism, is a positional and biological candidate gene for type 2 diabetes and the metabolic syndrome. Seven polymorphisms in the ADIPOQ gene $(\mathrm{a}-11426 \mathrm{~A}>\mathrm{G}$, a $-11391 \mathrm{G}>\mathrm{A}$, a $-11377 \mathrm{C}>\mathrm{G}$, a $4041 \mathrm{~A}>\mathrm{C}, \mathrm{a}+45 \mathrm{~T}>\mathrm{G}, \mathrm{a}+276 \mathrm{G}>\mathrm{T}$, and $\mathrm{a}+2019 \mathrm{del} \mathrm{A})$ have been reported to associate with type 2 diabetes, insulin resistance and/or circulating adiponectin levels. The aim of the present study was to examine the potential effect of these seven polymorphisms on susceptibility of type 2 diabetes, the metabolic syndrome, and related phenotypes in a large-scale study of Danish whites.

Materials and Methods: Seven polymorphisms of the $A D I P O Q$ gene were genotyped in the population-based Inter99 study cohort $(n=6,514)$ and in a group of type 2 diabetic patients $(n=1098)$ by analysis of PCR-generated primer extension products using high-throughput chipbased matrix-assisted laser desorption/ionisation time-offlight mass spectronomy.

Results: No association of these variants with type 2 diabetes or the WHO-defined metabolic syndrome were demonstrated. A meta-analysis including previously published case-control studies of the examined SNPs and our own results did not revealed any association with type 2 diabetes. However, we found an association of the $-11391 \mathrm{G}$ allele with higher fasting serum insulin levels $(\mathrm{p}=0.005)$ and higher HOMA insulin resistance index $(p=0.007)$ in a population based sample of 4,432 middle-aged glucose tolerant subjects.

Conclusion: Our data suggest that the $-11391 \mathrm{G}>\mathrm{A}$ polymorphism of the $A D I P O Q$ gene may contribute to the interindividual variation of serum insulin and insulin sensitivity, whereas the examined polymorphisms of the $A D I P O Q$ are not directly associated with type 2 diabetes or the metabolic syndrome in the Danish Caucasians.

The authors thank Birger Thorsteinsson, MD, DMSc for the collection of some of the type 2 diabetic patients. The study was supported by the Danish Medical Research Council and the Danish Diabetes Association.

0344

The A-11426G and G-11391A promoter variants of the $A C D C$ gene influence adiponectin levels in healthy subjects

S. Patel ${ }^{1}$ et al., A. Flyvbjerg ${ }^{2}$, J. Frystyk ${ }^{2}$, A. Mari ${ }^{3}$, M. Walker ${ }^{1}$ et al. and the RISC investigators,

${ }^{1}$ School of Clinical Medical Science, Diabetes, University of Newcastle, United Kingdom, ${ }^{2}$ Medical Research Laboratories, Clinical Institute and Medical Department (Diabetes \& Endocrinology), Aarhus University Hospital, Denmark, ${ }^{3}$ ISIB-CNR, National Research Council, Padova, Italy. 
Background and Aims: The $A C D C$ gene encodes the protein adiponectin and decreased circulating adiponectin levels have been observed in insulin resistance and type 2 diabetes. Single nucleotide polymorphisms (SNPs) in the promoter region of the $A C D C$ gene have been associated with adiponectin levels and type 2 diabetes. We investigated the role of the $A C D C$ SNPs A-11426G, G-11391A, C$11377 \mathrm{G}$ and their haplotypes with adiponectin levels and insulin sensitivity in a cohort of healthy subjects participating in the RISC study (Relationship between Insulin Sensitivity and Cardiovascular disease study).

Materials and Methods: Healthy subjects aged 30-60 yrs were recruited at 19 centres in 14 European countries. Each subject underwent anthropometric and metabolic assessment (standard OGTT), and whole body insulin sensitivity (M value) was assessed by euglycaemic hyperinsulinaemic (40 $\mathrm{mU} / \mathrm{m}^{2} / \mathrm{min}$ ) clamp. SNPs were genotyped using the TaqMan system (Applied Biosystems, Warrington, UK). Haplotypes were inferred using the PHASE program. Fasting plasma adiponectin levels were normalised by $\log$ transformation and the geometric means are presented.

Results: The study cohort consists of 1325 subjects (597 men and 728 women) aged $43.8 \pm 8.3$ yrs (mean \pm SD), with a mean BMI of $25.5 \pm 4.1 \mathrm{~kg} / \mathrm{m}^{2}$. All SNPs were in HardyWeinberg equilibrium and common (minor allele frequency>0.05). Fasting plasma adiponectin was significantly correlated with insulin sensitivity $(\mathrm{r}=0.33, \mathrm{p}<0.001)$ and inversely correlated with fasting serum insulin $(-0.34$, $\mathrm{p}<0.001$ ). Of the 3 SNPs, A-11426G and G-11391A were associated with fasting plasma adiponectin levels. For SNP A-11426G, subjects homozygous for the A allele had significantly higher plasma adiponectin levels compared to $\mathrm{G}$ allele carriers (geometric mean (interquartile range) 7.7 (5.6-10.5) vs. $7.2(5.4-9.6) \mathrm{mg} / \mathrm{l}, \mathrm{p}=0.015)$. This relationship remained significant after adjusting for age, sex, recruitment centre and $\mathrm{BMI}(\mathrm{p}=0.008)$. Carriers of the $\mathrm{A}$ allele of SNP -11391 had significantly higher plasma adiponectin levels compared to the G/G genotype (9.1 (6.9-12.2) vs. 7.4 (5.4-9.7) $\mathrm{mg} / \mathrm{l}, \mathrm{p}<0.0001)$ and remained significant after adjusting for the same factors and covariates $(p<0.0001)$. The haplotype pair (diplotype) containing the alleles $-11426 \mathrm{G},-11391 \mathrm{G}$ and $-11377 \mathrm{C}$ was significantly associated with lower adiponectin levels when compared to all other diplotypes $(6.8$ (5.2-8.7) vs. 7.6 (5.6-10.3) $\mathrm{mg} / \mathrm{l}, \mathrm{p}=0.008)$. The difference remained significant after accounting for the same factors and covariates. However, there were no associations between the A11426G and G-11391A SNPs (individually or as diplotypes) with the $M$ value and fasting insulin levels, before and after correction for the key covariates.

Conclusion: We confirm that variation in the $A C D C$ gene promoter at SNPs A-11426G and G-11391A influences circulating adiponectin levels, but not measures of insulin sensitivity.

RISC is supported by the European Union and AstraZeneca

\section{5}

The promoter region of adiponectin gene is a determinant in modulating insulin sensitivity in childhood obesity

S. Zavarella ${ }^{1}$, A. Petrone ${ }^{1}$, S. Zampetti ${ }^{1}$, M. Spoletini ${ }^{1}$, I. Alemanno ${ }^{1}$, G. Leto ${ }^{1}$, A. Vania ${ }^{2}$, R. Buzzetti ${ }^{1}$;

${ }^{1}$ Clinical Science, "La Sapienza" University, Rome, ${ }^{2}$ Paediatrics, "La Sapienza" University, Rome, Italy.

Background and Aims: Adiponectin (ACDC) is a recently discovered adipose tissue-specific cytokine that plays a role in modulating insulin sensitivity. Different single-nucleotide polymorphisms (SNPs) identified in the adiponectin gene, were found to be associated with the risk of diseases such as: obesity, insulin resistance, type 2 diabetes (T2DM) and metabolic disorders. The aim of our study was to assess the association of the $-11391 \mathrm{G}>\mathrm{A},-11377 \mathrm{C}>\mathrm{G},+45 \mathrm{~T}>\mathrm{G}$ and $+276 \mathrm{G}>\mathrm{T}$ adiponectin SNPs and expected haplotypes with the metabolic abnormalities of insulin resistance in 270 overweight/obese children and to distinguish the effect of each SNP independently on the investigated phenotypes. Materials and Methods: The SNPs were genotyped using the flurogenic $5^{\prime}$ nuclease assay application of the ABI PRISM 7900 HT Sequence Detection System. We performed ANOVA analysis to obtain initial overall p-values without assuming any specific genetic model. The effect of the polymorphisms on quantitative variables was investigated using multiple linear regression analysis. The values were adjusted for BMI-z, age and pubertal stage. Haplotype analysis was performed by using THESIAS software. Serum adiponectin levels were performed in 150 children and determined by radioimmunoassay (RIA Kit, LINCO Research Inc, USA).

Results: The GG genotype at the -11391 locus was associated with higher fasting insulin levels, HOMA-IR index and lower adiponectin levels compared to GA+AA genotypes $(\mathrm{p}=0.01, \mathrm{p}=0.002$ and $\mathrm{p}=0.03$, respectively). Children heterozygous or homozygous for $\mathrm{G}$ allele at the -11377 locus showed higher fasting glucose $(p=0.001$ for both), fasting insulin ( $\mathrm{p}=0.001$ for both), HOMA-IR index $(\mathrm{p}<0.001$ for both), triglyceride levels $(0.02$ and $\mathrm{p}=0.03$, respectively) and lower adiponectin levels ( $\mathrm{p}=0.002$ and $\mathrm{p}=0.02$, respectively) compared to $\mathrm{C}$ homozygous. The $+45 \mathrm{G}$ carriers showed higher fasting and $2 \mathrm{~h}$ glucose levels $(p=0.01$ for both) and lower adiponectin levels $(p=0.02)$ compared to non carriers. The relation between SNPs and insulin resistance phenotypes does not persist after adjust- 
ing for adiponectin levels ( $p>0.05$ ). The GGTG haplotype was associated with insulin resistance parameters $(p<0.05)$ compared to all the other haplotypes. Moreover the haplotype analysis suggested that considering the same haplotypic background, each of the three polymorphisms exerted an independent effect on investigated variables. The promoter haplotype GG, was strongly associated with a significant decrease of adiponectin levels and with higher degree of insulin resistance $(\mathrm{p}=0.01$ for both)

Conclusion: The $-11391 \mathrm{G}>\mathrm{A},-11377 \mathrm{C}>\mathrm{G}$ and $+45 \mathrm{~T}>\mathrm{G}$ SNPs seems to be associated with insulin resistance syndrome in overweight/obese Italian children. These polymorphisms independently influence the investigated variables. However the effect of $+45 \mathrm{~T}>\mathrm{G}$ SNP seems to be marginal compared to the promoter SNPs. A major finding of the present study is the association of the promoter SNPs haplotype with lower adiponectin levels and consecutively with insulin sensitivity. Thus the influence of the $-11391 \mathrm{G}>\mathrm{A},-11377 \mathrm{C}>\mathrm{G}$ and $+45 \mathrm{~T}>\mathrm{G}$ SNPs and related haplotypes on insulin resistance may be fully explained by their correlation on adiponectin levels.

Supported by MIUR 20032003061834 _004

\section{6}

The $-11377 C>G$ promoter variant of the adiponectin gene predicts vascular events in male coronary patients G. Hoefle ${ }^{1}$, A. Muendlein ${ }^{2}$, C. H. Saely ${ }^{1,2}$, L. Risch ${ }^{1}$, P. Rein ${ }^{2}$, L. Koch ${ }^{2}$, F. Schmid ${ }^{2}$, S. Aczel ${ }^{1,2}$, P. Langer ${ }^{2}$, H. Drexel ${ }^{1,2}$;

${ }^{1}$ Department of Internal Medicine, Academic Teaching Hospital Feldkirch, ${ }^{2}$ VIVIT Institute, VIVIT Institute, Feldkirch, Austria.

Background and Aims: Low serum levels of the adipocyte-derived peptide adiponectin are associated with obesity, type 2 diabetes, and cardiovascular disease. The -11377 $\mathrm{C}>\mathrm{G}$ promoter variant of the adiponectin gene recently has been linked to decreased adiponectin levels. No prospective data demonstrating an increased cardiovascular risk in individuals with the $\mathrm{G}$ versus the $\mathrm{C}$ allele are available. We therefore aimed at investigating the impact of the -11377 $\mathrm{C}>\mathrm{G}$ polymorphism on the incidence of vascular events in a high risk cohort of male coronary patients.

Materials and Methods: In a consecutive series of 402 men who had undergone coronary angiography for the evaluation of coronary artery disease, vascular events were recorded over $4.1 \pm 0.4$ years.

Results: The prevalence rates of the $-11377 \mathrm{CC}, \mathrm{GC}$, and GG genotypes among our patients were 56.5\%, 37.1\% and $6.5 \%$, respectively. At baseline, serum adiponectin levels decreased significantly from the CC over the GC to the GG genotype $\left(\mathrm{p}_{\text {trend }}=0.003\right)$, and the prevalence of significant coronary stenoses of at least $50 \%$ increased gradually from the $\mathrm{CC}$ over the GC to the GG genotype $(63.9 \%, 73.2 \%$, and $88.5 \%$, respectively; $\left.\mathrm{p}_{\text {trend }}=0.004\right)$. Prospectively, the presence of the $\mathrm{G}$ allele of the adiponectin gene -11377 $\mathrm{C}>\mathrm{G}$ polymorphism strongly and significantly predicted future vascular events (adjusted hazard ratio=1.703 [1.0742.699]; $\mathrm{p}=0.023$ ). Adjusted hazard ratios were 1.582 [0.971-2.577] and 2.403 [1.108-5.214] for patients with the $\mathrm{CG}$ and $\mathrm{GG}$ genotypes when compared to patients with the CC genotype ( $\left.p_{\text {trend }}=0.011\right)$.

Conclusion: Among male coronary patients the -11377 $\mathrm{C}>\mathrm{G}$ promoter variant of the adiponectin gene is i) associated with decreased serum adiponectin levels, ii) correlated with an increased prevalence of significant coronary stenoses and iii) strongly predictive of future vascular events.

\section{PS 10}

\section{Obesity genes}

\section{7}

Cannabinoid receptor 1 gene variation increases risk for obesity and type 2 diabetes and modulates phenotypes related to endocannabinoid-mediated physiological pathways

K. J. Ward ${ }^{1}$, P. Boutin ${ }^{1}$, J.-C. Chevre ${ }^{1}$, S. Lobbens ${ }^{1}$, C. Lecoeur ${ }^{1}$, C. Wachter ${ }^{1}$, C. Dina ${ }^{1}$, P. J. Larsen ${ }^{2}$, L. B. Tankó ${ }^{3}$, F. F. Horber ${ }^{4}$, G. Charpentier ${ }^{5}$, B. Balkau ${ }^{6}$, D. Meyre $^{1}$, P. Froguel ${ }^{1,7}$;

${ }^{1}$ CNRS 8090, Institut de Biologie de Lille, Institut Pasteur, France, ${ }^{2}$ Rheoscience, Rodovre, Denmark, ${ }^{3}$ Center for Clinical and Basic Research, Ballerup, Denmark, ${ }^{4}$ Klinik Hirslanden, Zürich, Switzerland, ${ }^{5}$ Endocrinology-Diabetology Unit, Corbeil-Essonnes Hospital, Corbeil-Essonnes, France, ${ }^{6}$ Inserm u780-ifr69, for the Data from an Epidemiologic Study on the Insulin Resistance Syndrome (D.E.S.I. R.) Study Group, Villejuif, France, ${ }^{7}$ Hammersmith Genome Centre and Genomic Medicine, Imperial College, London, United Kingdom.

Background and Aims: The endocannabinoid pathway contributes to the regulation of metabolism and food intake. To determine whether variation in the Cannabinoid Receptor gene (CNR1) modulates susceptibility to obesity, type 2 diabetes (T2DM) and related phenotypes we used large scale genetic epidemiology.

Materials and Methods: By sequencing all CNR1 coding regions, exon-intron boundaries and regulatory regions, 68 Single Nucleotide Polymorphisms were identified, 36 had a 
minor allele frequency (MAF) $\geq 5 \%$. Twenty-one tagged polymorphisms, which summarize CNR1 locus genetic variability were genotyped in French Caucasian obese adults and children $(n=2,016)$, T2DM cases $(n=1,519)$ and controls $(n=1,299)$. The seven polymorphisms showing evidence of an association in more than one case/control study, underwent further familial analysis. Potential modulation of metabolic, fat mass, bone mass density and behavioural traits were then analyzed in 819 obese children, 865 Swiss obese adults, 5,065 middle-aged adults from a general French population (D.E.S.I.R.), and 1,474 postmenopausal Danish women.

Results: We identified five polymorphisms associated with both obesity (Odds ratios (ORs) ranging from 1.21 to 1.37; $\mathrm{p}<0.03$ to $<0.0006$ ) and T2DM (ORs 1.21 to $1.28 ; \mathrm{p}<0.01$ to $<0.008$ ) and 4 with obesity only (ORs 1.17 to 1.36 ; $\mathrm{p}<0.05$ to $<0.00009$ ). Quantitative trait analysis in these studies showed that the CNR1 polymorphisms modulate food intake behaviour, smoking habits, alcohol consumption, fat mass, bone mass parameters, insulinaemia, glycaemia, and lipid and leptin levels.

Conclusion: Several CNRI polymorphisms increase the risk of obesity and T2DM and modulate body composition, glucose and lipid metabolisms and addictive behaviours, confirming a major role of the endocannabinoid system in the regulation of energy balance. This data from 12,238 European subjects provides physiological support for the use of cannabinoid receptor 1 antagonists for obesity and associated metabolic complications and further exploration of the endocannabinoid pathway using pharmaco-genomics is required.

\section{8}

An exon4 $+665 \mathrm{C}>\mathrm{T}$ polymorphism of the gene encoding Wingless-Type Mammary Tumor Virus Integration Site Family Member 3A (WNT3A) protects against obesity in a general population of whites

K. L. Andersen ${ }^{1}$, D. P. Jensen ${ }^{1}$, S. S. Torekov ${ }^{1}$, S. Maeda ${ }^{2}$, Y. Nakamura ${ }^{2}$, K. Borch-Johnsen ${ }^{1}$, T. Jørgensen ${ }^{3}$,

T. Hansen ${ }^{1}$, O. Pedersen ${ }^{1}$;

${ }^{1}$ Steno Diabetes Center, Gentofte, Denmark, ${ }^{2}$ SNP Research Center, RIKEN, Yokohama, Kanagawa, Japan, ${ }^{3}$ Copenhagen County, Glostrup University Hospital, Research Centre for Presention and Health, Glostrup, Denmark.

Background and Aim: Evidence suggests that WNTsignaling and genes related to the WNT-signaling pathway may be important regulators for adipogenesis. The WNT3A gene encodes Wingless-Type Mammary Tumor Virus Integration Site Family Member 3A, which in recent studies has been shown to possess an inhibitory effect on adipogenesis. The aim of this study was to examine a known $W N T 3 A$ gene polymorphism for an association with obesity and related quantitative metabolic traits.

Materials and Methods: One polymorphism (WNT3A exon 4+665 C>T, rs752107) was selected from the IMSJST (Institute of Medical Science-Japan Science and Technology Agency) Japanese SNP database for genotyping within the WNT3A gene locus. The WNT3A $665 \mathrm{C}>\mathrm{T}$ polymorphism was genotyped using the PCR-Invader technology in the population-based Inter99 study cohort of 5,846 middleaged and treatment-naïve subjects. Within this cohort a case-control study for obesity was undertaken in 963 obese (BMI $\geq 30 \mathrm{~kg} / \mathrm{m}^{2}$ ) subjects and 2,588 lean, glucose-tolerant control participants $\left(\mathrm{BMI}<25 \mathrm{~kg} / \mathrm{m}^{2}\right.$ ). Quantitative trait studies of metabolic variables were carried out in all 5,846 subjects of the Inter99 sample.

Results: The WNT3A $665 \mathrm{C}>\mathrm{T}$ T-allele frequency was lower in obese participants compared to control persons (30.9\% [95\% CI: $28.9-33.0]$ vs. $34.6 \%$ [95\% CI: $33.3-$ 35.9], $\mathrm{P}=0.003$; Odds ratio: 0.73 [CI: 0.56-0.94]). The difference in genotype distribution was CC/CT/TT 47/44/ $9 \%$ (cases) vs. $42 / 46 / 12 \%$ (control), respectively ( $\mathrm{P}=0.01$ ). Furthermore, in the quantitative trait studies, there was a significant difference in BMI between the different genotype carriers (mean $\pm \mathrm{SD}$ : BMI CC: $26.36 \pm 4.55$; CT: $26.12 \pm 4.46$ and TT: $25.76 \pm 4.38 \mathrm{~kg} / \mathrm{m}^{2}, \mathrm{P}=0.002$ ). There was no association between variation of the WNT3A gene and other quantitative anthropometric or metabolic traits.

Since it has been suggested that WNT signaling and genes related to the WNT signaling pathway are important regulators for adipogenesis we examined if there was a potential gene-gene interaction between the WNT3A 665 $\mathrm{C}>\mathrm{T}$ polymorphism and the PPARG Pro12Ala polymorphism (rs1801282). Using a linear model we observed a significant epistatic effect $(\mathrm{P}=0.03)$ between the two polymorphisms. This epistatic effect was more marked $(\mathrm{P}=0.006)$ when assuming equal effect for Pro/Ala and Ala/Ala. The 2-loci genotype with protective alleles from both polymorphisms $(\mathrm{n}=159)$ had lower BMI (mean \pm SE: $\left.24.7 \pm 0.6 \mathrm{~kg} / \mathrm{m}^{2}\right)$ compared to the other genotypes $(\mathrm{n}=5,620)\left(26.2 \pm 0.1 \mathrm{~kg} / \mathrm{m}^{2}, \mathrm{P}=0.00007\right)$.

Conclusion: The minor T-allele of the WNT3A $665 \mathrm{C}>\mathrm{T}$ polymorphism may protect against obesity in middle-aged whites. Also, the $W N T 3 A 665 \mathrm{C}>\mathrm{T}$ polymorphism interacts with the PPARG Pro12Ala polymorphism to influence BMI. 


\section{9}

$G A D 2-243 A>G$ polymorphism associates with measures of obesity and glycaemia and interacts with the $G C K-30 G>A$ polymorphism in a population-based sample of 5,857 middle-aged people

S. I. Castella ${ }^{1}$, T. W. Boesgaard ${ }^{1}$, G. Andersen ${ }^{1}$, A. Albrechtsen ${ }^{1}$, T. Spars $\varnothing^{1}$, K. Borch-Johnsen ${ }^{1,2}$, T. Jørgensen ${ }^{2}$, T. Hansen ${ }^{1}$, O. Pedersen ${ }^{1}$;

${ }^{1}$ Steno Diabetes Center, Gentofte, ${ }^{2}$ Research Centre for Prevention and Health, Glostrup University Hospital, Denmark.

Background and Aims: GAD2 encoding the glutamic acid decarboxylase enzyme GAD65 is located on chromosome $10 \mathrm{p} 12$, a region which has been linked to morbid obesity. A GAD2 $-243 \mathrm{~A}>\mathrm{G}$ polymorphism is reported to associate with morbid obesity among French people. The aim of the present study was in a population-based sample of middleaged white Danes to investigate the relationship between the GAD2 $-243 \mathrm{~A}>\mathrm{G}$ polymorphism and estimates of obesity and glycaemia, and to examine for epistatic interactions with other genetic variants known to influence obesity and glycaemia.

Materials and Methods: The GAD2 variant was genotyped in 5,857 treatment-naïve subjects from a population-based sample (Inter99) (2,920 men, 2,937 women, age 46 \pm 8 years (mean \pm SD), BMI $26.2 \pm 4.5 \mathrm{~kg} / \mathrm{m}^{2}$ ). All subjects underwent a standard $75 \mathrm{~g}$ OGTT. The GAD2 $-243 \mathrm{~A}>\mathrm{G}$ variant was genotyped using chip-based matrix-assisted laser desorption/ionization time-of-flight mass spectrometry.

Results: The G-allele frequency among 2,582 subjects with a BMI of $<25 \mathrm{~kg} / \mathrm{m}^{2}$ was $19.5 \%$ (95\% CI $18.4-20.6$ ) while among 968 subjects having BMI $\geq 30 \mathrm{~kg} / \mathrm{m}^{2}$ the frequency was $17.1 \%(15.5-18.8)(P=0.03)$, giving an odds ratio of $0.90(0.74-0.98)$ for the difference in allele frequencies. Also, the genotype distributions were different between the groups: BMI $<25 \mathrm{~kg} / \mathrm{m}^{2}$ AA $65 \%$, AG $31 \%$, GG $4 \%$; BMI $\geq 30 \mathrm{~kg} / \mathrm{m}^{2}$ AA $69 \%$, AG $29 \%$, GG 3\%, $P=0.01$. When analysing data in the total population of 5,857 treatmentnaïve subjects GG carriers had lower fasting and postOGTT plasma glucose: AA 5.6 \pm 0.8 , AG $5.5 \pm 0.8$, GG $5.5 \pm 0.8 \mathrm{mmol} / \mathrm{l}, P=0.008 ; 30$-min post-OGTT AA $8.7 \pm 1.9$, AG $8.6 \pm 1.9$, GG $8.6 \pm 2.0 \mathrm{mmol} / \mathrm{l}, P=0.04$. Similarly, in the subgroup of glucose-tolerant subjects $(n=4,431)$ GG carriers had lower fasting plasma glucose concentrations: AA $5.3 \pm 0.4$, AG 5.3 \pm 0.4 , GG $5.2 \pm 0.4 \mathrm{mmol} / 1, P=9 \cdot 10^{-5}$. No epistatic interactions between the GAD2 variant and variants in genes reported to associate with obesity (PPARGC1A, PPARGC1B, PPARG2, and NPY2R) were observed. Since GAD65 is also expressed in pancreatic $\beta$-cells we examined for a potential interaction between the GAD2 $-243 \mathrm{~A}>\mathrm{G}$ polymorphism and several variants in the $T C F 1 A, H N F 4 A, K C N J 11$, and $G C K$ genes with known impact on fasting plasma glucose. We observed a significant epistatic effect $(P=0.002)$ and a significant global statistic $\left(P=6 \cdot 10^{-9}\right)$ on fasting plasma glucose between a common $G C K-30 \mathrm{~A}>\mathrm{G}$ variant and the GAD2 $-243 \mathrm{~A}>\mathrm{G}$ polymorphism. Subjects homozygous for the $G C K$ A-allele and homozygous for the major GAD2 A-allele had higher fasting plasma glucose concentrations: AA/AA $5.8 \pm 1.3 \mathrm{mmol} / 1$ versus all remaining genotype combinations $5.5 \pm 0.8 \mathrm{mmol} / 1, P<10^{-5}$ when adjusted for sex, age, and BMI. Comparing glucose-tolerant subjects $(n=4,387$, AA/AA carriers $=54$ ) with hyperglycaemic subjects (IGT, IFG, and T2DM, n=1,531, AA/AA carriers $=38$ ) we found that subjects homozygous for the risk alleles of both polymorphisms had an estimated OR of 2.04 (1.31-3.16) for having hyperglycaemia $(P=0.001)$.

Conclusion: The GAD2 $-243 \mathrm{~A}>\mathrm{G}$ polymorphism associates with obesity and fasting and post-OGTT glycaemia in a population of middle-aged whites. Moreover, the GAD2 $243 \mathrm{~A}>\mathrm{G}$ polymorphism may interact with the $G C K-30 \mathrm{~A}>\mathrm{G}$ variant to influence the fasting plasma glucose levels.

0350

ENPP1 K121Q associates with severe obesity and T2D, and predicts overweight/class III obesity and familial glucose intolerance/T2D incidence in the prospective DESIR Study

D. Meyre ${ }^{1}$, C. Samson ${ }^{1}$, V. Vatin ${ }^{1}$, J. Tichet ${ }^{2}$, M. Marre ${ }^{3}$, B. Balkau ${ }^{4}$, P. Froguel ${ }^{1,5}$;

${ }^{1}$ UMR8090, CNRS, Lille, France, ${ }^{2}$ Institut Régional Pour la Santé, IRPS, Tours, France, ${ }^{3}$ U695, INSERM, Paris, France, ${ }^{4}$ U780, INSERM, Villejuif, France, ${ }^{5}$ Genome Centre and Genomic Medicine, Imperial College, London, United Kingdom.

Background and Aims: Variation in Ecto-Nucleotide Pyrophosphatase Phosphodiesterase 1 (ENPP1) gene associate with childhood and adult severe familial obesity and type 2 diabetes (T2D) in the French population. We analyzed the K121Q, IVS20delT-11 and A/G+1044TGA ENPP1 polymorphisms in a large French Caucasian cohort followed 9 years, D.E.S.I.R., to replicate previous results, and to assess the predictive value of ENPP1 polymorphisms on the risk of developing obesity and/or T2D in a French Caucasian general population.

Materials and Methods: We genotyped 5,153 middle-aged subjects (at baseline: men/women ratio: 49.6/50.4\%; age: $47.2 \pm 10.0$ y., body mass index (BMI): $24.7 \pm 3.8 \mathrm{~kg} / \mathrm{m}^{2}$ ). Genotyping used Taqman (Applied Biosystem) and Light Typer (Roche) technology. $\chi^{2}$ test and $\mathrm{P}$ values were empirically computed with the CLUMP program. 
Results: At baseline, the Q121Q genotype was associated with higher risk of class II obesity (BMI $\geq 35 \mathrm{~kg} / \mathrm{m}^{2}$; $\mathrm{OR}=3.37, \mathrm{p}=0.01$ ), class III obesity (BMI $\geq 40 \mathrm{~kg} / \mathrm{m}^{2}$; $\mathrm{OR}=6.23, \mathrm{p}=0.009), \mathrm{T} 2 \mathrm{D}(\mathrm{OR}=2.29, \mathrm{p}=0.04), \mathrm{T} 2 \mathrm{D}$ with familial history $(\mathrm{OR}=5.80, \mathrm{p}=0.00004)$, glucose intolerance (GI) or T2D with familial history of T2D $(\mathrm{OR}=2.89$, $\mathrm{p}=0.003$ ) when affected individuals were compared to the 1,245 lean normal glucose tolerant subjects (BMI $<25 \mathrm{~kg}$ / $\mathrm{m}^{2}$ ). Among normal weight participants at inclusion, the Q121 allele carriers had a trend towards an increased risk to develop overweight (BMI $\geq 27 \mathrm{~kg} / \mathrm{m}^{2} ; \mathrm{OR}=1.18, \mathrm{p}=0.06$ ) and class III obesity $(\mathrm{OR}=2.14, \mathrm{p}=0.07)$ during 9-year follow-up. In addition, 121Q allele increased the incidence of impaired glucose tolerance or frank T2D in context of familial T2D $(\mathrm{OR}=1.42, \mathrm{p}=0.04)$. IVS20delT-11 and $\mathrm{A} / \mathrm{G}+$ 1044TGA polymorphisms didn't associate with any trait at baseline and during the follow-up, and the [Q-delT-G] haplotype did not show more significant results than the K121Q polymorphism analyzed alone.

Conclusion: We confirmed in a general French population the association of K121Q polymorphism with severe adult obesity and T2D. Our results also suggest a potential predictive value of K121Q on the risk of developing overweight/class III obesity and familial impaired glucose tolerance or frank T2D.

Supported by a grant ALFEDIAM

\section{1}

Large-scale studies of association between the functional ADRB1 Arg389Gly polymorphism and obesity and hypertension

A. P. Gjesing ${ }^{1}$, G. Andersen ${ }^{1}$, C. Glümer ${ }^{1,2}$,

K. Borch-Johnsen ${ }^{1,2}$, T. Jørgensen ${ }^{2}$, T. Hansen ${ }^{1}$,

O. Pedersen ${ }^{1}$;

${ }^{1}$ Steno Diabetes Center, Gentofte, Denmark, ${ }^{2}$ Research Centre for Prevention and Health, Glostrup University Hospital, Denmark.

Background and Aims: The $\beta$-adrenergic receptors are expressed in several tissues and are activated by catecholamines as part of the sympathetic nervous system. Activation of the $\beta_{1}$-adrenergic receptor in adipocytes and cardiac myocytes causes increased lipolysis and enhanced cardiac output, respectively. Variants in the gene encoding this cell surface receptor, $\beta_{1}$-adrenergic receptor (ADRB1), have therefore been hypothesised to be involved in the pathogenesis of both obesity and hypertension. The frequent and functional $A D R B 1$ Arg389Gly variant influencing the adenylyl cyclase activity of the receptor has with ambiguous results been examined for association with both obesity and hypertension. For clarifying the potential impact of this variant in relation to obesity and hypertension we examined the Arg389Gly variant in a relatively large-scale study sample of middle-aged subjects.

Materials and Methods: The ADRB1 Arg389Gly polymorphism (rs1801253) was genotyped in a total of 7,677 Danish whites (3,886 men, 3,791 women) using a chip-based matrixassisted laser desorption/ionization time-of-flight mass spectrometry of PCR-generated primer extension products.

Results: A weak association between the codon 389 Glyallele and obesity defined as BMI $\geq 30 \mathrm{~kg} / \mathrm{m}^{2}$ was observed comparing Gly-allele frequencies (non-obese $n=6,108$, $25.2 \%$ [24.4-25.9] vs. obese $n=1,540,26.9$ [25.4-28.5], $p=0.05$ ) and genotype distribution (non-obese $\operatorname{Arg} / \operatorname{Arg}$ 56\%, Arg/Gly 37\%, Gly/Gly 6\% vs. obese Arg/Arg 53\%, Arg/Gly $41 \%$, Gly/Gly 7\%, $p=0.05$ ). No association was observed in quantitative trait analyses of BMI, waist circumference, waist-to-hip ratio, or fasting serum lipid concentrations (triglyceride, total and HDL cholesterol). Moreover, no association was observed for WHO-defined hypertension; however, in quantitative trait analyses carriers of the Gly-allele had a slightly lower diastolic blood pressure $(p=0.01)$, systolic blood pressure $(p=0.02)$, and mean arterial blood pressure $(p=0.008)$.

Conclusion: Despite the observed minor influence of the ADRB1 Arg389Gly polymorphism on BMI and blood pressure our results suggest that this common gene variant per se does not have any major impact in the pathogenesis of obesity and hypertension in the examined population of Danish whites.

\section{2}

Quantitative traits related to obesity: heritability, candidate genes and the EFPTS twins study

N. Y. P. Souren, R. G. J. Janssen, A. D. C. Paulussen; Department of Genetics and Cell Biology, Division Population Genetics, NUTRIM, Maastricht University, The Netherlands.

Background and Aims: Both lifestyle factors and genetic predisposition play a role in the development of obesity. In the present study the contribution of genetic and environmental factors on the phenotypic variation of several obesity-related traits were determined by making use of the classical twin study approach. In order to explain (part of) the genetic variance, sib-pair linkage analyses were performed using microsatellite markers located near $(<1.5 \mathrm{cM})$ five obesityrelated genes, consecutively leptin $(L E P)$, leptin receptor $(L E P R)$, adiponectin $(A P M 1)$, peroxisome proliferative activated receptor gamma $(P P A R \gamma)$ and resistin $(R E T N)$.

Materials and Methods: In the present study participated 480 monozygotic (MZ) and 276 dizygotic (DZ) healthy 
twins (388 men, mean age 25.6) recruited from the East Flanders Prospective Twin Survey (EFPTS). All twins have undergone a clinical investigation where several obesityrelated traits have been measured. Genetic (heritability) and environmental variance components were estimated using the structural equation modeling approach, which is implemented in the Mx package. Subsequently, variancecomponent linkage analyses were carried out using both the statistical programs $\mathrm{Mx}$ and Merlin. When appropriate, traits were adjusted for possible confounders including sex, (gestational) age, parity, height and adiposity.

Results: Heritabilities of body weight, body mass index (BMI), fat body weight, lean body weight, waist to hip ratio (WHR) and sum of four skin folds were high and ranged between 70 and $83 \%$. Total cholesterol, triglycerides, HDLcholesterol, LDL-cholesterol, total/HDL cholesterol ratio, leptin and free fatty acids concentrations had heritabilities varying from 37 to $79 \%$. Birth weight had a low heritability of $24 \%$. No sex differences in heritability estimates were observed. Marker D7S530 (LEP) showed suggestive linkage with BMI (Mx LOD=1.70; Merlin LOD=1.01), fat body weight $(\mathrm{Mx} L O D=1.33$; Merlin $\mathrm{LOD}=1.54)$ and WHR $(\mathrm{Mx}$ $\mathrm{LOD}=1.39$; Merlin $\mathrm{LOD}=1.33$ ) and in Merlin with birth weight (LOD=1.57). Marker D3S1602 (APMI) showed suggestive linkage with HDL (Mx $\mathrm{LOD}=1.59$; Merlin $\mathrm{LOD}=1.65)$ and leptin $(\mathrm{Mx} \mathrm{LOD}=2.16$; Merlin $\mathrm{LOD}=2.79$ ) and in $\mathrm{Mx}$ with triglycerides concentrations (LOD=1.02). Finally, marker D3S3686 (APM1) showed suggestive linkage with triglycerides levels (Mx LOD=1.24; Merlin LOD=1.02). Conclusion: This study illustrates that the genetic contribution to obesity-related intermediate traits is high. In addition, linkage analysis indicates that polymorphisms close to the $L E P$ and $A P M 1$ gene may be responsible for a part of this genetic contribution to several of these obesityrelated intermediate traits.

Supported by the Dutch Diabetes Research Foundation, Fund of Scientific Research, Flanders and the Association for Scientific Research in Multiple Births

\section{3}

Endothelial nitric oxide synthase (eNOS) E298D and T786C gene polymorphisms do not affect post challenge insulin levels in non diabetic hypertensive men

C. Fotino ${ }^{1}$, D. Lucchesi ${ }^{1}$, L. Pucci ${ }^{1}$, S. Triscornia ${ }^{1}$, G. Dell'Omo ${ }^{2}$, R. Pedrinelli ${ }^{2}$, R. Miccoli ${ }^{1}$, G. Penno ${ }^{1}$, S. Del Prato ${ }^{1}$;

${ }^{1}$ Endocrinology and Metabolism, University of Pisa, Italy,

${ }^{2}$ Cardiothorax, University of Pisa, Italy.

Background and Aims: The endothelial nitric oxide synthase (eNOS) gene has been suggested to modulate nitric oxide production and to regulate muscle blood flow by inducing vasodilation. Throughout this mechanism eNOS may play an important role in the regulation of insulin sensitivity and secretion altering supply of insulin to insulin-sensitive tissue and secretagogues to pancreatic islets. A decrease in local blood flow has been reported to arise insulin resistance. In non diabetic and type 2 diabetic subjects from Japan both the E298D (Glu298Asp) and the T-786C eNOS gene polymorphisms have been associated with insulin resistance. To our knowledge, no such date are available in Caucasian populations. Therefore we have examined the association of these polymorphisms with fasting and post-challenge glucose and insulin levels in 278 caucasian non diabetic men with essential hypertension.

Materials and Methods: A 75 g glucose tolerance test was performed and blood sampling were obtained at baseline and at $30^{\prime}, 60^{\prime}, 90^{\prime}, 120^{\prime}$ and $180^{\prime}$ after glucose loading. Blood glucose and insulin (RIA method) were measured. Genotypes of both Glu298Asp and T-786C mutations were examined by the PCR-RFLP method.

Results: The percentages of the EE298, ED298 and DD298 genotypes were $45.0,42.1$ and $12.9 \%$, respectively. The corrispective features for TT786, TC786 and CC786 were 21.4, 48.2 and $30.4 \%$. Genotypes did not differ in age, BMI, waist-to-hip ratio, systolic and diastolic blood pressure and lipid profile. Fasting and post-challenge glucose and insulin levels did not differ by genotypes. No significant differences by genotypes were observed in homeostasis model assessment for insulin resistance (HOMA-IR: E298D: EE 3.0 1.9; ED 3.6 \pm 3.2 ; DD $2.8 \pm 1.2, \mathrm{p}=0.10$; T786C: TT $3.3 \pm 2.7$; TC $3.3 \pm 2.7$; $\mathrm{CC} 3.2 \pm 1.7, \mathrm{P}=0.98$ ) as well as in areas under the curve (AUC) for both glucose and insulin, or in insulinogenic index (DeltaI30/DeltaG30: E298D: EE 98 \pm 58 ; ED 135 \pm 132 ; DD $100 \pm 69, \mathrm{p}=0.22$; T786C: TT $112 \pm 83$; TC $116 \pm 113$; CC $120 \pm 99, \mathrm{P}=0.91$ ). No interaction between the two polymorphisms was observed: fasting and post-challenge glucose and insulin, AUC for glucose and insulin, HOMA-IR and the insulinogenic index did not differ by haplotypes.

Conclusion: These data suggest that in non diabetic hypertensive caucasian men the eNOS Glu298Asp and T786C polymorphism did not affect insulin sensitivity or secretion as measured by oral glucose tolerance test.

\section{4}

A systematic review and meta-analysis of the relationship between lipoprotein lipase gene Asn291Ser variant and dyslipidaemia

W. Liu, Y. M. Hu;

Division of Endocrinology, Renji Hospital of Shanghai Jiaotong University, Shanghai, China. 
Aims: Several case-control studies have examined the association between the Asn291Ser variant in the lipoprotein lipase gene and dyslipidemia, but most had limited power and results could therefore be conflicting. This systematic review attempts to summarize the association between the Asn291Ser variant in the lipoprotein lipase gene and dyslipidemia.

Methods: We systematically reviewed the literature by means of a meta-analysis. Meta-analysis were performed on RevMan4.2.8. To check for publication bias, a funnel plot was constructed. Three-dimensional structure of the human lipoprotein lipase was reconstructed based on known crystal structure of human protein lipase.

Results: Thirty-three family or case-control studies including 19246 white subjects were selected for the meta-analysis. Funnel plots showed no asymmetry, neither visually nor in terms of statistical significance $(\mathrm{p}>0.05)$, indicating no publication bias. The summary standardised mean difference (SMD) of plasma triglyceride for carriers compared to noncarriers of the Asn291Ser variant was 3.23 (95\% C.I. 2.493.97, $\mathrm{p}<0.00001)$. The summary SMD of plasma HDL-C for carriers compared to non-carriers of the Asn291Ser variant was - 3.42 (95\% C.I. $-5.07-1.77$, $\mathrm{p}<0.0001)$. The summary SMD about the association of the Asn291Ser variant with plasma triglyceride increased with the age of cases. The SMD raised from 0.92 (95\% C.I. $0.20-1.64)$ for case subjects aged 35 to 45 years to 3.25 (95\% C.I. 1.83-4.67) for case subjects that were 65 to 75 years old.

Conclusions: This meta-analysis indicates that the Asn291Ser variant in the LPL gene is a risk factor for dyslipidemia, characterized by hypertriglyceridemia and low HDL-C levels. And the Asn291Ser variant in the LPL gene predispose to more severe dyslipidemia with the increasing age.

\section{5}

4G/5G polymorphism of plasminogen activator inhibitor (PAI-1) influences the relationship between Creactive protein and PAI-1 in type 2 diabetic patients

I. Testa ${ }^{1}$, A. R. Bonfigli ${ }^{1}$, M. Marra ${ }^{1}$, M. Boemi ${ }^{1}$, C. Sirolla ${ }^{2}$, D. Mari ${ }^{3}$, E. Sacchi ${ }^{4}$, A. Dolci ${ }^{5}$, A. Catalano ${ }^{6}$, A. Procopio ${ }^{6}$, A. Ceriello ${ }^{7}$, R. Testa ${ }^{1}$;

${ }^{1}$ Diabetology Unit, ${ }^{2}$ Center of Epidemiology and Biostatistic, IRCCS-INRCA, Ancona, Italy, ${ }^{3}$ Department of Internal Medicine, IRCCS-Maggiore Hospital, Milan, ${ }^{4}$ Department of Immunology, IRCCS-Sacco Hospital, Milan, Italy, ${ }^{5}$ Clinical Pathology, Multimedica Laboratories, Milan, ${ }^{6}$ Center of Citology, IRCCS-INRCA, Ancona, ${ }^{7}$ Department of Pathology and Medicine, University of Udine, Udine, Italy.
Background and Aims: C-reactive protein (CRP), in addition to being a proinflammatory biomarker, has been identified as a possible factor able to promote atherosclerosis. "In vitro" studies have demonstrated that CRP induces plasminogen activator inhibitor type 1 (PAI-1) expression, suggesting a hypofibrinolytic role for CRP. As CRP and PAI-1 levels increase in type 2 diabetic subjects, we decided to study the relationship between CRP and PAI-1, and the role of the $4 \mathrm{G} / 5 \mathrm{G}$ polymorphism of the PAI-1 gene on this relationship in a diabetic population without complications.

Materials and Methods: Two hundred and ninety five type 2 diabetic patients (age $60.9 \pm 10.5$ years) and 290 healthy controls (age 59.2 \pm 11.5 years) were enrolled and CRP, PAI-1 antigen and $4 \mathrm{G} / 5 \mathrm{G}$ polymorphism were detected.

Results: A significant correlation between PAI-1 and CRP in diabetic subjects was found $(\mathrm{r}=0.45, \mathrm{p}<0.001)$, whereas no relationship was evident in the control subjects between these inflammatory markers. Multiple regression analysis highlighted that CRP is the only one significant variable of PAI-1 levels in diabetic subjects (partial $\mathrm{r}=0.31, \mathrm{p}<0.01$ ). Stratifying by genotype, a positive correlation between PAI1 and CRP in $4 \mathrm{G} / 4 \mathrm{G}$ (partial $r=0.64 p<0.001$ ) and $4 \mathrm{G} / 5 \mathrm{G}$ (partial $r=0.47, p<0.001$ ) subjects was found, whereas no correlation in $5 \mathrm{G} / 5 \mathrm{G}$ was present. Multiple regression analysis confirmed the presence of this correlation in $4 \mathrm{G} /$ $4 \mathrm{G}$ (partial $\mathrm{r}=0.45, p<0.001$ ) and in $4 \mathrm{G} / 5 \mathrm{G}$ (partial $\mathrm{r}=0.34$, $p=0.007)$ diabetic patients.

Conclusions: These findings demonstrate that CRP plays an important role in the complex mechanism in the regulation of PAI-1 levels in 4G diabetic carriers, supporting the hypothesis that CRP may have a direct role in diabetic atherothrombosis.

\section{PS 11}

\section{Genetics of insulin resistance and cardiovascular disease}

\section{6}

Identification of functional protein kinase $\mathrm{C} \beta$ promoter polymorphisms related to insulin-resistance in nonobese humans

M. A. Osterhoff ${ }^{1,2}$, S. Richter ${ }^{1,2}$, M. Pfeiffer ${ }^{1,2}$, J. Tasic $^{1,2}$, S. Kaiser ${ }^{1,2}$, M. O. Weickert ${ }^{1,2}$, F. Isken ${ }^{1,2}$, M. Moehlig ${ }^{1,2}$, J. Spranger ${ }^{1,2}$, A. F. H. Pfeiffer ${ }^{1,2}$;

${ }^{1}$ Clinical Nutrition, German Institute of Human Nutrition, Nuthetal, ${ }^{2}$ Endocrinology, Diabetes and Nutrition, Charité Campus Benjamin Franklin, Berlin, Germany.

Background and Aims: Insulin-resistance is a marker of impaired glucose metabolism and a risk factor for the 
development of type 2 diabetes mellitus (T2DM). The alternative splicing of protein kinase $\mathrm{C} \beta(\mathrm{PKC} \beta)$ into subtypes $\beta 1$ and $\beta 2$ is affected by insulin and itself plays and important role in the regulation of insulin production in $\beta$-cells and the insulin depdendent glucose uptake in skeletal meuscle cells. We investigated five polymorphisms (SNPs) within the PKC $\beta$ gene (PRKCB1) regarding their influence on insulin-sensitivity in humans.

Materials and Methods: Promoter-assays were carried out by use of a promoter-fragment of PKC $\beta$ cloned into the pGL3-basic vector (Promega) containing either the frequent or rare allele of the polymorphism. We investigated 1068 caukasian individuals recruited in the region of Berlin and Potsdam, Germany. The cohort was categorized according to WHO-criteria into controls, patients with IFG, IGT and T2DM. The age was $52.2 \pm 14.3 \mathrm{a}$, the BMI $28.8 \pm 6.3 \mathrm{~kg} /$ sqrm. The subjects were characterized by OGTT, anthropometric and biochemical parameters. The genotype of the SNPs was determined by TaqMan technology.

Results: We identified 2 polymorphisms in the promoter of PKC $\beta$ related to an decreased protein-expression of the enzyme and to insulin-resistance in humans. PKC $\beta$ promoter assays with w.t. and at position -546 mutated constructs showed an up to 4-fold lower activation of the mutated PKC $\beta$ promoter. Consequently, we found a significantly 3.4-fold decreased protein-expression of $\mathrm{PKC} \beta$ in human subjects carrying the homozygously mutated variant at position -546 . The -546 PKC $\beta$ promoter polymorphism was in strong linkage with another polymorphism at position -1504 $\left(D^{\prime}=0.958, p<0.00001\right)$ and both were associated significantly with increased fasting insulin concentrations (FI) and HOMAIR in non-obese subjects (BMI $<30)$ with impaired glucose metabolism (IGM). The fasting glucose levels were not altered. In obese subjects $(B M I \geq 30)$ the FI levels and HOMA-IR were already increased and the polymorphisms had no additional effect.

Conclusion: The mutation of the $\mathrm{PKC} \beta$ promoter at positions -546 and -1504 might lead to a reduced expression of the enzyme leading to insulin resistance, because of an impaired signal transduction in skeletal muscle- and $\beta$-cells altering glucose uptake and insulin production.

Supported by the German Diabetes Association, German Ministry of Education and Science

\section{7}

The common $3^{\prime}$ UTR G $>$ A variant of $C T L A 4$ associates with reduced fasting and post- OGTT insulinaemia in the general population: a study of 5,762 middle-aged whites T. W. Boesgaard ${ }^{1}$, S. I. Castella ${ }^{1}$, G. Andersen ${ }^{1}$, A. Albrechtsen ${ }^{1}$, T. Spars $\varnothing^{1}$, K. Borch-Johnsen ${ }^{1,2}$, T. Jørgensen ${ }^{2}$, T. Hansen ${ }^{1}$, O. Pedersen ${ }^{1}$;
${ }^{1}$ Steno Diabetes Center, Gentofte, ${ }^{2}$ Research Centre for Prevention and Health, Glostrup University Hospital, Denmark.

Background and Aims: A widespread 3'UTR G>A polymorphism in the gene encoding the cytotoxic $\mathrm{T}$ lymphocyte antigen 4 (CTLA4), which acts as a potent negative regulator of T-cell responses, has been shown to associate with type 1 diabetes. This variant has been shown to influence the expression of the CTLA4 gene. We hypothesised that the A-allele of the CTLA4 variant rs3087243 modulates pancreatic $\beta$-cell function in the general population. Thus, the aim of the present study was to investigate the relationship between the CTLA4 3'UTR G $>$ A polymorphism and fasting and post-OGTT insulinaemia in a sample of Danish whites.

Materials and Methods: The CTLA4 3'UTR G>A rs3087243 variant was genotyped in a population-based sample (Inter99) of 5,762 middle-aged subjects (2,865 men, 2,897 women, age $46 \pm 8$ years (mean \pm SD), BMI $26.3 \pm 4.6 \mathrm{~kg} / \mathrm{m}^{2}$ ). All subjects underwent a standard $75 \mathrm{~g}$ oral glucose tolerance test (OGTT). Genotyping of the CTLA4 variant was performed using chip-based matrix-assisted laser desorption/ionization time-of-flight (MALDI-TOF) mass spectrometry.

Results: The minor A-allele of the CTLA4 3'UTR G>A variant associates with decreased fasting and 2-hour postOGTT serum insulin, and an increase in BMI (see table). In a case-control study the A-allele was less frequent among 2,548 subjects with BMI $<25 \mathrm{~kg} / \mathrm{m}^{2}$ (40.1; 95\%CI 38.7-41.4 (36\% GG; 48\% GA; $16 \%$ AA)) than among 952 participants having BMI $\geq 30 \mathrm{~kg} / \mathrm{m}^{2}$ (43.9; 95\%CI 41.6-46.1 (32\% GG; $49 \% \mathrm{GA} ; 20 \% \mathrm{AA}), P=0.005)$. Thus, the variant associates with obesity with an odds ratio of 1.2 (95\%CI 1.1-1.3).

\begin{tabular}{lllll}
\hline & G/G & G/A & A/A & $P$ \\
\hline N (men/ & 1,985 & 2,772 & 1,005 & \\
$\begin{array}{l}\text { women) } \\
\text { Age }(\text { years) }\end{array}$ & $46 \pm 8$ & $46 \pm 8$ & $46 \pm 8$ & \\
$\begin{array}{l}\text { BMI }\left(\mathrm{kg} / \mathrm{m}^{2}\right) \\
\text { Plasma glucose }\end{array}$ & $26.0 \pm 4.4$ & $26.3 \pm 4.5$ & $26.5 \pm 4.8$ & 0.003 \\
$\begin{array}{l}\text { Fasting } \\
\text { (mmol/l) }\end{array}$ & $5.5 \pm 0.8$ & $5.5 \pm 0.7$ & $5.6 \pm 1.0$ & 0.2 \\
$\begin{array}{l}\text { 120-min } \\
\text { post-OGTT } \\
\text { (mmol/l) }\end{array}$ & $6.3 \pm 2.2$ & $6.2 \pm 2.1$ & $6.3 \pm 2.1$ & 0.06 \\
$\begin{array}{l}\text { Serum insulin } \\
\text { Fasting } \\
\text { (pmol/l) }\end{array}$ & $43 \pm 29$ & $41 \pm 27$ & & \\
$\begin{array}{l}120-\text {-min } \\
\text { post-OGTT } \\
\text { (pmol/l) }\end{array}$ & $226 \pm 235$ & $211 \pm 203$ & $217 \pm 203$ & 0.002 \\
\hline
\end{tabular}

Data are mean \pm SD. A general linear model was applied. $P$ values were adjusted for sex, age, and BMI (where appropriate) and were calculated assuming a dominant model. 
Conclusion: The widespread CTLA4 3'UTR G>A polymorphism associates with significantly reduced fasting and 2hour post-OGTT serum insulin levels in middle-aged whites, suggesting that variation in a type 1 diabetes susceptibility gene may modulate insulinaemia in the general population of middle-aged people. Moreover, our findings suggest that the CTLA4 variant may associate with a modest increase in BMI. Further studies are needed to confirm this finding and to explore the mechanism by which variation in the CTLA4 locus may contribute to BMI regulation.

0358

The Q130E polymorphism in the Caveolin-2 gene is associated with biological features of hepatic steatosis and postprandial insulin levels

D. Rubin ${ }^{1}$, U. Helwig ${ }^{1}$, U. R. Foelsch ${ }^{2}$, J. Schrezenmeir ${ }^{1}$; ${ }^{1}$ Institute for Physiology and Biochemistry of Nutrition, Federal Research Centre for Nutrition and Food, Kiel, ${ }^{2}$ Department of General Internal Medicine, University Clinic Schleswig-Holstein, Campus Kiel, Germany.

Background and Aims: Type 2 diabetes in humans is associated with increased de novo lipogenesis, decreased fatty acid oxidation and hepatic steatosis. Caveolae are small membrane invaginations on the surface of cells that participate in membrane trafficking, sorting, transport and signal transduction. Caveolin-2 is a principal component of caveolae membranes in many cell types, with particular high levels in adipocytes, smooth muscle and the liver. The specific overexpression of Cav- $2 \beta$ results in the constitutive localization of $\mathrm{Cav}-2 \beta$ to the surface of lipid droplets, suggesting a role for this Cav-2 isoform in lipogenesis. As caveolin-2 could be an enhancer for hepatic lipogenesis, variations in this gene could lead to abnormal hepatic lipid accumulation and insulin resistance. We investigated the association between different caveolinpolymorphisms and hepatic and metabolic parameters in Metabolic Intervention Cohort Kiel (MICK) to elucidate this question.

Materials and Methods: A total of 716 male subjects from a postprandially characterised cohort (MICK) were genotyped for the Caveolin-2 polymorphism (rs8940). Subjects underwent an oral glucose tolerance test and a standardised mixed meal. Glucose, insulin, triglycerides were determined after the mixed meal, glucose and insulin after the glucose load. Increased liver enzymes were used as surrogates of liver steatosis.

Results: In comparison to homozygote subjects of the wild allele, carriers of the less common allele showed significantly lower levels of alanine aminotransferase and aspartat aminotransferase $(\mathrm{p}=0,006, \mathrm{p}=0,019 \mathrm{rsp}$.), lower postprandial insulin levels after a standardised mixed meal (OMTT) $(\mathrm{p}=0,018)$ and higher postprandial insulin sensitivity (HOMA AUC, $p=0,04$ ). BMI was not significantly different in both groups and adjustment for this confounder did not attain the results.

Conclusion: These results suggest that the rare allele of a Caveolin-2 gene polymorphism may be protective against insulin resistance and hepatic steatosis.

Supported by BMBF-Project "Fat and metabolism - gene variation, gene regulation and gene function"

0359

Single nucleotide polymorphisms in the procolipase gene are associated with insulin sensitivity and insulin secretion in humans

P. Weyrich, R. Lammers, F. Machicao, A. Fritsche, H.-U. Häring, N. Stefan;

Medizinische Klinik IV, University of Tuebingen, Germany.

Background and Aims: Procolipase (PCLPS) is secreted from the pancreas during fat digestion into the gastrointestinal tract. After removal of the signal peptide, PCLPS is proteolytically cleaved into colipase (CLPS) and the pentapeptide enterostatin (ES). CLPS is an indispensable activating cofactor for pancreatic lipase and therefore permits lipid digestion in the gut. By contrast, ES acts as a signaling molecule that inhibits insulin secretion and confers satiety signals to the brain, thereby restricting further food intake in animal models. As both high fat diet and obesity contribute to insulin resistance, we were interested whether single nucleotide polymorphisms (SNPs) of the human PCLPS gene are associated with insulin sensitivity, insulin secretion and serum lipids in humans

Materials and Methods: A total of 1500 base pairs of the promoter, all 3 exons, exon-intron boundaries and the 3'UTR of PCLPS were analyzed by bidirectional sequencing in 50 non-diabetic subjects. Among the identified SNPs, those with an allelic frequency $>5 \%$ were genotyped in 498 non-diabetic subjects.

Results: Two SNPs, $-3 A / G$ (rs3748050 in the Kozak sequence) and $+122 A / G$ (rs3748051 in intron 1) were in tight linkage disequilibrium. Both the $\mathrm{G}$ allele of the $-3 \mathrm{~A}$ / $G$ SNP (allele frequency 0.24 ) and the $\mathrm{G}$ allele of the +122 $A / G$ SNP (allele frequency 0.23 ) were associated with higher insulin sensitivity estimated from the oral glucose tolerance test [OGTT as estimated by Matsuda and DeFronzo, (e.g. $-3 A / G$, AA $14.68 \pm 0.69$, AG $15.86 \pm 0.81$ and GG $18.68 \pm 2.17$ arb. units, mean \pm SE, $p=0.02$ )], independent of age, gender and body fat. Moreover, they were 
associated with lower insulin secretion as determined by $\mathrm{C}$ peptide levels at minute 30 of the OGTT (e.g. $-3 A / G$, AA $1837 \pm 47$, AG $1789 \pm 53$ and $\mathrm{GG} 1587 \pm 98 \mathrm{pM}, \mathrm{p}=0.02$ ), after additional adjustment for insulin sensitivity. Further, haplotype analyses confirmed the relevance of both SNPs for these phenotypes. No significant associations with fasting total cholesterol (c), HDL-c, LDL-c, triglycerides and free fatty acids were found (all $\mathrm{p}>0.11$ ).

Conclusion: These data support that $P C L P S$, encoding both an enzyme (CLPS) and concomitantly a hormone (ES), has different functions on insulin sensitivity and insulin secretion. While the $G$ alleles of these SNPs may have beneficial effects on insulin sensitivity, particularly their inhibitory effects on insulin secretion may be important in subjects at high risk for the development of type 2 diabetes.

\section{0}

Heritability of serum resistin and its genetic correlation with insulin resistance-related features in non-diabetic Caucasians

C. Menzaghi ${ }^{1}$, A. $\mathrm{Coco}^{1}$, L. Salvemini ${ }^{1}$, R. Thompson ${ }^{2}$, S. De Cosmo ${ }^{1}$, A. Doria ${ }^{2}$, V. Trischitta ${ }^{1,3}$;

${ }^{1}$ Research Unit of Diabetology and Endocrinology, IRCCS, San Giovanni Rotondo, Italy, ${ }^{2}$ Research Division and Department of Medicine, Joslin Diabetes Center, Harvard Medical School, Boston, United States, ${ }^{3}$ Department of Clinical Sciences, University "La Sapienza", Roma, Italy.

Background and Aims: Resistin, a cysteine-rich peptide, is believed to play a role in several metabolic pathways and inflammatory responses. Serum levels of resistin are believed to modulate insulin resistance in humans.

The aim of this study was to investigate whether serum resistin levels are genetically controlled and whether this control is shared with other insulin resistance traits.

Materials and Methods: The study cohort included 264 non diabetic probands, Caucasian from Italy, and their 473 adult family members. Phenotypic characterization included anthropometric variables, blood pressure, fasting glucose and insulin, lipid profile and resistin levels. Genotypes were determined at position g.-420C $>\mathrm{G}$ (rs1862513), IVS2+ $181 \mathrm{G}>\mathrm{A}(\mathrm{rs} 3745367)$, and $\mathrm{GAT}_{(\mathrm{n})}$ polymorphisms of the resistin (RETN) gene.

Results: In the 264 unrelated probands, resistin levels were significantly $(p<0.01)$ correlated with adiposity, blood pressure, C-reactive protein, and the metabolic syndrome score. In a variance component analysis of the 264 probands and their 473 relatives, about $70 \%$ of the observed variation of serum resistin levels was heritable $(p<0.0001)$. A small, but significant $(p=0.004)$ proportion of this heritability was explained by the $\mathrm{G}>\mathrm{A}$ variation at position IVS $2+181$ of the RETN gene. Significant genetic correlations $(p<0.05)$ were observed between resistin and BMI $(\rho g=0.30)$, waist circumference $(\rho g=0.32)$, the insulin resistance index $\mathrm{HOMA}_{\mathrm{IR}}(\rho \mathrm{g}=0.28)$ and the metabolic syndrome score $(\rho g=0.35)$.

Conclusion: These data indicate that serum resistin is highly heritable and has some common genetic background with traits related to insulin resistance, reinforcing the hypothesis that this adipokine may play a pathogenic role in insulin resistance-related abnormalities, including type 2 diabetes and cardiovascular disease.

Supported by Italian Ministry of Health Ricerca Finalizzata 2004, Ricerca Corrente 2005

0361

Genetic association of Pro197Leu allele at GPX-1 gene to coronary artery calcification suggests importance of shifted redox balance for development of atherosclerosis in diabetes

M. Nemoto ${ }^{1,2}$, T. Sasaki ${ }^{1,2}$, Y. Hiki ${ }^{1,2}$, K. Fujimoto ${ }^{1,2}$, R. Nishimura ${ }^{1}$, Y. Miyashita ${ }^{1}$, T. Ohashi $^{2}$, N. Tajima ${ }^{1}$;

${ }^{1}$ Diabetes, Metabolism and Endocrinology, Jikei University School of Medicine, Tokyo, ${ }^{2}$ Department of Gene Therapy, Institute of DNA Medicine, Jikei University School of Medicine, Tokyo, Japan.

Background and Aims: Recent studies suggest that increase in reactive oxygen species (ROS), other than the classical Framingham factors, is a possible explanation for why diabetes mellitus promotes arterial sclerosis, specifically coronary artery disease. However, its mechanisms that shift the redox balance and determine individuals' susceptibility to coronary artery sclerosis in diabetes remain unknown. To know it, we analyzed SNPs of genes for enzymes that could scavenge and reduced ROS, as well as elements that could increase ROS and promote susceptibility to atherosclerosis in diabetes.

Materials and Methods: Eighty-six patients with type 2 diabetes (42-81 years olds, $\mathrm{M} / \mathrm{F}=60 / 26)$ were enrolled in this study, and were evaluated on coronary artery calcium score (Agaston CACS) by 16-detector Multi-Slice CT (MSCT). The patients were genotyped for ROS-scavenging enzymes; Glutathione peroxidase1 (GPx-1), Catalase, Mn$S O D$ and $C u / Z n-S O D$. The patients were also evaluated for ROS-promoting elements including SNPs of NADPH, age, blood pressure, BMI, glycemic control, lipid metabolism and duration of diabetes. Genotypes were determined by PCR-based methods. All clinical values are expressed by mean \pm SD. Statistical analyses were performed by t-test, $\chi^{2}$ test and multiple regression analysis using Dr.SPSS according to patients' groups divided based on CACS, or 
genotype. The genetic association test for deviation from the Hardy-Weinberg equilibrium was performed by the DeFinetti program.

Results: As shown in Table, only the CACS score of $G P x$ 1 gene was significantly disparate; CACS for Pro/Leu heterozygotes was higher than that of Pro/Pro homozygotes $(\mathrm{p}<0.002)$. Further analysis for $G P x-1$ gene showed that genotype frequency of Pro/Leu of patients' group with CACS over 1000 was significantly higher than that of CACS under 999 (45.5\% vs. $17.3 \%)$ when analyzed by $\chi^{2}$ test. Moreover the test for deviation from HardyWeinberg's equilibrium also confirmed the association $(\mathrm{OR}=3.97, \mathrm{CI}=1.05-15.01 ; \mathrm{p}=0.032)$. Finally, multiple regression analysis for CACS to patients' parameters including SNPs revealed presence of significant correlation with $G P x-1$ genotype and age to CACS.

\begin{tabular}{lllll}
\hline Gene & Genotype & frequencies & CACS & p value \\
GPx-1 & P/P & $68 / 86(79.1 \%)$ & $234 \pm 396$ & $0.002 *$ \\
(Pro197Leu) & P/L & $18 / 86(20.9 \%)$ & $824 \pm 1340$ & \\
Catalase & T/T & $20 / 83(24.1 \%)$ & $323 \pm 615$ & 0.755 \\
(SNP-89) & A/T \& A/A & $63 / 83(75.9 \%)$ & $384 \pm 787$ & \\
Mn-SOD & V/V & $46 / 83(55.4 \%)$ & $317 \pm 688$ & 0.483 \\
(Val16Ala) & V/A \& A/A & $37 / 83(44.6 \%)$ & $433 \pm 817$ & \\
Cu/Zn-SOD & C/C & $30 / 82(36.6 \%)$ & $388 \pm 825$ & 0.895 \\
(intron 3) & C/A \& A/A & $52 / 82(63.4 \%)$ & $365 \pm 711$ & \\
NADPH & $\mathrm{C} / \mathrm{C}$ & $72 / 83(86.7 \%)$ & $324 \pm 765$ & 0.16 \\
$(+242)$ & $\mathrm{T} / \mathrm{T} \& \mathrm{~T} / \mathrm{C}$ & $11 / 83(13.3 \%)$ & $666 \pm 544$ & \\
\hline
\end{tabular}

Conclusion: Presence of Pro197Leu variant allele of GPx-1 could determine genetic susceptibility to coronary artery sclerosis in type 2 diabetes by low scavenging ability of ROS.

0362

Prohormone convertase gene variants and proinsulin concentrations

B. Zethelius ${ }^{1}$, C. Berne ${ }^{2}$, L. Berglund ${ }^{3}$, T. Axelsson ${ }^{2}$,

A.-C. Syvänen ${ }^{2}$;

${ }^{1}$ Dept. of Public Health and Caring Sciences/Geriatrics,

${ }^{2}$ Dept. of Medical Sciences, ${ }^{3}$ Uppsala Clinical Research

Center, Uppsala University, Uppsala, Sweden.

Background and Aims: Recently, it was shown that proinsulin is a significant predictor of Type 2 diabetes (T2DM), independent of insulin resistance and insulin secretion. Proinsulin is converted to insulin by concerted action of two sequence-specific proteases termed prohormone convertase 1 (PC1) and 2 ( $\mathrm{PC} 2)$. PC1 initiates the sequential processing of proinsulin to insulin by cleaving the proinsulin molecule where the B-chain and C-peptide join. $\mathrm{PC} 2$ and Caboxypeptidase $\mathrm{E}(\mathrm{CPE})$ catalyze further reactions. Expressions of $\mathrm{PC} 1$ and insulin are co-ordinately regulated by glucose. Thus, PC1 plays a key role in regulating insulin biosynthesis.

To examine if SNPs in the PC1, PC2 and CPE genes are associated with proinsulin and insulin concentrations.

Materials and Methods: We investigated genotypes, SNPs, selected from public databases, using multiplex PCR at the WCN-Platform North, Uppsala in relation to proinsulin and insulin determinations using data from the ULSAM cohort.(www.pubcare.uu.se/ULSAM). Also haplotypes (HTs), estimated using the software PHASE 2.1, were investigated. Permutations were used to control for inflation of the alfa-error.

Results: A SNP in an exon of the PC1 gene showed 50\% higher proinsulin concentrations in homozygotes as compared to wildtype ( $\mathrm{p}$ for trend $<0.001$ ) and in a HT ( $\mathrm{n}=12$ / $545, \mathrm{p}$ for trend $<0.001)$. A SNP in an exon of the PC2 gene showed $25 \%$ higher proinsulin concentrations in homozygotes as compared to wildtype ( $\mathrm{p}$ for trend 0.021 ) and $150 \%$ higher proinsulin concentrations in a HT $(n=14 /$ 533, $\mathrm{p}$ for trend 0.002). This haplotype was borderline associated with an increased risk for T2DM $(\mathrm{OR}=3.1$, $\mathrm{p}=0.057)$. For CPE, results were non-significant.

Conclusion: These data suggest that genetic variation in the $\mathrm{PC} 1$ and $\mathrm{PC} 2$ genes is associated with proinsulin concentrations.

Supported by Wallenberg Consortium Nord, Sweden

0363

The ACE DD genotype is associated with a high risk for fatal myocardial infarction in people with type 2 diabetes and high cardiovascular risk S. Hadjadj ${ }^{1,2}$, R. Roussel ${ }^{3,4}$, F. Fumeron ${ }^{4}$, F. Pean ${ }^{4}$, G. Chatellier ${ }^{5}$, J. Menard ${ }^{6}$, F. Alhenc-Gelas ${ }^{7}$, M. Marre ${ }^{3,4}$; ${ }^{1}$ Endocrinology-Diabetology, ${ }^{2}$ Inserm e0324, Poitiers University Hospital, Poitiers, ${ }^{3}$ Endocrinology-Diabetology, Bichat Hospital, Paris, ${ }^{4}$ Inserm u695, Faculty of Medicine X. Bichat, Paris, ${ }^{5}$ Inserm u729, European Hospital Georges Pompidou, Paris, ${ }^{6}$ Public Health University Laboratory, Faculty of Medicine Paris 5, Paris, ${ }^{7}$ U652, INSERM, Paris, France.

Background and Aims: The insertion/deletion (I/D) polymorphism of the angiotensin converting enzyme (ACE) gene is a validated risk factor for diabetic nephropathy. The prognostic value of the $\mathrm{D}$ allele for acute myocardial infarction (AMI) was only established on case-control studies, seldom studying type 2 diabetic patients. The aim of our prospective study was to evaluate its prognostic value in a large population of type 2 diabetic patients. 
Materials and Methods: The DIABHYCAR trial is a randomised controlled trial comparing ramipril $1.25 \mathrm{mg} /$ day with placebo, for type 2 diabetic patients with high cardiovascular risk (micro- or macro-albuminuria, age $>50$ years). The main outcome was the occurrence of a cardiovascular event including fatal or non-fatal AMI. Here, the history of myocardial infarction at baseline, the occurrence of an AMI and the post-AMI mortality were examined in 3139 French subjects, taking part in this study, according to the ACE I/D polymorphism.

Results: At baseline, history of AMI was less frequently encountered in DD genotype subjects, compared with other participants : DD, 47/1119 (4.2\%), ID, 85/1468 (6.1\%) and II, $39 / 552(7.1 \%)(p=0.0384)$. This lower risk persisted after adjustment on sex, age, blood pressure, cholesterol, smoking, HbA1c (DD vs ID or II: adjusted odds ratio 0.64, 95\% CI: 0.44-0.94; $\mathrm{p}=0.02$ ). During the study follow-up (4 years), 95 AMI occurred (3\%) and this incidence was not associated with the I/D polymorphism. The post AMI mortality was significantly different according to the polymorphism : 7/28 DD genotype patients died (25\%) vs $3 / 49$ ID genotype subjects (6.1\%) and 1/18 II genotype subjects $(5.6 \%) \quad(p=0.03)$. This increased risk of DD subjects persisted after adjustment on the above cited risk factors (DD vs ID or II : adjusted hazard ratio $11.2,95 \% \mathrm{CI}$ 2.1-58.2; $\mathrm{p}=0.04)$.

Conclusion: The DD genotype of the ACE I/D is not a risk factor for AMI but is associated with an increased risk of post- AMI mortality, in type 2 diabetic subjects, which may lead to a survival bias that might account for spurious results in cross-sectional analyses.

\section{4}

\section{Effect of ENPP1 (PC-1) K121Q polymorphism on metabolic syndrome}

J. L. González-Sánchez, C. Zabena,

M. T. Martínez-Larrad, M. J. Martínez-Calatrava,

M. Serrano-Ríos;

Internal Medicine II, Hospital Clínico San Carlos, Madrid, Spain.

Background and Aims: Ectonucleotide pyrophosphatase/ phosphodiesterase 1 (ENPP1), also named plasma cell membrane glycoprotein (PC-1), is a promising candidate gene for type 2 diabetes because it inhibits autophosphorylation of the insulin receptor. The ENPP1 K121Q polymorphism has been associated with insulin resistance in some but not in all studies.

In this report we analyze in an adult (35-74 years) Caucasoid population $(n=794)$ from a cross-sectional population-based epidemiological survey in Central Spain
(Segovia, A.C Castille), including type 2 diabetics $(n=73$, $9.2 \%$ ), whether the K121Q polymorphism of the ENPP1 gene is associated with increased risk for the development of obesity, insulin resistance and other features of the metabolic syndrome as defined by the 2005 IDF criteria.

Materials and Methods: Anthropometric parameters: BMI, waist circumference. OGTT, fasting and $2 \mathrm{hrs}$ glucose; lipid profile, serum insulin, proinsulin, leptin, adiponectin levels. Insulin resistance was assessed by HOMA-IR. ENPP1 K121Q genotypes determined by PCR-RFLP.

Results: Glucose Tolerance: Normoglycemia 564 (71\%), Impaired Fasting Glucose+Impaired Glucose Tolerance 157 (19.8\%), Type 2 Diabetes 73 (9.2\%). ENPP1 genotype distribution: K121K 73.2\%, K121Q 24.8\%, Q121Q: 2.0\%. Overall prevalence of metabolic syndrome was $25.1 \% ; 24.3 \%$ in males and $25.7 \%$ in females. The $\mathrm{Q}$ allele was associated with higher $\mathrm{BMI}(31.3 \pm 3.0$ vs. $30.0 \pm 3.6, \mathrm{P}=0.027)$ and waist circumference $(101 \pm 7.2$ vs. $98 \pm 9.3, \mathrm{P}=0.043)$ among subjects with metabolic syndrome compared with K121K genotype. Those findings were also significant even after the adjustment for sex, age, and degree of glucose tolerance $(\beta=1.347$, $\mathrm{P}=0.017$ and $\beta=2.824, \mathrm{P}=0.046$; for $\mathrm{BMI}$ and waist circumference respectively).

Conclusion: The K121Q PC-1 polymorphism did not appear to be associated with insulin resistance in Caucasoid Spaniards although in subjects with metabolic syndrome the $\mathrm{Q}$ allele was associated with obesity related parameters such as a higher BMI and waist circumference.

\section{5}

The role of Leu72Met and $-604 \mathrm{C}>\mathrm{T}$ polymorphisms of the ghrelin gene on insulin resistance in Italian population

S. Zampetti ${ }^{1}$, S. Zavarella ${ }^{1}$, A. Petrone ${ }^{1}$, M. Spoletini ${ }^{1}$, M. Gueorguiev ${ }^{2}$, E. Garcia ${ }^{2}$, G. Leto ${ }^{1}$, S. Potenziani ${ }^{1}$, M. Capizzi ${ }^{1}$, F. Leonetti ${ }^{1}$, M. Korbonits ${ }^{2}$, R. Buzzetti ${ }^{1}$; ${ }^{1}$ Clinical Science, "La Sapienza" University, Rome, Italy, ${ }^{2}$ Endocrinology, William Harvey Research Institute, Bart's and the London Medical School, London, United Kingdom.

Background and Aim: Ghrelin (GHRL) is a circulating 28amino acid gastrointestinal and neuropeptide that is widely expressed in many tissues and primarily produced by the stomach. Circulating ghrelin levels are correlated with obesity and mutations of the gene could influence the function of this hormone and might alter the energy balance. Previous studies, suggested that polymorphisms in the coding region of the preproghrelin were involved in the aetiology of obesity and might modulate glucose induced insulin secretion.

The aim of our study was to evaluate the association of the three GHRL polymorphisms: $-604 \mathrm{C}>\mathrm{T}$, Leu72Met and 
Gln90Leu, with obesity and measures of insulin sensitivity in 1420 individuals.

Materials and Methods: The three SNPs were genotyped using ABI PRISM 7900 HT Sequence Detection System. We assumed specific co-dominant genetic model and we used multiple linear regression analysis for quantitative traits. Human plasma ghrelin was measured with a commercial radioimmunoassay (Phoenix) in 300 overweight/obese subjects. The plasma samples were collected under fasting conditions.

Results: We observed a significant decreasing trend from Leu/Leu homozygous, to Leu/Met heterozygous and to Met/ Met homozygous for triglycerides, fasting insulin levels and HOMA-IR index ( $\mathrm{p}=0.02, \mathrm{p}=0.01$ and 0.001 , respectively). Consistently with these findings a significant increasing trend was observed in ghrelin levels for three category genotypes (29.2 pg/ml, $32 \mathrm{pg} / \mathrm{ml}$ and $36.64 \mathrm{pg} / \mathrm{ml}, \mathrm{p}=0.007)$.

The $-604 \mathrm{~T}>\mathrm{C}$ SNP was analyzed in the whole cohort determining a trend of decrease from $\mathrm{CC}$ to $\mathrm{TC}$ and to $\mathrm{TT}$ for insulin levels and HOMA-IR index, however the data was not statistically significant ( $\mathrm{p}=0.08$ for both). Nevertheless the same analysis was performed in subjects homozygous for Leu72 and we noted a significant decreasing trend in insulin levels and HOMA-IR index ( $\mathrm{p}=0.001$ for both). No difference was observed in ghrelin levels between the groups. There were no significant associations between Gln90Leu polymorphism and investigated variables.

Conclusions: Our observations suggest that $-604 \mathrm{C}>\mathrm{T}$ and Leu72Met ghrelin gene polymorphisms modulate insulin sensitivity. Interestingly we found that the $-604 \mathrm{~T}$ allele influences insulin sensitivity only in subjects not carrying the Met72 variant.

Supported by MIUR 20032003061834 004

\section{PS 12}

\section{Cardiovascular risk factors}

\section{6}

Insulin resistance assessed by homeostasis model assessment predicts incident symptomatic cardiovascular disease in Caucasians subjects from the general population. The Bruneck Study

E. Bonora ${ }^{1}$, S. Kiechl ${ }^{2}$, J. Willeit ${ }^{2}$, R. C. Bonadonna ${ }^{1}$, M. Muggeo ${ }^{1}$;

${ }^{1}$ Endocrinology and Metabolic Diseases, University of Verona, Italy, ${ }^{2}$ Neurology, University of Innsbruck, Austria.

Background and Aims: Insulin resistance is related to several classic and non-traditional risk factors. Aim of the present study was to evaluate whether insulin resistance can independently predict incident cardiovascular disease (CVD) events in the general population.

Materials and Methods: A sample representative of the general population of Bruneck, Italy ( $\mathrm{n}=919$; age $40-79$ years) had an estimate of insulin resistance with the Homeostasis Model Assessment (HOMA-IR) and the measure of several candidate risk factors (smoking, BMI, blood pressure, $\mathrm{HbA1c}$, lipids, apoproteins, urate, adiponectin, inflammatory markers, endothelial adhesion molecules). Subjects were followed-up for 15 years. CVD was a composite end-point including fatal and non-fatal myocardial infarction and stroke, transitory ischemic attack, and any revascularization procedure. Subjects without insulin measurement $(n=31)$ or preexisting CVD ( $\mathrm{n}=49)$ were excluded from analyses.

Results: Insulin resistance was correlated to most classic and non-traditional risk factors also after adjusting for sex, age, BMI and smoking. During the 15 years of follow-up, 118 subjects experienced one or more symptomatic CVD events. Subjects of top quartile of HOMA-IR (i.e., insulin resistant subjects) had an increased risk of incident symptomatic CVD as compared to subjects belonging to other three quartiles (i.e., non-insulin-resistant subjects). This was found in the model including only demographic variables (sex and age), in the model including also physical activity and classic risk factors (smoking, HbA1c, LDL-cholesterol and hypertension), and in the model including also BMI, HDL-cholesterol and triglycerides. Moreover, when the model included also non-traditional risk factors (fibrinogen, oxidized LDL, hsCRP, E-selectin, adiponectin), the association between HOMA-IR and CVD remained significant and virtually unchanged (HR 2.4, 1.5-3.8, $\mathrm{p}<0.001$ ). Results were unchanged when subjects with IFG, IGT or diabetes were excluded. Results were similar in men and women.

Conclusion: Insulin resistance, as assessed by a simple method based upon the measurement of fasting plasma glucose and serum insulin, seems to be able to predict subsequent symptomatic CVD in the general population also independently of several classic and non-traditional risk factors related to this metabolic phenomenon.

0367

Screen detected subjects with type 2 diabetes and impaired glucose tolerance have more adverse cardiovascular risk than subjects with impaired fasting glucose

K. J. Gorter ${ }^{1}$, P. G. H. Janssen ${ }^{1}$, R. P. Stolk ${ }^{2}$,

G. E. H. Rutten ${ }^{1}$;

${ }^{1}$ Julius Center for Health Sciences and Primary Care, University Medical Center Utrecht, ${ }^{2}$ Department of Epidemiology, University of Groningen Medical Center, Groningen, The Netherlands. 
Background and Aims: Not only type 2 diabetic patients but also subjects with impaired glucose tolerance (IGT) and impaired fasting glucose (IFG) are at increased risk of cardiovascular diseases (CVD). However, there is debate regarding the strength of the association between IFG and CVD risk. In addition, differences in cardiovascular risk factors between different categories of impaired glucose regulation may have implications for screening strategies. Our aim was to assess and compare the cardiovascular risk profiles of subjects with type 2 diabetes, IGT and IFG identified in a screening programme.

Materials and Methods: From 2002 to 2003, 29,251 nondiabetic patients, aged 50-70 years, from 41 primary care practices, were invited to participate in a populationbased diabetes screening programme in the Netherlands. The screening was part of the ADDITION study (AngloDanish-Dutch Study in general practice of intensive treatment and complication prevention in type 2 diabetic patients identified by screening). Clinical characteristics were assessed of 285 detected diabetic patients, 175 subjects with IGT, 218 with IFG, and 72 subjects with normal glucose tolerance (NGT).

Results: Body mass index (BMI) and systolic (SBP) and diastolic (DBP) blood pressure were significantly different between subjects with NGT and IFG on the one hand and subjects with IGT and diabetes on the other hand (NGT: BMI $\left(\mathrm{kg} / \mathrm{m}^{2}\right) 26.7 \pm \mathrm{SD} 3.7$, SBP $(\mathrm{mmHg}) 150 \pm 25$, DBP (mmHg) $84 \pm 10$; IFG: BMI 27.3 \pm 4.4 , SBP 150 \pm 25 , DBP $84 \pm 12$; IGT: BMI 29.5 \pm 5.7 , SBP $161 \pm 24$, DBP $89 \pm 12$; type 2 diabetes: BMI 30.7 \pm 5.6 , SBP $162 \pm 23$, DBP 90 \pm 11 ). Poor glycaemic control was associated with elevated BMI, blood pressure and lipids. In subgroup analyses the age and gender adjusted association between glycaemic control and SBP, HDL-cholesterol, LDL-cholesterol and triglycerides were still significant in subjects with $\mathrm{BMI}>27 \mathrm{~kg} / \mathrm{m}^{2}$, but not any longer in those with BMI $<27 \mathrm{~kg} / \mathrm{m}^{2}$. Such effect modifying by BMI was not found regarding DBP. In subjects with $\mathrm{BMI}>27 \mathrm{~kg} / \mathrm{m}^{2} \mathrm{HbA} 1 \mathrm{c}$, blood pressure and lipids were more adverse than in those with BMI $<27 \mathrm{~kg} / \mathrm{m}^{2}$.

Conclusion: Both screen detected type 2 diabetic patients and subjects with IGT had an unfavourable cardiovascular risk profile. Blood pressure and weight in subjects with IFG were significantly lower than in subjects with type 2 diabetes and IGT suggesting IFG is a condition with less risk to develop cardiovascular diseases. The increased CVD risk of hyperglycaemia is notably present in obese persons. Our data indicate that it might be more appropriate to screen for overweight rather than for hyperglycaemia in order to detect people with increased cardiovascular risk.

\section{8}

Two-hour post-load glucose: an independent predictor of 5-year all-cause mortality - the AusDiab study

E. L. M. Barr ${ }^{1}$, P. Z. Zimmet ${ }^{1}$, T. A. Welborn ${ }^{2}$, J. E. Shaw ${ }^{1}$; ${ }^{1}$ Epidemiology, International Diabetes Institute, Melbourne,

${ }^{2}$ Department of Medicine, University of Western Australia, Nedlands, Australia.

Background and Aims: Hyperglycaemia, in particular elevated post-load glucose, has been identified as an important predictor of mortality. Some studies have indicated that even smaller elevations below the diabetic threshold are independently associated with mortality. However, few data exist from nationally representative cohorts which have incorporated an oral glucose tolerance test (OGTT). Therefore, the aim of this analysis was to investigate, in people without previously diagnosed diabetes, whether post-load plasma glucose is an independent predictor of all-cause mortality.

Materials and Methods: The Australian Diabetes, Obesity and Lifestyle Study (AusDiab) is a nationally representative population-based survey of adults aged $\geq 25$ years. Baseline (1999-2000) measurements for standardised $\mathrm{HbA}_{1 \mathrm{c}}$, fasting and post-load plasma glucose (from a 75 g OGTT) were available for 9935 people without previously diagnosed diabetes. In 2005, vital status was ascertained from the National Death Index.

Results: Over a median of 5.1 years, 247 deaths were identified. After adjusting for age and sex, the hazard ratios (HRs) across increasing quintiles for post-load plasma glucose were $1.5(95 \% \mathrm{CI} 0.9-2.7)$ for $4.7-5.4 \mathrm{mmol} / \mathrm{l}, 1.7$ (1.0-3.0) for 5.5-6.2 mmol/1, $1.4(0.8-2.4)$ for $6.3-$ $7.4 \mathrm{mmol} / \mathrm{l}$, and $1.9(1.1-3.1)$ for $7.5-28.7 \mathrm{mmol} / \mathrm{l}(P=0.04$ for trend). After further adjustment for other known risk factors, the hazard ratios (HRs) for increasing quintiles were $1.5(0.9-2.6), 1.8(1.0-3.0), 1.5(0.9-2.6)$, and 1.9(1.2-3.2) ( $P=0.025$ for trend). After fasting blood glucose and/or $\mathrm{HbA}_{1 \mathrm{c}}$ were included in the multivariate model, the HRs for increasing quintiles of post-load glucose were unchanged. However, while the overall trend for post-load glucose remained significant with the inclusion of fasting blood glucose $(P=0.038$ for trend), the trend showed only borderline significance when $\mathrm{HbA}_{1 \mathrm{c}}$ was included $(P=0.06)$.

Conclusion: Over a 5-year period, post-load plasma glucose, even at levels below the diabetic threshold, is an independent predictor of all-cause mortality in people without previously diagnosed diabetes. $\mathrm{HbA}_{1 \mathrm{c}}$ and/or fasting plasma glucose did not alter the mortality risk 
associated with post-load plasma glucose. The results of this study provide further support for the utility of post-load hyperglycaemia in predicting total mortality in people without diabetes.

Supported by National Health and Medical Research Council (NHMRC)/National Heart Foundation of Australia post graduate scholarship

\section{9}

Proinsulin predicts development of cardiovascular risk factors in a 7-years population-based follow-up study I. Toft ${ }^{1}$, J. Kronborg ${ }^{2}$, I. Njølstad ${ }^{3}$, B. O. Eriksen ${ }^{1}$, M. Solbu ${ }^{1}$, T. G. Jenssen ${ }^{1}$;

${ }^{1}$ Institute of Clinical Medicine, University of Tromsø, ${ }^{2}$ Department of Medicine, Lillehammer Hospital, Lillehammer, ${ }^{3}$ Institute of Community Medicine, University of Tromsø, Norway.

Background and Aims: Proinsulin is released in increased amounts in persons who are at cardiovascular risk or who develop type 2 diabetes. In fact, it has been speculated that proinsulin may have a pathogenetic role in the development of cardiovascular disease. Most studies have been performed in persons at increased risk of disease or in patients with established disease, and preferentially in men. We therefore posed the question whether proinsulin predicts development of cardiovascular risk in low-risk subjects, regardless of gender.

Materials and Methods: In a population-based study of 1.946 non-diabetic men (age 59.0 (9.2), average (SD)) and 1.926 non-diabetic women (age 59.6 (9.8)) we measured albumin:creatinine ratio (ACR) in three morning urine samples, as well as HbAlc, lipids, blood pressure and body weight, height and waist circumference. The measurements were performed both in 1994-5 and in 2001-2. Development of cardiovascular risk factors in relation to quartiles of proinsulin (as measured in 1994-5) was analyzed in a multiple logistic regression analysis. Cardiovascular risk factors were defined as: $\mathrm{HbA} 1 \mathrm{c}>6.0 \%$, dyslipidemia (triglycerides $>1.7 \mathrm{mmol} / \mathrm{l}$ and/or HDL-cholesterol $<0.9 \mathrm{mmol} / \mathrm{l}$ (men) or $<1.0 \mathrm{mmol} / \mathrm{l}$ (women), ACR $>0.7 \mathrm{mg} /$ mmol, and hypertension (blood pressure $\geq 140 / 90 \mathrm{mmHg}$ or pharmacological antihypertensive treatment). In each analysis the risk factor was not present in 1994-5.

Results: Over this 7-years period baseline proinsulin (highest vs. lowest quartile) predicted increase in $\mathrm{HbA} 1 \mathrm{c}>6.0 \%$ both in men and women (OR men 2.95, $95 \%$ CI: $1.44,6,06$; OR women $2.42,95 \%$ CI: $1.16,5.05)$ and dyslipidemia in both genders (OR men 1.65, 95\% CI:
1.04, 2,26; OR women 1.88, 95\% CI: 1.23, 2.90). Proinsulin predicted development of ACR $>0.7$ in men only (OR 2.08, 95\% CI: 1.24, 3.50). There was no relationship between proinsulin and development of hypertension in neither men nor women. The association between proinsulin and development of cardiovascular risk factors was no longer significant when proinsulin was corrected for by insulin (proinsulin:insulin ratio). Insulin:glucose ratio, a surrogate parameter for insulin resistance, gave similar results as proinsulin alone (data not shown).

Conclusions: Proinsulin predicts the development of cardiovascular risk factors in both genders, with the exception of microalbuminuria, which is predicted only in men. Since essentially the same results were found with the insulin: glucose ratio, and the associations were no longer present when the proinsulin:insulin ratio was calculated, we conclude that the relationship between proinsulin and cardiovascular risk factors is related to insulin resistance, and probably not by specific effects related to proinsulin per se.

Supported by Norwegian Council of Science

0370

The cardiovascular risk in patients with type 1 diabetes mellitus and metabolic syndrome

R. Z. Timar, V. Serban, L. Diaconu, V. Botea, A. Vlad, A. Sima, M. Rosu;

Diabetes Clinic, University of Medicine and Pharmacy "Victor Babes", Timisoara, Romania.

Background and Aims: The metabolic syndrome (MS) is an important risk factor for cardiovascular disease and premature mortality in patients with type 2 diabetes mellitus (DM) and nondiabetic persons. MS is a condition characterized by a clustering of clinical and biochemical proatherogenic abnormalities. The aims of our study were to estimate the prevalence of MS in patients with type $1 \mathrm{DM}$ and to compare the cardiovascular risk present in these individuals with that of type 1 diabetic patients without MS. In addition, we assessed whether the presence of MS in type $1 \mathrm{DM}$ is associated with impairment of renal function and poor glycemic control.

Materials and Methods: The study enrolled 724 patients with type $1 \mathrm{DM}, 382$ men (52.8\%) and 342 women (47.2\%), with the mean age of $42.3 \pm 7.8$ years and the mean duration of DM 23.4 \pm 7.2 years. The MS was defined according to the new IDF criteria. The absolute 10 years risk of developing 
coronary heart disease (CHD-Risk) was assessed using the Coronary Chart Euro '98. Glomerular filtration rate (GFR) was estimated with the Cockcroft-Gault equation. Glycemic control was classified as good $(\mathrm{HbA} 1 \mathrm{c}<6.5 \%)$, intermediate $(\mathrm{HbA} 1 \mathrm{c}=6.5-9 \%)$, or poor $(\mathrm{HbA} 1 \mathrm{c}>9 \%)$.

Results: The MS was present in 212 patients (29.28\%) with type $1 \mathrm{DM}$. The prevalence of MS was higher in women $(32.75 \%)$ than in men $(26.18 \%)$. The prevalence of MS increased with worsening of renal function: from $20.65 \%$, in patients with GFR $>60 \mathrm{ml} / \mathrm{min} / 1.73 \mathrm{~m}^{2}$, to $38.89 \%$, in those with GFR $=30-60 \mathrm{ml} / \mathrm{min} / 1.73 \mathrm{~m}^{2}$, and to $62.79 \%$, in persons with GFR $<30 \mathrm{ml} / \mathrm{min} / 1.73 \mathrm{~m}^{2}$. The prevalence of MS was $17.52 \%$ in patients with good glycemic control, $29.7 \%$ in those with intermediate and $46.03 \%$ in subjects with poor glycemic control. CHD-risk was $12.1 \%$ in patients with MS vs. $8.3 \%$ in those without MS.

Metabolic characteristics of type 1 diabetic patients with and without MS

\begin{tabular}{llll}
\hline & MS & Without MS & $\mathrm{p}$ \\
\hline $\begin{array}{l}\text { Number (M/F) } \\
\text { HbA1c (\%) }\end{array}$ & $100 / 112$ & $282 / 230$ & \\
$\begin{array}{l}\text { Systolic blood presure } \\
\text { (mmHg) }\end{array}$ & $14.4 \pm 2.5$ & $7.4 \pm 1.9$ & $<0.001$ \\
$\begin{array}{l}\text { Diastolic blood presure } \\
\text { (mmHg) }\end{array}$ & $92.4 \pm 10.7$ & $81.3 \pm 9.3$ & $<0.001$ \\
$\begin{array}{l}\text { Total cholesterol } \\
\text { (mg/dL) }\end{array}$ & $231.2 \pm 42.3$ & $203.4 \pm 38.2$ & $<0.001$ \\
$\begin{array}{l}\text { LDLc (mg/dL) } \\
\text { HDLc (mg/dL) }\end{array}$ & $121.2 \pm 26.1$ & $113.1 \pm 24.3$ & $<0.001$ \\
Triglycerides (mg/dL) & $274.6 \pm 8.7$ & $46.7 \pm 9.9$ & $<0.001$ \\
\hline
\end{tabular}

Data are mean \pm standard deviation. $P$ was calculated with unpaired Student's t test.

Conclusion: MS is more prevalent in individuals with type 1 DM than in general population and increases the cardiovascular risk of these patients. The prevalence of MS was higher in type 1 diabetic patients with renal function impairment and with poor glycemic control.

\section{1}

Does screening for diabetes identify a group of diabetics with high risk of coronary heart disease and is the risk modifiable? The ADDITION study

A. Sandbaek ${ }^{1}$, S. Griffin ${ }^{2}$, R. Stolk ${ }^{3}$, M. Davies ${ }^{4}$,

G. Rutten ${ }^{5}$, K. Khunti ${ }^{6}$, N. J. Wareham ${ }^{2}$,

K. Borch-Johnsen ${ }^{7,1}$, T. Lauritzen ${ }^{1}$;
${ }^{1}$ Department of General Practice, University of Aarhus, Denmark, ${ }^{2}$ MRC, Epidemiology Unit, Cambridge, United Kingdom, ${ }^{3}$ Department of Epidemiology, University Medical Centre Groningen, The Netherlands, ${ }^{4}$ Department of Cardiovascular Medicine, University of Leicester, United Kingdom, ${ }^{5}$ Julius Centre for Health Sciences and Primary Care, University of Utrecht, The Netherlands, ${ }^{6}$ Department of Health Sciences, University of Leicester, United Kingdom, ${ }^{7}$ Steno Diabetes Centre, Gentofte, Denmark.

Background and Aim: A key factor in assessing the balance between the costs and benefits of implementing policies for early detection of Type 2 diabetes is the magnitude of cardiovascular risk reduction following early detection and intensive therapy. The ADDITION study includes 3000 people with screen detected type 2 diabetes in the Netherlands, the United Kingdom and Denmark in order to address this issue. The study is a pragmatic randomised controlled trial of the impact of intensified multi-factorial treatment on 5 year cardiovascular event rate compared to standard care in people with screen-detected diabetes. Here we describe the baseline coronary heart disease (CHD) risk of the first 2000 enrolled individuals and analyse the extent to which that risk is potentially modifiable.

Material and Methods: Stepwise screening strategies varied between the centres but included risk questionnaires, routine general practice data, random blood glucose, HbAlc and fasting blood glucose. All individuals with Type 2 diabetes were diagnosed using the 1999 World Health Organization (WHO) criteria.

Results: In the period April 2001 to March 2005, 299 general practices were recruited and 58,736 people entered the first step of the screening process in the three countries combined. Demographic, clinical and biochemical characteristics of the first 2000 people with Type 2 diabetes included in the trial are shown in Table. The average 10year CHD risk (UKPDS engine) for the included women and men was respectively $13 \%(\mathrm{SD}=7.5)$ and $24 \%$ (11.7). The $25 \%$ and $75 \%$ quartiles in women and men were $7.6-$ $15.9 \%$ and $15.4-38.6 \%$ respectively. Ten percent of the women and men had a risk above $22 \%$ and $39 \%$ respectively. Of people with blood pressure $>140 / 90,57 \%$ were not in antihypertensive treatment. Correspondingly $91 \%$ of those with total cholesterol $>5 \mathrm{mmol} / \mathrm{l}$ were not in lipid lowering treatment.

Conclusion: The population identified by screening in the ADDITION trial has a CHD risk profile similar to populations with traditionally identified diabetes ${ }^{1}$. There seems to be a realistic possibility to reduce this risk through lifestyle intervention and medical therapy especially as a 
major proportion of people at risk is not on relevant pharmacological treatment.

${ }^{1}$ Song $\mathrm{SH}$ and Brown PM, Diabetic Medicine, 21, 238-245

Baseline characteristics. Standard deviations in brackets.

\begin{tabular}{|c|c|c|}
\hline & \multirow{2}{*}{$\begin{array}{l}\text { Men } \\
N=1153\end{array}$} & \multirow{2}{*}{$\begin{array}{l}\text { Women } \\
N=847\end{array}$} \\
\hline & & \\
\hline Age at diagnosis, $N=2000$ & 59.4 & 60.5 \\
\hline$\%$ White, $N=1938$ & 97.2 & 96.4 \\
\hline HbA1c (\%), $N=1885$ & $7.3(1.7)$ & $7.0(1.5)$ \\
\hline Systolic BP, mmHg, $N=1955$ & $154(23)$ & $153(23)$ \\
\hline Diastolic BP, $m m \mathrm{Hg}, N=1955$ & $89(12)$ & $86(12)$ \\
\hline BMI $\left(\mathrm{kg} / \mathrm{m}^{2}\right), N=1930$ & $\begin{array}{l}31.2 \\
(5.2)\end{array}$ & $\begin{array}{l}32.3 \\
(6.3)\end{array}$ \\
\hline Waist, cm, $N=1932$ & $110(12)$ & $103(14)$ \\
\hline Total Cholesterol, mmol/1, $N=1941$ & $5.5(1.1)$ & $5.8(1.1)$ \\
\hline Triglyceride, $\mathrm{mmol} / \mathrm{l}, N=1937$ & $2.1(1.7)$ & $1.9(1.1)$ \\
\hline $\begin{array}{l}\text { \% with microalbuminuria (UAC ratio }>3.4 \text { ), } \\
N=1799\end{array}$ & 18.0 & 13.7 \\
\hline
\end{tabular}

0372

The metabolic syndrome as predictor of coronary heart disease, all-cause mortality and insulin resistance - the influence of choice of criteria

C. Berne ${ }^{1}$, K. Jensevik ${ }^{2}$, L. Berglund ${ }^{2}$, B. Zethelius ${ }^{3}$;

${ }^{1}$ University Hospital, Department of Medical Sciences, Uppsala, ${ }^{2}$ University Hospital, Uppsala Clinical Research Centre, ${ }^{3}$ University Hospital, Public Health and Caring Scinces, Uppsala, Sweden.

Background and Aims: Controversy exists regarding the most appropriate definition of the metabolic syndrome (MS). In addition to the recent IDF criteria, earlier definitions from the WHO and NCEP ATP III have been widely used in their complete form or truncated, due to lack of full criteria, in epidemiological studies. In a populationbased investigation of elderly males, all three definitions could be applied, since data permitting the full definitions were collected at age 70, including measurements of insulin sensitivity with the euglycamic insulin clamp. We have compared the three definitions as predictors of coronary heart disease, all cause mortality, association with insulin sensitivity and consistency in defining the MS.

Materials and Methods: The subjects were participating in Uppsala Longitudinal Study of Adult Men. The baseline investigation was carried from August 1991 to May 1995 in
1221 men with a follow-up of up to 10 years using Swedish national registry data for CHD and all-cause mortality. The baseline investigation included anthropometry, OGTT, blood pressure, smoking, serum lipids, a euglycaemic insulin clamp and urinary albumin excretion. The outcome measure was defined as death or first time hospitalised for CHD. CHD was defined according to ICD 9 codes 410 to 414 . Associations were analyzed using Cox's proportional hazards regression, presented as hazard ratios (HRs) with $95 \%$ confidence intervals (CIs) for a one SD increase in the predictor variable. Results: In analyses adjusted for LDL-cholesterol and smoking, the the HR for CHD with MS defined according to the WHO, NCEP and IDF criteria at age 70, was 2.29 (95\% CI;1.56-3.38;p<0.0001), $2.06(1.37-3.10 ; \mathrm{p}<0.0005)$ and $1,42(0.95-2.13 ; \mathrm{p}=0.088)$ and for all-cause mortality 1.67 (1.28-2.17), 1.64(1.23-2.19) and 1.56 (1.17-2.09), respectively. The insulin sensitivity index $(\mathrm{M} / \mathrm{I})$ was 3.35 (SD;1,74), 3.21 (1.75), 3.44(1.78) $\mathrm{mg}^{*} \mathrm{~min}^{-1} * \mathrm{~kg}^{-1 /}$ (100 mU/l, respectively. The mean M/I in men not defined as having the metabolic syndrome ranged between 5.64 $6.20 \mathrm{mg}^{*} \mathrm{~min}^{-1} * \mathrm{~kg}^{-1} /(100 \mathrm{mU} / 1$.

Altogether $536(51.7 \%)$ men were defined as having the metabolic syndrome with either of the three definitions. Of those only 174 (32.5\%) were defined by all three.

Conclusion: In elderly males the metabolic syndrome, as defined by WHO, NCEP III ATP III and IDF criteria was associated with increased all-cause mortality. For CHD incidence WHO, NCEP III, but not IDF criteria, were associated with approximately 2 times higher incidence of CHD over 10 years of follow up, despite only moderate overlap between the three definitions, which all identified individuals with insulin resistance.

Supported by Swedish Diabetes Association

0373

Association of -1131T $>C$ and S19W APOA5 gene variants with dyslipidemia and coronary risk among type 2 diabetic patients

S. Charriere ${ }^{1}$, S. Bernard ${ }^{1}$, M. Aqallal ${ }^{2}$, P. Moulin ${ }^{1}$, C. Marcais ${ }^{2}$;

${ }^{1}$ Fédération d'Endocrinologie, Hospices Civils de Lyon, Bron, ${ }^{2}$ Laboratoire de Biochimie - Biologie Moléculaire, Hospices Civils de Lyon, Pierre Benite, France.

Background and Aims: Dyslipidemia in type 2 diabetes is an independent risk factor for cardiovascular diseases. Genetic factors favouring this dyslipidemia are poorly identified. We studied APOA5 gene as a candidate because APOA5 non-sense mutations cause familial hyperchylomicronemia and 2 common minor haplotypes are strong determinants of plasma TG variability across human populations. 
Materials and Methods: We studied the impact of 2 APOA5 gene polymorphisms, $-1131 \mathrm{~T}>\mathrm{C}$ and $\mathrm{S} 19 \mathrm{~W}$, on lipid profiles in 400 type 2 diabetic patients and on the occurrence of cardiovascular disease within a 5 years follow-up period in a subset of 196 patients. In group N, triglyceridemia was below the 90th percentile adjusted for age and sex $(n=130)$. 270 patients had moderate hypertriglyceridemia (group $\mathrm{T}>90$ th percentile). A third group (group $\mathrm{V}, \mathrm{n}=51$ ) of severely hypertriglyceridemic patients with $\mathrm{TG}$ over $15 \mathrm{mM}$ and hyperchylomicronemia was studied.

Results: $-1131 \mathrm{~T}>\mathrm{C}$ allele was more frequent in hypertriglyceridemic diabetics $(20.6 \%$ vs. $9.8 \%$ vs. $5.0 \%$, group $\mathrm{V}$ vs. $\mathrm{T}$ vs. $\mathrm{N}, \mathrm{p}<0.001)$. Hence $-1131 \mathrm{~T}>\mathrm{C}$ carriers were hypertriglyceridemic, either moderately $[\mathrm{OR}=2.10(1.09$ 4.01)] or severely $[\mathrm{OR}=4.50(1.99-10.18)]$. S19W allele was more frequent only in severely hypertriglyceridemic patients $(14.0 \%$ vs. $6.5 \%$ vs. $6.1 \%$, group V vs. T vs. N, $\mathrm{p}=0.001)$. Likewise $\mathrm{S} 19 \mathrm{~W}$ carriers were more at risk to undergo severe HTG [OR=3.22 (1.40-7.39)].

In group $(\mathrm{N}+\mathrm{T})$ the $-1131 \mathrm{~T}>\mathrm{C}$ allele was associated with higher TG $(+13 \%, \mathrm{p}=0.034)$ and lower HDLc $(-10 \%$, $\mathrm{p}=0.004)$. S19W allele was only associated with lower HDLc $(-10 \%, p=0.022)$. The risk of major coronary event was higher only in the carriers of the $-1131 \mathrm{~T}>\mathrm{C}$ allele $(\mathrm{OR}=3.52$, $\mathrm{p}=0.010$ and $\mathrm{OR}=3.51, \mathrm{p}=0.030$ after adjustment)

Conclusion: Overall these results strongly suggest that APOA5 polymorphisms may contribute to modulate dyslipidemia and cardiovascular risk in type 2 diabetes.

\section{4}

Proinsulin/insulin, but not insulin/glucose ratio, predicts progression of carotis plaques in women

J. P. B. Kronborg ${ }^{1}$, S. H. Johnsen ${ }^{2}$, T. Jenssen ${ }^{3}$,

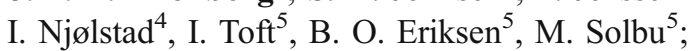

${ }^{1}$ Medical Department, Innlandet Hospital Trust,

Lillehammer, ${ }^{2}$ Neurology Department, University Hospital of North Norway, Tromsø, ${ }^{3}$ Medical Department, Innlandet Hospital Trust, Oslo, ${ }^{4}$ University Hospital of North Norway, Institute of Community Medicine, Tromsø, ${ }^{5} \mathrm{Nephrol-}$ ogy Department, University Hospital of North Norway, Tromsø, Norway.

Background and Aims: Proinsulin is increased among persons at cardiovascular risk and is also predictive for the development of diabetes. An increased secretion of proinsulin relative to insulin has been suggested as being a sign of defective proinsulin conversion associated with $\beta$-cell dysfunction. It has also been suggested that proinsulin has a more proatherogenic effect than insulin

In this prospective, population-based study we addressed whether the proinsulin/insulin or the insulin/glucose ratio predicted carotid plaque progression among non-diabetic men and women.

Materials and Methods: The study included 1886 men and 2028 women aged 25 to 82 years who were examined with B-mode high resolution ultrasound at baseline in 1994-1995 and at follow-up in 2000-2001. All plaque images were computer processed to yield a measure of plaque area in square millimetres. Proinsulin and insulin were measured at baseline. All analyses were stratified for gender

Results: Age, systolic blood pressure and tobacco smoking were associated with increments in plaque area in both genders, while cholesterol was associated with increased plaque areas in women only. After adjustment for age, baseline plaque area, waist circumference, cholesterol, HDLcholesterol, HbA1c, glucose, blood pressure and lifestyle factors (tobacco smoking, alcohol and physical activity), the proinsulin/insulin ratio was significantly associated with follow-up plaque size in women but not in men. For a 1SD (0.81) higher proinsulin/insulin ratio, mean plaque area was $0.92 \mathrm{~mm}^{2}$ larger in women. Insulin/glucose ratio was not associated with changes in plaque size.

Conclusion: The proinsulin/insulin, and not the insulin/ glucose ratio is an independent predictor of progression of arterial plaque size in non-diabetic women, but not in men. Supported by the Norwegian Research Council, the Norwegian Foundation for Health and Rehabilitation, Innlandet Hospital Trust and University Hospital of North Norway Trust

0375

Reduction of atherothrombosis for continued health (REACH) registry one-year results: cardiovascular event rates in patients with diabetes

M. Krempf ${ }^{1}$, P. Steg ${ }^{2}$, D. Bhatt ${ }^{3}$, M. Ohman ${ }^{4}$, J. Röther ${ }^{5}$, P. Wilson ${ }^{6}$, S. Goto ${ }^{7}$;

${ }^{1}$ Clinique d-Endocrinologie, CHRU Nantes, France, ${ }^{2}$ Department of Cardiology, AP-HP Hôpital Bichat-Claude Bernard, Paris, France, ${ }^{3}$ Department of Cardiovascular Medicine, Cleveland Clinic Foundation, Cleveland, United States, ${ }^{4}$ Duke University Medical Center, Duke Hospital North, Durham, United States, ${ }^{5}$ Neurologie, Klinikum Minden, Minden, Germany, ${ }^{6}$ Department of Endocrinology, Diabetes and Medical Genetics, Medical University of South Carolina, Charleston, United States, ${ }^{7}$ Department of Medicine, Tokai University School of Medicine, Kanagawa, Japan.

Background and Aims: The REACH Registry was set up to analyse cardiovascular disease (CVD) outcomes in high risk patients. We present the cardiovascular event rates in diabetic patients after one-year follow-up. 
Materials and Methods: More than 68,000 outpatients aged 45 years or older with documented arterial disease or $\geq 3$ risk factors for atherothrombosis were recruited from 44 countries all over the world, including 30,043 (44.03\%) patients with diabetes. The follow-up rate after one year was $92.5 \%$.

Results: In the diabetic patient population there were more females (39.03 vs 33.09\%, p<0.001) and greater BMI (29.4 vs $27 \mathrm{~kg} / \mathrm{m} 2, \mathrm{p}<0.001)$, waist circumference (101 vs $95 \mathrm{~cm}$, $\mathrm{p}<0.001$ ), microalbuminuria (129 vs $41.9 \mathrm{mg} / \mathrm{d}, \mathrm{p}<0.001)$ and blood glucose (146 vs $98.4 \mathrm{mg} / \mathrm{dl}, \mathrm{p}<0.001)$ compared with non-diabetic patients. The mean age of patients in both groups did not differ (68 years). HbA1c was not monitored. In the diabetic group $87.7 \%$ had high blood pressure, $79.4 \%$ had hypercholesterolemia and $14.2 \%$ were current smokers. At the start of the study half of the diabetic patients (51\%) had a history of coronary heart disease, 25\% neurovascular disease, $13 \%$ peripheral arterial disease and 30.7\% had $>3$ risk factors. Of patients with a history of atherosclerosis, $14 \%$ had two locations (mainly coronary and neurovascular) and $1.8 \%$ had three locations, similar to non-diabetic patients. Fewer diabetic patients were treated with antiplatelet drugs $(74$ vs $82 \%, \mathrm{p}<0.001)$ and more patients received antihypertensive treatments (93.3 vs 89.8\%, p<0.001), but there was no difference in statin treatment (70 vs 68\%). Further treatments included insulin (27\% of patients), metformine (42\%), sulfonylureas (45\%), glitazones $(18 \%)$, other drugs (11.5\%) and diet modifications (9.8\%).

After one-year follow-up, all cardiovascular event rates were higher $(p<0.001)$ in the diabetic patient population: cardiovascular death (1.82 vs $1.23 \%)$, non-fatal MI (1.33 vs $0.97 \%)$, non-fatal stroke $(1.67$ vs $1.46 \%)$ and total $\mathrm{CV}$ death/MI/stroke (3.80 vs 3.03\%). The CVD event rate was five times higher and cardiovascular death rate 7 times higher in diabetic patients with documented atherothrombosis ( 3 locations) compared with patients with only $>3$ risk factors. The non-fatal MI rate was higher in North America (1.79\%) and Eastern Europe compared with Western Europe $(\mathrm{p}<0.04)$ and Asia $(\mathrm{p}<0.001)$ but non-fatal stroke rate was higher in Asia (2.27\%) and Eastern Europe (2.21\%) compared with North America or West Europe (1.32 and $1.86 \%$, respectively).

Conclusion: After one-year follow-up, CVD event rate was $50 \%$ higher in diabetic patients compared with non-diabetic patients. This risk increased dramatically in patients with multiple atherothrombotic locations. The event rates and locations differed across the participating countries.

Supported by the REACH Registry is sponsored by sanofiaventis and Bristol-Myers Squibb

\section{PS 13}

\section{Metabolic syndrome}

0376

\section{Alanine aminotransferase and the 6-year risk of the metabolic syndrome in Caucasian men and women: the HOORN Study}

R. K. Schindhelm ${ }^{1}$, J. M. Dekker ${ }^{2}$, G. Nijpels ${ }^{2}$, C. D. A. Stehouwer ${ }^{3}$, L. M. Bouter ${ }^{2}$, R. J. Heine ${ }^{1}$, M. Diamant ${ }^{1}$;

${ }^{1}$ Department of Endocrinology/Diabetes Centre, VU University Medical Centre, Amsterdam, ${ }^{2}$ The EMGO Institute, VU University Medical Centre, Amsterdam, ${ }^{3}$ Department of Internal Medicine, Academic Medical Hospital, Maastricht, The Netherlands.

Background and Aims: The metabolic syndrome, characterised by abdominal obesity, hyperglycaemia, hypertension and dyslipidaemia, confers an enhanced risk for the development of type 2 diabetes mellitus and cardiovascular disease. Excessive deposition of fat in the liver, referred to as nonalcoholic fatty liver disease (NAFLD) is not only regarded as a risk factor but also as a feature of the metabolic syndrome. In the present study, we assessed the association between alanine aminotransferase (ALT), a marker of NAFLD, and the risk of developing the metabolic syndrome in a population-based study in Caucasian men and women.

Materials and Methods: The association of ALT with the 6-year risk of the metabolic syndrome in 1,097 subjects, aged 50-75 years, was assessed in the Hoorn Study with logistic regression analysis. Subjects with the metabolic syndrome at baseline, defined according to the Adult Treatment Panel III of the National Cholesterol Education Program, were excluded.

Results: After 6.4 (range: 4.4-8.1) years of follow-up, 226 subjects $(20.6 \%)$ had developed the metabolic syndrome. The odd ratio (95\% confidence interval) of metabolic syndrome, adjusted for age, sex, alcohol-intake and follow-up duration was 2.25 (1.50-3.37) for subjects in the upper tertile compared to those in the lower tertile of ALT. This association persisted after additional adjustment for all the baseline metabolic syndrome features [1.62 (1.02-2.58)]. Among the individual components of the metabolic syndrome, ALT was significantly associated only with fasting plasma glucose at follow-up.

Conclusion: ALT is associated with risk of the metabolic syndrome in a general population of middle-aged Caucasian men and women. These data indicate that NAFLD, 
reflected by ALT, may herald future metabolic derangements. Further studies should elucidate the role of liver fat accumulation in the pathogenesis of metabolic syndrome and the related complications.

Table. Odds ratios $(95 \% \mathrm{CI})$ of ALT for developing the metabolic syndrome after 6 years of follow-up

\begin{tabular}{llll}
\hline & Tertile 1 & Tertile 2 & Tertile 3 \\
\hline $\mathrm{n}$ & 329 & 418 & 350 \\
Model 1 & 1 & $1.43(0.96-2.13)$ & $2.25(1.50-3.37)$ \\
Model 2 & 1 & $1.40(0.90-2.18)$ & $1.62(1.02-2.58)$ \\
\hline
\end{tabular}

Model 1: adjusted for age, sex, alcohol-intake and followup duration; Model 2: Model 1+ additional adjustments for waist, glucose, HDL-cholesterol, triglycerides and systolic and diastolic blood pressure

\section{7}

10-year trends in prevalence of the metabolic syndrome among Finnish adults

G. Hu ${ }^{1,2}$, P. Jousilahti ${ }^{1}$, J. Tuomilehto ${ }^{1,2}$;

${ }^{1}$ Department of Epidemiology and Health Promotion, National Public Health Institute, Helsinki, ${ }^{2}$ Department of Public Health, University of Helsinki, Helsinki, Finland.

Background and Aims: Only few studies on the trend in prevalence of the metabolic syndrome have been reported. The aim of this study is to assess the trend between 1992 and 2002 in prevalence of the metabolic syndrome defined by the National Cholesterol Education Program (NCEP) and the International Diabetes Federation (IDF) among Finnish adults.

Materials and Methods: Two cross-sectional population surveys were carried out in Finland in 1992 and 2002. A total of 2120 participants aged 45-64 years in 1992 and 2002 participants aged 45-64 years in 2002 were included in the analysis.

Results: Between 1992 and 2002, the prevalence of the metabolic syndrome was $0.9 \%$ higher based on the NCEP definition (48.9 vs $49.8 \%$ ) and $1.8 \%$ higher based on the IDF definition (51.4 vs. 53.2\%) in men, however, the increase was not significant $(\mathrm{P}>0.4)$. Decreases in high blood pressure and low high-density lipoprotein cholesterol, and increase in high fasting glucose accounted for the non-significant increase in prevalence of the metabolic syndrome in men. In women, the prevalence of the metabolic syndrome was significantly higher in 2002 than in 1992. Between 1992 and 2002, the prevalence of the metabolic syndrome among women increased from 32.2 to $36.2 \%$ based on the NCEP definition ( $\mathrm{P}=0.045)$, and from 38.0 to $42.3 \%$ based on the IDF definition $(\mathrm{P}=0.036)$.
Increases in abdominal obesity and high fasting glucose accounted for the increase in prevalence of the metabolic syndrome in women.

Conclusion: The prevalence of the metabolic syndrome based on the NCEP and IDF definitions has increased significantly among Finnish women during the last 10 years. Supported by the Finnish Foundation for Cardiovascular Research

0378

Comparison of prevalence of the metabolic syndrome and its components in population from Denmark and Japan

F. Ebara ${ }^{1}$, K. Borch-Johnsen ${ }^{2}$, C. Glümer ${ }^{2,3}$, D. Vistisen ${ }^{2}$, T. Jørgensen ${ }^{3}$, M. Tominaga ${ }^{4}$, N. Tajima ${ }^{1}$;

${ }^{1}$ Division of Diabetes and Endocrinology Department of Internal Medicine, Jikei University School of Medicine, Tokyo, Japan, ${ }^{2}$ Epidemiology Group, Steno Diabetes Center, Gentofte, Denmark, ${ }^{3}$ Research Centre for Prevention and Health, Glostrup University Hospital, Denmark, ${ }^{4}$ Department of Laboratory Medicine, Yamagata University, Japan.

Background and Aims: The Metabolic Syndrome (MS), first proposed by Raeven is a cluster of risk factors for cardiovascular disease and diabetes.

The MS definition has been proposed by various academic societies including WHO, NECP, European Group for the Study of Insulin Resistance (EGIR) and IDF.

The EGIR proposed an epidemiological approach to the MS, where insulin resistance was defined based on fasting insulin.

We analyzed the prevalence of the MS and the characteristics of the MS positive subjects from Denmark (Caucasian) and Japan (Non-Caucasian).

Materials and Methods: Out of the patients enrolled in both the Funagata study (1995-1996) and the Inter99 study (1999-2001) which are characterized as population based studies, 6703 cases in total (5686 cases in Denmark and 1017 cases in Japan) of 35-60 years of age who were analyzed. These studies were participants between 3560 years of age who were not diagnosed with diabetes.

The MS positive subjects were defined according to the EGIR definition.

Presence of insulin resistance (upper quartile of fasting insulin level) together with at least two of the following components:

-Hyperglycemia: FPG $\geq 6.1 \mathrm{mmol} / 1$

-Hypertension: systolic blood pressure $\geq 140$ and/or diastolic blood pressure $\geq 90$ and/or treatment for hypertension.

-Dyslipidemia: p-triglyceride $\geq 2.0 \mathrm{mmol} / \mathrm{l}$ and/or $\mathrm{HDL}$ cholesterol $<1.0 \mathrm{mmol} / \mathrm{l}$ 
-Central obesity: waist circumference $\geq 94 \mathrm{~cm}$ in men and $\geq 80 \mathrm{~cm}$ in women

We divided the eligible subjects of 35-60 years of age for analysis into 5 groups based on 5 year age ranges and compared the prevalence in men and women from the two countries. Subsequently, a comparison was made to detect what kinds of characteristics existed in MS positive subjects from the two countries. All the statistical analysis was performed using SAS System Release 8.2.

Results: The mean age of the subjects was 48.1 years in Japan and 47.3 years in Denmark.

The prevalence of the MS based on the EGIR definition among males is $2.9-9.0 \%$ in Japan and $16.3 \%-22.9 \%$ in Denmark, while it is, among females, $1.0-9.4 \%$ in Japan and $6.0-14.5 \%$ in Denmark.

In all the age groups, the MS prevalence of the subjects of both sexes in Denmark was 2-6 times higher than those in Japan.

Comparison of the population characteristics of the MS positive subjects disclosed that obesity-related factors such as BMI and waist circumference, as well as diastolic blood pressure, were significantly lower in Japanese whereas total cholesterol levels were significantly higher in Japanese. There were no significant differences in HDL, triglyceride and diastolic blood pressure.

Conclusion: The prevalence of the MS according to the EGIR definition in Denmark was higher than those in Japan, while differences were observed in the characteristics of the MS positive subjects who were recognized as a high risk population susceptible to the onset of both cardiovascular diseases and diabetes.It is conceivable that comparative investigation of the fundamental items comprising the MS definition among races with different genetics and life-styles plays an important role in preparation of a plausible MS definition which can obtain international consensuses.

\section{9}

Prevalence of the metabolic syndrome in Germany assessed in a primary health care setting

S. Moebus ${ }^{1}$, J. Hanisch ${ }^{1}$, M. Neuhäuser ${ }^{1}$, P. Aidelsburger ${ }^{2}$, J. Wasem ${ }^{3}$, K.-H. Jöckel ${ }^{1}$, H. Hauner ${ }^{4}$;

${ }^{1}$ Institute for Medical Informatics, Biometry and Epidemiology, University Hospital Essen, University of DuisburgEssen, ${ }^{2}$ CAREM, GmbH, Sauerlach, Germany, ${ }^{3}$ Alfried Krupp von Bohlen und Halbach Foundation, Institute for Healthcare Management, University of Duisburg-Essen, ${ }^{4}$ Else Kroner-Fresenius-Centre for Nutritional Medicine of the Technical University, Klinikum Rechts der Isar, Munich, Germany.
Background and Aims: The Metabolic Syndrome describes the common occurrence of central obesity, hypertension, hyperglycemia, and dyslipidemia, and is considered a major risk factor for the development of cardiovascular disease. Due to differences in currently recommended definitions (e.g. IDF, WHO, NCEP ATP III), a clear picture about its prevalence in the whole population and in subgroups is still missing. For Germany, data on the prevalence of the syndrome is rare. Aim of this study was to assess the prevalence of the Metabolic Syndrome according to the NCEP ATP III definition among Germans aged $>18$ years attending a general practitioner. Material and Methods: The German-wide cross-sectional study ran two weeks in October 2005 with 1.511 randomly selected general practices from 397 out of 438 German cities and administrative districts. Blood samples were analyzed in a central laboratory, waist circumference and blood pressure assessed, data on smoking, life style, fasting status, and sociodemographic characteristics collected. Only pregnant or breast feeding women and patients with severe illness were excluded from the study. Non-responders information about gender, age and reasons for nonparticipation were collected. Since food intake has an impact on glucose and triglyceride levels, a two-stage approach was taken for blood sampling. First, all patients on a predetermined examination day were included, yielding about 35.000 random blood samples. In case the findings concerning fasting serum glucose or triglycerides were ambiguous due to a meal in the previous 12 hours, the patient was asked to come for a second appointment to give a fasting blood sample. Furthermore, a random sample $(30 \%)$ of participants were asked to come for a second fasting blood sample, regardless of the first test result. These data provide further useful information, e.g. estimation of intraindividual variations.

Results: In total, 35.869 patients were included (age range: 18-99, women $61,1 \%$ ). More than 50.000 blood samples were taken. Fasting blood samples are available for $49 \%$ of the participants ( $42 \%$ fasting $>12$ hours, $7 \%$ fasting $8-11$ hours). For another $49 \%$, random blood glucose and triglyceride samples as well as data on time and type of last meal are available. Prevalence of hypertension was $58,2 \%(95 \%$ CI: 57,7-58,7), abdominal obesity $39,5 \%$ (39,0-40,0), hyperglycemia $17,0 \%(17,3-18,1)$, triglyceridemia $16,9 \%(16,5-17,4)$ and low HDL-C $13,2 \%$ $(12,9-13,6)$. The prevalence of the Metabolic Syndrome according to the NCEP ATP III definition was 19,8\% (95\% CI: 19,4-20,2), with women aged $>60$ years showing the highest prevalence rate $(32,8 \%$; men: $31,5 \%)$, women $<45$ years showing the lowest rate $(6,6 \%$; men: $10,6 \%)$.

Conclusion: The findings of this study show a high prevalence of the Metabolic Syndrome in the German 
population attending a general practitioner. Prevalence rates increase with age in both genders.

Unrestricted grant from Sanofi-Aventis, Germany

\section{0}

Gender differences in metabolic features of type 2 diabetes in the AGES-Reykjavik Study

E. Olafsdottir ${ }^{1}$, T. Aspelund ${ }^{1}$, G. Sigurdsson ${ }^{2}$,

B. Thorsson ${ }^{1}$, R. Benediktsson ${ }^{2}$, T. B. Harris ${ }^{3}$,

L. J. Launer ${ }^{3}$, G. Eiriksdottir ${ }^{1}$, V. Gudnason ${ }^{1}$;

${ }^{1}$ Heart Preventive Clinic and Research Institute, Icelandic Heart Association, Kopavogur, Iceland, ${ }^{2}$ Faculty of Medicine, Landspitali University Hospital, Reykjavik, Iceland, ${ }^{3}$ NIA, NIH, Bethesda, United States.

Background and Aims: A population based study with data gathered in late life as well as midlife is used to compare the metabolic features of type 2 diabetes (T2D) in the genders, diagnosed in midlife vs. later life, and this related to cardiovascular risk levels.

Materials and Methods: Data are presented from 2279 participants (mean age 76 years) attending the population based AGES-Reykjavik Study and recruited over the period 2002-2004. T2D was identified by self-report of diabetes, use of diabetes medication, or fasting S-glucose of $\geq 7 \mathrm{mmol} / \mathrm{L}$. The T2D group is subdivided into three groups, two according to age at T2D diagnosis into midlife onset (age 40-66 years) and onset in later life (age $\geq 67$ years) and a third group diagnosed when recruited. Features of glucometabolic control and cardiovascular risk characteristics of the genders are compared.

Results: Of the 1313 women participating 9.2\% were diagnosed with T2D and $14.8 \%$ of the 966 men. Mean age at diagnosis was 68 years. In midlife onset T2D, 48\% report a positive family history, compared to $34 \%$ in the late onset group, and $19 \%$ of controls. Midlife BMI measured at the mean age of 52 years was greatest in those with midlife onset T2D. However, the largest increase in BMI with age was seen in the late onset group, especially in women. Men in midlife had a higher mean BMI than women but that is reversed later in life. Diabetic women as a whole had higher systolic blood pressure than diabetic men in spite of more intensive hypertensive medication. They also showed considerably greater increase in systolic BP from midlife values than men. Urinary albumin creatinine ratio is significantly higher in diabetic men than in women indicating greater prevalence of kidney damage in men. $90 \%$ of diabetic men receive medication affecting glucose metabolism but just over $50 \%$ of the women. Still the glucometabolic profile of diabetic men is worse than in women, showing both higher fasting glucose and higher HbAlc. Total and LDL cholesterol is higher in all subgroups of women than in corresponding groups of men, partly explained by statins being more commonly prescribed for men in general than women. Self reported overall prevalence of coronary events (MI, GABG, PCI) in women is $11.5 \%$ and $29.7 \%$ in men. The highest prevalence is in midlife onset T2D in both genders, but cases are few in number and the difference does not reach statistical significance.

Conclusion: Prevalence of T2D is higher in men than women. In both genders, midlife onset of T2D had a worse metabolic profile in old age than persons with late onset T2D. Midlife onset T2D was also more associated with positive family history and higher BMI. Diabetic women had higher systolic pressure, and higher LDL than diabetic men, but a more favourable urinary albumin creatinine ratio than men. This suggests that diabetes in old age although a heterogeneous disease, dependent on family history, duration of disease, and weight history, may present different effects on the genders as reflected in unfavourable cardiovascular risk levels in diabetic women.

\section{1}

National Cholesterol Educational Program (NCEP) and International Diabetes Federation (IDF) criteria for metabolic syndrome in an Italian cohort. Results from the FIBAR Study

G. Bardini ${ }^{1}$, E. Mannucci ${ }^{2}$, M. Monami ${ }^{2}$, I. Sposato ${ }^{3}$, A. Ognibene ${ }^{3}$, C. M. Rotella ${ }^{1}$;

${ }^{1}$ Section of Endocrinology, Diabetology and Metabolic Diseases, Clinical Pathophysiology, University of Florence, ${ }^{2}$ Geriatric Unit, Critical Care Dept., University of Florence, ${ }^{3}$ Central Laboratory of Clinical Biochemistry, Careggi University Hospital, University of Florence, Italy.

Background and Aims: The new IDF criteria for Metabolic Syndrome (MS), in comparison with NCEP, produces different changes in estimates of prevalence in several populations. Few data are available in Caucasian non-diabetic subjects. Aim of this study is to evaluate the prevalence of NCEP- and IDFdefined MS in a cohort of Caucasians subjects.

Materials and Methods: A sample of 2945 individuals (aged 55.2 \pm 11.5 years, BMI $26.0 \pm 4.2\left(\mathrm{~kg} / \mathrm{m}^{2}\right)$ with no history for diabetes, resident in Florence and Bagno a Ripoli municipalities enrolled in a screening program for diabetes. Association of different definitions of MS with glucose intolerance (120-min glucose $7.8 \mathrm{mmol} / \mathrm{l}$ after a 75 g-oral glucose load) and hyperuricaemia (uric acid $>0.38 \mathrm{mmol} / \mathrm{l})$ was also assessed.

Results: The diagnosis of metabolic syndrome was made in 489 (16.6\%), $680(23.1 \%)$ and 875 (29.7\%) subjects with NCEP, NCEP revised and IDF definitions, respectively. The prevalence of IDF-defined MS was higher than NCEP-MS 
through all age ranges; among those individuals aged $>60$ years, the prevalence of IDF-MS reached 52.8\% (vs. $33.1 \%$ for NCEP-MS). The prevalence of NCEPdefined MS in lean (BMI $<25 \mathrm{~kg} / \mathrm{m}^{2}$ ), overweight and obese subjects was $3.2 \%, 19.8 \%$ and $45.2 \%$, while applying IDF criteria was $8.9 \%, 36.8 \%$ and $62.4 \%$, respectively. The proportion of individuals with pathological waist circumference according to NCEP criteria in lean, overweight and obese subjects was $3.8 \%, 36.7 \%$, and $89.2 \%$, respectively, while the corresponding figures using IDF criteria for waist circumference (European population cut-points) were $36.7 \%, 89.2 \%$, and $99.2 \%$, respectively. Both NCEP- and IDF-MS criteria were associated with glucose intolerance and hyperuricaemia. Individuals fulfilling IDF, but not NCEP criteria for the diagnosis of MS, showed a prevalence of glucose intolerance $(22.7 \%)$ significantly $(\mathrm{p}<0.05)$ lower than those fulfilling NCEP criteria only $(31.6 \%)$ or both sets of criteria $(31.8 \%)$.

Conclusion: In Caucasian subjects without known diabetes, IDF criteria produce a relevant increase in estimates of prevalence of MS, particularly in older and lean subjects individuals, when compared with NCEP criteria. NCEP-MS seems to be more effective than IDF-MS in the identification of glucose intolerant subjects.

\section{2}

Low prevalence of the metabolic syndrome in diabetic African men by ATPIII and IDF definitions: a study in African, white and south Asian men and women

W. J. Kalk, B. I. Joffe;

Department of Medicine, University of the Witwatersrand, Johannesburg, South Africa.

Background and Aims: Type 2 diabetes is associated with a high prevalence of the Metabolic Syndrome (MS) in most populations. MS severity appears to be linked to coronary heart disease, which is rare in diabetic Africans. We have compared the prevalence and severity of the MS, defined by the ATPIII (ATPIII-MS) or IDF (IDF-MS) criteria, in African (A), white (W) and South Asian (S-A) men and women with diabetes, the latter populations at high risk for CHD.

Materials and Methods: The presence of the MS was assessed in 895 subjects with stable Type 2 diabetes: (A, 500; W, 254; S-A, 141) using ATPIII criteria (waist $>102 \mathrm{~cm}$ for men; $>88 \mathrm{~cm}$ for women) and IDF criteria (waist: men, $>94 \mathrm{~cm}$ for $\mathrm{A}$ and $\mathrm{W} ;>90 \mathrm{~cm}$ for $\mathrm{S}-\mathrm{A}$; women, $>88 \mathrm{~cm}$ ); other criteria were the same. Severe MS was defined as diabetes plus 3 or 4 additional elements of the MS.

Results: In men ATPIII-MS was found in $55.0 \%$ A, vs $74.1 \% \mathrm{~W}(\mathrm{p}<0.001)$ and $67.8 \% \mathrm{~S}-\mathrm{A}(\mathrm{p}=0.11)$; severe MS in $28.7 \%$, vs $51.1 \%(\mathrm{p}<0.001)$ and $33.5 \%(\mathrm{p}=0.27)$ respec- tively. IDF-MS was present in $65.1 \%, 82.2 \%$ and $89.8 \%$ $(p<0.001)$, and severe MS in $33.5 \%, 56,3 \%$ and $59.3 \%$ in $\mathrm{A}, \mathrm{W}$ ans $\mathrm{S}-\mathrm{A}$ respectively $(\mathrm{p}<0.001)$. Both $\mathrm{A}$ and $\mathrm{S}-\mathrm{A}$ had smaller waists than $\mathrm{W}(\mathrm{p}<0.001)$; triglycerides were lowest in $A(p<0.001)$. In the diabetic women the prevalence of both ATPIII-MS and IDF-MS was similar in each ethnic group (82.5-93.9\%); but severe MS by both definitions was lowest in the A group $(\mathrm{P}<0.007)$, explained by lower triglyceride levels $(\mathrm{p}<0.001)$.

Conclusion: The prevalence of MS and severe MS, by both definitions, was lowest in the diabetic African men, in whom a waist circumference of $90 \mathrm{~cm}$ may be appropriate for the IDF-MS, as in S-A men. In diabetic Africans the lower prevalence of the MS in men, and of severe MS in both sexes, might contribute to the previously observed low prevalence of CHD in this population.

Supported by the South African MRC

\section{3}

Are the patients with metabolic syndrome as defined by International Diabetes Federation criteria more insulin resistant than NCEP-ATP III criteria?

J.-H. Kim ${ }^{1}$, H.-S. Kwon ${ }^{1}$, Y.-M. Park ${ }^{2}$, S.-Y. Lim²,

J.-H. Cho ${ }^{1}$, S.-R. Kim ${ }^{1}$, S.-A. Chang ${ }^{1}$, K.-H. Song ${ }^{1}$,

K.-H. Yoon ${ }^{1}$, W. C. Lee ${ }^{2}$, B.-Y. Cha ${ }^{1}$, K.-W. Lee ${ }^{1}$,

S.-K. Kang ${ }^{1}$, H.-Y. Son ${ }^{1}$;

${ }^{1}$ Department of Internal Medicine, ${ }^{2}$ Department of Preventive Medicine, The Catholic University of Korea, Seoul, Republic of Korea.

Background and Aims: We analyzed how well International Diabetes Federation (IDF) definition of the metabolic syndrome reflect insulin resistance status in middle-aged adults in Korea and compared it with NCEP-ATP III criteria. Materials and Methods: This study was performed from February to April, 2003-2005 in adults over 40 years living in the Chungju city, South Korea, selected by the stratified random cluster sampling. We analyzed the data from total 8,395 subjects ( 3,436 men and 4,959 women) who completed the survey consisting of anthropometric measurement and blood chemistry. Metabolic syndrome was defined as by 2 different definitions, IDF and NCEP-ATP III. We analyzed how well these two criteria reflect insulin resistant status when we define the highest quartile of HOMA-IR in whole study population as insulin resistant (IR) group using sensitivity, positive likelihood ratio, negative predictive value and area under the curve (AUC) of ROC for detecting IR group.

Results: The age-adjusted prevalence of the metabolic syndrome using IDF definition was $30.2 \%$. According to NCEP-ATP III criteria, $34.5 \%$ of the study subjects had metabolic syndrome. There was no significant difference 
between the AUC value of IDF and NCEP-ATP III criteria in total study subjects $(\mathrm{p}=0.569)$. However, IDF definition did better reflect insulin resistance in non-diabetic population significantly $(p=0.001$ in total subjects, $p<0.001$ in women) except in men $(\mathrm{p}=0.668)$. Furthermore, positive likelihood ratio by IDF definition was somewhat higher than those by NCEP-ATP III criteria.

Conclusion: From our result, new IDF definition was more specific, but less sensitive than that of NCEP-ATP III criteria for detecting insulin resistance group. Overall diagnostic accuracy for insulin resistance group by IDF definition was somewhat superior than that by NCEP-ATP III criteria, but its difference was trivial.

\section{4}

Comparison of the prevalence of the metabolic syndrome in women with prior gestational diabetes mellitus using two definitions

I. Castro-Dufourny, L. Herranz, V. Martín,

P. Martín-Vaquero, L. F. Pallardo;

Servicio de Endocrinología y Nutrición, Hospital Universitario La Paz, Madrid, Spain.

Background and Aims: To estimate the prevalence of the metabolic syndrome (SM) in women with previous gestational diabetes mellitus using the Adult Treatment Panel III (ATPIII) and the International Diabetes Federation (IDF) definitions and to analyse the differences between both classifications.

Materials and Methods: We studied 1080 women (mean age: $33 \pm 4$ years; mean BMI: $25.6 \pm 4.7$ ) with GDM diagnosed between 1992 and 2004, who attended the postpartum follow-up visit (3-6 months postpartum). Assessment included: measurement of weight, height, waist circumference, blood pressure, fasting glucose level and lipid profile.

Results: The prevalence of the MS was $9.3 \%$ according to the ATP-III definition and $17.4 \%$ according to the IDF definition. Among all women, $8.7 \%$ had the MS under the two definitions, $0.6 \%$ had the MS under the ATP-III definition but not the IDF definition (due to the absence of abdominal obesity) and $8.7 \%$ had the MS under the IDF definition but not the ATP-III definition (due to the lower fasting glucose criteria in $17,02 \%$ of cases, to the lower abdominal circumference criteria in $13,82 \%$ of cases and to both criteria in $69,15 \%$ of cases). The prevalence of abdominal obesity was $19.2 \%$ using the ATP-III definition and $48.1 \%$ using the IDF definition. The prevalence of high fasting glucose was $8.7 \%$ using the ATP-III definition and $27,4 \%$ using the IDF definition. Among all women, 41.6\% under the ATP-III definition and $26.5 \%$ under the IDF definition had all five criteria below the cut-off value.
Conclusion: The prevalence of the MS in women with prior GDM using the IDF definition is almost two times higher than using the ATP-III definition. Only $26.5 \%$ of women with prior GDM have no risk factors under the IDF definition.

\section{5}

Identifying metabolic syndrome in type 2 diabetes: is it needed?

\section{S. H. Song;}

Diabetes Centre, Northern General Hospital, Sheffield, United Kingdom.

Background and Aims: The International Diabetes Federation (IDF) recently proposed a new world-wide definition of metabolic syndrome (MetS) emphasising the importance of central adiposity (as defined by ethnic-specific waist circumference) as a prerequisite to identify this condition. This differs from NCEP-ATPIII criteria which does not emphasise central adiposity as a prerequisite condition. These criteria function as clinical tools to identify patients at risk of developing cardiovascular disease and type 2 diabetes (T2DM). In T2DM, MetS is associated with higher incidence of cardiovascular disease. A recent study compared IDF with NCEP-ATPIII criteria in non-diabetic patients and demonstrated higher prevalence of MetS with IDF criteria $(\sim 40 \%)$. The aim of this study is to determine the prevalence of MetS with IDF and NCEP-ATPIII criteria in T2DM and its relevance in clinical practice.

Materials and Methods: Patients who attended hospital diabetes clinics were recruited. The following data were collected: age, sex, diabetes duration, body mass index, waist circumference, blood pressure, HDL cholesterol, triglyceride and HbAlc. IDF and NCEP-ATPIII criteria were used to define MetS.

Results: 252 patients were recruited. IDF and NCEP-ATPIII criteria identified $\sim 93 \%$ of T2DM patients to have MetS with good concordance between these two criteria $(\kappa=0.71$, $\mathrm{p}<0.0001$ ). Approximately $92 \%$ of patients have MetS by both criteria. Prevalence of MetS was higher in women ( $\sim 97 \%$ with both criteria) who had similar degree of central obesity as men (waist circumference; women vs men, 114.8 vs $114.0 \mathrm{~cm}, \mathrm{p}=\mathrm{NS}$ ). Among young T2DM patients ( $<40 \mathrm{yrs})$, MetS prevalence was $\sim 70 \%$. After the age of 40 , prevalence of MetS was higher and remained static ( $\sim 95 \%$ with both criteria) across different age groups. MetS was associated with higher prevalence of ischaemic heart disease (IDF; MetS vs no Mets, 33.1 vs $6.3 \%$, $\mathrm{p}=0.025$ and NCEP-ATPIII; MetS vs no MetS, 33.2 vs $5.9 \%, \mathrm{p}=0.019$ ) and cardiovascular disease (IDF; MetS vs no MetS, 41.5 vs $6.3 \%, p=0.005$ and NCEPATPIII; MetS vs no MetS, 41.7 vs $5.9 \%$, $\mathrm{p}=0.003$ ). 
Conclusion: IDF and NCEP-ATPIII criteria similarly identified patients with MetS in T2DM. Given its extremely high prevalence, these data support the concept of T2DM as a high risk condition and also suggest that identification tool for MetS is not needed in the clinical management of T2DM.

\section{PS 14}

\section{Obesity, abdominal obesity and insulin resistance}

0386

Is there any agreement between HOMA 1 and HOMA 2 models?

P. E. Lobo, M. Preiti, D. Colom, M. Huber, M. Quiros, C. Gonzalez, R. Urdaneta;

Diabetes y Nutrición, Hospital Universitario Austral, Buenos Aires, Argentina.

Background and Aims: To determine the cut off of HOMA 1 and HOMA 2 considering Impaired Glucose Tolerance and type 2 Diabetes as independent variable.

To evaluate concordance between HOMA 1 and HOMA 2 models.

Materials and Methods: Six hundred twenty one patients, age $43.1 \pm 12.2$ years old, male $313(50.4 \%$ ), female 308 (49.6\%), BMI $32.8 \pm 6.1$; euthyroids, without previous diagnosis of type 2 Diabetes (DM2) or Impaired Glucose Tolerance (IGT) or treated with metformin, thiazolidinediones, oral hypoglycemiant drugs, sibutramina and orlistat were studied. Patients with fasting insulinemia $\leq 2.9 \mathrm{mU} / \mathrm{ml}$ or $\geq 43.8 \mathrm{mU} /$ ml were excluded by HOMA 2 model's limitations.

Insulinemia was made by electrochemiluminiscense.

Diagnosis of IGT or DM2 were made with WHO criteria.

HOMA 1 model was calculated by original model from Matthieus et al.(1985) and HOMA 2 model by homa 2 calculator, Oxford.

The cut off of HOMA models were calculated by ROC curve, considering DM2 and IGT as independent variable. Statistical analysis were made through receiver operating curve (ROC), sensitivity, especificity, Bland \& Altman test and Kappa ( $\kappa$ ) coefficient (significative: $\mathrm{p}<0.05$ ).

Results: Patients were classified as IGT $n=39$ (6.3\%) and DM2 n=45 (7.25\%).

The cut off of HOMA 1 in DM2 and IGT was 3.04 (AUC: $0.845, \mathrm{p}<0.001$ ), sensitivity of 0.80 and especificity of 0.77 , and the cut off of HOMA 2 was 2.05 (AUC: 0.803, p<0.001), sensitivity and especificity of 0.73 and 0.77 respectively.
Table 1: Cut off of HOMA 1 and HOMA 2 in Impaired Glucose Tolerance and type 2 Diabetes

\begin{tabular}{lllllll}
$\begin{array}{l}\text { Dependent } \\
\text { variable }\end{array}$ & $\begin{array}{l}\text { Independent } \\
\text { variable }\end{array}$ & & & & & \\
\hline HOMA 1 & DM2+IGT & 3.04 & 0.845 & $\mathrm{p}<0.001$ & 0.80 & 0.77 \\
HOMA 2 & DM2+IGT & 2.05 & 0.803 & $\mathrm{p}<0.001$ & 0.73 & 0.77
\end{tabular}

The mean difference between HOMA 1 and HOMA 2 was $1.12 \pm 1.23$ by Bland \& Altman test (graphic 1 ).
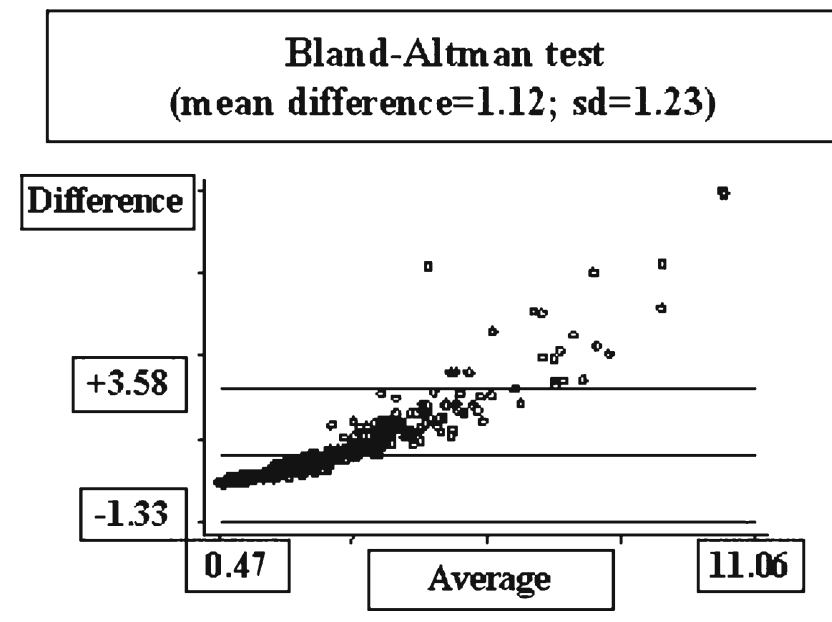

The $\kappa$ coefficient (concordance among HOMA 1 and HOMA 2 models) was $0.43, \mathrm{p}<0.05$. The agreement between different ranks of HOMA 1 and HOMA 2 can be found in table 2 .

Table 2: Concordance between different ranks of HOMA 1 and HOMA 2

\begin{tabular}{lllll}
\hline & HOMA & HOMA 2: $1.0-$ & HOMA 2: $2.0-$ & HOMA \\
& $2: \leq 0.99$ & 1.99 & 2.99 & $2: \geq 3.0$ \\
\hline HOMA 1: $\leq 0.99$ & $\mathbf{8 0}$ & 0 & 0 & 0 \\
HOMA 1: $1.0-1.99$ & 112 & $\mathbf{1 1 2}$ & 0 & 0 \\
HOMA 1: $2.0-2.99$ & 0 & 115 & $\mathbf{7}$ & 0 \\
HOMA 1: $: 3.0$ & 2 & 11 & 115 & $\mathbf{6 7}$ \\
\hline
\end{tabular}

The differences of cut off and concordance between HOMA 1 and HOMA 2 remain when they were adjusted by sex.

Conclusion: According to the selected independent variable (DM2 and IGT) the cut off of HOMA 1 and HOMA 2 models were 3.04 and 2.05 respectively.

We found a disagreement between HOMA 1 and HOMA 2 by Bland \& Altman test and $\kappa$ coefficient.

Supported by Fundación Cruz del Sur-Felix E. Puchulu MD 
0387

The impact of physical activity on estimates of insulin sensitivity and glucose-induced insulin release - the INTER99 study

S. Engberg ${ }^{1}$, C. Glümer ${ }^{1}$, T. Jørgensen ${ }^{2}$, D. Vistisen ${ }^{1}$, B. Carstensen ${ }^{1}$, O. Pedersen ${ }^{1}$, K. Borch-Johnsen ${ }^{1}$;

${ }^{1}$ Steno Diabetes Center, Gentofte, Denmark, ${ }^{2}$ Research Centre for Prevention and Health, Glostrup, Denmark.

Background and Aims: Deterioration of glucose tolerance is caused by a combination of increasing insulin resistance and impaired insulin secretion and can be postponed/ prevented by increased physical activity. The aim of this study was to analyse whether the level of physical activity is associated to insulin resistance.

Materials and Methods: The analysis is based on crosssectional data from the population-based Inter99 study $(\mathrm{N}=4,540$, aged $30-60 \mathrm{yrs})$. The history of physical activity (at work and leisure time) was assessed by questionnaires and categorised into 4 levels. All individuals underwent an OGTT including measurement of plasma glucose and serum insulin at time 0,30 and $120 \mathrm{~min}$. Insulin resistance was estimated by HOMAir and the Gutt index. Oral glucoseinduced insulin release was estimated by the insulinogenic index, IGI. The impact of physical activity on insulin sensitivity and insulin release was assessed using multiple linear regression analyses adjusted for sex, age and BMI.

Results: HOMAir was positively and the Gutt index was inversely associated (i.e. both estimates of insulin sensitivity were inversely associated) - and IGI was positively associated with decreasing level of physical activity.

Table1: Estimates on relative change (95\% CI) in outcome for the relationship between physical activity at work and leisure time and HOMAir, the Gutt index and IGI.

\begin{tabular}{|c|c|c|c|}
\hline & $\begin{array}{l}\text { HOMAir, } \\
\left(\mathrm{mmol} \cdot \mathrm{mIU} \cdot 1^{-2}\right) \text {, } \\
\dagger, \mathrm{a}\end{array}$ & $\begin{array}{l}\text { Gutt index, } \\
\left(\mathrm{mg} \cdot 1^{2} \cdot \mathrm{mmol}^{-1}\right. \\
\left.\mathrm{mIU}^{-1} \cdot \mathrm{min}^{-1}\right) \\
\dagger, \mathrm{a}\end{array}$ & $\begin{array}{l}\text { IGI, } \\
\left(\mathrm{pmol}^{-1} \mathrm{mmol}^{-1}\right) \text {, } \\
\dagger, \mathrm{a}\end{array}$ \\
\hline $\begin{array}{l}\text { Stronger/heavy } \\
\text { physical activity }\end{array}$ & $0.81(0.76-0.87)$ & $\begin{array}{l}1.20(1.15- \\
1.25)\end{array}$ & $\begin{array}{l}0.84(0.78- \\
0.92)\end{array}$ \\
\hline $\begin{array}{l}\text { Intermediate } \\
\text { physical activity }\end{array}$ & $0.88(0.84-0.92)$ & $\begin{array}{l}1.11(1.08- \\
1.15)\end{array}$ & $\begin{array}{l}0.90(0.85- \\
0.96)\end{array}$ \\
\hline $\begin{array}{l}\text { Light physical } \\
\text { activity }\end{array}$ & $0.93(0.90-0.96)$ & $\begin{array}{l}1.07(1.04- \\
1.10)\end{array}$ & $\begin{array}{l}0.94(0.89- \\
0.98)\end{array}$ \\
\hline $\begin{array}{l}\text { Physical } \\
\text { passivity }\end{array}$ & 1 & 1 & 1 \\
\hline
\end{tabular}

$\dagger$ ) The models used log-transformed dependent variables.

a) $\mathrm{p}<0.0001$.
Conclusion: Decreased levels of physical activity are positively associated with estimates of insulin resistance and positively associated with an estimate of insulin release after an oral glucose load.

Supported by the Danish Medical Research Council, the Danish Center for Evaluation and Health Technology Assessment, Novo Nordisk, Copenhagen County, the Danish Heart Foundation, the Danish Diabetes Association, the Danish Pharmaceutical Association

0388

\section{WITHDRAWN}

0389

Waist circumference and diabetes prevalence in European primary care patients: the IDEA study

J.-P. Després ${ }^{1}$, S. Haffner ${ }^{2}$, B. Balkau ${ }^{3}$ on behalf of the IDEA Study Group.

${ }^{1}$ Québec Heart Institute, Laval Hospital Research Center, Québec, Canada, ${ }^{2}$ Department of Medicine, University of Texas Health Science Center, San Antonio, United States, ${ }^{3}$ Epidemiology and Biostatistics, INSERM U780-IFR69, Villejuif, France.

Background and Aims: IDEA (the International Day for the Evaluation of Abdominal obesity) was an international, non interventional, cross-sectional study designed to estimate the prevalence of abdominal obesity (measured by waist circumference; WC) and its association with cardiometabolic risk factors in 168159 patients attending primary care physician (PCP) offices on two predefined half days in 63 countries worldwide. The aim of this analysis was to assess the prevalence of diabetes in 3 European regions and describe its relationship with anthropometric parameters (WC, and body mass index [BMI]).

Materials and Methods: WC, weight and height were measured by the PCPs; demographic data and the presence of diabetes were recorded. Data from 3 European regions were analysed (NW Europe: Austria, Belgium, Denmark, Finland, France, Germany, Ireland, The Netherlands, Norway, Sweden, Switzerland; S Europe: Greece, Italy, Portugal, Spain, Turkey; E Europe: Bulgaria, Czech Republic, Estonia, Hungary, Latvia, Lithuania, Poland, Russia, Slovakia, Slovenia, Ukraine). For each gender, diabetes prevalence was agestandardised with the entire European population as reference. Odds ratios (ORs) and 95\% confidence intervals (CIs) for the risk of having diabetes were estimated from multiple logistic regression models after adjusting for age.

Results: See table. 
Conclusions: In a primary care population, the prevalence of diabetes was higher in Southern Europe, compared with North Western and Eastern Europe. Across regions, the prevalence of diabetes was higher in men than women. Irrespective of geographical region, WC was strongly associated with diabetes independently from age and BMI, and this relationship was even stronger in women.

\begin{tabular}{|c|c|c|c|c|c|c|}
\hline & \multicolumn{3}{|l|}{ Men } & \multicolumn{3}{|l|}{ Women } \\
\hline & NW & $\mathrm{S}$ & $\mathrm{E}$ & NW & $\mathrm{S}$ & $\mathrm{E}$ \\
\hline & Europe & Europe & Europe & Europe & Europe & Europe \\
\hline & $(n=12$ & $(n=13$ & $(n=11$ & $(n=16$ & $(n=17$ & $(n=19$ \\
\hline & 796) & 465) & 176) & $786)$ & 824) & 199) \\
\hline Mean (SD) & 52.3 & 53.8 & 49.5 & 51.2 & 52.4 & 50.7 \\
\hline age, years & $(16.1)$ & $(15.9)$ & $(16.0)$ & $(16.5)$ & $(15.8)$ & (15.9) \\
\hline Mean (SD) & 97.8 & 99.4 & 96.9 & 88.3 & 91.3 & 89.7 \\
\hline $\mathrm{WC}, \mathrm{cm}$ & (13.5) & (12.9) & (13.7) & $(14.8)$ & (14.7) & (15.7) \\
\hline Mean (SD) & 27.2 & 28.2 & 27.5 & 26.4 & 27.9 & 27.6 \\
\hline BMI, $\mathrm{kg} / \mathrm{m}^{2}$ & (4.6) & (4.5) & $(4.8)$ & (5.6) & (5.6) & $(6.0)$ \\
\hline $\begin{array}{l}\mathrm{BMI} \geq 30 \\
\mathrm{~kg} / \mathrm{m}^{2}, \%\end{array}$ & 23.0 & 30.2 & 27.5 & 22.7 & 31.7 & 32.4 \\
\hline $\begin{array}{l}\text { Prevalence } \\
\text { of diabetes, \% }\end{array}$ & 12.8 & 14.5 & 12.4 & 8.8 & 11.5 & 10.7 \\
\hline WC, diabetes & 1.76 & 1.45 & 1.83 & 2.17 & 1.78 & 1.98 \\
\hline OR $(95 \% \mathrm{CI})^{*}$ & $\begin{array}{l}(1.67- \\
1.86)\end{array}$ & $\begin{array}{l}(1.38- \\
1.53)\end{array}$ & $\begin{array}{l}(1.72- \\
1.95)\end{array}$ & $\begin{array}{l}(2.05- \\
2.29)\end{array}$ & $\begin{array}{l}(1.69- \\
1.87)\end{array}$ & $\begin{array}{l}(1.88- \\
2.09)\end{array}$ \\
\hline WC, diabetes & 1.35 & 1.29 & 1.46 & 1.72 & 1.45 & 1.59 \\
\hline $\begin{array}{l}\text { OR }(95 \% \mathrm{CI})^{*} \\
\text { (adjusted } \\
\text { for BMI) }\end{array}$ & $\begin{array}{l}(1.24- \\
1.48)\end{array}$ & $\begin{array}{l}(1.20- \\
1.39)\end{array}$ & $\begin{array}{l}(1.32- \\
1.63)\end{array}$ & $\begin{array}{l}(1.58- \\
1.88)\end{array}$ & $\begin{array}{l}(1.35- \\
1.56)\end{array}$ & $\begin{array}{l}(1.46- \\
1.73)\end{array}$ \\
\hline
\end{tabular}

*Odds ratio $(95 \% \mathrm{CI})$ for diabetes for a $1 \mathrm{SD}$ increase in WC. SD, standard deviation.

Supported by sanofi-aventis

0390

Change in waist circumference and metabolic consequences over 9 years: the D.E.S.I.R. Study

B. Balkau ${ }^{1}$, P. Picard $^{1}$, S. Vol ${ }^{2}$, E. Eschwège ${ }^{1}$, D.E.S.I.R. Study Group ${ }^{2}$;

${ }^{1}$ U780-IFR69, INSERM, Villejuif, ${ }^{2}$ Epidemiology and Statistics, IRSA, La Riche, France.

Background and Aims: The long term effect on metabolic parameters, of gaining or loosing abdominal adiposity has been little analysed in longitudinal studies.

Materials and Methods: 1863 men and 1938 women, aged 30-65 years at inclusion in the French D.E.S.I.R. cohort (Data from an Epidemiological Study on the Insulin Resistance Syndrome) were studied for changes in metabolic syndrome parameters over nine years.
Results: At baseline, $8 \%$ of men and $13 \%$ of women had NCEP-ATP III defined abdominal adiposity: waist $>102$ / $88 \mathrm{~cm}$ (men/women) and $31 \%$ of men and $33 \%$ of women had IDF defined abdominal adiposity: waist $>94 / 80 \mathrm{~cm}$ (men/women); at 9 years, percentages were $16 \%$ and $25 \%$ for NCEP-ATP III and $43 \%$ and $50 \%$ for IDF. Over this period, $25 \%$ of the men and $34 \%$ of the women increased their waist by more than $7 \mathrm{~cm}$, while $14 \%$ and $13 \%$ lost more than $2 \mathrm{~cm}$. In subjects with a $7 \mathrm{~cm}$ or more increase in waist circumference, in comparison to those with a stable waist $( \pm 2 \mathrm{~cm})$, the age-adjusted odds ratios, for incident syndrome abnormalities are tabulated below. These results were little attenuated after adjusting on BMI.

Conclusion: Increasing waist circumferences had similar deleterious metabolic effects in men and women, which were accentuated when combined together into the metabolic syndrome: odds-ratios were high in both men and women whose waist circumferences increased by more than $7 \mathrm{~cm}$ over the 9 years, partly because the syndrome includes high waist circumference as a criterion.

Odds ratios, age adjusted, for incident abnormalities

\begin{tabular}{lll}
\hline & Men & Women \\
\hline $\begin{array}{lll}\text { hyperglycaemia }(>6.1 \mathrm{mmol} / \mathrm{l}) \\
\text { hyperglycaemia }(>5.6 \mathrm{mmol} / \mathrm{l})\end{array}$ & $1.7(1.0-2.8)$ & $2.7(1.4-5.0)$ \\
$\begin{array}{l}\text { high arterial blood pressure } \\
(\mathrm{SBP} / \mathrm{DBP}>130 / 85 \mathrm{mmHg})\end{array}$ & $1.5(0.9-2.4)$ & $1.5(1.0-2.2)$ \\
$\begin{array}{l}\text { hypo-HDL-cholesterolaemia } \\
(<1.03 / 1.29 \mathrm{mmol} / \mathrm{m} / \mathrm{w})\end{array}$ & $2.0(1.1-3.4)$ & $1.9(1.2-2.8)$ \\
$\begin{array}{l}\text { hypertriglyceridaemia } \\
(>1.7 \mathrm{mmol} / \mathrm{l})\end{array}$ & $2.3(1.5-3.5)$ & $2.2(1.4-3.6)$ \\
$\begin{array}{l}\text { NCEP-ATP III metabolic syndrome } \\
\text { IDF metabolic syndrome }\end{array}$ & $6.6(3.9-11.3)$ & $4.5(2.6-7.4)$ \\
\hline & $5.7(3.9-8.4)$ & $10.8(6.6-17.4)$ \\
\hline
\end{tabular}

Supported by Sanofi-Aventis France

0391

Optimal cut off value of waist circumference reflecting insulin resistance as diagnostic criteria of metabolic syndrome in nondiabetic Korean population: from CMS (Chungju Metabolic Syndrome) study

Y.-M. Park ${ }^{1}$, H.-S. Kwon ${ }^{2}$, S.-Y. Lim ${ }^{1}$, S.-R. Kim ${ }^{2}$, K.-H. Yoon $^{2}$, B.-Y. Cha ${ }^{2}$, H.-Y. Son ${ }^{2}$, D.-S. Kim ${ }^{3}$, W.-C. Lee ${ }^{1}$; ${ }^{1}$ Department of Preventive Medicine, ${ }^{2}$ Department of Internal Medicine, The Catholic University of Korea, Seoul, Republic of Korea, ${ }^{3}$ Chungju Public Health Center, Chungju, Republic of Korea.

Background and Aims: In 2005, the International Diabetes Federation proposed a new worldwide definition of 
metabolic syndrome that abdominal obesity is included as a prerequisite risk factor. Various cut-points of waist circumference that represent abdominal obesity were provided from different countries or ethnic groups. Insulin resistance plays the major role in the pathophysiology of metabolic syndrome, and a positive association between central obesity and insulin resistance has been consistently observed in many studies. So determining the cut off level of abdominal obesity to best predict insulin resistance will be more relevant to the explanation of pathophysiology of metabolic syndrome. We aimed to determine the cut off value of waist circumference with respect to its ability to reflect insulin resistance in nondiabetic Korean population. Materials and Methods: The CMS (Chungju metabolic syndrome) study is a community-based observational study of metabolic syndrome. 8,395 subjects aged over 40 years old were selected by stratified random cluster sampling between the year of 2002 and 2004, and 6,078 nondiabetic subjects (2,434 men and 3,644 women) without missing data about insulin, glucose, and other covariates were analyzed. Insulin resistant individuals were defined as the subjects with the highest quartile value of HOMA-IR which was determined among non-diabetic population. The associations of the waist circumference with insulin resistance were analyzed using multiple logistic regression analysis. The optimal sensitivity and specificity for predicting insulin resistance were the values producing maximum sums from receiver operating characteristic (ROC) curve in men and women separately.

Results: After controlling for age, systolic blood pressure, diastolic blood pressure, triglyceride, HDL cholesterol, and body mass index, the association was significant in the category of $>85-90 \mathrm{~cm}$ (OR 2.19, 95\%CI 1.07-4.51 form men; OR $1.89,95 \% \mathrm{CI} 1.25-2.87$ form women) when the category of $<70 \mathrm{~cm}$ of WC was taken as a reference, and the statistically significant association was also observed over the categories of $>85-90 \mathrm{~cm}$. And the cut off value of waist circumference for ROC analysis was $84.4 \mathrm{~cm}$ for men $(70.7 \%$ sensitivity and $58.3 \%$ specificity), and $84.7 \mathrm{~cm}$ for women $(55.4 \%$ sensitivity and $70.2 \%$ specificity).

Conclusion: Cutoff value for waist circumference predicting insulin resistance was considered to be $85 \mathrm{~cm}$ for men and women, respectively, suggesting that Asian-Pacific criteria of abdominal obesity might not be applicable for Korea.
0392

Serum uric acid is not a predictor of cardiovascular mortality in type 2 diabetes: the Casale Monferrato Study

C. Runzo ${ }^{1}$, G. Bruno ${ }^{1}$, G. Bargero ${ }^{2}$, I. Masi ${ }^{1}$, G. Novelli ${ }^{1}$, D. Melis ${ }^{1}$, A. Soddu ${ }^{1}$, M. Perotto ${ }^{1}$, G. Pagano ${ }^{1}$, P. Cavallo-Perin ${ }^{1}$;

${ }^{1}$ Internal Medicine, University of Torino, ${ }^{2}$ Diabetology, Hospital of Santo Spirito, Casale Monf.to, Italy.

Background and Aims: Studies have indicated that serum uric acid, the end product of purine catabolism, is associated with hypertension, obesity, hyperinsulinemia, dyslipidemia, suggesting that it could be a component of metabolic syndrome. Whether serum uric acid is an independent predictor of cardiovascular mortality in type 2 diabetes is actually unknown. Aims of this work were to assess in a population-based cohort of type 2 diabetes variables associated with serum uric acid and its role as an independent predictor of cardiovascular mortality, independently on other risk factors.

Materials and Methods: The study base was the population-based cohort of 1565 diabetic subjects (median age 68.9 yrs) of the Casale Monferrato Study. The predictive roles of serum uric acid on 11-yrs all-cause and cardiovascular mortality (ICD-9 codes 390-459) was assessed by using multivariate Cox proportional hazards modelling, adjusting for classical risk factor (age, sex, hypertension, smoking, CHD), novel risk factors (AER, fibrinogen), $\mathrm{HbA}_{1} \mathrm{C}$ cumulative average and referring physicians.

Results: Levels of serum uric acid were higher in males $\mathbf{( 5 . 5 5}$ vs $5.29 \mathrm{mg} / \mathrm{dl}, \mathrm{p}=0.003$ ), elderly ( 5.49 vs $5.34 \mathrm{mg} / \mathrm{dl}, \mathrm{p}=0.08)$, obese people $(\mathrm{p}<0.0001)$, those with diabetic nephropathy $(5.56 \mathrm{mg} / \mathrm{dl}$ for $\mathrm{AER} \geq 20 \mu \mathrm{g} / \mathrm{min}$ vs $5.25 \mathrm{mg} / \mathrm{dl}$ for $\mathrm{AER}$ $<20 \mu \mathrm{g} / \mathrm{min}$ ), hypertension (5.49 vs $4.93 \mathrm{mg} / \mathrm{dl}, \mathrm{p}<0.0001$ ), higher levels of serum creatinine $(>1.16 \mathrm{mg} / \mathrm{dl})$ vs lower $(<0.91 \mathrm{mg} / \mathrm{dl})(6.22 \mathrm{vs} 4.75, \mathrm{p}<0.001)$ and in people with the metabolic syndrome (WHO criteria) (5.57 vs $4.87 \mathrm{mg} / \mathrm{dl}$, $\mathrm{p}<0.0001)$; 685 death were identified in 10890.2 person-year of observations. All cause mortality was 63/1000 (95\% CI 59-68) and cardiovascular mortality was 31/1000 (95\% CI 28-35). In univariate analysis, mortality risk increased with increasing levels of serum uric acid (HR 1.36, 95\% CI 1.001.85 , in the upper vs lower quartile). HRs, however, were no more significant after adjustment for age and sex (HR 1.18, 95\% CI 0.86-1.61), for the above and for hypertension, apoB/ apoA1 ratio, and smoking (HR 1.00, 95\% CI 0.72-1.39), for the above and for AER, fibrinogen and individual cumulative HbA1c (HR 0.89, 95\% CI 0.63-1.24).

Conclusion: Our population-based study indicates that in Mediterranean diabetic population, serum uric acid is associ- 
ated with component of the metabolic syndrome but it is not an independent risk factor of cardiovascular mortality.

\section{3}

Genetic and environmental factors that modulate serum adiponectin concentrations in diabetic and non-diabetic participants in the PREDIMED trial

D. Godoy ${ }^{1}$, C. Ortega ${ }^{2}$, J. V. Sorli ${ }^{2}$, F. Frances ${ }^{2}$,

P. Carrasco ${ }^{2}$, E. Godoy ${ }^{2}$, D. Corella ${ }^{2}$;

${ }^{1}$ Internal Medicine, Consorcio Hospital General, Valencia,

${ }^{2}$ Preventive Medicine, University of Valencia, Spain.

Background and Aims: Many studies have reported that low plasma adiponectin concentrations associate closely higher adiposity, diabetes, hypertension and dyslipidaemia. Although evidence indicates that adiponectin is anti-inflammatory, some studies in diabetic patients have demonstrated that adiponectin is associated with impaired renal function and may be enhanced as a physiological counter-regulatory response to mitigate endothelial damage. The aim of our study was to compare adiponectin levels between diabetic and non diabetic subjects and to study genetic and environmental factors that may be associated with adiponectin concentrations in a high-cardiovascular risk population.

Materials and Methods: We conducted a cross-sectional study in 266 (110 men and 156 women) high-cardiovascular risk patients participating in the PREDIMED (PREvencion con DIeta MEditerranea) trial. They were men (age: 55-80) and women (age: 60-80) free of CHD but with either diabetes or at least three major cardiovascular risk factors (smoking, high blood pressure (HBP), high LDL-C, low HDL-C, overweight, and family history of premature $\mathrm{CHD}$ ), recruited in the Valencia region (Spain). Adiponectin, anthropometric, clinical, biochemical, and life style variables were determined. All subjects were genotyped for the c.331 $\mathrm{T}>\mathrm{C}$ mutation $(\mathrm{Y} 111 \mathrm{H})$ in exon 3.

Results: Prevalence of diabetes in this sample was $47 \%$. Adiponectin concentrations were statistically higher in women than in men $(11 \pm 5.1$ vs $6.9 \pm 3.3 \mu \mathrm{g} / \mathrm{mL} ; \mathrm{p}<0.001)$. Moreover adiponectin was statistically significant lower in diabetic than in non-diabetic patients $(7.8 \pm 4.6 \mathrm{vs} 11.4 \pm 4.8 \mu \mathrm{g} /$ $\mathrm{mL} ; \mathrm{p}<0.001)$. We did not find differences in adiponectin between obese and non-obese subjects. Although plasma adiponectin was slightly higher in non-smokers than in smokers, differences did not reach the statistical significance. Adiponectin was not associated with hypertension $(\mathrm{P}=0.214)$. We found statistically significant correlations between adiponectin and HDL-C $(\mathrm{r}=0.36 ; \mathrm{P}<0,001)$, triglycerides $(\mathrm{r}=-0.28$; $\mathrm{P}<0.001)$, glucose $(\mathrm{r}=-0.28$; $\mathrm{PC}$ polymorphism was very low (96.1\% TT, 3.9\% TC and 0\% CC). Although prevalence of the variant allele was two times lower in diabetics $(2.6 \%)$ than in non-diabetics $(5.2 \%)$, results were not statistical significance $(\mathrm{P}=0.179)$. Similarly, plasma adiponectin concentrations were non-significantly $(\mathrm{P}=0.29)$ higher in carriers of the variant allele $(11.7 \pm 7.3 \mu \mathrm{g} / \mathrm{mL})$ than in TT patients $(9.7 \pm 4.9 \mu \mathrm{g} / \mathrm{mL})$. Conclusion: In this high-cardiovascular risk population, diabetes and gender were the factors most related to adiponectin concentrations

Supported by G03/140

0394

Metabolic syndrome and adipocytokines in Japanese children aged 12-13 years old

R. Nishimura ${ }^{1}$, H. Sano ${ }^{1}$, A. Morimoto ${ }^{1}$, Y. Miyashita ${ }^{1}$, T. Shirasawa ${ }^{2}$, E. Takahashi ${ }^{2}$, T. Kawaguchi ${ }^{2}$, N. Tajima ${ }^{1}$; ${ }^{1}$ Department of Internal Medicine, Jikei University School of Medicine, Tokyo, ${ }^{2}$ Department of Public Health, Showa University School of Medicine, Tokyo, Japan.

Background and Aims: Obesity is a serious health issue for children around the world. In this study, we sought to determine the prevalence of metabolic syndrome (MetS) and the levels of biochemical markers including adipocytokines of children with/without MetS in Japanese children aged 12-13 years old.

Materials and Methods: As a population-based health promotion plan, 291 (138 boys and 152 girls) out of a total of 307 school children (participation rate: $94.8 \%$ ) in the first grade of all 3 junior high schools in Ina-town, Saitama, participated in this study in 2004. MetS in children was defined as having a waist circumference $\geq 90$ percentile of this group $(83 \mathrm{~cm}$ for boys and $75 \mathrm{~cm}$ for the girls) plus any two of the following four factors: raised TG level ( $\geq 150 \mathrm{mg} / \mathrm{dl})$, reduced HDL cholesterol ( $<40 \mathrm{mg} / \mathrm{dl}$ ), raised blood pressure ( $\mathrm{SBP} \geq 130 \mathrm{mmHg})$, or raised plasma glucose $(\geq 100 \mathrm{mg} / \mathrm{dl})$. The levels of the biochemical markers leptin, adiponectin, resistin, high-sensitivity $\mathrm{C}$ reactive protein (hCRP), insulin (IRI), aspartate aminotransferase (AST), alanine aminotransferase (ALT) and low density lipoprotein cholesterol (LDL) of children with MetS were compared to the levels of those without MetS. Statistical significance was examined by the Mann-Whitney test using the SPSS program. The study protocol was approved by two independent institutional review boards. Written informed consent was obtained from all participants and their parents.

Results: Six boys and one girl met MetS criteria, giving prevalence rates of MetS of $4.3 \%$ for boys and $0.7 \%$ for girls. Children with MetS have significantly higher median levels (intraquatile range) compared with those without MetS for leptin (MetS: 12.8 (10.6-14.9), non MetS: 3.4 (2.1-5.4) ng/ml, p<0.001), hCRP (MetS: 0.67 (0.37-1.5), non MetS: $0.11(0.07-0.26) \mathrm{mg} / \mathrm{dl}, \mathrm{p}=0.001)$, IRI (MetS: 37.6 (32.0-65.7), non MetS: 10.2 (6.0-15.7) $\mu \mathrm{U} / \mathrm{ml}$, 
$\mathrm{p}<0.001)$, AST (MetS: 29 (20-44), non MetS: 20 (18-23) $\mathrm{IU} / \mathrm{l}, \mathrm{p}=0.015)$ and ALT (MetS: 51 (30-70), non MetS: 12 $(10-14) \mathrm{IU} / 1, \mathrm{p}<0.001)$. The level of adiponectin in children with MetS tended to be lower than those without MetS (MetS: 6.1 (5.6-10.9), non MetS: 9.1 (7.5-11.6) $\mu \mathrm{g} / \mathrm{ml}$ ), however the difference did not reach statistical significance $(\mathrm{p}=0.051)$. The levels of resistin (MetS: 7.1 (5.8-10.6), non MetS: 6.5 (4.5-8.9) ng/ml, $\mathrm{p}=0.227$ ) and LDL (MetS: 87 (85-131), non MetS: 91 (79-107) mg/dl, $\mathrm{p}=0.348$ ) did not differ between children with MetS and those without MetS. Conclusion: The current study indicated that $2.4 \%$ of the Japanese children aged 12-13 years old had MetS, characterized by elevated leptin, hCRP, IRI, AST and ALT. It is indicated that lifestyle intervention should be initiated at least around puberty.

Supported by the grant from the Ministry of Education, Culture, Sports, Science and Technology, Japan, Basic Research (A) \#17209024

\section{PS 15}

\section{Treatment, metabolic control and outcome in diabetes}

\section{5}

Utilisation of antihyperglycaemic drugs in ten European countries: different developments and different levels A. Melander on behalf of DECADE and EuroMedStat, Public Health Research, Clinical Sciences, Malmö University Hospital, Sweden.

Aim: To compare secular trends and developments of the utilisation of antihyperglycæmic drugs (AHGD) in ten European countries.

Methods: Data on the yearly (1994-2003) utilisation of insulin and oral AHGD were collected from public registers in Denmark, Finland, Norway, Sweden, Belgium, England, Germany, Italy, Portugal and Spain, and were expressed in Defined Daily Doses per 1000 inhabitants per day.

Results: Total AGHD utilisation increased in each of the ten countries but at different rates and levels. Insulin utilisation doubled in England and Germany but hardly changed in Belgium, Portugal and Italy. Sulphonylurea utilisation doubled in Spain, England and Denmark but was reduced in Germany and Sweden. Metformin utilisation increased strongly everywhere. In 2003, there were two- to three-fold differences in AHGD utilisation even between neighbour countries, e.g. Denmark vs Finland, Denmark vs Sweden, Belgium vs Germany and Portugal vs Spain. In Finland, there were more users of both insulin $(+120 \%)$ and oral AHGD $(+80 \%)$ than in
Denmark, and also the daily oral AHGD doses were $20-25 \%$ higher. In Denmark and Sweden, AHGD utilisation was equal in subjects $<45$ but in those $>45$ both insulin and oral AHGD utilisation were twice as high in Sweden.

Conclusion: The ubiquitous increase in AHGD utilisation, especially of metformin, seems rational, considering the increasing prevalence of type 2 diabetes and the results of the UKPDS. However, the large differences even between neighbour countries appear less rational and suggest different habits and attitudes concerning screening and management of type 2 diabetes.

Supported by Grant no. 20033133 from the Health and Consumer Protection Directorate-General of the EU Commission

0396

The risk of cancer with antidiabetic agents: evidence from clinical trial and observational data C. E. Koro ${ }^{1}$, S. A. Oliveria ${ }^{2}$, M. Ulcickas Yood ${ }^{2}$, M. O. Sowell ${ }^{3}$, M. Heise ${ }^{4}$, M. Stender ${ }^{5}$;

${ }^{1}$ Worldwide Epidemiology, GSK, Collegeville, United States, ${ }^{2}$ Epidemiology, Episource, Hamden, United States, ${ }^{3}$ Global Clinical Safety and Pharmacovigilance, Glaxo SmithKline, Collegeville, United States, ${ }^{4}$ Biomedical Data Sciences, GSK, Upper Merion, PA, United States, ${ }^{5}$ Worldwide Epidemiology, GSK, Greenford, United Kingdom.

Background and Aims: Preliminary data suggest a beneficial role of PPAR gamma agonists as anticancer therapy. An increased incidence of some cancers has been associated with PPAR agonists in rodent carcinogenicity studies. It is difficult to place preclinical findings into context given that limited data have been gathered in clinical settings. This study evaluated the association between thiazolidinediones (TZDs) and colon, bladder, liver, bile duct, gall bladder, pancreatic cancer and melanoma using the integrated rosiglitazone clinical trial database (RSG CT) (14,359 subjects) and an administrative claims database (191,223 diabetes patients).

Materials and Methods: For RSG CT data, we compared cumulative incidence rates of various neoplasms for the RSG group $(\mathrm{N}=9,542)$ relative to other antidiabetic agents $(\mathrm{N}=4,062)$. We compared the incidence rate of neoplasms in RSG CT to that of the general population using SEER 2001 and calculated standardized incidence ratios (SIRs). For claims data, we used logistic regression to calculate relative risks (RR) of cancer (overall and site-specific) for patients ever exposed to each antidiabetic category compared to nonuse among a type 2 diabetes (T2D) and a general population cohort, adjusting for age, gender and cancer risk factors.

Results: In RSG CT the inidence rate per 100 patient years of exposure for any malignant, benign or unspecified neoplasm 
was similar for RSG and non-RSG treatment regimens. SIRs for cancers associated with diabetes were increased in RSG treated subjects relative to general population (table 1).

In claims data, the adjusted RR comparing risk of cancer in patients ever exposed to TZD monotherapy to nonuse in T2D cohort was 0.92 and 1.76 compared to nonuse in general population (table 2).

Conclusions: The overall risk of selected cancers was higher for all antidiabetic exposure categories, including TZDs compared to the general population; consistent with the literature on cancer and diabetes.

Table 1: Standardized Incidence Rates for Selected Cancers Using RSG CT Data and SEER 2001

\begin{tabular}{lll}
\hline Site & SIR $(95 \%$ C.I. $)$ & $\begin{array}{l}\text { Published data on relative } \\
\text { risk of cancer in diabetic } \\
\text { patients compared to the } \\
\text { general population }\end{array}$ \\
\hline Bladder & $1.35(0.49-2.93)$ & $1.5-2.7$ \\
Breast* & $0.76(0.35-1.44)$ & $0.8-1.4$ \\
Colorectal & $0.97(0.48-1.74)$ & $1.0-3.0$ \\
Kidney & $0.77(0.09-2.79)$ & $1.3-2.1$ \\
Liver & $4.48(1.46-10.46)$ & $1.4-4.3$ \\
Lung & $0.94(0.53-1.55)$ & 0.98 \\
Melanoma & $2.23(0.90-4.60)$ & Insufficient data \\
Pancreas & $2.12(0.69-4.94)$ & $1.4-4.9$ \\
Prostate** & $0.74(0.46-1.13)$ & $0.6-1.5$ \\
\hline
\end{tabular}

* Female patients ** Male patients

Table 2: Overall Incidence of Selected* Cancers Among Patients Ever Exposed to Selected Antidiabetic Regimens Compared to Non-Use in Diabetes Patient and General Population Sample using administrative claims data (01/01/2000-12/31/2004)

$\begin{array}{lll}\text { Cases } & \text { Total N } & \text { Adjusted** } \\ & & \text { RR }(95 \% \mathrm{CI})\end{array}$

TZD monotherapy

\begin{tabular}{llll}
\hline Ever Use & 153 & 40,797 & $0.92(0.77-1.11)$ \\
Non-use (diabetes population) & 625 & 139,448 & Referent \\
Ever Use & 153 & 40,797 & $1.76(1.44-2.14)$ \\
$\begin{array}{l}\text { Non-use (general population) } \\
\text { Sulfonylureas monotherapy }\end{array}$ & 356 & 251,489 & Referent \\
Ever use & 308 & 65,826 & $0.89(0.77-1.04)$ \\
Non-use (diabetes population) & 412 & 101,626 & Referent \\
Ever use & 308 & 65,826 & $1.75(1.49-2.07)$ \\
Non-use (general population) & 356 & 251,489 & Referent \\
Metformin monotherapy & & & \\
Ever use & 225 & 66,235 & $0.83(0.71-0.98)$ \\
Non-use (diabetes population) & 498 & 100,210 & Referent \\
Ever use & 225 & 66,235 & $1.67(1.40-1.99)$ \\
Non-use (general population) & 356 & 251,489 & Referent \\
\end{tabular}

* Includes colon, bladder, liver, bile duct, gall bladder, pancreas and melanoma

** Adjusted for age, gender and risk factors for cancers of interest
0397

Achivement of treatment goals in diabetes mellitus patients - pretension and reality. The CoRiMa-Study routine documentation of daily care

R. A. Bierwirth ${ }^{1}, \mathrm{~K} . \mathrm{Pels}^{2}, \mathrm{M}$. Prien $^{3}, \mathrm{U} . \mathrm{Keil}^{4}$, J. Bernarding ${ }^{5}$, B. Lippmann-Grob ${ }^{6}$, J. Hallauer ${ }^{7}$, H. P. Schultheiss ${ }^{2}$, M. Brosz ${ }^{8}$, W. Gerressen ${ }^{3}$, S. Kropf ${ }^{5}$, J. C. Geller';

${ }^{1}$ Diabetes Center at Elisabeth KH, Essen, ${ }^{2}$ Cardiology, Univ. Hospital Charite, Berlin, ${ }^{3}$ Pfizer $\mathrm{GmbH}$, Karlsruhe, ${ }^{4}$ Epidemiology, Univ. Hospital, Münster, ${ }^{5}$ Biometry, Univ. Hospital, Magdeburg, ${ }^{6}$ Diabetology, Diabetes Center, Offenburg, ${ }^{7}$ Health Care, Univ. Hospital Charite, Berlin, ${ }^{8}$ Biometry, StatConsult, Magdeburg, ${ }^{9}$ Cardiology, Zentralklinik, Bad Berka, Germany.

Background and Aims: As measured by prevalence and resulting expenses in public health systems, Diabetes mellitus (DM) is one of most important chronic diseases. The positive effect of a guideline conform glucose metabolism in combination with optimized blood pressure as well as cholesterol treatment is undisputed. The study objective was to investigate how well this consensus is transferred into daily practice.

Materials and Methods: Medical therapy data of patients with DM, treated between 1998 and 2005, were anonymously exported directly from patient management system in physician offices. To assess the quality of medical care, information about age, gender, blood pressure, cholesterol (Total-C, LDL-C, HDL-C) and other risk factors were evaluated. Pre-/post-analyses were carried out to compare rates of achievement of treatment goals between patients first and last office visit. The guidelines of the German Diabetes Society (DDG) were used as reference values.

Results: 83.252 patients with documented DM (ICD-10coding and/or $\mathrm{HbA} 1 \mathrm{c} \geq 6,5 \%$ and/or antidiabetic medication) were included. Most of them had at least one comorbidity: $54,9 \%$ hypertension, $55,9 \%$ hyperlipidemia, $21,8 \%$ confirmed coronary heart disease (CHD), and approximately $10,0 \%$ had all 3 comorbidities (Fig.). The rates of treatment goal achievement were as follows: LDL-C 28,6\%, Blood pressure 38,7\%, HbA1c 35,7\%. Considering the LDL-C distribution of patients with DM and CHD, about $66,2 \%$ showed LDL-C $\geq 100 \mathrm{mg} / \mathrm{dl}$ at the final visit.

Conclusion: Compared with DDG guidelines, remarkable deficiencies in medical care of patients with DM were detected. Possible causes could be underestimated cardiovascular risks, deficiency in patient's motivation as well as budget limits. 


\section{Diabetes and comorbidities}

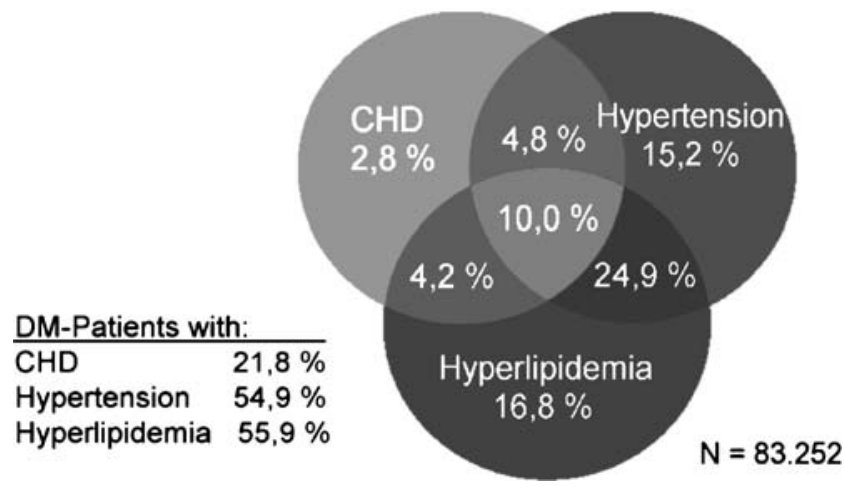

predictors $(\mathrm{p}<0.01)$ for all cause mortality in multivariate analysis; HbAlc, blood pressure, lipids, and a history of ischemic heart disease, were not.

Conclusion: This study shows a normal life expectancy in a non-smoking and well-supported T2DM population. Smoking, presence of PVD, and to a lesser degree albuminuria, however, reduce life expectancy. These results show that a normal life expectancy is achievable in T2DM-patients.

This study was supported by a grant from the Dutch Diabetes Research Foundation (project 2000.00.06) and the Medical Research Foundation Zwolle

0399

\section{8}

Normal life expectancy in non-smoking, well-controlled type 2 diabetes patients

H. L. Lutgers ${ }^{1,2}$, W. J. Sluiter ${ }^{3}$, L. J. Ubink-Veltmaat ${ }^{4}$, G. W. D. Landman ${ }^{1}$, T. P. Links ${ }^{3}$, R. O. B. Gans ${ }^{2}$, A. J. Smit ${ }^{2}$, H. J. G. Bilo ${ }^{1}$;

${ }^{1}$ Internal Medicine, Isala Clinics, Zwolle, ${ }^{2}$ Internal Medicine, University Medical Center Groningen, ${ }^{3}$ Endocrinology, University Medical Center Groningen, ${ }^{4}$ Family Practice, 't Veen, Hattem, The Netherlands.

Background and Aims: Studies of the 20th century reported increased mortality in type 2 diabetes (T2DM). Since there have been major changes in treatment regimes with more stringent goals for metabolic control and cardiovascular risk management, improvement of life expectancy in T2DM could be expected. In this study, we aimed to assess whether life expectancy of T2DM-patients has indeed changed.

Materials and Methods: In 2001, 973 primary care T2DM-patients, participating in a shared-care project, were included. This group (mean age $66 \mathrm{yrs}$ ) was well-controlled (mean $\mathrm{HbA} 1 \mathrm{c} 7.0 \%$ ) and had a relatively short diabetes duration (median $4.2 \mathrm{yrs}$ ). Vital status was assessed at January 2005. Life expectancy was assessed by transforming survival time to standardised survival time (SST) allowing adjustment for the baseline mortality rate of the general population.

Results: During a median follow-up of 3.2 years, 86 patients died (44 from cardiovascular disease). There was no reduction of median life expectancy in T2DM, compared to the general population ( $\mathrm{SST}=0.96$ vs. 1.0). However, mortality was increased in the smoking T2DMgroup compared to the general population at $\mathrm{SST}=0.25$ $(0.196$ vs. $0.070, \mathrm{p}<0.01)$ and $\mathrm{SST}=0.5(0.335$ vs. 0.169 , $\mathrm{p}<0.02$ ). Smoking (HR 2.0), peripheral vascular disease (PVD) (HR 2.2), and albuminuria (HR 1.2) were significant
Treatment and diagnosis of hyperlipidaemia in diabetes mellitus - awareness of an essential risk factor for cardiovascular disease - results from the German CoRiMa-Study

B. Lippmann-Grob ${ }^{1}$, K. Pels ${ }^{2}$, S. Cassens ${ }^{3}$, K. Schlueter ${ }^{3}$, M. Brosz ${ }^{4}$, R. A. Bierwirth ${ }^{5}$, H. P. Schultheiss ${ }^{2}$, U. Keil ${ }^{6}$, J. Bernarding ${ }^{7}$, J. F. Hallauer ${ }^{8}$, S. Kropf ${ }^{7}$, J. C. Geller ${ }^{9}$;

${ }^{1}$ Diabetes Center Offenburg, Offenburg, ${ }^{2}$ Cardiology, Univ. Hospital Charite, Berlin, ${ }^{3}$ Pfizer GmbH, Karlsruhe, ${ }^{4}$ Biometry, StatConsult, Magdeburg, ${ }^{5}$ Diabetes Center at Elisabeth KH, Essen, ${ }^{6}$ Epidemiology, Univ. Hospital, Münster, ${ }^{7}$ Biometry, Otto-von-Guericke Univ., Magdeburg, ${ }^{8}$ Health Care, Univ. Hospital Charite, Berlin, ${ }^{9}$ Cardiology, Zentralklinik, Bad Berka, Germany.

Background and Aims: Patients with diabetes mellitus (DM) have a high risk for cardiovascular disease, and they often have several additional risk factors. Identification of these risk factors is therefore important to develop adequate therapies to reduce morbidity and mortality. Thus, the present study focused on diagnostic accuracy and treatment of hyperlipidemia. Results according to total (TC) and LDL cholesterol (LDL-C) are compared to international guidelines.

Materials and Methods: In selected (general practitioners and internal medicine) physician offices in Germany, all patients with a diagnosis of DM seen between 1998 and 2005 were identified. The routine documentation was exported from the physician's patient management system into a project database. DM patients were selected based on 2 criteria: ICD-10 coding of DM and plausibility check for $\mathrm{DM}$ (i.e. use of anti-diabetic medication and/or $\mathrm{HbA}_{1 \mathrm{c}} \geq$ $6,5 \%$ ). The identification of hyperlipidemia was assessed using 3 criteria: ICD-10 coding, TC levels $\geq 190 \mathrm{mg} / \mathrm{dl}$ and/ or use of lipid-lowering medication. To evaluate the status of care, treatment, age, gender, serum lipids levels, and information regarding additional risk factors were analyzed. The last office visits were used for comparisons with 
treatment goals stated in current guidelines which were used as reference values for treatment evaluation.

Results: Overall, 83.252 patients with DM were identified: $88,7 \%$ by ICD- 10 coding, $9,1 \%$ by antidiabetic medication use and $2,2 \%$ based on $\mathrm{HbA}_{1 \mathrm{c}}$ values $\geq 6,5 \%$ at the last office visit without any documented antidiabetic medication. Adding plausibility checks, a total of 46.565 patients $(55,9 \%)$ with hyperlipidemia were identified, of whom only 22.931 had ICD-10 coding of hyperlipidemia. About $29,0 \%$ of patients without ICD-10 coding for hyperlipidemia were detected by use of lipid-lowering medication, and $70,1 \%$ by TC $\geq 190 \mathrm{mg} / \mathrm{dl}$ without lipid-lowering medication. Patients with (P1) and without (P2) documented hyperlipidemia (ICD-10), the following percentages achieved the goals of treatment: P1: TC 29,6\%; LDL-C 25,7\%; blood pressure $38,0 \%$; HbA1c 38,7\%; P2: TC 12,7\%; LDL-C 17,2\%; blood pressure $36,7 \%$; HbAlc $33,7 \%$. In addition to hyperlipidemia, hypertension and coronary heart disease were present in $54,9 \%$ and $21,8 \%$, respectively. At the last visit, $74,3 \%$ of patients in $\mathbf{P 1}$ and $82,9 \%$ in $\mathbf{P 2}$ had LDL-C $\geq 100 \mathrm{mg} / \mathrm{dl}$.

Conclusion: Compared with current guidelines, the majority of patients with DM does not achieve treatment goals for other relevant modifiable risk factors. Comparing ICD10 coding with hyperlipidemia diagnosed by plausibility checks, there is a significant prevalence of undetected hyperlipidemia in patients with DM. This lack of awareness of hyperlipidemia may be the cause for the high rates of LDL-C $\geq 100 \mathrm{mg} / \mathrm{dl}$.

\section{0}

Glycaemic control in type 2 patients starting insulin therapy - a retrospective study

I. C. Smith ${ }^{1}$, N. Richards ${ }^{2}$, G. H. Compion ${ }^{3}$;

${ }^{1}$ Pricing and Health Economics Unit, Novo Nordisk UK, Crawley, ${ }^{2}$ CompuFile Ltd, CompuFile Ltd, Surrey, ${ }^{3}$ Medical Information, Novo Nordisk UK, Crawley, United Kingdom.

Background and Aims: When using the traditional stepwise approach to treating type 2 diabetes, most patients progress from lifestyle interventions (e.g., diet and exercise) to oral anti-diabetic drugs (OADs), followed by insulin, with or without OADs. There is increasing recognition that this inherently reactive strategy to progressive treatment failure subjects patients to suboptimal glycaemic control. This study aimed to investigate at what stage of glycaemic control patients in real-life clinical practice began insulin therapy, and how the routine initiation of insulin affected their glycaemic control.

Materials and Methods: An anonymised computerised UK primary care database (DIN-LINK) was used to identify type 2 diabetes patients who were issued their first prescription of insulin between March 2002 and April 2005. Only patients who had a previous prescription for oral anti-diabetic therapy were included in the analysis to exclude type 1 patients and those who had moved from another district.

Results: The database identified 2,040 type 2 patients who were issued their first prescription of insulin, of whom 1,790 had an $\mathrm{HbA1c}$ reading before the prescription was issued. The mean age was 63.8 years $(\mathrm{SD}=12.8), 45 \%$ of patients were female with a mean $\mathrm{HbAlc}$ of $9.8 \%$ $(\mathrm{SD}=2.0 \%)$ before insulin initiation. After 6 months 774 patients had a second $\mathrm{HbAlc}$ recorded, mean $\mathrm{HbAlc}$ reduced by $1.4 \%(\mathrm{p}<0.001)$. This decrease was maintained at 1 year where 475 patients had a recorded $\mathrm{HbAlc}$.

Summary of absolute HbA1c (\%) for all newly initiated patients

\begin{tabular}{lllllllll}
\hline $\begin{array}{l}\text { Months from } 1^{\text {st }} \text { insulin } \\
\text { prescription }\end{array}$ & 0 & 6 & 9 & 12 & 15 & 18 & 21 & 24 \\
Number of patients & 1790 & 774 & 599 & 475 & 394 & 307 & 226 & 199 \\
Mean (\%HbA1c) & 9.75 & 8.31 & 8.19 & 8.22 & 8.29 & 8.36 & 8.27 & 8.26 \\
Median (\%HbA1c) & 9.40 & 8.10 & 7.90 & 8.00 & 7.90 & 8.20 & 8.10 & 8.20 \\
Standard deviation & 1.97 & 1.42 & 1.45 & 1.41 & 1.55 & 1.51 & 1.49 & 1.42 \\
\hline
\end{tabular}

Conclusion: Commencing insulin therapy in type 2 diabetes significantly improves glycaemic control; however $\mathrm{HbAlc}$ in patients starting insulin therapy is much higher than current recommended targets such as those set by NICE. These data indicate that more aggressive efforts are needed to initiate insulin at an earlier time point in the progression of type 2 diabetes.

Supported by an unrestricted grant from Novo Nordisk Ltd.

0401

Hypoglycaemia and self-monitoring blood glucose are predictive factors for achieving glycaemic control in people with diabetes

J.-J. Gagliardino ${ }^{1}$, P. Aschner ${ }^{2}$, S.-H. Baik ${ }^{3}$, J. Chan ${ }^{4}$, S. Ferreira ${ }^{5}$, N. Hancu ${ }^{6}$, H. Ilkova ${ }^{7}$, S. Twigg ${ }^{8}$,

A. Ramachandran ${ }^{9}$;

${ }^{1}$ Faculty of Medical Sciences, National University of La Plata, Buenos Aires, Argentina, ${ }^{2}$ Endocrinology Unit, Javeriana University, Bogota, Colombia, ${ }^{4}$ Department of Internal Medicine, Korea University Guro Hospital, Seoul, Republic of Korea, ${ }^{4}$ Department of Medicine and Therapeutics, Prince of Wales Hospital, Shatin, Hong Kong, ${ }^{5}$ Department of Preventive Medicine, Federal University of Sao Paulo, Brazil, ${ }^{6}$ Diabetes Center and Clinic, Iuliu Hatieganu University of Medicine, Cluj-Napoca, Romania, ${ }^{7}$ Department of Internal Medicine, Istanbul University Cerrahpasa Medical Faculty, Turkey, ${ }^{8}$ Department of Medicine, University of Sydney, Australia, ${ }^{9}$ Diabetes Research Centre, M.V. Hospital for Diabetes, Chennai, India. 
Background and Aims: To identify predictive factors for achieving a target of $\mathrm{HbA}_{1 \mathrm{c}}<7 \%$ in patients with type 1 (T1DM) and type 2 diabetes (T2DM) world wide, a logistic regression analysis was performed using initial data gathered in the International Diabetes Management Practices Study (IDMPS) in 11 countries (Eastern Europe, Asia, Latin America).

Materials and Methods: 1327 people with T1DM and 7543 people with T2DM were recruited; 798 T1DM patients and 3537 T2DM patients were available for the logistic regression analysis.

Results: In the overall population, the mean $\mathrm{HbA}_{1 \mathrm{c}}$ was $8.2 \pm 1.96 \%$ in $\mathrm{T} 1 \mathrm{DM}$ patients $\left(27 \%\right.$ had an $\left.\mathrm{HbA}_{1 \mathrm{c}}<7 \%\right)$ and was $7.7 \pm 1.75 \%$ in $\mathrm{T} 2 \mathrm{DM}$ patients $\left(38 \%\right.$ had an $\mathrm{HbA}_{1 \mathrm{c}}$ $<7 \%$ ). It was found that prior hypoglycemia or a fear of hypoglycemia largely decreases the chance that people with diabetes will achieve the $\mathrm{HbA}_{1 \mathrm{c}}$ target: people with T1DM have a 12 -times greater chance $(95 \%$ confidence interval [CI]: $5.106 ; 28.070)$ of achieving the target if they did not experience, or are not afraid of, hypoglycemia and the chance is 17 -times greater $(95 \% \mathrm{CI}$ : $6.118 ; 45.769)$ for people with T2DM. It was also found that self monitoring of blood glucose increased the likelihood of achieving the target of $\mathrm{HbA}_{1 \mathrm{c}}$ in both T1DM patients (2.2-times greater, 95\% CI: 0.265 ; 0.770 ) and T2DM patients (1.3-times greater, 95\% CI: $0.648 ; 0.886)$. The only other factors found to correlate with achieving the $\mathrm{HbA}_{1 \mathrm{c}}$ target were whether patients had a normal body mass index $\left(20-25 \mathrm{~kg} / \mathrm{m}^{2}\right)$, self-adjust their insulin dose, not having a macrovascular complication and had a shorter duration of disease (1-5 years).

Conclusion: Thus, the factors most strongly associated with achieving the recommended target of $\mathrm{HbA}_{1 \mathrm{c}}<7 \%$ in people with either T1DM or T2DM were whether people previously experienced, or were afraid of, hypoglycemia and self-monitoring of blood glucose.

Supported by Sanofi-Aventis

0402

Effect of orlistat on weight regain and development of type 2 diabetes following a very-low-energy diet in abdominally obese patients. A three-year-randomised placebo controlled study

B. Richelsen ${ }^{1}$, S. Tonstad ${ }^{2}$, S. Rössner ${ }^{3}$, A. Rissanen ${ }^{4}$;

${ }^{1}$ Medical Department C, Aarhus University Hospital, Denmark, ${ }^{2}$ Preventive Medicine, Ulleval Hospital, Oslo, Norway, ${ }^{3}$ Clinic of Obesity, Huddinge Hospital, Stockholm, Sweden, ${ }^{4}$ Obesity Research Unit, Helsinki Hospital, Finland.

Objective: To investigate the efficacy of orlistat on the maintenance of weight loss over three years following a major weight loss induced by very low energy diet (VLED) in abdominally obese patients with metabolic risk factors including impaired glucose tolerance.

Study design: Initially, weight loss was induced by an eight-week VLED (600-800 kcal/day) in 383 obese patients with a mean BMI of $37.5 \mathrm{~kg} / \mathrm{m}^{2}$ (range $30.0-45.2 \mathrm{~kg} / \mathrm{m}^{2}$ ). Those who lost $>5 \%$ of their body weight (309 of 383 patients) were then randomized to receive orlistat $120 \mathrm{mg} \mathrm{t}$. i.d. or matching placebo capsules and life style counselling for three years. Primary endpoints were the maintenance of $>5 \%$ weight loss and the absolute weight loss after three years. Additionally, we analysed differences in the development of type 2 diabetes between orlistat and placebo.

Results: The VLED induced a mean weight loss of $14.4 \pm 2.0 \mathrm{~kg}$ among the subsequently randomized patients. The mean weight gain after the three years was lower with orlistat than with placebo $(4.6 \pm 8.6 \mathrm{~kg}$ vs. $7.0 \pm 7.1 \mathrm{~kg}$; $\mathrm{P}<0.02)$. The number of participants who achieved $>5 \%$ weight loss favoured also orlistat (67\% vs. $56 \%, \mathrm{P}=0.037$ ). Waist circumference was significantly more reduced in the orlistat group $(\mathrm{P}<0.05)$ but no other differences in the risk factors were observed between the two groups. The incidences of new cases of type 2 diabetes were significantly reduced in the orlistat group $(5.2 \%)$ versus placebo $(10.9 \%)(\mathrm{P}=0.041)$.

Conclusion: The addition of orlistat to lifestyle intervention improved the maintenance of weight loss after VLED for up to three years in abdominally obese subjects. Even a modest extra weight loss induced by orlistat was associated with a reduced occurrence of type 2 diabetes.

Supported by Roche Scandinavia

\section{3}

The twenty-year outcomes - the 1984 insulin-treated diabetes cohort enrolled on the Canterbury diabetes registry

S. I. Dawson, J. A. Willis, C. M. Florkowski, R. S. Scott; Lipids and Diabetes Research Group, Christchurch, New Zealand.

Background and Aims: Despite improvements in the prognosis of individuals with diabetes, mortality rates in patients with Diabetes Mellitus are still two- to five-fold higher than in the general population despite many recent improvements in care. Most often this premature mortality is attributed to the long-term cardiovascular and renal complications of diabetes. Countless health initiatives have been put in place to reduce the heavy burden of disease in these patients by better recognition, earlier treatment and reduction of long-term complications. Many studies have assessed the success of these measures determining the accurate measurement of morbidity and mortality rates in 
diabetes. However these rates vary substantially from country to country and require long follow-up periods. If diabetes mortality, and changes in diabetes mortality, are to be studied accurately in New Zealand, then baseline mortality rates specific to the New Zealand population must be established.

Aims: To establish cause-specific death rates, by age and sex in insulin-treated diabetic individuals living in Canterbury, NZ. Materials and Methods: Insulin-treated diabetic subjects $(n=995)$ on the Canterbury Diabetes Registry were followed up over 20-years and vital status determined. Following notification of deaths during this period, age- and sexspecific mortality rates, standardised mortality rates and standardised mortality difference were calculated.

Results: Six subjects had left NZ leaving 989 diabetic subjects who contributed 13,706 person-years of follow-up. During follow-up 525 deaths occurred in subjects aged 17.3 to $96.7,261$ in females and 264 in males. At all ages mortality rates were considerably higher than expected mortality. The SMRs were higher for females than males at all ages, being 2.48 (95\% CI 2.18 to 2.78 ) for females and 2.17 (95\% CI 1.91 to 2.43 ) for males overall. Relative mortalities were increased for cardiovascular, renal, respiratory disease and malignancy with cardiovascular disease accounting for the single greatest cause of excess death at all ages.

A comparison of actual and expected mortality rates, by age at onset of diabetes, for the cohort per 1000 person-years is shown in Fig. 1. All-cause mortality rates increased with age, and, were higher in diabetic men than diabetic women at all ages except $\geq 80$ years. However numbers surviving to this age were very small ( $\mathrm{n}=2$ men and 4 women). Actual mortality rates were also higher at all ages in diabetic women than the expected deaths in non-diabetic men.

Conclusion: This is the longest follow-up study carried out in New Zealand and shows that mortality rates for diabetic individuals remain high relative to the general population. To reduce these death rates attention must be paid to the early detection and treatment of cardiovascular disease and associated risk factors.

Figure 1: Expected and actual mortality rates per 1000 person-years

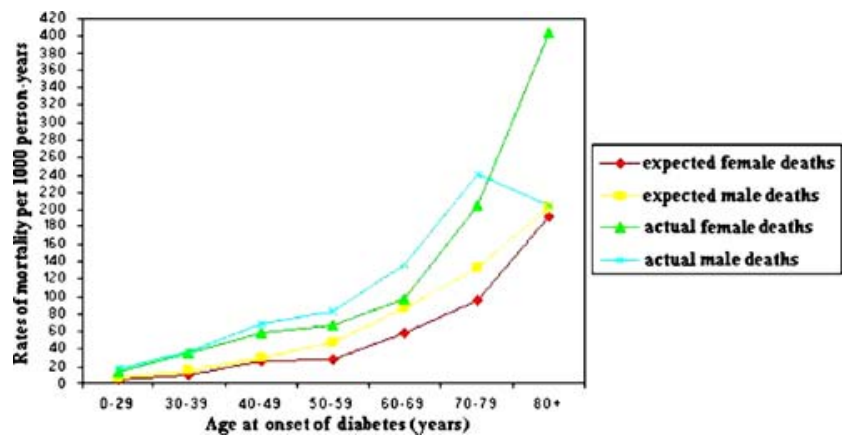

Supported by Canterbury Medical Research Foundation
0404

Unsatisfactory risk factor control in type 1 diabetes 1997-2004: results from the Swedish National Diabetes Register (NDR)

K. Eeg-Olofsson ${ }^{1}$, J. Cederholm ${ }^{2}$, P. M. Nilsson ${ }^{3}$,

S. Gudbjörnsdottir ${ }^{1}$, B. Eliasson ${ }^{1}$;

${ }^{1}$ Department of Medicine, Lundberglab for Diabetes Research, Göteborg, ${ }^{2}$ Department of Public Health and Caring Sciences, Family Medicine and Clinical Epidemiology Section, Uppsala, ${ }^{3}$ Department of Clinical Sciences Medicine, Devision of Medicine, Malmö, Sweden.

Background and Aim: In order to reduce micro- and macrovascular complications in type 1 diabetes (T1D), it is vital to improve the glycaemic control as well as other risk factors. In 2004 more than 100.000 type 1 and type 2 diabetic patients were reported to the NDR, which is one of the largest diabetes registries in the World. The aim of this survey was to describe the clinical characteristics, glycaemic and risk factor control in cross-sectional and longitudinal cohorts of T1D patients from 1997 to 2004.

Material and Methods: NDR was initiated as a tool of quality assurance in diabetes care in hospital outpatient clinics and primary care centres. Patients were reported annually to the NDR by physicians and nurses, via paper forms, computer software or via the Internet. Clinical characteristics, $\mathrm{HbAlc}$, treatment and risk factors were registered. The definition of $\mathrm{T} 1 \mathrm{D}$ was age of debut $<30$ years in combination with insulin treatment only. In 2004, 13.612 T1D patients (age $\geq 18$ years) were reported. This represents about half of the Swedish T1D population. Results: In 2004 the mean age of the T1D patients was $41.6 \pm$ SD13.5 years, the mean duration of diabetes was $26.1 \pm 14.1$ years and $46 \%$ of the patients were female. The mean HbA1c decreased from $8.2 \pm 1.3 \%$ in 1997 to $8.0 \pm 1.2 \%$ in $2004(\mathrm{p}<0.001)$. The proportion of patients reaching the national goal for HbA1c $(<7.3 \%$, DCCT standardized) increased form 24.8 to $29.6 \%$ ( $p<0.001)$. A slow but significant improvement in blood pressure levels was seen, but only $61.3 \%$ reached the blood pressure goal $(\leq 130 / 80)$. There was a slight increase in BMI from $24.9 \pm 3.5 \mathrm{~kg} / \mathrm{m}^{2}$ in 1997 to $25.3 \pm 3.9 \mathrm{~kg} / \mathrm{m}^{2}$. The use of antihypertensive and lipid lowering agents increased, whereas smoking habits were virtually unchanged. Logistic regression analyses showed that successful glycaemic control (HbA1c <7.3\% both in 1997 and in 2004) was predicted by low BMI in 1997, weight loss from 1997 to 2004, non-smoking and absence of microalbuminuria.

Conclusion: In this large cohort of Swedish T1D patients treated in everyday clinical practice, there was a slow improvement in glycaemic and risk factor control from 1997 to 2004, but the gaps between clinical results and 
treatment goals were still big. Only $17 \%$ of the T1D patients reached the treatment goals for $\mathrm{HbA1c}$, blood pressure and cholesterol. Risk factor control in T1D is still unsatisfactory and it is crucial that new measures are taken in order to improve diabetes care.

\section{PS 16}

\section{Acute and chronic complications in diabetes}

\section{5}

Diabetic ketoacidosis in Denmark. Incidence and mortality estimated from public health registries O. M. Henriksen ${ }^{1,2,3}$, M. E. Røder ${ }^{1}$, O. L. Svendsen ${ }^{1}$, J. B. Prahl ${ }^{4}$;

${ }^{1}$ Endocrine Section, Dept. Internal Medicine I, ${ }^{2}$ Dept. Clinical Physiology and Nuclear Medicine, Bispebjerg University Hospital, Copenhagen, ${ }^{3}$ Dept of Clinical Physiology and Nuclear Medicine, University Hospital Glostrup, ${ }^{4}$ Paediatrics Dept., University Hospital Gentofte, Denmark

Background and Aims: Previous studies have reported an increasing incidense of diabetic ketoacidosis (DKA) and a decreasing mortality from DKA in Denmark.

The aims of the present study were to estimate incidence of and mortality from DKA in Denmark and to characterise deaths associated with DKA using data from national health registries.

Materials and Methods: Hospital admissions in the period 1996-2002 with a discharge diagnosis of DKA were identified from the Danish National Patient Registry (NPR). Deaths associated with DKA in the period 1996-2000 were identified from the Danish Cause of Death Registry (CDR). Results: A total of 4807 admissions and 137 deaths with a diagnosis of DKA were identified. Annual incidence of DKA in the general population was estimated to 12,9 per 100.000. The annual incidence was higher in males than in females ( 14.4 vs. 11.4 per $100.000, p<0.0001) .12 \%$ of all patients were classified as Type $2 \mathrm{DM}$, and this percentage increased exponentially with age $>50$ years.

The mortality was $4 \%$. In patients $>70$ years the mortality was higher than in younger patients $(15 \%$ vs. $2 \%, \mathrm{p}<0.0001)$. One or more additional somatic diagnoses were stated on $77 \%$ of the death certificates, most often of a cardiovascular or infectious disease ( $47 \%$ and $30 \%$ respectively)

Conclusion: DKA is still a frequent complication to diabetes, but the number of admissions related to DKA has remained the same during the past 25 years despite an increasing prevalence of diabetes, however the incidence seems to have decreased in younger patients with Type $1 \mathrm{DM}$. Older patients with DKA differed from younger patients in having a higher mortality and a larger proportion of patients classified as Type 2 DM.

0406

Poor metabolic control in diabetic patients: a risk factor of shortsightedness

N. Jacobsen ${ }^{1}$, H. Jensen ${ }^{1}$, E. Goldschmidt ${ }^{2}$,

H. Lund-Andersen ${ }^{3}$;

${ }^{1}$ Ophthalmology, National Eye Clinic for the Visually Impaired, Hellerup, ${ }^{2}$ Ophthalmology, Danish Institute for Myopia Research, Hilleroed, ${ }^{3}$ Department of Ophthalmology, Herlev Hospital, University of Copenhagen, Herlev, Denmark.

Background and Aims: As a consequence of an increasing prevalence of shortsightedness (myopia) in parts of the world that has adopted western dietary patterns it has been hypothesized that hyperglycaemia and hyperinsulinaemia induce myopia. The purpose of this study was to evaluate the relation between glycosylated haemoglobin $\left(\mathrm{HbA}_{1 \mathrm{c}}\right)$, insulin dosage and myopia in diabetic patients.

Materials and Methods: A total of 383 type 1 diabetic patients aged 16-26 years (mean age 22.0 (SD 2.9)) attending the eye clinic at Steno Diabetes Center, Copenhagen, in 1995-97, were included in a retrospective cohort study. The following data were collected from the medical records from baseline to 2005: age at diabetes onset, age at baseline, sex, weight, $\mathrm{HbA}_{1 \mathrm{c}}$, insulin dosage, refractive error (automated refraction in cycloplegia), visual acuity and diabetes related complications.

Results: The prevalence of myopia $(\leq-0.5 \mathrm{D}$, spherical equivalent) was $53.3 \%$ (95\%CI (confidence interval) \pm 5.29$)$ at baseline and $65.6 \%(95 \% \mathrm{CI} \pm 5.88)$ at the last eye examination (mean follow-up 7.1 years (SD 2.2). In a logistic regression analysis, duration of diabetes (OR (Odds ratio) 0.918 (95\% CI 0.873;0.966), $\mathrm{p}=0.001)$, refraction at baseline (OR 0.858 (95\% CI 0.742; 0.992), $\mathrm{p}=0.038)$ and $\mathrm{HbA}_{1 \mathrm{c}}$ (OR 1.270 (95\% CI 1.058; 1.526), $\mathrm{p}=0.011$ ) were related to myopia, whereas insulin dosage was not (OR 1.833 (95\% CI 0.595; 5.647), $\mathrm{p}=0.291$ ).

Conclusion: This study suggests a relation between development and progression of myopia and poor metabolic control in diabetic patients. In addition, myopia was more frequent in the present study population than in non-diabetics.

Supported by VOS and the Velux Foundation 
0407

Increased risk of common infections in hospitalised patients with type 2 diabetes mellitus

P. S. Koureta ${ }^{1}$, N. G. Anastasopoulos ${ }^{1}$, A. Angelis ${ }^{1}$, A. Kramvis ${ }^{1}$, K. Makrilakis ${ }^{2}$, A. E. G. Alaveras ${ }^{1}$;

${ }^{1} 1$ st Medical Department, Red Cross Hospital, Athens, ${ }^{2} 1 \mathrm{st}$ Department of Propaedeutic Medicine, University of Athens Medical School, Greece.

Background and Aims: Many clinicians believe that people with diabetes are more susceptible to infections. Although certain rare infections are more common, risks of acquiring an infectious disease, as well as infection-related mortality, are not well studied for diabetic patients.

Materials and Methods: A retrospective cohort study was conducted, comparing all patients with any common infection hospitalized in our clinic during a decade, (1990-2000), to matched patients hospitalized for any reason other than infection. The risk ratios of having an infectious disease and of in-hospital death attributable to infectious disease between those with and without diabetes were calculated, using multiple regression analysis models. The results were adjusted for possible confounding factors (age, sex and history of cardiovascular disease).

Results: The cohort consisted of 2583 patients hospitalized for any common infection (491 (19.01\%) with diabetes and 2092 without) and 1408, matched for age and sex, patients hospitalized for any reason other than infection (199 (14.13\%) with diabetes and 1209 without). The risk of acquiring an infectious disease was $28 \%$ higher for diabetic patients compared to those without diabetes $(\mathrm{OR}=1.2895 \%$ CI $1.06-1.55 \mathrm{p}=0.010)$. There were 323 (12.50\%) deaths among the 2583 patients hospitalized with infection and $100(7.10 \%)$ deaths among the 1408 hospitalized for any other reason. Overall, the risk of death was about two times higher for patients hospitalized for any common infection $(\mathrm{OR}=2.36$ 95\% CI 1.81-3.07 $\mathrm{p}<0.001)$. Among patients hospitalized for any common infection, there were 68 (13.85\%) deaths among the 491 diabetic patients and 255 (12.19\%) deaths among the 2092 non-diabetic patients. The risk of death attributable to infectious disease did not differ between hospitalized diabetic and non-diabetic patients $(\mathrm{OR}=1.12$ 95\% CI $0.81-1.53 \mathrm{p}=0.497)$.

Conclusion: The risk of acquiring an infectious disease is higher among diabetic patients compared to their nondiabetic counterparts. On the other hand, infection-related mortality did not seem to differ between hospitalized diabetic and non-diabetic patients, although, overall, the risk of death attributable to infectious disease was quite high.
0408

Care and outcomes in 35,723 adults with diabetes mellitus in Germany 2005

N. Müller ${ }^{1}$, U. A. Müller ${ }^{1}$, W. Kerner ${ }^{2}$;

${ }^{1}$ Dept. Internal Medicine III, University Hospital Jena,

${ }^{2}$ Diabetology, Hospital Karlsburg, Germany.

Background and Aims: The German Diabetes Association provides a certificate of quality for hospitals (HO) and specialised private practices (PR). Candidates have to provide information on the staff, patient education and documentation of patient data. 27 items have to be submitted on $>50$ patients with type 1 diabetes (DM1) and 200 patients with type 2 diabetes (DM2). The aim of the analysis presented was to describe the quality of care of adult persons with diabetes mellitus in a large sample in Germany.

Materials and Methods: In 2005119 institutions (27 PR, $92 \mathrm{HO})$ applied for the certificate. 35,723 patients $(5,891$ DM1, 29,693 DM2, 100 pancreas diabetes) out of $26 \mathrm{PR}$ (1,664 DM1, age 43.7 y, years sime since diagnosis 15.7, BMI $25.9 \mathrm{~kg} / \mathrm{m}^{2}, 7,779$ DM2, $66.9 \mathrm{y}, 9.9 \mathrm{y}, 30 \mathrm{~kg} / \mathrm{m}^{2}$ ) and $92 \mathrm{HO}(4,227 \mathrm{DM}$, age $43.9 \mathrm{y}$, time since diagnosis 15.8 years, BMI $25.1 \mathrm{~kg} / \mathrm{m}^{2}, 21,914 \mathrm{DM} 2,64.3 \mathrm{y}, 9.9 \mathrm{y}$, $31.1 \mathrm{~kg} / \mathrm{m}^{2}$ ). HbA1c values were DCCT adjusted. The data from PR represent a cross section of care and outcome, in contrast to the data from hospitals which describe the quality of care at the time of admission.

\section{Results:}

Diabetologists' practices:

DM1 ( $=1664)$ age 43,7J; years since diagnosis 15.7; BMI $25.9 \mathrm{~kg} / \mathrm{m}^{2}$; HbA1c 7.22\% (HbAlc <7\%: 48.5\%; HbAlc $>7 \%$ : 51.5\%); RR 124/74 (RR sys. $>140$ or RR dia. >90: 16.4\%); severe hypoglycaemia 0.06/pat/y; severe ketoacidosis $0 / \mathrm{pat} / \mathrm{y}$.

IDDM 2: $(n=3664)$ age 65.3J; years since diagnosis 12.,6; BMI $30.6 \mathrm{~kg} / \mathrm{m}^{2}$; HbAlc 6.96\% (HbAlc <7\%: 59.8\%; HbA1c $>7 \%$ : 40.2\%); RR 135/77 mm hg (RR sys.>140 or RR dia.>90: 30.4\%); severe hypoglycaemia 0.01/pat/y; coma with hospitalisation $0 /$ pat/y

NIDDM 2: $(\mathrm{n}=4115)$ age $65.3 \mathrm{~J}$; years since diagnosis 6.8 ; BMI $31.6 \mathrm{~kg} / \mathrm{m}^{2}$; HbAlc 6.65\% (HbAlc <7\%: 69.7\%; HbAlc $>7 \%$ : 30.3\%); RR 135/79 (RR sys.>140 or RR dia. $>90$ : $31.7 \%$ ); severe hypoglycaemia 0/pat/y; coma with hospitalisation 0/pat/y.

Hospitals:

DM1 ( $\mathrm{n}=4227$ ) age $43.9 \mathrm{y}$; years since diagnosis 15.8 ; BMI $25.1 \mathrm{~kg} / \mathrm{m}^{2}$; HbA1c 8.2\% (HbA1c <7\%: 33,4\%; HbA1c $>7 \%$ : 66,6\%); RR126/76 mm hg (RR sys. $>140$ or RR dia.>90: 15.9\%); severe hypoglycaemia 0.3; severe ketoacidosis $0.11 / \mathrm{pat} / \mathrm{y}$

IDDM2 ( $\mathrm{n}=7523)$ age $66.9 \mathrm{y}$; years since diagnosis 12.0; BMI $30.3 \mathrm{~kg} / \mathrm{m}^{2}$; HbAlc 7.97\% (HbAlc <7\%: 36.6\%; 
HbA1c $>7 \%$ : 63.4\%); RR137/78 mm hg (RR sys. $>140$ or RR dia.>90: 31.5\%); severe hypoglycaemia 0.08/pat/y; coma with hospitalisation $0.02 / \mathrm{pat} / \mathrm{y}$.

NIDDM2 $(n=14391)$ age $66.9 \mathrm{y}$; years since diagnosis 6.0; BMI $29.5 \mathrm{~kg} / \mathrm{m}^{2}$; HbA1c 6.98\% (HbA1c <7\%: 63.0\%; HbAlc $>7 \%$ : 37.0\%); RR136/79 mm hg(RR sys. $>140$ or RR dia.>90: 30.1\%); severe hypoglycaemia 0.03/pat/y; coma with hospitalisation $0.01 / \mathrm{pat} / \mathrm{y}$.

Conclusion: In comparison to patients on continuous care in specialised private practice patients on admission to hospitals were older, diabetes duration was longer, BMI and $\mathrm{HbAlc}$ were higher and they had more acute complications. $50 \%$ of the patients with type 1 diabetes taken care of in practices of diabetologists have very good HbAlc-value $<7 \%$, respectively; $60 \%$ of patients with type 2 diabetes on insulin and $70 \%$ of patients with type 2 diabetes without insulin treatment. Acute complications occur very rarely altogether the data represent a very satisfactory quality of care. In patients on admission to hospitals Hbalc above 7\% is present in $67 \%$ of patients with DM1, $63 \%$ of patients with DM2 and insulin therapy and $37 \%$ of patients with DM2 without insulin therapy at admission to hospital - not unexpected since the patients were admitted to the hospitals due to problems of care. The general quality of documented blood pressure was excellent.

\section{9}

Frequent microangiopathy in fulminant type 1 diabetes - a nationwide, 5-year-study

Y. Murase $^{1}$, A. Imagawa ${ }^{1}$, T. Hanafusa ${ }^{1}$, H. Iwahashi ${ }^{2}$,

Y. Uchigata ${ }^{3}$, A. Kanatsuka ${ }^{4}$, E. Kawasaki ${ }^{5}$,

T. Kobayashi ${ }^{6}$, A. Shimada ${ }^{7}$, I. Shimizu ${ }^{8}$,

T. Maruyama ${ }^{9}$, H. Makino ${ }^{10}$;

${ }^{1}$ First Department of Internal Medicine, Osaka Medical College, Takatsuki, ${ }^{2}$ Department of Internal Medicine and Molecular Science, Graduate School of Medicine, Osaka University, ${ }^{3}$ Diabetes Center, Tokyo Women's Medical University School of Medicine, ${ }^{4}$ Diabetes Center, Chiba Central Medical Center, ${ }^{5}$ Department of Metabolism/Diabetes and Clinical Nutrition, Nagasaki University Hospital of Medicine and Dentistry, ${ }^{6}$ Third Department of Internal Medicine, Interdisciplinary Graduate School of Medical and Engineering, University of Yamanashi, Tamaho, ${ }^{7}$ Department of Internal Medicine, Keio University School of Medicine, Tokyo, ${ }^{8}$ Internal Medicine, Ehime Prefectural Imabari Hospital, Ehime, ${ }^{9}$ Department of Internal Medicine, Saitama Social Insurance Hospital, Japan, ${ }^{10}$ Department of Laboratory Medicine, Ehime University School of Medicine, Japan.
Background and Aims: Fulminant type 1 diabetes (FT1D) is a novel subtype of type 1 diabetes, which is characterized by a markedly acute onset of diabetes and an absence of islet-related autoantibodies. The aim of the present study was to assess the development of diabetic microangiopathy in FT1D.

Materials and Methods: Forty-one patients with FT1D (21 males and 20 females, age at onset; $38.7 \pm 15.1$ yrs) and ageand sex-matched 76 patients with type $1 \mathrm{~A}$ diabetes (T1AD) had been followed for 5 years in a nationwide survey in Japan. The following data were recorded every 12 months after the onset of diabetes: Seven point (preprandial and 120min postprandial for each meal and bedtime) blood glucose concentrations, $\mathrm{HbA}_{1 \mathrm{c}}$ level, urinary albumin excretion, fasting or postprandial serum C-peptide level, body weight, systolic and diastolic blood pressure, daily dosages of insulin, frequency of severe hypoglycaemic episodes, presence or absence of Achilles' tendon reflex, and the result of fundoscopic examination by ophthalmologist.

Results: Five-year cumulative incidence of microangiopathy was $24.4 \%$ in FT1D and $2.6 \%$ in T1AD. In longitudinal studies using Kaplan-Meier method, the incidence of each microangiopathy was significantly higher in FTID than in T1AD; retinopathy was $9.8 \%$ vs. $1.3 \%$ $(p=0.033)$, nephropathy $12.2 \%$ vs. $2.6 \% \quad(p=0.015)$, and neuropathy $12.2 \%$ vs. $1.3 \%(\mathrm{p}=0.010)$, respectively. Mean $\mathrm{HbA}_{1 \mathrm{c}}$ levels were similar in FT1D and T1AD group during the follow-up periods $(7.4 \pm 1.3 \%$ vs. $7.7 \pm 1.8 \%$, NS). However, mean insulin dosages, mean M-value, and the frequency of severe hypoglycaemic episodes were significantly higher in FT1D than in T1AD $(0.76 \pm 0.22$ vs. $0.60 \pm 0.21 \mathrm{U} / \mathrm{kg}, \mathrm{p}=0.0001,29.2 \pm 13.5$ vs. $23.0 \pm 15.3$, $\mathrm{p}=0.047$, and $0.30 \pm 1.04$ vs. $0.01 \pm 0.06$ episodes $/ \mathrm{yr} /$ person, $\mathrm{p}=0.018$, respectively). Blood pressure and body weight did not differ between the two groups. Significantly lower levels of mean postprandial C-peptide were observed in FT1D (0.26 \pm 0.12 vs. $0.71 \pm 0.44 \mathrm{ng} / \mathrm{ml}, \mathrm{p}=0.0007)$.

Conclusion: These data suggest that FT1D is a high-risk subgroup of diabetic microangiopathy, probably through brittleness in blood glucose levels due to the lack of endogenous insulin secretion from onset of diabetes.

\section{0}

Prevalence of diagnosed diabetic retinopathy and its severity in four European countries

A. Rubino ${ }^{1}$, M. D. Rousculp ${ }^{2}$, J. Wang ${ }^{1}$, K. Davis ${ }^{1}$,

A. Girach ${ }^{2}$;

${ }^{1}$ Health Outcomes, RTI-Health Solutions, Manchester, United Kingdom, ${ }^{2}$ Lilly Research Laboratories, Eli Lilly and Company, Indianapolis, United States. 
Background: Type 1 and type 2 diabetes mellitus are associated with a broad spectrum of microvascular complications, including DR. An understanding of the prevalence of DR and its severity is essential to an appreciation of the full burden of diabetes. The characteristics of DR are not well defined in the literature.

The primary objectives of this study were:

- To estimate the prevalence of diagnosed DR in patients with type 1 and type 2 diabetes mellitus; and

- To characterize and estimate the prevalence of the severity stages of DR.

Methods: This study was a cross-sectional survey of physicians who practice within the primary care (GP) setting in France, Italy, Spain and the United Kingdom (UK); additionally diabetes specialists were recruited in Italy and Spain. Information on the prevalence of diagnosed DR was obtained from existing physician records. Clinical details were collected from a random sample of DR patients using medical records.

Results: Forty-one clinics (mean) per country were included in the study (range: 34-49). The estimated prevalence of diagnosed DR amongst GP patient populations ranged from $9.6 \%$ in Spain to $19.6 \%$ in the UK. In Italy and Spain, prevalence among Diabetic Specialist (DS) patient populations was $20.2 \%$ and $32.7 \%$, respectively. In Spain, this was three-fold higher than prevalence in primary care, while no substantial difference was noted in Italy.

Prevalence of Diagnosed DR per 100 Diabetic Patients (95\% CI) per Country

\begin{tabular}{llll}
\hline France* $^{*}$ & Italy & Spain & UK* \\
11.4 & GP: 18.3 & GP: 9.6 & 19.6 \\
$(8.8-13.9)$ & $(6.9-29.8)$ & $(5.9-13.3)$ & $(16.0-23.1)$ \\
& DS: 20.2 & DS: 32.7 & \\
& $(14.01-26.4)$ & $(18.2-47.3)$ & \\
\hline
\end{tabular}

*Data collected only from GPs

Charts were abstracted for 752 patients with DR. Of these $53.9 \%$ were males and $66.9 \%$ had type 2 diabetes. Mean age (+SD) was $64.2+12.8$ years; HbA1c was $7.75+1.4 \%$ (Mean+SD). DR diagnosed as proliferative ranged from $19.7 \%$ in France to $31.5 \%$ in the UK. Consistently across countries, mild Non-Proliferative DR (NPDR) was the most common severity level of NPDR.

$\begin{aligned} & \text { Distribution of Severity Levels per } \\
& \text { Country }\end{aligned}$
\begin{tabular}{lcccc}
\hline DR Type & France & Italy & Spain & UK \\
NPDR & & & & \\
Mild & $40.9 \%$ & $39.2 \%$ & $43.2 \%$ & $42.0 \%$ \\
Moderate & $27.3 \%$ & $27.8 \%$ & $19.4 \%$ & $16.0 \%$ \\
Severe & $1.5 \%$ & $4.2 \%$ & $2.6 \%$ & $3.3 \%$ \\
Proliferative DR & $19.7 \%$ & $21.7 \%$ & $29.1 \%$ & $31.5 \%$ \\
\hline
\end{tabular}

Hypertension (70.1\%), dyslipidaemia (61.0\%) and neuropathy $(53.2 \%)$ were the most common co-morbidities. Nephropathy was present in $38.5 \%$ of patients with mild NPDR and in $64.4 \%$ of patients with proliferative DR.

Conclusion: In this multi-national study, country-specific prevalence estimates of diagnosed DR may reflect respective clinical practice in the management of diabetes and its complications. Consistently across countries, the majority of diagnosed NPDR is of mild to moderate severity.

Supported by Eli Lilly and Company

\section{1}

Decreasing prevalence of diabetic retinopathy in 1828 outpatients from the university diabetic clinic Jena in 2005 and 2006

A. Mandecka ${ }^{1}$, C. Kloos ${ }^{1}$, N. Müller ${ }^{1}$, S. Jurkutat ${ }^{2}$, G. Wolf ${ }^{1}$, U. A. Müller ${ }^{1}$;

${ }^{1}$ Endocrinology and Diabetes, ${ }^{2}$ Ophthalmology, University of Jena Medical School, Germany.

Background and Aims: The incidence of diabetic retinopathy (DR) has decreased due to improvements in diabetes care (Hovind, Diabetes care 2000). However, there is little knowledge concerning the incidence of retinopathy in Germany. The objectives of this study were to estimate the incidence and risk factors for DR using routinely collected data from a clinical information system at the University Jena (Germany) for type 1 and 2 diabetes.

Materials and Methods: We studied results of fundoscopy and clinical features of 1828 patients (943 male and 890 female) with diabetes mellitus (DM1 21.7\%, age 46, years since diagnosis 19; DM2 78.2\%, age 63.4, years since diagnosis 13.5) who presented in 2005 and 2006 at the outpatient clinic. Data were obtained from the electronic system EMIL ${ }^{\circledR}$. The mean age of the participants was 60 $( \pm 15)$ yr with a mean duration of diabetes of $14( \pm 10)$ yr. Ophthalmological examinations of 1229 patients $(67.2 \%)$ were available. The mean follow-up was $5( \pm 5)$ (range $0-20)$ yr.

Results: Diabetic retinopathy was found in $30.2 \%$ of patients (DM1 37.1\%; DM2 27.8\%): non-proliferative retinopathy (NPDR) 234 patients (19\%), proliferative (PDR) 11 (9,0\%), blindness $8(0,7 \%)$, clinically significant macular edeme (CSME) 29 (2.4\%). Regarding the type of diabetes:

DM1: no RD 62.8\%, NPDR 22.3\%, PDR 11.8\%, blindness $0.6 \%$, CSME $3.1 \%$. The incidence of RD depending on duration of diabetes was: $0 \leq 5$ years $0 \% ; 5 \leq 107.1 \% ; 10 \leq 15$ $17.7 \% ; 15 \leq 20 \quad 33.8 \% ; 20 \leq 25 \quad 52.7 \% ; 25 \leq 30 \quad 60.9 \% ; \geq 30$ $86.6 \%$.

DM2: no RD 72.2\%, NPDR 17.9\%, PDR 8.1\%, blindness $0.9 \%$, CSME $2.1 \%$. The incidence of RD depending on duration of diabetes was: $0 \leq 5$ years $5.4 \%$; $5 \leq 1015 \%$; 
$10 \leq 15 \quad 17.5 \% ; \quad 15 \leq 20 \quad 40.8 \% ; 20 \leq 25 \quad 59.6 \% ; 25 \leq 30$ $61.7 \% ; \geq 3054.4 \%$.

No patient with DM1 and duration of diabetes less than $5 \mathrm{yr}$ had retinopathy, but $3,0 \%$ of patients with DM2 exhibited NPDR and $1.8 \%$ had PDR. Among patients with DM1 after 5-10 years of the disease no patient had PDR, but NPDR was present in $7.1 \%$. Among patients with DM1 PDR was first detected by $10<15 \mathrm{yr}$ duration and was present in $3.7 \%$. $59 \%$ of patients with duration $>30 \mathrm{yr}$ had no retinopathy (DM1 13.3\%; DM2 45.7\%). In this group the PDR was present in $33.3 \%$ of DM1 and $17.4 \%$ of DM2. The major part of patients with duration $>30$ years had the NPDR. Of those patients with $\mathrm{RD}, 95.4 \%$ were insulin-treated, $4.5 \%$ with oral antiglycemic agents and $82.3 \%$ with antihypertensive medications. Of the patients without $\mathrm{RD}$ $83 \%, 19 \%$ and $74.7 \%$, respectively. The degree of DR was found to be related to the duration of diabetes, glycaemic control (HbAlc), systolic blood pressure and serum creatinine. In DM1, BMI, urinary albumin secretion and creatinine clearance were significantly higher among patients with PDR comparing to patients without RD.

Conclusion: Our study confirms the decreasing incidence of diabetic retinopathy. We provide contemporary data on the prevalence and risk factors for diabetic retinopathy among patients from outpatient diabetic clinic in Germany. Compared to a study from 1989 (Weimar, Germany) which used the register of the centralized diabetes care system in the former German Democratic Republic, we observed a decrease of diabetic retinopathy in patients with DM1 $(52.5 \%$ vs. $37.3 \%)$ and in patients with insulin-treated DM2 (50.3\% vs. $31.5 \%$ ). Diabetic retinopathy was found in $30 \%$ of diabetic patients, a lower rate than in Wisconsin Epidemiologic Study.

\section{2}

Prevalence of diagnosed Diabetic Peripheral Neuropathy (DPN) and its symptoms in four European countries

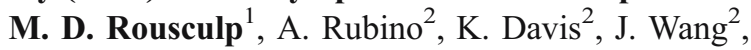
S. Tzivelekis ${ }^{1}$, E. J. Bastyr ${ }^{1}$, S. Tesfaye ${ }^{3}$;

${ }^{1}$ Eli Lilly and Company, Indianapolis, United States,

${ }^{2}$ Health Outcomes, RTI-Health Solutions, Manchester, United Kingdom, ${ }^{3}$ Medical Clinic, Royal Hallamshire Hospital, Sheffield, United Kingdom.

There is limited consensus on how best to diagnose DPN, yet patients are diagnosed and the magnitude of severity and clinical details are not well understood. A cross-sectional survey was administered to 125, general practitioners (GP) in France, Italy, Spain, and the United Kingdom (UK) to estimate the prevalence of diagnosed DPN within practices. Prevalence estimates ranged from $9.5 \%$ in Spain to $14.1 \%$ in the UK. Additional surveys of Diabetic Specialists in Italy and Spain found a prevalence that was two-fold higher in Italy (26.8\%) compared with GPs, while a smaller difference was noted in Spain (15.0\%).

Prevalence of Diagnosed DPN per 100 Patients (95\% CI) from GP Surveys

\begin{tabular}{llll}
\hline France & Italy & Spain & UK \\
$10.7(7.6-13.8)$ & $13.9(9.0-18.8)$ & $9.5(6.9-12.1)$ & $14.1(10.6-17.7)$ \\
\hline
\end{tabular}

Medical chart abstraction was conducted to collect data from a random sample of 913 DPN patients within the clinics. Of these, $55.0 \%$ were males and $78.5 \%$ had type 2 diabetes. Age was $64.5+12.5$ years and $\mathrm{HbAlc}$ was $7.67+1.4 \%$ (Mean $+\mathrm{SD})$. Approximately $27 \%$ of patients were diagnosed without a documented neurological exam. Hypertension $(72.7 \%)$ and dyslipidemia (64.8\%) were the most common co-morbidities. DPN diagnosis was mostly based on symptoms consistent with DPN. The most common DPN symptoms were numbness and prickling across all countries. Overall, approximately $24 \%$ of patients had no record of pain symptoms, while $26 \%$ had aching, burning and stabbing pain symptoms. Over $22 \%$ of patients were treated with anticonvulsants and $12.2 \%$ with tricyclic antidepressants.

Frequency of DPN-Related Symptoms Recorded in Medical Charts

\begin{tabular}{lllll}
\hline DPN Symptoms & France & Italy & Spain & UK \\
\hline Aching pain & $32.3 \%$ & $35.4 \%$ & $37.1 \%$ & $48.8 \%$ \\
Burning pain & $44.8 \%$ & $56.9 \%$ & $47.4 \%$ & $60.8 \%$ \\
Prickling & $81.0 \%$ & $68.0 \%$ & $73.7 \%$ & $49.8 \%$ \\
Numbness & $66.4 \%$ & $69.7 \%$ & $77.7 \%$ & $63.6 \%$ \\
Stabbing pain & $24.1 \%$ & $27.6 \%$ & $31.4 \%$ & $44.5 \%$ \\
Tenderness & $50.4 \%$ & $43.8 \%$ & $48.0 \%$ & $43.5 \%$ \\
\hline
\end{tabular}

Country-specific prevalence estimates of diagnosed DPN may reflect clinical management of diabetes and DPN. Consistently across countries, over a quarter of patients had a record of pain symptoms associated with DPN.

Supported by Eli Lilly and Company

\section{3}

Development of complications and costs after diagnosis of type 2 diabetes: results from the ROSSO study S. Martin ${ }^{1}$, W. Schramm ${ }^{2}$, B. Schneider ${ }^{3}$, K. Neeser ${ }^{2}$, C. Weber ${ }^{2}$, L. Heinemann ${ }^{4}$, W. A. Scherbaum ${ }^{1}$, H. Kolb ${ }^{1}$; ${ }^{1}$ German Diabetes Research Institute at the Heinrich-HeineUniversity, Düsseldorf, Germany, ${ }^{2}$ Institute for Medical Informatics and Biostatistics, Basel, Switzerland, ${ }^{3}$ Institute of Biometry, Medical University Hannover, Germany, ${ }^{4}$ Profil Institute for Metabolic Research, Neuss, Germany. 
Background and Aims: The German multi-centre, retrospective cohort study ROSSO observed a representative cohort of 3142 newly diagnosed type 2 diabetes patients with a public health insurance plan from randomly contacted 192 GP practices. The patients were followed over a mean of 6.5 years. The ROSSO cohort was used to analyse the development of complications and the total treatment costs.

Materials and Methods: The data of all patients of a practice with a type 2 diabetes diagnosis between 1995 1999 were taken directly from the physician's files. Monitoring assured the completeness and quality of the data collection. Based on these data, costs were attributed using the doctor's tariff, hospital DRGs and medication price lists for Germany.

Results: At diagnosis of type 2 diabetes previous diagnosis of myocardial infarction (MI) was present in $4 \%$ of the patients, stroke $3.2 \%$, congestive heart failure (CHF) $15.3 \%$, polyneuropathy (PNP) $5.0 \%$ and depression $7.3 \%$. During follow up over 7 years the complication rate rose linearly to $7.3 \%$ for $\mathrm{MI}, 8.6 \%$ for stroke, $33.4 \%$ for $\mathrm{CHF}$, $19.7 \%$ for PNP and $15.9 \%$ for depression.

Other diabetic endpoints as hemodialysis (1.4\%) amputation $(1.2 \%)$ and blindness $(0.6 \%)$ rose but stayed lower. Total cost of diabetes care was $1288 €$ for the first treatment year with diagnosed diabetes and amounted to $3845 €$ in the year seven. Even in the first years after diagnosis $58 \%$ of the cost was spent for the treatment of complications and this proportion increased to $83 \%$ in the seventh year. Average costs per patient and year were $3210 €$, while $6 \%$ were spend for medication and only less than $3 \%$ for monitoring systems.

Conclusion: The ROSSO is the first epidemiological cohort study, examining the longitudinal epidemiological data of the same patients over more than six years for type 2 diabetes mellitus in primary care practices. Stroke occurs more often than myocardial infarction, but even more patients suffer from congestive heart failure. Even in the first years after diabetes diagnosis an excess of costs for treating complications was observed. Costs for medication and monitoring systems represent only a small portion of the total costs.

Supported by an unrestricted grant from Roche Diagnostics

\section{PS 17}

\section{Cytokines, chemokines and beta cell death}

\section{4}

The role of $\mathrm{CBR} 1$ in the pathogenesis of type 1 diabetes C. Bang-Berthelsen, R. Bergholdt,, P. Heding, J. Nerup, F. Pociot;

Steno Diabetes Center, Gentofte, Denmark.
Background and Aims: Type 1 diabetes (T1D) is a genetically complex disease characterized by an immunemediated destruction of pancreatic beta-cells. We have recently fine mapped and characterized a T1D linked region (containing 32 genes) on chromosome $21(\mathrm{p}=0.0002)$ in a Danish family material. Interesting candidate genes emerged within this region, and several have been described to have modulating effects during oxidative stress in cells. Of these, the carbonyl reductase $1(C B R I)$ gene have additionally been demonstrated involved in regulation of apoptosis, with potential relevance for T1D pathogenesis. We have previously by two dimensional gel electrophoresis of IL-1 $\beta$ exposed, diabetes-prone Bio-Breeding rat islets demonstrated that the CBR1 protein was down regulated fivefold by IL-1 $\beta$ stimulation, and we have preliminary studies showing that the same is true on the mRNA level in human islets. The aim of the present study was to further characterize the $C B R 1$ gene and protein at functional/cellular levels.

Materials and Methods: Functional studies were performed in an insulinoma (INS-1) beta-cell line. Cells were stimulated with cytokines (IL-1 $\beta$ and/or IFN- $\gamma$ ) for different periods of time, as well as cultured under serum starving conditions. All conditions were analyzed in the absence or presence of a specific CBR1 inhibitor (Hydroxy-pp-met), previously reported to be able to inhibit serum starvation induced apoptosis in other cell types. Accumulated insulin content, nitric oxide (NO) generation and cellular viability (MTT) was measured for all conditions. Furthermore, apoptosis induction was estimated by a Caspase 3/7-assay and a cell death detection ELISA-assay. Cells treated with cytokines and serum starvation were evaluated using real time PCR and Western blot analyses for expression levels of mRNA and protein.

Results: Maximum down regulation of CBR1 on the mRNA level, was observed when INS-1 cells were stimulated with IL-1 $\beta(150 \mathrm{nM})$ for 12 hours. On the protein level we demonstrated a corresponding down regulation of the CBR1 protein level after 24 hours of IL-1 $\beta$ stimulation. We detected no differences in NO, insulin, MTT or Caspase $3 / 7$ activity in cells stimulated with cytokines in absence or presence of the CBR1 inhibitor. However, we observed that serum starvation induced beta-cell apoptosis can be suppressed by addition of the CBR1 inhibitor.

Conclusion: In the current study we have demonstrated CBR1 expression to be regulated by IL- $1 \beta$ on mRNA as well as the protein level. This down regulation by IL-1 $\beta$ may be an important mechanism, potentially making betacells more vulnerable towards oxidative stress. Furthermore we demonstrated an apoptosis modulating effect of CBR1 in beta-cells subjected to serum starvation. However, more studies are in demand to clarify the exact function of CBR1 
in beta-cells. Our findings support $C B R-1$ as a potentially interesting candidate gene/protein in T1D.

Supported by the Danish Diabetes Association

\section{5}

Proteomic analysis of cytokine-induced dysfunction and death in insulin producing INS-1E cells

W. D'Hertog ${ }^{1}$, L. Overbergh ${ }^{1}$, L. Schoofs ${ }^{2}$,

A. K. Cardozo ${ }^{3}$, L. Arckens ${ }^{2}$, E. Waelkens ${ }^{4}$,

D. L. Eizirik ${ }^{3}$, C. Mathieu ${ }^{1}$;

${ }^{1}$ LEGENDO, Catholic University Leuven, Leuven, Belgium, ${ }^{2}$ Animal Physiology and Neurobiology Section, Catholic University Leuven, ${ }^{3}$ Experimental medicine, Université Libre de Bruxelles, ${ }^{4}$ Biochemistry Section, Catholic University Leuven, Belgium.

Background and Aims: Type 1 diabetes is an autoimmune disease characterized by T-cell mediated destruction of the pancreatic beta-cells. Although the exact triggers initiating the disease need further unraveling, cell-destructive molecules, including cytokines (such as interleukin [IL]-1beta and interferon [IFN]-gamma) play a crucial role in beta-cell destruction. Previously performed time-course micro-array analysis of insulin producing INS-1E cells identified 698 genes as modified by cytokine exposure, many of which are related to apoptotic pathways. The aim of this study was to investigate the relevance of these findings at the protein level, as well as to detect translational and post-translational changes.

Materials and Methods: For this purpose quantitative changes in protein expression upon cytokine-treatment were investigated in INS-1E cells using 2-dimensional difference gel electrophoresis (2D-DIGE) and differentially expressed spots were identified by MALDI-TOF/ TOF. INS-1E cells were incubated during 1, 4 and 24 hours with IFN-gamma $(500 \mathrm{U} / \mathrm{ml})$ and IL-1beta $(10 \mathrm{U} /$ $\mathrm{ml}$ ). As expected, these cytokines induced nitric oxide (96 pmol/ $/ 10^{3}$ cells in cytokine-induced versus not detectable in control condition; $\mathrm{n}=4$ ) and apoptosis (Annexin/PI staining; $35.2 \%$ increase compared to control condition; $\mathrm{n}=4(\mathrm{p}<0.01))$. Differential protein expression was analyzed by 2D-DIGE, using 3 different fluorescent dyes (Cy2, Cy3 and Cy5). The samples were separated on $24 \mathrm{~cm}$ IPG strips in the first dimension (pH ranges 4-7 and 6-9) and run on a $12.5 \%$ polyacrylamide gel in the second dimension.

Results: Four independent experiments were performed, each detecting a total of approximately 2300 spots in the $\mathrm{pH}$ range 4-7 of which 107 spots were statistically significant regulated $(\mathrm{p}<0.01)$. For the $\mathrm{pH}$ range $6-9$, approximately 1500 spots were detected, of which 36 altered expression $(p<0.01)$. Nineteen differentially expressed spots have been identified up till now. The identified proteins can be classified in the following groups: 1) heat shock/chaperone proteins; 2) structural proteins; 3 ) proteins with various catalytic characteristics and 4) proteins with unknown or other characteristics. A comparison of this first set of differentially expressed proteins with the previously obtained micro-array data, revealed only 1 protein, ie. heat shock protein 70 , as also being regulated at the mRNA level. Interestingly, another protein involved in ER stress, ie. BiP, was significantly altered in its level of phosphorylation (2.68-fold downregulation of the native protein, but a 3.63-and 2.72-fold upregulation of the oneand two-times phosphorylated forms, respectively $(\mathrm{p}<0.01))$.

Conclusion: The present findings are complementary to the previously obtained micro-array data. A combination of the two techniques will provide a more detailed picture of the pathways involved in cytokine-mediated beta-cell destruction observed in type-1 diabetes.

\section{6}

In Silico identification of NF-кB-regulated genes in pancreatic beta-cells

N. Naamane ${ }^{1}$, J. Van Helden ${ }^{2}$, D. L. Eizirik ${ }^{1}$;

${ }^{1}$ Laboratory of Experimental Medicine, Université Libre de Bruxelles, ${ }^{2}$ Service de Conformation de Macromolécules Biologiques et de Bioinformatique, Université Libre de Bruxelles, Belgium.

Background and Aims: In type 1 diabetes mellitus (T1DM), pancreatic beta-cells are the target of an autoimmune-triggered destruction mediated in part by cytokines such as IL-1 $\beta$ and IFN- $\gamma$. These cytokines modify the expression of hundreds of genes, including genes related to stress responses and differentiated beta-cell functions. This leads to beta-cell dysfunction and death mainly by apoptosis. Several of these cytokine-induced genes are potentially regulated by the IL- $1 \beta$-activated transcription factor NF- $\mathrm{kB}$. Previous studies by our group have shown that cytokine-induced activation of NF- $\mathrm{\kappa B}$ is mostly proapoptotic in beta-cells. We presently used a discriminant analysis-based approach to predict NF-кB responding genes on the basis of putative regulatory elements, to identify NF-кB-regulated gene networks in beta-cells.

Material and Methods: The top matching scores of locally over-represented transcription factor binding site (TFBS) matrices in proximal promoters of known NF- $\mathrm{KB}$ target genes were used to characterize upstream sequences (1000 bp) of NF-кB-regulated and non-regulated genes (120 genes each). These genes were used for training and evaluation of two supervised classification methods (Linear 
and Quadratic Discriminant Analysis; LDA and QDA). The performance of the constructed discriminant classifiers was examined using a stratified cross-validation with an internal leave-one-out cross validation loop for automated feature selection and noise reduction. In order to predict potential NF-кB-regulated genes, the optimal discriminant classifier was used to analyse simultaneously the upstream sequences of rat and human beta-cell expressed genes and their homologous genes in other species. When possible, the reliability of the predictive results was assessed by comparison with available micro-array experimental data obtained by our group.

Results: As expected, 5 of the 6 TFBS matrices founded as enriched in the upstream sequences of known NF- $\mathrm{kB}$ regulated genes correspond to transcription factors from the Rel/NF-кB family. Thus, each gene was described by a $30-$ element matrix matching scores vector. In the calibration phase of the analysis, we observed that LDA discriminated NF-кB-responding genes with $61 \%$ sensitivity, $91 \%$ specificity and $87 \%$ positive predictive value; LDA performed slightly better than QDA and was then used in the prediction phase. We identified 234, 253 and $583 \mathrm{NF- \kappa B}$ target genes in insulin producing INS-1 cells, primary rat beta-cells and human pancreatic islets, respectively. Predicted NF- $\mathrm{KB}$ targets show a significant enrichment in genes regulated by cytokines (IL- $1 \beta$ or IL- $1 \beta+\mathrm{IFN}-\gamma$ ), double stranded RNA and a viral infection, as compared to genes not regulated by these NF-кB-dependent stimuli (Fisher exact probability test ; $<<0.005$ in all comparisons). Since groups of genes subject to specific regulation are assumed to be evolutionary stable, we increased confidence in our predictions by selecting only genes with homologs predicted as NF- $\mathrm{KB}$ targets in other organisms, namely rat, mouse, human and chimpanzee.

Conclusion: This work allowed us to identify novel regulatory targets of NF- $\mathrm{kB}$ using a supervised classification method based on putative binding motifs. This provided new insights into the gene networks regulating cytokine-induced beta-cell dysfunction and death.

Supported by EFSD/Novo Nordisk Research Grant

\section{7}

Cytokine-induced Fas and chemokine expression is inhibited by SOCS-3 in pancreatic $\beta$ cells

M. L. B. Jacobsen ${ }^{1}$, S. G. Rønn ${ }^{1}$, C. Bruun ${ }^{1}$, P. Heding ${ }^{1}$, A. E. Karlsen ${ }^{2}$, D. Eizirik ${ }^{3}$,

T. Mandrup-Poulsen ${ }^{1,4}$, N. Billestrup ${ }^{1}$;

${ }^{1}$ Steno Diabetes Center, Gentofte, Denmark, ${ }^{2}$ Novo Nordisk, Måløv, Denmark, ${ }^{3}$ Laboratory of Experimental Medicine, Université Libre de Bruxelles, Belgium, ${ }^{4}$ Department of Molecular Medicine, Karolinska Institute, Stockholm, Sweden.
Background and Aims: Type 1 diabetes mellitus is a disease characterized by an autoimmune attack of the insulin producing $\beta$-cells in the pancreas. The pro-inflammatory cytokines IL-1 $\beta$ and IFN- $\gamma$ are believed to be important effectors in the immune response leading to $\beta$-cell death. These cytokines can initiate the production of toxic free radicals and nitric oxide in the $\beta$-cell thereby directly causing its death. Moreover via activation of the NFkB and MAPK pathways they regulate the transcription of genes that may have deleterious effects in the $\beta$-cell. Among these is the gene encoding the pro-apoptotic receptor Fas as well as genes encoding various chemokines, many of these being NFkB dependent. Activation of the Fas receptor is initiated through binding to the Fas Ligand which is mainly expressed on the surface of activated Tcells and macrophages. The interaction results in apoptosismediated death of the Fas-expressing cell. Expression of chemokines enhances the inflammatory response as these proteins recruit activated immune cells. We have previously shown that the suppressor of cytokine signaling-3 (SOCS3) can inhibit IL-1 $\beta$ and IFN- $\gamma$ induced NO-production and apoptosis of the $\beta$-cell line INS-1. The aim of the present project is to investigate if SOCS-3 can influence cytokine induced Fas and chemokine expression in $\beta$-cells, as this could represent a new mechanism of SOCS-3 mediated protection of $\beta$-cells against inflammatory cytokines.

Materials and Methods: By means of a $\beta$-cell line with SOCS-3 inducible expression, it was investigated whether SOCS- 3 influences IL- $1 \beta$ and IL- $1 \beta+$ IFN- $\gamma$ induced Fas and chemokine mRNA production using real time RT-PCR analysis. Furthermore, the ability of SOCS-3 to influence the activity of a cytokine-responsive Fas promoter construct was measured by Luciferase analysis.

Results: IL-1 $\beta(150 \mathrm{pg} / \mathrm{ml})$ induced a 7 -fold increase in Fas mRNA expression after 6 hours of stimulation. This induction was inhibited by $40 \%$ in the presence of SOCS$3(\mathrm{p} \leq 0,05)$. After 24 hours of stimulation the induction was reduced to 2 -fold and not affected by SOCS-3. IL-1 $\beta+$ IFN$\gamma$ induced a 10-fold induction in the Fas mRNA expression after 6 hours and an 8-fold induction was seen after 24 hours. A reduction in the cytokine-mixture induced Fas mRNA expression by approximately $50 \%$ was seen both after 6 and 24 hours $(p \leq 0,01)$. The ability of SOCS-3 to influence a cytokine responsive Fas promoter was measured by Luciferase analysis. Transfected cells were exposed to IL-1 $\beta$ alone or to IL- $1 \beta+$ IFN- $\gamma$ for 6 hours. SOCS-3 inhibited both IL- $1 \beta$ and IL- $1 \beta+$ INF- $\gamma$-induced activity of the Fas promoter by $30-40 \%(p \leq 0,05)$. Moreover, preliminary data indicate that SOCS-3 is able to suppress cytokine induced chemokine mRNA expression in the $\beta$-cell.

Conclusions: The presented data indicate that SOCS-3 possibly via the blockade of the NFkB pathway can inhibit expression of the death receptor Fas, one of the major 
apoptotic genes induced by cytokines in the $\beta$-cell. Furthermore preliminary data suggests that SOCS-3 can inhibit cytokine-mediated chemokine expression in a $\beta$-cell line. These data represent a new explanation of the protective effect seen by SOCS- 3 in $\beta$-cells against cytotoxic cytokines.

\section{8}

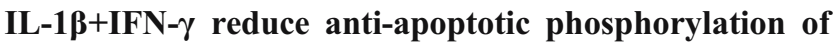
Bad, a proapoptotic Bcl-2 family member and induce cleavage of caspase-9 in the rat $\beta$ cell line INS-1E L. G. Petersen ${ }^{1}$, B. Kutlu ${ }^{2}$, J. Størling ${ }^{1}$, N. Billestrup ${ }^{1}$, D. Eizirik ${ }^{2}$, T. Mandrup-Poulsen ${ }^{1,3}$;

${ }^{1}$ Steno Diabetes Center, Gentofte, Denmark, ${ }^{2}$ Laboratory of Experimental Medicine, Université Libre de Bruxelles, Belgium, ${ }^{3}$ Dep. of Molecular Medicine, Karolinska Institute, Stockholm, Sweden.

Background and Aims: Interleukin (IL)- $1 \beta$ and Interferon (IFN) $-\gamma$ are pro-inflammatory cytokines proposed to be effectors in $\beta$-cell destruction in Type 1 Diabetes. The Bcl-2 protein family encompasses important regulators of the mitochondrial (intrinsic) pathway that controls cleavage of caspase-9 leading to cleavage of caspase- 3 and apoptosis. The family consists of both anti-apoptotic (e.g. Bcl-2 and Bcl- $\mathrm{X}_{\mathrm{L}}$ ) and pro-apoptotic members (e.g. Bid, Bax, Bak, and $\mathrm{Bad}$ ). In $\beta$-cells, overexpression of Bcl-2 and Bcl- $\mathrm{X}_{\mathrm{L}}$ inhibits the deleterious effects induced by cytokines and we have previously shown that IL- $1 \beta+\mathrm{IFN}-\gamma$ increase the mRNA, but not protein expression of Bid, Bax, Bak, and Bcl- $\mathrm{X}_{\mathrm{L}}$. Neither Bcl-2 mRNA nor protein levels were modulated by cytokines. It remains to be clarified if cytokines change the activity of $\mathrm{Bcl}-2$ proteins, e.g. by modulating the phosphorylation status. Aims: Since Bad in other cell types is a target of c-jun N-terminal kinase and Akt both of which are key signaling components in cytokine-induced $\beta$-cell death we investigated the effects of IL- $1 \beta+$ IFN- $\gamma$ on Bad protein expression and phosphorylation. Further, the cleavage of caspase- 9 and caspase-3 was investigated.

Material and Methods: Experiments were performed on the rat $\beta$-cell line INS-1E. Immunoblotting was performed to assess the protein expression and phosphorylation of Bad on Ser136 (anti-apoptotic phosphorylation) and cleavage of caspase- 9 and caspase-3. The protein expression of Bad and cleavage of the caspases were investigated after exposure to IL- $1 \beta+$ IFN- $\gamma$ for $4,8,12$, and 24 hours and the phosphorylation of Bad on Ser136 was investigated after exposure to IL- $1 \beta+\mathrm{IFN}-\gamma$ for $0.5,1,3,6,12$, and 24 hours.

Results: Cytokines induced time-dependent de-phosphorylation of Bad on ser136, significant after $1 \mathrm{~h}$. Dephosphorylation of Bad on Ser136 has been proposed to activate the pro-apoptotic activity of Bad by inhibiting 143-3 binding to Bad which under normal conditions retains Bad in the cytoplasm. By de-phosphorylating this residue, cytokines might induce a pro-apoptotic effect of Bad. To investigate this further we investigated if caspase- 9 was cleaved as a measure of mitochondrial pro-apoptotic signaling and found that both caspase-9 and -3 were cleaved after 12 and $24 \mathrm{~h}$ of cytokine exposure. Furthermore, cytokines were found to decrease the protein expression of Bad by $20 \%$ after 12 hours of exposure. Since bad de-phosphorylation was observed prior to the reduction in protein expression we speculate that dephosphorylation makes Bad more susceptible to degradation or negatively feeds-back on bad mRNA expression and/or protein synthesis.

Conclusion: Cytokines induced de-phosphorylation of Bad on ser136 followed by cleavage of caspase- 9 and -3 . Taken together, these data indicate that the mitochondrial pathway might be involved in cytokine-mediated $\beta$-cell death and that this process is mediated by activation of Bad.

Supported by the Danish Research Council, the Danish Diabetes Foundation, and the JDRF

\section{9}

Expression of a kinase deficient, dominant negative apoptosis signal-regulating kinase (ASK) 1 reduces cytokine- and endoplasmic reticulum stress-induced apoptosis in insulin-secreting INS-1 cells

J. Størling, N. Billestrup, T. Mandrup-Poulsen;

Steno Diabetes Center, Gentofte, Denmark.

Background and Aims: Apoptosis of insulin-secreting pancreatic $\beta$-cells is the underlying cause of type 1 diabetes. Pro-inflammatory cytokines including interleukin (IL)- $1 \beta$ are thought to be primary immune mediators of $\beta$-cell destruction. In vitro, IL-1 $\beta$ signals $\beta$-cell apoptosis via the c-jun N-terminal kinase (JNK) stress signaling pathway. Further, recent evidence points toward endoplasmic reticulum (ER) stress being an essential pro-apoptotic signal in $\beta$-cells exposed to cytokines. However, the molecular mechanisms behind cytokine- and ER stress-induced $\beta$-cell apoptosis remain unclear. One potential mechanism is via apoptosis signal-regulating kinase (ASK) 1, known to mediate stress-induced JNK activation and cell death. Thus, the aim of this study was to examine if transfection with an expression vector encoding a kinase deficient (KD), dominant negative form of ASK 1 protects against cytokine- and ER stress-induced apoptosis in insulin-secreting cells.

Materials and Methods: Rat INS-1 cells were stably transfected with KD-ASK 1 or empty vector (mock). Apoptotic cell death was evaluated by detection of nucleosomes present in the cytoplasm by ELISA. JNK 
activation was determined by immuno blotting using antibodies against the phosphorylated, activated forms of JNK $1 / 2$.

Results: Clones of INS-1 cells expressing KD-ASK 1 were less sensitive to IL- $1 \beta+$ interferon (IFN)- $\gamma$-induced apoptosis as compared to mock-transfected control cells. Further, apoptosis induced by thapsigargin (Tg), an inhibitor of sarco-endoplasmic reticulum calcium ATPase (SERCA) leading to ER stress, was reduced in cells expressing KDASK 1 compared to mock cells. Interestingly, neither cytokine-nor Tg-induced JNK phosphorylation was affected in cells expressing KD-ASK 1.

Conclusion: Our findings suggest that ASK 1 is involved in transducing cytokine-and ER stress-mediated apoptosis in insulin-secreting cells.

\section{0}

The chemokine IP-10 as a mediator of decreased $\beta$ cell mass in diabetes

F. T. Schulthess ${ }^{1}$, N. Sauter ${ }^{1}$, J. Oberholzer ${ }^{2}$, K. Maedler ${ }^{1}$; ${ }^{1}$ UCLA David Geffen School of Medicine, Larry L. Hillblom Islet Research Center, Los Angeles, United States, ${ }^{2}$ Medicine, Division of Transplantation, Chicago, United States.

Background and Aims: Both type 1 and type 2 diabetes result from reduced $\beta$-cell mass and increased apoptosis, secretion of pro-inflammatory cytokines may contribute to the disease. The aim of this study was to investigate the role of the chemokine Interferon-gamma-inducible protein-10 (IP-10) on $\beta$-cell function and survival.

Materials and Methods: Isolated human islets were exposed to IP-10, $\beta$-cell apoptosis (by the TUNEL assay), replication (by Ki-67 staining) and glucose stimulated insulin secretion (GSIS) were measured. Additionally, cell viability was determined using the MTT colorimetric assay. IP-10 secretion and mRNA were analyzed in isolated islets from healthy organ donors and donors with type 2 diabetes. Results: IP-10 secretion from diabetic islets was 825.55fold and IP-10 mRNA was 33.33-fold increased, compared to control $(p<0.05)$. Double staining for IP-10 and insulin in pancreatic sections from patients with type 2 diabetes revealed $\beta$-cells expressing IP-10, but no IP-10 staining was detected in control sections. $0.1 \mathrm{ng} / \mathrm{ml}$ IP-10 increased $\beta$-cell apoptosis $1.57 \pm 0.27$-fold (n.s.) after 1 day and $2.78 \pm 0.55$-fold $(\mathrm{p}<0.01)$ after 4 days of culture, compared to control.

Assessment of cell viability confirmed the proapoptotic effect of IP-10: Viabilities of cells treated with $0.1 \mathrm{ng} / \mathrm{ml}$ IP-10 for 1 day and 4 days were $91.52 \pm 1.98 \%$ and $85.33 \pm 2.34 \%$ of control value, respectively.
In parallel, GSIS was impaired in the IP-10 treated islets (1.48 \pm 0.37 -fold stimulatory index vs. $3.54 \pm 0.35$-fold in control, $\mathrm{p}<0.01)$ and insulin mRNA was 4 -fold decreased. IP-10 also induced $\beta$-cell proliferation (1.5 \pm 0.47 -fold at

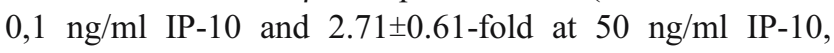
compared to control). Western Blot analysis for p-Akt (Ser473) revealed Akt activation within 10 min. of addition of IP-10, together with cleavage of PAK-2, which would initiate apoptosis. Inhibition of the PI3K/Akt pathway by Wortmannin decreased IP-10 induced Akt activation as well as IP-10 induced apoptosis.

Conclusion: Our data suggest that IP-10 induced proliferation is mediated by Akt activation, but this finally leads to apoptosis. This is in line with previous data showing that proliferating $\beta$-cells are more susceptible to apoptosis. Thus, IP-10 possibly contributes to the impaired $\beta$-cell function and increased apoptosis in diabetes.

\section{PS 18}

\section{Beta cell dysfunction and death in models of type 2 diabetes}

0421

\author{
Altered autophagy in the pancreatic beta cells of human \\ type 2 diabetes \\ M. Masini, M. Bugliani, L. Marselli, R. Lupi, \\ S. Del Guerra, S. Torri, M. Grupillo, R. Mancarella, \\ S. Del Prato, M. Pollera, P. Marchetti; \\ Endocrinology and Metabolism, Metabolic Unit, University \\ of Pisa, Italy.
}

Background and Aims: Decreased beta-cell functional mass is crucial to the development and progression of type 2 diabetes (T2D). Although enhanced apoptosis is a wellknown cause of increased beta-cell death, other mechanisms may play a role. Autophagy is the process which degrades long-lived proteins and cellular organelles, and may lead, if altered, to a form of programmed cell death morphologically distinct from apoptosis and necrosis.

Materials and Methods: We studied autophagic phenomena in the beta-cells from the pancreata of 18 multiorgan donors ( 8 non-diabetic, ND: age, $61 \pm 12$ yrs; BMI, $25.2 \pm 2.5 \mathrm{Kg} / \mathrm{m}^{2}$; and 10 with T2D: age, $67 \pm 8 \mathrm{yrs}$, BMI, $27.5 \pm 3.8 \mathrm{Kg} / \mathrm{m}^{2}$ ).

Results: By electron microscopy 3708 cells were evaluated, and the analysis showed a slight, but significant decrease of beta-cells in T2D islets $(62.0 \pm 2.2 \%$ vs $71.0 \pm 3.3 \%$, p $<0.05)$, with unchanged alpha- and delta-cell proportions. The percentage of dead cells was $14 \pm 2.4 \%$ in T2D and $5 \pm 1.4 \%$ in ND $(p<0.01)$, with apoptotic cells representing $7 \pm 4 \%$ and $2 \pm 1.3 \%$. respectively $(\mathrm{p}<0.05)$. The remaining proportions of 
$7 \pm 3 \%$ (in $\mathrm{T} 2 \mathrm{D}$ ) and $3 \pm 1 \%$ (in $\mathrm{ND})(\mathrm{p}<0.05)$ dead cells showed signs of autophagic cell death (membrane dysruption, extreme overload of vacuoles with/without electron-dense contents, no major chromatin condensation, no signs of inflammation). No cell with classical necrotic ultrastructure was found. In living beta-cells, the density volume ( $\mathrm{ml} \%)$ of autophagic vacuoles (containing still recognizable organelles, $0.6 \pm 0.1$ vs $0.1 \pm 0.05$ ) and of autophagosomes (containing lysosome degraded material, $7.7 \pm 1.1$ vs $4.7 \pm 0.3$ ) was significantly higher $(\mathrm{p}<0.05)$ in T2D than ND. Quantitative RTPCR showed a significant $(\mathrm{p}<0.01)$ decrease of the expression (ng/gene over ng/beta-actin) of the lysosomal degrading enzyme cathepsin D $(1.88 \pm 1.42$ vs $4.45 \pm 2.29)$ in T2D islets versus ND, with unchanged levels of cathepsin $F$ and $H$. These data were confirmed by microarray experiments performed on beta-cells captured by laser microdissection.

Conclusion: These results show that pancreatic beta-cells in human type 2 diabetes have signs of altered autophagy, possibly associated with reduced lysosomal activity, which may contribute to cause beta-cell functional and survival dysfunction.

\section{2}

Oxidative stress in isolated human pancreatic islets: possible role of frataxin

S. Del Guerra ${ }^{1}$, R. Lupi ${ }^{1}$, M. Bugliani ${ }^{1}$, R. Mancarella ${ }^{1}$, S. Torri ${ }^{1}$, M. Grupillo ${ }^{1}$, U. Boggi ${ }^{2}$, F. Mosca ${ }^{2}$,

S. Del Prato ${ }^{1}$, P. Marchetti ${ }^{1}$;

${ }^{1}$ Endocrinology and Metabolism - Metabolic Unit, ${ }^{2}$ Oncology, Transplantation and Advanced Technologies in Medicine, University of Pisa, Italy.

Background: Oxidative stress is a main mechanism leading to beta-cell damage in isolated islets. It has been suggested that the mitochondrial protein frataxin could be involved in the modulation of reactive oxygen and/or nitrogen species production. In fact, in animal models, disruption of the frataxin gene in pancreatic beta cells causes diabetes paralleled by an increase in reactive oxygen species. No information is currently available as for the role of frataxin in isolated human pancreatic islets.

Materials and Methods: We prepared human islets by enzymatic digestion and gradient separation from the pancreas of 18 non-diabetic (Ctrl, age $51.0 \pm 13.8$ yrs: sex 12M/6F: BMI $24.2 \pm 3.7 \mathrm{~kg} / \mathrm{m} 2)$ and 10 type 2 diabetic (T2DM) multiorgan donors (age $64.3 \pm 7.6$ yrs: sex $5 \mathrm{~F} / 5 \mathrm{M}$ : BMI $27.7 \pm 3.7 \mathrm{~kg} / \mathrm{m} 2$ ), measured frataxin mRNA (by Realtime RT-PCR) and protein (by Western-blotting) expression, and assessed nitrotyrosine levels (by an ELISA technique) as marker of oxidative stress; finally, we silenced frataxin gene expression by siRNA transfection method.

Results: In T2DM, frataxin mRNA $(-63.3 \pm 10.3 \%)$ and protein $(-36.4 \pm 14.1 \%)$ expressions were significantly lower than in Ctrl. $(\mathrm{p}<0.01)$. Moreover, in T2DM there was an increase of nitrotyrosine levels $(11.8 \pm 0.7$ vs $7.5 \pm 0.4 \mathrm{nmol} / 1$ of $\mathrm{Ctrl}, \mathrm{p}<0.01)$. When we silenced the frataxin gene in Ctrl $(-36.3 \pm 10.1 \%$ of mRNA expression), we found a significant $(\mathrm{p}<0.01)$ increase of nitrotyrosine levels $(+27.6 \pm 7.4 \%)$ and a reduction of insulin secretion $(-25.4 \pm 3.3 \%$ of S.I. ctrl), respect to non-transfected islets $(\mathrm{p}<0.01)$.

Conclusion: These results show a reduced expression of frataxin gene in T2DM islets, and indicate that a reduction of frataxin gene expression is associated with an increase of oxidative stress and reduced insulin secretory function. These data suggest a physiological role of frataxin in the modulation of human beta-cell function, and may be of help in developing strategies to improve survival of isolated islets.

0423

The role of hyperglycaemia in dexamethasone-induced apoptosis in insulin secreting INS-1 cells

F. Ranta ${ }^{1}$, D. Avram ${ }^{1}$, M. Düfer ${ }^{2}$, G. Drews ${ }^{2}$, F. Lang ${ }^{1}$, S. Ullrich ${ }^{1}$;

${ }^{1}$ Institute of Physiology, ${ }^{2}$ Institute of Pharmacy, University of Tuebingen, Germany.

Background and Aims: Glucocorticoids are known to induce apoptotic cell death in a variety of cells. In lymphocytes, glucocorticoids increase cytosolic $\mathrm{Ca}^{2+}$ by activation of $\mathrm{Ca}^{2+}$ release from IP3-sensitive intracellular stores. $\mathrm{Ca}^{2+}$ together with heat shock protein 90 (HSP90), released from the stimulated glucocorticoid receptor (GR), activates calcineurin (PP2B). Calcineurin activation contributes to mitochondrial mediated apoptosis by dephosphorylation of BAD. The aim of the present study was to examine whether activation of PP2B and dephosphorylation of BAD are involved in dexamethasone (dex)-induced apoptosis, and whether hyperglycaemia potentiates the cytotoxic effect of dex in insulin secreting cells.

Materials and Methods: INS- 1 cells were cultured under standard conditions in the presence of different glucose concentrations (2, 5, 11 and $20 \mathrm{mM})$. Apoptosis was quantified by DAPI-stained condensed nuclei and by TUNEL assay. Calcineurin activity was measured using pNPP (p-nitrophenylphosphate) or ${ }^{32} \mathrm{P}$-RII peptide as substrate. INS-1 cytosolic $\mathrm{Ca}^{2+}$ activity was measured using the fluorescent $\mathrm{Ca}^{2+}$-indicator Fura-2AM. BAD phosphorylation was quantified by Western blotting. 
Results: The synthetic glucocorticoid dex (100 nM) induced apoptosis in insulin secreting cells. RU486 $(1 \mu \mathrm{M})$, the glucocorticoid receptor (GR) antagonist, inhibited the dex effect. Two PP2B inhibitors, FK506 $(0.1 \mu \mathrm{M})$ and deltamethrin $(1 \mu \mathrm{M})$ reduced dex-mediated apoptosis by $34 \%$ and $72 \%$, respectively. The rate of dexinduced apoptosis was significantly higher at $20 \mathrm{mM}$ $(10.6 \%)$ than at $2 \mathrm{mM}$ glucose $(4.2 \%)$. Dex did not increase cytosolic $\mathrm{Ca}^{2+}$ activity while carbachol and glucose exerted their known effects. PP2B activity was significantly increased by dex in cells cultured at 11 and $20 \mathrm{mM}$ glucose, whereas dex did not enhance the enzyme activity at 2 and $5 \mathrm{mM}$ glucose. Thus, stimulation of PP2B by dex was glucose-dependent. Geldanamycin, an antagonist of HSP90, inhibited stimulation of PP2B by $80 \%$ in INS1 cells cultured at $11 \mathrm{mM}$ glucose. Dex decreased phosphorylation of BAD.

Conclusion: These data suggest that dex-induced apoptosis in INS-1 cells involves PP2B stimulation by HSP90 released from the stimulated GR complex and is enhanced by hyperglycaemia, probably through glucose-stimulated $\mathrm{Ca}^{2+}$ entry.

\section{4}

The role of renin-angiotensin system blockers in the protection of pancreatic beta cell function in diabetic rats

L. Yuan ${ }^{1}$, G. L. Xu ${ }^{1}$, X. $\mathrm{Li}^{1}$, C. J. Qi ${ }^{1}$, B. Zhang ${ }^{1,2}$, L. L. $\mathrm{Cao}^{3,4}$;

${ }^{1}$ Department of Endocrinology, Union Hospital, Tongji Medical College of Hua Zhong Science \&Technology University, Wuhan, ${ }^{2}$ Department of Endocrinology and Metabolism, Shanghai Changzheng Hospital, ${ }^{3}$ Department of Endocrinology and Metabolism, Union Hospital, Tongji Medical College of Hua Zhong Science \&Technology University, Wuhan, ${ }^{4}$ Department of Endocrinology and Metabolism, The 1st Republic Hospital of Jiujiang, China.

Background and Aims: Many large-scale clinical studies have shown that angiotensin converting enzyme inhibitor (ACEI) and $\mathrm{AT}_{1}$ receptor blocker (ARB) is associated with a low incidence of new-onset diabetes among high risk patients. This study aimed to determine if the block of local reninangiotensin system by ACEI and ARB play a role in the protection of pancreatic $\beta$-cell function in type 2 diabetes rats. Materials and Methods: Type 2 diabetic rat models were induced by intraperitoneal injection of a small dose of streptozotocin plus high fat high caloric laboratory chow. The diabetic rats were randomly divided into diabetic group, ACEI group and ARB group. 10 weeks treatment of perindopril or valsartan was given to ACEI group or ARB group. After an overnight fasting, intravenous glucose tolerance testing and insulin release testing were performed to assess the first-phase insulin response in diabetic rats. Serum glucose was analyzed by the glucose oxidase method. Plasma insulin was measured using radioimmunoassay. Gene expression of AGT, insulin, TGF- $\beta_{1}$ in pancreas extracts was determined using semiquantitative RT-PCR. We performed dual staining for proliferating cell nuclear antigen (PCNA) and insulin to assess intraislet proliferation, transferase-mediated dUTP nick-end labeling staining (TUNEL) to assess islet cell apoptosis, and staining for transforming growth factor- $\beta 1$ (TGF- $\beta 1$ ) as well as type I and type IV collagen to assess islet fibrogenesis.

Results: Both perindopril and valsartan treatment could significantly improve first-phase insulin secretion in diabetic rats $(\mathrm{P}<0.05)$. Compare with diabetic group, staining density for insulin in ACEI group and ARB group was increased by $45 \%, 37 \%$ respectively. Intraislet cell death, measured by TUNEL staining, was significantly decreased by $64 \%$ in islet of ACEI group, and by $52 \%$ in ARB group compared to diabetic group. The number of cells staining positively for PCNA was increased by $51 \%$ and $47 \%$ respectively in ACEI and ARB group. And the incremental area under the insulin curve between 0 and 10 min was negatively correlated with the islet cell apoptosis, and positively correlated with islet cell proliferation. TGF- $\beta 1 \mathrm{mRNA}$ and protein expression as well as type I and type IV collagen staining were decreased by $39 \%$ and $26 \%$ respectively in two groups with treatment. Gene expression of AGT was much greater in diabetic islet compared with control group, and both treatments had no significant effect on its expression.

Conclusion: In type 2 diabetic rats, local RAS block by ACEI and ARB could decrease islet $\beta$-cell apoptosis, increase hyperplasia, and decrease the islet fibrogenesis. Long-term treatment of ACEI and ARB could improve pancreatic $\beta$-cell architecture and increase the first-phase insulin secretion in type 2 diabetes. These findings support that local RAS block plays an important role in the protection the pancreatic $\beta$-cell architecture and function in type 2 diabetes.

Supported by Natural Science Study Foundation in Hubei China (2005ABA185)

\section{5}

Hepatocyte growth factor (HGF) protects pancreatic beta cells from fatty acid-induced apoptosis

E. Anastasi ${ }^{1}$, C. Santangelo ${ }^{2}$, P. Matarrese ${ }^{3}$,

M. Di Carlo', A. Di Lillo ${ }^{1}$, B. Scazzocchio ${ }^{2}$,

M. D' Archivio ${ }^{2}$, E. Vecci ${ }^{1}$, W. Malorni ${ }^{3}$, R. Masella ${ }^{2}$, R. Perfetti'; 
${ }^{1}$ Clinical Science, University "La Sapienza", Rome, Italy, ${ }^{2}$ National Centre for Food Quality and Risk Assesment, Istituto Superiore di Sanità, Rome, Italy, ${ }^{3}$ Drug Research, Istituto Superiore di Sanità, Rome, Italy, ${ }^{4}$ Diabetes, University of California, Los Angeles, United States.

Background and Aims: Type 2 diabetes is characterized by peripheral insulin resistance, pancreatic $\beta$-cell dysfunction and decreased $\beta$-cell mass with increased rates of beta cell apoptosis. Prolonged exposure to high level of free fatty acids (FFAs) has detrimental effects on $\beta$-cell function and survival. Hepatocyte growth factor (HGF) is a mesenchyme-derived multifunctional protein that plays a critical role in cell expansion and cell survival in various eukaryote cell types. While the action of HGF on the proliferation and differentiation of pancreatic beta cells is well documented, its role in antagonizing beta cell death in response to pro-apoptotic agents is not fully characterized. Materials and Methods: Rat insulin-producing cell RINm5F were cultured and exposed to FFA (long-chain fatty acid oleate:palmitate $2: 1$ ) for $72 \mathrm{~h}$ in order to induce apoptosis. Results: In the present study we demonstrated that HGF inhibits FFA-induced apoptosis of cultured beta cells, reducing DNA fragmentation and improving cell survival. This antiapoptotic action of HGF was mediated by the inhibition of oxidative stress that occurs in response to the prolonged exposure to high FFAs. HGF inhibited the formation of Reactive Oxygen Species and their negative effect on cell survival. The beneficial effect of HGF was mediated by an increase in bcl-2 level (mRNA and counterpart protein) and activity. Furthermore, investigation of the signaling pathway known to be activated HGF and to be associated with the regulation of bcl-2 showed that the protective effect of HGF required the phosphorylation of $\mathrm{PI} 3 \mathrm{~K}$.

Conclusion: The present study demonstrates that HGF protects beta cells from the pro-apoptotic action of FFAs, inhibiting mitochondrial damage and ROS production. The cell survival action of HGF occurred via a PI3K-dependent signaling pathway.

Supported by Istituto Superiore Sanità

\section{6}

PPARalpha plays a role in glucose stimulated insulin secretion and protects against lipotoxicity in INS-1E beta cells

F. Frigerio ${ }^{1}$, C. Bartley ${ }^{1}$, S. Mandrup ${ }^{2}$, P. Maechler ${ }^{1}$;

${ }^{1}$ Department of Cell Physiology and Metabolism, University of Geneva, Switzerland, ${ }^{2}$ Department of Biochemistry and Molecular Biology, University of Southern Denmark, Odense, Denmark.
Background and Aims: Among the molecular targets for the treatment of obesity and diabetes, great attention has been given to the three peroxisome-proliferator-activated receptor (PPAR) subtypes, nuclear lipid sensors, able to modulate metabolic events by regulating gene expression. In particular, PPARalpha plays a crucial role in metabolic adaptation by inducing key genes for fatty acid oxidation and ketogenesis in mitochondria, with a protective role under conditions of fatty acids challenge. Regarding betacells, PPARalpha function remains poorly investigated and controversial. In insulinoma cells overexpressing PPARalpha, glucose-stimulated insulin secretion (GSIS) was reported to be either reduced (J. Lipid Res. 2002, 43:93643.) or potentiated (Endocrinology 2005, 146: 3266-3276). The aim of our project was to investigate the molecular basis of apparent opposite effects of PPARalpha in insulin secreting cells. Specifically, we assessed the relative contributions of PPARalpha over-expression and fatty acid exposure to metabolism secretion coupling,

Materials and Methods: We transduced INS-1E beta-cells with adenoviruses expressing respectively PPARalpha and RXR, and then performed experiments 1 day and 3 days after transduction. Cells were cultured either in normal complete RPMI-1640 media or supplemented with $0.4 \mathrm{mM}$ oleate. At the end of the culture periods, we measured mitochondrial membrane potential (rhodamine-123 fluorescence), cellular calcium levels (Fura-2 fluorescence), and insulin secretory responses (analysed by radioimmunoassay) to stimulatory glucose concentrations.

Results: PPARalpha over-expression increased GSIS 1 day after transduction in normal conditions $(+26 \%)$ and did not affect the glucose response in oleate-treated cells. Interestingly, 3 days after transduction, PPARalpha increased GSIS in the presence of oleate $(+153 \%)$, showing strong protective effects against fatty acid induced toxicity. In order to understand the mechanisms implicated in PPARalpha mediated increase in GSIS, we quantified the mitochondrial membrane potential under the same conditions. After 1 day, mitochondrial hyperpolarization upon $15 \mathrm{mM}$ glucose stimulation was blunted in oleate-cultured cells $(-21 \%)$, an effect reinforced by PPARalpha overexpression $(-46 \%)$. No effects of PPARalpha were noticed in the absence of oleate. After 3 days, mitochondrial membrane potential was not affected by PPARalpha in normal conditions, but dramatically reduced in the presence of oleate, with or without PPARalpha over-expression ( $-42 \%$ and $-80 \%$ respectively). Potassium-induced calcium rises were blunted in the presence of oleate and neither protected nor aggravated by PPARalpha overexpression.

Conclusion: These data show complex relationship between the transcription factor PPARalpha and the nutrient state of cultured cells. Specifically, PPARalpha over- 
expression protected INS-1E beta-cells against oleate induced lipotoxicity. Current experiments measuring betaoxidation versus glucose oxidation, together with PPARalpha target gene expression, should help understanding how lipid and glucose metabolisms interact with the control of insulin secretion.

Supported by Swiss NF

\section{7}

Protect effects of fenofibrate and pioglitazone on islet $\beta$ cells of rats by corrected inflammatory and apoptotic regulation

B. Yang ${ }^{1}$, H. Tian ${ }^{2}, \mathrm{~J}_{\mathrm{Li}}{ }^{1}, \mathrm{X} . \mathrm{Chen}{ }^{1}$;

${ }^{1}$ Laboratory of Endocrinology and Metabolism, ${ }^{2}$ Department of Endocrinology, West China Hospital of Sichuan University, Chengdu, China.

Background and Aims: To explore the expressions of PPAR- $\alpha$, PPAR- $\gamma$, inflammatory mediators, apoptotic regulator caspase-3, and apoptotic level in pancreatic islets by establish obese rat models induced by high-fat or highsucrose diets. To investigate the effects of PPAR- $\alpha$ agonist fenofibrate and PPAR- $\gamma$ agonist pioglitazone on function and apoptosis of islets.

Materials and Methods: S-D obese rat models were established with high- carbohydrate diet (high-sucrose diet (CO group), high carbohydrate diet with fenofibrate (CF group) or pioglitazone (CF group) and high-fat diet high-fat diet (FO group), high-fat diet with fenofibrate (FF group) or pioglitazone (FP group), while the control rats were with normal diet (control group). Duration of intervention were 8-10 weeks,. Immunohistochemistry and western blot were performed to evaluate the expressions of insulin, glucagons, Somatostatin, PPAR- $\alpha$, PPAR- $\gamma$, nuclear factor kappa-B (NF-Éb), inhibitory protein $\kappa \mathrm{B}(\mathrm{I}-\kappa \mathrm{B}), \mathrm{P} 38$, extra cellular signal-regulated kinases1 (ERK1) and caspase-3 proteins in islets, in situ hybridization and RT-PCR were underwent to access the mRNA levels of PPAR- $\alpha$, PPAR- $\gamma$, NF- $\kappa$ B and caspase-3. At the same time, apoptotic level were evaluated by TUNEL, and islets mass were scored in tissue slides.

Results: 1) Islets mass enlarged in $\mathrm{CO}$ and FO groups. The compositions of islet cells were as same as the control. The expression of insulin was lower in $\mathrm{CO}$ and FO groups than the control, but after using pioglitazone, less islets mass and more insulin expression were found in $\mathrm{CP}$ and FP groups. 2) Comparing with the control group, expressions levels of PPAR- $\alpha$, PPAR- $\gamma$ protein and mRNA were reduced in $\mathrm{CO}$ and FO group, and the expression of PPAR- $\alpha$ protein and mRNA increased in CF and FF groups, the expression of PPAR- $\gamma$ protein and mRNA elevated in CP and FP groups. 3) The levels of NF-кB, P38, ERK1 proteins and mRNA increased significantly in FO group, the expressions of NF- $\kappa \mathrm{B}, \mathrm{P} 38$ decreased in FF and FP groups, the level of ERK1 only decreased in FP group. the protein level of I- $\kappa \mathrm{B}$ was no difference among control, $\mathrm{CO}$ and $\mathrm{FO}$ group, and I- $\mathrm{\kappa B}$ increased in $\mathrm{CF}$ and $\mathrm{FF}$ groups. 4) In control group, the expression of caspase-3 was lowest, and elevated gradually in $\mathrm{CO}$ and $\mathrm{FO}$ groups, the level of caspase-3 decreased in $\mathrm{CF}, \mathrm{CP}, \mathrm{FF}$ and $\mathrm{FP}$ groups comparing with $\mathrm{CO}$ and $\mathrm{FO}$ groups. Few TUNEL-position cells were found in all the groups.

Conclusion: In the obese rat models induced by highcarbohydrate and high-fat diets, islets mass enlarged, but insulin level decreased; The expressions of PPAR- $\alpha$ and PPAR- $\gamma$ in islets decreased significantly, and the level of several inflammatory mediators and caspase- 3 increased in islets of obese rat model. Though no evidence of inflammatory response and apoptosis were detected, the dysfunction of inflammatory and apoptotic redulators already existed in islets of obese rats. Fenofibrate and pioglitazone could protect $\beta$ cells function and survival by corrected inflammatory and apoptotic regulation.

\section{8}

Expression of islet inducible nitric oxide synthase and inhibition of glucose stimulated insulin release after longterm lipid infusion in the rat is counteracted by PACAP27 S. S. Qader ${ }^{1}$, J. Jimenez-Feltström ${ }^{1}$, S. Meidute-Abaraviciene ${ }^{1}$, M. Ekelund ${ }^{2}$, I. Lundquist ${ }^{3}$, A. Salehi ${ }^{1}$;

${ }^{1}$ Clinical Sciences, University of Lund, Malmö, Sweden, ${ }^{2}$ Clinical Sciences, ${ }^{3}$ Experimental Medical Sciences, University of Lund, Sweden.

Background and Aims:Chronic exposure of pancreatic islets to elevated plasma lipids (lipotoxicity) can lead to $\beta$-cell dysfunction. We examined, whether the expression of islet inducible nitric oxide synthase (iNOS) and the concomitant inhibition of glucose-stimulated insulin release seen after lipid infusion in rats was modulated by the islet neuropeptide PACAP27.

Materials and Methods:Confocal microscopy, HPLC and RIA were used.

Results: Lipid infusion for 8 days induced a strong expression of islet iNOS, which was mainly confined to $\beta$-cells, and was still evident after incubating islets at $8.3 \mathrm{mmol} / \mathrm{l}$ glucose. This was accompanied by a high iNOS-derived NO generation, a decreased insulin release and increased cyclic GMP accumulation. No iNOS expression was found in control islets. Addition of PACAP27 to incubated islets from lipid-infused rats resulted in loss of iNOS protein expression, increased cAMP, reduced cGMP and suppression of the activities of neuronal constitutive NOS (ncNOS) and iNOS and and enhanced glucose-stimulated insulin response. These effects 
were reversed by the PKA inhibitor H-89. The suppression of islet iNOS expression induced by PACAP27 was not affected by the proteasome inhibitor MG 132, which by itself induced the loss of iNOS protein making a direct proteasomic involvement unlikely.

Conclusion:Our results suggest that PACAP27 through its cAMP and PKA-stimulating capacity strongly suppresses not only ncNOS but importantly also the lipid-induced stimulation of iNOS expression by a non-proteasomic mechanism. Thus PACAP27 restores the impairment of glucose-stimulated insulin release and additionally might induce cytoprotection against deleterious actions of iNOSderived NO in $\beta$-cells.

\section{9}

Hyper-activation of liver $X$ receptor suppresses cell proliferation and induces apoptosis in pancreatic beta cells A. M. Efanov, M. B. Brenner, H. Zitzer, J. Gromada, W. Wente;

Endocrine, Lilly Research Laboratories, Hamburg, Germany.

Background and Aims: Liver X receptors (LXRs, alpha and beta isoforms) regulate cholesterol, lipid and glucose metabolism. Pancreatic beta-cells express only the LXRbeta. LXRs form functional heterodimers with the retinoid X receptors (RXR). We showed before that activation of LXR or LXR/RXR heterodimer modulates insulin secretion and biosynthesis via regulation of glucose and lipid metabolism in MIN6 cells and isolated pancreatic islets. In this study we investigated the effects of the LXR agonist TO901317 and the RXR agonist 9-cis-retinoic acid (9cRA) on cell proliferation and apoptosis in mouse insulinoma MIN6 cells.

Materials and Methods: Effects of TO901317 and 9cRA on cell proliferation in MIN6 cells were measured with a $\mathrm{BrdU}$ incorporation assay and flow cytometry. Apoptosis in MIN6 cell and isolated pancreatic islets was assessed with caspase assay, DNA fragmentation ELISA and annexin V assay.

Results: While the LXR agonist TO901317 shows no effect on proliferation of MIN6 cells, combination of T0901317 with 9cRA strongly inhibits cell proliferation. Flow cytometry analysis of cell cycle in cells treated with the compounds demonstrated that activation of LXR/RXR prevented cells from G0/G1 to G2 phase progression. Furthermore, combination of T0901317 and 9cRA produced a higher apoptosis rate and increased caspase 3/7 activity in MIN6 cells. Activation of neither RXR nor LXR alone was sufficient to bring the cells into an apoptotic state. However, LXR or LXR/RXR activation significantly increased the susceptibility of MIN6 cells to free fatty acidand cytokine- induced apoptosis. In isolated rat islets the apoptotic effect of palmitic acid was dramatically increased with TO901317 alone and even more with the combination of TO901317 and 9cRA.

Conclusion: Activation of LXR/RXR heterodimers inhibits cell proliferation and induces apoptosis in pancreatic betacells. Activation of LXR with T0901317 makes the betacell more sensitive to free fatty acid-induced apoptosis.

\section{PS 19}

\section{Beta cell damage and apoptosis}

\section{0}

\section{Effects of $\mathrm{IP}_{3} \mathrm{R}$ and $\mathrm{RyR} \mathrm{Ca}^{2+}$-release channels on beta} cell ER-stress

J. D. Johnson, D. S. Luciani, T. Yang, M. Frey,

T. B. Kalynyak, A. V. Sampaio, T. M. Underhill;

Cellular and Physiological Sciences, Diabetes Research Group, University of British Columbia, Vancouver, Canada.

Background and Aims: The endoplasmic reticulum (ER)stress response has been implicated in multiple animal models of diabetes and several forms of the human disease. This cellular response can be caused by blocking ER $\mathrm{Ca}^{2+}$ uptake at the SERCA pump. However, the effects of modulating $\mathrm{ER} \mathrm{Ca}^{2+}$ release on this cell death pathway remain unclear. In the present study, we examined the effects of stimulating or inhibiting two classes of ERresident $\mathrm{Ca}^{2+}$ channels, namely the inositol 1,4,5 trisphosphate receptor $\left(\mathrm{IP}_{3} \mathrm{R}\right)$ and the ryanodine receptor (RyR), on lumenal ER-Ca ${ }^{2+}$ dynamics, the induction of ER-stress and $\beta$-cell apoptosis.

Materials and Methods: The glucose-responsive MIN6 $\beta$-cell line has previously been used to model ER-stress and was used in this study. ER-stress and apoptosis was initiated with the commonly used SERCA inhibitor, thapsigargin and detected by western blot using antibodies to $\mathrm{CHOP} / \mathrm{GADD} 153$ and cleaved caspase-3. $\mathrm{IP}_{3} \mathrm{R}$ were inhibited using xestospongin $\mathrm{C}$ and activated following stimulation with carbachol. RyR were inhibited using high micromolar concentrations of ryanodine and activated with low-dose ryanodine or caffeine. Luminal $\mathrm{Ca}^{2+}$ levels in the ER were monitored in real-time using fluorescence resonance energy transmission (FRET) microscopy and the recently developed D1ER cameleon. Cell death was also quantified in real-time over a period of up to 48 hours by monitoring cellular propidium iodide incorporation using an automated Cellomics imaging system.

Results: First, we examined whether disrupting $\mathrm{Ca}^{2+}$ homeostasis by blocking ER $\mathrm{Ca}^{2+}$-release channels in the 
absence of SERCA inhibition would be sufficient to induce ER-stress. Although ryanodine is known to induce an alternate mode of programmed cell death in $\beta$-cells, neither $10-100 \mu \mathrm{M}$ ryanodine nor $10 \mu \mathrm{M}$ xestospongin $\mathrm{C}$, alone or in combination, led to a robust increase in CHOP expression. As a positive control, $1 \mu \mathrm{M}$ thapsigargin decreased luminal ER $\mathrm{Ca}^{2+}$ and induced marked ER-stress, caspase-3 activation and cell death (propidium iodidepositive $\beta$-cells). Next, we sought to determine whether inhibiting $\mathrm{Ca}^{2+}$ release via $\mathrm{IP}_{3} \mathrm{R}$ and $\mathrm{RyR}$ could blunt the ability of thapsigargin to deplete the $\mathrm{ER} \mathrm{Ca}^{2+}$ and induce ER-stress. In these experiments, inhibiting $\mathrm{Ca}^{2+}$ release through either channel led to a delay in thapsigargininduced ER-stress and cell death. Finally, we questioned whether stimulating $\mathrm{Ca}^{2+}$ release through $\mathrm{IP}_{3} \mathrm{R}$ or $\mathrm{RyR}$ would further deplete ER $\mathrm{Ca}^{2+}$ and accelerate ER-stress in the presence of thapsigargin. $100 \mu \mathrm{M}$ carbachol evoked a robust decrease in ER $\mathrm{Ca}^{2+}$ and potentiated the effects of thapsigargin on ER-stress and cell death.

Conclusions: The present study illustrates the interactions between SERCA, IP ${ }_{3} \mathrm{R}$ and RyR in the control of $\mathrm{ER} \mathrm{Ca}^{2+}$ levels. All three components of the $\mathrm{ER} \mathrm{Ca}^{2+}$ handling machinery can regulate distinct cell death pathways, although among these only SERCA-dependent depletion of the ER is sufficient to induce robust $\beta$-cell ER stress. Together, these studies indicate that $\beta$-cell ER-stress can be modulated at the level of the $\mathrm{Ca}^{2+}$ release channels, particularly the $\mathrm{IP}_{3} \mathrm{R}$.

Work was supported by funding from CIHR, CDA, JDRF, MSFHR and CFI.

\section{1}

FGF-21 is expressed in pancreatic islets and stimulates beta cell function and survival W. Wente ${ }^{1}$, A. M. Efanov ${ }^{1}$, M. Brenner ${ }^{1}$, A. Kharitonenkov' ${ }^{2}$, A. Koester ${ }^{2}$, G. E. Sandusky ${ }^{2}$, S. Sewing ${ }^{1}$, I. Treinies ${ }^{1}$, H. Zitzer ${ }^{1}$, J. Gromada ${ }^{1}$;

${ }^{1}$ Lilly Forschung GmbH, Hamburg, Germany, ${ }^{2}$ Lilly Research Laboratories, Indianapolis, United States.

Background and Aims: We have recently demonstrated that fibroblast growth factor 21 (FGF-21) stimulates glucose uptake in adipocytes and normalizes plasma glucose levels in diabetic animal models. In this study we examined expression and effects of FGF-21 in pancreatic beta-cells.

Materials and Methods: RT-PCR was used to examine expression of FGF-21 in islets and cell lines. BrdU proliferation ELISA, caspase assay and DNA fragmentation assay in INS-1E cells and isolated islets were used to assess effect of FGF-21 on beta-cell growth and survival. Insulin secretion and insulin content was measured in INS-1E cells and islets from healthy rodents or diabetic GK rats and $d b / d b$ mice.
Results: FGF-21 expression could be detected in rodent islets, purified beta-cells as well as insulinoma cell lines. In rat islets and INS-1E cells FGF-21 induced phosphorylation of the adapter protein FRS2alpha as well as activated Erk1/2 and Akt signaling pathways. While FGF-21 had no effect on proliferation of INS-1E cells, it could rescue cytokine treated cells from apoptosis. Treatment of isolated islets from healthy animals for 4-72 $\mathrm{h}$ with FGF-21 produced an increase in islet insulin content and islet insulin mRNA levels, while no significant effect of FGF-21 treatment on insulin secretion was observed. The FGF-21 induced increase in insulin production was blocked with a selective Erk1/2 inhibitor. Treatment of isolated islets from diabetic animals with FGF21 produced increases in both insulin secretion and islet insulin content. Moreover, infusion of FGF-21 to $d b / d b$ mice normalized plasma glucose levels. Insulin secretion and insulin content measured in islets isolated from FGF-21 treated mice were significantly elevated.

Conclusion: FGF-21 activates Erk1/2 and Akt signaling cascades in pancreatic beta-cells. FGF-21 increases insulin biosynthesis and protects the beta-cell from cytokine induced apoptosis. FGF-21 can lower plasma glucose in diabetic animals at least partially via its effects on pancreatic beta-cell function.

0432

Overexpression of IA-2 induces G2/M arrest and apoptosis in beta cells

S.-I. Harashima ${ }^{1,2}$, A. L. Notkins ${ }^{1}$;

${ }^{1}$ Experimental Medicine Section, NIDCR, National Institutes of Health, Bethesda, United States, ${ }^{2}$ Department of Clinical Research, Haradoi Hospital, Fukuoka, Japan.

Background and Aims: IA-2 is a major autoantigen in type 1 diabetes. Up to $70 \%$ of newly diagnosed patients have autoantibodies to IA-2. These autoantibodies appear years before the development of clinical disease and in individuals with autoantibodies to both IA-2 and glutamic acid decarboxylase (GAD65), the likelihood of developing type 1 diabetes within 5 years is $50 \%$ or greater. In spite of the existence of autoantibodies, it is still unclear whether IA-2 itself is involved in beta cell destruction. To address this question, we overexpressed or knocked down IA-2 expression in MIN-6, mouse beta cell line, and we investigated the role of IA-2 in beta cell destruction.

Materials and Methods: We established permanent IA-2 transfected MIN-6 cell lines with G418 resistant gene. IA-2 expression also was knocked down with IA-2 siRNA. Apoptosis was detected by flowcytometry, Western blot and DNA fragmentation analysis, and cell cycle also was determined by flowcytometry analysis. Phosphorylation of 
PDK-1 and the proteins of Akt signaling was analyzed by Western blot analysis.

Results: Overexpression of IA-2 puts MIN-6 beta cells into a pre-apoptotic state and exposure to a high concentration of glucose results in G2/M arrest and apoptosis. Treatment of the cells with IA-2 siRNA prevents both G2/M arrest and apoptosis. Analysis of the molecular events involved in preapoptotic cells revealed a decrease in phosphorylation of PDK-1 and the proteins of the Akt signaling pathway. Treatment of the cells with IA-2 siRNA increased Akt phosphorylation in both the IA-2 transfected pre-apoptotic cells and the non-transfected cells. In addition to being more susceptible to glucose-induced apoptosis, IA-2 preapoptotic cells are more susceptible to cytokine (TNF-alpha and INF-gamma) induced apoptosis.

Conclusion: Viewing the IA-2 system as a model, our studies suggest that overexpression of other still unidentified host proteins may put cells into a pre-apoptotic state which upon exposure to environment signals could trigger apoptosis and beta cell destruction.

\section{3}

SHIP2 inhibition improves insulin signal transduction in insulin producing INS1E cells: Potential anti-apoptotic effects?

R. Grempler, J. Rippmann, M. Tadayyon, N. Redemann; Boehringer-Ingelheim Pharma GmbH \& Co. KG, Biberach (Riss), Germany.

Background and Aims: The lipid phosphatase SH2containing inositol phosphatase 2 (SHIP2) is expressed in insulin target tissues, and functions to dephosphorylate the second messenger phosphatidylinositol-3,4,5-trisphosphate at the 5 -position. Polymorphisms of the SHIP2 gene promoter which lead to increased SHIP2 expression are associated with Type 2 diabetes. Adenoviral overexpression of a dominant negative SHIP2 mutant (dnSHIP2) improves insulin signaling and glucose uptake in differentiated L6 myocytes and 3T3-L1 adipocytes. Furthermore, liver specific expression of dnSHIP2 in $\mathrm{db} / \mathrm{db}$ mice decreases fasting glucose levels. Here we describe for the first time the effects of SHIP2 inhibition in INS1E insulinoma cells. Materials and Methods: C3A hepatoma cells or INS1E insulinoma cells were infected with recombinant adenoviruses expressing either $\beta$-galactosidase ( $\beta$-Gal) or dnSHIP2 at a multiplicity of infection (MOI) of 25 and incubated for $40 \mathrm{~h}(\mathrm{C} 3 \mathrm{~A})$ or $24 \mathrm{~h}$ (INS1E). Following serum free preincubation for $2 \mathrm{~h}$, cells were incubated for $15 \mathrm{~min}$ with insulin $(0.1 \mathrm{nM}$ to $1 \mu \mathrm{M})$. Phosphorylation of Akt was detected by phospho-Akt-ELISA or by Western blot.
Furthermore GSK3 $\alpha / \beta$ phosphorylation and Bad phosphorylation was measured by Western blot.

Results: To validate the functionality of the adenoviruses expressing $\beta$-galactosidase ( $\beta$-gal) or dnSHIP2 we infected $\mathrm{C} 3 \mathrm{~A}$ hepatoma cells with both viruses and analysed the phosphorylation of Akt in response to insulin. By using a phospho-Akt ELISA we showed that overexpression of dnSHIP2 shifted the insulin dose response curve to the left and significantly increased the maximum insulin effect. Consistent with the literature that human islets express SHIP2, we detected significant SHIP2 expression in the INS1E insulinoma cell line. Adenoviral infection resulted in $>90 \%$ infection of INS1E cells and in a 12 fold overexpression of dnSHIP2 over the endogenous protein. Overexpression of dnSHIP2 was accompanied by a significant increase in Akt phosphorylation under basal and insulin-stimulated conditions, as detected by phosphoAkt-ELISA. Similarly, we showed an increased basal and insulin stimulated phosphorylation of GSK $3 \alpha / \beta$ in INS1E cells following SHIP2 inhibition. Because increased Akt activity is directly linked to increased Bad phosphorylation and therefore to inhibition of apoptosis we analysed Bad phosphorylation after dnSHIP2 expression. As expected, the phosphorylation of $\mathrm{Bad}$ is significantly increased in comparison to the $\beta$-Gal expressing control.

Conclusion: These data show for the first time that dnSHIP2 acts as an insulin sensitizer/mimetic in in INS1E insulinoma cells with respect to early and also downstream insulin targets, namely Akt, Bad and GSK3 phosphorylation. Our data raise the interesting possibility that SHIP2 inhibition not only has an effect on glucose uptake in liver and muscle, but may also potentially exert important antiapoptotic effects in beta-cells.

\section{4}

Inducible nitric oxide synthase-nitric oxide plays an important role in hypoxic injury to pancreatic $\boldsymbol{\beta}$ cells K.-H. Song, S.-H. Ko, S. Kim, G. Ryu, H.-S. Kwon, S.-D. Moon, Y.-B. Ahn, B.-Y. Cha, K.-W. Lee;

Dpt of Internal Medicine, The Catholic University of Korea, Seoul, Republic of Korea.

Background and Aims: Islet transplantation is a potential strategy to cure type 1 diabetes mellitus. However, two or more donors are usually needed for one recipient because a substantial part of the graft becomes nonfunctional due to several factors including hypoxia. Though hypoxic exposure to pancreatic $\beta$ cells has been reported to induce apoptotic cell death, the molecular processes involved in hypoxiarelated cell death are poorly understood. Hypoxia is known to induce inducible nitric oxide synthase (iNOS)-derived 
nitric oxide (NO) production in many types of cells. The aim of the study was to investigate iNOS-NO signaling and its role in apoptosis in pancreatic $\beta$ cells under hypoxic conditions.

Materials and Methods: Mouse insulinoma cells (MIN6) and primary isolated islets from Sprague-Dawley rats were incubated in an anaerobic chamber for up to 12 hours. Cell viability was measured by acridine orange/propidium iodide staining. Caspase-3 activation was also determined. NO release into culture medium was measured using a Griess reagent. The expression of iNOS mRNA and protein was examined using real time RT-PCR and Western blot analysis. Results: Marked cell death $(\sim 50 \%)$ occured within 6 hour after hypoxic exposure to MIN6 and rat islets. Immunoreactivity to activated caspase-3 was observed. NO production was increased in a time dependent manner. Expression of iNOS mRNA and protein was significantly increased at 4 and 6 hour after hypoxia. iNOS expression was confirmed by immunostaining. Of note, Pdx-1 mRNA expression was markedly attenuated by hypoxia. The Pdx1 gene promoter activity in MIN6 was negatively regulated by hypoxia or a NO donor, DETA-NO. Pretreatment with a selective iNOS inhibitor, $1400 \mathrm{~W}$, significantly prevented apoptotic cell death by hypoxia.

Conclusion: Our data suggest that iNOS-NO plays an important role in hypoxic injury to pancreatic $\beta$ cells. Therefore, iNOS-NO might be a potential therapeutic target for improving engraftment of transplanted islets.

\section{5}

Controlled over expression of uncoupling protein 2 in INS-1 cells exerts minor effects on beta cell function and does not protect against cytokine-induced toxicity E. Pettersen ${ }^{1}$, I. Jorgensen ${ }^{2}$, F. Skorpen ${ }^{3}$, H. Ogata ${ }^{2}$, Z.-H. Ma ${ }^{4}$, A. Björklund ${ }^{4}$, K. Ekeberg ${ }^{2}$, V. Grill' ${ }^{2}$;

${ }^{1}$ Norwegian University of Science and Technology, Institute of Laboratory Medicine, Children's and Women's health, Levanger, ${ }^{2}$ Norwegian University of Science and Technology, Institute of Cancer Research and Molecular Medicine, Trondheim, ${ }^{3}$ Norwegian University of Science and Technology, Institute of Laboratory Medicine, Children's and Women's Health, Trondheim, Norway, ${ }^{4}$ Karolinska Institute, Department of Molecular Medicine and Surgery, Stockholm, Sweden.

Background and Aims: Uncoupling protein 2 (UCP-2) is proposed to have diabetogenic influence by decreasing efficiency of glucose metabolism to generate ATP and/or other signaling molecules for insulin secretion. However, UCP-2 may also exert a beneficial role, coupled to protection against radical oxygen formation (ROS). Studies in knockout or un-restricted overexpression models have given evidence for both the afore-mentioned negative and positive effect; however these extremes of regulation make interpretations difficult in physiological terms.

Material and Methods: We transfected INS-1 cells to obtain a tet-on, UCP-2 inducible cell line. Induction by 0.1 or $1.0 \mu \mathrm{g} / \mathrm{ml}$ doxycyclin for $48 \mathrm{~h}$ increased UCP-2 protein by respectively $178 \pm 29$ and $424 \pm 113 \%$.

Results: Mitochondrial membrane potential, ATP and insulin contents were not significantly changed. Induction by $0.1 \mu \mathrm{g} / \mathrm{ml}$ of doxycyclin failed to affect oxidation of C14 glucose and C14-oleate. Glucose $(27 \mathrm{mM})$-induced insulin secretion tended to be reduced in induced cells, $\mathrm{p}<0.08$. Secretion at $3.3 \mathrm{mM}$ glucose $+/-20 \mathrm{mM} \mathrm{KCl}$ was not affected. A protective effect against ROS was tested for $\mathrm{H} 2 \mathrm{O} 2$ and interleukin -1 beta (IL-1) by MTT assay. H202 exerted protection after induction with $1 \mu \mathrm{g} / \mathrm{ml}$ of doxycycline $(p=0,01)$ but not after $0.1 \mu \mathrm{g} / \mathrm{ml}$. No degree of induction prevented a decrease of MTT after $24 \mathrm{~h}$ exposure to IL-1. Instead, combined exposure to $0.2 \mathrm{mM}$ oleate and $500 \mathrm{ng} / \mathrm{ml} \mathrm{IL-1b}$ aggravated a reduction of MTT $(\mathrm{p}=0,02)$. Conclusion: Controlled, moderate over expression of UCP2 neither exerts major negative effects on beta cell function nor generalized protective effects against ROS. Thus, the functional role of UCP-2 may be mostly a permissive one.

0436

Search for molecules involved in the increased vulnerability of adult $\boldsymbol{\beta}$ cells after a maternal low protein diet. Prevention by taurine

K. J. R. Goosse, M. Balteau, B. Reusens, C. Remacle; Laboratory of Cell Biology, Université Catholique de Louvain, Belgium.

Background and Aims: We have shown that low protein diet induces long-term consequences on the endocrine pancreas of the progeny. Islets of adult rats that received a low protein diet during gestation and lactation but a normal diet after weaning, maintained a high vulnerability toward cytokines already observed at fetal age. Taurine is an amino acid of which concentration was reduced in the plasma of both mothers and fetuses fed a low protein diet. When malnourished dams are supplemented with taurine, a normal sensitivity toward cytokines was restored in the islets of the adult progeny.

Cytokines like IL-1 $\beta$, TNF- $\alpha$ or INF- $\gamma$ enhance the expression of genes like iNOS (inducible nitric oxide synthase), or chemokines such as MCP-1 (monocyte chemoattractant protein-1) and IP-10 (interferon inducible protein-10) in $\beta$ cells. Their products are known to contribute to $\beta$ cell death in type-1 diabetes. 


\begin{tabular}{|c|c|c|c|c|c|c|c|c|}
\hline & \multicolumn{2}{|l|}{$\mathrm{C}$} & \multicolumn{2}{|l|}{$\mathrm{R}$} & \multicolumn{2}{|l|}{$\mathrm{CT}$} & \multicolumn{2}{|l|}{ RT } \\
\hline & $\begin{array}{c}\text { No } \\
\text { Cytokines }\end{array}$ & Cytokines & $\begin{array}{c}\text { No } \\
\text { Cytokines }\end{array}$ & Cytokines & $\begin{array}{c}\text { No } \\
\text { Cytokines }\end{array}$ & Cytokines & $\begin{array}{c}\text { No } \\
\text { Cytokines }\end{array}$ & Cytokines \\
\hline Apoptosis (\%) & $0.5 \pm 0.1$ & $2.0 \pm 0.3$ & $1.6 \pm 0.4^{*}$ & $3.3 \pm 0.3 *$ & $1.1 \pm 0.2$ & $3.5 \pm 0.6^{\circ}$ & $0.7 \pm 0.1^{\#}$ & $2.9 \pm 0.2$ \\
\hline $\begin{array}{c}\mathrm{NO} \\
\text { (pmol/islet/ } \\
24 \mathrm{~h})\end{array}$ & $9.6 \pm 3.4$ & $57 \pm 5.8$ & $50 \pm 11 * * *$ & $92 \pm 10.4 *$ & $21 \pm 5.0$ & $85 \pm 8.3^{\circ}$ & $16.4 \pm 6.4^{\# \#}$ & $80 \pm 7.4$ \\
\hline
\end{tabular}

Effect of maternal diet and early taurine supplementation on adult islet apoptosis and NO secretion.

$* \mathrm{p}<0.05 \mathrm{R}$ vs. C, ${ }^{* * *} \mathrm{p}<0.001 \mathrm{R}$ vs. C, o p $<0,05$ CT vs. C, \# $\mathrm{p}<0.05 \mathrm{RT}$ vs. R and \#\# $\mathrm{p}<0.01 \mathrm{RT}$ vs. R.

Our aim is to determine if the expression of these molecules is modulated by early low protein diet and taurine supplementation.

Materials and Methods: Wistar rats were fed with a $20 \%$ (Control [C group]) or an isocaloric $8 \%$ protein diet (Recovery [R group]) supplemented or not with taurine (CT and RT group) during gestation and lactation. After weaning, offspring received a $20 \%$ protein diet. Islets from 3 month-old rats were isolated and cultured. On day 3, they were incubated or not with cytokines (IL-1 $\beta 50 \mathrm{U} / \mathrm{ml}$, TNF$\alpha 100 \mathrm{U} / \mathrm{ml}$ and INF- $\gamma 100 \mathrm{U} / \mathrm{ml}$ ) for a $24 \mathrm{~h}$ period. Apoptotic rate was analysed with TUNEL method. NO, MCP-1 and IP-10 secreted by islets were quantified.

Results: Compared to controls, islets from R group featured an enhanced apoptotic rate and secreted more NO. Both parameters increased with cytokines and remained higher in the $\mathrm{R}$ group. Maternal taurine supplementation to the LP diet normalized both apoptotic rate and NO secretion in the RT islets cultured in normal medium. In the presence of cytokines, the rate of apoptosis and NO production were lower in the RT group compared to the R group so that the difference between $\mathrm{C}$ islets and RT islets was no more significant. However, taurine added to the dam's control diet caused an increase in both apoptosis and NO secretion when the islets were cultured with cytokines. Neither the LP diet nor taurine affected the MCP-1 and IP-10 production, which were only released in the presence of cytokines.

Conclusion: The increased apoptotic rate in the adult islets of early malnourished rats may be partially due to an increase in NO production. There was no evidence that maternal malnutrition interfered with mechanisms leading to pre-insulitis, since MCP-1 and IP-10 were not modulated by the diet or taurine. Although the addition of taurine to protein-restricted diet protected adult islets from apoptosis and NO overproduction, unnecessary supplementation could have deleterious effect on the endocrine pancreas.

\section{7}

Role of beta cell NF- $\kappa$ B activation and anti-apoptotic preconditioning in Gleevec-mediated protection against diabetes in the mouse

N. Welsh, R. Hägerkvist, S. Sandler;

Dept. of Medical Cell Biology, Uppsala University, Sweden.

Background and Aims: It has recently been reported that tyrosine kinase inhibitor imatinib mesylate (Gleevec) improves Type 2 diabetes and protects against arteriosclerosis. The mechanisms that underlie this beneficial effect of Gleevec are, however, unclear. The aim of the present study was to establish whether Gleevec protects against diabetes in animal models for diabetes that are characterized by beta-cell destruction.

Materials and Methods: Gleevec was administered daily to non-obese diabetic (NOD) mice by gavage from 9 to 35 weeks of age. Gleevec was also given to NMRI mice one day before, the same day and one day after a $160 \mathrm{mg} /$ $\mathrm{kg}$ streptozotocin injection. Development of diabetes was followed by blood glucose measurements and beta-cell mass was assessed using immunohistochemical staining for insulin and morphometric analysis. For in vitro experiments the insulin producing cell line betaTC-6 and human islets were used.

Results: We demonstrate that Gleevec counteracted diabetes in the streptozotocin-injected mouse and the NOD mouse, and that this was paralleled by a partial restoration of the beta-cell mass. Gleevec affected neither insulitis nor splenocyte cytokine production in NOD mice. In addition, Gleevec decreased beta-cell death in vitro in response to streptozotocin, proinflammatory cytokines and a nitric oxide donor. The Gleevec effect was mimicked by knock-down of c-Abl using the RNAitechnique. Gleevec probably enhanced beta-cell survival by promoting a state similar to ischemic preconditioning, as evidenced by NF-kappaB activation, increased nitric oxide and hydrogen peroxide production and depolarization of the inner mitochondrial membrane. Pharmacological inhibition 
of NF-kappaB counteracted the benificial effects of Gleevec.

Conclusion: We conclude that Gleevec mediates beta-cell survival and that this could contribute to the beneficial effects observed in diabetes.

Supported by Swedish Reseach Council

\section{PS 20}

\section{Pancreas and islet transplantation}

\section{8}

Impaired stimulation of insulin secretion by GLP-1 after islet auto- and allo-transplantation in humans D. Elahi ${ }^{1}$, C. Rastellini ${ }^{2}$, G. Basadonna ${ }^{2}$, M. Uknis ${ }^{2}$, G. S. Meneilly ${ }^{3}$, M. Fung ${ }^{4}$, J. M. Egan ${ }^{5}$, D. Thompson ${ }^{4}$, G. Warnock ${ }^{4}$, M. J. Thompson ${ }^{6}$, D. K. Andersen ${ }^{1}$;

${ }^{1}$ Dept of Surgery, Johns Hopkins, Baltimore, ${ }^{2}$ Dept of Surgery, UMass, Worcester, MA, United States, ${ }^{3}$ Dept of Medicine, ${ }^{4}$ Dept of Surgery, UBC, Vancouver, BC, Canada, ${ }^{5}$ Diabetes Section, NIA, Baltimore, ${ }^{6}$ Dept of Medicine, UMass, Worcester, United States.

Background and Aims: Intrahepatic islet auto-transplantation (AUTO) following pancreatectomy for chronic pancreatitis would be expected to be more durable than allo-transplantation (ALLO) for treating type 1 diabetes because of avoidance of immunosuppression, but the control of insulin secretion in either tranplantation has not been studied. Our aims were to study insulin action and secretion in AUTO and ALLO patients and compare results to normal (NL) subjects.

Materials and Methods: Forty-six year old ALLO female (studied pre-pancreatectomy and 1 and 3 months after transplantation) who received 550,000 islet-like clusters, 10 ALLO patients (studied 1 month after transplantation) who received on average 910,000 islet-like clusters, and 6 NL subjects. We first performed hyperinsulinemic-euglycemic clamps $(80 \mathrm{mU} / \mathrm{kg} / \mathrm{min})$ for $2 \mathrm{~h}$. Following $1 \mathrm{~h}$ recovery, we then raised plasma glucose levels by $5.4 \mathrm{mmol} / \mathrm{l}$ with a primed infusion of $20 \%$ glucose from $0-120 \mathrm{~min}$ and infused glucagon-like peptide-1 (GLP-[7-36]amide, $1.5 \mathrm{pM} /$ $\mathrm{kg} / \mathrm{min}$ ) from 60-120 min. Mean plasma insulin levels (IRI, $\mathrm{pM}$ ) were calculated for each hour (means \pm SEM are shown).

Results: In both ALLO and AUTO patients peripheral tissue sensitivity to insulin was increased following transplantation. In NL subjects, hyperglycemia alone resulted in an IRI $(0-60 \mathrm{~min})$ of $138 \pm 24 \mathrm{pM}$ that was similar to that of the AUTO patient pre-op (74 pM). ALLO patients had a similar response to hyperglycemia alone $(185 \pm 30 \mathrm{pM})$, but had absent first-phase $(0$ $10 \mathrm{~min}$ ) response. One month after transplant, the AUTO patient showed a profound decrease overall in insulin secretion in response to hyperglycemia $(36 \mathrm{pM})$ and a similarly defective first-phase insulin secretion to ALLO. By 3 months post-op, the AUTO patient had regained IRI responsiveness to hyperglycemia $(90 \mathrm{pM})$ but still had absent first-phase secretion. GLP-1 infusion caused a 14-fold increase in glucose-stimulated IRI secretion in NL (IRI 60-120 min=1963 $\pm 350 \mathrm{pM}$ ) that was similar to the pre-op result in AUTO (1412 pM). In ALLO patients the GLP-1 augmentation of IRI was merely 3-fold (to $588 \mathrm{pM})$. The one month post-op IRI of the ALLO patient to GLP-1 was augmented only a 1.6-fold (to $57 \mathrm{pM}$ ) in reponse to hyperglycemia. Three months after auto-transplantation, the GLP-1 augmentation of glucosemediated IRI had increased 3-fold (to $280 \mathrm{pM}$ ).

Conclusion: Beta-cell responsiveness to glucose after islet transplantation is influenced by the quality and quantity of infused islets and the necessity for immuno-suppression, and may also be influenced in both allo- and autotransplants by the denervation status of the islet graft. First-phase insulin secretion is impaired after islet transplantation. Additionally, there appears to be a selective loss of responsiveness to GLP-1 in both auto- and allo-transplants. Three months after auto-transplantation, this deficit had lessened, and further studies will ascertain if the alterations in insulin release and responsiveness to GLP-1 persist long-term in intra-hepatic islet engraftment. The increased insulin sensitivity can be ascribed to insulinopenia following transplantation.

Supported by NIH

0439

Long-term follow-up of simultaneous islet-kidney transplantation

V. Pavlicek ${ }^{1}$, P. Gerber ${ }^{1}$, T. Pfammatter ${ }^{2}$, R. Wuethrich ${ }^{1}$, M. Weber ${ }^{3}$, G. A. Spinas ${ }^{1}$, R. Lehmann ${ }^{1}$;

${ }^{1}$ Internal Medicine, ${ }^{2}$ Radiology, ${ }^{3}$ Surgery, University Hospital, Zurich, Switzerland.

Background and Aims: Recently the beta-score was proposed as a simple clinical scoring system for islet function and diabetes control, since this integrated measure is more useful than the presence or absence of insulin independence in recipients of islet transplantation alone (ITA). We assessed beta-cell and renal function in recipients of simultaneous islet-kidney transplantation (SIK) with the beta-score and creatinine-clearance over a period of up to 6 years. 
Materials and Methods: 13 consecutive patients with type 1 diabetes received SIK with a median follow-up of 3.2 years (range 1.0-6.0 years). Two patients were lost to follow-up and, therefore, excluded from analysis. The betascore as a composite measure consists of glycated hemoglobin, stimulated C-peptide, need for insulin or oral antidabetic drugs, and fasting plasma glucose. For each of these variables 0,1 or 2 points were assigned (maximum score $=8$ and a score $>4$ can only be achieved with endogenous insulin production).

Results: The means of the following parameters over the whole follow-up period were calculated: Daily insulin dose decreased by $54 \%$ from $0.59+0.18 \mathrm{U} / \mathrm{kg}$ BW pretransplant to $0.27+0.18 \mathrm{U} / \mathrm{kg} \mathrm{BW}$ posttransplant. Although insulin independence in patients with $>2$ islet transplants decreased from $71 \%$ to $29 \%$ at the end of the follow-up period, glucose control was excellent as assessed by $\mathrm{HbAlc}$ and beta-score. HbAlc was 6.2 $+0.4 \%$ post- vs. $8.1+1.5 \%$ pretransplant and the betascore remained stable at $4.9+0.8$ vs. $1.3+0.3$ pretransplant, respectively. During follow-up there were no incidents of severe hypoglycemic episodes and creatinine and creatinine clearance were $136+16 \mathrm{~mol} / \mathrm{l}$ and $48+5 \mathrm{ml} /$ $\min / 1.73 \mathrm{~m}^{2}$, respectively.

Conclusion: We extend the applicability of the beta-score as a useful integrated measure of beta-cell function to recipients of SIK with a follow-up up to 6 years. Renal function and beta-score remained stable over time. Despite more advanced diabetes complications a betascore as good as reported in ITA could be achieved after SIK.

\section{0}

Viral transduction efficiency and gene expression in pseudo-islet aggregates with HIV-based lentiviral vectors: in vitro and in vivo analysis

H. Callewaert ${ }^{1}$, C. Gysemans ${ }^{1}$, A. K. Cardozo ${ }^{2}$, M. Elsner ${ }^{3}$, M. Tiedge ${ }^{3}$, D. Eizirik ${ }^{2}$, C. Mathieu ${ }^{1}$; ${ }^{1}$ Exp Geneeskunde, LEGENDO, Leuven, ${ }^{2}$ Univ Libre de Bruxelles, Lab Exp Med, Belgium, ${ }^{3}$ Medizinische Hochschule, Inst für Kl Biochemie, Hannover, Germany.

Background and Aims: Gene therapy using virus-based vectors is an appealing approach to target pancreatic islets to improve outcome in islet transplantation. Low transduction efficacy, viral-induced antigenicity and toxicity leading to increased apoptosis are limiting factors. In this study, we aimed at improving transduction efficacy with lentiviral transfer into pancreatic islets with acceptable cell loss and without cytotoxicity.
Materials and Methods: We studied transduction efficacy (scoring for green fluorescence) and viability (Hoechst/PI staining) in FACS-purified $\beta$-cells and pseudo-islet aggregates at different multiplicity of infection [(MOI) 5 and 50] and at different culture times [16 and 90 hours after infection with a replication-defective vesicular stomatitis virus (VSV-G) pseudo-typed lentiviral vector expressing enhanced green fluorescent protein (EGFP) under transcriptional control of the cytomegalovirus (CMV) promotor (LVpWPT-CMV-EGFP)].

Results: At 90 hours after transduction, transfection efficacy in whole islets was low, resulting in modest EGFP expression in the islet rim with no EGFP positive cells in the islet core (3.1 \pm 1.4 and $11.2 \pm 4.1 \%$ EGFP positive cells, at a MOI of 5 and 50 respectively). Viability 90 hours after transfection was similar to uninfected controls ( $>90 \%$ living cells), regardless of viral concentrations used. In contrast to whole islets, FACSpurified primary rat $\beta$-cells had $81.0 \pm 1.0 \%$ of $\beta$-cells expressing the reporter gene 90 hours post-transduction at a MOI of 5. No viral-related cytotoxic effects were observed $(92.3 \pm 1.8 \%$ viable cells at 90 hours culture, compared to $89.6 \pm 2.0 \%$ viable non-infected cells at 90 hours culture, NS). Pseudo-islet aggregates, prepared by further dissociation of islets with dispase and transduced at single cell level with LVpWPT-CMV-EGFP before re-aggregation to islet clusters, had transduction rates of $80.0 \pm 2.8 \%$ at MOI 5 already 64 hours post-infection with maintained viability $(83.5 \pm 12.0$ living cells). There was, however, an important cell loss during preparation of pseudo-islet aggregates, as evidenced by a 10fold decrease in insulin content. However, surviving cells did not show enhanced susceptibility to cytokine-induced cell death and their response to glucose was preserved. When using pseudo-islet aggregates (infected or not) for transplantation, at least $1,700,000$ cells were needed to correct hyperglycemia in alloxan-induced diabetic NOD-SCID mice. Graft-bearing kidneys (containing functioning LV-pWPTCMV-EGFP-transduced pseudo-islet aggregates) were strongly EGFP positive exclusively in the graft up to 90 days after transplantation. EGFP expression co-localized with insulin-positivity, confirming the presence of EGFP in $\beta$-cells and excluding viral spread to surrounding tissues. Moreover, lentiviral-transduced and non-transduced pseudo-islet aggregate groups had similar glucose responsiveness and immune susceptibility in vivo.

Conclusion: our data demonstrate that dispersion of islets prior to lentiviral transfection and re-aggregation prior to transplantation is an efficient way to introduce genes of interest into islets for transplantation purposes. However, important cell loss is the high price to pay for this improved transfection efficacy. 


\section{1}

Anti-angiogenic and immunomodulatory effects of rapamycin on pancreatic islet endothelial cells: relevance for islets engraftment after transplantation

G. Mauriello Romanazzi ${ }^{1}$, V. Cantaluppi ${ }^{1}$, L. Biancone ${ }^{1}$, F. Figliolini ${ }^{1}$, S. Beltramo ${ }^{1}$, F. Galimi ${ }^{2}$, R. Romagnoli ${ }^{3}$, A. Franchello ${ }^{3}$, M. Salizzoni ${ }^{3}$, C. Ricordi ${ }^{4}$, G. P. Segoloni ${ }^{1}$, P. Cavallo Perin ${ }^{5}$, G. Camussi ${ }^{1}$;

${ }^{1}$ Dpt Internal Medicine, Nephrology Division, Torino, ${ }^{2}$ University of Sassari, Department of Biomedical Sciences/INBB, Sassari, ${ }^{3}$ Liver Transplant Centre, San Giovanni Battista Hospital, Torino, Italy, ${ }^{4}$ University of Miami, Diabetes Research Institute, Miami, United States, ${ }^{5} \mathrm{Dpt}$ Internal Medicine, Diabetology Unit, Torino, Italy.

Background and Aims: Donor intra-islet endothelial cells contribute to islets neovascularization after tranplantation. Several factors may interfere with this process and ultimately influence islet engraftment. Rapamycin, a central immunosuppressant in islet transplantation, is a mTOR inhibitor that has been shown to inhibit cancer angiogenesis. The aim of this study was to evaluate the effects of rapamycin on the endothelium from freshly purified islets and on islet-derived endothelial cell lines.

Materials and Methods: The outgrowth of endothelial cells from freshly purified human islets and the formation of capillary structures on Matrigel were studied in vitro and in vivo. Human (hIECs) and mouse (mIECs) islet endothelial cell lines were isolated and analyzed for proliferation, apoptosis, motility and angiogenesis capacity in the presence or absence of rapamycin. In parallel, selected vascular growth factors and cell surface receptor expression were studied at gene and protein levels.

Results: Rapamycin inhibited the outgrowth of endothelial cells from freshly purified human islets and the formation of capillary-like structures in vitro on matrigel-coated surfaces and in vivo after subcateneous injection into SCID mice. Rapamycin decreased migration, proliferation and angiogenic properties of both hIECs and mIECs with appearance of apoptotic features in a dose-dependent manner. Gene-array analysis of angiogenesis-related genes on human islets revealed a decreased expression of several endothelial growth factors, CD31 (PECAM-1), alphaVbeta3 integrin and thrombin in presence of rapamycin. Western blot and FACS studies confirmed the down-regulation of angiogenic factors. On the other hand, rapamycin reduced the expression of molecules involved in immune processes such as ICAM-1 and CD40 and reduced the adhesion of T-cells to hIECs.

Conclusion: Our results suggest that rapamycin exerts dual effects on islet endothelium inducing a simultaneous inhibition of angiogenesis and a down-regulation of receptors involved in lymphocyte adhesion and activation.
Supported by the Italian Ministry of University and Research FIRB project to G.C. and COFIN 01 to L.B., G. C. and P.C.P., by Italian Ministry of Health to G.C. and G. P.S., and by Ricerca Finalizzata Regione Piemonte

0442

Adenoviral overexpression of insulin-like growth factor II increases beta cell replication and mass in transplanted islets

E. Estillles, N. Téllez, M. Montolio, S. Rodríguez-Mulero, J. Soler, E. Montanya;

Laboratory of Diabetes and Experimental Endocrinology, IDIBELL, Hospital Universitari Bellvitge, University of Barcelona, Spain.

Background and Aims: In the initial days after transplantation more than half of transplanted tissue is lost. Insulinlike growth factor-II (IGF-II) is a growth promoting peptide which is able to stimulate $\beta$-cell proliferation and survival. The aim of this study was to determine whether the IGF-II overexpression in rat islets could have a beneficial effect on transplanted islets.

Materials and Methods: Islets from Lewis rats were infected ex vivo with adenovirus encoding for IGF-II (Ad-IGF-II). Streptozotocin-diabetic Lewis rats were syngeneically transplanted with 500 Ad-IGF-II infected islets (Ad-IGF-II group) or 500 uninfected islets (control group) under the kidney capsule. 500 islets are an insufficient mass to restore normoglycemia; thus, we expected that all animals would remain hyperglycemic after transplantation. Grafts were removed 3, 10 and 28 days after transplantation and beta cell replication (BrdU incorporation) and mass (morphometry) were determined.

Results: All animals showed similar hyperglycemia after transplantation ensuring comparable metabolic conditions among groups throughout the study. In Ad-IGF-II group, beta cell replication was higher than in normal pancreas $(0.35 \pm 0.06 \% ; \mathrm{p}<0.001)$ and it was also higher than in control group on days $3(1.48 \pm 0.07 \%$ vs $0.59 \pm 0.02 \%$; $<<0.001), 10$ $(1.37 \pm 0.13 \%$ vs $0.93 \pm 0.15 \% ; \mathrm{p}<0.01)$ and $28(1.42 \pm 0.06 \%$ vs $0.67 \pm 0.04 \% ; \mathrm{p}<0.001)$. It is noteworthy that in control group beta cell replication was increased only on day 10 but not on days 3 and 28 after transplantation, despite the exposure to similar hyperglycemia in all time points. IGF-II overexpression was therefore able to overcome this initial limitation in beta cell replication (day 3), as well as that induced by long-term exposure to hyperglycemia (day 28). Beta cell mass was reduced (approx. 60\%) on day 3 after transplantation in control $(0.39 \pm 0.03 \mathrm{mg})$ and Ad-IGF-II group $(0.49 \pm 0.09 \mathrm{mg})$. Thus, IGF-II overexpression did not prevent the dramatic beta cell loss that took place in the 
initial days after transplantation. On day 10 after transplantation, beta cell mass was increased in Ad-IGF-II group $(1.39 \pm 0.18 \mathrm{mg} ; \mathrm{p}<0.05)$ and also in control islet grafts $(1.20 \pm 0.09 \mathrm{mg} ; \mathrm{p}<0.05)$ paralleling the increased beta cell replication in both groups. On day 28 , beta cell mass decreased in control $(0.31 \pm 0.07 \mathrm{mg})$ and Ad-IGF-II groups $(0.78 \pm 0.17 \mathrm{mg} ; \mathrm{p}<0.05)$, but it was partly preserved in IGFII group ( $p<0.05$ vs control group), in agreement with the increased beta cell replication in IGF-II group on day 28 , indicating a protective effect of IGF-II overexpression on beta cell mass.

Conclusion: IGF-II overexpression in transplanted islets was able to increase beta cell replication and mass in islets grafts, and partly protected them from the deleterious effects of chronic hyperglycemia on beta cells. IGF-II may be useful to design strategies aimed to preserve beta cell mass in transplanted islets.

Supported by JDFRI, IDIBELL Foundation; Instituto de Salud Carlos III, RCMN (C03/08)

\section{3}

Successful outcome after intraperitoneal transplantation of non-PLL high $G$ barium-alginate micro-encapsulated islets

S. Bohman ${ }^{1}$, A. Andersson ${ }^{1}$, A. King ${ }^{2}$;

${ }^{1}$ Department of Medical Cell Biology, Uppsala University, Uppsala, Sweden, ${ }^{2}$ Beta Cell Function and Development Group DHRED, Kings College, London, United Kingdom.

Background and Aims: Microencapsulation protects islets from the host immune system and thus allows for transplantation without use of immunosuppressive drugs. In type 1 diabetes, the transplanted cells need to be protected from immune rejection as well as the ongoing autoimmune reaction. Alginate is the most commonly used polymer for cell encapsulation. The properties of the alginate gel depend on the alginate composition. High guluronic acid (G) sequences are required for the alginate to form a stable and strong gel, but also make the gel more permeable than high mannuronic acid (M) gels. Traditionally, a poly-L-lysine (PLL) layer is added to the alginate core. However, PLL reduces the biocompatibility of implanted capsules by provoking an immune response and inducing a cellular reaction around the capsule. Most previous studies have been carried out on PLL capsules transplanted into animals with chemically induced diabetes. In this study, the polycation layer was omitted, and high $G$ barium-alginate capsules transplanted into mice with spontaneous diabetes.

Materials and Methods: $\mathrm{Balb} / \mathrm{c}$ or $\mathrm{C} 57 \mathrm{BL} / 6 \mathrm{~J}$ islets were encapsulated in high $\mathrm{G}$ alginate, using $\mathrm{BaCl}_{2}$ and $\mathrm{CaCl}_{2}$ as crosslinkers. Thereafter, 700 encapsulated islets were trans- planted into the peritoneal cavity of alloxan-diabetic C57BL/6J mice or spontaneously diabetic NOD mice. In addition, the same number of non-encapsulated islets and empty capsules were transplanted into diabetic C57BL/6J and NOD mice, respectively. Blood glucose concentrations were monitored once a week for up to 14 weeks after transplantation. Retrieved capsules were counted and visually evaluated for cellular overgrowth.

Results: Diabetic C57BL/6J mice transplanted with encapsulated syngeneic or allogeneic islets were normoglycemic for $23 \pm 7$ and $80 \pm 18$ days. Quite in contrast, both syngeneic and allogeneic non-encapsulated islets failed to reverse hyperglycemia. NOD mice grafted with encapsulated Balb/ $\mathrm{c}$ and $\mathrm{C} 57 \mathrm{BL} / 6 \mathrm{~J}$ islets remained normoglycemic for $67 \pm 14$ and $84 \pm 10$ days, respectively. The implanted empty capsules did not normalise blood glucose levels at all. In NOD mice, retrieval rates and cellular overgrowth on capsules containing islets on the one hand and empty capsules on the other did not differ.

Conclusions: As few as 700 intraperitoneally grafted encapsulated islets can reverse hyperglycemia in mice with alloxan-induced diabetes or in NOD mice. Non-encapsulated syngeneic and allogeneic islets lacked this property. This indicates that barium capsules without PLL protect the islets from immune rejection. Moreover, they also seem to serve as a matrix to support the encapsulated islet. The fact that the retrieved capsules had little or no cellular overgrowth indicates that this high $G$ barium-alginate capsule without PLL is highly biocompatible. In a previous study we found that retrieved encapsulated islets had low overall glucose oxidation and insulin release rates. This most probably reflected a decrease in beta cell mass of each islet. Despite that, the islets still responded well to an acute glucose challenge, indicating that the function of the beta cells was well preserved. Taken together these and previous data from our laboratory support the view that high $G$ capsules lacking a PLL-layer are superior to conventional PLL-covered alginate capsules.

\section{4}

Mesenchymal stem cells modulate allogenic response in pancreatic islet transplantation

B. Longoni ${ }^{1}$, G. T. Paoli ${ }^{1}$, E. Szilagyi ${ }^{1}$, T. Consani ${ }^{1}$,

R. Saccardi ${ }^{2}$, S. Urbani ${ }^{2}$, D. Campani ${ }^{1}$, P. Marzola ${ }^{3}$,

F. Mosca ${ }^{1}$;

${ }^{1}$ Department of Oncology, Transplantation and Advanced Technologies in Medicine, University of Pisa, ${ }^{2}$ Bone Marrow Transplantation Unit, Policlinico di Careggi, Florence, ${ }^{3}$ Department of Morphological and Biomedical Sciences, University of Verona, Italy. 
Background and Aims: Pancreas transplantation is currently the gold-standard therapeutic approach for type 1 diabetic patients which consistently provides insulin-independence. Intrahepatic human islet transplantation is a therapeutic option for diabetes mellitus (type I); however, the need for a substantial amount of islets and repeated transplantations indicate impaired survival or function of islets. The main obstacles for clinical islet transplantation are the need for pancreatic islets derived from multiple donors and the adverse effects of current immunosuppressive protocols. Mesenchymal stem cells (MSCs) are multipotent adult stem cells capable of differentiate in various cell lines like ostocytes, adipocytes, chondrocytes but also in neuronal and endothelial phenotypes. The MSCs escape from the immune system by suppression of lymphocyte proliferation and modulation of allogenic response in vitro. In vivo they prolong skin allograft survival and may decrease graft-versus-host disease after hematopoietic stem cell transplant. Therefore, we investigated the immunomodulatory effects of MSCs in an experimental model of marginal-mass islet transplantation.

Materials and Methods: Islets and bone marrow-derived MSCs were isolated from inbred Sprague-Dawley rats. Streptozotocin-induced diabetic Wistar rats underwent intraportal transplantation (700 IE) and simultaneous portal or systemic MSCs infusion $\left(2,5 \times 10^{4}, 7,5 \times 10^{4}, 15 \times 10^{4} / \mathrm{kg}\right.$ monodose). Blood glucose levels and insulin production (RIA) were continuously monitored, islet survival was followed by magnetic resonance imaging (MRI) and liver histology was assessed at 7 th post-transplant day.

Results: Portal infusion of $2,5 \times 10^{4}$ MSCs does not result in a significant enhancement in glycemic control, only a faster fall (2nd post-transplant day versus 4th post-transplant day) in the glycemic level was observed. Portal infusion of a higher number $\left(>2,5 \times 10^{4}\right)$ of MSCs causes serious thrombotic events in the liver also in presence of anticoagulative therapy. The systemic low dose $\left(2,5 \times 10^{4}\right.$ and 7,5 $\times 10^{4}$ ) infusion of MSCs does not improve allogenic islet function but $15 \times 10^{4} / \mathrm{kg}$ MSC increases significantly the normoglycemic period ( 5 days) after marginal mass islet transplant which is not able to normalize iperglycemia by itself. The histological analysis showed a lower lymphocyte infiltration around intrahepatic islet grafts in the presence of MSCs in both type of infusions.

Conclusion: Systemic high doses of MSCs improve glycemic control in our animal model and reduce lymphocyte infiltration in acute rejection. These data suggests that MSC mantain their in vitro properties of suppressing lymphocyte activation and proliferation also in vivo. The above described results of MSC need more investigation to define whether the observed effect is an allospecific process or part of a general antiinflammatory effect.

Supported by Fondazione Arpa and MIUR grants
0445

Effects of immunosuppressive drugs on human islet neogenesis in vitro

R. Gao ${ }^{1}$, J. Ustinov $^{1}$, O. Korsgren ${ }^{2}$, T. Otonkoski ${ }^{1,3}$; ${ }^{1}$ Program of Developmental and Reproductive Biology, Biomedicum Helsinki, University of Helsinki, Finland, ${ }^{2}$ Department of Clinical Immunology, University of Uppsala, Sweden, ${ }^{3}$ Hospital for Children and Adolescents, University of Helsinki, Finland.

Background and Aims: Immunosuppressive drugs may have deleterious effects on transplanted islets. In vitro studies have shown that these drugs have negative effects on beta-cell viability and insulin synthesis and secretion. In previous studies, we reported the generation of endocrine cells from human adult pancreatic ductal cell cultures. Assuming that neogenesis contributes to the long-term function of islet grafts, it is important to study the effects of immunosuppressive drugs on precursor cell proliferation and differentiation. We have now examined the effects of clinically used doses of immunosuppressive drugs on these processes in vitro.

Materials and Methods: Freshly isolated human pancreatic cells from 8 donors were used in this study. The culture consists of an expansion phase in serum-containing medium followed by Matrigel overlay and a differentiation phase in serum-free conditions. More than $90 \%$ of the proliferating cells were CK19-positive ductal cells. Immunosuppressive drugs, including sirolimus (10 ng/ml), tacrolimus (5 ng/ml), mycophenolate mofetil (MMF, $20 \mu \mathrm{g} / \mathrm{ml})$, daclizumab $(10 \mu \mathrm{g} / \mathrm{ml})$ and their combinations were tested in parallel culture wells through either the expansion phase (5-7 days) or the entire culture period (4-5 weeks).

Results: MMF, either alone or in combination with sirolimus or tacrolimus, severely hampered duct-cell proliferation by 8 -fold during the expansion period, and significantly reduced the total DNA content by about $40 \%$ after 5-week culture. After 4-5 week exposure to different drugs, only sirolimus and daclizumab showed no adverse effects on insulin content, whereas significant reductions of $30-60 \%$ in insulin content were seen in all other experimental groups. Only tacrolimus decreased the insulin content per DNA, as well as the proportion of insulinpositive cells by immunocytochemistry.

Conclusion: Based on these in vitro results, MMF has a potent inhibitory effect on human islet neogenesis primarily through an antiproliferative effect on the precursors, whereas tacrolimus mainly affects beta-cell differentiation. Sirolimus and daclizumab have no adverse effects on these parameters. The immunosuppressive protocol may be an important determinant of long-term clinical islet graft function. 
0446

Characterisation of a rat model of diabetes induced by prolonged treatment with cyclosporin A and FK-506

I. Hernández-Fisac;

Biochemistry, Complutense University of Madrid, Spain.

Background and Aims: The immunosuppressants cyclosporin A (CsA) and tacrolimus (FK-506) may induce "postransplant diabetes" with a relatively high prevalence in organ transplanted patients. We have developed a rat model of this type of diabetes with the aim of studying the diabetogenic mechanism of action of the two immunosuppressants.

Materials and Methods: Groups of 6 rats were injected (i. p.) twice a day $0.15 \mathrm{ml}$ of either saline (control), CsA (7.5 mg/Kg BW), or FK-506 (0.1 mg/Kg BW). Body weight and glycaemia were regularly recorded. Islets from either group of treated rats were isolated by collagenase digestion of the pancreas. Islet insulin was measured radioimmunologically and DNA fluorimetrically, after reaction with diaminobenzaldehyde.

Results: Rats (groups of 6) treated with CsA and FK-506 developed hyperglycaemia, as compared with control rats (175.5 \pm 12.4 and $159.8 \pm 8.9$, respectively, vs. $100.8 \pm 2.7 \mathrm{mg}$ / $\mathrm{dl} ; \mathrm{p}<0.0002$ and $\mathrm{p}<0.0001$ ), after 7 days and it persisted until day $17(204.5 \pm 28.1$ and $153.2 \pm 8.4$, vs. $97.3 \pm 2.1 \mathrm{mg} / \mathrm{dl}$; $\mathrm{p}<0.004$ and $\mathrm{p}<0.0001)$ in which the immunosuppressant treatment was withdrawn. Ten days later, the glycaemia started to return towards normality $(112.0 \pm 5.5$ and $103.8 \pm 2.9$, for CsA and FK-506, respectively, vs. $92.2 \pm 1.3 \mathrm{mg} / \mathrm{dl}$ in the controls; $p<0.006$ and $p<0.005$ ). The pancreatic insulin content was reduced by either CsA and FK-506 after 17 days of treatment $(40.0 \pm 3.6$ and $54.09 \pm 2.2$, respectively, vs. $104.6 \pm 8.3 \mathrm{ng} / \mathrm{mg}$ pancreas; $\mathrm{p}<0.001$ in both cases) but these differences were completely abolished 14 days after the withdrawal of treatment $(111.0 \pm 9.4$ and $104.3 \pm 10.8$, vs. $120.1 \pm 7.2 \mathrm{ng} / \mathrm{mg}$ pancreas). The immunossupressants retarded significantly (FK-506) or completely suppressed (CsA) the increase of body weight during the treatment period. Islets isolated from rats treated for three days with CsA or FK-506 did not show any modification of their insulin and DNA content, nor of their secretory response to $20 \mathrm{mM}$ glucose. However, in vivo treatment with CsA for 5 days strongly decreased the islet insulin content $(75.9 \pm 8.6, \mathrm{n}=6$, vs. $231.2 \pm 9.9 \mathrm{ng} /$ islet, $\mathrm{n}=6 ; \mathrm{p}<0.001)$ as well as the DNA content $(16.8 \pm 1.4, \mathrm{n}=4$, vs. $24.2 \pm 1.2 \mathrm{ng} /$ islet, $\mathrm{n}=5 ; \mathrm{p}<0.005)$ and the secretory response to $20 \mathrm{mM}$ glucose $(10.2 \pm 1.6, \mathrm{n}=6$, vs. $24.0 \pm 1.5 \mathrm{pg} /$ islet $\mathrm{x} \min , \mathrm{n}=6 ; \mathrm{p}<0.001)$. By contrast, 5 days of treatment with FK-506 did modify neither the DNA content nor the secretory response to $20 \mathrm{mM}$ glucose of islets but reduced their insulin content $(143.2 \pm 12.4, n=6$, vs. $231.2 \pm 9.9 \mathrm{ng} /$ islet, $\mathrm{n}=6 ; \mathrm{p}<0.001$ ). After 7 days of treatment with FK-506, the islet content of insulin was similarly suppressed within $50 \%(128.7 \pm 12.7, \mathrm{n}=6$,vs. $274.1 \pm 31.5 \mathrm{ng} /$ islet, $\mathrm{n}=6 ; \mathrm{p}<0.003$ ) but the islet insulin response to $20 \mathrm{mM}$ glucose was now diminished by more than $75 \%(8.0 \pm 2.2$, $\mathrm{n}=6$, vs. $33.9 \pm 6.0 \mathrm{pg} /$ islet $\mathrm{x} \min , \mathrm{n}=6$; $\mathrm{p}<0.01$ ).

Conclusion: Prolonged treatment of rats with CsA and FK506 induces hyperglycaemia and reduced insulin content of the islets, mimicking the post-transplant diabetes observed in patients. This "diabetic" state is easily reversible shortly after immunosuppressant withdrawal. The observed decrease of the islet insulin content is probably responsible of the diminished insulin response to glucose. We are currently investigating whether the reduction of islet insulin content might be attributed to a decrease of rat insulin genes' transcription and whether apoptosis is also contributing with a decreased $\beta$-cell mass.

Supported by Astellas Pharma S.A., Amgen S.A.

\section{PS 21}

\section{Immunopathogenesis of type 1 diabetes}

0447

The frequency and function of $\mathrm{CD} 4+\mathrm{CD} 25+\mathrm{T}$ regulatory cells in the diabetic initiation and progression of nonobese diabetic mice

M. Zhang, C. Liu, S. H. Xu, Y. Xu, C. P. Liu, X. D. Mao; Endocrinology, First Affiliated Hospital of Nanjing Medical University, Jiangsu, China.

Background and Aims: Type 1 diabetes is an autoimmune disease caused by selective destruction of islet beta cells in the pancreas mediated by pathogenic T cells. Some studies have found $\mathrm{CD} 4{ }^{+} \mathrm{CD} 25^{+} \mathrm{T}$ regulatory cells $\left(\mathrm{CD} 4^{+} \mathrm{CD} 25^{+}\right.$Treg cells) plays an important role in preventing the induction of several autoimmune diseases. Our studies investigated the frequency and function of $\mathrm{CD} 4{ }^{+} \mathrm{CD} 25^{+}$Treg cells in the diabetic initiation and progression of nonobose diabetic mice. Materials and Methods: Early-preclinical-stages(4W) female NOD mice were kept under specific pathogen-free conditions. The number of $\mathrm{CD} 4{ }^{+} \mathrm{CD} 25^{+}$Treg cells was observed per two weeks from 4 to 32 weeks of age. Cells from peripheral blood were stained with TC-CD3,FITCCD4 and PE-CD25 antibodys. Production of intracellular cytokine IL-4 and IFN- $\gamma$ were measured with flow cytometry. To assess their funtion, $\mathrm{CD} 4^{+} \mathrm{CD} 25^{+}$Treg cells were cocultured with $\mathrm{CD} 4^{+} \mathrm{CD} 25^{-} \mathrm{T}$ cells at 32 weeks of age. The expressions of Foxp3 were measured by RT-PCR. Pancreata were prepared for the histology. Insulitis severity were scored by hematoxylin and eosin.

Results: 1. The frequencies of $\mathrm{CD} 4{ }^{+} \mathrm{CD} 25^{+}$Treg cells of female NOD mice were progressively lower from 4 to 32 wekks of age $(\mathrm{p}<0.05)$. 
2. The numbers of $\mathrm{CD} 4^{+} \mathrm{CD} 25^{+}$Treg cells of diabetic NOD mice of 32-week-old was significantly lower than nondiabetic ones $(\mathrm{p}=0.01)$, and with increased secretion of IFN- $\gamma$ and decreased IL-4 production.

3. The suppressor function of the $\mathrm{CD} 4{ }^{+} \mathrm{CD} 25^{+}$Treg cells in vitro cocultures was reduced which was similar between diabetic and nondiabetic mice.

4. The insulitis of diabetic mice with $\geq 50 \%$ of infiltration of the islets at 32 weeks of age was more severe than nondiabetic ones.

Conclusion: 1. The progression to overt diabtes correlates with the age-dependent decrease in the number of and deficiency in the function of the $\mathrm{CD} 4{ }^{+} \mathrm{CD} 25^{+}$Treg cells, which cannot maintain the tolerance to islet autoantigens effectively. 2. This study give a direct and strong evidence to support that $\mathrm{CD}^{+} \mathrm{CD} 25^{+}$Treg cells contribute to the development of autoimmune diabetes. It maybe give us a new method to screen and treat the type 1 diabetes by Treg therapy. Supported by grant from Jiangsu Province Goverment

\section{8}

Insulitis severity and pancreatic expression of pathogenic lymphocytes markers CXCR3 and its chemokine ligands are reduced in probiotic treated NOD mice and is inversely correlated with IL-10 production

V. Bonato ${ }^{1}$, S. Dionisi ${ }^{2}$, M. Marinaro ${ }^{2}$, F. Vendrame ${ }^{1}$, C. Fondelli ${ }^{1}$, M. Boirivant ${ }^{2}$, F. Dotta ${ }^{1}$;

${ }^{1}$ Department of Internal Medicine and Endocrine Sciences, University of Siena, ${ }^{2}$ Infectious, Parasitic and Immunemediated Disease, Istituto Superiore di Sanità, Rome, Italy.

Background and Aims: In a previous study we have demonstrated that, in the NOD mouse, oral probiotic administration, via an immune-modulation of the gut associated lymphoid tissue (GALT) prevented autoimmune diabetes, reduced insulitis severity, and induced an upregulation of IL-10 production by lymphocytes in Peyer's Patches, spleen and pancreas. Recent studies focused on the role of proinflammatory chemokines in the pathogenesis of autoimmune disease. In particular in type 1 diabetes, it has been demonstrated the pathogenetic role of $\mathrm{CXCR}^{+}$ autoreactive lymphocytes, recruited in the pancreatic islet by IP-10/CXCL10 and MIG/CXCL9, secreted by islet cells. We aimed to further characterize the mechanisms of diabetes prevention induced by VSL\#3 probiotic compound administration.

Materials and Methods: Four weeks old, female NOD mice $(\mathrm{n}=18)$ were randomly divided into 2 groups to receive orally VSL\#3 or PBS, 3 times/week, for a total of 8 weeks. At 12 weeks of age the animals were sacrificed and pancreata were collected. IL-10, IFN- $\gamma$, TNF- $\alpha$, CXCL9, CXCL10 and CXCR3 mRNA expression was evaluated by Real Time PCR.
Histological and immuno-histochemical studies were performed to characterize the insulitis.

Results: In the VSL\#3 treated mice, a significant reduction of the insulitis score $(p<0.03)$ has been observed, together with an up-regulation of IL-10 expression $(p<0.0005)$, and a reduced expression of proinflammatory cytokines, IFN- $\gamma$ $(p<0.03)$ and TNF- $\alpha(p<0.05)$, and of proinflammatory chemokines, IP-10 $(p<0.0005)$ and MIG $(p<0.0005)$ and of their receptor, CXCR3 $(\mathrm{p}<0.0005)$. IP-10 was expressed by islet cells, as shown by immuno-histochemical staining. Confirming the protective role of IL-10, IL-10 pancreatic expression was inversely correlated with both insulitis severity $(p<0.02)$, as well as with the expression of the lymphocytes marker, CXCR3 $(\mathrm{p}<0.0005)$. As expected, a positive correlation existed between the pancreatic expression of the receptor CXCR3 and its ligands IP-10 $(\mathrm{p}<0.0001)$ and MIG $(\mathrm{p}<0.0001)$.

Conclusions: This study indicates that diabetes prevention induced by oral VSL\#3 administration is associated with local reduction of $\beta$-cell expression of proinflammatory chemokines and, as a consequence, reduced islet infiltration by pathogenic $\mathrm{CXCR}^{+}$lymphocytes as well as increased production of IL-10.

Supported by Italian Ministry of Health

\section{9}

Gimap/GTPase family is highly expressed in the lymphoid organs of the Bio-Breeding rat

D. H. Moralejo ${ }^{1}$, E. Rutledge ${ }^{2}$, B. Van Yserloo $^{2}$, J. Fuller ${ }^{2}$, R. Jensen ${ }^{3}$, J. Hoehna ${ }^{2}$, A. Lernmark ${ }^{2}$; ${ }^{1}$ Comparative Medicine, ${ }^{2}$ Medicine, ${ }^{3}$ Epidemiology, University of Washington, Seattle, United States.

Positional cloning of the lymphopenia (lyp) gene in diabetes prone (DP) Bio-breeding (BB) rats revealed a frameshift mutation in the Gimap5 (GTPase of immunity associated protein)(formerly called Ian5) a member of at least six other related genes comprising the Gimap family expanded in $150 \mathrm{~kb}$ on rat chromosome $4 \mathrm{q} 24$. To characterize this gene family and understand how it may function in lymphopenia and diabetes development, we cloned and sequenced each of the seven rat Gimap genes from our congenic BBDR.lyp line, both from the $+/+$ and the lyp/lyp rats. Several amino acid differences in this gene family were found between $+/+$ and lyp/lyp rats. In addition to the mutation in lyp/lyp rats in Gimap5 that causes a frameshift and premature truncation of the protein, Gimap1 from lyp/lyp rats has a threonine at position 251 instead methionine, and Gimap4 from lyp/lyp rats has a frameshift of three amino acids from the $\mathrm{C}$ terminus that results in a protein that is 18 amino acids longer than in the+/+rats. RNA expression analyses by quantitative real- 
time PCR, from each of these genes revealed unexpectedly, that in spleen and mesenteric lymph nodes the T cell subsets in each of the seven Gimap genes were reduced in the lyp/lyp compared with $+/+$ rats, even though only the mutation in Gimap5 is linked to lymphopenia and diabetes. To characterize further the specific cell types that show this coordinate regulation, we analyzed the Gimap gene expression in the thymus, spleen and mesenteric lymph nodes in sorted T CD4 ,+ CD $8+$ cell subsets and the T $(\mathrm{CD} 45+\mathrm{CD} 45 \mathrm{RA}-)$ and the $\mathrm{B}$ (CD45+CD45RA+) cell subsets in peripheral lymphoid organs. The entire Gimap family was found to be downregulated in the periphery of these cell subsets of the lyp/lyp compared with $+/+$ rats. Our data suggest that RNA Gimap gene family expression is controlled by the absence of the Gimap5 protein in the BB rat.

Supported by $A D A$ and $N I H$

0450

Introgression of F344 rat genomic DNA on BB rat chromosome 4 generates diabetes-resistant lymphopenic BB rats

J. M. Fuller ${ }^{1}$, A. E. Kwitek ${ }^{2}$, T. Hawkins ${ }^{1}$, D. Moralejo ${ }^{3}$, W. Lu ${ }^{1}$, T. Tupling ${ }^{1}$, S. Speros ${ }^{1}$, A. MacMurray ${ }^{1}$, G. Borchardt ${ }^{2}$, M. Hasinoff ${ }^{2}$, A. Lernmark ${ }^{1}$;

${ }^{1}$ Medicine, University of Washington, Seattle, United States, ${ }^{2}$ Physiology, Medical College of Wisconsin, Milwaukee, United States, ${ }^{3}$ Comparative Medicine, University of Washington, Seattle, United States.

Background and Aims: Failure to express the Gimap5 protein is associated with lymphopenia (lyp) and linked to spontaneous diabetes in diabetes prone (DP) BB rats. Gimap5 is a member of seven related genes located within $150 \mathrm{~Kb}$ on rat chromosome 4. All parental congenic DR.lyp/ lyp $\mathrm{BB}$ rats develop diabetes between 46-81 days of age (mean 61) while DR.lyp/+and+/+rats are non-lymphopenic and diabetes resistant (DR). In an intercross between F1(DR. lyp/lyp $\mathrm{x}$ F344) rats we identified a rat with a recombination event on chromosome 4, allowing us to fix 33Mb of F344 between D4Rat253 and D4Rhw6 in the congenic DR.lyp/lyp rats. Gimapl and Gimap5 were the only members of the Gimap family remaining lyp/lyp.

Our aim was to test whether the F344 introgression proximal to Gimap5 resulted in 1) no effect on diabetes development or 2) protection from diabetes. The first outcome would underscore lymphopenia and the Gimap5 mutation as a diabetogenic factor. The alternative outcome would identify a diabetogenic factor different from lymphopenia.

Materials and Methods: We secured introgression of the F344 recombination onto the congenic DR.lyp rat through a series of marker assisted crosses, intercrosses and backcrosses.
Results: Offspring homozygous F344 (f/f) for the recombination were lymphopenic $(85 / 85 ; 100 \%)$ but did not develop diabetes $(0 / 85)$. During rescue of the recombination, $(102 / 163 ; 63 \%)$ rats heterozygous $(b / f)$ for the recombination developed diabetes between 52-222 days of age (mean 88). In silico analysis confirms that the previously reported $I d d m 4$ locus is present within the $33 \mathrm{Mb}$ F344 DNA fragment.

Conclusion: This data demonstrates that introgression of F344 DNA proximal to the mutated Gimap5 gene renders the rat diabetes resistant despite being lymphopenic. Our data suggest that spontaneous diabetes in the BB rat is controlled, in part, by a diabetogenic factor(s) unrelated to the Gimap5 mutation on rat chromosome 4 . The molecular identification of this genetic factor(s) should prove critical to disclose the mechanisms by which diabetes develops in the BB rat.

Supported by NIH, JDRF

0451

The mutation leading to T1DM and altered T-cell content in LEW.1AR1-iddm rats is located at the telomeric end of chromosome 1

H. Weiss ${ }^{1,2}$, T. Arndt ${ }^{2}$, M. Tiedge ${ }^{1}$, S. Lenzen ${ }^{2}$, H.-J. Hedrich ${ }^{3}$, D. Wedekind ${ }^{3}$;

${ }^{1}$ University of Rostock, Institute of Medical Biochemistry and Molecular Biology, ${ }^{2}$ Hannover Medical School, Institute of Clinical Biochemistry, ${ }^{3}$ Hannover Medical School, Institute of Laboratory Animal Science, Hannover, Germany.

Background and Aims: The LEW.1AR1-iddm rat is an animal model of human Type 1 diabetes mellitus (T1DM), arose through a spontaneous mutation within the inbred strain LEW.1AR1. Genetic analyses using a (BN $x$ LEW.1AR1-iddm) x LEW.1AR1-iddm backcross population revealed three diabetes susceptibility loci (Iddm1, 8, 9). Additionally, we observed an altered T-cell content in peripheral blood lymphocytes (PBL) within the LEW.1AR1-iddm population in comparison to that of the coisogenic background strain. The mode of inheritance of this deviant phenotype is autosomal recessive. It was the aim of the study, (a) to investigate a different backcross population [(PAR x LEW.1AR1-iddm) x LEW.1AR1-iddm] to narrow the susceptibility regions and (b) to identify the gene locus responsible for the altered T-cell content.

Materials and Methods: T-cell content in PBLs (peripheral blood lymphocytes) was analysed using FACSTM analyses with monoclonal antibodies. 61 microsatellite markers were used for linkage analyses of 130 (PAR x LEW.1AR1-iddm) x LEW.1AR1-iddm backcross animals.

Results: Flow cytometry showed a significantly lower CD3 ${ }^{+}$ T-cell content in PBLs of diabetic PAR backcross animals in comparison to the LEW.1AR1 background strain $(39.6 \pm 1.28$ 
vs. 68.9 \pm 1.04$)$. This result approves the previous findings within the BN backcross. Linkage analyses of the (PAR x LEW.1AR1-iddm) x LEW.1AR1-iddm backcross population showed in opposite to the BN backcross (Iddml, 8,9) only two susceptibility loci, one on chromosome (RNO) 1 $(\operatorname{Iddm} 8)$ and one in the MHC region on RNO20 (Iddml) responsible for the diabetes disease. $\operatorname{Id} d m 9$ was missing in the PAR backcross. In accordance with previous findings, the locus responsible for alteration of the T-cell content within the PAR backcross population was also located at the distal end of RNO1 in immediate vicinity to $\operatorname{Iddm} 8$ (LOD>6) flanked by D1Ztm19 and D1Ztm17 (213Mb).

Conclusion: The findings of the linkage analyses show that the mutation of the LEW.1AR1-iddm rat maps within the $I d d m 8$ region at the telomeric end of RNO1. Additionally, this mutation is also responsible for alterations in the T-cell content of PBLs, which may also contribute to diabetes development. Interestingly, the centromeric part of $\operatorname{Iddm} 8$ (RNO1q42-43, D1Rat295 - D1Rat496) is homologues to HSA11q13 containing IDDM4. Thus, alterations of peripheral T-cell content are linked to the $\operatorname{Iddm} 8$ locus and may allow the elucidation of the network of genetic and immunological factors responsible for disease manifestation in this T1DM model.

\section{2}

Islet extracellular matrix stimulates inflammatory mediator secretion by macrophages

M. Armanet, A. Wojtusciszyn, P. Morel,

R. Baertschiger, T. Berney, D. Bosco;

Surgery, Geneva University Hospitals, Cell Isolation and Transplantation Center, Switzerland.

Background and Aims: Macrophages play a critical role in the inflammatory responses occurring after islet transplantation as well as in the onset of type I diabetes. Whether extracellular matrix (ECM) in islets affects inflammatory processes mediated by macrophages is still unknown. Laminin-5 gamma 2 subunit was detected by western-blotting and immunochemistry in islets. The aims of this study are i) to investigate whether laminin-5 (LN-5) is able to activate macrophages and ii) to elucidate the signaling pathways involved in this process.

Material and Methods: As source of LN-5, we used a matrix produced by a rat bladder carcinoma cell line that also contains fibronectin (804G matrix). Rat peritoneal macrophages were harvested after intraperitoneal injection of soluble starch and seeded either on non adherent plastic, collagen, fibronectin, 804G matrix or fibronectin-depleted $804 \mathrm{G}$ matrix. After a $24 \mathrm{~h}$-incubation in presence or absence of $1 \mathrm{microg} / \mathrm{ml}$ phorbol myristate acetate (PMA), we measured by ELISA TNF-alpha, IL-6 and nitrite (NO2-) secreted by macrophages. Activation of p38 MAPK, ERK1/ 2 MAPK, and FAK was analysed by western-blotting.

Results: We confirmed by immunofluorescence and western blotting that laminin-5 gamma 2 subunit is expressed in islets and is localized inalphacells. In absence ofPMA, macrophages cultured on $804 \mathrm{G}$ showed an increased secretion of TNF-alpha and NO2- compared to plastic, collagen and fibronectin. In presence of PMA, $804 \mathrm{G}$ matrix potentiated the secretion of all inflammatory mediators studied compared to plastic, collagen and fibronectin. Similar effects on TNF-alpha, IL-6 and NO2secretion were observed using fibronectin-depleted $804 \mathrm{G}$ matrix. After 30 min-incubation in the absence or presence of PMA, macrophages attached on $804 \mathrm{G}$ matrix showed an increased phosphorylation of 38 MAPK and ERK1/2 MAPK when compared to non adherent plastic. By contrast, phosphorylation of FAK was reduced under the same conditions.

Conclusion: LN-5 rich 804G matrix potentiated secretion of inflammatory mediators by peritoneal macrophages. These effects are likely to be mediated by activation of p38 MAPK and ERK1/2 MAPK.

Supported by the Swiss National Science Foundation

\section{PS 22}

\section{Immunology and prediction of type 1 diabetes}

\section{3}

Type 1 diabetes children without islet autoantibodies at the time of clinical diagnosis are more likely to have islet autoantibodies at birth

M. Elfving ${ }^{1}$, B. Lindberg ${ }^{2}$, K. Lynch $^{3}$, G. Sundkvist ${ }^{3}$, M. Månsson ${ }^{2}$, Å. Lernmark ${ }^{3}$, S. A. Ivarsson ${ }^{2}$;

${ }^{1}$ Pediatric Unit, Clinical Sciences, Lund, ${ }^{2}$ Pediatric Unit,

${ }^{3}$ Unit of Diabetes and Celiac disease, Clinical Sciences, Malmö, Sweden.

Background and Aims: A recent study suggested that fetal exposure to islet autoantibodies in children born to mothers with type 1 diabetes may be protective against future islet autoimmunity and diabetes. The possible induction of immune tolerance to islet autoantigen may reflect that islet autoimmunity is initiated already in utero.

The aim of this study was to investigate islet cell autoantibodies in an unselected population of children with type 1 diabetes, born to non-diabetic mothers, by comparing autoantibodies in their cord blood serum samples with a sample taken at the time of clinical diagnosis.

Materials and Methods: Serum samples at birth and at the time of diagnosis were available from 141 children who developed type 1 diabetes between 1.3-18.8 years of age (median 9.0 years; male/female ratio 83/58). Children born 
to mothers with type 1 diabetes or any other form of diabetes were excluded from the study. The samples were tested for autoantibodies against glutamic acid decarboxylase (GAD65Ab), islet-associated antigen 2 (IA-2Ab), insulin (IAA) by radiobinding assays as well as to islet cell antibodies (ICA) by indirect immunofluorescence

Results: The frequency of islets autoantibodies in the cord blood was 11\% (15/141), (ICA 4\%, GAD65Ab 6\%, IA2-Ab $1 \%$ and IAA $1 \%$ ) compared to $91 \%(129 / 141)$ (ICA $76 \%$, GAD65Ab 61\%, IA2-Ab 69\% and IAA 45\%) at diagnosis. The children who presented fewer islet autoantibodies (Ab's) at diagnosis were more likely to have had autoantibodies in their cord blood sample. Of children with no $\mathrm{Ab}^{\prime} \mathrm{s}$ at diagnosis $25 \%$ had autoantibodies in cord blood, $1 \mathrm{Ab}$ $21 \%, 2 \mathrm{Ab}$ 's $17 \%$ and $>3 \mathrm{Ab}$ 's $5 \%(\mathrm{p}<0.01)$ demonstrating a significant inverse relationship (trend, $\mathrm{p}<0.001$ ). Cord blood autoantibodies were most strongly associated with absence of ICA at diagnosis (OR 6.06 (95\%CI was 1.97-18.60) $\mathrm{p}=0.0016)$ even after adjusting for other islet autoantibodies at diagnosis (OR 4.65 (1.23-17.5).

Conclusion: Our data in type 1 diabetes children born to non-diabetic mothers support the notion that cord blood islet autoimmunity may induce immunological tolerance and explain why some type 1 diabetes children are islet autoantibody negative at the time of clinical diagnosis.

\section{4}

Autoantibodies specific to conformational epitopes of the $65 \mathrm{kDa}$ isoform of glutamate decarboxylase (GAD65) identify different types of autoimmune diabetes

\section{S. Hampe ${ }^{1}$, T. Hall ${ }^{1}$, T. Maruyama ${ }^{2}$;}

${ }^{1}$ Medicine, University of Washington, Seattle, United States, ${ }^{2}$ Internal Medicine, Saitama Social Insurance Hospital, Saitama, Japan.

Background and Aims: Autoimmune diabetes presents itself in different subtypes. Classical type 1 diabetes (T1D) is characterized by young age at onset and rapid progression, while patients with latent autoimmune diabetes in adults (LADA) present a slow onset form of autoimmune diabetes, where patients are initially insulin-independent and often diagnosed with type 2 diabetes, before developing insulin dependency. The autoimmune reaction in both subtypes is reflected in the presence of circulating autoantibodies directed to GAD65 (GAD65Ab). We investigated whether the underlying autoimmune reactions in these subtypes differ. Material and Methods: GAD65Ab-positive samples from Japanese T1D patients $(\mathrm{n}=111)$, and from LADA patients who were still insulin-independent $(\mathrm{n}=86)$, or had already progressed to insulin-dependency (slow onset) $(n=58)$ were analyzed for their GAD65Ab epitope specificity using seven recombinant Fab. These recombinant Fab were derived from human GAD65-specific monoclonal antibodies that recognize different epitopes of the molecule.

Results: We identified significant differences in the GAD65Ab epitope specificities between the three groups. Overall, the T1D patients recognized more epitopes (medi$a n=3)$ than both slow onset (median=2) or LADA patients (median $=2),(\mathrm{P}=0.0003$ and 0.0005 , respectively). In particular, binding to a middle epitope was significantly better recognized by GAD65Ab in T1D patients than in the slow onset $(\mathrm{p}=0.0002)$ or in the LADA patients $(\mathrm{p}<0.0001)$. This preferred binding was also observed for two N-terminal epitopes that were significantly better recognized in T1D patients than in slow onset $(\mathrm{P}=0.009$ and 0.005$)$, or LADA patients $(\mathrm{P}=0.01$ and $<0.0001)$. The same trend $(\mathrm{T} 1 \mathrm{D}>$ slow onset $>$ LADA) was observed for three of the remaining four epitopes, however the differences did not reach significance. While for six of the seven epitopes the slow onset patients recognized the epitope better than the LADA patients, these differences did not reach significance.

Conclusion: The autoimmune reaction in LADA patients differs from that in T1D patients. This is reflected in their GAD65Ab epitope specificities. It will be of interest to investigate whether the epitope pattern in the LADA patients change as they progress towards insulin dependency.

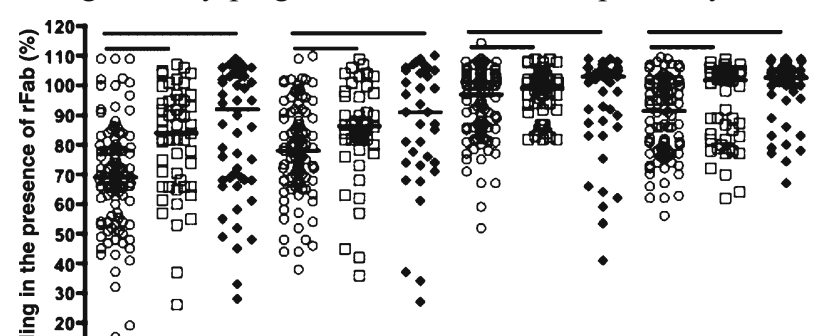

言 20

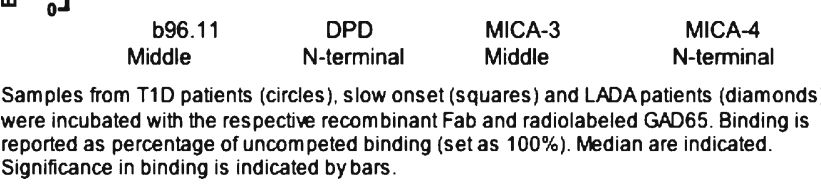

Supported by American Diabetes Association

0455

Comparison of islet cell autoimmunity in Japanese and Caucasian type 1 diabetes patients: Autoantibodies to the $65 \mathrm{kDa}$ isoform of glutamate decarboxylase recognize similar epitopes

R. Ettinger ${ }^{1}$, T. Maruyama ${ }^{2}$, E. Ortqvist ${ }^{3}$, T. Hall ${ }^{4}$, C. S. Hampe ${ }^{4}$;

${ }^{1}$ Department of Medicine, University of Washington, Seattle, United States, ${ }^{2}$ Internal Medicine, Saitama Social Insurance Hospital, Japan, ${ }^{3}$ Pediatric Diabetes Unit, Karolinska Institute, Stockholm, Sweden, ${ }^{4}$ Medicine, University of Washington, Seattle, United States. 
Background and Aims: The incidence of Type 1 diabetes (T1D) in the Japanese population is remarkably lower as compared with that in Caucasian populations. In both populations autoantibodies to the $65 \mathrm{kDa}$ isoform of glutamate decarboxylase (GAD65), insulin, and IA-2 are prevalent at onset of disease. However, the genetic susceptibility conferred by HLA class II alleles differs significantly between the two populations. The HLA class II genes which confer the highest risk in Caucasians (DRB1*0301DQB1*0201 and DRB1*0401-DQB1*0302), are rare in the Japanese population and instead the DRB $1 * 0405$ DQA $1 * 0302-\mathrm{DQB} 1 * 0401$, DRB1*0901-DQA 1*0302DQB1*0303 and DRB1*0802-DQB1*0302 emerge as the major susceptible haplotypes of T1D.

We compared the autoimmune islet cell reaction reflected in GAD65Ab epitope specificities in Japanese and Caucasian T1D patients.

Material and Methods: Japanese $(\mathrm{n}=111)$ and Caucasian $(\mathrm{n}=50)$ GAD65Ab-positive T1D patients were analyzed for their GAD65Ab epitope specificity. The sera were analyzed in a competitive radiobinding assay using six recombinant Fab ( $\mathrm{rFab}$ ) derived from GAD65-specific human monoclonal antibodies. Binding to GAD65Ab epitopes in both groups were compared using the nonparametric Mann Whitney $\mathrm{U}$ test. Binding to GAD65Ab epitopes was also tested for correlations with the HLA class II alleles.

Results: The overall GAD65Ab epitope pattern of Japanese T1D patients was remarkably similar to that observed in Caucasian T1D patients. Patients in both populations showed a strong binding to conformational epitopes located both in the middle and the N-terminus of GAD65, while a significantly lower reaction to a linear C-terminal epitope was observed for both populations. In the Japanese T1D patient groups we observed a significant correlation between the binding to a disease-specific epitope located in the middle of the molecule and the high risk HLA class II allele DRB $1 * 0802$-DQB $1 * 0302(\mathrm{p}=0.0008)$, while binding to this epitope in the Caucasian T1D patients is associated with DRB $1 * 0401 / \mathrm{DQB} 1 * 0302$.

Conclusions: While T1D susceptibility in Caucasian and Japanese populations is associated with different HLA class II alleles, we found that the GAD65Ab epitope specificity between the two populations is remarkably similar. The strong association between a GAD65 Ab epitope specificity that was identified by us earlier to be disease-specific and a high risk HLA class II alleles in both the Japanese and Caucasian populations leads to the speculation that high risk HLA class II alleles may shape GAD65Ab epitope specificities and lead to the generation of disease-specific autoantibodies.

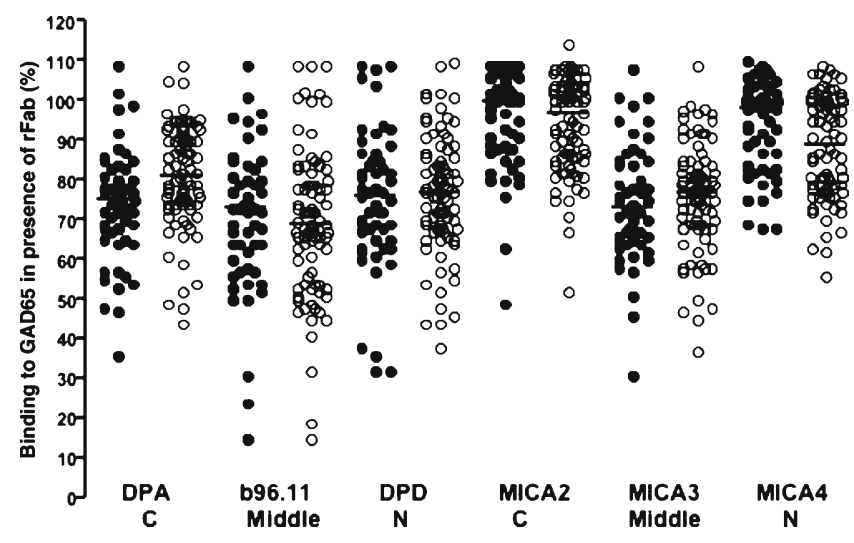

Supported by American Diabetes Association

0456

Detection of islet cellular autoimmunity in Chinese subjects initially diagnosed as idiopathic type 1 diabetes Y. Zhang, Z. Zhou, L. Yang;

Diabetes Center, Central South University, Changsha, Hunan Province, China.

Background and Aims: Idiopathic type 1 diabetes(Type 1B) was defined as islet-associated autoantibody-negativity together with unknown etiologies by ADA criteria. There was obvious heterogeneity in Type $1 \mathrm{~B}$ patients, some of the Chinese patients originally thought to have Type 1B was detected to be autoantibody positive to SOX13 or carboxypeptidase $(\mathrm{CPH})$ and genetic mutation, these patients may have a specific aetiology and should not be classified as Type 1B diabetes. In this study, we tried to demonstrate the existence of islet-associated antigen-specific T cells in Type 1B patients and abnormal T cell-mediated immunity.

Materials and Methods: Peripheral blood samples were obtained from 23 classical type 1diabetes(Type 1A) patients, 29 Type $1 \mathrm{~B}$ patients, 28 Type 2 diabetes(T2DM) and 16 healthy control subjects(Control). The diagnoses of Type 1B, Type 1A diabetes and T2DM were made based on the ADA criteria for diabetes. Islet-associated autoantibodies were determined by radioligand assay. Human peripheral blood mononuclear cells were isolated by Ficoll-Hypaque density gradients, enzyme linked immune spot (ELISPOT) assay was performed to measure the number of spots-forming (median, $75 \% \mathrm{CI}$ ). One spot-forming represents a GAD65-reactive IFN- $\gamma$-secreting T (IFN- $\gamma$-T) cell.

Results: The IFN- $\gamma$-spots number stimulated with GAD65 were 12(10.3 20.9)(median and 95\% CI) in Type 1A group, 3.5(3.0 5.7) in Type 1B group, 2.0(1.3 4.0) in T2DM group 
and 1.0(0.3 1.8) in Control group, respectively. The frequency of spots significantly increased in Type 1B group compared to Control group $(P<0.05)$, and there were significantly more spots number in Type $1 \mathrm{~A}$ group than Type $1 \mathrm{~B}$ or Control group(both $P<0.01$ ), and no significant difference was observed between T2DM and Control group concerning the spot number $(P>0.05)$. A positive response was scored when the spot number $\geq$ mean $+2 \mathrm{SD}$ of Control subjects. In case of Type $1 \mathrm{~B}$ patients 14 of $29(48.3 \%)$ had positive response to GAD65, and 18 of 23(78.3\%)Type $1 \mathrm{~A}$ patients and 1 of 28 (3.6\%)T2DM patient showed positive response. No subjects $(0 \%)$ positive for IFN- $\gamma$-spot were observed in Control group. It implied that approximately half(48.3\%)of the so-called "Type 1B" patients and very few (3.6\%)of T2DM patients were positive for GAD65-reactive IFN- $\gamma$-T cells. Whereas the number of IFN- $\gamma$-spots reactive to phytohemagglutinin (PHA) did not differ among the four groups(data not shown).

Conclusion: GAD65-reactive IFN- $\gamma$-T cells are found in some patients originally diagnosed as Type 1B with autoantibody-negative. This suggests, similar to Type 1A patients, that abnormal $\mathrm{T}$ cell-mediated immunity involved in the underlying aetiology and pathogenesis is also present in some Chinese patients originally diagnosed as Type 1B diabetes. More rigorous screening for these conditions is needed before classifying subjects as having Type 1B diabetes, and IFN- $\gamma$-T cells reaction against GAD65 detected by ELISPOT may contribute to the diagnosis of "autoimmune diabetes mellitus".

Supported by "Trans-Century Training Program Foundation for the Talents"

\section{7}

Markers associated with T-regulatory cells are induced at diagnosis of type 1 diabetes

M. Faresjö ${ }^{1}$, M. Hedman ${ }^{1}$, C. Cilio ${ }^{2}$, C.-O. Jonson ${ }^{1}$, J. Ludvigsson ${ }^{1}$, K. Stechova ${ }^{3}$;

${ }^{1}$ Department of Molecular \& Clinical Medicine, Division of Paediatrics \& Diabetes Research Centre, University of Linköping, Sweden, ${ }^{2}$ Department of Clinical Science/ Paediatrics, Cellular Autoimmunity Unit, University of Lund, Malmö, Sweden, ${ }^{3}$ Department of Paediatrics, Medical Faculty of Charles University, Prague, Czech Republic.

Background: Type 1 diabetes (T1D) is an autoimmune disease associated with a defective regulation of the immune system and imbalance of T-helper (Th) cells. Tregulatory (T-reg) cells are believed to control $\mathrm{T}$ cell reactivity and to maintain self-tolerance of the immune system. We hypothesise that the observed imbalance of subgroups of Th cells can be due to a dysregulation of T-reg cells in high-risk and T1D children.
Methods: Peripheral blood mononuclear cells (PBMC) were collected from T1D children four days post-diagnosis, from children with $35 \%$ risk of developing T1D within five years (ICA $>=20$ IJDF-U) and from healthy children carrying the HLA-risk haplotype DQB1*0302 or DQB1*0201 and DQA $1 * 0501$. Expression of genes associated with T-reg cells (FOXP3, CTLA-4, sCTLA-4 and TGF-beta mRNA) were detected together with endogenous rRNA by multiplex real-time RT-PCR. Secretion of TGFbeta, IL-10 and IFN-gamma were detected by protein micro array and IL-4 by sensitive ELISPOT technique from PBMC stimulated with glutamic acid decarboxylase (GAD65) and phytohaemagglutinin (PHA).

Results: Spontaneous mRNA expression of the T-reg associated genes FOXP3 $(\mathrm{p}<0.05)$, CTLA-4 $(\mathrm{p}<0.05)$ and TGF-beta $(\mathrm{p}<0.05)$ as well as spontaneous secretion of TGF-beta $(p=0.05)$ and IL-10 $(p=0.06)$ was more pronounced in newly diagnosed T1D children compared to high-risk children. Higher spontaneous mRNA expression of FOXP3 $(p=0.01)$ and CTLA-4 $(p<0.01)$ as well as secretion of TGF-beta $(p<0.05)$ was also observed in T1D compared to healthy children.

Secretion of TGF-beta, after in vitro stimulation with GAD65, was higher in T1D children compared to both high-risk $(p<0.05)$ and healthy $(p<0.01)$ children. Further, expression of sCTLA-4 $(\mathrm{p}<0.01)$ and TGF-beta $(\mathrm{p}=0.01)$ mRNA, induced by stimulation with PHA, was higher in T1D compared to high-risk children. PHA-induced mRNA expression of sCTLA-4 $(p<0.01)$, TGF-beta $(p=0.01)$ and FOXP3 $(p<0.05)$ as well as secretion of IL-10 $(p<0.05)$ was found to be low in high-risk compared to healthy children. In contrast, spontaneous secretion of IFN-gamma was less pronounced in newly diagnosed T1D children than in highrisk children $(\mathrm{p}<0.05)$ and secretion of IFN-gamma was inversely correlated to IL-10 secretion in high-risk children $(\mathrm{r}=-0.91, \mathrm{p}<0.05)$.

Conclusion: Increased expression and secretion of markers associated with T-reg cells was more pronounced in newly diagnosed T1D children than in high-risk and healthy children. These results suggest a disturbed interaction of Tcells with regulatory features to suppress the destruction of insulin-producing beta-cells mediated e $\mathrm{g}$ by Th1-like cells. Generously supported by the the Schelins Foundation and the Swedish Child Diabetes Foundation

\section{8}

Several novel characteristics of Treg cells in newlydiagnosed type 1 diabetics and controls: preliminary results

S. Paschou ${ }^{1}$, G. Vartholomatos ${ }^{2}$, L. Dova ${ }^{2}$, N. Kolaitis ${ }^{3}$,

E. Giotaki ${ }^{4}$, A. Tsatsoulis ${ }^{5}$, G. K. Papadopoulos ${ }^{6}$; 
${ }^{1}$ Endocrinology Clinic, ${ }^{2}$ Haematology Laboratory, Molecular Biology Unit, ${ }^{3}$ Haematology Laboratory, University of Ioannina Regional Hospital, Ioannina, Greece, ${ }^{4}$ Department of Nursing, Epirus Institute of Technology, Ioannina, ${ }^{5}$ Endocrinology Clinic, University of Ioannina Medical School, ${ }^{6}$ Laboratory of Biochemistry and Biophysics, Faculty of Agricultural Technology, Epirus Institute of Technology, Arta, Greece.

Background and Aims: One of the immunocyte populations that are pivotal to tolerance and autoimmunity is that of $\mathrm{CD}^{+}$Treg (regulatory cells). Our aim was to delineate the characteristics of Treg cells in newly-diagnosed type 1 diabetics and controls, especially in view of conflicting results in the literature regarding certain cell surface markers of these cells.

Materials and Methods: Peripheral blood from six newlydiagnosed type 1 diabetes patients (ages 1.5 to 35, mean 16) and ten controls (ages 25-45, mean 28) was analysed via triple colour flow cytometry for cell surface and intracellular characteristics of the Treg $\left(\mathrm{CD} 4^{\mathrm{hi}}\right)$, $\mathrm{CD} 4{ }^{+} \mathrm{CD} 25^{+}$and $\mathrm{CD} 4^{+} \mathrm{CD} 25^{-}$populations. The markers analysed for were CD28, CD45RO, CD95 (Fas), CD122 (IL-2R $\beta$ ), CD152 (CTLA-4) (cell surface and intracellular), CD220 (InsR), HLA-DR, HLA-DQ, TGF $\beta$ and TGF $\beta$ RII. The cut-off line between $\mathrm{CD} 25^{\mathrm{hi}}$ and $\mathrm{CD} 25^{+}$cells was either obvious from the CD4-CD25 dot plot, or was decided based on whether the highest expressing population in the latter group conformed to the bulk characteristics of that population or to those of the former population.

Results: Newly-diagnosed type 1 diabetics had a significantly lower percent of Treg compared to normal controls $(1.07+/-0.28$ vs. $2.27+/-0.75)$. All markers tested for were found on the membrane of Tregs from type 1 diabetics as well as normals. However, several of the markers were in a significantly different percent of Tregs from the normal group compared to the same cell type from newlydiagnosed type 1 diabetics (mean+/-SD): CD122 (40.5+/ -20.5 vs. $69.5+/-31.35), \mathrm{CD} 152(87.2+/-14.9$ vs. $78.2+/$ $-21.3)$, CD220 (82.8+/- 15.4 vs. $63.0+/-16.5)$, TGF $\beta$ $(88.7+/-6.4$ vs. $72.3+/-14.3)$, HLA-DR $(59.4+/-15.4$ vs. $74+/-35)$, HLA-DQ $(34.5+/-24.3$ vs. $63.5+/-36.6)$, in Treg cells. It seems that a higher percentage of Treg cells from newly-diagnosed type 1 diabetic patients have on their membrane several known markers of $\mathrm{T}$ cell activation (CD122, HLA-DR, HLA-DQ), but not CD220, compared to Tregs from normals. Furthermore, a lower percent of Treg cells from type 1 diabetic patients show surface markers associated with Treg suppressive activity (CD152, TGF $\beta$ ), and an even lower percent of Tegs from type 1 diabetics show very high expression of these two markers. Conclusion: These preliminary results from a small sample encompassing a wide spectrum of ages of onset, identifies a number of different markers not examined thus far in Tregs in human diabetes (membrane CD220, HLA-DQ, and TGF $\beta$ ), or establishes their presence where conflicting results had been obtained previously (very low percentage of Tregs with membrane CD152). Of these markers, HLADQ, TGF $\beta$ and CD152 have been associated with suppression/regulation of the immune response, and their role in type 1 diabetes pathogenesis requires further investigation. Supported by a grant (to GKP) of the programme ARCHIMEDES of the 3rd EU Framework for regional development in Greece

0459

The prognostic value of the immunological phenotype of lymphocytes in subjects positive for diabetes-associated autoantibodies

V. Popova ${ }^{1}$, K. Zak ${ }^{2}$, M. Gruzov ${ }^{2}$, V. Afanasyeva ${ }^{2}$, T. Simeonova ${ }^{2}$, B. Mankovsky ${ }^{1}$;

${ }^{1}$ Diabetology, Institute of Endocrinology, Kiev, ${ }^{2} \mathrm{Hematol}-$ ogy, Institute of Endocrinology, Kiev, Ukraine.

Background and Aims: The changes of the immunological phenotype of the lymphocytes were documented in the subjects with preclinical stages of type 1 diabetes mellitus. However, the prognostic value of these changes remains unknown. Therefore, the aim of this study was to investigate the immunological phenotype of lymphocytes in subjects positive for diabetes-associated autoantibodies who later developed the clinical type 1 diabetes.

Materials and Methods: We studied 366 normoglycemic children aged 8-15 year old who had first-degree relatives with type 1 diabetes. Fifty nine subjects were positive for autoantibodies against GAD and IA- 2 confirmed by two consecutive determinations by radioimmune assays. The control group consisted of 58 age-matched healthy subjects. The children were prospectively followed for 7 years. The immunological phenotype of lymphocytes was analyzed by fluorescent-activated flow cytometry.

Results: During the follow-up type 1 diabetes mellitus manifested in 29 out of 59 subjects positive for diabetesassociated autoantibodies. In this particular group of patients we found the most significant decrease of the number of $\mathrm{CD} 3+$ lymphocytes $\left(0.59+0.05 .10^{9} / \mathrm{L}\right), \mathrm{CD} 4+$ cells $\left(0.38+0.03 \cdot 10^{9} / \mathrm{L}\right)$ and ratio of CD4+/CD8+ lymphocytes $(1.42+0.06)$ compared to the entire group of subjects positive for diabetes-associated autoantibodies $(0.79$ $+0.05 .10^{9} / \mathrm{L} ; 0.52+0.03 .10^{9} / \mathrm{L}$, and $1.73+0.05$ for $\mathrm{CD} 3+$, $\mathrm{CD} 4+$, cells $\mathrm{CD} 4+/ \mathrm{CD} 8+$ ratio, respectively, $\mathrm{p}<0.05$ ) and compared to children negative for diabetes-associated autoantibodies $\left(1.02+0.06 \cdot 10^{9} / \mathrm{L} ; 0.67+0.04 \cdot 10^{9} / \mathrm{L}\right.$, and $1.82+0.04$ for $\mathrm{CD} 3+, \mathrm{CD} 4+$, cells $\mathrm{CD} 4+/ \mathrm{CD} 8+$ ratio, re- 
spectively, $\mathrm{p}<0.05)$ and healthy subjects $\left(1.39+0.09 \cdot 10^{9} / \mathrm{L}\right.$; $0.98+0.04 \cdot 10^{9} / \mathrm{L}$, and $1.98+0.10$ for $\mathrm{CD} 3+, \mathrm{CD} 4+$, cells $\mathrm{CD} 4+/ \mathrm{CD} 8+$ ratio, respectively, $\mathrm{p}<0.05$ ). The more decreased number of the lymphocytes measured the shorter was the term till the development of the clinical type 1 diabetes. The changes of CD56+between the groups were not statistically significant.

Conclusion: The determination of the immunological phenotype of the lymphocytes could have a prognostic value predicting the development of clinical type 1 diabetes mellitus in the predisposed subjects.

\section{0}

Different islet cell antibody titer at onset of type 1 diabetes: an association with changes in $\mathrm{CD} 4+\mathrm{T}$ cells subsets and clinical course of disease

A. Jotic ${ }^{1}$, N. Lalic ${ }^{1}$, T. Milicic ${ }^{1}$, M. Lukic ${ }^{1}$, I. Markovic ${ }^{2}$, B. Djuricic ${ }^{2}$, M. Zamaklar ${ }^{1}$, K. Lalic ${ }^{1}$, L. Lukic ${ }^{1}$,

N. Rajkovic ${ }^{1}$, M. Bukelica ${ }^{1}$;

${ }^{1}$ Diabetes Center, Institute for Endocrinology, Belgrade,

${ }^{2}$ Institute for Biochemistry, School of Medicine, Belgrade, Serbia and Montenegro.

Background and Aims: The predictive value of islet cell antibody (ICA) levels at onset of Type 1 diabetes (T1D) in respect to the clinical course of the disease and the changes in $\mathrm{T}$ cell subsets has not yet been elucidated. Therefore, the aim of this study was to compare the two groups of recentonset T1D showing (1) high ICA titer ( $\geq 80$ Juvenile Diabetes Foundation (JDF) units) (group A, N=33) and (2) low ICA titer ( $\leq 40$ JDF $\mathrm{U}$ ) (group $\mathrm{B}, \mathrm{N}=34$ ) at onset of the disease regarding the following parameters: (a) duration of clinical remission (CR) as a non-insulin requiring state during the clinical course of disease and (b) the changes in regulatory $\mathrm{T}$ cell subsets, memory $(\mathrm{CD} 45 \mathrm{R} 0+)$, naive $(\mathrm{CD} 45 \mathrm{RA}+)$ and suppressive $(\mathrm{CD} 25+) \mathrm{CD} 4+\mathrm{T}$ cells, tested both at the onset in insulin requiring state and in the state of $\mathrm{CR}$

Materials and Methods: ICA levels were determined by indirect immunofluorescence on human pancreatic tissue sections and expressed in Juvenile Diabetes Foundation (JDF) units. The percentages of the CD4+T cell subsets were analysed by using two-color immunofluorescence staining and flowcytometry. CR was defined as optimal metabolic control without insulin lasting $>30$ days.

Results: We found that in group A, the duration of CR was significantly shorter in group A than in group B (A:189 \pm 23 vs $B: 407 \pm 25$ days; $\mathrm{p}<0.05)$. Simultaneously, when the analysis of $\mathrm{CD} 4+\mathrm{T}$ cells was concerned, although there was no difference between the groups at onset of disease (CD45R0+:37.1+/-2.2 vs 36.8+/-3.3\%, CD45RA+: 23.2 $+/-2.2$ vs $24.9+/-2.0 \%, \mathrm{CD} 4+\mathrm{CD} 25+: 2.37 \pm 0.27$ vs
$2.40 \pm 0.31, \mathrm{p}=\mathrm{NS}$ ), in the state of CR the percentage of $\mathrm{CD} 4+\mathrm{CD} 45 \mathrm{R} 0+\mathrm{T}$ cells significantly decreased in group B and it became lower compared to group A (A:28.8+/-2.1 vs $\mathrm{B}: 24.0+/-2.0 \%, \mathrm{p}<0.05)$. Moreover, in the state of $\mathrm{CR}$, both the percentage of CD4+CD45RA+and CD4+CD25+T cells increased in group $\mathrm{B}$ and it became significantly higher than to group A (CD45RA+: A:20.5+/-1.7 vs B:27.9+/-1.4, CD25+: A:2.79+/- 0.30 vs B:3.82+/-0.44, $\mathrm{p}<0.05)$.

Conclusion: Our results have demonstrated that patients with recent-onset Type 1 diabetes and low ICA titer at onset of the disease have shown longer duration of CR accompanied with the increase in regulatory $\mathrm{T}$ cell subsets, naive $(\mathrm{CD} 45 \mathrm{RA}+)$ and suppressive $(\mathrm{CD} 25+)$, together with the decrease in memory $(\mathrm{CD} 45 \mathrm{R} 0+) \mathrm{CD} 4+\mathrm{T}$ cells in comparison to the patients with initially high ICA titer showing shorter CR. The results imply that the ICA levels at onset of recent-onset T1D might represent a useful predictive marker regarding the forthcoming duration of $\mathrm{CR}$ and the changes of regulatory $\mathrm{T}$ cell subsets.

\section{1}

Relation of serum cytokine levels and chemokine levels with $\mathrm{HbA}_{1 \mathrm{c}}$, C-peptide and proinsulin in patients with newly diagnosed type 1 diabetes mellitus. Results from the Hvidøre Study Group

C. Pfleger ${ }^{1}$, P. Hougaard ${ }^{2}$, L. Hansen ${ }^{3}$, R. Holl ${ }^{4}$, H. Kolb ${ }^{1}$, H. B. Mortensen ${ }^{5}$, N. C. Schloot ${ }^{1}$;

${ }^{1}$ Heinrich-Heine University, German Diabetes Clinic, Düsseldorf, Germany, ${ }^{2}$ University of Southern Denmark, Odense, Statistical Department, Denmark, ${ }^{3}$ Novo Nordisk A/S, Bagsværd, Denmark, ${ }^{4}$ University, Paediatrics, Ulm, Germany, ${ }^{5}$ University Hospital, Paediatrics Glostrup University Hospital, Glostrup, Denmark.

Background and Aims: Diabetes mellitus type 1 results from immune-mediated destruction of $\beta$-cells and is accompanied by altered immune responses. The course of disease after manifestation is characterized by various metabolic parameters. Cytokines and chemokines are thought to play an important role in the pathogenesis of type 1 diabetes and for $\beta$-cell function. The aim of the current study was to investigate circulating levels of the classical cytokines IFN $\gamma$, IL10 and $\mathrm{TNF} \alpha$ and the chemokines MIP-1 $\beta$ and RANTES regarding metabolic parameters 1,6 and 12 months after diagnosis in children and adolescents with newly diagnosed type 1 diabetes. Materials and Methods: Serum was obtained from 256 newly diagnosed patients with type 1 diabetes (122 males/ 134 females, aged 9.6 years, range $0.2-16.8$ years) recruited from 18 paediatric centers of the Hvidoere study group. HbA1c, stimulated C-peptide and proinsulin (Boost test) were determined by high pressure liquid chromatography 
(HbA1c), AutoDELFIA -fluoroimmunoassay (C-peptide) and Sandwich ELISA method (proinsulin). Serum cytokines and chemokines were measured by double sandwich ELISA and multiplex technology. Data were analysed by multiple regression analysis including gender, age, IFN $\gamma$, IL10, TNF $\alpha$ in one analysis and CCL4/MIP1 $\beta$, CCL5/RANTES in a separate analysis as co-variates and non parametric Wilcoxon test and Spearman's correlation analysis. All statistical analyses were performed using SAS version 8.6.

Results: One month after diabetes onset, $\mathrm{HbAlc}$ associated positively with CCL5/RANTES $(\mathrm{p}<0.0001)$ a similar trend was observed with $\operatorname{IFN} \gamma(\mathrm{p}=0.06)$. C-peptide levels related negatively with CCL4/MIP1 $\beta \quad(p=0.037)$ but not with cytokines. During follow up, HbAlc was not related to chemokines analysed but did relate to $\operatorname{IFN} \gamma(\mathrm{p}=0.048)$, IL$10(\mathrm{p}=0.041)$ and $\mathrm{TNF} \alpha(\mathrm{p}=0.037)$ at 12 months.

C-peptide associated positively with IFN $\gamma$ at $6(\mathrm{p}=0.06)$ and 12 months $(\mathrm{p}=0.04)$ and negatively with CCL4 $(\mathrm{p}=0.018)$. Proinsulin associated negatively with CCL4/MIP1 $\beta$ but not with cytokines. Prediction models for residual $\beta$-cell function ( $\mathrm{HbAlc}$, C-peptide, proinsulin) showed no association of cytokines but several associations with CCL4/ MIP1 $\beta$ and CCL5/RANTES (reported previously).

Conclusion: The present study shows a relation of IFN $\gamma$ and IL10 but not of TNF $\alpha$ with metabolic parameters 1, 6 and 12 months after disease onset. Chemokines did relate to metabolic parameters and were predictive of later C-peptide and proinsulin levels. From the cytokines tested, IL10 and IFN $\gamma$ correlated with other cytokines/chemokines but not with each other, demonstrating a complex regulation of the immune response possibly related to metabolic parameters and residual beta-cell function.

Supported by Stiftung "Das zuckerkranke Kind"

0462

Association of immune mediators at diagnosis of type 1 diabetes mellitus with later clinical remission

N. C. Schloot ${ }^{1}$, P. Hanifi-Moghaddam ${ }^{1}$,

N. Aabenhus-Andersen ${ }^{2}$, B. Z. Alizadeh ${ }^{3}$, M.-T. Saha ${ }^{4}$,

M. Knip ${ }^{5}$, D. Devendra ${ }^{6}$, T. Wilkin ${ }^{6}$, E. Bonifacio ${ }^{7}$,

B. O. Roep ${ }^{3}$, H. Kolb ${ }^{1}$, T. Mandrup-Poulsen ${ }^{8}$;

${ }^{1}$ Heinrich-Heine University, German Diabetes Clinic, Düsseldorf, Germany, ${ }^{2}$ Steno Diabetes Center, Gentofte, Denmark, ${ }^{3}$ University Medical Center, Dept. Immunohaematology \& Blood Transfusion, Leiden, The Netherlands, ${ }^{4}$ University of Tampere, Dept. of Pediatrics, ${ }^{5}$ University of Helsinki, Hospital for Children and Adolescents, Finland, ${ }^{6}$ Peninsula Medical School, Peninsula Medical School, Plymouth, United Kingdom, ${ }^{7}$ Istituto Scientifico San Raffaele, Telethon-JDRF Center for Beta Cell Replacement, Milan, Italy, ${ }^{8}$ Steno Diabetes Center, Gentofte, Denmark.
Background and Aims: We tested the hypothesis that systemic concentrations of cytokines, chemokines or soluble cytokine receptors predict or accompany (partial) clinical remission in type 1 diabetes (T1D).

Materials and Methods: In a prospective, multi-center study 48 patients with newly diagnosed T1D, and 55 agematched healthy control subjects were investigated. Blood was initially drawn 3-7 days after the diagnosis and then 34 months later. Partial clinical remission was defined by centrally measured $\mathrm{HbA} 1 \mathrm{c}$ with a maximum of $7.5 \%$ or below and daily insulin dose of maximum of $0.38 \mathrm{IU} / \mathrm{kg}$ or below. Systemic concentrations of 17 immune mediators were analyzed in serum or plasma. In addition, autoantibodies against insulin (IAA), IA-2 (IA-2A) and GAD65 (GADA) were quantified.

Results: All 17 immune mediators showed remarkable intraindividual stability in their systemic concentrations over time. As a consequence, remission was not accompanied by changes in mediator levels except for a moderate decrease of IL-1ra concentrations $(\mathrm{p}=0.02)$ and IL-10 concentrations $(\mathrm{p}=0.01)$ in non-remitters. Low $\operatorname{IFN} \gamma(\mathrm{p}=0.01)$, low IL-10 $(p=0.03)$ and low IL-R1 $(p=0.009)$ concentrations were associated with later remission. Lower autoantibody titers were also predictive of remission $(\mathrm{p}=0.007)$.

Conclusion: We conclude that the systemic immunoregulatory state at diagnosis of T1D is predictive of later partial remission. However, partial clinical remission does not appear to be accompanied by a general change in systemic immune reactivity.

Supported by European Union, PL963363: DiabMarker

\section{PS 23}

\section{Alpha and delta cell function}

\section{3}

IL-6 regulates alpha-cell mass and function

H. Ellingsgaard ${ }^{1}$, J. A. Ehses ${ }^{1}$, L. van Lommel $^{2}$, F. C. Schuit ${ }^{2}$, M. Y. Donath ${ }^{1}$;

${ }^{1}$ Division of Endocrinology and Diabetes, University Hospital Zürich, Switzerland, ${ }^{2}$ Campus Gasthuisberg, Gene Expression Unit, Leuven, Belgium.

Background and Aims: Increased alpha-cell mass and hyperglucagonemia contribute to hyperglycemia in type 2 diabetes. However, the reasons for this imbalance in alphacell mass remain unclear. IL-6 is elevated in the circulation of obese people, and is a strong predictor of the development of type 2 diabetes. The aim of the current study was to investigate the role of IL-6 in the regulation of pancreatic islet-cell mass and function.

Methods: Detection and localization of the IL-6 receptor (IL-6R) and the gp130 was done by gene array and 
immunohistochemistry. The effects of IL- 6 on cell turnover and function was assessed in human and mouse islets by Ki67, BrdU and TUNEL techniques and by RIA for insulin and glucagon. Morphometric quantification of endocrine cells was performed on pancreatic sections from wildtype and IL-6KO mice.

Results: Initial tissue gene array analysis revealed that the IL-6R is most highly expressed in the pancreatic islet. In human and mouse islets, we observed mRNA expression of both the IL-6R and the signaling component of this complex, the gp130 receptor. Further, IL-6 caused STAT3 phosphorylation in human and mouse islets, while immunocytochemistry localized this activation to the alpha-cell. In support of this, immunohistochemistry performed on pancreatic sections revealed expression of the IL-6R and the gp130 only in alpha-cells. Exposure of cultivated human and mouse islets to IL- 6 for 4 days caused a 3- to 6-fold increase in alpha-cell proliferation with no effect on apoptosis. Furthermore we found that IL-6 regulated glucagon secretion in human and mouse islets (IL-6KO islets were used to avoid influence from endogenous IL-6). High doses of IL- 6 resulted in a decrease in glucagon mRNA and secretion, whereas lower doses caused an increase in glucagon secretion. In addition, IL-6 impaired beta-cell secretory function as assessed by glucose stimulated insulin secretion (GSIS); an effect which was reversed using an IL-6 receptor antagonist, and partly by exogenous glucagon. Based on these in vitro findings we examined the role of IL-6 in regulating alpha-cell mass in vivo in WT and IL-6KO mice. Feeding of a high energy diet for 8 weeks induced a 3 -fold increase in alpha-cell area in WT mice compared to animals on chow diet. In contrast, alpha-cell area remained unchanged in IL-6KO mice following high energy diet, despite a similar increase in body weight.

Conclusion: Thus, IL-6 presents itself as a regulator of alpha-cell function and proliferation, and may be responsible for the increase in alpha-cell mass seen in patients with type 2 diabetes.

\section{4}

Regulation by glucose of $\mathrm{GABA}_{\mathrm{A}}$ receptor expression in mouse $\alpha$-cells: role in the intra-islet regulation of glucagon secretion

M. A. Ravier ${ }^{1}$, S. J. Bailey ${ }^{2}$, G. A. Rutter ${ }^{1}$;

${ }^{1}$ Biochemistry, University of Bristol, ${ }^{2}$ Pharmacy and Pharmacology, University of Bath, Bath, United Kingdom.

Background and Aims: The mechanism(s) by which glucose regulates the release of glucagon remain controversial. Whereas paracrine roles for $\beta$-cell factors including insulin or $\mathrm{Zn}^{2+}$ ions are implicated in the control of secretion from rat islets, we have recently reported that these are unlikely to be involved in the mouse. Moreover, isolated mouse $\alpha$-cells respond directly to elevated glucose concentrations with increases in ATP production and suppression of $\mathrm{Ca}^{2+}$ oscillations, questioning whether paracrine factors may be required at all to regulate glucagon release in this species. Here, we have investigated whether (a) $\gamma$-aminobutyric acid (GABA) plays a role in the glucoseinduced suppression of glucagon release from mouse islets or clonal $\alpha \mathrm{TC} 1-9$ cells, and (b) expression of the individual $\alpha, \beta$ or $\gamma$ subunits of the ionotropic $\mathrm{GABA}_{\mathrm{A}}$ receptor complex is regulated in $\alpha$-cells by glucose in the long term. Materials and Methods: CD1 mouse islets, isolated by collagenase digestion, or clonal $\alpha \mathrm{TC} 1-9$ cells, were cultured for $16 \mathrm{~h}$ at 10 or $16 \mathrm{mmol} / \mathrm{l}$ glucose respectively before assay of glucagon release during static incubations $(1 \mathrm{~h})$. aTC1-9 cells were incubated for a further $24 \mathrm{~h}$ in the presence of $1,3,10$ or $16 \mathrm{mmol} / 1$ glucose, prior to RNA extraction (Trizol reagent; Sigma). Levels of mRNA encoding $\mathrm{GABA}_{\mathrm{A}}$ receptor subunits were determined by quantitative real-time PCR (SYBR green), normalised to $18 \mathrm{~S}$ ribosomal RNA. Immunocytochemical analysis was performed after paraformaldehyde fixation and laser-scanning confocal microscopy. Data are expressed as meanSEM and statistical significance assessed by ANOVA and Newman-Keuls test.

Results: Exogenously added GABA (10 or $100 \mu \mathrm{mol} / \mathrm{l})$ inhibited glucagon release by $41 \pm 11$ or $56 \pm 5 \%(n=4, p<0.05)$ respectively, during incubation of isolated mouse islets in the presence of $0.5 \mathrm{mmol} / 1$ glucose, compared to $63 \pm 7 \%(\mathrm{p}<0.05)$ inhibition achieved at $10 \mathrm{mmol} / 1$ glucose alone. Treatment with the selective $\mathrm{GABA}_{\mathrm{A}}$ receptor antagonist SR95531 was without effect on glucagon release at $0.5 \mathrm{mmol} / 1$ glucose but substantially $(76.6 \pm 20 \%)$ reversed the inhibition of glucagon release in response to 10 (versus 0.5 ) $\mathrm{mmol} / 1$ glucose. Mouse islets expressed $\alpha_{4}, \beta_{3}$ and $\gamma_{2} \mathrm{GABA}_{\mathrm{A}}$ receptor subunit mRNAs, with the immunoreactivity of each largely confined within the islet to the plasma membrane of $\alpha$-cells. These subunits, plus low levels of $\alpha_{3}, \beta_{2}$ and $\delta$, were also present in $\alpha \mathrm{TC1}-9$ cells. After culture of $\alpha \mathrm{TC} 1-9$ cells for $24 \mathrm{~h}$ at 16 versus $1 \mathrm{mmol} / 1$ glucose, levels of mRNA encoding $\alpha_{4}$ were increased 2.7-fold, $\beta_{3}$ by 2.0 -fold and $\gamma_{2}$ by 1.6 -fold. Correspondingly, culture at 16 (versus 1 ) $\mathrm{mmol} / \mathrm{l}$ glucose augmented $\alpha_{4}$ immunoreactivity in $\alpha \mathrm{TC} 1-9$ cells by>twofold. These effects of high glucose concentration were mimicked at $1 \mathrm{mmol} / 1$ glucose by blockade of L-type $\mathrm{Ca}^{2+}$ channels with nimodipine. Conversely, stimulation of $\mathrm{Ca}^{2+}$ influx with $25 \mathrm{mmol} / \mathrm{l} \mathrm{KCl}$ reversed the activating effect of $16 \mathrm{mmol} / \mathrm{l}$ glucose.

Conclusions: (a) Release of GABA from neighbouring $\beta$-cells contributes $\sim 45 \%$ of the inhibitory response to glucose of glucagon secretion from mouse islets, whilst direct metabolic effects on $\alpha$-cells may explain the remaining 
effects of the sugar; (b) the expression of $\alpha$-cell $\mathrm{GABA}_{\mathrm{A}}$ receptors displays substantial plasticity. Increased expression of the $\mathrm{GABA}_{\mathrm{A}}$ receptor subunits by elevated glucose concentrations may provide a mechanism to suppress glucagon release chronically during sustained hyperglycemia. Supported by Juvenile Diabetes Research Foundation

\section{5}

The alpha cell in type 2 diabetes: studies in human pancreatic islets

R. Mancarella ${ }^{1}$, R. Lupi ${ }^{1}$, S. Del Guerra ${ }^{1}$, M. Bugliani ${ }^{1}$, S. Torri ${ }^{1}$, M. Grupillo ${ }^{1}$, U. Boggi ${ }^{2}$, F. Mosca ${ }^{2}$,

P. Marchetti ${ }^{1}$, S. Del Prato ${ }^{1}$;

${ }^{1}$ Endocrinology and Metabolism - Metabolic Unit, University of Pisa, ${ }^{2}$ Oncology, Transplantation and Advanced Technologies in Medicine, University of Pisa, Italy.

Background: In vivo studies have suggested that in Type 2 diabetes mellitus (T2DM) a relative hyperglucagonemia exists and that altered insulin:glucagon molar ratio, contributes to impairment of glucose homeostasis. Little is known, however, of the alpha cell activity within human pancreatic islets. Therefore, we evaluated cell composition as well as insulin and glucagon release from pancreatic islets obtained from normal and type 2 diabetic (T2DM) subjects.

Materials and Methods: Pancreatic islets were prepared by collagenase digestion and density gradient purification from 22 pancreata of multiorgan donors (14 non-diabetic, ND: $8 \mathrm{~F} / 6 \mathrm{M}$; age, $68 \pm 11 \mathrm{yrs}$; BMI $23.9 \pm 4.1 \mathrm{~kg} / \mathrm{m} 2$; and 8 T2DM: $6 \mathrm{~F} / 2 \mathrm{M}$; age, $66 \pm 9$ yrs; BMI: $27.0 \pm 3.2 \mathrm{~kg} / \mathrm{m} 2$ ). Isolated islets were cultured for $24 \mathrm{~h}$ with or without $22.2 \mathrm{mmol} / \mathrm{l}$ glucose or $1.0 \mathrm{mmol} / \mathrm{l} \mathrm{FFA} \mathrm{mixture} \mathrm{(oleic:}$ palmitic, 2:1). T2DM islets were studied within 3 days from isolation. At the end of each incubation, we determined insulin and glucagon release (IRMA or RIA technique), gene expression (qualitative and quantitative RT-PCR) and electron microscopy structure.

Results: With respect to ND, beta-cells were moderately though significantly reduced in T2DM islets $(69 \pm 3$ vs. $62 \pm 4 \%, \mathrm{p}<0.05$ ) whereas there was no difference in alphacell composition $(21 \pm 3$ vs. $23 \pm 4 \%)$ resulting in relative increase in alpha-cell population. Basal insulin release was reduced in T2DM (220 \pm 51 vs. $2531 \pm 776 \mu \mathrm{U} / \mathrm{ml} / 24-\mathrm{h})$, whereas glucagons was higher $(51.5 \pm 9.2$ vs. $7.3 \pm 1.3 \mathrm{pg} / \mathrm{ml} /$ 24-h; $\mathrm{p}<0.001$ ). In T2DM, glucagon and gene expression was not different with respect to ND. When expression of controlling factors for alpha-cell differentiation was determined, we found no difference in CDX2 while PAX-6 $(1.0 \pm 0.1$ vs. $0.46 \pm 0.06)$ and Foxa- $1(0.43 \pm 0.09$ vs. $0.24 \pm 0.08)$ were increased. $24 \mathrm{~h}$ exposure of ND to FFA, but not to glucose, caused an increase of glucagon release
$(47.3 \pm 12.5$ and $6.4 \pm 0.9 \mathrm{pg} / \mathrm{ml}$, respectively) that was associated with concomitant increase in PAX-6 $(0.7 \pm 0.02)$ and Foxa-1 (0.89 \pm 0.2$)$.

Conclusions: These data confirm relative increase in the alpha cell population and enhanced function in T2DM pancreatic islets and suggest, for the first, time an activation of the a-cell lineage both in T2DM pancreatic islets and in ND islets in response to FFA.

\section{6}

Adrenaline but not GLP-1 shifts the voltage-dependence of exocytosis in mouse pancreatic A-cells

L. Eliasson ${ }^{1}$, P. Rorsman ${ }^{2}$, A. Salehi ${ }^{1}$,

Y. Zhang Demarinis ${ }^{1}$;

${ }^{1}$ Division of Endocrinology, Department of Clinical Science, Malmö, Sweden, ${ }^{2}$ OCDEM, Oxford University, United Kingdom.

Background and Aim: Glucagon is released from the pancreatic A-cells through $\mathrm{Ca}^{2+}$-dependent exocytosis and it has been demonstrated that GLP-1 and adrenaline stimulate exocytosis in the A-cell through cAMP-dependent mechanisms. However when glucagon secretion is measured from whole islets adrenaline is stimulatory whereas GLP-1 is inhibitory. The aim of this study was to investigate the differences in the mechanisms by which GLP-1 and adrenaline stimulates exocytosis in pancreatic A-cells.

Methods: Single mouse A-cell exocytosis was monitored as increases in cell capacitance using the perforated wholecell configuration of the patch-clamp technique. Exocytosis was elicited by application of voltage-clamp depolarization of indicated durations from $-70 \mathrm{mV}$ to membrane potentials between $-30 \mathrm{mV}$ to $20 \mathrm{mV}$ to investigate the voltage-dependence of exocytosis. Glucagon secretion was measured using RIA.

Results: Adrenaline stimulated glucagon secretion in the presence of $1 \mathrm{mM}$ glucose $(1 \mathrm{G})$ from $33 \pm 1 \mathrm{pg} /$ islet/ $\mathrm{h}(\mathrm{n}=10)$ to $62 \pm 3 \mathrm{pg} / \mathrm{islet} / \mathrm{h}(\mathrm{P}<0.001, \mathrm{n}=10)$, whereas GLP-1 inhibited glucagon secretion to $16 \pm 1 \mathrm{pg} / \mathrm{ilset} /$ $\mathrm{h}(\mathrm{P}<0.001, \mathrm{n}=10)$. The same was observed under depolarizing conditions (in presence of $15 \mathrm{mM} \mathrm{K}$ ) and here adrenaline stimulated glucagon secretion 4-fold $(\mathrm{P}<0.001$, $\mathrm{n}=10$ ) whereas GLP-1 had neither a stimulatory or inhibitory effect. At even higher depolarizing conditions $\left(70 \mathrm{mM} \mathrm{K}{ }^{+}\right.$) adrenaline stimulated exocytosis 2-fold $(\mathrm{P}<0.001, \mathrm{n}=10)$ and GLP-1 had a slight stimulatory effect ( $25 \%$ increase, $\mathrm{P}<0.05, \mathrm{n}=10$ ). To further investigate these differences we investigated the voltage-dependence of exocytosis under control conditions and $4 \mathrm{~min}$ after application of either $10 \mathrm{nM}$ GLP-1 or $5 \mu \mathrm{M}$ adrenaline. Maximal exocytotic response under control conditions was 
observed when the membrane potential was depolarized to $0 \mathrm{mV}$ and amounted $39 \pm 9 \mathrm{fF}(\mathrm{n}=5)$. Addition of adrenaline was associated with a $>2$-fold $(n=5 ; P<0.01)$ increase in the exocytotic response. Interestingly, a significance increase in the exocytotic response $(13 \pm 5 \mathrm{fF}, \mathrm{P}<0.01$ vs control) was observed in the presence of adrenaline already at a voltage as negative as $-20 \mathrm{mV}$ whereas no capacitance increase was observed at control conditions. In contrast to adrenaline GLP-1 failed to stimulate exocytosis at $-20 \mathrm{mV}$. In the presence of GLP-1 exocytosis was first evoked at the $-10 \mathrm{mV}$ depolarization where it amounted $40 \pm 11 \mathrm{fF}(\mathrm{n}=7$; $\mathrm{P}<0.01$ vs control) Thus, adrenaline stimulates exocytosis at more negative membrane potentials than GLP-1. This might be of importance since small changes in the membrane potential are important in the control of glucagon secretion. The observed shift in the presence of adrenaline but not GLP-1 might be due to that adrenaline stimulate both PKAindependent (through cAMP-GEFII) and PKA-dependent pathways, whereas GLP-1 mainly acts through PKA-dependent stimulation. When testing the effects of the specific cAMP-GEFII-agonist 8CPT-2Me-cAMP using the standard whole-cell configuration it was found to stimulate exocytosis already at $-20 \mathrm{mV}$ (8-fold compared to control; $\mathrm{P}<0.01$, $\mathrm{n}=7-8$ ).

Conclusion: We suggest that 1) adrenaline is able to shift the voltage-dependence of exocytosis though activation of the cAMP-GEFII dependent pathway and 2) the fact that adrenaline stimulates exocytosis at a lower membrane potential than GLP-1 contributes to that adrenaline but not GLP-1 stimulates glucagon secretion from whole islets.

\section{7}

High concentrations of glucose unexpectedly stimulate glucagon secretion by a $\mathrm{Ca}^{2+}$ independent mechanism E. Vieira ${ }^{1}$, A. Salehi ${ }^{2}$, E. Gylfe ${ }^{1}$;

${ }^{1}$ Department of Medical Cell Biology, Uppsala University, Uppsala, ${ }^{2}$ Department of Clinical Science CRC (UMAS), Lund University, Malmö, Sweden.

Background and Aims: Diabetes is primarily due to insufficient secretion of insulin but is aggravated by a dysregulated release of glucagon. Glucagon secretion is not appropriately stimulated when the blood glucose concentration falls below the fasting level, making diabetic patients treated with insulin or sulphonylureas susceptible to dangerous hypoglycemia. Although glucose is an inhibitor of glucagon secretion patients with diabetic hyperglycemia have elevated levels of glucagon and even respond with additional secretion when blood glucose is raised further. The present study was undertaken to clarify the signal transduction underlying glucose regulation of glucagon secretion.
Materials and Methods: Glucagon and insulin secretion were measured in parallel from C57BL/6 mouse pancreatic islets and glucagon secretion from clonal hamster In-R1-G9 cells using radioimmunoassay. Single mouse islet cells were prepared and used for recording the cytoplasmic $\mathrm{Ca} 2+$ concentration with the indicator fura- 2 and digital imaging technique. After each experiment the $\alpha$-cells were identified by immunostaining.

Results: Glucose inhibited glucagon secretion from mouse islets with maximal effect at about $7 \mathrm{mM}$, which corresponded to the threshold for stimulation of insulin release. Higher concentrations of glucose stimulated insulin release and diminished the inhibition of glucagon secretion. At 25$30 \mathrm{mM}$ glucose there was a paradoxical stimulation of glucagon secretion coinciding with maximal insulin release. Also glucagon secretion from the In-R1-G9 cells showed a U-shaped dependence on glucose concentration with maximal inhibition at 7 and stimulation at $12-20 \mathrm{mM}$ of the sugar. Glucagon secretion stimulated by the absence of glucose was inhibited by diazoxide hyperpolarization but this agent tended to further enhance secretion evoked by high concentrations of the sugar. Studies of isolated mouse $\alpha$-cells indicated that glucose inhibited glucagon secretion by lowering the cytoplasmic $\mathrm{Ca} 2+$ concentration. However, stimulation of glucagon release by high glucose concentrations did not require elevation of $\mathrm{Ca} 2+$.

Conclusion: Glucose inhibition of glucagon secretion from mouse islets does not require release of factors from the $\beta$-cells. High glucose concentrations stimulate glucagon secretion by a novel $\mathrm{Ca} 2+$-independent mechanism. An U shaped dose-response relationship for glucose-regulated glucagon secretion may explain why diabetic patients with pronounced hyperglycemia display paradoxical hyperglucagonemia.

Support by Swedish Research Council 12X-6240

\section{8}

The $\alpha$-cell expresses GLP-2-receptors and GLP-2 stimulates glucagon secretion

J. de Heer ${ }^{1}$, J. Pedersen ${ }^{2}$, C. Orskov ${ }^{2}$, J. J. Holst ${ }^{1}$;

${ }^{1}$ Department of Medical Physiology, ${ }^{2}$ Department of Medical Anatomy, University of Copenhagen, Denmark.

Background and Aims: In spite of being encoded by the same gene and being secreted by the same type of endocrine cell, glucagon-like peptide-1 and -2 (GLP-1, -2) differ markedly in their effects. Unlike GLP-1, the actions of GLP-2 are regarded as being strictly confined to the gut, where GLP-2 influences intestinal growth, gastric transit and nutrient absorption, while no effect on the endocrine pancreas has been described so far. Recently however, 
GLP-2 was reported to stimulate glucagon secretion when given intravenously to healthy human beings, thereby evoking exactly the opposite effect of GLP-1, which potently inhibits glucagon secretion. Since the mechanisms underlying this effect have not been elucidated, our study aimed to explore the mode of action by which GLP-2 increases glucagon secretion. Furthermore, we investigated the effect of GLP-2 on insulin and somatostatin secretion and we examined the impact of simultaneous application of GLP-1 and GLP-2 on glucagon output.

Material and Methods: In order to characterize the GLP2-mediated effects on islet hormone secretion, the pancreata of male Wistar rats were perfused in situ at a glucose concentration of $3 \mathrm{mM}$. The glucagon, insulin and somatostatin responses to increasing concentrations of GLP-2 (0.1 to $10 \mathrm{nM}, \mathrm{n}=8)$ alone and to combined GLP-1 $(0.1$ and $1 \mathrm{nM})$ and GLP-2 $(10 \mathrm{nM}, \mathrm{n}=10)$ administration were analysed by radioimmunoassay. The localization of GLP-2 receptors (GLP-2r) in tissue samples from human and rat pancreas were studied by immunohistochemistry, employing validated antibodies reliably staining for the GLP-2r and proglucagon, respectively. Total RNA was obtained from isolated rat islets using Trizol and cDNA was created using Moloney Murine Leukemia Virus reverse transcriptase. The expression of the GLP-2r was analysed using primers designed with no crossreactivity with Beacon design Premierbiosoft. The PCR products were analysed on prestained ethidium bromide agarose gel.

Results: In the in situ perfused rat pancreas, infusion of $10 \mathrm{nM}$ GLP-2 caused a significant increase in glucagon secretion $(0.5 \pm 0.09$ vs. $0.27 \pm 0.03 \mathrm{pmol} / \mathrm{min}$ basal secretion, mean \pm SEM; $p<0.0001)$, whereas lower GLP-2-doses were ineffective $(0.3 \pm 0.05$ for $0.1 \mathrm{nM}$ and $0.36 \pm 0.06 \mathrm{pmol} / \mathrm{min}$ for $1 \mathrm{nM} ; \mathrm{p}=\mathrm{ns}$ ). Neither insulin nor somatostatin output were influenced by the administration of 0.1 to $10 \mathrm{nM}$ GLP-2. The glucagonotropic effect of GLP-2 was not attenuated by the simultaneous administration of 0.1 or $1 \mathrm{nM}$ GLP-1, which when given alone inhibited glucagon secretion by $35.3 \pm 7.5$ $(p=0.045)$ and $42.7 \pm 5.7 \% \quad(p=0.019)$, respectively. Using immunohistochemical double staining, we found that GLP2r-immunoreactivity was localized to cells which also were immunoreactive for proglucagon in both human and rat pancreas. Expression of GLP-2r transcript was confirmed by PCR on cDNA from isolated rat islets. The result was verified by a PCR-product of the expected length.

Conclusion: The glucagonotropic effect of GLP-2 on the endocrine rat pancreas is mediated by GLP-2r expressed by the $\alpha$-cell. Based on our analogous findings in human pancreas, this mode of action applies in all likelihood also to human beings. However, further in vivo studies are required in order to assess the physiological significance of the glucagonotropic effect of GLP-2 in humans, and to evaluate whether this contributes to the diabetic hyperglucagonemia.
0469

The insulin tolerance test as a method for in vivo assessment of $\alpha$-cell responsiveness in rats

C. Ammala, K. K. Brown;

Department of Metabolic Diseases, GSK, RTP, United States.

Background and Aims: Although there is evidence suggesting $\alpha$-cell dysfunction contributes to the pathogenesis of type 2 diabetes, the regulation of glucagon secretion remains little studied in vivo in experimental animals. In humans, the hyperinsulinemic, stepped hypoglycemic clamp is the most rigorous method for quantifying in vivo $\alpha$-cell responsiveness. This method is not readily adapted for rodents. As glucagon release from $\alpha$-cells serves to protect against hypoglycemia, we have therefore explored the possibility of using the insulin tolerance test as a simple provocative challenge to assess counter-regulatory $\alpha$-cell responses to hypoglycemic stress.

Materials and Methods: Normal Wistar rats (aged 1012 weeks) were instrumented with catheters in the jugular vein and carotid artery, which were used for intravenous insulin infusion and arterial blood sampling. Rats were allowed to recover for a week prior to experimentation. Animals were fasted either 14-16 hours overnight or 4-6 hours in the morning, before receiving an intravenous bolus of insulin (Novolin R; 0.1 or $0.05 \mathrm{U} / \mathrm{kg}$ ). Plasma glucose levels were measured using the glucose oxidase method (YSI) before giving insulin then every 2 minutes for the first 20 minutes and at 25, 30 and 45 minutes after the bolus injection. Blood samples $(0.4 \mathrm{ml})$ were collected at times 0 , $4,6,8,10,12,16$ and 30 minutes for human insulin and glucagon assays (LINCO).

Results: Both doses of intravenous insulin elicited robust decreases in plasma glucose and increased circulating glucagon levels. In animals fasted overnight the baseline glucagon levels were not different $(48 \pm 3$ and $45 \pm 2 \mathrm{pg} / \mathrm{ml})$, and 0.1 and $0.05 \mathrm{U} / \mathrm{kg}$ insulin elicited similar rates of glucose disposal, measured as the slope of plasma glucose between 2 and 8 minutes $(\mathrm{Kg},-6.3 \pm 0.1$ and $-5.5 \pm 0.4 \mathrm{mg} /$ $\mathrm{dl} / \mathrm{min}$ respectively, $\mathrm{p}=0.09)$, decreased plasma glucose $(-46 \pm 3$ and $-43 \pm 2 \mathrm{mg} / \mathrm{dl}, \mathrm{p}=0.52)$ and increased glucagon levels $(438 \pm 93$ and $374 \pm 95 \mathrm{pg} / \mathrm{ml}, \mathrm{p}=0.65)$. In contrast, when fasted for only 4-6 hours the rate of glucose disposal $(-8.2 \pm 0.9$ and $-6.0 \pm 0.5 \mathrm{mg} / \mathrm{dl} / \mathrm{min}$, respectively; $\mathrm{p}<0.05)$, fall in plasma glucose $(-58 \pm 3$ vs. $-44 \pm 2 \mathrm{mg} / \mathrm{dl}, \mathrm{p}<0.05)$ and peak glucagon response $(198 \pm 27$ vs. $95 \pm 10, \mathrm{p}<0.005)$ were significantly increased with the higher insulin dose. As for total glucagon output, the responses after the overnight fast were similar $(135 \pm 27$ and $117 \pm 30 \mathrm{pg} / \mathrm{ml} * \mathrm{hr}$, $\mathrm{p}=0.68$ ) for 0.1 and $0.05 \mathrm{U} / \mathrm{kg}$ insulin, whereas after the 
short term fast responses were significantly different $(60 \pm 6$ and $32 \pm 2 \mathrm{pg} / \mathrm{ml} * \mathrm{hr}, \mathrm{p}<0.001$ ).

Conclusion: The hypoglycaemia induced during the intravenous insulin tolerance test elicits an $\alpha$-cell response leading to a robust increase in circulating glucagon. The $\alpha$ cell response to, and the ability of glucagon to counteract, the insulin induced hypoglycemia is influenced by the duration of fasting. Overnight fasting led to a larger overall increase in glucagon independent of insulin dose; moreover plasma glucose levels failed to return to baseline and the glucagon levels remained elevated. We speculate the failure of glucagon to alleviate hypoglycemia is due to depletion of liver glycogen stores. Thus, the response following an overnight fast may reflect the maximal $\alpha$-cell response to hypoglycemia and could be useful to assess insufficient $\alpha$-cell function. With short term fasting it is possible to demonstrate a dose-dependent glucagon response reflected in both the rate and magnitude of fall in glucose, and the magnitude in the glucagon response, leaving a window for assessing mechanism(s) of enhanced $\alpha$-cell function in vivo.

\section{0}

Glucose stimulates glucagon release in children and adolescents with new onset type 1 diabetes 12 months after diagnosis - results from the Hvidøre Study Group S. Pörksen ${ }^{1}$, L. B. Nielsen ${ }^{1}$, R. W. Holl ${ }^{2}$, P. G. F. Swift ${ }^{3}$, C. Ørskov ${ }^{4}$, J. J. Holst ${ }^{5}$, S. Gammeltoft ${ }^{6}$, K. B. Plough ${ }^{7}$, I. Jansen-Olesen ${ }^{7}$, P. Hougaard ${ }^{8}$, L. Hansen ${ }^{9}$,

H. B. Mortensen ${ }^{1}$;

${ }^{1}$ Paediatric, Glostrup University Hospital, Denmark, ${ }^{2}$ Paediatric, University of Ulm, Germany, ${ }^{3}$ Paediatric, Royal Infirmery, Leicester, United Kingdom, ${ }^{4}$ Medical Anatomy, ${ }^{5}$ Medical Physiology, Panum Institute, Copenhagen, Denmark, ${ }^{6}$ Clinical Biochemistry, ${ }^{7}$ Clinical Experimental Research, Glostrup University Hospital, Denmark, ${ }^{8}$ Statistics, University of Southern Denmark, Odense, Denmark, ${ }^{9}$ Novo Nordisk A/S, Bagsværd, Denmark.

Background and Aims: Existance of a glucose-sensing insulin secretion-coupling mechanism similar to beta-cells including the ATP-sensitive $\mathrm{K}^{+}$- channel Kir6.2 and its regualtory subunit SUR1 was recently demonstrated in alpha-cells isolated from rat pancreatic islets: glucose, arginine, and the sulfonylurea compound tolbutamide all stimulated glucagon release. In humans and intact islets glucagon secretion in response to these agents is suppressed by the simultaneous intra-islet release of insulin. Type 1 diabetes, because of the loss of insulin producing beta cells, offers ideal conditions for an in vivo study of glucose-induced glucagon secretion, so the aim of the present study was to investigate the relationship between postprandial glucose and glucagon release in children during the first 12 months after diagnosis of type 1 diabetes. Furthermore we investigated the co-localization of Kir6.2 and SUR1 proteins with glucagon in the human islets. Materials and Methods: 257 children and adolescents aged $<16$ years from 22 centres in 18 countries with newly diagnosed type 1 diabetes were followed for 12 months. A 90-minutes Boost-test (mixed meal) was carried out in each patient at 1,6 , and 12 months after diagnosis to characterise the residual beta cell function (C-peptide, and rise in post-prandial blood glucose) and glucagon. All blood substances were centrally determined. Immunohistochemistry was carried out on formalin fixed material. Human pancreatic tissue $(n=6)$ was archival material collected during the 1970s according to the contemporary ethical guidelines. The antibodies used for immunohistochemistry were: monoclonal mouse anti-GLP-1 antibody code GLPb-17F1A37B22C4 (F1) diluted 1:3000, goat anti Kir6.2 code N-18 diluted 1:3000 (Santa Cruz Biotechnology), goat anti SUR1 code C-16 diluted 1:1000 (Santa Cruz Biotechnology)

Results: Multiple regression and compound symmetric repeated measurement models showed that postprandial (90 min post boost-test) glucagon increased over 1, 6, and 12 months $(p=0.005)$ independent of age, gender, stimulated C-peptide (residual beta-cell function), but highly dependent on the rise in postprandial glucose $(p=0.0004)$. Immunohistochemical staining of human pancreatic sections confirmed the co-expression of Kir6.2 and SUR1 proteins in glucagon immunoreactive cells.

Conclusion: The stimulatory effect of glucose on the alpha cells contrast with the suppressive effect of glucose in subjects with preserved insulin secretory capacity and highlights the primary importance of islet paracrine signaling in the regulation of glucagon release. The presence of Kir6.2/SUR1 on alpha-cells suggest that sulfonylureas administered at bedtime to C-peptide negative type diabetic 1 patients might prevent episodes with nocturnal hypoglycemia.

Support by Danish Diabetes Association

0471

R-type $\mathrm{Ca}^{2+}$ channel-evoked $\mathrm{Ca}^{2+}$-induced $\mathrm{Ca}^{2+}$ release regulates somatostatin secretion

Q. Zhang ${ }^{1,2}$, A. Salehi ${ }^{2}$, S. Collins ${ }^{1}$, C. Partridge ${ }^{1}$,

P. Rorsman ${ }^{1}$;

${ }^{1}$ Nuffield Department of Medicine, Oxford Centre for Diabetes, Endocrinology and Metabolism, United Kingdom, ${ }^{2}$ Department of Clinical Sciences, Clinical Research Centre, Malmö, Sweden. 
Background and Aims: Somatostatin is a powerful paracrine regulator of insulin and glucagon secretion in the islets but little is known about the control of its release from the $\delta$-cells. Here we have studied the regulation of somatostatin release from mouse pancreatic islets focusing on the role of ATP-regulated $\mathrm{K}^{+}$-channels, the $\mathrm{Ca}^{2+}$-channel dependence and the importance of intracellular $\mathrm{Ca}^{2+}$ stores in the process.

Materials and Methods: Membrane currents and exocytosis were recorded from superficial $\delta$-cells in intact pancreatic islets using the standard whole-cell configuration of the patch clamp technique. $\delta$-cells were identified based on the inactivation of voltage gated $\mathrm{Na}^{+}$current and lack of TEA resistant $\mathrm{K}^{+}$current. Exocytosis was detected as changes in cell capacitance. For ratiometric $\mathrm{Ca}^{2+}$ measurements, indo-1 was infused to the cytosol through the patch pipette. Hormone release from static incubations of groups of $\sim 10$ islets and was quantified by radioimmunoassay (RIA).

Results: The L-type $\mathrm{Ca}^{2+}$ channel blocker nifedipine $(25 \mu \mathrm{M})$ inhibited $\delta$-cells $\mathrm{Ca}^{2+}$ current by $40 \%(\mathrm{n}=5$, $\mathrm{p}<0.05$ ), while it had no effect on somatostatin secretion $(\mathrm{n}=10)$. The R-type $\mathrm{Ca}^{2+}$ channel blocker SNX482 $(100 \mathrm{nM})$ blocked $45 \%$ of the $\delta$-cell $\mathrm{Ca}^{2+}$ current $(\mathrm{n}=4$, $\mathrm{p}<0.05)$ and $80 \%$ of glucose-evoked somatostatin secretion $(\mathrm{n}=10, \mathrm{p}<0.05)$. In capacitance measurements, SNX482 blocked $41 \%$ of the exocytosis evoked by $500 \mathrm{~ms}$ depolarizations from $-70 \mathrm{mV}$ to $0 \mathrm{mV}(\mathrm{n}=4, \mathrm{p}<0.05)$. We explored the involvement of calcium-induced calciumrelease (CICR) in somatostatin secretion. Exocytosis was evoked by a depolarization of $200 \mathrm{~ms}$ from $-70 \mathrm{mV}$ to $0 \mathrm{mV}, 50 \mu \mathrm{M}$ Ryanodine, $25 \mu \mathrm{M}$ dantrolene and $10 \mu \mathrm{M}$ thapsigargin inhibited exocytosis by $96 \%(n=4, p<0.05)$, $61 \%(\mathrm{n}=7, \mathrm{p}<0.05)$ and $60 \%(\mathrm{n}=4, \mathrm{p}<0.05)$, respectively. Similar effects were observed on somatostatin secretion measured by RIA. Blocking CICR by applying ryanodine both intracellularly and extracellularly resulted in a shortening of the $\left[\mathrm{Ca}^{2+}\right]_{\mathrm{i}}$ transient triggered by $300 \mathrm{~ms}$ depolarization, and the time constant for the decay fell from $2.5 \mathrm{~s}$ to $1 \mathrm{~s}(\mathrm{n}=5, \mathrm{p}<0.05)$.

Conclusion: Based on our data, we conclude that Ltype $\mathrm{Ca}^{2+}$ channels contribute to the $\delta$-cell $\mathrm{Ca}^{2+}$ current without apparent function in $\delta$-cell exocytosis. The major $\mathrm{Ca}^{2+}$ sources for exocytosis in $\delta$-cells are influx through voltage gated R-type $\mathrm{Ca}^{2+}$ channels and calcium-induced calcium-release via ryanodine receptors in the ER.

Supported by the European Union (Biosim), the Wellcome Trust and Diabetes UK

\section{PS 24}

\section{Developmental biology of the endocrine pancreas}

0472

The long acting GLP-1 analogue, liraglutide, induces beta cell differentiation during normal human pancreas development

K. Piper Hanley, S. Gray, I. M. E. Dijkstra, T. Hearn, L. Williams, D. I. Wilson, N. A. Hanley;

Human Genetics Division, University of Southampton, United Kingdom.

Background and Aims: Glucagon-like peptide-1 (GLP-1) stimulates glucose-dependent insulin secretion; the primary reason long-acting analogues have been developed for use in Type 2 diabetes. In rodents and tumour cell lines, GLP-1 signaling has also increased beta cell 'mass' by promoting beta cell neogenesis, proliferation and inhibiting apoptosis. However, little is known about the role of GLP-1 during normal human beta cell differentiation. We have investigated GLP-1 signaling, via the long-acting analogue Liraglutide (Novo Nordisk A/S) in the developing human pancreas.

Materials and Methods: With ethical approval and informed consent, human fetal material was collected from first trimester termination and processed for fixed tissue, RNA and protein analysis. In vitro culture models were established to interrogate beta cell differentiation. Insulinpositive cells were expressed as a percentage of total epithelial cell number.

Results: Human pancreas was isolated at 8 weeks postconception (wpc), immediately prior to significant beta cell differentiation (Piper et al, J Endocrinology, 181, 11-23, 2004). GLP-1, along with its receptor, was expressed in the developing pancreas and duodenum. In 7-day explant culture, Liraglutide increased insulinpositive cell number by $68 \%(n=6)$, but had no effect on beta cell proliferation or apoptosis. Consistent with this implication of de novo differentiation, exposure to Liraglutide decreased epithelial progenitor cell proliferation by $24 \%$. The effects of Liraglutide on beta cell number were abrogated by co-incubation with 10 -fold molar excess of the GLP-1 receptor antagonist, Exendin 9-39, restoring insulin-positive cell number to just below $100 \%$ of control. The use of Exendin 9-39 alone reduced insulin-positive cells by $26 \%$.

Conclusion: Liraglutide increases human primary beta cell number, via GLP-1 receptor signaling that most likely enhances beta cell differentiation. For Exendin 939 to decrease insulin-positive cell number by itself 
suggests that native GLP-1 signaling is very important during early human development. Furthermore, our data suggest that altering the cell cycle status of progenitor cells may be an underlying mechanism that regulates human beta cell differentiation. Clinically, Liraglutide may increase human beta cell mass in Type 2 diabetes. In addition, modulating the GLP-1 pathway may be a potent mechanism for manipulating stem cells to beta cells as an ambitious transplantation therapy in Type 1 diabetes.

Supported by Novo Nordisk

\section{3}

Expression of the Notch-Hes1 pathway in adult pancreatic stem cells

J. Seissler ${ }^{1}$, U. Wohlrab ${ }^{2}$, D. Schmiegelt ${ }^{2}$, R. Ruetter ${ }^{2}$;

${ }^{1}$ Diabetes Center, Medical Clinic Innenstadt, Munich, ${ }^{2}$ Diabetes Clinic, German Diabetes Center, Duesseldorf, Germany.

Background and Aims: In previous studies we have demonstrated that stem cells can be isolated from adult pancreas (APSCs). The aim of the present study was to investigate the mechanisms of stem cell proliferation and differentiation into insulin-producing cells.

Materials and Methods: The expression of stem cell associated markers (BCRP1, nestin, Sox, CK19), markers of progenitor cells (Pdx1, Ngn3, Nkx6.1, Pax4) and proinsulin were analysed in two murine stem cell lines (mI5 and $\mathrm{mH} 1$ ) by flow cytometry and RT-PCR before and after the induction of differentiation. In addition, we studied gene expression programmes known to be involved in the regulation of proendocrine genes.

Results: Both the $\mathrm{mI} 5$ and the $\mathrm{mH} 1$ cells were positive for nestin, BCRP1, Sox1 and the beta cell progenitor marker Pdx1. By changing the cell culture conditions, it was possible to induce the expression of Ngn3, as well as proinsulin I and II. However, Ngn3 and insulin mRNA and protein expression was low as compared to primary isolated islet cells. Analysis of the Notch signaling pathway revealed that Notch1 and Hes1 were highly expressed under basal condition and were only slightly decreased after the differentiation phase.

Conclusion: We here demonstrate for the first time that the Notch-Hes1 pathway is activated in APSCs. It is known that Hes 1 strongly suppress Ngn3 expression. This could be one explanation for the incomplete in vitro differentiation and the low number of insulin positive cells. Thus, inhibition of Hes1 expression could be a target to further improve the in vitro generation of insulin-producing cells from adult stem cells.
0474

Human exocrine pancreas-derived mesenchymal stem cells and their potential to differentiate into beta cells C. Gonelle-Gispert ${ }^{1}$, R. M. Baertschiger ${ }^{1}$, P. Morel ${ }^{1}$, D. Bosco ${ }^{2}$, M. Armanet ${ }^{2}$, A. Wojtusciszyn ${ }^{2}$, V. Serre-Beinier ${ }^{1}$, T. Berney ${ }^{2}$, L. Buhler ${ }^{1}$; ${ }^{1}$ Dpt of Surgery, University Hospital Geneva, Surgical Research Unit, ${ }^{2}$ Dpt of Surgery, University Hospital Geneva, Cellular Isolation and Transplantation Laboratory, Switzerland.

Background: Transplantation of in vitro generated islets or insulin producing cells represents an attractive option for treatment of type 1 diabetes. Therefore, stem or progenitor cells with the capacity to differentiate into beta cells have to be identified. In this study we isolated and expanded mesenchymal stem cells (MSC) obtained from human exocrine pancreas and we investigated their potential to differentiate into beta cell.

Methods: We have cultured human exocrine pancreatic tissue obtained after isolation and purification of pancreatic islets for clinical transplantation in expansion media for human MSC (IMDM+10\% FCS+PDGF-BB). After 28 passages, these cells were characterized by FACS and compared to human MSC isolated from bone marrow. Mesenchymal differentiation potential was tested by culturing these cells in adipogenic and chondrogenic differentiation media. In order to induce endocrine differentiation, these cells were cultured at high density on non-adherent plastic in differentiation medium (high glucose DMEM containing nicotinamide, activin A and HGF). Differentiation was assessed by RT-PCR for insulin and early endocrine markers.

Results: In 14 of 18 human pancreatic exocrine fractions with a purity of $99 \%$, adherent fibroblast-like cells appeared and could be expanded. Cells were grown up to 40 population doublings (19 passages). These cells displayed a similar antigen surface expression as bone marrow MSC, i.e. they were negative for CD31, CD34, CD45, CD106, MHC class 1, CD54low, and positive for CD44, CD90, CD105. Culture of these cells in adipogenic and chondrogenic differentiation media allowed differentiation into adipocyte-like and chondrocyte-like cells, demonstrating mesenchymal phenotype and multipotentiality. Pancreatic MSC, when cultured in differentiation medium formed pseudo-islet-like clusters and after 15 days of differentiation expressed Pdx1, Nkx2.2, Nkx6.1, NeuroD, Isl-1, insulin, glucagon and glucokinase in 6 out of 10 experiments. However, these islet-like clusters were negative for Glucokinase and Glut2.

Conclusion: Our data show that MSC are present in the exocrine fraction of human pancreas and that they can be 
expanded extensively. These cells have the potential to form islets-like clusters expressing several early and late beta-and alpha-cell genes.

Supported by Swiss National Science Foundation

\section{5}

Endothelial cell involvement in proliferation and differentiation of human pancreatic duct cells

E. J. P. de Koning ${ }^{1,2}$, C. Nienaber ${ }^{1}$, S. Yatoh ${ }^{1}$, D. Patterson ${ }^{1}$, C. Rask-Madsen ${ }^{3}$, A.-J. van Zonneveld ${ }^{2}$, A. J. Rabelink ${ }^{2}$, A. Sharma ${ }^{1}$, S. Bonner-Weir ${ }^{1}$;

${ }^{1}$ Department of Islet Transplantation and Cell Biology, Joslin Diabetes Center, Boston, United States, ${ }^{2}$ Department of Nephrology, Leiden University Medical Center, The Netherlands, ${ }^{3}$ Department of Vascular Cell Biology, Joslin Diabetes Center, Boston, United States.

Background and Aims: Signals from vascular cells are required for normal pancreatic development. We hypothesized that endothelial cells modulate human duct cell proliferation and differentiation into a beta-cell phenotype.

Materials and Methods: To test this hypothesis we isolated human duct cells from tissue remaining after islet isolation. The islet-depleted tissue was dispersed by EDTA/ trypsin followed by immunomagnetic cell sorting using antibodies against the duct marker CA19-9. CA19-9+cells were expanded as monolayers during culture in DMEM medium plus $10 \%$ fetal bovine serum (expansion phase). After 5-7 days cells were dissociated and transferred into hydrophobic multiwell plates and aggregated in serum-free DMEM medium with ITS and nicotinamide for 5-6 days (differentiation phase). Bovine aortic endothelial cells (BAEC) were plated onto Transwell inserts and used at $50-70 \%$ confluency for co-culture experiments during the expansion and differentiation phases. Every other day new Transwell inserts were used. For qRT-PCR $10 \mathrm{ng}$ RNA equivalent was used.

Results: Ninety percent of dispersed cells were positive for CK19 and no insulin+cells were detected. Endothelial cells facilitated rapid attachment of dispersed CA19-9+cells and increased proliferation $(25.6 \pm 6.9$ vs $17.0 \pm 2.9 \% \mathrm{Ki} 67+$ cells after 2 days co-culture, mean \pm SD, $p<0.05 ; n=5$ ). All Ki67+ cells were positive for CK19 by double immunofluorescence assessment. Cycle threshold $\left(\mathrm{C}_{\mathrm{T}}\right)$ values for insulin and IPF1 gene expression were $30.3 \pm 3.7$ and $28.3 \pm 0.7$, respectively $(\mathrm{n}=5-8)$ in sorted CA19-9+cells by qRT-PCR. Insulin gene expression decreased 5.6 fold during expansion and increased 35.4 fold during aggregation compared to sorted CA19-9+cells. No significant change was observed in IPF1 gene expression during the expansion and differentiation phases $\left(C_{T} 28.2 \pm 1.5\right.$ and $28.6 \pm 0.6$, respectively). Endothe- lial cell co-culture attenuated the increased insulin gene expression during the differentiation phase (only 4.1 fold increase, $p<0.01 \mathrm{vs}$ control) and resulted in a non-significant decrease in IPF1 expression $\left(C_{T} 30.1 \pm 1.5 ; n=3\right)$. Endothelial-cell conditioned medium gave similar results.

Conclusion: Soluble factors from endothelial cells stimulate proliferation but attenuate differentiation of CA19-9+ human pancreatic duct cells.

Supported by EJP de Koning was supported by a Career Development Grant from the Dutch Diabetes Foundation and a Mary K Iacocca Senior Visiting Research Fellowship (Joslin Diabetes Center)

\section{6}

Identification of adult stem/progenitor cells in human exocrine pancreas

A. Fierabracci ${ }^{1}$, L. Giuliani ${ }^{1}$, R. Carsetti $^{2}$, O. Porzio $^{3}$, F. Bertuzzi ${ }^{4}$, G. Bottazzo ${ }^{5}$;

${ }^{1}$ Autoimmunity Laboratory, Children's Hospital Bambino Gesù, ${ }^{2}$ Cytofluorimetry Laboratory, Children's Hospital Bambino Gesù, ${ }^{3}$ Biochemistry Department, Tor Vergata University, Rome, Italy, ${ }^{4}$ Cell Therapy for Type 1 Diabetes Unit, St Raffaele Scientific Institute, Milan, ${ }^{5}$ Scientific Directorate, Children's Hospital Bambino Gesù, Rome, Italy.

Background and Aims: In recent years, evidence is provided that tissue-specific stem cells reside in certain adult organs; yet their specific properties remain elusive because of their rarity, technical difficulty in identifying them and characterizing their progeny. Nevertheless, while haematopoietic stem cells can be isolated nowadays using panels od surface markers, such strategies have not been convincingly applied to stem cells of most compact organs. With regard to the endocrine system, several potential adult islet/beta cell progenitors derived from pancreatic islets, liver and bone marrow have been studied, but, to date, no candidate cell has been fully characterized or is clinically applicable. Beside haematopoietic stem cells, neural stem cells, seem to be the cells most convincingly characterised. In this study, we aimed to device a method for isolating stem/progenitor cells from the human exocrine pancreas.

Materials and Methods: In initial experiments, tissue specimens from human exocrine pancreas were enzymatically digested; selected fragments, residual to digestion were plated and cultured in the presence of EGF and bFGF. Results: Spheroids with self replicative potential were obtained. Primary spheroids were harvested after 7-10 days; they were mechanically dissociated into single cells and replated for generating secondary cultures. The different 
lines of spheroids, generated from different pancreatic specimens, were characterised in flow cytometry (FACS). In addition to elements negative for pancreatic differentiation markers, a population of cells within spheroids was positive for insulin, glucagon, somatostatin, cytokeratin and pancreatic homeobox protein IDX-1. Stem cell markers identified a small percentage of CD34+CD45- cells. The percentage of positive cells varied within the different lines. Spheroids were also studied in RT-PCR; mRNA of nestin, a known marker of neurospheres, was detected. To verify whether spheroids could be induced to produce insulin, replicate cultures from the same spheroid lines were functionally evaluated in $3 \mathrm{D}$ collagen gel cultures. While in basal conditions (EGF, bFGF) a low level of insulin production was detectable in culture supernatants, progressively increasing levels of insulin were obtained in the presence of differentiating factors. We also attempted to verify whether spheroids obtained from the exocrine pancreas could acquire phenotypic characteristics of neuronal lineage. We therefore co-cultured CFDA labelled spheroids with the c-kit positive neuroblastoma cell line LAN-5. In FACS analysis, a population of cells expressing c-kit, was obtained. These cells were of a larger size compared with cells in the spheroidal population when kept in basal conditions, but smaller than neuroblastoma cells.

Conclusion: We conclude that a population of stem/ progenitor cells exists within the human exocrine pancreas. Our data support the concept of an intrinsic plasticity of the spheroids that showed in functional studies ability to generate insulin-producing cells and potentially non-pancreatic cells.

Supported by Italian Ministry of Health

\section{7}

Defective IGF-2 and IGF-1R protein expressions in pancreatic rudiment precede beta cell mass anomaly in GK rat

S. Calderari ${ }^{1}$, M.-N. Gangnerau ${ }^{1}$, M. Thibault ${ }^{1}$, M.-J. Meile ${ }^{1}$, C. Alvarez $^{2}$, B. Portha ${ }^{1}$, P. Serradas ${ }^{1}$;

${ }^{1}$ Université Paris 7, Lab. Physiopathol. Nutr. CNRS UMR 7059, Paris, France, ${ }^{2}$ Facultad de Farmacia, Universidad Complutense, Departemento de Bioquimica y Biologia Molecular, Madrid, Spain.

Background and Aims: The Goto-Kakizaki (GK) rat is a spontaneous model of type 2 diabetes with a defective betacell mass detectable in late fetal age. To determine the involvement of IGF-2 in this defect, we evaluated during GK pancreatic organogenesis: 1) the GK beta-cell development on embryonic day (E) 13.5 and E18.5; 2) IGF-2 and IGF-1 receptor (IGF-1R) pancreatic protein expressions at E13.5 and E18.5; 3) the in vitro development of E13.5
GK pancreatic rudiment; 4) the effect of IGF-2 addition on beta-cell mass in pancreatic rudiment in vitro.

Materials and Methods: GK rats were obtained from our local colony. Wistar (W) rats were used as control. Beta-cell quantitative analyses were determined by immunohistochemistry and morphometry at E13.5, E18.5 and in pancreatic rudiments after culture. IGF-2 and IGF-1R pancreatic protein expressions were evaluated using Western blot analyses at E13.5 and E18.5. For culture experiments, dorsal pancreatic rudiments were dissected at E13.5, separated from its surrounding mesenchyme and cultured for 7 days onto three-dimensional collagen gel at $5.5 \mathrm{mM}$ of glucose. Recombinant IGF-2 (100 ng/ml) was added every day to the culture medium. To label cells in the $\mathrm{S}$ phase, BrdU was added to the culture medium one hour before the end of the culture. Apoptosis in pancreatic rudiment was evaluated using the ApopTag kit.

Results: 1) The number of beta-cell was normal in GK pancreatic rudiment at E13.5. However, pancreatic beta-cell mass was decreased by $74 \%$ in E18.5 GK as compared to $\mathrm{W}$ control at the same age. 2) IGF-2 and IGF-1R protein expressions were respectively decreased by $27 \%$ and $42 \%$ in E13.5 GK pancreatic rudiments as compared to $\mathrm{W}$ controls. Again, IGF-2 and IGF-1R pancreatic protein expressions were decreased (by 58\% and 34\% respectively) in GK at E18.5 as compared to W values. 3) After 7 days of culture, GK pancreatic rudiments exhibited a beta-cell number reduced by $78 \%$. This beta-cell defect was similar to that observed in E18.5 GK. After culture, the total pancreatic-cell and the beta-cell proliferation rates in GK pancreatic rudiment were decreased respectively by $27 \%$ and by $40 \%$ as compared to $\mathrm{W}$ values. Moreover, the apoptotic rate of total cells was increased by $42 \%$ in GK pancreatic rudiment after culture. 4) The daily addition of IGF-2 to GK pancreatic rudiment increased the beta-cell number by $123 \%$, the total cell proliferation rate by $55 \%$, and the beta-cell proliferation rate by $111 \%$ as compared to GK pancreatic rudiment cultured without IGF-2.

Conclusion: Taken together these data show that while beta-cell mass is already decreased at E18.5, the differentiation of the first beta cells is in fact normal in E13.5 GK pancreas. Thus, a defective IGF-2 and IGF-1R protein expressions within the GK pancreatic rudiment represent a primary anomaly. The isolated GK pancreatic rudiment as maintained in vitro under the conditions here described, mimicks the GK beta-cell deficiency as observed in vivo. This in vitro approach was useful to highlight the effect of an IGF-2 supplementation on GK beta-cell growth. Moreover, it also suggests that, IGF signaling pathway seems to be functional in GK beta cells in response to IGF-2.

Supported by doctoral fellowships from the Ministère de l'Education Nationale and the Fondation pour la Recherche Médicale (S. Calderari) 
0478

Diabetes impairs ischaemia and cytokine-mediated mobilisation of progenitor cells in rats

G. Fadini ${ }^{1}$, S. Sartore ${ }^{2}$, I. Baesso ${ }^{1}$, C. Agostini ${ }^{1}$, A. Avogaro ${ }^{1}$;

${ }^{1}$ Dept Clinical and Experimental Medicine, ${ }^{2}$ Dept Biomedical Sciences, University of Padova, Italy.

Background and Aims: Endothelial progenitor cells (EPCs) represent a fraction of bone marrow-derived pluripotent stem cells which can be mobilized to the peripheral circulation in response to many stimuli, including tissue ischemia and cytokines. Once in the peripheral blood, EPCs are involved in cardiovascular homeostasis as they have the potential to repair the damaged endothelium and take part in compensatory neovascularization. Conversely, a decrease in the EPC pool is associated with established atherosclerosis and increased cardiovascular damage. Mechanisms involved in the pathogenesis of diabetic macrovascular complications include impaired angiogenic response to ischemia. Therefore, reduction of circulating EPC level may be a plausible cause of increased cardiovascular risk in diabetes mellitus. Causes of EPC decrease may include shortened peripheral survival and defective bone marrow stimulation. This study was undertaken to test the hypothesis that a weak ischemia- and cytokine-induced bone marrow mobilization is responsible for decrease of circulating EPCs in diabetes. Moreover, possible favorable effects of glucose lowering on EPC mobilization were also assessed.

Materials and Methods: We employed a model of hind limb ischemia-reperfusion (IR) injury in control and diabetic rats, 4 weeks after streptozotocin induction. Peripheral blood EPCs were defined as Sca- $1^{+} \mathrm{c}-\mathrm{kit}^{+}$cells and were counted by flow cytometry. Capillary density was quantified by vWF immunofluorescent staining of muscle sections. Plasma levels of stromal cell derived factor (SDF-1) and vascular endothelial growth factor (VEGF) were measured by ELISA. Protein levels of hypoxia-induced factor (HIF$1 \alpha$ ) were determined with Western Blot analysis.

Results: In control animals, EPCs increased after ischemia and described a mobilization curve within 7 days, while diabetic rats were completely unable to mobilize EPCs after IR injury. As a consequence, diabetic rats had no compensatory increase in muscle capillary density, while a $50 \%$ increase was found in control rats. Defective EPC mobilization in diabetic animals was associated with altered release of SDF-1 and VEGF and with inability to upregulate HIF- $1 \alpha$ in muscles subjected to the IR injury. Insulin administration to achieve near-normal glucose levels during ischemia and reperfusion periods increased VEGF and determined a partial recovery of EPC mobilization in diabetic rats.
Administration of a combination of $50 \mu \mathrm{g} / \mathrm{kg}$ granulocytecolony stimulating factor (G-CSF) and $200 \mu \mathrm{g} / \mathrm{kg}$ stem cell factor (SCF) daily for 5 days stimulated mobilization of EPC in diabetic rats, but to a lesser extent when compared to control rats. However, a premedication based on G-CSF plus SCF was able to promote EPC mobilization after IR injury in diabetic animals.

Conclusion: Defective ischemia- and cytokine-mediated bone marrow mobilization causes reduction of circulating EPCs in diabetes and impairs compensatory angiogenesis in ischemic tissues. Inability to up regulate hypoxia-sensing systems in the target tissue together with an intrinsic bone marrow deficit seems to be responsible for this pathophysiological alteration. Growth factor administration together with blood glucose control may represent in perspective a rational therapeutic strategy for diabetic ischemic syndromes.

This work was supported in part by a grant from Consortium Agreement European Community Heart Failure and Cardiac Repair-IP 018630

0479

Generalised immaturity of metabolic pathways in neonatal $\beta$ cells

A. Jermendy ${ }^{1}$, E. Toschi ${ }^{1}$, A. Koh ${ }^{1}$, T. Aye ${ }^{1}$, A. Sharma ${ }^{1}$, G. C. Weir ${ }^{1}$, D. C. Sgroi ${ }^{2}$, I. Leykin ${ }^{1}$, S. Bonner-Weir ${ }^{1}$;

${ }^{1}$ Islet Transplantation and Cell Biology, Joslin Diabetes Center, Boston, ${ }^{2}$ Molecular Pathology Unit, Massachusetts General Hospital, Boston, United States.

Mature $\beta$-cells are characterized by glucose stimulated insulin secretion (GSIS) that results from specialised metabolic pathways. Fetal and neonatal $\beta$-cells show an impaired insulin secretion in response to glucose but not to aminoacids. Only by 1 week after birth is glucose responsiveness established. The basis of this difference between neonatal and adult $\beta$-cells remains unclear. We hypothesize that this unresponsiveness is due to a generalised immaturity of the metabolic pathways found normally in $\beta$-cells. Our aim was to compare neonatal and adult $\beta$-cells by performing gene expression studies on laser capture microdissected $\beta$-cell enriched cores of neonatal (1-day-old) and adult (6-week-old) pancreas ( $\mathrm{n}=4$ each). RNA was extracted from the samples and amplified twice using T7 polymerase and hybridized to U34A Rat Genome microarrays. dChip software ( 2 fold differences, $\mathrm{p}<0.05$ ) was used to analyze the differences between the neonatal and adult samples; 215 genes/ESTs were significantly higher and 375 significantly lower in neonatal than adult $\beta$-cell-enriched cores. Among the genes poorly expressed in the neonatal $\beta$-cells were key metabolic genes including membrane mitochondrial shuttles (malate dehydrogenase, glycerolphosphate dehydrogenase, aspartate aminotransferase, malic enzyme), pyruvate carboxylase and carnitine palmitoyl 
transferase (CPT) II. Interestingly, CPT I was found to be higher in the neonates. These enzymes play key roles in the insulin secretion mechanism. These microarray findings were confirmed by real time PCR on 5 independent samples of isolated neonatal and adult islets (using the $\Delta \Delta \mathrm{C}_{\mathrm{T}}$ analysis, 313 fold lower in neonatal islets, $\mathrm{p}<0.01$ ). Thus, the lack of glucose responsiveness in neonatal islets may be due to a generalized immaturity of the metabolic specialization of pancreatic $\beta$-cells.

\section{PS 25}

\section{Transcriptional regulation in beta cells}

\section{0}

Mice lacking Hnf1 $\alpha$ (MODY3) reveal that gene activity is linked to nuclear compartmentalisation of histone modifications

R. F. Luco, M. A. Maestro, J. Ferrer; Endocrinology, IDIBAPS, Barcelona, Spain.

Background and Aims: Mutations in the transcriptional activator gene HNF1 $\alpha$ (also known as TCF1 or MODY3) are the major cause of monogenic diabetes, and represent a paradigm for a human transcriptional disease. HNF1 $\alpha$ is known to induce the acetylation of histones at target chromatin. Histone tail modifications such as acetylation and methylation are thought to be critically important in gene transcription. Recent evidence also indicates that gene regulation is associated with repositioning of loci within the nucleus. However, the possible relationship between histone modifications and nuclear topology concerning gene transcription is uncertain.

Material and Methods: Chromatin immunoprecipitation (ChIP) and confocal immunofluorescence-FISH analysis have been carried out in freshly isolated Hnfl $\alpha$-deficient and wild-type hepatocytes and islets.

Results: We show that the nucleosomes of genes that become silenced in Hnfl $\alpha$-deficient cells exhibit reduced methylated H3-Lys4 and increased methylated H3-Lys27. We also show that RNA Polymerase II and trimethylated H3-Lys27 are enriched in mutually exclusive nuclear domains. Remarkably, genes that become silenced in Hnfl $\alpha$-deficient cells reposition from nuclear domains enriched in methylated H3-Lys4 and RNA polymerase II to domains enriched in methylated H3-Lys27.

Conclusion: This study provides a novel insight into the defect underlying MODY3, and suggests that transcription is linked to histone code modifications not only locally, but also to its representation in nuclear space.

Supported by Ministerio de Educación y Ciencia and the European Commission
0481

Effect of aging on the expression of beta cell functionrelated genes in islets

S.-H. Ihm ${ }^{1}$, H. Moon ${ }^{1}$, J. Kang ${ }^{1}$, C. Park ${ }^{1}$, S. Park ${ }^{1}$, J. Ihm² ${ }^{1}$ Internal Medicine, Hallym University, Anyang, ${ }^{2}$ Biochemistry, Kyonggi University, Suwon, Republic of Korea.

Background and Aims: Age-related change in insulin secretory function may contribute to the alterations in glucose metabolism with age. Recently, the glucosestimulated insulin release of isolated human islets has been shown to deteriorate progressively with advancing donor age. This decline in beta cell function with aging may contribute to the increasing development of IGT and type 2 diabetes and also to the progressive nature of the disease. The aim of this study was to see whether there is any change in mRNA expression of beta cell-function-related genes in islets with aging.

Materials and Methods: Pancreatic islets were isolated from young (2 mo-old) and old (22-24 mo-old) LETO rats and $\mathrm{C} 57 \mathrm{BL} / 6 \mathrm{~N}$ mice. After overnight culture, in vitro glucose-stimulated insulin release was assessed by incubating in medium with either 1.7 or $16.7 \mathrm{mM}$ glucose for 1 hour. Analysis of mRNA levels of beta cell function-related genes was done with real-time RT-PCR with RNAs ( $\mathrm{n}=3-6 /$ group) isolated from pooled islets (3-5 animals/sample).

Results: The in vitro glucose-stimulated insulin release index was significantly lower in islets from old mice compared with young mice (2.7 vs. 1.3). PDX-1, insulin, GLUT2 and prohormone convertase 1/3 gene expression in islets was markedly lower in old rats $(33 \%, 13 \%, 20 \%$ and $34 \%)$ and old mice $(42 \%, 35 \%, 19 \%$ and $15 \%)$ compared with young animals: mRNA expression of Foxa2 and HNF1alpha in islets was similar regardless of mice age. On the other hand, genes not related to beta cell-specific function, such as caspase 3, ATP synthase and glycerol kinase were not significantly different in expression in islets according to age. UCP-2, known to suppress glucosestimulated insulin release when overexpressed in islets, was not increased in mRNA expression in old animal islets.

Conclusion: With increasing age, insulin secretory function of islets deteriorates accompanied with a decrease in expression of insulin synthesis/secretion-related genes, such as PDX-1, GLUT2 and prohormone convertase.

0482

Cytokines and dsRNA induce expression and activation of HIF- $1 \alpha$ in rat pancreatic beta cells

L. Ladrière, D. Flamez, F. Ortis, J. Rasschaert,

D. L. Eizirik; 
Laboratory of Experimental Medicine, Université Libre de Bruxelles, Belgium.

Background and Aims: Type 1 diabetes mellitus (T1D) is an autoimmune disease characterized by a progressive destruction of pancreatic beta cells. Viral infections and cytokines contribute to the pathogenesis of T1D. Exposure of primary rat beta cells to the viral product double stranded RNA (dsRNA; tested in the synthetic form PIC), in combination with interferon- $\gamma$ (IFN- $\gamma$ ), induces beta cell apoptosis. By microarray analysis, PIC and/or cytokines were shown to induce expression of several genes and transcription factors, including the hypoxia-inducible factor $1 \alpha(\mathrm{HIF}-1 \alpha)$ and several genes downstream of this transcription factor, such as Glut-1, VEGF and hexokinase II. HIF- $1 \alpha$ is induced during hypoxia and its activated form plays a key role in the regulation of genes implicated in metabolism, cellular proliferation and viability. We presently examined the regulation of HIF-1 $\alpha$ in primary rat beta cells and insulin-producing INS-1E cells. PC12 cells were used as positive controls.

Material and Methods: FACS-purified primary rat beta cells, INS-1E cells and PC12 cells were exposed for $30 \mathrm{~min}, 2,6,12$ and 24 hours to IL-1 $\beta$ (10-50 U/ml) and/ or IFN- $\gamma(100-1000 \mathrm{U} / \mathrm{ml})$ in the presence or absence of PIC $(100 \mu \mathrm{g} / \mathrm{ml})$ and/or the iNOS blocker NMA (1 mM). mRNA expression was examined by real-time RT-PCR and corrected for GAPDH expression. Activation of the HIF-1 promoter was studied by luciferase reporter assay. As positive control for HIF induction, we exposed the cells to cobalt chloride (CoCl2; $150 \mu \mathrm{M})$.

Results: Exposure of primary rat beta cells or INS-1E cells to IL-1 $\beta$ induced a $2-3$ fold increase in HIF-1 $\alpha$ mRNA expression after 24 hours $(\mathrm{P}<0.05 ; \mathrm{n}=8)$, while treatment with IL- $1 \beta+$ IFN- $\gamma$ induced an earlier and more pronounced increase in HIF-1 $\alpha$ mRNA expression, which was already significant after a 6-hour treatment $(\mathrm{P}<0.05 ; \mathrm{n}=4)$, and reached the highest levels after 24 hours (16.5 in cytokinetreated vs 5.2 in control beta cells; $\mathrm{P}<0.001 ; \mathrm{n}=5)$. In primary beta cells, PIC in combination with IFN- $\gamma$ also induced a significant increase in HIF-1 $\alpha$ mRNA expression after 24 hours (20.0 in cytokine-treated vs 6.0 in control cells; $\mathrm{P}<0.01 ; \mathrm{n}=5)$. The addition of NMA did not affect HIF- $1 \alpha$ induction, indicating that HIF- $1 \alpha$ expression does not depend on nitric oxide formation. In primary beta cells, exposure to IL- $1 \beta+\mathrm{IFN}-\gamma$ or to $\mathrm{CoCl} 2$ activated an HIF- $1 \alpha$ luciferase promoter-reporter after a 12-hour treatment $(2.5$ and 3.6 fold increase respectively ; $\mathrm{P}<0.05$ vs control; $\mathrm{n}=5$ ). In PC12 cells, a 12-hour exposure to $\mathrm{CoCl} 2$ also increased activity of the reporter construct ( 4.8 fold increase ; $\mathrm{P}<0.01$ vs control; $n=3$ ).

Conclusion: These data indicate that exposure of primary rat beta cells to either IL- $1 \beta$, IL- $1 \beta+\mathrm{IFN}-\gamma$ or PIC + IFN- $\gamma$ induces HIF-1 $\alpha$ mRNA expression. Exposure of INS-1E cells to IL- $1 \beta$ or to a combination of IL- $1 \beta+$ IFN- $\gamma$ also induces HIF-1 $\alpha$ mRNA. In primary rat beta cells, IL- $1 \beta+$ IFN- $\gamma$ activates an HIF- $1 \alpha$ promoter-reporter, suggesting that HIF- $1 \alpha$ is functional in these cells. Induction of HIF-1 $\alpha$ expression is probably part of the adaptive stress responses of primary rat beta cells exposed to agents that impair cell metabolism.

Supported by the Fonds National de la Recherche Scientifique and Actions de Recherche Concertées (ARC) de la Communauté française

\section{3}

Posttranslational modifications of the transcription factor Sp1 regulate glucose-induced $\mathrm{NF}_{\kappa} \mathrm{B}$ p65 expression Y. Seregin ${ }^{1}$, O. Voronko ${ }^{1}$, I. Konrade ${ }^{1}$, A. Yurochko ${ }^{2}$, P. P. Nawroth ${ }^{1}$, A. Bierhaus ${ }^{1}$;

${ }^{1}$ Department of Medicine I, University of Heidelberg, Germany, ${ }^{2}$ Department of Microbiology and Immunology, Louisiana State University Health Sciences Center, Shreveport, United States.

Background and Aims: The transcription factor NF-кB plays an important role in diabetes. The molecular mechanisms underlying the observed long-term activation of NF- $\mathrm{K}$ Bp65 mRNA transcription in diabetes are yet unknown. Detailed characteristics of the p65 promoter shows several binding sites for the transcription factors $\mathrm{Sp} 1 / \mathrm{Sp} 3$, implying that these proteins might act as transactivators of $\mathrm{p} 65$ de novo synthesis under hyperglycemic conditions. While Sp1 expression is not modulated by high glucose, we studied the role of glucose-mediated $\mathrm{Sp} 1$ posttranslational modifications. We characterized the influence of Sp1 O-glycosylation and phosphorylation for NF- $\mathrm{B}$ p65 gene transcription regulation under high glucose.

Materials and Methods: Binding capacity of Sp1 and Sp3 was measured in THP-1 and BAEC cells using electrophoretic mobility shift assay (EMSA). Full-length p65 promoter and its deletion mutants lacking Sp1 binding sites were cloned into luciferase reporter vector pGL3 and transfected into THP-1 and BAEC cells. Posttranslation modifications of Sp1 (O-glycosylation, phosphorylation and nitrotyrosine residues) were characterized by immunoprecipitation followed by immunoblotting. The transcription of NF-кB p65, O-GlcNAcase and O-GlcNAc transferase was determined by RT-PCR.

Results: High glucose (30 mM) induced a time- and dosedependent activation of $\mathrm{Sp} 1 / \mathrm{Sp} 3$ binding capacity to consensus Sp1 sequence. Transient transfection of p65 promoter constructs indicated that deletion of every $\mathrm{Sp} 1$ binding site significantly impaired NF-кB p65 expression. 
Consistently, Sp1-antisense blocked NF-кB p65 mRNA synthesis. Spl is constitutively phosphorylated and Oglycosylated, however, Sp1 O-glycosylation was dramatically increased immeadiately after high glucose induction, followed by continuous deglycosylation and increasing phosphorylation. Furthermore, the expression of O-GlcNAc transferase and O-GlcNAcase, enzymes, responsible for Sp1 O-glycosylation, was modulated by high glucose. Maximal expression of O-GlcNAcase, minimal Sp1 Oglycosylation and p65 mRNA de novo synthesis were achieved in the same time.

Conclusion: High glucose modulates posttranslational modifications and transactivation capacity of $\mathrm{Sp} 1$, which results in NF-кB p65 mRNA de novo synthesis in both, monocytic and endothelial cells.

Supported by SFB 405

\section{4}

Calcium entry is involved in 804G-ECM-induced NF$B$ activity and actin cytoskeleton remodelling E. B. Hammar ${ }^{1}$, G. Parnaud ${ }^{1}$, P. Ribaux ${ }^{1}$, D. Bosco ${ }^{2}$, P. A. Halban ${ }^{1}$;

${ }^{1}$ Genetic Medicine and Development, University Medical Center, Geneva, ${ }^{2}$ Surgery, University Hospital Geneva, Switzerland.

Background and Aims: Pancreatic beta cells cultured on a laminin-5 rich ECM (804G-ECM) display increased spreading and improved function (glucose-stimulated insulin secretion). It was shown previously that $\mathrm{Ca}^{2+}$ entry is involved in 804G-ECM induced cell spreading, that $804 \mathrm{G}$ ECM induces NF-кB transcriptional activity and that this activity is involved in $804 \mathrm{G}-\mathrm{ECM}$-induced beta cell spreading, cytoskeleton remodelling and beta cell function. The aim of this work was to study the involvement of $\mathrm{Ca}^{2+}$ entry in $804 \mathrm{G}-\mathrm{ECM}$-induced NF-кB activity and in the impact of $804 \mathrm{G}-\mathrm{ECM}$ on actin cytoskeleton remodelling.

Materials and Methods: Diazoxide (which impairs depolarization and $\mathrm{Ca}^{2+}$ entry) and SR-7037 (L-type $\mathrm{Ca}^{2+}$ channel blocker) were used to inhibit calcium entry. NF-кB nuclear translocation was assessed by immunofluorescence (after $1 \mathrm{~h}$ of culture), and expressed as the number of cells with dominant NF- $\mathrm{B}$ nuclear staining, normalized to control (804G-ECM=100\%). NF-кB transcriptional activity was analyzed by quantification of mRNA levels of $\mathrm{I} \kappa \mathrm{B} \alpha$, an established NF-кB target gene, relative to L3 RNA levels (normalized to $\mathrm{pLL}=1$ ) by real-time RT-PCR ( $4 \mathrm{~h}$ of culture). Actin cytoskeleton remodelling was analyzed by confocal immunofluorescence using phalloidin or antibodies specific for $\beta$ - or $\gamma$-actin isoforms. All experiments were performed with primary rat pancreatic beta cells purified by FACS. Data are means \pm SEM ( $\mathrm{n}=3$ independent experiments).

Results: Treatment of beta cells with $\mathrm{Ca}^{2+}$ entry blockers reduced $804 \mathrm{G}-\mathrm{ECM}$ induced $\mathrm{NF}-\kappa \mathrm{B}$ nuclear translocation significantly (804G-ECM control=100 \pm 37.31 , 804G-ECM+ diazoxide $=51.84 \pm 11.93(\mathrm{p}<0.02$ vs control); 804G-ECM+ SR-7037 $=61.43 \pm 10.63(\mathrm{p}<0.03$ vs control $))$. Furthermore, $804 \mathrm{G}-\mathrm{ECM}$-induced overexpression of the NF-кB target gene IкB $\alpha$ was significantly inhibited in cells treated with the $\mathrm{Ca}^{2+}$ entry blockers $(804 \mathrm{G}-\mathrm{ECM}$ control $=3.3 \pm 0.49$, $804 \mathrm{G}-\mathrm{ECM}+$ diazoxide $=1.5 \pm 0.16 \quad(\mathrm{p}<0.01$ vs control $)$, $804 \mathrm{G}-\mathrm{ECM}+\mathrm{SR}-7037=1.6 \pm 0.45$ ( $\mathrm{p}<0.03$ vs control)). Actin cytoskeleton remodelling was induced in cells cultured on 804G-ECM and use of actin isoform-specific antibodies revealed that $\gamma$-actin is the major remodelled isoform. Furthermore, $\mathrm{Ca}^{2+}$ entry blockers diazoxide and SR-7037 both perturb 804G-ECM-induced $\gamma$-actin and total actin reorganization.

Conclusion: These results show that $\mathrm{Ca}^{2+}$ entry is involved in 804G-ECM-induced NF-кB activity and in 804G-ECMinduced $\gamma$-actin cytoskeleton remodelling. Future work is aimed at identifying the link between $\mathrm{Ca}^{+2}$ entry and NF-K $B$ activity, that is involved in $804 \mathrm{G}-\mathrm{ECM}$-induced actin cytoskeleton remodelling and improved insulin secretion.

\section{5}

Insulin-stimulated c-fos gene transcription in pancreatic $\beta$ cells requires clathrin-dependent endocytosis of IR-B and signaling from early endosomes

B. Leibiger, T. Moede, S. Uhles, P.-O. Berggren,

I. B. Leibiger;

The Rolf Luft Research Center for Diabetes and Endocrinology, Karolinska Institutet, Stockholm, Sweden.

Background and Aims: Research from over the last seven years provides convincing evidence that beside liver, muscle and fat, also the insulin-producing $\beta$-cell is a target for positive insulin action. Here insulin has been shown to be involved in maintaining normal $\beta$-cell function by regulating processes such as transcription, translation, ion fluxes, cytoprotection and/or proliferation and, therefore, might be important for maintaining $\beta$-cell mass. By studying the insulin-dependent activation of three candidate genes, i.e. insulin, glucokinase (BGK) and c-fos genes, we have previously shown that the two insulin receptor (IR) isoforms IR-A and IR-B contribute to the selective action of insulin. Insulin activates its own gene by signaling through IR-A/PI3K Ia/p70s6k, it activates BGK via IR-B/PI3K$\mathrm{C} 2 \alpha / \mathrm{PKB}$ and c-fos via IR-B/Shc/MEK/ERK. While activation of the insulin and BGK genes occurs from receptors situated in different microdomains of the plasma 
membrane, c-fos gene transcription is sensitive to the action of dominant-negative dynamin-K44A suggesting the involvement of receptor endocytosis. The aim of this study was to identify the mechanisms that contribute to dynamindependent activation of c-fos gene transcription by insulin. Materials and Methods: We employed c-fos mRNA analysis, c-fos-promoter-driven reporter gene (GFP or DsRed) expression in online monitoring studies with transient coexpression of effector proteins. To discriminate between caveosome- and clathrin-dependent endocytosis, we studied the effect of co-expressed caveolin forms and that of the negative-interfering form of the AP2 subunit $\mu 2$, i.e. $\mu 2$ T165A. The involvement of the early and late endosome pools in IR-B-mediated signaling was analyzed by subcellular fractionation and the effect of co-expressed Rab5 and Rab7 variants, small GTP-binding proteins involved in the regulation of membrane trafficking in the endocytic pathway.

Results: Our data show that c-fos gene activation by insulin requires clathrin- but not caveosome-dependent endocytosis of IR-B with the integrity of the juxtamembrane GPLYmotif for receptor internalization. Preparation of early endosomes showed the presence of tyrosine-phosphorylated, i.e. activated, IR-B. The involvement of the early endosome pool but not that of late endosomes in IR-Bmediated activation of c-fos gene transcription was shown by the effect of dominant-negative Rab5a-S34N and Rab7$\mathrm{T} 22 \mathrm{~N}$, respectively.

Conclusion: Our data provide evidence that IR-B mediated activation of c-fos gene transcription in insulin-producing cells requires clathrin-dependent endocytosis of the receptor and signaling from the early endosome pool. These data lend further support to the concept that spatial segregation of selective signaling pathways originating from IR-A and IR-B allows the simultaneous activation of discrete signaling cascades that lead to specific insulin effects.

Supported by Karolinska Institutet, Swedish Research Council, the Family Stefan Persson Foundation and JDRF

\section{6}

Inhibition of the glucose-6-phosphatase gene expression by $\mathrm{TNF} \alpha$ requires the activation of IKK $\beta$

K. Scheffler ${ }^{1}$, A. Kienitz ${ }^{1}$, D. Schmoll ${ }^{2}$, R. Walther ${ }^{1}$, R. Grempler ${ }^{3}$;

${ }^{1}$ Department of Medical Biochemistry and Molecular Biology, University of Greifswald, ${ }^{2}$ Sanofi-Aventis, Frankfurt, ${ }^{3}$ Boehringer-Ingelheim GmbH\&CoKG, Biberach, Germany.

Background and Aims: Type II Diabetics exhibit fasting hyperglycaemia which is in part caused by permanent overexpression of the key gluconeogenic enzymes glucose6-phosphatase (G6Pase) and PEPCK due to insulin resistance of the liver. Proinflammatory cytokines like TNFa cause a strong inhibition of basal and induced G6Pase transcription and may contribute to the hypoglycaemia observed during the final stages of a sepsis. Recently, we have shown that $\mathrm{TNF} \alpha$ represses the G6Pase gene expression via $\mathrm{NF \kappa B}$ activation.

Here we analysed whether the IKK complex is necessary for the inhibition of G6Pase gene expression, but on the other hand leads to an insulin resistance in H4IIE rat hepatocytes.

Materials and Methods: Wild type IKK $\beta$ (IKK $\beta$-WT), a dominant negative (IKK $\beta$-S177/181A; IKK $\beta$-DN) and a constitutively active IKK $\beta$ (IKK $\beta$-S177/181D; IKK $\beta$-CA) were characterised by an in vitro kinase assay with IкB $\alpha$ as a substrate. The G6Pase promoter construct $(-1227 /+57)$ and a minimal thymidine kinase promoter containing two consensus $\mathrm{NF \kappa B}$ binding elements (TK-NFкB) were analysed in H4IIE rat hepatoma cells by promoter reporter gene assays. Endogenous G6Pase expression was assessed by Northern Blot. IKK $\beta$-DN which blocks the activation of the endogenous IKK complex was overexpressed using recombinant adenovirus. Phosphorylation of Proteinkinase $\mathrm{B}$ (Akt) in response to insulin with and without TNF $\alpha$ preincubation was detected by a specific phospho Ser473Antibody in western blots.

Results: To show that IKK $\beta$ activity is sufficient to suppress G6Pase gene expression we cotransfected the G6Pase promoter construct together with the wild type IKK $\beta$, IKK $\beta$ $\mathrm{DN}$ and IKK $\beta$-CA. In comparison to IKK $\beta$-DN the IKK $\beta$ WT showed a significant inhibition of the G6Pase promoter by $20 \%$. The IKK $\beta$-CA construct was more effective in the suppression of the promoter $(50 \%$ inhibition) which clearly shows that the activation of IKK is sufficient to suppress basal and induced G6Pase promoter activity. Catalytic activity of each constructs was confirmed by in vitro kinase assays. In these assays IKK $\beta$-CA showed the highest activity, IKK-WT showed modest activity whereas IKK $\beta$-DN was completely inactive. The specificity of the effect on G6Pase promoter was shown by using coexpression of the respective constructs with the TK-NFkB promoter construct. As expected, promoter activity corresponds to the activity of the respective IKK $\beta$ construct.

Adenovirus mediated overexpression of IKK $\beta$-DN showed a dose dependent inhibition of ІкB $\alpha$ degradation after TNF $\alpha$ stimulation. Downregulation of endogenous G6Pase mRNA after TNF $\alpha$ stimulation is significantly inhibited after adenoviral IKK $\beta$-DN overexpression. To further prove that IKK $\beta$ is necessary for inhibition of G6Pase gene transcription by TNF $\alpha$ experiments with a specific IKK $\beta$ inhibitor are ongoing. Additionally we could not detect a reduced phosphorylation of Akt in response to insulin after TNF $\alpha$ stimulation in H4IIE cells.

Conclusion: These results demonstrate that $\operatorname{IKK} \beta$ is not only sufficient but also necessary for the suppression of endogenous G6Pase gene transcription and promoter 
activity by TNF $\alpha$ in H4IIE cells. Furthermore, TNF $\alpha$ had no effect on the phosphorylation of Akt in response to insulin which indicates that IKK $\beta$ does not cause an insulin resistance in H4IIE cells.

\section{7}

Cluster analysis of co-regulated mRNAs of rat islets exposed to low, intermediate and high glucose M. Bensellam ${ }^{1}$, L. Van Lommel ${ }^{2}$, F. C. Schuit ${ }^{2}$, J.-C. Jonas ${ }^{1}$;

${ }^{1}$ Endocrinology and Metabolism, Université Catholique de Louvain, Brussels, ${ }^{2}$ Gene Expression Unit, Dept. of Molecular Cell Biology, Katholieke Universiteit Leuven, Belgium.

Background and Aims: Rat $\beta$-cell survival and function are best preserved by culture at $10 \mathrm{mM}$ glucose (G10) and deteriorate after a few days at low (G2-G5) or high (G30) glucose. To investigate the mechanisms by which glucose affects the $\beta$-cell phenotype, we tested the effects of increasing glucose concentrations on rat islet gene mRNA levels.

Materials and Methods: Male Wistar rat islets were cultured $18 \mathrm{~h}$ in the presence of G2, G5, G10 or G30 after 1 wk preculture in RPMI medium containing G10 and $5 \mathrm{~g} / \mathrm{L}$ BSA. Directly after culture, we measured acute glucoseinduced insulin secretion (RIA) and islet gene mRNA levels (Affymetrix Rat Genome 2302.0 oligonucleotide arrays + realtime RT-PCR for selected genes) $(n=4)$. GCOS software (Affymetrix) was used to calculate match/mismatch probe signal intensities and assess differences in mRNA abundance, expressed as absolute signal $\log _{2}$ ratios (SLR). Self-Organizing Map cluster analysis (GeneCluster2) was used to categorize groups of mRNAs with similar glucose concentration-dependent changes in RNA abundance. The function of glucose co-regulated genes within each cluster was analysed with GenMapp 2.

Results: Islet insulin secretory response was best preserved after culture in G10, and was reduced by $50-75 \%$ without changes in glucose sensitivity after culture in G2G5. Culture in G30 decreased both the threshold concentration of glucose activation and caused $\sim 30 \%$ reduction in maximal glucose stimulation. In parallel to these functional changes, large scale changes were observed in the islet mRNA profile. From the 31099 probe sets on the arrays, 18081 were reliably detected in at least one glucose concentration. The SLR for at least one of the comparisons between the different glucose concentrations was $\geq 2, \geq 1$ and $\geq 0.5$ in 170,1039 and 3530 probe sets respectively. Of the latter, 3120 were classified in 6 clusters with monophasic concentration-dependencies that differed in overall direction (increase/decrease) and glucose threshold. The remaining 410 probe sets were grouped into 4 small clusters with complex mRNA expression profiles (V-shaped or inverted V-shaped with minimum or maximum in G5 or G10). Analysis of gene function in each cluster suggests the presence of coregulated metabolic and signaling pathways in rat islets. Thus, genes involved in cholesterol and unsaturated fatty acid synthesis were up-regulated between G2-G5 and G10-G30. In contrast, ATF4 target genes (integrated stress response) and other pro-apoptotic genes were downregulated from G2-G5 to G10-G30. On the other hand, most glycolytic enzymes, XBP-1 target genes (unfolded protein response) and several proteasome subunits were mainly up-regulated between G10 and G30. Finally, several stress response genes had a V-shaped mRNA expression profile with a minimum in G10 and significant increases in both G2 and G30.

Conclusion: Assessment of the islet mRNA abundance after $18 \mathrm{~h}$ exposure to a large range of glucose concentrations reveals at least 10 distinct clusters of concentrationdependent co-regulated genes. We propose such level of analysis provides additional information to comparison of two glucose concentrations in a two-state model. Our results will help identifying key molecules that maintain the differentiated $\beta$-cell phenotype as well as those responsible for $\beta$-cell deterioration after exposure to chronic hypo- or hyperglycemia.

\section{PS 26}

\section{Intracellular mediators of insulin secretion}

\section{8}

Extracellular ATP modulates insulin secretion via functionally active $\mathrm{P} 2 \mathrm{X}$ and $\mathrm{P} 2 \mathrm{Y}$ receptors

A. Solini, E. Santini, S. Cuccato, S. Madec, D. Chimenti, E. Ferrannini;

Department of Internal Medicine, University of Pisa, Italy.

Background and Aims: Extracellular ATP (eATP) modulates several cell functions via specific receptors, named $\mathrm{P} 2 \mathrm{X}$ and $\mathrm{P} 2 \mathrm{Y}$ on the basis of their molecular structure. The role of these receptors in insulin release has been little explored, and available results are controversial.

Materials and Methods: We measured insulin release by INS-1e cells grown at $11 \mathrm{mM}$ glucose and incubated at 3.3, $7.5,11$ or $20 \mathrm{mM}$ glucose for $60 \mathrm{~min}$. After chronic (48 hours) challenge with different extracellular nucleotides (ATP, BzATP, a preferential $\mathrm{P}_{2} \mathrm{X}_{7}$ agonist, and UTP, a preferential $\mathrm{P} 2 \mathrm{Y}$ agonist), insulin release and total content 
were measured by RIA. Moreover, P2 receptors (P2Rs) expression was aestimated by RT-PCR and Western blot, intracellular ATP (AATP) and eATP concentrations by luminometric assay, $\left[\mathrm{Ca}^{2+}\right]_{i}$ by fluorimetry with the fluorescent indicator Fura2/AM, and apoptosis by FACS analysis.

Results: After exposure to $0.01-1.0 \mathrm{mM}$ eATP, insulin secretion (expressed as ratio between release and content) was significantly reduced at all the glucose concentration tested (eg, with $0.5 \mathrm{mM}$ ATP: from $5.3 \pm 0.1$ to $1.9 \pm 0.2 \%$ at $3.3 \mathrm{mM}$ glucose; from $8.6 \pm 0.1$ to $4.7 \pm 0.1 \%$ at $7.5 \mathrm{mM}$; from $8.0 \pm 0.2$ to $5.0 \pm 0.3 \%$ at $11 \mathrm{mM}$; from $11.5 \pm 0.2$ to $7.4 \pm 0.4 \%$ at $20 \mathrm{mM}$ ). This inhibitory effect of eATP was fully prevented by either oxidized ATP (which blocks all P2X receptors) or apyrase (which hydrolyses ATP). In control incubations, eATP release increased with increasing glucose concentrations $(37 \%$ and $58 \%$ above baseline with glucose 11 and $20 \mathrm{mM}$ ). BzATP, a preferential $\mathrm{P}_{2} \mathrm{X}_{7}$ agonist, did not significantly influence insulin release (eg, with $0.5 \mathrm{mM}$ BzATP: from $1.2 \pm 0.3$ to $1.8 \pm 0.1 \%$ at $3.3 \mathrm{mM}$ glucose; from $4.2 \pm 0.2$ to $5.9 \pm 0.5 \%$ at $7.5 \mathrm{mM}$; from $6.2 \pm 0.7$ to $6.8 \pm 0.4 \%$ at $11 \mathrm{mM}$; from $7.7 \pm 0.8$ to $7.2 \pm 1.2 \%$ at $20 \mathrm{mM}$ ), while UTP, at concentrations between $0.01-0.1 \mathrm{mM}$, stimulated insulin release (eg, with $0.1 \mathrm{mM}$ UTP: from $2.6 \pm 0.6$ to $2.8 \pm 0.5 \%$ at $3.3 \mathrm{mM}$ glucose; from $4.2 \pm 1.0$ to $8.6 \pm 0.8 \%$ at $7.5 \mathrm{mM}$; from $7.8 \pm 1.3$ to $9.3 \pm 0.9 \%$ at $11 \mathrm{mM}$; from $6.9 \pm 1.1$ to $9.8 \pm 0.8 \%$ at $20 \mathrm{mM}) . \mathrm{A}\left[\mathrm{Ca}^{2+}\right]_{\mathrm{i}}$ rise after acute challenge with extracellular nucleotides was observed $\left(\Delta\left[\mathrm{Ca}^{2+}\right]_{\mathrm{i}}\right.$ :180 $\pm 36 \mathrm{nM}$ after stimulation with ATP $1 \mathrm{mM}$ ); UTP was less effective. With regard to receptor(s) putatively involved in this process, both P2X (subtypes 1, 3, 4, 5, 6 and 7) and P2Y receptors (subtypes 1, 2, 4 and 6) were found to be expressed, but the level of expression was unchanged following nucleotide stimulation, except for $\mathrm{P}_{2} \mathrm{X}_{1}$ and $\mathrm{P}_{2} \mathrm{X}_{7}$ (the latter was present as a splicing form), whose expression was upregulated by ATP and BzATP, as shown by RT-PCR and WB. Neither early nor late apoptosis was enhanced by nucleotide stimulation (early apoptosis: after $3 \mathrm{hrs}$ : annexin-propidium positivity: from 1.30 to 1.10 with UTP $0.1 \mathrm{mM}$, to 1.71 with BzATP $0.5 \mathrm{mM}$, to $1.86 \%$ with ATP $0.5 \mathrm{mM}$; after $24 \mathrm{hrs}$ : from 1.09 to 1.81 with UTP $0.1 \mathrm{mM}$, to 1.20 with BzATP $0.5 \mathrm{mM}$, to $0.92 \%$ with ATP $0.5 \mathrm{mM}$; late apoptosis: after $24 \mathrm{hrs:}$ hypodiploid peak lower than $1 \%$ in both basal and stimulated cells).

Conclusion: In INS-1e cells pharmacologic P2X receptor stimulation downregulates, and $\mathrm{P} 2 \mathrm{Y}$ receptor stimulation upregulates, insulin synthesis and release. This effect could be intrinsically modulated by the enhanced eATP released by INS-1e cells in the presence of increasing glucose concentrations. Neither effect is explained by changes in apoptosis. $\mathrm{P} 2 \mathrm{X}_{7}$ expression was enhanced by ATP, but this was not coupled with changes in insulin release, suggesting that $\mathrm{P} 2 \mathrm{X}_{7}$ receptors in these cells may only be involved in cytokine release.

Supported by University of Pisa

0489

Involvement of Src in signal-transducing function of $\mathrm{Na}^{+} / \mathrm{K}^{+}$ATPase to decrease ATP production by generating reactive oxygen species in pancreatic islets

R. Kominato ${ }^{1}$, S. Fujimoto ${ }^{1}$, E. Mukai ${ }^{1}$, Y. Nakamura ${ }^{1}$, M. Hosokawa ${ }^{1}$, Y. Yamada ${ }^{1}$, Y. Seino ${ }^{2}$, N. Inagaki ${ }^{1}$;

${ }^{1}$ Department of Diabetes and Clinical Nutrition, Graduate School of Medicine, Kyoto University, ${ }^{2}$ Division of Diabetes and Clinical Nutrition, Kansai-Denryoku Hospital, Osaka, Japan.

Background and Aims: $\mathrm{Na}^{+} / \mathrm{K}^{+}$ATPase inhibition by ouabain suppresses mitochondrial ATP production by generating reactive oxygen species (ROS) that accounts for the late suppressive effect (after $30 \mathrm{~min}$ ) on glucoseinduced insulin secretion from pancreatic islets. As ouabain also suppresses the ATP level by generating ROS in the $\mathrm{Ca}^{2+}$-deprived condition in pancreatic islets, the signaltransducing function of $\mathrm{Na}^{+} / \mathrm{K}^{+}$ATPase is $\mathrm{Ca}^{2+}$-independent. Ouabain causes a rapid activation of Src that results in ROS generation in cardiomyocytes. To clarify the signaltransducing function of $\mathrm{Na}^{+} / \mathrm{K}^{+}$ATPase to decrease ATP production by generating ROS in pancreatic islets, the involvement of Src was examined.

Materials and Methods: After isolated islets from Wistar rats were cultured with RPMI medium containing a normal level of $\mathrm{Ca}^{2+}$, experiments were performed in $\mathrm{Ca}^{2+}$ deprived condition. ATP content was determined by luminometric method. ROS was measured by CM-DCF fluorescence using dispersed islet cells. After lysates were immunoprecipitated by anti-Src antibody, immunoblotting was performed using anti-Src, anti-Src[pY418], and antiSrc[pY529] antibodies.

Results: Ouabain $(1 \mathrm{mM})$ caused a rapid activation of Src in the presence of $16.7 \mathrm{mM}$ glucose. Maximum increase in $\mathrm{Tyr}^{418}$ phospholyration, which indicates activation of Src, was observed $3 \mathrm{~min}$ after ouabain exposure. Ouabain increased $\mathrm{Tyr}^{418}$ phospholyration but did not alter $\mathrm{Tyr}^{529}$ phospholyration, indicating inactivation of Src (fold increase relative to control at $3 \mathrm{~min}, \mathrm{pY} 418: 1.79 \pm 0.15$, $\mathrm{P}<0.01$; pY529: $1.09 \pm 0.04$, not significant; total Src: $0.97 \pm 0.03$, not significant). Ouabain suppressed the glucose-induced increase in ATP content at $60 \mathrm{~min}(2.8 \mathrm{mM}$ glucose, $9.1 \pm 0.5 ; 16.7 \mathrm{mM}$ glucose, $17.9 \pm 0.7 ; 16.7 \mathrm{mM}$ glucose plus ouabain, $12.3 \pm 0.5 \mathrm{pmol} /$ islet, $\mathrm{P}<0.01$ vs. 
$16.7 \mathrm{mM}$ glucose alone). However, in the presence of $10 \mu \mathrm{M}$ PP2, a specific Src inhibitor, ouabain did not affect ATP contents $(16.7 \mathrm{mM}$ glucose, $16.2 \pm 0.9 ; 16.7 \mathrm{mM}$ glucose plus ouabain, $17.2 \pm 0.9 \mathrm{pmol} /$ islet, not significant). The abolished decrease in ATP content by ouabain also was observed in the presence of other Src inhibitors including herbimycin A and SU6656. Ouabain increased ROS production in the presence of $16.7 \mathrm{mM}$ glucose (at $60 \mathrm{~min}$, $100 \pm 4$, control; vs. $128 \pm 6 \%$ of control, ouabain, $\mathrm{P}=0.01$ ). PP2 decreased ouabain-induced ROS production (ouabain plus $\mathrm{PP} 2,103 \pm 5 \%$ of control, $\mathrm{P}=0.02$ vs. ouabain).

Conclusion: $\mathrm{Na}^{+} / \mathrm{K}^{+}$ATPase inhibition by ouabain suppresses ATP content and increases ROS production by activating Src. Thus, Src plays a role in the signaltransducing function of $\mathrm{Na}^{+} / \mathrm{K}^{+}$ATPase to decrease ATP production by generating ROS in pancreatic islets.

\section{0}

L-Cysteine inhibits insulin release through the metabolic production of hydrogen sulfide, a novel gasotransmitter Y. Kaneko ${ }^{1}$, T. Kimura ${ }^{1}$, H. Kimura ${ }^{2}$, I. Niki ${ }^{1}$;

${ }^{1}$ Department of Pharmacology, Oita University Faculty of Medicine, Oita, ${ }^{2}$ Department of Molecular Genetics, National Institute of Neuroscience, Tokyo, Japan.

Background and Aims: L-Cysteine is a sulphur-containing amino acid distributed in a wide variety of tissues. Recent studies demonstrated that hydrogen sulfide $\left(\mathrm{H}_{2} \mathrm{~S}\right)$, a metabolite of L-cysteine, acts as an intracellular gasotransmitter in various types of cells including neural cells and smooth muscle cells. Moreover, increases of the L-cysteine contents have been reported in plasma and some tissues in diabetic subjects or model animals. Nevertheless, the roles of $\mathrm{H}_{2} \mathrm{~S}$ in the endocrine tissues remain unrevealed. The aim of the present study is to investigate the effect of L-cysteine and $\mathrm{H}_{2} \mathrm{~S}$ on insulin release from the mouse pancreatic $\beta$-cells.

Materials and Methods: Pancreatic islets were isolated from male ICR mice aged 8-12 weeks by the collagenase digestion method. Expression of cystathionine- $\beta$-synthase (CBS) and cystathionine- $\gamma$-lyase (CSE), both of which produce $\mathrm{H}_{2} \mathrm{~S}$ from L-cysteine, was determined by RT-PCR and immunoblot analysis. Insulin release from isolated islets was examined by batch incubation and insulin released into the media was measured by radioimmunoassay. For $\left[\mathrm{Ca}^{2+}\right]_{\mathrm{i}}$ measurement, pancreatic islets were dispersed by dispase and preloaded with the $\mathrm{Ca}^{2+}$-sensitive fluorescent dye, fura PE3. Real-time imaging of $\left[\mathrm{Ca}^{2+}\right]_{i}$ was carried out by a dual-wavelength microfluorometry system. The ATP content in the $\beta$-cell line, MIN6 cells, was measured by the luciferin-luciferase reaction and the mitochondrial membrane potential $\left(\Delta \Psi_{m}\right)$ in a single $\beta$-cell was monitored with rhodamine 123 , a fluorescent indicator of $\Delta \Psi_{m}$

Results: We found CBS and CSE were expressed in mouse pancreatic islets and MIN6 cells. L-cysteine and the $\mathrm{H}_{2} \mathrm{~S}$ donor NaHS inhibited glucose-induced insulin release from islets and MIN6 cells in a dose-dependent manner. These inhibitory effects were reproduced when insulin release was stimulated by $\alpha$-ketoisocaproate, tolbutamide or high $\mathrm{K}^{+}$. Both substances suppressed ATP production and attenuated glucose-induced hyperpolarization of the $\beta$-cell $\Delta \Psi_{m}$. These substances promptly and reversibly suppressed glucoseinduced $\left[\mathrm{Ca}^{2+}\right]_{i}$ oscillation in a single $\beta$-cell without obvious changes in the mean $\left[\mathrm{Ca}^{2+}\right]_{i}$ value, suggesting that L-cysteine and NaHS inhibit insulin release via their opening effects on the $\mathrm{K}_{\text {ATP }}$ channels and resultant $\left[\mathrm{Ca}^{2+}\right]_{\mathrm{i}}$ changes. In the presence of diazoxide, however, L-cysteine and NaHS still inhibited high $\mathrm{K}^{+}$-induced insulin release. Furthermore, both remained effective to inhibit $\mathrm{Ca}^{2+}$ induced insulin release when the islet cell membranes were permeabilized with streptolysin-O. Therefore, we suppose the inhibition of insulin release includes $\mathrm{K}_{\mathrm{ATP}}$-independent mechanisms.

Conclusion: $\mathrm{H}_{2} \mathrm{~S}$ produced by L-cysteine metabolism potently inhibits insulin release via multiple actions on the insulin secretory machinery. We suppose that increased L-cysteine contents and eventual $\mathrm{H}_{2} \mathrm{~S}$ production under the diabetic condition may affect the $\beta$-cell functions and that it may be involved in the deterioration of diabetes mellitus.

\section{1}

Intracellular GABA accumulation suppresses glucoseinduced insulin secretion in rat pancreatic islets as well as membrane depolarisation in $\beta$ cells

\section{J. Tamarit-Rodriguez;}

Biochemistry, Complutense University, Madrid, Spain.

Background and Aims: Overexpression of GAD65 in mice (Shi Y. et al, Am. J. Physiol. Endocrinol. Metab. 279, E684, 2000) or INS-1 cells (Rubí B. et al., J. Biol. Chem. $276,36391,2001)$ diminishes their secretory response to glucose. We have investigated whether increased intracellular GABA concentrations affect the mechanism of stimulation of insulin secretion in rat pancreatic islets and isolated $\beta$-cells.

Materials and Methods: Islet $\alpha$-amino acids were measured fluorometrically after precolumn derivatization with o-phtalaldehyde and HPLC separation, glucose utilization was measured as the amount of ${ }^{3} \mathrm{H}_{2} \mathrm{O}$-production from $20 \mathrm{mM} \mathrm{D}\left[5-{ }^{3} \mathrm{H}\right]$ glucose, ATP/ADP with the luciferase/ 
luciferin sytem, and insulin was radioimmunologically measured in batch-type incubations (1 hour). Membrane potential and whole-cell $\mathrm{K}_{\text {ATP }}$ currents in isolated $\beta$-cells were measured in the perforated patch whole-cell configuration of the patch-clamp technique, while single $\mathrm{K}_{\mathrm{ATP}}$-channel currents were recorded from inside-out or outside-out membrane patches.

Results: Islet incubations with a range of ethyl-GABA (eGABA, a membrane permeable GABA precursor) concentrations $(0.25,1,5$, and $10.0 \mathrm{mM})$ induced a linear increase of the islet GABA content that exceeded by 2- (at $5 \mathrm{mM}$ eGABA) and 4-fold (at $10 \mathrm{mM}$ eGABA) the level obtained at $1 \mathrm{mM} \mathrm{L}$-glutamine. No significant variation was observed in other islet $\alpha$-amino acids. $5 \mathrm{mM}$ eGABA suppressed by more than $50 \%$ the islet secretory response to $20 \mathrm{mM}$ glucose $(30.8 \pm 3.7, \mathrm{n}=5$, vs. $65.8 \pm 3.3 \mathrm{pg} / \mathrm{min} \mathrm{x}$ islet, $\mathrm{n}=12 ; \mathrm{p}<0.0001) .10 \mathrm{mM}$ eGABA decreased the rate of $20 \mathrm{mM}$ glucose utilization within 22 to $37 \%$ (with or without pre-exposure to eGABA, respectively) as well as the rate of oxidation $(17.9 \pm 0.6, \mathrm{n}=5$; vs. $24.5 \pm 0.9 \mathrm{pmol} / \mathrm{h} \mathrm{x}$ $\mu \mathrm{g}$ protein, $\mathrm{n}=6 ; \mathrm{p}<0.001)$ and the islet content of ATP (4.1 $\pm 0.1, n=6$, vs. $4.91 \pm 02$ pmol/islet, $n=6 ; p<0.01)$. In the presence of $10 \mathrm{mM}$ glucose, $5 \mathrm{mM}$ eGABA reversibly hyperpolarised isolated $\beta$-cells from $-19 \pm 3 \mathrm{mV}$ to $-49 \pm 4 \mathrm{mV}(\mathrm{n}=6 ; \mathrm{p}<0.001)$ and increased the whole-cell resting conductance by $213 \pm 127 \%(p<0.01)$. These effects were also completely reversed by tolbutamide $(100 \mu \mathrm{M}$, $\mathrm{n}=5)$. Extracellular application of GABA $(5 \mathrm{mM})$ had no effect on $\beta$-cell membrane potential or conductance $(n=3)$. Bath application of eGABA $(5 \mathrm{mM})$ did not activate $\mathrm{K}_{\mathrm{ATP}}$ single channel currents in isolated inside-out $(n=3)$ or outside-out ( $\mathrm{n}=7$ ) membrane patches.

Conclusion: 1) An increase of the intracellular GABA concentration above physiological values decreased insulin secretion in response to glucose with higher efficiency than islet metabolism. 2) eGABA reversed glucose-induced $\beta$-cell depolarization by increasing ATP-dependent $\mathrm{K}^{+}$channel activity. 3) It is hypothesized that GABA uptake into the synaptic like microvesicles of $\beta$-cells might compete for cytosolic ATP with the $\mathrm{K}^{+}$-channels.

Supported by Ministerio de Ciencia y Tecnología, Instituto de Salud Carlos III, Wellcome Trust

\section{2}

Inhibitors of phospholipase A2 rapidly suppresses glucose-induced $\mathrm{Ca}^{2+}$ oscillations in pancreatic $\beta$ cells G. Larsson-Nyrén ${ }^{1}$, E. Grapengiesser ${ }^{2}$, B. Hellman ${ }^{2}$;

${ }^{1}$ Integrative Medical Biology, Section for Histology and Cell Biology, Umea University, Sweden, ${ }^{2}$ Department of Medical Cell Biology, University of Uppsala, Sweden.
Background and Aims: Phospholipase A2 ( $\left.\mathrm{PLA}_{2}\right)$ generates arachidonic acid (AA) and lysophospholipids. Pancreatic $\beta$-cells express both cytoplasmic $\mathrm{cPLA}_{2} \alpha$ activated by $\mathrm{Ca}^{2+}$ and iPLA 2 activated by internal ATP. External ATP is a stimulator of $\mathrm{CPLA}_{2}$ in isolated mouse islets and $\mathrm{AA}$ has a suppressive action on KATP channels both in insulinsecreting HIT cells and in mouse $\beta$-cells. A role for AA in glucose-induced rhythmicity of cytoplasmic $\mathrm{Ca}^{2+}$ is suggested from observations that different external activators of $\mathrm{CPLA}_{2}$ induce $\mathrm{Ca}^{2+}$ oscillations. These observations, together with a report that $\mathrm{AA}$ induces $\mathrm{Ca}^{2+}$ oscillations in the exocrine pancreas, gave rise to the question whether AA participates in the generation of the $\mathrm{Ca}^{2+}$ oscillations responsible for pulsatile insulin release.

Materials and Methods: The cytoplasmic $\mathrm{Ca}^{2+}$ concentration $\left(\left[\mathrm{Ca}^{2+}\right] \mathrm{i}\right)$ was studied in single mouse $\beta$-cells (Umea $\mathrm{ob} / \mathrm{ob}$ ), using ratiometric fura-2 technique. Measurements were performed with an inverted microscope equipped for epifluorescence. The cells were illuminated only during the image capture and the excitation light was kept at a minimum. After raising the glucose concentration from 3 to $20 \mathrm{mM}$ the $\beta$-cells were exposed to the PLA $\mathrm{A}_{2}$ inhibitors $\mathrm{N}$-(p-amylcinnamoyl) anthranilic acid (ACA), arachidonyl trifluoromethyl ketone $\left(\mathrm{AACOCF}_{3}\right)$ and bromoenol lactone (BEL). For comparative purposes suppression of the AA formation with the diacylglycerol lipase inhibitor:1,6-bis(cyclohexyl-oximinocarbonylamino)-hexane (RHC 80267) was tested.

Results: Oscillations of $\left[\mathrm{Ca}^{2+}\right]$ i from a basal level were seen in most $\beta$-cells superfused with $20 \mathrm{mM}$ glucose. ACA at $25 \mu \mathrm{M}$ had often a suppressive action and $35 \mu \mathrm{M}$ was sufficient to remove the $\left[\mathrm{Ca}^{2+}\right]$ i oscillations in the $\beta$-cells. The action of ACA was reversible. Reappearance of oscillations in the presence of ACA was occasionally seen after addition of $100 \mu \mathrm{M}$ tolbutamide but not in the presence of $0.1 \mu \mathrm{M}$ BAY $\mathrm{K} 8644$ or $0.1 \mu \mathrm{M}$ ATP. $\mathrm{AACOCF}_{3}(10 \mu \mathrm{M})$ effectively removed the oscillations of $\left[\mathrm{Ca}^{2+}\right]$ i. However, omission of $25-35 \mu \mathrm{M}$ of this $\mathrm{CPLA}_{2}$ inhibitor did not result in a reappearance of the oscillations. The oscillations became lower and started from an elevated level at $10 \mu \mathrm{M}$ BEL. At a concentration of $15 \mu \mathrm{M}$, BEL induced complete loss of the oscillations, which often reappeared after omission of the inhibitor. The diacylglycerol lipase inhibitor RHC $(5-10 \mu \mathrm{M})$ effectively removed the $\left[\mathrm{Ca}^{2+}\right] \mathrm{i}$ oscillations, an effect reversed after omission of the substance or addition of $100 \mu \mathrm{M}$ tolbutamide.

Conclusion: Both low concentrations of inhibitors of PLA 2 and suppression of diacylglycerol lipase remove the $\left[\mathrm{Ca}^{2+}\right] \mathrm{i}$ oscillations in pancreatic $\beta$-cells. The results support the idea that $\mathrm{AA}$ is important for the $\left[\mathrm{Ca}^{2+}\right] \mathrm{i}$ rhythmicity responsible for pulsatile release of insulin. 
0493

Effects of glucose and tolbutamide on mitochondrial $\mathrm{Ca}^{2+}$ handling of insulin secreting cells

P. Krippeit-Drews ${ }^{1}$, M. Kelm ${ }^{1}$, D. Haspel ${ }^{1}$, M. Düfer ${ }^{1}$, R. Nitschke ${ }^{2}$, F. Ranta ${ }^{3}$, S. Ullrich ${ }^{3}$, G. Drews ${ }^{1}$;

${ }^{1}$ Pharmazeutisches Institut, Universität Tübingen, ${ }^{2}$ Life Imaging Center, Universität Freiburg, ${ }^{3}$ Physiologisches Institut, Universität Tübingen, Germany.

Background and Aims: Cytosolic $\mathrm{Ca}^{2+}$ concentration $\left(\left[\mathrm{Ca}^{2+}\right]_{\mathrm{c}}\right)$ and mitochondrial function of pancreatic B-cells are tightly coupled. Increases in $\left[\mathrm{Ca}^{2+}\right]_{\mathrm{c}}$ in response to glucose reduce mitochondrial ATP production thus contributing to a negative feedback mechanism which regulates B-cell oscillatory activity. Since mitochondrial $\mathrm{Ca}^{2+}\left(\left[\mathrm{Ca}^{2+}\right]_{\mathrm{m}}\right)$ was suggested to be the determinant for oscillatory ATP production, experiments were performed to evaluate whether $\left[\mathrm{Ca}^{2+}\right]_{\mathrm{m}}$ also fluctuates. Sulfonylureas are known to increase $\mathrm{Ca}^{2+}$ influx across the plasma membrane. We examined in this study whether sulfonylureas also interfere with $\mathrm{B}$-cell $\mathrm{Ca}^{2+}$ homeostasis by affecting $\mathrm{Ca}^{2+}$ storage into mitochondria.

Materials and Methods: Experiments were carried out with B-cells from C57BL/6 mice or INS-1 cells using fluorescence techniques. $\left[\mathrm{Ca}^{2+}\right]_{\mathrm{c}}$ was determined by fura2. Full width at half maximum of the $\mathrm{Ca}^{2+}$ peak (width $0.5 \mathrm{P}$ ) was used as an indicator for the amount of $\mathrm{Ca}^{2+}$ released from intracellular stores. $\left[\mathrm{Ca}^{2+}\right]_{\mathrm{m}}$ was estimated by ratiometric pericam targeted to mitochondria. INS-1 cells were transfected with the plasmid pcDNA3 by effectene.

Results: Increasing the glucose concentration from 0.5 to $15 \mathrm{mM}$ induced oscillations in $\left[\mathrm{Ca}^{2+}\right]_{\mathrm{m}}$ in INS-1 cells with a mean maximal amplitude of $56 \pm 3$ a.u. (arbitrary units, $\mathrm{n}=7$ ) supporting the hypothesis that $\left[\mathrm{Ca}^{2+}\right]_{\mathrm{m}}$ exhibits the link between $\left[\mathrm{Ca}^{2+}\right]_{\mathrm{c}}$ and fluctuations in mitochondrial metabolism. In the presence of $15 \mathrm{mM}$ glucose FCCP $(0.5 \mu \mathrm{M})$ induced a transient increase of $\mathrm{Ca}^{2+}$ in B-cells from $355 \pm 39 \mathrm{nM}$ to $606 \pm 74 \mathrm{nM}$ ( width $_{0.5 \mathrm{P}} 32 \pm 4 \mathrm{~s}, \mathrm{n}=6$ ). This release was completely suppressed by SERCA inhibition with cyclopiazonic acid (CPA, $10 \mu \mathrm{M})(\mathrm{n}=7)$. In the presence of tolbutamide $(1 \mathrm{mM}) \mathrm{FCCP}$ led to enhanced $\mathrm{Ca}^{2+}$ release (width $0.5 \mathrm{P} 89 \pm 10 \mathrm{~s}, \mathrm{n}=5$ ) that even persisted with CPA (width $0.5 \mathrm{P} 35 \pm 7 \mathrm{~s}, \mathrm{n}=4$ ). This observation suggests that $\mathrm{Ca}^{2+}$ is stored in non-ER $\mathrm{Ca}^{2+}$ pools as a consequence of sulfonylurea treatment. Indeed tolbutamide enhanced $\left[\mathrm{Ca}^{2+}\right]_{\mathrm{m}}$ to $271 \pm 59$ a.u. $(\mathrm{n}=6)$ which was about 5 fold larger than the increase observed with $15 \mathrm{mM}$ glucose $(\mathrm{P} \leq 0.002)$. This suggests that the non-ER $\mathrm{Ca}^{2+}$ stores recruited by tolbutamide are the mitochondria.

Conclusion: $\left[\mathrm{Ca}^{2+}\right]_{\mathrm{m}}$ is able to oscillate in response to glucose. We conclude that these oscillations trigger fluctuations of the mitochondrial membrane potential $\Delta \Psi$ and thus ATP production. It is demonstated that sulfonylureas influence mitochondrial $\mathrm{Ca}^{2+}$ handling. Compared to control cells where the ER is the predominent $\mathrm{Ca}^{2+}$ store $\mathrm{Ca}^{2+}$ uptake into mitochondria gains in importance in the presence of sulfonylureas. The latter effect may be involved in $\mathrm{Ca}^{2+}$-dependent apoptosis induced by sulfonylureas.

Supported by $D F G$

\section{4}

$K_{A T P}$ channel activity in pancreatic beta cells is regulated by an adenylate kinase

D. U. Schulze ${ }^{1}$, P. Krippeit-Drews ${ }^{1}$, M. Düfer ${ }^{1}$, E. Janssen ${ }^{2}$, B. Wieringa ${ }^{2}$, G. Drews ${ }^{1}$;

${ }^{1}$ Pharmakologie, Pharmazeutisches Institut, Tübingen, Germany, ${ }^{2}$ Dept. of Cell Biology, CMLS, UMC Radboud University, Nijmegen, The Netherlands.

Background and Aims: The cytosolic ATP concentration $[\mathrm{ATP}]_{\mathrm{c}}$ of pancreatic B-cells is about 30 fold higher than necessary for complete inhibition of $\mathrm{K}_{\mathrm{ATP}}$ channels in cellfree patches. Nevertheless, in intact cells insulin secretion in response to glucose is regulated by changes in $\mathrm{K}_{\text {ATP }}$ channel activity. It is known from cardiac myocytes that a metabolic barrier insulates $\mathrm{K}_{\mathrm{ATP}}$ channels from $[\mathrm{ATP}]_{\mathrm{c}}$. We suggest that an adenylate kinase (AK) may be involved in the regulation of $\mathrm{K}_{\mathrm{ATP}}$ channel activity in B-cells. This enzyme can form ADP in the presence of ATP and AMP and thus counterbalance channel inhibition by ATP.

Materials and Methods: Experiments were carried out with B-cells from C57BL/6 mice. Single $\mathrm{K}_{\mathrm{ATP}}$ channel current measurements were performed in the open cell attached (oca) or inside/out (i/o) mode of the patch-clamp technique. The AK isoform of B-cells was identified by Western blots and immuno staining experiments.

Results: ATP concentration response curves show a clear left-shift in the open probability (NPo) of $\mathrm{K}_{\mathrm{ATP}}$ channels when $\mathrm{i} / \mathrm{o}$ currents were compared to oca measurements $(\mathrm{Km}=6.6 \pm 0.7 \mu \mathrm{M}$ and $27.5 \pm 14.8 \mu \mathrm{M}$, respectively), pointing at an influence of cytosolic components on the ATP block. Accordingly, NPo of $\mathrm{K}_{\mathrm{ATP}}$ channels in the oca patch in the presence of $0.125 \mathrm{mM}$ ATP was increased by $595 \pm 107 \%$ $(\mathrm{n}=5)$ by addition of $0.1 \mathrm{mM}$ AMP. The same manoeuvre was without effect in $\mathrm{i} / \mathrm{o}$ patches $(\mathrm{n}=5)$ or in oca patches where $\mathrm{AK}$ was inhibited by $\mathrm{Ap}_{5} \mathrm{~A}(20 \mu \mathrm{M}, \mathrm{n}=6)$. Western blot analysis of whole islets exhibited a positive response for the $\mathrm{AK}$ isoform 1 (AK1). This was confirmed by immunofluorescence images showing a co-localization of AK1 with insulin. Conclusions: We conclude that an adenylate kinase, probably AK1, is part of a metabolic barrier shielding 
$\mathrm{K}_{\mathrm{ATP}}$ channels of pancreatic B-cells from the $[\mathrm{ATP}]_{\mathrm{c}}$ and thus allowing opening of $\mathrm{K}_{\mathrm{ATP}}$ channels despite the extremely high bulk ATP concentration.

Supported by DFG DR 225/6-3

\section{5}

Role of the Na:H exchanger in $\beta$ cell stimulus-secretion coupling: investigation in mice with a spontaneous inactivating mutation

P. Stiernet ${ }^{1}$, P. Moulin ${ }^{2}$, M. Nenquin ${ }^{1}$, J.-C. Henquin ${ }^{1}$; ${ }^{1}$ Unit of Endocrinology and Metabolism, ${ }^{2}$ Unit of Pathology, University of Louvain Faculty of Medicine, Brussels, Belgium.

Background and Aims: Pharmacological studies have suggested that both $\mathrm{Na}: \mathrm{H}$ and $\mathrm{Cl}: \mathrm{HCO}_{3}$ exchangers regulate cytosolic $\mathrm{pH}\left(\mathrm{pH}_{\mathrm{i}}\right)$ in $\beta$-cells, but their respective role has not been defined. This complicates the appraisal of models implicating $\mathrm{pH}_{\mathrm{i}}$ changes in stimulus-secretion coupling. Thus, the mechanisms underlying these changes and their possible influence on exocytosis of insulin granules remain unclear. Having identified NHE1 as the major isoform of the $\mathrm{Na}: \mathrm{H}$ exchanger in islets, we used mice bearing an inactivating mutation of NHE1 (Slc9A1 - swe/swe) (Swe mice) (Cell 91: 139-148, 1997) to investigate the role of $\mathrm{Na}: \mathrm{H}$ exchange in $\beta$-cell function.

Methods: Islets were isolated from genotypically identified, homozygous Swe mice of 6-8 weeks, as these animals do not live much longer because of ataxia and seizures. The islets were cultured for 1 day before measurement of insulin release, $\mathrm{pH}_{\mathrm{i}}$ and cytosolic $\mathrm{Ca}^{2+}\left(\left[\mathrm{Ca}^{2+}\right]_{\mathrm{i}}\right)(\mathrm{BCECF}$ and FURA-P3 methods). Experiments were performed in $\mathrm{HCO}_{3}$-buffer (gassed with $\mathrm{O}_{2}-\mathrm{CO}_{2}$ ) and $\mathrm{HCO}_{3}$-free, Hepes-buffer (gassed with $\mathrm{O}_{2}$ ), conditions under which both $\mathrm{Cl}: \mathrm{HCO}_{3}$ and $\mathrm{Na}: \mathrm{H}$ exchangers or only $\mathrm{Na}: \mathrm{H}$ exchanger can operate.

Results: Compared to age-matched controls, the body weight of Swe mice was 35\% smaller, their islet insulin content was $30 \%$ lower, but their blood glucose was not different. Basal $\mathrm{pH}_{\mathrm{i}}$ was lower in Swe islets $(\sim 6.7)$ than control islets $(\sim 6.9)$ in Hepes-buffer but not in $\mathrm{HCO}_{3}$ buffer. The ability of the islets to regulate $\mathrm{pH}_{\mathrm{i}}$ was tested by the $\mathrm{NH}_{4} \mathrm{Cl}$ pulse technique. In $\mathrm{HCO}_{3}$-buffer, Swe islets corrected the acidification slightly more slowly than controls whereas, unlike controls, they failed to correct the acidification in Hepes-buffer. These results indicate partial redundancy of the two exchangers in controls and absence of $\mathrm{pH}_{\mathrm{i}}$ regulators other than $\mathrm{Cl}: \mathrm{HCO}_{3}$ exchangers in Swe islets. In $\mathrm{HCO}_{3}$-buffer, stimulation with $15 \mathrm{mmol} /$ 1 glucose caused a similar small increase in $\mathrm{pH}_{\mathrm{i}}$, rise in $\left[\mathrm{Ca}^{2+}\right]_{\mathrm{i}}$ with oscillations in the steady-state, and biphasic release of insulin in control and Swe islets. After correction for differences in islet insulin content, the rate of insulin release was even higher in Swe islets. Stimulation with $30 \mathrm{mmol} / \mathrm{KCl}$ produced similar effects in both types of islets: a small decrease in $\mathrm{pH}_{\mathrm{i}}$ and large increases in $\left[\mathrm{Ca}^{2+}\right]_{\mathrm{i}}$ and insulin release. In Hepes-buffer, the behaviour of control and Swe islets was different. In controls, $15 \mathrm{mmol} / \mathrm{l}$ glucose now slightly lowered $\mathrm{pH}_{\mathrm{i}}$ while increasing $\left[\mathrm{Ca}^{2+}\right]_{\mathrm{i}}$ and insulin release as in $\mathrm{HCO}_{3}$-buffer. In contrast, a marked decrease in $\mathrm{pH}_{\mathrm{i}}$ occurred in glucose-stimulated Swe islets, accompanied by a progressive decrease in the insulin secretory rate. Similar alterations of $\mathrm{pH}_{\mathrm{i}}$ and insulin release were observed when Swe islets were stimulated with $\mathrm{KCl}$ in Hepes-buffer.

Conclusions: Loss of $\mathrm{Na}: \mathrm{H}$ exchange has no significant consequence on stimulus-secretion coupling in mouse $\beta$-cells as long as islets are maintained in physiological $\mathrm{HCO}_{3}$-buffer. A negative impact is only detected in Hepesbuffer, when $\mathrm{Cl}: \mathrm{HCO}_{3}$ exchangers cannot operate and $\mathrm{pH}_{\mathrm{i}}$ falls to very low values that are not normally observed. The results also suggest that the small changes in $\mathrm{pH}_{\mathrm{i}}$ occurring in control islets under physiological conditions are unlikely to influence insulin release directly.

\section{6}

Expression and role of sodium pump in insulin secreting cells

S. Ullrich, Y. Zang, F. Ranta, D. Avram, F. Lang; Institute of Physiology, University of Tuebingen, Germany.

Background and Aims: Stimulation of insulin secretion by $\mathrm{Ca}^{2+}$-influx depends on changes of membrane potential. The $\mathrm{Na}^{+} / \mathrm{K}^{+}$-ATPase creates the potassium gradient over the plasma membrane, the main gradient responsible for the resting membrane potential in insulin secreting cells. Due to the electrogenic nature of the $\mathrm{Na}^{+} / \mathrm{K}^{+}$-ATPase, stimulation of the enzyme generates a hyperpolarizing current, which may contribute to the stabilisation of the resting membrane potential and retarding $\mathrm{Ca}^{2+}$ entry. The aim of the present study was to examine the molecular composition of $\mathrm{Na}^{+} /$ $\mathrm{K}^{+}$-ATPase in insulin secreting cells and to analyse whether glucocorticoids which are known to inhibit insulin secretion alter its expression and activity.

Materials and Methods: Semiquantitative real time RT/ PCR was used to quantify mRNA levels of $\mathrm{Na}^{+} / \mathrm{K}^{+}$-ATPase subunits in insulin secreting INS-1 cells. Pump activity was estimated by the mean of outward current generated by the enzyme when $\mathrm{KCl}$ was added to an extracellular solution devoid in potassium using the whole cell configuration of the patch clamp method. 
Results: INS- 1 cells express $\alpha_{1-}, \beta_{1^{-}}$and $\beta_{3}$-subunits of rat $\mathrm{Na}^{+} / \mathrm{K}^{+}$-ATPase but not $\alpha_{2}, \alpha_{3}$ and $\beta_{2}$. Cell culture with $100 \mathrm{nM}$ dexamethasone for 1 day increased the mRNA levels of $\alpha_{1}$ - and $\beta_{1}$ - subunits six- and twofold, respectively. The addition of $5 \mathrm{mM} \mathrm{KCl}$ to a bath solution lacking potassium initiated an outward current of $0.55 \pm 0.02 \mathrm{pA} / \mathrm{pF}$ $(n=26)$ in INS-1 cells and of $0.77 \pm 0.17 \mathrm{pA} / \mathrm{pF}(\mathrm{n}=5)$ in isolated mouse islet cells. INS-1 cells were hyperpolarized by $-16.5 \pm 2.7 \mathrm{mV}$, mouse islet cells by $-15.7 \pm 3.1 \mathrm{mV}$. Ouabain $(1 \mathrm{mM})$ or omission of ATP from the pipette solution almost completely inhibited the effects of $\mathrm{KCl}$. Pretreatment of INS-1 cells with $100 \mathrm{nM}$ dexamethasone for 1 day increased outward current significantly by $25 \%$ to $0.72 \pm 0.05 \mathrm{pA} / \mathrm{pF}(\mathrm{n}=15)$, an effect which was antagonized by the glucocorticoid receptor antagonist RU486, $1 \mu \mathrm{M}$.

Conclusion: Insulin secreting INS-1 cells express the $\alpha_{1}$, $\beta_{1^{-}}$and $\beta_{3}$-subunits of $\mathrm{Na}^{+} / \mathrm{K}^{+}$-ATPase. The increased expression and pump activity observed after dexamethasone may stabilize the resting membrane potential and thus contribute to the known reduction of glucose-induced insulin secretion after glucocorticoid excess.

\section{PS 27}

\section{Mediators of insulin secretion}

0497

Functional heterogeneity in pancreatic beta cell subpopulations in Wistar rats

C. Tourrel-Cuzin, M. Karaca, J. Castel, C. Magnan; Laboratoire Physiopathologie de la Nutrition, CNRS UMR 7059, Paris, France.

Background and Aims: The sialylated form of the neural cell adhesion molecule (PSA-NCAM) which is present on the pancreatic beta-cell membrane is a good tool to sort different beta-cell subpopulations according to their functional activity. We previously showed a close correlation between abundance of PSA-NCAM and insulin response to glucose: high PSA-NCAM-labelled beta-cells (beta ${ }^{+}$-cells) secreted much more insulin in response to glucose than low PSA-NCAM-labelled beta-cells (beta'cells). The present study compared some aspects of the intracellular mechanisms that could explain this differential response to glucose in these two beta-cell subpopulations. For this purpose, we examined the extent to which the differences in the glucose-stimulated insulin secretion in the beta ${ }^{+}$and beta cells was related $1 /$ to the handling of cytoplasmic $\mathrm{Ca}^{2+}$ concentrations and 2/to the cAMP cellular levels in consideration of the role of cAMP in glucose-induced insulin secretion. In addition, because responsiveness might be variable according to the nature of the stimulus, we tested whether this different functional activity between the two subpopulations is glucose-specific or not.

Materials and Methods: Islet cells suspensions from Wistar rats were sorted by flow cytometry, according to their PSA-NCAM abundance using an anti-PSA-NCAM antibody. Insulin release was measured in sorted beta-cell subpopulations by a perifusion technique in a system allowing concomitant recording of intracytoplasmic $\mathrm{Ca}^{2+}$ movements $\left(\left[\mathrm{Ca}^{2+}\right] \mathrm{i}\right)$ by microfluorimetry. Using batchincubated cells, cAMP content and insulin release in response to glucose (5.5 and $16.7 \mathrm{mM}$ ) and/or $10 \mathrm{nM}$ GLP-1, $19 \mathrm{mM}$ arginine, $10 \mathrm{mM}$ leucine were studied.

Results: In beta $^{+}$-cells, insulin release, in response to $16.7 \mathrm{mM}$ glucose, was highly increased compared to beta ${ }^{-}$cells and was correlated with an increased in $\left[\mathrm{Ca}^{2+}\right]$ i. cAMP content, measured in presence of $16.7 \mathrm{mM}$ glucose without or with GLP-1 was increased compared to $5.5 \mathrm{mM}$ glucose. In parallel, insulin secretion in response to $16.7 \mathrm{mM}$ glucose was potentiated by GLP-1. By contrast, in beta ${ }^{-}$cells, insulin release and $\left[\mathrm{Ca}^{2+}\right] \mathrm{i}$ variations, in response to glucose $16.7 \mathrm{mM}$ were weak compared to beta $^{+}$-cells. Furthermore, beta ${ }^{-}$cells were not responsive to 16.7 glucose alone or glucose+GLP-1. Accordingly, their cAMP content was broken down. However, addition of $\mathrm{KCl}(50 \mathrm{mM})$, at the end of perifusion experiments showed that beta ${ }^{-}$cells were capable to secrete insulin to the same extent as beta ${ }^{+}$-cells. Moreover non-glucose secretagogues, arginine and leucine elicited the same high insulin release in both subpopulations.

Conclusion: Alteration of the functionality of beta cells could be explained by a blockage in the step(s) linking glucose metabolism to the insulin release process. The analysis of glucose-stimulated insulin release characteristics in beta-cell subpopulations with a different secretory activity is a prerequisite to identify factors able to ameliorate the functional status of beta-cells with low secretory capacities such as in advancing type 2 diabetes.

\section{8}

The direct effects of exendin-4 on human non-diabetic and type 2 diabetic islets

R. Lupi ${ }^{1}$, R. Mancarella ${ }^{1}$, S. Del Guerra ${ }^{1}$, M. Bugliani ${ }^{1}$, S. Torri ${ }^{1}$, M. Grupillo ${ }^{1}$, U. Boggi ${ }^{2}$, F. Mosca ${ }^{2}$, S. Del Prato ${ }^{1}$, P. Marchetti ${ }^{1}$;

${ }^{1}$ Endocrinology and Metabolism - Metabolic Unit, ${ }^{2}$ Oncology, Transplantation and Advanced Technologies in Medicine, University of Pisa, Italy.

Background and Aims: Exendin-4, a long-acting GLP-1 mimetic, has several beneficial metabolic effects, and its 
synthetic analogue Exenatide has been proposed in the therapy of Type 2 diabetes. However, little information is available on the direct actions of Ex-4 on human pancreatic islets (HI), in particular in the case of diabetes.

Materials and Methods: Islets were prepared from the pancreas of 18 multiorgan donors (11 non-diabetic, ND: 6F/ $5 \mathrm{M}$; age, $66 \pm 19$ yrs; BMI, $24.5 \pm 3.3 \mathrm{~kg} / \mathrm{m} 2$; and 7 with Type 2 diabetes, T2D: 5F/2M; age, $63 \pm 9$ yrs; BMI: $27.4 \pm 4.5 \mathrm{~kg} / \mathrm{m} 2$ ). Then dose-response (Ex-4 from 1 to $100 \mathrm{nmol} / \mathrm{l}$ ) and time-response (incubation from 1 to 7 days) experiments were performed, and $10 \mathrm{nmol} / 1 \mathrm{Ex}-4$ and 2 and 7 days of incubations were further investigated.

Results: Glucose-stimulated insulin secretion improved significantly $(\mathrm{p}<0.05)$ after incubation with $10 \mathrm{nmol} / \mathrm{l} \mathrm{Ex}$ 4 (stimulation index from $2.1 \pm 0.3$ to $2.55 \pm 0.25$ in ND and $1.27 \pm 0.3$ to $1.96 \pm 0.4$ in T2D). In both ND and T2D islets Ex-4 induced a significantly ( $<<0.05$ or less) higher gene expression (by quantitative Real-Time RT-PCR) of insulin (respectively $+93 \pm 38 \%$ and $+37.5 \pm 17.0 \%$ ), GLUT2 $(+283 \pm 41 \%$ and $+178.6 \pm 75.9 \%)$, glucokinase $(+117 \pm 45 \%$ and $+80.8 \pm 16.1 \%)$, PDX-1 $(+470 \pm 149 \%$ and $+157.5 \pm 78.0 \%)$ and Ki67 $(+217 \pm 55 \%$ and $+355.0 \pm 78.2 \%)$ after 2 days of incubation, which was maintained at 7 days. Furthermore, in ND Ex-4 caused a significantly $(\mathrm{p}<0.05$ or less $)$ increased transcription of several additional genes, including PAX4 $(+115 \pm 27 \%)$, NKX2.2 $(+45 \pm 33 \%)$ and NKX6.1 $(+176 \pm 22 \%)$, which, however, was not observed in T2D.

Conclusions: In conclusion, Ex-4 has beneficial effects on isolated human non-diabetic islets; most importantly, part of these positive actions are exerted also on Type 2 diabetic islets, supporting the concept that incretins are useful tool in the therapy of diabetes.

\section{9}

Combined effects of exenatide and glibenclamide on insulin secretion by the perfused rat pancreas J. Marco ${ }^{1}$, D. Parkes ${ }^{2}$, E. M. Egido ${ }^{1}$, R. Hernández ${ }^{1}$, R. A. Silvestre ${ }^{1}$;

${ }^{1}$ Experimental Endocrinology and Department of Physiology, Hospital Universitario Puerta de Hierro, Medical School, Universidad Autónoma de Madrid, Spain, ${ }^{2}$ Amylin Pharmaceuticals, Inc., San Diego, United States.

Background and Aims: Exenatide (exendin-4, Ex-4) is an incretin mimetic exerting glucose-dependent enhancement of insulin secretion similar to that of glucagon-like peptide 1. Ex-4 treatment has been shown to reduce $\mathrm{HbA}_{\mathrm{IC}}$ in type 2 diabetic patients unable to achieve adequate glycemic control under maximally effective doses of metformin and/or a sulfonylurea. In this work we have investigated the effects of $1 \mathrm{nM}$ Ex-4 and $0.1 \mu \mathrm{g} / \mathrm{ml}$ glybenclamide (Glyb), alone and in combination, on insulin secretion.

Materials and Methods: The study was performed in the perfused rat pancreas. Experiments were performed at high (9 mM), normal (5.5 mM) and low (3.2 mM) glucose levels. Results: At $9 \mathrm{mM}$ glucose, the insulin response to Ex-4 (1175 \pm 171 , SEM, ng/15 min) was greater than that to Glyb (596 $\pm 62 \mathrm{ng} / 15 \mathrm{~min} ; \mathrm{p}<0.01)$. When Ex-4 and Glyb were coinfused, an additive effect on insulin secretion was observed $(1681 \pm 363 \mathrm{ng} / 15 \mathrm{~min})$. At $5.5 \mathrm{mM}$ glucose, the insulin responses to Ex-4 $(128 \pm 39 \mathrm{ng} / 15 \mathrm{~min})$ and to Glyb $(215 \pm 39 \mathrm{ng} / 15 \mathrm{~min})$ were not significantly different. The

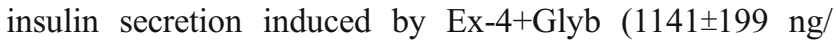
$15 \mathrm{~min}$ ) was greater than the sum of the individual responses to these two secretagogues, thus indicating a synergistic effect on the B cell. At $3.2 \mathrm{mM}$ glucose, Ex-4 did not affect basal insulin levels $(1.2 \pm 1.5 \mathrm{ng} / 15 \mathrm{~min})$ while Glyb stimulated insulin secretion $(143 \pm 44 \mathrm{ng} / 15 \mathrm{~min})$. Coinfusion of Ex-4 markedly potentiated the insulinotropic effect of Glyb ( $435 \pm 85 \mathrm{ng} / 15 \mathrm{~min} ; \mathrm{p}<0.025 \mathrm{vs}$. Glyb experiments).

Conclusion: Our findings confirm the glucose-dependent insulinotropic effect of Ex-4. Concerning the combined effects of Ex-4 and Glyb on insulin secretion, the synergism observed at normal $(5.5 \mathrm{mM})$ glucose levels would indicate that Ex-4 may exert a glucose-like priming effect of the B cell. Consequently, in the presence of Ex-4, sulfonylurea may induce an insulinotropic effect comparable to that found at high glucose concentration. In the isolated rat pancreas, this priming effect becomes more evident at low glucose levels as Ex-4 renders the B cell competent to the insulinotropic effect of Glyb. Importantly, our "in vitro" observations must be taken in context, as in the intact animal/human, incretin-mediated glucose-dependent stimulation of insulin is tightly regulated by multiple extrapancreatic (e.g. CNS) feedback control systems that would not exhibit any influence in the isolated preparation used in the present study.

Supported by Fondo de Investigación Sanitaria, Instituto de Salud Carlos III, Fundación Médica MMA, Ministerio de Educación y Ciencia and a grant-in-aid from Amylin Pharmaceuticals, Inc.

0500

Ghrelin attenuates glucose-induced insulin release in an autocrine/paracrine manner in pancreatic islets

K. Dezaki, H. Sone, T. Yada;

Physiology, Jichi Medical University, Shimotsuke, Japan.

Background and Aims: Ghrelin, isolated from the human and rat stomach, is the endogenous ligand for the growth 
hormone secretagogue receptor (GHS-R). We have reported that ghrelin and GHS-R were expressed in pancreatic islets and that ghrelin suppressed glucose-induced insulin release via activation of voltage-dependent delayed rectifier $\mathrm{K}^{+}$channels and attenuation of glucose-induced action potentials, which leads to suppression of glucose-induced $\mathrm{Ca}^{2+}$ signaling in $\beta$-cells. In this study, we aimed to examine (1) whether ghrelin is released from islet cells and (2) whether ghrelin in islets seves as a physiological regulator of insulin release.

Materials and Methods: To measure plasma ghrelin concentrations, blood samples were collected from the pancreatic arteries (celiac artery) and veins (splenic vein), and portal veins of anaesthetized rats or mice. Plasma concentrations of acylated- and desacyl-ghrelin were measured using enzymeimmunoassay. Ghrelin release from single islet cells were measured using single cell immunoblot assay. Insulin release was measured in rat perfused pancreas and isolated islets prepared by collagenase digestion, and determined using radioimmunoassay and enzymeimmunoassay, respectively.

Results: Plasma acylated- and desacyl-ghrelin concentrations were higher in pancreatic vein than in artery in rats. Single cell immunoblot analysis revealed ghrelin secretion from islet $\beta$ - and $\alpha$-cells. Both GHS-R blockade by a GHS$\mathrm{R}$ antagonist [D-Lys ${ }^{3}$ ]-GHRP-6 and ghrelin immunoneutralization with anti-ghrelin antiserum increased glucose (8.3 $\mathrm{mM})$-induced insulin release in rat perfused pancreas and isolated islets. Glucose-induced insulin release from isolated islets of ghrelin knockout (Ghr-KO) mice was significantly greater than that of wild-type control mice, while insulin content was unaltered. Ghr-KO mice displayed increased insulin and decreased glucose responses in glucose tolerance tests. The profiles of insulin tolerance test exhibited no significant differences between Ghr-KO and control mice.

Conclusion: Ghrelin is released at least from islet $\beta$ - and $\alpha$ cells and it serves as a physiological autocrine/paracrine regulator to downregulate glucose-induced insulin release in islets.

\section{1}

Stimulation of insulin release in MIN6 cells and isolated rodent islets by a small molecule glucokinase activator (GKA50)

D. Gill ${ }^{1}$, T. Gorman ${ }^{1}$, D. C. D. Hope ${ }^{1}$, K. J. Brocklehurst ${ }^{1}$, D. Johnson ${ }^{2}$, M. J. Dunne ${ }^{2}$, D. M. Smith ${ }^{1}$;

${ }^{1}$ AstraZeneca, Macclesfield, ${ }^{2}$ Faculty of Life Sciences, University of Manchester, United Kingdom.

Background and Aims: Glucokinase (GK) is the glucose sensor in the $\beta$-cell and acts to control the threshold for glucose-stimulated insulin secretion (GSIS). Several small molecule direct activators of GK have been discovered which increase GSIS in $\beta$-cells and stimulate hepatic glucose metabolism. We have previously reported on a series of activators of human GK which lower plasma glucose levels after oral glucose tolerance test in female high fat fed Zucker rats and are a potential treatment for Type 2 diabetes.

The aims here were to determine the effects of one of these compounds, GKA50, on pancreatic GK activity and GSIS in mouse insulinoma cells (MIN6) and in isolated rodent islets (rat and mouse). GK inhibitors were used in MIN6 cells to demonstrate that the effects of GKA50 on insulin secretion were due to activation of GK.

Materials and Methods: Recombinant human and rat pancreatic GK were cloned from cDNA by PCR, expressed in E. coli with a 6-His tag and assayed for glucose phosphorylation spectophotometrically. MIN6 cells were cultured under standard conditions in DMEM with $25 \mathrm{mM}$ glucose. Insulin release was measured by ELISA in media from $1 \mathrm{~h}$ incubations with $0-5 \mathrm{mM}$ glucose and $1 \mathrm{nM}-30 \mu \mathrm{M}$ GKA50. The effects of the GK inhibitors, mannoheptulose and 5-thioglucose (10 $\mathrm{mM})$, on insulin secretion were determined at $5 \mathrm{mM}$ glucose and $10 \mu \mathrm{M}$ GKA50 and compared with their effects on tolbutamide-stimulated $(50 \mu \mathrm{M})$ insulin release. Islets were prepared from male Wistar rats by Liberase digestion and insulin secretion measured by comparison of release at basal $(3 \& 5 \mathrm{mM})$ and stimulated $(8 \&$ $10 \mathrm{mM}$ ) glucose concentrations after $2 \mathrm{~h}$ in static incubations. Islets were prepared from male $\mathrm{C} 57 \mathrm{Bl} / 6$ mice by a similar method with insulin release measured at $3,10 \& 15 \mathrm{mM}$ glucose.

Results: GKA50 activated recombinant human and rat pancreatic $\mathrm{GK}$ with $\mathrm{EC}_{50}$ values of $0.039 \mu \mathrm{M}(\mathrm{n}=10)$ and $0.059 \mu \mathrm{M}(\mathrm{n}=5)$ respectively. The mechanism of activation in rat and human enzyme was a clear concentrationdependent leftward shift in $\mathrm{S}_{0.5}$ for glucose. In MIN6 cells at 2, 3 and $5 \mathrm{mM}$ glucose GKA50 increased insulin release with $\mathrm{EC}_{50}$ values of $7.2 \mu \mathrm{M}(\mathrm{n}=5), 2.5 \mu \mathrm{M}(\mathrm{n}=3)$ and $0.84 \mu \mathrm{M}(\mathrm{n}=5)$ respectively. The GK inhibitors, mannoheptulose and 5-thioglucose, completely blocked the effects of GKA50 on insulin secretion in MIN6 cells at $5 \mathrm{mM}$ glucose, but not those of tolbutamide. In Wistar rat islets incubated with $5 \mathrm{mM}$ glucose, $1 \mu \mathrm{M}$ GKA50 caused a 2.5 fold $(n=5)$ increase in insulin release and $10 \mu \mathrm{M}$ GKA50 caused a 4.4 fold $(n=4)$ increase in insulin release. At $8 \mathrm{mM}$ glucose GKA50 stimulated insulin release but this was not concentration-dependent. At $10 \mathrm{mM}$ glucose there was no effect of GKA50 on insulin secretion. GKA50 was shown to stimulate insulin secretion of mouse islets in the presence of $10 \mathrm{mM}$ glucose. 
Conclusion: GKA50 concentration-dependently and glucosedependently modulated insulin release from rat islets and MIN6 cells. Kinetically this represented a leftward shift in $\mathrm{S}_{0.5}$ for glucose. Effects on insulin secretion from MIN6 cells can be completely blocked by GK inhibitors, entirely consistent with GKA50 being a specific glucokinase activator.

\section{2}

Palmitic acid potentiates glucose induced insulin release in mouse islets via the GPR40 receptor

\section{J. A. Pierce;}

AstraZeneca Pharmaceuticals, Macclesfield, United Kingdom.

Background and Aims: GPR40 is a GPCR that is preferentially expressed in the pancreatic $\beta$-cells. Medium and long chain fatty acids have been shown to stimulate insulin secretion through the activation of GPR40. GPR40 null mice are protected against the detrimental effects of a high fat diet and have improved glucose tolerance compared with wild type mice fed a high fat diet; whilst overexpression of GPR40 in the $\beta$-cells of mice leads to impaired $\beta$-cell function, hypoinsulinaemia and diabetes. These data suggest that chronic fatty acid stimulation of insulin secretion is detrimental and that a GPR40 antagonist would be beneficial in the chronic treatment of type 2 diabetes. The aims of this study were two-fold. Firstly, to test whether fatty acid potentiation of glucose induced insulin secretion is reduced in islets from GPR40 null mice and secondly, to investigate whether a small molecule GPR40 antagonist is able to inhibit fatty acid stimulation of glucose induced insulin secretion.

Materials and Methods: Pancreatic islets were isolated from C57/BL6J mice and GPR40 null and wild type mice using collagenase digestion. Glucose induced insulin secretion was measured in a static assay, with 3 islets per well in a 96 well plate, at high (16.7-20 mM) glucose. Palmitic acid was pre-conjugated to bovine serum albumin at a ratio of $5: 1$. Following two hours incubation with the specified reagents insulin was measured using an ELISA assay.

Results: $0.5 \mathrm{mM}$ palmitic acid robustly ( $>2$-fold) potentiated glucose induced insulin secretion in islets from wild type mice but not in islets from GPR40 null mice. A small molecule GPR40 antagonist, at a concentration of $10 \mu \mathrm{M}$, completely inhibited the stimulatory effect of $0.5 \mathrm{mM}$ palmitic acid on glucose induced insulin secretion in C57/ BL6J mouse islets.

Conclusion: Palmitic acid robustly potentiates glucose induced insulin secretion in mouse islets and this effect is inhibited by GPR40 knock-out or a GPR40 antagonist. These data indicate that acute stimulation of glucose induced insulin secretion by palmitic acid occurs exclusively via GPR40 signaling.

\section{3}

A small molecule GPR40 antagonist inhibits fatty acid induced signaling and insulin secretion

J. Morrell, D. J. Masters;

Astrazeneca, Macclesfield, United Kingdom.

Background and Aims: GPR40 is a GPCR that is preferentially expressed in the pancreatic $\beta$-cells. Medium and long chain fatty acids have been shown to stimulate insulin secretion through the activation of GPR40. GPR40 null mice are protected against the detrimental effects of a high fat diet and have improved glucose tolerance compared with wild type mice fed a high fat diet; whilst overexpression of GPR40 in the $\beta$-cells of mice leads to impaired $\beta$-cell function, hypoinsulinaemia and diabetes. These data suggest that chronic fatty acid stimulation of insulin secretion is detrimental and that a GPR40 antagonist would be beneficial in the chronic treatment of type 2 diabetes. Here we present data detailing the in-vitro pharmacology of a novel, selective, small molecule GPR40 antagonist.

Materials and Methods: The HEK293 cell lines engineered to express the receptors (GPR40 or GPR10) were loaded with Calcium 3/Fluo 4 (Molecular Devices) in HBSS, $10 \mathrm{mM}$ HEPES with $0.01 \%$ BSA. The cells are incubated for one hour and loaded onto the FLIPR (Fluometric Imaging Plate Reader). Compounds were added and assessed for their ability to inhibit the response to the EC80 concentrations for elaidic acid or PrRP respectively. To examine the effect of the antagonist in $\beta$-cells, insulin secretion was measured in MIN6 cells in the presence of $20 \mathrm{mM}$ glucose. Following two hours incubation insulin was measured using an ELISA assay.

Results: The antagonist completely blocked the increase in intracellular calcium caused by stimulation with $22.5 \mu \mathrm{M}$ elaidic acid, in HEK293s cells expressing GPR40, with a mean IC50 of $60 \mathrm{nM}(\mathrm{n}=30)$. It had no effect in HEK293s cells expressing another Gaq receptor GPR10 or in null cells. It completely inhibited elaidic acid stimulated GTP $\gamma \mathrm{S}$ binding in GPR40 expressing membranes with a mean IC50 of $52 \mathrm{nM}(\mathrm{n}=11)$. In MIN6 cells, the antagonist completely inhibited the insulin secretion effects of a novel, small molecule GPR40 agonist and oleic acid.

Conclusion: Elaidic, oleic and the GPR40 agonist all robustly potentiated $\mathrm{Ca}++$ transients in the FLIPR assay and increase binding in the GTP $\gamma \mathrm{S}$ assay. These compounds increased GSIS in MIN6 cells. These effects were inhibited by the small molecule GPR40 antagonist. This compound had no effect on PrRP responses in GPR10 expressing cells. Supported by Astrazeneca 
0504

Glutamate is not co-secreted with insulin and glucagon in isolated rat beta and alpha cells

N. Feldmann, A. Wiederkehr, C. B. Wollheim;

Department of Cell Physiology and Metabolism, University

of Geneva, University Medical Centre, Switzerland.

Background and Aims: Glutamate release from rat islet $\alpha$ and $\beta$-cells has been suggested to regulate glucagon and insulin secretion by autocrine and paracrine mechanisms. In particular, it has been reported that glutamate is co-secreted with glucagon from secretory granules. To study the role of glutamate in the endocrine pancreas, we measured insulin secretion in intact islets and purified $\beta$-cells as well as glucagon secretion from isolated $\alpha$-cells and simultaneously monitored glutamate release.

Materials and Methods: Collagenase-isolated, free floating rat islets as well as FACS purified, attached rat $\alpha$-and $\beta$-cells were cultured over night in RPMI-1640 containing $2 \mathrm{mM}$ glutamine. All preparations were washed and incubated for 30 minutes in modified Krebs-Ringerbicarbonate-Hepes buffer $(0.05 \%$ BSA $)$ at $2.5 \mathrm{mM}$ glucose with various additions. Glutamate secretion was measured by a sensitive fluorescence enzyme cycling assay. Released glucagon and insulin were assessed by standard radioimmuno assays.

Results: In whole islets, $16.7 \mathrm{mM}$ glucose stimulated insulin secretion 2.5 fold, while glutamate release was not significantly affected. However, glutamate content was increased by $38 \%(\mathrm{p}<0.01)$. In isolated $\beta$-cells, insulin secretion was increased significantly by the following compounds: glucose $(16.7 \mathrm{mM}$; 44\%), leucine $(10 \mathrm{mM}$; $105 \%$ ), alpha-ketoisocarproic acid (KIC; $10 \mathrm{mM} ; 161 \%$ ) and monomethyl succinate (mmsucc; $5 \mathrm{mM} ; 142 \%$ ), which stimulate mitochondrial metabolism, as well as by the $\mathrm{K}_{\mathrm{ATP}}$ channel-closing agent tolbutamide (100 uM; 47\%). However, only glucose raised glutamate secretion (38\%; $\mathrm{p}<0.01)$. Epinephrine $(1 \mathrm{uM})$ abolished glucose-stimulated insulin and glutamate release but did not alter basal secretion rates. Consistent with our previous results, glucose, mmsucc and tolbutamide increased glucagon secretion from purified $\alpha$-cells by respectively $28 \%$ $(p<0.001), 37 \%$ and $44 \%$, while leucine and KIC had no effect. Again in this cell type, glutamate secretion was enhanced only by glucose $(47 \%$; $<<0.001)$. Remarkably, glutamate release was reduced by KIC $(40 \%$; $<<0.02)$ and by epinephrine $(32 \% ; \mathrm{p}<0.05)$. The latter agent stimulated glucagon release $(50 \% ; \mathrm{p}<0.01)$. Inhibition of the mitochondrial dicarboxylate carrier by phenylsuccinate $(10 \mathrm{mM})$ abolished glucose-induced glutamate release in $\beta$ - but not in $\alpha$-cells.
Conclusions: Glucose, but not other mitochondrial substrates, stimulates glutamate release, both in $\alpha$-and $\beta$-cells. This involves mitochondrial transport in $\beta$-cells. In $\alpha$-cells, glutamate production is markedly reduced by KIC, which drives the branched-chain amino acid transaminase reaction towards leucine, thereby resulting in glutamate depletion in these cells, in which glucose oxidation is less pronounced than in $\beta$-cells. It is unlikely that the reduction of glutamate release in both islet cell types by epinephrine results from inhibition of exocytosis, as even strong stimulation of hormone release is not associated with glutamate release. It remains to be established by which mechanism glutamate is released into the extra-cellular space from islet cells.

\section{PS 28}

\section{Novel approaches to restore beta cell mass}

\section{5}

Intracellular signaling in Reg-induced pancreatic beta cell regeneration: involvement of the $\mathrm{PI}(3) \mathrm{K} / \mathrm{ATF}-2$ / cyclin D1 signaling pathway in the $R$ eg/Reg receptor system

S. Takasawa ${ }^{1}$, K. Nata ${ }^{1}$, T. Ikeda ${ }^{1,2}$, N. Noguchi ${ }^{1}$, I. Takahashi ${ }^{1}$, A. Yamauchi ${ }^{1,3}$, T. Yoshikawa ${ }^{1}$, N. J. Shervani ${ }^{1}$, M. Unno ${ }^{4}$, H. Okamoto ${ }^{1}$;

${ }^{1}$ Advanced Biological Sciences for Regeneration (Kotobiken Medical Laboratories), Tohoku University, Sendai, ${ }^{2}$ Innovation Plaza Miyagi, JST, Sendai, ${ }^{3}$ Supramolecular Chemistry, ${ }^{4}$ Surgery, Tohoku University, Sendai, Japan.

Background and Aims: We have found an autocrine/ paracrine beta-cell regeneration factor, regenerating gene protein (Reg), that increases the beta-cell replication. Reg is induced in pancreatic beta-cells by inflammatory stimulation such as by IL-6/glucocorticoids and acts as an autocrine/paracrine growth factor for beta-cell regeneration via a cell surface Reg receptor to ameliorate experimental diabetes. Recently, we produced Reg transgenic mice. The islets from the transgenic mice expressing Reg in beta-cells showed increased the $\left[{ }^{3} \mathrm{H}\right]$ thymidine incorporation, and the development of diabetes in the non-obese diabetic (NOD) mice carrying the Reg transgene was significantly retarded. However, the manner by which Reg induces beta-cell regeneration was unknown.

Materials and Methods: RINm5F beta-cells were stimulated by Reg. Signal transduction pathways in pancreatic beta-cells by Reg stimulation were analyzed by using PathDetect in vivo trans-reporting systems. The levels of cyclin D1, phospho-ATF-2, and phospho-Rb were mea- 
sured by Western blot analyses. Characterization of Regstimulated cyclin D1 promoter was carried out by reporter, EMSA, and ChIP assays. $\mathrm{Reg}^{-/-}$mice were produced by homologous recombination.

Results: ATF-2, but not CREB, cJun, Elk1, CHOP or cFos, was activated by Reg stimulation. The phospho-ATF-2 bound to $-57 \sim-52$ of the cyclin D1 gene and the Reg-induced activation of the cyclin D1 promoter was inhibited by the cotransfection of the dominant-negative ATF-2 expression plasmid. The addition of PI(3)K inhibitors, LY294002 and wortmannin, attenuated the Reg/ATF-2-induced cyclin D1 promoter activity whereas SB203580, a p38 mitogenactivated protein (MAP) kinase inhibitor, PD98059, an inhibitor of the MAP kinase/ERK kinase-ERK pathway, $\mathrm{KN}-62$ and $\mathrm{KN}-93$, inhibitors of $\mathrm{Ca}^{2+} /$ calmodulin (CaM)dependent protein kinase II, K-252a, an inhibitor of $\mathrm{CaM}$ kinase IV, and H-89, an A-kinase inhibitor, showed a negligible inhibitory effect on the cyclin D1 promoter activity in beta-cells. The immunoprecipitated PI(3)K phosphorylated ATF-2 in a time-dependent and dose-dependent fashion, indicating that $\mathrm{PI}(3) \mathrm{K}$ directly phosphorylates ATF2 to activate the cyclin D1 promoter. Furthermore, We produced Reg-knockout mice by homologous recombination. In $\mathrm{Reg}^{-/-}$mice, no Reg expression was detected in the pancreas and therefore no Reg was secreted into the islet culture medium. The BrdU incorporation in $\mathrm{Reg}^{-/-}$islets in the culture medium was significantly reduced compared to that in $\mathrm{Reg}^{+/+}$islets. We then analyzed the levels of phospho-ATF-2, cyclin D1 protein, and phospho-Rb in the islets. $\mathrm{Reg}^{+/+}$islets secreted Reg in the culture medium and the phospho- $\mathrm{Rb}$ level in the islets was much higher than that in $\mathrm{Reg}^{-/-}$islets. As expected, both the levels of phosphoATF-2 and cyclin D1 were decreased in islets from Reg $^{-1-}$ mice, whereas the levels of other proteins such as PARP and CD38 as controls were unchanged.

Conclusion: These results indicate that the Reg-RegR system stimulates the PI(3)K/ATF-2/cyclin D1 signaling pathway to induce beta-cell regeneration.

\section{6}

Role of protein kinase $B$ isoforms in the regulation of functional $\beta$ cell mass

F. Buzzi ${ }^{1}$, L. Xu ${ }^{1}$, R. A. Zuellig ${ }^{1}$, S. B. Boller ${ }^{1}$, G. A. Spinas ${ }^{1}$, O. Tschopp ${ }^{2}$, B. Duemmler ${ }^{2}$,

B. A. Hemmings ${ }^{2}$, M. Niessen ${ }^{1}$;

${ }^{1}$ Endocrinology and Diabetes, University Hospital Zurich, Switzerland, ${ }^{2}$ Friedrich Miescher Institute for Biomedical Research, Basel, Switzerland.
Background and Aims: Total $\beta$-cell mass can adapt according to metabolic demand. This important mechanism often prevents the onset of hyperglycaemia in the face of persistent insulin resistance, a condition leading otherwise to type 2 diabetes. The IRS2/PI3K/PKB signaling axis is considered to be required for this compensatory regulation by promoting survival, growth and proliferation of $\beta$-cells as well as inhibiting apoptosis. The aim of the present study was to elucidate the role of PKB isoforms in regulation of islet phenotype.

Material and Methods: Phenotypic analysis of mice (between one and three months old) deficient for either of the three PKB isoforms and comparison with heterozygous and wild type littermates: assessment of body weight and random blood glucose levels, immunostaining of pancreatic sections for insulin and glucagon to determine islet size and number, $\beta$-cell size and number, $\alpha$-cell number.

Results: $P \mathrm{kba}^{-1-}$ mice were smaller (males $22.6 \pm 0.6 \mathrm{~g}$, $\mathrm{n}=6, P<0.05$; females $19.1 \pm 1.3 \mathrm{~g}, \mathrm{n}=3$, n.s.) and displayed lower blood glucose levels (males $6.5 \pm 0.8 \mathrm{mM}, P<0.05$; females $5.8 \pm 0.1 \mathrm{mM}$, n.s.) compared to control littermates (males $26.4 \pm 1.2 \mathrm{~g}$ and $9.45 \pm 0.35 \mathrm{mM}$, respectively, $\mathrm{n}=5$; females $21.5 \pm 0.8 \mathrm{~g}$ and $6.8 \pm 0.4 \mathrm{mM}, \mathrm{n}=5)$. $P k b \beta^{-1-}$ mice had significantly higher blood glucose (males $9.3 \pm 1.0 \mathrm{mM}$, $\mathrm{n}=11, P<0.05$; females $7.4 \pm 0.3 \mathrm{mM}, \mathrm{n}=6, P<0.05)$ compared to controls (males $7.4 \pm 0.6 \mathrm{mM}, \mathrm{n}=14$; females $6.3 \pm 0.3 \mathrm{mM}, \mathrm{n}=7$ ), whereas there was no difference in blood glucose levels between $P \mathrm{kb \gamma}^{-/-}$mice and controls. Morphologic analysis of the pancreas revealed increased islet number (1.27 fold, n.s.) and size (1.91 fold, $P<0.05)$ in $P k b \beta^{-1-}$ females $(\mathrm{n}=3$, controls $\mathrm{n}=4)$. A trend towards increased islet size (1.38 fold, n.s.) was also found in $P k b \alpha^{-1-}$ males ( $\mathrm{n}=5$, controls $\left.\mathrm{n}=5\right)$, whereas no differences were detected for the other genotypes.

Conclusions: Unlike in IRS2-deficient mice, which show islet hypoplasia (and develop diabetes), no decrease (or on the contrary even an increase) in $\beta$-cell mass was observed in PKB-deficient mice. Our results suggest that all three $\mathrm{PKB}$ isoforms are individually dispensable for the maintenance of islet mass. This could be due to redundancy between isoforms or could depend on other signaling elements downstream of IRS2. While $P k b \beta^{-/-}$mice revealed hyperglycaemia and compensatory islet hyperplasia/hypertrophy, $P \mathrm{kba}^{-/-}$mice displayed increased islet mass in combination with decreased blood glucose levels which might indicate deregulation of islet growth without need for compensation (insulin resistance). This suggests that $\mathrm{PKB} \alpha$ and $\beta$ isoforms are individually not necessary in $\beta$-cells to increase islet mass.

Supported by Takeda Research Foundation 
0507

A study of proliferation and prevention of cytokineinduced apoptosis in human beta cells

C. Bruun ${ }^{1}$, T. Mandrup-Poulsen ${ }^{1,2}$, N. Billestrup ${ }^{1}$;

${ }^{1}$ Steno Diabetes Center, Gentofte, Denmark, ${ }^{2}$ Dept. of Mol. Med., Karolinska Institute, Stockholm, Sweden.

Background and Aims: Type 1 diabetes mellitus (T1DM) is caused by a specific destruction of pancreatic beta cells. This pathogenic process is immune-mediated and it is wellknown that the pro-inflammatory cytokines IL-1 $\beta$, IFN $\gamma$ and TNF $\alpha$ are important mediators of this beta cell death. An effective curative therapy for T1DM could be islet transplantation, although, shortage of islet donors poses a major problem to this therapy. Induction of human beta cell proliferation in vitro could solve this problem, however, human beta cells hold a very low potential for proliferation in vitro under conventional culturing methods. It has been established that GH, STAT proteins and GLP-1 are able to induce proliferation and protection against apoptosis of beta cells from rats and mice. Similarly, it is known that suppressors of cytokine signaling (SOCS) proteins can protect rodent beta cells from cytokine mediated apoptosis. The aim of this study is to induce proliferation of human beta cells and protect human beta cells from cytokineinduced cell death by exposing free-floating human islets or human islets cultured on extracellular matrix $(\mathrm{ECM})$ to growth factors (including GLP-1 and GH) and/or by expression of SOCS- 3 and STAT- 5 proteins.

Materials and Methods: Isolated human islets with 40 $90 \%$ purity were used. Because of limited supply of human islets, neonatal rat islets were used for optimisation. GFPencoding Adenovirus was used to investigate and optimise transduction efficiency. Intact free-floating human islets or intact islets cultured on ECM (Biological Industries, Israel) were exposed to growth factors and/or transduced with STAT or SOCS-3 encoding Adenovira. Beta cells were identified by $\mathrm{Pdx}-1$ and/or insulin staining and proliferation was analysed by BrdU incorporation. Apoptosis was induced by a combination of IL-1 $\beta$, IFN $\gamma$ and TNF $\alpha$ and was detected by TUNEL assay. Immunostaining was analysed by use of confocal or conventional microscopy.

Results: In intact rat islets GH induced a 10 -fold increase in beta cell proliferation. Transduction of rat islets with constitutive active (CA) STAT-5 encoding Adenovirus induced a 3-fold increase in BrdU incorporation in beta cells. In free-floating intact human islets many BrdU positive cells were observed in islets transduced with CASTAT-5 Adenovirus or in islets stimulated with GLP-1 and
$\mathrm{GH}$, both in the periphery and in the center of the islets. However, these cells did not co-express Pdx-1 and their identity is presently unknown. In human islets cultured on ECM, BrdU incorporation was observed in beta cells following stimulation with GLP-1 and GH. The possible role of growth factors, STAT-5 and SOCS-3 on protection from cytokine-induced apoptosis is currently being investigated.

Conclusion: Although human beta cells have a low potential for regeneration these studies indicate that proliferation can be induced by the combined use of ECM, stimulation with growth factors and/or expression of growth controlling signaling molecules including STAT-5.

Supported by the Juvenile Diabetes Research Foundation International

\section{8}

IGF-1 protects insulin-secreting cells against glucocorticoid-mediated cell death via activation of MAP kinases but not PI3 kinase

D. Avram ${ }^{1}$, F. Ranta ${ }^{1}$, A. M. Hennige ${ }^{2}$, S. Berchtold ${ }^{1}$, H.-U. Häring ${ }^{2}$, F. Lang ${ }^{1}$;

${ }^{1}$ Institute of Physiology, ${ }^{2}$ Medicine IV, University of Tuebingen, Tuebingen, Germany.

Background and Aims: Patients under long term treatment with immunosuppressive glucocorticoids may develop diabetes mellitus. Glucocorticoids are known to inhibit insulin secretion. Previously, we have shown that dexamethasone (dex), a synthetic glucocorticoid, induces apoptosis in $\beta$-cells. IGF-1, a potent stimulator of cell growth and an inhibitor of cell death, has been described to support insulin secretion and to induce $\beta$-cell proliferation which maintains $\beta$-cell mass. The present study examines whether IGF-1 protects insulin secreting INS-1 cells against dex-induced inhibition of proliferation and activation of cell death.

Materials and Methods: INS-1 cells were cultured under standard conditions in the presence of test substances for 1 to 4 days. Apoptosis was quantified by DAPI-stained condensed nuclei, by TUNEL assay and by caspase-3 activity. Proliferation was measured by BrdU-staining. The amount and phosphorylation of proteins (Bcl-2, PKB and IRS-2) was quantified by Western blotting. To differentiate distinct pathways activated by IGF-1 the inhibitors LY294002 of PI3-kinase, SB203580 of p38ßMAP-kinase and PD98059 of p42/p44 MAP-kinase were used.

Results: Culture of INS- 1 cells in the presence of dex $(100 \mathrm{nM}, 24 \mathrm{~h})$ inhibited glucose (increased from 5 to 
$11 \mathrm{mM})$ - and IGF-1 (50 ng/ml)-induced cell proliferation. At the same time, dex reduced IGF-1 mediated phosphorylations of IRS-2 and PKB/Akt. Dex-stimulated cell death was reduced by IGF-1 by 75 and $58 \%$ after 1 and 4 days, respectively. While LY294002 abolished IGF-1 mediated phosphorylation of $\mathrm{PKB} / \mathrm{Akt}$ and proliferation, it did not counteract but improve the protective effect of IGF-1 on cell death. The addition of MAP-kinase inhibitors, SB203580 and PD98059 antagonized the protection of apoptosis by IGF-1. Dex reduced the anti-apoptotic protein $\mathrm{Bcl}-2$ an effect that persists in the presence of IGF-1.

Conclusion: These data suggest that IGF-1 stimulates proliferation of INS-1 cells via PI3-kinase pathway. The protective effect of IGF-1 against dex-induced apoptosis occurs in the absence of $\mathrm{PKB} / \mathrm{Akt}$ phosphorylation and is mediated via MAP-kinase pathways.

\section{9}

The anti-inflammatory cytokine interleukin-1 receptor antagonist protects from nutrition-induced hyperglycaemia and impaired glucose tolerance

N. S. Sauter ${ }^{1}$, F. T. Schulthess ${ }^{1}$, M. Donath ${ }^{2}$, K. Maedler ${ }^{1}$; ${ }^{1}$ David Geffen School of Medicine, UCLA, L. Hillblom Islet Research Center, Los Angeles, United States, ${ }^{2}$ Division of Endocrinology and Diabetes, University Hospital, Zurich, Switzerland.

Background and Aims: In type 2 diabetes, chronic hyperglycemia has been associated with impaired beta-cell function and beta-cell apoptosis. This is partly mediated by beta-cell secretion and production of the pro-inflammatory cytokine IL-1beta. Previous studies indicate that the interleukin-1 receptor antagonist (IL-1Ra) protects human islets from the deleterious effects of high glucose and IL-1beta. Therefore, in the present study we investigated a possible protective role of IL-1Ra in hyperglycemic mice in vivo.

Materials and Methods: Six-week-old C57Bl/6 mice were fed a high fat/high sucrose diet (HFD) or a control diet for 12 weeks. Mice were daily injected with $10 \mathrm{mg} / \mathrm{kg}$ body weight IL-1Ra or solvent. Body weight and food consumption were measured weekly, intra-peritoneal glucose and insulin tolerance tests (ipGTT/ipITT) were performed every 4 weeks. After 12 weeks, mice were sacrificed and their islets isolated.
Results: As expected, the HFD led to a rapid weight gain compared to normal chow ( $<<0.01$ after two weeks of diet). IL-1Ra injections did not significantly change body weights in either control or HFD mice. HFD feeding induced impaired glucose tolerance already after 4 weeks, which was inhibited in the IL-1Ra group after 4,8 and 12 weeks of $\operatorname{diet}(\mathrm{p}<0.05)$. After 8 and 12 weeks, the HFD fed control but not the IL$1 \mathrm{Ra}$ treated mice displayed hyperglycemia (blood glucose $11.75+/-0.83 \mathrm{mM}$ versus $7.52+/-0.38 \mathrm{mM}$ in control HFD versus IL-1Ra HFD mice after 12 weeks of diet, $\mathrm{p}<0.001)$. As predicted, serum insulin measurements revealed hyperinsulinemia induced by the HFD $(0.77+/-0.23 \mathrm{ug} / 1$ versus $2.97+/-0.46 \mathrm{ug} / 1$ insulin in control versus HFD mice). IL-1Ra increased basal as well as glucose stimulated insulin levels during an ipGTT in the normal diet group (4.11-fold increase in basal and 2.66-fold increase in stimulated serum insulin levels in normal diet mice, $\mathrm{p}<0.01)$. IPITT exhibited insulin resistance in the HFD group while IL-1Ra rendered them more insulin sensitive $(p<0.01$ after 12 weeks of diet). Glucose-stimulated insulin secretion (GSIS) assays of isolated islets showed that isolated islets from the HFD group lost glucose sensitivity compared to islets isolated from the normal diet group, which was improved by administering IL-1Ra (1.64+/0.58 stimulatory index in control HFD islets versus $2.16+/-0.36$ in the IL-1Ra HFD group).

Conclusion: These data show a protective role of IL-1Ra against nutrition-induced diabetes. This is in line with our previous study showing that IL-1Ra inhibits the deleterious effects of chronically elevated glucose or Interleukin- $1 \beta$ on isolated islets.Our results suggest a potential role for IL$1 \mathrm{Ra}$ in the treatment of diabetes.

0510

Augmented insulin secretion in the assessment of pancreatic $\beta$ cell mass in healthy volunteers and patients with type 2 diabetes

C. Chan ${ }^{1}$, D. Soon ${ }^{1}$, K. P. Yeo ${ }^{1}$, N. K. Porksen ${ }^{2}$, S. D. Wise ${ }^{1}$;

${ }^{1}$ Lilly-NUS Centre for Clinical Pharmacology, Singapore, ${ }^{2}$ Eli Lilly and Company, Indianapolis, United States.

Background and Aims: Insulin secretion, a product of $\beta$-cell mass and function, decreases with the progression of 
diabetes (DM), possibly due to decreasing $\beta$-cell mass. Methods used to investigate insulin secretion are often conducted on the background of changing insulin sensitivity, therefore failing to distinguish between improved function and increased mass. Previously, we developed a $\beta$-cell mass assessment procedure ( $\beta$-MAP) in healthy volunteers (HV) that appears to maximally stimulate $\beta$-cell insulin secretion and is robust to changes in peripheral insulin sensitivity (Porksen et al. 2005 ADA Scientific Sessions, 1536-P). The aim of this study was to assess this novel $\beta$-MAP and its reproducibility in $\mathrm{HV}$ and patients with type $2 \mathrm{DM}$ at various stages of disease severity.

Materials and Methods: Eight HV [median (range) age: 28(21-45 yr); BMI 24.8(19.7-27.5 $\left.\left.\mathrm{kg} / \mathrm{m}^{2}\right)\right]$ and patients from each of 3 investigator-defined grades of type $2 \mathrm{DM}$ severity [Grade 1: Dietary/exercise control $(n=8)$, Grade 2: Treated with oral medication(s) $(\mathrm{n}=8)$, Grade 3: Treated with insulin (n=7); age: 54(37-65 yr); BMI: 25.4(18.6$\left.29.9 \mathrm{~kg} / \mathrm{m}^{2}\right)$ ] participated in this open-label, 2-period, replicate study. The $\beta$-MAP consisted of the following sequence of manoeuvres; a loading glucose bolus ( $25 \mathrm{~g}$ of $50 \%$ dextrose); $45 \mathrm{~min}$ fixed-rate glucose infusion $(600 \mathrm{~mL} / \mathrm{h}$ of $20 \%$ dextrose) to induce hyperglycaemia (approx. $22 \mathrm{mmol} / \mathrm{L}$ ); and an i.v. glucagon bolus $(1 \mathrm{mg}$ ) administered $15 \mathrm{~min}$ after the start of glucose infusion. Glucose delivery was titrated lower if blood glucose levels exceeded $30 \mathrm{mmol} / \mathrm{L}$. Blood samples were collected for glucose, insulin, and C-peptide.

Results: Mean glucose levels at baseline and after administering glucagon, and mean baseline insulin levels were significantly higher for all DM groups compared to the healthy subjects. Prior to the glucagon bolus, the hyperglycaemic stimulus failed to increase insulin secretion in diabetics. The maximal insulin level and overall insulin exposure over $30 \mathrm{~min}$ after the glucagon bolus [insulin $\mathrm{C}_{\max }(15-45 \mathrm{~min})$ and $\left.\mathrm{AUC}(15-45 \mathrm{~min})\right]$ were significantly lower for the $3 \mathrm{DM}$ grades when compared to the HV. Insulin $\mathrm{C}_{\max }(15-45 \mathrm{~min})$ and $\mathrm{AUC}(15-45 \mathrm{~min})$ trended lower with increasing severity of the DM, with C-peptide levels demonstrating a similar trend. The intra-subject $\mathrm{CV} \%$ for insulin $\mathrm{C}_{\max }(15-45 \mathrm{~min})$ and $\mathrm{AUC}(15-45 \mathrm{~min})$ in all groups ranged from 8 to $33.3 \%$. Insulin $\mathrm{C}_{\max }(15-45 \mathrm{~min})$ correlated well with HOMA2\%B ( $\beta$-cell function index) $\left(\mathrm{R}^{2}=0.514, \mathrm{p}<0.001\right)$ but not HOMA IR (insulin resistance) $\left(\mathrm{R}^{2}=0.010\right)$.

Conclusion: The novel $\beta$-MAP demonstrated a clear separation of insulin response between HV and DM patients, and appears well correlated with an estimate of $\beta$-cell mass (HOMA2\%B) but not with insulin resistance, as indirectly estimated by HOMA IR. The low intra-subject variability suggests that the procedure is reproducible and may be a feasible tool in a clinical setting to monitor changes in $\beta$-cell mass.

\begin{tabular}{|c|c|c|c|c|c|}
\hline $\begin{array}{l}\text { Parameter } \\
\text { Group }\end{array}$ & $\begin{array}{l}\text { Control } \\
\text { Group } \\
\text { (Least- } \\
\text { squares } \\
\text { Geometric } \\
\text { Mean) }\end{array}$ & $\begin{array}{l}\text { DM Gro } \\
\text { (Least- } \\
\text { squares } \\
\text { Geometr } \\
\text { Mean) }\end{array}$ & & $\begin{array}{l}\text { Ratio to } \\
\text { Control } \\
(90 \% \\
\text { confidence } \\
\text { interval) }\end{array}$ & $\begin{array}{l}\text { P-value } \\
\text { for } \\
\text { Difference } \\
\text { to Control }\end{array}$ \\
\hline $\begin{array}{l}\text { Insulin } \\
\mathrm{C}_{\max }\end{array}$ & 4385 & $\begin{array}{l}\text { DM } \\
\text { Grade } 1\end{array}$ & 1418 & $\begin{array}{l}0.32 \\
(0.20,0.52)\end{array}$ & 0.001 \\
\hline $\begin{array}{l}(15- \\
45 \mathrm{~min})\end{array}$ & & $\begin{array}{l}\text { DM } \\
\text { Grade } 2\end{array}$ & 781 & $\begin{array}{l}0.18 \\
(0.09,0.34)\end{array}$ & 0.001 \\
\hline$(\mathrm{pmol} / \mathrm{L})$ & & $\begin{array}{l}\text { DM } \\
\text { Grade } 3\end{array}$ & 593 & $\begin{array}{l}0.14 \\
(0.07,0.26)\end{array}$ & 0.001 \\
\hline $\begin{array}{l}\text { Insulin } \\
\text { AUC }\end{array}$ & 1582 & $\begin{array}{l}\text { DM } \\
\text { Grade } 1\end{array}$ & 393 & $\begin{array}{l}0.25 \\
(0.15,0.41)\end{array}$ & $<0.001$ \\
\hline $\begin{array}{l}(15- \\
45 \mathrm{~min})\end{array}$ & & $\begin{array}{l}\text { DM } \\
\text { Grade } 2\end{array}$ & 208 & $\begin{array}{l}0.13 \\
(0.07,0.25)\end{array}$ & $<0.001$ \\
\hline$\left(\mathrm{pmol}^{*} \mathrm{~h} / \mathrm{L}\right)$ & & $\begin{array}{l}\text { DM } \\
\text { Grade } 3\end{array}$ & 184 & $\begin{array}{l}0.12 \\
(0.06,0.21)\end{array}$ & $<0.001$ \\
\hline $\begin{array}{l}\text { C-peptide } \\
\text { AUC }\end{array}$ & 2927 & $\begin{array}{l}\text { DM } \\
\text { Grade } 1\end{array}$ & 1278 & $\begin{array}{l}0.44 \\
(0.33,0.59)\end{array}$ & $<0.001$ \\
\hline $\begin{array}{l}(15- \\
45 \min )\end{array}$ & & $\begin{array}{l}\text { DM } \\
\text { Grade } 2\end{array}$ & 986 & $\begin{array}{l}0.34 \\
(0.22,0.51)\end{array}$ & 0.001 \\
\hline$\left(\mathrm{pmol}^{*} \mathrm{~h} / \mathrm{L}\right)$ & & $\begin{array}{l}\text { DM } \\
\text { Grade } 3\end{array}$ & 921 & $\begin{array}{l}0.31 \\
(0.22,0.44)\end{array}$ & $<0.001$ \\
\hline
\end{tabular}

Supported by Eli Lilly and Company

0511

Monoclonal autoantibodies in diabetes and their potential use in islet beta-cell Imaging: the BB-rat monoclonal IC2 further purified and fragmented for improved performance in non-invasive in vivo imaging

C.-H. Brogren, S. Petersen;

Department of Medical Biochemistry and Genetics, University of Copenhagen, Denmark.

Background and Aims: Since early 1980ths several monoclonal autoantibodies have been discovered from type-1 diabetic experimental animals (C57BLKsJ db/dbmice, NOD-mice, BB-rat) and from human diabetic patients. Among these autoantibodies are mainly insulin (K14D10), islet cell, neural and lymphoid cell autoantibodies described. Several hybridomas with specificity against gangliosides (A2B5, R2D6), sulfatides (IC2) and other cellular targets (3A4, 5C12, GAD and IA-2) have been described. Both target autoantigens and the immunogenic epitopes has been fully explored. In addition recombinant anti-GAD and anti-IA-2 have been made. 
Serum autoantibodies against these epitopes are seen across species, and increased titres of these specificities are used as prediagnostic tool for type-1 diabetes, however, without being strictly specific for type- 1 diabetes. Attempts have been done within the last 6 years to use some of these monoclonal autoantibody specificities for pancreatic betacell imaging. The K14D10 anti-insulin autoantibody failed to behave specific to the beta-cell in scintigraphy by gamma-imaging, but the IC2 monoclonal BB-rat autoantibody performed highly promising for a non-invasive in vivo imaging of the beta-cell mass with its pronounced specificity to the beta-cell surface. Being a strict beta-cell surface specific rat IgM-kappa autoantibody with a target molecule (sulfatide) known to be present also on islet cells innervating Schwann cells and other neural tissues, we still need fully to explain the unique beta-cell surface specificity of IC2.

Materials and Methods: To further explore the molecular specificity of IC2 we need large amount of purified IC2 IgM autoantibody for fluorochrome labelling, fragmentation, and radioisotope labelling. Affinity purified IC2 was obtained from several litres of IC2 culture supernatant using a column of MARK-1 (mouse anti-rat kappa) conjugated to Sepharose. The eluted rat IgM-kappa was further fragmented to create IC2 $\mathrm{F}(\mathrm{ab}) 2$ using standard protocols with pepsin digestion. Finally, the digest was affinity purified on Protein L-Sepharose to remove the Fc fragment. The entire IC2 IgM-kappa, and the F(ab) 2-kappa is radioisotope labelled by the DTPA-method, and the labelling efficiency of the antibody-amideDTPA-indium-111 conjugate was studied by autoradiography. In vivo non-invasive imaging experiments come next.

Results: The Integra bioreactor CELLine CL 1000 is used for large scale production of IC2 containing culture supernatant. After appropriate purification by MARK-1 (mouse anti-rat kappa) affinity chromatography, the purified IC2 is fragmented by pepsin digestion and the Fc and F(ab) 2 fragments further separated by Protein L-Sepharose affinity chromatography. These purified entire IgM and $\mathrm{F}$ (ab)2 is currently labelled with fluorochrome for immunofluorescence studies and Indium-111 for medical noninvasive imaging by gamma camera.

Conclusion: The monoclonal autoantibody IC2 is now fully characterized and made commercial available though contact with the presenting author. According to previous and recent studies the Indium-111-labelled IC2 is so far the only monoclonal autoantibody available for non-invasive beta-cell mass imaging. Its eventual reactivity to islet Schwann cells is currently under investigation by immunofluorescence microscopy.

Supported by the Danish Diabetes Society
0512 Effects of hyperglycaemia on human pancreatic islet
endothelial cells

E. Favaro, E. Beltramo, E. Berrone, F. Olivero,

A. Brescianini, M. Porta, P. Cavallo Perin, G. Camussi,

M. M. Zanone;

Department of Internal Medicine, University of Turin, Italy.

Background and Aims: High glucose induces pathological alterations in small and large vessels, responsible for micro and macrovascular complications in both type 1 and 2 diabetes and attributed to endothelial cell damage. The underlying mechanisms have not been fully elucidated and data are mainly derived from studies on HUVEC. In this study we aimed to analyse the effects of sustained hyperglycemia on human pancreatic islets microendothelial cells (MECs), themselves exposed to high glucose concentrations in the course of the disease. Islet MECs have been shown to have peculiar characteristics, including the expression of the transmembrane barrier protein nephrin, which has signaling functions, and possibly they are involved in fine-tuning islet blood glucose sensing and regulation. We analysed the effects on cell survival and proliferation, cell cycle, and apoptosis, with particular regard to the PI3K/Akt pathway.

Materials and Methods: Human pancreatic islet MECs were extracted and purified using anti-CD105 coated immunomagnetic beads, characterised and cultured in normal $(5.6 \mathrm{mmol} / \mathrm{L})$ or high $(28 \mathrm{mmol} / \mathrm{L})$ glucose in appropriate medium for up to approximately 30 days. Cell proliferation was assessed by cell count and DNA synthesis by $\mathrm{BrdU}$ incorporation assay. Apoptosis was evaluated at 3-6 days intervals, by enzyme immunoassay measuring mono- and oligo-nucleosomes in the cytoplasmic fraction of cell lysates, index of DNA fragmentation, and by detection of Caspase-3 activity. Moreover, by WB analysis, the antiapoptotic Akt activity and the P-Akt/Akt ratio, as well as the effects on expression and phosphorilation of nephrin were evaluated.

Results: Islet MECs cultured in high glucose retained endothelial characteristics, assessed by flow cytometric analysis but were significantly less numerous than those in physiological glucose concentration (proliferation was reduced of $12.7 \%$ after 2 days, $19 \%$ after 7 days, $23.8 \%$ after 12 days, $47.7 \%$ after 25 days, when compared to cells in normal glucose). In parallel experiments apoptosis progressively increased, from $7 \%$ after 2 days to $79 \%$ after 30 days in high glucose, compared to normal glucose culture. This was accompanied by increased caspase- 3 activity, up to $300 \%$, mainly during the first days of culture. Moreover, high glucose culture persistently reduced Akt 
activity and P-Akt/Akt ratio, accompanied by inhibition of nephrin tyrosine phosphorylation.

Conclusion: Hyperglycemia of several days duration induces apoptosis in islet MECs. Due to their emerging role in islet development and function, these effects might be relevant to beta cell mass and responsiveness and in the pathophysiological processes of type 1 and type 2 diabetes. The underlying mechanisms involve a reduction of the serine-threonine kinase Akt, which serves as a multifactorial cell regulator and that, in beta cells, has been shown to result in impaired glucose tolerance due to defective insulin secretory pathway. Impaired nephrin phosphorylation, that is a critical step in nephrin induced signaling and in stimulation of Akt-dependent pathways also appears to be relevant. Our findings suggest these mechanisms as target of potential intervention strategies in improvement of beta cell function.

Supported by Ricerca Sanitaria Finalizzata Regione Piemonte

\section{PS 29}

\section{Novel signaling molecules in beta cell function and dysfunction}

0513

\section{Analysis of the beta cell and insulin-containing vesicle proteome}

A. $\operatorname{Varadi}^{1}$, K. Sanders ${ }^{1}$, N. Avent ${ }^{1}$, E. Molnar ${ }^{2}$;

${ }^{1}$ Centre for Research in Biomedicine, University of the West of England, ${ }^{2}$ Department of Anatomy, University of Bristol, Bristol, United Kingdom.

Background and Aims: Beta-cells of the pancreatic islets of Langerhans act as glucose sensors, adjusting insulin secretion to the prevailing blood glucose concentration. Triggering of secretion involves increases in intracellular ATP concentrations and $\mathrm{Ca}^{2+}$ influx through voltage-gated $\mathrm{Ca}^{2+}$ channels which underlie the acute, or first-phase of insulin release. In contrast, the molecular basis of $\mathrm{K}_{\mathrm{ATP}}$-channel independent secretion is poorly understood while large dense core vesicle (LDCV) recruitment is thought to be a vital component of this process. Both microtubule and actin-dependent mobilisation of vesicles are required for sustained release of insulin through mechanisms which have yet to be fully elucidated. The aim of this study was to carry out systematic analysis of the insulin-granule proteome - which information is essential to determine the signaling pathways involved in glucose-induced LDCV recruitment and secretion.

Materials and Methods: MIN6 pancreatic beta-cells were homogenised and subcellular fractionation was performed using Optiprep ${ }^{\mathrm{TM}}$ gradients. Fractions were analysed for by immunoblotting using specific antibodies for organelle markers and by measuring their insulin content. Two complementary 2-dimensional gel electrophoresis methods were used: isoelectric focusing (IEF)/SDS-PAGE and benzyl hexadecylammonium chloride (16-BAC)/SDSPAGE to obtain the best coverage of the LDCV proteome. Proteins were excised from the gels, digested and peptides identified by MALDI-TOF mass spectrometry using the MASCOT database search.

Results: The density gradient centrifugation resulted in highly enriched LDCV fractions. The IEF/SDS-PAGE method provided superior resolution of soluble proteins while the 16-BAC/SDS-PAGE improved separation and resolution of integral membrane proteins. So far 24 proteins were identified including the heat shock cognate protein (HSc70) - involved in un-coating of clathrincoated vesicles; vacuolar ATP synthase (v-ATPase) subunits, which maintain a low intravesicular $\mathrm{pH}$, important for proinsulin conversion and storage of zincinsulin hexamers; valosin-containing protein - an NSF homologue involved in exocytosis; glucose regulated protein 54, 78 and 94. Several proteins not directly attached to vesicles but involved in vesicle cycle regulation copurified with vesicles for example actin and tubulin. Both actin and tubulin form core complex with synapsin proteins during vesicle docking thus explaining their presence on insulin vesicles.

Conclusion: Systematic proteomics analysis has revealed the molecular composition of insulin granules. Alteration in the composition and phosporylation status of LDCV proteome induced by glucose will provide fundamental information on the molecular basis and regulation of insulin-granule function. Better understanding of the downstream targets of glucose-derived metabolic signals on LDCVs likely to contribute to the development of a novel, more targeted therapeutic approach for type 2 diabetes.

Supported by Faculty of Applied Sciences, University of the West of England, Bristol

\section{4}

CART, a new player in the regulation of islet hormone secretion

N. Wierup ${ }^{1}$, M. Björkqvist ${ }^{1}$, H. Mulder ${ }^{1}$, M. J. Kuhar ${ }^{2}$, B. Ahrén ${ }^{3}$, F. Sundler ${ }^{1}$;

${ }^{1}$ Dept Experimental Medical Science, Lund University, Lund, Sweden, ${ }^{2}$ Yerkes National Primate Research Center, Emory University, Atlanta, United States, ${ }^{3}$ Dept of Clinical Science, Lund University, Lund, Sweden. 
Background and Aims: CART is an anorexigenic peptide widely expressed in the central and peripheral nervous systems as well as in islet endocrine cells, including beta cells during rat development and delta cells of adult rats. CART null mutant mice display impaired glucose-stimulated insulin secretion in vivo and in vitro, together with impaired glucose elimination and reduced expression of GLUT-2 and PDX-1. In addition, a mutation in the human CART gene cosegregates with obesity and type 2 diabetes. Materials and Methods: We examined the effect of CART 55-102 on islet hormone secretion, using INS-1 (832/13) cells and isolated rat islets. In addition, islet CART expression was examined in two rat models of type 2 diabetes: GotoKakizaki (GK) rats and dexamethasone treated rats.

Results: At high glucose CART potentiated cAMP stimulated insulin secretion via a further increase in cAMP and a subsequent stronger activation of the protein kinase $\mathrm{A}$ dependent pathway. In the absence of cAMP-elevating agents, CART was without effect on INS-1 cells but inhibited secretion of insulin, glucagon and somatostatin from isolated islets. CART was markedly upregulated in the beta cells of both diabetes models. Thus, in dexamethasone treated rats beta cells expressing CART mRNA and peptide were 10-fold increased compared with control rats. In GK rats the relative number of CART expressing beta cells was 30-fold higher than in Wistar control rats. Electron microscopy localised CART to the secretory granules of the beta cells, indicating that CART is co-secreted with insulin. In addition, GK-rats displayed a greater secretory response to CART than Wistar controls. Western blot analysis of CART in normal and diabetic islets revealed that islet CART had molecular weight similar to CART 55-102.

Conclusion: We conclude that 1) CART is a regulator of islet hormone secretion, acting through a receptor coupled to adenylate cyclase. 2) CART is upregulated in the beta cells of type 2 diabetic rats and is located to the secretory granules. 3) CART in normal and type 2 diabetic islets is biologically active. CART may therefore play important roles in glucose homeostasis and in the pathophysiology of type 2 diabetes. Supported by Novo Nordisk Foundation, the Swedish Medical Research Council, the Swedish Royal Physiographic Society

\section{5}

E-cadherin-mediated cell adhesion: a role in islet architecture, cell-to-cell coupling and insulin secretion? P. E. Squires, G. J. Rogers, M. N. Hodgkin; Biological Sciences, University of Warwick, Coventry, United Kingdom.
Background and Aims: The current drive towards islet transplantation as a viable cure for type 1 diabetes has focused attention on the characterization and development of substitute tissue that responds appropriately to elevations in glucose. It is widely accepted that the pancreatic islet and not individual beta-cells represent the unit of insulin secretion, yet no clear evidence exists to explain why homotypic populations of cells within islets respond more effectively than individual beta-cells. Furthermore, heterogeneous individual responses to nutrients are usurped by concerted, synchronised glucose-responsiveness when cells are configured as an islet. Close association between cells in the islet is facilitated by the expression of cell adhesion molecules, including E-cadherin (ECAD) and beta-catenin which binds the cytoplasmic domain of the cadherin. These molecules not only hold the 3-dimensional islet together, but may offer an explanation for improved glucoseresponsiveness. In the current study we have examined the importance of ECAD on synchronicity of activity and insulin secretion within model islets.

Materials and Methods: MIN6 were cultured either as monolayer (M) or pseudoislets (PI). Nutrient and nonnutrient-evoked changes in cytosolic calcium were determined from fura-2-loaded Ms and/or PIs cultured either in the presence or absence of the immuno-netralising ECADantibody or IgG control. Insulin secretion was measured via ELISA. Co-localization of beta-catenin and insulin was visualized using confocal microscopy, using Alexa-594/488 secondary antibodies. ECAD was over-expressed using Lipofectamine transfection with pcDNA3.1(-) vector containing the coding region of mouse ECAD gene. Expression levels were confirmed using Western blot analysis.

Results: MIN6 cells configured as PIs exhibit greater glucose $(20 \mathrm{mM})$-evoked increases in insulin secretion ( $802 \pm 73 \%$ over basal; $n=7)$ compared to those cultured as monolayers to $80 \%$ confluence $(147 \pm 14 \%$ over basal; $n=6)$. Western blot analysis suggests that MIN6 cells within a PI express more ECAD than cells within a monolayer. Fura-2 microfluorimetry data confirms synchronicity of glucoseevoked calcium oscillations in PIs compared to the heterogeneous response of individual MIN6-cells (data from 6 separate experiments). Both islet morphology and synchronicity of nutrient-evoked calcium oscillations are altered in the presence of immuno-neutralising anti-ECAD, which prevents homophilic ECAD ligation. Beta-catenin and insulin immunoreactivity were co-localised only at the cell-to-cell interface in MIN6 monolayers. Up-regulation of ECAD expression in our MIN6 cells was confirmed following transfection using Western blot and confocal microscopy (20-fold enhancement; $n=5)$. However, insulin secretion studies suggest that ECAD over-expression does not enhance glucose-stimulated insulin secretion from 
MIN6 cells (over-expressing cells: $171 \pm 20 \%$ basal; $n=3$; vector control cells: $175 \pm 17 \%$ basal; $n=3$ ).

Conclusion: Our data suggest that ECAD is important in the formation and maintenance of islet architecture and that loss of ECAD uncouples glucose-evoked signaling within the islet. Over-expression of ECAD fails to enhance insulin secretion, suggesting that ECAD may act to mediate appropriate nutrient-evoked signaling, rather than control insulin secretion per se.

Supported by the MRC

\section{6}

Mutant huntington impairs metabolism and GSIS in the 832/13 INS-1 cell line

K. Bacos ${ }^{1}$, N. Wierup ${ }^{1}$, F. Saudou ${ }^{2}$, M. Bjorkqvist ${ }^{1}$, P. Brundin ${ }^{1}$, H. Mulder ${ }^{1}$;

${ }^{1}$ Experimental Medical Science, Lund University, Lund, Sweden, ${ }^{2}$ Institut Curie, Centre National de la Recherche Scientifique, Orsay, France.

Background and Aims: Huntington's disease (HD) is a neurodegenerative disorder caused by an expansion of a CAG triplet repeat in the gene coding for the protein huntingtin. The disease develops when the resulting stretch of glutamine residues exceeds 36. One of the main pathological findings is formation of intracellular inclusions consisting of huntingtin and other proteins. In addition to neurological deficits, a relatively large proportion of HD patients suffer from diabetes, a condition that also develops in the most widely used animal model of the disease, the R6/2 mouse. We have previously found that R6/2 beta-cells exhibit intracellular inclusions, presumably causing impaired beta-cell replication and perturbed exocytosis. We now aim to further characterize the role of normal and mutant huntingtin in beta-cell function, using the highly glucose-responsive 832/13 cell line.

Materials and Methods: We overexpressed huntingtin with and without disease causing PolyQ lengths (17 and 69 Q, respectively) in 832/13 INS-1 cells and analysed the cells with regard to inclusion formation (immunocytochemistry), general metabolic activity (methyl-tetrazolium assay; MTS) and insulin secretion. In addition to the vectors mentioned above a control vector containing the gene for EGFP was also included in the study.

Results: Fluorescence microscopy showed that expression of 69Q huntingtin, but not 17Q huntingtin or EGFP, led to formation of intracellular inclusions $48 \mathrm{~h}$ after transduction in the 832/13 INS-1 cell line; inclusions were found both in the cytoplasm and in the nucleus, the latter form which is typically found in HD. This was accompanied by an overall reduction of metabolic activity, as measured by MTS-assay; 69Q huntingtin caused a $67 \%$ reduction relative to uninfected cells. This decrease is significantly larger than the one caused by expression of EGFP alone or 17Q huntingtin (47 and 45\% respectively, $\mathrm{p}<0.01)$. In addition to this, glucose stimulated insulin secretion (GSIS) was also markedly reduced in response to expression of mutant huntingtin. When stimulating the 69Q expressing cells with $16.7 \mathrm{mM}$ glucose, they displayed a dramatically reduced insulin secretion compared to cells expressing 17Q huntingtin (40.2 and $167.6 \mathrm{ng} / \mathrm{h} / \mathrm{mg}$ protein, respectively; $\mathrm{p}<0.001)$. The fold change relative to secretion at $2.8 \mathrm{mM}$ glucose was also reduced from 10.2 to 1.3. Conclusion: The results show that overexpression of huntingtin containing an HD-causing PolyQ stretch in beta-cells leads to formation of intracellular inclusions. Concomitant to this there were marked disruptions of cellular function, as both metabolism and insulin secretion were dramatically perturbed.

Supported by High Q Foundation

\section{7}

Molecular mechanism of $\boldsymbol{d} b$ gene induced pancreatic $\beta$ cell dysfunction: Evidence for compensatory mechanism to reduce susceptability to diabetes in $d b$ gene heterozygote

Y. Kanda, K. Tawaramoto, F. Kawasaki, K. Kotani, K. Nakashima, K. Kaku;

Diabetes and Endocrine Division, Kawasaki Medical School, Kurashiki-shi, Japan.

Background and Aims: The $d b$ gene is a splicing variant caused by a point mutation of the leptin receptor gene. The $d b$ gene homozygous BKS.Cg- $+\operatorname{Lepr}^{d b} /+\operatorname{Lepr}^{d b} / \mathrm{Jcl}(d b / d b)$ mice develop diabetes with a marked obesity, severe insulin resistance, and limited capacity of insulin secretion. The diabetes phenotype is not demonstrated in heterozygous mice $(d b /+)$. To clarify a molecular mechanism of pancreatic $\beta$ cell dysfunction related with the $d b$ gene, the cell function and gene expression profile of pancreatic islets were analyzed in $d b$ gene homozygous $d b / d b$, heterozygous $d b /+$, and lean littermates $(+/+)$. Laser Capture Microdissection (LCM) and real-time RT-PCR were applied to analyze islet cell specific gene expression.

Materials and Methods: The male $d b / d b, d b /+$ and $+/+$ were provided free access to standard feed. Body weight (BW), fasted blood glucose (FBG) and insulin (FIRI) were measured at the age of 8 and 12 weeks. To analyze gene expression profiles in $\beta$ cells, the islet core region was selectively obtained by LCM. Primer pairs encoding genes associated with pancreatic hormones, cell proliferation, cell differentiation, apoptosis, cell cycle, ER/oxidative stress, and organization construction were prepared, and real-time RT-PCR with Sybr Green was applied. Each gene expres- 
sion was relatively quantified by the comparative $\mathrm{Ct}$ method with each result in the $+/+$ mice as a control.

Results: BW, FBG and FIRI in 8 and 12 week-old $d b / d b$ mice were increased significantly compared with those in other groups. At 12 weeks of age, however, insulin content in the islet was markedly reduced in $d b / d b$ mice and increased significantly in $d b /+$ mice comapered with $+/+$ mice $(5.1 \pm 3.3$, $58.0 \pm 31.7,24.4 \pm 15.4 \mathrm{ng} /$ islet, respectively). A prominent increase of insulin 2 gene expression was demonstrated in $d b /+$ mice in comparison with $d b / d b$ mice at 8 weeks of age $(d b /+: 9.57 \pm 3.96, d b / d b: 1.72 \pm 0.96, \mathrm{p}<0.05)$, but any difference was not observed between both groups at 12 weeks of age $(d b /+: 7.86 \pm 3.56, d b / d b: 6.97 \pm 3.78)$. Expression of genes involved in cell proliferation (cyclin E \& ERK1) and apoptosis (CAD) was accelerated in 8 weekold $d b / d b$ mice. In 12 week-old $d b / d b$ mice, cyclin E \& ERK1 gene expressions were down-regulated and CAD gene expression was up-regulated. On the other hand, the bcl-2 gene expression was increased significantly in $d b /$ + mice. Expression of genes involved in anti-oxidation such as SOD2, catalase, and GSHPx were significantly decreased in $d b / d b$ mice. To examine a role of $d b$ gene on glucose tolerance, $d b /+$ and $+/+$ mice were fed high-fat-diet for 6 weeks from 10 weeks of age. A significant increase of BW was observed in mice fed high-fat diet compared with normal chow. The high fat diet did not impair glucose tolerance analyzed by ipGTT, and a significantly higher plasma insulin in response to glucose challenge was observed in $d b /+$ mice fed high fat diet, but not in $+/+$ mice. Conclusion: The islet gene expression profiles demonstrated that the $d b$ gene heterozygous mice have a protective mechanism against acceleration of apoptosis and reduction of cell proliferation observed in $d b$ gene homozygous mice. In addition, increase of islet insulin content and high response of insulin secretion to glucose load observed in $d b /+$ mice with high fat diet strongly suggest an existence of the compensatory mechanism in the $d b$ gene heterozygote to reduce a susceptibility to diabetes.

\section{8}

\section{Expression and function of GPR40 in Goto-Kakizaki rat islets \\ E. Flodgren, M. Sörhede Winzell, B. Ahrén; \\ Dept of Clinical Sciences, Medicine, Lund University, Lund, Sweden.}

Background and Aims: The recently characterized receptor GPR40 is abundantly expressed on pancreatic $\beta$-cells and is activated by medium to long chain fatty acids (FA). Receptor activation augments glucose-stimulated insulin secretion (GSIS) via the generation of the intracellular second messenger phosphatidyl inositol-3 phosphate ( $\left.\mathrm{IP}_{3}\right)$ and ensuing calcium release from the endoplasmatic reticulum. The Goto-Kakizaki (GK) rat is a commonly used model of spontaneous type 2 diabetes, with a number of established and proposed defects, created by repetitive inbreeding of hyperglycemic Wistar rats. The aim of this study was to investigate the expression and function of GPR40 in the islets of GK rats.

Materials and Methods: We used male Wistar and GK rats purchased from Taconic (Skensved, Denmark) and fed a normal diet. Pancreatic islets were isolated from the rats by collagense digestion. For mRNA expression analysis, islets from different individuals were kept separated and stored in $-80^{\circ} \mathrm{C}$ pending analysis. RNA was prepared and $2 \mu \mathrm{g}$ were used for cDNA synthesis and controls, after DNase treatment. Using real-time PCR, the expression of GPR40 mRNA between the different individuals was compared, after normalization against the islet mRNA expressions of three different house-keeping genes. In the phosphatidyl inositol (PI) hydrolysis experiments, islets from 2-4 animals were pooled and the divided into batches of 50, radioactively labelled with ${ }^{3} \mathrm{H}$-inositol, and incubated for 30 min with or without $100 \mu \mathrm{M}$ linoleic acid (LA). The islets were then lysed and the inositol phosphates (IPs) were collected using anion exchange chromatography. RIA analysis of FA augmentation of GSIS was performed on pooled islets in batches of 3 after $60 \mathrm{~min}$ of incubation at $3.3 \mathrm{mM}$ glucose $(0.1 \% \mathrm{BSA})$ in the presence or absence of a free LA concentration of $100 \mu \mathrm{M}$;

Results: On the mRNA level, the expression of GPR40 in the GK rat islets was markedly downregulated compared with that of the Wistar controls (GK GPR $40=40 \%$ of control, $\mathrm{p}=0.0001$ ). These results were corroborated in the functional analyses. In the IP production assay, LA stimulation of Wistar rats resulted in an $85 \%$ increase of IP levels compared to unstimulated islets $(\mathrm{p}=0.014)$, while no significant effect of the fatty acid challenge was detected in the GK model. The FA-induced augmentation of GSIS from GK islets was also significantly lower than that in the controls animals. The addition of LA caused a $240 \%$ increase in secreted insulin $(\mathrm{p}=0.013)$ from the Wistar islets, whereas the effect of LA on the GK islets was not statistically significant.

Conclusion: Our results show that the previously reported expression of the fatty acid receptor GPR40 in Wistar rats is significantly attenuated in the GK rat. This deficiency makes GK islets less responsive to in vitro FA stimulation, as exhibited both by a perturbed hydrolysis of radioactive PIs and by an abolished effect on GSIS. The conclusion is that a reduction of GPR40 expression and function can be added to the list of mutations in the GK rat model of type 2 diabetes.

Supported by the Royal Physiographic Society in Lund 


\section{9}

Identification of BACE ( $\beta$-site amyloid precursor protein cleaving enzyme) expression and activity in pancreatic cells

S. Casas ${ }^{1,2}$, S. Piquer ${ }^{2}$, M. Juliàa ${ }^{2}$ J. Altirriba ${ }^{2}$,

R. Gomis ${ }^{2}$, A. Novials ${ }^{1}$;

${ }^{1}$ Institute of Diabetes, Sardà Farriol Foundation, Barcelona,

${ }^{2}$ Endocrinology Unit and Experimental Diabetes Laboratory, IDIBAPS, Hospital Clínic, University of Barcelona, Spain.

Background and Aims: BACE ( $\beta$-site amyloid precursor protein cleaving enzyme) is a membrane-bound aspartyl protease that can process amyloid precursor protein (APP) at the $\beta$-secretase site, being responsible for amyloid $\beta$ protein $(A \beta)$ generation in brain. Although BACE mRNA expression is also found in pancreas, pancreatic BACE substrates are unidentified, and thus its pancreatic physiological role remains unknown. The aim of the study was to characterize expression and activity of BACE in pancreas, and testing influence of glucose estate.

Materials and Methods: Immunofluorescences against BACE were performed in paraffined pancreas sections. Cell lysates of $50 \mu \mathrm{g}$ protein from human pancreatic islets and pancreatic $\beta$ cell line MIN6 were immunoblotted for BACE expression, and tested for fluorometric BACE proteolytic activity assay with $20 \mu \mathrm{M}$ fluorometric BACE substrate (JMV2236). Human pancreatic islets and MIN6 cells were evaluated after 5 days culture at either 5.5 or $22.7 \mathrm{mM}$ glucose. Human pancreatic islets and MIN6 cells cultures were treated with $10 \mu \mathrm{M}$ cell permeable BACE inhibitor (Z-VLL-CHO) for $24 \mathrm{~h}$. Quantification of insulin transcript was performed by real time QRT-PCR using Taqman probe. Insulin peptide content was evaluated by immunoblot and quantified by ELISA.

Results: Islet cells showed higher expression levels of BACE compared to exocrine tissue in immunofluorescences of paraffined pancreatic sections. Protein extracts from human pancreatic islets exhibited BACE activity when these were tested in fluorometric BACE proteolytic activity assay $\left(n=5, K_{o b s}=0,166 \pm 0,02 \mathrm{sec}^{-1}\right)$. Human pancreatic islets expressed three major bands of approx 55, 70 and $140 \mathrm{KDa}$ in BACE immunoblots, identified as immature monomer, mature monomer and mature dimmer, respectively $(\mathrm{n}=6)$. Moreover, BACE was also expressed and catalytically active in protein extracts from MIN6 cells $\left(\mathrm{n}=8, \mathrm{~K}_{\mathrm{obs}}=0,029 \pm 0,001 \mathrm{sec}^{-1}\right)$. We then assessed whether $\mathrm{BACE}$ expression and activity could be regulated by glucose. Either human pancreatic islets or MIN6 cells cultured for 5 days at $22.7 \mathrm{mM}$ glucose showed a 4-fold increase in the expression level of $70 \mathrm{KDa} \mathrm{BACE}$ compared to those at $5.5 \mathrm{mM}(\mathrm{n}=10, p<0.001)$. Instead of being de novo peptide maturation, the increase was originated by dissociation of the mature peptide dimmer. When BACE proteolytic activity was tested, a significantly reduction was observed in protein extracts from cultures at $22.7 \mathrm{mM}$ compared to those at $5.5 \mathrm{mM}$ glucose with $\mathrm{K}_{\mathrm{obs}}=0,024 \pm 0,0004 \mathrm{sec}^{-1}$ and $\mathrm{K}_{\mathrm{obs}}=0,028 \pm 0,001 \mathrm{sec}^{-1}$, respectively $(\mathrm{n}=4, p<0.01)$. To investigate the effects of reduced BACE proteolytic activity, Z-VLL-CHO was administrated. A 1.3 to 3.8 fold-decrease in insulin transcript expression were identified in MIN6 cells from 2 to $24 \mathrm{~h}(\mathrm{n}=8, p<0.001)$, followed by a 1.4 to 2.2 foldreduction in insulin content from 4 to $24 \mathrm{~h}$ of treatment compared to control $(\mathrm{n}=4, p<0.05)$. Moreover, treated human pancreatic islets showed a 4 fold-change reduction in the expression level of insulin peptide compared to control $(\mathrm{n}=3, p<0.05)$.

Conclusion: BACE activity and expression in pancreatic islet cells is regulated by the glucose state. Reduced BACE activity downregulates both insulin transcript expression and peptide content. Further identification of pancreatic BACE substrates would be needed to interpret the present results.

0520

Islet endothelial cell signaling augment beta cell function

Å. Johansson, M. Johansson, P.-O. Carlsson; Medical Cell Biology, Uppsala University, Sweden.

Background and Aims: The proximity of islet endothelial cells and beta-cells in the pancreatic islets by necessity means that they are exposed to each others products. Islet endothelial cells require signals from the beta-cells to function properly, but little is known about how paracrine signals from the endothelial cells affect the beta-cells. The present study tested the hypothesis that islet endothelial cellderived products may affect adult beta-cell function.

Materials and Methods: Pancreatic islets from adult inbred Wistar-Furth rats were isolated. Islet endothelial cells from some of the islets were stimulated to proliferate and grow out on a collagen matrix, and were then purified with Dynabeads coated with the endothelial cell specific marker Bandeiraea simplicifolia, achieving a purity of the islet endothelial cells exceeding $90 \%$. Islet conditioned medium was obtained by incubating other islets in RPMI 1640 with $10 \%$ fetal calf serum for 24 hours. Endothelial cell conditioned medium was obtained by incubating islet endothelial cells in RPMI 1640 with $10 \%$ fetal calf serum for 12 hours. Insulin release, insulin content, glucose oxidation and insulin biosynthesis was measured in batch-type experiments following exposure 
of cultured islets incubated in these mediums, or control medium, for 24 hours.

Results: The islets incubated in endothelial cell conditioned medium released $50 \%$ more insulin than islets incubated in RPMI with $10 \%$ fetal calf serum alone when exposed to $16.7 \mathrm{mmol} / 1$ glucose ( $<00,001$; ANOVA). Moreover, the insulin content of islets exposed to endothelium conditioned medium was increased with $75 \%$ when compared to the same control group ( $<<0,01$; ANOVA). However, the glucose oxidation rates and (pro)insulin biosynthesis in response to $16.7 \mathrm{mmol} / 1$ glucose did not differ between the groups. Further studies are now undertaken to determine the endothelial-derived factor responsible for the beneficial effects on beta-cell function, and its exact mechanism.

Conclusions: The islet vascular system seems to have other effects besides its transport capacities to augment and sustain beta-cell insulin content and secretion.

Supported by grants from the Swedish Research Council (72XD-15043), The Juvenile Diabetes Research Foundation, the EFSD/Novo Nordisk Research Programme, the Swedish Diabetes Association, the Swedish Juvenile Diabetes Fund, the Novo Nordisk Insulin Fund, the Anér Foundation, the Ake Wiberg Foundation and the Family Ernfors Fund.

\section{PS 30}

\section{Lipid metabolism}

0521

Acyl CoA: diacylglycerol acyltransferase 2 (DGAT2)mediated triacylglycerol accumulation in glycolytic muscle promotes insulin resistance

M. C. Levin ${ }^{1}$, M. Monetti ${ }^{1}$, M. P. Sajan ${ }^{2}$, M. J. Watt ${ }^{3}$, R. V. Farese ${ }^{2}$, R. V. Farese, $\mathrm{Jr}^{1}$;

${ }^{1}$ UCSF, Gladstone Institute of Cardiovascular Disease, San Francisco, United States, ${ }^{2}$ ACOS-151, James A. Haley Veterans Hospital, Tampa, United States, ${ }^{3} \mathrm{NHMRC}$, St. Vincent's Institute of Medical Research, Fitzroy, Australia.

Background and Aims: Insulin resistance and type 2 diabetes are frequently accompanied by lipid accumulation in skeletal muscle. However, it is not known whether triacylglycerol (TG) deposition in skeletal muscle causes insulin resistance or whether the type of muscle fiberoxidative, red fibers or glycolytic, white fibers_is an important determinant of lipid-mediated insulin resistance. Oxidative muscle normally stores more TG and is more insulin-sensitive than glycolytic muscle, suggesting that increased TG content in oxidative muscle may not be detrimental. We hypothesized that $\mathrm{TG}$ accumulation in glycolytic muscle, which does not normally store large quantities of TG, would promote insulin resistance.

Materials and Methods: We generated transgenic mice that overexpress DGAT2, an enzyme that catalyzes triglyceride biosynthesis, in glycolytic muscle, by using the muscle creatine kinase (MCK) promoter.

Results: At 3 -months of age, MCK-DGAT2 mice had a fivefold increase DGAT2 mRNA expression and a twofold increase in TG accumulation in glycolytic muscle. Although fasting plasma levels of glucose and insulin were similar in MCK-DGAT2 and wild-type mice, MCK-DGAT2 mice exhibited impaired glucose tolerance. The insulinstimulated activity of PKC $\lambda$, a sensitive marker of insulin signaling that is highly correlated with glucose uptake in muscle, was severely reduced in glycolytic (but not oxidative) muscle in MCK-DGAT2 mice. Insulin-stimulated uptake of 2-deoxyglucose was also severely impaired in glycolytic (but not oxidative) muscle. The ceramide content was increased, and diacylglycerol content was reduced specifically in the glycolytic muscle of MCK-DGAT2 mice.

Conclusion: Our results indicate that the accumulation of moderate amounts of TG in glycolytic muscle impairs insulin sensitivity and glucose uptake and suggest that lipid deposition in nonoxidative, glycolytic muscle may contribute to insulin resistance and diabetes.

Supported by NIH grant DK 06559-02, Johan Throne Holst Foundation, the Larry L. Hillblom Foundation

0522

Heredity-independent effects of increased aerobic fitness on liver and adipose tissue FFA uptake

P. Nuutila ${ }^{1}$, J. Hannukainen ${ }^{1}$, R. Borra ${ }^{1}$, A. Viljanen ${ }^{1}$, T. Janatuinen ${ }^{1}$, O. J. Heinonen ${ }^{2}$, J. Kaprio ${ }^{3}$, U. Kujala ${ }^{4}$,

T. Rönnemaa ${ }^{5}$, T. Viljanen ${ }^{1}$, M. Haaparanta ${ }^{1}$, J. Knuuti ${ }^{1}$, K. K. Kalliokoski ${ }^{1}$;

${ }^{1}$ Turku PET Centre, ${ }^{2}$ Physiology, University of Turku, Finland, ${ }^{3}$ Public Health, University of Helsinki, Finland, ${ }^{4}$ Health Sciences, University of Jyväskylä, Finland, ${ }^{5}$ Medicine, University of Turku, Finland.

Background and Aims: Regular physical exercise decreases body adiposity and improves whole body lipid and glucose utilization although the effects of increased physical activity on liver and adipose tissue free fatty acid (FFA) uptake are largely unknown. In the present study we studied heredity-independent effects of moderately increased physical activity and aerobic fitness on liver and adipose tissue FFA uptake in monozygotic twins and compared FFA uptake rates in different tissues in vivo.

Materials and Methods: Nine young healthy adult male monozygotic twin pairs with $18 \%(50.1 \pm 5.1$ vs. $43.4 \pm 6.7 \mathrm{ml} /$ $\mathrm{kg} / \mathrm{min}, \mathrm{p}<0.001)$ mean difference in maximal oxygen uptake 
$\left(\mathrm{VO}_{2} \mathrm{max}\right)$ were recruited from a large twin cohort study. Abdominal subcutaneous and visceral fat, hepatic, and muscle FFA uptake were measured at fasting state with positron emission tomography and $\left[{ }^{18} \mathrm{~F}\right] \mathrm{FTHA}$ and fat masses were quantified using MRI. The co-twins with higher VO2max constituting the more active group were compared to the less active brothers with lower $\mathrm{VO}_{2}$ max.

Results: Both groups had similar BMI and similar lean body mass, but more active twins had $10 \%$ lower whole-body fat percent ( $17.6 \pm 5.5$ vs. $19.6 \pm 5.7 \%, \mathrm{p}<0.05)$. More active twins had $19 \%$ lower abdominal subcutaneous $(1.88 \pm 0.97$ vs. $2.37 \pm 1.06 \mathrm{~kg}, \mathrm{p}=0.02$ ) and $20 \%$ lower visceral fat mass $(0.81 \pm 0.44$ vs. $0.99 \pm 0.41 \mathrm{~kg}, \mathrm{p}=0.009)$ than less active twins. Fasting plasma glucose and serum FFA concentrations were similar between the groups. When expressed per kilogram of tissue, hepatic FFA uptake was highest $(7.23 \pm 5.43 \mu \mathrm{mol} /$ $100 \mathrm{ml} / \mathrm{min}$ ), visceral adipose tissue FFA uptake was $30 \%$ higher than subcutaneous uptake $(0.23 \pm 0.17$ vs. $0.19 \pm$ $0.15 \mu \mathrm{mol} / 100 \mathrm{~g} / \mathrm{min}, \mathrm{p}=0.006)$ and correlated with subcutaneous, hepatic and skeletal muscle FFA uptake $(\mathrm{p}<0.01$ for all). FFA uptake was not significantly different between more and less active twins in subcutaneous fat $(0.13 \pm 0.8$ vs. $0.23 \pm 1.19 \mu \mathrm{mol} / 100 \mathrm{~g} / \mathrm{min}, \mathrm{p}=0.19)$, visceral fat $(0.20 \pm 0.15$ vs. $0.26 \pm 0.18 \mu \mathrm{mol} / 100 \mathrm{~g} / \mathrm{min}, \mathrm{p}=0.38$ ), or in skeletal muscle ( $0.28 \pm 0.18$ vs. $0.38 \pm 0.23 \mu \mathrm{mol} / 100 \mathrm{~g} / \mathrm{min}, \mathrm{p}=0.3$ ), but it was lower in the liver in the more active twins as compared to the less active brothers $(5.5 \pm 4.3$ vs. $9.0 \pm 6.1 \mu \mathrm{mol} / 100 \mathrm{ml} / \mathrm{min}$, $\mathrm{p}=0.04$ ).

Conclusion: Without the confounding effects of genetic factors, moderately increased physical activity and aerobic fitness decreases visceral and subcutaneous adiposity in normal-weighted humans. Adipose tissue FFA uptake is higher in visceral than subcutaneous adipose tissue in the fasting state and not changed by exercise. In contrast, liver FFA uptake decreases by regular exercise, and this positive effect is observed even without changes in serum FFA concentrations.

\section{3}

Comparison of the effect of pioglitazone and insulin on postprandial lipoprotein composition and expression of genes affecting cholesterol absorption, synthesis and chylomicron assembly

G. H. Tomkin, S. M. Lally, D. Owens;

Diabetes and Endocrinology, Trinity College Dublin, Ireland.

Background and Aims: Abnormal postprandial lipoproteins may be an important cause of the increased atherosclerosis found in diabetes. Both insulin and pioglitazone have been shown to reduce postprandial lipoproteins but the mechanism may be different since the thiazolodinediones increase insulin sensitivity particularly in the liver. A recent human study has demonstrated that insulin sentisation with pioglitazone has a major effects in restoring postprandial lipaemia to normal. We have previously shown altered expression in diabetes of genes involved in cholesterol absorption and synthesis and lipoprotein assembly. Niemann Pick C1-like1(NPC1L1) which facilitates cholesterol absorption and 3-hydroxy-3-methylglutaryl coenzyme A reductase (HMGCoA reductase) the rate limiting enzyme for cholesterol synthesis were increased in diabetes as was microsomal triglyceride transfer protein (MTTP) which assembles the chylomicron and VLDL particles while the ATP binding cassette proteins (ABC) G5 and G8 which re-excrete absorbed cholesterol were reduced. The aim of the study was to examine the effect of insulin compared to pioglitazone on expression of genes in liver and intestine which are involved in cholesterol homeostasis in order to explain the mechanism whereby insulin sensitisation with pioglitazone restores postprandial lipaemia to normal.

Materials and Methods: Thirty zucker diabetic fatty fa/fa rats were examined. Animal experiments were carried out under licence from the Department of Health in Dublin. Diabetes was confirmed with a blood sugar $>7.5$. Ten of the diabetic rats were treated with insulin and 10 with pioglitazone for 2 weeks prior to saccrifice. Blood was collected at sacrifice and chylomicron and very low density lipoproteins (VLDL) isolated by sequential ultracentrifugation. mRNA expression of HMGCoA, MTTP, NPC1L1 and $\mathrm{ABC}$ G5 and $\mathrm{G} 8$ were determined in liver and intestine using real-time PCR.

Results: Blood glucose for untreated rats was $9.7 \pm$ $1.7 \mathrm{mmol} / 1$ this was significantly greater $(\mathrm{p}<0.005)$ than both insulin treated $(7.0 \pm 1.4)$ and pioglitazone treated $(7.9 \pm 0.4 \mathrm{mmol} / \mathrm{l})$ which were not significantly different from each other. While both insulin and pioglitazone significantly reduced chylomicron and VLDL cholesterol, triglyceride, apo B48 and apo B100 $(\mathrm{p}<0.001)$ the levels in pioglitazone-treated animals significantly lower than those in the insulin-treated rats $(\mathrm{p}<0.0001)$. Insulin and pioglitazone significantly reduced MTTP and in both liver $(p<0.001)$ and intestine $(p<0.001)$ and they both increased ABCG5 and G8 in the liver $(p<0.0001)$. Insulin significantly reduced hepatic HMGCoA reductase. In comparison to insulin pioglitazone significantly reduced hepatic, MTTP $(0.0001)$ and NPC1L1 mRNA (Insulin $15+3$ vs $8+3$ $\mathrm{p}<0.0001)$ and significantly increased hepatic ABCG5 $(21+6$ vs $73+29)$ and G8 mRNA $(20+4$ vs $63+6$ p $<0.0001)$. Conclusion: Although both insulin and pioglitazone reduced chylomicron and VLDL, pioglitazone was more effective for a similar decrease in blood sugar. This was due at least in part to the stronger effect of pioglitazone on 
mRNA of genes regulating cholesterol absorption and reexcretion in the liver but not the intestine and the resulting reduction in MTP. The study explains, at least in part, the improvement in postprandial lipaemia by the addition of pioglitazone to insulin treatment.

Supported by Takeda Pharmaceuticals

\section{4}

Triglyceride disposal and lipoprotein lipase-mediated free fatty acid spillover in the splanchnic bed of dogs R. H. Nelson ${ }^{1}$, R. Basu ${ }^{1}$, D. Edgerton ${ }^{2}$, J. C. Roesner ${ }^{1}$, A. D. Cherrington ${ }^{2}$, J. M. Miles ${ }^{1}$;

${ }^{1}$ Endocrine Research Unit, Mayo Clinic, Rochester, ${ }^{2}$ Molecular Physiology and Biophysics, Vanderbilt University, Nashville, United States.

Background and Aims: The action of lipoprotein lipase (LPL) on circulating triglyceride-rich lipoproteins generates fatty acids, some of which are known to "spill over" into the systemic circulation and contribute to the free fatty acid (FFA) pool. The contribution of the splanchnic bed, and the relative contribution of the liver and nonhepatic splanchnic tissues to this process, is not known. However, the liver is not generally believed to contain LPL. We have previously shown that a commercial lipid emulsion labeled with $\left[{ }^{3} \mathrm{H}\right]$ triolein is a suitable surrogate for the study of chylomicron triglyceride metabolism, and that the production of $\left[{ }^{3} \mathrm{H}\right]$ oleate therefrom can be quantified to estimate spillover. The present study was conducted to determine the efficiency of triglyceride storage in the splanchnic bed and the contribution of the splanchnic bed to whole-body triglyceride disposal.

Materials and Methods: Sampling catheters were surgically placed in the femoral artery, portal vein and hepatic vein of mongrel $\operatorname{dogs}(n=5)$. At the same time, Transonic flow probes were placed for subsequent measurement of hepatic artery and PV plasma flow. Two weeks after the procedure, the animals were studied under postabsorptive conditions. Infusions of tracer amounts of $\left[1-{ }^{14} \mathrm{C}\right]$ oleate $(\sim 0.2 \mu \mathrm{Ci} / \mathrm{min})$ and $\left[9,10-{ }^{3} \mathrm{H}\right]$ triolein $(\sim 1.4 \mu \mathrm{Ci} / \mathrm{min})$ were started, allowing $90 \mathrm{~min}$ to establish isotope equilibrium. Blood was taken from the three sampling sites every $7.5 \mathrm{~min}$ X5 for measurement of triglyceride radioactivity as well as the concentration and specific activity (SA) of plasma oleate.

Results: Plasma oleate concentration was higher in the portal vein than in the artery $(449 \pm 28$ vs $382 \pm 29 \mu \mathrm{mol} / \mathrm{L}$, $\mathrm{P}<0.005)$, whereas it was lower in the hepatic vein $(351 \pm 17 \mu \mathrm{mol} / \mathrm{L})$ was lower than in the portal vein, $\mathrm{P}<0.005$. Plasma ${ }^{14} \mathrm{C}$ oleate $\mathrm{SA}$ was lower in the portal vein than in the artery $(1.63 \pm .16$ vs $1.88 \pm .19 \mathrm{dpm} / \mathrm{nmol}$,
$\mathrm{P}<0.005)$, but the hepatic venous value $(1.66 \pm .17 \mathrm{dpm} /$ nmol) was similar to that in the portal vein. Plasma ${ }^{3} \mathrm{H}$ oleate SA was similar in the artery and portal vein $(6.33 \pm .31$ vs $6.00 \pm .17 \mathrm{dpm} / \mathrm{nmol}, \mathrm{P}=\mathrm{NS})$, whereas the value in the hepatic vein $(8.10 \pm .50 \mathrm{dpm} / \mathrm{nmol})$ was higher than the $\mathrm{PV}$ value, $\mathrm{P}<0.005$. Splanchnic uptake of the ${ }^{3} \mathrm{H}$ triglyceride was $32 \pm 7 \%$ of systemic triglyceride disappearance. Of the ${ }^{3} \mathrm{H}$ triglyceride uptake in the splanchnic bed, $26 \pm 5 \%$ occurred in nonhepatic tissues, and $74 \pm 5 \%$ in the liver. Fractional spillover of ${ }^{3} \mathrm{H}$ oleate was $59 \pm 11 \%$ and $40 \pm 9 \%$ in nonhepatic splanchnic tissues and liver, respectively. The splanchnic bed accounted for $15 \pm 3 \%$ of systemic oleate uptake and $8.7 \pm 2.0 \%$ of systemic oleate appearance.

Conclusion: These results indicate that the splanchnic bed makes a major contribution to systemic triglyceride disposal, and that the majority of splanchnic triglyceride uptake occurs in the liver. Significant spillover of fatty acids produced by triglyceride hydrolysis was observed in both the liver and in nonhepatic tissues. The enzymatic mechanism for triglyceride hydrolysis in the liver is not clear. The relevance of these observations to the fed state will require further study.

Supported by NIH RO1 HL67933 and DK18243

0525

Interaction of peroxisome proliferator-activated receptors with acyl-CoA binding protein in transgenic rats

S. Uotila ${ }^{1}$, T. Ahtialansaari ${ }^{1}$, A. Huotari ${ }^{1}$, M. Heinonen ${ }^{1}$, J. Idziak ${ }^{1}$, T. Mauriala ${ }^{2}$, S. Auriola ${ }^{2}$, K. Kiehne ${ }^{3}$,

U. R. Fölsch ${ }^{3}$, S. Wolffram ${ }^{4}$, J. Jänne ${ }^{1}$, L. Alhonen ${ }^{1}$, K.-H. Herzig ${ }^{1,5}$;

${ }^{1}$ Dept of Biotechnology and Molecular Medicine, ${ }^{2}$ Dept of Pharmaceutical Chemistry, University of Kuopio, Finland, ${ }^{3}$ Dept of Internal Medicine, ${ }^{4}$ Dept of Animal Nutrition and Physiology, University of Kiel, Germany, ${ }^{5}$ Dept of Internal Medicine, University of Kuopio, Finland.

Background and Aims: The metabolic syndrome (MetS) is characterized by central obesity, dysglycemia, dyslipidemia and/or elevated blood pressure. A central feature of the syndrome are elevated free fatty acids converted into acylCoAs in the cytosol and bound to storage proteins like acylCoA binding protein (ACBP). ACBP has previously been shown to affect insulin secretion and gene regulation. Important molecular targets for the treatment of the MetS are peroxisome proliferator-activated receptors (PPARs) lipid sensors regulating transcription of genes involved in lipid and glucose metabolism, and inflammation. Fatty acids are natural ligands of PPARs, whereas acyl-CoA esters act as antagonists and ACBP has been demonstrated 
to have a negative role in ligand-dependent PPAR transactivation in vitro. To determine the interaction of these two regulators in vivo under various physiological conditions we created a transgenic rat line over expressing mouse ACBP gene.

Materials and Methods: A rat line overexpressing the mouse ACBP gene was produced by standard techniques. Tissue levels of different acyl-CoAs were analysed by HPLC-ESI-MS/MS. Gene expression analysis was performed by quantitative RT-PCR from liver and adipose tissues under fasted or fed conditions. In addition, tg and wt rats were treated with PPAR- $\alpha$ agonist fenofibrate $(100 \mathrm{mg} /$ $\mathrm{kg} / \mathrm{d}$ gavage) for 3,5 days or received diets with either longchain (LC-diet; palm oil) or medium-chain (MC-diet; synthetic medium chain triglycerides) fatty acids for 1 month.

Results: RT-PCR and Western blot analysis demonstrated ACBP overexpression. Furthermore, in tg rats acyl-CoA levels were significantly elevated in adipose tissue. In fed state, PPAR- $\gamma$ and PPAR- $\delta$ gene expression levels in tg livers compared to wt rats were significantly reduced by $82 \%$ or $62 \%$, respectively, whereas PPAR- $\alpha$ expression was not affected. In adipose tissue of tg rats, both PPAR$\gamma$ and PPAR- $\delta$ were downregulated by $23 \%$ or $43 \%$, respectively. Fasting upregulated PPAR expression (compared to fed state) in livers of tg rats (7x PPAR- $\alpha, 7 x$ PPAR- $\gamma, 2,2 x$ PPAR- $\delta$ ) and $3 x$ PPAR- $\delta$ in adipose tissue, while PPAR- $\gamma$ was reduced but not signifcantly. Intragastric application of fenofibrate significantly stimulated ACBP expression $(4-5 x)$ in the liver. Neither MC-diet nor LC-diet affected PPAR expression in liver of wt rats. In contrast, tg rats had reduced PPAR expression levels in liver in response to MC-diet $(58 \%, 75 \%$, and $50 \%$ for PPAR- $\alpha$, PPAR- $\gamma$ and PPAR- $\delta$, respectively), but not to LC-diet. In adipose tissue, MC-diet induced PPAR- $\gamma$ expression $63 \%$ of $\mathrm{wt}$, but did not affect tg rats. Both diets reduced PPAR- $\delta$ expression of tg animals in adipose tissue $(55 \%$ and $27 \%$ for LC- and MC-diet, respectively).

Conclusion: Our results demonstrate that overexpression of ACBP gene elevates acyl-CoA levels and downregulates the expression of PPARs. This downregulation was reversed by fasting. In addition, diets affected PPAR levels of tg animals differently compared to wt littermates. Hence we conclude that ACBP might act as a negative regulator in vivo.

This study was supported in part by the German Federal Ministry of Education and Research
0526

Relationship between oxidized low density lipoprotein and insulin resistance in type 2 diabetes mellitus

M. Nakhjavani, F. Esfahanian, A. Esteghamati, M. Niafar, M. Abbasi, H. Pordal, R. Heshmat;

Endocrinolody and Metabolism Research Center, Tehran University of Medical Sciences, Islamic Republic of Iran.

Background and Aims: Both low-density lipoprotein oxidation (ox-LDL) and insulin resistance (IR) play important role in the endothelial dysfunction and atherosclerosis. Recent studies have demonstrated that the amount of ox-LDL in plasma is correlated with IR in non-diabetic population. The aim of this study was to evaluate correlation of ox-LDL and IR in type 2 diabetic patients, and to compare it with normal individuals.

Materials and Methods: A total of 150 individuals aged 40 to 65 years were studied including 81 type 2 diabetic patients and 69 non-diabetic sex and BMI matched healthy persons. Exclusion criteria included smoking, treatment with lipid lowering and antihypertensive drugs, positive history of cardiovascular and renal disease. Demographic characteristics and anthropometric data of participants were recorded. Levels of circulating ox-LDL were measured with 2 monoclonal antibody-based competition ELISA. Oxidized LDL antibodies (OLAB), glucose, insulin, and $\mathrm{HbA1C}$ were also determined in fasting blood samples. Insulin resistance was estimated according to homeostasis model assessment of insulin resistance (HOMA-IR).

Results: In diabetic patients ox-LDL concentration was significantly lower than normal controls $(11.8 \pm 5.6 \mathrm{mU} / 1 \mathrm{vs}$. $15.7 \pm 6.9 \mathrm{mU} / 1 \mathrm{p}<0.001)$. The difference in OLAB concentration in the two groups were not statistically significant. HOMA-IR was $2.77 \pm 1.96$ in diabetic patients and $1.41 \pm 0.91$ in controls $(\mathrm{p}<0.001)$. There was a significant correlation between ox-LDL with total $(\mathrm{r}=0.33, \mathrm{p}<0.005)$ and low density cholesterol $(\mathrm{r}=0.34, \mathrm{p}<0.005)$ in both patients and controls. After considering all the relevant factors including age, duration of diabetes, BMI, systolic and diastolic blood pressure, serum lipids, and HOMA-IR, regression analysis demonstrated that there was a significant correlation between ox-LDL and HOMA-IR in patients $(\mathrm{r}=0.39, \mathrm{p}<0.005)$ but not in healthy controls. OLAB had weak but significant correlation with $\mathrm{BMI}$ in both diabetic and non diabetic groups $(\mathrm{r}=0.23$ and $\mathrm{r}=0.24$ respectively, $\mathrm{p}<0.05$ ).

Conclusion: Our results suggest an association between insulin resistance and increased LDL oxidation independent of the effect of other contributing factors.

Supported by Endocrinology and Metabolism Research Center, Tehran University of Medical Sciences 
0527

The impaired regulation of LPL in adipose tissue contributes to the hypertriglyceridaemia in insulin resistance

M. Cahova, H. Vavrinkova, H. Dankova, L. Kazdova; Centrum of Diabetology, Institute for Clinical and Experimental Medicine, Prague, Czech Republic.

Background and Aims: Insulin resistance (IR) is associated with the disturbances in the metabolism of both glucose and lipids. In hereditary hypertriglyceridemic (HHTg) rats, the non-obese model of IR, the diet with high content of sucrose induces hypertriglyceridemia, elevation of serum FFA and substantial resistance of glucose metabolism to insulin action. The postprandial LPL activity is considered to be the rate-limiting factor determining the clearance of circulating triglycerides ( $\mathrm{Tg}$ ) but no less important is the ability of adipose tissue to store released FA as $\mathrm{Tg}$ via their esterification. The purpose of this study was to determine whether the postprandial modulation of LPL activity in adipose tissue may contribute to the elevated triglyceridemia in HHTg rats with resistance of glucose metabolism to insulin action induced by HS feeding.

Materials and Methods: Control (C) and HHTg rats (weight $300 \pm 20 \mathrm{~g}$ ) were fed either standard (SD) or high sucrose (HS, $70 \%$ of calories as sucrose) diet for two weeks. LPL activity was determined in the homogenates from distal parts of adipose tissue by the release of fatty acids from labeled substrate $\left({ }^{3} \mathrm{H}\right.$-trioleine). The sensitivity of glucose metabolism to insulin in adipose tissue was determined by ${ }^{14} \mathrm{C}$-glucose incorporation into lipids in vitro.

Results: The increased supply of sucrose induced in HHTg significantly higher postprandial insulinemia than in control rats $(780 \pm 29$ vs $440 \pm 23 \mathrm{pmol} / \mathrm{l})$. The HHTg rats exhibited elevated postprandial serum $\mathrm{Tg}$ compared to controls both on SD (by 180\%) and HS diet (by 290\%). The effect of insulin on in vitro glucose incorporation to $\mathrm{Tg}$ was similar in C and HHTg group on SD but HS feeding led in these groups to opposite effects - the insulin sensitivity in HHTg group decreased, in control rats it was potentiated. The postprandial LPL activity was lower in HHTg than in $\mathrm{C}$ rats on SD $(\mathrm{p}<0,01)$ but HS feeding led to its significant increase $(p<0,01)$. The LPL activity in control group tends to be lowered by the diet $(p=0,06)$. The activity of adipose tissue LPL of control rats decreased in fasting but in HHTg group this regulation was blunted and the LPL activity remains high what indicates the impairment of the regulation of synthesis and/or the activation of LPL. We found strong correlation $\left(r^{2}=0,72\right)$ between postprandial serum $\mathrm{Tg}$ content and adipose tissue LPL activity in HHTg rats both on standard and HS diet.

Conclusion: In contrast to controls, in HHTg rats HS feeding leads to the increase in LPL activity in adipose tissue probably as the result of higher insulinemia. In spite of higher LPL activity in HHTg groups the serum $\mathrm{Tg}$ content does not drop but significantly increased. We suppose that the impaired ability of HHTg adipose tissue to uptake glucose and to incorporate it into intracellular triglycerides leads to the increased escape of FFA derived from circulating lipoproteins by LPL. The released fatty acids remain in the circulation and are turned in liver into VLDL that contributes to the maintenance of hypertriglyceridemia.

Supported by IGA MH CR NR/7888-3

0528

Small dense LDL and oxidized LDL: differences between type 2 diabetes and familiar combined hyperlipidaemia

M. N. Zamaklar ${ }^{1}$, K. Lalic ${ }^{1}$, N. Rajkovic ${ }^{1}$, V. Kalimanovska ${ }^{2}$, A. Zeljkovic ${ }^{2}$, J. Stojanovic ${ }^{3}$;

${ }^{1}$ Center for Lipid Disorders, Institut for Endocrinology, ${ }^{2}$ Lipids, School of Pharmacy, ${ }^{3}$ Biochemistry Laboratory, Institut for Endocrinology, Belgrade, Serbia and Montenegro.

Background and Aims: Type 2 diabetes and combined hyperlipidemia $(\mathrm{CH})$ are associated with premature atherogenesis. In both diseases total and LDL cholesterol (LDL-C) are elevated as well as level of triglycerides. Obesity and insulin resistance are also present in both disorders. The present study analyze relationship between LDL particle size and lipids level, level of LDL oxidation, CRP and insulin sensitivity in diabetics and in patients with $\mathrm{CH}$.

\begin{tabular}{|c|c|c|c|c|c|c|c|c|}
\hline & \multicolumn{2}{|l|}{ C/SD } & \multicolumn{2}{|l|}{ C/HS } & \multicolumn{2}{|l|}{ HHTg/SD } & \multicolumn{2}{|l|}{ HHTg/HS } \\
\hline & fasting & fed & fasting & fed & fasting & fed & fasting & fed \\
\hline serum Tg mmol/l & $0,8 \pm 0,05$ & $0,9 \pm 0,1$ & $0,7 \pm 0,1$ & $1,35 \pm 0,2$ & $0,8 \pm 0,15$ & $2,5 \pm 0,3$ & $1,36 \pm 0,2$ & $5,3 \pm 0,2$ \\
\hline LPL activity umol FA/g/h & $\begin{array}{c}4,5 \pm 0,3 \\
- \text { insulin }\end{array}$ & $\begin{aligned} & 9 \pm 0,5 \\
+ & \text { Insulin }\end{aligned}$ & $\begin{array}{c}3 \pm 0,4 \\
- \text { insulin }\end{array}$ & $\begin{array}{l}6,9 \pm 0,45 \\
+ \text { insulin }\end{array}$ & $\begin{array}{c}3,7 \pm 0,3 \\
- \text { insulin }\end{array}$ & $\begin{array}{l}3,5 \pm 0,25 \\
+ \text { insulin }\end{array}$ & $\begin{array}{l}5,4 \pm 0,6 \\
- \text { insulin }\end{array}$ & $\begin{array}{r}6,9 \pm 0,7 \\
+ \text { insulin }\end{array}$ \\
\hline $\begin{array}{l}\text { glucose incorporation } \\
\text { into } \mathrm{Tg} \mathrm{umol} / \mathrm{g} / \mathrm{h}\end{array}$ & $1,3 \pm 0,12$ & $1,6 \pm 0,13$ & $1,6 \pm 0,1$ & $3,2 \pm 0,3$ & $1,7 \pm 0,06$ & $2,1 \pm 0,09$ & $1,2 \pm 0,09$ & $1,9 \pm 0,08$ \\
\hline
\end{tabular}


Materials and Methods: Investigation was performed in 60 type 2 diabetics: 27 without (group A) and 33 with dyslipidemia (group B), and in 62 non diabetics: 28 patients without dyslipidemia (group C) as well as in 34 patients with $\mathrm{CH}$ (group D). LDL size was measured by gradient gel electrophoresis, cut-off values was diameter $<25,5 \AA$. Oxidized LDL (ox-LDL) was measured by ELISA methods (Mercodia). Total cholesterol, HDL cholesterol (TC, HDL-C) and triglyceride (TG) were determined by chromatography, LDL-C and non HDL-C was calculated. Apolipoproteins, Lp (a) and CRP were measured by nephelometric methods. Insulin was assessed by RIA methods and insulin sensitivity was estimated by HOMA-IR.

Results: In our diabetics, 32 of all $(62,6 \%)$ had small dense LDL particle, but there was no significant differences between groups A and B. In group B patients had higher TC, LDL-C, non HDL-C, TG $(p<0.01)$, CRP $(p<0.05)$. Levels of HDL-C and Lp(a) were similar in both groups, as well as HOMA-IR. Ox-LDL was higher in group B (117, 12 vs. 91, $85 \mathrm{IU} / \mathrm{L}, \mathrm{p}<0.01)$. In both diabetic groups LDL particle size positive correlated with HDL-C, Apo AI, AII $(p<0.05)$, but negative correlated with levels of TG, Apo E and HOMA-IR $(p<0.05)$. The level of Ox-LDL correlated with TC, LDL-C, non HDL-C and Apo B 100 (p<0.001), as well as al level of TG, Apo AI, $(\mathrm{p}<0.05)$. In non diabetics, $\mathrm{CH}$ patients only 17 out of $62(25,9 \%)$ had small dense LDL particles. Patients from group D had higher TC, LDL$\mathrm{C}$, non HDL-C and TG $(\mathrm{p}<0.01)$. There was no significant differences in levels of HDL-C, Lp (a), but Apo B 100 and Apo E were higher in group $\mathrm{D}(\mathrm{p}<0.01)$. We have not found significant difference in the LDL particle size in these groups $(26,48$ vs. 26, $46 \AA$, p=NS), but dyslipidemic patients had higher ox-LDL levels $(111,83$ vs. 99,72 IU/L, $\mathrm{p}<0.05)$. Also, in non diabetic groups insulin sensitivity was similar. LDL particle size in all non diabetics positive correlated with TC $(p<0.05)$ and with $\operatorname{Lp}(a)(p<0.01)$. In these patients ox-LDL correlated with TC, LDL-C, non HDL-C and TG $(\mathrm{p}<0.01)$.

Conclusion: Diabetics predominantly have small dense LDL. LDL particle size negative correlate with insulin resistance (HOMA-IR) and triglyceride but positive with HDL-C, Apo AI and AII. In those patients with dyslipidemia oxidation of LDL was higher, but there was no correlation between small dense LDL particle size and oxLDL. In contrast, $\mathrm{CH}$ patients despite non dominantly small dense LDL, had higher level of LDL oxidation.

Supported by Mercodia
0529

Free fatty acids as mediators of adaptive compensatory responses to insulin resistance induced by dexamethasone treatment in rats

M. Novelli ${ }^{1}$, A. Pocai $^{1,2}$, C. Chiellini ${ }^{3}$, M. Maffei $^{3}$, P. Masiello ${ }^{1}$;

${ }^{1}$ Dip. Patologia Sperimentale, University of Pisa, Italy, ${ }^{2}$ Diabetes Research Center, Albert Einstein College of Medicine, Bronx, United States, ${ }^{3}$ Dulbecco Telethon Institute at Dept. Endocrinology \& Metabolism, University of Pisa, Italy.

Background and Aims: We previously showed that chronic low-dose dexamethasone (DEX) treatment in rats induces insulin resistance with compensatory hyperinsulinaemia and reduction in food intake. Here we test the hypotheses that DEX-induced hyperlipacidaemia mediates both insulin resistance and insulin hyperproduction and that a rise in circulating leptin is responsible for the anorectic effect of DEX.

Materials and Methods: DEX treatment $(0.125 \mathrm{mg} / \mathrm{kg} / \mathrm{day}$ dexamethasone phosphate s.c.) of Sprague-Dawley rats for 20 days; transient reduction of plasma free fatty acid (FFA) levels by repeated i.p. injections of the antilipolytic agent nicotinic acid, prior to intravenous glucose tolerance test and intraperitoneal insulin tolerance test; monitoring of plasma glucose, FFA, insulin and leptin levels during DEX treatment; evaluation of leptin expression by Northern blot in white adipose tissue (WAT) at the end of DEX treatment. Results: Upon DEX treatment, an early increase in circulating FFA followed by hyperinsulinaemia was observed, associated with normal or even slightly decreased glycaemia. Reduction in food intake and impairment in body weight gain was confirmed to occur. A transient pharmacological reduction of elevated circulating FFA reversed the DEX-induced insulin resistance and the compensatory post-loading hyperinsulinaemia, supporting the hypothesis that the chronic elevation in FFA is the common mediator of these changes. Despite enhanced leptin expression in WAT, DEX-treated rats showed no significant increase in plasma leptin levels, thus suggesting that the anorectic effect of DEX should be mediated, at least partially, by some other factor. Based on the reported anorectic effect of central FFA administration, we postulate that the chronic elevation of circulating FFA might be involved in the limitation of food intake in DEX-treated rats, probably acting through the accumulation of long chain fatty-acylCoAs in selective nutrient-sensing neurons. 
Conclusion: Our results support the idea that during DEX treatment chronic elevation of plasma FFA plays an important role in the adaptive regulation of both insulin action and energy balance, not only by potentiating insulin secretion, but also by providing a neuronal signal of "nutrient abundance" capable of restraining feeding behaviour. Supported by grants from University of Pisa to PM and Telethon Foundation to MM

\section{0}

A supervised exercise programme increases HDL ApoA-1 production rate in type 2 diabetes M. Stolinski ${ }^{1}, \mathrm{X} . \mathrm{Li}^{1}, \mathrm{~A} . \mathrm{Lo}^{2}, \mathrm{~F}$. Shojaee-Moradie ${ }^{1}$, S. Alam ${ }^{2}$, B. Wheeler ${ }^{2}$, C. Pentecost ${ }^{2}$, N. Jackson ${ }^{1}$, W. Jefferson ${ }^{2}$, M. Umpleby ${ }^{1}$;

${ }^{1}$ Diabetes and Endocrinology, Postgraduate Medical School, University of Surrey, Guildford, ${ }^{2}$ Diabetes and Endocrinology, St Thomas' Hospital, Kings College London, London, United Kingdom.

Background and Aims: Dyslipidaemia in type 2 diabetes is characterized by increased plasma triglycerides and decreased HDL cholesterol. Research indicates that the dyslipidaemia and insulin resistance of type 2 diabetes can be improved by aerobic exercise. Although patients are advised to exercise as a part of their therapy, unless this is part of a supervised exercise program this is usually unsuccessful. This study has measured the effect of exercise on HDL apoA-1 kinetics in two groups of patients following a 6 month exercise plan. One group had weekly meetings with an exercise trainer (supervised), the other group did not see an exercise trainer after their initial contact session (unsupervised).

Materials and Methods: Ten moderately obese type 2 diabetic patients who had failed to show improved glycaemic control with diet and tablets (HbA1c $>7.5 \%$ ) participated in this study. Those on oral hypoglycaemic agents did not change treatment throughout the study. All patients were given an initial training session and an individualised exercise programme and were asked to exercise for 20 $40 \mathrm{~min}$ at $60-85 \%$ of maximal oxygen uptake $\left(\mathrm{VO}_{2 \max }\right)$ four times per week, in a mode of aerobic activity of their choice, for a period of 6 months. Patients were then randomised into a supervised $\left(\mathrm{n}=6\right.$; BMI $28.54 \pm 1.75 \mathrm{~kg} / \mathrm{m}^{2}$, age $57.8 \pm 3.6$ years, $4 \mathrm{~F}: 2 \mathrm{M})$ or unsupervised $(\mathrm{n}=4$; BMI $32.6 \pm 1.83 \mathrm{~kg} / \mathrm{m}^{2}$, age $54.8 \pm 6.0$ years, $1 \mathrm{~F}: 3 \mathrm{M}$ ) group. HDL apoA-I metabolism was measured with an infusion of $1{ }^{13} \mathrm{C}$ leucine for 9 hours before and after the exercise programme. Patients did not exercise for 3 days prior to the metabolic study day. HDL was isolated by ultracentrifugation. ApoA-I enrichment was measured after separation from other apolipoproteins by polyacrylamide gel electrophoresis. ApoA-I concentration was measured by immunoturbidimetry. Insulin sensitivity was calculated by HOMA. The fractional secretion rate (FSR) of HDL apoA-I was calculated using a regression model. HDL apoA-I production rate (PR) was calculated from the product of the FSR and the pool size. The steady state enrichment of $\alpha$-ketoisocaproate was used as an estimate of the precursor pool enrichment. Results are presented as mean \pm SEM.

Results: Those in the supervised exercise group showed a significant decrease in \% HbAlc $(8.47 \pm 0.47$ v $7.45 \pm 0.51$; $\mathrm{p}<0.005)$ and plasma triglyceride $(1.06 \pm 0.23 \mathrm{v}$ $0.8 \pm 0.2 \mathrm{mmol} / \mathrm{l} ; \mathrm{p}<0.03$ ), an increase in insulin sensitivity (HOMA: $7.39 \pm 1.39$ v $3.69 \pm 0.29 ; \mathrm{p}<0.04$ ). There was an increase in HDL cholesterol $(1.05 \pm 0.09$ v $1.34 \pm 0.10 \mathrm{mmol} /$ $1 ; \mathrm{p}<0.03)$, apoA-1 concentration $(1.18 \pm 0.12 \mathrm{v} 1.42 \pm 0.11 \mathrm{~g} /$ $1 ; \mathrm{p}<0.05)$ and a significant decrease in HDL triglyceride $(0.16 \pm 0.02 \vee 0.10 \pm 0.01 \mathrm{mmol} / 1 ; \mathrm{p}<0.05)$. The increase in HDL was due to an increase in apoA-1 PR $(5.99 \pm 0.75 \mathrm{v}$ $8.79 \pm 1.04 \mathrm{mg} / \mathrm{kg} / \mathrm{day} ; \mathrm{p}<0.03)$. There was no significant change in HDL apoA-1 FSR $(0.15 \pm 0.01 \mathrm{v} 0.18 \pm 0.02$ pools/ day). The unsupervised group showed no significant changes in any of the parameters measured.

Conclusions: The study suggests that the increase in HDL cholesterol following the supervised exercise programme was due to an increase in HDL production rate. This may be due to an improvement in insulin sensitivity which was not apparent in the unsupervised group.

Supported by BHF

\section{PS 31}

\section{Cardiac metabolism}

0531

Cardiac pyruvate dehydrogenase kinase (PDK) 4 is enhanced by increased LXR signaling in the heart

M. C. Sugden ${ }^{1}$, C. G. Walker ${ }^{1}$, G. F. Gibbons ${ }^{2}$,

G. K. Greenwood ${ }^{1}$, M. J. Holness ${ }^{1}$;

${ }^{1}$ Diabetes and Metabolic Medicine, Barts and the London, London, ${ }^{2}$ Metabolic Research Laboratory, OCDEM, Oxford, United Kingdom.

Background and Aims: The liver X receptor (LXR) subfamily of nuclear receptors is activated upon binding of ligands, which probably comprise oxidised cholesterol derivatives. Improved glucose tolerance and insulin sensitivity in response to LXR activation has been reported in genetic and dietary models of type 2 diabetes: this may reflect decreased hepatic glucose output secondary to an LXR-led reduction in gluconeogenic capacity as LXR activation represses expression of hepatic gluconeogenic 
enzymes. However, activation of LXRs also causes a dramatic increase in hepatic triglyceride production and both increased lipid delivery and/or inappropriate induction of fatty acid synthesis in extrahepatic tissues could impair insulin action through excessive tissue lipid accumulation. The heart is particularly susceptible to adverse effects of lipid accumulation in diabetes. Increasing glucose oxidation and decreasing fat oxidation during cardiac hypertrophy and during reperfusion of ischemic hearts is believed to be beneficial in maintaining cardiac contractility and efficiency. Glucose oxidation is constrained by the activity of the pyruvate dehydrogenase kinases (PDKs), which inactivate pyruvate dehydrogenase complex (PDC) by phosphorylation. Cardiac PDK isoform 4 (PDK4) expression is upregulated in diabetes, resulting in PDC inactivation.

Materials and Methods: Male mice (4-6 months old, 25$30 \mathrm{~g})$ were allowed food and water ad libitum. TO901317 $(0.01 \%)$ was administered as a component of the diet for 5 days. Animals were killed in the absorptive state and hearts removed for analysis of PDK4 protein expression and triglyceride (TAG) content.

Results: LXR activation in vivo by the provision of TO901317 as a component of the diet (5 days, 0.01\%) causes a marked (3.6-fold; $\mathrm{P}<0.05)$ enhancement of cardiac PDK4 protein expression, in conjunction with cardiac TAG accumulation (a 3.8-fold increase) in mice. Protein expression of PDK1 and PDK2 was unchanged by LXR activation. Conclusion: As the heart has an optimal balance between the use of lipid and glucose as oxidative substrate, altering this balance through activation of LXR and increased PDK4 protein expression, despite improved insulin action, might be detrimental to cardiac function in diabetes.

Supported by Diabetes UK

\section{2}

Over-expression of CYP2J2 specifically augments cardiac pyruvate dehydrogenase kinase 4 expression M. J. Holness ${ }^{1}$, G. K. Greenwood ${ }^{1}$, S. Samsuddin ${ }^{1}$, D. C. Zeldin ${ }^{2}$, D. Bishop-Bailey ${ }^{3}$, M. C. Sugden ${ }^{1}$;

${ }^{1}$ Diabetes and Metabolic Medicine, Barts and the London, United Kingdom, ${ }^{2}$ Division of Intramural Research, NIEHS/NIH, Research Triangle Park, United States, ${ }^{3}$ Cardiac, Vascular \& Inflammation Research, Barts and the London, United Kingdom.

Background and Aims: Epidemiological studies reveal that people with diabetes and hypertension are at higher risk for the development of cardiovascular disease. The heart in uncontrolled diabetes is constrained from switching to glucose oxidation, and relies almost exclusively on lipid as an energy source. Peroxisome proliferator-activated receptor
(PPAR)- $\alpha$ is a nuclear receptor involved in regulation of lipid metabolism and the PPAR $\alpha /$ PGC- $1 \alpha$ axis is activated in the diabetic heart. Cardiac-specific over-expression of PPAR $\alpha$ results in enhanced sensitivity to ischemic injury and recapitulation of many of the metabolic abnormalities of the diabetic heart, including decreased glucose oxidation. The expression of cardiac pyruvate dehydrogenase isoform 4 (PDK4), a PPAR $\alpha$ target gene, is up-regulated in diabetes, resulting in pyruvate dehydrogenase complex (PDC) inactivation by phosphorylation and suppressed glucose oxidation. The expression of PDK1 is augmented by the HIF-1 transcription factor, which drives hypoxic gene expression. The biologically active cis-epoxyeicosatrienoic acids (EETs) that regulate blood pressure, and can activate PPAR $\alpha$ in vitro, are products of CYP2J2, an abundant epoxygenase in human cardiovascular systems. We investigated whether modification of CYP2J2 activity influences the cardiac PDK isoform profile.

Materials and Methods: Female cardiac-specific CYP2J2 transgenic mice and wild type controls (C57 black, 46 months old, 25-30 g) were allowed food and water ad libitum or fasted for $24 \mathrm{~h}$. Animals were then killed and hearts and kidneys removed for analysis of PDK4 protein expression in comparison to that of PDK1. Anti-PDK1 ann PDK4 antibodies, generated in rabbits against recombinant PDK4 protein, were generously provided by Professor Bob Harris (Indianapolis, U.S.A.).

Results: Upon fasting, PDK4 protein expression increased (3.7-fold; $\mathrm{P}<0.01$ ) in wild type mice compared to fed animals. This response was augmented (by2-fold; $\mathrm{P}<0.01$ ) in the hearts, but not kidneys, of CYP2J2 transgenic mice. Fasting had no significant effect on the levels of PDK1 in either wild type or CYP2J2 transgenic mice.

Conclusion: Thus, over-expression of CYP2J2 specifically augments cardiac PDK4 expression suggesting that the generation of EETs may facilitate PDK4 expression in heart, but not kidney, but that PDK1 is not a target for CYP2J2-generated compounds.

Supported by British Heart Foundation, Diabetes UK, William Harvey Research Foundation, European Community FP6 funding, Intramural Research Programs of the National Institutes of Health

\section{3}

Both subcutaneous and epicardial adipose tissue is a significant source of proinflammatory factors in cardiac surgery patients: possible role in postoperative hyperglycaemia and insulin resistance

M. Haluzik ${ }^{1}$, J. Kremen ${ }^{1}$, M. Dolinkova ${ }^{1}$, J. Blaha ${ }^{2}$, J. Krajickova ${ }^{1}$, K. Anderlova ${ }^{1}$, D. Haluzikova ${ }^{3}$, Z. Lacinova ${ }^{1}$, S. Svacina ${ }^{1}$; 
${ }^{1} 3$ Department of Medicine, ${ }^{2} 2$ Department of Anesthesia, Resuscitation and Intensive Medicine, ${ }^{3}$ Department of Sports Medicine, 1 Faculty of Medicine, Prague, Czech Republic.

Background and Aims: Critically ill patients frequently develop hyperglycemia and insulin resistance. Its detailed etiopatogenesis is still only partially understood. Here we studied the role of adipose tissue hormonal production in the development of postoperative insulin resistance in elective cardiac surgery patients.

Materials and Methods: Serum concentrations, subcutaneous (s.c.) and epicardial (epi.) adipose tissue mRNA expression of interleukin-6 (IL-6), monocyte chemoattractant protein-1 (MCP-1), tumor necrosis factor- $\alpha$ (TNF- $\alpha$ ), leptin, resistin and adiponectin were measured in 10 patients with elective cardiac surgery operation using commercial Lincoplex kits and real time PCR, respectively. Adipose tissue samples were taken at the beginning and at the end of operation. Blood samples were withdrawn before, at the end and 6,12, 24, 48 and 120 hours after the end of operation, respectively.

Results: The rate of insulin infusion required to maintain euglycemia increased up to 8-fold 12 hours after the operation indicating the development of insulin resistance. Serum IL-6 levels increased 43-fold and peaked 12 hours after operation. MCP-1 peaked 6-fold at the end of operation, while less pronounced peaks of TNF- $\alpha$ and leptin, 6 and 20 hours after operation, respectively were detected. Circulating resistin levels increased 2.7-fold 6 hours after operation and remained 3.5-fold elevated until 48 hours after operation. In contrast adiponectin levels were not significantly affected by the operation. TNF- $\alpha$ and resistin mRNA expression increased markedly during the operation in s.c. adipose tissue. IL-6 and MCP-1 mRNA expression increased in both s.c. and epi. adipose tissue. Neither leptin or adiponectin mRNA expression were significantly affected by the operation.

Conclusion: Elective cardiac surgery operation markedly activates production of proinflammatory cytokines in both subcutaneous and epicardial adipose tissue. We suggest that this production may significantly contribute to the development of postoperative insulin resistance in these patients. Supported by MSM 0021620814 and CLINICIP

\section{4}

Short-term exposure to polyunsaturated high fat diet preserves cardiac contractile function in rats

C. E. van den Brom ${ }^{1}$, R. Vlasblom ${ }^{2}$, M. Fodor ${ }^{3}$, C. Boer ${ }^{2}$, D. M. Ouwens ${ }^{4}$, M. Diamant ${ }^{1}$;
${ }^{1}$ Department of Endocrinology, Diabetes Center, ${ }^{2}$ Laboratory for Physiology, VUmc, Amsterdam, ${ }^{3}$ Department of Anatomy, ${ }^{4}$ Department of Molecular Cell Biology, LUmc, Leiden, The Netherlands.

Background: Cardiovascular complications are common in type 2 diabetes. Previously, we demonstrated cardiac functional, structural and molecular changes in Wistar rats after long-term exposure to high (saturated) fat diet (HSFD), resembling diabetic cardiomyopathy (DCM). The current study tests the hypothesis that polyunsaturated fatty acids (PUFA) may protect against HSFDinduced cardiac changes in rats, following short-term exposure.

Methods: Male Wistar rats (bodyweight, BW; $269 \mathrm{~g} \pm$ $14,0 \mathrm{~g})$ received HSFD $(\mathrm{n}=11)$, PUFA-enriched diet (PUFAD; $n=11$ ), or a low fat diet (LFD; $n=11)$ for four weeks. At day 25, all rats were subjected to an oral glucose (2 g/kg BW) tolerance test. Post-load blood glucose (BG) levels were determined at $\mathrm{t}=0,15,30,60,90,120 \mathrm{~min}$. At day 27 , echocardiography was performed to determine left ventricular end diastolic and end systolic wall thickness, lumen diameters, fractional shortening (FS\%) and epicardial thickness (EPI). At day 28, rats were decapitated and plasma insulin, non-esterified fatty acids (NEFA) and triglycerides (TG) levels were determined in trunk blood.

Results: Body weight and heart-to-tibia ratio were similar in the 3 groups. Fasting BG did not differ among groups, while post-load BG was increased in HSFD-treated rats at $60 \mathrm{~min}$ [LFD 8,5 vs. HSFD 10,3, $\mathrm{p}<0.05$ ] and at $90 \mathrm{~min}$ [LFD 7,2 vs. HSFD 8,9 $\mathrm{p}<0.05]$. Post-load $\mathrm{BG}$ at 60 and $90 \mathrm{~min}$ was comparable in HSFD- and PUFAD-treated rats. Reduced plasma NEFA and TG concentrations were observed in HSFD- and PUFAD-treated rats, relative to LFD-controls, whereas similar insulin levels and HOMA-IR were observed in all groups. Echocardiography revealed a significant decrease in FS\% in HSFD-rats, whereas no difference was found in PUFAD-treated rats, compared to LFD-controls [LFD: 48,9\% vs. HSFD: 42,0\% vs. PUFAD: 48,7\%, p <0.05 One-way ANOVA]. Both HSFD- and PUFAD-fed rats had increased EPI, as compared to LFD-rats [LFD: 0,05 cm vs. HSFD: 0,08 cm vs. PUFA: 0,07 cm].

Conclusions: A short-term exposure to a HSFD resulted in reduced glucose tolerance, contractile dysfunction and increased epicardial thickness, resembling DCM. Interestingly, PUFAD preserved cardiac contractile function, in spite of the presence of metabolic changes similar to those observed in HSFD-treated rats. The molecular mechanisms underlying the cardiac protective effects of PUFAD remain to be investigated.

Supported by DFN 2003.00.029 
0535

Abnormal left ventricular energy metabolism in obese men with preserved systolic and diastolic functions is associated with insulin resistance

G. Perseghin ${ }^{1}$, G. Ntali $^{1}$, F. De Cobelli ${ }^{2}$, G. Lattuada ${ }^{1}$, A. Esposito ${ }^{2}$, E. Belloni ${ }^{2}$, T. Canu ${ }^{2}$, F. Ragogna ${ }^{1}$, P. Scifo ${ }^{3}$, A. Del Maschio ${ }^{2}$, L. Luzi ${ }^{1}$;

${ }^{1}$ Internal Medicine, ${ }^{2}$ Diagnostic Radiology, ${ }^{3}$ Division of Nuclear Medicine, Istituto Scientifico H San Raffaele, Milano, Italy.

Background and Aims: It has been hypothesized that perturbations in cardiac energy metabolism might be among the earliest alterations in diabetes preceding functional and pathological changes. This study evaluated left ventricular (LV) structure and function in relation to energy metabolism and cardiovascular risk factors in overweight/obese men using magnetic resonance (MR) techniques.

Materials and Methods: We studied 81 healthy men (age range: $22-55$ years, body mass range: $19-35 \mathrm{~kg} / \mathrm{m}^{2}$ ) by means of cardiac MR imaging (MRI) and ${ }^{31} \mathrm{P}-\mathrm{MR}$ spectroscopy (MRS) in the resting and fasted conditions and stratified them in quartiles of BMI (cut offs: 23.2, 25.5 and $29.0 \mathrm{~kg} / \mathrm{m}^{2}$ ).

Results: The morphological features showed that the LV mass increased across increasing quartiles of BMI $(r=0.39$; $\mathrm{P}<0.002)$ meanwhile the chambers volume did not differ. Parameters of LV systolic (ejection fraction, stroke volume) and diastolic function (early/atrial peak ratio and deceleration time) were not different among quartiles. In contrast the phosphocreatine (PCr)/ATP ratio was reduced across increasing quartiles of BMI (2.25 $\pm 0.52,1.89 \pm 0.26$, $1.99 \pm 0.38$ and $1.79 \pm 0.29 ; \mathrm{P}<0.006)$ in association with insulin sensitivity (calculated using the updated computer HOMA2 model based on fasting plasma glucose and insulin concentrations) and this relation was independent of age, BMI, blood pressure, end diastolic wall mass, serum HDL-cholesterol, triglycerides, smoking habits and metabolic syndrome.

Conclusion: Abnormal LV energy metabolism is detectable in obese men in presence of normal LV function and these data support the hypothesis that metabolic remodeling in insulin resistant states precedes the functional and structural remodeling of the heart before the potential onset of frank hyperglycemia.

Supported by Italian Minister of Health and the Marie Curie Host Fellowship of the European Community
0536

In wild type mice long-term exposure to high-fat diet induces systemic insulin resistance without affecting cardiac function

R. Vlasblom ${ }^{1}$, M. Ouwens ${ }^{2}$, C. E. van den Brom ${ }^{3}$, C. Boer ${ }^{1}$, M. Diamant ${ }^{3}$;

${ }^{1}$ Laboratory for Physiology, VU University Medical Center, Amsterdam, ${ }^{2}$ Molecular Cell Biology, Leiden University Medical Center, Leiden, ${ }^{3}$ Department of Endocrinology; Diabetes Center, VU University Medical Center, Amsterdam, The Netherlands.

Background and Aims: Cardiac dysfunction is prevalent in diabetes mellitus type 2 (DM2), and in time, may progress from mild functional changes into overt heart failure. This study aims to reveal cardiac changes following 20 weeks exposure to high fat diet in wild type C57Bl/6J mice.

Materials and Methods: Sixteen male mice (17.0 g $\pm 0.9 \mathrm{~g}$ ) received either a high fat (HFD; $24 \%$ fat; $n=8)$ or a low fat diet (LFD; 4\% fat; $n=8$ ) diet. Fasting blood glucose (FBG) was determined each 2 weeks. After 18 weeks of diet, mice underwent an oral glucose $(2 \mathrm{mg} / \mathrm{g})$ tolerance test. $\mathrm{BG}$ levels were determined at $\mathrm{t}=0,15,30,60,90$, $120 \mathrm{~min}$ after the glucose load. In week 20, left ventricular (LV) end diastolic (EDD) and systolic diameters (ESD) and fractional shortening (FS\%) were measured by echocardiography. At decapitation, plasma insulin, nonesterified fatty acids and triglycerides were determined in trunk blood.

Results: At 20 weeks, HFD- vs. LFD-mice had higher body weight (38.6 g vs. $31.9 \mathrm{~g}, \mathrm{p}<0.05)$, but similar heartto-tibia ratio's. In HFD-mice, FBG tended to increase from week 14 on $(\mathrm{p}<0.05)$. Post-load BG was increased in HFDvs. LFD-mice at $30 \mathrm{~min}$ and at $90 \mathrm{~min}$ (both $\mathrm{p}<0.05$ ). In HFD- relative to LFD-mice, plasma insulin concentrations were increased 2.4-fold $(\mathrm{p}<0.05)$. HFD- vs. LFD-mice tended to be insulin resistant, as assessed by HOMA-IR $(\mathrm{p}=0.05)$. Cardiac parameters, including LVEDD, LVESD and FS\% were similar in both groups, however, HFD-mice showed increased epicardial fat pad $(0.07$ vs. $0.05 \mathrm{~cm}$; $\mathrm{p}<0.05$ ).

Conclusion: Long-term exposure to HFD in wild type C57B1/6J mice resulted in a DM2 phenotype, including obesity, hyperglycemia and insulin resistance, without affecting cardiac function. These data suggest that $\mathrm{C} 57 \mathrm{Bl} /$ $6 \mathrm{~J}$ mice may be resistant to HFD-induced cardiac dysfunction or that cardiac abnormalities may be a late event in HFD-related DM2 in these mice.

Supported by DFN2004.00.052 
Cardiac dysfunction in insulin resistant high-fat diet fed rats associates with elevated FAT/CD36-mediated fatty acid uptake and esterification

D. M. Ouwens ${ }^{1}$, M. Diamant ${ }^{2}$, M. Fodor ${ }^{3}$,

D. D. J. Habets ${ }^{4}$, M. M. A. Pelsers ${ }^{4}$, M. El Hasnaoui ${ }^{4}$, R. Vlasblom ${ }^{5}$, C. Boer ${ }^{5}$, S. L. M. Coort ${ }^{4}$, J. F. C. Glatz ${ }^{4}$, J. J. F. Luiken ${ }^{4}$;

${ }^{1}$ Molecular Cell Biology, LUMC, Leiden, ${ }^{2}$ Diabetes Centre/Endocrinology, VUMC, Amsterdam, ${ }^{3}$ Anatomy \& Embryology, LUMC, Leiden, ${ }^{4}$ Molecular Genetics, Maastricht University, ${ }^{5}$ Physiology, VUMC, Amsterdam, The Netherlands.

Background and Aims: Changes in cardiac substrate utilization leading to altered energy metabolism may underlie the development of diabetic cardiomyopathy (DCM). We studied cardiomyocyte substrate uptake and utilization and the role of fatty acid translocase (FAT) FAT/ CD36 in relation to in vivo cardiac function in a non-obese rat model of high-fat diet (HFD)-induced insulin resistance. Materials and Methods: Rats were exposed to HFD or lowfat diets (LFD) for 10 weeks. In vivo cardiac contractile function was monitored by echocardiography. Substrate uptake and utilization were determined in isolated cardiomyocytes.

Results: HFD- versus LFD-feeding induced left ventricular (LV) dilation, increased LV free wall thickness and decreased fractional shortening. Cardiomyocytes from HFD-hearts were insulin-resistant as reflected by $41 \%$ $(P<0.001)$ and $58 \%(P<0.02)$ decreases in insulin-mediated stimulation of glucose uptake and phosphorylation of glycogen synthase kinase 3, respectively. Fatty acid uptake was 1.4-fold increased in HFD- versus LFD-cardiomyocytes $(P<0.001)$, which could be ascribed to an increased abundance of FAT/CD36 at the sarcolemma rather than alterations in FAT/CD36 protein levels. Finally, in HFDcardiomyocytes, fatty acid oxidation rates were decreased by $22 \%(P<0.01)$, whereas fatty acid esterification into triacylglycerols and phospholipids were increased by 1.4 and 1.5 -fold, respectively (both $P<0.05$ ).

Conclusion: HFD-feeding induces cardiac contractile dysfunction that associates with a permanent relocation of FAT/ CD36 to the sarcolemma. This maladaptive presence of FAT/CD36 at the sarcolemma contributes to enhanced rates of fatty acid uptake, myocardial triacylglycerol-accumulation and accompanying insulin resistance, and may contribute to the development of DCM.

Supported by Dutch Diabetes Research Foundation, Netherlands Heart Foundation, Netherlands Organization for Health Research and Development
Cardio protective effect of glucose-insulin infusion on acute digoxin toxicity in rat

R. Oubaassine ${ }^{1}$, P. Bilbault ${ }^{2}$, J. Roegel ${ }^{3}$, E. Alexandre ${ }^{4}$, S. Sigrist ${ }^{1}$, T. Lavaux ${ }^{2}$, A. Jaeger ${ }^{2}$, M. Pinget ${ }^{1}$, L. Kessler ${ }^{1}$; ${ }^{1}$ Laboratoire de Thérapie Cellulaire et Génique du Diabète, Centre Européen d'Etude du Diabète, Strasbourg, ${ }^{2}$ Réanimation Médicale, Hôpitaux Universitaires, Strasbourg, ${ }^{3}$ Forenap Therapeutic Discovery, Institut de Pharmacologie, Strasbourg, ${ }^{4}$ Fondation Transplantation, Hôpitaux Universitaires, Strasbourg, France.

Background and Aims: Recently we observed a case of digoxin and insulin self-poisoning without cardiac repercussion. We raise the hypothesis that insulin may have a cardio-protective effect in case of digoxin toxicity. Therefore, we have evaluated the effect of glucose-insulin infusion on mortality and ECG abnormalities during acute digoxin toxicity in rats.

Materials and Methods: Before and after a hyperinsulinemia-euglycemia clamp, rats in glucose-insulin-digoxin (GID) group $(\mathrm{n}=10)$ received an intravenous infusion of $12 \mathrm{ml} / \mathrm{h}$ or $2,5 \mathrm{ml} / \mathrm{h}$ digoxin $(0.25 \mathrm{mg} / \mathrm{ml})$ respectively until death occured. Animals receiving digoxin or saline solution intravenously served as control $(\mathrm{n}=10)$. ECG recording was performed in all animals over the entire period. Serum insulin and digoxin concentrations were measured by ELISA method after digoxin administration.

Results: When digoxin was administered after the clamp, all animals in GID group were alive, whereas $80 \%$ of animals in digoxin group were dead $(p<0.001)$ after thirty minutes. Digoxin administration provoked rapid death of rats in digoxin group in $15 \pm 12$ min whereas in GID group the survival period was significantly increased to $38 \pm 3 \mathrm{~min}$ $(p<0.001)$. Twenty minutes after digoxin administration, $\mathrm{P}$ waves disappeared for $78 \%$ of animals in digoxin group while they were present in all rats of GID group $(p<0.0001)$. Animal death occurred after a digoxin infusion volume of 7.7 $+0.6 \mathrm{ml}$ and $3.0+2.4 \mathrm{ml}$ in GID and digoxin group respectively $(p<0.001)$. Five minutes after digoxin administration, potassium plasmatic level increased significantly in digoxin group as compared to GID group: $7.1 \pm 2 \mathrm{mmol} / \mathrm{l}$ vs. $4.4 \pm 0.4 \mathrm{mmol} /(\mathrm{p}<0.001)$. When digoxin was infused before the clamp, $40 \%$ of animals in GID group were alive after $180 \mathrm{~min}$ and the others $60 \%$ were died after $109+19 \mathrm{~min}$ whereas death of rats in digoxin group occured in $74+10$ minutes $(\mathrm{P}<0.001)$. Death of animals was preceded by the $\mathrm{P}$ waves disappearing. Volume of digoxin infusion in GID group was twice that of the digoxin group. Thirty minutes 
after digoxin administration, potassium plasmatic level increased significantly in digoxin group as compared to GID group: $6.9+0.5 \mathrm{mmol} / \mathrm{L}$ vs $4.9+0.3 \mathrm{mmol} / \mathrm{L}$ vs $(\mathrm{p}<0.001)$.

Conclusion: Glucose-insulin infusion delayed the abnormalities in cardiac conduction and improved rat survival after acute digoxin toxicity. These results suggest a cardioproctective effect of insulin in case of acute digoxin toxicity.

\section{PS 32}

\section{Brain metabolism}

\section{9}

5ptase IV inhibits inositide trisphosphate accumulation in hypothalamus and regulates food intake and body weight

L. A. Velloso, E. P. Araujo, D. F. Bertelli; Internal Medicine, State University of Campinas, Brazil.

Background and Aims: The lipid metabolizing enzyme, phosphatidylinositol 3-kinase (PI 3-kinase), exerts an important role in the transduction of the anorexigenic and thermogenic signals delivered by insulin and leptin to firstorder neurons of the arcuate nucleus in the hypothalamus. The termination of the intracellular signals generated by the activation of PI 3-kinase depends on the coordinated activity of specific inositol phosphatases, which are poorly characterized in hypothalamus.

Materials and Methods: All studies were performed with male Wistar rats $(\sim 200 \mathrm{~g})$. The investigation followed the University guidelines for the use of animals in experimental studies and conforms to the NIH publication No. 85-23 revised 1996. By sequence homology with the classical $5^{\prime}$ - phosphatases SHIP-1 and SHIP-2 we identified the enzyme phosphoinositidespecific inositol polyphosphate $5^{\prime}$ - phosphatase IV (5ptase IV) in hypothalamic regions involved with the control of feeding and thermogenesis. The expression, distribution and activity of this enzyme were evaluated by immunohistochemistry, immunoprecipitation, real time-PCR, in situ hybridizaton and knock down by antisense oligonucleotide.

Results: Phosphoinositide-specific inositol polyphosphate 5-phosphatase IV (5ptase IV) is highly expressed in neurons of the arcuate and lateral nuclei of the hypothalamus. Upon intracerebroventicular (ICV) treatment with insulin, 5ptase IV undergoes a time-dependent tyrosine phosphorylation, which follows the same patterns of canonical insulin signaling through the insulin receptor, IRS2, and PI 3-kinase. This tyrosine phosphorylation activates 5ptase IV catalytic activity towards $5^{\prime}$ phosphorylated inositide phosphate. To evaluate the participation of 5ptase IV in insulin action in hypothalamus, we employed a phosphorthioate modified antisense oligonucleotide specific for this enzyme. The treatment of rats with this oligonucleotide for four days reduced the hypothalamic expression of 5 ptase IV by $\sim 80 \%$. This was accompanied by a $\sim 70 \%$ reduction of insulin-induced tyrosine phosphorylation of 5ptase IV and by an increase in basal accumulation of phosphorylated inositols in the hypothalamus. Finally, the inhibition of hypothalamic 5ptase IV expression by the antisense approach resulted in reduced daily food intake, body weight loss and decreased $12 \mathrm{~h}$ spontaneous food intake.

Conclusion: 5ptase IV is a powerful regulator of signaling through PI 3-kinase in hypothalamus and may become an interesting target for therapeutics of obesity and related disorders.

Supported by FAPESP

0540

HPA axis regulation reaches a new setpoint in acute and chronic type 1 diabetes: implications for brain pathology Y. Revsin ${ }^{1,2}$, D. van Wijk ${ }^{1}$, F. E. Saravia ${ }^{2}$,

A. F. De Nicola ${ }^{2}$, M. S. Oitzl ${ }^{1}$, E. R. de Kloet ${ }^{1}$;

${ }^{1}$ Medical Pharmacology, Leiden University, The Netherlands, ${ }^{2}$ Neuroendocrine Biochemistry, IBYME, Buenos Aires, Argentina.

Background and Aims: The influence of diabetes mellitus on brain pathology is increasingly recognized. Previous contributions of our laboratory demonstrated in one month experimental type 1 diabetes (streptozotocin-STZ-treated mice) hippocampal disturbances, such as astrogliosis and increased oxidative stress.Diabetic animals also show aberrant activity of the glucocorticoid system, including increased circulating corticosterone and lack of periodicity. In our model the hypothalamus-pituitary-adrenal (HPA) axis reached a new setpoint characterized by higher circulating corticosterone. Based on these results and the involvement of the hippocampus in stress-system regulation via the HPA axis, we determined markers of HPA axis activation in acute and chronic diabetes, at 2 and 11 days of disease, respectively.

Materials and Methods: Three-months-old C57BL/6J male mice were injected with STZ (170 mg/kg ip) or vehicle.Two or 11 days after the onset of the disease the mice were sacrificed by decapitation and blood samples were taken in order to determine CORT and ACTH plasma levels. At the same time, glycemia and body, thymus and adrenal weight were measure. Brains and adrenal glands were processed for in situ hybridization and RT-PCR respectively.

Results: Two days diabetic mice show increased plasma corticosterone, while plasma ACTH is decreased, and AVP 
mRNA in the paraventricular nucleus (PVN) of the hypothalamus is elevated. In addition, 11-days diabetic mice express less hippocampal MR mRNA compared to controls. The expressions of neither GR mRNA in the hippocampus and PVN, nor CRH in the PVN were changed in diabetic mice at any time point studied. The AVP and MR mRNA alterations in parallel with decreased ACTH, but in the face of exaggerated corticosterone secretion mark a profound disturbance in HPA axis regulation, most likely including sensitization of the adrenals to ACTH.Indeed, RT-PCR revealed the increased expression of ACTH receptors MC2 and MC5 in the adrenal glands in acute and chronic diabetes.

Conclusion: The fast upregulation of ACTH receptors in the adrenal glands is a likely mechanism that allows low plasma ACTH concentrations to trigger and maintain corticosterone hypersecretion. A better understanding of these mechanisms may explain how diabetic pathophysiology causes adaptation in the CNS leading to damage and cognitive impairment.

Supported by NWO-WOTRO

\section{1}

Differences in regional brain activation response to changes in plasma glucose according to route of administration: seeking the mechanisms of appetite control in man

Y. P. Samarasinghe ${ }^{1}$, S. Lee ${ }^{2}$, F. Zelaya ${ }^{2}$, L. J. Reed ${ }^{2}$, M.-M. Teh ${ }^{1}$, P. Choudhary ${ }^{1}$, A. Pernet ${ }^{1}$, B. Wilson ${ }^{1}$, M. Brammer ${ }^{2}$, S. Williams ${ }^{2}$, S. A. Amiel ${ }^{1}$;

${ }^{1}$ Diabetes Research Unit, King's College London School of Medicine, ${ }^{2}$ Centre for Neuroimaging Sciences, King's College London, United Kingdom.

Background: Type 2 diabetes (T2DM) and obesity are lifestyle diseases, exacerbated by imbalances in matching energy intake and expenditure. Central homeostatic mechanisms maintain energy balance. We hypothesise that the cortical response to nutrient intake may also be important in regulating energy intake. Glucose is the brain's primary fuel and major biochemical disturbance in T2DM. Its neural effects when ingested, as demonstrated by our group using fMRI, reveal activation of cortical centres involved in food seeking behaviour and achieving reward. However whether this response is directly related to the rise in blood glucose per se, or to more generic processes of oral nutrient intake is not known. We have thus compared regional brain responses to oral intake with intravenous (IV) infusion of glucose to discriminate the neural correlates of glucose interoception from oral ingestion.
Methods: 4 healthy volunteers (median(range)age 22(1955)yrs) having fasted overnight ( $8 \mathrm{hrs})$, underwent a 40minute continuous fMRI scan (gradient echo EPI, 56 axial slices, $2.5 \mathrm{~mm}$ thickness, $\mathrm{TR}=10 \mathrm{~s}$ ) on 2 separate occasions. During each scan subjects either drank a $75 \mathrm{~g}$ glucose solution or had a continuous IV glucose infusion that mimicked the rate of change in plasma concentration of the glucose ingestion. Arterialised blood samples were obtained from matched studies.

Results: MR signal changes, indicating changes in neuronal activation, were identified across whole brain in response to both stimuli with a cluster level $p=0.0003$. At this cluster-level we expect $<1$ false positive cluster per brain. Major amplitude change occurred around 10(+/-5) minutes. There was no significant difference between mean glucose concentrations of oral $\mathrm{v}$ IV glucose at this time (6.0v5.7 mmol/1 $[\mathrm{p}=0.10]$ ). Regions showing similar changes in signal with oral and IV glucose were located in the orbitomedial frontal cortex (OMF), anterior cingulate cortex (ACC), right insula and the amygdala/hippocampus. Changes in signal where oral glucose only occurred were in visual cortex, parietal lobe and the uncus. Changes in signal where IV glucose alone was detected were in left insula, cerebellar vermis and the sensorimotor cortex.

Conclusion: Changes in plasma glucose concentration (or the associated insulin response), activated brain regions subserving reward (amygdalae, hippocampus), hedonic value of food (OMF) and interoception (ACC). Brain regions responding to a gastric signal only were most noted in insula cortex, which is increasingly recognised to exhibit extensive regional subspecialisation to gustatory stimuli. Activations seen with IV glucose alone, perhaps indicate responses to mismatches between expectation and changes in the body's internal environment (interception). The brains response to changes in plasma glucose occurring as a result of oral ingestion or IV infusion, revealed a complex neural response involving regions controlling ingestive behaviour, hedonic value of nutrients, interoception and reward, and not solely homeostatic control centres. These data provide a firm basis for extension of these studies to people predisposed to obesity and T2DM, seeking differences that may contribute to the development of these conditions.

Supported by the Wellcome Trust

0542

Pre-labeling: a novel approach towards measuring absolute glycogen concentration changes with NMR

F. D. Morgenthaler ${ }^{1}$, S. Laus ${ }^{2}, H$. Lei $^{3}$, R. Gruetter ${ }^{1}$; 
${ }^{1}$ EPFL, CIBM and Laboratory for Functional and Metabolic Imaging (EPFL), Lausanne, Switzerland, ${ }^{2}$ Radiology Department, University of Geneva, ${ }^{3}$ Radiology Department, University of Lausanne, Switzerland.

Background and Aims: ${ }^{13} \mathrm{C}$ Nuclear Magnetic Resonance (NMR) spectroscopy has been recently established as the only method to measure brain glycogen non invasively. However, all ${ }^{13} \mathrm{C}$ NMR studies of brain glycogen to date relied on observing the incorporation of ${ }^{13} \mathrm{C}$ label to overcome sensitivity limitations. We previously showed that derived changes in glycogen concentration were within experimental limits in excellent qualitative agreement with biochemical measurements. Given the fact that only the ${ }^{13} \mathrm{C}$-labeled part of the glycogen molecule is measured, we sought to develop a method allowing absolute concentration measurement using NMR. The rationale for this method was to achieve near steady-state enrichment using long-term ${ }^{13} \mathrm{C}$ administration, to measure this enrichment in vivo which can then be matched by subsequent glucose infusion, thereby minimizing turnover as potential confound. Materials and Methods: Male Sprague-Dawley rats $(n=6)$ were fasted overnight with free access to water and then fed ad libitum for $48 \mathrm{~h}$ with a $50 \%$ enriched Glc solution $(10 \%$ $\mathrm{w} / \mathrm{v})$ only. Then they were anesthetized using isoflurane, intubated, ventilated (for $1 \mathrm{~h} 45 \mathrm{~min}$ ), placed into the magnet (9.4T) and indirect measurement of ${ }^{13} \mathrm{C}-\mathrm{NAA}$ enrichment began. To minimize postmortem degradation, rats were sacrificed using a focused microwave fixation device, brains dissected and assayed for tissue glycogen and Glc concentrations using biochemical measurements. By labeling the Glc molecule in its first $\mathrm{C}$ position with ${ }^{13} \mathrm{C}$, we expected the enrichment of acetyl-CoA (NAA's precursor) to be half of the ${ }^{13} \mathrm{C}-\mathrm{Glc}$ enrichment. The method consisted of in vivo measurement of ${ }^{13} \mathrm{C}$ enrichment in NAA using ${ }^{1} \mathrm{H}$ spectroscopy. ${ }^{13} \mathrm{C}$ enrichment of NAA and Glc (as well as digested glycogen) was also evaluated in vitro by high field spectroscopy (14.1T, $600 \mathrm{MHz}$ ). Rats that did not drink a significant amount of ${ }^{13} \mathrm{C}$-Glc $(\mathrm{n}=1)$, resulting in ${ }^{13} \mathrm{C}$-NAA enrichment $<5 \%$, were excluded from the analysis.

Results: The biochemical measurements of brain glycogen (ave \pm SEM, 4.8 \pm 0.1 ) and brain Glc $(3.8 \pm 0.3)$ were in excellent agreement with our previous results. In vitro NMR measurements of ${ }^{13} \mathrm{C}$-labeled-NAA on brain extracts was within experimental error identical with the concomitant in vivo NMR measurements ( $\mathrm{t}$-test, $\mathrm{P}>0.05$ ). The average ${ }^{13} \mathrm{C}$-glycogen enrichment was $24 \pm 1 \%$ and NAA was approximately half of that of glycogen (Glycogen ${ }^{13} \mathrm{C}$ enrichment/NAA ${ }^{13} \mathrm{C}$-enrichment: $2.2 \pm 0.2$ ).
Conclusion: The study demonstrates that after extended ${ }^{13} \mathrm{C}$-labeled-Glc administration, glycogen is labeled, that this degree of labeling can be inferred from the in vivo enrichment of NAA. Therefore, we conclude that studies where the enrichment of the infusate nearly matches that of glycogen are feasible.

Supported by NIH R01NS42005 and JDRF 1-2002-722; supported by CIBM and Jeantet-Leenard Foundations

\section{3}

ER stress in the hypothalamus causes central leptin and insulin resistance in rodents

M.-S. Kim ${ }^{1}$, J.-C. Won ${ }^{2}$, C. Namkoong ${ }^{3}$, P.-G. Jang ${ }^{3}$, E.-H. Cho ${ }^{1}$, J.-Y. Park ${ }^{1}$, K.-U. Lee ${ }^{1}$;

${ }^{1}$ Internal Medicine, University of Ulsan College of Medicine, Seoul, ${ }^{2}$ Internal Medicine, Soonchunhyang University College of Medicine, Bucheon, ${ }^{3}$ Asan Instituite for Life Sciences, University of Ulsan College of Medicine, Seoul, Republic of Korea.

Background and Aims: Recent study has demonstrated that endoplasmic reticulum (ER) stress is increased in the liver of obese animals and causes insulin resistance by inhibiting insulin signaling pathway. In the present study, we investigated whether ER stress may also involves a central resistance to insulin and leptin, major appetite regulating hormones, in the hypothalamus.

Materials and Methods: We measured ER stress markers (GRP78, CHOP) and JNK phosphorylation in the hypothalamus of high fat diet ( $60 \%$ fat, 7 weeks) induced obese mice. ER stress in the hypothalamic neurons wasinduced by intra-cerebroventricular (ICV) or intra-hypothalamic (arcuate nucleus) administration of thapsigargin or tunicamycin. We monitored food intake and body weight following ICV administration of insulin and leptin with or without administration of ER stress inducer.

Results: In the hypothalamus of high fat diet-fed mice, ER stress marker (GRP78, CHOP) and phospho-JNK expression was increased compared to low fat diet-fed controls. ICV administration of thapsigargin and tunicamycin prior to ICV leptin and insulin completely inhibited leptin and insulin-induced anorexia and weight loss. Bilateral injection of thapsigargin or tunicamycin into the hypothalamic arcuate nucleus also caused a similar inhibition on leptin and insulin-induced anorexia, suggesting that these effects are mediated via the hypothalamus.

Conclusion: The levels of ER stress markers are increased in the hypothalamus of obese animals. Induction of ER stress in the hypothalamus inhibits the effects of leptin and insulin and on food intake and body weight. These data may suggest 
that increased ER stress in the hypothalamus of obese animals may cause central leptin and insulin resistance.

This work is supported by grants from Korea Research Foundation and Korean Ministry of Health \& Welfare (03PJ1-PG1-CH05-0005, A05-0513).

0544

Alzheimer-like tau phosphorylation induced by dysregulated insulin signal transduction and impaired glucose metabolism in type 1 diabetes mellitus

Y. Yan, H. Shu-hong, Z. Mu-xun; Department of Endocrinology, Tongji Hospital,Tonji Medical College, Wuhan, China.

Background and Aims: Abnormal hyperphosphorylation of tau plays a critical role in the pathogenesis of Alzheimer disease $(\mathrm{AD})$ and diabetes is a known risk factor of $\mathrm{AD}$. We therefore studied the roles of dysregulation of insulin signaling and glucose metabolism in hyperphosphorylation of tau in type 1 diabetic rats.

Materials and Methods: Wistar rats were randomized into 3 groups, pancreatectomy (PX), type 1 diabetes mellitus (T1DM) and control (CTL) group. The model of PX group characterized by low plasma insulin was made by partial (90\%) pancreatectomy, and the model of T1DM group was made by single injection of STZ. The plasma insulin level was measured by RIA method, and the plasma glucose by glucose-oxidase method. Total tau level, the phosphorylation level of tau at individual phosphorylation sites (Ser199, Thr212, Ser214, Ser396 and Ser422) and glucose transport 3 (GLUT3) on neuronal membrane fractions were analyzed by Western blots. The activity of glycogen synthase kinase $3 \beta$ (GSK-3 $\beta$ ), a key component of insulin signal transudation pathway and a known tau kinase, in the hippocampus of rats was determined by using $\gamma^{32}$-P-ATP and the specific peptide substrate.

Results: We found that the plasma insul level was remarkedly decreased in T1DM and PX group as compared to CTL group, and plasma glucose level in T1DM was increased to $\sim 3$ times of the CTL, but that in PX group was only marginally increased. Neither pancreatectomy nor STZ injection changed the total level of tau protein in the hippocami of rats. However, Tau was found to be hyperphosphorylated at several AD-related phosphorylation sites (Ser199,Thr212, Ser214, Ser396 and Ser422) in T1DM group. In PX group, Ser199,Thr212 and Ser396 of tau protein were hyperphosphorylated, but no significant changes at Ser214 and Ser422. The activity of GSK-3 $\beta$ was found to be increased dramatically in the hippocampi of rats in both PX and T1DM groups. The level of GLUT3 was also found to be decreased in both PX and T1DM groups, with a more dramatic decrease in T1DM group.

Conclusion: These findings suggest that impaired insulin signaling could facilitate hyperphosphorylation of tau via both upregulation of GSK-3 $\beta$ and decreased intracellular glucose metabolism.

\section{PS 33}

\section{Mitochondria}

\section{5}

Decreased mitochondrial respiration impairs insulin action in cultured human skeletal muscle cells

A. E. Brown ${ }^{1}$, M. Elstner ${ }^{2}$, S. J. Yeaman ${ }^{3}$,

D. M. Turnbull ${ }^{2}$, M. Walker ${ }^{1}$;

${ }^{1}$ Clinical Medical Sciences, ${ }^{2}$ Neurology, Neurobiology and Psychology, ${ }^{3}$ Cell and Molecular Biosciences, Newcastle University, Newcastle upon Tyne, United Kingdom.

Background and Aims: Skeletal muscle insulin resistance is a characteristic feature of type 2 diabetes. Insulin resistant type 2 diabetic patients have been shown to have impaired skeletal muscle mitochondrial respiratory function. A key question is whether the impaired mitochondrial function contributes directly to the decreased insulin action. Materials and Methods: Human skeletal muscle cell cultures were used to develop a model of impaired cellular respiratory function to examine the effects upon insulin action. Human skeletal muscle cells were incubated with the mitochondrial inhibitor sodium azide, an inhibitor of complex IV of the mitochondrial respiratory chain. Cellular respiration was measured polarographically. By titrating the dose of the mitochondrial inhibitor, we aimed to mimic the decrease in skeletal muscle mitochondrial oxidation observed in the insulin resistant patients. Skeletal muscle cell cultures from 5 healthy subjects were incubated with increasing concentrations of sodium azide for $24 \mathrm{~h}$ before examining the effects of this inhibitor on respiration and glucose uptake.

Results: Incubation of human skeletal muscle cells with $50 \mu \mathrm{M}$ and $75 \mu \mathrm{M}$ sodium azide resulted in a $48 \pm 8.3 \%$ and $56 \pm 3.2 \%$ decrease in respiration compared to untreated cells. After incubation with $50 \mu \mathrm{M}$ and $75 \mu \mathrm{M}$ sodium azide, basal rates of glucose uptake were increased 2.1 and 2.7 fold, (both $\mathrm{p}<0.0001$ ) respectively, compared to the untreated cells. In response to $100 \mathrm{nM}$ insulin, glucose uptake was impaired in cells treated with sodium azide $(\mathrm{n}=6$ for each culture). In untreated cells, the fold increase over basal in response to insulin was $1.49 \pm 0.05$ (mean \pm SEM: vs basal $\mathrm{p}=0.0003$ ). This was significantly decreased in cells treated with $50 \mu \mathrm{M}$ azide $(1.14 \pm 0.04$ fold increase: vs 
untreated $\mathrm{p}<0.0001)$ and $75 \mu \mathrm{M}$ azide $(1.25 \pm 0.02$ fold increase: vs untreated $\mathrm{p}=0.0001$ ). To investigate the underlying cause of the increased basal glucose uptake, Western blotting was performed using a phospho-AMPK $\left(\mathrm{Thr}^{172}\right.$ ) antibody. It was found that phosphorylation, and thus activation, of AMPK was substantially increased under conditions of impaired cellular respiration.

Conclusion: In conclusion, suppression of cellular respiration to levels comparable to those reported in insulin resistant subjects results in decreased responsiveness of glucose uptake to insulin in cultured human muscle cells. This decreased insulin action was accompanied by increased rates of basal glucose uptake and increased phosphorylation of AMPK.

Supported by Diabetes UK

\section{6}

Muscle fatty acid oxidative capacity and PGC-1 $\alpha$ expression are upregulated in mouse models of insulin resistance and type 2 diabetes

N. Turner ${ }^{1}$, T. So ${ }^{2}$, C. R. Bruce ${ }^{1}$, M. S. Rolph ${ }^{2}$, G. J. Cooney ${ }^{1}$;

${ }^{1}$ Diabetes and Obesity Program, ${ }^{2}$ Arthritis and Inflammation Program, Garvan Institute of Medical Research, Darlinghurst, Australia.

Background and Aims: Decreased muscle mitochondrial number as well as a reduced capacity for fatty acid oxidation by muscle mitochondria, have been suggested as key factors in the development of obesity, insulin resistance and type 2 diabetes. It is unclear however, whether these defects are due to genetic factors or are acquired (e.g. through dietary lipid excess or physical inactivity). In the current study we have investigated parameters of mitochondrial function and lipid metabolism in skeletal muscle of high-fat fed $\mathrm{C} 57 \mathrm{Bl} / 6 \mathrm{~J}$ mice and compared the results to those obtained from a genetic model of type 2 diabetes, the $d b / d b$ mouse.

Material and Methods: C57B1/6J mice were fed a high-fat diet (Fat $45 \%$, Protein 20\%, CHO 35\%) for either $5 \mathrm{wk}$ or $20 \mathrm{wk}$ and an ipGTT $(2 \mathrm{~g} / \mathrm{kg})$ was conducted to confirm glucose intolerance. The activity of key enzymes involved in fat metabolism (citrate synthase (CS), $\beta$-hydroxyacylCoA dehydrogenase ( $\beta H A D)$ and medium chain acyl-CoA dehydrogenase (MCAD)), as well as the ${ }^{14} \mathrm{C}$-palmitate oxidation rate were assessed in skeletal muscle homogenates. Immunoblotting was used to determine the expression levels of proteins involved in mitochondrial biogenesis and fatty acid metabolism, including PGC- $1 \alpha$, UCP3 and subunits of mitochondrial respiratory chain complexes.
Results: There was a highly significant $(P<0.001)$ increase in epididymal fat mass as a result of the high-fat diet after both $5 \mathrm{wk}(150 \%)$ and $20 \mathrm{wk}(350 \%)$, with a significant $(P<0.05)$ impairment in glucose tolerance also observed at both timepoints. A significant increase $(P<0.01)$ in the activity of CS (25-60\%), BHAD (30-70\%) and MCAD $(60-120 \%)$ was observed in the fat-fed animals compared with chow-fed controls. In muscle from $d b / d b$ mice there was also a significant $(P<0.01)$ elevation in the activity of CS (25\%), $\beta$ HAD (25\%) and MCAD (55\%) compared with lean controls $(d b /+)$. Fat-feeding substantially altered the expression of proteins involved in mitochondrial fatty acid metabolism with a significant $(P<0.05)$ increase observed for PGC-1 $\alpha$ (100-250\%), UCP3 (80-240\%), Complex III (65-110\%) and Complex IV (50-140\%). These results were paralleled by those observed in $d b / d b$ mice, where there was a significant $(P<0.05)$ elevation in the protein expression of PGC-1 $\alpha$ (45\%), UCP3 (120\%) and Complex III (35\%) compared with $d b /+$ controls. To gain a direct measure of fatty acid oxidative capacity we assessed palmitate oxidation rate in tissue homogenates from a separate group of fat-fed animals (20 wk), and found a marked improvement in fatty acid oxidative capacity compared with chow-fed controls $(3.60 \pm 0.14$ vs $2.34 \pm$ $0.16 \mu$ moles palmitate $/ \mathrm{g} / \mathrm{hr}, P<0.001)$.

Conclusions: In summary, our results show that in both high-fat fed mice and $d b / d b$ mice there is an improved fatty acid oxidative capacity and an upregulated expression of proteins related to mitochondrial function. We therefore conclude that defects in muscle mitochondrial function are not responsible for the obesity, insulin resistance and type 2 diabetes observed in these mouse models.

Supported by National Health and Medical Research Council of Australia

\section{7}

Expression of mitochondrial genes related to energy homoeostasis are dysregulated in obesity and type 2 diabetes

P. G. McTernan ${ }^{1}$, F. J. Hardo ${ }^{1}$, A. R. Baker ${ }^{1}$, K. C. McGee ${ }^{1}$, G. Tripathi ${ }^{1}$, S. J. Creely ${ }^{1}$,

N. F. da Silva ${ }^{1}$, S. Kumar ${ }^{1}$, J. C. Clapham ${ }^{2}$;

${ }^{1}$ Department of Clinical Sciences, University of Warwick, Coventry, United Kingdom, ${ }^{2}$ AstraZeneca R\&D, Molndal, Sweden.

Background and Aims: The integrity of mitochondria in adipose tissue in obesity and type 2 diabetes (T2DM) has recently been investigated in animal models but to date limited data is available in humans. The primary defect(s) causing bioenergetic dysfunction may reside in a non- 
bioenergetic pathways such as signaling between mitochondria and nucleus or in overall mitochondrial biogenesis or degradation pathways impacted by inflammation. Therefore we investigated a number of mitochondrial genes including PPAR $\gamma$ co-activator 1 (PGCl $\alpha$, mitochondrial biogenensis), UCP-2 (fatty acid oxidation), COX4 (flux-generating reactions in the electron transfer chain), fatty acid synthase (FAS, catalysing the synthesis of long chain saturated fatty acids) and SIRT3 (implicated in inhibition of PGC1 $\alpha$ ).

Materials and Methods: Utilising real time PCR and Western blot for SIRT3, we examined the effect of obesity and T2DM status, as well as the relative influence of adipose tissue (AT) depots (abdominal subcutaneous (Abd $\mathrm{Sc}$ ) versus omental $(\mathrm{Om}) \mathrm{AT})$. For this study, we collected a lean non-diabetic cohort (BMI: $22.2 \pm\left(\right.$ Mean \pm SD) $2.1 \mathrm{~kg} / \mathrm{m}^{2}$, age: $39.8 \pm($ Mean $\pm S D) 8.7$ yrs, $n=12)$, obese non-diabetic cohort (BMI:30.1 $\pm 2.81 \mathrm{~kg} / \mathrm{m}^{2}$, age: $47.3 \pm 11.5 \mathrm{yrs}, \mathrm{n}=9$ ) and T2DM cohort (BMI: $59.9 \pm 7.5 \mathrm{~kg} / \mathrm{m}^{2}$, age: $37.15 \pm 7.8 \mathrm{yrs}$, $\mathrm{n}=7$ ) of human adipose tissue (AT).

Results: Our findings determined that in Abd Sc AT increasing adiposity significantly reduced FAS $(\mathrm{p}=0.013)$, COX4 $(p=0.0003)$ and UCP2 $(p<0.001)$ mRNA, whilst $\mathrm{PGC} 1 \alpha(\mathrm{p}=0.003)$ mRNA was increased. In addition, in Abd Sc AT from T2DM subjects there was a reduction in FAS, COX4, UCP-2 mRNA ( $<<0.05)$; whilst PGC1 $\alpha$ mRNA $(p=0.0004)$ was again increased. In Om fat, FAS, PGC1 $\alpha$, COX4 and UCP2 mRNA were all reduced $(\mathrm{p}<0.001)$. Depot specific differences were also observed. SIRT 3 protein expression highlighted that there was no significant effect with adiposity in either depot, with a reduction in T2DM Abd Sc AT protein expression (Abd Sc Lean vs T2DM, p=0.002). Conclusion: In summary, these studies highlight that in Abd Sc AT, whilst PGC1 $\alpha$ mRNA (related to biogenesis of mitochondria) continues to increase in obesity, the genes related to energy metabolism appears to reduce with a similar pattern observed in Abd Sc AT from T2DM subjects. In Om AT, PGC1 $\alpha$ mRNA also reduces along with genes related to mitochondrial metabolism. Finally, SIRT 3 implicated in inhibiting PGC1 $\alpha$ in rodent models is also reduced in AT from T2DM subjects. In conclusion, these studies suggest a role for mitochondria in human adipose tissue appears dysregulated in obesity and diabetes. The causes of mitochondrial dysregulation are unclear, however candidate factors may include oxidant stress as well as lipotoxicity. Supported by AstraZeneca

\section{8}

TNF $\alpha$ impairs mitochondrial metabolism in 3T3-L1 adipocytes

S. Dubois ${ }^{1}$, N. Konstantapoulos ${ }^{1}$, C. Swinton ${ }^{1}$,

T. Connor ${ }^{1}$, K. Walder ${ }^{1,2}$, G. Collier ${ }^{1,2}$;
${ }^{1}$ Metabolic Research Unit, Deakin University, Waurn ponds, Australia, ${ }^{2}$ ChemGenex Pharmaceuticals, Geelong, Australia.

Background and Aims: The adipose tissue is now recognized as an endocrine/paracrine organ which can influence whole body metabolism not only through the release of non-esterified free fatty acids but also via many adipokines such as tumor necrosis factor $\alpha(\mathrm{TNF} \alpha)$. TNF $\alpha$ is a proinflammatory cytokine, which has been implicated as a contributing cause of insulin resistance. Although the effects of TNF $\alpha$ are well known, the mechanisms by which $\mathrm{TNF} \alpha$ impairs insulin action are not well understood. A number of recent studies support the concept that insulin resistance is linked to mitochondrial dysfunction. The aim of this study was to investigate whether TNF $\alpha$ may affect pathways involved in mitochondrial biogenesis and fatty acid oxidation in 3T3-L1 adipocytes.

Materials and Methods: Differentiated 3T3-L1 adipocytes were treated with TNF $\alpha(3 \mathrm{ng} / \mathrm{ml}, 72 \mathrm{hrs})$ and in the final $24 \mathrm{hrs}$ of that incubation, cells were also treated with troglitazone (TGZ; $10 \mu \mathrm{M}$ ), and aspirin (ASA; $5 \mathrm{mM}$ ). Insulin sensitivity was determined by 2-deoxyglucose uptake and phosphorylation of key proteins in insulin signaling pathway. Following treatments, RNA was extracted and hybridised to custom-made cDNA microarrays containing $\sim 12,000$ elements. Real-Time PCR of mitochondrial-specific target genes was also performed to confirm microarray data. Results: Insulin-stimulated glucose uptake was inhibited by $30-40 \%(p \leq 0.0002$ compared to vehicle, $n \geq 10)$ when 3T3$\mathrm{L} 1$ adipocytes were exposed to TNF $\alpha$ for $72 \mathrm{hrs}$. Treatment with TGZ and/or ASA alleviated TNF $\alpha$-induced inhibition of insulin-stimulated glucose uptake $(\mathrm{p} \leq 0.007$ compared to TNF $\alpha$ alone, $n \geq 6$ ). Microarray analysis of 3T3-L1 adipocytes treated with $\mathrm{TNF} \alpha \pm \mathrm{TGZ}$ and ASA ( $\mathrm{n}=20$ replicates per treatment) identified significant changes in the expression of several genes encoding key mitochondrial proteins such as citrate synthase (CS), CPT-1, PGC1 $\alpha$. CPT1, PGC1 $\alpha$ and CS gene expression were all decreased following TNF $\alpha$ treatment and restored to basal levels with TGZ/ASA treatment. Real time PCR analysis confirmed the microarray results and showed that PGC1 $\alpha$ mRNA levels were markedly decreased following TNF $\alpha$ treatment $\left(\mathrm{p}<10^{-5}\right)$. Treatment with TGZ/ ASA was reversed the suppressed PGC1 $\alpha$ gene expression following TNF $\alpha$ treatment $\left(\mathrm{p}<10^{-4}\right)$.

Conclusion: Our results showed that TNF $\alpha$ not only impairs insulin-stimulated glucose uptake but also genes encoding key mitochondrial proteins, particularly those involved in the regulation of fatty acid oxidation (PGC1 $\alpha$, CPT1, CS, PPAR $\alpha$ ) but not genes involved in mitochondrial biogenesis (mtTFA, NRF1, PPAR $\gamma$ ). 
Reduced expression of nuclear-encoded genes involved in mitochondrial oxidative metabolism in skeletal muscle of obese women with polycystic ovary syndrome (PCOS)

V. Skov ${ }^{1}$, D. Glintborg ${ }^{2}$, T. A. Kruse ${ }^{1}$, S. Knudsen ${ }^{3}$, T. Jensen ${ }^{3}$, Q. Tan ${ }^{1,4}$, H. Beck-Nielsen ${ }^{2}$, K. Højlund ${ }^{2}$;

${ }^{1}$ Department of Biochemistry, Pharmacology and Genetics,

${ }^{2}$ Department of Endocrinology, Odense University Hospital, Odense, Denmark, ${ }^{3}$ R\&D Department, Medical Prognosis Institute Aps, Hørsholm, Denmark, ${ }^{4}$ Institute of Public Health, University of Southern Denmark, Odense, Denmark.

Background and Aims: Recently, abnormalities in mitochondrial oxidative phosphorylation (OXPHOS) have been implicated in the pathogenesis of skeletal muscle insulin resistance in type 2 diabetes. In the present study, we hypothesized that decreased expression of OXPHOS genes could be of similar importance for insulin resistance in the polycystic ovary syndrome (PCOS).

Materials and Methods: Using the HG-U133 Plus 2.0 expression array from Affymetrix, we analyzed gene expression in skeletal muscle from obese women with $\operatorname{PCOS}(\mathrm{n}=16)$ and age- and body mass index-matched control women $(n=13)$ metabolically characterized by euglycemic-hyperinsulinemic clamp and indirect calorimetry. First, Welch's two sample t-test was applied to examine the significance of OXPHOS genes separately. The overall significance of the OXPHOS genes was assessed by calculating a sum statistic and conducting a permutation test. Subsequently, we performed biological pathway analysis using Gene Set Enrichment Analysis (GSEA) and Gene Microarray Pathway Profiler (GenMAPP).

Results: Women with PCOS were characterized by fasting hyperinsulinemia and impaired insulin-stimulated glucose disposal - caused by reduced glucose oxidation and storage as well as impaired suppression of lipid oxidation (all $\mathrm{P}<0.01$ ). These abnormalities were associated with a significant decrease in the selected subset of nuclear-encoded genes involved in mitochondrial OXPHOS ( $\mathrm{P}=0.025)$. GSEA and GenMAPP both revealed the same set of genes involved in OXPHOS, which was also the most downregulated biological pathway $(\mathrm{P}<0.01)$. These findings are currently being validated by quantitative real-time PCR and immunoblot analysis.

Conclusion: Our results, for the first time, provide evidence for an association between insulin resistance and impaired mitochondrial oxidative metabolism in skeletal muscle in women with PCOS. This may contribute to the increased risk of type 2 diabetes observed in these women. This study was supported by grants from the Novo Nordisk Foundation
The autonomic nervous system could influence the skeletal muscle metabolic activity by regulating the expression of PGC-1alpha and Mfn-2

G. G. Gastaldi ${ }^{1}$, A. P. Russell ${ }^{2}$, A. Golay ${ }^{3}$,

E. Bobbioni-Harsch ${ }^{3}$;

${ }^{1}$ Internal medicine, Geneva University Hospital, Geneva, ${ }^{2}$ SUVA Care, Clinique Romande de Réadaptation, Sion, ${ }^{3}$ Division of Therapeutic Education for Chronic Diseases, Geneva University Hospital, Switzerland.

Background and Aims: Imbalanced autonomic nervous system (ANS), reduced glucose uptake as well as impaired mitochondrial function at the level of skeletal muscle are part of the metabolic alterations observed in obesity. Peroxisome proliferator-activated receptor gamma coactivator (PGC-1alpha) is a nuclear transcriptional co-activator that regulates mitochondrial biogenesis and adaptive thermogenesis. Mitofusin-2 (Mfn-2) is an other key factor involved in the formation and maintenance of the mitochondrial network. Decreased expression of PCG-1alpha and $\mathrm{Mfn} 2$ has been suggested to participate in the pathogenesis of insulin resistance in obese and/or diabetic patients. In turn, insulin resistance could contribute to the development of ANS dysfunction. We therefore designed a study aimed at investigating possible links between the mRNA expression of PGC-1alpha and Mfn2, insulin resistance in muscle and the cardiac autonomic balance.

Materials and Methods: 23 morbidly obese women (BMI: $46 \pm 4 \mathrm{~kg} / \mathrm{m}^{2}$, age: $37 \pm 9$ y) exempt from diabetes were investigated. Cardiac autonomic balance was investigated by power spectral analysis of Heart Rate Variability (HRV) during a 30-minute period of continuous ECG record; insulin sensitivity was assessed by an euglycaemichyperinsulinaemic clamp; RT-PCR was used to measure mRNA expression of PGC-1alpha and Mfn-2 in skeletal muscle biopsies.

Results: Glucose uptake was $3,5 \pm 0.4 \mathrm{mg} / \mathrm{kg} \mathrm{LBM} / \mathrm{min}$, indicating a reduced insulin sensitivity. The HRV parameters were: Low Frequency $=53 \pm 4 \mathrm{NU}$, High frequen$c y=35 \pm 4 \mathrm{NU}$, Low-to-High ratio $=2.0 \pm 0.3$, suggesting a sympathetic prevalence at the heart level. PGC-1alpha value was $0.51 \mathrm{AU}$ and Mfn-2 $0.37 \mathrm{AU}$. A significant, positive correlation linked the mRNA expression of PGC1alpha and the cardiac autonomic parameters mostly influenced by the sympathetic activity: Low Frequency $(p=0.003)$, Low-to-High Frequency ratio $(p=0.001)$. Similar results were found with the mRNA expression of Mfn-2. A strong positive relationship linked the mRNA expression of PGC-1alpha and Mfn-2 $(\mathrm{p}<0.0001)$. However, glucose uptake did not show any significant relationship with the 
cardiac autonomic parameters, nor with the expression of PGC-1alpha and Mfn-2.

Conclusion: In morbidly obese subjects, an elevated mRNA expression of PGC-1alpha and Mfn-2 in skeletal muscle corresponds to a high sympathetic tone at the cardiac level. The knowledge that genes participating in carbohydrate and lipid metabolism are regulated by epinephrine in human skeletal muscle support the hypothesis that ANS activity could be concomitantly and in parallel regulated in the cardiac and muscle district. Furthermore, the ANS could influence the skeletal muscle metabolic activity by regulating the expression of PGC-1alpha and Mfn-2.

Supported by Fond national, Fondation pour la promotion de la recherche sur la nutrition en suisse, Fondation Barbour, Fondation Ousseimi

\section{PS 34}

\section{Effects of glitazones on insulin sensitivity}

\section{1}

Long-term pioglitazone treatment prevent age-related impairment of tissues insulin sensitivity and adiponectin secretion in sucrose fed SHR rats

H. Malinska ${ }^{1}$, M. Cahova ${ }^{1}$, L. Kazdova ${ }^{1}$, M. Pravenec ${ }^{2}$;

${ }^{1}$ Department of Metabolism and Diabetes, Institute for Clinical and Experimental Medicine, Prague, ${ }^{2}$ Institute of Physiology, Czech Academy of Sciences, Prague, Czech Republic.

Background and Aims: Thiazolidinediones (TZDs) are peroxisome proliferator activated receptor-gamma (PPAR- $\gamma$ ) agonists used for treatment of type 2 diabetes. TZDs improve glycemic control by reducing insulin resistance in target tissues, however, mechanisms of their action are not fully understood. The aim of the current study was to analyze long-term effects of pioglitazone on insulin and adiponectin secretion, muscle lipid accumulation a peripheral tissue insulin sensitivity in an animal model of the metabolic syndrome, the spontaneously hypertensive rats.

Materials and Methods: Groups of adult male rats were fed a high carbohydrate diet ( $70 \mathrm{cal} \%$ sucrose) from the age of 4 to 8 months without or with pioglitazone $(300 \mathrm{mg} / \mathrm{kg})$. The insulin sensitivity of adipose tissue and skeletal muscle tissues was measured by incorporation of ${ }^{14} \mathrm{C}-\mathrm{U}$ glucose into lipids and glycogen, respectively. Serum insulin concentrations were determined using Mercodia Rat Insulin ELISA kit (Mercodia AB, Uppsala, Sweden) and adiponectin concentrations by using Rat Adiponektin ELISA kit (B-Bridge International, CA, USA).

Results: Body weight gains were similar in control and experimental groups. Pioglitazone treatment markedly reduced serum concentrations of triglycerides $(0.73 \pm 0.05$ vs $0.52 \pm 0.01 \mathrm{mmol} / 1, \mathrm{P}<0.005)$, fasting serum glucose $(5.1 \pm 0.2$ vs $4.6 \pm 0.1 \mathrm{mmol} / \mathrm{l}, \mathrm{P}<0.05)$ and prevented agerelated development of hyperinsulinemia $(0.454 \pm 0.040$ vs $0.135 \pm 0.018, \mathrm{nmol} / 1, \mathrm{P}<0.0005)$. In addition, pioglitazone treated rats exhibited significantly increased concentrations of serum adiponectin $(5.8 \pm 0.6$ vs $8.9 \pm 0.1 \mu \mathrm{g} / \mathrm{ml}, \mathrm{P}<0.005)$. Compared to controls, pioglitazone treatment was associated with redistribution of fat stores, with significantly increased epididymal fat pad weight $(1.41 \pm 0.057$ vs $1.65 \pm 0.076 \mathrm{~g}, \mathrm{P}<0.05)$ and muscle triglycerides $(2.25 \pm$ 0.12 vs $3.83 \pm 0.33 \mu \mathrm{mol} / \mathrm{g}, \mathrm{P}<0.005)$ and with a parallel amelioration of hepatic steatosis $(7.75 \pm 0.31$ vs $6.21 \pm$ $0.16 \mu \mathrm{mol} / \mathrm{g}, \mathrm{P}<0.005)$. Remodeled adipose tissue contained higher percentage of protein $(1.17 \pm 0.03$ vs $1.48 \pm 0.06 \%, \mathrm{P}<0.005$ ) and was metabolically more active, exhibiting significantly increased basal and adrenalinestimulated lipolysis $(\mathrm{P}<0.005)$ and elevated sensitivity to insulin action as estimated by insulin stimulated ${ }^{14} \mathrm{C}$ glucose incorporation into lipids $(967 \pm 74$ vs $679 \pm 63 \mathrm{nmol}$ glucose $/ \mathrm{g} / 2 \mathrm{~h}, \mathrm{P}<0.01)$. Surprisingly, pioglitazone treated rats exhibited increased sensitivity of muscle tissue to insulin stimulated glucose incorporation into glycogen (774 \pm 58 vs $1288 \pm 63$ nmol glucose/g/ $2 \mathrm{~h}, \mathrm{P}<0.0001$, $\mathrm{P}<0.005)$ despite increased muscle triglyceride accumulation. Conclusion: Our results provide evidence for long-term beneficial therapeutical effects of pioglitazone due to its effects on adipose tissue remodeling and on modulation of adiponectin secretion. Amelioration of insulin resistance in skeletal muscles was independent on reduction of muscle triglyceride storage. We conclude that pioglitazone due to lower insulin secretory demand might preserve beta-cell function and slow down age-related progression of insulin resistance.

Study was supported by grant NR/7888-3 and NR/8495 from IGA MH CR

0552

First-line therapy with fixed-dose combination of rosiglitazone plus glimepiride improves insulin sensitivity and reduces cardiovascular risk markers in patients with type 2 diabetes

D. Seidel ${ }^{1}$, T. Segiet ${ }^{2}$, C. Ferreira-Cornwell ${ }^{3}$, J. B. Krebs ${ }^{3}$, H. S. $\mathrm{Chou}^{3}$, B. J. Goldstein ${ }^{4}$;

${ }^{1}$ GlaxoSmithKline, Munich, Germany, ${ }^{2}$ Diabetes Center Speyer, Germany, ${ }^{3}$ GlaxoSmithKline, King of Prussia, United States, ${ }^{4}$ Division of Endocrinology, Diabetes and Metabolic Disease, Jefferson Medical College of Thomas Jefferson University, Philadelphia, United States. 
Background and Aims: The long-term complications observed in patients with type 2 diabetes (T2D) are caused by a number of conditions, including insulin resistance and vascular inflammation, as well as poor glycaemic control. It has been suggested that early and aggressive treatment with an effective antihyperglycaemic therapy that also has beneficial effects on insulin resistance and vascular inflammation may have long-term benefits. The aim of this study was to evaluate the effect of a fixed-dose combination (FDC) of rosiglitazone (RSG) and glimepiride (GLIM), in comparison with RSG and GLIM monotherapies, on insulin resistance, $\beta$-cell function and biomarkers of atherosclerotic cardiovascular risk in drug-naive individuals with T2D.

Materials and Methods: Patients were globally randomized to receive double-blind treatment with either GLIM monotherapy [1-4 mg once daily (OD)], RSG monotherapy (4-8 mg OD), RSG/GLIM FDC A (4 mg/1-4 mg OD) or RSG/GLIM FDC B (4-8 mg/1-4 mg OD) for 28 weeks. Treatment was started on the lowest dose and uptitrated at 4-week intervals to achieve a mean daily glucose level of $<6.1 \mathrm{mmol} / \mathrm{l}$. Insulin sensitivity [as estimated by homeostasis model assessment (HOMA)-S], $\beta$-cell function (as estimated by HOMA-B) and the levels of cardiovascular biomarkers were determined at the end of the study.

Results: A total of 901 patients were involved in the study. Increased adiponectin levels correlated with improvements in insulin sensitivity (HOMA-S) with both FDC regimens and with RSG monotherapy but not with GLIM monotherapy. Treatment with either FDC regimen resulted in greater increases in $\beta$-cell function (HOMA-B) than did either GLIM or RSG monotherapy. There were greater reductions in the level of $\mathrm{C}$-reactive protein (CRP) and the activity of plasminogen activator inhibitor-1 (PAI-1) following treatment with either FDC or with RSG compared with GLIM monotherapy. There was a smaller increase in LDL cholesterol (LDL-C) in both FDC treatment groups compared with RSG monotherapy and the level of HDL cholesterol (HDL-C) was increased following treatment with either FDC regimen or with RSG monotherapy.

\begin{tabular}{lllll}
\hline$\% \Delta$ from & GLIM & RSG & FDC A & FDC B \\
Baseline & $\mathrm{n}=221$ & $\mathrm{n}=227$ & $\mathrm{n}=221$ & $\mathrm{n}=214$ \\
Adiponectin & -5.3 & 128.5 & $65.7^{*, \dagger}$ & $116.46^{*}$ \\
HOMA-S & -3.2 & 36.3 & $21.9^{*, *}$ & $23.0^{*, \S}$ \\
HOMA-B & 58.7 & 46.1 & $73.4^{\circ, *}$ & $105.8^{*, \dagger}$ \\
CRP & -7.9 & -39.8 & $-43.6^{*, \dagger}$ & $-50.7^{*, \dagger}$ \\
PAI-1 & -4.5 & -22.5 & -20.1 & $-19.1^{¥}$ \\
LDL-C & -2.1 & 9.4 & $2.6^{\ddagger}$, & $4.2^{¥}$ \\
HDL-C & -1.2 & 5.2 & $3.8^{¥}$ & 2.8 \\
\hline
\end{tabular}

${ }^{*} \mathrm{p}<0.0001$ vs. GLIM; ${ }^{\dagger} \mathrm{p}<0.0001$ vs. RSG; ${ }^{\dagger} \mathrm{p}<0.01$ vs. RSG; ${ }^{\S} \mathrm{p}<0.05$ vs. RSG; ${ }^{\ddagger} \mathrm{p}<0.05$ vs.GLIM; ${ }^{\circ} \mathrm{p}<0.001$ vs. GLIM;
Conclusion: These results suggest that early aggressive treatment with RSG/GLIM FDC may reduce vascular inflammation and enhance insulin sensitivity in drug-naive patients with T2D.

0553

Effects of pioglitazone on glucose metabolism and blood pressure in type 2 diabetic patients in relation to physical activity - The IRIS III Study

C. Hohberg ${ }^{1}$, G. Lübben ${ }^{2}$, T. Forst ${ }^{1,3}$, A. Pfützner ${ }^{1}$, M. M. Weber ${ }^{3}$, E. Karagiannis ${ }^{2}$;

${ }^{1}$ Institute for Clinical Research and Development, Mainz, ${ }^{2}$ TAKEDA Pharma, Aachen, ${ }^{3}$ Endocrinology, University Mainz, Germany.

Background and Aims: Improvement of insulin sensitivity by physical activation or pharmaceutical intervention with PPAR $\gamma$-agonists in type 2 diabetic patients was shown to improve glucose metabolism and to reduce several cardiovascular risk factors. There is only little information available about the interactions of physical activity and PPAR $\gamma$ stimulation on insulin resistance, glucose metabolism, and blood pressure in type 2 diabetic patients. The aim of our investigation was to explore the effect of pioglitazone treatment in type 2 diabetic patients according to different levels of physical activity.

Materials and Methods: One-thousand-nine-hundred-thirty patients with type 2 diabetes (1020 male, 910 female; age $63.1 \pm 10.4$ years; duration of diabetes $6.1 \pm 5.0$ years; mean \pm SD) were included in this observational trial and received treatment with $45 \mathrm{mg}$ Pioglitazone. At baseline and after twenty weeks of treatment with Pioglitazone, fasting glucose, HbA1c, systolic and diastolic blood pressure, and the body mass index were documented. According to the frequency and extend of their physical activity, the patients were classified in three different groups with no physical activity, occasional physical activity and frequent physical activity.

Results: As shown in table 1, patients reporting occasional or frequent physical activity were presented with significantly lower fasting glucose, HbAlc, BMI, and blood pressure measurements at baseline compared with type 2 diabetic patients without physical activity. Despite the absolute reduction in fasting glucose, HbAlc and diastolic blood pressure was somewhat lower in the patient groups with occasional or frequent physical activity compared with those without physical activity $(\mathrm{p}<0.05$, respectively), treatment with pioglitazone resulted in a significant improvement in fasting glucose, HbAlc, systolic and diastolic blood pressure in all three patient groups. No 


\begin{tabular}{lccccccccc}
\hline Physical Activity & Without & Without & Without & Occasional & Occasional & Occasional & Frequent & Frequent & Frequent \\
\hline $\mathrm{n}=$ & 478 & 478 & 478 & 1136 & 1136 & 1136 & 316 & 316 & 316 \\
& Baseline & 20 weeks & delta & Baseline & 20 weeks & delta & Baseline & 20 weeks & delta \\
Fasting Glucose (mmol/L) & $9.5 \pm 2.6$ & $7.9 \pm 2.5 \$$ & $-1.6 \pm 2.9$ & $9.1 \pm 2.6 \S$ & $8.8 \pm 2.3 \$$ & $-1.3 \pm 2.6$ & $9.1 \pm 2.6 \S$ & $8.0 \pm 2.5 \$$ & $-1.0 \pm 2.8 \S$ \\
HbA1c (\%) & $8.0 \pm 1.4$ & $7.1 \pm 1.3 \$$ & $-0.9 \pm 1.3$ & $7.7 \pm 1.3 \S$ & $7.0 \pm 1.2 \$$ & $-0.7 \pm 1.2$ & $7.8 \pm 1.2 \S$ & $7.0 \pm 1.1 \$$ & $-0.7 \pm 1.1$ \\
$\mathrm{RR}$ syst. (mmHg) & $142 \pm 16$ & $137 \pm 14 \$$ & $-4.45 \pm 14.4$ & $140 \pm 15 \S$ & $136 \pm 14 \$$ & $-2.7 \pm 12.2 \S$ & $140 \pm 16$ & $139 \pm 15$ & $-1.5 \pm 14.4 \$$ \\
$\mathrm{RR}$ diast. (mmHg) & $82.1 \pm 8.6$ & $82.2 \pm 7.9$ & $0.1 \pm 8.7$ & $81.3 \pm 7.7 \S$ & $80.9 \pm 7.2$ & $-0.4 \pm 7.4$ & $80.9 \pm 8.9 \S$ & $81.5 \pm 7.2$ & $0.8 \pm 8.3$ \\
BMI $\left(\mathrm{kg} / \mathrm{m}^{2}\right)$ & $31.7 \pm 6.0$ & $31.8 \pm 6.4$ & $-0.2 \pm 1.3$ & $30.3 \pm 5.1 \S$ & $30.1 \pm 5.2$ & $0.0 \pm 1.7$ & $29.6 \pm 4.6 \S$ & $29.7 \pm 4.8$ & $0.1 \pm 1.2 \S$ \\
& & & & & & & & &
\end{tabular}

change in BMI could be observed during Pioglitazone treatment in any of the different physical activity groups.

Conclusion: As expected, in our observational trial type 2 diabetic patients without physical activity presented with worse glucose control and higher blood pressure measurements at baseline. Nevertheless, also in type 2 diabetic patients at a high physical activity level treatment with pioglitazone still evolves additional clinical effects on glucose control and blood pressure.

Supported by TAKEDA

\section{4}

Metabolic effects of telmisartan and irbesartan in type 2 diabetic patients with metabolic syndrome treated with rosiglitazone: a double-blind, randomised clinical trial G. Derosa ${ }^{1}$, A. D'Angelo ${ }^{1}$, P. D. Ragonesi ${ }^{2}$, L. Ciccarelli ${ }^{1}$, M. N. Piccinni ${ }^{1}$, F. Pricolo ${ }^{1}$, S. A. T. Salvadeo ${ }^{1}$,

A. Gravina ${ }^{1}$, I. Ferrari ${ }^{1}$, E. Fogari ${ }^{1}$, A. F. G. Cicero ${ }^{3}$, R. Fogari ${ }^{1}$;

${ }^{1}$ Internal Medicine and Therapeutics, University of Pavia, IRCCS Policlinico S. Matteo, Pavia, ${ }^{2}$ Diabetes Care Unit, S. Carlo Hospital, Milano, ${ }^{3}$ Clinical Medicine and Applied Biotechnology, University of Bologna, Italy.

Background and Aims: Angiotensin II receptor blockers represent a class of effective and well tolerated orally active antihypertensive drugs, both in general population and diabetic patients. The aim of our study was to investigate the metabolic effect of telmisartan and irbesartan in subjects treated with rosiglitazone, a well-known insulin sensitizing drug, in order to discriminate eventual direct metabolic effects of the studied molecules.

Materials and Methods: Patients were enrolled, evaluated, and followed at 3 Italian Centres. We evaluated 188 type 2 diabetic patients with metabolic syndrome (94 males and 94 females; 49 males and 46 females, aged $56 \pm 5$ with telmisartan; 45 males and 48 females, aged $55 \pm 4$ with irbesartan). All were required to have been diagnosed as being diabetic for at least 6 months, and did not have glycemic control with diet and oral hypoglycemic agents, both to the maximum tolerated dose. All patients took a fixed dose of rosiglitazone, $4 \mathrm{mg} /$ day. We administered telmisartan $(40 \mathrm{mg} /$ day $)$ or irbesartan $(150 \mathrm{mg} /$ day $)$ in a randomized, controlled, double-blind clinical study. We evaluated BMI, glycemic control $\left(\mathrm{HbA}_{1 \mathrm{c}}, \mathrm{FPG}, \mathrm{FPI}\right.$, and Homa-index), lipid profile (TC, LDL-C, HDL-C, and Tg), SBP and DBP, adiponectin, resistin, TNF- $\alpha$, and leptin during 12 months of this treatment.

Results: No BMI change was observed after 6, and 12 months in both groups. Significant $\mathrm{HbA}_{1} \mathrm{c}$ and FPG decrease was observed after 6 months in telmisartan group, and after 12 months in both groups. $\mathrm{HbA}_{1} \mathrm{c}$ and $\mathrm{FPG}$ decrease was significantly lower after 12 months in telmisartan group. Significant FPI decrease was obtained at 12 months in both groups, and this decrease was significantly lower in telmisartan group. Significant Homa index decrease was obtained at 6 , and 12 months in both groups, and Homa index lowering was significant in telmisartan group after 12 months. Significant SBP, DBP, TC, and LDL-C change was observed after 6, and 12 months in both groups. Adiponectin concentration was increased after 6 months in telmisartan group, and after 12 months in both groups and this difference was significant after 12 months in telmisartan group. Significant resistin decrease was obtained after 6 months, and 12 months in telmisartan group, whereas it was observed a significant change at 12 months in irbesartan group and this difference was significant after 12 months in telmisartan group. Significant TNF- $\alpha$ and leptin levels decrease was observed after 6 months in telmisartan group, and after 12 months in both groups.

Conclusion: In this study of patients with type 2 diabetes mellitus and metabolic syndrome, telmisartan seemed to improve glycemic and lipid control and metabolic parameters regarding metabolic syndrome, compared to irbesar$\tan$. These observed metabolic effects of different AT(1) receptor blockers could be relevant in the therapy choice for the correct management of patients affected by metabolic syndrome and diabetes. 
0555

Effects of short-term metformin and rosiglitazone treatment on GLP-1, leptin and adiponectin levels in patients with type 2 diabetes mellitus

Z. Alphan ${ }^{1}$, S. Gullu ${ }^{1}$,

${ }^{1}$ Department of Endocrinology and Metabolic Diseases, Ankara University, School of Medicine, Ankara, Turkey.

Background and Aims: Type 2 diabetes mellitus (DM) is a chronic progressive disorder with an increasing prevalance. Decreased insulin secretion and peripheral insulin resistance are the two major pathogenetic factors in the disease. Disordered glucagon like peptide (GLP)-1 production have been shown in diabetic patients. GLP-1 is an incretin that increases insulin secretion after food intake and plays role in the regulation of plasma glucose levels. Increased leptin and decreased adiponectin levels have been reported in patients with insulin resistance. Metformin and rosiglitazone are used in the treatment of type 2 DM. These drugs act by improving insulin sensitivity.

The aim of this study was to evaluate the short-term effects of metformin and rosiglitazone on serum levels of GLP-1, leptin and adiponectin in type 2 diabetic patients.

Materials and Methods: Forty-one patients with type 2 DM were included to the study. Serum GLP-1, leptin, adiponectin, glucose and insulin levels were measured before and 30, 60, 90, 120 minutes after ingestion of a mixed meal at the initiation of the study. After basal evaluations patients were divided into three groups and either metformin ( $\mathrm{n}=15,1700 \mathrm{mg} /$ day $)$ or rosiglitazone $(\mathrm{n}=15,4 \mathrm{mg} /$ day $)$ or placebo $(\mathrm{n}=11)$ was started. All above mentioned parameters were measured after 14 days of drug treatments.

Results: Insulin resistance which was calculated as HOMAIR decreased significantly in both metformin and rosiglitazone groups $(6.4 \pm 2.8$ vs. $2.3 \pm 0.8 ; \mathrm{p}<0.01$ and $6.8 \pm 3.5$ vs. $3.2 \pm 1.1 ; \mathrm{p}<0.01$; respectively). No changes were observed in GLP-1 levels in the groups. Basal and peak leptin levels decreased in both drug groups $(38.5 \pm 16.6$ vs. $35.0 \pm 14.1 \mathrm{ng} /$ $\mathrm{ml} ; \mathrm{p}<0.05$ and $36.3 \pm 14.8$ vs. $33.3 \pm 15.5 \mathrm{ng} / \mathrm{ml} ; \mathrm{p}<0.05$ in metformin group; and $37.8 \pm 19.7$ vs. $35.4 \pm 18.1 \mathrm{ng} / \mathrm{ml}$; $\mathrm{p}<0.05$ and $40.1 \pm 17.5$ vs $35.3 \pm 15.6 \mathrm{ng} / \mathrm{ml} ; \mathrm{p}<0.05$ in rosiglitazone group). Adiponectin levels increased significantly in rosiglitsone group $(4.0 \pm 2.0$ vs $5.6 \pm 1.3 \mathrm{ng} / \mathrm{ml}$; $\mathrm{p}<0.05$ and $4.6 \pm 1.8$ vs $7.4 \pm 2.1 \mathrm{ng} / \mathrm{ml} ; \mathrm{p}<0.05$ basal and peak levels, respectively). An increase in adiponectin levels was also observed in the metformin group but it was not statistically significant. HOMA-IR, leptin and adiponectin levels did not change in the placebo group.

Conclusion: In conclusion, both rosiglitazone and metformin decreased leptin levels and increased adiponectin levels by improving insulin resistance in this short-term study. No effect on GLP-1 levels could be found in the present study but more long-term longitudinal studies are needed to confirm this finding.

0556

Genome-wide investigation of genes regulated in different cell fractions of adipose tissue from insulin-resistant subjects by short-term treatment with pioglitazone

X. Yang, P.-A. Jansson, V. Rotter Sopasakis,

A. Hammarstedt, S. Gogg, U. Smith;

The Lundberg Laboratory for Diabetes Research, Sahlgrenska Academy, Göteborg University, Sweden.

Background and Aims: Insulin resistance is a major predictor for the development of type 2 diabetes. Thiazolidinediones (TZDs) are commonly used insulin sensitizing drugs that activate nuclear receptor PPARs, leading to adipogenesis and improvement in systemic insulin sensitivity. The genes regulated by TZD treatment may therefore potentially contribute to the development of insulin resistance and hence type 2 diabetes.

Materials and Methods: We treated five insulin-resistant first-degree relatives of type 2 diabetic patients (age 51.2+ $2.6 \mathrm{yrs}$, BMI $28.1+1.5 \mathrm{~kg} / \mathrm{m}^{2}$ ) with pioglitazone at $30 \mathrm{mg} /$ day for three weeks, and investigated gene expression in isolated adipocytes and adipose tissue using Affymetrix U133 GeneChip ${ }^{\circledR}$ for human genome. The microarray measurements were verified by TaqMan real-time PCR. This study design is thought to provide two advantages; 1 ) the short-term treatment eliminates the potential confounding effects resulting from significant body weight gain that is often associated with long-term treatment, 2) the GeneChip ${ }^{\circledR}$ detection algorithm permits segregation of genes into those expressed in adipocytes, in non-adipocytes, or in the both cell fractions of adipose tissue.

Results: Body weight and BMI were not altered after the treatment. Fasting plasma glucose $(5.1+0.2$ vs. $4.7+$ $0.2 \mathrm{mM}, \mathrm{P}=0.05)$ and insulin $(12.9+1.2 \mathrm{vs} .11 .0+0.9 \mathrm{mU} /$ L) were numerically decreased. Glucose disposal rate was increased from $6.9+0.5$ before to $8.1+0.6 \mathrm{mg} / \mathrm{kg} \mathrm{LBM} / \mathrm{min}$ after the treatment $(\mathrm{P}<0.01)$. There were 325 transcripts upregulated and 142 down-regulated in the adipocytes and 109 up-regulated and 90 down-regulated in the adipose tissue. Functional classification of these transcripts suggested a transcriptional activation of pathways essential for adipocyte energy storage, prominently fatty acid esterification and glucose metabolism (Table). Many genes enrolling in cell division or differentiation and in tissue remodeling were also found to be expressed differentially after the treament. Approximately 30 genes were induced (absent before the treatment and present after) or suppressed (present before the treatment and absent after) in either adipocytes or non-adipocytes by the treatment. In addition, 
37 genes, mostly stress-responding genes, were induced (absent in adipose tissue but present in isolated adipocytes) by the standard adipocyte isolation procedure.

Conclusion: Short-term treatment with pioglitazone improves systemic insulin sensitivity by increasing energy partitioning to the adipose tissue before significant adipogenesis occurs (estimated from the number and scope of adipogenic gene induction and body weight gain). Further study of the genes responding to TZDs at the early stage of treatment before significant body weight gain may enable the identification of gene candidates that predispose to insulin resistance and/or type 2 diabetes.

Table. Selected genes up-regulated in adipocytes by shortterm treatment with pioglitazone

\begin{tabular}{ll}
\hline Genes & Fold Increase \\
Acetyl-CoA carboxylase & 1.7 \\
Acetyl-CoA synthetase & 1.4 \\
Acyl-CoA synthetase long-chain & 1.5 \\
Acetyl-CoA acyltransferase & 1.3 \\
Acyglycerol-3-phosphate O-acyltransferase & 2.5 \\
Diacylglycerol O-acyltransferase & 1.2 \\
Phosphoenolpyruvate carboxykinase & 2.0 \\
Hexokinase & 1.3 \\
Pyruvate carboxylase & 1.4 \\
Pyruvate dehydrogenase & 1.4 \\
Fatty acid binding protein 5 & 1.8 \\
\hline
\end{tabular}

Supported by the Swedish Research Council, the European Community, the Swedish Diabetes Association, the Novo Nordisk Foundation

0557

Depot-specific regulation of perilipin by rosiglitazone in a diabetic animal model

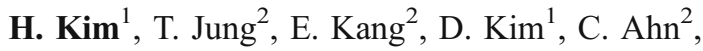
K. Lee ${ }^{1}$, H. Lee ${ }^{2}$, B. Cha'

${ }^{1}$ Endocrinology and Metabolism, Ajou University School of Medicine, Suwon, ${ }^{2}$ Internal Medicine, Yonsei University College of Medicine, Seoul, Republic of Korea.

Background and Aims: Rosiglitazone, an insulin sensitizer of thiazolidinedione (TZD) class, is known as potent agonist for the peroxisome proliferator-activated receptor- $\gamma$ (PPAR $\gamma$ ) and promote differentiation of pre-adipocytes into adipocytes, resulting in lipid storage coupled with reduced release of free fatty acids into the circulation. Many studies reported that TZDs increase subcutaneous adiposity but have no effect on the visceral fat mass. Perilipin, a family of phosphoproteins located around lipid droplets in adipocytes, is essential for the enlargement of lipid droplets and lipolytic reaction by hormone-sensitivie lipase. Recently, a functional PPAR-responsive element (PPRE) was identified within the murine perilipin gene. We hypothesized that the depot-specific regulation of perilipin by rosiglitazone may be associated with the fat redistribution and insulin sensitizing effects of rosiglitazone.

Materials and Methods: We examined the change of adiposity, triglyceride contents in liver and muscle, morphological evaluation of pancreas after 6 weeks of treatment with placebo or rosiglitazone in OLETF rats, a type 2 diabetic animal model. Perilipin mRNA and protein expression were assessed in subcutaneous and epididymal fat using semi-quantitative RT-PCR and Western blotting.

Results: Rosiglitazone increased subcutaneous adiposity and decreased triglyceride contents of liver and muscle. Rosiglitazone decreased plasma free fatty acids (2107.3 \pm 507.4 in placebo group vs. $823.9 \pm 148.3$ in rosiglitazone group, $\mathrm{p}<0.05)$ and triglyceride concentration $(164.0 \pm 37.9$ in placebo group vs. $56.8 \pm 14.7$ in rosiglitazone group, $\mathrm{p}<0.05)$ and improved insulin resistance. The islets of placebo-treated rats showed hypertrophy and destruction, while the islets of rosiglitazone-treated rats showed hypertrophy but architecture intact. Perilipin mRNA and protein expression increased in subcutaneous fat, but did not change in visceral fat by rosiglitazone. In 3T3-L1 cell, rosiglitazone pretreatment decreased lipolysis and increased perilipin protein.

Conclusion: From the results, the increased perilipin expression in subcutaneous fat after rosiglitazone treatment is likely to be a mediator of reduced lipolysis, resulting in lipid storage in subcutaneous fat, the fat redistribution and insulin sensitizing effect.

\section{PS 35}

\section{Pathophysiology, metabolism}

0558

The influence of PPAR- $\alpha$ agonist treatment on insulin sensitivity in patients with type 2 diabetes: the role of resistin and adiponectin

R. Dolezalova, K. Anderlova, J. Housova, L. Bosanska, J. Skrha, M. Haluzik;

3 Department of Medicine, 1 Faculty of Medicine, Prague, Czech Republic.

Background and Aims: Treatment with PPAR- $\alpha$ agonists fibrates markedly improves insulin sensitivity in different rodent models of obesity and diabetes. Its influence on insulin sensitivity in humans is less clear. The aim of this study was to evaluate the influence of 3-months administration of PPAR- $\alpha$ agonist fenofibrate $(200 \mathrm{mg}$ /day) on 
insulin sensitivity in obese patients with type 2 diabetes. In addition, possible involvement of the changes of circulating levels of adipose tissue-derived hormones adiponectin and resistin were also studied.

Materials and Methods: Basic anthropometric and biochemical parameters, insulin sensitivity and serum concentrations of adiponectin and resistin were measured in 12 obese female patients with type 2 diabetes (DM) and 12 lean healthy control women before and after 3 months of treatment with fenofibrate $(200 \mathrm{mg} /$ day $)$. Insulin sensitivity was measured by isoglycaemic-hyperinsulinaemic clamp. Serum concentrations of resistin and adiponectin were assessed by commercial ELISA kits (BioVendor, CZ and Linco Research, USA, respectively).

Results: Body mass index, blood glucose, serum triglycerides, glycated hemoglobin and atherogenic index were significantly higher in DM relative to control group. In contrast, serum adiponectin and HDL cholesterol were lower in DM than in controls, while no difference between the two groups was found in serum resistin levels. Fenofibrate treatment significantly decreased serum triglycerides $(2.07 \pm 0.30$ vs. $3.12 \pm 0.50 \mathrm{mg} / \mathrm{dl}$, $\mathrm{p}<0.01$ ), but did not affect any other parameters including BMI and diabetes compensation. 3-months of fenofibrate treatment did not affect either adiponectin or resistin levels. Insulin sensitivity measured by isoglycaemic-hyperinsulinaemic clamp was markedly decreased in DM relative to control group. 3-months of fenofibrate treatment significantly improved glucose disposal rate ( $4.20 \pm 0.70$ vs. $\left.3.43 \pm 0.68 \mu \mathrm{mol} \cdot \mathrm{kg}^{-1} \cdot \mathrm{min}^{-1}, \mathrm{p}<0.05\right)$ but did not significantly affect either metabolic clearance rate of glucose or insulin sensitivity index in patients with type 2 diabetes mellitus.

Conclusion: In contrast to experimental studies, 3-months treatment of obese patients with type 2 diabetes mellitus with PPAR- $\alpha$ agonist fenofibrate had only minor effects on insulin sensitivity. Metabolic effects of PPAR- $\alpha$ agonists were not mediated by the changes of circulating levels of resistin or adiponectin.

Supported by IGA 8302-5 and Research project 64165

0559

Pharmacological PPAR $\alpha$ activation induces substantial alterations in amino acid metabolism in fat fed, insulin resistant rats

K. Sheikh ${ }^{1}$, G. Camejo ${ }^{2}$, B. Lanne ${ }^{3}$, T. Halvarsson ${ }^{1}$, M. R. Landergren ${ }^{1}$, N. D. Oakes ${ }^{2}$;

${ }^{1}$ Medicinal Chemistry, CV/GI, Mölndal, Sweden, ${ }^{2}$ Integrative Pharmacology, CV/GI, Mölndal, Sweden, ${ }^{3}$ Molecular Pharmacology, CV/GI, Mölndal, Sweden.
Background and Aims: Effects of PPAR $\alpha$ agonists have been characterized largely in terms of their influence on lipids and glucose metabolism, but little has been reported about amino acid metabolism. The aim of this study was to provide further insight into effects of the PPAR $\alpha$ agonist WY 14,643 with particular emphasis on treatment related changes in amino acid metabolism in fat-fed rats, a model of dyslipidaemia and insulin resistance.

Materials and Methods: WY 14,643 $(30 \mu \mathrm{mol} / \mathrm{kg} / \mathrm{d}$ for 4 wk) was administered to Sprague-Dawley rats fed a saturated fat diet. An initial metabonomics study was conducted, involving ${ }^{1} \mathrm{H}$ NMR analysis of plasma and urine samples. In addition, plasma amino acid levels were measured using HPLC and hepatic gene expression was assessed by measurement of mRNA levels.

Results: The high fat diet elevated plasma levels of insulin and triglycerides and WY 14,643 treatment ameliorated this hyperinsulinaemia and hypertriglyceridaemia. In addition, treatment decreased body weight gain, without altering cumulative food intake and increased liver mass. WY 14,643 increased plasma levels of 12 out of 22 amino acids compared to fat-fed controls, most noticeably for serine $(+66 \%)$, glycine $(+82 \%)$ and threonine $(+78 \%)$, while arginine was significantly decreased. There was no alteration in branched chain amino acid levels. Compared with the fat fed control animals, WY 14,643 treated animals had raised plasma urea and ammonia levels. The methyl carriers, N-methylnicotinamide and dimethylglycine, were also elevated in urine of treated animals. WY 14,643 increased hepatic expression of a number of key genes involved in amino acid and fatty acid catabolism, including genes involved in ketone body formation.

Conclusion: The results show that in rodents pharmacological PPAR $\alpha$ activation increases whole body amino acid mobilisation and catabolism thus extending the role of this transcription factor beyond the control of lipid and carbohydrate metabolism.

0560

Effects of physiological vs non-physiological restoration of postprandial insulin secretion on glucose and lipid metabolism in type 2 diabetes

V. Lambadiari ${ }^{1}$, G. Dimitriadis ${ }^{1}$, E. Boutati ${ }^{1}$, P. Mitrou ${ }^{1}$, E. Maratou ${ }^{2}$, S. A. Raptis ${ }^{1,2}$;

${ }^{1}$ 2nd Department of Internal Medicine and Research Institute, Athens University Medical School, ${ }^{2}$ Hellenic National Diabetes Center, "Attikon" University Hospital, Athens, Greece. 
Background and Aims: The early increase in insulin secretion has a critical role in the regulation of postprandial glucose and lipid responses. Early in the course of type 2 diabetes (T2D), the increase in insulin secretion after a meal is delayed. However, the impact of this defect on postprandial glucose and lipid metabolism is questionable. This was investigated in T2D patients by giving nateglinide or glibenclamide (a rapid-acting and slower-acting insulin secretagogue respectively) prior to a meal. To avoid the confounding effects of chronic amelioration of hyperglycaemia on the parameters investigated, the experiments were performed after a single administration of these drugs. Materials and Methods: 24 newly-diagnosed T2D (age $54 \pm 2$ yrs, BMI $25 \pm 1$, HbA1c $7.5 \pm 0.3 \%$ ) rendered euglycaemic with i.v. insulin, were studied on two different occasions under the following conditions: (1) Ingestion of a standard mixed meal with no drug administration, and (2) same as (1) but following randomized administration of a single dose of either $120 \mathrm{mg}$ nateglinide $(\mathrm{n}=12)$ or $5 \mathrm{mg}$ glibenclamide $(\mathrm{n}=12)$ before the meal. Plasma samples for glucose (G), insulin (I), glycerol (GLY), triglycerides (TAG), and non-esterified fatty acids (NEFA), were obtained at standard intervals for $360 \mathrm{~min}$ from an arterialized hand vein (A), and from a vein (V) draining the anterior abdominal subcutaneous adipose tissue (AD). Blood flow (BF) in $\mathrm{AD}$ was measured with ${ }^{133} \mathrm{Xe}$. Calculations: $\mathrm{NEFA}_{\text {Output }}=\left[\mathrm{NEFA}_{(\mathrm{V}-\mathrm{A})} \mathrm{x}(\mathrm{BF})\right], \quad \mathrm{LPL}=$ $\left[\mathrm{TAG}_{(\mathrm{A}-\mathrm{V})} \mathrm{x}(\mathrm{BF})\right], \quad \mathrm{HSL}=\left[\left(\mathrm{GLY}_{\text {flux }}\right)-(\mathrm{LPL})\right]$. Postprandial increases are presented as absolute values or areas under the curve (AUC, 0-360 min).

Results: Preprandial levels of $\mathrm{G}$ and I in A were comparable in both groups $(5.7 \pm 0.2$ vs $5.9 \pm 0.2 \mathrm{mM}$ and $5.0 \pm 0.3$ vs $6.0 \pm 0.4 \mathrm{mU} / \mathrm{L}$ respectively). After the meal: (a) the responses of $\mathrm{G}$ were similarly reduced by nateglinide (49+ $\left.3 \mathrm{mM}^{*} \mathrm{~h}\right)$ and glibenclamide $\left(41 \pm 4 \mathrm{mM}^{*} \mathrm{~h}\right)$; (b) secretion of I during the first $30 \mathrm{~min}$ was more prominent in the nateglinide $\left(13 \pm 1 \mathrm{mU} / \mathrm{L}^{*} \mathrm{~h}\right)$ than in the glibenclamide group $\left(9 \pm 1 \mathrm{mU} / \mathrm{L}^{*} \mathrm{~h}, \mathrm{p}<0.05\right)$; in contrast, overall I secretion $(0-$ $360 \mathrm{~min}$ ) was more prominent after glibenclamide (337 +30 mU/1*h) than after nateglinide $\left(264 \pm 20 \mathrm{mU} / \mathrm{l}^{*} \mathrm{~h}\right.$, $\mathrm{p}<0.05$ ); (c) TAG levels in A and LPL action were in agreement after nateglinide $\left(4285 \pm 436 \mu \mathrm{mol} / \mathrm{l}^{*} \mathrm{~h}\right.$ and $373+$ $50 \mathrm{nmol}$ glycerol/100 ml tissue ${ }^{*} \mathrm{~h}$ respectively) and glibenclamide $\left(4983 \pm 1004 \mu \mathrm{mol} / \mathrm{l}^{*} \mathrm{~h}\right.$ and $464 \pm 74 \mathrm{nmol}$ glycerol/ $100 \mathrm{ml}_{\text {tissue }}{ }^{*} \mathrm{~h}$ respectively); (d) NEFA output and HSL action over the first $90 \mathrm{~min}$ after the meal were less after nateglinide $\left(167 \pm 36 \mathrm{nmol} / 100 \mathrm{ml}_{\text {tissue }}{ }^{*} \mathrm{~h}\right.$ and $85 \pm 10 \mathrm{nmol}$ glycerol/100 $\mathrm{ml}_{\text {tissue }} * \mathrm{~h}$ respectively, $\left.\mathrm{p}<0.05\right)$ than after glibenclamide $\left(345 \pm 33 \mathrm{nmol} / 100 \mathrm{ml}_{\text {tissue }}{ }^{*} \mathrm{~h}\right.$ and $157+$ $18 \mathrm{nmol}$ glycerol $/ 100 \mathrm{ml}_{\text {tissue }} * \mathrm{~h}$ respectively, $\mathrm{p}<0.05$ ).

Conclusion: The near-physiological insulin secretory profile achieved with nateglinide was more efficient in controlling postprandial hyperglycaemia and in suppressing endogenous lipolysis after the meal, than that achieved with the administration of glibenclamide. These results emphasize the importance of the impairment in insulin secretion pattern in the development of postprandial hyperglycaemia and hyperlipidaemia in type 2 diabetes.

\section{1}

The second meal effect results from priming of glycogen synthesis in muscle and liver and can be mimicked by administration of arginine

A. Jovanovic ${ }^{1}$, J. Gerrard ${ }^{1}$, B. Solanky ${ }^{2}$, E. Leverton ${ }^{2}$, J. E. M. Snaar ${ }^{2}$, P. G. Morris ${ }^{2}$, R. Taylor ${ }^{1}$;

${ }^{1}$ SCMS Diabetes, University of Newcastle, ${ }^{2}$ Sir Peter Mansfield Magnetic Resonance Centre, University of Nottingham, United Kingdom.

Background and Aims: The rise in blood glucose after lunch is less if breakfast has been eaten. We have shown that this second meal effect is present in both health and type 2 diabetes. To determine the metabolic basis and potential therapeutic application of the second meal effect we studied 6 healthy subjects on a three separate days.

Material and Methods: Metabolic changes after a lunch, labeled with $3 \mathrm{~g}$ of ${ }^{13} \mathrm{C}$ - labeled $(99 \%)$ glucose, were examined on Day A with breakfast; Day B without breakfast; and day $\mathrm{C}$ without breakfast but with intravenous arginine one hour before lunch. Changes in muscle and liver glycogen were measured on Days A and B using magnetic resonance spectroscopy and meal carbohydrate oxidation was measured using breath ${ }^{13} \mathrm{CO}_{2}$ enrichment.

Results: Despite comparable post-lunch insulin and C-peptide increments, the rise in plasma glucose after lunch was significantly less if breakfast had been taken (Day A: $0.8 \pm 0.5 \mathrm{mmol} / \mathrm{l}$; Day B: $2.8 \pm 0.2 \mathrm{mmol} / \mathrm{l}, \mathrm{p}<0.02$ ). Pre-lunch NEFA were suppressed when breakfast had been eaten $(0.11 \pm 0.02$ vs $0.58 \pm 0.04 \mathrm{mmol} / \mathrm{l})$. Muscle glycogen storage was greater 5 hours after lunch on a breakfast day $(155.3 \pm 16.1$ vs $40.3 \pm 1.9 \mathrm{U} \mathrm{p}<0.02)$. On the same day the liver glycogen storage was significantly greater 1.5 hours after lunch $(171.7 \pm 42.8$ vs $43.8 \pm 18.6 \mathrm{U}, \mathrm{p}<0.04)$. We observed no difference in carbohydrate oxidation $(248.27 \pm 107.76$ vs $247.98 \pm 62.24 \mathrm{U}$ at one hour). Arginine administration brought about a significantly smaller rise in plasma glucose after lunch in these normal subjects $(2.1 \pm 0.2$ vs $2.9 \pm 0.2 \mathrm{mmol} / 1$ at one hour, $\mathrm{p}<0.05$ ) and this was associated with suppression of pre lunch NEFA $(0.21 \pm 0.05$ vs $0.58 \pm 0.04 \mathrm{mmol} / \mathrm{l})$.

Conclusions: The second meal effect is a result of priming of glycogen synthesis in liver and muscle. This phenomenon is associated with sustained suppression of plasma 
NEFA. Pre meal administration of arginine mimicks the second meal effect, resulting in lower postprandial glucose rise suggesting a novel therapeutic approach to improving glucose control in type 2 diabetes.

Supported by Wellcome Trust

\section{2}

$\mathrm{N}$-terminal pro brain natriuretic peptide levels increases during weight loss in obese subjects

C. Kistorp ${ }^{1}$, H. Bliddal ${ }^{2}$, P. Hildebrandt ${ }^{1}$, J. Faber ${ }^{3}$;

${ }^{1}$ Cardiology and Endocrinology, Frederiksberg University Hospital, Copenhagen, ${ }^{2}$ The Parker Institute, Frederiksberg University Hospital, Copenhagen, ${ }^{3}$ Endocrinology, Herlev University Hospital, Copenhagen, Denmark.

Background and Aims: The cardiac hormone brain natriuretic peptide (BNP), including the $\mathrm{N}$-amino terminal (NT-proBNP), are very strong prognostic biomarkers in patients with cardiovascular disease and diabetes. Recent cross-sectional studies have demonstrated that B-type natriuretic peptides are inversely and independently associated with body mass index (BMI), the mechanism behind this relation is unknown. A possible role of both adipose and muscle tissue in clearance and degradation, of these peptides have been suggested. The aims of the present study was to investigate the impact of weight loss on plasma levels of NT-proBNP in obese individuals without heart disease. A second aim was to address the association between NT-proBNP levels, fat mass and lean tissue mass, as measured by DEXA scanning.

Material and Methods: A total of 63 obese individuals, 59 women and 4 men, age $61.7( \pm 6.6)$ year, BMI $36.1(5.5) \mathrm{kg} /$ $\mathrm{m}^{2}$ (mean \pm SD) were included. Subjects were without known cardiovascular disease, and NT-proBNP levels were below $125 \mathrm{pg} / \mathrm{mL}$, which is the recommended cut-off value for rule out a diagnosis of heart failure. The participants were randomized 2:1 to either an intervention group, which followed an intensive weight reduction program, including a low energy diet between $3.4-5 \mathrm{MJ} /$ day for 1 year, or a control group which was offered diet counseling. Body weight, fat mass and lean mass, as well as plasma levels of NT-proBNP were measured at baseline and after 52 weeks. Results: In the intervention group $(n=50)$ total weight loss was $14.7 \%( \pm 1.1 \%)(\mathrm{P}<0.001)$ after 52 weeks, and DEXA scan demonstrated a loss in body fat of $26.1 \%( \pm 1.9 \%)$ $(\mathrm{P}<0.001)$, with a decrease in lean mass of $2.4 \%( \pm 0.7 \%)$ $(\mathrm{P}=0.001)($ mean $\pm \mathrm{SE})$. Plasma NT-proBNP levels increased from $51.6(39.8-80.1) \mathrm{pg} / \mathrm{mL}$ at baseline to 89.1 (49.0 114.9) $\mathrm{pg} / \mathrm{mL}$ after 52 weeks, $(\mathrm{P}<0.001)$ median (interquartile range). In the control group $(n=13)$ a modest weight loss of $4.2 \%(1.3 \%)(\mathrm{P}=0.003)$ was observed, the decrease in fat mass was $6.2 \%(2.3 \%)(\mathrm{P}=0.015)$, while lean mass was unchanged during follow-up. Decrease in total weight, fat and lean tissue mass were significantly higher in the intervention group as compared with the control group $(\mathrm{P}<0.001)$. Furthermore, no change in plasma NT-proBNP levels were observed in the control group 64.9 (25.3-91.1) vs. 55.7 (38.8-118.6) $\mathrm{pg} / \mathrm{mL}(\mathrm{P}=0.42)$. Linear regression analyses demonstrated that higher lean mass was associated with lower NT-proBNP levels $\mathrm{r}=-0.46(\mathrm{P}<0.001)$ at baseline, whereas no association between fat mass and NT-proBNP levels were observed $(\mathrm{P}=0.28)$.

Conclusions: The present intervention study demonstrate that plasma levels of NT-proBNP increases significantly during weight loss, including both loss in fat and lean mass. Furthermore, the association between higher BMI and lower B-type natriuretic peptide levels may be mediated by lean mass rather than fat tissue mass.

\section{3}

Oxidative stress and autologous IgG binding to band 3 dimers in erythrocytes of diabetic subjects

V. Rossi ${ }^{1}$, I. Tanganelli ${ }^{2}$, S. Leoncini ${ }^{1}$, C. Signorini ${ }^{1}$, D. Totagiancaspro ${ }^{2}$, L. Ciccoli ${ }^{1}$;

${ }^{1}$ Pathophysiology, Experimental Medicine and Public Health, University of Siena, ${ }^{2}$ Odontostomatology - Diabetology Section, University of Siena, Italy.

Background and Aims: Oxidative stress is involved in the pathogenesis of long term diabetic complications. Oxidative stress, exacerbated by hyperglycemia, can damage red blood cells (RBCs) given their high content of iron and polyunsaturated fatty acids compared to other cells. Reported abnormalities include altered membrane lipid composition, decreased deformability, increased membrane microviscosity, erythrocyte aggregation, glycated hemoglobin and accumulation of advanced glycation endproducts (AGEs) on the membrane with consequent modifications of rheological properties of RBCs, of their capacity to yield oxygen to tissues and of more rapid RBCs removal from the blood stream by macrophagic system. Our previous studies have shown a link among oxidative stress, intraerythrocyte iron release, alterations of $\mathrm{RBC}$ membrane proteins, in particular of band 3, autologous IgG binding and RBC ageing. In the present study we investigated whether diabetic RBCs exposure to oxidative stress results in the binding of autologous IgG to band 3 dimers in a higher percentage of diabetic subjects as compared to health subjects (controls). The iron release and methemoglobin (MetHb) levels in RBCs and the occurrence of total F2-isoprostanes (IsoPs) and of advanced oxidation protein products (AOPPs) in plasma, as markers of oxidative stress, were also studied. 
Material and Methods: Our study included 46 diabetic subjects, type 1 and 2 and 20 controls. The IgG binding to band 3 dimers was determined by western blot. The intraerythrocyte iron release was determined as desferroxaminechelatable iron (DCI) by HPLC and MetHb by spectrophotometer. F2-isoprostanes were determined by gascromatography-mass spectrometry and AOPPs were measured by absorbance of acetic acid-acidified plasma at $340 \mathrm{~nm}$.

Results: The results showed the presence of IgG binding in $47,8 \%$ of diabetics and in $15 \%$ of controls (Fisher's Exact Test $\mathrm{p}=0.0137$ ); no differences between diabetics type 1 and type 2 was observed. MetHb levels were significantly higher in diabetic than in control RBCs $(\mathrm{p}<0.001)$. DCI was higher in diabetic RBCs showing IgG binding to band 3 dimers, with significant correlation between DCI and IgG binding (optical density obtained by scanning densitometry, $\mathrm{r}=0.639, \mathrm{p}<0.001$ ). Plasma IsoPs and AOPP levels were significantly higher in diabetic subjects than in controls ( $<<0.01$ and $\mathrm{p}<0.05$, respectively).

Conclusion: In conclusion our data suggest a link between oxidative stress, iron release and $\operatorname{IgG}$ binding to band 3 dimers in RBCs of diabetic subjects. In these subjects a large part of RBCs is ready to be eliminated by macrophagic system, with an accelerated RBCs turnover. The increased erythrophagocytosis and the possible increased exocytosis of iron can have an important role in the LDL oxidation, mechanism underlying atherosclerotic injuries and long term diabetic complications.

Supported by Grant from University of Siena (research project 2005)

\section{4}

Impact of short term high-fat overfeeding on hepatic glucose production and peripheral insulin action in young healthy men

A. Vaag $^{1}$, C. Brøns ${ }^{1}$, C. B. Jensen ${ }^{1}$, J. S. Appel ${ }^{1}$, H. Storgaard ${ }^{1}$, A. V. Astrup ${ }^{2}$;

${ }^{1}$ Steno Diabetes Center, Gentofte, ${ }^{2}$ Department of Human Nutrition, The Royal Veterinary and Agricultural University, Frederiksberg, Denmark.

Background and Aims: Patients with overt type 2 diabetes (T2D) are characterized by multiple defects of glucose homeostasis with two of the major players being elevated hepatic glucose production (HGP) and peripheral (muscle) insulin resistance. While the relative roles of the liver versus muscle insulin resistance in overt T2D is currently debated, there is a general agreement that muscle insulin resistance is present decades before abnormal HGP and overt T2D in most pre-diabetic states and individuals including first degree relatives, subjects with low birth weight and obese subjects. It has been speculated that insulin resistance in obesity to some extent may be due to the concomitant overeating necessary to maintain an elevated body weight per se. However, little is known concerning the individual role of liver versus muscle in the abnormal glucose metabolism induced by short-term overfeeding in healthy subjects. The aim of this study was to determine the sequential events, and relative roles of liver versus muscle in the putative abnormal glucose homeostasis and insulin action induced by short term overfeeding of a high fat diet in young, lean and healthy subjects.

Materials and Methods: Nineteen healthy 24-year-old, non-obese male volunteers without a family history of diabetes were included in the study. The subjects were randomized to two study periods separated by $4-6$ weeks; a 5 -day period on a high-fat $(60 \mathrm{E} \%)$, high-energy $(+50 \%)$ overfeeding-diet, and a control period on an iso-energetic control diet. At the end of each period, the participants were studied using the hyperinsulinemic euglycemic clamp technique $\left(80 \mathrm{mU} / \mathrm{m}^{2} / \mathrm{min}\right)$ combined with indirect calorimetry and a primed, continuous infusion of $\left[3-{ }^{3} \mathrm{H}\right]$ glucose to study the partitioning of glucose metabolism between liver and periphery. Intravenous glucose tolerance tests were performed prior to the clamps to measure first phase insulin secretion.

Results: Five days of high-fat overfeeding resulted in an increase in the mean fasting blood glucose levels $(4.59 \pm 0.44$ vs. $5.00 \pm 0.48 \mathrm{pmol} / \mathrm{L}, \mathrm{P}=0.01)$. Furthermore, fasting (basal) HGP was increased by overfeeding ( $2.05 \pm 0.33$ vs. $2.58 \pm 0.32 \mathrm{mg} / \mathrm{kgFFM} / \mathrm{min}, \mathrm{P}=0.01)$. HGP was fully suppressed during insulin infusion both before and after overfeeding, and overfeeding did surprisingly not influence peripheral (muscle) glucose disposal (14.05 \pm 2.37 vs. $13.96 \pm 3.42 \mathrm{mg} / \mathrm{kgFFM} / \mathrm{min}, \mathrm{P}=0.86$ ). First phase insulin secretion capacity as well as basal and insulin stimulated oxidative- and non-oxidative glucose metabolism was not significantly influenced by overfeeding.

Conclusion: Short term overfeeding increases fasting plasma glucose levels in healthy, young men. This effect is partly - or fully - mediated by a rise in basal hepatic glucose production. In contrast, insulin secretion capacity as well as peripheral insulin action was not significantly affected by overfeeding. The data suggest that elevated HGP in overt T2D patients to some extent may be due to overeating. In contrast, peripheral insulin resistance in obese subjects or in patients with overt T2D, is caused by factors other than short term overfeeding per se.

Supported by European Foundation for the Study of Diabetes, Diabetesforeningen, Aase og Ejnar Danielsens Fond 


\section{5}

High fat and lipid induced insulin resistance in rats: the comparison of glucose metabolism, plasma resistin and adiponectin levels

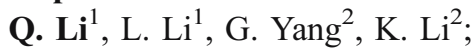

${ }^{1}$ Department of Clinical Biochemistry, ${ }^{2}$ Department of Endocrinology, Chongqing University of Medical Sciences, Chongqing, China.

Background and Aims: In animal models, both acute elevation of plasma FFA via intravenous infusion of lipid emulsion and chronic elevation of plasma FFA via high-fat feeding have been shown to induce skeletal muscle and liver insulin resistance. However, very few studies have compared the effects of high fat and lipid induced insulin resistance on glucose metabolism and adipocytokine in the same experiments in vivo. In the current study, we used a lipid infusion and high fat feed in combination with hyperinsulinemic-euglycemic clamps to assess the impact of acute and chronic elevated FFA levels on overall glucose metabolism and insulin action, two adipocytokines resistin and adiponectin.

Materials and Methods: Male S-D rats were divided into 3 groups: control( $n=10), \mathrm{HF}$ (high fat diet, $n=10)$, lipid groups(lipid emulsion was infused, $n=10$ ). Five to seven days before the study, rats receivered a surgery. A silastic catheter was inserted into the right internal jugular vein and extended to the level of the right atrium. Another catheter was advanced through the left carotid artery until its tip reached the aortic arch. After an overnight fast, euglycemic-hyperinsulinemic clamps were performed with awake and unrestrained rats. glucose concentrations were clamped at euglycemic levels. $3-{ }^{3} \mathrm{H}$ glucose was infused through the jugular vein catheter starting at $60 \mathrm{~min}$. A lipid emulsion was infused at a rate of $5 \mathrm{ml} / \mathrm{kg} / \mathrm{h}$ after $60 \mathrm{~min}$ (lipid group). Controls and high-fat group (HF group) received saline infusions instead of lipid. Insulin was infused from 180 to $300 \mathrm{~min}$. Blood samples were obtained from the carotid artery at $0,60,260,280,290$ and $300 \mathrm{~min}$ for determination of insulin, resistin, adiponectin, free fatty acids and specific activity of tritiated glucose.

Results: In baseline, plasma free fatty acids (FFA) levels were significantly increased in high fat rats (HF group) compared with controls $(P<0.05)$. During clamp steadystate, the FFA levels were reduced by $\sim 77$ and $\sim 56 \%$ in control and HF groups, respectively, but there was a significant increase in plasma FFA level in lipid-infused rats (lipid group). The glucose infusion rates (GIR) in HF and lipid groups were obviously lower than in the controls (all $P<0.01$ ). Moreover,GIR was lower in lipid group compared with HF group $(P<0.05)$. The rate of glucose disappearance $\left(\mathrm{G}_{\mathrm{Rd}}\right)$ was significantly lower in lipid group compared with controls. The endogenous glucose produc- tion (EGP) in controls was suppressed by $\sim 85 \%$, but only suppressed by $\sim 29$ and $\sim 19 \%$ in HF and lipid groups, respectively. The resistin levels of muscle tissues in Lipid group was significantly higher compared with control and HF groups (Both $P<0.05$ ). After the insulin clamp, circulating adiponectin levels were significantly decreased in the lipid group compared with the control and HF rats. $(P<0.05)$.

Conclusion: Both lipid infusion and high fat feed can induce peripheral and hepatic insulin resistance in rats, and the former was more significantly, it might be associated with elevated resistin.

0566

Low efficiency of n-3 polyunsaturated fatty acids in the reversal of insulin resistance induced by a high-fat diet in mice

Z. Jilkova, M. Rossmeisl, P. Flachs, J. Kopecky; Dept. of Adipose Tissue Biology, Institute of Physiology, Prague, Czech Republic.

Background and Aims: Previous studies including ours established that n-3 polyunsaturated fatty acids (n-3 PUFAs) from marine fish oils, potent natural hypolipidemics, could prevent obesity and insulin reistance (IR) induced by high-fat (HF) diets in laboratory rodents while counteracting proliferation of adipose tissue cells. Considerably less is known about the efficiency of n-3 PUFAs in the reversal of IR associated with an already established obesity. Therefore, we analyzed the effects of n-3 PUFAs, namely eicosapentaenoic (EPA) and docosahexaenoic (DHA) acids, in the obese C57BL/6N (B6) mice.

Materials and Methods: Male B6 mice, fed a standard chow from weaning, were assigned a HF diet $(60 \%$ calories from fat, mainly corn oil) at the age of 3 mo. Obesity was induced during 3 mo of HF feeding (mean body weigth $\sim 46.3 \pm 0.8$ ). At this stage, some mice were randomly assigned to a HF diet, in which $9 \%$ of dietary lipids was replaced by EPA and DHA, added as a $60 \%$ concentrate (EPAX1050TG; Pronova Biocare, Norway), while the rest of mice was maintained on a HF diet $(n=10)$. Body weight gain and food intake were monitored during 2 mo of feeding. No differences in food intake were noted. Intraperitoneal glucose tolerance test (IPGTT; D-glucose as a bolus of $1 \mathrm{mg} / \mathrm{g}$ body weight) was used to assess glucose tolerance before and after EPA/DHA feeding. Plasma lipids were measured by enzymatic methods, insulin by RIA (Linco), and blood glucose by glucometers (OneTouch Ultra; LifeScan). DNA content in epididymal fat was measured by fluorometry. Statistical analysis was performed using One way ANOVA or t-Test. 
Results: Compared to mice maintained on a HF diet, 2 mo of EPA/DHA feeding resulted in a significantly lower body weight gain $(5.2 \pm 0.3$ vs. $2.4 \pm 0.9 \mathrm{~g})$ and plasma levels of triacylglycerols $(2.00 \pm 0.29$ vs. $1.21 \pm 0.17 \mathrm{mmol} / \mathrm{L})$, nonesterified fatty acids $(0.70 \pm 0.05$ vs. $0.44 \pm 0.03 \mathrm{mmol} / \mathrm{L})$, and cholesterol $(5.58 \pm 0.26$ vs. $4.24 \pm 0.23 \mathrm{mmol} / \mathrm{L})$ in the fed state. Differences in fasted blood glucose $(103 \pm 6$ vs. $89 \pm 5 \mathrm{mg} / \mathrm{dL})$ were of borderline significance $(P=0.07)$. Fasted plasma insulin, blood glucose and plasma insulin in the fed state, as well as weights of the liver, white subcutaneous and epididymal fat, and interscapular brown adipose tissue did not differ between the groups. Cellularity of epididymal fat, assessed as a DNA content, did not change. Comparison of IPGTT data obtained before and after the treatment with EPA/DHA showed significant improvements in blood glucose levels during the first $60 \mathrm{~min}$ of the test, while no significant changes were observed in the control group maintained on a HF diet.

Conclusion: In contrast to a potent effect of EPA and DHA in the prevention of HF diet-induced obesity and IR, these n-3 PUFAs exerted only modest effects on body weight and glucose tolerance once obesity and IR had been already established. However, the strong lipid-lowering effect was retained even in the obese animals. The potency of EPA and DHA to affect adiposity and insulin sensitivity during the development or reversal of obesity correlates with their differential effect on cellularity of white adipose tissue. Prevention of adipose cell proliferation by EPA and DHA may be essential for the potent and beneficial effects of these $n-3$ PUFAs on adiposity and insulin sensitivity.

\section{PS 36}

\section{Other hormones I}

\section{7}

Angiotensin II modulates $\mathrm{Na}^{+} / \mathrm{K}^{+}$ATPase subunits in diabetic rat kidney

K. Rosta ${ }^{1}$, A. Fekete ${ }^{2}$, E. Végh ${ }^{1}$, É. Ruzicska ${ }^{3}$, A. Somogyi ${ }^{3}$, Á. Vér ${ }^{1}$;

${ }^{1}$ Department of Medical Chemistry, Molecular Biology and Pathobiochemistry, ${ }^{2} 1$ st Department of Pediatrics, ${ }^{3} 2$ nd Department of Internal Medicine, Semmelweis University, Budapest, Hungary.

Background and Aims: Angiotensin II (AII) is synthesized in the kidney and is a progressive mediator of renal injury in diabetic nephrosclerosis. Previous studies demonstrated that AII directly stimulates $\mathrm{Na}^{+} / \mathrm{K}^{+}$ATPase (NKA) activity via angiotensin 1 receptor in the proximal tubules, thereby affecting blood pressure and contributing to hypertension. The clinical efficacy of angiotensin II receptor blockers has been widely implicated in the therapy of diabetic nephropathy, but the exact mechanism is still unknown. Thus we investigated the direct effects of AII treatment on renal $\mathrm{Na}^{+} / \mathrm{K}^{+}$ ATPase, a key enzyme of kidney function in diabetic rats. The present study evaluates renal mRNA, protein expression, phosphorylation and enzyme activity of NKA $\alpha-1$ and $\beta-1$ subunits in AII treated streptozotocin (STZ) diabetic rats.

Materials and Methods: Six-week-old, male Wistar rats control (C) and STZ (65 mg/kg) induced diabetic after 3 weeks (D3) and 7 weeks (D7) from the onset of diabetes were divided into diabetic and insulin treated (2 times daily 2-4 $\mathrm{U}$ insulin (Humulin U Lilly) according to the blood glucose levels), reverted diabetic (D3R and D7R) groups. All five groups were further subdivided into untreated and AII treated $(24 \mathrm{~h}, 33 \mu \mathrm{g} / \mathrm{kg} / \mathrm{h}$ via osmotic pump) subgroups (CA, D3A, D7A, D3AR, D7AR). The minipump was implanted under anesthesia (40 mg pentobarbital sodium/ kg ip.) Kidney cortex was homogenized and specific mRNA levels were quantified by RT-PCR. The protein expression of the $\alpha 1$ and the $\beta 1$ subunits of NKA as well as the phosphorylation status were analyzed using Western blotting technique and specific antibodies. The enzyme activity was measured in a coupled reaction. The data were analyzed on STATISTICA.6 software $\left(\right.$ StatSoft ${ }^{\circledR}$ Inc., USA). Data are presented as means \pm S. $\mathrm{D}$, and were tested for normal distribution with Kolmogorov-Smirnov test. Criterion for significance was $\mathrm{P}<0.05$ in all experiments.

Results: In D7 rats the mRNA expression and protein level of $\alpha 1$ subunit of NKA was significantly elevated. This change was not observable in D3 animals. Administration of insulin reverted the changes observed in the D7 animals by lowering the level of the $\alpha 1$ subunit mRNA and protein. AII administration alone elevated the mRNA and protein expression of the $\alpha 1$ subunit in C, D3 and in D7 animals. This effect was less pronounced when the diabetes was reverted with insulin. A rise in the mRNA and protein levels of the $\beta$ subunit of NKA was also observable in reponse to AII treatment, but to a lesser extent. Both diabetic condition and AII treatment increased NKA enzyme activity measured in D7 rats, while D3 rats did not exhibit the same change in enzyme activity. Both diabetic condition and AII administration changed the serin phosphorylation level of the $\alpha 1$ subunit.

Conclusion: Diabetes and AII administration both elevate the level of mRNA and protein of the $\alpha 1$ subunit and increase the activity of NKA. AII effect is more pronounced, when the animal suffers from long term (D7) untreated diabetes. Diabetic condition seems to potentiate the effect of AII on the activity of NKA. This might 
contribute to the development of hypertension in diabetes mellitus and explain the beneficial effect of AII blockers.

\section{8}

Prolactin modulates the association and phosphorylation of SNARE and kinesin/MAP-2 proteins in neonatal rat islets

D. A. Cunha, L. P. Roma, A. C. Boschero;

Physiology, Unicamp, Campinas, Brazil.

Background and Aims: During pregnancy and the perinatal period of life, prolactin induces maturation of the stimulus-secretion coupling mechanism in pancreatic $\beta$-cells. However, the mechanisms of mobilization of vesicles as well as the extrusion of the insulin containing granules are not yet completely understood. We investigated here whether the improved secretory response to glucose by prolactin, involves alteration in the association and phosphorylation of several proteins that participate in these processes.

Materials and Methods: The association and phosphorylation of proteins was analyzed by immunoprecipitation followed by Western blotting, after acute exposure to prolactin.

Results: Association between SNARE proteins and kinesin/MAP-2 was increased by prolactin while the association of munc-18/syntaxin 1A was decreased. Serine phosphorylation of SNAP-25, syntaxin 1A, munc-18, MAP-2 was significantly higher while kinesin phosphorylation was decreased in prolactin-treated islets. We observed an increase in SNARE complex formation in islets stimulated by prolactin, $22 \mathrm{mM}$ glucose, $40 \mathrm{mM}$ of $\mathrm{K}^{+}$, Carbachol and PMA. The prolactin-induced increase in the formation of SNARE complex and syntaxin 1A phosphorylation was inhibited by PD098059, a blocker of MAPK pathway.

Conclusion: These findings indicate that prolactin prime the pancreatic $\beta$-cells to release insulin by increasing the phosphorylation/association of proteins implicated in the secretory machinery, probably involving the MAPK/PKC pathway.

Supported by CAPES, FAPESP

\section{9}

Splanchnic cortisol production in dogs occurs primarily in the liver with only a small contribution from viscera and visceral fat: Evidence for tissue specific $11 \beta$ hydroxysteroid dehydrogenase type 1 activity

R. Basu ${ }^{1}$, D. Edgerton ${ }^{2}$, R. Singh ${ }^{3}$, A. Cherrington ${ }^{2}$, R. Rizza ${ }^{1}$;
${ }^{1}$ Endocrinology,Metabolism \& Nutrition, Mayo Clinic College Of Medicine, Rochester, ${ }^{2}$ Molecular Biology \& Biophysics, Vanderbilt University Medical Center, Nashville, ${ }^{3}$ Laboratory Medicine and Pathology, Mayo Clinic College Of Medicine, Rochester, United States.

Background and Aims: Glucocorticoids are potent regulators of protein, fat and carbohydrate metabolism. We have shown in humans that the splanchnic bed produces cortisol at rates approximating extra-adrenal tissues by converting cortisone to cortisol via the $11 \beta$-hydroxysteroid dehydrogenase type1 (11 $\beta$-HSD-1) pathway. Since portal vein catheterization is invasive and impractical in humans and in vitro studies indicated that $11 \beta$-HSD-1 activity is present in both visceral fat and liver, the present studies were undertaken to determine the relative contribution of the viscera and the liver to splanchnic cortisol production in the dog.

Materials and Methods: Seven dogs underwent the combined hepatic/portal vein catheterization method and an infusion of cortisol (F) labeled with 4 deuteriums (4DF). Total body, portal and hepatic D-3 cortisol production (an index of $11 \beta$-HSD-1 activity) were measured.

Results: Hepatic artery (HA), portal vein (PV) and hepatic vein (HV) cortisol concentrations did not differ $(0.33 \pm 0.12$ vs. $0.31 \pm 0.11$ vs. $0.29 \pm 0.10 \mu \mathrm{g} / \mathrm{dl})$ indicating zero net cortisol balance. However, 4DF concentrations progressively decreased ( $p<0.0001$ ANOVA) from the HA to PV to HV $(2.6 \pm 0.09$ vs. $2.4 \pm 0.08$ vs. $1.7 \pm 0.06 \mu \mathrm{g} / \mathrm{dl})$ resulting in $8.0 \pm 2.0 \%$ and $30.3 \pm 2.0 \%$ extraction across the viscera and liver indicating $0.07 \pm 0.03$ and $0.33 \pm 0.03 \mu \mathrm{g} / \mathrm{min}$ cortisol uptake ( $p<0.04$ and $p<0.0001$ vs. zero ; $p<0.0002$ vs. one another). Since net cortisol balance was zero, this meant that the viscera was producing cortisol at a rate of $0.02 \pm 0.01 \mu \mathrm{g} /$ $\min (\mathrm{p}<0.04$ vs zero) and the liver $1.0 \pm 0.0 \mu \mathrm{g} / \mathrm{min}(\mathrm{p}<0.001$ vs. zero; $p<0.001$ vs. viscera). $4 \mathrm{DF}$ loses a deuterium during conversion via $11-\beta$ HSD type 2 to $3 \mathrm{D}$ cortisone (3DE) which in turn yields $3 \mathrm{DF}$ when $3 \mathrm{DE}$ is converted to cortisol via $11-\beta$ HSD type 1 . Therefore $3 \mathrm{DF}$ production provides an index of cortisone to cortisol conversion via the 11- $\beta$ HSD type 1 pathway. $3 \mathrm{DE}$ concentrations did not differ in $\mathrm{PV}$ and HA $(0.46 \pm 0.04$ vs. $0.40 \pm 0.06 \mu \mathrm{g} / \mathrm{dl})$ but were markedly lower $(\mathrm{p}<0.002)$ in the HV $(0.02 \pm 0.002)$ indicating extensive uptake of the precursor cortisone by the liver but not by the viscera. $3 \mathrm{DF}$ concentrations were lower $(\mathrm{p}<0.01) \mathrm{PV}$ vs. HA $(0.45 \pm 0.03$ vs $0.49 \pm 0.02)$ but did not differ in the HV indicating net uptake across the viscera but zero balance across the liver. 3DF uptake averaged $0.01 \pm 0.00$ indicating a visceral 3DF production rate of $0.02 \pm 0.01 \mathrm{ug} / \mathrm{min}(\mathrm{p}<0.05$ vs. zero). In contrast, $3 \mathrm{DF}$ uptake across the liver averaged $0.08 \pm 0.01 \mu \mathrm{g} / \mathrm{min}$ indicating liver $3 \mathrm{DF}$ production rates of $0.09 \pm 0.01 \mu \mathrm{g} / \mathrm{min}$ ( $\mathrm{p}<0.0001 \mathrm{vs}$. zero). $3 \mathrm{DF}$ production was greater $(\mathrm{p}<0.0002)$ in the liver than the viscera indicating 
greater flux via the 11- $\beta$ HSD type 1 pathway. Total body cortisol production rates in extra splanchnic tissues (e.g. the adrenals) averaged $1.7 \pm 0.2 \mu \mathrm{g} / \mathrm{min}$.

Conclusions: We conclude that in dogs a) the viscera and liver both take up cortisol with extraction being greater in the liver, b) small amounts of cortisol may be made by the viscera (D3F data), however c) in excess of $80 \%$ of splanchnic cortisol production occurs within the liver suggesting a potentially important role in the regulation of hepatic glucose, fat and protein metabolism.

Supported by NIDDK 29953

\section{0}

Progesterone-induced inhibition of glucose utilisation in rat skeletal muscle: involvement of a receptor located on the cell surface?

B. Brunmair, F. Gras, L. Quarré, W. Waldhäusl,

C. Fürnsinn;

Dept. Med. III, Div. Endocrinol. Metab., Medical University of Vienna, Austria.

Background and Aims: Late pregnancy is characterised by loss of insulin sensitivity, which can lead to deterioration of glucose homeostasis and gestational diabetes. This has been attributed, at least in part, to increased circulating concentrations of progesterone. The present study was to examine, if and how progesterone directly affects glucose metabolism of skeletal muscle, which is the predominant tissue for insulin-induced glucose uptake.

Materials and Methods: Freshly isolated rat soleus muscle strips were incubated with progesterone or other steroids for $4 \mathrm{~h}$ and insulin-stimulated $(25 \mathrm{nmol} / \mathrm{l})$ rates of glucose metabolism were measured during the subsequent hour. Furthermore, the expression of a progesterone binding protein in skeletal muscle was examined by semiquantitative PCR using specific primers.

Results: Exposure to $1 \mu \mathrm{M}$ progesterone, a concentration reached in plasma of pregnant rats and humans, impaired insulin-stimulated glycogen synthesis in isolated rat muscle $(-14 \pm 5 \%, \mathrm{p}<0.01$; all results given as $\%$ change vs. intraindividual control), which resulted in decreased muscle glycogen content $(-13 \pm 5 \%, \mathrm{p}<0.02)$. This became more distinct at higher concentrations of progesterone with an almost complete suppression of glycogen storage by $50 \mu \mathrm{M}$ progesterone (glycogen synthesis: $3 \mu \mathrm{M},-33 \pm 6 \% ; 10 \mu \mathrm{M}$, $-73 \pm 3 \% ; 50 \mu \mathrm{M},-87 \pm 6 \%$; glycogen content: $3 \mu \mathrm{M}$, $-28 \pm 5 \% ; 10 \mu \mathrm{M},-52 \pm 4 \% ; 50 \mu \mathrm{M},-91 \pm 7 \% ; \mathrm{p}<0.001$ each). Impaired glycogen storage was accompanied by reductions in the rates of glucose transport $(50 \mu \mathrm{M}$ progesterone; $-15 \pm 4 \% ; \mathrm{p}<0.005)$ and glucose oxidation $(-80 \pm 3 \% ; \mathrm{p}<0.001)$. Pharmacological inhibitors of tran- scription, translation, or the cytosolic progesterone receptor failed to block progesterone-induced impairments of glycogen synthesis (effect of $50 \mu \mathrm{M}$ progesterone in the presence of $1 \mathrm{mg} / 1$ actinomycin $\mathrm{D},-61 \pm 10 \%$; of $1 \mathrm{mg} /$ 1 cycloheximide, $-68 \pm 7 \%$; and of $10 \mu \mathrm{M}$ RU486, $-83 \pm 2 \% ; \mathrm{p}<0.001$ each) and glucose oxidation $(-52 \pm 10 \%,-53 \pm 4 \%$, and $-79 \pm 3 \%$, respectively; $\mathrm{p}<0.001$ each), suggesting that progesterone acted via a non-genomic mechanism that could involve a receptor located on the cell surface. Two progesterone binding proteins on the cell surface have been described and are regarded as mediators of non-genomic progesterone actions (i.e. putative progesterone receptors). Whereas one of them is not expressed in skeletal muscle, we found that the other, which is believed to mediate the progesterone-induced acrosome reaction of sperm, is abundant in rat skeletal muscle. The relative potentials of various steroids to inhibit glycogen synthesis showed similarities with their reported binding activities to this progesterone binding protein. This included that testosterone was a relatively potent inhibitor of glycogen synthesis $(-61 \pm 6 \%, p<0.001)$ while dexamethasone was rather weak $(-13 \pm 8 \%, \mathrm{~ns})$, and that corticosterone $(-36 \pm 4 \%$, $\mathrm{p}<0.001)$ was clearly superior to cortisol $(-12 \pm 6 \%$, $\mathrm{p}<0.01$; steroids compared at $50 \mu \mathrm{M}$ ).

Conclusion: Progesterone distinctly reduces glucose utilisation by isolated rat skeletal muscle via a non-genomic mechanism of action. This mechanism could contribute to impaired glucose homeostasis in late pregnancy and could serve to shift more fuel substrate towards the fetus. Furthermore, our findings make it tempting to speculate that this effect could be mediated by a progesterone binding protein located on the cell surface, which also mediates the acrosome reaction of sperm.

0571

Physiological concentrations of C-peptide increase Na, K-ATPase expression via PKC- and MAP kinasedependent activation of transcription factor ZEB in human renal tubular cells

A. V. Chibalin, Z. Zhong, O. Kotova, I. Ehrén,

K. Ekberg, J. Wahren;

Department of Molecular Medicine and Surgery, Karolinska Institutet, Stockholm, Sweden.

Background and Aims: Accumulating evidence indicates that replacement of proinsulin C-peptide in type 1 diabetes ameliorates nerve and kidney dysfunction. These disorders are associated with decreases in Na,K-ATPase activity. C-peptide shows specific binding to a G-protein coupled membrane receptor resulting in $\mathrm{Ca}^{2+}$ influx, activation of a PKC and 
MAP kinase signaling pathways and stimulation of $\mathrm{Na}, \mathrm{K}$ ATPase activity. We determined the molecular mechanism by which long-term exposure to C-peptide stimulates $\mathrm{Na}, \mathrm{K}$ ATPase expression and activity in primary human renal tubular cells (HRTC) in normo- and hyperglycemic conditions.

Materials and Methods: Human renal tubular cells were cultured from the outer cortex of renal tissue obtained from patients undergoing elective nephrectomy. Cells were cultured in $5 \mathrm{mM}$ or $25 \mathrm{mM}$ glucose in absence or in presence of $1 \mathrm{nM}$ or $10 \mathrm{nM}$ of C-peptide. Ouabain-sensitive rubidium $\left({ }^{86} \mathrm{Rb}^{+}\right)$uptake and $\mathrm{Na}, \mathrm{K}$-ATPase activity were determined. Abundance of Na,K-ATPase was determined by Western blotting in intact cells or isolated basolateral membranes (BLM). DNA binding activity of transcription factors was determined by electrical mobility shift assay (EMSA).

Results: Culturing of HRTCs with $1 \mathrm{nM}$, but not $10 \mathrm{nM}$, of human C-peptide for 5 days resulted in $62 \%(\mathrm{p}<0.05)$ increase in $\mathrm{Na}, \mathrm{K}$-ATPase $\alpha_{1}$-subunit protein expression, accompanied by $37 \%(\mathrm{p}<0.05)$ increase in ${ }^{86} \mathrm{Rb}^{+}$uptake, both in normo- and hyperglycemic conditions. High glucose concentrations did not affect Na,K-ATPase $\alpha_{1}$ subunit expression but $\mathrm{Na}, \mathrm{K}$-ATPase activity was reduced in BLM isolated from cells cultured in presence of high glucose in a PKC-dependent manner, suggesting that during hyperglycemia $\mathrm{Na}, \mathrm{K}$-ATPase may have internalized and sequestered in intracellular stores. Exposure to high glucose resulted in significant $(\mathrm{p}<0.01)$ increase in phosphorylation of ERK1/2, PKC $\alpha / \beta$ and decrease in I $\mathrm{B} \alpha$ abundance in the absence or presence of $\mathrm{C}$-peptide. In parallel with augmented $\alpha_{1}$-subunit expression and stimulation of ouabainsensitive ${ }^{86} \mathrm{Rb}^{+}$uptake, exposure to $1 \mathrm{nM}$ of $\mathrm{C}$-peptide increased DNA binding activity of ZEB (AREB6), a transcription factor involved in regulation of $\mathrm{Na}, \mathrm{K}$-ATPase $\alpha_{1}$-subunit expression. ZEB DNA binding activity was not affected by exposure to $10 \mathrm{nM}$ C-peptide or high glucose concentrations. Effects of $1 \mathrm{nM} \mathrm{C}$-peptide on Na,K-ATPase $\alpha_{1}$-subunit expression and ZEB DNA binding activity in HRTC were abolished by overnight incubation with PKC inhibitor $(1 \mu \mathrm{M}$ GF109203X) or MEK1/2 inhibitor $(10 \mu \mathrm{M}$ PD98059), suggesting involvement of conventional and novel PKCs and ERK1/2 in the regulation C-peptide induced sodium pump expression.

Conclusion: Our results indicate that C-peptide in physiological concentration stimulates sodium pump expression via activation of ZEB, a transcription factor not previously implicated in C-peptide-mediated signaling. The results suggest that despite activation of ERK1/2 and PKC by hyperglycemia, C-peptide influences a distinct pool of PKCs and ERK1/ 2 in the regulation of $\mathrm{Na}, \mathrm{K}$-ATPase expression and activity.

Supported by Swedish Research Council and Creative Peptides $A B$
0572

Mechanisms by which the Amylin analogue pramlintide reduces postprandial glucose (PPG) excursions in humans

H.-J. Woerle ${ }^{1}$, M. Albrecht ${ }^{1}$, R. Linke ${ }^{2}$, C. Neumann ${ }^{3}$, S. Zschau ${ }^{3}$, M. Nicolaus ${ }^{1}$, M. Storr ${ }^{1}$, J. Limmer ${ }^{4}$,

B. Goeke ${ }^{1}$, J. Schirra ${ }^{1}$;

${ }^{1}$ 2nd Medical Department, ${ }^{2}$ Department of Nuclear Medicine, Klinikum Grosshadern, LMU University, Munich, Germany, ${ }^{3}$ Diabetes Outpatient Clinic, Munich, Germany, ${ }^{4}$ Amylin Pharmaceuticals, Inc., San Diego, United States.

Background and Aims: Pramlintide helps to reduce PPG excursions by slowing gastric emptying (GE). PPG increases occur due to increases in the rate of glucose appearance. The latter is the sum of endogenous glucose production and the appearance of ingested glucose not been sequestered by the liver. Thus PPG reduction could result from greater suppression of endogenous glucose production or greater meal splanchnic sequestration

Materials and Methods: Nine healthy subjects were studied (4M, 5F, $413 \mathrm{y}, \mathrm{BW} 843 \mathrm{~kg}$, BMI 27.8土1.1 kg/ $\mathrm{m}^{2}$ ). After $3-\mathrm{h}$ primed continuous $13-\mathrm{C}$ glucose infusion, $30 \mathrm{~g}$ pramlintide or placebo (PBO) were injected sc and subjects ate a mixed meal, $50 \mathrm{~g}$ glucose, $25 \mathrm{~g}$ fat and $20 \mathrm{~g}$ protein, enriched with $3 \mathrm{~g}$ 6,6-dideuteroglucose. Also, the meal was labelled to measure GE scintigraphically.

Results: Starting with comparable glucose conc. $(4.5 \pm 0.1$ vs $4.4 \pm 0.1 \mathrm{mM}$, ns, PBO vs verum), PPG excursions were greater over the initial $120 \mathrm{~min}$ with $\mathrm{PBO}(5.2 \pm 0.1 \mathrm{vs}$ $4.8 \pm 0.1 \mathrm{mM}, \mathrm{p}<0.001 \mathrm{PBO}$ vs verum). Insulin concentrations followed a similar pattern and were greater in $\mathrm{PBO}$ $(48.0 \pm 4.3$ vs $23.9 \pm 4.2 \mathrm{U} / \mathrm{ml}, \mathrm{p}<0.001)$.

No differences in glucagon were found $(53.7 \pm 4.7$ vs $52.8 \pm 5.2 \mathrm{pg} / \mathrm{ml}$, ns). Despite lower PP insulin, PPG excursions were lower in the verum, corresponding to significant reduction in total plasma glucose appearance (24.6 \pm 1.3 vs $18.5 \pm 1.4 \mathrm{~mol} / \mathrm{kg} / \mathrm{min}, \mathrm{p}=0.007)$. Endogenous glucose trended lower with PBO $(6.2 \pm 0.6$ vs $8.7 \pm 0.9 \mathrm{~mol} /$ $\mathrm{kg} / \mathrm{min}, \mathrm{p}=0.05)$. Slowed $\mathrm{GE}$ in the verum arm $(67.6 \pm 3.1 \mathrm{vs}$ 78.22 .7 retention at $120 \mathrm{~min} \mathrm{p}<0.05)$ led to a reduction in the rate of appearance of the oral glucose $(18.4 \pm 1.2 \mathrm{vs}$ $9.9 \pm 1.5 \mathrm{~mol} / \mathrm{kg} / \mathrm{min}, \mathrm{p}<0.05)$ consistent with greater splanchnic sequestration of the meal despite lower insulin concentrations. Pramlintide was well tolerated.

Conclusion: Pramlintide reduced PPG excursions by slowing GE which led to greater splanchnic sequestration of the meal reduced pp plasma glucose concentrations.

Supported by Amylin Pharmaceuticals, Inc 
0573

Nicotinic receptors on pancreatic beta cells are regulated by nicotine and glucose

H. Ogata ${ }^{1}$, Z. $\mathrm{Ma}^{2}$, A. Björklund ${ }^{2}$, V. Grill ${ }^{1}$;

${ }^{1}$ NTNU.Faculty of Medicine, Institute of Cancer Research and Molecular Biology, Trondheim, Norway, ${ }^{2}$ Dept of Molecular Medicine and Surgery, Karolinska Institute, Stockholm, Sweden.

Background and Aims: We previously documented for the first time in beta cells specific binding and mRNA expression for different subunits of acetylcholine nicotinic receptors of the neuronal type (nAchr). We have presently tested for protein expression, regulation and functional importance.

Materials and Methods: Rat pancreatic islets and clonal INS-1 cells were used.

Results: Western immunoblotting detected strong signals for the alpha 7 subunit of the nAchr. both in pancreatic islets and INS-1 cells. Culture at 27 vs. $5.5 \mathrm{mM}$ glucose down-regulated the protein expression by $81+/-3$ percent, $\mathrm{p}<0.001$. Exposure to nicotine $(10-6 \mathrm{M})$ for $48 \mathrm{~h}$ upregulated the alpha7 protein by $19+/-5$ percent, $p<0.01$. Mecamylamine which inhibits nAchr. reduced the viability of INS-1 cells 92.2 percent by MTT assay. This negative effect was abolished by pre-exposure to nicotine. On the other hand pre-exposure to nicotine did not affect the impact of toxicity tested by exposure to $\mathrm{H}_{2} \mathrm{O}_{2}, 400$ mikroM, or Interleukin-1beta $0.5 \mathrm{ng} / \mathrm{ml}$ for $24 \mathrm{~h}$ in INS-1 cells.

Conclusion: Subunits of nAchr are strongly expressed and regulated in beta cells. Activation of nAchr may play a positive role for viability of beta cells.

0574

The peroxisome proliferator-activated receptor $-\alpha$ and $\gamma$ agonists modulate regulation and metabolism of glucocorticoids through different mechanisms

X. Chen ${ }^{1}$, J. Li $^{1}$, H. Tian ${ }^{2}$;

${ }^{1}$ Laboratory of Endocrinology and Metabolism, ${ }^{2}$ Department of Endocrinology, West China Hospital of Sichuan University, Chengdu, China.

Background and Aims: Glucocorticoid (GC) is one of main hormonal antagonistics to the physiological function of insulin in vivo and executes its function by glucocorticoid receptor (GR). The increased GC concentrations in circulation or at local tissues have been found closely related with insulin resistance. 11 $\beta$-hydroxysteroid dehydrogenase type 1 (11 $\beta$-HSD1) converts inactive GCs to active GCs at local tissues and the increased gene expression and enzyme activity of 11ß-HSD1 especially in adipose tissue have been found associated with obesity and insulin resistance. The peroxisome proliferator-activated receptor (PPAR)- $\alpha$ and $-\gamma$ agonists are widely used for their roles on regulating lipid and glucose homeostasis. Some researches suggested both PPAR- $\alpha$ and $-\gamma$ agonists could downregulate $11 \beta$-HSD1 gene expression in liver and adipose tissue respectively. Therefore, this study was designed to investigate whether the metabolism of GCs at pre-receptor and receptor levels took part in the development of insulin resistance induced by high fat and high starch diets and whether PPAR- $\alpha$ and $-\gamma$ agonists were involved in the metabolism and regulation of GCs.

Materials and Methods: We observed that gene expression of 11 $\beta$-HSD1 was increased in liver and adipose tissue in rats fed on high fat and high starch diets and was attenuated by PPAR- $\gamma$ agonists Pioglitazone and PPAR- $\alpha$ agonist Fenofibrate.

Results: To our surprising, we first found that Fenofibrate could increase GR isoforms GR $\beta / \mathrm{GR} \alpha$ protein ratio especially in liver tissue resulting in glucocorticoid resistance at receptor level and leading to increased circular corticosterone level. We concluded that the increased expression level of 11 $\beta$-HSD1 was associated with insulin resistance and PPAR- $\alpha$ and PPAR- $\gamma$ agonists modulated regulation and metabolism of glucocorticoids through different mechanisms.

Conclusion: We first discovered that Fenofibrate could induce glucocorticoid resistance and a complex feedback loop may exist between PPAR- $\alpha$ and glucocorticoid receptor.

\section{PS 37}

\section{Other hormones II}

0575

Postprandial hyperglucagonaemia contributes to hyperglycaemia in somatostatin receptor subtype 2 deficient mice with diet-induced obesity and insulin resistance

M. Z. Strowski, V. Singh, S. Zacharias, E. Göncz,

I. Eichhorn, B. Wiedenmann, C. Grötzinger, U. Plöckinger; Hepatologie, Gastroenterologie \& Interdisziplinäres Stoffwechsel-Centrum/Endokrinologie und Diabetes, Charité Universitätsmedizin Berlin, Germany.

Background and Aims: Somatostatin (SST) inhibits glucagon and insulin secretion. Five receptor subtypes for SST are known (SSTR1-SSTR5), all of which are expressed in the endocrine pancreas. SSTR2 inhibits glucagon secretion in vitro. Glucagon increases blood glucose levels through increased hepatic glucose production. Excessive circulating glucagon increases the rate of 
hepatic gluconeogenesis in type 2 diabetes, thereby contributing to hyperglycemia. Although the role of SSTR2 at inhibiting glucagon secretion has been demonstrated in vitro, there are no studies characterizing the role of SSTR2 in type 2 diabetes.

Here, we characterize the role of SSTR2 in regulating glucose homeostasis in a mouse model of type 2 diabetes.

Materials and Methods: Male SSTR2-deficient (SSTR2 ${ }^{-1}$ ) and age matched control mice $\left(\mathrm{SSTR}_{2}{ }^{+/+}\right)$were fed high fat diet (HFD) $(60 \% \mathrm{kcal} \mathrm{w} / \mathrm{w})$ for 14 weeks and the parameters of glucose homeostasis were monitored. Hepatic glycogen and lipid content was quantified enzymatically and visualized by histomorphology. RNA levels of enzymes regulating glycogen synthesis and breakdown were measured by a real time PCR and their activity by Western blot. Insulin, somatostatin and glucose tolerance tests were performed. Glucagon secretion from isolated islets was measured by RIA.

Results: Despite a similar body weight gain in response to HFD, the weight of the visceral fat of SSTR2deficient mice was decreased. Postprandial glucagon, glucose as well as fasting glucose, and free fatty acid levels were increased in SSTR2-deficient mice. Glucose disappearance rate following ip administration of glucose, insulin or SST was delayed in SSTR2 ${ }^{-1-}$ mice. In the postprandial state, SSTR2-deficient mice had decreased hepatic glycogen content. Furthermore, SSTR2-deficient mice had decreased mRNA levels of glucokinase. The activity of glycogen synthase of SSTR2 ${ }^{-1-}$ mice was decreased while glycogen synthase kinase- 3 was increased. The phosphorylation state and mRNA levels of glycogen phosphorylase, phosphorylase-kinase, and CREB were increased. The hepatic lipid content of SSTR2-deficient mice was decreased. Glucose less potently suppressed glucagon secretion from islets isolated from SSTR2deficient mice.

Conclusion: This study for the first time demonstrates the SSTR2 dependent regulation of glucagon secretion in mouse model of type 2 diabetes. Deletion of SSTR2 accounts for the postprandial hyperglucagonemia. Increased glucose concentration may be due to decreased hepatic glucose utilization, lipid accumulation, and increased glycogen breakdown. SSTR2 may provide a valuable therapeutic target at improving hyperglycemia in type 2 diabetes.

Supported by Deutsche Forschungsgemeinschaft

EGR-1 and CREB are target molecules for orexin-A dependant inhibition of glucagon gene expression C. Grötzinger ${ }^{1}$, E. Göncz ${ }^{1}$, S. Mergler ${ }^{1}$, I. Eichhorn ${ }^{1}$, M. Theodoropoulou ${ }^{2}$, G. K. Stalla ${ }^{2}$, B. Wiedenmann ${ }^{1}$, U. Plöckinger ${ }^{1}$, M. Z. Strowski ${ }^{1}$;
${ }^{1}$ Medizinische Klinik Hepatologie, Gastroenterologie, Charité - Universitätsmedizin Berlin, ${ }^{2}$ Klinische Neuroendokrinologie, Max-Planck-Institut für Psychiatrie, München, Germany.

Background and Aims: Glucagon plays a major role in regulating glucose homeostasis. Recently it has been demonstrated that the early growth response-1 transcription factor (egr-1) upregulates glucagon gene expression in response to treatment with gastrin. Orexin-A (OXA) is a hypothalamic neuropeptide that reduces glucagon secretion in vivo, however the effects of OXA on pancreatic A-cells on the cellular level have not yet been demonstrated. Aim of our study was therefore to characterise the effects of OXA on glucagon producing cells (InR1-G9) and the underlying signal transduction pathway.

Materials and Methods: OXR-1 expression in InR1-G9 cells was detected by western blot and the intra-cellular distribution pattern by immunofluorescence. Intracellular concentration of cAMP, the activity of cAMP response element binding protein $\mathrm{B}$ (CREB) and egr-1 were measured by ELISA, western blots and real-time PCR. Changes of the intracellular calcium $\left(\mathrm{Ca}^{2+}\right)$ were monitored by Fura- 2 measurements.

Results: InR1-G9 cells express OXR-1 on mRNA and protein level. OXR-1 was co-localized with glucagon at the cytoplasmatic compartment. OXA reduced glucagon secretion and proglucagon gene expression. Short time OXA treatment (up to $20 \mathrm{~min}$.) decreased intracellular cyclic AMP as well as $\mathrm{Ca}^{2+}$ levels. OXA reduced CREB mRNA and dephosphorylated CREB protein. Furthermore, OXA reduced the expression of egr-1.

Conclusion: Our study provides the first in vitro evidence for OXA as an inhibitor of glucagon secretion and gene expression. In addition we identify cAMP and $\mathrm{Ca}^{2+}$ as intracellular target molecules for OXA action. Furthermore our results demonstrate two different transcription factors CREB and egr- 1 that act as downstream targets for OXA signaling, suggesting a role in mediating the inhibitory effects of OXA on glucagon gene expression. We have now increasing evidence that OXA affects glucagon homeostasis. The inhibition of glucagon biosynthesis and secretion by OXA may provide a potential use in therapy of glucagon hypersecretion in type 2 diabetes.

0577

Orexin-A inhibits glucagon secretion and gene expression by foxo1 dependant pathway

E. Göncz ${ }^{1}$, C. Grötzinger ${ }^{1}$, S. Mergler ${ }^{1}$, B. F. El-Zayat ${ }^{2}$, M. Theodoropoulou ${ }^{3}$, G. K. Stalla ${ }^{3}$, B. Wiedenmann ${ }^{1}$, M. Z. Strowski ${ }^{1}$, U. Plöckinger ${ }^{1}$; 
${ }^{1}$ Medizinische Klinik Hepatologie, Gastroenterologie, Endokrinologie und Stoffwechsel, Charité - Universitätsmedizin Berlin, ${ }^{2}$ Klinik für Unfall-, Wiederherstellungs- und Handchirurgie, Philipps-Universität Marburg, ${ }^{3}$ Klinische Neuroendokrinologie, Max-Planck-Institut für Psychiatrie, München, Germany.

Background and Aims: Orexin-A (OXA) regulates food intake and energy homeostasis. In vivo, OXA increases insulin secretion and inhibits glucagon secretion, suggesting a role of OXA in regulating glucose homeostasis. However, it is not known whether the effects of OXA are mediated through a direct interaction with the pancreatic Acells. Aim of the study was therefore to provide the evidence for the direct interaction of OXA with glucagon producing cells, describe the biological effects, and characterize the underlying intracellular mechanisms.

Materials and Methods: The effects of OXA on glucagon secretion were evaluated using an in situ perfused rat pancreas model and clonal pancreatic A-cells (InR1-G9). Glucagon was measured by RIA. OXR-1 expression in InR1-G9 cells was detected by western blot and immunofluorescence. The effects of OXA on intracellular cyclic AMP, AKT, PDK-1 were measured by ELISA and western blots, changes of the intracellular calcium $\left(\mathrm{Ca}^{2+}\right)$ by Fura-2 measurements. Proglucagon and foxo1 mRNA levels were quantified by real-time PCR. Silencing of foxo1 in InR1G9 cells was performed by transfection with short interfering RNA (siRNA).

Results: OXR-1 expression in InR1-G9 cells was detected by western blots and confirmed by immunofluorescence. OXA inhibited glucagon secretion from perfused rat pancreas and InR1-G9 cells, and proglucagon gene expression in InR1-G9 cells. OXA decreased intracellular cyclic AMP and $\mathrm{Ca}^{2+}$ concentrations, and increased the phosphorylation of AKT und PDK-1. PI-3 kinase inhibitor blocked the effects of OXA on proglucagon gene transcription. Silencing of foxo1 had no effects on basal proglucagon gene expression; however the inhibitory effect of OXA on glucagon gene expression was reversed.

Conclusion: Our study provides the first in vitro evidence for the interaction of OXA with pancreatic A-cells. OXA inhibits glucagon secretion and proglucagon gene expression. In addition we identify cAMP/AKT/PDK-1 and $\mathrm{Ca}^{2+}$ as intracellular target molecules for OXA action. Furthermore, we demonstrate that foxol mediates the inhibition of proglucagon gene expression in response to OXA treatment. Inhibition of glucagon secretion by OXA may have potential implication at lowering hyperglucagonemia frequently encountered in type 2 diabetes.
0578

Metabolic evaluation of FGF-21 transgenic mice

A. Koester, C. L. Broderick, Y. Chen,

A. Kharitonenkov, A. Ford;

${ }^{1}$ Eli Lilly and Company, Indianapolis, United States.

Background and Aims: Fibroblast Groth Factor 21 (FGF21 ) is a secreted $20 \mathrm{KDa}$ protein and a novel member of the FGF family that is predominantly expressed in the liver and to a lower extent in the asinar cells of the pancreas. While the majority of the 22 known members of FGF family have been primarily associated with mitosis, transformation, angiogenesis, and survival, recent data shows that they may play important roles in defining and regulating functions of some endocrine-relevant tissues and organs, as well as modulating various metabolic processes. We have recently discovered FGF-21 as a potent regulator of glucose homeostasis.

Materials and Methods: To further investigate the FGF-21 biology we generated and explored FGF-21 transgenic mice. Results: FGF-21 transgenic mice that express the protein from the liver are viable, lean, resistant to diet induced obesity, and overall have a favorable metabolic phenotype.

Conclusion: While these mice have a well characterized phenotype, little is known about the underlying mechanisms by which FGF-21 over-expression modulates metabolism. Indirect calorimetry and extensive gene expression data of several important metabolic tissues will be presented that provide further insight in the biology of FGF-21.

0579

Effect of acute hyperinsulinaemia and AT-1 receptor blocade on activity of $5 \alpha$-reductase and aromatase in healthy subjects and subjects with type 2 diabetes

K. Zidkova ${ }^{1}$, E. Krusinova ${ }^{1}$, J. Vrbikova ${ }^{2,1}$, M. Hill ${ }^{2}$, T. Pelikanova ${ }^{1}$;

${ }^{1}$ Diabetes Center, Institute for Clinical and Experimental Medicine, Prague, ${ }^{2}$ Department for Steroid Hormones, Institute of Endocrinology, Prague, Czech Republic.

Background and Aims: Several components of reninangiotensin system, including AT-2 and AT-1 receptor were detected within the testis. Hyperinsulinaemia/insulin resistance is another factor interacting with steroidogenesis. Connection between lower testosterone levels and type 2 diabetes in men was delineated, however the effect of hyperinsulinaemia and acute AT-1 receptor blockade on testosterone metabolism in vivo was not investigated till now. The aim of the study was to study the effect of acute hyperinsulinaemia with and without acute AT-1 receptor 
blockade (AT) on the activity of $5 \alpha$ - reductase and aromatase in men with type 2 diabetes (D) and healthy subjects $(\mathrm{H})$.

Materials and Methods: Serum concentrations of testosterone (T), dihydrotestosterone (DHT) and estradiol (E2) were measured before and at 240 minutes of hyperinsulinaemic (1 mU.kg $\left.{ }^{-1} \cdot \mathrm{min}^{-1}\right)$ euglycaemic (5 mmol. $1^{-1}$ ) clamp (HIC), and during HIC after acute AT-1 receptor blockade (cumulative dose of losartan was $200 \mathrm{mg}$ ) using the same protocol (AT-HIC) in $8 \mathrm{H}$ and in $11 \mathrm{D}$. Enzymatic activities were computed as product/precursor ratios (DHT/ $\mathrm{T}$ for 5 alpha reductase $(5 \alpha \mathrm{R})$ and E2/T for aromatase (A)). ANOVA with repeated measures, followed by Bonferroni multiple comparisons were used.

Results: D had significantly lower insulin sensitivity than $\mathrm{H}$, as measured by metabolic clearance rate of glucose (MCR) $(\mathrm{p}<0.001)$, without any significant changes during AT-HIC in either group. DHT was lower in D than in H $(p<0.02)$ and declined during HIC $(p<0.001)$ in all subjects; the decrease was more pronounced in $\mathrm{H}(\mathrm{p}<0.006$; ANOVA). The AT increased the DHT levels $(p<0.01)$ in all subjects. $5 \alpha \mathrm{R}$ activity was lower in $\mathrm{D}$ than in $\mathrm{H}$ $(p<0.006)$ and declined after HIC $(p<0.001)$. The decrease was more pronounced in $\mathrm{H}(\mathrm{p}<0.02$; ANOVA). After losartan administration, $5 \alpha \mathrm{R}$ declined only in $\mathrm{H}(\mathrm{p}<0.02$; ANOVA) and similarly A activity declined in $\mathrm{H}$, but not in D $(p<0.006$; ANOVA).

Conclusion: In type 2 diabetes, lower DHT levels and $5 \alpha \mathrm{R}$ activity was found compared to healthy subjects. Acute hyperinsulinaemia declines DHT concentrations; the decrease is more pronounced in $\mathrm{H}$, in $\mathrm{D}$ the reaction is mittigated. Losartan administration results in an increase in DHT, also more pronounced in H. AT-1 receptor blockade could thus prevent untoward decline of DHT during acute hyperinsulinaemia.

Serum concentrations of DHT and activity of $5 \alpha \mathrm{R}$ in D and H.

\begin{tabular}{llllll}
\hline & & $\begin{array}{l}\text { D } \\
\text { DHT } \\
\left(\mathrm{nmol} .1^{-1}\right)\end{array}$ & $\begin{array}{l}5 \alpha \mathrm{R} \\
(\mathrm{DHT} / \mathrm{T})\end{array}$ & $\begin{array}{l}\text { KHT } \\
\left(\mathrm{nmol} .1^{-1}\right)\end{array}$ & $\begin{array}{l}5 \alpha \mathrm{R} \\
(\mathrm{DHT} / \mathrm{T})\end{array}$ \\
\hline \multirow{2}{*}{ HIC } & 0 min. & $3.27 \pm 0.40$ & $0.284 \pm 0.031$ & $2.25 \pm 0.34$ & $0.191 \pm 0.035$ \\
& 240 min. & $2.45 \pm 0.35$ & $0.203 \pm 0.031$ & $2.19 \pm 0.38$ & $0.151 \pm 0.027$ \\
AT-HIC & 0 min. & $3.54 \pm 0.64$ & $0.268 \pm 0.050$ & $2.59 \pm 0.53$ & $0.184 \pm 0.049$ \\
& 240 min. & $2.52 \pm 0.55$ & $0.201 \pm 0.029$ & $2.47 \pm 0.65$ & $0.183 \pm 0.057$ \\
\hline
\end{tabular}

Data are expressed as median \pm standard error. Evaluated with ANOVA with repeated measures, statistic significance in text.

Supported by IGA MH CZ No.NR/7880-3 and No.NR/8525-5
0580

Glucocorticoids impair glucose metabolism of isolated rat skeletal muscle via a direct non-genomic mechanism C. Fürnsinn, F. Gras, B. Brunmair, V. Marian, W. Waldhäusl;

Dept. Med. III, Div. Endocrinol. Metab., Medical University of Vienna, Austria.

Background and Aims: Increased concentrations of circulating glucocorticoids are known to impair glucose homeostasis and are considered to contribute to the development of type 2 diabetes. This has been attributed to the activation of the cytosolic glucocorticoid receptor, which affects pancreatic insulin secretion and visceral fat accumulation. Although skeletal muscle is the most important tissue for insulin-induced glucose lowering, the possible contribution of glucocorticoid effects on muscle has hardly been studied. To close this gap in knowledge, we examined the direct effects of glucocorticoids on glucose handling by skeletal muscle.

Materials and Methods: Freshly isolated specimens of rat soleus muscle were exposed to corticosterone, which is the predominant glucocorticoid in rats and was used at concentrations ranging from $1 \mu \mathrm{M}$ (corresponding to diurnal peak plasma levels) to $50 \mu \mathrm{M}$. During the last hour of incubation, rates of glucose metabolism were measured under stimulation with $25 \mathrm{nM}$ insulin (if not stated otherwise). Pharmacological blockers and other steroids were used to analyse the mechanims underlying the observed effects.

Results: $5 \mathrm{~h}$ of corticosterone exposure dose-dependently reduced insulin-stimulated glycogen synthesis in the muscle specimens (control: $3.5 \pm 0.1 \mu \mathrm{mol}$ glucose incorporated into glycogen $\mathrm{g}^{-1} * \mathrm{~h}^{-1}$; changes induced by corticosterone: $1 \mu \mathrm{M},-0.4 \pm 0.2 ; 10 \mu \mathrm{M},-0.5 \pm 0.2 ; 50 \mu \mathrm{M},-1.1 \pm 0.2$; $\mathrm{p}<0.05$ each), which resulted in decreased glycogen content (control: $11.7 \pm 0.7 \mu \mathrm{mol}$ glucosyl units/g; changes induced by corticosterone: $1 \mu \mathrm{M},-1.4 \pm 0.3 ; 10 \mu \mathrm{M},-1.6 \pm 0.7$; $50 \mu \mathrm{M},-1.7 \pm 0.8 ; \mathrm{p}<0.05$ each). Rates of glucose oxidation were reduced in parallel (control: 1.03 $\pm 0.04 \mu \mathrm{mol}$ glucose $* \mathrm{~g}^{-1} * \mathrm{~h}^{-1}$; changes induced by corticosterone: $1 \mu \mathrm{M},+0.10 \pm 0.05$, ns; $10 \mu \mathrm{M},-0.15 \pm 0.04, \mathrm{p}<0.05$; $50 \mu \mathrm{M},-0.43 \pm 0.07 ; \mathrm{p}<0.001)$. Relative impairment of glycogen synthesis was similar in the presence and absence of various concentrations of insulin and, hence, could not be attributed to blunted insulin sensitivity ( $\%$ decrease induced by $50 \mu \mathrm{M}$ corticosterone: no insulin, $-34 \pm 11 \%$; $0.3 \mathrm{nM}$ insulin, $-27 \pm 7 \% ; 3 \mathrm{nM}$ insulin, $-34 \pm 11 \% ; 30 \mathrm{nM}$ insulin, $-34 \pm 11 \%$; $<0.02$ each). Furthermore, inhibition of glycogen synthesis by $50 \mu \mathrm{M}$ corticosterone occurred rapidly ( $\mu \mathrm{mol}$ glucose $* \mathrm{~g}^{-1} * \mathrm{~h}^{-1}$ : after $30 \mathrm{~min}, 8.0 \pm 0.6 \mathrm{vs}$. 
$5.7 \pm 0.3$; after $90 \mathrm{~min}, 6.1 \pm 0.2$ vs. $2.3 \pm 0.5 ; \mathrm{p}<0.01$ each) and was not affected by concomitant exposure to an inhibitor of transcription ( $5 \mathrm{~h}$, without vs. with $1 \mathrm{mg} /$ 1 actinomycin D: $-1.5 \pm 0.2$ vs. $-1.5 \pm 0.4$; ns), which indicates a non-genomic mechanism of action. Glycogen synthesis was also reduced by $50 \mu \mathrm{M} 11$-dehydro-corticosterone $(-1.7 \pm 0.2 ; \mathrm{p}<0.001)$, but was hardly affected by $50 \mu \mathrm{M}$ dexamethasone $(-1.0 \pm 0.4, \mathrm{p}=0.06, \mathrm{~ns})$, which contrasts with the agonistic activities of these compounds at the cytosyolic glucocorticoid receptor (dexamethasone >corticosterone $>>11$-dehydro-corticosterone).

Conclusion: Glucocorticoids directly impair glycogen storage and glucose oxidation in isolated rat skeletal muscle, which is mediated by a non-genomic mechanism of action and does not involve the "classic" cytosolic glucocorticoid receptor. This non-genomic pathway could contribute to glucocorticoid-induced impairment of glucose metabolism in vivo.

\section{1}

Effects of dehydroepiandrosterone (DHEA) on AMPK activity and gene expression in fat tissue

K. Kajita, T. Ikeda, I. Mori, M. Matsumoto, K. Matsubara, Y. Uno, H. Morita, T. Ishizuka; General Medicine, Gifu University, Japan.

Background and Aims: Numerous evidences indicate DHEA reduce adipose tissue in men and animals, however precise mechanism has been unclear. In this study, we investigated the effects of DHEA treatment on gene expression in adipose tissue. Furthermore, we measured AMP-activated kinase (AMPK) activity, which is the target of leptin and adiponectin, in fat tissue.

Materials and Methods: Epididymal fat was obtained from male C57BL mice after breeding with or without (control) $0.5 \%$ DHEA containing food for 7 day. Approximately 30000 genes were analyzed with Acegene Oligo Chip (HitachiSoft, Japan). AMPK activity was determined by the amount of phospho-AMPK (pAMPK) and phosphoacetyl CoA carboxylase (pACC) levels.

Results: DHEA decreased adipose tissue weight by $22 \%$. It elevated p-ACC levels significantly. Three hundred four genes were upregulated more than $200 \%$ (vs control) with DHEA administration, whereas 361 genes were reduced less than $50 \%$. Although few genes involved in energy metabolism such as malonyl-CoA decarboxylase were affected, genes involved in intracellular signal transduction, such as Rho GTPase activating protein 23 , integrin $\alpha 7$, protein phosphatase 1, ras homolog gene family were increased, and genes of phosphatidylinositol 3-kinase, regulatory subunit, (p85a), ADP ribosylation like 6, rho-associated coiled-coil forming kinase 2 decreased. Many genes regulat- ing transcription and cell cycle, such as foxal, telometic repeat binding factor 2 , interacting protein, retinoblastoma protein. Although apoptosis-related genes, such as caspase 3, were upregulated, no evidences of apoptosis, such as cleaved caspase 3 and cleaved PARP, were detected.

Conclusion: These results suggested that DHEA regulates the amount of adipose tissue through the expression level of genes involved in intracellular signaling and transcription.

\section{2}

Glucagon hypersecretion underlies glucose intolerance in CAPS-deficient mice

D. Speidel ${ }^{1,2}$, I. Lundquist ${ }^{3}$, N. Brose ${ }^{2}$, P. Rorsman ${ }^{1,4}$, E. Renström ${ }^{1}$;

${ }^{1}$ Clinical Sciences Malmoe, Lund University, Malmoe, Sweden, ${ }^{2}$ Molecular Neurobiology, Max-Planck-Institute for Experimental Medicine, Goettingen, Germany, ${ }^{3}$ Experimental Medical Science, Lund University, Sweden, ${ }^{4}$ Centre for Diabetes, Endocrinology and Metabolism, University of Oxford, United Kingdom.

Background and Aims: CAPS1 $\left(\mathrm{Ca}^{2+}\right.$-dependent activator protein for secretion) is thought to play an essential role in mediating exocytosis from transmitter storing large densecore vesicles (LDCVs) in neuroendocrine cells. Antibody inhibition studies in chromaffin cells, melanotrophs and mouse pancreatic beta-cells indicate that CAPS1 may be required for a late release phase of LDCV. However, our studies on perinatal lethal CAPS1-knockout mice (CAPS1 $-/-$ mice) indicated that CAPS1 rather influences the uptake or storage of transmitters in LDCVs than their exocytosis. The second isoform, CAPS2, is encoded by a separate gene, but much less is known about its function. Here we have investigated the roles of CAPS1 and CAPS2 in adult pancreatic alpha- and beta-cells.

Materials and Methods: Animal models used are: Wildtype (WT), single homozygous CAPS2 knockout mice (CAPS2 - /-), and double heterozygous CAPS1 and homozygous CAPS2 knockout mice (CAPS1+/-; CAPS2 $-/-)$. These mouse models have been investigated by immunocytochemistry, single cell capacitance measurements of exocytosis, as well as in vitro and in vivo islet hormone release.

Results: Immunocytochemistry with CAPS1- or CAPS2specific antibodies revealed that both CAPS isoforms are widely co-expressed in wild-type islets of Langerhans. Life expectancy and fertility is unaffected in CAPS $2-/-$ and CAPS1+/-; CAPS2 - /- mice. Glucose tolerance is impaired in CAPS1+/-; CAPS2 - - mice compared to CAPS2 $-/-$ mice $(12.6 \pm 0.9$ vs $10.3 \pm 0.4 \mathrm{mmol} / \mathrm{l} ; \mathrm{p}<0.04)$. Interestingly, in vivo and in vitro insulin secretion was not different in CAPS1+/-; CAPS2 $-/-$ and CAPS2 - /- 
mice. By contrast in vitro glucagon release data clearly indicate hypersecretion of glucagon in CAPS1+/-; CAPS2 $-/-$ mice compared to CAPS $2-/-$ mice at $1 \mathrm{mM}$ glucose (100.4 \pm 11.4 vs. $64.3 \pm 8.3 \mathrm{pg} / \mathrm{islet} / \mathrm{h} ; \mathrm{p}<0.001)$, as well as $20 \mathrm{mM}$ glucose $(92.8 \pm 11.3$ v. $56.8 \pm 12.0 \mathrm{pg} /$ islet $/ \mathrm{h}$; $\mathrm{p}<0.03)$. In addition, overall morphology of the pancreas is affected by CAPS deficiency.

Conclusion: We conclude that a) CAPS1 and CAPS2 protein function is (at least in part) overlapping; b) CAPS deficiency results in glucose intolerance, and c) CAPS deficiency specifically affects the secretory capacity of pancreatic alpha-cells. These effects may be secondary to pancreatic development.

Supported by postdoctoral fellowship from the "Deutsche Forschungsgemeinschaft" and by a grant from the "Kungliga Fysiografiska Sällskapet, Lund"

\section{3}

Acute effects of the CB1 receptor antagonist AVE1625 on metabolic biomarkers in wistar rats

M. Gossel, G. Haschke, G. Jaehne, W. Kramer,

A. W. Herling;

Sanofi-Aventis Deutschland GmbH, TD Metabolism, Frankfurt, Germany.

Background and Aims: In acute preclinical studies CB1 receptor antagonists unequivocally demonstrated a powerful reduction of food consumption and body weight, which was more pronounced during starved-to-refed conditions. In multiple dose studies CB1 receptor antagonists showed sustained reductive effects on body weight, but only a transient anorectic action. This indicates, that (i) the effect of $\mathrm{CB} 1$ receptor antagonists must also be present during normal fed conditions, and (ii) that $\mathrm{CB} 1$ receptor antagonists should decrease food effectiveness or in other words increase energy expenditure. The objective of the present study was to investigate mechanistically in fed Wistar rats, whether AVE1625 causes primary effects on metabolic blood and tissue parameters as well as energy balance, which are independent of reduced caloric intake.

Materials and Methods: AVE1625 was administered to normal fed male Wistar rats at doses of 10 and $30 \mathrm{mg} / \mathrm{kg}$. Blood was collected $2 \mathrm{~h}, 5 \mathrm{~h}$ and $6 \mathrm{~h}$ after compound administration for determination of metabolic blood parameters. After $6 \mathrm{~h}$ the rats were killed, the liver was removed for determination of hepatic glycogen content. In the setting of indirect calorimetry the effects of acutely administered $30 \mathrm{mg} / \mathrm{kg}$ of AVE1625 on $\mathrm{O}_{2}$-consumption and $\mathrm{CO}_{2}$ production was measured; energy expenditure, RQ, the oxidation rates of fat and carbohydrates were calculated.
Results: AVE1625 caused a slight dose-dependent increase in basal lipolysis. In spite of increased free fatty acids there was a tendency for a time-dependent decrease of triglycerides within the study period. $6 \mathrm{~h}$ after a single administration of the drug liver glycogen content was dose-dependently reduced to $40-50 \%$ compared to that of untreated controls. These findings demonstrate a primary acute effect of AVE1625 on induction of (i) lipolysis from fat tissue (increased FFA) and (ii) glycogenolysis from the liver (reduced hepatic glycogen). Measured by indirect calorimetry, AVE1625 caused an immediate increase in total energy expenditure, a transient increase of glucose oxidation and a long-lasting increase of fat oxidation, which were consistent with the acute findings on metabolic blood and tissue parameters.

Conclusion: We conclude that in addition to the wellinvestigated effect to reduce body weight by CB1 receptor antagonists due to reduced caloric intake, AVE1625 increased lipid oxidation induced by persistently increased lipolysis from fat tissues, which might significantly contribute to the weight reducing effect of AVE1625. Furthermore, the improvement of serum triglyceride levels appeared to be caused by an additional primary effect of AVE1625 on intermediary metabolism rather than a secondary effect due to reduced body weight.

\section{PS38}

\section{Glucose transport}

\section{4}

Insulin and IGF1 effects on glucose transporter expression in monocytes from obese subjects

A. Kollias ${ }^{1}$, E. Maratou ${ }^{2}$, G. Dimitriadis ${ }^{1}$, E. Boutati ${ }^{1}$, P. Mitrou ${ }^{1}$, V. Lambadiari ${ }^{1}$, V. Komesidou ${ }^{3}$, A.

Papakonstantinou $^{4}$, S. A. Raptis ${ }^{1,2}$;

${ }^{1}$ 2nd Department of Internal Medicine and Research Institute, Athens University Medical School, ${ }^{2}$ Hellenic National Diabetes Center, "Attikon" University Hospital, Athens, ${ }^{3}$ Department of Nutrition, ${ }^{4} 1$ st Department of Surgery, "Evangelismos" Hospital, Athens, Greece.

Background and Aims: IGF1 exhibits metabolic effects similar to those of insulin. Administration of IGF1 to subjects with insulin resistance reduces hyperglycaemia and insulin requirements, implying that this peptide may compensate for the defect in insulin action. Obesity is associated with insulin resistance and is a major predisposing factor for type 2 diabetes. The aim of our study was to examine the effects of IGF1 and insulin on the translocation of GLUT3 and GLUT4 glucose transporter isoforms on the plasma membrane of monocytes from obese subjects. 
Materials and Methods: Blood was withdrawn from 7 nonobese ([NOB], BMI $24+1 \mathrm{Kg} / \mathrm{m}^{2}$, HOMA $\left.1.5+0.5\right)$ and 6 obese subjects ([OB], BMI $37+1 \mathrm{Kg} / \mathrm{m}^{2}$, HOMA $3.3+0.6$ ). Circulating mononuclear cells from blood were obtained by Histopaque gradient centrifugation. Cells were incubated for 1 hour at various concentrations of insulin $(0,0.07,0.17$, $0.7 \mathrm{nM})$ and $\operatorname{IGF} 1(0,0.07,0.14,0.7 \mathrm{nM})$ to determine the expression of GLUT3 and GLUT4 isoforms on the plasma membrane. After termination of the incubation, monocytes were stained with CD14-PE antibody and anti-human GLUT3 or GLUT4 antisera conjugated with Alexa488 and anti-IgG-Alexa488 as an isotype control. Flow cytometry was performed utilizing a FACS Calibur (Becton-Dickinson). The data were analyzed using Cell Quest software and presented as Mean Fluorescence Intensity (MFI). Statistical analysis of insulin and IGF1 dose-response curves has been done with repeated-measures analysis of variance.

Results: (A) Monocytes from NOB: Insulin and IGF1 increased plasma membrane GLUT4 and GLUT3 levels in a dose-dependent manner, with a maximal response at $0.7 \mathrm{nM}$ for each hormone. Insulin increased GLUT4 levels by $42 \%(211+19$ and $299+24 \mathrm{MFI}$ at 0 and $0.7 \mathrm{nM}$ respectively, $\mathrm{p}<0.0001)$ and GLUT3 levels by 39\% (302+ 21 and $419+22$ MFI at 0 and $0.7 \mathrm{nM}$ respectively, $\mathrm{p}<0.0001)$. The respective increases for IGF1 were $21 \%$ in GLUT4 (207+27 and 251+34 MFI, p=0.0005) and 25\% in GLUT3 $(308+27$ and $384+44$ MFI, $\mathrm{p}=0.0005)$. (B) Monocytes from $O B$ : Insulin did not increase the levels of GLUT4 on the plasma membrane $(197+27$ and $216+$ $0.5 \mathrm{MFI}$, at 0 and $0.7 \mathrm{nM}$ respectively, $\mathrm{p}=0.2$ ) but increased those of GLUT3 $(301+9$ and $348+7 \mathrm{MFI}$, at 0 and $0.7 \mathrm{nM}$ respectively, $\mathrm{p}=0.0016)$; the latter increase was significantly less $(p=0.02)$ than that observed in NOB. IGF1 increased both GLUT4 $(197+27$ and $232+21$ MFI, $\mathrm{p}=0.0016)$ and GLUT3 $(298+12$ and $354+5$ MFI, $p=0.0016)$ and these responses were not different from those in NOB.

Conclusion: In obese subjects, translocation of GLUT4 and GLUT3 on the plasma membrane in response to insulin is impaired whilst that in response to IGF1 is retained. The results suggest that although insulin and IGF1 share common signaling pathways, their actions are distinct. This may imply a plausible compensatory role of IGF1 on glucose homeostasis in obesity and insulin resistance.

\section{5}

Activation of p38 MAP kinase is essential for oxidant stress-induced glucose transport in rat skeletal muscle

E. J. Henriksen, J. S. Kim, V. Saengsirisuwan,

J. A. Sloniger;

Department of Physiology, University of Arizona, Tucson, United States.
Background and Aims: We have previously demonstrated that an oxidant stress (hydrogen peroxide) can acutely activate the glucose transport system in isolated rat skeletal muscle. In addition, this oxidant stress simultaneously engages the stressactivated protein kinase $\mathrm{p} 38$ mitogen-activated protein kinase (p38 MAPK). Previous investigations from other research groups indicate that activation of p38 MAPK can modulate the glucose transport system in insulin-sensitive cells and tissues. However, it is currently unclear whether the activation of p38 MAPK is mechanistically involved in the stimulatory effect of hydrogen peroxide on the mammalian skeletal muscle glucose transport system. Therefore, the purpose of the present study was to assess the role of p38 MAPK activation in oxidant stress-stimulated glucose transport in type IIb (epitrochlearis) and type I (soleus) skeletal muscle of insulin-sensitive lean Zucker rats.

Materials and Methods: Isolated muscles were incubated in $8 \mathrm{mM}$ glucose for $2 \mathrm{hr}$ in the absence or presence of $100 \mathrm{mU} / \mathrm{ml}$ glucose oxidase to produce hydrogen peroxide (60-90 $\mu \mathrm{M})$. In addition, muscles were incubated without or with A304000, a selective inhibitor of p38 MAPK. Glucose transport activity was assessed by 2-deoxyglucose uptake, and phosphorylation of p38 MAPK and Akt was performed using standard immunoblotting methods.

Results: Hydrogen peroxide activated glucose transport by $53-66 \%(p<0.05)$ in both muscle types, and this oxidant stress increased thr180/tyr182 phosphorylation of p38 MAPK by $40-50 \%$. Exposure to $10 \mu \mathrm{M}$ A304000 in the presence of hydrogen peroxide had the desired effect of substantially decreasing p38 MAPK phosphorylation without diminishing activation of other kinases associated with glucose transport stimulation, such as Akt ser473 phosphorylation. Importantly, co-treatment with the p38 MAPK inhibitor A304000 essentially abolished the oxidant stressinduced increase in glucose transport in both epitrochlearis and soleus muscles.

Conclusion: Collectively, these data underscore the critical role of p38 MAPK in the acute activation of the glucose transport system by an oxidant stress. The oxidant stressinduced activation of p38 MAPK was essential in both type I and type IIb muscles for stimulation of glucose transport. Further studies are needed to define these interactions between p38 MAPK and the muscle glucose transport system.

0586

Insulin signaling and glucose transport in skeletal muscle from first-degree relatives of type 2 diabetic patients

H. A. Koistinen ${ }^{1}$, H. K. R. Karlsson ${ }^{2}$, M. Ahlsén ${ }^{3}$, J. R. Zierath ${ }^{2}$, H. Wallberg-Henriksson ${ }^{2}$; 
${ }^{1}$ Department of Medicine, Division of Cardiology, Helsinki University Central Hospital, Finland, ${ }^{2}$ Department of Molecular Medicine and Surgery, Karolinska Hospital, ${ }^{3}$ Department of Physiology and Pharmacology, Karolinska Institute, Stockholm, Sweden.

Background and Aims: Aberrant insulin signaling and glucose metabolism in skeletal muscle from type 2 diabetic patients may arise from genetic defects and an altered metabolic milieu. To avoid the influence of hyperglycaemia, we determined insulin action on signal transduction and glucose transport in isolated vastus lateralis skeletal muscle from normoglycaemic subjects at high risk for developing type 2 diabetes: first-degree relatives of type 2 diabetic patients.

Materials and Methods: Normal glucose tolerant firstdegree relatives of type 2 diabetic patients $(n=8,41 \pm 3 \mathrm{yrs}$, BMI $\left.25.1 \pm 0.8 \mathrm{~kg} / \mathrm{m}^{2}\right)$ and healthy control subjects $(\mathrm{n}=9$, $40 \pm 2$ yrs, BMI $23.4 \pm 0.7 \mathrm{~kg} / \mathrm{m}^{2}$ ) with no family history of diabetes were studied. Smaller muscle strips were prepared from open muscle biopsy specimens, and exposed to different concentrations of insulin. Insulin signaling intermediates were determined by Western Blotting. mRNA of key transcriptional factors and co-regulators of mitochondrial biogenesis were determined by Real-Time PCR.

Results: Basal and sub-maximal insulin-stimulated (0.6 and $1.2 \mathrm{nM}$ ) glucose transport was comparable between groups, whereas the maximal response $(120 \mathrm{nM})$ was $38 \%$ lower $(\mathrm{P}<0.05)$ in the relatives. Insulin increased phosphorylation of Akt and its substrate AS160 in a dose-dependent manner, with comparable responses between groups. AS160 phosphorylation and glucose transport were positively correlated in controls $\left(\mathrm{R}^{2}=0.97, \mathrm{P}=0.01\right)$, but not in relatives $\left(\mathrm{R}^{2}=0.46, \mathrm{P}=0.32\right)$. Skeletal muscle mRNA expression of PGC- $1 \alpha$, PGC-1 $\beta$, PPAR $\delta$, NRF-1 and UCP-3 was comparable between first-degree relatives and control subjects.

Conclusion: The uncoupling of insulin action on Akt/ AS160 signaling and glucose transport implicates defective GLUT4 trafficking may constitute an early event in the pathogenesis of type 2 diabetes.

Supported by Swedish Research Council, Finnish Cultural Foundation and Novo Nordisk Foundation

\section{7}

hSGLT5 (SLC5A10)- a novel human sodium-dependent sugar transporter in the kidney

S. Leicht ${ }^{1}$, S. Grohmann ${ }^{1}$, K. Page ${ }^{2}$, R. Streicher ${ }^{1}$, M. Mark ${ }^{1}$, P. Eickelmann ${ }^{1}$;

${ }^{1}$ Boehringer-Ingelheim Pharma GmbH \& Co.KG, Biberach, Germany, ${ }^{2}$ Molecular Expression Profiling, Asterand, Royston, United Kingdom.
Background and Aims: Monosaccharides are absorbed by the small intestine and are reabsorbed in the kidney from primary urine back into the blood. Sodium-dependent sugar transporters are essential to transport the sugars across the plasma membranes. The aim of the present study was to examine the expression and function of a novel member of the human sodium/glucose cotransporter family, the sodium-dependent sugar transporter hSGLT5 (SLC5A10).

Materials and Methods: The localization of the hSGLT5 gene expression in 20 human tissues was determined by the quantitative RT-PCR and compared with the expression pattern of hSGLT2 in these tissues. Concrete localization of hSGLT5 was determined by in-situ hybridization in the kidney.

hSGLT5 cDNA isolated from human kidney RNA was cloned and transfected in HEK293 cells. The function of hSGLT5 was characterized in the FLIPR $^{\circledR}$ membrane potential assay by detecting the $\mathrm{Na}^{+}$-dependent transport activity of hSGLT5 in the presence of different sugars in the transfected cells. Furthermore $\left[{ }^{14} \mathrm{C}\right] \mathrm{AMG}(\alpha$-methyl-Dglucose) and $\left[{ }^{14} \mathrm{C}\right]$ galactose uptake into the HEK/hSGLT5 cells was measured. Monosaccharides and SGLT-inhibitors were added to examine their inhibitory activity towards the $\left[{ }^{14} \mathrm{C}\right] \mathrm{AMG}$ uptake.

Results: It could be shown by quantitative expression analysis that hSGLT5 is selectively expressed in the kidney. The expression pattern corresponds to that of hSGLT2 which is essential for the reabsorption of glucose in the kidney.

The in-situ hybridization revealed the coexpression of hSGLT2 and hSGLT5 mainly in epithelial tissues of the glomerulus and distal and proximal tubulus, a low expression could be shown for the collecting ducts.

In an membrane potential assay the monosaccharides galactose, glucose and fructose lead to a $\mathrm{Na}^{+}$-dependent membrane depolarization whereas AMG and mannose only showed a smaller effect.

The examination of the $\left[{ }^{14} \mathrm{C}\right] \mathrm{AMG}$ uptake into HEK/ hSGLT5 cells revealed a $\mathrm{Na}^{+}$-dependent transport of $\left[{ }^{14} \mathrm{C}\right]$ AMG into the cells. This transport could be inhibited by hexoses, galactose being the most potent one. It could also be shown that hSGLT5 actively transports $\left[{ }^{14} \mathrm{C}\right]$ galactose. Furthermore the $\left[{ }^{14} \mathrm{C}\right] \mathrm{AMG}$ and $\left[{ }^{14} \mathrm{C}\right]$ galactose uptake could be inhibited by Phlorizin and T1095A in a concentration dependent manner.

Conclusion: These results suggest that hSGLT5 is a sodium-dependent sugar transporter, which is mainly expressed in the kidney and serves for the reabsorption of different hexoses. 


\section{8}

Impaired glucose uptake and overexpression of glucotransporter 1 (GLUT1) and glucotransporter 3 (GLUT3) are characteristic of natural killer (NK) cells from subjects in the prediabetic state

P. J. Piatkiewicz ${ }^{1}$, A. Czech ${ }^{1}$, J. Taton ${ }^{1}$, M. Nowaczyk ${ }^{2}$; ${ }^{1}$ Chair and Department of Internal Diseases and Diabetology, ${ }^{2}$ Department of Clinical Immunology, Medical University, Warsaw, Poland.

Background and Aims: Diabetic hyperglycemia is associated with impaired cellular glucose transport. Moreover, fluctuations in blood glucose levels are known to affect peripheral blood cells. This relation can be particularly expressed by glucose transporter proteins and glucose uptake by cells of the peripheral blood. Studies of the glucose transport activity of NK cells in the prediabetic state may contribute to the better understanding of the pathogenetic phenomenon in the natural history of Type 2 diabetes. The earlier results revealed significant differences in glucose transport between NK cells of healthy subjects and Type 2 diabetic patients. Therefore the aim of this study was to determine the quantitative expression of glucotransporters GLUT1 and GLUT3 and to evaluate potential abnormalities of glucose uptake in NK cells obtained from prediabetic persons in comparison with healthy subjects.

Materials and Methods: The study group included 10 patients with a diagnosis of the prediabetic state, naive to any hypoglycaemic drugs. As a control group 9 carefully matched healthy subjects were enrolled. Lymphocytes of the peripheral blood were obtained by Ficoll-Isopaque gradient centifugation. NK cells were isolated and removed by anti-CD16 antibody treatment. Glucose transport was monitored with deoxy-D-glucose $\left(2-\left[{ }^{3} \mathrm{H}(\mathrm{G})\right]\right)$. At previously assigned time points (15, 30, 60 minutes of incubation) deoxy-D-glucose uptake was stopped and its concentration in the cells was measured by scintillation counting. The expression of GLUT1 and GLUT3 was investigated by flow cytometry, which was performed utilizing a FACSCalibur (Becton-Dickinson). Indirect immunofluorescence was applied as a staining technique. Cells were stained by using anti-human GLUT1,3 antibodies (R\&D Systems) and FITC-conjugated immunoglobulin. The data was analyzed using Cell Quest software and presented as a percentage of cells revealing expression of the determined receptor protein.

Results: The study revealed the significant decrease of deoxy-D-glucose transport in NK cells obtained from prediabetic subjects $(\mathrm{P}<0.001)$. The mean values of 4260 ccpm at $15 \mathrm{~min} .8538 \mathrm{ccpm}$ at $30 \mathrm{~min}$. and $10925 \mathrm{ccpm}$ at $60 \mathrm{~min}$. were obtained in NK cells of healthy subjects. The glucose uptake was, respectively, 2076 ccpm, 4151 ccpm and $7907 \mathrm{ccpm}$ in NK cells of prediabetic persons. The decrease of glucose uptake correlated with the higher levels of GLUT1 and GLUT3 in the studied cells. Flow cytometry showed the substantial increase in GLUT1 and GLUT3 proteins level in NK cells of prediabetic patients $(43 \pm 3,7 \%$ and $55 \pm 4,3 \%$ respectively) in comparison with the expression of $21 \pm 1,9 \%$-GLUT1 and $24 \pm 2,3 \%$-GLUT3 in NK cells of healthy subjects.

Conclusion: Cellular glucose transport in NK cells is significantly impaired in the prediabetic state. The high expression of GLUT1 and GLUT3 in NK cells of prediabetic patients is associated with a different pattern of glucose transport in these cells. Overexpression of glucotransporters may become a significant factor in evaluation of glucose metabolic disturbances induced by insulin deficiency in type 2 diabetes. Alterations of GLUT proteins expression in NK-cells seem to be an interesting model system which may be used in recognition of the prediabetic state and evaluation of preventive actions.

0589

Effects of the SGLT2-inhibitor AVE2268 on urinary glucose excretion (UGE) and blood glucose in mice, rats and dogs

M. Bickel, H. Brummerhop, H. Glombik, W. Frick, H. Heuer, O. Plettenburg, U. Werner, W. Kramer; Sanofi-Aventis Pharma Deutschland GmbH, Frankfurt, Germany.

Background and Aims: $\mathrm{Na}^{+}$-dependent glucose transporters (SGLT's) exist in at least two forms. The SGLT1 is present in the brush border membrane of the intestine and transports glucose into the enterocytes. Both, SGLT1 and SGLT2 are found in the kidney and transport glucose from the primary urine into the tubular cells. Inhibition of glucose re-absorption in the kidney by phlorizin has been known for a long time. In diabetic rodents phlorizin have beneficial effect in experimental type 2 diabetes. However, phlorizin is not absorbed from the gut. The search for oral active inhibitors of SGLT2 led to the development of AVE2268, a substituted glucopyranosid. In vitro studies on isolated brush border membrane vesicles showed that AVE2268 inhibited SGLT2 with an $\mathrm{IC}_{50}$ of $6 \mu \mathrm{M}$, the affinity to SGLT1 was lower $\left(\mathrm{IC}_{50}=36 \mu \mathrm{M}\right)$. The aim of the study was, to validate the proof of principle (SGLT2activity) of AVE2268.

Materials and Methods: Dose and time-dependency of urinary glucose excretion (UGE) was tested in mice, rats and dogs. The effect of AVE2268 on blood glucose after a glucose load was tested in mice and rats. Postprandial blood 
glucose was measured in Zucker diabetic fatty (ZDF) rats after a meal.

Results: AVE2268 stimulated UGE in a dose-dependent fashion in mice, rats and dogs. Mice were the most sensitive species, rats being 5 -fold less sensitive. In the time-course experiment in rats, UGE increased from the 1st $\mathrm{h}$ and peaked after 6 to $8 \mathrm{~h}$. AVE2268 decreased blood glucose in mice and rats in a dose-dependent fashion, following an oral or intraperitoneal glucose tolerance test. Postprandial blood glucose was dose-dependently lowered in ZDF rats after a meal.

Conclusion: The present experiments clearly demonstrate that AVE2268 is a preferential inhibitor of SGLT2 in the kidney. Blood glucose after a glucose challenge was lowered in healthy and diabetic rodents. AVE2268 is a new antidiabetic drug that may represent an innovative approach for the treatment of type 2 diabetes.

\section{0}

Loss of detection of sugar abundance in GLUT2 expressing tissues deeply alters mouse glucose homeostasis

E. Stolarczyk ${ }^{1}$, M. Le Gall ${ }^{1}$, P. Even ${ }^{2}$, P. Serradas ${ }^{1}$, V. Tobin ${ }^{1}$, E. Brot-Laroche ${ }^{1}$, A. Leturque ${ }^{1}$;

${ }^{1}$ UMR505, INSERM/UPMC, Paris, ${ }^{2}$ Umr inra 914, INAPG, Paris, France.

Background and Aims: We are investigating the mechanisms by which cells sense nutrients and adapt to their nutritional environment. Glucose is not only a substrate for most mammalian cells but it also generates a signal to the nucleus that regulates gene transcription. In hepatic cells, we can block the stimulation of glucose sensitive gene transcription by inhibiting metabolism or by expressing a GLUT2 loop domain that leaves unaffected glucose metabolism. Our aim was to evaluate in vivo, the importance of the detection of sugar abundance triggered by GLUT2

Materials and Methods: We took advantage of the tool that we developed in vitro, to produce transgenic mice that expressed the GLUT2 loop domain driven in most tissues by an actin promoter. We expected thus to modulate the detection of sugar in GLUT2 expressing tissues i.e. liver, kidney, pancreas, intestine and brain. We measured the perturbations induced by the transgene on glucose homeostasis and on sugar-sensitive gene expression.

Results: Transgenic mice presented growth retardation, which was correlated to transgene expression levels. This occurred despite normal daily food intake and blood glucose concentrations. In response to an oral glucose challenge, we observed pronounced glucose intolerance. Moreover, during OGTT, significantly reduced plasma insulin concentrations and 2 fold increased glycosuria underlined defects in pancreas and kidney functions. Nevertheless, insulin tolerance tests were unchanged suggesting that peripheral tissues that are not expressing GLUT2, were unaffected by the transgene. By indirect calorimetry, we recorded that mice favored lipid over glucose oxidation that was delayed by 3 hours after a mixed meal. A poor central control of satiety in transgenic mice is suspected because meal consumption was not reduced appropriately after a glucose injection. Importantly, in liver, stimulation of gene expressions by sugar diet was almost abolished, but their basal levels were unchanged. These transgenic mice appeared thus unable to detect sugar abundance, but kept the functions displayed by GLUT2 expressing tissues at basal levels. The molecular role of GLUT2 loop domain might be either to anchor proteins of sugar signaling pathway or to mediate transcription activation.

Conclusion: Taken together, these data suggest that the detection of sugar abundance mediated by GLUT2 in liver pancreas, kidney and brain, controls multiple aspects of glucose homeostasis. Perturbations of this physiological process might participate to metabolic diseases.

Supported by INSERM/UPMC

0591

Regulating cell surface GLUT4 arrival and fusion in L6 skeletal muscle cells

V. K. Randhawa ${ }^{1,2}$, M. Ishiki ${ }^{1}$, A. Klip ${ }^{1,2}$;

${ }^{1}$ Programme in Cell Biology, Hospital for Sick Children, Toronto, Canada, ${ }^{2}$ Department of Biochemistry, University of Toronto, Canada.

Background and Aims: Skeletal muscle and adipose are the main insulin-responsive tissues to facilitate glucose uptake via the GLUT4 glucose transporter. Recently, phosphorylation of an Akt substrate and putative RabGAP, AS160, was implicated in GLUT4 traffic in cultured 3T3L1 adipocytes. Insulin-stimulated AS160 phosphorylation was also shown to be impaired in skeletal muscle of type 2 diabetic patients. However, little is known of the mechanism(s) by which AS160 regulates GLUT4 traffic in skeletal muscle. We have recently shown that GLUT4 arrival and/or fusion leading to glucose uptake can be differentially modulated by insulin signaling molecules and phospholipids. Thus, our aim in the present study was to determine whether AS160 regulates GLUT4 arrival and/or fusion in L6 skeletal muscle cells.

Materials and Methods: To this end, we explored the effects of transient expression of wild type (WT) or a nonphosphorylatable mutant (4P) of AS160 using an L6 skeletal muscle cell line stably expressing GLUT4myc. 
By transient transfection of cDNA encoding tetanus toxin light chain (TeTx), which cleaves the toxin-sensitive VAMP2, we also examined its role in mediating surface GLUT4 arrival or fusion. Immunostaining intact or permeabilized "rounded" L6 cells with antibodies directed to the transporter's cytosolic C-terminus or exofacial myc epitope respectively allows detection of GLUT4 arrival or fusion at the plasma membrane.

Results: We show transient expression of AS160 4P prevents insulin-stimulated plasma membrane fusion but not arrival of GLUT4myc. WT AS160 over-expression slightly inhibits insulin-mediated surface GLUT4myc fusion, but not its arrival. Similarly, cleavage of VAMP2 by TeTx prevents GLUT4myc fusion without perturbing cell surface transporter arrival. In contrast, cytoskeletal disruption by cytochalasin D diminishes largely insulin-induced plasma membrane GLUT4myc fusion and partially arrival. Interestingly, AS160 4P also appears to 'cluster' the intracellular GLUT4 in the perinucleus.

Conclusion: Altogether, these data suggest AS160 is an unlikely RabGAP for modulating GLUT4 vesicle movement along cytoskeletal tracks, which partially facilitate insulin-stimulated transporter arrival to the cell surface. We propose that AS160 may regulate the plasma membraneassociated docking and/or fusion of GLUT4 vesicles. It will be interesting to note which Rabs are required for these AS160-dependent events. VAMP2 has a major role in fusion of GLUT4 rather than arrival of GLUT4 at the cell periphery.

Supported by Canadian Institutes of Health Research and Canadian Diabetes Association

0592

Atorvastatin treatment inhibits differentiation and glucose transporter 4 expression in 3T3-L1 adipocytes and impairs glucose tolerance in diabetic NSY mice

T. Yada, M. Nakata;

Physiology, Jichi Medical School, Shimotsuke, Japan.

Background and Aims: HMG-CoA reductase inhibitors (statins) are widely used for controlling hypercholesterolemia and hyperlipidemia, which often occur in patients with type 2 diabetes mellitus. Therefore, type 2 diabetic patients are often treated with statins. However, disadvantageous influence of statins on glucose metabolism has also been reported. Adipocytes have been shown play an important role in glucose metabolism. This study aimed to explore possible effects of statins on the differentiation and glucose handling in adipocytes.

Materials and Methods: Effects of atorvastatin, simvastatin and pravastatin on 3T3-L1 cells in the period during differentiation (culture days 1-7) and on those after differentiation (culture days 8-10) were examined. Adipocyte maturation was determined morphologically. Protein levels and mRNA expressions of glucose transporter 4 (GLUT4) and adipocyte marker proteins were determined by immunoblotting and real-time RT-PCR, respectively. In type 2 diabetic NSY mice, following treatment with atorvastatin for 15 weeks, glucose and insulin tolerance tests were performed and GLUT4 levels in white adipose tissue were examined.

Results: Treatment with atorvastatin $(1 \mathrm{ng} / \mathrm{ml})$ inhibited adipocyte maturation, reduced protein levels and mRNA expressions of GLUT4 and C/EBP $\alpha$, and inhibited insulininduced glucose uptake in 3T3-L1 cells. In differentiated 3T3-L1 adipocytes, atorvastatin also reduced, though to a lesser extent, GLUT4 and $\mathrm{C} / \mathrm{EBP} \alpha$ protein levels and mRNA expressions and insulin-induced glucose uptake. These effects were reversed by L-mevalonate or geranylgeranylpyrophosphate. Neither simvastatin nor pravastatin even at higher doses exerted these effects. In NSY mice, atorvastatin accelerated glucose intolerance due to insulin resistance and decreased GLUT4 in white adipose tissue.

Conclusion: Atorvastatin attenuates adipocyte differentiation and GLUT4 expression by inhibiting isoprenoid biosynthesis and impairs glucose tolerance in type 2 diabetic NSY mice.

\section{PS 39}

\section{Insulin action - human studies}

0593

The study on insulin secretion and insulin action in obese Chinese diabetic patients with unprovoked ketosis

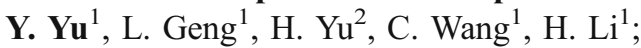
${ }^{1}$ Department of Endocrinology, ${ }^{2}$ Lab. of Endocrinology, West China Hospital of Sichuan University, Chengdu, China

Background and Aims: The frequency of newly diagnosed obese diabetic patients with unprovoked ketosis was increased in recent years in China. The mechanisms of unprovoked ketosis in these subjects were not quite clear. The aim of the present study was to evaluate the peripheral insulin sensitivity and pancreatic $\beta$ cell insulin secretion function in obese Chinese diabetic patients with unprovoked ketosis, and to explore the possible mechanism of unprovoked ketosis in obese diabetic subjects.

Materials and Methods: Two groups of newly diagnosed diabetic patients were involved in this study: OB-DK group: 10 obese diabetic patients with unprovoked ketosis; 
T2DM group: 10 non-obese, non-ketosis type 2 diabetic patients. 10 healthy lean volunteers (C) and 10 obese persons with normal glucose tolerance $(\mathrm{OB})$ were enrolled as control. The ICA, IAA, and GAD-Ab were negative in all subjects. The serum insulin, C-peptide, free fatty acids (FFAs) levels were measured at baseline. Then subjects underwent a euglycemic-hyperinsulinemic clamp study (insulin infusion rate: $120 \mathrm{mU} / \mathrm{min} / \mathrm{kg}$ ), intravenous glucose tolerance test (IVGTT) and hyperglycemic clamp study to evaluate the insulin action and insulin secretion.

Results: In OB-DK group, the area under curve $\left(\mathrm{AUC}_{10} \mathrm{~min}\right)$ was smallest $(\mathrm{C}: 352.9 \pm 125.8 \mathrm{mU} / \mathrm{L}, \mathrm{OB}$ : 672.6 $\pm 262.1 \mathrm{mU} / \mathrm{L}, \mathrm{T} 2 \mathrm{DM}: 187.2 \pm 75.8 \mathrm{mU} / \mathrm{L}, \mathrm{OB}-\mathrm{DK}$ : $79.9 \pm 28.4 \mathrm{mU} / \mathrm{L}, \mathrm{p}<0.05$ when compared with $\mathrm{C}, \mathrm{OB}$, T2DM). The second-phase insulin response and glucose infusion rate during steady state of hyperglycemiac clamp were lower in OB-DK when compared with T2DM (2nd Ins: $10.8 \pm 3.7 \mathrm{mU} / \mathrm{L}$, vs. $44.7 \pm 18.8 \mathrm{mU} / \mathrm{L}, \mathrm{p}<0.05$; GIR: $1.14 \pm 0.57 \mathrm{mg} / \mathrm{Kg} . \mathrm{min}$ vs. $7.62 \pm 2.57 \mathrm{mg} / \mathrm{Kg} . \mathrm{min}, \mathrm{p}<0.05)$. The glucose disposal rates during steady state of euglycemic- hyperinsulinemic clamp were very low in DK-OB group (C: $12.8 \pm 1.5 \mathrm{mg} / \mathrm{kg} . \mathrm{min}, \mathrm{OB}: 6.1 \pm 1.2 \mathrm{mg} / \mathrm{kg} . \mathrm{min}$, T2DM: $9.7 \pm 1.1 \mathrm{mg} / \mathrm{kg} . \mathrm{min}$; DK-OB: $4.3 \pm 1.6 \mathrm{mg} / \mathrm{kg} . \mathrm{min})$. The serum FFAs levels were highest in OB-DK group $(\mathrm{C}$ : 364.3 $\pm 158.6 \mu \mathrm{mol} / \mathrm{L}$, OB: $887.9 \pm 157.9 \mu \mathrm{mol} / \mathrm{L}, \mathrm{T} 2 \mathrm{DM}$ : $603.4 \pm 289.1 \mu \mathrm{mol} / \mathrm{L}$, OB-DK: $1019.4 \pm 372.8 \mu \mathrm{mol} / \mathrm{L}$, $\mathrm{p}<0.05$ when compared with $\mathrm{C}, \mathrm{OB}, \mathrm{T} 2 \mathrm{DM})$.

Conclusion: Severely peripheral insulin resistance and markedly impaired $\beta$-cell insulin secretion were observed in obese diabetic patients with unprovoked ketosis. The potential mechanism of ketone over-production in newly diagnosed obese diabetic patients may associate with glucolipotoxicity which severely damaged the B-cell function. It is logical to expect that these diabetic patients may belong to type 2 diabetes.

This work was supported by National Natural Science Foundation of China grants and a research grant from Ministry of Education of the People's Republic of China

0594

Differences in insulin signaling kinetics and glucose transport of human visceral and subcutaneous adipocytes differentiated in vitro from precursor stromal cells

S. Perrini ${ }^{1}$, L. Laviola ${ }^{1}$, A. Cignarelli ${ }^{1}$, F. De Stefano ${ }^{1}$, C. Caccioppoli ${ }^{1}$, A. Natalicchio ${ }^{1}$, A. Leonardini ${ }^{1}$, M. Cignarelli ${ }^{2}$, R. Giorgino ${ }^{1}$, F. Giorgino ${ }^{1}$;

${ }^{1}$ Internal Medicine, Endocrinology and Metabolic Diseases, University of Bari, ${ }^{2}$ Endocrinology, University of Foggia, Italy.
Background and Aims: visceral fat accumulation is a strong determinant of the metabolic and cardiovascular abnormalities that develop in the metabolic syndrome. The objective of this study was to identify signaling and biological differences in adipocytes differentiated in vitro from precursor stromal cells isolated from abdominal subcutaneous (SC) and visceral omental (O) fat biopsies.

Materials and Methods: preadipocytes were obtained from paired $\mathrm{SC}$ and $\mathrm{O}$ biopsies from 6 non-obese subjects with normal insulin sensitivity, and were differentiated into mature adipocytes in vitro. Phosphorylation levels of multiple insulin signaling intermediates were analyzed under basal conditions and after insulin stimulation (10 nM).

Results: Akt phosphorylation on Ser473 and Thr308 peaked at $6 \mathrm{~min}$ and tended to decrease at later time points in $\mathrm{O}$ adipocytes, whereas a more progressive activation of Akt by insulin was evident in SC adipocytes. No difference was found in Akt protein content in the two fat cell populations. Similarly, GSK-3 phosphorylation by insulin was characterised by a 6-min peak in $\mathrm{O}$ adipocytes, which tended to decrease at $30 \mathrm{~min}$, whereas a more persistent phosphorylation was observed in SC cells. Furthermore, in $\mathrm{O}$ adipocytes, insulin stimulation induced a rapid increase in Erk-1/2 phosphorylation at $6 \mathrm{~min}$, which returned to basal levels at $30 \mathrm{~min}$. By contrast, insulin did not induce any significant Erk-1/2 phosphorylation in SC adipocytes. Protein content of both Erk-1 and Erk-2 was found to be increased in $\mathrm{O}$ compared to $\mathrm{SC}$ adipocytes. To verify whether the observed signaling differences translate into changes in biological endpoints, we assessed 2-deoxy-Dglucose uptake in the two fat cell types. Importantly, $\mathrm{O}$ adipocytes showed approximately 2-fold higher glucose transport rates compared to SC adipocytes, both in the basal state and following insulin stimulation.

Conclusion: in conclusion, $\mathrm{O}$ and $\mathrm{SC}$ adipocytes, even when differentiated in vitro from precursor stromal cells, display intrinsic differences in the kinetics of insulin signaling activation. Greater glucose transport responses in $\mathrm{O}$ adipocytes may be explained by more prominent Akt activation by insulin in this fat cell population.

0595

\section{Insulin sensitivity in anorexia nervosa}

A. Prioletta, A. Antonelli, G. Muscogiuri, E. Salomone,

A. Pavone, I. Malandrucco, N. Galiano, A. Pontecorvi,

S. Della Casa, A. Giaccari;

Policlinico Gemelli, Endocrinology UCSC, Rome, Italy.

Background and Aims: In anorexia nervosa, the correct measure of insulin sensitivity could constitute a precise marker of the metabolic state, however, due to the different 
methodologies utilized in literature, results are not concordant. Our aim was to obtain a method to estimate the variations of insulin sensitivity in anorexic patients by comparing four different indices of insulin sensitivity compared with the gold standard euglycemic hyperinsulinemic clamp.

Materials and Methods: Twelve patients affected by

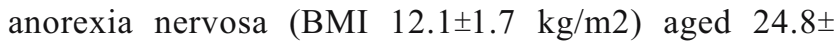
2.12 yrs. and 12 control subjects matched for age but with normal BMI (18-25 kg/m2), underwent OGTT and dosage of FT3, FSH, LH, estradiol and cortisol. With the obtained results we calculated insulin sensitivity indexes according to HOMA-R, McAuley, Matsuda and the insulinogenic index. Results: Although the patients were anorexic, all the surrogate indexes for insulin sensitivity suggested the presence of an increase of this parameter: HOMA-R 0.66 vs. $1.07, \mathrm{p}<0,05$, Matsuda 15.02 vs. $7.35, \mathrm{p}<0.002$; McAuley 3.05 vs. $1.79 \mathrm{p}<0.016$. On the contrary, the sensitivity measured by the glucose clamp demonstrated the presence of insulin-resistance M: 5.35 vs $7.16 \mathrm{mg} / \mathrm{kg} / \mathrm{min}$ $\mathrm{p}<0.02$, even though with a wide range of results. None of the surrogate indexes correlated with the clinical parameters of the disease (FT3; LH; Cortisol, Estradiol). On the contrary, within the group of the anorexic patients, we found a negative correlation between $\mathrm{M}$ and FT3 and Estradiol (clamp-FT3: -0.93; clamp-Estradiol:-0.76).

Conclusion: In anorexia nervosa, surrogate indexes usually derived from OGTT or fasting values are not reliable, probably due to the absorptive and secretive alterations present in these patients. The euglicemic-hyperinsulinemic clamp, although difficult to perform, especially in these patients, remains the only reliable method and not replaceable at the moment. The correlation between insulinresistance and the indexes of remission of the disease seem to suggest (possibly due to remission of the disease and subsequent accumulation of fat in adipocytes) a possible increase of adipokines and may contribute to the difficulty of these patients to recover a normal weight.

\section{6}

Insulin action in adipose tissue and skeletal muscle in hypothyroid patients

P. Mitrou ${ }^{1}$, G. Dimitriadis ${ }^{1}$, V. Lambadiari ${ }^{1}$,

E. Maratou ${ }^{2}$, E. Boutati ${ }^{1}$, E. Koukou ${ }^{3}$, M. Tzanela ${ }^{4}$,

N. Thalassinos ${ }^{4}$, S. A. Raptis ${ }^{1,2}$;

${ }^{1}$ 2nd Department of Internal Medicine and Research Institute, Athens Medical School, Athens, ${ }^{2}$ Hellenic National Diabetes Center, "Attikon" University Hospital, Athens, ${ }^{3}$ Department of Endocrinology, "Elena Venizelou" Hospital, Athens, ${ }^{4}$ Department of Endocrinology, "Evangelismos" Hospital, Athens, Greece.
Background and Aims: Although insulin resistance in hyperthyroidism is well documented, information on insulin action in muscle (M) and adipose tissue (AD) in hypothyroidism is limited. This study was undertaken in patients with hypothyroidism to investigate insulin action on glucose disposal and lipolysis.

Materials and Methods: A mixed meal was given to 11 hypothyroid (HO) (age $45+3$ yrs, BMI $24+1 \mathrm{~kg} / \mathrm{m}^{2}$, T4 $1.6+0.4 \mu \mathrm{U} / \mathrm{dl}$, TSH $72+10 \mu \mathrm{U} / \mathrm{ml}$ ) and 10 euthyroid (EU) subjects (age $42+4 \mathrm{yrs}$, BMI $24+1 \mathrm{~kg} / \mathrm{m}^{2}$, T4 9+1 $\mu \mathrm{U} / \mathrm{dl}$, TSH $1+0.08 \mu \mathrm{U} / \mathrm{ml}$ ). Plasma samples for measurements of glucose (G), insulin (I), triglycerides, glycerol (GLY) and non-esterified fatty acids (NEFA) were taken for $360 \mathrm{~min}$ from veins $(\mathrm{V})$ draining the anterior abdominal subcutaneous $\mathrm{AD}$ depot and forearm $\mathrm{M}$ and from the radial artery (A). Blood flow (BF) in $\mathrm{AD}$ and $\mathrm{M}$ was measured with ${ }^{133} \mathrm{Xe}$ and strain-gauge plethysmography respectively. Calculations: Glucose uptake $(\mathrm{GU})=\left[\mathrm{G}_{(\mathrm{A}-\mathrm{V})}\right] \mathrm{x}[\mathrm{BF}]$; Fractional glucose uptake $(\mathrm{FGU})=\mathrm{G}_{(\mathrm{A}-\mathrm{V})} / \mathrm{G}_{\mathrm{A}} ; \mathrm{LPL}=\left[\mathrm{TAG}_{(\mathrm{A}-\mathrm{V})}\right]$ $\mathrm{x}[\mathrm{BF}] ;$ AD lipolysis $=\left[\mathrm{GLY}_{(\mathrm{V}-\mathrm{A})} \mathrm{x}(\mathrm{BF})\right]-[\mathrm{LPL}]$.

Results: Postprandial changes (area under curve 0-360 min) in HO vs EU: (1) Arterial G was not different $(2295+159$ vs $2063+36 \mathrm{mMmin})$, but I was increased $(15+2$ vs $10+0.7 \mathrm{U} /$ Lmin, $\mathrm{p}<0.05)$. (2) BF $(1290+79$ vs $1579+106 \mathrm{ml} / 100 \mathrm{ml}$ tissue in $\mathrm{M}$ and $706+105 \mathrm{vs} 1340+144 \mathrm{ml} / 100 \mathrm{ml}$ tissue in $\mathrm{AD})$ and $\mathrm{GU}(464+74 \mathrm{vs} 850+155 \mu \mathrm{mol} / 100 \mathrm{ml}$ tissue in $\mathrm{M}$ and $208+42$ vs $406+47 \mu \mathrm{mol} / 100 \mathrm{ml}$ tissue in $\mathrm{AD}$ were decreased $(\mathrm{p}<0.05)$, but FGU was not impaired $(28+6$ vs 33 $+6 \%$ per min in $\mathrm{M}$ and $17+4$ vs $14+3 \%$ per min in $\mathrm{AD}$ ). (3) Suppression of lipolysis by insulin was similar in both groups. (4) Arterial triglyceride levels were increased (489 +91 vs $264+36 \mathrm{nmol} / \mathrm{Lmin}, \mathrm{p}<0.05$ ); LPL was similar in both groups $(42+11 \mathrm{vs} 80+21 \mu \mathrm{mol} / 100 \mathrm{ml}$ tissue in $\mathrm{AD}$ and $48+10$ vs $42+9 \mu \mathrm{mol} / 100 \mathrm{ml}$ tissue in $\mathrm{M})$.

Conclusion: In hypothyroidism: (1) Glucose uptake in muscle and adipose tissue is resistant to insulin; this may be due, at least in part, to the lower blood flow rates in these tissues. (2) Suppression of lipolysis by insulin is not impaired. (3) Hypertriglyceridaemia is not due to decreased triglyceride clearance by adipose tissue or muscle.

0597

Effect of insulin on serum CD40L in subjects with normal or impaired glucose tolerance

E. Muscelli, A. Solini, E. Santini, S. Camastra, F. R. Femia, S. Pinnola, A. Casolaro, E. Ferrannini; Department of Internal Medicine, University of Pisa, Italy.

Background and Aims: Inflammation is an important pathogenic factor in atherosclerosis and coronary heart 
disease, particularly in the context of diabetes, obesity and the metabolic syndrome. The pro-inflammatory mediator, soluble CD40 ligand (sCD40L), which interacts with CD40L expressed on vascular cells, plays an important role in the cascade of inflammatory and proatherothrombotic functions. High serum sCD40L levels have been reported in patients with type 2 diabetes, hypertension, and unstable angina; moreover, its levels increase immediately following an acute myocardial infarction. No information is available on the effects of acute glucose and insulin changes on this marker of atherosclerosis. Aims of the present study were to assess the acute effect of glucose and insulin on SCD40L levels and their relationship with insulin sensitivity in subjects with different degrees of glucose tolerance.

Materials and Methods: 24 subjects with normal glucose tolerance (NGT) and 18 with impaired glucose tolerance (IGT) underwent a 3-h OGTT with measurement of serum sCD40L levels at time 0,90 and $180 \mathrm{~min}$. Insulin sensitivity was assessed by the Oral Glucose Sensitivity Index (OGIS). To distinguish the role of glucose and insulin, 10 other NGT subjects underwent a 2-h euglycaemic hyperinsulinaemic clamp (insulin infusion: $40 \mathrm{mU} \cdot \mathrm{m}^{-2} \cdot \mathrm{min}^{-1}$ ), while a 3 -h time-control study (TC) was performed in 10 healthy volunteers.

Results: In comparison with NGT subjects, IGT subjects were heavier $\left(\mathrm{BMI}=35.9 \pm 2.2\right.$ vs $\left.28.5 \pm 1.5 \mathrm{~kg}^{-2}{ }^{-2}, \mathrm{p}=0.006\right)$, more insulin resistant $\left(319 \pm 12\right.$ vs $374 \pm 12 \mathrm{ml}^{-1} \mathrm{~min}^{-1} \mathrm{~m}^{-2}$, $\mathrm{p}=0.003$ ) and more hyperinsulinaemic both at baseline (122 \pm 19 vs $\left.86 \pm 16 \mathrm{pmolml}^{-1}, \mathrm{p}=0.05\right)$ and during the OGTT (AUC: $102 \pm 11$ vs $69 \pm 8 \mathrm{nmol}^{-1} \cdot 180 \mathrm{~min}, \mathrm{p}=0.02$ ). Fasting sCD40L was $6.64 \pm 0.68 \mathrm{ngml}^{-1}$ in NGT and $3.00 \pm 0.73 \mathrm{ngml}^{-1}$ in IGT $(\mathrm{p}<0.001$ vs NGT). A significant reduction of fasting $\mathrm{sCD} 40 \mathrm{~L}$ concentrations was observed during the OGTT in NGT subjects (from 6.64 \pm 0.68 to $\left.2.48 \pm 0.50 \mathrm{ngml}^{-1}, \mathrm{p}<0.0001\right)$ but not in IGT $(3.00 \pm 0.73$ to $2.58 \pm 0.88 \mathrm{ngml}^{-1}, \mathrm{p}=\mathrm{ns}$ ). This reduction was not due to time-related changes since sCD40L levels did not change during the TC study. During the clamp, insulin induced a marked drop in sCD40L levels (from 10.26 \pm 2.01 to $\left.1.81 \pm 0.58 \mathrm{ng} \cdot \mathrm{ml}^{-1}, \mathrm{p}<0.0001\right)$. In the pooled data from all studies, fasting $\mathrm{sCD} 40 \mathrm{~L}$ was reciprocally related to the area under the OGTT glucose curve $(r=-0.32, \mathrm{p}=0.04)$ and the incremental area under the insulin curve $(r=-0.31, \mathrm{p}=0.05)$, and directly related to insulin sensitivity $(r=0.30, \mathrm{p}=0.05)$.

Conclusion: Fasting circulating sCD40L levels are reduced in IGT. Acute hyperinsulinaemia, whether induced by oral glucose administration or by euglycaemic insulin infusion, reduces sCD40L levels only in NGT. The lack of such response in IGT may be related to their insulin resistance and/or to the chronic hyperinsulinaemia.

Supported by University of Pisa
0598

Possible polycystic ovary syndrome: Any difference
from normal needs to be considered?
M. E. Ertorer ${ }^{1}$, I. Anaforoglu ${ }^{1}$, E. Bozkirli ${ }^{1}$,
O. Bakiner ${ }^{1}$, N. B. Tutuncu ${ }^{2}$, N. G. Demirag ${ }^{2}$;
${ }^{1}$ Endocrinology and Metabolism, Baskent University,
School of Medicine, Adana Medical Center, ${ }^{2}$ Endocrinol-
ogy and Metabolism, Baskent University, School of
Medicine, Ankara Medical Center, Turkey.

Background and Aims: Recently, the term of 'possible' polycystic ovary syndrome (PCOS) has been used for defining cases whose biochemical evaluations are incomplete, but clinical phenotypes are suggestive of PCOS. In this study, by evaluating medical records of the cases admitted with complaints and symptoms suggestive of PCOS, we aimed to detect the rate of possible PCOS and compare their characteristics and insulin sensitivity status with confirmed PCOS cases.

Materials and Methods: Medical records of 118 women, admitted with complaints and symptoms of PCOS were evaluated retrospectively. Menstrual regularity, hirsutismus, age and body mass index (BMI) on admission were recorded. Insulin sensitivity status of all cases were calculated by Homeostasis Model Assesment of Insulin Resistance (HOMA-IR). Hirsutismus was defined if Modified Ferriman-Gallwey score was found to be over 8 by the same three physicians. Diagnosis of PCOS was performed according to Rotterdam 2003 criteria. Considering medical data regarding complaints of patients and clinical evaluations of physicians on admission, cases were subgroupped as: a. Cases with no hirsutism and menstrual dysfunction. (They were evaluated depending on their persistent statements reporting change in body hair.). b. Cases with hirsutism only. c. Cases with menstrual dysfunction only. d. Cases with both hirsutism and menstrual dysfunction. Cases fulfilling Rotterdam 2003 criteria were defined as confirmed PCOS and indeterminate subjects as possible PCOS.

Results: Confirmed PCOS was detected in 70 (59.3\%), possible PCOS in $48(40.7 \%)$ cases. Confirmed PCOS was most prevalent in group d; confirmed PCOS vs possible PCOS; 4 cases $(28.6 \%)$ vs $10(71.4 \%)$ in group a, $8(34.8 \%)$ vs $15(65.2 \%)$ in group b, $26(63.4 \%)$ vs 15 $(36.6 \%)$ in group c, $32(80.0 \%)$ vs $8(20 \%)$ in group d, $(p=0.000)$. Body mass index and HOMA-IR values did not differ between groups, confirmed PCOS vs possible PCOS; $25.46 \pm 5.55 \mathrm{~kg} / \mathrm{m}^{2}$ vs $26.75 \pm 7.55 \mathrm{~kg} / \mathrm{m}^{2}, 3.37 \pm 4.12 \mathrm{vs}$ $3.21 \pm 2.50,(p>0.05)$, subjects with possible PCOS were older $(22.04 \pm 5.28$ years vs $24.87 \pm 6.56, p=0.011)$. Thirtysix $(51.4 \%)$ cases in confirmed PCOS group and 27 
$(56.3 \%)$ in possible PCOS group had family story of type 2 diabetes mellitus $(p>0.05)$.

Conclusion: Possible PCOS group exhibited similar insulin sensitivity status, family story of diabetes and BMI values with confirmed PCOS group. Considering data demonstrating the role of insulin resistance in PCOS and predominance of pancreatic $\beta$-cell dysfunction in PCOS cases who have type 2 diabetic first-degree relatives, we propose that possible PCOS patients may belong to confirmed PCOS group. High rates of confirmed PCOS in group d obviously underlines the compliance of patients to medical care as clinical signs become more severe. Their younger age may be explained with their earlier attempts for medical search with full-blown symptoms. Detection of confirmed PCOS in group a points at the importance of affected individuals' capability of self-assesment for diagnosis. Changes in body hair recognized only by the patient may better be regarded as serious, even though physical examination does not support. Many PCOS patients seem to be undiagnosed due to inadherence to diagnostic work-up. In addition to Rotterdam 2003 criteria, recognition of any difference from normal needs to be considered.

0599

Different relationship between insulin resistance and body fat in young type 2 diabetic patients of Pakistani and Norwegian ethnicity

C. Wium ${ }^{1,2}$, H. B. Eggesboe ${ }^{3}$, I. F. Larsen ${ }^{4}$, T. Sandem ${ }^{5}$, R. Jensen ${ }^{5}$, P. M. Thorsby ${ }^{1,6}$, K. I. Birkeland ${ }^{1,7}$;

${ }^{1}$ Faculty Division, ${ }^{2}$ Dept. of Medicine, ${ }^{3}$ Dept. of Radiology, Aker University Hospital, Oslo, ${ }^{4}$ Dept. of Medicine, Lovisenberg Deaconess Hospital, Oslo, ${ }^{5}$ Faculty of Medicine, University of Oslo, ${ }^{6}$ Hormone Laboratory, ${ }^{7}$ Dept. of Endocrinology, Aker University Hospital, Oslo, Norway.

Background and Aims: Immigrants from South Asia to Western countries have a high prevalence of T2DM. We wanted to study the relationship between insulin sensitivity and BMI, abdominal visceral (VAT) and subcutaneous (SAT) adipose tissue, and fatty infiltration in liver, in young patients with T2DM from Norway and Pakistan living in Oslo.

Materials and Methods: Fifteen patients of Pakistani and 15 of Norwegian ethnicity, with mean \pm SD age $40.3 \pm 3.7$ and $37.3 \pm 6.3$, were studied with anthropometric measurements, fasting blood samples, abdominal CT scans and 2 step (40 and $400 \mathrm{mU} / \mathrm{m}^{2} / \mathrm{min}$ ) euglycemic, hyperinsulinemic clamp, measuring the glucose infusion rate (GIR) in $\mu \mathrm{mol} / \mathrm{m}^{2} / \mathrm{min}$.

Results: Mean BMI, WHR and fasting pl-glucose were similar in the two groups, but the Pakistani group had a significantly higher $\mathrm{HbA1c}(8.9 \pm 1.8$ vs $7.3 \pm 1.1, \mathrm{p}=0.007)$. A tendency towards lower GIR during clamp was observed in the Pakistani group $(320 \pm 274$ vs $726 \pm 743, \mathrm{p}=0.057)$. A significant negative correlation was observed between GIR and BMI in the Norwegian group $(\mathrm{r}=-0.68, \mathrm{p}=0.006)$, but not in the Pakistani group $(\mathrm{r}=-0.43, \mathrm{p}=0.114)$. No differences were observed between the groups in the amount of VAT, SAT, or fatty infiltration in liver, measured by Liver/ Spleen (L/S) ratio. In the Pakistani group, a significant negative correlation was observed between GIR and VAT $(\mathrm{r}=-0.76, \mathrm{p}=0.001)$, and a significant positive correlation between $\mathrm{GIR}$ and $\mathrm{L} / \mathrm{S}$ ratio $(\mathrm{r}=0.64, \mathrm{p}=0.01)$. There was no significant correlation between GIR and VAT $(r=-0.39$, $\mathrm{p}=0.15)$, and GIR and $\mathrm{L} / \mathrm{S}$ ratio $(\mathrm{r}=0.43, \mathrm{p}=0.13)$ in the Norwegian group.

Conclusion: All patients of Pakistani ethnicity were insulin resistant, and strong associations were observed between insulin resistance and measures of visceral fat. Patients of Norwegian ethnicity showed larger heterogeneity with respect to insulin resistance.

\section{0}

A $50 \%$ overweight reduction using a very low calorie diet, significantly improves insulin-stimulated glucose disposal and skeletal muscle insulin signaling in obese type 2 diabetic patients

I. M. Jazet ${ }^{1}$, G. Schaart ${ }^{2}$, A. Gastaldelli ${ }^{3}$, E. Ferrannini ${ }^{4}$, M. K. Hesselink ${ }^{2}$, P. Schrauwen ${ }^{2}$, J. A. Maassen ${ }^{5}$, J. A. Romijn ${ }^{1}$, H. Pij1 ${ }^{1}$, D. M. Ouwens ${ }^{5}$, A. E. Meinders ${ }^{1}$; ${ }^{1}$ Endocrinology and General Internal Medicine, Leiden University Medical Centre, ${ }^{2}$ Nutrition and Toxicology Research Institute Maastricht, Maastricht University, The Netherlands, ${ }^{3}$ IFCNR, University of Pisa, ${ }^{4}$ Internal Medicine, University of Pisa, Italy, ${ }^{5}$ Molecular Cell Biology, Leiden University Medical Centre, The Netherlands.

Background and Aims: Energy restriction per sé and weight loss improve blood glucose levels in obese insulintreated type 2 diabetic patients. Short-term energy restriction decreases basal endogenous glucose production (EGP) with no effect on insulin sensitivity. The blood-glucose lowering mechanism of substantial weight loss has not been fully elucidated in these patients. Especially the effect of weight loss on the disturbed insulin-signaling pathway, leading to impaired translocation of GLUT-4 to the cell membrane, is unknown. The aim of this study was to investigate the effect of considerable weight loss on glucose metabolism, both at the whole-body and at the myocellular level, in obese insulin-treated type 2 diabetic patients.

Materials and Methods: 10 obese (BMI $40.2 \pm 1.6 \mathrm{~kg} / \mathrm{m} 2$ [mean $\pm \mathrm{SEM}]$ ) insulin-treated (mean $94 \pm 14 \mathrm{U} /$ day) type 2 diabetic patients (HbA1c $7.7 \pm 0.4 \%$, FPG $11.1 \pm 0.8 \mathrm{mmol} / \mathrm{L}$ ) were studied during a very low calorie diet (VLCD, 
$450 \mathrm{kCal} /$ day) on day 2 and again after losing $50 \%$ of their overweight $(50 \%$ OWR). Oral blood glucose-lowering agents and insulin were discontinued 3 weeks prior to and at the start of the VLCD, respectively. EGP and wholebody glucose disposal $\left(\left[6,6-{ }^{2} \mathrm{H}_{2}\right]\right.$-glucose $)$, lipolysis $\left(\left[{ }^{2} \mathrm{H}_{5}\right]\right.$ glycerol) and substrate oxidation rates were measured on both study days in basal and hyperinsulinaemic euglycaemic conditions. Skeletal muscle biopsies were obtained from the vastus lateralis muscle in the basal situation and $30 \mathrm{~min}$ after the start of the insulin infusion for determination of insulin signaling, insulin-mediated expression of GLUT-4 and FAT/CD36 at the sarcolemma and intramyocellular triglyceride content.

Results: Weight reduction $(-20.3 \pm 2.2 \mathrm{~kg}$ from day 2 to day $50 \%$ OWR) normalised basal EGP $(20.0 \pm 0.9$ to $16.4 \pm 1.2 \mu$ mol.kgfatfreemass $[\mathrm{FFM}]^{-1} \cdot \mathrm{min}^{-1}$ ) and improved insulin sensitivity, especially insulin-stimulated glucose disposal $\left(18.8 \pm 2.0\right.$ to $39.1 \pm 2.8 \mu$ mol.kgFFM $^{-1}$. min-1, $\mathrm{p}=0.001$ ). Insulin-stimulated phosphatidylinositol 3'-kinase (PI3K)-activity over basal was significantly higher after weight loss and the down-stream PKB/Akt substrates AS160 and PRAS40 showed absolute increases, both in the basal and the insulin-stimulated situation. Insulin-mediated GLUT-4 content at the sarcolemma was increased in 7 out of 10 patients. Intramyocellular triglyceride content decreased, without an alteration of sarcolemmal FAT/CD36 content. Time required to lose $50 \%$ overweight was negatively correlated with the amount of type I fibres at baseline and there was a tendency for an increased percentage type 1 muscle fibres after weight loss.

Conclusion: Considerable weight loss in obese type 2 diabetic patients improves basal EGP and insulin sensitivity, especially insulin-stimulated glucose disposal. This is associated with improved insulin signaling at the level of PI3K, AS160 and PRAS40. The observed decrease in intramyocellular triglyceride content might have contributed to this effect.

\section{PS 40}

\section{Insulin signaling}

\section{1}

\section{Impaired insulin action despite up-regulation of proximal insulin signaling: novel insights into skeletal muscle insulin resistance in liver cirrhosis \\ N. Jessen ${ }^{1}$, E. S. Buhl ${ }^{1}$, O. Schmitz ${ }^{2}$, S. Lund ${ }^{1}$; \\ ${ }^{1}$ Medical Research Laboratory and Medical Department M, Aarhus University Hospital, Aarhus, Denmark, ${ }^{2}$ Department of Clinical Pharmacology, Aarhus University, Denmark.}

Background and Aims: Disturbance in glucose metabolism is a common feature in diseases of the liver. This is reflected by a high prevalence of glucose intolerance among patients with hepatic cirrhosis. Hyperinsulinemic euglycemic clamps studies of cirrhotic patients have shown that insulin resistance in skeletal muscles is a major component of the impaired glucose tolerance but the underlying molecular mechanisms are unclear. Treatment with the somatostatin-analogue octreotide suggests that the insulin resistance may be due to inadequate clearance of insulin by the liver, a pathogenesis that is unlike other common forms of insulin resistance. To further characterize skeletal muscle insulin resistance associated with liver disease we examined isolated muscles from an animal model of liver cirrhosis.

Materials and Methods: Cirrhosis was induced by obstruction of the Common Bile Duct. Clinical findings, elevated plasma levels of liver enzymes and histological examinations confirmed cirrhosis. Whole body insulin sensitivity was assessed with an oral glucose tolerance test. Insulin stimulated glucose transport and insulin signaling was measured in isolated muscles incubated in vitro.

Results: Cirrhotic animals were insulin resistant on a whole body level and this was at least partly explained by reduced insulin stimulated glucose transport into muscle cells. Interestingly, activity in the proximal part of the insulin signaling cascade in skeletal muscles from cirrhotic animals was increased. This was shown both by increased activity of the IRS1 associated PI3 kinase and by increased expression and phosphorylation of PKB/Akt, key enzymes in the signal to glucose transport. Expression of the insulin sensitive glucose transporter, GLUT4, was normal so together these results indicate that signaling downstream of PKB/Akt and/or the translocation of GLUT4 is impaired in skeletal muscle for cirrhotic animals.

Conclusion: In conclusion, in an animal model of liver cirrhosis whole body insulin resistance is associated with insulin resistance in skeletal muscles. Unlike other common forms of insulin resistance muscles from cirrhotic animals have increased activity in the proximal insulin signaling cascade. This emphasizes that skeletal muscle insulin resistance associated with liver cirrhosis is a unique entity.

Supported by the University of Aarhus Research Foundation and the Danish Diabetes Association 


\section{2}

Investigation of the role of Kv1.3 as a potential regulator of peripheral insulin sensitivity

A. S. Gillings ${ }^{1}$, J. Yang ${ }^{2}$, A. E. Marley ${ }^{3}$, G. D. Holman ${ }^{2}$, M. Peckham ${ }^{4}$, E. Kilgour ${ }^{3}$, S. A. Baldwin ${ }^{1}$;

${ }^{1}$ Institute of Membrane and Systems Biology, University of Leeds, ${ }^{2}$ Department of Biology and Biochemistry, University of Bath, ${ }^{3}$ AstraZeneca, Alderley Park, Cheshire, ${ }^{4}$ Institute of Molecular and Cellular Biology, University of Leeds, United Kingdom.

Background and Aims: Kv1.3, a shaker-related voltagegated potassium channel, is highly expressed in the olfactory bulb, and is also expressed in a number of other tissues, including fat and skeletal muscle. Kv1.3 knockout mice weigh significantly less than wild-type littermates, have significantly lower fasting and non-fasting blood insulin levels and are protected from diet induced obesity. Channel inhibition has been reported to facilitate the translocation of the insulin sensitive glucose transporter, GLUT4, to the plasma membrane and increase glucose uptake in adipose and skeletal muscle tissues. Furthermore, a polymorphism in the human Kv1.3 gene has been associated with decreased sensitivity to insulin. This channel is therefore an interesting potential target for the development of novel anti-diabetic therapies. The aim of this study was to further investigate the link between channel inhibition and increased insulin sensitivity, using Kv1.3 channel inhibitors in both primary and cultured cells.

Materials and Methods: Work was carried out using the $\mathrm{H}-2 \mathrm{~K}^{\mathrm{b}}$-tsA58 cell line, a potential model of skeletal muscle cells, the HL-1 atrial cardiomyocyte cell line, NIH 3T3 cells (American Type Culture Collection number CRL1658), 3T3-L1 cells (American Type Culture Collection number CL-173) and isolated primary rat cardiomyocytes and adipocytes. Kv1.3 mRNA expression in cultured and primary cells was measured using real-time RT-PCR. The potencies of three Kv1.3 blockers were tested against Chinese Hamster Ovary cells expressing human Kv1.3 using IonWorksHT, an automated patch clamp instrument. Glucose transport into cells was monitored using $\left[{ }^{3} \mathrm{H}\right]$ deoxy-D-glucose and GLUT4 translocation was followed by immunocytochemical techniques.

Results: Kv1.3 expression at the mRNA level was detected at similar levels in primary rat cardiomyocytes and adipocytes $(0.043 \pm 0.012$ units and $0.044 \pm 0.004$ units, respectively). Low expression levels of Kv1.3 mRNA were observed in $\mathrm{H}-2 \mathrm{~K}^{\mathrm{b}}$-tsA58 myoblasts $(0.008 \pm 0.003$ units $)$ and expression increased following differentiation into myotubes $(0.078 \pm 0.009$ units $)$. Expression was also detected in NIH 3T3 cells but not in the HL-1 cardiomyocyte cell line or 3T3-L1 cells. The Kv1.3 channel blockers, Margatoxin, PAP-1 and Psora-4 were found to be active against human Kv1.3 expressed in Chinese Hamster Ovary cells, with $\mathrm{IC}_{50} \mathrm{~s}$ of $0.9 \pm 0.9 \mathrm{nmol} / 1,22.6 \pm 8.5 \mathrm{nmol} / 1$ and $36.5 \pm 14.8 \mathrm{nmol} / 1$, respectively. Surprisingly, however, we saw minimal effects of these channel inhibitors, both in terms of glucose uptake and GLUT4 translocation, in cells where Kv1.3 mRNA was detected.

Conclusion: Work using transgenic animals has implicated a role for Kv1.3 in regulating peripheral insulin sensitivity. However, despite confirmation of inhibitor activity and channel expression, our present studies, within the limitations of currently available Kv1.3 blockers, have shown only small effects of these inhibitors on cells from insulin sensitive tissues.

Supported by the Biotechnology and Biological Sciences Research Council, with AstraZeneca as an industrial partner in the studentship

\section{3}

Ras associated with diabetes (RAD) modulates inducible nitric oxide synthase (iNOS) activity in vascular smooth muscle cells (vSMC) from diabetic rats

A. Giardinelli ${ }^{1}$, P. Di Tomo ${ }^{2}$, S. Di Silvestre ${ }^{2}$, G. Formoso ${ }^{3}$, P. Di Fulvio ${ }^{3}$, F. Capani ${ }^{3}$, A. Consoli ${ }^{3}$, A. Pandolfi ${ }^{1}$;

${ }^{1}$ Department of Biomorphology, Ce.S.I., University G d'Annunzio, Chieti, Italy, ${ }^{2}$ Ce.S.I., University G d'Annunzio, Chieti, ${ }^{3}$ Department of Medicine and Aging Science, Ce.S.I., University G d'Annunzio, Chieti, Italy.

Background and Aims: In diabetes, inflammation and increased oxidative stress could induce iNOS expression contributing to vascular damage. Rad, a $\mathrm{G}$ protein overexpressed in diabetic animals, binds calmodulin (CaM) in a $\mathrm{Ca}^{2+}$-dependent manner. Since $\mathrm{Ca}^{2+}-\mathrm{CaM}$ can modulate iNOS activity, aim of this study was to investigate the relationships between iNOS activity and Rad expression in vSMC from DR.

Methods and Results: We measured Rad mRNA levels (Real Time PCR), iNOS expression (RT-PCR, Western Blot) and activity (radiometric technique) and nitrotyrosine levels (immunofluorescence) in cultures of aortic vSMC from 10 diabetic (90\% pancreatectomy, DR) and 10 control (sham surgery, CR) rats, after $24 \mathrm{hrs}$ incubation with $20 \mathrm{microg} / \mathrm{ml}$ LPS. LPS increased iNOS mRNA and protein levels to the same extent in CR and DR, while iNOS activity was about 7 folds greater in DR. Notwithstanding increased iNOS activity, cGMP levels were not different between DR and CR cells: DR cells, however, 
exhibited increased nitrotyrosine levels. As to the effect of LPS on RAD expression, exposure to LPS was followed in $\mathrm{CR}$ by a rapid increase in RAD, whereas in DR RAD mRNA levels decreased significantly.

Conclusions: In DR increased CaM availability, possibly due to inhibition of RAD expression, may increase iNOS activity. However, the lack of i.c. cGMP increase and the enhanced nitrotyrosine generation in DR suggest that most of the NO produced in pro-oxidant milieu gets converted into peroxinytrite in these cells. Rad potential role in modulating iNOS activity in diabetes might provide new insight in the mechanisms linking diabetes and atherosclerosis.

Supported by an Italian Government Grant: PRIN 2004 n. 2004050541 to $A P$

\section{4}

Modulation of hENPP1/PC1 gene expression by heat shock protein $\mathbf{7 0}$

R. Di Paola ${ }^{1}$, A. Marucci ${ }^{1}$, G. Miscio ${ }^{1}$, V. Trischitta ${ }^{1,2}$; ${ }^{1}$ Research Unit of Diabetology and Endocrinology, IRCCS "Casa Sollievo della Sofferenza", San Giovanni Rotondo, ${ }^{2}$ Department of Clinical Sciences, Univerity La Sapienza, Roma, Italy.

Background and Aims: The ectonucleotide pyrophosphatase phosphodiesterase 1 (ENPP1, also known as plasma cell glycoprotein 1, PC-1) is a class II transmembrane glycoprotein that directly interacts with and inhibits insulin receptor phosphorylation, thus decreasing insulin signaling and action. ENPP1/PC1 is over-expressed in skeletal muscle, adipose tissue and cultured skin fibroblasts of insulin resistant individuals who are not yet obese nor diabetic, thus suggesting that ENPP1/PC1 over-expression is an early, intrinsic defect in human insulin resistance. Previous data indicate that $3^{\prime}$ untraslated region (3'UTR) regulatory elements may modulate gene expression through the regulation of mRNA stability of ENPP1/PC1. The aim of the present study was to investigate whether potential trans-acting cytosolic factors bind to the 3'UTR of ENPP1/ PC1, thus modulating its expression

Materials and Methods: RNA probes and REMSA analyses.

The most conserved region of the in vitro transcribed ENPP1/PC1 3'UTR, identified by in silico analysis, was used as DNA template for transcribing the RNA probe. Cytoplasmic extract of HEK293 cells and 32P-radiolabeled RNA were incubated in appropriate conditions and the reactions were subjected to $5 \%$ nondenaturing PAGE and visualized by autoradiography.
Isolation of ENPP1/PC1 RNA binding protein. High molecular weight complexes were successively excised and eluted and separated on 10\% SDS-PAGE: The identified band was eluted and analyzed with tandem mass spectrometry. Analysis of mRNA stability in HEK293 cells. HEK293 cells were treated with antisense Hsp70 oligonucleotide at different time and the occurred silencing was analyzed by western blot. ENPP1/PC1 mRNA half-life (after transcription inhibition by Actinomycin-D) in HEK293 cells treated or not with antisense oligonucleotides was evaluated by RT-PCR on ABI Prism SDS 7000 after total RNA extraction at different time points. HEK293 cells were transfected with $\mathrm{pENPP} 1 / \mathrm{PC} 1 \mathrm{cDNA}$ and its mRNA content was measured after treating or not the cells with Hsp70 antisense oligonucleotide.

Results: REMSA using the most conserved region of the ENPP1/PC1 3'UTR, demonstrated the formation of an abundant high molecular mass RNA-protein complex, thus suggesting the presence of mRNA-binding protein $/ \mathrm{s}$ in HEK293 cell cytosolic. Tandem mass spectrometry analysis of the high molecular weight complex identified the heat shock protein 70 (Hsp70), as a ENPP1/PC1 3' UTR binding protein. This result was confirmed by supershift analysis with two different antibody recognizing two different epitopes of HSP70 protein.

The role of Hsp70 in the regulation of ENPP1/PC1 expression was investigated using siRNA-based suppression of $\mathrm{Hsp} 70$ and then evaluating ENPP1/PC1 mRNA stability in HEK293 cells after transcription inhibition. Down-regulation of Hsp70 expression by siRNA was paralleled $d$ by a significant decrease of ENPP1/PC1 mRNA stability $(p<0.01)$. Moreover, in HEK293 cells transfected with ENPP1/PC1 cDNA, Hsp70 siRNA-based suppression caused a significant reduction $(80 \% ; p=0.001)$ in the ENPP1/PC1 mRNA levels as compared to compared to Hsp70 expressing cells.

Conclusion: We identified Hsp70 as ENPP1/PC1 3'UTR binding protein. This specific binding increases ENPP1/ PC1 mRNA stability and levels. Our findings suggest a pathogenic role of Hsp70 in insulin resistance through the modulation of ENPP1/PC1 expression.

Supported by Italian Ministry of Health Ricerche Correnti 2004, 2005

0605

Human insulin increases the outgrowth of circulating progenitor cells via IGF-1 receptor dependent MAPKinase activation

Z. Djuric, P. Humpert, K. Laine, P. P. Nawroth,

A. Bierhaus;

Internal Medicine I, University Clinic Heidelberg, Germany. 
Background and Aims: It has been shown that patients with both diabetes type 1 and 2 have impaired outgrowth and function of endothelial progenitor cells (EPC), which are known to take part in angiogenesis and vascular repair. In patients with poorly controlled type 2 diabetes insulin therapy leads to an increased number of circulating EPC. Therefore, we studied the effect of human insulin and its analogues on outgrowth and cellular signaling cascades in $\mathrm{EPC}$, isolated from healthy volunteers.

Materials and Methods: EPC derived from peripheral blood mononuclear cells (PBMC) of healthy volunteers were grown in medium supplemented with different concentrations of human insulin and insulin analogues in the absence or presence of either IGF-1 receptor- and insulin receptor-inhibitors or specific signal transduction inhibitors. EPC outgrowth was studied using a standardized proliferation assays. Underlying signaling cascades were identified by PCR- based microarrays, RT-PCR and Western Blot assays.

Results: Human insulin significantly increased the formation of EPC colony forming units in a dose-dependent manner with a half maximal effect at $0,25 \mathrm{mU} / \mathrm{mL}$. The only insulin analogue affecting the outgrowth of EPC to a similar extent as insulin was glargine. Incubation of EPC with neutralizing IGF-1 receptor antibodies suppressed EPC proliferation below baseline levels, while inhibition of the insulin receptor had no effect on EPC outgrowth. Insulin dependent EPC proliferation was mediated by IGF1 receptor dependent downstream signals via ERK1/2, p38 MAPK and JK 2.

Conclusion: Human insulin stimulates the outgrowth of circulating EPC via IGF-1 receptors with downstream involvement of MAPKinase pathways. This might imply a potential role of these signaling cascades as therapeutic targets for modulation of EPC-based vascular regeneration in diabetes.

\section{6}

Binding kinetics of insulin and Insulin-like Growth Factor-II to the insulin receptor isoform $A$ and the dynamics and affinity of interaction between the receptor and She after stimulation with these ligands

S. Versteyhe ${ }^{1}$, C. Blanquart ${ }^{2}$, J. N. Larsen ${ }^{1}$, S. G. Gray ${ }^{1}$, T. Issad ${ }^{2}$, P. De Meyts ${ }^{1}$;

${ }^{1}$ Receptor Systems Biology Laboratory, Hagedorn Research Institute, Gentofte, Denmark, ${ }^{2}$ Department of Cellular Biology, Institut Cochin, Paris, France.

Background and Aims: Insulin and the insulin-like growth factors (IGFs)-I and -II bind to and activate receptor tyrosine kinases, thereby triggering a network of signaling pathways. Physiological and genetic evidence suggests that insulin is primarily a metabolic regulator, while the IGFs are primarily growth promoters.

The insulin receptor exists under two isoforms, A (IR-A) and B (IR-B). Recent studies have shown that IGF-II, besides binding to the IGF-I receptor, also binds with high affinity to the $\mathrm{A}$ isoform of the insulin receptor. This binding leads primarily to mitogenic (=growth) effects while insulin binding leads primarily to metabolic effects. This is associated with differential recruitment and activation of intracellular substrates. We and others have shown that insulin analogues with slow dissociation kinetics from the insulin receptor have enhanced mitogenicity. These analogues cause a differential activation of the intracellular substrate She in comparison to insulin. She is the first step in a mitogenic pathway.

We examined, by Bioluminescence Resonance Energy Transfer, in real time and in living human embryonic kidney cells, the dynamics and affinity of interaction between the IR-A and the receptor substrate Shc, after stimulation with insulin, IGF-I and IGF-II.

These data will be correlated with the binding kinetics of insulin and IGF-II on the IR-A, measured by direct 125Iligand binding studies on whole cells. For that we are using a fibroblast cell line derived from cation-independent mannose-6-phosphate receptor/IGF-II knock-out mice (gift from Dr. von Figura). We have now stably transfected this cell line to overexpress the IR-A.

Materials and Methods: HEK 293 cells were co-transfected with IR-A-Renilla Luciferase and EYFP-Shc cDNA. Two days later and after addition of coelenterazine, BRET measurements were performed in 96-well microplates and at room temperature, using a Fusion microplate analyzer.

Results: The first results show that the three ligands dosedependently stimulate the interaction between the receptor and Shc. The maximum BRET signal after stimulation with insulin is reached with $100 \mathrm{nM}$. For IGF-I $2000 \mathrm{nM}$ was necessary and for IGF-II $500 \mathrm{nM}$. This is in accordance with the data on the relative binding affinities of the ligands for this receptor.

No difference in affinity between the receptor and Shc was detected after stimulation with the above mentioned maximum concentrations of the three ligands. From the kinetic measurements it could be seen that the time to reach maximal BRET signal with these concentrations is approximately the same for the three ligands and the signals continue at this level for at least 30 minutes.

Conclusion: Neither the kinetics nor the affinity of interaction between the receptor and Shc were different after stimulation with $100 \mathrm{nM}$ insulin, $2000 \mathrm{nM}$ IGF-I or $500 \mathrm{nM}$ IGF-II. These results can therefore not explain the different biological effects after stimulating the receptor with insulin or IGF-II. 
However, BRET experiments with EC50 concentrations of insulin, IGF-I and IGF-II, are planned. The time the interaction stays intact after removal of the ligands, after stimulation, will also be investigated.

Supported by the Danish Ministry of Science, Technology and Innovation

\section{7}

Function of the novel protein kinase $\mathrm{C}$-dependent serine 357 phosphorylation of insulin receptor substrate-1 on insulin action in skeletal muscle cells

R. Sanaullah Wariach, C. Weigert, A. Beck, W. Voelter, E. D. Schleicher, H. U. Häring, R. Lehmann;

Department of Internal Medicine IV, Eberhard-Karls University, Tuebingen, Germany.

Background and Aims: The function of insulin receptor substrate-1 (IRS-1), a key molecule of insulin signaling, is modulated by phosphorylation at multiple Ser/Thr residues. The present work was aimed to study the (patho)physiological function and interplay of novel Ser/Thr phosphorylation sites in IRS-1.

Materials and Methods: To identify Ser/Thr phosphorylation sites in IRS-1, in vitro phosphorylation assays were performed using GST-IRS-1 protein fragments and protein kinase $\mathrm{C}$ isoforms (PKC). The samples were analyzed by SDS 7.5\% PAGE and visualized by autoradiography. Identification of specific sites was done by mass-spectrometry. To monitor phosphorylation of Ser-357 in cellular extracts we prepared a polyclonal phospho-site specific antibody. Furthermore, Ala-357 and Ala-318 mutants were made by oligonucleotide mediated sitedirected mutagenesis.

Results: Upon incubation of recombinant IRS-1 fragments with various PKC-isoforms we identified Ser-357 of rat IRS-1 (Ser-362 in human IRS-1) as an in vitro $\mathrm{PKC}$ phosphorylation site. Using the phospho-Ser-357 specific antibody we observed in $\mathrm{C} 2 \mathrm{C} 12$ skeletal muscle cells that Ser-357 is strongly phosphorylated by phorbolester (TPA) and to a lesser extent by insulin stimulation. This phosphorylation is mediated, at least partially, by PKC- $\delta$. Moreover, investigating the physiological function of this site on a key molecule in downstream signaling we found after 5 and $10 \mathrm{~min}$ of insulin stimulation, i.e. in the early phase of insulin action, that the activation of $\mathrm{Akt} / \mathrm{PKB}$ was more pronounced in IRS-1 Ala-357 expressing skeletal muscle cells (simulating the unphosphorylated state) compared to cells expressing wild type IRS-1. But, after $60 \mathrm{~min}$ of stimulation, i.e. in the continuing insulin action, no difference in the down regulation of $\mathrm{Akt} / \mathrm{PKB}$ activity between IRS-1 Ala-357 and wild type IRS-1 expressing cells was detectable. Additionally, the phosphorylation of Ser-357 at the early time points of insulin stimulation (5 and $10 \mathrm{~min}$ ) is more pronounced if Ser-318 is in the unphosphorylated state (represented by the Ala-318 mutation), giving a hint for an interplay of these two PKC phosphorylation sites in IRS-1.

Conclusion: In summary we could show phosphorylation of the novel Ser-357 site of IRS-1 after stimulation with PKC activators TPA and insulin in skeletal muscle cells. Moreover, this site is, at least partially, phosphorylated by PKC- $\delta$. Phosphorylation of Ser-357 could exert negative effects on insulin signal transduction. In addition we have evidence for an interplay of the PKC phosphorylation sites Ser-357 and Ser-318 of IRS-1.

Supported by Higher Education Commission, Pakistan \& German Academic Exchange Service (DAAD), Germany for support of Doctoral studies of Rizwana S. Wariach

\section{PS 41}

\section{Insulin action}

\section{8}

Fetal and neonatal carnitine deficiency influences development and induces insulin resistance in rat pups R. Ricciolini ${ }^{1}$, A. Peschechera ${ }^{1}$, M. Scalibastri ${ }^{1}$, F. Russo ${ }^{1}$, M. Giarrizzo ${ }^{1}$, D. Pesce $^{2}$, A. Onali ${ }^{2}$, A. Arduini ${ }^{3}$, P. Carminati ${ }^{1}$, F. Giannessi ${ }^{1}$;

${ }^{1}$ Endocrinology and Metabolism, ${ }^{2}$ Toxicology, Sigma-Tau, Pomezia, ${ }^{3}$ Iperboreal Pharma, Pescara, Italy.

Background and Aims: During normal development, the successful adaptation of neonates to modifications in nutritional state and to various physiological functions requires important changes in glucose and fatty acid metabolism. Carnitine is an important cofactor in fat metabolism and is crucial in the neonatal period, since mitochondrial beta-oxidation is essential for energy production in newborns. Carnitine deficiency was induced in neonates by administration of sodium pivalate to female rats during pregnancy and lactation to evaluate if carnitine deficiency leads to developmental adaptations in various tissues of the offspring, linked to the insulin resistance observed in adult life.

Materials and Methods: Thirty Sprague-Dawley female rats were divided into three groups of ten animals each, treated from day 14 of gestation until weaning (day 21 after birth) with: 1) $20 \mathrm{mM}$ pivalate in drinking water; 2) $20 \mathrm{mM}$ pivalate plus $40 \mathrm{mM}$ carnitine in drinking water 3) vehicle $\left(\mathrm{NaHCO}_{3} 20 \mathrm{mM}\right.$ in drinking water). Carnitine levels were determined in different tissues of fetuses, dams and 
neonates by HPLC-MS. Twelve-day-old rats were orally force-fed a solution containing either glucose $(25 \%)$ or intralipid (10\%), collecting blood samples at 0 and $30 \mathrm{~min}$ after load. Glucose, insulin, peptide $\mathrm{C}$, triglyceride and NEFA levels were determined in serum; on days 12 and 21, glycogen and triglycerides were also measured in the liver, with specific histopathology on 21st day only. Pancreas morphology was assessed in tissues from 3-, 12- and 21day-old pups.

Results: A large reduction of carnitine levels in dams, fetuses and in different neonatal tissues was observed in pivalate group (about $60-80 \%$ of controls). On day 12 basal insulin levels were significantly increased in carnitinedepleted pups compared to controls $(1.09 \pm 0.34$ vs $0.69 \pm 0.20 \mathrm{ng} / \mathrm{ml} \mathrm{p} \leq 0.01$ ); the unchanged peptide $\mathrm{C}$ levels in pivalate group support the hypothesis of a decrease in liver insulin clearance rather than an increase in insulin secretion. Moreover, an increase in triglyceride levels and a decrease in glycogen content were also observed in the liver of suckling rats. Thirty minutes after glucose load an increase in glucose levels was evidenced in pivalate group $(282 \pm 5$ vs $233 \pm 27 \mathrm{mg} / \mathrm{dl} ; \mathrm{p} \leq 0.01)$ with no significant increase in insulin levels. After intralipid load, carnitinedepleted pups showed a reduction in insulin secretion with respect to controls, as evidenced by peptide $\mathrm{C}$ levels $(0.82 \pm 0.20$ vs $1.37 \pm 0.36 \mathrm{ng} / \mathrm{ml}, \mathrm{p} \leq 0.05)$. In pivalate group, an increase in both insulin content and Langerhans islets size was observed only in pancreas from 21- day-old animals, mostly related to increased intracytoplasmic insulin secretory granules and beta cell hypertrophy. Carnitine supplementation restored carnitine levels in pivalate-treated female rats and pup tissues, preventing a majority of above-mentioned alterations.

Conclusion: This work provides evidence of an insulin resistance status during suckling period in carnitine-depleted rats. Carnitine depletion calls for, in the neonate, additional adaptive strategies, both metabolic and organspecific, which may be at the base of the insulin resistance observed in adult rats, as previously reported.

\section{9}

The effect of HMG-CoA reductase inhibitor on glucose tolerance and insulin sensitivity in type 2 diabetes mellitus animal model

H.-S. Kwon, O.-K. Hong, S.-H. Ko, Y.-H. Choi, S.-S. Lee, T.-S. Son, S.-D. Moon, S.-J. Yoo, H.-S. Son, K.-H. Yoon, B.-Y. Cha, H.-Y. Son;

Department of Internal Medicine, The Catholic University of Korea, Seoul, Republic of Korea.
Background and Aims: We examined the effects of early treatment with pravastatin on the progression of glucose intolerance and insulin sensitivity in the type 2 diabetes mellitus (DM) animal model.

Materials and Methods: Ten week-old Otsuka LongEvans Tokushima Fatty (OLETF) rats were used as a model of DM and age-matched Long-Evans Tokushima Otsuka (LETO) rats were used as a normal control. The treated OLETF group was divided into 2 groups, receiving low dose $(20 \mathrm{mg} / \mathrm{kg}$ ) pravastatin (OLETF-Low) and high dose $(100 \mathrm{mg} / \mathrm{kg})$ pravastatin (OLETF-High) in drinking water from 10 weeks to 40 weeks of age. At 10, 20, and 30 weeks, we examined body weight and the amount of food intake in all experimental animals. At the same time, glucose tolerance test (GTT) was performed and various biochemical analyses was done. We also performed insulin tolerance test (ITT). Lipid profiles, free fatty acids, adiponectin, leptin and insulin were also checked.

Results: The GTT revealed that OLETF groups showed higher glucose levels and AUCg (area under the curve of glucose) than LETO group at 30 weeks. At 20 weeks, 50\% and $50 \%$ of untreated OLETF rats were impared glucose tolerance (IGT) and DM, but $75 \%$ and $8.3 \%$ of treated OLETF rats were IGT and DM ( $\mathrm{P}<0.01)$. At 30 weeks, $30 \%$ and $70 \%$ of untreated OLETF rats were IGT and DM, but $50 \%$ and $50 \%$ of treated OLETF rats were IGT and DM $(\mathrm{P}<0.01)$. At 30 weeks, the free fatty acid level was lower in the treated OLETF groups than OLETF-Control group $(\mathrm{P}<0.05)$. The HDL-cholesterol level was higher in the OLETF-High group than the OLETF-Control group. The OLETF-Low group showed improved insulin sensitivity than the OLETF-Control group in the ITT. Adiponectin, leptin and insulin level between groups was not significantly different.

Conclusion: Early treatment with pravastatin retarded the progression of glucose intolerancein OLETF rats. The favorable effects were associated with the reduction of serum free fatty acid, elevated HDL-cholesterol, and the improvement of insulin sensitivity.

0610

Insulin delivery to muscle is sensitive to tissue perfusion M. G. Clark, G. C. Vollus, M. K. Roberts,

S. M. Richards, S. Rattigan;

Biochemistry, University of Tasmania, Hobart, Australia.

Background and Aims: Muscle at rest is only partly perfused and insulin acts in vivo to increase capillary recruitment in muscle of rats and human subjects. In normally responsive rats in vivo, insulin's microvascular effect occurs at physiological doses, independent of total 
blood flow, and prior to changes in Akt phosphorylation or glucose uptake in muscle. To test the possibility that muscle's response to insulin is limited by its delivery, we have partly occluded the rat hindlimb vasculature with $15 \mu \mathrm{m}$ MS in a constant-flow perfused hindlimb preparation. We then determined insulin-mediated phosphorylation of Akt on Ser 473 and the increase in glucose uptake due to insulin. Assessment was also made of total available nutritive capacity, oxygen and glucose uptake. Oxygen, glucose and insulin have different diffusibility into muscle and therefore we hypothesize that insulin delivery is the most sensitive to limited perfusion of muscle.

Materials and Methods: $15 \mu \mathrm{m}$ microspheres (MS) were introduced to partially occlude the microvasculature of the surgically isolated pump-perfused rat hindlimb maintained at constant flow. Muscle samples from MS-injected or control perfusions (vehicle only) were assayed for energy charge (EC), phosphocreatine/creatine ratio $(\mathrm{PCr} / \mathrm{Cr})$, Akt Ser 473 phosphorylation (P-Akt/total Akt) and muscle 2deoxyglucose uptake ( $\left.\mathrm{R}^{\prime} \mathrm{g}\right)$. Basal oxygen uptake, as well as nutritive capacity (defined as the difference in oxygen uptake during vasoconstriction by maximally vascular recruiting and derecruiting vasoconstrictors), were determined by arterial-venous difference.

Results: MS $\left(0,9,15\right.$ and $30 \times 10^{6}$ per 15 g hindlimb muscle) into the pump-perfused $\left(0.5 \mathrm{ml} \cdot \mathrm{min}^{-1} . \mathrm{g}^{-1}\right)$ rat hindlimb led to increased pressure $(-0.5 \pm 0.8,15.9 \pm 2.1$, $28.7 \pm 4.6$ and $60.3 \pm 9.4 \mathrm{mmHg}$, respectively) with minimal changes in oxygen uptake. Nutritive capacity was decreased from $10.6 \pm 1.0$ to $3.8 \pm 0.9 \mu \mathrm{mol} . \mathrm{h}^{-1} . \mathrm{g}^{-1}(P<0.05)$ with $30 \times 10^{6} \mathrm{MS}$. EC was unchanged, but $\mathrm{PCr} / \mathrm{Cr}$ was decreased dose dependently to $61 \%$ of basal at $30 \times 10^{6}$ MS. Insulinmediated increases in P-Akt/total Akt decreased from $2.15 \pm 0.35$ to $1.41 \pm 0.23(P<0.05)$ and $\mathrm{R}^{\prime} \mathrm{g}$ from $130 \pm 19$ to $80 \pm 12 \mu \mathrm{g} \cdot \mathrm{min}^{-1}$.g dry wt ${ }^{-1}(P<0.05)$ with $15 \times 10^{6} \mathrm{MS}$; basal P-Akt/total Akt and R'g in the absence of insulin were unaffected.

Conclusion: Partial occlusion of the hindlimb muscle by a defined number and size of MS has no effect on basal glucose uptake, marginally impacted on basal oxygen uptake but markedly decreased the total available nutritive capacity. Insulin delivery to muscle was impaired, indicated by decreased Akt phosphorylation, and resulted in reduced insulin-mediated glucose uptake in muscle. Glucose delivery was sufficient in basal conditions but may be limited during insulin stimulation additionally contributing to the reduced insulin-mediated glucose uptake. Thus insulinmediated glucose uptake by skeletal muscle cells is critically dependent on tissue perfusion that determines insulin delivery.

Supported by NHMRC and National Heart Foundation of Australia
0611

DAPIT, a novel protein up-regulated in insulin-sensitive tissues in streptozotocin-induced diabetes

H. Kontro;

Pediatric Research Center, University of Tampere, Finland.

Background and Aims: We have previously identified a novel gene product DAPIT (Diabetes Associated Protein in Insulin-sensitive Tissues) differentially expressed in insulin-sensitive tissues in streptozotocin-induced diabetes. DAPIT transcript was homologous with wide range of species and ESTs. DAPIT located inside Usmg5 gene in rat chromosome 1 and in unidentified areas of chromosomes 8 and 10. The amino acid sequence of DAPIT was similar to that of a putative protein of Drosophila melanogaster and to a cyclic AMP (cAMP)-generating peptide isolated from the flesh fly Neobellieria bullata. Our aim was to study DAPIT expression in the insulin-sensitive tissues and in the cell culture systems. We also studied DAPIT effect on cAMP level in DAPIT-transfected cells.

Materials and Methods: By immunohistochemistry and Western blot was studied the DAPIT tissue expression in insulin-sensitive tissues of control and streptozotocindiabetic rat. By Western blot was studied DAPIT expression in serum free culture medium of rat (L6) and mouse (C2C12) muscle cells, human hepatocytes (HepG2) and kidney cells (HEK293T) stably transfected by DAPIT. By enzyme immunoassay was measured the cellular effect of DAPIT on cellular cAMP level in DAPIT-transfected cells. Results: The immunohistochemical studies revealed notable DAPIT expression in skeletal and cardiac muscle, adipose tissue, liver and various structures of brain of normal subjects. The protein expression in insulin-sensitive tissues exhibited clear up-regulation of DAPIT in diabetic myocardium, skeletal muscle and adipose tissue but downregulation in liver. The cell culture studies revealed that native mouse and rat muscle cells, human hepatocytes and DAPIT-transfected cells secrete DAPIT into culture medium. The cellular DAPIT did not affect in cellular cAMP level in over-expression system.

Conclusion: DAPIT shows the expected differential expression in control and in diabetic insulin-sensitive tissues. The cell culture studies and immunohistochemistry let us believe that DAPIT is secreted. The secreted DAPIT effect in secreted cAMP level remains still unknown.

Supported by Medical Research Fund of Tampere University Hospital, University of Tampere and the Foundation for Diabetes Research in Finland 
0612

Antioxidative therapy improving insulin resistance caused by elevated FFAs concentration

Z. $\mathbf{L u}^{1}, \mathrm{Y}$. $\mathrm{Yu}^{2}$;

${ }^{1}$ Lab. of Endocrinology, ${ }^{2}$ Department of Endocrinology, West China Hospital of Sichuan University, Chengdu, China.

Background and Aims: Elevated circulating FFAs concentration, a characteristic of metabolic syndrome, may induce systemic oxidative stress. Here we report that increased oxidative stress caused by elevated blood FFAs concentration is an important pathogenic mechanism of insulin resistance and antioxidative treatment can improve insulin sensitivity via restoration of normal reactive oxygen (ROS) production.

Materials and Methods: Male S-D rats, 6 8 weeks in age, were assigned to three groups and all underwent $6 \mathrm{hr}$ iv infusions in each group: Controls $(C, n=6)$ : saline; FFA $(\mathrm{n}=6): 20 \%$ intralipid1.8 $\mathrm{uL} / \mathrm{min}+$ heparin $0.72 \mathrm{IU} / \mathrm{min}$; Intervention ( $\mathrm{I}, \mathrm{n}=6)$ : GSH $(1.2 \mathrm{mg} / \mathrm{kg} / \mathrm{min})$ and lipid/ heparin. Serum FFAs level and GSH/GSSG ratio both in blood and erythrocyte were measured at baseline and after infusion. The euglycemic hyperinsulinemia clamp study was conducted at the last 2 hours of infusion to evaluate the peripheral insulin sensitivity.

Results: Serum FFAs levels at baseline were similar in the various groups and they were $3 \sim 5$ times higher in FFA group after infusion when compared with $\mathrm{C}$ group and they were intermediate between $\mathrm{C}$ and FFA group in $\mathrm{I}$ group $(\mathrm{C}$ : $340.2 \pm 103.6 \mu \mathrm{mol} / \mathrm{L}$, FFA: $1146.4 \pm 336.6 \mu \mathrm{mol} / \mathrm{L}, \mathrm{I}$ : $620.3 \pm 101.4 \mu \mathrm{mol} / \mathrm{L}, \mathrm{P}<0.05)$. In FFA group the $\mathrm{GSH} /$ GSSG ratio both in serum $(92.1 \pm 10.2$ vs. $62.3 \pm 7.8 \mathrm{p}<0.05)$ and erythrocytes $(74.8 \pm 8.4$ vs. $38.2 \pm 4.5 \mathrm{p}<0.01)$ were remarkably decreased after infusion. In I group the serum GSH/GSSG ratio also reduced after infusing (89.7 \pm 9.3 vs. $75.8 \pm 9.3 \mathrm{p}<0.05)$, but it only had little change in erythrocytes $(71.3 \pm 7.3$ vs. $72.5 \pm 7.1 \mathrm{p}>0.05)$. The glucose infusion rates (GIR) during clamp study were $28.6 \pm 2.7 \mathrm{mg} / \mathrm{kg} / \mathrm{min}(\mathrm{C}$ group), $21.6 \pm 2.7 \mathrm{mg} / \mathrm{kg} / \mathrm{min}$ (FFA group, vs. $\mathrm{C}, \mathrm{p}<0.01$ ) and $25.3 \pm 3.1 \mathrm{mg} / \mathrm{kg} / \mathrm{min}$ (I group, vs. FFA, $\mathrm{p}<0.01$ ) respectively. The values of GIR were positively correlated with GSH/ GSSG ratio in erythrocyte $(\mathrm{r}=0.92, \mathrm{p}<0.01)$.

Conclusion: The elevated circulating FFAs levels can decrease GSH production through interrupting cells pentose phosphate pathway and lead to insulin resistance. Since there is positive correlation between GIR and GSH/GSSG ratio in erythrocytes and supplement with GSH has beneficial effects on insulin sensitivity and FFAs concentration, the present study suggests that antioxidative treatment may improve insulin resistance through enhancing mitochondria function to deal with elevated FFAs concentration. This work was supported by a National Natural Science Foundation of China grants 30370679 and 30570874

0613

Defects of neuronal NO synthase activity in skeletal muscle of obese insulin resistant Zucker fa/fa rats is due to abnormal expression of the enzyme and its protein inhibitor PIN

A.-D. Lajoix, K. Mezghenna, S. Gervais, P. Petit, R. Gross; Faculte de Pharmacie, CNRS UMR 5160, Montpellier, France.

Background and Aims: Skeletal muscle is known to express a variant of neuronal NO synthase (nNOS), nNOS $\mu$, which modulates contractile force and glucose utilization in the myocytes. The importance of such a control is suggested by the muscle and liver insulin resistance occurring in mice lacking nNOS and eNOS genes. Skeletal muscles also express the protein inhibitor of nNOS, PIN, that inhibits nNOS dimerization and catalytic activity. If a defect of the NOS pathway seems to be responsible for insulin resistance in type 2 diabetes, the reasons for remain to be determined. Our aim is thus to identify the nNOS abnormalities present in the skeletal muscle of a rat model of insulin resistance, the obese Zucker fa/fa rat. We therefore studied nNOS expression at the mRNA and proteic levels, its dimerization state as well as PIN expression in the gastrocnemius skeletal muscle of $\mathrm{fa} / \mathrm{fa}$ rats.

Materials and Methods: nNOS and PIN expression has been analysed by quantitative RT-PCR as well as by Western blotting using specific monoclonal antibodies. Dimerization of nNOS has been studied by low temperature SDS-PAGE, followed by a Western blotting.

Results: We observed a $50 \%$ decrease in nNOS expression, in Zucker fa/fa versus control fa/+rats, that affects the whole protein as well as the two splice variants of the enzyme expressed in skeletal muscle. Surprisingly, no changes in nNOS mRNA were observed by quantitative RT-PCR, suggesting that an abnormal nNOS degradation could occur in the skeletal muscle of fa/fa rats. In addition, no changes in the dimeric state of nNOS could be detected. Concerning PIN, we also observed a $75 \%$ increase of PIN mRNA, and a $100 \%$ increase in the protein level.

Conclusion: The decreased catalytic activity of nNOS observed in skeletal muscle of obese insulin resistant fa/fa rats, is related to a decreased level of nNOS as well as an increased expression of its inhibitor PIN. 
0614

Endothelin-1 infusion in vivo decreases insulin clearance and causes acute muscle insulin resistance through impaired capillary perfusion

R. M. Ross, S. Rattigan, M. G. Clark;

Biochemistry, University of Tasmania, Hobart, Tasmania, Australia.

Background and Aims: Elevated endothelin-1 (ET-1) levels are a common characteristic of many disease states including hypertension, artherosclerosis, peripheral vascular disease, obesity and type 2 diabetes. ET-1 is a potent vasoconstrictor mediating its effects through the $\mathrm{ET}_{\mathrm{A}}$ receptor found on smooth muscle cells and may also have metabolic effects through similar receptors on skeletal muscle and adipocytes. The aim of this study was to test the effect of ET-1 infusion (both alone and in conjunction with insulin infusion) in vivo, on skeletal muscle blood flow and capillary perfusion, insulin mediated glucose uptake, glucose infusion rate (GIR) and insulin clearance.

Materials and Methods: Anaesthetised Hooded Wistar rats were infused with ET-1 (50 pmol. $\left.\mathrm{min}^{-1} \cdot \mathrm{kg}^{-1} \times 2.5 \mathrm{hr}\right)$ alone (ET), or 30 minutes prior to a hyperinsulinaemic euglycemic insulin clamp $\left(10 \mathrm{mU} \cdot \mathrm{min}^{-1} \cdot \mathrm{kg}^{-1} \times 2 \mathrm{hr}\right.$, ETINS10). $10 \mathrm{mU} \cdot \mathrm{min}^{-1} \cdot \mathrm{kg}^{-1}$ (INS10) or $15 \mathrm{mU} \cdot \mathrm{min}^{-1}$. $\mathrm{kg}^{-1}$ (INS15) insulin clamps were performed after 30 minutes of saline infusion as reference experiments. Capillary perfusion was measured via 1-methylxanthine metabolism and muscle glucose uptake by 2-deoxy-D$\left[1-{ }^{14} \mathrm{C}\right]$ glucose $\left(\mathrm{R}^{\prime} \mathrm{g}\right)$. Insulin clearance was determined from the rate of clearance of fluorescein isothiocyanate (FTIC) labelled insulin administered as a bolus 45 minutes before the end of a saline or ET-1 experiment.

Results: ET-1 infusion alone and during insulin infusion significantly $(P<0.05)$ increased plasma endothelin concentrations by approximately seven fold to $129 \pm 7$ and $140 \pm 6$ pmol. $\mathrm{L}^{-1}$ respectively and blood pressure by approximately $20 \%(P<0.05)$. ET-1 alone had no effect on femoral blood flow (FBF), capillary perfusion or glucose uptake, but did cause a significant $(P=0.02)$ decrease in insulin clearance from $0.35 \pm 0.6$ to $0.19 \pm 0.02 \mathrm{ml} . \mathrm{min}^{-1}$. ET-1 infusion during insulin clamp resulted in significantly higher plasma insulin levels (INS10: $2120 \pm 190$ pmol. $\mathrm{L}^{-1}$; ETINS10: $4740 \pm 910$ pmol. $\left.\mathrm{L}^{-1}\right)$, equivalent to a $15 \mathrm{mU}$. $\min ^{-1} \cdot \mathrm{kg}^{-1}$ clamp $\left(4920 \pm 190\right.$ pmol.L $\left.{ }^{-1}\right)$. C-peptide concentrations remained unchanged throughout the experiments. Both insulin doses significantly $(P<0.05)$ increased FBF, muscle capillary perfusion and glucose uptake. The presence of ET-1 blocked the stimulatory effect of insulin on FBF, and capillary perfusion $(P<0.05)$. GIR decreased (compared to $15 \mathrm{mU} \cdot \mathrm{min}^{-1} \cdot \mathrm{kg}^{-1}$ infusion) from a steady state infusion of $31.2 \pm 1.7$ to $22.4 \pm 1.2 \mathrm{mg} \cdot \mathrm{min}^{-1} \mathrm{~kg}^{-1}$
$(P<0.05)$ and muscle glucose uptake (represented by the calf muscle R'g) decreased from $31.5 \pm 1.7$ to $15.4 \pm 2.7 \mu \mathrm{g}$. $\mathrm{g}^{-1} \cdot \min ^{-1}(P<0.05)$.

Conclusion: This study shows that ET-1 decreases whole body insulin clearance thereby augmenting plasma insulin levels during insulin clamps. At similar levels of hyperinsulinaemia, ET-1 blocks insulin's haemodynamic effects, particularly capillary perfusion and decreases muscle glucose uptake and whole body glucose clearance. Based on previous studies, it would seem likely that the impairment of capillary perfusion accounts for the acute muscle insulin resistance

Supported by NHMRC and Heart Foundation Australia

0615

Trans fatty acid feeding leads to insulin resistance and the induction of obesity phenotypes in rats

S. Dorfman, T. E. Hughes, D. Laurent, F. Sari-Sarraf, X. Li, T. Mullarkey, E. Rocheford, J. Gounarides,

S. R. Commerford;

Novartis Institute of Biomedical Research, Cambridge, United States.

Background and Aims: In rodents, high fat diets induce body weight (BW) gain, fat mass accumulation, and insulin resistance (IR) relative to diets lower in fat. The extent to which these phenomena are observed differ with respect to dietary fat type. Trans fatty acids (TFA) have a negative impact on blood lipid profiles, but their effects on BW and insulin action are equivocal. Here we test the hypothesis that even a low fat diet enriched with TFA would induce an increase in BW gain and worsen insulin action through effects on tissue lipid profiles.

Materials and Methods: Male 8 wk old Sprague-Dawley rats were fed a $10 \%$ saturated fat (LSF), $10 \%$ TFA (LFT), or $45 \%$ saturated fat (HFS) diet for $8 \mathrm{wk}$. At 0,3 and $6 \mathrm{wk}$, in vivo magnetic resonance spectroscopy (MRS) assessed intramyocellular lipid (IMCL) and intrahepatic lipid (IHL) content. During wk 8, euglycemic-hyperinsulinemic clamps determined whole body insulin sensitivity. Ex vivo MRS determined tissue biochemistry following clamp studies.

Results: At wk 6, LFT and HFS groups weighed significantly more than LFS group $(510 \pm 13,534 \pm 15$, $479 \pm 16 \mathrm{~g}$; respectively, $P<0.05$ ). We used $\Delta_{6}$ wk-baseline total fat mass/body weight ratio as a marker of obesity. Adiposity increased most in HFS, then LFT (LFT $0.07 \pm 0.01$, HFS $0.08 \pm 0.02$, LFS $0.04 \pm 0.01 ; P<0.05$ ). When considered by fat depot, visceral fat mass increased significantly more in LFT and HFS (LFT 0.042 \pm 0.01 ; HFS $0.045 \pm 0.01$; LFS $0.024 \pm 0.003 ; P<0.05$ ), but subcutaneous fat increased significantly more in HFS (LFT 
$0.03 \pm 0.004$; HFS $0.05 \pm 0.01$; LFS $0.02 \pm 0.005 ; P<0.05)$. At $6 \mathrm{wk}, \%$ IHL in LFT and HFS was $10.6 \pm 1.4,10.3 \pm 1.2$, respectively, $\sim 6$-fold higher than LFS $(P<0.001)$. At $8 \mathrm{wk}$, whole body insulin action among groups were similar (LFT glucose infusion rate (GIR) 12.5 \pm 1.7 ; HFS GIR, 12.3 \pm 2.6 , LFS GIR $\left.16.5 \pm 2.7 \mathrm{mg} \cdot \mathrm{kg}^{-1} \cdot \mathrm{min}^{-1} ; P=0.3\right)$. Basal glucose $\mathrm{R}_{\mathrm{d}}$ was similar among diets, while insulin-stimulated glucose $\mathrm{R}_{\mathrm{d}}$ was significantly lower $(P<0.05)$ in HFS group relative to the other groups and there was a trend $(P<0.06)$ for LFT to have lower $\mathrm{R}_{\mathrm{d}}$ than LFS group (LFT 12.7 \pm 1.6 ; HFS 11.2 \pm 1.7 ; LFS $\left.17.4 \pm 1.8 \mathrm{mg} \cdot \mathrm{kg}^{-1} \cdot \mathrm{min}^{-1}\right)$. These suggest that both LFT and HFS diets induced peripheral IR. The diet- time interaction for IMCL/tCr was significant in tibialis anterior $(T A)(\mathrm{P}<0.001)$ such that HFS diet had an approximate 2 -fold increase in $\mathrm{IMCL} / \mathrm{tCr}$ ratio at both 3 and 6 wk (3 wk: LFT 1.2 \pm 0.4 ; HFS 2.9 \pm 1.3 ; LFS 1.2 \pm 0.7 ; 6 wk: LFT 1.5 \pm 0.8 ; HFS 2.6 \pm 1.1 ; LFS 1.3 \pm 0.5 ). Diacylglycerol (DAG) concentration in the TA muscle trended $(P=0.06)$ to be higher in LTF and HFS relative to the LFS $(0.03 \pm 0.007,0.03 \pm 0.003,0.009 \pm 0.002 \%$ total fatty acid content; respectively).Thus, decreased glucose $\mathrm{Rd}$ in the LFT group was independent of an increase in IMCL but was related to increased DAG.

Conclusion: We have shown for the first time that a low fat diet enriched with TFA increases visceral adiposity and intrahepatic lipid accumulation and decreases skeletal muscle insulin sensitivity. Thus a diet low in fat but enriched in TFA provides a model to understand the early physiologic processes implicated in the development of skeletal muscle IR involving increased DAG concentration, prior to the onset of whole body IR. The removal of even small quantities of TFA from the diet would therefore be prudent when considering the etiology of IR and obesity.

\section{6}

Nitric oxide resistance in insulin resistant states: the model of vascular smooth muscle cells from the obese Zucker rats

G. Doronzo, I. Russo, P. Del Mese, L. Mattiello,

A. De Salve, G. Anfossi, M. Trovati;

Department of Clinical and Biological Sciences of the Turin University, Diabetes Unit, San Luigi Gonzaga Hospital, Orbassano-Turin, Italy.

Background and Aims: Obesity, both in the absence and in the presence of diabetes, is characterized by a reduced synthesis and action of nitric oxide (NO), playing a role in the pathogenesis of arterial hypertension and atherosclerosis, because NO induces vasodilation and inhibits multiple steps of the atherogenic process. NO mainly acts by inducing the synthesis of guanosine $3^{\prime}, 5^{\prime}$ cyclic monophosphate (cGMP), which exerts a wide spectrum of biological actions by activating the cGMP-dependent protein kinase (PKG).

It has been demonstrated that insulin, via the NO/cGMP pathway, increases Vascular Endothelial Growth Factor (VEGF) synthesis in aortic Vascular Smooth Muscle Cells (VSMC) from lean Zucker fa/+rats by activating both the Phosphatidylinositol-3-Kinase (PI3-K/Akt) and the Mitogen Activated Protein Kinase (MAPK) pathways, and that this NO-mediated insulin effect on VEGF is blunted in VSMC from obese Zucker fa/fa rats, with the possible consequence of a reduced collateral vessel formation. In the present study, we aimed at evaluating whether the $\mathrm{NO}$ / cGMP signaling through PI3-K and MAPK pathways is impaired in VSMC from obese Zucker fa/fa rats, a classical animal model of insulin-resistance.

Materials and Methods: In aortic VSMC from lean, insulin-sensitive Zucker fa/+rats and from obese, insulinresistant Zucker fa/fa rats, obtained in primary culture in our laboratory, we measured the influence of the NO donor DETA NONOate and of the cGMP analog 8-Bromo-cGMP on the activation of the PI3-K/Akt pathway -by evaluating Akt phosphorylation- and of the MAPK pathway -by measuring the phosphorylation of ERK-1 and ERK-2 (western blots, $\mathrm{n}=6, \mathrm{~m} \pm \mathrm{SEM}$ ).

Results: In VSMC from Zucker fa/+rats DETA NONOate (500 microM) and 8-Bromo-cGMP (500 microM) increased the phosphorylation of Akt (at 2 hours of incubation, $p=0.0001$ and $p=0.007$ ) and of ERK-1 and ERK-2 (at 2 hours of incubation, $p=0.0001$ and $p=0.013$ for ERK-1; $\mathrm{p}=0.0001$ and $\mathrm{p}=0.019$ for $E R K-2)$. A significant activation of PI-3K and MAPK pathways was induced by DETA NONOate and 8-Bromo cGMP also in VSMC from Zucker fa/fa rats $(\mathrm{p}=0.03-0.007)$, but the extent of activation was significantly lower than in Zucker $\mathrm{fa} /+$ rats. In particular, a) at 2 hours of VSMC incubation with DETA NONOate: i)phosphorylation of Akt was $149.1 \pm 12.9 \%$ of baseline in lean vs $119.56 \pm 4 \%$ in obese Zucker rats $(\mathrm{p}=0.0001)$; ii)phosphorylation of ERK-1 was $226.0 \pm 15.8 \%$ of baseline in lean vs $118.9 \pm 6.9 \%$ in obese Zucker rats $(\mathrm{p}=0.0001)$; iii) phosphorylation of ERK-2 was $164.8 \pm 5.6 \%$ of baseline in lean vs $119.2 \pm 6.8 \%$ in obese Zucker rats $(p=0.0001)$; b)at 2 hours of VSMC incubation with 8-Bromo-cGMP: i)phosphorylation of $\mathrm{Akt}$ was $174.4 \pm 8.5 \%$ of baseline in lean vs $115.4 \pm 8.6 \%$ in obese Zucker rats $(\mathrm{p}=0.0001)$; ii)phosphorylation of ERK1 was $169.2 \pm 19.8 \%$ of baseline in lean vs $118.9 \pm 6.4 \%$ in obese Zucker rats ( $\mathrm{p}=0.0001)$; iii)phosphorylation of ERK2 was $168.2 \pm 21.4 \%$ of baseline in lean vs $128.5 \pm 8.6 \%$ in obese Zucker rats $(\mathrm{p}=0.0001)$.

Conclusions: The NO and cGMP- induced activation of PI3-K and MAPK pathways is impaired in VSMC from a classical animal model of insulin resistance, the obese 
Zucker fa/fa rat, providing new insights into the molecular mechanisms involved in the resistance to NO occurring in the insulin-resistant states, which likely plays a role in their increased cardiovascular risk.

Supported by University of Turin

\section{PS 42}

\section{Insulin secretion I}

0617

Insulin secretion and insulin action in individuals with different categories of glucose tolerance. The GENFIEV study

S. Del Prato ${ }^{1}$ on behalf of GENFIEV Study Group ${ }^{2}$;

${ }^{1}$ Department of Endocrinology and Metabolism, Pisa University, ${ }^{2}$ Italian Society of Diabetology, FoRiSID, Roma, Italy.

Background and Aims: The GENFIEV (Genetics, PHYsiopathology and Evolution of Type 2 diabetes) is a multicenter study recruiting individuals with IFG and/or IGT to identify phenotypic and genotypic features allowing identification of subjects at high risk for Type 2 diabetes.

Materials and Methods: 1017 subjects have been recruited over 2-yrs. Subjects underwent $75 \mathrm{~g}$ OGTT for assessment of plasma glucose (PG) and C-peptide (CP). Fasting insulin was determined as well.

Results: At OGTT, $50 \%$ of individuals had NGT, 15\% were diabetics, $4 \%$ had IFG (110-125 mg/dl), 23\% IGT, and $8 \%$ IFG+IGT. HOMA-IR increased and the insulinogenic index (DeltaCP30 min/DeltaG30 min) declined as a function of glucose tolerance (ANOVA $\mathrm{p}<0.0001$ ). IFG, IGT, and IFG+IGT subjects all had lower insulinogenic index as compared to NGT and greater than diabetics. To further explore the relationship between glucose tolerance and insulin secretion and sensitivity the population was analyzed based on 2-hrPG as $20 \mathrm{mg} / \mathrm{dl}$ glucose increment above an arbitrary value of $100 \mathrm{mg} / \mathrm{dl}$. When the area under the curve (AUC) of CP was considered, a "horse-shoe" shaped relationship was found with a peak AUC for individuals with $160-180 \mathrm{mg} / \mathrm{dl} 2$-hrPG. On the contrary, the AUCCP:AUCG ratio decreased across the 2-hrPG range $(\mathrm{r}=-0.37 ; \mathrm{p}<0.001)$, while HOMA-IR increased $(\mathrm{r}=0.28 ; \mathrm{p}<0.001)$. The ratio between the two parameters, a surrogate of the disposition index, showed a linear decline with increasing 2-hrPG $(r=-0.44 ; \mathrm{p}<0.001)$. Changes were detected for individuals with NGT, as AUCCP:AUCG ratio $(0.54+/-0.02$ vs. $0.49+/-0.02, \mathrm{p}<0.075)$ and HOMA-IR $(2.01+/-1.2$ vs. $2.8+/-1.7, \mathrm{p}<0.0001)$ were different between individuals with 2 -hrPG $<100 \mathrm{mg} / \mathrm{dl}$ than those with $120-140 \mathrm{mg} / \mathrm{dl}$. Amongst NGT, the ratio between the two parameters dropped by $16 \%$ from 2 -hrPG $<100$ to 100 $120 \mathrm{mg} / \mathrm{dl}$ and by $34 \%$ for $2-\mathrm{hr}$ glucose $120-140 \mathrm{mg} / \mathrm{dl}$.

Conclusion: These data, though requiring confirmation in longitudinal studies, suggest that changes in insulin sensitivity and secretion occur in a linear fashion across the different categories of glucose tolerance, and emphasize that major changes in beta-cell performance occur even with modest increases in 2-hrPG within the NGT category. Supported by FoRiSID and Eli Lilly-Italy

0618

The European C-Peptide Trial CPT: comparison of liquid mixed meal and glucagon stimulation assays for the analysis of residual $\beta$ cell function in type 1 diabetes

T. R. Mandrup-Poulsen ${ }^{1,2}$, T. Battelino ${ }^{3}$, B. Haastert ${ }^{4}$, J. Ludvigsson ${ }^{5}$, P. Pozzilli ${ }^{6}$, N. M. Lalic ${ }^{7}$, M. T. Martinez-Larrad ${ }^{8}$, D. Mauricio ${ }^{9}$, M. Philip ${ }^{10}$, N. C. Schloot ${ }^{4}$, E. Schober ${ }^{11}$, G. Spinas ${ }^{12}$, K. Vondra ${ }^{13}$, G. Giani ${ }^{4}$, H. Kolb ${ }^{4}$; CPT Study Group,

${ }^{1}$ Steno Diabetes Center, Gentofte, Denmark, ${ }^{2}$ Dept. of Molecular Medicine, Karolinska Institute, Stockholm, Sweden, ${ }^{3}$ Dept. of Pediatric Endocrinology, Diabetes and Metabolism, University Children's Hospital, Ljubljana, Slovenia, ${ }^{4}$ German Diabetes Center, Heinrich Heine Universität Düsseldorf, Germany, ${ }^{5}$ Division of Pediatrics and Diabetes Research Center, Linköping University, Sweden, ${ }^{6}$ Endocrinology and Diabetes, University Campus BioMedico, Rome, Italy, ${ }^{7}$ Diabetes and Metabolic Diseases, Institute for Endocrinology, Belgrade, Serbia and Montenegro, ${ }^{8}$ Dept. of Internal Medicine, Hospital Clinico San Carlos, Madrid, ${ }^{9}$ Dept. Endocrinology, Hospital de Sant Pau, Barcelona, Spain, ${ }^{10}$ Institute for Endocrinology and Diabetes, Schneider Children's Medical Center of Israel, Petah Tikva, Israel, ${ }^{11}$ University Children's Hospital, University of Vienna, Austria, ${ }^{12}$ Dept. for Endocrinology and Diabetes, University of Zürich, Switzerland, ${ }^{13}$ Endocrinology, Institute of Endocrinology, Prague, Czech Republic.

Background and Aims: The European multicentre CPeptide Trial (CPT) was conducted to compare key characteristics of glucagon and liquid mixed meal stimulated $\beta$ cell responses in people with type 1 diabetes since residual $\beta$-cell function is an important surrogate parameter for $\beta$-cell mass in trials aimed at its protection.

Materials and Methods: A total of 118 patients were randomised to three $\beta$-cell function tests, each 3-10 days apart. 113 patients $8-40$ years of age, $41 \%$ female, with diabetes duration $0.75-5$ years were included in the analysis. Two baseline blood samples were followed by one sample at $6 \mathrm{~min}$ after $1 \mathrm{mg}$ glucagon i.v. (GST, 
glucagon stimulation test), or at 10,20,30,60, 90 and $120 \mathrm{~min}$ after ingestion of a liquid standard meal (MMTT, mixed meal tolerance test with Boost HP, $6 \mathrm{ml} / \mathrm{kg}$, maximum $360 \mathrm{ml}$ ).

Results: The peak C-peptide response to MMTT (geometric mean $0.13 \mathrm{nmol} / 1$, range $\leq 0.01-2.1$ ) was significantly higher than 6 min after glucagon $(0.09 \mathrm{nmol} / \mathrm{l}$, range $\leq 0.01-1.37$, $\mathrm{p}<0.0001)$. The overall intra-individual correlation of $6 \mathrm{~min}$ GST with peak MMTT C-peptide concentrations was strong $(\mathrm{r}=0.90, \mathrm{p}<0.0001)$. MMTT elicited a higher C-peptide response compared to GST in both the tertile with highest basal C-peptide levels as well as in the tertile with lowest basal C-peptide levels (geometric means 0.54 vs $0.38 \mathrm{nmol} /$ 1 [relative difference $43 \%$ ] and 0.031 vs. $0.019 \mathrm{nmol} /$ 1 [relative difference $59 \%, \mathrm{p}=0.46]$ ). A higher C-peptide response to MMTT was also seen in the groups with short as well as with long diabetes duration (9-18 months or 2.55 years, geometric means higher by $40 \%$ or $27 \%$, $\mathrm{p}=0.45$ ).

Conclusion: Consistently higher C-peptide responses were observed during MMTT than GST, the close correlation being independent of diabetes duration or basal C-peptide levels. Different kinetics in the loss of $\beta$-cell function during the first years after diagnosis of type 1 diabetes between many trials applying either MMTT or GST cannot be accounted for by the method of determining residual $\beta$-cell function.

Supported by EASD and Novo Nordisk

\section{9}

Several defects of $\beta$ cell function characterise changes in glucose homeostasis: the GENFIEV Study

R. C. Bonadonna ${ }^{1}$ on behalf of the GENFIEV

Study Group ${ }^{2}$;

${ }^{1}$ Dept of Biomedical and Surgical Sciences, University of Verona School of Medicine, Verona, ${ }^{2}$ GENFIEV Study Group, Research Foundation of the Italian Society of Diabetology, Roma, Italy.

Insulin (I) secretion (Isec) in vivo during an OGTT is the sum of 2 components: 1. derivative control ( $\mathrm{sec} 1$ : pmol ${ }^{-2}$ BSA per $\mathrm{mM}$ of glucose $(\mathrm{G})$ ): Isec in response to the rate of increase in $\mathrm{G} ; 2$. proportional control $(\sec 2)$ : stimulus (G: $\mathrm{mM}$ )- response (Isec: pmol $\mathrm{min}^{-1} \cdot \mathrm{m}^{-2}$ BSA) curve. The role of sec1 and sec2 in the early changes in $G$ homeostasis still is under debate.

We aimed at assessing sec1 and sec2 in the early changes in $\mathrm{G}$ homeostasis, by exploiting part of the baseline cross-sectional data base of GENFIEV, a multicenter national prospective study of the determinants of the progression from pre-diabetes to type 2 diabetes (DM2). We measured sec1 and sec2 during an OGTT (G and C-peptide curves analyzed by an established mathematical model of G-induced Isec) in $366(49.5 \% \mathrm{M})$ subjects (age: $50.0 \pm 0.6$ yrs; BMI: $29.0 \pm 0.4 \mathrm{~kg} / \mathrm{m}^{2}$ ), divided according to fasting $\mathrm{G}$ (normal: NFG; impaired: IFG; diabetic: DFG) and to 2-hour G (normal: NGT; impaired: IGT; diabetic: DGT) in 9 groups. Diabetic subjects were newly daignosed and treatment naive. The DFG/NGT group $(\mathrm{n}=2)$ is not included in this analysis. Comparisons were adjusted by age, gender, BMI and fasting insulin, as a proxy of I-resistance. Sec1 was similar in NFG/NGT $(1390 \pm 98, \mathrm{n}=87)$, IFG/NGT $(1267 \pm 147$, $\mathrm{n}=39)$ and NFG/IGT $(1595 \pm 121, \mathrm{n}=59)$, declined in IFG/ IGT $(1038 \pm 98, n=86, p<0.01)$, and fell further in NFG/ DGT $(n=10)$, IFG/DGT $(n=51)$, DFG/IGT $(n=8)$ and DFG/ DGT $(n=24)$ (value ranging between $697 \pm 185$ and $808 \pm 286, \mathrm{p}<0.01$ ). In comparison to NFG/NGT (Isec: $105 \pm 4.5,197 \pm 9,545 \pm 21,982 \pm 42$ at $\mathrm{G}$ levels of 4.0, 5.5, 8.0 , and $11 \mathrm{mM}$, respectively), sec 2 declined $(\sim 22 \%$, $\mathrm{p}<0.03)$ in IFG/NGT and NFG/IGT, was further decreased $(\sim 41 \%, \mathrm{p}<0.01)$ in IFG/IGT and NFG/DGT, and fell even more (between $56 \%$ and $65 \%, \mathrm{p}<0.01$ ) in IFG/DGT, DFG/ IGT and DFG/DGT.

Conclusions: Across the spectrum from normal G homeostasis to newly diagnosed DM2, sec1 shows at least 3 and sec2 shows at least 4 distinct levels of $\beta$-cell function, suggesting that the evolution to DM2 crosses several stages of ${ }_{1}^{2}$-cell function, the last of which implies invariably a joint decline in both $\sec 1$ and $\sec 2$.

Supported by FORISID

0620

Characterisation of insulin secretory defect in Bangladeshi type 2 diabetic subjects

L. Ali ${ }^{1}$, R. Zinnat ${ }^{1}$, R. Karim ${ }^{2}$, S. Islam ${ }^{2}$;

${ }^{1}$ Dept of Biochemistry \& Cell Biology, BIRDEM, Dhaka,

${ }^{2}$ Dept of Biochemistry and Molecular Biology, University of Chittagong, Bangladesh.

Background and Aims: It still remains controversial whether insulin deficiency or insulin resistance is the earliest abnormalities in the natural history of the disease. Considerable heterogeneity seems to exist regarding these abnormalities among various populations. Previous studies suggest that insulin secretory dysfunction is the relatively more prominent defect in Bangladeshi nonobese type 2 diabetic population. The characterization of the defect may provide valuable tools for further understanding of the role of insulin secretory defect in the pathogenesis of type 2 diabetes. The aim of the present study was to investigate the nature of insulin secretory defect in type 2 diabetic subjects in various phases of secretion. 
Materials and Methods: Forty-four type 2 diabetic subjects, along with 30 Age-and BMI-matched control subjects, were studied. The diabetic subjects were never treated with insulin. Insulin was measured by chemiluminiscent ELISA. Insulin secretory capacity (\%HOMA B) and insulin sensitivity (\%HOMA S) were calculated by homeostasis model assessment (HOMA) using HOMA software. First and second phases of insulin secretion were calculated by the following equations: 1 st phase est $=1283+$ 1.829. Ins $_{30}-138.7 . \mathrm{Glu}_{30}+3.772 . \mathrm{Ins}_{0} ; 2$ nd phase $_{\mathrm{est}}=287+$ 0.4164. Ins $_{30}-26.07 . \mathrm{Glu}_{30}+0.9226 . \mathrm{Ins}_{0}$

Results: The mean age (years, $\mathrm{M} \pm \mathrm{SD}$ ) of the patients was $44 \pm 7$ and that of controls $41 \pm 7$. BMI $\left(\mathrm{kg} / \mathrm{m}^{2}, \mathrm{M} \pm \mathrm{SD}\right)$ was $25.46 \pm 3.26$ in patients vs $25.31 \pm 3.61$ in controls. Fasting serum insulin [Median (range); 53.96 (6.67-162.58) vs 74.55 (6.25-216.89), $\mathrm{p}=0.0001]$; and $30 \mathrm{~min}$ after glucose insulin [Median (range); 132.44 (17.78-422.19) vs 544.59 (33.27-1168.64), $\mathrm{p}=0.013$ ] levels were significantly lower in diabetic as compared to control subjects. The B-cell function was significantly lower in diabetic subjects as compared to the controls [\%HOMA B, Median (range); $53.90(10.40-162.90)$ vs 163.60 (48.40-381.70), $\mathrm{p}=0.0001]$, but no significant difference was observed in case of insulin sensitivity [\%HOMA S, Median (range); 79.10 (26.40-226.90) vs 65.20 (21.90-259.90), $\mathrm{p}=0.088]$. The 1 st and 2 nd phases of insulin secretion were also significantly lower in diabetic subjects compared to control [Phase I: Median (range); 208.49 (-1412-1230) vs $1655.56(-109-2831), \mathrm{p}=0.0001$, Phase II: Median (range); 101.23 (-217-341) vs 418.56 (51-679), $\mathrm{p}=0.0001]$. A significant positive correlation was found between BMI and Phase I $(r=0.315 ; \mathrm{p}=0.037)$ and Phase II $(r=0.320 ; p=0.034)$ of insulin secretion in case of diabetic subjects. No such correlation was observed in case of the control subjects. A significant negative correlation was also observed between insulin sensitivity and BMI $(r=-0.439$; $\mathrm{p}=0.003$ ) in the diabetic subjects.

Conclusion: Nonobese type 2 diabetic patients in Bangladesh have insulin secretory dysfunction and they do not seem to have insulin resistance as a primary pathophysiologic characteristics. The B-cell secretory dysfunction in type 2 diabetics involves both fasting and poststimulatory states; poststimulatory defect, however, is much prominent in comparison to the fasting state. Both 1 st and 2 nd phases of insulin secretion are affected in type 2 diabetes, but the deficiency in 1 st phase secretion seems to be predominant in these subjects.
0621

Pitfalls of the disposition index as an index of beta cell function

A. Mari $^{1}$, G. Pacini ${ }^{1}$, A. Natali ${ }^{2}$, B. Balkau ${ }^{3}$, J. M. Dekker ${ }^{4}$, S. Coppack ${ }^{5}$, M. Walker ${ }^{6}$, E. Ferrannini ${ }^{2}$, \&. the RISC Investigators ${ }^{7}$;

${ }^{1}$ Institute of Biomedical Engineering, National Research Council, Padova, Italy, ${ }^{2}$ Department of Internal Medicine, University of Pisa, Italy, ${ }^{3} \mathrm{U}$ 258, INSERM, Villejuif, France, ${ }^{4} \mathrm{EMGO}$ Institute, Vrije Universiteit Amsterdam, The Netherlands, ${ }^{5}$ Department of Diabetes, The Royal London Hospital, ${ }^{6}$ Department of Medicine, The Medical School, University of Newcastle upon Tyne, United Kingdom, ${ }^{7}$ RISC Coordinating Center, The RISC Consortium, Pisa, Italy.

Background and Aims: It is generally agreed that, in healthy subjects some parameters of insulin secretion are reciprocally related to insulin sensitivity. This notion has led to a general paradigm whereby beta-cell function is correctly assessed by calculating the "disposition index" (DI), i.e., the product of insulin secretion and insulin sensitivity. However, the validity of this approach is critically dependent on the assumption that the relationship between insulin secretion and insulin sensitivity is hyperbolic. To test whether this assumption is correct, we used the database of the RISC study, which includes measures of insulin sensitivity and secretion.

Materials and Methods: Insulin sensitivity was measured by the euglycemic clamp and insulin secretion was assessed by two typical parameters, i.e., fasting plasma insulin concentration and the insulinogenic index (i.e, the ratio of incremental insulin to incremental glucose concentration at $30 \mathrm{~min}$ into an OGTT). All subjects (506 men/633 women, age $44 \pm 8$ years, BMI $25 \pm 4 \mathrm{~kg} \mathrm{~m}^{-2}$, mean \pm SD) had normal glucose tolerance on the OGTT. The validity of the hyperbolic assumption was tested by verifying the constancy of DI across the insulin sensitivity span. Subjects were stratified into deciles of insulin sensitivity and the constancy of DI in the groups tested by ANOVA.

Results: DI increased with insulin sensitivity across the entire sensitivity range $(\mathrm{p}<0.0001)$, both when DI was calculated using the fasting insulin concentration $(\sim 2$-fold increase from the lowest to the highest insulin sensitivity decile) and when using the insulinogenic index ( $\sim 3$-fold increase). This artifact derives from the fact that the relationships between insulin sensitivity and secretion are not hyperbolic, but rather power functions with an 
exponent $>-1$. Furthermore, when fasting plasma glucose concentration was stratified into deciles, the DI using fasting insulin was positively related to fasting glycemia $(p<0.00001)$, whereas the DI using the insulinogenic index was negatively related to fasting glucose $(\mathrm{p}<0.00005)$. Thus, DI neither correctly estimates beta-cell function across the insulin resistance range nor is unequivocally predictive of fasting plasma glucose concentrations.

Conclusion: This study shows that the hyperbola paradigm and the DI are not generally valid, and their use may produce significant artifacts if validity is not tested.

Supported by European Union and AstraZeneca

\section{2}

Pancreatic islet blood flow is selectively enhanced by captopril, irbesartan and pravastatin, and suppressed by palmitate

Z. Huang ${ }^{1}$, L. Jansson ${ }^{2}$, A. Sjoholm ${ }^{3}$;

${ }^{1}$ Research Centre(foc), Stockholm South Hospital, Karolinska Institutet, Stockholm, ${ }^{2}$ Department of Medical Cell Biology, University of Uppsala, ${ }^{3}$ Department of Internal Medicin, Stockholm South Hospital, Karolinska Institutet, Sweden.

Background and Aims: The systemic renin-angiotensin system (RAS) plays a crucial role in the regulation of arterial blood pressure. In the pancreas of several species, mRNA encoding angiotensinogen and renin, as well as substantial levels of angiotensin II, have been detected. Ang II has been shown to adversely influence pancreatic and islet blood flow through vasocontractive effects. Also, high affinity binding sites for Ang II were recently localized specifically to islet $\beta$-cells by double immunostaining and Ang II was found to block glucose-stimulated insulin secretion, an event fully reversible by losartan. It is thus conceivable that pancreatic Ang II, locally produced by intrinsic RAS, may adversely influence insulin secretion in vivo, either directly by suppressing $\beta$-cell insulin exocytosis or indirectly through inhibitory effects on islet blood perfusion. This may be of particular importance in diabetic patients since hypertension is markedly overrepresented in these individuals, and angiotensinogen expression seems to be upregulated by hypertension. Hence, many diabetic patients are treated with ACE inhibitors or angiotensin receptor antagonists against their hypertension or as part of a renal protection strategy. An additional hallmark of diabetic cardiovascular risk is hyperlipidemia and elevated serum levels of free fatty acids; consequently many diabetic patients use lipid lowering drugs, most notably statins. Interestingly, both ACE inhibitors or angiotensin receptor antagonists and certain statins have been reported to decrease the risk of developing diabetes in large clinical trials. However, the mechanisms behind these antidiabetic effects remain elusive. We now studied whether these drugs and the fatty acid palmitate influence insulin secretion in vivo in rats through effects on islet blood perfusion.

Materials and Methods: Male Wistar rats weighting 300$350 \mathrm{~g}$, with free access to tap water and pelleted food, were used in all experiments. The microsphere technique in combination with a freeze-thawing technique was performed for measurements of pancreatic blood flow and islet blood flow.

Results: Whole pancreatic blood flow was markedly increased by captopril and irbesartan and decreased by palmitate. Islet blood flow was significantly and preferentially enhanced by captopril, irbesartan and pravastatin, and suppressed by palmitate. Both captopril and irbesartan raised serum insulin concentrations significantly. However, glycemia was not affected in any group.

Conclusion: The present study suggests that a local pancreatic RAS and pravastatin may be selectively controlling pancreatic islet blood flow and thereby influencing insulin secretion. The antidiabetic actions of statins and RAS inhibitors noted in clinical trials might in part occur through the beneficial direct islet effects shown here. Conversely, free fatty acids that are elevated in type 2 diabetic patients may contribute to an impaired nutritive islet blood flow and thereby further aggravate the diabetic state by limiting the supply of insulin needed to curb hyperglycemia.

0623

Relationships between insulin secretion after acute intravenous and oral glucose administration from normal glucose tolerance to diabetes

E. Ferrannini ${ }^{1}$, A. Tura ${ }^{2}$, G. Pacini ${ }^{2}$, A. Kautzky-Willer ${ }^{3}$, A. Mari ${ }^{2}$;

${ }^{1}$ Department of Internal Medicine, University of Pisa, ${ }^{2}$ Institute of Biomedical Engineering, National Research Council, Padova, Italy, ${ }^{3}$ Clinic of Internal Medicine III, Medical University of Vienna, Austria.

Background and Aims: It has long been known that firstphase insulin secretion in response to acute intravenous glucose injection (IVGTT) can be abolished in diabetic subjects at a time when their response to oral glucose is maintained. However, the mechanisms underlying this phenomenon are poorly understood.

Materials and Methods: We measured the acute insulin response (AIR) to intravenous glucose and the secretory response to an oral glucose tolerance test (OGTT) in 221 subjects (194 women/27 men; age $37 \pm 11$ years, BMI $28 \pm 5 \mathrm{~kg} / \mathrm{m}^{2}$; mean $\pm \mathrm{SD}$ ) spanning the range from normal glucose tolerance (NGT, $\mathrm{n}=147$ ) to impaired fasting glycaemia/impaired glucose tolerance (IFG/IGT, $\mathrm{n}=28$ ) to 
overt diabetes (T2D, n=46). Beta-cell function parameters from the OGTT, and, in particular, beta-cell glucose sensitivity, were obtained by modelling analysis.

Results: Both AIR and beta-cell glucose sensitivity declined as glucose tolerance worsened $(\mathrm{p}<0.0001$ by ANOVA). However, AIR reached a value near zero at a fasting glycaemia of $\sim 7 \mathrm{mmol} / \mathrm{L}$, while beta-cell glucose sensitivity fell continuously throughout the fasting plasma glucose range. Therefore, the relationship between AIR and beta-cell glucose sensitivity, which was strongly linear in NGT $(r=0.58, \mathrm{p}<0.0001)$, became flat at $\sim 7 \mathrm{mmol} / \mathrm{L}$ glucose. In addition, for fasting glucose levels above $7 \mathrm{mmol} / \mathrm{L}$, AIR was no longer related to fasting glucose, while a strong relationship between fasting glucose and glucose sensitivity was preserved $(r=0.71, \mathrm{p}<0.0001)$. Similar results were obtained when using 2-hour plasma glucose levels instead of fasting glucose, the glucose threshold being $\sim 13 \mathrm{mM}$.

Conclusion: AIR vanishes at thresholds of fasting or postglucose hyperglycaemia before beta-cell glucose sensitivity is severely compromised. Consequently, AIR misjudges beta-cell function in hyperglycaemic states.

\section{4}

Beta cell function and insulin sensitivity in subjects with non-alcoholic fatty liver disease and normal glucose regulation

H. M. $\operatorname{Yan}^{1}$, X. Gao ${ }^{1}, \mathrm{M} . \mathrm{Liu}^{1}, \mathrm{~J} . \mathrm{Gao}^{2}$;

${ }^{1}$ Endocrinology and Metabolism, ${ }^{2}$ Nutrition, Zhongshan Hospital, Shanghai, China.

Background and Aims: Ever-increasing evidences has suggested that non-alcoholic fatty liver disease(NAFLD) is associated with type 2 diabetes mellitus and insulin resistance(IR). The relationship of NAFLD and beta cell function, however, was seldom presented. This study was to investigate the impact of liver fat content on beta cell function and insulin sensitivity in subjects with NAFLD and normal glucose regulation (NGR).

Materials and Methods: Twenty seven subjects aged 20 to 60 years with NGR (assessed by OGTT) were recruited. They had no history of excessive drinking (less than $40 \mathrm{~g}$ per day in males and $20 \mathrm{~g}$ per day in females) and were negative in serological analysis of HBsAg and HBC-Ab. Anthropological parameters(BMI, waist circumference, and waist-to-hip ratio) of all participants were collected and liver fat content was evaluated by semi-quantitive CT scan technique. According to liver fat content, subjects with NAFLD were divided into three groups. Liver fat content in subjects of group $\mathrm{A}(\mathrm{n}=6)$ was no more than $5.0 \%$, group $\mathrm{B}$ $(n=7) 5.1 \% \sim 9.9 \%$ and group $C(n=6) 10.0 \% \sim 20.0 \%$. Eight healthy subjects were enrolled as normal control group. Beta cell function and insulin sensitivity were assessed by hyperglycemic clamp technique. Area under first phase insulin release curve $\left(\mathrm{AUC}_{1}\right)$ and area under second phase insulin release curve $\left(\mathrm{AUC}_{2}\right)$ were calculated to analyze beta cell function. Insulin sensitivity was demonstrated by insulin sensitivity index (ISI). All participants signed written informed consents.

Results: There were no significant differences in age, sex, waist circumference, and waist hip ratio among Group A, B, and C. BMI of Group C $\left(30.80 \pm 3.43 \mathrm{~kg} / \mathrm{m}^{2}\right)$ were higher than that of Group A $\left(25.86 \pm 0.92 \mathrm{~kg} / \mathrm{m}^{2}\right)$ and $\mathrm{B}\left(26.42 \pm 2.28 \mathrm{~kg} / \mathrm{m}^{2}\right)$ $(p<0.05)$ while no significant difference was observed between group $\mathrm{A}$ and group $\mathrm{B}$. The $\mathrm{AUC}_{1}$ was $2.60 \pm 0.12 \mathrm{~min} . \mathrm{mU} / \mathrm{L}$ in group $\mathrm{A}$, it increased to $2.81 \pm 0.18 \mathrm{~min} . \mathrm{mU} / \mathrm{L}$ in group $\mathrm{B}(p>0.05)$, and returned to $2.70 \pm 0.23$ min.mU/L $(p>0.05)$ in group $\mathrm{C}$. The $\mathrm{AUC}_{2}$ was $4.03 \pm 0.28 \mathrm{~min} . \mathrm{mU} / \mathrm{L}$ in group $\mathrm{A}$, increased to $4.20 \pm 0.17 \mathrm{~min}$. $\mathrm{mU} / \mathrm{L}$ in group $\mathrm{B}$, and further increased to $4.41 \pm 0.17 \mathrm{~min} . \mathrm{mU} /$ $\mathrm{L}$ in group $\mathrm{C}(\mathrm{ANVOA}$ : Group A vs Group $\mathrm{B}, p>0.05$; Group $\mathrm{C}$ vs Group A, $p<0.05$; Group C vs Group B, $p>0.05$ ).ISI was $25.14 \pm 14.77 \mathrm{mg} . \mathrm{L} /(\mathrm{kg} . \mathrm{min} . \mathrm{mU})$ in group $\mathrm{A}$, it decreased to $8.31 \pm 2.24 \mathrm{mg} . \mathrm{L} /(\mathrm{kg} . \mathrm{min} . \mathrm{mU})$ in group $\mathrm{B}$, and further descended to $4.57 \pm 2.49 \mathrm{mg} . \mathrm{L} /(\mathrm{kg} . \mathrm{min} . \mathrm{mU})$ in group $\mathrm{C}$ (ANVOA: Group A vs Group B, $p<0.05$; Group C vs Group A, $p<0.05$; Group C vs Group B, $p>0.05$ ).

Conclusion: The results suggest that there is a positive correlation between the extent of IR and the level of liver fat content in patients with NAFLD and NGR when their liver fat contents are no more than $20 \%$. A positive correlation may also occur in first phase insulin release of beta cells and liver fat contents in patients with NAFLD and NGR when their liver fat contents are no more than $10 \%$. Beta cell dysfunction, however, may appear in such patients when their liver fat contents increase from $10 \%$ to $20 \%$. Controlling liver fat content may benefit improvement of IR and beta cell dysfunction in patients with NAFLD.

Supported by the technical support from Endocrine \& Metabolism Department Shanghai Jiao Tong University affiliated Sixth People Hospital and the help from my colleages

\section{5}

Beta cell dysfunction in IGT and early type 2 diabetes: comparison of surrogate markers with first phase insulin secretion from an intravenous glucose tolerance test

A. Festa $^{1,2}$, S. M. Haffner ${ }^{3}$, K. Williams ${ }^{3}$;

${ }^{1}$ Eli Lilly, Vienna, Austria, ${ }^{2}$ University of Texas, San Antonio, United States, ${ }^{3}$ Dept of Medicine, University of Texas Health Science Center at San Antonio, United States. 
Background and Aims: Methods to assess $\beta$-cell function in clinical studies are limited. The aim of the current study was to compare a direct measure of insulin secretion with indirect markers in relation to glucose tolerance status.

Materials and Methods: In $n=1380$ individuals from the IRAS (Insulin Resistance Atherosclerosis Study), $\beta$-cell function was assessed using a frequently sampled intravenous glucose tolerance test (first phase insulin secretion; AIR), HOMA-B (using the formula, 1985; HOMA-B or the computer program, 1998; HOMA-\%B), proinsulin (PI) levels and the $\mathrm{PI} /$ insulin ratio $(\mathrm{P} / \mathrm{I}-\mathrm{R})$. $\beta$-cell function was cross-sectionally analysed by glucose tolerance categories [NGT; $n=712$, IGT; $n=353$; newly diagnosed diabetes by $2 \mathrm{~h}$ glucose criteria from an OGTT; $\mathrm{n}=80(\mathrm{DM} 2 \mathrm{~h})$ or by fasting glucose criteria; $\mathrm{n}=135$ (DMf), or established diabetes on diet/exercise only; $\mathrm{n}=100(\mathrm{DM})]$.

Results: In Spearman correlation analyses (all p-values $<0.0001$ ), proinsulin levels were moderately related to HOMA-B and HOMA- $\% \mathrm{~B}(\mathrm{r}=0.17$ and 0.13 ), and inversely related to AIR ( $r=-0.21)$. The PI/I-R was inversely related to HOMA-B, HOMA-\%B and AIR $(-0.49,-0.51$, and -0.27 , resp.). AIR was related to HOMA-B and HOMA- $\%$ $\mathrm{B}(\mathrm{r}=0.43$ and $\mathrm{r}=0.47)$. The table shows $\beta$-cell function (normalized to NGT) by glucose tolerance category.

\begin{tabular}{llllll}
\hline & NGT & IGT & DM2 h & DMf & DM \\
\hline $\begin{array}{l}\text { Fasting glucose } \\
\text { (mg/dl) }\end{array}$ & 95.3 & 103.8 & 110.4 & 157.8 & 168.0 \\
Proinsulin & 100 & 148 & 220 & 331 & 323 \\
1/PI & 100 & 67 & 45 & 30 & 31 \\
P/I-Ratio & 100 & 112 & 133 & 173 & 179 \\
1/PIR & 100 & 89 & 75 & 58 & 56 \\
HOMA-B; 1985 & 100 & 106 & 111 & 65 & 58 \\
*HOMA-B; 1985 & 100 & 81 & 71 & 30 & 27 \\
HOMA-\%B; 1998 & 100 & 104 & 106 & 63 & 56 \\
*HOMA-\%B; 1998 & 100 & 87 & 78 & 41 & 38 \\
AIR & 100 & 79 & 65 & 38 & 43 \\
*AIR & 100 & 60 & 40 & 23 & 25 \\
\hline
\end{tabular}

* adjusted for insulin sensitivity, as assessed by HOMA or MINMOD, respectively

Conclusions: Indirect markers of $\beta$-cell function were modestly related to each other and to directly measured (first phase) insulin secretion. Markers based on proinsulin levels better discriminated between glucose tolerance categories than HOMA-B, particularly when unadjusted for insulin resistance. All markers markedly underestimated the magnitude of the $\beta$-cell defect across declining glucose tolerance status compared to a direct measure of insulin secretion (AIR). Subjects with IGT and early stage type 2 diabetic patients may have markedly more pronounced $\beta$-cell defects than estimated from epidemiological studies using indirect markers of $\beta$-cell function.

Supported by National Heart, Lung and Blood Institute (Grants HL47887, HL47889, HL47890, HL47892, HL47902, HL55208, and R01 HL58329) and the General Clinic Research Centers Program (Grants NCRR GCRC, M01 RR431 and M01 RR01346)

0626

Effects of exercise on the function and morphology of pancreatic islet cell in insulin resistant rats

X. Li, L. Yuan, B. Zhang, Z. S. Tang;

Endocrinology and Metabolism, Union Hospital, Wuhan, China.

Background and Aims: Insulin resistance is a common pathogenetic feature of type 2 diabetes mellitus. Nondiabetic subjects have the ability to secrete enough insulin as a compensate for insulin resistance,whereas in subjects with type 2 diabetes mellitus, the adaptation is insufficient. Islet dysfunction is a key factor promoting the progression from insulin resistant state to overt type 2 diabetes mellitus. Previous studies have provided evidence that swimming is an effective intervention to improve body's insulin sensitivity. The aim of the study is to observe the effects of swimming on the function and morphology of pancreatic islet cell in insulin resistant rats, and to investigate the relationship between the improvement of insulin sensitivity and the protection of pancreatic islet function.

Materials and Methods: Normal male Wistar rats were randomly allocated into 3 groups: a normal-chow $(15.36 \mathrm{kcal} / \mathrm{g}, 17 \%$ fat) group (NC, $\mathrm{n}=15)$, a high-caloric and high-fat diet (22.39 kcal/g, 55\%fat) group (HF, $\mathrm{n}=15)$, and a swimming (50 60 mins/d, 5 days $/ \mathrm{w}$ ) group with high-caloric and high-fat $\operatorname{diet}(\mathrm{SW}, \mathrm{n}=15)$. Insulin resistance was assessed by euglycemic hyperinsulinemic clamp technique. The insulin secretory function of islet was evaluated by intravenous insulin releasing test (IVIRT). Morphological and quantitative analysis was performed on pancreatic tissues stained by double-label insulin and glucagon through immunohistochemistry approach. Proinsulin mRNA was detected by RT-PCR.

Results: Rats taking HF had severe visceral obesity and developed remarkable insulin resistance compared with $\mathrm{NC}$ rats (GIR60 120: $7.60 \pm 1.29$ vs $5.83 \pm 0.79, \mathrm{P}<0.05$ ), whereas the insulin sensitivity in SW rats had significantly improved $(7.40 \pm 1.60, \mathrm{P}<0.05)$. Immunohistochemistry showed the insulin relative concentration of $\beta$ cells in HF decreased,as compare with $\mathrm{NC}$ rats $(\mathrm{P}<0.01)$. And islet size had an 1.42-fold increased in HF rats $(\mathrm{P}<0.01)$. The islet relative $\beta$-cell volume was decreased by $33.68 \%$ in $\mathrm{HF}$ 
$(\mathrm{P}<0.01)$, whereas the relative $\alpha$ cell volume increased by $57.96 \%(p<0.01)$. So there was a $60.47 \%$ decrease of the ratio of $\beta$ to $\alpha$ cells in HF. As compared with HF rats, SW rats had a higher insulin relative concentration of $\beta$ cells $(\mathrm{P}<0.01)$ and lower islet size $(\mathrm{P}<0.05)$. Swimming intervention resulted in $34.87 \%$ increase in the islet relative $\beta$ cell volume, and have a $86.32 \%$ increase in the ratio of $\beta$ to $\alpha$ cells. The peak of insulin secretion in IVIRT in NC and SW was on $5 \mathrm{~min}$, whereas that in HF rats was on $10 \mathrm{~min}$. AUC (area under curve) 10 60 of insulin in HF was higher than $\mathrm{NC}$ and $\mathrm{SW}$ rats $(\mathrm{P}<0.05)$. Proinsulin mRNA level had no significant difference among 3 groups.

Conclusion: Long-term high-caloric and high-fat diet results in early impairment of islet morphology and function, and significant insulin resistance as well. It shows in the early course of Type 2 diabetes mellitus the adaptation of islet has already been impaired. By meliorating insulin resistance, exercise can reverse the early change of morphology and function of pancreatic islet, maintain and increase the islet compensation to insulin resistance.

\section{PS 43}

\section{Insulin secretion II}

0627

Characterisation of a non-genetic non-obese model of type 2 diabetes in mice given streptozotocin and nicotinamide and its application for testing the antidiabetic properties of a new antioxidant compound P. Masiello ${ }^{1}$, V. D'Aleo ${ }^{1}$, R. Lupi ${ }^{2}$, M. Paolini ${ }^{3}$, A. Soleti ${ }^{4}$, P. Marchetti ${ }^{2}$, M. Novelli ${ }^{1}$;

${ }^{1}$ Dip. Patologia Sperimentale, ${ }^{2}$ Dip. Endocrinologia e Metabolismo, University of Pisa, ${ }^{3}$ Dip. Farmacologia, University of Bologna, Bologna, ${ }^{4}$ Medestea Research, Medestea, Torino, Italy.

Background and Aims: On the basis of a diabetic syndrome with reduced beta-cell mass previously obtained in rats, we intended to establish an original model of type 2 diabetes in mice, i.e. in a species of small size, suitable for long-term experimentation of scarcely available or expensive new potential antidiabetic agents. Furthermore, as oxidative stress is considered a major mechanism of progressive beta cell damage in diabetes, we tested in this model the protective effects of a new antioxidant drug.

Materials and Methods: Male and female C57B1/6 mice were given diabetogenic doses of streptozotocin (STZ) combined with different partially protective doses of nicotinamide (NA) and monitored for glycaemia, insulinaemia, glucose tolerance and pancreatic insulin content. These parameters as well as plasma nitrotyrosine as an index of oxidative stress were also evaluated in a cohort of male STZ-NA mice during a 5-week treatment with a novel antioxidant compound (IACVITA, Medestea).

Results: The combined administration of appropriate doses of STZ and NA to adult mice, in the absence of genetic alterations, resulted in a diabetic syndrome characterized by moderate hyperglycaemia ( $200 \mathrm{mg} / \mathrm{dl}$ in non-fasting animals, which remained stable for approximately two months, progressively increasing thereafter), hypoinsulinaemia, glucose intolerance, growth impairment and markedly reduced pancreatic insulin content $(20-30 \%$ as compared to controls). Upon treatment with the antioxidant drug, STZNA diabetic mice showed significant reduction but not normalization of plasma glucose levels, a slight increase in circulating insulin and a better preservation of residual pancreatic insulin content (controls: $126 \pm 5.4 \mathrm{ng} / \mathrm{mg}$ pancreas; STZ-NA: $28 \pm 4.6 \mathrm{ng} / \mathrm{mg}$; STZ-NA+antioxidant: $56 \pm 7.1 \mathrm{ng} / \mathrm{mg}$ ). Plasma nitrotyrosine levels, markedly enhanced in untreated diabetic mice, were significantly reduced although not fully reversed by the treatment (controls: $0.29 \pm 0.03 \mathrm{nM}$; STZ-NA: $0.79 \pm 0.04 \mathrm{nM}$; STZNA+antioxidant: $0.55 \pm 0.06 \mathrm{nM}$ ). Highly significant correlations were found between plasma nitrotyrosine values and either plasma glucose $(\mathrm{r}=0.59, \mathrm{p}<0.01)$ or pancreatic insulin content $(\mathrm{r}=0.70, \mathrm{p}<0.01)$ of all experimental groups.

Conclusion: We have established a novel non-obese nongenetic murine model of type 2 diabetes with reduced pancreatic insulin stores, particularly suitable for pharmacological research in diabetes. In this model, the new antioxidant compound IACVITA was able to partially reverse diabetic metabolic alterations, likely by counteracting beta cell dysfunction and loss associated with oxidative stress.

\section{8}

Regulation of pancreatic beta cell function and viability by adiponectin

J. E. P. Brown ${ }^{1}$, J. E. Digby ${ }^{2}$, K. L. Ward ${ }^{1}$, H. S. Randeva ${ }^{3}$, S. J. Dunmore ${ }^{1}$;

${ }^{1}$ RIHS, University of Wolverhampton, ${ }^{2}$ Warwick Medical School, University of Warwick, Coventry, ${ }^{3}$ Department of Biological Sciences, University of Warwick, Coventry, United Kingdom.

Background and Aims: Obesity is a risk factor for the metabolic syndrome and type 2 diabetes. Adiponectin, a $30 \mathrm{kDa}$ adipocyte secreted peptide has been shown to be anti-atherogenic, and anti-inflammatory, as well as affecting insulin resistance and pancreatic beta-cell function. This study investigated the effects of full length and globular 
adiponectin on pancreatic beta-cell viability, and expression of UCP-2, PDX-1 and LPL genes, as well as studying levels of expression of the adiponectin receptor subtypes 1 and 2 (AdR-1 and AdR-2) and their regulation in the pancreatic BRIN-BD11 beta-cell line.

Materials and Methods: Cells were cultured in RPMI1640 and treated with full length (fAd) or globular (gAd) adiponectin $(0-100 \mathrm{nM})+/-50 \mathrm{ng} / \mathrm{ml}$ leptin to test effects on cell viability, where an MTS assay was used. For gene expression experiments, $100 \mathrm{nM}$ adiponectin concentrations were used. For AdR expression experiments, cells were treated with rosiglitazone (10 micromolar), leptin $(10 \mathrm{ng} / \mathrm{ml}$ and $50 \mathrm{ng} / \mathrm{ml}$ ), oleic acid (50 micromolar), palmitic acid (50 micromolar), elaidic acid (50 micromolar) and the PPAR alpha agonist WY14643 (10 micromolar) for 24 hours. Total cellular RNA/protein was extracted and expression assessed by real-time PCR/Western blotting respectively.

Results: Both fAD and gAD caused significant increases in cell viability, however fAd only was reversed by addition of $50 \mathrm{ng} / \mathrm{ml}$ leptin. Expression of AdR- 1 mRNA was 70-fold higher than that of AdR-2 $(p<0.001)$ however this was reversed at protein level. Rosiglitazione treatment resulted in a $32 \%$ decrease in AdR-2 mRNA expression $(\mathrm{p}=0.029)$ but had no effect on AdR-1, with palmitic acid having similar effects. Oleic acid treatment conversely resulted in a decrease in expression of both receptor subtypes $(p<0.05)$ with WY14643 having similar effects. Leptin had no effect on expression of either receptor type. Neither fAd or gAd affected UCP-2 mRNA expression, however gAd increased LPL expression and PDX-1 expression.

Conclusion: These observations confirm a differential expression of AdR-1 and - 2 in pancreatic beta-cells, and suggest a role for adiponectin in beta-cell function and viability which may be altered in obesity and insulin resistant state.

\section{9}

$\mathrm{L}-\left[\mathrm{U}-{ }^{14} \mathrm{C}\right]$ glutamine and $\left[\mathrm{U}^{14}{ }^{14} \mathrm{C}\right]$ palmitate metabolism in islets from rats depleted in long-chain polyunsaturated $\omega 3$ fatty acids

Y. Zhang ${ }^{1}$, K. Louchami ${ }^{1}$, Y. A. Carpentier ${ }^{2}$, A. Sener ${ }^{1}$, W. J. Malaisse ${ }^{1}$;

${ }^{1}$ Lab of Experimental Hormonology, ${ }^{2}$ Lab of Experimental Surgery, Brussels Free University, Belgium.

Background and Aims: Rats depleted in long-chain polyunsaturated $\omega 3$ fatty acids ( $\omega 3$ rats) present several features of the metabolic syndrome, including obesity, ectopic fat accumulation and insulin resistance. This coincides with an alteration of D-glucose metabolism in pancreatic islets characterized by an impaired response to increases in hexose concentration and low oxidation/ utilization ratio. The specificity and possible mechanism of such an alteration were now investigated by comparing the metabolism of L-[U- $\left.{ }^{14} \mathrm{C}\right]$ glutamine and $\left[\mathrm{U}_{-}-{ }^{14} \mathrm{C}\right]$ palmitate in islets from normal and $\omega 3$ rats.

Materials and Methods: The production of ${ }^{14} \mathrm{CO}_{2}$ by islets from female normal and $\omega 3$ rats was measured upon either exposure to exogenous $\mathrm{L}-\left[\mathrm{U}-{ }^{14} \mathrm{C}\right]$ glutamine and $\left[\mathrm{U}-{ }^{14} \mathrm{C}\right]$ palmitate or after prelabelling of the islets with these nutrients.

Results: Despite comparable uptake of the amino acid, the oxidation of exogenous L-[U- $\left.{ }^{14} \mathrm{C}\right]$ glutamine (pmol/islet per $30 \mathrm{~min})$ was higher $(\mathrm{p}<0.001)$ in $\omega 3$ rats $(21.5 \pm 0.8 ; \mathrm{n}=83)$ than in normal rats $(16.7 \pm 0.7 ; \mathrm{n}=30)$. The output of ${ }^{14} \mathrm{CO}_{2}$ from prelabelled islets, as measured in the presence of either D-glucose $(8.3 \mathrm{mM})$ or metabolic poisons, when expressed relative to the corresponding value found in the absence of these agents, was comparable in $\omega 3$ and normal rats, with mean respective values of $110.8 \pm 3.9 \%$ ( $n=74$; $D$ glucose) and $24.6 \pm 1.7 \%(\mathrm{n}=72$; metabolic poisons). Over 90 min incubation, both the oxidation and uptake of exogenous $\left[\mathrm{U}-{ }^{14} \mathrm{C}\right]$ palmitate were significantly higher in $\omega 3$ than normal rats. Even the ratio between these two variables remained higher $(\mathrm{p}<0.05)$ in the $\omega 3$ rats $(3.14 \pm 0.30 \% ; \mathrm{n}=39)$ than in normal rats $(2.26 \pm 0.26 \%$; $\mathrm{n}=26$ ). Moreover, relative to the value found in the absence of exogenous nutrients, the output of ${ }^{14} \mathrm{CO}_{2}$ from prelabelled islets incubated in the presence of D-glucose $(8.3 \mathrm{mM})$ was higher $(\mathrm{p}<0.001)$ in $\omega 3$ rats $(117.9 \pm 6.2 \%$; $\mathrm{n}=29)$ than in normal rats $(78.5 \pm 5.5 \% ; \mathrm{n}=18)$.

Conclusion: These findings document that the impaired catrabolism of D-glucose in islets from $\omega 3$ rats represents a specific feature, contrasting with increased oxidation of L$\left[\mathrm{U}_{-}{ }^{14} \mathrm{C}\right]$ glutamine and $\left[\mathrm{U}_{-}{ }^{14} \mathrm{C}\right]$ palmitate. The increased uptake and oxidation of $\left[\mathrm{U}_{-}{ }^{14} \mathrm{C}\right]$ palmitate by the islets of $\omega 3$ rats is consistent with both an accelerated clearance of circulating fatty acids in these animals and the proposal that the impairment of D-glucose metabolism in the islets of $\omega 3$ rats may be attributable to altered glucokinase kinetics as a result of a high acyl-CoA content of islet cells.

0630

Effect of increased L- alanine, L- glutamine and Laspartate concentrations on INS- $1 \mathrm{E}$ cells over- expressing Aralar1

K. Bender ${ }^{1}$, P. Maechler ${ }^{2}$, P. Newsholme ${ }^{1}$;

${ }^{1}$ Conway Institute, UCD School of Biomolecular and Biomedical Research, Dublin, Ireland, ${ }^{2}$ Department of Cell Physiology and Metabolism, University Medical Centre, University of Geneva, Switzerland. 
Background and Aims: NADH shuttles transport reducing equivalents from the cytosol to the mitochondria. In pancreatic beta cells, the NADH shuttle system plays an essential role in coupling glucose metabolism to insulin secretion by delivering glycolysis- derived electrons to the electron transport chain in the mitochondria, which then, through ATP production and subsequent calcium rise, initiates insulin exocytosis. In beta cells, the NADH shuttle system generally consist of the glycerophosphate and the malate- aspartate shuttle. In beta cells, the latter functionally predominates and plays a crucial role in both mitochondrial metabolism and cytosolic redox state. One key constituent of the malate- aspartate shuttle is Aralar1, a $\mathrm{Ca}^{2+}$ sensitive isoform of the aspartate/ glutamate carrier 1 (AGC1) known to have a potentiating effect on glucose- stimulated insulin secretion. We overexpressed Aralar1 by means of a recombinant adenovirus in the INS- $1 \mathrm{E}$ beta cell line and investigated cell metabolism and nutrient- stimulated insulin secretion, in particular the impact of L- alanine, L- aspartate and Lglutamine.

Materials and Methods: INS- 1E cells were cultured for 72 hours prior to transduction with AdCA- Aralar1 and AdCA- LacZ and then incubated with L- alanine (2 mM), L- aspartate $(2 \mathrm{mM})$ and L- glutamine $(10 \mathrm{mM})$ respectively. Cell viability was determined using a WST-1 assay. Insulin secretion was measured by radioimmunoassay. Mitochondrial membrane potential was determined upon stimulation with $15 \mathrm{mM}$ glucose using 123-rhodamine as a probe. NADH levels were measured as autofluorescence.

Results: Insulin secretion measured in Aralar1 overexpressing cells cultured with L- alanine was enhanced compared to control cells cultured without L- alanine, resulting in a $20 \%$ increase upon $15 \mathrm{mM}$ glucose stimulation and a $25 \%$ increase at $2.5 \mathrm{mM}$ glucose (for both conditions $\mathrm{p}<0.05$ ). Both $\mathrm{L}$ - alanine and control cells gave a 5.5- fold increase in insulin secretion relative to basal $2.5 \mathrm{mM}$ glucose levels. Insulin secretion in Aralar1 over- expressing cells was decreasedby $2 \mathrm{mM}$ L- aspartate to almost $50 \%$ of the control cells $(\mathrm{p}<0.05)$. Insulin secretion in transduced cells remained unaltered by Lglutamine. Cell metabolism, as assessed by mitochondrial membrane potential and NADH levels, was also potentiated by L- alanine $(\mathrm{p}<0.05)$, inhibited by L- aspartate.

Conclusion: Cell metabolism and insulin secretion in INS$1 \mathrm{E}$ cells over- expressing Aralar1 was enhanced by $2 \mathrm{mM}$ L- alanine but decreased by L- aspartate. Our results indicate beneficial effects of $\mathrm{L}$ - alanine for metabolic secretion coupling.

Supported by the Health Research Board Ireland
0631

The ability of non-glucose nutrients to modulate postprandial insulin and glucagon secretion is intact in people with pre-diabetes

G. M. Bock ${ }^{1}$, C. Dalla Man ${ }^{2}$, E. Chittilapilly ${ }^{1}$, M. Campioni ${ }^{2}$, R. Basu ${ }^{1}$, G. Toffolo ${ }^{2}$, C. Cobelli ${ }^{2}$, R. Rizza ${ }^{1}$;

${ }^{1}$ Division of Endocrinology, Diabetes, Metabolism \& Nutrition, Mayo Clinic College of Medicine, Rochester, United States, ${ }^{2}$ Department of Electronics and Informatics, University of Padova, Italy.

Background/Aim: Although there is extensive data indicating that insulin secretion is impaired in subjects with pre-diabetes, it is not known if the ability of non-glucose nutrients (e.g. protein and fat) to modulate glucose induced insulin and glucagon secretion also is impaired.

Methods: 32 subjects (16F/16M; $54 \pm 1$ years; BMI: $31.3 \pm 0.9 \mathrm{~kg} / \mathrm{m}^{2}$ ) with impaired fasting glucose (IFG: $5.5-$ $6.9 \mathrm{mmol} / \mathrm{l})$ and 17 subjects $(11 \mathrm{~F} / 6 \mathrm{M} ; 50 \pm 2$ years; $27.7 \pm 0.8 \mathrm{~kg} / \mathrm{m}^{2}$ ) with normal fasting glucose (NFG: $<5 \mathrm{mmol} / \mathrm{l}$ ) ingested in random order on separate occasions 75 grams of glucose (OGTT) or a mixed meal (45\% CHO, $15 \%$ protein and $40 \%$ fat) also containing 75 grams of glucose. Plasma glucose, insulin, C-peptide and glucagon concentrations were measured at frequent intervals for 240 minutes.

Results: Fasting plasma glucose concentrations did not differ on the OGTT and meal study days in either the IFG $(5.7 \pm 0.1$ vs. $5.8 \pm 0.1 \mathrm{mmol} / \mathrm{l})$ or $\mathrm{NFG}(4.9 \pm 0.1 \mathrm{vs}$. $4.9 \pm 0.1 \mathrm{mmol} / \mathrm{l})$ groups. In addition, although 2 hour glucose concentrations were lower after meal than OGTT in the NFG $(6.1 \pm 0.3$ vs. $6.9 \pm 0.1 \mathrm{mmol} / \mathrm{l} ; \mathrm{p}=0.01)$ and IFG $(8.9 \pm 0.5$ vs. $9.7 \pm 0.4 \mathrm{mmol} / \mathrm{l} ; \mathrm{p}=0.01)$ groups, the glucose area above basal did not differ in either the IFG (522 \pm 51 vs. $562 \pm 54 \mathrm{mmol} / 4 \mathrm{hrs})$ or NFG $(353 \pm 23 \mathrm{vs} .348 \pm 45 \mathrm{mmol} /$ $4 \mathrm{hrs}$ ) groups. Despite comparable glucose areas above basal, insulin concentrations were higher $(p<0.01)$ after the mixed meal than the OGTT in both the IFG $(62.9 \pm 4.9$ vs. $42.0 \pm 4.3 \mathrm{nmol} / 4 \mathrm{hrs}$ ) and NFG groups (50.2 $\pm 6.2 \mathrm{vs}$. $23.8 \pm 2.6 \mathrm{nmol} / 4 \mathrm{hrs}$ ) indicating more insulin was required to achieve the same postprandial glycemic response in the presence of non-glucose nutrients. On the other hand, Cpeptide concentrations also were higher $(p<0.05)$ after the mixed meal than the OGTT in the IFG $(384 \pm 22$ vs. $296 \pm 22 \mathrm{nmol} / 4 \mathrm{hrs})$ and NFG (355 \pm 40 vs. $238 \pm 22 \mathrm{nmol} /$ $4 \mathrm{hrs}$ ) groups indicating that nutrients caused a compensatory increase in insulin secretion in both groups. Of particular interest, the nutrient induced delta (meal minus OGTT) in plasma insulin ( $21 \pm 3$ vs. $26 \pm 7 \mathrm{nmol} / 4 \mathrm{hrs})$ and C-peptide ( $88 \pm 18$ vs. $119 \pm 33 \mathrm{pmol} / 4 \mathrm{hrs})$ concentrations 
did not differ in the IFG and NFG groups respectively. Similarly, while fasting plasma glucagon concentrations tended to be higher $(\mathrm{p}=0.06)$ in the IFG than NFG group ( $75 \pm 6$ vs. $59 \pm 5 \mathrm{pg} / \mathrm{ml}$ ), glucagon concentrations were comparably suppressed during the OGTT $(-3197 \pm 601$ vs. $-2385 \pm 1160 \mathrm{pmol} / 4 \mathrm{hrs}$ ) and if anything increased less $(p<0.05)$ in the IFG than NFG group after mixed meal ingestion ( $1162 \pm 763$ vs. $4925 \pm 1446 \mathrm{pmol} / 4 \mathrm{hrs})$ resulting in a comparable nutrient induced delta in glucagon $(4359 \pm 842$ vs. $7310 \pm 2099 \mathrm{pmol} / 4 \mathrm{hrs})$ in the IFG and NFG groups.

Conclusion: When the same amount of glucose is ingested a) more insulin is required to achieve a comparable postprandial glycemic response in the presence than in the absence of non-glucose nutrients, b) this increase in insulin requirement is compensated by a nutrient induced increase in insulin secretion in both IFG and NFG, and c) although glucose induced insulin secretion is impaired in IFG, the ability of nutrients to enhance glucose induced insulin secretion and to modulate postprandial glucagon release is intact.

Supported by NIDDK 29953 and ADA

0632

Association of neurogenin 3 polymorphisms with insulin secretion

B. Bendlova ${ }^{1}$, J. Vcelak ${ }^{1}$, M. Vankova ${ }^{1}$, P. Lukasova ${ }^{1}$, D. Vejrazkova ${ }^{1}$, A. Zemanova ${ }^{1}$, K. Andela ${ }^{2}$, H. Kvasnickova ${ }^{1}$, K. Vondra ${ }^{1}$;

${ }^{1} \mathrm{OME}$, Institute of Endocrinology, Praha, ${ }^{2}$ Prenatal Clinic, Institute for Mother and Child Care, Praha, Czech Republic.

Background and Aims: Mutations in the transcription factors which play a role in the development of endocrine pancreas are associated with diabetes mellitus (DM). Neurogenin 3 belongs to a group of cell specific transcription factors which are expressed during embryogenesis. In mice with functionless Neurogenin 3, the endocrine pancreas is not developed and these mice die of diabetes early in postnatal period. In human, rare mutations in this gene are associated with MODY (maturity diabetes of the young). Inconsistent results of association studies dealing with the relation of Neurogenin 3 polymorphisms to insulin secretion in patients with common forms of diabetes mellitus type 2 (DM2) are published.

Materials and Methods: DM2 $(\mathrm{n}=246$; age 58,81 $\pm 6,98$; BMI $\left.25,85 \pm 4,14 \mathrm{~kg} / \mathrm{m}^{2}\right)$, offspring of DM2 $(\mathrm{n}=120$; age $34,15 \pm 10,93$; BMI $24,56 \pm 5,02 \mathrm{~kg} / \mathrm{m}^{2}$ ), healthy controls non-diabetic subjects without family history of DM2 $(n=238$; age $32,23 \pm 10,67$; BMI $23,21 \pm 3,67 \mathrm{~kg} / \mathrm{m}^{2}$ ), women with gestational diabetes ( $\mathrm{n}=112$; age $31,6 \pm 4,6$; BMI $24,7 \pm 4,0 \mathrm{~kg}$ / $\mathrm{m}^{2}$ ) entered the study. All subjects were anthropometrically and biochemically well characterized. In groups of controls and offspring, the oral glucose tolerance test (oGTT) and insulin tolerance test (ivITT) were performed and more than 20 indices of insulin secretion and insulin sensitivity were calculated. Neurogenin 3 gene polymorphisms were identified by SSCP method and the results were confirmed in all subjects by RFLP (enzyme SmaI - Gly167Arg; enzyme Eam1104I - Ser199Phe).

Statistical methods: Chi-Square test, ANCOVA (adjustments for age and BMI). Anthropometric parameters were evaluated for men and women separately.

Results:

\begin{tabular}{llllll}
\hline Genotype & DM2 & $\begin{array}{l}\text { Offspring } \\
\text { of DM2 }\end{array}$ & Controls & $\begin{array}{l}\text { Gestational } \\
\text { diabetics }\end{array}$ & $\mathrm{p}$ \\
\hline $\begin{array}{l}\text { Gly167Arg } \\
\text { Gly/Gly }\end{array}$ & $89.55 \%$ & $93.60 \%$ & $91.67 \%$ & $90.63 \%$ & n.s. \\
Gly/Arg & $8.96 \%$ & $4.80 \%$ & $6.77 \%$ & $9.38 \%$ & \\
Arg/Arg & $1.49 \%$ & $1.60 \%$ & $1.56 \%$ & $0 \%$ & \\
Ser199Phe & & & & & \\
Ser/Ser & $41.53 \%$ & $48.97 \%$ & $40.50 \%$ & $45.30 \%$ & n.s. \\
Ser/Phe & $44.76 \%$ & $41.58 \%$ & $43.73 \%$ & $47.01 \%$ & \\
Phe/Phe & $13.71 \%$ & $9.45 \%$ & $15.77 \%$ & $7.69 \%$ & \\
\hline
\end{tabular}

We did not find any significant differencies in the allelic and genotypic frequencies of two screened Neurogenin 3 polymorphisms among our study groups. However, statistical analysis revealed significantly lower basal as well as stimulated C-peptide levels in Phe allele carriers in comparison to Ser homozygotes (Ser199Phe).

Conclusion: We suggested that Neurogenin 3 variants could contribute to the deterioration of beta-cell secretion and thus influence the risk of DM2 development.

Supported by IGA MHCR NR/7809-5

0633

Glucolipotoxicity severely damage insulin secretion and cause ketone overproduction

N. Zhao ${ }^{1}, \mathrm{Y} . \mathrm{Yu}^{1}, \mathrm{H} . \mathrm{Tan}^{2}, \mathrm{X} . \mathrm{Zhang}^{2}$;

${ }^{1}$ Department of Endocrinology, ${ }^{2}$ Lab. of Endocrinology, West China Hospital of Sichuan University, Chengdu, China.

Background and Aims: We have found in a previous study that obese diabetic patients with unprovoked ketosis have severely insulin resistance and markedly impaired $\beta$-cell insulin secretion. To explore the roles of elevated glucose and FFAs in the pathogenesis of ketone overproduction in obese diabetes, here we test the effect of FFAs in 
the presence or absence of elevated glucose on insulin secretion and ketone production.

Materials and Methods: Diet-induced obese Wistar rats were assigned to three groups and all underwent $48 \mathrm{hr}$ iv infusions in each group: Controls $(\mathrm{NS}, \mathrm{n}=11)$ : saline alone; FFA ( $\mathrm{n}=11): 20 \%$ intralipid+heparin, $0.5 \mathrm{ml} / \mathrm{h}$ to maintain circulating FFA concentration at 1000 $2000 \mu \mathrm{M}$; GS-FFA group $(\mathrm{n}=12): 25 \%$ glucose $+20 \%$ intralipid+heparin in amounts required to maintain circulating glucose and FFAs concentration at $15-20 \mathrm{mM}$ and $1000-2000 \mu \mathrm{M}$ respectively. Serum FFA and $\beta$-hydroxybutyrate $(\beta$-HBA) were measured before and after infusion. Ten minutes after the end of the infusion, an IVGTT was performed in each group to evaluate the glucose-stimulated insulin secretion (GSIS) in vivo. The isolated rat pancreases preparations were applied to test the GSIS in vitro. Pancreases were perfused with Krebs-Ringer bicarbonate buffer contained glucose $(16.7 \mathrm{mM})$ or arginine $(19 \mathrm{mM})$ respectively.

Results: Serum $\beta$-HBA levels, which were similar at baseline in various groups, were markedly elevated at the end of infusion in GS-FFA group (NS: $0.290 \pm 0.170 \mathrm{mM}$, FFA: $0.526 \pm 0.107 \mathrm{mM}$, GS-FFA: $1.257 \pm 0.344 \mathrm{mM}$, p value was less than 0.05). The glucose/insulin ratio during IVGTT was significantly increased in GS-FFA group (Table $1, \mathrm{p}$ value was less than 0.05 ). Insulin secretions from perfused pancreases were similar in each group during the basal period ( $3 \mathrm{mM}$ Glucose). The glucose and arginine stimulated insulin secretions were obviously declined in GS-FFA group when compared with FFA and NS group (Table 2, $\mathrm{p}$ value was less than 0.01 ).

Conclusion: A marked synergistic effect of high glucose and FFAs on impaired insulin secretions and ketone overproduction was demonstrated in this study. It is suggested that the pathogenesis of ketone overproduction in obese diabetes may be associated with dramatically declined $\beta$-cell insulin secretion, as a result of synergistic effect of high glucose and FFAs. The insulin deficiency results in long-chain Fatty acyl CoA accumulated in cytosol of hepatocyte transport into mitochondrion in large amount and cause ketone overproduction.

Table 1. Serum glucose/insulin concentration during IVGTT in three groups

\begin{tabular}{llll}
\hline $\begin{array}{l}\text { Time NS group } \\
(\mathrm{min})\end{array}$ & \multicolumn{1}{l}{ FFA group } & GS-FFA group \\
\hline 0 & $5.7 \pm 0.6 / 31.3 \pm 2.4$ & $5.5 \pm 0.3 / 31.2 \pm 4.1$ & $7.3 \pm 0.3 / 34.3 \pm 7.4$ \\
5 & $14.3 \pm 1.3 / 135.4 \pm 11.2$ & $14.6 \pm 1.2 / 129.1 \pm 10.1$ & $17.2 \pm 1.1^{*} / 65.3 \pm 4.5^{*}$ \\
10 & $11.3 \pm 0.9 / 112.6 \pm 5.3$ & $12.4 \pm 0.8 / 107.1 \pm 6.1$ & $14.8 \pm 0.8^{*} / 75.6 \pm 5.2^{*}$ \\
15 & $9.1 \pm 0.8 / 94.8 \pm 10.3$ & $11.0 \pm 0.6 / 87.3 \pm 6.6$ & $13.2 \pm 1.1^{*} / 66.4 \pm 4.7^{*}$ \\
20 & $8.3 \pm 0.4 / 70.0 \pm 5.4$ & $9.3 \pm 0.9 / 64.7 \pm 1.9$ & $11.2 \pm 0.8^{*} / 56.8 \pm 5.9^{*}$ \\
\hline
\end{tabular}

Table 2. Glucose and arginine stimulated insulin secretion from perfused pancreases $(\mu \mathrm{U} / \mathrm{min})$

\begin{tabular}{llll}
\hline & NS group & FFA group & GS-FFA group \\
\hline Baseline & $123.4 \pm 52.9$ & $122.3 \pm 45.9$ & $106.7 \pm 39.6$ \\
Glucose & $386.7 \pm 64.8$ & $356.4 \pm 47.1$ & $158.3 \pm 48.1^{*}$ \\
$(16.7 \mathrm{mM})$ & & & \\
Arginine $(19 \mathrm{mM})$ & $601.1 \pm 106.2$ & $548.5 \pm 64.3$ & $233.1 \pm 61.6^{*}$ \\
\hline
\end{tabular}

Supported by a National Science Foundation of China grants 30370679 and 30570874

0634

The functional maturation of insulin secretion during late stage of pancreatic development in rat

C. Liu ${ }^{1}$, Q. X. Yuan ${ }^{1}$, W. De ${ }^{2}$;

${ }^{1}$ Department of Endocrinology, The First Affiliated Hospital of Nanjing Medical University, ${ }^{2}$ Department of Biochemistry and Molecular Biology, Nanjing Medical University, China.

Background and Aims: Defect of insulin synthesis, secretion and sensitivity in target cells plays an important role in the pathogenesis of diabetes mellitus. Insulin exocytosis is essential for blood insulin level, though relatively less studied. According to the hypothesis of thrifty phenotype, it is considered that adult diabetes has a close relation to abnormal embryonic development of pancreas. Therefore it is important to study the pancreatic development and pancreatic gene expression among this complex process. We have investigated gene expression profiles during various stages of embryonic pancreatic development of rats in our previous work. The aim of the present study is to define key genes relevant to insulin synthesis and exocytosis, and to investigate the improved process of insulin secretion.

Materials and Methods: Pancreata of SD rat at embryonic day 12.5(E12.5),E15.5,E18.5 and newborn,21 days after birth,adult rats were dissected respectively. Genechips from Affymetrix company were applied to explore gene expression profiles. Some genes related to insulin synthesis and secretion were verified by Realtime PCR. Intraperitoneal glucose tolerance tests (IPGTT) and ELISA were done to detect the function of pancreatic islet.

Results: Among genes related to insulin synthesis and release, insulin,proinsulin and relevant transcription factors such as PDX-1 began to be expressed from E12.5; Munc13-1,syntaxin-1a,Rab3a,Vamp-2,Glut-2 began to be expressed from E15.5, while GCK was expressed at late stage of embryonic day. The result of IPGTT revealed that newborn rats before a week after birth were significantly 
glucose intolerant showing an increased excursion in blood glucose in response to a glucose challenge. Basic blood insulin level was detected at E18.5 and increased rapidly thereafter. However, in response to a glucose load, only rats older than a week after birth began to show normal insulin secretory response gradually.

Conclusion: Potential ability of insulin synthesis and release in rat occurs from early and middle stage of pancreatic development. But the normal insulin secretion response to glucose challenge starts later.

Supported by funds of Jiangsu Province government

0635

Beta cell glucolipotoxicity induced by a diet high in saturated fat during pregnancy is reversed by PPARalpha activation

M. J. Holness, G. K. Greenwood, M. C. Sugden;

Diabetes and Metabolic Medicine, Barts and the London, United Kingdom.

Background and Aims: Glucose is the major physiological regulator of insulin secretion; however, chronically elevated levels of glucose contribute to worsening beta cell function in type 2 diabetes (glucotoxicity), particularly when in combination with elevated lipids (glucolipotoxicity). Pregnancy causes increased glucose transport into beta cells, and a decreased glucose threshold for glucosestimulated insulin secretion (GSIS); hence adverse effects of chronic hyperglycemia on beta cell function may be exacerbated in pregnancy, particularly as late pregnancy is also associated with hyperlipidemia.

Materials and Methods: Pregnant rats were maintained on either a control (low-fat) diet or a high-saturated-fat diet from the onset of pregnancy and sampled at day 19 of pregnancy (term $=22-23$ days). The PPARalpha agonist WY14,643 was administered ( $50 \mathrm{mg} / \mathrm{kg}$ body wt.; i.p.) at day 18 of pregnancy (i.e. $24 \mathrm{~h}$ before sampling). Glucosestimulated insulin secretion (GSIS) was assessed in vivo after acute glucose bolus injection, and in vitro using stepup and step-down islet perifusions

Results: Maintenance of rats on a high-saturated fat diet throughout pregnancy caused post-absorptive hyperglycemia (by 26\%) and glucose intolerance at day 19 of pregnancy (term $=23$ days), with an elevated $\Delta \mathrm{G}_{0-30}(38 \%$; $\mathrm{P}<0.05)$ and decreased glucose disappearance rates by $41 \%$; $\mathrm{P}<0.001)$ after intravenous glucose challenge. Although GSIS in vivo was increased (a $59 \%$ increase in $\Delta \mathrm{I}_{0-30}$ following glucose challenge; $\mathrm{P}<0.05)$, isolated islets showed markedly impaired GSIS (a 45\% decline in total insulin secretion; $\mathrm{P}<0.01$ ) during step-up and step-down islet perifusionss, with an elevated glucose threshold for
GSIS as well as decreased glucose responsiveness. To test whether impaired GSIS ex vivo reflected glucolipotoxicity, pregnant rats maintained on high-saturated fat diet were treated for $24 \mathrm{~h}$ with the PPARalpha agonist WY14,643 to augment lipid clearance via oxidation. This manipulation reversed fasting hyperglycemia. Although glucose tolerance remained impaired, GSIS with islet perifusions was significantly improved (by $63 \% ; \mathrm{P}<0.05$ ), with complete restoration of glucose responsiveness and a substantial lowering of the glucose threshold for GSIS.

Conclusion: It is concluded that beta-cell glucolipotoxicity is induced by a diet high in saturated fat through increased lipid delivery, in the absence of a corresponding increase in lipid oxidation, in combination with its consequence, chronic fasting hyperglycemia.

Supported by Diabetes UK

\section{PS 44}

\section{Human liver metabolism}

0636

Elevated plasma free fatty acids exacerbate underlying hepatic insulin resistance in type 2 diabetes P. Shah ${ }^{1}$, R. Basu ${ }^{2}$, O. Cohen ${ }^{3}$, A. Basu' ${ }^{2}$, R. A. Rizza ${ }^{2}$; ${ }^{1}$ Endocrine Neoplasia and Hormonal Disorders, Univ Texas MD Anderson Cancer Ctr, Houston, ${ }^{2}$ Endocrine Research Unit, Mayo Clinic, Rochester, United States, ${ }^{3}$ Endocrinology, Chaim Sheba Med Ctr, Tel-Hashomer, Israel.

Background and Aims: Elevated FFAs stimulate gluconeogenesis in people with type 2 diabetes mellitus (DM), however their effects on endogenous glucose production (EGP) are less certain due to autoregulation or presence of insulin concentrations that result in near maximal suppression of glycogenolysis.

Materials and Methods: Twentythree DM subjects and 13 matched non-diabetic controls (ND) were randomly studied twice. Intralipid-heparin (ILH) or glycerol (Gly) were infused for 11 hours. DM subjects were kept euglycemic during the night with a variable insulin infusion, thereafter insulin was infused in all subjects at rate of $0.6 \mathrm{mU} / \mathrm{kg}$ total body weight along with somatostatin, glucagon and growth hormone thereby maintaining equal portal concentrations. ${ }^{2} \mathrm{H}_{2} \mathrm{O}$ was given the evening before for measuring gluconeogenesis; $\left[3-{ }^{3} \mathrm{H}\right]$ glucose was infused to measure EGP; plasma glucose was clamped at $5.2 \mathrm{mM}$.

Results: Plasma FFA was higher $(\mathrm{p}<0.01)$ ILH vs Gly both before and during clamp in the DM $(1.3 \pm 0.3$ vs $0.3 \pm 0.0$; $1.3 \pm 0.3$ vs $0.3 \pm 0.1 \mathrm{mmol} / \mathrm{L}$ respectively) and ND $(0.9 \pm 0.4$ vs $0.1 \pm 0.0 ; 0.4 \pm 0.0$ vs $0.0 \pm 0.0 \mathrm{mmol} / \mathrm{L}$ respectively). 
Plasma glucose $(5.2 \mathrm{mM})$, insulin $(180 \mathrm{pmol} / \mathrm{L})$, and Cpeptide $(0.02 \mathrm{ng} / \mathrm{mL})$ did not differ ILH vs. Gly or between groups. Less $(p<0.01)$ glucose was required to maintain euglycemia during ILH than Gly both in DM and ND $(9.2 \pm 2.2$ vs $16.5 \pm 2.9$ and $32.6 \pm 4.5$ vs $42.4 \pm 3.7 \mathrm{~mol} / \mathrm{kg}$ / min). In DM this was due to both lower $(\mathrm{p}<0.001)$ glucose utilization $(16.0 \pm 1.5$ vs $22.1 \pm 2.5 \mathrm{~mol} / \mathrm{kg} / \mathrm{min})$ and higher $(\mathrm{p}<0.05)$ EGP $(6.8 \pm 0.8$ vs $5.7 \pm 0.7 \mathrm{~mol} / \mathrm{kg} / \mathrm{min})$. In $\mathrm{ND}$, whereas glucose utilization was lower $(\mathrm{p}<0.001)$ on ILH vs Gly $(37.2 \pm 3.7$ vs $45.3 \pm 3.8 \mathrm{~mol} / \mathrm{kg} / \mathrm{min})$, EGP did not differ $(3.6 \pm 1.2$ and $2.8 \pm 1.0 \mathrm{~mol} / \mathrm{kg} / \mathrm{min})$. Despite lower $(\mathrm{p}<0.01)$ plasma FFA, EGP was higher $(\mathrm{p}<0.05)$ during the clamp in DM on Gly day vs.ND on ILH day implying an additional abnormality in hepatic insulin action.

Conclusion: People with type 2 diabetes have hepatic insulin resistance that is further exacerbated by elevated plasma FFA.

Supported by NIDDK DK 29953

0637

Liver fat accumulation and isolated islets function in non-diabetic and type $\mathbf{2}$ diabetic subjects

S. Torri ${ }^{1}$, S. Petruccelli ${ }^{2}$, M. Bugliani ${ }^{1}$, R. Lupi ${ }^{1}$, S. Del Guerra ${ }^{1}$, M. Grupillo ${ }^{1}$, R. Mancarella ${ }^{1}$, N. Funel ${ }^{2}$, E. Pollina ${ }^{2}$, U. Boggi ${ }^{2}$, F. Filipponi ${ }^{2}$, F. Mosca ${ }^{2}$,

S. Del Prato ${ }^{1}$, D. Campani ${ }^{2}$, P. Marchetti ${ }^{1}$;

${ }^{1}$ Endocrinology and Metabolism, Metabolic Unit, ${ }^{2}$ Oncology, Transplantation and Advanced Technologies in Medicine, University of Pisa, Italy.

Background and Aims: Overall adiposity, and visceral fat (VF) tissue accumulation in particular, is associated with insulin resistance and type 2 diabetes. However, the specific role of VF in beta-cell function is still unclear.

Materials and Methods: We determined liver fat (LF) accumulation (as a definite measure of VF) by histology and assessed isolated pancreatic islets insulin secretion and survival of 45 non-diabetic (ND; age: $61 \pm 14 \mathrm{yrs}$; M/F: $27 /$ 18; BMI: $24.5 \pm 2.7 \mathrm{Kg} / \mathrm{m}^{2}$; waist circumference: $93.0 \pm 12.7 \mathrm{~cm}$ ) and 12 type 2 diabetic (T2DM; age: $68 \pm 7 \mathrm{yrs}$; M/F: 7/5; BMI 27.6 $\pm 2.6 \mathrm{Kg} / \mathrm{m}^{2}, \mathrm{p}<0.01$ vs ND; waist circumference $103.2 \pm 8.9 \mathrm{~cm}, \mathrm{p}<0.01$ vs ND) multiorgan donors.

Results: Average fat infiltration tended to be higher in T2DM (10.9 $\pm 3.1 \%$, range 0 to $35 \%)$ than in ND $(6.2 \pm 8.9 \%$, range 0 to $50 \%$ ), and subjects with $>20 \%$ LF were $25 \%$ in T2DM and 9\% in ND. Correlation of LF was strong with waist circumference $(p<0.001)$, weak with BMI $(p<0.05)$ and absent with age. Isolated islets insulin release (IR, $\mu \mathrm{U} /$ islet $/ \mathrm{min}$ ) was $0.036 \pm 0.013$ in $\mathrm{ND}$ and $0.033 \pm 0.008$ in
T2DM in response to $3.3 \mathrm{mmol} / 1$ glucose $(\mathrm{G})$, and $0.076 \pm 0.057$ in ND $(\mathrm{p}<0.01$ vs $3.3 \mathrm{mmol} / \mathrm{l} \mathrm{G})$ and $0.044 \pm 0.021$ in T2DM (NS vs $3.3 \mathrm{mmol} / 1 \mathrm{G}$ and $\mathrm{p}<0.05$ vs ND) in response to $16.7 \mathrm{mmol} / \mathrm{l} \mathrm{G}$. Apoptotic rate, as assessed by determination of cytoplasmic histone-associated DNA fragments, was $0.57 \pm 0.54$ and $1.17 \pm 0.87$ optical density units in ND and T2DM islets, respectively $(\mathrm{p}<0.05)$. In ND, glucose-stimulated IR and apoptotic rate did not correlate with $\mathrm{LF}(\mathrm{R}=0.1, \mathrm{p}=0.5$ and $\mathrm{R}=0.1, \mathrm{p}=0.6$, respectively); in $\mathrm{T} 2 \mathrm{DM}$, insulin release tended to be inversely correlated to $\mathrm{LF}(\mathrm{R}=0.5, \mathrm{p}=0.06)$.

Conclusion: We found therefore that histologically determined LF amount tended to be higher in T2DM and correlated mainly with waist circumference; however, exvivo pancreatic islet experiments failed to show strong associations between glucose-stimulated IR or islet cell apoptosis with LF, suggesting that if such a correlation occurs in- vivo, this is probably due to circulating factors.

0638

Noninvasive analysis of hepatic gluconeogenic and Krebs cycle fluxes in type 1 a glycogen storage disease T. C. Delgado ${ }^{1}$, L. Diogo ${ }^{2}$, P. García ${ }^{2}$, C. Barosa ${ }^{1}$, J. G. Jones ${ }^{1}$;

${ }^{1}$ NMR Research Unit, Dept. of Biochemistry, Center for Neurosciences and Cell Biology, Coimbra, ${ }^{2}$ Dept. of Metabolic Diseases, Pediatric Hospital of Coimbra, Coimbra, Portugal.

Background and Aims: In Type 1a glycogen storage disease (GSD), G6P-ase activity is absent resulting in no glucose or net glycogen synthesis from gluconeogenic precursors. To determine the effects of this block on hepatic gluconeogenic and Krebs cycle fluxes, GSD patients and healthy controls were given oral $\left[\mathrm{U}-{ }^{13} \mathrm{C}\right]$ glycerol, Paracetamol and phenylbutyric acid. On entry of $\left[\mathrm{U}-{ }^{13} \mathrm{C}\right]$ glycerol into hepatic Triose-P, UDPglucose ${ }^{13} \mathrm{C}$-enrichment can be noninvasively analyzed via urinary Paracetamol glucuronide (PG). Likewise, ${ }^{13} \mathrm{C}$-enrichment of Krebs cycle metabolites can be measured via phenylacetylglutamine (PAGN).

Materials and Methods: Five GSD patients (3M, 2F; $14 \pm 6 \mathrm{yr} ; 35 \pm 9 \mathrm{~kg}$ ) and 5 controls $(1 \mathrm{M}, 4 \mathrm{~F} ; 20 \pm 4 \mathrm{yr}$; $53 \pm 13 \mathrm{~kg}$ ) were studied. At 08:00, 11:00 and 14:00, subjects ingested $1 \mathrm{~g} / \mathrm{kg}$ body wt of cornstarch. Paracetamol (10$15 \mathrm{mg} / \mathrm{kg}$ ) was ingested at 11:00 and phenylbutyric acid $(5 \mathrm{mg} / \mathrm{kg})$ plus [U- ${ }^{13} \mathrm{C}$ ]glycerol $(25-30 \mathrm{mg} / \mathrm{kg})$ at 14:00. Blood $(10 \mathrm{ml})$ was collected at 17:00 and urine was collected from 16:45-18:45. Urinary PG and PAGN were isolated by ion-exchange chromatography and ${ }^{13} \mathrm{C}$-enrichment and isotopomer distributions analyzed by ${ }^{13} \mathrm{C}$ NMR. 
Relative metabolic fluxes by $13 \mathrm{C}$ isotopomer analysis: ${ }^{*} \mathrm{p}<0.05 ;{ }^{*} \mathrm{p}<0.01$ vs controls

\begin{tabular}{|c|c|c|c|c|c|c|c|}
\hline $\begin{array}{l}\text { Subject } \\
\text { Group }\end{array}$ & $\begin{array}{c}\text { Citrate } \\
\text { synthase flux }\end{array}$ & $\begin{array}{c}\text { Pyruvate } \\
\text { dehydrogenase flux }\end{array}$ & $\begin{array}{c}\text { Pyruvate } \\
\text { carboxylase flux }\end{array}$ & $\begin{array}{l}\text { Pyruvate } \\
\text { kinase flux }\end{array}$ & $\begin{array}{c}\text { Net } \\
\text { anaplerotic } \\
\text { flux }\end{array}$ & $\begin{array}{c}\text { Plasma glucose from } \\
\text { G6P-ase (\%) }\end{array}$ & $\begin{array}{l}\text { Triose-P to } \\
\text { UDPG flux }\end{array}$ \\
\hline Controls & 1.0 & $0.3 \pm 0.1$ & $9.9 \pm 3.3$ & $8.7 \pm 2.8$ & $1.3 \pm 0.5$ & $56 \pm 13$ & $100 \pm 31$ \\
\hline $\begin{array}{l}\text { GSD } \\
\text { Patients }\end{array}$ & 1.0 & $0.5 \pm 0.2 * *$ & $16.6 \pm 3.5^{* *}$ & $14.4 \pm 3.8^{*}$ & $2.2 \pm 0.8$ & not detected & $8 \pm 8^{* *}$ \\
\hline
\end{tabular}

Plasma glucose was analyzed by ${ }^{13} \mathrm{C}$ NMR following deproteinization of plasma. The minimum fraction of plasma glucose derived via G6P-ase was estimated from the ratio of plasma glucose to $\mathrm{PG}{ }^{13} \mathrm{C}$-enrichment. Triose-P to glycogen flux in GSD patients compared to controls was estimated from relative $\mathrm{PG}{ }^{13} \mathrm{C}$-enrichment levels. Krebs cycle and pyruvate fluxes were estimated by analysis of PAGN ${ }^{13} \mathrm{C}$ NMR data.

Results: In controls, both plasma glucose and glucuronide were enriched with ${ }^{13} \mathrm{C}$ indicating active hepatic glucose production. At least half of the plasma glucose was derived via G6P-ase (Table 1). GSD patients had no glucose ${ }^{13} \mathrm{C}$ enrichment and minimal $\mathrm{PG}$ enrichment indicating no G6Pase flux and little flux into glycogen. In GSD patients, pyruvate oxidation, carboxylation and recycling were all significantly elevated compared to controls. Surprisingly, net anaplerotic fluxes tended to be higher despite the blockage of gluconeogenesis, the principal anaplerotic outflow pathway in liver. Plasma lactate was enriched in GSD patients despite the absence of ${ }^{13} \mathrm{C}$-glucose. Isotopomer analysis indicates that the ${ }^{13} \mathrm{C}$-lactate was formed via the anaplerotic pathways of the hepatic Krebs cycle rather than by glycolysis.

Conclusion: In GSD, there was no gluconeogenic outflow to glucose and glycogen but pyruvate utilization by the Krebs cycle was increased. There was a redirection of anaplerotic outflow from gluconeogenesis to lactate production. Unlike glycolysis, lactate synthesis from anaplerotic precursors does not generate metabolic acidosis, hence this route may allow the safe production of lactate as an alternative oxidizable substrate to glucose for use by peripheral tissues.

0639

Quantifying direct and indirect pathways of hepatic glycogen synthesis with deuterated water and paracetamol J. Jones ${ }^{1}$, A. Fagulha ${ }^{2}$, M. Bastos ${ }^{2}$, C. Baptista ${ }^{2}$,

L. Barros $^{2}$, M. Caldeira ${ }^{3}$, C. Barosa ${ }^{1}$, M. Carvalheiro ${ }^{2}$;

${ }^{1}$ Dept of Biochemistry, Center for Neurosciences, Coimbra,

${ }^{2}$ Endocrinology and Diabetes, University Hospital of Coimbra, ${ }^{3}$ Department of Chemistry, University of Coimbra, Portugal.
Background: Hepatic glycogen is synthesized by direct and indirect pathways (Fig. 1). Normally, the majority of glycogen is synthesized by direct pathway, but in Type 1 diabetes (T1D) patients, the indirect pathway predominates. The indirect pathway has futile metabolic cycles while the direct pathway is energetically more efficient. The direct pathway is further facilitated by the association of glucokinase with the glycogen synthesis enzymes - a process that is stimulated by the presence of insulin. In T1D patients, intensive insulin therapy does not restore direct pathway activity to normal levels. Novel interventions that target insulin delivery to hepatocytes, such as islet transplantation, may prove more effective. To effectively address such questions, we present a highly practical method for quantifying direct and indirect pathway activities in the clinical setting.

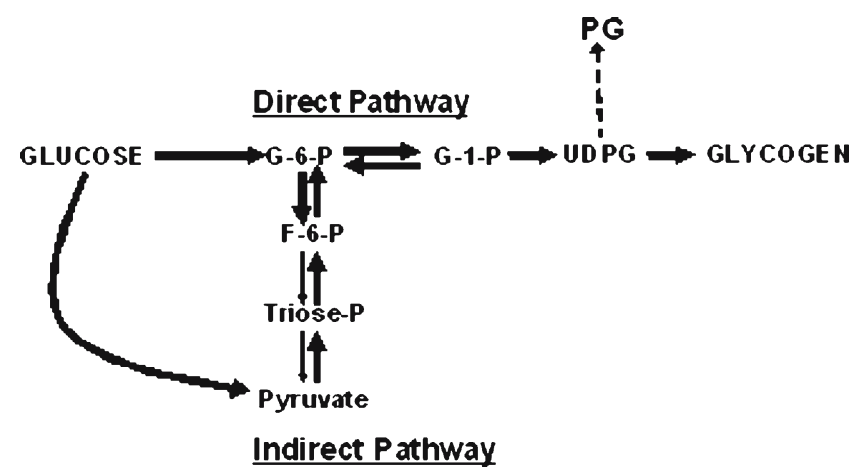

Figure 1. Direct and indirect pathways of hepatic glycogen synthesis and the sampling of UDPG as Paracetamol glucuronide $(P G)$. In the presence of ${ }^{2} \mathrm{H}_{2} \mathrm{O}$, glucuronide formed by direct pathway is labeled in position 2 as a result of G6P-F6P exchange. Glucuronide synthesized by indirect pathway is labeled in both positions 2 and 5 as a result of triose-P isomerase and other exchange reactions.

Methods: 9 healthy subjects $(3 \mathrm{~F} / 6 \mathrm{M}, 21 \pm 1 \mathrm{yr}, 67 \pm 4 \mathrm{~kg}$, $\left.\mathrm{Hb}_{1 \mathrm{Ac}} 4.9 \pm 0.2 \%\right)$ and $8 \mathrm{~T} 1 \mathrm{D}$ subjects $(3 \mathrm{~F} / 5 \mathrm{M}, 20 \pm 1 \mathrm{yr}$, $65 \pm 3 \mathrm{~kg}, \mathrm{Hb}_{1 \mathrm{Ac}} 8.1 \pm 0.6 \%$ ) were studied. Subjects began fasting at 20:00. Paracetamol $(1000 \mathrm{mg})$ and ${ }^{2} \mathrm{H}_{2} \mathrm{O}(3.0 \mathrm{~g} /$ $\mathrm{kg}$ body-water) were taken orally at 02:00. At 08:00, subjects had breakfast $(720 \mathrm{kcal}, 60 \% \mathrm{CHO}, 20 \%$ fat, $20 \%$ protein) and $500 \mathrm{mg}$ Paracetamol. Urine was collected at 
10:00-12:00 and ${ }^{2} \mathrm{H}$-enrichment of $\mathrm{PG}$ and body water was quantified by ${ }^{2} \mathrm{H}$ NMR. Indirect pathway contribution (\%) was estimated from glucuronide hydrogen 5 enrichment divided by that of body water $(\mathrm{G} 5 / \mathrm{bw}) \times 100$ and percent direct pathway contribution was estimated as 100 - indirect contribution. The percent of glucosyl units that did not undergo G6P-F6P exchange during glycogen synthesis, a putative marker for targeted glycogen synthesis via glucokinase, was estimated as $100 \times[1-(\mathrm{G} 2 / \mathrm{bw})]$ where $\mathrm{G} 2$ is the glucuronide hydrogen 2 enrichment. Data are reported as means \pm SE.

Results: Body water enrichment was $0.27 \pm 0.01 \%$ for controls and $0.27 \pm 0.01 \%$ for T1D patients and was constant between 08:00 and 12:00 for both groups. G5/bw was significantly higher in TID patients compared to controls $(0.53 \pm 0.02$ vs. $0.41 \pm 0.02, \mathrm{p}<0.03)$, indicating that a significantly smaller fraction of glycogen had been synthesized by the direct pathway $(47 \pm 2 \%$ versus $59 \pm 2 \%, \mathrm{p}<0.03)$. For both groups, glucuronide hydrogen 2 enrichment was significantly lower than that of body water $(0.21 \pm 0.01$ for controls; $\mathrm{p}<10^{-4}$ and $0.22 \pm 0.01$ for T1D patients, $\left.\mathrm{p}<0.01\right)$. This indicates that a substantial portion of glucosyl units did not undergo G6P-F6P exchange during conversion to glycogen ( $22 \pm 3 \%$ for controls and $18 \pm 5 \%$ for T1D patients).

Conclusion: A simple and noninvasive method for quantifying the pathways of hepatic glycogen synthesis in humans was demonstrated. The measurement revealed significant differences in the direct pathway contribution to glycogen synthesis between healthy and T1D subjects.

Supported by JDRFI 5-2004-306

\section{0}

Indices of insulin sensitivity of glucose production and disposal from double-tracer oral glucose tolerance test K. Thomaseth ${ }^{1}$, A. Pavan ${ }^{1}$, R. Berria ${ }^{2}$, L. Glass ${ }^{2}$, R. E. DeFronzo ${ }^{2}$, A. Gastaldelli ${ }^{3}$;

${ }^{1}$ Institute of Biomedical Engineering, CNR, Padova, Italy, ${ }^{2}$ Diabetes Division, University of Texas Health Science Center, San Antonio, United States, ${ }^{3}$ Institute of Clinical Physiology, CNR, Pisa, Italy.

Background and Aims: The oral glucose tolerance test (OGTT), used with only two glucose concentration measurements for the diagnosis of glucose intolerance and diabetes, is increasingly employed as dynamic test in more complex experiments for the study of metabolic disorders. In fact, in addition to its simplicity of execution, OGTT elicits a physiological secretory response from splancnic organs of glucose regulating hormones including gastrointestinal incretins. Aim of this study was the development of a simplified method for the analysis of data from double- tracer OGTT experiments, which are used for reconstructing rates of absorption, elimination and hepatic production (HGP) of glucose.

Materials and Methods: The proposed approach is based on a new mathematical model, derived from the minimal model of glucose disappearance proposed by RN Bergman and C Cobelli, which quantifies univocally the disappearance of both cold and tracer glucose by means of glucose clearance at zero insulin (GEZI) (dl/min) and insulin sensitivity of glucose disappearance $\mathrm{S}_{\mathrm{I}}(\mathrm{dl} / \mathrm{min} /(\mu \mathrm{U} / \mathrm{ml}))$. The novelties consist in the description of: (i) inhibition of HGP by insulin through a parameter, $I_{50}(\mu \mathrm{U} / \mathrm{ml})$, which quantifies the deviation of insulin concentration from basal required to inhibit HGP by $50 \%$; and (ii) gastrointestinal glucose absorption as a function of only two parameters. Model parameter estimates were obtained by means of the statistical non-linear mixed effects population approach. Twelve type 2 diabetic patients (D) (7 males and 5 females, age $=53.6 \pm 2.5$ yrs, $B W=81.9 \pm 3.6 \mathrm{~kg}, \mathrm{BMI}=30.5 \pm 1.1 \mathrm{~kg}$ $\mathrm{m}^{-2}$ ) and 10 control subjects with normal glucose tolerance (NGT) and similar BMI (5 M and $5 \mathrm{~F}$, age $=39.6 \pm 3.7$, $\mathrm{BW}=90.1 \pm 3.9, \mathrm{BMI}=31.1 \pm 0.9$ ), underwent a double-tracer OGTT with primed-continuous infusion of $0.25 \mu \mathrm{Ci} / \mathrm{min}$ $\left[3-{ }^{3} \mathrm{H}\right]$-glucose starting at $\mathrm{T}=-180 \mathrm{~min}$ in $\mathrm{D}$ and -120 in NGT, and with oral load at $\mathrm{T}=0$ of $75 \mathrm{~g}$ glucose dissolved in water together with $75 \mu \mathrm{Ci}\left[1-{ }^{14} \mathrm{C}\right]$-glucose. Blood samples for measurement of concentrations of cold and tracer glucose and insulin were collected every 15 minutes between $\mathrm{T}=-30$ and $240 \mathrm{~min}$.

Results: Model parameter estimates indicate a population average glucose clearance at zero insulin independent of the diabetic state and gender with GEZI $=0.72 \mathrm{dl} / \mathrm{min}(9.1 \%$ $\mathrm{CV})$ at a reference $\mathrm{BW}$ of $85 \mathrm{~kg}$ that increases with $\mathrm{BW}$ by $+2.1 \% / \mathrm{kg}(0.7 \% \mathrm{CV})$; an insulin sensitivity of glucose disappearance in NGT subjects $\mathrm{S}_{\mathrm{I}}(\mathrm{NGT})=0.098 \mathrm{dl} / \mathrm{min} /(\mu \mathrm{U} /$ $\mathrm{ml})(15 \% \mathrm{CV})$ about 20 times higher than in diabetic patients $\mathrm{S}_{\mathrm{I}}(\mathrm{D})=0.0048(18 \% \mathrm{CV})$; and an inhibition of HGP already at small suprabasal insulin levels $\mathrm{I}_{50}=16 \mu \mathrm{U} / \mathrm{ml}$ $(24 \% \mathrm{CV})$, which was not significantly dependent on the diagnosis or gender.

Conclusion: This study suggests that type 2 diabetes is characterized predominantly by a reduction of insulin sensitivity of glucose disappearance, and supports the usefulness of a simplified mathematical modelling approach to assess glucose absorption, production and disposition during double-tracer OGTT experiments. The population analysis allows the assessment of factors, such as BW and diagnosis, that influence between-subject variability of these processes, and provides a means for planning additional experiments for validating the present results and for designing simplified protocols for a broader use in clinical studies. 
High hepatic stearoyl-CoA desaturase-1 (SCD-1) index is associated with low liver fat and high insulin sensitivity in obese humans

N. Stefan ${ }^{1}$, A. Cegan ${ }^{1}$, J. Machann ${ }^{2}$, F. Schick ${ }^{2}$, A. Fritsche ${ }^{1}$, H.-U. Häring ${ }^{1}$, E. Schleicher ${ }^{1}$;

${ }^{1}$ Department of Internal Medicine, ${ }^{2}$ Section of Experimental Radiology, University of Tübingen, Germany.

Background and Aims: SCD-1 catalyzes the synthesis of monounsaturated fatty acids (FA) from saturated FA. It is imperative for the assembly of very low density lipoprotein (VLDL) particles, which transport FA from liver to adipose tissue and other sites. Studies in animals suggest that SCD1 deficiency is beneficial for metabolism. However, in humans data are conflicting.

Materials and Methods: We applied an elaborate method to measure an index of hepatic SCD-1 activity. First, we determined the pattern of FA in the triglyceride fraction of the VLDLs by ultracentrifugation followed by thin layer separation and gas chromatographic quantification in plasma samples from 54 healthy subjects. The oleate $(\mathrm{C} 18: 1, \mathrm{n} 9) /$ stearate $(\mathrm{C} 18: 0)$ ratio was used as an index of hepatic SCD-1 activity, thereafter. Insulin sensitivity (IS) was estimated from an oral glucose tolerance test (OGTT) and determined during a euglycemic clamp $(n=48)$. Liver fat was measured by $1 \mathrm{H}$ magnetic resonance spectroscopy.

Results: Gender distribution, mean age and body fat were as follows: $26 \mathrm{~m} / 28 \mathrm{f}, 44 \pm 2 \mathrm{y}, 31 \pm 1 \%$. The hepatic SCD-1 activity index was negatively associated with liver fat $(\mathrm{r}=-0.28, \mathrm{p}=0.049)$, independently of age, gender and body fat. It was positively associated with IS(OGTT) $(\mathrm{r}=0.42, \mathrm{p}=0.002)$ and weakly with IS(clamp) $(\mathrm{r}=0.27$, $\mathrm{p}=0.07)$. Importantly, these relationships were dependent on overall adiposity. When subjects were divided in a lean $(n=27,26 \pm 5 \%)$ and in an obese $(n=27,35 \pm 6 \%)$ group by the median body fat, the significant relationships were absent in the lean group (liver fat: $\mathrm{p}=0.34$, IS(OGTT): $p=0.75$, IS(clamp): $p=0.24)$. However, they were strengthened in the obese group $(p=0.004, p=0.0002, p=0.006)$.

After a 9 month lifestyle intervention with diet and increase in physical activity, a high SCD-1 activity index at baseline was associated with a larger increase in IS (clamp, adjusted) in the obese $(p=0.04)$ but not in the lean $(\mathrm{p}=0.35)$ group.

Conclusion: We conclude that hepatic SCD-1 is an important regulator of liver fat and insulin sensitivity in humans. In addition, this interaction is modulated by adiposity.

Supported by Deutsche Forschungsgemeinschaft (KFO 114/1)
Loss of hepatic clearance of insulin with resulting hyperinsulinaemia after orthotopic liver transplantation in humans

D. K. Andersen ${ }^{1}$, J. B. Hanks ${ }^{2}$, T. L. Pruett ${ }^{2}$, B. A. Clark ${ }^{3}$, R. L. Gingerich ${ }^{4}$, G. S. Meneilly ${ }^{5}$, D. Elahi ${ }^{1}$;

${ }^{1}$ Dept. of Surgery, Johns Hopkins Medical School, Baltimore, United States, ${ }^{2}$ Dept. of Surgery, University of Virginia, Charlottesville, United States, ${ }^{3}$ Dept. of Medicine, Alleghaney General Hospital, Pittsburgh, United States, ${ }^{4}$ Dept. of Assay Development, Linco, St. Charles, United States, ${ }^{5}$ Dept. of Medicine, University of British Columbia, Vancouver, Canada.

Background and Aims: The regulation of carbohydrate metabolism is presumed to be restored by Orthotopic liver transplantation (OLT) in liver disease patients, but is likely to be affected by immunosuppression and the possible effects of hepatic denervation. The aim of our study was to evaluate regulation of hepatic glucose production following OLT.

Materials and Methods: To assess this further, we performed hyperinsulinemic, euglycemic clamp studies for 2 hours in 10 patients (47-66 yrs, BMI 19-38) who had undergone OLT 5 to 12 months earlier, and in 4 patients (32-57 yrs, BMI 27-33) who had undergone renal transplantation (RT) 3-6 months earlier and were on the same immunosuppression regimens as the OLT patients, and in 6 normal (NL) subjects (19-25 yrs, BMI 20-24). A primed-constant infusion of $3{ }^{3} \mathrm{H}$-glucose was begun $2.5 \mathrm{hr}$ before the start of the clamp and continued throughout the hyperinsulinemic clamp. Insulin $\left(10 \mathrm{mU} / \mathrm{m}^{2} / \mathrm{min}\right)$ was infused from $0-120 \mathrm{~min}$ and glucagon $(3.5 \mathrm{ng} / \mathrm{kg} / \mathrm{min}$ ) was infused from $60-120 \mathrm{~min}$. The $20 \%$ glucose solution used to maintain euglycemia was spiked with ${ }^{3} \mathrm{H}$-glucose (hot $\mathrm{G}_{\text {inf }}$ ). Rates of hepatic glucose appearance $(\mathrm{Ra}, \mathrm{mg} / \mathrm{kg} /$ $\mathrm{min}$ ), of overall glucose disappearance ( $\mathrm{Rd}, \mathrm{mg} / \mathrm{kg} / \mathrm{min}$ ), and insulin levels (IRI, pM) were measured every 10 minutes from -30 to 120 minutes.

Results:

\begin{tabular}{lllll}
\hline & & BASAL & $\mathbf{0 - 6 0}$ min & $\mathbf{6 0 - 1 2 0}$ min \\
\hline OLT & Ra & $2.0 \pm 0.16$ & $0.8 \pm 0.23$ & $2.9 \pm 0.31$ \\
& Rd & $2.0 \pm 0.16$ & $2.5 \pm 0.23 \dagger$ & $2.4 \pm 0.31 \dagger$ \\
& IRI & $154+28 \dagger$ & $316 \pm 44 \dagger$ & $479 \pm 57 \dagger$ \\
RT & Ra & $2.4 \pm 0.45$ & $2.0 \pm 0.30 \dagger$ & $2.2 \pm 0.14$ \\
& Rd & $2.4 \pm 0.45$ & $3.1 \pm 0.20$ & $2.2 \pm 0.15 \dagger$ \\
& IRI & $48+17$ & $115 \pm 23$ & $120 \pm 27$ \\
NL & Ra & $2.2 \pm 0.17$ & $1.3 \pm 0.51$ & $2.9 \pm 1.13$ \\
& Rd & $2.2 \pm 0.20$ & $3.1+0.31$ & $3.7 \pm 0.64$ \\
& IRI & $35 \pm 7$ & $133 \pm 11$ & $135 \pm 12$ \\
\hline
\end{tabular}

Mean values \pm SEM are shown $(\dagger \mathrm{p}<0.05$ vs NL by t-test $)$. 
Hepatic glucose production after OLT is normal despite striking hyperinsulinemia. Basal C-peptide levels are elevated in OLT (3.3 ng/ml vs. 1.6 in RT and 1.5 in NL), but hepatic clearance of insulin is profoundly impaired in OLT (metabolic clearance rate of insulin: $36 \pm 4.3 \mathrm{ml} / \mathrm{m}^{2} /$ min) compared to NL $(87 \pm 1.9)$ and RT $(75 \pm 0.9)$. Hepatic sensitivity to insulin is decreased after both OLT and RT, which suggests a drug effect, but peripheral sensitivity to insulin is impaired only after OLT.

Conclusion: 1) OLT results in significant impairments in hepatic sensitivity to, and clearance of, insulin; 2) systemic insulin levels increase 3-4 fold largely due to a decrease in hepatic clearance of insulin; 3) peripheral insulin sensitivity is down-regulated by the hyperinsulinemic state; and 4) the loss of hepatic clearance of insulin appears independent of drug effects, and is probably secondary to interruption of CNS regulation of hepatic glucoregulatory functions.

Supported by NIH

\section{PS 45}

\section{Liver metabolism - animal models}

0643

AMPK regulates HNF4alpha transcription by the inhibition of Sp1 activity

Y. Joe ${ }^{1}$, S.-J. Kang ${ }^{1}$, E.-H. Seo ${ }^{1}$, M.-K. Park ${ }^{2}$, J.-Y. Park ${ }^{2}$, D. Kim ${ }^{2}$, H.-J. Lee ${ }^{1}$;

${ }^{1}$ Pharmacology, ${ }^{2}$ Internal Medicine, Dong-A University, Busan, Republic of Korea.

Background and Aims: The transcription factor hepatic nuclear factor $4 \alpha(\mathrm{HNF} 4 \alpha)$ is a regulator of the expression of genes involved in energy metabolism in the liver, intestine, and endocrine pancreas. It has been shown recently to play an important role in the regulation of hepatic glucose output, a key component of the maintenance of plasma glucose levels. Previous study reported that HNF4 $\alpha$ is a downstream target of AMPK and that AMPK activation is decreasing protein stability of HNF $4 \alpha$. It has been also studied that $\mathrm{Sp} 1$ binds to the promoter region of HNF4 $\alpha$. In this study we investigated whether the regulation of Sp1 by AMPK could affect HNF4a expression.

Materials and Methods: AMPK was overexpressed with adenoviral -mediated AMPK gene(Ad.AMPK) in HepG2 cells for 2 days after virus infection. Adenoviral -mediated AMPK -kinase dead gene(Ad.KD) was used for inhibition of AMPK kinase activity. To identify in vivo, SD rats were adminstered intravenously with Ad.AMPK and Ad.lacZ as control. The activation of AMPK was confirmed by Western blot analysis with HepG2 lysates and extracts of liver tissues. The expression levels of HNF4 $\alpha$ were evaluated with anti-HNF4 $\alpha$ antibody. The phosphorylation of Sp1 by AMPK was determined by in vitro kinase assay or with anti-phospho-serine antibody.

Results: Activated AMPK by Ad.AMPK infection decreased protein level of HNF4 $\alpha$. Protein level of HNF4 $\alpha$ was not decreased in Ad.KD infected cells or Ad.LacZ injected rats. Phosphorylation of Sp1 was increased in Ad. AMPK infected HepG2 cells. Ad.KD infection or treatment with AMPK inhibitor did not show the phosphorlyation of Sp1. Phosphorylation of $\mathrm{Sp} 1$ inhibited the transcription of HNF4 $\alpha$. These results were confirmed by decreased protein levels of HNF4 $\alpha$ in Ad.AMPK administrated SD rats.

Conclusion: Activated AMPK by Ad.AMPK increased Sp1 phosphorylation and decreased protein levels of HNF4 $\alpha$. Sp1 phosphorylation may play an inhibitory role in transcription of HNF4 $\alpha$ gene. Conclusively, overexpression of AMPK may down-regulate HNF4 $\alpha$ transcription by inhibitory Sp1 phosphorylation. Down-regulated HNF4 $\alpha$ by AMPK activation may diminish the interaction of HNF4 $\alpha$ with PGC-1and may suppress gene expression related to gluconeogenesis.

0644

Adenoviral-mediated AMPK gene expression ameliorates hyperglycaemia and hyperlipidaemia in overt type 2 diabetic rats

H.-J. Lee ${ }^{1}$, M.-K. Park ${ }^{2}$, E.-H. Seo ${ }^{1}$, E.-J. Park ${ }^{1}$, Y. Joe ${ }^{1}$, S.-J. Kang ${ }^{1}$, J.-Y. Park ${ }^{2}$, D. Kim ${ }^{2}$;

${ }^{1}$ Pharmacology, ${ }^{2}$ Internal Medicine, Dong-A University, Busan, Republic of Korea.

Background and Aims: AMP-activated protein kinase (AMPK) has been proposed to regulate the activities of key enzymes of lipid synthesis and metabolism and to improve insulin sensitivity. The Otsuka Long-Evans Tokushima Fatty (OLETF) rat is an established animal model of human type 2 diabetes that exhibits chronic and slowly progressive hyperglycemia and hyperlipidemia and is accompanied by progressive fibrosis in the islets. We have investigated the effects of AMPK in overt type 2 diabetic rats via adenoviral-mediated AMPK gene expression.

Materials and Methods: 42 weeks of aged overt type 2 diabetic rats $(\mathrm{n}=10)$ were administered intravenously with adenoviral-mediated AMPK gene (Ad.AMPK). Adenoviral-mediated lacZ gene was used for control group $(n=10)$. Expression of Ad.AMPK was confirmed by 
RT-PCR and Western blot analysis. AMPK activity was measured by phosphorylation of acetyl CoA carboxlyase (ACC) as a substrate of AMPK. Blood samples were collected from the tail vein for measurement of insulin, glucose, triglyceride, cholesterol and free fatty acid just before injection of Ad.AMPK for vehicles and on the 3rd and 7th days after Ad.AMPK administration. To investigate the changes of gene expression related glucose and lipid metabolism, quantitative real-time PCR was performed with liver tissues.

Results: Administration of Ad.AMPK showed high expression of AMPK in liver for 1 week and then decreased to level of control rats. Activity of AMPK in Ad.AMPK injected rats confirmed by phosphorylation of ACC. For transiently high expressed period (usually 1 week), Ad.AMPK injected rats improved hyperglycemia and hyperlipidemia. Plasma concentration of metabolic parameters is presented on the attached table. However, circulating level of free fatty acid did not change significantly on 3 or 7 days in Ad.AMPK administrated rats. The changes of metabolic gene expression showed that gluconeogenesis-related genes, phosphoenol pyruvate carboxykinase (PEPCK) and G6Pase were down-regulated in Ad.AMPK injected rats, and that lipogenesis-related gene, fatty acid synthase (FAS), stearoyl-CoA desaturase-1 (SCD-1) and glycerol-3-phosphate acyltransferase (GPAT) were also decreased in Ad. AMPK injected rats.

Conclusion: Adenoviral-mediated AMPK gene was successfully expressed in the liver of Ad.AMPK injected rats and the expressed AMPK phosphorylated its substrate. The effects of AMPK were improvement of hyperglycemia and hyperlipidemia and suppression of gene expression related with gluconeogensis and lipogenesis. These results suggest that intravenous administration of Ad.AMPK may play important role in liver and the effects of AMPK be directed to metabolism of glucose and lipid. Even though the expression period of Ad.AMPK was so short that the effects of AMPK showed transiently, long-term expressed Ad.AMPK would be promising therapeutic modality for treatment of type 2 diabetes.

Plasma levels of metabolic parameters in diabetic rats 0,3 and 7 days postinjection with Ad.AMPK

\begin{tabular}{llll}
\hline Parameter & 0 day & 3 day & 7 day \\
\hline Glucose $(\mathrm{mg} / \mathrm{dl})$ & $291.5 \pm 45.0$ & $257.4 \pm 56.3$ & $212.6 \pm 34.3^{*}$ \\
Insulin $(\mathrm{ng} / \mathrm{ml})$ & $1.022 \pm 0.252$ & $1.456 \pm 0.211$ & $2.212 \pm 0.346^{*}$ \\
Triglyceride $(\mathrm{mg} / \mathrm{dl})$ & $182.0 \pm 35.9$ & $104.7 \pm 25.4^{*}$ & $180 \pm 45.2$ \\
Total cholesterol $(\mathrm{mg} / \mathrm{dl})$ & $399.2 \pm 78.3$ & $246.9 \pm 45.2 *$ & $431 \pm 54.5$ \\
Free fatty acid $(\mathrm{uEq} / \mathrm{L})$ & $454.8 \pm 87.3$ & $389 \pm 56.9$ & $488 \pm 77.6$ \\
\hline
\end{tabular}

0645

Profile of rhythmic gene expression in the liver of obese diabetic KK-A $\mathbf{A}^{\mathbf{y}}$ mice

H. Ando ${ }^{1,2}$, H. Yanagihara ${ }^{1}$, Y. Oshima ${ }^{1}$, Y. Hayashi ${ }^{1}$, T. Takamura ${ }^{2}$, S. Kaneko ${ }^{2}$, A. Fujimura ${ }^{1}$;

${ }^{1}$ Clinical Pharmacology, Jichi Medical University, Shimotsuke, ${ }^{2}$ Diabetes and Digestive Disease, Kanazawa University Graduate School of Medical Science, Kanazawa, Japan.

Background and Aims: In vivo effect of type 2 diabetes on gene expression is usually studied only at one scheduled time of day. However, such a result might be affected by an observation time, because the expression of many genes exhibits circadian, 24-h rhythmicity in various tissues, including liver. At present, it remains to be determined whether the influence of type 2 diabetes on the gene expression is greater than that of the circadian time. To address this issue, we investigated the effects of experiment time on hepatic mRNA levels of various genes in genetically obese, diabetic $\mathrm{KK}-\mathrm{A}^{\mathrm{y}}$ and control KK mice.

Materials and Methods: Female 8-week-old KK and KK$\mathrm{A}^{\mathrm{y}}$ mice ( $n=12$ in each strain) were maintained under a 12h light/12-h dark cycle for 2 weeks, and thereafter the liver samples were obtained at the following zeitgeber times (ZT): $0,6,12$, and 18, where ZT 0 is defined as lights on and ZT 12 as lights off. Transcript levels of various genes in each sample were determined by microarray (GeneChip Mouse Expression Array 430A) and real-time quantitative PCR analysis.

Results: Severe obesity, obvious hyperglycemia and hyperinsulinemia were detected in $\mathrm{KK}-\mathrm{A}^{\mathrm{y}}$ but not in KK mice. A Gene tree clustering using the samples obtained from both strains at ZT 0 and 12 suggested the greater effects of the ZT on gene expression than those of obese diabetes as a whole. The real-time PCR of the genes whose microarray expression levels differed markedly $(>5$-fold, $P<0.05)$ between ZT 0 and 12 in either strain revealed that 22 of 23 genes showed circadian expression rhythms even in $\mathrm{KK}-\mathrm{A}^{\mathrm{y}}$ mice. Obese diabetes did not affect the phases, but reduced the peak levels in half of the genes. Although the mRNA levels of most (16 of 22) genes significantly differed between KK and $\mathrm{KK}-\mathrm{A}^{\mathrm{y}}$ mice at one or more $\mathrm{ZT}$, only 3 of these 16 genes showed the significant differences throughout the day.

Conclusion: Because obese diabetes alters the expression levels of various genes only at some observation time points, the consideration of expression rhythmicity could be critical in interpreting gene expression data. 


\section{6}

Undetectability of intestinal gluconeogenesis in fed and fasted rats: In vitro carbon 13 NMR spectroscopy and in vivo evidence

G. Baverel, B. Ferrier, M. Martin, A. Conjard, F. Saadé, M. Boghossian, R. Nazaret, G. Martin;

Metabolic and Renal Pathophysiology, INSERM U499, Lyon, France.

Background and Aims: It is classically admitted that only the liver and the kidney have the capacity to synthesize glucose from non-carbohydrate precursors, mainly lactate, alanine, glutamine and glycerol. However, recent studies have reported that the small intestine of fasted and diabetic rats substantially contributes in vivo to systemic glucose production via gluconeogenesis. Given the potential pathophysiological importance of intestinal gluconeogenesis, we have re-examined this subject.

Materials and Methods: For this, metabolically viable cells prepared from the small intestine of fed and fasted adult Wistar rats were incubated in Krebs-Henseleit medium under well-oxygenated conditions in the presence of $5 \mathrm{mM}\left[3-{ }^{13} \mathrm{C}\right]$-glutamine without and with unlabelled glucose. At the end of incubation, substrate removal and product formation were measured by enzymatic and ${ }^{13} \mathrm{C}$ NMR spectroscopy methods. Arterio-portal concentration difference measurements for substrate removal or production were also performed in $72 \mathrm{~h}$-fasted rats.

Results: The intestinal cells from both fed and $72 \mathrm{~h}$-fasted rats removed glutamine and synthesized glutamate, lactate, alanine, citrulline and ${ }^{13} \mathrm{CO}_{2}$ at high rates; despite the high rate of substrate removal, the synthesis of ${ }^{13} \mathrm{C}$-labelled glucose remained undetectable both in the absence and the presence of unlabelled glucose during the incubation. By contrast, hepatic and renal cells isolated from fasted rats and incubated with $5 \mathrm{mM}\left[3-{ }^{13} \mathrm{C}\right]$-glutamine synthesized large amounts of glucose labelled on its carbons 1, 2, 5 and 6 to approximately the same extent. Similarly to what was observed with glutamine as substrate, no glucose synthesis was observed in intestinal cells isolated from $72 \mathrm{~h}$-fasted rats and incubated with $5 \mathrm{mM}$ glycerol or fructose despite a substantial rate of utilization of these substrates.

Arterio-portal concentration difference measurements for glucose, lactate, pyruvate, alanine, glutamine, glutamate, glycerol, ketone bodies and ammonia in anesthetized $72 \mathrm{~h}$ fasted rats showed that the small intestine did not produce but rather removed glucose in net amounts. Only a small fraction of the circulating glutamine and glycerol, the precursors of the glucose reported to be synthesized in vivo, was taken up by the small intestine of these rats. Assuming the complete conversion of the latter fraction into glucose, the circulating radioactive glucose would be diluted by only approximaltely $1 \%$. This means that, under these conditions, intestinal gluconeogenesis from glutamine and glycerol is not demonstrable in vivo because the hardly measurable dilution of circulating radioactive glucose would be smaller than the margin of errors of the methods used.

Conclusion: The existence of gluconeogenesis in the rat small intestine, which has been reported to explain up to $35 \%$ of endogenous glucose production in $72 \mathrm{~h}$-fasted rats, is seriously challenged by the present results and, in our opinion, remains to be demonstrated.

Supported by INSERM

0647

Sustained activation of the mTOR nutrient sensing pathway associates with hepatic insulin resistance but not with steatosis per se in mice

E. V. Korsheninnikova ${ }^{1}$, G. C. M. van der Zon ${ }^{1}$, P. J. Voshol' ${ }^{2}$, G. M. C. Janssen ${ }^{1}$, L. M. Havekes ${ }^{3}$,

A. Grefhorst ${ }^{4}$, F. Kuipers ${ }^{4}$, D.-J. Reijngoud ${ }^{4}$,

J. A. Romijn ${ }^{2,3}$, D. M. Ouwens ${ }^{1}$, J. A. Maassen ${ }^{1,5}$;

${ }^{1}$ Molecular Cell Biology, ${ }^{2}$ Endocrinology, ${ }^{3}$ General Internal Medicine, Leiden University Medical Centre, ${ }^{4}$ Center for Liver, Digestive, and Metabolic Diseases, University Medical Centre Groningen, ${ }^{5}$ Endocrinology/Diabetes Centre, VU University Medical Centre, Amsterdam, The Netherlands.

Background and Aims: Increased activation of nutrient sensing through mammalian traget of rapamycin (mTOR) has been linked to the pathogenesis of insulin resistance. We examined activation of mTOR-signaling in relation to insulin resistance and hepatic steatosis in mice.

Materials and Methods: Insulin resistance and chronic hepatic steatosis were induced by high-fat (HF) feeding of male C57/BL/Jico mice for 6 weeks. Acute hepatic steatosis in the absence of insulin resisance was induced by pharmacological blockade of b-oxidation at the level of carnitine palmitoyltransferase I using tatradecylglycidic acid. Mitochnondrial function and mTOR signaling were examined in liver homogenates.

Results: HF feeding caused increased body weight $(\mathrm{p}<0.001)$ and hepatic insulin resistance $(\mathrm{p}<0.05)$. The phosphorylation of mTOR and its downstream targets p70S6 kinase and S6 ribosomal protein were 2-fold higher in HF- versus chow-fed mice (all $\mathrm{p}<0.05$ ), and associated with enhanced rates of protein synthesis. Acute induction of hepatic steatosis had no effect on mTOR activity. The increased activity of the mTOR pathway in livers from HF-fed mice could not be ascribed to diet-induced alterations in known modulators of mTOR activity, such as circulating plasma leucine levels, phosphor- 
ylation of protein kinase B, AMP-activated protein kinase and changes in mitochondrial function.

Conclusion: HF-diet leading to insulin resistance, but not hepatic lipid accumulation per se, induces increased activation of the mTOR nutrient sensing pathway. Supported by the Dutch Diabetes Research Foundation

\section{8}

Characterisation of the human Ped-Pea/15 promoter: identification of a functional HNF4 $\alpha$ response element P. Ungaro ${ }^{1}$, R. Teperino ${ }^{2}$, A. Lombardi ${ }^{2}$, R. Valentino ${ }^{1}$, A. Perfetti ${ }^{2}$, I. Esposito ${ }^{2}$, A. T. Alberobello ${ }^{2}$, G. Lupoli ${ }^{2}$, C. Miele ${ }^{1}$, P. Formisano ${ }^{2}$, F. Beguinot ${ }^{2}$;

${ }^{1}$ IEOS, CNR, Naples, ${ }^{2}$ Dipartimento di Biologia e Patologia Cellulare e Molecolare, Università degli Studi di Napoli "Federico II", Naples, Italy.

Background and Aims: Type 2 diabetes is a heterogeneous disease caused by both environmental and genetic factors. The pathophysiology of type 2 diabetes results from insulin resistance coupled with functional defects in insulin secretion by pancreatic beta cells. However, the precise factors leading to this disease are not known.

PED/PEA-15 is a ubiquitously expressed multifunctional protein whose gene has been shown to be over-expressed in skeletal muscle and adipose tissue from individuals with type 2 diabetes. Studies in transgenic mice also indicate that over-expression of PED/PEA-15 causes insulin-resistance and impairs glucose tolerance. The aim of our study is to characterize the molecular mechanism involved in the regulation of Ped-Pea/15 gene expression.

Materials and Methods: We have investigated this issue in cultured Hela and HepG2 cells.

Results: We started our study by identifying the promoter region driving the transcription of the gene. A $4 \mathrm{~Kb}$ fragment of the 5 -flanking region of Ped-Pea15 was cloned in front of the luciferase gene. Transfection of the construct into Hela cells produced an increase in the luciferase activity. Deletion analysis of this construct led to the identification of a $288 \mathrm{bp}$ fragment as a core promoter sufficient for the transcriptional activation of the gene. In Silico studies first indicated the presence of a consensus binding site for the Hepatocyte Nuclear Factor 4 alpha (HNF4alpha) between nucleotides -477 and -367 , suggesting that HNF4alpha could be involved in the regulation of Ped-Pea-15 gene expression. Co-transfection experiments with the HNF4alpha expression vector led to a $60 \%$ reduction of the luciferase activity associated with Ped-Pea-15 promoter fragment carrying HNF4alpha binding site. This effect is specific, since it is lost when the cells were transfected with a $5^{\prime}$ deletion construct lacking
HNF4alpha site. As expected increasing concentrations of this nuclear receptor proportionally reduced the luciferase activity of the reporter gene. Consistent with these data Real Time PCR assay on total RNA extracted from HNF4alpha transfected Hela cells confirmed a decrease in Ped-Pea 15 expression level.

Finally, chromatin immunoprecipitation (ChIP) experiment conducted in HepG2 cells evidenced the presence of a functional HNF4alpha response element between nucleotides -477 to -367 on the Ped-Pea/ 15 promoter.

Conclusion: Our results indicate that HNF4alpha downregulate Ped-Pea/15 mRNA expression through a direct or indirect action on HNF4alpha responsive element present on Ped-Pea/15 promoter sequence.

\section{9}

PPAR- $\alpha$ activation upregulated liver $X$ receptor- $\alpha$ and downregulated sterol regulatory element-binding protein-1c in liver of high-fat-fed and high-starch-fed rats J. Li ${ }^{1}$, H. Tian ${ }^{1}$, J. Cheng ${ }^{2}$, X. Chen ${ }^{3}$, Y. Wang ${ }^{4}$, Y. Long ${ }^{3}$, J. Liang ${ }^{1}$;

${ }^{1}$ Department of Endocrinology, ${ }^{2}$ Laboratory of Transplant Engineering and Immunology, ${ }^{3}$ Laboratory of Endocrinology and Metabolism, West China Hospital of Sichuan University, ${ }^{4}$ Biomedical Engineering Department, West China Basic Medical School of Sichuan University, Chengdu, China.

Background and Aims: Recent data suggested that liver X receptor (LXR)- $\alpha$ played a dynamic role in the regulation of fatty acid metabolism. And sterol regulatory elementbinding protein (SREBP)-1c activated transcription of the genes required for fatty acid synthesis in the liver. The present study was to examine the potential preventive effect of PPAR- $\alpha$ activition on the development of insulin resistance and on the expressions of both LXR- $\alpha$ and SREBP-1c in liver of high-fat-fed and high-starch-fed rats. Materials and Methods: High-starch-fed and high-fat-fed rats were treated with PPAR- $\alpha$ activator fenofibrate for 8 weeks. Insulin sensitivity was evaluated using a hyperinsulemic-euglycemic clamp technique. The expressions of LXR- $\alpha$ and SREBP-1c were determined by real-time PCR and western blotting.

Results: Fenofibrate decreased body weight and visceral fat, but increased plasma adiponectin level. Furthermore, fenofibrate significantly decreased the triglyceride content in the liver and improved insulin sensitivity in both highfat-fed and high-starch-fed rats. High fat feeding and high starch feeding stimulated the expression of SREBP-1c in liver. Fenofibrate induced the expression of LXR- $\alpha$ and decreased the level of SREBP-1c significantly. 
Conclusion: The PPAR- $\alpha$ agonist prevented the development of insulin resistance and hepatic fat deposition in high-fat-fed and high-starch-fed rats, which might be related to the upregulation of LXR- $\alpha$ and downregulation of SREBP-1c in liver.

\section{0}

Splanchnic and liver glucose metabolism: an insulin dose-response study

P. Iozzo $^{1}$, A. Gastaldelli ${ }^{2}$, M. J. Jarvisalo ${ }^{3}$, M. Pettiti $^{2}$,

J. Kiss ${ }^{3}$, E. Buzzigoli ${ }^{2}$, L. Guiducci ${ }^{1}$, A. Viljanen ${ }^{3}$,

R. Borra ${ }^{3}$, S. Frascerra ${ }^{4}$, S. Baldi ${ }^{4}$, G. A. Naum ${ }^{3}$,

P. A. Salvadori ${ }^{1}$, E. Ferrannini ${ }^{4}$, P. Nuutila ${ }^{3}$;

${ }^{1}$ PET Laboratory, IFC-CNR, Pisa, ${ }^{2}$ Metabolism Unit, IFCCNR, Pisa, Italy, ${ }^{3}$ Turku PET Centre, University of Turku, Finland, ${ }^{4}$ Dept of Internal Medicine, University of Pisa, Italy.

Background and Aims: The present study was undertaken to investigate the response of splanchnic (pre-hepatic, SPL) and liver (LIV) glucose and gluconeogenic substrate fluxes to different levels of insulin.

Methods: Eighteen pigs underwent catheterization of the carotid artery, portal and hepatic veins for the measurement of lactate, pyruvate, alanine, beta-hydroxybutyrate, glycerol, glucose, free fatty acids and insulin. Blood flow was measured by Doppler flow probes. Animals were studied during fasting $(\mathrm{n}=10), 1 \mathrm{mU} / \mathrm{kg} / \mathrm{min}(\mathrm{n}=4)$ and $5 \mathrm{mU} / \mathrm{kg} /$ $\min (n=4)$ euglycemic hyperinsulinemic clamp. Glucose production (EGP) and gluconeogenesis (GNG) were assessed by stable tracer methods.

Results: In SPL, glucose uptake increased in proportion with insulin levels $(\mathrm{r}=0.61, \mathrm{p}=0.01)$; hyperinsulinemia switched alanine and pyruvate balance from release to uptake $(p<0.05)$ while no change was observed in the other substrates. In LIV, hyperinsulinemia diminished net glucose release by decreasing EGP by $60 \%$ during the low and 94\% during the high insulin infusion $(p=0.05$ and $0.02 v s$ fasting, respectively); $\mathrm{GNG} \%$ accounted for $80 \%$ of EGP at fasting, and was suppressed by $>50 \%$ during the clamp $(\mathrm{p}<0.03)$. SPL insulin uptake went from release in the fasting state to uptake during the $1 \mathrm{mU}$ clamp, and was further doubled during the 5 mUclamp $(p<0.02)$; LIV insulin uptake was increased by 7 - and 45 fold from fasting to physiological and supraphysiological hyperinsulinemia, respectively $(\mathrm{p}<0.0001)$. The SPL uptake of insulin was inversely correlated with GNG $(\mathrm{r}=-0.68, \mathrm{p}=0.02)$. When fluxes were calculated considering LIV and SPL as a whole compartment, release of GNG precursors and glucose uptake by the pre-hepatic SPL were fully masked, due to opposite higher LIV fluxes.
Conclusions: SPL substrate balance documented an insulin dose-dependent switch of GNG precursors from release to uptake, contributing to the suppression of GNG during hyperinsulinemia.

Supported by Academy of Finland, Finnish Diabetes Foundation

0651

Novel mechanistical aspects in action of glycogen phosphorylase inhibitors on glycogen metabolism in primary rat hepatocytes

D. Zibrova, R. Streicher, S. G. Kauschke;

Metabolic Diseases, Boehringer Ingelheim Pharma $\mathrm{GmbH}$ \& Co. KG, Biberach an der Riss, Germany.

Background and Aims: Hepatic glucose output is elevated in type 2 diabetes. Glycogen breakdown (glycogenolysis) contributes significantly to the abnormally high glucose production by the liver. Glycogen phosphorylase (GP) catalyzes glycogenolysis and several pharmacological agents inhibiting this enzyme have been described as potential anti-diabetic drugs attenuating hyperglycemia. Since glycogenolysis is in dynamic interplay with glycogen synthesis, it is important to explore the consequences of inhibiting GP for glycogen metabolism as a whole. Therefore, the aim of the study was to investigate the effects of different GP inhibitors on proteins involved in glycogen metabolism to better understand the mechanistic basis for their pharmacological profile.

Materials and Methods: Hepatocytes were isolated from the livers of male Wistar rats by collagenase digestion. The cells were maintained in William's E medium or DMEM supplemented with different glucose concentrations depending on the experimental design and for all studies treatment with compounds was performed $24 \mathrm{~h}$ after cell isolation. Glycogen synthesis was assessed by determination of $\left[{ }^{14} \mathrm{C}\right]$ glucose incorporation into glycogen within $2 \mathrm{~h}$ at suitable glucose concentrations (e.g. $10 \mathrm{mM}$ ). Glycogen content was measured as glucose residues by amyloglucosidase method. To investigate the effects of GP inhibition on glycogen metabolism at the molecular level, glycogenenriched fractions were prepared by differential centrifugation. Cellular distribution and phosphorylation status of proteins regulating glycogen metabolism were determined by immunoblotting.

Results: Administration of GP inhibitors not only inhibited GP activity but also stimulated glycogen synthesis using rat hepatocytes. This correlated with profound increase in glycogen levels (by 2 fold) upon application of the compounds at hyperglycemic conditions (20 mM glucose). 
Compared to untreated controls, compound effects on glycogen amounts were even more pronounced (up to 5 fold increase) at conditions of complete glucose deprivation (0 $\mathrm{mM}$ glucose) suggesting prevention of glycogen store mobilization by GP inhibitors. With regard to proteins involved in glycogen metabolism, in cells treated with compounds at both high $(10 \mathrm{mM})$ and low $(2.5 \mathrm{mM})$ glucose, most of glycogen synthase (GS) associated with glycogen was in its less phosphorylated (more active) state as revealed by phospho(Ser641)-immunoblotting of glycogen-enriched fractions. Surprisingly, the amounts of glycogen-bound GP were dramatically reduced in compound-treated samples at high glucose. At low glucose this effect was somewhat blunted, albeit not abolished. Correspondingly, more GP was recovered in the cytosolic fractions obtained from compound-treated cells.

Conclusion: In the present study, GP inhibition increased glycogen levels in primary rat hepatocytes at both high and low glucose conditions suggesting increased energy pools a protective measure to control diabetic hyperglycemia. Furthermore, the present study provides the evidence that, in addition to inhibiting GP enzymatic activity, cellular localization of GP and activation of GS are influenced. Thorough understanding of underlying mechanistic principles could help to design compounds with different pharmacological profiles.

\section{2}

Estrogen improves glucose tolerance and hepatic insulin sensitivity in ob/ob mice

A. Khan ${ }^{1}, \mathrm{H} . \mathrm{Gao}^{2}$, G. Bryzgalova ${ }^{3}$, E. Hedman ${ }^{2}$, J.-A. Gustafsson ${ }^{2}$, K. Dahlman-Wright ${ }^{2}$, S. Efendic ${ }^{3}$;

${ }^{1}$ Molecular Medicine and Sugery, Karolinska Institute, Stockholm, Sweden, ${ }^{2}$ Biosciences and Nutrition, Karolinska Institute, Novum, Stockholm, ${ }^{3}$ Molecular Medicine and Surgery, Karolinska Institute, Stockholm, Sweden.

Background and Aims: Recent studies have demonstrated that estrogen plays an important role in glucose homeostasis. This estrogen effect is mediated via estrogen receptor- $\alpha$ $(\mathrm{ER} \alpha)$. Thus, in estrogen receptor- $\alpha$ knockout (ERKO) mice, impaired glucose homeostasis was observed which was mainly due to decreased hepatic insulin sensitivity resulting from the upregulation of lipogenic genes.

The aim of the present study is to investigate the mechanisms behind the effect of estrogen on glucose tolerance and hepatic insulin sensitivity in ob/ob mice.

Materials and Methods: ob/ob mice were treated with $17 \beta$ - estradiol ( $100 \mu \mathrm{g} / \mathrm{kg} /$ day) for 30 days, while control $\mathrm{ob} / \mathrm{ob}$ mice received vehicle. In both groups, we performed intraperitoneal glucose tolerance tests and insulin tolerance tests. Furthermore, genome-wide expression profiles were analyzed in the liver, using high-density oligonucleotide microarrays. Pathway analysis was performed to detect coordinated changes in the expression of groups of functionally related genes. Real-time PCR was used to confirm the expression of several genes which were found to be regulated by estradiol treatment.

Results: Estradiol treatment improved glucose and insulin tolerance and decreased basal blood glucose $(8.0 \pm 0.48$ vs $4.9 \pm 0.29 \mathrm{mM}, \mathrm{p}<0.01$; control vs treated) and plasma insulin (204.6 \pm 19.1 vs $138.0 \pm 10.4 \mu \mathrm{U} / \mathrm{ml}, \mathrm{p}<0.01$; control vs treated) levels in ob/ob mice. Estradiol decreased the expression of gene categories involved in fatty acid and lipid biosynthesis. Among the genes involved in these categories, expression levels of fatty acid synthase, stearoyl-coenzymeA desaturase1, and glycerol-3-phosphate acyltransferase were significantly decreased. Further searches for genes directly targeted by estrogen revealed increased hepatic mRNA expression of signal transducer and activator of transcription 3 (Stat3). Furthermore, levels of Stat3 protein and phosphorylated Stat3 protein were increased in the liver of estradiol treated ob/ob mice. Finally, Stat3 expression was rapidly induced by estradiol in the mouse liver.

Conclusion: In conclusion, estradiol treatment improves glucose tolerance and insulin sensitivity in ob/ob mice. We propose that this may be mediated, at least partially, via estrogen stimulation of the hepatic expression of Stat3, leading to decreased expression of hepatic lipogenic genes and thereby to the observed antidiabetic effects.

Supported by Swedish Research Council and Karo Bio AB

\section{PS 46}

\section{Regulation of beta cell function by incretin hormones}

0653

Sitagliptin, a dipeptidyl peptidase-4 inhibitor, improved $\beta$ cell function in patients with type 2 diabetes: a modelbased analysis

L. Xu ${ }^{1}$, C. Dalla Man ${ }^{2}$, C. Cobelli ${ }^{2}$, D. Williams-Herman ${ }^{1}$, G. Meininger ${ }^{1}$, H. Khatami ${ }^{1}$, P. Stein ${ }^{1}$;

${ }^{1}$ Merck \& Co. Inc., Rahway, United States, ${ }^{2}$ Department of Information Engineering, University of Padova, Italy.

Background and Aims: $\beta$-cell dysfunction occurs early in type 2 diabetes and is characterized by abnormalities such as depressed $\beta$-cell sensitivity to glucose with respect to insulin release. Incretins have been shown to enhance $\beta$-cell function in patients with type 2 diabetes and in animal models of 
diabetes. Since sitagliptin increases active incretin levels, $\beta$-cell function was assessed using frequently sampled (9points) meal tolerance tests (MTT) in sub-studies from 3 Phase III trials (2 monotherapy studies and an add-on to metformin study) using a model-based analysis.

Methods: For the MTT, blood was collected at $-10,0,10$, 20, 30, 60, 90, 120, and $180 \mathrm{~min}$ relative to start of the meal. Parameters for $\beta$-cell function were assessed using the $\mathrm{C}$ peptide minimal model, which allows for the estimation of the insulin secretion rate and for characterization of the insulin secretion response into basal $\left(\Phi_{\mathrm{b}} ; \beta\right.$-cell responsiveness to basal glucose concentrations), static ( $\Phi_{\mathrm{s}} ; \beta$-cell responsiveness to above-basal glucose concentrations following a meal), and dynamic $\left(\Phi_{\mathrm{d}} ; \beta\right.$-cell responsiveness to the rate of increase in above-basal glucose concentrations following a meal) components. Insulin sensitivity was assessed with a validated composite index (ISI). The disposition indices, which assess insulin secretion in the context of changes in insulin sensitivity, were also assessed.

Results: When used as monotherapy or added to on-going metformin therapy, sitagliptin produced improvements in $\Phi_{\mathrm{s}}$ and in $\Phi_{\mathrm{b}}$ and overall responsiveness of the $\beta$-cell to glucose $(\Phi)$. The parameter describing dynamic sensitivity of the $\beta$-cell to glucose $\left(\Phi_{\mathrm{d}}\right)$ showed numerical, but not statistically significant, improvements with sitagliptin. Disposition indices were also broadly improved with sitagliptin treatment relative to placebo. Despite enhanced $\beta$-cell sensitivity to glucose, the very low rate of hypoglycemia observed in clinical trials with sitagliptin indicated that the increase in $\beta$-cell function remained glucose-dependent.

Conclusions: In this model-based analysis, sitagliptin improved $\beta$-cell function in both fasting and postprandial states in patients with type 2 diabetes.

Supported by Merck \& Co. Inc

\section{4}

Differential effects of vildagliptin on islet function in patients with type 2 diabetes receiving no additional agent or concomitant metformin

J. E. Foley ${ }^{1}$, M. Ligueros-Saylan ${ }^{1}$, C. A. Watson ${ }^{1}$, Y.-L. He ${ }^{1}$, B. E. Dunning ${ }^{2}$, R. E. Pratley ${ }^{3}$, D. A. D’Alessio ${ }^{4}$; ${ }^{1}$ Novartis, E Hanover, ${ }^{2}$ PharmaWrite, Princeton, ${ }^{3}$ Diabetes/ Metab Trans Med, Univ. of Vermont, Burlington, ${ }^{4}$ Endo, Univ. of Cincinnati, United States.

Background and Aims: Vildagliptin is a DPP-4 inhibitor that improves glycemic control in patients (pts) with type 2 diabetes (T2DM) by increasing plasma levels of intact (active) incretin hormones. The aim of this study was to characterize the effects of 12-wk treatment (Rx) with vildagliptin on islet function in pts with T2DM and to explore durability of actions by repeating assessments after washout from study drug.

Methods: This was a double-blind, randomized, placebo (PBO)-controlled, parallel-group study of 39 pts with T2DM who were maintaining glycemic control $\left(\mathrm{HbA}_{1 \mathrm{c}}=6.5-7.5 \%\right)$ with diet and exercise or with a stable dose of metformin (Met). Islet function tests (iv glucose tolerance test, glucose ramp to $25 \mathrm{mM}$ glucose and arginine (Arg) stimulation test at $25 \mathrm{mM}$ glucose) were conducted before (Wk 0 ) and after 12-wk Rx with vildagliptin $50 \mathrm{mg}$ bid or PBO and after 2-3 wk washout.

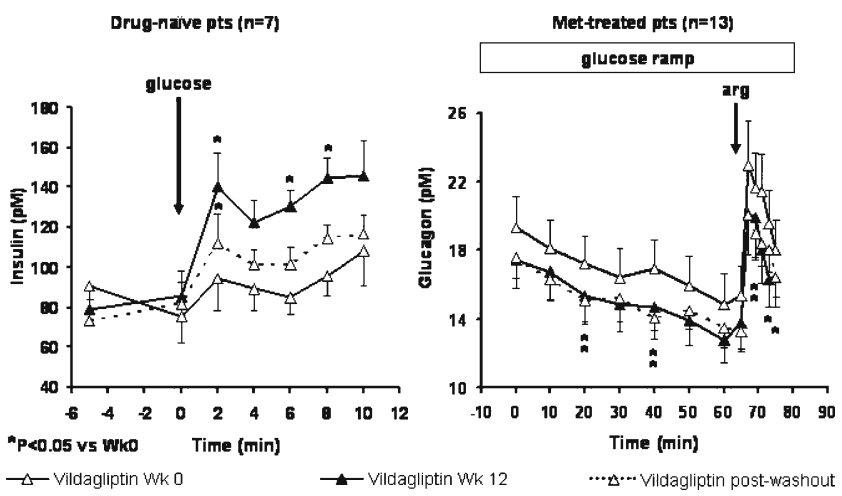

Results: Islet function did not change in pts receiving $\mathrm{PBO}$; these data are not shown. Of 20 pts randomized to vildagliptin, 7 were drug-naïve and 13 received concomitant Met. $\mathrm{HbA}_{1 \mathrm{c}}$, BMI and disease duration were similar in drug-naïve and Met-treated pts, averaging $6.7 \pm 0.4 \%$, $30.6 \pm .5 \mathrm{~kg} / \mathrm{m}^{2}$ and $36.5 \pm 20.9 \mathrm{mo}$ in all pts randomized to vildagliptin. As shown below, in drug-naïve pts, vildagliptin improved $\beta$-cell function. The acute insulin response to glucose (AIRg) increased from $16 \pm 7$ to $54 \pm 9$ pM $(+238 \%$, $\mathrm{P}<0.001$ vs Wk 0 or $\mathrm{PBO}$ ); part of this effect remained after washout. Insulin sensitivity ( $\mathrm{Si}$ ) also increased significantly in drug-naïve pts, thus the disposition index ( $\mathrm{Si} \times \mathrm{AIRg}$ ) increased by $326 \%(\mathrm{P}<0.05$ vs Wk 0 or $\mathrm{PBO})$. Measures of $\alpha$-cell function were unaffected by vildagliptin in drugnaïve pts. In Met-treated pts, $12-w k \mathrm{Rx}$ with vildagliptin improved $\alpha$-cell function, decreasing glucagon levels during glucose ramp and the glucagon $\mathrm{AUC}_{0-10}$ after $\mathrm{Arg}$ given at $25 \mathrm{mM}$ glucose (from $204 \pm 20$ at Wk 0 to $175 \pm 17$ $\mathrm{pM} \bullet \mathrm{min}$ at $\mathrm{Wk} 12, \mathrm{P}<0.01$ vs $\mathrm{Wk} 0$ or $\mathrm{PBO})$. Part of this effect remained after washout.

In the combined cohort of vildagliptin-treated pts, relative to Wk 0 , AIRg increased by $111 \%(\mathrm{P}<0.05)$, the slope of insulin vs glucose increased by $34 \%(\mathrm{P}<0.05)$ and measures of insulin secretory capacity also increased (eg, 16\% increase in insulin $\mathrm{AUC}_{0-15}$ after $\mathrm{Arg}$ given at $25 \mathrm{mM}$ glucose, $\mathrm{P}<0.01)$. These changes were not maintained after washout.

Conclusions: Twelve-week $\mathrm{Rx}$ with vildagliptin improves both $\alpha$ - and $\beta$-cell function in pts with T2DM. The 
mechanisms underlying the more pronounced improvement in $\beta$-cell function in drug-naïve pts and more pronounced improvement in $\alpha$-cell function in Met-treated pts may relate to differential effects on GLP-1 and GIP and remain to be more fully explored, as do possible disease-modifying effects of vildagliptin suggested by the maintenance of some effects after drug washout.

Supported by Novartis

0655

The insulinotropic effect of vildagliptin is revealed in patients with type 2 diabetes when oral glucose tolerance tests impose a large glucose load

Y.-L. He ${ }^{1}$, C. F. Deacon ${ }^{2}$, J. J. Holst ${ }^{2}$, B. E. Dunning ${ }^{3}$, M. Ligueros-Saylan ${ }^{4}$, J. E. Foley ${ }^{4}$;

${ }^{1}$ Novartis, Cambridge, United States, ${ }^{2}$ Panum Inst, Univ of Copenhagen, Denmark, ${ }^{3}$ PharmaWrite, Princeton, United States, ${ }^{4}$ Novartis, E Hanover, United States.

Background and Aims: Although vildagliptin improves $\beta$-cell function and reduces postprandial glucose (PPG) in patients with type 2 diabetes (T2DM), it does not increase post-meal insulin levels during 4-12 wk treatment in patients with T2DM, nor after a single dose given before a 75-g oral glucose tolerance test (OGTT) in healthy subjects. The aim of this work was to explore this apparent apparent conundrum by comparing the hormone and metabolic responses to OGTT and a standardized meal test after a single $100 \mathrm{mg}$ dose of vildagliptin in diet-treated patients with T2DM.

Methods: Two single-center, randomized, placebo (PBO)controlled studies were conducted. In Study 1, sixteen patients received vildagliptin $100 \mathrm{mg}$ or PBO thirty minutes before before a 75-g OGTT in an open-label, randomized, crossover manner, with $\geq 72 \mathrm{~h}$ interdose interval. In Study 2, nine patients received $\mathrm{PBO}$ on Day -1 and vildagliptin $(100 \mathrm{mg}$ ) on Day 1, thirty minutes before a breakfast meal test, during a 28-day, double-blind, randomized, PBOcontrolled trial.

Results: Patients in Study 1 had a mean BMI $=30.0 \mathrm{~kg} / \mathrm{m}^{2}$; $\mathrm{HbA}_{1 \mathrm{c}}=8.0 \%$; $\mathrm{FPG}=8.3 \mathrm{mM}$ and fasting insulin $=96 \mathrm{pM}$. Patients in Study 2 had a mean $\mathrm{BMI}=31.8 \mathrm{~kg} / \mathrm{m}^{2}$; $\mathrm{HbA}_{1 \mathrm{c}}=7.5 \%$; $\mathrm{FPG}=8.9 \mathrm{mM}$ and fasting insulin=212 $\mathrm{pM}$. The table below summarizes the $\%$ inhibition of plasma DPP-4 activity measured at 2-h post-dose, the mean (AUC/ time) post-load plasma levels of intact (active)GLP-1 and GIP, plasma glucose levels at $1.5 \mathrm{~h}$ post-load (mean time of glucose peak) and mean post-load plasma insulin levels. A single $100 \mathrm{mg}$ dose of vildagliptin produced essentially complete inhibition of DPP-4 and significantly increased post-load plasma levels of intact GLP-1 and GIP during
OGTT or meal test. The mixed meal was a stronger stimulus than OGTT for GIP release, but the glucose load imposed by OGTT, as reflected by peak glucose levels, was nearly twice that of the mixed meal. Vildagliptin significantly increased plasma insulin levels after OGTT but not after the mixed meal.

Table 1

Study 1: OGTT $(n=16) \quad$ Study 2: Breakfast $(n=9)$

PlaceboPBO Vildagliptin PlaceboPBO Vildagliptin

\begin{tabular}{lcccc}
\hline $\begin{array}{l}\text { DPP-4 (\% } \\
\text { inhibition @ 2 h) }\end{array}$ & $4.6 \pm 1.1$ & $97.5 \pm 0.3^{*}$ & $\mathrm{ND}$ & $98.2 \pm 0.5$ \\
$\begin{array}{l}\text { Active GLP-1 } \\
\text { (pM) }\end{array}$ & $9 \pm 3$ & $13 \pm 3 *$ & $8 \pm 3$ & $18 \pm 4 *$ \\
$\begin{array}{l}\text { Active GIP (pM) } \\
\text { Glucose @ 1.5 h }\end{array}$ & $21.4 \pm 1.1$ & $18.7 \pm 1.1^{*}$ & $10.9 \pm 2.1$ & $9.9 \pm 2.1^{*}$ \\
(mM) & & $24 \pm 3 *$ & $40 \pm 4$ & $79 \pm 10^{*}$ \\
Insulin (pM) & $206 \pm 35$ & $278 \pm 48^{*}$ & $384 \pm 85$ & $402 \pm 91$ \\
\hline
\end{tabular}

$* \mathrm{p}<0.05$ vs placeboPBO

Conclusion: An acute insulinotropic effect of vildagliptin is observed in patients with T2DM only when the glucose load provides a strong independent stimulus for insulin secretion.

Supported by Novartis

0656

Treatment of obese Zucker diabetic fatty rats with novel GLP-1 receptor agonist AVE0010 improves oral glucose tolerance and glycaemic control without risk of hypoglycaemia

U. Werner, M. Gerlach, M. Hofmann, A. W. Herling;

Sanofi-Aventis Deutschland GmbH, Frankfurt, Germany.

Background and Aims: During recent years the beneficial effects of glucagon-like peptide-1 (GPL-1) in type 2 diabetes have been investigated extensively. It was demonstrated that GLP-1 improves glycemic control by reducing appetite and food intake and by augmentation pancreatic responsiveness to meals. However, due to rapid degradation the half-life of active GLP- in vivo is only 1-2 $\mathrm{min}$. Therefore native GLP-1 is only of limited value for diabetes therapy and degradation-resistant GLP-1 mimetics are needed. In the present study we evaluated the anti-diabetic activity of AVE0010, a novel synthetic GLP-1 agonist with an extended biological activity, in the Zucker Diabetic Fatty (ZDF) rat, a well-established animal model of type 2 diabetes.

Materials and Methods: Male obese ZDF (ZDF/Gmi-fa/ fa) and age-matched lean controls (ZDF/Gmi-Fa/?) 6- 
7 weeks of age were obtained from Charles River and maintained under standardized animal house conditions on standard rodent diet until study start. An acute oral glucose tolerance test (OGTT) with $2 \mathrm{~g} / \mathrm{kg}$ glucose was performed in overtly diabetic animals of 11 weeks of age 30 min after single dose injection of $0.2-2 \mathrm{nmol} / \mathrm{kg}$ sc AVE0010 and blood glucose excursion was determined in comparison to saline-treated obese and lean controls. In a 2nd study 8 weeks old ZDF rats were implanted with osmotic minipumps for constant subcutaneous delivery of AVE0010 (0.1-10 nmol/ $\mathrm{kg}^{*}$ day) or saline. Progression of diabetes was monitored by regular determination of blood glucose for 12 weeks. OGTTs were performed after 1 and 11 weeks of treatment.

Results: Following an oral glucose challenge blood glucose rose by $\sim 5 \mathrm{mmol} / \mathrm{L}$ in obese ZDF controls while in insulinsensitive lean controls the mean blood excursion was only $1.6 \mathrm{mmol} / \mathrm{L}$. Single sc injection of 1,5 and $10 \mu \mathrm{g} / \mathrm{kg}$ AVE0010 dose-dependently decreased the area under the blood glucose curve by $41 \%, 70 \%$ and $81 \%$ of the lean control AUC, respectively. Chronic treatment of prediabetic obese ZDF rats for 12 weeks with $10 \mathrm{nmol} / \mathrm{kg}^{*}$ day AVE0010 delivered via subcutaneously implanted osmotic minipumps markedly decreased basal blood glucose by $64 \%$ and resulted in $1.7 \% \mathrm{HbAlc}$ reduction compared to saline infusion at the end of the study. In contrast, the same dose of AVE0010 had no hypoglycemic effect and did not change $\mathrm{HbA1c}$ levels in normoglycemic lean controls.

Conclusion: AVE0010 very effectively and dose-dependently improved oral glucose tolerance in obese, diabetic ZDF rats both acutely and after chronic treatment. Furthermore, chronic treatment starting in prediabetic animals improved basal blood glucose and overall glycemic control resulting in an $\mathrm{HbAlc}$ difference of $1.7 \%$ compared to placebo-treated diabetic obese control animals. In normoglycemic lean control animals the same dose of AVE0010 did not exert any blood glucose lowering activity. In the ZDF rat, a widely used animal model of type 2 diabetes, it could be demonstrated that AVE0010 lowers blood glucose under conditions of hyperglycemia but not at normoglycemia. It is therefore concluded that AVE0010 is a novel, effective anti-diabetic agent for the treatment of type 2 diabetes with no risk of hypoglycemia.

Supported by Sanofi-Aventis

\section{7}

The incretin effect is preserved in glucose tolerant patients with chronic pancreatitis - but reduced in patients with chronic pancreatitis and secondary diabetes mellitus

T. Krarup ${ }^{1}$, T. Vilsbøll ${ }^{1}$, S. Madsbad ${ }^{2}$, S. Larsen ${ }^{3}$, J. J. Holst ${ }^{4}$, P. V. Højberg ${ }^{1}$, F. K. Knop ${ }^{1,4}$;
${ }^{1}$ Department of Internal Medicine $\mathrm{F},{ }^{2}$ Department of Endocrinology, ${ }^{3}$ Department of Internal Medicine M, ${ }^{4}$ Department of Medical Physiology, University of Copenhagen, Denmark.

Background and Aims: The incretin effect (the enhancement of glucose-induced insulin secretion following oral glucose tolerance test (OGTT) compared to isoglycemic intravenous glucose tolerance test (IVGTT)) is markedly reduced in patients with type 2 diabetes mellitus (T2DM). We aimed to investigate whether the reduced incretin effect is a consequence of the diabetic state per se or pathognomonic for T2DM.

Materials and Methods: The incretin effect was measured over 2 days by means of 4-hours OGTT ( $50 \mathrm{~g}$ of glucose) and isoglycemic IVGTT in four matched (age, gender and BMI) groups of volunteers: 1) Eight patients with chronic pancreatitis (CP) and normal glucose tolerance (NGT) $\left(\mathrm{HbA}_{1} \mathrm{c}: 5.7\right.$ (5.4-5.9)\% (mean(range)); 2) Eight patients with $\mathrm{CP}$ and secondary diabetes mellitus (DM) $\left(\mathrm{HbA}_{1} \mathrm{c}: 6.9\right.$ $(6.2-8.0) \%)$; 3) Eight patients with T2DM $\left(\mathrm{HbA}_{1} \mathrm{c}: 6.7\right.$ $(5.7-8.7) \%)$; and 4) Eight healthy subjects $\left(\mathrm{HbA}_{1} \mathrm{c}: 5.5\right.$ $(5.1-5.8) \%)$.

Results: The incretin effect $\left(100 \% \times\left(\mathrm{AUC}_{\mathrm{ins}}\right.\right.$, OGTT $\mathrm{AUC}_{\text {ins, }}$ IVGTT $\left.) / \mathrm{AUC}_{\text {ins, OGTT }}\right)$ was significantly $(\mathrm{P} \leq 0.05)$ reduced in patients with $\mathrm{CP}$ and secondary DM $(45 \pm 6 \%$ (mean \pm SEM)) compared to patients with $\mathrm{CP}$ and NGT $(68 \pm 7 \%)$ and healthy subjects $(70 \pm 7 \%)$, respectively. In the group of patients with $\mathrm{T} 2 \mathrm{DM}$ the incretin effect was significantly $(\mathrm{P} \leq 0.05)$ lower than the group of patients with $\mathrm{CP}$ and NGT and the group of healthy subjects, respectively.

Conclusion: Our results suggest that the reduced incretin effect is a consequence of the diabetic state and not a primary pathogenetic defect unique to T2DM.

Supported by the Danish Diabetes Association

\section{8}

Differential anti-diabetic efficacy of incretin agonists vs. DPP-4 inhibition in high fat fed mice

B. J. Lamont, D. J. Drucker;

BBDC, Medicine, University of Toronto, Canada.

Background and Aims: GLP-1R agonists exemplified by Ex-4 (exendin-4) exhibit both classic incretin actions on the islet $\beta$-cell and non-incretin actions including inhibition of glucagon secretion, gastric emptying and food intake. In contrast, GIPR agonists such as D-Ala 2 -GIP (a glucosedependent insulinotropic polypeptide analogue) lower blood glucose predominantly through effects on the $\beta$-cell. Less well understood are the actions of the DPP-4 
inhibitors, which stabilize the postprandial levels of both GLP-1 and GIP following food ingestion. In this study, the relative efficacy and contrasting mechanisms of action of DPP-4 inhibition vs. Ex-4 or D-Ala 2 -GIP was studied in diabetic mice.

Materials and Methods: C57BL/6 mice were fed a $(60 \%)$ high fat diet for a 4 wk basal period, followed by an 8 wk treatment period with twice daily injections of either: 24 nmol. $\mathrm{kg}^{-1} \mathrm{Ex}-4$ (Ex-4 group), 24 nmol. $\mathrm{kg}^{-1} \mathrm{D}$ $\mathrm{Ala}_{2}$-GIP (GIP group), vehicle (PBS group) or vehicle with the addition of a DPP-4 inhibitor (Sitagliptin analogue-DPP-4i) in the food at $11 \mathrm{~g} / \mathrm{kg}$ (DPP-4i group). Body weight was monitored weekly, and body composition was assessed by MRI. Non-fasting blood glucose levels were determined in tail-vein samples. Glucose tolerance was assessed by oral (OGTT, $1.5 \mathrm{~g} . \mathrm{kg}^{-1}$ ) and intra-peritoneal glucose tolerance tests (IPGTT, $1.5 \mathrm{~g} . \mathrm{kg}^{-1}$ ). $\mathrm{HbA1c}$ levels were measured at the end of the treatment period.

Results: Body weight was significantly reduced in mice treated with either Ex-4 or the DPPi, but not in mice treated with GIP (Ex-4: $36.2 \pm 1.2$, DPP-4i: $36.1 \pm 1.1$, GIP: $42.3 \pm 1.5$, PBS: $43.9 \pm 2.0$ g). Fat mass assessed by MRI was significantly reduced in the Ex-4 and DPP-4i groups compared to mice treated with GIP or PBS. Acute glucose tolerance (IPGTT), was improved in the Ex-4 and GIP groups, but not in mice treated with the DPP4i. In contrast, Ex-4 and DPP-4i but not GIP reduced the AUC for glycemic excursion following OGTT (Ex-4: 909 \pm 51 , DPP-4i: $1240 \pm 25$, GIP: $1472 \pm 43$ vs PBS: $1653 \pm 69 \mathrm{mM} . \mathrm{min})$. Insulin:glucose ratios were significantly improved following therapy with the DPP-4i. Acute administration of Ex-4 and D-Ala - -GIP reduced non-fasting hyperglycaemia $1 \mathrm{hr}$ following peptide injection (Ex-4: 5.1 \pm 0.1 ; GIP: $5.0 \pm 0.3$, vs PBS: $7.5 \pm 0.4 \mathrm{mM})$. Blood glucose in mice treated with the DPP-4i was also significantly reduced $1 \mathrm{hr}$ after "sham injection" $(6.5 \pm 0.22 \mathrm{mM})$; when random glucose was assessed $9 \mathrm{hr}$ after the last injection (Ex-4 or GIP or saline), blood glucose levels were significantly lower in the DPP-4i group relative to mice treated with either PBS, Ex-4 or GIP (DPP-4i: $6.4 \pm 0.2$, Ex-4: $8.0 \pm 0.2$, GIP: $7.3 \pm 0.2$, vs PBS: $7.5 \pm 0.3 \mathrm{mM}$ ). Furthermore, only mice treated with the DPP-4i exhibited significant reductions in levels of $\mathrm{HbAlc}$ at study termination (DPP-4i: $3.33 \pm 0.08$, Ex-4: $3.53 \pm 0.06$, GIP: $3.56 \pm 0.05$, vs PBS: $3.59 \pm 0.07 \%)$.

Conclusion: Although acute pharmacological administration of Ex-4, and to a lesser extent, GIP markedly improved glucose tolerance, DPP-4 inhibition exhibited beneficial effects on glucoregulation for more prolonged time periods, in part through apparent improvement in $\beta$-cell function. Hence, sustained activation of the incretin axis appears critical for optimizing the anti-diabetic efficacy of incretinbased therapies.

Supported in part by a grant from Merck Frosst Canadal $M R L$

0659

The GLP-1 receptor agonist AVE0010 preserves beta cell function and insulin secretion after a 6 week treatment in male obese Zucker diabetic fatty rats - an isolated perfused pancreas study

G. Haschke, S. Haag-Diergarten, U. Werner, W. Kramer, A. W. Herling;

Sanofi-Aventis Deutschland GmbH, Frankfurt am Main, Germany.

Background and Aims: Glucagon-like petide-1 (GLP-1) is a secretagogue of the pancreatic beta cells and enhances glucose-induced insulin secretion. In addition GLP-1 suppresses glucagon secretion. The improved insulin-toglucagon-ratio might contribute to suppress hepatic glucose production during a meal. In type 2 diabetes insulin secretion and its secretion pattern is altered as well as glucagon secretion is increased. The male obese Zucker Diabetic Fatty rat (ZDF rat) is an animal model of insulin resistance and demonstrates a spontaneous progression to overt type 2 diabetes starting at the age around 10 weeks, which reflects the altered state in insulin secretion during the progression of the disease. The pattern of insulin secretion from isolated pancreas was studied in male ZDF rats after prolonged treatment with the GLP-1 agonist AVE0010 (ex vivo study).

Materials and Methods: The pancreas was isolated from starved (16 h) ZDF rats (obese: ZDF/Gmi-fa/fa; lean: ZDF/ $\mathrm{Gmi}-\mathrm{Fa} /$ ?) of various ages and perfused with a modified Krebs Ringer buffer containing $5.6 \mathrm{mM}$ glucose and $1 \%$ BSA for 10 minutes (basal). Thereafter insulin secretion was induced by switch of the perfusion medium to a glucose concentration of $16.5 \mathrm{mM}$ (stimulation). The effluent was collected in intervals of 2 minutes and the concentrations of insulin as well as glucagon were determined. Insulin secretion was investigated in untreated (A) and AVE0010 treated (B) lean and obese ZDF rats up to ages of 22 and 12 weeks, respectively. (A) In untreated male lean and obese ZDF rats at ages of 8,12 and 22 weeks insulin secretion pattern was investigated. (B) Treated male ZDF rats were continuously infused by subcutaneously implanted osmotic minipumps with AVE0010 (10 nmol/kg/ day) or saline starting at the age of 6 weeks and their insulin secretion profile was investigated at the age of 12 weeks. 
Results: (A) In untreated male obese ZDF rats insulin secretion was increased during basal conditions $(5.6 \mathrm{mM})$ compared to lean control rats at all studied ages. Glucose stimulated (16.5 mM) insulin secretion demonstrated a biphasic pattern in lean rats at all ages as well as in 8 weeks old obese ZDF rats. In older obese ZDF rats the glucose stimulatory response on insulin secretion declined and the biphasic secretion pattern was no longer present. (B) In male obese ZDF rats treated with AVE0010 at the age of 12 weeks, insulin secretion of isolated pancreas remained preserved and showed a physiological biphasic pattern similar to that of lean control. In treated rats glucagon secretion during high glucose perfusion was decreased.

Conclusion: The GLP-1 agonist AVE0010 preserved the physiological biphasic pattern of insulin secretion in male obese ZDF rats after a treatment period of 6 weeks (age: 6.12. week). In control groups the untreated male obese ZDF rats showed a decreased and unregulated insulin secretion. Therefore, AVE0010 is a potent new agent in the treatment of diabetes to prevent the loss of insulin biphasic secretion pattern and thereby preserve the function of the endocrine pancreas.

\section{PS 47}

\section{Metabolic effects of incretin hormones I}

0660

Effects of vildagliptin on postprandial glucose homeostasis in type 2 DM

Z. Radikova ${ }^{1}$, K. Azuma ${ }^{1}$, E. Thomas ${ }^{1}$, F. G. S. Toledo ${ }^{1}$, N. D. Lubowsky ${ }^{1}$, C. O. Kangani ${ }^{1}$, J. M. Mancino ${ }^{1}$, C. A. Kelley ${ }^{1}$, J. J. Holst ${ }^{2}$, C. F. Deacon ${ }^{2}$, D. Serra ${ }^{3}$, Y. L. $\mathrm{He}^{3}$, M. Ligueros-Saylan ${ }^{3}$, J. E. Foley ${ }^{3}$, D. E. Kelley ${ }^{1}$; ${ }^{1}$ Division of Endocrinology and Metabolism, Department of Medicine, School of Medicine, University of Pittsburgh, Pittsburgh, ${ }^{2}$ Department of Medical Physiology, Panum Institute, University of Copenhagen, Denmark, ${ }^{3}$ Novartis Pharmaceuticals, Corp., East Hanover, United States.

Background and Aims: Vildagliptin (V), a DPP-4 inhibitor that delays degradation of endogenous incretin hormones (e.g. GLP-1 and GIP), improves fasting and postprandial hyperglycemia in type $2 \mathrm{DM}$. Improvement of beta-cell dysfunction in type $2 \mathrm{DM}$ is an anticipated mechanism of action for a DPP-4 inhibitor. In a parallel group, randomized, placebo $(\mathrm{P})$ controlled, study of $\mathrm{V}$ in type 2 DM, Ahren et al observed that postprandial insulin concentrations were similar with V vs. P, but with improved hyperglycemia during $\mathrm{V}$. This is consistent with improved glucose sensing by $\beta$-cells. Another effect was a reduction in glucagon, suggesting this contributed to improved glucose homeostasis additional to insulin secretion. The current study used a cross-over, within-subject design with doubleblinded $\mathrm{P}$ control to examine postprandial $\beta$-cell, $\alpha$-cell, and extra-pancreatic effects of V (50 mg BID) in type 2 DM.

Materials and Methods: 16 Type 2 DM, drug naïve $(n=5)$ or metformin treated $(\mathrm{n}=11)$; F7M9, age $55.7 \pm 1.7$ years, BMI: $31.3 \pm 0.9 \mathrm{~kg} / \mathrm{m}^{2}$, baseline HbAlc: $7.1 \pm 0.2 \%$ participated and received $\mathrm{P}$ or $\mathrm{V}$ (50 $\mathrm{mg} \mathrm{BID})$ in random order for 6 wks. A meal tolerance test (MTT), $7 \mathrm{kcal} / \mathrm{kg}$ (50\% CHO, $35 \%$ fat, $15 \%$ protein) was performed on day 41 . On day 42 of each period, potential effects on IR were assessed using an insulin infusion glucose clamp.

Results: Fasting hyperglycemia (P: $148.3 \pm 5.4$ vs. V: $125.5 \pm$ $3.8 \mathrm{mg} / \mathrm{dl}$; mean $\pm \mathrm{SE})$ and prandial glucose $\left(\mathrm{AUC}_{0-300}: \mathrm{P}\right.$ : $50189 \pm 2361$ vs. V: $41618 \pm 1430 \mathrm{mg} / \mathrm{dl} .300 \mathrm{~min}^{-1}$ ) were improved with $\mathrm{V}(\mathrm{p}<0.001)$. Prandial insulin was quite similar across arms $\left(\mathrm{AUC}_{0-300}\right.$ : P: $8671 \pm 1554$ vs. V: $8588 \pm 1813 \mathrm{mU} /$ $1.300 \mathrm{~min}^{-1}$; NS), including during the initial $120 \mathrm{~min}$ $\left(\mathrm{AUC}_{0-120}\right.$ : P: $4441 \pm 821$ vs. $\mathrm{V}: 4339 \pm 869 \mathrm{mU} / 1.300 \mathrm{~min}^{-1}$; NS); similar data were found for C-peptide. Postprandial glucagon rose $\sim 20 \%$ above fasting during $\mathrm{P}$ but remained nearly flat during $\mathrm{V}$, with different $\mathrm{AUC}_{0-300}(\mathrm{P}: 37315 \pm 3176$ vs. V: $31054 \pm 2186 \mathrm{pg} / \mathrm{ml} .300 \mathrm{~min}^{-1} ; \mathrm{p}=0.002$ ). As expected there was higher intact GIP and GLP-1 (both $\mathrm{p}<0.001$ ) during $\mathrm{V}$. The kinetics of ingested glucose appearance into systemic circulation was examined by labeling the meal with $\left[1-{ }^{13} \mathrm{C}\right]$ glucose; this did not reveal significant differences between treatments indicating little effect of delayed gastric emptying during treatment with a DPP4 inhibitor. Postprandial insulin sensitivity was greater (3 h-OGIS: P: $294 \pm 10$ vs. V: $345 \pm$ $\left.14 \mathrm{ml} . \mathrm{min}^{-1} . \mathrm{m}^{-2} ; \mathrm{p}<0.001\right)$ during V. The two-step clamp (20 $\mathrm{mU}$ and $80 \mathrm{mU} / \mathrm{min} \cdot \mathrm{m}^{-2}$ ) revealed greater insulin sensitivity at both insulin levels, by $\sim 15 \%$ with $\mathrm{V}(\mathrm{p}<0.01)$. Conclusion: In summary, DPP-4 inhibition by vildagliptin, which increases activities of GIP and GLP-1, lowers circulating glucagon and improves insulin sensitivity. We conclude that extra-pancreatic as well as pancreatic effects contribute substantially to the mechanisms for improved glucose control in type 2 DM induced by DPP-4 inhibition with vildagliptin.

Supported by Novartis Pharmaceuticals

\section{1}

Cellular and metabolic evaluation of (hydroxyproline ${ }^{3}$ )GIP as new stable GIP receptor antagonist with antidiabetic potential

F. P. M. O'Harte ${ }^{1}$, K. Hunter ${ }^{1}$, V. A. Gault ${ }^{1}$, N. Irwin ${ }^{1}$, P. Harriott ${ }^{2}$, P. R. Flatt ${ }^{1}$; 
${ }^{1}$ School of Biomedical Sciences, University of Ulster, Coleraine, ${ }^{2}$ School of Biology and Biochemistry, The Queen's University of Belfast, United Kingdom.

Aims: The therapeutic potential of the novel glucosedependent insulinotropic polypeptide (GIP) analogue, $\left(\mathrm{Hyp}^{3}\right)$ GIP, was investigated in vitro and in obese diabetic $(o b / o b)$ mice with comparison to $\left(\mathrm{Pro}^{3}\right)$ GIP.

Methods: Peptide stability to dipeptidylpeptidase IV (DPPIV) was tested by incubation with enzyme up to $8 \mathrm{~h}$ followed by analysis by HPLC. Cyclic AMP production was monitored in Chinese hamster lung (CHL) cells transfected with the human GIP receptor. Acute insulin secretion studies were performed in pancreatic BRIN-BD11 cells at $5.6 \mathrm{mmol} / \mathrm{l}$ glucose. A range of peptide concentrations $\left(10^{-13}\right.$ to $\left.10^{-7} \mathrm{M}\right)$ were tested in the presence of native GIP $\left(10^{-7} \mathrm{M}\right)$. Additionally, longer-term studies were performed in obese diabetic mice $(n=8)$ given $\left(\mathrm{Hyp}^{3}\right)$ GIP or ( Pro $\left.^{3}\right)$ GIP (25 nmoles/kg body weight) by intraperitoneal (i.p.) injection once daily for 14 days. Glucose tolerance $(18 \mathrm{mmol} / \mathrm{kg}$ body $\mathrm{wt})$ and insulin sensitivity ( $50 \mathrm{U} / \mathrm{kg}$ body wt) tests were performed at the end of the study period. Responses to acute 15 min feeding in $18 \mathrm{~h}$ fasted mice were also assessed.

Results: Both $\left(\mathrm{Hyp}^{3}\right)$ GIP and $\left(\mathrm{Pro}^{3}\right)$ GIP were completely resistant to DPP-IV degradation and showed considerable ability to antagonise GIP stimulated cAMP formation by 34 $44 \%(\mathrm{P}<0.01)$. Furthermore, $10^{-7} \mathrm{M}$ GIP-induced insulin secretion in BRIN-BD11 cells was reduced by $29 \%(\mathrm{P}<0.05)$ by $\left(\mathrm{Hyp}^{3}\right)$ GIP and $36 \%(\mathrm{P}<0.01)$ by $\left(\mathrm{Pro}^{3}\right)$ GIP. Once daily treatment of diabetic mice with $\left(\mathrm{Hyp}^{3}\right)$ GIP or $\left(\mathrm{Pro}^{3}\right)$ GIP produced significant $23-27 \%$ reductions in plasma glucose concentrations $(\mathrm{P}<0.05)$ on day 12 and 14. $\left(\mathrm{Pro}^{3}\right)$ GIP improved glycaemic response to i.p. glucose after 14 days, with area under the curve (AUC, $0-60 \mathrm{~min}$ ) values $45 \%$ lower $(\mathrm{P}<0.01)$ than saline treated mice and $37 \%$ lower than $\left(\mathrm{Hyp}^{3}\right)$ GIP treated mice $(\mathrm{P}<0.05)$. Both $\left(\mathrm{Pro}^{3}\right) \mathrm{GIP}$ and $\left(\mathrm{Hyp}^{3}\right)$ GIP treated groups had substantially lower overall insulin responses to i.p. glucose compared with controls $(\mathrm{P}<0.01)$. Following $15 \mathrm{~min}$ feeding on day 14 no differences were observed in glucose excursion between the treatment groups but the plasma insulin AUC (0-90 min) responses were $35 \%$ and $27 \%$ lower $(\mathrm{P}<0.001$ and $\mathrm{P}<0.01)$ for the $\left(\mathrm{Pro}^{3}\right)$ GIP and $\left(\mathrm{Hyp}^{3}\right)$ GIP treated groups compared to saline controls. Consistent with these observations administration of insulin on day 14 was associated with a significant improvement of glucose lowering in $\left(\mathrm{Pro}^{3}\right) \mathrm{GIP}$ and $\left(\mathrm{Hyp}^{3}\right)$ GIP treated $(o b / o b)$ mice compared to saline treated controls corresponding to 1.3 -fold to 3.0 -fold reductions ( $\mathrm{P}<0.01$ to $\mathrm{P}<0.001)$, respectively.

Conclusions: Substitution of GIP with hydroxyproline at position 3 provides an novel stable GIP receptor antagonist $\left(\left(\mathrm{Hyp}^{3}\right) \mathrm{GIP}\right)$ that decreased hyperglycaemia and enhanced insulin sensitivity following longer-term administration in obese diabetic $(o b / o b)$ mice. $\left(\mathrm{Hyp}^{3}\right)$ GIP, and particularly the sister analogue $\left(\mathrm{Pro}^{3}\right) \mathrm{GIP}$, has impressive potential for improving glucose tolerance and insulin resistance in obesity-related type 2 diabetes.

Supported by Diabetes UK

0662

Pharmacological profile of GRC 8011, a novel dipeptidyl peptidase-IV (DPP-IV) inhibitor

R. Anupindi ${ }^{1,2}$, L. Gharat ${ }^{3}$, D. M. Shah ${ }^{1}, K$. Varanasi ${ }^{1}$, S. V. S. Vakkalanka ${ }^{1}$, U. K. Majumdar' ${ }^{2}$, M. Gupta ${ }^{2}$,

S. Narayanan ${ }^{1}$;

${ }^{1}$ Biological Research Division, Glenmark Research Centre, Navi Mumbai, ${ }^{2}$ Dept. of Pharmaceutical Technology, Jadavpur University, Kolkata, ${ }^{3}$ Discovery Chemistry, Glenmark Research Centre, Navi Mumbai, India.

Background and Aims: Inhibition of DPP-IV enzyme is a novel approach for the treatment of type 2 diabetes as it results in the increase in levels of endogenous active GLP-1 by prolonging its half-life. Glucagon-like peptide-1 (GLP1), a gut incretin hormone released from the L-cells of the intestine in response to nutrient intake augmenting insulin secretion, is rapidly degraded by DPP-IV enzyme. GRC 8011, a selective DPP-IV inhibitor, showed promising glucose lowering activity in oral glucose tolerance tests (OGTT) in different animal models administered alone and in combination with metformin, supporting its potential utility in the treatment of type 2 diabetes.

Materials and Methods: DPP-IV activity was determined in a fluorescence based assay by the cleavage rate of 7 amino-4-methyl coumarin (AMC) from substrate GlycylProlyl-AMC using human or rat enzyme. Glucose tolerance tests (OGTT) were done in C57BL/6J mice of either sex. The animals were fasted for $18 \mathrm{~h}$ and were administered orally either vehicle or different doses of GRC 8011 along with a glucose load of $2 \mathrm{gm} / \mathrm{kg}$. Whole blood glucose (WBG) measurement was done at $0,30,60,90$ and $120 \mathrm{~min}$ by tail cut method using a hand-held glucometer. DPP-IV levels were also estimated for correlation with blood glucose. To substantiate these findings in diabetic conditions, OGTT was carried out in 8 week old wistar rats which were treated with streptozocin $(100 \mathrm{mg} / \mathrm{kg}$, i.p.) on the day of birth (n0-STZ). Pharmacokinetic studies were performed on SD rats of either sex weighing between 150$180 \mathrm{gm}$. Statistical analyses were performed using one-way ANOVA, followed by Dunnett's test.

Results: GRC 8011 showed an $\mathrm{IC}_{50}$ of $57.19,157.9$ and $97.5 \mathrm{nM}$ in human recombinant enzyme, human plasma and rat plasma systems respectively and exhibited a competitive 
reversible inhibition of DPP-IV enzyme in kinetic studies. In specificity studies, GRC 8011 showed good specificities against DPP-II (88 fold), PPCE (55 fold). The compound did not produce inhibition of Leu-aminopeptidase and elastase at $1 \mathrm{mM}$ concentration. In an OGTT in C57BL/6J mice, GRC 8011 produced significant dose-dependent reduction in $\mathrm{AUC}_{0-120}$ of WBG levels at 1,3 and $10 \mathrm{mg} /$ $\operatorname{kg}(31 \pm 7,37 \pm 6$ and $50 \pm 7$ respectively). In a separate OGTT experiment in the same model, GRC 8011 ( $3 \mathrm{mg} / \mathrm{kg}$ ) showed $>80 \%$ reduction in $\mathrm{AUC}_{0-120}$ of DPP-IV levels $(p<0.001)$. In a repeated dose study in n0-STZ model, chronic dosing of the combination of GRC $8011(1 \mathrm{mg} /$ $\mathrm{kg}$, b.i.d.) and metformin $(100 \mathrm{mg} / \mathrm{kg}$, b.i.d.) produced a greater reduction in $\mathrm{AUC}_{0-120}$ of $\mathrm{WBG}$ levels than either treatment given alone on day 18 of the study $(31 \pm 5,31 \pm 5$, $46 \pm 3$, in GRC 8011, metformin and combination respectively). On oral administration GRC 8011 exhibited a favorable pharmacokinetic profile in $\mathrm{SD}$ rats with a bioavailability of $78 \%, \mathrm{C}_{\max }$ of $1.46 \mu \mathrm{g} / \mathrm{mL}$ at $0.25 \mathrm{~h}$. It has a clearance of $10.96 \mathrm{~L} / \mathrm{h} / \mathrm{kg}$ and volume of distribution of $4.67 \mathrm{~L} / \mathrm{kg}$.

Conclusion: GRC 8011 is a potent, orally active inhibitor of rodent and human DPP-IV, and is being used as a research tool.

\section{3}

Lack of suppression of glucagon during OGTT contributes to the reduced incretin effect in type 2 diabetes

F. K. Knop ${ }^{1,2}$, T. Vilsbøll ${ }^{1}$, S. Madsbad ${ }^{3}$, J. J. Holst ${ }^{2}$,

T. Krarup ${ }^{1}$;

${ }^{1}$ Department of Internal Medicine F, ${ }^{2}$ Department of Medical Physiology, ${ }^{3}$ Department of Endocrinology, University of Copenhagen, Denmark.

Background and Aims: We aimed to investigate the role of glucagon suppression during oral glucose tolerance test (OGTT) and isoglycemic intravenous glucose tolerance test (iIVGTT), respectively, in order to further elucidate the mechanisms behind the reduced incretin effect observed in patients with type 2 diabetes mellitus (T2DM).

Materials and Methods: Eight patients (6 males) with T2DM (Age: 62(51-75) years; BMI: 24(21-26) $\mathrm{kg} / \mathrm{m}^{2}$; $\mathrm{HbA}_{1} \mathrm{c}:$ 6.7(5.7-8.7)\% (mean (range)), and 8 matched (gender, age, BMI) healthy control subjects were studied. Blood was sampled on two separate days following a 4 hour $50 \mathrm{~g}$-OGTT and an iIVGTT, respectively.

Results: Isoglycemia during the two days was obtained in both groups. The incretin effect was significantly reduced in patients with $\mathrm{T} 2 \mathrm{DM}$ compared to healthy controls ( $34 \pm 18 \%$ vs. $70 \pm 5 \%$ (mean \pm SEM); $\mathrm{p}=0.05$ ). No significant differences in the secretion of glucagon-like peptide-1
(GLP-1) or glucose-dependent insulinotropic polypeptide (GIP) during OGTT or iIVGTT were observed between the two groups. Normal glucagon suppression during OGTT and IIVGTT, respectively, was observed in the group of healthy control subjects $(\mathrm{p}=\mathrm{NS})$, whereas significant glucagon suppression only occurred following iIVGTT in the group of patients with T2DM $(-247 \pm 47 \mathrm{mM} \times 2 \mathrm{~h}$ vs. $-20 \pm 45 \mathrm{mM} \times 2 \mathrm{~h} ; \mathrm{p}=0.002$ ).

Conclusion: These results suggest that the lack of glucagon suppression during OGTT in combination with a normal suppression of glucagon during iIVGTT contributes to the reduced incretin effect in patients with T2DM.

0664

4-weeks of near-normalisation of blood glucose has no effect on postprandial GLP-1 secretion but enhances beta cell responsivenes during a meal in patients with type 2 diabetes

P. V. Højberg ${ }^{1}$, T. Vilsbøll ${ }^{2}$, M. Zander ${ }^{1}$, F. K. Knop ${ }^{2}$, A. Vølund ${ }^{3}$, T. Krarup ${ }^{2}$, J. J. Holst ${ }^{4}$, S. Madsbad ${ }^{1}$; ${ }^{1}$ Endokrinology, Hvidovre Hospital, ${ }^{2}$ Internal Medicine, Gentofte Amtssygehus, Hellerup, Denmark, ${ }^{3}$ Novo Nordisk A/S, Bagsværd, ${ }^{4}$ Medical Physiology, University of Copenhagen, Denmark.

Background and Aims: Postprandial GLP-1 secretion is reduced in patients with type 2 diabetes mellitus (T2DM). We have shown that beta-cell responsiveness to GLP-1, but not to intraveneous (IV) glucose, improved after 4 weeks of near-normalization of blood glucose. The aim of the present study was to investigate whether 4 weeks of nearnormalization of blood glucose (BG) improved GLP-1 secretion and beta-cell function during a meal.

Materials and Methods: Nine patients with T2DM in poor glycemic (HbA1c: $8.0 \pm 0.4 \%$, mean $\pm \mathrm{SEM}$ ) were investigated before and after 4 weeks of near-normalization of BG (mean BG: $6.4 \pm 0.3 \mathrm{mmol} / \mathrm{l}$ ) using insulin treatment. HbAlc after insulin treatment was $(6.6 \pm 0.3 \%)$. Nine healthy subjects were also studied. Postprandial GLP-1 response was evaluated from total area under the curve (AUC) during a test meal. Beta-cell function was evaluated from total AUC C-peptid(CP) $)^{\prime} \mathrm{AUC}(\mathrm{BG})$ ratio and beta-cell responsiveness to glucose and incretins was evaluated from the slope of the linear regression between insulin secretion rate and concommitant plasma glucose during the meal.

Results: $\mathrm{AUC}_{\mathrm{GLP}-1}$ were; $5.50 \pm 1.07$ vs. $5.91 \pm 1.11 \mu \mathrm{mol}$ * $4 \mathrm{~h}$ before and after insulin treatment, respectivly ( $\mathrm{p}=\mathrm{NS}$ ), compared to $7.56 \pm 0.83 \mu \mathrm{mol} * 4 \mathrm{~h}$ in healtht subjects. Total AUC ${ }_{\mathrm{CP}} / \mathrm{AUC}_{\mathrm{BG}}$ ratio increased from $70.7 \pm 18.3$ to $82.3 \pm 9.8 \mathrm{pmol} / \mathrm{mmol}$ after insulin treatment $(\mathrm{p}<0.05)$, compared to $123 \pm 10 \mathrm{pmol} / \mathrm{mmol}$ in healthy subjects. Beta- 
cell responsiveness in patients with $\mathrm{T} 2 \mathrm{DM}$ increased from $0.59 \pm 0.16$ to $0.94 \pm 0.13 \mathrm{pmol} / \mathrm{min} / \mathrm{mmol},(p=0.05)$, compared to $3.15 \pm 0.51 \mathrm{pmol} / \mathrm{min} / \mathrm{mmol}$ in healthy subjects $(\mathrm{p}<0.01)$.

Conclusion: Four weeks of near-normalization of BG had no effect on postprandial GLP-1 secretion. Nevertheless, beta-cell function improved significantly during the meal, which may be explained by an increased beta-cell responsiveness to incretin hormones.

\section{5}

Effect of GLP-1 and exendins upon glucose uptake and cellular mechanism, in human adipose tissue

V. Sancho, B. Nuche-Berenguer, L. Arnés,

P. Tornero-Esteban, J. Cancelas, I. Valverde,

M. L. Villanueva-Peñacarrillo;

Metabolism, Nutrition and Hormones, Fundación Jiménez Díaz, Madrid, Spain.

Background and Aims: Exendin $4(\mathrm{Ex}-4)$ and its truncated form exendin 9 (Ex-9) stimulate, like insulin, glucose transport in rat fat, in which effect PI3K has been implicated. In rat muscle and liver, Ex-9 antagonizes the GLP-1 and Ex-4 increasing action upon parameters of the glucose metabolism, while Ex-9 mimics the effect of GLP1 and Ex-4 in human myocytes. We have studied, in human adipocytes 1) the effect of GLP-1, Ex-4 and Ex-9, compared with that of insulin, on glucose uptake, 2) their capability to increase the activity of kinases -PI3K, PKB, p42/44 MAPKs and p70s6k- proposed to be involved in the GLP-1 and insulin actions in other extrapancreatic tissues, and 3 ) the possible implication of PI3K in the action of GLP-1 and exendins on glucose transport.

Materials and Methods: Adipocytes were isolated by enzymatic digestion from subcutaneous fat tissue obtained, previously informed consent given, from 15 normal subjects (6F/9M; age: $59.4 \pm 3.5 \mathrm{yr}$; fasting plasma glucose: $93.7 \pm 4.4 \mathrm{mg} / \mathrm{dl}$ ) undergoing inguinal hernia or plastic surgery. We measured glucose transport -2-deoxi-D-[1,2- $\left.{ }^{3} \mathrm{H}\right]$ glucose uptake- in cells $\left(10^{5}\right)$ after 18 min incubation in KRB-Hepes, without (basal) and with $10^{-9}$ M GLP-1, Ex-4, Ex-9, insulin or glucagon, and in the absence and presence of $10^{-6} \mathrm{M}$ wortmaninn (PI3K inhibitor). The activity of PI3K -PIP 2 to $\mathrm{PIP}_{3}$, by TLC-, PKB, p42/44 MAPKs and p70s6k -by Western blotting- was measured in the cells after $3 \mathrm{~min}$ incubation without (basal) and with $10^{-9} \mathrm{M}$ either peptide. Statistical significance of results (mean \pm SEM) was assessed by ANOVA test.

Results: In adipocytes from 6 subjects, basal glucose uptake $\left(14.1 \pm 2.1 \mathrm{fmol} / 10^{5}\right.$ cells $)$ was similarly increased by GLP-1 $(151 \pm 8 \%$ basal, $p<0.001)$, Ex-4 $(156 \pm 12 \%$, $p<0.001)$ and insulin $(168 \pm 9 \%, p<0.001)$, but was not affected by Ex-9 or glucagon. In 3-5 subjects, basal PI3K activity was increased $(p<0.05)$ by all hormones/peptides tested (GLP-1: $134 \pm 7 \%$ basal; Ex-4: 145 $\pm 8 \%$; Ex-9: $164 \pm 26 \%$; insulin: $133 \pm 10 \%$ ), and so it was that of $\mathrm{p} 42$ and p44 MAPKs (GLP-1: 145 $\pm 15 \%$ basal and 123 $\pm 4 \%$, p42 and p44, respectively; Ex-4: $158 \pm 16 \%$ and $150 \pm 7 \%$; insulin: $141 \pm 7 \%$ and $140 \pm 8 \%$, all $p<0.05$ ), except in the case of Ex-9, which did not modified the respective basal value (p42: $107 \pm 13 \%$; p44: $111 \pm 11 \%$ ); the degree of PKB phosphorylation was affected only by insulin $(231 \pm 34 \%$ basal, $p<0.05$ ), while that of p70s6k was stimulated by Ex$4(137 \pm 6 \%$ basal, $p<0.01)$ but apparently not by GLP-1 $(140 \pm 20 \%)$, insulin $(121 \pm 10 \%)$ or Ex-9 (109 $\pm 11 \%)$. Inhibition of PI3K activity by wortmaninn abolished the stimulatory action of GLP-1, Ex-4 and insulin upon glucose transport ( $70 \pm 3 \%$ basal, $84 \pm 6 \%$ and $107 \pm 13 \%$, respectively, $\mathrm{n}=3$ and $p<0.05$, in all cases), to the same levels as that obtained with the inhibitor alone $(86 \pm 14 \%$ basal).

Conclusion: GLP-1 and Ex-4, like insulin, but not Ex-9, stimulate glucose uptake in human adipocytes, and in their action activation of PI3K is required. As PI3K is also stimulated by Ex-9, this enzyme should be participating in other effects of the peptide in the adipose tissue.

Supported by BFI 2003-07399; SCIII PI050363 and PI050369

0666

Characteristics of the action of exendins upon glucose uptake by the skeletal muscle, in normal rat and in a type 2 diabetic model

L. Arnés, N. González, V. Sancho, B. Nuche, P. Tornero-Esteban, J. Cancelas, I. Valverde,

M. L. Villanueva-Peñacarrillo;

Metabolism, Nutrition and Hormones, Fundación Jiménez Díaz, Madrid, Spain.

Background and Aims: Exendin-4 (Ex-4), insulinotropic peptide of non-mammalian nature, is under current investigation because of its structural analogy with GLP-1 and antidiabetic properties. We studied, in the skeletal muscle of normal and streptozotocin (STZ)-induced type 2 diabetic rats, the effect of Ex-4, compared to that of GLP-1, on glucose transport; in normal rats, we also searched for the action of Ex-4 upon the activity of kinases known to be involved in GLP-1 and insulin action - PI3K, PKB, p70s6k and p44/p42 MAPKs - , and their possible role in its effect on glucose transport. Exendin-9 (Ex-9), truncated form of Ex-4 and antagonist of its stimulatory action upon glucose metabolism in rat liver and muscle but not in human myocytes, was also studied. 
Materials and Methods: For glucose transport $-{ }^{3} \mathrm{H}$ 2DOG uptake-, paired soleus muscle (two per rat) were incubated for $60 \mathrm{~min}$ in KRB-HEPES containing $0.5 \mathrm{M} 2$ deoxi-D-[1,2- $\left.{ }^{3} \mathrm{H}\right]$ glucose (Sp. Ac. $\left.40 \mathrm{nCi} / \mu \mathrm{mol}\right)$ and $2 \mathrm{M}$ $\left[{ }^{14} \mathrm{C}\right]$ sorbitol (Sp. Ac. $\left.5 \mathrm{nCi} / \mu \mathrm{mol}\right)$, in the absence (basal) and presence of $10^{-9} \mathrm{M}$ GLP-1, insulin, Ex-4 or Ex-9; in normal rats, two additional conditions were studied: i) in the presence of Ex-4 or Ex-9 with inhibitors $-10^{-6} \mathrm{M}$ wortmannin (W), $10^{-7} \mathrm{M}$ rapamycin (RAP), $2.5 \times 10^{-5} \mathrm{M}$ PD98059 (PD), and $10^{-4} \mathrm{M} \mathrm{H}-7-$ of respective kinases -PI3K, p70s6k, MAPKs, and PKC-, and ii) in the sole presence of each inhibitor. Data (mean \pm SEM) were normalized and referred to the basal value. The activity of PI3K - measured as $\mathrm{PIP}_{2}$ phosphorylation to $\mathrm{PIP}_{3}$, by TLC - PKB, p70s6k and p44/p42 MAPKs - phosphorylated/total form, by Western blotting-, were assayed in muscle strips (two per muscle) treated during 3 min without (basal) and with each exendin.

Results: In normal rats, the basal glucose uptake value $(0.367 \pm 0.017 \mathrm{nmol} / \mathrm{mg}$ per $\min , \mathrm{n}=27)$ was significantly increased by Ex-4 $(129 \pm 5 \%$ basal, $n=13, p<0.005)$, similarly to previously observed with GLP-1(129 $\pm 6 \%, n=9$, $p<0.001)$, while it was not affected by Ex-9 $(92 \pm 5 \%, \mathrm{n}=14)$; the stimulatory effect of Ex-4 was not modified by the concomitant presence of Ex-9 (Ex-4+Ex-9: 141 $\pm 11 \%, n=6$, $p<0.005)$; in muscle samples from 3-5 rats, both Ex-4 and Ex-9 increased $(p<0.05)$ the activity of PI3K, p70s6k and p44/p42 MAPKs, as previously detected with GLP-1; Ex-4, like GLP-1, also stimulated PKB phosphorylation (153 $\pm 15 \%$ basal, $p=0.034)$, while no effect was detected with Ex-9 (97 $\pm 7 \%$ ); Ex-4-induced stimulation of glucose uptake was blocked by the respective inhibitor of PI3K $(66 \pm 5 \%)$, P70S6K $(78 \pm 3 \%)$, PKC $(83 \pm 7 \%)$ and MAPK $(68 \pm 9 \%)$ activity, to values similar to those obtained in the sole presence of the inhibitory agents (W: $76 \pm 7 \%$ basal, $p<0.05$; RAP: $81 \pm 3 \%$; H7: $91 \pm 8 \%$; PD: $74 \pm 8 \%, p<0.05)$. In STZ-type2 diabetic rats, the basal glucose uptake value $(0.345 \pm 0.016 \mathrm{nmol} / \mathrm{mg}$ per $\min , \mathrm{n}=47)$ was indistinct of that in normal animals; yet, the muscle did not respond to GLP-1 or Ex-4, while insulin induced a similar increment ( $142 \pm 5 \%$ basal, $\mathrm{n}=11, p<0.001)$ to that in normal rats.

Conclusion: In normal rat skeletal muscle, Ex-4 mimics GLP-1 and insulin in their stimulatory effect upon glucose transport through, apparently, the same kinases as those involved in the GLP-1 action; as Ex-9 does not modulate glucose uptake, its increasing effect upon the activity of some of the kinases should be correlated with other actions of this peptide. The muscle glucose uptake process in the STZ-type 2 diabetic model seems to be altered in response to GLP-1 or Ex-4.

Supported by BFI 2003-07399; SCIII PI050363 and PI050369
0667

Activity of signaling kinases involved in the GLP-1 metabolic actions, in skeletal muscle of type 2 diabetes and/or obese patients

P. Tornero-Esteban ${ }^{1}$, V. Sancho ${ }^{1}$, N. González ${ }^{1}$, A. Martín-Duce ${ }^{2}$, L. Arnés ${ }^{1}$, B. Nuche-Berenguer ${ }^{1}$, J. Cancelas ${ }^{1}$, I. Valverde ${ }^{1}$, M. L. Villanueva-Peñacarrillo ${ }^{1}$;

${ }^{1}$ Metabolism, Nutrition and Hormones, Fundación Jiménez Díaz, Madrid, ${ }^{2}$ Surgery, Hospital Príncipe de Asturias, Alcalá de Henares, Spain.

Background and Aims: Changes in the activity of several kinases by GLP-1 have been implied in their increasing effect upon glucose transport and glycogen synthase $a$ activity in normal human myocytes. Moreover, an altered response of some kinases to insulin could account for the impaired glucose uptake by insulin in muscle cells of type 2 diabetic and obese patients. In this work we have studied, in myocytes from morbidly obese patients and also in obese suffering of type 2 diabetes, the effect of insulin and GLP-1, upon the activity of kinases known to be involved in their insulin actions in the muscle.

Materials and Methods: Myotubes were established from satellite cells of dissociated skeletal muscle samples obtained, previously informed consent given, from five morbidly obese patients (4F/1M; age: $45 \pm 4$ yr; BMI: $49 \pm 2 \mathrm{~kg} / \mathrm{m}^{2}$ fasting plasma glucose: $110 \pm 7 \mathrm{mg} / \mathrm{dl}$; HDL: $52 \pm 2 \mathrm{mg} / \mathrm{dl}$; cholesterol: $177 \pm 19 \mathrm{mg} / \mathrm{dl}$; triglycerides: $119 \pm 10 \mathrm{mg} / \mathrm{dl}$ ) and three obese also suffering of type 2 diabetes (3F, age: $48 \pm 3 \mathrm{yr}$; BMI: $47 \pm 2 \mathrm{~kg} / \mathrm{m}^{2}$ fasting plasma glucose: $160 \pm 8 \mathrm{mg} / \mathrm{dl}$; HDL: $55 \pm 6 \mathrm{mg} / \mathrm{dl}$; cholesterol: $210 \pm 41 \mathrm{mg} / \mathrm{dl}$; triglycerides: $164 \pm 29 \mathrm{mg} / \mathrm{dl}$ ), undergoing bariatric surgery; for purpose of comparison of basal values, muscle samples from three normal subjects (2F/1M; age: $83 \pm 3 \mathrm{yr}$; fasting plasma glucose: $107 \pm 16 \mathrm{mg} / \mathrm{dl}$ ), undergoing orthopedic surgery, were also included. The activity of p44/42 MAPKs, p70s6k and PKB - phosphorylated/total form, by Western blot- was assayed in 20.000 cells after 3-min incubation without (basal) and with $10^{-9} \mathrm{M}$ insulin or GLP-1. Data are expressed as mean $\pm \mathrm{SEM}$, and statistical significance was assessed by the Student $t$-test.

Results: The basal activity of p70s6k, PKB and p42/44 MAPKs in myocytes from the obese patients, diabetic or not, was diminished $(p<0.0001)$, the overall mean value averaging $67 \pm 3 \%(n=8)$ of that found in the normal control group $(100 \pm 7 \%, \mathrm{n}=3)$. In the cells from the non-diabetic obese subjects, both insulin and GLP-1 induced an increment in the phosphorylation of MAPKs (p44: $167 \pm 18 \%$ basal and $136 \pm 13 \%$, respectively, $p<0.05$; $\mathrm{p} 42$ : $135 \pm 8 \%, p<0.05$ and $112 \pm 4 \%$, respectively, all $n=3$ ), but 
the activity of PKB and p70s6k was increased only by GLP-1 $(136 \pm 2 \%$ basal and $161 \pm 21 \%$, respectively, both $\mathrm{n}=3$ and $p<0.05$ ). In the obese patients with type 2 diabetes, insulin did not stimulate $\mathrm{p} 44$ or $\mathrm{p} 42$ MAPKs, while GLP-1 exerted a clear increase in both enzymes (p44: $177 \pm 12 \%$ basal and $\mathrm{p} 42$ : $135 \pm 15 \%$, both $\mathrm{n}=3$ and $p<0.05$ ); insulin, but not GLP-1, stimulated the myocyte PKB activity to $165 \pm 21 \%(\mathrm{n}=3, p<0.05)$.

Conclusion: In morbid obesity, alteration of the insulininduced stimulation of PKB and p70s6k in the muscle, and that of MAPKs when type 2 diabetes is present, could account for the impaired insulin-induced glucose transport, whereas the effect of GLP-1 upon the activity of these enzymes remains normal.

Supported by BFI 2003-07399; SCIII PI050363 and PI050369

\section{PS 48}

\section{Metabolic effects of incretin hormones II}

0668

Vildagliptin increases liver glucose uptake in the conscious dog

D. S. Edgerton ${ }^{1}$, K. Stettler ${ }^{1}$, D. Neal ${ }^{1}$, M. Scott ${ }^{1}$, C. Hobbs $^{2}$, X. Zhang ${ }^{3}$, A. Duttaroy ${ }^{3}$, A. D. Cherrington ${ }^{1}$; ${ }^{1}$ Molecular Physiology and Biophysics, Vanderbilt University Medical Center, Nashville, ${ }^{2}$ Toxicology, Loveless Respiratory Research Institute, Albuquerque, ${ }^{3}$ Diabetes \& Metabolism, Novartis Institutes for Biomedical Research Inc, Cambridge, United States.

Background and Aims: Glucagon-like peptide-1 (GLP-1) is an important incretin hormone released by the gut in response to nutrient ingestion. The incretin effect accounts for up to $60 \%$ of the postprandial insulin secretion response, but is diminished in patients with $\mathrm{T} 2$ diabetes. Therapeutic treatment with GLP-1 is limited because it is rapidly degraded by dipeptidyl peptidase-4 (DPP-4). This study tested the effect of a DPP-4 specific inhibitor, vildagliptin, on DPP-4 activity, GLP-1 concentration, and the associated effects on pancreatic hormone levels and glucose metabolism in the $18 \mathrm{~h}$ fasted conscious dog.

Materials and Methods: Vildagliptin $(1 \mathrm{mg} / \mathrm{kg} ; \mathrm{n}=6)$ or vehicle (VEH; $\mathrm{n}=6$ ) were given orally at $\mathrm{t}=-20 \mathrm{~min}$. At $\mathrm{t}=0 \mathrm{~min}$ constant portal infusions of glucose $(4 \mathrm{mg} / \mathrm{kg} / \mathrm{min})$ and GLP-1 (1 pmol/kg/min) were started, and glucose was infused into a peripheral vein to maintain arterial (art) plasma glucose at $160 \mathrm{mg} / \mathrm{dl}$.
Results: The art and portal vein DPP-4 activities were fully suppressed during the experimental period (240 min; $\mathrm{P}<0.001)$. The mean art plasma GLP-1 levels were $30 \pm 3$ vs $47 \pm 2 \mathrm{pM}(\mathrm{P}<0.001)$ in the VEH and vildagliptin groups during the last $3 \mathrm{~h}$, while portal levels were $49 \pm 3$ vs $58 \pm 2 \mathrm{pM}$ $(\mathrm{P}<0.001)$, respectively. The peripheral glucose infusion rates (GIR) were $6.1 \pm 1.2$ vs $8.6 \pm 1.5 \mathrm{mg} / \mathrm{kg} / \mathrm{min}(\mathrm{P}<0.001)$ in the VEH and vildagliptin groups. The art plasma insulin levels were $26 \pm 4$ vs $36 \pm 8 \mu \mathrm{U} / \mathrm{ml}(\mathrm{P}<0.001)$, in the 2 groups, and the hepatic plasma insulin levels were $73 \pm 10$ vs $88 \pm 15 \mu \mathrm{U} /$ $\mathrm{ml}(\mathrm{P}=0.088)$. Hepatic glucagon levels fell equally in both groups. Net hepatic glucose uptake was $0.7 \pm 0.1$ vs $2.0 \pm 0.7 \mathrm{mg} / \mathrm{kg} / \mathrm{min}(\mathrm{P}<0.001)$, respectively, and \% hepatic glucose extraction was $2.4 \pm 0.5$ vs $5.6 \pm 1.4 \%(\mathrm{P}<0.001)$.

Conclusion: In summary, acute treatment with vildagliptin fully inhibited DPP-4 activity over $4 \mathrm{~h}$, resulting in a $57 \%$ increase in art GLP-1 levels, $40 \%$ increases in art insulin and GIR, and a $186 \%$ increase in net hepatic glucose uptake. Thus, improvement of postprandial glucose with DPP-4 inhibitor vildagliptin in part reflects an increase in net hepatic glucose uptake.

Supported by Novartis Institutes for Biomedical Research Inc

0669

Effect of the DPP-4 inhibitor vildagliptin on fasting plasma levels of intact GLP-1 and GIP in type 1 or type 2 diabetes

M. Ligueros-Saylan ${ }^{1}$, D. E. Kelley ${ }^{2}$, Y.-L. He ${ }^{3}$, C. A. Watson ${ }^{3}$, D. Serra ${ }^{3}$, B. E. Dunning ${ }^{4}$, C. F. Deacon ${ }^{5}$, J. J. Holst ${ }^{5}$, J. E. Foley ${ }^{3}$;

${ }^{1}$ Novartis Pharmaceuticals Corporation, East Hanover, United States, ${ }^{2}$ Medicine, Univ of Pittsburgh, ${ }^{3}$ Novartis, East Hanover, ${ }^{4}$ PharmaWrite, Princeton, United States, ${ }^{5}$ Panum Institute, Univ. of Copenhagen, Denmark.

Background and Aims: Vildagliptin increases post-meal plasma levels of intact (active) GLP-1 and GIP in patients (pts) with type 2 diabetes (T2DM) by inhibiting DPP-4, the enzyme that degrades and inactivates these incretin hormones. Because incretin hormone release is dependent on nutrient ingestion, it might be expected that DPP-4 inhibition would have little influence on GLP-1 or GIP in the fasting state. The aim of this work was to assess the effect of vildagliptin on fasting levels of the active form of GLP-1 and GIP in pts with T2DM and its effects in pts with type 1 diabetes (T1DM).

Methods: Two 4-wk randomized, double-blind, placebocontrolled studies were conducted. The study in pts with T1DM had a crossover design, and 11 insulin pump-treated pts received vildagliptin $100 \mathrm{mg}$ bid. The study in pts with 
T2DM had a parallel-group design and 9 drug-naïve pts were randomized to receive vildagliptin $100 \mathrm{mg}$ bid. Plasma levels of active GLP-1 and GIP were measured with ELISA and RIA, respectively, using N-terminally directed antisera. Samples were obtained after an overnight fast before the morning dose of vildagliptin.

Results: PBO had no effect on fasting levels of intact GLP-1 or GIP, and to allow comparison between the two studies with different designs, only data obtained before (BL) and after 4-wk treatment during the active-treatment phase/arm are presented. Mean age and BMI of the pts with T1DM were $39 \mathrm{y}$ and $24 \mathrm{~kg} / \mathrm{m}^{2}$, respectively. Mean age and BMI of the pts with T2DM were $45 \mathrm{y}$ and $32 \mathrm{~kg} / \mathrm{m}^{2}$, respectively. As reported below, both at BL and after 4-wk treatment, fasting levels of intact GIP were significantly higher in obese pts with T2DM than in lean pts with T1DM. Four-wk treatment with vildagliptin significantly increased fasting levels of active GLP-1 (by $\sim 9$ pM in pts with T1DM and by $\sim 14$ pM in T2DM) and GIP ( $>2$-fold in both T1DM and T2DM). The magnitude of change (delta) in GLP-1 and GIP associated with 4-wk vildagliptin administration did not differ significantly between T1DM and T2DM.

\begin{tabular}{lllllll}
\hline & \multicolumn{2}{l}{ Intact (active) GLP-1 (pM) } & \multicolumn{2}{l}{ Intact (active) GIP (pM) } \\
\cline { 2 - 7 } & T1DM & T2DM & $\begin{array}{l}\text { p vs } \\
\text { T1DM }\end{array}$ & T1DM & T2DM & $\begin{array}{l}\text { p vs } \\
\text { T1DM }\end{array}$ \\
\hline $\begin{array}{l}\text { Baseline } \\
\text { (BL) }\end{array}$ & $0.7 \pm 0.5$ & $4.0 \pm 2.5$ & 0.160 & $6.0 \pm 2.3$ & $18.1 \pm 5.0$ & 0.030 \\
Wk 4 & $9.8 \pm 2.0$ & $18.1 \pm 4.8$ & 0.106 & $16.0 \pm 3.1$ & $46.4 \pm 13.1$ & 0.024 \\
$\begin{array}{l}\text { Delta } \\
\text { p vs BL }\end{array}$ & $9.1 \pm 1.9$ & $14.2 \pm 3.5$ & 0.217 & $10.1 \pm 3.1$ & $28.3 \pm 12.2$ & 0.137 \\
\hline
\end{tabular}

Conclusions: Four-wk treatment with vildagliptin increases fasting plasma levels of intact (active) GLP-1 and GIP in the absence of an acute (meal) stimulus for incretin hormone release in patients pts with T1DM and those with T2DM. This effect may contribute to the efficacy of vildagliptin to decrease FPG.

Supported by Novartis

0670

Co-localisation of the ATP-sensitive $\mathrm{K}^{+-}$channel complex, Kir6.2/SUR1, with GLP-1- and GIP-expressing Land $\mathrm{K}$-cells in the human small intestine

L. B. Nielsen ${ }^{1}$, K. B. Plough ${ }^{2}$, I. Jansen-Olesen ${ }^{2}$,

S. Poerksen ${ }^{1}$, J. J. Holst ${ }^{3}$, S. Gammeltoft ${ }^{4}$,

H. B. Mortensen ${ }^{1}$, L. Hansen ${ }^{5}$, C. Ørskov ${ }^{6}$;
${ }^{1}$ Department of Paediatrics, ${ }^{2}$ Department of Clinical Experimental Research, Amtssygehuset Glostrup, ${ }^{3}$ Department of Medical Physiology, The Panum Institute, Copenhagen, ${ }^{4}$ Department of Clinical Biochemistry, Amtssygehuset Glostrup, ${ }^{5}$ Novo Nordisk A/S, Bagsværd, ${ }^{6}$ Department of Medical Anatomy, The Panum Institute, Copenhagen, Denmark.

Background and Aims: A common genetic variant, Glu23Lys in the ATP-sensitive $\mathrm{K}^{+}$-channel (K-ATP) has previously been associated with impaired glucose-induced insulin release in healthy subjects and patients with type 2 diabetes, possibly due to a hyperactive state of the $\mathrm{K}^{+}$channel. Recently, we found an association between this variant and reduced postprandial glucagon-like peptide 1 (GLP-1) levels, enhanced glucagon levels and impaired blood glucose control in children with new onset type diabetes during the first 12 months after diagnosis. To clarify a possible common glucose-sensing regulatory mechanism in the pancreas and the small intestine the present study aimed at investigating the expression and co-localization of the K-ATP channel, Kir6.2/SUR1 protein complex, with glucagon and insulin in the human islets and glucose-dependent insulinotrophic polypeptide (GIP) and GLP-1 in the gut K- and L-cells.

Materials and Methods: All immunohistochemical analysis was carried out on formalin fixed material. Human intestinal and pancreatic tissue $(n=6)$ was archival material collected during the 1970s according to the contemporary ethical guidelines. The antibodies used for immunohistochemical analysis were: monoclonal mouse anti-GLP-1 antibody code GLPb-17F1A37B2245C4 (F1) diluted 1:3000, goat anti Kir6.2 code N-18 diluted 1:3000 (Santa Cruz Biotechnology), goat anti SUR1 code C-16 diluted 1:1000 (Santa Cruz Biotechnology) and monoclonal mouse anti-insulin antibody code HUI 001 (Novo Nordisk) diluted $1: 5000$.

Results: By western blot analysis the expression of both the pore-forming and regulatory subunits of the K-ATP channel, Kir6.2 and SUR1, was found in primary tissue from human ileum. Moreover, antibody staining demonstrated that the Kir6.2/SUR1 complex co-localized with the GLP-1-expressing L-cells in the distal part of the gut and with the GIP-expressing K-cells in the proximal part of the gut. In addition the alpha- and beta-cells in the pancreas exhibited Kir6.2/SUR1 staining co-localizing with glucagon and insulin.

Conclusion: The L- and K-cells are equipped with the same ATP-sensitive $\mathrm{K}^{+}$-channel complex as alpha- and beta-cells - indicating common glucose-sensing regulatory machinery for these cell-types in humans. These findings 
support the interpretation of the clinical results, that carriers of the hyperactive variant, the Lys allele, have an impaired blood glucose regulation due to a systemic channeldysfunction.

Supported by the Danish Diabetes Association

\section{1}

Rapid improvement in glucose tolerance after Roux-en$Y$ gastric bypass surgery is not associated with change in fasting plasma glucagon-like peptide-1 (GLP-1) concentration

M. Pawlowski ${ }^{1}$, L. Czupryniak ${ }^{1}$, J. Strzelczyk ${ }^{2}$, D. Szymanski ${ }^{2}$, M. E. Nowicki ${ }^{2}$, M. Saryusz-Wolska ${ }^{1}$, A. Borkowska ${ }^{1}$, J. Loba ${ }^{1}$;

${ }^{1}$ Diabetology \& Metabolic Diseases Department, ${ }^{2}$ General and Transplant Surgery Department, Medical University of Lodz, Poland.

Background and Aims: Roux-en-Y gastric bypass (RYGB) surgery is an effective method of achieving permanent body weight reduction in severely obese individuals. In obese subjects with diabetes treated with RYGB glucose tolerance improves significantly. However, as number of gastrointestinal as well as adipose tissue hormones regulate insulin sensitivity and satiety feeling, and because RYGB changes physiological intestinal passage by forming gastrojejunal and duodenojejunal anastomoses, it may be suspected that RYGB may eventually affect incretin regulation of glucose metabolism. The aim of the study was to assess the early effect of RYGB on fasting levels of insulin, adiponectin, leptin, ghrelin and glucagon-like peptide-1 (GLP-1).

Materials and Methods: The study group comprised 17 obese subjects (mean $[ \pm \mathrm{SD}]$ body weight $132 \pm 12 \mathrm{~kg}$, BMI $43.2 \pm 5.4 \mathrm{~kg} / \mathrm{m}^{2}$ ) with mild glucose intolerance (fasting plasma glucose $6.2 \pm 0.4 \mathrm{mmol} / \mathrm{l}$ ) who were treated with RYGB. Fasting serum insulin, adiponectin, leptin, and ghrelin were measured with ELISA method and GLP-1 with immunofluorescent method. The measurements were performed before the surgery and one week after discharge from hospital which was $3 \pm 1$ weeks after RYGB.

Results: Body weight at $3 \pm 1$ weeks after RYGB decreased significantly by $10.3 \pm 3.8 \mathrm{~kg}$ ( $\mathrm{p}<0.05$ vs baseline) as did fasting plasma glucose was $4.9 \pm 0.2 \mathrm{mmol} / 1 \quad(\mathrm{p}<0.05)$. Fasting serum insulin before and after RYGB was $201 \pm 74$ and $144 \pm 50 \mathrm{pmol} / \mathrm{l}$, respectively $(\mathrm{p}<0.01)$, leptin $56.3 \pm 32.6$ and $31.9 \pm 20.5 \mathrm{pg} / \mathrm{ml}(\mathrm{p}<0.01)$, ghrelin $20.1 \pm 5.7$ and $26.0 \pm 4.7 \mathrm{ng} / \mathrm{ml}(\mathrm{p}<0.01)$. Fasting serum GLP-1 did not change significantly: $6.25 \pm 0.6$ and $6.4 \pm 0.8 \mathrm{pmol} / 1$ ( $\mathrm{p}>0.05)$, and neither did adiponectin concentration: $3276 \pm 1718$ and $3224 \pm 1057 \mathrm{ng} / \mathrm{ml}(\mathrm{p}>0.05)$.

Conclusion: In severely obese subjects treated with RYGB glucose tolerance significantly improved shortly after RYGB, with concomitant decrease in fasting insulin concentration. However, no changes in fasting GLP-1 concentrations were noted which may indicate that RYGB does not substantially affect entero-insular axis at the basal state in the early period after the surgery. To fully elucidate RYGB effect on incretin secretion a postprandial GLP-1 assessment must be performed.

Supported by Medical University Grant No 502-18-306

0672

Characterisation of novel glucose-dependent insulinotropic polypeptide (GIP) antagonists generated through amino acid substitution at position $\mathbf{G l u}^{3}$

V. A. Gault, K. Hunter, N. Irwin, B. D. Green, F. P. M. O'Harte, P. R. Flatt;

School of Biomedical Sciences, University of Ulster, Coleraine, United Kingdom.

Background and Aims: Glucose-dependent insulinotropic polypeptide (GIP) is a 42 amino acid hormone which regulates insulin release and glucose homeostasis, but is rapidly degraded by the enzyme dipeptidylpeptidase-IV (DPP-IV). In the present study, five novel $\mathrm{Glu}^{3}$-substituted analogues of GIP, namely $\left(\mathrm{Ala}^{3}\right) \mathrm{GIP},\left(\mathrm{Lys}^{3}\right) \mathrm{GIP},\left(\mathrm{Phe}^{3}\right) \mathrm{GIP}$, $\left(\operatorname{Trp}^{3}\right)$ GIP and $\left(\mathrm{Tyr}^{3}\right)$ GIP were synthesised and tested for DPP-IV resistance and biological activity both in vitro and in vivo.

Materials and Methods: GIP and GIP analogues were synthesised by Fmoc solid-phase peptide synthesis, purified by high-performance liquid chromatography (HPLC) and characterised by electrospray ionisation-mass spectrometry (ESI-MS). Stability of each peptide $(15 \mu \mathrm{g})$ was assessed by incubation $\left(0,2,4\right.$ and $8 \mathrm{~h}$ at $\left.37^{\circ} \mathrm{C}\right)$ in the presence of DPP-IV ( $5 \mathrm{mU} ; \mathrm{n}=3$ ) and peptide degradation quantified using HPLC. Cyclic-AMP production $\left(10^{-13}\right.$ to $10^{-7} \mathrm{~mol} / \mathrm{l}$; $\mathrm{n}=3$ ) was measured in Chinese hamster lung (CHL) cells transfected with the human GIP receptor. The insulinreleasing activity of each analogue was assessed following acute incubation ( $20 \mathrm{~min} ; 5.6 \mathrm{mmol} / \mathrm{l}$ glucose $)$ in the clonal pancreatic beta-cell line, BRIN-BD11 $(\mathrm{n}=8)$. In vivo potency was examined following intraperitoneal injection of GIP analogues $(25 \mathrm{nmol} / \mathrm{kg} \mathrm{bw})$ in 14-18 week old obese diabetic $(o b / o b)$ mice $(\mathrm{n}=8)$, monitoring plasma glucose and insulin responses. 
Results: GIP and GIP analogues were purified to $>95 \%$ purity and measured molecular masses correlated well with predicted theoretical masses, thereby confirming identity. Native GIP was rapidly hydrolysed by DPP-IV with only $20.9 \pm 1.6 \%$ remaining intact after $2 \mathrm{~h}$ incubation $\left(\mathrm{t}_{1 / 2}=1.3 \mathrm{~h}\right)$. From each of the analogues tested, only $\left(\mathrm{Lys}^{3}\right) \mathrm{GIP}$ demonstrated moderately enhanced resistance to DPP-IV $\left(\mathrm{t}_{1 / 2}=1.7 \mathrm{~h} ; \mathrm{P}<0.05\right.$ to $\left.\mathrm{P}<0.01\right)$ compared to native GIP. In transfected CHL cells, all analogues demonstrated a decreased potency in cyclic AMP production $\left(\mathrm{EC}_{50} 1.47\right.$ to $11.02 \mathrm{nmol} / \mathrm{l} ; \mathrm{P}<0.01$ to $\mathrm{P}<0.01)$ with $\left(\mathrm{Lys}^{3}\right) \mathrm{GIP}$ and $\left(\mathrm{Phe}^{3}\right)$ GIP significantly inhibiting GIP-stimulated $\left(10^{-7} \mathrm{~mol} / \mathrm{l}\right)$ cyclic AMP production (maximal inhibition $63 . \pm 4 \%$ and $62.0 \pm 3 \%$, respectively; $\mathrm{P}<0.05)$. In BRIN-BD11 cells, $\left(\mathrm{Lys}^{3}\right)$ GIP, $\left(\mathrm{Phe}^{3}\right)$ GIP, $\left(\mathrm{Trp}^{3}\right)$ GIP and $\left(\mathrm{Tyr}^{3}\right)$ GIP $\left(10^{-13}\right.$ to $\left.10^{-6} \mathrm{~mol} / \mathrm{l}\right)$ did not stimulate insulin secretion. Both $\left(\mathrm{Lys}^{3}\right)$ GIP and $\left(\mathrm{Phe}^{3}\right)$ GIP significantly inhibited GIP-stimulated insulin secretion with maximal inhibitions of $78.0 \pm 4 \%$ and $73.0 \pm 3 \%$, respectively $(\mathrm{P}<0.05)$. In $(o b / o b)$ mice, injection of each GIP analogue together with glucose $(18 \mathrm{mmol} / \mathrm{kg}$ bw) significantly increased the glycaemic excursion compared to control $\left(\mathrm{AUC}_{0-60}\right.$ min values 1.3 to 1.7 -fold; $\mathrm{P}<0.05$ to $\mathrm{P}<0.001$ ). This was associated with a lack of significant insulin responses. To test for receptor antagonist activity in vivo, GIP analogues were administered in combination with native GIP $(25 \mathrm{nmol} / \mathrm{kg}$ bw) to $(o b / o b)$ mice. $\left(\mathrm{Ala}^{3}\right)$ GIP, $\left(\mathrm{Phe}^{3}\right)$ GIP and $\left(\mathrm{Tyr}^{3}\right)$ GIP, when administered together with GIP, significantly reduced plasma insulin $(1.2$ to 1.5 -fold; $\mathrm{P}<0.05)$ and impaired the glucoselowering ability $(1.4$-fold; $\mathrm{P}<0.01)$ of the native peptide.

Conclusions: These data demonstrate that position 3 amino acid substitution of GIP with $\left(\mathrm{Ala}^{3}\right)$, $\left(\mathrm{Phe}^{3}\right)$ or $\left(\mathrm{Tyr}^{3}\right)$ provides a novel class of functional GIP receptor antagonists. DPP-IV resistance and GIP antagonism observed with these analogues were similar but less pronounced than the already well-established GIP-receptor antagonist, $\left(\mathrm{Pro}^{3}\right)$ GIP.

\section{3}

The effect of GIP on glucose absorption in small intestine O. Eiichi ${ }^{1}$, M. Hosokawa ${ }^{1}$, K. Fukuda ${ }^{1}$, K. Tsukiyama ${ }^{1}$, K. Yamada ${ }^{1}$, Y. Fujita ${ }^{1}$, Y. Yamada ${ }^{1}$, Y. Seino ${ }^{2}$, N. Inagaki ${ }^{1}$; ${ }^{1}$ Diabetes and Clinical Nutrition, Kyoto University, ${ }^{2}$ Division of Diabetes and Clinical Nutrition, Kansai Denryoku Hospital, Kyoto, Japan.

Background and Aims: Glucose-dependent insulinotropic polypeptide (GIP) plays an important role in glucose dependent regulation of postprandial glycemia. GIP is released from the small intestine upon meal ingestion, but the mechanism of its extra-pancreatic effects is unclear. We investigated the effect of GIP on glucose absorption in mouse intestine.

Materials and Methods: C57/BL6J mice and GIP receptor-deficient Imice were compared (8 weeks). Intestinal glucose absorption was measured by the single-pass perfusion method. The abdominal wall was opened, and the jejunum was ligated $1 \mathrm{~cm}$ below the ligament of Treitz. A Silastic perfusion catheter bearing two silicone swelling $3 \mathrm{~mm}$ apart was inserted into the gut just below the ligature and secured in place by a silk ligature between the two swelling. A collecting cannula was inserted into the lumen of the jejunum $10 \mathrm{~cm}$ further down to collect the perfusion fluid. To evaluate the effect of GIP on sodium-glucose cotranspoters, short-circuit current (Isc) in intestinal mucosa was measured using an Ussing chamber. Stripped jejunum mucosa was mounted vertically between temperature $\left(37^{\circ} \mathrm{C}\right)$-controlled Ussing chambers. Ringer's solution containing $10 \mathrm{mM}$ glucose was used as the standard bathing solution. All bathing solutions were gassed with a mixture of $95 \% \mathrm{O}_{2} / 5 \% \mathrm{CO}_{2}$, resulting in $\mathrm{pH} 7.4$. Motility of the small intestine was evaluated by measuring intestinal transit by oral administration of a non-absorbed marker. After twenty minutes, the animals were killed by cervical dislocation, and the entire gastrointestinal tract was removed. The distance from the pylorus to the front of the charcoal bolus and the ileocecal junction was measured. The rate of transit was determined as [(distance to charcoal front)/(length of small intestine) $] \times 100(\%)$.

Results: In controls, glucose absorption was inhibited by intraperitonial administration of GIP, (control: $-15.2 \pm$ $30.6 \mu \mathrm{mol} / \mathrm{cm}$ intestine $/ 15 \mathrm{~min}, 50 \mathrm{nmol} / \mathrm{kg}$ GIP: $-90.5 \pm$ $73.6 \mu \mathrm{mol} / \mathrm{cm}$ intestine $/ 15 \mathrm{~min})$. Electrophysiologically, GIP did not evoke any change in Isc incubated in bicarbonate-free Ringer solution in intestinal mucosa. After intraperitonial administration of GIP, intestinal transit was inhibited (control: $68.4 \pm 3.6 \%, 10 \mathrm{nmol} / \mathrm{kg}$ GIP: $44.7 \pm 7.8 \%$ ). GIP administration did not affect intestinal transit in GIP receptor-deficient mice.

Conclusion: GIP inhibits intestinal glucose absorption by reducing intestinal motility, thus decreasing the blood glucose level independent of its pancreatic action.

\section{4}

Development of chimeric GLP-1 analogues with improved potency and stability

H. Y. Kim ${ }^{1}$, M. J. Moon ${ }^{2}$, H. J. Yoo ${ }^{1}$, S. Y. Park ${ }^{1}$, Y. J. Lee ${ }^{1}$, O. H. Ryu ${ }^{1}$, K. W. Lee ${ }^{1}$, J. A. Seo ${ }^{1}$, D. H. $\operatorname{Shin}^{3}$, S. G. Kim ${ }^{1}$, N. H. Kim ${ }^{1}$, K. M. Choi ${ }^{1}$, S. H. Baik ${ }^{1}$, D. S. Choi ${ }^{1}$, J. Y. Seoung ${ }^{2}$; 
${ }^{1}$ Division of Endocrinology and Metabolism, Department of Internal Medicine, Korea University Medical Center, Seoul, ${ }^{2}$ Graduate School of Medicine, Korea University, Seoul, ${ }^{3}$ Department of Internal Medicine, Bundang Jesaeng General Hospital, Seongnam, Republic of Korea.

Background and Aims: Glucagon-like peptide-1 (GLP-1) is an incretin hormone derived from the proglucagon gene at the intestinal L-cells. In the pancreas, GLP-1 stimulates meal-induced insulin secretion, promotes insulin biosynthesis, and improves insulin sensitivity. Because of its insulinotropic activity, GLP-1 is considered as a good candidate drug for diabetes treatment. The clinical use of GLP-1 however, has been limited by short half life due to rapid degradation by dipeptidyl peptidase-IV (DPP-IV). The purpose of this study is to evaluate the potency and stability of newly designed GLP-1 analogs base on the amino acid sequence of Xenopus GLP-1, exendin-4, and human GIP (Glucosedependent insulinotropic polypeptide).

Materials and Methods: The GLP-1 receptor is a member of G-protein-coupled receptor (GPCR) that induces the protein kinase A signaling pathway. The GLP-1 receptor cDNA was co-transfected into HEK293 with cAMP-dependent protein kinase A (PKA)-specific (CRE-luciferase) reporter. Various concentrations of chimeric GLP-1 agonists were treated to the cells for 6 hours and luciferase activities were determined. The stability of agonist was evaluated by determining the activity of the agonist that was preincubated in the presence of fetal bovine serum (FBS) which naturally contains DPP-IV activities.

Results: In this study, we observed GLP-1 was able to induce cAMP responsive element (CRE)-driven transcriptional activities. A chimeric GLP-1 analog with the sequence of Xenopus GLP-1 and exendin-4 (termed xGLP-E4) was as effective as exendin-4 (EC50 values of $0.17 \mathrm{nM}, 0.11 \mathrm{nM}$, and $0.44 \mathrm{nM}$ for xGLP-E4, Exendin-4, GLP-1, respectively). When incubated with FBS for 72 hours, the potency of GLP1 was rapidly decreased to $15 \%$ of initial activity, but the activity of xGLP-E4 was maintained up to $85 \%$ of initial activity. The chimeric peptides with GLP-1 and GIP activated both the GLP-1 receptor and GIP receptors.

Conclusion: The addition of $\mathrm{C}$-terminal ten amino acid sequences from exendin 4 to Xenopus GLP-1 may improve the potency to activate the GLP-1 receptor. Substitution of serine for alanine at the second position of the GLP-1 may improve the stability of the peptide against the action of DPP-IV. Combination of GIP and GLP-1 sequence allows activating both GLP-1 and GIP receptors.
0675

Pharmacological intervention against diabetic dyslipidaemia confers additive antidiabetic efficacy of insulinotropic GLP-1 analogue, liraglutide

P. J. Larsen ${ }^{1}$, K. Lykkegaard ${ }^{1}$, E. M. Wulff ${ }^{2}$, L. Bjerre Knudsen ${ }^{3}$

${ }^{1}$ Rheoscience, Rødovre, ${ }^{2}$ NovoNordisk A/S, Måløv, ${ }^{3}$ NovoNordisk A/S, Bagsværd, Denmark.

Background and Aims: Type 2 diabetes is frequently associated with secondary dyslipidaemia and hence with marked increased risk of cardiovascular disease.

Materials and Methods: We have used severely insulin resistant male Zucker Diabetic Fatty (ZDF) rats as a model for progressed human type 2 diabetes to assess therapeutic intervention with 42 days administration of the GlucagonLike Peptide-1 (GLP-1) analogue liraglutide (L) and HMGCoA inhibitor atorvastatin (A).

Results: Treatment with $\mathrm{L}$ either alone or in combination with A significantly reduced cumulated food intake with resulting loss of body weight, whilst A alone had no impact on feeding and body weight. Compared to vehicle, combination of $\mathrm{L}+\mathrm{A}$ synergistically reduced fasting plasma glucose $(21.4 \pm 2.1$ vs. $29.5, \pm 0.9 \mathrm{mmol} / \mathrm{l})$. Long term glycaemic control as reflected by $\mathrm{HbA1C}$ was only marginally affected by $\mathrm{L}$ alone, whereas $\mathrm{L}+\mathrm{A}$ significantly reduced $\mathrm{HbA} 1 \mathrm{C}$ by $1.5 \%$ after 28 days of treatment (vehicle: $9.7 \pm 0.3 \%$; liraglutide: $8.8 \pm 0.6 \%$; atrovastatin: $9.2 \pm 0.2 \%$; liraglutide+atorvastatin: $8.2 \pm 0.6^{*}$ ). At a terminal oral glucose tolerance test, treatment with $\mathrm{A}+\mathrm{L}$ combination as well as treatment with liraglutide alone significantly improved glycaemic control. At day 34, 24-hour plasma glucose and insulin profiles were obtained by sampling every 4 hours. Both $\mathrm{L}+\mathrm{A}$ and $\mathrm{L}$ alone significantly reduced 24-hour plasma glucose profile, whereas plasma insulin levels were significantly increased only with $\mathrm{L}+\mathrm{A}$ treatment. Compared to vehicle, all treatment paradigms significantly lowered plasma levels of triglycerides and free fatty acids (vehicle: $0.81 \pm 0.05$; liraglutide: $0.58 \pm 0.05^{* *}$; atorvastatin: $0.65 \pm 0.04 *$ liraglutide+atorvatstatin: $0.57 \pm 0.07 * *$ $\mathrm{mmol} / \mathrm{l})$. Plasma cholesterol was significantly lowered only by $\mathrm{L}+\mathrm{A}$ treatment (vehicle: $4.5 \pm 0.3 \mathrm{mmol} / \mathrm{l}$; liraglutide: $4.1 \pm 0.2 \mathrm{mmol} / \mathrm{l}$; atorvastatin: $4.8 \pm 0.1 \mathrm{mmol} / \mathrm{l}$; liraglutide+ atorvastatin: $3.9 \pm 0.2 * \mathrm{mmol} / \mathrm{l})$. It is concluded that therapeutic intervention against diabetes related secondary dyslipidaemia improves glycaemic control above and beyond therapeutic benefits of the insulinotropic actions of liraglutide.

Conclusion: As atorvastatin alone has no impact on glycaemic control, it can be speculated that be speculated that its therapeutic benefits are mediated indirectly via lowering lipotoxicity in insulin sensitive tissues. 


\section{PS 49}

\section{Inflammation and insulin resistance}

0676

\section{Signaling specificity of IL6 action on glucose and lipid metabolism in skeletal muscle}

L. Al-Khalili ${ }^{1}$, K. Bouzakri ${ }^{1}$, S. Glund ${ }^{1}$, F. Lönnqvist ${ }^{2}$, H. A. Koistinen ${ }^{3}$, A. Krook ${ }^{1}$;

${ }^{1}$ Molecular Medicine and Surgery, Karolinska Institutet, ${ }^{2}$ Cell Biology, Biovitrum, Stockholm, Sweden, ${ }^{3}$ Department of Medicine, University Central Hospital, Helsinki, Finland.

Background and Aims: Physical exercise and muscle contraction leads to increased production and release of IL6 from skeletal muscle, and this has been suggested to enhance substrate metabolism and whole body glucose homeostasis. Our aims is to determine the effect of IL6 on glucose and fat metabolism.We hypothesize that PI3-kinase and AMPK pathways may play a role in IL6 action on muscle cell.

Materials and Methods: Primary human skeletal muscle cells were exposed to IL6 ( $25 \mathrm{ng} / \mathrm{ml}$ for $20 \mathrm{~min}$ to 8 days) and AMPKal/2siRNA gene-silencing was applied to measure glucose and fat metabolism.

Results: Chronic IL6 exposure increased myotube fusion and formation and expression of GLUT4, peroxisome proliferator activated receptor (PPAR) $\alpha$, PPAR, PPAR $\gamma$, PPAR coactivator 1 , glycogen synthase, myocyte enhancer factor $2 D$, uncoupling protein 2 , fatty acid transporter 4 and IL6 $(\mathrm{p}<0.05)$, whereas GLUT1, CEBPa and UCP3 was decreased. IL6 increased glucose incorporation to glycogen and fatty acid uptake and oxidation, concomitant with increased phosphorylation of AMP-activated protein kinase (AMPK), STAT3 and ERK1/2. IL6 also increased PI3 kinase activity $(200 \%$; $p<0.05)$ which was blunted by subsequent insulinstimulation $(50 \% ; \mathrm{p}<0.05)$. IL6-mediated glucose metabolism was suppressed, but lipid metabolism was unaltered, by inhibition of PI3 kinase with LY294002. siRNA-directed depletion of AMPK reduced IL6-mediated fatty acid oxidation and palmitate uptake, but not glycogen synthesis.

Conclusion: IL6 increases glycogen synthesis via a PI3kinase dependent mechanism and enhances lipid oxidation via an AMPK-dependent mechanism in skeletal muscle. Thus, IL6 directly promotes skeletal muscle differentiation and regulates muscle substrate utilization, promoting glycogen storage and lipid oxidation.

Supported by the Swedish Research Council, Swedish Medical Association, the Novo-Nordisk Foundation, the Swedish Diabetes Association, EXGENESIS LSHM-CT2004-005272 and Network of Excellence EUGENE2 no. LSHM-CT-2004-512013
0677

Inflammation is the primary signature of gene expression profile in leukocytes of insulin-resistant subjects D. Ardigò ${ }^{1,2}$, B. Byers ${ }^{2}$, S. Valtueña ${ }^{3}$, L. Franzini ${ }^{3}$, T. Quetermous ${ }^{2}$, I. Zavaroni ${ }^{3}$;

${ }^{1}$ Dept. Internal Medicine and Biomedical Sciences, Parma University, Italy, ${ }^{2}$ Cardiovascular Medicine, Stanford University, Stanford, United States, ${ }^{3}$ Internal Medicine and Biomedical Sciences, University of Parma, Italy.

Aims and Methods: Insulin resistance is a risk factor for diabetes, cardiovascular diseases (CVD) and metabolic syndrome, and is associated with systemic inflammation. To characterize the cellular phenotype of the inflammatory response related to insulin resistance, we performed a comprehensive evaluation of gene expression in circulating leukocytes of 53 subjects [ 28 insulin sensitive (IS) and 25 resistant (IR)] using a customized 22,000 features Agilent oligo-microarray. The study population was selected from a larger cohort in the first (IS) and third (IR) tertiles of 2hours insulinemic response during OGTT. Enrolled subjects were free of diabetes, CVD and chronic inflammatory diseases, and the two groups were matched for age (mean \pm SD of IR vs IS: $60 \pm 5$ vs $60 \pm 6$ years), gender and whole body adiposity, assessed by BMI $(26.7 \pm 2.8$ vs $\left.27.1 \pm 2.9 \mathrm{~kg} / \mathrm{m}^{2}\right)$, waist $(94.1 \pm 8.7$ vs $95.2 \pm 9.6 \mathrm{~cm})$, and total-body DXA (fat mass $30.3 \pm 7.0$ vs $28.3 \pm 8.1 \%$ ).

Results: Besides matching criteria, the 2 selected groups resulted also similar for blood pressure, total and LDL cholesterol, triglycerides, uric acids, and markers of subclinic systemic inflammation (white blood cells count, highsensitivity C-Reactive Protein). Despite an equal clinical and laboratory phenotype, a total of 632 genes were significantly $(>1.5$ fold; fdr $2.5 \%$ ) over-expressed in IR compared to IS, including genes of intracellular response to cytokines (IL1, $2,4,6,18$, and 30, chemokines, TNF-alpha and gammaIFN), antigen processing and MHC activity, NFkB pathway activation, insulin metabolism (IRS1, MAPK family), and cell survival. A systematic classification of genes overexpressed in IR was performed on the basis of functional similarity using gene ontology (GO) annotation. EASE analysis on GO groups revealed significant ( $p$ for EASE score) activation of genes related to innate, as well as cellmediated, immune response ["immune response" $(<0.000)$, "defense response" $(<0.000)$, "antigen processing" $(<0.000)$, "response to external stimulus" (0.001), "antigen presentation" (0.001), "T-cell activation" (0.002), "lymphocyte activation" (0.003), "MHC class II receptor activity" (0.003), "immune cell activation" (0.003)] and insulin metabolism ["protein-tyrosine kinase activity" $(<0.000)$, "protein serine/ threonine kinase activity" $(0.001)]$. 
Conclusion: In apparently healthy subjects, the presence of insulin resistance is associated to significantly different gene expression profile in circulating leukocytes, being proinflammatory response and MAPK-pathway activation its primary expression signature. Inflammation associated to insulin resistance seems to involve both innate and cellularmediated immunitary networks.

\section{8}

Regulation of skeletal muscle chemokine receptor expression and its role in skeletal muscle insulin resistance

H. Sell, U. Kaiser, J. Eckel; Institute for Clinical Biochemistry and Pathobiochemistry, German Diabetes-Center, Duesseldorf, Germany.

Background and Aims: Adipocyte-conditioned medium (CM) contains a variety of chemokines that induce insulin resistance in skeletal muscle cells. Chemokines such as MCP-1 are also able to induce insulin resistance on their own. It has already been demonstrated that adipocytes express a wide range of chemokine receptors including CCR2, CCR4 and CCR10. However, the expression of chemokine receptors in skeletal muscle where chemokines might play a role in the development of diabetes was not yet established. In addition, this study aimed to analyse the regulation of these receptors in the insulin resistant state in human skeletal muscle cells.

Materials and Methods: Expression of chemokine receptors in human skeletal muscle cells and adipocytes was analysed by RT-PCR and Western Blot. Differentiated skeletal muscle cells were incubated for $24 \mathrm{~h}, 48 \mathrm{~h}$ und $72 \mathrm{~h}$ with high glucose/high insulin (GI) or TNF $\alpha$ in order to induce insulin resistance. In addition to these treatment regimens, skeletal muscle cells were co-stimulated with TNF $\alpha$ and MCP-1 or CM for 24 and $48 \mathrm{~h}$ to analyse possible synergistic effects of different situations of skeletal muscle insulin resistance.

Results: Human skeletal muscle cells and adipocytes expressed the CC chemokine receptors CCR1/2/4/5/10 and the $\mathrm{CXC}$ chemokine receptors CXCR1/2. While the expression at the protein level of CCR4 and CCR10 was similar in muscle cells and adipocytes the expression of CCR2 was significantly higher in skeletal muscle cells. Treatment of skeletal muscle cells with GI induced insulin resistance on the level of Akt and GSK $3 \alpha / \beta$ as soon as after $24 \mathrm{~h}$. TNF $\alpha$ reduced insulin-stimulated Akt phosphorylation in myotubes after $48 \mathrm{~h}$ and $72 \mathrm{~h}$ without having any effect on GSK3 phosphorylation. Treatment with $\mathrm{CM}$ and MCP-1 also lead to skeletal muscle insulin resistance, that could however not be increased by co-stimulation with
TNF $\alpha$. CCR2 was downregulated by TNF $\alpha(24 \mathrm{~h})$ and MCP-1 (48 h) and upregulated by GI (24-72 h) and TNF $\alpha$ (72 h). CCR4 was decreased after treatment with MCP-1 (48 h) and TNFa (72 h). MCP-1 (24-48 h) and TNFa (48 h) also downregulated the expression of CCR10.

Conclusion: Human skeletal muscle cells and adipocytes express different chemokine receptors that may play a role in the negative crosstalk between adipose tissue and skeletal muscle and may be involved in the development of skeletal muscle insulin resistance by mediating adipocytokine-induced insulin resistance. CCR2 is highly expressed in skeletal muscle cells compared to adipocytes possibly explaining the prominent sensitivity of skeletal muscle cells towards MCP-1. Upregulation of CCR2 by high glucose/high insulin and $\mathrm{TNF} \alpha$ may sensitize human skeletal muscle cells towards adipocytokine-induced insulin resistance.

0679

Elevated serum monocyte chemoattractant protein-4 (MCP-4) levels in overweight subjects and its correlation with chronic inflammation

I. Hashimoto ${ }^{1}$, J. Wada ${ }^{1}$, A. Hida ${ }^{1}$, M. Baba ${ }^{1}$, N. Miyatake ${ }^{2}$, J. Eguchi ${ }^{1}$, K. Shikata ${ }^{1}$, H. Makino ${ }^{1}$;

${ }^{1}$ Department of Medicine and Clinical Science, Okayama University Graduate School of Medicine, Okayama, ${ }^{2}$ Internal Medicine, Okayama Southern Institute of Health, Okayama, Japan.

Background and Aims: Chronic inflammation observed in obesity has been reported to be implicated in the development of atherosclerosis; however its mechanism has not been fully elucidated. We screened candidate chemokines which link chronic inflammation and obesity.

Materials and Methods: Japanese overweight $(n=39$, BMI $\left.28.7 \pm 0.65 \mathrm{~kg} / \mathrm{m}^{2}\right)$ and normal weight subjects $(\mathrm{n}=24$, BMI $22.3 \pm 0.45 \mathrm{~kg} / \mathrm{m}^{2}$ ) were enrolled into cross-sectional study. Using antibody-based protein microarray, spot intensities of monocyte chemoattractant protein-4 (MCP-4), eotaxin and eotaxin-2 correlated with anthropometric parameters. We further measured serum concentration of these chemokines and mRNA levels in adipose tissues obtained from volunteers.

Results: Serum MCP-4 levels showed positive correlation with BMI $(r=0.318, p=0.014)$, waist $(r=0.316, p=0.018)$ and waist hip ratio $(\mathrm{r}=0.264, \mathrm{p}=0.049)$. Furthermore, MCP-4 correlated with HOMA-IR $(\mathrm{r}=0.392, \mathrm{p}=0.002)$ and highsensitivity C-reactive protein (hsCRP) $(\mathrm{r}=0.350, \mathrm{p}=0.006)$, and HDL cholesterol $(r=-0.409, p=0.001)$. In stepwise multiple regression analyses, hsCRP independently correlated with MCP-4 levels. Using real time RT-PCR, the 
expression of MCP-4 mRNA in visceral adipose tissue positively correlates with BMI. Serum eotaxin levels correlate with $\mathrm{BMI}(\mathrm{r}=0.262, \mathrm{p}=0.045)$ and waist hip ratio $(r=0.383, p=0.003)$. Serum eotaxin-2 levels correlated with BMI $(\mathrm{r}=0.464, \mathrm{p}<0.001)$, waist $(\mathrm{r}=0.333, \mathrm{p}=0.017)$ and waist hip ratio $(\mathrm{r}=0.278, \mathrm{p}=0.048)$. However eotaxin and eotaxin-2 levels did not show significant correlation with hsCRP.

Conclusion: Serum levels of MCP-4, eotaxin and eotaxin2, which belong to $\mathrm{CC}$ chemokine family and share $\mathrm{CC}$ chemokine receptor 3, correlated with BMI. MCP-4 further correlated with degree of chronic inflammation. These chemokines, especially MCP-4, may be critical molecules which link obesity and chronic inflammation.

\section{0}

Tumour necrosis factor (TNF)alfa and interleukin (IL)6 impair differentiation of preadipocytes and promote an inflammatory phenotype

B. Gustafson, U. Smith;

The Lundberg Laboratory for Diabetes Research, Department of Internal Medicine, Göteborg University, Sweden.

Background and Aims: Obesity is a central and causal part of insulin resistance and type 2 diabetes. Enlargement of adipocytes induces inflammation and macrophage recruitment into the adipose tissue with production of a variety of inflammatory cytokines such as interleukin (IL)-6, TNFalfa, plasminogen activator inhibitor (PAI)-1 and monocyte chemoattractant protein (MCP)-1. These agents can exert local effects in the adipose tissue or systemic effects in other tissues such as liver and muscle. Both IL-6 and TNFalfa have been shown to impair adipocyte differentiation of 3T3-L1 preadipocytes. In this study, we examined particularly how TNFalfa prevents preadipocyte differentiation.

Materials and Methods: 3T3-L1 preadipocytes were incubated with or without TNFalfa $(1.5 \mathrm{ng} / \mathrm{mL})$ or IL-6 (20 $\mathrm{ng} / \mathrm{mL}$ ) from the induction of differentiation and for 8 days. RNA was extracted and analysed with real-time PCR and immunoblots were performed on cell lysates.

Results: Both IL-6, and in particular, TNFalfa prevented preadipocyte differentiation and promoted an inflammatory phenotype. IL-6 did not prevent the initiation of differentiation but prevented the cells from entering the terminal differentiation through maintaining the Wnt signaling with low levels of axin and increased levels of beta-catenin. TNFalfa did not prevent the induction of either $\mathrm{C} / \mathrm{EBPbeta}$ or $\mathrm{C} / \mathrm{EBPdelta}$ nor the activation of the cAMP response element binding protein (CREB). During induction of differentiation, the inhibitory $\mathrm{C} / \mathrm{EBP}$ homologue protein (CHOP)-10 is temporarily induced but this protein was not affected by TNFalfa and was rapidly down-regulated. The cyclin D kinase (CDK) inhibitor, p27, was also degradaded in the presence of TNFalfa and this is necessary for the cells to normally enter the S-phase during mitotic clonal expansion. However, TNFalfa maintained the Retinoblastoma protein $(\mathrm{pRb})$ during differentiation and totally prevented the induction of PPARgamma and C/EBPalfa. In addition, TNFalfa induced the expression of GATA2, which is a critical transcription factor that can suppress adipogenesis and promote an inflammatory phenotype of the cells.

Conclusion: These data show that the presence of TNFalfa does not alter the early induction of differentiation with a decrease in CHOP-10 and p27 expression, induction of $\mathrm{C} /$ EBPbeta and C/EBPdelta and activation of CREB. However, TNFalfa totally prevented induction of PPARgamma and C/ EBPalfa and, thereby, differentiation towards an adipocyte phenotype and, instead, induced an inflammatory phenotype. The induction of GATA2 by TNFalfa is likely to be an important mediator of the altered phenotype of the cells.

0681

CD166 (ALCAM) modulates inflammatory responses upon RAGE-ligand activation in vivo

D. Petrov ${ }^{1}$, I. K. Lukic ${ }^{1}$, J. C. Igwe ${ }^{1}$, M. Fabbi ${ }^{2}$, S. Ferrini ${ }^{2}$, P. P. Nawroth ${ }^{1}$, A. Bierhaus ${ }^{1}$;

${ }^{1}$ Department of Medicine I, University of Heidelberg, Germany, ${ }^{2}$ Terapia Immunologica, Istituto Nationale per la Ricerca sul Cancro, Genova, Italy.

Background and Aims: RAGE is a multiligand receptor known to play a role in chronic inflammatory diseases. Deletion of RAGE provided protection from the lethal effects of septic shock caused by cecal ligation and puncture (CLP), while it did not protect from inflammatory reactions in delayed type hypersensitivity (DTH). DTH, however, could be blocked by sRAGE in both, WT and RAGE-/--mice, implying that other receptors binding RAGE-ligands might compensate for RAGE-deficiency. ALCAM (CD166) shares significant structural homology to RAGE. This implies that ALCAM is a candidate receptor able to bind one or more of RAGE ligands.

We studied whether ALCAM is a receptor able to bind RAGE ligands and compensate for RAGE deficiency in settings of adaptive immune response. Therefore, we identify the effects of SALCAM and ALCAM neutralizing antibodies in the delayed type hypersensitivity (DTH) model in wild type (WT) and RAGE -/-- mice.

Materials and Methods: 8 weeks old male WT and RAGE-/-- mice were sensitized by injection of $100 \mu \mathrm{l}$ $25 \mathrm{mg} / \mathrm{ml}$ methylated BSA (mBSA), $50 \mathrm{mg} / \mathrm{ml}$ Dextran, $50 \%$ Freunds incomplete adjuvant, $0.9 \% \mathrm{NaCl}$ over the left 
inguinal lymph node. 21 days later DTH reaction was induced (footpad injection $50 \mu l \quad 0.25 \mathrm{mg} / \mathrm{ml} \mathrm{mBSA}$ ). sRAGE, sALCAM and ALCAM antibody were applied i.p. (100 $\mu \mathrm{g} /$ mouse) $6 \mathrm{~h}$ before and 6 and $12 \mathrm{~h}$ after mBSA injection. $24 \mathrm{~h}$ after footpad injection mice were sacrificed and the footpads examined morphologically for signs of swelling and inflammation.

Results: In vitro studies in $\mathrm{CHO}$ cells overexpressing ALCAM confirmed that treatment with the RAGE-ligand $\mathrm{S} 100 \mathrm{~B}$ led to activation of transcription factor NF- $\mathrm{KB}$. That effect was reversed by administration of both, sRAGE and sALCAM. In addition, RAGE-ligand induced NF- $\mathrm{B}$ binding activity in S100B stimulated RAGE -/- - fibroblasts was inhibited by sRAGE, sALCAM and ALCAM neutralizing antibodies (ab) While administration of sRAGE led to significant decrease of inflammation in the footpads of both WT and RAGE -/- - animals, sALCAM and ALCAM neutralizing antibodies reduced inflammation only in RAGE -/--, but not in WT mice. Furthermore, ALCAM $\mathrm{ab}$ and SALCAM treatment led to the reduction of expression of TNF $\alpha$, IL-1 $\beta$, IL-6 and IFN $\gamma$ mRNA in the footpad homogenates of RAGE -/-- animals.

Conclusion: ALCAM is a RAGE homologue receptor that modulates inflammatory responses upon RAGE-ligand activation in vivo.

Supported by DFG SFB 405

\section{2}

Relationships between serum adiponectin, IL-10 and IL18 concentrations and muscle lipid fractions in healthy humans

M. Straczkowski ${ }^{1}$, I. Kowalska ${ }^{1}$, A. Nikolajuk ${ }^{1}$, M. Baranowski ${ }^{2}$, A. Adamska ${ }^{1}$, M. Karolczuk-Zarachowicz ${ }^{1}$, A. Blachnio ${ }^{2}$, J. Gorski ${ }^{2}$, M. Gorska ${ }^{1}$;

${ }^{1}$ Department of Endocrinology, Diabetology and Internal Medicine, ${ }^{2}$ Department of Physiology, Medical University of Bialystok, Poland.

Background and Aims: Intramuscular lipids, including ceramide, might be responsible for the development of insulin resistance. Numerous studies also indicate, that insulin action might be inversely associated with circulating proinflammatory cytokines, like IL-18 and positively related to antiinflammatory factors, like adiponectin and IL-10. Interrelationships between the above circulating factors and intramuscular lipids remain unclear. The aim of the present study was to estimate the relationships between serum adiponectin, IL-10 and IL-18 concentrations and muscle lipid fractions in healthy humans.
Materials and Methods: The study group consisted of 37 male subjects with normal glucose tolerance, without morbid obesity or other serious medical problems. Euglycemic hyperinsulinemic clamp and a biopsy of vastus lateralis muscle were performed. To avoid contamination of extracellular fat, muscles were lyophilized. After lipid extraction, ceramides were hydrolyzed and then were estimated as the sphingosine content with HPLC system. Muscle free sphingosine and sphinganine conent and the activities of the enzymes: neutral and acid sphingomyelinase, neutral and alkaline ceramidase and serine palmitoyltransferase was also measured. Muscle free fatty acid (FFA), diacylglycerol (DAG) and triacylglycerol (TG) were separated with thin-layer chromatography. The content of particular fatty acids in these fractions was determined by gas-liquid chromatography.

Results: Insulin sensitivity was related to circulating cytokines (adiponectin, $\mathrm{r}=0.38, \mathrm{p}=0.021$; IL-10, $\mathrm{r}=0.47$, $\mathrm{p}=0.0034$; IL-18, $\mathrm{r}=-0.37, \mathrm{p}=0.023)$ and to muscle lipids (ceramide, $\mathrm{r}=-0.45, \mathrm{p}=0.024 ; \mathrm{DAG}, \mathrm{r}=-0.43, \mathrm{p}=0.031$; TG, $r=-0.52 ; p=0.01)$. It was also associated with the activities of the enzymes regulating ceramide metabolism (serine palmitoyltransferase, $\mathrm{r}=-0.58, \mathrm{p}=0.002$; alkaline ceramidase, $\mathrm{r}=-0.37, \mathrm{p}=0.025)$. The relationship between insulin action and neutral ceramidase activity approached the level of significance $(\mathrm{r}=-0.32, \mathrm{p}=0.056)$. Adiponectin was negatively related to muscle ceramide content $(\mathrm{r}=-0.44, \mathrm{p}=0.027)$ and to serine palmitoyltransferase activity $(\mathrm{r}=-0.35, \mathrm{p}=0.032)$. IL-10 and IL-18 were associated, in an opposite manner, with muscle DAG (IL-10, $\mathrm{r}=-0.46, \mathrm{p}=0.022$; IL-18, $\mathrm{r}=0.40, \mathrm{p}=0.049$ ) and muscle TG (IL-10, $\mathrm{r}=-0.50, \mathrm{p}=0.014$; IL-18, $\mathrm{r}=0.46, \mathrm{p}=0.026$ ). IL-10 was also related to muscle FFA pool $(\mathrm{r}=-0.51, \mathrm{p}=0.026)$.

Conclusion: There are multiple associations between circulating cytokines and muscle lipid pool, which possibly might influence insulin sensitivity. Adiponectin is related to muscle ceramide content, mostly through an association with de novo synthesis pathway (as indicated by serine palmitoyltransferase activity). In contrast, IL-10 and IL-18 are associated with muscle FFA-DAG-TG pathway.

\section{3}

Iron overload in type 2 diabetes and its relationship to chronic inflammatory process

F. Pervin ${ }^{1}$, F. Nasrin ${ }^{2}$, F. Jebunnesa ${ }^{1}$, L. Ali ${ }^{1}$;

${ }^{1}$ Dept of Biochemistry \& Cell Biology, BIRDEM, Dhaka,

${ }^{2}$ Dept Biochemistry and Molecular Biology, University of Dhaka, Bangladesh. 
Background and Aims: Iron overload has been implicated in the etiopathogenesis of diabetes mellitus, but limitations of the existing markers and involvement of confounding variables have made it difficult to ascertain its precise role in diabetes. Chronic subclinical inflammation is claimed to be present in both diabetes and iron overload is. The objective of the present study was to explore the body iron status and chronic inflammation in type 2 diabetic subjects and also to investigate the interrelationship between these two variables in the context of the hyperglycemic state.

Materials and Methods: Eighty-nine diabetic subjects as case and 54 healthy individuals as control, matched by BMI [mean \pm SD (kg/m2: $25 \pm 4.62$ in diabetic vs $24.43 \pm 4.01$ in control, $\mathrm{p}<0.451,95 \% \mathrm{CI}-2.07-0.93)]$ were included in the study. Serum ferritin and serum sTfR were used as markers of body iron status and those were measured by MEIA and ELISA methods respectively. Serum C-reactive protein (CRP) was used as a marker of inflammation and it was estimated by FPIA technology. Serum fasting glucose and lipid profile were measured by enzymatic-colorimetric method.

Results: The diabetic subjects had higher systolic blood pressure [mean \pm SD (mmHg: $127 \pm 12.57$ in diabetic vs120 \pm 4.86 in control; $\mathrm{p}<0.001,95 \%$ CI $-9.06-3.17)]$, fasting glucose [median (range): \{mmol/1: 8.72 (4.12-26.96) in diabetic vs $4.98(3.71-6)$ in control; $\mathrm{p}<0.001\}$ ] and serum triglyceride levels [median (range): $\{\mathrm{mg} / \mathrm{dl}: 164.5$ (59.98$801)$ in diabetic vs $121(50-276)$ in control; $\mathrm{p}<0.001\}$ ] when compared to control. The diabetic subjects had higher serum ferritin levels than the control subjects [median (range): \{ng/ml: $103(28.7-605.3)$ in diabetic vs 73.47 (12.18-189) in control; $\mathrm{P}<0.019\}$ ]. However, no difference in sTfR concentrations were observed between diabetic and control subjects [median (range): \{nmol/1: 21.6 (10.3-44.3) in diabetic vs. 19.76 (13.58-38.89) in control, $\mathrm{P}<0.151\}]$. Regarding CRP level, the diabetic subjects showed considerably higher value when compared to control subjects [median (range): $\{\mathrm{mg} / \mathrm{dl}: 1.35(0-3.68)$ in diabetic vs $0(0$ $1.48)$ in control, $\mathrm{p}<0.001\}]$. In control subjects log-sTfR levels showed significant negative correlation with logferritin $(\mathrm{r}=-0.42, \mathrm{p}<0.001)$. In contrast diabetic subjects $(\mathrm{r}=0.033, \mathrm{p}<0.755)$ did not show any significant association. Significant positive correlation was observed between log-ferritin and CRP in diabetic subjects $(r=0.254$; $\mathrm{p}<0.016$ ), but in the control subjects no association was found $(\mathrm{r}=0.04, \mathrm{p}<0.78)$. In diabetic subjects fasting glucose level was not significantly associated with log-ferritin $(p<0.59)$ and CRP levels $(p<0.22)$. In multiple regression analysis, an independent inverse relationship between logferritin and log-sTfR was observed in controls $(\mathrm{p}<0.001$, 95\% CI -2.26-0.74). However, there was no such relationship between log-ferritin and log-sTfR in diabetic subjects. There was a positive association of log-ferritin with CRP in the diabetic subjects $(\mathrm{p}<0.053)$.
Conclusion: The present study demonstrates that (a) body iron store is elevated in type 2 diabetes mellitus; (b) diabetes is associated with a subclinical chronic inflammatory process; (c) hyperglycemia does not have a straightforward relationship with iron overload or with inflammation; and (d) chronic inflammation is associated with excess body iron store in diabetes.

Supported by IPICS and Diabetic Association of Bangladesh (DAB)

\section{4}

Plasma CD36 is related to obesity and low grade inflammation

A. Handberg ${ }^{1}$, A. López-Bermejo ${ }^{2}$, C. Gubern ${ }^{2}$, J. Vendrell $^{3}$, W. Ricart ${ }^{2}$, J. M. Fernández-Real ${ }^{2}$;

${ }^{1}$ Dept. of Clinical Biochemistry, Aarhus University Hospital, Denmark, ${ }^{2}$ Section of Diabetes, Endocrinology and Nutrition, University Hospital of Girona "Dr. Josep Trueta", Spain, ${ }^{3}$ Endocrinology and Research Unit., Hospital Universitari Joan XXIII from Tarragona, Rovira i Virgili University, Spain.

Background and Aims: Recently we have demonstrated the presence of soluble CD36 (sCD36) in cell free plasma, and in a small population of type 2 diabetic patients we found markedly increased levels of sCD36. Furthermore, sCD36 was tightly correlated with insulin resistance and BMI. We aimed to evaluate if this relationship was also present in a larger population of glucose intolerant males, and furthermore to study the relationship between markers of low grade inflammation and sCD36.

Materials and Methods: 147 apparently healthy men, 90 normotolerant and 57 glucose-intolerant subjects were included in the study. Insulin sensitivity was assessed by the minimal model, and sCD36 was measured by ELISA.

Results: When all subjects were considered as a whole, circulating sCD36 concentration was significantly associated with fat-free mass, glycosylated hemoglobin, plasma triglycerides, platelet number, plasma interleukin 6 (IL-6) and with glucose effectiveness ( $\mathrm{p}$ between 0.04 and 0.001). However, the association with fat-free mass, triglycerides, platelet number and IL-6 was mainly observed in subjects with glucose intolerance but not in normotolerant subjects. In subjects with glucose intolerance, sCD36 was positively associated with fasting glucose and negatively with insulin sensitivity.

In a multiple linear regression analysis to predict circulating sCD36, among subjects with glucose intolerance, IL-6 $(p=0.0035)$ and fat mass $(p=0.025)$ were independently associated with plasma sCD36 concentration, contributing to $12 \%$ and $9 \%$, respectively, of $\mathrm{sCD} 36$ variance after 
adjusting for age, fasting glucose, plasma triglycerides, platelet count and insulin sensitivity.

Conclusion: We propose that levels of sCD36 in men reflect obesity related low grade inflammation and insulin resistance. Supported by the Novo Nordisk Foundation

\section{5}

Increased circulatory metalloproteinase-2 and metalloproteinase-9 levels and activities in patients with type 1 diabetes mellitus

M.-Y. Shiau ${ }^{1}$, Y.-T. Hsu ${ }^{1}$, Y.-C. Huang ${ }^{2}$, Y.-H. Chang ${ }^{3}$; ${ }^{1}$ College of Medicines and Nursings, Hang Kuang University, Sha Lu, Taichung, ${ }^{2}$ Institute of Biochemistry and Biotechnology, ${ }^{3}$ School of Medical Laboratory and Biotechnology, Chung Shan Medical University, Taichung, Taiwan.

Background and Aims: Type 1 diabetes mellitus (T1DM) is an autoimmune disorder with unknown etiology. The incidence of T1DM in Taiwanese is among the lowest range of diabetic incidence worldwide. Metalloproteinases (MMPs) are suggested to play important roles in the development and complications of autoimmune disorders. The present study is to examine if the MMPs expression or activities are altered in Taiwanese T1DM patients.

Materials and Methods: Blood samples were collected from 201 T1DM and 189 non-diabetic control subjects in Division of Endocrinology \& Metabolism, Veterans General Hospital-Taipei. Levels and activities of plasma MMP-2 and MMP-9 in T1DM patients were investigated and compared to those of control subjects by enzymelinked immunosorbant assay and zymorgraphy, respectively. The demographic characteristics and clinical manifestations of each patient were filed for statistical analysis. Written consent was obtained from all the study subjects after the nature of the procedure was explained. The study has been approved by the Ethics Committee of Chung Shan University Hospital.

Results: The zymagraphy data showed that both MMP-2 and MMP-9 activities in sera from patients with T1DM were significantly higher than that from control subjects. When the MMP-2 and MMP-9 expression levels were quantitatively measured by ELISA, MMP-2 level in control subjects and T1DM patients was $146.1 \pm 34.2$ and 241.5 \pm $73.7 \mathrm{ng} / \mathrm{mL}$, respectively. The MMP-9 level in control subjects and T1DM patients was $51.4 \pm 57.1$ and $305.3 \pm 180.6 n \mathrm{~g} / \mathrm{mL}$, respectively. Both MMP-2 and MMP-9 levels were significantly higher in sera of T1DM patients, compared to that from control subjects. No significant correlation between MMPs levels or activities with patients' demographic or clinical data was found.

Conclusions: MMPs expression and activities are significantly increased in patients with T1DM. Our results not only document plasma MMPs levels and activities in Taiwanese population with possibly the lowest type 1 diabetic incidence, but also suggest that MMPs expression and activity are elevated before the onset of complications in diabetic patients.

\section{PS 50}

\section{Metabolic effects of adipocytokines}

\section{6}

Pattern of expression of adiponectin receptors in human adipose tissue depots and its relation to metabolic state M. Nannipieri ${ }^{1}$, F. Cecchetti ${ }^{1}$, M. Anselmino ${ }^{2}$, E. Mancini ${ }^{1}$, A. Bonotti ${ }^{1}$, S. Baldi ${ }^{1}$, F. Santini ${ }^{3}$, M. Rossi ${ }^{2}$, E. Ferrannini ${ }^{1}$;

${ }^{1}$ Internal Medicine, University of Pisa, ${ }^{2}$ IV Unit of SurgeryBariatric Surgery Division, Santa Chiara Hospital, Pisa, ${ }^{3}$ Endocrinology, University of Pisa, Italy.

Background and Aims: The adiponectin receptors, APMR1 and APMR2, are thought to mediate the insulinsensitizing effects of adiponectin. We examined whether Adipo R1 and R2 expression in human subcutaneous (SAT) and visceral (VAT) adipose tissue correlates with adiponectin gene (APM1) expression and in vivo metabolic parameters in morbid obesity with or without diabetes.

Materials and Methods: Total RNA was extracted from SAT and VAT tissue obtained during abdominal surgery from 7 lean non-diabetic women (C) $(60 \pm 10$ years, BMI $25.1 \pm 3.1 \mathrm{~kg} / \mathrm{m} 2), 15$ obese diabetic women (DM2-Ob) $(49 \pm 9$ years, BMI $45.0 \pm 7.5 \mathrm{~kg} / \mathrm{m} 2)$ and 30 obese women with normal glucose tolerance $(\mathrm{Ob})(38.6 \pm 11$ years, BMI $47.6 \pm 8.1 \mathrm{~kg} / \mathrm{m} 2$ ). cDNA was synthesised and relative quantifications of adiponectin receptors mRNA (target gene) was performed by Real-Time PCR. Results were expressed as the target to reference (hypoxantine-phosphorybosyltransferase) ratio for each sample.

Results: In SAT, APMR1 was significantly more expressed in $\mathrm{C}$ than $\mathrm{Ob}$ subjects $(1.14 \pm 0.74$ vs $0.60 \pm 0.50$ vs $0.90 \pm 0.72$, respectively, $\mathrm{p}=0.04$ for $\mathrm{C}$ vs $\mathrm{Ob})$. Likewise, APMR2 was more expressed in $\mathrm{C}$ compared to $\mathrm{Ob}$ or $\mathrm{DM} 2-\mathrm{Ob}(11.6 \pm 8.1$ vs $5.8 \pm 6.1$ and $5.8 \pm 5.5, \mathrm{p} \leq 0.05)$. In VAT, APMR1 was more abundantly expressed in $\mathrm{C}$ than DM2-Ob ( $1.1 \pm 0.8$ vs $0.7 \pm 0.4$ and $0.6 \pm 0.3, p=0.02)$. Furthermore, APMR2 was again more expressed in $\mathrm{C}$ than DM2-Ob (1.1 \pm 1.0 vs $0.9 \pm 0.8$ and $0.6 \pm 0.5, p=0.05)$. A 
positive correlation was found between APMR1 and APMR2 expression in SAT ( $\left.\leq 0.0001, \mathrm{r}^{2}=0.25\right)$ as well as VAT $\left(p \leq 0.0001, r^{2}=0.76\right)$, but in SAT APMR2 was about ten times higher than APMR1. APMR1 expression in SAT was inversely related to BMI $\left(\mathrm{r}^{2}=0.10, \mathrm{p}=0.02\right)$ and serum HDL-cholesterol $\left(\mathrm{r}^{2}=0.10, \mathrm{p}=0.04\right)$. Both APMR1 and APMR2 expression in VAT were strongly related to fasting plasma insulin $\left(\mathrm{r}^{2}=0.16, \mathrm{p}=0.015\right.$ and $\mathrm{r}^{2}=0.14, \mathrm{p}=0.028$, respectively) and serum triglycerides $\left(\mathrm{r}^{2}=0.17, \mathrm{p}=0.007\right.$ and $\mathrm{r}^{2}=0.16, \mathrm{p}=0.009$, respectively). In both SAT and VAT, a direct correlation was found between the expression of either receptor and APM1 expression.

Conclusion: In conclusion, severe obesity is associated with a lower expression of both APMR1 and APMR2 in SAT, whereas expression of both receptors in VAT is reduced only when diabetes were present. The pattern of association with the clinical variables suggests that, in SAT APMR receptor downregulation (predominantly APMR2) is related to body fat mass, while in VAT it is linked with hyperglycaemia, hyperinsulinaemia and dyslipidaemia.

\section{7}

Adiponectin and insulin resistance modulate the gene expression of adiponectin receptors in human adipose tissue

C. Invitti ${ }^{1}$, L. Alberti ${ }^{1}$, L. Gilardini ${ }^{1}$, A. Girola ${ }^{1}$, A. Conti ${ }^{1}$, G. Redaelli ${ }^{1}$, G. Micheletto ${ }^{2}$;

${ }^{1}$ Unit of Metabolic Diseases and Diabetes, Istituto Auxologico Italiano, Milan, ${ }^{2}$ Department of Surgical Science, University of Milan, Italy.

Background and Aims: Two adiponectin (AD) receptors have been described, ADIPOR1 ubiquitously expressed and ADIPOR2 more expressed in liver and skeletal muscle. Their putative role as insulin sensitizer has been proved in rodents but it is uncertain in humans. The gene expression of ADIPORs in human adipose tissue and its relation with adiposity and insulin sensitivity has been poorly studied. We investigated 1) the mRNA expression of AD, ADIPOR1 and ADIPOR2 in subcutaneous (SAT) and visceral adipose tissue (VAT), 2) their relation with adiposity, $\mathrm{AD}$ levels in culture medium and serum and with circulating levels of insulin, fasting and post load glucose and lipids.

Materials and Methods: Abdominal SAT was obtained in 60 obese subjects $(86 \%$ women, $44.7 \pm 11.2 \mathrm{yr}$, BMI $37.6 \pm 5.1 \mathrm{~kg} / \mathrm{m}^{2}$, waist $\left.112.1 \pm 13.1 \mathrm{~cm}\right) .11 / 60$ subjects underwent bariatric surgery and VAT was also taken. ADIPO and ADIPORs mRNA was measured using RT-PCR. Insulin resistance was defined with HOMA-IR $\geq 2.5$. AD release was measured from SAT and VAT minced and placed into culture medium for $24 \mathrm{~h}$.
Results: Family history of diabetes did not influence the expression of AD and ADIPORs. The expression levels of $\mathrm{AD}$ and ADIPORs were lower in men than in women $(p=0.05)$. We therefore analysed the women only. In 52 obese women, ADIPOR2 expression was 4-fold higher than that of ADIPOR $1(2.0 \pm 1.1$ vs $0.4 \pm 0.3$ in SAT and $2.0 \pm 0.7$ vs $0.5 \pm 0.3$ in VAT, $p<0.0001$ for both). The mRNA expression levels of ADIPORs were highly correlated with each other ( $\mathrm{r} 0.781, \mathrm{p}<0.0001$ in SAT and $\mathrm{r} 0.541,0.05$ in VAT) and in SAT with that of AD ( $\mathrm{r} 0.816, \mathrm{p}<0.0001$ for R1 and $\mathrm{r} 0.890, \mathrm{p}<0.0001$ for $\mathrm{R} 2$ ). The gene expression of $\mathrm{AD}$ and ADIPOR2 was not related to AD release from SAT and VAT. The SAT expression of $A D$ and ADIPOR2 was positively correlated with serum $\mathrm{AD}(\mathrm{r} 0.274, \mathrm{p}<0.05$ and $\mathrm{r}$ $0.288, \mathrm{p}<0.05)$ and negatively with waist $(\mathrm{r}-0.552$, $\mathrm{p}<0.0001$ and $\mathrm{r}-0.558, \mathrm{p}<0.0001)$, insulin $(\mathrm{r}-0.341$, $\mathrm{p}<0.05$ and $\mathrm{r}-0.283, \mathrm{p}<0.05)$ and HOMA-IR $(\mathrm{r}-0.321$, $\mathrm{p}<0.05$ and $\mathrm{r}-0.265 \mathrm{p}<0.05$ ). In the multivariate analysis with ADIPOR2 as dependent variable, only AD remained positively and independently correlated with ADIPOR2 ( $\beta 0.895, \mathrm{p}<0.0001$ ). $48 \%$ of women had insulin resistance. These women compared to those without insulin resistance had significantly lower levels of serum AD $(4.4 \pm 1.9$ vs $6.3 \pm 2.5, \mathrm{p}<0.01)$ and lower expression levels in SAT of AD $(15.2 \pm 6.8$ vs $23.1 \pm 14.3, \mathrm{p}<0.05)$ and ADIPOR2 $(1.7 \pm 0.8$ vs $2.3 \pm 1.3, \mathrm{p}<0.05)$. Low HDL and high triglycerides were associated with lower levels of serum $\mathrm{AD}$, but not of $\mathrm{AD}$ and ADIPOR2 expression in adipose tissue.

Conclusion: 1) Human adipose tissue express more abundantly ADIPOR2 than ADIPOR 1, 2) The strict relation between $\mathrm{AD}$ and ADIPORs expression suggests autocrine/ paracrine effects for $\mathrm{AD}$ in the adipose tissue, 3) Serum AD reflects the levels of expression of $\mathrm{AD}$ in SAT, 4) $\mathrm{AD}$ and ADIPOR2 expression in SAT as well as serum AD are negatively affected by insulin resistance.

\section{8}

Adiponectin does not mediate obesity-related insulin resistance in young children: a longitudinal study A. N. Jeffery ${ }^{1}$, M. J. Murphy ${ }^{2}$, B. S. Metcalf ${ }^{1}$, J. Hosking ${ }^{1}$, D. S. Gardner ${ }^{1}$, N. Sattar ${ }^{3}$, T. J. Wilkin ${ }^{1}$; ${ }^{1}$ Department of Endocrinology and Metabolism, Peninsula Medical School, Plymouth, ${ }^{2}$ Department of Biochemical Medicine, Ninewells Hospital, Dundee, ${ }^{3}$ University of Glagow, BHF Glasgow Cardiovascular Research Centre, United Kingdom.

Background and Aims: Adiponectin, an adipokine secreted exclusively by adipose tissue, is present in abundant concentrations in the circulation. It has both insulin sensitising and anti-atherogenic properties. In adults, 
adiponectin has independent inverse associations with obesity and hyperinsulinaemia. Cross-sectional studies of predominantly obese, pubertal individuals indicate that adiponectin may also play an important role in glucose regulation in children. To date, limited data are available in healthy young children. Our aim was to characterise adiponectin levels in prepubertal children and their relationship to BMI, body fat and insulin resistance over time. Materials and Methods: Participants: healthy children (114 boys, 86 girls) from stratified randomly-selected schools enrolled in The EarlyBird Study measured at 5,6,7 and 8 years. Measures: adiponectin, insulin resistance (HOMA-IR), BMI, adiposity measured by sum of 5 skinfolds (SSF) (correlation with total body fat measured by DEXA $r=0.9, p<0.001$ in the children aged 7 and 8 years).

\section{Results:}

1. Both BMI sds and SSF rose progressively from age 5 to 8 y $(+0.1$ sd and $+30 \%, \mathrm{p}<0.001)$.

2. HOMA-IR nevertheless fell progressively $(-24 \%$, $\mathrm{p}<0.001)$.

3. Adiponectin levels correlated strongly year-on-year $(\mathrm{r}=0.71$ to $0.79, \mathrm{p}<0.001)$.

4. In boys, adiponectin fell $11.1 \%(\mathrm{p}<0.001)$ from $5 \mathrm{y}-8 \mathrm{y}$ $(13.8-12.9-12.7-12.5 \mathrm{ug} / \mathrm{ml})$, and in girls it fell $6.5 \%$ $(\mathrm{p}=0.02)(13.7-12.6-13.2-12.6 \mathrm{ug} / \mathrm{ml})$.

5. HOMA-IR was directly related to BMI at all ages $(\mathrm{r}=0.25$ to $0.47, \mathrm{p}<0.001)$ and to SSF from age 6 in girls and age 7 in boys $(\mathrm{r}=0.22$ to $0.55, \mathrm{p}<0.05)$.

6. Adiponectin did not correlate with BMI at any age, and with SSF only weakly 8 year old girls $(\mathrm{r}=-0.22, \mathrm{p}<0.05)$. 7. There was no correlation of adiponectin with HOMA-IR in girls of any age, and in boys only at 5 and $6 \mathrm{y}$ (both $\mathrm{r}=-0.19, \mathrm{p}<0.05)$.

Conclusion: Adiponectin rank order was maintained from year to year (suggesting the measure was precise and the levels systematic), and it fell (as expected) as adiposity rose. The behaviour of insulin resistance in the face of rising adiposity was unexpected. Insulin resistance and adiposity were correlated, as expected, but the relationships of adiponectin with adiposity and insulin resistance were weak and inconsistent. We found little evidence that circulating adiponectin mediates obesity-related insulin resistance in young children, although relationships may become apparent during the pubertal years.

Supported by Diabetes UK, GSK, Abbott Laboratories, Astra-Zeneca, Ipsen, Smith's Charity, Child Growth Foundation
0689

Low adiponectin and high resistin plasma levels predict 5-year mortality after first-ever ischaemic stroke in type 2 diabetic patients

A. G. Tsiakou ${ }^{1}$, S. P. Efstathiou ${ }^{2}$, D. I. Tsioulos ${ }^{1}$, Y. E. Gratsias ${ }^{2}$, T. N. Panagiotou ${ }^{1}$, A. D. Achimastos ${ }^{1}$, T. D. Mountokalakis ${ }^{2}$;

${ }^{1}$ Third University Department of Medicine, Sotiria General Hospital, Athens, ${ }^{2}$ Center for the Prevention of Cardiovascular Disease, Hygeias Melathron, Athens, Greece.

Background and Aims: The adipocytokines resistin (RS) and adiponectin (ADPN) are implicated - by contradictory actions - in insulin sensitivity, inflammation and cardiovascular disease. Data on the prognostic significance of these proteins in diabetic patients with cerebrovascular events are lacking. The aim of the present study was to investigate the association between i) plasma RS and ADPN levels and ii) stroke severity and long-term outcome in type 2 diabetic patients with first-ever ischemic stroke.

Materials and Methods: RS, ADPN and C-reactive protein (CRP) in the first 24 hours after the onset of stroke were measured by sandwich ELISA. Initial infarct volume and clinical severity were assessed by brain CT and the National Institute of Health Stroke Scale (NIHSS), respectively, whereas insulin sensitivity was estimated using the homeostasis model assessment (HOMA). A multivariate Cox's proportional hazards model was used to evaluate 5-year survival.

Results: Data from 60 individuals were analyzed (mean age $67.1 \pm 10.4$ years, body mass index [BMI] $27.1 \pm 5.3 \mathrm{~kg} / \mathrm{m}^{2}$, 32 men, mean $\mathrm{HbA}_{1 \mathrm{c}} 8.1 \%$, fasting glucose $168 \mathrm{mg} / \mathrm{dl}$, fasting insulin $19.2 \mu \mathrm{U} / \mathrm{ml}$, HOMA 8.2). Twenty-eight deaths $(46.7 \%)$ occurred within 5 years after stroke. Mean ADPN and RS values were $5.8 \pm 3.1 \mu \mathrm{g} / \mathrm{ml}$ and $28.3 \pm 5.9 \mathrm{ng} / \mathrm{ml}$, while median values of infarct volume, NIHSS score and CRP were 45 (interquartile range 9108) $\mathrm{cm}^{3}, 15(6-30)$ and 10 (4-17) $\mathrm{mg} / \mathrm{L}$, respectively. There was a significant inverse correlation of ADPN with RS $(\mathrm{r}=-0.72 ; \mathrm{p}<0.001)$, CRP $(\mathrm{r}=-0.61 ; \mathrm{p}<0.001)$, BMI $(\mathrm{r}=-0.39 ; \mathrm{p}<0.05)$, mean arterial pressure $(\mathrm{r}=-0.43$; $\mathrm{p}<0.05)$, infarct volume $(\mathrm{r}=-0.34 ; \mathrm{p}<0.05)$ and NIHSS score $(\mathrm{r}=-0.41 ; \mathrm{p}<0.05)$. RS showed a negative association with HDL-cholesterol $(\mathrm{r}=-0.33 ; \mathrm{p}<0.05)$, whereas it was positively related to CRP $(\mathrm{r}=0.61 ; \mathrm{p}<0.01)$, BMI $(\mathrm{r}=0.59$; $\mathrm{p}<0.01)$, fasting insulin $(\mathrm{r}=0.38 ; \mathrm{p}<0.05)$, fasting glucose $(\mathrm{r}=0.42 ; \mathrm{p}<0.05)$ and HOMA $(\mathrm{r}=0.55 ; \mathrm{p}<0.01)$. In multivariate analysis, the risk of death within 5 years after index stroke was significantly associated with the presence of 
coronary heart disease (hazard ratio [HR] 8.7, 95\% confidence intervals $[\mathrm{CI}] 3.6,20.4)$, NIHSS score in the highest tertile (HR 7.1, 95\% CI 3.2, 18.4), CRP in the highest tertile (HR 5.6, 95\% CI 3.1, 16.2), age $>70$ years (HR 4.4, 95\% CI 2.2, 14.3), ADPN in the lowest tertile (HR $3.5,95 \%$ CI 1.5, 9.8) and RS in the highest tertile (HR 2.1, $95 \%$ CI 1.2, 6.9).

Conclusion: Low ADPN and high RS plasma levels on admission of first-ever ischemic stroke in type 2 diabetic patients appear to reflect the extent of brain injury, the stroke severity and the risk of 5-year mortality, independently of the impact of acute phase reaction as estimated by CRP concentration. The above findings provide additional support to the view that there is an adipo-vascular axis linking insulin resistance, obesity and atherosclerosis.

\section{0}

Serum adipocytokine visfatin at first trimester is an early predictor of the insulin sensitivity index (ISI) of the second trimester in normal pregnancy

G. D. Valsamakis ${ }^{1}$, D. Papatheodorou ${ }^{2}$, I. Barlas ${ }^{1}$, I. Kouskouni ${ }^{1}$, I. Papasotiriou ${ }^{3}$, N. Vitoratos ${ }^{1}$, A. Papadimitriou ${ }^{2}$, G. Creatsas ${ }^{1}$, G. Mastorakos ${ }^{1}$;

${ }^{1}$ 2nd Department of Obstetrics and Gynecology, Endocrine Unit, Aretaieion Hospital, Athens, ${ }^{2}$ Department of Obstetrics and Gynecology, Nikaia General District Hospital, Athens, ${ }^{3}$ Biochemistry, Aghia Sophia Paediatric University Hospital, Athens, Greece.

Background and Aims: Adipose tissue is an active endocrine organ producing adipocytokines and is involved in glucose metabolism, inflammation, and cardiovascular disease. Throughout pregnancy there is an increase and redistribution of fat deposition. During the second trimester of pregnancy the regulatory effects of insulin on fat and carbohydrate metabolism become impaired so that a state of relative insulin resistance occurs. Recently it has been found adipocytokine visfatin to be expressed and secreted from splachnic fat in humans.

Aim of this study is to examine the role of adipocytokines in the process of insulin resistance in normal pregnancy.

Materials and Methods: 40 pregnant women with mean BMI (mean+SD) $24.7+4.2 \mathrm{~kg} / \mathrm{m} 2$ before pregnancy, and age of $29.5+4.5$ years, with no history of type 2 Diabetes Mellitus were followed. After informed consent pregnant women did 3 visits between 10th and 12th week, 24th to 26th and 35th $\mu \varepsilon$ 36th where a single observer did anthropometric measurements (weight, height), and a 75 gr OGTT with blood serum collection at $0,5,15,30$, 60, 90 к 120 minutes. From the above tests the insulin sensitivity index (ISI) and HOMA-R (insulin resistance index) were estimated during each trimester. None of the above pregnant women developed Gestational Diabetes during pregnancy.

Results: During analysis with ANOVA-one way there was a statistically significant difference in the change of maternal weight between 1 st and 2 nd trimester $(p=0.005)$ and between 2 nd and $3 \mathrm{rd}(p=0.0005)$. ISI index was statistically significant decreased between 2 nd and $3 \mathrm{rd}$ trimester $(p=0.02)$ but also between 1 st and 3rd. HOMA-R showed statistically significant difference between 1 st and 3 rd trimester $(p=0.03)$. Forward multiple regression analysis showed first trimester serum visfatin levels (beta $=1.01$, $\mathrm{p}=0.01$ ) as the only predictor of 2 nd trimester ISI among other metabolic parameters of first trimester like adiponectin, hs-CRP, IL-6, leptin, maternal weight. Finally the main predictors of serum visfatin levels during 2 nd trimester were adiponectin (beta $=-0.81, \mathrm{p}=0.01$ ) basal insulin (beta $=-1.55 \mathrm{p}=0.02$ ) and maternal weight (beta $=1.98$ $\mathrm{p}=0.03$ ).

Conclusion: The study shows for the first time that adipocytokine visfatin has a role in insulin sensitivity in normal pregnancy in contrast with other adipocytokines produced by adipose tissue (adiponectin, hsCRP, leptin, IL6). Its correlation with insulin at 0 min confirms its possible insulinmimetic action.

Supported by Greek Endocrine Society

0691

Effect of acute hyperinsulinaemia with and without AT1 receptor blockade on plasma concentrations of leptin and adiponectin and their expressions in adipose tissue in diabetes mellitus

E. Krusinova ${ }^{1}$, J. Kopecky, Jr. ${ }^{1}$, P. Mlejnek ${ }^{2}$, P. Wohl ${ }^{1}$, L. Kazdova ${ }^{1}$, M. Pravenec ${ }^{2}$, T. Pelikanova ${ }^{1}$;

${ }^{1}$ Diabetes Center, Institute for Clinical and Experimental Medicine, Prague, ${ }^{2}$ Institute of Physiology, Academy of Sciences of the Czech Republic, Prague, Czech Republic.

Background and Aims: Recently it was suggested, that AT-1 receptor blockers (ARB) have distinct antidiabetic and insulin-sensitising properties, however the underlying mechanisms are not clear. The effect of ARBs on leptin and adiponectin that are involved in insulin resistance, has not been clarified in humans till now.

The aims of our study were to measure the plasma concentrations of leptin and adiponectin and their expressions in abdominal subcutaneous adipose tissue and to test their response to acutely-induced hyperinsulinaemia with and without acute AT-1 receptor blockade in type 2 diabetes (D) and in healthy subjects (H). 
Materials and Methods: Plasma concentrations of leptin and adiponectin were measured: 1$)$ before (0 $\mathrm{min})$, at $30 \mathrm{~min}$ and $240 \mathrm{~min}$ of hyperinsulinaemic $\left(1 \mathrm{mU} \cdot \mathrm{kg}^{-1}\right.$. $\min ^{-1}$ ) euglycaemic (5 mmol. $\left.{ }^{-1}\right)$ clamp (HIC), and 2) during HIC after acute AT-1 receptor blockade (losartan cumulative dose $200 \mathrm{mg}$ ) (AT-HIC) using the same protocol, in $11 \mathrm{D}$ and $8 \mathrm{H}$. Needle biopsy of abdominal subcutaneous adipose tissue was performed before (0 min), at $30 \mathrm{~min}$ and $240 \mathrm{~min}$ of both clamps to asses the relative expressions of leptin and adiponectin using the real-time PCR method (expression of adipokine mRNA relative to cyclophilin mRNA).

Results: Insulin sensitivity, expressed as metabolic clearence rate of glucose, was significantly higher in $\mathrm{H}$ compared to $\mathrm{D}(\mathrm{p}<0.001)$, without any changes during AT-HIC in both groups. Plasma concentrations of leptin and adiponectin were comparable during HIC and AT-HIC in both groups and were not affected by insulin. The relative expressions of adiponectin in abdominal subcutaneous adipose tissue were higher after losartan administration in $\mathrm{D}$, but not in $\mathrm{H}$ (see the table; $\mathrm{p}<0.05$; ANOVA), without any dynamic changes during HIC and AT-HIC in both groups. The expressions of leptin were not changed by hyperinsulinaemia with or without losartan administration both in $\mathrm{D}$ and $\mathrm{H}$.

Conclusion: In type 2 diabetes and healthy subjects, acute hyperinsulinaemia does not influence plasma levels and adipose tissue expressions of adiponectin and leptin.

In type 2 diabetes, but not in healthy subjects, losartan administration stimulates the expression of adiponectin in subcutaneous adipose tissue, leptin expression is not affected. The results support the hypothesis, that the changes in adiponectin expression might be involved in the improvement of insulin sensitivity connected with ARB treatment.

Relative expressions of adiponectin (AdipoQ mRNA/ HCYC mRNA)

\begin{tabular}{lllllll}
\hline & \multicolumn{2}{l}{ Type 2 diabetes } & \multicolumn{4}{c}{ Healthy subjects } \\
\cline { 2 - 7 } & $0 \mathrm{~min}$ & $30 \mathrm{~min}$ & $240 \mathrm{~min}$ & $0 \mathrm{~min}$ & $30 \mathrm{~min}$ & $240 \mathrm{~min}$ \\
\hline HIC & $43.97 \pm$ & $50.91 \pm$ & $45.72 \pm$ & $23.99 \pm$ & $31.03 \pm$ & $40.82 \pm$ \\
& 20.03 & 18.17 & 15.71 & 5.70 & 9.13 & 11.15 \\
AT-HIC & $55.14 \pm$ & $53.09 \pm$ & $54.07 \pm$ & $43.92 \pm$ & $31.67 \pm$ & $28.26 \pm$ \\
& 24.74 & 19.90 & 13.63 & 22.91 & 3.83 & 7.74 \\
\hline
\end{tabular}

Data are expressed as mean \pm standard error.

For type 2 diabetes: HIC vs. AT-HIC: $\mathrm{p}<0.05$; ANOVA.

Supported by IGA MH CZ No. NR/7880-3
0692

Increased oxidative stress and decreased plasma levels of adiponectin are associated with insulin resistance in Japanese metabolically obese, normal-weight men M. Suematsu ${ }^{1}$, A. Katsuki ${ }^{1}$, K. Matsumoto ${ }^{1}$, H. Akatsuka ${ }^{1}$, K. Nakatani ${ }^{2}$, Y. Yano ${ }^{1}$, Y. Sumida ${ }^{1}$;

${ }^{1}$ Department of Internal Medicine, Divison of Diabetology and Endocrinology, ${ }^{2}$ Department of Laboratory Medicine, Mie University School of Medicine, Tsu, Mie, Japan.

Background and Aims: Recently, we described metabolically obese, normal-weight (MONW) subjects [BMI $<25 \mathrm{~kg} / \mathrm{m}^{2}$ and visceral fat area (VFA) evaluated by abdominal computed CT scanning $\geq 100 \mathrm{~cm}^{2}$ ] in the Japanese population, and demonstrated that insulin resistance together with visceral fat accumulation play an important role in the pathophysiology of metabolic obesity. The roles of oxidative stress and adiponectin in the pathophysiology of obesity have been the focus of many investigations. Oxidative stress is significantly correlated with decreased plasma levels of adiponectin in subjects with obesity, whereas both of them are significantly correlated with insulin resistance. However, the relationship between oxidative stress, plasma levels of adiponectin and insulin resistance in MONW subjects have not been as yet clarified. In the present study, we investigated the relationship between oxidative stress, circulating levels of adiponectin and insulin resistance in Japanese MONW men.

Material and Methods: We measured the plasma levels of free 8-epi-prostaglandin F2 $\alpha$ (8-epi-PGF2 $\alpha$ ), a marker of oxidative stress, and adiponectin in $28 \mathrm{MONW}$ (age $35.7 \pm 1.0$ years old, BMI $23.7 \pm 0.2 \mathrm{~kg} / \mathrm{m}^{2}$, VFA $130.6 \pm$ $3.7 \mathrm{~cm}^{2}[$ mean $\pm \mathrm{SE}]$ ) and 23 normal (age $33.4 \pm 1.4$ years old, BMI $21.0 \pm 0.2 \mathrm{~kg} / \mathrm{m}^{2}$, VFA $57.1 \pm 4.2 \mathrm{~cm}^{2}$ ) men. The plasma levels of free 8-epi-PGF2 $\alpha$ were measured using a commercially available enzyme immunoassay (EIA) kit (Cayman Chemical, Ann Arbor, MI) and the plasma levels of adiponectin using a radioimmunoassay kit (LINCO Research, St. Charles, MO). Insulin resistance was evaluated by the euglycemic hyperinsulinemic clamp technique using an artificial pancreas (Nikkiso STG-22, Tokyo, Japan).

Results: The plasma level of 8 -epi-PGF2 $\alpha(30.4 \pm 4.0 \mathrm{pg} / \mathrm{ml}$ vs. $8.1 \pm 1.3 \mathrm{pg} / \mathrm{ml}, P<0.01)$ in MONW subjects was significantly increased, whereas that of adiponectin $(8.6 \pm 0.9 \mu \mathrm{g} / \mathrm{ml}$ vs. $11.6 \pm 0.7 \mu \mathrm{g} / \mathrm{ml}, P<0.01)$ was significantly decreased in MONW subjects as compared to normal subjects. Glucose infusion rate (GIR) during the euglycemic hyperinsulinemic clamp study in MONW men (8.8 \pm $0.5 \mathrm{mg} / \mathrm{kg} / \mathrm{min}$ ) was significantly decreased compared to normal subjects $(11.5 \pm 0.4 \mathrm{mg} / \mathrm{kg} / \mathrm{min}, P<0.01)$. Significant correlations were observed between VFA and the plasma levels of 8-epi-PGF2 $\alpha(\mathrm{r}=0.434, P<0.05)$ and adiponectin 
$(\mathrm{r}=-0.535, P<0.01)$ in MONW men. The plasma levels of 8 -epi-PGF2 $\alpha$ and adiponectin were significantly correlated in MONW subjects $(\mathrm{r}=-0.617, P<0.01)$. Both plasma levels of 8-epi-PGF2 $\alpha$ and adiponectin were significantly associated with GIR in MONW men (8-epi-PGF2 $\alpha$; $\mathrm{r}=-0.481, P<0.01$, adiponectin; $\mathrm{r}=0.687, P<0.01$ ).

Conclusion: The present study showed that systemic increase in oxidative stress and decrease in circulating levels of adiponectin are significantly correlated with insulin resistance in Japanese MONW men. Although correlation does not prove causation, these observations suggest that increased oxidative stress may cause decreased plasma levels of adiponectin and that both abnormalities may contribute to development of insulin resistance in Japanese MONW men.

\section{3}

The relationship between visfatin levels and anthropometric and metabolic parameters in healthy subjects C.-C. Chen ${ }^{1}$, T.-C. $\mathrm{Li}^{2}$, C.-I. $\mathrm{Li}^{3}$, C.-S. Liu ${ }^{4}$, W.-Y. Lin ${ }^{4}$, M.-T. Wu ${ }^{5}$, C.-C. $\mathrm{Lin}^{4}$;

${ }^{1}$ Department of Medicine, China Medical University Hospital, Taichung, ${ }^{2}$ Graduate Institute of Chinese Medicine Science, ${ }^{3}$ Department of Medical Research, ${ }^{4}$ Department of Family Medicine, ${ }^{5}$ Department of Family Medicine and Occupational Medicine, China Medical University Hospital, Taichung, Taiwan

Background and Aims: Adipose tissue has been identified as an endocrine organ. Visfatin is a novel adipocytokine predominantly secreted from visceral adipocytes. Visceral obesity is an important component of metabolic syndrome; however, the relationship between visfatin levels and metabolic syndrome is not clear. The aim of this study was to investigate the association between visfatin levels and parameters of metabolic syndrome and anthropometry in a healthy population.

Materials and Methods: We recruited 198 healthy participants ( 70 men and 128 women), between the age of 40 to 70 years old. Healthy subjects were defined as individuals with a blood pressure of $<140 / 90 \mathrm{mmHg}$, normal renal and liver function, a fasting plasma glucose level of $<6.1 \mathrm{mmol} / \mathrm{L}$; none of them were taking medication at the time of the study, and all of them were disease free. Anthropometric measurements included height, weight, body mass index, waist and hip circumferences, waist-tohip ratio and blood pressure. Metabolic parameters including fasting serum visfatin, insulin and plasma glucose, lipid profiles and uric acid levels were measured. Insulin resistance was determined by homeostasis model assessment (HOMA).
Results: Serum visfatin levels in both men and women did not correlate with any markers for adiposity, fasting plasma glucose, fasting serum insulin levels, HOMA or uric acid levels. Visfatin correlated negatively with diastolic pressure in men only after adjusting for age; however, this correlation disappeared after adjusting for age and waist circumference. Visfatin only correlated positively with HDL-C levles in women; this correlation remained unchanged even after adjusting for age. However, this correlation disappeared after adjusting for age and waist circumference. Multiple linear regression analysis using the logarithm of visfatin as a dependent variable revealed that, except for fasting insulin levels and HDL levels in female subjects, non of the anthropometric or metabolic parameters correlated independently with visfatin in either male and female subjects.

Conclusions: Visfatin is not related to anthropometric and most parameters of metabolic syndrome in healthy subjects. The physiologic roles of visfatin in metabolic syndrome warrants further investigation.

Supported by the National Science Council (NSC 93-2314B-039-025 and NSC 94-2314-B-039-024) of the Republic of China

\section{4}

Regulation of white adipose tissue-released factors by insulin and glucose in healthy young men

M. Faraj ${ }^{1}$, G. Beauregard ${ }^{1}$, A. Tardif ${ }^{2}$, E. Loizon ${ }^{3}$, K. Cianflone ${ }^{4}, \mathrm{H}$ Vidal $^{3}$, R. Rabasa-Lhoret ${ }^{1}$;

${ }^{1}$ Nutrition, Faculty of Medicine, Université de Montréal, Canada, ${ }^{2}$ Endocrinology, Centre de Recherche du Centre Hospitalier de L'Université de Montréal, Canada, ${ }^{3}$ Inserm u.449/inra u.1235, ifr 62, René Laennec Faculty of Medicine, Lyon, France, ${ }^{4}$ Anatomy and Physiology, Laval Université, Ste Foy, Canada.

Background and Aims: Type 2 diabetes is associated with the dysregulation of white adipose tissue (WAT)-released factors as plasma leptin, ASP, TNF $\alpha$ and TNF $\alpha-\mathrm{R} 1$ are elevated while those of adiponectin are decreased in diabetic subjects. However, it is unknown whether this dysregulation is a precursor to, or a consequence of, the diabetic state as studies examining the effect of hyperglycemia (HG) and hyperinsulinemia (HI) on WAT-released factors are scarce in human. Our aim was thus to investigate the role of $\mathrm{HG}$ and/or $\mathrm{HI}$ in the regulation of WAT-released factors in vivo in a healthy subjects.

Population and Methods: We investigated the regulation of plasma concentrations and mRNA expression levels of the following WAT-released factors in response to 3-hour HG and/or HI in healthy lean young men; leptin, adiponectin, ASP (mRNA for which is $\mathrm{C} 3$ ), TNF $\alpha$, TNF $\alpha$ - 
$\mathrm{R} 1$ as well as mRNA expression of HKII and p85 $\alpha$ PI3K (as positive controls for activation of insulin signaling). Hyperglycemia euinsulinemia clamp ( $\mathrm{HG}, \mathrm{N}=8$ ) was induced by constant infusion of $20 \%$ dextrose during which endogenous insulin was suppressed by somatostatin and insulin was replaced at baseline levels. Hyperinsulinemia euglycemia clamp ( $\mathrm{HI}, \mathrm{N}=10$ ) was induced by a primedconstant exogenous insulin infusion at $75 \mathrm{mU} / \mathrm{m}^{2} / \mathrm{min}$ during which glucose was maintained at baseline levels. Hyperglycemia hyperinsulinemia clamp (HGHI, N=7) was a combination of both above clamps. As somatostatin was utilized in both HG clamps, a control somatostatin clamp was also carried out $(\mathrm{N}=4)$. All target mRNA expression levels are expressed relative to those of the reference gene HPRT, significance is at $\mathrm{p}<0.05$.

Results: Preliminary data indicates that $\mathrm{HG}$ clamps (glucose $=9.8 \pm 1.2 \mathrm{mM}$, insulin $=15.5 \pm 4 \mu \mathrm{U} / \mathrm{mL}$ ) significantly decreased plasma leptin by $20 \%(1.8 \pm 1.2 v s 1.5 \pm 1.3 \mathrm{ng} / \mathrm{mL})$ while increased mRNA expression levels of $\mathrm{p} 85 \alpha$ PI3K by $46 \%(10.2 \pm 3.3$ vs $14.4 \pm 4.8)$. HI clamps (glucose $=5.2 \pm$ $0.4 \mathrm{mM}$, insulin $=138.2 \pm 53.9 \mu \mathrm{U} / \mathrm{mL}$ ) decreased plasma adiponectin by $15 \%(11.6 \pm 4.8$ vs $10.0 \pm 4.5 \mu \mathrm{g} / \mathrm{mL})$ while increased plasma leptin by $11 \%(3.8 \pm 2.9$ vs $4.2 \pm 3.1 \mathrm{ng} / \mathrm{mL})$ and increased mRNA expression of HKII by $147 \%(20.2 \pm$ 16.8 vs $41.7 \pm 23.8)$ and of p $85 \alpha$ PI3K by $150 \%(20.3 \pm 17.2$ vs 39.1 \pm 24.5 ). HGHI clamps (glucose $=10.0 \pm 1.2 \mathrm{mM}$, insulin $=154.3 \pm 25.6 \mu \mathrm{U} / \mathrm{mL}$ ) increased plasma ASP by $21 \%(30.0 \pm 5.7$ vs 35.6 \pm 7.0$)$, and increased mRNA expression of leptin by $130 \%(8.9 \pm 5.5$ vs $14.0 \pm 6.5)$, of C3 by $27 \%$ $(10.2 \pm 1.9$ vs $12.8 \pm 2.6)$ and of HKII by $90 \%$ (12.9 \pm 7.1 vs $26.9 \pm 13.8)$. Somatostatin clamp decreased plasma leptin by $-17 \%$, which may explain the equivalent suppression in plasma leptin with the HG clamp. There was no significant effect of the HG and/or HI clamps on plasma TNF $\alpha$ or TNF $\alpha-R 1$.

Conclusion: In healthy young lean men, HI suppresses plasma adiponectin and increases plasma leptin while HGHI increases plasma ASP and the WAT-mRNA expression levels of leptin and C3. Thus in diabetes, we hypothesize that elevated plasma leptin and ASP and depressed plasma adiponectin may be a consequence of, rather than a precursor to, $\mathrm{HG}$ and HI. On the other hand, the upregulation of the $\mathrm{TNF} \alpha$ system that exists in the diabetic subjects may proceed diabetes as $\mathrm{HG}$ and/or $\mathrm{HI}$ per se had no effect on $\mathrm{TNF} \alpha$ or its receptor 1 .

Supported by the Canadian Institute for Health Research and Fonds de Recherche en santé du Québec
0695

Changes and relations of circulating visfatin, apelin, and resistin levels in normal, impaired glucose tolerance, and type 2 diabetic subjects

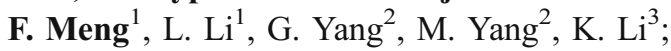

${ }^{1}$ Department of Clinical Biochemistry, ${ }^{2}$ Department of Endocrinology, The Second Affiliated Hospital, ${ }^{3}$ Department of Endocrinology, Chongqing University of Medical Sciences, Chongqing, China.

Background and Aims: Visfatin and apelin are two novel adipocyte- secreted hormone proposed to link obesity with insulin resistance. In this study we investigated whether or not plasma visfatin and apelin levels were altered in in normal, impaired glucose tolerance(IGT), and type 2 diabetic(T2DM) subjects. We also assessed the association between plasma visfatin, or apelin and body composition, metabolic parameters, and resistin concentrations in these subjects.

Materials and Methods: Thirty subjects with T2DM, 26 subjects with IGT,37 subjects with obesity, 55 subjects with no obesity and 36 control subjects participated in the study. Anthropometric measurement was performed in the morning, before breakfast, with the subject wearing light clothing, without shoes. Blood samples were drawn at $14 \mathrm{~h}$ of fasting and $2 \mathrm{~h}$ of glucose overload for determination of plasma glucose, free fatty acid, insulin, resistin, visfatin, apelin and Glycosylated hemoglobin, blood fat levels.Visfatin, apelin as well as resistin were evaluated using a commercially available ELISA. Plasma insulin was measured in deproteinized serum by radioimmunoassay using human insulin as standard. Plasma glucose was assayed using the glucose oxidase method. Glycosylated hemoglobin was measured by isoelectric focusing. Plasma triglyceride and cholesterol concentrations were determined enzymatically. We evaluated for associations between plasma Visfatin, apelin levels and anthropometric (BMI, WHR), metabolic (fasting and overload $2 \mathrm{~h}$ glucose and insulin, HbA1c, FFA, HOMA-IR, lipid profile) parameters, as well as resistin, all expressed as continuous variables.

Results: Fasting and glucose overload $2 \mathrm{~h}$ visfatin levels were found to be significantly decreased in the IGT and obesity subjects compared with the controls $(P<0.05)$, and visfatin levels were even lower in diabetics than the IGT and obesity subjects $(P<0.05)$. In contrast, basal apelin levels were significantly increased in the IGT and diabetic subjects compared with the obesity and controls $(P<0.05$ and $P<0.01$ ). The apelin levels of glucose overload $2 \mathrm{~h}$ were significantly higher than the basal levels in every group (all 
$P<0.05)$. Fasting plasma visfatin was found to correlate positively and significantly with BMI,WHR, and fasting plasma resistin, but negatively with $\mathrm{HbA1c}$ and the blood glucose of overload $2 \mathrm{~h}$. Multiple regression analysis showed that WHR,HbA1c,2hPBG were independent related factors influencing plasma visfatin levels. Fasting plasma apelin levels correlated positively with HOMA-IR,BMI,TC, LDL-C,FBG and Fasting plasma insulin. Multiple regression analysis showed that HOMA-IR,BMI,and TC were independent related factors influencing plasma apelin levels. Conclusion: These results indicate that visfatin and apelin may play a role in the pathogenesis of insulin resistance and T2DM.

This work was supported by a research grant from the National Natural Science Foundation of China, "Chung Huei program" of China Education Ministry

\section{PS 51}

\section{Adipokines - in vitro studies}

\section{6}

The novel myokine musclin/osteocrin is induced in human skeletal muscle cells by troglitazone but not by $\mathbf{W y}$ 14,643

H. Staiger, C. Haas, F. Machicao, H.-U. Häring; Internal Medicine IV, Medical Clinic, Eberhard-KarlsUniversity Tübingen, Germany.

Background and Aims: Recently, a novel myokine named musclin/osteocrin was identified in mice and shown to inhibit insulin action in murine $\mathrm{C} 2 \mathrm{C} 12$ myocytes. In this study, we asked whether musclin is expressed in human in vitro differentiated skeletal muscle cells (myotubes) as well, and whether agonists of the peroxisome proliferatoractivated receptors (PPAR) $\alpha$ and $\gamma$ modulate musclin mRNA expression.

Materials and Methods: Human myotubes were treated with 5 and $10 \mu \mathrm{mol} / 1$ troglitazone, 5 and $10 \mu \mathrm{mol} / 1 \mathrm{Wy}-$ 14,643 , or $0.1 \%(\mathrm{v} / \mathrm{v})$ dimethylsulfoxide for carrier control. Myocellular mRNA contents were quantified by real-time RT-PCR.

Results: Untreated myotubes expressed $1.51 \pm 0.82 \mathrm{fg}$ musclin $\mathrm{mRNA} / \mu \mathrm{g}$ total RNA (mean $\pm \mathrm{SD}, \mathrm{n}=5$ ). These levels were about 3.5-fold higher than that found in a representative biopsy from human skeletal muscle. 20$\mathrm{h}$ treatment of human myotubes with the PPAR $\gamma$ agonist troglitazone dose-dependently increased musclin mRNA expression up to 2.8-fold over control. By contrast, the
PPAR $\alpha$ agonist Wy-14,643 did not significantly modulate musclin mRNA expression.

Conclusion: In conclusion, we show here that musclin is indeed expressed in human myotubes and that PPAR $\gamma$ activation up-regulates musclin expression.

The study was supported by a grant from the German Research Foundation and the European Community's FP6 EUGENE 2

0697

The role of visfatin in type 2 diabetes mellitus: the in vivo and in vitro effects of rosiglitazone, TNF $\alpha$ and IL-6 on visfatin expression in human abdominal subcutaneous adipocytes

K. C. McGee $^{1}$, A. L. Harte ${ }^{1}$, N. F. da Silva ${ }^{1}$, M. V. Chittari ${ }^{1}$, M. Khanolkar ${ }^{2}$, S. J. Creely ${ }^{1}$, M. Evans ${ }^{2}$, S. Kumar ${ }^{1}$, P. G. McTernan ${ }^{1}$;

${ }^{1}$ Unit for Diabetes and Metabolism, University of Warwick, Coventry, ${ }^{2}$ Heart Research Institute, Cardiff University, Wales, United Kingdom.

Background and Aims: Visfatin, a novel adipose tissue protein, has been shown to exert insulin mimetic effects in humans and mouse models. However, in hyperinsulinaemic/insulin resistant conditions, visfatin is increased suggesting its' potential role in the pathogenesis of type 2 diabetes mellitus (T2DM). The observed increase may occur directly, via insulin action, or through inducers of insulin resistance, such as TNF $\alpha$ and IL-6, which have been implicated in visfatin regulation. To date, no human studies have explored the role of the insulin sensitiser, rosiglitazone (RSG), TNF $\alpha$ and IL-6 in visfatin regulation. Therefore the aims of these studies were to examine (1) visfatin serum levels in non-diabetic (ND) and T2DM patients; (2) the effect of RSG treatment on circulating visfatin in newly diagnosed T2DM patients; (3) the influence of insulin and RSG on visfatin protein expression in isolated abdominal subcutaneous (AbSc) adipocytes; (4) the role of human recombinant (hr)TNF $\alpha$ and hr IL-6 as regulators of visfatin protein in $\mathrm{AbSc}$ adipocytes.

Materials and Methods: Fasting serum samples were obtained from $23 \mathrm{ND}$ and 36 T2DM subjects (ND: BMI: 26.0 (mean $\pm \mathrm{SD}) \pm 2.7 \mathrm{~kg} / \mathrm{m}^{2}$; Age: $38.9 \pm 12.9$ yrs; T2DM: BMI: $35.9 \pm 7.2 \mathrm{~kg} / \mathrm{m}^{2}$; Age: $\left.56.0 \pm 9.8 \mathrm{yrs}\right)$ and T2DM patients pre and post 10 week RSG treatment (Age: $57.8 \pm 4.0$ yrs, BMI: $\left.36.1 \pm 2.5 \mathrm{~kg} / \mathrm{m}^{2 ;}, \mathrm{n}=8\right)$. AbSc and omental (Om) adipose tissue (AT) was obtained from human ND subjects (BMI: $26.5 \pm 5.9 \mathrm{~kg} / \mathrm{m}^{2}$, Age $45.7 \pm 9.2 \mathrm{yrs}$ ) undergoing elective surgery. AbSc adipocytes 
were treated for $48 \mathrm{hrs}$ with $100 \mathrm{nM}$ insulin alone or in combination with $10 \mathrm{nM}$ RSG, protein extracted and conditioned media collected. Adipocytes were also treated with hr TNF- $\alpha(1-10 \mathrm{ng} / \mathrm{mL}, \mathrm{n}=4)$ and hr IL-6 (1-10 ng/ $\mathrm{mL}, \mathrm{n}=4$ ).

Results: T2DM subjects exhibited higher serum visfatin levels (ND: $13.9($ mean \pm SEM) $\pm 1.5 \mathrm{ng} / \mathrm{mL}$ vs T2DM: $21.8 \pm 3.6 \mathrm{ng} / \mathrm{mL}, \mathrm{p}<0.05)$ and TNF $\alpha$ levels $(\mathrm{TNF}-\alpha \mathrm{ND}$ : $11 \cdot 87 \pm 4.3 \mathrm{pg} / \mathrm{mL}$ vs T2DM: $16.83 \pm 7.0 \mathrm{pg} / \mathrm{mL} ; \mathrm{p}=0 \cdot 007)$ compared with ND. Post-RSG treated T2DM showed a significant reduction in serum visfatin and insulin compared to pre-RSG treatment (pre-RSG: $10.77 \pm 1.47 \mathrm{ng} / \mathrm{mL}$ vs post-RSG: $7.45 \pm 1.60 \mathrm{ng} / \mathrm{mL}, \mathrm{p}=0.04$ ). $\mathrm{RSG} 10 \mathrm{nM} /$ insulin $100 \mathrm{nM}$ treated adipocytes showed a significant reduction in visfatin and $\mathrm{TNF} \alpha$ protein expression compared with insulin alone $(p<0.05)$, whilst both TNF- $\alpha$ and IL-6 upregulated visfatin protein expression in these cells (hrTNF$\alpha$ : Control: $1.0 \pm 0.02$ (relative fold expression) $\mathrm{hr} T N F \alpha$, $10 \mathrm{nM}: 1.24 \pm 0.06, \mathrm{p}<0.05$; IL-6: Control: $1.0 \pm 0.01,10 \mathrm{nM}$ : $1.13 \pm 0.09, \mathrm{p}<0.05)$. Analysis of visfatin protein expression in human AT showed visfatin was significantly higher in omental AT compared to AbSc AT ( $<<0.001 ; n=6)$.

Conclusion: Circulating visfatin was elevated in T2DM subjects compared to non-diabetic controls. Furthermore, treatment with RSG for 10 weeks lowered circulating visfatin levels in T2DM patients. RSG treated adipocytes reduced visfatin protein expression and $\mathrm{TNF} \alpha$ secretion, whilst both $\mathrm{hr}$ TNF- $\alpha$ and hr IL-6 increased visfatin expression in adipocytes. Taken together, these data highlight that visfatin is elevated in states where insulin sensitivity is reduced and, as such, visfatin may act as a biomarker for impaired insulin sensitivity.

Supported by the Department of Health

\section{8}

Newly identified adipokines secreted by visceral adipose tissue and their deregulation in obesity

E. Maury ${ }^{1}$, K. Ehala-Aleksejev ${ }^{1}$, Y. Guiot ${ }^{2}$, M.-L. Delporte ${ }^{1}$, R. Detry ${ }^{3}$, S. M. Brichard ${ }^{1}$;

${ }^{1}$ Endocrinology and metabolism, University of Louvain, Brussels, ${ }^{2}$ Pathology, University of Louvain, Brussels, ${ }^{3}$ Surgery, University of Louvain, Brussels, Belgium.

Background and Aims: Obesity, and more particularly central obesity, is strongly associated with chronic disorders such as type 2 diabetes, cardiovascular disease and cancer. Adipokines are involved in the pathogenesis of these obesity-linked disorders. However, adipokines secreted by visceral adipose tissue (AT), unlike those secreted by other fat depots, are still poorly characterised. In this study, we searched for novel adipokines secreted by visceral AT, compared their production in lean versus obese subjects and examined the relative contribution of the different cell types to this production.

Subjects and Methods: Visceral AT was obtained from 7 obese (age, $46 \pm 7 \mathrm{yr}$; body mass index (BMI), $42 \pm 3 \mathrm{~kg} / \mathrm{m}^{2}$ ) and 7 non-obese (age, $51 \pm 7 \mathrm{yr}$; BMI, $24 \pm 1 \mathrm{~kg} / \mathrm{m}^{2}$ ) men undergoing elective abdominal surgery. AT was digested by collagenase treatment into isolated adipocytes (A) and stromal-vascular cells (SVC). These cellular fractions were cultured separately for $24 \mathrm{~h}$. In another experiment, cultured preadipocytes were differentiated over 12 days into mature adipocytes.

Results: We screened culture medium of A and SVC using a commercial array allowing the detection of 120 cytokines. We identified 16 cytokines secreted by each cellular fraction of visceral AT in lean and obese subjects. Out of them, 6 adipokines were newly identified as secretory products of visceral AT. These adipokines include 3 chemokines, 1 interleukin, 1 tissue inhibitor of matrix metalloproteinases and 1 hematopoietic growth factor. A and SVC secreted rather similar amounts of these adipokines, when data were normalised on a cellular basis. However, for each fraction, this secretion was enhanced in obese subjects compared to lean ones (from 2- up to 8- fold, $P<0.05$ ). Qualitatively similar results were obtained when mRNA levels of these adipokines were analysed by realtime RTQ-PCR. The mRNA concentrations of 5 of these 6 adipokines markedly increased during in vitro adipogenesis (6- to 18 - fold, $P<0.05$ or less versus preadipocyte state).

Conclusion: Six adipokines were newly identified in visceral AT. The expression and secretion of these adipokines were increased in obesity, with a relatively similar contribution of $\mathrm{A}$ and $\mathrm{SVC}$ to this enhanced production. Because of their already known involvement in cardiovascular disease, cell proliferation or insulin resistance, these adipokines may play a role in obesitylinked adverse health outcomes.

0699

Protein array reveals changed levels of several cytokines in adipose tissue of obese subjects with features of metabolic syndrome

D. Gasperikova ${ }^{1}$, M. Skopkova ${ }^{1}$, A. Penesova ${ }^{2}$, M. Vlcek ${ }^{2}$, Z. Radikova ${ }^{2}$, J. Koska ${ }^{2}$, H. Sell ${ }^{3}$, J. Eckel ${ }^{3}$, I. Klimes ${ }^{1}$;

${ }^{1}$ Diabetes and Nutrition, ${ }^{2}$ Human Endocrinology, Institute of Experimental Endocrinology, Bratislava, Slovakia, ${ }^{3}$ Institute of Clinical Biochemistry and Pathobiochemistry, German Diabetes Centre, Duesseldorf, Germany. 
Background and Aims: The role of adipokines and inflammatory cytokines of adipose tissue for development of obesity-related insulin resistance has not yet been completely understood. Therefore, the aim of the study was 1) screened the protein level of 120 cytokines in subcutaneous adipose tissue (ScAT) of lean and obese subjects and 2) compared the gene expression for selected cytokines in ScAT in basal state and after euglycemic hyperinsulinemia (EHI).

Materials and Methods: All recruited subjects initially underwent an oGTT, and those with IFG, IGT and DM2 were excluded. Thus, $18 \mathrm{drug}$ naive Caucasian males (12 obese (OB): BMI $33.5 \pm 0.9 \mathrm{~kg} / \mathrm{m}^{2}$, age $26.8 \pm 2$ yrs and 6 lean control (C): BMI 21.5 \pm 0.5 , age $28.7 \pm 1$ ) were included into the study. OB had increased fasting plasma glucose, insulin, hsCRP, Tg and HDL-Ch levels, decreased in vivo insulin action (assessed by euglycemic hyperinsulinemic clamp [EHC]) $(\mathrm{p}<0.01)$. ScAT biopsies were performed after an overnight fast and in the 3rd hour of EHC. Protein levels in basal state were assessed in tissue lysates using the RayBio ${ }^{\circledR}$ Human Cytokine Antibody Array C Series 1000.

Results: From 120 cytokines measured, 27 presented higher (leptin, HGF, EGF-R, FGF-6, IGF-1sR, Fas, ENA-78, PARC, lymphotactin, HCC-4, IL-10, IL-1a, IL1R1, IL-1R4, IL-12p70, angiopoietin-2, Axl, Dtk, MIF, MIP-1a, -1b, -3b, MSP-a, OPG, TECK, TIMP-1, -2) and 1 (RANTES) presented lower levels in ScAT of OB subjects in comparison with $\mathrm{C}(\mathrm{p}<0.05)$. Furthemore in the OB, leptin had increased also ScAT mRNA (real-time RT-PCR) and plasma levels; TNF $\alpha$ had increased mRNA only, and IL-6 and adiponectin did not differ at any level studied. EHI led to further elevation of the mRNA levels for leptin and IL-6 in $\mathrm{OB}$ and $\mathrm{C}(\mathrm{p}<0.05)$.

Conclusion: Our data provide new evidence on a very early dysregulation of cytokine ScAT levels in clinically yet healthy OB subjects. Further amplification of the changes by EHI may indicate toward a possible perturbation of the situation in response to endogenous hyperinsulinemia in obesity.

Supported by STAA No. 51-040602, COSTB17, Slovak Diabetes Society

\section{0}

Comparison of super-coiled minicircle with conventional plasmid in systemic non-viral adiponectin gene therapy to ameliorate insulin resistance in diet induced obese mice

M. K. Kim ${ }^{1}$, M. Lee ${ }^{2}$, S. W. Kim ${ }^{3}$, J. H. Park ${ }^{4}$;
${ }^{1}$ Department of Internal Medicine, Maryknoll General Hospital, Busan, Republic of Korea, ${ }^{2}$ Department of Bioengineering, Hanyang University, Seoul, Republic of Korea, ${ }^{3}$ Department of Pharmaceutics \& Pharmaceutical Engineering, University of Utah, SLC, United States, ${ }^{4}$ Molecular Therapy Lab, Division of Endocrinology \& Metabolism, Department of Internal Medicine, Paik Memorial Institute for Clinical Research, Pusan Paik Hospital, College of Medicine, Inje University, Busan, Republic of Korea.

Background and Aims: Gene therapy is an attractive tool to be used for the treatment of various human diseases. However, several serious harmful side effects of widely used viral gene carrier limit its clinical usages. Non-viral gene delivery might be an alternative to avoid the immune response and the illegitimate DNA integration to the host chromosome. Despite of the superior biological safety, non-viral gene delivery has suffered from its weak transfection efficiency as compared with the viral gene therapy. Adiponectin is a very famous adipocytokine that can improve many biological markers of insulin resistance. But its blood concentration is so high to be increased by conventional non-viral gene therapy technique. To increase the transfection efficiency of non-viral gene delivery, we performed this study.

Materials and Methods: We cloned small sized novel super-coiled minicircle plasmid pMINI-beta-ADN(adiponectin) devoid of bacterially originated $\mathrm{CpG}$ motifs. We injected this plasmid complexed with the cationic polymeric carrier into the tail veins of $\mathrm{DIO}$ (diet induced obese) C57BL/6J mice.

Results: Tail vein injection of pMINI-beta-ADN showed 1.5-2.0 fold increase of blood adiponectin level. Blood glucose, free fatty acid and HOMA-IR values were normalized. RT-PCR revealed that the adiponectin gene expression mainly took place at the liver and spleen, and the main oligomer form of the increased adiponectin in blood was HMW(high molecular weight) form which was verified by Western blot analysis. On the contrary, the injection of conventional plasmid pBETA-ADN did not increase the blood adiponectin level.

Conclusion: These data might suggest that the non-viral gene therapy using minicircle plasmid could be successfully employed to increase the blood level of therapeutic peptides including adiponectin, one of the most abundant proteins in the human blood. 
Elevated expression of visfatin in streptozocin-induced diabetic rats

Y. Lu, W.-B. Xu, M. Luo;

Study Group of Diabetic Complications, Shanghai Institute of Endocrine and Metabolic Diseases, Shanghai, China.

Background and Aims: Visfatin, also known as pre-B cell colony-enhancing factor (PBEF), was identified as a novel adipocytokine exerting putative antidiabetogenic properties. Previous studies have shown that PBEF is not only expressed in many important tissues, but the protein lacks a signal sequence, so it is necessary to clarify the origin of serum visfatin. This study was to investigate the relationship between visfatin serum level and its expression in tissues in a model of streptozotocin (STZ)-induced diabetic rats.

Materials and Methods: 8 week old male Sprague-Dawley (SD) rats were chosen. Hyperglycaemia was induced by intravenous STZ injection at $65 \mathrm{mg} / \mathrm{kg}$ bodyweight $(\mathrm{n}=12)$. citrate buffer-injected rats were chosen as parallel control $(\mathrm{n}=12)$. Blood glucose and bodyweight were monitored. Rats were sacrificed at indicated time, tissues (heart, epididymal adipose tissue, skeletal muscle, liver, thoracic aorta) and serum were harvested. Expression of visfatin in various tissues was investigated by Northern blotting, and serum protein level was measured by immunoblotting.

Results: Diabetic rats remained hyperglycemic (10.8 \pm $3.4 \mathrm{mmol} / \mathrm{L}$ at 4 th week and $19.7 \pm 4.1 \mathrm{mmol} / \mathrm{L}$ at 12 th week; $\mathrm{n}=6$, respectively, $\mathrm{P}<0.01$ vs control) after confirmation of hyperglycemia $(>10 \mathrm{mmol} / \mathrm{L})$. Visfatin $\mathrm{mRNA}$ expression was observed in all the tissues tested, with the maximum in liver and the least in thoracic aorta. Its expression was significantly high in all tissues of diabetic rats as compared to parallel control ones $(\mathrm{P}<0.05)$, and a remarkable increase was observed in liver and epididymal adipose tissue of diabetic rats (3- and 4-fold, respectively; $\mathrm{P}<0.01$ vs control). Serum visfatin protein level mirrored its mRNA expression in tissues both in diabetic rats and normal control ones $(r=0.31, P<0.05)$. Furthermore, diabetic course seemed have no influence on visfatin expression in various tissues and serum level as well. Conclusion: Visfatin is expressed universally and constantly in vivo with a highest level in liver under physical condition. Its Increased circulating level in parallel with upregulated expression in tissues under diabetic milieu probably indicates that visfatin, through its insulin-mimetic metabolic effects, might play a compensative role in the course of diabetes and total body tissues, besides adipose, may contribute to the change in serum visfatin level.

This study was supported by the Major State Basic Research Development Program of the People's Republic of China (No. 2002BA711A05) and the National Nature Science foundation of China (No. 30470817).
Pioglitazone effect on plasma, visceral and subcutaneous adipose tissue adiponectin of fatty Zucker rats

J. Pita, A. Panadero, E. Rodríguez, M. Gonzalo,

A. Rovira;

Laboratory of Endocrinology, Fundación Jimenez Diaz, Madrid, Spain.

Background and Aims: Adiponectin is an insulin-sensitizing hormone with anti-inflammatory properties which is reduced in insulin resistance states like obesity and type 2 diabetes.

Thiazolidindiones are PPAR- $\gamma$ agonists that improve insulin resistance and upregulate adiponectin levels in plasma and adipose tissue. It has been proposed that adiponectin upregulation could be the mechanism used by thiazolidinediones to improve insulin resistance linked to obesity.

The aim of this study is to know if the amelioration of insulin resistance produced by pioglitazone is associated with changes in the adiponectin content in plasma, visceral and subcutaneous adipose tissue in Zucker fatty rats, an animal model that associates extreme obesity, insulin resistance and dysregulation of lipidic metabolism.

Materials and Methods: Fatty Zucker rats were treated with pioglitazone $(3 \mathrm{mg} / \mathrm{kg} / \mathrm{d}, \mathrm{n}=10)$ or placebo $(\mathrm{n}=10)$ for 12 weeks. Blood was collected weekly, and at the end of the treatment rats were sacrificed by intraperitoneal injection of sodium penthotal, visceral and subcutaneous adipose tissue, and blood were collected.

Measurements: Plasma insulin by RIA, plasma cholesterol and triglycerides by enzymatic assays, plasma and visceral and subcutaneous adipose tissue adiponectin content by Western-blot.

Results: Compared to placebo, rats treated with pioglitazone had a significant decrease $(\mathrm{p}<0.05)$ of plasma insulin levels $(16.8 \pm 2.2$ vs $3.4 \pm 0.4 \mathrm{ng} / \mathrm{ml})$, plasma cholesterol $(147.1 \pm 5.0$ vs $110.7 \pm 5.7 \mathrm{mg} / \mathrm{dl})$ and plasma triglycerides $(235 \pm 14.1$ vs $141.7 \pm 9.3 \mathrm{mg} / \mathrm{dl})$. Pioglitazone treatment, compared to placebo, produced a significant increase $(\mathrm{p}<0.05)$ in plasma adiponectin levels $(1.34 \pm 0.12$ vs $0.60 \pm 0.10 \mathrm{AU})$, and in adiponectin protein content of visceral adipose tissue $(1.12 \pm 0.13$ vs $0.51 \pm 0.06$ AU) and subcutaneous adipose tissue $(1.54 \pm 0.28$ vs $0.79 \pm$ $0.08 \mathrm{AU})$.

Placebo treated rats presented lower adiponectin protein content in visceral adipose tissue compared to subcutaneous adipose tissue $(\mathrm{p}<0.05)$, whereas pioglitazone treated rats had similar adiponectin protein content in both tissues. 
Plasma adiponectin correlated negatively $(\mathrm{p}<0.05)$ with HOMA value $(\mathrm{R}=-0.71)$, cholesterol $(\mathrm{R}=-0.58)$ and triglycerides $(\mathrm{R}=-0.71)$.

Conclusion: Pioglitazone produces an increase of adiponectin in plasma and adipose tissue that is associated with amelioration of insulin sensitivity and lipidic metabolism.

Study supported by Fondo de Investigación Sanitaria, Spain

\section{3}

Acute and chronic treatments with a new recombinant globular domain of adiponectin: improvement of insulin sensitivity, glucose tolerance and FFA metabolism in the high fat diet-fed diabetic mouse

T. Sulpice ${ }^{1}$, C. Molveaux ${ }^{1}$, C. Knauf ${ }^{2}$, P. Vitte $^{3}$,

P. Graber $^{3}$, M. Dreano ${ }^{3}$, R. Burcelin ${ }^{2}$;

${ }^{1}$ Metabolic Diseases, Physiogenex S.A.S, Labège Innopole, France, ${ }^{2}$ Functional Genomics, Inserm, Toulouse, France, ${ }^{3}$ Discovery, Serono International, Geneva, Switzerland.

Background and Aims: Adiponectin increases glucose transport, reduces inflammation, and controls vascular function. Hence, we propose that treatments with a recombinant globular domain of adiponectin (aa 110-244), designated AS100-244, exhibit significant therapeutic potential for hyperglycemic syndromes.

Materials and Methods and Results: Mice were fed for three months a high fat-carbohydrate free diet (HFD) to induce diabetes and mild obesity. In the first set of experiments, the mice were infused intravenously with different doses of AS100-244 (0.12; 0.4 and $1.2 \mu \mathrm{g} / \mathrm{kg} / \mathrm{min})$ for 5 hours. Basal and insulin sensitive glucose utilization rates were assessed by the use of a continuous tritated glucose infusion and pharmacological dose of insulin $(18 \mathrm{mU} /$ $\mathrm{kg} / \mathrm{min}$ ) in the awake free moving mouse. The data showed that AS100-244 dose dependently reduces insulin resistance by increasing whole body glucose utilization $(35 \%$ at the highest dose) and glycolysis rate of the HFD mice. Similarly, the reduction of FFA concentration by insulin was dramatically improved and fastened. However, in the basal fasting state hepatic glucose production was unchanged by AS100-244 infusion. This acute improved glucose homeostasis was associated with an increased content of muscle phospho-Akt and AMP-activated kinase. In a second set, HFD mice were chronically treated with a twice daily s.c AS100-244 injection at three doses (10, 30 and $100 \mu \mathrm{g} / \mathrm{kg}$ ). Fasted glycemia, glucose tolerance as well as insulin sensitive glucose utilization were improved by AS100-244 at the highest dose after 3 weeks of treatment with concomitant decrease in body weight gain when compared to vehicle treated mice. The metabolic improve- ments were similar to those obtained by a metformin treatment.

Conclusion: Our data show that AS100-244, a recombinant globular domain of adiponectin, represents an efficient therapeutic strategy for the treatment of hyperglycemic and insulin resistant syndromes.

0704

Serum adiponectin levels in relation to the tissue insulin resistance in obese and non-obese models of metabolic syndrome

O. Oliyarnyk, M. Cahova, M. Hubova, L. Kazdova; Department of Metabolism and Diabetes, Institute for Clinical and Experimental Medicine, Prague, Czech Republic.

Background and Aims: Adiponectin is adipocytokines with antidiabetic properties. Its plasma concentration is decreased in obesity and type 2 diabetes. Adiponectin is believed to positively influence insulin sensitivity but information concerning regulation of its secretion in insulin resistance which is not associated with obesity is still missing. We investigated the relationship between serum concentration of adiponectin and visceral adiposity, triglyceridemia and the peripheral tissue insulin sensitivity in obese and non-obese experimental models of insulin resistance. Further the effect of high fructose diet on adiponectin levels was examined.

Materials and Methods: The experiments were carried on adult male a) non-obese hereditary hypertriglyceridemic rats (HHTg) 4- and 18- month old, b) obese Zucker rats and c) non-obese spontaneously hypertensive rats (SHR) fed a high carbohydrate diet $(60 \%$ of fructose) for two weeks. Age- and strain-matched non-obese rats served as controls. The adiponectin was determined by ELISA kit (B-Bridge Inc., USA). The resistance of muscles and adipose tissue to insulin was estimated according to the insulin stimulated ${ }^{14} \mathrm{C}$-U-glucose incorporation into glycogen and lipids.

Results: In obese rats, the low adiponectin levels in serum were associated with higher epidydimal fat pad weight, with significant hypertriglyceridemia a nearly complete resistance of adipose and skeletal tissue to the insulin stimulated glucose utilization for lipid and glycogen synthesis. In contrast, in HHTg and control rats the serum adiponectin concentration were not different at the age 4 and 18 months in spite of higher triglyceridemia and impaired insulin sensitivity of skeletal and adipose tissue in HHTg rats. The age-related drop in adiponectin serum concentrations by $30 \%$ was similar in 18 -month-old HHTg and control rats although icreased hypertriglyceridemia and muscle and adipose tissue insulin resistance in HHTg rats. High fructose feeding increased triglyceridemia and periph- 
eral insulin resistance in HHTg rats, and against expectation serum adiponectin levels were increased both in 4-mo old HHTg rats by $96 \%$ and in 18 -mo old rats by $190 \%$. This diet increased serum adiponectin similarly in 4-mo old control rats by $66 \%$ and in 18 -mo old rats by $190 \%$. Fructose-induced hypertriglyceridemia and insulin resistance in SHR rats had no effect on serum adiponectin concentration.

Conclusion: Our results suggest contribution of downregulation of adiponectin secretion to the development of age-dependent deterioration of insulin sensitivity. The correlation between epididymal fat pad mass and serum adiponectin concentration $\left(\mathrm{r}^{2}=-0,82 ; \mathrm{p}<0,02\right)$ supports the hypothesis about the relationship between adiponectin secretion and the adipose tissue mass. In contrast to this neither inherited hypertriglyceridemia nor nutritionally induced insulin resistance were not associated with different adiponectin serum concentration.

\begin{tabular}{llll}
\hline Group & $\begin{array}{l}\text { Epididymal fat } \\
\text { pads, g/10 }\end{array}$ & $\begin{array}{l}\text { Serum TG } \\
\mathrm{mmol} / 1\end{array}$ & $\begin{array}{l}\text { Serum } \\
\text { adiponec, } \mu \mathrm{g} / \mathrm{ml}\end{array}$ \\
\hline $\begin{array}{l}\text { Zucker obese, } \\
\text { 4-mo old }\end{array}$ & $2.75 \pm 0.09$ & $2.81 \pm 0.26$ & $1.86 \pm 0.17$ \\
$\begin{array}{l}\text { K-Wistar, } \\
\text { 4-mo old }\end{array}$ & $1.55 \pm 0.07$ & $0.85 \pm 0.07^{\mathrm{a}}$ & $3.17 \pm 0.31^{\mathrm{a}}$ \\
$\begin{array}{l}\text { K-Wistar, } \\
\text { 18- mo old }\end{array}$ & $1.72 \pm 0.07$ & $1.17 \pm 0.07^{\mathrm{a}}$ & $2.25 \pm 0.12^{\mathrm{a}}$ \\
$\begin{array}{l}\text { HHTg, 4-mo old } \\
\text { HHTg, 18-mo }\end{array}$ & $1.39 \pm 0.06^{\mathrm{b}}$ & $2.95 \pm 0.21^{\mathrm{b}}$ & $3.32 \pm 0.18^{\mathrm{b}}$ \\
$\begin{array}{l}\text { old } \\
\text { SHR, 4-mo old }\end{array}$ & $1.05 \pm 0.09^{\mathrm{b}}$ & $3.83 \pm 0.41^{\mathrm{b}}$ & $2.44 \pm 0.20^{\mathrm{b}}$ \\
$\begin{array}{l}\text { SHR 4-mo old }+ \\
\text { fructose }\end{array}$ & $1.05 \pm 0.04$ & $0.54 \pm 0.09^{\mathrm{c}}$ & $6.16 \pm 0.61$ \\
\hline
\end{tabular}

The same symbols represent statistical significance $\mathrm{p}<0.05$.

Supported grant MZ 00023001 and NR 8495-3

\section{PS 52}

\section{Adipose tissue metabolism}

\section{5}

\section{Modulation of lipolysis, lipogenesis and glucose uptake through human adipocyte differentiation: evaluation of insulin sensitivity}

S. Fernandez-Veledo, I. Nieto, R. Vila, M. Lorenzo; Bioquimica y Biologia Molecular II, Universidad Complutense, Madrid, Spain.

Background and Aims: Currently there is strong evidence that dysfunction of adipose tissue plays a crucial role in the development of insulin resistance associated to Obesity and Type 2 diabetes. Adipose tissue not only responds to afferent signal from traditional hormone systems but also expresses and secretes factors with important endocrine functions, a fact that is emphasized by the adverse metabolic consequences of fat excess. Human adipocyte research is hampered by the lack of human fat cell lines. So most studies are performed on immortalized rodent cell lines such as $3 \mathrm{~T} 3-\mathrm{L} 1$, murine cells with several metabolic and signal transduction differences compared with human cells; or primary cultures of human preadipocytes, which are obtained from fibroblast-like cells isolated form the stromal portion of human fat tissue. These cells cannot be expanded and display only a limited life span. Moreover, the reduced availability of human adipose tissue is a limitation for more detailed studies. Therefore, the purpose of the present study was to characterize a novel human adipocyte cell line that expresses differentiated functions, particularly referred to glucose and lipid metabolism.

Materials and Methods: LiSa-2 is a stable cell line derived from a poorly differentiated human liposarcoma. In serum-containing medium they are fibroblastoid and rapidly dividing but when grown in adipogenic medium, LiSa-2 cells differentiate into adipocyte-like cells with several phenotypic, biochemical and functional characteristics of mature human fat cells. In addition to the protein expression pattern, and insulin signaling pathway, representative metabolic functions of adipose tissue such as glucose uptake, lipogenesis and lipolysis were investigated. Studies were performed on undifferentiated ( 0 days of culture) and confluent differentiated cells (7, 14 and 21 days of culture). Moreover, the effect of cytokines (TNF- $\alpha$ and IL6) and agonists of different nuclear receptors (PPAR $\gamma$, LRX, RAR, and RXR) on glucose uptake and lipolysis were also analyzed.

Results: Differentiation of LiSa-2 cells was associated with expression of several adipocyte markers and sensitivity to lipolysis and glucose uptake induced by insulin, reaching maxim levels at 14 days of differentiation. However, prolonged cell cultures gradually lose the expression of some proteins such as GLUT4 glucose transporter. These changes in gene expression contribute to the development of insulin resistance in LiSa-2 cells and mimic the insulin-resistant phenotype in adipose tissue in vivo. On the other hand, in differentiated and insulin sensitive cells TNF- $\alpha$ but not IL6 induce lipolysis and caused insulin resistance. Treatment with different nuclear receptor agonists differentially regulate glucose and lipid metabolism under physiological and insulin-resistance conditions.

Conclusion: LiSa-2 is a novel human adipocyte cell line that may serve as a well-established in vitro cell model as an alternative to primary cultures. Studies with this human cell line is likely to reveal further insight into the endocrine 
function of adipose tissue and the molecular mechanisms involved in insulin resistance states associated to Obesity. This knowledge may permit more rational approaches to treatment of the metabolic consequences of excess of adipose tissue.

Supported by BFU2005-03054 from MEC

\section{6}

WISP-2, a Wnt-signaling protein, is increased in obesity and adipocyte enlargement

A. Hammarstedt ${ }^{1}$, P. Isakson ${ }^{1}$, J. Pihlajamäki ${ }^{2}$, P.-A. Jansson ${ }^{1}$, M. Laakso ${ }^{2}$, U. Smith ${ }^{1}$;

${ }^{1}$ The Lundberg Laboratory for Diabetes Reserach, Internal Medicine, Göteborg University, Sweden, ${ }^{2}$ Medicine, University of Kuopio, Finland.

Background and Aims: In recent years the importance of the adipose tissue for the development of type 2 diabetes and its complications has become evident. type 2 diabetes and insulin resistance has been shown to correlate with different markers of adipocyte differentiation and, thus, function. The Wnt-signaling pathway is a well known regulator of cell cycle progression and an inhibitor of cell differentiation. Recent studies in our laboratory have shown that exposure of 3T3-L1 preadipocytes to cytokines results in maintenance of Wnt-signaling and inhibition of adipocyte differentiation. In this study, we have focused on the gene expression of WISP (Wnt-1 inducible signaling protein) -2 , a Wnt-signaling responsive gene, and its relation to adipocyte differentiation and risk factors for type 2 diabetes, such as obesity and circulating IL-6 levels.

Material and Methods: To study the expression of WISP2 in human adipocytes, we obtained abdominal subcutaneous adipose tissue by surgical incision from 10 type 2 diabetic subjects before and after treatment with the insulin sensitizer, pioglitazone. To study WISP-2 in pre-diabetic patients, we also obtained adipose tissue biopsies from 36 well-characterized first-degree relatives to type 2 diabetic patients. Furthermore, we have cultured and differentiated human preadipocytes from 6 donors to study WISP-2 expression during adipogenesis.

Results: The expression of WISP-2 in adipose tissue from type 2 diabetic patients correlated with several risk factors for type 2 diabetes such as BMI ( $r=0.85, \mathrm{p}=0.002)$ WHR $(\mathrm{r}=0.71, \mathrm{p}=0.02)$ and circulating IL-6 levels $(\mathrm{r}=0.61$, $\mathrm{p}=0.06$ )but not to insulin sensitivity. The expression of WISP-2 was reduced by $20 \%$ after pioglitazone treatment $(p=0.06)$, but the reduction did not correlate with the improvement of the insulin resistance. In the pre-diabetic group, WISP-2 expression in adipose tissue was also positively correlated to waist $(r=0.34, p=0.04)$, WHR $(\mathrm{r}=0.30, \mathrm{p}=0.07)$ and cell size $(\mathrm{r}=0.42, \mathrm{p}=0.01)$, but not to insulin sensitivity. In the human preadipocytes, we found that the expression of WISP-2 was significantly reduced during adipocyte differentiation, while the presence of IL-6 counteracted this decrease. This is in agreement with the positive correlation between circulating IL-6 levels and the WISP-2 expression in adipose tissue seen in the diabetic group.

Conclusion: Taken together, these data suggest that Wntsignaling is increased in adipocytes from obese subjects and subjects with enlarged adipocytes. Furthermore, the expression of the wnt-signaling responsive gene WISP-2 correlates with known risk factors for type 2 diabetes.

Supported by the Swedish Research Council, the European Community's FP6 EUGENE, the Swedish Diabetes Association, the Inga Britt and Arne Lundberg Foundation, the Novo Nordisk Foundation

0707

Mesenchymal stem cell differentiation into adipocytes is equally induced by insulin and proinsulin in vitro A. Pfützner ${ }^{1,2}$, A. Pansky ${ }^{3}$, A. Maiworm ${ }^{3}$, M. Matthey $^{3}$, K. Lückerath ${ }^{3}$, B. Roitzheim ${ }^{3}$, T. Forst ${ }^{1,4}$, E. Tobiasch ${ }^{3}$; ${ }^{1}$ Medical, Institute for Clinical Research and Development, Mainz, ${ }^{2}$ Clinical Research, University of Applied Science, Rheinbach, ${ }^{3}$ Stem Cell Research, University of Applied Sciences, Rheinbach, ${ }^{4}$ Endocrinology, University Mainz, Germany.

Background and Aims: In advanced $\beta$-cell dysfunction stage III, proinsulin (PI) is increasingly replacing insulin (INS) as major component of the secretion product. Proinsulin has only $10-20 \%$ of the glucose lowering activity of INS, but it has been speculated that PI has at least the same adipogenetic potency than INS, leading to an increased tendency of lipid tissue formation in patients with late stage $\beta$-cell dysfunction. Since the hormonal secretion of the growing adipose tissue is a driver for insulin resistance, the increased PI amounts required to lower glucose may be strongly promoting the further deterioration of the metabolic situation. The purpose of this experiment was to investigate the adipogenetic effects of PI in comparison to that of INS.

Materials and Methods: Mesenchymal stem cells obtained from liposuction material were grown in differentiation media containing INS $(0.01 \mu \mathrm{Mol})$, PI $(0.01 \mu \mathrm{Mol})$ or PI + INS (each $0.005 \mu \mathrm{Mol}$ ). Cell culture supernatants were taken from these experiments and an untreated control at weeks 1,2 , and 3 , and were stored at $-80^{\circ} \mathrm{C}$ until analysis. Cell differentiation was microscopically supervised and confirmed by Oilred-O staining, and adiponectin concentrations 
were measured as marker for differentiation into mature lipid cells. This experiment was repeated three times.

Results: No growth of lipid cells and no change in adiponectin values was observed in the negative control group $(3.2 \pm 0.5 / 3.3 \pm 0.1 / 4.4 \pm 0.5 \mathrm{ng} / \mathrm{ml})$. A continuous differentiation into mature adipocytes (also confirmed by Oilred-O-staining) and a corresponding increase in adiponectin values was observed in the experiments with INS (3.6 $\pm 1.9 / 5.1 \pm 1.4 / 13,3 \pm 1.5 \mathrm{ng} / \mathrm{ml} ; \mathrm{p}<0.05$ week 1 vs. week $3)$ and PI $(3.3 \pm 1.2 / 3.5 \pm 0.3 / 12.2 \pm 1.2 \mathrm{ng} / \mathrm{ml} ; \mathrm{p}<0.05)$. Comparable effects were also seen with the INS+PI combination $(3.5 \pm 0.7 / 4.0 \pm 0.2 / 14.6 \pm 3.0 \mathrm{ng} / \mathrm{ml} ; \mathrm{p}<0.05)$.

Conclusion: Proinsulin has the same adipogenetic potential than insulin in vitro. Since the blood glucose lowering potency of PI is much lower than that of INS, much more PI needs to be secreted to control blood glucose in vivo. It can be speculated that the adipogenetic potential of proinsulin may be a large contributor to the increased body weight problems in patients with type 2 diabetes and advanced $\beta$-cell dysfunction.

\section{8}

High subcutaneous interstitial levels and trivial spillover into the circulation implicate monocyte chemoattractant protein-1 as a paracrine regulator

G. Murdolo ${ }^{1,2}$, A. Hammarstedt ${ }^{1}$, M. Sandqvist ${ }^{1}$, M. Schmelz ${ }^{3}$, U. Smith ${ }^{1}$, P.-A. Jansson ${ }^{1}$;

${ }^{1}$ Department of Internal Medicine, The Lundberg Laboratory for Diabetes Research, Gothenburg, Sweden, ${ }^{2}$ Department of Internal Medicine, Division of Endocrinology and Metabolic Sciences, DiMISEM, Perugia, Italy, ${ }^{3}$ Department of Anesthesiology, University of Heidelberg, Mannheim, Germany.

Background and Aims: The chemokine monocyte chemoattractant protein-1 (MCP-1), known to be produced by the adipose tissue (AT), has been strongly implicated in the obesity-associated macrophage infiltration into AT, insulinresistance, and inflammation. The aims of the present study were: 1) to evaluate the contribution of adipose-derived MCP-1 to the systemic levels by combining the in situ calibrated microdialysis technique with serum measurements; 2) to characterize the gene expression pattern in AT before and after a euglycemic hyperinsulinemic glucose clamp.

Material and Methods: Nine lean (L) (Age: $34.6 \pm 3.4 \mathrm{yr}$; BMI: $22.5 \pm 0.8 \mathrm{~kg} / \mathrm{m}^{2}$ ) and 9 uncomplicated obese (OB) (Age: $32.8 \pm 2.6$ yr; BMI: $34.7 \pm 1 \mathrm{~kg} / \mathrm{m}^{2}$ ), male subjects were studied in the fasting state, and during a 4-hr euglycemic hyperinsulinemic glucose clamp $\left(40 \mathrm{mU} / \mathrm{m}^{2} / \mathrm{min}\right)$. Moreover, serum (s) and interstitial (i) MCP-1 measurements were estimated. A needle biopsy from the abdominal subcutaneous AT was taken at baseline and after 4-hr of insulin infusion to examine the gene expression profiles.

Results: OB showed slightly higher basal sMCP-1, as compared with L ( $352 \pm 38$ vs $271 \pm 22 \mathrm{pg} / \mathrm{ml}, \mathrm{p}=0.07)$. Basal iMCP-1 was $\sim 4-$ and $\sim 7$-fold higher than sMCP-1 $(p<0.001)$ in OB and L, respectively. During the clamp no changes of sMCP-1 occurred. Among the OB, basal AT gene expression pattern revealed an up-regulation of MCP$1(p<0.01)$ and CD68 ( $p=0.02)$, coupled with a downregulation of GLUT-4. Insulin infusion increased MCP-1 and GLUT-4 in the L $(\mathrm{p}<0.05)$ but not in OB, showing a differential regulation by insulin in these groups.

Conclusion: These observations show: 1) high subcutaneous interstitial MCP-1 levels and trivial spillover into the bloodstream implicating adipose-derived MCP-1 as a paracrine/autocrine modulator; 2) acute hyperinsulinemia does not affect circulating MCP-1 concentrations, but increases GLUT-4 and MCP-1 gene expression in the adipose tissue in lean but not in obese subjects.

Supported by FoRI SIE; Novo Nordisk Foundation; Swedish Diabetes Association

0709

Systemic activation of the endocannabinoid system in adipose tissue of obese and type 2 diabetic patients

K. Kempf ${ }^{1}$, B. Rose ${ }^{1}$, C. Herder ${ }^{1}$, S. Martin ${ }^{1}$,

P. Algenstaedt ${ }^{2}$;

${ }^{1}$ German Diabetes Clinic, Düsseldorf, Germany, ${ }^{2}$ Department of Internal Medicine, University Hamburg-Eppendorf, Germany.

Background and Aims: Obesity is one of the main risk factors for type 2 diabetes mellitus (T2DM). Activation of the endocannabinoid system (ECS) promotes appetite and weight gain. This process can be inhibited by blocking of the cannabinoid receptor 1 (Cb1), which is expressed in neuronal and immune cells as well as in adipose tissue (subcutaneous and visceral). In obesity activation of the ECS was demonstrated by elevated serum levels of endocannabinoids and reduced expression of $\mathrm{Cb} 1$ and fatty acid amide hydrolase (FAAH) in subcutaneous adipose tissue. FAAH plays a significant role in degradation of circulating endocannabinoids and is therefore known as a negative regulator of the ECS. So far it is unknown if the expression of $\mathrm{Cb} 1$ and FAAH is only reduced in subcutaneous adipose tissue or if the reduction also is present in visceral adipose tissue and if metabolic dysregulation like T2DM affects their expression.

Materials and Methods: Using quantitative RT-PCR we analyzed the expression of $\mathrm{Cb} 1$ and FAAH in visceral and 
subcutaneous adipose tissue of non-diabetic subjects with normal (visceral: $\mathrm{n}=10$, BMI $24.4 \pm 1.1$; subcutaneous: $\mathrm{n}=18$, BMI 23.8 \pm 1.9 ) and with strongly elevated BMI (visceral and subcutaneous: $n=11$, BMI $37.6 \pm 13.6)$ as well as in subcutaneous adipose tissue of T2DM patients $(n=5$, BMI $25.0 \pm 2.0$ and $\mathrm{n}=8$, BMI 35.0 \pm 5.2 ).

Results: In non-diabetic obese subjects the expression of $\mathrm{Cb} 1(30 \%)$ and FAAH (60\%) was reduced in visceral adipose tissue compared to normal weight controls. Moreover, we could show that in comparison to nondiabetic subjects the expression of $\mathrm{Cb} 1(70 \%)$ and FAAH $(45 \%)$ was strongly reduced in subcutaneous adipose tissue of T2DM patients, with further reduction of mean $\mathrm{Cb} 1$ expression by obesity in the non-diabetic $(80 \%)$ as well as in the diabetic $(50 \%)$ group.

Conclusion: Our results suggest that obesity as well as T2DM independently reduce the expression of $\mathrm{Cb} 1$ and FAAH, which might be explained by systemic activation of the ECS in adipose tissue.

0710

Lipin (Lpin1) expression is decreased in subcutaneous adipose tissue in human obesity

M. Miranda ${ }^{1}$, M. R. Chacón ${ }^{1}$, C. Gutiérrez ${ }^{1}$, L. Gallart ${ }^{1}$, J. M. Fernández-Real ${ }^{2}$, W. Ricart ${ }^{2}$, C. Richart ${ }^{1}$,

J. Vendrell ${ }^{1}$;

${ }^{1}$ Research Unit, University Hospital of Tarragona Joan XXIII, ${ }^{2}$ Endocrinology and Diabetes Unit, University Hospital Josep Trueta from Girona, Spain.

Background and Aims: Lpin1 gene is emerging as an important determinant of adipose tissue differentiation and has been related to lipodystrophy and to obesity in animal models. The purposes of this analysis were to compare lipin adipose tissue gene expression levels in obese subjects and patients with type 2 diabetes and to assess if its expression parallels to animal models in human adipocyte differentiation. Materials and Methods: Subcutaneous adipose tissue samples were obtained from 44 healthy lean and obese subjects and from 48 patients with type 2 diabetes matched for body mass index (BMI), whose clinical and anthropometrical variables were recruited. Real Time PCR was used to measure gene expression of Lpin1, PPAR $\gamma$ and SREBP1c in whole adipose tissue and of Lpin1 in in vitro differentiated subcutaneous- and visceral-isolated preadipocytes. Results:In non-diabetic subjects, Lpin1 mRNA expression levels were significantly reduced in the obese group ( $7.57 \pm 0.11$ vs $7.23 \pm 0.07 ; p=0.013$ ), but no differences with type 2 diabetes patients were observed. In the multiple regression analysis, after adjusting for confounding variables only BMI was negatively associated with Lpin 1 expression $(\beta=-0.354, p=0.01)$. Lpin1 mRNA expression increased with preadipocyte differentiation but the increment was higher in subcutaneous than in visceral adipose tissue.

Conclusion: Human obese subjects express lower Lpin1 mRNA levels than lean subjects in subcutaneous adipose tissue and its expression is not altered by the presence of type 2 diabetes mellitus. Lpin 1 expression is induced during human adipocyte differentiation in vitro.

Supported by the following grants: Marató TV3, Red de Centros C03/08, Red de Grupos G03/212, FIS 05/1994

\section{1}

\section{WITHDRAWN}

\section{2}

Preadipocyte factor 1 (Pref-1/DLK1/FA1) in obesity and insulin resistance

J. J. Vendrell ${ }^{1}$, M. R. Chacón ${ }^{1}$, C. H. Jensen ${ }^{2}$, M. Miranda ${ }^{1}$, J. Fernández-Real ${ }^{3}$, A. Megia ${ }^{1}$, J. Gómez ${ }^{4}$, E. Caubet ${ }^{5}$, C. Masdevall ${ }^{6}$, N. Vilarrasa ${ }^{4}$, W. Ricard ${ }^{3}$, C. Richart ${ }^{1}$;

${ }^{1}$ Endocrinology and Diabetes, Hospital Universitari Joan XXIII de Tarragona, Spain, ${ }^{2}$ Immunology and Microbiology Winsloparken, University of Southern Denmark., Odense, Denmark, ${ }^{3}$ Endocrinology and Diabetes, University Hospital of Girona. "Dr. Josep Trueta", Spain, ${ }^{4}$ Endocrinology and Diabetes Unit, University Hospital of Bellvitge, Barcelona, Spain, ${ }^{5}$ Surgery Service, Hospital "St Pau i StaTecla", Tarragona, Spain, 'Surgery Service, University Hospital of Bellvitge, Barcelona, Spain.

Background and Aims: Preadipocyte differentiation occurs during distinct periods of human development and is a key determinant of body mass. Preadipocyte factor 1/ Delta like factor 1 (Pref-1/DLK-1) is a gene encoding a transmembrane protein that undergoes proteolitic cleavage to generate a soluble form called in humans, Fetal antigen 1 (FA1). Pref-1/DLK-1 constitutive expression in vitro led to inhibition of osteoblast and adipocyte differentiation on human stromal cells an on 3T3L-1 mouse cells inhibits its differentiation to adipocyte.

Our aim was to investigate if there was variation in committed preadipocytes between individuals with different degree of obesity. We also aimed to find any correlation between circulating levels (FA1) and its local adipose tissue expression and insulin resistance.

Materials and Methods: FA1 were measured by ELISA technique in $n=134$ consecutive healthy male subjects to 
whom insulin sensitivity $\left(S_{\mathrm{i}}\right)$ test was performed. Circulating levels of sCD14, sTNFR2, sIL-6 and adiponectin were also measured in this population.

Subcutaneous adipose tissue samples were obtained from a different cohort in non-diabetic $(n=40)$ and patients with Type 2 diabetes $(\mathrm{n}=16)$ with different degrees of obesity. Real Time PCR was used to measure gene expression of pref-1. FA1 plasma levels were also measured in this group. Results: In healthy male cohort, after multiple regression analysis, $\mathrm{S}_{\mathrm{i}}(\beta=-0.180, p<0.05)$ and HDLCholesterol $(\beta=-0.191, p<0.039)$ appeared as independent determinants of FA1 plasma levels.

Pref-1 mRNA expression levels in subcutaneous adipose tissue did not show differences between obesity groups or with the presence of Type 2 diabetes. In this cohort, FA1 levels correlate positively with age, waist and hip ratio (WHR), soluble fraction of tumor necrosis alpha receptors TNFR 1 and TNFR2 $(r=0.287, \mathrm{p}=0.009 ; \mathrm{r}=0.276, \mathrm{p}=0.027$; $\mathrm{r}=0.356, \mathrm{p}=0.004$ and $\mathrm{r}=0.354, \mathrm{p}=0.003$, respectively). After controlling for confounding variables, only WHR $(\beta=0.375, p=0.002)$ and sTNFR2 $(\beta=0.352, p=0.004)$ remained as the independent determinants of FA1 levels.

Conclusions: Increased FA1 circulating levels may be representatives of an increased cardiovascular risk, including low insulin sensitivity. Adipose tissue expression does not contribute in a relevant manner to FA1 plasma concentration.

Supported by a grant from Fondo de Investigación Sanitaria (FIS 04/0377)

\section{3}

Increased glucose tolerance despite low adiponectin levels in obesity-resistant aP2-Ucp1 transgenic mice fed a high-fat diet

M. Rossmeisl ${ }^{1}$, T. Jelenik ${ }^{1}$, N. C. Ogston ${ }^{2}$, K. Slamova ${ }^{1}$, V. Mohamed-Ali ${ }^{2}$, J. Kopecky ${ }^{1}$;

${ }^{1}$ Department of Adipose Tissue Biology, Institute of Physiology, Prague, Czech Republic, ${ }^{2}$ Adipokines and Metabolism Research Group, Department of Medicine, University College London, United Kingdom.

Background and Aims: Transgenic mice with ectopic expression of mitochondrial uncoupling protein 1 (UCP1) in white fat (aP2-Ucp1 mice) show partial resistance to obesity induced by a high-fat (HF) diet, which is associated with a preferential reduction of subcutaneous fat depots, low plasma triacylglycerols, and reduced lipid content in the muscle and liver. Despite these beneficial effects, transgenic mice are hyperglycemic in the fasted state, especially when fed a standard chow. In order to understand the mechanims involved in the control of glucose metab- olism in the aP2-Ucp1 mice, we analyzed glucose homeostasis, plasma concentrations of adipokines, as well as insulin sensitivity in the aP2-Ucpl transgenic mice and their C57BL/6J (B6) littermate controls.

Materials and Methods: Male transgenic aP2-Ucp1 and their B6 littermate controls were fed a standard chow until 3 mo of age, when some mice were assigned to a HF diet $(60 \%$ calories as fat). Basal hepatic glucose production (HGP) was measured by continuous D- $\left(3-{ }^{3} \mathrm{H}\right)$ glucose infusion into fasted mice fed a standard chow. Glucose turnover (GTO) was assessed by hyperinsulinemic-euglycemic clamps (a primed continuous infusion of human insulin $\sim 4 \mathrm{mU} / \mathrm{kg} . \mathrm{min}$, blood glucose clamped at $5.5 \mathrm{mM}$ ) in overnight fasted mice after a short-term (3 wks) HF feeding. Glycemic control in mice fed a HF diet for 2 mo was assessed by intraperitoneal glucose tolerance test and expressed as area under the curve (AUC). Plasma levels of adiponectin and leptin were measured by ELISA, while gene expression in the skeletal muscle by quantitative realtime RT-PCR.

Results: Fasted glycemia ( $\sim 15 \mathrm{hr}$ fast $)$ in the chow-fed transgenic mice was in a range of 109 to $135 \mathrm{mg} / \mathrm{dL}$, while in B6 controls the range was $87-103 \mathrm{mg} / \mathrm{dL}$. However, basal HGP was only slightly elevated in the transgenic mice compared to controls $(15.1 \pm 1.3$ vs. $12.8 \pm 0.8 \mathrm{mg} / \mathrm{kg} . \mathrm{min})$, while GTO was not different between the genotypes (aP2Ucp 1, $60 \pm 3$ vs. B6, $70 \pm 9 \mathrm{mg} / \mathrm{kg} . \mathrm{min}$ ). Plasma levels of adiponectin, a negative regulator of HGP, were decreased by $\sim 30 \%$ in the aP2-Ucp 1 mice. Short-term HF feeding significantly lowered GTO in both strains to the same extent (aP2-Ucp 1, 48.3 \pm 3.8 vs. B6, $51.5 \pm 6.0 \mathrm{mg} / \mathrm{kg} . \mathrm{min}$ ). Despite the absence of detectable differences in insulin sensitivity after a short-term HF diet, glucose tolerance was significantly higher in the transgenics compared to B6 controls after 2 mo of HF feeding (AUC: aP2-Ucp1, $1105 \pm 97$ vs. B6, $1625 \pm 127 \mathrm{mM} . \mathrm{min})$. Better glycemic control of transgenic mice compared to B6 controls was associated with a higher expression of Glut4 in the skeletal muscle (by $\sim 44 \%$ ) despite lower plasma levels of adiponectin (aP2-Ucp1, $4.5 \pm 0.4$ vs. B6, $8.2 \pm 0.4 \mu \mathrm{g} / \mathrm{mL}$ ) and leptin $(3.3 \pm 0.5$ vs. $6.2 \pm 1.7 \mathrm{ng} / \mathrm{mL})$.

Conclusion: Respiratory uncoupling in white fat of the aP2-Ucp1 transgenic mice was associated with low adiponectin levels that could be responsible for elevated HGP in these mice. UCP1 over-expression in white fat did not affect the impairment of whole-body insulin sensitivity induced by a short-term HF feeding, however it partially protected against glucose intolerance and down-regulation of Glut4 in skeletal muscle induced by a prolonged HF feeding, likely due to its ability to protect non-adipose tissues against the accumulation of lipids. 


\section{4}

Significance of white adipose tissue metabolism for whole body nutrition partitioning

P. Janovska, M. Rossmeisl, P. Flachs, O. Kuda,

J. Kopecky;

Adipose Tissue Biology, Institute of Physiology, Acadamy of Sciences of the Czech Republic, Prague, Czech Republic.

Background and Aims: Studies on various transgenic mice indicated that accumulation of body fat can be decreased by specific modulation of metabolism of white adipose tissue (WAT), in spite of the fact that WAT contributes marginally to whole body energy expenditure. We used the model of mice resistant to obesity due to ectopic expression of mitochondrial uncoupling protein 1 (UCP1) in WAT (aP2-Ucp1 mice) to study the effect of respiratory uncoupling in WAT mitochondria on whole body energy expenditure and nutrition partitioning. The effect of n-3 polyunsaturated fatty acids of marine origin, eicosapentaenoic (EPA) and docosahexaenoic (DHA) acids, which limit accretion of body fat while up-regulating mitochondrial biogenesis and increasing fatty acid oxidation in WAT was also studied.

Materials and Methods: To characterize the effect of ectopic UCP1 in WAT, aged male hemizygous aP2-Ucp 1 mice and their nontransgenic littermates on C57BL/6J background fed chow diet ad libitum and maintained at $30^{\circ} \mathrm{C}$ were used. Body weight was lower in transgenic than nontransgenic mice $(34.1 \pm 0.7$ vs. $38.1 \pm 0.9 \mathrm{~g} ; \mathrm{n}=8)$. To study the effect of EPA and DHA, adult male C57BL/6J were maintained at $20^{\circ} \mathrm{C}$ and fed for 2 wk either high-fat (HF) diet ( $60 \%$ calories as fat, mainly corn oil) or a HF diet in which $9 \%$ of dietary lipids was replaced by EPA and DHA (using EPAX 1050TG; Pronova Biocare, Norway). Whole body oxygen consumption, $\mathrm{CO}_{2}$ production, and deep body temperature were analyzed in resting animals, using an indirect calorimetry system INCA (Somedic; Sweden) and telemetry system E-Mitter (Mini Mitter Co. Inc.; USA). Gene expression was assessed by qRT-PCR.

Results: In the $30^{\circ} \mathrm{C}$-acclimated mice, whole body oxygen consumption was 2 -fold higher at $20^{\circ} \mathrm{C}$ than at $30^{\circ} \mathrm{C}$ and it was not affected by the ectopic UCP1 in white fat. Mean values of $\mathrm{CO}_{2} / \mathrm{O}_{2}$ ratio ( $\mathrm{RQ}$ value) were higher in the nontransgenic than in aP2-Ucp 1 mice, both at $20^{\circ} \mathrm{C}$ $(0.883 \pm 0.016$ vs. $0.847 \pm 0.017)$ and at $30^{\circ} \mathrm{C}(0.941 \pm 0.015$ vs. $0.918 \pm 0.027)$ and the differences between genotypes were confirmed by the plots of RQ vs. percent relative cumulative frequency (PRCF). In accordance with the known atrophy of brown adipose tissue in the aP2-Ucp 1 mice, minimum deep body temperature $\left({ }^{\circ} \mathrm{C}\right)$ was lower in the transgenic mice than in nontransgenic mice if measured at $20^{\circ} \mathrm{C}(32.7 \pm 0.4$ vs. $34.3 \pm 0.2)$ but not at $30^{\circ} \mathrm{C}(34.7 \pm 0.7$ vs. $34.8 \pm 0.2$ ). Study of the effects of $n-3$ polyunsaturated fatty acids indicated strong induction by EPA and DHA of the genes engaged in lipid oxidation, CPT-1 and PPARalpha, in adipocytes in epididymal WAT (2.1- and 2.5-fold induction, respectively) but not in skeletal muscle or liver. In this experiment, no significant effect of the diet on either whole body oxygen consumption or RQ values was observed.

Conclusion: Modulation of WAT metabolism by ectopic UCP1 is likely to result in increased in situ lipid oxidation that can help to limit fat accumulation in animals exposed to HF diet and that could be detected at the whole organism level by the indirect calorimetry. However, even lower induction of fatty acid oxidation in WAT (and possibly other tissues) by dietary EPA and DHA, undetectable by indirect calorimetry, may contribute to the anti-obesity effect of these compounds.

\section{PS 53}

\section{Inflammation and adipose tissue}

\section{5}

\section{Serum visfatin increases with progressive $\beta$ cell deterioration \\ A. López-Bermejo ${ }^{1}$, B. Chico-Julià ${ }^{1}$, M. Fernàndez- Balsells $^{1}$, M. Recasens ${ }^{1}$, E. Esteve ${ }^{1}$, R. Casamitjana ${ }^{2}$, W. Ricart ${ }^{1}$, J.-M. Fernández-Real ${ }^{1}$; \\ ${ }^{1}$ Endocrinology, Dr. Josep Trueta Hospital, Girona, ${ }^{2}$ Endo- crine Laboratory, University Clinical Hospital, Barcelona, Spain.}

Background and Aims: Visfatin has shown to be increased in type-2 diabetes mellitus (T2DM), but to be unrelated to insulin sensitivity. We hypothesized that visfatin is associated with insulin secretion in humans.

Materials and Methods: A cross-sectional study was conducted in 103 non-diabetic (non-DM) men and 57 (31 male and 26 female) T2DM patients. Type-1 diabetic (T1DM) patients with long-standing disease $(n=24 ; 11$ male and 13 female) were also studied.

Results: In non-DM subjects, circulating visfatin (ELISA) was independently associated with insulin secretion [acute insulin response to glucose (AIRg), from intravenous glucose tolerance tests], but not with insulin sensitivity $\left(S_{\mathrm{I}}\right)$ or other metabolic or anthropometric parameters, and AIRg alone explained between $4 \%$ and $8 \%$ of visfatin variance $(\beta=-0.31, P=0.007)$. Circulating visfatin was increased in T2DM [mean (95\% CI): 19 (18-21) $\mathrm{ng} / \mathrm{ml}$ vs. $16(15-17) \mathrm{ng} / \mathrm{ml}$, for T2DM and non-DM subjects, 
respectively; $P=0.005$ (adjusted for sex, age and BMI)], although this association was largely attenuated after accounting for HbAlc (Figure). Finally, circulating visfatin was found to be increased in patients with long-standing T1DM, even after adjusting for HbA1c values [mean $(95 \%$ CI): 26 (22-29) ng/ml; $P<0.005$ and $P<0.0001$ (adjusted for sex, age, BMI and HbA1c), compared to either T2DM or non-DM subjects, respectively; Figure].

Conclusion: Circulating visfatin is increased with progressive $\beta$-cell deterioration. The study of the regulation and role of visfatin in diabetes mellitus merits further consideration.

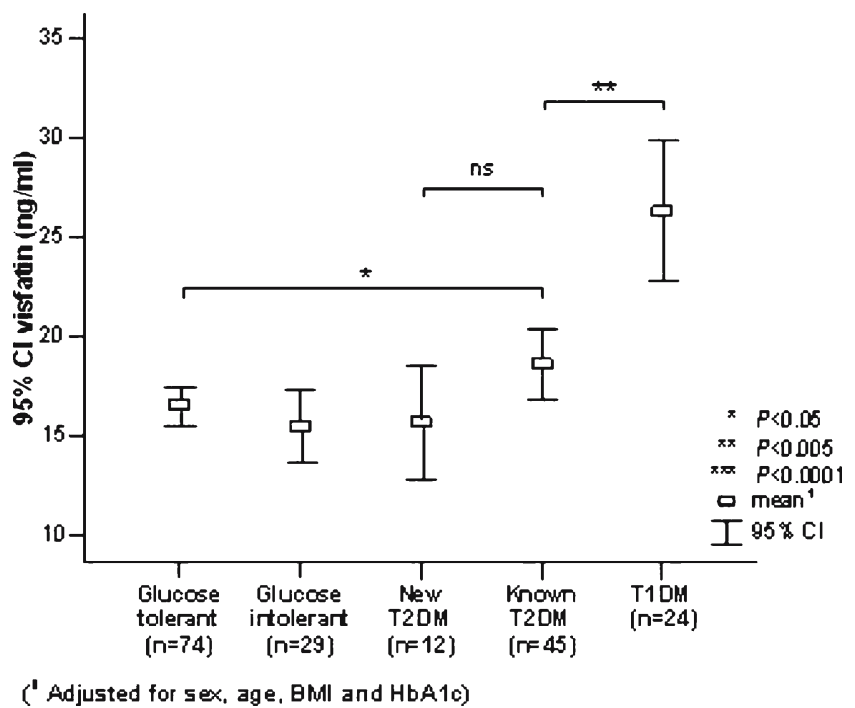

A. L-B. is a Research Investigator of the Fund for Scientific Research "Ramon y Cajal" (Ministry of Education and Science, Spain).

\section{6}

The expression of visfatin in viceral fat of obese rats and the regulation of blockade of renin-angiotensin-system (RAS)

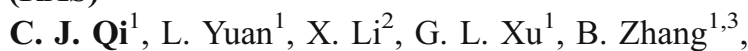
Z. S. Tang ${ }^{1}$, Y. Zhang ${ }^{1}$, L. L. Cao ${ }^{1,4}$, H. Y. Qiu ${ }^{1}$;

${ }^{1}$ Enocrinology and Metabolism, Union Hospital, Tongji Medical College, Huazhong University of Science \& Technology, Wuhan, ${ }^{2}$ Enocrinology and Metabolism, Union Hospital, Tongji Medical College,Huazhong University of Science \& Technology, Wuhan, ${ }^{3}$ Endocrinology and Metabolism, Shanghai Changzheng Hospital, Shanghai, ${ }^{4}$ Endocrinology and Metabolism, The 1st Republic Hospital of Jiujiang, China.
Background and Aims: Adipose tissue secretes several proteins (adipokines) that may influence insulin sensitivity and atherogenesis. Visfatin is a newly identified adipokine, highly expressed in human visceral fat. Visfatin's plasma levels are increased with expanding obesity and it is thought to promote accumulation of visceral fat, most propably acting through the insulin receptor pathway. It has been demonstrated that renin-angiotensin system (RAS) relate to insulin resistance state. RAS blockade promotes the recruitment and differentiation of pre-adipocytes and that increased formation of small insulin-sensitive adipocytes counteracted ectopic deposition of lipids, thereby improving insulin sensitivity. Angiotensin-converting enzyme inhibitors and angiotensin II receptor blockers have been reported to improve insulin sensitivity, but there are no reports on the relationship between visfatin concentrations and blockade of the renin-angiotensin system (RAS). We therefore examined the association between insulin sensitivity assessed by the euglycemic-hyperinsulinemic glucose clamp technique and visfatin mRNA levels as well as the RAS blockade on visfatin mRNA expression in high-fat diet induced insulin resistance rats.

Materials and Methods: 24 6-week-old male rats were randomly devided into 3 groups: normal control group, insulin resistance group, intervention group. Insulin resistance rat model was created by high fat high caloric laboratory chow for 8 weeks. Intervention treatment was performed by AT2 receptor antagonist Valsartn $(15 \mathrm{mg} / \mathrm{kg}$, 4 weeks). Insulin sensitivity was measured with euglycemic, hyper-insulinemic clamp technique. AGT, GLUT-4, visfatin mRNA expression levels in epididymal fat were examined by the RT-PCR.

Results: 1.comparison between insulin resistance and normal group: The percentages of visceral fat (epididymal fat as example) in whole body weight have a striking increase $(3.5 \%$ vs. $2 \%)$. Visfatin and AGT mRNA levels were significantly increased in high-fat diet induced insulin resistance rats when compared to normal control group, whereas GLUT-4 mRNA and insulin-stimulating glucose disposal rates (GDR) during the clamp were significantly decreased $\left(5.5 \mathrm{mg} / \mathrm{kg}^{-1} * \mathrm{~min}^{-1}\right.$ vs. $\left.12 \mathrm{mg} / \mathrm{kg}^{-1} * \mathrm{~min}^{-1}\right)$. Fasting insulin levels were also increased compared to control group $(87.78 \mathrm{uIU} / \mathrm{ml}$ vs. $15.24 \mathrm{uIU} / \mathrm{ml})$. The fasting serum glucose levels didn't have notable difference. 2 . intervention group compared to the above two groups: After 6 wks treatment with Valsartn, the mean fasting insulin levels reduced to $30.28 \mathrm{uIU} / \mathrm{ml}$, GDR increased to $9 \mathrm{mg} / \mathrm{kg}^{-1} * \mathrm{~min}^{-1}$. There was also a decrease of the visceral fat percentage $(2.9 \%)$, yet not have significantly difference. AGT and visfatin mRNA were significantly reduced, GLUT-4 mRNA were also increased.

Conclusion: Visfatin mRNA levels were significantly increased in obese insulin resistant state and this may 
related to their increased adipose tissue mass. RAS blockade can improve insulin resistance state effectively and can reduce the visfatin levels in adipose tissue.

Supported by a grant of Hubei Provincial Natural Science Foundation (2005ABA158)

\section{7}

High-fat diets do not provoke adipose tissue inflammation in obese mice when including $n-3$ polyunsaturated fatty acids

J. Todoric, M. Loeffler, W. Waldhaeusl, T. M. Stulnig; Department of Internal Medicine III, Division of Endocrinology and Metabolism, Medical University Vienna, Austria.

Background and Aims: Inflammatory changes in white adipose tissue of obese individuals appear to have a causal role in development of insulin resistance and diabetes mellitus type 2. Based on beneficial effects of $n-3$ polyunsaturated fatty acids (PUFAs) on inflammatory diseases and diabetes development, we aimed at investigating possible effects of PUFAs on white adipose tissue inflammation in obese diabetic mice.

Materials and Methods: We treated obese diabetic $(\mathrm{db} / \mathrm{db})$ as well as lean non-diabetic mice $(\mathrm{db} /+)$ with either low-fat standard diet (LF) or high-fat diets enriched in saturated and monounsaturated fatty acids (HF/S), n-6 PUFA (HF/6) and the latter including $30 \%$ of fatty acids as n-3 PUFA $(\mathrm{HF} / 3)$ for 6 weeks. Gene expression was evaluated from $\mathrm{db} / \mathrm{db}$ and $\mathrm{LF} \mathrm{db} /+$ animals by oligonucleotide microarrays ( 3 per group) and by quantitative RT-PCR (4-5 per group). Results: We found increased expression of 244 genes in $\mathrm{db} /$ $\mathrm{db}$ animals treated with HF/S compared to those on LF, many of which were involved in inflammatory responses indicating macrophage infiltration (e.g., CCL2, CD68, Itgam). N-3 PUFA reverted most HF-induced changes in $\mathrm{db} / \mathrm{db}$ animals. Gene expression profiles of $\mathrm{db} /+$ mice revealed only few HF-induced changes. Histological analyses revealed considerable mononuclear infiltrates mostly expressing the macrophage markers $\mathrm{F} 4 / 80$ and/or MAC-2 in white adipose tissue from $\mathrm{db} / \mathrm{db}$ mice fed $\mathrm{HF} / \mathrm{S}$ compared to those on LF. Inclusion of n-3 PUFA in the high-fat diet completely prevented macrophage infiltration induced by high-fat diets. Feeding HF/S resulted in a twofold borderline-significant increase in JNK phosphorilation that was almost completely prevented by the n-3 PUFA containing diet in $\mathrm{db} / \mathrm{db}$ mice.

Conclusion: We conclude that protective effects of $n-3$ PUFAs on development of complications of human obesity could be mediated by their anti-inflammatory effect in white adipose tissue.
Supported by the CeMM-Center of Molecular Medicine, a basic research institute within the companies of the Austrian Academy of Sciences and the Skoda Award of the Austrian Association for Internal Medicine

0718

Lamin $\mathrm{C}$ isoform expression is increased in subcutaneous adipose tissue in human obesity and type 2 diabetes. Relationship with inflammatory markers

A. García-España ${ }^{1}$, M. Miranda ${ }^{1}$, C. Richart ${ }^{1}$, M. R. Chacón ${ }^{1}$, L. Gallart ${ }^{1}$, J. M. Fernández-Real ${ }^{2}$, F. Vidal ${ }^{1}$, W. Ricart ${ }^{2}$, J. Vendrell ${ }^{1}$;

${ }^{1}$ Research Unit, University Hospital of Tarragona Joan XXIII, ${ }^{2}$ Endocrinology and Diabetes Unit, University Hospital Josep Trueta from Girona, Spain.

Background and Aims: Variations of gene expression in adipose tissue may be involved in obesity and related metabolic abnormalities. Recently, lamins have been associated with proteins involved in adipocyte differentiation in lipodystrophy syndromes associated with insulin resistance. However, little is known about the role of lamin in adipose tissue of obese and of type 2 diabetic (T2D) patients. In this work, we studied the expression of lamin A/C (LMNA) and adipogenic and inflammatory markers in subcutaneous adipose tissue of lean, obese, and type 2 diabetic subjects.

Materials and Methods: Subcutaneous adipose tissue samples were obtained from 52 non diabetic subjects with different degrees of obesity and from 25 subjects with T2D. Clinical and anthropometrical variables were obtained. Real Time PCR was used to measure mRNA gene expression of Lamin A and C, PPAR $\gamma$, SREBP1c, CD14 and CD68 in whole adipose tissue. Likewise, variation of Lamin A and $\mathrm{C}$ in response to an endotoxin inflammatory stimulus was measured in subcutaneous-isolated preadipocytes and in the human monocytic THP-1 cell line.

Results: In non-diabetic subjects, Lamin C mRNA expression levels were significantly increased in the morbidly obese group compared with the lean group $(8.47 \pm 0.63 \mathrm{vs}$ $7.72 \pm 0.57 ; \mathrm{p}<0.01)$. In the multiple linear regression analysis, Lamin $\mathrm{C}$ was found positively associated with BMI $(\beta=0.576, p<0.001)$ and negatively associated with CD68 $(\beta=-0.442, p<0.01)$. In T2D patients, Lamin C mRNA expression was increased compared with non diabetic subjects $(8.67 \pm 0.44$ vs $7.91 \pm 0.55 ; \mathrm{p}<0.001)$. Regression analysis showed that Lamin $\mathrm{C}$ was positively associated with gender $(\beta=0.262, \mathrm{p}<0.05)$ and PPAR $\gamma$ $(\beta=0.317, p<0.01)$ and negatively associated with CD68 $(\beta=-0.652, p<0.001)$. Lamin A was found more expressed in stromovascular fraction than in mature adipocytes. Treatment of preadipocytes and in vitro differentiated adipocytes 
with endotoxin (LPS) did not affect Lamin A/C mRNA or protein expression. On the contrary, treatment of THP-1 monocytic cell line with the endotoxin significantly increased Lamin A/C mRNA and protein expression in a dose-dependent manner.

Conclusion: Obesity and type 2 diabetes are associated with increased expression levels of Lamin $\mathrm{C}$ in subcutaneous adipose tissue. Our data provide for the first time evidence that LMNA mRNA expression is modulated in human obesity and T2D and lamin $\mathrm{C}$ is related to adipose inflammatory environment.

Supported by the following grants: Marato TV3, FIS 05 1994, Red de Centros C03/08, and Red de Grupos G03/212

\section{9}

Adipose tissue is infiltrated with macrophages around dead fat cells in subjects with high liver fat content, independent of obesity

J. Westerbacka ${ }^{1}$, M. Kolak ${ }^{2}$, D. Wågsäter ${ }^{2}$, A. Rissanen ${ }^{3}$, A.-M. Häkkinen ${ }^{4}$, P. Eriksson ${ }^{2}$, R. M. Fisher ${ }^{2}$,

H. Yki-Järvinen ${ }^{1,2}$;

${ }^{1}$ Department of Medicine, University of Helsinki, Finland, ${ }^{2}$ Karolinska Institutet, King Gustaf V Research Institute, Stockholm, Sweden, ${ }^{3}$ Obesity Research Unit, University of Helsinki, Helsinki, Finland, ${ }^{4}$ Department of Oncology, University of Helsinki, Finland.

Background and Aims: The causes of insulin resistance related hepatic fat accumulation are unknown. Adipose tissue of obese mice and men is characterized by increased number of macrophages potentially regulating liver fat via adipose tissue-derived factors. It is unknown whether i) number of macrophages, ii) gene expression of macrophage marker CD68 or chemokines attracting monocytes/macrophages or iii) expression of genes related to inflammation and/or insulin resistance differ in adipose tissue of equally obese humans with low or high liver fat content (LFAT). We determined in human adipose tissue: i) number of macrophages and macrophage aggregates suggested to engulf dead adipocytes (crown-like structures, CLS) ii) expression of monocyte-chemoattractant protein 1 (MCP-1) and macrophage inflammatory protein $1 \alpha$ (MIP-1 $1 \alpha$ ) and iii) adiponectin, PPAR $\gamma$, TNF $\alpha$ and PAI-1 in BMI-matched subjects with low and high LFAT.

Materials and Methods: 20 non-diabetic apparently healthy overweight/obese women were divided into low and high LFAT groups based on their median LFAT ( $2.6 \pm 0.4$ vs. $15.3 \pm 3.1 \%$ for low vs. high LFAT, mean \pm SEM, $\mathrm{p}<0.001)$ measured using magnetic resonance proton spectroscopy. Surgical adipose tissue biopsies were obtained and adipose tissue mRNA concentrations of
CD68, MCP-1, MIP-1 $\alpha$, PPAR $\gamma$, TNF $\alpha$ and PAI-1 and of adiponectin were measured using real-time PCR. Dead adipocytes were identified by negative perilipin staining and the number of macrophages by immunohistochemistry using antibodies against the macrophage marker CD68. In addition, fasting serum lipids, insulin and adiponectin were measured.

Results: The groups had similar BMI $(35.5 \pm 1.0$ vs. $36.8 \pm 0.9 \mathrm{~kg} / \mathrm{m}^{2}$, low vs. high LFAT) and age $(42 \pm 3 \mathrm{vs}$. $38 \pm 2$ yrs). The high LFAT group had higher insulin $(\mathrm{p}=0.004)$ and lower HDL cholesterol $(\mathrm{p}=0.010)$ concentration than the low LFAT group. Expression of the macrophage marker and monocyte/macrophage chemokines were significantly higher in adipose tissue in high LFAT group as compared to low LFAT group: (CD68: $3.3 \pm 0.4$ vs. $2.0 \pm 0.1, \mathrm{p}=0.007, \mathrm{MCP}-1: 0.55 \pm 0.08$ vs. $0.34 \pm 0.03$, $\mathrm{p}=0.037, \mathrm{MIP}-1 \alpha: 1.0 \pm 0.2$ vs. $0.37 \pm 0.06, \mathrm{p}=0.007) . \mathrm{CD} 68$ expression was significantly correlated with LFAT (Spearman's $\mathrm{r}=0.57, \mathrm{p}=0.009)$ but not with BMI $(\mathrm{r}=0.02$, $\mathrm{p}=0.945$ ). Adipose tissue expression or serum concentration of adiponectin did not differ between the groups. The CLSs were identified around dead adipocytes (i.e. absence of perilipin) and their quantitative analyses are ongoing.

Conclusion: Adipose tissue is inflamed in those obese subjects who have increased liver fat content. Dead adipocytes may be the signal for macrophage accumulation in adipose tissue. Lack of difference in serum or adipose tissue adiponectin between subjects with high and low liver fat content does not negate a role for adiponectin in regulating liver fat but suggests additional contribution of adipose tissue-dependent or -independent mechanisms.

Supported by Biovitrum and EVO funds

0720

Gene expression profiles and roles of leukocyte cell adhesion molecules in obese-related inflammation in adipose tissue

C. Sato, K. Shikata, A. Tone, M. Sasaki, S. Ohga,

S. Nishishita, K. Sarai, Y. Shikata, H. Makino;

Department of Medicine and Clinical Science, Okayama University Graduate School of Medicine, Dentistry and Pharmaceutical Sciences, Okayama, Japan.

Background and Aims: Obesity increases the risk of developing diabetes and cardiovascular disease. Recently, obesity has been described as a "low-grade"systemic inflammatory condition. It has been reported that macrophages are increased in adispose tissues of obese people suggesting that adipose tissue itself is a source and site of 
inflammation. Leukocyte recruitment into inflammatory sites is promoted by chemokines and cell adhesion molecules. Moocyte chemoattractant protein-1(MCP-1), one of the major chemokines, is upregulated in adipose tissues of obese individuals suggesting that MCP-1 contributes to the recruitment of macrophage infiltration. However, the adhesion pathway of monocytes into adipose tissue has not been clarified. We aimed to clarify the adhesion molecules which contiributes to the process of macrophage infiltration into adipose tissue using obese mouse models.

Materials and Methods: We examined glucose and lipid metabolism of 8 week-old female C57/BL6 $(n=10)$ and $\mathrm{db} /$ $\mathrm{db}$ mice $(\mathrm{C} 57 \mathrm{BL} / \mathrm{KsJ}-\mathrm{db} / \mathrm{db})(\mathrm{n}=10)$. We carried out microarray gene profiling of RNA isolated from visceral adipose tissues from these mice using Affymetrix Gene Chip Mouse Genome 430 2.0 Array. After data analyses, we measured the transcript levels of selected genes based on their biological functions using quantitative real-time PCR. In addition, the distribution was examined by immunohistochemistry.

Furthermore, 7 week-old male C57/BL6 mice were fed with regular diet $(n=9)$ and high-fat diet (HFD) $(n=11)$ respectively for 12 weeks. We performed insulin tolerance test (ITT) and intraperitoneal glucose tolerance test (IPGTT), and then evaluated the visceral adipose tissues of these mice by microarray, real-time PCR and immunohistochemistry as described above.

Results: The $\mathrm{db} / \mathrm{db}$ mice and HFD mice showed significant increase of body weight, weight of visceral adipose tissue, triglyceride, free fatty acid, blood glucose and insulin level as compared with C57/BL6 control mice. Hemoglobin A1c was not different in all groups. HFD mice showed glucose intolerance and insulin resistance by ITT and IPGTT. Microarray profilling showed that more than twice upregulated genes consisted of 1001 genes in $\mathrm{db} / \mathrm{db}$ mice and 566 genes in HFD mice. We classified these genes into Gene Ontology categories. The analyses indicated the upregulation of cell adhesion-related genes including P-selectin, Pselectin glycoprotein ligand-1 (PSGL-1) and L-selectin as well as immune response-related genes in $\mathrm{db} / \mathrm{db}$ mice and HFD mice. However, expression of E-selectin was not detected in all groups. Quantitative real-time PCR and immunostaining confirmed the transcriptional and protein level upregulation of these adhesion molecules. Macrophagerelated genes such as CD14 were also increased in adipose tissues of $\mathrm{db} / \mathrm{db}$ and HFD mice.

Conclusion: Current results indicate that low grade inflammation is occurred in visceral fat of obese mice with insulin resistance. PSGL-1 is expressed on leukocyte surface and acts as a ligand for P-selectin which is expressed on endothelial cells. PSGL-1 also binds to E- and L-selectin. Selectin-PSGL-1 pathway may promote the recruitment of macrophages into adipose tissue. Selectin and PSGL-1 might be a novel target for the prevention of diabetes and atherosclerosis in obese people.

0721

Obesity and role of adipose tissue (AT) in inflammation and insulin sensitivity - the GENOBIN study

T. J. Lappalainen ${ }^{1}$, M. Kolehmainen ${ }^{2}$, U. Schwab ${ }^{1,3}$, L. Pulkkinen ${ }^{1}$, R. Kouki ${ }^{4}$, R. Rauramaa ${ }^{4}$, M. Uusitupa ${ }^{1}$, H. Gylling ${ }^{1,3}$;

${ }^{1}$ Department of Clinical Nutrition, ${ }^{2}$ Food and Health Research Centre, University of Kuopio, ${ }^{3}$ Department of Medicine, Kuopio University Hospital, Kuopio, ${ }^{4}$ Department of Physiology, Kuopio Research Institute of Exercise Medicine, Finland.

Background and Aims: Obesity is characterized as a lowgrade inflammation state shown as increased circulating concentrations of several cytokines and acute phase proteins. AT has been illustrated to secrete a wide number of inflammatory factors. Recently serum amyloid A (SAA) was found to be expressed in AT. There is also the growing view that the obesity-associated inflammatory state plays a causal role in the development of type 2 diabetes. The aim of the present study was to examine circulating inflammatory markers and their gene expressions in AT in obese subjects with features of metabolic syndrome.

Materials and Methods: Seventy-five overweight to obese subjects (BMI 32.9 $\pm 2.8 \mathrm{~kg} / \mathrm{m} 2$, mean $\pm \mathrm{SD}$ ) with impaired glucose tolerance and other features of metabolic syndrome, aged $60 \pm 7$ years participated in the lifestyle modification intervention for $33.3 \pm 1.1$ weeks. Fasting blood samples were drawn before the intervention to determine serum glucose, lipids, lipoproteins, cytokines, CRP and SAA. SAA gene expression was studied from mRNA extracted from abdominal subcutaneous AT using real-time PCR. The study was approved by the Ethics Committee of the Kuopio University Hospital and was performed in accordance with the standards of the Helsinki Declaration.

Results: In women the serum SAA concentration $(17.6 \pm 15.7 \mathrm{ug} / \mathrm{ml}$ in women vs. $8.7 \pm 4.9 \mathrm{ug} / \mathrm{ml}$ in men, $\mathrm{p}=0.002)$ and mRNA expression in AT $(108 \pm 71$ in women vs. $26 \pm 19$ in men, $\mathrm{p}=0.001$ ) were significantly higher than in men. In the whole study population serum SAA concentrations correlated positively with percentage of body fat $(\mathrm{r} 2=0.502, \mathrm{p}=0.001)$, body fat mass $(\mathrm{r} 2=0.443$, $\mathrm{p}=0.001)$ and BMI $(\mathrm{r} 2=0.448, \mathrm{p}=0.001)$ in baseline. Interestingly, we found also significant correlation between leptin and fS-SAA $(\mathrm{r} 2=0.518, \mathrm{p}=0.0001)$ and CRP $(\mathrm{r} 2=0.309, \mathrm{p}=0.007)$. 
In women CRP and fS-SAA were negatively associated with insulin sensitivity $(\mathrm{r} 2=-0.388, \mathrm{p}=0.021 ; \mathrm{r} 2=-0.355$, $\mathrm{p}=0.039$, respectively). Serum levels of CRP also correlated highly with fS-SAA $(\mathrm{r} 2=0.529, \mathrm{p}=0.001)$. Women were also characterized by larger adipocytes than men $(\mathrm{p}=0.0001)$, but the size of adipocyte did not correlate with inflammatory markers.

Conclusion: The present study shows that elevated serum concentrations of inflammatory markers are involved in the obesity-induced inflammatory state. The relationships between leptin and inflammatory markers indicate that leptin may have a role in the regulation of the obesity-associated inflammation. Furthermore, the associations of inflammatory markers with insulin sensitivity confirm their contribution to the development of type 2 diabetes.

Supported by the Academy of Finland, the Ministry of Education in Finland, the Finnish Foundation for Diabetes Research and the Sigrid Juselius Foundation

\section{2}

Mesothelial cells as a source of the pro-inflammatory cytokine IL-18 in human omental fat

C. Darimont ${ }^{1}$, P. Leone-Vautravers ${ }^{1}$, R. Mansourian ${ }^{2}$, F. Blancher ${ }^{1}$, O. Avanti ${ }^{1}$, M. A. Roberts ${ }^{1}$, V. Giusti ${ }^{3}$, K. Macé ${ }^{1}$;

${ }^{1}$ Nutrition \& Health, ${ }^{2}$ Bioanalytical Science, Nestlé Research Center, Lausanne, ${ }^{3}$ Internal Medicine, University Hospital CHUV, Lausanne, Switzerland.

Background and Aims: In obesity, the production of proinflammatory cytokines by adipose tissue is proposed to participate to the impairment of insulin sensitivity. The objective of this study was to analyze the expression profile of pro-inflammatory cytokines and cofactors in the visceral and the subcutaneous fat of obese subjects.

Materials and Methods: Omental (OAT) and subcutaneous adipose (SAT) samples were collected during a gastroplasty surgery in 14 obese female patients $\left(\mathrm{BMI}=47.5 \pm 1.6 \mathrm{~kg} / \mathrm{m}^{2}\right)$ having no associated co-morbidities. RNA from each samples were prepared and cRNA were hybridized on Affymetrix GeneChip containing almost 39,000 transcripts. Tissue samples were fixed in paraformaldehyde for protein detection by immonuhistochemistry.

Results: Among the 24 pro-inflammatory cytokines and cofactors found to be differentially regulated in both tissues, 19 were up regulated in OAT as compared to SAT. The interleukin 18 (IL-18) was the pro-inflammatory cytokine the most up regulated in OAT as compared to SAT ( +6 fold). This cytokine was mostly expressed in the stromavascular fraction of OAT suggesting that other cells than adipocytes preferentially expressed IL-18. The analysis of cellular location of IL-18 protein expression demonstrated that this interleukin was expressed in neutrophils and in mast cells, both in OAT and SAT, but not in macrophages. In OAT, mesothelial cells were identified as an additional source of IL-18. Expression of this pro-inflammatory cytokine in mesothelial cells, known to be absent in SAT, may explain the higher level of IL-18 expression in OAT.

Conclusion: Altogether, our data demonstrate that 1) in obese subjects the omental fat depot had a higher expression level of pro-inflammatory cytokines than in SAT, and 2) in OAT, mesothelial cells, by secreting IL-18, participate to the inflammatory process associated to obesity.

0723

TNF-alpha activates the expression of inflammatory cytokines in undifferentiated human pre-adipocytes

P. Isakson, A. Hammarstedt, U. Smith; Department of Internal Medicine, The Lundberg Laboratory for Diabetes Research, The Sahlgrenska Academy at Göteborg University, Sweden.

Background and Aims: The adipose tissue is not only important for lipid storage and release; it is also the largest endocrine and secretory organ of the body releasing a number of important adipokines like leptin, adiponectin and retinol-binding protein-4 (RBP-4) as well as cytokines like Interleukin-6 (IL-6) and monocyte chemo-attractant protein-1 (MCP-1) that are related to inflammation and the innate inflammatory response. Moreover, the adipokines cross-talk with other tissues of the body to modulate insulin sensitivity and action. Type 2 diabetes and obesity are characterized by low-grade inflammation with elevated levels of acute phase-reactants like CRP and serum amyloid A. This inflammatory response is believed to be initiated in the adipose tissue and also to play an important role for the development of cardiovascular disease.Several studies have shown that it is not the primary adipocytes, which are the major producers of cytokines in the adipose tissue but rather the stromal cell fraction containing preadipocytes, fibroblasts, endothelial and inflammatory cells. During the development of obesity and insulin resistance, macrophages are recruited into the adipose tissue and this seems to induce a sequence of events, which are important for the development of insulin resistance and probably also the inflammation.Here we examined if the undifferentiated preadipocytes in human adipose tissue are responsive to inflammatory cytokines, in particular TNF alpha, since this potent cytokine is released by infiltrating macrophages.

Material and Methods: Abdominal subcutaneous adipose tissue was obtained from six different donors by needle biopsy. From the biopsy, the stromal vascular fraction was 
retrieved following collagenase digestion and removal of the primary adipocytes. The inflammatory cells were also removed by magnetic bead eluation of CD 14 and CD 45 positive cells. The remaining part of the fraction was seeded and grown in six-well plates. The cells were either left untreated or grown in the presence of TNF-alpha, IL-6 or Resistin for 10 days. RNA was extracted with the QIAGEN RNEasy mini kit. The gene expression was analyzed with real-time RT-PCR (Taqman).

Results: The undifferentiated preadipocytes expressed several inflammatory cytokines also in the non-stimulated state. However, the expression of TNF-alpha itself as well as IL-6 and MCP-1 were markedly and significantly increased in the presence of TNF-alpha. Interestingly, CCR-2, the receptor for MCP-1, was not expressed in human preadipocytes indicating that these cells produce MCP-1, as well as other cytokines, but they are not target cells of MCP-1. Both IL-6 and Resistin had only minor inflammatory effects when compared to TNF-alpha.

Conclusion: These data show that the presence of TNFalpha, which is released by macrophages, leads to a marked inflammatory phenotype in human undifferentiated preadipocytes. This finding provides a mechanism for the increased production of cytokines found in the stromal vascular cells of the adipose tissue in obesity. Furthermore, since TNF-alpha is not released by adipocytes, these data support a key role for invading macrophages in inducing the inflammatory response in human adipose tissue in obesity.

Supported by the Swedish Research Council, the European Community's FP6 EUGENE2, the Swedish Diabetes Association, the Inga Britt and Arne Lundberg Foundation, the Novo Nordisk Foundation

\section{4}

Insulin exerts an anti-inflammatory effect in adipose cells by antagonising IL-6 signaling

C. X. Andersson, E. Wallerstedt, V. Rotter Sopasakis, U. Smith;

Lundberg Laboratory for Diabetes Research, Internal Medicine, Göteborg, Sweden.

Background and Aims: Both obesity and insulin resistance correlate with a low-grade chronic inflammation that probably originates in the adipose tissue. Several studies have in recent years shown that cytokines, such as interleukin-6 (IL-6), secreted by the adipose tissue constitute an inhibitory effect on the insulin signaling, suggesting a role in the development of insulin resistance. To further explore the cross-talk between IL- 6 and insulin signaling pathways we have studied the effect of insulin on the IL-6 signaling pathway.
Materials and Methods: The mouse pre-adipocyte cell line, 3T3-L1, was differentiated to mature adipocytes and incubated with insulin, IL-6 and IL-6 together with insulin. The same experiments were performed in MEF cells lacking functional SHP2. Protein and RNA samples were purified and analyzed by western blotting and real-time PCR (TaqMan) analysis, respectively.

Results: Here we show a mechanism whereby insulin reduces the tyrosine phosphorylation and induces the serine phosphorylation of STAT3 and, thereby, reduces its nuclear localization and transcriptional activity. As a consequence, expression of the inflammatory markers, serum amyloid A 3 (SAA3) and haptoglobin, are significantly decreased in cells stimulated with both IL-6 and insulin compared to cells treated with IL- 6 alone. We further show that the protein tyrosine phosphatase, SHP2, is activated upon insulin signaling. Since SHP2 is required for dephosphorylation of STAT3, this finding provides a mechanism for the effect of insulin on the tyrosine phosphorylation of STAT3. Additionally, we present that the effect of insulin on the IL-6 signaling pathway is mediated through the MAPK pathway, since treatment with the MEK inhibitor, PD98059, reduces all of the observed effects. We, therefore, conclude that insulin exerts an important anti-inflammatory effect on IL-6 signaling.

Conclusion: We conclude that insulin, through the MAPKsignaling pathway also involving SHP2, exerts an antiinflammatory effect by anagonizing IL-6 signaling.

Supported by the Swedish Research Council, the European Community, Inga Britt and Arne Lundberg Foundation and Sonya Hedenbratt Memorial Fund

\section{PS 54}

\section{Prediction of risk in insulin resistance and diabetes}

0725

Simple risk factors for developing type 2 diabetes mellitus as identified in the STOP-NIDDM trial

J. Tuomilehto ${ }^{1,2}$, M. Peltonen ${ }^{1,2}$, Q. Qiao ${ }^{1,2}$,

J.-L. Chiasson ${ }^{3}$, M. Hellmich ${ }^{4}$, W. Lehmacher ${ }^{4}$, T. Evers ${ }^{5}$; ${ }^{1}$ Department of Public Health, University of Helsinki, Finland, ${ }^{2}$ Department of Epidemiology and Health Promotion, National Public Health Institute, Helsinki, Finland, ${ }^{3}$ CHUM-Hôtel-Dieu, L'Université de Montréal, ${ }^{4}$ Institute of Medical Statistics, Informatics and Epidemiology, University of Cologne, Germany, ${ }^{5}$ Bayer Healthcare AG, Wuppertal, Germany. 
Background and Aims: Interventions to prevent or delay type 2 diabetes mellitus (DM) should be available for subjects at increased risk. The risk may be assessed by specific characteristics, so called risk factors that are associated with the development of the disease. Ideally, such risk factors are causal, and may favorably be altered by interventions and are relatively easy to determine even in a general practice setting. Materials and Methods: The STOP-NIDDM trial showed that acarbose treatment reduced the individual risk of developing DM in patients with impaired glucose tolerance (IGT). The trial database $(n=1160)$ was used to select important predictors by means of Cox regression for the time to onset of DM. The final model was externally validated using DECODE study data.

Results: Sex, body height ( $>168$ vs. $\leq 168 \mathrm{~cm}$ ), waist circumference ( $\leq 88$ vs. $89-102$ vs. $>102$ ) and diagnosed hypertension were identified as both important and readily available risk factors. The 2.5-year risk of developing DM for each combination of these four risk factors was calculated based on the full Cox model and is presented in the following table.

Risk chart for 2.5-year risk of developing type 2 diabetes mellitus

\begin{tabular}{|c|c|c|c|c|c|c|}
\hline \multirow{2}{*}{$\begin{array}{l}\text { Body height } \\
(\mathrm{cm})\end{array}$} & \multicolumn{6}{|l|}{ Women } \\
\hline & \multicolumn{3}{|l|}{$>168$} & \multicolumn{3}{|l|}{$\leq 168$} \\
\hline $\begin{array}{l}\text { Waist } \\
\text { circumference } \\
(\mathrm{cm})\end{array}$ & $\leq 88$ & 89-102 & $>102$ & $\leq 88$ & 89-102 & $>102$ \\
\hline $\begin{array}{l}\text { Diagnosed } \\
\text { hypertension }\end{array}$ & no yes & no yes & no yes & no yes & no yes & no yes \\
\hline \multirow[t]{2}{*}{$\begin{array}{l}\text { 2.5-year-risk } \\
(\%)\end{array}$} & 1519 & $17 \quad 23$ & 2431 & $22 \quad 29$ & $27 \quad 34$ & 3644 \\
\hline & Men & & & & & \\
\hline $\begin{array}{l}\text { Body height } \\
\text { (cm) }\end{array}$ & $>168$ & & & $\leq 168$ & & \\
\hline $\begin{array}{l}\text { Waist } \\
\text { circumference } \\
(\mathrm{cm})\end{array}$ & $\leq 88$ & 89-102 & $>102$ & $\leq 88$ & 89-102 & $>102$ \\
\hline $\begin{array}{l}\text { Diagnosed } \\
\text { hypertension }\end{array}$ & no yes & no yes & no yes & no yes & no yes & no yes \\
\hline $\begin{array}{l}\text { 2.5-year-risk } \\
(\%)\end{array}$ & 1722 & $20 \quad 26$ & $28 \quad 35$ & 2633 & $31 \quad 38$ & 4150 \\
\hline
\end{tabular}

Conclusions: Based on simple risk factors such as sex, body height, waist circumference and diagnosed hypertension patients may be screened at low costs. Patients at increased risk for developing DM may be offered further diagnostic procedures and/or specific DM prevention strategies, such as lifestyle modification with or without pharmaceutical intervention (i.e. acarbose or metformin). Supported by research grants from Bayer Healthcare $A G$, Wuppertal
0726

Use of clinical parameters to identify insulin resistance, measured by euglycaemic hyperinsulinaemic clamp, in an Italian population of $\mathbf{3 0 0}$ subjects

D. Bracaglia ${ }^{1}$, M. Perna ${ }^{1}$, A. Baroni ${ }^{1}$, M. Marini ${ }^{1}$, M. Cardellini ${ }^{1}$, S. Gambardella ${ }^{1}$, G. Sesti ${ }^{2}$, S. Frontoni ${ }^{1}$; ${ }^{1}$ Dept. of Internal Medicine, University of Rome Tor Vergata, ${ }^{2}$ Dept. of Experimental and Clinical Medicine, University of Catanzaro "Magna Graecia”, Italy.

Background and Aims: Insulin resistance is associated with adverse outcomes, such as cardiovascular disease and type 2 diabetes mellitus. However, the methodologies currently available for the measurement of insulin resistance are cumbersome to administer and expensive. Given the relative ease of measuring plasma glucose and insulin, numerous alternative indexes, both fasting and during oral glucose tolerance test (OGTT), have been proposed. The EGIR database, "enlarged" with the insulin resistant population of PIMA Indians and Mexican Americans, is the only one presently existing. Aim of the present study was to investigate the reproducibility of these data in a population of about 300 Italian subjects, evaluated by OGTT and euglycemic hyperinsulinaemic clamp study $\left(40 \mathrm{mU} / \mathrm{m}^{2}\right.$. $\left.\mathrm{min}\right)$.

Materials and Methods: 101 males and 214 females, mean age: $37.7+4.2$ years, 10 unknown diabetic subjects, $31 \mathrm{IFG}$ and/or IGT, 254 normo-tolerant subjects). The proposed definition (Diabetes 2005, 333) of $\mathrm{M}<5.04 \mathrm{mg} / \mathrm{min} . \mathrm{kg}$ of lean mass identified as insulin-resistant only $5 \%$ of normal and $25 \%$ of diabetic subjects, whilst the surrogate formula (BMI $>28.9 \mathrm{~kg} / \mathrm{m}^{2}$ or HOMA-IR $>4.65$ o $\mathrm{BMI}>27.5$ and HOMA-IR $>3.60$ ) identified as insulin-resistant the $44 \%$ of normo-tolerant subjects.

Results: In order to modify these indexes for the Italian population, we considered all diabetic and IFG/IGT subjects as insulin-resistant, and an $\mathrm{M}$ value equal to mean+ 1DS (6.4 mg/kg.min) of the subjects was chosen as cut-off value for insulin resistance. We therefore calculated which index, among those proposed in the literature, (Matsuda, Quicki, BMI, fasting insulin, HOMA, metabolic syndrome, according to NCEP-ATPIII criteria) is able to predict insulin resistance, measured by euglycemic clamp, with the best sensitivity and specificity. We found that Matsuda index, combined with metabolic syndrome, displays a good sensitivity (63.4\%) and an excellent specificity (91.3\%); among the fasting indexes, fasting insulin combined with metabolic syndrome criteria, showed the best sensitivity and specificity (67.1\% and $83.1 \%$, respectively). 
Conclusion: In conclusion, parameters determined on EGIR enlarged with an insulin resistant population seems irreproducible on Italian population. On the contrary, Matsuda index combined with NCEP criteria for metabolic syndrome has the best predictive value for insulin resistance, evaluated by insulin clamp. In the absence of OGTT, the most predictive index is the combination of fasting insulin and metabolic syndrome.

\section{7}

Elevated concentrations of asymmetrical dimethylarginine predict deterioration of glucose tolerance in women with previous gestational diabetes mellitus

F. Mittermayer ${ }^{1}$, C. Winzer ${ }^{2}$, K. Krzyzanowska ${ }^{3}$, T. Prikoszovich ${ }^{2}$, S. Demehri ${ }^{1}$, A. Tura ${ }^{4}$, G. Pacini ${ }^{4}$, O. Wagner ${ }^{5}$, A. Luger ${ }^{2}$, M. Wolzt ${ }^{1}$, A. Kautzky-Willer ${ }^{2}$; ${ }^{1}$ Department of Clinical Pharmacology, Medical University Vienna, ${ }^{2}$ Department of Internal Medicine III, Division of Endocrinology and Metabolism, Medical University Vienna, ${ }^{3}$ Department of Internal Medicine I, Rudolfstiftung Hospital, Vienna, Austria, ${ }^{4}$ Metabolic Unit, Institute of Biomedical Engineering, CNR, Padua, Italy, ${ }^{5}$ Institute for Medical Laboratory Diagnostics, Medical University Vienna, Austria.

Background and Aims: Women with previous gestational diabetes (GDM) have a high risk for type 2 diabetes mellitus. The endogenous nitric oxide synthase inhibitor asymmetric dimethylarginine (ADMA) could be related to disorders of glucose metabolism such as insulin resistance. To evaluate if ADMA predicts a deterioration of glucose tolerance in women with previous GDM and to assess the time course of concentration changes we analysed ADMA in women with previous GDM 14-16 weeks after delivery and after a median follow-up of 2.8 years (interquartile range: 1.5-4.6). Materials and Methods: ADMA and L-arginine were determined by high performance liquid chromatography in 77 women with previous GDM who underwent also an oral glucose tolerance test. Survival analysis was performed using the Kaplan-Meier method and Cox regression analysis.

Results: Deterioration of glucose tolerance was observed in $36 \%$ of women with ADMA above and $11 \%$ of those with ADMA below the median of $0.56 \mu \mathrm{mol} / 1(\mathrm{P}=0.008, \log$ rank test). ADMA correlated significantly with mean arterial blood pressure (MAP) but not with other parameters. The fully adjusted hazard ratio for a decline of glucose tolerance during follow-up was 4.06 (95\% CI: 1.13-14.54; $\mathrm{P}=0.031$; adjusted for age, BMI, fasting glucose, insulin sensitivity, MAP, high sensitivity C-reactive protein [hsCRP] and creatinine). Apart from ADMA, hsCRP also predicted deterioration of glucose tolerance [hazard ratio
3.69 (1.01-13.45, $\mathrm{P}=0.048)]$. L-arginine was not associated with changes of the glucose tolerance status. ADMA decreased significantly during follow-up.

Conclusion: High ADMA concentrations after delivery predict deterioration of glucose tolerance in women with previous GDM.

Supported by the Austrian Science Fund

0728

Do patients with elevated blood glucose and the metabolic syndrome have increased risk of diabetes type 2 according to Finnish Diabetes Risk Score criteria?

A. Gilis-Januszewska ${ }^{1}$, R. Topór-Mądry ${ }^{2}$, A. Pająk ${ }^{1}$;

${ }^{1}$ Dept of Endocrinology, ${ }^{2}$ Dept of Epidemiology, Jagiellonian University, Krakow, Poland.

Background and Aim: Finnish Diabetes Risk Score (FRS), simple, and quastionaire based tool was developed to identify individuals at high risk of diabetes. This tool has not been used and validated in Polish population yet. Similarly the relation between the Metabolic Syndrome (MS) - cluster of metabolic disorders known to be related to diabetes and CVD, and FRS has not been studied.

The aim was to study the association of elevaed blood glocose, the MS and the risk of development of diabetes type 2 according to FRS.

Sample and Methods: The studied population were 977 men and 983 women aged 45-64, randomly selected from the Krakow's population. All analyses were performed after exclusion of subjects with known diabetes. The MS was calculated according to the Interational Diabetes Federation criteria. Categories of FRS were made according to definition. The chi square test was used for estimation of the relation of the elevated glucose and the MS in FRS categories.

Results: FRS higher that 7 was present in $66,8 \%$ men and $74,1 \%$ women. Fasting blood glucose over $6,1 \mathrm{mmol} / 1$ was observed in 16,8\% men and 9,7\% women. Fasting blood glucose over $7 \mathrm{mmol} / 1$ had 4,8\% men and 3,0\% women. The metabolic syndrome was present in $30,3 \%$ men and $32,4 \%$ women. The frequency of low and high FRS in groups with elevated glucose and MS are presented in the table.

\begin{tabular}{|c|c|c|c|c|c|c|}
\hline & \multicolumn{2}{|c|}{ Glucose $>6,1 \mathrm{mmol} / 1$} & \multicolumn{2}{|c|}{ Glucose $>7,0 \mathrm{mmol} / 1$} & \multicolumn{2}{|c|}{ MS (IDF) } \\
\hline & Men* & Women* & Men* & Women* & Men* & Women* \\
\hline$>7$ & $3,4 \%$ & $2,3 \%$ & $0 \%$ & $0 \%$ & $1,5 \%$ & $0,7 \%$ \\
\hline $15-20$ & $37,7 \%$ & $62,1 \%$ & $45,2 \%$ & $74,1 \%$ & $29,7 \%$ & $34,9 \%$ \\
\hline
\end{tabular}

* $\mathrm{p}<0,05$ 
Conclusion: Low FRS (less than 7) is very rare in the subjects with elevated glucose and with the MS. Only one third of men and women with the MS have high FRS (1520). The subjects with elevated glucose had high incidence of FRS between 15-20, however this association was stronger in women than in men. Further investigations on the defition of the MS and prognostic tool for prediction of diabetes in everyday practice should be carried on.

\section{9}

Reduced antioxidative defence and prothrombogenic factors in children and adolescents with metabolic syndrome

V. S. Dimitrijevic Sreckovic ${ }^{1}$, E. Colak $^{2}$, P. B. Djordjevic ${ }^{1}$, D. Gostiljac ${ }^{1}$, S. Popovic ${ }^{1}$, B. M. Sreckovic ${ }^{3}$,

T. Novakovic ${ }^{1}$, R. Obrenovic ${ }^{2}$, F. Canovic ${ }^{1}$, M. Ilic ${ }^{1}$, D. Nikolic ${ }^{1}$, G. Milic ${ }^{1}$, T. Nisic ${ }^{1}$, V. Panasiuk ${ }^{1}$, G. Pejcic ${ }^{1}$; ${ }^{1}$ Diabetology, Institute for Endocrinology, Diabetes and Metabolic Diseases, ${ }^{2}$ Diabetology, Institute of Medical Biochemistry, ${ }^{3}$ Urgent Cardiology, Institute of Cardiovascular Diseases, Belgrade, Serbia and Montenegro.

Background and Aims: Since early glycoregulation disorders, impaired fasting glucose (IFG) and impaired glucose tolerance (IGT) are associated with metabolic syndrome (MS), the aim of this study was to determine the frequency of metabolic syndrome, examine the extent of disorders, and analyze insulin sensitivity, secretion, lipid status, prothrombogenic factors and antioxidative status. Three of the following five criteria were used for MS diagnosis in children and adolescents: waist circumference $\geq 90$ percentile for age and sex; triglycerides $>1.7 \mathrm{mmol} / \mathrm{l}$; HDL cholesterol $<1.0$; hypertension $\geq 90$ percentile for height, age and sex; glycaemia $>6.0$. Materials and Methods: The study included 24 obese individuals (age 10-20 years, BMI or waist circumference $\geq 90$ percentile). Oral glucose tolerance test (OGTT) was used to evaluate the extent of disorder. Insulin sensitivity was determined by HOMA IR, insulin secretion by HOMA $\beta$, lipid status by spectrophotometry (total cholesterol, HDL-cholesterol, LDL-cholesterol, triglycerides). Prothrombogenic factors analyzed were the plasma plasminogen activator PAI-1 (by plasminogen substrate assay) and fibrinogen. Erythrocyte dismutase (SOD) and erythrocyte glutation peroxide (GPX) were markers of antioxidative defense.

Results: Metabolic syndrome was found in one third of patients (BMI $33.9 \pm 0.74 \mathrm{~kg} / \mathrm{m}^{2}$; waist circumference $98 \pm 0.55 \mathrm{~cm}$; blood pressure $123.7 \pm 4.8 / 78.7 \pm 13.1 \mathrm{mmHg}$; insulinemia mean value $57.7 \pm 20.3 \mathrm{mlU} / \mathrm{l}$; HOMA IR elevated in all patients (6.5 \pm 3.2$)$; HOMA $\beta$ showing insulin secretion disorder $(246 \pm 55.6)$; increased triglycerides
$(1.77 \pm 0.05 \mathrm{mmol} / \mathrm{l})$; decreased HDL $(0.72 \pm 0.12 \mathrm{mmol} / \mathrm{l}))$. These patients had increased values of PAI-1 (6.4 $\pm 0.08 \mathrm{U} /$ $\mathrm{ml}$ ), normal values of fibrinogen, and reduced antioxidative

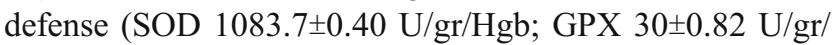
$\mathrm{Hgb}$ ). The other two thirds showed 1-2 metabolic

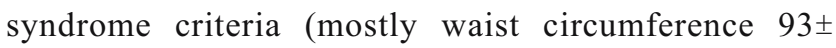
$21.6 \mathrm{~cm}$; decreased HDL $1.0 \pm 0.15 \mathrm{mmol} / 1)$, insulin resistance parameters (HOMA IR 6.2 \pm 4.7 , insulin mean value $70.0 \pm 37.9 \mathrm{mlU} / \mathrm{l})$, normal prothrombogenic status and reduced antioxidative defense (SOD 932 $\pm 0.81 \mathrm{U} / \mathrm{gr} / \mathrm{Hgb}$; GPX 30.3 $\pm 0.8 \mathrm{U} / \mathrm{gr} / \mathrm{Hgb}$ ).

Conclusion: These results indicate a growing occurrence of MS in children and adolescents and therefore an increased risk of obtaining diabetes mellitus type 2. Increased HOMA IR and HOMA $\beta$ and insulinemia mean value confirm hyperinsulinism, insulin resistance and decreased insulin sensitivity, all these correlating with waist circumference and lipid status. Increased values of plasminogen and reduced antioxidative defense, all resulting in accelerated atherosclerosis and coronary disease in MS patients.

\section{0}

Leisure-time physical activity is associated with the metabolic syndrome in type 1 diabetes; interaction with the Pro12Ala variant of the PPAR $\gamma$ Gene (The FinnDiane Study)

J. Wadén, C. Forsblom, L. Thorn, J. Fagerudd,

K. Pettersson-Fernholm, H. Tikkanen, J. Turunen,

M. Wessman, M. Rosengård-Bärlund, O. Heikkilä,

M. Saraheimo, P.-H. Groop;

Dept of Medicine, University of Helsinki, Folkhälsan Genetic Institute, Helsinki, Finland.

Background and Aims: Recently, we showed that the metabolic syndrome (MetS) is frequently found in type 1 diabetes (T1D). Additionally, the MetS is associated with micro- and macrovascular diabetic complications. Physical activity has been shown to reduce the risk for MetS in the general population. Further, the Pro12Ala variant of the PPAR $\gamma$ gene has been linked to components of the MetS (hyperglycaemia, dyslipidaemia and obesity). The aim of our study was to investigate if leisure-time physical activity (LTPA) and/or PPAR $\gamma$ Pro12Ala genotype are associated with MetS in T1D.

Materials and Methods: MetS was defined according to the NCEP ATPIII criteria. A MetS-score (1-5) was established according to the number of fulfilled criteria, all patients having hyperglycaemia. A minimum of three criteria out of five indicated MetS. LTPA was assessed with a validated, quantitative questionnaire and expressed as $\mathrm{MET}^{*} \mathrm{~h} /$ week (MET=metabolic equivalent). LTPA $<10$ 
MET*h/week indicated sedentary LTPA habits. All T1D patients in the FinnDiane study with complete data on LTPA and MetS were studied. Excluding patients with endstage renal disease and/or major cardiovascular events, 1104 patients remained (male/female:520/584, age $36.1 \pm 11.5 \mathrm{yrs}$, duration of diabetes $20.8 \pm 11.9 \mathrm{yrs}$, BMI $25.0 \pm 3.2 \mathrm{~kg} / \mathrm{m}^{2}, \mathrm{HbA}_{1 \mathrm{c}} 8.3 \pm 1.4 \%$ ). Genotyping of the Pro12Ala variant of PPAR $\gamma$ was performed using TaqMan ${ }^{\circledR}$ technology; 881 patients with data on LTPA and MetS were genotyped.

Results: MetS was found in $29.6 \%$ of the patients (male/ female $=28.1 / 31.0 \%, \mathrm{P}=0.29$ ). The genotype distribution of PPAR $\gamma$ Pro12Pro:Pro12Ala:Ala12Ala was 598:261:22 patients. $31.4 \%$ of Ala-carriers and $30.4 \%$ with the Pro12Pro genotype had MetS ( $\mathrm{P}=0.76)$. In sedentary vs. more physically active patients, MetS was present in 35.1\% vs. $27.9 \%$, respectively ( $\mathrm{P}=0.033$ adjusted for age). Level of LTPA was inversely associated with the MetS-score. Stratifying for $P P A R \gamma$ genotype, the association was apparent only in Ala-carriers (see table: LTPA values are presented as $M E T * \mathrm{~h} /$ week, median[IQR]).

Conclusion: LTPA is associated with features of the metabolic syndrome in T1D. The data further suggest a gene-environment interaction as Ala-carriers of the PPAR gene may respond better to physical activity than patients with the Pro12Pro genotype. Whether higher level of LTPA may lead to a reduction in the risk for diabetic late complications has to be established.

LTPA (MET*h/week, median[IQR]) by MetS-score.

\begin{tabular}{llll}
\hline MetS-score & All Patients & Pro12Pro & $\begin{array}{l}\text { Pro12Ala }+ \\
\text { Ala12Ala }\end{array}$ \\
\hline 1 & $20.0[11.0-35.0]$, & $21.0[11.0-37.0]$, & $23.0[13.0-35.3]$, \\
& $\mathrm{N}=259$ & $\mathrm{~N}=135$ & $\mathrm{~N}=66$ \\
2 & $22.0[10.0-35.0]$, & $21.0[9.5-34.0]$, & $22.0[11.0-35.0]$, \\
& $\mathrm{N}=518$ & $\mathrm{~N}=281$ & $\mathrm{~N}=128$ \\
3 & $18.0[10.0-35.0]$, & $19.0[10.0-36.0]$, & $16.0[9.0-26.0]$, \\
& $\mathrm{N}=239$ & $\mathrm{~N}=132$ & $\mathrm{~N}=67$ \\
4 & $14.0[7.0-33.0]$, & $14.5[4.3-39.8]$, & $10.0[4.0-16.5]$, \\
& $\mathrm{N}=71$ & $\mathrm{~N}=40$ & $\mathrm{~N}=17$ \\
5 & $12.0[5.5-20.5]$, & $12.5[7.3-23.5]$, & $9.0[1.5-31.0]$, \\
& $\mathrm{N}=17$ & $\mathrm{~N}=10$ & $\mathrm{~N}=5$ \\
P-value & 0.017 & 0.356 & 0.0011 \\
P-value adj. & 0.002 & 0.429 & 0.0000036 \\
for age & & & \\
\hline
\end{tabular}

Supported by Wilhelm och Else Stockmanns Stiftelse
0731

Independent measures of insulin secretion and insulin sensitivity during the same test for clinical purposes: The Glucagon-Insulin Tolerance Test (GITT) M. Dorkhan ${ }^{1}$, D. Tripathy ${ }^{1}$, G. Malm ${ }^{1}$, Y. Wessman ${ }^{1}$, T. Tuomi ${ }^{2}$, L. Groop ${ }^{1}$;

${ }^{1}$ Department of Clinical Sciences, Lund University, Malmö, Sweden, ${ }^{2}$ Department of Medicine, Helsinki University Hospital, Finland.

Background and Aims: To validate a test for the independent assessment of insulin secretion and insulin sensitivity during the same occasion for future use in phenotyping studies for clinical use, i.e. a glucagon-Cpeptide test followed by an insulin tolerance test (GITT).

Materials and Methods: The GITT measures C-peptide response to $0.5 \mathrm{mg}$ of glucagon i.v. followed $30 \mathrm{~min}$ later by a $0.05 \mathrm{U} / \mathrm{kg}$ insulin tolerance test (ITT). Nine subjects with normal glucose tolerance participated on different days in an ITT, glucagon-C-peptide test, ITT followed by a glucagon-C-peptide test and glucagon-C-peptide test followed by an ITT (GITT) to establish whether and how the two tests can be combined. The test was then repeated to investigate the reproducibility and was validated against Botnia clamp in patients with normal and abnormal glucose tolerance and in patients with type 2 diabetes. The Botnia clamp measures the first phase insulin response to $0.3 \mathrm{~g} / \mathrm{kg}$ glucose i.v. (FPIR) and insulin sensitivity (M-value) from a $2 \mathrm{hr}$ euglycemic clamp started $60 \mathrm{~min}$ after the glucose bolus.

Results: The $\mathrm{K}_{i t t}$ from the GITT was reproducible (CV $13 \%$ in NGT, $22 \%$ in T2D) and correlated with the M-value from the Botnia clamp $(\mathrm{r}=0.86 ; \mathrm{p}=0.01$, in $\mathrm{T} 2 \mathrm{D}+\mathrm{NGT}+\mathrm{IGT}$, $\mathrm{n}=20$ ). The relative $\mathrm{C}$-peptide response to glucagon correlated with FPIR $(r=0.78, p=0.02)$.

Conclusion: The Botnia clamp provides reproducible and independent estimates of insulin sensitivity and insulin secretion on the same occasion for metabolic studies. The GITT is a pheasible and reproducible test, which provides similar information for clinical use.

Supported by grants from the Sigrid Juselius Foundation, JDF-Wallenberg, Academy of Finland, Swedish Medical Research Council, Finnish Diabetic Research Foundation, Swedish Diabetic Research Foundation, EEC GIFT, and the Novo Nordisk Foundation 
0732

A robust simple clinical measurement to diagnose partial lipodystrophy

S. G. I. Suliman, G. D. Tan, L. Dennis, F. Karpe;

Oxford Centre for Diabetes, Endocrinology and Metabo-

lism, University of Oxford, Oxford, United Kingdom.

Background and Aims: Subjects with partial lipodystrophy (PLD) display the full spectrum of symptoms of the metabolic syndrome, including severe insulin resistance (IR), hypertension, hypertriglyceridaemia, low HDL-cholesterol, and hyperglycaemia. These subjects exhibit a substantial reduction in peripheral fat mass, yet show similar and often more extreme metabolic characteristics than obese individuals.

PLD is considered a rare cause of IR and type 2 diabetes, with the exception of subjects with human immunodeficiency virus, particularly those on highly active antiretroviral therapy. However, we recently started actively identifying subjects with PLD within our clinics. It appears that the frequency of PLD is possibly under-reported, as we have identified 23 subjects with irrefutable clinical characteristics of PLD amongst subjects attending T2DM, Lipid, and/or polycystic ovarian syndrome clinics within 18 months.

The difficulty in detecting subjects with PLD has, at least in part, been due to lack of simple clinical tests to aid diagnosis. In view of the clear phenotype of PLD, we hypothesized that a simple ratio of central to peripheral skin fold measurements would distinguish PLD from healthy individuals.

Materials and Methods: The sum of two trunk skinfolds measurements (subscapular+suprailiac) divided by the sum of two peripheral (triceps+biceps) were measured in 5 subjects with $L M N A / C$ PLD, 14 subjects with PLD awaiting genetic diagnosis, and 835 healthy populationbased controls aged 30-50 yrs from the Oxford Biobank.

Results:

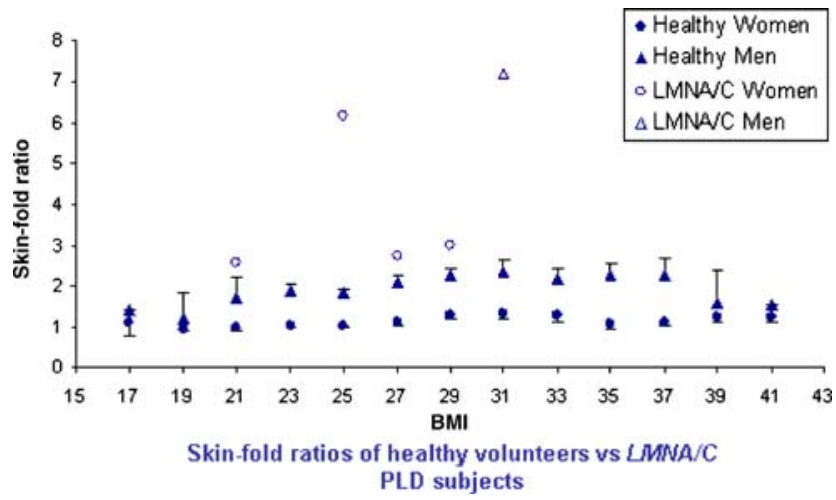

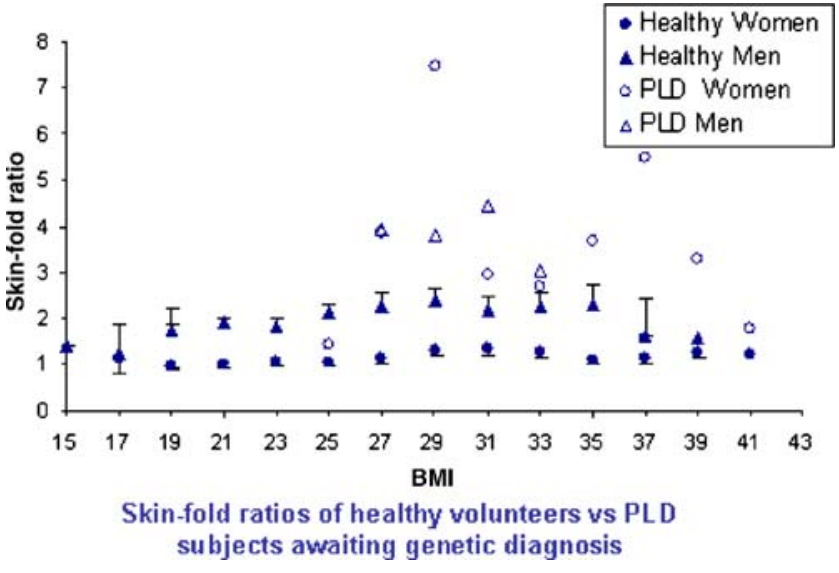

All PLD subjects were well outside (above) the 95\% confidence interval of body mass index normalised healthy subjects.

Conclusion: We demonstrate that the measurement of skin fold ratios is a robust and simple clinical tool to identify PLD. This will simplify the detection of subjects with PLD.

0733

Preservation of beta cell secretion in T1DM subjects with low risk HLA genotype

M. Spoletini ${ }^{1}$, A. Petrone ${ }^{1}$, S. Zampetti ${ }^{1}$, I. Alemanno ${ }^{1}$, M. Capizzi ${ }^{1}$, S. Zavarella ${ }^{1}$, C. Bizzarri ${ }^{2}$, IMDIAB Group ${ }^{3}$, P. Pozzilli ${ }^{3}$, R. Buzzetti ${ }^{1}$;

${ }^{1}$ Clinical Science, "La Sapienza" University, Rome,

${ }^{2}$ Scientific Institute, Bambino Gesù Hospital, Rome, ${ }^{3}$ Endocrinology, Campus Biomedico, Rome, Italy.

Background and Aims: Type 1 diabetes (T1DM) is a autoimmune disease leading to the destruction of beta cells and consequently to the need of insulin therapy. HLA complex constitutes the most relevant genetic region contributing $50 \%$ of the inherited risk for T1DM.

Although previous studies demonstrated that HLA susceptible genotypes are important in determining of residual beta cell function as measured by C-peptide levels, the relationship between HLA class II and beta cell function did not lead to conclusive reports. The aim of this study was to investigate in T1DM patients the relation between HLA and differences in C-peptide levels at diagnosis and twelve months thereafter following intensive insulin therapy.

Materials and Methods: A total of 120 T1DM consecutive subjects (age 1-49; M 53\%, F 47\%) were studied. These subjects included those diagnosed in childhood, adolescence and young adult age. HLA class II (DRB1 and DQB1) genes were typed using a SSO (Sequence Specific 
Oligonucleotide). HLA genotypes were classified in three risk categories based on the absolute risk values (AR) previously estimated in the Italian population: high risk ( $\mathrm{RA}=1: 23)$, moderate risk $(\mathrm{RA}=1: 150)$ and low risk $(\mathrm{RA}=1: 1100)$. C-peptide levels at diagnosis and at $12^{\circ}$ month were measured by a radioimmunoassay validated trough the international $\mathrm{C}$-peptide workshop. The normal range of fasting $\mathrm{C}$-peptide established in 150 control subjects 5-40 years old, median 18 years, was $0.35-1 \mathrm{~nm} / \mathrm{L}$ with intra-coefficient and inter-coefficients varying between 10 and $15 \%$, respectively.

Statistical analysis was performed using multiple linear regression. $\triangle-\mathrm{CP}$, calculated as $\mathrm{CP} 12^{\circ}$ month - $\mathrm{CP} 0^{\circ}$ month, was $\log _{10}$ transformed to normalize its distribution. Results: We observed that the $\Delta$-CP showed an increasing linear trend with lower values in patients with high risk genotype, intermediate in moderate risk genotype and higher values in low risk genotype $(\Delta-\mathrm{CP}=-0.25,-0.09$ and 0.13 respectively; $p$ for trend $=0.009)$. Moreover, we did not observe any statistically significant difference when we analysed the relation between $\Delta-\mathrm{CP}$ and age of onset $(\mathrm{p}=0.569)$.

Conclusion: The results of this study indicate that a preservation of beta-cell secretion occurs in T1DM subjects with low risk HLA genotype compared to the other two HLA risk categories irrespective of age at disease of diagnosis. This finding is of particular relevance for designing trials of prevention of beta cell destruction in T1DM.

Supported by FIRB 2001 RBNE01C5S2_004

\section{PS 55}

\section{Markers of insulin resistance and metabolic syndrome}

0734

Inflammatory parameters in type 2 diabetics and in the metabolic syndrome

A. Grosz ${ }^{1}$, E. Nagy ${ }^{2}$, T. Halmos ${ }^{3}$;

${ }^{1}$ Internal Department, Polyclinic of the Hospitaler Brothers of St.John, Budapest, ${ }^{2}$ Clinical Laboratory, Polyclinic of the Hospitaler Brothers of St.John, Budapest, ${ }^{3}$ Diabet. Dept., National Institute Korányi, Budapest, Hungary.

Background and Aims: to investigate which inflammatory parameters seem to prove the inflammatory origin of the metabolic syndrome.

Materials and Methods: 1./19 patients who fit at laest 3 criteria - due to IDF - of the metabolic syndrome, but were glucose tolerant ( 9 females, 10 males: mean age:
$43 \pm 16$ years, BMI:32 $\pm 4,8$, waist circumference: in males: $110,4 \pm 11,5 \mathrm{~cm}$, in females: $93 \pm 8,5 \mathrm{~cm}) .2 . / 113$ type 2 noninsulin treated diabetics, (males: 65, females: 48, mean age:55 \pm 10 years, BMI: $29 \pm 4.5$, waist circumference: $105 \pm$ $13 \mathrm{~cm}$ ). Clinical data: family anamnesis, waist circumference, RR, therapy. Laboratory investigations: se insulin level, HbA1c, hsCRP with immun turbidity, ferritin by sandwich-immunoassay, TNF $\alpha$, IL-6, IL-6sR by ELISA. Statistical methods: Pearson correlation, ANOVA variance analysis, Mann-Whitney test, significance: $p<0,05$.

Results: In the metabolic syndrome group: we found significant correlation $(\mathrm{p}<0,05)$ between BMI and following inflammatory markers: hsCRP, IL-6sR, IL-6 and TNF $\alpha$. Similar significant correlation could be found between blood sugar, HbA1c and IL-6sR. However we couldn't prove any significant link between metabolic parameters and ferritin levels, but the tendency $(160 \pm 122 \mu \mathrm{g} / \mathrm{l})$ was increased. HbAlc in the type2diabetic group presented correlation with hsCRP, IL-6sR and with the number of white blood cells. The blood sugar level showed correlation with the IL-6sR level. To compare these two patient groups, the level of TNF $\alpha$ did not presented any significant difference. The IL-6 was significantly higher in the diabetic group $(p=0,02)$. However the level of IL-6sR was higher in the metabolic syndrome group $(499 \pm 150 \mathrm{pg} / \mathrm{ml})$ but significant difference could not be demonstrated $(p=0,332)$.

Conclusion: To analyse our results we can state that among normal glucose tolerant metabolic syndrome patients the inflammatory markers- hsCRP, IL-6,IL-6sR, TNF $\alpha$-prove the correlation between metabolic alterations and inflammation. The ferritin values on a significant level could not prove this correlation but there was a strong elevated tendency in the metabolic syndrome group. For more objective evaluation further investigations are needed with enrolling numerous patients mainly in the normal glucose tolerant metabolic syndrome group.

0735

C-reactive protein levels in subjects at risk for diabetes and its association with metabolic parameters

N. E. Gulcelik ${ }^{1}$, Y. Aral' ${ }^{2}$, M. Ozkaya ${ }^{2}$;

${ }^{1}$ Endocrinology and Metabolism, Ankara Oncology Hospital, Ankara, ${ }^{2}$ Endocrinology and Metabolism, Ankara Training Hospital, Ankara, Turkey.

Background and Aims: Subclinical inflamation is considered as a nontraditional risk factor for cardiovascular disease. By this time, there are several reports that type 2 diabetic subjects have higher serum levels of C-reactive protein (CRP) than those of non-diabetic subjects. We 
evaluated CRP levels and its association with metabolic parameters in a group of patients at high risk for diabetes. Materials and Methods: In this study we evaluated 46 patients at risk for diabetes, classified using the criteria described by Diabetes Prevention Program Research Group, and 22 control subjects matched for age, sex and body mass index. Anthropometric measurements, biochemical analyses and insulin and CRP levels were performed in each subject. Insulin resistance was assessed using the homeostasis model assessment of insulin resistance (HOMA).

Results: CRP levels were significantly higher in subjects at risk for diabetes than control subjects $(3.9 \pm 1.0$ vs. $2.1 \pm 0.8 \mathrm{mg} / \mathrm{L}, \mathrm{p}<0.001)$. Subjects at risk for diabetes were more insulin resistant than control subjects $(2.8 \pm 0.6$ vs. $1.09 \pm 0.4 \mathrm{mg} / \mathrm{L}, \mathrm{p}<0.001)$. CRP levels were correlated positively with systolic blood pressure $(\mathrm{r}=0.256, \mathrm{p}=0.035)$, fasting blood glucose $(r=0.417, p=0.001)$, postprandial glucose levels $(\mathrm{r}=0.367, \mathrm{p}=0.002)$, total cholesterol levels $(\mathrm{r}=0.383, \mathrm{p}=0.001)$, LDL levels $(\mathrm{r}=0.398, \mathrm{p}=0.001)$, triglyceride levels $(r=0.240, p=0.049)$ and HOMA score $(\mathrm{r}=0.326, \mathrm{p}=0.007)$, and correlated negatively with HDL levels $(r=-0.256, p=0.035)$. These correlations were still of significant importance after adjustment for body mass index. There was no correlation between CRP and diastolic blood pressure $(r=0.160, p=0.193)$ and waist circumference $(\mathrm{r}=0.160, \mathrm{p}=0.193)$.

Conclusion: These findings support that serum CRP level is higher in subjects at risk for diabetes than subjects wihout known risks. CRP level as an indicator of subclinical inflammation is correlated with risk factors of cardiovascular diseases and may be useful to predict accelerated atherosclerotic process in subjects at risk for diabetes.

\section{6}

Gender as an important determinant of elevated Creactive protein levels in subjects with the metabolic syndrome

M. Schulz, W. Leonhardt, C. Koehler, S. Gallo,

M. Hanefeld;

Centre for Clinical Studies, GWT-TUD, Dresden, Germany.

Background and Aims: Although there are a lot of studies on the relationship between the metabolic syndrome (MS) and C-reactive protein (CRP), a sensitive systemic marker of inflammation, little is known about gender differences. The aim of this study was to investigate the relationship between the MS and CRP in men and women.

Materials and Methods: The study population included 720 patients (354 men and 366 women) aged 40-75 years recruited from the Risk Factor in Impaired Glucose
Tolerance for Atherosclerosis and Diabetes (RIAD) study. CRP serum levels were measured by an immunoturbidimetric assay. MS was defined using the IDF-criteria (2005) and was present when a person have central obesity (defined as waist circumference $\geq 94 \mathrm{~cm}$ for men and $\geq 80 \mathrm{~cm}$ for women) plus any two of the following factors: triglyceride levels $\geq 1.7 \mathrm{mmol} / 1$, HDL cholesterol $<1.03 \mathrm{mmol} / 1$ in men and $<1.29 \mathrm{mmol} / 1$ in women, blood pressure $\geq 130 / 85 \mathrm{mmHg}$, and fasting plasma glucose $\geq 5.6 \mathrm{mmol} / 1$ or previously diagnosed type 2 diabetes. Subjects with CRP levels greater than $10 \mathrm{mg} / \mathrm{l}$ were excluded from the analysis.

Results: Both men and women with the MS had elevated CRP levels compared to those without the MS (geometric mean (standard error) CRP: men 1.3 (0.15) vs 1.86 (0.14)mg/l, women 1.49 (0.13) vs $2.69(0.14) \mathrm{mg} / \mathrm{l}$; both $\mathrm{p}<0.001)$. There was no significant difference in CRP levels between men and women without the MS, but compared to men with the MS women with the MS had significant higher CRP levels (1.86 (0.14) vs 2.69 (0.14) $\mathrm{mg} / \mathrm{l}, \mathrm{p}=0.001)$. All geometric means were adjusted for age, smoking status and alcohol consumption. Excluding all women taking hormone replacement therapy did not change these findings. Thirty-one percent of the women who had MS also had CRP levels $>3 \mathrm{mg} / \mathrm{l}$, characterizing subjects with high cardiovascular risk, in contrast to only $15.5 \%$ of the men. The odds ratio for CRP levels $>3 \mathrm{mg} /$ 1 was 1.37 (95\%CI: $0.74-2.55)$ for men and 3.57 (95\%CI: 2.04-6.25) for women with the MS.

Conclusion: These results indicate that the MS is associated with elevated CRP levels in both genders. However there exists a strong gender difference with higher CRP levels in women with the MS whereas no gender difference was found in people without MS.

0737

C-reactive protein, degree of glucose tolerance and metabolic syndrome

M. T. Martínez-Larrad, M. J. Martínez-Calatrava, J. L. González-Sánchez, C. Zabena, C. Fernández-Pérez, M. Serrano-Ríos;

Internal Medicine II, Hospital Clínico San Carlos, Madrid, Spain.

Background and Aims: To investigate the relationship between CRP, degree of glucose tolerance and Metabolic syndrome (MS).

Materials and Methods: Population based, cross sectional nationwide survey $(\mathrm{n}=1933$ individuals, aged 35-74 years, 47\% males (M), 53\% females (F)). Anthropometric parameters: Body mass Index (BMI), Waist Circumference (WC), 
Waist/Hip ratio $(\mathrm{W} / \mathrm{H})$, Systolic (SBP)/Diastolic Blood Pressure (DBP). Laboratory parameters: Oral glucose tolerance, Lipid Profile, CRP (nephelometry). MS: ATPIII criteria.

Results: CRP levels $\geq 1 \mathrm{mg} / \mathrm{l}$ a) Normoglycemics (NG) $65.2 \%$, impaired fasting glucose (IFG) $6.7 \%$, Impaired glucose tolerance (IGT) 15\% and type 2 Diabetes Mellitus (DM2) $13.1 \%$.

Overall MS prevalence 20.4\%, F: $23.6 \% / \mathrm{M}: 16.7 \%$ $(\mathrm{p}<0.001)$. MS in NG: 10.5\%, IFG: $57.8 \%$, IGT: $25.7 \%$; DM2: $59.5 \%(p<0.001)$. Obesity $(B M I \geq 30) \quad N G: 23.3 \%$, IFG:34.4\%, IGT: $35.6 \%$ and DM2: $42.6 \%$.

Correlations CRP/Components of MS by sex: M/F statistically significant unadjusted positive correlation with CRP: FG, glucose 2 hs (G2hs), BMI (strongest in F), WC, TG, SBP, DBP $(\mathrm{p}<0.003)$; significant negative correlation CRP/ HDL-C $(p<0.001)$. Strongest correlation observed: NG, IGT and DM was with : CRP/BMI ( $\mathrm{r}=0.29,0.39$ and 0.30 respectively)and in IFG: CRP with $\mathrm{WC}$ and BMI were $\operatorname{similar}(\mathrm{r}=0.38$ and 0.35 respectively).

In subjects with CRP levels $\geq 1 \mathrm{mg} / 1$ prevalence of MS increased a) NG OR:1.53 (IC 95\% 1.03-2.29), b) IFG OR: 5.55 (IC 95\% 2.48-12.40), c) IGT OR: 2.95 (IC 95\%1.177.49), d) DM2 OR: 3.64 (1.74-7.63). Prevalence of MS in NG was significant after adjusting by fasting glucose/ G2hs., in IFG after adjusting by BMI/WC/TG/HDL-C/BP/ FG; in IGT after adjusting for all abnormalities including BMI/G2hs, in DM2 only after adjusting by WC. Higher prevalence of MS adjusted by the abnormalities of MS presented in IFG.

CRP levels increased with number ( 2 or more) abnormalities of MS $(p<0.001)$ and degree of glucose tolerance $(\mathrm{p}<0.001)$.

The prevalence of MS increased 1.68 (IC 95\% 1.21-2.35) fold in individuals with $\mathrm{CRP} \geq 1.0 \mathrm{mg} / 1 \mathrm{age} / \mathrm{sex} / \mathrm{BMI} / \mathrm{IGT}$ adjusted by logistic regression model.

Conclusion: It appears that CRP levels over $\geq 1.0 \mathrm{mg} / 1$ are associated with criteria (ATPIII) of Metabolic Syndrome; obesity and glucose intolerance in a stepwise dependent manner.

\section{8}

Excretion of catecholamines and serum uric acid levels in obese patients with type 2 diabetes mellitus and metabolic syndrome

V. Skybun, N. Gurina, A. Shuprovich, V. Hovaka,

V. Korpachev;

Pharmacology and Pharmacotherapy of Endocrine Diseases, Institute of Endocrinology and Metobolism, Kiev, Ukraine.
Background and Aims: Metabolic syndrome (MS) is associated with increased activity of sympathoadrenal system (SAS). Elevated level of serum uric acid $\left(U_{\mathrm{s}}\right)$ is a sign of MS. Uric acid can be consider an activator of SAS because it has a structural similarity with methylxantines. The aim of the present investigation is to study association between activity of SNS and uricemia, inslin resistance (IR), insulinemia in patients with type 2 diabetes mellitus (DM2).

Materials and Methods: 49 subjects (M/F 27/22, aged $55.9 \pm 1.5 \mathrm{yr}$ ) with DM2 and signs of MS: abdominal obesity, hypertension, fasting hyperglycemia, cholesterolemia, and 17 age- and BMI- matched non-diabetic controls were studied. In 23 diabetics and 9 controls, content of catecholamines (CA) was measured in fractions of urea collected during 24 hours in periods: I - 8:00-12:00, II - 12:00-16:00, III - 16:00-18:00, IV - 20:00-8:00.

Results: $\mathrm{UA}_{\mathrm{s}}$ in diabetic subjects was $326 \pm 21 \mu \mathrm{mol} / \mathrm{L}$ (in women) and $361 \pm 15$ (in men); HOMA-IR was $4,06 \pm 0,6$ and $4,01 \pm 0,5$, respectively, with no difference with control $(\mathrm{p}>0.05) . \mathrm{UA}_{\mathrm{s}}$ correlated with BMI $(\mathrm{r}=0.45 ; 0.58)$, insulin $(\mathrm{r}=0.62 ; 0.42)$, HOMA-IR $(\mathrm{r}=0.69 ; 0.45), \mathrm{p}<0.01$. Positive correlation of CA urinary excretion $\left(\mathrm{CA}_{\mathrm{u}}\right)$, blood pressure and heart rate $(\mathrm{p}<0.05)$ indicates that SNS activity may be increased in the patients. Correlation between diabetes duration and $\mathrm{CA}_{\mathrm{u}}$ : adrenaline, noradrenaline, dopamine in I period $(\mathrm{r}=-0,48 ;-0,43 ;-0,42)$ and in III period $(\mathrm{r}=0,32 ; 0,31 ; 0,33$, resp.) was found, so the patients were divided in 2 groups: A - duration of $\mathrm{DM}<5 \mathrm{yr}(\mathrm{n}=12)$, and $B->5$ yr $(n=11)$. Different shapes of day's $C_{u}$ profiles in the groups were revealed. In cohort $\mathrm{A}$ of diabetics, as well as in control subjects, decreased $\mathrm{CA}_{u}$ in III period (comparing to I period) was found. In contrast, $\mathrm{CA}_{\mathrm{u}}$ in $\mathrm{B}$ group was growing constantly. These data suggest that patients of group B develop disorder of day's CA excretion rhythm. This may be in parallel with development of DM: level of C-peptide in B cohort was decreased comparing with A $(2,62 \pm 0,6$ vs $5,61 \pm 1,1 \mathrm{ng} / \mathrm{ml})$. $\mathrm{UA}_{\mathrm{s}}$ in IV period negatively correlated with $\mathrm{C}$-peptide in group $\mathrm{A}(\mathrm{r}=-0.70)$ and in $\mathrm{B}(\mathrm{r}=-0.36) \mathrm{p}<0.05$. A strong positive correlation of $\mathrm{UA}_{\mathrm{s}}$ with $\mathrm{CA}_{\mathrm{u}}$ in IV period $(\mathrm{r}=0.72 ; 0.62 ; 0.82, \mathrm{p}<0.05)$ in patients of group $\mathrm{A}$, and negative correlation with adrenaline and dopamine excretion in $\mathrm{B}(\mathrm{r}=-0.61 ;-0.80$, $\mathrm{p}<0.05)$ was found. $\mathrm{CA}_{\mathrm{u}}$ in diabetic patients exceeded normal rate, this suggested hyperproduction of UA. In group $\mathrm{A}_{\mathrm{SA}}$ was increased in comparison with $\mathrm{B}$ (11.2 \pm 0.9 vs $5.8 \pm 0.5 \mathrm{mmol})$.

Conclusion: The results demonstrate that relationship between activity of SNS (assessed by urinary excretion of CA) and characteristics of MS (IR, insulinemia, uricemia) in obese patients with type $2 \mathrm{DM}$ was more prominent in subjects with duration of diabetes less then $5 \mathrm{yrs}$, under conditions of relatively high serum levels of insulin and 
uric acid. This may be accounted for hypouricosuric action of insulin and stimulating effect of UA on SNS activity. In patients with $\mathrm{DM}>5$ yrs disorders of daily rhythm of $\mathrm{CA}$ excretion were revealed.

0739

\section{Insulin resistance and hypertension}

A. R. Esteghamati, M. Abbasi, M. Nakhjavani,

L. Novin, A. Yousefizadeh;

Endocrine, Endocrinology and Metabolism Research Center, Tehran, Islamic Republic of Iran

Background and Aims: The association between insulin resistance (IR), hyperinsulinemia and essential hypertension is controversial. The aim of this study was to examine the IR estimated by homeostasis model assessment (HOMAIR) in diabetic and non diabetics with and without hypertension.

Materials and Methods: A total of 607 subjects between November 2004 and December 2005 who were referred to a general hospital were included in a cross sectional study. We analyzed data of 150 non-diabetics ( 75 normal, 75 hypertensive) and 150 diabetic (75 normotensive, 75 hypertensive) age-BMI matched patients. Demographic data, anthropometric measurements were recorded. Smokers and patients on insulin therapy were excluded. Fasting blood samples were collected and FBS, serum creatinine, electrolytes, lipids, insulin (FPI), C-peptide, HbAlc were measured. HOMA-IR was calculated as FBS $(\mathrm{mg} / \mathrm{dl}) \times \mathrm{FPI}$ $(\mathrm{mU} / \mathrm{l}) / 405$ and HOMA-B was calculated as $(20 \times \mathrm{FPI}) /$ [(FBS/18)-3.5)]. Regression analysis was done to determine the association between HOMA-IR and various components of metabolic syndrome.

Results: The age, BMI, waist circumference, fasting insulin, C-peptide, HOMA-IR and HOMA-B values are summarized in table 1. These data show that HOMA-IR and HOMA-B were statistically different in diabetic patients versus non diabetic individuals with and without hypertension $(p<0.001$ for both), however, there was not significant difference between hypertensive versus control groups. The relative contribution of history of DM, HTN, sex, age, BMI, waist circumference, serum lipids, and mean arterial pressure (MAP) was analyzed to predict HOMA-IR by multiple regression analysis. History of DM, HTN, age, waist circumference, MAP, TG, and HDL levels were significantly independent predictor variables $(\mathrm{r}=0.46$, $P=0.001)$. The best predictors of HOMA-IR were the age and waist circumference in addition to DM.

Conclusion: These results do not support the evidence that insulin resistance and insulinemia are increased in hyper- tensive patients. IR is associated with the history of DM, age, and central obesity.

Demographic and insulin indice of participants

\begin{tabular}{|c|c|c|c|c|c|c|c|}
\hline Group & $\begin{array}{l}\text { Age } \\
\text { (mean } \pm \\
\text { SD) }\end{array}$ & $\begin{array}{l}\text { BMI } \\
(\text { mean } \pm \\
\text { SD) }\end{array}$ & $\begin{array}{l}\text { Waist } \\
\text { (mean } \pm \\
\text { SD) }\end{array}$ & $\begin{array}{l}\begin{array}{l}\text { Insulin } \\
(\text { mean } \pm \\
\text { SD) }\end{array}\end{array}$ & $\begin{array}{l}\text { C- } \\
=\text { Peptide } \\
\text { (mean } \pm \\
\text { SD) }\end{array}$ & $\begin{array}{l}\text { HOMA- } \\
\text { IR } \\
\text { (mean } \pm \\
\text { SD) }\end{array}$ & $\begin{array}{l}\text { HOMA- } \\
\text { B } \\
(\text { mean } \pm \\
\text { SD) }\end{array}$ \\
\hline Normal & $55 \pm 8$ & $\begin{array}{l}29.89 \pm \\
4.55\end{array}$ & $97 \pm 9$ & $\begin{array}{l}6.52 \pm \\
3.15\end{array}$ & $\begin{array}{l}2.39 \pm \\
1.1\end{array}$ & $\begin{array}{l}1.57 \pm \\
0.81\end{array}$ & $\begin{array}{l}74.7 \pm \\
40.96\end{array}$ \\
\hline HTN & $56 \pm 8$ & $\begin{array}{l}29.65 \pm \\
4.51\end{array}$ & $100 \pm 11$ & $\begin{array}{l}7.45 \pm \\
4.13\end{array}$ & $\begin{array}{l}3.04 \pm \\
1.38\end{array}$ & $\begin{array}{l}1.82 \pm \\
1.01\end{array}$ & $\begin{array}{l}85.15 \pm \\
69.03\end{array}$ \\
\hline DM & $55 \pm 8$ & $\begin{array}{l}29.5 \pm \\
4.79\end{array}$ & $98 \pm 12$ & $\begin{array}{l}8.62 \pm \\
6.73 *\end{array}$ & $\begin{array}{l}3.21 \pm \\
1.75^{*}\end{array}$ & $\begin{array}{l}3.54 \pm \\
3.15^{*}\end{array}$ & $\begin{array}{l}35.09 \pm \\
28.29 *\end{array}$ \\
\hline $\begin{array}{l}\text { DM+ } \\
\text { HTN }\end{array}$ & $56 \pm 7$ & $\begin{array}{l}29.76 \pm \\
4.94\end{array}$ & $99 \pm 9$ & $\begin{array}{l}6.22 \pm \\
3.39\end{array}$ & $\begin{array}{l}2.91 \pm \\
1.21\end{array}$ & $\begin{array}{l}2.51 \pm \\
1.47 *\end{array}$ & $\begin{array}{l}26.17 \pm \\
18.44^{*}\end{array}$ \\
\hline
\end{tabular}

Supported by TUMS

0740

Which plays more important role in pathogenesis of metabolic syndrome, the fat accumulation in visceral cavity or in the liver?

J. Ohya ${ }^{1,2}$, T. Nakagami ${ }^{2}$, S. Jimba ${ }^{2}$, N. Tanaka ${ }^{1,2}$, Y. Iwamoto ${ }^{2}$, T. Wasada ${ }^{1}$;

${ }^{1}$ Internal Medicine, Saitamaken Saiseikai Kurihashi Hospital, Saitamaken, ${ }^{2}$ Diabetes Center, Tokyo Women's Medical University, Tokyo, Japan.

Background and Aims: Metabolic syndrome (MetS) has been highlighted as an important risk for cardiovascular disease. Visceral adiposity (VA) is generally thought to be a prerequisite to the diagnosis of MetS. However, it has been shown that the percent liver fat, but not the visceral fat, is a parameter best correlated with a hepatic insulin resistance and the risk of hepatic steatosis increases exponentially with each addition of a component of the MetS, such as impaired glucose tolerance, hypertension (HT), and dyslipidemia. The aim of this study was to investigate which is more etiologically important for MetS, the fat accumulation in visceral cavity or in the liver.

Subjects and Methods: The study subjects were 962 men (age: mean $\pm \mathrm{SD}, 50 \pm 8$ years) and 495 women ( $50 \pm 8$ years) in a cohort of middle-aged apparently healthy adults who took medical examinations including measurement of waist circumference $(\mathrm{Wc})$ at the height of umbilicus, the surrogate measure of visceral adiposity and B-mode ultrasonography of the liver. The Japanese diagnostic criteria for MetS are as follows; Wc $\geq 85 \mathrm{~cm}$ (men), $\geq 90 \mathrm{~cm}$ (women) plus any two of the following three: $\mathrm{BP} \geq 130 / 85$ or under antihypertensive treatment as HT, TG $\geq 150 \mathrm{mg} / \mathrm{dl}$, HDL-C $<40 \mathrm{mg} / \mathrm{dl}$ or under 
lipid lowering agents as dyslipidemia, FBS $\geq 110 \mathrm{mg} / \mathrm{dl}$ or under treatment of diabetes as hyperglycemia. The sex specific prevalence of nonessential components of MetS was calculated by tertile of Wc and Body Mass Index (BMI). Further stratified analysis was made by the presence or absence of fatty liver (FL). The independent impact of FL on the presence of MetS was analysed by the logistic regression model, after adjusting for age.

Results: 1) The prevalence of the MetS was $22.6 \%$ for men and $4.4 \%$ for women. 2) The aggregation rate of each component of MetS was almost identical when compared with the stratification by BMI and Wc (each $\mathrm{p}<0.0001$ for linear trend). 3) The prevalence of FL was $13.7 \%$ for men and $2.4 \%$ for women. 4 ) In men, through the three strata by Wc, all of the prevalence of HT, Dyslipidemia (high TG, low HDL-C) were insignificantly higher in the FL group than in the non-FL group, but that of FBS was significantly higher in the FL group of the lowest and middle Wc group (Table 1). In women, a similar tendency was found without a statistical significance. 5) The age-adjusted odds ratios of having MetS associated with FL was 2.04 (95\% CI 1.50-2.78) for men and $1.44(0.60-3.49)$ for women.

Conclusions: These results suggest but do not confirm that FL is more important than visceral adiposity in generating MetS.

Prevalence of components of the MetS by status of the liver and $\mathrm{Wc}$ in men

\begin{tabular}{lllllll}
\hline & $\begin{array}{l}\text { Lowest Wc } \\
(<82.5 \mathrm{~cm})\end{array}$ & $\begin{array}{l}\text { Middle Wc } \\
(82.5-88.4 \mathrm{~cm})\end{array}$ & $\begin{array}{l}\text { Highest Wc } \\
(\geq 88.5 \mathrm{~cm})\end{array}$ \\
\cline { 2 - 7 } & $\begin{array}{l}\text { normal } \\
(\mathrm{n}=126)\end{array}$ & $\begin{array}{l}\mathrm{FL} \\
(\mathrm{n}=176)\end{array}$ & $\begin{array}{l}\text { normal } \\
(\mathrm{n}=148)\end{array}$ & $\begin{array}{l}\text { FL } \\
(\mathrm{n}=169)\end{array}$ & $\begin{array}{l}\text { normal } \\
(\mathrm{n}=178)\end{array}$ & $\begin{array}{l}\mathrm{FL} \\
(\mathrm{n}=165)\end{array}$ \\
\hline HT & 43.8 & 42.9 & 53.8 & 57.4 & 53.3 & 59.0 \\
Dyslipidemia & 20.5 & 29.4 & 39.1 & 50.7 & 46.7 & 56.2 \\
High TG & 11.9 & 17.5 & 27.8 & 40.5 & 36.4 & 44.4 \\
Low HDL & 7.4 & 11.1 & 15.4 & 15.5 & 21.2 & 24.7 \\
High FBS & 10.2 & 24.6 & 14.8 & 20.9 & 23.0 & 25.8 \\
\hline
\end{tabular}

0741

Insulin sensitivity and fasting insulin: association with indicators of cardiovascular disease in healthy subjects. The RISC Study

J. M. Dekker ${ }^{1}$, A. Kok ${ }^{2}$, M.-T. Guillanneuf ${ }^{3}$, B. Balkau ${ }^{3}$, S. Coppack ${ }^{4}$, M. Walker ${ }^{5}$, A. Mari ${ }^{6}$, M. Kozakova ${ }^{7}$, S. Hills ${ }^{7}$, A. Natali ${ }^{7}$, E. Ferrannini ${ }^{7}$

and the RISC Investigators,
${ }^{1}$ EMGO Institute, ${ }^{2}$ Clinical Chemistry, VU University Medical Center, Amsterdam, The Netherlands, ${ }^{3}$ Unite 780, Inserm, Paris, France, ${ }^{4}$ Dept. of Diabetes, London Royal Hospital, United Kingdom, ${ }^{5}$ Dept. of Medicine, Universtiy of Newcastle upon Tyne, United Kingdom, ${ }^{6}$ ISIB, CNR ISIB, Padova, Italy, ${ }^{7}$ Department of Internal Medicine, University of Pisa, Italy.

Background and Aims: Insulin resistance is believed to underlie the clustering of cardiovascular disease (CVD) risk factors. However, elevated insulin levels per se may be also implicated in the pathogenesis of CVD. Previous studies have not distinguished between insulin level and insulin sensitivity. We studied this question in a European study of healthy subjects.

Methods: In the RISC Study (Relationship between Insulin Sensitivity and Cardiovascular disease), 1538 men and women were recruited in 18 European centres. Inclusion criteria were: age 30-60 years, no history of CVD, blood pressure $<140 / 90 \mathrm{~mm} \mathrm{Hg}$, serum total cholesterol $<7.8 \mathrm{mmol} / \mathrm{l}$, serum triglycerides $<4.6 \mathrm{mmol} / 1$, and plasma glucose (fasting/postload glucose) $<7.0 / 11.1 \mathrm{mmol} / \mathrm{l}$. A euglycaemic hyperinsulinaemic $\left(40 \mathrm{mU} \cdot \mathrm{min}^{-1} \cdot \mathrm{m}^{-2}\right)$ clamp was performed to assess insulin sensitivity ( $\mathrm{M}$ value). Low insulin sensitivity was defined by values below the first quartile of the population $M$ value. High insulin was defined by values above the upper quartile of fasting plasma insulin. CVD risk factors were assessed using a standardised protocol. Intima media thickness (IMT) was assessed as an indicator of atherosclerosis, and IMT above the upper quartile was considered as high IMT. A resting 12-lead electrocardiogram (ECG) was made to assess subclinical heart disease. ECG abnormality was defined as the presence of Minnesota Codes 1.1 and 1.2, any 4 and 5 or 7.1. Urinary albumin and creatinine concentrations were assessed, and microalbuminuria, defined as albumin to creatinine ratio $\geq 2$, was considered a marker of impaired endothelial function. We computed odds ratios for the presence of the metabolic syndrome (NCEP criteria), high IMT, ECG abnormalities or microalbuminuria in subjects with high insulin and subjects with low insulin sensitivity, adjusted for age, gender and body mass index (BMI).

Results: Information on all variables was available for 1089 subjects. High fasting insulin was more strongly associated with the metabolic syndrome and microalbuminuria than low insulin sensitivity, but insulin sensitivity was associated with ECG abnormalities (table).

Conclusion: These results support the hypothesis that other mechanisms besides insulin resistance contribute to the clustering of CVD risk factors in the metabolic syndrome, 
but that insulin resistance may be related to sub-clinical heart disease.

Odds Ratios (95\% CI) of low insulin sensitivity and high fasting insulin for indicators of CVD

\begin{tabular}{|c|c|c|c|}
\hline & Prevalence & $\begin{array}{l}\text { Low Insulin } \\
\text { Sensitivity }\end{array}$ & $\begin{array}{l}\text { High Fasting } \\
\text { Insulin }\end{array}$ \\
\hline $\begin{array}{l}\text { NCEP Metabolic } \\
\text { Syndrome }\end{array}$ & $7 \%$ & $\begin{array}{l}2.9 \\
(1.6-5.1)\end{array}$ & $\begin{array}{l}5.0 \\
(2.7-9.1)\end{array}$ \\
\hline $\begin{array}{l}\text { IMT in upper } \\
\text { quartile }\end{array}$ & $25 \%$ & $\begin{array}{l}1.0 \\
(0.6-1.5)\end{array}$ & $\begin{array}{l}1.4 \\
(0.9-2.3)\end{array}$ \\
\hline ECG abnormality & $10 \%$ & $\begin{array}{l}3.2 \\
(1.5-7.0)\end{array}$ & $\begin{array}{l}1.9 \\
(0.8-4.3)\end{array}$ \\
\hline Micro-albuminuria & $3 \%$ & $\begin{array}{l}2.2 \\
(1.5-3.4)\end{array}$ & $\begin{array}{l}3.3 \\
(2.1-5.1)\end{array}$ \\
\hline
\end{tabular}

RISC is supported by the European Union and by AstraZeneca

0742

Relationship between insulin-resistance, metabolic syndrome and altered glucose tolerance. The GENFIEV Study

R. Miccoli $^{1}$ on behalf of the GENFIEV Study Group ${ }^{2}$;

${ }^{1}$ Department of Endocrinology and Metabolism, Pisa University, Italy, ${ }^{2}$ Italian Society of Diabetology, FoRiSID, Rome, Italy.

Background and Aims: Insulin-resistance (IR) is a characteristic finding in prediabetic individuals, predicts the development of type 2 diabetes and is the defect underlying metabolic syndrome (MS), but the relationship between IR and the traits of the MS is not yet well defined. Aim of this study was to evaluate the association between IR and MS among subjects with abnormal glucose regulation (AGR).

Materials and Methods: From the database of GENFIEV (GENetics PHYsiopathology and EVolution of type 2 Diabetes), an Italian nationwide study designed to recruit individuals with AGR, we studied 1017 subjects (589 men/ 428 women, age 49 \pm 11 , BMI $29,2 \pm 5,3 \mathrm{Kg} / \mathrm{m} 2)$. In all individuals we performed an OGTT with measurement of glycemia, c-peptide and fasting insulin and evaluated anthropometric and biochemical parameters to define MS by NCEP-ATP III criteria.

Results: The most represented MS trait was abdominal obesity (59\%), followed by hypertension (53\%), low HDL (33\%), high triglycerides (33\%) and hyperglycemia (27\%).

The prevalence of MS in the population study was 35\% ( $31 \%$ in men and $40 \%$ in women); $21,5 \%$ in subjects with normal glucose tolerance (NGT), 43,5\% in those with impaired fasting glucose (IFG), 33,3\% in those with impaired glucose tolerance (IGT), 75\% in IFG+IGT subjects, $63 \%$ in newly diagnosed diabetic patients. The prevalence of IR (HOMA $>75^{\circ}$ percentile of controls) was $61 \%$ in subjects with MS (Chi-Square: $\mathrm{p}<0,0001$ ) and increased according to the traits number: $52 \%, 69 \%$ and $91 \%$ in presence of 3,4 or 5 traits, respectively. Among subjects with ARG, 42\% was IR (Chi-Square: $\mathrm{p}<0,0001$ ) and those with IFG+IGT showed an higher frequency of IR than those with the isolated defect ( $28 \%$ vs $10 \%$ in IFG and $13 \%$ in IGT; Chi-Square: $\mathrm{p}<0,0001)$. The $70 \%$ of subjects with MS was identified by 3 traits; the association of obesity, hypertriglyceridemia, low HDL and hypertension was present in more than $50 \%$ of the cases. The combinations of 3 traits including hyperglycemia and/or obesity were those with the highest predictive power to identify subjects with MS (PPV 50-100\%).

Conclusion: This study showed that in subjects with abnormal glucose regulation the prevalence of MS is high (44\%) and is generally associated to IR, and some combinations of traits of the MS may significantly contribute to identify subjects with IR.

Supported by FoRiSID and Eli Lilly Italy

\section{PS 56}

\section{Metabolic syndrome and lipid lowering in type 2 diabetes}

0743

Metabolic syndrome in young adults with type 1 diabetes mellitus

A. Szadkowska ${ }^{1}$, I. Pietrzak ${ }^{1}$, J. Bodalska-Lipiñska ${ }^{2}$,

E. Czerniawska ${ }^{1}$, M. Kuberska-Kędzierska ${ }^{2}$, M. Włodarczyk ${ }^{1}$, L. Markuszewski ${ }^{2}$, J. Bodalski ${ }^{1}$;

${ }^{1}$ Department of Pediatrics, ${ }^{2}$ Department of Interventional Cardiology, Cardiodiabetology and Cardiac Rehabilitation, Medical University of Lodz, Poland.

Background and Aims: Metabolic syndrome (MS) is a risk factor for vascular complications. The prevalence of MS in type 1 diabetic patients is unknown. The aim of the study was to estimate the prevalence of metabolic syndrome diagnosed according to the definitions of International Diabetes Federation (IDF) in own modification, in young adults with type 1 diabetes.

Materials and Methods: 142 patients with type 1 diabetes mellitus (62 females and 80 males) aged from 18 to 35 years (mean: $23.0 \pm 4,0$ years) were included into the study. The diabetes duration ranged from 4 to 26 years (mean: $14.1 \pm 5.4$ years). The height, weight, waist circum- 
ference and blood pressure were measured. HbA1c (HPLC method) and plasma lipids (enzymatic method) concentrations were examined. Because in type 1 diabetics hyperglycemia is mainly caused by insulin deficiency, the criterion of hyperglycemia was replaced with the criterion of insulin resistance. Euglycemic-hyperinsulinemic clamp was performed to establish insulin resistance. In patients with glucose disposal rate below $6.5 \mathrm{mg} / \mathrm{kg} / \mathrm{min}$ insulin resistance was diagnosed. Body mass index (BMI) and daily dose of insulin were calculated.

Results:The presence of MS was found in 15 patients $(10.6 \%)$. Abdominal obesity was observed in 27 patients (19.0\%). Lowered plasma HDL-cholesterol levels were noted in 12 individuals from the study group (8.5\%), and hypertriglicerydemia - in $12(8.5 \%)$ of them. Blood pressure exceeding $130 / 85 \mathrm{mmHg}$ was observed in 45 patients $(31.7 \%)$. Glucose disposal rate ranged from 2.0 to $15.5 \mathrm{mg} / \mathrm{kg} / \mathrm{min}$. Insulin resistance was diagnosed in 62 individuals $(43,7 \%)$. In the study group mean BMI was $24.5 \pm 4.1 \mathrm{~kg} / \mathrm{m}^{2}, \mathrm{HbA} 1 \mathrm{c}-7,6 \pm 1.2 \%$, daily dose of insulin $-0.86 \pm 0.2 \mathrm{IU} / \mathrm{kg}$ and $33.5 \pm 8.5 \mathrm{IU} / \mathrm{m}^{2}$. Logistic regression analysis showed association between prevalence of MS and patients age (OR: 1.27; $\mathrm{p}=0.002)$ and BMI (OR: 1.9, $\mathrm{p}<0.001)$. No statistically significant relationship was found between MS prevalence and $\mathrm{HbAlc}$, diabetes duration or sex. In patients with MS daily dose of insulin calculated per unit of body surface area was higher than among patients without MS (41.7 vs. $\left.32.5 \mathrm{IU} / \mathrm{m}^{2}, \mathrm{p}<0.0001\right)$.

Conclusion: Metabolic syndrome, as defined by IDF criteria in own modification, is not a very frequent finding in young type 1 diabetic subjects. The elder age and higher BMI were associated with prevalence of MS.

Supported by Medical University of Lodz No 503-109-3

\section{4}

Insulin secretion and insulin sensitivity in subjects with impaired glucose regulation

M. H. Rahman ${ }^{1}$, R. M. Hafizur ${ }^{2}$, F. Huq ${ }^{2}$, Q. Nahar $^{2}$, T. Tazib ${ }^{2}$, A. R. Khan ${ }^{3}$, L. Ali ${ }^{2}$;

${ }^{1}$ Department of Endocrinology, ${ }^{2}$ Department of Biochemistry and Cell Biology, ${ }^{3}$ Department of Medicine, BIRDEM, Dhaka, Bangladesh.

Background and Aims: Substantial progress in understanding the underlying defects in diabetes mellitus has led to an etiological classification of the disorder. However, the same has not yet been possible for the prediabetic stages of impaired fasting glucose (IFG) and/or impaired glucose tolerance (IGT) collectively termed as Impaired Glucose
Regulation (IGR) by WHO study Group. There are still incompatibilities between the clinical classifications of IFG and/or IGT proposed by WHO Study Group and ADA Expert Committee and, in particular, the phenotype of combined IFG and IGT has not been adequately addressed. Understanding of the pathophysiology is a prerequisite for further characterization of these conditions. The present study was undertaken to explore the pancreatic B cell function and insulin sensitivity of the clinical types of IGR in a Bangladeshi population.

Materials and Methods: Following ADA 1997 and WHO 1999 guidelines 13 IFG and 112 IGT subjects, along with 39 healthy controls, aged $>20$ years, were selected purposively from the out-patient department of BIRDEM (the tertiary hospital of Diabetic Association of Bangladesh). During analysis, we also categorized IGT subjects into 91 isolated IGT (I-IGT) and 21 combined IFG-IGT subjects. Fasting and 2 h-plasma glucose and insulin (by chemiluminescent immunoassay) were measured. Insulin secretory capacity $(\% \mathrm{~B})$ and insulin sensitivity $(\% \mathrm{~S})$ were assessed by homeostasis model assessment (HOMA) using HOMACIGMA software.

Results: There was significantly higher fasting and $2 \mathrm{~h}-$ plasma insulin in IGT subjects compared to Control. HOMA $\% \mathrm{~B}$ was significantly decreased in IFG compared to IGT and Control [Median (range); IFG, 60 (34-164); IGT, 83 (36235); Control, 90 (41-251)], but there was no difference between IGT and Control. There was significantly compromised HOMA \%S in IGT compared to Control [IGT, 88 (22266); Control, 121 (37-371)]. When IGT subjects were categorized into I-IGT and IFG-IGT, it was found that plasma insulin was raised in I-IGT and IFG-IGT compared to control. HOMA \%B did not differ significantly in I-IGT [92 (36-235)] compared to Control, but it was significantly higher compared to IFG and IFG-IGT [75 (36-138)]. The IFG-IGT had significantly lower value compared to Control. HOMA \%S was significantly compromised in both categories of IGT [I-IGT, 91 (22-266); IFG-IGT, 71 (31-165)]. HOMA $\% \mathrm{~S}$ was decreased but just outside the range of statistical significance in IFG compared to Control $(\mathrm{p}=0.084)$ and in IFG-IGT to I-IGT ( $\mathrm{p}=0.069)$.

Conclusion: The pathophysiological mechanisms differ in IFG (primary defect of B-cell dysfunction with a tendency to insulin resistance) and isolated IGT (primarily an insulin resistant condition). The pathophysiology in the clinical entity of combined IFG-IGT (both B-cell dysfunction and insulin resistance) seems to characterise it as a different clinical entity and, thus, it may not be included in IGT as done by ADA and WHO.

Supported by the Biomedical Research Group (BMRG), IPICS 


\section{5}

Evaluation of islet beta cell function in newly diagnosed type 2 diabetic patients

Y. $\mathbf{L i}^{1}$, D. L. Zhu ${ }^{2}$, H. M. Tian ${ }^{3}$, L. X. $\mathrm{Shi}^{4}$, Z. J. Luo ${ }^{5}$, L. Yan ${ }^{6}$, L. Y. Zeng ${ }^{7}$, Z. G. Zhou ${ }^{8}$, L. Y. Yang ${ }^{9}$, J. Liu ${ }^{1}$, M. Li ${ }^{1}$, J. P. Weng ${ }^{1}$;

${ }^{1}$ Department of Endocrinology, The First Affiliated Hospital of Sun Yat-Sen University, Guangzhou, ${ }^{2}$ Department of Endocrinology, Gulou Hospital of Nanjing University, Nanjing, ${ }^{3}$ Department of Endocrinology, West China Hospital of Sichuan University, Sichaun, ${ }^{4}$ Department of Endocrinology, the Affiliated Hospital of Guiyang Medical University, Guiyang, ${ }^{5}$ Department of Endocrinology, the First Affiliated Hospital of Guangxi Medical University, Guangxi, ${ }^{6}$ Department of Endocrinology, the Second Affiliated Hospital of Sun Yat-Sen University, Guangzhou, ${ }^{7}$ Department of Endocrinology, the Third Affiliated Hospital of Sun Yat-Sen University, Guangzhou, ${ }^{8}$ Department of Endocrinology, Xiangya Second Affiliated Hopital of central South University, Hunan, ${ }^{9}$ Department of Endocrinology, the First Affiliated Hospital of Fujian Medical University, Fujian, China.

Background and Aims: Clinical studies have shown that short-term intensive insulin treatment in newly diagnosed type 2 diabetic patients yielded prolonged remission. To investigate the effects of different treatments (oral antihyperglycemic agents, multiple daily injection or continuous subcutaneous insulin infusion) on $\beta$ cell function and prognosis of newly diagnosed type 2 diabetes mellitus, we conducted this multicenter, perspective clinical trial. Here we evaluated the baseline function of islet $\beta$ cell in recruited subjects and compared the different indexes assessing insulin secretion capacity.

Materials and Methods: 352 newly diagnosed type 2 diabetic patients and 48 subjects with normal glucose tolerance (NGT) underwent intravenous glucose tolerance tests (IVGTT). Serum samples were obtained before and 1, 2, 4,6 , and $10 \mathrm{~min}$ after injection for insulin determination. The acute insulin response (AIR) and the area under the curve of insulin (AUC) were used to evaluate the insulin secretion. HOMA B was another indicator of insulin secretion. HOMA IR was used to assess insulin resistance. To represent another profile of $\beta$ cell dysfunction, the fasting plasma proinsulin level was measured and the ratio of fasting proinsulin to fasting insulin (PI/I), an index representative of reduced conversion of proinsulin to insulin, was calculated.

Results: Both AUC and AIR during IVGTT were evidently reduced in type 2 diabetic patients compared with NGT subjects. In particular, as FPG was above $7.0 \mathrm{mmol} / \mathrm{l}$, AIR had been evidently reduced significantly. And AIR subsequently disappeared when FPG was above $9.0 \mathrm{mmol} / 1$.
After adjusted by HOMA IR, HOMA B of the type 2 diabetic patients was reduced compared with the NGT subjects (about $30 \%$ of the normal level). Both fasting proinsulin concentration and the ratio of PI/I were significantly higher in T2DM than that in NGT subjects $(22.6 \pm 14.7 \mathrm{pmol} / 1$ vs. $11.5 \pm 7.1 \mathrm{pmol} / \mathrm{l}, \mathrm{p}<0.001 ; 30.1 \pm$ $20.5 \%$ vs. $12.1 \pm 9.6 \%, \mathrm{P}<0.001$, respectively). In diabetic population, AIR, AUC and HOMA B were significantly negatively correlated with FPG $(\mathrm{P}<0.001)$, and $\mathrm{PI} / \mathrm{I}$ positively correlated with FPG $(\mathrm{P}<0.001)$.

Conclusion: The dysfunction of islet $\beta$ cell in newly diagnosed type 2 diabetic patients was mainly represented by the disappearance of AIR and the evident decline of AUC and HOMA B, companied with qualitative decreasing of insulin secretion.

Supported by NCET04 0807 (Jianping Weng) and Guangdong Natural Science Foundation 2005 (Yanbing Li)

\section{6}

Effects of co-administered ezetimibe and fenofibrate in mixed dyslipidaemic patients with metabolic syndrome M. J. Davies ${ }^{1}$, H. E. Bays ${ }^{2}$, A. Shah ${ }^{1}$, G. Macdonell ${ }^{1}$, B. Gumbiner ${ }^{1}$;

${ }^{1}$ Merck \& Co. Inc., Rahway, ${ }^{2}$ Clinical Research, L-MARC, Louisville, United States.

Background and Aims: Metabolic syndrome (METS) is a clustering of atherosclerotic CHD risk factors including abdominal obesity, decreased HDL-C, and increased fasting plasma glucose, triglycerides (TG), and blood pressure. According to NCEP ATP III, the dyslipidemia defined by the METS is a secondary treatment target after LDL-C. This post-hoc analysis evaluated the treatment response of an LDL-C lowering therapy, ezetimibe (EZE), co-administered with a TG-lowering/HDL-C raising therapy, fenofibrate (FENO) in patients with both mixed dyslipidemia and metabolic syndrome.

Materials and Methods: Data were derived from a cohort of 625 patients with mixed dyslipidemia that were randomized in a 1:3:3:3 ratio to 1 of 4 treatments for 12 weeks: placebo; EZE $10 \mathrm{mg}$; FENO $160 \mathrm{mg}$; or EZE $10 \mathrm{mg}+\mathrm{FENO} 160 \mathrm{mg}$. Patients were 18-75 years old, with LDL-C $3.36-5.69 \mathrm{mmol} /$ $\mathrm{L}$ [2.59-4.65 $\mathrm{mmol} / \mathrm{L}$ for patients with type 2 diabetes], TG 2.26-5.65 mmol/L, with no history of CHD or CHD risk $>20 \%$, or other CHD risk equivalent disease (except for type 2 diabetes). Metabolic syndrome status was determined in 622 evaluable patients by NCEP ATP III criteria.

Results: At baseline, patients with metabolic syndrome were more obese, had higher TG, and lower HDL-C, and tended to have LDL size pattern B compared to those without metabolic syndrome. Treatment with EZE and 
FENO alone or when co-administered produced comparable improvements in lipid parameters irrespective of the presence of metabolic syndrome (Table). Treatment with EZE+FENO and FENO also shifted more patients away from the smaller, more dense LDL size pattern B after 12 weeks, which was similar between the subgroups.

Conclusions: In this post-hoc analysis, co-administration of EZE+FENO had similar lipid profile benefits in patients with mixed dyslipidemia and with or without the presence of metabolic syndrome.

\begin{tabular}{llllll}
\hline \multicolumn{5}{c}{$\begin{array}{l}\text { LS Mean Percent Change } \\
\text { from Baseline }\end{array}$} & \multicolumn{2}{l}{$\begin{array}{l}\text { LDL Size Pattern B, } \\
\mathrm{n}(\%)\end{array}$} \\
\hline Treatment & LDL-C & TG* & HDL-C & Baseline & Week 12 \\
\hline Metabolic Syndrome (n=450) & & & \\
Placebo & 1.1 & -6.9 & 3.3 & $34(76)$ & $30(67)$ \\
EZE & -14.0 & -9.8 & 3.0 & $96(80)$ & $74(62)$ \\
FENO & -3.0 & -44.2 & 20.4 & $93(79)$ & $24(20)$ \\
EZE+FENO & -20.4 & -45.1 & 19.0 & $90(77)$ & $21(18)$ \\
No Metabolic Syndrome (n=172) & & & \\
Placebo & -5.1 & -13.7 & 2.0 & $6(46)$ & $6(46)$ \\
EZE & -10.5 & -20.7 & 7.2 & $32(73)$ & $24(55)$ \\
FENO & -11.3 & -42.0 & 15.0 & $34(67)$ & $10(20)$ \\
EZE+FENO & -21.4 & -41.4 & 18.8 & $33(69)$ & $8(17)$ \\
\hline
\end{tabular}

*median \% change; With ANCOVA, treatment by subgroup interaction term was not significant for lipid parameters, except LDL-C $(\mathrm{p}=0.02)$.

Supported by Merck \& Co. Inc.

\section{7}

Effects of ezetimibe/simvastatin on atherogenic ratios ApoB/ApoA1 and LDL-C/HDL-C

L. Ose ${ }^{1}$, E. Veltri ${ }^{2}$, C. M. McCrary $\operatorname{Sisk}^{3}$, A. K. Shah ${ }^{3}$, D. L. Maccubbin ${ }^{3}$;

${ }^{1}$ Department of Medicine, The Lipid Clinic, Rikshospitalet, Oslo, Norway, ${ }^{2}$ Research Institute, Schering-Plough, Kenilworth, ${ }^{3}$ Merck \& Co. Inc., Rahway, United States.

Background: The ratios of low density lipoprotein-cholesterol to high density lipoprotein-cholesterol (LDL-C/HDLC) and apolipoprotein B to apolipoprotein A-1 (Apo B/Apo A-1) have been shown to be good predictors of cardiovascular risk and plaque progression. This analysis compared the effects of ezetimibe (EZE), simvastatin (SIM) and ezetimibe/simvastatin (EZE/SIM) on Apo B/Apo A-1 and LDL$\mathrm{C} / \mathrm{HDL}-\mathrm{C}$ in patients with primary hypercholesterolemia.

Methods: This post hoc analysis was performed on data collected from 3 similar randomized, placebo (Pbo)-controlled, double-blind studies. After a 6- to 8-wk washout and 4-wk diet/
Pbo run in, 3083 hypercholesterolemic patients (LDL-C: 145$250 \mathrm{mg} / \mathrm{dL} ; \mathrm{TG} \leq 350 \mathrm{mg} / \mathrm{dL}$ ) were randomized equally to placebo (Pbo), EZE 10 mg, EZE/SIM (10/10, 10/20, 10/40, 10/ $80 \mathrm{mg}$ ), or SIM $(10,20,40,80 \mathrm{mg})$ for 12 weeks. Mean percent change from baseline in Apo B/Apo A-1 and LDL-C/HDL-C were examined across individual and pooled treatment groups. Results: Relative to Pbo, EZE alone significantly reduced Apo B/Apo A-1 and LDL-C/HDL-C ( $<<0.001$ for both comparisons; see Table). When pooled across all doses, SIM and EZE/SIM produced greater reductions in Apo B/ Apo A-1 and LDL-C/HDL-C compared to EZE alone $(p<0.001$ for all comparisons). The Apo B/Apo A-1 and LDL-C/HDL-C lowering efficacy of EZE/SIM was greater than that observed with SIM alone when pooled across doses and at each of the corresponding dose comparisons $(\mathrm{p}<0.001$ for all comparisons). The enhanced Apo B/Apo A-1 and LDL-C/HDL-C lowering effects of EZE/SIM versus SIM were consistent across subgroups based on age $(<65$ vs $\geq 65$ years), gender, body mass index $(<30$ $\mathrm{vs} \geq 30 \mathrm{~kg} / \mathrm{m}^{2}$ ), and patient history of coronary heart disease, diabetes, and metabolic syndrome status.

Conclusions: Compared to Pbo, EZE alone produced significantly greater improvements in lipoprotein ratios in patients with primary hypercholesterolemia. Treatment with EZE/SIM significantly reduced these ratios relative to EZE and SIM alone.

\begin{tabular}{lllll}
\hline \multicolumn{5}{c}{ Mean \% change from baseline \pm SE } \\
\cline { 2 - 5 } & Pbo & EZE 10 & Pooled SIM & \\
& & & $\begin{array}{l}\text { Pooled } \\
\text { EZE/SIM }\end{array}$ \\
\hline$\frac{A p o B}{A p o ~ A-1}$ & $0.31 \pm 0.88$ & $-16.31 \pm 0.71^{*}$ & $-33.41 \pm 0.43$ & $-43.92 \pm 0.43^{* *}$ \\
$(\mathrm{~N})$ & $(296)$ & $(287)$ & $(1177)$ & $(1167)$ \\
$\frac{L D L-C}{H D L-C}$ & $-0.38 \pm 0.91$ & $-22.59 \pm 0.73^{*}$ & $-41.55 \pm 0.45$ & $-55.47 \pm 0.45^{* *}$ \\
$(\mathrm{~N})$ & $(307)$ & $(297)$ & $(1218)$ & $(1226)$ \\
\hline
\end{tabular}

${ }^{\dagger}$ Treatment (LS) means are based on the ANOVA model with term for treatments (10).

$* p$-value $<0.001$ versus Pbo.

** $p$-value $<0.001$ versus pooled SIM.

Supported by Merck \& Co. Inc.

0748

Efficacy of ezetimibe/simvastatin $10 / 20 \mathrm{mg}$ and $10 / 40 \mathrm{mg}$ compared to atorvastatin $20 \mathrm{mg}$ in type 2 diabetic patients

C. Constance ${ }^{1}$, S. Westphal ${ }^{2}$, N. Chung ${ }^{3}$, M. Lund ${ }^{4}$, A. JohnsonLevonas ${ }^{4}$, R. Massaad ${ }^{4}$, C. Allen ${ }^{4}$; 
${ }^{1}$ Centre de recherche en cardiologie, Hôpital MaisonneuveRosemont, Montréal, Canada, ${ }^{2}$ Institut fuer Klinische Chemie und Pathobiochemie, Otto-von-Guericke-Universitaet, Magdeburg, Germany, ${ }^{3}$ Yonsei University College of Medicine, Yonsei Cardiovascular Center, Seoul, Republic of Korea, ${ }^{4}$ Merck \& Co. Inc., Rahway, United States.

Background and Aims: This multi-center, randomized, double-blind study evaluated the efficacy and safety of switching from atorvastatin $10 \mathrm{mg}$ to ezetimibe/simvastatin (EZE/SIM) 10/20, EZE/SIM 10/40 mg or doubling the dose of atorvastatin (ATV) to $20 \mathrm{mg}$ in patients with Type II diabetes (T2DM).

Materials and Methods: Eligible patients had $\mathrm{HbA}_{1 \mathrm{C}} \leq$ $10 \%$, were aged $\geq 18$ yrs and were taking ATV $10 \mathrm{mg}$ for $\geq 6$ wks before study entry. Following 4 wks of open-label ATV $10 \mathrm{mg} /$ day, patients were randomized 1:1:1 to EZE/ SIM $10 / 20 \mathrm{mg}$ /day $(\mathrm{n}=220)$, EZE/SIM $10 / 40 \mathrm{mg} /$ day $(\mathrm{n}=222)$, or ATV $20 \mathrm{mg} /$ day $(\mathrm{n}=219)$ for 6 wks. The primary endpoint was mean \% reduction in low-density lipoprotein cholesterol (LDL-C).

Results: Treatment with EZE/SIM 10/20 and 10/40 mg produced significant reductions in LDL-C, total cholesterol (TC), non-high-density lipoprotein cholesterol (non-HDLC), and apolipoprotein B (Apo B) relative to ATV $20 \mathrm{mg}$ (Table). There were no significant differences in HDL-C and triglycerides (TG) between EZE/SIM and ATV. Significantly more patients achieved LDL-C goal $<2.6 \mathrm{mmol} / \mathrm{L}$ with EZE/ SIM 10/20 and 10/40 mg than with ATV (91\%, 87\%, and $70 \%$, respectively; $\mathrm{p} \leq 0.001$ ). Both EZE/SIM doses were generally well tolerated, with an overall safety profile similar to ATV. There were no differences in the incidences of liver transaminase or creatine kinase elevations between groups. Conclusions: EZE/SIM 10/20 and 10/40 mg, dual inhibition treatment strategies targeting both cholesterol synthesis and absorption, were well tolerated and provided greater lipid-altering efficacy than doubling the dose of ATV in T2DM patients.

\begin{tabular}{llll}
\hline Parameter $\dagger$ & $\begin{array}{l}\text { EZE/SIM } \\
10 / 20 \mathrm{mg}\end{array}$ & $\begin{array}{l}\text { EZE/SIM } \\
10 / 40 \mathrm{mg}\end{array}$ & $\begin{array}{l}\text { ATV } \\
20 \mathrm{mg}\end{array}$ \\
\hline LDL-C (\%) & $-26.2^{*}$ & $-30.1^{*}$ & -8.5 \\
TC (\%) & $-14.2^{*}$ & $-16.8^{*}$ & -5.5 \\
HDL-C (\%) & $2.4^{* *}$ & $1.3 * *$ & 1.6 \\
TG (\%) $\dagger \dagger$ & $-9.7 * *$ & $-8.4^{* *}$ & -5.5 \\
Non-HDL-C (\%) & $-20.9^{*}$ & $-23.8^{*}$ & -7.4 \\
Apo B (\%) & $-14.9^{*}$ & $-19.5^{*}$ & -6.7 \\
\hline
\end{tabular}

$\dagger$ Least-squares mean percent change from baseline to endpoint $\dagger \dagger$ Median percent change from baseline to endpoint ${ }^{*} \mathrm{p} \leq 0.001$ vs. ATV $20 \mathrm{mg}$

**not significant, $\mathrm{p} \geq 0.117$ vs. ATV $20 \mathrm{mg}$

Supported by Merck \& Co. Inc.
0749

Elevated serum retinol binding protein 4 levels with triglycerides in obesity and type 2 diabetes mellitus

W. Jia, H. Wu, L. Wei, J. Lu, Y. Bao, K. Xiang;

Endocrinology and Metabolism, Shanghai Diabetes Institute, China.

Background and Aims: Retinol binding protein 4 (RBP4) is a cytokine that is mainly expressed in hepatocytes. Recent studies in animal models have shown that RBP4 is also expressed in adipocytes and elevated RBP4 levels in the plasma may contribute to the pathogenesis of Type 2 diabetes mellitus $\left(\mathrm{T}_{2} \mathrm{DM}\right)$. However, the pathophysiological roles of RBP4 in humans remain largely unknown. In this study, we investigated whether the human serum RBP4 levels are altered in patients with obesity and $\mathrm{T}_{2} \mathrm{DM}$ and the relationship between RBP4 and lipid metabolism.

Materials and Methods: Enrolled subjects $(\mathrm{n}=131$, age $44.7 \pm 11.6$ years old) were divided into 4 groups: normal control (NC) $(n=31)$, normal glucose regulation with overweight or obesity (OW/OB-NGR) $(n=31)$, type 2 diabetes mellitus with normal weight $\left(\mathrm{NW}-\mathrm{T}_{2} \mathrm{DM}\right)(\mathrm{n}=33)$, and type 2 diabetes mellitus with overweight or obesity $\left(\mathrm{OW} / \mathrm{OB}-\mathrm{T}_{2} \mathrm{DM}\right)(\mathrm{n}=36)$. Serum RBP4 and insulin were measured by radioimmunology.

Results: After age and sex adjusted, serum RBP4 levels in OW/OB-NGR and OW/OB- $\mathrm{T}_{2} \mathrm{DM}$ were significantly elevated compared to the NC group $(29.85 \pm 6.60 \mathrm{vs}$ $25.47 \pm 6.84 \mu \mathrm{g} / \mathrm{ml}, \quad 30.86 \pm 6.11 v s \quad 25.47 \pm 6.84 \mu \mathrm{g} / \mathrm{ml}$, $P<0.01, P<0.01)$. However, there is no significant difference between $\mathrm{NC}$ and $\mathrm{NW}-\mathrm{T}_{2} \mathrm{DM}(29.85 \pm 6.60 \mathrm{vs}$ $28.35 \pm 5.42 \mu \mathrm{g} / \mathrm{ml}$ ). The serum RBP4 level in OW/OB$\mathrm{T}_{2} \mathrm{DM}$ is significantly higher than that of NW- $\mathrm{T}_{2} \mathrm{DM}(30$. $86 \pm 6.11 v s \quad 28.35 \pm 5.42 \mu \mathrm{g} / \mathrm{ml} \quad P<0.05)$. Multiple linear regression analyses reveal that WHR $\left(\mathrm{r}^{2}=0.166, P<0.001\right)$, triglyceride (TG) $\left(\mathrm{r}^{2}=0.188, P<0.001\right)$ and age $\left(\mathrm{r}^{2}=0.205\right.$, $P<0.001)$ are independent variables for RBP4. Because there was no correlation between the fasting plasma glucose and RBP4 levels, total of 131 subjects were re-divided into 4 groups: normal TG $(\mathrm{TG}<1.7 \mathrm{mmol} / \mathrm{L})$ with normal WHR (WHR $\leq 0.90$ in male, $W H R \leq 0.85$ in female) group ( $n=39$ ), high TG with normal WHR group ( $\mathrm{n}=11)$, normal TG with large WHR group $(n=43)$ and high TG with large WHR group $(\mathrm{n}=38)$. After age, sex and WHR adjusted, serum RBP4 levels in the high TG group were significantly higher than that of the normal or large WHR groups with normal TG $(P<0.05)$. However, there was no significant difference in the RBP4 levels between the normal WHR group and large WHR group $(P<0.05)$.

Conclusion: Serum RBP4 levels are significantly increased in obese Chinese subjects. The elevated RBP4 levels are 
correlated with visceral obesity and, more importantly, lipid metabolism.

Supported by Shanghai Science and Technology Development Foundation

0750

ACE inhibition and insulin sensitivity in obese insulin resistant subjects: a double-blind placebo-controlled randomized trial to examine underlying mechanisms

G. H. Goossens ${ }^{1,2}$, E. E. Blaak ${ }^{1,2}$, P. M. Schiffers ${ }^{3}$,

W. H. M. Saris ${ }^{1}$, M. A. van Baak ${ }^{1}$;

${ }^{1}$ Department of Human Biology (NUTRIM), Maastricht University, ${ }^{2}$ Wageningen Centre for Food Sciences, WCFS, Wageningen, ${ }^{3}$ Department of Pharmacology and Toxicology, Maastricht University, The Netherlands.

Background and Aims: Recent clinical trials have demonstrated that long-term angiotensin-converting enzyme (ACE) inhibitor treatment reduces the incidence of type 2 diabetes. This study was designed to investigate the effect of shortterm ACE inhibitor treatment on insulin sensitivity and to examine for the first time possible underlying metabolic and hemodynamic effects in obese insulin resistant subjects.

Materials and Methods: A double-blind placebo-controlled randomized trial was performed in 18 obese insulin resistant men (age, $53 \pm 2$ yr; BMI, $32.6 \pm 0.8 \mathrm{~kg} / \mathrm{m}^{2}$; HOMAIR, 5.6 \pm 0.5 ; SBP, $140.8 \pm 3.2 \mathrm{mmHg}$; DBP, $88.8 \pm$ $1.6 \mathrm{mmHg}$ ), free of any medication, to examine the effects of 2-week ACE inhibitor treatment (ramipril, $5 \mathrm{mg} / \mathrm{d}$ ) on insulin sensitivity, forearm blood flow, substrate fluxes across the forearm, whole-body substrate oxidation, and intramuscular triacylglycerol (IMTG) content.

Results: Ramipril treatment significantly decreased ACE activity compared with placebo $(-22.0 \pm 1.7 v$ s. $0.2 \pm 1.1 \mathrm{U} / 1$, respectively, $P<0.001)$, resulting in a significantly reduced blood pressure (SBP, $-10.8 \pm 2.1$ vs. $-2.7 \pm 2.0 \mathrm{mmHg}$, respectively, $P=0.01$; DBP, $-10.1 \pm 1.3$ vs. $-4.2 \pm 2.1 \mathrm{mmHg}$, respectively, $P=0.03$ ). Ramipril treatment had no significant effect on whole-body insulin-mediated glucose disposal (before: $17.9 \pm 2.0 v s$. after: $19.1 \pm 2.4 \mu \mathrm{mol} \cdot \mathrm{kg} \mathrm{bw}^{-1} \cdot \mathrm{min}^{-1}$, $P=0.44$ ), insulin-mediated glucose uptake in skeletal muscle (before: $1.82 \pm 0.39 v s$. after: $1.92 \pm 0.29 \mu \mathrm{mol} \cdot 100 \mathrm{ml}$ forearm tissue $^{-1} \cdot \mathrm{min}^{-1}, P=0.81$ ), and IMTG content (before: $45.4 \pm 18.8 v s$. after: $48.8 \pm 27.5 \mu \mathrm{mol} \cdot \mathrm{mg}$ dry muscle $^{-1}$, $P=0.92)$. Furthermore, the increase in carbohydrate oxidation $(P<0.001)$ and forearm blood flow $(P<0.01)$, and the decrease in fat oxidation $(P<0.001)$ during insulin stimulation were not significantly different between treatments.

Conclusion: Short-term ramipril treatment adequately reduced ACE activity and blood pressure, but had no significant effects on insulin sensitivity, forearm blood flow, substrate fluxes across the forearm, whole-body substrate oxidation, and IMTG content in obese insulin resistant subjects. The present data suggest that the reduced incidence of type 2 diabetes after long-term ACE inhibition can be explained by long-term effects of these agents that affect insulin sensitivity or insulin secretion.

This study was funded by the Wageningen Centre for Food Sciences, an alliance of major Dutch food industries, TNO Nutrition and Food Research, and Wageningen University and Research Center, with financial support from the Dutch government.

\section{1}

BGP-15, a new insulin sensitizer targeting mitochondria K. Tory ${ }^{1}$, A. Kolonics ${ }^{1}$, B. Peitl ${ }^{2}$, Á. Bajza ${ }^{1}$, L. László ${ }^{3}$, T. Horváth ${ }^{4}$, L. Vigh ${ }^{5}$, S. Bernáth ${ }^{1}$, L. Korányi ${ }^{4}$, Z. Szilvássy ${ }^{2}$, P. Literati-Nagy ${ }^{1}$;

${ }^{1}$ Molecular Pharmacology, N-Gene Research Laboratrory, Budapest, ${ }^{2}$ Department of Pharmacology and Pharmacotherapy, University of Debrecen, ${ }^{3}$ Department of General Zoology, Eötvös Loránd University, Budapest, ${ }^{4}$ Metabolic Disease, DRC, Balatonfüred, Hungary, ${ }^{5}$ Deparment of Biochemistry, Biological Research Center, Szeged, Hungary.

Background and Aims: Validation of the experimental and clinical insulin sensitizing activity of BGP-15, a hydroxylamine drivative.

Materials and Methods: Insulin sensitizing effect of BGP15 was tested both in animals and in humans. Hypercholesterolemic, insulin resistant rabbits and Goto-Kakizaki diabetic rats were used to demonstrate experimental insulin sensitizing activity. In humans, clinical efficacy was evaluated in 42 insulin resistant patients selected upon HOMA IR, waist-to-hip ratio, and BMI. The insulin sensitizing efficacy was evaluated by hyperinsulinemic euglycemic clamp and by iv glucose tolerance test. The effect of treatment on expression of heat shock proteins (HSP72, HSP90) and constitutive nitric oxide synthases (eNOS, nNOS) was evaluated by Western blotting both in cultured endothelial cells and in tissues of Goto-Kakizaki rats. Mitochondrial mass was evaluated by MitoTracker staining in cell cultures and by the amount of COX IV protein in animal tissues. Nitric oxide production was analyzed by DAF staining and by isotope labelled arginine conversion assay.

Results: BGP-15 increased glucose utilization by 237 and $175 \%$, respectively at dose of $10 \mathrm{mg} / \mathrm{kg}$, p.o in hypercholesterolemic insulin resistant rabbits and Goto-Kakizaki diabetic rats. The 28-day treatment with 200 and $400 \mathrm{mg}$ daily oral doses of BGP-15 increased whole body glucose utilization ( $\mathrm{M}$ value) by 1,6 and $1,75 \mathrm{mg} / \mathrm{kg} / \mathrm{min}$, respectively as compared to the placebo group (ANOVA, $\mathrm{p}=0,032$ ). 
BGP-15 treatment increased the levels of heat shock proteins and restored the expression and function of constitutive nitric oxide synthases both in tissues of GotoKakizaki rats and in endothelial cells exposed to hyperglycemia. Restored cNOS activity was accompanied with improved mitochondrial function and increased mitochondrial mass both in cultured endothelial cells and in tissues of diabetic rats. In endothelial cells, BGP-15 alleviated hyperglycaemia-induced increased mitochondrial membrane potential and protected against mitochondrial structural damage. The mitochondrial effect of BGP-15 was greatly reduced when the NO-cGMP pathway was inhibited. BGP-15 did not show any PPAR $\gamma$ agonist activity.

Conclusion: Results show that BGP-15 exerts its insulin sensitizing effect by a novel mechanism that is linked with stimulation of the HSP/cNOS systems and is mediated by multiple downstream pathways including primarily mitochondrial, cellular and NO-mediated neuronal effects. Based on our data BGP-15 represents a novel promising insulin sensitizer drug candidate that affects causal mechanisms of insulin resistance.

Supported by research grants from the Hungarian government

0752

Sex difference in the effect of pioglitazone on anthropometric and metabolic parameters in type 2 diabetic patients

S. Park ${ }^{1}$, J. Moon ${ }^{1}$, H. Kim ${ }^{2}$, S. Kim ${ }^{3}$, W. Shim ${ }^{1}$, E. Kang ${ }^{1}$, J. Lee ${ }^{4}$, C. Ahn ${ }^{1}$, H. Lee ${ }^{1}$, B. Cha ${ }^{1}$;

${ }^{1}$ Department of Internal Medicine, Yonsei University College of Medicine, Seoul, ${ }^{2}$ Department of Internal Medicine, Ajou University College of Medicine, Kyonggido, ${ }^{3}$ Department of Internal Medicine, Pochon CHA University College of Medicine, Kyonggi-do, ${ }^{4}$ Department of Internal Medicine, Catholic University of Daegu School of Medicine, Daegu, Republic of Korea.

Background and Aims: The purpose of the present study was to evaluate and explain the sex difference of the clinical effect of pioglitazone on arthropometric and metabolic parameters in type 2 diabetes patients.

Materials and Methods: A total 93 type 2 diabetes patients (men, $\mathrm{n}=47$; women, $\mathrm{n}=46$; mean age, men : 48.5 \pm 10.0 , women $51.5 \pm 11.5$ years) were studied before and after 3 months, 6 months, and 12 months of pioglitazone treatment. Anthropometric and metabolic parameters were measured.

Results: In both men and women, fasting plasma glucose (FPG) and glycosylated hemoglobin (HbAlc) decreased after 6 months of pioglitazone treatment $(P<0.01)$. The decreases in FPG and HbAlc levels after pioglitazone treatment relative to the baseline were more prominent in women than in men (FPG -11.9 vs. $-33.3 \pm 41.9 \mathrm{mg} / \mathrm{dL}$, $P<0.01 ; \mathrm{HbAlc},-0.6 \pm 1.1$ vs. $-1.0 \pm 1.2 \%, P=0.062)$. The decrease in the homeostasis model assessment of insulin resistance(HOMA-IR) index tended to more prominent in women than in men $(-0.7 \pm 1.6$ vs. $-1.4 \pm 1.3, P=0.09)$. Body weight and body mass index (BMI) significantly increased after 6 months of pioglitazone treatment only in women. The increases of body weight and BMI was prominent in women than in men (body weight, $0.5 \pm 2.4$ vs. $2.6 \pm 2.1 \mathrm{~kg}, P<0.01$; BMI, $0.2 \pm 0.8$ vs. $1.1 \pm 0.9 \mathrm{~kg} / \mathrm{m}^{2}$ ). Serum leptin level were increased after 6 months of pioglitazone treatment only in female patient population $(14.0 \pm 8.0$ vs. $17.2 \pm 10.1 \mu \mathrm{g} / \mathrm{mL}, P<0.01)$, and serum high sensitivity $\mathrm{C}$ reactive protein (hsCRP) level were decreased only in women $(2.2 \pm 3.6$ vs. $0.8 \pm 0.7 \mathrm{mg} / \mathrm{dL}, P<0.05)$. Serum high density lipoprotein (HDL) cholesterol increased after pioglitazone treatment in both men and women $(P<0.01)$ and, on the other hand, serum triglyceride decreased only in female patient populations $(225.5 \pm 226.3$ vs. $148.3 \pm 89.9 \mathrm{mg} / \mathrm{dL}, P<0.05)$

Conclusions: Pioglitazone acts not only on lowering glucose but also on other metabolisms, anti-inflammatory effect and improvement of lipid metabolism, more effectively in women than in men.

\section{PS 57}

\section{Life style interventions in metabolic syndrome and obesity}

0753

Self reported physical activity after a pregnancy complicated with gestational diabetes (GDM) is associated with less features of the metabolic syndrome

J. Lauenborg ${ }^{1}$, E. R. Mathiesen ${ }^{2}$, T. Hansen ${ }^{3}$, C. Glümer ${ }^{3}$, T. Jørgensen ${ }^{4}$, K. Borch-Johnsen ${ }^{3}$, O. Pedersen ${ }^{3}$, P. Damm ${ }^{1}$;

${ }^{1}$ Department of Obstetrics, Rigshospitalet, JMC, Copenhagen ${ }^{2}$ Department of Endrocrinology, ${ }^{3}$ Rigshospitalet, Copenhagen Steno Diabetes Center, Gentofte, ${ }^{4}$ Research Center for Prevention and Health, Glostrup, Denmark.

Background and Aim: We have previously shown a markedly increased prevalence of diabetes and the metabolic syndrome in women with previous GDM. Physical activity is known to have a positive impact on both glucose tolerance and the other components of the metabolic syndrome. The aim of the present study was to examine the level of physical activity and other lifestyle habits among women with prior gestational diabetes mellitus compared with a control group. 
Material and Methods: Three-hundred-eighthy-four women of Nordic Caucasian origin diagnosed with gestational diabetes (93\% diet treated) in the years 1978-1996, examined at the centre for diabetes and pregnancy, Rigshopitalet, Copenhagen University Hospital at a median of 10 (range 4-23) years after index pregnancy. All women were during pregnancy advised to continue a healthy lifestyle after pregnancy. An age-matched control group of 950 women from a population based cohort study (Inter99) was included. Women with prior GDM not reporting diabetes at follow-up underwent a 2-hour 75 g OGTT (WHO1999), the components of the metabolic syndrome were investigated and the participants completed a self-administered questionnaire regarding physical activity and other lifestyle habits. Physical activity was evaluated by self-estimated physical activity at work and leisure time according to Saltin 1968: Physical inactive: predominantly sedentary at work and leisure time. Light physical activity: Minor activity at work and/or leisure time. Physical activity: At least moderate activity at work and/or leisure time.

Results:

\begin{tabular}{|c|c|c|c|c|c|c|}
\hline & \multicolumn{3}{|c|}{ Prior - GDM } & \multicolumn{3}{|l|}{ Control } \\
\hline & $\begin{array}{l}\text { Physical } \\
\text { active }\end{array}$ & $\begin{array}{l}\text { Minor or } \\
\text { inactive }\end{array}$ & & $\begin{array}{l}\text { Physical } \\
\text { active }\end{array}$ & $\begin{array}{l}\text { Minor or } \\
\text { inactive }\end{array}$ & $p$ \\
\hline $\mathrm{n}$ & $\begin{array}{l}59 \\
(15 \%)\end{array}$ & $\begin{array}{l}325 \\
(85 \%)\end{array}$ & $\begin{array}{l}126 \\
(13 \%)\end{array}$ & $\begin{array}{l}824 \\
(87 \%)\end{array}$ & $\begin{array}{l}\text { NS (GDM } \\
\text { vs.control) }\end{array}$ & \\
\hline Age, years & $\begin{array}{l}40.5 \\
(6.5)\end{array}$ & $\begin{array}{l}43.2 \\
(7.3)\end{array}$ & 0.010 & $\begin{array}{l}44.9 \\
(8.1)\end{array}$ & $45.1(8.0)$ & NS \\
\hline BMI, $\mathrm{kg} / \mathrm{m}^{2}$ & $\begin{array}{l}25.3 \\
(5.3)\end{array}$ & $\begin{array}{l}28.1 \\
(6.5)\end{array}$ & 0.013 & $\begin{array}{l}24.3 \\
(5.0)\end{array}$ & $24.6(5.2)$ & NS \\
\hline $\begin{array}{l}\text { Obesity } \\
(\mathrm{BMI} \geq \\
\left.30 \mathrm{~kg} / \mathrm{m}^{2}\right)\end{array}$ & $20 \%$ & $39 \%$ & 0.006 & $18 \%$ & $17 \%$ & NS \\
\hline Diabetes & $32 \%$ & $39 \%$ & NS & $1 \%$ & $4 \%$ & NS \\
\hline $\begin{array}{l}\text { Metabolic } \\
\text { syndrome } \\
(\mathrm{WHO})\end{array}$ & $20 \%$ & $41 \%$ & 0.009 & $10 \%$ & $14 \%$ & NS \\
\hline Dyslipidaemia & $17 \%$ & $36 \%$ & 0.017 & $15 \%$ & $17 \%$ & NS \\
\hline Hypertension & $15 \%$ & $32 \%$ & 0.015 & $27 \%$ & $31 \%$ & NS \\
\hline $\begin{array}{l}\text { Insulin } \\
\text { resistance }\end{array}$ & $34 \%$ & $60 \%$ & 0.002 & $18 \%$ & $26 \%$ & NS \\
\hline $\begin{array}{l}\text { Abdominal } \\
\text { obesity }\end{array}$ & $29 \%$ & $54 \%$ & 0.004 & $27 \%$ & $27 \%$ & NS \\
\hline $\begin{array}{l}\text { Glucose } \\
\text { intolerance } \\
\text { or diabetes }\end{array}$ & $54 \%$ & $68 \%$ & NS & $9 \%$ & $19 \%$ & 0.007 \\
\hline $\begin{array}{l}N \text { alcoholic } \\
\text { beverages }> \\
\text { 14/week }\end{array}$ & $2 \%$ & $8 \%$ & NS & $11 \%$ & $13 \%$ & NS \\
\hline $\begin{array}{l}\text { Current } \\
\text { smoker }\end{array}$ & $34 \%$ & $41 \%$ & NS & $33 \%$ & $38 \%$ & NS \\
\hline
\end{tabular}

Data presented as percentages or median (SD)
Conclusion: The percentage of physically active women with prior GDM was low and did not differ from the background population. Physically active women with previous GDM had a more favourable health profile regarding the following components of the meatbolic syndrome: dyslipidaemia, hypertension, insulin resistance and abdominal obesity compared to the minor or inactive women. Although our data are cross-sectional they support the notion that women with a history of GDM should be advised to be physically active to improve their overall health profile and reduce their risk of cardiovascular disease. Supported by the Copenhagen University, the Danish Medical Research Council, the Danish Diabetes Association, Handelsgartner Ove Villiam Buhl Olesen og agtefalle Edith Buhl Olesens Mindelegat, Dagmar Marshalls Fond

\section{4}

The role of lifestyle modification in preventing type 2 diabetes in subjects with impaired glucose homeostasis E. Shishko, T. Mokhort, D. Garmaev; Endocrinology Department, Belarusian State Medical University, Minsk, Belarus.

Background and Aims: Leptin is proposed to be involved in regulation of body weight. Only little information is available how lifestyle modification influences on leptin concentrations in individuals with impaired glucose tolerance. The aim of our study was to assess the efficacy of lifestyle modification including altered diet composition and physical activity in preventing diabetes mellitus type 2 (DM 2) and their influence on serum leptin levels in individuals with impaired glucose homeostasis.

Materials and Methods: The study included 327 patients (69m258f) 25-65 y.o. with impaired glucose tolerance/ impaired fasting glucose (IGT/IFG) and newly diagnosed DM 2. Patients were divided into 2 groups matched by sex, age, weight, body mass index (BMI), waist-to-hip ratio (WHR). Research group included 183 patients (33m150f) who received and carried out individual recommendations of a balanced diet and physical activity. Control group included 144 patients (36m108f) who made no lifestyle modifications. The study lasted for 48 weeks. We measured fasting plasma glucose (FPG), 2-hour plasma glucose concentrations (2-h PG) following a 75-g oral glucose tolerance test and related to fasting leptin (FL) concentrations by sensitive ELISA. Comparisons among groups were performed using ANOVA/MANOVA.

Results: Patients of the research group demonstrated reduction of body weight $(-4.6 \pm 5.2 \mathrm{~kg})$, BMI $(-2.3 \pm$ $\left.3.1 \mathrm{~kg} / \mathrm{m}^{2}\right)$ and WHR $(-0.02 \pm 0.025)(\mathrm{p}<0.01$ for all $)$. They had positive dynamics of FPG and 2-h PG concentrations 
also $(-0.5 \pm 0.8$ and $-0.7 \pm 0.9 \mathrm{mmol} / 1$ respectively; $\mathrm{p}<0.001)$. Persons of the control group had significant increase in weight, BMI and WHR as well as FPG and 2h PG concentrations elevation $(p<0.05)$. The main novel finding was that median serum leptin in the research group decreased by $23.9 \%$ (from 34.7 to $26.4 \mathrm{ng} / \mathrm{ml}, \mathrm{p}<0.01$ ) and increased in the control group by $27.6 \%$ (from 37.9 to $44.7 \mathrm{ng} / \mathrm{ml}, \mathrm{p}<0.01$ ). Among subjects with IGT from the research group, glucose levels normalized in $49.3 \%$ $(p<0.001)$ and serum leptin levels decreased by $-8.5 \mathrm{ng} /$ ml, i.e. $26.9 \%(p<0.01)$ from initial. In the control group glucose levels normalized in $4.5 \%(\mathrm{p}<0.01)$ persons with IGT at baseline and combined with FL concentration increase by $7.9 \mathrm{ng} / \mathrm{ml}(22.9 \%, \mathrm{p}<0.01)$. By the end of the study $12 \%$ of non-diabetic obese subjects of the control group have developed DM 2, 28\% subjects - IFG and 48\% - IGT, while in the research group there were no changes in glucose concentrations among subjects with normal glucose tolerance $(p<0.001)$. Among patients of the research group there was a reduction of DM 2 by $11.9 \%$ and an increase in the control group by $35.1 \%$ but FL in the research group did not change significantly.

Conclusion: Thereby, lifestyle modifications lead to reduction not only fasting plasma glucose, 2-hour plasma glucose concentrations but also fasting leptin concentrations in individuals with impaired glucose tolerance.

\section{5}

A lifestyle intervention improves the incretin response during an OGTT

S. A. Schaefer ${ }^{1}$, O. Tschritter ${ }^{1}$, C. Thamer ${ }^{1}$, K. Müssig ${ }^{1}$, J. J. Holst ${ }^{2}$, H.-U. Häring ${ }^{1}$, A. Fritsche ${ }^{1}$, B. Gallwitz ${ }^{1}$;

${ }^{1}$ Department of Endocrinology, Metabolism, Pathobiochemistry, Vascular Medicine and Nephrology, Medical University Hospital, Tuebingen, Germany, ${ }^{2}$ Medical Physiology, The Panum Institute, University of Copenhagen, Denmark.

Background and Aims: The incretin hormones glucosedependent-insulinotropic peptide (GIP) and glucagon-like peptide-1 (GLP-1) postprandially enhance the glucoseinduced insulin response. GLP-1 also plays an important role in controlling appetite, energy intake and satiety. Life style intervention trials with reduced caloric intake and increased physical activity resulted in an improvement of glucose tolerance. Whether incretin secretion is affected by lifestyle intervention is not yet clear. The aim of this study was to investigate the effect of changes in life style on GLP-1 and GIP secretion.

Materials and Methods: $47(28 \mathrm{f} / 19 \mathrm{~m})$ prediabetic persons (age $47 \pm 1.6$ years, BMI $30.1 \pm 0.9 \mathrm{~kg} / \mathrm{m}^{2}, 35 \mathrm{NGT}, 12$ IGT) underwent oral glucose tolerance tests (OGTT) before (T0) and 9 months (T1) after life style intervention (calorie restricted diet and regular physical activity). GLP-1 and GIP concentrations $(0 ; 30 ; 120 \mathrm{~min}$ of the OGTT) were analysed with regard to changes of glucose tolerance, insulin secretion and insulin sensitivity estimated by validated indices from the OGTT.

Results: After 9 months of life style intervention GLP-1 concentrations in the OGTT (AUC GLP-1) were significantly higher than in the basal OGTT (T0: AUC GLP-1 $3291 \pm 262 \mathrm{pmol} / \mathrm{l} / \mathrm{min}, \mathrm{T} 1:$ AUC GLP-1 $4171 \pm 207 \mathrm{pmol} / \mathrm{l} /$ $\min ; \mathrm{p}=0.006)$. Furthermore, the increase in total GLP-1 secretion (AUC GLP-1) was positively correlated with a rise in insulin secretion relative to insulin sensitivity (disposition index) $(\mathrm{r}=0.32, \mathrm{p}=0.03)$ as well as an improvement in glucose tolerance $(\mathrm{r}=-0.43, \mathrm{p}=0.003)$. Also, a positive correlation was found between the increase in AUC GLP-1 and mean fibre content in the diet $(\mathrm{r}=0.38$, $\mathrm{p}=0.01$ ). In contrast to GLP-1 no significant changes in total GIP secretion after life style intervention were observed (T0: AUC GIP 7917 \pm 403 pmol/1/min, T1: AUC GIP $8840 \pm 395 \mathrm{pmol} / \mathrm{l} / \mathrm{min}, \mathrm{p}=0.10$ ).

Conclusion: Life style intervention results in an increased GLP-1 response in the OGTT accompanied by an increase in insulin secretion relative to insulin sensitivity and an improvement in glucose tolerance. Moreover, higher fibre intake in the diet correlated with a stronger rise in GLP-1 secretion. The underlying mechanisms are not yet completely understood. Stimulation of gastric receptors, vagal afferent outputs and neural mechanisms might be involved. Supported by Deutsche Forschungsgemeinschaft KFO 114

0756

Impact of a school intervention in physical activity or diet on insulin resistance, body mass index, and arterial pressure in adolescents

G. Ramírez-López ${ }^{1}$, J. Salmerón ${ }^{2}$, V. Valles-Sánchez ${ }^{3}$, B. Hernández-Prado ${ }^{4}$, L. Neufeld ${ }^{5}$;

${ }^{1}$ Unidad de Investigación Epidemiológica y en Servicios de Salud del Adolescente, Instituto Mexicano del Seguro Social, Tonalá, Jal, ${ }^{2}$ Unidad de Investigación Epidemiológica y en Servicios de Salud, Instituto Mexicano del Seguro Social, Cuernavaca, Mor, ${ }^{3}$ Departamento de Endocrinología, Instituto Nacional de Ciencias Médicas y Nutrición "SZ", Mexico, ${ }^{4}$ Centro de Investigación en Salud Poblacional, Instituto Nacional de Salud Pública, Cuernavaca, Mor., ${ }^{5}$ Centro de Investigación en Nutrición y Salud, Instituto Nacional de Salud Pública, Cuernavaca, Mor., Mexico. 
Background and Aims: Type 2 diabetes mellitus has increased in adults and adolescents due to obesity, physical inactivity and inadequate food intake. Successful school interventions aimed to reduce risk factors are needed. The effects on these factors were evaluated after interventions in either nutrition or physical activity in high school adolescents from Guadalajara, Jal., Mexico

Materials and Methods: 682 adolescents were assigned to either group with physical activity, diet or control. Variables studied before and after one year intervention were: insulin, glucose, insulin resistance (HOMA-IR), HDL-C, LDL-C, systolic and diastolic blood pressure, BMI, Tanner sexual development, physical activity, diet and tobacco use. Interventions were based on the social cognitive and constuctivist theories aimed to obtain significant learning and consisted of a course in either nutrition or physical activity, monthly counseling, workshops with parents and posters aimed to promote changes in behaviour. Homogeneity between groups was evaluated with ANOVA or $X^{2}$ and the effect of interventions with multiple linear regressions and a model of differences in differences.

Results: 248 adolescents comprised the control group, 227 the physical activity group, and 207 the diet group. Followup losses were $21.6 \%$. Mean age at the start of the study was 15.8 years (range 14-19 years), 51.5\% were female, $71.2 \%$ in low socieconomic status and $26.4 \%$ overweight. Initial insulin was $11.9 \pm 5.9 \mu \mathrm{U} / \mathrm{ml}$, glucose: $85.6 \pm 7.2 \mathrm{mg} /$ dl, LDL-C: $103.2 \pm 29.1 \mathrm{mg} / \mathrm{dl}$, HDL-C: $48.0 \pm 12.0 \mathrm{mg} / \mathrm{dl}$, systolic blood pressure: $114.3 \pm 10.6 \mathrm{mg} / \mathrm{dl}$ and diastolic blood pressure: $75.6 \pm 9.5 \mathrm{mg} / \mathrm{dl}$. Physical activity reduced insulin concentration $(\beta=-1.62, p<0.01)$, glucose $(\beta=-8.01, p<0.01)$, HOMA-IR $(\beta=-0.59, p<0.01)$, BMI $(\beta=-0.25, \quad p<0.01)$, systolic blood pressure $(\beta=-3.70$, $\mathrm{p}<0.01)$ and diastolic blood pressure $(\beta=-3.34, \mathrm{p}<0.01)$. Diet reduced BMI $(\beta=-0.39, \mathrm{p}<0.01)$ and diastolic blood pressure $(\beta=-3.83, \mathrm{p}<0.01)$.

Conclusion: Interventions in diet or physical activity are able to reduce risk factors for chronic diseases in highschool adolescents.

This research was supported by grants CONACyT 37951-M

\section{7}

Identification of cardiovascular risk factors and longterm treatment of children and adolescents with overweight and obesity - a prospective trial with telemedical support (TeleObe)

R. Schiel, W. Beltschikow, S. Radón, G. Kramer; Department of Diabetes and Metabolic Diseases, Inselklinik Heringsdorf GmbH, Seeheilbad Heringsdorf, Germany.
Background and Aims: During the last decades in children and adolescents the prevalence of overweight and obesity increased dramatically. It was the goal of the prospective trial to examine childrens' and adolescents' cardiovascular risk factors and to establish an effective strategy not only to reduce body weight, but also to induce a long-term stabilisation.

Materials and Methods: Out of a cohort of 228 over-

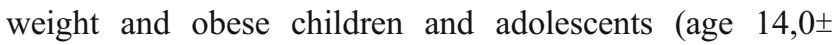
$2,4 \mathrm{ys}$, BMI $30,6 \pm 5,2 \mathrm{~kg} / \mathrm{m}^{2}$ ), addmitted to hospital to participate in a structured treatment and teaching programme (STTP) aiming weight reduction, 30 patients $(13.2 \%)$ were screened positive with a cluster of metabolic and cardiovascular risk factors. All patients screened positive were included in a long-term treatment programme with telemedical support. It integrates data collection, visualization, and recommendations for handling by using mobile phone and Internet services. Its core is a module, which visualizes a summary of the patients' clinical data. A feedback from the physician to the patient is given periodically. At the end of the trial a questionnaire on patients experiences and satisfaction was applied.

Results: In children and adolescents (age 13.4 \pm 2.3 ys, BMI $32.1 \pm 6.0 \mathrm{~kg} / \mathrm{m}^{2}$ ) screened positive for cardiovascular risk factors the following parameters were identified: 11 patients (37\%) had systolic blood pressure levels $>120 \mathrm{mmHg}, 5$ (17\%) had diastolic levels $>90 \mathrm{mmHg}$ (mean systolic/diastolic RR 119.5 $\pm 12.9 / 75.2 \pm 9.9 \mathrm{mmHg}), 12(40 \%)$ had values of Creactive proteine $(\mathrm{CRP})>0.5 \mathrm{mg} / \mathrm{dl}$ (median $0.5 \mathrm{mg} / \mathrm{dl}), 22$ $(73 \%)$ had elevated uric acid levels ( $>381$, median $449 \mu \mathrm{mol} /$ 1), $4(13 \%)$ had a dyslipidemia and in 18 patients $(60 \%)$ the carotis intima media thickness was above the cut-off $(>0.47$, median $0.50 \mathrm{~mm})$. During the first phase of intervention, over 35.2 \pm 7.1 days patients participated in the STTP. BMI decreased to $29.9 \pm 4.7 \mathrm{~kg} / \mathrm{m}^{2}(\mathrm{p}<0,001)$. Afterwards the telemedical support programme was started: During the follow-up of 12 months further modifications were registered in respect of eating habits in $73 \%$ of the patients, in physical exercise and sports in 60\%. Parallel, there was still a tendency to further reduction of body weight $(p=0.079)$. Using the telemedical support programme motivational and psychological problems were identified in $28 \%$ of the patients followed by feedback and specific interventions. All in all about $77 \%$ of participants considered telemedical support helpful for the everyday management and to improve empowerment and motivation.

Conclusion: In Western industry countries 10-20\% of children and adolescents are overweight or obese. A high percentage has excessive risk factors for diabetes and cardiovascular diseases. Early intervention is mandadory. Hence, the telemedical support programme TeleObe seems to be a highly effective tool. It guarantees not only an effective initial weight reduction, but a stabilization over a long period 
of time. Via feedback the identification and specific intervention is possible in respect of motivational, psychological or other problems in everyday management. TeleObe is highly accepted by children, adolescents and their parents. TeleObe was supported by a grant from the National Ministry of Education and Science (BMBF, DISCO-Innoregio)

0758

Long-term aerobic exercise can potentiate the effect of rosiglitazone on islet preservation and insulin resistance in diabetic prone OLETF rat model M. Nam ${ }^{1}$, I.-S. Park ${ }^{2}$, S.-H. Park ${ }^{1}$, J. Earm ${ }^{2}$, W. Shim ${ }^{1}$, S. Hong ${ }^{1}$, Y. Kim ${ }^{1}$;

${ }^{1}$ Internal Medicine, Inha University College of Medicine, Incheon, ${ }^{2}$ Anatomy, Inha University College of Medicine, Incheon, Republic of Korea.

Background and Aims: Steady aerobic exercise is recommended for diabetes treatment with anti-diabetic medications, particularly thiazolidinediones in obese type 2 diabetes.

Materials and Methods: We evaluated the effects of aerobic exercise $(20 \mathrm{~m} / \mathrm{min}, 30$ minutes/day) and rosiglitazone $(3 \mathrm{mg} / \mathrm{kg} /$ day) for 24 weeks on islet morphology and insulin resistance in obese diabetic prone animal model, OLETF rat.

Results: Increased lean body mass, decreased epididymal fat, and improved intraperitoneal glucose tolerance test were observed in both rosiglitazone plus exercise and exercise only groups (each group $n=15$ ). And the onset of diabetes was also delayed in both exercise and rosiglitazone group compared with control. On the chronological changes of islet morphology, more significant decrease of beta cell destruction was shown in rosiglitazone plus exercise group compared with exercise or rosiglitazone only. And exercise led to extension of normal expressions of insulin and GLUT-2 to the level of rosiglitazone group with western blot analysis and confocal microscopic finding, indicating comparable preventive or therapeutic effects of exercise with rosiglitazone treatment. The measured NOx (nitrite and nitrate) in pancreatic tissue were recovered in rosiglitazone only and plus exercise groups compared with control OLEFT rats.

Conclusion: We suggest the exercise is an essential regimen for islet preservation and improving insulin resistance in obese type 2 diabetes, particularly for antidiabetic treatment with rosiglitazone.
Supported by a grant of the Korean Health $21 R \& D$ Project, Ministry of Health \& Welfare, Republic of Korea (A050463)

0759

Pharmacological and lifestyle interventions to prevent or delay type 2 diabetes mellitus in individuals with impaired glucose tolerance: a systematic review and meta-analysis

K. Khunti, C. L. Gillies, P. C. Lambert, N. J. Cooper, R. Hsu, A. J. Sutton, K. R. Abrams;

Department of Health Sciences, University of Leicester, United Kingdom.

Background and Aims: Individuals diagnosed with impaired glucose tolerance (IGT) have a high risk of developing type 2 diabetes (T2DM) and therefore many trials of interventions for prevention of T2DM have focused on such high risk individuals. Interventions assessed have been diverse but form two main categories, pharmacological or lifestyle. The aim of this study was to conduct a systematic review and meta-analysis of the effectiveness of pharmacological and lifestyle interventions, to prevent or delay T2DM, in individuals with IGT.

Materials and Methods: Medline and Embase databases were searched up to July 2005, for eligible randomised controlled trials (RCT). The reference lists of any studies that fitted our inclusion criteria and any relevant published reviews were checked. We selected RCTs published in English language that evaluated interventions to delay or prevent T2DM in individuals with IGT. Teams of reviewers independently extracted relevant data and assessed study quality using the Jadad score.

Results: Fifteen trials met the inclusion criteria for the systematic review: 13 trials comprising 7904 individuals with IGT, reported results in sufficient detail for inclusion in the meta-analyses. Due to the diversity of the interventions three separate meta-analyses were undertaken, all showed statistically significant benefit of intervention to delay or prevent T2DM. Results of the meta-analyses were, lifestyle interventions compared to standard advice, pooled hazard ratio 0.50 (95\% confidence interval [CI]: 0.42 , 0.59 ), oral hypoglycaemic agents compared to placebo, 0.66 (95\% CI: $0.53,0.81)$ and anti-obesity agent compared to placebo, 0.44 (95\% CI: $0.28,0.69)$.

Conclusion: Both lifestyle and pharmacological interventions significantly reduce the rate of progression to T2DM in individuals with IGT with lifestyle interventions proving just as effective as pharmacological.

Supported by MRC and ESRC 


\section{0}

Changes in insulin sensitivity in response to exercise in overweight Swedish and Arabian women with type 2 diabetes

F. Haghanifar ${ }^{1}$, K. F. Eriksson ${ }^{1}$, A. Segerström ${ }^{2}$, P. Wollmer ${ }^{3}$, O. Thorsson ${ }^{3}$, Y. Wessman ${ }^{1}$, P. Nyberg ${ }^{4}$, J. Apelqvist ${ }^{1}$, L. Groop ${ }^{1}$;

${ }^{1}$ Clinical Sciences Division of Diabetes and Endocrinology, ${ }^{2}$ Department of Health Sciences, Division of Physiotherapy, ${ }^{3}$ Department of Clinical Sciences, Division of Clinical Physiology, Lund University, Lund, ${ }^{4}$ Department of Health Sciences, Lund University, Malmö, Sweden.

Background and Aims: Physical inactivity is directly linked to the rising incidence of insulin resistance and could contribute to the epidemic increase of type 2 diabetes in certain ethnic populations. Although exercise is frequently prescribed to patients with type 2 diabetes, there are few studies assessing the effect of exercise on insulin sensitivity in different populations. The purpose of this study was thus to determine the effects of exercise on insulin sensitivity and maximal aerobic capacity in previously sedentary, obese Swedish and Arabian women with type 2 diabetes.

Material and Methods: The study included 14 Swedish and 18 Arabian women (age 49.8 \pm 6.8 ) with type 2 diabetes (11 treated with insulin, 19 with oral agents and 2 with only diet) who participated in a supervised 6-month structured aerobic and resistance training program 2 times a week of moderateintensity with the aim to increase insulin sensitivity by at least $20 \%$ and maximal oxygen uptake by approximately $10 \%$. Insulin sensitivity (M-value) was measured by euglycemic hyperinsulinemic clamp combined with indirect calorimetry before and after 3 and 6 month intervention. Vo2max was estimated from submaximal ergometer test.

Results: All patients were markedly insulin resistant at entry to the study (M value approx. 30\% of controls). Eleven Swedish and 9 Arabian completed the intervention. Vo2max at baseline was $(19.9 \pm 5.1 \mathrm{ml} / \mathrm{kgxmin})$ and increased by $13 \%$ after 3 months $(22.7 \pm 4.8 \mathrm{ml} / \mathrm{kgxmin}, \mathrm{p}=0.001)$ and $9 \%$ after 6 months $(21.8 \pm 5.3 \mathrm{ml} / \mathrm{kgxmin}, \mathrm{p}=0.018)$. M-value at the baseline was $(2.75 \pm 1.6 \mathrm{mg} / \mathrm{kgxmin})$ and increased by $10 \%$ after 3 month $(3.03 \pm 2 \mathrm{mg} / \mathrm{kgxmin}, \mathrm{p}=0.1)$ and $25 \%$ after 6 month $(3.47 \pm 2 \mathrm{mg} / \mathrm{kgxmin}, \mathrm{p}=0.007)$. This effect was primarily due to the Scandinavian group $(\mathrm{p}=0.001)$. BMI (32.6 \pm 3.8 vs. $32.5 \pm 4.1), \mathrm{HbA} 1 \mathrm{c}(6.49 \pm 1.3$ vs. $6.39 \pm 1 \%$ ), triglycerides $(1.9 \pm 1.2$ vs. $1.7 \pm 0.7 \mathrm{mmol} / \mathrm{l})$, diastolic blood pressure $(75.3 \pm 12$ vs. $73.6 \pm 8.9 \mathrm{mmHg})$ were not significantly improved by training.

Conclusion: Exercise without weight loss increased modestly insulin sensitivity and maximal oxygen uptake in previously sedentary women with type 2 diabetes. However, the $25 \%$ enhancement in insulin sensitivity was not associated with a significant improvement in metabolic control suggesting that a longer or more intense training period and/or additional means are needed as treatment for type 2 diabetes.

\section{PS 58}

\section{Mechanisms of obesity and weight loss}

\section{1}

Candidate gene polymorphisms for obesity and DM2: association with the somatotypes

D. Vejrazkova ${ }^{1}$, J. Vcelak ${ }^{1}$, M. Vankova ${ }^{1}$, P. Lukasova ${ }^{1}$, H. Kvasnickova ${ }^{2}$, P. Blaha ${ }^{3}$, K. Vondra ${ }^{2}$, B. Bendlova ${ }^{1}$;

${ }^{1}$ Dept. of Molecular Endocrinology, ${ }^{2}$ Lab. of Functional Diagnostics, Institute of Endocrinology, Praha, ${ }^{3}$ Dept. of Anthropology and Human Genetics, Faculty of Science, Charles University, Praha, Czech Republic.

Background and Aims: Type 2 diabetes mellitus and obesity are complex diseases characterized by the interaction of the genetic and environmental factors. The aim of the study was to identify the possible associations of the selected candidate gene polymorphisms with somatotypes. Materials and Methods: We genotyped polymorphisms in 7 different candidate genes for obesity or type 2 diabetes INS VNTR (HphI), FABP2 (Ala54Thr), PPARgamma2 (Pro12Ala), B2AR (Gln27Glu), B3AR (Trp64Arg), UCP1 (A-3826G), Kir6.2 (Glu23Lys) in 301 unrelated well biochemically characterized non-diabetic subjects who underwent the oGTT and ITT tests. Detailed anthropometric data were taken using standard anthropometric technique according to Martin and Saller: 11 height measures, 14 circumferences, 12 width measures and 14 skin folds. Body composition (\%fat mass, \%muscles, \%bone mass) was calculated using the ANTROPO programme. The anthropometric parameters were statistically analyzed after adjustment for age, men and women separately (NCSS 2004). To reduce the number of anthropometric variables, factor analysis was performed. This analysis revealed three main somatotype determining factors which were used in the following cluster analysis (K-Means).

Results: Three clusters of probands corresponding well to ectomorph, endomorph, and mesomorph somatotypes were obtained (number of subjects; BMI $\left(\mathrm{kg} / \mathrm{m}^{2}\right)$; WHR; muscles/fat mass; presented as a mean $\pm \mathrm{SD}$ ):

Ectomorphs women: $(94 ; 23,26 \pm 4,21 ; 0,728 \pm 0,067 ; 1,74 \pm$ 0,76); Ectomorphs men: (46; 26,40 $\pm 3,40 ; 0,781 \pm 0,064$; $2,49 \pm 0,98)$; Mesomorphs women: $(25 ; 21,64 \pm 2,51 ; 0,721 \pm$ $0,034 ; 3,04 \pm 0,43)$; Mesomorphs men: (31; 22,56 $\pm 2,41$; $0,767 \pm 0,028 ; 5,27 \pm 1,55)$; Endomorphs women: $(65 ; 29$, 
$84 \pm 4,54 ; 0,839 \pm 0,051 ; 1,37 \pm 0,61)$; Endomorphs men: (40; $32,94 \pm 5,37 ; 0,892 \pm 0,060 ; 2,51 \pm 0,71)$ With respect to cluster distribution, both genders were analyzed together according to genotypic frequencies. Among the assessed gene polymorphisms, UCP1 (A-3826G) displayed significant association with the defined somatotypes. The genotype frequencies: Ectomorphs AA(53,15\%), AG(40,54\%), GG(6,31\%); Endomorphs AA(44,83\%), AG(45,98\%), GG(9,20\%); Mesomorphs $\mathrm{AA}(60,9 \%), \mathrm{AG}(29,0 \%), \mathrm{GG}(10,1 \%)$ - Chi-square test, $\mathrm{p}=0,046$.

Conclusion: We have demonstrated that the AA genotype of the UCP1 promoter polymorphism A-3826G is more frequent in the groups of lean probands (ectomorphs, mesomorphs) in comparison with probands who have abundant body fat content (endomorphs). It is in agreement with literature data reporting the $\mathrm{G}$ allele as obesity risk factor.

Supported by IGA MH CR NR/7809-5

\section{2}

Neuromedin beta: the polymorphism P73T in Czech diabetic and nondiabetic populations

P. Lukasova, J. Spalova, J. Vcelak, M. Vankova,

K. Vondra, V. Hainer, B. Bendlova;

Laboratory of Molecular Endocrinology, Institute of Endocrinology, Prague, Czech Republic.

Background and Aims: Neuromedin beta $(N M B)$ is a member of the bombesin-like peptides widely expressed in brain, pancreas, adrenals and gastrointestinal tract. It regulates exocrine and endocrine secretions, smooth muscle contraction, feeding, blood pressure, glycemia, body temperature and cell growth. Recently, the significant association of the missense polymorphism P73T (c.217C $>$ A) with the score of disinhibition and susceptibility to hunger was published. Homozygous mutation TT was associated with increased body weight, BMI, waist girth and body fat. The aim of our study was to determine the genotypic distribution of the $N M B$ gene in the Czech diabetic and nondiabetic populations and to assess possible association of the minor allele $\mathrm{T}$ carriership with biochemical and anthropometric parameters.

Materials and Methods: Detailed biochemical and anthropometric characteristics of 288 patients with diabetes mellitus type 2 (DM2) (M/F 108/180; age 60 18.2 years; BMI $31.1 \pm 5,66 \mathrm{~kg} / \mathrm{m}^{2}$ ) and 367 nondiabetic subjects (ND) (M/F 132/235; age $32 \pm 11.0$ years; BMI $23.7 \pm 3.82 \mathrm{~kg} / \mathrm{m}^{2}$ ) were collected. In healthy population the oGTT and insulin tolerance tests (ITT) were performed. For screening of P73T (c.217C $>\mathrm{A}$ ) variant in the exon 2 of the $N M B$ gene the single strand conformation polymorphism (PCR-SSCP) method was used. The positive controls (PP, PT and TT genotypes) were used in every run. Statistical analysis: Chisquare test, Mann-Whitney test and ANCOVA (adjustment for age) (NCSS 2004).

Results: The genotypic distribution (PP/PT/TT) in diabetic and nondiabetic populations was not significantly different: DM2 - 54.2/37.1/8.7 and ND - 51.0/41.1/7.9 [\%]. In ND group the homozygotes for allele $\mathrm{T}$ had significantly higher level of glycosylated proteins $(p=0.0007)$ that could be considered as an indicator of the eating behaviour. It is in agreement with previously observed relation of $\mathrm{T}$ allele to score of disinhibition and susceptibility to hunger. In DM2 patients the level of glycosylated proteins could be affected by diet and drug treatment. Nevertheless, we did not evidence the higher body weight, BMI, waist girth, body fat or any difference in other anthropometric parameters in $\mathrm{T}$ allele carriers. We noticed that $\mathrm{T}$ allele is associated with higher insulinemia. In DM2 the ratio fasting glycemia/ fasting insulin $\left(\mathrm{G}_{0} / \mathrm{IRI}_{0}\right)$ was significantly lower $(\mathrm{p}=0.0456)$ in T allele carriers. In ND the sum of fasting and stimulated insulin levels (ÓIRI0,60,120) and meanIRI0,60,120 were significantly higher $(\mathrm{p}=0.0424)$ in TT homozygotes and the area under the insulin curve in ITT (AUCITT) was significantly higher $(\mathrm{p}=0.0292)$ in $\mathrm{T}$ allele carriers.

Conclusion: Our study suggests that the polymorphism P73T of the $N M B$ gene is associated with higher levels of glycosylated proteins and with higher insulin levels. $N M B$ seems to be the candidate gene for the eating behaviour. However, we did not uphold its influence on the predisposition to obesity.

Supported by IGA MHCR NR/7809-5 and NR/7800-4

\section{3}

Changes of appetite regulation systems activity under a chronic social stress and experimental metabolic syndrome A. L. Zagayko ${ }^{1}$, L. N. Voronina ${ }^{1}$, N. S. Krasova ${ }^{2}$, E. V. Strel'chenko ${ }^{1}$, V. V. Poltorak ${ }^{2}$;

${ }^{1}$ Department of biological chemistry, National University of Pharmacy, Kharkiv, ${ }^{2}$ Pathophysiology laboratory, Institute of Endocrine Pathology Problems, Kharkiv, Ukraine.

Background and Aims: Changes of appetite caused by action of unfavorable factors (including stress and unbalanced diet) evokes the increasing of the fat tissue mass of the body, which can lead to obesity, insulin resistance (IR) and metabolic syndrome (MS). The hormones of fat tissue adipocytokines and endocannabinoid system plays the key role in appetite regulation. The aim of the present study was 
to characterize the changes of appetite regulation systems activity under a chronic social stress and experimental MS in Golden Syrian hamsters.

Materials and Methods: 164 1-y-old Golden Syrian hamsters (males/females: 1/1) were randomized into 3 groups. A control group $(\mathrm{n}=12)$ was submitted to a standard diet. The 2 nd group ( $\mathrm{n}=76)$ was submitted for 5 weeks to a diet containing $29 \%$ vegetable- and animal oils and fructose ( $2 \mathrm{~g} / 100 \mathrm{~g} \mathrm{~b}$. w./day) - MS group. The 3 rd group ( $\mathrm{n}=76)$ was subjected for 5 weeks to the chronic social stress. For the final 3 days, 1, 2, 3, 4 and 5 weeks plasma leptin and adiponectin levels (by ELISA), anandamide (endogenic cannabinoid receptors ligand) levels in hypothalamus (by HPLC) were determined. Fasting blood glucose and insulin (by RIA) were measured. Homeostasis Model Assessment (HOMA) was used to estimate IR. Statistical analyses were carried out using SPSS and Microsoft Excel. Values are expressed as means \pm SEM.

Results: It has been shown, that both MS and stress are accompanied by body mass rising, but under stress the weight enhances after its fall. In both models were shown 2-phase changes of the leptin content: decrease during the first week and increase in 3 weeks. Inability of high dose of leptin to block the body mass increase indicated the leptinresistance development. It was detected the strong correlation between leptin concentration and body mass rising in group 3 (males: $r=0.70, \mathrm{p}=0.045$; females: $r=0.86$, $\mathrm{p}=0.018$ ). IR was observed both in the models of MS and chronic stress. The plasma adiponectin level was decreased in the all time points of consideration. It was detected the strong correlation between adiponectin level and IR (group 2 males: $r=-0.86, \mathrm{p}=0.031$; females: $r=-0.73, \mathrm{p}=0.044$; group 3 males: $r=-0.79, \mathrm{p}=0.042$; females: $r=-0.92$, $\mathrm{p}=0.026$ ) also between body mass rising and IR under all experimental impacts. The orexigenic factor anandamide was significantly increase in hypothalamus of hamsters both under chronic social stress and experimental MS.

Conclusion: The hyperphagia against the background of body mass increase can be linked to leptin-resistance development and arrest of activation by leptin of orexigenic anandamide degradation. Our results suggest that changes of appetite which lead to obesity and insulin resistance development under stress and experimental metabolic syndrome have similar mechanisms.

\section{4}

Assessment of abdominal subcutaneous and visceral adipose tissue volumes with multi detector-row computed tomography

P. Maurovich-Horvat ${ }^{1,2}$, J. Massaro ${ }^{3}$, C. S. Fox ${ }^{3}$, F. Moselewski ${ }^{1}$, C. J. O’Donnell ${ }^{3,4}$, U. Hoffmann ${ }^{1}$;
${ }^{1}$ Department of Radiology, Massachusetts General Hospital, Boston, United States, ${ }^{2}$ Department of Cardiovascular Surgery, Semmelweis University, Budapest, Hungary, ${ }^{3}$ Framingham Heart Study, National Heart, Lung, and Blood Institute, Framingham, United States, ${ }^{4}$ Department of Medicine, Massachusetts General Hospital, Boston, United States.

Background and Aims: Abdominal obesity is associated with insulin resistance, type 2 diabetes mellitus (T2DM) and cardiovascular diseases. Cross sectional imaging may enable accurate localization and quantification of subcutaneous and visceral adipose tissue. The reproducibility of multidetector-row computed tomography (MDCT) based volumetric quantification of abdominal adipose tissue and the ability to depict age and gender related characteristics of adipose tissue deposition have not been reported.

Materials and Methods: We evaluated a random subset of 100 subjects (age range: $37-83$ years; $49 \%$ female) of the Framingham Heart Study offspring cohort who underwent MDCT scanning. Two readers measured subcutaneous and visceral adipose tissue volumes ( $\mathrm{SAV}$ and $\mathrm{VAV} ; \mathrm{cm}^{3}$ ) and areas (VAA and SAA $\mathrm{cm}^{2}$ ) as well as abdominal sagital diameter (SD) and waist circumference (WC).

Results: Inter-reader reproducibility was excellent (relative difference: $-0.34 \% \pm 0.52 \%$ for SAV and $0.59 \% \pm 0.93 \%$ for $\mathrm{VAV}$, intra class correlation (ICC) $=0.99$ each). The mean SAA/VAA ratio was significantly different from the mean SAV/VAV ratio $(2.0 \pm 1.2$ vs. $1.7 \pm 0.9 ; p<0.001)$. The ratio of $\mathrm{SAV} / \mathrm{VAV}$ was only weakly inversely associated with SD $(\mathrm{ICC}=-0.32, \mathrm{p}=0.01)$ and not significantly associated with WC $(\mathrm{ICC}=-0.14, \mathrm{p}=0.14)$ or BMI $(\mathrm{ICC}=-0.17, \mathrm{p}=0.09)$. The mean SAV/VAV ratio was significantly different between participants $<60$ years vs. $>60$ years $(1.9 \pm 1.0$ vs. $1.5 \pm 0.7 ; \mathrm{p}<0.001)$ and between men and women $(1.2 \pm 0.5$ vs. $2.2 \pm 0.9 ; \mathrm{p}<0.001)$.

Conclusion: This study demonstrates that MDCT-based volumetric quantification of abdominal adipose tissue is highly reproducible. In addition, our results suggest that volumetric measurements can depict age and gender related differences of visceral and subcutaneous abdominal adipose tissue deposition. Further research is warranted to assess whether volumetric measurements may substantially improve the predictive value of obesity measures for insulin resistance, T2DM and other diseases.

0765

Osteoprotegerin declines in parallel with insulin resistance and inflammatory biomarkers in morbidly obese patients after weight loss

H.-P. Kopp ${ }^{1}$, G. H. Schernthaner ${ }^{2}$, R. Koppensteiner ${ }^{2}$,

G. Schernthaner ${ }^{1}$; 
${ }^{1}$ Department of Medicine I, Rudolfstiftung Hospital, Vienna, Austria, ${ }^{2}$ Department of Angiology, Medical University of Vienna, Austria.

Background and Aims: Morbid Obesity is associated with chronic inflammation, insulin resistance and an increased risk for development of diabetes and cardiovascular mortality. Recent studies suggest that osteoprotegerin (OPG) may play an important pathogenetic role in the atherothrombotic complications in cardiovascular disease as well as in diabetes. Thus, it was of interest to study OPG in insulin resistant patients with morbid obesity before and after massive weight loss induced by bariatric surgery.

Materials and Methods: A total of 32 patients (mean age $41 \pm 12$ years) with morbid obesity was studied before and $26.3 \pm 11.4$ months after bariatric surgery. High sensitivity assays were used to measure concentrations of fasting OPG, monocyte-chemoattractant-protein-1 (MCP-1), Interleukin18 (IL-18), and high-sensitive C-reactive protein (hsCRP).

Results: After a mean weight loss of $34.1 \pm 17.0 \mathrm{~kg}$, circulating OPG decreased significantly from $4.7 \pm 1.6$ to $2.7 \pm 1.4 \mathrm{ng} / \mathrm{ml}(\mathrm{p}<0.001), \mathrm{hsCRP}$ from $0.8(0.3-1.4)$ to 0.1 $(0.1-0.2) \mathrm{mg} / \mathrm{dl} \quad(\mathrm{p}<0.001), \mathrm{MCP}-1$ from $576 \pm 185$ to $314 \pm 149 \mathrm{pg} / \mathrm{ml}(\mathrm{p}<0.001)$ and IL-18 from $1478 \pm 631$ to $981 \pm 591 \mathrm{pg} / \mathrm{ml}(\mathrm{p}=0.001)$. The decline in OPG after weight loss correlated significantly with the decrease in bodymass-index, fasting insulin, HOMA-IR, and the inflammatory biomarkers MCP-1 and hsCRP.

Conclusion: This is the first intervention study demonstrating a significant decrease in circulating OPG in association with an improvement of both insulin resistance and chronic inflammation in morbidly obese patients after weight loss. Since high OPG was shown to predict cardiovascular death and myocardial infarction in several prospective studies, the observed marked lowering of OPG might be of clinical relevance in morbidly obese insulin-resistant patients.

\section{6}

Visceral but not subcutaneous fat reduction is responsible for metabolic improvements (MI) after weight loss (WL)

G. Formoso ${ }^{1}$, M. Taraborrelli ${ }^{1}$, E. Ciccarone ${ }^{1}$, A. Pandolfi ${ }^{2}$, S. Martinotti ${ }^{3}$, M. T. Guagnano ${ }^{1}$, A. Tartaro ${ }^{4}$, A. Consoli ${ }^{1}$;

${ }^{1}$ Department of Medicine and Aging Science, ${ }^{2}$ Department of Biomorphology, ${ }^{3}$ Oncology and Neurosciences, Center of Excellenze on Aging, Chieti, ${ }^{4}$ Clinical Science and Bioimaging, Institute of Advanced Biomedical Technologies, Chieti, Italy.
Background and Aims: Visceral adiposity associates with insulin resistance (IR) and increased cardiovascular risk (CVR). Inflammatory cytokines levels are increased in obese subjects and might be responsible for IR and increased CVR. WL is associated with improved insulin sensitivity (IS) and decreased inflammatory cytokines levels, however, little data exist about the relative role of visceral and subcutaneous fat loss on the MI brought about by WL. Aim of this study was to investigate whether, after caloric restriction, changes in IS and in TNF and IL6 plasma levels correlate with visceral and/or subcutaneous fat loss.

Materials and Methods: We determined in 10 obese subjects (BMI $33.82 .5 \mathrm{Kg} / \mathrm{mq}$ ) visceral and subcutaneous abdominal fat volume (VAV and SCV) by MRI. Subjects were enrolled in a WL program based on dietary and life style counselling and MRI was repeated after each subject had achieved a WL equal to at least $5 \%$ of the original BW. Fasting plasma glucose, insulin, TNF, IL6 and leptin were measured before and after WL.

Results: After WL, IS significantly improved $(\mathrm{p}<0.05)$ while TNF, IL6 and leptin levels decreased (by 40\%, 45\% and $30 \%$ respectively, $\mathrm{p}<0.05$ for all). We observed a significant correlation $(r=0.64, p<0.01)$ between the amount of VA lost and the improvement in IS, between the percent reduction in VAV and the percent reduction in both TNF $(\mathrm{r}=0.71, \mathrm{p}<0.01)$ and IL6 $(\mathrm{r}=0.61, \mathrm{p}<0.01)$ plasma levels. No correlation was observed between the investigated parameters and either the amount or the percentage of SCV lost.

Conclusion:The reduction in VAV but not in SCV may be responsible for $\mathrm{MI}$ after WL in obese subjects.

\section{7}

Increase of visfatin after weight loss induced by gastroplastic surgery

G. Mitteregger ${ }^{1}$, K. Krzyzanowska ${ }^{1}$, F. Mittermayer ${ }^{2}$, W. Krugluger ${ }^{3}$, H. Kopp ${ }^{1}$, G. Schernthaner ${ }^{1}$;

${ }^{1}$ Department of Internal medicine 1, Rudolfstiftung Hospital, Vienna, ${ }^{2}$ Department of Clinical Pharmacology, Medical University Vienna, ${ }^{3}$ Central Laboratory, Rudolfstiftung Hospital, Vienna, Austria.

Background and Aims: The recently described adipocytokine visfatin is produced in visceral fat and has been suggested to influence insulin resistance. For a further investigation of the relationship between visfatin and insulin resistance this adipocytokine was measured in insulin resistant morbidly obese patients before and after gastroplastic surgery.

Materials and Methods: Visfatin, interleukin (IL)-6, high sensitivity C-reactive protein (hsCRP), homeostasis model assessment insulin resistance (HOMA-IR) and other clinical 
parameters were assessed in 36 morbidly obese subjects (28 female, mean age 43 yrs.) with a median body mass index (BMI) of $44.3 \mathrm{~kg} / \mathrm{m} 2$.

Results: Median (95\% confidence interval) visfatin concentrations increased significantly after weight loss (70.9 [61.4-75.6] ng/ml vs. 86.4 [79.4-89.8] ng/ml; p<0.0005). This increase correlated with the decrease of insulin and HOMA-IR and was paralleled by a reduction of IL-6 and hsCRP plasma concentrations after gastroplastic surgery.

Conclusion: Massive weight loss after gastroplastic surgery is accompanied by an increase of the novel adipocytokine visfatin. This increase correlates with the decrease of insulin plasma concentrations and HOMA-IR.

\section{8}

Comparision of the effects of peroxisome proliferatoractivated receptor- $\gamma$ with $-\alpha$ on energy homeostasis and fat distribution in high-starch-fed rats

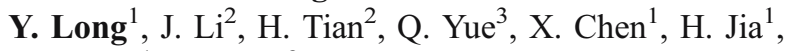
X. Zhang ${ }^{1}$, J. Liang ${ }^{2}$;

${ }^{1}$ Laboratory of Endocrinology and Metabolism, ${ }^{2}$ Department of Endocrinology, ${ }^{3}$ Department of Radiology, West China Hospital of Sichuan University, Chengdu, China.

Background and Aims: Peroxisome proliferator-activated receptor (PPAR)- $\alpha$ activation enhances free fatty acid oxidation and PPAR- $\gamma$ activation is involved in adipocyte differentiation, fatty acid uptake and storage. This study focused on the effects of PPAR- $\alpha$ and $-\gamma$ activation on energy homeostasis and fat distribution in high-starch-fed rats.

Materials and Methods: Weight gain, food intake, feeding efficiency, and metabolic surrogate markers were analyzed in high-starch-fed rats treated for 8-10 weeks with either PPAR- $\alpha$ activator fenofibrate or PPAR- $\gamma$ activator pioglitazone, and compared with high-fat-fed rats. Body fat distribution was evaluated in subcutaneous and visceral fat using a computed tomography scan at upper margin of pelvis level.

Results: High-starch feeding significantly raised the level of TG and high-fat feeding raised the level of TC. Pioglitazone increased and fenofibrate decreased caloric intake. Body weight gain increased in pioglitazone-treated rats and decreased in fenofibrate-treated rats. PPAR- $\gamma$ activator markedly increased feeding efficiency, whereas PPAR- $\alpha$ agonist treatment decreased feeding efficiency. Furthermore, PPAR- $\gamma$ activator tended to increase subcutaneous fat area/visceral fat area (SFA/VFA) ratio.

Conclusion: PPAR- $\gamma$ activation is associated with weight gain due to both increased food intake and improved feeding efficiency, whereas PPAR- $\alpha$ activation leads to opposite effects. The actions were independent of dietary content. There was a redistribution of fat from intra-abdominal visceral region to abdominal subcutaneous region by pioglitazone.
0769

Weight reduction decreases TNF- $\alpha$ and increases IL-6 gene expression in lymphocytes in subjects with metabolic syndrome - the GENOBIN study

V. D. F. Mello ${ }^{1,2}$, M. Kolehmainen ${ }^{1}$, U. Schwab ${ }^{1,3}$, U. Mager ${ }^{1}$, D. E. Laaksonen ${ }^{3}$, L. Pulkkinen ${ }^{1}$, H. Gylling ${ }^{1,3}$, M. Uusitupa ${ }^{1}$;

${ }^{1}$ Division of Clinical Nutrition/Food and Health Research Center, Kuopio University, Finland, ${ }^{2}$ Department of Internal Medicine, Federal University of Rio Grande do Sul, Porto Alegre, Brazil, ${ }^{3}$ Department of Medicine, Kuopio University Kuopio, Finland.

Background and Aims: Obesity has been considered as a proinflammatory state. Inflammation has been implicated in metabolic syndrome (MS), type 2 diabetes and cardiovascular diseases. Weight loss may have an effect on expression of genes related to inflammation in adipose tissue, and genes like tumor necrosis factor-alpha (TNF- $\alpha$ ) and interleukin (IL)-6 have been found to be increased in the adipose tissue of obese subjects. However, expression of these genes in immune cells is poorly described. We sought to evaluate how long-term weight reduction affects expression of genes related to inflammation in lymphocytes.

Materials and Methods: We carried out a randomized clinical trial in 75 40-70 year-old overweight or obese men and women with the MS. The subjects entered one of three lifestyle interventions - weight reduction (WR), aerobic training or resistance training - or the control group. Subjects randomized to WR participated in an intensive 12 wk weight loss period in which they met a clinical nutritionist for 5 times. During the following weight maintenance phase 13-34 wks, they met the clinical nutritionist twice. Blood samples were taken at the beginning, at wk 12 and at the wk 34. Lymphocytes from 24 subjects from the WR group intervention were isolated with the use of Lymphoprep kit. Total RNA was extracted and converted to cDNA for quantitative real-time PCR gene expression analysis. The Ethics Committee of Kuopio University Hospital approved the protocol according with the standards of the Helsinki Declaration.

Results: A significant reduction in weight $(\mathrm{P}<0.001)$, waist circumference $(\mathrm{P}<0.001)$, body fat $(\mathrm{BF} ; \mathrm{P}<0.001), \mathrm{BF} \%$ $(\mathrm{P}<0.001)$ and fasting plasma glucose $(\mathrm{FPG} ; \mathrm{P}<0.001)$ was observed in the WR group at wk 34 compared with wk 0 . Gene expression levels of TNF- $\alpha$ were lower at wk 34 than at wk 0 [median 23.4\% (95\% CI: 1-24), $\mathrm{P}<0.05$ ]. Conversely, IL-6 expression increased at wk 34 compared with wk 0 [median 9\% (95\% CI: -5-35), $\mathrm{P}<0.05$ ]. Changes in both genes were significantly correlated between each other $(\mathrm{r}=0.61, \mathrm{P}=0.002)$. Change in IL-6 expression was significantly correlated to changes in $\mathrm{BF}$ 
$(\mathrm{r}=-0.42, \mathrm{P}=0.042)$, in $\mathrm{BF} \%(\mathrm{r}=-0.49, \mathrm{P}=0.014)$ and in FPG $(r=-0.44, P=0.035)$. Even when adjusting for changes in weight or waist circumference, correlations between changes in IL-6 expression with FPG and BF changes remained significant.

Conclusion: Weight loss in subjects with MS decreased TNF- $\alpha$ gene expression and paradoxically increased IL-6 gene expression in lymphocytes. The increase of IL-6 expression was correlated with decreases in body fat and fasting plasma glucose levels. Although the literature seems to support a decrease in gene expression of proinflammatory cytokines such as IL-6 with weight reduction, the immunomodulatory effects of weight loss on gene expression in lymphocytes may be more complex.

This work has been financially supported by grants from the Academy of Finland (n. 211034 to M.Kolehmainen, n. 211497 to M. Uusitupa), and from the Sigrid Juselius Foundation. V.D.F. Mello is supported by CNPq, Brazil.

\section{PS 59}

\section{Metabolic effects of exercise}

0770

Effect of short-term exercise training on insulin action and muscle energy metabolism

G. Kacerovsky ${ }^{1,2}$, M. Chmelik ${ }^{3}$, R. Pokan ${ }^{4}$, M. Farukuoye ${ }^{2}$, S. Gruber ${ }^{3}$, J. Szendroedi ${ }^{5}$, A. Brehm ${ }^{1,2}$, P. Nowotny ${ }^{5}$, A. Schmid ${ }^{3}$, E. Moser ${ }^{3}$, S. Trattnig ${ }^{3}$,

G. Pacini ${ }^{6}$, G. Smekal ${ }^{4}$, M. Roden ${ }^{1,2}$;

${ }^{1}$ 1. Medical Department, Hanusch Hospital, Vienna, ${ }^{2}$ Endocrinology and Metabolism, Karl-Landsteiner Institute, Vienna, ${ }^{3}$ MR Centre of Excellence, Medical University of Vienna, ${ }^{4}$ Department of Sports Physiology, University of Vienna, ${ }^{5}$ Department of Internal Medicine 3, Medical University of Vienna, Austria, ${ }^{6}$ Institute of Biomedical Engineering, National Research Council, Padova, Italy.

Background: Exercise training increases whole body insulin sensitivity due to increased muscle glucose transport in healthy and insulin resistant humans. In insulin resistant humans, such as elderly or 1 st degree relatives of type 2 diabetic (T2D) patients, reduced flux through ATP synthase mainly contributes to impaired muscle glucose transport and to elevated hepatocellular fat content (HCL). Thus, we hypothesized that exercise training should increase ATP synthetic flux in parallel with improvement of glucose metabolism.

Materials and Methods: Healthy humans ( $\mathrm{n}=7$; age: $40 \pm 5$ a, body mass index, BMI: $22 \pm 1 \mathrm{~kg} / \mathrm{m}^{2}$ ) without a family history of T2D underwent a 75 -g oral glucose tolerance test
(OGTT) and in vivo magnetic resonance spectroscopy (MRS). Thereafter, they performed incremental exercise testing $(+10-20 \mathrm{~W} / \mathrm{min}$ until exhaustion on a cycle ergometer, respiratory gas exchange measured in breathby-breath mode using an open-air spirometry system) and further two bouts of exercise ( $40 \mathrm{~min}, 90 \%$ of power output at the respiratory compensation point, rcp, on a cycle ergometer) within one week. At $48 \mathrm{~h}$ after the last bout of exercise, OGTT and MRS were repeated. Areas under the time curves (AUC) of glucose, insulin and C-peptide (cp) concentrations and model analyses were performed to derive parameters of insulin action and secretion (OGIS, adaptation index). Rates of muscle ATP synthesis were measured with the saturation magnetisation transfer experiment using ${ }^{31} \mathrm{P}$ MRS $(\mathrm{n}=6)$. HCL were quantified with ${ }^{1} \mathrm{H}$ MRS. Lactate concentrations in arterialized blood were measured with an enzymatic-amperometric method to allow for determination of rcp (Wasserman, 1984).

Results: Incremental exercise testing revealed VO2max and VO2rcp of $30.8 \pm 3.1$ and $23.0 \pm 2.2 \mathrm{ml} \cdot \mathrm{kg}^{-1} \cdot \mathrm{min}^{-1}$. Fasting glucose $(88 \pm 3$ vs. $87 \pm 3 \mathrm{mg} / \mathrm{dl})$ and insulin concentrations ( $3.6 \pm 0.5$ vs. $3.4 \pm 0.3 \mathrm{mU} / \mathrm{l})$ were comparable before and after exercise training. Short-term exercise training augmented the initial C-peptide release (AUCcp30, before vs. after: $119 \pm 9$ vs. $132 \pm 6 \mathrm{ng} / \mathrm{ml} .30 \mathrm{~min} ; \mathrm{p}<0.01)$. The adaptation index markedly increased (\{OGIS x dAUCcp30/ dAUCg30 , before vs after: $183 \pm 33$ vs. $277 \pm 69$; $\mathrm{p}<0.05$ ). Insulin sensitivity only tended to increase (OGIS, before vs after: $451 \pm 9$ vs. $\left.475 \pm 17 \mathrm{ml} . \mathrm{min}^{-1} . \mathrm{m}^{-2} ; \mathrm{p}<0.076\right)$. Exercise training increased muscle ATP synthesis by $22 \%$ from $11.5 \pm 0.9$ to $14.0 \pm 0.9 \mu \mathrm{mol} . \mathrm{g}^{-1} \cdot \mathrm{min}^{-1}(\mathrm{p}<0.005)$, whereas HCL remained unchanged (before vs. after: $1.44 \pm 0.38 \%$ vs. $1.29 \pm 0.29 \%$, ns)

Conclusion: Short-term exercise training at near maximal exercise capacity increases $\beta$-cell function and adaptation of $\beta$ cell function to insulin sensitivity. Flux through muscle ATP synthase rises before marked changes in insulin sensitivity suggesting that exercise training primarily improves mitochondrial function.

Supported by the Austrian National Bank (ÖNB), Austrian Diabetes Association (ÖDG), Hochschuljubiläumsstiftung Stadt Wien

0771

Substrate source utilization in master athletes compared to healthy sedentary controls

H. Boon ${ }^{1}$, R. A. M. Jonkers ${ }^{1}$, R. Koopman ${ }^{1}$, E. E. Blaak ${ }^{1}$, W. H. M. Saris ${ }^{1}$, A. J. M. Wagenmakers ${ }^{2}$,

L. J. C. van Loon ${ }^{3}$; 
${ }^{1}$ Human Biology, Maastricht University, The Netherlands, ${ }^{2}$ School of Sport and Exercise Science, University of Birmingham, United Kingdom, ${ }^{3}$ Movement Sciences, Maastricht University, The Netherlands.

Background and Aims: Endurance exercise training has been associated with increased skeletal muscle fat oxidative capacity. In accordance, greater whole-body fat oxidation rates have been reported at rest and/or during exercise conditions in the endurance trained state. However, data on the specific substrate sources that are being utilized to a greater or lesser extent in the endurance trained state (i.e. plasma derived glucose, muscle glycogen, plasma free fatty acids (FFA), or intramuscular triacylglycerol (IMTG)) are inconclusive.

Materials and Methods: In the present study, we applied contemporary stable isotope methodology to determine whole-body substrate source utilization rates at rest, during moderate intensity exercise $(50 \% \mathrm{Wmax})$ and recovery in 10 master athletes and 10 healthy, age-matched sedentary controls (age: $57 \pm 1$ and $60 \pm 2$ y; BMI: $26 \pm 1$ and $28 \pm 1 ; \mathrm{VO}_{2^{-}}$ max: $48.8 \pm 1.8$ and $36.8 \pm 2.0 \mathrm{ml} \mathrm{kg}^{-1}$ body weight, respectively). $\left[\mathrm{U}_{-}{ }^{13} \mathrm{C}\right]$ palmitate and $\left[6,6-{ }^{2} \mathrm{H}_{2}\right]$ glucose tracers were applied to assess plasma FFA and glucose oxidation rates, and to estimate the use of muscle and/or lipoprotein derived triacylglycerol and muscle glycogen. Muscle biopsies were taken before and after exercise to assess net changes in muscle fiber type specific lipid and glycogen content.

Results: At rest, plasma palmitate rate of appearance (Ra), rate of disappearance (Rd) and rate of oxidation (Rox) were not significantly different between groups. During moderateintensity exercise $(150 \pm 5(\mathrm{~T})$ and $101 \pm 8 \mathrm{~W}(\mathrm{C}))$, plasma palmitate $\mathrm{Ra}$, $\mathrm{Rd}$ and Rox, were greater in trained subjects compared to the control group $(\mathrm{P}<0.001)$. The latter was accompanied by a significantly greater total fat oxidation rate $\left(0.52 \pm 0.03\right.$ g.min ${ }^{-1}$ and $0.35 \pm 0.03$ g. $\mathrm{min}^{-1}$ in $\mathrm{T}$ and $\mathrm{C}$, resp., $\mathrm{P}<0.001$ ), contributing $43 \pm 2$ and $39 \pm 3 \%$ to total energy expenditure during exercise $(\mathrm{P}=0.19, \mathrm{NS})$. Elevated fat oxidation rates were mainly attributed to a greater plasma FFA oxidation rate, both from an absolute $(\mathrm{P}<0.002)$ as well as a relative point of view $(\mathrm{P}=0.06)$. Muscle plus lipoprotein derived TG use contributed only $10 \pm 2$ and $11 \pm 3 \%$ in the trained and control group during exercise (NS). No significant changes in net type I or II muscle fiber lipid content were observed following exercise in both groups. Concomitantly, total carbohydrate oxidation rates during exercise were reduced in trained subjects as compared to sedentary controls $\left(1.70 \pm 0.07\right.$ g.min ${ }^{-1}$ and $1.37 \pm 0.08$ g. $\min ^{-1}$ in $\mathrm{T}$ and $\mathrm{C}$, resp., $\mathrm{P}<0.01$ ). The reduced carbohydrate oxidation rates were mainly attributed to a decline in plasma glucose utilization rate, averaging $8 \pm 0$ and $14 \pm 1 \%$ in trained vs. control subjects $(\mathrm{P}<0.001)$, whereas the relative contri- bution of muscle glycogen did not differ between groups (49 \pm 2 and $46 \pm 3 \%$ in $T$ and $C$, resp.).

Conclusion: In conclusion, master athletes oxidize significantly more plasma FFA and less plasma glucose as compared to sedentary, age-matched controls, at the same relative exercise intensity. IMTG stores do not seem to represent a major substrate source during moderate intensity exercise in either sedentary or endurance trained middle-aged males.

Supported by the Dutch Diabetes Research Foundation

\section{2}

Response of insulin-treated long-term type 2 diabetic subjects to a 5-month exercise training programme J. J. Prompers ${ }^{1}$, H. M. M. De Feyter ${ }^{1}$, S. F. Praet ${ }^{2}$, N. M. A. van den Broek ${ }^{1}$, L. J. C. van Loon ${ }^{2}$, K. Nicolay ${ }^{1}$; ${ }^{1}$ Biomedical NMR, Eindhoven University of Technology, ${ }^{2}$ Department of Movement Sciences, Maastricht University, Maastricht, The Netherlands.

Background and Aims: Insulin resistance is the key player in the pathogenesis and progression of type 2 diabetes (T2D), leading to a severely impaired blood glucose homeostasis. Recently, mitochondrial dysfunction has been hypothesised to play a role in the development of skeletal muscle insulin resistance, leading to a reduced ability to oxidize fatty acids and to an accumulation of intramyocellular lipids (IMCL). Exercise training, in various forms, can influence IMCL, mitochondrial function, as well as insulin sensitivity and has the potential to be an efficient intervention in the prevention as well as the treatment of T2D.

We studied this triad of IMCL, skeletal muscle mitochondrial function and glucose homeostasis in insulin-dependent T2D patients before and after 5 months of combined strength and endurance exercise training.

Materials \& Methods: Eleven male T2D patients were selected (mean \pm SE: age: $59.1 \pm 2.3$ years, BMI: $32.2 \pm 1.2 \mathrm{~kg} /$ $\mathrm{m}^{2}$ ). Subjects were diagnosed with T2D over 5 years and were on exogenous insulin treatment for at least 24 months. Subjects participated in a supervised 5-month progressive circuit-training program consisting of 45-60 $\mathrm{min}$ of resistance and interval exercises for 3 times a week. Before and after the 5-month training program the following parameters were measured: fasting plasma glucose and $\mathrm{HbA}_{1} \mathrm{c}$ concentration, maximal whole-body oxygen capacity $\left(\mathrm{VO}_{2 \max }\right)$ and maximal workload $\left(\mathrm{P}_{\max }\right)$ on a cycle ergometer, body composition (DEXA), IMCL using ${ }^{1} \mathrm{H}$ MR spectroscopy (MRS) and post-exercise phosphocreatine (PCr) and ADP recovery time constants $\left(\tau_{\mathrm{PCr}}\right.$ and $\left.\tau_{\mathrm{ADP}}\right)$ applying dynamic ${ }^{31} \mathrm{P}$ MRS in the $m$. vastus lateralis. 
Table 1: Body composition, blood glucose homeostasis, IMCL content and ${ }^{31} \mathrm{P}$ parameters before and after 5 months of exercise training

\begin{tabular}{|c|c|c|c|c|c|c|c|c|c|c|c|}
\hline & BM & WC & $\mathrm{HbA}_{1} \mathrm{c}$ & Fat & FFLM & $\begin{array}{l}\text { Fasting } \\
\text { plasma } \\
\text { glucose }\end{array}$ & $\mathrm{VO}_{2 \max }$ & $\mathbf{P}_{\max }$ & IMCL & $\tau \mathrm{p}_{\mathrm{Cr}}$ & $\tau_{\mathrm{ADP}}$ \\
\hline & $\mathrm{kg}$ & $\mathrm{cm}$ & $\%$ & $\%$ & $\mathrm{~kg}$ & $\mathrm{mmol} / 1$ & $\mathrm{ml} / \mathrm{min} / \mathrm{kg}$ & Watt/kg BM & $\begin{array}{c}\% \text { of water } \\
\text { signal }\end{array}$ & $\mathrm{s}$ & $\mathrm{s}$ \\
\hline $\begin{array}{l}\text { PRE } \\
\text { training }\end{array}$ & $97.5 \pm 4.9$ & $112.6 \pm 3.7$ & $7.6 \pm 0.3$ & $27.0 \pm 0.8$ & $20.6 \pm 1.0$ & $10.4 \pm 0.9$ & $24.3 \pm 1.4$ & $1.6 \pm 0.1$ & $2.05 \pm 0.28$ & $49.44 \pm 5.48$ & $22.47 \pm 2.86$ \\
\hline $\begin{array}{l}\text { POST } \\
\text { training }\end{array}$ & $97.5 \pm 4.8$ & $113.2 \pm 4.0$ & $7.2 \pm 0.2$ & $25.9 \pm 0.9$ & $21.2 \pm 0.9$ & $8.6 \pm 0.7$ & $24.2 \pm 1.5$ & $1.9 \pm 0.2$ & $2.40 \pm 0.43$ & $45.56 \pm 5.60$ & $21.19 \pm 2.45$ \\
\hline p-value & 0.95 & 0.69 & 0.04 & 0.009 & 0.033 & 0.059 & 0.95 & 0.0004 & 0.107 & 0.088 & 0.434 \\
\hline
\end{tabular}

Results: Table 1 shows the parameters measured before and after the exercise-training program. Body mass (BM) or waist circumference (WC) of the subjects remained unchanged after 5 months of exercise training. However, whole body fat percentage and fat free leg mass (FFLM) both changed significantly. The plasma marker of glucose homeostasis, $\mathrm{HbA}_{1} \mathrm{c}$, was significantly improved and fasting plasma glucose levels tended to be lower after the exercise-training period. $\mathrm{VO}_{2 \max }$ did not change due to the exercise training, whereas $\mathrm{P}_{\max }$ increased. ${ }^{1} \mathrm{H}$ MRS measurements revealed no differences between IMCL levels before and after the training program. Also ${ }^{31} \mathrm{P}$ parameters of oxidative capacity, $\tau_{\mathrm{PCr}}$ and $\tau_{\mathrm{ADP}}$, determined from the dynamic ${ }^{31} \mathrm{P}$ MRS measurements showed no improvement. Conclusion: In these T2D patients suffering from severe muscle deconditoning, the exercise training did result in an improved glucose homeostasis, indicated by the lower $\mathrm{HbA}_{1} \mathrm{c}$ and a trend towards lower fasting plasma glucose. This was not accompanied by an amelioration of mitochondrial function $\left(\mathrm{VO}_{2 \max }, \tau_{\mathrm{PCr}}\right.$ and $\tau_{\mathrm{ADP}}$ did not change) or a decrease in IMCL content, although maximal workload per $\mathrm{kg} \mathrm{BM}$ did increase.

\section{3}

Leptin and adiponectin responses following acute resistance training in aged males

S. Tournis ${ }^{1}$, I. G. Fatouros ${ }^{2}$, D. Leontsini ${ }^{2}$,

A. Z. Jamurtas ${ }^{3}$, K. Margonis ${ }^{2}$, M. Manousaki ${ }^{4}$,

A. Galanos ${ }^{1}$, K. Taxildaris ${ }^{2}$, G. Mastorakos ${ }^{5}$, A. Mitrakou ${ }^{1}$;
${ }^{1}$ Diabetes Unit, Henry Dunant Hospital, Athens, ${ }^{2} 2$ Department of Physical Education and Sports Science, Democritus University of Thrace, Komotini, ${ }^{3}$ Department of Physical Education \& Sports Sciences, University of Thessaly, Trikala, ${ }^{4}$ Biochemistry Department, Henry Dunant Hospital, Athens, ${ }^{5}$ Endocrine Unit, Areteion Hospital, University of Athens, Athens, Greece.

Background and Aims: Adiponectin and leptin are closely related to weight control and insulin sensitivity. Exercise affects elderly metabolic regulation and functional capacity. The purpose of this study was to investigate leptin and adiponectin responses in elderly males following acute resistance exercise training.

Materials and Methods: Forty inactive men (65-82 years) were randomly assigned to a control $(C, n=10)$, lowintensity training $(\mathrm{LI} / \mathrm{n}=10)$, moderate-intensity training $(\mathrm{MI} / \mathrm{n}=10)$, and high-intensity training $(\mathrm{HI} / \mathrm{n}=10)$ group. Strength, exercise energy cost (EEC), $\mathrm{VO}_{2} \max$, resting metabolic rate (RMR), plasma leptin and adiponectin, were determined at baseline, immediately following exercise, and at 12-, 24-, 48-, and 72-hours post-exercise. Statistical analysis was performed using repeated measures ANOVA.

Results: Following exercise leptin decreased in all active groups, effect being significant only in the HI group $(P<0.05)$. Furthermore both \% (HI: $-10.62 \pm 1.99$, MI: $-6.68 \pm 6$, LI: $-4.94 \pm 3.75$, C: $11.4 \pm 5.7, \mathrm{p}=0.014)$ and absolute decrease of leptin in all active groups was significantly different compared with controls. Adiponectin levels increased significantly only in HI group $(p<0.001)$, effect preserved even $12 \mathrm{~h}$ following exercise. Furthermore both $\%$ (HI: 71.2 \pm 13.8 , MI: $13 \pm 9$, LI: 9.6 \pm 11.6 , C: $-0.89 \pm 11.4$, $\mathrm{p}=0.003$ ) and absolute increase of adiponectin in the HI 
group was significantly higher compared with all other groups.

Conclusions: Acute resistance exercise training may alter leptin and adiponectin levels in an intensity-dependent manner.

\section{4}

Hyperinsulinaemia during exercise does not suppress hepatic glycogen utilisation in patients with type 1 diabetes

K. Chokkalingam ${ }^{1,2}$, K. Tsintzas ${ }^{2}$, A. Snaar ${ }^{3}$, L. Norton ${ }^{2}$, B. Solanky ${ }^{3}$, E. Leverton ${ }^{3}$, P. Morris ${ }^{3}$, P. Mansell ${ }^{1}$,

I. Macdonald ${ }^{2}$;

${ }^{1}$ Diabetes \& Endocrinology, Queen's Medical Centre, Nottingham, ${ }^{2}$ School of Biomedical Sciences, University of Nottingham, ${ }^{3}$ Sir Peter Mansfield Magnetic Resonance Centre, University of Nottingham, United Kingdom.

Background and Aims: Patients with type 1 diabetes run the risk of hypoglycaemia during exercise. It has been suggested that this may be due to impaired mobilisation of liver glycogen associated with relative hyperinsulinaemia. The aim of this study was to compare the dynamic in-vivo changes in liver glycogen and whole body substrate metabolism in patients with type 1 diabetes and healthy volunteers during exercise using ${ }^{13} \mathrm{C}$ magnetic resonance spectroscopy and indirect calorimetry.

Methods: 7 male patients with uncomplicated type 1 diabetes [Group D, Mean (SEM) diabetes duration 10 (2) yr, age 33 (3) yr, BMI 24 (1) kg/m², $\mathrm{HbA}_{1 \mathrm{C}} 8.2(0.2) \%$ and $\mathrm{VO}_{2 \max } 49$ (2) $\left.\mathrm{ml} \mathrm{kg}^{-1} \cdot \mathrm{min}^{-1}\right]$ and 5 non diabetic controls [Group C, age 30 (3) yr, BMI 22 (1) kg/m², $\mathrm{HbA}_{1 \mathrm{c}} 5.4$ $(0.1) \%$ and $\mathrm{VO}_{2 \max } 58$ (4) $\left.\mathrm{ml} \cdot \mathrm{kg}^{-1} \cdot \mathrm{min}^{-1}\right]$ were studied before and after a standardised breakfast $(2.5 \mathrm{~g}$ carbohydrate $/ \mathrm{kg}$ ) and after 3 bouts (EX1, EX2, EX3) of $40 \mathrm{~min}$ cycling at $60 \% \mathrm{VO}_{2 \max }$. The interval between breakfast and the first bout of exercise was $\sim 212 \mathrm{~h}$. Patients were admitted overnight, having omitted their long acting insulin, and euglycaemia was maintained with a variable low dose insulin infusion. Patients subcutaneously injected 0.5 units of rapid acting insulin analogue (Novorapid) per $10 \mathrm{~g}$ of carbohydrate with breakfast. Furthermore, insulin was infused throughout the trial at a rate of $5 \mathrm{mU} / \mathrm{m}^{2} / \mathrm{min}$ for basal requirements. ${ }^{13} \mathrm{C}$ glycogen measurements were acquired in a 3.0T magnet using a surface coil. The $\mathrm{C} 1$ glycogen peak at 100.4 ppm was analysed. Liver glycogen concentrations were quantified using a phantom of known glycogen concentration. Whole body substrate oxidation was determined during a separate visit under identical conditions.
Results: Fasting glucose [6.1(0.9) vs. $3.7(0.2) \mathrm{mmol} / \mathrm{l}]$ and insulin [26 (8) mU/1 vs.7 (2)] concentrations were higher in $\mathrm{D}$ than $\mathrm{C}$ respectively $(\mathrm{P}<0.05)$. Glucose and insulin concentrations were higher $(\mathrm{P}<0.01)$ during $\mathrm{EX} 1$ in $\mathrm{D}[11$ (2) $\mathrm{mmol} / \mathrm{l}$ and 34 (7) $\mathrm{mU} / 1$ respectively] compared with $\mathrm{C}$ [3.0 (0.1) $\mathrm{mmol} / 1$ and 5 (1) $\mathrm{mU} / 1$ respectively]. Insulin concentrations declined during the remaining two exercise sessions in D [EX2: 25 (3) mU/1 and EX3: 22 (2)] but were still greater $(\mathrm{P}<0.01)$ than $\mathrm{C}$. There were no differences in fasting liver glycogen between groups [D: 178 (58) $\mathrm{mmol} /$ 1 vs. C: 212 (84)]. Post-prandial glycogen repletion was also similar [D: 589 (169) vs. C: 463 (117)]. Liver glycogen concentrations after each exercise session in D were comparable to C [EX1: 298 (87) vs. 303 (64), EX2: 267 (142) vs. 210 (65) and EX3: 195 (98) vs. 145 (66) respectively] despite the relative hyperinsulinaemia. Whole body carbohydrate oxidation during exercise in $\mathrm{D}$ was similar to C [2.1(0.2) g/min vs $2.3(0.2)]$.

Conclusion: Hyperinsulinaemic conditions during exercise in patients with type 1 diabetes do not suppress hepatic glycogen utilisation. This does not support the perception that exercise-induced-hypoglycaemia in patients with type 1 diabetes is secondary to suppressed hepatic glycogen utilisation.

Supported by Special Trustees for Nottingham University Hospital

0775

Metabolic effects of physical exercise started at an advanced age

M. Lechleitner ${ }^{1}$, A. Dzien ${ }^{2}$, C. Dzien-Bischinger ${ }^{2}$, F. Hoppichler ${ }^{3}$;

${ }^{1}$ Internal Medicine, Hospital Hochzirl, Zirl, ${ }^{2}$ Internal Medicine, Medical Center Hentschelhof, Innsbruck, ${ }^{3}$ Internal Medicine, Hospital Barmherzige Brueder, Salzburg, Austria.

Background and Aims: The proportion of elderly increases in Westernized societies, which is associated with an increase in type 2 diabetes and cardiovascular disorders. Weight reduction and physical exercise are effective strategies for the prevention of these diseases, but only few data are available about the efficiency of life-style programs when started at an advanced age.

Materials and Methods: We have restrospectively evaluated clinical and laboratory data of 580 patients older than 50 years of age, who were admitted to our medical center for a bicycle exercise test. Beside the results of this test (percentage of maximal physical capability), patients were asked about the performance of regular physical exercise, 
and whether they have started before or after the age of 50 years. Medication was recorded in all patients

Results: 301 patients (mean age $68.6+/-22.0$ years) reported about regular physical exercise, 106 (mean age $67.8+/-7.0$ years) had started after an age of 50 years. 279 patients (mean age $69.8+/-18.0$ years) denied the performance of physical exercise. The corresponding maximal capability in the exercise test was $94+/-23 \%$ in the active and $80+/-22 \%$ in the inactive group $(\mathrm{p}<0.001)$. Body mass index (BMI) $(25.3+/-3.1 \mathrm{~kg} / \mathrm{m} 2)$ and the percentage of body fat $(30.4+/-6.5 \%)$ were significantly lower in the active than in the inactive group (BMI $27.7+/-3.7 \mathrm{~kg} / \mathrm{m} 2$; body fat $35.1+/-6.3 \%$; $<0.001$ ). This was paralleled by a more favourable metabolic risk profile. C-reactive protein values were $0.24+/-0.09 \mathrm{mg} / \mathrm{dL}$ in inactive patients and $0.22+/-0.08 \mathrm{mg} / \mathrm{dL}$ in active $(\mathrm{p}<0.001)$. Between patients, who had started regular physical exercise before or after the age of 50 years, only the percentage of body fat revealed a significant difference $(29.6+/-6.6 \%$ and $31.3+/-6.1 \%$; $\mathrm{p}<0.010$ ). The frequency of cardiovascular and metabolic medication was significantly lower in the active group, but did not differ between patients who started exercise before or after the age of 50 years. Among the 135 diabetic patients, 58 (mean age $75.0+/-7.8$ years) reported to be physically active and 78 (mean age $74.6+/-8.0$ years) to be inactive. Active diabetic patients revealed significantly lower HbAlc values $(6.7+/-1.2 \%)$, systolic $(144+/-21.5)$ and diastolic $(83+/-11)$ blood pressure values than inactive patients (HbA1c 7.1+/-1.3\%; $<0.001$; systolic blood pressure 149+/-20; $<<0.001$ : diastolic blood pressure $86+/-11$; $\mathrm{p}<0.010$ ).

Conclusion: Regular physical exercise, also when started beyond an age of 50 years, is related to a more favourable metabolic risk profile and lower rate of medication compared to inactive patients at the same age.

\section{6}

Effects of exercise on inflammatory markers, insulin resistance, and arterial stiffness

O. Ryu ${ }^{1}$, H. Kim ${ }^{2}$, D. Shin ${ }^{3}$, H. Yoo ${ }^{1}$, S. Park ${ }^{1}$, Y. Lee ${ }^{1}$, H. Kim ${ }^{1}$, K. Lee ${ }^{1}$, J.-A. Seo ${ }^{1}$, K. Choi ${ }^{1}$, S. Kim ${ }^{1}$, N. Kim ${ }^{1}$, K. Choi ${ }^{1}$, D. Choi ${ }^{1}$, S. Baik ${ }^{1}$;

${ }^{1}$ Division of endocrinology and metabolsim, Department of internal medicine, Korea University College of Medicine, Seoul, ${ }^{2}$ Orthopedic Surgery, Kwang-Jin Orthopedic Clinic, Seoul, ${ }^{3}$ Department of Internal Medicine, Bundang Jesaeng General Hospital, Seoul, Republic of Korea.

Backgrounds: Physical exercise exerts many beneficial effects. It protects against diseases associated with chronic low-grade inflammation, such as insulin resistance and atherosclerosis. But acute bouts of intense or prolonged strenuous exercise such as marathon running can stimulate inflammatory response. It may trigger acute myocardial infarction, sudden cardiac death, immunosuppression, oxidative stress, and biochemical and hormonal changes.

Aims: The objective of this study was to ascertain whether exercise in ultra-marathon runners affects inflammatory markers, insulin sensitivity, and arterial stiffness.

Methods: Study populations were ultra-marathon hobby runners who participated in $622-\mathrm{km}$ ultra-marathon foot race held July 10 to July 16, 2005 in Korea. Twenty ultramarathon runners consented to this study. Only 13 participants were studied pre-race, immediately after the race, and 4 weeks after the race. We also compared 20 ultra-marathon runners with age-, sex-, and BMI-matched 20 healthy control subjects. We measured lipid profiles, glucose, insulin, adiponectin, and inflammatory markers. Insulin resistance was calculated by Homeostasis Model Assessment (HOMA). Total body fat mass was measured using a multiple frequency bioelectrical impedance method. We also measured brachial-ankle pulse wave velocity (baPWV).

Results: Ultra-marathon foot race elevated plasma glucose ( $\mathrm{P}=0.007)$, insulin $(\mathrm{p}=0.001)$, hsCRP $(\mathrm{P}=0.039)$, IL-6 $(\mathrm{P}=0.016)$, HOMA-IR $(\mathrm{P}=0.003)$, liver enzyme and muscle enzyme. It reduced total cholesterol $(\mathrm{P}<0.001)$, triglyceride $(\mathrm{P}<0.001)$, LDL-C $(\mathrm{P}<0.001)$ and body weight $(\mathrm{P}=0.003)$. But all these parameters were returned to the baseline values within 4 weeks after the race except insulin and HOMA-IR. Ba-PWV and body fat mass were not changed significantly. In a multiple regression analysis, the elevation of liver enzymes in the pre-race predicted the elevation of muscle enzymes after ultra-marathon race.

Compared with the healthy controls, there were no difference in adiponectin, TNF- $\alpha$, hs-CRP, total cholesterol, triglyceride, and HDL-cholesterol. But there were difference in body fat mass, the amount of exercise, IL-6, insulin, HOMA-IR, LDL-cholesterol, and ba-PWV between the two groups.

Conclusions: The present study shows that regular exercise decrease body fat mass. Also it significantly increases IL-6. But TNF- $\alpha$ is insignificantly decreased. The changes of body fat mass, inflammatory markers, and lipid profiles by regular exercise affect insulin sensitivity and arterial stiffness. It also shows that extreme exercise influences biochemical parameters, hormonal levels, inflammatory markers, and insulin resistance. Insulin resistance particularly is related to the elevation of inflammatory cytokines and sub-clinical damages of muscle and liver. But the influences of ultra-marathon foot race are transient and reversible within 4 weeks after the race except insulin and HOMA-IR.

This study is supported by a grant of the Korean Health 21 $R \& D$ Project, Ministry of Health \&Welfare, Republic of Korea (A050463). 


\section{7}

At high insulin levels, intravenous AICA-riboside infusion does not affect whole body nor forearm skeletal muscle glucose uptake in vivo in humans

M. Bosselaar ${ }^{1}$, P. Smits ${ }^{2}$, C. Tack ${ }^{1}$;

${ }^{1}$ Department of Medicine, 541, ${ }^{2}$ Department of Pharmacology/Toxicology, 233, Radboud University Nijmegen Medical Centre, The Netherlands.

Background and Aims: AMPK is a potential pharmacological target for the treatment of type 2 diabetes. 5Aminoimidazole-4-carboxamide-riboside (AICA-riboside) activates AMPK. Animal studies have shown that AICAriboside stimulates glucose uptake in skeletal muscle and improves insulin sensitivity. In a previous human in vivo study, we showed that intra-arterial AICA-riboside is a potent vasodilator and decreases systemic glucose and free fatty acid levels, but it has no direct local effect on skeletal muscle glucose uptake. In the present human in vivo study we investigated whether AICA-riboside infusion increased whole body glucose uptake during hyperinsulinemia.

Materials and Methods: Studies were performed in healthy subjects ( $\mathrm{n}=15$, age: $21.6 \pm 2.2 \mathrm{yr}$, BMI $21.4 \pm 1.5 \mathrm{~kg} / \mathrm{m}^{2}$ ). They underwent two euglycemic hyperinsulinemic $\left(60 \mathrm{mU} / \mathrm{m}^{2} /\right.$ min) clamps with and without systemic co-infusion of AICAriboside, in a single-blind, randomized order. After $60 \mathrm{~min}$ of the clamp, insulin was combined with AICA-riboside $(66.7 \mathrm{mg} / \mathrm{kg}$ ) or placebo (=normal saline) infusion for an additional $60 \mathrm{~min}$. Forearm blood flow (plethysmography) and plasma $\mathrm{AV}$ glucose difference were used for calculation of Forearm Glucose Uptake (FGU). In addition, (intraarterial) blood pressure and heart rate were measured. We measured AICA-riboside plasma concentrations and AICAriboside uptake and phosphorylation to ZMP in erythrocytes using HPLC technique.

Results: During insulin with AICA-riboside or placebo infusion, insulin levels increased almost equally $(474 \pm 25$ and $471 \pm 17 \mathrm{pmol} / \mathrm{l}$ resp.). Glucose infusion rates (95$120 \mathrm{~min})$ were also similar $(48.5 \pm 7.7$ and $45.5 \pm 4.3 \mu \mathrm{mol} /$ $\mathrm{kg} / \mathrm{min}$ resp., $P=\mathrm{NS}$ between both groups). Insulin with AICA-riboside had no effect on FGU versus insulin with placebo $(0.2 \pm 0.1$ to $2.2 \pm 0.6$ and $0.4 \pm 0.1$ to $2.3 \pm 0.4 \mu \mathrm{mol} /$ $\mathrm{min} / \mathrm{dl}$ resp., $P=\mathrm{NS}$ ). AICA-riboside slightly increased FBF (from $1.2 \pm 0.2$ to $1.6 \pm 0.3 \mathrm{ml} / \mathrm{min}$ per $\mathrm{dl}$ of forearm tissue) compared to placebo $(1.4 \pm 0.2$ to $1.2 \pm 0.2 \mathrm{ml} / \mathrm{min} / \mathrm{dl}$, $P<0.05)$. AICA-riboside increased heart rate from $60 \pm 4$ to $74 \pm 3$ versus $62 \pm 4$ to $66 \pm 4 \mathrm{bpm}$ during placebo $(P<0.05)$. Mean arterial blood pressure tended to decrease during AICA-riboside compared to placebo $(83 \pm 2$ to $80 \pm 2$ and $83 \pm 2$ to $84 \pm 2 \mathrm{mmHg}$ resp., $P=0.09$ ). The AICA-riboside plasma concentration increased from $0 \pm 0$ to $203.6 \pm 5.8 \mu \mathrm{M}$ during AICA-riboside infusion $(n=3)$. There was also a significant increase in AICA- riboside uptake and phosphorylation to ZMP in erythrocytes.

Conclusion: During hyperinsulinemia, AICA-riboside does not increase insulin-stimulated whole body nor local skeletal muscle glucose uptake in humans. AICA-riboside induces systemic vasodilation in skeletal muscle resulting in an increase in heart rate and decrease in blood pressure. These results are in line with our earlier findings, and suggest that in humans, AICA-riboside may not reach the subendothelial compartment (skeletal muscle) and thus may not be able to stimulate glucose uptake. AICA-riboside is probably taken up and phosphorylated in the endothelium, resulting in profound changes in vascular tone. This current study does not allow to determine whether AICA-riboside acts in the liver. Because here the endothelial junctions are less tight, AICA-riboside may be able to inhibit hepatic glucose production and hence lower plasma glucose levels. Supported by Dutch Diabetes Foundation

\section{8}

Effect of body composition on the validity and reliability of an electronic pedometer

A. F. Kirk, C. Gardner, J. Herd;

Institute of Sport and Exercise, University of Dundee, United Kingdom.

Background: Pedometer based activity recommendations such as the 10,000 steps programme, are increasingly being used to promote physical activity and subsequently control weight in people with Type 2 diabetes. While pedometer validity has been confirmed in individuals with a normal body composition, there is conflicting evidence about pedometer validity in overweight and obese individuals.

Aims: 1) examine the effect of body composition on the validity and reliability of the Yamax Digiwalker SW 401 pedometer while walking at three self-selected walking speeds; 2) examine the effect of different pedometer placement sites on the validity and reliability of the pedometer.

Material and Methods: 52 adults (24F, 28M) participated with Body Mass Index (BMI) ranging from 21 to $52 \mathrm{~kg} / \mathrm{m}^{2}$. Body composition was measured by BMI, waist to hip ratio and $\%$ body fat (Harpenden skin fold callipers, 4 placement sites) and categorised as follows: BMI (category 1-20$24.9 \mathrm{~kg} / \mathrm{m}^{2} \quad(\mathrm{n}=11)$, category 2-25-29.9 kg/m ${ }^{2} \quad(\mathrm{n}=15)$, category 3-30-34.9 kg/m ${ }^{2}(\mathrm{n}=13)$, category $4-35 \mathrm{~kg} / \mathrm{m}^{2}$ and over $(n=13)$; waist/hip ratio (category $1<0.8$ for women, 0.9 for men $(n=17)$, category $2>0.8$ for women, 0.9 for men $(n=12)$, category $3>0.85$ for women, 1 for men $(n=23)$. Percent body fat was categorised using reference table adapted from Durnin and Womersley (1974) (category 
1 - average $(n=11)$, category 2 - overweight, $(n=15)$, category 3 - obese, $(n=26)$. Participants were fitted with 4 pedometers (two in position $1=$ midline of thigh (front), one in position $2=$ midaxiallary line (side), one in position $3=$ posterior midline of thigh (back) on the right side of their body at waist level. Participants completed a 40 metre walking course at 3 self selected walking paces of (slow, moderate and brisk) on two occasions. Pedometer steps in each position were compared to steps recorded by direct observation and analysed across body composition categories using repeated measures analysis of variance.

Results: Significant main effect for position $(p<0.001)$ and the interaction of position with all body composition groupings $(p<0.001)$ were found. Multiple comparison analysis identified differences between observed counts and counts recorded from the pedometers in the side position in BMI category $4(p<0.001)$, waist/hip ratio category $3(p<0.001)$ and percentage body fat category $3(\mathrm{p}<0.001)$, and from pedometers in the back position in BMI category $2(\mathrm{p}<0.001)$ and waist/hip ratio category $3(\mathrm{p}<0.001)$. Significant correlations $(\mathrm{p}<0.001)$ were found at all walking speeds (slow $\mathrm{r}=0.84$, moderate $\mathrm{r}=0.92$, brisk $\mathrm{r}=0.96$ ) for steps recorded from pedometers in position 1 and 2, indicating high interinstrument reliability. No significant differences $(\mathrm{p}>0.05)$ were found between test 1 and test 2 across body composition groupings, pedometer position and walking speed.

Conclusions: The Digiwalker electronic pedometer is valid and reliable across variable body composition and walking speeds when worn in the front (manufacturer recommended) position.

\section{PS 60}

\section{GLP-I analogues and incretin effect}

0779

Plasma ghrelin concentrations in type 1 diabetic patients with chronic autoimmune gastritis N. Alonso ${ }^{1}$, I. Salinas ${ }^{1}$, A. Cantón ${ }^{1}$, B. Soldevila ${ }^{1}$, Y. Alba $^{2}$, M. L. Granada ${ }^{2}$, I. Ojanguren ${ }^{3}$, A. Sanmartí ${ }^{1}$; ${ }^{1}$ Endocrinology and Nutrition, ${ }^{2}$ Clinical Chemistry, ${ }^{3}$ Pathology, Hospital Universitari Germans Trias i Pujol, Badalona, Spain.

Background and Aims: Type 1 diabetic patients (DM1) show an increased prevalence of pernicious anemia whose hystological substrate is an atrophic chronic gastritis located in the corpus of the stomach, the main source of ghrelin.

Aims: To evaluate if DM1 patients with chronic autoimmune gastritis (CAG) $\left(\mathrm{DM}_{1 \mathrm{CAG}}{ }^{+}\right.$) have lower concentrations of ghrelin compared with patients with DM1 without CAG (DM1CAG-) and a control group, all of them matched for age, sex and body mass index (BMI). In addition, we seek to evaluate the correlation between ghrelin and a biochemical marker of gastric mucosa atrophy (pepsinogen I) and of hypo/achlorhydria (gastrin).

Materials and Methods: $15 \mathrm{DM}_{1 \mathrm{CAG}}{ }^{+}$patients $(9$ women, $36.6 \pm 12.8$ years, BMI $\left.24.3 \pm 2.8 \mathrm{Kg} / \mathrm{m}^{2}\right) ; 15 \mathrm{DM}^{2} \mathrm{CAG}^{-}$ $\left(37.4 \pm 12.6\right.$ years, BMI $\left.24 \pm 2.7 \mathrm{Kg} / \mathrm{m}^{2}\right)$ and 15 control $\left(35.6 \pm 13.4\right.$ years, BMI $\left.23,1 \pm 2,5 \mathrm{Kg} / \mathrm{m}^{2}\right)$. Pepsinogen I (ELISA), gastrin (RIA), parietal cell antibodies (PCA) (indirect immunofluorescence) and ghrelin (RIA) were determined in all patients. $\mathrm{HbAl}_{\mathrm{c}}$ was only measured in DM1. In all $15 \mathrm{DM} 1 \mathrm{CAG}^{+}$an esophagogastroduodenoscopy was performed to take biopsy samples of gastric mucosa. Chromogranin A was the antibody used for the immunohistochemical study of gastric neuroendocrine cell proliferation. Results: The histological samples of biopsed patients showed different grades of gastric mucosa atrophy and in some cases (10/15) neuroendocrine cell hyperplasia. No differences were found in ghrelin concentrations between groups (DM1CAG ${ }^{+}: 651.1 \pm 282.9 \mathrm{pg} / \mathrm{mL}$; DM1CAG ${ }^{-}$: $515.1 \pm 148.2 \mathrm{pg} / \mathrm{mL}$; Control:670.9 $\pm 224.6 \mathrm{pg} / \mathrm{mL}$ ).Gastrin concentrations were higher in $\mathrm{DM} 1 \mathrm{CAG}^{+}$than $\mathrm{DM}^{\mathrm{C} C A \mathrm{C}^{-}}$ and the control group (DM1CAG ${ }^{+}: 469(225-708) \mathrm{pg} / \mathrm{mL}$; DM1CAG 26 (22-30) pg/mL; Control 21 (18.5-42) pg/mL; $\mathrm{p}<0.001)$. Pepsinogen I was lower in $\mathrm{DM}_{1 C A G}{ }^{+}$than in $\mathrm{DM}_{1 C A G^{-}}$and the control (DM1CAG ${ }^{+}: 18.52 \pm 15.05 \mu \mathrm{g} / \mathrm{L}$; DM1CAG-: $113.72 \pm 32.9 \mu \mathrm{g} / \mathrm{L}$; Control $125.46 \pm 32.65 \mu \mathrm{g} / \mathrm{L}$; $\mathrm{p}<0.001$ ). No correlation was found between ghrelin and pepsinogen I. Ghrelin correlated positively with gastrin $(\mathrm{r}=0.52 ; \mathrm{p}=0.006)$ in DM1 (with and without $\mathrm{ACG})$. In $\mathrm{DM}_{1 C A G^{+}}$, gastrin levels correlated with the presence of hyperplastic neuroendocrine lesions $(159.66 \mathrm{pg} / \mathrm{mL}$ vs $690.6 \mathrm{pg} / \mathrm{mL} ; \mathrm{p}=0.05$ ). No differences were found in ghrelin concentrations regardless of the presence or not of hyperplastic neuroendocrine lesions in $\mathrm{DM}_{1} \mathrm{CAG}^{+}$patients.

Conclusion: No differences were found in plasma ghrelin concentrations between $\mathrm{DM} 1 \mathrm{CAG}^{+}$and other studied groups, thus ghrelin does not seem to be a good marker of gastric mucosa atrophy in patients with DM1 and chronic autoimmune gastritis.

\section{0}

Exploring the safety of substituting exenatide for insulin in patients with type 2 diabetes who were using insulin in combination with oral antidiabetic agents

R. Brodows ${ }^{1}$, D. Maggs ${ }^{2}$, D. Johns ${ }^{1}$, J. Northrup ${ }^{1}$, H. $\mathrm{Xu}^{1}$, S. Davis ${ }^{3}$; 
${ }^{1}$ Eli Lilly and Company, Indianapolis, ${ }^{2}$ Amylin Pharmaceuticals, Inc., San Diego, ${ }^{3}$ School of Medicine, Vanderbilt University, Nashville, United States.

Background and Aims: Exenatide is a first in class incretin mimetic indicated for use in the United States with metformin (MET) and/or a sulphonylurea (SU) in patients with type 2 diabetes, but is not a substitute for insulin in insulin-requiring patients. We explored the safety of substituting exenatide $(5 \mu \mathrm{g}$ BID for 4 wks, $10 \mu \mathrm{g}$ BID thereafter) for insulin in patients with type 2 diabetes who were using insulin in combination with MET, SU, or both. Materials and Methods: $\mathrm{HbA}_{1 \mathrm{c}}$, fasting serum glucose (FSG), C-peptide, and body weight were measured during this 16-wk pilot study; successful maintenance of glycaemic control, after substituting exenatide for insulin, was predefined as an $\mathrm{HbA}_{1 \mathrm{c}}$ increase $<0.5 \%$. 49 patients (mean $\pm \mathrm{SD}$ : age $54 \pm 8$ y, BMI $34 \pm 4 \mathrm{~kg} / \mathrm{m}^{2}, \mathrm{HbA}_{1 \mathrm{c}}$ $8.1 \pm 1.1 \%$, FSG $8.8 \pm 2.6 \mathrm{mmol} / \mathrm{L}$, daily insulin $45 \pm 30 \mathrm{U}$, duration on insulin $3 \pm 3 \mathrm{y}$, duration of diabetes $11 \pm 7 \mathrm{y}$ ) were randomised 2:1 to either substitute exenatide for insulin or remain on their current regimen. Evaluable patients $(n=45)$ completed $\geq 8$ wks of the study or discontinued earlier due to loss of glycaemic control; 4 exenatide patients withdrew for reasons precluding assessment of glycaemic control.

Results: $62 \%$ of exenatide-treated patients $(18 / 29)$ were able to maintain glycaemic control (mean \pm SEM: $\mathrm{HbA}_{1 \mathrm{c}}$ change $-0.5 \pm 0.7 \%, \mathrm{p}=.003)$. The majority $(14 / 18)$ of the exenatide-treated patients that successfully maintained glycemic control were also taking SU or a combination of SU/MET; 4 of 12 patients in the MET only group maintained control. The remaining 38\% (11/29) unable to maintain glycaemic control $\left(\mathrm{HbA}_{1 \mathrm{c}}\right.$ change $+1.6 \pm 1.5 \%$, $\mathrm{p}=.001)$ had elevated FSG levels by Wk $2(+3.9 \pm$ $2.7 \mathrm{mmol} / \mathrm{L}, \mathrm{p}=.005$ ) that increased throughout the study; excess hyperglycaemia was observed in 1 patient. In the exenatide group overall, body weight was decreased at endpoint $-4.2 \pm 3.0 \mathrm{~kg}(\mathrm{p}<0.001)$ independent of glycaemic response and median $\mathrm{C}$-peptide was increased $\sim 20 \%$. The overall adverse event profile was consistent with previous exenatide trials. In the insulin reference group, mean insulin doses were similar at baseline $(53.8 \mathrm{U} / \mathrm{d})$ and endpoint $(51.5 \mathrm{U} / \mathrm{d})$. Body weight remained relatively stable $(+0.5 \pm 1.7 \mathrm{~kg})$ and $81 \%$ of insulin-treated patients $(13 / 16)$ maintained glycaemic control $\left(\mathrm{HbA}_{1 \mathrm{c}}\right.$ change $\left.-0.3 \pm 0.6 \%\right)$. Conclusion: This pilot study suggests that it is feasible for some patients with type 2 diabetes to sustain glycaemic control when substituting exenatide for insulin; however, deterioration in glycaemic control can occur. Background therapy with SU and FSG levels measured after exenatide initiation may predict treatment success. Further studies are warranted to better evaluate insulin-using patients with type 2 diabetes who may benefit from exenatide treatment.
Supported by Eli Lilly and Company and Amylin Pharmaceuticals, Inc.

0781

Exenatide maintained glycaemic control with associated weight reduction over two years in patients with type 2 diabetes

R. Henry ${ }^{1}$, R. Ratner ${ }^{2}$, A. Stonehouse ${ }^{3}$, X. Guan ${ }^{3}$, T. Poon ${ }^{3}$, J. Malone ${ }^{4}$, D. Kim ${ }^{3}$, D. Kendall ${ }^{3}$;

${ }^{1}$ VA Healthcare System, UCSD, San Diego, ${ }^{2}$ Scientific Affairs, Medstar Res. Inst., Hyattsville, ${ }^{3}$ Amylin Pharmaceuticals, Inc., San Diego, ${ }^{4}$ Eli Lilly and Company, Indianapolis, United States.

Background and Aims: Exenatide is an incretin mimetic that improves glycaemic control with associated weight reduction in patients with type 2 diabetes when used in combination with metformin (MET) and/or sulphonylurea (SU).

Materials and Methods: We analysed data from type 2 diabetes patients not achieving glycaemic control with MET and/or SU enrolled in phase 3 trials followed by open-label extensions such that at the time of this analysis, patients had received $\geq 2$ years of exposure to exenatide. The effects of twice-daily treatment of exenatide (10 $\mu \mathrm{g}$ BID) on glycaemic control $\left(\mathrm{HbA}_{1 \mathrm{c}}\right.$ and fasting plasma glucose [FPG]), HOMA-B, body weight, safety, and tolerability were assessed. At the time of this analysis, 283 patients entering the open-label extension $(177 \mathrm{M}, 106 \mathrm{~F}$; age $57.3 \pm 9.7$ yr; BMI $33.7 \pm 5.5 \mathrm{~kg} / \mathrm{m}^{2}$; mean $\pm \mathrm{SD}$; baseline $\mathrm{HbA}_{1 \mathrm{c}} 8.3 \pm 1.0 \%$; baseline FPG $9.7 \pm 0.1 \mathrm{mmol} / \mathrm{L}$; meanSE) had completed 2 years of follow-up.

Results: The 2-year completer population had reductions from baseline $\mathrm{HbA}_{1 \mathrm{c}}, \mathrm{FPG}$, and body weight of $-1.1 \pm 0.1 \%,-1.2 \pm 0.2 \mathrm{mmol} / \mathrm{L}$, and $-2.4 \pm 0.2 \mathrm{~kg}$ (meanSE), respectively at Week 30 of the placebo-controlled trial. After 2 years of treatment, changes in glycaemic control were similar, demonstrating sustained reductions in $\mathrm{HbA}_{1 \mathrm{c}}$ and FPG from baseline of $-1.1 \pm 0.1 \%(\mathrm{P}<0.05)$ and $-1.4 \pm 0.2 \mathrm{mmol} / \mathrm{L}, \quad(\mathrm{P}<0.05) \quad($ mean $\pm \mathrm{SE})$, respectively. Weight reduction from baseline after 2 years was progressive at $-4.7 \pm 0.3 \mathrm{~kg},(\mathrm{P}<0.05)$ (mean $\pm \mathrm{SE})$. After 2 years, $50 \%$ and $31 \%$ of patients achieved $\mathrm{HbA}_{1 \mathrm{c}}$ of $\leq 7 \%$ and $\leq 6.5 \%$, respectively. Similar results were obtained for the ITT population. In a subgroup of the 2-year completer population (patients who received MET or $\mathrm{SU}, \mathrm{N}=112$ ), HOMA-B improved from baseline by $11 \%(\mathrm{P}<0.05)$ after 2 years of exenatide exposure. Exenatide was generally well-tolerated during 2 years of exposure. The most frequent adverse events were gastrointestinal in nature. 
Conclusion: In this open-label extension study, exenatide exerted sustained improvements in glycaemic control and progressive reduction in body weight out to 2 years in patients with type 2 diabetes.

\section{2}

A comparison of exenatide and insulin glargine in patients using a single oral antidiabetic agent

A. H. Barnett ${ }^{1}$, M. Trautmann ${ }^{2}$, J. Burger ${ }^{3}$, D. Johns ${ }^{3}$,

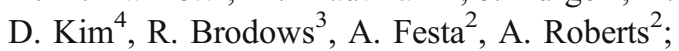

${ }^{1}$ Department of Diabetes/Endocrinology, Birmingham Heartlands Hospital, United Kingdom, ${ }^{2}$ Eli Lilly and Company, Hamburg, Germany, ${ }^{3}$ Eli Lilly and Company, Indianapolis, United States, ${ }^{4}$ Amylin Pharmaceuticals, Inc., San Diego, United States.

Background and Aims: Insulin glargine is a common treatment for type 2 diabetes patients when oral medications no longer provide adequate glycaemic control. Exenatide is an incretin mimetic for the treatment of type 2 diabetes inadequately controlled with metformin (MET) and/or a sulfonylurea (SFU).

Materials and Methods: This randomised, two-period, open-label, crossover study compared effects between exenatide $(5 \mu \mathrm{g}$ BID for $4 \mathrm{wks}$, then $10 \mu \mathrm{g}$ BID for 12 weeks) and insulin glargine (QD titrated to fasting blood glucose $\leq 5.6 \mathrm{mmol} / \mathrm{L}$ ). Either treatment was added to ongoing single oral agent therapy (MET 56\%; or SFU $44 \%$ ) during two, 16-wk treatment periods. Patients (mean \pm SD) (age, $54 \pm 9$ y; weight, $86 \pm 16.4 \mathrm{~kg}$; HbAlc, $8.9 \pm 1.1 \%$; fasting glucose, $12.09 \pm .31 \mathrm{mmol} / \mathrm{L}$ ) continued their oral agent at maximal dose.

Results: Similar reductions in $\mathrm{HbAlc}$ from baseline $(\mathrm{n}=114$, completers) were observed during treatment periods with exenatide $(-1.43 \pm 0.09 \%)$ and glargine $(-1.41 \pm 0.09 \%)$. A similar percentage of patients achieved HbAlc $\leq 7 \%$ with exenatide $(40 \%)$ and glargine treatment (41\%). HbA $1 \mathrm{c} \leq 6.5 \%$ was achieved by $24 \%$ of exenatidetreated patients compared with $14 \%$ of glargine-treated patients $(\mathrm{P}=.056)$. Both treatments maintained lowered HbAlc from the first treatment period through the second treatment period. Weight reduction during the first treatment period of exenatide $(-2.35 \mathrm{~kg})$ was reversed by glargine $(+2.3 \mathrm{~kg}, \mathrm{n}=55)$, and weight gain during the first treatment period by glargine $(+0.75 \mathrm{~kg})$ was followed by weight reduction on exenatide $(-2.3 \mathrm{~kg}, \mathrm{n}=59)$. Overall weight change from baseline was significantly different $(\mathrm{P}<0.001)$ between exenatide $(-1.95 \mathrm{~kg})$ and glargine treatments $(+0.35 \mathrm{~kg})$. Both treatments significantly decreased fasting glucose from baseline (exenatide, $-3.04 \pm 0.23 \mathrm{mmol} / \mathrm{L}$; glargine, $-4.17 \pm 0.23 \mathrm{mmol} / \mathrm{L}$; $\mathrm{P}<0.0001$, within- and between-treatment groups). Exena- tide injections prior to the morning and evening meals significantly decreased 2 -hr post-meal glucose excursions (both $\mathrm{P}<0.001$ ) compared with glargine injections. Combined $2 \mathrm{hr}$ glucose excursions after all 3 meals were also significantly lower in exenatide-treated patients compared with glargine-treated patients $(\mathrm{P}=.036)$. Overall efficacy results were similar between the MET- and SFU-treated subgroups, except for greater weight reduction in patients treated with exenatide and MET $(-2.97 \pm 4.28 \mathrm{~kg})$ compared with patients treated with exenatide and SFU $(-0.61 \pm 2.86 \mathrm{~kg})$. Hypoglycemia occurred in a greater percentage of patients treated with SFU (30\% exenatide, $35 \%$ glargine) compared with patients treated with MET ( $3 \%$ exenatide, $17 \%$ glargine; between groups, $\mathrm{P}=.01$ ). The most common adverse events potentially related to study drug were nausea (33\%) and headache $(8.7 \%)$ during exenatide and insulin glargine treatments, respectively.

Conclusion: In patients receiving ongoing treatment with MET or an SFU, while both exenatide and insulin glargine improved $\mathrm{HbAlc}$ and significantly decreased fasting glucose, only exenatide significantly reduced body weight and combined 2 hour post-meal glucose excursions.

Supported by Eli Lilly and Company and Amylin Pharmaceuticals, Inc.

0783

Patient reported outcomes in patients with type 2 diabetes treated with exenatide compared to biphasic insulin aspart

N. Yurgin, K. Secnik, C. Hayes, D. Johns, M. Trautmann; Eli Lilly and Company, Indianapolis, United States.

Background and Aims: Although exenatide and biphasic insulin aspart appear to have similar clinical efficacy, there are several differences between the two treatments that could influence outcomes from the patient's perspective. Therefore, the purpose of this study was to conduct a secondary analysis of clinical trial data to examine whether the two drugs were comparable as assessed by patientreported outcomes.

Materials and Methods: Patients with type 2 diabetes $(n=505)$ who had inadequate glycaemic control despite treatment with a sulphonylurea and metformin were randomised to receive either twice daily exenatide or biphasic insulin aspart during the 52-week, non-inferiority trial. At baseline, mean age was $59 \pm 9$, BMI mean $30 \pm 4$, and $\mathrm{HbA1c}$ mean $8.6 \% \pm 1.1 \%$. At baseline and endpoint patients were administered the EuroQol instrument (EQ5D) and the SF-36 Vitality Scale. Where validated language translations were available, the Diabetes Symptom Checklist-Revised (DSC-R) and the Treatment Flexibility Scale 
were administered at baseline and endpoint. Change from baseline to endpoint was compared for per protocol patients within each treatment, and then between treatment groups with analysis of covariance models - controlling for country and baseline scores.

Results: At endpoint, exenatide and biphasic insulin aspart achieved similar HbAlc reductions $(-0.98 \%$ and $-0.88 \%$, respectively). Exenatide-treated patients exhibited statistically significant improvements from baseline to endpoint in the DSC-R $(-0.13, \mathrm{p}=.0356)$, the EQ-5D Index Score (0.03, $\mathrm{p}=.0399)$, the EQ-5D VAS score $(3.39, \mathrm{p}=.0009)$, and SF-36 Vitality Scale $(3.89, \mathrm{p}=.0019)$ scores - no significant difference in the Treatment Flexibility Scale was observed for exenatide patients. There were no statistically significant changes from baseline to endpoint in the biphasic insulin aspart treatment group for any of the four patient-reported outcomes. Exenatide-treated patients demonstrated significant improvement from baseline compared to biphasic insulin aspart-treated patients in the DSC$\mathrm{R}(-0.13$ vs. $0.05, \mathrm{p}=.0218)$, but not in other patientreported outcome instruments.

Conclusion: Exenatide-treated patients showed improvement from baseline to endpoint in many patient-reported outcomes. In contrast, biphasic insulin aspart-treated patients demonstrated no similar improvement in patient reported outcomes despite similar glycaemic improvements compared with exenatide. These data indicate that exenatide may be associated with improvements in patient reported outcomes and quality of life in patients with type 2 diabetes.

Supported by Eli Lilly and Company

\section{4}

Exenatide treatment for 82 weeks reduced C-reactive protein, $\mathrm{HbA}_{1 \mathrm{C}}$, and body weight in patients with type 2 diabetes mellitus

D. Kendall ${ }^{1}$, D. Bhole ${ }^{1}$, X. Guan ${ }^{1}$, L. Nielsen ${ }^{1}$, M. Trautmann ${ }^{2}$, M. Wintle ${ }^{1}$, D. Kim ${ }^{1}$;

${ }^{1}$ Amylin Pharmaceuticals, Inc., San Diego, United States,

${ }^{2}$ Lilly Forschung, Hamburg, Germany.

Background and Aims: Cardiovascular disease (CVD) is a major cause of morbidity and mortality in patients with type 2 diabetes mellitus (T2DM). Elevated C-Reactive Protein (CRP), a proinflammatory peptide, is associated with increased risk of CVD. In T2DM patients not achieving glycemic control with MET and/or SFU and enrolled in phase 3 trials followed by open-label extensions, the incretin mimetic exenatide improved glycemic control, diastolic blood pressure, serum HDL-C, and serum triglycerides; reduced body weight, and was generally well tolerated with mild-to-moderate nausea the most common adverse event. We investigated whether there were CRP changes after 82 wks of exenatide treatment in a subset of patients with evaluable serum samples $(66 \% \mathrm{M}$, age $57 \pm$ $10 \mathrm{y}$, wt $97 \pm 20 \mathrm{~kg}$, BMI $33 \pm 5 \mathrm{~kg} / \mathrm{m}^{2}, \mathrm{HbA}_{1 \mathrm{c}} 8.2 \pm 1.0 \%$ $[$ mean $\pm \mathrm{SD}]$ ).

Materials and Methods: A post hoc interim analysis of serum high-sensitivity CRP was performed $(\mathrm{n}=178)$. CRP data was $\log$ transformed for correlation and regression analyses due to non-normal data distribution. Patients with $\mathrm{CRP} \geq 10 \mathrm{mg} / \mathrm{L}$ at any visit were excluded from the analysis to avoid confounding the results by including patients with generalized inflammatory disease.

Results: Patients completing 82 wks on exenatide with evaluable serum samples had reductions in $\mathrm{HbA}_{1 \mathrm{c}}$ $(-1.2 \pm 0.1 \%)$ and body weight $(-4.5 \pm 0.4 \mathrm{~kg})$ from baseline (mean $\pm \mathrm{SE}$ ). Median serum CRP concentrations decreased significantly by $-1.35 \mathrm{mg} / \mathrm{L}$ from baseline median $3.21 \mathrm{mg} / \mathrm{L}$ $(p<0.0001)$. In multiple regression analysis adjusting for baseline CRP, both $\mathrm{HbA}_{1 \mathrm{c}}$ change and weight change were significantly associated with Week 82 CRP values $(p=0.0244$ and $\mathrm{p}<0.0001$, respectively). Subgroup analysis stratifying by baseline $\mathrm{CRP} \leq 3 \mathrm{mg} / \mathrm{L}$ (low/moderate CVD risk) or $>3 \mathrm{mg} / \mathrm{L}$ (high CVD risk) revealed a marginal decrease in median CRP for the low risk group $(-0.43 \mathrm{mg} / \mathrm{L}$; $-22 \%$; $p=0.0617$ ), contrasted with a significant reduction in median CRP of $-2.25 \mathrm{mg} / \mathrm{L}(-50 \%$; $<<0.0001)$ for the high risk group.

Conclusion: Exenatide treatment for 82 weeks was associated with a reduction in CRP, and this was correlated with reduced $\mathrm{HbA}_{1 \mathrm{c}}$ and body weight in T2DM patients. The greatest CRP decrease occurred in the high CVD risk group, suggesting further studies are warranted into whether exenatide can improve CVD risk in this patient population.

\section{5}

Safety and efficacy of exenatide over 16 weeks in patients with type 2 diabetes mellitus using a thiazolidinedione with or without metformin

B. Zinman ${ }^{1}$, B. Hoogwerf ${ }^{2}$, S. Duran Garcia ${ }^{3}$, D. Milton ${ }^{4}$, J. Giaconia ${ }^{4}$, D. Kim ${ }^{5}$, M. Trautmann ${ }^{6}$, R. Brodows ${ }^{4}$;

${ }^{1}$ Sinai Centre for Diabetes, Mount Sinai Hospital, Toronto, Canada, ${ }^{2}$ Department of Endocrinology, Cleveland Clinic Foundation, Cleveland, United States, ${ }^{3}$ Cátedra de Endocrinología, Hospital de Valme, Sevilla, Spain, ${ }^{4}$ Eli Lilly and Company, Indianapolis, United States, ${ }^{5}$ Amylin Pharmaceuticals, Inc., San Diego, United States, ${ }^{6}$ Eli Lilly and Company, Hamburg, Germany.

Background and Aims: Exenatide, an incretin mimetic for the treatment of type 2 diabetes mellitus (T2DM), mimics 
several glucose-lowering actions of GLP-1 including glucose-mediated insulin secretion. Adjunctive therapy of exenatide and a TZD with or without metformin may reduce glucose levels by targeting the central defects of T2DM: beta cell dysfunction and insulin resistance.

Materials and Methods: This study was a randomised, placebo-controlled, parallel, double-blind trial in 233 patients with elevated $\mathrm{HbA}_{1 \mathrm{c}}$ (range: $7.1-10.0 \%$ ) in spite of therapy with a TZD alone $(20 \%)$ or a TZD with metformin $(80 \%)$. Patients (129M, 56 \pm 10 y, BMI $34 \pm 5 \mathrm{~kg} / \mathrm{m}^{2}, \mathrm{HbA}_{1 \mathrm{c}} 7.9 \pm 0.9 \%$ [mean $\left.\left.\pm \mathrm{SD}\right]\right)$ received subcutaneous injections of placebo or exenatide BID for 16 weeks. Patients that received exenatide received $5 \mu \mathrm{g}$ BID for 4 weeks followed by $10 \mu \mathrm{g}$ BID for 12 weeks. Endpoint measures included $\mathrm{HbA}_{1 \mathrm{c}}$, fasting serum glucose (FSG), body weight, 7-point self-monitored blood glucose (SMBG), safety, and tolerability.

Results: For exenatide, $71 \%$ of patients completed the study versus $86 \%$ for placebo. Exenatide decreased mean $\mathrm{HbA}_{1 \mathrm{c}}$ by $-0.8 \pm 0.9 \%$ from baseline (treatment effect, $-0.9 \%$, $\mathrm{p}<0.0001)$. With exenatide, $62 \%$ of patients achieved $\mathrm{HbA}_{1 \mathrm{c}} \leq 7 \%$ versus $16 \%$ with placebo $(\mathrm{p}<0.0001) ; 29 \%$ achieved $\mathrm{HbA}_{1 \mathrm{c}} \leq 6.5 \%$ with exenatide versus $8 \%$ with placebo $(p=0.0002)$. Mean FSG for patients who received exenatide was $-1.5 \mathrm{mmol} / \mathrm{L}$ lower than placebo $(p<0.0001)$. Exenatide reduced mean body weight from baseline versus placebo $(-1.5 \pm 3.1 \mathrm{~kg}$ vs. $-0.2 \pm 2.5 \mathrm{~kg}$, respectively, $\mathrm{p}<0.001$ ). Mean daily $2-\mathrm{h}$ SMBG postprandial excursions for exenatide were lower than baseline $(-1.4 \pm 1.4 \mathrm{mmol} / \mathrm{L}, \mathrm{p}<0.0001)$ with greatest differences after breakfast $(-1.9 \pm 2.9 \mathrm{mmol} / \mathrm{L}, \mathrm{p}<0.0001)$ and dinner $(-1.9 \pm 2.5 \mathrm{mmol} / \mathrm{L}, \mathrm{p}<0.0001)$. Placebo-treated patients showed no significant reductions in postprandial glucose excursions from baseline at any meal but demonstrated a reduction in the daily mean glucose concentration $(p=0.0408)$. Exenatide reduced the proinsulin/insulin ratio over placebo $(\mathrm{p}=0.0337)$ and increased HOMA-B versus placebo $(\mathrm{p}=0.0032)$. No statistically significant difference in HOMA-S existed between groups. The most frequent adverse event was nausea $(40 \%$ exenatide vs. $15 \%$ placebo); there was no significant difference in incidence of hypoglycaemia.

Conclusion: These findings support the potential use of exenatide as adjunctive therapy for patients with T2DM who inadequately control glucose levels with a TZD alone or in combination with metformin.

Supported by Eli Lilly and Company and Amylin Pharmaceuticals, Inc.
0786

Liraglutide significantly improves glycaemic control, and lowers body weight without risk of either major or minor hypoglycaemic episodes in subjects with type 2 diabetes

T. Vilsbøll ${ }^{1}$, M. Zdravkovic ${ }^{2}$, T. Le-Thi ${ }^{2}$, T. Krarup ${ }^{1}$, O. Schmitz ${ }^{3}$, J. P. Courreges ${ }^{4}$, R. Verhoeven ${ }^{5}$,

I. Buganova ${ }^{6}$, S. Madsbad ${ }^{7}$;

${ }^{1}$ Internal Medicine, Gentofte Hospital, Hellerup, Denmark, ${ }^{2}$ Novo Nordisk A/S, Bagsværd, Denmark, ${ }^{3}$ Internal Medicine (M), Aarhus University Hospital, Aarhus, Denmark, ${ }^{4}$ Service de Médecine A, Centre Hospitalier de Narbonne, ${ }^{5}$ Poli Interne Geneeskunde, Gelre Ziekenhuizen, Apeldoorn, The Netherlands, ${ }^{6}$ Diabetologická Ambulancia, ilina, Slovakia, ${ }^{7}$ Endocrinology, Hvidovre University Hospital, Denmark.

Background and Aims: The objective of this 14-week study was to assess the efficacy and safety of liraglutide, a once-daily GLP-1 analogue.

Materials and Methods: 165 subjects with type 2 diabetes with an $\mathrm{HbA}_{1 \mathrm{c}}$ at randomization of $8.1-8.5 \%$ were studied. Subjects treated with diet $(19 \%)$ or single oral antidiabetic agents (after 4 weeks washout) were randomized to one of three once-daily doses of liraglutide $(0.65 \mathrm{mg}, 1.25 \mathrm{mg}$ or $1.9 \mathrm{mg})$ or placebo $(1: 1: 1: 1)$.

Results: Significant improvement in $\mathrm{HbA}_{1 \mathrm{c}}$ was achieved in all actively treated groups vs. placebo $(p<0.0001)$, with an estimated difference vs. placebo of $-1.74 \%$ at the highest dose level (last observation carried forward LOCF). The fraction of subjects achieving an $\mathrm{HbA}_{1 \mathrm{c}}$ level $\leq 7.0 \%$ was between 0.43 and 0.50 in the three liraglutide arms versus 0.08 in the placebo group. Improvement in glycemic control was achieved without any major or minor hypoglycemic episodes. Dose dependent weight reduction was achieved. In the high-dose liraglutide treatment group, estimated change in body weight was $-2.99 \mathrm{~kg}$ from baseline and $-1.21 \mathrm{~kg}$ compared to placebo $(p=0.039) \quad(L O C F)$. Liraglutide was well tolerated; four subjects withdrew due to adverse events in the three liraglutide treated groups vs. three in the placebo treated group. Main adverse events were from the gastrointestinal (GI) system; diarrhea was the most frequent, with an incidence of $19.5 \%$ and $12.5 \%$ in the high-dose liraglutide group and in the placebo group, respectively. In the highdose group $10 \%$ of the subjects experienced nausea. The frequency of GI events decreased over time.

Conclusion: Liraglutide used as monotherapy significantly improved glycemic control without risk of major or minor hypoglycemia, was well tolerated, and lowered body weight in subjects with type 2 diabetes.

This study was sponsored by Novo Nordisk A/S 
0787

Cost-effectiveness of exenatide versus insulin glargine for the treatment of type 2 diabetes in a UK setting: a long-term health economic study

J. A. Ray ${ }^{1}$, K. Secnik ${ }^{2}$, N. Yurgin ${ }^{2}$, W. J. Valentine ${ }^{1}$, S. Roze ${ }^{1}$, J. McKendrick ${ }^{3}$, V. Foos ${ }^{1}$, A. J. Palmer ${ }^{1}$;

${ }^{1}$ CORE, IMS, Basel, Switzerland, ${ }^{2}$ Eli Lilly \& Company, Indianapolis, United States, ${ }^{3}$ Eli Lilly \& Company, Basingstoke, United Kingdom.

Background and Aims: A recent randomised open-label, parallel group, clinical trial in 549 patients with type 2 diabetes unable to achieve adequate glycaemic control using sulphonylurea and metformin was performed to compare exenatide to insulin glargine. After 26-weeks, exenatide and insulin glargine were associated with a decrease in $\mathrm{HbA} 1 \mathrm{c}$ values $(-0.99 \%$ vs $-1.07 \%)$. The most notable difference was a reduction in body weight in patients treated with exenatide $(-2.32 \mathrm{kgs})$ compared with an increase in body weight for the insulin glargine treatment arm $(+1.75 \mathrm{kgs})$. Although exenatide is currently available in the US, it has not yet been approved in the UK. The aim of the present analysis was to determine in the UK, over a range of prices, the incremental cost-effectiveness ratio (ICER) for exenatide compared to insulin glargine.

Materials and Methods: A previously published model of type 2 diabetes was used to project the clinical and economic outcomes from the clinical trial over patient lifetimes. The model simulates disease progression by combining 15 Markov-based sub-models to determine the costs and clinical outcomes associated with the occurrence of disease-related complications. Baseline cohort characteristics (mean age 58.9 yrs; $55.7 \%$ male; mean $\mathrm{HbA1c}$ $8.21 \%$; duration of diabetes $9.56 \mathrm{yrs}$ ) and treatment effects were taken from the clinical trial. The study assumed the perspective of the UK national health service payer using treatment costs presented in 2004 British Pounds(£). As no price is currently available for exenatide in the UK, the US price ( $\$ 5.75$ per day, approximately £3.28) was used as a point estimate, with the price reduced in increments of $20 \%$ in exploratory scenarios. Medication costs and utilities/ disutilities based on weight loss and weight gain and other significant product attribute differences were included in the model. A discount rate of $3.5 \%$ was applied to costs and clinical outcomes.

Results: Exenatide was associated with a higher life expectancy (10.05 vs 9.99 years) and quality-adjusted life expectancy (7.01 vs 6.00 QALYs) compared to insulin glargine, primarily attributed to a reduction in CV complications, as determined by the CORE Diabetes Model that uses projective risk equations. Lifetime direct medical costs were higher in the exenatide group for prices at 40 to $100 \%$ of the US price with ICERs between $£ 1124$ and $£ 8722$ per QALY gained, compared to insulin glargine.

Conclusion: Using the CORE Diabetes Model to simulate long-term outcomes, treatment with exenatide was associated with a higher life expectancy and an improvement in quality-adjusted life expectancy compared to insulin glargine. Using the US daily price, exenatide was estimated to have an ICER of $£ 8722$ per QALY gained compared to insulin glargine, well below the thresholds commonly referred to as representing good value for money in the UK.

\begin{tabular}{lllll}
\hline & $\begin{array}{l}\text { Exenatide } \\
\text { medical } \\
\text { costs }\end{array}$ & $\begin{array}{l}\text { Insulin } \\
\text { Glargine } \\
\text { medical } \\
\text { costs }\end{array}$ & $\begin{array}{l}\text { Difference } \\
\text { in costs }\end{array}$ & $\begin{array}{l}\text { ICER } \\
\text { (Costs per } \\
\text { QALY) }\end{array}$ \\
$100 \%$ of US price & $£ 31,877$ & $£ 23,073$ & $£ 8,804$ & $£ 8,722$ \\
$80 \%$ of US price & $£ 29,320$ & $£ 23,073$ & $£ 6,247$ & $£ 6,189$ \\
$60 \%$ of US price & $£ 26,764$ & $£ 23,073$ & $£ 3,691$ & $£ 3,657$ \\
$40 \%$ of US price & $£ 24,208$ & $£ 23,073$ & $£ 1,135$ & $£ 1,124$ \\
$20 \%$ of US price & $£ 21,651$ & $£ 23,073$ & $-£ 1,422$ & Dominant \\
\hline
\end{tabular}

Supported by Eli Lilly and Company

\section{PS 61}

\section{DPP-4 inhibitors}

\section{8}

Vildagliptin displays slow tight-binding to dipeptidyl peptidase (DPP)-4, but not DPP-8 or DPP-9

B. F. Burkey ${ }^{1}$, M. Russell ${ }^{1}$, K. Wang ${ }^{1}$, J. Trappe ${ }^{2}$, T. E. Hughes ${ }^{1}$;

${ }^{1}$ Novartis Institutes for Biomedical Research Inc., Cambridge, United States, ${ }^{2}$ Novartis Institutes for Biomedical Research Inc., Basel, Switzerland.

Background and Aims: Vildagliptin [(S)1-[(3-hydroxyadamant-1-ylamino)acetyl]pyrrolidine-2-carbonitrile] is an inhibitor of DPP-4 in late stage clinical development for treatment of patients with type 2 diabetes. Vildagliptin potently inhibits DPP-4 with an $\mathrm{IC}_{50}$ of approximately $3 \mathrm{nM}$. The chirality of the nitrile functionality of vildagliptin (which is the S-enantiomer) was found to be critical to its inhibitory action, as the $\mathrm{IC}_{50}$ of the R-antipode for inhibition of DPP-4 was $>1100$-fold higher than that of vildagliptin. Vildagliptin is highly selective for DPP-4 despite the similarity of substrate specificities among many physiologically important proteases. When tested at concentrations up to 100 microM, vildagliptin elicited little or no inhibition of human aminopeptidase $\mathrm{P}$, human leukocyte elastase, porcine trypsin, rabbit angiotensin converting enzyme or rat neutral 
endopeptidase 24.11. Vildagliptin was found to be a weak inhibitor of human post-proline cleaving enzyme, with an $\mathrm{IC}_{50}$ of 206 microM. The current study evaluates the selectivity and binding characteristics of vildagliptin for four closely related serine proteases: DPP-2, DPP-8, DPP-9 and fibroblast activation protein alpha (FAP alpha; seperase). Materials and Methods: The kinetics of inhibition of Caco-2 DPP-4 and recombinant human DPP-2, DPP-4, DPP-8, DPP-9 and FAP alpha by vildagliptin were evaluated to establish the $\mathrm{K}_{\mathrm{i}}$ and the half-lives of dissociation of the enzyme-inhibitor complexes after 100fold dilution. Kinetic measurements were derived from progression curves of AMC formation from the substrate H-Gly-Pro-AMC at 25 degrees C. Furthermore, the potential modification of vildagliptin by DPP-4 was assessed by LC/MS analysis of incubation reactions.

Results: Kinetic analysis of vildagliptin-induced inhibition of DPP-4 from Caco-2 cells indicated that, although vildagliptin is a substrate analog that displays apparent single-site binding behavior, it does not exhibit a simple competitive inhibitor pattern. Rather, the kinetics of vildagliptin-induced inhibition of DPP-4 suggest a slow tight-binding mechanism. The $\mathrm{K}_{\mathrm{i}} \mathrm{s}$ of vildagliptin for DPP-2, DPP-4, DPP-8, DPP-9 and FAP alpha were $>20,000,3,810,95$, and 9,900 nM, respectively. Further, the very slow dissociation of the vildagliptin/DPP-4 complex $\left(\mathrm{t}_{1 / 2}=55 \mathrm{~min}\right)$ is in contrast with the very rapid dissociation of vildagliptin from DPP-8 or DPP-9 $(<10 \mathrm{sec})$. The high potency and slow dissociation kinetics of vildagliptin for DPP-4 are due to the formation of a transient high-affinity complex that represents a transitory imidate intermediate which leads to cleavage of the carbonnitrogen bond in the nitrile moiety of the compound, generating an inactive carboxylic acid byproduct.

Conclusion: Vildagliptin is a substrate for DPP-4 that displays slow tight-binding kinetics with very slow dissociation rates. This contrasts with vildagliptin's rapid dissociation kinetics for the related proteases DPP-8 and DPP-9. These differences in dissociation rates for DPP-4 relative to DPP-8 and DPP-9 infer greater selectivity among these related proteases than the simple ratio of $\mathrm{K}_{\mathrm{i}}$. would suggest, reducing the potential of vildagliptin to inhibit these enzymes at pharmacologic doses.

\section{9}

Vildagliptin decreases $\mathrm{HbA}_{1 \mathrm{c}}$ after 12 week treatment in Japanese patients with type 2 diabetes

M. Kikuchi ${ }^{1}$, N. $\mathrm{Abe}^{2}$, M. Kato ${ }^{3}, \mathrm{~S} . \mathrm{Terao}^{4}$,

D. Holmes ${ }^{4}$, N. Mimori ${ }^{4}$;

${ }^{1}$ Int Med, Asahi Life Foundation, Tokyo, ${ }^{2}$ Int Med, Abe

Diabetes Clinic, Oita, ${ }^{3}$ Int Med, Kato Clinic, Tokyo,

${ }^{4}$ Novartis, Tokyo, Japan.
Background and Aims: Vildagliptin is a potent and selective DPP-4 inhibitor that improves glycemic control by increasing pancreatic $\alpha$ - and $\beta$-cell responsiveness to glucose. The purpose of the present study was to assess the efficacy and tolerability of vildagliptin monotherapy in Japanese patients with type 2 diabetes (T2DM)

Methods: This was a 12-wk, randomized, double-blind, placebo (PBO)-controlled study of vildagliptin $(10 \mathrm{mg}$ bid, $25 \mathrm{mg}$ bid or $50 \mathrm{mg}$ bid) in 219 drug-naïve Japanese patients with T2DM. A standard meal test (SMT, Japanese meal, $\mathrm{kcal}=470, \mathrm{CHO}=73 \%$, protein $=12 \%$, fat $=15 \%$ ) was conducted at baseline (BL) and Wk 12 in a subset of patients.

Results: In the randomized population, the mean \pm SD age and BMI were $59 \pm 8.4 \mathrm{y}$ and $24.4 \pm 2.8 \mathrm{~kg} / \mathrm{m}^{2}$, respectively. As reported below, vildagliptin decreased $\mathrm{HbA}_{1 \mathrm{c}}$ and FPG in a dose-related manner whereas glycemic control deteriorated slightly in patients receiving PBO.

\begin{tabular}{lllll}
\hline & \multicolumn{2}{c}{$\mathrm{HbA}_{1 \mathrm{c}}(\%)$} & \multicolumn{2}{c}{$\mathrm{FPG}(\mathrm{mM})$} \\
Mean \pm SE & Baseline & $\Delta$ & Baseline & \multicolumn{1}{c}{$\Delta$} \\
\hline PBO (n=72) & $7.4 \pm 0.8$ & $0.3 \pm 0.1$ & $9.0 \pm 1.7$ & $0.1 \pm 0.2$ \\
$\begin{array}{l}\text { Vildagliptin } 10 \mathrm{mg} \text { bid } \\
(\mathrm{n}=71)\end{array}$ & $7.4 \pm 0.8$ & $-0.5 \pm 0.1 *$ & $8.9 \pm 1.9$ & $-0.6 \pm 0.2^{*}$ \\
$\begin{array}{l}\text { Vildagliptin } 25 \mathrm{mg} \text { bid } \\
(\mathrm{n}=72)\end{array}$ & $7.4 \pm 0.9$ & $-0.7 \pm 0.1 * 9.0 \pm 1.8$ & $-0.8 \pm 0.2^{*}$ \\
$\begin{array}{l}\text { Vildagliptin } 50 \mathrm{mg} \text { bid } \\
(\mathrm{n}=76)\end{array}$ & $7.4 \pm 0.8$ & $-0.9 \pm 0.1 * 8.9 \pm 1.8$ & $-1.4 \pm 0.2^{*}$ \\
\hline
\end{tabular}

$* \mathrm{P}<0.001$ vs $\mathrm{PBO}$

During the SMT, peak prandial GLP-1(7-36) increased about 2-fold at Wk 12 relative to $\mathrm{BL}$ in vildagliptin-treated patients. In PBO-treated patients, the 2-hr prandial glucose level increased by $0.2 \mathrm{mM}$ and it decreased by $3.5,3.2$ and $3.4 \mathrm{mM}$ in patients receiving vildagliptin 10,25 and $50 \mathrm{mg}$ bid respectively $(\mathrm{p}<0.001$ for all vs. $\mathrm{PBO})$. Adverse events (AEs) were reported by $74 \%$ of patients receiving PBO and by $62 \%$ in each of the vildagliptin treatment groups. There was a similar incidence of gastrointestinal AEs in all groups. Hypoglycemia was reported by 1.4, 4.2, 0 and $2.6 \%$ of patients receiving PBO and vildagliptin 10, 25, $50 \mathrm{mg}$ bid, respectively.

Conclusions: In Japanese patients with T2DM, 12-wk treatment with vildagliptin significantly decreases $\mathrm{HbA}_{1 \mathrm{c}}$ in a clinically meaningful and dose-dependent manner. This reflects decreases in both FPG and postprandial glucose. Vildagliptin is an effective and well-tolerated approach to achieving glycemic control in Japanese patients with T2DM.

Supported by Novartis 
0790

Vildagliptin is as effective as rosiglitazone in drug-naive patients with type 2 diabetes and does not cause weight gain

A. Schweizer ${ }^{1}$, S. Dejager ${ }^{1}$, M. A. Baron ${ }^{1}$, D. Mills ${ }^{1}$, Y. Amiour ${ }^{1}$, J. Rosenstock ${ }^{2}$;

${ }^{1}$ Novartis, Basel, Switzerland, ${ }^{2}$ Endo, Dallas Diabetes \& Endo Ctr, Dallas, United States.

Background and Aims: Vildagliptin is a potent and specific DPP-4 inhibitor that improves glycemic control by increasing pancreatic $\alpha$ - and $\beta$-cell responsiveness to glucose. The purpose of this study was to compare the efficacy and tolerability of vildagliptin and rosiglitazone (Rosi) in drug-naïve patients (pts) with type 2 diabetes (T2DM).

Methods: This was a 24-wk, multicenter, randomized, double-blind, parallel group, active-controlled study comparing vildagliptin $50 \mathrm{mg}$ bid $(\mathrm{n}=459)$ to Rosi $8 \mathrm{mg} \mathrm{qd}$ $(n=238)$ in drug-naïve pts with T2DM. The primary endpoint was the between-group difference and associated $95 \%$ confidence interval in the adjusted mean change $(\mathrm{AM} \Delta)$ in $\mathrm{HbA}_{1 \mathrm{c}}$ from baseline (BL) to Wk 24 or study endpoint.

Results: The two groups were well balanced at BL. Mean age $=54.4 \mathrm{y} ; \mathrm{BMI}=32.4 \mathrm{~kg} / \mathrm{m}^{2}$; duration of diabetes $=2.4 \mathrm{y}$ and $\mathrm{HbA}_{1 \mathrm{c}}=8.7 \%$. As shown below, the two agents decreased $\mathrm{HbA}_{1 \mathrm{c}}$ progressively and to a similar extent, although the onset of action of vildagliptin appeared to be more rapid than that of Rosi. Thus, the mean change at Wk 4 was $-0.6 \pm 0.0 \%$ in pts receiving vildagliptin and $-0.3 \pm 0.0 \%$ in those receiving Rosi. The $\mathrm{AM} \Delta \mathrm{HbA}_{1 \mathrm{c}}$ from $\mathrm{BL}$ to endpoint in pts receiving vildagliptin $50 \mathrm{mg}$ bid was $-1.1 \pm 0.1 \%$, and that in Rosi-treated pts was $-1.3 \pm 0.1 \%$. The study established non-inferiority of vildagliptin $50 \mathrm{mg}$ bid to Rosi 8 mg qd.

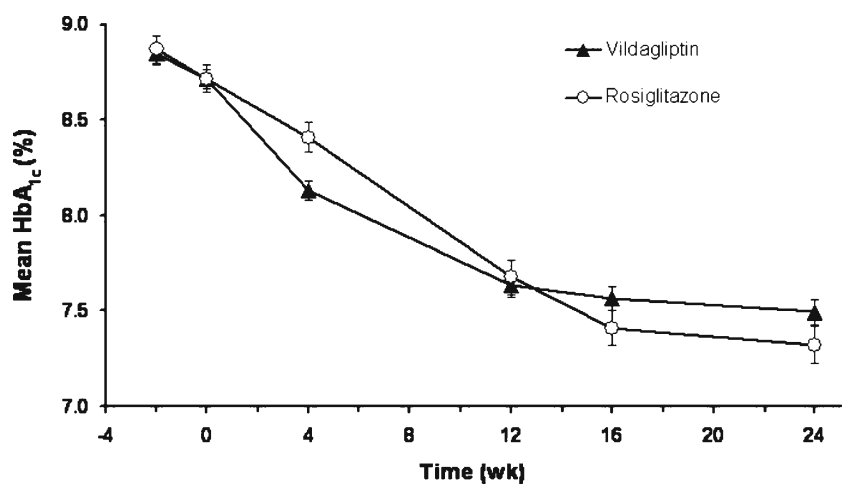

In pts with $\mathrm{BL} \mathrm{HbA}_{1 \mathrm{c}}>9.0 \%$, the $\Delta \mathrm{HbA}_{1 \mathrm{c}}$ was $-1.8 \pm 0.1 \%$ in the vildagliptin group $(n=166)$ and $-1.9 \pm 0.2 \%$ in the
Rosi group ( $\mathrm{n}=88$ ). In non-obese pts $\left(\mathrm{BMI}<30 \mathrm{~kg} / \mathrm{m}^{2}\right.$ ) vildagliptin appeared to be somewhat more effective $\left(\Delta \mathrm{HbA}_{1 \mathrm{c}}=-1.3 \pm 0.1 \%, \mathrm{n}=184\right)$ than Rosi $\left(\Delta \mathrm{HbA}_{1 \mathrm{c}}=\right.$ $-1.1 \pm 0.2 \%, \mathrm{n}=83$ ). Relative to Rosi, vildagliptin improved the lipid profile, producing significant decreases in triglycerides $(-9 \%, \mathrm{P}=0.010)$, total $(-14 \%, \mathrm{P}<0.001)$, LDL $(-16 \%, \mathrm{P}<0.001)$, and non-HDL cholesterol $(-16 \%$, $\mathrm{P}<0.001)$, but a smaller increase in HDL-cholesterol $(+3.9$ vs $+8.9 \%,-5 \%, \mathrm{P}=0.003)$. Vildagliptin did not affect body weight (BW, $\mathrm{AM} \Delta=-0.3 \pm 0.2 \mathrm{~kg}$ ) but $\mathrm{BW}$ increased in Rosi-treated pts $(\mathrm{AM} \Delta=+1.6 \pm 0.3 \mathrm{~kg}, \mathrm{P}<0.001$ vs vildagliptin). The overall incidence of side effects was $61.4 \%$ in pts receiving vildagliptin and $64.0 \%$ in those receiving Rosi. The incidence of edema-related events was greater with Rosi $(4.9 \%)$ than with vildagliptin $(2.5 \%)$. One patient in each group experienced one mild hypoglycemic episode.

Conclusions: Vildagliptin $50 \mathrm{mg}$ bid 1) is efficacious and well-tolerated in drug-naïve pts with T2DM; 2) is noninferior to Rosi $8 \mathrm{mg} \mathrm{qd}$; 3) does not affect BW and is associated with less edema and an improved lipid profile relative to Rosi $8 \mathrm{mg}$ qd.

Supported by Novartis

0791

Efficacy of vildagliptin in drug-naive patients with type 2 diabetes

S. Dejager, M. A. Baron, S. Razac, J. E. Foley, S. Dickinson, A. Schweizer;

Novartis, E Hanover, United States.

Background and Aims: Vildagliptin is a DPP-4 inhibitor shown in Phase 2 studies to improve glycemic control by increasing pancreatic islet responsiveness to glucose. The purpose of the present trial was to assess the efficacy and tolerability of three vildagliptin monotherapy regimens in drug-naïve patients (pts) with type 2 diabetes (T2DM).

Materials and Methods: This was a 24-wk, multicenter, randomized, double-blind, parallel-group study of vildagliptin $50 \mathrm{mg}$ qd ( $\mathrm{n}=104), 50 \mathrm{mg}$ bid ( $\mathrm{n}=90), 100 \mathrm{mg}$ qd $(\mathrm{n}=92)$ and placebo (PBO, $\mathrm{n}=94)$ in drug-naïve pts with $\mathrm{HbA}_{1 \mathrm{c}}$ of $7.5-10 \%$. The primary endpoint was the betweengroup difference in the adjusted mean change $(A M \Delta)$ in $\mathrm{HbA}_{1 \mathrm{c}}$ from baseline (BL) to Wk 24 or study endpoint, comparing each active treatment to PBO by ANCOVA.

Results: The four treatment groups were well-balanced. At $\mathrm{BL}$, mean age $=53.6 \mathrm{y}, \mathrm{BMI}=32.8 \mathrm{~kg} / \mathrm{m}^{2}$, disease duration=2.0 y and $\mathrm{HbA}_{1 \mathrm{c}}=8.4 \%$. The between-group difference in $\mathrm{AM} \Delta \mathrm{HbA}_{1 \mathrm{c}}$ was $-0.5 \pm 0.1 \% \quad(\mathrm{P}=0.006)$, $-0.5 \pm 0.1 \%(\mathrm{P}=0.006)$ and $-0.6 \pm 0.1 \%(\mathrm{P}=0.001$ vs $\mathrm{PBO})$ in pts receiving vildagliptin $50 \mathrm{mg} \mathrm{qd}, 50 \mathrm{mg}$ bid and $100 \mathrm{mg}$ qd respectively. An unusual study effect of PBO 
was observed ( $\mathrm{AM} \Delta=-0.3 \pm 0.1 \%)$, largely driven by $16 \%$ of patients who were enrolled within 2 wks of diagnosis (Dx). In this small newly-diagnosed cohort, $\mathrm{HbA}_{1 \mathrm{c}}$ decreased by more than $1 \%$ in all treatment groups, with no significant difference between vildagliptin and PBO. However, in pts diagnosed $>2$ wks prior to enrollment, vildagliptin elicited significant decreases in $\mathrm{HbA}_{1 \mathrm{c}}$ and little PBO effect was observed. Since clinical trials have traditionally recruited patients with a $\mathrm{Dx}>3$ months, and physicians often employ this time frame prior to initiating pharmacotherapy (presumably for a trial of diet and exercise), data were evaluated using this criterion. In patients who were diagnosed $>3$ mos before enrollment $(60 \%)$ there was a small increase in $\mathrm{HbA}_{1 \mathrm{c}}$ in the $\mathrm{PBO}$ group $(\mathrm{AM} \Delta=0.2 \pm 0.2 \%)$ and the between-group difference in $\mathrm{AM} \triangle \mathrm{HbA}_{1 \mathrm{c}}$ was $-0.8 \pm 0.2 \%$ in the $50 \mathrm{mg}$ qd group $(\mathrm{P}<0.001),-0.7 \pm 0.2 \%$ in the 50 bid group $(\mathrm{P}=0.003)$ and $-0.9 \pm 0.2 \%$ in pts receiving $100 \mathrm{mg}$ qd $(\mathrm{P}<0.001$ vs $\mathrm{PBO})$. Overall, vildagliptin was well-tolerated. Mild hypoglycemia was reported by $1.2 \%, 0 \%, 0.6 \%$ and $0 \%$ of pts randomized to $50 \mathrm{mg} \mathrm{qd}, 50 \mathrm{mg}$ bid, $100 \mathrm{mg}$ qd and $\mathrm{PBO}$, respectively. There was a modest decrease in mean BW in each treatment group, ranging from an $\mathrm{AM} \Delta$ body weight of $-0.3 \mathrm{~kg}$ in the $50 \mathrm{mg}$ bid group to $-1.8 \mathrm{~kg}$ in the $50 \mathrm{mg}$ qd group.

Conclusions: 1) Vildagliptin produces clinically meaningful and statistically significant decreases in $\mathrm{HbA}_{1 \mathrm{c}}$ in drugnaïve patients; 2) Vildagliptin monotherapy is well tolerated; it does not cause weight gain and is associated with a low risk of hypoglycemia.

Supported by Novartis

0792

\section{WITHDRAWN}

0793

Vildagliptin added to metformin improves glycaemic control by decreasing fasting and postprandial glucose in patients with type 2 diabetes

E. Bosi ${ }^{1}$, C. Collober ${ }^{2}$, E. Rochotte ${ }^{2}$, R.-P. Camisasca ${ }^{2}$, A. J. Garber ${ }^{3}$;

${ }^{1}$ General Medicine, San Raffaele Univ Hosp, Milan, Italy,

${ }^{2}$ Novartis, Basel, Switzerland, ${ }^{3}$ Endo, Baylor, Houston, United States.

Background and Aims: Vildagliptin is a potent and selective DPP-4 inhibitor that improves glucose metabolism by increasing $\alpha$ - and $\beta$-cell responsiveness to glucose. The purpose of this study was to assess the efficacy and tolerability of vildagliptin when added to metformin treatment in patients (pts) with type 2 diabetes (T2DM).

Materials and Methods: This was a 24-wk, multicenter, double-blind, randomized, parallel group study comparing vildagliptin (50 $\mathrm{mg}$ qd or bid) to placebo (PBO) in 416 patients with $\mathrm{T} 2 \mathrm{DM}$ continuing a previous stable metformin regimen $(\geq 1500 \mathrm{mg} / \mathrm{d})$. The primary endpoint was the between-group difference in the adjusted mean change $\left(\mathrm{AM} \Delta\right.$ ) in $\mathrm{HbA}_{1 \mathrm{c}}$ from baseline (BL) to $\mathrm{Wk} 24$ or study endpoint, comparing both active treatments to $\mathrm{PBO}$ by ANCOVA.

Results: The 3 treatment groups were well-balanced. At BL mean age $=54.2 \pm 9.8 \mathrm{y}, \mathrm{BMI}=32.8 \pm 5.5 \mathrm{~kg} / \mathrm{m}^{2}$, disease duration $=6.2 \pm 5.2 \mathrm{y}, \mathrm{HbA}_{1 \mathrm{c}}=8.4 \pm 1.0 \%$, metformin dose $=$ $2109 \pm 315 \mathrm{mg} / \mathrm{d}$ and duration of metformin use $=17.2 \pm$ 21.3 mo. As shown below, in pts receiving vildagliptin, the mean $\mathrm{HbA}_{1 \mathrm{c}}$ and FPG decreased rapidly and progressively during the first $12 \mathrm{wks}$ of treatment, and appeared to reach a steady state at $\mathrm{Wk} 16$. The between-group difference in the $\mathrm{AM} \Delta \mathrm{HbA}_{1 \mathrm{c}}$ was $-0.7 \pm 0.1 \%$ in pts receiving vildagliptin $50 \mathrm{mg}$ qd $(\mathrm{P}<0.001 \mathrm{vs} P B O)$ and $-1.1 \pm 0.1 \%$ in pts receiving $50 \mathrm{mg}$ bid $(\mathrm{P}<0.001 \mathrm{vs} \mathrm{PBO})$. The proportion of pts reaching target $\mathrm{HbA}_{1 \mathrm{c}}<7.0 \%$ was 34-fold higher in the vildagliptin groups than in the $\mathrm{PBO}$ group $(\mathrm{P}<0.001)$. The between-group difference in the $\mathrm{AM} \Delta$ in $\mathrm{FPG}$ was $-0.8 \pm 0.3 \mathrm{mM}(\mathrm{P}=0.003$ vs $\mathrm{PBO})$ and $-1.7 \pm 0.3 \mathrm{mM}(\mathrm{P}<0.001$ vs $\mathrm{PBO})$ in pts receiving vildagliptin $50 \mathrm{mg}$ qd and $50 \mathrm{mg}$ bid, respectively.

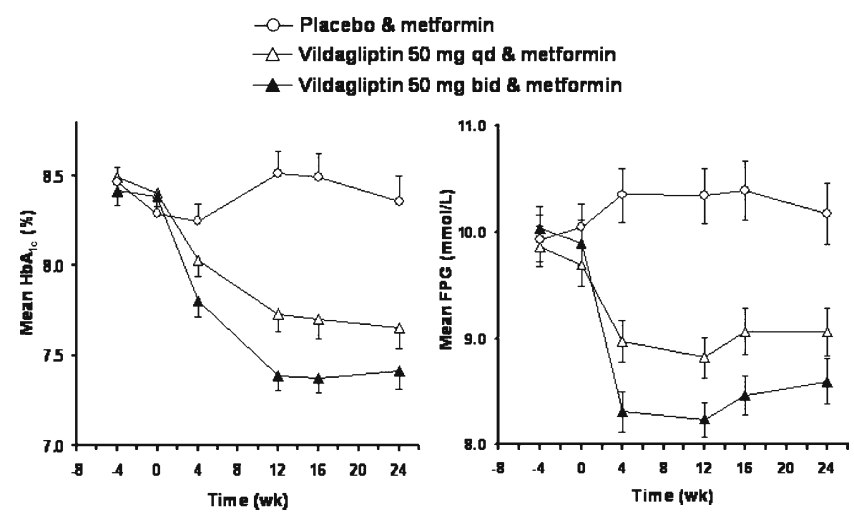

A standard meal test was performed in pts volunteering to participate $(40 \%, \sim 55 \mathrm{pts} /$ group). Relative to $\mathrm{PBO}$, the $2-$ $\mathrm{h}$ postprandial glucose (PPG) level was significantly decreased in vildagliptin-treated pts. The between-group difference in $\mathrm{AM} \triangle \mathrm{PPG}$ was $-1.9 \pm 0.6 \mathrm{mM}$ in the $50 \mathrm{mg} \mathrm{qd}$ group $(\mathrm{P}=0.001 \mathrm{vs} \mathrm{PBO})$ and $-2.3 \pm 0.6 \mathrm{mM}$ in the $50 \mathrm{mg}$ bid group ( $\mathrm{P}<0.001$ vs $\mathrm{PBO}$ ). This was associated with an improvement in several measures of $\beta$-cell function. There was no weight gain in the vildagliptin groups and the incidence of side effects did not differ between groups (63.3, 65.0 and $63.5 \%$ of pts randomized to vildagliptin 
$50 \mathrm{mg} \mathrm{qd}, 50 \mathrm{mg}$ bid and PBO, respectively). Notably, $9.6 \%, 14.8 \%$ and $18.2 \%$ of pts receiving vildagliptin $50 \mathrm{mg}$ $\mathrm{qd}, 50 \mathrm{mg}$ bid and $\mathrm{PBO}$ experienced $\geq 1$ gastrointestinal side effect and the incidence in the vildagliptin $50 \mathrm{mg}$ qd group was significantly lower $(\mathrm{P}=0.022)$ than in the $\mathrm{PBO}$ group. One hypoglycemic event occurred in each group.

Conclusions: Vildagliptin is well-tolerated and produces clinically meaningful, dose-related decreases in FPG, PPG and $\mathrm{HbA}_{1 \mathrm{c}}$ in metformin-treated patients with T2DM.

Supported by Novartis

\section{4}

Efficacy and safety of sitagliptin over 18 weeks in patients with type 2 diabetes

I. $\mathbf{R a z}^{1}$, M. Hanefeld ${ }^{2}$, L. Xu ${ }^{3}$, C. Caria ${ }^{3}$,

A. G. Meehan ${ }^{3}$, D. Williams-Herman ${ }^{3}$, H. Khatami ${ }^{3}$;

${ }^{1}$ Diabetes Research Center, Hadassah University Hospital, Jerusalem, Israel, ${ }^{2}$ Centre for Clinical Studies, Dresden Technical University, Germany, ${ }^{3}$ Merck \& Co. Inc., Rahway, United States.

Background and Aims: Sitagliptin is a new, orally-active, potent and selective dipeptidyl peptidase-4 (DPP-4) inhibitor currently in development for the treatment of type 2 diabetes. Sitagliptin acts by blocking DPP-4-mediated inactivation of active incretin hormones that are released into circulation following ingestion of a meal. Incretins increase insulin release and lower glucagon secretion in a glucose-dependent manner, leading to a suppression of post-meal increases in glucose concentration and a reduction in fasting glucose concentrations. In addition to improving glycemic control, sitagliptin treatment may improve pancreatic $\beta$-cell function and may also lead to $\beta$-cell preservation. The present randomized, double-blind, placebo(PBO)-controlled, Phase III study assessed the efficacy and safety of once-daily sitagliptin in patients with type 2 diabetes.

Material and methods: After a washout period for patients on an antihyperglycemic agent, patients with $\mathrm{HbA}_{1 \mathrm{c}} \geq 7 \%$ and $\leq 10 \%$ entered a 2 -week single-blind run-in period, at the completion of which, 521 patients ages 27 to $76 \mathrm{yrs}$ were randomized in a 1:2:2 ratio to $\mathrm{PBO}$, sitagliptin $100 \mathrm{mg}$ q.d., or $200 \mathrm{mg}$ q.d., for 18 weeks. Patients not meeting pre-specified glycemic thresholds were provided rescue therapy (metformin) until study completion. Mean baseline $\mathrm{HbA}_{1 \mathrm{c}}$ for the study population was $8.1 \%$. The efficacy analysis was based on an all-patients-treated population using an ANCOVA and excluded data obtained after glycemic rescue.

Results: After 18 weeks, $\mathrm{HbA}_{1 \mathrm{c}}$ was significantly reduced with sitagliptin $100 \mathrm{mg}$ and $200 \mathrm{mg}$ compared with PBO (mean PBO-subtracted change from baseline in $\mathrm{HbA}_{1 \mathrm{c}}$ : $-0.60 \%$ and $-0.48 \%$, respectively). Patients with higher baseline $\mathrm{HbA}_{1 \mathrm{c}}(\geq 9 \%)$ experienced greater PBO-subtracted reductions in $\mathrm{HbA}_{1 \mathrm{c}}$ with sitagliptin treatment $(-1.20 \%$ for $100 \mathrm{mg}$ and $-1.04 \%$ for $200 \mathrm{mg}$ ) than those with baseline $\mathrm{HbA}_{1 \mathrm{c}}<8 \%(-0.44 \%$ and $-0.33 \%$, respectively) or $8 \%$ to $8.9 \%(-0.61 \%$ and $-0.39 \%$, respectively). Sitagliptin significantly decreased fasting plasma glucose (FPG) relative to $\mathrm{PBO}$ after 18 weeks (mean $\mathrm{PBO}$-subtracted change from baseline in FPG: $-1.09 \mathrm{mmol} / \mathrm{L}$ for $100 \mathrm{mg}$ and $-0.94 \mathrm{mmol} / \mathrm{L}$ for $200 \mathrm{mg}$ ). In a subset of patients who completed a meal tolerance test $(n=150), 2$-hour post-meal glucose (PPG) was significantly reduced with with both doses of sitagliptin compared with PBO (mean PBOsubtracted change from baseline in 2-hour PPG: $-2.57 \mathrm{mmol} / \mathrm{L}$ and $-2.92 \mathrm{mmol} / \mathrm{L}$, respectively). There were no significant differences between the two sitagliptin doses for their effects on $\mathrm{HbA}_{1 \mathrm{c}}$, FPG, or PPG. HOMA- $\beta$ was significantly increased for both sitagliptin doses, and the fasting proinsulin/insulin ratio was significantly decreased with sitagliptin $100 \mathrm{mg}$. Sitagliptin was well tolerated with no significant increased incidence of hypoglycemia or gastrointestinal adverse experiences with sitagliptin compared to PBO. Mean body weight was similarly reduced with sitagliptin $100 \mathrm{mg}(-0.6 \mathrm{~kg})$, $200 \mathrm{mg}(-0.2 \mathrm{~kg})$, and PBO $(-0.7 \mathrm{~kg})$ after 18 weeks.

Conclusion: In this study, sitagliptin monotherapy improved glycemic control as well as measures of $\beta$-cell function and was well-tolerated over 18 weeks in patients with type 2 diabetes.

Supported by Merck \& Co. Inc.

\section{5}

Sitagliptin, a DPP-4 inhibitor: an overview of the pharmacokinetic (PK) profile and the propensity for drug-drug interactions (DDI)

G. Herman, A. Bergman, J. A. Wagner;

Merck \& Co. Inc., Rahway, United States.

Background and Aims: Patients with type 2 diabetes are usually on polypharmacy to treat hyperglycemia and other co-mordib conditions. The PK profile and propensity for DDI were extensively examined during the development of sitagliptin.

Materials: Twenty-seven Phase I studies that characterized the PK and pharmacodynamic characteristics and initial safety and tolerability of sitagliptin were conducted in healthy subjects and patients with type 2 diabetes.

Results: Sitagliptin exposure (AUC) increases dose proportionally with increasing dose. At 100-mg dose, apparent terminal elimination half-life is $\sim 10$ to $12 \mathrm{hrs}$. Steady-state 
is generally reached by 3 days and accumulation is slight ( $\sim 14 \%$ following 100-mg dose). There is no effect of food on PK. Sitagliptin is well absorbed, with an absolute bioavailability of $87 \%$. Sitagliptin is primarily renally eliminated as unchanged drug ( $\sim 75 \%$ of oral dose excreted unchanged in urine), with metabolism playing only a minor role (primarily cytochrome P-450 (CYP) 3A4 mediated). Given a renal clearance of $\sim 350 \mathrm{~mL} / \mathrm{min}$, active tubular secretion appears to be involved in the elimination of sitagliptin, but it is not definitively known which transporters are involved in this process in vivo. In vitro studies suggest that the organic anionic transporter-3 may play a role, but organic cationic transporters (OCT) and peptide transporter-1 transporters do not transport sitagliptin. Sitagliptin is a human p-glycoprotein (Pgp) substrate, and thus Pgp may influence the absorption and elimination of sitagliptin. However, the observed modest effects of supratherapuetic doses of cyclosporine A (AUC increased $\sim 29 \%$ ), a potent probe Pgp inhibitor, on sitagliptin PK suggest that the potential for clinically meaningful Pgpmediated drug interactions is limited. In vitro, sitagliptin is not an inhibitor of CYP enzymes and is not an inducer of CYP3A4. In clinical studies, sitagliptin did not meaningfully alter the PK of metformin, rosiglitazone, glyburide, simvastatin, warfarin, or oral contraceptives providing further in vivo evidence for a low propensity for perpetrating drug interactions with substrates of hOCT, CYP3A4, CYP2C8, and CYP2C9. Multiple doses of sitagliptin 100 and $200 \mathrm{mg}$ slightly increased plasma immunoreactive digoxin concentrations $\sim(11 \%$ and $\sim 18 \%$ increase in AUC, respectively), however, these increases were not considered likely to be clinically meaningful. Sitagliptin concentrations are not meaningfully altered by metformin. Sitagliptin exposure is increased by approximately $2.3,3.8$, and 4.5 fold relative to healthy subjects for patients with moderate renal insufficiency $[\mathrm{RI}]$ (creatinine clearance $[\mathrm{CrCl}]=30$ to $<50 \mathrm{~mL} / \mathrm{min})$, severe $\mathrm{RI}(\mathrm{CrCl}<30 \mathrm{~mL} / \mathrm{min})$, and ESRD (on dialysis), respectively. At doses of $100 \mathrm{mg}$ or higher, weighted average plasma DPP-4 inhibition was $80 \%$ or greater and produced 2 to 3 -fold increases in active glucagon-like peptide-1 and glucose-dependent insulinotropic peptide levels over 24 hours postdose. Sitagliptin was generally well-tolerated in the various patient cohorts studied. In a population PK analysis of Phase I and IIb studies, RI was the only factor found to have a potentially clinically relevant effect on PK. Concomitant medications $(\mathrm{n}=83)$ were also screened for potential effects on sitagliptin PK; no clinically relevant DDIs were detected.

Conclusion: Sitagliptin has the PK profile consistent with once-daily dosing and a low propensity to be involved in DDI as either a perpetrator or a victim.

Supported by Merck \& Co. Inc.
0796

Insulinotropic actions of nateglinide are accompanied by inhibition of dipeptidyl peptidase-IV activity

A. M. McKillop ${ }^{1}$, N. A. Duffy ${ }^{1}$, J. R. Lindsay ${ }^{2}$, B. D. Green ${ }^{1}$, F. P. M. O'Harte ${ }^{1}$, P. M. Bell ${ }^{2}$, P. R. Flatt ${ }^{1}$; ${ }^{1}$ School of Biomedical Sciences, University of Ulster, Coleraine, ${ }^{2}$ Regional Centre for Endocrinology and Diabetes, Royal Victoria Hospital, Belfast, United Kingdom.

Background and Aims: Nateglinide restores early phase insulin secretion to feeding and reduces postprandial hyperglycaemia in type 2 diabetes. Gastric inhibitory polypeptide (GIP) and glucagon-like peptide-1(7-36)amide (GLP-1), natural mediators of enhanced postprandial insulin secretion, are rapidly cleaved by dipeptidyl peptidase (DPP-IV) resulting in rapid clearance from the circulation. This study examined the effect of nateglinide on DPP-IV activity and incretin hormone degradation in type 2 diabetic patients.

Materials and Methods: DPP-IV activity in type 2 diabetic subjects $\left(\mathrm{n}=10\right.$, age $57.1 \pm 1.9$ y, BMI $34.7 \pm 2.6 \mathrm{~kg} / \mathrm{m}^{2}$, fasting glucose $9.3 \pm 1.2 \mathrm{mmol} / \mathrm{l}$ ) was measured following administration of oral nateglinide $(120 \mathrm{mg})$ or placebo at time $\mathrm{t}-10 \mathrm{~min}$ followed by a $75 \mathrm{~g}$ oral glucose load at t0 $\mathrm{min}$ in a randomised cross-over design. Other parameters measured included $\mathrm{HbA}_{1 \mathrm{c}}$, plasma glucose, insulin and Cpeptide concentrations. Effects of nateglinide on GIP degradation were further investigated in pooled human plasma using reverse-phase HPLC and electrospray ionization mass spectrometry.

Results: The mean activity of DPP-IV in the placebo group $\left(\mathrm{HbA}_{1 \mathrm{c}} 7.3 \pm 0.6 \%\right.$, glucose $9.6 \pm 1.4 \mathrm{mmol} / \mathrm{l}$, insulin $99.8 \pm$ $17.7 \mathrm{pmol} / \mathrm{l})$ and in the nateglinide group $\left(\mathrm{HbA}_{1 \mathrm{c}} 7.9 \pm 0.9 \%\right.$, glucose $9.1 \pm 1.0 \mathrm{mmol} / 1$, insulin $108.9 \pm 19.8 \mathrm{pmol} / \mathrm{l}$ ) prior to treatment was $27.4 \pm 0.7$ and $29.6 \pm 2.3 \mathrm{nmol} / \mathrm{ml} / \mathrm{min}$, respectively. Following nateglinide, DPP-IV activity was reduced after $10 \mathrm{~min}(19.9 \pm 1.6 \mathrm{nmol} / \mathrm{ml} / \mathrm{min}, \mathrm{p}<0.05)$, reaching a minimum of $1.9 \pm 0.1 \mathrm{nmol} / \mathrm{ml} / \mathrm{min}$ at $120 \mathrm{~min}(\mathrm{p}<0.001)$. Mean DPP-IV activity in the placebo group was $22.5 \pm 0.6 \mathrm{nmol} / \mathrm{ml} / \mathrm{min}$ and decreased to a minimum at $40 \mathrm{~min}(18.9 \pm 0.6 \mathrm{nmol} / \mathrm{ml} / \mathrm{min})$ and $120 \mathrm{~min}$ $(18.3 \pm 0.4 \mathrm{nmol} / \mathrm{ml} / \mathrm{min})$. Circulating insulin and C-peptide levels increased in the nateglinide group to $637.6 \pm$ $163.9 \mathrm{pmol} / \mathrm{l}(\mathrm{p}<0.05)$ and $11.8 \pm 1.4 \mathrm{mg} / \mathrm{l}(\mathrm{p}<0.01)$, respectively, compared to placebo. Plasma glucose reached $18.2 \pm 1.7 \mathrm{mmol} / \mathrm{l}$ and $16.7 \pm 1.7 \mathrm{mmol} / 1$ at $90 \mathrm{~min}$ in the placebo and nateglinide groups, respectively $(\mathrm{p}<0.001)$. Percentage intact GIP(1-42) remaining in nateglinidetreated type 2 diabetic plasma at 2,4 and $8 \mathrm{~h}$ incubations was $93 \pm 2.2 \%, 83 \pm 0.0 \%(p<0.05)$ and $64 \pm 1.0 \%(p<0.01)$, and was higher compared with placebo $(91 \pm 0.4 \%, 72 \pm 1.4 \%$ $(p<0.05)$ and $38 \pm 0.7 \%(p<0.01))$, respectively. Nateglinide 
inhibited the degradation of $\operatorname{GIP}(1-42)$ at 2,4 and $8 \mathrm{~h}$ in human plasma, at concentrations of $62.5 \mu \mathrm{M}$ by $79.1 \pm 1.6 \%$ $(\mathrm{p}<0.05), 64.7 \pm 1.5 \% \quad(\mathrm{p}<0.05), 48.0 \pm 3.1 \% \quad(\mathrm{p}<0.001)$, $125 \mu \mathrm{M}$ by $81.5 \pm 2.2 \% \quad(\mathrm{p}<0.05), 73.0 \pm 1.7 \% \quad(\mathrm{p}<0.01)$, $64.0 \pm 2.0 \%(\mathrm{p}<0.001)$ and $250 \mu \mathrm{M}$ by $100 \pm 0 \%(\mathrm{p}<0.001)$, $100 \pm 0 \%(\mathrm{p}<0.001), 70.0 \pm 1.7 \%(\mathrm{p}<0.001)$, respectively.

Conclusion: DPP-IV activity was inhibited by oral nateglinide in type 2 diabetic subjects with reduced degradation of GIP(1-42) to its inactive N-terminally truncated form, GIP (3-42). This action may play a role in the activity of prandial insulin-releaser agents.

\section{PS 62}

\section{Oral agents: new drugs and new combinations}

0797

\section{Metformin gum: an alternate method for delivery of biguanides \\ M. Guevara-Aguirre, J. Guevara-Aguirre, \\ D. Saldarreaga, J. Saavedra; \\ IEMYR, Instituto de Endocrinologia, Quito, Ecuador.}

Background and Aims: Metformin (Met) is widely used in the treatment of Diabetes Mellitus due to its efficacy, comparable to that of sulfonylureas, but devoid of side effects such as weight gain and hypoglycemia. Weight loss seen with the use of Met suggests an anoretic mechanism and has been associated to decreases in caloric expenditure, reductions of leptin levels and other findings. Despite its beneficial actions, approximately $30 \%$ of Met users develop gastrointestinal (GI) adverse effects. The aim of this study was to compare the pharmacokinetic (PK) profiles of Met administered by both the traditional manner (tablets) and by an alternative presentation (Met-Gum), that can be slowly chewed, ingested and that supposedly diminish the GI adverse effects seen with present preparations.

Materials and Methods: We studied 10 healthy volunteers (6 M, 4 F) mean age 30 and 29.8; mean BMI 23.9 and 21.49. Met tablets (Glucophage $850 \mathrm{mg}$ Merck) were purchased; Met-Gum (214.5 mg/unit) were donated by Generex Biotechnology, Canada. Met concentrations were sequentially measured in: a) a 12-hour period after administration of an 850 Met tablet; b) a 24-hour period after 429 Met-Gum (2 units); and c) a 24-hour period after 429 tablet (fraction of the larger tablet) administration. Plasma Met concentrations were determined by HPLC (HPLC time: 6 minutes; retention time of Met and internal standard atenolol: 3.4 and 2.9 minutes respectively. Frozen $\left(-70^{\circ}\right)$ experimental specimens were unthawed for 1 hour before the assay. Removal of proteins and lipids from the biological matrix were achieved prior to HPLC.

Results: Results are shown in the graph.

Conclusion: The Metformin Gum displays a PK profile similar to that of tablets and shows promise as an alternative method for delivery of this biguanide.

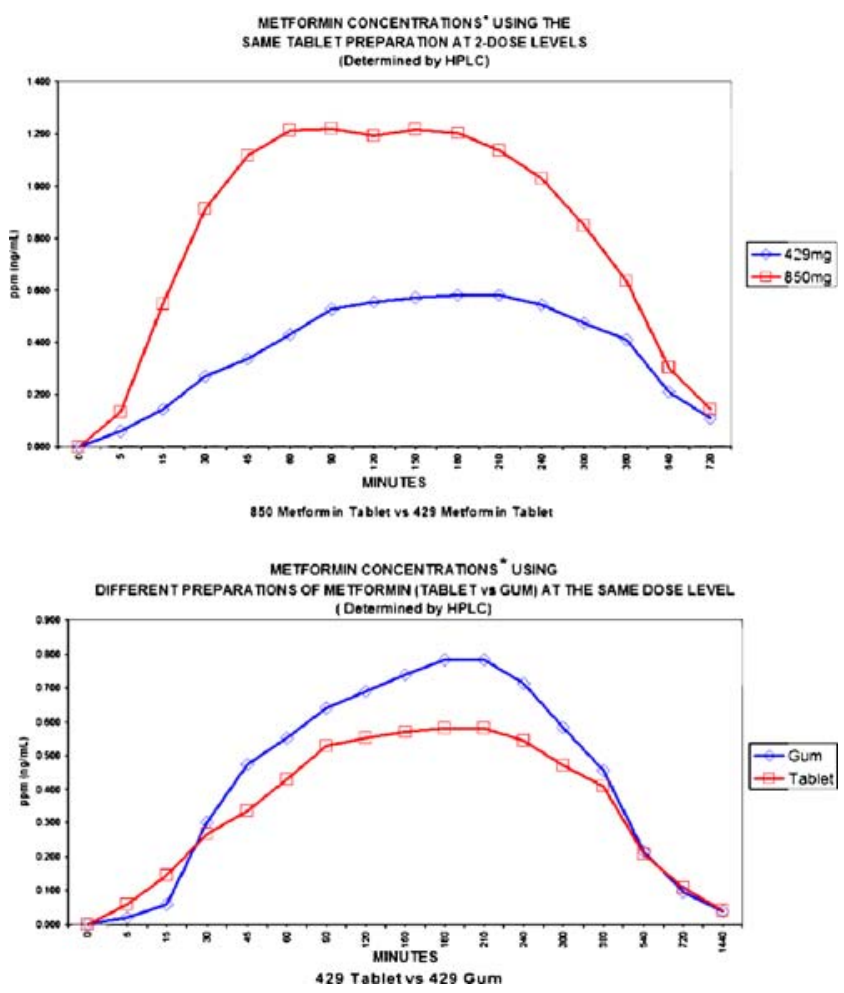

Supported by Generex Biotechnology Corp.

0798

Rimonabant improves cardiometabolic risk factors in overweight/obese patients with poorly controlled type 2 diabetes $\left(\mathrm{HbA}_{1 \mathrm{c}} \geq \mathbf{8 \%}\right)$ on monotherapy with metformin or sulfonylureas

A. Scheen ${ }^{1}$, N. Finer ${ }^{2}$, M. Jensen ${ }^{3}$, P. Hollander ${ }^{4}$, L. Van $\mathrm{Gaal}^{5}$;

${ }^{1}$ Division of Diabetes, Nutrition \& Metabolic Disorders, CHU Sart Tilman, Liège, Belgium, ${ }^{2}$ Addenbrooke's Hospital, University of Cambridge, United Kingdom, ${ }^{3}$ Endocrine Research Unit, Mayo Clinic, Rochester, United States, ${ }^{4}$ Ruth Collins Diabetes Center, Baylor University Medical Center, Dallas, United States, ${ }^{5}$ Dept of Diabetology, Antwerp University Hospital, Belgium. 
Background and Aims: Rimonabant, the first selective $\mathrm{CB}_{1}$ receptor blocker, significantly improved $\mathrm{HbA}_{1 \mathrm{c}}$, fasting glucose, triglycerides, HDL-cholesterol, body weight and waist circumference in a randomised clinical trial in overweight/obese patients with type 2 diabetes treated with metformin $(65 \%)$ or sulfonylureas $(35 \%)$ (RIO-Diabetes [N=1047]). Baseline $\mathrm{HbA}_{1 \mathrm{c}}$ was $7.3 \%$ in this study. At 1 year, changes in mean $\mathrm{HbA}_{1 \mathrm{c}}$ were $-0.1 \%$ and $-0.6 \%$ with rimonabant 5 and $20 \mathrm{mg} /$ day vs $+0.1 \%$ for placebo $(\mathrm{p}=0.034$ and $\mathrm{p}<0.001$ vs placebo, respectively). In this analysis, the benefits of rimonabant in the subset of patients with poor glycaemic control $\left(\mathrm{HbA}_{1 \mathrm{c}} \geq 8 \%\right)$ were assessed.

Material and Methods: We evaluated the efficacy and tolerability of rimonabant therapy in patients with $\mathrm{HbA}_{1 \mathrm{c}}$ $<8 \%(\mathrm{~N}=833)$ or $\geq 8 \%(\mathrm{~N}=188)$ at baseline. All patients received a hypocaloric diet $(600 \mathrm{kcal} /$ day deficit) throughout the study in addition to study treatment with either placebo or rimonabant $(5 \mathrm{mg} /$ day or $20 \mathrm{mg} /$ day).

Results: Compared with placebo, rimonabant $20 \mathrm{mg} / \mathrm{day}$ significantly decreased body weight and waist circumference from baseline to 1 year in both patient subgroups, as well as improving glycaemic and lipid risk factors (Table). In the $\mathrm{HbA}_{1 \mathrm{c}} \geq 8 \%$ group, $30.2 \%$ of patients receiving rimonabant $20 \mathrm{mg}$ /day had $\mathrm{HbA}_{1 \mathrm{c}}<7 \%$ at 1 year compared with $11.1 \%$ of those receiving placebo $(\mathrm{p}=0.012)$. The corresponding proportions of patients with $\mathrm{HbA}_{1 \mathrm{c}}<6.5 \%$ were $14.3 \%$ and $1.9 \%(\mathrm{p}=0.02)$. Within the $\mathrm{HbA}_{1 \mathrm{c}}<8 \%$ and $\geq 8 \%$ groups, respectively, $5.4 \%$ and $6.6 \%$ of patients receiving placebo discontinued therapy as a result of a treatment-emergent adverse events compared with $15.6 \%$ and $13.6 \%$ of patients receiving rimonabant $20 \mathrm{mg} /$ day. Among the adverse events leading to discontinuation with rimonabant $20 \mathrm{mg} /$ day, those already reported in the previous papers, i.e. psychiatric disorders, nervous system disorders and gastrointestinal disorders, were reported with the following frequency in patients with $\mathrm{HbA}_{1 \mathrm{c}}<8 \%$ or $\geq 8 \%$, respectively: $6.3 \%$ vs $1.5 \% ; 1.9 \%$ vs $4.5 \%$ and $3.7 \%$ vs $0 \%$.

Conclusion: Rimonabant $20 \mathrm{mg}$ /day improved glycaemic control irrespective of patients' baseline $\mathrm{HbA}_{1 \mathrm{c}}$ levels. In patients with $\mathrm{HbA}_{1 \mathrm{c}} \geq 8 \%$ (who were already receiving metformin or a sulfonylurea) reduction from baseline in $\mathrm{HbA}_{1 \mathrm{c}}$ of $1.1 \%$ was observed with rimonabant $20 \mathrm{mg} /$ day, along with significant improvements in HDL-cholesterol and triglycerides. Rimonabant $20 \mathrm{mg} /$ day was generally well tolerated. These findings support the use of rimonabant $20 \mathrm{mg}$ /day for improving glycaemic control and reducing cardiometabolic risk in overweight/obese patients with type 2 diabetes.

\begin{tabular}{|c|c|c|c|c|}
\hline & \multicolumn{2}{|c|}{$\mathrm{HbA}_{1 \mathrm{c}}<8 \%$} & \multicolumn{2}{|c|}{$\mathrm{HbA}_{1 \mathrm{c}} \geq 8 \%$} \\
\hline & Placebo & $\begin{array}{l}\text { Rimonabant } \\
20 \mathrm{mg} / \text { day }\end{array}$ & Placebo & $\begin{array}{l}\text { Rimonabant } \\
20 \mathrm{mg} / \text { day }\end{array}$ \\
\hline $\begin{array}{l}\text { Mean change } \\
\text { from baseline } \\
\text { at } 1 \text { year in: }\end{array}$ & $(\mathrm{N}=279)$ & $(\mathrm{N}=269)$ & $(\mathrm{N}=61)$ & $(\mathrm{N}=66)$ \\
\hline Body weight (kg) & -1.4 & $-5.5 * * *$ & -1.6 & $-4.9 * * *$ \\
\hline $\begin{array}{l}\text { Waist circumference } \\
(\mathrm{cm})\end{array}$ & -1.9 & $-5.5^{* * *}$ & -2.0 & $-4.3^{*}$ \\
\hline $\mathrm{HbA}_{1 \mathrm{c}}(\%)$ & 0.2 & $-0.5^{* * *}$ & -0.3 & $-1.1 * * *$ \\
\hline $\begin{array}{l}\text { Fasting glucose } \\
(\mathrm{mmol} / \mathrm{L})\end{array}$ & 0.5 & $-0.4 * * *$ & -0.3 & $-1.7^{*}$ \\
\hline $\begin{array}{l}\text { Fasting insulin } \\
(\mu \mathrm{IU} / \mathrm{mL})\end{array}$ & 0.6 & -0.5 & 0.4 & -1.3 \\
\hline Triglycerides (\%) & 8.9 & $-6.2 * * *$ & -0.4 & $-21.3^{* * *}$ \\
\hline $\begin{array}{l}\text { HDL-cholesterol } \\
(\%)\end{array}$ & 7.2 & $15.4 * * *$ & 7.1 & $16.3^{*}$ \\
\hline
\end{tabular}

Data are for ITT population, last observation carried forward.

${ }^{*} \mathrm{p}<0.05 ;{ }^{* * *} \mathrm{p} \leq 0.001$ vs placebo.

Supported by sanofi-aventis

0799

\section{Rosiglitazone stimulates AMP-activated protein kinase mediated nitric oxide synthesis in cultured human aortic endothelial cells}

J. G. Boyle, S. A. Ritchie, J. M. C. Connell, S. J. Cleland, I. P. Salt;

The Henry Wellcome Laboratory of Cell Biology and the Division of Cardiovascular \& Medical Sciences, University of Glasgow, United Kingdom.

Endothelial dysfunction, as assessed by reduced nitric oxide (NO) bioavailability, is associated with Type 2 diabetes and vascular disease. Thiazolidinediones improve insulin resistance and are used in the management of Type 2 diabetes. Incubation of cultured endothelial cells with thiazolidinediones has been reported to stimulate NO production but the precise mechanism remains uncertain. It has been recently demonstrated that AMP-activated protein kinase (AMPK) regulates NO synthesis in cultured endothelial cells via phosphorylation of Ser1177 on eNOS. We have determined the effects of thiazolidinediones on AMPK activity and NO synthesis in cultured human aortic endothelial cells. Incubation of endothelial cells with the thiazolidinedione, rosiglitazone led to a rapid, dose-dependent stimulation of NO synthesis. Moreover, rosiglitazone stimulated AMPK activity, phosphorylation of eNOS at Ser1177 and phosphorylation of acetyl-CoA carboxylase. Rosiglitazone stimulated NO synthesis was inhibited in 
cells infected with adenovirus expressing a dominantnegative AMPK mutant. Rosiglitazone-stimulated AMPK activity was not inhibited by the PPAR $\gamma$ inhibitor, GW9662, indicating that the effect of rosiglitazone on AMPK activity was independent of PPAR $\gamma$. These studies demonstrate that thiazolidinedione drugs stimulate NO synthesis through an AMPK-dependent mechanism in cultured human aortic endothelial cells. Further investigation of the way in which thiazolidinediones affect intracellular signaling in vascular endothelial cells may facilitate more selective treatments for the management of diabetes and vascular disease.

Supported by British Heart Foundation

0800

Tesaglitazar improves glucose and lipid responder rates and goal attainment in patients with type 2 diabetes

B. Goldstein ${ }^{1}$, J. Rosenstock ${ }^{2}$, D. Anzalone ${ }^{3}$, C. Tou ${ }^{3}$, P. Öhman ${ }^{3}$;

${ }^{1}$ Department of Medicine, Jefferson Medical College, Thomas Jefferson University, Philadelphia, United States,

${ }^{2}$ Dallas Diabetes and Endocrine Center, Medical City, Dallas, United States, ${ }^{3}$ AstraZeneca, Wilmington, United States.

Background and Aims: Tesaglitazar (GALIDA ${ }^{\mathrm{TM}}$ ) is a dual PPAR $\alpha / \gamma$ agonist in development for the treatment of glucose and lipid abnormalities associated with type 2 diabetes and insulin resistance. GLAD (Glucose and Lipid Assessment in Diabetes) was a randomized, double-blind, placebo-controlled dose-finding study of tesaglitazar in type 2 diabetes which included a pre-planned analysis to determine responder rates and goal attainment for fasting plasma glucose (FPG) and lipid variables.

Materials and Methods: Patients received once-daily tesaglitazar $(0.1,0.5,1.0,2.0$ or $3.0 \mathrm{mg})$, placebo or open-label pioglitazone $45 \mathrm{mg}$ (as a therapeutic benchmark) for 12 weeks. Responder rates were defined a-priori as the proportion of patients achieving minimum changes in FPG $(-1.7 \mathrm{mmol} / \mathrm{L})$, triglycerides $(\mathrm{TG} ;-30 \%)$ and HDL-C $(+15 \%)$ from baseline to treatment end. The proportion of FPG responders in each tesaglitazar dose and placebo were compared using a 2-sided Fisher's Exact test at the 5\% significance level while lipid responders were summarized descriptively. The goal attainment target for FPG was based on the level for diagnosis of type 2 diabetes (FPG $<7.0 \mathrm{mmol} / \mathrm{L}$ ) and lipid goals were $\mathrm{TG}<1.7 \mathrm{mmol} / \mathrm{L}$ and HDL-C $>1.0 \mathrm{mmol} / \mathrm{L}$. Goal attainment data were summarized descriptively.

Results: Baseline characteristics for randomized patients $(\mathrm{n}=500)$ were similar between treatment groups: FPG 9.5 (SD 1.9) mmol/L; TG 2.1 (1.1) mmol/L; HDL-C $1.1(0.3)$
mmol/L; BMI 30.7 (4.7) kg/m². Tesaglitazar $1.0 \mathrm{mg}$ resulted in statistically significant reductions in FPG of $2.28 \mathrm{mmol} / \mathrm{L}$, in TG of $32.9 \%$ and increases in HDL-C of $15.0 \%$. At baseline $3.7 \%, 41.0 \%$ and $53.8 \%$ of patients were already within FPG, TG and HDL-C goals, respectively. Tesaglitazar dose-dependently increased FPG responder rates, which were significantly greater for tesaglitazar $\geq 0.5 \mathrm{mg}$ vs placebo $(P<0.0001)$ (Table). Dosedependent improvements in responder rates were also shown for TG and HDL-C following treatment with tesaglitazar and these improvements were greater than placebo for tesaglitazar doses of $0.5 \mathrm{mg}$ and above (Table). Greater proportions of tesaglitazar-treated patients attained glucose and lipid treatment goals than placebo recipients (Table). Responder and goal attainment rates were numerically similar for FPG and higher for lipid variables with tesaglitazar $1.0 \mathrm{mg}$ than with pioglitazone $45 \mathrm{mg}$.

Conclusions: Tesaglitazar treatment produced marked, dose-dependent increases in responder rates and in goal attainment for glucose and lipid measures in diabetic patients. The $0.5-$ and $1.0-\mathrm{mg}$ doses of tesaglitazar are being studied in Phase III trials in patients with type 2 diabetes.

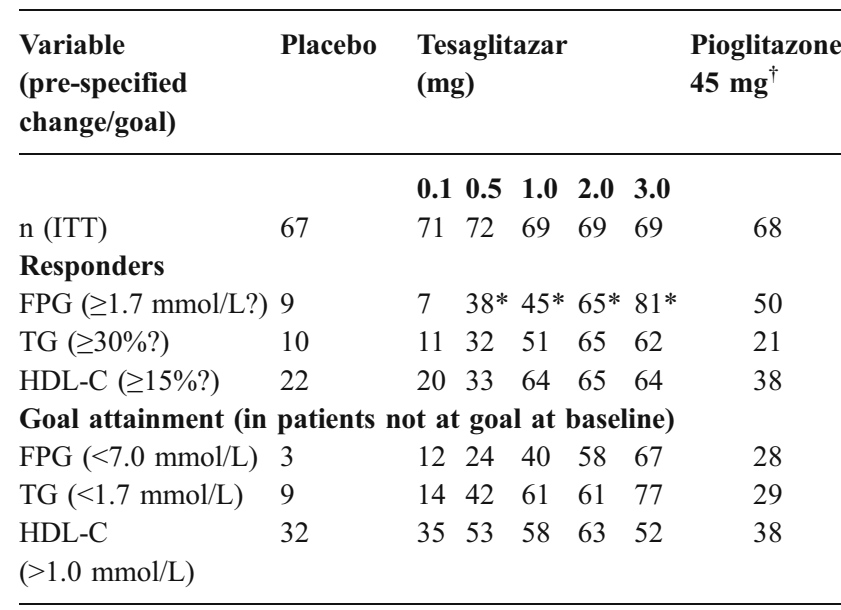

$* P<0.0001$

${ }^{\dagger}$ No statistical comparisons were made between pioglitazone and other groups

Supported by AstraZeneca

0801

Efficacy of vildagliptin combined with pioglitazone in patients with type 2 diabetes

M. A. Baron ${ }^{1}$, J. Rosenstock ${ }^{2}$, B. Bassiri ${ }^{3}$, E. Rochette ${ }^{3}$, F. $\mathrm{Cressier}^{3}$, Y. Amiour ${ }^{3}$, C. $\mathrm{Hsu}^{3}$, R.-P. Camisasca ${ }^{3}$, S. Dejager ${ }^{3}$, A. LeBeaut ${ }^{3}$; 
${ }^{1}$ Diabetes/Endocrinology, Dallas Diabetes \& Endocrine Center, Dallas, United States, ${ }^{2}$ Novartis, Basel, Switzerland.

Background and Aims: Vildagliptin is a DPP-4 inhibitor that improves glycemic control by increasing pancreatic $\alpha$ and $\beta$-cell responsiveness to glucose. Vildagliptin is effective and well-tolerated when given as monotherapy or in combination with metformin, but its effects when combined with a thiazolidinedione (TZD) are unknown. The aim of the present study was to assess the efficacy and tolerability of vildagliptin combined with pioglitazone (Pio) in patients with type 2 diabetes (T2DM)

Materials and Methods: Two 24-wk, multicenter, randomized, double-blind, parallel group studies were performed. Study 1 compared vildagliptin $100 \mathrm{mg}$ qd, Pio $30 \mathrm{mg} \mathrm{qd}$, vildagliptin $100 \mathrm{mg}$ qd+Pio $30 \mathrm{mg}$ qd and vildagliptin $50 \mathrm{mg}$ qd+Pio $15 \mathrm{mg}$ qd in drug-naïve patients with T2DM. Study 2 compared vildagliptin $50 \mathrm{mg}$ qd or bid and placebo (PBO) in patients with inadequate glycemic control $\left(\mathrm{HbA}_{1 \mathrm{c}}=7.5\right.$ $11 \%$ ) on TZD monotherapy. All patients in Study 2 received Pio $45 \mathrm{mg}$ for $\geq 4 \mathrm{wk}$ prior to randomization and continued Pio $45 \mathrm{mg}$ qd throughout the study.

Results: At baseline (BL), $\mathrm{HbA}_{1 \mathrm{c}}$ averaged $8.6-8.8 \%$ in all treatment groups. As shown below, vildagliptin produced a statistically significant and clinically meaningful improvement in glycemic control in combination with Pio, whether combined with lower doses ( 15 or $30 \mathrm{mg}$ qd) as initial pharmacotherapy as in Study 1, or added to a maximum dose (45 mg qd) as in Study 2.

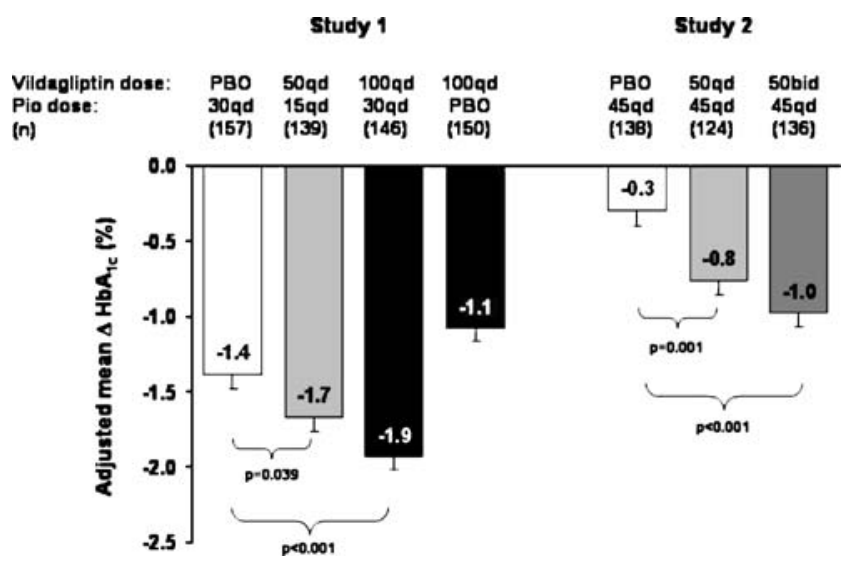

In drug-naïve patients with $\mathrm{BL} \mathrm{HbA}_{1 \mathrm{c}}>9.0 \%$ (in Study 1), the change from $\mathrm{BL}$ (mean=10.0\%, $\mathrm{n}=54$ ) in patients receiving initial combination therapy with vildagliptin $100 \mathrm{mg}$ qd+Pio $30 \mathrm{mg}$ qd averaged $-2.8 \pm 0.2 \%$ (ie, a mean reduction in $\mathrm{HbA}_{1 \mathrm{c}}$ of $2.8 \%$ ). All treatments were welltolerated, with a similar number of patients in each treatment group reporting any adverse event (ranging from $46 \%$ of those receiving vildagliptin $50 \mathrm{mg} \mathrm{qd}+$ Pio $15 \mathrm{mg}$ qd to $56 \%$ of those receiving vildagliptin $50 \mathrm{mg} \mathrm{qd}+\mathrm{Pio}$ $45 \mathrm{mg} \mathrm{qd})$.

Conclusions: Vildagliptin is highly effective and welltolerated when combined with pioglitazone.

Supported by Novartis

0802

Sustained efficacy and reduced hypoglycaemia with vildagliptin added to insulin in patients with type 2 diabetes

V. Fonseca ${ }^{1}$, S. Dejager ${ }^{2}$, D. Albrecht ${ }^{2}$, Q. Shao ${ }^{2}$, A. Schweizer ${ }^{2}$;

${ }^{1}$ Endo, Tulane Univ, New Orleans, United States, ${ }^{2}$ Novartis, E Hanover, United States.

Background and Aims: Vildagliptin is a DPP-4 inhibitor that lowers $\mathrm{HbA}_{1 \mathrm{c}}$ in patients (pts) with type 2 diabetes (T2DM) when given as monotherapy or combined with metformin, but its efficacy in pts with more advanced disease requiring insulin (Ins) is unknown. The aim of this study was to assess the efficacy and tolerability of one-year treatment with vildagliptin added to Ins in pts with T2DM. Methods: This was a 28-wk extension of a 24-wk, randomized, double-blind, parallel-group study in pts with T2DM inadequately controlled $\left(\mathrm{HbA}_{1 \mathrm{c}} 7.5-11 \%\right)$ by Ins monotherapy $(>30 \mathrm{U} / \mathrm{d})$. In the 24 -wk core study pts received vildagliptin $50 \mathrm{mg}$ bid $(\mathrm{n}=125)$ or placebo (PBO, $\mathrm{n}=131$ ) added to Ins. In the 28 -wk extension, pts who received $\mathrm{PBO}$ during the core study switched to vildagliptin $50 \mathrm{mg}$ qd ( $\mathrm{n}=104)$, while vildagliptin-treated pts from the core study maintained the $50 \mathrm{mg}$ bid regimen $(\mathrm{n}=96)$. All pts maintained Ins therapy, but adjustments to dose regimen were allowed.

Results: At baseline $(\mathrm{BL})$ mean age $=59 \mathrm{y}, \mathrm{BMI}=33.0 \mathrm{~kg} /$ $\mathrm{m}^{2}$, disease duration $=14.6 \mathrm{y}, \mathrm{FPG}=9.3 \mathrm{mM}$ and $\mathrm{HbA}_{1 \mathrm{c}}=8.5 \%$. Mean duration of Ins use was $76 \mathrm{mo}$ and mean dose was $82 \mathrm{U} / \mathrm{d}$. Eighty-three percent of pts completed the core study and $87.5 \%$ of pts in the extension completed 52-wk treatment. In the core study, the adjusted mean change $(\mathrm{AM} \Delta)$ in $\mathrm{HbA}_{1 \mathrm{c}}$ in vildagliptin-treated pts was $-0.5 \pm 0.1 \%$ and a modest PBO effect was noted (AM $\Delta$ $\mathrm{HbA}_{1 \mathrm{c}}=-0.2 \pm 0.1 \%$ ), with the between-group difference being significant $(\mathrm{P}=0.022)$. Greater efficacy was seen in the $>30 \%$ of pts age $\geq 65$ y for whom the between-group difference $(-0.7 \pm 0.1 \%)$ was highly significant $(\mathrm{P}<0.001)$. During the core study, hypoglycemic events were less common and less severe in vildagliptin-treated pts (33 pts, 113 events, 0 severe) than in those receiving PBO (45 pts, 185 events, 6 severe). As depicted below, the efficacy of vildagliptin $50 \mathrm{mg}$ bid added to Ins was maintained throughout the extension and in pts switched from PBO to 
vildagliptin $50 \mathrm{mg} \mathrm{qd}, \mathrm{HbA}_{1 \mathrm{c}}$ decreased promptly and the improvement was maintained through $\mathrm{Wk} 52$. The mean $\Delta \mathrm{HbA}_{1 \mathrm{c}}$ from Wk 24 to endpoint in these pts was $-0.4 \pm 0.1 \%(\mathrm{P}<0.001$ vs $\mathrm{Wk} 24)$.

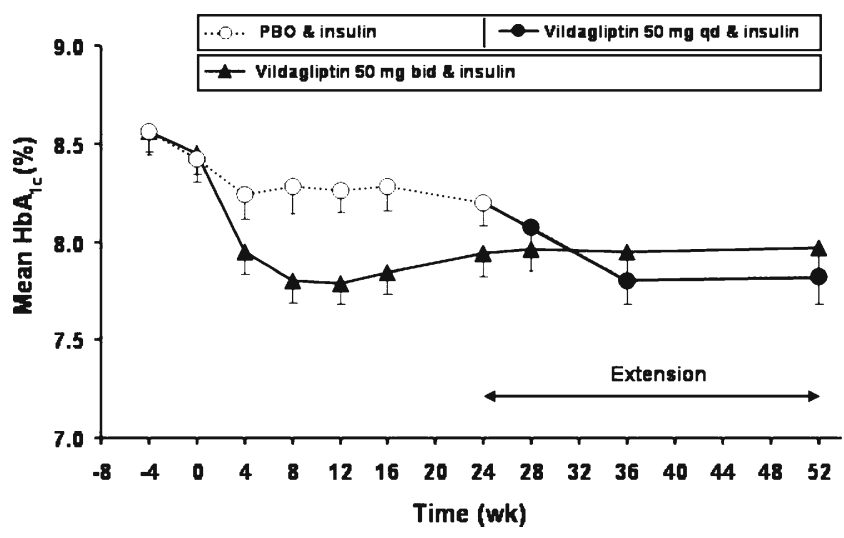

In the $32 \mathrm{pts}$ aged $\geq 65 \mathrm{y}$ in the vildagliptin $50 \mathrm{mg}$ bid arm of the extension, the mean $\Delta \mathrm{HbA}_{1 \mathrm{c}}$ from $\mathrm{BL}$ to endpoint was $-0.9 \pm 0.1 \%$. The protective effect of vildagliptin against hypoglycemia was also maintained in the extension. During the whole study, the incidence of hypoglycemia, expressed as events/pt/y was $1.78,1.80$ and 2.66 in the vildagliptin $50 \mathrm{mg} \mathrm{qd}, 50 \mathrm{mg}$ bid and PBO groups, respectively. The overall incidence of side effects was similar in pts receiving vildagliptin vs $\mathrm{PBO}$.

Conclusion: Adding vildagliptin to Ins in pts with T2DM is well-tolerated and improves glycemic control, with reduced hypoglycemia, particularly in older individuals. Both the greater efficacy in older pts and the protective effect against hypoglycemia are maintained through 52 -wk treatment. The mechanism(s) underlying these observations may reflect improved $\alpha$-cell glucose sensing and merit further study. Supported by Novartis

\section{3}

Over $75 \%$ of patients with type 2 diabetes reached $\mathrm{HbA}_{1 \mathrm{c}}<7 \%$ by adding biphasic insulin aspart 30 to optimized metformin and pioglitazone treatment P. Raskin ${ }^{1}$, R. Braceras ${ }^{2}$, S. Schwartz ${ }^{3}$, L. Chaykin ${ }^{4}$, P.-L. $\mathrm{Chu}^{2}$, A. Wynne';

${ }^{1}$ Department of Internal Medicine, University of Texas Southwestern Medical Center, Dallas, United States, ${ }^{2}$ Novo Nordisk, Inc., Princeton, United States, ${ }^{3}$ Endocrinology, Diabetes \& Glandular Disease Research Associates, P.A., San Antonio, United States, ${ }^{4}$ Endocrinology, Medical Research Unlimited, Aventura, United States, ${ }^{5}$ Diabetes Department, Cotton-O'Neil Diabetes and Endocrinology Center, Topeka, United States.
Physicians often are reluctant to initiate insulin therapy in subjects with type 2 diabetes particularly when oral therapies are not optimized. This randomized, treat-totarget, 34-week, parallel-group study of BIAsp $30(30 \%$ soluble and $70 \%$ protaminated insulin aspart) was conducted in insulin-naive, type 2 diabetes patients with $\mathrm{HbA}_{1 \mathrm{c}}$ $7.5-12.0 \%$ who were taking any two oral antidiabetic agents. BIAsp30 was compared for efficacy and safety when added to an optimized treatment of metformin and pioglitazone (met + pio) to optimized treatment with met +pio without insulin. During an 8-week run-in, treatment was changed to met and pio, and doses were adjusted to $2500 \mathrm{mg} /$ day and 30 or $45 \mathrm{mg} /$ day, respectively. After randomization, BIAsp 30 was initiated at $6 \mathrm{U}$ BID (prebreakfast and presupper) and titrated to target blood glucose (BG) $(4.4-6.1 \mathrm{mmol} / \mathrm{L})$ by an algorithm-directed forced titration. At the end of the study, subjects treated with BIAsp $30+$ met+pio $(\mathrm{n}=93)$ had a mean $\mathrm{HbA}_{1 \mathrm{c}}$ reduction that was significantly greater than the mean reduction for subjects treated with met+pio $(n=88)(1.5 \%$ vs. $0.2 \%, p<0.0001$ between groups). Subjects treated with BIAsp 30+met+pio were more likely to reach $\mathrm{HbA}_{1 \mathrm{c}}$ targets of $\leq 6.5 \%$ and $<7.0 \%$ than with met+pio only (odds ratios $=12.3$ and 11.1, respectively). Final $\mathrm{HbA}_{1 \mathrm{c}}$ and fasting plasma glucose (FPG) values were significantly lower for the BIAsp 30 group, as compared to the met+pio group (Table 1). More subjects treated with BIAsp 30 were able to reach the aggressive $\mathrm{HbA}_{1 \mathrm{c}}$ goals of $\leq 6.0 \%$ and $\leq 5.5 \%$.

Table 1.

\begin{tabular}{llll}
\hline & & BIAsp 30+Met+Pio & Met+Pio \\
$\mathrm{HbA}_{1 \mathrm{c}}($ mean $\pm \mathrm{SD})$ & $\begin{array}{l}\text { Baseline } \\
\text { End of Study }\end{array}$ & $6.1 \pm 1.0$ & $7.9 \pm 0.9$ \\
& (EOS) & & $7.8 \pm 1.2$ \\
$\%$ with $\mathrm{HbA}_{1 \mathrm{c}}<7.0 \%$ & EOS & 76.3 & \\
$\%$ with $\mathrm{HbA}_{1 \mathrm{c}} \leq 6.5 \%$ & EOS & 59.1 & 24.1 \\
$\%$ with $\mathrm{HbA}_{1 \mathrm{c}} \leq 6.0 \%$ & EOS & 33.3 & 11.5 \\
$\%$ with $\mathrm{HbA}_{1 \mathrm{c}} \leq 5.5 \%$ & EOS & 14.0 & 2.3 \\
$\mathrm{FPG}, \mathrm{mmol}^{\mathrm{L}}$ & EOS & $7.2 \pm 2.8$ & 0 \\
\hline
\end{tabular}

The rate of minor hypoglycemia (defined as $\mathrm{BG}$ $<3.1 \mathrm{mmol} / \mathrm{L}$ ) in the BIAsp 30 group was significantly greater than in the met+pio group (8.3 vs. 0.1 events/year, $\mathrm{p}<0.001)$. Both groups gained weight during treatment (BIAsp 30, 4.6 $\pm 4.3 \mathrm{~kg}$; met+pio, $0.8 \pm 3.2 \mathrm{~kg}, \mathrm{p}<0.05$ ). Peripheral edema was reported by $10 \%$ and $12 \%$ of subjects in the BIAsp 30 and met+pio groups respectively.

In conclusion, more patients with type 2 diabetes, poorly controlled by 2 oral agents, could achieve currently recommended $\mathrm{ADA}$ and $\mathrm{IDF} / \mathrm{ACE} \mathrm{HbA}_{1 \mathrm{c}}$ targets using BIAsp 30+met+pio than using met+pio alone. 
0804

Insulin (INS) add-on to rosiglitazone/metformin (RSG/ MET) fixed-dose combination therapy (FDCT) vs. INS switch from RSG/MET FDCT: satisfaction with treatment

R. Horblyuk ${ }^{1}$, B. Arondekar ${ }^{1}$, J. Donaldson ${ }^{2}$, H. Chen ${ }^{3}$; ${ }^{1}$ GSK, Philadelphia, United States, ${ }^{2} \mathrm{GSK}$, Greenford, United Kingdom, ${ }^{3}$ GSK, King of Prussia, United States.

Background and Aims: Greater treatment satisfaction may predict improved medication adherence and long-term glycemic control in type 2 diabetes (T2D). This 24-week, randomized, double-blind study assessed treatment satisfaction in patients with T2D insufficiently controlled on a maximum daily dose of RSG/MET FDCT $(8 \mathrm{mg} / 2000 \mathrm{mg}$ ). Following 8-week max dose RSG/MET FDCT run-in, patients received INS supplemental to RSG/MET (INS add-on to RSG/MET) ( $\mathrm{n}=163)$ or were switched to an INSonly regimen (INS switch) $(\mathrm{n}=161)$.

Materials and Methods: The Diabetes Treatment Satisfaction Questionnaire (DTSQ) assessed patient satisfaction with treatment (range 0-36), perceived frequency of hyperglycemia (range 0-6) and perceived frequency of hypoglycemia (range 0-6) in INS add-on to RSG/MET vs. INS switch groups.

Results: INS add-on to RSG/MET FDCT was superior to INS switch in reducing $\mathrm{HbAlc}$ (mean adjusted treatment difference $-0.65 \%, p<0.0001$ ) while requiring less INS. At study end, mean treatment differences for change from screening and baseline in treatment satisfaction were significant (1.4 and 1.5 points, respectively, $p<0.05)$ and favored INS add-on to RSG/MET. Perceived frequency of hyperglycemia decreased (improved) during the study in two groups. Mean treatment differences for change from screening and baseline in perceived frequency of hyperglycemia were significant $(0.7$ points, $p<0.0006$ and 0.8 points, $p<0.0001$, respectively) showing greater improvements in INS add-on to RSG/MET. There were no betweengroup differences in perceived frequency of hypoglycemia. Conclusion: In patients with T2D, significant positive changes in treatment satisfaction and perceived frequency of hyperglycemia along with stable perceived frequency in hypoglycemia scores add value to superior glycemic control afforded by lower doses of INS added to RSG/MET FDCT compared to switch to higher doses of INS alone. Increased satisfaction with treatment could benefit long-term medication adherence and sustainable glycemic control.

Supported by GSK
PS 63

\section{Thiazolidinediones in combination therapy}

0805

Long-term glycaemic effects of pioglitazone in triple oral therapy: Results from PROactive

B. Charbonnel ${ }^{1}$, A. Scheen $^{2}$ on behalf

of the PROactive investigators,

${ }^{1}$ Hotel Dieu, Clinique d'Endocrinologie, Nantes, France,

${ }^{2}$ Division of Diabetes, Nutrition, and Metabolic Disorders, University of Liege, Belgium.

Background and Aims: Type 2 diabetes is a progressive disease and its treatment eventually requires multiple-agent therapy, including insulin. PROactive was a cardiovascular outcome study, which examined the effects of pioglitazone on patients whose type 2 diabetes had been diagnosed on average 9.5 years before study entry. Little data exists on the benefits of using triple oral therapy (metformin +sulfonylurea+thiazolidinedione) in those patients failing dual oral treatment. This subanalysis evaluates the longterm glycaemic effects of pioglitazone add-on therapy in patients with type 2 diabetes and macrovascular disease who entered on metformin plus sulfonylurea.

Materials and Methods: PROactive randomised patients to either pioglitazone or placebo, in addition to other glucose-lowering and cardiovascular medication, which was adjusted as necessary to treat to IDF target. Pioglitazone doses were force-titrated from $15 \mathrm{mg}$ to $45 \mathrm{mg}$. Mean follow-up was 34.5 months. In total, 1314 patients entered the study on metformin plus sulfonylurea. Within this cohort, mean baseline $\mathrm{HbA}_{1 \mathrm{c}}$ values were similar between groups (pioglitazone: $8.16 \%$; placebo: $8.14 \%$ ).

Results: Significantly greater reductions in $\mathrm{HbA}_{1 \mathrm{c}}$ were seen with pioglitazone $(-0.9 \%)$ compared with placebo $(-0.4 \%, \mathrm{P}<0.0001)$. The significant improvement in $\mathrm{HbA}_{1 \mathrm{c}}$ with pioglitazone versus placebo was shown with the following changes in associated glucose-lowering medication: more pioglitazone patients had either metformin or sulfonylurea dropped from their regimen (16\%) and fewer had insulin added to their regimen (16\%) than did placebo patients ( $8 \%$ and $31 \%$, respectively). The metformin dose increased by $19 \mathrm{mg}$ with pioglitazone versus $228 \mathrm{mg}$ with placebo $(\mathrm{P}<0.0001)$. Sulfonylurea doses decreased or were unchanged in the pioglitazone group $(-1.4 \mathrm{mg}$ for glibenclamide versus $-0.2 \mathrm{mg}$ for placebo, $\mathrm{P}=0.013 ;-33 \mathrm{mg}$ for gliclazide versus $-23 \mathrm{mg}$ for placebo, $\mathrm{P}=0.270 ; 0 \mathrm{mg}$ for glimepiride versus $+0.6 \mathrm{mg}$ for placebo, $\mathrm{P}=0.009$ ). Oedema occurred in $29 \%$ of patients in the pioglitazone group versus $17 \%$ in the placebo group $(\mathrm{P}<0.0001)$ and hypoglycaemia occurred in $27 \%$ in the pioglitazone group 
versus $20 \%$ in the placebo group $(\mathrm{P}=0.0013)$. There was a weight increase of $4.1 \mathrm{~kg}$ in the pioglitazone group and a decrease of $0.7 \mathrm{~kg}$ in the placebo group $(\mathrm{P}<0.0001)$.

Changes in glycaemic control (as measured by $\mathrm{Hb}_{\mathrm{lc}}$ ) over time with pioglitazone (solid lines) or placebo (dashed lines) in patients receiving metformin plus sulfonylurea

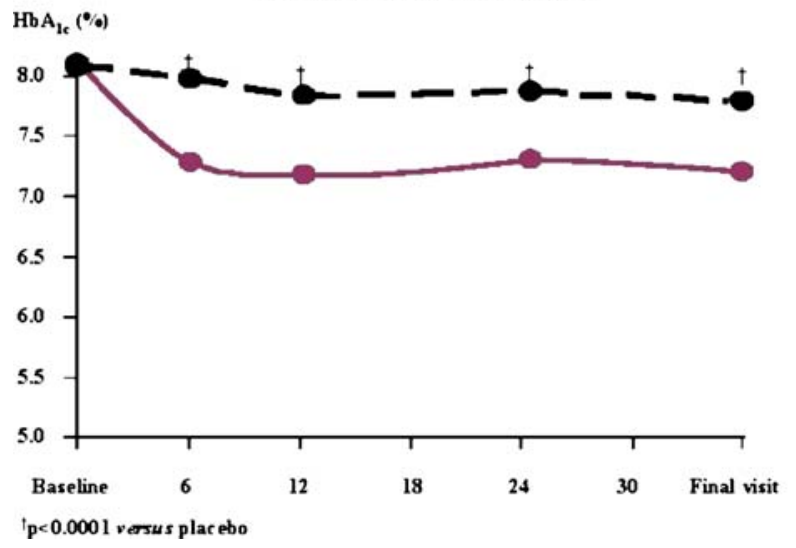

Conclusion: Little data exist on the benefits of triple oral therapy in patients with type 2 diabetes. Adding pioglitazone to a dual oral therapy regimen (metformin+sulfonylurea), thus resulting in a triple oral regimen, resulted in a sustained improvement in glycaemic control and a reduced need for insulin, with a good overall safety profile.

\section{6}

Pioglitazone reduces insulin requirements and improves glycaemic control in insulin-treated patients with type 2 diabetes: results from PROactive

O. Schmitz ${ }^{1}$, B. Charbonnel ${ }^{2}$, A. Scheen ${ }^{3}$

on behalf of the PROactive investigators;

${ }^{1}$ Department of Clinical Pharmacology, University of Aarhus, Denmark, ${ }^{2}$ Hotel Dieu, Clinique d'Endocrinologie, Nantes, France, ${ }^{3}$ Division of Diabetes, Nutrition, and Metabolic Disorders, University of Liege, Belgium.

Background and Aims: Type 2 diabetes is a progressive disease with associated insulin resistance and diminished insulin production. Treatment typically requires multiple agents, including insulin. PROactive was a study designed to evaluate the effects of pioglitazone on cardiovascular outcomes in 5,238 high-risk patients with type 2 diabetes. We examined insulin requirements and glycaemic control in the one-third of patients ( $\mathrm{n}=864$ in the pioglitazone group; $\mathrm{n}=896$ in the placebo group) who were treated with insulin at baseline in PROactive.
Materials and Methods: PROactive was a randomised, double-blind, placebo-controlled outcome study in patients with type 2 diabetes and macrovascular disease randomised to pioglitazone or matching placebo, in addition to their existing glucose-lowering and cardiovascular medication. Doses of pioglitazone were force-titrated to a dose of $45 \mathrm{mg}$, if tolerated. Mean follow-up was 34.5 months. Within the cohort of patients receiving insulin at baseline, baseline mean daily insulin doses and $\mathrm{HbA}_{1 \mathrm{c}}$ values were similar between treatment groups (pioglitazone: $47 \mathrm{U} / \mathrm{d}$ and 8.4\%; placebo: $47 \mathrm{U} / \mathrm{d}$ and $8.5 \%$ ). In both groups at baseline, insulin was combined with oral monotherapy with metformin in $53 \%$ and sulfonylurea in $24 \%$ of patients and with dual therapy with metformin+sulfonylurea in $12 \%$.

Results: There was a rapid and sustained decrease in insulin dose with pioglitazone, whereas there was a progressive increase with placebo. By study end, the mean insulin dose was lower with pioglitazone ( $42 \mathrm{U} / \mathrm{d})$ than with placebo (55 U/d; $\mathrm{P}<0.0001)$; nevertheless, a greater decrease in $\mathrm{HbA}_{1 \mathrm{c}}$ was observed with pioglitazone $(-0.93 \%)$ compared with placebo $(-0.45 \% ; \mathrm{P}<0.0001)$. At final visit, insulin had been discontinued in $9 \%$ of patients in the pioglitazone group versus $2 \%$ in the placebo group $(\mathrm{P}<0.0001)$. The distribution of patients on oral/ insulin combined therapy remained similar in both groups: metformin alone in $47 \%$ versus $52 \%$, sulfonylurea alone in $16 \%$ versus $16 \%$, and metformin+sulfonylurea in $10 \%$ versus $11 \%$, in the pioglitazone group versus the placebo group, respectively $(\mathrm{P}=\mathrm{NS})$. There were differences in oedema (pioglitazone: $31 \%$; placebo: $18 \% ; \mathrm{P}<0.0001$ ) and hypoglycaemia (pioglitazone: $41 \%$; placebo: $29 \%$; $\mathrm{P}<0.0001$ ), but there were no other differences in the safety profiles between the pioglitazone and placebo groups (with or without other treatments).

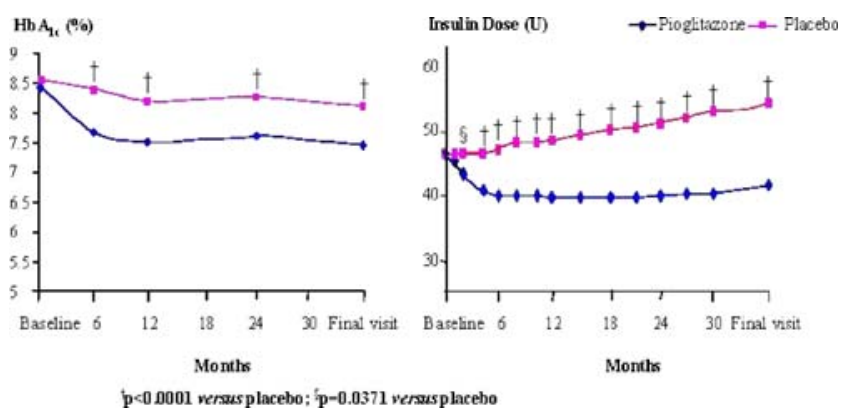

Conclusion: Pioglitazone reduced the number of patients on insulin and the mean daily insulin dose while providing better glycaemic control than placebo. 
0807

Glycaemic control in patients with type 2 diabetes: the effects of pioglitazone vs glyburide in a 3-year randomized double-blind trial

A. Perez ${ }^{1}$, R. Spanheimer ${ }^{2}$, M. Khan ${ }^{2}$;

${ }^{1}$ Takeda Global Research and Development Center, Lincolnshire, United States, ${ }^{2}$ Takeda Pharmaceuticals North America, Inc., Lincolnshire, United States.

Background and Aims: Little long-term data comparing the effects of a sulfonylurea to a thiazolidinedione on glycemic control have been published. UKPDS has shown that because of the progressive nature of type 2 diabetes (T2D), many patients fail initial monotherapy for glycemic control after 3 to 4 years as noted by the terms "sulfonylurea burnout" and "metformin failure." We examined the long-term glycemic benefits of pioglitazone (PIO) compared to glyburide (GLY) in one of the longest studies evaluating the effects of PIO.

Materials and Methods: In this US multicenter, doubleblind, randomized comparator study, 2120 patients (18$80 \mathrm{yrs}$ ) with T2D were enrolled. Patients on metformin at study entry were allowed to continue this therapy (up to $850 \mathrm{mg}$ bid); all sulfonylurea therapy was discontinued. During the 36-month treatment period, PIO was titrated to $45 \mathrm{mg}$ (in $15 \mathrm{mg}$ intervals) and GLY was titrated to $15 \mathrm{mg}$ (in $5 \mathrm{mg}$ intervals). At the investigator's discretion, metformin was either maximized or added to achieve A1C $<7.5 \%$. If A1C goals were not achieved after 3 months, insulin therapy was added.

Results:

Least Squares Mean Change From Baseline in A1C

\begin{tabular}{llllll}
\hline & & $\begin{array}{l}\text { PIO } \\
\text { N=1051 }\end{array}$ & & $\begin{array}{l}\text { GLY } \\
\text { N=1046 }\end{array}$ & \\
& $\mathrm{n}$ & $\begin{array}{l}\text { LS Mean } \\
\text { \% (SD) }\end{array}$ & $\mathrm{n}$ & $\begin{array}{l}\text { LS Mean } \\
\text { \% (SD) }\end{array}$ & $\begin{array}{l}\text { Between- } \\
\text { groups } \\
\text { P-Value }\end{array}$ \\
& & & & & \\
\hline Baseline & 782 & $9.41(2.07)$ & 850 & $9.34(2.01)$ & 0.492 \\
Week 16 & 683 & $-0.29(2.27)$ & 739 & $-1.12(1.98)$ & $<0.0001$ \\
Week 32 & 574 & $-1.11(2.24)$ & 601 & $-1.21(2.07)$ & 0.3522 \\
Week 48 & 512 & $-1.59(2.11)$ & 559 & $-1.54(2.11)$ & 0.6167 \\
Week 72 & 447 & $-2.07(2.07)$ & 495 & $-1.67(2.10)$ & 0.0002 \\
Week 96 & 403 & $-2.17(2.05)$ & 450 & $-1.83(2.07)$ & 0.0008 \\
Week 120 & 360 & $-2.08(1.87)$ & 396 & $-1.65(2.13)$ & $<0.0001$ \\
Week 144 & 335 & $-2.39(2.11)$ & 355 & $-1.91(2.22)$ & $<0.0001$ \\
Week 156 & 323 & $-2.34(2.08)$ & 333 & $-1.85(2.07)$ & $<0.0001$ \\
\hline
\end{tabular}

Conclusion: Treatment groups had similar mean baseline A1C levels (PIO=9.41\%, GLY=9.34\%). PIO treatment resulted in significantly greater reductions in A1C compared to GLY starting at Week 72 and continued through
3 years of treatment. In conclusion, subjects treated with PIO showed sustained and greater long-term glycemic control compared to GLY.

0808

Evaluation of changes of glycaemic control and lipid parameters in patients with T2DM after one year of treatment with pioglitazone in combination with metformin or sulfonylurea in a Greek observational study A. A. Ginis ${ }^{1}$, B. Karamanos ${ }^{2}$, E. Charalampidou ${ }^{1}$, A. Saridaki ${ }^{1}$, V. Drossinos ${ }^{1}$; The Hellenic Ecla Study Group; ${ }^{1}$ Pharmaserv-Lilly SACI, Kifisia, Greece, ${ }^{2}$ 2nd Medical Department, Diabetes Center, Hippokratio Hospital, Athens University, Greece.

Background and Aims: Type 2 diabetes mellitus (T2DM) is associated with hypertriglyceridemia, low HDL-c and an increase of small dense LDL-c. This feature of diabetic dyslipidemia is the most well characterized risk factor for increasing atherosclerosis in patients(pts) with T2DM. Evaluate the changes of the glycemic control and of the lipid profile in pts with T2DM treated with Pioglitazone (Pio) in combination with Metformin (Met) or Sulfonylurea (Su).

Materials and Methods: The Clinical Evaluation of the Lipid profile in T2DM treated with oral Agents in combination (ECLA) is a one-year, multicenter Phase IV, open label, prospective study in 2388 T2DM pts poorly controlled with monotherapy and according to the physician's judgement required combined oral treatment. They were assigned in 3 treatment groups: (1) $\mathrm{Pio}+\mathrm{Su}$ (2) Pio +Met and (3) $\mathrm{Su}+$ Met. We report here the data analysis performed after 12 months of follow-up in 2116 (88.6\%) pts who completed the study without changing their treatment[ $\mathrm{Pio}+\mathrm{Su}(791), \mathrm{Pio}+\mathrm{Met}(705), \mathrm{Su}+\mathrm{Met}(620)]$. $77.3 \%$ from $\mathrm{Pio}+\mathrm{Su}, 72.1 \%$ from Pio + Met and $72 \%$ from $\mathrm{Su}+$ Met were not receiving hypolipidemic treatment.

Results: Fasting plasma glucose $(\mathrm{mg} / \mathrm{dl})$ decreased significantly from baseline with $\mathrm{Pio}+\mathrm{Su}$ (198 to 134) and Pio +Met (194 to 131) compared to Su+Met (189 to 138), representing a decrease of $31.1,32$ and $26.3 \%$ respectively $(\mathrm{p}<0.0001$ comparing Pio groups to $\mathrm{Su}+\mathrm{Met})$. HbAlc decreased significantly in all groups $(\mathrm{p}<0.0001)$ but more in the Pio groups $(8.23$ and $8.1 \%$ becoming 6.65 and $6.61 \%)$, compared to $\mathrm{Su}+\mathrm{Met}(7.96$ to $6.93 \%)(\mathrm{p}<0.0001)$. Total cholesterol (TC) $(\mathrm{mg} / \mathrm{dl})$ baseline mean values were similar in all cohorts (237). After 12 months TC was decreased significantly in all groups $(\mathrm{p}<0.0001)$, but significantly more in the Pio groups (207) representing a reduction of $12.8 \%$ vs $9.9 \%$ in the $\mathrm{Su}+\mathrm{Met}$ (212) $(\mathrm{p}<0.0001)$. The HDL-c $(\mathrm{mg} / \mathrm{dl})$ mean values were 43.8 for $\mathrm{Pio}+\mathrm{Su}, 43.1$ for $\mathrm{Pio}+\mathrm{Met}$ and 43.5 for $\mathrm{Su}+\mathrm{Met}$ at 
baseline. After 12 months an increase was observed in all groups $(\mathrm{p}<0.0001)$ but significantly more in the Pio groups $8.3-9.5 \%$ vs $4.6 \%$ in Su+Met $(\mathrm{p}=.0003, \mathrm{p}<0.0001$ correspondingly). Triglycerides $(\mathrm{mg} / \mathrm{dl})$ decreased in all groups $(p<0.0001)$ but significantly more for the $\mathrm{Pio}+\mathrm{Su}$ and Pio +Met groups (177 to 141 and 184 to 142 ) representing a reduction of 20.7 and $21.6 \%$ vs $15.1 \%$ in the $\mathrm{Su}+\mathrm{Met}(178$ to150) $(\mathrm{p}<0.0001)$. A significant reduction of the LDL $(\mathrm{mg} /$ dl) from baseline to 12 months was detected with $\mathrm{Pio}+\mathrm{Su}$ (157 to 133) and Pio+Met (153 to 132) compared to $\mathrm{Su}$ + Met (154 to 137), representing a decrease of 15.2, 14.6 and $11.3 \%$ respectively $(\mathrm{p}=.0001$ and $\mathrm{p}=.005$ comparing $\mathrm{Pio}+\mathrm{Su}$ and $\mathrm{Pio}+\mathrm{Met}$ to $\mathrm{Su}+\mathrm{Met})$. The atherogenic index i. e. $\log$ [TG/HDL-c] decline after 12 months in the Pio+Su and $\mathrm{Pio}+$ Met groups (22.6 and 23.3\%) vs $15 \%$ in $\mathrm{Su}+\mathrm{Met}$ $(p<0.0001)$. The differences observed in the lipid profile, were independent of concomitant administration of hypolipidemic medication and were also documented in the analysis of the 6 months results.

Conclusion: Combined treatment with $\mathrm{Pio}+\mathrm{Su}$ and $\mathrm{Pio}$ +Met over one year, compared with $\mathrm{Su}+$ Met in a daily clinical practice setting, resulted in significantly greater improvement in glycemic control and led to a less atherogenic lipid profile, confirming the value of Pioglitazone for the combined treatment of T2DM.

Supported by Pharmaserv-Lilly SACI

\section{9}

Dose dependent improvement of $\beta$ cell dysfunction by addition of pioglitazone to existing oral treatments under daily routine conditions

E. Karagiannis ${ }^{1}$, M. Flannery ${ }^{2}$, W. Roth ${ }^{2}$, T. Schöndorf ${ }^{2}$, G. Lübben ${ }^{1}$, T. Forst ${ }^{2}$, A. Pfützner ${ }^{2}$;

${ }^{1}$ Takeda Pharma, Aachen, Germany, ${ }^{2}$ IKFE Institute for Clinical Research and Development, Mainz, Germany.

Background and Aims: The degree of $\beta$-cell dysfunction in orally treated patients with type 2 diabetes has been shown to correlate with cardiovascular risk and prevalence of macrovascular disease. The ratio of correctly processed secretion product (C-peptide) and the uncleaved intact proinsulin is a good marker for cell function. This study was performed to investigate the effect of addition of different doses of pioglitazone to the existing therapy on $\beta$-cell dysfunction under daily life conditions.

Materials and Methods: In total 402 patients were included (223 women, 179 men, age $($ mean \pm SD): $61 \pm$ 11 years, disease duration: $6 \pm 5$ years, BMI: $31.4 \pm 5.5 \mathrm{~kg} / \mathrm{m}^{2}$, HbAlc: $7.6 \pm 1.1 \%$ ). Decision about the dose was left to the discretion of the treating physician. Before and after 5 months of treatment, $\beta$-cell function was assessed by measurement of fasting $\mathrm{C}$-peptide $(\mathrm{CP})$ and intact proinsulin (iPi) and formation of the fasting $\mathrm{CP} / \mathrm{iPi}$ ratio. Other observation parameters included $\mathrm{HbAlc}$, fasting glucose, and blood pressure.

Results: Patients who received only $15 \mathrm{mg}$ of Pio $(\mathrm{n}=32)$ had a better fasting $\mathrm{CP} / \mathrm{PI}$ ratio and a lower blood pressure at baseline compared to those receiving $30 \mathrm{mg}(\mathrm{n}=227)$ or $45 \mathrm{mg}(\mathrm{n}=93)$. While no statistically significant difference between the groups was observed regarding reduction of HbAlc (mean change: $-0.7 \pm 1.1 \%, \mathrm{p}<0.001$ vs. baseline) and fasting glucose $(-31 \pm 43 \mathrm{mg}, \mathrm{p}<0.001)$, dose dependent improvements could be observed for $\beta$-cell dysfunction and the blood pressure assessments as shown in the table.

Conclusion: Addition of pioglitazone to existing oral antidiabetic treatments under daily routine conditions, leads to improvement of glucose control and additional dose dependent improvements of $\beta$-cell dysfunction and blood pressure. Dose dependent improvement by pioglitazone in $\beta$-cell function $(\mathrm{CP} / \mathrm{iPi})$ and blood pressure $(\mathrm{BP})$

\begin{tabular}{llllllll}
\hline Parameter & \multicolumn{2}{c}{$15 \mathrm{mg}$} & & \multicolumn{2}{c}{$30 \mathrm{mg}$} & & $45 \mathrm{mg}$ \\
\cline { 2 - 3 } $\begin{array}{l}\mathrm{a}: \mathrm{p}<0.05 \\
\mathrm{~b}: \mathrm{p}<0.001\end{array}$ & baseline & endpoint & & baseline endpoint & & baseline endpoint \\
\hline $\mathrm{CP} / \mathrm{iPi}$ & $118 \pm 105$ & $112 \pm 54$ & $107 \pm 73$ & $123 \pm 82^{\mathrm{b}}$ & $106 \pm 70$ & $131 \pm 84^{\mathrm{b}}$ \\
ratio & & & & & & & \\
$\begin{array}{l}\mathrm{BP} \text { syst } \\
{[\mathrm{mmHg}]}\end{array}$ & $136 \pm 17$ & $136 \pm 17$ & $140 \pm 15$ & $135 \pm 13^{\mathrm{b}}$ & $142 \pm 17$ & $134 \pm 14^{\mathrm{b}}$ \\
$\begin{array}{l}\mathrm{BP} \text { diast } \\
{[\mathrm{mmHg}]}\end{array}$ & $78 \pm 8$ & $79 \pm 7$ & $82 \pm 9$ & $81 \pm 8^{\mathrm{a}}$ & $84 \pm 10$ & $81 \pm 7^{\mathrm{b}}$ \\
\hline
\end{tabular}

Supported by Takeda Pharma

0810

Effects of the addition of pioglitazone on insulin resistance, beta cell function and proinsulin/insulin ratio in patients with type 2 diabetes inadequately controlled on metformin and a sulphonylurea

S. Halimi ${ }^{1}$, G. Charpentier ${ }^{2}$ on behalf of the Study

F-PIO-10 Investigators,

${ }^{1}$ Diabetology Nutrition, Centre Hospitalier Universitaire de Grenoble, ${ }^{2}$ Service de Diabetologie, Centre Hospitalier Sud Francilien, Corbeil-Essonnes, France.

Background and Aims: Pioglitazone belongs to the class of thiazolidinediones that reduces insulin resistance in the liver, adipose tissue and peripheral muscle. In addition, pioglitazone (PIO) has shown improvements in markers of beta-cell function. We evaluated the effect on endogenous insulin production and markers of beta-cell function of 
adding PIO to metformin/sulphonylurea (MET+SU) dual therapy in inadequately controlled, insulin-naïve patients with Type 2 diabetes.

Materials and Methods: This prospective double-blind, placebo-controlled study randomised 299 patients with Type 2 diabetes previously treated with MET $(\geq 1700 \mathrm{mg} /$ day) and SU (maximal dose) for more than 3 months and $\mathrm{HbA}_{1 \mathrm{c}}$ between $7.0 \%$ and $9.5 \%$. After a 4-week run-in, patients received either PIO $30 \mathrm{mg}$ once daily or placebo. After 3 months, if $\mathrm{HbA}_{1 \mathrm{c}}>6.5 \%$, PIO or placebo was uptitrated to $45 \mathrm{mg}$ for the remaining 4 months. Endogenous insulin production was assessed by measuring fasting plasma insulin (I), intact proinsulin (PI) and C-peptide at baseline and month 7 . Insulin resistance and beta-cell function were evaluated using the homeostasis model assessment for insulin resistance (HOMA-IR) and beta-cell function (HOMA-B). The analyses were conducted on data from patients who had received at least 3 months' study treatment.

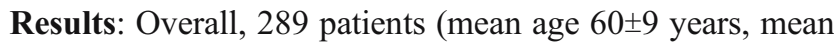
duration Type 2 diabetes $12 \pm 8$ years) were included in the full analysis population.

Conclusion: The addition of PIO to MET+SU dual therapy significantly improved glycaemic control, markers of insulin resistance and beta-cell function in patients with Type 2 diabetes inadequately controlled on dual therapy. Moreover, this double-blind, randomised controlled study has shown for the first time that the addition of PIO to MET + SU decreases PI and improves PI/I ratio, representing a potential additional benefit by reducing this independent cardiovascular risk factor.

Adjusted mean changes \pm S.E. from baseline at 7 months

\begin{tabular}{llll}
\hline & Pioglitazone & Placebo & $\begin{array}{l}\text { Treatment } \\
\text { difference }\end{array}$ \\
\hline Insulin, & $-14.49 \pm 2.57$ & $-6.67 \pm 2.54$ & $-7.82 \pm 3.62 ;$ \\
mmol/L & $\mathrm{n}=127$ & $\mathrm{n}=130$ & $\mathrm{p}=0.032$ \\
Proinsulin, & $-4.44 \pm 2.74$ & $11.29 \pm 2.68$ & $-15.75 \pm 3.84 ;$ \\
pmol/L & $\mathrm{n}=133$ & $\mathrm{n}=139$ & $\mathrm{p}<0.001$ \\
Proinsulin/insulin & $0.63 \pm 0.05$ & $0.77 \pm 0.05$ & $-0.14 \pm 0.07 ;$ \\
ratio & $\mathrm{n}=126$ & $\mathrm{n}=128$ & $\mathrm{p}<0.04$ \\
C-peptide, & $-0.20 \pm 0.08$ & $0.13 \pm 0.07$ & $-0.33 \pm 0.11 ;$ \\
Mg/L & $\mathrm{n}=129$ & $\mathrm{n}=137$ & $\mathrm{p}=0.002$ \\
HOMA-IR & $-2.01 \pm 0.19$ & $-0.43 \pm 0.18$ & $-1.58 \pm 0.26 ;$ \\
& $\mathrm{n}=115$ & $\mathrm{n}=119$ & $\mathrm{p}<0.001$ \\
HOMA-B & $24.24 \pm 7.03$ & $-2.26 \pm 6.88$ & $26.50 \pm 9.84 ;$ \\
& $\mathrm{n}=114$ & $\mathrm{n}=119$ & $\mathrm{p}=0.008$ \\
\hline
\end{tabular}

Supported by Laboratoires Takeda, France
0811

Fixed-dose combination of rosiglitazone and glimepiride provides superior glycaemic control compared with glimepiride or rosiglitazone monotherapy in drug-naïve individuals with type 2 diabetes mellitus

H. S. Chou ${ }^{1}$, T. Segiet ${ }^{2}$, A. R. Jones-Leone ${ }^{1}$, J. P. Palmer ${ }^{3}$; ${ }^{1}$ GlaxoSmithKline, King of Prussia, United States, ${ }^{2}$ Diabetes Center Speyer, Germany, ${ }^{3}$ Division of Endocrinology, Metabolism and Nutrition, University of Washington, Seattle, United States.

Background and Aims: Conventional management of type 2 diabetes (T2DM) continues to involve maximizing the use of a monotherapy before introducing a second agent, despite the high incidence of therapeutic failure with this strategy. Recent evidence suggests that the earlier use of combination therapy may improve long-term glycemic control. The aim of this study was to investigate whether combination therapy with rosiglitazone (RSG) and glimepiride (GLIM) was more effective than either agent alone in drug-naïve individuals with T2DM.

Materials and Methods: In this multi-centre study, drugnaïve participants with T2DM were randomized to receive 28 weeks of double-blind treatment with GLIM [1-4 mg once daily (OD)], RSG (4-8 mg OD), RSG/GLIM fixeddose combination (FDC) A (4 mg/1-4 mg OD) or RSG/ GLIM FDC B (4-8 mg/1-4 mg OD). Treatment was started on the lowest dose and uptitrated at 4-week intervals up to 12 weeks unless mean daily glucose level of $<6.1 \mathrm{mmol} / \mathrm{l}$. Efficacy was assessed by determining $\mathrm{HbA}_{1 \mathrm{c}}$ levels at the end of the study.

Results: A total of 901 patients were involved in the study, and 883 patients constituted the efficacy population. Baseline characteristics of mean age (53.96 \pm 10.99 years), BMI $\left(31.68 \pm 6.41 \mathrm{~kg} / \mathrm{m}^{2}\right), \mathrm{HbA}_{1 \mathrm{c}}(9.07 \pm 1.31 \%)$ and duration of T2DM (2.89 \pm 3.87 years) were comparable between treatment groups. Both FDC regimens gave superior glycemic control compared with RSG or GLIM alone. A statistically greater proportion of participants achieved the ADA target of $\mathrm{HbA}_{1 \mathrm{c}}<7 \%$ with FDC A $(75 \%)$ or FDC B $(72 \%)$ compared with GLIM (49\%) or RSG (46\%) monotherapy. Furthermore, the more aggressive target of $<6.5 \%$ was also achieved by more patients with FDC A $(56 \%)$ or FDC B (54\%) compared with GLIM $(32 \%)$ or RSG (30\%) alone. Rapid FPG reductions were observed in both FDC regimens, with a statistically greater reduction compared with either GLIM or RSG monotherapy. Both FDCs were generally safe and well tolerated, with adverse event profiles as expected from their component therapies. Despite improved glycemic control, there was no increase in hypoglycemic events compared with GLIM alone (FDC A $3.6 \%$, FDC B 5.5\%, GLIM 4.1\%). 


\begin{tabular}{llll}
\hline GLIM & RSG & FDC A & FDC B \\
$\mathrm{n}=221$ & $\mathrm{n}=227$ & $\mathrm{n}=221$ & $\mathrm{n}=214$ \\
\hline
\end{tabular}

\begin{tabular}{lllll}
\hline $\mathbf{H b A}_{\mathbf{1 c}} \%$ & & & & \\
$\begin{array}{l}\text { Baseline } \\
\text { Mean } \Delta \text { from } \\
\text { baseline }\end{array}$ & $-1.7 \pm 1.4$ & $-1.8 \pm 1.5$ & $-2.4 \pm 1.4^{* \dagger}$ & $-2.5 \pm 1.4^{* \dagger}$ \\
$\begin{array}{l}\text { FPG mmol/l } \\
\text { Baseline }\end{array}$ & $11.7 \pm 3.9$ & $11.8 \pm 3.7$ & $11.5 \pm 3.2$ & $11.9 \pm 3.4$ \\
$\begin{array}{l}\text { Mean } \Delta \text { from } \\
\text { baseline }\end{array}$ & $-2.3 \pm 3.7$ & $-3.2 \pm 3.2$ & $-3.9 \pm 3 . *^{\dagger}$ & $-4.4 \pm 3.2 *^{\dagger}$ \\
\hline
\end{tabular}

${ }^{*} p<0.0001$ vs. GLIM; ${ }^{\dagger} p<0.0001$ vs. RSG

Conclusion: This study shows that the use of FDC (RSG/ GLIM) is an effective and well tolerated initial therapy for drug-naïve T2DM individuals. These data also demonstrate the superior efficacy of FDC (RSG/GLIM) compared with RSG or GLIM monotherapy in this patient population, with more individuals achieving glycemic targets.

\section{2}

Glycaemic response of pioglitazone in dual combination and triple therapy in patients with type 2 diabetes mellitus

H. Heddaeus ${ }^{1}$, E. Karagiannis ${ }^{2}$, G. Lübben ${ }^{2}$;

${ }^{1}$ Diabetes Centre, Würselen, ${ }^{2}$ Takeda Pharma $\mathrm{GmbH}$, Aachen, Germany

Background and Aims: Type-2-Diabetes mellitus (T2DM) is a disease with progressively deteriorating glycaemic control. Insulin resistance (IR) and interrelated declining betacell function (BCF) are believed to be the core defects of the disease. Pioglitazone (PIO) is a PPAR $\gamma$ _agonist offering unique and sustained glucose-lowering benefits via ameliorating IR and BCF. Clinical studies revealed that combination therapy of PIO with Metformin (MET) could be the preferred treatment option of choice offering additional metabolic control combined with a favourable safety profile. Current evidence suggests that early and aggressive combination therapy will slow the progression of the disease and its complications. There are limited data available for the effects of PIO in triple therapy. In addition data are lacking whether the glycaemic response is related to the dose of the concomitant antihyperglycaemic therapy at baseline. Therefore we studied the glucose lowering effect of PIO in a "real world situation" in a cohort of rather unselected patients with T2DM.

Materials and methods: open-label, non-controlled observational study performed according to the guidelines of the German Federal Health Ministry. Combination therapy with PIO $30 \mathrm{mg}$ qd was initiated for a mean observation time of $17 \pm 5$ weeks. PIO therapy was initiated in 2,341 patients as add-on to MET and in 820 patients as third agent. PIO and MET dosage were aimed to keep stable throughout the observation period. Primary parameters were for different BL levels adjusted changes of $\mathrm{HbA} 1 \mathrm{C}$ and fasting Glucose levels (FG) compared to that at BL. All results are expressed as means \pm SE. Wilcoxon-test was used to test statistical significant difference for HbAlc and blood sugar levels. KruskalWallis-test was used to test intergroup differences concerning the used dosages of concomitant antidiabetic medication.

Results: HbA1C declined from $8.4 \% \pm 1.4$ to $7.0 \% \pm 1.0$ $(p<0,001)$ and $8.8 \% \pm 1.5$ to $7.3 \% \pm 1.1 \quad(p<0,001)$ in dual combination therapy (DT) and triple therapy (TT), respectively. Compared to BL FG levels could be reduced by $50.8 \mathrm{mg} / \mathrm{dl}(\mathrm{DT})$ and $53.5 \mathrm{mg} / \mathrm{dl}(\mathrm{TT})$.

Overall $51.9 \%$ (DT) and $40.4 \%$ (TT) reached a $\mathrm{HbA1C}$ goal of $<7 \%$. Only $1(0.03 \%)$ reported hypoglycaemia. A mean weight gain of $1.1 \mathrm{~kg}$ was observed.

Conclusion: PIO revealed significant benefits for glycaemic control in DT as well as TT. These benefits could be achieved independent of MET doses at BL. The combination approach offers also a favourable safety profile which could be relevant for patient acceptance in the long-term.

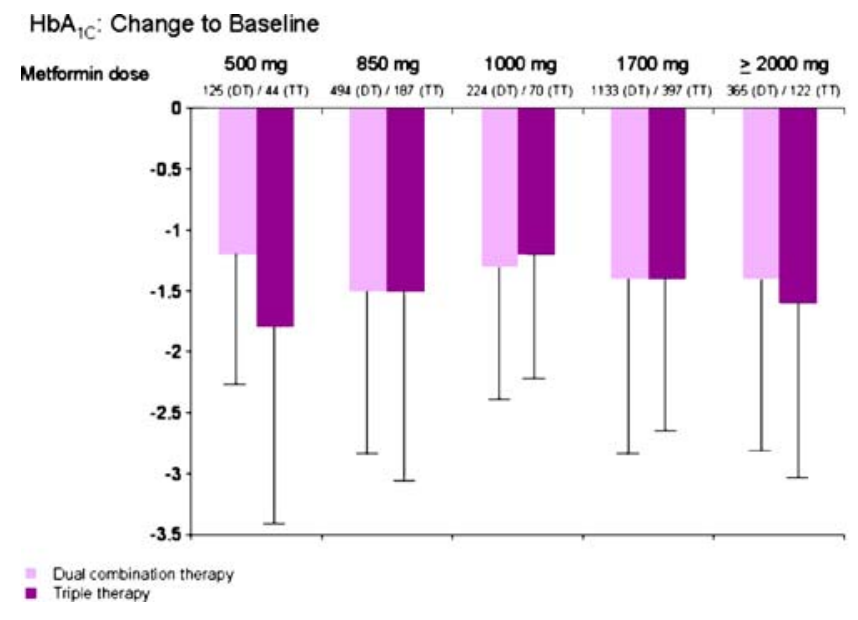

Supported by Takeda Pharma

0813

The efficacy and safety of pioglitazone in addition to SU or metformin vs fixed combination of metformin plus glibenclamide on diabetic dyslipidaemia

E. Torre ${ }^{1}$, P. Ponzani ${ }^{1}$, F. Menozzi ${ }^{1}$, S. Mariz ${ }^{2}$,

A. Corsi ${ }^{1}$, M. Comaschi ${ }^{3}$;

${ }^{1}$ Dept. of Internal Medicine Westend Hospital Genoa Italy, Diabetes Unit, Health District Genoa, Arenzano - Genoa, Italy,

${ }^{2}$ Takeda International, London, United Kingdom, ${ }^{3}$ Dept. of Public Health Genoa Italy, Health District Genoa, Italy. 
Background and Aims: The effects of co-administration of pioglitazone with either metformin or sulphonylurea on diabetic dyslipidemia have been described in recent publications. Little or no data exists on the comparative effects between a fixed combination of metformin +sulphonylurea and the co-administration of pioglitazone and metformin or sulphonylurea. This study aims to present data on the comparative effectiveness of these different treatment regimes on diabetic dyslipidemia.

Materials and Methods: This was a prospective open, three arm comparative randomised study in patients with Type 2 diabetes previously treated with maximal permitted/ tolerated does of metformin or sulphonylurea for at least 3 months and $\mathrm{HbAlc}$ between $7.5 \%$ and $11 \%$. After a 1 week run-in period, patients received either pioglitazone $15 \mathrm{mg}$ once daily as add-on in patients receiving metformin or sulphonylurea or a fixed dose combination of metformin $400 \mathrm{mg}$ and glibenclamide $2.5 \mathrm{mg}$ twice a day. Pioglitazone could be increased to $30 \mathrm{mg} /$ day and the fixed combination could be increased to 3 times a day at 15 days, 2 or 4 months if the plasma glucose was $>140 \mathrm{mg} / \mathrm{dl}$ or $\mathrm{HbAlc}$ was $>7.5 \%$. HDL-cholesterol, total cholesterol and triglycerides were measured at baseline and 6 months.

Results: 398 patients were screened and 250 were randomised into the trial. 103 patients were put Ùnto the pioglitazone + metformin group, 77 into the pioglitazone +sulponylurea group and 80 into the fixed dose metformin + glibenclamide group. HDL-cholesterol was significantly raised by $2.3 \mathrm{mg} / \mathrm{dl}$ from baseline in the pio+met group whereas there was a significant decrease of $-3.3 \mathrm{mg} / \mathrm{dl}$ in the fixed dose combination group at 6 months. There was a significant difference in favour of both pio + met and pio $+\mathrm{SU}$ vs fixed dose regarding HDL change. There were no differences between the groups for total cholesterol. A significant drop in triglycerides was only seen in the pio +SU group $(28.3 \mathrm{mg} / \mathrm{dl})$.

Conclusion: This study showed that using pioglitazone in co-administration with either metformin or sulphonylurea effectively improved diabetic dyslipidemia as compared with a worsening of HDL and no effect on other lipid parameters with the fixed dose combination. All three treatments were well tolerated with an increase in body weight seen in all three groups.

Supported by Takeda Italy

\section{PS 64}

\section{Diabetes in the "real world"}

\section{4}

Frequency and motives of blood glucose self-monitoring in type 1 diabetes: association with glycaemic control, complications, and patient characteristics

M. V. Hansen ${ }^{1}$, U. Pedersen-Bjergaard ${ }^{1}$, S. R. Heller ${ }^{2}$, T. M. Wallace ${ }^{3}$, Å. K. Rasmussen ${ }^{4}$, H. V. Jørgensen ${ }^{4}$, D. R. Matthews ${ }^{3}$, P. Hougaard ${ }^{5}$, S. Pramming ${ }^{5}$, B. Thorsteinsson ${ }^{1}$;

${ }^{1}$ Division of Internal Medicine I, Hillerød Hospital, Denmark, ${ }^{2}$ Clinical Sciences Centre, Northern General Hospital, Sheffield, United Kingdom, ${ }^{3}$ Endocrinology and Metabolism, The Oxford Centre for Diabetes, United Kingdom, ${ }^{4}$ Steno Diabetes Center, Gentofte, Denmark, ${ }^{5}$ Novo Nordisk A/S, Bagsværd, Denmark.

Background and Aims: Recommendations for self-monitoring of blood glucose (SMBG) from the DCCT have not been implemented with the same intensity as recommendations for insulin therapy. We assessed frequency and motives of SMBG and the relationship between SMBG and clinical, behavioural and demographic characteristics.

Material and Methods: Cross-sectional Danish-British multicentre survey of 1076 consecutive patients with type 1 diabetes who completed a detailed questionnaire on SMBG and related issues. The key variables were test frequency and motive.

Results: SMBG was performed daily by $39 \%$ of the patients, in series one or more days per week by $20 \%$, as single tests more than once a week by $17 \%$ and less than weekly by $24 \%$. The incentives to test were suspicion of hypo- or hyperglycaemia $(33 \%)$, routine checks $(44 \%)$, or a combination (23\%). The following parameters remained significant in a stepwise multivariate analysis and were related with daily SMBG: higher age, female gender, high educational level, non-smoking, 4-dose insulin regimen, low HbA1c, more feelings in the preceding week, unawareness, autonomic neuropathy, and long duration of diabetes. Routine testing was associated with higher age, longer duration of diabetes, and lower HbAlc in the multivariate analysis.

Conclusion: Patient compliance regarding SMBG is suboptimal and too many patients do not perform SMBG routinely. Patient groups needing more attention are young men with a short duration of diabetes, who have a lower educational level, do not think much of their diabetes, and are smokers. 
0815

Self-monitoring of blood glucose in type 2 diabetic patients not on insulin treatment: waist of money or tool to reach the glycaemic targets?

K. Bonomo, A. De Salve, S. Pignatelli, E. Fiora,

E. Mularoni, F. Cavalot, M. Trovati;

Department of Clinical and Biological Sciences of the Turin University, Diabetes Unit, Orbassano (Torino), Italy.

Background and Aims: There is no agreement whether self-monitoring of blood glucose (SMBG) is a tool to improve clinical outcomes in type 2 diabetic patients not on insulin. For this reason, limitations in BG strips reimbursement are applied: in our Region, 4 strips/month are routinely admitted. In this study, we aimed at investigating whether frequency and completeness of SMBG play a role in the attainment of the glycemic targets.

Materials and Methods: We enrolled 273 consecutive type 2 diabetic patients not on insulin, with $\mathrm{HbA} 1 \mathrm{c}$ stable in the last 6 months, already on $\mathrm{SMBG}$, and randomized them into 3 groups: Group A $(n=96)$ was invited to continue the usual SMBG by performing one BG profile a month with fasting, $2 \mathrm{~h}$ after breakfast, $2 \mathrm{~h}$ after lunch and $2 \mathrm{~h}$ after dinner BG measurement; Group B1 $(n=89)$ was invited to perform one BG profile every 15 days with fasting, $2 \mathrm{~h}$ after breakfast, before lunch, $2 \mathrm{~h}$ after lunch, before dinner, 2 hours after dinner BG measurement; Group B2 $(n=88)$ was invited to perform BG profiles as group B1, with the recommendation to call the Diabetes Clinic when BG targets were not reached. The three groups did not differ for gender, age, known diabetes duration, BMI, waist circumference, type of therapy and $\mathrm{HbA}$ 1c. Patients were followed-up by the same team at 3 month intervals and received the same education and treatment protocol. At 3 and 6 months, SMBG profiles were evaluated and HBA1c was measured.

Results: SMBG was carried out as recommended by $73 \%$ of Group A patients and 44\% of Groups B1 and B2 patients, which were finally considered together (Group B) since a very few patients of Group B2 called the Diabetic Clinic. Compliant and not compliant patients did not differ for the parameters mentioned in Materials and Methods. Thus, BG and $\mathrm{HbAlc}$ were evaluated in the compliant patients of Group A $(n=70 / 96)$ and B $(n=78 / 177)$, which presented no significant differences in gender (M/F 41/29 vs 47/31), age $(63.1 \pm 9.44$ vs $65.8 \pm 9.2$ years), known diabetes duration (10.6 \pm 8.9 vs $10.6 \pm 8.5$ years), BMI $(29.1 \pm 4.9$ vs $28.1 \pm 3.5)$, waist circumference $(103.4 \pm 12.1$ vs $102.1 \pm 8.8 \mathrm{~cm}), \mathrm{HbAlc}$ $(7.97 \pm 0.72$ vs $8.1 \pm 0.8 \%)$. At 6 months, HbA1c was $7.78 \pm 1.05$ vs $7.97 \pm 0.72 \%$ (ns) in Group $A$ and $7.6 \pm 0.73$ vs $8.08 \pm 0.84 \%(p=0.001)$ in Group $B$; no significant change in BG was observed in Group A, whereas in Group B the BG values at 6 months vs baseline were: i)fasting $129.61 \pm 27.33$ vs $137.5 \pm 28.29 \mathrm{mg} / \mathrm{dl}, \mathrm{p}=0.013$; ii) 2 hours after breakfast $147.62 \pm 29.4$ vs $158.3 \pm 35.86 \mathrm{mg} / \mathrm{dl}, \mathrm{p}=0.004$; iii)before lunch, $124.56 \pm 25.71$ vs $134.99 \pm 34.84 \mathrm{mg} / \mathrm{dl}, \mathrm{p}=0.003$; iv) 2 hours after lunch $157.32 \pm 27.93$ vs $163.9 \pm 34.38 \mathrm{mg} / \mathrm{dl}$, ns; v) before dinner, $120.27 \pm 27.06$ vs $129.42 \pm 32.8 \mathrm{mg} / \mathrm{dl}$, $\mathrm{p}=0.037$; vi) 2 hours after dinner $153.22 \pm 29.42$ vs $162.88 \pm$ $30.08 \mathrm{mg} / \mathrm{dl}, \mathrm{p}=0.002$. Therapeutical changes prescribed at the 3 month visit were more frequent in Group B (44.9\%) vs Group A (32.8\%).

Conclusion: $\mathrm{SMBG}$ is a tool to improve BG control in type 2 diabetic patients not on insulin only when performed with an appropriate frequency/completeness, because only in this case it has a sufficient educational value and reduces the therapeutical "inertia". Only $44 \%$ of patients, however, are able to do it carefully. Furthermore, this study shows that type 2 diabetic patients not on insulin seldom utilize the availability of the diabetes team to be contacted out of the scheduled visits.

Supported by Piedmont Region

\section{6}

Self monitoring of blood glucose is common in German people with non-insulin treated type 2 diabetes and associated with better long term outcome

H. Kolb ${ }^{1}$, B. Schneider ${ }^{2}$, L. Heinemann ${ }^{3}$, V. Lodwig ${ }^{4}$, W. A. Scherbaum ${ }^{1}$, S. Martin ${ }^{1}$;

${ }^{1}$ German Diabetes Clinic, Düsseldorf, Germany, ${ }^{2}$ Institute of Biometrics, Medical University of Hannover, Germany, ${ }^{3}$ Profil, Institute of Metabolic Research, Neuss, Germany, ${ }^{4}$ IMIB, Institute for Medical Informatics and Biostatistics, Basel, Switzerland.

Background and Aims: Randomised controlled trials of self-monitoring of blood glucose (SMBG) for long term outcome in non-insulin treated type 2 diabetes are not feasible. Therefore information from epidemiological cohort studies should be considered.

Materials and Methods: In the German multicentre, retrospective cohort study ROSSO people with type 2 diabetes were followed from diagnosis between 1995 and 1999 until the end of 2003. In order to minimise any bias in patient selection, all diabetic patients of 192 randomly picked practices involved in primary care were screened and included in the analysis if $\geq 45$ years of age at diagnosis and treated for at least one year. Patients who received oral antidiabetic drugs (OAD) for at least one year, but no insulin during the observation period or until a clinical endpoint were analysed.

Results: During a mean follow-up of 6.5 years 742 of a total of 1912 patients in the OAD cohort $(38.8 \%)$ began with SMBG. Initiation of SMBG was preceded by worsening of 
metabolic control. People performing SMBG were younger by a mean of 3 years and more often male (56\% versus $47 \%$ in the no SMBG cohort), but did not differ for most other characteristics including smoking. Persons in the SMBG cohort changed OAD therapy more often $(66.3 \%$ of patients versus $48.3 \%, \mathrm{p}<0.001)$. SMBG was not accompanied by more medication for treating hypertension, hypercholesterinaemia or thrombocyte aggregation risk. The composite clinical endpoint comprised diabetes-related morbidity (myocardial infarction, stroke, foot amputation, blindness, or haemodialysis) and all-cause mortality. In the SMBG cohort, significantly fewer persons had an endpoint $(9.2 \%)$ compared to the cohort without SMBG $(13.2 \%, \mathrm{p}=0.038$ after multivariate adjustments for baseline differences, confirmed by Kaplan-Meier survival analysis, $\mathrm{p}=0.002$ ). Stratification of patients by metabolic control, blood lipids or BMI did not reveal a subgroup in which the use of SMBG was not associated with better outcome.

Conclusion: In this retrospective study of patients treated in German primary care centres, more than one third of non-insulin treated type 2 diabetes patients began with SMBG during the first 6 years after diagnosis, apparently in response to deteriorating metabolic control. The use of SMBG was associated with better long-term outcome.

Unrestricted Research Grant by Roche Diagnostics

\section{7}

\section{ASIAD PROJECT: $A$ lphabet $S$ trategy Indian $A$ pplication for Diabetes: Effective application of European strategy in outpatient setting in Asia \\ P. Kumaran ${ }^{1,2}$, L. Varadhan ${ }^{1}$, A. Gopinath ${ }^{1}$, D. James ${ }^{1}$, J. Morrissey ${ }^{1}$, V. Patel ${ }^{1}$; \\ ${ }^{1}$ Diabetes Centre, George Eliot Hospital NHS Trust, Nuneaton, United Kingdom, ${ }^{2}$ Diabetes Centre, Shenbagam Hospital, Madurai, India.}

Background and Aims: The Alphabet Strategy is an evidence based template that focuses around seven essential bits of effective diabetes care: $\boldsymbol{A}$ dvice, $\boldsymbol{B}$ lood pressure control, Cholesterol control, Diabetes control, Eye care, Foot care, Guardian drugs eg. Aspirin, ACE-inhibitors. Onset of diabetes in Asians occurs atleast a decade earlier, thereby increasing the risk of complications. An effective, target driven diabetes care strategy is required to combat the economic burden.

The aim of our study was

1. To assess the application of the Alphabet Strategy of diabetes care to improve health care delivery in outpatient setting in India.
2. To compare the health care delivery standards of care achieved in India by application of the Alphabet Strategy with that of outpatient care in UK.

Materials and Methods: 100 outpatient records in an outpatient setting were assessed for the quality of diabetes care based on the Alphabet Strategy. The Alphabet Strategy of diabetes care was slightly modified by using Fasting and post prandial plasma glucose instead of HbAlc and proteinuria for microalbuminuria to suit Indian economic standards. A prospective audit was done using this modified strategy on further 100 patients with diabetes. The template based on the strategy prompted the medical team to verify the previous year outpatient record and deliver the essential components of diabetes care if needed. Data were collected and compared against 100 case notes randomly picked at the UK Diabetes centre, where the strategy is in use for 6 years.

Results: With the implementation of the modified Alphabet Strategy, the recording of BMI, Blood pressure and smoking at the Indian centre was $99 \%$ before and after vs. $100 \%$ in UK; the recording of cessation advice was $100 \%$ in the both centre. The recording of total cholesterol was $60 \%$ before and $99 \%$ after $(p<0.0001)$ vs. $97 \%$ in UK. The recording of lipid profile also improved from $10 \%$ to $64 \%$ $(\mathrm{p}<0.0001)$ vs. $97 \%$ in UK. The record of creatinine improved from $5 \%$ to $49 \%(\mathrm{p}<0.0001)$ vs. $99 \%$ in UK and the record of proteinuria improved from $48 \%$ to $93 \%$ $(p<0.0001)$ after applying the template $(56 \%$ in UK). $41 \%$ of the patients had both fasting and postprandial glucose which improved to $97 \%$ after implementation of strategy (HbAlc measured $100 \%$ in UK). The Indian centre achieved $100 \%$ on recording the status of eye and feet examination during the audit which was $98 \%$ and $95 \%$ respectively before.

The usage of guardian drugs in India before and after the strategy compared to UK was: Aspirin 6\% vs.71\% (UK $71 \%$ ), ACE inhibitors or ARB 7\% vs. 57\% (UK 75\%) and Statin $5 \%$ vs. $38 \%$ (UK $73 \%$ ) and all these were statistically significant. $20 \%$ of the Indian patients were on all 3 compared to $52 \%$ in UK. The overall score, based on the GMS contract system of UK, improved from 45 to 61 in India compared to 76 in UK out of a maximum possible 99. Conclusion: The Alphabet strategy of diabetes care can potentially be easily and effectively adopted for any setup of diabetes care, with a few modifications, to suit any healthcare economy. This strategy is an useful model that ensures that comparable standards of diabetes care are achieved in a cost effective way. 


\section{8}

Glycaemic control after initiation of oral antidiabetic therapies

A. Oglesby ${ }^{1}$, S. Shetty ${ }^{2}$, M. Wintle ${ }^{3}, \mathrm{~K}$. Stockl ${ }^{3}$;

${ }^{1}$ Eli Lilly and Company, Indianapolis, United States,

${ }^{2}$ i3 Innovus, Eden Prairie, United States, ${ }^{3}$ Amylin Pharmaceuticals, San Diego, United States.

Background and Aims: Currently available oral antidiabetic agents (OADs) are reportedly unable to sustain good glycemic control over time, often requiring the addition of new therapies in order to maintain glycemic control. The objective of this study was to analyze the duration of commonly used OAD therapies before loss of glycemic control $\left(\mathrm{HBbA}_{1 \mathrm{c}} \geq 7.0 \%\right)$ occurs. Secondary analysis assessed the time taken before therapy intensification occurred in subjects with type 2 diabetes (T2D) who were treated with OADs.

Materials and Methods: A retrospective analysis was performed using eligibility, laboratory, medical and pharmacy claims data from a large US health care organization. Subjects were included if they had a claim for T2D, no claim for $\mathrm{T} 1 \mathrm{D}$ and at least one $\mathrm{HbA}_{1 \mathrm{c}}$ measure recorded 90 days \pm the index date (date of first OAD claim) during the period 07/01/01 through 06/30/02. Study subjects were grouped as monotherapy cohorts (SU, MET, TZD) and combination therapy cohorts (SU+MET, SU+TZD, MET $+\mathrm{TZD}, \mathrm{SU}+\mathrm{MET}+\mathrm{TZD}$ ) irrespective of treatment order and based on prescription claim history for OADs during the baseline period and within 90 days of index date in the follow-up period. Subjects were followed for a minimum of 24 months and a maximum of 42 months. Demographic and clinical variables (comorbid conditions, baseline $\mathrm{HbA}_{1 \mathrm{c}}$, creatinine, and cholesterol values), and prescribing clinician specialty were assessed for each subject. Cox proportional hazards model was used to compare time to reach $\mathrm{HbA}_{1 \mathrm{c}} \geq 7.0 \%$ and time to therapy intensification among the different treatment cohorts.

Results: Of the 6,131 identified subjects, $69 \%$ had an $\mathrm{HbA}_{1 \mathrm{c}} \geq 7.0 \%$ in the follow-up period. $82 \%$ of subjects in the SU+MET+TZD cohort had an $\mathrm{HbA}_{1 \mathrm{c}} \geq 7.0 \%$ in the follow-up and had the shortest period of time (323 days) until $\mathrm{HbA}_{1 \mathrm{c}}$ became $\geq 7.0 \%$. 49\% subjects in the TZD monotherapy cohort had an $\mathrm{HbA}_{1 \mathrm{c}} \geq 7.0 \%$ in the follow-up and had the longest time (418 days) until $\mathrm{HbA}_{1 \mathrm{c}} \geq 7.0 \%$. After controlling for demographic and clinical characteristics and clinician specialty, the Cox model revealed that the SU+MET and SU+MET+TZD cohorts were $16 \%$ and $31 \%$ more likely to reach an of $\mathrm{HbA}_{1 \mathrm{c}} \geq 7.0 \%$ compared to the SU monotherapy cohort. In contrast, patients in the MET and TZD monotherapy cohorts were $18 \%$ and $38 \%$ less likely reach an $\mathrm{HbA}_{1 \mathrm{c}} \geq 7.0 \%$ compared to the $\mathrm{SU}$ monotherapy cohort. On average, subjects remained on their current therapy for $499.1 \pm 294.3$ days before a new therapy was added. A higher percentage of subjects in the SU monotherapy cohort (34\%) had an intensification of therapy while the percentage was smallest in the SU+MET +TZD combination cohort. Subjects in the SU+MET cohort had the longest time to therapy intensification (531.4 \pm 300.6 days). Time to therapy intensification was shortest in the TZD cohort (444.6 \pm 299.1 days). After controlling for baseline demographic and clinical variables and clinician specialty, the results of the Cox model indicate that the hazard of therapy intensification was greater in the MET (58\%), TZD (31\%) and SU+MET $(22 \%)$ cohorts than in the SU cohort.

Conclusion: Results of this analysis suggest that about two-thirds of the study population on OADs did not have their $\mathrm{HbA}_{1 \mathrm{c}}$ under good control. Although there were differences between treatment cohorts, subjects in all OAD cohorts remained on therapy an average of 1.4 years before intensification of therapy regimen occurred.

Supported by Eli Lilly and Company and Amylin Pharmaceuticals

\section{9}

The importance of weight changes on the economic burden of type 2 diabetes treatment

S. Liatis, N. Apostolopoulos, P. Thomakos,

G. Georgakopoulos, P. Tsapogas, N. Vrizidis,

N. Katsilambros;

1st Internal Medicine Department, Athens University Medical School Laiko Hospital, Athens, Greece.

Background and Aims: Obesity and type 2 diabetes are considered as epidemics of the modern societies. In addition, both conditions are closely related to each other and their prevalence increase in parallel. It is well known that these two conditions raise a heavy economic burden on the health care systems of all societies. The well-documented need for a decent glycemic control of type 2 diabetic patients is often accomplished with the use of multiple antidiabetic medications. On the other hand, weight management remains a cornerstone on the overall type 2 diabetes treatment and is often neglected due to the chronic strain of both patients and health care proffessionals.

Aim of this study was to examine the influence of weight loss change on the economic cost of type 2 diabetes treatment with oral hypoglycemic medications and/or insulin.

Subjects and Methods: The files of 1112 consecutive type 2 diabetic patients who referred to the diabetes outpatient clinic of our hospital form January 2000 to December 2003 were reviewed. Only those patients who have been 
followed regularly in our clinic (having at least 3 visits per year for a minimum of 24 months) were included. All patients attending the clinic were given dietary advise regularly by specialized dieticians. The cost of treatment was measured in Euros, using the 01-01-2006 official greek market prices (as indicated in every medication's label) of all licenced antidiabetic medications in Greece. Skewed data were log-transormed to improve normality for statistical testing.

Results: Data from 264 patients (115 men [43.6\%], mean age $61.8 \pm 10.8$ years, mean duration of diabetes $9.0 \pm$ 8.2 years) who fullfilled the inclusion criteria were analyzed. The mean duration of follow-up was $42.4 \pm$ 16.6 months and during that period, mean $\mathrm{HbA}_{1} \mathrm{c}$ decreased by $1.47 \%$ (from $8.54 \pm 2.0 \%$ to $7.07 \pm 1.13 \%$ ). At the same time, body mass index (BMI) increased by $0.75 \mathrm{Kg} / \mathrm{m}^{2}$ (from $29.36 \pm 4.97 \mathrm{~kg} / \mathrm{m}^{2}$ to $30.11 \pm 5.13 \mathrm{Kg} / \mathrm{m}^{2}$ ). At the end of the study period, the mean cost of antidiabetic drug treatment per patient/month was $28.22 \pm 26.40$ Euros. In multivariate linear regression analysis, after adjustment for age, gender, duration of diabetes, BMI, type of antidiabetic treatment, drug choice and $\mathrm{HbA}_{1} \mathrm{c}$ achieved, the final cost of antidiabetic medication was positively associated to the degree of weight gain (standardized $\beta$-regression coefficient: $\left.0.181, \mathrm{P}<0.0001, \mathrm{R}^{2}=51.1\right)$.

Conclusion: Weight loss, or even less weight gain, is associated with a reduction of the economic burden of antidiabetic medications used for glycemic control of type 2 diabetic patients.

0820

Achievement of clinical targets for type $\mathbf{2}$ diabetes is variable accross primary care populations in England, Scotland and Wales

P. D. Ambery ${ }^{1}$, M. W. Savage ${ }^{2}$;

${ }^{1}$ Glaxosmithkline, Uxbridge, ${ }^{2}$ Department of Diabetes, North Manchester General Hospital, United Kingdom.

Background and Aims: The GMS contract has encouraged intensification of glycaemic lowering therapy, blood pressure lowering and treatment to cholesterol targets in the Type 2 diabetes population in the UK. Here we examine target achievement in typical English, Welsh and Scottish primary care populations

Materials and Methods: Data was exported from General Practices who consented to electronic audit in pooled anonomised format to a central server. Results presented represent latest patient data exported in the period MarchOctober 2005.HbA1c results were available on 57427 English patients, 3412 Welsh patients and 23694 Scottish patients.
Results: Average HbAlcs were 7.84\% England, 7.44\% Wales and $7.86 \%$ in Scotland. Patients in Wales displayed the best HbA1c control, odds ratio for meeting 7\% target: 1.33 vs England, 1.64 vs Scotland $(p<0.0001)$. There was a similar trend for worse glycaemic control for monotherapy patients in Scotland, odds ratio 1.17 for $7 \% \mathrm{HbA} 1 \mathrm{c}$ target achievement England vs Scotland, $\mathrm{p}<0.0001$. BP target achievement was also better in Wales, odds ratio for 140/80 target achievement 1.12 Wales vs England, 1.89 Wales vs Scotland, $\mathrm{p}<0.0001$. Total cholesterol target $<5$ was met by a significantly higher number of Scottish patients, odds ratio Scotland vs England 1.22, Scotland vs Wales 1.34.

Conclusion: Whilst no correction is made for duration of diabetes or population age this is not likely to have impacted significantly on the results of this audit. There is clear evidence of differential control of blood glucose, blood pressure and cholesterol in the Type 2 diabetes population between the three countries and it is important all patients are managed aggresively to treatment targets. This is particularly important for patients managed with oral monotherapy for blood glucose lowering. As the largest treated patient group they represent an important opportunity for early intervention.

0821

Health related quality of life of body mass index for people with type 2 diabetes in the general adult population in England

M. Hammer ${ }^{2}$, T. L. Christensen ${ }^{2}$, S. Gough ${ }^{1}$

${ }^{1}$ Department of Medicine, University of Birmingham, United Kingdom, ${ }^{2}$ Novo Nordisk A/S, Bagsværd, Denmark.

Background and Aims: The risk of developing cardiovascular and other life threatening diseases is strongly linked to increased body weight. This is particularly relevant to people with type 2 diabetes (T2D) in whom over $80 \%$ are overweight or obese. However, limited data exist on the impact on Health Related Quality of Life (HRQOL) of Body Mass Index (BMI) for people with type 2 diabetes.

The aim of this study was to characterize the impact of BMI on HRQOL for people with and without T2D in England.

Materials and Methods: Data from the Health Survey for England 2003 (HSE03) commissioned by the Department of Health was evaluated. Data was collected during the period January 2003 to March 2004. HSE03 consisted of a stratified random sample designed to provide data about the general population living in private households in England. Observations for 13,233 non-diabetic subjects and 461 patients with $\mathrm{T} 2 \mathrm{D}$ (age $\geq 18$ years) were included in the analyses. 
HSE03 involved a questionnaire-based interview followed by a nurse visit, where measurements and blood samples were taken. HRQOL was measured using the EQ-5D questionnaire. EQ-5D is a generic measure of HRQOL that generates a single index score based on a descriptive system that defines health in terms of 5 dimensions (mobility, selfcare, usual activities, pain/discomfort and anxiety/depression). An individual who had no problems in any domain would score 1, that is, a health state of perfect health. Death is regarded as zero.

Overall HRQOL differences were investigated using t-tests. Analyses of variance (ANOVA) were used to investigate relations between EQ-5D scores and different categories of BMI (underweight $(<18.5)$, normal $(18.5-<25)$, overweight $(25-<30)$, obese $(30-<40)$ and extremely obese $(\geq 40)$.

Results: The overall HRQOL was significantly lower for people with T2D compared to non-diabetic subjects, with EQ-5D scores of 0.795 versus 0.867 , respectively $(p<0.01)$. An ANOVA showed that the non-diabetic obese subgroup (BMI $\geq 30$ ) had a significant reduced HRQOL compared to others $(p<0.05)$. Similarly, the HRQOL for people with T2D was significantly $(\mathrm{p}<0.05)$ lower for the morbidly obese $(B M I \geq 40)$ compared to people in the normal weight band (BMI of $18.5-<25$ ). The HRQOL was lower for people with T2D across all BMI categories compared to non-diabetics; however, due to sample size some of these findings were not significant.

Conclusion: These findings suggest that obesity in the general adult population in England has a significant impact on HRQOL. The impact of obesity on HRQOL is particularly evident in people with T2D who are further burdened by the impact of their disease.

Supported by Novo Nordisk A/S

\section{PS 65}

\section{Liver disease and transplantation-induced diabetes}

\section{2}

Metabolic syndrome in long-term survivors of haematopoietic stem cell transplantation: evidence for a role of TNF- $\alpha$

L. Airaghi ${ }^{1}$, P. Usardi ${ }^{2}$, M. Baldini ${ }^{1}$, A. Branchi ${ }^{1}$,

V. Giunta ${ }^{1}$, A. Orsatti ${ }^{1}$, G. Lambertenghi Deliliers ${ }^{2}$,

C. Annaloro ${ }^{2}$;

${ }^{1}$ Medicina Interna I, ${ }^{2}$ Ematologia I - Centro Trapianti di Midollo, Ospedale Maggiore Policlinico, Mangiagalli \& Regina Elena, Milano, Italy.
Background and Aims: The success of haematopoietic stem cell transplantation (HSCT) has resulted in a large number of patients becoming long-term survivors. In adults the late effects of HSCT include graft versus host disease and its sequelae, effects of long-term immune deficiency, end-organ damage, development of secondary malignancies, and endocrine disorders, including gonadal function and fertility. Until recently, the metabolic disorders following HSCT in adults were unknown.

The purpose of this study is to assess the prevalence of metabolic syndrome (MS) among HSCT long-term survivors and to investigate the potential clinical and biological risk factors for cardiovascular disease (CVD) in this group of patients.

Materials and Methods: The study was carried out in 50 out-patients (27 men and 23 women, mean age $45.4 \pm$ $9.7 \mathrm{yrs}$ ) of the Bone Marrow Transplantation Centre, defined long-term survivors because continuous complete remission lasted at least five years. Clinical characteristics were assessed by data collection concerning waist circumference, blood pressure, and current use of medications for hypertension and/or type 2 diabetes. Blood samples were taken from patients after an overnight fast to evaluate serum concentration of glucose, total and HDL cholesterol, triglycerides, insulin, adiponectin, resistin, leptin, and TNF- $\alpha$. Differences between the means in the groups were evaluated using paired t-test in the case of normal distribution of data sets and Mann-Whitney Rank Sum test when at least in one of the data sets the normal distribution was excluded.

Results: The MS, as defined by the International Diabetes Federation (IDF) criteria, was present in 20 percent of the patients. This subgroup was characterized by elevated serum TNF- $\alpha$ concentrations when compared with the other patients $(11.8 \pm 3.3$ vs $9.6 \pm 3.6 \mathrm{pg} / \mathrm{mL} ; \mathrm{p}<0.05)$. No difference was found in the prevalence of autologous $v s$ allogeneic HSCT.

Using the TNF- $\alpha$ median observed in the patients with MS $(11 \mathrm{pg} / \mathrm{mL})$ as a cut-off value for all the patients, two groups were obtained without significant differences in clinical characteristics. Serum insulin $(19.9 \pm 3.3$ vs $12.8 \pm$ $1.1 \mathrm{mIU} / \mathrm{mL} ; \mathrm{p}<0.01)$, leptin $(26.1 \pm 8.5$ vs $14.9 \pm 1.9 \mathrm{ng} / \mathrm{ml}$; $\mathrm{p}<0.001)$, resistin $(8.9 \pm 1.7$ vs $5.7 \pm 0.5 \mathrm{ng} / \mathrm{mL} ; \mathrm{p}<0.01)$, and triglycerides $(164.5 \pm 18.6$ vs $101.5 \pm 10.2 \mathrm{mg} / \mathrm{dL} ; \mathrm{p}<0.001)$ were significantly higher in the patients with TNF- $\alpha \geq 11 \mathrm{pg} /$ $\mathrm{mL}$ than in the others. Moreover, serum HDL-cholesterol concentrations were significantly lower in the first investigated group ( $54.0 \pm 3.5 v s 67.5 \pm 4.0 \mathrm{mg} / \mathrm{dL} ; \mathrm{p}<0.01$ ).

Conclusion: The prevalence of MS is high in patients who have had HSCT in adulthood. TNF- $\alpha$ increase is detectable either in patients with MS or in patients with some of the biochemical abnormalities observed in MS, i.e. lipid profile and serum concentrations of insulin, leptin, and resistin. So, the proinflammatory cytokine TNF- $\alpha$, implicated in insulin 
resistance, could be a biological marker of the inflammatory state predisposing to MS. In conclusion, MS could be considered an adverse long-term effects of HSCT, affecting the quality of life of patients surviving the procedure and making this growing population prone to CVD.

\section{3}

Carbohydrate metabolism disorders in patients after renal transplantation

B. Brzezińska ${ }^{1}$, R. Junik ${ }^{1}$, A. Bronisz ${ }^{1}$, M. Pujanek ${ }^{1}$, Z. Włodarczyk ${ }^{2}$, A. Adamowicz ${ }^{2}$, J. Manitius ${ }^{3}$;

${ }^{1}$ Endocrinology and Diabetology, ${ }^{2}$ Transplantology and General Surgery, ${ }^{3}$ Nephrology, Hypertension and Internal Medicine, Nicolaus Copernicus University in Torun, L. Rydygier Collegium Medicum in Bydgoszcz, Poland.

Background and Aims: Post-transplantation diabetes mellitus is a complication occurring relatively often after renal transplantation. It significantly decreases prognosis as to the patient's survival and graft functioning. The aim of the study was to analyze the carbohydrate metabolism disorders in patients with chronic renal failure treated by renal transplantation and to estimate diabetogenic effect of immunosuppressive medications.

Materials and Methods: Ninety one patients after renal transplantation were investigated (55 men and 36 women). Average age of the study population was 48 , with the mean weight of $76.9 \mathrm{~kg}$ and the mean post-transplantation period was about 3.8 years. Patients had been dialysed before transplantation for mean time of 28.7 months. Acute graft rejection were observed in 21 patients $(23 \%)$. The metylprednisolon pulses (Solu-Medrol) were administered in 22 patients. The average creatinine concentration at the time of screening was $1.61 \mathrm{mg} / \mathrm{dl}$, BUN - 27,41 mg/dl, total cholesterol concentration $-211.7 \mathrm{mg} / \mathrm{dl}$, triglycerides concentration $-174 \mathrm{mg} / \mathrm{dl}$. The mean systolic blood pressure was $117.5 \mathrm{mmHg}$ and diastolic blood pressure $-82.3 \mathrm{mmHg}$ in analyzed group. The patients were treated with following therapeutic regimen: cyclosporin/tacrolimus combined with azatioprine/mycophenolate mophetil (MMF) and prednisone. The standard oral glucose tolerance test (OGTT) and $\mathrm{HbA}_{1 \mathrm{C}}$ level were performed in the tested group.

Results: The following carbohydrate disorders were observed in the analyzed group: normal glucose tolerance (NGT) - in 31 patients $(34.4 \%)$, impaired fasting glucose (IFG) - in 21 patients (23.3\%), impaired glucose intolerance (IGT) - in 18 patients (20\%), post-transplantation diabetes mellitus (PTDM) - in $15(16.4 \%)$, diabetes as the cause of chronic renal failure - in $2(2.2 \%)$, and diabetes of type 2 diagnosed before transplantation and not being the cause of renal failure - in 3 patients (3.3\%). The significantly higher percentage of PTDM was found in patients taking tacrolimus $(50 \%)$ as compared with $16 \%$ of patients treated with cyclosporin The average $\mathrm{HbA}_{1 \mathrm{C}}$ concentration was the highest in patients treated with tacrolimus combined with MMF and prednisone $(6.69 \%)$. In patients treated with azatioprine and prednisone the $\mathrm{HbA}_{1 \mathrm{C}}$ level was $6.38 \%$. In patients treated with cyclosporin the $\mathrm{HbA}_{1 \mathrm{C}}$ level was $6.26 \%$ although not reaching statistical significance.

Conclusion: It is necessary to control the glycemia level carefully in the renal graft recipients so as to make an early diabetes diagnosis and to begin the treatment as soon as possible. Post-transplantation diabetes was more frequently found in the group treated with tacrolimus.

\section{4}

New-onset diabetes impaired fasting glucose after renal transplantation: results of a prospective, randomised trial comparing cyclosporine versus tacrolimus

P. Marchetti ${ }^{1}$, F. Vincenti ${ }^{2}$, S. Friman ${ }^{3}$,

E. Scheuermann ${ }^{4}$, L. Rostaing and on behalf of the DIRECT Study Group ${ }^{5}$;

${ }^{1}$ Metabolic Unit, Department of Endocrinology and Metabolism, University of Pisa, Italy, ${ }^{2}$ Kidney Transplant Service, University of California San Francisco, United States, ${ }^{3}$ Department of Transplantation and Liver Surgery, Sahlgrenska University Hospital, Goteburg, Sweden, ${ }^{4}$ Department of Transplantation, Medizinische Klinik IV, Frankfurt, Germany, ${ }^{5}$ Service de Néphrologie, Unité de Transplantation d'Organes, CHU Rangueil, Toulouse, France.

Background and Aims: New-onset diabetes mellitus (NODM) and other categories of glucose intolerance (OCGI) after solid organ transplantation are associated with increased morbidity and mortality. Among several risk factors predisposing to NODM or OCGI, the type of immunosuppression plays a major role. Here we describe the results of DIRECT, a 6-month, open-label, randomized, prospective multicenter study which used the ADA/WHO criteria to define glucose abnormalities, and compared cyclosporine versus tacrolimus in kidney graft recipients.

Materials and Methods: De novo renal transplant patients were stratified pre-transplant as (1) diabetic (2) nondiabetic/Caucasian or (3) non-diabetic/non-Caucasian, and were randomized to cyclosporine microemulsion (CsA-ME) with $\mathrm{C}_{2}$ monitoring or tacrolimus. All patients received mycophenolic acid therapy, steroids, and basiliximab. The primary safety endpoint was a composite of NODM or impaired fasting glucose (IFG) at 6 months in patients who were non-diabetic at baseline; the primary efficacy endpoint was a composite of biopsy-proven acute rejection (BPAR), graft loss or death at 6 months in all patients. 
Results: The population analyzed comprised 682 patients (336 CsA-ME, 346 tacrolimus): 115 were diabetic at baseline, 483 were non-diabetic/Caucasian and 84 were non-diabetic/non-Caucasian. Patient characteristics, diabetes risk factors and steroid doses were similar between treatment groups. NODM or IFG occurred in $73 \mathrm{CsA}-\mathrm{ME}$ patients $(26.0 \%)$ and 96 tacrolimus patients (33.6\%, $\mathrm{p}=0.046$ ) at 6 months. In the Caucasian and non-Caucasian subpopulations, the 6-month incidence was $24.8 \%$ and $32.6 \%$ with CsA-ME and $32.2 \%$ and $39.0 \%$ with tacrolimus, respectively. At month $6,12.5 \%$ of CsA-ME patients and $18.0 \%$ of tacrolimus patients who were non-diabetic at baseline required hypoglycemic treatment $(\mathrm{p}<0.05)$. The incidence of BPAR, graft loss or death was $12.8 \%$ with CsA-ME and $9.8 \%$ with tacrolimus $(\mathrm{p}=0.211)$. Blood pressure and renal function were similar. An increase in both median LDL- and HDL-cholesterol occurred in the CsA-ME group (LDL $+0.42 \mathrm{mmol} / \mathrm{L}, \mathrm{p}<0.0001$; HDL $+0.23 \mathrm{mmol} / \mathrm{L}, \mathrm{p}<0.0001)$. The ratio of total cholesterol/ HDL remained unchanged (4.03 vs 3.98 at month 6, $\mathrm{p}=0.43$ ). Adverse events were similar between groups.

Conclusion: The incidence of NODM or IFG at 6 months after renal transplantation is significantly lower with CsAME than tacrolimus with no difference in terms of efficacy. Supported by Novartis Pharma AG

\section{5}

\section{Intermediate clinical phenotype of diabetes in $\mathrm{HCV}$ patients}

M. Skowronski ${ }^{1}$, D. Zozulinska ${ }^{1}$, J. Juszczyk ${ }^{2}$, B. Wierusz-Wysocka ${ }^{1}$;

${ }^{1}$ Department of Internal Medicine and Diabetology, ${ }^{2}$ Department of Infectious Diseases, Poznan University of Medical Sciences, Poznan, Poland.

Background and Aims: There is mounting evidence to indicate the genuine link between diabetes mellitus (DM) and hepatitis $\mathrm{C}$ virus infection (HCV). In the majority of studies diabetes was classified as type 2. Apparently, both insulin resistance (IR) and islet cell dysfunction have been reported to contribute to the development of diabetes in $\mathrm{HCV}$ patients. Moreover, the predictors for this association such as older age, obesity, family history, Black ethnicity are well accepted as the classical risk factors for type 2 diabetes in the general population. However, several questions concerning the true character of diabetes in this group of patients must be underlined. Direct cytopathic effect of $\mathrm{HCV}$ at the islet cell level as well as viral-induced IR may be pathogenic mechanisms causing DM in HCV patients. Thus we propose that the diagnosis of type 2 diabetes in this special group of patients should not be so straightforward. Our aim was to establish the clinical phenotype of diabetes in non-cirrhotic HCV patients.

Materials and Methods: 38 non-cirrhotic HCV (positive anti-HCV antibodies and HCV-RNA) diabetic patients (23 men and 15 women, aged: $47.5 \pm 11.8$, duration of diabetes: $5.1 \pm 4.4$ years, HCV infection: $4.1 \pm 3.0$ years; $\mathrm{HbA} 1 \mathrm{c}$ $8.0 \pm 2.3 \%)$ were compared with patients with type 2 $(n=25,18$ men and 7 women, aged: $49.6 \pm 6.4$, duration of diabetes: $9.0 \pm 5.9$ years; HbAlc $8.3 \pm 1.4 \%$ ) and type 1 $(n=32,19$ men and 13 women, aged: $44.8 \pm 7.1$, diabetes: $16.1 \pm 10.6$ years; $\mathrm{HbA} 1 \mathrm{c} 8.2 \pm 1.3 \%$ ) and with non-diabetic non-cirrhotic HCV patients $(\mathrm{n}=23,14$ men and 9 women, aged: $41.0 \pm 12.4$ ). In all the study groups selected metabolic variables such as systolic and diastolic blood pressure, blood lipids and body-mass-index (BMI) were measured.

Results: HCV diabetic patients presented with intermediate clinical phenotype in comparison with both type 1 and type 2 diabetic controls (Table).

\begin{tabular}{lllll}
\hline & $\begin{array}{l}\text { HCV } \\
\text { diabetes }\end{array}$ & $\begin{array}{l}\text { Diabetes } \\
\text { type 2 }\end{array}$ & $\begin{array}{l}\text { Diabetes } \\
\text { type } 1\end{array}$ & HCV \\
\hline BMI $\left(\mathrm{kg} / \mathrm{m}^{2}\right)$ & $26.5 \pm 4.8$ & $30.9 \pm 6.3^{*}$ & $24.0 \pm 3.4^{\#}$ & $25.0 \pm 3.2$ \\
$\mathrm{SBP}(\mathrm{mmHg})$ & $133.9 \pm 14.0$ & $142.9 \pm 25.6^{*}$ & $128.8 \pm 17.0^{\#}$ & $123.8 \pm 16.6^{\#}$ \\
$\mathrm{DBP}(\mathrm{mmHg})$ & $84.4 \pm 10.2$ & $88.1 \pm 16.0^{*}$ & $82.2 \pm 11.6^{\#}$ & $79.5 \pm 8.9^{\#}$ \\
$\mathrm{TC}(\mathrm{mmol} / \mathrm{l})$ & $3.5 \pm 0.7$ & $4.7 \pm 0.7^{*}$ & $4.3 \pm 0.8^{*}$ & $1.7 \pm 0.5$ \\
$\mathrm{LDL}-\mathrm{C}$ & $1.9 \pm 0.5$ & $2.7 \pm 0.8^{*}$ & $2.3 \pm 0.7^{*}$ & $2.4 \pm 0.6$ \\
$(\mathrm{mmol} / \mathrm{l})$ & & & & \\
$\mathrm{HDL}-\mathrm{C}(\mathrm{mmol} / \mathrm{l})$ & $1.0 \pm 0.3$ & $1.0 \pm 0.2$ & $1.4 \pm 0.4^{*} *^{\#}$ & $1.1 \pm 0.1$ \\
$\mathrm{TG}(\mathrm{mmol} / \mathrm{l})$ & $1.4 \pm 0.8$ & $2.6 \pm 1.9^{*}$ & $1.2 \pm 0.6^{\#}$ & $0.8 \pm 0.1$ \\
\hline
\end{tabular}

${ }^{*} \mathrm{p}<0.05$ vs HCV diabetes; ${ }^{\#} \mathrm{p}<0.05$ vs diabetes type

Conclusion: The current study indicates that a distinct diabetic phenotype is associated with $\mathrm{HCV}$ infection. Therefore, it should be emphasized that the complex pathogenesis of diabetes in HCV patients together with the observed differences in the clinical phenotype cast doubt on the diagnosis of type 2 diabetes in that group of patients.

0826

High incidence of de novo diabetes mellitus type 1 during or after treatment with pegylated interferon in patients with chronic hepatitis $\mathbf{C}$ virus infection

J. Hoekstra ${ }^{1}$, T. Schreuder ${ }^{2}$, H. Gelderblom ${ }^{2}$, C. Weegink ${ }^{2}$, H. Reesink ${ }^{2}$, J. DeVries ${ }^{1}$, P. Jansen ${ }^{2}$; ${ }^{1}$ Internal Medicine, ${ }^{2}$ AMC Liver Center, Academic Medical Center, Amsterdam, The Netherlands.

Background and Aims: Previous reports have shown the occurrence of diabetes mellitus (DM) during treatment with interferon in patients chronically infected with hepatitis $\mathrm{C}$ 
virus (HCV). Both type 1 and type $2 \mathrm{DM}$ have been described. We prospectively assessed the incidence of DM during or shortly after treatment with pegylated-interferon (PEG-IFN) and ribavirin (RBV) and the type of DM.

Material and Methods: After observing a first case of hyperglycaemia during PEG-IFN/RBV treatment in our hospital, blood glucose levels (PGL) of patients with HCV have been routinely assessed at all outpatient visits during and after treatment with PEG-IFN/RBV since January 2003. In those patients who developed DM, autoantibodies to glutamic acid decarboxylase (anti-GAD65), protein tyrosine phosphatase (anti-IA2) and pancreas islet cells (ICA) were investigated by standard immunoassays.

Results: Between January 2003 and October 2005, 150 patients were treated with PEG-IFN/RBV of which 8 developed hyperglycaemia $(5,3 \%)$. Classical symptoms of DM were present in only 2 patients who both developed DM shortly after cessation of PEG-IFN/RBV. One of these patients has achieved a sustained virologic response (SVR). In all other patients, DM was diagnosed by elevated PGL, and symptoms of DM were either indistinguishable from sideeffects provoked by PEG-IFN/RBV or not present. Retrospective analysis revealed that in 2 patients anti-GAD65 became positive after 11 and 22 weeks administration of PEG-IFN (table 1). One patient was positive for anti-GAD65 before treatment. Two patients tested weakly positive for antiIA2 during treatment. One patient became positive for ICA during treatment. Finally, 2 of 8 patients achieved an SVR.

Conclusion: In our population the incidence of diabetes mellitus during therapy with PEG-IFN/RBV is higher than previously reported by others. Classical symptoms associated with DM may be masked by or mistaken for sideeffects provoked by treatment with PEG-IFN/RBV. Most patients were identified by routine assessment of PGL. We therefore suggest routine assessment of PGL at all outpatient visits before, during and after PEG-IFN/RBV treatment for patients infected with $\mathrm{HCV}$.

Autoantibodies before and during treatment with PEG-IFN

\begin{tabular}{lllllll}
\hline Patient & $\begin{array}{l}\text { anti- } \\
\text { GAD65 } \\
\text { before }\end{array}$ & $\begin{array}{l}\text { anti- } \\
\text { GAD65 } \\
\text { during }\end{array}$ & $\begin{array}{l}\text { anti- } \\
\text { IA2 } \\
\text { before }\end{array}$ & $\begin{array}{l}\text { anti- } \\
\text { IA2 } \\
\text { during }\end{array}$ & $\begin{array}{l}\text { ICA } \\
\text { before }\end{array}$ & $\begin{array}{l}\text { ICA } \\
\text { during }\end{array}$ \\
\hline 1 & neg & neg & neg & neg & neg & neg \\
2 & neg & neg & neg & $\begin{array}{l}\text { weakly } \\
\text { nos }\end{array}$ & neg & neg \\
3 & neg & neg & neg & neg & neg & neg \\
4 & weakly & positive & neg & neg & neg & neg \\
& pos & & & & & \\
5 & neg & neg & neg & neg & neg & neg \\
6 & neg & positive & neg & neg & neg & neg \\
7 & neg & positive & neg & neg & neg & neg \\
8 & positive & positive & neg & weakly & weakly & positive \\
& & & & pos & pos &
\end{tabular}

0827

Exenatide improved elevated hepatic alanine transaminase (ALT) and reduced weight in patients with type 2 diabetes mellitus

J. Buse ${ }^{1}$, D. Klonoff ${ }^{2}$, L. Nielsen ${ }^{3}$, X. Guan ${ }^{3}$, C. Bowlus ${ }^{4}$, J. Holcombe ${ }^{5}$, D. Maggs ${ }^{3}$, M. Wintle ${ }^{3}$;

${ }^{1}$ Diabetes, UNC School of Med., Chapel Hill, United States, ${ }^{2}$ Mills-Peninsula Diabetes Res. Inst., UCSF, United States, ${ }^{3}$ Amylin Pharmaceuticals, Inc., San Diego, United States, ${ }^{4}$ Gastro. \& Hep., UC Davis, Davis, CA, United States, ${ }^{5}$ Eli Lilly and Company, Indianapolis, United States.

Background and Aims: Type 2 diabetes mellitus (T2DM), obesity, and nonalcoholic fatty liver disease (NAFLD) are emerging public health concerns that often co-exist; with diabetes and obesity the two most important risk factors for NAFLD. In T2DM patients not achieving glycemic control with MET and/or SFU and enrolled in 30-wk, placebocontrolled, phase 3 trials followed by open-label extensions $\left(63 \% \mathrm{M}\right.$, age $57 \pm 10 \mathrm{y}$, wt $100 \pm 19 \mathrm{~kg}$, BMI $34 \pm 6 \mathrm{~kg} / \mathrm{m}^{2}$, A1C $8.3 \pm 1.0 \%[$ mean $\pm \mathrm{SD}])$, the incretin mimetic exenatide improved glycemic control, reduced body weight, and was generally well tolerated. We evaluated whether these patients had ALT changes that might indicate improved NAFLD.

Materials and Methods: A post hoc interim analysis of the liver biomarker ALT was performed, with a subanalysis by weight change quartiles at 2 years. Patients were stratified by normal baseline ALT values: $\leq 19 \mathrm{IU} / \mathrm{L}(\mathrm{F}), \leq 30 \mathrm{IU} / \mathrm{L}$ (M; Prati 2002). An alternate analysis was done using the older guideline: normal $\leq 37$ IU/L (F), $\leq 48$ Üé/¥ (M).

Results: Patients completing 2 years on exenatide $(\mathrm{n}=283)$ had reduced A1C $(-1.1 \pm 0.1 \% ; \mathrm{p}<0.05)$ and weight $(-4.7 \pm 0.3 \mathrm{~kg} ; \mathrm{p}<0.05)$ from baseline $(\operatorname{mean} \pm \mathrm{SE})$. Patients with normal baseline ALT $(n=132)$ had minimal ALT change: $+1 \pm 1 \mathrm{IU} / \mathrm{L}$ from baseline $21 \pm 0.4 \mathrm{IU} / \mathrm{L}$ (NS) with $-3.9 \pm 0.5 \mathrm{~kg}$ from baseline weight $99 \pm 2 \mathrm{~kg}$. In contrast, patients with elevated ALT at baseline $(n=151)$ had reductions in ALT: $-11 \pm 1 \mathrm{IU} / \mathrm{L}$ from baseline $38 \pm 1 \mathrm{IU} / \mathrm{L}$ $(\mathrm{p}<0.05)$ with $-5.3 \pm 0.5 \mathrm{~kg}$ from baseline weight $100 \pm 2 \mathrm{~kg}$. Patients in the high baseline ALT $(41 \pm 3 \mathrm{IU} / \mathrm{L}) /$ highest weight loss quartile had $-18 \pm 4 \mathrm{IU} / \mathrm{L}$ ALT reduction and $-13.5 \pm 0.7 \mathrm{~kg}$ weight change $(\mathrm{n}=38)$. Patients with elevated ALT at baseline tended to lose more weight than patients with normal ALT at baseline $(\mathrm{p}=0.04)$, however weight change was minimally correlated with baseline ALT $(r=-0.09)$. Similar results were obtained for the ITT and alternate ALT-range analyses.

Conclusion: Exenatide-treated T2DM patients with elevated baseline ALT had ALT reductions, suggesting exenatide may improve NAFLD in this patient population. 
0828

Effects of pioglitazone on liver function in triple oral therapy: a 42-week, placebo-controlled randomised study

C. Le Dévéhat ${ }^{1}, \mathrm{G}$. Charpentier ${ }^{2}, \mathrm{~S}$. Halimi ${ }^{3}$

on behalf of the Study F-PIO-10 Investigators;

${ }^{1}$ Service de Diabétologie, Centre Hospitalier, Nevers,

${ }^{2}$ Service de Diabetologie, Centre Hospitalier Sud Francilien, Corbeil-Essonnes, ${ }^{3}$ Diabetology Nutrition, Centre Hospitalier Universitaire de Grenoble, France.

Background and Aims: Currently there are no safety data from randomised, double-blind studies that have examined the effects of triple oral anti-diabetic therapy (combination of metformin [MET], a thiazolidinedione [TZD] and a sulphonylurea [SU]) on liver function tests. Previous studies of pioglitazone (PIO) have demonstrated favourable effects on liver enzymes when either used as monotherapy or in combination with either MET or an SU. We evaluated the effect on liver function of adding in PIO to MET plus an SU in inadequately controlled, insulin-naïve patients with Type 2 diabetes.

Materials and Methods: This prospective, double-blind, placebo-controlled study randomised 299 patients with Type 2 diabetes previously treated with MET ( $\geq 1700 \mathrm{mg} /$ day) and $\mathrm{SU}$ (maximal dose) for more than 3 months and $\mathrm{HbA}_{1 \mathrm{c}}$ between $7.0 \%$ and $9.5 \%$. After a 4-week run-in, patients received either PIO $30 \mathrm{mg}$ once daily or placebo (PBO). After 3 months, if $\mathrm{HbA}_{1 \mathrm{c}}>6.5 \%$, $\mathrm{PIO}$ or $\mathrm{PBO}$ was up-titrated to $45 \mathrm{mg}$ for the remaining 4 months. Liver function tests were conducted at baseline and at final visit ( 7 months) for alanine aminotransferase (ALT), aspartate aminotransferase (AST), and gamma glutamyltransferase (GGT).

Results: Mean changes (SD) from baseline at Month 7 for ALT (U/L) were $-6.8 \pm 11.6$ with PIO and $-0.2 \pm 10.5$ with $\mathrm{PBO}$, for AST $(\mathrm{U} / \mathrm{L})-1.2 \pm 6.0$ with $\mathrm{PIO}$ and $-0.7 \pm 6.8$ with PBO and for GGT (U/L) $-9.5 \pm 20.1$ with PIO and $3.8 \pm 19.9$ with $\mathrm{PBO}$. Of the 37 patients with high baseline ALT values in the PIO group, 48.6\% (18/37) were normalised at Month 7 vs. 40.7\% (11/27) in the PBO group. Of the $13 \mathrm{PIO}$ patients with elevated baseline AST, $76.9 \%(10 / 13)$ were normalised at Month 7 vs. and $66.7 \%$ (6/9) with PBO. Of the 43 patients in the PIO group with elevated GGT at baseline, 48.8\% (21/43) were normalised at Month 7 vs. $10.8 \%$ (4/37) in the PBO group.

Conclusion: Triple oral anti-diabetic therapy with PIO plus high doses of MET and SU was associated with liver function improvement after 7 months. There was no evidence of hepatotoxicity, consistent with previous findings with pioglitazone.
Shift in the $\%$ of patients with high or normal baseline values to high or normal values at Month 7

\begin{tabular}{|c|c|c|c|c|}
\hline \multirow[b]{2}{*}{ Baseline value: } & \multicolumn{2}{|c|}{ Pioglitazone } & \multicolumn{2}{|c|}{ Placebo } \\
\hline & High & Normal & High & Normal \\
\hline ALT & $n=37$ & $\mathrm{n}=97$ & $\mathrm{n}=27$ & $n=109$ \\
\hline $\operatorname{High}(>30 / 40 \mathrm{U} / \mathrm{L})$ & 51.4 & 5.2 & 59.3 & 11.0 \\
\hline Normal & 48.6 & 94.8 & 40.7 & 89.0 \\
\hline AST & $\mathrm{n}=13$ & $\mathrm{n}=121$ & $\mathrm{n}=9$ & $\mathrm{n}=127$ \\
\hline High $(>32 / 38 \mathrm{U} / \mathrm{L})$ & 23.1 & 0.8 & 33.3 & 1.6 \\
\hline Normal & 76.9 & 99.2 & 66.7 & 98.4 \\
\hline GGT & $\mathrm{n}=43$ & $\mathrm{n}=92$ & $\mathrm{n}=37$ & $\mathrm{n}=99$ \\
\hline High $(>32 / 49 \mathrm{U} / \mathrm{L})$ & 51.2 & 3.3 & 89.2 & 8.1 \\
\hline Normal & 48.8 & 96.7 & 10.8 & 91.9 \\
\hline
\end{tabular}

'High' values are female/male; $n=$ number of patients with data at baseline and Month 7.

Supported by Laboratoires Takeda, France

0829

Enalapril attenuates hepatocytes apoptosis in diabetic rats: role of oxidative stress

S. Bakhti ${ }^{1}$, S. Payabvash ${ }^{2}$;

${ }^{1}$ Department of Cardiology, Shahid Beheshti University of Medical Sciences., Tehran, ${ }^{2}$ Department of Pharmacology, Tehran University of Medical Sciences., Tehran, Islamic Republic of Iran.

Background and Aims: Oxidative stress is involved in both the pathogenesis and complications of diabetes. It has been shown that ACE inhibitors can slow reduce oxidative stress and tissue injury in the streptozotocin-induced diabetic rats. The aim of the present study was to determine the role of the enalapril treatment in the reduction of hepatocytes apoptosis of diabetic rats.

Materials and Methods: The rats were divided into the following groups: STZ group, received streptozotocin (65 mg/kg, single intraperitoneal dose); STZ-ENL group that received both streptozotocin injection and enalapril (20 mg/L drinking water), and Control (intraperitoneal saline). Five months after streptozotocin injection, rats were sacrificed and the liver samples were obtained for evaluation of hepatocytes apoptosis index and measurement of glutathione concentration and antioxidant enzymes activities for assessment of oxidative stress.

Results: Five months after injection of streptozotocin, the number of apoptotic hepatocytes was increased significantly in diabetic rats $(3.12 \pm 0.21 \%)$ compared to control animals $(0.91 \pm 0.13 \%)(\mathrm{P}<0.001)$. Treatment of diabetic rats with enalapril significantly reduced the number of apoptotic 
hepatocytes in STZ-ENL group $(2.04 \pm 0.19 \%)$, compared to STZ group $(\mathrm{P}<0.01)$; however percentage of apoptotic hepatocytes was still higher than control group $(\mathrm{P}<0.01)$. Streptozotocin-induced diabetes was associated with a decrease in hepatic glutathione concentrations $(6.95 \pm 0.24$ vs. $4.78 \pm 0.35, \mathrm{P}<0.001)$, which was significantly attenuated following chronic treatment with enalapril $(5.41 \pm 0.28)(\mathrm{P}<0.01$ vs. Control and $\mathrm{P}<0.05$ vs. STZ). Liver catalase, glutathione peroxidase, and superoxide dismutase activies were significantly reduced in rats of STZ group compared to control. Chronic treatment with enalapril raised the levels of hepatic antioxidant enzyme activities in diabetic animals significantly to levels that were not different from control values.

Conclusion: For the first time, our findings demonstrate that chronic enalapril treatment is protective against hepatic damage in streptozotocin-induced diabetic rats. These results suggest that the protective action of enalapril might be mediated, at least in part, by its effect on tissue oxidant/ antioxidant balance.

\section{0}

Associates of the change in liver fat content after weight loss

C. H. Thompson ${ }^{1}$, M. L. Phillips ${ }^{1}$, M. C. Lewis ${ }^{2}$,

J. P. Slavotinek ${ }^{3}$, R. Valentine ${ }^{3}$, S. Wahlroos ${ }^{1}$, L. Kow $^{2}$,

J. Toouli ${ }^{2}$;

${ }^{1}$ General Medicine, ${ }^{2}$ General and Digestive Surgery, ${ }^{3}$ Medical Imaging, Flinders Medical Centre, Bedford Park, Australia.

Background and Aims: Non-alcoholic fatty liver disease (NAFLD) is a common manifestation of obesity and type 2 diabetes. Known predictors of NAFLD include serum ALT, visceral obesity and insulin resistance. NAFLD improves following weight loss. The degree of resolution of NAFLD following weight loss is not routinely assessed. The aims of this study were to identify simple anthropometric or biochemical correlates of liver fat content (LFAT) at baseline and the correlates of the change in LFAT following weight loss in a group of morbidly obese (BMI $31-52 \mathrm{~kg} / \mathrm{m}^{2}$ ) women. To assess any effect of the type of weight loss intervention on correlates of change in LFAT, subjects were divided into a non-surgical arm utilising only a very low calorie diet (VLCD) or a surgical arm using laparoscopic adjustable gastric banding (LAGB). The duration of intervention was determined by the estimated time to achieve $8 \%$ weight loss. Materials and Methods: LFAT and abdominal subcutaneous (SAT) and visceral adipose tissue volumes (VAT) were measured using magnetic resonance (MR) spectroscopy and MR imaging before $(n=48)$ and, where possible, after 6 weeks of Optifast VLCD $(n=14)$ or 3 months after LAGB $(n=24)$. Anthropometry (body weight, BMI and waist circumference) and fasting blood tests (liver enzymes, free fatty acids, insulin, glucose, ferritin, leptin and adiponectin) were performed before and after each intervention. Insulin sensitivity was estimated by homeostasis model assessment (HOMA). Pearson's correlation coefficient was used to relate variables. $\mathrm{P} \leq 0.05$ indicated statistical significance.

Results: LFAT content in both groups was similar at baseline (0.14 \pm 0.12 SD AU VLCD; $0.11 \pm 0.11$ AU LAGB). Eleven patients in the VLCD arm and 15 patients in the LAGB arm had hepatic steatosis at baseline (LFAT> $0.05 \mathrm{AU})$. As expected, in the group as a whole at baseline, LFAT was best correlated to ALT $(r=0.707, p<0.01)$, VAT $(\mathrm{r}=0.691, \mathrm{p}<0.01)$ and HOMA $(\mathrm{r}=0.502, \mathrm{p}<0.01)$. Mean $A L T=29 \pm 14 \mathrm{U} / \mathrm{L}$ (normal $<50 \mathrm{U} / \mathrm{L}$ ). Neither plasma ferritin, leptin nor adiponectin related to LFAT at baseline. LFAT related to waist circumference at baseline $(\mathrm{r}=0.396$, $\mathrm{p}=0.005$ ) but not to weight or BMI. Similar relative weight loss occurred after VLCD $(8 \pm 3 \%)$ and LAGB $(9 \pm 4 \%)$. LFAT content reduced to $0.08 \pm 0.08 \mathrm{AU}$ and $0.08 \pm 0.11 \mathrm{AU}$ respectively after weight loss but the change was only significant in the VLCD group ( $\mathrm{p}=0.016)$. After VLCD, the change in LFAT was best related to the change in body weight $(\mathrm{r}=0.728, \mathrm{p}=0.003)$. The change in ALT was the only significant correlate of the change in LFAT after LAGB $(\mathrm{r}=0.573, \mathrm{p}=0.003)$.

Conclusion: In the morbidly obese female, LFAT relates to visceral adiposity and insulin sensitivity and relates closely to "normal" levels of ALT. After 6 weeks VLCD intervention, the success of LFAT removal can be best gauged by assessing the patient's weight loss. The effect on LFAT of the more gradual weight loss induced by LAGB can be better assessed by the extent of change in ALT. The differing associates of change in LFAT following these two interventions may reflect differing daily caloric or fat intakes and the differing time periods.

Supported by Novartis Consumer Health Australia Pty Ltd and Diabetes Australia Research Trust

\section{PS 66}

\section{Hypoglycaemia}

0831

The Arg16Gly polymorphism of the $\beta_{2}$-adrenergic receptor and impaired hypoglycaemia awareness in patients with type 1 diabetes

B. J. J. Schouwenberg ${ }^{1}$, B. A. J. Veldman ${ }^{2}$,

B. E. De Galan², C. J. Tack ${ }^{2}$, P. Smits ${ }^{1}$;

${ }^{1}$ Pharmacology-Toxicology, ${ }^{2}$ General Internal Medicine, Radboud University Nijmegen Medical Centre, The Netherlands. 
Background and Aims: Reduced $\beta$-adrenergic sensitivity has been reported in type 1 diabetic patients with impaired hypoglycaemia awareness, presumably as a result of a desensitization process. Desensitization of the $\beta_{2}$-adrenergic receptor is in part determined by the Arg16Gly polymorphism at codon 16 of the gene encoding the $\beta_{2}$-adrenergic receptor. We tested the hypothesis that impaired hypoglycaemia awareness is more common among patients homozygous Gly16.

Materials and Methods: We studied the effect of the Arg16Gly polymorphism of the $\beta_{2}$-adrenergic receptor in 85 Caucasian patients with type 1 diabetes. All subjects were characterized for basic demographic and clinical parameters. Hypoglycaemia awareness status was assessed using a standardized and well-validated questionnaire.

Results: A total of 45 patients (53\%) were homozygous Gly16, 8 patients (9\%) were homozygous Arg16 and 32 patients (38\%) were heterozygous Arg16Gly. Nineteen of the homozygous Gly16 patients (42\%) were classified as having impaired hypoglycaemia awareness, compared to 2 $(25 \%)$ and $5(16 \%)$ of the patients who were homozygous Arg16 or heterozygous Arg16Gly, respectively $(\mathrm{p}=0.04)$. Patients who were homozygous Gly16 were 3 times more likely to have impaired hypoglycaemia awareness than patients without this polymorphism (OR 3.4; 95\%CI 1.39.4; $\mathrm{p}=0.01$ ).

Conclusion: The results of this study indicate that type 1 diabetic patients homozygous Gly16 for the $\beta_{2}$-adrenergic receptor are at increased risk for having impaired hypoglycaemia awareness. Future studies are required to confirm these data in larger and other populations.

Supported by the Dutch Diabetes Foundation

0832

Symptomatic episodes of hypoglycaemia recorded by CGMS in patients with type 1 diabetes

T. Høi-Hansen, U. Pedersen-Bjergaard, B. Thorsteinsson; Dept. Internal Medicine I, Hillerød Hospital, Denmark.

Background and Aims: We analyzed symptomatic episodes of hypoglycaemia recorded by the Medtronic MiniMed Continuous Glucose Monitoring System (CGMS).

Materials and Methods: A cohort of 119 patients with type 1 diabetes (age 46 years $+12($ mean $+\mathrm{SD})$; duration of diabetes 21 years +12 ; HbA1c $8.5 \%+1.0 ; 86 \%$ on multiple injections; $35 \%$ women) participated in 6 days of continuous subcutaneous glucose monitoring with CGMS. Selfmonitored blood glucose (SMBG) measured with HemoCue 201+was used to calibrate the CGMS four times daily. Participants kept a diary of meals, insulin dose, and episodes of symptomatic hypoglycaemia.
Results: A total of 2.6 symptomatic episodes/patient/week were reported by the patients: $2.3(89 \%)$ in the daytime and $0.3(11 \%)$ in the night $(\mathrm{p}<0.001)$. Episodes were less frequent in the night $(11 \%)$ compared to mornings $(30 \%)$, afternoons $(34 \%)$ and evenings $(25 \%) \quad(p<0.001)$. One hundred and sixtyfive (63\%) symptomatic episodes were accompanied by SMBG. The median values of SMBG and CGMS at these episodes were $2.7 \mathrm{mM}$ (range 0.8-8.0) and $3.6 \mathrm{mM}(2.2-11.9)(\mathrm{p}<0.001)$. In $15 \%$ of the episodes a blood glucose concentration $>3.5 \mathrm{mM}$ was measured with SMBG versus $51 \%$ by CGMS. The most frequently reported reason for episodes was physical activity $(36 \%)$. However, in $43 \%$ of the events no reason could be given. In the diaries, household chores were the most frequently selfreported activity during episodes.

Conclusion: In conclusion, most episodes of symptomatic hypoglycaemia occur in the daytime. The variation in blood glucose is wide whether measured by SMBG or CGMS. Physical activity is the most frequent reason for symptomatic episodes, however most events were unexplained.

\section{3}

Effects of amino-acid stimulation on glucagon secretion in non-diabetic and type 1 diabetic subjects during hyperinsulinaemic eu-, hypo and post-hypoglycaemic hyperglycaemia

P. Rossetti, F. Porcellati, S. Pampanelli, C. G. Fanelli,

G. B. Bolli;

Internal Medicine, University of Perugia, Italy.

Background and Aims: Response of glucagon (G) to hypoglycemia $(\mathrm{H})$, is usually lost in type 1 diabetes mellitus (T1 DM). Aim of the present study was to establish if responses of $\mathrm{G}$ in $\mathrm{T} 1 \mathrm{DM}$ are selectively defective during $\mathrm{H}$ as compared to eu- and hyperglycemia.

Materials and Methods: We thus compared responses of $G$ to alanine (A), an amino-acid (AA) known to stimulate $G$ secretion, in a group of T1 DM subjects with those of nondiabetic (N-DS) during hyperinsulinemic eu-hypo- and

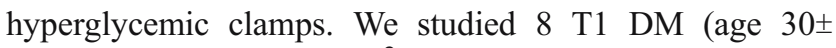
$11 \mathrm{yrs}$, BMI $23.4 \pm 4.3 \mathrm{~kg} / \mathrm{m}^{2}, \mathrm{HbA}_{1 \mathrm{c}} 6.9 \pm 0.9 \%$ ) and $6 \mathrm{~N}-\mathrm{DS}$ on 3 different, randomized, occasions for $370 \mathrm{~min}$. On 2 occasions, i.v. 1-A was infused either at the rate of 5.6 (low dose LDA) or 28 (high dose HDA) $\mu \mathrm{mol} / \mathrm{kg} / \mathrm{min}$ from -120 to $250 \mathrm{~min}$. On a third occasion, saline (S) was infused throughout. In all studies, insulin at the rate of $0.5 \mathrm{mU} / \mathrm{Kg} / \mathrm{min}$ was infused from 0 to $250 \mathrm{~min}$. Variable glucose was infused to clamp plasma glucose (PG) at $2.8 \mathrm{mmol} / 1$ from 90 to $130 \mathrm{~min}$, to restore euglycemia (5.5 mmol/1) from 150 to190 $\mathrm{min}$, and to produce hyperglycemia (10 $\mathrm{mmol} / \mathrm{l})$ from 210 to $250 \mathrm{~min}$. 
Results: PG and insulin concentrations did not differ among studies $(\mathrm{p}>0.2)$. Plasma A increased similarly in T1 DM and N-DS. Glucose infusion rates (GIR) at clamped $\mathrm{H}$ were different in $\mathrm{T} 1 \mathrm{DM}$ : HDA $(0.7 \pm 0.3)$, LDA $(2.02 \pm 0.5)$ and $\mathrm{S}(3.03 \pm 0.6 \mathrm{mg} / \mathrm{kg} / \mathrm{min})(\mathrm{p}<0.05)$. In $\mathrm{N}$ DS, GIR was lower after HDA $(0.9 \pm 0.2)$ as compared to LDA $(1.69 \pm 0.4)$ and $S(1.63 \pm 0.5)(p<0.05)$. In $S, G$ increased in N-DS $(121 \pm 23 \mathrm{ng} / \mathrm{l})$ whereas it did not in T1 DM (51 \pm 5.6$)$. G increased further after LDA $(177 \pm 29)$ and HDA $(228 \pm 36)$ in N-DS, but only after HDA $(111 \pm 11)$ in T1 DM. G decreased after recovering euglycemia and following hyperglycemia both in N-DS and T1 DM.

Conclusion: In T1 DM, deficient response of $\mathrm{G}$ to $\mathrm{H}$ can be recovered by increasing plasma AA concentration. Nevertheless $\mathrm{G}$ response remains reduced in $\mathrm{T} 1 \mathrm{DM}$ as compared to $\mathrm{N}$-DS. AA-induced secretion of $\mathrm{G}$ to $\mathrm{H}$ is largely suppressed by restoration of euglycemia and induction of hyperglycemia. Supported by JDRF

\section{4}

Subcutaneous infusion of very low dose-glucagon averts insulin induced hypoglycaemia in patients with type 1 diabetes mellitus

S. V. Edelman ${ }^{1}$, W. Isley ${ }^{2}$, S. Schwartz ${ }^{3}$, V. Fonseca ${ }^{4}$, S. Mudaliar ${ }^{1}$, R. Henry ${ }^{1}$;

${ }^{1}$ Medicine, University of California San Diego, San Diego,

${ }^{2}$ Endocrinology and Metabolism, Mayo Clinic, Rochester,

${ }^{3}$ Clinical Research Unit, Diabetes and Glandular Disease Clinic, San Antonio, ${ }^{4}$ Medicine, Tulane University Medical Center, New Orleans, United States.

Background and Aims: Insulin intensification to achieve optimal glycemic control and prevent microvascular complications of type 1 diabetes mellitus (t1DM) is associated with increased rates of hypoglycemia, the critical factor limiting optimized glycemic control. We studied the effects of continuous subcutaneous (SC) infusion of Very Low Dose-Glucagon (VLDG) (doses of $<16 \mathrm{ng} / \mathrm{kg} / \mathrm{min}$ ) in patients undergoing insulin intensification in order to induce hypoglycemia.

Materials and Methods: Patients with the following baseline characteristics were enrolled: t1DM of $>10$ years duration, use of insulin pump therapy, BMI $<29 \mathrm{~kg} / \mathrm{m}^{2}$ and HbAlc level $<8.0 \%$. After overnight fasting, patients underwent acute insulin intensification in increments of $50 \%$ every 90 minutes (up to $300 \%$ of basal insulin rates) to induce hypoglycemia (blood glucose $(\mathrm{BG})<50 \mathrm{mg} / \mathrm{dL}$ ). Under the same conditions of insulin intensification for a given patient, VLDG was then administered at doses of 4 , 8,12 or $16 \mathrm{ng} / \mathrm{kg} / \mathrm{min}$ for up to 9 hours or until hypoglycemia was reached.
Results: Plasma glucagon levels reached peak concentrations within 1 hour of initiating the infusion and remained near peak levels for the infusion duration, returning to basal levels $<1$ hour after infusion cessation. VLDG administration induced an initial rise in BG levels that generally peaked within 1 to 2 hours and then declined. All VLDG doses studied either delayed or completely averted the onset of the insulin-induced hypoglycemia in a dose-dependent fashion; however these effects did not reach statistical significance.

\begin{tabular}{llllll}
\hline $\begin{array}{l}\text { VLDG } \\
\text { Dose } \\
\text { ng/kg/ } \\
\text { min }\end{array}$ & $\begin{array}{l}\text { Mean } \\
\text { Plasma } \\
\text { Glucagon* } \\
(\mathrm{pg} / \mathrm{mL})\end{array}$ & $\begin{array}{l}\text { Peak } \\
\text { Blood } \\
(\mathrm{mg} /\end{array}$ & $\begin{array}{l}\text { Time to BG } \\
<70 \mathrm{mg} / \mathrm{dL} \text { (in hours) }\end{array}$ & $\begin{array}{l}<60 \mathrm{mg} / \mathrm{dL} \text { (in hours) } \\
\text { (iL) }\end{array}$ & $\begin{array}{l}<50 \mathrm{mg} / \mathrm{dL} \\
\text { (in hours) }\end{array}$ \\
\hline $0(\mathrm{~N}=14)$ & 56 & 122 & 5.0 & 5.6 & 6.2 \\
$4(\mathrm{~N}=6)$ & 58 & 139.2 & 5.9 & 6.3 & 7.2 \\
$8(\mathrm{~N}=9)$ & 90 & 207 & 7.1 & 7.4 & 7.8 \\
$12(\mathrm{~N}=9)$ & 104 & 214 & 7.0 & 7.1 & 7.5 \\
$16(\mathrm{~N}=8)$ & 124 & 217 & 7.1 & 7.4 & 7.9 \\
\hline
\end{tabular}

*reference range in non-diabetic individuals $50-150 \mathrm{pg} / \mathrm{mL}$

Conclusion: In summary, continuous SC infusion of VLDG is well tolerated, results in a moderate increase in both glucose and glucagon levels, and is able to avert or delay the onset of hypoglycemia induced acutely by increasing basal insulin by 50 to $300 \%$. VLDG will be studied further as a therapeutic intervention to avert hypoglycemia in association with insulin intensification.

0835

PREDICTIVE ${ }^{\mathrm{TM}}$ : A global, prospective, observational study to evaluate insulin detemir treatment in type 1 and 2 diabetes - baseline characteristics and predictors of hypoglycaemia in the European cohort*

A. Dornhorst ${ }^{1}$, J. Copeland Kristensen ${ }^{2}$, K. Horn Rosenberg ${ }^{2}$, H.-J. Lüddeke ${ }^{3}$;

${ }^{1}$ Dept Metabolic Medicine, Imperial College, London, United Kingdom, ${ }^{2}$ Novo Nordisk A/S, Bagsværd, Denmark, ${ }^{3}$ Praxis Lüddeke/Trauner, Munich, Germany.

Background and Aims: Observational studies are valuable in confirming clinical trial outcomes in clinical practice. The objective of PREDICTIVETM (Predictable Results and Experience in Diabetes through Intensification and Control to Target: An International Variability Evaluation) - one of the largest observational studies in diabetes to date - is to assess the safety and efficacy of insulin detemir in a realworld setting. The primary endpoint is incidence of serious 
adverse drug reactions, including major hypoglycaemia. Hypoglycaemia, traditionally associated with insulin therapy, is a key concern for patients. Secondary endpoints are change in: glycosylated haemoglobin $\mathrm{A} 1 \mathrm{c}\left(\mathrm{HbA}_{1 \mathrm{c}}\right)$; weight; fasting blood glucose (FBG); within-subject FBG variability; and incidence of minor hypoglycaemia. Here we report baseline patient characteristics from the European cohort and, in insulin-treated patients, assess the association of hypoglycaemia with baseline parameters.

Materials and Methods: A total of 19,911 patients (15,919 insulin-treated, 3,529 OADs only and 463 treatment naïve), with type 1 or 2 diabetes and requiring basal insulin, were prescribed insulin detemir and will be followed up for 12, 26 and/or 52 weeks. Demography and efficacy parameters were collected from patient records. Hypoglycaemic episodes were collected from patient records or recall in the 4 weeks before initiation of insulin detemir, and negative binomial distribution was used for hypoglycaemic modelling.

Results: Baseline characteristics are shown in the table. The incidence of hypoglycaemia in type 1 and 2 insulintreated patients was positively correlated with duration of diabetes, total number of insulin injections and FBG variability ( $\mathrm{P}<0.05$ for all). The incidence of hypoglycaemia was inversely related to $\mathrm{HbA}_{1 \mathrm{c}}, \mathrm{FBG}$ and body mass index (BMI; $\mathrm{P}<0.05$ for all). Female patients had a higher incidence of hypoglycaemia than males.

Table: Baseline demographic, safety and efficacy measures

\begin{tabular}{lll}
\hline Parameter & $\begin{array}{l}\text { Type 1 } \\
(\mathbf{n}=\mathbf{7 , 0 6 7 )}\end{array}$ & $\begin{array}{l}\text { Type 2 } \\
(\mathbf{n = 1 2 , 7 2 0})\end{array}$ \\
\hline Gender M/F, \% patients & $49.3 / 50.7$ & $47.1 / 52.9$ \\
Mean age, years \pm SD & $41.7 \pm 16.8$ & $60.8 \pm 10.9$ \\
Mean diabetes duration, years \pm SD & $16.5 \pm 12.5$ & $11.1 \pm 7.5$ \\
Mean BMI, kg/m ${ }^{2} \pm \mathrm{SD}$ & $25.2 \pm 4.5$ & $30.9 \pm 5.9$ \\
Mean HbA $1 \mathrm{c}, \% \pm \mathrm{SD}$ & $8.3 \pm 1.7$ & $8.5 \pm 1.6$ \\
Mean FBG, mmol/L $\pm \mathrm{SD}$ & $9.7 \pm 3.4$ & $10.6 \pm 3.3$ \\
Mean within-subject FBG variability & $2.8 \pm 1.9$ & $1.6 \pm 1.3$ \\
(calculated as the SD of FBG), & & \\
mmol/L $\pm \mathrm{SD}$ & & \\
Mean hypoglycaemic episodes, & & \\
in the 4 weeks prior to study start & & \\
All hypoglycaemic events $\pm \mathrm{SD}$ & $3.7 \pm 6.3$ & $0.7 \pm 2.4$ \\
Major hypoglycaemic events \pm SD & $0.2 \pm 1.3$ & $0.1 \pm 0.4$ \\
Pre-study/new therapy, \% patients & & \\
OAD only & $1 / 0$ & $27 / 0$ \\
Insulin & $96.8 / 100$ & $70.6 / 100$ \\
\hline
\end{tabular}

Conclusion: Baseline data from the PREDICTIVETM European cohort show that insulin-treated patients enrolled in this study, in particular type 1 patients, were experiencing substantial hypoglycaemia. Based on observation of this large patient cohort, some interesting associations can be noted; the rate of hypoglycaemia increased with treatment intensification and degree of glycaemic control. Additionally, hypoglycaemia was positively correlated with duration of diabetes and FBG variability, and inversely related to BMI. Follow-up data from the PREDICTIVETM study will provide valuable insights on the safety and efficacy of insulin detemir in the management of diabetes. *Austria, Czech Rep., Denmark, Finland, Germany, Ireland, Israel, Netherlands, Sweden, Turkey and UK

0836

Sensitivity and specificity of CGMS for identifying clinially relevant symptomatic hypoglycaemia

P. Choudhary ${ }^{1}$, C. M. Davies ${ }^{2}$, C. J. Emery ${ }^{3}$, J. V. Freeman ${ }^{4}$, S. R. Heller ${ }^{3}$;

${ }^{1}$ Medical School Building, Kings College London, ${ }^{2}$ Department of Diabetes, Northern General Hospital, Sheffield, ${ }^{3}$ Department of Diabetes, ${ }^{4}$ ScHARR, University of Sheffield, United Kingdom.

Background and Aims: Continuous Glucose monitoring (CGMS) is used in both the clinical and research setting to identify and to quantify hypoglycaemia. Interstitial glucose measured by CGMS differs physiologically from capillary glucose, so it is important to determine the optimal sensor glucose level which predicts clinically relevant symptomatic hypoglycaemia.

Materials and Methods: We analysed CGMS data from 381 patients ( $72 \%$ type 2 diabetic) who underwent a total of 10 days CGMS each over 2 periods separated by $8_{-}^{-}$ 10 months. During CGMS monitoring, they recorded capillary glucose readings during all symptomatic hypoglycaemia. We determined the corresponding sensor glucose value and analysed the relationship between sensor and capillary glucose during these events. We used ROC curve analysis to determine the sensitivity and specificity of different sensor glucose levels for identifying a capillary glucose of $<3 \mathrm{mM}$.

Results: A total of 373 events of symptomatic hypoglycaemia were reported and corresponding sensor and capillary glucose were available for 234 of these. There were 7 episodes of severe hypoglycaemia with mean capillary glucose of 2.3 (range $(1.2-3.5) \mathrm{mM}$ ). During these, corresponding sensor glucose was below the lower limit of detection $(2.2 \mathrm{mM})$ in all but two episodes when the readings were 2.5 and $3.5 \mathrm{mM}$. Mean capillary glucose during 63 symptomatic events when the sensor glucose was below the lower limit of detection $(2.2 \mathrm{mM}$ ) was 2.6 (range 1.2-3.9)mM. There were 173 symptomatic events with a sensor glucose above $2.2 \mathrm{mM}$ and mean (sd) capillary and sensor glucose were $3.2 \mathrm{mM}$ and $3.4 \mathrm{mM}$ respectively. 
Sensor glucose was a mean (sd) of 0.3(0.9) $\mathrm{mM}$ higher than capillary glucose and this difference was greater in type 2 than type 1 diabetic patients.

There were 121 events with a capillary glucose of $<3 \mathrm{mM}$ during which corresponding sensor glucose was also $<3 \mathrm{mM}$ in $73 \%$ and was $<4 \mathrm{mM}$ in $94 \%$ events. Sensitivity and specificity of different sensor glucose levels for identifying a capillary glucose $<3 \mathrm{mM}$ are shown in the table.

Conclusion: CGMS reliably identifies clinically relevant hypoglycaemia. For clinical use a sensor glucose of $3 \mathrm{mM}$ appears optimal in identifying hypoglycaemia. However in the research setting, which requires greater specificity, we would recommend a cut off of $2.2 \mathrm{mM}$.

Sensitivity and Specificity of different sensor glucose cut offs for hypoglycaemia

\begin{tabular}{lll}
\hline Sensor glucose $\mathrm{mM}$ & Sensitivity $\%$ & Specificity \% \\
\hline 4.0 & 93.7 & 22.4 \\
3.5 & 82.9 & 40.8 \\
3.0 & 64.9 & 68 \\
2.5 & 46 & 80 \\
2.2 & 39.6 & 86.4 \\
\hline
\end{tabular}

Supported by the Department for Transport, UK

0837

Experience of hypoglycaemia is associated with lower quality of life (QoL) in German patients with type 2 diabetes on metformin (MF)+PPAR $\gamma$ agonist or MF+ sulphonylurea (SU) combination therapies

D. D. Yin ${ }^{1}$, P. Mavros ${ }^{1}$, R. R. Lyu ${ }^{1}$, E. Seignobos ${ }^{2}$,

E. Trudeau ${ }^{2}$;

${ }^{1}$ Merck \& Co., Inc., Whitehouse Station, United States,

${ }^{2}$ Mapi Values, Lyon, France.

Background and Aims: Hypoglycemia is associated with some glucose-lowering medications. Experience of hypoglycemia is expected to have a significant impact on patients' QoL and treatment compliance. This study was undertaken to evaluate the experience of hypoglycemia and its impact on QoL among diabetic patients treated with MF in combination with a PPAR $\gamma$ agonist or a SU in Germany. Materials and Methods: This is an observational, cross sectional study conducted in 92 clinical centers in Germany in 2005. Patients ( $\geq 35$ years old) with type 2 diabetes who had been treated with MF in combination with a PPAR $\gamma$ agonist or a SU for at least 6 months prior to study enrollment were included. Excluded were patients receiving insulin, treated for hepatitis or HIV, or having gestational diabetes. Case report forms were developed for collection of patients' medical record, experience of hypoglycemia, and general QoL. Both the EQ-5D VAS score and the un-weighted scoring rule for EQ-5D (100 point scale) were used to obtain QoL scores. Adjusted linear regression analysis was used to evaluate the effect of hypoglycemia on patients' QoL controlling for demographic and medical characteristics.

Results: Of the 392 patients included in the study, 29\% (115) received MF in combination with a PPAR $\gamma$ agonist, and the remaining 277 metformin in combination with a SU. The average patient age was 62 years, with patients on $\mathrm{MF}+\mathrm{SU}$ combination therapy being slightly older than those on MF+PPAR $\gamma$ agonist combination therapy (63 vs. 59 years, respectively). Forty three percent of the patients were female, and $45 \%$ had been diagnosed with type 2 diabetes for at least 7 years. Twenty seven percent (105) patients reported at least one episode of hypoglycemia during the previous 6 months. There were more patients on $\mathrm{MF}+\mathrm{SU}$ combination therapy than MF+PPAR $\gamma$ agonist combination therapy $(29 \%$ vs. $23 \%$; p $>0.05)$ that experienced hypoglycemia symptoms. Among patients with episodes of hypoglycemia, 103 (98\%) experienced mild/ moderate hypoglycemic symptoms, $54(51 \%)$ had severe symptoms, with $51 \%$ reported experience of both mild/ moderate and severe symptoms. More than half of those reporting hypoglycemic symptoms had at least 3 episodes during the previous 6 months. Relative to patients not reporting any hypoglycemic symptoms, patients with episodes of hypoglycemia reported lower EQ-5D VAS scores (71.7 vs. $65.5 ; \mathrm{p}=0.0072)$ and lower un-weighted EQ-5D scores (82.4 vs. $71.5 ; \mathrm{p}=0.0003$ ). Hypoglycemia was associated with reductions in both EQ-5D VAS and the un-weighted EQ-5D scores (6.4, $\mathrm{p}=0.0053$ and 11.5 $\mathrm{p}<0.0001$ respectively) after adjusting for basic demographic and medical characteristics (age, gender, marital status, education, co-morbid conditions).

Conclusion: More than $27 \%$ of the study patients treated with metformin $+\mathrm{PPAR} \gamma$ agonist or $\mathrm{MF}+\mathrm{SU}$ combination therapies experienced symptoms of hypoglycemia. This study found that experience of symptoms of hypoglycemia is significantly associated with lower QoL as measured by both the EQ-5D VAS score and the un-weighted EQ-5D score.

0838

The effect of an education programme (HyPOS) to treat hypoglycaemia problems in insulin dependent diabetes patients

N. Hermanns ${ }^{1}$, B. Kulzer ${ }^{1}$, T. Kubiak ${ }^{2}$, M. Krichbaum ${ }^{1}$, T. Haak ${ }^{1}$;

${ }^{1}$ FIDAM, Research Institute of Diabetes Academy Mergentheim, Bad Mergentheim, Germany, ${ }^{2}$ Institute of Psychology, University of Greifswald, Germany. 
Background and Aims: In a randomised, prospective multi-centre trial the effect of an education programme (HyPOS) for patients with hypoglycaemia problems was studied. HyPOS consists of 5 lessons. It focussed on the topics: causes and improvement of hypoglycaemia unawareness, modification of dysfunctional glycaemic targets and health beliefs

Materials and Methods: Patients with hypoglycaemia problems were eligible. 164 patients were randomised either to a control group (CG), receiving a standardised training aiming at intensive insulin therapy $(n=80)$, or to HyPOS $(n=84)$. Hypoglycaemia awareness was measured by the hypoglycaemia awareness questionnaire (HAQ) and by a visual analogue scale (VAS). Mild hypoglycaemic episodes (HE) were assessed by data provided by blood glucose memory meters (\% of blood glucose $<3.9 \mathrm{mmol} / \mathrm{l}$ ). Results: At baseline there were no differences between HyPOS and CG (age: $46.2 \pm 11.6$ yrs. vs. $45.9 \pm 13.3$ yrs.; diabetes duration: $20.3 \pm 10.7$ yrs. vs. $22.1 \pm 10.9$ yrs.; HbAlc: $7.1 \pm 0.9 \%$ vs. $7.4 \pm 1.1 \%$; HAQ: $4.8 \pm 1.6$ vs. $5.0 \pm 1.7$; VAS: $4.3 \pm 2.4$ vs. $4.3 \pm 2.3$; HE: $17.6 \pm 9.8 \%$ vs. $16.0 \pm 9.2 \%$; severe hypoglycaemic episodes (SHE) treated with glucose or glucagon injection: $0.93 \pm 2.2$ vs. $1.07 \pm 2.1$ events per patient and year). After a 6 months Follow-up the drop out rate was $10.3 \%$. Hypoglycaemia awareness significantly improved in HyPOS compared to the CG (VAS- $\Delta:+1.8 \pm 2.1$ vs. $+1.3 \pm 2.1$, $\mathrm{p}<0.05$; HAQ $-\Delta$ : $-2.7 \pm 1.9$ vs. $-2.4 \pm 2.1 \mathrm{p}=.01)$. The number of HE was significantly reduced $(\mathrm{p}=.015)$ by $3.3 \pm 7.4 \%$ in HyPOS compared to $0.3 \pm 6.4 \%$ in the CG. Also the number of SHE dropped to $0.28 \pm 1.0$ in HyPOS compared to $0.45 \pm 1.3$ events per patient and year in the $\mathrm{CG}$ $(\mathrm{p}=.12)$.

Conclusion: HyPOS improved hypoglycaemia awareness significantly. Furthermore HyPOS reduced significantly the occurrence of HE. Hypoglycaemia unawareness and the occurrence of mild hypoglycaemic episodes play an important role in the pathogenesis of hypoglycaemia associated autonomic failure. Thus HyPOS could contribute to an improvement of hypoglycaemic associated autonomic failure. Regarding the rather short follow up period the reduction of SHE in HyPOS is also remarkable. HyPOS seems to be effective to treat hypoglycaemia unawareness.

\section{9}

\section{Accuracy of the new HemoCue ${ }^{\circledR}$ Monitor Glucose Measuring System assessed using patient samples and an isotope dilution mass spectrometry reference method U. Hannestad, E. Theodorsson; \\ Faculty of Health Science, Division of Clinical Chemistry, Linköping, Sweden.}

Background and Aims: HemoCue ${ }^{\circledR} \mathrm{AB}$, Ängelholm, Sweden, has developed a new glucose measuring system, the HemoCue Monitor (HCM). This new system has been developed and is designed primarily as a glucose meter for self-monitoring of blood glucose. The instrument has an extended measuring range compared to previous HemoCue glucose systems, with an upper concentration limit of $27.8 \mathrm{mmol} / \mathrm{L}(500 \mathrm{mg} / \mathrm{dL})$ for whole blood or an upper limit of $30.9 \mathrm{mmol} / \mathrm{L}(555 \mathrm{mg} / \mathrm{dL})$ for the plasma equivalent version of the system. Moreover, the microcuvettes of the HCM system have a new design and are stable at room temperature. In this study the accuracy of the new system is compared to an isotope dilution mass spectrometry (ID GCMS) method using venous patient samples.

Material and Methods: One hundred thirty six venous EDTA whole blood samples were collected over a wide concentration range, mainly from diabetics. Glucose concentrations were measured by split-sample technique using both the HCM instrument and the ID GC-MS method. To measure the between-run variation in the ID GC-MS method, controls samples at three concentration levels were analyzed together with the patient samples. Also, a control sample supplied by HemoCue was analyzed with the HCM system. The HCM microcuvette requires $4 \mu \mathrm{L}$ of whole blood which is hemolyzed in the cuvette and the glucose is measured by means of the glucose dehydrogenase method. A chromogen in the cuvette results in an absorption proportional to the glucose concentration in the sample. For the ID GC-MS method $50 \mu \mathrm{L}$ of whole blood was taken and pipetted into $200 \mu \mathrm{L}$ of internal standard solution. Glucose was quantified as its aldononitrile pentaacetate on a HP MSD 5970 mass spectrometer (Clinical Chemistry 43:794$800,1997)$. The glucose used as calibrator in the ID GCMS method was SRM 917b from NIST.

Results: In total 136 whole blood samples were analyzed and no results were excluded. The glucose concentration in the samples ranged from $1.6 \mathrm{mmol} / \mathrm{L}(29 \mathrm{mg} / \mathrm{dL})$ to $27.1 \mathrm{mmol} / \mathrm{L}(488 \mathrm{mg} / \mathrm{dL})$. Linear regression analysis comparing the HCM and the ID GC-MS results, showed a slope of 0.986 and an intercept of 0.038 . The correlation coefficient was $r=0.998$. The mean glucose concentration of the 136 samples analyzed by the ID GC-MS method was $10.556 \mathrm{mmol} / \mathrm{L}(190 \mathrm{mg} / \mathrm{dL})$, and $10.364 \mathrm{mmol} / \mathrm{L}(187 \mathrm{mg} /$ dL) for the HCM method. Thus the bias for the HCM instrument was $-0.192 \mathrm{mmol} / \mathrm{L}(-1.8 \%)$ compared to the reference method. No proportional error was found. The ID GC-MS method had a between-run relative variation of $0.86 \%$ ((mean concentration $4.45 \mathrm{mmol} / \mathrm{L}(80 \mathrm{mg} / \mathrm{dL}))$; $2.11 \%$ (mean concentration $1.87 \mathrm{mmol} / \mathrm{L}(34 \mathrm{mg} / \mathrm{dL})$ ) and $1.34 \%$ (mean concentration $11.16 \mathrm{mmol} / \mathrm{L}(201 \mathrm{mg} / \mathrm{dL})$ ) for the control samples Seronorm, Pathonorm Low and Pathonorm High, respectively. The total variation for the HCM system measured with the HCM control sample was 
$1.3 \%$. Of all the 136 glucose results from the HCM system, $89.0 \% \quad(\mathrm{n}=121)$ differed less than $\pm 10 \%, 96.3 \% \quad(\mathrm{n}=131)$ differed less than $\pm 15 \%$ and $98.5 \%(n=134)$ results differed less than $\pm 20 \%$ from the ID GC-MS reference method. These comparison results means that all glucose results of the HCM system compared to ID GC-MS are well in agreement of the demands from the International Standardization Organization.

Conclusion: The accuracy of the glucose analyzer HemoCue Monitor compared to the ID GC-MS reference method was very good and fulfilled the requirements for in vitro blood glucose monitoring systems stated of the International Standardization Organization.

\section{PS 67}

\section{Diet and natural remedies}

0840

Improvement of endothelial function in diabetic subjects after consumption of flavanol-rich cocoa J. Balzer ${ }^{1}$, P. Brouzos ${ }^{1}$, P. Kleinbongard ${ }^{1}$, T. Rassaf ${ }^{1}$, T. Lauer ${ }^{1}$, H. Schroeter ${ }^{2}$, M. Kelm ${ }^{1}$;

${ }^{1}$ Division of Cardiology, Pulmonology, Vascular Diseases, Medical Clinic I, University Hospital RWTH Aachen, Germany, ${ }^{2}$ Department of Nutrition, University of California, Davis, United States.

Background and Aims: Endothelial dysfunction characterized by a reduced bioavailability of nitric oxide (NO) is a key event in arteriosclerosis, and Diabetes mellitus is associated with rapidly progressive arteriosclerosis in humans. Recently, it could be shown that endothelial function in smokers was substantially improved after ingestion of a flavanol-rich diet. The present study was aimed to investigate the effects of a cocoa drink rich in flavanols on endothelial function in diabetic subjects.

Materials and Methods: Ten diabetic patients received orally administered cocoa containing 90,385 and $975 \mathrm{mg}$ of total flavanols during a randomized, double-blind, crossover study. Endothelial function was measured before and up to 6 hours after cocoa ingestion by flow-mediated dilation (FMD) of the brachial artery using high resolution ultrasound. In parallel circulating NO-species in plasma (RNO, Nitrite, Nitrate) were characterized by reductive gasphase chemiluminescence.

Results: Endothelial dysfunction was found in every diabetic individual under control conditions (FMD $3,71 \pm 0,27 \%$ ). Ingestion of flavanols dose-dependently increased FMD to a maximum of $5,54 \pm 0,35 \% \quad(p<0,01)$ after two hours. This increase was accompanied by significant augmentations in plasma concentrations of nitrite (baseline: $173 \pm 26 \mathrm{nM}$; maximum post ingestion: $293 \pm 38 \mathrm{nM}$ ), indicating an improvement of NO-bioavailabillity. Endothelium-independent arterial dilation was unaffected by the intervention.

Conclusion: This study for the first time provides evidence that cocoa rich in flavanols can improve endothelial function and is associated with an increase in circulating NO-species in diabetic patients. These findings may implicate a potential use of flavanols in dietary or therapeutic interventions aimed at treating Diabetes-associated vascular diseases, including peripheral and coronary artery disease that are characterized by impaired regional NO production.

Supported by MARS Incorporated

\section{1}

Corosolic acid reduces hypertension, oxidative stress and inflammation in a rat model of metabolic syndrome K. Yamada ${ }^{1}$, Y. Yamaguchi ${ }^{2}$, N. Yoshikawa ${ }^{2}$, A. Harada ${ }^{2}$, J. Haginaka ${ }^{3}$, K. Nakamura ${ }^{2}$, M. Kunitomo ${ }^{2}$;

${ }^{1}$ Diabetes and Clinical Nutrition, Use Techno Corporation, Kyoto, ${ }^{2}$ Pharmacology, ${ }^{3}$ Analytical Chemistry, Faculty of Pharmaceutical Sciences, Mukogawa Women's University, Hyogo, Japan.

Background and Aims: Banaba leaves (Lagerstroemia speciosa LINN.) tea is a popular drink in Southeast Asia and it has traditionally been used to treat diabetes mellitus. Corosolic acid (CRA) is one of the most pharmacologically important compounds contained in Banaba leaves. We previously found that CRA exerts its anti-diabetic effects by reducing hepatic glucose output in vitro and improving insulin sensitivity in vivo. The metabolic syndrome (MS) is characterized by a group of multiple metabolic risk factors, such as obesity, insulin resistance and elevated blood pressure. It is also known that MS is a proinflammatory state with excessive oxidative stress. In this study, we investigated the protective effects of CRA on MS using SHR/ND mcr-cp(cp/cp) (SHR-cp) rats, an animal model of MS, which develop obesity, hyperinsulinemia, hyperglycemia, hyperlipidemia and hypertension.

Materials and Methods: 6-week-old male SHR/cp rats were fed high fat diet containing $0.072 \%$ CRA for 14 weeks. We measured the plasma levels of thiobarbituric acid-reactive substances (TBARS), and 8-hydroxydeoxyguanosine levels (8-OHDG) as oxidative stress biomarkers and 3-nitorotyrosine, 3-chlortyrosine, and high-sensitive C-Reactive Protein (hsCRP) as inflammatory biomarkers, as well as body weight, blood pressure, and plasma free fatty acid.

Results: Body weight gain was slightly smaller in the CRA-treated group than in the untreated group during the 
experiment, although it was not significant. Blood pressure levels at 8 weeks was significantly lower in the CRAtreated group (CRA, 180 \pm 2 : Control, $200 \pm 5 \mathrm{~mm} \mathrm{Hg}$, $\mathrm{p}<0.05$ ), which was also observed at 12 weeks (CRA, $188 \pm 5$ :Control, 209 $\pm 3 \mathrm{~mm} \mathrm{Hg}, \mathrm{p}<0.05)$. Free fatty acid was significantly decreased in the in CRA-treated group at 2 weeks (CRA, 1.0 \pm 1.2 : Control, $1.3 \pm 1.5 \mathrm{mEq} / \mathrm{L}, \mathrm{p}<0.05$ ). CRA decreased oxidative stress markers as early as at 2 weeks; TBARS (CRA, $2.7 \pm 0.1$ :Control, $3.7 \pm 0.1 \mathrm{nmol} / \mathrm{ml}$,

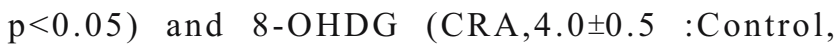
$9.8 \pm 0.3 \mathrm{pmol} / \mathrm{ml}, \mathrm{p}<0.05)$, which continued throughout the experimental period. CRA also reduced inflammatory markers; 3-Nitrotyrosine (CRA,21.1 \pm 1.2 :Control, $34.0 \pm 2.5 \mathrm{pmol} / \mathrm{mg}$ protein, $\mathrm{p}<0.05)$ and 3 -Chlorotyrosine (CRA, 17.4 \pm 0.6 :Control, $28.3 \pm 1.3 \mathrm{pmol} / \mathrm{mg}$ protein, $\mathrm{p}<0.05$ ) at 10 weeks, and hsCRP (CRA,222+14:Control, $268 \pm 16 \mu \mathrm{g} / \mathrm{ml}$ )at 12 weeks.

Conclusion: These data demonstrate that CRA ameliorates hypertension, lipid metabolism, oxidative stress and inflammation in SHR-cp rats. Together with its insulin sensitizing effect, CRA will show beneficial effects on metabolic syndrome, which has become a growing healthcare problem in the world.

Supported by Grant-in-Aid for Scientific Research from the Ministry of Education, Science, Sports and Culture of Japan. Open Research Center Project of Mukogawa Women's University for studying lifestyle-related diseases

\section{2}

Natural sweetener stevioside ameliorates metabolic disturbances and attenuates hypertension in rats with fructose-induced insulin resistance

N. Gorbenko ${ }^{1}$, V. Poltorack ${ }^{2}$, O. Ivanova ${ }^{2}$,

K. Gorbenko ${ }^{3}$, E. Khudyakova ${ }^{1}$;

${ }^{1}$ Pharmacology, Institute of Endocrine Pathology Problems, Kharkiv, ${ }^{2}$ Pathophysiology, Institute of Endocrine Pathology Problems, Kharkiv, ${ }^{3}$ Pathophysiology, Kharkov Medical University, Kharkiv, Ukraine.

Background and Aims: It has been shown that stevioside (S), a sweet-tasting diterpene glycoside isolated from Stevia rabaudiana Bertoni leaves, exerts antihyperglycaemic, antihypertensive effects and improves lipid profiles and oxidative status in diabetic animals. The aim of the study was to explore the impact of $\mathrm{S}$ on changes in metabolism and blood pressure in rats with fructose-induced insulin resistance.

Materials and Methods: Male Wistar rats were divided into three groups: the control group $(\mathrm{C}, \mathrm{n}=8)$, the high fructose-fed group $(\mathrm{F}, \mathrm{n}=8)$, which had free access to 250 $\mathrm{g} / \mathrm{L}$ solutions of fructose for 8 weeks and fructose-fed group treated with stevioside $(\mathrm{F}+\mathrm{S})$ for 8 weeks $(0.2 \mathrm{~g} / \mathrm{kg} /$ day per os). At the end of the study fasted rats were subjected to the glucose tolerance test (GTT, $3 \mathrm{~g} / \mathrm{kg}$ i.p.). Indexes of insulin resistance (IR) were determined using the homeostasis model assessment methods (HOMA) and quantitative insulin sensitivity check index (QUICKI). Serum levels of non-esterified fatty acids (NEFA), triglycerides (TG) and alanine aminotransferase (ALT) were measured as parameters of insulin resistance. Activity of hepatic glucose-6phosphatase (G6P) was also measured in all experimental groups. Oxidative status was estimated by lipid peroxidation intermediates - plasma thiobarbituric acid reactive substances (TBARS) and total antioxidant activity (TAA).

Results: Fructose feeding induced insulin resistance, as indicated by higher HOMA-IR index (F: $4.71 \pm 0.70$ vs $\mathrm{C}$ : $1.49 \pm 0.01, \mathrm{p}<0.001)$ and lower QUICKI $(\mathrm{p}<0.01)$ indexes. The fructose group also developed glucose intolerance, hypertriglyceridemia, significantly elevated NEFA levels, ALT and G6P activities $(\mathrm{p}<0.01)$. Administration of $\mathrm{S}$ protected against the forming of fructose-induced glucose intolerance. Area under curve over the GTT was F+S: $1064 \pm 49 \mathrm{mM} x$ hour vs F: $1403 \pm 90, \mathrm{p}<0.01 ; \mathrm{C}: 847 \pm 25$. The treatment with $\mathrm{S}$ decreases insulin resistance (HOMA-IR index $\mathrm{F}+\mathrm{S}$ : $2.49 \pm 0.18, \mathrm{p}<0.01)$, triglyceride concentration $(\mathrm{F}+\mathrm{S}: 0.593 \pm 0.058$ vs $\mathrm{F}: 0.934 \pm 0.93 \mathrm{mmol} / \mathrm{l}, \mathrm{p}<0.01), \mathrm{NEFA}$ levels $(2.03 \pm 0.13 \mathrm{vs} \mathrm{F}: 4.10 \pm 0.39 \mathrm{mmol} / 1, \mathrm{p}<0.01)$ and serum ALT activity $(\mathrm{p}<0.02)$ in compared with fructose-fed rats treated with placebo. Activity of G6P was reduced by $42 \%$ $(p<0.01)$ in liver of fructose-fed animals after administration of $\mathrm{S}$. In addition, the use of $\mathrm{S}$ was associated with decrease in TBARS concentration 1.5 -fold $(\mathrm{p}<0.02)$ and increase in TAA by $25 \%(p<0.01)$ compared to control fructose-fed rats. $S$ administration resulted in a significant decrease in mean of arterial pressure in fructose-fed rats $(\mathrm{F}+\mathrm{S}: 123 \pm 7.0 \mathrm{vs} \mathrm{F}: 155 \pm$ $8.0 \mathrm{~mm} \mathrm{Hg} ; \mathrm{P}<0.05)$.

Conclusion: These data demonstrate that stevioside administration can ameliorate the deleterious effects of high-fructose diet such as hypertension, insulin resistance, hypertriglycerdemia, increased gluconeogenesis and oxidative stress.

\section{3}

Efficacy and tolerability of oral stevioside in patients with type 2 diabetes: a long-term, randomized, doubleblinded, placebo-controlled study

P. B. Jeppesen ${ }^{1}$, L. Barriocanal ${ }^{2}$, M. T. Meyer ${ }^{3}$, M. Palacios ${ }^{2}$, F. Canete ${ }^{2}$, S. Benitez ${ }^{2}$, S. Logwin ${ }^{2}$, Y. Schupmann ${ }^{3}$, G. Benitez ${ }^{2}$, J. T. Jimenez ${ }^{2}$;

${ }^{1}$ Dept. of Endocrinology and Metabolism C, Aarhus University Hospital, Denmark, ${ }^{2}$ Dept. of Endocrinology and Metabolism, 3rd Internal Medicine Unit, Clinical Hospital, National University of Asuncion, Paraguay, ${ }^{3}$ Laboratorio de Analisis Clinicos, MeyerLab, Asuncion, Paraguay. 
Background and Aims: Stevioside, a natural glycoside, have in animal and human studies shown to possesses a dual beneficial action i.e. reduces blood glucose as well as blood pressure and may have a potential role in the treatment of type 2 diabetes mellitus and the metabolic syndrome. The aim of the study was to carry out a randomised, placebo-controlled, double blind, parallel, long-term trial in type 2 diabetic subjects studying the impact of stevioside on the glycaemic control, insulin sensitivity, hormone responses, lipids, weight development, and diurnal blood pressure i.e. the most important features of the metabolic syndrome.

Materials and Methods: 55 type 2 diabetic subjects aged 40-70 years were included. The subject received $500 \mathrm{mg}$ stevioside or placebo (maize starch) thrice daily in a period of thee months. A twenty four hour blood pressure measurement and a meal test were carried out in the beginning and at the end of the study. The patients were monitored during the study according the protocol, re. BW, fasting blood glucose, blood pressure and lipids. At the end of the study, an IV glucose tolerance test was performed. The diabetic subjects stopped to take there p.o.antidiabetic agents two week before the onset of the study and until its end.

Results: The HbA1C was significant increased for the placebo group $(\mathrm{p}<0.01)$, as the stevioside group was unchanged $(p<0.14)$ during the 3 months of treatment. In the placebo group an increase in the fasting blood glucose was detected $(p<0.007)$, but not for the stevioside group $(p<0.1)$. Compared to placebo, stevioside did not reduced the incremental area under the glucose response curve (IAUC) $(\mathrm{p}<0.1)$ either the meal test or the IVGTT test as well as BW. The insulin response was reduced by (45\%) for the placebo group but maintained for the stevioside group. In the IVGTT test the stevioside group increased the insulin responses after injection of glucose about $21 \%$, but it was not significant compared to the placebo group. Lipids like TG, FFA and Cholesterol did not change between the two groups, as well as the blood pressure, which may be due to the fact that the subject in both groups continue there normal hypertensive treatment during the study. Conclusion: We found that the group treated with stevioside did not have a decrease of insulin level in the meal test, as it was observed for the placebo group after 3 months of treatment. Insulin response was higher for the stevioside group in the GTT test compared to placebo. HbA1C and fasting blood glucose was significant increased for the placebo group, but unchanged for the stevioside group. No difference in lipids or blood pressure was observed. The patients included in this study may already have developed a late stage of diabetes, and thereby a limited beta cell function, which may explain the difference in data found in animal studies. A group of newly diagnosed type 2 diabetes patients, medicine naïve, would be preferable to confirm the anti-diabetic action of stevioside.

Supported by the Research Council for Development Research, DANIDA, Denmark

0844

Differential acute effects of two different isocaloric meals, one rich in saturated fat and one rich in monounsaturated fat, on endothelial function in subjects with type 2 diabetes mellitus

C. Arapostathi, N. Tentolouris, D. Perrea,

A. Papazafiropoulou, G. Argyrakopoulou, A. Kokkinos,

N. Katsilambros;

1st Department of Propaedeutic Medicine, Laiko Hospital, Athens, Greece.

Background and Aims: Data on the effect of monounsaturated fatty acids (MUFA) on endothelial function in subjects with Type 2 Diabetes Mellitus (T2DM) are limited and controversial. The aim of this study was to examine the acute effects of two different meals, one rich in MUFA and one rich in saturated fatty acids (SAFA) on endothelial function in individuals with T2DM.

Materials and Methods: A total of 19 participants with T2DM (13 men and 6 women, mean age 58.6 \pm 10.3 years, mean duration of diabetes $4.0 \pm 3.4$ years), were examined before and after consumption of two different isocaloric meals (about 560 Kcal each), one MUFA-rich and one SAFA-rich meal. The MUFA-rich meal contained $100 \mathrm{~g}$ toast bread and $33 \mathrm{~g}$ extra virgin olive oil and the SAFArich meal $100 \mathrm{~g}$ toast bread and $40 \mathrm{~g}$ butter. The two meals were given on two different occasions, 7-10 days apart, and in random order. Endothelial function was assessed by measurement of flow mediated dilatation (FMD) by the use of high resolution ultrasonography at baseline, $2 \mathrm{~h}, 4 \mathrm{~h}$ and $6 \mathrm{~h}$ after the test meals. In addition, plasma lipids and glucose levels were measured at the same time intervals.

Results: FMD did not change significantly over time after consumption of the MUFA-rich meal (baseline: $7.2 \pm 0.8 \%$; 2 h: $5.0 \pm 0.9 \%$; 4 h: $6.8 \pm 1.1 \%$; and 6 h: $7.1 \pm 1.3 \%$, ANOVA for repeated measurements $\mathrm{P}=0.27$ ). A progressive significant decline, however, was observed after consumption of the SAFA-rich meal (baseline: $7.6 \pm 1.5 \% ; 2 \mathrm{~h}: 5.5 \pm 1.3 \%$; $4 \mathrm{~h}: 1.8 \pm 0.8 \%$; $6 \mathrm{~h}: 3.9 \pm 1.1 \%$, ANOVA for repeated measurements $\mathrm{P}=0.002)$, reaching a nadir $(\mathrm{P}$ value in comparison with the baseline value $<0.001$ ) at $4 \mathrm{~h}$. The FMD throughout the experiment, expressed as area under the curve (AUC) after adjustment for the baseline values, increased after the MUFA-rich meal $(+5.2 \pm 2.5 \%)$ and decreased after the SAFA-rich meal $(-16.7 \pm 6.0 \%)$ $(\Delta=-11.5 \pm 6.4 \%$, P value for the comparison of the AUCs 
of the FMD change between the test meals $=0.008$ ). Plasma glucose and triglycerides concentrations increased postprandially to the same degree after both test meals.

Conclusion: In subjects with T2DM, the consumption of a SAFA-rich meal exerts a detrimental effect on endothelial function. On the contrary, a MUFA-rich meal does not result in an impairment of the endothelial function.

\section{5}

Improvement of insulin resistance after diet with a whole grain based dietary product: results of a randomised, controlled cross-over study in obese subjects with elevated fasting blood glucose

K. Roggen ${ }^{1}$, K. Rave ${ }^{1}$, S. Dellweg ${ }^{1}$, T. Heise ${ }^{1}$,

H. tom Dieck ${ }^{2}$;

${ }^{1}$ Profil, Institut für Stoffwechselforschung $\mathrm{GmbH}$, Neuss,

${ }^{2}$ Degussa, Food Ingredients GmbH, Freising, Germany.

Background and Aims: Subjects with obesity and elevated fasting blood glucose are at high risk of developing type 2 diabetes. This risk may be reduced by a dietary intervention that leads to an improvement of insulin resistance.

Materials and Methods: We investigated the potential of a whole grain based dietary product (WG) with reduced starch content derived from double-fermented wheat (Degussa Food Ingredients $\mathrm{GmbH}$ ) to positively influence body weight, fasting blood glucose, insulin resistance and lipids in comparison to a leading, commercially available nutrient-dense meal replacement product (MR). Thirty-six obese subjects (BMI $>29$ and $<40 \mathrm{~kg} / \mathrm{m}^{2}$ ) with elevated fasting blood glucose $(>6.1$ and $\leq 7.1 \mathrm{mmol} / \mathrm{l})$ were enrolled in a randomized two-way cross-over study with two 4-week treatment periods separated by a 2 -week wash out with habitual diet. Subjects were instructed to replace at least two meals (preferentially breakfast and dinner) with WG or MR, respectively, targeting for a daily consumption of $200 \mathrm{~g}$ of either product. During diet periods total daily calorie intake was limited to $1700 \mathrm{kcal}$.

Results: Thirty-one subjects (age $51 \pm 13$ y, BMI $33.9 \pm$ $2.7 \mathrm{~kg} / \mathrm{m}^{2}$, fasting blood glucose $6.4 \pm 0.3 \mathrm{mmol} / \mathrm{l}$; mean \pm SD) completed the study. Both, after diet with WG and with MR body weight $(-2.5 \pm 2.0$ vs. $-3.2 \pm 1.6 \mathrm{~kg})$, fasting blood glucose $(-0.4 \pm 0.5 \mathrm{vs}$. $-0.5 \pm 0.8 \mathrm{mg} / \mathrm{dl})$, total cholesterol $(-0.5 \pm 0.7$ vs. $-0.6 \pm 0.7 \mathrm{mmol} / \mathrm{l})$, LDL-cholesterol $(-0.7 \pm 0.6$ vs. $-0.3 \pm 0.5 \mathrm{mmol} / \mathrm{l})$ and triglycerides $(-0.3 \pm 0.2$ vs. $-0.3 \pm 0.1 \mathrm{mmol} / \mathrm{l})$ decreased significantly (ANOVA) as HOMA insulin resistance score $(-0.7 \pm 1.2$ vs. $-1.1 \pm 2.3 \mu \mathrm{U} / \mathrm{ml} \times \mathrm{mmol} / \mathrm{l})$ improved. However, after adjustment for body weight loss (COANOVA), the improvement of fasting blood glucose and HOMA-IR score remained statistically significant only for WG but not for MR - indi- cating an effect of WG on fasting blood glucose and insulin resistance independent from changes in body weight.

Conclusions: We conclude that a diet with WG beneficially influences metabolic risk factors for type 2 diabetes thus potentially contributing to postpone the development of the disease.

Supported by Degussa Food Ingredients GmbH

0846

Fiber diet ameliorates glucose homeostasis in a rat model of fructose-induced insulin resistance

J. Cancelas ${ }^{1}$, P. G. Prieto ${ }^{1}$, P. Moreno ${ }^{1}$,

M. L. Villanueva-Peñacarrillo ${ }^{1}$, W. J. Malaisse ${ }^{2}$,

I. Valverde ${ }^{1}$;

${ }^{1}$ Metabolism, Nutrition and Hormones, Fundación Jiménez Díaz, Madrid, Spain, ${ }^{2}$ Laboratory of Experimental Hormonology, Brussels Free University, Belgium.

Background and Aims: The long-term administration for up to 50 days of either an olive oil- or guar-enriched diet to normal rats was recently found to improve glucose tolerance and increase the secretory response of insulinproducing cells to oral glucose. The influence of these two diets on insulin sensitivity was now explored in a rat model of fructose-induced insulin resistance.

Materials and Methods: Four groups of 8-13 male Wistar rats each were given free access to tap water and a standard diet (C) or fructose dissolved in tap water $(20 \%, w / v)$ and either the standard diet (F) or a diet containing 15\% (w/w) olive oil (FO) or $20 \%(\mathrm{w} / \mathrm{w})$ guar (FG). After 65 days, a hyperinsulinemic-euglycemic clamp was performed over $90 \mathrm{~min}$ in overnight starved condition, insulin being infused at $0.4 \mathrm{U}$ per hour and per $\mathrm{g}$ body weight.

Results: Compared to C, F displayed decreased food intake $(-37 \% ; \mathrm{p}<0.0002)$, lower gain in body weight $(-17 \%$; $\mathrm{p}<0.004)$, increased overnight fasting plasma glucose $(p<0.03)$ and insulin $(p<0.04)$ concentrations, and higher $(\mathrm{p}<0.04)$ HOMA (insulin resistance). During the hyperinsulinemic-euglycemic clamp, the amount of infused glucose was $18 \%$ lower $(\mathrm{p}<0.0003)$ in $\mathrm{F}$ than in C. In the FO rats, the daily food intake and gain in body weight were restored to normal values; however, none of the other metabolic variables differed significantly in $\mathrm{F}$ and $\mathrm{FO}$. When comparing FG to $\mathrm{F}$, the food intake remained reduced ( $\mathrm{p}<0.0001 v s \mathrm{C}$ ), and the overnight fasting plasma glucose concentration was restored to a normal level. No other significant differences in metabolic variables were found between FG and F.

Conclusions: The present results indicate that, in a rat model of fructose-induced insulin resistance, the long-term administration of an olive oil-enriched diet opposes the 
lowering effect of the ketohexose on food intake and body weight gain. The long-term administration of a guarenriched diet prevents the fructose-induced increase in glycemia otherwise recorded after overnight starvation, whilst maintaining a low food intake. These findings argue in favour of guar, rather than olive oil, intake to oppose the effect of exogenous fructose on glucose homeostasis. Supported by FIS 02/0967; ISCIII PI050363 and PI050365

\section{7}

Partial substitution of proteins of animal origin by soy drink in the diet of persons with type 2 diabetes. Effect on metabolic control, inflammatory markers, lipid profile and albumin excretion rate

J. Silva-Nunes ${ }^{1,2}$, M. Barradas ${ }^{3,2}$, E. Dionisio ${ }^{2}$, C. Moreira ${ }^{2}$, L. Mendes ${ }^{2}$, L. Gardete-Correia ${ }^{1}$, J. M. Boavida ${ }^{1}$;

${ }^{1}$ Diabetes Department, Portuguese Diabetes Association, Lisbon, ${ }^{2}$ Dietetics Department, High School for the Health Technology of Lisbon, Lisbon, ${ }^{3}$ Nutrition Department, Portuguese Diabetes Association, Lisbon, Portugal.

Background and Aims: Soybean is a high-quality protein product rich in isoflavones. Consumed at quantities around the $25 \mathrm{~g} /$ day of soy protein, it has been associated with several health beneficial effects, namely cardiovascular risk reduction. The aim of our study was to evaluate the effect of consuming moderate quantities of soy in the metabolic control, inflammatory markers, lipid profile and albumin excretion rate (AER) of persons with type 2 diabetes (T2D). Materials and Methods: We studied 63 persons with T2D (35 males; 28 females) randomized by two dietetic interventions: Group I $(\mathrm{n}=26)$ with a diet according to ADA guidelines and Group II $(\mathrm{n}=37)$ following a diet with the same macronutrient composition but with partial substitution in proteins of animal origin by soy drink $(2 \mathrm{~L}$ per week $=75 \mathrm{~g}$ of protein). Visits were made at months $0,1,2$, 4, 6, 9 and 12 for dietetic monitoring and for the determination of $\mathrm{HbAlc}$, high-sensitivity $\mathrm{C}$ reactive proteín (hs-CRP), erythrocyte sedimentation rate (ESR), leucocytes, fibrinogen, total cholesterol, HDL-c, LDL-c, triglycerides, apolipoprotein B (apoB), urea, creatinin and AER (night period). For each parameter, we determined the variation between basal value and that obtained in the month considered and compared delta values between the two intervention groups. Patients with inflammatory/infectious episodes were excluded as well as those with pharmacological changes or dietetic non-compliance during follow-up.

Results: Patients presented with mean age $=61 \pm 9$ yrs, $\mathrm{BMI}=$ $30.5 \pm 5.9 \mathrm{~kg} / \mathrm{m}^{2}, \mathrm{HbAl}=7.1 \pm 0.7 \%$, hs-CRP $=4.1 \pm 4.3 \mathrm{mg} / \mathrm{l}$, $\mathrm{ESR}=15.6 \pm 13.8 \mathrm{~mm} / \mathrm{h}$, leucocytes $=7.2 \pm 1.9 \times 10^{6} / \mathrm{ml}$, fibrinogen $=363.7 \pm 81.3 \mathrm{mg} / \mathrm{dl}$, total cholesterol $=188.7 \pm$
$32.6 \mathrm{mg} / \mathrm{dl}$, HDL-c $=47.8 \pm 10.2 \mathrm{mg} / \mathrm{dl}, \quad$ LDL-c $=122.4 \pm$ $26.1 \mathrm{mg} / \mathrm{dl}$, triglycerides $=146.4 \pm 70.7 \mathrm{mg} / \mathrm{dl}$, apoB=90.8 \pm $15.9 \mathrm{mg} / \mathrm{dl}$, urea $=41 \pm 12.2 \mathrm{mg} / \mathrm{dl}$, creatinin $=1 \pm 0.2 \mathrm{mg} / \mathrm{dl}$ and AER $=55.4 \pm 175 \mu \mathrm{g} / \mathrm{min}$. At baseline AER was abnormal in 15 patients $(23.8 \%) ; 13$ patients $(20.6 \%)$ were on insulin therapy and $32(50.8 \%)$ on lipid lowering drugs. Globally we found a progressive increase in $\mathrm{HbAlc}$ throughout the study. That was significantly lower in Group II: month 1 $(\mathrm{p}=0.075)$, month $2(\mathrm{p}=0.023)$, month $6(\mathrm{p}=0.014)$, month 9 $(\mathrm{p}=0.003)$ and month $12(\mathrm{p}=0.047)$. A tendency for increase in triglycerides and reduction in inflammatory markers was observed in Group II comparatively to Group I: triglycerides $(\mathrm{p}=0.073$ and $\mathrm{p}=0.046$; respectively in month 1 and 6$)$, ESR $(p=0.008)$ in month 6 ; leucocytes $(p=0.041)$ and fibrinogen $(\mathrm{p}=0.069)$ in month 12 .

Conclusion: Partial substitution of proteins of animal origin by soy, for short/intermediate periods, does not induce any reliable effect in lipid profile, inflammatory markers or kidney function. However, moderate consumption of soy is associated with a consistent beneficial effect in metabolic control of persons with T2D.

0848

High fibre intake, but not low energy intake, promotes the reduction in visceral adipose tissue mass and hepatic lipids during weight loss

C. Thamer ${ }^{1}$, J. Machann ${ }^{2}$, M. Graf ${ }^{1}$, F. Schick ${ }^{2}$, H. Häring ${ }^{1}$, A. Fritsche ${ }^{1}$;

${ }^{1}$ Innere Medizin IV, Medizinische Universitätsklinik, ${ }^{2}$ Experimentelle Radiologie, Radiologische Universitätsklinik, Tuebingen, Germany.

Background and Aims: Weight loss reduces visceral adipose tissue mass (VAT) and improves insulin sensitivity. It is unknown whether diet composition during weight loss provides beneficial effects on body composition and ectopic fat storage in the liver beyond the effects of energy restriction alone.

Materials and Methods: 99 subjects (38 males, 61 females, age $45 \pm 11$ years, BMI $29.2 \pm 4.5 \mathrm{~kg} / \mathrm{m} 2$ ) were included in a 9 months interim analysis of the Tuebingen Lifestyle Intervention Program (TULIP). Insulin sensitivity was estimated from the oral glucose tolerance test (oGTT). In all subjects body fat depots were quantified using magnetic resonance imaging and spectroscopy. Food intake was estimated from dietary records of $15 \pm 1$ representative days during the study period per subject. Nutrient intake was analyzed using a validated computer program (DGEPC 3.0, Deutsche Gesellschaft für Ernährung).

Results: During lifestyle intervention subjects ingested $1820 \pm 30$ calories and $25 \pm 6 \mathrm{~g}$ of total fibre per day. BMI (from $29.2 \pm 0.5$ to $28.2 \pm 0.4 \mathrm{~kg} / \mathrm{m} 2, \mathrm{p}<0.0001$ ) and VAT 
(from $3.5 \pm 0.2$ to $3.1 \pm 0.2 \%$ total body weight, $\mathrm{p}<0.0001$ ) and hepatic lipids (HL) (from $5.5 \pm 0.6$ to $3.7 \pm 0.4 \%$ signal, $\mathrm{p}<0.0001)$ were reduced. In a multivariate regression model the relative reduction in BMI was negatively and independently associated with both, total energy $(p=0.002)$ and the total amount of fibre $(\mathrm{p}<0.0001)$ ingested per day. In contrast, the reduction in VAT was associated only with the total amount of fibre $(p<0.0001)$ ingested per day but not with ingested energy $(p=0.17)$. The same results were obtained for hepatic lipids $(p=0.02$ for the effect of fibre intake). In addition, the relative reductions in BMI $(p=0.01)$, VAT $(p=0.01)$ and HL $(p<0.01)$ were associated with the increase in insulin sensitivity.

Conclusion: Both, energy restriction and high fibre intake independently promote weight loss. High intake of dietary fibre results in beneficial effects on body composition and ectopic fat storage in the liver independent and beyond the effects of energy restriction alone.

Supported by DFG KFO 114

0849

Postprandial mononuclear NF-kB activation is independent of the AGE-content of a single meal

S. Schiekofer ${ }^{1}$, F. Sybille ${ }^{2}$, M. Andrassy ${ }^{3}$, J. Chen $^{4}$, G. Rudofsky ${ }^{4}$, J. Schneider ${ }^{4}$, M. von Eynatten ${ }^{5}$,

T. Wendt ${ }^{4}$, M. Morcos ${ }^{4}$, B. Böhm ${ }^{1}$, G. Stein ${ }^{2}$,

E. Schleicher ${ }^{6}$, P. Nawroth ${ }^{4}$, A. Bierhaus ${ }^{4}$;

${ }^{1}$ Department of Internal Medicine I, Ulm University, ${ }^{2}$ Department of Internal Medicine IV, Jena University, ${ }^{3}$ Department of Internal Medicine III, ${ }^{4}$ Department of Internal Medicine I, Heidelberg University, ${ }^{5}$ Department of Internal Medicine I, Würzburg University, ${ }^{6}$ Department of Internal Medicine IV, Tübingen University, Germany.

Background and Aims: Dietary uptake of Advanced Glycation Endproducts (AGE) is supposed to potentially contribute to inflammatory reactions linked to vascular dysfunction and late diabetic complications. One mechanism by which dietary AGE might exert these effects is by activation of the proinflammatory transcription factor NF$\mathrm{kB}$. The aim of this study was to analyze the postprandial effects of a casein meal with low or high AGE content on postprandial NF-kB activation in peripheral blood mononuclear cells (pBMC) of healthy volunteers.

Materials and Methods: Casein was heated for $40 \mathrm{~h}$ at $50^{\circ} \mathrm{C}$ in the presence of sorbitol or glucose, resulting in either minimal (Sorbitol (S)-casein) or large (glucose (G)casein) amounts of AGE-modified casein. 9 healthy volunteers ate $250 \mathrm{~g}$ of both types of casein, whereas both meals were separated at least by 2 weeks. Plasma and pBMC were taken before and $2 \mathrm{~h}$ after each meal.
Thereafter, the defined AGE carboxymethyllysine (CML) was determined by ELISA and Western blot. NF-kB activation in pBMC was assayed using Electrophoretic Mobility Shift Assays (EMSA) and Western blot analysis.

Results: S-casein contained only minor amounts of CML and no pentosidine, while G-casein contained large amounts of both. $2 \mathrm{~h}$ after ingestion, the S-casein or the G-casein-meal, both, resulted in a non-significant increase in plasma CML and in the intracellular CML-content of pBMC. This was paralleled by a highly significant increase in postprandial mononuclear NF-kB binding activity. Remarkably, neither the extent of NF-kB induction (178\% for S-casein, $188 \%$ for G-casein), nor the composition of the NF-kB heterodimer were significantly different after intake of S-casein or G-casein. Consistently, western blots confirmed an increased NF-kBp65 nuclear translocation and a decrease of NF-kBp65 in the cytoplasm, while no difference in postprandial NF-kB nuclear translocation was observed following intake of S-casein or G-casein.

Conclusion: Postprandial mononuclear NF-kB activation after a single meal is independent of the AGE-content of the ingested protein.

Supported by DFG-NA 138 and SFB405 to PN, German Diabetes Association and European Foundation for the Study of Diabetes to $A B$

\section{PS 68}

\section{Novel therapies (preclinical)}

0850

Inhibiting glycosphingolipid synthesis improves glycaemic control and insulin sensitivity in animal models of type 2 diabetes

N. S. Yew, H. Zhao, M. Przybylska, I.-H. Wu, J. Zhang, C. Siegel, S. Komarnitsky, R. Rubenstein, S. Boule, D. P. Copeland, C. Jiang, D. Armentano, C. M. Arbeeny, R. K. Scheule, S. H. Cheng;

Applied Discovery Research, Genzyme Corporation, Framingham, United States.

Background and Aims: Gangliosides are sialic acidcontaining glycosphingolipids that can modulate the activity of various cell surface receptors, including the insulin receptor (IR). Previous studies have suggested that elevated ganglioside levels may lead to insulin resistance. We have developed a small molecule inhibitor of glucosylceramide synthase, the enzyme involved in the first step in the synthesis of gangliosides and other glycosphingolipids. We investigated the effects of this inhibitor (Genz-123346) on glucose homeosta- 
sis and insulin sensitivity in the Zucker Diabetic Fatty (ZDF) rat and the Diet-induced Obese (DIO) mouse.

Materials and Methods: ZDF rats, 6 weeks old, were gavaged daily with Genz-123346 (75 mg/kg/day) for 6 weeks. Body weight, food consumption, glucose, glucose tolerance, hemoglobin A1c (HbA1c) and insulin levels were measured during the course of treatment. At the end of the study, various tissues including the pancreas were excised and examined histologically. For the second model, C57BL/6 mice were placed on a high fat ( $45 \%$ of calories) diet for 8 weeks. Obese, hyperglycemic mice were selected and gavaged daily with Genz-123346 (125 mg/kg/day) for 10 weeks.

Results: In ZDF rats, drug treatment did not have any significant effect on weight gain compared to the placebo control. Genz-123346 decreased GL1 levels in liver and GM3 levels in muscle. Genz-123346 significantly lowered glucose and $\mathrm{HbA} 1 \mathrm{c}$ levels, improved glucose tolerance, and partially preserved pancreatic islet structure and function. In DIO mice, drug treatment normalized $\mathrm{HbA} 1 \mathrm{c}$ levels and improved glucose tolerance. Increased activation of the IR was observed in the muscles of both the ZDF rats and DIO mice, indicating that drug treatment increased insulin signaling.

Conclusion: The glucosylceramide synthase inhibitor Genz-123346 lowered blood glucose, improved glucose tolerance, and increased insulin sensitivity in two animal models of type 2 diabetes. In ZDF rats, treatment with Genz-123346 also partially preserved pancreatic beta-cell function. The results suggest that modulating glycosphingolipid synthesis may be a novel and effective approach to treat type 2 diabetes.

\section{1}

The effect of novel antioxidant L-2264 on glucose homeostasis and lipids peroxidation in rats with noninsulin dependent diabetes mellitus

O. Ivanova ${ }^{1}$, V. Poltorak ${ }^{1}$, N. Gorbenko ${ }^{2}$, A. Gladkich ${ }^{1}$, V. Lipson ${ }^{3}$;

${ }^{1}$ Pathophysiology, ${ }^{2}$ Pharmacology, ${ }^{3}$ Medical Chemistry, Institute of Endocrine Pathology Problems, Kharkiv, Ukraine.

Background and Aims: We have previously reported that a new derivative of 1,2,4-triazolopropionamide (L-2264) decreases lipid peroxidation and corrects impairment of insulin sensitivity in rats with dexamethasone-induced insulin resistance. The aim of this study was to determine the effect of L-2264 on the glucose homeostasis and oxidative status in rats with non-insulin dependent diabetes mellitus.

Materials and Methods: Male Wistar rats (3-mo-old) were made diabetic by streptozotocin (STZ, $65 \mathrm{mg} / \mathrm{kg}$, i.v.) given in $15 \mathrm{~min}$ after nicotinamide injection $(230 \mathrm{mg} / \mathrm{kg}$, i.p). One group of diabetic animals received L-2264 (100 mg/kg/day per os) for 4 weeks. Control diabetic (D) and intact groups (C) have been included in the study. At the end of the experiment rats were subjected to a glucose tolerance test (GTT, $3 \mathrm{~g} / \mathrm{kg}$ i.p.) and insulin tolerance test $(0.3 \mathrm{U} / \mathrm{kg}$ i.v.) as an index of insulin sensitivity (IS). Oxidative status of experimental animals was assessed by determination of malonic dialdehyde (MDA), hydroperoxides (triene, oxidiene and tetraene conjugates) contents in liver homogenate, serum total antioxidant activity (TAA) and nonetherified fatty acids (NEFA) levels in plasma.

Results: In 4 weeks after diabetes induction D rats exhibited a moderate elevation of fasting plasma glucose levels $(7.4 \pm 0.3$ vs $C: 4.4 \pm 0.1 \mathrm{mmol} / 1, \mathrm{p}<0.01)$, impaired glucose tolerance (area under curve (AUC) over GTT was $1502 \pm 30$ vs $\mathrm{C}: 760 \pm 48 \mathrm{mmol} / \mathrm{l} / \mathrm{min}, \mathrm{p}<0.01)$ and decreased IS $(\mathrm{p}<0.01)$. L-2264-administration reduced basal hyperglycaemia $(6.1 \pm 0.1 \mathrm{mmol} / 1, \mathrm{p}<0.05)$, increased IS by $47 \%$ $(p<0.02)$ and corrected impairment of glucose tolerance (AUC over GTT was $990 \pm 49 \mathrm{mmol} / \mathrm{l} / \mathrm{min}, \mathrm{p}<0.05$ ) compared to D group. Diabetic rats had a greater lipid peroxidation, as indicated by the higher level of MDA $(502.9 \pm 44.6$ vs $\mathrm{C}: 243.6 \pm 20.6 \mu \mathrm{mol} / \mathrm{g}, \mathrm{p}<0.01)$; triene (47.8 \pm 3.4 vs $\mathrm{C}: 27.6 \pm 1.9 \mathrm{mmol} / \mathrm{g}, \mathrm{p}<0.01)$, oxidiene $(17.5 \pm 2.1$ vs $\mathrm{C}: 11.7 \pm 2.5 \mathrm{mmol} / \mathrm{g}, \mathrm{p}<0.01)$ and tetraene $(68.4 \pm 7.6$ vs $\mathrm{C}: 40.1 \pm 3.5 \mathrm{E} / \mathrm{g}, \mathrm{p}<0.01)$ conjugates in liver homogenates and lower serum TAA $(\mathrm{p}<0.001)$ in comparison with $\mathrm{C}$ group. Furthermore, NEFA levels in plasma diabetic animals was increased 3 -fold $(\mathrm{p}<0.01)$ compared to C. L-2264-supplementation decreased NEFA levels 2-fold $(p<0.01)$ and suppressed oxidative stress in diabetic animals reducing MDA and hydroperoxides contents 1.5-2-fold compared to $\mathrm{C}(\mathrm{p}<0.05)$. Moreover, TAA was increased $(40.36 \pm 2.15 \%$ vs D: $29.85 \pm 1.56 \%, \mathrm{p}<0.01)$ after administration of L-2264.

Conclusion: Treatment with L-2264 corrects glucose homeostasis and oxidative status decreasing hyperglycemia, NEFA levels, attenuating lipid peroxidation and improving free radicals defense system in rats with STZ-induced noninsulin dependent diabetes mellitus. This data justify the perspectivity of L-2264's future studies as agent for the treatment of diabetes and its complications.

0852

Combination therapy with pioglitazone and a fructose 1,6-bisphosphatase inhibitor (MB06322) improves glycaemic control and lactate homeostasis in male Zucker diabetic fatty (ZDF) rats

P. D. van Poelje, S. C. Potter, E. Topczewski, J. Hou,

D. L. Linemeyer, M. D. Erion;

Biology, Metabasis Therapeutics Inc., La Jolla, United States. 
Background and Aims: MB06322 (CS-917) is a prodrug of a potent inhibitor of fructose 1,6-bisphosphatase, a regulatory enzyme in gluconeogenesis (GNG), that improves glycemic control in a broad spectrum of animal models of T2DM. In male ZDF rats with advanced disease ( $>10$ weeks of age), MB06322 treatment increases blood levels of lactate, the predominant precursor of GNG. This is not observed in younger male ZDF rats, high fat-fed female ZDF rats, or male $\mathrm{db} / \mathrm{db}$ mice. Thiazolidinediones (TZDs) such as pioglitazone (PIO) are known to improve insulin sensitivity and overall metabolic state. The aim of this study was to explore the therapeutic potential of the combination of PIO and MB06322 in male ZDF rats and to evaluate potential benefits with regards to lactate homeostatasis.

Materials and Methods: Male ZDF rats ( $\sim 11$ weeks old) were divided into blood glucose-matched groups $(n=8 /$ group; blood glucose $\sim 600 \mathrm{mg} / \mathrm{dL}$ ) and fed Teklad 5008 chow or an admixture of this chow with MB06322 $(0.2 \%)$, PIO $(0.03 \%)$, or the combination of MB06322 and PIO $(0.2 \%$ and $0.03 \%$, respectively) for 3 weeks. A panel of physiological and biochemical parameters was measured at least weekly. On Day 16, satellite groups of control and PIOtreated animals were subjected to an IV lactate tolerance test $(0.1 \mathrm{~g} / \mathrm{kg})$. On Day 21, leg muscle samples were harvested under anesthesia and analyzed for the expression of pyruvate dehydrogenase kinase-4 (PDK4) by RT-PCR.

Results: Combination therapy with PIO and MB06322 resulted in markedly improved glycemic control relative to the control and monotherapy groups (Table 1). Blood lactate levels increased progressively in the MB06322 monotherapy group during the first treatment week and remained $\sim 2.5$-fold elevated thereafter. Lactate elevations also occurred with combination therapy but were fully attenuated within 7 days of treatment. On Day 16, IV lactate tolerance was significantly improved in PIO-treated rats (lactate AUC 69.7 \pm 9.1 and 43.1 $\pm 4.5 \mathrm{mM} *$ min; controls vs. PIO). PIO monotherapy and combination therapy, but not MB06322 monotherapy, increased body weight. The expression of PDK4 in leg muscle was $>60 \%$ reduced in the PIO monotherapy and combination groups relative to the control and MB06322-treated groups.

Conclusion: Two important benefits of combination therapy with a TZD and an FBPase inhibitor were noted in male ZDF rats with advanced diabetes: improved glycemic control and the attenuation of FBPase inhibitor-induced elevation of blood lactate. PDK4 is a kinase that regulates the clearance of lactate and pyruvate by inhibiting flux through the pyruvate dehydrogenase $(\mathrm{PDH})$ complex. The reduced expression of PDK4 in muscle in PIO-treated rats suggests that increased flux through muscle PDH may be responsible in part for the normalization of FBPase inhibitor-induced lactacemia and improved IV lactate tolerance. The combination of FBPase inhibitors and TZDs merits clinical evaluation.
Table 1. Biochemical and physiological parameters in male ZDF rats on Day 21.

\begin{tabular}{lllll}
\hline Group & $\begin{array}{l}\text { Blood } \\
\text { glucose, } \\
\mathrm{mg} / \mathrm{dL}\end{array}$ & $\begin{array}{l}\text { Blood } \\
\text { lactate, } \\
\mathrm{mM}\end{array}$ & $\begin{array}{l}\text { Body } \\
\text { weight, g }\end{array}$ & $\begin{array}{l}\text { PDK4 } \\
\text { mRNA, } \\
\text { relative } \\
\text { units }\end{array}$ \\
\hline Control & $778 \pm 48^{* *}$ & $2.2 \pm 0.2$ & $393 \pm 8$ & $1 \pm 0.1$ \\
MB06322 & $388 \pm 20^{* *}$ & $5.7 \pm 0.3 * *$ & $390 \pm 7$ & $1.2 \pm 0.3$ \\
Pioglitazone & $577 \pm 61^{* *}$ & $1.9 \pm 0.2$ & $467 \pm 13^{*+}$ & $0.2 \pm 0.3^{*+}$ \\
Combination & $212 \pm 17^{* *}$ & $2.0 \pm 0.2$ & $472 \pm 8^{*+}$ & $0.4 \pm 0.2^{*+}$ \\
\hline
\end{tabular}

Data were analyzed by repeated measures or 1-way ANOVA with an appropriate post hoc test. ${ }^{*} \mathrm{p}<0.05$ vs. control, $+\mathrm{p}<0.05$ vs. MB06322, $* * \mathrm{p}<0.05$ vs. all other groups.

\section{3}

Effects of niacin on plasma lipids of wild-type and PPAR-alpha-KO mice

R. Hanf ${ }^{1}$, A. Rubenstrunk ${ }^{1}$, F. Contard ${ }^{2}$, J. Fruchart ${ }^{3}$;

${ }^{1}$ Research and Development, GENFIT, Loos, ${ }^{2}$ Merck-Sante, Chilly-Mazarin, ${ }^{3}$ Atherosclerosis Department, Institut Pasteur Lille, INSERM U545, Université Lille 2, France.

Background and Aims: In human, the effects of niacin on plasma lipids and lipoproteins share numerous features with that of fibrates: both increase HDL-C levels and plasma apoAI, reduce LDL-C levels and small dense LDL fractions and reduce VLDL-C and triglycerides. In this study, we addressed the contribution of $\operatorname{PPAR} \alpha$ receptors activation in the niacin actions on plasma lipids.

Materials and Methods: To assess the potential role of PPAR $\alpha$, wild-type C57/B16 (WT) and PPAR $\alpha-K O$ mice were treated once daily for 14 days with niacin at 250, 500 or $1000 \mathrm{mg} / \mathrm{kg} / \mathrm{d}, 200$ or $300 \mathrm{mg} / \mathrm{kg} / \mathrm{d}$ fenofibrate or vehicle. At the end of the treatment period, plasma total cholesterol (TC), triglycerides (TG), free-glycerol (FG) and free-fatty acids (FFA) were measured. WT animals were sacrificed and livers were collected to isolate mRNA for gene expression using Q-PCR.

Results: In three independent experiments, in WT mice, niacin provoked moderate dose-dependent reductions in TG using total glycerol determination. In the most representative experiment, in animals treated with $500 \mathrm{mg} / \mathrm{kg} / \mathrm{d}$ of niacin, TG was $49 \pm 20 \mathrm{mg} / \mathrm{dL}$ vs $67 \pm 14 \mathrm{mg} / \mathrm{dL}$ in vehicle treated group $(\mathrm{p}<0.05)$. Concomitantly, a 10-20\% decrease in FFA concentration was also observed. The lowering effects on TG resembled that obtained with $300 \mathrm{mg} / \mathrm{kg} / \mathrm{d}$ fenofibrate $(32.06 \pm 7.8 \mathrm{mg} / \mathrm{dL}$ vs $66.78 \pm 14 \mathrm{mg} / \mathrm{dL}, \mathrm{p}<0.001)$. A chromatographic analysis revealed that niacin decreased $\mathrm{FG}$ by $50 \%$ without changing the glycerol content of the VLDL-TG 
fraction. Conversely, fenofibrate decreased glycerol in the VLDL-TG fraction by $50 \%$ without modifying FG. Accordingly, fenofibrate but not niacin reduced plasma ApoCIII and ApoB concentrations (respectively $42 \%$ and $39 \%$ reduction in fenofibrate- vs vehicle-treated animals). In addition, the well-known PPAR $\alpha$ mediated hepatomegaly in rodent was observed with fenofibrate but not with niacin. A role for PPAR $\alpha$ in the lipid lowering action of niacin could be suspected since no effect was observed in PPAR $\alpha$-KO mice treated with niacin and fenofibrate. Thus we performed QPCR experiments on key genes involved in lipid metabolism in WT mice.

Q-PCR analysis of mRNA from liver of WT mice revealed that the expression of ACO, PDK4, UCP2, SCD1 and CD36 were not modified in the niacin treated groups. This contrasted with the large up-regulation of these PPARa target genes after fenofibrate treatments (up to 20X for PDK4). Further experiments revealed that fenofibrate reduced the hepatic expression of ApoCIII, fibrinogen beta, FAS and ACC genes in WT mice. Interestingly, such a decrease in the expression of ApoCIII, FAS and ACC could be also observed in the liver of niacin treated animals.

Conclusion: These data rule out any direct role of PPARa activation in the mechanism of action of niacin in WT mice although a cross talk with a PPAR $\alpha$ dependent pathway cannot be excluded. Indeed, niacin as fenofibrate reduced the expression of key genes involved in hepatic triglyceride synthesis such as FAS and ACC. Whether this hepatic effect can be linked to a decrease in VLDL-TG output in dyslipidemic animals should be specifically addressed in models such as the ApoE2/E2 mice.

Supported by Merck-Sante

\section{4}

MP-513, a highly potent and highly selective noncyanopyrroridine DPP-IV inhibitor, achieves glucose lowering effect all day in Zucker fatty rats

Y. Takashina, S. Ishii, Y. Abe, J. Anabuki,

K. Yoshida, K. Sakai;

Mitsubishi Pharma Corporation, Yokohama, Japan.

Background and Aims: Inhibition of DPP-IV is a promising approach to improve hyperglycemia in type 2 diabetes. In particular, long-lasting DPP-IV inhibitors as well as long-acting GLP-1 analogs are expected to bring the incretin effects maximally. In addition, the selectivity of DPP-IV inhibition against other peptidases, especially DPP8/9 is one of the key issues in the clinical usage. A non-cyanopyrrolidine DPP-IV inhibitor, MP-513 is currently under development for the treatment of type 2 diabetes. The aim of this study was to evaluate the enzyme inhibition profile of MP-513 in vitro. In addition, to clarify the beneficial effect of the long-lasting inhibition, we investigated whether once-a-day treatment of MP-513 improves glucose tolerance for a whole day in Zucker Fatty (ZF) rats. Materials and Methods: In in vitro study, the inhibition property and potency of MP-513 were investigated using recombinant human DPP-IV (rhDPP-IV). The selectivity was also evaluated using crude DPP8 and DPP9. In in vivo study, 13 weeks old male ZF rats were subjected to two successive oral carbohydrate tolerance tests at a 12-hour interval following a single oral administration of MP-513 or vildagliptin. The concentrations of plasma glucose, insulin and DPP-IV activity were measured.

Results: MP-513 showed a competitive-type inhibition on rhDPP-IV with a Ki value of $0.41 \mathrm{nmol} / \mathrm{L}$, and exhibited an excellent selectivity against DPP8/9 with IC50 rations of 300-700 fold. The DPP-IV inhibition of MP-513 was about 10 times more potent than that of vildagliptin, and also about 7 times more potent than sitagliptin. MP-513 at doses of 0.3 and $3 \mathrm{mg} / \mathrm{kg}$ inhibited DPP-IV activity more than $50 \%$ around 15 hours after single dosing, and clearly improved glucose tolerance accompanied with increasing insulin secretion at both 1 st and 2nd carbohydrates loading in a 12-hour interval in ZF rats, i.e. the plasma glucose $\triangle \mathrm{AUC}$ at both 1 st and 2 nd loading in MP-513 treatment was significantly lower than that in vehicle control. On the other hand, $3 \mathrm{mg} / \mathrm{kg}$ of vildagliptin treatment did not clearly improved plasma glucose $\triangle A U C$ in the 2nd carbohydrates loading, even though decreased in the 1st carbohydrates loading.

Conclusion: MP-513, a novel non-cyanopyrrolidine derivative showed a highly potent and highly selective inhibition on DPP-IV. Once-a-day treatment of MP-513 ameliorated hyperglycemia all day long, accompanied with long-lasting inhibition of DPP-IV and with increasing plasma insulin after carbohydrate loading. Thus, long-lasting DPP-IV inhibitor, MP-513 is expected to control plasma glucose all day long for once daily treatment, and to achieve a maximum of incretin effects in type 2 diabetes patients.

0855

The effect of vardenafil (a type 5 phosphodiesterase inhibitor) on renal function in diabetic rabbits

D. H. W. Lau ${ }^{1}$, F. H. Mumtaz ${ }^{2}$, D. P. Mikhailidis ${ }^{1}$, C. S. Thompson ${ }^{1}$;

${ }^{1}$ Clinical Biochemistry, Royal Free Hospital, London, ${ }^{2}$ Urology, Chase Farm Hospital, Enfield, United Kingdom.

Background and Aims: Diabetic nephropathy, a common cause of chronic kidney disease, can affect $20-30 \%$ of the diabetic population. The limitations of the existing treatments for diabetic renal insufficiency point to the need to develop new treatment strategies. We investigated the effect oral 
treatment of the type 5 phosphodiesterase inhibitor vardenafil, has on established diabetic renal impairment in the rabbit. Materials and Methods: Blood samples were taken from 4 and 6-month control $(\mathrm{n}=8)$ and alloxan-induced diabetic animals $(n=8)$ and serum biochemical variables pertaining to renal function determined. A further 7-month sample was taken for analysis after giving control and diabetic animals either acid water vehicle ( $\mathrm{n}=4$ in each group) or vardenafil $(3 \mathrm{mg} / \mathrm{kg}$ made up in $120 \mathrm{ml} \mathrm{HCl}$ acid water $\mathrm{pH} 4.5, \mathrm{n}=4$ in each group) to drink for 4 weeks. Spot urine samples were collected at 6 months from control and diabetic animals for the measurement of total protein and creatinine concentrations. At 7 months a 24 h-urine sample was collected to also measure the glomerular filtration rate (GFR).

Results: Renal impairment was established 4 months after the initiation of diabetes and persisted at 6 months. This was highlighted by a significant increase in serum creatinine concentration, which was significantly reduced by vardenafil treatment ( 6 month diabetic serum creatinine; $97+/-12.7 \mathrm{mmol} / \mathrm{l} ; \mathrm{n}=4$ vs 7 month diabetic serum creatinine following vardenafil; $87+/-12.2 \mathrm{mmol} / \mathrm{l} ; \mathrm{n}=4$, $\mathrm{p}=0.015$ ), paired Student's t-test.

The total protein/creatinine ratio (TP/C) from the 6 month spot urine samples from the diabetic rabbits were significantly elevated compared to controls, indicating the presence of proteinuria, a feature of diabetic nephropathy and a predictor of renal failure. Control TP/C: $\mathrm{n}=8,0.0116(0.0091-0.0191)$ vs diabetic TP/C: $\mathrm{n}=8,0.0227(0.0096-0.1632), \mathrm{p}<0.038$ (Mann Whitney, unpaired test). Vardenafil treatment caused a normalisation of TP/C $(n=3)$. Diabetic animals treated with vardenafil showed a significant improvement in GFR, when compared to diabetic animals given acid water only; GFR: diabetic acid water-treated; $6.10+/-3.68 \mathrm{ml} / \mathrm{min}, \mathrm{n}=4$ vs diabetic vardenafil-treated; $11.28+/-1.04 \mathrm{ml} / \mathrm{min}, \mathrm{n}=4$, $\mathrm{p}=0.035$ (unpaired Student's t-test). Similar results were also recorded when GFR was expressed $/ \mathrm{kg}$.

Conclusion: One possible explanation for our results is that vardenafil induces renal vasodilatation through the formation of cyclic GMP, which improves diabetic renal function. These findings highlight a potential role for vardenafil in the treatment of diabetic nephropathy.

Supported by Bayer plc

\section{6}

CS-917, a potent and selective gluconeogenesis inhibitor, was well tolerated and not associated with hypoglycaemia in normal male volunteers

S. Freudenthaler ${ }^{1}$, J. Walker ${ }^{2}$, J. Triscari ${ }^{2}$,

C. Dmuchowski ${ }^{2}$, T. Kaneko ${ }^{2}$, S. R. Bruce ${ }^{2}$;

${ }^{1}$ Sankyo Pharma GmbH, Munich, Germany, ${ }^{2}$ Sankyo Pharma Dev., Edison, United States.
Background and Aims: CS-917 is the prodrug of a potent and specific inhibitor of the enzyme fructose 1,6-bisphosphatase, being investigated as a potential treatment for type 2 diabetes. The proposed mechanism of action is through direct suppression of abnormally elevated hepatic gluconeogenesis. Phase I clinical trials were conducted in healthy volunteers to determine the acute safety, tolerability and clinical pharmacology of this novel agent.

\section{Materials and Methods:}

Single Ascending Dose Study (SAD): Ninety-six healthy male subjects were treated with single doses of CS-917 $(\mathrm{N}=72)$ or placebo $(\mathrm{N}=24)$, administered in the morning after an overnight fast. Blinded safety data review was conducted prior to each dose escalation. In each dose panel, 8 subjects were randomized to receive double-blinded CS$917(\mathrm{n}=6)$ or placebo $(\mathrm{n}=2)$. Dose panels included CS-917 at $2.5,5,10,25,50,100,200,400,600,800$ and $1000 \mathrm{mg}$. Subjects were fasted for an additional four hours post-dose. Safety and tolerability were assessed and areas under the curves (AUC-0-4 hours) for glucose and lactic acid levels were calculated.

Multiple Ascending Dose Study (MAD): Thirty-three healthy male subjects were randomized in sequential escalating dose panels (6 active and 2 placebo subjects per panel) to receive once daily dosing of CS-917, administered at dosages of $100,200,400$ and $800 \mathrm{mg}$, or placebo, for 14 days. CS-917 was administered after an overnight fast and fasting continued for an additional 6 hours. Safety and tolerability were assessed. Calculation and exploratory analysis of fasting AUC(0-6 hour) and 24hour profiles for glucose and lactic acid were performed on Day -1 and Day+14.

\section{Results:}

SAD: No subject dropped out or was discontinued. Single doses of CS-917 were generally well tolerated. No serious adverse events occurred. Gastrointestinal adverse events were not evident at lower doses but included nausea at $400 \mathrm{mg}$, and vomiting at doses of 800 and $1000 \mathrm{mg}$. Glucose levels were not affected. No subject experienced hypoglycemia. A dose dependent effect on the area under the curve for fasting lactic acid levels was suggested at doses of $200 \mathrm{mg}$ and above.

MAD: One subject withdrew consent due to personal reasons in the placebo group. No other subjects dropped out or were discontinued. Multiple doses of CS-917 were generally well tolerated. No serious adverse events occurred. Transient nausea occurred frequently in the 400 and $800 \mathrm{mg}$ groups and vomiting occurred in $4 / 6$ subjects in the $800 \mathrm{mg}$ group only. Fasting glucose concentrations were not clearly affected. The area under the curve for fasting plasma lactic acid was increased in the $800 \mathrm{mg}$ dose group. Mean fasting lactate levels remained within the normal range in all groups. 
Conclusion: Single and multiple doses of CS-917 were safely administered to normal volunteers in the fasted state across a broad dose range from 2.5 to $1000 \mathrm{mg}$. CS-917 was well tolerated without serious adverse events or hypoglycemia. Dose limiting nausea and vomiting appeared at doses of 800 and $1000 \mathrm{mg}$. Glucose concentrations were not affected in healthy subjects. Small increases in mean lactic acid concentrations were seen only at the highest doses. It remains to be determined if potent and selective gluconeogenesis inhibitors can ameliorate the hyperglycemia of patients with type 2 diabetes. These initial safety results support further testing of CS-917.

Supported by Sankyo Pharma Development

0857

The effects of pinitol on glycaemic control and adipocytokines level in type 2 diabetes patients

H.-J. Kim ${ }^{1}$, B. Ku ${ }^{2}$, J. Lee ${ }^{1}$, S.-K. Lee ${ }^{1}$, Y. Kim ${ }^{2}$, K. Park ${ }^{1}$; ${ }^{1}$ Internal Medicine, Eulji university, Daejeon, ${ }^{2}$ Internal Medicine, Chungnam National University, Daejeon, Republic of Korea.

Background and Aims: Pinitol(3-O-methyl-D-chiro-inositol, DCI) is thoght to mediate the insulin action and improve glycemic control. We studied to assess the effects of pinitol on glycemic control and adipocytokines level, and the safety.

Materials and Methods: A total 66 patients with type 2 diabetes who had been taken oral hypoglycemic agents for least 3 months were enrolled in the study. They were randomly assigned to two groups and were given $400 \mathrm{mg}$ pinitol or placebo three times daily for 12 weeks(Placebo, $\mathrm{n}=33$, DCI, $\mathrm{n}=33$ ). All subjects continued their current medication during the study.

Results: After pinitol therapy, $\mathrm{HbA} 1 \mathrm{c}(\mathrm{P}<0.05)$, fasting glucose level $(\mathrm{P}<0.05)$ and HOMA-IR $(\mathrm{P}<0.05)$ decreased significantly, compared with placebo group. Such lowering effect on HbAlc was evident in subjects with poorly controlled type 2 diabetes. In the group of patietns that had $\mathrm{HbAl}$ c higher than $8.0 \%$ showed a greater level of reduction $(\mathrm{P}<0.05)$ than those lower than HbA1c $8.0 \%(\mathrm{P}=0.16)$. Also in the group of patients with HOMA-IR higher than 2.5, there was significant decrease in $\mathrm{HbAl} \mathrm{c}(\mathrm{P}<0.01)$, compared with group of patients with HOMA-IR lower than 2.5 $(\mathrm{P}=0.26)$. There is no significant difference in change of adiponectin, leptin, free fatty acid, resistin and C-reactive protein within groups before and after treatment. No toxicity due to the pinitol was observed during the study.

Conclusion: These data support the view than pinitol can medicate the insulin action to improve glycemic control and insulin sensitivity, especially in patients with insulin resistance. There is need for further studies to elucidate the mechanism of improvement of glycemic control and insulin resistance.

0858

Insulin secretory effect of plant extracts PE080 and PE081 in perfused pancreas and BRIN-BD11 cells

B. Rokeya ${ }^{1}$, M. M. Rahman ${ }^{2}$, J. M. A. Hannan ${ }^{1}$, M. Akter ${ }^{1}$, M. Lamin ${ }^{3}$, A. K. Azad Khan ${ }^{4}$;

${ }^{1}$ Dept of Pharmacology, BIRDEM, Dhaka, Bangladesh,

${ }^{2}$ Dept of Biochemistry and Cell Biology, BIRDEM, Dhaka, Bangladesh, ${ }^{3}$ School of Biomedical Sciences, University of Ulster, Northern Ireland, United Kingdom, ${ }^{4}$ Dept of Gastroenterology, BIRDEM, Dhaka, Bangladesh.

Background and Aims: PE080 (an ethanol extract) and PE081 (a choloroform extract) from the seeds of a tropical medicinal plant have previously been shown to reduce blood glucose level in Long-Evans rats. This plant is used extensively in Ayurvedic and Unani medicine for the treatment of sciatica, rheumatism and many other diseases. In the present study, the effects of these extracts were evaluated on insulin secretion together with exploration of their mechanism of action in isolated perfused pancreas and BRIN-BD11 insulin secreting cell line.

Materials and Methods: Ripe seeds of the plant were dried in an oven at $40^{\circ} \mathrm{C}$ and ground into a fine powder. PE080 was prepared by $80 \%$ ethanol and PE081 with nhexane and chloroform. To study the effects of the extracts on insulin secretion Long-Evans rats (200-220 g) were anesthetized with sodium pentobarbital $(50 \mathrm{mg} / \mathrm{kg}$, ip) and the pancreas was isolated and perfused through mesenteric and celiac vessels of whole pancreas at $37^{\circ} \mathrm{C}$. Both the extracts (PE080 and PE081) were dissolved at a dose of $0.1 \mathrm{mg} / \mathrm{ml}$ in Krebs-Ringer bicarbonate buffer (KRB) with BSA and dextran containing $2.8 \mathrm{mM} \mathrm{D}$ glucose. The perfusate was continuously gassed with a mixture of $\mathrm{O}_{2}: \mathrm{CO}$ (95:50). After 20 min equilibration period the composition of the perfusate was changed every ten min with plant extracts and other reagents. Effluent samples were frozen and stored at $-20^{\circ} \mathrm{C}$ for insulin assay by ELISA. Clonal BRIN-BD11 cells were also used to evaluate the action of PE080 and PE081 on insulin secretion. Cells were seeded in the multiwell plates at a conc of $0.15 \times 10^{6}$ and allowed to attach overnight. Following preincubation for $40 \mathrm{~min}$ at KRB supplemented with $1.1 \mathrm{mM}$ glucose, cells were incubated for $20 \mathrm{~min}$ at $37^{\circ} \mathrm{C}$ with $\mathrm{KRB}$, supplemented with glucose, with different concentrations of plant extracts. Aliquots were removed from each well and stored at $-20^{\circ} \mathrm{C}$ for insulin measurement by radioimmunoassay. Cell viability was evaluated by modified neutral red assay. Results are expressed as Mean+SD for a given number of 
observations(n) and compared using unpaired student t-test and Man Whitney U test where appropriate.

Results: PE080 stimulated insulin secretion in isolated perfused rat pancreas which caused a significant increase in insulin release during 10 minute perfusion with almost a 6-fold increase above basal level (Insulin, M+SD ng/ml; 0.204+0.02 basal vs 1.22+0.12 PE080, Peak value; $\mathrm{p}<0.001)$. PE081 did not evoke a significant increase on insulin secretion. The stimulatory effect of the PE080 in the perfused pancreas was inhibited by diazoxide $(300 \mu \mathrm{M})$ (Insulin $\mathrm{M}+\mathrm{SD}, \mathrm{ng} / \mathrm{ml}$; $1.22+0.12$ PE080 vs 0.42+0.05 PE080+diazoxide; $\mathrm{p}<0.01)$. In BRIN-BD11 cell line PE080 stimulated insulin release in both the concentrations (50 and $100 \mu \mathrm{g} / \mathrm{ml}$ ) compared to control (5.6 mM glucose). The higher concentration did not affect cell viability. PE081 in $100 \mu \mathrm{g} / \mathrm{ml}$ concentration stimulated insulin release which was also not associated with a significant reduction in cell viability.

Conclusion: These findings reveal that PE080 and PE081 exert stimulatory effects on insulin secretion mediated through a physiological insulinotropic pathway. Future work directed towards the identification of active principle (s) from PE080 and PE081 may provide interesting possibilities against diabetes.

Supported by IPICS and DAB

\section{9}

Beneficial effects of Momordica charantia fruit juice on lipid and free fatty acid levels in type 2 diabetic model rats

S. Murshed ${ }^{1}$, B. Rokeya ${ }^{2}$, L. Ali ${ }^{1}$, N. Nahar ${ }^{3}$, A. K. Azad Khan ${ }^{4}$, M. Mosihuzzaman ${ }^{3}$;

${ }^{1}$ Dept of Biochemistry \& Cell Biology, ${ }^{2}$ Dept of Pharmacology, BIRDEM, Dhaka, ${ }^{3}$ Dept of Chemistry, Dhaka University, Dhaka, ${ }^{4}$ Dept of Gastroenterology, BIRDEM, Dhaka, Bangladesh.

Background and Aims: Momordica charantia fruit is used as a traditional remedy for diabetes mellitus throughout the world. The hypoglycemic activity of its various extracts has been widely reported; but, its effects on lipids and, particularly on individual fatty acids, have not been adequately studied. Importance of studying the effect of plant extracts on individual fatty acids is exemplified by the report that palmitic acid has an adverse effect on pancreatic $\beta$-cell proliferation whereas palmitoleic acid has a beneficial effect. The present study was undertaken to assess the effect of Momordica charantia fruit juice on plasma FFA and lipid levels in type 2 diabetic model rats.

Materials and Methods: Momordica charantia fruit was separated from seed and crushed. The juice was squeezed out from it and freez-dried (MC-J). The rats were made type 2 diabetic by intraperitonial injection of streptozotocine to $48 \mathrm{~h}$ old pups and experiments were carried out 3 months after the injection. The rats were fed with MC-J at a dose of $1.25 \mathrm{~g} / \mathrm{kg}$ body weight $/ 10 \mathrm{ml}$ water once a day for 28 days. The diabetic control rats were fed with deionized water only. After 28 days the rats were sacrificed and blood was collected for estimation of plasma glucose, free fatty acids and lipid profiles. Plasma glucose was measured by glucose oxides (GOD-PAP) method; triglyceride and cholesterol by enzymatic-colorimetric method and plasma free fatty acids were measured by gas chromatographic method. Significance of difference was analyzed by t-test.

Results: After 28 days fasting plasma glucose of MC-J treated rats was significantly lowered as compared to the control rats (plasma glucose in mmol/1: $9.40+0.43$ in control vs $7.58+0.45$ in $\mathrm{MC}-\mathrm{J}$ treated rats, $\mathrm{M}+\mathrm{SD}$, $\mathrm{p}=0.001)$. MC-J treated rats also showed significantly lower plasma TG $(p=0.001)$; cholesterol $(p=0.04)$ and LDL-cholesterol $(\mathrm{p}=0.001)$ as compared to the control rats. Compared to control, MC-J treated rats showed a significantly lower plasma saturated FFA ( $\%$ composition, $\mathrm{M}+\mathrm{SD}$, control vs treated- palmitic acid: $41.06 \pm 2.51$ vs $31.01 \pm 2.17$ $\mathrm{p}=0.001$; stearic acid: $20.33 \pm 4.28$ vs $14.67 \pm 3.92 \mathrm{p}=0.01$; arachidic acid: $4.39 \pm 1.19$ vs $0.37 \pm 0.18 \mathrm{p}=0.001$ and behenic acid: $8.42 \pm 1.50$ vs $1.52 \pm 0.50, \mathrm{p}=0.001)$. In contrast, plasma MUFA of MC-J treated rats were significantly raised as compared to the control rats (control vs treatedpalmitoleic acid: $0.89 \pm 0.11$ vs $1.68 \pm 0.98 \mathrm{p}=0.05$; oleic acid: $13.32 \pm 2.29$ vs $19.90 \pm 2.27 \mathrm{p}=0.001)$. Plasma PUFA of MC-J treated rats were also significantly raised as compared to the control rats (control vs treated- linoleic acid: $9.49 \pm 2.03$ vs $24.58 \pm 2.55 \mathrm{p}=0.0001$; linolenic acid: $0.50 \pm$ 0.26 vs $1.95 \pm 01.74 \mathrm{p}=0.0001$; arachidonic acid: $0.45 \pm 0.17$ vs $1.14 \pm 0.35 \mathrm{p}=0.001)$. Plasma $\omega-3$ fatty acid eicosapentaenoic acid and docosahexaenoic acid of MC-J treated rats were also significantly ( $p=0.001$ and $p=0.05$ respectively) raised as compared to the control rats.

Conclusion: The data suggest that Momordica charantia fruit juice may have a beneficial effect through improvement of the fatty acid as well as lipid profiles in type 2 diabetes. Supported by IPICS and DAB

\section{PS 69}

\section{Psychological complications of diabetes}

\section{0}

Sexual dysfunction in women with diabetes

B. Kalra ${ }^{1}$, S. Kalra ${ }^{2}$, K. Chawla ${ }^{3}$; ${ }^{1}$ Gynaecology, ${ }^{2}$ Endocrinology, ${ }^{3}$ Psychology, Bharti Hospital, Karnal, India. 
Background and Aims: Sexual function is a neglected aspect of health in women with diabetes, though it contributes greatly towards quality of life and feeling of well being.

Materials and Methods: This study was designed to assess sexual function of married premenopausal diabetic women and ascertain the effects of glycemic control and other variates on it. 177 married premenopausal female patients of diabetes (average age 33.14 \pm 8.09 years) were administered a pre tested questionnaire, designed as a 5 point Likert scale, to assess function in the domains of sexual desire, arousal, orgasm and pain. It also assessed medical and psychological aspects of sexuality as separate domains. The control group was 100 premenopausal non-diabetic women attending the obstetric clinic in the same hospital.

Results: Women with diabetes scored highly on domain of desire (3.17 \pm 0.83$)$. Scores on the domains of arousal and orgasm were poor $(2.01 \pm 1.57$ and $2.81 \pm 1.57)$ implying a high incidence of sexual dysfunction in women with diabetes. Scores were high for medical health related to sexuality $(4.47 \pm 0.57)$ but comparatively low for psychological health $(2.82 \pm 0.94)$.

$15.25 \%$ of the patients experienced orgasm most or all of the time while $44.07 \%$ had never experienced it. Only $10.17 \%$ patients complained of vaginal dryness/itching or foul discharge. The incidence of painful coitus/dyspareunia was high $(20.38 \%$ ). $3.38 \%$ patients had history of premarital or extramarital sexual contact while $54.24 \%$ had frequent contact ( $>2-3$ times a week). $83.05 \%$ patients reported that they would appreciate sexual counseling as a part of routine diabetes care services. Sexual dysfunction was not related to duration of diabetes, insulin therapy or glycemic control. Desire, arousal, orgasm, and pain disorders were more common in women $>40$ years. The self reported incidence of sensory neuropathy in this group was $33.89 \%$.

Conclusion: This study reveals the low incidence of sexual desire dysfunction, along with a comparatively higher incidence of arousal, orgasm and pain disorders, and psychological dysfunction in premenopausal diabetic women. This underscores the need for psychological counseling to improve sexual, as well as general, health in women with diabetes.

\section{1}

Physicians' and patients' perception of sexuality in diabetic men with erectile dysfunction. The PREDICT study G. G. R. T'Sjoen ${ }^{1}$, R. Opsomer ${ }^{2}$, M. P. Hermans ${ }^{3}$, F. Duyck ${ }^{4}$, G. Krzentowski ${ }^{5}$, L. Van Gaal ${ }^{6}$, D. Vanderschueren ${ }^{7}$;
${ }^{1}$ Department of Endocrinology, University Hospital Gent, ${ }^{2}$ Urology, ${ }^{3}$ Department of Endocrinology, Cliniques Universitaires Saint Luc, Brussels, ${ }^{4}$ Department of Endocrinology, Heilig Hart Ziekenhuis, Roeselaere, ${ }^{5}$ Department of Endocrinology, Centre Hospitalier Universitaire de Charleroi, Charleroi, ${ }^{6}$ Department of Diabetology, University Hospital Antwerpen, Gent, ${ }^{7}$ Department of Endocrinology, Katholieke Universiteit, Leuven, Belgium.

Objectives: The aim of the survey was to provide crosssectional data on sexual functioning in diabetic men, and to evaluate general practitioners (GP), diabetologists and patients' appraisal on erectile dysfunction (ED) and its therapy. Design and methods: Type 1 (21\%) and type 2 (79\%) diabetes patients were recruited by their GPs $(n=189)$ or diabetologists $(n=24)$. A self-administered questionnaire designed by the study group was dispensed by an independent market research company (Medistrat N.V.).

Results: In all, 737 men with a mean age of $59 \mathrm{y}$ participated to the study. Known diabetes duration was 17 years for type 1 and 8 years for type 2 diabetic subjects. Most participants (93\%) had a stable relationship with their partner, lasting for $>10$ years in $71 \%$. Sexuality was considered an important aspect of quality of life by $87 \%$ of participants.

About $50 \%$ of diabetic men with ED stated that their erectile capacity was severely impaired $(40 \%$ at age $<55 \mathrm{y}$, $44 \%$ at age $55-65 \mathrm{y}$, and $69 \%$ at age $>65 \mathrm{y}$ ), while $10 \%$ reported minor ED. Only $31 \%$ of men with ED were previously treated with an oral PDE-V inhibitor.

In $53 \%$ of patients, medical doctors had initiated discussion on ED within a frame of potential complications arising from poorly controlled diabetes. At consultation, $21 \%$ of diabetologists and up to 55\% of GP's admitted not to routinely include sexual history in regular diabetes followup. A more passive attitude was displayed by GP's, since they expected patients themselves to mention any sexual dysfunction. In their advice physicians stressed the importance of better metabolic control (48\%), while as erectogenic agent tadalafil (67\%), sildenafil (15\%) and vardenafil (14\%) were prescribed.

Forty-three $\%$ of patients in all age groups felt uncomfortable to discuss ED with their doctors. Forty-eight \% of diabetic patients with ED considered that improving metabolic control could better or solve ED. Fast-acting PDE V inhibitors were considered to be of lesser relevance with increasing age $(71 \%<55 \mathrm{y}, 64 \% 55-64 \mathrm{y}, 44 \%>65 \mathrm{y})$. Both physicians and patients considered sufficiently penile rigidity as the key component of efficacy during oral treatment of ED.

Conclusion: ED remains largely undiscussed, undiagnosed and maybe under-treated in men with diabetes, possibly 
because both physicians and patients feel reluctant to discuss this problem.

Supported by Eli Lilly Belgium

0862

The role of patients' representations of foot complications in guiding adherence to foot self-care: a longitudinal study

L. Vileikyte ${ }^{1}$, J. S. Gonzalez ${ }^{2}$, H. Leventhal ${ }^{3}$, M. Peyrot ${ }^{4}$, A. Garrow ${ }^{1}$, R. Rubin ${ }^{4}$, J. Ulbrecht ${ }^{5}$, P. Cavanagh ${ }^{6}$,

A. Boulton ${ }^{1}$;

${ }^{1}$ Medicine, University of Manchester, United Kingdom, ${ }^{2}$ Psychiatry, Harvard Medical School, Boston, United States, ${ }^{3}$ Psychology, Rutgers University, New Brunswick, United States, ${ }^{4}$ Medicine, Johns Hopkins University, Baltimore, United States, ${ }^{5}$ Medicine, Penn State University, State College, United States, ${ }^{6}$ Biomechanics, The Cleveland Clinic, Cleveland, United States.

Background and Aims: Structural equation modelling examined the ways patients' common-sense beliefs about foot complications combine with medical information and foot ulcer experience in predicting foot self-care behaviours during an 18 month follow-up.

Materials and Methods: 495 patients with diabetic peripheral neuropathy, DN (mean age $=63 \mathrm{y} ; 71 \%$ male; $72 \%$ type 2 diabetes) attending UK and USA diabetes centres (325 UK), completed self-report psychological and foot self-care behaviour questionnaires. DN was diagnosed by the Neuropathy Disability Score (NDS; mean=7.4), Patients' common-sense beliefs and interpretation of foot complications were assessed with the Neuropathy Psychosocial Inventory, NPI; foot self-care behaviour scales were based on international guidelines and covered preventive foot self-care (12 items) and potentially foot-damaging behaviours (9 items).

Results: Patients' common-sense beliefs predicted less preventive foot self-care ("good circulation=healthy feet", standardized coefficient, $\mathrm{sc}=-.10, \mathrm{p}<0.05)$ and more potentially foot-damaging behaviours ("foot ulcer=pain" $\mathrm{sc}=.14$, $\mathrm{p}<0.01)$. Accurate interpretation of medical information ("DN=nerve damage" and "foot ulcer causes") enhanced preventive foot self-care $(\mathrm{sc}=.11, \mathrm{p}<0.05 ; \mathrm{sc}=.13, \mathrm{p}<0.01)$, increased perceived foot ulcer unpredictability $(\mathrm{sc}=.11$, $\mathrm{p}<0.01 ; \mathrm{sc}=.21, \mathrm{p}<0.001)$ and anticipation of DN consequences ( $\mathrm{sc}=.18, \mathrm{p}<0.001 ; \mathrm{sc}=.23, \mathrm{p}<0.001$ ), which, in turn, predicted preventive foot self-care $(\mathrm{sc}=12, \mathrm{p}<0.01)$. Foot ulcer history directly impacted preventive foot self-care $(\mathrm{sc}=.25, \mathrm{p}<0.001)$ and potentially foot-damaging behaviours $(\mathrm{sc}=-.23, \mathrm{p}<0.001)$ and indirectly enhanced preventive foot self-care by "correcting" patients' common-sense beliefs "good circulation=healthy feet" $(\mathrm{sc}=-.16, \mathrm{p}<0.001)$ and "foot ulcer=pain" $(\mathrm{sc}=-.38, \mathrm{p}<0.001)$. It also increased perceived ulcer unpredictability $(\mathrm{sc}=.31, \mathrm{p}<0.001)$ and facilitated better understanding about foot ulcer causes $(\mathrm{sc}=.25, \mathrm{p}<0.001)$. The model accounted for $30 \%$ of the variance in preventive foot self-care and $25 \%$ of the variance in potentially foot-damaging behaviours.

Conclusion: These findings highlight the importance of patients' common-sense beliefs about diabetic foot complications in shaping foot self-care and provide the basis for the development of more efficacious interventions to improve the patients' ability to manage their foot ulcer risks.

Supported by Diabetes UK and ADA

0863

A psychometric validation of a new measure of treatment satisfaction with oral antidiabetic medication: the satisfaction with oral antidiabetic agents scale (SOADAS)

D. Wild ${ }^{1}$, C. Donatti ${ }^{1}$, R. Horblyuk ${ }^{2}$, B. Arondekar ${ }^{2}$, E. Brohan ${ }^{1}$;

${ }^{1}$ Psychometrics, Oxford Outcomes, Botley, United Kingdom, ${ }^{2}$ GSK, Philadelphia, United States.

Background and Aims: The objective of this study was to conduct a psychometric validation of the SOADAS having initially conducted patient focus groups and a comprehensive literature review.

Materials and Methods: Adults with type 2 diabetes (T2D), aged between 21 and 70, taking 1-3 OAD agents were eligible. 105 patients were recruited from sites across the US. $50 \%$ of the patients were re-tested 7 days after the initial assessment. Exploratory factor analysis (EFA) was used to assess the factor structure of the SOADAS. Reliability and validity of the SOADAS was examined. To validate the SOADAS, ancillary measures were administered to assess medication complexity and medical adherence (Morisky Medication Adherence Scale; MMAS), health status (SF-12) and treatment satisfaction (Treatment Satisfaction with Medication Questionnaire; TSQM). Clinical data were collected. Results: The EFA presented a one-factor solution for the SOADAS. In addition, a RASCH analysis of the SOADAS was conducted to assess the uni-dimensional structure of the scale. The scale was invariant, with a non-significant chisquare $(p=0.249)$. That is, all items were fitting the model at a Bonferroni adjusted 0.05 significance level. Internal consistency, using Cronbach's alpha, was 0.856, and indicated good internal consistency. The difference between scores at test and retest was not significant $(\mathrm{t}=1.127$, df 46 , $\mathrm{p}=.265$ ). Spearman's $\mathrm{r}$ for total SOADAS score at initial assessment and retest was $0.711(\mathrm{p}<0.001)$, indicating test- 
retest reliability. The correlation between the total SOADAS score and the global treatment satisfaction subscale of the TSQM was high $(\mathrm{r}=.707, \mathrm{p}<0.001)$ indicating convergent validity. The SF-12 Physical Component Scale and the Mental Components Scale correlated moderately with the SOADAS total score $(\mathrm{r}=-0.354, \mathrm{p}<0.001, \mathrm{r}=-0.237$, $\mathrm{p}<0.01$ ), indicating divergent validity.

Conclusion: In summary, the SOADAS demonstrated good reliability and validity, and is ready for use to assess treatment satisfaction across a wide spectrum of OAD therapies.

Supported by GSK

\section{4}

\section{Determinants of psychological well-being in insulin treated persons with diabetes \\ M. Pibernik-Okanovic ${ }^{1}$, R. Besenic ${ }^{2}$, Z. Metelko ${ }^{1}$; ${ }^{1}$ Outpatient Clinic, Vuk Vrhovac Institute, Zagreb, ${ }^{2}$ Diabe- tes Care Unit, Medilab, Zagreb, Croatia.}

Background and Aims: Psychological well-being is an important, patient-centered endpoint of diabetes care. This study was aimed at examining psychological well-being in insulin-treated patients and determining its associations with disease-related variables and coping with diabetes as assessed by diabetes care providers.

Materials and Methods: Psychological well-being was assessed by using the WHO-5 Well-being index in 828 insulin-treated patients ( $49 \%$ female, aged $59 \pm 13$ yrs, $13 \%$ with type 1 , diabetes duration $11 \pm 8$ yrs, HbA1C $8.8 \% \pm 1.8$, BMI $27 \mathrm{~kg} / \mathrm{m} 2 \pm 4$, with neuropathy in $53 \%$, nephropathy in $13 \%$ and retinopathy in $38 \%$ cases). The diabetes care providers were instructed to classify the patients' coping with diabetes, taking account of the prevailing emotions (positive vs negative), and approach towards information (active vs passive). Five different patterns were described as «Empowered and in control» (positive; active), «Fearful information seeker» (negative; active), «Tell me what to do» (neutral; passive), «Resentful victim $\gg$ (negative; passive) or $\ll$ Feeling fine and uninvolved» (positive; passive). Analysis of variance and multiple regression analysis were used to define demographic, disease- and coping-related factors affecting psychological well-being.

Results: The well-being scores in the examined subjects differed with respect to gender (male vs female $14 \pm 6$ vs $13 \pm 6 ; p=0.01$ ), type of diabetes (type 1 vs type $215 \pm 7$ vs $13 \pm 6$; $\mathrm{p}=0.0001$ ), presence of neuropathy (yes vs no $11 \pm 5$ vs $15 \pm 6 ; p<0.0001$ ), presence of retinopathy (yes vs no $11 \pm 5$ vs $14 \pm 5 ; p<0.0001$ ), family support (yes vs no $14 \pm 6$ vs $12 \pm 6 ; \mathrm{p}=0.0002$ ), and coping as assessed by diabetes care providers. «Empowered and in control» subjects obtained the highest well-being scores $(15 \pm 6)$ followed by $\ll$ Tell me what to do» $(13 \pm 6)$, «Fearful information seeker» $\gg(12 \pm 5), \ll$ Feeling fine and uninvolved $\gg(11 \pm 5)$ and «Resentful victim» $(9 \pm 5)$ groups. The empowered group scored better than all other groups (all $\mathrm{p}<0.01$ ), while the «Fearful information seeker» and «Tell me what to do» groups scored better than «Resentful victim» group ( $<<0.0001$ and $p<0.0001$, respectively). The subjects described as $\ll$ Feeling fine and uninvolved» scored their well-being to be low. Seventy-one percent of patients from this group obtained WHO- 5 scores $<13$, indicating possibly serious emotional problems (compared to $25 \%$ of subjects with scores $<13$ in the empowered group, $36 \%$ in the fearful group, $49 \%$ in the group relying on their physicians, and $74 \%$ in the $\ll$ Resentful victim» group).

Multiple regression analysis indicated that psychological well-being could be predicted by gender, age, type and duration of diabetes, neuropathy, retinopathy, HbA1C, family support, and coping-related characteristics as assessed by health care providers ( $\mathrm{R} 2=0.195 \mathrm{~F}=13.93$; $\mathrm{p}=0.0001$ ). Being younger, free of complications, supported by family, with better glycaemic control, and approaching diabetes positively/actively independently predicted better psychological well-being.

Conclusion: Psychological well-being was found to be associated with age, gender, family support, glycaemic control, diabetes complications and coping with the disease. The self-assessed well-being seemed to be interrelated with the providers' assessments. However, poor well-being remained unrecognized in patients labeled as $\ll$ Feeling fine and uninvolved». Self-assessments of psychological wellbeing should be incorporated into routine diabetes care.

Supported by BAYER Diabetes Care Division

\section{5}

The association between stages of change and initiation of insulin treatment in Japanese patients with type 2 diabetes

H. Ishii ${ }^{1}$, S. Oikawa ${ }^{2}$, M. Odawara ${ }^{3}$, J. Kishimoto ${ }^{4}$, Y. Saito ${ }^{5}$, H. Seino ${ }^{6}$, N. Tajima ${ }^{7}$, Y. Hayashi ${ }^{8}$, H. Makino ${ }^{9}$, T. Yamazaki ${ }^{4}$, N. Yoshioka ${ }^{10}$, Y. Iwamoto ${ }^{11}$;

${ }^{1}$ Diabetes Dept, Tenri Yorozu Hospital, Nara, ${ }^{2}$ Diabetes, Nippon Medical School, Tokyo, ${ }^{3}$ Diabetes Dept, Tokyo Medical University, ${ }^{4}$ School of Medicine, University of Tokyo, ${ }^{5}$ School of Medicine, Chiba University, ${ }^{6}$ Diabetes, Ohta Nishinouchi Hospital, Fukushima, ${ }^{7}$ School of Medicine, Jikei University, Tokyo, Japan, ${ }^{8}$ School of Medicine, Nihon University, Tokyo, ${ }^{9}$ School of Medicine, Okayama University, ${ }^{10}$ Medicine, Hokkaido University, ${ }^{11}$ Diabetes, Tokyo Women's Medical University, Tokyo, Japan. 
Background and Aims: The Diabetes Attitudes Wishes and Needs (DAWN) study was designed to give a global perspective on patient and provider perceptions of diabetes management, and to identify opportunities to improve psychosocial aspects of diabetes care. Results suggest that psychological hindrance to starting insulin treatment exists in patients and healthcare professionals. In response to the DAWN study, we initiated a survey to identify psychological factors related to insulin treatment in Japan. The prospective association between stages of change in patient attitudes towards insulin and success of insulin initiation after patients were advised to start insulin was also assessed.

Materials and Methods: Patients with type 2 diabetes $(n=117)$ reported their attitudes towards starting insulin treatment using a self-administered questionnaire at the time of receiving a recommendation to initiate insulin. The questions evaluated the patients' attitudes towards change. Agreement with each question indicated whether they had a 'preparation', 'contemplation' or 'precontemplation' attitude. The percentage of patients who had commenced insulin was assessed twice, 1 month after recommendation and at 6 months.

Results: At the time of insulin recommendation, 37 patients (31\%) responded "I am thinking of taking insulin immediately" (preparation stage), 47 (40\%) responded "I am wondering whether to take insulin" (contemplation stage) and $33(28 \%)$ responded "I have no intention of taking insulin" (precontemplation stage).

At 1 and 6 months, there was a significant difference in the number of patients receiving insulin dependent on their response, both $p<0.001$ (Table). At 6 months, insulin initiation had been successful in almost all of the patients in the preparation stage.

Table. Patients using insulin 1 and 6 months after recommendation

\begin{tabular}{|c|c|c|}
\hline $\begin{array}{l}\text { Patient attitude } \\
\text { at time of insulin } \\
\text { recommendation }\end{array}$ & $\begin{array}{l}\text { Percentage of pat } \\
\text { insulin: } \\
1 \text { month after } \\
\text { recommendation }\end{array}$ & $\begin{array}{l}\text { tients using } \\
6 \text { months after } \\
\text { recommendation }\end{array}$ \\
\hline $\begin{array}{l}\text { "I am thinking } \\
\text { of taking insulin } \\
\text { immediately" }(\mathrm{n}=37) \\
\text { Preparation stage }\end{array}$ & $87 \%$ & $97 \%$ \\
\hline $\begin{array}{l}\text { "I am wondering } \\
\text { whether to take insulin" } \\
(n=47) \text { Contemplation stage }\end{array}$ & $26 \%$ & $38 \%$ \\
\hline $\begin{array}{l}\text { "I have no intention of taking } \\
\text { insulin" ( } \mathrm{n}=33) \\
\text { Precontemplation stage }\end{array}$ & $9 \%$ & $30 \%$ \\
\hline
\end{tabular}

Conclusions: The degree of patient willingness to start insulin at the time of their healthcare professional's recommendation directly influenced the success rate of insulin initiation. Furthermore, patient willingness to start insulin treatment increased with time, following recommendation from their doctor. When insulin is recommended, it could be useful to assess patient willingness to initiate treatment, so that personalised interventions to change patients' attitudes towards insulin may be implemented if needed.

Supported by Novo Nordisk Pharma Ltd

0866

Japanese physicians' attitudes towards insulin therapy may influence prescribing behaviour

Y. Iwamoto ${ }^{1}$, H. Ishii ${ }^{2}$, S. Oikawa ${ }^{3}$, M. Odawara ${ }^{4}$, J. Kishimoto $^{5}$, Y. Saito ${ }^{6}$, H. Seino ${ }^{7}$, N. Tajima ${ }^{8}$, Y. Hayashi $^{9}$, H. Makino ${ }^{10}$, T. Yamazaki ${ }^{5}$, N. Yoshioka ${ }^{11}$; ${ }^{1}$ Diabetes, Tokyo Women's Medical University, Tokyo, ${ }^{2}$ Diabetes, Tenri Yorozu Hospital, Nara, ${ }^{3}$ Diabetes, Nippon Medical School, Tokyo, ${ }^{4}$ Diabetes, Tokyo Medical University, Tokyo, ${ }^{5}$ School of Medicine, University of Tokyo, ${ }^{6}$ School of Medicine, Chiba University, ${ }^{7}$ Diabetes, Ohta Nishinouchi Hospital, Fukushima, ${ }^{8}$ School of Medicine, Jikei University, Tokyo, ${ }^{9}$ School of Medicine, Nihon University, Tokyo, ${ }^{10}$ School of Medicine, Okayama University, ${ }^{11}$ School of Medicine, Hokkaido University, Japan.

Background and Aims: The worldwide Diabetes Attitudes, Wishes and Needs (DAWN) study investigated psychosocial and behavioural barriers to insulin treatment experienced by patients and healthcare professionals. Nearly $50 \%$ of doctors did not begin insulin treatment until it was absolutely necessary and suggested that readiness to prescribe insulin is related to doctors' diabetes experience. In response to the DAWN study, the relationship between physicians' attitudes towards prescribing insulin and their prescribing behaviour was investigated in Japan.

Materials and Methods: Japanese physicians $(n=132)$ participated: 75 Japan Diabetes Society (JDS)-certified specialists (treated the most patients with diabetes); 30 JDS-affiliated specialists; and 27 doctors not affiliated with JDS (treated the least patients with diabetes). JDS-certified specialists had more patients receiving insulin (34\%) than JDS-affiliated specialists $(23 \%)$ and non-JDS doctors $(10 \%)$. Attitudes towards prescribing insulin were evaluated with a questionnaire (27 attitude statements). High scores indicate agreement with attitude statements. The association between doctors' attitudes and recommendation for insulin was assessed by multiple regression analysis.

Results: Scores for 4 attitude statements correlated with physicians' level of JDS affiliation. Non-JDS-affiliated doctors obtained the highest scores for all of the statements $(p<0.001)$, Table. JDS-certified specialists had the lowest scores for three of the statements, positively correlating with level of affiliation. However, scores for the statement "It is 
troublesome for me to explain about handling injectors" were negatively correlated with level of JDS affiliation.

Table. Attitude scores for different physician groups

\begin{tabular}{|c|c|c|c|c|c|}
\hline $\begin{array}{l}\text { Attitude } \\
\text { statements }\end{array}$ & $\begin{array}{l}\beta \\
\text { (multiple } \\
\text { regression } \\
\text { analysis) }\end{array}$ & $p$ & $\begin{array}{l}\text { Scores } \\
\text { JDS- } \\
\text { certified } \\
\text { specialists }\end{array}$ & $\begin{array}{l}\text { JDS- } \\
\text { affiliated } \\
\text { specialists }\end{array}$ & $\begin{array}{l}\text { Non- } \\
\text { JDS } \\
\text { doctors }\end{array}$ \\
\hline $\begin{array}{l}\text { "I am not } \\
\text { familiar with } \\
\text { insulin therapy" }\end{array}$ & 0.34 & $p<0.001$ & 1.20 & 1.90 & 2.93 \\
\hline $\begin{array}{l}\text { "There are no } \\
\text { in-house } \\
\text { support staff" }\end{array}$ & 0.45 & $p<0.001$ & 1.35 & 2.03 & 3.26 \\
\hline $\begin{array}{l}\text { "If insulin } \\
\text { therapy is } \\
\text { necessary the } \\
\text { patient has } \\
\text { to be referred } \\
\text { to a specialist" }\end{array}$ & 0.24 & $p<0.01$ & 1.39 & 1.97 & 3.33 \\
\hline $\begin{array}{l}\text { "It is } \\
\text { troublesome } \\
\text { for me to explain } \\
\text { about handling } \\
\text { injectors" }\end{array}$ & -0.33 & $p<0.001$ & 1.97 & 1.90 & 3.07 \\
\hline
\end{tabular}

Doctors recommended insulin for only $23 \%$ of patients who were defined as candidates for insulin therapy $\left(\mathrm{HbA}_{1 \mathrm{c}} \geq 8 \%\right.$, receiving high-dose sulphonylureas).

Conclusions: Treating fewer diabetes patients and insulin therapy-related psychological factors may affect doctors' attitudes towards insulin treatment, delaying prescribing for patients who would benefit. Conversely, treating the most patients may also result in attitudes which delay insulin treatment. Addressing these attitudes and behaviours may enable more patients to receive timely recommendations to initiate insulin.

Supported by Novo Nordisk

0867

Treatment satisfaction in type 2 patients: Evaluating the impact of clinical outcomes, patient and disease characteristics

M. Brod ${ }^{1}$, D. Cobden ${ }^{2}$, M. Lammert ${ }^{3}$, D. M. Bushnell ${ }^{4}$; ${ }^{1}$ Health Outcomes Research, The BROD GROUP, Mill Valley, United States, ${ }^{2}$ Novo Nordisk Inc., Princeton, United States, ${ }^{3}$ Novo Nordisk A/S, Copenhagen, Denmark, ${ }^{4}$ Data Management and Analysis, Health Research Associates, Inc, Mountlake Terrace, United States.

Background and Aims: Treatment satisfaction (TS) differs between therapy options and impacts patient compliance, self-management behaviors and costs. Clinical wisdom assumes that TS with insulin therapy improves with fewer injections and side effects. However, little is known about the combined impact of treatment outcomes and patient characteristics on TS. This study examined the relationships between TS, patient characteristics (age, gender, ethnicity, weight, co-morbidities, diabetes duration) and treatment outcomes $\left(\mathrm{HbA}_{1 \mathrm{c}}\right.$, number of daily injections, weight gain, hypoglycemic events).

Material and Methods: Baseline and 28 week end of study data from a 25 site (US) RCT comparing efficacy and safety of BID biphasic insulin aspart 70/30 (BIAsp 70/30) vs. QD glargine in insulin naïve type 2 patients failing oral medication $(\mathrm{n}=240)$ were analyzed. Subjects completed the validated Insulin Treatment Satisfaction Questionnaire (ITSQ), assessing overall TS and 5 domains (Lifestyle Flexibility, Hypoglycemic Control, Glycemic Control, Device Satisfaction, Inconvenience of Regimen). Independent regression analyses examined the relationship between TS, baseline patient/disease characteristic and treatment outcomes. All significant factors were then examined together by multivariate regression analyses.

Results: A major predictor of overall TS, Lifestyle Flexibility, Hypoglycemic and Glycemic Control, was the presence of diabetes-related co-morbidities, notably neuropathy $(p<0.05)$. Retinopathy significantly impacted Device Satisfaction, and age was related to Lifestyle Flexibility $(p<0.05)$. No other baseline patient/disease characteristic impacted TS. Reduction of $\mathrm{HbA}_{1 \mathrm{c}}$ significantly impacted overall TS, Device Satisfaction, Glycemic Control, and Inconvenience of Regimen $(\mathrm{p}<0.05)$. Number of hypoglycemic events impacted Hypoglycemic Control satisfaction, whereas the timing of the event (day vs. night) was related to overall TS and all domains ( $\mathrm{p}<0.001$, negative impact for daytime events). Weight gain, number of daily injections and treatment group did not impact TS despite the presence of significant treatment group differences (BIAsp 70/30 significantly reduced $\mathrm{HbA}_{1 \mathrm{c}}$, with increased minor hypoglycemia and weight gain). When all significant factors were examined together, $\mathrm{HbA}_{1 \mathrm{c}}$ reduction and neuropathy maintained their previously identified significant impacts on TS. The number of hypoglycemic events remained significant only for satisfaction with Hypoglycemic Control, and timing of the hypoglycemic event significantly impacted overall TS and $3 / 5$ domains $(\mathrm{p}<0.01)$.

Conclusion: Contrary to clinical wisdom, the number of daily injections and the occurrence of weight gain or minor hypoglycemia may not negatively impact TS when balanced by significant $\mathrm{HbA}_{1 \mathrm{c}}$ treatment benefits. Further, reduced $\mathrm{HbA}_{1 \mathrm{c}}$ and timing of hypoglycemia, rather than number of events, may have more broad impact on TS. Factors impacting TS when examined independently did not always exert the same significant influence when 
assessed in combination with other factors. Thus, clinicians should judge the balance between positive and negative treatment outcomes before considering treatment options. Supported by Novo Nordisk

\section{8}

Newly diagnosed patients with type $\mathbf{2}$ diabetes mellitus are satisfied with insulin treatment

I. Lingvay, P. F. Kaloyanova, P. Raskin;

Endocrinology, University of Texas Southwestern Medical Center, Dallas, United States.

Background and Aims: Insulin treatment in type 2 diabetes mellitus (T2DM) is usually deferred until oral hypoglycemic agents fail because physicians fear side effects (mainly hypoglycemia and weight gain) and believe that patients would not accept an injectable therapy. Thus, as glycemic control deteriorates, treatment is intensified in a stepwise manner resulting in a longer exposure to hyperglycemia which increases the risk of complications and exposes the beta-cells to the potential damaging effects of hyperglycemia. Early insulin treatment could prevent progressive glycemic deterioration and might preserve the beta-cell function. The study evaluates the feasibility, acceptance, and effectiveness of insulin in combination with metformin in newly-diagnosed, treatment-naive, patients with T2DM.

Materials and Methods: Eligible patients were started on biphasic insulin aspart 30/70 (30\% soluble and 70\% protaminated insuln aspart) delivered by $\mathrm{FlexPen}^{\circledR}$ and metformin $1000 \mathrm{mg}$ twice daily. Biochemical evaluation was done at baseline and end of the 3-month study period. Patients were seen monthly for evaluation of side effects, compliance, and insulin dose adjustments, which were based on BG logs and historical information. Patient quality of life and acceptance of insulin treatment was evaluated with the Diabetes Quality of Life Questionnaire (DQoL) at the end of the study.

Results: Of 63 patients enrolled (age 44.7, BMI 35.4, 43 males) $8 \%$ dropped out for reasons unrelated to the study intervention. HbAlc decreased from 10.8 to $5.9 \%$, with $100 \%$ of subjects achieving $\mathrm{HbA} 1 \mathrm{c} \leq 7 \%$, and $90 \%$ a $\mathrm{HbA} 1 \mathrm{c} \leq 6.5 \%$. The weight increased from 100 to $101.5 \mathrm{~kg}$ $(p=0.006)$, but if we account for an average of $7.2 \mathrm{~kg}$ lost in the 6-month preceding the diagnosis of diabetes, the net weight change was $-5.55 \mathrm{~kg}(\mathrm{p}<0.001)$, so not all the weight lost prior to diagnosis was regained. The rate of hypoglycemia was 1.5 episodes/patient-month, with a liberal definition of hypoglycemia (BG $<3,9 \mathrm{mmol} / \mathrm{l}$ with symptoms). Only one severe hypoglycemic event occured. At the end of the study $84.5 \%$ of the patients rated their general health good or above and $88 \%$ reported improvement in their general health compared to baseline. $93 \%$ of the patients were satisfied with their insulin treatment, and $88 \%$ were willing to continue insulin. $81 \%$ reported they felt their diabetes was well controlled on the current treatment. Mean score for the following subscales of the DQoL was: treatment satisfaction 1.83 , disease impact 1.81 , impact of hypoglycemia 1.75 , lifestyle flexibility 2.15 , and social impact 1.18 . Compliance rate with insulin was $96.7 \%$ and metformin $95.2 \%$.

Conclusion: Outpatient initiation of insulin in combination with metformin at the time of diagnosis of T2DM is an effective, safe, and feasible strategy to rapidly achieve normoglycemia. All patients got excellent glycemic control within 3 months, had a low rate of hypoglycemia with minimal weight gain. Insulin therapy was very well accepted by the patients, refuting the misconception of low satisfaction and compliance with such treatment.

Supported by NovoNordisk, Inc

\section{9}

Physician beliefs in efficacy of insulin glargine (GLAR) are associated with glucose control in type 2 diabetes (T2DM)

W. Herman ${ }^{1}$, Q. Zhang ${ }^{2}$, L. Kennedy ${ }^{3}$;

${ }^{1}$ Dept of Med, U of Michigan Med Center, Ann Arbor,

${ }^{2}$ sanofi-aventis U.S., Bridgewater, United States, ${ }^{3}$ Dept of Med, U of FL, Gainesville, United States.

Background and Aims: Goal A1C (Glycemic Optimization with Algorithm and Labs At Polnt of Care) was a randomized, parallel group, four-arm, open-label, 24-week study to assess the impact of active (weekly monitored) vs usual monitoring of algorithmic insulin titration and pointof-care (POC) vs laboratory A1C in patients with T2DM $(\mathrm{N}=7893$; mean age $57 \mathrm{y}$, weight $98 \mathrm{~kg}$, diabetes duration $8.4 \mathrm{y}$, fasting blood glucose $211 \mathrm{mg} / \mathrm{dL}$, A1C $8.9 \%$ ) who failed oral antidiabetic therapy and started GLAR treatment. The objective of the present analysis was to determine if physicians' knowledge of, attitudes toward, and expectations of GLAR and its effects might be associated with glycemic control.

Material and Methods: At baseline, physicians $(\mathrm{N}=2871)$ were surveyed about their demographics, GLAR knowledge, reasons for prescribing GLAR, and expected efficacy and safety of GLAR. Physicians were asked to predict the percentage of patients they expected to achieve A1C $<7.0 \%$, and the percent decrease in mean $\mathrm{A} 1 \mathrm{C}$ their patients would achieve by week 12 and week 24 . They were also asked to rate their level of agreement with factors that may affect success of treatment with GLAR (1-strongly disagree to 5 -strongly agree), and to rank 4 reasons for prescribing 
GLAR from highest (1) to lowest (4). Reasons to be ranked were "improves blood sugar control", "flexibility of use", "fewer hypoglycemic episodes", and "positive feedback from patients".

Results: Of physicians surveyed 14\% were women; $11 \%$ were endocrinologists; $79 \%$ were primary care/internal medicine and $10 \%$ were of other specialties; and $33 \%$ practiced in urban, $44 \%$ in suburban, and $23 \%$ in rural areas. Average patient A1C over the study did not differ between physician subspecialties. When patient A1C was evaluated against physician responses to the survey at baseline, we found that lower average A1C was associated with higher physician expectation in goal achievement $(<7 \%)$ by week $12(P=0.005)$. Patients whose physicians gave lower rankings to "fewer hypoglycemic events" and "positive feedback from patients" as reasons for prescribing GLAR achieved lower (better) A1C averaging over the study period ( $P=0.02$ and 0.04 , respectively). Physician practices that reported seeing more than 10 patients per week were also associated with better mean patient A1C $(P=0.007)$. Regardless of $\mathrm{A} 1 \mathrm{C}$ treatment group, physicians who "strongly" or "somewhat agreed" that the ease of use of GLAR will make patients more receptive to its use was associated with a higher mean patient A1C $(P=0.02)$. As previously reported, more patients in POC A1C active titration group achieved A1C $<7 \%$ (41\% for POC vs $36 \%$ for laboratory testing; $\mathrm{P}<0.0001)$. Results of the current analysis showed further A1C differentiation between groups. In the POC A $1 \mathrm{C}$ active titration group, patients of physicians who ranked "GLAR improves blood sugar control" as the most important reason for prescribing it achieved lower mean A1Cs than those who gave lower rankings to this reason $(P=0.047)$. The opposite was true for laboratory testing conditions.

Conclusion: This study presents novel evidence that physician beliefs in efficacy of GLAR are an important determinant of actual glucose control in patients with type 2 diabetes treated with GLAR.

Supported by sanofi-aventis U.S.

\section{PS 70}

\section{Psychiatric complications of diabetes}

0870

Depression and treatment choice in type 2 diabetes patient failing oral antidiabetic treatment F. Petrak $^{1}$, E. Stridde ${ }^{2}$, I. v. Toerne ${ }^{3}$, A. Pfützner ${ }^{4}$;
${ }^{1}$ Psychosomatic Medicine and Psychotherapy, Westfaelische Clinic Dortmund/Ruhr-University of Bochum, Dortmund, ${ }^{2}$ Pfizer Pharma GmbH, Karslruhe, ${ }^{3}$ TNS Healthcare, Muenchen, ${ }^{4}$ IKFE Institute for Clinical Research \& Development, Mainz, Germany.

Background and Aims: The aim of this cross-sectional study was to assess the interaction between depression and treatment preferences regarding outcome parameters in type 2 diabetes patients failing oral antidiabetic treatment (OAD). Materials and Methods: Patients with type 2 diabetes $(\mathrm{N}=532)$ on OADs (Male $66.9 \%$ ) with a mean age of 56,5 yrs $(\mathrm{sd} 8,8)$ and a mean $\mathrm{HbA} 1_{\mathrm{c}}$ of $8.34 \%$ (sd 1.5 ) were recruited in study sites across Germany. Participants discussed treatment optimization options with a physician and were asked to make a theoretical choice about future diabetes therapy. In Step One options were to continue with OADs or to move on to subcutaneous (SC) insulin. In Step Two an additional treatment option with inhaled insulin (INH) was offered. Questionnaires were used to assess depressive disorders (PHQ), diabetes-specific stress (PAID, higher values indicates more stress), and health related quality of life (HRQoL; SF-36, z-values).

Results: Taken both steps together, $16.0 \%$ opted for SC, $27.4 \%$ opted for INH, and $56.6 \%$ for OAD. For the whole sample a $10.0 \%$ prevalence of depression was observed. Patients reported moderate diabetes related stress (mean 23.51; sd 17.23) and HRQoL were clearly within the normal range of the general German population for the physical (mean -0.3; sd 1,0) and mental (mean -0.3 ; sd 1,1) component. Significant main effects for depression were observed in analyses of variances for physical $(\mathrm{F}=87.64$; $\mathrm{df}=1 ; \mathrm{p}<0.001)$ and mental HRQoL $(\mathrm{F}=137.00 ; \mathrm{df}=1$; $\mathrm{p}<0.001)$. There were two significant interactions between treatment choice and depression. First, HbAlc was higher in patient who opted for SC insulin, but only for depressed patients $(\mathrm{F}=3.91 ; \mathrm{df}=2 ; \mathrm{p}<0.05)$. Second, diabetes-specific stress was higher in patients with depression but only for those who opted for OAD or INH $(\mathrm{F}=6.04 ; \mathrm{df}=2 ; \mathrm{p}<0.01)$.

Conclusion: A view at the whole sample gives the impression of relatively low diabetes related stress and normal HRQoL despite the poor metabolic control of the patients. In contrast, the subgroup of patients with depression reports strongly impaired HRQoL. The consideration of depression in interaction with the treatment choice revealed that patients with depression and extremely poor metabolic control were more likely to choose SC insulin. Participants who preferred OADs or inhaled insulin described themselves as strongly impaired by diabetes-specific stress when depression was present whereas those who opted for SC insulin seems to have very low perceived burdens of their diabetes even in the presence of depression.

Supported by Pfizer Pharma GmbH 
Interaction of treatment choice, depression and diabetes outcome (mean, SD)

\begin{tabular}{lccccc}
\hline Treatment Choice & Depression PHQ & HbA1 $\mathbf{c} \%$ & PAID (0-100) & Physical HRQoL (z-values) & Mental HRQoL (z-values) \\
\hline OAD & No Depression N=276 & $8.1 \pm 1.4$ & $20.8 \pm 15.2$ & $-0.0 \pm 0.8$ & $-0.1 \pm 0.9$ \\
& Depression N=25 & $8.6 \pm 1.5$ & $41.6 \pm 26.4$ & $-1.6 \pm 0.7$ & $-2.2 \pm 0.8$ \\
\multirow{5}{*}{ SC insulin } & Sum N=301 & $8.2 \pm 1.4$ & $22.5 \pm 17.3$ & $-0.17 \pm 0.9$ & $-0.3 \pm 1.0$ \\
& No Depression N=76 & $8.5 \pm 1.6$ & $22.3 \pm 15.5$ & $-0.4 \pm 1.0$ & $-0.3 \pm 1.2$ \\
& Depression N=9 & $10.4 \pm 1.9$ & $20.4 \pm 14.2$ & $-1.5 \pm 1.0$ & $-1.5 \pm 1.0$ \\
Inhaled Insulin & Sum N=85 & $8.7 \pm 1.7$ & $22.1 \pm 15.3$ & $-0.5 \pm 1.1$ & $-0.4 \pm 1.2$ \\
& No Depression N=127 & $8.5 \pm 1.4$ & $23.9 \pm 16.1$ & $-0.2 \pm 0.9$ & $-0.1 \pm 0.9$ \\
& Depression N=19 & $8.7 \pm 1.5$ & $42.8 \pm 20.3$ & $-1.4 \pm 1.0$ & $-2.0 \pm 1.0$ \\
Sum Depression & Sum N=146 & $8.5 \pm 1.4$ & $23.5 \pm 17.2$ & $-0.3 \pm 1.0$ & $-0.4 \pm 1.1$ \\
& No Depression N=479 & $8.3 \pm 1.5$ & $21.9 \pm 15.5$ & $-0.1 \pm 0.9$ & $-0.1 \pm 0.9$ \\
& Depression N=53 & $8.9 \pm 1.7$ & $38.4 \pm 23.7$ & $-1.5 \pm 0.9$ & $-2.0 \pm 1.1$ \\
\hline
\end{tabular}

0871

\section{Depression in type 2 diabetes in primary care H. Yaman ${ }^{1}$, O. Göktas ${ }^{2}$; \\ ${ }^{1}$ Family Medicine, Akdeniz University, Antalya, ${ }^{2}$ Ertuğrul- gazi Family Medicine Center, Ertuğrulgazi Family Medi- cine Center, Bursa, Turkey.}

Background and Aims: Diabetes mellitus is a chronic health problem that might also influence the affective status of patients. Besides its effects on lifestyle, it has important implications for a patient's well-being and social life. The aim of this study was to evaluate the level of depressive condition in a primary care population and to investigate any relationship with socio-demographic and health outcome measure.

Materials and Methods: The intervention method was a prospective, cross-sectional survey, which was performed randomly during clinical open access encounter.The family medicine centre (FMC) is located in Bursa/West Turkey.

The sample size of the study population for type 2 diabetes was calculated as 263 (confidence level 95\%, confidence interval $5+/-1 \%)$. Three hundred diabetes patients (3287 years; $69.7 \%$ female, $30.3 \%$ male; $75.4 \%$ married and $24.6 \%$ widowed) filled out the Turkish Beck Depression Inventory (BDI) and the Turkish SF-36 health outcome measure. Information on age, sex, marital status, social security, duration of diabetes, other health problems, type of management, physical activity, perception on their quality of life and perception on their diabetes specific quality of life have been gathered with a questionnaire. Chi-Square, Student $t$ test and logistic regression methods have been applied.

Results: Participants had type 2 diabetes since 1-37 years (mean $8.2 \pm 7.2$ years). The internal validity of BDI and SF-
36 revealed medium-high results (Cronbach alpha 0.70 $0.89)$. BDI scores in $24(8 \%)$ participants had scores of 17 and above (cut-off value for depression disorder). Female $(20 ; 83 \%)$ suffered more than male of depression [x2 $(300,1)=4,2 ; p=0.04]$. Marital and occupational status showed significant differences $[\mathrm{x} 2(300,1)=11,4$ and 4,2 ; $\mathrm{p}<0.05]$. Physically active participants had lower BDI scores $[\mathrm{t}(300,298)=2,5 ; \mathrm{p}=0.01]$. Patients having higher BDI scores were suffering of lower health outcome measure dimension scores (i.e. physical functioning, physical role, pain, general health, vitality, social functioning, mental health) $[\mathrm{t}(300,298)=2.3-5.5 ; \mathrm{p}<0.05]$. The age, perception of general quality of life, perception of diabetes specific quality of life, physical functioning sub-dimension and general health sub-dimension of SF-36 were explaining the higher BDI scores $(>16)$ according to the logistic regression model (inclusion method).

Conclusion: Depression was prevalent in $8 \%$ of type 2 diabetics and women were suffering more in primary care setting. The age, the perception of quality of life and diabetes specific quality are predictors of depression in type 2 diabetics. It seems advisable that depressive disorders should be considered during primary diabetes care in special risk groups (i.e. women, single-divorced, elderly, perception of low quality of life).

0872

Differential effects of neuropathic symptoms on anxiety and depression: a longitudinal study

J. S. Gonzalez ${ }^{1,2}$, L. Vileikyte ${ }^{1}$, M. Peyrot ${ }^{3}$, R. Rubin ${ }^{3}$, A. Garrow ${ }^{1}$, J. Ulbrecht ${ }^{4}$, H. Leventhal ${ }^{5}$, P. Cavanagh ${ }^{6}$, A. Boulton ${ }^{1}$; 
${ }^{1}$ Medicine, University of Manchester, United Kingdom, ${ }^{2}$ Psychiatry, Harvard University, Boston, United States, ${ }^{3}$ Medicine, Johns Hopkins University, Baltimore, United States, ${ }^{4}$ Medicine, Penn State University, State College, United States, ${ }^{5}$ Psychology, Rutgers University, New Brunswick, United States, ${ }^{6}$ Biomechanics, The Cleveland Clinic, Cleveland, United States.

Background and Aims: Structural equation modelling was used to examine longitudinally the role of neuropathic symptoms and foot ulceration in generating anxiety and depression.

Materials and Methods: 338 patients (mean age $=63 \mathrm{y}$; $71 \%$ male) with diabetic peripheral neuropathy (DN) completed the Hospital Anxiety and Depression Scale (HADS). DN was diagnosed by the Neuropathy Disability Score (NDS; mean=7.4). Neuropathic symptoms (pain, unsteadiness and reduced feeling in feet) were assessed with a neuropathy-specific quality of life scale, NeuroQoL. Measures were completed at baseline, 9 and 18 months. To account for confounding between anxiety (HADS/A) and depression (HADS/D), and to assess factors associated with change in HADS over time, both baseline HADS/A and HADS/D scores were included as predictors of 18-month HADS/A and HADS/D.

Results: Controlling for demographic and disease variables, baseline NDS predicted 18-month HADS/A (standardized coefficient, $\mathrm{sc}=.13, \mathrm{p}=.011)$ and HADS/D $(\mathrm{sc}=.15$, $\mathrm{p}<0.001$ ). Preliminary models showed that neither baseline nor changes in symptoms of reduced feeling from baseline to 9 months predicted 18-month HADS/A or HADS/D. Likewise, neither foot ulceration at baseline nor new foot ulcers during follow-up predicted 18-month HADS scores. Thus, only symptoms of pain and unsteadiness were included in the final model. The final model fitted the data well $\left[x^{2}(29)=36.64, p=.16, \mathrm{CFI}=.99, \mathrm{RMSEA}=.02\right.$, $\mathrm{SRMR}=.03$ ] and accounted for approximately $58 \%$ of the variance in 18-month HADS/A and HADS/D. Baseline and change in unsteadiness from baseline to 9 months predicted both 18-month HADS/A (baseline: $\mathrm{sc}=.14, \mathrm{p}<0.001$; change: $\mathrm{sc}=.09, \mathrm{p}<0.05$ ) and HADS/D (baseline: $\mathrm{sc}=.20$, $\mathrm{p}<0.001$, change: $\mathrm{sc}=.14, \mathrm{p}<0.001)$. Change in pain also was predictive of both 18-month HADS/A $(\mathrm{sc}=.08, \mathrm{p}<0.05)$ and HADS/D ( $\mathrm{sc}=.12 \mathrm{p}<0.001)$. However, baseline level of pain was predictive only of 18 -month HADS/A ( $\mathrm{sc}=.09, \mathrm{p}<0.05)$. Conclusion: DN predicts depression and anxiety through symptoms of pain and unsteadiness. Depression and anxiety are associated with changes in both pain and unsteadiness. However, depression is not associated with baseline levels of pain while anxiety is. This suggests that depression accommodates to pain over time, while anxiety does not. Clinicians should monitor levels of both anxiety and depression in patients who have experienced recent increases in pain and unsteadiness, as well as persistent unsteadiness. Anxiety should be monitored in patients with painful DN even in the absence of recent increases in pain. Supported by Diabetes $U K$ and ADA

0873

Depressive disorders, diabetic foot ulcers and death: results from a population based cohort of people with diabetes and their first foot ulcer

K. Ismail, K. Winkley, S. Daniel, T. Chalder, M. Edmonds; Psychological Medicine, Institute of Psychiatry, London, United Kingdom.

Background and Aims: The outcome for people with diabetic foot ulcers is very poor despite intensive medical regimens. The role of psychological factors in contributing to poor outcomes has not been prospectively studied. Our aim was to test whether depressive disorders increased the risk of death and morbidity in people with diabetes following the onset of their first foot ulcer over an 18 month period.

Materials and Methods: We used a prospective cohort design. We recruited a population-based cohort of people presenting with their first diabetic foot ulcer from all the community podiatry and hospital diabetic foot clinics within 5 health authorities in South London, United Kingdom representing a population of 6 million between August 2000 to October 2002. The baseline diabetic foot ulcer was defined as: i) the first ulceration in the anatomical foot (the largest ulcer if several ulcers) and ii) a full thickness break in the epithelium with a minimum width of $5 \mathrm{~mm}$ and iii) ankle:brachial ratio $\geq 0.5$ at either the dorsalis pedis or anterior tibial sites using Doppler pressure readings. We used the World Health Organization's Schedule for Clinical Assessment in Neuropsychiatry 2.1 face to face interview to generate Diagnostic and Statistical Manual for Mental Disorders-IV (DSM-IV) major depressive disorder at baseline. We measured the following outcomes: number of deaths of first amputation, of first recurrence and whether the ulcer had heal and time taken for each of these events to occur, censoring the event time at the time a person died or otherwise at 18 months. We measured at baseline age, sex, marital status, socioeconomic status, smoking, glycosylated haemoglobin $\left(\mathrm{A}_{1 \mathrm{c}}\right)$ and Texas severity of ulcer as potential confounders.

Results: 262 people presented with their first foot ulcer; 260 met the study criteria and of these 253 consented. The majority of the cohort were male, had an average age of 62 years, from social classes III-V and had type 2 diabetes. The average duration (years) of diabetes was 30.1 (SD 15.4) and 11.4 (SD 10.1) in people with type 1 and type 2 diabetes respectively. One sixth of the study population was current 
smokers. The prevalence of DSM-IV major depression was $25 \%(\mathrm{n}=60) .10$ people were receiving an antidepressant. There were 41 (16.2\%) deaths (all from natural causes), 36 (15.5\%) first amputations, and 99 (43.2\%) first recurrences. In the Cox regression analysis at time 18 months, DSM-IV major depression was associated with around a two-fold increased risk of mortality at 18 months (adjusted hazards ratio $2.1,95 \%$ confidence interval 1.1 to 4.1 ), amputation (adjusted hazards ratio $1.7,95 \%$ confidence interval 0.9 to 3.5 ) but not with healing (adjusted hazard ratio 1.3, 95\% confidence interval 0.9 to 1.9 ) or recurrence (adjusted hazards ratio $1.2,95 \%$ confidence interval 0.7 to 1.9 ).

Conclusion: Depressive disorders are very common in people who develop their first diabetic foot ulcer and is prospectively associated with an increased risk of mortality after biological confounders such as severity of ulcer are accounted for. The mediating mechanisms are as yet unclear. Depression is a reversible condition and its identification and treatment could improve the life expectancy and morbidity of people with diabetic foot disease.

Supported by Wellcome Trust

\section{4}

What is the evidence for an increased risk of diabetes mellitus in people with schizophrenia who are prescribed atypical antipsychotics compared to those prescribed typical antipsychotics?

M. A. Smith ${ }^{1}$, D. Hopkins ${ }^{2}$, R. C. Peveler ${ }^{3}$, R. I. G. Holt ${ }^{4}$, M. Woodward ${ }^{5}$, K. Ismail ${ }^{1}$;

${ }^{1}$ Department of Psychological Medicine, Institute of Psychiatry, London, ${ }^{2}$ Department of Diabetic Medicine, Kings College Hospital, London, ${ }^{3}$ Community Clinical Sciences Division, University of Southampton, ${ }^{4}$ Endocrinology \& Metabolism Sub-division, University of Southampton, United Kingdom, ${ }^{5}$ Biostatistics, George Institute for International Health, University of Sydney, Australia.

Background and Aims: There is increasing concern that atypical antipsychotics may have an adverse effect on increasing the risk of developing diabetes mellitus in people with schizophrenia perhaps by inducing weight gain. There are numerous alternative explanations such age, ethnicity, obesity, polypharmacy and definition and type of psychiatric disorder, as well as selection bias in screening for diabetes which have not been consistently accounted for in the literature. Our aim was to conduct a systematic review and meta-analysis of the pooled relative risk of diabetes in people with schizophrenia who were prescribed atypical antipsychotics compared to those prescribed typical antipsychotics. Materials and Methods: We used the Meta-analysis of Observational Studies in Epidemiology (MOOSE) guide- lines on reporting of descriptive studies. Studies eligible for inclusion were case series, cross-sectional, case-control studies, cohort or controlled trial designs of children and adults with schizophrenia or related illness. The main outcome was diabetes mellitus. Using established Cochrane Collaboration search strategies and definitions for schizophrenia, antipsychotics and diabetes mellitus, we searched MEDLINE, PsychINFO, EMBASE, International pharmaceutical abstracts, CINAHL, and Web of knowledge from their inception to 31 st July 2005 for studies in all languages. Studies were excluded if data could not be stratified into schizophrenia and related disorders versus other psychiatric disorders. Data extracted included the number of subjects with and without diabetes, type of antipsychotic medication, age, ethnicity, body mass index, and diagnostic classification used (if any) for schizophrenia and related disorders and for diabetes. The main outcome was diabetes mellitus. Corresponding authors were contacted for additional information and non-English papers were translated. The quality of the studies was assessed using the MOOSE checklist.

Results: Our electronic search found 1546 abstracts of which 230 articles were identified for full data extraction. Of these, 83 were cross-sectional, cohort or controlled studies, 69 case reports/case series and 78 review papers. To date 7 studies fulfil the criteria for inclusion in the systematic review; 4 are cross-sectional and 3 are cohort studies.

In our preliminary analysis, in the first 4 studies we identified, the unadjusted pooled relative risk of a diagnosis of diabetes in people with schizophrenia prescribed an atypical antipsychotic was 1.41 (95\% confirdence interval 0.92 to 2.16 ) compared to being prescribed a typical antipsychotic. Further analyses of all identified studies will be presented.

Conclusion: There is a lack of high quality epidemiological studies and at present there is insufficient evidence for an increased risk of diabetes in people with schizophrenia who are prescribed atypical antipsychotics compared to those on typical antipsychotics.

0875

Alexithymia in type 1 diabetes and coeliac disease S. Manfrini ${ }^{1}$, R. Bruni ${ }^{1}$, N. Terminio ${ }^{1}$, M. Khazrai ${ }^{1}$, G. Sandri ${ }^{2}$, D. Benevento ${ }^{1}$, S. Spera ${ }^{1}$, M. Ganz ${ }^{3}$, P. Pozzilli ${ }^{1}$;

${ }^{1}$ Endocrinology, Università Campus Bio-Medico, Roma, ${ }^{2}$ Ospedale Sant'Eugenio, Roma, Italy, ${ }^{3}$ Roche Diagnostics, Mannheim, Germany.

Background and Aims: Type 1 diabetes (T1D) and coeliac disease (CD) are chronic conditions characterized by a careful attention to diet with consequent implications on 
psychological and social life. The strength to maintain a correct way of eating for lifetime is a relevant stress factor leading to psychosomatic or psychiatric disorders such as depression anxiety. In subjects with T1D the quality of life is influenced by several individual factors like personal attitude and psychopathological traits such as alexithymia, a psychological construct linked to failure in psychological self-regulation and deficit in experiencing emotional life.

It has been shown that a relationship exists between the alexithymic construct and perceived stress so that alexithymic features may interfere with the affected subject's disease-coping strategies because of a higher perceived stress. We previously reported that in T1D alexithemia is frequent, however it is not clear whether this is a specific feature of T1D or consequence of a chronic disease at onset in childhood irrespective of the type. In the present study we tested the differences in the emotive and cognitive function between subjects affected by T1D and CD through the evaluation of the alexithymic construct.

Materials and Methods: We studied 50 consecutive subjects affected by T1D (mean age 29 years, 17 males and 23 females, mean disease duration 10.8 years) and 31 subjects with CD (14 males and 17 females, mean age 31 years, mean disease duration 6.7 years) attending out patient clinics in two different hospitals. Alexithymia was assessed using a validated translated version of TAS-20 (Bagby 1994), a self-report measure of the alexithymia construct. Alexithymia was used as a continuous variable. Perceived stress was assessed by Perceived Stress Scale (PSS), a 14-item self report tool used to provide a global measure of perceived stress in daily life.

Results: According to standard evaluation parameters (alexithymia score $>51$ ), alexithymia was observed in $48 \%$ of T1D and 45.3 of $\mathrm{CD}$ subjects ( $\mathrm{p}=\mathrm{NS}$ ). A positive correlation was also found between TAS and PSS (linear regression $\mathrm{r}=0.69$, $\mathrm{p}<0.0001)$ in TD1 but not in CD. Evaluation of the three different subscales of the alexithymic construct (1=difficulty identifying and describing feelings; $2=$ difficulty distinguishing between feelings and body sensations; $3=$ constricted imaginal processes) in subjects with high and low levels of alexithymia showed a different prevalence of the three relative specific constructs both in TD1 and in CD patients.

Conclusion: This is the first study investigating whether alexithymia is a specific feature of T1D. We demonstrated that a clear relationship occurs between the alexithymic construct and perceived stress in T1D but not in CD affected subjects suggesting that disease occurrence and duration irrespective of the type of the disease cause alexithymia, however alexithymia is not the major factor that determines stress in CD. Nevertheless differences exist in the prevalence of three subtypes of this construct in TD1 and in CD patients. An early psychological therapeutic approach to the subject affected by TD1 showing high levels of alexithymia should be designed taking into account the three different subscales of the alexithymic construct.

Supported by Roche Diagnostics

0876

Needle phobia, anxiety, and self blood glucose monitoring A. Moir, M. D. Feher;

Beta Cell Diabetes Centre, Chelsea \& Westminster Hospital, London, United Kingdom.

Background and Aims: The management of diabetes often involves individuals testing their own blood glucose by capillary blood glucose measurement. This method is labeled "home" or "self" blood glucose monitoring and requires the use of a lancet device (finger prick) to obtain the appropriate aliquot of blood for glucose measurement. By contrast, needle phobia traditionally describes phobia to the insulin injection, rather than the finger prick testing method. There is accumulating evidence on phobia and anxiety relating to insulin injection, however to date there is limited evidence relating to phobia due to the finger prick method. The aims, therefore, of the present study were to assess the incidence of, and factors relating to, injection anxiety specifically relating to the finger prick method of measuring glucose.

Materials and Methods: Individuals with type 1 or type 2 diabetes, attending a London teaching hospital diabetes centre for routine follow up, participated in a self administered questionnaire on needle phobia and anxiety. In addition to demographic details, Injection Anxiety was assessed by using questions derived from the validated DSM-1V. General Anxiety was assessed by using the Anxiety subsection of the Hospital Anxiety and Depression Scale. Considerable Injection Anxiety was defined by an anxiety score $>3$ and high levels of General Anxiety was defined by a score $>8$. The following statistical methods were used for the data: Kendall's tau_b for rank correlations and Chi-square.

Results: Over a two month period 315 individuals completed the questionnaire: $93 \%$ reported previous self blood glucose monitoring of whom $50.0 \%$ test at least twice a day, $22.8 \%$ once a day, $19.7 \%$ twice a week, while only $7.5 \%$ less than once per week. Infrequent or complete avoidance of testing due to fear of the finger prick device was reported in $13.7 \%$ of the group. Nearly a quarter of the group (22.5\%) reported other reasons (including - pain, forgetfulness, and time pressures) apart from needle phobia as reasons why they tested less frequently. Considerable Injection Anxiety (IAS $>3$ ) was observed in $30.2 \%$ and high levels of anxiety (GAS $>8$ ) was observed in $33.3 \%$. Women compared to men reported higher Injection Anxiety and General Anxiety and ethnic minority groups reported 
higher Injection Anxiety and General Anxiety. Significant positive correlations were found for avoidance of testing with Injection Anxiety and Injection Anxiety with General Anxiety. Significant negative correlations were found for age with General Anxiety and for age with testing times. Significant associations were found for gender with needle phobia, ethnicity with needle phobia, gender with anxiety, and for ethnicity with avoidance of testing.

Conclusion: A large proportion of the clinic patients appeared to have severe degrees of phobia and anxiety relating to the finger prick device which is more frequently reported in women and ethnic minorities. This observation is clearly important for the patient but also for the health care professional in identifying important management issues relating to self blood glucose monitoring.

\section{PS 71}

\section{Education in diabetes}

0877

Is patient education helpful in type $\mathbf{2}$ diabetes?

A. K. Sigurdardottir ${ }^{1}$, R. Benediktsson ${ }^{2}$, H. Jonsdottir ${ }^{3}$; ${ }^{1}$ Department of Health, University of Akureyri, ${ }^{2}$ Department of Endocrinology and Metabolism, University Hospital Iceland, Reykjavik, ${ }^{3}$ Faculty of Nursing, University of Iceland, Reykjavik, Iceland.

Introduction: Systematic reviews indicate that diabetes education increases knowledge among people with type 2 diabetes, enhances positive changes in self-reported diet, reduces body weight and enhances metabolic control. However it is unclear which factors exactly contribute to these outcomes.

Aim: To analyze educational interventions in type 2 diabetes (T2DM) reported in randomized controlled trials (RCT) with regard to content, type and effectiveness.

Methods: Papers were extracted from Medline and Scopus from 2001 to October week 3, 2005 using e.g. educational intervention as keywords. Inclusion criteria were RCT design and adult T2DM. Papers regarding gestational diabetes and where results were not analyzed according to type of diabetes were excluded. WEKA data mining was used to explore the data.

Results: Of 464 titles extracted, only 19 articles met the inclusion criteria. The most common reason for exclusion was a non-interventional study. Data mining (WEKA) suggested that for initial HbAlc level $\leq 7.9 \%$, the intervention achieved only a small change in HbAlc level. For initial $\mathrm{HbA} 1 \mathrm{c} \geq 8.0 \%$, a significant drop in HbA1c level of $0.8 \%$ to $1.7 \%$ was observed. Data mining failed to identify duration, educational content or intensity of education as significant predictors of outcome.

Conclusion: Initial HbAlc level is the single most important factor predicting improvement in glycaemic control in response to patient education. The methodological details revealed in papers on patient education in T2DM is poor which hampers the utilization of results and dissemination of effective educational practices.

\section{8}

Impact of a novel diabetes inpatient specialist nurse (DISN) service in the UK

D. Stanisstreet ${ }^{1}$, K. Mahaffey ${ }^{2}$, A. Ainsworth ${ }^{3}$, P. H. Winocour ${ }^{2}$;

${ }^{1}$ Lister Hospital, ${ }^{2} \mathrm{QE} 2$ Hospital, ${ }^{3}$ Clinical Audit, East and North Herts NHS Trust, Hertfordshire, United Kingdom.

Background and Aims: A newly implemented DISN service was evakuated in order to calculate the number of patients seen, to assess the efficacy of and adherence to referral criteria, to identify whether the refferal was the result of a diabetes management incident and to determine the number of incidents encountered by the DISNs.

Materials and Methods: Data was collected on a proforma divided into 14 sections and a unique index of 34 diabetes incidents was used to obtain objective information.

Results: This study highlighted a vast workload for the DISNs, with a total of 890 patient visits across two hospital sites over a six month period. A large proportion of this workload involved the management of acutely ill patients with complicated diabetes. 308 patients were reffered with mean age of 64 yrs. 14\% had Type 1 diabetes, $1.5 \%$ newly diagnosed and 68.5\% had Type 2 diabetes, 16\% newly diagnosed. $13 \%$ of patients with Type 2 diabetes required conversion to insulin therapy and education regarding self blood glucose monitoring. $33 \%$ of patients were referred due to acute illness resulting in unstable blood glucose levels, $10 \%$ because of hypoglycaemia, $14 \%$ following acute coronary syndrome, 5\% active foot problems and $27 \%$ required education. Although $7 \%$ of referrals resulted directly from an incident, $36 \%$ of DISN visits identified one or more adverse incident. $63.5 \%$ of the incidents detected by the DISNs were attributed to insulin prescribing/administration errors resulting in preventable hypo/ hyperglycaemia and the inappropriate treatment of hypo/ hyperglycaemia.

Conclusion: Many diverse diabetes issues will go undetected without the DISNs' proactive approach. This study emphasises the importance of a proactive DISN service in identifying and managing common and preventable adverse incidents relating to diabetes inpatient care and providing 
education and support for all non specialist health care professionals working in the hospital setting.

0879

The higher knowledege of diabetes, the better metabolic control and lower risk of microangiopathy in type 1 diabetic patients

A. Araszkiewicz, M. Trepinska, D. Zozulinska,

B. Wierusz-Wysocka;

Department of Internal Medicine and Diabetology, Poznan University of Medical Sciences, Poland.

Background and Aims: The DCCT and its follow up EDIC study demonstrated that improvement of metabolic control and intensive insulin therapy reduces the risk of development and progression of late diabetic complications. The aim of this study was to assess the association between the knowledge of diabetes and intensive functional insulin therapy (IFIT) and prevalence of microangiopathy in type 1 diabetic patients treated with intensive functional insulin therapy from the onset of the disease.

Materials and Methods: 100 patients with newly diagnosed type 1 diabetes (43 female, 57 male, aged 24.1 \pm 6.1 ) were recruited to the study (between years 1994-1999). All subjects underwent a five-day teaching programme providing the skills in basal-bolus insulin management. We assessed at baseline and every one year: knowledge about diabetes and IFIT (test including 20 questions, maximum score: 20 points), number of hypoglycaemic episodes, quality of life, eye fundus, urine albumin excretion and parameters of metabolic control. The study group was divided into 3 subgroups according to scores achieved in the knowledge test: group A $>16$ points, group B: $12-16$ points, group $\mathrm{C}:<12$ points.

Results: During mean follow-up $7.1 \pm 1.5$ years $18(20 \%)$ subjects have developed diabetic nephropathy and retinopathy. Some results are presented in the table below.

\begin{tabular}{llll}
\hline At baseline & Group A & Group B & Group C \\
\hline Knowledge (points) & $17.4 \pm 0.9^{*}$ & $13.8 \pm 1.4$ & $9.2 \pm 1.9$ \\
FPG (mmol/l) & $8.6 \pm 3.9$ & $9.8 \pm 3.2$ & $11.1 \pm 4.1$ \\
2 h PPG (mmol/l) & $8.1 \pm 4.2$ & $9.9 \pm 4.2$ & $11.1 \pm 5.6$ \\
HbA1c (\%) & $7.6 \pm 1.5^{*}$ & $7.7 \pm 1.6$ & $9.2 \pm 1.9$ \\
LDL-cholesterol (mmol/1) & $2.9 \pm 1.0$ & $3.1 \pm 1.0$ & $3.3 \pm 0.7$ \\
HDL-cholesterol (mmol/l) & $2.0 \pm 0.3^{*}$ & $1.8 \pm 0.4$ & $1.7 \pm 0.3$ \\
Triglycerides (mmol/l) & $1.0 \pm 0.4$ & $1.1 \pm 0.6$ & $1.2 \pm 0.9$ \\
BMI (kg/m $\left.{ }^{2}\right)$ & $21.8 \pm 3.1 *$ & $23.5 \pm 2.6$ & $23.9 \pm 3.2$ \\
SBP (mmHg) & $120.0 \pm 15.9$ & $124.9 \pm 16.4$ & $127.5 \pm 15.6$ \\
DBP (mmHg) & $75.7 \pm 8.0$ & $76.5 \pm 9.3$ & $78.6 \pm 7.7$ \\
\hline
\end{tabular}

${ }^{*} \mathrm{p}<0.05$ Group A vs Group C
We noticed higher risk associated with low diabetic knowledge (score: $<12$ vs $>16$ points) for microangiopathy $(\mathrm{RR}=3.71$; 95\%CI: $1.15-12.01, \mathrm{p}=0.02)$

Conclusion: The higher knowledge about diabetes at the baseline, the better metabolic control and lower risk for microangiopathy in Type 1 diabetic patients treated with intensive functional insulin therapy from the onset of the disease.

0880

The Poole diabetes education programme for newly diagnosed type 2 diabetes: an audit of outcome at 3 and 12 months

W. Gatling ${ }^{1}$, M. Wong ${ }^{1}$, D. Clark ${ }^{1}$, P. Thomas ${ }^{2}$;

${ }^{1}$ Dept of Diabetes, ${ }^{2}$ Research \& Development Support Unit, Poole Hospital NHS Trust, Poole, United Kingdom.

Background and Aims: Group education for newly diagnosed type 2 diabetic patients has been established for over 25 years in the Poole area. It involves three 2-hour sessions over a 6 week period (Diabetes Education Session DES 1-3). The programme is facilitated by a diabetes nurse specialist and dietitian and involves a range of educational methods including interactive discussions, case studies and a food quiz. In 2002, we have updated the programme to encourage patient empowerment by involving patients in decision-making about diet and need for oral hypoglycaemic agents when reviewing home monitoring results and laboratory tests. We also started using community sites to run the programme making access easier for patients in the rural areas around Poole. After the education programme, the majority of patients are followed by the GP or practice nurse in primary health care. We undertook an audit to assess the effectiveness of the education programme and on going care for all the patients who attended in 1 year.

Materials and Methods: 897 patients attended the education sessions between 1 June 2003 and 31 May 2004. Using routinely collected data, we looked for any significant change in glycaemic control (HBA1c), cholesterol levels and weight between the first and the final session, and if this was maintained at 1 year.

Results: Following diagnosis, mean HBA1c at DES1 was $8.0 \%$ falling to $7.3 \%$ at DES3. Overall, mean HBA1c was $6.6 \%$ at 1 year. Mean reduction in HBA1c using the paired t-test was $0.8 \%(95 \%$ CI: $0.7,0.9, p<0.001)$ between first and final sessions with a mean reduction of $1.4 \%(95 \% \mathrm{CI}$ : $1.3,1.5, \mathrm{p}<0.001)$ at 1 year. Mean reduction in weight between DES1 and DES3 was $1.9 \mathrm{~kg}(98 \% \mathrm{CI}: 1.7,2.1$; $\mathrm{p}<0.001)$. Mean reduction in cholesterol was $0.7 \mathrm{mmol} /$ $1(95 \%$ CI: $0.6,0.9, \mathrm{p}<0.001)$ from DES1 to 1 year. At DES3, $68 \%$ of patients achieved HbAlc $<7.5 \%$ (primary 
care QOF target) improving to $87 \%$ by 12 months. Our local target for patients is $\mathrm{HbA} 1 \mathrm{c}<7.1 \%$ and $57 \%$ achieved this by DES3 improving to $77 \%$ at 12 months.

Conclusion: In newly diagnosed type 2 diabetic patients, our group education programme with emphasis on patient empowerment and patient involvement in clinical decisionmaking followed by on-going care in primary care achieved significant improvement in glycaemic control, weight loss and reduction in cholesterol levels.

\section{1}

Effects of a structured education programme on illness beliefs, QoL and physical activity in individuals newly diagnosed with type 2 diabetes: the DESMOND pilot study

M. J. Davies ${ }^{1}$, M. E. Carey ${ }^{2}$, H. M. Dallosso ${ }^{2}$, S. Heller ${ }^{3}$, K. Khunti ${ }^{4}$, T. C. Skinner ${ }^{5}$;

${ }^{1}$ Dept of Cardiovascular Sciences, University of Leicester, ${ }^{2}$ Diabetes Research, University Hospitals of Leicester NHS Trust, ${ }^{3}$ Dept of Medicine, University of Sheffield, ${ }^{4}$ Dept of Health Sciences, University of Leicester, ${ }^{5}$ School of Psychology and Medicine, University of Southampton, United Kingdom.

Aim: Pilot study evaluating effects of the DESMOND structured education programme.

Method: 226 subjects newly diagnosed with T2D attended a DESMOND course in primary care, consisting of 6 hours of structured group education delivered to patients within 4-6 weeks of diagnosis, by 2 trained health professional educators. Patients completed measures of illness beliefs (IPQ-R, DIRQ) physical activity (IPAQ) quality of life (WHOQOL-BREF) prior to \& 3, 6 \& 12 mnths post attendance. Biomedical data collected at the same intervals. Results: At 3 mnths subjects more likely to agree they understood their diabetes $(\mathrm{t}=-7.92 ; \mathrm{df}=99 ; \mathrm{p}<0.001)$ that it is a serious health risk $(\mathrm{t}=2.89 ; \mathrm{df}=99 ; \mathrm{p}<0.005)$ a chronic illness $(\mathrm{t}=2.09 ; \mathrm{df}=99 ; \mathrm{p}<0.05)$, that they can affect its course $\mathrm{t}=1.9$; $\mathrm{df}=99 ; \mathrm{p}<0.05)$. Changes sustained at $6 \& 12 \mathrm{mnths}$ for perceived understanding, impact \& duration of diabetes $(\mathrm{F}=34.7 ; \mathrm{p}<0.001 ; \mathrm{F}=3.8 ; \mathrm{p}<0.05 \mathrm{~F}=2.7 ; \mathrm{p}<0.05)$, at $6 \mathrm{mnths}$ for perceived seriousness, personal responsibility(Fquadratic $=10.0 ; \mathrm{p}<0.005 ; \mathrm{F}=44.8 ; \mathrm{p}<0.005 ; \mathrm{Fquadratic}=28.6$; $\mathrm{p}<0.005)$. Frequency of walking showed linear increase baseline to 12 mnths (Flinear $=7.0 ; p<0.05$ ). Physical domain of QoL increased baseline to 3 mnths $(\mathrm{t}=2.03 ; \mathrm{df}=131 ; \mathrm{p}<0.05)$ sustained at 12 mnths. Complete biomedical data in 60/109 patients showed significant reduction in $\mathrm{A} 1 \mathrm{c}(\mathrm{p}<0.01) \mathrm{BP}$ $(p<0.01)$ weight $(p<0.05) \&$ waist circumference $(p<0.05)$ baseline to12 mnths, and in lipids baseline to $6 \mathrm{mnths}$ $(\mathrm{p}<0.01)$. Subjects agreeing more strongly they understood their diabetes $(\beta=.18 ; \mathrm{p}<0.05)$ and that it is a chronic illness $(\beta=.20 ; p<0.05)$ reported greater increase in frequency of physical activity. Greater belief in understanding diabetes $(\mathrm{r}=.21 ; \mathrm{p}<0.05)$ the better QoL. Greater belief in ability to control diabetes $(\mathrm{r}=.24 ; \mathrm{p}=.05)$ greater $\mathrm{A} 1 \mathrm{c}$ reduction.

Conclusion: Pilot data indicates the DESMOND course facilitates changes in illness beliefs, sustained at follow-up and predictive of metabolic control. A full scale randomised controlled trial is now underway in 17 primary care organisations and 238 practices in England and Scotland. Recruitment is completed with 823 patients randomised, and is due to report early 2007 . HbA1c is the primary outcome. Supported by Diabetes UK

\section{2}

Changes in dietary practices of type 2 diabetic subjects during Ramadan fasting

S. J. Mumu ${ }^{1}$, K. R. Ahmed ${ }^{2}$, S. Akter ${ }^{1}$, S. Shahid ${ }^{3}$, S. Akter ${ }^{3}$, A. Hossain ${ }^{3}$, S. Hossain ${ }^{3}$, F. Saleh ${ }^{1}$, L. Ali ${ }^{4}$; ${ }^{1}$ Dept of Human Nutrition, BIRDEM, Dhaka, ${ }^{2}$ Health Economics Unit, Diabetic Association of Bangladesh, Dhaka, ${ }^{3}$ Dept of Food and Nutrition, National College of Home Economics, Dhaka, ${ }^{4}$ Dept of Biochemistry \& Cell Biology, BIRDEM, Dhaka, Bangladesh.

Background and Aims: Fasting during the month of Ramadan is one of the most important religious practices of millions of Muslims in the world. Ramadan fasting involves major changes in dietary pattern and metabolic state. Dietary measures comprise the first line therapy for controlling metabolic changes during Ramadan. The aim of the study was to explore the dietary practice of Bangladeshi type 2 diabetic subjects during Ramadan Fasting.

Materials and Methods: Ninety two type 2 diabetic subjects (male: female ratio 61:31, age $47 \pm 9$ years, mean \pm SD) were selected from the Out-Patient Department of BIRDEM (the tertiary care hospital of Diabetic Association of Bangladesh). The first survey was conducted 14 weeks before Ramadan and the second interview was done during 3-4 weeks of Ramadan. The dietary intake of macro- and micronutrients by the study subjects was calculated by a specific food frequency questionnaire (FFQ). Data were expressed as Mean+SD. Difference between during Ramadan and before Ramadan values were calculated using paired t-test. Spearman's coefficient correlation analysis was performed for skewed data.

Results: BMI of the study subjects was significantly reduced during Ramadan compared to before Ramadan $(24.5+3.4$ vs $24.1+3.4$, Mean+SD, $\mathrm{p}<0.001)$. No significant difference was found between during and before Ramadan in terms of per day intake of total energy (calorie, 2424+ 
678 vs $2418+1137)$ carbohydrate (g, 363+128 vs $385+205)$, protein $(g, 95.68+42$ vs $97.1+69.9)$ and fiber $(g, 6.2+2.4$ vs $5.9+3.2$ ) among the study subjects. However, daily fat intake was significantly higher during Ramadan compared to before Ramadan ( $g, 65.61+15.67$ vs $54.5 \pm 15.3$; $\mathrm{p}<0.05$ ). In terms of proportion the fat intake increased by about $26 \%$ during Ramadan. The major source of this increased fat was traditional oily foods. A positive correlation was found between total cholesterol and fat intake $(r=0.046$, $\mathrm{p}=0.673$ ) during Ramadan. Iron and calcium intake were found significantly higher during Ramadan compared to before Ramadan (mg/day, $31.89 \pm 17.83$ vs $25.3 \pm 14.06$ and $747 \pm 469$ vs $588 \pm 349$, respectively, $\mathrm{p}<0.05$ ). Total thiamin intake of the subjects was significantly lower during Ramadan compared to before Ramadan value (mg/day, $1.72 \pm 0.91$ vs $2.11 \pm 1.75, \mathrm{p}<0.05)$. But riboflavin, vitamin-C, beta-carotene and retinol intake remained unchanged during Ramadan.

Conclusion: In Bangladeshi population, Ramadan fasting is accompanied by weight loss without any change in overall energy balance indicating a possible dehydration in this condition. Substantially increased fat intake during Ramadan fasting may lead to dyslipidemia and it warrants educational efforts among the population to promote a balanced and healthier diet during the Holy Month.

Supported by IPICS and DAB

\section{3}

Meta-analysis do not allow appraisal of education programmes of diabetes or hypertension self-management - a systematic review

M. Lenz, A. Steckelberg, I. Mühlhauser;

Unit of Health Sciences and Education, University of Hamburg, Germany.

Background and Aims: Patient education programmes are complex interventions. Their evaluation is difficult because of problems in identifying and assessing the effects of their various components. The U.K. Medical Research Council proposed a framework for the design and evaluation of complex interventions. Five sequential phases of 'increasing evidence' have been defined including development, efficacy and implementation trials. The aim of the present review was 1) to systematically explore problems of current methodologies in systematic reviews that evaluate patient education programmes in the field of diabetes care and hypertension; and 2) to propose a systematic approach allowing an appropriate evaluation.
Materials and Methods: A systematic search has been performed in the Cochrane Database, DARE, PubMed, Cinahl (3/2006) and HTA-databases (11/2005). The selected reviews were analysed by two independent investigators using standard extraction forms. It was analysed 1) if the 'increasing evidence' was detected and included; 2) if the active components were identified and analysed; 3) if and why the programmes had been allocated into categories of interventions; 4) if all relevant outcomes had been assessed and if interactions between them had been analysed.

Results: Our data base searches yielded 1441 results. All titles were screened; 1311 publications were excluded, they were not systematic reviews or not relevant. The remaining 130 abstracts were assessed for inclusion. A total of 15 reviews in 16 publications were considered eligible. Metaanalysis was performed in 8 reviews; 7 reviews used qualitative data-synthesis. In 6 reviews only controlled trials were included; other types of publication concerning the 'increasing evidence' were not considered. In 8 reviews experts in the field were contacted. Only one review reported author contact. Theoretical funding was discussed in 10 reviews. 12 reviews reported that the included programmes had been multidimensional. The impact of active components was analysed in 10 reviews. The approach of 'increasing evidence' was cited in 3 reviews. Interdependencies between outcome measures were considered in 3 reviews.

Conclusion: Most of the included reviews discussed methodological challenges to appraise complex interventions. However, these considerations did not have any impact on the methods used. We therefore propose the following approach: 1) all studies referring to the phases of 'increasing evidence' should be considered; 2) searching should not be limited to certain types of studies, authors should be contacted; 3) interdependencies between the active components should be taken into account, components should only be examined separately if they are independent and should not be disassembled if they work interdependently; 4) education programmes should not be allocated into categories referring to interdependent components; 5) all relevant outcome parameters should be included; 6) pooling of outcome measures across different programmes is inappropriate; the interdependency between treatment goals and outcomes has to be considered. Information necessary for the evaluation of education programmes is difficult or impossible to identify. Therefore, we propose to create an electronic database which provides available programmes together with all relevant background information or to implement such a system into existing databases. 


\section{4}

Improving diabetes education using Alphabet Strategy. Use of printed educational materials versus electronically stored information. The GEDI Project (George Eliot Diabetes Information Project)

Y. Thankey, A. Gopinath, L. Varadhan, D. James, P. Sear, J. Wilson, J. Morrissey, V. Patel;

Diabetes Centre, George Eliot Hospital NHS Trust, Nuneaton, United Kingdom.

Background and Aims: There is an increasing need for effective education in diabetes to empower the patient to better self manage their own care. Traditionally printed material is used but can be expensive to produce. Now electronic devices are becoming cheaper

Our aim was to compare the effectiveness of using computer assisted learning using a portable memory stick and printed form.

Materials and Methods: A baseline survey of 100 patients revealed that there was most interest in knowing about the following (scores out of 4 maximum):

- Diabetes Control (3.52)

- Risk factors (3.54)

- Emergency situations (3.36)

$82 \%$ of patients expressed an interest in having copies of their clinic letters with $49 \%$ expressing a very strong interest. 100 patients were included in the study. All patients had to have access to a computer and agree to participate in this project. Participants were requested to complete a questionnaire on diabetes, risk factors for complications and daily management. This is based on the Alphabet Strategy: $A$ dvice (diet, exercise, smoking cessation, concordance with treatment), Blood pressure $(<145 / 85 \mathrm{mmHg})$, Cholesterol $(\leq 5 \mathrm{mmol} / \mathrm{l}), \boldsymbol{D}$ iabetes control $(<7.5 \%)$, Eye care and $\boldsymbol{F}$ oot care (annual screen and treatment), Guardian drugs, e.g., Aspirin, ACE-I. There are 35 questions with multiple choice and short answer format. Previously we have shown the Alphabet Strategy to be enthusiastically accepted by patients as a method of education.

Patients were then randomised to receive educational materials on a portable memory device $(n=50$, GEDI, George Eliot Diabetes Information stick) or in printed format $(n=50)$. GEDI contained information including their clinical letters (last 3 years), their diabetes care plan and an overview and summary of the Alphabet Strategy. The paper format had exactly the same materials. The GEDI device costs $€ 2.60$ versus $€ 8$ for the paper format.

Results: The average age of the patients with was 37.1 (range 14-76). This included patients with type 1 diabetes or type 2 diabetes. Baseline education score was $61.5 \% \pm 22.1 \%$ in the GEDI group versus $61.5 \% \pm 20.7 \%$ in the paper format group (NS). After a period of 12 to 16 weeks there was a significant improvement in education scores in both groups (GEDI to $80.0 \% \pm 15.8 \%$. Paper format $84.7 \% \pm 16.3 \%$ ) $(\mathrm{p}<0.001)$. There was no difference in increase in improvement comparing the two different methods. However, only $31 / 50$ returned the second questionnaire and 23/50 in the paper format group. After a cross over-period, when all patients had both GEDI and the paper format, the education scores remained stable at above $80 \%$ in both group

Conclusion: Computer assisted learning using a portable memory stick was as effective as the more expensive printed materials. We also demonstrated a significant improvement in education scores amongst our patients using the locallycreated but freely available Alphabet Strategy.

\section{5}

Field glucose variability index is related to laboratory measures of insulin sensitivity and hypoglycaemia counterregulation

B. P. Kovatchev ${ }^{1}$, S. M. Anderson ${ }^{2}$, E. Otto ${ }^{3}$;

${ }^{1}$ Psychiatric Medicine, ${ }^{2}$ Internal Medicine, University of Virginia, Charlottesville, ${ }^{3}$ LifeScan Inc, Milipitas, United States.

Background and Aims: Contemporary studies increasingly support the idea that blood glucose (BG) variability is a major determinant of diabetes complications; therefore $\mathrm{BG}$ variability, along with $\mathrm{HbA}_{1 \mathrm{c}}$, becomes a major marker of glycemic control in Type 1 and Type 2 diabetes (T1DM, T2DM). However, quantifying glucose variability is not straightforward - traditional variability measures, such as standard deviation (SD), M-value, etc. appear to be mostly dependent on hyperglycemic excursions, ignoring hypoglycemic BG fluctuations and being insensitive to hypoglycemia-related characteristics of T1DM.This study introduces a new variability measure computed from routine self-monitoring $\mathrm{BG}$ (SMBG) data, the Average Daily Risk Range (ADRR), which is equally sensitive to hypoglycemic and hyperglycemic excursions, and investigates its relationship with laboratory parameters of glucose dynamics in T1DM. Materials and Methods: Twenty-five adults with T1DM, average age $=42.5 \pm 12$ years, duration of diabetes $=$ $21.6 \pm 9.4$ years, $\mathrm{HbA}_{1 \mathrm{c}}=7.4 \pm 0.8,16$ males, performed routine SMBG for 2 months, 4-5 times/day. The ADRR was computed from routine SMBG data taking into account the value and the time of each SMBG reading. SD of BG and M-value were computed as well. Subjects were then hospitalized and their $\mathrm{BG}$ was controlled overnight at $\sim 6 \mathrm{mmol} / \mathrm{l}$. Hyperinsulinemic clamp (1 $\mathrm{mU} / \mathrm{kg} / \mathrm{minute})$ was initiated in the morning, beginning with 2-hour euglycemia at $\sim 5.5 \mathrm{mmol} / 1$, followed by 1 -hour descent into hypoglycemia with a target level of $2.2 \mathrm{mmol} / \mathrm{l}$. BG 
was sampled every 5 minutes (Beckman glucose analyzer); epinephrine was sampled twice at euglycemia and every 10 min during descent in hypoglycemia. Glucose uptake during euglycemia was computed in $\mathrm{mg} / \mathrm{kg} / \mathrm{min}$ as a marker of insulin sensitivity.

Results: Within the field SMBG data the ADRR correlated approximately equally well with significant hypoglycemic $(\mathrm{BG}<2.2 \mathrm{mmol} / \mathrm{l})$ and hyperglycemic $(\mathrm{BG}>22.2 \mathrm{mmol} / \mathrm{l})$ episodes, correlations of $r=0.42$ and $r=0.51$ respectively, $\mathrm{p}<0.05$. In contrast, the $\mathrm{SD}$ of $\mathrm{BG}$ and the $\mathrm{M}$-value had zero correlation with hypoglycemic events and $\mathrm{r}=0.63, \mathrm{r}=0.54$ with hyperglycemic events respectively, confirming previous observations that these measures are primarily related to hyperglycemic excursions. The ADRR, computed from SMBG, was highly predictive of subsequent hospital laboratory results: its correlation with subjects' glucose uptake during euglycemia was 0.59 ; its correlation with [the logarithm of] peak epinephrine during induced hypoglycemia was $-0.59, \mathrm{p}=0.002$, indicating that the ADRR was equally related to both insulin sensitivity and degree of counterregulation. No other measure of glucose variability achieved statistically significant relationship with these hospital parameters.

Conclusion: A new field glucose variability measure called ADRR that is calculated from regular time-stamped SMBG data, specifically designed to be equally sensitive to hypoglycemia and hyperglycemia, is more closely related to both increased glucose uptake and reduced counterregulatory response than any other measures of variability tested. Further studies will determine whether the active reduction of field glucose variability, as measured by ADRR, will improve counterregulatory response and insulin sensitivity in T1DM.

Supported by NIH/NIDDK RO1 DK 51562, University of Virginia General Clinical Research Center M01 RR00847, LifeScan Inc., Milpitas, CA

\section{6}

The periodontium condition in men with type 1 diabetes O. R. Grigoryan ${ }^{1}$, K. R. Grigoryan ${ }^{2}$;

${ }^{1}$ Institution of Diabetes Mellitus, Endocrinology Research Center RAMS, Moscow, ${ }^{2}$ Preventive Dentistry, Moscow State University of Medicine and Dentistry, Moscow, Russian Federation.

Background and Aims: Diabetes has been implicated in the development of significant periodontal disease and with other significant local effects on the oral cavity that contribute to periodontal disease. The aim of thus investigation was to relate the effects of the metabolic control of adult type 1 diabetes to the periodontal health of these patients. It has been suggested that long-term poorly controlled insulin dependent diabetic (PIDD) subjects are at risk for periodontal disease. We compared the periodontal health status of PIDD subjects with controlled insulindependent diabetic (CIDD) subjects in cross-sectional and longitudinal studies.

Materials and Methods: At basic examination, 18 PIDD, $20 \mathrm{CIDD}$ and 20 subjects of controls were examined. The subjects were male, mostly long-term ( $>10$ years) type 1 diabetics with an age range from 18 to 40 years. Mean glycosylated hemoglobin and blood glucose levels were significantly higher in the PIDD (HbAlc $>9 \%)$ than the CIDD (HbA1c - 6,5-7,5\%) subjects at baseline ( $<<0,001)$. Results: The one-year longitudinal study revealed that the PIDD subjects showed greater tooth loss, more gingival inflammation and bleeding after probing than did the CIDD subjects. Despite similar oral hygiene conditions, at the first and at the two subsequent examinations the PIDD subjects exhibited more gingival recession than the CIDD subjects $(p<0,05)$, more loss of attachment $(p 0,05)$.

Conclusion: These data may suggest that poorly controlled diabetes accelerates the progression of periodontitis lesion in the active phase of the disease.

\section{PS 72}

\section{Social and economic implications of diabetes treatment}

0887

Trends in outpatient prescription drug costs in diabetic patients in Germany, 1994-2004

W. Rathmann, B. Haastert, A. Icks, G. Giani;

Institute of Biometrics and Epidemiology, German Diabetes Center, Düsseldorf, Germany.

Background and Aims: New antidiabetic medications have been introduced during the last decade, but their impact on health care cost is largely unknown. Therefore, prescription costs in diabetic patients and nondiabetic controls in primary care were evaluated (Germany: 1994-2004).

Material and Methods: Data were derived from a nationwide pharmaceutical database (IMS Health, Disease Analyzer). Primary care data of all 46,017 diabetic patients and 46,017 controls in 417 nationwide practices (2004) were compared to 29,956 diabetic patients and 13,226 controls (361 practices) in 1994. Age- and sex-standardised prescription prevalences, costs and cost ratios (2004/1994), using drug company sales prices, adjusted for inflation (German Consumer Price Index) were calculated. 
Results: A 50\% prevalence increase for insulin prescriptions $(28.9 \%$ vs $19.3 \%)$, a 4 fold increase for biguanide use (35.2\% vs 9.4\%), and a threefold higher prevalence of blood glucose self-monitoring prescriptions (BGSM) $(28.7 \%$ vs $9.9 \%$ ) was observed over 10 years (all $\mathrm{p}<0.01$ ). A decline was found for sulphonlyureas $(-37 \%)$ and acarbose $(-50 \%)$. Mean total annual drug costs (company sales prices) per diabetic patient were 523 Euro in 2004 (1994, inflation-adjusted: 335 Euro) equalling a 56\% increase $(p<0.01)$. Average costs for antidiabetic medication were 192 Euro in 2004, a 100\% increase $(p<0.01)$. The major antidiabetic cost factors were insulins and analogues (mean costs per patient, 2004 vs 1994: 113.9 vs 58.1 Euro, Ratio: 2.0, $<<0.01$ ), followed by oral antidiabetics (44.0 vs 20.9 Euro, 2.1, p<0.01), and BGSM (33.9 vs 14.8 Euro, $2.3, \mathrm{p}<0.01)$. In 2004, insulins accounted for $22 \%$ of total drug costs among diabetes patients (1994: 17\%), all oral antidiabetics combined captured 8.4\% (1994: 6.2\%), and BGSM accounted for 6.5\% (1994: 4.4\%). Although glitazones were only prescribed in $3.6 \%$ in 2004 , average expenses (12.0 Euro) in the entire diabetic population were already comparable to biguanides (10.9 Euro) and sulphonylureas (9.8 Euro) due to its high annual treatment costs (332 Euro per treated). Overall, newly launched antidiabetics (insulin analogues, glitazones, glinides) accounted for $40 \%$ of antidiabetic drug costs in diabetic patients in 2004. The $38 \%$ increase (331 vs 240 Euros) in non-diabetes related drugs over the 10-year period among diabetic patients was mainly due to cardiovascular drugs. In nondiabetic patients, a comparable drug cost increase (33\%) was found (222 vs 167 Euro, $\mathrm{p}<0.01$ ).

Conclusions: Prescription drug costs among diabetic patients in primary care in Germany increased about $60 \%$ during the last decade, which was about twofold higher than in nondiabetic patients. In particular, an overproportional increase $(100 \%)$ was found for costs of diabetes treatment (insulin, oral antidiabetics) and blood glucose self-monitoring test. New types of antidiabetic drugs accounted for a substantial cost share in 2004. Thus, pharmacological advance is a key driver of drug expenditure growth in diabetes care, indicating the need of costeffectiveness analyses of new agents.

\section{8}

Ambulatory $\mathrm{HbA}_{1 \mathrm{c}}$ determination is cost-effective in the fol low-up of type 1 diabetic patients

F. J. Ampudia-Blasco ${ }^{1}$, J. Girbes ${ }^{2}$, M. Catala ${ }^{1}$,

R. Carmena ${ }^{1}$;

${ }^{1}$ Endocrinology, Clinic University Hospital Valencia,

${ }^{2}$ Endocrinology Unit, Arnau de Vilanova Hospital, Valencia, Spain.
Background and Aims: Although ambulatory $\mathrm{HbA}_{1 \mathrm{c}}$ determination is preferred by most patients and reduces their time wasted for sampling, it is unknown if this technique is also cost-effective when indirect costs are taken into account. The aim of the study was to compare direct and indirect costs between two different follow-up strategies of type 1 diabetic (T1D) patients.

Materials and Methods: 87 T1D patients were randomized in a 6-month prospective study in two groups using either (1) ambulatory $\mathrm{HbA}_{1 \mathrm{c}}$ determination by means of a DCA 2000 meter (ambulatory, AMB group, Bayer Diagnostics) or (2) central laboratory $\mathrm{HbA}_{1 \mathrm{c}}$ determination (control, CON group, HPLC) in addition to other routine biochemical parameters (glucose, creatinine, uric acid, lipids, AST and ALT). CON patients were instructed to estimate the working-time lost due to traveling, waiting and sampling time in the hospital as well as public transportation costs, both at 3 and 6 months. The costs of time lost for each patient (indirect costs) were evaluated using individual income data or alternatively the minimum salary for not active-working patients (2004, Spain). The estimated sample to achieve a statistical difference was 41 patients per group with a 0.05 alfa and a 0.1 beta error, considering $10 \%$ of patient lost in the follow-up, a $5.00 €$ standard deviation and a $4.02 €$ difference to be detected. Statistical analysis was made using the t-Student test.

Results: 42 T1D patients were included in the AMB (age $33 \pm 8$ years-old, 20 women, BMI $24.9 \pm 3.8 \mathrm{~kg} / \mathrm{m}^{2}$, diabetes duration $12 \pm 9 \mathrm{y}$, basal HbAlc $7.4 \%$ ) and 45 in the $\mathrm{CON}$ group (age $37 \pm 12$ years-old, 14 women, BMI $24.1 \pm 2.6 \mathrm{~kg} /$ $\mathrm{m}^{2}$, diabetes duration $16 \pm 11 \mathrm{y}$, basal HbA1c $7.7 \%$ ). Educational level and mean income were comparable between the groups. In comparison with the HPLC method, we found that ambulatory $\mathrm{HbA}_{1 \mathrm{c}}$ determination was $+2.50 €$ more expensive (direct costs). Working time lost due to sampling in the hospital was $80 \pm 36 \mathrm{~min}$ in the control group. Total costs (direct+indirect costs) were $6.90 €(95 \% \mathrm{CI}$, 5.90-8.00 $€, p<0.0001$ ) higher in the CON vs AMB group. Conclusion: Ambulatory $\mathrm{HbA}_{1 \mathrm{c}}$ determination is a costeffective approach when used in T1D patients. This strategy is associated with a diminution of indirect and total costs and, additionally, may help patients to save an important amount of time due to sampling prior to medical follow-up visits.

\section{9}

The association of socioeconomical status and the risk of diabetes type 2 estimated by Finnish Diabetes Risk Score criteria in Polish population

R. Topór-Mądry ${ }^{1}$, A. Gilis-Januszewska ${ }^{2}$, A. Pająk ${ }^{1}$; 
${ }^{1}$ Dept of Epidemiology, Institute of Public Health, Krakow,

${ }^{2}$ Dept of Endorinology, Jegiellonian University, Krakow, Poland.

Background and Aim: The socioeconomic status (SES) has the impact on many lifestyle factors like physical activity, obesity and nutritional patterns. The association of SES with elevated blood glucose and diabetes type 2 risk assessed by Finnish Diabetes Risk Score (FRS) has not been well studied yet.

Aim: The aim of the study was to examine the relation between the socioeconomic status and and the risk of diabetes type 2 according to FRS criteria.

Sample and Methods: The studied population were 977 men and 983 women aged 45-64, randomly selected from the Krakow's population. All analyses were performed after exclusion of subjects with known diabetes. Education level, marital status, professional position and economical status data were collected from the standardized questionnaire. Chi square test was used for estimation of relation between the SES in FRS criteria and elevated blood glucose.

Results: The FRS below 7 was present in $33.2 \%$ men and $25,9 \%$ of women $(\mathrm{p}<0,05))$, and the FRS between $15-20$ was present in $11,8 \%$ men and $15,6 \%$ of women $(\mathrm{p}<0,05))$. University education had $30,2 \%$ men and $26,0 \%$ women. The risk of having diabetes estimated by FRS is lower in university educated women compared to less educated group. The same observation was found for elevated blood glucose. There were no assotiation between FRS and elevated blood glucose with education in men and with other SES variables in both sex.

\begin{tabular}{llll}
\hline & & $\begin{array}{l}\text { Education level } \\
\text { Elementary } \\
\text { and high school }\end{array}$ & high school \\
\hline Findrisk score $>7$ & Men & $67,9 \%$ & $64,1 \%$ \\
Glucose $>6,1 \mathrm{mmol} / 1$ & Women* & $77,8 \%$ & $63,7 \%$ \\
& Men & $16,9 \%$ & $16,8 \%$ \\
Glucose $>7,0 \mathrm{mmol} / 1$ & Women* & $11,0 \%$ & $6,0 \%$ \\
& Women* & $4,5 \%$ & $4,8 \%$ \\
& $3,8 \%$ & $0,9 \%$ \\
\hline
\end{tabular}

$* \mathrm{p}<0,05$

Conclusion: The higher education was related to lower risk of type 2 diabetes according to FRS criteria in women but not in men. The same was observed for elevated blood glucose. This findings might confirm the impact of education on lifestyle factors associated with development of diabetes in women found in previous studies.
0890

Assessing the economic burden of needlestick injuries associated with insulin delivery amongst healthcare professionals in community settings in the UK

N. Twena ${ }^{1}$, M. Taylor ${ }^{2}$, P. Trueman ${ }^{2}$, B. Chubb ${ }^{1}$;

${ }^{1}$ Novo Nordisk, Crawley, ${ }^{2}$ York Health Economics Consortium, University of York, United Kingdom.

Background and Aims: Needlestick injuries to healthcare professionals represent an avoidable economic burden to the health service and can cause significant anxiety and distress to healthcare workers. The National Audit Office suggests that such injuries are the second leading cause of staff absence in the NHS in the UK. Research has identified that the use of unfamiliar sharps and the removal or re-sheathing of needles increase the risk of injury. Healthcare workers who administer insulin may be at elevated risk as they often use a patients' own insulin delivery device. Research involving healthcare professionals estimated that between $10-20 \%$ of all needlestick injuries are associated with insulin delivery. This research aimed to quantify the burden of needlestick injuries to healthcare professionals associated with insulin delivery in community settings in the UK.

Materials and Methods: Data on the number of people with diabetes in the UK, the rate of third party insulin delivery in the community, the rate and cost of needlestick injuries were drawn from published sources. Where data were unavailable from the literature, assumptions based on expert input were applied. Simple modelling techniques were used. No account has been made to take into account injuries that occur in hospital settings. As such, any estimates should be regarded as conservative

Results: Current estimates suggest that there are 1.8 million people diagnosed with diabetes in the UK. Approximately $25 \%$ of these are believed to be insulin dependent. Our model assumes that $2 \%$ of insulin dependent diabetics rely on a third party for insulin administration.

Based on a previously published study, the average number of injections per day is estimated to be 1.68 per person. The rate and cost of needlestick injuries are drawn from studies conducted by the Scottish Executive. The rate of injuries used is 250 per million needles. Based on published research, an average weighted cost of $£ 362$ per needlestick injury was derived. This covers all post-exposure management including prophylaxis, laboratory testing, counselling and where necessary treatment. Based on the above data, our analysis estimates the total burden to the NHS of needlestick injuries associated with insulin delivery in the community to be $£ 499,614$.

Conclusion: Needlestick injuries associated with insulin delivery in community settings are estimated to cost the NHS almost $£ 500,000$ per annum. This takes no account of 
under-reporting or injuries occurring in other care settings and is likely to be an under-estimate of the true cost. The analysis also takes no account of the costs which may be associated with litigation which is increasing.

Many of these injuries are avoidable through improved professional practice or the use of safety devices designed to reduce the risk of injury. Best practice guidelines incorporating recommendations on the use of safety devices should be promoted to reduce this unnecessary burden on health care workers and NHS resources.

Supported by Novo Nordisk

\section{1}

Continuous subcutaneous insulin infusion versus multiple daily injections for type 1 diabetes: a cost-effectiveness analysis for Sweden

L. Nicklasson ${ }^{1}$, K. Zakrzewska ${ }^{2}$, S. Roze ${ }^{3}$, V. Voss ${ }^{3}$, S. Attvall ${ }^{4}$, A. Palmer ${ }^{3}$, D. M. D. Tucker ${ }^{3}$;

${ }^{1}$ Medtronic AB, Järfälla, Sweden, ${ }^{2}$ Medtronic AG, Switzerland, ${ }^{3}$ Health Economics and Outcomes Research, CORE a Unit of IMS, Basel, Switzerland, ${ }^{4}$ Sahlgrenska University Hospital, Sweden.

Background and Aims: A recently published metaanalysis of insulin pump therapy in patients with diabetes identified some important differences between continuous subcutaneous insulin infusion (CSII) and multiple daily injection (MDI). The analysis took into account 52 studies and over 1,500 patients and reported that CSII was associated with improved HbAlc, compared to MDI. The aim of this study was to project the long-term clinical and cost outcomes of CSII versus MDI in patients with type 1 diabetes in Sweden, based on this meta-analysis.

Material and Methods: The CORE Diabetes Model, a published and validated, interactive computer model was adapted to simulate the long-term incidence and progression of diabetes-related complications in the Swedish setting. The model utilized Markov modeling and Monte Carlo simulation techniques, and disease progression was simulated in a hypothetical cohort of patients with type 1 diabetes and baseline characteristics defined in terms of demographics and management and complication status. Baseline cohort characteristics included mean age 27 years, duration of diabetes 6 years and mean $\mathrm{HbA1c} 8.875 \%$. Basecase intervention effects were based on a meta-analysis by Weissberg-Benchall et al. who reported a mean reduction in baseline HbA1c of $1.2 \%$-points with CSII compared to MDI. This intervention effect was set to $0.51 \%$-points in a sensitivity analysis, according to data reported by Pickup et al. Costs were accounted from a societal perspective (treatment, complication and lost-productivity costs) and expressed in year 2005 Swedish Kroner (SEK) values. Clinical and cost outcomes were projected over a lifetime horizon and discounted at a rate of 3\% per annum.

Results: In the basecase, treatment with CSII was associated with improved mean life expectancy ( 0.844 life years) $(17.55 \pm 0.21$ versus $16.71 \pm 0.20)$ and quality adjusted life expectancy (QALE) (0.850 quality-adjusted life years [QALYs]) (12.06 \pm 0.14 versus $11.17 \pm 0.13)$, compared to MDI. Mean direct lifetime costs were SEK 348,582 higher with CSII treatment compared to MDI, but incremental total lifetime costs were offset to SEK 193,078 for CSII versus MDI after indirect costs were accounted; CSII was assumed to be associated with greater workplace productivity through fewer diabetes-related complications. This produced an incremental cost-effectiveness ratio (ICER) of SEK 227,066 $(\sim € 24,300)$ per QALY gained with CSII versus MDI. In the sensitivity analysis, using the results from Pickup et al., the relative outcomes were unchanged, with CSII associated with increased life expectancy $(0.370$ life years) and QALE (0.505 QALYs) compared to MDI, with an ICER of 482,920 ( €51,600) per QALY gained.

Conclusions: CSII was associated with increased life expectancy and QALE, and a reduced incidence of diabetes-related complications, compared to MDI. ICERs indicated that CSII represented good value for money from a societal perspective by current standards in Sweden, compared to MDI.

Supported by Medtronic AG, Switzerland

0892

Evaluating improvements in life expectancy and diabetes-related complications associated with reaching $\mathrm{HbA}_{1 \mathrm{c}}$ targets: a modelling study of type 2 diabetes in the UK setting

B. Chubb ${ }^{1}$, W. J. Valentine ${ }^{2}$, N. Twena ${ }^{1}$, J. Ray ${ }^{2}$, S. Roze ${ }^{2}$, V. Foos ${ }^{2}$, C. J. Curry ${ }^{3}$, P. McEwan ${ }^{4}$,

A. J. Palmer ${ }^{2}$;

${ }^{1}$ Novo Nordisk Ltd., Crawley, United Kingdom, ${ }^{2}$ Health Economics and Outcomes Research, CORE - A Unit of IMS, Basel, Switzerland, ${ }^{3}$ Department of Medicine, University Hospital of Wales, Cardiff, ${ }^{4}$ School of Mathematics, University Hospital of Wales, Cardiff, United Kingdom.

Background and Aims: To determine the long-term health economic impact of treatment to target HbA1c levels in patients with type 2 diabetes in the UK.

Materials and Methods: A validated and published model for type 2 diabetes was used to estimate the impact of treating patients to target $\mathrm{HbAlc}$ levels on complication rates, life expectancy (LE), quality-adjusted life expectancy (QALE) and complication costs in a 'typical' UK cohort of 
type 2 diabetes patients. Four hypothetical reductions in mean $\mathrm{HbA} 1 \mathrm{c}$ were simulated (versus no reduction): 1) $12 \%$ to $9.5 \%$; 2) $9.5 \%$ to $8 \%$; 3) $8.0 \%$ to $7.0 \%$; and 4$) 7.0 \%$ to $6.5 \%$. All other clinical parameters (blood pressure, body weight and lipid levels) remained constant. Patient characteristics were derived from the HODaR database which included over 20,000 patients with type 2 diabetes in England and Wales. Complication costs, inflated to 2004 British pounds (£) were discounted at $3.5 \%$ annually in line with NICE recommendations.

Results: In poorly controlled patients, decreasing $\mathrm{HbA} 1 \mathrm{c}$ from $12 \%$ to $9.5 \%$ delayed the onset and reduced the cumulative incidence of complications, particularly retinopathy and nephropathy, increased LE and QALE, and reduced costs of treating diabetes-related complications. Improvements were also projected in well controlled patients (reduction from $7.0 \%$ to $6.5 \%$ ). Sensitivity analysis showed these results to be consistent when variation in the time horizon and discount rates were applied.

Conclusion: It is well established that improving glycemic control improves outcomes. Our study shows that improving $\mathrm{HbAlc}$ leads to substantial clinical and economic benefits (increased in LE, QALE and a reduction in the costs of treating diabetes-related complications) even in patients who are not close to recommended $\mathrm{HbA} 1 \mathrm{c}$ targets, supporting the hypothesis that any improvement in $\mathrm{HbAlc}$ is beneficial in patients with type 2 diabetes.

Summary of Clinical and Cost Outcomes

\begin{tabular}{llll}
\hline & & \multicolumn{3}{c}{$\begin{array}{l}\text { Difference } \\
\text { (HbA1c reduction - } \\
\text { no reduction) }\end{array}$} & $\begin{array}{l}\text { Undiscounted } \\
\text { LE (years) }\end{array}$ & $\begin{array}{l}\text { Undiscounted } \\
\text { QALE (QALYs) }\end{array}$ & $\begin{array}{l}\text { Lifetime cost of } \\
\text { complications (£) }\end{array}$ \\
\hline $\begin{array}{l}\text { Scenario } 1 \\
(12.0 \text { to } 9.5 \%)\end{array}$ & 1.24 & 0.91 & $-3,946$ \\
$\begin{array}{l}\text { Scenario } 2 \\
(9.5 \text { to } 8.0 \%)\end{array}$ & 0.65 & 0.50 & $-1,802$ \\
$\begin{array}{l}\text { Scenario } 3 \\
(8.0 \text { to } 7.0 \%)\end{array}$ & 0.45 & 0.35 & -947 \\
$\begin{array}{l}\text { Scenario } 4 \\
(7.0 \text { to } 6.5 \%)\end{array}$ & 0.19 & 0.15 & -443 \\
\hline
\end{tabular}

Supported by Novo Nordisk Ltd., UK

\section{3}

External validation of the economic assessment of glycaemic control and long-term effects of diabetes (EAGLE) model

S. Walzer $^{1}$, R. Bergemann ${ }^{1}$, N. Freemantle ${ }^{2}$, C. Mathieu ${ }^{3}$, M. Kvasz ${ }^{4}$, N. Marchant ${ }^{5}$, E. Mueller ${ }^{1}$;
${ }^{1}$ Analytica International, Loerrach, Germany, ${ }^{2}$ Department of Primary Care and General Practice, University of Birmingham, Edgbaston, United Kingdom, ${ }^{3}$ Endocrinology Clinic, University Hospital Gasthuisberg, Leuven, Belgium, ${ }^{4}$ Pfizer Inc, New York, United States, ${ }^{5}$ Pfizer Inc, Walton Oaks, United Kingdom.

Background and Aims: The Economic Assessment of Glycemic Control and Long-term Effects of diabetes (EAGLE) model version 2.0 was developed to provide a flexible and comprehensive tool for the simulation of the long-term effects of diabetes treatment and related costs in type 1 and type 2 diabetes. EAGLE simulations are based on risk equations, developed using published data from several large studies including the Diabetes Control and Complications Trial, the United Kingdom Prospective Diabetes Study, and the Wisconsin Epidemiology Study of Diabetic Retinopathy. We previously demonstrated that EAGLE is an internally valid model; EAGLE was able to reproduce the results of the published studies used to build the model within an acceptable range of $\pm 10 \%$. The aim of the present study was to demonstrate the EAGLE model's ability to predict the results of studies that were not used to build the model, using standard methodologies for performing external validation.

Materials and Methods: External validation included the following steps: 1) A systematic literature search to identify suitable publications related to glycemic control. Publications were rejected if they did not contain sufficient details of the main influence parameters $\left(\mathrm{HbA}_{1 \mathrm{c}}\right.$, systolic blood pressure, age, and gender) and if they did not define events in a similar way to the definitions used in EAGLE; 2) Simulation sets were created in EAGLE based on the data contained in the selected articles. Patient numbers were set as 50,000 and iterations as 5,000; 3) Simulations were run. The results obtained with EAGLE were compared with the outcomes reported in the selected publications.

Results: Thirteen publications met the inclusion criteria and were selected for the external validation. Our analyses demonstrated that every mean event rate simulated by EAGLE deviated from the published event rates within an acceptable range of $0 \%$ to $30 \%$, and always overlapped with the confidence intervals of the published event data. For example, in type 2 diabetes, EAGLE successfully predicted the heart failure event rate with only $6 \%$ deviation in comparison to rates published by Torffvit and Agardh ( $\mathrm{J}$ Diabet Comp. 2001;15:307-313).

Conclusion: The EAGLE model is an externally valid tool for making predictions about the long-term effects of diabetes treatment in type 1 and type 2 diabetes patients. The external validation of EAGLE showed that the model is able to predict event rates, reported in studies that were not used to build the model, within a reasonable range of deviation.

Supported by Pfizer Inc 
0894

Health-related quality of life and costs of receiving addon therapy with insulin glargine or pioglitazone in patients with type 2 diabetes

L. Meneghini ${ }^{1}$, S. Schwartz ${ }^{2}$, P. Strange ${ }^{3}$, G. Oster ${ }^{4}$;

${ }^{1}$ Miller School of Medicine, University of Miami, United States, ${ }^{2}$ Diabetes, Diabetes \& Glandular Disease Research Associates, San Antonio, United States, ${ }^{3}$ US Medical Affairs, sanofi-aventis U.S., Bridgewater, ${ }^{4}$ Scientific, Policy Analysis Inc, Brookline, United States.

Background and Aims: Diabetes and its treatment can impact health-related quality of life (HRQoL). We examined HRQoL in patients with type 2 diabetes who received insulin glargine (GLAR) versus pioglitazone (PIO) as addon therapy in a randomized clinical trial. Additionally, we assessed the economic cost associated with these therapies. Materials and Methods: Patients, inadequately controlled on sulfonylurea or metformin (MET), were randomized to GLAR $(n=189)$ or PIO $(n=199)$ for 24 weeks of treatment, and follow-up was subsequently extended to 48 weeks. HRQoL was assessed at baseline and each of 10 follow-up visits, with the 34-item Diabetes Symptom ChecklistRevised (DSC-R), the Emotional Well-Being, and General Health Perceptions scales from the Medical Outcomes Study 36-item Short-Form Health Survey. Using the DSC-R, patients were asked to indicate whether or not they experienced a particular diabetes symptom, such as numbness in the feet, and to rate the intensity of that symptom from 1 (not at all) to 5 (extremely). The cost calculation included components of study medication, other antidiabetic agents, syringes, test strips, and lancets for self-monitoring of blood glucose, and liver function test costs according to PIO label. Average wholesale price was used for drugs and supplies; costs of liver function tests were determined using fee schedules from Medicare's Resource-Based Relative Value Scale. The frequency of self-monitoring of blood glucose was based on the study protocol.

Results: The average daily dose was 47 IU for GLAR and $33 \mathrm{mg}$ for PIO. GLAR provided better glycemic control compared with PIO [mean A1C change from baseline: $-2.6 \%$ (GLAR) vs $-2.3 \%$ (PIO); $P \leq 0.05$ ]. 230 subjects completed the baseline and week-48 HRQoL assessments $(\mathrm{n}=118$, GLAR; $\mathrm{n}=112$, PIO). GLAR had greater improvement in 17 of 20 HRQoL measurements from baseline to week 48 relative to PIO; findings were statistically significant $(P<0.05)$ for the domains measuring psychological distress related to hyperglycemic symptoms, fatigue, and total symptom-related distress. In multivariate repeatedmeasures analysis, GLAR was associated with significantly better outcomes for hyperglycemic $(P \leq 0.001)$ and ophthalmologic symptoms $(P=0.004)$ and with psychological distress related to fatigue $(P=0.005)$, hyperglycemic $(P<0.001)$, hypoglycemic $(P=0.014)$, ophthalmologic $(P<0.001)$, and cardiovascular symptoms $(P=0.025)$. The estimated mean total cost of glycemic control over 48 weeks was $\$ 379$ lower for insulin glargine subjects $(\$ 1721$ vs $\$ 2100$ for pioglitazone).

Conclusions: Insulin glargine, when added to oral monotherapy, resulted in improved glycemic control and diabetes-related quality of life with lower costs compared with pioglitazone.

Supported by sanofi-aventis U.S.

\section{5}

Cost effectiveness of SMBG for the treatment of non insulin dependent diabetes mellitus

C. Weber, K. Erny-Albrecht, K. Neeser;

Medical Affairs, Institute for Medical Informatics and Biostatistics, Basel, Switzerland.

Background and Aims: Despite a number of randomized controlled trials having been conducted to assess the value of SMBG in Type 2 diabetics not using insulin, controversy remains. Whilst it would be premature to restrict use of SMBG (self measurement of blood glucose) based upon current evidence, a clear appreciation of the cost associated with use of SMBG is required before any decision on interim policies can be made. Reports on the cost effectiveness of SMBG are lacking.

Materials and Methods: A Markov model of diabetes management and complications was used to perform a cost effectiveness analysis of SMBG in non-insulin requiring type 2 diabetics treated over a 10 year period.

We assumed that the sole impact of SMBG was on glycaemia as reflected by changes in HbAlc. The impact of SMBG on HbAlc was extacted from the recent metaanalysis of Sarol et al. (Curr.Med.Res.Opin., 2005) in which both random and fixed effects estimates were calculated for a frequency of testing of 5-7 times per week.

Results: At a frequency of SMBG testing of 7 times per week and assuming a reduction in $\mathrm{HbA} 1 \mathrm{c}$ of $0.42 \%$ the CLYG (cost per life-year gained) was within the limits of acceptability with both Metformin and sulphonylurea treatment, being respectively $€ 28,171$ and $€ 27,062$. The reduction in diabetes related complications was of greatest clinical impact in retinopathy and nephropathy for which reductions of between 10-15\% were calculated. To test the impact of variable patient responsiveness to SMBG we recalculated the associated costs when the change in $\mathrm{HbAlc}$ approached the lower and upper confidence limits defined in the meta analysis of Sarol et al. The result of this was a change in CLYG to $€ 63,404$ and $€ 19,351$ for the lower and upper limits respectively. 
Conclusion: We have shown in our analysis that if the recently reported and significant $0.4 \%$ reduction in $\mathrm{HbA} 1 \mathrm{c}$ calculated by pooling data from recent RCT studies is assumed, then SMBG is cost effective. Using a Markov model of diabetes and its complications we determined that a reduction in $\mathrm{HbAlc}$ of $0.4 \%$ would result in modest improvements particularly in the event rates for retinopathy and nephropathy in the order of $10-15 \%$, and that the cost of achieving this via SMBG was within the economic limits of acceptability, at a CLYG of $€ 28,171$.

Supported by sanofi-aventis U.S.

\section{6}

\section{Higher medical care costs accompany the IDF metabolic syndrome}

G. A. Nichols, A. C. Feldstein, J. B. Brown;

Center for Health Research, Kaiser Permanente, Portland, United States.

Background and Aims: The EASD and ADA recently issued a joint statement calling for a critical appraisal of the metabolic syndrome. We sought to contribute to that appraisal from an economic perspective. Specifically, our objective was to compare costs among patients with 1) type 2 diabetes; 2) no diabetes but who otherwise met International Diabetes Federation (IDF) criteria for the metabolic syndrome; and 3) neither diabetes nor IDF metabolic syndrome. Materials and Methods: We identified 33,054 members of a large group model HMO located in the Western United States who, between 1 July 2003 and 30 June 2004, had all clinical measures necessary to ascertain IDF metabolic syndrome status. However, waist circumference was not available, so we substituted body mass index (BMI) for the central obesity criterion, using a cut-point of $27.0 \mathrm{~kg} / \mathrm{m}^{2}$. Subjects with a diagnosis of type 2 diabetes $(n=8,573)$ were assigned to the diabetes group regardless of metabolic syndrome status. Of the remainder, 10,419 met IDF metabolic syndrome criteria, and 14,062 did not. We calculated and compare age-and sex-adjusted medical care costs (in 2005 US dollars) among these three groups for the one-year period of July 2004 through June 2005. In addition, we estimated a multivariable model regressing total costs on age, sex, and dichotomous variables for each of the five metabolic syndrome criteria among patients without diabetes. Results: Although subjects with IDF metabolic syndrome incurred only about $56 \%$ of the total costs incurred by subjects with diabetes ( $\$ 3663$ vs. $\$ 6488, \mathrm{p}<0.0001$ ), their costs were $25 \%$ greater than subjects with neither diabetes nor metabolic syndrome $(\$ 2926, \mathrm{p}<0.0001)$. Among those without diabetes, pharmaceutical costs were $45 \%$ higher when metabolic syndrome was present (\$1123 vs. \$776, $\mathrm{p}<0.0001)$, and outpatient costs were $22 \%$ greater $(\$ 1777$ vs. $\$ 1461, \mathrm{p}<0.0001)$. These differences remained significant even after removing the cost of treating risk factors, such as hypertension, dyslipidemia and obesity, that accompany the metabolic syndrome. Inpatient costs were nonsignificantly (11\%) higher. In the multivariate model of total costs, meeting the hypertension metabolic syndrome criterion was associated with $\$ 1134$ in additional costs $(\mathrm{p}<0.0001)$, while meeting the HDL criterion added $\$ 458$ $(p<0.0001)$. Coefficients for the other three metabolic syndrome criteria were not statistically significant.

Conclusion: Medical costs among patients with IDF metabolic syndrome are moderately elevated relative to patients without the syndrome, but do not approach the level of expense incurred by patients with diabetes. Hypertension and low HDL are the metabolic syndrome criteria most strongly associated with cost, probably because of their link to cardiovascular disease, which is expensive.

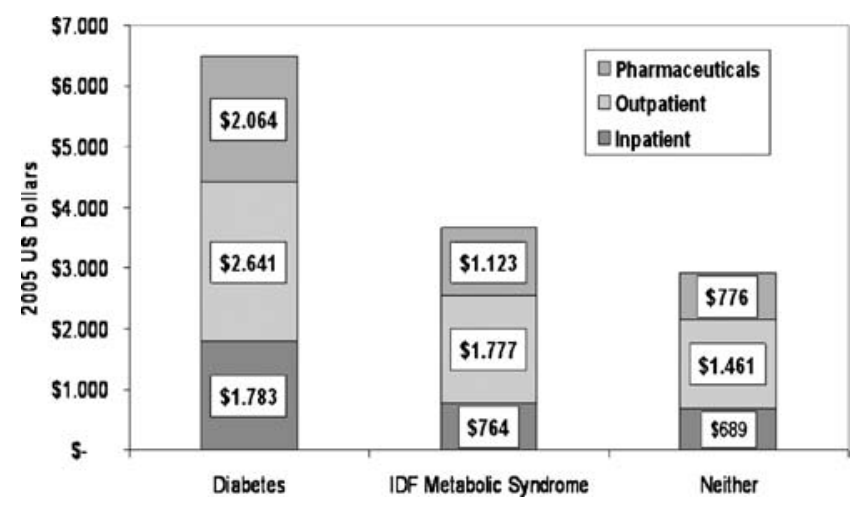

PS 73

\section{Quality of care}

0897

$\mathrm{HbA}_{1 \mathrm{c}}$ levels exceed recommended targets prior to initiation or intensification of insulin therapy in clinical practice: baseline data from the PREDICTIVETM European cohort*

S. Sreenan ${ }^{1}$, M. Yenigun ${ }^{2}$, A. Robinson ${ }^{3}$, P. Kozlovski ${ }^{4}$, R. W. Ackermann ${ }^{4}$, A. Dornhorst ${ }^{5}$;

${ }^{1}$ Dept of Endocrinology, James Connolly Memorial Hospital, Dublin, Ireland, ${ }^{2}$ Fourth Internal Clinic \& Diabetes Dept, Haseki Training and Research Hospital, Aksaray/ Istanbul, Turkey, ${ }^{3}$ Dept Diabetes and Endocrinology, The Royal Oldham Hospital, Oldham, United Kingdom, ${ }^{4}$ Novo Nordisk A/S, Bagsværd, Denmark, ${ }^{5}$ Dept Metabolic Medicine, Imperial College, London, United Kingdom. 
Background and Aims: Although the benefits of good glycaemic control are well established, as reflected in clinical guidelines, many patients with diabetes remain sub-optimally controlled. The European cohort of PREDICTIVETM - a multi-national, prospective, observational, study of the safety and efficacy of insulin detemir - provides the opportunity to assess glycaemic control in a large patient group.

Table 1: Physician reasons for commencing insulin detemir therapy

\begin{tabular}{lcc}
\hline $\begin{array}{l}\text { Reason for starting insulin } \\
\text { detemir** } \mathbf{( \% )}\end{array}$ & $\begin{array}{l}\text { Type 1 } \\
(\mathbf{n = 7 , 0 6 7 )}\end{array}$ & $\begin{array}{l}\text { Type 2 } \\
(\mathbf{n = 1 2 , 7 2 0 )}\end{array}$ \\
\hline Improve glycaemic control & 75 & 87 \\
Reduce plasma glucose variability & 53 & 50 \\
Reduce risk of hypoglycaemia & 52 & 20 \\
Unstable diabetes & 27 & 17 \\
Patient dissatisfaction with current & 25 & 27 \\
therapy & 18 & \\
Try new insulin & 15 & 19 \\
Improve weight control & 5 & 35 \\
Change due to insulin pen & 2 & 4 \\
Other & 2 & 4 \\
Side effects from current therapy & & 3 \\
\end{tabular}

**more than one reason may have been stated
Materials and Methods: The PREDICTIVETM European cohort includes 19,911 patients with type 1 or 2 diabetes. Physician reasons (more than one could be stated) for commencing insulin detemir therapy were recorded, and baseline $\mathrm{HbA}_{1 \mathrm{c}}$ evaluated by pre-study therapy in patients prescribed insulin detemir for the reason to 'improve glycaemic control'. The approach to initiation or intensification with insulin detemir, as reported on the baseline CRF, was explored.

Results: The primary reason reported by physicians for commencing insulin detemir therapy was to 'improve glycaemic control' (Table 1). This subgroup of 16,423 patients, comprised 5,298 (32\%) type 1, 11,042 (67\%) type 2 and $83(1 \%)$ other classified. $\mathrm{HbA}_{1 \mathrm{c}}$ levels according to pre-study therapy group are given in Table 2 .

Of patients initiated on insulin detemir for reasons other than to 'improve glycaemic control', $58 \%$ had $\mathrm{HbA}_{1 \mathrm{c}}>7.0 \%$. Overall, on enrolment, physicians chose to intensify insulin therapy. The proportion of patients intensified to a basalbolus regimen, pre-study vs current therapy, were: Group 1, $1.0 \%$ vs $54.0 \%$; Group 3, $0.0 \%$ vs $84.5 \%$; Group 5, $0.0 \%$ vs 10.6\%; and Group 6, 0.0\% vs 39.6\%. In Group 4 - OADs only - OADs were continued with the addition of insulin

Table 2: Baseline measures of patients initiated on insulin detemir to improve glycaemic control

\begin{tabular}{|c|c|c|c|c|c|c|c|}
\hline & \multirow{3}{*}{$\begin{array}{l}\text { Type } 1 \\
1 \times \text { and } \\
2 \times \text { daily } \\
\text { insulin } \\
\text { Group } 1\end{array}$} & \multirow{3}{*}{$\begin{array}{l}\geq 3 \text { daily } \\
\text { insulin } \\
\text { Group } 2\end{array}$} & \multirow{3}{*}{$\begin{array}{l}2 \times \text { daily } \\
\text { premix } \\
\text { insulin } \\
\text { Group } 3\end{array}$} & \multicolumn{3}{|l|}{ Type 2} & \multirow{3}{*}{$>3$ daily \pm OADs } \\
\hline & & & & OADs only & $\begin{array}{l}1 \times \text { bedtime } \\
\text { insulin } \pm \text { OADs }\end{array}$ & $\begin{array}{l}2 \times \text { daily } \\
\text { insulin } \pm \text { OADs }\end{array}$ & \\
\hline & & & & Group 4 & Group 5 & Group 6 & \\
\hline $\begin{array}{l}\text { Number of } \\
\text { patients }(\mathrm{N})\end{array}$ & 702 & 4,444 & 431 & 3,257 & 2,080 & 1,709 & 5,005 \\
\hline $\begin{array}{l}\text { Mean age, } \\
\text { years }( \pm \text { SD) }\end{array}$ & $38.4( \pm 18.3)$ & $41.9( \pm 16.6)$ & $36.3( \pm 19.8)$ & $60.0( \pm 11.2)$ & $60.1( \pm 9.9)$ & $61.1( \pm 11.0)$ & $61.1( \pm 10.5)$ \\
\hline $\begin{array}{l}\text { Mean diabetes } \\
\text { duration, years }( \pm \mathrm{SD})\end{array}$ & $13.5( \pm 11.1)$ & $17( \pm 12.2)$ & $12.4( \pm 11.3)$ & $8.2( \pm 5.8)$ & $12.4( \pm 7.0)$ & $12.1( \pm 7.0)$ & $12.9( \pm 7.8)$ \\
\hline $\begin{array}{l}\text { Mean BMI, } \\
\mathrm{kg} / \mathrm{m}^{2}( \pm \mathrm{SD})\end{array}$ & $24.6( \pm 4.7)$ & $25.4( \pm 4.5)$ & $24.4( \pm 5.0)$ & $29.5( \pm 5.1)$ & $33.0( \pm 6.4)$ & $30.6( \pm 6.0)$ & $32.1( \pm 6.1)$ \\
\hline $\begin{array}{l}\text { Mean } \mathrm{HbA}_{1 \mathrm{c}} \\
\%( \pm \mathrm{SD})\end{array}$ & $8.8( \pm 1.8)$ & $8.4( \pm 1.6)$ & $9.3( \pm 1.8)$ & $9.0( \pm 1.6)$ & $8.4( \pm 1.4)$ & $8.9( \pm 1.6)$ & $8.4( \pm 1.4)$ \\
\hline $\begin{array}{l}\text { Proportion with } \\
\mathrm{HbA}_{1 \mathrm{c}}>7.0 \%\end{array}$ & $85 \%$ & $84 \%$ & $94 \%$ & $95 \%$ & $87 \%$ & $94 \%$ & $86 \%$ \\
\hline $\begin{array}{l}\text { Proportion with } \\
\mathrm{HbA}_{1 \mathrm{c}}>8.0 \%\end{array}$ & $62 \%$ & $54 \%$ & $77 \%$ & $70 \%$ & $56 \%$ & $69 \%$ & $55 \%$ \\
\hline $\begin{array}{l}\text { Proportion with } \\
\mathrm{HbA}_{1 \mathrm{c}}>9.0 \%\end{array}$ & $36 \%$ & $27 \%$ & $50 \%$ & $40 \%$ & $29 \%$ & $38 \%$ & $27 \%$ \\
\hline
\end{tabular}


detemir in $70 \%$ of patients ( $74 \%$ with a once-daily injection at bedtime or before dinner).

Conclusion: The results show that a significant number of type 1 and 2 patients are not achieving glycaemic targets, confirming a gap between guidelines and clinical reality. Insulin treatment is initiated or intensified at a mean $\mathrm{HbA}_{1 \mathrm{c}}$ much higher than the currently recommended targets.

*Austria, Czech Rep., Denmark, Finland, Germany, Ireland, Israel, Netherlands, Sweden, Turkey and UK

Supported by Novo Nordisk A/S

\section{8}

Clinical and social factors affecting the quality of diabetes care in Poland - a multicenter Prospective Diabetes Care Quality Study (PDCQ Study)

I. Glowania, A. Czech, J. Taton;

Department of Internal Diseases and Diabetology, Warsaw Medical University, Poland.

Background and Aims: Diabetes mellitus prognosis and costs are closely related to the quality of care. Improvement of medical care for 2 millions of diabetics in Poland is therefore very crucial and practical problem. As based on this assumption, the aim of the study was to assess the quality of care in patients with diabetes mellitus and to identify clinical, organizational and social factors acting as barriers for delivering high care quality.

Materials and Methods: The study was consisted with prospective, multicenter, randomized evaluation of medical, organizational and social factors in a sample of randomly selected diabetic patients from 18 centers ( 9 primary care clinics and 9 diabetes clinics) in 3 areas of Poland. The quality indicators were collected with standardized questionnaires for both patients and doctors. Study included 1294 adults with type $1(17.9 \%)$ or type $2(78.5 \%)$ diabetes. The mean age was $60.6 \pm 14.5$ years with a greater proportion of females than males $(54.9 \%$ vs $45.1 \%)$. The average diabetes duration was $12.1 \pm 8.6$ years.

Results: The study revealed the following data: physical examination was performed in $90.7 \%$, foot and eye examinations in $91.7 \%$ and $87.6 \%$ of patients, respectively. HbAlc was measured in $79.3 \%$, lipids in $56.3 \%$ and microalbuminuria only in $18.1 \%$ of patients. The percentage of tested patients who were at goals in accordance to EASD recommendations was: $21.3 \%$ for $\mathrm{HbA1C}, 17.8 \%$ for glycemia, $11.1 \%$ for lipids, and $12.8 \%$ for blood pressure. The mean level of HbA1c was $7.53 \%$. Retinop- athy was present in $59.2 \%$ of patients, nephropathy in $10.7 \%$, neuropathy in $41.4 \%$ and coronary heart diseases in $35.9 \%$. Multiple logistic regression analysis showed that patients who had been examined at least once in the last year were above 2 times more likely to receive optimal diabetes care. Advising patients on diet, at least secondary education and earnings $>760$ PLN were also the predictors of high quality of diabetes care. However, older patients, with longer diabetes duration, with insulin treatment, males and living in the northern area of Poland were less likely to receive optimal diabetes care. Likelihood of optimal care in the general outpatient clinics was about $95 \%$ lower than in the specialist outpatient clinics.

Conclusion: Sex, age, diabetes duration, insulin therapy, at least one during last year physical examination, advising patients on diets, level of health care, region of Poland, education and earnings are factors affecting the quality of diabetes care. Attending primary care was associated with the worst quality of diabetes care. These results indicate for necessity of urgent improvement of diabetes care in general practice. Evidence based quality indicators included in questionnaire - a new, useful tool for monitoring and improvement of diabetes care in Polish outpatient clinics.

0899

Four years of voluntary quality management in diabetes care in Austria: effects on process quality and intermediate outcome

I. Rakovac ${ }^{1}$, P. Beck ${ }^{1}$, P. Mrak ${ }^{2}$, B. Bauer ${ }^{2}$, W. Habacher ${ }^{1}$, S. Seereiner ${ }^{1}$, K. Jeitler ${ }^{1,3}$, T. R. Pieber ${ }^{1,3}$;

${ }^{1}$ Inst of Medical Technologies and Health Management, Joanneum Research, Graz, Austria, ${ }^{2}$ Department of Internal Medicine, LKH Hoergas, Gratwein, Austria, ${ }^{3}$ Department of Internal Medicine, Medical University of Graz, Austria.

Background and Aims: Forum for Quality Systems in Diabetes Care Austria (FQSD-A) is a voluntary Quality Management (QM) initiative in Austria. In this abstract, we describe the impact of 4 years of QM in FQSD-A on process and intermediate outcome.

Materials and Methods: The intervention consisted of continuous medical education workshops organized twice yearly with involvement of local opinion leaders, structured documentation of patient examinations, feedback on medical performance and continuous quality improvement in quality circles. Anonymized patient data was collected on annual examinations either in paper form or using an internet-based 
application. Non-anonymized (with identifiable centers) quality of care benchmarks were sent to all participants quarterly and could be done anytime online. It was hypothesized that processes of care and the outcomes would be significantly linearly related to increased duration of participation in FQSDA. We used multilevel logistic regression models with random center and patient intercept to compute average half year trends. Results: In the first 4 years of continuous participation in FQSD-A, 25 health care centers collected data on 7447 diabetic patients on 9912 visits. At the first visit, patients were $61.3 \pm 15.1$ years old, had an diabetes duration of $9.7 \pm 9.7$ years, $56 \%$ were female and $82 \%$ had Type 2 diabetes. Processes of care and outcomes plotted against the half years of participation are given in Figure.

A - Proportion of examinations with recorded: HbA1c ( $p$ n. s.)=white circles, RR (p n.s.)=black circles, lipid profile $(p<0.001$, OR 1.96)=white squares, urinary protein or albumin excretion $(p<0.001$, OR 1.12)=black squares, smoking habits ( $\mathrm{p}$ n.s.) $=$ white triangles, erectile dysfunction $(p<0.001$, OR 1.16) $=$ black triangles .

B - Proportion of examinations with: eye examinations ( $p$ n.s. $)=$ white circles, feet examinations (p n.s.)=black circles, consecutive antihypertensive medication prescription if $\mathrm{RR}>140 / 90 \mathrm{~mm} \mathrm{Hg}(\mathrm{p}<0.001$, OR 1.35)=white squares, prescription of antiplatelet medication in patients with CHD (p n.s.)=black squares, prescription of statins in patients with $\mathrm{CHD}$ and $\mathrm{TC}>200 \mathrm{mg} / \mathrm{dl}$ (p n.s.)=white triangles, proportion of patients with structured patient education $(p<0.001$, OR 1.47)=black triangles .

C - Proportion of visits with recorded: $\mathrm{RR}<140 / 90 \mathrm{mmHg}$ $(p<0.001$, OR 1.09)=white circles, HbAlc $<8 \%(p<0.001$, OR 1.08)=black circles, TC $<200 \mathrm{mg} / \mathrm{dl}(\mathrm{p}<0.001$, OR $1.08)=$ white squares and LDL $<130 \mathrm{mg} / \mathrm{dl}(\mathrm{p}=0.009, \mathrm{OR}$ 1.07) $=$ black squares.

Conclusion: Centres that continuously participated in QM for 4 years significantly improved numerous processes of care and all considered patient outcome measures. Due to the absence of control group we can not estimate the improvement attributable to QM.
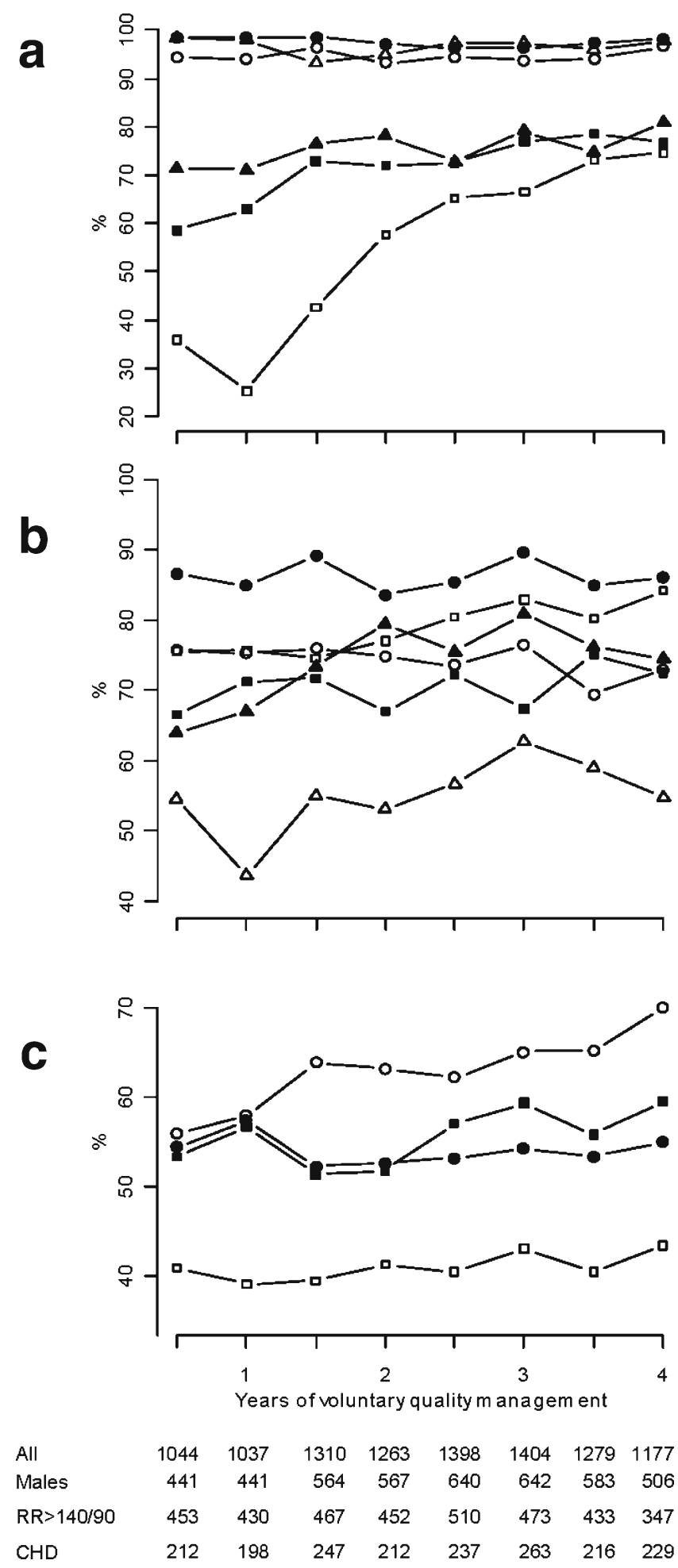
0900

Pay per results. Does it improve diabetes care? A model from the UK

A. A. Tahrani ${ }^{1}$, M. McCarthy ${ }^{2}$, J. Godson ${ }^{2}$, P. Moulik ${ }^{1}$, H. Slater ${ }^{3}$, A. F. Macleod ${ }^{1}$;

${ }^{1}$ Diabetes, Royal Shrewsbury Hospital, ${ }^{2}$ Diabetes, Shropshire PCT, Shrewsbury, ${ }^{3}$ Diabetes, Telford and Wrekin PCT, Shrewsbury, United Kingdom.

Background and Aims: The new General Medical Service (nGMS) contract was implemented in the UK after April 2004. The nGMS gives financial rewards to general practitioners (GPs) for delivering enhanced clinical and organisational quality care and for improving patients' experience. Organisational standards include records and information about/for patients, whilst clinical standards include variables such as HbA1c, cholesterol levels, and blood pressure (BP) (Table).

The aim of our project is to assess the impact of the nGMS on the delivery of diabetes care in our area and whether the implementation of a pay per result system has improved the care provided to people with diabetes.

Material and Methods: We collected data from the two Primary Care Trusts (PCTs) in Shropshire, UK. Data included the number of patients with diabetes, and the numbers of patients achieving each indicator of the nGMS contract, in each practice. Shropshire covers a wide area that includes both rural and urban population. We included data from all GP practices in Shropshire (66 in total), comprising 16,858 people with diabetes.

Data were collected at four time-points: April 2004, October 2004, March 2005 and October 2005. The data for April 2004 were collected form the National Diabetes Audit (NDA). The data for the rest of the time-points were obtained from the PCTs in Shropshire.

In the analysis we have compared the mean percentage of patients achieving each indicator in each GP practice at each of the time-points.

Results: The results including means \pm SD, $p$ values and $95 \%$ confidence interval for the difference in means for each of the time-points are shown in the Table.

The results show improvement in all organisational as well as the clinical standards between April 2004 and October 2005. All these improvements achieved statistical significance $(p<0.001)$. Scrutiny of the data reveals that the majority of the organisational indicators improved rapidly after implementing the nGMS (October 2004), compared to April 2004, and then stabilised. Clinical indicators, i.e. control of HBA1c, BP and cholesterol, showed a sustained

Summary of indicator data at three time points. Mean(SD), 95\%CI for the difference in means.

\begin{tabular}{|c|c|c|c|c|c|c|c|c|c|c|c|c|}
\hline \multirow{2}{*}{$\begin{array}{l}\text { Indicator } \\
\text { BMI record }\end{array}$} & \multirow{2}{*}{$\begin{array}{l}\text { NDA } \\
\text { (Apr 04) } \\
.73(.1)\end{array}$} & \multirow{2}{*}{$\begin{array}{l}\text { October } \\
04 \\
.76(.11)\end{array}$} & \multicolumn{2}{|c|}{$\begin{array}{c}95 \% \text { CI } \\
\text { (NDA-Oct04) }\end{array}$} & \multirow{2}{*}{$\begin{array}{l}\text { P } \\
\text { (NDA- } \\
\text { Oct04) }\end{array}$} & \multirow{2}{*}{$\begin{array}{l}\text { October } \\
05 \\
.78(.10)\end{array}$} & \multicolumn{2}{|c|}{$\begin{array}{c}95 \% \text { CI } \\
\text { (Oct04-Oct05) }\end{array}$} & \multirow{2}{*}{$\begin{array}{l}\mathrm{P} \\
(\text { Oct04- } \\
\text { Oct05) }\end{array}$} & \multicolumn{2}{|c|}{$\begin{array}{c}95 \% \mathrm{CI} \\
\text { (NDA-Oct05) }\end{array}$} & \multirow{2}{*}{$\begin{array}{l}\begin{array}{l}\mathrm{P} \\
\text { (NDA- } \\
\text { Oct05) }\end{array} \\
.000\end{array}$} \\
\hline & & & -.07 & -.01 & & & -.04 & .01 & & -.08 & -.03 & \\
\hline Smoking record & $.44(.14)$ & $.89(.08)$ & -.48 & -.41 & .000 & $.87(.07)$ & -.003 & .04 & .103 & -.47 & -.39 & .000 \\
\hline HbA1c record & $.75(.15)$ & $.85(.07)$ & -.14 & -.06 & .000 & $.86(.07)$ & -.03 & -.0005 & .042 & -.16 & -.08 & .000 \\
\hline $\mathrm{HbA} 1 \mathrm{c}<7.5$ & $.53(.18)$ & $.48(.12)$ & .01 & .10 & .030 & $.66(.09)$ & -.20 & -.16 & .000 & -.17 & -.08 & .000 \\
\hline $\mathrm{HbA} 1 \mathrm{c}<10.1$ & $.90(.06)$ & $.87(.12)$ & -.003 & .06 & .080 & $.94(.02)$ & -.10 & -.04 & .000 & -.06 & -.03 & .000 \\
\hline $\begin{array}{l}\text { Retinal screening } \\
\text { record }\end{array}$ & $.47(.21)$ & $.58(.11)$ & -.17 & -.06 & .000 & $.60(.12)$ & -.06 & .01 & .113 & -.19 & -.08 & .000 \\
\hline $\begin{array}{l}\text { Peripheral pulses } \\
\text { record }\end{array}$ & $.22(.23)$ & $.56(.13)$ & -.40 & -.28 & .000 & $.59(.12)$ & -.062 & .004 & .092 & -.43 & -.31 & .000 \\
\hline $\begin{array}{l}\text { Neuropathy } \\
\text { testing record }\end{array}$ & $.22(.23)$ & $.55(.12)$ & -.39 & -.26 & .000 & $.59(.12)$ & -.07 & -.01 & .018 & -.42 & -.30 & .000 \\
\hline $\mathrm{BP}$ record & $.87(.06)$ & $.91(.06)$ & -.05 & -.02 & .000 & $.91(.05)$ & -.02 & .01 & .274 & -.06 & -.03 & .000 \\
\hline $\mathrm{BP}<=145 / 85$ & $.53(.11)$ & $.62(.10)$ & -.11 & -.07 & .000 & $.66(.01)$ & -.06 & -.01 & .003 & -.14 & -.10 & .000 \\
\hline $\begin{array}{l}\text { Microalbuminuria } \\
\text { record }\end{array}$ & $.07(.12)$ & $.50(.20)$ & -.48 & -.37 & .000 & $.57(.15)$ & -.11 & -.02 & .006 & -.54 & -.45 & .000 \\
\hline $\begin{array}{l}\text { Serum creatinine } \\
\text { record }\end{array}$ & $.81(.09)$ & $.83(.07)$ & -.043 & -.002 & .029 & $.85(.07)$ & -.03 & .003 & .105 & -.06 & -.02 & .001 \\
\hline $\begin{array}{l}\text { ACEi or A2 } \\
\text { antagonist }\end{array}$ & & $.01(.02)$ & & & & $.03(.03)$ & -.02 & -.01 & .000 & & & \\
\hline $\begin{array}{l}\text { Cholesterol } \\
\text { record }\end{array}$ & $.78(.08)$ & $.80(.07)$ & -.04 & -.004 & .019 & $.82(.06)$ & -.04 & -.01 & .008 & -.07 & -.03 & .000 \\
\hline Cholesterol $<=5$ & $.60(0.1)$ & $.66(.07)$ & -.07 & -.04 & .000 & $.74(.06)$ & -.010 & -.07 & .000 & -.16 & -.12 & .000 \\
\hline
\end{tabular}

The data represent the mean percentage of patients who achieved the indicator in each GP practice. 
improvement. For example the mean percentage of patients with HbAlc $<7.5$ and HbAlc $<10.1$ in each GP practice failed to improve initially between April 2004 and October 2004, but improved and achieved statistical significance by October $2005(\mathrm{p}<0.001)$.

Conclusions: The implementation of the nGMS contract has been associated with improvement in the care delivered to patients with diabetes, whether this care was organisational or clinical, in Shropshire. Although the more progressive improvement in clinical indicators might suggest the influence of improved practice in general, it is likely that the nGMS has had an impact. This improvement in Shropshire is likely to reflect a wider improvement in diabetes care across England.

\section{1}

Quality of diabetes care in 35 centers in 25 countries using a European primary care clinical audit tool. Global Alphabet Strategy Implementation Audit (The GAIA Project)

N. Warsi ${ }^{1}$, R. Goldspring ${ }^{1}$, A. Gopinath ${ }^{2}$, S. Poulter ${ }^{3}$, J. Morrissey ${ }^{2}$, V. Patel ${ }^{2}$;

${ }^{1}$ University of Warwick, Warwick Medical School, Coventry, ${ }^{2}$ Diabetes Centre, George Eliot Hospital NHS Trust, Coventry, ${ }^{3}$ Human Science, University College London, United Kingdom.

Background and Aims: The Alphabet Strategy is a patientcentred, evidence-based clinical strategy based on the seven most important aspects of diabetes care ; Advice (diet, exercise, smoking cessation, concordance with treatment), Blood pressure $(<145 / 85 \mathrm{mmHg})$, Cholesterol ( $\leq 5 \mathrm{mmol} / \mathrm{l})$, Diabetes control $(<7.5 \%), \boldsymbol{E}$ ye care and $\boldsymbol{F}$ oot care (annual screen and treatment), Guardian drugs, e.g., Aspirin, ACEI. Previously it has been shown that the Alphabet Strategy has produced clinical audit outcomes comparable to UKPDS and Steno-2 intensively treated patients.

Our aim was to investigate the quality of diabetes care internationally and assess the feasibility of using the Alphabet Strategy in diabetes centres in other countries particularly outside of Europe.

Materials and Methods: Medical students from Warwick University collected data in the Alphabet Strategy format during their electives around the world in partnership with the GAIA Collaborators. The UK Primary Care Quality outcomes Framework (QoF) provides a template for clinical audit in diabetes care (maximum points 99). Audit parameters included smoking cessation advice, blood pressure and cholesterol target attainment, diabetes control, eye and feet screening, micro-albuminuria and proteinuria screening and treatment. We analysed data on 3275 patients from 35 centres, in 25 countries, in all continents bar Antarctica. The results were organised into quintiles (Q1Q5) according to QoF points.

Results: - Overall average points 45.2 \pm 24.9 , Europe $66.8 \pm 26.5$ vs rest of the world $39.0 \pm 21.0(p=0.002)$.

- Large variation in management of diabetes care throughout the world (QoF points range 10.2 to 87.5).

- There is a significant correlation between socio-economic parameters and quality points

- The more \% GDP (Gross Domestic Product) spent on healthcare, the better diabetes care $(\mathrm{r}=0.87, \mathrm{p}<0.01)$.

- The GDP correlated with quality points $(\mathrm{r}=0.80, \mathrm{p}<0.01)$.

- The country's life expectancy correlated with quality points $(\mathrm{r}=0.72, \mathrm{p}<0.01)$

- Overall $57.5 \%$ of 146 healthcare professionals questioned felt that they were likely to adopt the Alphabet strategy. A similar proportion of patients were positive about the Alphabet Strategy

Conclusion: There is large variation in standards of diabetes care internationally as assessed using the UK Primary Care QoF points system. This correlated with adverse socio-economic circumstances. The Alphabet Strategy was deemed to be useful in helping to implement an evidence-based, patient education focussed strategy for delivering diabetes care. The level of support for the Alphabet Strategy was independent of economic factors and has the potential to help deliver effective diabetes care.

0902

Development of an insulin dose selection device supporting tool for visually impaired patients with insulintreated diabetes mellitus for: FlexPen

T. Kenichi ${ }^{1}$, Y. Sako ${ }^{2}$, N. Sekiguchi ${ }^{2}$, K. Okadome ${ }^{2}$;

${ }^{1}$ Department of Pharmacy, ${ }^{2}$ Department of Internal Medicine, Saiseikai Fukuoka General Hospital, Fukuoka, Japan.

Background and Aims: The dose selection of insulin selfinjection practice is difficult for visually impaired patients, but there is no specific insulin injection device for them. Though insulin injection device dose selection assisting device exists for InnoLet, which is contributing to QOL improvement of visually impaired patients, treatment range is narrower as InnoLet has no ultra rapid acting insulin.

Objective here is to develop an insulin injection device dose selection assisting tool attachable to FlexPen which enables dose selection by patients with visual impairment.

Materials and Methods: This is a tool with two rectangle rubber poles with a hole in each pole. An user pinches FlexPen with the rubber poles and listens to the click sound generated near the unit display section of FexPen while rotating the dial, through air type receivers connected to the poles. 
Use Precision Sound Level Meter Type 2003 (Node) to measure, in anechoic room, the click sound from FlexPen from a distance of $30 \mathrm{~cm}$ and the sound through earphone of air type receiver when the assist tool is attached to FlexPen. Results: Sound intensity was 28 and 29 decibels respectively.

Intensity of sound from receiver was 1.13 times higher than that at the distance of $30 \mathrm{~cm}$. The clicking sound will be heard louder due to the effect of excluding ambient noise by applying receivers to both ears.

Conclusion: There has been no insulin-injecting device available for visually impaired patients needing ultra rapid acting insulin. An insulin-injection device dose selectionassisting tool for FlexPen has been developed enabling visually impaired patients to use FlexPen, and expected to contribute to the improvement of patients QOL.

\section{3}

The efficacy of self blood glucose monitoring in type 2 diabetes: The ESMON Study

M. J. O'Kane ${ }^{1}$, V. E. Coates ${ }^{2}$, B. Bunting ${ }^{3}$

on behalf of the ESMON Study Group,

${ }^{1}$ Clinical Chemistry, Altnagelvin Hospital, Londonderry,

${ }^{2}$ Institute of Nursing Research, University of Ulster, Coleraine, ${ }^{3}$ School of Psychology, University of Ulster, Londonderry, United Kingdom.

Background and Aims: Although widely advocated by healthcare professionals there is uncertainty about the value of self-monitoring of blood glucose [SMBG] in type 2 diabetes [T2DM]. There is conflicting evidence as to whether monitoring is associated with improved diabetes control. SMBG might contribute to diabetes management by providing instant feedback to patients on glycaemic control thus positively reinforcing beneficial lifestyle habits. Conversely SMBG might have a negative effect on patient experience by increasing anxiety levels. The aim of this study was to investigate in a prospective randomised controlled trial the effect of SMBG on glycaemic control and psychological indices over a 1 year period in a cohort of patients with newly diagnosed T2DM.

Materials and Methods: Patients with newly diagnosed T2DM were randomised to a SMBG group or a non monitoring [control] group in 4 Northern Ireland Diabetes Clinics. All patients underwent a comprehensive diabetes education programme and an identical structured treatment regimen for glycaemic control based on HbAlc targets was followed in each group. Each patient was reviewed at 3 monthly intervals for 1 year. $\mathrm{HbAlc}$ was measured at each clinic site using a DCCT aligned HPLC assay. Patients in the SMBG group were asked to perform 4 fasting and 4 postprandial measurements per week using a single meter type provided by the investigators. Monitoring patients were instructed in the interpretation of glucose readings and advised on appropriate responses to high and low readings. Psychological wellbeing [satisfaction, locus of control, attitudes to diabetes, anxiety and depression] was evaluated by a validated questionnaire tool at each time point. The predeterminded primary endpoints reported are $\mathrm{HbAlc}$ and measures of psychological well being.

Results: 184 patients were randomised to the SMBG group [55 males/41 females; 57.7 (11.04) years, mean (SD)] or non monitoring [control] group [56 males/32females; 60.9 (11.5) years. There was no difference in baseline HbA1c or BMI between the groups [HbAlc 8.76(2.12)\% v 8.6 (2.27)\%, BMI $32(6.23) \mathrm{kg} / \mathrm{m}^{2}$ v. 34 (6.98); mean (SD) monitoring $\mathrm{v}$ non monitoring, $\mathrm{p}=\mathrm{NS}]$. Although $\mathrm{HbAlc}$ levels decreased in both the monitoring and non monitoring groups there was no significant difference in $\mathrm{HbAlc}$ between the groups at any timepoint with 12 month $\mathrm{HbA} 1 \mathrm{c}$ values of $6.87(0.76) \% \mathrm{v} 6.94(1.16) \%$, monitoring $\mathrm{v}$ non monitoring, $p=N S$.There was no significant difference in BMI between the 2 groups at any time point with 12 month values of $31.8(6.03) \mathrm{v} 33.1(6.32)$ monitoring $\mathrm{v}$ non monitoring, $p=N S$. There was a trend towards greater anxiety levels in the monitoring group when corrected for age and gender. There was no difference in other psychological indices between the groups.

Conclusions: In this cohort of patients with newly dignosed T2DM, SMBG was not associated with improved glycaemic control. There was a trend towards increased levels of anxiety in the self monitoring group.

This study was funded by the N Ireland Research and Development Office

\section{4}

Treatment of diabetic ketoacidosis in Denmark: a national survey

O. L. Svendsen ${ }^{1}$, O. M. Henriksen ${ }^{1,2,3}$, J. B. Prahl ${ }^{4}$, M. E. Røder ${ }^{1}$;

${ }^{1}$ Endocrine Section, Dept. of Internal Medicine I, ${ }^{2}$ Dept. Clinical Physiology and Nuclear Medicine, Bispebjerg University Hospital, Copenhagen, ${ }^{3}$ Dept of Clinical Physiology and Nuclear Medicine, University Hospital Glostrup, ${ }^{4}$ Paediatrics Dept., University Hospital Gentofte, Denmark

Background and Aims: The aim of the present was to examine management routines of diabetic ketoacidosis (DKA) in adult patients in departments of internal medicine in Denmark.

Materials and Methods: A questionnaire was sent to all departments of internal medicine in Denmark responsible of 
managing DKA. The questionnaire requested information on management routines of DKA and a copy of the departments current management protocol.

Results: A questionnaire was sent to 71 departments of which 9 were excluded from the survey. Of the remaining 62 departments 59 returned the questionnaire and/or a copy of their management protocol, giving a response rate of $95 \%$.

At 19 departments (32\%), all patients with DKA were managed in an intensive care unit. Routines of insulin therapy varied significantly in terms of doses and routes of administration. 24 different regimens were identified.

21 different fluid protocols were identified. Isotonic saline was the preferred hydration solution by 58 departments (97\%). Potassium supplements were provided as a separate infusion of either isotonic potassium-sodium-chloride $(73 \%)$ or isotonic potassium-chloride $(13 \%)$. Volume administered during the first eight hours of treatment could be estimated in 20 protocols (median $4750 \mathrm{ml}$, range 3750 to $7700 \mathrm{ml}$ ). Use of bicarbonate was endorsed by $80 \%$.

Conclusion: This study shows significant variations in management routines of DKA in Denmark.

In many cases, the treatment routines employed are not supported by evidence from clinical trials. We recommend implementation of national and/or European guidelines for management of DKA in adult patients.

\section{5}

Perceptions of seriousness and management of diabetes. Findings from the SHARED study (Survey comparing Healthcare professionals and patients to Assess REal perceptions of Diabetes issues)

\section{F. J. Snoek ${ }^{1}$, M.-P. Dain ${ }^{2}$, W. H. Polonsky ${ }^{3}$;}

${ }^{1}$ Department of Medical Psychology, VU University Medical Centre, Amsterdam, The Netherlands, ${ }^{2}$ Sanofi-Aventis, Paris, France, ${ }^{3}$ Behavioral Diabetes Institute, University of California, San Diego, United States.

Background and Aims: There is growing appreciation of the shared responsibilities between patients and healthcare professionals (HCPs) in diabetes management. A better understanding of patients' views may enhance communication and clinical outcomes. SHARED is an international survey designed to investigate the perceptions and expectations of patients with diabetes, HCPs (physicians and nurses) and the general public on the seriousness and management of diabetes.

Materials and Methods: The survey was conducted between August and October 2005 and involved >12,000 individuals from 8 countries (France, Germany, Italy, Netherlands, Spain, Sweden, UK and the USA). These included the general public $(n=8,024)$, diabetologists/endocrinologists $(\mathrm{n}=697)$, general practitioners $(\mathrm{n}=818)$, nurses $(\mathrm{n}=814)$ and people with Type $1(\mathrm{n}=404)$ and Type 2 diabetes $(\mathrm{n}=1,609)$. The survey employed the "mirror principle" (asking the same question to patients and HCPs). Participants were questioned by: telephone survey (general public [sample representative of the national population aged $\geq 15$ years] and HCPs) or online survey (patients). Data for patients, physicians and in some cases nurses are reported.

Results: Mean age for patients was 50 years with $58 \%$ male. $46 \%$ of patients thought that diabetes was an extremely or very serious condition, while physicians anticipated that only $25 \%$ of their patients would think so. $90 \%$ of patients expressed an interest in learning more about their diabetes, while physicians believed this to be the case for only $65 \%$ of their patients. $78 \%$ of patients reported that they search for more information about their diabetes, while physicians estimated that only $56 \%$ of their patients are doing so. A majority of patients lacked knowledge about $\mathrm{HbA}_{1 \mathrm{c}}$, with only $44 \%$ of patients having heard about the $\mathrm{HbA}_{1 \mathrm{c}}$ test. $47 \%$ of patients who were not taking insulin said they would be willing to take insulin if their physician recommended it. $62 \%$ of patients on insulin felt that their condition had improved since they had started taking insulin and $90 \%$ of those on insulin said they would be willing to adjust their own insulin doses; however, physicians and nurses thought that only $42 \%$ and $39 \%$, respectively, of their patients would be willing to do so. In total, $91 \%$ of patients taking oral medication would like to take part in decision-making about their treatment, while physicians believed only $44 \%$ of their patients would be willing to do so.

Conclusion: Almost half of insulin-naïve patients are willing to consider insulin therapy. Contrary to the perceptions of their HCPs, those patients who are on insulin are largely positive about their treatment and are willing to self-titrate in order to improve their blood glucose levels and prevent long-term complications. There is a broad lack of knowledge among patients about $\mathrm{HbA}_{1 \mathrm{c}}$. This study was supported by sanofi-aventis

\section{6}

Parameters affecting postprandial blood glucose: effects of errors - the diabetes error test model

T. Koschinsky ${ }^{1}$, S. Heckermann ${ }^{2}$, L. Heinemann ${ }^{2}$;

${ }^{1}$ Heinrich-Heine-University of Duesseldorf, Germany, ${ }^{2}$ Profil Institute for Metabolic Research, Neuss, Germany.

Background and Aims: Diagnostic and therapeutic tools for people with diabetes are characterized by rather large and varying margins of error, which increase the risk for hypoand hyperglycemia. The Diabetes Error Test Model (DETM) 
has been developed to characterize the clinical relevance of the margins of error of parameters affecting blood glucose (BG) and their relative contribution to postprandial BG.

Materials and Methods: The DETM is based on a treatment concept aimed at normoglycemia after meals by pre-prandial injections of insulin in adult people with diabetes. The model includes as parameters: a) preprandial BG measurement by patient self-monitoring (SM), b) patient estimate of carbohydrate amounts (CARB) in food, c) effect of CARB on maximum BG-increase, d) effect of insulin on maximum BG-decrease, e) insulin dosage. Covering the relevant range of pre-prandial BG (30-330 mg/dl) the DETM simulates the maximum effect of these parameters and their margins of error on postprandial $\mathrm{BG}$ values as outcome according to standard textbook guidelines. If the postprandial outcome is not normoglycemia but turns into hyper- or hypoglycemia, we define this as the Critical Point. If this postprandial outcome exceeds the BG-range of $50-200 \mathrm{mg} / \mathrm{dl}$ for $>4.5 \%$ of all SMBG-values (sample size and range: ISO-NORM $15197: 2003 / E$ ), this device is classified as unacceptable (Error Acceptance Analysis). At first, the effects of SMBG measurement errors are evaluated while all other parameters are kept at $0 \%$ error. In a second step, the combined effects of 2 parameter errors are examined as an example of daily life experience of people with diabetes.

Results: A SMBG error of $+20 \%$ (classified e.g. by the Error Grid Analysis/EGA as acceptable and within the Zone A) results as postprandial outcome in normoglycemia (BG range: $60-160 \mathrm{mg} / \mathrm{dl}$ ) if pre-prandial $\mathrm{BG}$ values are in the range of $30-130$ or $260-330 \mathrm{mg} / \mathrm{dl}$, but results unexpectedly in hypoglycemia (BG-range: $50-60 \mathrm{mg} / \mathrm{dl}$ ) if pre-prandial BG values are between $131-259 \mathrm{mg} / \mathrm{dl}$. In this range the Critical Point for hypoglycemia is already reached at a SMBG error of $+11 \%$. If the SMBG error of $+20 \%$ is combined with an error of CARB estimate in the food of $+20 \%$ hypoglycemia as postprandial outcome is worsened (BG-range: $<50 \mathrm{mg} / \mathrm{dl}$ ). In contrast, the combination of the SMBG error of $+20 \%$ with an error of CARB estimate of $-20 \%$ results in normoglycemia as postprandial outcome. The calculated error acceptance areas for SMBG values (Error Acceptance Analysis) are different and smaller than the combined areas of EGA-Zone A and B. SMBG devices that are still acceptable by EGA criteria (within Zone B limits) are classified already as clinically unacceptable by the Error Acceptance Analysis.

Conclusion: The DETM simulates the effects of potential errors of parameters affecting postprandial BG within the clinically relevant $\mathrm{BG}$ range. It analyzes the relative error contribution not only of SMBG but also of therapeutic parameters like carbohydrates and insulin per se as well as combined. The DETM evaluates the clinical relevance of these errors, distinguishes between acceptable and unacceptable parameter errors and presents a detailed risk assessment with the focus on postprandial BG outcome. No other available model for the clinical evaluation of SMBG errors has a comparable detailed analysis and hypothesis generating potential.

Supported by Disetronic Medical Systems, Burgdorf, Switzerland

\section{PS 74}

\section{Diabetes management in outpatient care}

0907

Interactive diary for diabetes: a useful and easy-to-use new telemedicine system to support the decision-making process in type 1 diabetes

G. Vespasiani ${ }^{1}$, M. C. E. Rossi ${ }^{2}$, A. Nicolucci ${ }^{2}$, D. Bruttomesso ${ }^{3}$, P. Di Bartolo ${ }^{4}$, G. Marelli ${ }^{5}$, M. Dal Pos ${ }^{3}$, M. Galetta ${ }^{1}$, D. Horwitz ${ }^{6}$;

${ }^{1}$ Diabetes Unit, "Madonna del Soccorso" Hospital, S. Benedetto del Tronto (AP), ${ }^{2}$ Clinical Pharmacology and Epidemiology, Consorzio Mario Negri Sud, S. Maria Imbaro $(\mathrm{CH}),{ }^{3}$ Department of Clinical and Experimental Medicine, University of Padova, ${ }^{4}$ Diabetes Unit, Ravenna Local Health Unit, Ravenna, ${ }^{5}$ Diabetes Unit, Civil Hospital, Desio (MI), Italy, ${ }^{6}$ Lifescan, Inc, Milpitas, United States.

Background and Aims: A widespread use of carbohydrates $(\mathrm{CHO})$ counting is limited by its complex patient educational approach. Telemedicine can represent an important tool to help patients to adjust the insulin bolus according to the amount of $\mathrm{CHO}$ ingested in a meal, while avoiding the use of complex calculations. The aim of this pilot study was to evaluate the applicability and acceptability of the Interactive Diary, a Bolus Calculator and a telemedicine system based on communication between patient and specialist by SMSs through mobile phones.

Materials and Methods: The Interactive Diary for Diabetes (IDD) was set up in patients' mobile phones and allowed: 1. to record the blood glucose values; 2 . to quantify the total $\mathrm{CHO}$ assumed during a meal, by choosing from a list of pictures the specific food and the amount ingested; 3 . to suggest the most appropriate bolus insulin in relation to the patient's $\mathrm{CHO} /$ Insulin ratio, the factor of insulin sensitivity, the blood glucose goal, and the physical activity done; 4 . to send the data filed in the mobile phone as short messages (SMSs) to the physician. Four Italian Diabetes Outpatient Clinics enrolled 50 patients with type 1 diabetes, aged between 18 and 65 years, and familiar with the use of mobile phones. Socio-demographic and clinical information was collected and questionnaires investigating quality of life (SF-36 Health Survey Instrument and WHO- 
DTSQ) and satisfaction with the IDD were filled in at baseline and after 12 weeks.

Results: At the end of the study, $67 \%$ of patients found the system good and $27 \%$ excellent; $66 \%$ stated the system was very or extremely helpful; $90 \%$ judged it easy to use. CHO counting and insulin bolus calculation were considered by the patients as the most useful functions of the IDD. No significant changes were documented in clinical parameters and in DTSQ-WHO and SF-36 scores after 12 weeks (table). No serious hypoglycemic episodes requiring medical intervention were reported during the study, although patients could choose food they wished, modifying their pre-meal insulin doses as suggested by the IDD.

Conclusion: This pilot study showed that the IDD was safe, easy to use and well accepted by all the patients.

\begin{tabular}{llll}
\hline Clinical data & Baseline & Follow-up & p \\
\hline HbA1c & $7.2 \pm 0.8$ & $7.2 \pm 0.9$ & 1.0 \\
Systolic blood pressure & $117.9 \pm 14.4$ & $119.6 \pm 9.3$ & 0.6 \\
Diastolic blood pressure & $77.4 \pm 6.7$ & $77.6 \pm 5.1$ & 0.6 \\
BMI & $23.4 \pm 3.2$ & $23.6 \pm 3.4$ & 0.8 \\
DTSQ-WHO score & $29.6 \pm 4.5$ & $30.5 \pm 3.6$ & 0.4 \\
SF-36: & & & \\
Physical Functioning & $87.0 \pm 14.0$ & $90.3 \pm 10.8$ & 0.6 \\
Role Limitations due & $85.7 \pm 30.6$ & $78.3 \pm 37.6$ & 0.5 \\
to Physical Problems & & & \\
Bodily Pain & $74.3 \pm 28.5$ & $77.4 \pm 28.8$ & 0.8 \\
General Health Perception & $51.9 \pm 20.0$ & $51.1 \pm 20.7$ & 1.0 \\
Energy/vitality & $51.4 \pm 20.4$ & $58.1 \pm 18.5$ & 0.4 \\
Social Functioning & $63.3 \pm 23.8$ & $71.7 \pm 21.9$ & 0.3 \\
Role Limitations due & $71.1 \pm 35.3$ & $68.9 \pm 46.2$ & 0.7 \\
to Emotional Problems & & & \\
Mental Health & $65.4 \pm 18.4$ & $68.8 \pm 15.7$ & 0.6 \\
PHYSICAL COMPONENT SCORE & $49.9 \pm 7.7$ & $49.9 \pm 6.5$ & 0.9 \\
MENTAL COMPONENT SCORE & $72.6 \pm 10.4$ & $44.6 \pm 11.3$ & 0.4 \\
\hline
\end{tabular}

Data are expressed as means \pm std; * Wilcoxon test

Supported by Lifescan Inc. Milpitas, United States

\section{8}

Internet diabetic patient management system using short messaging service automatically produced by knowledge matrix system

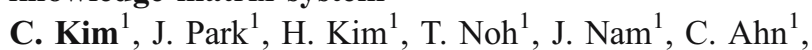
K. Kim ${ }^{1}$, Y. Song ${ }^{2}$, K. Kim ${ }^{3}$, K. Kim ${ }^{3}$, S. Han ${ }^{3}$, H. $\mathrm{Ahn}^{3}$; ${ }^{1}$ Department of Internal Medicine, Yongdong Severance Hospital, Seoul, ${ }^{2}$ Department of Internal Medicine, National Health Insurance Corporation Ilsan Hospital, Goyang, ${ }^{3}$ Ubquitous Healthcare team, ISU UBCare Co., Ltd., Seoul, Republic of Korea.
Background and Aims: Several internet-based diabetic management systems have been developed, and through these systems, physicians are able to manage a lot of patients wherever internet is available. However, in previous systems, it took a lot of time for physicians to look over each patient's data, formulate an appropriate message to each patient, and send it to the patient. In our study, we developed a knowledge matrix system based on Korea Staged Diabetes Management Guideline, which is used to formulate the appropriate message through an automated algorithm, and did a clinical trial to see the effectiveness of this system in the management of diabetic patients.

Materials and Methods: We developed a knowledge matrix system for proper diet and exercise for diabetic patients based on Korea Staged Diabetes Management Guideline. Also, we invented a device that has dual function of blood glucose meter and pedometer. By simply connecting this device to the patient's cellular phone, the patient's measured data is automatically sent to his/her personal data sheet on the internet web site (http://yds. healthkorea.net). In addition, patients are to record the amount and type of food they have taken on the internet. When these data are sent to the main system, our knowledge matrix system automatically composes a message that corresponds to the patient's current status concerning diet and exercise, and this message is sent to each patient after the physician confirms this message on line. Therefore, the patient receives a regular medical recommendation through the cellular phone. And, we applied this system for 6 weeks in 32 diabetic patients who visited our hospital from January to March, 2006. Then, we compared fasting and postprandial blood glucose levels and $\mathrm{HbA} 1 \mathrm{c}$ before and after 6 week trial.

Results: After 6 week trial of this Internet diabetic patient management system, the daily amount of exercise was significantly increased, and fasting and postprandial blood glucose levels were significantly decreased $(133.87 \pm 40.97$ to $121.37 \pm 26.80 \mathrm{mg} / \mathrm{dL}, p=0.019 ; 184.62 \pm 38.03$ to $144.31 \pm$ $32.51 \mathrm{mg} / \mathrm{dL}, p=0.000$, respectively). Moreover, HbA1c level was improved from $7.88 \pm 0.92$ to $7.28 \pm 0.59 \%$ ( $p=0.041$ ), and hypoglycemic event was not reported during the trial.

Conclusion: We propose this internet diabetic patient management using short messaging service automatically produced by knowledge matrix system' as a new tool for communication between diabetes care group and patients.

0909

Locus of control in patients with type 2 and 1 diabetes after long-term management by group care M. Trento ${ }^{1}$, M. Tomelini ${ }^{1}$, E. Borgo ${ }^{1}$, P. Passera ${ }^{1}$, V. Miselli ${ }^{2}$, M. Tomalino ${ }^{1}$, F. Cavallo ${ }^{3}$, M. Porta ${ }^{1}$; 
${ }^{1}$ Internal Medicine, University of Turin, ${ }^{2}$ Internal Medicine, Scandiano Hospital, Scandiano, ${ }^{3}$ Public Health and Microbiology, University of Turin, Italy.

Background: The locus of control describes the relationship between patients' behaviour and various daily life situations. We have developed and validated a model to manage type 2 and type 1 diabetes by systemic group education (Group Care $=$ GC) which, over 5 years, improved health conducts, knowledge of diabetes and quality of life in all patients but improved clinical variables only in type 2 patients.

Aims: to investigate the locus of control in patients with type 2 and 1 diabetes followed for at least 5 years by GC, compared with control patients followed by traditional one-to-one care.

Methods: Two questionnaires were administered to 83 patients followed by GC (56 Type 2 and 27 Type 1) and 79 controls (51 Type 2 and 28 Type 1): the Peyrot and Rubin tool, specific for diabetes, and the Wallston and Wallston, generic for chronic diseases. Both questionnaires include 18 items assessing 3 areas: Internal Control of disease, the role of Chance in changing the disease, trust in Others (family members, health operators). The mean scores in each test for the two groups and for the different diabetes patients were compared; then a multivariate model was fitted, where the score of each test represented the dependent variable and group care, type of diabetes, sex, age and duration of diabetes the independent ones.

Results: with the Peyrot and Rubin questionnaire, GC increased Internal Control independently of type of diabetes $(p<0.001)$, whereas the Wallston and Wallston tool showed lower Internal Control among patients with Type 1 diabetes. Patients with Type 1 and 2 diabetes followed by GC had reduced fatalistic attitudes, as shown by both questionnaires $(\mathrm{p}<0.001)$. Both the Peyrot and Rubin $(\mathrm{p}<0.001)$ and Wallston and Wallston $(p<0.002)$ questionnaires showed lower trust in Others among patients with Type 1 diabetes, which was not modified by GC. The differences of both questionnaires associated with GC were independent of sex, age and duration of diabetes.

Conclusions: Patients with type 1 diabetes may have lower Internal Control and trust in family and health operators than those with type 2. GC may increase Internal Control and reduce fatalistic attitudes without modifying trust in Others in patients with both type 2 and 1 diabetes. Supported by Compagnia di San Paolo, Turin, Italy

0910

\section{Lifestyle intervention: comparison among different strategies}

N. Visalli, S. Abbruzzese, M. Altomare, S. Carletti, C. Suraci, S. Leotta;

Diabetologia, Hospital S.Pertini, Rome, Italy.
Background and Aims: The ADA recommends assessment of self-management skills and knowledge of diabetes at least annually, and the provision or encouragement of continuing diabetes education.Educational techniques have shifted from educational presentations to interventions involving "empowerment" with patient participation and collaboration. The objective of this study was to compare the efficacy on glycaemic control of different educational strategies in obese adults with type 2 diabetes. We focused our behavioural intervention on weight loss and physical activity.

Materials and Methods: 152 obese T2DM patients (90M,62F), duration of disease yrs 9,5 $\pm 8,5$, age $63,4 \pm$ $11,3 \mathrm{yrs}$, were randomly assigned to one of three intervention groups 1st group: received a short, written leaflet given by doctor. Every month a physician visits patients and gives reinforcing compliant message on life style. 2nd group: received individual dietary instructions by a dietitian. Every 15 days, a dietitian meets the patient to individualize the dietary prescription. 3rd group: received group counselling for behaviour modifications to help make healthy food choices and understanding how food affects blood glucose level. Patients learn different meal-planning approaches (pyramid and exchange list). They meet the diabetology team every two week for 4 months. Glycaemic control, lipid levels, body weight, knowledge about diabetes, medication requirements, and symptoms were monitored during the 4 months.

Results: At the end of 4 months, there were no significant reductions in total cholesterol, and low-density lipoprotein cholesterol (LDL-C) values in the 3 intervention groups. In the third group there was a significant weight loss $(p=0,01)$, a significant improvement in glycosylated haemoglobin $(p=0,01)$. In the third group there was a higher number of correct answers about the knowledge of diabetes.

Conclusion: The educational strategy with creation of motivation for proper diet and exercise was effective not only in weight reduction but also in better control of diabetes and in a reduction of OHA usage.

\section{1}

Employer-based diabetes disease management: assessing the health outcome and economic benefits J. Marquess ${ }^{1}$, W. Valentine ${ }^{2}$, D. Cobden ${ }^{3}$, A. Palmer $^{2}$, S. Roze ${ }^{2}$;

${ }^{1}$ Institute for Wellness and Education Inc., Woodstock, United States, ${ }^{2}$ Health Economics, CORE - Center for Outcomes Research, Binningen, Switzerland, ${ }^{3}$ Novo Nordisk Inc., Princeton, United States.

Background and Aims: Disease management initiatives aimed at improving health outcomes for diabetes patients 
through enhanced self-management education and disease awareness may help reduce the growing economic burden impacting employers and health insurers. To this end, programs utilizing face-to-face, targeted, and sustained measures provided by trained caregivers with knowledge of a patient's history have been shown to reduce hyperglycemia, cholesterol and blood pressure among employee populations. The goal of this analysis was to estimate the potential for clinical benefits and cost savings through implementing a workplace diabetes wellness plan.

Materials and Methods: Clinical parameters and patient demographics were derived from results observed among a cohort of 47 patients completing a Diabetes Intensive Management Program at 9 employer groups in the US. Patients were evaluated at baseline and end-of-study for HbAlc, diabetes knowledge, medication adherence and blood glucose monitoring compliance. Those at high risk for or with confirmed diabetes were enrolled in an intensive 1-on-1 education program, with access to several interactive learning sessions. Patients' antidiabetic medication regimens were not altered during the study period: $74.5 \%$ received orals only, $6.4 \%$ insulin only, $8.5 \%$ insulin plus orals, and $10.6 \%$ did not take medication. A validated and peer-reviewed health economic model was used to project long-term clinical and cost outcomes for intensive management versus those modelled not to receive intervention based on the results of this disease management program. The model utilizes Markov structure and Monte Carlo simulation techniques to simulate the progression of diabetes-related complications. Life expectancy (LE), quality-adjusted life expectancy (QALE), incidence rates of complications and total costs were projected over a lifetime horizon. A societal perspective was adopted, with direct costs (treatment and complication) and indirect costs (workplace productivity) accounted. Costs and clinical outcomes were discounted at 3\% per annum.

Results: Patients receiving intensive disease management training (mean period of 9 months) considerably improved glycemic control, LDL cholesterol and BP (absolute HbA1c: $-0.5 \%$; LDL: $-33 \mathrm{mg} / \mathrm{dL}$; BP: $140 / 86$ to $124 /$ $83 \mathrm{~mm} / \mathrm{Hg}$ ). Such benefits were projected to increase LE by 0.63 years and QALE by 0.52 quality-adjusted life years compared to non-intervention. Projected cumulative incidence rates of complications were lower for those on intensive management, including $14.6 \%$ less diabetic retinopathy, $24.9 \%$ less end-stage renal disease, $10.9 \%$ less neuropathy and $9.3 \%$ less myocardial infarction events. Lifetime direct and indirect cost savings were projected to be $\$ 4,975$ and $\$ 14,347$ per patient, respectively, (\$552 total savings per patient per year) for intensive versus noninterventional management.

Conclusion: These data illustrate the potential for clinical and economic benefits through implementing an intensive diabetes management program in the workplace. Sustained intensive training on nutritional, educational, and behavioral modification has the potential to save costs and improve patient quality of life, with benefits gained by employees, employers and health insurance groups.

Supported by Novo Nordisk Inc.

0912

Beyond good intentions: a proactive self-management intervention for patients recently diagnosed with type 2 diabetes during the ADDITION-Netherlands study

B. Thoolen ${ }^{1}$, D. de Ridder ${ }^{1}$, J. Bensing ${ }^{2}$, K. Gorter ${ }^{3}$, G. Rutten ${ }^{3}$;

${ }^{1}$ Health Psychology, Utrecht University, ${ }^{2}$ Netherlands Institute for Health Services Research, Nivel, Utrecht, ${ }^{3}$ Julius Center for Health Sciences and Primary Care, Utrecht University Medical Center, The Netherlands.

Background and Aims: to evaluate the effectiveness of a behavioral intervention for screen-detected patients with type-2 diabetes (DM2) on top of and apart from an intensive pharmacological intervention. The theoretical background of this approach lies in the concept of proactive coping, which comprises the elements of anticipation, timing and planning of adequate behavior to deal with future threatening situations.

Materials and Methods: Patients were recruited from the Dutch ADDITION study, a screening trial which examines the effects of an intensive multi-factorial pharmacological intervention on cardiovascular risk factors in screen-detected DM2 patients. Participants were subsequently randomized to a usual care or experimental condition in which they received the course "Beyond good intentions". Both groups were followed for one year, assessing psychological outcomes (proactive coping, self-efficacy, diabetes distress and selfcare behavior) and medical outcomes (weight, bloodpressure, Hba1c) at baseline (T1), at 3 months (T2) and at one year (T3). The final sample included 102 control patients and 78 experimental patients. A $2 \times 2$ factorial design was employed to evaluate the behavioral intervention (experimental versus usual care) nested within the pharmacological intervention (intensive versus usual care).

Results: Compared to controls, course participants improved significantly with regard to proactive coping, self-efficacy and self-care between T1 and T2 $(\mathrm{p}<0.01)$ and they were able to maintain these changes at T2 $(\mathrm{p}<0.05)$. Participants profited equally from the course, regardless of their medical treatment condition. The full set of medical outcomes data is expected in April 2006 but initial analyses of weight change at T2 reveals that patients in the intervention condition lost significantly more weight than controls $(-2.0$ versus $+0.5 \mathrm{~kg})$. 
Conclusion: An intervention based on proactive coping is effective in helping newly diagnosed patients to manage their diabetes and it appears also to be feasible, regardless of the intensity of treatment that patients are receiving. Proactive coping theory is relevant to self-management in that it helps patients both initiate and maintain self-care behavior.

Supported by Diabetes Fonds

\section{3}

Public awareness of the importance of cardiovascular risk factors characteristic for the metabolic syndrome T. Hidvégi ${ }^{1}$, J. Nádas ${ }^{2}$, Z. Putz ${ }^{2}$, G. Jermendy ${ }^{2}$;

${ }^{1}$ Metabolism\&Diabetes, Petz County Teaching Hospital, Gyór, ${ }^{2} 3$ rd Medical Department, Bajcsy-Zsilinszky Hospital, Budapest, Hungary.

Background and Aims: The epidemic of the metabolic syndrome poses a major challenge throughout the world. Despite the current debate in the literature, the metabolic syndrome offers a simple public health concept and an easily identified starting point for clinical interventions known to reduce risk for the obesity-related type 2 diabetes and cardiovascular diseases. To prevent the metabolic syndrome, a wide social collaboration is needed and, therefore, the public awareness of the metabolic syndrome is of great importance.

Materials and Methods: To evaluate the public awareness of the metabolic syndrome, a cross-sectional survey was performed among randomly selected adult subjects aged 35 years or more in Hungary. Simple questionnaires were used by representatives of a communication bureau in 20 cities. People on the street were asked to stop for a moment to answer 14 written questions voluntarily and anonymously. A total of 1001 questionnaires (601 women, 400 men with different professions; 335 aged 35-44 years, 333 aged 4554 years and 333 aged 55 years or more) were summarised. Results: The term of the metabolic syndrome was known by 286 subjects (29\%). In this group, subjects were informed about the syndrome from acquaintances (40\%), media (29\%), physicians $(26 \%)$ or others $(5 \%)$. In the total cohort $(n=1001)$ clinical abnormalities were reported as follows: hypertension $323(32 \%)$, obesity 206 (21\%), diabetes mellitus 113 (11\%) and lipid abnormalities 182 (18\%) subjects. Forty-eight percent of the total cohort was regularly cared by general practitioners. Medical information about the metabolic syndrome provided by general practitioners were considered insufficient and superficial by more than half $(52 \%)$ of the subjects questioned. The severe consequences of the clinical abnormalities were known by the majority of subjects. In a scale from 1 (neglected) to 5 (considered seriously) the mean score for hypertension was
4.19, it was 3.92 for lipid abnormalities, 4.35 for diabetes and 3.71 for obesity. As for the metabolic syndrome itself, its significance was well known (mean score 4.6 using the same scoring system). Unfortunately, 19\% of subjects reported neither regular physical activity nor keeping a healthy diet.

Conclusion: In order to increase the public awareness, extensive public information campaigns should be implemented by health care providers as well as by written and electronic media focusing on the metabolic syndrome.

0914

Understanding of diabetes prevention studies: questionnaire survey of professionals in diabetes care

I. Mühlhauser ${ }^{1}$, J. Kasper ${ }^{1}$, G. Meyer ${ }^{1}$, FEND $^{2}$;

${ }^{1}$ Health Sciences and Education, University of Hamburg, Germany, ${ }^{2}$ Federation of European Nurses in Diabetes, FEND, London, United Kingdom.

Background and Aims: Framing of data is a well recognised cause of misconceptions about efficacy of health interventions. Diabetes prevention studies are reported to have shown reductions of diabetes risk by up to $60 \%$. Since the underlying metabolic changes are small the clinical significance of this effect may be overestimated. The present survey explores the extent to which different formats of presenting study results may influence diabetes health care professionals' perceptions of the importance of intervention effects on diabetes risk.

Materials and Methods: Participants of three European diabetes conferences in autumn 2005 (160 nurse educators, 112 diabetologists or physicians with a special interest in diabetes, 27 other professionals) were presented a questionnaire that included nine items with different presentations of results of three diabetes prevention studies (Finish Diabetes Prevention Study, Diabetes Prevention Program, Women's Health Initiative). Results were presented as relative risk reductions, proportion of subjects with diabetes, fasting glucose values, HbAlc values, probability of remaining free of diabetes, and graphs with truncated scale or scale with complete range from 0.0 to 1.0. Almost identical wording was used in all items: "Looking at this result (these results, this graph), what is your impression about the effect of this (preventive) intervention on diabetes risk?" Participants were asked to mark their ratings as either "very important $(++)$ ", “important $(+)$ ", "not very important $(-)$ " or "not important at all (-)". In addition, the UKPDS' main results on the primary endpoint were used to evaluate understanding of natural frequencies.

Results: Participation rate was 96\%. Effects were interpreted as important or very important by $92 \%(255 / 276)$ when results were presented as proportions of subjects with 
diabetes (14\% intervention group, $29 \%$ control group), by $87 \%(248 / 285)$ when communicated as a risk reduction of $57 \%$, by $39 \%(110 / 284)$ when the corresponding fasting plasma glucose values were presented (mean difference $0.3 \mathrm{mmol} / \mathrm{L})$, and by $18 \%(52 / 283)$ when glycosylated haemoglobin values were used $(6.0 \%$ versus $6.1 \%)$. About $73 \%(202 / 278)$ rated the study effect on diabetes risk as more important when the graph of the original publication with a truncated scale narrowed to the range of results $(3.5 \%$ versus $4.2 \%)$ was presented rather than a graph with a scale ranging from 0.0 to 1.0. Corresponding results of the 3 diabetes prevention studies were rated as of identical importance by only $23 \%, 13 \%$, and $16 \%$ of participants, respectively. Understanding of natural frequencies was poor. The intervention effect was substantially overestimated by $47 \%$ of participants. Only 2 to 6 participants answered correctly.

Conclusion: Health care professionals view the benefit of preventive interventions substantially higher when changes in diabetes risk are communicated rather than related glycaemic parameters. Transformation of continuous metabolic data into diagnostic categories may interfere with understanding of study effects.

\section{5}

Basic characteristics and treatment regimens in people with type 1 diabetes in the international diabetes management practices study (IDMPS)

S. Ferreira ${ }^{1}$, A. Ramachandran ${ }^{2}$, P. Aschner ${ }^{3}$, J. Chan ${ }^{4}$, N. Hancu ${ }^{5}$, S.-H. Baik ${ }^{6}$, H. Ilkova ${ }^{7}$, S. Twigg ${ }^{8}$,

J.-J. Gagliardino 9 ;

${ }^{1}$ Preventive Medicine Department, Sao Paulo Medical School, Brazil, ${ }^{2}$ Diabetes Research Centre, M.V. Hospital for Diabetes, Chennai, India, ${ }^{3}$ Endocrinology Unit, Unidad Médica de Diagnóstico, Bogotá, Colombia, ${ }^{4}$ Department of Medicine and Therapeutics, Prince of Wales Hospital, Shatin, Hong Kong, ${ }^{5}$ Diabetes Center and Clinic, Iuliu Hatieganu University of Medicine, Cluj-Napoca, Romania, ${ }^{6}$ Department of Internal Medicine, Korea University Guro Hospital, Seoul, Republic of Korea, ${ }^{7}$ Department of Internal Medicine, Istanbul University Cerrahpasa Medical Faculty, Istanbul, Turkey, ${ }^{8}$ Department of Medicine, University of Sydney, Australia, ${ }^{9}$ Faculty of Medical Sciences, National University of La Plata, Argentina.

Background and Aims: This multinational observational study is being performed to gather data that reflect current practices in the management of people with diabetes in different areas of the world and to evaluate compliance with international guidelines.
Materials and Methods: Data are being collected from type 1 diabetic patients ( $\geq 18$ years) in 1 -year cycles (a 2week cross-sectional period followed by a 9-month longitudinal period) for 5 years.

Results: Data from the first cross-sectional period are available from 1327 people (51\% men) in 11 countries in Eastern Europe, Asia, and Latin America. The mean age was $36 \pm 13$ years, mean BMI $23 \pm 4 \mathrm{~kg} / \mathrm{m}^{2}$ (for both men and women) and mean disease duration 11 years. Mean $\mathrm{HbA}_{1 \mathrm{c}}$ was $8.2 \pm 1.96 \%$ and $27 \%$ of people had an $\mathrm{HbA}_{1 \mathrm{c}}<7 \%$. Mean FBG (laboratory) was $161 \pm 75 \mathrm{mg} / \mathrm{dL}$. Mean systolic/ diastolic blood pressure was $122 / 75 \mathrm{mmHg}$. Target treatment levels were achieved for LDL $(<100 \mathrm{mg} / \mathrm{dL})$ in $41 \%$ of people, for HDL ( $>40 \mathrm{mg} / \mathrm{dL})$ in $81 \%$, and for triglycerides $(<150 \mathrm{mg} / \mathrm{dL})$ in $72 \%$ of people. A basal+prandial insulin regimen was used by $51 \%$ of people with an average daily dose of $52 \mathrm{IU}$ insulin and a mean $\mathrm{HbA}_{1 \mathrm{c}}$ of $8.1 \%$. Premixed insulin was used by $27 \%$ of people with an average dose of $38 \mathrm{IU}$ insulin and a mean $\mathrm{HbA}_{1 \mathrm{c}}$ of $8.5 \%$. Only $9 \%$ of people were using a basal insulin alone (average dose $31 \mathrm{IU}$ insulin and mean $\mathrm{HbA}_{1 \mathrm{c}} 8.5 \%$ ) and $4 \%$ a prandial insulin alone (average dose $29 \mathrm{IU}$ insulin and mean $\mathrm{HbA}_{1 \mathrm{c}}$ 7.6\%).

Conclusion: As can be seen, despite the findings of the DCCT and the recommendations of the ADA, the majority of people recruited have not achieved the recommended treatment goals for $\mathrm{HbA}_{1 \mathrm{c}}$ and FBG. It is thus apparent that more efficient guidance and education be made available to improve treatment outcomes in type 1 diabetic patients world wide.

Supported by Sanofi-Aventis

0916

Lipid profile modification is the most effective cardiovascular intervention in the Steno-2 Study J. D. Lee ${ }^{1}$, O. Pedersen ${ }^{2}$, H.-H. Parving ${ }^{2}$, P. Gaede ${ }^{2}$, A. Venkataraman ${ }^{1}$, J. R. Morrissey ${ }^{3}$, V. Patel ${ }^{3}$; ${ }^{1}$ Cardiology, George Eliot Hospital NHS Trust, Nuneaton, United Kingdom, ${ }^{2}$ Steno Diabetes Centre, Copenhagen, Denmark, ${ }^{3}$ Diabetes, George Eliot Hospital NHS Trust, Nuneaton, United Kingdom.

Background and Aims: The majority of trials studying the impact of cardiovascular risk factor modification in diabetes care have been limited to the intervention of only one risk factor. The landmark Steno-2 Study (S2S) demonstrated the benfits of a multifactorial approach to reducing cardiovascular events and mortality in a population of patients with type 2 diabetes mellitus. We employed cardiovascular risk calculators in a mathematical model to determine the most effective risk factor intervention in the S2S. 
Materials and Methods: 10-year absolute coronary artery disease risk using the Framingham based risk function (FRF) and the UKPDS Risk Engine (UKPDS) was calculated from cardiovascular data at baseline (T0) and the end of the S2S (Tfu). Instead of averaging the risk scores of individual subjects at these times, the average of each risk factor in the subgroups of male and female smokers, and male and female non-smokers were inputted into each risk estimator. Separate calculations were made for these subgroups as "average smoking" and "average sex" does not exist. To estimate the contribution made by improvement in a paricular risk factor, its average value was inputted at $\mathrm{T} 0$, then the exercise was repeated with its average value at $\mathrm{Tfu}$, leaving the other risk variables inchanged. As ageing alone increases risk, to make a valid comparison an age-adjusted risk score was calculated by using risk variables at $\mathrm{T} 0$ and age at Tfu. Knowing the proportions of individuals in these subgroups allows the estimation of overall risk score improvement due to improvement in this particular risk factor. This improvement was expressed as a percentage of the total risk reduction between $\mathrm{T} 0$ and $\mathrm{Tfu}$.

Results: Conventional arm using UKPDS - total risk reduction $38.79-33.86=4.93 \%$. Percentage contribution of risk factors to this reduction:

- HDL increase $51.67 \%$

- Total cholesterol rection (TC) $13.82 \%$

- Stopping smoking $12.27 \%$

- Systolic blood pressure reduction (SBP) 5.64\%

- Glycaemic control (HbA1c) 0\%

Conventional arm using FRF - total risk reduction 29.44$24.32=5.11 \%$. Percentage contribution of risk factors to this reduction:

- HDL $61.47 \% \%$

- Stopping smoking $14.68 \%$

- TC $12.11 \%$

- SBP $11.74 \%$

Intensive arm of using UKPDS - total risk reduction 36.08 $21.39=14.69 \%$. Percentage contribution of risk factors to this risk reduction:

- TC $46.51 \%$

- HDL $27.65 \%$

- HbA1c $10.77 \%$

- SBP 9.95\%

- Stopping smoking $5.12 \%$

Intensive arm sing FRF - total risk reduction 27.25$15.48=11.77 \%$. Percentage contribution of risk factors to this reduction:

- TC $45.41 \%$

- HDL $26.06 \%$

- SBP $22.59 \%$

- Stopping smoking $5.94 \%$
Conclusion: Lipid profile modification appeared to be the most effective cardiovascular intervention in both treatment arms of S2S.

\section{PS 75}

\section{Diabetes in childhood}

0917

Intensified insulin therapy in children and adolescents with type 1 diabetes mellitus - comparison of treatment with regular versus analogue insulin

G. Kramer, R. Schiel;

Department of Diabetes and Metabolic Diseases, Inselklinik Heringsdorf, Seebad Heringsdorf, Germany.

Background and Aims: Today, more than two third of all children and adolescents with intensified insulin therapy were treated with short and long acting insulin analogues. However, up to the present, in children and adolescents after starting treatment with regular and NPH insulin, the change to insulin analogues is mostly the second step. Hence, it was the aim of the trial to analyse the quality of diabetes control, the incidence of acute complications, quality of life and possible benefits of treatment with insulin analogues versus regular and NPH insulin.

Materials and Methods: Over a period of 23 months all patients $(n=350)$ with type 1 diabetes mellitus and intensified insulin therapy admitted to our hospital were included in the trial and underwent clinical examination, analysis of selfmonitoring, frequency of acute complications and an extensive test battery (including standardised analyses of diabetes self-management, handling, diabetes-related knowledge, psychological status, well-being and motivation).

Results: Mean age of patients was $12.3 \pm 3.8$ ys, diabetes duration was $4.3 \pm 3.5$ ys and $\mathrm{HbAlc}$ was $8.0 \pm 1.4 \%$, with only $25.7 \%$ (90/350 patients) having levels $\leq 7.0 \%$ (DCA 2000 , mean normal $4.0-6.4 \%$ ) (number of severe hypoglycaemia [glucose i.v., glucagons injection] 5). Out of the total group 243 patients $(69.4 \%)$ were treated with insulin analogues. In comparison to patients with regular and NPH insulin, patients treated with insulin analogues were older $(12.7 \pm 3.7 \mathrm{vs} 11.3 \pm 3.7 \mathrm{ys}, \mathrm{p}=0.001)$, had a longer diabetes duration ( $4.6 \pm 3.5$ vs $3.7 \pm 3.4 \mathrm{ys}, \mathrm{p}=0.021)$ and a higher BMI $\left(20.5 \pm 4.2\right.$ vs $\left.19.2 \pm 3.8 \mathrm{~kg} / \mathrm{m}^{2}, \mathrm{p}=0.003\right)$. There were no differences between the groups in respect of $\mathrm{HbA} 1 \mathrm{c}$ $(8.0 \pm 1.1$ vs $7.8 \pm 1.3, \mathrm{p}=0.2)$, insulin dosage $(0.8 \pm 0.3$ vs $0.8 . \pm 0.3 \mathrm{I} . \mathrm{U} . \mathrm{kg}$ bd wt, $\mathrm{p}=0.72$ ) and the number of mild hypoglycaemia $(10.8 \pm 7.0$ vs $10.5 \pm 5.9, \mathrm{p}=0.7)$. There was a difference between the groups regarding diabetes-related knowledge (scores: $67.0 \pm 16.3$ vs $61.4 \pm 18.4$ points, 


\begin{tabular}{ccccccccc}
\hline $\begin{array}{c}\text { Type of } \\
\text { basal } \\
\text { insulin }\end{array}$ & $\begin{array}{c}\text { HbAlc } \\
\text { after } \\
4 \mathrm{mths}\end{array}$ & $\begin{array}{c}\text { HbA1c } \\
\text { after } \\
6 \%]\end{array}$ & $\begin{array}{c}\text { Mean daily BG Mean daily BG } \\
\text { after } 4 \mathrm{mths} \\
{[\%]}\end{array}$ & $\begin{array}{c}\text { [mg/dl] } \\
\text { after } 6 \mathrm{mths} \\
{[\mathrm{mg} / \mathrm{dl}]}\end{array}$ & $\begin{array}{c}\text { Mean BG before } \\
\text { breakfast, after } \\
4 \mathrm{mths}[\mathrm{mg} / \mathrm{dl}]\end{array}$ & $\begin{array}{c}\text { Mean BG before } \\
\text { breakfast, after } \\
6 \mathrm{mths}[\mathrm{mg} / \mathrm{dl}]\end{array}$ & $\begin{array}{c}\text { Total DID } \\
\text { after } 6 \mathrm{mths} \\
{[\mathrm{U} / \mathrm{kg} / \mathrm{day}]}\end{array}$ & $\begin{array}{c}\text { Glargine or NPH } \\
\text { dose after } 6 \mathrm{mths} \\
{[\mathrm{U} / \mathrm{kg} / \mathrm{day}]}\end{array}$ \\
\hline Glargine & $7,1 \pm 0,6$ & $7,6 \pm 0,9$ & $153 \pm 22$ & $161 \pm 23$ & $148 \pm 23$ & $143 \pm 25$ & $0,72 \pm 0,25$ & $0,35 \pm 0,13$ \\
NPH & $7,8 \pm 0,9$ & $7,7 \pm 0,9$ & $160 \pm 15$ & $165 \pm 20$ & $155 \pm 38$ & $168 \pm 26$ & $0,80 \pm 0,11$ & $0,44 \pm 0,11$ \\
P & 0,03 & 0,76 & 0,47 & 0,66 & 0,65 & 0,03 & 0,34 & 0,07 \\
$95 \%$ CI of & 0,07 to & - & - & - & - & 2,37 to 46,23 & - & $-0,01$ to 0,18
\end{tabular}

$\mathrm{p}=0.02$ ). Analysing reasons for changing therapy from therapy with regular and NPH insulin to short and/or longacting insulin analogues the following indications were overt (more than $100 \%$ because in some patients there was more than one indication): 1 . improvement of the quality of diabetes control $(71 \%), 2$. instability of blood glucose profiles $(48 \%)$, 3. Dawn-Phenomenon (10\%) 3. frequent hypoglycaemia $(5 \%)$.

Conclusion: At present, in Germany most of the children and adolescents with type 1 diabetes mellitus and intensified insulin admitted to hospital are under treatment with insulin analogues. There were no differences between patients treated with regular/NPH and short and/or long acting insulin analogues in respect of quality of diabetes control and the incidence of acute complications. Hence, it seems, that treatment with insulin analogues is the second step. Mostly patients changed treatment strategy due to severe problems (improvement of quality of diabetes control, instability of blood glucose profiles, Dawn-Phenomenon or/and frequent hypoglycaemia) In children and adolescents insulin analogues seems to be a very worthful tool to overcome specific problems. They should used individually.

\section{8}

A randomized cross-over trial comparing glargine and NPH insulin in preadolescent type 1 diabetic children B. Mianowska, A. Szadkowska, E. Czerniawska, I. Pietrzak, J. Bodalski;

Department of Pediatrics, Medical University, Lodz, Poland.

Background and Aims: Glargine is a long-acting insulin analog with flat action profile. Although it is more and more widely used as basal insulin replacement therapy, also in young diabetics, results of scrupulous trials conducted in children are sparse. The aim of this cross-over study was to compare glycemic control on Glargine and on NPH insulin (Humulin $\mathrm{N}$ ) in type 1 preadolescent children.

Materials and Methods: 14 children ( 7 boys and 7 girls) aged 6-12 years (mean 10,4 years) with diabetes duration of
2-11 years (mean 6,9 years) were included into the study. The study protocol comprised for each patient (i) a 6 months' period of therapy with NPH insulin as basal insulin and (ii) a 6 months' period of therapy with Glargine as basal insulin. Patients were randomized to start either with Glargine or with NPH insulin. Patients used Humalog and/or rapid acting human insulin (Humulin R) for meal and correction boluses during all the study period. All patients measured blood glucose regularly (at least 3-4 times a day). They used the same type of memory provided blood glucose meters, that cooperated with the outpatients' clinic's computers. Results of HbAlc and 2 weeks' mean blood glucose (BG) measurements (all day and before breakfast), data concerning daily insulin doses (DID), insulin injections at 3.00-4.00 AM, weight and height as well as hypoglycemia and ketoacidosis history were recorded 4 and 6 months after starting each of two 6 month's periods of the cross-over study protocol (i.e. either on Glargine or on NPH insulin). T-test was used for statistical analysis of data.

Results: Mean values and standard deviations for main variables after 4 months and after 6 months on Glargine and on NPH respectively are presented in the Table below. Mean BMI after 6 months was $18,7 \mathrm{~kg} / \mathrm{m} 2$ on Glargine and $18,5 \mathrm{~kg} / \mathrm{m} 2$ on NPH (NS). Three patients on NPH insulin but none on Glargine were injected insulin at 3.00-4.00 AM. No severe hypoglycemia or ketoacidosis occurred during the study period.

Conclusion: In preadolescent children Glargine constitues an useful and safe alternative to NPH insulin providing better early morning and (at least for a short-term) good total glycemic control, not increasing the risk of severe hypoglycemia or excessive weight gain. As such, it should be reimbursed by national health services.

\section{9}

Insulin glargine (GLAR) vs intermediate-acting insulin in adolescents with type 1 diabetes (T1DM) using multiple daily injection (MDI) therapy

H. P. Chase ${ }^{1}$, S. Arslanian ${ }^{2}$, N. White ${ }^{3}$, K. Usiskin ${ }^{4}$, W. Tamborlane ${ }^{5}$; 
${ }^{1}$ Barbara Davis Ctr, U of CO, Aurora, ${ }^{2}$ Peds, Children's Hosp of Pittsburgh, Pittsburgh, ${ }^{3}$ Peds, Wash U School of Med, St. Louis, ${ }^{4}$ sanofi-aventis U.S., Bridgewater, ${ }^{5}$ Dept Ped \& Children's Clin Res Ctr, Yale U School of Med, New Haven, United States.

Background and Aims: The Diabetes Control and Complications Trial demonstrated that improvements in A1C reduced complications in adults and adolescents with T1DM. However, improved A1C was also associated with increased hypoglycemia. Increased variability in blood glucose (BG) may contribute to the inability to reduce $\mathrm{A} 1 \mathrm{C}$ without increased hypoglycemia. The aim of the current study was to compare efficacy (A1C, hypoglycemia, and BG variability) of once-daily GLAR vs twice-daily intermediate-acting insulin (neutral protamine Hagedorn [NPH] or lente) as basal therapy in pediatric pts with T1DM treated with an MDI regimen including premeal rapid-acting analog (lispro).

Materials and Methods: This was a 24-wk, multicenter, randomized, open-label, parallel-group study of 175 adolescents with T1DM (GLAR, $n=85$; NPH/lente, $n=90$ ) who had $\mathrm{A} 1 \mathrm{C} \geq 7.0 \%$ and $\leq 9.5 \%$ on any insulin regimen. Basal doses began at $\sim 40-50 \%$ of total daily dose and were titrated to target fasting BG ( $\geq 70$ and $\leq 100 \mathrm{mg} / \mathrm{dL})$. In a subset of pts $(n=90)$, BG variability was assessed using the Continuous Glucose Monitoring System (Medtronic Mini$\operatorname{Med}^{\mathbb{B}}$ ). Measures included SDs of BG values and measures of glycemic excursion, such as MAGE (mean amplitude of glycemic excursion) and $\mathrm{M}$ value (?/N absval[10log $\{$ SMBG $/ 120\}]^{3}+[\{$ Max-MinSMBG/20 $\}]$ ).

Results: Treatment groups had similar demographics: mean age of 13 years; diabetes duration 5 years; body mass index $23 \mathrm{~kg} / \mathrm{m}^{2}$; A1C $7.9 \%$; $82 \%$ of pts were white. There was a small reduction in mean $\mathrm{A} 1 \mathrm{C}$ from baseline to endpoint in both groups $(\mathrm{GLAR}=-0.18 \%$; NPH/lente $=-0.03 \%)$. However, there was a significant interaction between treatment effect and baseline A1C; GLAR pts with high baseline A1C (mean $\mathrm{A} 1 \mathrm{C}$ of $\geq 7.9 \%$ ) had greater $\mathrm{A} 1 \mathrm{C}$ reduction than $\mathrm{NPH} /$ lente pts (Fig.). Mean daily basal insulin dose at 24 wks was significantly greater $(P=.0064)$ for $\mathrm{NPH} /$ lente $(50.7 \pm 2.1 \mathrm{U})$ vs GLAR $(42.4 \pm 2.1 \mathrm{U})$. Mean total daily insulin dose was similar between groups $(P=\mathrm{NS})$.

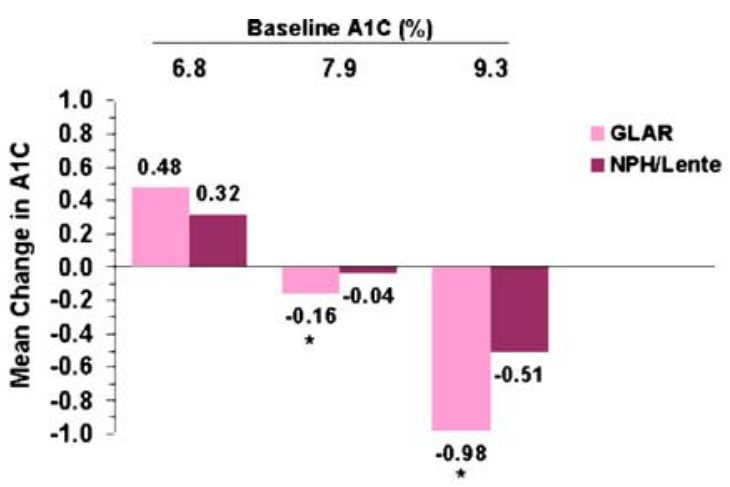

${ }^{*} P<.05$. between groups. Baseline values represent modeled effect of baseline A1C

Number of pts with confirmed clinically relevant hypoglycemia ( $\mathrm{BG}<36 \mathrm{mg} / \mathrm{dL}$ ) [GLAR 28 vs NPH/lente 30] and severe hypoglycemic events (GLAR 9 vs NPH/lente 4) and possibly related adverse events (AEs) was comparable between groups $(P=\mathrm{NS})$. BG variability was significantly less for GLAR vs NPH/lente at 24 wks as demonstrated by $\mathrm{SD}$ and MAGE (SD, -13.4 vs $-2.4, P=.0147$; MAGE, -34.4 vs $-2.5, P=.0055)$. The $\mathrm{M}$ value also tended to be lower with GLAR vs NPH/lente $(P=.0631)$.

Conclusion: These modeled effects show that in adolescents with high baseline A1C values, treatment with GLAR results in greater A1C improvement compared with NPH/ lente despite significantly lower doses of GLAR and comparable rates of hypoglycemia and AEs. Pts treated with GLAR also have less variability in BG.

Supported by sanof-aventis U.S.

0920

The impact of insulin regimens and dose adjustment on glycaemic control in 21 centres

P. G. F. Swift ${ }^{1}$, C. E. de Beaufort ${ }^{2}$, T. Danne ${ }^{3}$,

C. T. Skinner ${ }^{4}$, E. Schoenle ${ }^{5}$

on behalf of the Hvidore Study Group;

${ }^{1}$ Paediatrics, Children's Hospital Leicester Royal Infirmary, United Kingdom, ${ }^{2}$ DECCP, Clinique pediatrique, Luxembourg, ${ }^{3}$ Paediatrics, Kinderkrankenhaus Auf der Bult, Hannover, Germany, ${ }^{4}$ Dept of Psychology, University of Southampton, United Kingdom, ${ }^{5}$ Paediatrics, University Childrens Hospital, Zurich, Switzerland.

Objective: To evaluate the impact of different insulin regimens and insulin adjustment strategies on metabolic control in a large international cohort of adolescents with type 1 diabetes.

Methodology: Questionnaires were completed by adolescents and their parents/carers attending sequentially in 21 international centres. Questionnaires investigated age, gen- 
der, diabetes duration, insulin regimens, insulin adjustments and glycaemic targets. HbA1c (DCCT adjusted) was measured centrally.

Results: Questionnaires were completed by 2062 adolescents (age $14.4 \mathrm{y}^{+/}-2.3 \mathrm{y}$, male $50.6 \%$, diabetes duration $6.1 \mathrm{y} \pm 3.5)$.

Different insulin regimens were used both within and between the 21 different centres. Mean $\mathrm{HbA} 1 \mathrm{c}=8.2 \%$ SD 1.4 , with significant influence of age $(\mathrm{r}=0.1 ; \mathrm{p}<0.001)$, gender (females $8.3 \% \pm 1.5$, males $8.1 \% \pm 1.3 ; \mathrm{t}=3.0$; $\mathrm{p}<0.005)$, diabetes duration $(r=0.29 ; \mathrm{p}<0.001)$. Insulin regimens used and analysed were pre-mixed insulin twice daily (7\%), free mixing insulin twice daily (7\%), basal bolus (39\%), pump treatment (15\%). For these four groups there are significant differences between groups $(\mathrm{F}=13.9$; $\mathrm{df}=3$; $\mathrm{p}<0.001)$. Individuals free mixing had significantly better glycaemic control than all other groups (mean $\mathrm{HbAlc}$ 7.6\%; SD 1.3). There was no difference between pump users (HbA1c 8.1\%; SD 1.3) and individuals on basal bolus regimens (HbA1c $8.3 \%$; SD 1.5) with both these groups having significantly lower $\mathrm{HbAlc}$ than individuals on $\mathrm{BD}$ pre-mix regimens (HbAlc $8.6 \%$; SD 1.6). Free mixing was mainly used in two centres and within those centres no difference was observed between the free mixing individuals and the remaining group.

Adolescents stating that they adjust insulin therapy based on blood sugar levels $(\mathrm{F}=4.4 ; \mathrm{p}<0.004)$ and their intended food intake had significantly lower $\mathrm{HbAlc}$ levels than those who do not adjust $(\mathrm{F}=6.0 ; \mathrm{p}<0.001)$ as did those who give extra doses for additional snacks $(\mathrm{F}=3.01 ; \mathrm{p}<0.05)$. Also centres with lower $\mathrm{HbAlc}$ had more individuals making insulin adjustments. Young people who test their blood glucose when managing hypos have better $\mathrm{HbAl} \mathrm{c}$ than those who eat until they feel better $(\mathrm{F}=3.9 ; \mathrm{p}<0.01)$. Both the target for ideal $\mathrm{HbAlc}$ perceived by adolescents and the result they would be happy with in clinic were associated with the actual HbAlc achieved (ideal $\mathrm{r}=0.24$; happy $\mathrm{r}=0.41$ ).

Conclusion: Insulin regimens per se have less impact on metabolic control than adjustment strategies and perceived targets. Further analysis of data in this study will look at which other factors including education are most influential in diabetes management and outcome.

Supported by Novo Nordisk

\section{1}

Quality of care of children and adolescents with type 1 diabetes mellitus in new EU accessing countries (the DEPAC Study)

L. Madacsy ${ }^{1}$, W. Greszczak ${ }^{2}$, J. Michalek ${ }^{3}$, M. Medvescek ${ }^{4}$, A. Norkus ${ }^{5}$, M. Niewada ${ }^{6}$, B. Kaminsky ${ }^{7}$, M. Andel ${ }^{8}$;
${ }^{1}$ I.Dept. of Pediatrics, Semmelweis University, Budapest, Hungary, ${ }^{2}$ Dept. of Diabetology and Nephrology, Klinika Chorób Wewnetrznych, Katowice, Poland, ${ }^{3}$ Dept. of Endocrinology, National Diabetes Center, Lubochna, Slovakia, ${ }^{4}$ Dept. of Diabetes, Klinicni Center Ljubljana, Slovenia, ${ }^{5}$ Dept. of Diabetology, Insitute of Endocrinology, Vilnius, Lithuania, ${ }^{6}$ Dept. of Clinical and Experimental Pharmacology, Warsaw Medical University, Poland, ${ }^{7}$ Dept. of Decision Analysis, Insitute of Econometrics, Poland, ${ }^{8}$ Dept.of Intern. Medicine, University of Prague, Czech Republic.

Background and Aims: According to an IDF-Europe initiative, a baseline evaluation of diabetes care of 11085 diabetic patients has been performed in the Czech Republic, Estonia, Hungary, Latvia, Lithuania, Poland, Slovakia and Slovenia in 2005 (the DEPAC Study). We assessed separately the data of children and adolescents with Type 1 diabetes mellitus.

Materials and Methods: Data of 281 patients, $<18$ yrs of age with Type 1 diabetes mellitus (boys: $48.45 \%$, girls 51.55\%) were analyzed (mean age boys: 13.04 yrs, girls: $12.70 \mathrm{yrs}$ ). The mean time from diagnosis was $5.27 \mathrm{yrs}$. Patients were randomly enrolled. Questionnaires for each patient were completed by a diabetologist. Questions included data about age, sex, duration of diabetes, type of treatment, metabolic control $(\mathrm{HbA} 1 \mathrm{c})$, lipid data (total cholesterol, LDL cholesterol, triglycerides and HDL cholesterol), blood pressure (BP) and data about short and long term complications. Data were analyzed centrally in the University of Warsaw.

Results: The mean $\mathrm{HbAlc}$ was $8.78 \%$, and the percentage of patients with an $\mathrm{HbAlc}<7.0 \%$ was $16.66 \%$. Self monitoring of blood glucose was available in $93.47 \%$ of patients. Human insulin was used for treatment in $67 \%$ and analogue insulin in 57\%.Two third of patients were treated on the basal-bolus regimen, and $28 \%$ of them with an insulin pump (CSII). Targets for total cholesterol levels $(1.1 \mathrm{mmol} / \mathrm{L})$ in $86.57 \%$-; targets for LDL cholesterol $(<2.5 \mathrm{mmol} / \mathrm{L})$ in $62.79 \%$-; and targets for triglyceride levels $(<1.7 \mathrm{mmol} / \mathrm{L})$ were achieved in $90.50 \%$ of patients. The target for systolic BP $(<120 \mathrm{~mm} \mathrm{Hg})$ was achieved in $97.12 \%$-, and for diastolic BP $(<80 \mathrm{mmHg})$ in $98.52 \%$ of patients. The prevalence of severe hypoglycaemia - within the last six months- was $4.47 \%$; and of ketoacidotic coma was $2.41 \%$.We found that the prevalence of non-proliferative diabetic retinopathy to be $2 \%$, and of microalbuminuria to be $4 \%$. The prevalence of diabetic neuropathy was $5 \%$. Unexpectedly, peripheral arterial disease was reported in two boys, and other forms of CHD was found in two girls. The percentage of smokers among adolescents was $9.97 \%$. No significant differences between boys and girls has been found except in the mean HDL cholesterol level and in the percentage of smokers ( $p<0.02$ and $p<0.05$, respectively). 
Conclusions: Despite a good access to insulin and diabetes supplies, the average level of metabolic control and of serum lipids is far from desirable in young diabetics. The results should be analyzed in national health services for the options of improved care and education of patients.

Supported by Novo Nordisk

0922

Adolescents with type 1 diabetes and risky behaviours A. E. Scaramuzza, S. Riboni, A. De Palma, G. Zuccotti; Paediatriacs, Ospedale, Milano, Italy.

Background and Aims: A growing body of literature indicates that adolescents with chronic conditions are as likely, or more likely, to take risky behaviours than their healthy peers. Very few data exists about adolescents with type 1 diabetes mellitus (T1DM). The aim of our study was to assess whether adolescents with T1DM in Northern Italy differ from their healthy peers in risky taking behaviours.

Materials and Methods: Data were drawn from 187 patients attending 4 summer camps, aged 12-20 yrs (mean $14 \pm 2 \mathrm{yrs}$ ) and with a mean disease duration of $7 \pm 5$ yrs (range 1-19 yrs). During the camp each of them filled an anonimous confidential questionnaire to determine the prevalence of sexual behaviour, drug use (tobacco, alcohol, cannabis, cocaine, and synthetic drugs), and only in T1DM group the frequency of mismanagement related to diabetes care. The control group comprised 464 healthy adolescents recruited among high school students. Personal, family and school related variables were analysed to ensure comparatibility between groups. Analysis was carried out separately by gender. Chi-square, Fisher's and Student's tests were used to compare categorical and continuous variables.

Results: The T1DM group showed similar rates of sexual intercourse among males and lower rates among females (34.1\% vs $35.5 \%$, p NS, and $27.3 \%$ vs $41.4 \%, \mathrm{p}<0.05$, respectively). For most studied drugs, T1DM males reported higher rates of use than control group only about tobacco, while females showed similar or higher rates for every drug studied (see table). Among T1DM group, the patients who engaged in risky behaviours showed higher rates in tratment mismanagement $(76 \%$ vs $34 \%, \mathrm{p}<0.01)$.

Conclusion: Adoelscents with T1DM in our sample are as likely, or more likely, to take risky behaviours than their healthy peers, and should receive the same anticipatory guidance, also because this could lead to a worsening of glycemic control.

\section{3}

Factors of low grade inflammation in type 1 diabetes children and adolescents

E. Pankowska, A. Szypowska, M. Lipka, L. Groele; Pediatric Diabetology and Birth Defects, Medical University, Warsaw, Poland.

Background and Aims: Fibrinogen and C-reactive protein are the markers of inflammation and its slightly elevated plasma level in subject with type 2 diabetes indicates a risk for cardiovascular events. Pathogenesis of subclinical inflammation in type 1 diabetes it has still been poorly investigated.

The aim of study was to determine the CRP and fibrinogen plasma level in metabolically stable, free from microangiopathy type 1 young diabetics and analysis of significant factors low grade inflammation.

Materials and Methods: We analyzed 190 type 1 diabetes (92 female/98 male), mean age 11.54 SD $4.13 \mathrm{y}$; mean diabetes duration 4.47 SD 3.96 y $(0,1-17,6)$ mean $\mathrm{HbA1}_{\mathrm{c}} 8.46$ SD 2.18\% (4.9-19). During schedules visits clinical and biochemical parameters: CRP, Fibrinogen, $\mathrm{HbA1}{ }_{\mathrm{c}}$, Total-, LDL-, HDL-cholesterol, trigliceride, Cpeptide, antibody TPO, TG, IgA $\mathrm{EmA}_{2}, \mathrm{IA}_{2}, \mathrm{GAD}$ were assessed. After examinations subjects with severe hypoglycaemia, DKA (ketoacidosis), infections or endocrine diseases were excluded.

Results: $17 \%$ of study population had plasma fibrinogen level above age-related range, this group presents significantly higher C-reactive protein $(0.5 \mathrm{mg} / \mathrm{dl}$ vs 0.167 respectively $\mathrm{p}=0.019$ ), but metabolic control, BMI, diabetes duration and age was comparable to group with normal fibrinogen range. After adjustment for age, diabetes duration, gender, BMI, total-, LDL-, HDL -cholesterol, positive fibrinogen correlation with antibody TPO $(\mathrm{r}=0.22 \mathrm{p}=0.0045), \mathrm{TG}$ $(\mathrm{r}=0.12 \mathrm{p}=0.0022)$ was found. Additional analyzes in group with increased risk of microvascular complications ( $\mathrm{HbA} 1 \mathrm{c}>8,5 \%$ and diabetes duration $>5$ years) did not revel correlation with fibrinogen and CRP plasma concentrations. Conclusion: This preliminary analysis indicates that low grade inflammation parameters correlate with non metabolic and diabetes related factors in children with type 1 
diabetes. Autoimmune thyroid process is a significant factor involved into this pathology.

\section{4}

3-month safety and efficacy of an oral insulin (Orallyn ${ }^{\mathrm{TM}}$ ) administered at lunchtime in juvenile type-1 DM subjects maintained on basal glargine insulin and prebreakfast and pre-dinner regular insulin

J. Guevara-Aguirre, M. Guevara-Aguirre, J. Saavedra; IEMYR, Institute of Endocrinology, Quito, Ecuador.

Background and Aims: Participating subjects in this 6month phase are 24 adolescents $(12 \mathrm{M} ; 12 \mathrm{~F})$ and 5 young adults $(2 \mathrm{M} ; 3 \mathrm{~F})$ referred to us in various degrees of metabolic control as reflected by altered baseline measurements of glucose 236.6 (116.6) $\mathrm{mg} / \mathrm{dL}$; fructosamine 472.6 (126.6) umol/L; and glycosylated hemoglobin (HbA1c) 9.8 (2.3) $\mathrm{g} / \mathrm{dL}$. Baseline demographics of the 24 adolescents are: Age 14.7 y (2.1); Bone age 14.1 (2.2); CA/BA 1.0 (0.1); Height $153.8 \mathrm{~cm}$ (9.4); Weight $51.0 \mathrm{~kg}$ (10.2); BMI 21.7 (3.2); DM since onset 6.7 (2.8). Baseline demographics of the 5 young adults are: Age 20.6 y (2.2); Bone age (BA) 18.8 (0.4); CA/BA 1.1 (0.1); Height $161.1 \mathrm{~cm}$ (12.8); Weight $62.5 \mathrm{~kg}$ (9.3); BMI 23.0 (1.8); DM since onset 7.0 (1.7). Mean age for the entire group is $15.7 \mathrm{y}(3.0)$; BA 14.9 (2.7); CA/BA 1.1 (0.1); DM since onset is 6.8 (2.6).

Materials and Methods: After a stabilization phase using basal s.c. glargine insulin and 3 pre-prandial s.c. regular insulin injections, standard therapy continued for 2 additional weeks for comparison. Immediately thereafter, split doses of Oral-lyn ${ }^{\mathrm{TM}}$ immediately before and after lunch replaced the lunch-time injection of regular insulin. Patientcollected glucose values in the initial phase, fructosamine and HbAlc were compared weekly. Values are reported at the end of each phase.

Results: Results are shown in the chart and graph.

Conclusion: Fructosamine and $\mathrm{HbA} 1 \mathrm{c}$ results at the 12th week of treatment demonstrated a trend similar to that obtained with standard therapy. In summary, successful replacement of regular s.c insulin for Oral-lyn ${ }^{\mathrm{TM}}$ at lunchtime during the initial 3 months of this 6-month trial was achieved.

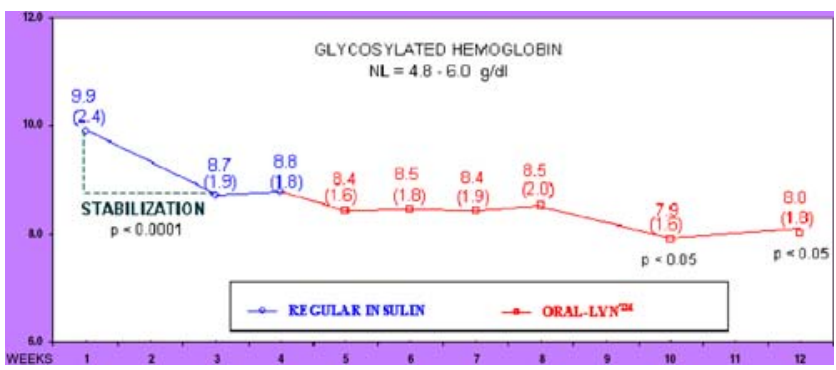

Supported by Generex Biotechnology Corp.

\section{PS 76}

\section{Gestational diabetes}

0925

Insulin secretion is markedly reduced in women with previous gestational diabetes

G. Seghieri, F. Tesi, A. De Bellis, R. Anichini, G. Fabbri, L. Alviggi;

Internal Medicine, Spedali Riuniti, Pistoia, Italy.

Background and Aims: Gestational diabetes (GDM) constitutes a risk factor for progressive glucose metabolism deterioration throughout the post-pregnancy years. To explain this aspect, the hypothesis tested by this study was that a previous GDM modifies insulin sensitivity or insulin secretion even in women who remain glucosenormotolerant after the preganancy.

Materials and Methods: Gestational diabetes (GDM) constitutes a risk factor for progressive glucose metabolism deterioration throughout the post-pregnancy years. To explain this aspect, the hypothesis tested by this study was that a previous GDM modifies insulin sensitivity or

\begin{tabular}{lccccc}
\hline & FRUCTOSAMINE & FRUCTOSAMINE & HbA1C & $\begin{array}{c}\text { HbA1C } \\
\text { GLUCOSE }\end{array} \begin{array}{c}\text { GLUCOSE } \\
\text { (BASAL) }\end{array}$ \\
\hline PHASE & MEAN & SD & MEAN & SD & MEAN \\
BASELINE & 476.89 & 130.22 & 9.9 & 2.4 & $\mathbf{2 3 6 . 6}$ \\
REGULAR INSULIN (end of week 6) & 368.2 & 91 & 8.4 & 1.6 & 140.4 \\
ORAL-LYN (end of week 9) & 379.1 & 133 & 8.5 & 2.1 & 143.3 \\
COMPARED TO BASELINE & & $<\mathbf{0 . 0 0 0 1}$ & $<\mathbf{0 . 0 0 0 1}$ & $<\mathbf{0 . 0 0 0 5}$ & 39.5 \\
ORAL-LYN (end of week 12) & 390.6 & 98.9 & 8.0 & 1.8 & \\
COMPARED TO REG. INSULIN & & $\mathbf{N S}$ & $<\mathbf{0 . 0 0 5}$ & & \\
\hline
\end{tabular}


insulin secretion even in women who remain glucosenormotolerant after the preganancy.

Results: In spite of similar mean values of BMI, age, time from pregnancy and glucose-area-under-curve, ISIogtt (mg/ dl per $\min \pm \mathrm{SD})$ was significantly lower $(3.75 \pm 1.73)$ in the IGT group than in those with GDM $(4.91 \pm 2.45)$ and with NGT (5.25 $\pm 2.71 ; p<0.05$, after ANOVA), while the median value (range) of insulin secretion $(\mathrm{mU} / \mathrm{mmol}$,) was greatly decreased in GDM group: $18.6(35-45)$ compared to IGTgroup: 33.2 (9-109) or NGT: $26(10-86)$; $\mathrm{p}<0.05$ after ANOVA. Median disposition index (range) was consequently markedly lower in GDM-group: 76 (15-460) than in IGT-group: 98 (27-423) or in NGT-group:130 (31-465). These differences remained unchanged after adjusting for age, BMI and post-partum lag-time.

Conclusion: Even in presence of a normal glucose tolerance, the women with previous GDM present a modest reduction in insulin sensitivity and a marked decrease in insulin secretion, and consequently of the disposition index, as compared to those who had normal or impaired glucose tolerance during a previous pregnancy.

Supported by Fondazione Cassa Risparmio Pistoia

0926

First and second trimester insulin resistance and subsequent development of pregnancy induced hypertension R. Helal ${ }^{1}$, S. Saha ${ }^{2}$, F. Jebunnesa ${ }^{1}$, F. Pervin ${ }^{1}$, A. Akhter ${ }^{1}$, L. Ali ${ }^{1}$;

${ }^{1}$ Dept of Biochemistry and Cell Biology, ${ }^{2}$ Dept of Physiology and Molecular Biology, BIRDEM, Dhaka, Bangladesh.

Background and Aims: The association of insulin resistance and pregnancy induced hypertension (PIH) is now widely accepted but their causal relationship is not yet established. Prospective studies are necessary to establish this relationship; these are relatively scarce and still inconclusive. Also technical and ethical issues in measuring insulin sensitivity in pregnancy are major obstacles in conducting studies in this area. The present study was undertaken to investigate insulin sensitivity in first and second trimesters of pregnancy simultaneously by homeostasis model assessment and plasma sex hormone binding globulin (SHBG) and to explore the causal relationship of insulin resistance on the subsequent development of PIH.

Materials and Methods: A total of 216 pregnant women participated in the study up to their end of pregnancy. Among them 24 women developed PIH. By a nested case control design, 53 age- and BMI-matched normotensive pregnant women as control were randomly selected from the Non-PIH subjects. PIH was diagnosed by standard criteria; proteinuria was measured by urinary protein-creatinine ratio. Plasma
Insulin level was measured by Microparticle Enzyme Immunoassay (MEIA). Insulin secretory capacity (\%HOMA B) and insulin sensitivity (\%HOMA S) were assessed by Homeostasis Model Assessment (HOMA). Plasma SHBG (Sex Hormone Binding Globulin) was measured by Chemiluminescent Immunometric Assay.

Results: The two study groups were age-and BMImatched [age, median (range); in years: 25(22-32) in Control and 25 (20-34) in PIH; BMI, median (range); kg/ $\mathrm{m}^{2}: 21.78(15.22-27.89)$ in control and 21.71(16.44-32.05) in PIH groups. There was no significant difference in fasting insulin level between the two groups [median (range); $\mu \mathrm{U} / \mathrm{ml}: 5.7(1.5-70)$ in control and 6.1(1.4-29.8) in $\mathrm{PIH}, \mathrm{P}<0.71$ ]; SHBG [median (range); nmol/1: 133.0 (17.3-180) in control and 139.5(10.5-180) in PIH, $\mathrm{P}<0.72$ ]; and HOMA S [median (range) 146.9(12.4442.5) in control and 136.8(28.1-294.6) in $\mathrm{PIH}, \mathrm{P}<0.74]$. \%HOMA B showed significantly higher value in $\mathrm{PIH}$ group compared to control. [ median (range) 111.2(36.2325.9) in control and 141.4(49-381.6) in $\mathrm{PIH}, \mathrm{P}<0.05]$. When the data was analyzed separately in 1 st and 2 nd trimesters of pregnancy, there was no significant difference between the two groups in any trimester. Using 25-75th percentile of SHBG of the control the reference range of SHBG was $84.5-180 \mathrm{nmol} / \mathrm{l}$. Among the PIH cases $6.5 \%$ had SHBG values below lower cut -off point and $24.7 \%$ of the PIH cases had values above the lower cut- off point (Odds ratio 1.11; 95\% CI, 0.34,3.60).

Conclusion: The present data demonstrates that early pregnancy insulin resistance, with HOMA S or SHBG as markers, may not be predictive for development of PIH in later stages of pregnancy.

Supported by BMRG, IPICS

0927

Resistin levels are associated with insulin sensitivity in pregnant women with and without gestational diabetes A. Megia ${ }^{1}$, I. Simón ${ }^{1}$, M. Broch ${ }^{2}$, J. Fernandez-Real ${ }^{3}$, R. Miralles ${ }^{4}$, W. Ricart ${ }^{5}$, C. Richart ${ }^{6}$, J. Vendrell ${ }^{1}$; ${ }^{1}$ Endocrinology and Diabetes, ${ }^{2}$ Research Unit, Hospital Universitari Joan XXIII, Tarragona, ${ }^{3}$ Endocrinology and Diabetes, Hospital Universitari Josep Trueta, Girona, ${ }^{4}$ Obstetrics and Gynecology, Hospital Universitari Joan XXIII, Tarragona, ${ }^{5}$ Endocrinology and Diabetes, Hospital Universitari Josep Trueta, Girona, ${ }^{6}$ Internal Medicine, Hospital Universitari Joan XXIII, Tarragona, Spain.

Background and Aims: Resistin, which has been proposed to play a role in obesity-mediated insulin resistance and diabetes, is expressed in placental tissue. It is known that resistin levels increase in the second half of normal 
pregnancy, although the relevance and physiological role that it could play in the pathogenesis of the insulin resistance observed remains unknown. To study the relationship between resistin and adiponectin levels and insulin sensitivity in pregnant women with and without gestational diabetes mellitus (GDM).

Materials and Methods: Fifty-eight pregnant women, 23 with GDM and 35 with normal pregnancy (NP) were studied. At the time of the OGTT (26-30 weeks of gestation), anthropometric and clinical characteristics were recorded. Baseline resistin and adiponectin levels were determined. Glucose and insulin levels were measured at baseline and 60', $120^{\prime}$ and $180^{\prime}$ postload. Insulin sensitivity was calculated using the ISIogtt (1). Statistical Analysis: Independent samples ttest, chi-square test and multiple regression analysis.

Results: There were no differences between both groups in age, week of gestation at the time of the OGTT, prepregnancy and current BMI, neonatal birth weight and the week of delivery. Resistin levels were significantly lower in GDM women than in NP group $(4,7 \pm 2,29$ vs $9,62 \pm 2,43 \mathrm{ng} /$ $\mathrm{ml}$, respectively; $\mathrm{p}<0.001$ ), but adiponectin levels were similar in both groups $(11,62 \pm 3,46 \mathrm{vs} 12,40 \pm 5,04 \mu \mathrm{g} / \mathrm{L})$. Insulin sensitivity was significantly higher in the NP group than in the GDM group $(9,97 \pm 4,17$ vs $3,23 \pm 1,07$, respectively; $\mathrm{p}<0,001)$. The ISIogtt index correlated with resistin levels ( $\mathrm{r}: 0.587 ; \mathrm{p}<0,001)$ but a negative correlation was found between Insulin sensitivity and pre-pregnancy BMI $(\mathrm{r}:-0,364 ; \mathrm{p}<0,05)$ and current BMI $(\mathrm{r}:-0.414 ; \mathrm{p}<0.001)$. When a multiple linear regression analysis was developed including ISIogtt as dependent variable and age, current BMI, adiponectin and resistin as independent variables, all the variables except age were independently associated with ISI ogtt $\left(r^{2}: 0.633, p<0.001\right)$.

Conclusion: Resistin levels are lower in GDM women compared with women with a Healthy Pregnancy, and resistin levels are independently associated with insulin sensitivity in pregnancy.

(1).- Kirwan JP, Huston-Presley H, Kalhan SC, Catalano PM: Clinically useful estimates of insulin sensitivity during pregnancy. Diabetes Care 24:1602-1607, 2001

Supported by PI03/1322 and G03/212

\section{8}

\author{
Serum concentrations of retinol binding protein 4 in \\ women with gestational diabetes \\ K. Krzyzanowska ${ }^{1}$, W. Krugluger ${ }^{2}$, F. Mittermayer ${ }^{3}$, \\ R. Rahman ${ }^{1}$, J. Schnabler ${ }^{1}$, G. Schernthaner ${ }^{1}$; \\ ${ }^{1}$ Internal Medicine I, ${ }^{2}$ Central Laboratory, Rudolfstiftung \\ Hospital, Vienna, ${ }^{3}$ Clinical Pharmacology, Medical Univer- \\ sity Vienna, Austria.
}

Background and Aims: Gestational diabetes mellitus (GDM) is characterized by various features of the metabolic syndrome. Different mechanisms leading to insulin resistance in GDM have been discussed. Recently, the adipocyte derived retinol binding protein 4 (RBP4) has been shown to cause insulin resistance. However, clinical data on RBP4 are limited. We hypothesized that RBP4 is elevated in women with GDM who are in a temporary state of insulin resistance. Materials and Methods: According to a cross sectional study design RBP4 serum concentrations were measured in 48 women with GDM (mean age: 32 yrs.) and in 56 pregnant controls (mean age: 29 yrs.). An oral glucose tolerance test and anthropometric measurements were performed in all subjects. Insulin resistance was estimated by Homeostasis Model Assessment (HOMA-IR).

Results: RBP4 serum concentrations were comparable between women with GDM and controls (median: 32.2 [interquartile range: $17.3-50.5] \mu \mathrm{g} / \mathrm{ml}$ vs. 28.0 [IQR: $13.6-$ 48.9] $\mu \mathrm{g} / \mathrm{ml} ; \mathrm{P}=0.382$ ). Women with GDM had higher body mass index (BMI), HOMA-IR, insulin concentrations and fasting as well as post load blood glucose levels compared to controls (all $\mathrm{P}<0.05$ ). In a correlation analysis RBP4 was associated with $\mathrm{BMI}(\mathrm{R}=0.29 ; \mathrm{P}=0.047)$ and fasting glucose ( $\mathrm{R}=0.33 ; \mathrm{P}=0.024)$ but not with other clinical parameters in subjects with GDM. In contrast, RBP4 only correlated with insulin $(\mathrm{R}=0.37 ; \mathrm{P}=0.005)$ and HOMA-IR $(\mathrm{R}=0.37$; $\mathrm{P}=0.006$ ) in controls.

Conclusion: The adipokine RBP4 was neither elevated nor related to insulin resistance in women with GDM. Therefore RBP4 is unlikely to play a role for insulin resistance in patients with GDM.

0929

Plasma taurine in women with history of gestational diabetes

F. Franconi ${ }^{1}$, F. Tesi ${ }^{2}$, L. Bianchi ${ }^{3}$, A. Loizzo ${ }^{4}$, G. Saccomanni ${ }^{5}$, G. Ghirlanda ${ }^{6}$, G. Seghieri ${ }^{2}$;

${ }^{1}$ Pharmacology, Univerity of Sassari, ${ }^{2}$ Internal Medicine, Spedali Riuniti, Pistoia, ${ }^{3}$ Clinical Chemistry, Laboratory, Pistoia, ${ }^{4}$ Pharmacology, Istituto Sup. Sanità, Rome, ${ }^{5}$ Pharmaceutical Sciences, Univerity of Pisa, ${ }^{6}$ Internal Medicine, Catholic University, Rome, Italy.

Background and Aims: Taurine is the most abundant amino acid in the human body and seems to play an important role in increasing glucose-mediated insulin secretion, as well as in programming beta-cell maturation during the prenatal life in utero. Aim of this study was therefore to test the hypothesis that plasma taurine is related to glucose tolerance, insulin sensitivity and insulin secre- 
tion in subjects with history of $\beta$-cell dysfunction such as women with history of gestational diabetes (GDM).

Materials and Methods: We studied 72 non-diabetic women with history of GDM $(n=43)$, impaired glucose tolerance (IGT; $n=7$ ), and normal glucose tolerance (NGT; $\mathrm{n}=22$ ) as previously classified by a $100 \mathrm{~g}-3 \mathrm{hr}-$ OGTT performed between the 24th and the 28th gestational week. Insulin sensitivity (ISIogtt, calculated through MatsudaDeFronzo index) and a proxy for insulin secretion (basal plasma C-peptide/fasting plasma glucose; CP/FPG) were measured during and after pregnancy. Plasma taurine was measured after a median period of $6 \mathrm{yr}(2-11 \mathrm{yr})$ from the index pregnancy, when glucose tolerance was retested by a 75 g-2 hr-OGTT.

Results: Plasma taurine $(\mu \mathrm{mol} / 1 \pm \mathrm{SD})$ was significantly lower in women who had experienced GDM $(88.4 \pm 30.5)$ as compared to IGT $(110.1 \pm 34.2)$ and NGT (119.8 \pm 52.1$)$, and was unrelated to ISIogtt. Moreover plasma taurine was inversely related to previous gestational area-under-curve of glucose and directly related to post-gestational $\mathrm{CP} / \mathrm{FPG}$, as well to CP/FPG measured during the pregnancy $(p<0.05$ for both). The relative risk of altered glucose metabolism during previous pregnancies (IGT+GDM) was higher as plasma taurine decreased, even after adjusting for age, timelag from pregnancy, body mass index and family history of diabetes (OR: 0.980;CI95\%:0.963-0.999, p=0.003).

Conclusion: Plasma taurine seems to be a fair predictor of altered glucose metabolism during past pregnancies in women with antecedent GDM and appears to be inversely related to the previous as well as to the actual insulin secretion in these subjects.

Supported by Fondazione Cassa Risparmio Pistoia

\section{0}

Comparison of high sensitive CRP levels and serum selenium concentrations during gestational diabetic and uncomplicated pregnancy and after delivery: a follow-up study

J. Molnár ${ }^{1}$, Z. Garamvölgyi ${ }^{2}$, B. Sármán ${ }^{1}$, J. Rigó Jr. ${ }^{2}$, A. Somogyi ${ }^{1}$;

${ }^{1}$ 2nd Department of Medicine, ${ }^{2} 1$ st Department of Obstetrics and Gynaecology, Semmelweis University, Budapest, Hungary.

Background and Aims: There is increasing evidence on the importance of oxidative stress in gestational diabetic pregnancy. The essential trace element selenium, apart from its pivotal functions in several antioxidant enzymes, has also insulin-mimic properties in vitro and in vivo. In a previous study a highly significant inverse correlation $(p<0.01)$ was observed between inflammatory biomarker
hsCRP values and serum selenium concentrations in both gestational diabetic and uncomplicated pregnancy. The aim of our present study was to compare hsCRP levels and serum selenium concentrations during pregnancy and 810 months after delivery. The glycaemic status of previously gestational diabetic women was also re-assessed.

Materials and Methods: The hsCRP concentrations and serum selenium levels of gestational diabetic pregnant women (n: 20), control pregnant women (n: 20) and nonpregnant controls (n: 20) were compared. Blood was taken between the 24th and 28th week of pregnancy when the oral glucose tolerance test was performed according to WHO guidelines. The oral glucose tolerance test was repeated in former gestational diabetic study participants 8-10 months after delivery. HsCRP was measured by immunturbidimetry. Selenium was determined by hydride generation atomic absorption spectrometry.

Results: HsCRP values of both gestational diabetics $(8.9 \pm 6.0 \mathrm{mg} / \mathrm{l})$ and control pregnant women $(6.3 \pm 5.8 \mathrm{mg} / \mathrm{l})$ were significantly higher than in controls $(2.7 \pm 3.7 \mathrm{mg} / \mathrm{l})$. After delivery, hsCRP values returned to normal in both former gestational diabetics and control pregnant women $(2.4 \pm 2.2$ and $4.6 \pm 5.8 \mathrm{mg} / 1$, respectively). The serum selenium concentrations of gestational diabetics $(52.0 \pm 10.7 \mu \mathrm{g} / 1)$ and non-diabetic pregnant women $(40.5 \pm 8.0 \mu \mathrm{g} / \mathrm{l})$ were significantly lower than in non-pregnant controls $(77.4 \pm 14.8 \mu \mathrm{g} / \mathrm{l})$. Cholesterol, HDL, LDL and triglyceride concentrations were significantly higher in pregnant women compared to controls, with no difference between the two pregnant subgroups. Differences in serum selenium and lipid concentrations disappeared after delivery. When the oral glucose tolerance test was repeated 8-10 months after delivery, 14\% of former gestational diabetics were diagnosed with IFG and $14 \%$ with IGT. After delivery, serum selenium concentrations no longer correlated significantly with hsCRP values.

Conclusion: The higher hsCRP in gestational diabetic and control pregnant women may indicate increased subclinical inflammation and lipid peroxidation during pregnancy. The highly significant inverse correlation between lower selenium concentrations and hsCRP values in pregnancy disappears after delivery, when serum selenium levels spontaneously increase and hsCRP values decrease. The marked decrease in serum selenium observed during pregnancy may be attributed to an increased requirement for this element in pregnancy.

0931

C-reactive protein (CRP) and insulin resistance in gestational diabetes mellitus (GDM)

Y. Liu ${ }^{1}$, J. Du ${ }^{2}$, D. Zhao ${ }^{1}$, X. Song ${ }^{1}$, L. Zhou ${ }^{2}$, P. Wang ${ }^{1}$, Z. Xu ${ }^{1}$; 
${ }^{1}$ Endocrinology, Department of Endocrinology, ${ }^{2}$ Obstetrics, 306 Hospital of PLA, Beijing, China.

Background and Aims: To investigate the changes and the effects of CRP in GDM.

Materials and Methods: We investigated serum sCRP in 135 GDM, and the correlation with their gestational weeks, body mass index (BMI), blood pressure(BP), heart beating rate(HBR), serum insulin levels and glucose levels during $75 \mathrm{~g}$ OGTT, their serum lipids and urine albumin/creatinine (Alb/Cr).

Results: Ninety nine GDM had normal sCRP levels (group 1 , G1) which were $1.34 \pm 0.83 \mathrm{mmol} / 1$ and $36 \mathrm{GDM}$ had increased sCRP levels (group 2, G2) which were $5.73 \pm 3.14 \mathrm{mmol} / \mathrm{l}$. There was no difference of gestational weeks between the two groups. Body weight of both prepregnancy and pregnancy were significantly higher in $\mathrm{G} 2$ as well as their BMI respectively. Significant higher glucose levels of 0 (G2 $5.38 \pm 1.87 \mathrm{vs} \mathrm{G1} 4.49 \pm 0.77 \mathrm{mmol} / \mathrm{l}, \mathrm{p}=0.041)$ and $120 \mathrm{~min}$ (G2 $9.07 \pm 1.87$ vs G1 $7.86 \pm 1.21 \mathrm{mmol} / \mathrm{l}$, $\mathrm{p}=0.012), \mathrm{HbA1c}(\mathrm{G} 25.79 \pm 0.58$ vs G1 $5.30 \pm 0.57 \%, \mathrm{p}=)$ as well as $30 \mathrm{~min}$ (G2 $132.61 \pm 44.95$ vs G1 $70.96 \pm 37.97 \mathrm{mmol} /$ $1, \mathrm{p}=0.020)$ and $120 \mathrm{~min}(\mathrm{G} 2110.59 \pm 43.34 \mathrm{vs} \mathrm{G} 1$ $68.25 \pm 37.71 \mathrm{uIU} / \mathrm{ml}, \mathrm{p}=0.049$ ) insulin levels during OGTT were found in G2. TG (G2 $3.13 \pm 1.22$ vs G1 $2.39 \pm 1.36 \mathrm{mmol} / \mathrm{l}, \mathrm{p}=0.047)$ and HBR (G2 110.19 \pm 13.34 vs G1 $86.72 \pm 14.23 / \mathrm{min}, \mathrm{p}=0.000$ ) was also higher in $\mathrm{G} 2$. But no differences of $\mathrm{Alb} / \mathrm{Cr}$ and $\mathrm{BP}$ between the two groups were found. Significant positive correlations of sCRP with gestational week, pre-pregnant and pregnant body weight and BMI, HBR and insulin injection doses. No correlation was found between maternal CRP and infant birth weight.

Conclusion: Body weight and BMI of both pre-pregnant and pregnant may play an important role in the sCRP increasing in GDM. GDM with increased CRP may have higher glucose, TG and insulin levels. They look like having severe insulin resistance.

0932

Circulating concentrations of asymmetric dimethylarginine and endothelial function in women with gestational diabetes

B. Telejko, A. Zonenberg, A. Modzelewska, K. Niedziolko-Bagniuk, A. Nikolajuk, M. Gorska; Department of Endocrinology, Diabetology and Internal Medicine, Medical University of Bialystok, Poland.

Background and Aims: An increase of circulating asymmetric dimethylarginine (ADMA), an endogenous inhibitor of the nitric oxide synthase, has been reported in women with previous gestational diabetes (GDM) and other patients at risk of endothelial dysfunction and atherosclerosis, but has never been studied in the course of GDM. Therefore, the aim of our study was to compare ADMA level and other markers of endothelial function between subjects with GDM and healthy pregnant women.

Materials and Methods: The group studied consisted of 56 patients with GDM, 68 pregnant women with normal glucose tolerance (NGT) and 36 healthy non-pregnant women. Plasma concentrations of ADMA, endothelin-1 (ET-1), soluble vascular cell adhesion molekule-1 (sVCAM-1) and sE-selectin were measured by enzyme linked immunoassay (ELISA).

Results: ADMA concentrations were significantly lower in pregnant women with NTG $(68.2 \pm 21.2 \mathrm{ng} / \mathrm{ml})$ in comparison to GDM subjects $(75.7 \pm 33.7 \mathrm{ng} / \mathrm{ml})$ and healthy controls $(76.5 \pm 21.6 \mathrm{ng} / \mathrm{ml}, \mathrm{p}<0.005)$. In contrast, ET-1 concentrations were comparable between GDM $(0.79 \pm 0.29 \mathrm{pg} / \mathrm{ml})$ and NGT pregnant women $(0.79 \pm 0.34 \mathrm{pg} / \mathrm{ml})$, and markedly higher than in non-pregnant subjects $(0.62 \pm 0.18 \mathrm{pg} / \mathrm{ml}$, $\mathrm{p}<0.01$ ). There were not significant differences in SVCAM1 and sE-selectin levels between the groups studied. Endothelin-1 levels correlated slightly with fasting plasma glucose $(r=0.163, p=0.04)$ and glycaemia $60^{\prime}$ after glucose load $(\mathrm{r}=0.215, \mathrm{p}=0.016)$. In a multiple regression analysis ADMA concentrations were associated with gestational week (beta $=0.384, p=0.019$ ) and glycaemia $120^{\prime}$ after glucose load (beta $=0.505, \mathrm{p}=0.008$ ), whereas ET-1 levels were associated with gestational week (beta $=0.480$, $\mathrm{p}=0.015$ ) and insulin level $120^{\prime}$ after glucose load (beta $=-0.457, \mathrm{p}=0.038$ ).

Conclusion: The results suggest that in pregnancy maternal vascular function is modulated, at least in part, by a reduction of ADMA - the mechanism, which seems to be altered in GDM subjects, and that ET-1 may play a role in maintaining peripheral vascular tone in pregnancy, both normal and complicated by GDM.

\section{3}

Peripheral monocyte visfatin gene expression is increased in normal and gestational diabetes pregnancy P. C. Tsiotra ${ }^{1}$, C. Tsigos $^{1}$, A. Koukourava ${ }^{1}$, E. Anastasiou ${ }^{2}$, S. A. Raptis ${ }^{1}$;

${ }^{1}$ Molecular Biology Lab., Division of Basic Sciences, Hellenic National Diabetes Center (H.N.D.C.), ${ }^{2}$ First Endocrine Section and Diabetes Centre, "Alexandra" Hospital, Athens, Greece.

Background and Aims: Visfatin is a newly identified adipokine, highly expressed in human visceral fat. Visfatin's plasma levels are increased with expanding obesity as well as in type 2 diabetes, and it is thought to 
promote accumulation of visceral fat, most propably acting through the insulin receptor pathway. We examined whether visfatin/PBEF mRNA production by peripheral monocytes is altered in normal and in gestational diabetes (GDM) pregnancy and whether this might relate to monocyte $\mathrm{TNF} \alpha$ expression and to indices of insulin resistance.

Materials and Methods: We studied 18 women with normal pregnancy (Gestation), 15 with gestational diabetes (GDM), and 26 non-pregnant controls, all premenopausal. All pregnant women were studied during the 3rd trimester. We developed a real-time quantitative RT-PCR using fluorecently labelled hybridization probes to detect relative visfatin/PBEF and TNF $\alpha$ mRNA levels in isolated blood monocyte-enriched mononuclear cells (Light Cycler, Roche). Visfatin/PBEF and TNF $\alpha$ mRNA levels were calculated as the relative ratio of the fluorescence acquisition of visfatin or TNF $\alpha$ mRNA divided by that of $\beta$-actin, using the Relative Quantification Software (Roche). We also measured fasting and 2 hour post-OGTT plasma glucose and insulin (RIA) levels.

Results: Visfatin/PBEF mRNA levels were significantly higher in peripheral monocytes from pregnant (normal and GDM) women compared to controls (Table) and correlated with monocyte TNF $\alpha$ mRNA $(\mathrm{r}=0.478, \mathrm{p}<0.001)$, which was also higher in pregnancy. We did not observe any correlation of visfatin/PBEF mRNA levels with indices of insulin resistance overall or among pregnant women. Visfatin/PBEF mRNA expression was comparable between normal pregnant and gestational diabetic women.

\begin{tabular}{|c|c|c|c|c|c|}
\hline & BMI & $\begin{array}{l}\text { Insulin } \\
\text { fasting }\end{array}$ & HOMA-IR & $\begin{array}{l}\text { Visfatin/ } \\
\text { PBEF }\end{array}$ & $\mathrm{TNF} \alpha$ \\
\hline & $\mathrm{kgr} / \mathrm{m}^{2}$ & $\mathrm{mU} / \mathrm{L}$ & & mRNA & mRNA \\
\hline Control & $27.3 \pm 1.3$ & $9.3 \pm 1.2$ & $2.1 \pm 0.3$ & $0.6 \pm 0.2$ & $0.03 \pm 0.006$ \\
\hline Gestation & $26.9 \pm 1.4$ & $11.0 \pm 1.3$ & $2.2 \pm 0.3$ & $1.8 \pm 0.2 *$ & $0.06 \pm 0.02 *$ \\
\hline GDM & $25.7 \pm 1.0$ & $12.6 \pm 1.3^{*}$ & $2.9 \pm 0.9$ & $2.3 \pm 0.8^{*}$ & $0.09 \pm 0.04$ \\
\hline
\end{tabular}

$*, \mathrm{p}<0.05$ vs control

Conclusion: Peripheral mononuclear visfatin/PBEF and TNF $\alpha$ mRNA production is elevated in normal and gestational diabetes pregnancy. This might contribute to the insulin resistance that characterizes these conditions and might increase their atherogenic risk.
0934

\section{Gestational diabetes is negatively associated with HLA DQ6}

A. Katsarou, K. Lynch, B. Lernmark, Å. Lernmark; Department of Clinical Sciences/Malmö, Lund University, Malmö, Sweden.

Background and Aims: The aim of this study was to investigate the association between HLA genotypes and gestational diabetes, as well as to compare the distribution of HLA genotypes and islet autoantibodies in mothers with type 1 diabetes (T1D) and gestational diabetes (GDM) and their offspring.

Materials and Methods: HLA genotypes were determined in dried blood spots of cord blood taken from 32,663 children born to non-diabetic mothers and 1179 children born to diabetic mothers in DiPiS (Diabetes Prediction in Skåne) study. In addition, 1046 diabetic mothers were HLA typed. Out of them, 208 reported having type 1 diabetes and 838 had gestational diabetes. GAD65, IA-2 and insulin autoantibodies were determined both in cord blood and in mothers' plasma taken at delivery in standard radioligand binding assays. GDM mothers positive for islet autoantibodies were excluded from the HLA distribution analysis.

Results: 1) As expected, T1D was strongly associated with the high risk HLA DQB1 genotypes and the association was passed on to the offspring $(\mathrm{p}<0.0001$ for DQB1*0201/ 0302, 0302/X and 0302/0604). There was no positive association between HLA and GDM. 2) The HLA DQB1*0602 allele, that is considered protective for type 1 diabetes, was confirmed to be negatively associated with T1D ( $\mathrm{p}=0.04$ for $\mathrm{DQB} 1 * 0602-3-4 / \mathrm{X})$, but was also negatively associated with GDM $(p=0.01$ for $\mathrm{DQB} 1 * 0602 / 02, \mathrm{p}=0.04$ for $\mathrm{DQB} 1 * 0602 / 0301$ and $\mathrm{p}=0.0008$ for $\mathrm{DQB} 1 * 0602 / \mathrm{X})$, as was also the case for the offspring $(\mathrm{p}=0.0008$ for $\mathrm{DQB} 1 * 0602 / 02$ and $\mathrm{p}=0.02$ for DQB $1 * 0602 / \mathrm{X}) 3$ ) A positive association was found between maternal age and GDM $(\mathrm{p}<0.0001) 4)$ T1D and GDM mothers gave birth prematurely $(<37$ th week) in a higher frequency than the control population $(\mathrm{p}<0.0001)$ 5) Approximately $61 \%$ and $6 \%$ of children born to T1D and GDM mothers respectively, were antibody positive in cord blood. No significant association was found between islet cell autoantibodies and high risk HLA alleles in both mothers and offspring.

Conclusion: Maternal age is a risk factor for GDM. High risk HLA genotypes for T1D are not associated with GDM. The HLA DQB1*0602 allele is negatively associated with GDM and may protect from the disease. 
Association between T1D, GDM and HLA DQB1 high risk genotypes in DiPiS mothers and children

\begin{tabular}{lllll}
\hline & & $\mathbf{0 2 0 1} / \mathbf{0 3 0 2}$ & $\mathbf{0 3 0 2} / \mathbf{X}$ & $\mathbf{0 3 0 2 / 0 6 0 4}$ \\
\hline T1D mothers & $\mathbf{p}$ value & $<0.0001$ & $<0.0001$ & $<0.0001$ \\
& OR & 12.2 & 4.5 & 9.1 \\
\multirow{4}{*}{ Offspring } & $95 \% \mathrm{CI}$ & {$[8.3-18]$} & {$[3-6.7]$} & {$[5-16.6]$} \\
& $\mathbf{p}$ value & 0.002 & $<0.0001$ & $<0.0001$ \\
& OR & 2.3 & 2.3 & 5.1 \\
GDM mothers & 95\%CI & {$[1.4-4.1]$} & {$[1.6-3.5]$} & {$[2.7-9.5]$} \\
& $\mathbf{p}$ value & 0.8 & 0.9 & 0.8 \\
& OR & 1 & 1 & 1.1 \\
Offspring & $95 \% \mathrm{CI}$ & {$[0.7-1.5]$} & {$[0.8-1.3]$} & {$[0.6-1.9]$} \\
& $\mathbf{p}$ value & 0.2 & 0.8 & 0.6 \\
& OR & 1.2 & 0.9 & 1.2 \\
& $95 \% \mathrm{CI}$ & {$[0.9-1.7]$} & {$[0.7-1.2]$} & {$[0.7-2.1]$} \\
\hline
\end{tabular}

Association between T1D, GDM and HLA DQB1 genotypes protective for T1D in DiPiS mothers and children

\begin{tabular}{|c|c|c|c|c|c|}
\hline & & $0602-3-4 / X$ & $0602 / 02$ & $0602 / 0301$ & $0602 / \mathrm{X}$ \\
\hline \multirow[t]{3}{*}{ T1D mothers } & $p$ value & 0.04 & 0.3 & none & 0.3 \\
\hline & OR & 0.3 & 0.5 & & 0.6 \\
\hline & $95 \% \mathrm{CI}$ & {$[0.1-0.9]$} & {$[0.2-1.5]$} & & {$[0.2-1.4]$} \\
\hline \multirow[t]{3}{*}{ Offspring } & $p$ value & 0.01 & 0.3 & 0.8 & 0.05 \\
\hline & OR & 0.3 & 1.3 & 0.8 & 0.4 \\
\hline & $95 \% \mathrm{CI}$ & {$[0.1-0.7]$} & [0.7-2.3] & [0.4-1.7] & {$[0.2-0.9]$} \\
\hline \multirow[t]{3}{*}{ GDM mothers } & $p$ value & 0.4 & 0.01 & 0.04 & 0.0008 \\
\hline & OR & 0.9 & 0.6 & 0.6 & 0.5 \\
\hline & $95 \% \mathrm{CI}$ & [0.6-1.1] & [0.4-0.9] & [0.4-0.9] & {$[0.4-0.7]$} \\
\hline \multirow[t]{3}{*}{ Offspring } & $P$ value & 0.07 & 0.0008 & 0.9 & 0.02 \\
\hline & OR & 0.7 & 0.5 & 1 & 0.7 \\
\hline & $95 \% \mathrm{CI}$ & {$[0.6-1]$} & {$[0.3-0.7]$} & {$[0.7-1.3]$} & {$[0.5-0.9]$} \\
\hline
\end{tabular}

Supported by Lund University

PS 77

\section{Type 1 diabetes in pregnancy}

\section{5}

Respective contributions of pregnancy planning in type 1 diabetic women and insulin treatment regimen to glucose control and fetal malformations

C. Brunet ${ }^{1}$, D. Carles ${ }^{1}$, C. Barusko ${ }^{1}$, P. Boulot ${ }^{2}$,

J. Bringer ${ }^{1}$, E. M. Renard ${ }^{1}$;

${ }^{1}$ Endocrinology, Lapeyronie Hospital, Montpellier, ${ }^{2}$ Obstetrics \& Gynaecology, Arnaud de Villeneuve Hospital, Montpellier, France.

Background and Aims: Beside planning of pregnancy in type 1 diabetic women, the effectiveness of insulin treatment regimen is a potential determinant of fetal malformations. Our study assessed their respective relationships with glucose control during pregnancy and occurrence of fetal anomalies.

Materials and Methods: We analyzed 107 consecutive pregnancies in type 1 diabetic women followed in our clinic between 1994 and 2004. 59 (55\%) had been planned. Insulin therapy at conception time and during pregnancy included multiple daily injections of regular and NPH human insulins (group I, $\mathrm{n}=35$ ), or lispro and NPH insulins (II, $\mathrm{n}=11$ ), insulin pump infusing human regular insulin (III, $n=32$ ) or infusing lispro insulin (IV, $\mathrm{n}=29$ ). Rates of pregnancy planning were 26 (I), 54 (II), 75 (III) et 69 (IV), respectively. Results: Mean HbAlc (\%) at conception time and at the end of each quarter was significantly different between groups (p<0.0001): 8.66/8.08/6.98/7.06 (I), 7.67/6.62/6.31/6.43 (II), 7.44/7.08/6.68/6.72 (III), 7.46/7.10/6.65/6.82 (IV). Numbers of fetal malformations were $9(28 \%), 1(9 \%), 2(7 \%)$ and 0 in groups I, II, III and IV, respectively. Pregnancy planning was independently associated with $\mathrm{HbAlc}$ levels $(\mathrm{p}<0.0001)$, whereas pump use or insulin type were not $(\mathrm{p}=0.07$ et 0.16$)$. However, insulin pump use was independently associated with a minor risk of malformations (OR 0.116 [0.024-0.561], $\mathrm{p}=0.0074$ ), as well as use of lispro insulin but with a lower significance ( $\mathrm{p}=0.046$ by univariate analysis).

Conclusion: Our data confirm the benefits of pregnancy planning on glucose control at conception time and during pregnancy in type 1 diabetic patients, while use of insulin pump by itself appears as a mean to reduce dramatically the risk of fetal malformations. Use of insulin lispro shows no deleterious outcome. Including insulin pump use in pregnancy planning looks as an option to be promoted to improve pregnancy outcomes in type 1 diabetic women.

0936

Results of pre-pregnancy care in diabetic women

A. García-Patterson ${ }^{1}$, J. M. Adelantado ${ }^{2}$, G. Ginovart ${ }^{3}$, B. Biagetti ${ }^{1}$, E. Martín ${ }^{1}$, A. de Leiva ${ }^{1}$, R. Corcoy ${ }^{1}$; ${ }^{1}$ Servei d'Endocrinologia, ${ }^{2}$ Servei de Ginecologia i Obstetricia, ${ }^{3}$ Servei de Pediatria, Hospital de Sant Pau, Barcelona, Spain.

Background and Aims: Prepregnancy clinics (PPC) in women with pregestational diabetes $(\mathrm{DM})$ aim to improve perinatal outcome; a meta-analysis has established that they are associated to better $\mathrm{HbAl} \mathrm{c}$ at the beginning of pregnancy and lower rates of congenital malformations (CM).

The aim of this study was to analyze the results of a PPC. Materials and Methods: We studied the characteristics and outcome of diabetic patients attending the PPC initiated in March 1986 and pregnant women with DM without prepregnancy care delivering in the same period. Chisquare 
tests were used to compare distributions and non-parametric tests to compare quantitative variables. Significance was set at $\mathrm{p}<0,05$.

Results: In the PPC, 282 women (92.9\% Type 1 DM), have been attended; $64.5 \%$ achieved a condition deemed adequate for pregnancy and 75.5\% got pregnant. Median follow-up lasted 40 weeks and significant modifications were observed in HbAlc $(-0.713 \%)$ and body mass index $(+0.215 \mathrm{~kg} /$ $\mathrm{m} 2$ ). Four hundred and sixty-eight pregnancies (86\% Type 1 DM) have been attended. Pregnant women with and without PPC attendance were different in: rate of Type $1 \mathrm{DM}$ (92.9 vs 78\%), age (30 vs 29 years), DM duration (11 vs 9 years), gestational age at initiation of gestational care (1 vs 9 weeks), first HbA1c (6.0 vs 6.8\%), smoking habit (36 vs 52\%) and caesarean section (64.3 vs $53.8 \%$ ); they did not differ in DM severity (White classification), initial body mass index, miscarriages, final HbAlc, hypertension, preterm delivery, major CM (5.2 vs 7.9\%), minor CM (5.8 vs $9.1 \%$ ), macrosomia and perinatal mortality.

Conclusion: In women with DM and acceptable metabolic control, PPC attendance is associated with better intermediate indicators but differences in perinatal outcome do not reach signficance.

0937

Audit of an integrated care pathway for pregnant women with diabetes; improved delivery of care and outcome in a UK hospital

G. M. Russell ${ }^{1}$, R. Paterson ${ }^{1}$, P. Lambert ${ }^{1}$, R. Fox ${ }^{2}$, J. M. Watson ${ }^{1}$;

${ }^{1}$ Diabetes and Endocrinology, ${ }^{2}$ Obstectrics and Gynaecology, Taunton and Somerset NHS Trust, Taunton, United Kingdom.

Background and Aims: A national audit of diabetes and obstetric care for women with pre-gestational diabetes (CEMACH- Confidential Enquiry into Maternal and Child Health) was carried out in the UK between 2002 and 2003. Key Findings from the CEMACH report (2005):

1.Women with diabetes are poorly prepared for pregnancy

2.All mothers should be entering pregnancy with substantially better glycaemic control

3.Women with diabetes are at greater risk of experiencing preterm delivery and caesarean section

4. Women with diabetes gave birth to larger babies

5.One third of term babies were admitted to a neonatal unit; $10 \%$ in general population

As a participating centre in this audit, we realised that our own documentation could be improved and the Integrated Care Pathway (ICP) for the Management of Diabetic Women during pregnancy was developed.
Materials and Methods: Between 2000 and 2003, 55 pregestational diabetic pregnancies were identified. An audit was performed comparing care; first before and then after introduction of the ICP, and second against the national data. Results:

Comparison of Pre-ICP and ICP Pregnancies to the CEMACH Report

\begin{tabular}{|c|c|c|c|}
\hline Key Finding & СЕМАСН & $\begin{array}{l}\text { Pre-ICP: } \\
\text { n/total }(\%)\end{array}$ & $\begin{array}{l}\text { ICP: } \\
\text { n/total (\%) }\end{array}$ \\
\hline Total no. of pregnancies & 3808 & 25 & 30 \\
\hline Early loss $(<24 w)$ & $326(8 \%)$ & 4 & 0 \\
\hline Perinatal death rate $[\mathrm{CI}]$ & $32[23-40]$ & 40 & 33 \\
\hline $\begin{array}{l}\% \text { pre-pregnancy } \\
\text { counselling }\end{array}$ & $35 \%$ & $5 / 25(20 \%)$ & $9 / 30(30 \%)$ \\
\hline $\begin{array}{l}\% \mathrm{HbAlc} \text { within } \\
6 \mathrm{~m} \text { of conception }\end{array}$ & $37 \%$ & $22 / 25(88 \%)$ & $21 / 30(70 \%)$ \\
\hline $\begin{array}{l}\% \mathrm{HbA} 1 \mathrm{c} \text { measured } \\
\text { by } 13 \mathrm{w}\end{array}$ & $72 \%$ & $19 / 21(92 \%)$ & $\mathrm{N}=26(100 \%)$ \\
\hline$\% \mathrm{HbA} 1 \mathrm{c}<7 \%$ by $13 \mathrm{w}$ & $38 \%$ & $3 / 21(14 \%)$ & $9 / 26(34 \%)$ \\
\hline$\%$ preterm delivery rate & $36 \%$ & $10 / 21(48 \%)$ & $11 / 30(37 \%)$ \\
\hline$\%$ caesarean section & $67 \%$ & $14 / 21(67 \%)$ & $26 / 30(90 \%)$ \\
\hline$\%$ babies born $>4000 \mathrm{~g}$ & $21 \%$ & $5 / 20(25 \%)$ & $8 / 28(28 \%)$ \\
\hline $\begin{array}{l}\% \text { term admissions } \\
\text { to neonatal unit }\end{array}$ & $33 \%$ & $16 / 20(80 \%)$ & $26 / 28(93 \%)$ \\
\hline Average stay (days) & 3 & 12 & 3 \\
\hline
\end{tabular}

Conclusion: The results demonstrate that there remains a significant deficit in the care of women with diabetes, both nationally and locally, such that the St Vincent Declaration will remain unmet in the near future. This audit demonstrates specifically where our service differs from the national standards and where we need to invest greater efforts through education and/or resources. The introduction of the ICP as a single, comprehensive record of care for all health care professionals has improved the standards of care for these women. Whilst there is a much higher caesarean section rate in the ICP group, there was a shift from emergency to elective procedures following the introduction of the ICP (elective caesarean sections as a proportion of total caesareans $30 \%$ vs $8 \%$ following introduction of the ICP). Transfer to neonatal unit policy reflect local services at this time. This audit is a stimulus to improve care for women with diabetes in pregnancy.

0938

Effect of glycaemia control initiated at different stages of pregnancy on complications and pregnancy outcomes N. G. Asatiani ${ }^{1}$, R. Kurashvili ${ }^{1}$, M. Dundua ${ }^{1}$, E. Shelestova ${ }^{1}$, K. Pagava ${ }^{1}$, M. Hod ${ }^{2}$, S. Smirnov ${ }^{3}$, V. Vlasov ${ }^{3}$; 
${ }^{1}$ Georgian Diabetes Center, Georgian Diabetes Center, Tbilisi, Georgia, ${ }^{2}$ Rabin Medical Center, Dep.of Obstetrics and Gynecology, Tel-Aviv, Israel, ${ }^{3}$ Novo Nordisk A/S, Copenhagen, Denmark.

Background and Aims: Glycemic targets, achieved by appropriate treatment of type 1 diabetes (T1DM), should mimic those of normal pregnancies, and metabolic state should be such that the fetus does not recognize its mother as diabetic. Our aim was to assess the effect of glycemia control, that was initiated at different stages of pregnancy, on complications and pregnancy outcomes.

Materials and Methods: In total, 118 women with T1DM were enrolled in the study (mean age $-26 \pm 7 \mathrm{yrs}$; diabetes duration $-10.2 \pm 5.4 \mathrm{yrs}$ ); they were separated into 3 groups (Gr.): Gr.1-38 patients (pts), who received preconception care (PC), Gr.2-36 pts enrolled in the study at $<10$ weeks of gestations, Gr.3-44 pts enrolled in the study at $>10$ weeks of gestation. Pts with nephropathy were excluded. Strict metabolic control and fetal surveillance were performed throughout the pregnancy.

Results: We obtained following HbAlc levels(\%): before PC $-8.7 \pm 2.36(\mathrm{Gr} .1)$; 1 st trimester(tr.) $-6.23 \pm 0.99$ (Gr.1), $9.41 \pm 2.62$ (Gr.2), $(\mathrm{P}=0.000)$; $2 \mathrm{nd}$ tr.- these values dropped to $5.78 \pm 1.02$ (Gr.1) and $6.47 \pm 1.09$ (Gr.2), and were $8.07 \pm 2.28$ for Gr.3, being statistically higher, than in Gr.1 and $2(\mathrm{P}=0.000, \mathrm{P}=0.001)$; 3rd tr. $-5.18 \pm 0.64$ (Gr.1), $6.62 \pm 1.04$ (Gr.2), 6.58 \pm 1.12 (Gr.3) ( $\mathrm{P}=0.000$ - Gr.1-2; $\mathrm{P}=0.000$ - Gr.1-3). Microalbuminuria had 2 (5.2\%) - Gr.1, $5(13.9 \%)$ - Gr.2, and $7(20.45 \%)$ - Gr.3 pts. In the 3rd tr. their number increased to $6(15.8 \%), 9(25 \%)$, and 12 $(20.45 \%)$ respectively. Preproliferative retinopathy was found in $9.09 \%$-Gr.1, 33.3\% -Gr.2 and in $29.4 \%-\mathrm{Gr} .3$ pts, proliferative retinopathy was found in $18.1 \%$-Gr.1, $25.9 \%$ - Gr.2 and in $20.5 \%$-Gr.3 pts. There was no retinopathy progression after delivery in Gr.1 and 2, while in two $(4.5 \%)$ Gr.3 pts retinopathy progression was observed. In Gr.1, 2 and 3 following pathologies were observed: pre-eclampsia $13.5 \%, 19.4 \%$ and $47.7 \%$; eclampsia $-0 \%, 8.3 \%$ and $13.3 \%$; preterm delivery $-2.6 \%$, $11.1 \%$ and $20.4 \%$; macrosomia $-18.4 \%, 13.8 \%$ and $15.9 \%$ respectively. Mean infants' birth weight $(\mathrm{g})$ was 3 $562 \pm 568$ (Gr.1); $3473 \pm 518$ (Gr2), and $3349 \pm 637$ (Gr.3); Cesarean section was performed in 59.09\%- Gr.1, 77.7\% Gr.2 and $61.7 \%$ - Gr.3 pts; perinatal deaths were registered in $2(5.5 \%)$ - Gr.1, and $6(13.6 \%)$ - Gr.3 pts; spontaneous abortions had 2 (5.2\%) - Gr.1, and 4 (11.1\%) - Gr.2 pts; fetus malformations were registered in $2(4.5 \%)$ - Gr.3 pts. Conclusion: Good glycemia control achieved prior to and during pregnancy not always prevents macrosomia, though significantly reduces the risk of spontaneous abortions, fetus malformations and perinatal deaths, prevents preeclampsia and eclampsia. When preconception care is carried out, pre-eclampsia and preterm delivery are less possible, than when supervision is initiated after conception. No retinopathy progression is observed, when women are well-controlled throughout the pregnancy.

0939

Type 1 diabetic pregnant women may develop postpartum thyroditis indepently of the presence of anti-thyroid antibodies

G. Philippou ${ }^{1}$, V. Sarandopoulou ${ }^{1}$, M. Alevizaki ${ }^{2}$, P. Lymperi ${ }^{3}$, E. Anastasiou ${ }^{1}$;

${ }^{1} 1$ st Endocrine Section-Diabetes Center, Alexandra Hospital, Athens, ${ }^{2}$ Medical Therapeutics, Athens University, ${ }^{3}$ Immunology Dept., Pasteur Institute, Athens, Greece.

Background and Aims: Postpartum thyroiditis (PPTD), which is a disorder of autoimmune nature, occurs with increased frequency in women with type 1 diabetes mellitus (DM1). No conclusive data exist for gestational diabetes mellitus (GDM). In a previous study we showed that the prevalence of PPTD in DM1 women was $73 \%$ higher compared to controls, and that GDM was not associated with increased prevalence of PPTD. The aim of this study is to evaluate the presence of anti-thyroid antibodies (antiTPO and anti-TG), during pregnancy and after delivery, as a risk factor for the appearance of the PPTD in normal, GDM and DM1 pregnant women.

Materials and Methods: We prospectively examined 148 pregnant women attending our outpatients over a period of 4 years during pregnancy, as well as during the first postpartum year. All women were of Greek ethnic origin. PPTD was defined as the de novo appearance of TSH levels outside the normal range $0.2-4.36 \mu \mathrm{U} / \mathrm{ml}$. Women with pre-existing autoimmune thyroid disease, or taking thyroxin were not included. 44 of them were normal, (group A), 55 presented with GDM (group B), and 49 with DM1 (group C).

Results: The distribution of the anti-TPO abs before and after delivery in the three groups was as follows: group A $13.6 \%$ vs. $43.2 \%(\mathrm{p}<0.01)$, group B $27.3 \%$ vs. $40 \%$ $(\mathrm{p}<0.05)$, while for group C was $36.7 \%$ vs. $42.9 \%$ (NS) respectively. Regarding the anti-TG abs the distribution was: group A $15.9 \%$ vs. $56.8 \%(\mathrm{p}<0.01)$, group B $14.5 \%$ vs. $54.5 \%(p<0.01)$, while for group $C$ it was $49.0 \%$ vs. 44.9 (NS). PPTD was found in $13.6 \%$ of group A, $10.9 \%$ of group B, and $24.6 \%$ of group C. From among the women with positive ant-TPO abs during pregnancy, PPDT was found in $50 \%$ of group A, $20 \%$ of group B, and $39 \%$ of group $\mathrm{C}$, while from among those with negative anti-TPO abs, PPDT was found in $8 \%$ of group A, $7.5 \%$ of group B, and $16.1 \%$ of group $C$. With respect to the presence of positive anti-TG abs during pregnancy, PPDT was found in 
$28.6 \%$ of group A, $0 \%$ of group B, and $37.5 \%$ of group C, while of those with negative anti-TG abs, PPDT was found in $10.8 \%$ of group A, $12.8 \%$ of group B, and $12 \%$ of group C. Taking groups A and B together, the presence of DM1 gave an OR 2.9 for the development of PPDT in pregnant women with negative anti-TPO abs and an OR 6.8 in those with positive anti-TPO abs. Respectively, the presence of DM1 in pregnant women with negative anti-TG abs gave an OR 7.5 for the development of PPDT and 24.6 in those with positive anti-TG abs.

Conclusion: DM1 pregnant women appeared with higher anti-thyroid antibodies during pregnancy, compared to normal and GDM women. However, after delivery, in the DM1 group there was no further increase in anti-thyroid antibodies, in contrast to the other two groups (GDM and controls) in which a significant increase in both anti-TPO and anti-TG abs was observed. Finally, DM1 pregnant women present a higher risk for the development of PPDT compared to normal and GDM women, regardless of the presence or not of anti-thyroid antibodies.

\section{0}

Concentration of sE-selectine and SVCAM-1 in early pregnancy as predictors of fetal growth in pregnancy complicated with pre-gestational diabetes

A. Zawiejska, E. Wender-Oegowska, J. Brazert;

Dept of Obst. and Women's Diseases, University School of Med Sciences, Poznań, Poland.

Background and Aims: Pregestational diabetes (PGDM) is known factor influencing fetal growth. Pregnancy related hyperglycemia induces excessive fetal growth, whereas impaired function of placental vasculature result in intruterine fetal restriction. Macrosomia defined as birth weight exceeding $4000 \mathrm{~g}$ complicates up to $40 \%$ of all diabetic pregnancies and diagnostics of this complication still needs an improvement.The aim of this study was to check if altered concentration of markers of diabetic endothelial dysfunction can be of any use in prediction of fetal growth disturbances in diabetic pregnancy.

Materials and Methods: 97 pregnant women with PGDM and singleton pregnancy were enrolled into the study. Average age in the study group was: $26,5 \pm 5,5$ years, average age at diabetes onset: $17 \pm 8,5$ years, average diabetes duration: 9,5 $\pm 6,5$ years, average $\mathrm{HbA1C}$ concentration at booking: $7,3 \pm 1,8 \%$.In the study group venous blood was taken twice: at booking and in term pregnancy. Following parameters were assessed: sE-selectine, sVCAM-1, $\mathrm{HbA1c}$, lipid profile, as well as average daily glycemia and 24-h creatinine clearance.After delivery all patients were retrospectively allocated to LGA, SGA,or AGA group according to birth weight of newborn. Statystical analysis of data was pwerformed using Kruskal-Wallis test and Wilcoxon test, as well as ROC curve analysic, p value $<0,05$ was considered statistically significant.

Results: No significant differences in $\mathrm{HbA} 1 \mathrm{C}$, average daily glucemia and lipid parameters both in early and in term pregnancy were found comparing AGA, LGA and SGA group. Significant differences between groups were noted, comparing early pregnancy concentrations of sEselectine and sVCAM-1: 39,2 (8,6-160,4) ng/ml, 53,1 $(12,5-139,1) \mathrm{ng} / \mathrm{ml}, 39,0(9,9-64,8) \mathrm{ng} / \mathrm{ml}$ (for sE-selectine in AGA, LGA and SGA group, respectively) and 576,8 $(249,9-1666,3) \mathrm{ng} / \mathrm{ml}, 524,1(224,7-1931,9) \mathrm{ng} / \mathrm{ml}, 846,2$ $(361,3-1846,0) \mathrm{ng} / \mathrm{ml}$ (for sVCAM-1 in AGA, LGA and SGA group, respectively). In the ROC curve analysis following markers of endothelial dysfunction were predictors for macrosomia: sE-selectine concentration in early pregnancy $(\mathrm{AUC}=0,67$, cut-off value $=42,2 \mathrm{ng} / \mathrm{ml}$, sensitivity $=38,7 \%$, specificity $=60,7 \%$ ), sVCAM- 1 concentration in early pregnancy $(\mathrm{AUC}=0,63$, cut-off value $=348,4 \mathrm{ng} / \mathrm{ml}$, sensitivity $=34,4 \%$, specificity $=85,5 \%$ ), change in sVCAM1 concentration during pregnancy $(\mathrm{AUC}=0,35$, cut-off value $=605,9 \mathrm{ng} / \mathrm{ml}$, sensitivity $=31 \%$, specificity $=61 \%$ ).

Conclusion: 1/Measurements of sE-selectine and sVCAM1 concentrations in early pregnancy can be useful in prediction of fetal growth abnormalities in pregnant women with PGDM.

Supported by National Science Comittee Poland

0941

Platelet disaggregation and intracellular levels cGMP and cAMP in pregnant women with gestational diabetes mellitus and type 1 diabetes mellitus

Z. V. Zabarovskaj ${ }^{1}$, V. D. Bichan ${ }^{2}$, A. B. Samal ${ }^{2}$, S. M. Shulyakovskaya ${ }^{3}$;

${ }^{1}$ General Medicine, ${ }^{2}$ Biophysics, ${ }^{3}$ Chemistry of Nucleotides, Belarussian State Medical University, Minsk, Belarus.

Background and Aims: The diabetes mellitus (DM) of any type already at early stages is characterized by abnormality of platelets. At the patients with DM the rising intravascular activation of platelets resulting in to occurrence in a vascular channel platelet aggregates and dysfunction of microcirculation is observed. It is known, that volume of NO synthesis in platelet at the patients with Type 1 and Type 2 DM make no more than halves from recorded at the healthy persons. However now is shown, that at the pregnant women with gestational diabetes mellitus (GDM) the activity NO-synthase is much higher, than in group of the healthy pregnant women. We have shown that the partial decay of stable platelet aggregates (disaggregation) 
can be induced by activity of some exogenous substances increases concentration of cGMP and cAMP in the platelets of the healthy donors. The aim of this study was to measure the velocity of platelet disaggregation and the concentration of the platelet second messengers cGMP and cAMP by sodium nitroprusside (SNP) and ATP, respectively, in vitro in pregnant women with GDM.

Materials and Methods: We compared 14 pregnant women with GDM $(24.68 \pm 5.12$ years and gestational age (24-26 weeks)), 11 pregnant women with Type 1 DM $(24.31 \pm 3.68$ years) and 20 healthy pregnant women (23.96 \pm 5.18 years). There were no significant differences in fasting glycaemia $(5.38 \pm 0.41) \mathrm{mmol} / \mathrm{l}$ for GDM, $(6.27 \pm 0.21) \mathrm{mmol} /$ for pregnant women with Type $1 \mathrm{DM}$ and $(4.61 \pm 0.17) \mathrm{mmol} / \mathrm{l}$ for healthy pregnant women) or in glycated hemoglobin levels $(6.78 \pm 0.21 \%, 6.94 \pm 0.13 \%$ and $5.83 \pm 0.61 \%$ accordingly). The study was performed in accordance with the principles of the Declaration of Helsinki as revised in 1996. Platelet disaggregation was determined by light transmission using an AP 2110 computerized analyzer of platelet aggregation (SOLAR, Belarus). Concentrations of cAMP and cGMP were determined radioimmunologically with a $\gamma$-counter (LKB Wallac, Finland).

Results: The nonreversible aggregation was induced by $1.5 \mu \mathrm{mol} / 1 \mathrm{ADP}$, thus the intracellular concentration cGMP in each group has made $0.4 \pm 0.1 \mathrm{pmol}$. The donor NO - NP in concentration $400 \mu \mathrm{mol} / 1$ added to aggregating under activity ADP to platelets for 3 minutes of aggregation, in this case level cGMP was increaseds in 4 times in the first and second groups and in 5.5 times in the third group of the donors. However differences between groups were inappreciable (1.6 $\pm 0.1 \mathrm{pmol}, 1.5 \pm 0.1 \mathrm{pmol}$ and $2.2 \pm 0.1 \mathrm{pmol}$ accordingly). Thus the velocity of induced disaggregation at the pregnant women with GDM and with Type $1 \mathrm{DM}$ was higher than in control group in 1.8 and 3.4 times accordingly. There was no significant difference between ATP-induced disaggregation and cAMP levels in pregnant women with GDM, pregnant women with Type $1 \mathrm{DM}$ and healthy pregnant women.

Conclusions: Is assumed that the NP-induced platelet disaggregation in investigation groups of the donors is not linked to cGMP-signaling systems and under activity of ADP are formed less stable platelet aggregates, than in control group. ATP-cAMP pathways is not activated in investigation group.

\section{PS 78}

\section{Diagnosis and treatment of diabetes in pregnancy}

0942

Performance of the 1 hour glucose value as a diagnostic test for gestational diabetes (World Health Organization Procedure)

M. A. A. Campos, A. J. Reichelt, M. I. Schmidt;

Department of Social Medicine, Posgraduate Program in Epidemilogy, Federal University of Rio Grande do Sul, Porto Alegre, Brazil.

Background and Aims: Diagnostic criteria for gestational diabetes are based on glucose tolerance tests performed in 2 or 3 hours. The objective of the study is the evaluation of the ability of the 1 hour glycemia after a $75 \mathrm{~g}$ glucose oral load to predict gestational diabetes (WHO criteria) and selected pregnancy outcomes.

Materials and Methods: A cohort of 4998 pregnant women with 20 years or more, no previous diabetes, who attended prenatal care clinics in Brazil answered a questionnaire and had a $2 \mathrm{~h}$ glucose test between their 24th and 28th gestational weeks.

Results: For the diagnosis of gestational diabetes the area under the ROC curve of the $1 \mathrm{~h}$ glucose was $0,903(0,886-$ $0,919)$. The cut point that jointly maximized sensitivity $(83 \%)$ and specificity (83\%) for detection of gestational diabetes was $141 \mathrm{mg} / \mathrm{dl}$; for macrosomia detection it had sensitivity of $33 \%$ and specificity of $78 \%$. Specificity of $99 \%$ for gestational diabetes and of $97 \%$ for macrosomia was reached with values of $180 \mathrm{mg} / \mathrm{dl}$. An intermediate cut point with sensitivity of $62 \%$ and specificity of $94 \%$ in the prediction of gestational diabetes was $160 \mathrm{mg} / \mathrm{dl}$; for macrosomia its specificity was $90 \%$. The prediction of adverse gestational outcomes was similar to that reached with the $2 \mathrm{~h}$ glucose.

Conclusion: It is possible to simplify the detection of gestational diabetes with the $1 \mathrm{~h}$ glucose, simultaneously as a screening $(141 \mathrm{mg} / \mathrm{dl})$ and as a diagnostic $(180 \mathrm{mg} / \mathrm{dl})$ test. A small proportion of women will require confirmation, but the diagnosis will have been made earlier for those with higher glucose values. 
0943

New threshold for impaired fasting glucose: impact on postpartum reevaluation of glucose metabolism disorders in women with gestational diabetes mellitus

I. Capel, I. Vinagre, A. García-Patterson, S. Torrejón,

J. Úbeda, M. María, A. de Leiva, R. Corcoy;

Endocrinology, Hospital de Sant Pau, Barcelona, Spain.

Background and Aims: In 2003 the American Diabetes Association (ADA) decreased the cut-off value of fasting plasma glucose (FPG) for the diagnosis of impaired fasting glucose (IFG) to $\geq 5,6 \mathrm{mmol} / \mathrm{l}$. The aim was to increase the sensitivity in the diagnosis of glucose metabolism disorders and the agreement between abnormal categories according to FPG and plasma glucose after an oral glucose tolerance test (OGTT). Our aim was to measure the impact of the adoption of new criteria (WHO1998 $\rightarrow$ ADA2003) in the postpartum reevaluation of women with gestational diabetes mellitus (GDM). We measured the influence on the prevalence of glucose metabolism disturbances and the agreement between fasting and post-OGTT categories.

Material and Methods: 1168 women with GDM were reevaluated with an OGTT within 18 months after delivery. $55 \%$ had required insulin treatment during pregnancy. At evaluation, age was 33.4 years $(18.1-45.6)$ and body mass index was $24.28 \mathrm{~kg} / \mathrm{m}^{2}(16.6-50.17)$. OGTT was performed 4.8 months $(0.12-17.9)$ after delivery. The distribution of glucose tolerance according to the different criteria was compared with a $\chi^{2}$ test and the agreement between fasting and post-OGTT categories with the kappa index.

Results: The prevalence of glucose metabolism disorders was $16.9 \%$ according to WHO1998 criteria vs $28.8 \%$ according to ADA2003 criteria. ( $70 \%$ increase; $\mathrm{p}<0.01)$. The agreement between fasting and post-OGTT categories was bad with both criteria (kappa index 0.247 with OMS1998 vs 0.200 with ADA2003).

Conclusions: In women with GDM, the reduction in the threshold for IFG diagnosis increases substantially the prevalence of glucose metabolism disorders at the postpartum reevaluation. There is a bad agreement between basal and post-OGTT categories with both criteria, suggesting a different physiopathology.

\section{4}

Standard breakfast validity in the diagnosis of gestational diabetes

B. Pascual ${ }^{1}$, A. Rufo ${ }^{2}$, J. Mateo ${ }^{3}$, A. García ${ }^{1}$, M. Hernández. ${ }^{4}$, I. Fernández ${ }^{1}$;
${ }^{1}$ Research Unit, Servicio Andaluz de Salud, Camas Sevilla, ${ }^{2}$ Midwife, Servicio Andaluz de Salud, Camas Sevilla, ${ }^{3}$ Clinical Laboratory, Servicio Andaluz de Salud., Hospital Virgen Macarena - Sevilla, ${ }^{4}$ Midwife, Servicio Andaluz de Salud, Mairena del Aljarafe - Sevilla, Spain.

Background and Aims: The diagnosed of Gestational diabetes(GD) is based on an oral glucose tolerance test (OGTT). This test commonly induces vomiting, nausea or unspecific unpleasant state and is recognized as a not very physiological test. Our aims is to analyse the criterion validity of a standard breakfast for the Gestational Diabetes diagnosis in pregnant women, using as gold standard the100 g oral glucose tolerance test and National Diabetes Data Group criteria.

Material and Methods: Design: Multicenter observational study about diagnostic test in Primary Care. Measurements: A SB $(711 \mathrm{Kcal}, 98 \mathrm{~g}$ carbohydrates, $29 \mathrm{~g}$ of fat, $15 \mathrm{~g}$ of proteins and $3 \mathrm{~g}$ of fibber) in pregnant women was carried out in 24-28 gestation weeks, determining venous plasma glycaemia (VPG) in 0, 1st, 2nd and 3rd hours. We analyse sensibility(Se), specificity (Sp), positive and negative predictive values (PPV and NPV). We use the ROC curve to study the diagnostic performance and to seek the best cutoff values.

Results: 637 pregnant women accepted the study: 506 were included (vomit during OGTT test was the more frequency exclusion criteria). The prevalence of GD was $11.4 \%$. The ROC curves analysis shows: the best area under curve (AUC) is for 1st VPG measurement $(126 \mathrm{mg} / \mathrm{dl}$, AUC 0.885 , Se $81.67 \%$, Sp $81.84 \%$, PPV $35.72 \%$, NPV 97.31\%), 2nd VPG measurement (102 mg/dl, AUC 0.830, Se $79.31 \%$, Sp 66.07\%, PPV 21.54\%, NPV 96.54\%).

Conclusion: Diagnosing GD through SB would be a usefulness alternative for women with OGTT intolerance: 1st and 2nd VPG present an adequate Se and Sp. We will have a most physiological and better tolerated test to apply in the pregnancy to diagnose the gestational diabetes.

Supported by semFYC

0945

Maternal gestational diabetes and the manifestations of metabolic syndrome in adolescent offspring

A. Pouta ${ }^{1,2}, M$. Vääräsmäki ${ }^{2}$, P. Tapanainen ${ }^{3}$, A.-L. Hartikainen ${ }^{2}$, U. Sovio ${ }^{4}$, A. Ruokonen ${ }^{5}$, P. Elliott ${ }^{4}$, M. I. McCarthy ${ }^{6}$, M.-R. Järvelin ${ }^{4,7}$; 
${ }^{1}$ Life Course Studies Unit, National Public Health Institute, Oulu, Finland, ${ }^{2}$ Dept of Obstetrics and Gynecology, ${ }^{3}$ Dept of Pediatrics, University of Oulu, Finland, ${ }^{4}$ Dept of Epidemiology and Public Health, Imperial College London, United Kingdom, ${ }^{5}$ Dept of Clinical Chemistry, University Hospital of Oulu, Finland, ${ }^{6}$ Oxford Centre for Diabetes, Endocrinology \& Metabolism, University of Oxford, United Kingdom, ${ }^{7}$ Dept of Public Health Science and General Practise, University of Oulu, Finland.

Background and Aims: The increasing epidemiological evidence suggests that the prenatal environment has an important role in the development of chronic adult diseases, like diabetes and cardiovascular disorders. Maternal gestational diabetes (GDM) has been found to associate with subsequent early morbidity of the offspring but there are no population-based studies on the incidence of traits of metabolic syndrome in later life among offspring, and practically no data on gender differences.

Materials and Methods: The general population-based data were obtained from the prospective Northern Finland 1986 Birth Cohort comprising all singleton pregnancies of the 9247 mothers and their offspring born in 1985-86. The latest clinical follow-up was performed at age 16 for the offspring with $74 \%$ response rate $(\mathrm{N}=6798)$. The risk factor based screening of GDM was performed during pregnancy according to national criteria (glucosuria, prior GDM or macrosomic infant, current macrosomia, BMI $>25 \mathrm{~kg} / \mathrm{m}^{2}$, age $>40 \mathrm{yr}$ ). The diagnosis of GDM was confirmed by $75 \mathrm{~g}$ OGTT performed after overnight fasting. The study population with written consent consists of offspring of mothers from three groups: 1) GDM diagnosed by OGTT ( $\mathrm{n}=95), 2)$ mothers with normal results of OGTT ( $\mathrm{n}=742$, screen indicated), and 3) the reference group consisting of mothers without GDM or the above risk factors used for screening $(n=3938)$. Women without OGTT despite indications $(\mathrm{n}=1942)$ and women with pre-pregnancy diabetes $(\mathrm{n}=27)$ were not included in the analyses. The distributions of metabolic traits were skewed and logarithmic transformations were applied. The results are reported in geometric means and percentage differences between exposure groups with $95 \% \mathrm{CI}$.

Results: The prevalence of overweight (BMI $>25 \mathrm{~kg} / \mathrm{m}^{2}$ ) was significantly higher among offspring of GDM-mothers than in the reference group (18.1 vs. $8.4 \%$ ), and their mean BMI was higher (Table). Waist circumference, insulin and HOMA-S were significantly higher in offspring of GDMmothers than in the reference group. Interestingly, these findings seem to be stronger in girls than in boys.

Conclusion: The features of metabolic syndrome were clearly seen in offspring of GDM mothers. This risk group should be noted by planning preventive and therapeutic strategies already before potential clinical manifestation of metabolic syndrome.
Outcomes at age 16

\begin{tabular}{|c|c|c|c|c|c|c|c|}
\hline & \multirow{2}{*}{\multicolumn{2}{|c|}{$\begin{array}{l}\text { GDM } \\
\text { offspring } \\
\mathrm{n}=95\end{array}$}} & \multicolumn{5}{|c|}{$\begin{array}{l}\text { Reference } \\
\text { group }\end{array}$} \\
\hline & & & $\mathrm{n}=393$ & & & & \\
\hline & Mean & $95 \% \mathrm{CI}$ & Mean & $95 \% \mathrm{CI}$ & $\begin{array}{l}\text { Percentage } \\
\text { difference }\end{array}$ & $95 \%$ CI & $\mathrm{p}$-value \\
\hline BMI kg/m² & 21.9 & $\begin{array}{l}(21.3 \\
22.6)\end{array}$ & 20.6 & $\begin{array}{l}(20.5, \\
20.7)\end{array}$ & 6.4 & $(3.5,9.5)$ & $<0.0001$ \\
\hline $\begin{array}{l}\text { Waist } \\
\text { circumference, } \\
\mathrm{cm}\end{array}$ & 76.7 & $\begin{array}{l}(75.1 \\
78.3)\end{array}$ & 72.5 & $\begin{array}{l}(72.3 \\
72.7)\end{array}$ & 5.7 & $(3.5,8.0)$ & $<0.0001$ \\
\hline SBP, mmHg & 117 & $\begin{array}{l}(115, \\
120)\end{array}$ & 114 & $\begin{array}{l}(114, \\
115)\end{array}$ & 2.5 & $(0.3,4.8)$ & 0.028 \\
\hline DBP, $\mathrm{mmHg}$ & 68 & $(66,69)$ & 67 & $(67,67)$ & 1.3 & $\begin{array}{l}(-0.9, \\
3.7)\end{array}$ & 0.251 \\
\hline $\begin{array}{l}\text { Insulin, } \\
\mathrm{mU} / \mathrm{L}\end{array}$ & 10.9 & $\begin{array}{l}(10.0 \\
11.9)\end{array}$ & 9.4 & $\begin{array}{l}(9.3 \\
9.6)\end{array}$ & 15.2 & $\begin{array}{l}(5.6, \\
25.6)\end{array}$ & 0.002 \\
\hline $\begin{array}{l}\text { Glucose, } \\
\mathrm{mmol} / \mathrm{L}\end{array}$ & 5.2 & $\begin{array}{l}(5.1, \\
5.3)\end{array}$ & 5.1 & $\begin{array}{l}(5.1, \\
5.2)\end{array}$ & 1.7 & $\begin{array}{l}(-0.3, \\
3.8)\end{array}$ & 0.101 \\
\hline HOMA-S & 475 & $\begin{array}{l}(435, \\
519)\end{array}$ & 548 & $\begin{array}{l}(540, \\
555)\end{array}$ & -13.3 & $\begin{array}{l}(-20.6 \\
-5.3)\end{array}$ & 0.002 \\
\hline
\end{tabular}

Supported by Academy of Finland

0946

First trimester sex hormone binding globulin and subsequent development of gestational diabetes mellitus R. Zinnat ${ }^{1}$, S. Yasmin ${ }^{2}$, T. A. Chowdhury ${ }^{2}$, L. Ali ${ }^{1}$; ${ }^{1}$ Dept of Biochemistry and Cell Biology, ${ }^{2}$ Dept of Gyne and Obs, BIRDEM, Dhaka, Bangladesh.

Background and Aims: Sex hormone binding globulin (SHBG) has been shown to have an inverse association with insulin sensitivity and, thus, its lower value has been suggested as a useful tool for investigating insulin resistance. The association of reduced SHBG with gestational diabetes mellitus (GDM) is now fairly well recognized, but the causal relationship between the two events is yet debated. The present study was undertaken to test the hypothesis that first trimester SHBG may predict the subsequent development of GDM in later stages of pregnancy.

Materials and Methods: A total of 103 subjects, collected from various hospitals and clinics in Dhaka city, were followed from their 1st to 3rd trimesters of pregnancy. Detailed history, clinical examination and anthropometry (height and weight) were recorded. GDM was diagnosed following the WHO guidelines. SHBG level was estimated by Chemiluminescent Immunometric Assay.

Results: Out of the 103, $62(60 \%)$ patients showed normal glucose tolerance upto the end of the pregnancy (the Control 
Group). A number of 22 subjects (21\%) subsequently developed GDM (the GDM Group) and 19 (18\%) patients already showed evidence of glucose intolerance at the first trimester. This last group was designated as Positive Control. Taking 25-75th percentile levels of SHBG in the Control as the range, the reference values for SHBG in our 1st trimester pregnant population was found to be $80-157 \mathrm{nmol} / \mathrm{l}$. The GDM group showed less than $50 \%$ of SHBG levels compared to control. [SHBG, nmol/1; median (range): Control, 125 (25-180) vs GDM 52 (15-180), p <0.0001]. About $82 \%$ of GDM cases showed SHBG values lower than the lowest limit of the reference range $(80 \mathrm{nmol} / \mathrm{l})$ and there was high probability of developing GDM in subjects having low SHBG in the first trimester (Odds Ratio; 14: 95\% CL 4.12-48.21). In contrast to GDM the Positive Control Group showed no significant difference of SHBG levels as compared to Control group. Only 36\% of the Positive Control subjects showed low SHBG values (ie $<80 \mathrm{nmol} / \mathrm{l}$ ) as compared to $25 \%$ in the Control.

Conclusion: The data indicate that insulin resistance has a causal relation with GDM and the abnormality can be detected at the early stages of pregnancy using SHBG as a marker.

Supported by the International Program in the Chemical Sciences (IPICS) and Diabetic Association of Bangladesh

0947

Safety and efficacy of short-acting insulin aspart and lispro analogues in patients with gestational diabetes mellitus (GDM)

A. Ghio, L. Volpe, C. Lencioni, I. Cuccuru, V. Resi, S. Del Prato, G. Di Cianni;

Department of Endocrinology and Metabolism, University of Pisa, Italy.

Background and Aim: Because of increased risk of maternal and neonatal complications, insulin treatment is often necessary in GDM women. Although both Aspart (Asp) and Lispro (Lis) have been used systematic comparison with respect to regular insulin (HI) is still scanty. The aim of this study was to compare the pregnancy outcome in GDM women treated with Asp, Lis, or HI.

Material and Methods: In 96 women (age $34.8 \pm 4.7$ years, 70 primiparous, pre-pregnancy BMI $25.8 \pm 5.4 \mathrm{Kg} / \mathrm{m} 2$ ) with GDM (Carpenter and Coustan's criteria at 27.5 \pm 2.1 week of gestation) and on $30 \mathrm{Kcal} / \mathrm{Kg} / \mathrm{die}$ (CHO $50 \%$, proteins $20 \%$, fat $30 \%$ ) insulin therapy was begun (median value: 29th week of gestation) for fasting/pre-prandial and/or 1 -h postprandial glucose $>90$ and $>130 \mathrm{mg} / \mathrm{dl}$ (therapy targets), respectively. GDM women were randomly allocated to pre-prandial injection of $\mathrm{HI}(\mathrm{n}=32)$, Asp $(\mathrm{n}=31)$, or Lis $(n=33)$ treatment. Blood glucose profiles (5 points/day) were recorded and weekly contact with the diabetologist ensured for treat-to-target modification of insulin dose, including addition of bed-time NPH insulin if necessary.

Results: All 3 groups were comparable for age, prepregnancy BMI, weight gain, and HbAlc. NPH insulin was required in $23 \mathrm{HI}(5.6 \pm 4 \mathrm{IU}), 18 \mathrm{Lis}(5.2 \pm 5 \mathrm{IU})$ and 16 Asp (4.7 $\pm 6 \mathrm{IU})(\mathrm{p}=\mathrm{NS})$. At the end of the pregnancy, total insulin dose (HI 18.4 \pm 11 , Lis $22.4 \pm 13$, Asp $22.3 \pm 18 \mathrm{UI} /$ day), weight gain (HI 10.6 \pm 5.2 , Lis $11.2 \pm 5.9$, Asp $10 \pm 4 \mathrm{Kg}$ ), fasting plasma glucose (HI $89.6 \pm 11.3$, Lis 86.1 \pm 10.6 , Asp $82.6 \pm 8.9 \mathrm{mg} / \mathrm{dl}$ ), and HbAlc (HI 5.45 \pm 0.4 , Lis $5.35 \pm 0.4$, Asp $5.45 \pm 0.3$ ) were not different. On the contrary, 1-h post-prandial glucose as detrmined after standardized breakfast was higher in HI $(135 \pm 24 \mathrm{mg} / \mathrm{dl})$ than Lis $(119 \pm 19 \mathrm{mg} / \mathrm{dl}, \mathrm{p}<0.03)$ and Asp $(121.5 \pm 20.3$; $\mathrm{p}=0.06$ ). No hypoglycaemic episode was recorded for any of the 3 treatments. Delivery occurred at 38th week of gestation in all groups. Birth weight was greater in $\mathrm{HI}$ $(3359 \pm 393$ vs. Lis $3218 \pm 458$ and Asp $3066 \pm 494 \mathrm{~g}$; ANOVA $\mathrm{p}<0.04$ ); incidence of macrosomia (LGA) was $15.6 \%$ in $\mathrm{HI}, 12.1 \%$ in Lis, and $9.6 \%$ in Asp ( $p=? ?)$; in newborns of HI-treated women cranial/thoracic $(\mathrm{cc} / \mathrm{ct})$ ratio was lower than Lis and Asp (1.01 \pm 0.39 vs. Lis $1.04 \pm 0.3$; Asp $1.03 \pm 0.35$; ANOVA $p<0.03$ ) with $18 \%$ of them with cc/ct $<1$ (Lis $0 \%$, Asp 3.2\%; $<0.02$ ).

Conclusions: In spite of similar overall glycaemic control, use of short-acting insulin analogues in GDM women was ensured better prandial glucose tolerance. This effect was associated with more proportionated foetal growth as compared to use of regular insulin.

0948

Maternal hypoglycaemia and glycaemic control in pregnancy: a randomised trial comparing insulin aspart with human insulin in 322 subjects with type 1 diabetes S. R. Heller ${ }^{1}$, E. Mathiesen ${ }^{2}$, B. Kinsley ${ }^{3}$, D. McCance ${ }^{4}$, S. Duran ${ }^{5}$, S. Bellaire ${ }^{6}$, A. Raben ${ }^{6}$; ${ }^{1}$ Academic Unit of Diabetes, Endocrinology and Metabolism, University of Sheffield, United Kingdom, ${ }^{2}$ Dept of Endocrinology, University of Copenhagen, Denmark, ${ }^{3}$ Dept of Endocrinology and Diabetes Mellitus, Mater Misericordiae University Hospital, Dublin, Ireland, ${ }^{4}$ Metabolic Unit, Royal Victoria Hospital, Belfast, United Kingdom, ${ }^{5}$ Catedra De Endocrinologica, Hospital Universitario Nuestra Senora De Valme, Seville, Spain, ${ }^{6}$ Novo Nordisk A/S, Copenhagen, Denmark

Background: Although outcomes during pregnancy in type 1 diabetes have improved due to intensive insulin therapy and achieving near normoglycaemia, treatment is often complicated by the associated risk of severe and occasion- 
ally life-threatening maternal hypoglycaemia. We measured the effect of a rapid-acting insulin analogue in a parallel group, randomised, controlled, multi-centre trial comparing Insulin Aspart (IAsp) and Human Insulin (HI) as meal time insulin in a basal-bolus regimen with NPH.

Material and Methods: Pregnant women with type 1 diabetes, aged 19-43 yr, HbAlc $<8 \%$ at confirmation of pregnancy, were treated with IAsp $(n=157)$ or HI $(n=165)$. Evaluations included measurements of severe hypoglycaemia (requiring assistance), HbA1c, 8-point plasma glucose (PG) profiles and perinatal outcome.

Results: The estimated risk of severe night-time hypoglycaemia was 52\% lower for IAsp than HI (Relative Risk (RR): $0.48,95 \%$ CI $[0.20 ; 1.14] ; \mathrm{p}=0.10$ (gamma frailty model)). Observed rates of severe hypoglycaemia (episodes per year) tended to be lower for IAsp (1.4) than for HI (2.1) and the estimated risk was $28 \%$ lower for IAsp than HI (RR: 0.72 ; $95 \%$ CI $[0.36 ; 1.46] ; \mathrm{p}=0.36)$. Prandial glucose increments (mean of breakfast, lunch and dinner) were significantly lower with IAsp than with HI both in the 1 st $(0.73$ vs. $1.49 \mathrm{mM} ; \mathrm{p}<0.01)$ and 3 rd trimester $(1.10$ vs. $1.50 \mathrm{mM}$; $\mathrm{p}=0.04)$. No differences were observed for the average 24hour PG or HbAlc (HbA1c (\%): IAsp: 6.0 and 6.18, HI: 6.04 and 6.26 in 2nd and 3rd trimester, respectively). Most patients in either treatment group showed no progression in retinopathy. Perinatal outcome was generally good, without large differences between IAsp and HI.

Conclusion: The risk of severe hypoglycaemia tended to be lower in the Insulin Aspart treated than Human insulin treated women, especially during the night.

Supported by Novo Nordisk A/S Denmark

0949

Safety and perinatal outcome in pregnancy: a randomised trial comparing insulin aspart with human insulin in 322 subjects with type 1 diabetes

R. Kaaja ${ }^{1}$, M. $\operatorname{Hod}^{2}$, G. Wisser ${ }^{3}$, P. Damm ${ }^{4}$, F. Dunne ${ }^{5}$, A. Hansen ${ }^{6}$, H. Mersebach ${ }^{6}$;

${ }^{1}$ Obsterics and Gynecology, Helsinki University Hospital, Finland, ${ }^{2}$ Department of High Risk Pregnancy, Rabin Medical Center, Beilinson Petah Tikva, Israel, ${ }^{3}$ Obsterics and Gynecology, UMC, Utrecht, The Netherlands, ${ }^{4}$ Clinical Biochemistry, Rigshospitalet, Copenhagen, Denmark, ${ }^{5}$ Medicine, Galway University College Hospital, Ireland, ${ }^{6}$ Novo Nordisk, Copenhagen, Denmark,

Background: Pregnancy in diabetes is associated with increased complications for mother and offspring, and strict blood glucose control is vital. This parallel group, randomised, controlled, multi-centre trial studied the pregnancy outcome (maternal and foetal) after treatment of pregnant women with Insulin Aspart (IAsp) or Human Insulin (HI). Materials and methods: Pregnant women with type 1 diabetes, aged 19-43 $\mathrm{yr}, \mathrm{HbA}_{1 \mathrm{c}} \leq 8 \%$ at confirmation of pregnancy, were treated with IAsp $(n=157)$ or $H I(n=165)$ as meal time insulin in a basal-bolus regimen with NPH. Evaluations included pregnancy outcome, obstetric complications, severe maternal hypoglycaemia and glycaemic control.

Results: Overall, 268 live births and 35 foetal losses (mostly spontaneous miscarriages) were reported (IAsp: 14, HI: 21). No difference in foetal mortality or major congenital malformations was observed between the two groups. The percentage of children who were macrosomic ( $>4000 \mathrm{~g}$ at birth) or large for gestational age was similar in the two groups. Median gestational age was 37.9 and 37.7 weeks, respectively, for IAsp and HI. The most frequent obstetric complications were emergency caesarean section (IAsp: 15.9\%, HI: 12.7\%), pre-eclampsia (IAsp: 8.3\%, HI: $6.7 \%$ ) and imminent premature labour (IAsp: $3.8 \%$, HI: $4.2 \%$ ). Preterm delivery was reported in $20 \%$ and $30 \%$ of live births for IAsp and HI, respectively. Treatmentrequiring neonatal hypoglycaemia was reported in 98 of the live births (IAsp: 46, HI: 52). Mean birth weight was $3467 \mathrm{~g}$ and $3555 \mathrm{~g}$ for IAsp and HI, respectively. The estimated risk of severe maternal hypoglycaemia was $28 \%$ lower for IAsp than $\mathrm{HI}$ with similar $\mathrm{HbA}_{1 \mathrm{c}}$ values. The lower estimated risk of hypoglycaemic episodes for IAsp was more pronounced for nightly (52\%lower) than for daytime ( $15 \%$ lower) episodes; however none of these risk differences were statistically significant.

Conclusion: Treatment with Insulin Aspart was at least as safe as Human Insulin regarding perinatal outcome.

Supported by Novo Nordisk

0950

Congenital anomaly rate in offspring of Italian mothers with type 1 diabetes treated with insulin lispro during pregnancy

A. Lapolla ${ }^{1}$, M. Dalfrà ${ }^{1}$, R. Spezia ${ }^{2}$, R. Anichini ${ }^{3}$, M. Bonomo ${ }^{4}$, G. Di Cianni ${ }^{5}$, I. Franzetti ${ }^{6}$, A. Galluzzo ${ }^{7}$, G. Mello ${ }^{8}$, G. Menato ${ }^{9}$, A. Napoli ${ }^{10}$, C. Noacco ${ }^{11}$, E. Parretti ${ }^{8}$, C. Santini ${ }^{12}$, E. Scaldaferri ${ }^{13}$; L. Scaldaferri ${ }^{1}$, M. Songini ${ }^{14}$, L. Tonutti ${ }^{11}$, E. Torlone ${ }^{15}$, R. Gentilella ${ }^{16}$, A. Rossi ${ }^{16}$, D. Valle ${ }^{16}$, 
${ }^{1}$ Medical and Surgical Sciences, University of Padova, Italy, ${ }^{2}$ STATEAM, Milano, Italy, ${ }^{3}$ Diabetic Center, Hospital of Pistoia, Italy, ${ }^{4}$ Diabetic Center, Hospital Niguarda, Milano, Italy, ${ }^{5}$ Endocrinology and Metabolism, University of Pisa, Italy, ${ }^{6}$ Diabetic Center, Hospital of Varese, Italy, ${ }^{7}$ Endocrinology, University of Palermo, Italy, ${ }^{8}$ Gynecology Perinatology and Human Reproduction, University of Firenze, Italy, ${ }^{9}$ Gynecology and Obstetrics, University of Torino, Italy, ${ }^{10}$ Diabetic Center, University "La Sapienza", Roma, Italy, ${ }^{11}$ Diabetic center, Hospital of Udine, Italy, ${ }^{12}$ Diabetic Center, Hospital of Cesena, Italy, ${ }^{13}$ Diabetic Center, Hospital of Treviso, Italy, ${ }^{14}$ Hospital of Brotzu, Cagliari, Italy, ${ }^{15}$ University of Perugia DIMISEM, Italy, ${ }^{16}$ Eli Lilly Italia spa.

Background and Aims: Some studies indicate that insulin Lispro can be used in pregnant diabetic women based on similar fetal outcomes as those observed in pregnant women using regular insulin. This study aims to analyze Italian data of a multinational, multicenter, retrospective study that included mothers with prepregnancy type 1 diabetes mellitus, who were treated with insulin lispro(L) for at least 3 months before conception and during at least the first trimester of pregnancy, with respect to prepregnant type 1 diabetes mellitus women treated with regular insulin (C) followed from the same centers.

Materials and Methods: 12 Italian centers collected data from pregnant women with diabetes, followed from 1998 2001, including: HbAlc at conception during the first and 3rd trimester,frequency of hypoglycemic episodes, spontaneous abortions, mode and timing of delivery, fetal malformations and/or death. Fisher's Exact test and Wilcoxon test were used to compare proportions and continuous data respectively.

Results: 72 L pregnancies and 298 controls (C), matched for age $(31.1+4.8$ vs $30.6+4.6)$, were included in the analysis. $50 \%$ of L pregnant had prepregnancy counseling vs $38 \%$ of C. During pregnancy, a numerically lower percentage of $\mathrm{L}$ patients experienced hypoglycemic episodes than $\mathrm{C}$ ones $(2.8 \%$ vs $5.4 \%, \mathrm{p}=\mathrm{ns})$. Gestational hypertension and preeclampsia were similar in both groups. HbA1c was similar at conception in both groups, improved significantly only in L patients $(p<0.005)$ in the first trimester and was similar at the end of pregnancy in both groups. Frequency of cesarean section was similar in both groups $(68.1 \mathrm{~L} ; 65.4 \% \mathrm{C})$. The rate of malformations was similar in both groups $(4.2 \% \mathrm{~L} ; 4.5 \% \mathrm{C})$. Conclusion: The rate of major congenital anomalies was $4.3 \%[95 \% \mathrm{CI}(0.9 \%, 12 \%)]$ for offspring of Italian mothers treated with insulin lispro before and during pregnancy.This rate was not statistically different from a comparable group treated with regular insulin(4.5\%). A lower percentage of patients treated with insulin lispro reported hypoglycemic episodes, even with improved glycemic control, as shown by the significant reduction of $\mathrm{HbAlc}$.
These data shows that also in our population the rate of congenital malformations in pregnant women treated with insulin lispro is not different from that observed in pregnant women treated with regular insulin.

0951

Glycaemic control during pregnancy in women with long duration type 1 diabetes: lessons learnt using continuous glucose monitoring systems

R. C. Temple ${ }^{1}$, K. Duffield ${ }^{1}$, K. Lewis ${ }^{2}$, H. R. Murphy ${ }^{2}$; ${ }^{1}$ Department of Diabetes and Endocrinology, Norfolk and Norwich University Hospital NHS Trust, Norwich, ${ }^{2}$ Department of Diabetes, Ipswich Hospital NHS Trust, Ipswich, United Kingdom.

Background and Aims: Recent nationwide studies of women with diabetes show that rates of complications including macrosomia, preeclampsia and prematurity remain very high compared to women without diabetes. Tight glycaemic control throughout pregnancy is important to reduce the risk of complications and current guidelines suggest target fasting glucose levels below $5.4 \mathrm{mmol} / \mathrm{L}$ and peak postprandial glucose levels below $7.9 \mathrm{mmol} / \mathrm{L}$. The aim of our study is to use continuous glucose monitoring systems (CGMS) to examine blood glucose levels achieved throughout pregnancy by women with type 1 diabetes of long duration, in particular studying day to day variability and hypoglycaemic excursions.

Materials and Methods: Eight women, with type 1 diabetes of greater than 15 years duration, wore blinded CGMS for up to 7 days at monthly intervals throughout pregnancy during a randomised controlled study of CGMS intervention. For each CGMS study, we determined mean glucose level, percentage readings above $7.8 \mathrm{mmol} / \mathrm{L}$, percentage readings below $3.9 \mathrm{mmol} / \mathrm{L}$ and the day to day variability, defined as the difference between highest and lowest 24 hour mean glucose during a CGMS study. HbAlc was measured monthly.

Results: Mean duration of diabetes was 25.6 (16-33) years. The women had 6 (5-8) CGMS studies during pregnancy and mean duration of each study was 4 (1-6) complete days. Seven women were managed with basal/bolus regimens using intermediate insulin once or twice daily as basal insulin. One woman used an insulin pump. Comparing the first CGMS study with the last CGMS study, there was a significant fall in mean blood glucose (8.2(1.9) vs 6.3 (1.4) $\mathrm{mmol} / \mathrm{L}, \mathrm{p}=0.04)$. Hyperglycaemic excursions were seen frequently but between first and last CGMS studies there was a significant fall in percentage glucose readings above $7.8 \mathrm{mmol} / \mathrm{L}(43.6(15.2)$ vs $25.2(18.0) \%$, $\mathrm{p}<0.05)$. In contrast, there was no significant difference in percentage 
glucose readings below $3.9 \mathrm{mmol} / \mathrm{L}$ between first and last CGMS studies (17.1(10.5) vs 21.4(13.3)\%, NS). Great day to day variability was observed during CGMS studies and there was no significant difference in day to day variability between the first and last CGMS studies (3.4(1.0) vs 2.8 (1.1) $\mathrm{mmol} / \mathrm{L}, \mathrm{NS}$ ). The highest day to day variability observed was $5.4 \mathrm{mmol} / \mathrm{L}$. There was a significant decrease in mean $\mathrm{HbA} 1 \mathrm{c}$ between first and last studies $(7.3(1.0) \mathrm{vs}$ $5.7(0.5) \%, \mathrm{p}=0.002)$.

Conclusion: These results show hyperglycaemic excursions remain common throughout pregnancy in women with long duration type 1 diabetes. The data shows that day to day variability of blood glucose is high throughout pregnancy and there is no change in day to day glucose variability or percentage hypoglycaemic readings as pregnancy progresses. The results suggest it is difficult to achieve blood glucose targets with current treatments and may explain the continuing high rate of complications during pregnancy in women with type 1 diabetes.

\section{PS 79}

\section{Continuous glucose monitoring}

0952

\section{Continuous subcutaneous glucose monitoring in healthy subjects during daily life conditions}

G. Freckmann ${ }^{1}$, S. Hagenlocher ${ }^{1}$, A. Baumstark ${ }^{1}$, N. Jendrike ${ }^{1}$, C. Krauß $\beta^{1}$, R. Gillen ${ }^{1}$, D. Brandt ${ }^{2}$, C. Haug ${ }^{1}$; ${ }^{1}$ Institute for Diabetes-Technology, at the University of Ulm, Germany, ${ }^{2}$ Disetronic Medical Systems, Burgdorf, Switzerland.

Background and Aims: In recent years, subcutaneous glucose monitoring devices have been introduced into clinical application, and continuous glucose profiles in patients with type 1 diabetes have been investigated in numerous studies. However, therapeutic target ranges for subcutaneous continuous glucose profiles have not yet been defined. Since only little data in healthy persons are available, this study aimed to assess continuous subcutaneous glucose traces in healthy subjects under daily life conditions.

Materials and Methods: In 24 healthy volunteers (12 f, 12 $\mathrm{m}$; age 27.1 (19.4-34.5) years; BMI $22.6 \pm 1.7 \mathrm{~kg} / \mathrm{m} 2$; HbAlc $5.0 \pm 0.2 \%$ [mean $\pm \mathrm{SD}]$ ) an oral glucose tolerance test was performed to exclude diabetes mellitus or IGT. Two continuous monitoring devices based on microdialysis technique (subcutaneous continuous glucose monitoring (SCGM) device: in development by Roche Diagnostics) were applied to the abdominal subcutaneous tissue for $\sim 98$ hours; for calibration and verification of tissue glucose values 15 capillary blood glucose measurements were performed per day. During the first two days the subjects stayed in house, while on days 3 and 4 they followed their daily life conditions with free choice meals at fixed times (7:30, 12:30 and 18:00). The continuous monitoring data were calibrated over all reference values. The results of the two glucose monitoring devices were merged to one curve, 37 of 48 twenty-four hour glucose traces recorded during daily life conditions (days 3 and 4) could be evaluated.

Results: The mean $24 \mathrm{~h}$ glucose concentration was $88.3 \pm 9.5 \mathrm{mg} / \mathrm{dl}$. Mean glucose concentrations in the daytime (6 a.m. to 10 p.m.) and during the night (10 p.m. to 6 a.m.) were $91.0 \pm 10.5 \mathrm{mg} / \mathrm{dl}$ and $82.8 \pm 1.9$, respectively (Figure 1 ). The $3 \mathrm{~h}$ and $5 \mathrm{~h}$ areas under the curve above baseline were 2908 and $2920 \mathrm{mg} / \mathrm{dl}^{*} \mathrm{~min}$ after breakfast, 3007 and $3931 \mathrm{mg} / \mathrm{dl} * \min$ after lunch and 3317 and $4275 \mathrm{mg} / \mathrm{dl} * \min$ after dinner respectively. Time to maximum glucose was 44 , 49 and 50 minutes after breakfast, lunch and dinner. Mean preprandial glucose concentrations were $80.8 \pm 7.9,78.3 \pm 7.9$ and $80.5 \pm 10.8 \mathrm{mg} / \mathrm{dl}$, maximum glucose concentrations were $121.2 \pm 19.7,111.3 \pm 19.3$ and $116.8 \pm 20.8 \mathrm{mg} / \mathrm{dl}$ for breakfast, lunch and dinner, respectively.

Conclusion: The continuous glucose monitoring data obtained in healthy subjects, provide interesting additional information on fasting and postprandial tissue glucose levels in healthy subjects under daily life conditions. These data may also be used as target continuous glucose traces in the development of closed loop systems using subcutaneous monitoring and algorithm-controlled subcutaneous insulin application.

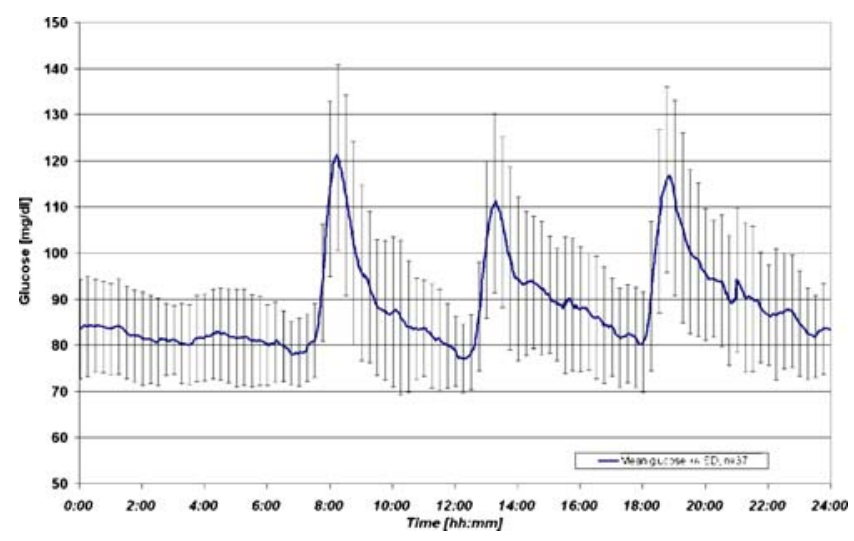

Supported by Disetronic Medical Systems 
0953

Insulin detemir shows a lower glucose variability compared to NPH insulin in subjects with type 1 diabetes undergoing continuous glucose monitoring D. R. Jones ${ }^{1}$, P. Clauson ${ }^{2}$, S. Ong ${ }^{3}$, C. Mathieu ${ }^{4}$; ${ }^{1}$ Department of Diabetes and Endocrinology, Royal Surrey County Hospital, United Kingdom, ${ }^{2}$ Novo Nordisk, Copenhagen, Denmark, ${ }^{3}$ Novo Nordisk, Singapore, ${ }^{4}$ Medical Department, Katholieke Universiteit, Leuven, Belgium.

Background and Aims: The aim of the study was to compare the glycaemic profiles of subjects with Type 1 diabetes mellitus treated with either insulin detemir (Detemir) or NPH insulin in a randomised, multi-national, openlabel, parallel group trial.

Materials and Methods: Subjects (88 Detemir: $37 \mathrm{NPH}$ ) using continuous glucose monitoring system (CGMS), aged $\geq 18$ yrs (Detemir: $39.3 \pm 10.3$ yrs, NPH: $40.9 \pm 13.7 \mathrm{yrs})$, diabetes duration $\geq 1$ yrs $(17.5 \pm 12.1$ yrs vs $18.7 \pm 10.0 \mathrm{yrs})$ and $\mathrm{HbA}_{1 \mathrm{c}} \leq 12 \%(8.3 \pm 1.1 \%$ vs $8.2 \pm 1.3 \%)$ were assessed. They were treated once daily with either Detemir or NPH at bedtime for six months, in combination with human soluble insulin (HSI) at main meals. $\mathrm{HbA}_{1 \mathrm{c}}$, FPG, body weight and within-subject variation were assessed. During the last month of treatment, all subjects wore CGMS for three days and mean amplitude of glycaemic excursions (MAGE) was determined in excess of $1 \mathrm{SD}$ (peak-to-nadir and nadir-to-peak) of the respective 24-hr mean glucose value. Data were presented as mean \pm SD and frequency (percentage).

Results: After six months, FPG was significantly reduced in the Detemir group $(-1.97 \pm 6.30 \mathrm{mmol} / \mathrm{L}, \mathrm{p}=0.0059)$ than in the NPH group $(-1.30 \pm 6.56 \mathrm{mmol} / \mathrm{L}, \mathrm{p}=0.2364)$. However, the improvement was comparable between treatment groups $(-1.38 \pm 0.077 \mathrm{mmol} / \mathrm{L}, 95 \%$ C.I. $2.92 ; 0.152)$. As for $\mathrm{HbA}_{1 \mathrm{c}}$, the changes from baseline were not significant for both groups (Detemir: $-0.13 \pm 0.88 \%$, $\mathrm{p}=0.1739$; NPH: $0.12 \pm 1.19 \%, \mathrm{p}=0.5569)$ and no treatment difference was apparent $(-0.2126 \pm 0.16 \%, 95 \%$ C.I. $0.526 ; 0.10)$. Mean daily insulin dose was $0.27 \mathrm{U} / \mathrm{kg}$ for Detemir and $0.36 \mathrm{U} / \mathrm{kg}$ for NPH. Within-subject variation in self-measured PG was significantly lower for Detemir vs $\mathrm{NPH}$ (SD of 2.8 vs $4.1 \mathrm{mmol} / \mathrm{L}, \mathrm{p}<0.001$ ). MAGE was significantly lower with Detemir vs NPH $(-1.08 \pm$ $0.43 \mathrm{mmol} / \mathrm{L}, 95 \%$ C.I. $-1.94 ;-0.227, \mathrm{p}=0.0136)$. The same proportion of patients (76 Detemir: 32 NPH subjects, $86 \%$ ) reported nocturnal hypoglycaemic events (major, minor, symptoms), 442 vs 219 events.

Conclusion: Subjects with Type 1 diabetes treated for six months with Detemir+HSI experienced comparable glycaemic control, but significantly lower within-subject variabil- ity and lower glucose excursions compared to subjects treated with NPH+HSI.

Supported by Novo Nordisk A/S

0954

Diabetes self-management decisions guided by continuous glucose monitoring: Results of an in-clinic pilot study

P. A. Gottlieb;

Pediatrics and Medicine, Barbara Davis Center for Childhood Diabetes, Aurora, United States.

Background and Aims: Continuous glucose monitoring (CGM) devices currently available in the United States are approved for use as adjuncts to standard self-monitoring of blood glucose (SMBG). Use of CGM with the STS System (DexCom, Inc.) as a primary means for directing diabetes self-management decisions (SMD) was evaluated in this pilot study.

Materials and Methods: Fifteen (15) subjects with type 1 diabetes participated in staff supervised 10-hour (7 am$5 \mathrm{pm}$ ) in-clinic sessions on 3 consecutive days. During all 3 sessions, self-monitoring of blood glucose (SMBG) was performed just prior to each SMD. Session 1: CGM blinded, decisions were guided by SMBG. Sessions 2 and 3: CGM unblinded (continuous glucose values, trends, and alerts/alarms provided) and was the only data used by subjects to guide decisions (SMBG blinded). Every SMD made by the subjects was verified with staff who had access to $\mathrm{SMBG}$ data.

Results: Two types of SMD were analyzed: bolus of fastacting insulin $(\mathrm{n}=154)$, and correction of hypoglycemia with carbohydrates $(n=43)$. Of the former, $39(25.3 \%)$ were adjusted by staff prior to delivery, the remaining 115 (74.7\%) were unchanged. SMD guided by SMBG (Session 1) vs. CGM (Sessions 2+3) were compared. Each decision was treated independently and deemed appropriate if 3hours post-decision, the sensor glucose value was within a target range of 80-200 mg/dL (per protocol CGM low/high glucose alert settings). Of SMBG-guided decisions, 45.7\% were appropriate; this percentage increased to $64.6 \%$ when SMD was guided by CGM ( $\mathrm{p}=0.02)$. Subjects experienced a median increase of 2.0 hours per session (10 hours) spent in target range during Sessions $2+3$, as compared to Session $1 \quad(p=0.003)$; and reduced time spent $>200 \mathrm{mg} / \mathrm{dL}$ without increasing time spent $<55 \mathrm{mg} / \mathrm{dL}$. No episodes of severe hypoglycemia were reported during this study.

Conclusion: In this pilot study, real-time availability of CGM resulted in an increased frequency of appropriate diabetes self-management decisions with a significant improvement in target range glycemia. 
Median Change in Time Spent in Various Glucose Ranges

\begin{tabular}{lll}
\hline $\begin{array}{l}\text { Glucose } \\
\text { Range }(\mathrm{mg} / \mathrm{dL})\end{array}$ & $\begin{array}{l}\text { Session 1 vs. Sessions } \\
2+3(\mathrm{Hrs} / \text { Session) }\end{array}$ & p-value \\
\hline$<55$ & -0.01 & \\
$55-79$ & +0.52 & 0.6377 \\
$80-200$ & +1.99 & 0.1937 \\
$201-300$ & -2.18 & 0.0026 \\
$>300$ & -0.19 & 0.0398 \\
\hline
\end{tabular}

0955

Evaluation of a non-invasive continuous glucose monitoring device in a home use setting

O. Amir ${ }^{1}$, A. Kononenko ${ }^{1}$, E. Gabis ${ }^{1}$, A. Karasik ${ }^{2,3}$;

${ }^{1}$ OrSense, Nes Ziona, ${ }^{2}$ Endocrinology, Sheba Medical Center, Ramat Gan, ${ }^{3}$ Medicine, Tel Aviv University, Israel.

Background and Aims: A growing body of work supports the benefit of continuous glucose monitoring (CGM) in improving the treatment and health of diabetic patients. Primarily, invasive subcutaneous devices are used to perform such monitoring. The purpose of this study is to evaluate the feasibility of the fully non-invasive NBM-100 (OrSense Ltd.) device for continuous blood glucose (BG) monitoring in type 1 and 2 diabetes subjects.

Materials and Methods: The NBM-100 uses a finger-based sensor shaped as a ring, located at the finger's root. The configuration of this novel sensor enables the detection of red/ near-infrared light transmitted through the finger via Occlusion Spectroscopy. Analysis of the resulting spectrum renders the subjects $\mathrm{BG}$ levels, $\mathrm{Hb} / \mathrm{Hct}$ concentrations, and $\mathrm{SpO}_{2}$ levels. Specifically, the NBM-100 utilizes an enhanced optical signal resulting from a temporary over-systolic occlusion, produced by a finger-based pneumatic cuff. The resulting changes in the optical absorption and scattering create the sensitivity needed for measuring BG and other analyte concentrations. The study consisted of three phases. (1) A hyperinsulinemic hypoglycemic clamp was performed at a clinic in 4.5 hour sessions $(2 \mathrm{M}, 3 \mathrm{~F}$, aged $21-40, \mathrm{DM} 1)$ with reference BG measured every 5 minutes (Freestyle, Abbot). (2) A home use trial conducted on 17 subjects (8 DM1, 9 DM2 10M, 7F, ages 21-74) with 2-10 days of 816 hour sessions. Reference BG readings were collected every 30-60 minutes (Glucose 201+, HemoCue). (3) A 24 hour use trial with 4 subjects (2M, 2F, aged 21-40, DM1), conducted in a clinic with venous reference $\mathrm{BG}$ readings collected every 20 minutes (Olympus AU 2700). In all three trial settings the non-invasive data was collected every 10 minutes. In each session, glucose predicting parameters were calibrated using four BG reference points taken within a three hour period. Based on a predetermined inclusion criterion from the calibration phase, 88 of the 101 sessions were included in the above analysis.

Results: A prospective analysis was performed on the NBM-100 readings for all 88 patient sessions to calculate the standard error values for the total of 1202 paired data points (NBM-100 vs. Reference Gl). The glucose range was $38-488 \mathrm{mg} / \mathrm{dl}$. The resulting median relative absolute error (RAE) was $12.6 \%$, the median absolute error (MAE) was $21.2 \mathrm{mg} / \mathrm{dl}$, and the overall correlation was $\mathrm{r}=0.87$. A Clark error grid analysis showed that $94.8 \%$ of the measurements fall within zones A and B.

Conclusion: This study indicates the potential use of the NBM-100 as a non-invasive sensor for continuous BG evaluation. In all three clinical settings user compliance was positive. The NBM-100 is currently under evaluation in broader clinical settings.

\section{6}

Clinical trial of a seven-day continuous glucose sensor demonstrates the importance of fasting hyperglycaemia and the dawn phenomenon

S. K. $\operatorname{Garg}^{1}$, L. Jovanovic ${ }^{2}$;

${ }^{1}$ Pediatrics and Medicine, Barbara Davis Center for Childhood Diabetes, Aurora, ${ }^{2}$ Sansum Diabetes Research Institute, Santa Barbara, United States.

Background and Aims: Relative contributions of fasting and post-prandial hyperglycaemia differ at different levels of glycaemic control (HbAlc values). Previous studies have documented higher contributions of post-prandial elevations at lower HbAlc values based on self home blood glucose monitoring (SMBG). No data is available on continuous home glucose monitoring (CHGM) and its relationship to different $\mathrm{HbAlc}$ values. We evaluated this relationship in this study.

Materials and Methods: Sixty-nine subjects with type 1 diabetes and 17 with insulin-requiring type 2 diabetes participated at five centers. Baseline HbAlc was measured within 30 days of enrollment. Subjects inserted and wore a transcutaneous glucose sensor (STS System, DexCom, Inc.) for 7 days over 3 consecutive periods during normal activities. Sensors were BLINDED for period 1. During periods 2 and 3 (DISPLAY), glucose values, trend graphs, and high/low alerts and alarms were provided.

Results: Of 6357 paired sensor-SMBG points prospectively analyzed, 97.2\% fell in Clarke Error Grid zones A+B with none in zone $\mathrm{E}$; mean absolute relative difference was $15.7 \%$ with stable performance over 7 days. Continuous glucose values were pooled for subjects within 6 groups of baseline HbAlc (from $<6 \%$ to $>10 \%$, increments of $1 \%$ ). Modal day graphs for each $\mathrm{HbA} 1 \mathrm{c}$ group were generated by 
plotting mean hourly glucose values during BLINDED and DISPLAY device use (Figure). During BLINDED use, distinctive glycaemic patterns were observed at varying baseline HbA1c levels. Of note, mean glucose values from 4-7AM (dawn phenomenon and fasting) were only normal for subjects with $\mathrm{HbA} 1 \mathrm{c}<6 \%$, and mean glucose values between 4PM and 12AM were higher by $\sim 20-40 \mathrm{mg} / \mathrm{dL}$ per $1 \%$ increase in baseline HbA1c $(\mathrm{p}<0.05$ when comparing the $\mathrm{HbA} 1 \mathrm{c}<6 \%$ group to each of the 3 highest HbAlc groups at these hourly time points). Glycaemic excursions were improved during the DISPLAY periods.

Conclusion: We conclude that 7-day use of the STS System was safe, accurate, stable, and resulted in improved glycaemic excursions. Continuous glucose monitoring, with a focus on fasting and evening hyperglycaemia and the dawn phenomenon, may help achieve lower HbA1c values.

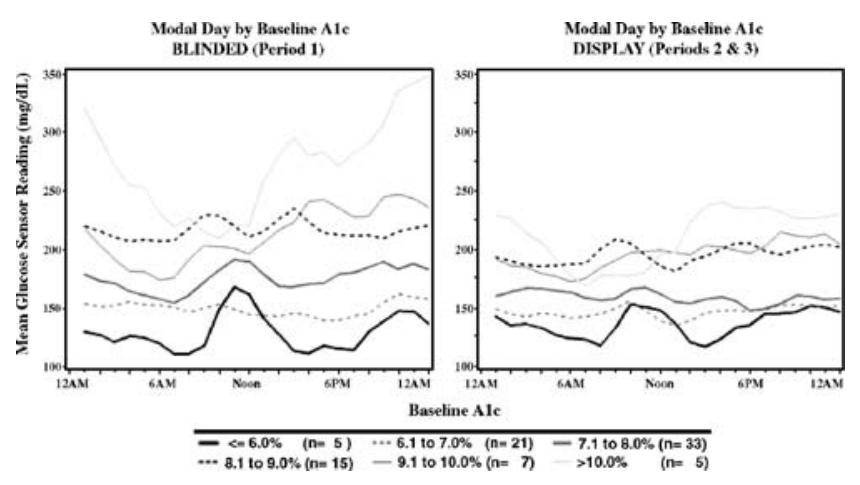

Supported by DexCom, Inc.

0957

Home use evaluation of the freestyle navigator continuous glucose monitoring system

J. R. Bugler ${ }^{1}$, R. M. Bergenstal ${ }^{2}$, B. W. Bode ${ }^{3}$,

R. L. Weinstein ${ }^{4}$, S. L. Schwartz ${ }^{5}$, R. M. Bernstein ${ }^{6}$,

S. S. ElDeiry ${ }^{7}$;

${ }^{1}$ Abbott Diabetes Care, Alameda, ${ }^{2}$ International Diabetes Center, Minneapolis, ${ }^{3}$ Atlanta Diabetes Associates, Atlanta,

${ }^{4}$ Diablo Clinical Research, Walnut Creek, ${ }^{5}$ Clinical, Diabetes \& Glandular Disease Research Associates, San Antonio, ${ }^{6}$ Clinical, Regional Endocrinology Associates, Santa Fe, ${ }^{7}$ The Endocrine Group, Albany, United States.

Background and Aims: The FreeStyle Navigator Continuous Glucose Monitoring System, an investigational continuous glucose monitoring system, was tested by 137 subjects with type 1 and type 2 diabetes to validate its use in the home environment at 6 clinical sites. Its usability was assessed by continuous use for 40 days, followed by a user questionnaire on ease and potential benefit of use. The system includes a sensor (worn up to 5 days), a reusable transmitter, and a handheld receiver with a built-in FreeStyle ${ }^{\circledR}$ blood glucose (BG) monitoring system. Each sensor requires calibration against capillary BG at approximately 10, 12, 24, and 72 hours after insertion.

Materials and Methods: Sensor insertions at either arm or abdomen (minimum of 8 ) and calibrations were performed by the subjects. Real-time data and alarms were not available for the first 20 days (blinded phase). Real-time data, trend arrows and threshold alarms for hypo- and hyperglycemia (programmed at $65 \mathrm{mg} / \mathrm{dL}$ and $300 \mathrm{mg} / \mathrm{dL}$, respectively) were unblinded for the next 20 days. For the entire study, subjects were instructed to make all treatment decisions based on capillary BG tests only and to perform BG tests with the built-in FreeStyle meter in duplicates prior to meals, at bedtime, and when symptomatic for hypoor hyperglycemia at a minimum.

Results: Summary data represents 123 subjects (mean \pm SD, age: $48.6 \pm 13.0$ years, duration of diabetes: $22.0 \pm 11.9$ years, daily insulin use: $51.7 \pm 28.7$ IU) who completed the protocol. Based on 11,487 paired FreeStyle Navigator system/BG data points, the mean absolute relative difference was $14.4 \pm 13.4 \%$. A total of 14 subjects withdrew during the first phase of the study due to non-compliance with protocol demands $(n=8)$ or technical difficulties handling the device $(n=6)$. The majority of subjects reported that system functions were easy to learn: $90.5 \%$ age $18-34,84.8 \%$ age $35-54$, and $93.0 \%$ age $55+$. Overall, $93.7 \%$ of the subjects found the information provided by the FreeStyle Navigator system helped them understand their blood glucose, and $93.6 \%$ felt glucose alarms were easy to interpret. When asked about the benefit of continuous glucose data, $100 \%$ of subjects age 18-34, $95.4 \%$ age $35-54$, and $93.0 \% 55$ and over responded that it was simple to understand glucose changes.

Although not considered a primary endpoint, subjects spent significantly more time in euglycemia in the unblinded phase. Subjects with type 1 spent significantly less time ( 0.8 hours versus 1.4 hours, or $42 \%$ less) in the hypoglycemic range, defined as $<70 \mathrm{mg} / \mathrm{dL}$, whereas subjects with type 2 shortened significantly the time spent in hyperglycemia (8.0 hours versus 9.6 hours, or 17\% less), defined as $>300 \mathrm{mg} / \mathrm{dL}$, with no increase in hypoglycemia.

Conclusion: Accurate sensor performance coupled with general ease of use and interpretation of continuous glucose data demonstrate safe and efficient use of the FreeStyle Navigator system in the home environment. The observed improvements in glycemic control reinforce the clinical utility of the FreeStyle Navigator system.

Supported by Abbott Diabetes Care, Abbott Laboratories 


\section{8}

Clarke Error Grid and Continuous Clarke Error Grid analysis of the accuracy of the 5-day FreeStyle navigator continuous glucose monitoring system

R. L. Weinstein ${ }^{1}$, S. L. Schwartz ${ }^{2}$, R. L. Brazg ${ }^{3}$, J. R. Bugler ${ }^{4}$, T. A. Peyser ${ }^{4}$, G. V. McGarraugh ${ }^{4}$;

${ }^{1}$ Diablo Clinical Research, Inc., Walnut Creek, ${ }^{2}$ Diabetes \& Glandular Disease Research Associates, P.A., San Antonio, ${ }^{3}$ Ranier Clinical Research, Renton, ${ }^{4}$ Abbott Diabetes Care, Alameda, United States.

The FreeStyle Navigator Continuous Glucose Monitoring System is an investigational continuous glucose monitoring system based on Wired Enzyme ${ }^{\mathrm{TM}}$ glucose transduction technology. The FreeStyle Navigator system includes a disposable subcutaneous sensor that can be worn up to 5 days, a radio transmitter connected to the sensor, and a small handheld receiver.

The accuracy and performance of the sensor were evaluated in a multi-center, prospective, single-arm study. Fifty-eight subjects aged 18 to 64 were enrolled, and each wore two sensors, one on the arm and one on the abdomen. Sensors were calibrated with capillary BG measurements using the built-in FreeStyle ${ }^{\circledR}$ blood glucose (BG) monitoring system at approximately $10,12,24$, and 72 hours after insertion. The FreeStyle Navigator system measurements were collected at one-minute intervals for the entire study and were not displayed to patients or investigators. Venous blood glucose measurements were taken using a standard laboratory reference (YSI 2300) every 15 minutes for 50 hours distributed across the 5-day sensor wear.

The primary endpoint was accuracy as determined by pairwise comparisons of the FreeStyle Navigator system values and laboratory reference values. These measurements were compared using mean and median absolute relative difference, standard Clarke Error Grid Analysis, and Continuous Glucose Error Grid Analysis (CG-EGA), a method of evaluation that assesses both accuracy for point values and for measurements of direction and rate of change.

Regarding precision, the matched sensors worn on the arm and abdomen had a coefficient of variation of $10 \%$ $(n=312,953)$. The analysis yielded a mean absolute relative difference of $12.8 \%$ and a median absolute relative difference of $9.3 \%$ between the FreeStyle Navigator system values and YSI.

On the Clarke Error Grid (CEG) $81.7 \%$ were in zone A and $16.7 \%$ were in zone B. The data included periods of high rates of change of blood glucose during IV glucose and insulin challenges with periods of hyperglycemia and hypoglycemia. When the rate of change of blood glucose was between $-1 \mathrm{mg} / \mathrm{dL} / \mathrm{min}$ and $+1 \mathrm{mg} / \mathrm{dL} / \mathrm{min}$, the percentage in zone A increased to $88 \%$. The accuracy performance remained high through all five days of use, with $82.5 \%$ of data in zone A on the first day and $80.9 \%$ in zone A on the fifth day.

Continuous Glucose Error Grid Analysis (CG-EGA) yielded $81.1 \%$ in the rate error grid zone A, $14.4 \%$ in the rate error grid zone $\mathrm{B}$ and $1.5 \%, 2.3 \%$ and $0.7 \%$ in the rate error grid zones $\mathrm{C}, \mathrm{D}$ and $\mathrm{E}$ respectively. Point analysis using the CGEGA yielded $85.7 \%$ in point error grid zone A, $13.6 \%$ in point error grid zone $\mathrm{B}, 0.0 \%, 0.7 \%$ and $0.0 \%$ in point error grid zones $\mathrm{C}, \mathrm{D}$ and $\mathrm{E}$ respectively. The CG-EGA analysis combining rate and point information revealed that accuracy, measured as a percentage of accurate readings plus benign errors, was $97.5 \%$ (94.1\% accurate, $3.4 \%$ benign).

The study results support the accuracy and clinical performance of the FreeStyle Navigator continuous glucose monitoring system over five days of sensor wear.

Supported by Abbott Diabetes Care

\section{9}

An innovative approach to non invasive glucose monitoring A. Gal ${ }^{1}$, I. Harman-Boehm ${ }^{2}$;

${ }^{1}$ Management, Integrity Applications Ltd., Ashkelon, ${ }^{2}$ Internal Medicine and the Diabetes Unit, Soroka University Medical Center, Beer-Sheva, Israel.

Background and Aims: Attempts to develop an accurate non-invasive glucose monitoring device have been only partially successful. Depending on the particular method, the readings might vary with changes in glucose level, but might also be affected by changes in other components in the blood. In order to achieve an accurate reading, there is a need for a mechanism which will idle the impact of the non-glucose components and isolate the changes caused by the glucose per se.

Materials and Methods: An innovative approach to the problem is the simultaneous utilization of three different technologies and comparison of the separate results by using a unique algorithm. For each glucose reading, achieved by one method, a tolerance window of $\pm \mathrm{N} \%$ is opened around the original value. The three tolerance windows are then correlated and compared, while overlapping is expected. If there is a full correlation, meaning three overlaps are achieved, then the final result will be a weighted average of the three measured values. If only two overlaps occur, then the tolerance windows are shrunken, $(0.75 * \mathrm{~N})$ and an overlapping between the two readings is then checked. Failure of overlapping causes a reading error and auto repeat of the measurement. Three trials are allowed. Failure in all three attempts brings an error message, requesting a new personal calibration.

Results: GlucoTrack ${ }^{\mathrm{TM}}$ uses Ultra Sound, Conductivity and Heat Capacity technologies for measuring the glucose level. 
A basic tolerance window of $\mathrm{N}=20 \%$ was used in 278 measurements. As a reference we used the FreeStyle ${ }^{\circledR}$ invasive glucose measurement device. The results are shown below: raw glucose readings of the three different technologies are shown in the left Clarke Error Grid (CEG), while the final results, after using the algorithm are demonstrated in the right CEG. The enhancement in precision is clearly shown in the right CEG. The device has been used by 69 patients. A total of 174 points were measured: $83.3 \%$ fell in Zone A of CEG and $16.7 \%$ in Zone B.

Conclusion: Using a combination of three different technologies and comparing the results by the suggested algorithm improves the overall accuracy level of the glucose measurement, thus GlucoTrack is a novel non-invasive device for the accurate measurement of blood glucose.
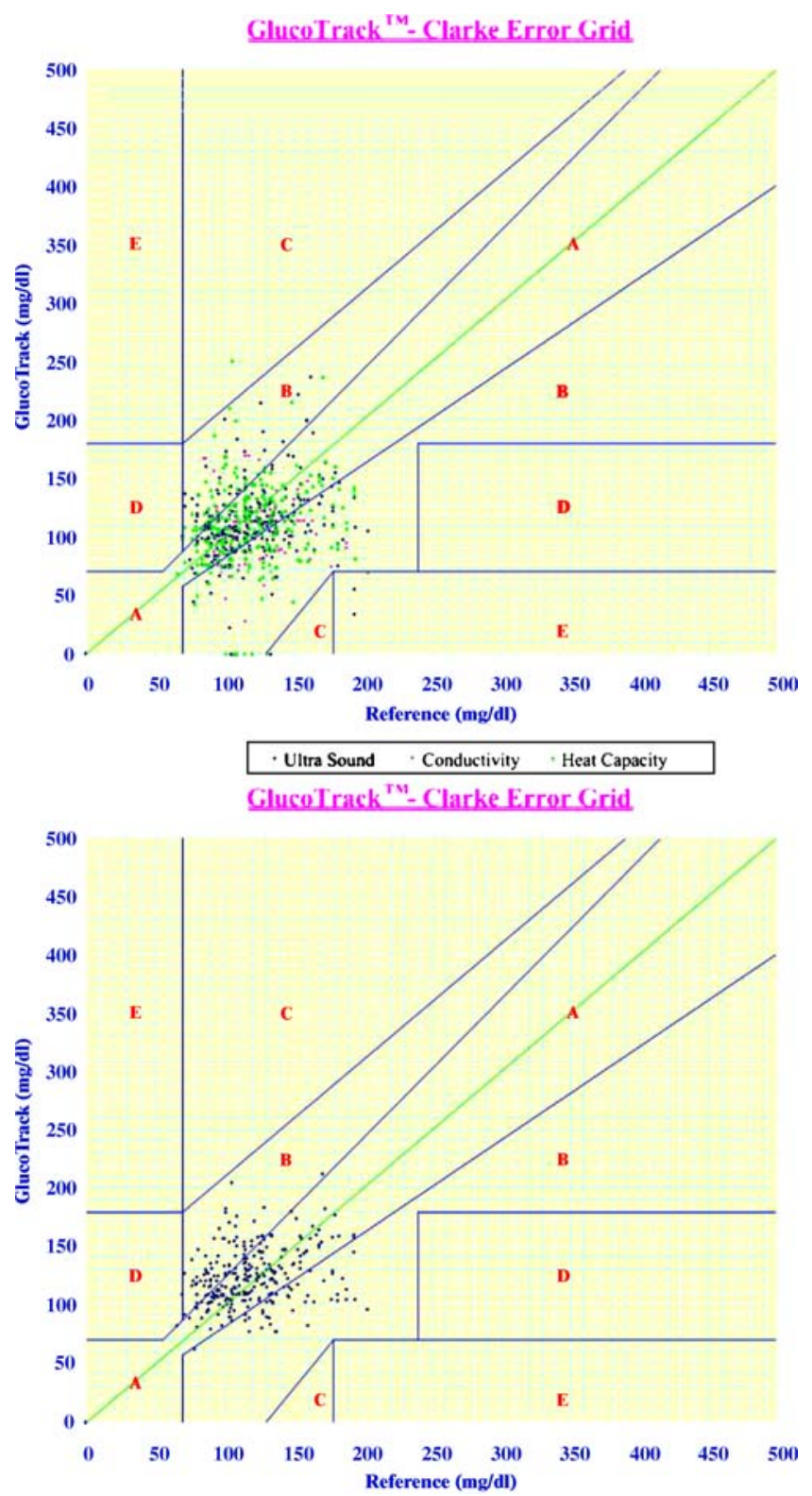

0960

Fiberoptic hybrid sensor for continuous glucose monitoring H. Koehler ${ }^{1}$, A. Pasic ${ }^{2}$, I. Klimant ${ }^{2}$, L. Schaupp ${ }^{1}$, T. R. Pieber ${ }^{3}$;

${ }^{1}$ Institute of Medical Technologies and Health Management, Joanneum Research, Graz, ${ }^{2}$ Institute of Analytical Chemistry and Radiochemistry, University of Technology Graz, ${ }^{3}$ Department of Internal Medicine, Diabetes and Metabolism, Medical University Graz, Austria.

Background and Aims: Continuous glucose monitoring (CGM) is beneficial for tight glycaemic control in diabetic as well as in critically ill patients. Most of the sensors used for CGM are based on the glucose oxidase reaction and electrochemical detection of $\mathrm{H}_{2} \mathrm{O}_{2}$. Such sensors often suffer from poor selectivity and effects caused by changing $\mathrm{pO}_{2}$-levels. This is of particular importance in the case the measurement is performed in critically ill patients (high doses of drugs) and in the interstitial fluid (low and varying $\mathrm{pO}_{2}$-levels). The objective was therefore to design and test a new sensor approach using optical hybrid sensors for simultaneous measurement of oxygen and glucose minimizing the effects of interfering substances and changing $\mathrm{pO}_{2}$-levels.

Materials and Methods: The hybrid sensor consists of two oxygen optodes (each $125 \mu \mathrm{m}$ in diameter) whereof one optode acts as glucose sensitive probe and the other one as reference - measuring the surrounding oxygen tension. Both measurements are based on the phase shift method for the determination of the luminescence decay time, which is insensitive to changes of the absorption of the luminescence. Long-term stability, accuracy and precision were determined, sensor response to interfering substances and different physiological pO2 levels were investigated in-vitro.

In-vivo a flow through configuration of the sensor was combined with the microdialysis technique. This approach was tested in 16 (12 healthy, 4 diabetic) subjects over a period of 24 hours. Meals were standardized; blood readings were taken in 15 minutes to hourly intervals and compared with the sensor signals.

Results: During in-vivo trials sensor drift was negligible; in Clark-Error Grid analysis more than $96 \%$ of the measured values were in region A or B. $20 \%$ of the sensor data had to be discarded for technical reasons (mainly fluidic problems).

In-vitro sensor accuracy was between $2 \%$ and $15 \%$, precision $<2 \%$ and drift within 72 hours $<5 \%$. 
Sensor response to 6 most common interferences:

\begin{tabular}{llll}
\hline $\begin{array}{l}\text { Interfering } \\
\text { Compound }\end{array}$ & $\begin{array}{l}\text { Therapeutic/ } \\
\text { Physiological } \\
\text { Level }[\mathrm{mg} / \mathrm{dl}]\end{array}$ & $\begin{array}{l}\text { Test } \\
\text { Concentration } \\
{[\mathrm{mg} / \mathrm{dl}]}\end{array}$ & $\begin{array}{l}\text { Interference } \\
\text { Effect at } \\
\mathbf{5 ~ m M} \\
\text { glucose }[\mathrm{mM}]\end{array}$ \\
\hline Ascorbic Acid & $0.8-1.2$ & 3 & $<0.1$ \\
Acetaminophen & $1-2$ & 20 & $<0.1$ \\
Acetylsalycylat & $15-30$ & 50 & $<0.1$ \\
Uric Acid & 7 & 20 & $<0.1$ \\
Mannit & not available & 200 & $<0.2$ \\
Dopamine & $0.4-1.6$ & 13 & $<0.1$ \\
\hline
\end{tabular}

Conclusion: The new sensor approach enables simultaneous measurement of oxygen and glucose which results in stable glucose measurements. In addition the optical measurement is very insensitive to interferences which turns out to be beneficial in critically ill patients.

Supported by the European Community, EC-Clinicip FP6 IST 506965

\section{PS 80}

\section{Inpatient glycaemia/diabetes management}

\section{1}

A comparison of capillary and venous blood glucose concentrations measured during steady and dynamic glycaemic states

K. Kempe ${ }^{1}$, C. Bowden ${ }^{2}$, K. Win ${ }^{2}$, K. Kuschma ${ }^{2}$,

D. Budd ${ }^{1}$, M. Hompesch ${ }^{2}$;

${ }^{1}$ LifeScan, Inc., Milpitas, United States, ${ }^{2}$ Profil Institute for Clinical Research, Chula Vista, United States.

Background and Aims: A February 2006 AACE/ADA consensus conference on inpatient diabetes and glycemic control identified glucose sampling sites and rates as an area needing further research. We compared capillary and venous blood glucose concentrations during euglycemic, hypoglycemic, and hyperglycemic steady states and transition periods (dynamic states) between the steady states.

Materials and Methods: Forty (40) patients with Diabetes Mellitus (28 with Type 1 and 12 with Type 2 Diabetes, 25 male, 15 female, age $38 \pm 12$, BMI $27 \pm 4.1$ ) participated in this glucose clamp study. Experimental intervention began with the subject in a euglycemic steady state $(\sim 120 \mathrm{mg} / \mathrm{dL})$. A Biostator was used as glucose clamp device over a 38 hour period to achieve hypoglycemic $(\sim 50 \mathrm{mg} / \mathrm{dL})$, hyperglycemic $(\sim 250 \mathrm{mg} / \mathrm{dL})$, and hypoglycemic $(\sim 50 \mathrm{mg} / \mathrm{dL})$ plateaus in each subject and to induce the dynamic states between the plateaus. Plateaus were kept for approximately 30 minutes, the rate of change during the dynamic states was at least $2 \mathrm{mg} / \mathrm{dl} / \mathrm{min}$. Capillary (fingertip) and venous (forearm) samples were drawn at the beginning of the experimental intervention, at the beginning and end of each plateau, and at the approximate midpoint of each transition period (total of 10 sampling points). Plasma glucose concentrations of the capillary and venous samples were determined with the YSI 2300 Blood Glucose Analyzer. Results were analyzed in two ways: a) for each of the sampling points, the mean capillary and venous glucose concentrations were calculated, and b) for each subject and sampling point, the difference between capillary and venous concentrations was plotted against the venous concentration (BlandAltman plot).

Results: Mean glucose concentrations followed the pattern of 1) decrease, 2) steady state (hypo), 3) increase, 4) steady state (hyper), 5) decrease, and 6) steady state (hypo). Mean capillary and venous concentrations were nearly identical during phases 1, 2, and 6; during phases 3 and 4, capillary glucose exceeded venous glucose by approximately 6$14 \%$, and during phase 5, venous glucose exceeded capillary glucose by $5 \%$. Bland-Altman analysis (see table below) shows a similar relationship: during the periods of decreasing blood glucose, venous concentrations tended to be higher, and during the period of increasing blood glucose, venous concentrations tended to be lower.

Conclusion: Our data suggests that capillary or venous blood may be used for sampling under steady state conditions. Samples collected when glucose levels are rapidly changing frequently show large site-to-site differences. Protocols to guide inpatient mangement and assess glucose meter performance should account for these potential differences.

\begin{tabular}{|c|c|c|c|c|c|c|}
\hline \multirow[t]{2}{*}{$\begin{array}{l}\text { Diff } \\
\text { [Cap-Ven] } \\
(\mathrm{mg} / \mathrm{dL})\end{array}$} & \multicolumn{2}{|c|}{$\begin{array}{l}\text { Steady } \\
\text { State } \\
(n=270)\end{array}$} & \multicolumn{2}{|c|}{$\begin{array}{l}\text { Dynamic } \\
\text { State [decr.] } \\
(n=75)\end{array}$} & \multicolumn{2}{|c|}{$\begin{array}{l}\text { Dynamic } \\
\text { State [incr] } \\
(n=39)\end{array}$} \\
\hline & $\mathrm{n}$ & $\%$ & $\mathrm{n}$ & $\%$ & $\mathrm{n}$ & $\%$ \\
\hline$>+15$ & 31 & 11.5 & 8 & 10.7 & 23 & 59.0 \\
\hline+10 to +15 & 18 & 6.7 & 1 & 1.3 & 3 & 7.7 \\
\hline+5 to +10 & 40 & 14.8 & 8 & 10.7 & 1 & 2.6 \\
\hline-5 to +5 & 155 & 57.4 & 26 & 34.7 & 6 & 15.4 \\
\hline-5 to -10 & 15 & 5.6 & 9 & 12.0 & 2 & 5.1 \\
\hline-10 to -15 & 8 & 3.0 & 6 & 8.0 & 1 & 2.6 \\
\hline$<-15$ & 3 & 1.1 & 17 & 22.7 & 3 & 7.7 \\
\hline
\end{tabular}

Supported by LifeScan, Inc. 


\section{2}

Assessment and validation of a novel critical pathway for the management of hyperglycaemia in cardiac patients admitted to the cardiac care units

N. Mezitis ${ }^{1}$, E. Aziz ${ }^{2}$, S. Croitor ${ }^{2}$, D. Musat ${ }^{3}$, O. Frankenberger ${ }^{3}$, E. Herzog ${ }^{3}$;

${ }^{1}$ Endocrinology, ${ }^{2}$ Internal Medicine, ${ }^{3}$ Cardiology, St. Luke's-Roosevelt Hospital Center, New York City, United States.

Background and Aims: Morbidity and mortality in hospitalized patients is increased in hyperglycemia. Normalization of blood glucose levels using intensive insulin infusion protocols has been shown to improve clinical outcomes. Although Insulin infusion algorithms have recently been published, a major obstacle in their implementation is their complexity. We have developed a straightforward, novel pathway for the management of hyperglycemia which overcomes these technical difficulties. Materials and Methods: This pathway incorporates two new concepts - the "WHEEL" concept for insulin dosing, complemented by the notion of "Catch-up Insulin". The WHEEL which serves as a treatment guideline is made up of four circles depicting the range of blood glucose on the innermost circle and three tiers of intravenous insulin infusion rates on the outer circles. The graphic WHEEL is then translated into a nursing work sheet. Cardiac Care Unit (CCU) nurses are able to follow the protocol without any further assistance. We tested the protocol on 10 patients who were admitted to the CCU at St. Luke's-Roosevelt Hospital of Columbia University in New York, aiming for tight blood glucose $(\mathrm{BG})$ control with a $\mathrm{BG}$ range between 80 and $120 \mathrm{mg} / \mathrm{dl}$ measured by finger sticks (FS). Secondarily we aimed to show that the implementation of the WHEEL concept would allow us to achieve target range BG control without the risk of hypoglycemia. The control group consisted of 10 patients managed with "standard" insulin drips adjusted based on physician determination prior to the pathway implementation.

Results: 10 patients in the Control group (CG) (60.8 16.9 years, $40 \%$ males) and 10 patients in the On-Protocol group (PG) (63.6 \pm 12.5 years, $60 \%$ males), $p=0.67$, were compared. The mean initial glucose was $(369 \pm 162 \mathrm{mg} / \mathrm{dl}) \&$ $(329 \pm 157 \mathrm{mg} / \mathrm{dl})$ in the CG and PG respectively. The mean blood glucose on treatment in the CG was $(157 \pm 53 \mathrm{mg} / \mathrm{dl})$ compared to $(117 \pm 17.5 \mathrm{mg} / \mathrm{dl})$ in the $\mathrm{PG}, p=0.06$. Target blood glucose levels were reached in $30 \%$ of the $\mathrm{CG}$ and $70 \%$ of the PG $p<0.001$. The lowest $\mathrm{BG}$ readings in the CG were $(46.2 \pm 21 \mathrm{mg} / \mathrm{dl})$ vs. $(82 \pm 10.4 \mathrm{mg} / \mathrm{dl})$ in $\mathrm{PG}, p=0.0003$. The incidence of symptomatic hypoglycemia was $(0 \%)$ in the PG compared to $(90 \%)$ in the CG, $p<0.0001$.
Conclusion: This pilot study shows that our concept and implementation of the WHEEL is an easily applicable IV insulin protocol that can be managed by a CCU nurse and will facilitate the accomplishment of intensive glycemic control in all cardiac critical care patients without causing a significant number of hypoglycemic episodes.

\section{3}

Algorithm changes in computerized IV insulin infusion protocol using the clarian glucostabilizer $\left(\mathrm{CGS}^{\odot}\right)$ result in marked reduction in incidence of hypoglycaemia C. Roudebush ${ }^{1}$, R. Juneja ${ }^{2}$, A. Golas ${ }^{2}$, J. Carroll ${ }^{1}$, D. Nelson ${ }^{1}$, S. Flanders ${ }^{1}$;

${ }^{1}$ Clarian Health Partners, Indianapolis, ${ }^{2}$ Medicine, Indiana University, Indianapolis, United States.

Background and Aims: We have previously shown the effectiveness of a computerized IV Insulin (IVI) protocol in achieving and maintaining Blood Glucose (BG) targets. Once the physician orders IV insulin with a target BG range, the IVI program recommends an insulin infusion rate followed by an alarm for BG checks every 60-120 minutes. Once a new BG is entered, the program recommends change/ no change in the insulin infusion until target BG is reached. Materials and Methods: This study focused on cardiovascular surgery patients, whose target BGs generally are 80 $110 \mathrm{mg} / \mathrm{dl}$ in our institution. With this protocol, average hypoglycemia rate $(\mathrm{BG} \leq 70 \mathrm{mg} / \mathrm{dl})$ was $4.25 \%$, with $0.42 \%$ of BGs being $<=50 \mathrm{mg} / \mathrm{dl}$. To attempt to further decrease hypoglycemia while on the protocol, we instituted the following changes in the algorithms used in the Clarian Glucostabilizer $\left(\mathrm{CGS}^{\odot}\right)$ Protocol: If a patient had 3 out of the last 8 blood glucose values $\leq 70 \mathrm{mg} / \mathrm{dl}$, the infusion rate was cut in half and the target blood glucose range was raised by $10 \mathrm{mg} / \mathrm{dl}$ at the lower and upper limits (e.g. target of 80 $110 \mathrm{mg} / \mathrm{dl}$ was changed to $90-120 \mathrm{mg} / \mathrm{dl}$ ). These changes were instituted in late Dec 2005 (data point 2005-52).

Results: For \% $\mathrm{BG} \leq 70 \mathrm{mg} / \mathrm{dl}$, with the above process modification, we were able to show a statistically significant $30 \%$ reduction in the hypoglycemia rate from $4.25 \%$ to $2.99 \%$ (see "p" Control Chart; \%BGs < $<70$ ). For \% $\mathrm{BG} \leq 50 \mathrm{mg} / \mathrm{dl}$, the hypoglycemia rate was reduced by $43 \%$, from $0.42 \%$ to $0.24 \%$. There were some readings in week 2006-8, when there were a total of 8 events with $B G \leq 50 \mathrm{mg} /$ $\mathrm{dl}$, which skewed the statistical significance for this BG level, but overall the trend appears to be towards marked reduction in the hypoglycemia frequency (see "p" chart, $\%$ BGs $<=50$ ). Conclusion: Programming an algorithm change of a reduction in the infusion rate and slightly less aggressive BG targets in patients on IV insulin, results in a marked decrease in the frequency of hypoglycemia thus increasing 
the safety of the Clarian Glucostabilizer ${ }^{\mathcal{C}}$, a computerized IV insulin Protocol.
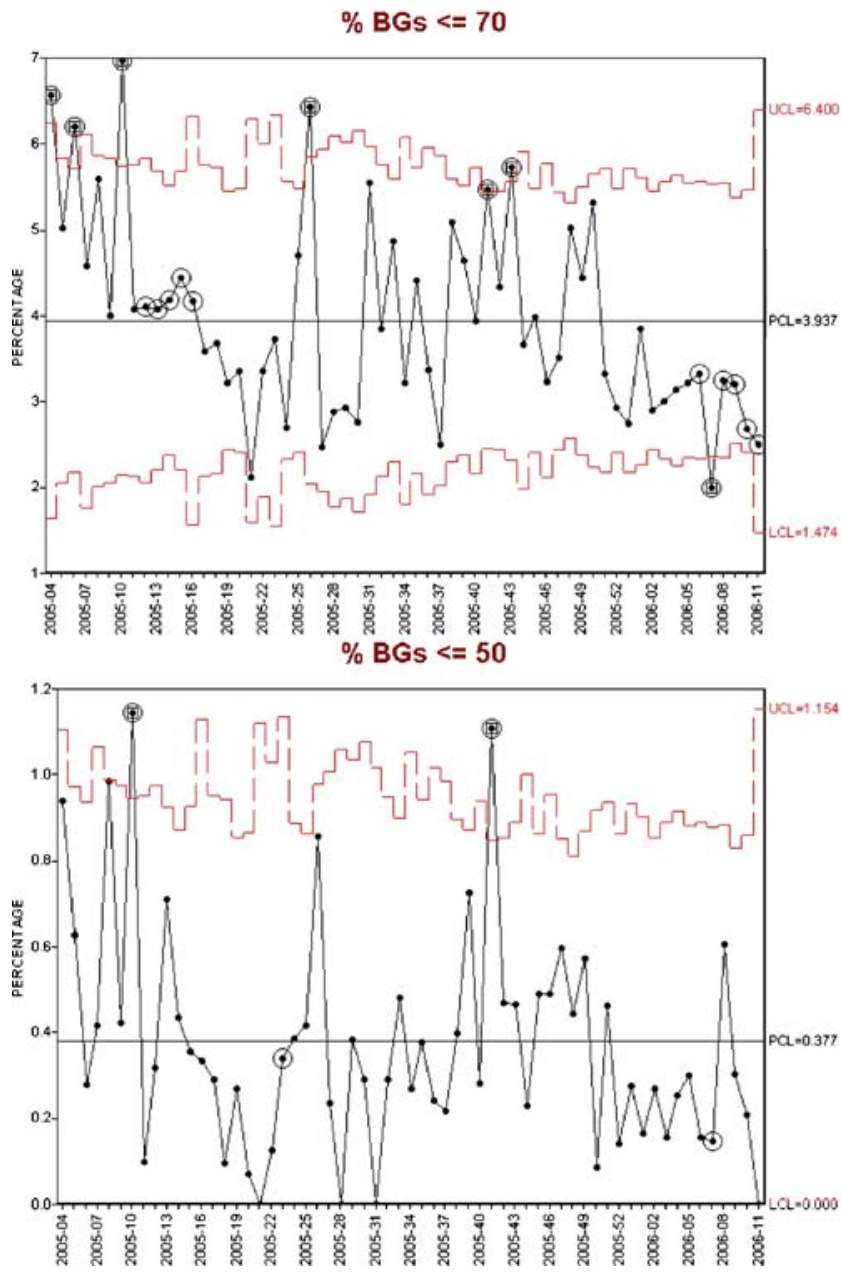

0964

Use of Glucommander following cardiovascular surgery resulted in ideal control in $96 \%$ of patients and 1.1 day reduction in LOS

P. C. Davidson ${ }^{1}$, L. Prevosti ${ }^{2}$, H. Hebblewhite ${ }^{1}$,

V. Cheekati ${ }^{3}$, R. Steed ${ }^{1}$, B. Bode ${ }^{1}$;

${ }^{1}$ Atlanta Diabetes Associates, Atlanta, ${ }^{2}$ Piedmont Hospital, Atlanta, ${ }^{3}$ Atlanta Medical Center, Atlanta, United States.

Background and Aims: Because the IHI has set a standard of keeping all perioperative BGs $<200 \mathrm{mg} / \mathrm{dl}$ following CV surgery and because our CVsurgery unit was only achieving this goal in $56 \%$ of patients during the past year, we have initiated a new protocol for BG management.

Materials and Methods: The protocol was developed by a committee of surgeons, nurses, and endocrinologists. The basis of this protocol is the Glucommander. This is a computer program advising IV insulin dosing which we have developed. Glucommander has been extensively studied in various clinical situations. The goal of the protocol is a mean $\mathrm{BG}$ of $105 \mathrm{mg} / \mathrm{dl}$ with minimal hypoglycemia and post-op BG's $<200 \mathrm{mg} / \mathrm{dl}$.

Results: At this point $182 \mathrm{CV}$ surgery patients in Piedmont Hospital have been studied. The mean A1c of all patients was $6.4 \% .31 \%$ were recognized as having diabetes on admission. The criteria for treating patients with IV insulin was a history of diabetes, $\mathrm{BG}>140 \mathrm{mg} / \mathrm{dl}$, or two BG's post-op $>110 \mathrm{mg} / \mathrm{dl}$. $91 \%$ of patients were placed on IV insulin. They stabilized at a $B G<120 \mathrm{mg} / \mathrm{dl}$ in an average of $3.2 \mathrm{hrs}$. IV insulin was continued for an average of $29 \mathrm{hrs} .96 \%$ were controlled so that no BG was $>200 \mathrm{mg} / \mathrm{dl}$ in the 48 hours following surgery. Only $8 \%$ had transient BG $<60 \mathrm{mg} / \mathrm{dl}(51-58 \mathrm{mg} / \mathrm{dl})$. $13 \%$ of patients were on insulin on admission. $53 \%$ were transitioned to sc insulin while in the hospital. $34 \%$ were discharged on insulin. All were on basal-bolus insulin with glargine and aspart following IV insulin and following discharge.

The LOS post-op in this population was 6.2 days. This compares to the LOS in the year prior to initiation of this protocol of 7.3 days and projects to a saving of $\$ 2.1$ millions per year in one 450 bed hospital.

Conclusions: This study has shown that it is possible to safely achieve and maintain normoglycemia following CV surgery in patients who have been on cardiopulmonary bypass and treated with 250-500 mg methylprednisolone and ionotropes. This excellent BG control is expected to reduce post-op infections and complications to levels comparable to non-diabetic persons and not only reduce LOS but decrease readmissions.

0965

Effect of nutritional protocol and measurement error on automated glucose control in the ICU

M. E. Wilinska, L. J. Chassin, R. Hovorka;

Department of Paediatrics, University of Cambridge, United Kingdom.

Background and Aims: Tight glycaemic control has been shown to reduce mortality and morbidity in critically ill patients. Using in silico computational approach, the objective of this study was to evaluate the effect of nutrition and the measurement error (ME) on the quality of glucose control with fully automated computer-based algorithm.

Materials and Methods: In silico simulation environment was used to simulate a $48 \mathrm{~h}$ clinical trial with a computer-based control algorithm in the intensive care unit (ICU). The simulation environment represented all components of a clinical trial such as the experimental protocol, synthetic subjects (a glucoregulatory model with patient-specific parameters), a glucose meter with a defined ME, an insulin pump, a glucose controller, and finally, statistical measures to describe 
the results. A virtual ICU population of 21 subjects was used to simulate clinical trials using two types of nutritional protocols, simple and complex, and various levels of the ME. The simple protocol consisted of a constant parenteral glucose infusion of $4 \mathrm{~g} / \mathrm{h}$ and a $40 \mathrm{~g} \mathrm{CHO}$ meal at $44 \mathrm{~h}$. The complex protocol consisted of varied parenteral and enteral glucose infusions, a parenteral glucose bolus of $12 \mathrm{~g}$ at $15 \mathrm{~h}$ and a meal of $40 \mathrm{~g}$ $\mathrm{CHO}$ at $44 \mathrm{~h}$. The parenteral glucose infusion commenced at a rate of $4 \mathrm{~g} / \mathrm{h}$ at the beginning of the study. The rate was increased to $8 \mathrm{~g} / \mathrm{h}$ at $12 \mathrm{~h}$ and dropped back to $4 \mathrm{~g} / \mathrm{h}$ at $24 \mathrm{~h}$. At $30 \mathrm{~h}$ the infusion rate was increased to $12 \mathrm{~g} / \mathrm{h}$ and continued until the end of the study. The enteral glucose infusion commenced at $33 \mathrm{~h}$ and $20 \mathrm{~min}$ at a rate of $8 \mathrm{~g} / \mathrm{h}$ and continued until the end of the study. Glucose was sampled hourly. The coefficient of variation $(\mathrm{CV})$ of the $\mathrm{ME}$ ranged from 2.5 to $15 \%$. A number of clinical and statistical measures were used to assess the quality of glucose control.

Results: The mean glucose, time spent in target glucose band, the hyperglycaemic index and the number of hypoglycaemia episodes versus the two types of nutritional protocol and the selected levels of measurement error are listed in Table 1. The measures deteriorated when moving from the simple to the complex protocol. A significant deterioration was also noted with the increasing level of the ME. Severe hypoglycaemia episodes $(<2.8 \mathrm{mM})$ were observed with the ME $>10 \%$.

Conclusion: With automated glucose control, nutritional protocol should be kept simple to facilitate an effective control of glucose. The measurement error of the glucose measuring device should not be higher than $10 \%$.

Table 1 Control measures for simple and complex protocols and selected levels of $\mathrm{ME}(\mathrm{N}=21)$

\begin{tabular}{llllllllll}
\hline & \multicolumn{3}{l}{ Simple Protocol } & \multicolumn{5}{c}{ Complex Protocol } \\
\cline { 2 - 9 } & $2.5 \% *$ & $5 \%$ & $10 \%$ & $15 \%$ & $2.5 \%$ & $5 \%$ & $10 \%$ & $15 \%$ \\
\hline $\begin{array}{l}\text { Mean } \\
\text { Glucose }\end{array}$ & 6.00 & 5.92 & 5.97 & 5.94 & 6.08 & 6.12 & 6.25 & 6.53 \\
{$[\mathrm{mM}]$} & & & & & & & & \\
$\begin{array}{l}\text { Time in } \\
\text { Band [\%]* }\end{array}$ & 80 & 69 & 61 & 51 & 53 & 51 & 38 & 29 \\
$\begin{array}{l}\text { HGI [mM] } \\
\text { Hypo }\end{array}$ & 0.23 & 0.24 & 0.33 & 0.49 & 0.50 & 0.48 & 0.60 & 0.89 \\
$\begin{array}{l}\text { Episodes } \\
(<3.3 \mathrm{mM})\end{array}$ & 0 & 1 & 2 & 11 & 1 & 0 & 0 & 3 \\
{$\left[\begin{array}{l}\text { [unitless] } \\
\text { Severe Hypo }\end{array}\right.$} & 0 & 0 & 0 & 2 & 0 & 0 & 0 & 0 \\
$\begin{array}{l}\text { Episodes } \\
(<2.8 \mathrm{mM})\end{array}$ & & & & & & & & & \\
{$[$ unitless] } & & & & & & & & & \\
\hline
\end{tabular}

* ME expressed as CV

**Target glucose band 4.4 to $6.1 \mathrm{mM}$

\# Hyperglycaemic index

Supported by EU Clinicip Project IST-2002-506965
0966

Structured approaches to the evaluation and treatment of inpatient hyperglycaemia and length of stay in the hospital

R. Juneja ${ }^{1}$, A. Golas ${ }^{1}$, N. Kumar ${ }^{2}$, J. Carroll ${ }^{3}$, D. Nelson ${ }^{3}$, S. Flanders ${ }^{3}$, C. Roudebush ${ }^{1}$;

${ }^{1}$ Medicine, Indiana University, Indianapolis, ${ }^{2}$ Department of Internal Medicine, Medical College of Wisconsin, Milwaukee, ${ }^{3}$ Medicine, Clarian Health Partners, Indianapolis, United States.

Background and Aims: The Systematic Utilization of $\boldsymbol{G}$ lucose $\boldsymbol{A}$ ssessment and $\boldsymbol{R}$ esponse (SUGAR) Program was implemented in a staged manner to reduce inpatient hyperglycemia at Clarian Health Partner Hospitals in Indianapolis, USA.

Materials and Methods: The program electronically tracks all in-hospital Blood Glucose (BG) measurements and triggers intervention (if $\mathrm{BG}$ is $>180 \mathrm{mg} / \mathrm{dl} ;>110 \mathrm{mg} / \mathrm{dl}$ in the ICU). In the ICU, target BG is achieved with IV insulin (IVI) driven by a computer program prompting $\mathrm{BG}$ monitoring and IVI adjustment to target. In non-ICU settings, BG control is driven by ongoing programs of increasing awareness of inpatient hyperglycemia through education (morning reports/computer training modules) for physicians/nursing staff by inpatient diabetes nurses and standardization of $\mathrm{s} / \mathrm{c}$ insulin orders.

Results: We have previously reported significant increase in the numbers of patients reaching target BG from $75 \%$ $\mathrm{BG}<180 \mathrm{mg} / \mathrm{dl}$ to $83 \%(\mathrm{p}<0.001)$ with a simultaneous increase in the number of BG tests from 40,000 to over $65,000 /$ month. The main impact has been with ICU targets being achieved through our IVI program (started Oct 2004) with over $60 \%$ now achieving target BG (up from 26\%). In non-ICU patients we have not seen substantive reduction in overall BG targets, although there has been a sharp increase in endocrinology consults for diabetes. This study analyzes the length of stay (LOS), charges and mortality data from the hospital database during this time (data from patients $\geq 18$ years of age and with $>$ one BG measurement during hospitalization).

See Table 1 data for ALL patients ( $p<0.001$ :Jan-Jun 2005 vs Jul-Dec 2005 for LOS and charges; mortality $p=0.013$.Other time points mortality $\mathrm{p}<0.001$ compared to Jul-Dec 2005).

See Table 2 data for patients with at least one day in ICU ( $p<0.001$ :Jan-Jun 2005 vs Jul-Dec 2005 for LOS and charges; $\mathrm{p}=\mathrm{ns}$ for mortality)

Conclusion: Our analysis shows that LOS decreased during the period when tight glycemic control (TGC) measures were introduced in the hospital accompanied by a reduction in charges despite a sharp increase in the number of glucose measurements. However, since overall BG control has improved only in ICU's, further studies are 
required to evaluate the direct effects of glucose reduction versus other factors that might be contributing to this reduction in LOS.

Table 1: LOS All Patients

\begin{tabular}{lllll}
\hline Months & \# of Patients & $\begin{array}{l}\text { Mean } \\
\text { LOS } \\
\text { (Days) }\end{array}$ & $\begin{array}{l}\text { Charges } \\
(\$)\end{array}$ & $\begin{array}{l}\text { Mortality } \\
(\%)\end{array}$ \\
\hline Jan-Jun 04 & 13078 & 8.7 & 34661 & 4.5 \\
Jul-Dec 04 & 13706 & 8.5 & 34900 & 4.4 \\
Jan-Jun 05 & 13882 & 8.4 & 37836 & 4.2 \\
Jul-Dec 05 & 13139 & 7.7 & 35538 & 3.6 \\
TOTAL & $\mathbf{5 3 8 0 5}$ & $\mathbf{8 . 3}$ & $\mathbf{3 5 7 3 4}$ & $\mathbf{4 . 2}$ \\
\hline
\end{tabular}

Table 2: LOS ICU Patients

\begin{tabular}{lllll}
\hline Months & \# of Patients & $\begin{array}{l}\text { Mean } \\
\text { LOS } \\
(\text { Days })\end{array}$ & $\begin{array}{l}\text { Charges } \\
(\$)\end{array}$ & $\begin{array}{l}\text { Mortality } \\
(\%)\end{array}$ \\
\hline Jan-Jun 04 & 3209 & 13.2 & 70652 & 11.0 \\
Jul-Dec 04 & 3220 & 13.5 & 72762 & 11.8 \\
Jan-Jun 05 & 3105 & 13.2 & 80478 & 11.6 \\
Jul-Dec 05 & 2865 & 11.5 & 72421 & 10.4 \\
TOTAL & $\mathbf{1 2 3 9 9}$ & $\mathbf{1 2 . 9}$ & $\mathbf{7 4 0 7 8}$ & $\mathbf{1 1 . 2}$ \\
\hline
\end{tabular}

Supported by Novo Nordisk Pharmaceuticals

0967

Effectiveness of acute diabetes complications management in day hospital care in new onset type 1 diabetes mellitus patients

J. J. Chillarón ${ }^{1}$, M. J. Carrera ${ }^{1}$, J. F. Cano Pérez ${ }^{1}$,

J. Flores ${ }^{1}$, M. Cerdà ${ }^{1}$, A. Goday ${ }^{1}$, M. Fernández ${ }^{1}$,

R. Güerri ${ }^{2}$, E. Corominas ${ }^{1}$, J. Puig ${ }^{1}$;

${ }^{1}$ Endocrinology and Nutrition, ${ }^{2}$ Internal Medicine, Hospital del Mar, Barcelona, Spain.

Background and Aims: Acute metabolic complications in new onset type 1 diabetes have been treated in a conventional hospitalization ward. The inhospital management is an expensive way of care, but there are no studies comparing effectiveness and feasibility of Day Hospital treatment versus conventional hospitalization for adults with type 1 diabetes. Aims: 1.To assess the effectiveness of treating acute complications of diabetes (ACD) in a day hospital (DH) versus conventional hospitalization $(\mathrm{CH})$. 2. To evaluate long term (1 year) glycemic control in both groups. 3. To compare short term re-admissions (3-6 months) due to ACD between study groups.
Material and Methods: Study design: prospective, non randomized, cohorts study with 1 year follow-up.

We included all adults with new onset type 1 diabetes (positive GAD Ab, C-peptide $<1.0 \mathrm{ng} / \mathrm{mL}$ ) consecutively admitted in our hospital, with ACD (ketosis/ketoacidosis or acute hyperglycemia $>300 \mathrm{mg}$./dl).

We excluded patients presenting $\mathrm{HCO} 3<10 \mathrm{mEq}$, glycemia $>600 \mathrm{mg} / \mathrm{dl}$, severe intercurrent illness (infectious, $\mathrm{CV}$ disease....) and social isolation.

Patients were assigned to $\mathrm{DH}$ or $\mathrm{CH}$ according to time of admission to the emergency-room. If patients were admitted out of DH opening hours (Monday through Friday 08:0017:00 h) they were assigned to $\mathrm{CH}$. All patients admitted in $\mathrm{CH}$ cohort could have been assisted in DH if they had arrived during the DH-opening hours. Investigators did not participate in group assignment.

The cost calculation included structural cost, and all visits (emergency ward, Day hospital, ambulatory consultations).

Results: During the period studied (2000-2004), fifty patients met the inclusion criteria, and in all cases the ACD was the first manifestation of new onset type 1 diabetes. The average age was 29,98 years (range 15-57 years), ketoacidosis/ketosis was present in $74 \%$ of the patients (37 cases) and $26 \%$ (13 cases) had acute hyperglycemia.

\begin{tabular}{llll}
\hline & Day hospital & Hospitalization & \\
\cline { 2 - 3 } $\mathrm{n}$ & 32 & 18 & \\
\hline Age (years) & $31,8+10,6$ & $26,8+9,2$ & $\mathrm{n} . \mathrm{s}$ \\
Gender (M/F) & $19 / 13$ & $12 / 6$ & $\mathrm{n} . \mathrm{s}$ \\
Blood Glucose (mg/dL) & $362,5+111,8$ & $379,39+99,5$ & $\mathrm{n} . \mathrm{s}$ \\
PH & $7,34+0,07$ & $7,34+0,06$ & $\mathrm{n} . \mathrm{s}$ \\
HCO3 (mmol/L) & $24,1+7,4$ & $24,4+4,8$ & $\mathrm{n} . \mathrm{s}$ \\
Length of stay (days) & 0 & $6,83+1,30$ & $<0,001$ \\
HbA1C (baseline) & $11,61+2,40$ & $11,72+2,2$ & $\mathrm{n} . \mathrm{s}$ \\
HbA1C (3 months) & $6,56+0,85$ & $6,95+0,88$ & $\mathrm{n} . \mathrm{s}$ \\
HbA1C (6 months) & $6,61+1,17$ & $6,48+0,77$ & $\mathrm{n} . \mathrm{s}$ \\
HbA1C (1 year) & $6,93+1,43$ & $6,70+1,21$ & $\mathrm{n} . \mathrm{s}$ \\
Hypoglycemic & $4,79+2,28$ & $4,75+4,35$ & $\mathrm{n} . \mathrm{s}$ \\
episodes at 3 months & & & \\
\hline
\end{tabular}

There were no differences in the number of short term readmissions (at 3-6 months) when comparing $\mathrm{DH}$ and $\mathrm{CH}$ cohorts (1 patient in each group). After discharge the mean number of ambulatory visits per patient over a six months period was similar in both groups $(7,5 \pm 1,5$ in $\mathrm{DH}$ and $6,8 \pm 4,1$ in $\mathrm{CH})$. The median cost per patient and year was $584,9 €$ for the DH cohort and $1.796,0 €$ for the $\mathrm{CH}$ group.

Conclusions: Our study seems to confirm DH management shows a clear advantage. Results show a net economic saving per assistance of $1.211,1 €$ with no differences in short and long term glycemic control nor in the number of re-admissions.

Supported by FIS 020402 (Spain Department of Health) 


\section{PS 81}

\section{Continuous insulin infusion - subcutaneous or intraperitoneal}

\section{8}

\begin{abstract}
Alternate site (like) phenomenon: rapid changes from hypoglycaemic to hyperglycaemic glucose levels in a clinical-experimental study in patients with type 1 diabetes
\end{abstract}

S. Dellweg ${ }^{1}$, L. Nosek ${ }^{1}$, C. Kapitza ${ }^{1}$, D. Brandt ${ }^{2}$, M. Essenpreis, T. Koschinsky ${ }^{3}$, L. Heinemann ${ }^{1}$;

${ }^{1}$ Profil Inst. für Stoffwechselforschung, Neuss, Germany, ${ }^{2}$ Disetronic Medical AG, Burgdorf, Switzerland, ${ }^{3}$ HeinrichHeine University of Düsseldorf, Germany

Background and Aims: Monitoring of glucose changes by SMBG and by continuous glucose monitoring (CGM) systems is prone to certain delays during rapid changes in glycemia if the blood sample is collected at non-glabrous skin or in deeper layers of the skin. Aim of this study was to evaluate the magnitude of such delays.

Materials and Methods: We performed a clinical experimental study with a glucose-clamp day and a day with a standardized meal in 15 patients with type 1 diabetes (6 female/9 male; age $31 \pm 8$ years (mean $\pm \mathrm{SD}$ ); BMI $26,5 \pm$ $2,9 \mathrm{~kg} / \mathrm{m}^{2}$; HbAlc $7,1 \pm 0,9 \%$; duration of diabetes $4 \pm$ 3 years). Venous blood glucose levels were measured continuously by means of the Biostator and in sc interstitial fluid by means of a microdialysis technique. In parallel, venous blood glucose levels were measured discontinuously by means of a laboratory method (reference values) and in capillary blood samples collected at 3 measurement sites (finger, lower arm, and abdomen) by means of three blood glucose meters (one for each site; measured in duplicate). On the first study day blood glucose was clamped at a level of $100 \mathrm{mg} / \mathrm{dl}$ for $120 \mathrm{~min}, 300 \mathrm{mg} / \mathrm{dl}$ for $120 \mathrm{~min}$ at this level, $50 \mathrm{mg} / \mathrm{dl}$ for $60 \mathrm{~min}, 100 \mathrm{mg} / \mathrm{dl}$ for another $120 \mathrm{~min}$, with rapid changes in blood glucose between the plateaus. On the next day, the second study day, slower changes in glycemia were induced by means of a carbohydrate-rich standardized meal (starting BG $90-150 \mathrm{mg} / \mathrm{dl}$ ).

Results: Venous blood glucose values measured with the lab system were comparable with the continuous recording with the Biostator. In contrast, the capillary measurement results are higher at all three measurement sites. This study shows that ISF glucose levels are higher in comparison to venous blood values during hyperglycemia on both study days but are identical to capillary blood glucose values measured in samples collected at the fingertips. Glucose levels in samples collected at the forearm and abdomen are identical to those collected at the fingertips during stable glycemic levels but run behind during rapid changes in glycemia: max. $30 \mathrm{mg} / \mathrm{dl}$ after $60 \mathrm{~min}$.

Conclusion: The previously described AST phenomenon could be reproduced in relatively young patients with Type 1 diabetes. No pronounced AST-like phenomenon could be observed with CGM despite considerable rates of change of glycemia.

Supported by Disetronic/Roche Diagnostics

0969

Comparison between CSII with multiple basal lispro infusion rates and MDI with lispro and glargine D. Bruttomesso ${ }^{1}$, D. Crazzolara ${ }^{1}$, S. Costa ${ }^{1}$, A. Girelli ${ }^{2}$, G. Lepore ${ }^{3}$, M. Aragona ${ }^{4}$, U. Valentini ${ }^{2}$, R. Trevisan ${ }^{3}$, S. Del Prato ${ }^{4}$, A. Buhr ${ }^{5}$, A. Tiengo ${ }^{1}$, A. Maran ${ }^{1}$;

${ }^{1}$ Cattedra di Malattie del Metabolismo, Università di Padova, Italy, ${ }^{2}$ Unità Operativa di Diabetologica, Spedali Civili, Brescia, Italy, ${ }^{3}$ USC Diabetologia, Ospedali Riuniti di Bergamo, Italy, ${ }^{4}$ Dipartimento di Endocrinologia e Metabolismo, Università di Pisa, Italy, ${ }^{5}$ Disetronic Medical Systems AG, Burgdorf, Switzerland.

Background and Aims: To compare the effectiveness of insulin lispro and glargine (MDI-glargine) vs CSII in type 1 diabetic patients in good metabolic control.

Materials and Methods: After a one month run-in period 39 type 1 diabetics (age $38 \pm 9$ years, duration of diabetes $17 \pm 8$ years, $\mathrm{HbA1c} 7.6 \pm 0.8 \%, \mathrm{M} \pm \mathrm{DS}$ ), treated with CSII for at least 6 months, were randomly assigned to CSII with lispro or to MDI with lispro and glargine. After 4 months of therapy patients were crossed-over to the alternate treatment. We evaluated HbA1c, blood glucose levels and number of hypoglycaemic episodes. During the last month of each treatment patients underwent 48 hours of continuous glucose monitoring. The satisfaction was evaluated with the DTSQ questionnaire.

Results: HbA1c did not change with both treatments. Compared to CSII, MDI- glargine was associated to higher preprandial glycaemic values $(153 \pm 71$ vs $147 \pm 67 \mathrm{mg} / \mathrm{dl}$; $\mathrm{P}<0.005)$, higher fasting glycaemic variability $(66 \pm 15$ vs $59 \pm 16 \mathrm{mg} / \mathrm{dl}, \mathrm{P}<0.05$ ), higher total daily insulin dose $(0.63 \pm 0.15$ vs $0.54 \pm 0.12 \mathrm{UI} / \mathrm{Kg}, \mathrm{P}<0.05)$. Continuous glucose monitoring profiles in 11 patients (mean duration $34 \pm 3$ hours) showed a lower glucose exposure with CSII (mean glycaemia $147 \pm 10 \mathrm{mg} / \mathrm{dl}$ vs $189 \pm 11 \mathrm{mg} / \mathrm{dl}, \mathrm{p}<0.02$; glycaemia $>65 \mathrm{mg} / \mathrm{dl}$ and $<180 \mathrm{mg} / \mathrm{dl} \quad 1568 \pm 154$ vs $929 \pm 150 \mathrm{~min}, \mathrm{p}<0.02$, glycaemia $>180 \mathrm{mg} / \mathrm{dl} 519 \pm 122$ vs $1144 \pm 205$ min, $p<0.02$ ). During the night patients using CSII were exposed to glycaemic values $>65 \mathrm{mg} / \mathrm{dl}$ and $<180 \mathrm{mg} / \mathrm{dl}$ for a longer period than patients using MDI $(371 \pm 52$ vs $232 \pm 48$ min, $p<0.02$, mean glycaemia $135 \pm 11$ 
vs $186 \pm 12 \mathrm{mg} / \mathrm{dl}, \mathrm{p}<0.02$ ). Patients using CSII had greater satisfaction.

Conclusion: In patients treated with CSII, preprandial glycemia, glycaemic variability and insulin dose were lower and the satisfaction higher. The reduction of glycaemic variability could be important in the prevention of diabetic complications.

Supported by Disetronic Medical Systems AG, Switzerland

\section{0}

Type 1 diabetes. Modalities of external insulin pump treatment initiation in France

B. Guerci $^{1}$, V. Lassmann-Vague ${ }^{2}$, H. Leblanc ${ }^{3}$, M. Mihaileanu ${ }^{4}$, E. Leutenegger ${ }^{5}$;

${ }^{1}$ Diabetology, Endocrinology \& Nutrition Department, Jeanne d'Arc Hospital (CHU Nancy), Toul, ${ }^{2}$ Nutrition, Endocrinology \& Metabolic Disorders Department, La Timone Hospital (CHU Marseille), ${ }^{3}$ Diabetology and Endocrinology Department, St Louis Hospital, Paris, ${ }^{4}$ Medical Department, Vitalaire, Gentilly, ${ }^{5}$ ABR Pharma, Paris, France.

Background and Aims: The efficiency of insulin pump treatment on glycaemic control and patient quality of life has been clearly demonstrated. Thus, the number of insulin pump treated patients should keep on increase in the future. In this context, it is pertinent to describe the medical practices in insulin pump initiation in patients with type 1 diabetes, and to compare them to official guidelines (Alfediam, France).

Materials and Methods: A transectional study was conducted in France in 2004/2005 with 110 diabetologists from 68 centres (general public hospitals: 48\%, university hospitals: $35 \%$, private hospitals: $11 \%$, others: $6 \%$ ). Each participating physician had to select 3 type 1 diabetic patients $>15$ years old, whose insulin pump treatment was initiated at the inclusion visit or less than 3 months before.

Results: Among the 349 diabetic patients included, 62\% were women. Their mean age was $37 \pm 14$ and the mean duration of diabetes was $15 \pm 10$ years. Before pump initiation, $73 \%$ of the patients were treated by rapid-acting insulin analogues; $54 \%$ by long-acting insulin analogues. Severe hypoglycaemia and ketoacidosis had occurred for respectively $17 \%$ and $6 \%$ of the patients in the six months preceding pump initiation. Before being treated by pump, patients were used to perform $4.3 \pm 1.8$ capillary blood glucose measurements every day and they reported 3.1 \pm 2.6 symptomatic hypoglycaemia episodes per week. Mean number of insulin injections per day was $3.8 \pm 0.8$ but $24 \%$ of the patients had less than 4 . When pump was initiated, the mean HbA1c value was $8.44 \pm 1.58 \%$. HbAlc was above
$8 \%$ (guidelines indication) for $56 \%$ of the patients, of which $19 \%$ were women waiting for pregnancy. HbA1c was $>10 \%$ for $14 \%$ of the patients, $<8 \%$ for $42 \%$, and $<7 \%$ for $14 \%$. Two thirds of the patients with $\mathrm{HbA} 1 \mathrm{c}<8 \%$ were waiting for a pregnancy, presenting recurrent severe hypoglycaemia or ketoacidosis, or having irregular lifestyle (guidelines indication). The prescribed treatment consisted in rapid insulin analogs for most of the patients $(86 \%)$, with 6 home blood glucose measurements or more per day (76\%) and an urinary $(77 \%)$ or capillary $(35 \%)$ ketone bodies monitoring. The pump initiation was mainly motivated by the uncontrolled status of diabetes (75\%) and patient's demand (43\%). Therapeutic goals declared by the physicians were to get an $\mathrm{HbA} 1 \mathrm{c}$ value below $7 \%$ ( $88 \%$ of cases), to improve patient's quality of life $(70 \%)$, and to prevent diabetes complications (69\%).

Conclusion: Practices in insulin pump treatment of type 1 diabetes are mostly consistent with French official guidelines, especially concerning HbAlc values at initiation and therapeutic goals. However, pump initiation is not always preceded by an intensification of insulin treatment using multiple insulin injections. Frequent hypoglycaemia and patient's demand are increasing motivation factors for pump prescribing.

0971

Using insulin pump therapy together with real time continuous glucose monitoring in the therapy of type 1 diabetic patients: the impact on glycaemic excursion - a pilot study

J. Broz, M. Andel;

3rd Medical Faculty, Charles University, Prague, Czech Republic.

Background and Aims: to confirm the efficacy of a new therapeutic system - sensor augmented insulin pump Paradigm $^{\circledR}$ RT (Medtronic Inc), which displays updated subcutaneous glucose readings every five minutes and sounds the alarm when levels become over or below previously determined values.

Materials and Methods: 7 patient (type 1 diabetic men, treated with CSII, older Paradigm type). Each patient was continuously monitored for 5 days (CGMS Gold) and was not allowed to see the results. After detailed training the older Paradigm type pump was replaced by Paradigm ${ }^{\circledR}$ RT and each patient used this system for another 5 days. We compared the frequency of symptomatic hypoglycaemias and the total duration of glycaemic excursions over $10 \mathrm{mmol} / 1$ and below $3,5 \mathrm{mmol} / 1$ between those two 5 day periods (Monday - Friday). 
Results: we found a decrease in the average number of symptomatic hypoglycaemias per 5 days using Paradigm ${ }^{\circledR}$ RT: $2,1 \pm 0,78$ v.s. $3,33 \pm 0,81$ (ns) in the previous period, an average total duration of hypoglycaemic excursion per 24 hours decrease to 21 minutes v.s. 105 minutes (ns) previously and the duration of hyperglycaemic excursion decrease to 202 minutes v.s. 352 minutes (ns) in the previous period.

Conclusion: the first experience using the Paradigm ${ }^{\circledR}$ RT indicates an important influence on the stabilization of glycaemic levels in type 1 diabetic patients. More patients are to undergo the study to confirm these results definitively. Supported by IGA NR/8226-3 and MSM 0021620814

\section{2}

Treatment satisfaction and metabolic control in type 2 patients using CSII: results from an international observational study

M. A. Schweitzer ${ }^{1}$, T. Pieber ${ }^{2}$, L. Heinemann ${ }^{3}$,

S. Grunder ${ }^{4}$, A. Buhr ${ }^{4}$;

${ }^{1}$ Roche Diagnostics GmbH, Mannheim, Germany, ${ }^{2}$ JOANNEUM RESAERCH Forschungsgesellschaft mbH, Graz, Austria, ${ }^{3}$ Profil Institute for Metabolic Research, Neuss, Germany, ${ }^{4}$ Disetronic Medical Systems AG, Burgdorf, Switzerland.

Background and Aims: The progressive character of type 2 diabetes with an increasing loss of $\beta$-cell function often results in the need for insulin treatment to recover good metabolic control. Intensification of insulin treatment including CSII is also an attractive option for such patients if basal insulin supply needs to be optimised and hypoglycemic episodes shall be minimised. However, few systematic data exist on patients with type 2 diabetes using insulin pumps. We documented data from those patients in routine daily life.

Materials and Methods: A structured questionnaire was distributed to 36,450 patients treated with CSII across Europe and North America to determine patient characteristics, medical data, treatment complications and satisfaction. In total, there were 25 structured questions with additional information on socio-demographic background, insulin pump indication, initiation and follow-up of treatment. We report the analysis of the sub-set of data of patients with type 2 diabetes on CSII.

Results: A 38.4\% response rate was achieved with 14,015 questionnaires returned, out of which 729 (5.2\%) were patients with type 2 (64\% males). They had a mean $( \pm \mathrm{SD})$ age of $56.4 \pm 11.9$ years, a BMI of $29.9 \pm 5.9 \mathrm{~kg} / \mathrm{m}^{2}$, a duration of diabetes of $17.2 \pm 8.9$ years, a duration of insulin use of $10.8 \pm 7.8$ years and were already $3.4 \pm 3.2$ years on CSII. Theses patients detailed multiple reasons for selecting CSII therapy out of choice of 12 indications: The most common was poor glycemic control with s.c. insulin therapy (76.5\%), uncontrolled blood glucose fluctuations (44.4\%) and high insulin requirements with syringe/pen therapy (34.4\%). Initiation of CSII was carried out either at hospital as in- $(44.3 \%)$ or out-patient $(5.5 \%)$, in diabetes specialist's office $(46.4 \%)$ or in a general practitioner's office $(2.9 \%)$. The average total daily insulin dose applied was $0.84 \pm 0.6 \mathrm{IU} / \mathrm{kg}$ body weight whereas $49.3 \%$ was given as basal insulin reflecting a close to 50:50 ratio. Patients achieved a HbAlc of $7.0 \pm 1.2 \%$ (non-standardized), with the majority of patients $(67.2 \%)$ being below $7.5 \%$. Patients were highly satisfied with CSII therapy. They left their infusion set in place for $2.7 \pm 1.2$ days.

Conclusion: This international observational study provides real world data about a relatively "unknown" patient group, i.e. patients with type 2 diabetes on CSII. Patients are relatively young and have already a significant duration of diabetes and insulin use before starting insulin pump treatment. They achieve good glycemic control with a 50:50 ratio basal/bolus insulin for the average patients with high treatment satisfaction. Clearly, the evidence for the use of CSII in such patients should be studied in a randomised controlled clinical trial.

Supported by Disetronic Medical Systems AG, Switzerland

0973

Glycaemic control, health status and treatment satisfaction in subjects with diabetes mellitus treated with Continuous IntraPeritoneal Insulin Infusion (CIPII). A single centre experience

S. J. J. Logtenberg, E. van Ballegooie,

H. Israel-Bultman, A. van Linde, H. J. G. Bilo;

Department of Internal Medicine, Isala Clinics, Zwolle, The Netherlands.

Background and Aims: Continuous intraperitoneal insulin infusion (CIPII) has been in use now for over 20 years. High costs and technical problems have prevented a widespread use, with most intraperitoneal (IP) pumps implanted in France and the USA. In the Netherlands, the Isala Clinics in Zwolle is the centre with the most experience with CIPII. Its use is aimed at improving glycaemic control with less hypoglycaemic events, and thus improving health related quality of life in patients with poorly controlled diabetes despite intensified insulin treatment with multiple subcutaneous injections or an external pump. 
The aim of this study is to assess effects of CIPII on glycaemic control, health status and treatment satisfaction in all subjects treated with CIPII within the Isala Clinics.

Material and methods: Retrospective longitudinal analysis of clinical data in 48 patients initiated on CIPII between 1983 and 2005. HbA1c levels at baseline, after one year, and at present assessment or at end of pump use were applicable. Cross-sectional assessment of health status using the Short Form 12 item health survey (SF-12), of well-being using the Well-Being Index (WHO-5) and of treatment satisfaction using the Diabetes Treatment Satisfaction Questionnaire (DTSQ).

Results: 33 of 48 patients were still treated with CIPII at the moment of assessment (fourth quarter 2005). Over $90 \%$ returned the questionnaire. 5 patients died whilst on CIPII (4 diabetes related), none died of hypoglycaemia. Reasons for cessation of CIPII were; pump malfunction (3), inadequate glycaemic control (4), kidney-pancreas transplantation (2) and recurrent infections (1). HbAlc (mean \pm standard deviation) decreased from $9.3 \pm 1.7 \%$ to $8.8 \pm 1.7 \%$ at one year and $8.7 \pm 1.6 \%$ at long term follow-up ( $\mathrm{p}=0.004$ and $p=0.001$ respectively as compared to baseline). Patients reported less hypoglycaemic events with CIPII. The Physical Component Summary score of the SF-12 was $37.4 \pm 12.1$, the Mental Component Summary score was $47.2 \pm 11.1$ (scores range from 0 to 100 with scores in general population standardised to $50 \pm 10$ ). Mean score on the WHO-5 was $52.7 \pm 28.6$ (score ranges from 0 to 100 ). Thirteen patients scored below the cut-off point of the WHO-5 for further testing for depression. Median treatment satisfaction score was $32\left(\mathrm{P}^{25}-\mathrm{P}^{75}: 28-36\right)$ (score ranges from 0 to 36 ).

Conclusion: Use of CIPII results in improved glycaemic control with less self-reported hypoglycaemic events in patients with poorly controlled diabetes despite intensified subcutaneous insulin treatment. Treatment satisfaction is high, and comparable to that with other treatment modalities. Mental health status and well-being scores are low however, with nearly 1 out of 3 patients in need of further assessment for depression, at least according to WHO-guidelines.

\section{4}

Modelization of glucose tracking performance of IV sensors and insulin pharmacokinetics by IP delivery during standard meals to improve algorithms used in a closed loop system

E. M. Renard ${ }^{1}$, A. E. Panteleon ${ }^{2}$, M. E. Miller ${ }^{2}$, G. M. Steil'

${ }^{1}$ Endocrinology, Lapeyronie Hospital, Montpellier, France,

${ }^{2}$ Medtronic MiniMed, Glucose Sensor R\&D, Los Angeles, United States.
Background and Aims: Effective glucose control at meal times represents an important challenge for the clinical development of closed loop insulin delivery systems. The aim of this study was the modelization of glucose tracking by an intravenous (IV) sensor and insulin kinetics by intraperitoneal (IP) delivery from implanted pumps, in view of more effective algorithms used for automated glucose control by a model of implanted artificial pancreas that combines both devices.

Materials and Methods: IV glucose sensor performance and delays associated with glucose sensing and IP insulin delivery were quantified in 6 type 1 diabetic patients $(4 \mathrm{M} /$ $2 \mathrm{~F}$, BMI $22.5+1.0 \mathrm{~kg} / \mathrm{m} 2$ (mean+SEM), duration of diabetes $46.8+1.9 \mathrm{y}$, age $62.5+1.9 \mathrm{y}$ ). IV sensors had been implanted for $\sim 10$ months prior to performing experiments and were linked to IP pumps (both Medtronic MiniMed). Following an overnight fast during which insulin delivery was kept constant $(0.6+0.1 \mathrm{U} / \mathrm{h})$, subjects received a meal (100 g carbohydrate) and a bolus of insulin $(4.7+0.6 \mathrm{U})$. Blood was sampled every $5 \mathrm{~min}$ until completion of the first $30 \mathrm{~min}$ after the meal, every $10 \mathrm{~min}$ for the remaining 1 st hour, every $20 \mathrm{~min}$ for the 2 nd hour and every $30 \mathrm{~min}$ for the 3rd and 4th hour.

Results: Sensor kinetics were described using a first order model described by a transport delay $\left(\mathrm{T}_{\mathrm{D}}\right)$ and a time constant $\mathrm{t}: \mathrm{R}^{2}=0.84+0.1, \mathrm{~T}_{\mathrm{D}}=21.8+6.1 \mathrm{~min}, \mathrm{t}=20.2+6.3$ $\mathrm{min}$. Sensor glucose was well correlated with reference and generated an unbiased estimate of the reference $\left(\mathrm{R}^{2}=0.85+\right.$ $0.03, \mathrm{SG}=1.02 \times$ reference -25.7 , slope not different than 1 , $\mathrm{P}<0.05)$. IP insulin kinetics were well described $\left(\mathrm{R}^{2}=0.84\right.$ +0.1 ) using a bi-exponential model with time delays $\mathrm{t}_{1}=60.0+8.7 \mathrm{~min}$ and $\mathrm{t}_{2}=27.2+9.3 \mathrm{~min}$, and insulin clearance $(3600+759.2 \mathrm{ml} / \mathrm{min})$.

Conclusion: Besides demonstrating accuracy of IV sensors in tracking glycemic excursions ten months after implantation, the proposed models accurately describe the dynamics associated with IV glucose sensing and IP insulin delivery. Integration of these models with algorithms modulating IP insulin delivery according to IV glucose sensing are expected to allow more effective glucose control at meal times in future closed loop trials with our implanted system.

0975

Automated intensive diabetes management based on continuous glucose monitoring.

A. E. Panteleon, M. Loutseiko, V. Stafekhina,

G. Kopelyan, G. M. Steil;

Glucose Sensor R\&D, Medtronic MiniMed, Los Angeles, United States. 
Background and Aims: Intensive diabetes therapy has been shown to lead to improved glucose control and reduced long-term complications. Nonetheless, adjustments in pump therapy are typically performed empirically. The aim of this study was to demonstrate the feasibility of automating these adjustments using a proportional-integralderivative (PID) algorithm variation.

Materials and Methods: The proposed algorithm was evaluated over a 12-week period in 4 pancreatectomized canines $(1 \mathrm{M}, 3 \mathrm{~F} 29.9 \pm 3.5 \mathrm{~kg})$, fed once per day $(6: 00 \mathrm{am})$. During the initial 3 weeks, daytime glucose control was established using Multiple Daily Injection (MDI) therapy. A long acting insulin injection was given with the 6:00 AM meal. Blood samples were drawn daily at 6 AM, 8 AM, 10 AM, 12 PM and 2 PM. The total daily dose (TDD) of insulin was adjusted to achieve good daytime glucose (TDD $=46.3 \pm 3.3 \mathrm{U}$ ). Thereafter, 24-hour glucose control was assessed using a continuous glucose monitoring system (CGMS; Medtronic MiniMed) over six days. At week 4, Continuous Subcutaneous Insulin Infusion (CSII) therapy was initiated (Medtronic MiniMed Paradigm pump). A fixed basal rate (24 hour), Carbohydrate-to-Insulin Ratio (CIR) and Insulin Sensitivity Factor (ISF), were calculated based on the TDD established during MDI therapy (basal $=\mathrm{TDD} / 48, \mathrm{CIR}=800 / \mathrm{TDD}$ grams covered per U; ISF $=2200 / T D D)$. Continuous glucose monitoring was performed throughout the CSII period. Insulin infusion sets were replaced 2 to 3 times per week, and sensors were replaced twice per week. While using CSII, a meal bolus was calculated using the CIR but delivered as a dual wave $(50 \%$ immediate; and $50 \%$ over $3 \mathrm{~h})$. CIR was adapted based on a 3-hour postprandial target of $180 \mathrm{mg} / \mathrm{dl}$. Basal rates were adapted in three-hour blocks (starting at $12 \mathrm{AM}$ ) based on target glucose of $120 \mathrm{mg} / \mathrm{dl}$. Correction boluses were given at $10 \mathrm{AM}$ and $12 \mathrm{PM}$, with the ISF adapted using 2-hour post correction target values. Adaptation was performed using a discrete proportional integral derivative algorithm with changes (recommendations) confirmed by study staff.

Results: Therapy adjustments were made every $4.2 \pm$ 0.1 days during CSII therapy. Insulin requirement significantly decreased after 10 weeks $(46.3 \pm 3.3$ vs. $31.4 \pm 2.6 \mathrm{U}$, $\mathrm{P}<0.05$ ). HbAlc decreased during the CSII period, albeit the decrease was not significant $(3.9 \pm 0.2 \%$ vs. $3.7 \% \pm 0.1$, $\mathrm{P}=\mathrm{NS})$. Overnight glucose converged to the target glucose concentration approximately 50 days after CSII initiation (see Figure for all $6 \mathrm{AM}$ glucose values; final midnight, 3 $\mathrm{AM}$, and $6 \mathrm{AM}$ glucose levels were $141.0 \pm 9.4 \mathrm{mg} / \mathrm{dl}$, $125.1 \pm 13.0 \mathrm{mg} / \mathrm{dl}, 124.0 \pm 6.6 \mathrm{mg} / \mathrm{dl}$ respectively). Postprandial glucose converged to the 3-hour ( $9 \mathrm{AM}$ ) postprandial setpoint of $180 \mathrm{mg} / \mathrm{dl}(174.9 \pm 7.7 \mathrm{mg} / \mathrm{dl})$ with $12 \mathrm{PM}$ glucose at $157.7 \pm 10.1 \mathrm{mg} / \mathrm{dl}$ and $3 \mathrm{PM}$ glucose at $115.8 \pm$
$7.6 \mathrm{mg} / \mathrm{dl}$. Overnight control was stable $(129.0 \pm 14.2$ at 12:00 AM; $130.6 \pm 13.1 \mathrm{mg} / \mathrm{dl}$ at $9 \mathrm{PM}, \mathrm{P}=\mathrm{NS}$ ).

Conclusion: We conclude that automating pump therapy adjustments is, in principal, a feasible strategy during pump therapy.

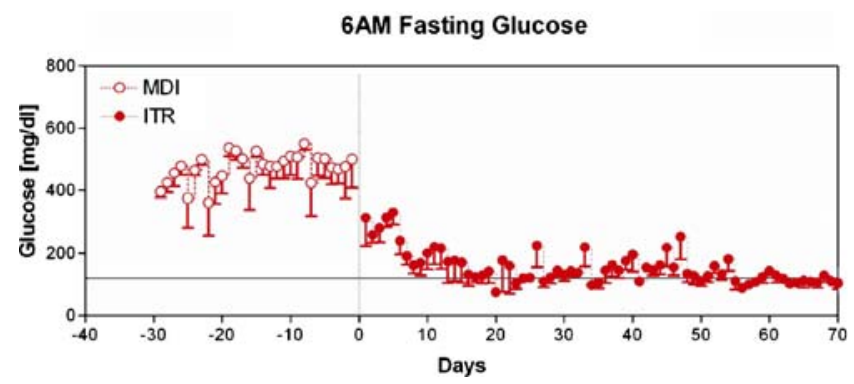

Supported by NIH/DK 64567

\section{PS 82}

\section{Insulin treatment modalities}

0976

Metabolic control and hypoglycaemia in 5,891 type 1 diabetic patients on human insulin (HI) or insulin analogues (AI) in routine care in Germany 2005 U. A. Müller ${ }^{1}$, N. Müller ${ }^{1}$, W. Kerner ${ }^{2}$, H. Hauner ${ }^{3}$, B. Osterbrink ${ }^{4}$, R. Schiel ${ }^{5}$, P. Beyer ${ }^{6}$;

${ }^{1}$ Endocrinology/Diabetes, University of, Jena, ${ }^{2}$ Diabetology, Clinic, Karlsburg, ${ }^{3}$ Else Kröner-Fresenius-Centre of Medical Nutrition, Clinic of TU, München, ${ }^{4}$ Mathias, Spital, Rheine, ${ }^{5}$ Kinder und Jugendmedizin/Stoffwechselerkrankungen, Inselklinik, Heringsdorf, Germany, ${ }^{6}$ Evangelisches Krankenhaus, Oberhausen, Germany.

Background and Aims: Insulin analogues are increasingly prescribed aiming at improving $\mathrm{HbA}_{1 \mathrm{c}}$, lowering incidence of hypoglycaemia or increasing quality of life. However, a recent Cochrane review described only minor benefits of short acting analogues in type 1 diabetes. Results of trials with long acting analogues are controversial concerning $\mathrm{HbA}_{1 \mathrm{c}}$ and demonstrated a beneficial effect on hypoglycaemia in some studies. Results from large samples representing the "real life situation" are not available. We analysed 5,891 pat. on human insulin or analogues in the database of the German Diabetes Association.

Materials and Methods: The GDA provides a certificate of quality for hospitals and specialised private practices. Candidates have to provide information on staff, teaching programmes and documentation of pat. data. 27 items have to be submitted on $>50$ pat. with type 1 diab. (and 200 pat. 
with type 2 diab. - data not shown). Hospitals collect data of consecutive pat. on admission, in private practice data are taken from a random sample. Data include: age, years since diagnosis, $\mathrm{BMI}, \mathrm{IU} /$ day, injections/day, $\mathrm{HbA}_{1 \mathrm{c}}$, severe hypoglycaemia (requiring i.v. glucose or glucagon), use of analogue insulin (yes/no). In 2005 data of 5,891 type 1 diabetic pat. were submitted from 26 private practices $(n=1,664)$ and 92 hospitals $(n=4,227)$. Information about use of short and/or long acting analogues was available in 5,888 pat.

Results: $58 \%$ of 5,888 pat. were treated with insulin analogues.

In private practice $(\mathrm{n}=1661) 63 \%$ were on AI. Compared with pat. on HI they had a longer duration of diabetes (16.1 vs 14.8 years)*, more injections per day (4.6 vs 4.4$)^{*}$ and higher $\mathrm{HbA}_{1 \mathrm{c}}$ (7.29 vs $7.12 \%$, DCCT adjusted)*. Not signif. different were: age (43.2 vs $44.7 \mathrm{y}$ ), BMI (25.8 vs $26.1 \mathrm{~kg} / \mathrm{m}^{2}$ ) insulin units per day (47.3 vs 45.5$)$, severe hypoglycaemia ( $0.06 \mathrm{vs} 0.07 / \mathrm{pat} / \mathrm{year}$ ), incidence of comata or severe ketoacidosis ( $0.01 \mathrm{vs} 0.00 /$ pat/year).

In hospitals $(\mathrm{n}=4,227)$ on admission $56 \%$ of the pat. were treated with AI. Compared with pat. on HI they were younger (42.8 vs $45.5 \mathrm{y})^{*}$, they injected more frequently (4.3 vs $4.2 /$ day)*, severe hypoglycaemia had occurred more frequently ( 0.34 vs $0.24 /$ pat/year)* and the insulin dosage was higher (46.2 vs $43.8 \mathrm{IU} /$ day)*. Not signif. different were: years since diagnosis (16.1 vs 15.4$)$, BMI (25.2 vs $\left.25.1 \mathrm{~kg} / \mathrm{m}^{2}\right), \mathrm{HbA}_{1 \mathrm{c}}(8.18$ vs $8.23 \%)$, coma or severe ketoacidosis $(0.12$ vs $0.11 /$ pat/year $) .\left({ }^{*}=p<0.05\right)$.

Conclusion: Preceding admission to hospitals type 1 diab. pat. on HI had experienced significantly less episodes of severe hypoglycaemia compared to treatment with AI. In ambulatory care the use of AI was associated with slightly higher $\mathrm{HbA}_{1 \mathrm{c}}$ compared to pat. on HI. It cannot be excluded that pat. with more severe diabetes problems were treated with AI. The results of our observational data cannot challenge the results of properly designed controlled trials. Nevertheless, in the "real life" situation problems may have occurred, which were not foreseeable by trials. Research is imperative to continuously monitor the various strategies using specific short and long acting analogues and their impact on outcome.

0977

Modelling study comparing complications in type 1 diabetes patients treated with either human or analogue insulin in a basal-bolus regimen

T. L. Christensen ${ }^{1}$, W. J. Valentine ${ }^{2}$, J. A. Ray ${ }^{2}$,

S. Roze ${ }^{2}$, V. Foos ${ }^{2}$, A. J. Palmer ${ }^{2}$;

${ }^{1}$ Novo Nordisk A/S, Copenhagen, Denmark, ${ }^{2} \mathrm{CORE}$, Center for Outcomes Research, Binningen/Basel, Switzerland.
Background and Aims: Long-term epidemiological studies in type 1 diabetes patients have shown that poor glycaemic control is associated with an increased risk of disease-related complications. In a recent multi-center randomized controlled trial of basal/bolus therapy with insulin detemir plus insulin aspart (analogue) versus neutral protamine Hagedorn (NPH) insulin plus human soluble insulin (human), the analogue regimen was associated with an improved $\mathrm{HbA} 1 \mathrm{c}$ profile $(0.22 \%$-points lower, $\mathrm{p}=$ $0.0004)$, a reduced risk of hypoglycemic events $(R R=0,79$, $\mathrm{p}=0.036)$ and decreased BMI $\left(-0.3 \mathrm{~kg} / \mathrm{m}^{2}, \mathrm{p}<0.001\right)$ compared to human insulin after 18 weeks.

The aim of this study was to estimate the time to onset of diabetes related complications for type 1 diabetes patients treated with analogue and human insulin based basal-bolus regimens.

Materials and Methods: A published and validated model of type 1 diabetes, which combines Markov sub-models and Monte Carlo simulation to simulate the progression of the disease, was used project the long-term impact of treatment with analogue and human insulin. Baseline cohort characteristics (mean age 39 years, HbAlc 8.38\%-points, BMI $24.9 \mathrm{~kg} / \mathrm{m}^{2}$, duration of diabetes 15 years) and treatment effects observed over the 18 -week trial were used in the model to project the time to onset of diabetesrelated complications over a 35-year time horizon.

Results: Treatment with analogue insulin delayed the time to onset of any diabetes-related complication by 0.09 years (1.06 versus 0.97 years). Notably, analogue insulin postponed the onset of end-stage renal disease by a mean of 0.34 years (over 4 months) per patient (20.61 versus 20.27 years). Similarly, analogue insulin was projected to delay the mean time to onset of proliferative retinopathy (by 0.48 years), lower extremity amputation ( 0.32 years), myocardial infarction $(0.33$ years $)$ and stroke $(0.30$ years $)$ compared to human insulin.

Conclusion: Improvements in glycemic control, hypoglycemic event rates and BMI associated with analogue insulin compared to human insulin treatment as part of a basal-bolus regimen were projected to delay the onset of diabetes-related complications, which are associated with a substantial clinical and economic burden.

Supported by Novo Nordisk A/S

0978

Basal-bolus therapy with insulin detemir/insulin aspart improves glycaemic control and reduces hypoglycaemia: data from the German cohort of the PREDICTIVE study R. W. Ackermann ${ }^{1}$, J. B. Hansen ${ }^{1}$, M. Nauck ${ }^{2}$;

${ }^{1}$ Novo Nordisk A/S, Bagsvaerd, Denmark, ${ }^{2}$ Abt. Innere Medizin, Diabeteszentrum Bad Lauterberg, Germany. 
Background and Aims: Basal-bolus (BB) regimens comprising analogue insulins have the potential to offer clinical advantages over BB regimens with human insulins, including improved glycaemic control and reduced risk for hypoglycaemia. The safety and efficacy of insulin detemir, a basal analogue insulin, is currently being evaluated in actual clinical practice in a large, multi-centre, prospective observational study - The Predictable Results and Experience in Diabetes through Intensification and Control to Target: An International Variability Evaluation (PREDICTIVE) study. This report evaluates the safety and efficacy of treatment with an all analogue BB regimen, comprising insulin detemir/insulin aspart in patients switched from a conventional regimen of NPH insulin/ human soluble insulin.

Materials and Methods: Data were compared between baseline and 3 months from 179 patients in the German cohort of the PREDICTIVE study, who switched from NPH insulin/human soluble insulin to insulin detemir/insulin aspart. This cohort was part of the 1811 intensively-treated type 1 patients in the PREDICTIVE study. Key baseline data for the 179 patients were: 54\% female; mean age 44.1 years; diabetes duration 17.7 years; $\mathrm{HbA}_{1 \mathrm{c}} 8.1 \%$; $\mathrm{BMI}$ $25.4 \mathrm{~kg} / \mathrm{m}^{2}$.

Results: Switching BB therapy to insulin detemir/insulin aspart was associated with a marked reduction in the rate of overall hypoglycaemia to 11 events/patient year in the 4 weeks prior to the follow-up visit, as compared with 55 events/patient year in the 4 weeks prior to study start. No major hypoglycaemia was observed at follow-up (reduced from 1.2 events/patient year at study start). The rate of nocturnal hypoglycaemia was also reduced from 18.3 to 1.7 events patient/year. Overall, the proportion of patients reporting all hypoglycaemic episodes fell to $23 \%$ from $59 \%$ on switching to the all analogue regimen, while the proportion of patients experiencing nocturnal hypoglycaemia was reduced from $39 \%$ to $8 \%$. Switching to all analogue BB therapy was also associated with improved glycaemic control: $\mathrm{HbA}_{1 \mathrm{c}}$ was reduced by $0.65 \%$ from prestudy values to $7.45 \%$; fasting blood glucose (FBG) was reduced by $39.4 \mathrm{mg} / \mathrm{dl}$. Within-subject $\mathrm{FBG}$ variability, calculated as SD of FBG, was also reduced by $13.4 \mathrm{mg} / \mathrm{dl}$. Throughout the observation period, body weight remained stable and unchanged from pre-study values and total insulin dose remained constant (pre-study, $0.68 \mathrm{IU} / \mathrm{kg}$; follow-up $0.71 \mathrm{IU} / \mathrm{kg}$ ).

Conclusion: The data support that changing BB regimen from human insulin to an all analogue regimen, consisting of insulin detemir and insulin aspart, improves metabolic control and reduces the rate of hypoglycaemia, without increasing body weight, in patients with type 1 diabetes.

Supported by Novo Nordisk A/S
0979

An evaluation of insulin detemir treatment in type 1 and type 2 diabetes: German cohort data from the PREDICTIVE study

H.-J. Lüddeke ${ }^{1}$, J. B. Hansen ${ }^{2}$, M. Meriläinen ${ }^{2}$,

B. Gallwitz ${ }^{3}$;

${ }^{1}$ Praxis Lüddeke/Trauner, Munich, Germany, ${ }^{2}$ Novo Nordisk A/S, Copenhagen, Denmark, ${ }^{3}$ Med. Klinik und Poliklinik, Universitätsklinikum Tübingen, Germany.

Background and Aims: Observational studies provide an effective method for validating data from clinical trials in actual clinical practice. The Predictable Results and Experience in Diabetes through Intensification and Control to Target: An International Variability Evaluation (PREDICTIVE) study is a large, multi-centre, prospective, observational study in people with type 1 and type 2 diabetes. The aim of the study is to provide an insight into treatment patterns, and the efficacy and safety associated with use of insulin detemir in everyday clinical practice.

Materials and Methods: Data from a cohort of 10,276 patients from Germany participating in the PREDICTIVE study were evaluated before and after (mean follow-up period $=14.5$ weeks) initiation of insulin detemir. Baseline characteristics of the cohort were: $26 \%$ type 1 diabetes, $73 \%$ type 2 diabetes, $1 \%$ other classification; $52 \%$ female. Type 1 diabetes: mean age 45 years, mean duration of diabetes, 17 years; type 2 diabetes, mean age 62 years, mean duration of diabetes, 10 years. The primary endpoint of the study was safety. Secondary endpoints included changes in the following parameters from baseline: $\mathrm{HbA}_{1 \mathrm{c}}$, weight change, fasting blood glucose (FBG) and FBG variability.

Results: During the follow-up period, 28 serious adverse drug reactions (SADRs) were reported. Most SADRs (79\%) were hypoglycaemic episodes. In patients with type 1 diabetes, treatment with insulin detemir was associated with a significant reduction in the rate of major hypoglycaemic episodes from 108 events/100 patient years in the 4 weeks before treatment start to 12 events $/ 100$ patient years in the 4 weeks prior to follow-up $(p<0.001)$. For patients with type 2 diabetes, the rate of major hypoglycaemia was reduced from 30 to 6 events $/ 100$ patient years $(p<0.001)$. Glycaemic control also improved during the follow-up period with mean $\mathrm{HbA}_{1 \mathrm{c}}$ values reduced by $-0.54 \%$ and $-0.89 \%$ from baseline values in patients with type 1 and type 2 diabetes, respectively ( $p<0.001$ vs. baseline for both). Values for FBG and within-subject FBG variability were also significantly reduced from baseline at follow-up. FBG was reduced by $33 \mathrm{mg} / \mathrm{dl}$ in patients with type 1 diabetes and by $44 \mathrm{mg} / \mathrm{dl}$ in patients with type 2 diabetes $(p<0.001$ for both). Within-subject FBG variability (SD of FBG) was reduced by 13 and $8 \mathrm{mg} / \mathrm{dl}$, respectively 
$(p<0.001$ for both). Treatment with insulin detemir was not associated with weight gain in patients with type 1 diabetes. Mean weight reduction of $-0.6 \mathrm{~kg}$ from baseline $(p<0.001)$ was reported in patients with type 2 dabetes.Insulin detemir was injected once daily by $29 \%$ of type 1 and $75 \%$ of type 2 diabetes patients.

Conclusions: Data from the German cohort of the PREDICTIVE study support that insulin detemir improves glycaemic control, reduces the incidence of hypoglycaemia, and maintains weight neutrality in clinical practice.

Supported by Novo Nordisk A/S

\section{0}

Predictors of weight and FBG variability in insulintreated patients: data from the European Cohort* of the PREDICTIVETM Study

H. Gydesen ${ }^{1}$, B. Gallwitz ${ }^{2}$, P. G. Clauson ${ }^{1}$, S. Maxeiner ${ }^{3}$, H.-J. Lüddeke ${ }^{4}$;

${ }^{1}$ Novo Nordisk A/S, Bagsværd, Denmark, ${ }^{2}$ Medizinische Klinik IV, Universitätsklinikum Tübingen, Germany, ${ }^{3}$ Diabetologische Schwerpunktpraxis Bosenheim, Bad Kreuznach, Germany, ${ }^{4}$ Praxis Lüddeke/Trauner, Munich, Germany.

Background and Aims: Insulin therapy has traditionally been associated with weight gain, which can result in greater insulin resistance, increased cardiovascular risk and poor compliance. Another concern with earlier insulins was the high within-subject variability of action that can lead to an increased risk of hypoglycaemia. Here we examine predictors of bodyweight and within-subject fasting blood glucose $(\mathrm{FBG})$ variability in insulin-treated patients from the European cohort of PREDICTIVETM - a large multinational, prospective, observational study.

Materials and Methods: Baseline data collected from 15,919 insulin-treated patients (6,839 [42.96\%] type 1, 8,974 [56.37\%] type 2 and 106 [0.67\%] other classified) from the European cohort were analysed using multifactorial analysis of covariance (ANCOVA), to assess the association of current bodyweight and within-subject FBG variability (calculated as the standard deviation of FBG) with: age; gender; diabetes duration; pre-study therapy (OAD plus insulin, basal only, premix, bolus only, basalbolus); incidence of total hypoglycaemia in the 4 weeks prior to study; and baseline values of $\mathrm{HbA}_{1 \mathrm{c}}$, mean $\mathrm{FBG}$, FBG variability (in weight model only), BMI (in FBG variability model only), total number of injections and total insulin dosage per $\mathrm{kg}$ bodyweight (IU/kg).

Results: In both type 1 and type 2 diabetes patients, weight showed a significant positive association with gender (male $>$ female) and total insulin dosage (IU/kg; $\mathrm{P}<0.0001$ for all). Weight was also positively associated with FBG variability $(\mathrm{P}<0.0001)$ in type 1 patients, and age $(\mathrm{P}<0.0001)$, total number of injections $(\mathrm{P}<0.0001)$ and pre-study therapy $(\mathrm{P}<0.01)$ in type 2 patients. Neither weight nor FBG variability were associated with the incidence of total hypoglycaemia. The proportion of patients with type 1 diabetes using once versus twice-daily basal insulin in their treatment regimen was $59.1 \%$ vs $40.9 \%$ respectively; in type 2 patients this proportion was $75.8 \%$ vs $24.2 \%$. There was no difference in the FBG variability between the once- and twice-daily basal insulin users in either type of diabetes.

Conclusion: The analysis shows that, except for gender and total insulin dosage, weight was associated with different variables in type 1 versus type 2 patients. In type 1 diabetes there was a strong association with FBG variability. In type 2 diabetes there was an association with pre-study treatment regimen. Three-quarters of insulin-treated type 2 patients received once-daily injection of basal insulin.

*Austria, Czech Rep., Denmark, Finland, Germany, Ireland, Israel, Netherlands, Sweden, Turkey and UK

Supported by Novo Nordisk A/S

\section{1}

Glucose control among patients using and not using insulin: a systematic review of $\mathrm{HbA}_{1 \mathrm{c}}$ at entry in 93 clinical trials, 2000-2004

A. de Leiva ${ }^{1}$, A. D. Toft ${ }^{2}$, K. B. Frandsen ${ }^{2}$;

${ }^{1}$ Dept of Endocrinology, Universitat Autonoma de Barcelona, Spain, ${ }^{2}$ Novo Nordisk A/S, Bagsvaerd, Denmark.

Background and Aims: The importance of good glycaemic control has been well-established by landmark clinical trials, such as the DCCT and the United Kingdom Prospective Diabetes Study. Subsequent studies continue to document the efficacy of more intensive therapeutic regimens, including the benefits of early and aggressive use of insulin. Nevertheless, clinicians are intuitively aware that many patients continue to have suboptimal glycaemic control. This study used existing data from published clinical trials to assess glycaemic control at trial entry across a broad spectrum of patients. We also compared glycaemic control among those using and not using insulin. Materials and Methods: We searched MEDLINE using Medical Subject Headings (MeSH) terms 'type 2 diabetes', 'clinical trial', and 'insulin' for the period of January 1, 2000-January 10, 2005, to identify papers reporting data on baseline $\mathrm{HbA}_{1 \mathrm{c}}$ in people entering clinical trials. We also scanned the reference lists of any studies retrieved to complement the original search. So as not to artificially inflate the mean $\mathrm{HbA}_{1 \mathrm{c}}$ value at trial entry, we excluded 
studies indicating either that enrolment was limited to patients with $\mathrm{HbA}_{1 \mathrm{c}} \geq 8.5 \%$, or that $\mathrm{HbA}_{1 \mathrm{c}}$ values were determined after a washout period between oral antidiabetic therapy and starting insulin. An overall $\mathrm{HbA}_{1 \mathrm{c}}$ was calculated by weighting the mean $\mathrm{HbA}_{1 \mathrm{c}}$ from each trial by the number of patients enrolled.

Results: A total of 121 relevant publications were identified; 28 were excluded because insulin naïve and insulin-treated patients were not reported separately, the trial was limited to patients with $\mathrm{HbA}_{1 \mathrm{c}} \geq 8.5 \%, \mathrm{HbA}_{1 \mathrm{c}}$ was obtained after washout period, or baseline $\mathrm{HbA}_{1 \mathrm{c}}$ was not reported. This left 93 trials involving 15,833 people with type 2 diabetes in the final analysis. The weighted mean $\mathrm{HbA}_{1 \mathrm{c}}$ at baseline was $9.07 \%$ for 7684 insulin-naïve patients and $8.51 \%$ for 8149 insulin-treated patients. The distribution of mean $\mathrm{HbA}_{1 \mathrm{c}}$ for these trials is shown in the figure.

Conclusion: Data from a large sample of clinical trials in diverse groups of patients with type 2 diabetes demonstrate that mean $\mathrm{HbA}_{1 \mathrm{c}}$ values of groups of patients at trial entry frequently exceed recommended glycaemic targets by a clinically significant margin (1.5-2.0 absolute percentage points). Since mean $\mathrm{HbA}_{1 \mathrm{c}}$ values reflect a distribution of individual patient values above and below that value, these results indicate that the discrepancy between target values and glycaemic control in clinical reality was substantially worse for many patients. Although it was not unexpected that, at the time of enrolment in a clinical trial, patients would have suboptimal glycaemic control, it was surprising that the weighted mean $\mathrm{HbA}_{1 \mathrm{c}}$ for patients taking insulin was only about $0.5 \%$ better than insulin-naïve-patients. This suggests that either titration of insulin was insufficient, or adherence to prescribed therapy was poor.

Figure: Distribution of mean $\mathrm{HbA}_{1 \mathrm{c}}$

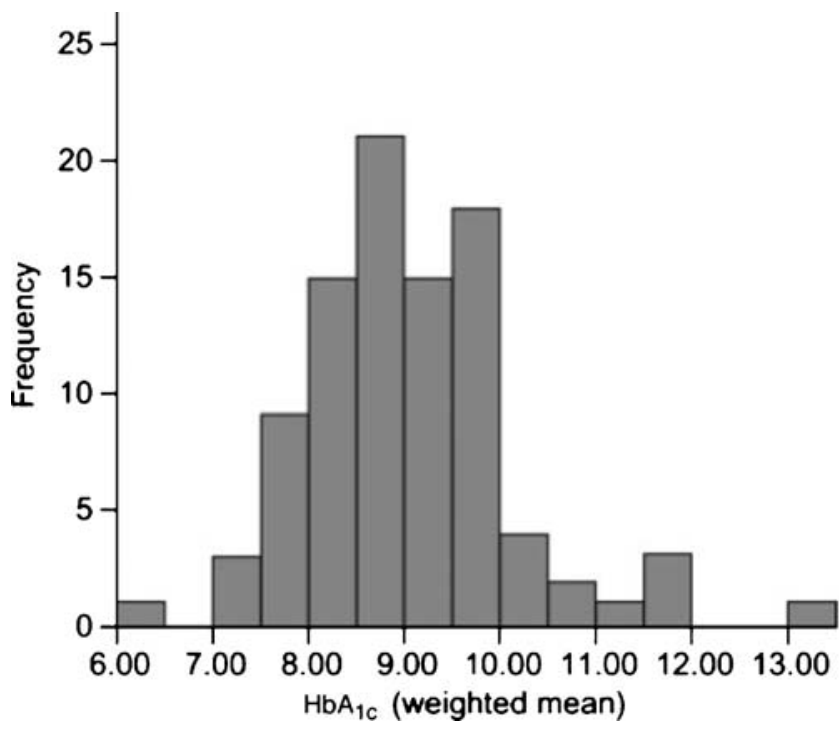

Supported by Novo Nordisk A/S
0982

Failure of insulin monotherapy in patients with type 2 diabetes: a population-based study

S. Gough ${ }^{1}$, K. B. Frandsen ${ }^{2}$, A. D. Toft ${ }^{2}$;

${ }^{1}$ Institute of Biomedical Research, University of Birmingham, United Kingdom, ${ }^{2}$ Diabetes Department, Novo Nordisk, Copenhagen, Denmark.

Background and Aims: Clinical trials have shown that insulin can be used to help patients with type 2 diabetes reach recommended glycaemic targets. Theoretically, insulin therapy should have comparable efficacy when initiated outside of the structured environment of a clinical trial. Despite the prevalence of its use, the extent to which insulin users in the general population reach these glycaemic targets is largely unknown. In the UK and Germany, we assessed glycaemic control in a clinical setting via a population-based analysis of last recorded $\mathrm{HbA}_{1 \mathrm{c}}$ levels, using data from adult patients who were prescribed insulin and who had not been prescribed oral antidiabetic drugs (OADs) within the previous 3 months.

Materials and Methods: Data were obtained from IMS Health, Inc, who collected longitudinal population-based data for up to 14 years from computerised primary care practices in the EU. These data were sampled from 6 million patient records from 1045 physicians in 983 practices in Germany and 3.6 million patient records from 630 physicians in 200 practices in the UK.

Results: Overall mean and median $\mathrm{HbA}_{1 \mathrm{c}}$ were $8.42 \%$ and $8.10 \%$, respectively, for 3658 patients with type 2 diabetes (Figure). Insulin has the potential to enable patients to achieve targets, and given the difficulty of persuading patients to initiate insulin, it is discouraging that many insulin users remained above recommended glycaemic targets. About one-third (32.3\%) had $\mathrm{HbA}_{1 \mathrm{c}} \geq 9.0 \%$ and almost one-fifth $(18.2 \%)$ had $\mathrm{HbA}_{1 \mathrm{c}} \geq 10.0 \%$. Similar proportions of patients could be categorised as poorly controlled in both countries (ie, $30.1 \% \mathrm{HbA}_{1 \mathrm{c}} \geq 9.0 \%$ and $18.6 \% \mathrm{HbA}_{1 \mathrm{c}} \geq 10.0 \%$ from Germany, and $34.0 \%$ $\mathrm{HbA}_{1 \mathrm{c}} \geq 9.0 \%$ and $17.9 \% \quad \mathrm{HbA}_{1 \mathrm{c}} \geq 10.0 \%$ from the UK), suggesting a robustness to the findings.

Having excluded from the sample patients prescribed OADs in the previous 3 months, it was not clear how well the analysis reflected the level of control of people using insulin plus OADs. Although it might be logical to assume that patients using OADs may have better control, ultimately the direction and magnitude of any bias resulting from omission of OAD users is unknown. Comparison of the results from the UK and Germany must be made with caution, as the proportion of laboratories standardising their results to the DCCT measurements (i.e., those who are DCCT-aligned) are not the same. Most labs in the UK are 
DCCT aligned, whereas this is not the case in Germany, where at least 4 different methods are used. Thus, normal ranges may differ somewhat.

Conclusions: These data suggest that glycaemic control may be suboptimal $\left(\mathrm{HbA}_{1 \mathrm{c}}>7.0 \%\right)$ for up to three-quarters of patients on insulin monotherapy. Identifying and overcoming obstacles preventing optimal insulin therapy, including adequate titration, is essential if the gap between recommended glycaemic targets and control in the clinical setting is to be closed.

Figure. Distribution of $\mathrm{HbA}_{1 \mathrm{c}}$ for 3658 patients with type 2 diabetes in Germany $(n=1536)$ and the UK $(n=2122)$ prescribed insulin, 2004

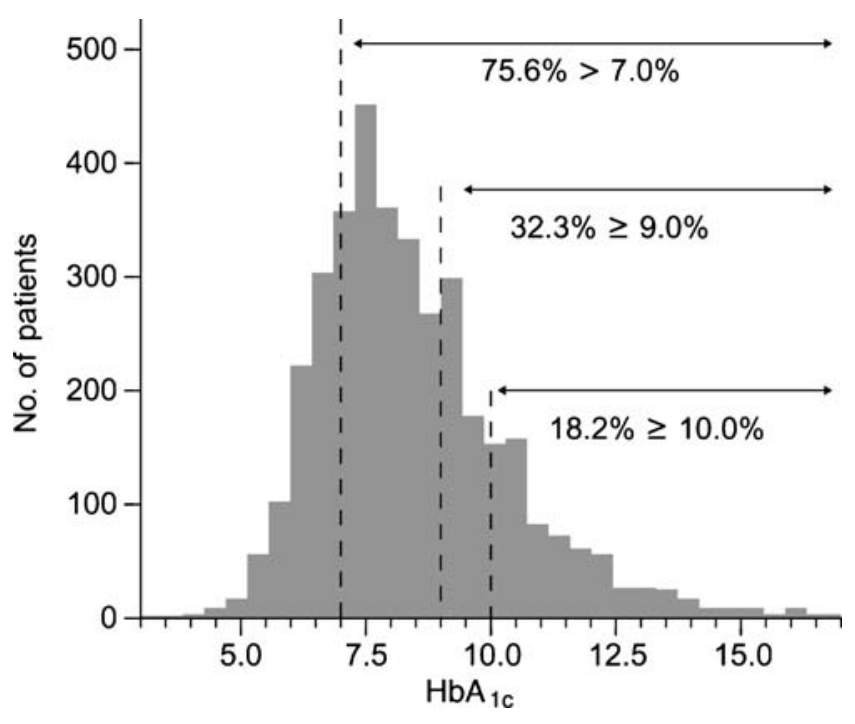

Supported by Novo Nordisk

\section{PS 83}

\section{Insulin trials in type 2 diabetes}

\section{3}

Effect of biphasic insulin aspart 30/70 (BIAsp30) in combination with metformin on glycaemic control in subjects with type 2 diabetes not optimally controlled on oral antidiabetic agents

P. Naiker ${ }^{1}$, H. A. Makan ${ }^{2}$, M. A. K. Omar ${ }^{3}$, T. Kedijang ${ }^{4}$, L. L. L. Kong ${ }^{5}$ and the BIAsp-1579 study group;

${ }^{1}$ St Augustine's Hosp, Durban, South Africa, 2Johannesburg, South Africa, ${ }^{3}$ Durban, South Africa, ${ }^{4}$ Novo Nordisk, Johannesburg, South Africa, ${ }^{5}$ Novo Nordisk, Singapore.
Background and Aims: Biphasic insulin aspart 30/70 (BIAsp30) is a new premixed insulin analogue comprising $30 \%$ soluble and $70 \%$ protamine-crystallised insulin aspart. Addition or substitution with twice-daily premixed insulin analogue/human insulin is a common treatment approach in subjects with type 2 diabetes (T2DM) inadequately controlled on oral antidiabetic agents (OADs). There is growing evidence that addition of once-daily BIAsp30 to OADs provides significant clinical benefit to subjects with T2DM. This trial was designed to evaluate whether oncedaily BIAsp30 at dinner in combination with metformin (BIAsp30+Met) provides glycaemic control that is at least as efficacious as twice-daily BIAsp30 alone (BIAsp30).

Materials and Methods: This 24-week, multicentre, openlabelled, parallel, randomised (1:1) comparison trial included 140 subjects with T2DM (BIAsp30+Met vs BIAsp30: mean T2DM duration 8.2 vs 9.7 years, $\mathrm{HbA}_{1 \mathrm{c}} 10.1$ vs $10.2 \%$, BMI $28.8 \mathrm{vs} 29.5 \mathrm{~kg} / \mathrm{m}^{2}$ ) in South Africa inadequately controlled on one or two OADs. At randomisation, OADs were discontinued and subjects received BIAsp30+Met or BIAsp30. Efficacy was assessed by $\mathrm{HbA}_{1 \mathrm{c}}$, prandial plasma glucose (PG) increment and plasma lipids. $\mathrm{HbA}_{1 \mathrm{c}}$ at 24 weeks was analysed using ANOVA with adjustment to baseline, and comparison between treatments was performed using a predefined non-inferiority criterion of $0.6 \%$. Safety was evaluated by incidence of hypoglycaemic episodes and adverse events.

Results: After 24 weeks of treatment, glycaemic control was improved in both treatment groups: $\mathrm{HbA}_{1 \mathrm{c}}$ was reduced by $1.90 \pm 1.44 \%$ in the BIAsp30+Met group and $1.62 \pm 1.74 \%$ in the BIAsp30 group and non-inferiority between treatments was confirmed (diff $-0.31 \%$; 95\% CI $[-0.752 ; 0.129], \mathrm{p}=0.164)$. Consistent with the trend in $\mathrm{HbA}_{1 \mathrm{c}}$, improvement was observed in change from baseline in average prandial (breakfast, lunch and dinner) PG profiles in both the BIAsp30+Met group $(-1.18 \pm$ $2.47 \mathrm{mmol} / \mathrm{L}, \mathrm{p}=0.0183)$ and BIAsp30 group $(-0.86 \pm$ $2.41 \mathrm{mmol} / \mathrm{L}, \mathrm{p}=0.0054)$. Improvement in glycaemic control was associated with slight weight changes from baseline in the BIAsp30+Met group $(0.50 \pm 3.33 \mathrm{~kg})$ and BIAsp30 group $(3.69 \pm 4.91 \mathrm{~kg})$. Total cholesterol decreased by $0.12 \pm 1.02 \mathrm{mmol} / \mathrm{L}$ in the BIAsp30+Met group and increased by $0.21 \pm 0.88 \mathrm{mmol} / \mathrm{L}$ in the BIAsp30 group (diff $-0.34 \mathrm{mmol} / \mathrm{L} ; 95 \%$ CI $[-0.678 ;-0.004])$. Other parameters (LDL-, HDL-cholesterol and triglycerides) were clinically comparable in both treatments. Mean total daily dose of BIAsp30 was $0.36 \mathrm{U} / \mathrm{kg}$ in the BIAsp30+Met group (with daily metformin dose of $2324 \mathrm{mg}$ ) and $0.65 \mathrm{U} / \mathrm{kg}$ in the BIAsp30 group. A total of 94 hypoglycaemic episodes were reported by 28 subjects on BIAsp30+Met and 147 hypoglycaemic episodes by 30 subjects on BIAsp30, of which all were minor or symptomatic, except for one major event in the BIAsp30+Met group. Adverse events were similar between treatments. 
Conclusion: In subjects with T2DM inadequately controlled on OADs, addition of once-daily BIAsp30 to metformin therapy or substitution with twice-daily BIAsp30 offer significant clinical benefit. The barrier to insulin initiation can be overcome with BIAsp30 once-daily with metformin, which offers a convenient, safe and effective approach to treat T2DM.

Supported by Novo Nordisk

\section{4}

Effect of BIAsp 30 (Biphasic insulin aspart 30) in combination with oral hypoglycaemic agents on glycaemic regulation in non-obese patients with type 2 diabetes

S. S. Lund, L. Tarnow, B. B. Nielsen, B. V. Hansen, O. Pedersen, H.-H. Parving, A. A. Vaag;

Steno Diabetes Center, Gentofte, Denmark.

Background and Aims: Biphasic insulin aspart (BIAsp30, $30 \%$ soluble and $70 \%$ protaminated insulin aspart) is a premixed insulin analogue targeting both postprandial and basal glucose regulation. The objective was in a randomized double masked trial to study the effect of BIAsp30 in combination with oral hypoglycaemic agents (OHA) on glycaemic regulation in non-obese patients with type 2-diabetes (T2DM). A dose titration algorithm with increasing numbers of insulin injections was applied to reach the glycaemic target.

Materials and Methods: 102 non-obese $\left(\mathrm{BMI} \leq 27 \mathrm{~kg} / \mathrm{m}^{2}\right)$ patients with $\mathrm{T} 2 \mathrm{DM}$ and poor glycaemic regulation $\left(\mathrm{HbA}_{1 \mathrm{c}} \geq 6.5 \%\right.$; normal range: 4.1-6.4\%) were included after a 4 months run-in on combination therapy with repaglinide plus metformin. At baseline ( 0 months) patients were randomized to either repaglinide $6 \mathrm{mg}$ daily or metformin $2 \mathrm{~g}$ daily (double-masked), both in combination with BIAsp30 (once daily, pre-dinner) for 12 months. Patients self-adjusted insulin dose according to an algorithm aiming for fasting plasma-glucose of $4.0-6.0 \mathrm{mmol} / \mathrm{l}$ and $\mathrm{HbA}_{1 \mathrm{c}}<6.5 \%$. Patients intensified to 2 or 3 daily injections after 3, 6 or 9 months if target glycaemic goals were not reached, or earlier if hypoglycaemia prevented an increase in insulin dose. Predefined endpoints were assessed at 0,3 , 6, 9 and 12 months. Concomitant OHA therapies remain double-masked until June 2006, and the extent to which combination of insulin with metformin or repaglinide treatment influenced $\mathrm{HbA}_{1 \mathrm{c}}$ will be determined immediately thereafter.

Results: $\mathrm{HbA}_{1 \mathrm{c}}$ (mean $\left.\pm \mathrm{SD}\right)$ was significantly reduced $(7.56 \% \pm 0.91 ; 7.02 \% \pm 0.67 ; 6.96 \% \pm 0.67$ and $6.79 \% \pm 0.66)$ at $3,6,9$ and 12 months, respectively, versus baseline $(8.10 \pm 1.40), \mathrm{p}<0.001$ for all comparisons versus baseline. $\mathrm{HbA}_{1 \mathrm{c}}$ decreased significantly between all successive visits $(\mathrm{p}<0.001)$, except from 6 until 9 months where $\mathrm{HbA}_{1 \mathrm{c}}$ remained stable. Target $\left(\mathrm{HbA}_{1 \mathrm{c}}<6.5 \%\right)$ was reached in $11 \%$ $(n=11), 20 \%(n=20), 23 \%(n=23)$ and $35 \%(n=36)$ of all patients after 3, 6, 9 and 12 months, respectively. During the 12 months intervention period $43 \%(n=44)$ of all patients obtained at least one measurement of $\mathrm{HbA}_{1 \mathrm{c}}$ $<6.5 \%$ and $25 \%(n=25)$ of all patients obtained at least two measurements of $\mathrm{HbA}_{1 \mathrm{c}}<6.5 \%$, respectively. Among patients with $\mathrm{HbA}_{1 \mathrm{c}} \geq 7.0 \%$ at baseline $(\mathrm{n}=77), 64 \%(\mathrm{n}=49)$ achieved $\mathrm{HbA}_{1 \mathrm{c}}<7.0 \%$ after 12 months. After 3 months $10 \%(\mathrm{n}=10)$ of all patients achieved $\mathrm{HbA}_{1 \mathrm{c}}<6.5 \%$ with once daily BIAsp 30 injection compared to $6 \%(\mathrm{n}=6)$ of all patients after 12 months. The percentage of patients who received 1,2 and 3 daily injections of BIAsp30 was $14 \%$ $(\mathrm{n}=14), 42 \%(\mathrm{n}=41)$ and $43 \%(\mathrm{n}=42)$, respectively after 12 months. Median (range) dose of BIAsp30 was $30 \mathrm{U}$ (6108) after 12 months. During the 12 months intervention period only $5 \%(\mathrm{n}=5)$ of all patients dropped out.

Conclusion: BIAsp30 in combination with OHA results in long-lasting near-optimal glycaemic regulation in nonobese patients with T2DM uncontrolled by OHA combination-therapy. A treatment algorithm using increasing number of insulin injections and patient self-adjustment of BIAsp30 dose can obtain glycaemic targets in many patients while maintaining a high level of adherence to therapy. BIAsp30 in combination with OHA can be initiated once daily pre-dinner, but most non-obese patients with T2DM need more than one daily injection to reach and maintain glycaemic regulation within targets after one year.

Supported by Novo Nordisk A/S; Novo Nordisk Scandinavia $A B$

0985

Insulin glargine-based therapy improves glycaemic control in patients with type 2 diabetes suboptimally controlled on premixed insulin therapies

F. Storms ${ }^{1}$, M. Davies ${ }^{2}$, R. Gomis ${ }^{3}$ on behalf

of the AT.LANTUS study group;

${ }^{1}$ Mesos Diabetes Centrum, Utrecht, The Netherlands, ${ }^{2}$ Department of Diabetes, University Hospital of Leicester, United Kingdom, ${ }^{3}$ Hospital Clinic Universitari, Barcelona, Spain.

Background and Aims: The AT.LANTUS study compared insulin glargine initiation and titration via one of two algorithms in poorly controlled type 2 diabetes (T2DM) patients. We report results of a subanalysis of patients transferring from premixed insulin \pm oral hypoglycaemic agents (OHAs) to a once-daily (OD) glargine-based regimen ( \pm OHAs). 
Materials and Methods: In a multicentre $(\mathrm{n}=611)$, multinational $(\mathrm{n}=59)$, open-label, 24-week, randomized trial in 4961 suboptimally controlled T2DM patients, glargine dose was titrated to the target fasting blood glucose (FBG) $5.5 \mathrm{mmol} / \mathrm{L}(\leq 100 \mathrm{mg} / \mathrm{dL}$ ) according to Algorithm 1 (clinic driven) or Algorithm 2 (patient driven). The primary outcome measure was incidence of severe hypoglycaemia. Results: Patients previously on premixed insulin (mainly two injections/day in all subgroups) at baseline $(\mathrm{n}=952)$ transferred to OD glargine \pm OHAs without $(\mathrm{n}=534 ; 56.1 \%$ ) or with prandial insulin OD $(\mathrm{n}=47 ; 4.9 \%)$, twice-daily (BD; $\mathrm{n}=178 ; 18.7 \%)$ or $>\mathrm{BD}(\mathrm{n}=193 ; 20.3 \%)$. Baseline $\mathrm{HbA}_{1 \mathrm{c}}$ was $8.87 \pm 1.27 \%, 8.94 \pm 1.31 \%, 9.11 \pm 1.23 \%, 9.15 \pm 1.14 \%$, respectively. When transferred onto glargine, the incidence of severe hypoglycaemia was low $(2.71,0.00,3.47,1.05$ events/patient-century, respectively), with significant decreases in $\mathrm{HbA}_{1 \mathrm{c}}$ (Figure; $\mathrm{p}<0.001$ for baseline-endpoint change; $\mathrm{p}=\mathrm{n} / \mathrm{s}$ for between algorithms) and FBG $(-3.1$, $3.3,-3.2,-3.8 \mathrm{mmol} / \mathrm{L}[-55.2,-58.7,-57.5$, $-67.6 \mathrm{mg} / \mathrm{dL}]$, respectively; $\mathrm{p}<0.001$ for baseline-endpoint change; $\mathrm{p}=\mathrm{n} / \mathrm{s}$ for between algorithms) and small weight changes $(+0.4,+1.4,+1.7,+1.5 \mathrm{~kg}$, respectively; $\mathrm{p} \leq 0.005)$.

Conclusion: This study showed that both physician-led and patient-led treatment algorithms were associated with similar improvements in glycaemic control (FBG and $\mathrm{HbA}_{1 \mathrm{c}}$ ) within each of the four sub-groups. Furthermore, T2DM patients suboptimally controlled on premixed insulin-based regimens can safely and effectively transfer to a glargine-based regimen \pm the addition of prandial insulin. Prandial insulin confers added benefit over glargine + OADs alone, with the biggest improvement seen after addition of the first prandial dose. The concept of basal plus one injection of prandial insulin warrents formal evaluation.

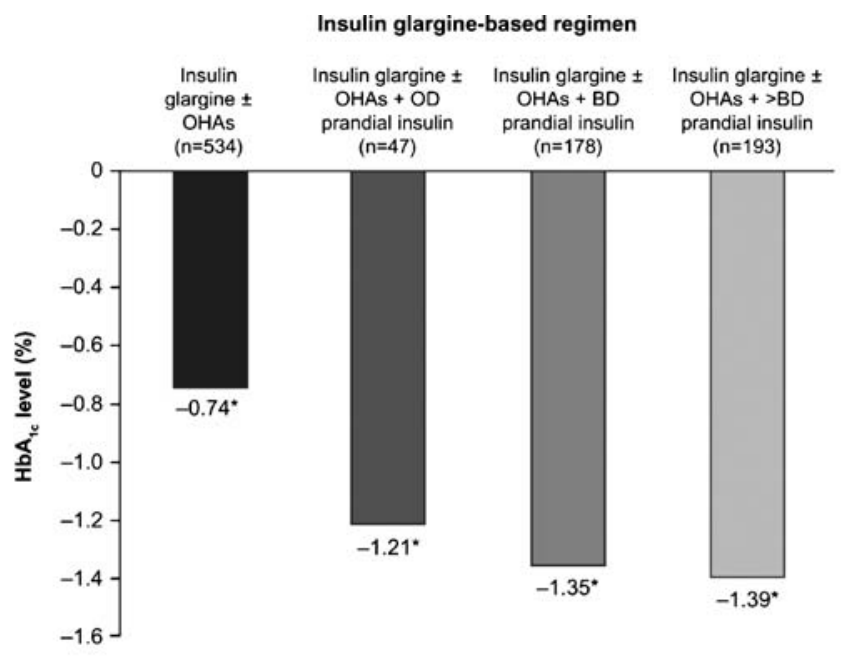

" $p<0.001$ from baseline to endpoint: $O H A=$ oral antihyperglycemic agent; $O D=$ once daily; $B D=t w i c e$ daily

Supported by sanofi-aventis
0986

Glycaemia optimization treatment (GOT): Glycaemic control and rate of severe hypoglycaemia for 5 different dosing algorithms of insulin glargine (GLAR) in patients with type 2 diabetes mellitus (T2DM)

R. J. Tanenberg ${ }^{1}$, A. Zisman ${ }^{2}$, J. A. Stewart ${ }^{3}$;

${ }^{1}$ Division of Endocrinology, Brody School of Medicine, Greenville, United States, ${ }^{2}$ Endocrinology, Diabetes and Metabolism, University of Miami School of Medicine, United States, ${ }^{3}$ sanofi-aventis U.S., Laval, Canada.

Background and Aims: This 24-week, randomized, openlabel study compared the rate of severe hypoglycemic events (primary endpoint) for 5 dosing algorithms of GLAR, as well as the proportion of patients reaching A1C levels $<7.0 \%$.

Materials and Methods: The intent-to-treat population included 4824 patients with $\mathrm{T} 2 \mathrm{DM}$ and $\mathrm{A} 1 \mathrm{C} \geq 7.0 \%$, who were not currently on insulin therapy. Patients continued prior oral agents (except thiazolidinediones, which were discontinued) and began GLAR $10 \mathrm{U} / \mathrm{d}$, which was actively titrated weekly according to the algorithm group (defined by fasting blood glucose [FBG] goals of $80,90,100,110$, or $120 \mathrm{mg} / \mathrm{dL}$, respectively).

Results: Demographics (median age 56 y, 48\% male, 70\% white, mean diabetes duration $8.3 \mathrm{y}$, baseline characteristics (mean A1C 9.3\%, BMI $34.7 \mathrm{~kg} / \mathrm{m}^{2}$ ) and study completion rates $(88.4 \%-90.5 \%)$ were similar among groups. A1C values improved in all groups in a linear progression from $1.7 \%$ to $1.9 \%$ below baseline. Despite low overall hypoglycemic rates, severe hypoglycemia was associated with increasingly aggressive dose titration. More aggressive FBG targets required higher doses of GLAR, resulting in greater improvements in $\mathrm{A} 1 \mathrm{C}$ and a higher proportion of patients achieving $\mathrm{A} 1 \mathrm{C}<7.0 \%$. The number of patients achieving $\mathrm{A} 1 \mathrm{C}<7.0 \%$ at the expense of a severe hypoglycemic event was lower with less aggressive dose titration. However, within each dosing algorithm, the risk of severe hypoglycemia was not higher among patients who achieved target $\mathrm{A} 1 \mathrm{C}<7.0 \%$.

Conclusion: Comparison of results in the different target groups shows that targeting FBG of $110 \mathrm{mg} / \mathrm{dL}$ yielded the best balance between resulting A1C levels and the risk for hypoglycemia. Aiming at this goal should allow more patients to achieve target A1C $(<7.0 \%)$ without encountering more hypoglycemia. 


\begin{tabular}{|c|c|c|c|c|c|c|c|}
\hline $\begin{array}{l}\text { Treatment } \\
\text { Group } \\
\text { (Goal FBG)* }^{\text {(Gol }}\end{array}$ & $\begin{array}{l}\text { GLAR } \\
\text { Daily Dose, } \\
\text { IU Mean } \\
\text { (SD), } \\
\text { Endpoint }\end{array}$ & $\begin{array}{l}\text { A1C, } \\
\text { \% Mean } \\
\text { (SD), } \\
\text { Endpoint }\end{array}$ & $\begin{array}{l}\text { Proportion } \\
\text { of Patients } \\
\text { With A1C } \\
<7.0 \%, \%\end{array}$ & $\begin{array}{l}\text { Observed } \\
\text { Rate of } \\
\text { Severe } \\
\text { Hypoglycemia }^{\dagger}\end{array}$ & $\begin{array}{l}\text { Modeled }^{\dagger} \\
\text { Rate of } \\
\text { Severe } \\
\text { Hypoglycemia } \\
\text { (SE) }\end{array}$ & $\begin{array}{l}\text { A1C } \\
<7.0 \% \\
\text { Observed } \\
\text { Rate of } \\
\text { Severe } \\
\text { Hypoglycemia }{ }^{\dagger}\end{array}$ & $\begin{array}{l}\text { A1C } \\
\geq 7.0 \% \\
\text { Observed } \\
\text { Rate of } \\
\text { Severe } \\
\text { Hypoglycemia }\end{array}$ \\
\hline $\begin{array}{l}120 \mathrm{mg} / \mathrm{Dl} \\
\mathrm{N}=952\end{array}$ & $59.2(36.6)$ & $7.58(1.1)$ & $\begin{array}{l}31.5 \\
298 / 945\end{array}$ & 0.02 & $\begin{array}{l}0.02(0.007) \\
(0.01,0.04)\end{array}$ & 0.02 & 0.02 \\
\hline $\begin{array}{l}110 \mathrm{mg} / \mathrm{dL} \\
\mathrm{N}=974\end{array}$ & $62.2(37.3)$ & $7.52(1.1)$ & $\begin{array}{l}32.2 \\
812 / 969\end{array}$ & 0.07 & $\begin{array}{l}0.03(0.008) \\
(0.02,0.05)\end{array}$ & 0.02 & $0.08^{\S}$ \\
\hline $\begin{array}{l}100 \mathrm{mg} / \mathrm{dL} \\
\mathrm{N}=973\end{array}$ & $69.6(41.0)$ & $7.41(1.1)$ & $\begin{array}{l}37.5 \\
363 / 967\end{array}$ & 0.04 & $\begin{array}{l}0.05(0.009) \\
(0.04,0.07)\end{array}$ & 0.04 & 0.05 \\
\hline $\begin{array}{l}90 \mathrm{mg} / \mathrm{dL} \\
\mathrm{N}=950\end{array}$ & $74.9(50.3)$ & $7.36(1.1)$ & $\begin{array}{l}41.1 \\
388 / 943\end{array}$ & 0.10 & $\begin{array}{l}0.08(0.012) \\
(0.06,0.11)\end{array}$ & 0.08 & 0.12 \\
\hline $\begin{array}{l}80 \mathrm{mg} / \mathrm{dL} \\
\mathrm{N}=975\end{array}$ & $78.1(42.9)$ & $7.32(1.2)$ & $\begin{array}{l}44.3 \\
428 / 966\end{array}$ & 0.15 & $\begin{array}{l}0.13(0.023) \\
(0.09,0.18)\end{array}$ & 0.11 & 0.19 \\
\hline
\end{tabular}

*Intent-to-treat subjects.

${ }^{\dagger}$ Rate $=$ events/patient-year. Severe hypoglycemia defined as self-monitored blood glucose $<36 \mathrm{mg} / \mathrm{dL}$ or patient required assistance and responded promptly to treatment with glucagon or glucose.

* Means based on unadjusted data using a Poisson regression model.

${ }^{\S} \mathrm{A} 1 \mathrm{C}<7.0 \%$ rate differs significantly from $\mathrm{A} 1 \mathrm{C} \geq 7.0 \%$ rate $(P<0.05)$

Supported by sanofi-aventis U.S.

0987

Glycaemic control and safety of insulin glargine (GLAR) in elderly patients with type 2 diabetes mellitus (T2DM): The GOAL A1C Study

J. Halter ${ }^{1}$, L. Kennedy ${ }^{2}$, M. Miller ${ }^{3}$, W. Herman ${ }^{4}$;

${ }^{1}$ Division of Geriatric Medicine, University of Michigan Geriatrics Center, Ann Arbor, United States, ${ }^{2}$ Dept of Medicine, University of Florida, Gainesville, United States, ${ }^{3}$ sanofi-aventis U.S., Bridgewater, United States, ${ }^{4}$ Dept of Medicine, Univ of Michigan Med Ctr, Ann Arbor, United States.

Background and Aims: GOAL A1C (Glycemic Optimization with $A$ lgorithms and Labs $A$ t Polnt of Care), the primary results of which were previously reported, assessed the impact of active vs usual monitoring of insulin titration and point-of-care vs lab A1C testing on glycemic control. This 24-wk multicenter (2164 US sites) open-label study conducted mainly by primary care physicians evaluated the addition of GLAR in 7893 T2DM patients uncontrolled on oral agents (A1C>7.0\%).

Material and Methods: To assess the safety and efficacy of GLAR in the elderly, this analysis used all subjects from the primary efficacy cohort (all patients were treated with GLAR; $\mathrm{n}=5721$ ), and subdivided patients into those aged $<65,65-74$, and $>75$ years.

Results: Among patients with comparable baseline FPG, older patients (65-74 years and $>75$ years) achieved significantly greater FPG reductions by week 24 . However, among patients with comparable baseline A1C, older patients achieved smaller A1C reductions by week 24 . Lower FPG, hence, lower glargine doses may have prevented further reductions in $\mathrm{A} 1 \mathrm{C}$ in the older patients. There were significant differences among the 3 age groups in the unadjusted rates of hypoglycemia; however, these differences were explained primarily by differences in BMI. Weight gain was greatest in patients $<65 \mathrm{y}$ and lowest in patients $>75 \mathrm{y}$, even after controlling for baseline factors.

Conclusion: Adding GLAR to oral therapy improves glycemic control with low overall rates of hypoglycemia in patients $65-75$ and $>75$ with $\mathrm{T} 2 \mathrm{DM}$ in primary care settings. 
Study Population Age Subgroup Unadjusted Means

\begin{tabular}{|c|c|c|c|c|}
\hline & & $\begin{array}{l}\text { Age }<65 \text { y } \\
(n=4251)\end{array}$ & $\begin{array}{l}\text { Age 65- } \\
74 \mathbf{y} \\
(n=1086)\end{array}$ & $\begin{array}{l}\text { Age } \geq 75 \text { y } \\
(n=384)\end{array}$ \\
\hline $\begin{array}{l}\text { GLAR daily dose, } \\
\text { U }\end{array}$ & Week 24, Mean (SE) ${ }^{1}$ & $58.3(0.5)$ & $45.3(1.1)$ & $37.1(2.1)$ \\
\hline FPG, mg/dL & $\begin{array}{l}\text { Baseline, mean (SE) } \\
\text { Week } 24, \text { mean }(\mathrm{SE}) \\
\Delta, \text { mean }(\mathrm{SE})^{2}\end{array}$ & $\begin{array}{l}215.0(1.1) \\
127.1(0.7) \\
-87.9(1.2)\end{array}$ & $\begin{array}{l}200.2(1.8) \\
115.8(1.0) \\
-84.4(2.1)\end{array}$ & $\begin{array}{l}197.9(3.5) \\
110.8(1.4) \\
-87.1(3.8)\end{array}$ \\
\hline $\mathrm{A} 1 \mathrm{C}, \%$ & $\begin{array}{l}\text { Baseline, mean (SE) } \\
\text { Week } 24, \text { mean }(\mathrm{SE}) \\
\Delta, \text { mean }(\mathrm{SE})^{3}\end{array}$ & $\begin{array}{l}9.00(0.02) \\
7.46(0.02) \\
-1.54(0.02)\end{array}$ & $\begin{array}{l}8.56(0.04) \\
7.43(0.03) \\
-1.13(0.04)\end{array}$ & $\begin{array}{l}8.45(0.07) \\
7.44(0.05) \\
-1.01(0.07)\end{array}$ \\
\hline Hypoglycemia (events/patient-years) & $\begin{array}{l}\text { SMBG }<70 \mathrm{mg} / \mathrm{dL}(\mathrm{SE})^{4} \\
\mathrm{SMBG}<50 \mathrm{mg} / \mathrm{dL}(\mathrm{SE})^{4} \\
\text { Severe }(\mathrm{SE})^{4}\end{array}$ & $\begin{array}{l}4.5(0.12) \\
0.55(0.04) \\
0.08(0.01)\end{array}$ & $\begin{array}{l}6.4(0.4) \\
0.59(0.07) \\
0.16(0.05)\end{array}$ & $\begin{array}{l}8.1(1.0) \\
0.68(0.15) \\
0.21(0.08)\end{array}$ \\
\hline
\end{tabular}

FPG $=$ fasting plasma glucose; $\mathrm{SE}=$ standard error

${ }^{1}$ All pairwise comparisons statistically significant ( $p<0.001$, adjusting for study arm, BMI, use of sulfonylurea, metformin, and TZD discontinuation).

${ }^{2}$ Percent reduction from baseline was largest in the elderly groups ( $\mathrm{p}<0.0005<65$ vs $65-74 \mathrm{y} ; \mathrm{p}<0.0005<65$ vs $>75$, adjusting for study arm, BMI, baseline FPG, use of sulfonylurea, metformin, and TZD discontinuation).

${ }^{3} \mathrm{p}<0.01<65$ vs $65-74$ y and $<65$ vs $>75$ y, adjusting for study arm, BMI, baseline A1C, use of sulfonylurea, metformin, and TZD discontinuation.

${ }^{4}$ No statistically significant differences found between age groups after adjusting for study arm, BMI, gender, use of sulfonylurea, metformin, and TZD discontinuation ( $p>0.05$ for all pairwise comparisons).

Supported by sanofi-aventis U.S.

0988

Comparison of insulin lispro mid mixture (MM) plus metformin (Met) with glargine (G) plus met on $\mathrm{HbA}_{1 C}$ (A1C) and blood glucose (BG) profiles in patients with type 2 diabetes (T2D)

D. C. Robbins ${ }^{1}$, P. J. Beisswenger ${ }^{2}$, R. G. Moses ${ }^{3}$,

A. Ceriello ${ }^{4}$, Z. Milicevic ${ }^{1}$, S. Sarwat ${ }^{1}$, C. A. Jones ${ }^{1}$, M. H. Tan ;

${ }^{1}$ Eli Lilly and Company, Indianapolis, United States, ${ }^{2}$ Section of Endocrinology, Diabetes, and Metabolism, Dartmouth Medical School and Dartmouth-Hitchcock Medical Center, Hanover, United States, ${ }^{3}$ SESIAHS, Illawarra Diabetes Service, Sydney, Australia, ${ }^{4}$ Department of Clinical Sciences, Warwick Medical School, United Kingdom.

Background and Aims: Various insulin regimens used to attain improved glycemia in people with $\mathrm{T} 2 \mathrm{D}$, including basal+prandial insulin and basal insulin only, may differ in their outcomes. We compared the effects of MM $(50 \%$ lispro, $50 \%$ NPL [prandial+basal])+Met with G (basal only) +Met on A1C and mean daily and pre-/post-meal BG levels and their excursions in patients with $\mathrm{T} 2 \mathrm{D}$ previously treated with oral antihyperglycemic medications and $0-2 \times$ daily insulin in a prospective, multinational, open-label, randomized, parallel group study.

Materials and Methods: After the 6-wk lead-in period during which all patients received insulin lispro low mixture (25\% lispro, $75 \%$ NPL [LM]) bid+Met bid $(1000-2000 \mathrm{mg} /$ day $)$, patients $(\mathrm{n}=315)$ were randomized to MM (ac meal tid)+Met or G (hs) + Met and treated for 24 wks. Insulins were titrated to achieve fasting [F] BG $<6.7 \mathrm{mM}$ in both groups, and the MM group also targeted 2-hr postprandial (pp) $\mathrm{BG}<8.0 \mathrm{mM}$. Patients on $\mathrm{MM}$ unable to reach FBG target could switch to LM before dinner. Patients measured 7-point BG profiles $3 \times$ during the 2 wks before each monthly visit. Chi-square and ANCOVA models (treatment and country as a fixed effect and baseline as a covariate) were used to compare treatment differences.

Results: At baseline, both groups had similar mean A1C (7.8\% vs $7.8 \%)$, age, sex, BMI, T2D duration, pre-/postmeal BG levels, and mean amplitude of glucose excursion (M) value. One-third (34\%) of MM patients 
switched to LM before dinner. End-point results (mean $\pm \mathrm{SD})$ were:

\begin{tabular}{|c|c|c|c|}
\hline & MM+Met & G+Met & p-value \\
\hline & $(\mathrm{n}=157)$ & $(n=158)$ & \\
\hline A1C (\%), $24 \mathrm{wk}$ & $7.1 \pm 0.9$ & $7.5 \pm 1.0$ & $<0.0001$ \\
\hline A1C $(\%)$, change at $24 \mathrm{wk}$ & $-0.7 \pm 0.9$ & $-0.4 \pm 0.9$ & $<0.0001$ \\
\hline $\mathrm{A} 1 \mathrm{C} \leq 7.0(\mathrm{n}[\%]), 24 \mathrm{wk}$ & $85(56.3)$ & $58(39.7)$ & 0.005 \\
\hline Daily insulin dose $(\mathrm{u} / \mathrm{kg})$ & $0.7 \pm 0.3$ & $0.6 \pm 0.3$ & $<0.0001$ \\
\hline $\begin{array}{l}\text { Overall hypoglycemia } \\
\text { (episodes/patient/30 d) }\end{array}$ & $0.7 \pm 1.7$ & $0.3 \pm 0.8$ & 0.02 \\
\hline $\begin{array}{l}\text { Nocturnal hypoglycemia } \\
\text { (episodes/patient/30 d) }\end{array}$ & $0.2 \pm 0.9$ & $0.2 \pm 0.6$ & 0.62 \\
\hline $\begin{array}{l}\text { Daytime (non-nocturnal) } \\
\text { hypoglycemia (episodes/patient/ } \\
30 \mathrm{~d} \text { ) }\end{array}$ & $0.5 \pm 1.2$ & $0.1 \pm 0.4$ & 0.002 \\
\hline Severe hypoglycemia (n[\%]) & $4(2.5)$ & $4(2.5)$ & 1.00 \\
\hline \multicolumn{4}{|l|}{ BG levels (mM) } \\
\hline Daily 7-point BG & $8.2 \pm 1.3$ & $8.7 \pm 2.0$ & 0.002 \\
\hline Fasting & $8.1 \pm 1.8$ & $6.5 \pm 1.6$ & $<0.0001$ \\
\hline Pre-lunch & $7.4 \pm 1.9$ & $7.9 \pm 2.1$ & 0.03 \\
\hline Pre-supper & $8.3 \pm 2.0$ & $8.9 \pm 2.8$ & 0.04 \\
\hline $3 \mathrm{AM}$ & $7.6 \pm 1.9$ & $7.7 \pm 2.4$ & 0.25 \\
\hline \multicolumn{4}{|l|}{ 2-hr pp BG (mM) } \\
\hline Breakfast & $8.7 \pm 2.2$ & $9.2 \pm 2.6$ & 0.03 \\
\hline Lunch & $8.4 \pm 1.9$ & $9.8 \pm 2.6$ & $<0.0001$ \\
\hline Supper & $8.7 \pm 2.2$ & $10.7 \pm 3.2$ & $<0.0001$ \\
\hline \multicolumn{4}{|c|}{ Change from baseline: 2-hr pp BG excursion (mM) } \\
\hline Daily & $-1.0 \pm 1.6$ & $0.6 \pm 1.6$ & $<0.0001$ \\
\hline Breakfast & $-1.1 \pm 2.7$ & $0.8 \pm 2.7$ & $<0.0001$ \\
\hline Lunch & $-1.4 \pm 2.8$ & $-0.4 \pm 3.0$ & $<0.0001$ \\
\hline Supper & $-0.4 \pm 2.8$ & $1.3 \pm 3.0$ & 0.005 \\
\hline $\mathbf{M}$ (intraday variability in $\mathrm{BG}$ ) & $17.3 \pm 13.8$ & $25.1 \pm 24.8$ & 0.0004 \\
\hline Change in $\mathrm{M}$ from baseline & $-13.6 \pm 21.7$ & $-7.0 \pm 26.9$ & 0.0004 \\
\hline
\end{tabular}

Conclusion: Premeal $\mathrm{MM}+\mathrm{Met}$, compared with $\mathrm{G}+\mathrm{Met}$, resulted in better glycemic control in many ways including lower A1C, lower mean daily and pre- (lunch \& supper only) and post-meal BG levels and their excursions, similar 3 AM BG, and lower $M$ value, despite higher FBG levels. More patients attained $\mathrm{A} 1 \mathrm{C} \leq 7 \%$ in the $\mathrm{MM}+$ Met group. Nocturnal and severe hypoglycemia were similar in both groups, though overall hypoglycemia (mild) was higher in the MM+Met group due to a higher rate of mild hypoglycemia during the day. A pre-meal $\mathrm{MM}+\mathrm{Met}$ regimen is clinically useful for glycemic management of T2D.

Funded by Eli Lilly and Company
0989

Postprandial increase in hs-CRP in Patients with type 2 diabetes (T2D): comparison of lispro mid mixture (MM) plus metformin (Met) with glargine (G) plus met M. H. Tan $^{1}$, N.-A. Le ${ }^{2}$, R. B. Goldberg ${ }^{3}$, W. V. Brown ${ }^{2}$, A. Ceriello ${ }^{4}$, P. J. Beisswenger ${ }^{5}$, S. Sarwat ${ }^{1}$, C. A. Jones ${ }^{1}$, Z. Milicevic ${ }^{1}$, D. C. Robbins ${ }^{1}$;

${ }^{1}$ Eli Lilly and Company, Indianapolis, United States, ${ }^{2}$ Dept of Medicine, Emory University, Atlanta, United States, ${ }^{3}$ School of Medicine, Division of Endocrinology, Metabolism, and Diabetes, University of Miami, United States, ${ }^{4}$ Department of Clinical Sciences, Warwick Medical School, United Kingdom, ${ }^{5}$ Section of Endocrinology, Diabetes, and Metabolism, Dartmouth Medical School and Dartmouth-Hitchcock Medical Center, Hanover, United States.

Background and Aims: Inflammatory markers, specifically hs-CRP, are reported to increase after a meal. In T2D the timing and postprandial (pp) change in insulin and/or blood glucose (BG) may affect pp changes in hs-CRP. We compared the effects of basal insulin $\mathrm{G}$ with prandial+ basal insulin MM (50\% lispro, 50\% NPL), both plus Met, on post-breakfast BG, serum insulin, and hs-CRP in patients (pts) with T2D before and after 24 wks of treatment in a prospective, multinational, open-label, randomized, parallel group (parent) study.

Materials and Methods: After a 6-wk lead-in period of lispro low mixture (25\% lispro, $75 \%$ NPL) bid+Met bid (1000-2000 mg/day), pts were randomized to $\mathrm{G}$ (hs)+Met or MM (ac meal tid)+Met. Both groups titrated insulins to achieve FBG $<6.7 \mathrm{mM}$ and also $2-\mathrm{hr}$ pp $\mathrm{BG}<8.0 \mathrm{mM}$ in the MM+Met group. A subgroup of US patients [G+Met, $n=27 ; M M+M e t, n=29]$ participated in the test meal. The night before, pts ate their supper, took their assigned insulin, and fasted overnight. Met was taken $30 \mathrm{~min}$ and MM was injected $5 \mathrm{~min}$ before a standardized McDonald's breakfast [fat $39 \mathrm{~g}$, CHO $78 \mathrm{~g}$, protein $24 \mathrm{~g}$ ] followed by sampling for $8 \mathrm{hrs}$. Change from $0-\mathrm{hr}$ value was used for within- and between-treatment comparisons. ANCOVA models included treatment as a fixed effect and change from $0 \mathrm{hr}$ from the pre-randomization visit as a baseline covariate for all corresponding blood draw timepoints.

Results: Pre-randomization, the changes in BG, insulin, and hs-CRP levels before and after breakfast were similar 
in both groups. At endpoint mean levels of BG, insulin, and hs-CRP were:

\begin{tabular}{|c|c|c|c|c|c|c|}
\hline \multirow[t]{2}{*}{$\begin{array}{l}\text { Time } \\
\text { (hrs) }\end{array}$} & \multicolumn{2}{|l|}{$\begin{array}{l}\text { BG } \\
{[\mathrm{mM}]}\end{array}$} & \multicolumn{2}{|l|}{$\begin{array}{l}\text { Insulin } \\
\text { [pM] }\end{array}$} & \multicolumn{2}{|l|}{$\begin{array}{l}\text { hs-CRP } \\
{[\mathrm{ng} / \mathrm{mL}]}\end{array}$} \\
\hline & $\mathrm{MM}+\mathrm{Met}$ & G+Met & $\mathrm{MM}+\mathrm{Met}$ & $\mathrm{G}+\mathrm{Met}$ & $\mathrm{MM}+\mathrm{Met}$ & $\mathrm{G}+\mathrm{Met}$ \\
\hline 0 & 6.9 & 5.8 & 163 & 156 & 1947 & 1797 \\
\hline 1 & $9.1^{\mathrm{a} *}$ & $9.5^{*}$ & $440^{\mathrm{a} *}$ & $244 *$ & $2856^{*}$ & $2979 *$ \\
\hline 2 & $8.3^{\mathrm{a} *}$ & $9.6^{*}$ & $411^{\mathrm{a} *}$ & $255^{*}$ & $2635^{\mathrm{a}} *$ & $3175^{*}$ \\
\hline 3 & $7.1^{\mathrm{a}}$ & $9.0^{*}$ & $329 *$ & $271 *$ & $2381^{\mathrm{a}} *$ & $3402 *$ \\
\hline 4 & $5.9^{\mathrm{a} *}$ & $7.7 *$ & $243 *$ & $222 *$ & $1953^{\mathrm{a}}$ & $2735^{*}$ \\
\hline 6 & $5.3^{\mathrm{a} *}$ & 6.0 & 164 & 173 & 1738 & 2070 \\
\hline 8 & $5.5^{\mathrm{a}} *$ & 5.3 & 141 & 144 & 1595 & 1898 \\
\hline
\end{tabular}

${ }^{a}$ Between groups changes from 0 -hr significantly different (p-value $<0.05$ )

* Within group significantly different from $0-\mathrm{hr}$

Conclusions: In T2D postprandial glycemia is associated with a significant increase in hs-CRP which depends on insulin therapy. At end-point, with $\mathrm{MM}+\mathrm{Met}$, peak hs-CRP occurred at $1 \mathrm{hr}$ concomitant with peak BG and insulin; with $\mathrm{G}+$ Met, peak hs-CRP occurred later at $3 \mathrm{hr}$ with peak insulin. Compared with the pre-breakfast (0-hr) value, an increase in hs-CRP post-breakfast was seen, with levels increasing 1-4 hrs for the G+Met group versus 1-3 hrs for the MM+Met group. Postprandially, for pts on $\mathrm{G}+\mathrm{Met}$ vs those on MM+Met, hs-CRP were higher at 2-4 hours and BG were higher at 1-6 hrs and these elevations were associated with lower insulin at 1-2 hrs after breakfast. Whether these differences in this surrogate $\mathrm{CV}$ risk marker are associated with decreased CV disease risk is not known. Funded by Eli Lilly and Company

\section{0}

The effects of insulin and OHA therapy on beta cell function in newly diagnosed type 2 diabetes mellitus

Z. Yan, Y. Li;

Endocrinology, Huazhong University of Science and Technology, Wuhan, China.

Background and Aims: To compare the effect of insulin therapy and OHA therapy on plasma glucose and beta cell function in newly diagnosed type 2 diabetic patients.

Materials and Methods: sixty newly diagnosed type 2 diabetic patients with FPG $>11.1 \mathrm{mmol} / 1$ were randomized into two groups. The subjects in insulin group received intensive or regular insulin treatment. The subjects in OHA group received one sulfonylurea and metformin treatment.
All the subjects keep the therapy for three months after fasting plasma glucose(FPG) below the $6 \mathrm{mmol} / \mathrm{l}$. The two group have intravenous glucose tolerance test (IVGTT) before and after treatment. Fasting plasma glucose, 2 hours postprandial glucose (2 hPG), glycosylated hemoglobin A1c (GHbA1C), fasting plasma insulin(Fin), insulin and $\mathrm{C}$ peptide were measured. Compare the mean area under the curve (AUC0-10,AUC10-60) of insulin and C peptide, the insulin peak, the increment of the insulin peak ( $\triangle \mathrm{INS}$ ), the ratio of total amount insulin to total amount glucose, the ratio of $\Delta \mathrm{INS} / \Delta \mathrm{G}$, Homaßand Homa IR.

Results: Both patients in two groups were achieved the excellent control of FPG, 2hPG and GHbA1C $(\mathrm{P}<0.01$, $\mathrm{P}<0.05$ ), but no significant differents between the groups. The time for two groups achieved FPG below $5.6 \mathrm{mmol} / \mathrm{l}$ was $5.57 \pm 2.3$ days and $7.85 \pm 1.34$ days $(\mathrm{P}<0.05)$. The AUCI0-10 of two group increased 98 percent and 74 percent respectively, AUCI10-60 increased 70 percent and 34 percent respectively the insulin peak increased 150 percent and 86 percent respectively. The increment of the insulin peak $(\Delta \mathrm{INS})$, the ratio of total amount insulin to total amount glucose, the ratio of $\Delta \mathrm{INS} / \Delta \mathrm{G}, \mathrm{AUC} 0-10$ of $\mathrm{C}$ peptide were all increased in two group after treatment, but only the insulin group were significantly increased $(\mathrm{P}<0.01$ or $\mathrm{P}<0.05)$. Moreover,there were more significantly improvement of AUCI0-10, the insulin peak, the increment of the insulin peak $(\Delta \mathrm{INS})$ in insulin group than OHA group. After treatment the Homa $\beta$ were significantly increased and Homa IR were decreased,but on difference between the group. The increase of Homa $\beta$ and decrease of Homa IR were positively correlated with the decrease of plasma glucose.

Conclusion: Both insulin treatment and OHA treatment can effectively control the glycemic in newly diagnosed type 2 diabetic patients with high fasting plasma glucose through eliminating the glucose toxicity. There were shorter time to get blood glucose achieving standard by insulin treatment than OHA treatment. Moreover the insulin therapy can improve the $\beta$ cell function by partly restoring the first phase of insulin secretion, and facilite to regulate insulin secretion mode of the beta cell. This suggests that the insulin treatment might be used as primary approach in the management of newly diagnosed type 2 diabetes patients.

0991

Diabetes treatment satisfaction as an independent factor contributing to glycaemic control in patients with type 2 diabetes mellitus

Q. Zhang ${ }^{1}$, P. A. Levin ${ }^{2}$;

${ }^{1}$ sanofi-aventis US, Bridgewater, ${ }^{2}$ Model Clinical Research, Baltimore, United States. 
Background and Aims: Health-related quality of life (HRQoL) and treatment satisfaction are typically reported directly by patients without physician interpretation and can be uniquely informative of the patient's perspective on effectiveness of therapy. We included patient-reported outcomes (PROs) in a recent clinical trial evaluating two dosing regimens of insulin glulisine (GLU) to assess whether PRO measures uniquely contribute to glycemic control in patients with type 2 diabetes mellitus (DM).

Materials and Methods: A multicenter, randomized, parallelgroup, 24-wk study compared glycemic control in 273 patients with type $2 \mathrm{DM}$ receiving basal insulin glargine (GLAR) and either 1) premeal GLU doses based on carbohydrate counting (Carb Count group; $\mathrm{n}=137$ ) or 2) premeal GLU doses adjusted weekly to target according to a simple algorithm based on preprandial glucose patterns (ALG group; $\mathrm{n}=136$ ). At baseline (BL), wk12, and wk24, patients were assessed on treatment satisfaction and HRQoL using the Diabetes Treatment Satisfaction Questionnaire (DTSQ), the Audit of Diabetes Dependent Quality of Life (ADDQoL) scale, and the Well-Being Questionnaire (W-BQ12).

Results: Patient characteristics at BL included a mean age of $55 \mathrm{y}, 56 \%$ female, $81 \%$ white, $11 \%$ black, and mean $\mathrm{A} 1 \mathrm{c}=8.16 \%$. Only BL BMI was significantly different between groups, higher in the ALG group (37.73) than in the Carb Count group (35.56; $\mathrm{p}=0.019)$. Both treatment arms achieved target A1c at study end (ALG: 6.70\%; Carb Count: $6.54 \%$ ). The ALG group used higher daily doses of GLU (110.2 vs 94.3U; p=0.04), GLAR (103.4 vs $87.0 \mathrm{U} ; \mathrm{p}<0.0001)$, and total insulin $(212.2$ vs $181.1 \mathrm{U}$; $\mathrm{p}=0.002$ ). The ALG group had less symptomatic hypoglycemia $<50 \mathrm{mg} / \mathrm{dL}$ than the Carb Count group (4.9 vs 8.0 events/pt-y; p=0.02). Patients' self-reported treatment satisfaction improved in both groups $(\mathrm{p}<0.0001)$. Treatment satisfaction contributed independently to glycemic control; patients who were dissatisfied at study midpoint (wk12) were significantly more likely than satisfied patients to have higher average A1c values $(p=0.0073)$. This contribution was above and beyond the effects of patient demographics, BL A1c, history of diet and carb counting, titration group, visits, and status of early study withdrawal. In addition, we found that patients in the ALG group who had a prior history of maintaining a healthy diet and practicing carb counting had the lowest mean A1c $(6.46 \%$, interaction with $\mathrm{p}=0.0174)$ over the course of the study.

Conclusions: In these patients with type $2 \mathrm{DM}$, treatment satisfaction with insulin therapy was independently related to glycemic control above and beyond other clinical variables such as baseline A1c and treatment arm. Greater diabetes treatment satisfaction was associated with improved A1c in patients receiving basal:bolus insulin therapy. Therefore, insulin therapy might be most effica- cious for improving glycemic control when there is direct concern for patient satisfaction throughout treatment. Supported by sanofi-aventis US

0992

Hyperglycaemia and its effect after acute myocodial infraction on cardiovascular (CV) outcomes in patients type 2 diabetes mellitus (HEART2D): Baseline characteristics

Z. Milicevic ${ }^{1}$, I. Raz ${ }^{2}$, S. Sarwat ${ }^{1}$, V. Bozikov ${ }^{3}$, P. Naiker ${ }^{4}$, K. Strojek ${ }^{5}$, D. Oakley ${ }^{1}$, D. C. Robbins ${ }^{1}$, M. Tan ${ }^{1}$;

${ }^{1}$ Eli Lilly and Company, Indianapolis, United States, ${ }^{2}$ Diabetic Unit, Hadassah Hebrew University Hospital, Jerusalem, Israel, ${ }^{3}$ Department of Internal Medicine, Dubrava University Hospital, Zagreb, Croatia, ${ }^{4}$ Chelmsfotd Medical Centre 3, St. Augustines Hospital, Durban, South Africa, ${ }^{5}$ Department of Internal Diseases, Diabetology and Nephrology, Silesian Medical Academy, Zabrze, Poland.

Background and Aims: Cardiovascular disease (CVD) is the major cause of morbidity and mortality in patients with type 2 diabetes mellitus (T2DM). Epidemiological studies indicate that the risk of CVD is associated with postprandial hyperglycemia, independently from fasting hyperglycemia, hemoglobin A1c (A1C), or other CV risk factors. The present study is designed to assess the effects of two different insulin regimens, one targeting postprandial (pp) blood glucose (BG) and the other targeting fasting/pre-meal $\mathrm{BG}$, on $\mathrm{CV}$ outcomes up to 5 years after enrollment in patients with $\mathrm{T} 2 \mathrm{DM}$ and acute myocardial infarction (AMI). Here we present characteristics of the study population at baseline.

Materials and Methods: 1120 patients from 105 study centres in 17 countries were randomised to 1 of 2 treatment strategies: (1) prandial strategy: premeal insulin lispro with basal insulin (NPH insulin) at bedtime if needed, targeting 2-h pp BG $<7.5 \mathrm{mM}$ or (2) basal strategy: NPH twice-daily or insulin glargine once-daily, followed by premixed human insulin twice-daily if needed, and targeting fasting and premeal $\mathrm{BG}(<6.7 \mathrm{mM})$. Both groups aim for a target $\mathrm{AlC}$ of $<7 \%$. The anticipated difference in pp BG (2.0 to $2.5 \mathrm{mM})$ between strategies is expected to demonstrate approximately $18.5 \%$ relative $\mathrm{CV}$ risk reduction in the prandial strategy group.

Results: At baseline patients' mean $( \pm \mathrm{SD})$ for age was 61.0 $( \pm 9.8)$ years, body mass index was $29.1( \pm 4.8) \mathrm{kg} / \mathrm{m}^{2}$, duration of diabetes was $9.1( \pm 7.2)$ years, $63 \%$ were male, and $\mathrm{A} 1 \mathrm{C}$ was $8.3( \pm 1.5) \%$. Treatment for diabetes prior to AMI included: diet and exercise (9\%), oral antihyperglycemic medications (sulfonylurea 26\%, metformin + sulfonylurea $15 \%$, biguanides $7 \%$ ) and insulin (conven- 
tional regimens 22\%, MDI 6\%). $15.7 \%$ patients had background retinopathy (proliferative retinopathy 4\%), $21 \%$ had peripheral neuropathy, 9\% had nephropathy, and $1.6 \%$ had a history of amputation. After entry AMI, reperfusion was immediately attempted in $58 \%$ of patients: primary percutaneous transluminal coronary angiopathy (PTCA) (43\%), fibrinolysis (18\%) and/or coronary artery bypass grafting $\mathrm{CABG}(3 \%)$. The mean left ventricular ejection fraction for the study population was $50.8( \pm 10) \%$. Conclusion: When compared to the baseline demographic and clinical characteristics of patient populations from other interventional trials, HEART2D study population shows many typical features of patients with T2DM and AMI. Epidemiological evidence indicates that these individuals are at very high risk of recurrent $\mathrm{CV}$ events and death. Therefore, results of HEART2D may have important implications for the management of a broader population of people with T2DM complicated by coronary heart disease.

Funded by Eli Lilly and Company

\section{PS 84}

\section{Insulin trials in type 1 or type 2 diabetes}

0993

Transfer of patients with type 1 diabetes from NPH insulin to insulin glargine as basal insulin: a multicentre, randomised, parallel-group, open-label study

G. Bolli ${ }^{1}$, M. Songini ${ }^{2}$, M. Trovati ${ }^{3}$, S. Del Prato ${ }^{4}$,

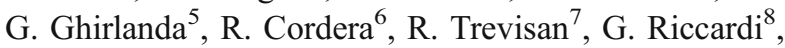
C. Noacco ${ }^{9}$ on behalf of HOE901/4019 Italian study group;

${ }^{1}$ Department of Internal Medicine, Endocrinology and Metabolism, University of Perugia, ${ }^{2}$ Diabetes Unit, Ospedale S. Michele, Cagliari, ${ }^{3}$ Department of Clinical and Biological Sciences, Turin University, ${ }^{4}$ Department of Endocrinology and Metabolism, University of Pisa, ${ }^{5}$ Inst. Internal Medicine and Geriatrics, Catholic University, Rome, ${ }^{6}$ Department of Endocrinology and Metabolism, University of Genova, ${ }^{7}$ Department of Medicine, Ospedali Riuniti di Bergamo, Bergamo, ${ }^{8}$ Department of Clinical Medicine, University of Naples, ${ }^{9}$ Diabetes Unit, Hospital Saint Maria della Misericordia, Udine, Italy.

Background and Aims: Once-daily (OD) basal insulin glargine can be used as part of a simple multiple daily injection (MDI) regimen in type 1 diabetes (T1DM) patients. This randomized, multicentre study compared the use of glargine and NPH insulin to normalize fasting plasma glucose (FPG; target 5.0-6.6 mmol/L [90-120 mg/ dL]) during the last month (Month 6) of treatment.

Materials and Methods: Patients with T1DM were treated for 6 months with prandial insulin lispro plus either OD basal glargine $(n=85)$ or twice-daily (BD) NPH $(n=90)$ in MDI regimens (baseline characteristics given in Table). The primary outcome measures were FPG and mean amplitude of glycaemic excursion (MAGE).

Results: $\mathrm{HbA}_{1 \mathrm{c}}$ decreased from baseline to endpoint with glargine (mean $\pm \mathrm{SD} ; 7.9 \pm 0.7$ to $7.3 \pm 0.7 \%$ ) and NPH $(7.9 \pm 0.6$ to $7.3 \pm 1.0 \%)(p=N / S$ between glargine and NPH). During the last month of treatment, decreases in mean daily FPG from baseline were significantly greater with glargine versus NPH (mean \pm coefficient of variation; $10.2 \pm 1.3$ to $8.7 \pm 1.1 \mathrm{mmol} / \mathrm{L}$ [184.3 \pm 24.3 to $156.3 \pm 20.0 \mathrm{mg} / \mathrm{dL}]$ vs $10.4 \pm 1.3$ to $9.9 \pm$ $1.3 \mathrm{mmol} / \mathrm{L}[188.1 \pm 24.1$ to $178.3 \pm 24.0 \mathrm{mg} / \mathrm{dL}] ; \mathrm{p}<0.05)$. Similarly, the decrease in MAGE was significantly greater with glargine versus NPH (mean \pm coefficient of variation; $8.2 \pm 1.5$ to $7.1 \pm 1.9 \mathrm{mmol} / \mathrm{L}[148.3 \pm 26.6$ to $128.1 \pm 33.9 \mathrm{mg} /$ $\mathrm{dL}]$ vs $7.9 \pm 1.9$ to $7.7 \pm 2.0 \mathrm{mmol} / \mathrm{L}[143.0 \pm 34.2$ to $139.5 \pm$ $35.8 \mathrm{mg} / \mathrm{dL}] ; \mathrm{p}<0.05)$. In contrast, there was no significant difference with glargine versus NPH with respect to confirmed serious hypoglycaemic events (blood glucose $<2.3 \mathrm{mmol} / \mathrm{L}[<41 \mathrm{mg} / \mathrm{dL}]$ ) per patient-week over the last month of treatment ( 0.15 vs 0.17 events). The low incidence of severe hypoglycaemia in both groups prevented meaningful comparison.

Conclusion: In this study of patients with T1DM, where basal insulin was insufficiently titrated to target, OD glargine was still superior to BD NPH with lower FPG and lower glucose variability despite similar $\mathrm{HbA}_{1 \mathrm{c}}$.

Baseline characteristics

\begin{tabular}{|c|c|c|c|c|}
\hline & $\begin{array}{l}\text { Insulin } \\
\text { glargine }\end{array}$ & $(\mathrm{n}=85)$ & $\begin{array}{c}\text { NPH } \\
\text { insulin }\end{array}$ & $(n=90)$ \\
\hline & median & $(\min -\max )$ & median & $(\min -\max )$ \\
\hline Male/female (\%) & $59 / 41$ & & $54 / 46$ & \\
\hline Age (years) & 35.5 & $18.0-57.0$ & 36.3 & $19.0-58.0$ \\
\hline BMI $\left(\mathrm{kg} / \mathrm{m}^{2}\right)$ & 23.4 & $18.9-27.9$ & 23.5 & $18.9-27.6$ \\
\hline $\begin{array}{l}\text { Duration of } \\
\text { diabetes (years) }\end{array}$ & 13.1 & $1.2-37.6$ & 14.3 & $2.4-48.1$ \\
\hline $\begin{array}{l}\mathrm{FBG}(\mathrm{mmol} / \mathrm{L}) \\
{[\mathrm{mg} / \mathrm{dL}]}\end{array}$ & $\begin{array}{l}10.2 \\
{[184]}\end{array}$ & $\begin{array}{l}5.0-16.8 \\
{[91-303]}\end{array}$ & $\begin{array}{l}10.3 \\
{[186]}\end{array}$ & $\begin{array}{l}5.4-16.8 \\
{[97-302]}\end{array}$ \\
\hline $\mathrm{HbA}_{1 \mathrm{c}}(\%)$ & 7.8 & $5.9-9.8$ & 7.8 & $6.4-9.0$ \\
\hline
\end{tabular}

Data are medians with minimum-maximum values except male/female ratio $(\%)$

Supported by sanofi-aventis 


\section{4}

Time of evening administration of insulin detemir can be fitted to individual needs and lifestyle

T. R. Pieber ${ }^{1}$, E. Draeger ${ }^{2}$, C. B. Pedersen ${ }^{3}$, P. D. Home ${ }^{4}$; ${ }^{1}$ Internal Medicine, Diabetes and Metabolism, Medical University, Graz, Austria, ${ }^{2}$ Medical Affairs, Novo Nordisk Region Europe, Zürich, Switzerland, ${ }^{3}$ Novo Nordisk, Bagsvaerd, Denmark, ${ }^{4}$ Department of Medicine, University of Newcastle upon Tyne, United Kingdom.

Background and Aims: Studies of blood glucose control with insulin detemir (detemir) vs. NPH insulin (NPH) with insulin aspart at meal-times have been subjected to metaanalyses.

Materials and Methods: Data were included from two 16week randomised, multi-national, parallel, open-label trials in 808 people with Type 1 diabetes (mean age $40 \mathrm{yr}, \mathrm{HbA}_{1 \mathrm{c}}$ $8.3 \%$, BMI: $25.2 \mathrm{~kg} / \mathrm{m}^{2}$ and diabetes duration $16 \mathrm{yr}$ ). Both $\mathrm{NPH}$ and detemir were given morning+bedtime (NPH, detemir $_{\text {bed }}$ ) but additionally detemir was given morning+ evening (morning+dinner or at $12-\mathrm{h}$ intervals), (detemir ${ }_{\text {eve }}$ ). Results: Baseline-adjusted $\mathrm{HbA}_{1 \mathrm{c}}$ was significantly lower with detemir eve $(7.71 \%)$ than with NPH $(7.85 \%)$ (Table 1$)$ at 16 weeks, and possibly lower with detemir bed $_{\text {b. }}(7.72 \%)$. The two detemir arms were comparable. Clinic fasting plasma glucose (FPG) was lower with detemir ${ }_{\text {bed }}$ than with detemir $_{\text {eve }}(9.0$ vs. $9.8 \mathrm{mmol} / \mathrm{L})$, and both were lower compared to NPH $(11.2 \mathrm{mmol} / \mathrm{L})$. Within-participant variation in self-measured FPG was significantly lower with detemir (both arms) than with NPH (SD 2.7 vs. $3.3 \mathrm{mmol} / \mathrm{L}, \mathrm{p}<0.001)$. Self-measured plasma glucose during the night was higher with detemir bed $_{\text {than with }}$ detemir $_{\text {eve }}$ and the risk of nocturnal hypoglycaemia was 29 and $26 \%$ lower with detemir bed than with detemir eve $_{\text {bnd }}$ $\mathrm{NPH}$ respectively.

Table 1: Estimated treatment differences

\begin{tabular}{|c|c|c|c|}
\hline $\mathrm{HbA}_{1 \mathrm{c}}(\%)$ & Difference & $95 \% \mathrm{CI}$ & $\mathrm{p}$ \\
\hline detemir $_{\text {eve }}-\mathrm{NPH}$ & -0.13 & {$[-0.26 ;-0.00]$} & 0.043 \\
\hline detemir $_{\text {bed }}-\mathrm{NPH}$ & -0.13 & {$[-0.25 ; 0.00]$} & 0.053 \\
\hline $\begin{array}{l}\text { detemir }_{\text {eve }}-\text { detemir }_{\text {bed }} \\
\text { FPG }(\mathrm{mmol} / \mathrm{L})\end{array}$ & -0.00 & {$[-0.13 ; 0.12]$} & NS \\
\hline detemir $_{\text {eve }}-\mathrm{NPH}$ & -1.35 & {$[-1.83 ;-0.87]$} & $<0.001$ \\
\hline detemir $_{\text {bed }}-\mathrm{NPH}$ & -2.14 & {$[-2.62 ;-1.65]$} & $<0.001$ \\
\hline $\begin{array}{l}\text { detemir }_{\text {eve }} \text { - detemir } \text { bed }_{\text {be }} \\
\text { Night-time hypoglycaemia } \\
\text { (Relative Risk) }\end{array}$ & 0.78 & {$[0.31 ; 1.26]$} & 0.001 \\
\hline $\mathrm{NPH} /$ detemir $_{\text {eve }}$ & 0.96 & {$[0.74 ; 1.25]$} & NS \\
\hline detemir $_{\text {bed }} / \mathrm{NPH}$ & 0.74 & {$[0.57 ; 0.96]$} & 0.026 \\
\hline detemir $_{\text {bed }} /$ detemir $_{\text {eve }}$ & 0.71 & {$[0.55 ; 0.92]$} & 0.011 \\
\hline
\end{tabular}

Conclusion: The time of evening administration of insulin detemir can be varied to match the needs and wishes of the individual patient.

Sponsored by Novo Nordisk A/S

0995

Insulin detemir achieves lower, less variable fasting glucose and a reduced risk of nocturnal hypoglycaemia and weight gain compared to NPH insulin in basal bolus therapy of Japanese patients with type 1 diabetes M. Kobayashi ${ }^{1}$, Y. Iwamoto ${ }^{2}$, R. Kawamori ${ }^{3}$, N. Tajima ${ }^{4}$, T. Nishida ${ }^{5}$, K. Kaku

${ }^{1}$ Diabetes, University of Toyama, ${ }^{2}$ Diabetes Center, Tokyo Women's Medical University, ${ }^{3}$ School of Medicine, Juntendo University, Tokyo, ${ }^{4}$ School of Medicine, Jikei University, Tokyo, ${ }^{5}$ Novo Nordisk Pharma Ltd, Tokyo, ${ }^{6}$ Diabetes and Endocrine Division, Kawasaki Medical School, Okayama, Japan.

Background and Aims: Insulin detemir has been associated with reductions in fasting plasma glucose (FPG) variability, risk of hypoglycaemia and weight gain in comparison with NPH insulin in a series of phase 3 studies. The present investigation sought to assess the clinical profile of insulin detemir in a Japanese cohort, and to test for non-inferiority in comparison with NPH insulin.

Materials and Methods: This 48-week, multi-centre, openlabel, parallel-group trial asymmetrically randomised and exposed 396 patients with type 1 or type 2 diabetes (body mass index $[\mathrm{BMI}]<30 \mathrm{~kg} / \mathrm{m}^{2}$ ) previously established on basal-bolus therapy, to receive mealtime insulin aspart with either insulin detemir or NPH insulin, each given once or twice daily, with continuous titration. The majority of patients had type 1 diabetes (insulin detemir, $n=196$; NPH insulin, $n=98$ ), hence the outcomes of this subgroup were assessed independently, and the key data are presented here. Key demographic characteristics of this group included: mean age, 42.2 years; mean duration of diabetes, 13.3 years; mean $\mathrm{HbA}_{1 \mathrm{c}}, 7.41 \%$; mean BMI $22.4 \mathrm{~kg} / \mathrm{m}^{2}$. Demographic characteristics were well matched by treatment.

Results: $\mathrm{HbA}_{1 \mathrm{c}}$ at endpoint did not differ between treatments, the baseline-adjusted means at endpoint being $7.33 \%$ and $7.29 \%$ for insulin detemir and NPH insulin, respectively (point estimate $[95 \% \mathrm{CI}]$ for detemir $\mathrm{NPH}=0.03 \% \quad[-0.14 ; 0.21]$, confirming non-inferiority). There were small respective decreases in $\mathrm{HbA}_{1 \mathrm{c}}$ of $-0.08 \%$ and $-0.12 \%$ from baseline values. FPG at endpoint was lower with insulin detemir than NPH insulin, with baselineadjusted means at endpoint being $146.5 \mathrm{mg} / \mathrm{dl}$ and $157.0 \mathrm{mg} / \mathrm{dl}(p=0.0263)$, respectively $(8.14 \mathrm{mmol} / \mathrm{l}$ and $8.72 \mathrm{mmol} / \mathrm{l})$. There were respective decreases in FPG of 
$-24.4 \mathrm{mg} / \mathrm{dl}$ and $-17.4 \mathrm{mg} / \mathrm{dl}(1.36 \mathrm{mmol} / \mathrm{l}$ and $0.97 \mathrm{mmol} / \mathrm{l}$ ). Within-subject variability (SD) of FPG was also lower at endpoint with insulin detemir $(53.4 \mathrm{mg} / \mathrm{dl}$ vs. $63.6 \mathrm{mg} / \mathrm{dl}, p<0.0001 ; 2.97 \mathrm{mmol} / 1$ vs. $3.53 \mathrm{mmol} / \mathrm{l})$. The overall incidence of hypoglycaemia was $\sim 51$ episodes/ patient-year in both groups, but the risk for nocturnal hypoglycaemia was lower with insulin detemir (Relative Risk, 0.69, $p=0.0464$ ). Baseline-adjusted weight was lower in insulin detemir-treated patients at endpoint (point estimate for detemir-NPH: $-0.92 \mathrm{~kg}, p=0.0024)$. There were no between-group differences in other safety parameters. The mean daily basal insulin dose at end of trial was significantly lower with insulin detemir $(p=0.0001)$, the ratio (insulin detemir/NPH insulin) being 0.869 . However, the dose of bolus insulin was slightly higher in the insulin detemir group (NS), hence the ratio for total daily insulin dose at end of trial was 0.983 .

Conclusion: Insulin detemir, as the basal component of basal-bolus therapy, was associated with several clinical advantages in comparison with NPH insulin in Japanese patients with type 1 diabetes. These advantages included better fasting glucose control, with lower variability, fewer nocturnal hypoglycaemic episodes and reduced weight gain. Supported by Novo Nordisk Pharma Ltd

\section{6}

Once-daily insulin detemir added to oral antidiabetic drugs results in less weight gain and a trend for reduced hypoglycaemia in comparison to NPH insulin in Japanese patients with type 2 diabetes

N. Tajima ${ }^{1}$, Y. Iwamoto ${ }^{2}, \mathrm{~K} . \mathrm{Kaku}^{3}$, R. Kawamori ${ }^{4}$, T. Nishida ${ }^{5}$, M. Kobayashi ${ }^{6}$;

${ }^{1}$ School of Medicine, Jikei University, Tokyo, ${ }^{2}$ Department of Medicine, Tokyo Women's Medical University, ${ }^{3}$ Diabetes and Endocrine Division, Kawasaki Medical School, Okayama, Japan, ${ }^{4}$ Department of Medicine, Juntendo University, Tokyo, ${ }^{5}$ Novo Nordisk Pharma Ltd, Tokyo, ${ }^{6}$ Diabetes, University of Toyama, Japan.

Background and Aims: Patients with type 2 diabetes usually benefit from addition of insulin therapy when hyperglycaemia is no longer adequately controlled by oral antidiabetic drugs (OADs). A popular, simple regimen for initiating insulin is to add a once daily injection of a long acting insulin preparation to existing therapy, titrating dose towards guideline targets. This study compared efficacy and tolerability of once-daily insulin detemir with NPH insulin used in this way in Japanese patients, seeking to confirm non-inferiority of insulin detemir.

Materials and Methods: This 36 week, multi-centre, openlabel, parallel group trial randomised and exposed insulin naïve adults with type 2 diabetes diagnosed $\geq 1$ year, with $\mathrm{BMI}<30 \mathrm{~kg} / \mathrm{m}^{2}$, and $\mathrm{HbA}_{1 \mathrm{c}} \geq 7.5 \%,<10.0 \%$, to receive a once-daily bedtime injection of insulin detemir $(n=180)$ or NPH insulin $(\mathrm{n}=183)$, in addition to their existing 1-3 OADS. All patients were on sulphonylureas, $+/-$ biguanides and/or alpha glucosidase inhibitors, with all doses within approved Japanese labelling. Insulin was titrated against pre-breakfast plasma glucose (FPG), the dose increased in small steps if this remained $\geq 120 \mathrm{mg} / \mathrm{dl}$ and decreased if it was below $70 \mathrm{mg} / \mathrm{dl}$. The goal was to aim for Japanese Diabetes Association guidelines for 2002-2003 for good or excellent glycaemic control $\left(\mathrm{HbA}_{1 \mathrm{c}} \leq 6.4 \%\right.$, $\mathrm{FPG} \leq 119 \mathrm{mg} / \mathrm{dl}$ ), without hypoglycaemia.

Results: Glycaemic control was similar for both basal insulins at end of trial. Adjusted endpoint mean (SE) values for $\mathrm{HbA}_{1 \mathrm{c}}$ were $7.81 \%(0.07)$ and $7.74 \%(0.07)$ for insulin detemir and NPH insulin, respectively (point estimate $[95 \%$ $\mathrm{CI}]$, detemir-NPH, $=0.07 \%[-0.07 ; 0.21]$ ), confirming noninferiority of insulin detemir. There were respective mean decreases in $\mathrm{HbA}_{1 \mathrm{c}}$ of $-0.59 \%$ and $-0.67 \%$. The adjusted endpoint mean (SE) values for FPG were $125.38 \mathrm{mg} / \mathrm{dl}$ (2.97) and $123.32 \mathrm{mg} / \mathrm{dl}$ (2.90) for insulin detemir and NPH insulin $(6.97 \mathrm{mmol} / \mathrm{l}$ and $6.85 \mathrm{mmol} / \mathrm{l})$, respectively (point estimate $[95 \% \mathrm{CI}]$, detemir-NPH, $=2.06 \mathrm{mg} / \mathrm{dl}[-3.95$; 8.07]). There were respective mean decreases in FPG of $-41.88 \mathrm{mg} / \mathrm{dl}$ and $-43.48 \mathrm{mg} / \mathrm{dl}(2.33 \mathrm{mmol} / \mathrm{l}$ and $2.42 \mathrm{mmol} / \mathrm{l}$ ). There was a trend for a reduced incidence of hypoglycaemia in recipients of insulin detemir, compared with NPH insulin: 4.51 vs. 6.46 events/subject/year, relative risk $0.71(p=0.0636)$. Similarly, respective incidences for nocturnal hypoglycaemia were 1.12 vs. 2.10 events/ subject/year, relative risk $0.60(p=0.0754)$. Weight gain was lower with insulin detemir $(0.78 \mathrm{~kg})$ than NPH insulin $(1.18 \mathrm{~kg})$, adjusted endpoint means (SE): $61.26 \mathrm{~kg}(0.18)$ and $61.64(0.18)$ respectively $(p=0.0392)$. No betweengroup differences in other safety parameters were apparent. Mean daily insulin dose was slightly, but statistically significantly, higher in recipients of insulin detemir than NPH insulin (ratio, 1.19).

Conclusion: Once-daily insulin detemir added to OAD therapy in Japanese patients with poorly controlled type 2 diabetes improves glycaemic control to the same extent as NPH insulin, but with reduced weight gain and a tendency for fewer hypoglycaemic episodes.

Supported by Novo Nordisk Pharma Ltd 
0997

The PREFER Study: a comparison of outcomes in patients using insulin detemir once daily or twice daily in a basal bolus regimen with mealtime insulin aspart A. Garber ${ }^{1}$, K. Binz ${ }^{2}$, R. Bergenstal ${ }^{3}$, A. Liebl ${ }^{4}$;

${ }^{1}$ Methodist Hospital, Baylor College of Medicine, Houston, United States, ${ }^{2}$ Diabetes Department, Triemli City Hospital, Zurich, Switzerland, ${ }^{3}$ Diabetes Department, International Diabetes Center, Minneapolis, United States, ${ }^{4}$ Diabetes Department, Center for Diabetes and Metabolism, Bad Heilbrunn, Germany.

Background and Aims: IDet is a long-acting basal insulin analogue often used in basal-bolus regimens. At present it is unclear what proportion of patients need IDet twice daily (BID), rather than once daily (OD), and whether or not they achieve the same clinical outcomes. In this study designed to compare a basal-bolus regimen with a BID regimen of premixed analogue BIAsp 30, dosing and outcome data in the basal-bolus arm were analysed for patients that received IDet OD or BID in addition to mealtime IAsp.

Methods and Materials: Poorly controlled $\left(7 \leq \mathrm{HbA}_{1 \mathrm{c}} \leq 12 \%\right)$ patients with type 2 diabetes previously treated with OADs alone, or in combination with basal insulin (glargine or $\mathrm{NPH}$ ), were included in this 26-week, multi-centre, openlabel, parallel-group trial. In the IDet/IAsp group $(n=537)$, IDet was initiated once daily plus IAsp three times daily (distributed 3:1:2 breakfast:lunch:dinner). In patients pretreated with basal insulin, the previous dose was switched 1:1 unit-for-unit for IDet, while in insulin-naïve patients, initial IDet dose was 10-14 U. OADs were discontinued. Insulin doses were titrated to predefined plasma glucose (PG) targets using an algorithm. IDet was titrated to $\leq 7.0 \mathrm{mmol} / 1$ prebreakfast and IAsp to $90 \mathrm{~min}$ postprandial $\mathrm{PG} \leq 10 \mathrm{mmol} / \mathrm{l}$. If predinner PG exceeded $7.0 \mathrm{mmol} / 1$ on $>5$ consecutive days and/or nocturnal hypoglycaemia prevented adequate titration of the dinner IDet dose, a second IDet dose was added prebreakfast and titrated to predinner $\mathrm{PG} \leq 7.0 \mathrm{mmol} / \mathrm{l}$.

Results: In the IDet/IAsp group, $87 \%$ of patients remained on once-daily IDet throughout the trial. End-of-trial IDet doses were $0.353 \mathrm{U} / \mathrm{kg}$ when used once daily, and $0.557 \mathrm{U} /$ $\mathrm{kg}$ when used twice daily $(0.201 \mathrm{U} / \mathrm{kg}$ at breakfast, $0.356 \mathrm{U} / \mathrm{kg}$ at dinner). End-of-trial (EOT) total daily insulin dose was $0.86 \mathrm{U} / \mathrm{kg}$ ( $0.38 \mathrm{U} / \mathrm{kg}$ IDet, $0.48 \mathrm{U} / \mathrm{kg}$ IAsp). For IAsp, mean initial dose was $20.2 \mathrm{U}$ and final dose split was: breakfast $35 \%(0.173 \mathrm{U} / \mathrm{kg})$, lunch $29 \%(0.140 \mathrm{U} / \mathrm{kg})$ and dinner $36 \%(0.176 \mathrm{U} / \mathrm{kg})$. Glycaemic control $\left(\mathrm{HbA}_{1 \mathrm{c}}\right)$ was similar for patients receiving IDet OD or BID: $6.93 \%$ and $6.97 \%$, respectively; $\mathrm{p}=0.697$. Fasting plasma glucose (FPG) measured during the last week of the trial was $7.46 \mathrm{mmol} / \mathrm{l}$ for OD IDet and $8.00 \mathrm{mmol} / \mathrm{l}$ for BID IDet.
The respective FPG coefficients of variation were $12.91 \%$ and $15.34 \%$. At EOT there was no significant difference in body weight between OD and BID IDet-treated patients: 91.7 vs. $92.2 \mathrm{~kg}$, respectively; $\mathrm{p}=0.305$. EOT BMI was also similar: 31.8 (OD IDet) vs. 32.0 (BID IDet); $\mathrm{p}=0.316$. The proportion of patients that experienced any hypoglycaemia was $48.5 \%$ for OD and $42.2 \%$ for BID IDet. Minor nocturnal hypoglycaemia was similar for OD vs. BID IDet: $7.3 \%$ vs. $6.3 \%$ of patients.

Conclusions: OD dosing of IDet was effective for the majority of patients in a treat-to-target basal-bolus regimen with IAsp at mealtimes. Patients using IDet OD or BID achieved similar glycaemic control, body weight and BMI, and experienced similar rates of hypoglycaemia.

Supported by Novo Nordisk

\section{8}

The PREFER study: both biphasic insulin aspart 30 twice-daily and basal-bolus using insulin detemir and insulin aspart enabled patients with type 2 diabetes to achieve $\mathrm{HbA}_{1 \mathrm{c}}$ target $<\mathbf{7 . 0 \%}$

A. Liebl ${ }^{1}$, R. Prager ${ }^{2}$, M. Kaiser ${ }^{3}$, K. Binz ${ }^{4}$, B. Gallwitz ; ${ }^{1}$ Diabetes Department, Center for Diabetes and Metabolism, Bad Heilbrunn, Germany, ${ }^{2}$ Diabetes Department, L. Boltzman Institute of Metabolic Diseases and Nutrition, Vienna, Austria, ${ }^{3}$ Novo Nordisk Pharma GmbH, Mainz, Germany, ${ }^{4}$ Diabetes Department, Triemli City Hospital, Zurich, Switzerland, ${ }^{5}$ Diabetes Department, Eberhard-Karls University, Tübingen, Germany.

Background and Aims: Insulin analogues are widely used in type 2 diabetes management but rarely compared in clinical trials. This study evaluated two insulin analogue regimens in patients with type 2 diabetes: biphasic insulin aspart 30 (BIAsp 30, 30\% insulin aspart, 70\% protaminated aspart) and basal-bolus (BB) therapy using insulin detemir (IDet) and insulin aspart (IAsp).

Materials and Methods: This was a 26-week, treat-totarget, multicentre, randomised, open-label, parallel-group trial of 715 subjects $\left(7 \leq \mathrm{HbA}_{1 \mathrm{c}} \leq 12 \%\right)$ previously treated with OADs alone $(72 \%)$ or in combination with glargine or NPH insulin. BIAsp $30(\mathrm{n}=178)$ was initiated twice daily $(0.2 \mathrm{U} / \mathrm{kg}$ at breakfast, $0.1 \mathrm{U} / \mathrm{kg}$ at dinner). BB therapy $(\mathrm{n}=537)$ was initiated with IDet once daily (10U or $14 \mathrm{U}$ if BMI $>32 \mathrm{~kg} / \mathrm{m}^{2}$ ) plus IAsp with meals (dosed individually in ratio 3:1:2 breakfast:lunch:dinner). OADs were discontinued in both arms. BIAsp 30 was titrated to plasma glucose (PG) target $\leq 7.0 \mathrm{mmol} / 1$ prebreakfast and predinner. IDet (administered predinner) was titrated to $\mathrm{PG}$ $\leq 7.0 \mathrm{mmol} / \mathrm{l}$ prebreakfast and IAsp to $90 \mathrm{~min}$ postprandial $\mathrm{PG} \leq 10 \mathrm{mmol} / \mathrm{l}$. If pre-dinner $\mathrm{PG}$ was elevated for 5 
consecutive days and/or nocturnal hypoglycaemia prevented adequate titration of the IDet dose, a second IDet dose was added prebreakfast and titrated to $P G \leq 7.0 \mathrm{mmol} / \mathrm{l}$ predinner.

Results: In the IDet/IAsp group, initial mean dose for IAsp was $20.2 \mathrm{U}$. At end of trial, mean total daily doses were $0.63 \mathrm{U} / \mathrm{kg}$ for BIAsp 30 and $0.86 \mathrm{U} / \mathrm{kg}$ for IDet $/$ IAsp. Of the IDet/IAsp patients, $87 \%$ remained on once-daily IDet. Reduction in $\mathrm{HbA}_{1 \mathrm{c}}$ was significantly greater in patients on IDet/IAsp $(8.52 \%$ to $6.96 \%)$ than on BIAsp30 (8.40\% to 7.17\%); baseline-adjusted $\mathrm{HbA}_{1 \mathrm{c}}$ difference (IDet/IAspBIAsp 30) $0.23 \%(p=0.0052)$. More than half of all patients reached $\mathrm{HbA}_{1 \mathrm{c}} \leq 7.0 \%: 50 \%$ of the BIAsp 30 group and $60 \%$ of the IDet/IAsp group. Patients previously treated with insulin had greater $\mathrm{HbA}_{1 \mathrm{c}}$ reduction with IDet/IAsp than with BIAsp $30(1.21 \%$ vs $0.75 \%$; $p=0.0129)$, while insulin naive patients had similar $\mathrm{HbA}_{1 \mathrm{c}}$ reductions $(1.69 \%$ vs $1.42 \% ; \mathrm{p}=0.106)$. Mean fasting PG (FPG) was similar between groups: $8.05 \mathrm{vs} 8.27 \mathrm{mmol} / \mathrm{l}$ (BIAsp $30 \mathrm{vs}$. IDet/ IAsp; $\mathrm{p}=0.345$ ). Intrasubject coefficients of variation for FPG were low for both regimens: BIAsp 30, 12.6\%; IDet/ IAsp, $13.3 \%$. No patients on BIAsp 30 experienced major hypoglycaemia, compared with 5 patients $(0.9 \%)$ on IDet/ IAsp. Minor hypoglycaemia $(\mathrm{PG}<3.1 \mathrm{mmol} / \mathrm{l})$ occurred in $28 \%$ of BIAsp 30 and $31 \%$ of IDet/IAsp patients. Nocturnal minor hypoglycaemia occurred in $7.3 \%$ and $7.4 \%$ of patients, respectively. Both groups showed a similar increase in body weight from baseline (BIAsp 30: $2.1 \mathrm{~kg}$; IDet/IAsp: $2.4 \mathrm{~kg}$ ). Conclusions: Overall, in poorly-controlled patients with type 2 diabetes, premixed and $\mathrm{BB}$ insulin analogue regimens enabled $58 \%$ to reach $\mathrm{HbA}_{1 \mathrm{c}} \leq 7 \%$. $\mathrm{BB}$ treatment with IDet/IAsp resulted in a significantly greater $\mathrm{HbA}_{1 \mathrm{c}}$ reduction than twice-daily BIAsp 30 in previously insulintreated patients, but insulin-naive patients achieved equal glycaemic control on each regimen. Hypoglycaemia rates were low and similar between treatments.

Supported by Novo Nordisk

\section{9}

The PREFER Study: Dosing recommendations for biphasic insulin aspart 30, insulin detemir and insulin aspart using treat-to-target titration

B. Gallwitz ${ }^{1}$, R. Prager ${ }^{2}$, M. Kaiser ${ }^{3}$, K. Binz ${ }^{4}$, A. Liebl $1^{5}$; ${ }^{1}$ Department of Medicine, Eberhard-Karls University, Tuebingen, Germany, ${ }^{2}$ Department of Endocrinology and Metabolism, L. Boltzman Institute of Metabolic Diseases and Nutrition, Vienna, Austria, ${ }^{3}$ Novo Nordisk Pharma GmbH, Mainz, Germany, ${ }^{4}$ Diabetes Department, Triemli City Hospital, Zurich, Switzerland, ${ }^{5}$ Department of Diabetes, Centre for Diabetes and Metabolism, Bad Heilbrunn, Germany.
Background and Aims: Premixed and basal-bolus insulin analogue regimens are widely prescribed for the treatment of type 2 diabetes but few data are available on dose distribution. In this treat-to-target study, dosing data were analysed from 715 patients receiving premixed analogue, biphasic insulin aspart 30/70 (BIAsp 30; 30\% insulin aspart, $70 \%$ protaminated aspart), or all-analogue basalbolus therapy using insulin detemir and insulin aspart (IDet/IAsp).

Materials and Methods: Prior to randomisation in this 26week, multi-centre, open-labelled, parallel-group trial, poorly controlled $\left(7 \leq \mathrm{HbA}_{1 \mathrm{c}} \leq 12 \%\right)$ patients with type 2 diabetes were treated with OADs alone or in combination with basal insulin (insulin glargine or NPH insulin). In the BIAsp 30 group ( $\mathrm{n}=178$ ), insulin was initiated twice daily at $0.3 \mathrm{U} / \mathrm{kg}: 2 / 3$ of dose at breakfast, $1 / 3$ at dinner, irrespective of pre-trial treatment. In the basal-bolus group $(n=537)$, IDet was initiated once daily plus IAsp three times daily with meals (in ratio 3:1:2 breakfast:lunch:dinner). In patients previously treated with basal insulin, the IDet dose was a 1:1 unit-for-unit switch, while in insulin-naïve patients, initial IDet dose was $10 \mathrm{U}$ or $14 \mathrm{U}$ if BMI $>32 \mathrm{~kg} / \mathrm{m}^{2}$. All OADs were discontinued in both groups. Insulin dose adjustment was algorithm-driven to predefined plasma glucose (PG) targets. BIAsp 30 was titrated to $\leq 7.0 \mathrm{mmol} / \mathrm{l}$ prebreakfast and predinner. IDet was titrated to $\leq 7.0 \mathrm{mmol} / \mathrm{l}$ prebreakfast and IAsp to $90 \mathrm{~min}$ postprandial PG $\leq 10 \mathrm{mmol} / \mathrm{l}$. If pre-dinner $\mathrm{PG}$ exceeded $7.0 \mathrm{mmol} / \mathrm{l}$ on $>5$ consecutive days and/or nocturnal hypoglycaemia prevented adequate titration of the dinner IDet dose, a second IDet dose was added pre-breakfast and titrated to pre-dinner PG $\leq 7.0 \mathrm{mmol} / 1$.

Results: Mean total daily insulin doses after 26 weeks were $0.63 \mathrm{U} / \mathrm{kg}$ for BIAsp30 and $0.86 \mathrm{U} / \mathrm{kg}$ for IDet $/ \mathrm{IAsp}$ $(0.38 \mathrm{U} / \mathrm{kg}$ IDet, $0.48 \mathrm{U} / \mathrm{kg}$ IAsp; initial mean IAsp dose was $20.2 \mathrm{U}$ ). Titration of BIAsp 30 resulted in a 50:50 dose split between breakfast and dinner: 0.315 and $0.316 \mathrm{U} / \mathrm{kg}$, respectively. For IAsp, the final dose split between meals was: breakfast $35 \%(0.17 \mathrm{U} / \mathrm{kg})$, lunch $29 \%(0.14 \mathrm{U} / \mathrm{kg})$ and dinner $36 \%(0.18 \mathrm{U} / \mathrm{kg})$. In the IDet/IAsp group, $87 \%$ of patients remained on once-daily IDet throughout the trial. End-of-trial IDet doses were $0.35 \mathrm{U} / \mathrm{kg}$ when used once daily, and $0.56 \mathrm{U} / \mathrm{kg}$ when used twice daily $(0.20 \mathrm{U} /$ $\mathrm{kg}$ at breakfast, $0.36 \mathrm{U} / \mathrm{kg}$ at dinner). After 26 weeks, $\mathrm{HbA}_{1 \mathrm{c}}$ was $6.96 \%$ for the IDet/IAsp group and $7.17 \%$ for BIAsp 30, baseline-adjusted $\mathrm{HbA}_{1 \mathrm{c}}$ difference (IDet/IAspBIAsp 30): $0.23 \%$; $\mathrm{p}=0.0052$. Glycaemic control $\left(\mathrm{HbA}_{1 \mathrm{c}}\right)$ was similar for patients receiving IDet once or twice daily: $6.97 \%$ and $6.93 \%$, respectively; $\mathrm{p}=0.697$.

Conclusions: Titration of twice-daily BIAsp 30 and three-times-daily IAsp (in an analogue basal-bolus regimen) resulted in equal distribution of doses between meals. Only $13 \%$ of IDet/IAsp patients required twice- 
daily IDet, showing once-daily dosing was effective for most patients.

Supported by Novo Nordisk A/S

1000

Pharmacokinetics and pharmacodynamics of biphasic insulin aspart 50 and biphasic insulin aspart 70 in healthy Japanese subjects

S. Irie ${ }^{1}$, Y. Matsumura ${ }^{2}$, H. Furuie ${ }^{1}$, K. Matsuguma ${ }^{1}$;

${ }^{1}$ Clinical Pharmacology, Kyushu Clinical Pharmacology

Research Clinic, Fukuoka, ${ }^{2}$ Novo Nordisk Pharma Ltd, Tokyo, Japan.

Background and Aims: Biphasic insulin aspart (BIAsp) is a premixed insulin analogue formulation comprising a proportion of soluble insulin aspart (IAsp) in association with protamine-retarded IAsp. The soluble IAsp fraction is rapidly absorbed after subcutaneous injection, providing prandial glucose control, while the delayed absorption of the protamine-retarded fraction provides basal insulin coverage. In clinical trials, BIAsp $30(30 \%$ soluble IAsp; $70 \%$ protamine-retarded IAsp) provides effective glycaemic control when administered twice-daily before meals. The rationale for developing BIAsp 50 and BIAsp 70 (50\% and $70 \%$ soluble IAsp; $50 \%$ and $30 \%$ protamine-retarded IAsp, respectively) is to provide the possibility of premixed insulin analogues for administration twice or three times daily in patients with additional need for controlling postprandial glucose or varying diets and lifestyles. The present investigation compared the pharmacokinetics and pharmacodynamics of BIAsp 50 and BIAsp 70.

Materials and Methods: A randomised, open-labelled, two-period crossover trial involving 24 healthy Japanese male subjects (age: 20-50 years; body mass index: 19$27 \mathrm{~kg} / \mathrm{m}^{2}$; fasting blood glucose: $3.8-6.0 \mathrm{mmol} / \mathrm{L}$ ). Subjects received a single dose $(0.8 \mathrm{U} / \mathrm{kg})$ of BIAsp 50 or BIAsp 70 at the start of the first treatment period. Subjects then received the same dose of the alternate trial drug at the start of the second treatment period, scheduled 6-12 days after the first. A 28-point (24-hour) serum insulin aspart (IAsp) profile and a 21-point (10-hour) serum glucose profile were determined after dosing. Endpoint analysis was performed using ANOVA.

Results: Maximum insulin concentration $\left(\mathrm{C}_{\max }\right.$ (ins, $\left.0-24 \mathrm{~h}\right)$ was higher for BIAsp 70 than for BIAsp 50 (214.1 vs $153.7 \mathrm{pmol} / \mathrm{L} ; \mathrm{p}<0.0001)$. Correspondingly, the maximum glucose-lowering effect for BIAsp 70 was significantly greater than for BIAsp 50 (delta $\mathrm{C}_{\min }$ (glu, 0-10 h), 1.664 vs.
$1.250 \mathrm{mmol} / \mathrm{L} ; \mathrm{p}<0.0001)$. Minimum glucose levels were achieved 45 minutes earlier with BIAsp 70 than with BIAsp $50(\mathrm{p}<0.05)$. Furthermore, mean residence time was statistically shorter for BIAsp 70 than for BIAsp 50 ( 1.986 vs. $3.683 \mathrm{~h} ; \mathrm{p}=0.0001$ ). Overall exposure to IAsp was similar between treatments $\left(\mathrm{AUC}_{\mathrm{ins}}, 0-\infty\right.$, BIAsp 50 , 442.0; BIAsp70, $435.7 \mathrm{pmol} / \mathrm{h} / \mathrm{L})$ although exposure to IAsp over the first 4 hours after dosing $\left(\mathrm{AUC}_{\mathrm{ins}}, 0-4 \mathrm{~h}\right)$ was greater for BIAsp 70 than for BIAsp 50 (379.0 vs. $304.2 \mathrm{pmol} / \mathrm{h} / \mathrm{L} ; \mathrm{p}<0.0001)$. The overall glucose-lowering effect was similar between treatment groups AOC $_{\text {(glu, } 0-}$ $10 \mathrm{~h}$ ), BIAsp 70, 6.747; BIAsp 50, $6.514 \mathrm{mmol} / \mathrm{h} / \mathrm{L}$; n.s.).

Conclusion: Maximum IAsp levels were higher for BIAsp 70. This could be predicted due to the greater proportion of soluble IAsp in BIAsp 70. Accordingly, the increased proportion of soluble IAsp in BIAsp 70 than in BIAsp 50 resulted in a significantly greater initial glucose-lowering effect with BIAsp 70. However, overall exposure to IAsp and the overall glucose-lowering effect was similar between treatments. In summary, the pharmacokinetic and pharmacodynamic properties of BIAsp 50 and BIAsp 70 reflected the proportions of soluble and protamine-retarded IAsp in the formulations. In clinical practice, the enhanced initial glucose-lowering effect of BIAsp 70 may offer improved control of postprandial blood glucose levels as compared with BIAsp 50.

Supported by Novo Nordisk Pharma Ltd

\section{1}

Early study termination is related to patient-reported outcomes during therapy with mealtime insulin glulisine in patients with type 2 diabetes mellitus

P. A. Levin ${ }^{1}$, Q. Zhang ${ }^{2}$;

${ }^{1}$ Model Clinical Research, Baltimore, ${ }^{2}$ sanofi-aventis US, Bridgewater, United States.

Background and Aims: Participants may terminate early from a clinical trial for reasons such as "do not wish to continue," "lost to follow-up," and "study-related or unrelated adverse events." While study-specific factors related to early termination may impact study completion rates and trial success, the effect of more stable factors such as measures of clinical and patient-reported outcomes (PROs) may have implications beyond the trial for reallife treatment success.

Materials and Methods: A multicenter, randomized, parallel-group, 24-wk study compared glycemic control in 273 patients with type 2 diabetes mellitus (DM) who, in 
addition to receiving basal insulin glargine (GLAR) throughout, received either 1) premeal insulin glulisine (GLU) at doses based on carbohydrate counting (Carb Count group; $\mathrm{n}=137$ ) or 2) premeal GLU at doses adjusted weekly to target according to a simple algorithm based on preprandial glucose patterns (ALG group; $n=136$ ). At baseline (BL), wk12, and wk24, patients were assessed on treatment satisfaction and health-related quality of life (HRQoL), using the Diabetes Treatment Satisfaction Questionnaire (DTSQ), the Audit of Diabetes Dependent Quality of Life (ADDQoL) scale, and the Well-Being Questionnaire (W-BQ12).

Results: Patient characteristics at BL included a mean age of $55 \mathrm{y}, 56 \%$ female, $81 \%$ white, $11 \%$ black, and mean $\mathrm{A} 1 \mathrm{c}=8.16 \%$. Only BL BMI was significantly different between groups, higher in the ALG group (37.73) than in the Carb Count group (35.56; $\mathrm{p}=0.019)$. Both treatment arms achieved target A1c at study end (ALG: 6.70\%; Carb Count: $6.54 \%$ ). The ALG group had less symptomatic hypoglycemia $<50 \mathrm{mg} / \mathrm{dL}$ than the Carb Count group (4.9 vs 8.0 events $/ p t-y ; p=0.02$ ). Twelve ALG patients and 28 Carb Count patients withdrew from the study before the last visit. Patients who stayed in the study reported greater treatment satisfaction than patients who withdrew $(p=0.0002)$. Specifically, early termination was more likely among patients who were randomized to Carb Count $(p=0.0361)$, younger $(p=0.0003)$, had higher BL A1c $(\mathrm{p}=0.0012)$, or no history of a healthy diet prior to the study $(p<0.0001)$. Furthermore, the relationships between HRQoL and treatment satisfaction and early study termination were largely dependent on the timing of assessment. Patients reporting greater negative impact of diabetes on HRQoL at BL were less inclined to withdraw from the study $(p=0.002)$. However, if such impact was reported at wk12, patients were more likely to withdraw $(\mathrm{p}=0.0015)$. Patients reporting dissatisfaction at wk12 due to hyperglycemic events were more inclined to withdraw if in the Carb Count group $(p=0.0027)$. Treatment satisfaction at wk12 was unrelated to early termination in the ALG group.

Conclusions: Early study termination is correlated with baseline A1c and patient-reported outcomes, beyond studyspecific factors. We found that patient reports of poor HRQoL at baseline are associated with a higher chance of study completion, while poor HRQoL at mid-study increases the chance of early termination. It appears that patients may have attributed HRQoL to past or present treatment success, which is, in turn, related to early study termination.

Supported by sanofi-aventis US
1002

Safe and effective control with insulin in patients with type 2 diabetes mellitus using two different methods of titrating mealtime glulisine

R. Bergenstal ${ }^{1}$, M. Johnson ${ }^{1}$, M. Powers ${ }^{1}$, A. Wynne ${ }^{2}$, K. Bradbury ${ }^{2}$, G. Graf $f^{2}$, A. Vlajnic ${ }^{3}$, P. Hollander ${ }^{4}$;

${ }^{1}$ International Diabetes Center, Minneapolis, ${ }^{2}$ CottonO’Neil Clinic, Topeka, ${ }^{3}$ sanofi-aventis US, Bridgewater, ${ }^{4}$ Baylor Endocrine Center, Dallas, United States.

Background and Aims: This open-label, multicenter, randomized, 24-wk study tested two methods of titrating mealtime insulin glulisine (GLU): (ALG; $n=136$ ) titration based on preprandial blood glucose (BG) patterns (number of $B G$ values above or below the premeal target) with dosing adjustments based on a simple algorithm method (adding 1, 2, or 3 units (U) depending on specific mealtime dose) vs (Carb Count; $\mathrm{n}=137$ ) titration based on preprandial BG patterns and insulin to carbohydrate (I:C) ratio changes with dosing adjustments based on the carbohydrate content of a meal.

Materials and Methods: Patients had type 2 diabetes mellitus (DM) uncontrolled on 2 or more insulin injections (age $55.1 \mathrm{y}$; duration $13 \mathrm{y}$; BMI $36.7 \mathrm{~kg} / \mathrm{m}^{2}$; Alc 8.2\%). Patients were switched to basal:bolus therapy with daily glargine (GLAR), titrated to fasting $\mathrm{BG}<95 \mathrm{mg} / \mathrm{dL}$, and GLU before meals, \pm metformin, with targets of $<100 \mathrm{mg} / \mathrm{dL}$ prelunch/dinner and $<130 \mathrm{mg} / \mathrm{dL}$ at bedtime. Premeal GLU was adjusted weekly. ALG added 1, 2, or $3 \mathrm{U}$ based on premeal glucose patterns; Carb Count adjusted the I:C ratio. Results: At wk24, A1c was significantly reduced in both groups $(\mathrm{p}<0.0001)$; ALG and Carb Count did not differ in change from baseline A1c $(-1.46 \%$ vs $-1.62 \% ; \mathrm{p}=0.15)$, proportion of patients achieving $\mathrm{A} 1 \mathrm{c}<7.0 \%(73.0 \%$ vs $69.2 \%$; $=0.7$ ), and weight gain (3.7 vs $2.4 \mathrm{~kg} ; \mathrm{p}=0.06$ ). The ALG group used higher doses of GLU (110.2 vs 94.3U; $\mathrm{p}=0.04)$ and GLAR (103.4 vs $87.0 \mathrm{U} ; \mathrm{p}<0.0001)$, and had less symptomatic hypoglycemia $<50 \mathrm{mg} / \mathrm{dL}$ ( 4.9 vs 8.0 events/pt-y; $\mathrm{p}=0.02$ ) than the Carb Count group. Both groups ended with a basal:bolus ratio of close to 50:50 and used 1.8-2 U/kg of insulin/day. Adverse events in each group were similar.

Conclusions: Both titration methods used for adjustments of mealtime GLU based on preprandial glucose patterns are safe and effective. GLU adjustment based on a simple algorithm method provides a new simplified alternative to the carb counting method. 


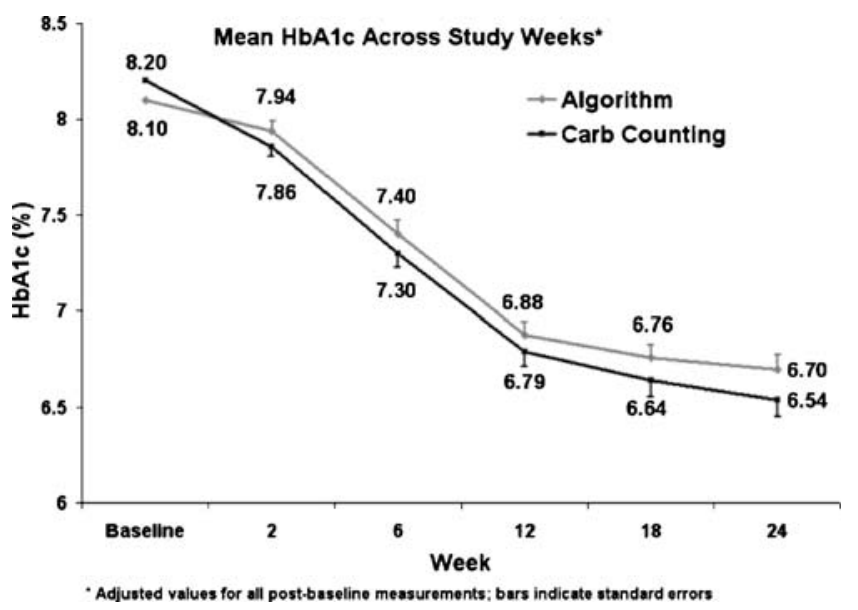

Supported by sanofi-aventis US

\section{PS}

\section{Inhaled insulin}

1003

Body weight changes associated with insulin therapy: A retrospective pooled analysis of inhaled human insulin $\left(\right.$ Exubera $^{\circledR}$ ) versus subcutaneous insulin in $\mathbf{5}$ controlled phase 3 trials

P. A. Hollander ${ }^{1}$, A. Krasner ${ }^{2}$, S. Klioze ${ }^{2}$, P. Schwartz ${ }^{2}$, W. Duggan ${ }^{2}$;

${ }^{1}$ Endocrinology Center, Baylor University Medical Center, Dallas, ${ }^{2}$ Pfizer Global Research and Development, Pfizer Inc, Groton, United States.
Background and Aims: Weight gain is a frequent effect of insulin therapy and recent evidence suggests that fear of weight gain is associated with reduced adherence to diabetes medication. The aim of this study was to compare the effect of inhaled human insulin (INH, Exubera ${ }^{\circledR}$ (insulin human [rDNA origin]) Inhalation Powder) administered in combination with intermediate or long-acting subcutaneous (SC) insulin versus SC insulin regimens on body weight after 6 months of treatment in adult patients with type 1 (T1DM) or type 2 (T2DM) diabetes mellitus.

Materials and Methods: Retrospective analysis of pooled 6-month data from 5 controlled Phase 3 clinical trials conducted in the Americas.

Results: Less weight gain was observed with INH therapy compared to SC insulin in adult patients with T1DM or T2DM while changes in glycosylated hemoglobin $\left(\mathrm{HbA}_{1 \mathrm{c}}\right)$ were comparable in both treatment groups (Table). Hypoglycemia (T1DM [n=1048]: INH 6.981 vs SC 6.823 events/ subject-month; T2DM [n=912]: INH 1.812 vs SC 2.077 events/subject-month) and severe hypoglycemia (T1DM: INH 5.829 vs SC 5.225 events/100 subject-months; T2DM: INH 0.781 vs SC 1.051 events/100 subject-months) were also comparable.

Conclusion: Less weight gain was observed with INH therapy compared to $\mathrm{SC}$ insulin in adult patients with T1DM or T2DM. It is possible that the pharmacokinetic profile of INH more closely approximates near physiologic prandial insulin replacement resulting in reduced hyperinsulinemia and, thus, less weight gain in the setting of comparable $\mathrm{HbA}_{1 \mathrm{c}}$ and incidence of hypoglycemia. Reduction in the fear of weight gain may be one factor that helps to encourage the adoption of insulin, especially in adult patients with T2DM who are frequently reluctant to accept insulin therapy despite the failure of oral agent therapy. Supported by Pfizer Inc

\begin{tabular}{|c|c|c|c|c|c|c|}
\hline \multirow[t]{2}{*}{ 6-Month Data } & \multicolumn{3}{|l|}{ T1DM } & \multicolumn{3}{|l|}{$\mathrm{T} 2 \mathrm{DM}$} \\
\hline & $\mathrm{INH}$ & $\mathrm{SC}$ & $\begin{array}{l}\text { Treatment group } \\
\text { difference }(95 \% \mathrm{CI})\end{array}$ & INH & $\mathrm{SC}$ & $\begin{array}{l}\text { Treatment group difference } \\
\qquad(95 \% \mathrm{CI})\end{array}$ \\
\hline $\begin{array}{l}\text { Mean } \pm \text { SD (n) body } \\
\text { weight ( } \mathrm{kg} \text { ) at baseline }\end{array}$ & $76.0 \pm 14.0(496)$ & $75.0 \pm 13.4(510)$ & - & $\begin{array}{l}88.5 \pm 14.9 \\
(423)\end{array}$ & $\begin{array}{l}88.5 \pm 15.0 \\
(428)\end{array}$ & - \\
\hline $\begin{array}{l}\text { Mean (SE) adjusted } \\
\text { change from baseline } \\
\text { in body weight }(\mathrm{kg}) \dagger\end{array}$ & $0.2(0.1)$ & $1.1(0.1)$ & $-0.87(-1.24,-0.50)$ & $0.7(0.2)$ & $1.6(0.2)$ & $-0.93(-1.39,-0.48)$ \\
\hline $\begin{array}{l}\text { Mean } \pm \text { SD }(n) H b A_{1 c} \\
(\%) \text { at baseline }\end{array}$ & $7.6 \pm 1.0(500)$ & $7.7 \pm 1.0(509)$ & - & $7.8 \pm 1.1(425)$ & $7.9 \pm 1.1(437)$ & - \\
\hline $\begin{array}{l}\text { Mean (SE) adjusted } \\
\text { change from baseline } \\
\mathrm{HbA}_{1 \mathrm{c}}(\%) \dagger\end{array}$ & $-0.2(0.03)$ & $-0.3(0.03)$ & $0.09(0.00,0.18)$ & $-0.7(0.05)$ & $-0.6(0.04)$ & $-0.07(-0.19,0.05)$ \\
\hline
\end{tabular}

†least squares means based on the primary model with terms for baseline, treatment, and center. 


\section{4}

Sustained efficacy and tolerability of inhaled human insulin $\left(\right.$ Exubera $\left.^{\mathbb{B}}\right)$. Therapy over 2 years: patients with type 2 diabetes

W. Cefalu ${ }^{1}$, J. Rosenstock ${ }^{2}$, S. Klioze ${ }^{3}$, H. Foyt ${ }^{4}$, M. Ogawa ${ }^{4}$, L. St Aubin ${ }^{3}$, W. Duggan ${ }^{3}$;

${ }^{1}$ Division of Nutrition and Chronic Diseases, Pennington Biomedical Research Center, Baton Rouge, ${ }^{2}$ Dallas Diabetes and Endocrine Center, Dallas, ${ }^{3}$ Pfizer Inc, Research and Development, Groton, ${ }^{4}$ Pfizer Inc, Research and Development, Ann Arbor, United States.

Background and Aims: The availability of inhaled human insulin (INH; Exubera ${ }^{\circledR}$ (insulin human [rDNA origin]) Inhalation Powder) may increase patient acceptance of insulin, improve glycemic control, and potentially reduce the burden of insulin therapy in diabetes. In patients with type 2 diabetes mellitus (T2DM), one previous 24-week study showed comparable efficacy between INH and subcutaneous (SC) insulin, and another 104-week study demonstrated superior glycemic control of INH compared with oral agents (OAs). Small treatment group differences in lung function (measured using routine pulmonary function testing $[\mathrm{PFT}]$ ) were observed in both studies, but long-term studies are critical to better characterize pulmonary safety.The aim of this study was to investigate the long-term (2-year) efficacy and safety profile of INH therapy in patients with T2DM.

Materials and Methods: An open-label, parallel-group, multicenter study where patients (aged between 35 and 75 years) were randomized to INH $(n=319)$ or $\mathrm{SC}$ insulin $(n=316)$. Primary end point was pulmonary function measured using highly standardized PFT. Secondary end points included glycosylated hemoglobin $\left(\mathrm{HbA}_{1 \mathrm{c}}\right)$, hypoglycemia, fasting plasma glucose (FPG), and body weight. Results: Treatment group differences in changes from baseline in forced expiratory volume in 1 second $\left(\mathrm{FEV}_{1}\right)$ and carbon monoxide diffusing capacity $\left(\mathrm{DL}_{\mathrm{co}}\right)$ were small $(<1.5 \%$ of mean baseline), occurred early (Month 3 data: $-0.043 \mathrm{~L}[90 \%$ confidence interval $(\mathrm{CI}),-0.065,-0.020]$ for $\mathrm{FEV}_{1}$ and $-0.194 \mathrm{~mL} / \mathrm{min} / \mathrm{mm} \mathrm{Hg}[-0.437,0.050]$ for $\mathrm{DL}_{\mathrm{co}}$ ), and remained stable with no progression for up to 2 years $(-0.023[-0.047,0.002]$ and $0.165[-0.102$, 0.432], for $\mathrm{FEV}_{1}$ and $\mathrm{DL}_{\mathrm{co}}$, respectively). Glycemic control was maintained in both groups with $\mathrm{HbA}_{1 \mathrm{c}}$ improvements from $7.7 \%$ and $7.8 \%$ to $7.3 \%$ and $7.3 \%$ in INH and SC, respectively, and the percentage of patients who achieved target $\mathrm{HbA}_{1 \mathrm{c}}<7 \%$ was comparable between groups (INH, $47.5 \%$ and SC, $45.2 \%$ ). The incidence of hypoglycemic events was lower with INH than with SC insulin ( 0.8 vs 1.0 events/subject-month, respectively), and the incidence of severe hypoglycemic events was comparable in the 2 groups ( 0.4 vs 0.6 events/100 subject-month, respectively). After 24 months, INH resulted in greater reductions in FPG compared with SC insulin (from 8.4 to 7.5 vs 8.2 to $8.2 \mathrm{mmol} / \mathrm{L}$ [151.2 to $135.6 \mathrm{vs} 148.2$ to $147.1 \mathrm{mg} / \mathrm{dL}$ ], respectively), and less body weight gain (mean $\pm \mathrm{SD}$, $1.7 \pm 4.7 \mathrm{~kg}$ vs $3.0 \pm 5.2 \mathrm{~kg}$ ). Adverse events were generally comparable with the exception of cough in the INH group, which was mild in most patients, nonproductive, and closely associated with dosing.

Conclusion: This study demonstrated that INH is well tolerated, provides comparable glycemic control, less weight gain, and greater FPG reductions when compared with SC insulin over 2 years in adult patients with type 2 diabetes.

This study was funded by Pfizer Inc

\section{5}

Single-dose pharmacokinetics of inhaled human insulin (Exubera ${ }^{\circledR}$ ) after acute passive cigarette smoke exposure A. Milton ${ }^{1}$, R. Fountaine ${ }^{2}$, G. Wei ${ }^{2}$, J. G. Teeter ${ }^{2}$, R. Jaeger ${ }^{3}$, T. Checchio ${ }^{2}$, M. Stolar ${ }^{2}$, D. A. Fryburg ${ }^{2}$;

${ }^{1}$ Clinical Pharmacology, Pfizer Research and Development, Groton, ${ }^{2}$ Pfizer Inc, Research and Development, Groton, ${ }^{3}$ Environmental Medicine Incorporated, Environmental Medicine Incorporated, Westwood, United States.

Background and Aims: The bioavailability of inhaled human insulin (INH; Exubera ${ }^{\circledR}$ (insulin human [rDNA origin]) Inhalation Powder) is increased in chronic smokers compared with nonsmokers. As INH could be used in circumstances where patients are passively exposed to cigarette smoke, this study investigated the effect of a single acute passive cigarette smoke exposure (PSE) on INH bioavailability.

Materials and Methods: In this open-label, randomized, crossover study, healthy, nonsmoking subjects $(n=28)$ received a single INH dose $(3 \mathrm{mg})$ with no PSE, or after 2 hours of PSE. To simulate PSE, a commercial smoking machine burning full flavor cigarettes generated environmental nicotine levels typical of social settings (75-125 $\mu \mathrm{g} /$ $\mathrm{m}^{3}$ ). There was a $\geq 2$ week washout between study phases. Blood samples were collected at specified times up to 6 hours after INH administration to assess insulin pharmacokinetics.

Results: The mean plasma insulin area under the curve $\left(\mathrm{AUC}_{0-360}\right)$ decreased from $5703 \mu \mathrm{U} \cdot \mathrm{min} / \mathrm{mL}$ with $\mathrm{INH}$ alone to $4718 \mu \mathrm{U} \cdot \mathrm{min} / \mathrm{mL}$ after PSE exposure; ratio PSE+ INH/INH alone 82.7 (95\% confidence interval [CI], 68.8, 99.5). The mean maximum concentration $\left(\mathrm{C}_{\max }\right)$ also decreased after PSE: $41.0 \mu \mathrm{U} / \mathrm{mL}$ with $\mathrm{INH}$ alone to $28.9 \mu \mathrm{U} / \mathrm{mL}$; ratio $\mathrm{PSE}+\mathrm{INH} / \mathrm{INH}$ alone $70.5(95 \% \mathrm{CI}$, $59.8,83.1)$. The median time to maximum concentration 
$\left(\mathrm{T}_{\max }\right)$ occurred 15 minutes later (75 vs $60 \mathrm{~min}$ ), after PSE relative to no PSE. INH was generally well tolerated by volunteers in this study.

Conclusion: In contrast to the effects of active smoking, investigations using technetium labeled diethylenetriaminepentaacetate (DTPA) and findings from this study suggest that acute PSE causes a decrease in lung permeability. This decrease in INH bioavailability after acute PSE does not create a risk of hypoglycemia.

This study was funded by Pfizer Inc

1006

Absorption of inhaled human insulin (Exubera ${ }^{\circledR}$ ) after 3 and 13 weeks of active smoking cessation

R. Fountaine ${ }^{1}$, A. Milton ${ }^{1}$, G. Wei ${ }^{1}$, S. Willavize ${ }^{1}$,

J. G. Teeter ${ }^{1}$, S. Sha ${ }^{2}$, D. A. Fryburg ${ }^{1}$;

${ }^{1}$ Pfizer Inc, Research and Development, Groton, ${ }^{2}$ Pharmaceutical Research and Development, LLC, Johnson and Johnson, Raritan, United States.

Background and Aims: Previous studies have demonstrated that bioavailability of inhaled human insulin (INH; Exubera $^{\circledR}$ (insulin human [rDNA origin]) Inhalation Powder) is greater in chronic smokers relative to nonsmokers. The effects of smoking cessation for 3 and 13 weeks on INH pharmacokinetics were investigated in this open-label, randomized, crossover study.

Materials and Methods: A total of 68 nondiabetic subjects were included in the study: 38 smokers $(\mathrm{Sm})$ (21 cigarettes/ d) and 30 nonsmokers (NSm). At baseline, all subjects received either INH (2 mg) or subcutaneous (SC) insulin (6U) in a randomized, crossover design. In Sm, the second (INH2) and third (INH3) doses of INH were administered 3 and 13 weeks after smoking cessation, respectively. Plasma carboxyhemoglobin and serum cotinine concentrations were used to measure smoking status.

Results: At baseline (INH1), Sm had significantly higher mean maximum insulin concentration $\left(\mathrm{C}_{\max }\right)$ and area under the curve $\left(\mathrm{AUC}_{0-360}\right)$, and a significantly shorter time to mean maximum concentration ( $\left.\mathrm{T}_{\max }\right)$ compared with NSm (72.3 vs $15.8 \mu \mathrm{U} / \mathrm{mL} ; 4850$ vs $1410 \mu \mathrm{U} \cdot \mathrm{min} / \mathrm{mL}$; and 31 vs $53 \mathrm{~min}$, respectively) ( $P$-value $<0.05$ for all 3 variables). After 3 weeks cessation, $\mathrm{C}_{\max }$ and $\mathrm{AUC}_{0-360}$ were significantly reduced to $35.7 \mu \mathrm{U} / \mathrm{mL}$ and $2850 \mu \mathrm{U} \cdot \mathrm{min} / \mathrm{mL}$ ( $P$-value $<0.05$ vs INH1 for both variables), and $\mathrm{T}_{\max }$ increased to 41 minutes. No further reductions were observed after 13 weeks cessation: $C_{\max } 43.1 \mu \mathrm{U} / \mathrm{mL}$, $\mathrm{AUC}_{0-360} 3260 \mu \mathrm{U} \cdot \mathrm{min} / \mathrm{mL}$ and $\mathrm{T}_{\max } 40$ minutes. Sm had a higher rate of hypoglycemia versus NSm, but most events were mild. There were no serious adverse events or discontinuations due to safety concerns.

Conclusion: INH absorption was reduced by almost $50 \%$ after 3 weeks of smoking cessation. This reduction was maintained after 13 weeks cessation, but the bioavailability of INH still remained greater than that of NSm. These findings concur with previous investigations that absorption of INH and other small molecules is increased in Sm compared with NSm.

This study was funded by Pfizer Inc

1007

Effect of intercurrent respiratory tract infections on inhaled human insulin (Exubera ${ }^{\circledR}$ ) therapy: a retrospective pooled analysis of controlled phase 2 and 3 trials P. Camus;

Department of Pulmonary and Intensive Care, Centre Hospitalier et Universite de Bourgogne, Dijon, France.

Background and Aims: The availability of inhaled human insulin (INH; Exubera ${ }^{\mathbb{B}}$ (insulin human [rDNA origin]) Inhalation Powder) may increase patient acceptance of insulin, improve glycemic control and reduce the burden of diabetes. INH has been administered to over 1900 adult type 1 (T1DM) and type 2 (T2DM) diabetes patients in controlled clinical trials. Initial premeal INH doses were recommended based on patient body weight. Doses were subsequently adjusted using premeal fingerstick glucose values. The aim of this study was to assess the incidence and potential clinical implications of intercurrent respiratory tract infections (iRTIs*) on INH therapy.

Materials and Methods: Retrospective analysis of pooled data from 14 controlled Phase 2 and 3 clinical trials ( 3 to 24 months duration).

Results: Intercurrent RTIs were common in all treatment groups with $\leq 1.5 \%$ of events leading to permanent discontinuation (Table). There were no apparent changes in glycosylated hemoglobin levels (\%) or overall hypoglycemic rates (events/subject-month) with iRTIs in any treatment group. In T1DM patients, the mean fasting plasma glucose $(\mathrm{mg} / \mathrm{dL})$ and rates of severe hypoglycemia (events/100 subject-month) increased during iRTIs in both INH (161.5 vs 170.6 [9.0 vs $9.5 \mathrm{mmol} / \mathrm{L}] ; 3.77$ vs 7.72 , respectively) and $\mathrm{SC}$ groups (171.6 vs 175.8 [9.5 vs $9.8 \mathrm{mmol} / \mathrm{L}] ; 4.43$ vs 8.58 , respectively); no changes were seen in T2DM patients. Conclusion: In summary, INH is well tolerated, safe, and efficacious in adult diabetes patients during iRTIs. During iRTIs, close monitoring of blood glucose concentrations and dose adjustment may be required on an individual basis. 
Table

\begin{tabular}{lllllll}
\hline & T1DM & \multicolumn{5}{l}{ T2DM } \\
\cline { 2 - 7 } & INH & SC & INH & SC & OAs \\
\hline Evaluable subjects (n) & 698 & 705 & 1277 & 488 & 644 \\
Subjects with iRTIs* (\%) & 62.2 & 57.2 & 44.7 & 51.8 & 30.1 \\
iRTIs (n) & 1010 & 889 & 1123 & 501 & 328 \\
iRTI leading to temporary & $19 ; 1.9$ & $0 ; 0$ & $22 ; 2.0$ & $0 ; 0$ & $0 ; 0$ \\
study drug discontinuation (n; \%) & & & & & \\
iRTI leading to permanent & $6 ; 0.6$ & $0 ; 0$ & $17 ; 1.5$ & $0 ; 0$ & $0 ; 0$ \\
discontinuation (n; \%) & & & & & \\
\hline
\end{tabular}

$\mathrm{INH}=$ inhaled human insulin; $\mathrm{SC}=$ subcutaneous insulin; $\mathrm{OA}=$ oral agents $* \mathrm{iRTIs}=\mathrm{COSTART}$ preferred terms of all causality: bronchitis, laryngitis, pharyngitis, pneumonia, rhinitis, asthma, RTI, and/or sinusitis.

This study was funded by Pfizer Inc

\section{8}

Can the human insulin inhalation powder (HIIP) delivery system be used during an upper respiratory infection (URI)? The effect of URI on the pharmacokinetics (PK) and glucodynamics (GD) of HIIP in healthy subjects

M. Nakano ${ }^{1}$, J. Gern ${ }^{2}$, C. Stone ${ }^{2}$, F. Tibaldi ${ }^{3}$,

A. de la Peña ${ }^{1}$, A. Suri ${ }^{1}$, D. Soon ${ }^{4}$, D. Muchmore ${ }^{1}$;

${ }^{1}$ Eli Lilly and Company, Indianapolis, United States, ${ }^{2}$ Clinical Science Center, University of Wisconsin Medical School, Madison, United States, ${ }^{3}$ Eli Lilly and Company, Mont-Saint-Guibert, Belgium, ${ }^{4}$ Eli Lilly and Company, Singapore.

Background and Aims: Inhaled insulin is being tested as an alternative to subcutaneous (sc) injectable insulin. One concern is the potential for URIs to affect the delivery of dry powder insulin, potentially causing changes in both its bioavailability and variability. This may result in inconsistent blood glucose control, causing either hyper- or hypoglycaemia. Our study assessed the effect of symptomatic rhinovirus 16 (RV16) infections on the PK, GD, and safety and tolerability of HIIP delivered to healthy nondiabetic patients using the Lilly/Alkermes HIIP delivery system.

Materials and Methods: Twenty-one healthy, non-smoking male and female subjects (age 20.3 \pm 2.1 years; BMI $22.8 \pm 1.7 \mathrm{~kg} / \mathrm{m}^{2}$ ) participated in a single-centre, singlesequence, 2-period, euglycaemic glucose clamp study. Subjects received 1 dose of HIIP equivalent to $12 \mathrm{U}$ of sc insulin $(5.2 \mathrm{mg})$, and underwent a clamp procedure. On a different day, subjects received a nasal inoculation with the RV16 virus. Two to 4 days later, symptomatic subjects received a second dose of HIIP (equivalent to 12U) and underwent a second clamp. Blood samples were collected to determine serum insulin immunoreactive concentrations for PK analysis. Glucose infusion rates and times were recorded for GD analysis.

Results: Sixteen subjects completed both doses/clamps. Pharmacokinetic and GD parameters, before or during infection, were comparable as shown in the table. The variability of PK and GD parameters did not increase during URI as indicated by the $\mathrm{CV} \%$. There were no serious adverse events reported, and no subjects had difficulty using the system. No significant change in mean FEV1 and FVC was observed following HIIP administration or during infection.

\begin{tabular}{|c|c|c|c|}
\hline \multirow[t]{2}{*}{ Parameter (Unit) } & \multicolumn{2}{|c|}{$\begin{array}{l}\text { RV16 Infection } \\
\text { Geometric LS Mean (CV\%) }\end{array}$} & \multirow[t]{2}{*}{$\begin{array}{l}\text { Ratio (\%) } \\
{[90 \% \mathrm{CI}]}\end{array}$} \\
\hline & Before $(n=21)$ & During $(n=16)$ & \\
\hline $\mathrm{AUC}_{\left(0-\mathrm{t}^{\prime}\right)}(\mathrm{pmol} \cdot \mathrm{min} / \mathrm{L})$ & $46300(51)$ & $52600(32)$ & $114[96,135]$ \\
\hline $\mathrm{C}_{\max }(\mathrm{pmol} / \mathrm{L})$ & $248(45)$ & $260(36)$ & $105[89,124]$ \\
\hline $\mathrm{G}_{\mathrm{tot}}(\mathrm{mg})$ & $61800(104)^{*}$ & $68700(80)$ & $111[89,138]$ \\
\hline $\mathrm{R}_{\max }(\mathrm{mg} / \mathrm{min})$ & $327(84)^{*}$ & $325(50)$ & $99[77,128]$ \\
\hline
\end{tabular}

$*_{\mathrm{n}}=19$

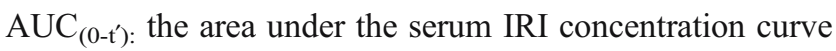
from time of insulin administration to time insulin level returns to baseline; $\mathrm{C}_{\max }$ : maximal serum IRI concentration; $\mathrm{CV}$ : coefficient of variation; $\mathrm{G}_{\text {tot }(0-600)}$ amount of glucose infused from time of insulin administration to $600 \mathrm{~min}$; LS mean: least squares mean; $n$ : number of subjects; $\mathrm{R}_{\max }$ : maximal glucose infusion rate; CI, confidence interval.

Conclusion: These findings suggest that the presence of URI does not significantly alter the PK and GD of insulin delivered by the Lilly/Alkermes HIIP delivery system. Further, HIIP was safe and well-tolerated in subjects during URI. Patients should be able to continue to use this delivery system during a moderate URI.

This study was funded by Eli Lilly and Company

1009

Dose interchangeability between human insulin inhalation powder (HIIP) 2U- and 6U-equivalent Capsules

A. de la Peña ${ }^{1}$, S. Irie ${ }^{2}$, M. Nakano ${ }^{1}$, L. Zhang ${ }^{1}$,

E. Yuen ${ }^{3}$, K. Poo Yeo ${ }^{3}$, D. Muchmore ${ }^{1}$;

${ }^{1}$ Eli Lilly and Company, Indianapolis, United States, ${ }^{2}$ Kyushu Clinical Pharmacology Research Clinic, Fukuoka, Japan, ${ }^{3}$ Eli Lilly and Company, Singapore. 
Background and Aims: The Lilly/Alkermes inhaled insulin delivery system is designed to be patient- and provider-friendly, and to include the ability to freely exchange dosage strengths as needed.

Material and Methods: A phase I, open-label, randomised, dose-escalation, 5 period complete block design, euglycaemic glucose clamp study was conducted in healthy, nonsmoking, male, Japanese subjects $(\mathrm{n}=16)$ at a single center. Among other objectives, the relationship between the administration of three $2 \mathrm{U}$-equivalent $(0.9 \mathrm{mg}$ each $)$ and one $6 \mathrm{U}$-equivalent $(2.6 \mathrm{mg})$ HIIP capsules delivered by Lilly/Alkermes HIIP delivery system, was evaluated based on insulin pharmacokinetic (PK) and glucodynamic (GD) parameters. Each subject was randomly assigned to 1 of 4 possible dosing sequences, and received four single doses of HIIP: one $6 \mathrm{U}$ capsule; two $6 \mathrm{U}$ capsules; three $6 \mathrm{U}$ capsules; three $2 \mathrm{U}$ capsules and one dose of subcutaneous insulin lispro (12 U, $0.4 \mathrm{mg}$ ). Serum immunoreactive insulin (IRI) concentrations were measured for PK evaluation using a validated radioimmunoassay. Glucodynamics were assessed based on glucose infusion rate data collected during the glucose clamp procedure for up to 10 hours.

Results: PK and GD parameters after administration of three $2 \mathrm{U}$ capsules and one $6 \mathrm{U}$ capsule and the ratios using the $6 \mathrm{U}$ capsule as reference, are listed in the table below. AUC represents the total exposure of HIIP, and $\mathrm{C}_{\max }$ is the highest exposure. $G_{\text {tot }}$ represents the total effect of HIIP, and $R_{\max }$ is the highest effect.

\begin{tabular}{llll}
\hline Parameter (unit) & Treatment & $\begin{array}{l}\text { Geometric } \\
\text { Mean }\end{array}$ & $\begin{array}{l}\text { Ratio }(3 \times 2 \mathrm{U} / 1 \times 6 \mathrm{U}) \\
\text { and }[90 \% \mathrm{CI}]\end{array}$ \\
\hline $\mathrm{AUC}_{\left(0-\mathrm{t}^{\prime}\right)}(\mathrm{pmol} \cdot \mathrm{min} / \mathrm{L})$ & $3 \times 2 \mathrm{U}$ & 23355 & $0.93[0.75,1.14]$ \\
& $1 \times 6 \mathrm{U}$ & 25142 & \\
$\mathrm{C}_{\max }(\mathrm{pmol} / \mathrm{L})$ & $3 \times 2 \mathrm{U}$ & 120 & $0.95[0.82,1.11]$ \\
& $1 \times 6 \mathrm{U}$ & 126 & \\
$\mathrm{G}_{\text {tot }(0-600)}(\mathrm{g})$ & $3 \times 2 \mathrm{U}$ & 29.6 & $1.01[0.69,1.49]$ \\
& $1 \times 6 \mathrm{U}$ & 29.3 & \\
$\mathrm{R}_{\max }(\mathrm{mg} / \mathrm{min})$ & $3 \times 2 \mathrm{U}$ & 132 & $0.96[0.79,1.17]$ \\
& $1 \times 6 \mathrm{U}$ & 137 & \\
\hline
\end{tabular}

*AUC $\left(0-t^{\prime}\right):$ the area under the serum IRI concentration curve from time of insulin administration to time of insulin level returns to baseline; $\mathrm{C}_{\max }$ : maximal serum IRI concentration; $G_{\text {tot }(0-600)}$ amount of glucose infused from time of insulin administration to $600 \mathrm{~min}$; $\mathrm{R}_{\max }$ : maximal glucose infusion rate; $\mathrm{CI}$, confidence interval.

The ratio of geometric mean between the two dose strengths for $A U C\left(0-t^{\prime}\right) C_{\max }, R_{\max }$ and $G_{\text {tot }}$ were close to 1 and the $90 \%$ confidence intervals (CI) included 1 . Bioequivalence based on the $(0.80-1.25)$ reference range for the $90 \%$ CI could only be statistically concluded for $\mathrm{C}_{\max }$.
Conclusion: Given the typical variability in PK and GD after insulin administration, and the fact that the study was not specifically powered to detect the bioequivalence between these two dose strengths, patients should be able to use three $2 \mathrm{U}$-equivalent capsules and one $6 \mathrm{U}$-equivalent capsule interchangeably in the clinical setting.

This study was funded by Eli Lilly and Company

1010

The availability of inhaled human insulin may lead to a greater acceptance of insulin therapy in European type 2 diabetes patients failing on diet and/or oral agent therapy

N. Freemantle ${ }^{1}$, L. Blonde ${ }^{2}$, F. D. R. Hobbs ${ }^{1}$, M. Kvasz ${ }^{3}$, N. Marchant ${ }^{3}$, S. A. Ross ${ }^{4}$;

${ }^{1}$ Department of Primary Care and General Practice, University of Birmingham, Edgbaston, United Kingdom, ${ }^{2}$ Ochsner Diabetes Clinical Research Unit, Ochsner Clinic Foundation, New Orleans, United States, ${ }^{3}$ Outcomes Research, Pfizer Inc, New York, United States, ${ }^{4}$ Departments of Medicine and Community Health Sciences, University of Calgary, Canada.

Background and Aims: Despite the proven benefits of intensive glycemic control, over $50 \%$ of patients with type 2 diabetes mellitus (T2DM) suboptimally controlled on oral agents (OAs) delay initiating subcutaneous (SC) insulin therapy for at least 4 to 6 years. Furthermore, a Canadian study found that insulin-naïve T2DM patients appear willing to sacrifice adequate glycemic control to reduce the number of daily insulin injections - a disconcerting phenomenon given the rapidly increasing prevalence of T2DM worldwide and increasing burden of diabetes-related complications on healthcare resources. We recently reported that the theoretical availability of inhaled human insulin $\left(\mathrm{INH}\right.$, Exubera $^{\circledR}$ (insulin human [rDNA origin]) Inhalation Powder) significantly increased the proportion of T2DM patients who accept insulin therapy. The aim of this substudy was to examine the potential impact of INH availability on the T2DM patient acceptance of insulin therapy in the European setting, where insulin is predominantly administered with a pen.

Materials and Methods: T2DM patients failing on diet and/or OAs $\left(\mathrm{HbA}_{1 \mathrm{c}} \geq 8 \%\right)$ were randomized into 2 groups. Both groups ( $\mathrm{A}$ and $\mathrm{B}$ ) received educational information on currently licensed treatment options (including OAs and SC insulin); Group B received the same information with the exception that INH was offered as an additional treatment option. Subjects were then asked to make a theoretical choice of therapy, including no change in their current treatment. Patients consulted with their physician/diabetes 
nurse on treatment options and completed questionnaires concerning their choice. The primary outcome was the proportion of T2DM patients choosing insulin.

Results: In total, 576 T2DM patients $(\mathrm{A}=288, \mathrm{~B}=288)$ were recruited in 5 European countries (France, Germany, Italy, Spain, and Sweden). In Group B, 127 patients (44\%) chose a treatment option that included insulin compared with 48 (17\%) in Group A (odds ratio 3.9, 95\% CI [2.7, 5.8]; $P<0.0001)$. In Group B, INH was chosen by 101 patients (35\%) whereas $26(9 \%)$ patients chose SC insulin. Despite a mean $\mathrm{HbA}_{1 \mathrm{c}}$ of $9.1 \%$ and the availability of insulin pens, significantly more patients in Group A (132 [46\%]) chose to make no change to their therapy compared to patients in Group B patients (86 [30\%]; odds ratio $0.50,95 \%$ CI [0.36, $0.71], P<0.0001)$. In a subgroup of T2DM patients uncontrolled on 2 OAs - those most likely to require insulin to achieve glycemic control -83 patients $(50 \%)$ in Group B chose a treatment option that included insulin compared with $32(21 \%)$ of those in Group A. No systematic differences between countries were found.

Conclusion: In European T2DM patients failing to achieve target glycemic control with diet and/or OA therapy, the availability of INH as a potential treatment option encourages more patients to accept insulin therapy than SC insulin, even where insulin pens are predominantly used. The availability of INH could result in increased insulin acceptance by European T2DM patients, which would lead to improved glycemic control and better short- and longterm health outcomes.

Supported by Pfizer Inc

\section{PS 86}

\section{Pharmacodynamics of new insulin}

\section{1}

Expected additive effects of combining insulin detemir and human insulin on glucose metabolism in rats

J. Sturis ${ }^{1}$, C. L. Brand ${ }^{1}$, A. R. Sørensen ${ }^{1}$, A. Vølund ${ }^{2}$, U. Ribel ${ }^{1}$;

${ }^{1}$ Insulin Pharmacology, Novo Nordisk A/S, Måløv, Denmark,

${ }^{2}$ Biostatistics, Novo Nordisk A/S, Bagsværd, Denmark.

Background and Aims: Insulin detemir (IDet) is a longacting acylated desB30 human insulin designed to cover basal insulin requirements in diabetic patients. Co-administration of short acting insulin at mealtime is frequently used to obtain adequate glycemic control.

Materials and Methods: To study the clinical relevant pharmacodynamic interactions between human insulin (HI) and IDet on glucose disposal in vivo, the two insulins were tested separately and in combinations during steady state hyperinsulinemic-euglycemic clamp conditions in Sprague Dawley (SD) rats instrumented with permanet carotic and jugular chateters. HI and/or IDet was infused intravenously at a constant rate for 5 hours into conscious, unrestricted rats and the mean glucose infusion rates (GIR) during the last hour of the clamp was used as measure of efficacy. Three pair-wise equi-efficacious doses $(1 \mathrm{D}, 2 \mathrm{D}, 4 \mathrm{D})$ of $\mathrm{HI}(1.5,3,6 \mathrm{mIU} / \mathrm{kg} / \mathrm{min})$ and IDet $(2.5,5,10 \mathrm{mU} / \mathrm{kg} / \mathrm{min})$ and two HI+IDet combination doses $(1 \mathrm{D}+1 \mathrm{D}$ and $2 \mathrm{D}+2 \mathrm{D})$ were tested. Furthermore, lipogenesis via ${ }^{3} \mathrm{H}$-glucose was measured in primary rat adipocytes incubated for 2 hours at 37 Celsius with mixed concentrations of HI and IDet.

Results: Clamp data are presented in the table.

Steady state GIR ( $\mathrm{mg} / \mathrm{kg} / \mathrm{min})$.

\begin{tabular}{lllll}
\hline Dose & HI & ID & $\begin{array}{l}\text { HI+IDet } \\
(1 \mathrm{D}+1 \mathrm{D})\end{array}$ & $\begin{array}{l}\text { HI+IDet } \\
(2 \mathrm{D}+2 \mathrm{D})\end{array}$ \\
\hline $1 \mathrm{D}$ & $9.6 \pm 1.9$ & $5.7 \pm 1.0$ & - & - \\
2D & $17.5 \pm 1.6$ & $15.9 \pm 2.3$ & $16.6 \pm 1.9$ & - \\
$4 \mathrm{D}$ & $29.4 \pm 2.3$ & $25.7 \pm 1.6$ & - & $27.0 \pm 2.0$ \\
\hline
\end{tabular}

The lipogenic response in vitro showed full agonism of IDet compared to HI, and the interaction of the two ligands was primarily additive with a trend towards synergy.

Conclusion: The effects of combining IDet and HI are additive on glucose disposal in vivo and and on lipogenesis in vitro as expected for drugs acting via the same receptor.

1012

\section{WITHDRAWN}

1013

Equivalent metabolic effects of insulin detemir and human insulin on target tissues during hyperinsulinaemic -euglycaemic clamp conditions in Zucker obese rats

C. L. Brand, J. Sturis, U. Ribel;

Novo Nordisk A/S, Måløv, Denmark.

Background and Aims: Insulin detemir (IDet) is a longacting acylated desB30 human insulin designed to cover basal insulin requirements in diabetic patients. Theoretically, the effects of IDet on glucose metabolism in various insulin sensitive tissues may differ from that of human insulin (HI).

Materials and Methods: To investigate possible differential effects, we undertook series of 5 hour intravenous hyperinsulinemic-euglycemic clamp studies in conscious 
Zucker obese rats instrumented with permanent carotic and jugular catheters. Whole body glucose disposal assessed by the glucose infusion rates (GIR) to maintain euglycemia and ${ }^{3} \mathrm{H}$-glucose-determined rates of endogenous glucose production (EGP) and glucose utilization (GU) were compared at lower, medium and higher doses (LD, MD, HD) of IDet $(10,20,40 \mathrm{mU} / \mathrm{kg} / \mathrm{min})$ and $\mathrm{HI}(5,10$, $20 \mathrm{mIU} / \mathrm{kg} / \mathrm{min}$ ) (table 1). In a separate clamp study (table 2), ${ }^{14} \mathrm{C}$-2-deoxyglucose (2DG) uptake was measured in white/red gastrocnemius (WGM/RGM) and soleus (SM) muscle and in subcutaneous/epididymal fat (SCF/EPF) tissue at $\mathrm{HI}$ and IDet doses resulting in matched rates of GU (10 $\mathrm{mIU} / \mathrm{kg} / \mathrm{min}$ and $25 \mathrm{mU} / \mathrm{kg} / \mathrm{min}$, respectively). Data given as mean \pm SE for $\mathrm{n}=5$-6/group.

Results:

Table 1. Glucose turnover $(\mathrm{mg} / \mathrm{kg} / \mathrm{min})$.

\begin{tabular}{lllllll}
\hline & $\mathrm{IDet}_{\mathrm{LD}}$ & $\mathrm{IDet}_{\mathrm{MD}}$ & $\mathrm{IDet}_{\mathrm{HD}}$ & $\mathrm{HI}_{\mathrm{LD}}$ & $\mathrm{HI}_{\mathrm{MD}}$ & $\mathrm{HI}_{\mathrm{HD}}$ \\
GIR & $3.9 \pm 1.2$ & $5.7 \pm 0.7$ & $15.1 \pm 0.8$ & $3.7 \pm 0.5$ & $8.3 \pm 1.3$ & $13.5 \pm 1.4$ \\
Delta- & $-1.7 \pm 1.0$ & $0.0 \pm 0.6$ & $-5.0 \pm 1.6$ & $-1.3 \pm 0.9$ & $-3.8 \pm 0.5$ & $-4.0 \pm 0.9$ \\
EGP & & & & & & \\
$\begin{array}{l}\text { Delta- } \\
\text { GU }\end{array}$ & $2.2 \pm 2.0$ & $5.4 \pm 1.2$ & $9.7 \pm 1.0$ & $2.4 \pm 1.5$ & $4.7 \pm 1.4$ & $9.5 \pm 1.0$ \\
\hline
\end{tabular}

Table 2. GU and tissue specific 2DG uptake ( $\mathrm{mg} / \mathrm{kg} / \mathrm{min})$.

\begin{tabular}{lllllll}
\hline & Clamp GU & WGM & RGM & SM & SCF & EPF \\
IDet & $18.8 \pm 1.0$ & $6.5 \pm 1.7$ & $23.5 \pm 5.8$ & $58.2 \pm 14.1$ & $4.6 \pm 0.4$ & $3.7 \pm 0.5$ \\
HI & $18.8 \pm 0.8$ & $11.7 \pm 3.1$ & $31.9 \pm 3.5$ & $68.0 \pm 8.0$ & $4.4 \pm 0.3$ & $3.4 \pm 0.3$ \\
\hline
\end{tabular}

Conclusion: IDet has similar effects compared to $\mathrm{HI}$ on suppression of EGP and stimulation of GU. Furthermore, at matched rates of whole body GU, IDet stimulates 2DG uptake in various muscle and fat tissues to the same extent as HI. These findings indicate that IDet and $\mathrm{HI}$ have equivalent effects on glucose metabolism in vivo.

\section{4}

Intra-dermal insulin lispro application with a new microneedle delivery system led to a substantially more rapid insulin absorption than subcutaneous injection L. Heinemann ${ }^{1}$, M. Hompesch ${ }^{2}$, C. Kapitza ${ }^{1}$, N. G. Harvey ${ }^{3}$, B. H. Ginsberg ${ }^{3}$, R. J. Pettis ${ }^{3}$;

${ }^{1}$ Profil Inst. für Stoffwechselforschung, Neuss, Germany, ${ }^{2}$ Profil Institute for Clinical Research, San Diego, United States, ${ }^{3}$ Becton Dickinson, Research Triangle Park, United States.

Background and Aims: We investigated the pharmacokinetic and pharmacodynamic effects of insulin Lispro applied intra-dermally with a new microneedle delivery system. Effective and strictly intra-dermally administration was achieved by the special design and shortness of a needle as part of the system.

Materials and Methods: Ten healthy male volunteers (age $31 \pm 6$ years, BMI $23.7 \pm 1.7 \mathrm{~kg} / \mathrm{m}^{2}$ (mean $\left.\pm \mathrm{SD}\right)$ ) received $10 \mathrm{U}$ insulin Lispro s.c. on one study day and the same dose intra-dermally via 3 different microneedles lengths: 1.25 , 1.5 and $1.75 \mathrm{~mm}$ under euglycemic glucose clamp conditions (target level $5.0 \mathrm{mmol} / \mathrm{L}$, continuous i.v insulin infusion of $0.15 \mathrm{mU} / \mathrm{kg} / \mathrm{min}$, clamp duration $360 \mathrm{~min}$ postdosing).

Results: Intra-dermally applied LIS was absorbed more rapidly compared to sc application as indicated by lower values for $\mathrm{t}_{\mathrm{INS}-\max }(1.25 \mathrm{~mm}: 40,1.5 \mathrm{~mm}: 36,1.75 \mathrm{~mm}$ : 47 min vs. sc 56 min; $\mathrm{p}<0.05)$ and $\mathrm{AUC}_{\mathrm{INS}-0-1} \mathrm{~h}(3752$, 3841,3817 vs. $\left.2546 \mathrm{~h}^{*} \mu \mathrm{U} / \mathrm{mL} ; \mathrm{p}<0.05\right)$. The PD measures confirm the more rapid onset of action with intra-dermal injection ( $\mathrm{t}_{\mathrm{GIR}-\max } 28,29,21$ vs. $\left.41 \mathrm{~min} ; \mathrm{p}<0.05\right)$, but with a comparable maximal metabolic effect $\left(\mathrm{GIR}_{\max }\right.$ 9.4, 8.9, 8.6 vs. $9.6 \mathrm{mg} / \mathrm{kg} / \mathrm{min}$; NS). In contrast to other alternative routes of insulin delivery, the relative bioavailability (147, $155,150 \%)$ and the relative bioeffect $(142,137,124 \%)$ of intra-dermally applied insulin was higher than with sc. There were no local skin reactions at any of the injection sites.

Conclusion: Intra-dermal injection of insulin Lispro by means of the microneedle system studied is a safe and effective method of delivering insulin rapidly and completely. Because of the rapid onset of the metabolic effect this application form appears to offer a great potential for prandial insulin administration.

Supported by Becton Dickinson

1015

Viaject $^{\mathrm{TM}}$ insulin has faster onset of action and less intra-individual variability than regular human insulin in patients with type 1 diabetes

M. Hompesch ${ }^{1}$, C. Bowden ${ }^{1}$, L. McManus ${ }^{1}$, R. Pohl ${ }^{2}$, P. Simms ${ }^{2}$, A. Pfuetzner ${ }^{3}$, L. Heinemann ${ }^{4}$, S. S. Steiner ${ }^{2}$; ${ }^{1}$ Profil Institute for Clinical Research, Inc., Chula Vista, United States, ${ }^{2}$ Biodel Inc., Danbury, United States, ${ }^{3}$ IKFE $\mathrm{GmbH}$, Mainz, Germany, ${ }^{4}$ Profil Institut fuer Stoffwechselforschung, Neuss, Germany.

Background and Aims: Viaject ${ }^{\mathrm{TM}}$ Insulin (VJ) is a formulation of recombinant human insulin and Generally Regarded as Safe excipients, with significantly more rapid absorption kinetics than regular human insulin (RI). We investigated the pharmacodynamic (PD) and pharmacokinetic (PK) properties of VJ and RI by means of the glucose clamp technique. The aim was to evaluate the intra-subject 
variability of the PD and PK properties of $\mathrm{VJ}$ and RI in patients with Type 1 diabetes.

Materials and Methods: Fourteen patients with Type 1 diabetes participated in six, 10-hour euglycaemic glucose clamps (blood glucose target $90 \mathrm{mg} / \mathrm{dl}$ ). During a 2 hour run in before dosing, the target level was achieved by means of a basal insulin infusion. The basal insulin infusion was shut off before dosing. Patients were 4 females and 10 males, age $35 \pm 11$ years (mean $\pm \mathrm{SD}$ ), and were randomly assigned to a sequence of 2 experimental blocks. Each block consisted of 3 doses $0.1 \mathrm{IU} / \mathrm{kg}$ of $\mathrm{VJ}$ or RI, respectively, administered by abdominal s.c. injection in this double-blind, cross-over study. The glucose clamps were continued for 8 hours after dosing or terminated when blood glucose exceeded $270 \mathrm{mg} / \mathrm{dl}$. The time to maximal plasma insulin concentration (Tmax) and the time to maximal glucose infusion rate (GIR Tmax) were used as outcome measures. In addition the within-patient standard deviation was calculated for these parameters and compared with a paired two-tailed t-test.

Results: Pharmacodynamic and pharmacokinetic data of VJ and RI indicated that VJ has an earlier onset of action and shorter time to maximal plasma insulin concentration when compared to RI. The GIR Tmax was $94.4 \pm 44.9$ min for VJ and 144.2 $\pm 66.6 \mathrm{~min}$ for RI ( $\mathrm{p}=0.01)$. Plasma insulin Tmax was $31.1 \pm 14.3 \mathrm{~min}$ for $\mathrm{VJ}$ and $112.3 \pm 48.9 \mathrm{~min}$ for $\mathrm{RI}$, $(p=0.00001)$. Additionally, the within subject variability of Tmax $(p=0.04)$ and the within subject variability of GIR Tmax $(\mathrm{p}=0.015)$ was significantly less for VJ than for RI as measured by standard deviation. No clinically significant adverse events occurred.

Conclusion: Viaject ${ }^{\mathrm{TM}}$ is a novel formulation of recombinant human insulin that replicated its initial finding of significantly more rapid onset and faster absorption kinetics in patients with Type I diabetes. The results also show that VJ has significantly less intra-individual variability than RI. Supported by Biodel Inc.

\section{6}

\section{Insulin glulisine: dose response relationship in subjects with type 1 diabetes \\ L. Nosek ${ }^{1}$, R. H. A. Becker ${ }^{2}$, A. D. Frick ${ }^{2}$, L. Heinemann ${ }^{1}$, K. Rave ${ }^{1}$; \\ ${ }^{1}$ Profil Institut für Stoffwechselforschung, Neuss, Germany, ${ }^{2}$ sanofi-aventis, Frankfurt/Main, Germany.}

Background and Aims: There is sparse information on the dose response relationship of insulin analogues in patients with diabetes. This randomized, complete crossover study in Type 1 diabetes (T1DM) subjects $(n=18)$ assessed pharmacokinetic and pharmacodynamic systemic insulin exposure and the metabolic effect of $0.075,0.15$ and $0.3 \mathrm{U}$. $\mathrm{kg}^{-1}$ of subcutaneously injected insulin glulisine (GLU) or regular human insulin (RHI) during a BIOSTATOR-based euglycaemic glucose clamp.

Materials and Methods: Bioequivalence (BE) criteria, with $0.15{\mathrm{U} . \mathrm{kg}^{-1}}^{-1}$ as reference dose, were applied for doseproportionality testing in insulin exposure and glucose utilization.

Results: Insulin overall exposure (INS-AUC total) and metabolic effect (GIR-AUC total $_{\text {) }}$ were comparable for each dose of GLU and RHI. However, GLU was twice as rapidly absorbed (INS- $\left.\mathrm{T}_{\max } ; \mathrm{p}<0.05\right)$ and had a higher metabolic effect during the 2 hours post-injection $\left(\mathrm{GIR}-\mathrm{AUC}_{2 \mathrm{~h}}\right.$; $\mathrm{p}<0.05)$ versus RHI. GLU and RHI showed a doseproportional increase in overall insulin exposure (INS$\left.A U C_{\text {total }}\right)$ and maximum insulin $\left(\mathrm{C}_{\max }\right)$ for each dose. The overall metabolic effect (GIR-AUC $\mathrm{Ctal}_{\text {tol }}$ ) was proportional for 0.075 and $0.15{\mathrm{U} . \mathrm{kg}^{-1}}^{-1}$ of GLU. Dose-separation was observed for any subject with GLU and RHI, with regards to INS-AUC total $_{1}, \mathrm{C}_{\max }$ and essentially in GIR-AUC total $_{\text {; }}$; however, none was observed for the metabolic effect during the 2 hours post-injection (GIR-AUC ${ }_{2}$ h). Absorption (INS$\mathrm{T}_{90 \%}$ ) and activity (GIR-T $\mathrm{T}_{90 \%}$ ) completion were $\sim 120$ minutes less for GLU than RHI; doubling the dose added $\sim 60$ minutes to the action of both insulins.

Conclusions: In T1DM subjects, GLU displays doseproportionality in insulin exposure from $0.075-0.3 \mathrm{U}$. $\mathrm{kg}^{-1}$, which is associated with dose-proportional metabolic

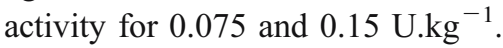

\begin{tabular}{|c|c|c|c|c|c|c|}
\hline & GLU & & & RHI & & \\
\hline Dose $\left(\mathrm{U}^{\mathrm{kgg}}{ }^{-1}\right)$ & 0.075 & 0.15 & 0.3 & 0.075 & 0.15 & 0.3 \\
\hline $\mathrm{C}_{\max }\left(\mu \mathrm{U} \cdot \mathrm{mL}^{-1}\right)$ & $42 *$ & 72 & $140 * *$ & $22 * *$ & 38 & $69^{*}$ \\
\hline $\mathrm{INS} \mathrm{T}_{\max }(\min )$ & 47 & 57 & 72 & 82 & 104 & 119 \\
\hline $\begin{array}{l}\text { INS-AUC }_{2} \text { h } \\
\left(\mu \mathrm{U} \cdot \min \cdot \mathrm{mL}^{-1}\right)\end{array}$ & $3792 * *$ & 6676 & $12992 * *$ & 2211 & 3448 & $5792 *$ \\
\hline $\begin{array}{l}\text { INS-AUC }_{\text {total }} \\
\left(\mu \mathrm{U} \cdot \min \cdot \mathrm{mL}^{-1}\right)\end{array}$ & $5341 * *$ & 11196 & $24891 * *$ & $5969 * *$ & 10608 & $21226^{* *}$ \\
\hline $\mathrm{INS} \mathrm{T}_{90 \%}(\mathrm{~min})$ & 149 & 205 & 242 & 313 & 348 & 410 \\
\hline $\begin{array}{l}\mathrm{GIR}_{\max }(\mathrm{mg} \\
\left.\mathrm{kg}^{-1} \min ^{-1}\right)\end{array}$ & 4.1 & 6.4 & 6.7 & $2.5^{*}$ & 4.6 & 5.4 \\
\hline GIR-T $_{\max }(\min )$ & 87 & 114 & 121 & 178 & 169 & 207 \\
\hline $\begin{array}{l}\text { GIR-AUC } 2 \mathrm{~h} \\
\left(\mathrm{mg} \cdot \mathrm{kg}^{-1}\right)\end{array}$ & 314 & 491 & 536 & 127 & 219 & 294 \\
\hline $\begin{array}{l}\text { GIR-AUC }{ }_{\text {total }} \\
\left(\mathrm{mg} \mathrm{kg}^{-1}\right)\end{array}$ & $499 *$ & 1090 & 1476 & 416 & 1076 & 1555 \\
\hline GIR-T $_{90 \%}(\mathrm{~min})$ & 180 & 238 & 275 & 278 & 330 & 423 \\
\hline
\end{tabular}

*Point estimate and upper or lower limit within BE limits; **dose-proportionality within $\mathrm{BE}$ limits with reference to $0.15 \mathrm{U} / \mathrm{kg}$ dose

\section{Supported by sanofi-aventis}




\section{7}

Variability in concentration and glucodynamic profiles of insulin glargine in healthy volunteers

R. H. A. Becker ${ }^{1}$, A. D. Frick ${ }^{1}$, L. Nosek ${ }^{2}$, L. Heinemann ${ }^{2}$, T. Heise ${ }^{2}$, K. Rave'

${ }^{1}$ sanofi-aventis, Frankfurt/Main, Germany, ${ }^{2}$ Profil Institut für Stoffwechselforschung, Neuss, Germany.

Background and Aims: Traditional long-acting insulin preparations, such as NPH insulin and insulin ultralente, are associated with a peak-action profile and large day-to-day variability in absorption following administration, which can result in periods of unwanted hypo- or hyperglycaemia. Insulin glargine has a predictable flat time-action profile with lower intra-subject variability in comparison to the above mentioned insulin formulations in systemic insulin exposure and glucodynamic effects.

Materials and Methods: Using the Biostator supported euglycaemic clamp technique, two formulations of insulin glargine (reference: R; Test: T) were tested for equivalence in exposure (insulin [INS]) and efficacy (glucose infusion rate [GIR]) over 30 hours in 24 healthy male volunteers (mean age 31 years [range 20-48 years]; BMI $24 \mathrm{~kg} / \mathrm{m}^{2}$ [20-27 kg/m ${ }^{2}$ ]). Subjects received replicate single subcutaneous injections of insulin $0.4 \mathrm{IU} / \mathrm{kg}$ body weight in a four-way complete crossover trial, randomized for RTRT or TRTR sequences.

Results: Both R and T produced flat INS and GIR profiles, superimposable within and between preparations, establishing equivalence ( $\mathrm{T} / \mathrm{R}$ ratio within $80-125 \%$ ) in INS- $\mathrm{AUC}_{0}$ $30 \mathrm{~h}$ and GIR-AUC ${ }_{0-30 \mathrm{~h}}$, and secondary metrics (INS-C $\mathrm{C}_{\max }$, $\left.\mathrm{GIR}_{\max }\right)$. Overall, intra-subject variabilities in INS-AUC, assessed as coefficient of variation ( $\mathrm{CV} \%$ ) and derived from mean square error terms of ANOVA were $18 \%$; the intrasubject variabilities for GIR-AUC values was $32 \%$. Individual variability in INS-AUC did not completely correspond to individual variability in GIR-AUC, highlighting the greater variability in glucodynamic efficacy in general. See Table.

\begin{tabular}{|c|c|c|c|}
\hline & Reference $\mathbf{R}$ & Test $\mathbf{T}$ & $\begin{array}{l}\text { Confidence interval } \\
\text { T/R }(95 \% \text { CI })\end{array}$ \\
\hline $\begin{array}{l}\text { INS-AUC }_{0 \text {-end }} \\
\left(\mu \mathrm{IU} \cdot \mathrm{h} \cdot \mathrm{ml}^{-1}\right)\end{array}$ & 425 & 414 & $97(92 ; 103)$ \\
\hline $\begin{array}{l}\mathrm{CV} \% \\
\left(\mathrm{INS}-\mathrm{AUC}_{0-30 \mathrm{~h}}\right)\left[{ }^{*}\right]\end{array}$ & 13 & 19 & $146(117 ; 213)$ \\
\hline $\begin{array}{l}\text { GIR-AUC }{ }_{0-30 \mathrm{~h}} \\
\left(\mathrm{mg} \cdot \mathrm{kg}^{-1}\right)\end{array}$ & 2743 & 2796 & $102(91 ; 115)$ \\
\hline $\begin{array}{l}\mathrm{CV} \% \\
\left(\mathrm{GIR}^{-A U C_{0-30 h}}\right)^{[+]}\end{array}$ & 34 & 30 & $88(75 ; 96)$ \\
\hline & \multicolumn{2}{|c|}{ R \& T Combined } & $R \& T(95 \%$ CI $)$ \\
\hline $\begin{array}{l}\mathrm{CV} \% \\
\left(\mathrm{INS}-\mathrm{AUC}_{0-30 \mathrm{~h}}\right)\left[{ }^{*}\right]\end{array}$ & \multicolumn{2}{|c|}{18} & $13 ; 22$ \\
\hline $\begin{array}{l}\mathrm{CV} \% \\
\left(\text { GIR-AUC }{ }_{0-30 \mathrm{~h}}\right)[+]\end{array}$ & \multicolumn{2}{|l|}{32} & $23 ; 41$ \\
\hline
\end{tabular}

Chinchilli \& Esinhardt on ln-transformed $\left[{ }^{*}\right]$ or raw data $[+]$
Conclusions: This study establishes equivalence of the two insulin glargine formulations, the superimposable flat concentration and glucodynamic profiles of insulin glargine, and attests to the low intra-subject variability of insulin glargine.

Supported by sanofi-aventis

1018

Pharmacokinetics and -dynamics of therapeutic doses of the "long-acting" insulin analogues glargine and detemir at steady-state in type 1 diabetes mellitus

F. Porcellati ${ }^{1}$, P. Rossetti ${ }^{1}$, N. Ricci Busciantella ${ }^{1}$, S. Marzotti ${ }^{1}$, P. Lucidi ${ }^{1}$, C. G. Fanelli ${ }^{1}$, D. R. Owens ${ }^{2}$, S. D. Luzio ${ }^{2}$, G. B. Bolli ${ }^{1}$;

${ }^{1}$ Internal Medicine, University of Perugia, Italy, ${ }^{2}$ Diabetes Research Unit, Llandough Hospital, Cardiff, United Kingdom.

To compare pharmacokinetics and -dynamics (PD) of the "long-acting" insulin analogues glargine (GLA) and detemir (DET), 24 subjects with T1DM naïve to GLA and DET were studied twice (randomized, double-blind, cross-over study). 14 subjects completed, $39 \pm 13$ years, BMI $22 \pm 1.7$, $\mathrm{kg} / \mathrm{m}^{2}, \mathrm{HbA}_{1 \mathrm{c}} 7.0 \pm 0.4 \%$ (means $\pm \mathrm{SD}$ ). Plasma glucose (PG) was clamped at target of $5.5 \mathrm{mmol}$ for $24-\mathrm{h}$ after sc injection of $0.35 \mathrm{UI} / \mathrm{kg}$ GLA or $0.35 \mathrm{U} / \mathrm{Kg}$ DET in the abdomen at 19:00 h after 2 week treatment with either GLA or DET (o.d. at 19:00 h). Primary endpoints were onset of action (time at which iv G was initiated after sc GLA or DET); minimal duration of action (time at which PG was $>$ $6.5 \mathrm{mmol} / 1$ in absence of $\mathrm{G}$ infusion). Results are given referred to insulin A and B (data locked). With A, PG remained at $5.7 \pm 0.2 \mathrm{mmol} / \mathrm{l}$ until $24 \mathrm{~h}$ (all 14 subjects completed study). With B, PG started to increase after $11 \mathrm{~h}$ and was $10.5 \pm 2.17 \mathrm{mmol} / \mathrm{l}$ by $24 \mathrm{~h}$ (only 2 out of 14 subjects completed study because PG increased $>11.1 \mathrm{mmol} / 1$ before $24 \mathrm{~h}$ ). G infusion rate (GIR) was constant over $24 \mathrm{~h}$ with A, but decreased markedly with $\mathrm{B}$. Total activity (GIR-AUC ${ }_{0-}$ end of GIR) was $1623 \pm 792$ and $832 \pm 252 \mathrm{mg} / \mathrm{Kg}$, whereas the end of GIR was $23.4 \pm 1.4$ and $15 \pm 4.2 \mathrm{~h}$ for insulin A and B, respectively $(\mathrm{p}<0.01)$. Other $\mathrm{pD}$ and clinical endpoints are shown below:

\begin{tabular}{|c|c|c|c|c|c|c|}
\hline & $\begin{array}{l}\text { Onset } \\
\text { of } \\
\text { action } \\
\text { (h) }\end{array}$ & $\begin{array}{l}\text { Minimal } \\
\text { duration } \\
\text { of action } \\
\text { (h) }\end{array}$ & $\begin{array}{l}\text { GIR } \\
(0- \\
12 \mathrm{~h}) \\
(\mathrm{mg} / \mathrm{Kg} / \\
\mathrm{min})\end{array}$ & $\begin{array}{l}\text { GIR } \\
(12-24) \\
(\mathrm{mg} / \mathrm{kg} / \\
\mathrm{min})\end{array}$ & $\begin{array}{l}\text { GIR } \\
\max (\mathrm{mg} / \\
\mathrm{kg} / \mathrm{min})\end{array}$ & $\begin{array}{l}\text { GIR } \\
\text { Tmax } \\
\text { (h) }\end{array}$ \\
\hline Insulin A & $1.5 \pm 0.7$ & $24 \pm 0.6$ & $1.2 \pm 0.2$ & $1.1 \pm 0.2$ & $2.0 \pm 0.9$ & $10.6 \pm 7.0$ \\
\hline Insulin B & $1.5 \pm 0.6$ & $16.4 \pm 3.3^{*}$ & $1.0 \pm 0.1$ & $0.2 \pm 0.1 *$ & $1.7 \pm 0.5$ & $7.7 \pm 2.0$ \\
\hline
\end{tabular}

$* P<0.05$ vs A. 
Conclusions: Insulin A (likely GLA) and B (likely DET) at steady-state, have different total activity despite identical nominal units, and different $\mathrm{pD}$ which should be taken into consideration for dosing, number and timing of daily administrations in T1DM.

\section{PS 87}

\section{Genetics of complications}

\section{9}

\section{Proliferative retinopathy clusters in type 1 diabetic} siblings

K. Hietala ${ }^{1,2}$, C. Forsblom ${ }^{1}$, P. Summanen ${ }^{2}$,

P.-H. Groop ${ }^{1}$;

${ }^{1}$ FinnDiane -Study, Folkhalsan Research Center, Helsinki, ${ }^{2}$ Department of Ophthalmology, Helsinki University Hospital, Finland.

Background and Aims: Diabetic nephropathy clusters in families suggesting that genetic factors play a role in the pathogenesis of this complication. Whether proliferative retinopathy, a complication frequently seen in conjunction with nephropathy, also clusters in families is less clear. Therefore the aim was to study the presence and potential clustering of proliferative retinopathy (PDR) in type 1 diabetic sibpairs.

Materials and Methods: As part of the ongoing FinnDiane Study we have so far collected all available data on the development of diabetic retinopathy from almost 2000 patients including over 26000 pages of ophthalmic records. In addition 23931 fundus photographs from 1438 patients have been scanned at $300 \mathrm{dpi}$ and saved in our digital image library. The images have been graded using a modified ETDRS-scale by an ophthalmologist unaware of the demographic data and the presence or absence of other diabetic complications. The first $714(400 \mathrm{M} / 314 \mathrm{~F})$ completely classified patients were used in the present analysis.

Results: There were 70 sibpairs (140 patients) and in 12 of them (24 patients) both had PDR (17\%). Out of all the 714 patients studied 242 patients had PDR (34\%). In order to detect potential clustering of PDR, a group of control sibpairs was constructed from the available 218 agematched unrelated PDR patients. The difference in the time interval from diagnosis of diabetes to onset of PDR within sibpairs was shorter in related $(4.8 \pm 3.1$ years $)$ than unrelated $(7.6 \pm 6.5$ years $)$ patients $(\mathrm{p}=0.01)$. This observation is suggestive of familial clustering of PDR and is further supported by the positive correlation between related sibpairs $\left(\mathrm{r}^{2}=0.352, \mathrm{p}=0.04\right)$. The duration to incident
PDR was similar in both groups, $20.6 \pm 7.3$ years in the unrelated and $18.6 \pm 7.3$ years in the related sibpairs $(\mathrm{p}=0.21)$. The sex distribution was equal between $(\mathrm{p}=0.41)$ and within $(\mathrm{p}=0.25)$ the related and unrelated sibpairs. Furthermore, the related and unrelated sibpairs were no different with regards to age at onset $(\mathrm{p}=0.31)$, $\mathrm{HbA}_{1 \mathrm{c}}(\mathrm{p}=0.10)$, or blood pressure $(\mathrm{p}=0.98)$. Notably nephropathy status showed a remarkable concordance in related as compared to unrelated sibpairs $(\mathrm{p}=0.002)$.

Conclusion: PDR seems to cluster in related sibpairs suggesting that genetic factors may play a role in the pathogenesis of PDR.

Supported by Silmäsäätiö

1020

Polymorphisms in the 5'-flanking region of the PEDF gene are associated with diabetic retinopathy and diabetic macular oedema in type 2 diabetes

T. Awata $^{1,2}$, H. Iizuka ${ }^{1}$, S. Kurihara ${ }^{2}$, K. Inoue ${ }^{2}$, K. Mori ${ }^{3}$, S. Yoneya ${ }^{3}$, S. Katayama ${ }^{2}$;

${ }^{1}$ Division of RI Laboratory, Biomedical Research Center, ${ }^{2}$ Division of Endocrinology and Diabetes, Department of Medicine, ${ }^{3}$ Department of Ophthalmology, Saitama Medical School, Saitama, Japan.

Background and Aims: PEDF (pigment epithelium-derived factor), a member of the serpin superfamily of proteins, is an important neuromodulatory factor that can provide effective neuroprotection. In the retina, PEDF protects retinal neurons against insults such as oxidative stress and glutamate excitotoxicity. PEDF is also antiangiogenic and can inhibit the growth of blood vessels in the eye. Thus, PEDF is an attractive candidate gene for development of diabetic retinopathy (DR) and diabetic macular edema (ME).

Materials and Methods: In the present study, subsequent to the VEGF and eNOS genes, we examined three polymorphisms of the PEDF gene, C-811T, A-379G and Met72Thr. Among 416 Japanese patients with type 2 diabetes studied, 229 patients had no retinopathy, 101 had non-proliferative diabetic retinopathy (NPDR), and 86 had proliferative diabetic retinopathy (PDR). Macular edema was present in 17 patients with NPDR and 49 patients with PDR.

Results: Genotype and allele distribution of C-811T and A379G, but not Met72Thr, were significantly different between patients with and without diabetic retinopathy. Logistic regression analysis revealed that patients with the C-811T genotype had an increased risk of developing DR $(\mathrm{p}=0.0002)$, with the $-811 \mathrm{C}$ allele increasing the risk. The C-811T and A-379G polymorphism were in tight linkage 
disequilibrium, and the A-379G genotype was also had an increased risk of DR $(p=0.0003)$. Furthermore, the C-811T or A-379G genotype was a significant risk factor for $\mathrm{ME}$, independently from severity of DR.

Conclusion: These results demonstrate that C-811T and A379G polymorphisms of the PEDF gene is a novel genetic risk factor for DR and ME.

\section{1}

EURAGEDIC study: analyses of 144 candidate genes for diabetic nephropathy

N. Vionnet ${ }^{1}$, G. Kazeem ${ }^{2}$, I. Gut ${ }^{3}$, P.-H. Groop ${ }^{4,5}$, C. Forsblom ${ }^{4,5}$, L. Tarnow ${ }^{6}$, H.-H. Parving ${ }^{6}$, S. Hadjadj $^{7}$, M. Marre ${ }^{8,9}$, D. Gauguier ${ }^{2}$, R. Cox ${ }^{10}$, M. Farrall ${ }^{2}$, D. A. Tregouët ${ }^{11}$, F. Cambien ${ }^{11}$, M. G. Lathrop ${ }^{3}$;

${ }^{1}$ U525, INSERM, EVRY, France, ${ }^{2}$ University of Oxford, Wellcome Trust Centre for Human Genetics, United Kingdom, ${ }^{3} \mathrm{CNG}$, Centre National de Génotypage, Evry, France, ${ }^{4}$ Department of Medicine Division of Nephrology, Helsinki University Central Hospital, Finland, ${ }^{5}$ Folkhälsan Institute of Genetics, Biomedicum Helsinki, Finland, ${ }^{6}$ Steno Diabetes Centre, Copenhagen, Denmark, ${ }^{7}$ Department of Diabetology, Poitiers University Hospital, Poitiers, France, ${ }^{8}$ Department of Diabetology, Bichat University Hospital, Paris, France, ${ }^{9}$ U695, INSERM, Paris, France, ${ }^{10}$ Mammalian Genetics Unit, Mammalian Research Council, Oxfordshire, United Kingdom, ${ }^{11}$ U525, INSERM, Paris, France.

Background and Aims: Diabetic nephropathy (DN) is likely to be a complex genetic trait. The objective of the EURAGEDIC study is to identify DN susceptibility genes. We report molecular genetic studies for 144 candidate genes for DN.

Materials and Methods: More than 4000 DNAs have been assembled from 3 European populations in France, Denmark and Finland including type 1 diabetic patients with $(n=1057)$ and without ( $n=1127)$ DN for case/control study, and 532 parent-offspring trios with $(n=244)$ or without $(n=288)$ DN for transmission disequilibrium testing. Candidate genes were selected through assessment of linkage studies, knowledge of metabolic pathways, and animal models. After re-sequencing of the genes, 439 polymorphisms tagging the haplotypes with a frequency greater than $5 \%$ in at least one population or non-synonymous polymorphisms were identified and further sought for association with DN. Association was first tested in each population by single-point analysis and in the absence of heterogeneity across the 3 populations, a combined OR was provided. Any suggestive association $(\mathrm{P}<0.1)$ was checked for confirmation through TDT analysis in trios with DN and in the absence of heterogeneity across the two studies results were combined to provide an overall estimate of the OR.

Results: Fifty SNPs showed suggestive association with DN in single-point analysis, for 6 of which results were heterogeneous across the 3 populations. Results of the TDT-analysis for the 44 SNPs further analysed were not significant but were consistent with the results of the case/ control study for 31 of them. Overall estimate of the OR for the combined analyses was significant at the 5\% level for 13 SNPs in 10 genes: ACE_DI $(\mathrm{OR}=0.87$ [0.78-0.98], $\mathrm{p}=0.018), \mathrm{ACE} 17944 \mathrm{CT} \quad(\mathrm{OR}=1.17 \quad[1.05-1.31]$, $\mathrm{p}=0.006), \quad \mathrm{ATP} 1 \mathrm{~B} 2$ 3208GA $(\mathrm{OR}=1.20 \quad[1.05-1.37]$, $\mathrm{p}=0.007)$, ATP1B2_3623CT $(\mathrm{OR}=0.88 \quad$ [0.798-0.98], $\mathrm{p}=0.025), \mathrm{ENO} 2 \_8908 \mathrm{CT}(\mathrm{OR}=0.85[0.73-0.97], \mathrm{p}=0.02)$, GLO1_20729AT $(\mathrm{OR}=1.20 \quad[1.01-1.43], \mathrm{p}=0.042)$, IGF2_rs4320932CT $(\mathrm{OR}=1.16$ [1.02-1.32], $\mathrm{p}=0.021)$, NFATC1_133215TC $(\mathrm{OR}=1.18$ [1.03-1.36], $\mathrm{p}=0.018)$, PRKWNK2_63213GA $(\mathrm{OR}=0.57$ [0.34-0.97], $\mathrm{p}=0.036)$, UBA52_2351CT $(\mathrm{OR}=0.89[0.80-0.98], \mathrm{p}=0.025)$, VEGF 7541CA $(\mathrm{OR}=0.89 \quad[0.79-1.00], \mathrm{p}=0.046)$, TIMP1_2544GA $(\mathrm{OR}=0.867 \quad[0.76-0.98], \mathrm{p}=0.022)$, TIMP1_4418TC $(\mathrm{OR}=0.85$ [0.76-0.95], $\mathrm{p}=0.006)$. Haplotype analysis as well as gene-gene interaction analysis for the whole dataset are still ongoing.

Conclusion: We found suggestive association between DN and markers in 10 genes. Further investigations are needed to clarify the role of those genes in the pathogenesis of diabetic nephropathy.

Supported by EC grant QLG2-CT-2001-01669

1022

Combinational effect of the genes encoding components of renin-angiotensin system on conferring susceptibility to diabetic nephropathy in Japanese subjects with type 2 diabetes

S. Maeda ${ }^{1}$, N. Osawa ${ }^{1,2}$, M. Kamiyama ${ }^{1}$, A. Kashiwagi ${ }^{2}$, R. Kikkawa ${ }^{2}$;

${ }^{1}$ Laboratory for Diabetic Nephropathy, SNP Research Center, RIKEN, Yokohama, ${ }^{2}$ Department of Medicine, Shiga University of Medical Science, Otsu, Japan.

Background and Aims: To evaluate a role of reninangiotensin system (RAS) in conferring susceptibility to diabetic nephropathy, we examined the association between polymorphisms within the genes encoding angiotensin converting enzyme (ACE), angiotensinogen (AGT), angiotensin II receptor type 1 (AGTR1) and diabetic nephropathy in a large cohort of Japanese type 2 diabetic patients.

Materials and Methods: Single nucleotide polymorphisms (SNPs) within the ACE, AGT, and AGTR1 genes were selected from Japanese SNP database, and analyzed by 
invader assay in 747 nephropathy cases and 557 control subjects.

Results: Ten SNPs within ACE gene were successfully genotyped, and 8 SNPs were significantly associated with diabetic nephropathy $(\mathrm{P}<0.05)$. Among them, 5SNPs were almost in complete linkage disequilibrium to the I/D polymorphism $(\Delta=1)$, and the allele corresponding to the $\mathrm{D}$ allele for ACE I/D polymorphism was considered to be a risk allele for diabetic nephropathy in this population $(\mathrm{P}=0.01$, Odds ratio=1.34 95\% CI: 1.07-1.69). Regarding the AGT gene, six SNPs including M235T polymorphism could be determined, and M235T and two other SNPs were significantly associated with diabetic nephropathy (M235T: $\mathrm{P}=0.01$, Odds ratio $=0.74$, 95\% CI: 0.59-0.94). In the AGTR1 gene, twenty-six SNPs could be genotyped, and 1SNP within the second intron was associated with nephropathy $(\mathrm{P}=0.029)$. We further examined the combinational effect of these genes by a step-wise logistic regression analysis. The result indicated that each gene might contribute to the susceptibility to diabetic nephropathy independently, and there was no significant synergistic interaction among these genes, but a significant additional effect of these three genes (final empirical $\mathrm{P}$ value= 0.00005). Subsequent logistic regression analysis revealed that the increased number of risk alleles (corresponding allele of D allele, $C$ allele for rs2242638 in the ACE, M allele for the AGT, and C allele of rs388915 for the AGTR1) was an independent risk for the development and progression of diabetic nephropathy $(\mathrm{P}=0.0017, \mathrm{OR}=1.42$, 95\% CI; 1.14-1.76). We could not find any SNPs within the RAS gene associated with hypertension in this cross sectional study.

Conclusion: We concluded RAS gene polymorphisms might contribute to the susceptibility to diabetic nephropathy in type 2 diabetes, and the combination of these genes is useful for the prediction of the development and progression of the disease.

Result of logistic regression analysis

\begin{tabular}{|c|c|c|c|c|c|c|c|}
\hline & $\begin{array}{l}\text { Sex } \\
\text { (male 1, } \\
\text { female } \\
\text { 2) }\end{array}$ & Age & BMI & $\begin{array}{l}\text { Duration } \\
\text { of } \\
\text { diabetes }\end{array}$ & HbA1c & $\begin{array}{l}\text { Hyper- } \\
\text { tension }\end{array}$ & $\begin{array}{l}\text { Number } \\
\text { of risk } \\
\text { genotype }\end{array}$ \\
\hline $\begin{array}{l}\text { regression } \\
\text { coefficient }\end{array}$ & 0.821 & -0.040 & 0.016 & 0.060 & 0.011 & 2.169 & 0.348 \\
\hline $\begin{array}{l}\text { standard } \\
\text { error }\end{array}$ & 0.177 & 0.008 & 0.024 & 0.010 & 0.031 & 0.215 & 0.111 \\
\hline $\mathrm{P}$ & $<0.0001$ & $<0.0001$ & 0.51 & $<0.0001$ & 0.73 & $<0.0001$ & 0.0017 \\
\hline
\end{tabular}

1023

Role of genetic variability in antioxidant and DNA reparation enzymes in diabetic nephropathy

L. Pacal, K. Kankova, A. Stejskalova;

Department of Pathophysiology, Faculty of Medicine, Brno, Czech Republic.

Background and Aims: Diabetic nephropathy (DN) develops as a result of hemodynamic and metabolic changes accompanying diabetes. Hyperglycemia-induced overproduction of reactive oxygen species (ROS) is an important pathogenic determinant of DN. (In)efficient cellular protection by antioxidant enzymes is reflected in the overall extent of oxidative damage. Genetic variability in genes encoding antioxidant enzymes belong to functional candidates potentially modulating susceptibility to DN. Subsequently, oxidative DNA damage due to ROS overproduction activates DNA reparation. Unfortunately, this further aggravates cellular dysfunction/damage due to consumption of energy substrates and metabolic cofactors. The aim of the study was to investigate relationship between genetic variability in genes encoding poly-(ADP-ribose) polymerase (ADPRT), manganese and extracellular superoxide dismutase (SOD2, EC-SOD), catalase (CAT), glutathion peroxidase (GPx), glutathione S-transferases (GST) and susceptibility to DN.

Materials and Methods: Case-control study comprised a total of 421 unrelated Caucasian subjects, 236 type 2 diabetics with DN and 185 type 2 diabetics without DN. DN was classified according to the albumin excretion rate and glomerular filtration rate; diabetics with minimally persistent proteinuria were classified as DN. Genotypes of ADPRT C410T, C1362T and G1672A, SOD2 T245C, ECSOD C760G, GSTM1 deletion, GSTP1 deletion, CAT 262C/T and GPx C593T were detected by PCR-based methodology. Differences in allele frequencies were tested by Fisher-exact test. Differences in genotype distributions and Hardy-Weinberg equilibrium were tested by $\chi 2$ test. Estimation of haplotype frequencies was performed in silico by PHASE software and differences in haplotype distributions tested by permutation test. Bonferroni correction was used to correct for multiple comparisons.

Results: No significant differences in allele, genotype or haplotype distribution of any of the SNPs studied were found between groups of subjects with and without DN $(\mathrm{P}>0.05)$. Solely the frequency of CAT-262C/T protective allele $\mathrm{T}$ was marginally significantly higher $(\mathrm{P}=0.06)$ in diabetics without DN. 
Conclusion: Our results suggest that despite of pathophysiological importance of antioxidant defensive system its genetic variability does not constitute major genetic risk for the development of diabetic nephropathy in type 2 diabetics, at least in Czech population.

Supported by grants 310/06/0827 from Czech Science Foundation and 10/II NR/7996-3 from The Internal Grant Agency of The Ministry of Health of Czech Republic.

\section{4}

Evidence for association of the NPY Leu7Pro polymorphism with type 1 diabetes and diabetic nephropathy in Swedish Caucasians

J. Ma ${ }^{1}$, A. Möllsten ${ }^{2}$, H. Falhammar ${ }^{1}$, K. Brismar ${ }^{1}$, G. Dahlquist ${ }^{2}$, S. Efendic ${ }^{1}$, H. F. Gu ${ }^{1}$;

${ }^{1}$ Department of Molecular Medicine and Surgery, Karolinska Institute, Stockholm, ${ }^{2}$ Department of Clinical Sciences, Paediatrics, Umeå University, Sweden.

Background and Aims: Neuropeptide Y (NPY) is present in the central and peripheral nervous system, several organs and plasma. NPY has been shown to regulate renal blood flow. A recent report indicates that the Leu7Pro (T1128C) polymorphism in this gene confers the susceptibility risk to diabetic nephropathy and coronary heart disease (CHD) in Finnish type 1 diabetic (T1D) patients (PetterssonFernholm et al. 2004). The present study evaluates whether this polymorphism is associated with T1D and diabetic nephropathy among Swedish Caucasians.

Materials and Methods: Genotyping of the NPY Leu7Pro polymorphism was performed by using dynamic allele specific hybridization (DASH). A total of 423 (female 230/ male 193) T1D patients and 187 (116/71) non-diabetic control subjects were included. Among the patients, 174 (98/76) had diabetic nephropathy.

Results: The risk $\mathrm{C}$ allele was strongly associated with the development of T1D ( $\mathrm{P}=0.042, \mathrm{OR}=2.32395 \%$ CI 1.028 5.246) and diabetic nephropathy $(\mathrm{P}=0.011, \mathrm{OR}=2.61495 \%$ CI 1.249-5.467) in women, but no significant association was seen in men.

Conclusion: The present study provides evidence that the NPY Leu7Pro polymorphism confers a significant genetic risk to the development of both T1D and diabetic nephropathy in Swedish women.

Supported by Novo Nordic Consortium, Swedish Research Council, Vetenskapligt arbete inom diabetologi foundation, Loo and Hans Osterman foundation, Family S Persson foundation and Swedish Diabetes association.

\section{PS 88}

\section{Cardiovascular disease - clinical interventions}

1025

Complications and cardiovascular risk factors in people with type 1 diabetes in the International Diabetes Management Practices Study (IDMPS)

A. Ramachandran ${ }^{1}$, P. Aschner ${ }^{2}$, J. Chan ${ }^{3}$, N. Hancu ${ }^{4}$, S.-H. Baik ${ }^{5}$, S. Ferreira ${ }^{6}$, H. Ilkova ${ }^{7}$, S. Twigg ${ }^{8}$,

J.-J. Gagliardino';

${ }^{1}$ Diabetes Research Centre, M.V. Hospital for Diabetes, Chennai, India, ${ }^{2}$ Endocrinology Unit, Unidad Médica de diagnóstico, Bogotá, Colombia, ${ }^{3}$ Department of Medicine and Therapeutics, Prince of Wales Hospital, Shatin, Hong Kong, ${ }^{4}$ Diabetes Center and Clinic, Iuliu Hatieganu University of Medicine, Cluj-Napoca, Romania, ${ }^{5}$ Department of Internal Medicine, Korea University Guro Hospital, Seoul, Republic of Korea, ${ }^{6}$ Department of Preventive Medicine, Federal University of Sao Paulo, Brazil, ${ }^{7}$ Department of Internal Medicine, Istanbul University Cerrahpasa Medical Faculty, Istanbul, Turkey, ${ }^{8}$ Department of Medicine, University of Sydney, Australia, ${ }^{9}$ Faculty of Medical Sciences, National University of La Plata, Argentina.

Background and Aims: Preliminary data from the IDMPS were used to assess the frequency of chronic complications and associated cardiovascular risk factors in people with type 1 diabetes ( $\geq 18$ years) from 11 countries in Eastern Europe, Asia, and Latin America.

Materials and Methods: This observational study enrolls patients seen in current medical practice by physicians managing diabetic patients.

Results: Data are currently available for 1327 people (51\% men) with type 1 diabetes who had a mean age of $36 \pm 13$ years and a mean disease duration of 11 years. $18 \%$ were smokers. Mean $\mathrm{HbA}_{1 \mathrm{c}}$ was $8.2 \pm 1.96 \%$ and $27 \%$ of people had an $\mathrm{HbA}_{1 \mathrm{c}}<7 \%$. 98\% of people had microvascular complications (56\% retinopathy, $45 \%$ nephropathy, $72 \%$ neuropathy) and $19 \%$ had macrovascular complications $(10 \%$ coronary artery disease, $3 \%$ cerebrovascular disease, $11 \%$ peripheral vascular disease). Mean systolic/diastolic blood pressure (BP) was $122 / 75 \mathrm{mmHg}$. Antihypertensive medication was taken by $20 \%$ of people and $47 \%$ had achieved a BP below $130 / 80 \mathrm{mmHg}$. Target treatment values were achieved for LDL $(<100 \mathrm{mg} / \mathrm{dL})$ in $41 \%$ of people ( $11 \%$ receiving lipid lowering treatment), for HDL $(>40 \mathrm{mg} / \mathrm{dL})$ in $81 \%$ of people $(18 \%$ receiving lipid lowering treatment), and for triglycerides $(<150 \mathrm{mg} / \mathrm{dL})$ in 
$72 \%$ of people ( $10 \%$ receiving lipid lowering treatment). Only $3 \%$ of people reached all 3 recommended targets for $\mathrm{HbA}_{1 \mathrm{c}}$, BP and lipids.

Conclusion: Thus, although patients have wide access to medications that specifically treat these conditions, it appears that more suitable guidance and education are necessary to ensure their effective use by people with type 1 diabetes world wide.

Supported by sanofi-aventis

\section{6}

Integration of diabetes care improves general risk factor control in patients with type 2 diabetes attending comprehensive cardiac rehabilitation - the randomised DANSUK study

A. M. B. Soja ${ }^{1}$, A.-D. O. Zwisler ${ }^{2}$, M. Frederiksen ${ }^{3}$, T. Melchior ${ }^{4}$, E. Hommel ${ }^{5}$, C. Torp-Pedersen ${ }^{6}$, M. Madsen ${ }^{7}$; ${ }^{1}$ Medical Department II, Hilleroed County Hospital, Denmark, ${ }^{2}$ Medical Department, Holbaek County Hospital, Denmark, ${ }^{3}$ Department of Cardiology, Bispebjerg University Hospital, Copenhagen, Denmark, ${ }^{4}$ Department of Cardiology, Roskilde County Hospital, Roskilde, Denmark, ${ }^{5}$ Steno Diabetes Center, Gentofte, Denmark, ${ }^{6}$ Department of Cardiology, Bispebjerg University Hospital, Copenhagen, Denmark, ${ }^{7}$ Department of Public Health, National Institute of Public Health, Copenhagen, Denmark.

Background and Aims: Mortality and readmission rates for ischemic events are unacceptable high in patients with type 2 diabetes (T2DM). The DANSUK study was designed to test if integration of diabetes care in the settings of hospital-based comprehensive cardiac rehabilitation (CCR), would result in a further improvement of risk factor control in patients with T2DM compared to usual care (UC).

Materials and Methods: 201 patients eligible for CCR were randomized after being discharged from a department of cardiology. Thirty-four percent in each study group had T2DM. Patients allocated to UC received treatment in specialized outpatient clinics. Patients randomized to $\mathrm{CCR}$ received 6 weeks of exercise training, psychosocial support, patient education and intensified systematic risk factor control after 3, 6 and 12 months.

Results: After one year, the mean change of HbAlc was $-0.7 \pm 0.9 \%$ (from mean $7.13 \pm 1.2 \%$ to mean $6.48 \pm 0.8 \%$ ) in the CCR group compared to a mean change of $-0.1 \pm 0.7 \%$ (from mean $6.95 \pm 1.1$ to mean $6.87 \pm 1.2 \%$ ) in the UC group $(\mathrm{p}<0.05)$. Systolic and diastolic blood pressure was improved $5 \% / 7 \%$ in the CCR group (from mean $132 \pm 21$ / $75 \pm 10 \mathrm{mmHg}$ to mean $125 \pm 16 / 70 \pm 7 \mathrm{mmHg}$ ) compared to an improvement of $1 \% / 1 \%$ in UC (from mean $131 \pm 21$ /
$72 \pm 9 \mathrm{mmHg}$ to mean $129 \pm 23 / 71 \pm 11 \mathrm{mmHg})(\mathrm{p}<0.01)$. Insulin resistance was improved $7 \%$ in the CCR group compared to a deterioration of $15 \%$ in the UC group $(p<0.05)$. Sixty-six percent of the patients in the CCR group obtained a treatment goal of HbA1c below $6.5 \%$ and more patients attending CCR were in treatment with cardio-protective agents at the end of the study $(\mathrm{p}<0.05)$.

Conclusion: In patients with T2DM, intensified CCR with an integrated diabetes module was more efficient in further improving risk factor control than UC. Diabetes care should be considered a core component in future settings of CCR.

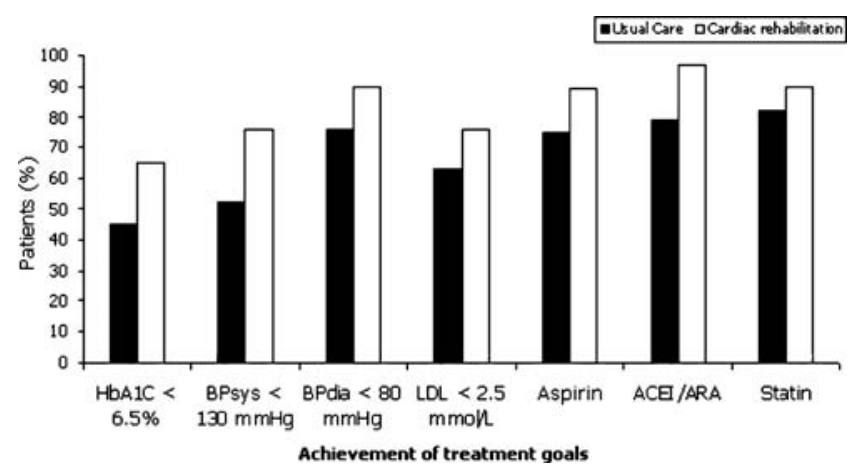

Supported by Henry and Eva Frankel's Memorial Foundation, The Danish Heart Foundation, Builder LP Christensen's Foundation, and the Danish Pharmacy Foundation of 1991

1027

Three years follow-up of screen detected diabetic and non-diabetic subjects: who is better off? The ADDITION Netherlands study

P. G. H. Janssen ${ }^{1}$, K. J. Gorter ${ }^{1}$, R. P. Stolk ${ }^{2}$, G. E. H. Rutten ${ }^{1}$;

${ }^{1}$ Julius Center for Health Sciences and Primary Care, University Medical Center Utrecht, ${ }^{2}$ Department of Epidemiology, University of Groningen Medical Center, The Netherlands.

Background and Aims: As part of the ADDITION study (Anglo-Danish-Dutch Study in general practice of intensive treatment and complication prevention in type 2 diabetic patients identified by screening), we performed a stepwise population-based screening in 2002-2004 in 79 general practices in the Netherlands. The first step of the screening consisted of a cardiovascular risk questionnaire. All subjects who had an elevated score ( $>6$ points, range $0-29)$ on the questionnaire were invited for further diagnostic testing. Screen detected type 2 diabetic patients underwent subsequent treatment for cardiovascular risk factors but we do not know whether this also applies for screened non- 
diabetic subjects with an elevated risk score. Our aim was to determine the risk of cardiovascular disease (CVD) and the extent of health care use of screened non-diabetic subjects with an elevated cardiovascular risk score compared to screen detected diabetic patients after three years of follow-up.

Materials and Methods: For the present study, 64 subjects with type 2 diabetes, 62 with impaired glucose tolerance (IGT), 86 with impaired fasting glucose (IFG) and 142 subjects with normal glucose tolerance (NGT), from 24 practices, screened from June to October 2002, were compared with regard to development of cardiovascular events, health care consumption, use of medication, and biochemical and antropometric parameters after three years follow-up.

Results: In total $16.1 \%$ of subjects with IGT, $9.3 \%$ of those with IFG and $4.2 \%$ of subjects with NGT developed diabetes during the follow-up period. Most cardiovascular events occurred in subjects with IFG (32.6 per 1,000 person-years), least in diabetic patients (15.7 per 1,000 person-years). The number of visits to care providers in the follow-up period was significantly higher in people with diabetes (33.5; interquartile range 22.3-46.0) compared to subjects with IGT $(26.0 ; 15.0-43.5)$, IFG $(23.0 ; 9.0-34.0)$ and NGT $(20.0 ; 11.0-34.0)$. Also glucose, lipids, and blood pressure measurements were performed most frequently in diabetic patients. During follow-up, in all categories cardiovascular medication was prescribed more frequently with the strongest increase in diabetic patients. Blood pressure improved in all categories except NGT.

Conclusion: In three years of follow-up, screened nondiabetic subjects with an elevated risk score developed similar CVD risk as subjects with diabetes likely because of lacking adequate treatment for cardiovascular risk factors. These results underline that screened non-diabetic subjects with an elevated cardiovascular risk score should not be reassured only because of not having diabetes.

\section{8}

Age at onset is an important factor of the severity of type 2 diabetes mellitus: CoDiC-analysis of clinical data obtained at multiple institutions across Japan

A. Kanatsuka ${ }^{1}$, M. Kobayashi ${ }^{2}$; the Japan Diabetes Clinical Data Management Study Group (JDDM);

${ }^{1}$ Diabetes Center, Chiba Central Medical Center, Chiba,

${ }^{2}$ The First Department of Internal Medicine, School of Medicine, Toyama University, Japan.

Background and Aims: We now have the chance to treat type 2 diabetic (T2D) patients with a variety of antihyperglycemic drugs according to the patient's lifestyle and the underlying pathophysiology. However, it is difficult to get good glycemic control in many T2D patients and thus sufficiently protect diabetic complications. To clarify the factors for the severity of the disease, we analyzed clinical data in Japanese T2D patients treated with the drugs.

Materials and Methods: Clinical data were collected from 41 institutes across Japan, using the $\mathrm{CoDiC}^{\circledR}$ database:a diabetes data collection and management information system developed by The Japan Diabetes Clinical Data Management Study Group (JDDM). A total of 13,921 T2D patients treated with the drugs had been registered in 2004. The data were collected in the central analytical center. Analyses were carried out using Microsoft Excel and SPSS. Glycemic control was evaluated by hemoglobin A1c (HbAlc) which was measured using the HPLC method and the normal range was defined as $4.3-5.8 \%$. The standardization of the HbAlc level was carried out in each institute according to the criteria recommended by the Committee of Japan Diabetes Society. The study protocol was approved by the JDDM ethics committee.

Results: In the patients, $37 \%$ were treated with monotherapy of oral hypoglycemic agent (OHA), 33\% with combination therapy of OHAs, $11 \%$ with combination therapy of OHA plus insulin (INS) and 19\% with monotherapy of INS. Patients with OHA plus INS and INS had a longer disease duration. Body mass index (BMI) was lower in patients with INS. Post-prandial plasma CPR was lower in the patients with OHA plus INS and markedly lower in the patients with INS. $28.3 \%$ of the patients had good glycemic control $(\mathrm{HbAl}<6.5 \%), 49.8 \%$ had fair contol $(6.5 \%<=\mathrm{HbA} 1 \mathrm{c}<8.0 \%)$ and $21.8 \%$ had poor control $(\mathrm{HbAl}>\mathrm{c}=8.0 \%)$. Clinical data were analysed between the patients with good control and the patients with poor control. Age at onset was younger in the patients with poor control than that in the patients with good control (47.2 years vs 53.6 years, $p<0.000)$ and also was same in the cases of patients with OHA, OHAs and INS, respectively. The age was the youngest in the patients who received intensive conventional insulin treatment and had poor control (42.6 years). Duration of the disease was longer and BMI, blood pressure (BP), total cholesterol (T-Cho) and fasting IRI were higher in the patients with poor control than those in the patients with good control. Logistic regression analyses showed that odds ratio for glycemic control was strong for age at onset $(0.969,95 \%$ CI: $0.964-0.974, p<0.000)$ as well as other clinical characteristics: duration of the disease, BMI, BP and TCho.

Conclusion: Age at onset is an important factor of the severity of T2D patients. We have to start intensive antihyperglycemic treatment in T2D patients as possible as earlier. Insulin-sensitizing agents should be positively added in T2D patients diagnosed at a younger age, with 
high fasting IRI level, high BMI, high BP and dyslipidemia. Insulin therapy should be actively introduced in T2D patients diagnosed at a younger age, with relatively low BMI and with low postprandial plasma CPR level. Also, this result led us to promote the research for the factors attribute to the earlier onset of T2D: genetic and environmental factors.

Supported by a grant from the Japan Diabetes Foundation

\section{9}

Utility of amlodipine/atorvastatin single-pill therapy in patients with diabetes mellitus or metabolic syndrome: Results from the JEWEL programme

F. D. R. Hobbs ${ }^{1}$, G. Gensini ${ }^{2}$, G. B. J. Mancini ${ }^{3}$,

A. J. Manolis ${ }^{4}$, B. Bauer ${ }^{5}$, J. Genest ${ }^{6}$, R. Feldman ${ }^{7}$,

P. Harvey ${ }^{8}$, T. G. Jenssen ${ }^{9}$, M. Metcalfe ${ }^{10}$,

P. Marques Da Silva ${ }^{11}$ on behalf of the JEWEL

Study Group.

${ }^{1}$ University of Birmingham, United Kingdom, ${ }^{2}$ University of Florence, Italy, ${ }^{3}$ University of British Columbia, Vancouver, Canada, ${ }^{4}$ Tzanio Hospital, Piraeus, Greece, ${ }^{5}$ Pfizer Canada Inc, Kirkland, Canada, ${ }^{6} \mathrm{McGill}$ University, Montreal, Canada, ${ }^{7}$ University of Western Ontario, London, Canada, ${ }^{8}$ Crouch Oak Family Practice, Addlestone, United Kingdom, ${ }^{9}$ Department of Medicine, University Hospital of Tromso, Norway, ${ }^{10}$ Pfizer Ltd, Surrey, United Kingdom,

${ }^{11}$ Núcleo de Investigação Arterial Hospital de Santa Marta, Lisboa, Portugal.

Background and Aims: Patients with diabetes and/or metabolic syndrome (MetS) are at high risk of cardiovascular (CV) disease. The prevalence of concomitant hypertension (HTN) and dyslipidaemia (DYS) is high among patients with these conditions who often have difficulty in reaching their aggressive therapeutic goals. This analysis of the JEWEL studies evaluated the utility of amlodipine/ atorvastatin single-pill therapy in patients with HTN/DYS and diabetes or MetS.

Materials and Methods: The JEWEL programme consisted of two 16-week, international, open-label, multicentre, titration-to-goal studies in patients with HTN and DYS. The 2 study designs differed mainly on country of enrolment (JEWEL I: UK and Canada; JEWEL II: 11 European countries) and country/region-specific BP and LDL-C targets. Eight dosage strengths of amlodipine/ atorvastatin single-pill $(5 / 10-10 / 80 \mathrm{mg})$ were administered and electively uptitrated to reach targets as necessary. This planned analysis included patients with diabetes or those with MetS, as defined by the National Cholesterol Education Program Adult Treatment Panel III (NCEP ATP III) or International Diabetes Foundation (IDF) criteria. The percentage of these patients attaining goals for BP (diabetic patients, $<130 / 80 \mathrm{~mm} \mathrm{Hg}$ [UK: $140 / 80 \mathrm{~mm} \mathrm{Hg}$; nondiabetic patients, $<140 / 90 \mathrm{~mm} \mathrm{Hg}$ [UK: $150 / 90 \mathrm{~mm} \mathrm{Hg}$ ]) and LDL-C (2.5-4.5 mmol/L depending on risk level and country goal) at end point was assessed.

Results: Across JEWEL I and II, 2245 patients received study medication and 2219 were included in the intent-totreat (ITT) population. Overall, $696(31 \%)$ of these ITT patients had diabetes while $1056(47 \%)$ were classified with MetS according to NCEP ATP III and 1648 (74\%) patients by IDF criteria. At the end of the studies, $37.2 \%$ of diabetic patients reached both BP and LDL-C therapeutic goals, $42.2 \%$ reached their BP goal alone, and $83.2 \%$ reached LDL-C goal alone. This compared with $65.9 \%, 77.2 \%$, and $83.0 \%$ respectively, for patients without diabetes. Over half of the patients with MetS (50.9\% [NCEP ATP III]; 53.9\% [IDF]) achieved both BP and LDL-C goals, whereas almost two-thirds of patients without MetS (62.3\% [NCEP ATP III]; 65.3\% [IDF]) achieved both targets. Amlodipine/ atorvastatin single-pill therapy was well tolerated in all patients and most adverse events were mild or moderate in intensity.

Conclusion: An additional 592 patients (an increase of $56 \%$ ) were classified with MetS when IDF criteria were used versus NCEP criteria. Single-pill amlodipine/atorvastatin was effective and safe for reducing BP and LDL-C to target levels in patients with diabetes and/or MetS. These high-risk patients traditionally have difficulty obtaining BP and LDL-C goals, therefore it is important to apply novel treatment strategies to improve their overall $\mathrm{CV}$ risk factor profile.

Supported by Pfizer Inc

1030

Secondary prevention of cardiovascular disease using Alphabet Strategy in type 2 diabetes

V. Patel, J. D. Lee, A. Venkataraman, J. Morrissey;

Diabetes centre, George Eliot Hospital NHS Trust, Nuneaton, United Kingdom.

Background and Aims: Diabetes mellitus predicts a poor prognosis following a cardiovascular event. There is now a large evidence base demonstrating the benefit of secondary prevention measures. The Alphabet Strategy is diabetes care based around the mnemonic: A - Advice regarding smoking cessation, ideal weight, exercise; B - Blood pressure aiming for $<140 / 80 \mathrm{mmHg}$; $\mathrm{C}$ - Cholesterol aiming $\mathrm{TC}<5, \mathrm{LDL}<3, \mathrm{HDL}>1$; D - Diabetes care aiming HbA1c $<7 \%$; E - yearly fundoscopic exam; F - yearly foot exam; $\mathrm{G}$ - Guardian drugs with respect to aspirin, ACE inhibitors, and lipid lowering therapy. We present data from the 
POEM (Practice of Evidence-based Medicine) Audit database showing the impact of the Alphabet Strategy on secondary prevention in those with type 2 diabetes mellitus.

Materials and Methods: The POEM Audit database of 400 consecutive patients with diabetes mellitus contains diabetes and cardiovascular parameters at time 0 (T0, referral) and time follow-up (Tfu). Of these, 86 had established cardiovascular disease. Statistical analysis was performed using Student's paired t-test and chi-squared test. Results: Mean age of patients with atherosclerotic disease at T0 was 64 years. $60 \%$ were male. Mean duration of diabetes was 9 years. Average follow-up through the POEM database was 5 years. Alphabet Strategy results T0 vs Tfu (excluding eye and foot exams):

- Advice: Smokers $12.8 \%$ vs 9.3\% ( $\mathrm{p}=0.33$ )

- Blood pressure: SBP $144 \mathrm{mmHg}$ vs $139 \mathrm{mmHg}$ $(\mathrm{p}=0.037)$; DBP $79 \mathrm{mmHg}$ vs $74 \mathrm{mmHg}(\mathrm{p}<0.001)$

- Cholesterol: TC $5.7 \mathrm{mmol} / \mathrm{l}$ vs $4.7 \mathrm{mmol} / \mathrm{l}(\mathrm{p}<0.0001)$; HDL $1.01 \mathrm{mmol} / \mathrm{l}$ vs $1.20 \mathrm{mmol} / \mathrm{l}(\mathrm{p}<0.0001)$

- Diabetes control: HbA1c $7.7 \%$ vs $8.0 \%(p=0.15)$

- Guardian drugs: Aspirin $68 \%$ vs $93 \%$; ACEI or ARB $39 \%$ vs $88 \%$; Lipid lowering therapy $29 \%$ vs $81 \%$ (of which $97 \%$ were statins)

Conclusion: Use of the Alphabet Strategy resulted in significantly better control of blood pressure and lipid profile, and uptake of aspirin, ACE inhibitors and statin therapy. The Alphabet Strategy optimises secondary prevention in subjects with type 2 diabetes mellitus.

\section{1}

Association between cardiac autonomic neuropathy and hypertension and its potential effects on diabetic complications

F. Ayad ${ }^{1}$, P. Valensi ${ }^{2}$, J. Pariès ${ }^{2}$, J.-R. Attali ${ }^{2}$, M. Belhadj ${ }^{1}$; ${ }^{1}$ Internal Medicine, University Hospital Oran, Algeria, ${ }^{2}$ Endocrinology-Diabetology-Nutrition, Jean Verdier Hospital, Bondy, France.

Background and Aim: The association of cardiac autonomic neuropathy (CAN) with hypertension has not been well studied in diabetes. This cross-sectional study aimed to examine this association and its potential role in diabetes complications.

Patients and Methods: We included 310 diabetic patients, 138 type 1 and 172 type 2, 62 of whom were hypertensive and 38 with known coronary artery disease. CAN was assessed on 3 standard tests (deep breathing, lying-tostanding, Valsalva) after withdrawal of drugs able to change heart rate variability, and interpreted according to age.
Results: CAN as defined by at least one abnormal test was found in 123 patients. The prevalence of hypertension was significantly higher in the patients with CAN $(31.7 \%$ vs $12 \% ; \mathrm{p}<0.0001$ ) and increased from $12 \%$ to $23 \%, 35 \%$ and $58 \%$ in patients with $0,1,2$ or 3 abnormal CAN function tests respectively $(\mathrm{p}<0.0001)$, in the whole series as well as in type 1 and type 2 diabetic patients taken separately. $\mathrm{CAN} /$ hypertension combination was twice more frequent in type 2 than in type 1 diabetic patients $(16 \%$ vs $8 \%)$. The prevalence of this combination increased with age, diabetes duration, and was higher in patients with coronary artery disease, retinopathy, nephropathy, peripheral neuropathy, and postural hypotension $(\mathrm{p}<0.0001$ for all), and this was found also in type 1 and type 2 diabetic patients taken separately. There was no significant association between CAN or hypertension independently with diabetes complications.

Conclusions: The present data strongly suggest that in diabetic patients CAN, defined by defects mainly in cardiac parasympathetic control, may contribute to hypertension, and that the CAN/hypertension combination may be an important contributor to diabetes complications.

1032

Investigation of cardiac autonomic and peripheral sensory neuropathy in long-standing hypertension with and without type 2 diabetes mellitus

P. Legrady ${ }^{1}$, D. Bajcsi ${ }^{1}$, T. T. Varkonyi ${ }^{1}$, C. Lengyel ${ }^{1}$, P. Kempler ${ }^{2}$, G. Abraham ${ }^{1}$;

${ }^{1} 1$ st Dept. of Int. Med., Univ. of Szeged, Hungary, ${ }^{2} 1$ st Dept. of Int. Med., Semmelweis Medical Univ., Budapest, Hungary.

Background and Aims: The aim of this study was to evaluate cardiac autonomic neuropathy (CAN) and/or peripheral sensory neuropathy (PSN) in long-standing hypertension (HT) with and without type 2 diabetes mellitus (DM). We also tried to find correlations between the duration of HT and DM, the systolic and diastolic blood pressure (BP), the CAN and the PSN.

Materials and Methods: 28 treated hypertensive patients were divided into 2 groups: 18 non-diabetic with normal oral glucose tolerance test (NDHT, age: $54.4 \pm 3.3$ yrs, HbA1c: $5.74 \pm 0.09 \%$, duration of HT: $10.6 \pm 2.3$ yrs, BP: 134.9/84.2 $\pm 4.2 / 2.8 \mathrm{~mm} \mathrm{Hg}$, BMI: $28.2 \pm 1.1 \mathrm{~kg} / \mathrm{m}^{2}$; meanSEM), and 10 type $2 \mathrm{DM}$ (DHT, age: $51.0 \pm 2.7 \mathrm{yrs}, \mathrm{HbAlc}$ : $6.8 \pm 0.4 \%$, duration of HT: $12.3 \pm 3.1$ yrs, duration of DM: 6.1 \pm 2.4 yrs, BP: 131.6/85.6 $\pm 3.9 / 2.6 \mathrm{~mm} \mathrm{Hg}$, BMI: $28.5 \pm$ $0.9 \mathrm{~kg} / \mathrm{m} 2$; mean \pm SEM). 11 healthy controls (C, age: $44.9 \pm$ 2.2 yrs, BP: $116.8 / 64.1 \pm 3.5 / 1.9$ mm Hg, BMI: $23.7 \pm 1.2 \mathrm{~kg} /$ $\mathrm{m}^{2}$; mean \pm SEM) were also studied. The peripheral sensory 
nerve function was characterized via detection of current perception thresholds (CPTs) measured with a Neurometer (Neurotron Inc.) on the median and the peroneal nerves. CAN was assessed by means of five standard cardiovascular reflex tests and a CAN score was calculated (from 0 to 10).

Results: There were no statistical differences between the two HT groups in BMI, duration of HT and BP. The CAN score was close in the two HT groups but they also statistically differed form group C $(4.6 \pm 0.6 v s$. $0.46 \pm 0.28$, $3.9 \pm 0.6$ vs. $0.46 \pm 0.28$ in order NDHT vs. C, DHT vs. C; $p<0.001$, mean $\pm \mathrm{SEM}$,). All the parasympathetic tests and the lying-to-standing test were impaired in both HT groups compared to group C. In the NDHT group the duration of HT positively correlated with the CAN score $(p=0.003$, $\mathrm{r}=0.66$ ). In both HT groups higher CPT values indicating hypaesthesia were found at the peroneal nerve: in group NDHT at $250 \mathrm{~Hz}(238.2 \pm 67.7$ microA; mean \pm SEM, ) and in group DHT at $250 \mathrm{~Hz}(221.8 \pm 88.2$ microA; mean \pm SEM, and $5 \mathrm{~Hz}(173.5 \pm 92.2$ microA, mean $\pm \mathrm{SEM}$,$) . Both the$ duration of HT and DM positively correlated with CPTs measured on the lower extremities at $250 \mathrm{~Hz}$ (HT: $p=0.03$, $\mathrm{r}=0.7$; DM: $p=0.02, \mathrm{r}=0.7)$ and $5 \mathrm{~Hz}(\mathrm{HT}: p=0.03, \mathrm{r}=0.7$; DM: $p=0.008, \mathrm{r}=0.8)$ in group DHT. Neither the systolic nor the diastolic BP not correlated with the CAN.

Conclusion: Severity of CAN and PSN was similar in NDHT and DHT patients. In the case of HT complicated with DM the PSN can affect more types of neurofibers. Hypertension should be considered as pathogenetic factor for the development of nerve dysfunction. Our data may provide further support, that vascular factors may play an essential role in the development of neuropathy.

\section{3}

Impairment of peripheral nerve function in obese subjects without diabetes. Influence of hypertension

M. Rivero Luis, S. Chiheb, E. Cosson, J.-R. Attali,

P. Valensi;

Diabetology, Jean Verdier Hospital, Bondy, France.

Backgrounds and Aims: Impairment in nerve function has been reported in recently-diagnosed type 2 diabetic patients, and some reports also suggest such impairment in non diabetic obese subjects. The aim was to evaluate extensively clinical symptoms, peripheral sensory function and cardiac autonomic neuropathy (CAN) in patients with obesity but without diabetes.

Materials and Methods: We explored 41 obese patients ( 34 women, $7 \mathrm{men}$; body mass index $41.1 \pm 6.4 \mathrm{~kg} / \mathrm{m}^{2}$ ) without diabetes according to $75-\mathrm{g}$ oral glucose tolerance test (mean fasting blood glucose $4.73 \pm .63 \mathrm{mM}, 2-\mathrm{hr}$ blood glucose $6.60 \pm 1.78 \mathrm{mM})$. Seventeen of them were hypertensive. The symptoms and signs of peripheral neuropathy were graded using Neuropathy Total Symptom Score-6 (NTSS-6) and the Michigan Neuropathy Screening Instrument (MNSI). Current perception threshold for the upper and lower limbs was evaluated using the Neurometer ${ }^{\circledR}$ device at three frequencies $(2000,250,5 \mathrm{~Hz})$, that assess large and small myelinated and small unmyelinated sensory nerve fiber function (A $\beta, A \delta$ and $C$, respectively). The Medoc $^{\circledR}$ system (TSA II thermo analyser and WSA3000 vibratory analyser) was used to detect cold, warm and vibration perception thresholds. CAN was defined as at least one abnormal out of 3 heart rate variation standard tests. Sympathetic and parasympathetic activities were investigated using Finapress ${ }^{\circledR}$ device.

Results: NTSS-6 and MNSI identified 2 (4.9\%) and 10 (24.4\%) patients with clinical neuropathy. According to Neurometer and Medoc tests, $\mathrm{C}$ fibers were the most often impaired, with Neurometer 1 or 2 out of 12 parameters recorded were abnormal in $51.3 \%$ or $25.6 \%$ of the patients, respectively and with Medoc it was in $77.1 \%$ or $74.3 \%$. Both 2 Neurometer parameters were abnormal in $23 \%$ of the patients. CAN was identified in $45.5 \%$. The predictors for one abnormal Neurometer parameter were hypertension (76.5\% vs $31.8 \%, \mathrm{p}<0.01)$, male gender $(100 \%$ vs $40.6 \%$, $\mathrm{p}<0.01)$ and low sympathetic activity $(\mathrm{p}<0.05)$.

Conclusions: Peripheral and autonomic nerve function is often altered in obese people without diabetes. Hypertension might contribute to peripheral nerve dysfunction.

\section{4}

Higher fasting glucose levels within the normal range are associated with sensory nerve dysfunction in patients with essential hypertension

K. Keresztes, I. Istenes, P. Vargha, P. Kempler; 1st Department of Medicine, Semmelweis University, Budapest, Hungary.

Background and Aims: On the one hand, increased cardiovascular risk factors are associated with neuropathy in diabetic patients. On the other hand, autonomic and peripheral sensory neuropathy may be present in patients with essential hypertension, but their prevalence and risk factors has not been identified up to now. We aimed to assess the prevalence and risk factors for autonomic and peripheral sensory nerve dysfunction in patients with essential hypertension.

Materials and Methods: Seventy-two patients with essential hypertension and 31 age-matched healthy control subjects were examined. The five standard cardiovascular reflex tests and heart rate variability (HRV) analysis were performed to define autonomic function. HRV was charac- 
terized both by HRV triangular index (HRVTI) and spectral parameters of frequency domain methods. Peripheral sensory nerve function on median and peroneal nerves was characterized by current perception threshold (Neurometer (PT), measured by a neuroselective diagnostic stimulator (Neurotron, Baltimore, MD).

Results: $37.5 \%$ of patients had at least one abnormal cardiovascular reflex test, while at least one abnormal HRV parameter was observed in $87 \%$ of patients. The prevalence of sensory neuropathy was $15 \%$. Elevation of fasting glucose within the normal limits correlated with higher CPT values indicating hypaesthesia at median nerve testing $(2000 \mathrm{~Hz}$ : $\mathrm{p}=0.002 ; 250 \mathrm{~Hz}: \mathrm{p}=0.03$; $5 \mathrm{~Hz}: \mathrm{p}=0.01)$. Classifying patients into subgroups by fasting glucose $(<5.2 ; 5.2-5.5 ; 5.6$ $6.0 \mathrm{mmol} / \mathrm{l})$ higher CPT values were associated with increasing fasting glucose levels even after adjustment for other cardiovascular risk factors (ANCOVA: N. medianus $2000 \mathrm{~Hz}$ : $\mathrm{p}=0.003$; $5 \mathrm{~Hz}$ : $\mathrm{p}=0.002$ ). Peripheral sensory nerve dysfunction was also associated with weight, elevated 24hour mean blood pressure, and triglycerides (all $\mathrm{p}<0.05$ ). Lower heart rate response to deep breathing $(\mathrm{p}<0.0001)$, standing and Valsalva manoeuvre (both $\mathrm{p}<0.05$ ) was found in patients with essential hypertension compared to control subjects. Compared to healthy controls, hypertensive patients had lower HRVTI $(\mathrm{p}<0.05)$, lower total power $(\mathrm{p}=0.01)$, and low frequency power $(\mathrm{p}<0.01)$. Autonomic dysfunction was independently associated with age $(\mathrm{p}<0.001)$, weight $(\mathrm{p}<0.05)$, and female gender $(\mathrm{p}=0.001)$.

Conclusion: Autonomic and sensory neuropathy are frequent complications among patients with essential hypertension. Age, weight and female sex were independent determinants of autonomic impairment. In these patients peripheral sensory neuropathy was independently associated with higher fasting glucose levels within the normal range, just as with weight, higher 24-hour mean blood pressure and triglycerides. Similarly to observation among diabetic patients, traditional cardiovascular risk factors are associated with sensory nerve dysfunction among patients with essential hypertension as well.

\section{PS 89}

\section{Hypertension}

\section{5}

Ambulatory blood pressure monitoring in normotensive and normoalbuminuric type 1 diabetic patients

E. Aguilera, L. Flores, S. Pellitero, C. Joaquin,

B. Soldevila, I. Salinas, A. Sanmartí;

Endocrinology and Diabetes Unit, Hospital Universitari

Germans Trias i Pujol, Badalona, Barcelona, Spain.
Background and Aims: Elevated blood pressure (BP) levels are frequently observed in patients with type 1 diabetes mellitus (DM1). Clinical nephropathy is preceded by microalbuminuria (MAL) which is associated with increased BP and/or abnormalities in circadian rhythm. Ambulatory blood pressure monitoring (ABPM) is a sensitive method to detect early increases in BP in DM1 patients and is a better predictor of vascular events and mortality than casual BP.

The aim of the study was to examine patterns of BP in normotensive and normoalbuminuric DM1 patients during 24 h. using a ABPM (Spacelabs 90207) device.

Materials and Methods: 94 normotensive and normoalbuminuric DM1 patients (age 32.8 \pm 7.8 yr., 37 males, $14.8 \pm$ $6.0 \mathrm{yr}$. of evolution of the disease, BMI $24.7 \pm 3.1 \mathrm{~kg} / \mathrm{m}^{2}$, $\mathrm{HbA}_{1 \mathrm{c}} 7.6 \pm 1.0 \%$, MAL $8.4 \pm 6.6 \mathrm{mg} / 24 \mathrm{~h}$., insulin dose $0.7 \pm 0.2 \mathrm{U} / \mathrm{kg}$ ) were evaluated. Systolic BP (sBP) and diastolic BP (dBP) were recorded for $24 \mathrm{~h}$., daytime and nightime. Night/day BP ratios and percent nightime BP fall were determined. Subjects with a nocturnal fall in either sBP or dBP of less than $10 \%$ of daytime values were classified as non-dippers.

Results: Mean \pm SD ABPM (systolic/diastolic in $\mathrm{mmHg}$ ) measurements were: 24 h.: $114.5 \pm 8.4$ and $69.9 \pm 6.2$; daytime: $116.9 \pm 8.9$ and $72.6 \pm 6.7$ and nightime: $106.5 \pm 8.7$ and 60.9 \pm 7.0. Mean night/day sBP and dBP ratios were $1.1 \pm 0.1$ and $1.2 \pm 0.1 \mathrm{mmHg}$ respectively. Mean percent nightime sBP fall and dBP fall were $-9 \pm 5$ and $-16 \pm 7 \%$ respectively. $48(51 \%)$ of the patients were dippers and 46 nondippers. There were no statistically significant differences in relation to the evolution of diabetes (patients $>15 \mathrm{yr}$. vs. $<15$ yr.) concerning $\mathrm{HbA}_{1 \mathrm{c}}$, MAL, $\mathrm{BP}$ or percent of nondippers. Comparing dippers with non-dippers, there were no differences in terms of age, evolution of the disease, BMI, $\mathrm{HbA}_{1 \mathrm{c}}$ or MAL. Patients were also analyzed according to percentiles of MAL: below the 25th percentile (<4.1 mg): 23 patients; 25 th-50th percentile (4.1-8.4): 38 patients and above the 50th percentile (>8.4): 29 patients. Patients with MAL below the 25th percentile displayed significantly lower $\mathrm{sBP}$ and $\mathrm{dBP}(\mathrm{p}<0.05)$ than patients with higher percentiles. There were no differences between percentile groups in relation to the other parameters studied. Conclusion: There is a high prevalence of alterations in the patterns of BP in normotensive and normoalbuminuric DM1 patients. Patients with the lowest percentile of MAL showed significantly lower BP levels.

1036

Arterial stiffness and microalbuminuria in subjects with type 2 diabetes

N. Tentolouris, A. Papazafiropoulou, D. Perrea,

N. Katsilambros; 
1st Department of Propaedeutic Medicine, Laiko University Hospital, Athens, Greece.

Background and Aims: Microalbuminuria is a risk factor for atherosclerosis in subjects with type 2 diabetes (T2D). Pulse wave velocity (PWV) represents a marker of arterial stiffness and it is increased in T2D. However, little information exists regarding the relationship between microalbuminuria and arterial stiffness in subjects with T2D. In this study we examined the association between PWV and microalbuminuria in diabetic subjects.

Materials and Methods: A total of 84 males with T2D [mean \pm SD age: $62.3 \pm 9.5$ years; duration of diabetes $9.7 \pm 8.2$ years] were studied. All patients underwent clinical and biochemical study. Urinary albumin secretion was assessed by measurement of the 24-hour urine albumin excretion. Arterial stiffness was assessed by measurement of aortic and radial PWV (elastic and muscular artery, respectively). All analyses were preformed using the SPSS statistical package.

Results: Patients with microalbuminuria had higher aorticPWV values $(\mathrm{P}=0.02)$ as well as radial-PWV $(\mathrm{P}<0.001)$ in comparison with patients without microalbuminuria. Univariate linear regression analysis showed that aortic-PWV was positively associated with age, duration of diabetes, systolic arterial pressure, pulse pressure, plasma creatinine and urea, history of hypertension and negatively with glomerular filtration rate. Multivariate analysis after adjustment for these associated factors, demonstrated an independent relationship between aortic-PWV and duration of diabetes $(b=0.24, P=0.04)$ and degree of microalbuminuria $(b=0.26, P=0.02)$. Univariate linear regression analysis showed positive associations between radialPWV and plasma glucose and triglycerides, smoking status and the degree of microalbuminuria. After multivariate adjustment only plasma triglycerides $(b=0.20$, $\mathrm{P}=0.06)$, smoking status $(\mathrm{b}=0.27, \mathrm{P}=0.01)$ and degree of microalbuminuria $(\mathrm{b}=0.28, \mathrm{P}=0.007)$ were associated with radial-PWV.

Conclusion: Patients with T2D and microalbuminuria have stiffer arteries than diabetic subjects without microalbuminuria. In addition, microalbuminuria is independently associated with arterial stiffness in both elastic and muscular arteries. Therefore, microalbuminuria may represent an early marker of a widespread arterial dysfunction in diabetic subjects. Interventions aiming at preventing or reducing microalbuminuria might result in improvement of the elastic properties of the arteries and the subsequent high risk for macrovascular complications in subjects with T2D.
1037

Endothelial function and glucagon-like peptide-1 (GLP-1) in normotensive normoalbuminuric type 2 diabetes subjects with impaired circadian blood pressure variation L. Czupryniak ${ }^{1}$, M. Pawlowski ${ }^{1}$, M. Saryusz-Wolska ${ }^{1}$, P. Grzelak ${ }^{2}$, L. Stefanczyk ${ }^{2}$, A. Borkowska ${ }^{1}$, J. Loba ${ }^{1}$;

${ }^{1}$ Diabetology \& Metabolic Diseases Dept, Medical University of Lodz, Poland, ${ }^{2}$ Radiology Dept, Medical University of Lodz, Poland.

Background and Aims: In individials with diabetes loss of circadian blood pressure variation (i.e. lack of nocturnal blood pressure dip by at least $10 \mathrm{mmHg}$, 'non-dipping') is associated with an increased mortality rate. Non-dipping phenomenon is often found in essential hypertension, and in diabetes patients it additionally might be associated with autonomic neuropathy and renal function impairment. Little is known on endothelial function in normotensive diabetes subjects with blunted blood pressure rhythm ("nondippers'). Recently, glucagon-like peptide-1 (GLP-1), an incretin hormone with therapeutic potential in diabetes, has been suggested to increase blood pressure and heart rate through sympathetic stimulation. However, it has never been studied in non-dipper subjects with diabetes.

Materials and Methods: The study group comprised 27 normotensive normoalbuminuric type 2 diabetes patients (19 women, 13 men, mean [ \pm SD] age $58 \pm 7$ years, BMI $29.3 \pm 2.7 \mathrm{~kg} / \mathrm{m}^{2}$, diabetes duration $7.3 \pm 4.7$ years, $\mathrm{HbA}_{1 \mathrm{c}}$ $8.1 \pm 1.2 \%$ ) with impaired blood pressure variation, the controls were 18 normotensive normoalbuminuric type 2 diabetes subjects (11 women, 8 men, mean $[ \pm \mathrm{SD}]$ age $61 \pm 9$ years, BMI $30.9 \pm 3.4 \mathrm{~kg} / \mathrm{m}^{2}$, diabetes duration $6.8 \pm 2.8$ years, $\mathrm{HbA}_{1 \mathrm{c}} 7.8 \pm 0.9 \%$ ) with normal blood pressure rhythm. $\mathrm{HbA}_{1 \mathrm{c}}$ values in the study group and controls were $7.8 \pm 0.8$ and $8.0 \pm 1.1 \%$, respectively $(\mathrm{p}>0.05)$. Fasting serum endothelin-1, sE-selectin, intercellular (ICAM) and vascular cell adhesion molecules (VCAM) as well as tumor necrosis factor- $\alpha$ (TNF- $\alpha)$ and aldosteron were assessed with ELISA method, GLP-1 concentration was measured with immunofluorescence method.

Results: In the study group and controls fasting serum endothelin- 1 was $7.5 \pm 1.2$ and $6.7 \pm 0.9 \mathrm{pg} / \mathrm{ml}(\mathrm{p}<0.05)$, sEselectin $24.1 \pm 10.8$ and $17.9 \pm 10.5 \mathrm{ng} / \mathrm{ml}(\mathrm{p}<0.05)$, ICAM $1266 \pm 548$ and $676 \pm 512 \mathrm{ng} / \mathrm{ml}(\mathrm{p}<0.01)$, VCAM $1792 \pm 691$ and $1359 \pm 548 \mathrm{ng} / \mathrm{ml}(\mathrm{p}<0.05)$, and GLP-1 5.9 \pm 0.9 and $5.1 \pm 0.8 \mathrm{pmol} / 1 \quad(\mathrm{p}<0.05)$, respectively. No statistically significant differences were found in serum TNF- $\alpha$ $(78.1 \pm 91.1$ and $74.3 \pm 81.9 \mathrm{pg} / \mathrm{ml}, \mathrm{p}>0.05)$ and aldosteron 
( $167 \pm 36$ and $161 \pm 27 \mathrm{pg} / \mathrm{ml}, \mathrm{p}>0.05)$ concentrations between the groups.

Conclusion: Normotensive normoalbuminuric type 2 diabetes patients with impaired blood pressure variation present with marked endothelial dysfunction and elevated fasting GLP-1 levels when compared to the subjects with normal blood pressure rhythm. Further studies are vital to elucidate causal associations between these findings. Supported by Medical University Grant No 502-18-306

\section{8}

Inverse correlation between serum free IGF-1 and IGFBP-3 levels and blood pressure in type 1 diabetes. A possible explanation of pathogenesis of diabetic complications

E. Capoluongo ${ }^{1}$, D. Pitocco ${ }^{2}$, A. Manto ${ }^{2}$, P. lulli ${ }^{1}$,

F. Zaccardi ${ }^{2}$, T. Musella ${ }^{2}$, B. Giardina ${ }^{1}$, F. Ameglio ${ }^{1}$,

C. Zuppi ${ }^{1}$, G. Ghirlanda ${ }^{2}$;

${ }^{1}$ Biochemistry, ${ }^{2}$ Internal Medicine, Catholic University, Rome, Italy.

Background: Although in mouse model the gene coding for IGF-1 is considered one of the most important to regulate blood pressure, few discordant data are available in the literature regarding a possible relationship between blood pressure and serum free IGF-1 values in humans. No information is available on type 1 diabetes patients.

Aim: To analyze the relationship between systolic and diastolic blood pressure and serum free IGF-1 and IGFBP-3 levels in subjects in 246 patients with type 1 diabetes without anti-hypertensive therapies. Following the recent guidelines of International Diabetes Federation we decided to use systolic pressure $\leq 130 \mathrm{mmHg}$ and diastolic pressure $\leq 80 \mathrm{mmHg}$ for the cut-offs to be considered in this study.

Results: A highly significant inverse correlation has been observed between serum free IGF-1 levels and both systolic $(\mathrm{p}<0,0001)$ and diastolic blood pressure $(\mathrm{p}=0,002)$ in type 1 diabetes. Similar significant relationships were observed for IGFBP-3 $(p=0,005)$, whose levels were also significantly and directly correlated with those of free IGF-1. This correlation was confirmed by analysis of the free IGF-1 and IGFBP-3 levels after age and gender correction, in subjects with normal or high systolic or diastolic blood pressure. In patients with diabetic rethinopathy ( $\mathrm{pts}=32$ ) IGF-1, IGFBP3 and their ratio were significantly lower

Conclusion: Lower levels of Free IGF-1 and IGFBP-3 are linked to an increase of blood pressure in type 1 diabetes. This linkage could influence significantly and explain the presence of diabetic complications.
1039

Soluble CD40 ligand and C-reactive protein concentrations in essential hypertension: effects of hyperglycaemia and hyperinsulinaemia.

G. Penno ${ }^{1}$, L. Pucci ${ }^{1}$, D. Lucchesi ${ }^{1}$, R. Pedrinelli ${ }^{2}$, E. Santini ${ }^{3}$, G. Dell'Omo ${ }^{2}$, E. Ferranini ${ }^{3}$, S. Del Prato ${ }^{1}$, A. Solini ${ }^{3}$;

${ }^{1}$ Endocrinology and Metabolism, ${ }^{2}$ Cardiothorax, ${ }^{3}$ Internal Medicine, University of Pisa, Italy.

Background and Aims: Subclinical inflammation and platelets activation are involved in the pathophysiology of cardiovascular disease (CVD). Recently both sCD40L (a molecule released by activated platelets) and hs-CRP (an inflammatory marker) have been reported higher among hypertensive patients, but factors accounting for the large variability of both parameters in hypertensive subjects are poorly understood. To measure hs-CRP and SCD40L in 89 CVD event-free men with essential hypertension.

Materials and Methods: We studied 89 patients (mean age $49 \pm 11$ yrs, range of BMI: $18.4-34.5 \mathrm{~kg} / \mathrm{m} 2$, serum creatinine below $1.2 \mathrm{mg} / \mathrm{dl}$ and cystatin-C concentration ranging from 0.53 to $1.18 \mathrm{mg} / \mathrm{l}$ ). All subjects underwent a 24 hour ABPM and an OGTT. Lipid and lipoprotein concentrations were measured in a fasting blood sample. Specific immunoassay were used for sCD40L and hs-CRP. Among these subjects, 18\% had IFG, 8\% IGT, 5\% both conditions. Metabolic syndrome (MS, NCEP/ATPIII criteria) was present in 30 out of 89 subjects (34\%). Data were analyzed by a SPSS package and were expressed as mean \pm sd or range.

Results: Mean levels of hs-CRP were $2.4 \pm 2.3$ (range 0.4 to 8.5 ) $\mathrm{ng} / \mathrm{ml}$; mean levels of sCD40L $8.8 \pm 5.0$ (range 0.8 to $18.5) \mathrm{ng} / \mathrm{ml}$. No correlation was observed between hs-CRP and $\mathrm{sCD} 40 \mathrm{~L}(\mathrm{p}=0.97)$ and no differences in both parameters emerged in subjects with and without MS. Neither hsCRP and sCD40L did correlate with age, BMI, systolic and diastolic $24 \mathrm{~h}$ ABPM parameters, renal function indexes or urinary albumin excretion. Within lipid parameters, a weak positive regression was observed between total cholesterol and $\mathrm{sCD} 40 \mathrm{~L}(\mathrm{p}=0.047)$. hs-CRP strongly correlated with fibrinogen levels $(p<0.0001)$. $s C D 40 L$ positively correlated with fasting glucose $(\mathrm{r}=0.23$, $\mathrm{p}=0.029)$, basal insulin levels $(\mathrm{r}=0.34, \mathrm{p}=0.002)$, and HOMA-IR ( $\mathrm{r}=0.37, \mathrm{p}=0.001)$, while hs-CRP weakly correlated with the insulinogenic index $(r=0.25, \mathrm{p}=0.05)$ and the area under the OGTT curve for insulin $(\mathrm{r}=0.32$, $\mathrm{p}=0.027$ ). In a multiple regression model, HOMA-IR emerged as the main determinant of the variability of sCD40L levels, while AUC insulin was the main determinant of the variability of hs-CRP concentrations. 
Conclusion: Minor changes in glucose and insulin metabolism might account at least in part for the inflammatory disorders related to essential hypertension.

\section{0}

Exogenous insulin and hypertension in patients with type 2 diabetes

P. Lazzari ${ }^{1}$, A. Zoppi ${ }^{2}$, R. Fogari ${ }^{2}$;

${ }^{1}$ Internal Medicine, Diabetes Center, Cremona, ${ }^{2}$ Departement of Internal Medicine, University of Pavia, Italy.

Background and Aims: The influence of exogenous insulin and consequent peripheral hyperinsulinemia on blood pressure are still controversial.Aim of this study was to investigate the relations between insulin therapy and hypertension in patients with type 2 diabetes.

Materials and Methods: We studied 466 consecutive type 2 diabetics(236 M,230 F,age 64.9+-8.8 yrs).Presence of hypertension $(\mathrm{H})$ (defined as blood pressure $>140 / 90 \mathrm{mmHg}$ or taking hypotensive therapy),glycemic control(in terms of fasting glycemia and $\mathrm{HbAlc}$ over the last 10 years), duration of diabetes,body mass index(BMI), and the presence of microvascular(nephropathy and retinopathy) and macrovascular(coronary heart disease and peripheral vascular disease) complications were recorded.According to the type of therapy,patients were divided in insulin treated (IT)(treated with insulin alone or associated with oral agents) and non insulin treated(NIT)(treated with oral agents or only diet).

Results: 88 patients(18.8\%) were IT,being the remaining fraction $(81.2 \%)$ NIT.The prevalence of $\mathrm{H}$ was similar in the 2 groups $(78.4 \%$ vs $82.8 \%, p=N S)$.Systolic blood pressure was similar in IT and NIT, whereas diastolic blood pressure was lower in the former than in the latter(91.3+ -10.2 vs $93.9+-10.8 \mathrm{mmHg}, \mathrm{p}=0.04)$. No correlation was found between insulin daily dosage and systolic or diastolic blood pressure (respectively $r=-0.07$ and $r=0.13$ ). Insulin daily dosage was lower in hypertensives than in normotensives $(31.2+-18.5$ vs $45.7+-29 \mathrm{U} / \mathrm{die}, \mathrm{p}=0.01)$. H frequency was similar in IT and NIT in normoweight $(\mathrm{BMI}<25)$,overweight(BMI 25- 29.9) and obese (BMI> $30)$ patients. Stratifying subjects by fasting glycemia $(<140$; $141-180 ;>180 \mathrm{mg} / \mathrm{dl})$ and $\mathrm{HbA} 1 \mathrm{c}(<7 ; 7-8 ;>8 \%) \mathrm{H}$ frequency was not significantly different in any stratum in IT and NIT; the same result was observed stratifying subjects by duration of diabetes $(<5 ; 6-10 ;>10$ yrs $)$.H frequency was similar in IT and NIT in patients with normo- $(<30 \mathrm{mg} /$ $24 \mathrm{~h}$ ), micro(30-299 $\mathrm{mg} / 24 \mathrm{~h})$ or macroalbuminuria $(>=300 \mathrm{mg} / 24 \mathrm{~h}$ ), as well as in those with or withouth diabetic retinopathy(background and proliferative).Similarly,the proportion of hypertensive and normotensive subjects was not significantly different in relation to the presence or not of coronary heart disease or peripheral vascular disease in IT and NIT.

Conclusion:The results of this study show no relation between insulin therapy and hypertension, and therefore they are not supportative of the hypothesis that exogenous insulin may increase blood pressure in type 2 diabetics.

1041

Candesartan improves insulin sensitivity as compared to standard treatment with hydrochlorothiazide in hypertensive subjects with abdominal obesity - the MEDICA study

J. W. Eriksson ${ }^{1,2}$, P.-A. Jansson ${ }^{3}$, B. Carlberg ${ }^{1}$, A. Hägg ${ }^{4}$, L. Kurland ${ }^{4}$, M. Svensson ${ }^{1}$, L. Lind ${ }^{4,2}$;

${ }^{1}$ Dept of Medicine, Umeå University Hospital, Sweden, ${ }^{2}$ AstraZeneca, Mölndal, Sweden, ${ }^{3}$ Dept of Medicine, Sahlgrenska University Hospital, Göteborg, ${ }^{4}$ Dept of Medicine, Uppsala University Hospital, Sweden.

Background and Aim: Several studies have indicated that blockers of the type 1 angiotensin II receptor (ARBs), e.g. candesartan, are favorable in comparison to standard antihypertensive therapy with beta blockers and thiazide diuretics with respect to metabolic effects. In particular, this has been shown for the risk for development of type 2 diabetes. The MEDICA study was performed to identify underlying mechanisms for the diabetes-preventing effect of candesartan. The primary objective was to compare insulin sensitivity during candesartan (C) and hydrochlorothiazide (H) treatment. Comparisons were also made with placebo (P).

Patients and Methods: 26 abdominally obese (waist $>102 \mathrm{~cm} \mathrm{M},>88 \mathrm{~cm} \mathrm{~F}$ ), non-diabetic, hypertensive patients were randomized to 12 -wk treatment periods, in a 3-way crossover-design, with C (16-32 mg), H (25-50 mg) and P, respectively. Mean age was $54.8 \pm 10.4$ (SD) yr and BMI $30.2 \pm 3.0 \mathrm{~kg} / \mathrm{m}^{2}$. A euglycaemic hyperinsulinaemic $(56 \mathrm{mU} /$ $\mathrm{m}^{2} / \mathrm{min}$ ) clamp and other assessments were performed at the end of each treatment period. 22 subjects $(10 \mathrm{M}, 12 \mathrm{~F})$ completed the study and were available for analysis.

Results: Insulin sensitivity following $\mathrm{C}$ was higher than following $\mathrm{H}(\mathrm{p}=0.004)$ but not significantly different from $\mathrm{P}$, and $\mathrm{M}$-values were: $\mathrm{C} 6.63 \pm 2.04, \mathrm{H} \quad 6.07 \pm 2.05$ and $\mathrm{P}$ $6.90 \pm 2.10 \mathrm{mg} / \mathrm{kg} \mathrm{BW} / \mathrm{min}$. HbA1c was higher $(\mathrm{p}=0.035)$ following $\mathrm{H}$ than $\mathrm{P}$ (C $4.5 \pm 0.4, \mathrm{H} 4.6 \pm 0.6$, P $4.3 \pm 0.4 \%$ ). There were no statistically significant differences between treatments in body weight, serum total, HDL and LDL cholesterol, triglycerides or in 1st phase insulin secretion (AUC for insulin during the first $10 \mathrm{~min}$ of an IVGTT). Systolic and diastolic blood pressures were reduced by $\mathrm{C}$ 
and $\mathrm{H}$ compared to $\mathrm{P}(135 \pm 15 / 88 \pm 9,138 \pm 8 / 91 \pm 7$ vs $150 \pm 9 /$ $96 \pm 7 \mathrm{mmHg}$, respectively, $\mathrm{p}<0.01)$. In addition, DBP was significantly lower for $\mathrm{C}$ than $\mathrm{H}(\mathrm{p}=0.009)$. Additional analyses in this study will address other mechanisms, e.g. vascular and autonomic nerve regulation and adipose tissue distribution and function, that may have an impact on glucose and lipid metabolism.

Conclusions: Insulin sensitivity was higher following candesartan than hydrochlorothiazide treatment. This may partly explain previously demonstrated favorable metabolic outcomes with candesartan and other ARBs as compared to antihypertensive regimens that include thiazide diuretics. Supported by AstraZeneca Sweden and AstraZeneca $R \& D$

\section{2}

Elevated blood pressure during exercise is related to insulin resistance and central obesity in offspring of type 2 diabetic patients

P. Jokela ${ }^{1}$, T. Laitinen ${ }^{1}$, T. Lyyra-Laitinen ${ }^{1}$, T. Lakka ${ }^{2}$, E. Vanninen ${ }^{2}$, U. Salmenniemi ${ }^{3}$, E. Ruotsalainen ${ }^{3}$, S. Kainulainen ${ }^{4}$, M. Laakso ${ }^{3}$;

${ }^{1}$ Department of Clinical Physiology and Nuclear Medicine, Kuopio University Hospital, ${ }^{2}$ Department of Physiology, University of Kuopio, ${ }^{3}$ Department of Medicine, ${ }^{4}$ Department of Clinical Radiology, Kuopio University Hospital, Kuopio, Finland.

Background and Aims: We hypothesize that insulin resistance and related metabolic disorders are associated with augmented sympathetic activation which results in elevation of blood pressure and heart rate during physical exercise. Thus, we studied cardiovascular responses to exercise in offspring of type 2 diabetic patients and in control subjects.

Materials and Methods: According to the distribution of insulin sensitivity assessed by the hyperinsulinemic, euglycemic clamp in a population of 119 healthy offspring of type 2 diabetic patient, we selected 1st and 4th quartiles to represent insulin resistant (IR, $\mathrm{n}=30$ ) and insulin sensitive (IS, $n=31)$ groups. Control group $(n=19)$ consisted of healthy subjects without family history of type 2 diabetes. Blood pressure and heart rate were measured at rest (1 MET $=\mathrm{VO}_{2}$ of $3.5 \mathrm{ml} * \mathrm{~kg}^{-1} * \mathrm{~min}^{-1}$ ), during exercise at intensities corresponding to 3 MET, 5 MET, 7 MET, 9 MET and recovery phase of the cardiopulmonary exercise test. Other main examinations were anthropometric measurements, and an oral glucose tolerance test to exclude diabetes. Furthermore, abdominal fat distribution was evaluated by computed tomography.

Results: Blood pressure and heart rate were comparable between study groups at rest. Compared to the control group the IR-group had higher systolic and diastolic blood pressure at 5 MET and 9vMET exercise levels and during recovery $(\mathrm{P}<0.05$ for all). In addition, the IR-group had higher heart rate at 5 MET level as compared with that in the IS-group $(\mathrm{P}<0.05)$ and in the control group $(\mathrm{P}<0.01)$. The only difference between the IS-group and the control group was higher diastolic blood pressure in the IS-group at 9 MET level $(\mathrm{P}<0.05)$. Other factors associated with exercise blood pressure response were body mass index and waist circumference for systolic blood pressure $(\mathrm{P}<0.01$ for both) and intra-abdominal fat area and waist circumference for diastolic blood pressure $(\mathrm{P}<0.01$ and $\mathrm{P}<0.05$, respectively). After adjustment for these confounding factors the differences between the groups disappeared with respect to hemodynamic responses to exercise.

Conclusion: Our results suggest that in insulin resistant offspring of type 2 diabetic patients the cardiovascular system becomes strained due to the hyperdynamic state which is induced by physical exercise already at moderate intensity corresponding to some daily activities. The higher pressor response to physical activity in offspring of type 2 diabetic patients with insulin resistance may be attributed to extra load caused by overweight and central obesity.

Supported by Kuopio University Hospital (EVO grant 5031316) and Paavo and Eila Salonen's foundation

1043

Effectiveness of nurse led hypertension clinic in Wolverhampton Diabetes Centre - a report of 4-year clinical experience

P. K. Singh, P. Beach, N. Iqbal, H. N. Buch, B. M. Singh; Medicine, Wolverhampton Diabetes Centre, United Kingdom.

Background and Aims: An effective blood pressure (BP) control remains difficult to achieve in patients with Diabetes Mellitus (DM) despite its undisputed benefits. Possible explanations include poor patient education, noncompliance, the need for multiple antihypertensive agents, and failure to titrate their dose within a reasonable time period. A nurse led, protocol driven hypertension clinic was established in Wolverhampton Diabetes Centre to address these issues. We report the results of a retrospective audit undertaken to assess the implementation of the protocol and its effectiveness in optimising blood pressure control.

Material and Methods: Patients with established complications of DM with suboptimal BP control despite antihypertensive treatment, were referred to this clinic. The referral form included the intended sequence of antihypertensive therapy according to a protocol which is based on the compelling indications and contraindications for clinical 
characteristics of the individual. During the clinic visits BP was measured in a standardised manner and antihypertensive therapy was initiated or titrated according to the protocol. Adverse events were reported to the referring clinicians. Patients were discharged when the intended sequence of therapy was completed or target BP was achieved.

Results: 131 patients were referred to the clinic between August 2001 and September 2005. 2 patients defaulted, 1 died and 6 were considered inappropriate referrals. The majority had type 2 DM, 94\% were insulin treated, $57 \%$ patients were obese, $50 \%$ had retinopathy and $76 \%$ had proteinuria. There was a significant reduction in systolic $(186 \pm 24$ to $139 \pm 12 \mathrm{~mm} \mathrm{Hg}$, mean $\pm \mathrm{SD} \mathrm{p}<0.05)$ and diastolic ( $85 \pm 13$ to $67 \pm 11 \mathrm{~mm} \mathrm{Hg}) \mathrm{BP}$ after a mean of 3.3 clinic visits (range 2-13). 64\% achieved systolic $\mathrm{BP}<140 \mathrm{~mm} \mathrm{Hg}$ at the time of discharge. $51(62 \%)$ of the 83 patients who had ambulatory BP monitoring prior to discharge had a mean systolic BP of $<140 \mathrm{~mm} \mathrm{Hg}$. The total number of antihypertensive agents used increased from $2.5 \pm 1.2$ to $3.1 \pm 1.1$ and the number for which the dose was maximised increased from $1.2 \pm 1.1$ to $2.1 \pm 1.2$. In 65 (49\%) patients number of antihypertensive agents used remained the same or were reduced. Improvements in total cholesterol $(4.8+/-1.3$ to $4.3+/-0.9)$ and glycaemic control $(8.4+/-1.8$ to $8.0+/-1.7)$ were also noticed.

Conclusions: A systematic approach in a nurse led hypertension clinic led to an effective BP control in a significant proportion of patients. In approximately half the patients, same or fewer numbers of tablets were used due to a more effective dose titration. A more favourable biochemical profile may have been a result of opportunistic interventions and education.

\section{4}

Blood pressure control in diabetic patients in the eight newly accessed EU countries

M. Medvešček ${ }^{1}$, W. Grzeszczak ${ }^{2}$, J. Michalek ${ }^{3}$, L. Madacsy ${ }^{4}$, A. Norkus ${ }^{5}$, M. Niewada ${ }^{6}$, B. Kaminski ${ }^{7}$, M. Andel ${ }^{8}$;

${ }^{1}$ Dept. of Endocrinology, University Medical Centre Ljubljana, Slovenia, ${ }^{2}$ Dept. of Internal Medcine, Silesian School of Medicine, Zabrze, Poland, ${ }^{3}$ Dept. of Diabetes, National Center for Endocrinology and Diabetes, Lubochna, Slovakia, ${ }^{4}$ Dept. of Pediatrics, 1st Semmelweis University, Budapest, Hungary, ${ }^{5}$ Institute of Endocrinology, Medical University Kaunas, Lithuania, ${ }^{6}$ Dept. of Clinical and Experimental Pharmacology, Warsaw Medical University, Poland, ${ }^{7}$ Division of Decision Analysis and Support, Warsaw School of Economics, Poland, ${ }^{8}$ 2nd Dept. of Internal Medicine, University of Prague, Prague, Czech Republic.
Background and Aims: To determine blood pressure (BP) control in diabetic patients in the Czech Republic, Estonia, Hungary, Lithuania, Latvia, Poland, Slovakia and Slovenia.

Materials and Methods: 11.084 patients (type 1, $\mathrm{n}=2.497$, type $2, \mathrm{n}=8.231$, other types, $\mathrm{n}=356$ ) were randomly enrolled. Data on BP values, diagnosed arterial hypertension, use of antihypertensive drugs, and chronic diabetes complications were collected in a survey by diabetologist or endocrinologist at regular visits to diabetes centres.

Results: Only $8.6 \%$ of type 2 (T2), and $41.9 \%$ of type 1 (T1) patients had $\mathrm{BP}<130 / 80 \mathrm{mmHg}$. Hypertension was diagnosed in $31.1 \%$ of $\mathrm{T} 1$ and $81.0 \%$ of $\mathrm{T} 2$ patients. Percentages of drug-treated hypertensive patients under/over the following combined SBP and DBP cut-off values were:

\begin{tabular}{llll}
\hline BP mmHg & All & T1 & T2 \\
\hline $\mathrm{n}$ & 7488 & 762 & 6543 \\
\hline$<130 / 80$ & 5.9 & 11.8 & 5.2 \\
$<140 / 90$ & 30.5 & 40.9 & 29.3 \\
$<160 / 90$ & 57.0 & 64.9 & 56.1 \\
$\geq 160 / 90$ & 13.5 & 9.4 & 13.9 \\
\hline
\end{tabular}

The respective results in patients with micro- and macrovascular complications were:

\begin{tabular}{lllllll}
\hline BP mmHg & \multicolumn{2}{l}{ Nephropathy } & \multicolumn{4}{c}{ Retinopathy } \\
& All & T1 & T2 & All & T1 & T2 \\
$\mathrm{n}$ & 2837 & 694 & 2074 & 3626 & 992 & 2547 \\
\hline$<130 / 80$ & 11.3 & 28.5 & 5.6 & 11.4 & 26.5 & 5.6 \\
$<140 / 90$ & 41.9 & 66.5 & 33.6 & 42.3 & 65.2 & 33.3 \\
$<160 / 90$ & 65.5 & 81.1 & 60.4 & 65.0 & 81.5 & 58.5 \\
$\geq 160 / 90$ & 10.3 & 4.4 & 12.2 & 11.8 & 5.5 & 14.2 \\
\hline
\end{tabular}

\begin{tabular}{lllllllll}
\hline \multicolumn{1}{c}{ Stroke/TIA } & \multicolumn{3}{c}{ Coron. art. disease } & \multicolumn{2}{c}{ Periph. art. disease } \\
\hline All & T1 & T2 & All & T1 & T2 & All & T1 & T2 \\
\hline 639 & 27 & 595 & 3550 & 198 & 3285 & 1470 & 150 & 1282 \\
6.3 & 14.8 & 5.9 & 8.0 & 13.4 & 7.0 & 8.0 & 13.0 & 7.4 \\
31.4 & 33.3 & 31.1 & 35.5 & 42.2 & 34.9 & 34.7 & 49.3 & 32.6 \\
58.3 & 66.6 & 57.7 & 60.4 & 68.0 & 60.0 & 62.2 & 76.7 & 60.4 \\
14.2 & 18.5 & 14.2 & 12.8 & 12.8 & 12.8 & 11.8 & 8.2 & 12.3 \\
\hline
\end{tabular}

Conclusion: The level of BP control is highly unfavourable in diabetic patients attending diabetes centres in the eight newly accessed EU countries. Only a small proportion of diabetics treated for hypertension and those with chronic complications requiring strict BP control eventually achieve BP target values, and a considerable proportion of them are in a very poor BP control. The results from the survey call 
for a much more intensive BP lowering to achieve target values.

Supported by Novo Nordisk

\section{PS 90}

\section{Nephropathy - clinical interventions}

\section{5}

Effect of the oral PKC $\beta$ post hoc analysis of renal safety data from two diabetic retinopathy trials

J. B. McGill ${ }^{1}$, K. R. Tuttle ${ }^{2}$, D. Haney ${ }^{3}$, T. E. Lin ${ }^{3}$, P. W. Anderson ${ }^{3}$;

${ }^{1}$ Department of Medicine, Washington University School of Medicine, St. Louis, ${ }^{2}$ Sacred Heart Medical Center, The Heart Institute, Spokane, United States, ${ }^{3}$ Lilly Research Laboratories, Eli Lilly and Company, Indianapolis, United States.

Background and Aims: Ruboxistaurin (RBX), an oral PKC $\beta$ inhibitor, reduced visual loss in two long-term phase 3 diabetic retinopathy studies. A one-year diabetic nephropathy pilot trial demonstrated that RBX improved albuminuria and was not associated with significant decline in estimated glomerular filtration rate (eGFR). This post hoc analysis of the PKC-DRS and PKC-DRS2 diabetic retinopathy trials $(n=813)$ evaluates the long-term impact of $\mathrm{RBX}$ on renal outcomes.

Materials and Methods: We performed a post hoc analysis of combined data from two three-year, randomized, doubleblind, parallel, multi-center, placebo-controlled, phase 3 studies of RBX $32 \mathrm{mg} /$ day. Patients (placebo: $n=401, \mathrm{RBX}$ $32 \mathrm{mg} / \mathrm{d}: \mathrm{n}=412$ ) had moderate to severe (AAO severity scale; equivalent to ETDRS score $\geq 47 \mathrm{~A}$ and $\leq 53 \mathrm{E}$ ) nonproliferative diabetic retinopathy and well-preserved baseline renal function. Renal outcomes were defined as: 1) doubling of serum creatinine; 2) progression to advanced (stage 4: eGFR $\leq 30 \mathrm{~mL} / \mathrm{min} / 1.73 \mathrm{~m}^{2}$ ) chronic kidney disease $(\mathrm{CKD})$ from an earlier stage (Note: Patients in the PKC-DRS2 study had few ESRD events. Thus, low GFR was used instead of ESRD as a renal outcome); or 3) death. The Modification of Diet in Renal Disease formula was used to calculate eGFR.

Results: At baseline, the mean eGFR $( \pm \mathrm{SD})$ was $83.3 \pm$ $27.9 \mathrm{~mL} / \mathrm{min} / 1.73 \mathrm{~m}^{2}$ and $20.7 \%$ of patients had CKD (eGFR $\leq 60 \mathrm{~mL} / \mathrm{min} / 1.73 \mathrm{~m}^{2}$ ). Renal outcomes in the RBXtreated group did not differ from those receiving placebo. During the trials, $6.8 \%$ of patients experienced doubling of serum creatinine (RBX: 6.1\%, placebo: $7.5 \%, \mathrm{p}=\mathrm{NS}$ ), $5.0 \%$ progressed to advanced CKD (RBX: $4.9 \%$, placebo: $5.0 \%$, $\mathrm{p}=\mathrm{NS}$ ), and $5.0 \%$ died (RBX: $4.4 \%$, placebo: $5.7 \%, \mathrm{p}=\mathrm{NS}$ ). At least one renal outcome occurred in $13.2 \%$ of patients
(RBX: $12.1 \%$, placebo: $14.2 \%, p=N S$ ). From baseline to endpoint of the three-year studies, eGFR decreased by 10.6 $\pm 20.7 \mathrm{~mL} / \mathrm{min} / 1.73 \mathrm{~m}^{2}$ (RBX: $-10.1 \pm 20.7$, placebo: $-11.1 \pm 20.8, \mathrm{p}=\mathrm{NS})$. Decline in eGFR was related to five baseline variables: diabetes duration, diabetes type, alcohol use, blood pressure, and eGFR.

Conclusion: Post hoc analysis of long-term trial data demonstrates that $32 \mathrm{mg} / \mathrm{d}$ RBX has no negative impact on renal outcomes or eGFR in diabetic retinopathy patients with well-preserved baseline renal function. The effect of RBX on renal outcomes must be confirmed using largescale prospective trials powered to evaluate the efficacy of RBX in this population.

Supported by a grant from Eli Lilly and Company.

\section{6}

Antiproteinuric effects of telmisartan versus valsartan in patients with type 2 diabetes and overt nephropathy J. Galle ${ }^{1}$, S. Quispe Bravo ${ }^{2}$, C. Wanner ${ }^{1}$;

${ }^{1}$ Department of Internal Medicine, University Hospital, Wuerzburg, ${ }^{2}$ Medical Department, Boehringer Ingelheim, Biberach, Germany.

Background and Aims: Proteinuria is a key indicator of renal and cardiovascular health. Reducing proteinuria is a central aim to prevent progression in patients with type 2 diabetes mellitus (T2D) and nephropathy. Telmisartan is an angiotensin receptor blocker (ARB) with 24-h plasma halflife that has demonstrated efficacy in slowing glomerular filtration rate decline in T2D. This study (VIVALDI) directly compared the efficacy of telmisartan with that of valsartan in this patient population.

Materials and Methods: This 1-year, prospective, doubleblind, parallel-group study randomized 885 patients with T2D, hypertension (seated SBP/DBP >130/80 $\mathrm{mmHg}$ ), macroalbuminuria ( $\geq 900 \mathrm{mg} / 24 \mathrm{~h}$ ) and a serum creatinine of 97-265 $\mu \mathrm{mol} / \mathrm{L}$ in women and 115-265 $\mu \mathrm{mol} / \mathrm{L}$ in men to either telmisartan $80 \mathrm{mg} /$ day or valsartan $160 \mathrm{mg} /$ day. To ensure blood pressure control $(<130 / 80 \mathrm{mmHg}), \alpha$ - or $\beta$ blockers, calcium channel blockers, or diuretics were allowed. The primary analysis was a non-inferiority test of 24-h urinary protein excretion rate (UPER).

Results: In the full analysis set, geometric mean UPER (95\% CI) was reduced by $33 \%(27 \%, 39 \%)$ of baseline with telmisartan $(n=431)$ and by $33 \%(27 \%, 38 \%)$ with valsartan $(n=431)$, demonstrating non-inferiority. Results were similar for the per-protocol analysis. No significant differences were seen in changes in serum creatinine, creatinine clearance or estimated glomerular filtration rate. In the two groups, a trend towards greater efficacy was seen among patients with better blood pressure and diabetes control, and 
in patients who had not previously received ARBs or ACE inhibitors, indicating the importance of these modalities for improving outcomes in this patient population.

Conclusion: In patients with $\mathrm{T} 2 \mathrm{D}$ and proteinuria, telmisartan provides similar reductions in proteinuria to valsartan. Treatment with telmisartan is an effective therapeutic option in these patients.

Supported by Boehringer Ingelheim $\mathrm{GmbH}$

\section{7}

Low-dose aspirin does not affect of antiproteinuric effect of enalapril in microalbuminuric type 2 diabetic patients. A randomised, double-blind, crossover study

E. G. Camargo, L. Weinert, J. Lavinsky, J. L. Gross,

S. P. Silveiro;

Endocrine Unit, Universidade Federal do Rio Grande do Sul, Porto Alegre, Brazil.

Background and Aims: An interaction between aspirin and angiotensin-converting-enzyme (ACE) inhibitors is pharmacologically plausible through their opposite effects on vasodilators prostaglandins. The aim of this study was to analyze the effect of aspirin on urinary albumin excretion (UAE) and glomerular filtration rate (GFR) of microalbuminuric type 2 diabetic patients treated with the ACE inhibitor enalapril.

Materials and Methods: In this randomized, double-blind, crossover, placebo-controlled study, 18 microalbuminuric (UAE $=30-300 \mathrm{mg} / 24 \mathrm{~h}$ ) type 2 diabetic patients received aspirin $(300 \mathrm{mg} / \mathrm{d})$ or identical placebo for 8 weeks, with a 6 -week washout period. The patients were aged $56 \pm 9$ years, with a diabetes duration of $16 \pm 7.5$ years; 11 (61\%) were female, and were all using enalapril $10 \mathrm{mg}$ bid. GFR was measured by ${ }^{51} \mathrm{Cr}$-EDTA single-injection method, UAE by imunoturbidimetry and expressed as median (range). Sample-size calculation disclosed a minimum of 17 patients to detect a $30 \%$ change in UAE, greater than the mean coefficient of variation of UAE (alfa 0.05 and beta 0.20 ).

Results: There were no significant statistical differences at baseline between placebo or aspirin groups, regarding GFR $\left(113 \pm 35\right.$ vs. $\left.112 \pm 34 \mathrm{ml} / \mathrm{min} / 1.73 \mathrm{~m}^{2} ; \mathrm{P}=0.91\right)$ and $\mathrm{UAE}[58.6$ (6.8-216) vs. $64(8.2-268) \mathrm{mg} / 24 \mathrm{~h}$; $\mathrm{P}=0.66]$, respectively. After 8 weeks of treatment there were no significant differences between the period using placebo or aspirin, respectively, regarding GFR $\left(108 \pm 34\right.$ vs. $111 \pm 47 \mathrm{ml} / \mathrm{min} / 1.73 \mathrm{~m}^{2}$; $\mathrm{P}=0.90)$ and UAE $[57.7(8.9-420.0)$ vs. 63 (8.2-272.0) $\mathrm{mg} / 24 \mathrm{~h} ; \mathrm{P}=0.45]$ (Fig. 1). There were also no significant differences between blood pressure $(133 \pm 16 / 79 \pm 7$ vs. $134 \pm 11 / 77 \pm 7 \mathrm{mmHg} ; \mathrm{P}=0.41)$, capillary glycemia $(127 \pm 46$ vs. $131 \pm 39 \mathrm{mg} / \mathrm{dl} ; \mathrm{P}=0.67)$ and $\mathrm{A} 1 \mathrm{c}$ test $(8.0 \pm 1.7$ vs. $8.6 \pm 1.7 \%$ ), respectively, after placebo and aspirin. There were no period $(\mathrm{P}=0.41)$ or carry-over effects $(\mathrm{P}=0.49)$.
Conclusion: Low-dose aspirin $(300 \mathrm{mg} / \mathrm{d})$ does not interfere with UAE levels of microalbuminuric type $2 \mathrm{DM}$ while concomitantly using an ACE inhibitor.

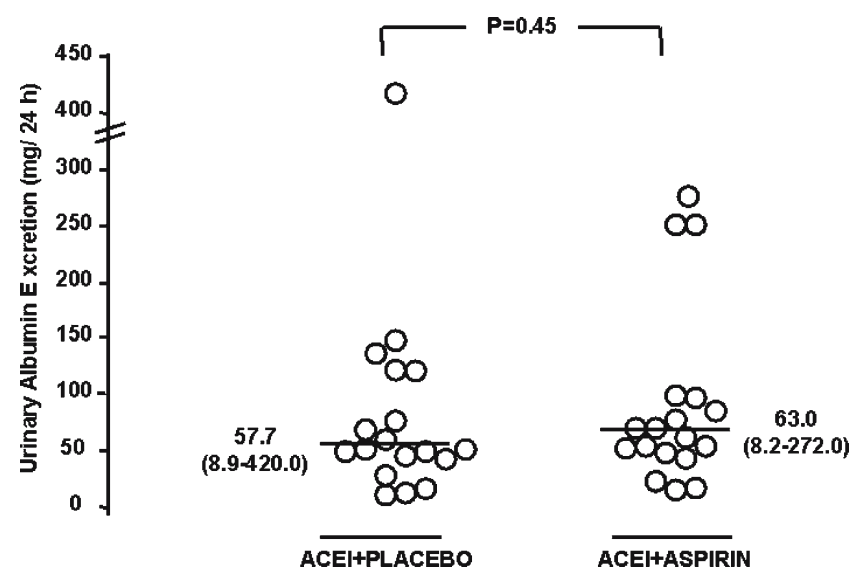

Figure 1 - Urinary albumin excretion (UAE) values after 8 weeks of angiotensin-converting-enzyme inhibitor (ACEI) plus placebo or aspirin

Supported by FIPE - Hospital de Clínicas de Porto Alegre

1048

Irbesartan treatment reduces biomarkers of inflammatory activity in patients with type 2 diabetes and microalbuminuria: an IRMA 2 substudy

F. Persson ${ }^{1}$, P. Rossing ${ }^{1}$, P. Hovind ${ }^{1}$, C. D. A. Stehouwer ${ }^{2}$, C. Schalkwijk ${ }^{2}$, L. Tarnow ${ }^{1}$, H.-H. Parving ${ }^{1,3}$;

${ }^{1}$ Steno Diabetes Center, Gentofte, Denmark, ${ }^{2}$ Department of Medicine, University Hospital, Maastricht, The Netherlands, ${ }^{3}$ Faculty of Health Science, Aarhus University, Denmark.

Background and Aims: To evaluate the impact of irbesartan treatment on biomarkers of low-grade inflammation, endothelial dysfunction, growth factors and advanced glycation end products (AGE peptides) during the Irbesartan in Patients with Type 2 diabetes and Microalbuminuria (IRMA 2) study. The study was a two-year multicenter, randomised, double-blind trial in patients with type 2 diabetes mellitus and microalbuminuria comparing irbesar$\tan (150$ or $300 \mathrm{mg}$ once daily) versus placebo. Primary endpoint was onset of overt nephropathy.

Materials and Methods: A subgroup $(\mathrm{n}=269,68 \%)$ was analysed for biomarkers at baseline and after one and two years. We assessed high sensitivity CRP (hs-CRP), interleukin 6 (IL-6), fibrinogen, soluble vascular cell adhesion molecule-1 (sVCAM-1), soluble inter cellular adhesion 
molecule-1 (sICAM-1), sE-selectin, transforming growth factor-beta (TGF-beta) and AGE-peptides.

Results: Treatment with irbesartan yielded significant changes in hs-CRP (based on GEE regression coefficient) -24.9 $(-35.6$ to -12.3$) \%(95 \% \mathrm{CI})$ during follow-up with a $5.4 \%$ decrease per year versus the $10 \%$ increase per year in the placebo group $(\mathrm{p}<0.001))$, fibrinogen (change, $-0.16(-0.30$ to -0.018$) \mathrm{g} / \mathrm{L}$ with treatment with a $-0.059 \mathrm{~g} / \mathrm{L}$ change per year from baseline versus placebo $0.059 \mathrm{~g} / \mathrm{L}$ increase per year $(\mathrm{p}=0.027)$, and IL-6 (treatment effect, $-13.6 \%(-22.0$ to -4.3 ) with $1.8 \%$ increase per year compared to placebo $6.5 \%$ increase per year $(\mathrm{p}=0.005))$. Changes in IL-6 were associated with changes in albumin excretion $(\mathrm{p}=0.04)$. There was no significant treatment effect on the other biomarkers.

Conclusion: $300 \mathrm{mg}$ irbesartan once daily reduces lowgrade inflammation in this high-risk population and this may reduce the risk of cardiovascular disease.

\section{9}

Impact of carvedilol on microalbuminuria is independent of the statin effect

D. S. H. Bell ${ }^{1}$, J. B. McGill ${ }^{2}$, V. A. Fonseca ${ }^{3}$;

${ }^{1}$ Department of Medicine, University of Alabama, Birmingham, United States, ${ }^{2}$ Department of Medicine, Washington University School of Medicine, St. Louis, United States, ${ }^{3}$ Department of Medicine, Tulane University Medical Center, New Orleans, United States.

Introduction: Both carvedilol and statins by their antiinflammatory effects improve endothelial function and microalbuminuria. To assess if carvedilol's effect was independent of the statin effect we preformed this study.

Methods: The GEMINI study compared the beta-blocker metoprolol with the combined alfa-beta-blocker carvedilol in a randomized, double-blind, parallel-group, multi-center US trial with 1210 hypertensive type 2 diabetic patients. Microalbuminuria was measured in all patients by radioimmunoassay.

Results: In 388 subjects on carvedilol microalbumin was decreased by $14.0 \%$, and in 542 subjects on metoprolol non-significantly increased by $2.5 \%$ resulting in a $16 \%$ relative reduction $(95 \%$ CI $6,2.5 \mathrm{p}=0.003)$. Over the course of the trial $6.4 \%$ of subjects on carvedilol and $10.6 \%$ of subjects on metoprolol developed microalbuminuria (OR $0.53,95 \%$ CI $0.30,0.93 \mathrm{p}=0.03$ ). At baseline 183 of the carvedilol group and 247 of the metoprolol group were taking statins. In the carvedilol group there was a $16.8 \%$ reduction $(\mathrm{p}=0.01)$ and in the metoprolol group a $6.8 \%$ increase $(\mathrm{p}=0.32)$ in microalbuminuria. Overall there was a $22.2 \%$ treatment difference (95\% CI 34.8-7.2 $\mathrm{p}=0.005$ ).

Conclusion:The effect of carvedilol on microalbuminuria was not ameliorated by concurrent statin use. Therefore, it is likely that separate anti-inflammatory pathways are stimulated by statins and carvedilol resulting in improved endothelial function.

Supported by Research Grant from GlaxoSmithKline

1050

Combined antihypertensive drugs and antihypertensive/ statin combination seem to be superior in reducing blood pressure and inflammation

S. Ljubic ${ }^{1}$, J. Bozikov ${ }^{2}$, M. Vucic Lovrencic ${ }^{3}$,

I. Pavlic Renar ${ }^{1}$, Z. Metelko ${ }^{4}$;

${ }^{1}$ Diabetology Department, Vuk Vrhovac University Clinic, Zagreb, ${ }^{2}$ Public Health Department, Zagreb University Medical School, ${ }^{3}$ Department for Laboratory Medicine, Vuk Vrhovac University Clinic, Zagreb, ${ }^{4}$ Hospital Directorate, Vuk Vrhovac University Clinic, Zagreb, Croatia.

Background and Aims: Hypertension and inflammation facilitate the progression of nephropathy, particularly when albuminuria is present. The aim of the study was to compare the effect of renin-angiotensin system (RAS) inhibitors and statins alone and in combination on blood pressure, albuminuria, and C-reactive protein (CRP) and fibrinogen (FIB) as pro-inflammatory markers.

Materials and Methods: A total of 328 hypertensive type 2 diabetics (56.48 \pm 10.9 yrs. old; diabetes duration $11.2 \pm 6.7$ yrs.) were studied over a $1-y r$ follow-up period. Patients were randomized to receive either angiotensinconverting enzyme (ACE) inhibitor lisinopril (group I, $\mathrm{n}=65$ ), angiotensin receptor blocker (ARB) losartan (group II, $\mathrm{n}=52$ ) and atorvastatin (group III, $\mathrm{n}=64$ ), or lisinopril/ losartan (group IV, $\mathrm{n}=45$ ) and atorvastatin/lisinopril combination (group $\mathrm{V}, \mathrm{n}=58$ ). The control group included 44 patients. CRP, FIB, atherogenic index of plasma (AIP), systolic (SBP), diastolic (DBP) and pulse pressure (PP) and albumin excretion rate (AER) were determined at the beginning and at the end of the study. The patients were assigned to groups based on AER/mg/24 $\mathrm{h} /(<15,15-30$, $30-300,>300)$ and PP $(<45,45-50,50-65,>65)$. Wilcoxon signed ranks test and Kruskall-Wallis test were used in the statistical analysis.

Results: Initial SBP, DBP, PP and AER were significantly reduced in the groups treated with RAS-inhibitors (generally $\mathrm{p}<0.01$ ), but $\mathrm{PP}$ also fell in the group treated with atorvastatin $(\mathrm{p}=0.009)$. CRP decreased significantly in all groups $(p<0.01)$, with the exception of Group II $(p=0.232)$, whereas FIB was significantly reduced in the groups I, III and $\mathrm{V}(\mathrm{p}=0.040, \mathrm{p}=0.001$ and $\mathrm{p}=0.023$, respectively). AIP was significantly reduced in the atorvastatin-treated group $(p<0.001)$. Among-group comparison revealed that the reduction in PP and SBP was most pronounced in Group 
IV (both $\mathrm{p}<0.001$ ), while a difference in DBP reduction was not observed $(\mathrm{p}=0.147)$. Group III, followed by Group I, was the most effective in AER reduction. Group I and III did not differ in their effect on CRP and FIB ( $p=0.134$ and $p=0.256$, respectively), whereas the reduction of CRP and FIB was more pronounced in Groups III and V, mainly in the group treated with the atorvastatin/lisinopril combination $(\mathrm{p}<0.01)$. Significant differences were found in initial CRP, FIB and AIP ( $p=0.01, p=0.004$ and $p=0.041$, respectively) according to AER, and in FIB and AIP (both $\mathrm{p}<0.001$ ) according to PP. Conclusion: Blood pressure, AER, AIP and inflammatory markers are among risk factors for the development of nephropathy. ACE-inhibitor/ARB and ACE-inhibitor/atorvastatin combinations seem to be more efficient than these drugs used separately in the reduction of BP and AER, and inflammatory markers, respectively.

\section{PS 91}

\section{Nephropathy - clinical}

\section{1}

Microalbuminuria is common in Japanese type 2 diabetes: a nationwide survey from Japan Diabetes Clinical Data Management Study Group

H. Yokoyama ${ }^{1}$, K. Kawai ${ }^{2}$, M. Kobayashi ${ }^{3}$ on behalf of JDDM Study Group;

${ }^{1}$ Internal Medicine, Jiyugaoka Clinic, Obihiro, ${ }^{2}$ Internal Medicine, Kawai Clinic, Tsukuba, Japan, ${ }^{3}$ First Dept. of Medicine, Toyama Med. Phar. Univ., Toyama, Japan.

Background and Aims: To elucidate the prevalence of microalbuminuria and clinical nephropathy in a nationwide survey in patients with T2DM.

Materials and Methods: We performed a cross-sectional, multi-center study which consists of 22 medical clinics (from different areas in Japan) using the same software to incorporate patients' record. All consecutive patients who visited from Jan. 2004 to July 2005 and whose diabetes was diagnosed before 2003 were included.Main Outcome and Measurements were urinary albumin to creatinine ratio (ACR, $\mathrm{mg} / \mathrm{gcr}$ ) and serum concentration of creatinine (Scr, $\mathrm{mg} / \mathrm{dl}$ ). Nephropathy stage was defined as follows: I, normoalbuminuria $(\mathrm{ACR}<30)$; II, microalbuminuria ( $\mathrm{ACR}$ $>=30$ and $<300$ ); III, overt nephropathy (ACR $>300$ and $\mathrm{Scr}<2.0$ ); IV, renal failure (Scr $>=2.0$ ); V, end-stage renal failure (treated with dialysis or renal transplantation).

Results: A total of 8897 patients were included. More than $30 \%$ of patients were microalbuminuric, and $10 \%$ were suffering from clinical nephropathy and/or renal failure. In patients with normo- or microalbuminuria, ACR was significantly higher in female than in male, and muliple regression analysis revealed that significant determinants of ACR were SBP, HbAlc, age, femaleness, BMI, duration, and triglyceride (F-value 53.9, $\mathrm{p}<0.0001$ ).

Conclusion: In a nationwide survey of patients with T2DM in Japan, microalbuminuria is common. The result may account for the increasing incidence of end-stage renal failure in Japan.

Clinical characteristics of the subjects

\begin{tabular}{llllll}
\hline Stage & I & II & III & IV & V \\
\hline N(\%) & $5152(58)$ & $2812(32)$ & $657(7)$ & $242(2.6)$ & $34(0.4)$ \\
Male\% & 66 & 63 & 72 & 70 & 82 \\
BMI & $24.1 \pm 3.7$ & $24.7 \pm 4.0$ & $25.1 \pm 4.1$ & $23.8 \pm 3.9$ & $23.9 \pm 4.0$ \\
HbA1c (\%) & $6.9 \pm 1.1$ & $7.3 \pm 1.3$ & $7.4 \pm 1.3$ & $6.7 \pm 1.2$ & $6.5 \pm 2.5$ \\
Diet/Tablet/Insulin\% & $23 / 59 / 18$ & $15 / 59 / 26$ & $9 / 49 / 42$ & $14 / 36 / 50$ & $15 / 12 / 73$ \\
SBP (mmHg) & $127 \pm 15$ & $133 \pm 16$ & $137 \pm 19$ & $139 \pm 22$ & $139 \pm 16$ \\
DBP (mmHg) & $75 \pm 10$ & $76 \pm 11$ & $76 \pm 12$ & $74 \pm 12$ & $75 \pm 10$ \\
LDL-chol(mg/dl) & $122 \pm 58$ & $121 \pm 52$ & $133 \pm 82$ & $121 \pm 66$ & $103 \pm 49$ \\
HDL-chol (mg/dl) & $55 \pm 15$ & $54 \pm 15$ & $50 \pm 14$ & $47 \pm 14$ & $53 \pm 20$ \\
\hline
\end{tabular}

1052

Course of microalbuminuria in type 2 diabetic patients: a 5-year follow-up study

M. Murussi, N. Campagnolo, A. Coester, M. O. Beck, J. L. Gross, S. P. Silveiro;

Endocrine Division, Hospital de Clínicas de Porto Alegre, Porto Brazil.

Background and Aims: The aim of this study was to analyze the clinical course of microalbuminuria in a cohort of type 2 diabetes mellitus (DM) patients, evaluating risk factors related to progression to macroalbuminuria and mortality.

Materials and Methods: In this prospective study, 83 microalbuminuric (urinary albumin excretion [UAE] 20$199 \mu \mathrm{g} / \mathrm{min})$ type 2 DM patients, 51 men $(61 \%), 70(84 \%)$ white and $13(16 \%)$ mixed or black, aged $61 \pm 8$ years, with diabetes duration of $13 \pm 7$ years, and median UAE of $47.2 \mu \mathrm{g} / \mathrm{min}$ (range 20-193), were followed for a mean period of $5.2 \pm 2.5$ years. UAE was measured by immunoturbidimetry, and glomerular filtration rate (GFR) was estimated by the four-component Modification of Diet in Renal Disease equation, at baseline and at follow-up.

Results: Six patients (7\%) were lost to follow-up. Three (4\%) died from coronary artery disease before renal status re-evaluation. Among the $74(89 \%)$ re-examined patients, $18(24.3 \%)$ progressed to macroalbuminuria (MA), and 6 of them developed kidney failure, 41 (55.4\%) remained microalbuminuric (MI), and $15(20.3 \%)$ regressed to normoalbuminuria (NO). There were no differences among the groups concerning the number of patients receiving 
angiotensin-converting-enzyme inhibitors at baseline $(\mathrm{P}=0.31)$ or at follow-up $(\mathrm{P}=0.10)$. Cox proportional hazards analysis (Hazard Ratio [95\% Confidence Interval]) showed that baseline UAE above the median, $\geq 47.2 \mu \mathrm{g} / \mathrm{min}$, (HR: 3.5 [1.05-11.65], $P=0.042$ ) was related to the progression to macroalbuminuria, higher baseline GFR (0.96 [0.94-0.99], $P=0.002)$ was a protector factor for progression, while age $(P=0.20)$, and diabetic retinopathy $(P=0.31)$ were not significant. Seventeen $(23 \%)$ patients developed GFR values $<60 \mathrm{ml} / \mathrm{min} / 1.73 \mathrm{~m}^{2}$ at follow-up (11 in MA, 5 in MI, and 1 in NO group [ $P=0.0001])$. A Cox proportional hazards analysis disclosed that higher baseline GFR levels (0.95 [0.93-0.97], $P=0.001$ ) protected against the development of GFR values $<60 \mathrm{ml} / \mathrm{min} / 1.73 \mathrm{~m}^{2}$ at follow-up, while UAE $\geq$ $47.2 \mu \mathrm{g} / \mathrm{min}(P=0.20)$, and age $(P=0.27)$ were not significant in the model. The mortality rates were not different among the patients who progressed or not (log rank, $P=0.46$ ).

Conclusion: In microalbuminuric type 2 DM patients, the progression to later stages of kidney disease was observed in only $25 \%$ of the patients and was mainly related to a greater involvement of renal function, expressed by higher baseline UAE levels, and lower baseline GFRs, which also predicted the development of GFR values $<60 \mathrm{ml} / \mathrm{min} /$ $1.73 \mathrm{~m}^{2}$ at follow-up.

Supported by grants from Programa de Apoio a Núcleos de Excelência, Hospital de Clínicas de Porto Alegre, and Fundação de Coordenação de Aperfeiçoamento de Pessoal de Ensino Superior (CAPES).

\section{3}

Long-term prevention of diabetic nephropathy: an audit K. J. Schjoedt ${ }^{1}$, P. Rossing ${ }^{1}$, H. P. Hansen ${ }^{1}$, L. Tarnow ${ }^{1}$, H.-H. Parving ${ }^{1,2}$;

${ }^{1}$ Steno Diabetes Center, Gentofte, Denmark, ${ }^{2}$ Faculty of Health Science, Aarhus University, Denmark.

Background and Aims: A risk for developing diabetic nephropathy of 3-30\%/year has been reported in type 1 diabetic patients with microalbuminuria not receiving antihypertensive treatment. In 1995 international guidelines recommended angiotensin converting enzyme (ACE) inhibitor treatment in patients with microalbuminuria, as randomised studies demonstrated a risk reduction of $62 \%$ for development of diabetic nephropathy in patients treated with ACE inhibitors. The aim of the present 11-year observational follow-up study was to audit the effect of RAS-blocking treatment on progression of microalbuminuria and development of diabetic nephropathy.

Materials and Methods: In 1995 ACE inhibitors were recommended for treatment of microalbuminuric type 1 diabetic patients in our outpatient clinic at Steno Diabetes
Center. For audit purpose we consecutively identified all type 1 diabetic patients with persistent microalbuminuria (30-300 mg/24 hours) ( $\mathrm{n}=227)$. During follow-up, patients visited our outpatient clinic 2-4 times/year, where albuminuria, BP and HbA1c was measured. Development of diabetic nephropathy was defined as urinary albumin excretion rate $>300 \mathrm{mg} / 24 \mathrm{~h}$ in 2 of 3 urine samples. Patients were followed until December 31, 2005, death, or emigration.

Results: At end of follow up, a total of 134 patients were still followed at Steno Diabetes Center, 58 patients $(26 \%)$ had died, and 35 patients (15\%) had moved to another part of the country. Mean follow-up time was 8.5 (3.5) years. Throughout the study $78 \%$ were treated with a RAS-blocking agent (ACE inhibitor or angiotensin II receptor blocker). In 200529 patients were not receiving RAS-blocking treatment, of whom 19 had spontaneously regressed to normoalbuminuria, all of the remaining patients had been recommended RASblocking treatment, but did not comply with the treatment, mostly because of adverse effects. Overall there was a mean decline in UAER of 4\%/year. During follow-up 60 patients (26\%) progressed to overt diabetic nephropathy, corresponding to $3 \% /$ year. Of these patients 26 regressed to microalbuminuria and 5 to normoalbuminuria on RAS blocking agents. Median (range) number of antihypertensive agents prescribed was $2(0-5), 60 \%$ of patients with elevated urinary albumin excretion rate received low-dose aspirin and $63 \%$ received statins. Mean (SE) systolic BP was 141 (1) mm $\mathrm{Hg}$ during follow-up as compared to 142 (1) at baseline (NS). In contrast, diastolic BP was reduced from 80 (1) $\mathrm{mm} \mathrm{Hg}$ to 77 (1), $P<0.001$. There was a small decrease in HbAlc of 0.2 (95\% CI: 0.1 to 0.3 )\%-points from $8.8 \%$ at baseline, $P<0.01$.

Conclusion: In an outpatient clinic, the implementation of RAS-blocking treatment in type 1 diabetic patients with microalbuminuria successfully reduced long-term progression to overt diabetic nephropathy comparably to what has previously been reported in intervention trials of shorter duration.

Supported by the Clinical Development Foundation at Steno Diabetes Center

\section{4}

Is the decrement in renal function in older compared to younger patients with type 2 diabetes independent of albuminuria status?

E. A. Premaratne ${ }^{1}$, R. J. MacIsaac ${ }^{1}$, C. Tsalamandris ${ }^{1}$, S. Panagiotopoulos ${ }^{1}$, D. Power ${ }^{2}$, G. Jerums ${ }^{1}$;

${ }^{1}$ Endocrinology Unit, Austin Health/Department of Medicine, University of Melbourne, Heidelberg, Victoria, Australia, ${ }^{2}$ Nephrology and Dialysis Unit, Austin Health/Department of Medicine, University of Melbourne, Heidelberg, Victoria, Australia. 
Background and Aims: Diabetic nephropathy is the leading cause of renal failure in the developed world. We aimed to characterize the association between age, albuminuria status, blood pressure and renal function in a cohort of subjects with Type 2 diabetes.

Materials and Methods: In a cross sectional study, glomerular filtration rate (GFR) was measured in 656 unselected subjects with Type 2 diabetes, (age 28-86 years) attending a tertiary referral centre and 49 non-diabetic healthy subjects (potential kidney donors, age 31-65 years) using plasma disappearance of ${ }^{99 \mathrm{~m}} \mathrm{Tc}-\mathrm{DTPA}$.

Results: For subjects aged $>55$ years (Figure) mean GFR was similar in those with normo-and microalbuminuria, but reduced in those with macroalbuminuria $(\mathrm{p}<0.01)$. For subjects aged $<55$ years, GFR was similar regardless of albuminuria status.

Across different age groups, the decrement in GFR in the non-diabetic subjects was $1.03 \mathrm{ml} / \mathrm{min} / 1.73 \mathrm{~m}^{2} /$ year, consistent with the age related decline of GFR reported in previous studies. In subjects with diabetes, a greater decrement in GFR was observed beyond that expected with ageing alone in all of the albuminuria categories $(1.8$, 1.6 and $1.8 \mathrm{ml} / \mathrm{min} / 1.73 \mathrm{~m}^{2} /$ year in the normo-, micro- and macroalbuminuric groups respectively).

Decrements in GFR with age in subjects on antihypertensive therapy (AHT) were similar to the above rates in each albuminuria group. There was also a correlation between age and pulse pressure in all albuminuria categories $(\mathrm{r}=0.36, \mathrm{p}<0.001$ in normo- and microalbuminuric groups and $\mathrm{r}=0.27, \mathrm{p}<0.001$ in macroalbuminuric group). Mean $\mathrm{HbA1c}$ values were similar among the albuminuria categories and the age groups.

Mean GFR was significantly lower in the group treated with AHT compared to the group not on AHT in the normoalbuminuria $\left(83+2\right.$ vs $101+3 \mathrm{ml} / \mathrm{min} / 1.73 \mathrm{~m}^{2}$, $\mathrm{p}<0.01)$ and microalbuminuria $(76+3$ vs $101+7 \mathrm{ml} / \mathrm{min} /$ $\left.1.73 \mathrm{~m}^{2}, \mathrm{p}<0.01\right)$ categories. However, the subjects on AHT were significantly older, had a longer disease duration and higher systolic blood pressure and pulse pressure. AHT was associated with a lower GFR even when adjusted for age.

Conclusions: This study shows a strong association between increasing age and GFR in Type 2 diabetes, above and beyond that expected with ageing alone which may be related to increasing pulse pressure with age. Similar decrements in GFR with age were observed in normo-, micro- and macroalbuminuric patients, suggesting that this apparent age related GFR decline is independent of the albuminuria status. However, as increased albumin excretion and low GFR are each related to increased cardiovascular risk, longitudinal studies free from possible survival bias are required to further delineate the true decline in GFR with ageing.
Figure: Mean GFR across age groups in Type 2 diabetic subjects

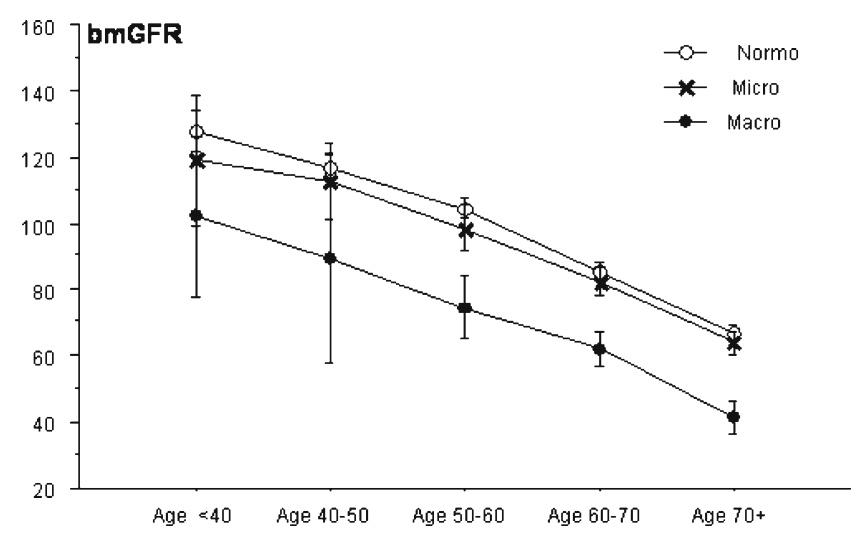

Supported by NHMRC grants, Australia

1055

Relationship between homocysteine and incipient diabetic nephropathy in type $\mathbf{2}$ diabetes mellitus

Y. Yue Hong, J. Liu;

Endocrinology, Shanxi Provencial People Hospital, Taiyuan, China.

Back ground and Aims: To investigate the ralationship betwee Homocysteine (Hcy)and incipient diabetic nephropathy in type 2 diabetes mellitus(T2DM), and explor the factors influencing plasma Hcy in cases of T2DM.

Materials and Methods: Two hundred and fifty-two cases of T2DM and 30 cases of normal control(CON)were enrolled in the study. The cases of T2DM were divided into two groups: one group without complications (NDC) and another group with incipient diabetic nephropathy(DN).Plasma Hcy, folic acids(FA), vitaminB $\mathrm{B}_{12}\left(\mathrm{VitB}_{12}\right), \mathrm{HbA}_{1} \mathrm{c}$, fasting blood glucose (FBG),postprandial blood glucose(PBG), creatinine(Cr),cholesterol(CHO),triglyceride(TG),transforming growth factor $\beta_{1}$ (TGF- $\beta_{1}$ ) levels, urinous $N$-acetylglucosaminidase(NAG) and microalbumin/creatinine (MAlb/cr) levels were measured.The mutation of the C677T transition of methylenetetrahydrofolate(MTHFR) was determined by PCR-RFLP.

Results: The patients with T2DM had higher Hcy levels and higher prevalence of hyperhomocysteinemia (Hhcy) than normal group,while incipient diabetic nephropathy higher than those without this complication. The Hcy levels of group NDC versus CON is (11.91 \pm 7.29$)$ versus (9.72 \pm $2.20)$, group $D N$ versus NDC is $(16.60 \pm 12.59)$ versus (11.91 \pm 7.29), $(\mathrm{P}<0.05)$. The incidence rate of Hhcy in group $\mathrm{DN}$ is $45.4 \%$, NDC is $22.9 \%$, while $\mathrm{CON}$ is $3.3 \%,(\mathrm{P}<0.05)$. The spearman's correlation analysis between Hcy and other 
variables showed that the Hcy levels in T2DM were positively related to TT genetype of MTHFR(r $=0.654)$, $\operatorname{BMI}(\mathrm{r}=0.214), \operatorname{FBG}(\mathrm{r}=0.141), \operatorname{PBG}(\mathrm{r}=0.171), \operatorname{HbA} 1 \mathrm{c}(\mathrm{r}=$ $0.170), \mathrm{NAG}(\mathrm{r}=0.178), \mathrm{MA} 1 \mathrm{~b} / \mathrm{cr}(\mathrm{r}=0.3)$, course $(\mathrm{r}=0.150)$, smoking $(\mathrm{r}=0.166)$ and metformin treatment $(\mathrm{r}=0.157)$, $(\mathrm{P}<0.05)$, and was negtively related to plasma $\mathrm{FA}(\mathrm{r}=$ $-0.133)$ and $\mathrm{VitB}_{12}$ levels $(\mathrm{r}=-\mathrm{O} .273),(\mathrm{P}<0.05)$. Multiple logistis regression analysis showed that course, diastolic blood preasure (DBP), FPG, NAG and plasma Hcy levels were the independent risk factors for incipient diabetic nephropathy, their OR value is $1.074,1.054,1.206,1.043$, 1.057 , and $\mathrm{P}$ value is $0.045,0.001,0.003,0.000$ and 0.005 respectingly.

Conclusion: Hhcy was a independent risk for incipient diabetic nephropathy; TT genetype of MTHFR, FA, VitB 12 , MA1b/cr,NAG level and metabolic disorder influenced the plasma Hcy level in T2DM.

\section{6}

Systemic inhibition of nitric oxide synthase unmasks differences in renal vascular function between African and Caucasian patients with type 2 diabetes without nephropathy

K. Zitouni ${ }^{1,2}$, J. Barron ${ }^{3}$, D. Harry ${ }^{1,2}$, M. Mitra ${ }^{1}$,

K. A. Earle ${ }^{1,2}$;

${ }^{1}$ Medicine, Royal Free and University College Medical School, London, ${ }^{2}$ Cellular and Molecular Medicine, St George's Hospital University of London, ${ }^{3}$ Carshalton, St Helier Hospital, Surrey, United Kingdom.

Background and Aims: Systemic hypertension promotes renal disease and is associated with altered nitric oxide bioavailability. We considered that prior to the onset of nephropathy, variations in this pathway may be evident between racial groups with differing susceptibility diabetic microangiopathy.

Materials and Methods: We studied 18, age normotensive, normoalbuminuric patients with type 2 diabetes of African and Caucasian origin of similar age and duration of diabetes. Renal blood flow was measured using a constant infusion of paraminohippurate, in response to an amino acid mixture and $\mathrm{N}^{\mathrm{g}}$-monomethyl-1-arginine (L-NMMA) under euglycaemic conditions.

Results: There were no differences in basal renal plasma flow (RPF) rates, systolic or diastolic blood pressure in the African and Caucasians groups (533.7[174.7] vs 565.3 [260.8] $\mathrm{mls} / \mathrm{min} / 1.73 \mathrm{~m}^{-2} ; \mathrm{p}=0.78,124.9[23.7]$ vs 121.6 [12.3] $\mathrm{mmHg} ; \mathrm{p}=0.29$ or $77.1[9.5]$ vs $76.3[5.7] \mathrm{mHg}$; $\mathrm{p}=0.81$ ). Addition of L-NMMA to the amino acid infusion resulted in a significant mean [95\% confidence interval] increase in systemic blood pressure in the African $(\Delta 7.3$
[1.0-13.7] $\mathrm{mmHg} ; \mathrm{p}=0.03$ ) but not in the Caucasian group $(\Delta 2.4[-3.5-8.3] \mathrm{mmHg} ; \mathrm{p}=0.38)$. There were mean falls in RPF of 13.3 and $46.0 \mathrm{mls} / \mathrm{min} / 1.73 \mathrm{~m}^{-2}$ ( $\mathrm{p}<0.05$ and $\mathrm{p}=0.25$ ), whilst renal vascular resistance (RVR) was unchanged at $0.11(0.02) \mathrm{mmHg} / \mathrm{ml} / \mathrm{min} / 1.73 \mathrm{~m}^{-2} ; \mathrm{p}=0.61$ but had risen to $0.14(0.04) \mathrm{mmHg} / \mathrm{ml} / \mathrm{min} / 1.73 \mathrm{~m}^{-2}$; $\mathrm{p}=0.036$ in Caucasian and African groups respectively. Systemic mean arterial pressure was associated with RVR in the African $\left(r^{2}=0.77 ; p=0.004\right)$ but not in the Caucasian group $\left(\mathrm{r}^{2}=0.03 ; \mathrm{p}=0.68\right)$.

Conclusion: In these patients with type 2 diabetes without nephropathy, systemic inhibition of nitric oxide synthase caused a greater pressor response and decrement in renal blood flow in patients of African compared with Caucasian origin. This suggests that NO bioavailability plays a more critical role in preserving renal haemodynamic integrity in African than Caucasian patients. These observations may be relevant to the racial differences in vulnerability to diabetic renal disease.

1057

Prevalence of ischaemic nephropathy in type 2 diabetes I. I. Klefortova ${ }^{1}$, M. S. Shamkhalova ${ }^{1}$, N. B. Smirnova ${ }^{2}$, M. V. Shestakova ${ }^{1}$, V. A. Alpenidze ${ }^{3}$, E. F. Tugeeva ${ }^{3}$, U. I. Buziashvili;

${ }^{1}$ Diabetic Nephropathy, ${ }^{2}$ Ophtalmology, Endocrinology Research Center, Moscow, ${ }^{3}$ Klinical Diagnostic, Bakoulev Scientific Center of Cardiovascular Surgery, Moscow, Russian Federation.

Background and Aims: The aim of study was to evaluate the prevalence of renal artery stenosis (RAS) in subjects with type 2 diabetes and hypertensive aged 50 and above by using duplex sonography, to assess clinical, biochemical and hemodynamic predictors of ischemic nephropathy.

Materials and Methods: 156 patients (62 males and 94 females) with type 2 diabetes were studied by color-flow duplex sonography of renal artery and abdominal aorta.

Results: 55 (35.3\%), cases had significant RAS (26.3\% monolateral, $8.97 \%$ bilateral). Men more frequently had RAS (43.5\% of all men, $29.8 \%$ of all female). $39.7 \%$ had diabetic retinopathy, $47.4 \%$ had hypertonic angioretinopathy. RAS was present in $41.3 \%$ of patients in the age group $50-59,30.7 \%$ in the $60-69$ and $40.9 \%$ at age 70 and above. RAS-negative and RAS-positive groups were similar in duration of diabetes, aged, hyperlipidemia, 24-h blood pressure, diabetic management and glomerular filtration rate (GFR). The comparison between patients with and without RAS revealed significant difference in the incidence of previous myocardial infarction $(\mathrm{p}=0.027)$, prevalence of renal dimensions asymmetry $(>11 \mathrm{~mm})(\mathrm{p}=0.02)$, cardiac 
interventricular septum hypertrophy $(\mathrm{p}=0.05)$, hyperfibrinogenaemia $(\mathrm{p}=0.001)$, smoking $(\mathrm{p}=0.003)$.

Conclusions: Color-duplex ulnrasonography-detected RAS is common in type 2 diabetic patients (specially in smoking males) with myocardial dysfunction and rheological abnormalities.

\section{8}

Metabolic control and insulin analogues influence erythropoietin levels in type 2 diabetic patients with nephropathy

C. Hasslacher, S. Sanden, D. Raupp;

Innere Medizin, St. Josefskrankenhaus, Heidelberg, Germany.

Background and Aims: Diabetic patients with nephropathy $(\mathrm{N})$ show a disturbed synthesis of erythropoietin (EPO) even at moderate degree of kidney dysfunction compared to nondiabetic patients. Possible consequences are an earlier onset of anemia and/or cardiovascular complications, since EPO - aside from its stimulating influence on erythropoesis - posses protective effects on development of ischemic damage. There is only little information on factors which influence levels of erythropoetin in type 2 diabetic patients with and without nephropathy

Materials and Methods: The following parameters were determined in 248 type 2 diabetic patients $(54 \%$ men, age $<75$ years) of our outpatient clinic: erythropeotin (EPO) levels, hemoglobin $(\mathrm{Hb})$, calculated creatinine clearance (CCL), albumin excretion (AE), HbAlc, high sensitive CRP (hs CRP), NT-proBNP, serum lipids, kind of therapy. Patients were divided in two group:,,without nephropathy“" (=N- ; CCL $>90 \mathrm{ml} / \mathrm{min}$, no increased AE; $\mathrm{n}=86$ ) and, with nephropathy" (=N+: CCL $<90 \mathrm{ml} / \mathrm{min}$ with or without AE; $\mathrm{n}=148$ ). Apparently healthy nondiabetic people served as controls $(\mathrm{n}=61)$.

Results: EPO levels were 10,9 U/1 in nondiabetic C, $14.5 \mathrm{U} / 1$ in group $\mathrm{N}-$ and $13.0 \mathrm{U} / 1$ in group $\mathrm{N}+$. In $\mathrm{N}$-, EPO showed a negative correlation to $\mathrm{Hb}(\mathrm{r}=-0,357$; $\mathrm{p}<0,001$ ), no corresponding correlation could be found in $\mathrm{N}+$. Patients with poor metabolic control $(\mathrm{HbAlc}>9 \%)$ exhibited lower EPO levels (12 U/l) compared to patients with better metabolic control (HbAlc $<7 \% ; 15 \mathrm{U} / \mathrm{l})$ in both groups. In $\mathrm{N}$ - higher EPO levels were associated with betablocker therapy, in $\mathrm{N}+$ with insulin therapy, especially with insulin analogs. Positive correlation could be found between EPO and levels of NT-proBNP ( $\mathrm{r}=0.324$, $\mathrm{p}<0.002)$ and hsCRP $(r=0,222 ; \mathrm{p}<0.04)$. A multiple linear regression analysis showed that in group N- EPO levels were primarily influenced by hemoglobin concentration and betablocker therapy. In group $\mathrm{N}+$ important influencing factors were $\mathrm{HbA1c}$ - levels, use of insulin analogs and RAS- inhibiting substances.

Conclusion: EPO levels correlated with quality of metabolic control and kind of antidiabetic therapy in type 2 diabetics with nephropathy. These findings may have important therapeutic implications for the treatment of diabetic patients with renal insufficiency with respect to impaired synthesis of EPO.

Supported by Lautenschläger Stiftung Diabetes and Roche Pharma Germany

1059

Haematocrit lowering effect and anaemia due to inactivation of renin-angiotensin system in type 2 diabetic patients with normal renal function

A. E. Raptis ${ }^{1}$, D. Vlahakos ${ }^{1}$, C. Kourkouti ${ }^{1}$, M. Panagiotidou ${ }^{1}$, T. Economopoulos ${ }^{1}$, S. A. Raptis ${ }^{1,2}$;

${ }^{1}$ 2nd Dept of Internal Medicine-Propaedeutic,Research Institute and Diabetes Center,Attikon Hospital, Athens University, Athens, ${ }^{2}$ Hellenic National Diabetes Center, HNDC, Athens, Greece.

Background and Aims: An intriguing interrelationship has been revealed between Renin Angiotensin System (RAS) and erythropoesis. Decrease in hematocrit (Hct) and anemia has been reported in patients with renal, cardiac and pulmonary diseases treated with Angiotensin Converting Enzyme Inhibitors (ACEI) and/or Angiotensin Receptor Blockers (ARBs). Anemia is a common finding in patients with diabetic nephropathy, and identifies a subset of highrisk patients for premature death. The aim of this study was to explore the Hct lowering effect of ACEI and/or ARBs in type 2 diabetic (T2D) patients with normal renal function. Materials and Methods: From a cohort of 1750 pts with T2D followed in our tertiary diabetic clinic, we selected 180 patients (group Rx), with normal renal function $(\mathrm{Cr} \leq 1.5 \mathrm{mg} \%)$ treated with ACEI and/or ARBs. These patients where matched for age, gender, renal function and duration of T2D with 180 patients (group C) who did not received ACEI or ARBs. Exclusion criteria included: malignancy, chronic inflammation, hepatic disease, gastrointestinal blood loss, abnormal MCV or therapy with PPRA- $\gamma$ agents. Hct and anemia (Hct $<36 \%$ female/ Hct $<39 \%$ male) where recorded in group $\mathrm{Rx}$, prior to ACEI or ARBS therapy and 1 and 3 years after RAS inactivation. Corresponding values were also recorded in group $\mathrm{C}$ patients.

Results (Mean \pm SD): The rate of complications, were higher in group $\mathrm{Rx}$ than in group C: hypertension $(91.6 \%$ vs. $23.9 \%, \mathrm{p}=0.0001)$, hyperlipidemia $(43.3 \%$ vs. $32.7 \%$, $\mathrm{p}=0.039)$, protein in urine $(9.4 \%$ vs. $1.1 \%, \mathrm{p}=0.0001)$, 
coronary artery disease $(6.1 \%$ vs. $0.5 \%, p=0.03)$. This reflects the high-risk group, which warranted RAS inactivation. In fact patients in group $\mathrm{Rx}$ had greater RAS activation than group $\mathrm{C}$, as indicated by ACE polymorphisms (DD/ID/II was 53\%/38\%/9\% group Rx 33\%/56\%/ $11 \%$ group $\mathrm{C}, \mathrm{p}=0.01)$. Glycocylated hemoglobin (HbA1c) values were comparable at baseline between the two groups (7.6 \pm 2 group $\mathrm{Rx}$ vs. $7.13 \pm 2.4$ group $\mathrm{C}$ ). $\mathrm{HbA1c}$ values remained stable in group $\mathrm{C}$ after 1 and 3 years of observation but decreased in group $\operatorname{Rx}(6.6 \pm 1.3$ the 1 st year, $p=0.003,6.7 \pm 1.4$ the 3rd year, $p=0.005$ ).

Both Hct values and prevalence of anemia did not differ between the groups at baseline (Hct: $43.3 \pm 3.3 \%$ group $\mathrm{Rx}$ vs. $42.6 \pm 3.3 \%$ group $\mathrm{C}$, and anemia: $5.3 \%$ group $\mathrm{Rx}$ vs. $3.4 \%$ group C). No significant difference in Hct and prevalence of anemia was observed in group $\mathrm{C}$ patients after 1 and 3 years of follow up. In contrast in group Rx Hct decreased (to $41.8 \pm 3.5 \%, \mathrm{p}=0.003$ ) after 1 year and remained unchanged for at least 3 years. Moreover, at the end of the 3rd year the prevalence of anemia in group Rx was higher than group C ( $7.9 \%$ vs. $2.8 \%, p=0.037)$.

Conclusion: Reduction in Hct is often found in diabetic patients with normal renal function, after treatment with ACEI and/or ARBs. Anemia is seen three times more often in patients treated with ACEI and/or ARBs compared to controls. Therefore the use of these drugs should be considered when other causes of anemia are excluded.

\section{PS 92}

\section{Erythropoietin and nephropathy}

\section{0}

Human cell line derived erythropoietin (epoetin delta) administered subcutaneously is effective in the management of anaemia associated with chronic kidney disease

J. T. C. Kwan ${ }^{1}$, R. D. Pratt ${ }^{2}$;

${ }^{1}$ South West Thames Renal \& Transplantation Unit, St Helier Hospital, Carshalton, United Kingdom, ${ }^{2}$ Clinical Medicine, Shire Pharmaceuticals, Wayne, United States.

Background and Aims: Epoetin delta (Shire Pharmaceuticals) is an erythropoietin produced by gene-activation technology in a human cell line and has a human-type carbohydrate profile, unlike recombinant erythropoietins produced in Chinese Hamster Ovary cell lines. In this study (carried out between 1998-2000), we assessed the efficacy and safety of subcutaneous (sc) epoetin delta in patients with chronic kidney disease (CKD) and anaemia.

Materials and Methods: Patients with CKD who had previously received sc epoetin for at least 30 days and had a haemoglobin level between 9.6 and $10.4 \mathrm{~g} / \mathrm{dL}$ at baseline, were eligible for this multicentre, open-label study. Patients who entered the study received sc epoetin delta at an initial dose identical to their previous epoetin treatment for up to 52 weeks. Treatment was given one, two or three times per week (depending on previous epoetin regimen) and was titrated to maintain haemoglobin levels at $10.0-12.0 \mathrm{~g} / \mathrm{dL}$. Primary objective was to assess mean haemoglobin levels over Weeks 12, 16, 20 and 24 in the study population who had at least one haemoglobin measure during this period.

Results: In total, 478 patients entered the study, 411 (288 on haemodialysis, 91 peritoneal dialysis and 32 who did not require dialysis) of whom were included in the primary efficacy analysis. Diabetic nephropathy was one of the most prevalent primary causes of CKD (23.6\% of patients). Most patients $(319,66.7 \%)$ completed the full 52 weeks of treatment. The most common reason for withdrawal was 'other' (38 patients, 7.9\%). One specific reason for this was a sterility test failure of one batch of study drug. As no immediate replacement batch was available, 19 patients were withdrawn and the study was terminated in the UK. Subsequent investigation found no contamination. Requirement for red blood cell transfusion (a per protocol withdrawal event) was the next most common withdrawal reason (27 patients, 5.6\%). The mean \pm SD haemoglobin level over Weeks 12, 16, 20 and 24 was $11.31 \pm 1.11 \mathrm{~g} / \mathrm{dL}$. Haemoglobin levels $>10.0 \mathrm{~g} / \mathrm{dL}$ were recorded in $83.9 \%$ of patients over this period. Mean haemoglobin levels were $>11.0 \mathrm{~g} / \mathrm{dL}$ in all analysed subgroups (e.g. dialysis requirements, age, sex, race). Mean dose of epoetin delta was $45.2 \mathrm{IU} / \mathrm{kg}$ and there was no evidence that dosing frequency had an effect on efficacy (mean haemoglobin levels, three-times weekly $v s$. once weekly: $11.16 \pm 1.09 \mathrm{~g} / \mathrm{dL}$ vs. $11.50 \pm 1.16 \mathrm{~g} / \mathrm{dL}$ ). Control of anaemia was maintained over the 52 weeks of the study, with mean haemoglobin levels from Week 37 to Week 52 of $11.16 \pm 1.14 \mathrm{~g} / \mathrm{dL}$. Overall, $86.8 \%$ of patients experienced at least one adverse event during the study, with $12.3 \%$ of patients having an event that was considered possibly related to treatment. The most common event thought possibly related to treatment was hypertension. Serious adverse events occurred in $49.4 \%$ of patients, with $4.6 \%$ of patients experiencing a serious event that was thought possibly related to treatment. 
Conclusion: Subcutaneous epoetin delta is effective in the management of patients with anaemia resulting from CKD, many of whom have diabetic nephropathy. It is effective in maintaining haemoglobin levels at $10.0-12.0 \mathrm{~g} / \mathrm{dL}$ regardless of dialysis requirements and can maintain haemoglobin control at a dosing frequency of once per week. The adverse event profile was as expected in this patient group. Supported by Hoechst Marion Roussel

\section{1}

Epoetin delta, erythropoietin produced by a human cell line, is as effective as epoetin alfa in patients with renal anaemia, including those with diabetic nephropathy

R. D. $\operatorname{Pratt}^{1}$, K. J. Martin ${ }^{2}$;

${ }^{1}$ Clinical Medicine, Shire Pharmaceuticals, Wayne, ${ }^{2}$ Department of Internal Medicine, Nephrology Division, Saint Louis University School of Medicine, St Louis, United States.

Background and Aims: Recombinant erythropoietins are used extensively in the management of anaemia associated with chronic kidney disease. At present, all of these agents are produced in Chinese Hamster Ovary (CHO) cell lines. Epoetin delta is an erythropoietin produced by geneactivation technology in a human cell line.

Materials and Methods: In this double-blind, activecomparator study, patients requiring haemodialysis and previously receiving intravenous erythropoietin therapy were randomized in a $3: 1$ ratio to receive epoetin delta or epoetin alfa. Eligible patients (haemoglobin level at baseline: $9.6-12.4 \mathrm{~g} / \mathrm{dL}$ ) received a starting dose equivalent to that they were receiving before randomization, given intravenously three-times per week. Doses were titrated to maintain haemoglobin at $10-12.0 \mathrm{~g} / \mathrm{dL}$. The double-blind treatment lasted for 24 weeks and was followed by a 28week, open-label phase during which all patients received epoetin delta. Primary efficacy endpoint was to assess the difference in mean haemoglobin levels (over Weeks 12, 16, 20 and 24) between the two groups. Subgroup analyses included patients with diabetic nephropathy.

Results: In total, 752 patients were randomized of whom 666 (363 men, 303 women) had a recorded baseline haemoglobin value and at least one haemoglobin measurement between Weeks 12 and 24. The epoetin delta group included 491 patients (mean age: $56.3 \pm 15.6$ years), with 175 in the epoetin alfa group (mean age: $56.8 \pm 15.1$ years). Primary diagnosis was diabetic nephropathy in $29.5 \%$ of the epoetin delta group and in $37.1 \%$ of the epoetin alfa group. The double-blind period was completed by 583 patients $(77.5 \%)$ and all of these entered the open-label phase. Mean haemoglobin levels between Weeks 12 and 24 were $11.57 \mathrm{~g} / \mathrm{dL}$ (95\% CI: 11.47-11.66) and $11.56 \mathrm{~g} / \mathrm{dL}$ (95\% CI: 11.41-11.70) in the epoetin delta and epoetin alfa groups, respectively. The difference between the two groups was $0.01 \mathrm{~g} / \mathrm{dL}$ (90\% CI: $-0.13-0.15)$ demonstrating equivalence between the two study agents. Mean dose was similar in the two groups. Analysis of results from Week 12 to study completion showed that epoetin delta maintained control of haemoglobin, with a mean level of $11.31 \mathrm{~g} / \mathrm{dL}$. Similar efficacy results were seen in the subgroup of patients with diabetic nephropathy (mean haemoglobin levels Weeks 12-24, epoetin delta $v s$. epoetin alfa: $11.47 \mathrm{~g} / \mathrm{dL}$ vs. $11.47 \mathrm{~g} / \mathrm{dL})$. Adverse events possibly related to treatment were reported in $9.2 \%$ of patients receiving epoetin delta and $8.4 \%$ of those receiving epoetin alfa. Serious adverse events occurred in $47.3 \%$ of patients $(1.7 \%$ thought possibly related to treatment) receiving epoetin delta over 52 weeks of treatment and in $33.0 \%$ of patients receiving epoetin delta over 24 weeks. The latter value compared with $26.7 \%$ in the epoetin alfa group over the same period. No deaths were thought to have any possible relation to study treatment. The overall frequency of adverse events was similar in diabetic and non-diabetic patients.

Conclusion: Epoetin delta is effective in maintaining haemoglobin levels of $10-12 \mathrm{~g} / \mathrm{dL}$ over 24 weeks in patients requiring haemodialysis (including patients with diabetic nephropathy), and is able to maintain control of haemoglobin over 52 weeks. Results are similar to those with epoetin alfa.

Supported by Hoechst Marion Roussel

1062

Anaemia correction with epoetin beta in patients with diabetes and chronic kidney disease - primary results of the Anaemia CORrection in Diabetes (ACORD) study E. Ritz ${ }^{1}$, R. W. Bilous ${ }^{2}$, F. de Alvaro ${ }^{3}$, M. Laville ${ }^{4}$, D. O'Donoghue ${ }^{5}$;

${ }^{1}$ Department of Internal Medicine, University of Heidelberg, Germany, ${ }^{2}$ Education Centre, James Cook University Hospital, Middlesbrough, United Kingdom, ${ }^{3}$ Servicio Nefrología, Hospital Universitario La Paz, Madrid, Spain, ${ }^{4}$ Department of Nephrology, Edouard Herriot Hospital, Lyon, France, ${ }^{5}$ Department of Renal Medicine, Hope Hospital, Salford, United Kingdom. 
Background and Aims: Diabetes is a major risk factor for cardiovascular (CV) disease, with the risk amplified by the presence of diabetic nephropathy (DN) and anaemia. Anaemia is associated with the incidence of $\mathrm{CV}$ events and a predictor for left ventricular hypertrophy. ACORD is the first prospective study examining whether attaining a target $\mathrm{Hb}$ of $13-15 \mathrm{~g} / \mathrm{dL}$ in patients with early diabetic nephropathy and moderate anaemia can reduce $\mathrm{CV}$ risk, preserve renal function, improve clinical outcomes and quality of life (QoL).

Material and Methods: ACORD was an open-label, multi-centre, randomised trial in patients with diabetes and stage II-III chronic kidney disease (CKD) comparing the effect of epoetin beta treatment to attain a target $\mathrm{Hb}$ of 13-15 g/dL (Group $1-\mathrm{G} 1$ ), vs maintaining a $\mathrm{Hb}$ range of 10.5-11.5 g/dL (Group 2 - G2). The primary endpoint was change from baseline in left ventricular mass index (LVMI) at 15 months. Additional echocardiographic and clinical variables, renal function and quality of life (QoL) were also examined.

Results: A total of 170 patients were randomized into two treatment arms (G1: 88; G2: 82). Study groups were well matched at baseline. The majority of patients had diabetic retinopathy (G1: 84\%; G2: 83\%) and pre-existing $\mathrm{CV}$ disease (G1: 90\%; G2: 94\%). Mean age was 56 years, HbA1c $8.1 \%$, systolic and diastolic blood pressure (SBP/ DBP) $131 / 76 \mathrm{mmHg}$. Change in $\mathrm{Hb}$ from baseline to study end differed significantly between study groups in accordance with the relevant target windows (G1: 11.8 to $13.4 \mathrm{~g} / \mathrm{dL}$; $\mathrm{G} 2: 11.7$ to $11.9 \mathrm{~g} / \mathrm{dL} ; \mathrm{p}<0.001)$ supporting the efficacy of once weekly SC epoetin beta treatment. At study end there was no significant difference in LVMI $(p=0.8811)$ between study groups. Multivariate analysis, however, showed a trend $(p=0.1539)$ towards a reduction in LVMI in G1 compared to G2. Secondary echocardiographic variables did not differ between treatment groups. Anaemia correction with epoetin beta was safe without clinically significant difference between G1 and G2. Blood pressure (BP) was well controlled during the study with no difference in mean BPs at study end (SBP/DBP in G1: 136/75 mmHg; G2: $134 / 77 \mathrm{mmHg}$ ). The annual $\mathrm{CV}$ event rate was low $(\sim 4 \%)$. There were significant benefits in QoL in favour of G1 (by SF-36; $p=0.039$ ). There was no difference in renal disease progression between study groups. Mean creatinine clearance decreased by $3.8 \mathrm{~mL} / \mathrm{min} / \mathrm{yr}$ in $\mathrm{G} 1$ and $3.9 \mathrm{~mL} /$ $\mathrm{min} / \mathrm{yr}$ in $\mathrm{G} 2$.

Conclusion: In diabetic patients with early $\mathrm{DN}$ and anaemia once weekly treatment with SC epoetin beta resulted in a significant increase in $\mathrm{Hb}$ and related significant improvements in quality of life. In contrast to clinical expectation,
LVMI was maintained stable in both study groups with no difference in favour of higher $\mathrm{Hb}$ targets, despite a trend towards regression of LVMI in multivariate analysis. Treatment with epoetin beta to avoid severe anaemia was safe. In this study the annual CV event rate was considerably lower than reported in previous studies.

Supported by Hoffman La-Roche Ltd

1063

The influence of erythropoietin on immunity in patients with diabetic nephropathy and anaemia

O. V. Ivanchenko, K. Zak, M. Gruzov, A. Butenko,

S. Melnichenco, A. Efimov;

Diabetology, V.P. Komissarenko Institute of Endocrinology and Metabolism, Kiev, Ukraine.

Background and Aims: The biological effect of erythropoietin (EPO) is more widely. Erythropoietin not only restores erythropoiesis in patients with EPO deficiency anemia but improves the quality of life and survival in patients with cardiovascular disease. The influence of EPO on the other systems is not well known. The aim of this study was to investigate the influence of EPO on immunity in patients with diabetic nephropathy (DN) treated with recombinant $[\mathrm{r}] \mathrm{EPO}$.

Materials and Methods: Patients with DN and EPO deficiency anemia and those with DN without anemia were examined. Control group was consisted of age- and sexmatched healthy persons. Fasting plasma endogen [e]EPO levels were measured using ELISA. Hemoglobin $(\mathrm{Hb})$, erythrocyte $(\mathrm{RBC})$, mean content of hemoglobin $(\mathrm{MCH})$, mean concentration hemoglobin in erythrocyte (MCHC), mean volume erythrocyte (MCV) were measured with hematological analyzer (Coulter). Lymphocyte (CD3+, CD4+, CD8+, CD20+, and CD56+cells) immunophenotype was determined by the method of flow cytometry on FACStar Plus. rEPO-beta (Roche) was injected subcutaneously at a dose of 2000 IU twice a week.

Results: Our data are shown in table. The level of eEPO in patients with DN and anemia was significant lower compared with patients with DN without anemia and healthy subjects. However, there was no direct correlation between degree of anemia and plasma eEPO level. Absolute content of $\mathrm{CD} 3+, \mathrm{CD} 4+, \mathrm{CD} 56+$ cells was decreased in DN patients with and without anemia before treatment. Anaemia was significantly improved after 8 weeks of rEPO administration, there were no significant differences in $\mathrm{MCV}, \mathrm{MCH}, \mathrm{MCHC}$ before and after 
treatment. At the same time absolute content of $\mathrm{CD} 3+, \mathrm{CD} 4+$, CD56+cells and CD4/CD8 index normalized.

\begin{tabular}{|c|c|c|c|c|}
\hline \multirow[t]{2}{*}{ Characteristic } & \multirow[t]{2}{*}{$\begin{array}{l}\text { Control } \\
\text { group }\end{array}$} & \multirow{2}{*}{$\begin{array}{l}\text { Patients with } \\
\text { DN without } \\
\text { anemia }\end{array}$} & \multicolumn{2}{|c|}{$\begin{array}{l}\text { Patients with DN } \\
\text { and anemia }\end{array}$} \\
\hline & & & $\begin{array}{l}\text { Before } \\
\text { treatment }\end{array}$ & $\begin{array}{l}\text { After } \\
\text { treatment }\end{array}$ \\
\hline $\mathrm{n}$ & 30 & 30 & 20 & 20 \\
\hline eErythropoietin & $13,48 \pm 2,08$ & $9,92 \pm 1,03 *$ & $3,82 \pm 0,94 *$ & \\
\hline $\mathrm{Hb}(\mathrm{g} / \mathrm{dl})$ & $14,2 \pm 0,31$ & $13,1 \pm 0,29$ & $7,51 \pm 0,28$ & $8,85 \pm 0,39 * *$ \\
\hline $\mathrm{RBC}\left(10^{12} 1\right)$ & $4,7 \pm 0,41$ & $4,2 \pm 0,37$ & $2,59 \pm 0,10$ & $3,24 \pm 0,35^{* *}$ \\
\hline Ht \% & $39,0 \pm 1,28$ & $33,1 \pm 1,29$ & $24,4 \pm 1,17$ & $32,33 \pm 1,24 *$ \\
\hline $\begin{array}{l}\text { Lymphocyte } \\
\left(10^{9} 1\right)\end{array}$ & $2,12 \pm 0,08$ & $1,83 \pm 0,1$ & $1,39 \pm 0,09$ & $1,62 \pm 0,10$ \\
\hline $\begin{array}{l}\text { CD3+cells } \\
\left(10^{9} 1\right)\end{array}$ & $1,39 \pm 0,09$ & $1,07 \pm 0,05$ & $1,02 \pm 0,05$ & $1,31 \pm 0,06^{* *}$ \\
\hline $\begin{array}{l}\text { CD4+cells } \\
\left(10^{9} 1\right)\end{array}$ & $0,98 \pm 0,04$ & $0,73 \pm 0,03$ & $0,65 \pm 0,04$ & $0,88 \pm 0,05 * *$ \\
\hline $\begin{array}{l}\text { CD8+cells } \\
\left(10^{9} 1\right)\end{array}$ & $0,48 \pm 0,04$ & $0,42 \pm 0,03$ & $0,39 \pm 0,03$ & $0,40 \pm 0,04$ \\
\hline $\begin{array}{l}\text { CD20+cells } \\
\left(10^{9} 1\right)\end{array}$ & $0,25 \pm 0,02$ & $0,24 \pm 0,02$ & $0,19 \pm 0,01$ & $0,18 \pm 0,02$ \\
\hline $\begin{array}{l}\text { CD56+cells } \\
\left(10^{9} 1\right)\end{array}$ & $0,29 \pm 0,02$ & $0,21 \pm 0,02$ & $0,18 \pm 0,02$ & $0,25 \pm 0,02 * *$ \\
\hline $\begin{array}{l}\text { CD4/CD8 } \\
\text { index }\end{array}$ & $1,98 \pm 0,1$ & $1,73 \pm 0,1$ & $1,66 \pm 0,1$ & $2,20 \pm 0,09 * *$ \\
\hline
\end{tabular}

${ }^{*} \mathrm{p}<0,05$ for difference between control group and diabetic patients with and without anemia

${ }^{* *} \mathrm{p}<0,05$ for difference between patients with $\mathrm{DN}$ and anemia before and after treatment

Conclusion: We showed for the first time that increase of the main parameters of erythropoiesis in patients with DN and anemia leads to normalization parameters of innate and acquired immunity, improves the quality of patients' life.

\section{4}

In patients with diabetes on dialysis, subcutaneous C.E. R.A. (continuous erythropoietin receptor activator) maintains Hb levels when administered up to once monthly

I. C. Macdougall ${ }^{1}$, J. P. Ryckelynck ${ }^{2}$, A. P. Maxwell ${ }^{3}$, F. C. Dougherty ${ }^{4}$;

${ }^{1}$ Department of Renal Medicine, King's College Hospital, London, United Kingdom, ${ }^{2}$ Department of Nephrology, CHU Clémenceau, Caen, France, ${ }^{3}$ Regional Nephrology Unit, Belfast City Hospital, Belfast, United Kingdom, ${ }^{4}$ F. Hoffmann-La Roche, Basel, Switzerland.
Background and Aims: Chronic kidney disease (CKD) is a major complication of diabetes, and around $70-80 \%$ of patients with CKD have anaemia. C.E.R.A., an innovative agent with unique erythropoietin receptor activity and a prolonged half-life, is currently in development to provide correction of anaemia and stable control of haemoglobin $(\mathrm{Hb})$ levels at extended administration intervals in patients with CKD on dialysis and not on dialysis. The present study examined the efficacy of subcutaneous (SC) C.E.R.A. in maintaining $\mathrm{Hb}$ levels at administration intervals up to $1 \times / 4 \mathrm{wk}$ in patients on dialysis with and without diabetes who were previously maintained on SC epoetin alfa or beta $1-3 \times /$ wk. The effect of the switch in therapy on $\mathrm{Hb}$ levels was compared in patients with and without diabetes.

Material and Methods: This large Phase III, multicentre, open-label study included dialysis patients $\geq 18$ years of age receiving stable SC epoetin alfa or beta $(1-3 \times / \mathrm{wk})$ maintenance therapy. Patients received adequate dialysis and had stable baseline $\mathrm{Hb}(10.5-13.0 \mathrm{~g} / \mathrm{dL})$ and adequate iron status. After a 4-week baseline period, patients $(n=572)$ were randomised (1:1:1) to receive SC C.E.R.A. $1 \times / 2 \mathrm{wk}$ $(n=190)$ or $1 \times / 4 \mathrm{wk}(\mathrm{n}=191)$ or to continue the same SC epoetin regimen $(1-3 \times / w k ; n=191)$ for 52 weeks. Dosage was adjusted to maintain $\mathrm{Hb}$ within $\pm 1.0 \mathrm{~g} / \mathrm{dL}$ of the baseline level and between 10.0-13.5 g/dL. Here we present an analysis of change in mean $\mathrm{Hb}$ levels between baseline and the evaluation period (weeks 29-36) according to diabetic status.

Results: Patients with/without diabetes were randomised to each treatment group as follows: $62 / 128$ (C.E.R.A. $1 \times 1$ $2 \mathrm{wk}$ ), 52/138 (C.E.R.A. $1 \times / 4 \mathrm{wk}$ ) and 63/126 (epoetin). Mean $\mathrm{Hb}$ levels at baseline were similar across treatment groups and in patients with and without diabetes: C.E.R.A. $1 \times / 2$ wk (11.8 and $11.7 \mathrm{~g} / \mathrm{dL}$, respectively); C.E.R.A. $1 \times /$ 4 wk (11.7 and $11.6 \mathrm{~g} / \mathrm{dL})$; epoetin (11.6 and $11.7 \mathrm{~g} / \mathrm{dL})$. During the evaluation period, mean $\mathrm{Hb}$ levels in patients with and without diabetes were: C.E.R.A. $1 \times / 2$ wk $(11.8$ and $11.7 \mathrm{~g} / \mathrm{dL}$, respectively); C.E.R.A. $1 \times / 4 \mathrm{wk}(11.7$ and $11.3 \mathrm{~g} / \mathrm{dL})$; epoetin $(11.5$ and $11.4 \mathrm{~g} / \mathrm{dL})$. Hb was maintained between the baseline and evaluation periods in all treatment groups and in patients with and without diabetes. Mean changes in $\mathrm{Hb}$ between baseline and the evaluation period (weeks 29-36) in patients with and without diabetes were: C.E.R.A. $1 \times / 2$ wk $(-0.38$ and $-0.06 \mathrm{~g} / \mathrm{dL}$, respectively); C.E.R.A. $1 \times / 4$ wk $(-0.19$ and $-0.30 \mathrm{~g} / \mathrm{dL})$; epoetin $(-0.04$ and $-0.24 \mathrm{~g} / \mathrm{dL})$. C.E.R.A. was well tolerated, with a safety profile characteristic of the patient population. The most frequent adverse events were hypertension, procedural hypotension and nasopharyngitis.

Conclusion: These Phase III results indicate that SC C.E.R. A effectively maintained stable $\mathrm{Hb}$ levels in dialysis 
patients who converted from epoetin $1-3 \times /$ wk to C.E.R.A. $1 \times / 2$ wk or $1 \times / 4$ wk, irrespective of diabetic status. Maintaining adequate $\mathrm{Hb}$ within guideline levels is an important goal of anaemia management. The possibility of achieving this with C.E.R.A. administered at extended intervals up to once monthly could provide benefits for both patients and healthcare providers.

Supported by F. Hoffmann-La Roche

\section{5}

Hb levels in patients with diabetes and chronic kidney disease receiving dialysis are maintained with C.E.R.A. (Continuous Erythropoietin Receptor Activator) administered once monthly

F. Locatelli ${ }^{1}$, J. Mann ${ }^{2}$, I. C. Macdougall ${ }^{3}$,

F. C. Dougherty ${ }^{4}$;

${ }^{1}$ Division of Nephrology and Dialysis, Osperdale A. Manzoni, Lecco, Italy, ${ }^{2}$ Department of Nephrology, KfH Dialysezentrum, Munich, Germany, ${ }^{3}$ Department of Renal Medicine, King's College Hospital, London, United Kingdom, ${ }^{4}$ F. Hoffmann-La Roche, Basel, Switzerland.

Background and Aims: Chronic kidney disease (CKD) is a major consequence of diabetes and diabetes is the leading cause of CKD in many countries. Anaemia is often associated with both diabetes and CKD and anaemia is often more severe in patients with diabetes than in those without diabetes at similar levels of kidney function. C.E.R. A., an innovative agent with unique erythropoietin receptor activity and a prolonged half-life, is currently in development to provide correction of anaemia and stable control of haemoglobin $(\mathrm{Hb})$ levels at extended administration intervals in patients with CKD on dialysis and not on dialysis. Here, we present pooled data from two Phase III studies in dialysis patients where C.E.R.A. $1 \times / 4$ wk was administered either by subcutaneous (SC) or intravenous (IV) injection. The efficacy of C.E.R.A. in maintaining $\mathrm{Hb}$ levels in patients who converted from epoetin $1-3 \times /$ wk directly to C.E.R.A. $1 \times / 4 \mathrm{wk}$ in patients with and without diabetes who were previously maintained on IV or SC epoetin alfa or beta was evaluated.

Material and Methods: Two large Phase III, multicentre, open-label studies were conducted in dialysis patients $\geq 18$ years of age receiving stable epoetin alfa or beta (1$3 \times /$ wk) maintenance therapy. Patients with adequate dialysis and iron status who had stable baseline $\mathrm{Hb}(10.5$ $13.0 \mathrm{~g} / \mathrm{dL}$ ) were included. After a 4-week baseline period, patients $(n=1245)$ were randomised $(1: 1: 1)$ to receive IV or SC C.E.R.A. $1 \times / 2$ wk $(n=413)$ or $1 \times / 4$ wk $(n=415)$ or to continue the same IV or SC epoetin regimen $(1-3 \times / \mathrm{wk}$; $\mathrm{n}=417$ ) for 52 weeks. Dosage was adjusted to maintain $\mathrm{Hb}$ within $\pm 1.0 \mathrm{~g} / \mathrm{dL}$ of the baseline level and between 10.0 $13.5 \mathrm{~g} / \mathrm{dL}$. Here we present an analysis of $\mathrm{Hb}$ data stratified by the presence or absence of diabetes. The primary endpoint was the mean change in $\mathrm{Hb}$ between baseline and the evaluation period (weeks 29-36).

Results: $144 / 415$ (35\%) of the patients randomised to the C.E.R.A. $1 \times / 4$ wk group were diabetic. Mean $\mathrm{Hb}$ levels at baseline in the C.E.R.A. $1 \times / 4$ wk group were similar in patients with and without diabetes: 11.8 and $11.7 \mathrm{~g} / \mathrm{dL}$, respectively. During the evaluation period, mean $\mathrm{Hb}$ levels in patients with and without diabetes were also similar: 11.6 and $11.5 \mathrm{~g} / \mathrm{dL}$, respectively. C.E.R.A. $1 \times / 4$ wk maintained $\mathrm{Hb}$ between the baseline and evaluation periods in patients with and without diabetes. Mean $\mathrm{Hb}$ changes during this period were -0.23 and $-0.21 \mathrm{~g} / \mathrm{dL}$ in diabetic and nondiabetic patients, respectively. C.E.R.A. was well tolerated, with a safety profile characteristic of the patient population. The most frequent adverse events were hypertension, nasopharyngitis and diarrhoea.

Conclusion: These pooled data from two large Phase III studies where C.E.R.A was administered either IV or SC indicate that stable $\mathrm{Hb}$ levels were maintained in dialysis patients with or without diabetes who converted directly from epoetin $1-3 \times /$ wk to C.E.R.A. $1 \times / 4$ wk. The feasibility of maintaining adequate $\mathrm{Hb}$ levels within guideline ranges with C.E.R.A. administered once monthly could offer potential benefits to patients regardless of their diabetic status.

Supported by F. Hoffmann-La Roche

\section{6}

Intravenous C.E.R.A. (continuous erythropoietin receptor activator) administered up to once monthly maintains $\mathbf{H b}$ levels in patients with diabetes on dialysis J. F. Mann ${ }^{1}$, C. Combe ${ }^{2}$, S. M. Donnelly ${ }^{3}$, F. C. Dougherty ${ }^{4}$;

${ }^{1} \mathrm{KfH}$ Nierenzentrum, Munich, Germany, ${ }^{2}$ Department of Nephrology, Hôpital Pellegrin, Bordeaux, France, ${ }^{3}$ Department of Medicine, St Michael's Hospital, Toronto, Canada, ${ }^{4}$ F. Hoffmann-La Roche, Basel, Switzerland.

Background and Aims: Anaemia often manifests early in the course of chronic kidney disease (CKD) in patients with diabetes. C.E.R.A., an innovative agent with unique erythropoietin receptor activity and a prolonged half-life, is currently in development to provide correction of anaemia and stable control of haemoglobin $(\mathrm{Hb})$ levels at extended administration intervals in patients with CKD on dialysis and not on dialysis. In the present study, we examined the efficacy of intravenous (IV) C.E.R.A. in maintaining $\mathrm{Hb}$ levels at administration intervals up to once 
monthly in patients on dialysis with and without diabetes previously maintained on IV epoetin alfa or beta $1-3 \times / \mathrm{wk}$. The effect of the switch in therapy on $\mathrm{Hb}$ levels was compared in patients with and without diabetes.

Material and Methods: This large Phase III, multicentre, open-label study included dialysis patients $\geq 18$ years of age receiving stable IV epoetin alfa or beta $(1-3 \times /$ wk $)$ maintenance therapy. Patients received adequate dialysis and had stable baseline $\mathrm{Hb}(10.5-13.0 \mathrm{~g} / \mathrm{dL})$ and adequate iron status. After a 4-week baseline period, patients $(n=673)$ were randomised $(1: 1: 1)$ to receive IV C.E.R.A. $1 \times / 2 \mathrm{wk}$ $(n=223)$ or IV C.E.R.A. $1 \times / 4 \mathrm{wk}(\mathrm{n}=224)$ for 52 weeks or to continue the same IV epoetin regimen $(1-3 \times / w k ; n=226)$. Dosage was adjusted to maintain $\mathrm{Hb}$ within $\pm 1.0 \mathrm{~g} / \mathrm{dL}$ of the baseline level and between 10.0-13.5 g/dL. Here we present an analysis of change in mean $\mathrm{Hb}$ levels between baseline and the evaluation period (weeks 29-36) according to diabetic status.

Results: Patients with/without diabetes were randomised as follows: 107/116 (C.E.R.A. $1 \times / 2$ wk), 92/132 (C.E.R.A. $1 \times / 4 \mathrm{wk}$ ) and 114/112 (epoetin). Mean Hb levels at baseline were similar across treatment groups and in patients with and without diabetes: C.E.R.A. $1 \times / 2 \mathrm{wk}(12.0 \mathrm{~g} / \mathrm{dL}$ for both groups); C.E.R.A. $1 \times / 4$ wk (11.9 and $11.8 \mathrm{~g} / \mathrm{dL})$; epoetin (11.8 and $12.0 \mathrm{~g} / \mathrm{dL})$. At the end of the evaluation period (week 36), mean $\mathrm{Hb}$ levels in patients with and without diabetes were: C.E.R.A. $1 \times / 2$ wk $(11.7$ and $11.9 \mathrm{~g} / \mathrm{dL}$, respectively); C.E.R.A. $1 \times / 4$ wk $(11.5$ and $11.8 \mathrm{~g} / \mathrm{dL})$; epoetin (11.7 and $11.8 \mathrm{~g} / \mathrm{dL})$. Hb was maintained between the baseline and evaluation periods in all treatment groups and in patients with and without diabetes. Mean changes in $\mathrm{Hb}$ between baseline and the evaluation period (weeks 2936 ) in patients with and without diabetes were: C.E.R.A. $1 \times / 2$ wk $(-0.36$ and $-0.08 \mathrm{~g} / \mathrm{dL}$, respectively); C.E.R.A. $1 \times / 4$ wk $(-0.25$ and $-0.11 \mathrm{~g} / \mathrm{dL})$; epoetin $(-0.13$ and $-0.32 \mathrm{~g} / \mathrm{dL}$ ). C.E.R.A. was well tolerated, with a safety profile characteristic of the patient population. The most frequent adverse events were diarrhoea, nasopharyngitis and hypertension.

Conclusion: These Phase III results indicate that IV C.E.R. A. effectively maintained stable $\mathrm{Hb}$ levels in dialysis patients who converted from epoetin $1-3 \times /$ wk to C.E.R. A. $1 \times / 2 \mathrm{wk}$ or $1 \times / 4 \mathrm{wk}$ irrespective of the presence of diabetes. Adequate maintenance of $\mathrm{Hb}$ levels within guideline ranges is an important goal of anaemia management. The possibility of achieving this with C.E.R.A. administered at extended intervals up to once monthly could provide benefits for both patients and healthcare providers.

Supported by F. Hoffmann-La Roche

\section{PS 93}

\section{Nephropathy - experimental}

1067

Increased glomerular cell (podocyte) apoptosis in rats with streptozotocin-induced diabetes mellitus: relation to increased p53 expression

G. Pugliese ${ }^{1}$, C. Iacobini $^{1}$, G. Oddi ${ }^{1}$, C. Ricci $^{1}$, F. Pugliese ${ }^{1}$, M. Grattarola ${ }^{2}$, C. Pesce $^{2}$, S. Menini ${ }^{1}$; ${ }^{1}$ Clinical Sciences, "La Sapienza" University, Rome, ${ }^{2}$ DISTBIMO, University of Genoa, Genoa, Italy.

Background and Aims: Glomerular cell apoptosis is considered as a major mechanism for resolution of glomerular/mesangial hypercellularity, but it may also play a pathogenic role in glomerular injury by promoting tissue repair leading to matrix deposition. This study was aimed at assessing glomerular cell death rate and time course in experimental diabetic nephropathy and to identify the cell type(s) and the molecular mechanisms(s) involved, in parallel with renal/glomerular structural changes and expression of apoptosis-related proteins in experimental diabetes.

Materials and Methods: Male Sprague-Dawley rats were rendered diabetic (D) with streptozotocin $(55 \mathrm{mg} / \mathrm{kg})$ and killed 2, 4 or 6 months later, together with age-matched nondiabetic controls (ND). Apoptosis was assessed by immunohistochemistry for active caspase-3, followed by staining for specific glomerular cell type markers or counterstaining with PAS, renal/glomerular structure by morphometric analysis, the glycoxidation product $\mathrm{N} \varepsilon$ carboxymethyllysine (CML), the AGE-receptors RAGE and galectin-3, p21 and phosphorylated p53 (in serine 15) by immunohistochemistry and p53 mRNA levels by competitive RT-PCR.

Results: Mean glomerular area, fractional mesangial area and glomerular sclerosis index increased progressively with age in both groups and were significantly higher $(p<0.001)$ in D vs. ND (at 6 months, $17008 \pm 822$ vs. $13384 \pm 710 \mu \mathrm{m}^{2}$; $30.7 \pm 3.1$ vs. $18.4 \pm 1.4 \%$ and $1.2 \pm 0.2$ vs. $0.5 \pm 0.1$, respectively). Glomerular cell number and density decreased with age and were significantly lower in D vs. ND (70.0 7.1 vs. $80.9 \pm 5.8$ cells/glomerulus, $\mathrm{p}<0.01$, and $4.1 \pm 0.5$ vs. $6.1 \pm 0.5$ $1 / \mathrm{mm}^{2}, \mathrm{p}<0.001$, respectively, at 6 months). This was accounted for by a progressive increase in glomerular cell apoptosis, which was significantly higher in D vs. ND rats, particularly at 6 months $(1.5 \pm 0.4$ vs. $0.3 \pm 0.1 \%, \mathrm{p}<0.001)$. Apoptosing cells were mainly podocytes, though endothe- 
lial and mesangial cells were not spared; moreover, the expression of the podocyte marker synaptopodin was markedly reduced in D vs. ND already at 2 months of disease duration, when apoptosis was only slightly increased, and further decreased thereafter. In glomeruli from $\mathrm{D}$ rats, CML, RAGE and galectin-3 were progressively upregulated, whereas p 21 showed a peak at 4 months and phosphorylated p53 became detectable at 6 months; mRNA levels for $\mathrm{p} 53$ also increased progressively in D rats $(+78 \%$ vs. ND, $\mathrm{p}<0.001$ at 6 months).

Conclusion: These data indicate that, in parallel with the development of glomerular damage, significant apoptosis occurs in glomeruli from experimental diabetic rats, particularly in podocytes, and correlates with increased expression and activation of proapototic protein p53. Apoptosis is preceded and accompanied by a reduced expression of podocyte-specific proteins.

\section{8}

Diabetes-induced up-regulation of uncoupling protein-2 in the kidney cortex results in increased oxygen consumption unrelated to active transport

M. Friederich ${ }^{1}$, P.-O. Carlsson ${ }^{1}$, A. Fasching ${ }^{1}$, P. Liss ${ }^{2}$, P. Hansell ${ }^{1}$, F. Palm ${ }^{1}$;

${ }^{1}$ Medical Cellbiology, Uppsala University, ${ }^{2}$ Diagnostic Radiology, University Hospital, Uppsala, Sweden.

Background and Aims: Up-regulation of uncoupling protein 2 (UCP-2) is a possible protective mechanism against hyperglycaemia-induced mitochondrial formation of reactive oxygen species (ROS), the latter believed to be crucial for the development of diabetic nephropathy. This study evaluated the renal UCP-2-levels in control and streptozotocin-induced diabetic rats, and related this to changes in oxygen consumption.

Materials and Methods: UCP-2 in renal cortex and medulla were detected by Western Blot. Oxygen consumption was measured in vitro using isolated renal tubular cells placed in a sealed chamber and the oxygen consumption measured as the disappearance of oxygen corrected for protein content. Ouabain was used to inhibit transportrelated oxygen consumption.

Results: UCP-2 protein levels (normalized to $\beta$-actin) in the renal cortex were increased 3.7 -fold in diabetic animals compared to controls $(0.56 \pm 0.08 ; n=5$ vs. $0.15 \pm 0.05 ; n=5)$, and in the medulla the levels were increased 3 -fold $(0.39 \pm 0.09 ; n=5$ vs. $0.13 \pm 0.04 ; n=5)$. Total oxygen consumption by isolated cortical cells from diabetic animals was not significantly different compared to controls $(24.3 \pm 4.3 ; \mathrm{n}=6$ vs. $20.6 \pm 2.5 \mathrm{nmol} / \mathrm{min} / \mathrm{mg}$; $\mathrm{n}=5)$. However, oxygen consumption not related to active transport (i.e. ouabain-insensitive) was increased 1.7 -fold in cells from the diabetic animals $(13.1 \pm 1.9 ; n=6$ vs. $7.5 \pm 1.4 ; n=5)$.

Conclusion: Diabetic animals show an increase in ouabaininsensitive oxygen consumption. Concomitantly, the diabetic animals have up-regulation of UCP-2 protein levels, suggesting that the increased oxygen consumption is due to increased mitochondrial uncoupling. The increased UCP-2 levels might be a protective mechanism against hyperglycaemia-induced mitochondrial ROS formation which warrants further studies.

Support for these studies was obtained from the Swedish Medical Research Council and Swedish Diabetes Foundation

1069

Diabetes-induced hyperfiltration in adenosine A1-receptor deficient mice lacking the tubuloglomerular feedback mechanism

J. Sällström ${ }^{1}$, P.-O. Carlsson ${ }^{1,2}$, B. Fredholm ${ }^{3}$, E. Larsson ${ }^{4}$, A. E. G. Persson ${ }^{1}$, F. Palm ${ }^{1}$;

${ }^{1}$ Department of Medical Cell Biology, ${ }^{2}$ Department of Medical Sciences, Uppsala University, ${ }^{3}$ Department of Physiology and Pharmacology, Karolinska Institute, Stockholm, ${ }^{4}$ Department of Genetics and Pathology, Uppsala University, Uppsala, Sweden.

Background and Aims: Glomerular hyperfiltration is commonly found in diabetic patients early after the onset of the disease. This is also one of the first indications of the development of progressive diabetic nephropathy. It has been proposed that glomerular hyperfiltration is caused by a decreased delivery of electrolytes to the macula densa due to the increased sodium and glucose reabsorption in the proximal tubule, which would increase glomerular filtration rate (GFR) via the tubuloglomerular feedback (TGF) mechanism. In this study, we investigated the role of TGF in the development of diabetes-induced glomerular hyperfiltration by inducing diabetes in adenosine A1-receptor knock-out ( $\mathrm{A} 1 \mathrm{AR}^{-/-}$) mice lacking the TGF and wild-type $\left(\mathrm{A}_{1 \mathrm{AR}^{+/+}}\right)$.

Materials and Methods: Diabetes was induced by a single dose of alloxan $(75 \mathrm{mg} / \mathrm{kg} \mathrm{bw})$ injected into the tail vein. The 24-hour urinary electrolyte excretion was measured in metabolism cages, GFR was determined by inulin clearance under isoflurane-anesthesia and histological changes were evaluated.

Results: All alloxan-treated animals developed a sustained hyperglycemia ( $\geq 20 \mathrm{mM}$ ). The diuresis was increased about 8 -fold in both diabetic groups compared to normoglycemic controls. Normoglycemic animals had similar GFR independent of genotype $\left(\mathrm{A}_{1} \mathrm{AR}^{+/+} 9.3 \pm 0.5, \mathrm{n}=10 \mathrm{vs}\right.$. $\mathrm{A} 1 \mathrm{AR}^{-/-}$ $10.1 \pm 0.8 \mu \mathrm{l} / \mathrm{min} / \mathrm{g} \mathrm{bw}, \mathrm{n}=5)$ and the onset of diabetes 
resulted in the development of similar glomerular hyperfiltration in both genotypes $\left(\mathrm{A} \mathrm{AR}^{+/+} 14.0 \pm 1.7, \mathrm{n}=9\right.$ vs. $\mathrm{A} 1 \mathrm{AR}{ }^{-/-} 15.3 \pm 1.9 \mu \mathrm{l} / \mathrm{min} / \mathrm{g}$ bw, $\mathrm{n}=6 ; \mathrm{p}<0.05 \mathrm{vs}$. corresponding normoglycemic control group). The glomerular area was similar in both genotypes, and was unaltered by the onset of diabetes.

Conclusion: This study shows that the $\mathrm{A}_{1 \mathrm{AR}}{ }^{-1-}$ mice develop diabetes-induced glomerular hyperfiltration, suggesting that the TGF mechanism is not involved in the development of hyperfiltration. Furthermore, the hyperfiltration in the present study was not related to alterations in the glomerular filtration area.

This study was supported by the Swedish Medical Research Council

\section{0}

Renal RhoA activity in experimental diabetes (DM) J. Zdychova ${ }^{1}$, A. Lodererova ${ }^{2}$, D. Lecian ${ }^{3}$, R. Komers ${ }^{1}$; ${ }^{1}$ Diabetes Center, ${ }^{2}$ Pathology, Institute for Clinical and Experimental Medicine, ${ }^{3}$ Transplant Center, Nephrology, Institute for Clinical and Experimental Medicine, Prague, Czech Republic.

Introduction: RhoA is a member of Ras family of Gproteins involved in postreceptor signaling of growth factors, cytokines, and vasoactive hormones. Rho activation is dependent on its binding to GTP and subsequent translocation to the plasma membrane. Rho activation has been associated with growth signaling, cytoskeleton reorganization and cell contractility, and implicated in the pathophysiology atherosclerosis, hypertension and nondiabetic glomerulosclerosis (GS). However, the effects of DM and its treatment on renal RhoA have not been elucidated. Methods: RhoA protein expression (western blotting) was determined in crude membrane and cytosolic fractions of renal cortical homogenates obtained from three groups of streptozotocin-diabetic rats (60 mg/kg, n=6/group) after 4weeks duration of DM, and different metabolic control achieved by variable insulin treatment: severe hyperglycemia (DM-S, no insulin treatment); moderate hyperglycemia (DM-M, 4 IU of insulin/day); and tight metabolic control (DM-I, 12 IU/day). Renal RhoA localization was determined by immunohistochemistry (IHC). Data in diabetic rats were compared to age-matched non-diabetic controls (C). To elucidate effects of insulin per se on renal RhoA activity, further studies compared RhoA activation in $\mathrm{C}$ rats and in non-diabetic rats treated with $6 \mathrm{IU}$ of insulin/day (C-I).

Results: Membrane RhoA expression was increased in all groups of diabetic rats $(+50-90 \%, \mathrm{p}<0.01$ vs. C), whereas a decrease in cytosolic RhoA was observed in DM-S $(-35 \%$, $\mathrm{p}<0.01$ vs. C). However, the ratio of membrane/cytosolic fractions, determined as the major characteristic of RhoA activity was markedly increased in all groups of diabetic rats irrespective of level of metabolic control (DM-S $+767 \%$; DM-M+654\%; DM-I+491\%; p $<0.01$ vs. C). Similar to DM rats, C-I demonstrated enhanced membrane RhoA translocation $(+230 \%, \mathrm{p}<0.01)$ as compared to $\mathrm{C}$. IHC localized RhoA into the glomeruli, in particular in podocytes, and in luminal aspects of proximal tubules.

Conclusion: Early stages of diabetic nephropathy are associated with renal RhoA activation, which is not ameliorated by improved metabolic control, possibly due to independent effects of insulin. Glomerular localization of RhoA suggests its role in the development of proteinuria in DM.

Supported by MZO 00023001, Czech Republic

1071

Reduced nitric oxide in the renal medulla of streptozotocin-induced diabetic rats is due to limited availability of arginine. A crucial role for hepatic cationic amino acid transporter-2A?

F. Palm ${ }^{1}$, A. Fasching ${ }^{1}$, P. Liss ${ }^{2}$, T. Teerlink ${ }^{3}$, P. Hansell ${ }^{1}$; ${ }^{1}$ Department of Medical Cell Biology, ${ }^{2}$ Department of Oncology, Radiology and Clinical Immunology, Uppsala University, Uppsala, Sweden, ${ }^{3}$ Department of Clinical Chemistry, VU University Medical Center, Amsterdam, The Netherlands.

Background and Aims: Altered concentrations of reactive oxygen species (ROS) and nitric oxide (NO) are suggested to mediate the development of diabetic nephropathy. We hypothesized that diabetes-induced generation of ROS changes renomedullary NO concentration, which alters local oxygen tension $\left(\mathrm{PO}_{2}\right)$ and blood flow.

Materials and Methods: Control and streptozotocin-induced diabetic animals, with or without $\alpha$-tocopherol treatment, were administered L-arginine followed by LNAME. NO concentrations were measured by haemoglobintrapping using microdialysis probes, $\mathrm{PO}_{2}$ by microsensors, blood flow by laser-Doppler flowmetry and plasma arginine and asymmetric dimethylarginine (ADMA) by HPLC. Liver arginase activity was measured in vitro. Cationic amino acid transporter (CAT)-2A and renal cortical CAT-1 mRNA levels were determined by real time PCR.

Results: Renomedullary NO concentrations were lower in diabetic animals $(59 \pm 4 \mathrm{nmol} / \mathrm{l}, \mathrm{n}=10)$ compared to control animals $(122 \pm 7 \mathrm{nmol} / \mathrm{l}, \mathrm{n}=8)$, and unaffected by $\alpha$-tocoph- 
erol. L-arginine increased renomedullary $\mathrm{NO}$ in diabetic animals, and totally restored $\mathrm{NO}$ in those fed $\alpha$-tocopherol. L-arginine selectively increased medullary $\mathrm{PO}_{2}$ in diabetics, although the blood flow increased equally in all groups. Diabetes decreased plasma arginine $(-49 \%)$ and ADMA $(-15 \%)$ independently of treatment. No differences were detected in either hepatic arginase activity or mRNA levels of CAT-1 in the kidney cortex. However, hepatic CAT-2A mRNA levels were increased about 3.5-fold in both diabetic groups.

Conclusion: We conclude that the reduced renomedullary NO concentration in diabetic animals is due to reduced arginine availability. ROS inhibition augments the NO response to arginine administration, but does not restore basal NO deficiency per se. The reduced plasma levels of L-arginine correlated with increased expression of hepatic CAT-2A, suggesting increased hepatic uptake of L-arginine. Increased NO concentration following L-arginine administration increases local $\mathrm{PO}_{2}$ in diabetic animals. These changes may be important factors for the onset and progression of diabetes-induced renal dysfunction.

Supported by Swedish Research Council and Swedish Diabetes Foundation

1072

p38 Mitogen-Activated Protein Kinase (MAPK) expression and activity in early and advanced diabetic nephropathy in rats

R. Komers ${ }^{1,2}$, J. N. Lindsley ${ }^{2}$, T. T. Oyama ${ }^{2}$, S. Anderson ${ }^{2}$; ${ }^{1}$ Diabetes Center, Institute for Clinical and Experimental Medicine, Prague, Czech Republic, ${ }^{2}$ Division of Nephrology and Hypertension, Oregon Health and Science University, Portland, United States.

Background and Aims: Studies have suggested that diabetes is associated with activation of p38 MAPK in the kidney. This alteration may have impact on renal morphology and function in diabetes. Available experimental evidence has suggested that $\mathrm{p} 38$ activation is a feature of early nephropathy in STZ-diabetic rats. However, whether p38 activation could be detected in advanced stages of the disease associated with proteinuria and glomerulosclerosis remains to be elucidated. In the present studies, we determined renal cortical p38 activity and expression in a rat model of Type 1 diabetes with early and advanced nephropathy.

Methods: Streptozotocin-diabetic rats (DM, $60 \mathrm{mg} / \mathrm{kg}$ bwt) were studied 4 weeks $(n=8)$ and 12 months $(n=4)$ after induction of DM. Diabetic rats were treated with subopti- mal dose of insulin (2-4 U/day) to achieve moderate hyperglycemia. Age-matched non-diabetic rat were studied as controls (C). Renal cortical activity of $\mathrm{p} 38$ (in vitro kinase pull-down assay with ATF-2 used as a substrate), and protein expression of phosphorylated (active) and total p38 (P-p38,t-p38) were determined by western blotting in nuclear-free cortical homogenates. Renal localization of active p38 was determined by immunohistochemistry.

Results: Compared with age-matched controls, 4-week diabetic rats demonstrated increases in renal cortical p38 activity $(81 \pm 19 \%, \mathrm{p}<0.05)$, as assessed by in vitro kinase assay. Furthermore, expression of active p38 and P-p38/ total $\mathrm{p} 38$ ratio, determined as another indicator of p38 activity, were also increased DM rats (P-p38 expression: $70 \pm 26 \%$; P-p38/t-p38: $40 \pm 19 \%$, p $<0.05$ vs. C). There were no differences in t-p38 between DM and $\mathrm{C}$ rats. In all rats, P-p38 was localized in macula densa (MD). In addition, DM rats had P-p38-positive cells in adjacent portions of the distal tubule and in glomeruli.

After 12 months of diabetes, the rats developed markedly elevated albuminuria and substantial glomerulosclerosis. Similar to 4-week diabetic rats, p38 kinase activity assay and determination of expression of P-p38 revealed marked increases in these parameters in DM as compared to $\mathrm{C}$ rats (p38 activity: 79 $\pm 20 \%$; P-p38 expression: $60 \pm 17 \%$; P-p38/ t-p38: $148 \pm 41 \%$; $\mathrm{p}<0.01)$. Immunohistochemical localization of active $\mathrm{p} 38$ showed similar distribution of the protein in the renal cortex as observed in 4-week diabetic rats with most intensive stain found in macula densa cells and in distal tubules. However, no glomerular P-p38 immunoreactivity was detected in diabetic rats with established nephropathy. Conclusions: Increases in renal cortical p38 MAPK activity are detectable not only early after onset of experimental diabetes, but also in diabetic rats with established nephropathy. Active p38 is predominantly localized in macula densa and distal tubules throughout the disease, whereas glomerular P-p38 is detectable only at early stages of diabetes.

Supported by JDRF; NIH; Grant \# MZO-00023001 Czech Republic

1073

Deranged transcriptional regulation of SGK and ENaC: a link between hyperglycaemia and diabetic nephropathy C. E. Hills ${ }^{1}$, R. Bland ${ }^{1}$, P. M. Ronco ${ }^{2}$, P. E. Squires ${ }^{1}$; ${ }^{1}$ Biological Sciences, University of Warwick, Coventry, United Kingdom, ${ }^{2}$ Unite INSERM 702, Hopital Tenon (AP-HP), Paris, France. 
Background and Aims: Alterations in the resorptive capacity of renal epithelia to sodium can have severe implications for the normal functioning of the nephron and are most likely to be prominent in the development and pathogenesis of secondary hypertension, a condition associated with a number of renal diseases including diabetic nephropathy and glomerulonephritis. One of the major regulators of sodium reabsorption in the nephron is the serum and glucocorticoid induced kinase (SGK), an aldosterone regulated gene, which mediates sodium reabsorption via its actions on the epithelial sodium channel (ENaC). Recent reports have identified SGK as a key signaling element whose level of expression appears to be upregulated in kidneys of diabetic humans and diabetic nephropathy. Hyperglycamia is believed to be the key pathophysiological component in promoting intercellular changes ultimately leading to the deranged transcriptional regulation of SGK.

Materials and Methods: RT-PCR, immunocytochemistry and western blot analysis confirmed that human cortical collecting duct (HCD) cells express SGK and alpha ENaC. For glucose, experiments, HCD cells were treated with (25 mM) glucose for 24 and 48 hours. For TGF-beta $(2 \mathrm{~nm})$ and Ionomycin $(1 \mu \mathrm{m})$ experiments, HCD cells were treated for 4,6,8,12 and $24 \mathrm{hrs}$. A functional correlate for these increased levels of expression was provided by sodium microfluorimetry.

Results: Incubation of HCD cells with high glucose (25 mM) for 24 and 48 hours increased expression of SGK and Alpha ENaC protein (SGK, 261.7 $\pm 14.9 \%$ of control $(5 \mathrm{mM})$ at 48 hours; $\mathrm{n}=3, \mathrm{p}<0.01 \%$; alpha $\mathrm{ENaC}$, $316.5 \% \pm 9.9 \%$ of control $(5 \mathrm{mM})$ at 48 hours $n=6, p<0.01)$. Hyperglycaemia has been linked to both raised calcium and TGF-beta levels. Application of TGF-beta or Ionomycin alone induced a significant increase in SGK protein expression (TGF-beta, $125.5 \% \pm 7.5 \%$ of control at 8 hours; $\mathrm{n}=3 \mathrm{p}<0.001 \%$; Ionomycin $263.7 \% \pm 8.6 \%$ of control at 8 hours; $n=3 p<0.001$ ) suggesting a possible role for both TGF-beta and calcium in mediating downstream effects of hyperglycaemia. Intracellular sodium levels were found to be significantly elevated following $24(107 \% \pm 0.88 \%)$ and $48(114 \% \pm 0.93 \%)$ hours exposure to $25 \mathrm{mM}$ glucose, as compared to cells cultured in low $(5 \mathrm{mM})$ glucose.

Conclusion: This data taken in conjunction with that described in previous literature suggests that increases in intracellular sodium in response to high glucose after 48 hours may be mediated via the increased expression of both SGK and alpha-ENaC. This data highlights a possible link between hyperglycaemia and the deranged sodium reabsorption observed in cases of diabetic nephropathy, and may lead to identification of potential therapeutic targets. Supported by BBSRC
1074 Renal oxidative stress in normotensive and hypertensive
model of early experimental diabetes mellitus

S. K. Biswas, J. B. Lopes de Faria;

Division of Nephrology, Department of Internal Medicine, University of Campinas (UNICAMP), Campinas, Sao Paulo, Brazil.

Background and Aims: Oxidative stress plays important role in the pathogenesis of nephropathy resulting from both diabetes and hypertension. These last 2 conditions frequently coexist in humans, and individually both of them can induce oxidative stress in the kidney. Recently oxidative stress was demonstrated in the renal cortex in hypertensive, but not in normotensive, model of early experimental diabetes. This finding prompted us to search for the mechanism of renal protection against oxidative stress in normotensive model, and the mechanism of renal oxidative stress in hypertensive model of early experimental diabetes mellitus.

Materials and Methods: Diabetes (D) was induced in spontaneously hypertensive rats (SHR) and their genetically normotensive control Wistar Kyoto (WKY) rats by streptozotocin at 12 weeks of age. After 10 days, rats were sacrificed and kidneys were collected. Renal cortical nitrotyrosine, receptor for advanced glycation end products (RAGE), and nicotinamide adenine dinucletide phosphate (NADPH) oxidase isoform Nox4, and p47phox subunit were assessed by Western blot. 8-hydroxy-2'-deoxyguanosine $(8-\mathrm{OHdG})$ positive cells were evaluated by immunohistochemistry. Superoxide generation was assessed by lucigenin-enhanced chemiluminescence, and reduced glutathione (GSH) levels were quantified by chemical method. Results: Plasma glucose levels were similar in WKY-D and SHR-D groups. Systolic blood pressure was higher $(\mathrm{p}<0.001, \mathrm{n}=10)$ in control $(\mathrm{C})$ and diabetic SHR than the WKY groups. Nitrotyrosine and $8-\mathrm{OHdG}$, markers of oxidative stress-induced protein and DNA modification, respectively, were found similar in the kidney in WKY-C and WKY-D, but they were elevated $(p=0.068$ in case of nitrotyrosine, $\mathrm{p}=0.016$ in case of $8-\mathrm{OHdG} ; \mathrm{n}=5$ ) in SHR-D as compared with SHR-C. However, RAGE, Nox4 or p47phox levels were found similar between control and diabetic groups of both WKY and SHR strains. Nonenzymatic antioxidant GSH level was slightly increased in IWKY-D (4.60 $\pm 0.33 \mu \mathrm{M} / \mathrm{g}$ tissue) than WKY-C (4.34 \pm $0.36 \mu \mathrm{M} / \mathrm{g})$, but it was significantly decreased $(\mathrm{p}=0.033$, $\mathrm{n}=10)$ in SHR-D $(3.70 \pm 0.25 \mu \mathrm{M} / \mathrm{g})$ than SHR-C $(4.20 \pm$ $0.26 \mu \mathrm{M} / \mathrm{g})$. On the other hand, we have observed an elevation of NADPH-induced superoxide generation in the renal cortex in both WKY-D and SHR-D groups as 
compared with their respective controls in our initial experiments.

Conclusion: We conclude that the kidney of normotensive model, but not of hypertensive model, is apparently protected from oxidative damage in the very early stage of experimental diabetes. Although the significant depletion of the antioxidant GSH level in the kidney occurs only in hypertensive model, the pro-oxidant superoxide generation in the renal cortex appears to be elevated in both normotensive and hypertensive model of early experimental diabetes mellitus. Taken together, our data suggests that the impaired antioxidant defence mechanism may be responsible for the early susceptibility to renal oxidative stress in hypertensive model of diabetes. Currently we are evaluating enzymatic antioxidant status, and searching for the mechanism and source of superoxide generation in normotensive and hypertensive model of early experimental diabetes.

Supported by FAPESP, CAPES

\section{5}

Possible involvement of a VEGF receptor subtypedependent mechanism in the pathogenesis of diabetic nephropathy in $\beta$ cell-specific calmodulin overexpressing mice

Y. Yuzawa ${ }^{1}$, T. $\operatorname{Kosugi}^{1}$, H. Hayashi ${ }^{1}$, S. Maruyama ${ }^{1}$, F. Yoshida ${ }^{2}$, Y. Tagawa ${ }^{3}$, M. Takeda ${ }^{3}$, S. Matsuo ${ }^{1}$, I. Niki ${ }^{4}$; ${ }^{1}$ Department of Nephrology, Nagoya University Graduate School of Medicine, Nagoya, ${ }^{2}$ Department of Internal Medicine, Aichi Prefectural Colony Central Hospital, Kasugai, ${ }^{3}$ Central Research Laboratory, Sanwa Kagaku Kenkyusho Co., Ltd., Inabe, ${ }^{4}$ Department of Parmacology, Oita University, Oita, Japan.

Background and Aims: Although diabetic nephropathy (DN) is a serious and life-threatening complication, there are very few proper animal models with distinct glomerular lesions mimicking human DN. We here characterized kidney lesions in $\beta$ cell-specific calmodulin over-expressing (CaMTg) mice which experienced early-onset, marked and long-lasting hyperglycemia. Possible mechanisms underlying its pathogenesis were also investigated.

Materials and Methods: CaMTg mice were prepared using a transgene where the calmodulin gene was placed downstream the insulin promoter leading to pancreatic $\beta$ cell-specific calmodulin overexpression in the ICR strain. CaMTg mice and non Tg mice were sacrificed at 3, 6 and 9 month-old (M) age. Renal functions of these animals were evaluated biochemically and histologically. Expression levels of type III, type IV collagens and $\alpha$ smooth muscle actin ( $\alpha$ SMA) were examined immunohistochemi- cally, and those of vascular endothelial growth factor (VEGF), VEGF receptor 1 and 2 (VEGFR-1, 2), and endothelial nitric oxide synthase (eNOS) were detected by Western blot analysis.

Results: CaMTg mice showed consistent and evident hyperglycemia, and increases of urinary protein excretion of at all the ages examined. Morphometrical analysis resulted in considerable increases of the glomerular and mesangial areas with Type III and IV collagen depositions as well as accumulation of $\alpha \mathrm{SMA}$ in the CaMTg kidneys. Nodular lesions were seen in CaMTg mice at 6M and 9M, and even exudative lesions occasionally appeared in CaMTg mice at 9M. Vascular pole hyalinosis was also observed at 6M and 9M. In immunoblot analysis, expression levels of eNOS were decreased and those of VEGF were elevated in the $6 \mathrm{M} \mathrm{CaM} \mathrm{Tg} \mathrm{kidney.} \mathrm{VEGFR-1} \mathrm{was}$ less expressed in the $6 \mathrm{M}$ CaM kidney than nonTg one. Such difference between the CaM Tg and non Tg kidneys were not seen for VEGFR-2.

Conclusion: CaMTg mice developed multiple distinct lesions of the hallmark of human DN; i.e. nodular lesions, exudative lesions and vascular pole hyalinosis. Elevated VEGF levels and diminished eNOS expression may result in preferential activation of VEGFR-2, which is considered to cause endotherial cell proliferation. We expect this model is very useful in the elucidation of the pathogenesis of DN and in the development of its treatment.

Supported by Grant-in-Aid from the Ministry of Education, Science, Sports and Culture in Japan

\section{PS 94}

\section{Retinopathy}

1076

Effect of the oral protein kinase $C \beta$ inhibitor ruboxistaurin on visual acuity in the PKC-DRS2 study

L. Vignati ${ }^{1}$, M. J. Sheetz ${ }^{1}$, A. Girach ${ }^{2}$, X. Zhi ${ }^{1}$, M. D. Davis ${ }^{3}$, R. C. Milton ${ }^{4}$, L. P. Aiello ${ }^{5}$;

${ }^{1}$ Lilly Research Laboratories, Eli Lilly and Company, Indianapolis, United States, ${ }^{2}$ Eli Lilly and Company, Erl Wood, United Kingdom, ${ }^{3}$ Department of Ophthalmology, University of Wisconsin, Madison, United States, ${ }^{4}$ Diabetes Research, EMMES Corporation, Rockville, United States, ${ }^{5}$ Joslin Diabetes Center, Harvard Medical School, Boston, United States.

Background and Aims: Patients with advanced nonproliferative diabetic retinopathy (NPDR) are at increased risk of vision loss. In the PKC-DRS2 study, orallyadministered ruboxistaurin (RBX) mesylate, a protein 
kinase $\mathrm{C}$ (PKC) $\beta$ inhibitor, reduced the occurrence of sustained moderate visual loss (SMVL: $\geq 15$ ETDRS letter loss sustained over the last 6 months of study participation) by $40 \%$ compared to placebo (from $9.1 \%$ to $5.5 \%$ of patients, $\mathrm{p}=0.034)$. The PKC-DRS2 also evaluated the effect of RBX $32 \mathrm{mg} /$ day on categorical measures of visual acuity (VA).

Materials and Methods: The PKC-DRS2 study was a 36month, randomized, double-masked, parallel, placebo-controlled, phase 3 study of 685 patients in 70 centers. Patients (placebo, n=340; RBX 32 mg/d, n=345) had moderately severe to very severe NPDR (ETDRS retinopathy score $\geq 47 \mathrm{~A}$ and $\leq 53 \mathrm{E}$ ), no prior panretinal photocoagulation, and a bestcorrected VA of $\geq 45$ letters $(\sim 6 / 38,0.16$ Snellen) in a study eye. Results: VA of RBX eyes was better than that of placebo eyes after 12 months of therapy. The mean change in VA from baseline-to-endpoint (LOCF) was -0.8 letters, RBX, vs. -2.6 letters, placebo $(\mathrm{p}=0.014)$. During the study, $4.9 \%$ of RBX eyes gained 15 letters in VA, compared to $2.4 \%$ of placebo eyes $(p=0.027)$. In addition to its positive effect on SMVL, RBX reduced sustained VA loss of 10 letters $(30 \%$ reduction, $\mathrm{p}=0.043)$ and 5 letters $(23 \%$ reduction, $\mathrm{p}=0.033)$ compared to placebo. There was a positive effect of RBX on VA loss both in eyes that did and did not receive focal photocoagulation during the study.

Conclusion: Compared to placebo, RBX reduced sustained 5-, 10-, and 15-letter loss in visual acuity and improved visual acuity more frequently over 36 months of treatment. Supported by a grant from Eli Lilly and Company.

\section{7}

Clinical safety of the selective PKC $\beta$ inhibitor, ruboxistaurin, in patients with diabetic microvascular complications

G. L. King ${ }^{1}$, J. B. McGill ${ }^{2}$, D. Hyslop ${ }^{3}$, P. H. Berg ${ }^{3}$, K. L. Price ${ }^{3}$, K. A. Kles ${ }^{3}$, E. J. Bastyr ${ }^{3}$, L. Vignati ${ }^{3}$;

${ }^{1}$ Vascular Cell Biology, Joslin Diabetes Center, Boston,

${ }^{2}$ Department of Medicine, Washington University School of Medicine, St. Louis, ${ }^{3}$ Eli Lilly and Company, Indianapolis, United States.

Background and Aims: Ruboxistaurin (RBX;LY333531) mesylate is a selective protein kinase $\mathrm{C} \beta(\mathrm{PKC} \beta)$ inhibitor that has been demonstrated to reduce the risk of vision loss in patients with moderately severe to severe nonproliferative diabetic retinopathy. In preclinical animal studies, RBX at high and non-isoform-selective doses has been reported to be associated with reversible immune system dysfunction, cataractous lens changes, and hepatobiliary inflammation. We report here the safety profile from patients with diabetic complications treated with $\mathrm{RBX}$
(32 mg per day) for up to 3 years, which is critical information for the consideration of $\mathrm{RBX}$ as a treatment for diabetic retinopathy.

Materials and Methods: Data from patients with diabetes (1396 RBX; 1408 placebo [PL]) were combined from 11 Phase 2 and 3 placebo-controlled, double-masked studies. The patients enrolled had $\mathrm{HbA}_{1 \mathrm{c}} \leq 13 \%$, blood pressure $<190 / 105$ and were diagnosed with at least one of the following diabetic complications: retinopathy, neuropathy, nephropathy, erectile dysfunction or peripheral vascular disease.

Results: Patients who were enrolled in the studies were predominantly caucasian (79\%), males (70\%), had type 2 diabetes $(77 \%)$, a mean patient age of 54.7 years and a mean duration of diabetes of 14.8 years. Twenty three point two percent (327) of the PL-treated patients experienced $\geq 1$ serious adverse event versus $20.8 \%$ (290) of the RBXtreated patients. There were 51 deaths (21 RBX; $30 \mathrm{PL}$ ) reported; none of the deaths was attributed to study drug by the investigators. The common $(\geq 1 / 100$ to $<1 / 10)$ reported adverse drug reactions in the RBX treated patients were dyspepsia and increased blood creatine phosphokinase (CPK). Uncommon $(\geq 1 / 1000$ to $\leq 1 / 100)$ reported adverse drug reactions included atrioventricular first degree block (0.6\%RBX; $0.1 \% \mathrm{PL})$, thrombosis (0.4\%RBX; $0 \% \mathrm{PL})$, chalazion $(0.4 \% \mathrm{RBX} ; 0 \% \mathrm{PL})$, posterior capsule opacification $(0.5 \% \mathrm{RBX} ; 0.1 \% \mathrm{PL})$, micturition urgency, $(0.4 \%$ RBX; $0 \% \mathrm{PL})$, and skin discolouration (0.5\%RBX; $0.1 \%$ PL). Specific analyses based on the animal toxicology findings were conducted. No increased incidence of hepatobiliary disease, bacterial infections and cataracts (AREDs Lens Grading) was observed in the RBX group. RBX had no effect on glycemic control, blood pressure, incidence of severe hypoglycemia, or incidence of diabetic ketoacidosis. Incidence of patients reporting serious cardiac disorders was similar between the PL- $(8.6 \%)$ and RBX(7.6\%) groups. No clinically relevant effects were observed on serial ECGs.

Conclusion: In controlled randomized clinical trials, RBX, a selective inhibitor of the PKC $\beta$ isoform, did not alter glycemic control, had an AE profile comparable to PL and was well tolerated. RBX is the first member of a new class of therapeutics for the treatment of diabetic retinopathy. Supported by a grant from Eli Lilly and Company.

\section{8}

Automated identification of change in retinal images N. R. Hill ${ }^{1}$, M. Brady ${ }^{2}$, D. R. Matthews ${ }^{1}$;

${ }^{1}$ Oxford Centre for Diabetes, Endocrinology and Metabolism, Oxford University, ${ }^{2}$ Robotics Research Group, Oxford University, Oxford, United Kingdom. 
Introduction: Annual screening for retinopathy is recommended for diabetic patients. The optimal way is to use digital imaging after pupil dilatation. These images can easily be stored, retrieved and catalogued. However, in the UK about 2 million images annually have to be analysed.

The aim was to create an automated software solution which could establish no-change criteria in images and allow only grading of retinal images where change of retinopathy had occurred.

Methods: We developed a computer system that could perform registration of retinal images after automatically adjusting to rotational, magnification, astigmatic, clouding and colour changes. Differencing was performed to allow the differences to become apparent. The change was then enhanced. A set of 30 randomly selected retinal image pairs was used for validation.

Results: The software adjusted to all changes, registered and differenced the image pairs in a mean time of 23.5 seconds with a success rate of $93 \%$. The identification of common features in images had a success rate of $100 \%$. Shadowing was found to be the cause of all misalignments. Conclusion: The automated software allowed longitudinal changes to become apparent to the users and could detect the direction of the change (regression, progression). Additionally it could demonstrate where no change had occurred.

\section{9}

Use of broadband technology and remote diagnosis of diabetic retinopathy

M. Kato ${ }^{1}$, N. Kato ${ }^{1}$, T. Nakano ${ }^{2}$, H. Fujii ${ }^{3}$, B. T. Smith ${ }^{3}$, T. Miyakawa ${ }^{3}$;

${ }^{1}$ Internal Medicine, Kato Clinic of Internal Medicine, Takasago, Katsushika, ${ }^{2}$ Internal Medicine, Tama-Hokubu Medical Center, Higashimurayama, ${ }^{3}$ Internal Medicine, Tama-Minami Clinic, Nagayama,Tamacity, Japan.

Background and Aims: Transmission and receiving of high-resolution images has become easy with the widespread adoption of IT technology and broadband. As a result, remote diagnosis of images has been attempted in the medical field. It would be ideal for all patients with diabetes mellitus to periodically undergo ophthalmological examination, but this is difficult in reality, especially in remote areas where transportation and access to a specialist is limited. However, patients without symptoms may be more motivated to undergo examination in their primary care office. The other appeal is that findings can be displayed to the patient directly.
Retinal images were digitalized and a network filing system was introduced at our clinic. A remote diagnosis support system for the retinal images was also established to allow for the use of the broadband service. After repeated clinical trials an effective system was established.

Materials and Methods: Among the patients who underwent retinal imaging at our clinic, radiographs were interpreted for 106 patients who mainly suffered from diabetes mellitus. Retinal images were taken by a nurse using an EOS-D60 digital camera mounted on the CR6$45 \mathrm{NM}$ non-mydriatic retinal camera. The images were stored in a computer as DICOM files for real-time transmission to the computers of physicians. Current HbA1c and B.P etc. were recorded in the comments. The images were then transmitted via e-mail together with a referral letter. These files were encrypted for transmission to the ophthalmologist via a secured mail server made by Canon. The ophthalmologist interpreted the images after opening the attached file, recorded his/her findings on a form designed for this purpose, and sent the form back to our clinic.

Results: This system facilitated processes such as taking retinal images, transmitting them, and receiving reports. The images were recorded as 2048*1360 pixel full color JPEG/ DICOM files and were rapidly transmitted via CATV broadband. The return e-mail from the ophthalmologist contained the assessment and the classification of retinal findings. The ophthalmologists determined that consultation had been necessary or desirable for $45 \%$ of the patients referred from our clinic. The ophthalmologist also commented that the $2048 * 1360$ pixel images were of good quality for interpretation, and the functions such as zooming or altering brightness and contrast were useful for evaluation. Conclusion: This system made it possible to accurately assess the changes of the retina in the asymptomatic diabetic patient. Concerns raised by non-specialist about abnormal findings being overlooked ware alleviated. In addition, it became possible to provide an accurate explanation with pictures of the findings to each patient. Ophthalmologist provided interpretations of the images, which was very informative for the physicians and expanded their knowledge. In conclusion, this system is useful because it can avoid the tragedy of progressive undiagnosed diabetic retinopathy in a patient with diabetes even though he/she is regularly seeing her primary care doctor. This system is also considered worth reporting because it can be introduced in any country and makes consultation with an ophthalmology specialist available even for patients living in remote areas or with limited health care facilities. 
Prevalence of retinopathy and eye lesions in a regional pilot project to monitor diabetes related complications 2002-2004 - the "Diabetes-TÜV" of the Deutsche BKK C. S. Kloos ${ }^{1}$, N. Müller ${ }^{1}$, T. Tschauner ${ }^{2}$, R. Berner ${ }^{3}$, B. Bertram ${ }^{4}$, U. A. Müller ${ }^{1}$;

${ }^{1}$ Internal Medicine, Klinikum für Innere Medizin III, Jena, Germany, ${ }^{2}$ Health Insurance, Deutsche BKK, Wolfsburg, ${ }^{3}$ Controlling und Versorgungsmanagment, Deutsche BKK, Wolfsburg, ${ }^{4}$ Ophthalmology Practice, Aachen, Germany.

Background and Aims: In population based studies up to $30 \%$ of patients with diabetes manifestation already have diabetic retinopathy (DR). The prevalence of DR in Germany of all diabetic patients insured in a public health insurance (Deutsche BKK) was examined in the context of a general diabetes check-up ("Diabetes-TÜV") in a regional pilot project in one German Federal State (Lower-Saxony) from 2002 to 2004.

Materials and Methods: Patients of 351 primary care physicians were screened by ophthalmologists. The prevalence of diabetes related eye diseases documentated according to the screening sheet developed by the initiative group early diagnosis of diabetic eye diseases (Prof. Kroll, Marburg) was assessed. 6500 screening sheets were analysed (mean age 65 $\pm 12,3 \mathrm{y}, 48.5 \%$ female, diabetes duration 10.2 $\pm 9.0 \mathrm{~J}, 10,3 \%$ Diabetes Type 1). 14.5\% multiple documentations occured.

Results: $84.5 \%$ of the patients had no DR. The mean diabetes duration was 9.8 years. In $4.4 \%$ of the patients a retina examination was impossible due to impaired visibility of internal eye structures. $11.2 \%$ of the patients had DR, mostly early stages (DR/diabetes duration: mild DR $8.8 \%$ $15.6 \mathrm{y}$; severe non-proliferative DR: $1.8 \% / 18, \mathrm{y}$; proliferative DR: $0.6 \% / 17.6$ y.). A rubeosis iridis had $0.1 \%$, macular oedema $0.9 \%$, vitreous haemorrhage $0.3 \%$, retinal detachment with or without macular lesion $0.1 \%$, cataract or posterior capsular opacification $25.7 \%$, an artificial lens $8.3 \%$. Diagnostic and therapeutic actions recommended were fluorescent angiography $0.6 \%$, a focal laser coagulation $0.1 \%$, a panretinal laser coagulation $1.3 \%$, a cryocoagulation $0.1 \%$ and vitrectomy $0.2 \%$.

Conclusion: Documentation and completeness of the eye examinations in the "Diabetes-TÜV" of the Deutsche BKK were excellent. The prevalence of DR, even after long diabetes duration, is low compared with earlier population based studies in the USA (Wisconsin study) or GreatBritain (UKPDS) and slightly lower than published in Germany before. The better outcome could be due to improved diabetes care in Germany.
Incidence of retinopathy in a cohort of type 2 diabetics during 10-year follow-up

P. Luźniak, A. Wojciechowska-Luźniak, A. Czech, J. Tatoń; Department of Internal Medicine and Diabetology, Warsaw Medical University, Poland.

Background and Aims: Diabetic retinopathy remains the main cause of blindness in developed countries in spite of marked progress in the treatment of diabetes mellitus and wide use of lasertherapy. This fact points to the significance of many other circumstances. Therefore the undertaken study was aimed at a determination of incidence of diabetic retinopathy in a cohort of type 2 diabetics during a 10-year follow-up and to a delineation of the selected risk factors (age, sex, diabetes duration, BMI, fasting and postprandial glycemia, systolic and diastolic pressure, daily proteinuria, cholesterol, triglycerides, creatinine levels and insulintherapy). This could improve the pertinent prevention programs.

Materials and Methods: The study involved 1334 patients from an out-patient diabetological clinic. Diabetic retinopathy was diagnosed on the basis of ophthalmoscopy. Persons with diabetic retinopathy at the baseline examination were excluded from analysis of incidence. The cohort under study was submitted to the continuous programmed clinical observation.

Results: At baseline diabetic retinopathy was present in 419 persons (31.4\%). During the 10-year follow-up diabetic retinopathy was found in 230 patients $(25.7 \%)$ from a group of 915 patients without retinopathy at baseline. Therefore the cumulative incidence of retinopathy in the whole cohort was $49.0 \%$. The incidence of retinopathy during follow-up was 38.9 per 1000 person-years. Mean age at the moment of diagnosis of new retinopathy was $63.8 \pm 9.7$ years and mean diabetes mellitus duration was $12.0 \pm 5.5$ years. 17 cases of proliferative retinopathy were diagnosed at the baseline, 35 additional cases developed during the 10-year follow-up. In the majority (94.3\%) of patients with proliferative retinopathy diagnosed during follow-up, background retinopathy was present at baseline. The cumulative incidence of proliferative retinopathy in the whole cohort was $3.9 \%$. Fasting and postprandial glycemia were higher in persons with retinopathy diagnosed during follow-up in comparison with those without any retinopathy. The former were characterized by longer duration of diabetes mellitus. A multifactorial regression analysis of Cox found that the predictors of retinopathy were: duration of diabetes and fasting glycemia. The statistical significance of several other risk factors could not be confirmed. During the 10-year follow-up 6 new cases of blindness occurred. Diabetic retinopathy was responsible for 5 of them (4 cases of proliferative retinopathy, 1 case with maculopathy). 
Conclusion: During 10-year observation the newly diagnosed diabetic retinopathy developed in $25.7 \%$ of type 2 diabetics; the cumulative incidence reaches the level $49.0 \%$. This is firmly connected as shown by multifactorial analysis to the diabetes mellitus duration and fasting glycemia. The significance of other factors could not be confirmed statistically in the studied cohort. The relation between the statistical and pathophysiological assessments is therefore also discussed.

\section{2}

Optical coherence tomography findings in patients with diabetes mellitus type 1 , with no or minimal diabetic retinopathy

J. H. DeVries ${ }^{1}$, C. Biallosterski ${ }^{2}$,

M. E. J. van Velthoven ${ }^{2}$, R. P. J. Michels ${ }^{1}$,

F. D. Verbraak ${ }^{2}$;

${ }^{1}$ Internal Medicine, ${ }^{2}$ Ophthalmology, Academic Medical Center, Amsterdam, The Netherlands.

Background and Aims: Optical Coherence Tomography (OCT) is a non-invasive, non-contact, high resolution imaging technique, that acquires scans of the retina, providing information on morphology and pathological changes in the retina. OCT has enabled ophthalmologists to reliably detect and measure small changes in retinal thickness (RT), which can be presented in a topographic map. This allows for quantitative evaluation of RT in patients and monitoring of the efficacy of different therapies. The topographic maps are obtained from 6 radial, cross-sectional scans centered on the fovea. RT is calculated automatically by the OCT analysis software in macular areas centered around the fovea. Recent studies in mixed type 1 and type 2 diabetes mellitus (DM) populations suggest that the retina is thickened, even in a preclinical phase of diabetic retinopathy (DRP) and especially in a circular area directly surrounding the fovea. The first aim of this study is to evaluate the RT measured with OCT exclusively in patients with DM type 1, with no or minimal DRP, and compare this to a healthy control group. Secondly, we evaluated the possible association of metabolic control and RT.

Materials and Methods: Patients with DM type 1, with minimal or no DRP on biomicroscopy and stereo fundus photography, seen at the outpatient clinic of the department of internal medicine at the AMC, were included and underwent a full ophthalmologic examination, fundus photography and OCT scanning of the macula. Mean RT measurements were taken in the pericentral area around the fovea (donut shaped ring with inner diameter of $1 \mathrm{~mm}$ and outer diameter of $3 \mathrm{~mm}$ ) and the peripheral area around the fovea (inner diameter of $3 \mathrm{~mm}$ and outer diameter of $6 \mathrm{~mm}$ ). RT measurements in patients with minimal DRP were compared to RT measurements in patients without DRP and age and gender matched healthy subjects. Parameters of metabolic control (blood pressure, HbA1c, cholesterol, triglycerides, TSH, creatinine clearance) were recorded simultaneously and correlated to the RT measurements.

Results: Fifty-two patients were included in the study. 23 patients had minimal DRP and 29 showed no signs of DRP. Mean RT in the pericentral area was $268 \mu \mathrm{m}( \pm 19 \mu \mathrm{m})$ in patients with minimal DRP, $278 \mu \mathrm{m}( \pm 16 \mu \mathrm{m})$ in patients without DRP compared to $281 \mu \mathrm{m}( \pm 13 \mu \mathrm{m})$ in healthy subjects $(n=29)$. The difference in pericentral RT between the patients with minimal DRP compared to the controls was statistically significant ( $p$-value $=0.008$ ). No significant difference was found between the groups for the RT in the peripheral area. No correlation was found between RT measurements in the patients and any of the metabolic parameters.

Conclusion: In contrast to earlier reports we could not demonstrate an increased RT of the pericentral or peripheral area in patients with DM type 1 with no or minimal DRP. Instead, we found a decreased pericentral RT in patients with minimal DRP compared to healthy controls. This could be explained by a loss of intraretinal neurons in the earliest stage of DRP. The potential role of loss of retinal thickness as an early marker of incipient diabetic retinopathy deserves prospective study.

\section{3}

The effect of activated inflammatory cells in diabetic retinopathy

A. M. C. Serra ${ }^{1}$, J. M. Waddell ${ }^{1}$, L. Duncan ${ }^{1}$, M. A. Cotter ${ }^{2}$, J. V. Forrester ${ }^{1}$;

${ }^{1}$ Department of Ophthalmology, ${ }^{2}$ Department of Biomedical Sciences, Institute of Medical Sciences, Aberdeen, United Kingdom.

Background and Aims: Some studies postulated that inflammation might play an important role in the development of Diabetic Retinopathy (DR). It was stated that monocytes/macrophages were involved in the initiation process of microvascular pathology characteristics of DR. In addition, several studies have reported that diabetic leukocytes are activated shown by increased expression of adhesion molecules and by greater adhesion to endothelial cells. Furthermore, up regulation of adhesion molecules are confirmed in leukocytes of DR patients.

The aim was to study the expression of adhesion, activation, chemokine receptors molecules, to measure the 
cytokines levels and to correlate the type of expression with the adhesiveness of leukocytes to retinal vessels in vivo.

Materials and Methods: Diabetes was induced in mice by intraperitoneal injection of streptozotocin (STZ) and mice were analysed after 2 weeks. Immunofluorescence staining was performed in fixed whole flat mount retina and stained leukocytes were counted using a fluorescence microscope and recorded images were taken under a confocal laser scanning microscope. Myeloid bone marrow and leukocyte blood cells of wild-type and diabetic-induced animals were analysed by flow cytometry to quantify monocyte (CD11b), activation cell surface (CD40) and chemokine receptor (CCR5) markers. Cytometric bead assay and ELISA methods were realized in blood serum and supernatant of monocytes culture cells of these mice in order to measure the cytokine secretion. The data were expressed as mean \pm SD. Statistical significance was determined by using a One-Way Analysis of Variance followed by Bonferroni's Multiple Comparision test and Paired Student T-test.

Results: Identification and statistically significant increased numbers of adherent CD11b cells were localized inside of the endothelial capillaries of diabetic retinea. The flow cytometric analyses resulted in a significant enhanced of CD40 and CCR5 expression on slightly increased number of diabetic myeloid bone marrow cells. Unchanged number of leukocyte blood cells shown a tendency to increased expression of CD40 in diabetic comparing to wild type animals.

Conclusion: The STZ induced-diabetic mice presented adhesive and activated myeloid cells inside of the retinal vessels. In summary, the data suggest that diabetes-induced leukocytes capillary occlusion in Diabetic Retinopathy. Supported by Development Trust of University of Aberdeen, Astra Zeneca and Miltenyi Biotec Ltd

\section{4}

Inflammation but not oxidative stress gets elevated in the retina in a model that combines genetic hypertension and experimental diabetes

C. C. Pinto, K. C. Silva, S. K. Biswas,

J. B. Lopes de Faria, J. M. Lopes de Faria;

Faculty of Medical Sciences, State University of Campinas, Brazil.

Background and Aims: Hypertension is the main secondary factor associated with diabetic retinopathy (DR). The mechanisms by which hypertension increase the risk for DR are poorly understood. Recent data have revealed that oxidative stress and inflammatory responses are involved in pathogenesis of DR activating the transcription factor nuclear kappa beta (NF-kB). But the crosstalk between the redox status, inflammation and RAGE (AGE-receptor) system in diabetic retinopathy is not understood. The aims of this study were to investigate the role of inflammation and oxidative stress in experimental model that combines hypertension and diabetes.

Materials and Methods: Four week old spontaneously hypertensive rats (SHR) and their normotensive counterpart Wystar Kyoto (WKY) rats were rendered diabetic by intravenous injection of streptozotocin. After 20 days, rats were sacrificed and retinas were collected.

Results:The number of immunohistochemically detected ED1/microglial positive cells and the expression of ICAM1 in the retina were significanthy higher in diabetic SHR than in the control $(\mathrm{p}<0.0001)$. The levels of reduced glutathione were lower in diabetic SHR in comparison with WKY groups $(\mathrm{p}<0.04)$ but the NADPH oxidase, evaluated by expression of NOX-4 subunit, did not differ in groups. NF-kB p65 levels were higher in SHR compared with WKY groups $(\mathrm{p}<0.005)$.

Conclusion: The concomitance of diabetes and hypertension leads to exuberant inflammatory response but not oxidative stress in the retina. Perhaps the RAGE system can be involved in the activation of NF-kB. This system and its blockage are under investigation.

Supported by FAPESP

1085

Diabetes mellitus and the eye: axial length

B. Huntjens, C. O'Donnell;

Optometry and Neuroscience, The University of Manchester, United Kingdom.

Background and Aims: The refractive error of the eye is dependent on its axial length. Refractive error is known to fluctuate significantly in poorly-controlled diabetic patients. Recently it has been reported that human eyes fluctuate in axial length during the day. However, this change is not detectable in all subjects, suggesting physiological influences such as diet. The purpose of this study was to investigate fluctuations in axial length and blood glucose levels (BGLs) in diabetic patients and control subjects, using partial coherence interferometry.

Material and Methods: Periodic axial length measurements were taken with an IOLMaster (Zeiss, Oberkochen, Germany) in 21 type 2 diabetic subjects (age 56 \pm 11 years), 19 type 1 diabetic subjects (age $38 \pm 15$ years) and 18 nondiabetic controls (age 46 \pm 22 years). Additionally, BGLs were measured using a HemoCue Beta glucose analyser (HemoCue, Ängelholm, Sweden). Measurements were taken between 8AM and 8PM at approximately two hourly intervals. The ocular length variability was mapped against 
time of day and related to BGLs, age, and the degree of diabetic retinopathy.

Results: Axial length variations were found to be significant with time (ANOVA, $\mathrm{P}=.003$ ) and there was a significant effect for the interaction between group and time of day (ANOVA, $\mathrm{P}<0.0005$ ). DM type 1 and control subjects showed a significantly increased axial length in the afternoon compared to in the morning (t-test, $\mathrm{P}<0.0005$ ). No significant change was observed among DM type 2 subjects. Axial length fluctuated sinusoidally with time of day. A three-parameter sinusoidal curve was used to fit each set of axial length measurements, which resulted in a correlation coefficient $\mathrm{R}^{2} \geq 0.7$ in $62 \%$ of type 2 diabetic subjects, $78 \%$ of type 1 diabetic subjects and $72 \%$ of control subjects. Multiple regression analysis established that neither BGLs, age, nor the degree of diabetic retinopathy had a significant effect on the fluctuations of the axial length $(\mathrm{P}>.05)$

Conclusion: Axial length varies throughout the day in diabetic and control subjects; however, this change does not appear to be significant in every individual. The data suggest a maximum axial length value in the afternoon. Changes in axial length from baseline did not correlate with BGLs, age, or the degree of diabetic retinopathy. Therefore, blood glucose levels may not be the main physiological influence in axial length fluctuations.

Supported by a grant awarded by Lein Applied Diagnostics

\section{PS 95}

\section{Somatic neuropathy}

\section{6}

RAGE-dependent impairment of glyoxalase-1 contributes to functional deficits in diabetic neuropathy

I. Konrade ${ }^{1}$, S. Stoyanov ${ }^{1}$, G. M. Haag ${ }^{1}$, Y. Seregin ${ }^{1}$, P. M. Humpert ${ }^{1}$, M. $\operatorname{Morcos}^{1}$, S. R. Thorpe ${ }^{2}$,

P. Thornalley ${ }^{3}$, P. P. Nawroth ${ }^{1}$, A. Bierhaus ${ }^{1}$;

${ }^{1}$ Department of Internal Medicine I, University Clinic Heidelberg, Germany, ${ }^{2}$ Department of Chemistry and Biochemistry, Medical University of South Carolina, United States, ${ }^{3}$ Department of Biological Sciences, University of Essex, United Kingdom.

Background and Aims: The Glyoxalase I (GO1)-system is a central part of the cellular defense detoxifying precursors of Advanced Glycation Endproducts (AGEs) such as methylglyoxal, glyoxal and other alpha-oxoaldehydes. Since AGEs, binding of AGEs to the receptor RAGE and subsequent RAGE dependent cellular activation are supposed to contribute to the development and progression of late diabetic complications we studied the interaction of RAGE and GO1 in vitro and in experimental animal models of diabetic neuropathy (DN).

Materials and Methods: GO1-transcription (Real time PCR), -expression (Western Blot) and -activity (enzyme assay) and its modulation by RAGE-ligands, determined in transient transfection studies using murine GO1-promoter plasmids, was measured in RAGE bearing (WT) and RAGE deficient ( $\mathrm{RAGE}^{-/-}$) fibroblasts, endothelial cells and dorsal root ganglia (DRG). In vitro studies were paralleled in vivo by immunhistochemistry of sciatic nerves and experimental models of DN including, pain perception assays and determination of neuronal blood flow in healthy and diabetic WT- and RAGE ${ }^{-1-}$ - mice.

Results: Methylglyoxal and AGEs, but also other RAGEligands such as S100B significantly reduced GO1-transcription, expression and activity in WT derived fibroblasts, endothelial cells and dorsal root ganglia cells (DRG). In $\mathrm{RAGE}^{-/-}$cells, however, RAGE ligands induced a significant upregulation of GO1 implying that engagement of RAGE directly suppressed GO1. Consistently, GO1-expression was increased in WT cells treated with the RAGE antagonist sRAGE $(25 \mu \mathrm{g} / \mathrm{ml})$ for $48 \mathrm{~h}$. To study a potential role of RAGE dependent GO1-reduction in DN, GO1transcription and expression was determined in sciatic nerves from healthy and diabetic WT and $\mathrm{RAGE}^{-/-}$mice. Compared to $\mathrm{RAGE}^{-/-}$mice, GO1 was significantly lower in peripheral nerves of WT-mice and further attenuated in diabetes. The functional significance of GO1 deficiency for DN was studied in diabetic mice, kept in the diabetic state for 4 months in the absence or presence of an inhibitor of GO1. GO1-inhibitor treatment resulted in significant increased pain perception in early stages of DN and in impaired neuronal blood flow (NBF) in both, diabetic WT and $\mathrm{RAGE}^{-/-}$mice, whereas impairment was even more pronounced in $\mathrm{RAGE}^{-/-}$mice.

Conclusion: These data provide firm evidence that the diabetes dependent reduction in GO1, which contributes to functional deficits in $\mathrm{DN}$, is at least in part caused by RAGE-ligand interaction.

Supported by Albert Renold Fellowship (I.K.) European Foundation for the Study of Diabetes (A.B.)

1087

Metabolic syndrome in type 1 diabetes: a potential risk factor for the development of diabetic neuropathy after 9 years

S. M. Rajbhandari, D. Selvarajah, M. E. Plater, M. T. Dent, N. D. Harris, J. D. Ward, S. Tesfaye; Diabetes Research Unit, Royal Hallamshire Hospital, Sheffield, United Kingdom. 
Background and Aims: Cardiovascular risk factors such as hypertension, lipids, smoking have been well recognised as a risk factor of diabetic neuropathy (DN). Metabolic syndrome (MS) is one of the important cardiovascular risk factor and can be present even in type 1 diabetes. However, the association of MS to the development of DN is still not clear. In Sheffield Prospective Diabetes Study we investigated if the presence of MS predicts the future development of DN. Our aim was to follow up a well-defined cohort of subjects with newly diagnosed type 1 diabetes and to investigate the relationship between the presence of MS to the development of DN.

Materials and Methods: 66 newly diagnosed type 1 diabetic subjects (mean age $31+/-9$ (SD) duration (3 years $+/-2$ ) were identified and followed for 9 years. They had detailed neurological assessment (symptoms and signs score, nerve conduction, vibration perception threshold, warm thermal discrimination threshold and autonomic function tests) and blood tests performed at base line, 3 years and 9 years. Metabolic syndrome was defined using NCEP Adult Treatment Panel III criteria. DN was defined as per Dyck's criteria

Results: At the 9 years, 51 patients were followed up and there were no difference between these subjects and those who were lost to follow up.Overall presence of MS was $21.2 \%$ at baseline which rose to $31.4 \%$ by 9 years follow up. In subjects with MS, DN was present in $60 \%$ in compariosn to $29.3 \%$ in subjects without $\mathrm{MS}(\mathrm{p}=0.03) .9$ subjects already had DN at baseline and were excluded from further analysis. Of the remaining 42 subjects $33.3 \%$ subjects with MS at baseline developed new onset DN, whereas only $19.4 \%$ without MS developed DN ( $p=0.001$ ). Conclusion: This prospective study suggests that as other cardiovascular risk factors, MS may also be an important risk factor for the development of DN. Aggressive management of the risk factors of MS is essential to reduces cardiovascular mortality and possoibly morbidity due to DN.

Supported by Diabetes UK

\section{8}

Metabolic syndrome includes augmented, not impaired, insulin-mediated vasodilation of the cutaneous microcirculation

X. T. Tigno ${ }^{1}$, S. Ding ${ }^{2}$, F. L. Rice ${ }^{3}$, B. C. Hansen ${ }^{1}$;

${ }^{1}$ Physiology, College of Medicine, Tampa, ${ }^{2}$ Physiology, College of Medicine, Baltimore, ${ }^{3}$ Center for Neuropharmacology and Neurosciences, Albany Medical College, Albany, United States.
Background and Aims: Insulin is reported to improve capillary flow, increasing both arteriolar diameters and erythrocyte flow velocity. However, hyperinsulinemia may also exert a negative effect on erythrocyte deformability, thus impeding flow. While capillary perfusion is thought to be impaired among diabetic subjects, we have previously described that thermally- induced hyperemia in the skin microcirculation, based on percent increase in perfusion over baseline, was significantly greater among prediabetic compared to normal monkeys, and that this finding was significantly correlated with circulating levels of hsCRP. The study was conducted to better understand the interactions between insulin, the metabolic status and tissue perfusion.

Materials and Methods: Twenty normoglycemic (N) $(\mathrm{FPG}<110 \mathrm{mg} / \mathrm{dl})$ non human primates, 8 of which were prediabetic with characteristics of the metabolic syndrome (PreDM/MetSyn), underwent the hyperinsulinemic, euglycemic clamp procedure. Microvascular flow was assessed using a laser Doppler flowmeter. Baseline and thermallystimulated perfusion levels were measured before and at 10 minute intervals following insulin infusion for a period of 60 minutes. Immunohistological analyses using various neuronal markers were performed on skin biopsies.

Results: Mean basal perfusion improved over pre-clamp levels 60 minutes after insulin infusion (from $36.7 \pm 7.9$ to $104.9 \pm 20.2$ P.U., $p=.0002$ ) and heat- induced peak flow was also markedly enhanced during the hyperinsulinemic period (from $87.4 \pm 13.8$ to $157.8 \pm 25.6 \mathrm{PU}, \mathrm{p}<0.0004$ ) in all monkeys. The per cent increase in perfusion during heating, corresponding to microvascular recruitment evoked by the axon flare response, was significantly higher among PreDM/MetSyn subjects compared to normal similar aged monkeys. Extensive neurovascular remodeling, including proliferation of capillaries in the dermis and an increase in CGRP/SP - expressing C- fiber innervation to the epidermis during the prediabetic/metabolic syndrome phase of the disease were also observed.

Conclusion: The alterations in the cutaneous angioarchitecture and its innervation are likely to provide the morphological basis for the increased neurogenic vasodilation seen in prediabetes, a prodrome to the later deterioration in heat-stimulated and flow -mediated dilation seen in the more advanced stages of diabetes in humans and in monkeys. Thus augmented insulin-stimulated flow is likely to be a marker of progressing cutaneous pathology leading to the distal micorvascular complications of diabetes.

Supported by NIA/NIH NO1-AG-3-1012 
Cardiovascular disease, diabetic polyneuropathy and ACE gene polymorphisms in the North Catalonia Diabetes Study (NCDS)

J. Jurado ${ }^{1}$, M. Pastoret ${ }^{1}$, J. Ybarra ${ }^{2}$, I. Gich ${ }^{3}$, J. M. Pou ${ }^{4}$; ${ }^{1}$ Primary Care, Institut Catala Salut, Olot, ${ }^{2}$ Research Institute, Hospital Sant Pau, Barcelona, ${ }^{3}$ Clinical Pharmacology, Hospital Sant Pau, Barcelona, ${ }^{4}$ Endocrinology and Nutrition, Hospital Sant Pau - Autonomous University of Barcelona, Spain.

Background and Aims: We observed several relationship between cardiovascular disease prevalence (CVD) and polymorphisms of Renin-Angiotensin axis gene regulation. The aim was to evaluate the possible association between diabetic polineuropathy (DPN) and ACE gen polymorphism (insertion/delection (I/D) in a random sample of diabetic population obtained from the North Catalonia Diabetes Study (NCDS).

Materials and Methods: Forty type 2 diabetic patients (T2DM) with CVD and thirty one without CVD (matched for gender an age) were selected. Previouly, they were selected for evaluation of non classical cardiovascular risk factors.

DPN was assessed by a clinical neurological evaluation (bilateral affectation of vibration perception thresholds with neurothesiometer and tuning fork, sensory tests to touch, pinprick and cold perception, Semmes-Weinstein Monofilament 5.07, muscle strength and reflexes according to the Michigan Diabetic Neuropathy Score and the items of modified Neuropathy Symptom Score). CVD includes stroke, coronary and peripheral ischemia

Results: Diabetic polyneuropathy (DNP) was related to cardiovascular disease (CVD) prevalence $(p=0.036)$ in the NCDS Study. In the evaluation of T2DM with CVD, patients with DPN manifested less D/I heterozygous genotype than patients without DPN $(p=0.012)$.

In logistic regression analysis using D/I-additive model, the $\mathrm{D} / \mathrm{I}$ allele combination manifested some protective effect on diabetic polyneuropathy prevalence (odds ratio: $0.081 ; 95 \%$ confidence interval, 0.009-0.720). T2DM patients without CVD did not observe any significant difference only a tendency $(p=0.08)$. In contrast, the distribution of other homocygous genotypes [D/D or I/I allele combination] did not differed in both T2DM patients with or without DPN.

Conclusion: Our results suggest that the heterozygous genotype, combination of $\mathrm{D} / \mathrm{I}$ allele, could be a protector factor for DPN presence in this previous study of T2DM with CVD.

Supported by Fundacio Gol $i$ Gurina and FIS 01/0846 Instituto Carlos III, Spain
The new indicator test (Neuropad) in the assessment of the staged severity of neuropathy

N. $\operatorname{Papanas}^{1}$, G. Giassakis ${ }^{2}$, K. Papatheodorou ${ }^{1}$,

D. Papazoglou ${ }^{1}$, C. Monastiriotis ${ }^{1}$, D. Christakidis ${ }^{3}$,

H. Piperidou ${ }^{2}$, E. Maltezos ${ }^{1}$;

${ }^{1}$ Second Department of Internal Medicine, ${ }^{2}$ Department of Neurology, Democritus University of Thrace, Alexandroupolis, ${ }^{3}$ Diabetic Department, University Hospital of Alexandroupolis, Alexandroupolis, Greece.

Background and Aims: The new indicator test for sudomotor function $\left(\right.$ Neuropad $\left.^{\circledR}\right)$ has been shown to be a highly sensitive and reproducible tool for the diagnosis of diabetic peripheral neuropathy. The aim of this study was to examine the utility of the indicator test in the assessment of the staged severity of diabetic neuropathy.

Patients and Methods: This study included 120 type 2 diabetic patients (58 men) with a mean age of $67.3 \pm 5.9$ years and a mean diabetes duration of $13.1 \pm 3.2$ years. Neuropathy was diagnosed and staged by clinical examination and nerve conduction study, according to the Michigan classification system (Feldman et al, 1994). Patients were also examined with the indicator test, applied on the plantar aspect of the feet. Time until complete colour change of the test was recorded and stratified into deciles according to the spread of measurements in the study population.

Results: Neuropathy was staged as class 0 in 37 patients, class 1 in 44 patients, class 2 in 28 patients and class 3 in 11 patients. Time until complete colour change was $436.5 \pm$ $62.9,740 \pm 88.1,1192.5 \pm 161$ and $1817.3 \pm 127.4$ seconds in patients staged as class $0,1,2$ and 3 respectively $(p=0.001)$. Use of a threshold lower than 530 seconds until complete colour change had $97.3 \%$ sensitivity and $100 \%$ specificity for diagnosis of class 0 . Use of a threshold lower than 1000 seconds until complete colour change had 100\% sensitivity and $97.4 \%$ specificity for class 1 neuropathy. A threshold lower than 1440 seconds had $92.9 \%$ sensitivity and $100 \%$ specificity for class 2 neuropathy. A threshold above 1440 seconds had 100\% sensitivity and 99\% specificity for class 3 neuropathy. A highly significant (Kendall's tau- $b=0.848$, $\mathrm{p}=0.001$ ) correlation was shown between time until complete colour change of the test and Michigan class of neuropathy.

Conclusions: It appears that the indicator test contributes substantially to the assessment of the staged severity of neuropathy. There is excellent agreement between the indicator test and the Michigan classification system. These results suggest a role for the indicator test in the assessment of diabetic neuropathy. 
Frequence modulated electromagnetic neural stimulation enances cutaneous microvascular perfusion in patients with diabetic neuropathy

M. Conti ${ }^{1}$, E. Peretti ${ }^{1}$, G. Cazzetta ${ }^{2}$, L. Folini ${ }^{1}$, C. Vermigli ${ }^{2}$, G. Galimberti ${ }^{1}$, L. Scionti ${ }^{2}$, E. Bosi ${ }^{1}$;

${ }^{1}$ Endocrinology, San Raffaele Scientific Institute and VitaSalute University, Milano, ${ }^{2}$ Internal Medicine, Monteluce Hospital, University of Perugia, Italy.

Background and Aims: As a non pharmacological treatment, Frequency Modulated Electromagnetic Neural Stimulation (FREMS) has recently demonstrated, in a placebo-controlled trial, to be a safe and effective treatment of painful diabetic neuropathy, leading to amelioration of symptoms and improvement of some parameters of peripheral nerve function. We here present the results of an ancillary study of this trial, investigating the effects on microvascular function.

Materials and Methods: Patients $(n=31)$ with painful neuropathy associated with decreased motor and/or sensory nerve conduction velocity $(<40 \mathrm{~m} / \mathrm{s})$ in at least one nerve trunk of the lower limbs and increased vibration perception threshold $(>25 \mathrm{~V})$ were enrolled in a randomised, doubleblind, crossover FREMS vs placebo study. Each patient received two series of ten treatments of either FREMS or placebo in random sequence, each lasting no more than 3 weeks. Measures at baseline, end of FREMS and placebo series and after 4 months of follow up included: cutaneous blood flow by laser doppler flowmetry, partial tissue tension of oxygen (TcpO2) and carbonic anhydride (TcpCO2) by oxymetry (Clark electrode) at the lower extremities in basal resting conditions and as incremental response after a thermal stimulation.

Results: Cross-over analysis showed no differences between FREMS and placebo as cutaneous blood flow and TcpCO2 and a significant reduction of TcpO2 after FREMS in resting conditions $(\mathrm{p}=0.007)$, but not as incremental flow after warming. At 4 months, a significant increase of resting cutaneous blood flow was observed ( $p=0.0033$ vs baseline), with no differences as incremental flow after warming; no differences were observed for TcpO 2 and $\mathrm{TcpCO} 2$ either in resting conditions or as response to thermal stimulation.

Conclusion: These findings indicate that ten treatments with FREMS induce an enhancement of microvascular blood flow measurable up to 4 months of follow-up, and a short term reduction of TcpO2, possibly due to increased epidermal oxygen consumption. The increased cutaneous blood flow could reflect an increased endoneural blood flow and this effect might contribute to explain the improvements of some parameters of peripheral nerve function induced by FREMS.
Numbness in both toes and soles and disproportional sweating restricted on face and chest are reliable and useful symptoms for diagnosis of diabetic polyneuropathy H. Sasaki, M. Nakatani, H. Yamasaki, K. Ogawa, T. Shono, S. Matsuno, H. Wakasaki, H. Furuta, M. Nishi, T. Nakao, K. Nanjo;

First Department of Medicine, Wakayama University of Medical Science, Japan.

Background and Aims: Though symptoms and signs of diabetic symmetric polyneuropathy (DPN) are well known experientially, the reports which examined the specific symptom of DPN based on multiple objective neurological tests are very rare. We aimed to reconfirm the specific symptoms and signs of DPN using 7 objective nerve function tests in 593 diabetic patients. Furthermore, the prevalence of the characteristic symptoms in Japanese type 2 diabetic outpatients under primary-care physicians was examined using a nationwide questionnaire survey.

Materials and Methods: I. Specific symptoms and sign of $\underline{D P N}$ Subjective symptoms: "numbness in toes and soles", "pain in feet", "numbness in hands"; "pain in hands"; "coldness in both feet", "frequent leg cramp", "orthostatic dizziness", "sweating restricted on face and chest", "frequent constipation/diarrhea" were investigated by interview. "Achilles tendon reflex (ATR)" and "grasping calf pain" were examined as neurological physical signs. The relationships between symptoms, signs and 7 objective nerve function tests such as: motor nerve, sensory nerve and Fwave conduction velocity, sensory nerve action potential, coefficient of variation of R-R intervals in electrocardiogram, fall in systolic blood pressure during head-up tilt and quantitative vibratory perception threshold were evaluated. II. Prevalence of the neurological symptoms The questionnaire survey which was a part of the nationwide survey focused on self-management of the diabetes and erectile dysfunction was performed on 1524 male diabetic outpatients of 119 hospitals or clinics. Additional survey was also carried out on 93 outpatients of the local public hospital (Shingu municipal medical center: SMC) to confirm the absence of gender difference in symptoms.

Results: I. Specific symptoms and sign of DPN ;1) bilateral not unilateral "numbness in toes and soles" and "diminished ATR" were significantly related to the all nerve functions, 2) bilateral "pain in feet", "grasping calf pain" and "sweating restricted on face and chest" were significantly related to 6 nerve functions, 3) "numbness in hands", "frequent leg cramp" and "coldness in feet" were related to only a few nerve function. II. Prevalence of the neurological symptoms; There was no gender difference in all symptoms and signs in SMC survey, and the 
prevalence of symptoms and signs were almost same between national and SMC surveys. The prevalence of bilateral "numbness in toes and soles", "pain in feet" and "sweating restricted on face and chest" were $25 \%, 10 \%$ and $15 \%$, respectively.

Conclusion: Bilateral "numbness in toes and soles" and disproportional "sweating restricted on face and chest" seem to be reliable and useful symptoms for diagnosis of DPN. Bilateral "diminished ATR" and "grasping calf pain" also seem to be characteristic signs of DPN.

\section{3}

A provisional evaluation of the validity of the modified clinical neuropathy examination (mCNE)

S. G. Mengoulis ${ }^{1}$, H. M. Rathur ${ }^{2}$, A. P. Garrow ${ }^{3}$, A. J. M. Boulton ${ }^{2}$;

${ }^{1}$ Department of Medicine, 251 Hellenic Air Force Hospital, Athens, Greece, ${ }^{2}$ Medicine, Manchester Royal Infirmary, ${ }^{3}$ Disablement Services Centre, Withington Hospital, Manchester, United Kingdom.

Introduction: Diabetic neuropathy is a common and serious complication of diabetes. Various methods are used to assess the severity of neuropathy. In this study we aimed to evaluate the inter-observer repeatability and criterion validity of the mCNE.

Methods: Forty six diabetic patients, aged 32 to 70 years, $35 \%$ Type 1, mean duration of diabetes 16.7 years, were examined at baseline and followed up after $\sim 1$ week. Different methods of assessing neuropathy were used by 2 examiners. These were the mCNE (cut off for normality $=>4=$ neuropathy), Vibration Perception Threshold (VPT), modified Neuropathy Disability Score (mNDS), Nerve Conduction Studies at right peroneal nerve, [(NCS) cut off for normality $>42 \mathrm{~m} / \mathrm{s}=$ neuropathy].

We analysed correlation between mCNE \& VPT as well as NCS and NDS.

The inter-observer agreement between baseline and follow up was assessed using a kappa statistic. Sensitivity and specificity values were calculated comparing $\mathrm{mCNE}$ with $\mathrm{NCV}=>42 \mathrm{~m} / \mathrm{s}$.

Results: The sample population had different levels of neuropathy with a mean VPT $25 \pm 15.9$ volts, mNDS 5 (IQR $1-8)$ and NCV of $41.2 \pm 6.1 \mathrm{~m} / \mathrm{s}$. mCNE correlated well with VPT [correlation coefficient (CC) 0.86]; NCV [CC: -0.76 ], NDS [CC: 0.92]. The inter-observer agreement was the same at baseline and and follow (kappa 0.91; $0.62,1.2)$. When compared with the gold standard NCS, the sensitivity of the mCNE was: $0.87 ; 95 \%$ CI $(0.64,0.97)$ while specificity was: $0.93 ; 95 \% \mathrm{CI}(0.72,0.98)$.
Conclusions: These results suggest that the modified Clinical Neuropathy Examination is a good method of assessment of diabetic peripheral neuropathy for research and clinical use.

1094

Decreased vibration perception threshold in type 1 but not type 2 patients with proliferative retinopathy

D. S. Tesic ${ }^{1}$, P. Pantelinac ${ }^{1}$, A. Oros ${ }^{2}$, M. Mitrovic ${ }^{1}$,

V. Dzinic ${ }^{2}$, R. Pejin ${ }^{1}$, J. Radosavljevic ${ }^{1}$;

${ }^{1}$ Clinic of Endocrinology, Diabetes and Metabolic Diseases, Institut for Internal Diseases, Clinical Centre, Novi Sad, ${ }^{2}$ Clinic of Ophthalomology, Clinical Centre, Novi Sad, Serbia and Montenegro.

Background and Aims: The aim of this study was to examine the prevalence of neuropathy and nephropathy in patients with type 1 (T1DM) and type 2 (T2DM) diabetes complicated by proliferative retinopathy (PDR).

Materials and Methods: Comparisons were made between 18 patients with T1DM and PDR and 19 age and diabetes duration matched controls without PDR, and also between 24 patients with T2DM with PDR and 20 diabetes duration matched controls. Clinical examination of the eyes was performed through dilated pupils using a slit lamp and a magnifying lens. Vibration perception threshold (VPT) was measured by semiquantitative tuning fork $\mathrm{C} 128$ (grade 0-8) and ankle reflexes were recorded. Body weight $(\mathrm{Kg})$, serum creatinine, albumin, fibrinogen and urine albumin concentration were measured, and the presence of macrovascular (coronary, cerebrovascular and peripheral arterial) complications was also documented.

Results: VPT was significantly worse in patients T1DM and PDR compared with controls $(4.7 \pm 1.9$ vs. $7.3 \pm 0.73$, $\mathrm{p}<0.01)$, and ankle reflex score was also worse $(3.56 \pm 1.04$ vs. $1.9 \pm 1.74, p<0.01)$. Vibration perception was negatively correlated with body weight $(\mathrm{r}=-0.37, \mathrm{p}=0.02)$, proteinuria $(\mathrm{r}=-0.52, \mathrm{p}<0.01)$, creatinine $(\mathrm{r}=-0.50, \mathrm{p}<0.01)$, albuminaemia $(r=-0.41, \mathrm{p}=0.01)$, fibrinogen $(\mathrm{r}=-0.39, \mathrm{p}=0.02)$. After multiple regression analysis the correlations with age $(\mathrm{p}=0.02)$, proteinuria $(\mathrm{p}=0.01)$ and creatinine $(\mathrm{p}<0.01)$ remained significant. In T2DM patients with PDR were younger than controls $(60.7 \pm 8.6$ vs. $67.5 \pm 6$ years, $p<0.01)$, but VPT was not significantly different between the two groups ( $5 \pm 2.8$ vs. $6.1 \pm 1.6, \mathrm{p}=0.13$ ). VPT was negatively correlated with duration of diabetes $(r=-0.30, p=0.05)$, duration of insulin therapy $(r=-0.33, p=0.03)$ in T2DM. A positive history of macrovascular disease was more common in T2DM without DPR than among T2DM with PDR $\left(20.8 \%\right.$ vs. $55 \%, X^{2} 5.5, \mathrm{p}=0.02 ; 33.3 \%$ vs. $70 \%, X^{2}$ $5.88, \mathrm{p}=0.02)$. 
Conclusion: Worsening VPT in T1DM is strongly associated with diabetic nephropathy and diabetic proliferative retinopathy. In T2DM it is possible that endogenous insulin may exert a protective effect on microvascular complications while also having an adverse effect on the incidence of macrovascular complications.

Thanks for suggestions to William Jeffcoate

\section{PS 96}

\section{Somatic neuropathy - pathogenesis and treatment}

\section{5}

Activation of $\mathrm{Na}^{+} / \mathrm{H}^{+}$- exchanger-1: a novel pathogenetic mechanism in peripheral diabetic neuropathy (PDN) I. G. Obrosova ${ }^{1}$, O. Ilnytska ${ }^{1}$, V. Lyzogubov ${ }^{1}$, N. Mashtalir ${ }^{1}$, M. A. Yorek ${ }^{2}$, V. R. Drel ${ }^{1}$;

${ }^{1}$ Division of Nutrition and Chronic Disease, Pennington Biomedical Research Center, Baton Rouge, ${ }^{2}$ Internal Medicine, University of Iowa, Iowa City, United States.

Background and Aims: Overall hypothesis: Enhanced $\mathrm{Na}^{+} / \mathrm{H}^{+}$-exchange (NHE) results in cytosol alkalinization and activation of the upper part of glycolysis which, under conditions of diabetes-associated inhibition or insufficient upregulation of glyceraldehyde 3-phosphate dehydrogenase, causes diversion of the glycolytic flux towards several pathways implicated in diabetic complications. The purpose of our study was to explore the role for $\mathrm{Na}^{+} / \mathrm{H}^{+}$-exchanger1 (NHE-1), the most abundant NHE isoform, in PDN.

Material and Methods: In vivo experiments were performed in 1) control and STZ-diabetic rats treated with/ without the NHE-1 inhibitor cariporide; 2) control and STZ-diabetic NHE-1+/+and+/- mice. In vitro studies were performed in human Schwann cells (HSC) cultured in $5 \mathrm{mM}$ or $30 \mathrm{mM}$ glucose. Nerve blood flow was measured by microelectrode polarography and hydrogen clearance, thermal algesia by paw withdrawal and tail-flick tests, mechanical algesia by von Frey anesthesiometer/rigid filament and Randall-Sellito tests, and tactile allodynia by flexible von Frey filament test. Oxidative stress was assessed by immunohistochemistry [nerve nitrotyrosine and poly(ADP-ribose)] and lucigenin test (aortic superoxide production). In vitro studies employed real-time PCR, Western blot analysis and BCECF test of NHE-1 activity.

Results: NHE-1 is abundantly expressed in rat and mouse sciatic nerves and DRG neurons and its expression is increased in diabetes. Nerve blood flow (microelectrode polarography/hydrogen clearance) and sensory nerve conduction velocity (SNCV) deficits were prevented, and motor nerve conduction velocity (MNCV) deficit, thermal hyperalgesia, mechanical hyperalgesia, tactile allodynia, nerve nitrotyrosine and poly(ADP-ribose) accumulation, and aortic superoxide production alleviated in STZ-diabetic rats treated with cariporide compared with untreated diabetic rats. MNCV deficit, thermal and mechanical hypoalgesia and tactile allodynia were less severe in $\mathrm{NHE}+/-$ than in NHE-1+/+STZ-diabetic mice. High glucose-induced NHE-1 mRNA and protein overexpression and NHE activation were clearly manifest in HSC, and were mediated via poly (ADP-ribose) polymerase-1 (PARP-1) and MAPK (ERK, SAPK/JNK)-involved mechanisms.

Conclusion: NHE-1 activation is a novel fundamental mechanism in the pathogenesis of PDN, and is likely to be implicated in neuropathy in human subjects with diabetes.

\section{6}

Neuronal mitochondrial degeneration in diabetic rats and ameliorative effects of pioglitazone

T. Inoue ${ }^{1}$, E. K. Kim ${ }^{1}$, J. W. Russell ${ }^{1,2}$;

${ }^{1}$ Neurology, University of Michigan, Ann Arbor, United States,

${ }^{2}$ Neurology and GRECC, Veterans Affairs Medical Center, Ann Arbor, United States.

Background and Aims: Mitochondrial degeneration and induction of oxidative stress has been considered to play a role in the progression of diabetic complications. Peroxisome proliferator-activated receptor gamma coactivator- $1 \alpha$ $(\mathrm{PGC1} \alpha)$ is a co-activator that increases mitochondrial number and proteins by coactivating and inducing transcriptional factors such as Nrf-1 and Tfam. The aim of this study was to investigate 1) mitochondrial degeneration in neuronal tissues of diabetic animals, 2) the changes in PGC1 $\alpha$ and its related factors, and 3) the effects of pioglitazone, a PPAR $\gamma$ agonist, in normalizing these changes.

Materials and Methods: Adult rats were made diabetic with $45 \mathrm{mg} / \mathrm{kg}$ streptozotocin (STZ). Pioglitazone (20 mg/ $\mathrm{kg})$ or vehicle $(0.5 \%$ carboxymethylcellulose $)$ was administered once daily by gastric gavage within one week of the onset of diabetes. For neuropathy measurement, tactile allodynia and nerve conduction were examined for 3 months after STZ injection. For tissue analysis, dorsal root ganglion (DRG) neurons were collected at 1 and 3 months. To determine the mitochondrial DNA copy numbers, total DNA was extracted and NADH dehydrogenase 1 (mitochondrial genome) was measured using a real-time PCR technique, which was then normalized against the amount of lipoprotein lipase (nuclear genome). For gene expression 
analysis, PGC1 $\alpha$ and related genes involved in mitochondrial homeostasis were measured using real-time PCR.

Results: Both motor and sensory nerve conductions were slowed at 1 and 3 months by diabetes. Tactile allodynia was seen only after 2 months of diabetes. Mitochondrial DNA was decreased at both 1 and 3 months (11 and 27\% decrease respectively) and loss was greater in animals with more severe neuropathy. PGC1 $\alpha$ was not changed at 1 month but increased at 3 months by $11 \%$, a change that is associated with upregulation of Nrfl and Tfam (23 and $26 \%$ increase respectively at 3 months). All changes were statistically significant. Pioglitazone treatment improved neuropathy and prevented the loss in mitochondrial DNA in diabetic animals $(P<0.05)$. Pioglitazone also reduced gene expression changes in $\mathrm{PGC} 1 \alpha$ and downstream transcriptional factors.

Conclusion: In DRG from chronic diabetic rodents, there is loss of mitochondrial DNA. In response, PGC1 $\alpha$, Nrf-1 and Tfam are upregulated. Pioglitazone prevents mitochondrial DNA loss and normalizes gene expression changes suggesting protection against mitochondrial degeneration under diabetic conditions.

Supported in part by NIH NS42056, JDRF, Office of Research Development (Medical Research Service), Department of Veterans Affairs

\section{7}

\section{Effect of pioglitazone in experimental diabetic neuropa-} thy

J. W. Russell ${ }^{1,2}$, T. Inoue ${ }^{1}$, E. K. Kim ${ }^{1}$;

${ }^{1}$ Neurology, University of Michigan, Ann Arbor, United States, ${ }^{2}$ Neurology and GRECC, Veterans Affairs Medical Center, Ann Arbor, United States.

Background and Aims: Generation of oxidative stress from mitochondria plays an important role in the progression of diabetic complications. Uncoupling proteins (UCPs) may be preventing excess amount of oxidative stress generation in mitochondria and recently was shown that its polymorphism is associated with human diabetic neuropathy. In dorsal root ganglion (DRG) of diabetic rats, UCP-3 proteins decrease suggesting the loss of antioxidative defense. It has been also shown that overexpression of UCPs protects DRG neurons from oxidative injury under high-glucose conditions. These factors regulating mitochondrial homeostasis may be controlled by key gene regulators that include Peroxisome Proliferator-Activated Receptor $\gamma$ (PPAR $\gamma$ ). The aim of this study was to investigate the neuroprotective effects and mechanisms of pioglitazone, a PPAR $\gamma$ agonist, in diabetic rats.
Materials and Methods: The study was designed with four animal experimental groups; 1) non-diabetic control, 2) non-diabetic+pioglitazone, 3) diabetic control, 4) diabetic +pioglitazone. Adult rats were made diabetic with $45 \mathrm{mg} /$ $\mathrm{kg}$ streptozotocin. Pioglitazone $(20 \mathrm{mg} / \mathrm{kg})$ or vehicle $(0.5 \%$ carboxymethylcellulose) was administered once daily by gastric gavage within one week of the onset of diabetes. For neuropathy measurement, tactile allodynia and nerve conduction were examined for 3 months after STZ injection. In tissue analysis, DRGs were collected at 3 months and gene expression was analyzed using realtime PCR technique.

Results: Tail motor nerve conduction was slowed by diabetes $(42.2+/-1.0$ in non-diabetic control vs $39.0+/-$ 0.8 in diabetic control, $\mathrm{m} / \mathrm{s}, P<0.05)$, which was recovered by pioglitazone treatment $(42.7+/-1.2$ in diabetic + pioglitazone, $P<0.05)$. Tactile allodynia was observed in diabetic group and it was improved by pioglitazone treatment. In diabetic animals, PPAR $\gamma$ expression was increased by $58 \%$, UCP- 2 and -3 was increased by $31 \%$ and $43 \%$ respectively. There was also an increase in antioxidative enzyme genes, manganese superoxide dismutase (SOD2) and glutathione peroxidase. These changes were considered a cell response mechanism against diabetic oxidative stress and to compensate the loss of UCP proteins under diabetic conditions. Pioglitazone increased UCP-3 but not UCP-2 in the non-diabetic conditions showing it enhances UCP-3 expression in DRG neurons. Pioglitazone further upregulated UCP-3 in diabetic conditions.

Conclusion: Pioglitazone ameliorated diabetic neuropathy. The mechanism was considered at least in part through UCP-3 upregulation and increased antioxidant defense.

Supported in part by NIH NS42056, JDRF, Office of Research Development (Medical Research Service), Department of Veterans Affairs

1098

Are patient characteristics predictive of a response to pregabalin? Analysis from 5 randomized, placebo-controlled trials in painful diabetic peripheral neuropathy

A. J. M. Boulton ${ }^{1}$, R. Freeman ${ }^{2}$, E. D’Urso De $\mathrm{Cruz}^{3}$, B. $\mathrm{Emir}^{3}$;

${ }^{1}$ Medicine, Manchester Royal Infirmary, Manchester, United Kingdom, ${ }^{2}$ Harvard Medical School, Beth Israel Deaconess Medical Center, Boston, ${ }^{3}$ Pfizer Global Pharmaceuticals, Pfizer Inc, New York, United States.

Background and Aims: Pregabalin has consistently demonstrated rapid and robust efficacy for the treatment of 
peripheral neuropathic pain syndromes and associated sleep interference. We investigated whether selected patient characteristics might be predictive of responder rates greater than that for the total population of patients from 5 pregabalin RCTs (of 5-12 weeks) in painful diabetic peripheral neuropathy (DPN).

Materials and Methods: In these 5 trials, pregabalintreated patients received dosages of $150(n=175), 300$ $(\mathrm{n}=252)$, or $600(\mathrm{n}=347) \mathrm{mg} / \mathrm{d} ; 421$ patients received placebo. Patients were considered responders if they had $\geq 30 \%$ reduction from baseline to endpoint in mean pain score (11-point scale). Using ARF (data-mining trees) software, we searched for subregions of treatment group, age, gender, baseline creatinine clearance $\left(\mathrm{CL}_{\mathrm{cr}}\right)$, pain score or sleep interference score, and Patient Global Impression of Change (PGIC) that had high responder rates.

Results: The overall responder rate for the entire population (including placebo patients) was 33\%. Sixteen subsets of patients demonstrated significantly greater responder rates than the overall responder rate. Treatment with 300 or $600 \mathrm{mg} / \mathrm{d}(\mathrm{n}=568)$ as opposed to treatment with $150 \mathrm{mg} / \mathrm{d}$ or placebo was associated with a responder rate of $42 \%$. Across treatment groups, a PGIC rating of "much improved" or "very much improved" was consistently associated with greater percentages of patients meeting the definition of responder. Baseline characteristics eg, gender, age, $\mathrm{CL}_{\mathrm{cr}}$, and pain and sleep scores were not consistently predictive of greater likelihood of response to pregabalin.

Conclusion: Pregabalin particularly at dosages of 300 or $600 \mathrm{mg} / \mathrm{d}$ provided clinically meaningful pain relief for patients with painful DPN. Response to pregabalin appeared to be largely independent of baseline characteristics. Supported by Pfizer Inc

\section{9}

Pregabalin safely and efficaciously treats painful diabetic peripheral neuropathy without affecting nerve conduction: a double blind, placebo controlled trial

J. A. Barrett ${ }^{1}$, J. Rosenstock ${ }^{2}$, J. Arezzo ${ }^{3}$, L. Pauer ${ }^{4}$, U. Sharma ${ }^{4}$, L. LaMoreaux ${ }^{4}$;

${ }^{1}$ Pfizer Global Pharmaceuticals, New York, ${ }^{2}$ Endocrinology, Dallas Diabetes \& Endocrine Research Center, Dallas, ${ }^{3}$ Neuroscience, Albert Einstein College of Medicine, New York, ${ }^{4}$ Pfizer Global R \& D, Pfizer Inc, Ann Arbor, United States.

Background and Aims: Pregabalin is approved in the EU for the treatment of peripheral neuropathic pain. This 13 week trial evaluated nerve conduction and the efficacy and safety of pregabalin $600 \mathrm{mg} / \mathrm{d}$ (BID) for relieving neuropathic pain associated with diabetic peripheral neuropathy (DPN).
Materials and Methods: Amplitude and conduction velocity were assessed at baseline (BL), endpoint (EP), and 2 week follow up for 4 nerves: median motor, median sensory, ulnar sensory, and peroneal motor. The study's primary efficacy measure was EP mean pain score (MPS) on an 11 point numeric rating scale $(0=$ "no pain" to $10=$ "worst possible pain") derived from patients' daily pain diaries. Secondary efficacy measures included weekly MPS and proportion of responders (patients with $\geq 50 \%$ reduction in MPS from BL to EP). Eighty two patients were randomized to pregabalin and 85 to placebo. Most were male $(62 \%)$ and white $(74 \%)$; mean age was 58 years (range, 31-86); 92\% had type 2 diabetes; mean durations of diabetes and painful DPN were 10 and 5 years, respectively. After a 1-week titration, pregabalin patients received $300 \mathrm{mg}$ (BID) for 12 weeks.

Results: For all 4 nerves, 95\% CIs for median differences in amplitude and velocity from BL to EP and to follow-up included 0. At EP, pregabalin patients' MPS was significantly lower than placebo patients' (mean difference, $-1.28 ; P<0.001)$. Significant pain improvement among pregabalin patients was evident at Week 1 and was sustained at every weekly timepoint. Forty-nine percent of pregabalin-treated patients vs $23 \%$ of placebo patients were responders $(P<0.001)$.

Conclusion: Pregabalin had no statistically significant or clinically meaningful effect on nerve conduction. BID dosing at the upper end of pregabalin's effective dosing range $(150-600 \mathrm{mg} / \mathrm{d})$ showed robust efficacy and was well tolerated.

Supported by Pfizer Inc

1100

Amelioration of diabetic peripheral neuropathy by implantation of haematopoietic mononuclear cells in streptozotocin-induced diabetic rats

A. Kosaki, T. Hasagawa, H. Matsubara, N. Toyoda, M. Inoue-Shibata, M. Nishikawa, T. Iwasaka;

Department of Medicine II, Kansai Medical University, Hirakata, Osaka, Japan.

Background and Aims: This study was performed in order to evaluate the angiogenic effect of implantation of either peripheral blood mononuclear cells (PBMNCs) or bone marrow mononuclear cells (BMMNCs) on diabetic peripheral neuropathy.

Materials and Methods: Streptozotocin $(50 \mathrm{mg} / \mathrm{kg})$ was injected intravenously into six-week-old male Lewis rats. Four weeks after the induction of diabetes, $6 \times 10^{7}$ of PBMNCs or $1 \times 10^{8}$ of BMMNCs were implanted into the left hindlimb muscle. Motor nerve conduction velocity 
(MNCV) was monitored before and after implantation. At the end of the experiment, bilateral nerve blood flow (NBF) was measured by laser Doppler and the number of vessels in the sciatic nerves quantified by Factor VIII staining of the sections.

Results: Diabetes resulted in an approximately $20 \%$ reduction $(\mathrm{p}<0.01)$ in sciatic MNCV. Four weeks after implantation, MNCV was improved by $54 \%$ with PBMNCs, and by $67 \%$ with BMMNCs (both $\mathrm{p}<0.01$ ). Moreover, the effects of implantation were almost abolished by administration of VEGF-neutralizing antibody. Sciatic NBF was reduced by approximately $50 \%$ by diabetes $(\mathrm{p}<0.05)$. This reduction in perfusion was improved by $74 \%$ by implantation of PBMNCs and by $62 \%$ by implantation of BMMNCs $(\mathrm{P}<0.05$ and $\mathrm{p}<0.01$, respectively). These effects were observed only in the implanted limb. Immunohistochemical staining of sciatic nerve sections for Factor VIII showed no significant increase in the number of vessels in the sciatic nerve following implantation of either PBMNCs or BMMNCs. These data suggest that implantation of hematopoietic mononuclear cell fractions is associated with an improvement in MNCV as a result of arteriogenic effects in the sciatic nerve and that VEGF may contribute to this effect. This improvement occurred in the absence of angiogenesis.

Conclusion: Implantation of these cell fractions may therefore be a potential new therapeutic method for treating diabetic peripheral neuropathy.

\section{1}

Single treatment, phase 1 trial of a zinc finger DNAbinding protein (ZFP) activator (SB-509) of vascular endothelial growth factor (VEGF) in subjects with diabetic neuropathy

D. G. Ando ${ }^{1}$, D. R. Tomlinson ${ }^{2}$;

${ }^{1}$ Therapeutic Development, Sangamo BioSciences, Richmond, United States, ${ }^{2}$ Faculty of Life Sciences, University of Manchester, United Kingdom.

Background and Aims:: This is a single treatment, dose escalation trial for evaluation of the clinical and laboratory safety of SB-509, a formulation of a plasmid DNA expressing an engineered zinc finger DNA-binding protein transcription factor (ZFP TF) activator of VEGF, in subjects with diabetic neuropathy (DN).This ZFP TF for the VEGF gene has been developed to induce expression of all isoforms of VEGF. VEGF is a growth factor for both neurons and glial cells. Pre-clinical studies in the Rat Streptozotocin diabetic neuropathy model with SB-509 has shown improved nerve conduction.
Materials and Methods: Patients with mild to moderate diabetic neuropathy were enrolled. The clinical trial design was a dose escalation Phase 1 trial with 4 dose levels, $1 \mathrm{mg}$, $5 \mathrm{mg}, 15 \mathrm{mg}$ and $30 \mathrm{mg}$. SB-509 was given intramuscularly in one leg and placebo intramuscular injections were given in the other leg. In addition, the trial was amended to include patients treated with either placebo or SB-509 in both legs at dose levels of 30 and $60 \mathrm{mg}$. Patients were followed for 6 months for clinical and laboratory safety, Total Neuropathy Score (combining neurologic sensory, motor, reflex exam, QST and NCV measurements), visual analog scales for pain (VAS), Quantitative Sensory testing by Vibratron II (QST).

Results:: Eighteen patients have been enrolled and evaluablel for safety and twelve patients are evaluable for clinical endpoints. Adverse events were mild and primarily related to injection site reactions. No dose limiting toxicities (DLT) or serious adverse events (SAE's) were seen. Improvements in symptoms of pain (2 points VAS) were seen in 6/12 patients and numbness in 6/12 patients. TNS composite scores improvements were seen in 6/12 patients and a decrease in vibration threshold sensitivity by QST (2 point) were seen in $7 / 12$ patients.

Conclusion: Single dose treatment of SB-509 was well tolerated to doses of $60 \mathrm{mg}$ in both legs with no DLT or SAE's. Encouraging anecdotal improvements in symptoms of pain and numbness, measured TNS scores and QST measurement were noted and suggest further evaluation in placebo controlled trials. Improvements in pain symptoms suggest a novel endpoint for ZFP TF VEGF activator treatment in Diabetic Neuropathy. A Phase 2 controlled trial of SB-509 versus placebo is planned for 2006.

\section{2}

Duloxetine in the management of diabetic peripheral neuropathic pain: Response profile

H. Schmitt ${ }^{1}$, Y. L. Pritchett ${ }^{2}$, B. H. McCarberg ${ }^{3}$, J. G. Watkin ${ }^{2}$, M. J. Robinson ${ }^{2}$;

${ }^{1}$ Eli Lilly Benelux S.A., Brussels, Belgium, ${ }^{2}$ Eli Lilly and Company, Indianapolis, ${ }^{3}$ School of Medicine, Kaiser Permanente, San Diego, United States.

Introduction: Analyses examine the response rate at endpoint, as well as time course of response, in patients receiving duloxetine for management of diabetic peripheral neuropathic pain (DPNP).

Methods: Data were pooled from 3 double-blind, randomized, placebo-controlled 12-week trials in patients with DPNP $\geq 6$ months duration, and without depression. Study $1(\mathrm{~N}=457)$ compared duloxetine $20 \mathrm{mg}$ once daily (QD), $60 \mathrm{mg}$ QD, $60 \mathrm{mg}$ twice daily (BID), and placebo; Studies 2 
$(\mathrm{N}=334)$ and $3(\mathrm{~N}=348)$ compared duloxetine $60 \mathrm{mg} \mathrm{QD}$ and $60 \mathrm{mg}$ BID with placebo. Ethics committees approved the study protocol in accordance with Declaration of Helsinki principles. Patients provided written informed consent prior to study participation. Treatment response was defined a priori as $30 \%$ reduction in the primary efficacy measure, 24hour average pain severity. Analysis was replicated using alternative criteria (50\% reduction or 2-point reduction).

Results: Endpoint response rates were significantly higher among patients receiving duloxetine $(60 \mathrm{mg}$ QD or $60 \mathrm{mg}$ BID) than those receiving placebo, regardless of chosen response criterion. The proportion of patients responding (30\% reduction in 24 -hour average pain severity) to duloxetine was statistically greater than to placebo Week 1 and all subsequent visits. Similar results were obtained for the visitwise sustained response rate. Within the group with a sustained response at Week 12, the proportion of patients first exhibiting a response at Weeks 1 or 2 was higher in the duloxetine groups (60 mg QD, 65.0\%; $60 \mathrm{mg}$ BID, 62.0\%) when compared with the placebo group (40.2\%).

Conclusion: Patients with DPNP receiving duloxetine $60 \mathrm{mg}$ QD or $60 \mathrm{mg}$ BID had significantly higher treatment response rates compared with patients receiving placebo, regardless of response criterion. Response to duloxetine treatment tended to occur early, with approximately $30 \%$ of patients responding at Week 1. Among duloxetine-treated patients with a sustained treatment response at Week 12, over $60 \%$ had maintained responder status for 10 to 11 weeks.

Funding provided by Eli Lilly and Company and Boehringer Ingelheim

\section{3}

Duloxetine's long-term effects on glycaemic control in patients with diabetic peripheral neuropathic pain

E. J. Bastyr, J. Hall, F. Wang, B. Pangallo,

L. Vignati, A. S. Chappell, J. F. Wernicke;

Eli Lilly and Company, Indianapolis, United States.

Duloxetine has been shown to be safe and effective in the management of diabetic peripheral neuropathic pain (DPNP). We report the impact of long-term treatment with duloxetine on fasting blood glucose (FBG), glycosylated hemoglobin (HbA1C), lipids and weight.

In three long-term studies, 867 patients were randomized at $2: 1$ ratio to duloxetine $60 \mathrm{mg} \mathrm{BID}(\mathrm{N}=580)$ or routine care $(\mathrm{N}=287)$ for 52 weeks after completing 12-week placebocontrolled, acute phase studies. Pooled data from the study extensions were analyzed.

After long-term treatment with duloxetine of up to 52 weeks, there was an increase in $\mathrm{HbAlC}$ in both the duloxetine and routine care groups, but the mean increase was greater in the duloxetine-treated group $(0.5 \%$ vs $0.2 \%$, respectively, $\mathrm{p}<0.001)$. There was also a modest increase in FBG and a small increase in total cholesterol in duloxetinetreated patients while the routine care group showed a slight decrease (mean change FBG: 0.67 vs $-0.64 \mathrm{mmol} / \mathrm{L}$, $\mathrm{p}<0.001$; cholesterol: $0.06 \mathrm{vs}-0.16 \mathrm{mmol} / \mathrm{L}, \mathrm{p}=.005$, respectively). A small decease in HDL cholesterol was seen in both groups, although the decline was significantly smaller with duloxetine than with routine care (mean change: -0.014 vs $-0.078 \mathrm{mmol} / \mathrm{L}, \mathrm{p}=.002$ respectively). In these trials, after 52 weeks of therapy, the weight increase was smaller in the duloxetine-treated patients, compared to routine care group, but the difference was not statistically significant. The frequency of diabetes-related adverse events was similar between groups.

Long-term duloxetine treatment appears to be associated with a slight worsening of metabolic control in patients with DPNP. Patients managed with duloxetine for DPNP should continue to have their glycemic control closely monitored.

Funding provided by Eli Lilly and Company and Boehringer Ingelheim

\section{4}

Treatment of painful diabetic neuropathy with Sativex (a cannabis based medicinal product) - results of a randomised placebo controlled trial

D. Selvarajah, R. A. Gandhi, D. Witte, H. Bowler,

C. Emery, S. Tesfaye;

Diabetes Research Unit, Royal Hallamshire Hospital, Sheffield, United Kingdom.

Background: Painful diabetic neuropathy (PDN) affects $10 \%$ of subjects with diabetes and about a third with diabetic neuropathy. Adjuvant drug therapies to alleviate painful symptoms are often ineffective and associated with side-effects. Cannabis in its various guises has been consumed for its pain relieving properties for many centuries. Sativex is an oromucosal spray preparation containing the therapeutic components of cannabis $(2.7 \mathrm{mg} / \mathrm{ml}$ tetrahydrocannabinol, $2.5 \mathrm{mg} / \mathrm{ml}$ cannabidiol per actuation) and has provided symtomatic relief in other conditions of chronic pain.

Aims: To assess the efficacy of a 12 week multiple daily dosing of Sativex added to standard therapies in improving primary (pain) and secondary (quality of life) end points in adult subjects with PDN.

Methods: In this randomised, double-blind, placebo controlled trial, 30 subjects with intractable PDN unresponsive to currently available treatments were recruited. They were randomised to receive either Sativex or placebo as an oromucosal spray. The primary outcome measure was mean 
daily pain intensity in subjects receiving a maximum tolerated dose. Pain symptoms was assessed using a multi-dimensional daily pain diary. The secondary outcome measure was quality of life as assessed by 1) the McGill Pain and Quality of Life Questionnaires, 2) the SF-36 Health survey and 3) the EuroQOL Health Utilities Index. Results: There was a substantial and significant improvement in pain scores in both groups over the course of the study. There was, however, no significant difference between the two treatment arms for the primary outcome measures. The change in pain from baseline (mean+SD), based on a 0 $10 \mathrm{~cm}$ visual analogue scale for deep pain, muscular pain and superficial pain, at the maximum tolerated dose of the study drug is shown in Table 1. There was also no significant differences between Sativex and placebo in the secondary outcome measures for quality of life.

Table 1 . Change in pain scores during study

\begin{tabular}{|c|c|c|c|}
\hline & Sativex $($ mean $(\mathrm{SD})$ ) & Placebo (mean $(\mathrm{SD})$ ) & P-value \\
\hline Total Pain & $-1.66(2.4)$ & $-1.91(3.0)$ & 0.50 \\
\hline Superficial Pain & $-1.56(1.9)$ & $-1.52(3.2)$ & 0.14 \\
\hline Deep Pain & $-2.00(2.9)$ & $-2.21(2.9)$ & 0.97 \\
\hline Muscular Pain & $-1.50(3.0)$ & $-2.00(3.3)$ & 0.49 \\
\hline
\end{tabular}

Conclusion: This first ever randomised placebo controlled trial assessing the efficacy of a cannabis based medicinal product (Sativex) has shown it to be no more efficacious than placebo in the treatment of painful diabetic neuropathy. The larger than expected placebo response, which has also been demonstrated in other recent studies, may provide us with a important clue as to the nature of pain in diabetic neuropathy. It may also have important implications for the design of future trials in the treatment of painful diabetic neuropathy. Supported by Diabetes UK

\section{PS 97}

\section{Autonomic neuropathy - cardiovascular}

\section{5}

Prospective evaluation of myocardial sympathetic innervation defects and autonomic function testing in relation to glycaemic control over 12 years in type 1 diabetic patients

D. Ziegler ${ }^{1}$, T. D. Poeppel ${ }^{2}$, A. Heinzel ${ }^{2}$, K.-J. Langen ${ }^{2}$;

${ }^{1}$ German Diabetes Clinic, Düsseldorf, ${ }^{2}$ Department of Nuclear Medicine, Research Centre Jülich, Germany.

Background and Aims: Reduced or absent myocardial $\left[{ }^{123} \mathrm{I}\right]$ metaiodobenzylguanidine (MIBG) uptake is a fre- quent feature of diabetic cardiovascular autonomic neuropathy (CAN), but the long-term progression of myocardial adrenergic innervation defects in relation to changes in autonomic function tests (AFTs) and glycaemic control is not known. We aimed to determine the role of glycaemic control in the long-term progression of these defects in a 12-year prospective study.

Materials and Methods: We examined left ventricular myocardial sympathetic innervation in 12 long-term Type 1 diabetic patients (age [mean $\pm \mathrm{SD}$ ]: $41.4 \pm 10.4$ years, diabetes duration: 22.6 \pm 8.8 years) using MIBG scintigraphy. Global and regional relative MIBG uptake were assessed and MIBG defects at $2 \mathrm{~h}$ p.i. were scored by 3 scorers at baseline and after 4 and 12 years, respectively. AFTs were performed using a standardized test battery including time domain and frequency domain measures of heart rate variability. Two patients died during follow-up. Among the remaining patients 6 were well controlled (mean $\mathrm{HbA}_{1 \mathrm{c}}$ over 12 years: $7.1 \pm 0.4 \%$; Group 1 ), whereas 4 patients were poorly con-

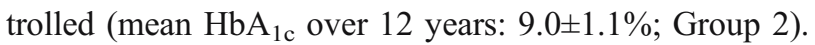

Results: In the entire cohort both myocardial MIBG uptake defects of all 9 regions summed to a score did not change significantly from baseline to 12 years $(13.1 \pm 2.8$ vs $12.4 \pm$ 4.8) and, likewise, the relative MIBG uptake remained unchanged $(78.8 \pm 8.0 \%$ vs $79.2 \pm 6.0 \%)$. No significant differences were noted between the well and poorly controlled patients. In contrast, regional MIBG uptake score in the posterior wall increased by $0.28 \pm 0.48$ from baseline to 12 years in Group 1 and decreased by $-0.23 \pm$ 0.15 in Group $2(\mathrm{p}<0.05)$. Among the AFTs, measures of parasympathetic activity such as the mean circular resultant during deep breathing declined significantly from $0.021 \pm$ 0.014 to $0.015 \pm 0.011(\mathrm{p}<0.05)$ and the maximum $/$ minimum $30: 15$ ratio tended to progress from $1.16 \pm 0.19$ to $1.05 \pm 0.03$ $(p=0.05)$ in the entire cohort, without significant differences between the two groups of glycaemic control.

Conclusion: Global myocardial adrenergic innervation defects do not progress over 12 years in Type 1 diabetic patients, despite continuing progression of vagal dysfunction. However, regional progression of sympathetic dysinnervation in the posterior wall may be slowed by long-term near-normoglycaemia.

\section{6}

Cardiac autonomic dysfunction and inflammation in type 1 diabetic patients. Effect of beta-blockade

D. Pitocco $^{1}$, G. Sgueglia ${ }^{2}$, E. Navarese ${ }^{2}$, A. Manto ${ }^{1}$, T. Musella ${ }^{1}$, A. Sestito ${ }^{2}$, F. $\mathrm{Crea}^{2}$, G. A. Lanza ${ }^{2}$, G. Ghirlanda ${ }^{1}$;

${ }^{1}$ Internal Medicine, Catholic University, Rome, ${ }^{2}$ Cardiology, Catholic University, Rome, Italy. 
Background: Recent studies suggest a relation between abnormal nervous autonomic function and inflammation. Impaired cardiac autonomic activity and inflammation have both been found to be associated with a worse outcome in diabetic patients. However, whether the two abnormalities are related in these patients is unknown.

Methods and Results: We studied 40 patients with type 1 diabetes mellitus (age 50.6 \pm 10 years, 25 men). Serum levels of high-sensitivity C-reactive protein (CRP) and 24hour Holter monitoring to assess frequency-domain heart rate variability (HRV), as a measure of cardiac autonomic function, were obtained in all patients. Seventeen patients with depressed HRV were subsequently randomized to receive atenolol (50 $\mathrm{mg}$ daily) or no beta-blockade. HRV and CRP were reassessed after 4 weeks from randomization. A significant inverse correlation was found between CRP levels and HRV parameters, with the highest $r$ coefficient shown with low frequency (LF) amplitude $(\mathrm{r}=-0.47$.; $\mathrm{P}=0.008)$. On multivariate logistic regression analysis only low LF amplitude $(\mathrm{P}=0.037)$ and high body mass index $(\mathrm{P}=0.05)$ were independent predictors of high (>3 mg/L) CRP serum levels. HRV parameters improved significantly in patients treated with atenolol, but not in the no-atenolol group. CRP also tended to decrease in the former group $(\mathrm{P}=0.10)$, but not in the latter.

Conclusion: In type 1 diabetic patients, serum CRP levels are significantly associated with depressed HRV; the favourable effects of beta-blockade on both HRV parameters and CRP serum levels suggest that autonomic nervous system can be an important modulator of inflammation.

\section{7}

Validation of a new diabetic autonomic neuropathy bedside test (plaster) versus the cardiovascular reflex tests

T. P. Didangelos, I. Zografou, A. Papageorgiou,

M. Koukourikou, V. Athyros, K. Kontotasios,

D. Karamitsos;

Diabetes Center, Hippocration Hospital of Thessaloniki, Aristotle University of Thessaloniki, Greece.

Background and Aims: Autonomic neuropathy is a serious and fatal complication of Diabetes Mellitus (DM). Perspiration is a feature of Diabetic Autonomic Neuropathy (DAN) and the Indicator Plaster (IP) could provide an easy and accurate way to diagnose DAN especially for general practitioners. The aim of the present study was to evaluate indicator's specificity, sensitivity and accuracy in detecting DAN.

Materials and Methods: We studied 174 patients (79 with DM type 1, 88 women), with mean age $49.8+16.1$ years and mean duration of DM. 17.3+7.7 (range 4-45 years). We performed the following four Cardiovascular Reflex Tests (CRT): R-R variation during deep breathing [assessed by Expiration/Inspiration ratio (E/I), Mean Circular Resultant (MCR, vector analysis) and Standard Deviation (SD)], Valsalva maneuver, 30:15 ratio and postural hypotension in all patients. The presence of DAN was established if 2 or more 4 CRT were abnormal. One test abnormal considered as early DAN According to the change of color of the sticker IP or not patients divided in two groups: Group A $(n=82$, completed change in color from pink to blue, normal perspiration), and Group B $(n=92$, uncompleted or no change, abnormal perspiration).

Results: According to the number of abnormal tests, established DAN detected in 81 patients. E/I index was abnormal in 118 patients and postural hypotension in 73 . The sensitivity, specificity and accuracy of IP versus CRT of the above measured parameters appear in the following table.

\begin{tabular}{llll}
\hline Measured Parameter & Sensitivity & Specificity & Accuracy \\
\hline Established DAN & $89 \%$ & $78 \%$ & $84 \%$ \\
E/I index & $73 \%$ & $89 \%$ & $85 \%$ \\
Postural Hypot & $95 \%$ & $77 \%$ & $85 \%$ \\
\hline
\end{tabular}

Conclusion: Indicator has a high sensitivity, specificity and accuracy in detecting established DAN versus CRT. Neuropathy of parasympathetic origin was observed with IP with low sensitivity, but high specificity. Neuropathy of sympathetic origin was detected with IP with high sensitivity and specificity. Accuracy of the IP test was high in all measurements.

\section{8}

How two different diagnostic criteria of orthostatic hypotension relate to the presence of cardiac autonomic neuropathy or nondipping in type 1 and type 2 diabetes V. Spallone, R. Morganti, S. Mandica, M. R. Maiello; Internal Medicine, Tor Vergata University, Rome, Italy.

Background and Aims: There is not yet a widely accepted approach to the diagnosis of orthostatic hypotension $(\mathrm{OH})$ in diabetes. Thus, we evaluated the diagnostic accuracy for autonomic neuropathy (AN) and for blood pressure (BP) nondipping of $\mathrm{OH}$ defined according to two distinct criteria, i.e. a fall in systolic BP $>30 \mathrm{mmHg}(30-\mathrm{OH})$ or $>20 \mathrm{mmHg}(20-\mathrm{OH})$.

Materials and Methods: We studied 84 type 1 (age 36 \pm 11 , duration $17 \pm 10$ years, serum creatinine $0.76 \pm 0.18 \mathrm{mg} / \mathrm{dl}$, 
casual BP $119 / 73 \pm 14 / 9 \mathrm{mmHg}$ ), and 80 type 2 diabetic patients (age $51 \pm 11$, duration $11 \pm 8$ years, serum creatinine $0.74 \pm 0.19 \mathrm{mg} / \mathrm{dl}$, casual BP $127 / 77 \pm 15 / 9 \mathrm{mmHg}$ ), all with normal renal function and without current antihypertensive treatment. We assessed the presence of orthostatic symptoms and performed $24 \mathrm{~h}$ BP monitoring and 4 cardiovascular tests (deep breathing, lying to standing, Valsalva manoeuvre, and postural hypotension test measuring BP supine and following standing at 1 and $2 \mathrm{~min}$ ).

Results: Among type 1 patients, $30-\mathrm{OH}$ and $20-\mathrm{OH}$ were present in $9.5 \%$ and $20.2 \%$ of patients, respectively. Orthostatic symptoms were present in $29.6 \%$ without a complete overlapping with $\mathrm{OH}$ (for $30-\mathrm{OH}: \mathrm{Chi}^{2}=8.8$, $\mathrm{p}=0.007$; for $\left.20-\mathrm{OH}: \mathrm{Chi}^{2}=10.3, \mathrm{p}=0.004\right)$. Both $30-\mathrm{OH}$ and $20-\mathrm{OH}$ were associated with $\mathrm{AN}$, defined as the presence of at least 1 abnormal heart rate test $\left(\mathrm{Chi}^{2}=6.6\right.$, $\mathrm{p}=0.009$, and $\mathrm{Chi}^{2}=5.5, \mathrm{p}=0.03$, respectively), and with nondipping, defined as a nocturnal fall in systolic or diastolic $\mathrm{BP}<0 \%\left(\mathrm{Chi}^{2}=13.4, \mathrm{p}=0.003\right.$, and $\mathrm{Chi}^{2}=5.3$, $\mathrm{p}=0.03$, respectively). Using ROC analysis, systolic BP fall on standing showed a rather low diagnostic accuracy in discriminating between patients with and without AN (AUC $0.62 \pm 0.06,95 \%$ C.I. $0.50-0.73$ ), and a fair accuracy in discriminating between patients with and without nondipping (AUC $0.75 \pm 0.08,95 \%$ C.I. 0.58-0.88). Both 30$\mathrm{OH}$ and 20-OH showed low sensitivity and high specificity for $\mathrm{AN}$ [for 30-OH: sensitivity $17 \%$, specificity $100 \%$, positive predictive value (PPV) $100 \%$ (95\% C.I. 100-100), negative predictive value (NPV) $47 \%$ (95\% C.I. $36-58$ ); for $20-\mathrm{OH}$ : sensitivity $29 \%$, specificity $92 \%$, PPV $82 \%(95 \%$ C.I. 64-100), NPV 49\% (95\% C.I. 37-61)]. 30-OH showed a slightly better PPV for nondipping [sensitivity 36\%, specificity $96 \%$, PPV $62 \%$ (95\% C.I. 29-96), NPV $88 \%$ (95\% C.I. $81-95)$ ] than $20-\mathrm{OH}$ [sensitivity $43 \%$, specificity $84 \%$, PPV $35 \%$ (95\% C.I. $13-58$ ), NPV $88 \%$ (95\% C.I. $80-96)$ ]. Among type 2 patients $30-\mathrm{OH}$ and $20-\mathrm{OH}$ were present in $12.5 \%$ and $18.7 \%$ of patients, respectively. Orthostatic symptoms were present in $24 \%$ of patients with weak or no association with $30-\mathrm{OH}\left(\mathrm{Chi}^{2}=4.2, \mathrm{p}=0.05\right)$ or $20-\mathrm{OH}\left(\mathrm{Chi}^{2}=2.6, \mathrm{p}=0.18\right)$. Also in type 2 patients $30-\mathrm{OH}$ and $20-\mathrm{OH}$ were associated with $\mathrm{AN}\left(\mathrm{Chi}^{2}=8.8, \mathrm{p}=0.005\right.$, and $\mathrm{Chi}^{2}=12.1, \mathrm{p}=0.001$, respectively), or nondipping $\left(\mathrm{Chi}^{2}=17.9, \mathrm{p}=0.0003\right.$, and $\mathrm{Chi}^{2}=12.8, \mathrm{p}=0.001$, respectively), with values of AUC, sensitivity, specificity, PPV, and NPV similar to those observed in type 1 patients.

Conclusion: There is no strict overlapping between orthostatic symptoms and $\mathrm{OH}$, in particular in type 2 patients. Both $30 \mathrm{mmHg}$ and $20 \mathrm{mmHg}$ criteria display low sensitivity and high specificity in diagnosing cardiac AN. The $30 \mathrm{mmHg}$ criterion has a better predictive value for nondipping as opposed to $20 \mathrm{mmHg}$. Since the presence of postural hypotension is burdened by a further increased adverse prognostic impact with regard to $\mathrm{AN}$, the more restrictive $30 \mathrm{mmHg}$ criterion could be likely preferred in diabetic patients.

1109

Disturbances of spectral heart rate variability during tilting in patients with diabetes mellitus type 1

A. P. Bissinger ${ }^{1}$, A. Szadkowska ${ }^{2}$, J. Bodalska ${ }^{1}$,

L. Markuszewski ${ }^{1}$;

${ }^{1}$ Department of Interventional Cardiology, Cardiodiabetology and Cardiac Rehabilitation, University Hospital No. 2, ${ }^{2}$ Department of Pediatric Diabetology, University Hospital No. 4, Lodz, Poland.

Background and Aims: The autonomic regulation is impaired in patients with diabetes mellitus. Ortostatic hypotension $(\mathrm{OH})$ in patients with diabetes is one of the sign of autonomic dysfunction. Analysis of heart rate variability (HRV) is regognized method to evaluate cardiac autonomic neuropathy. The aim of this study was to assess blood pressure and spectral HRV. parameters and evaluate during 5 minutes tilting in patients with type 1 diabetes mellitus (DM1).

Materials and Methods: We studied 48 normotensive patients with DM1 aged 18-28 years, with 9-23 years of DM1. Control group consisted of 32 healthy persons, matched to the DM1 group. We performed $24 \mathrm{~h}$ Holter ECG monitoring (SUPRIMA 12, DMS, USA) and ABPM (Spacelab 90207, Datex-Ohmeda, USA). We initialized manually measurements every minute during 5 minutes standing up, preceded by 15 minutes of rest in supine position. Furthermore we analyzed spectral HRV parameters: total power (TP), very low frequency (VLF), low frequency (LF) and high frequency (HF) - in 5 minutes periods in supine and standing position.

Results: According to the adopted diagnostic criteria of orthostatic hypotension $(\mathrm{OH})$ we noted systolic blood pressure (sBP) fall in 19\% patients with DM1 and 3\% persons in control group $(\mathrm{p}=0.001)$ and diastolic blood pressure $(\mathrm{dPB})$ fall in $23 \%$ patients with $\mathrm{DM}$ and $0 \%$ in control group ( $\mathrm{p}=0.001)$. In rest conditions (lying position) patients with $\mathrm{DM}$ and $\mathrm{OH}$, in comparison to healthy persons and patients with diabetes without co-existent $\mathrm{OH}$, showed significant lower TP and VLF spectrum $(\mathrm{p}=0.001)$.

After tilting we observed lower values of TP, VLF, LF and $\mathrm{HF}$ in group of patients with $\mathrm{DM}$ and $\mathrm{HO}$ versus control $(\mathrm{p}=0.001)$. Moreover in standing position we registered lowering of LF component, measured in normalized unit (nu) in patients with $\mathrm{DM}$ (with and without $\mathrm{OH}$ ) in contrast to healthy people, in which LF spectrum increased. 
Conclusions: $\mathrm{OH}$ is a frequent pathology in young patients with long-lasting DM1. Patients with DM and $\mathrm{OH}$ have more advanced of autonomic dysfunction then DM patients without $\mathrm{OH}$, manifested of lower spectral HRV components during tilting. DM patients show pathological fall LF (nu) component during tilting.

\section{0}

Correlation between autonomic dysfunction and arterial stiffness in patients with long-standing diabetes mellitus C. Lengyel ${ }^{1}$, H. Gavallér ${ }^{2}$, A. Nemes ${ }^{2}$, R. Takács ${ }^{1}$, T. T. Várkonyi ${ }^{1}$, G. Ábrahám ${ }^{1}$, P. Kempler ${ }^{3}$, J. Lonovics ${ }^{1}$, M. Csanády ${ }^{2}$, T. Forster ${ }^{2}$;

${ }^{1} 1$ st Department of Medicine, ${ }^{2}$ 2nd Department of Medicine and Cardiology Centre, University of Szeged, ${ }^{3} 1$ st Department of Medicine, Semmelweis University, Budapest, Hungary.

Background and Aims: The augmentation index (AIx80), a ratio calculated from the first pulse wave generated in the aorta after left ventricular contraction and the second pulse wave reflected from the aortic bifurcation, provides information regarding both the aortic elasticity and the peripheral arteriolar resistance. A higher than normal value of AIx80 is mainly indicative of an elevated peripheral resistance. On the other hand, an accelerated pulse wave velocity (PWVS35) is primarily characteristic of aortic stiffness. It is well known that the level of arterial stiffness is elevated in diabetic patients. The aim of the present study was to evaluate the correlations between the arterial stiffness characterized by AIx 80 and PWVS35 and the results of standard cadiovascular reflex tests (CRTs) in patients with long-standing diabetes mellitus (DM).

Patients and Methods: 22 patients with long-standing DM (16 women and 6 men, age: $47.5 \pm 3.3$ years, duration of DM: $15.3 \pm 2.0$ years, BMI: $26.2 \pm 1.0 \mathrm{~kg} / \mathrm{m}^{2}$, HbAlc: $8.1 \pm 0.4 \%$, systolic BP: $147.6 \pm 3.5 \mathrm{~mm} \mathrm{Hg}$, diastolic BP: $84.1 \pm 2.2 \mathrm{~mm}$ $\mathrm{Hg}$; mean $\pm \mathrm{SEM}$ ) were enrolled into the study. The autonomic function was assessed by means of five standard CRTs, the results of which were utilized to create a score with which to express the severity of autonomic neuropathy (AN score). AIx80 and PWVS35 were measured non-invasively with a TensioClinic TL1 arteriograph and PC-based software. Pearson correlation coefficients $(r)$ were evaluated between AIx80, PWVS35 and the CRT parameters.

Results: AIx 80 revealed a close correlation with the AN score $(r=0.72, p=0.00015)$, the $30 / 15$ ratio $(r=-0.60$, $p=0.003)$, the heart rate response to deep breathing $(r=-0.64, p=0.0012)$, the Valsalva ratio $(\mathrm{r}=-0.56$, $\mathrm{p}=0.006$ ) and the systolic blood pressure response to standing ( $r=0.56, p=0.007)$. PWVS35 correlated with the AN score $(r=0.44, p=0.038)$ and the heart rate response to deep breathing $(r=-0.53, p=0.011)$. There was no significant relationship between AIx80, PWVS35 and HbA1c.

Conclusion: Our results suggest the existence of a close relationship between the enhanced arterial stiffness characterized by the augmentation index and by the pulse wave velocity and the degree of autonomic dysfunction in patients with long-standing diabetes mellitus.

Supported by OTKA F 61224

\section{1}

Continous variations of glucose levels in type 2 diabetic patients are accompanied by corresponding changes in heart rate variability

G. A. Lanza $^{1}$, D. Pitocco ${ }^{2}$, E. Navarese ${ }^{1}$, G. Sgueglia ${ }^{1}$, A. Sestito ${ }^{1}$, A. Manto ${ }^{2}$, T. Musella ${ }^{2}$, F. Zaccardi ${ }^{2}$, F. $\mathrm{Crea}^{2}$, G. Ghirlanda ${ }^{2}$;

${ }^{1}$ Cardiology, ${ }^{2}$ Internal Medicine, Catholic University, Rome, Italy.

Background: Heart rate variability (HRV), a marker of autonomic dysfunction, is known to be impaired in type 2 diabetic patients and tight blood glucose control has been reported to improve HRV parameters in these patients. Recent data deriving from continuous glucose measurement in animal models of diabetes mellitus have suggested tha HRV may phasically influenced by blood glucose fluctuations. Whether this potentiallity relevant dynamic is present in humans is unknown.

Methods: Twelve patients with type 2 diabetes mellitus (mean age $65 \pm 4$ years, 2 women) underwent 48-hour Holter monitoring and continuous interstitial glucose measurement by amperometric method with a needle electrode (Medtronic/MiniMed) placed subcutaneously in the abdomen and acquiring data every 5 minutes. The higher and lower glucose levels for each 3-hour segment of the day and their time of detection were identified and RR interval and HRV parameters were calculated on a 5-minute interval centred on these times. HRV was assessed both in the time-domain (average value of the 5-minute standard deviations [SDNNI], root-mean squared successive difference [r-MSSD], the percentage of RR intervals differing by $\geq 50 \mathrm{msec}$ from the previous interval [pNN50] and in the frequency-domain (low-frequency amplitude [LF], high-frequency amplitude [HF], and LF/HF ratio).

Results: Overall, 303 glucose levels were available for analysis. A significant correlation was found between glucose levels and several HRV parameters as r-MSSD $(p<0.003), p N N 50 \quad(p<0.001)$, LF $(p<0.03)$, and HF $(\mathrm{p}<0.01)$. 
Conclusions: Our data suggest that, in type 2 diabetic patients, daily fluctuations in glucose levels are associated with parallel changes in HRV parameters. Specifically, HRV changes towards decrease in vagal activity, with adrenergic predominance, are associated with lower glucose levels. The pathophysiologic and clinical implications of these findings deserve further investigation.

\section{2}

Acute effects of sildenafil on flow mediated dilatation, haemodynamic parameters and cardiovascular autonomic function in type $\mathbf{2}$ diabetic men

A. O. Stirban ${ }^{1}$, D. Sander ${ }^{2}$, D. Laude ${ }^{3}$, J.-L. Elghozi ${ }^{3}$, B. Haastert ${ }^{2}$, D. Ziegler ${ }^{2}$;

${ }^{1}$ Diabetes Clinic, Heart and Diabetes Center North RhineWestphalia, Bad Oeynhausen, ${ }^{2}$ German Diabetes Clinic, Düsseldorf, Germany, ${ }^{3}$ Clinic, Hôpital Necker-Enfants Malades, Paris, France.

Background and Aims: Sildenafil, a selective inhibitor of the cGMP-specific phosphodiesterase type 5 (PDE5) isoenzyme, is widely used in patients with type 2 Diabetes mellitus (T2DM) in the treatment of erectile dysfunction (ED). Evidence has emerged that sildenafil may exert effects on various vascular and autonomic nervous systems other than the corpus cavernosum. Because both endothelial function and the autonomic nervous system integrity are frequently disturbed in Type 2 diabetic patients, we aimed to assess the effects of sildenafil on flow mediated dilatation (FMD) and cardiovascular autonomic nerve function in men with T2DM.

Materials and Methods: A double-blind, placebo-controlled cross-over trial has been conducted in 35 men with T2DM without known cardiovascular disease (CVD). Patients' characteristics were (mean \pm SEM): age: $55.1 \pm$ 1.17 yrs; diabetes duration: $6.4 \pm 0.8$ yrs, BMI: $30.8 \pm$ $0.8 \mathrm{~kg} / \mathrm{m}^{2}$; HbA1c: $7.9 \pm 0.3 \%$. Diabetes complications were present as follows: ED: 91\%; peripheral neuropathy: 34\%, cardiac autonomic neuropathy: 17\%, microalbuminuria: $17 \%$, and retinopathy: $14 \%$. We assessed in these patients the acute effect of $100 \mathrm{mg}$ Sildenafil on FMD of the brachial artery as a measure of endothelial dysfunction, blood pressure (BP) at rest and its response to standing up, heart rate (HR), heart rate variability (HRV) using time domain and frequency domain indices, and spontaneous baroreflex sensitivity (BRS).

Results: Sixty minutes after a single oral administration of sildenafil, a decrease in supine systolic $(-5.41 \pm 1.87$ $\mathrm{mmHg}$, ) and diastolic $(-4.46 \pm 1.13 \mathrm{mmHg}) \mathrm{BP}$, as well as systolic $(-7.41 \pm 2.35 \mathrm{mmHg})$ and diastolic $(-5.65 \pm$ $1.45 \mathrm{mmHg}$ ) BP on standing were observed, accompanied by an increase in heart rate $(+1.98 \pm 0.69$ beats $/ \mathrm{min})$ (all $\mathrm{p}<0.01$ vs. placebo). No acute effects of sildenafil were noted on parasympathetic and sympathetic nervous activity as assessed by HRV and BRS. FMD variations from baseline to $60 \mathrm{~min}$ after medication intake were not statistically significant after any intake: $4.13 \pm 0.28 \%$ to $3.67 \pm 029 \%$ (sildenafil) and $4.08 \pm 0.31 \%$ to $4.42 \pm 0.31 \%$ (placebo) $\mathrm{p}=\mathrm{NS}$ vs. corresponding baseline). There was no significant difference between sildenafil and placebo regarding the changes in FMD.

Conclusion: In men with T2DM without clinical evidence of CVD, a single oral administration of $100 \mathrm{mg}$ sildenafil leads to a decrease in blood pressure and an increase in heart rate, but not to an adverse, potentially arrhythmogenic effect on cardiovascular autonomic function with increased sympathetic and decreased vagal activity. However, no acute effect of sildenafil on endothelial dysfunction could be demonstrated.

Supported by an unrestricted grant from Pfizer.

\section{3}

Erectile dysfunction and cardiovascular risk in male patients with type 2 diabetes in primary care

F. G. W. Cleveringa, M. W. van der Linden, K. J. Gorter, G. E. H. Rutten;

General Practice, Julius Centre for Health Science and Primary Care, Utrecht, Netherlands.

Background and Aims: The prevalence of questionnairediagnosed erectile dysfunction (ED) in primary care is 30 $50 \%$. The prevalence in diabetic men varies from $20-70 \%$ and increases with age. ED questionnaires are too time consuming in daily practice. However, the ED prevalence rate in primary care diagnosed by a single question is unknown.

Patients with Diabetes Mellitus, cardiovascular disease (CVD) or markers of poor cardiovascular prognosis have more often ED. ED is a strong predictor of silent coronary artery disease in diabetic patients.

We aim to study the single question prevalence of ED in men with type 2 diabetes (DM2) in general practice (GP) and the association with cardiovascular risk-factors.

Materials and Methods: In a cross-sectional study in 54 practices, all male DM2 patients treated by their GP were asked by a female practice nurse if they had erection problems (yes/no). Gender, ethnicity, smoking, duration of diabetes, HbA1c, systolic blood pressure, cholesterol and HDL-cholesterol were used to calculate the 10 year UKPDS coronary heart disease risk score. Medication, Simm's-classification for the diabetic foot as a sign of microvascular complications (Simm's 2 and 3 vs 0 and 1), 
cardiovascular complaints and diabetic complications (yes/ no) were co-determinants. Prevalence of ED in DM2 men and its univariate associations with risk factors were analysed by frequency, cross-tabulation and MannWhitney's test. In a multivariate logistic regression analysis we adjusted for the co-determinants.

Results: 1675 DM2 men consulted the practice nurse, ED was registered in 1476 patients. The prevalence of ED was $41.3 \%$, increasing with age. These men had more often cardiovascular complaints (odds ratio, OR 1.6; 95\% confidence interval, 95\% CI 1.3-2.0), diabetic complications (OR 2.9; 95\%CI 2.3-3.7) and foot complications (OR 1.6; 95\% CI 1.1-2.2). The mean 10 year UKPDS risk score was $29.5 \%$ and $24.0 \%$ for men with and without ED, respectively $(\mathrm{p}<0.001)$. The mean $\mathrm{HbAlc}$ in both groups was $7.1 \%$ and $7.0 \%(\mathrm{p}=0.01)$, systolic blood pressure $150 \mathrm{mmHg}$ and $146 \mathrm{mmHg}(\mathrm{p}=0.003)$, and for duration of diabetes 6.3 years and 5.4 years $(\mathrm{p}=0.001)$, respectively. For smoking, total cholesterol, HDL cholesterol, BMI and diastolic blood pressure the difference was not statistically significant.

Conclusion: In general practice $41.3 \%$ of DM2 men have $\mathrm{ED}$ as diagnosed by a single question, this is comparable to questionnaire based prevalence. These men have a $5.5 \%$ higher 10 year UKPDS cardiovascular risk estimate and more cardiovascular complaints, diabetic complications and microvascular foot complications, irrespective of cholesterol, HDL-cholesterol, BMI and diastolic blood pressure. Time consuming questionnaires may not be necessary for identifying DM2 men with ED. Asking for ED identifies patients at high cardiovascular risk.

Supported by Pfizer b.v.

\section{PS 98}

\section{Autonomic neuropathy - gastrointestinal}

\section{4}

Determination of gastric emptying and neuropathy status in patients with different durations of type 1 diabetes

T. T. Várkonyi ${ }^{1}$, Ü. Börcsök ${ }^{1}$, R. Takács ${ }^{1}$, R. Róka ${ }^{1}$, C. Lengyel ${ }^{1}$, M. Lázár ${ }^{2}$, M. Papós ${ }^{2}$, L. Pávics ${ }^{2}$, P. Kempler ${ }^{3}$, J. Lonovics ${ }^{1}$;

${ }^{1} 1$ st Department of Internal Medicine, ${ }^{2}$ Department of Nuclear Medicine, University of Szeged, Hungary, ${ }^{3} 1$ st Department of Internal Medicine, Semmelweis University, Budapest, Hungary.

The roles of the disease duration and age in the pathogenesis of impaired gastric emptying in type-1 diabetes (DM) are still not clearly explored. The background and aims of this study were to determine the postprandial gastric emptying, the autonomic and the sensory neuropathy in groups of diabetic patients (pts) with different durations and age.

Patients, methods: 17 patients had a shorter, 23 pts had a longer history of type-1 DM (age: $30.7 \pm 2$ vs $44.6 \pm 2.6 \mathrm{yrs}$, $\mathrm{p}<0.001$; duration: $8 \pm 1.4$ vs $25.3 \pm 2.3$ yrs, $\mathrm{p}<0.0001$; mean \pm $\mathrm{SE}$ ). The emptying of the stomach was evaluated by a scintigraphic gastric emptying procedure. Autonomic neuropathy (AN) was assessed by means of the five cardiovascular reflex tests. The sensory nerve function was studied with a Neurometer (Neurotron Inc. Baltimore, Md) using transcutaneous electrical stimulation.

Results: There was a tendency to a longer half-time of gastric emptying (HTE) in type-1 DM pts with longer duration, but the difference between the groups did not reach the level of significance (HTE: $93.5 \pm 10.1$ vs $67.8 \pm$ 6.7 min., $\mathrm{p}>0.05$; long-standing $v s$ short-standing type$1 \mathrm{DM})$. The overall severity of AN and the results of three reflex tests differed between the groups (AN score: $4.3 \pm 0.5$ vs $2.5 \pm 0.4, \mathrm{p}<0.05$; heart rate response to breathing: $12.7 \pm$ 1.5 vs $19.4 \pm 2.2$ beats/min., $\mathrm{p}<0.05$; Valsalva ratio: $1.3 \pm 0.05$ vs $1.75 \pm 0.1, \mathrm{p}<0,0001$; orthostatic hypotension: $23 \pm 3.4$ vs $11 \pm 3.7 \mathrm{~mm} \mathrm{Hg}, \mathrm{p}<0.05$ ). A positive correlation was observed between HTE and the AN score in long-standing $\mathrm{DM}(\mathrm{r}=0.57, \mathrm{p}<0.01)$. The correlations between heart rate response to breathing and HTE $(r=-0.57, p<0.01)$ and between the Valsalva ratio and HTE $(r=-0.43, p<0.05)$ in this group were negative, while a positive association was found between the orthostatic hypotension and HTE $(\mathrm{r}=0.51, \mathrm{p}<0.01)$. The current perception thresholds $(\mathrm{CPT})$ detected at $2 \mathrm{kHz}$ frequency differed between the groups at the upper and lower limbs (CPT at the median nerve: $3.66 \pm$ 0.35 vs $1.84 \pm 0.25 \mathrm{~mA}, \mathrm{p}<0,001$; $\mathrm{CPT}$ at the peroneal nerve: $5.39 \pm 0.71$ vs $3.70 \pm 0.68 \mathrm{~mA}, \mathrm{p}<0.05)$.

Conclusions: Impaired gastric emptying was found more frequently in pts with long-standing type-1 DM, but this difference was not significant between the groups. Both the parasympathetic and the sympathetic systems and the sensory function of the large myelinated fibres were more seriously altered in long-standing DM. The severe autonomic dysfunction exhibited a correlation with the slower gastric emptying. These data support the hypothesis, that the most progressive manifestations of diabetic neuropathy might be responsible for the development of gastroparesis. Supported by MTA Bolyai János Kutatási Ösztöndíj

\section{5}

Intensive management with CSII can prevent hospital admissions of patients with gastroparesis

G. Morrison, T. S. Purewal, P. J. Weston;

Diabetes Centre, Royal Liverpool University Hospital, Liverpool, United Kingdom. 
Introduction: Gastroparesis is a difficult management problem that can have a significant impact on quality of life (QOL) and due to glycaemic instability it often leads to repeated hospital admissions.

A combination of hyperglycaemia, multiple motor and sensory abnormalities of the upper gut results in variable absorption of nutrients which leads to difficulties in controlling blood glucose levels with multiple dose injections (MDI). Individualised insulin delivery via continuous subcutaneous insulin infusion (CSII) may provide greater glycaemic stability in this group, which in turn may prevent acute complications and the necessity for in-patient management.

Aims: Prevent hospital admissions and improve QOL by stabilising glycaemic control in patients with gastroparesis by conversion from MDI onto CSII.

Methods: Seven female patients mean Age 32 years SD+/ - 6.8 (range $24-41$ ) with a mean duration of type 1 diabetes of 20 years $\mathrm{SD}+/-6.3$ (range 12 -29) and symptomatic gastroparesis were converted from MDI onto CSII. All were on an optimised MDI regimen, which incorporated an average total daily insulin (TDI) of 44 units SD+/- 18.5 (range 24-78). Despite no educational deficits all had sub-optimal glycaemic control (mean HbA1c 10.5\% $\mathrm{SD}+/-1.3$ (range $8.8-12.2$ )), and erratic control which incorporated frequent episodes of hypoglycaemia, often requiring third party assistance, 5 describing total unawareness. Further autonomic complications included, 1 oesophageal, 3 bowel, 2 postural hypotension and 2 neurogenic bladder. Other established complications included, 5 retinopathy, 3 nephropathy (1 requiring dialysis), 2 hypertension, and 4 painful peripheral neuropathy. One hundred and seventy-four in-patient days in the preceding twelve months prior to CSII initiation were recorded. All reported low mood and 3 were treated with antidepressants.

Results: Mean duration of treatment with CSII was 15.7 months $\mathrm{SD}+/-20.4$ (range 2-60) and resulted in a reduction in the mean value of $\mathrm{HbA} 1 \mathrm{c}$ by $1.9 \%$ and in TDI to a mean of 41.5 units $\mathrm{SD}+/-9.2$ (range 34.6-57.9). Hypoglycaemia was reported as infrequent and accountable and all episodes were associated with awareness. There was improvement of gastroparesis related symptoms and weight increased on average by $6.6 \mathrm{~kg}$ (mean BMI 23.3 SD+/- 3.5 (range 17-27.5). Well being and QOL was heightened with one patient discontinuing antidepressants. In-patient episodes related to glycaemic management or gastroparesis have not been required since conversion onto CSII.

Conclusion: CSII is an effective means of overcoming glycaemic instability caused by gastroparesis. Hospital admissions are reduced, symptom impact and QOL issues improve.
1116

Gastric neurostimulation ameliorates symptoms of diabetic gastroparesis

P. Rask $^{1}$, J. Froekjaer ${ }^{2}$, P. Funch-Jensen ${ }^{1}$, J. S. Christiansen ${ }^{3}$, A. M. Drewes ${ }^{4}$, N. Ejskjaer ${ }^{3}$;

${ }^{1}$ Department of Surgical Gastroenterology L, Aarhus University Hospital, ${ }^{2}$ Department of Radiology, Aalborg University Hospital, ${ }^{3}$ Department of Endocrinology M, Aarhus University Hospital, ${ }^{4}$ Center for Visceral Biomechanics and Pain, Aalborg University Hospital, Aalborg, Denmark.

Background: In its most unremitting form diabetic gastroparesis may present with abdominal pain and continous nausea and vomiting leading to hospitalisation for fluid substitution and blood glucose control. Fortunately the condition is often more limited, although frequently resistant to standard pharmacological and symptomatic treatment.

Method: Selected Type 1 diabetes patients underwent standard clinical examination excluding other causes of dyspepsia. All were before and after implantation of a gastric stimulator subjected to scintigraphic gastric emptying studies, visceral-biomechanical studies, 24 h-antroduodenal motility testing and qualitative symptomatic testing using a validated questionnaire.

Patients: Five patients, four males and one female, all Type 1 diabetes all long duration, all presenting with an array of late diabetic complications and attending the out-patient clinic at Department of Endocrinology M, Aarhus University Hospital. All had suffered symptoms of diabetic gastroparesis over several years resistant to standard treatment measures. All were frequently admitted to hospital for this condition.

Results: Three male patients have been relieved nearly totally of symptoms. One male patient is completely relieved of nausea and vomiting, but suffers abdominal cramps of unknown origin. One female demonstrates halving of admission frequency and is admitted for treatment of competing diabetic late complications. Some have not been admitted to hospital after implantation of the gastric neurostimulator, whereas they were frequently so before.

Conclusion: We conclude that the patients included in our study were significantly relieved of severe and disabling of diabetic gastroparesis. 
Acute exposure to palmitic acid damages autonomic enteric nerve in rat: preventive effects of protein kinase C inhibitor

Y. Fujita ${ }^{1}$, M. Hosokawa ${ }^{1}$, K. Fukuda ${ }^{1}$, K. Nagashima ${ }^{1}$, S. Fujimoto ${ }^{1}$, Y. Yamada ${ }^{1}$, Y. Seino ${ }^{2}$, N. Inagaki ${ }^{1}$;

${ }^{1}$ Department of Diabetes and Clinical Nutrition, Kyoto University, Kyoto, ${ }^{2}$ Division of Diabetes and Clinical Nutrition, Kansai Denryoku Hospital, Osaka, Japan.

Background and Aims: Diabetes mellitus is often accompanied by elevated plasma free fatty acid levels. To determine if free fatty acid is an independent factor in the progression of diabetic gastroenteropathy, we evaluated functional changes in submucosal nerve plexus by acute exposure of rat colon to palmitic acid for representative substrate of free fatty acids. It is well known that protein kinase $\mathrm{C}$ (PKC) inhibitor prevents diabetic somatic nerve dysfunction. We evaluated the preventive effects of PKC inhibitor against damage of enteric nerve caused by palmitic acid.

Materials and Methods: Male Wister rats weighing about $200 \mathrm{~g}$ were used. Short-circuit current (Isc) in colonic mucosa was measured using an Ussing chamber. Stripped colonic mucosa (submucosal plexus intact) was mounted vertically between temperature $\left(37^{\circ} \mathrm{C}\right)$-controlled Ussing chambers. Ringer's solution containing $10 \mathrm{mM}$ glucose was used as the standard bathing solution. All bathing solutions were gassed with a mixture of $95 \% \mathrm{O}_{2} / 5 \% \mathrm{CO}_{2}$, resulting in $\mathrm{pH}$ 7.4. All drugs were applied to the serosal bathing solution from the stock solution. The Isc response to electric-field-stimulation (EFS) was measured 0 and 30 minutes after exposure to $0.02 \mathrm{mM}$ to $0.14 \mathrm{mM}$ palmitic acid. To investigate the effect of PKC inhibitor, the colonic mucosa was pretreated with PKC inhibitor at a dose of $0.5 \mathrm{mM}$ in the Ussing chamber before the application of $0.1 \mathrm{mM}$ palmitic acid, and the Isc response to EFS was measured after 30 minutes.

Results: The Isc in response to EFS was dose-dependently decreased by exposure to palmitic acid, due to suppression of neuron-mediated $\mathrm{Cl}^{-}$secretion $\left(\mathrm{EC}_{50}\right.$ value and maximal decrease were $0.07 \mathrm{mM}$ and $40 \%$ ). Treatment with PKC inhibitor mitigated the suppression by exposure to $0.1 \mathrm{mM}$ palmitic acid $\left(4.9 \pm 0.6\right.$ vs. $7.1 \pm 1.1 \mu \mathrm{A} / 0.38 \mathrm{~cm}^{2}$ at 30 minutes after treatment, $P<0.05$ ).

Conclusion: Acute exposure to palmitic acid clearly damaged autonomic enteric nerve, suggesting that palmitic acid is an independent factor in the progression of diabetic autonomic neuropathy, and indicates that PKC inhibitor may have a preventive effect in diabetic gastroenteropathy.
Diabetic autonomic neuropathy and changes in oesophageal and duodenal contractility: neuronal and structural abnormalities

N. Ejskjaer ${ }^{1}$, H. Gregersen ${ }^{2}$, J. S. Christiansen ${ }^{1}$, A. M. Drewes ${ }^{2}$, J. Froekjaer ${ }^{3}$;

${ }^{1}$ Department of Medicine M (Endocrinology and Diabetes), Aarhus University Hospital, ${ }^{2}$ Center for Visceral Biomechanics and Pain, Aalborg University Hospital, ${ }^{3}$ Department of Radiology, Aalborg University Hospital, Denmark.

Background and Aims: The pathogenesis of gastrointestinal symptoms in diabetes mellitus is complex in nature, multifactorial and not understood in detail. Diabetes induced neuronal changes are of major importance but also structural changes and remodeling of the gastrointestinal tract may be relevant.

Methods: The esophageal and duodenal activity to standardized bag distension was assessed in 12 diabetic patients with autonomic neuropathy and severe gastrointestinal symptoms and in 12 healthy controls using a specialized ultrasound based probe. the geometry and biomechanical parameters (strain, tension/stress, stiffness) were assessed.

Results: The diabetic patients had increased reactivity to esophagela distensions, e. g. increased frequency og distension-induced contractions $(\mathrm{p}<0.001)$. Impaired coordination of the contractions (dysfunction of local reflex arcs) was verified as ambalance between the time needed to evoke the first contraction at the distension site and proximal to it $(\mathrm{p}<0.025)$. The duration of the disease was associated with the increased activity. The esophageal wall thickness including all layers except the muscle layer was increased $(p<0.001)$, longitudinal shortening decreased $(p<0.001)$, and the radial compressibility increased $(p<0.001)$ in the diabetic patients. The esophageal and duodenal wall stiffness and circumferential deformation induced by bag distensions were not affected by diabetes.

Conclusion: The impaired contractile acitivity observed may reflect neuronal abnomalities due to autonomic neuropathy but structural changes and remodeling of the GI tract may also interfere could explain the gastrointestinal dysfunction and symptoms observed in diabetes. This may have impact on the future management of diabetic patients with gastrointestinal symptoms. 
1119

Diabetic autonomic neuropathy: aspects of differentiated visceral hypersensititation

J. Froekjaer ${ }^{1}$, A. M. Drewes ${ }^{2}$, J. S. Christiansen ${ }^{3}$, H. Gregersen ${ }^{2}$, N. Ejskjaer ${ }^{3}$;

${ }^{1}$ Department of Radiology, ${ }^{2}$ Center for Visceral Biomechanics and Pain, Aalborg University Hospital, ${ }^{3}$ Department of Endocrinology M, Aarhus University Hospital, Denmark.

Background: The pathogenesis of gastrointestinal symptoms in diabetes mellitus is complex in nature, multifactorial and is not well understood. Diabetes induced neuronal changes (peripheral and central) may be of importance.

Methods: The visceral sensitivity to mechanical, thermal and electrical in the esophagus and duodenum was assessed in 15 diabetes patients with proven autonomic neuropathy and severe gastrointestinal symptoms and in 12 healthy controls using a specialized multimodal ultrasonic testing device. The sensory response and the somatic referred pain areas were recorded.

Results: An overall hyposensitivity to the combination of all visceral stimulations was found in the duodenum $(P=0.01)$, and in the esophagus and duodenum combined $(P=0.02)$. The visceral hyposensitivity was accompanied by an increase in the somatic referred pain areas $(P=0.04)$ indicating central neuronal changes. Subanalysis revealed hyposensitivity to mechanical stimulations in the esophagus $(P=0.006)$ and duodenum ( $P$ hyperexcitability) may be involved in the pathogenesis of the often severe and neglected gastrointestinal symptoms in longstanding diabetes. This can be of importance since future targets in the treatment of gastrointestinal symptoms in diabetic patients with autonomic neuropathy could be modulation=0.002), and to thermal $(P<0.001)$ and electrical $(P=0.005)$ stimulations in the esophagus and duodenum combined.

Conclusion: This multimodal and multisegmental sensory testing approach indicates that complex neuronal (interaction between peripheral hypoalgesia and central excitability)may be involved in the pathogenesis of the often severe and neglected gastrointestinal symptoms in longstanding diabetes. These finding may be of importance since future targets in the treatment of gastrintestinal symptoms in patients with diabetic autonomic neuropathy could be modulation of the central nervous system by receptor specific drugs or gastric electrical stimulation.
1120

Glucose metabolism improvement by gastric electrical stimulation in morbidly obese type 2 diabetic patients interim results

C. Rosak $^{1}$, R. Weiner ${ }^{2}$, G. Prager ${ }^{3}$, B. Ludvik ${ }^{4}$;

${ }^{1}$ Dept of Endocrinology, ${ }^{2}$ Sachsenhausen Hospital, Frankfurt a. Main, Germany, ${ }^{3}$ Dept of Surgery, ${ }^{4}$ Dept. of Internal Medicine, Medical University of Vienna, Austria.

Background and Aims: The stomach plays a key role in the regulation of nutrient intake and disposition through neural, hormonal and mechanical functions. Previous work suggests that enhancement of gastric contractions during meals can induce weight loss in morbidly obese subjects. The TANTALUS ${ }^{\mathrm{TM}}$ System (MetaCure N.V.), a minimally invasive, non-restrictive, non-malabsorptive and non-excitatory gastric stimulation modality, may achieve improved glucose homeostasis and weight loss in obese diabetic subjects.

Materials and Methods: In an ongoing study, 24 adult obese T2DM subjects with different degrees of glycemic control and varied treatments (combinations of oral agents alone or with various insulin preparations) were implanted laparoscopically with the system, comprised of three bipolar electrodes stitched onto the stomach, connected to an implantable device. The device automatically detects food intake and delivers electrical signals synchronized to the stomach's natural rhythm. Mean baseline BMI and $\mathrm{HbA} 1 \mathrm{c}$ in the patients were $41.7 \pm 0.9 \mathrm{~kg} / \mathrm{m}^{2}$ and $8.0 \pm 0.2 \%$. Results: Patients who were receiving insulin therapy had inconsistent responses. In the sub group of 9 patients inadequately controlled (HbAlc in the range of $7 \%-9.5 \%$ at baseline) on oral anti-hyperglycemic agents only, mean $\mathrm{HbA} 1 \mathrm{c}$ at 20 weeks post-operative was reduced from $8.21 \pm$ $0.28 \%$ to $7.04 \pm 0.25 \%(\mathrm{p}<0.05)$ and fasting glucose decreased from $197.3 \pm 16.0$ to $142.3 \pm 11.6 \mathrm{mg} / \mathrm{dl} \quad(\mathrm{p}<0.05)$. Weight loss was $6.4 \pm 1.5 \%$ of the baseline body weight $(\mathrm{p}<0.01)$. No significant device related complications or side effects were observed in the 24 patients.

Conclusion: Twenty week data indicate that gastric electrical stimulation initiated upon food intake detection improves glycemic control in morbidly obese T2DM subjects who are inadequately controlled with conservative oral medication. Improved glycemic control was accompanied by significant weight loss. The TANTULUS System appears to be a promising implantable device for the treatment of the obese type 2 diabetic patient inadequately controlled on oral anti-hyperglycemic agents.

Supported by MetaCure N.V 


\section{1}

A subclinical enteropathy precedes the clinical onset of type 1 diabetes

L. Molteni ${ }^{1}$, L. Folini ${ }^{2}$, M. G. Radaelli ${ }^{2}$, I. Fermo ${ }^{2}$, R. Paroni ${ }^{3}$, M. R. Pastore ${ }^{2}$, E. Bosi' ${ }^{2}$;

${ }^{1}$ Diabetes and Endocrinology Unit, Vita-Salute San Raffaele University and Hospital General Medicine, Milano, ${ }^{2}$ General Medicine, Diabetes and Endocrinology Unit, VitaSalute San Raffaele University and Hospital, Milan, ${ }^{3}$ Department of Medicine, Surgery and Dental Science, University of Milan, San Paolo Hospital, Milan, Italy.

Background and Aims: A number of recent studies indicate the presence of several intestinal abnormalities both in experimental and human autoimmune diabetes. In the $\mathrm{BB}$ rat increased intestinal permeability, mucosal lymphocytic infiltration, altered expression of mucosal associated mucins and intestinal intraluminal zonulin upregulation have been reported. In patients with type 1 diabetes, increased intestinal permeability, intestinal biopsydocumented signs of enhanced immune activation and ultra-structural changes have also been shown. In human type 1 diabetes it remains to be clarified whether these alterations are related to an underlying pathogenetic process or simply reflect alterations secondary to the associated metabolic abnormalities. In order to elucidate this issue, we investigated the intestinal permeability to sugars in subjects at the different stages of type 1 diabetes. The aim was to further characterize the intestinal dysfunction associated with human type 1 diabetes and clarify its natural history in parallel with that of the underlying autoimmune process.

Materials and Methods: We investigated the permeability of intestine to sugars (monosaccharide Mannitol and disaccharide Lactulose) in subjects at different stages of type 1 diabetes: pre-clinical ( 17 relatives with $\geq 2$ autoantibodies, median age 22 , range $8-42 \mathrm{yr}$ ), clinical onset (21 patients, median age 28 , range $6-56 \mathrm{yr}$ ), long-standing (33 patients, median age 41 , range $22-66 \mathrm{yr}$, median duration of disease $19 \mathrm{yr}$, range 4-40 yr) and healthy controls (32 subjects, median age 26, range 9-54 yr). All subjects underwent a lactulose-mannitol test, consisting in oral administration of the two sugars and measurement by capillary electrophoresis of their urine excretion during the following 5 hours. Alterations of intestinal integrity may result in reduced passage of small molecules as mannitol (reflecting a reduced area of integral mucosa), and/or increased uptake of large molecules as lactulose (reflecting a damaged barrier).

Results: Intestinal permeability to lactulose was uniformly increased in all groups with islet autoimmunity, including pre-clinical, new onset and long standing type 1 diabetes (all $\mathrm{P}<0.002$ vs healthy controls). Intestinal permeability to mannitol was significantly decreased only in long standing type 1 diabetes $(\mathrm{P}=0.0185$ vs healthy controls).

Conclusion: These data provide evidence for the presence of a subclinical enteropathy associated with type 1 diabetes. Hallmarks of this enteropathy are: increased intestinal permeability to macromolecules (lactulose), already detectable before clinical onset, most likely associated with the autoimmune process; reduced permeability to small molecules (mannitol), detectable only in long standing disease, possibly secondary to diabetes. These findings bring to attention the possible participation of the small intestine in the pathogenesis of type 1 diabetes.

\section{PS 99}

\section{Diabetic foot}

\section{2}

Simple clinical characteristics can identify diabetic foot patients at risk of poor outcome: the Eurodiale experience L. Prompers ${ }^{1}$, M. E. Edmonds ${ }^{2}$, M. Huijberts ${ }^{3}$, E. Jude ${ }^{4}$, D. Mauricio ${ }^{5}$, L. Uccioli ${ }^{6}$, V. Urbancic ${ }^{7}$, K. Van Acker ${ }^{8}$, N. Schaper ${ }^{3}$;

${ }^{1}$ Eurodiale Study Group, University Hospital Maastricht, The Netherlands, ${ }^{2}$ King's College Hospital, London, United Kingdom, ${ }^{3}$ University Hospital Maastricht, The Netherlands, ${ }^{4}$ Tameside General Hospital, Ashton-UnderLyne, United Kingdom, ${ }^{5}$ Hospital de la Santa Creu I Sant Pau, Barcelona, Spain, ${ }^{6}$ Universita di Roma Tor Vergata, Rome, Italy, ${ }^{7}$ University Medical Centre, Ljubljana, Slovenia, ${ }^{8}$ St Jozefs Ziekenhuis, Bornem, Belgium.

Background and Aims: Comprehensive data on clinical characteristics and outcome in diabetic foot disease is scarce; most information comes from single centres and from short-term studies. The Eurodiale study group has brought together 14 European centres specialized in diabetic foot care; one of its aims is to study the outcome of diabetic foot ulceration in relation to the clinical characteristics at presentation.

Materials and Methods: Data were obtained on 1229 consecutive patients with diabetes and a new foot ulcer. Data on patient characteristics, including co-morbidity, as well as foot and ulcer characteristics were obtained using a standardised case record form. Foot disease was categorised in 4 stages according to the presence of peripheral arterial disease (PAD) and infection: A (PAD -/infection -); B (PAD-/infection+); C (PAD+/infection-); $\mathrm{D}(\mathrm{PAD}+$ /infection+). The patients were followed up until final outcome 
(healing, major amputation or death) or up to one year from study entry.

Results: Outcome data are available on 1053 patients (mean age $65 \pm 12 \mathrm{yrs}$ ). In the whole cohort $77 \%$ healed; major amputation was performed in $4 \%$ and $7 \%$ of the patients died. Final outcome according to disease stage is shown in the table below. There was a significant difference for each outcome in relation to stage at presentation $(p<0.05)$. Patient outcome was also related to the presence of disabling co-morbidities (end-stage renal disease, heart disease NYHA III/IV, severe visual impairment, inability to stand or walk without help): in such patients with one or more of these co-morbidities healing was significantly lower at $69 \%$ vs. $81 \%$ in patients without co-morbidities; amputation was performed more frequently (6\% vs. $3 \%$ ) and death occurred in $12 \%$ vs. $5 \%$ (all $\mathrm{p}<0.05$ ). Ulcer characteristics (size, depth and location) were also associated with poorer outcome.

Conclusion: In this large European cohort healing rate was higher than expected. However, this study has demonstrated that the risk of poor outcome can be identified by using simple clinical parameters, including PAD, infection, comorbidities and ulcer characteristics. Awareness of the usefulness of these clinical indicators should be promoted amongst all health care professionals working in the field of diabetes.

Outcome according to disease stage

\begin{tabular}{llll}
\hline Stage & Healing & Amputation & Death \\
\hline A $(\mathrm{n}=270)$ & $\mathbf{8 6 \%}$ & $\mathbf{2 \%}$ & $\mathbf{3 \%}$ \\
B $(\mathrm{n}=\mathbf{3 0 5})$ & $\mathbf{8 4 \%}$ & $\mathbf{1} \%$ & $\mathbf{5 \%}$ \\
C $(\mathrm{n}=\mathbf{2 0 5})$ & $\mathbf{7 8 \%}$ & $\mathbf{3 \%}$ & $\mathbf{6 \%}$ \\
D $(\mathrm{n}=\mathbf{3 4 7})$ & $\mathbf{6 5 \%}$ & $\mathbf{8 \%}$ & $14 \%$ \\
\hline
\end{tabular}

Supported by EC 5th Framework Programme

\section{3}

The prevalence of foot ulcer risk factors in Egyptian diabetic patients

M. EL-Nahas, H. Gawish, M. Tarshoby, O. State; Diabetes and endocrinology Unit, Internal Medicine Department, Mansoura University, Egypt.

Background and Aims: The effect of ethnicity on diabetic foot ulceration (DFU), neuropathy and peripheral arterial disease (PAD) remains controversial. Contradictory results about the prevalence of DFU and amputation had been especially reported in diabetic patients of African descent living in UK or USA. The large nature of Africa continent that include diverse populations together with the possible effect of environmental and cultural factors on racial differences, make it plausible to study African patients in their original countries. Egypt is geographically located in Africa, however, it is also considered as a Mediterranean country. Few studies had reported the prevalence of DFU as part of diabetes related complications but, to our knowledge, there has not been any previous survey on DFU risk factors in Egypt. Objective: To study the prevalence of DFU and their risk factors in a cohort of patients presented to outpatient diabetes clinic at Mansoura University Hospital.

Materials and Methods: One thousand two hundred and twenty diabetic patients were screened over one year for DFU, neuropathy, PAD, foot deformities, skin and nail abnormalities. Patients were also questioned about receiving any previous education about foot problems or foot care.

Results: The mean age of our patients was $50.5+10.9$ years and $36.8 \%$ were male. The mean duration of diabetes was 7.9+5.9 years and mean BMI was 34.5+6.7. The prevalence of active foot ulceration or past history of DFU was $1.2 \%$ and $5.7 \%$ respectively; so, the overall DFU prevalence was 6.9\%. 5.07 SW monofilament insensitivity was found in 113 patients (9.3\%). Only 38 patients (3.1\%) had absent foot pulses that proved by doppler ultrasound to have PAD. The prevalence of Hallux valgus, hammer toe and flat foot was $23.4,10.2$ and $2.1 \%$ respectively. Dry skin and calluses were recorded in 544 patients $(44.6 \%)$ and 69 patients (5.7\%) respectively. Tinea pedis was found in 532 patients (43.6\%). Thick nails and badly cut nails were found in 215 $(17.6 \%)$ and $376(30.8 \%)$ respectively. The shoes were inappropriate in 751 patients $(61.6 \%)$ and 1145 patients (93.8\%) didn't receive any prior foot education.

Conclusion: Despite the low prevalence of insensate neuropathy and PAD in our diabetic patients the prevalence of DFU is still high. The lack of knowledge regarding diabetic foot problems, the inappropriate foot wears and the high prevalence of skin and nail pathology in Egypt could explain this paradox. It is suggested that regional differences in the risk factors for DFU should be considered when preventative strategies for DFU are planed. Education of patients and healthcare providers together with establishment of podiatric care may have better outcome in our country.

\section{4}

Diabetic foot care in French hospitals between 1997 and 2003

I. Got ${ }^{1}$, P. Oberlin ${ }^{2}$, M.-C. Mouquet ${ }^{3}$;

${ }^{1}$ Diabetes Center, CHU Nancy, Toul, ${ }^{2}$ Department of Surgery, Centre Hospitalier, Villeneuve Saint-Georges, ${ }^{3}$ DREES, Direction de la Recherche, des Etudes, de l'Evaluation et des Statistiques, Paris, France. 
Background and Aims: There is a lack of any published data in France on the extent of hospital admissions for diabetic foot ulcerations, lower limb amputations and revascularizations. Yet these are important markers to evaluate the quality of foot care in our country and its advances.

Materials and Methods: We have used the national database of the French Hospital Information System (PMSI) for public and private hospitalizations from 1997 to 2003 . The selection of the ICD-10 codes for diabetes and its complications was crossed with codes for foot ulceration, procedures of revascularization, lower limb amputations, operations on stumps and debridements of wounds.

Results: Between 1997 and 2003, the French Health Care has registered a growing number of new treated diabetic patients, aged 18 years or more, on a rythm of $+9 \%$ per year, with a total of 1.967 .000 subjects on the $01 / 01 / 04(3.27 \%$ of the French population). During the same period, hospital admissions for adult diabetic patients increased by $5.6 \%$ per year. We observed a progression of admissions for diabetic foot ulcerations (age and gender-adjusted rates :+7.3\% per year), with a sharp increase of "non surgical" hospitalizations $(+15.9 \%$ per year $)$, while surgical stays grew slower $(+2.9 \%$ per year). Each year, diabetic foot lesions were the cause of $8 \%$ of all diabetic admissions and accounted for $16 \%$ of total hospitalization days. The procedures realized during the "surgical"stays were, depending on the year, debridement in 14 to $18 \%$ of cases, revascularization in 40 to $43 \%$, amputation in 40 to $42 \%$. Amputation level was limited to toes or foot in $2 / 3$ of cases, while major amputations (leg or thigh) occurred in $1 / 3$ of cases. There was a development of the day cases, specially for non surgical care or debridements, growing from $17 \%$ in 1997 to $42 \%$ in 2003 . For the other surgical stays ( $>24$ hours), their duration shortened by 3 days in case of a revascularization, by 2 days when a minor amputation was performed, and increased by half a day after a major amputation. Mean length of stay decreased from 19.6 to 18.1 days. Hospital admission rate for 1000 diabetic persons grew from less than $13 \%$ for patients under 55 years to $30 \%$ o for those above 84 years. In 2003, the 35.900 hospital admissions for diabetic foot wounds concerned 24.400 patients. Diabetic men were more often hospitalized than women $(68 \%)$ and were younger (mean age of 66.6 vs 70.3). They were more often implicated in stays leading to a revascularization or a limited amputation (75\%). More than $80 \%$ patients had only one hospitalization during the year, $12 \%$ needed two stays and $7 \%$ three or more stays. The more the stays were numerous, the more the frequency of amputation was high. When a revascularization was associated to an other procedure, it was a minor one in $80 \%$ of cases. A high amputation occured in spite of a revascularization in $20 \%$ of cases. Regional rates of hospitalization for diabetic foot were not related with the prevalence of diabetes. Regional variations in the incidence of diabetes-related amputations were noted, while revascularization rates were very similar between the different French regions.

Conclusion: In spite of the development of conservative surgery during the last years, more than 4 "surgical" admissions for diabetic foot on 10 still lead to a lower limb amputation in France. Amputation rate for men is still 3 times more than for women.

\section{5}

Bad news? Many patients with diabetic foot disease in Europe present with ischaemia, infection, and serious co-morbidity

M. Huijberts ${ }^{1}$, L. Prompers ${ }^{2}$, J. Apelqvist ${ }^{3}$,

S. van $\mathrm{Baal}^{4}$, K. Bakker ${ }^{5}$, P. Holstein ${ }^{6}$, A. Jirkovska ${ }^{7}$, F. van Merode ${ }^{8}$, A. Piaggesi ${ }^{9}$, G. Ragnarson Tennvall ${ }^{10}$, H. Reike ${ }^{11}$, M. Spraul ${ }^{12}$, N. Schaper ${ }^{2}$;

${ }^{1}$ Eurodiale Study Group, University Hospital Maastricht, The Netherlands, ${ }^{2}$ University Hospital Maastricht, The Netherlands, ${ }^{3}$ Diabetic Foot Centre, Lund, Sweden, ${ }^{4}$ Twenteborg Ziekenhuis, Almelo, The Netherlands, ${ }^{5}$ IDF Consultative Section on the Diabetic Foot, Heemstede, The Netherlands, ${ }^{6}$ Bispebjerg Wound Healing Centre, Copenhagen, Denmark, ${ }^{7}$ Institute for Clinical and Experimental Medicine, Prague, Czech Republic, ${ }^{8}$ University of Maastricht, The Netherlands, ${ }^{9}$ Azienda Ospedaliera Universitaria Pisana, Pisa, Italy, ${ }^{10}$ Institute for Health Economics, Lund, Sweden, ${ }^{11}$ Mariannen Hospital, Werl, Germany, ${ }^{12}$ MathiasSpital, Rheine, Germany.

Background and Aims: Foot ulcers in diabetic patients have a variable clinical presentation and prognosis which is related to differences in underlying pathology, such as neuropathy, peripheral arterial disease (PAD) and infection. Large clinical studies describing the typical clinical presentation of diabetic foot ulcers are limited. In this study we assessed the characteristics of diabetic patients with a foot ulcer who were treated in 14 European hospitals.

Materials and Methods: Data were obtained on 1229 consecutive patients with diabetes and a new foot ulcer. Data on patient characteristics, including co-morbidity, as well as foot and ulcer characteristics were obtained using a standardized case record form. Foot disease was categorised in 4 stages according to the presence of peripheral arterial disease (PAD) and infection.

Results: At study entry $27 \%$ percent of the patients were admitted. Disabling co-morbidity was present in 32\%. PAD was diagnosed in $49 \%$, infection in $58 \%$. The majority of the ulcers were located on the non-plantar surface of the 
foot $(52 \%)$. The frequency of the 4 stages was: A) PAD -/ infection-: 24\%, B) PAD-/infection+: 27\%, C) PAD $+/$ infection-: $18 \%$, D) PAD+/infection+: $31 \%$. Patients in this latter group had a distinct profile: they were older (age > 70 years $56 \%$ in stage D vs. $22 \%$ in A), had more nonplantar ulcers (65 vs. 36\%), more extensive tissue loss (deep ulcers 64 vs. 20\%; size $>5 \mathrm{~cm}^{2} 20 \%$ vs. $4 \%$ ) and more serious co-morbidity ( 38 vs. $23 \%$ ), all $\mathrm{p}<0.05$.

Conclusion: We conclude that the clinical presentation of patients with diabetic foot ulcers in Europe is more severe than previously reported, in view of the presence PAD and infection in one third of the patients. Non-plantar foot ulcers are more common than plantar ulcers, especially in patients with severe disease, and serious co-morbidity increases significantly with increasing severity of foot disease.

Supported by EC 5th Framework Programme

\section{6}

What happens to the initial bone marrow oedema in the natural history of Charcot osteoarthropathy?

M. E. Edmonds ${ }^{1}$, N. L. Petrova ${ }^{1}$, A. E. Edmonds ${ }^{1}$, D. A. Elias ${ }^{2}$;

${ }^{1}$ Diabetic Foot Clinic, ${ }^{2}$ Dept of Diagnostic Radiology, King's College Hospital, London, United Kingdom.

Background and Aims: The aim of the study was to monitor what happens to the bone marrow oedema that is seen on Magnetic Resonance Imaging (MRI) in the early stages of Charcot osteoarthropathy.

Materials and Methods: We studied 12 diabetic patients with Charcot osteoarthropathy presenting with a hot swollen foot, hot isotope bone scan and normal radiograph. Sites of increased radionuclide uptake were metatarsaltarsal (9), metatarsal-phalangeal (2) and ankle (1). Mean age was $55 \pm 10.6$ years (mean \pm SD) and mean duration of diabetes was $32 \pm 13.7$ years. There were 8 females and 4 males; type 1 diabetes (8) and type 2 diabetes (4). MRI was performed at presentation and at a median time of followup of 6 months (range 3-25 months). Offloading consisted of total contact casting in 8 patients and Aircasts in 4 .

Results: The patients were divided into 3 groups; group 1 consisted of 6 patients with bone marrow oedema alone. On follow-up, 3 of these patients showed resolution of bone marrow oedema. In the remaining 3 , one progressed to subchondral cysts, one to subchondral fracture and one to bony erosion with final reduction of oedema in all 3 .

Group 2 comprised 3 patients with bone marrow oedema already associated with subchondral fracture. On follow-up, all 3 patients showed reduction of oedema and healing of fractures.
Group 3 comprised 3 patients with bone marrow oedema already associated with subchondral cystic change. On follow-up, definite subchondral cysts were seen with resorption of surrounding bone marrow oedema.

On follow-up of all patients, radiographs showed no subluxation and there was no clinical deformity.

Conclusion: We conclude that in the Charcot foot presenting with no radiological changes, bone marrow oedema, seen as the first lesion on MRI, may resolve completely or lead to subchondral fracture, subchondral cysts or erosions with eventual reduction of marrow oedema. This study gives new information on the natural history of the Charcot foot.

Supported by Diabetes UK BDA 05/0003025

1127

Propolis, the anti-inflammatory bee hive protectant improves wound healing in experimental diabetes

S. V. McLennan ${ }^{1,2}$, J. Jia ${ }^{1}$, S. Milne ${ }^{1,2}$, L. Lo ${ }^{1}$,

J. Bonner ${ }^{2}$, D. K. Yue ${ }^{1}$, S. M. Twigg ${ }^{1,2}$;

${ }^{1}$ Discipline of Medicine, University of Sydney, ${ }^{2}$ Department of Endocrinology, Royal Prince Alfred Hospital, Sydney, Australia.

Background and Aims: Up to $25 \%$ of patients with diabetes will develop a foot ulcer at some stage, leading in a minority to amputation. Consequently, treatments aimed at improving diabetic ulcer healing are of great importance. The naturally derived anti-inflammatory agent, Propolis has not been investigated in diabetic wound healing. This study examined the effect of a single topical application of Propolis in a standardised rodent model of diabetic wound healing. The main end-points determined were epithelial closure rate, total wound volume, and inflammatory cell infiltration.

Materials and Methods: Diabetes was induced in Sprague Dawley rats $(\mathrm{n}=16)$ using streptozocin (i.p. $65 \mathrm{mg} / \mathrm{kg}$ ). Animals were maintained on 2 nd daily insulin (2-4 IU Protophane). After 6 weeks of diabetes, six full thickness skin wounds were created on the backs of diabetic and control rats $(\mathrm{n}=16)$, using a $6 \mathrm{~mm}$ biopsy punch. Three wounds in each animal were treated at the time of wounding with $20 \mu \mathrm{l}$ of either Propolis, or sterile saline control. Epithelial closure was monitored by progressive calliper measurement of lesion diameter. At days 6 and 12 after wounding, animals were sacrificed and the skin was removed, fixed and embedded. Tissue was serially sectioned for analysis of wound volume by polarised light microscopy, and neutrophil and macrophage number (mean of 20 fields) identified by morphology following PAS staining. Data are expressed as mean \pm SD. 
Results: Compared with controls, diabetes caused a mean $27 \%$ decrease in epithelial closure rate. The delay in wound closure at day 6 and day 12 (shown in the table) was reversed by topical Propolis. Wound volume did not differ across the groups and the impaired tissue macrophage infiltration in diabetic wounds was not altered by Propolis. In contrast, persistent neutrophil infiltration observed at day 12 in diabetic rats, was abolished by Propolis.

\begin{tabular}{lllll}
\hline & $\begin{array}{l}\text { Epithelial } \\
\text { closure }(\mathrm{mm} / \\
\text { day) }\end{array}$ & $\begin{array}{l}\text { Wound } \\
\text { volume } \\
\left(\mathrm{mm}^{3}\right)\end{array}$ & $\begin{array}{l}\text { Macrophage } \\
\text { (number/field) }\end{array}$ & $\begin{array}{l}\text { Neutrophil } \\
\text { (number/ } \\
\text { field) }\end{array}$ \\
\cline { 2 - 5 } Control & $0.37 \pm 0.05$ & $3.22 \pm 0.13$ & $1.49 \pm 0.09$ & $0.22 \pm 0.19$ \\
$\begin{array}{l}\text { Diabetic } \\
\text { Control }\end{array}$ & $0.27 \pm 0.05^{*}$ & $3.86 \pm 0.21$ & $0.25 \pm 0.14^{*}$ & $1.33 \pm 0.81^{*}$ \\
+ Propolis & $0.43 \pm 0.06$ & $3.36 \pm 0.71$ & $0.89 \pm 0.39$ & $0.17 \pm 0.17$ \\
$\begin{array}{l}\text { Diabetic } \\
+ \text { Propolis }\end{array}$ & $0.34 \pm 0.06$ & $3.98 \pm 1.25$ & $0.33 \pm 0.25^{*}$ & $0.23 \pm 0.19$ \\
\hline
\end{tabular}

$* \mathrm{p}<0.05$ different from untreated control values

Conclusion: This data indicates for the first time that a single application of Propolis has utility in accelerating ulcer healing rate in diabetes. As neutrophil infiltration is normalised, its mechanism may be through anti-inflammatory actions. This result and the established safety profile of Propolis provide a rationale for studying topical application of this agent in a clinical setting.

Supported by Innovative grant from JDRFI

\section{8}

Prospective randomized controlled study of non-ischaemic diabetic foot ulcers dressed with Hydrofiber ${ }^{\circledR}$ containing ionic silver or calcium alginate dressings

E. Jude ${ }^{1}$, J. Apelqvist ${ }^{2}$, M. Spraul ${ }^{3}$, J. Martini ${ }^{4}$;

${ }^{1}$ Department of Diabetic Medicine, Tameside General Hospital, Ashton-Under-Lyne, United Kingdom, ${ }^{2}$ Department of Endocrinology, Malmo University Hospital, Malmo, Sweden, ${ }^{3}$ Diabetologie, Mathias Hospital, Rheine, Germany, ${ }^{4}$ Service Diabetologie \& Endocrinologie, Hopital de Rangueil, Toulouse, France.

Background and Aims: Diabetic foot ulcers (DFU) risk infection and impaired healing, placing patients at risk of lower extremity amputation. Debridement and dressings are required for DFU care. Hydrofiber ${ }^{\circledR}$ dressings retain more absorbed wound fluid and bacteria than alginate dressings and with ionic silver, persistently kill local pathogens. A prospective, multi-center study compared clinical efficacy and safety of AQUACEL ${ }^{\circledR}$ Hydrofiber ${ }^{\circledR}$ dressings containing ionic silver $\left(\mathrm{AQ}^{*}\right)$ to those of calcium alginate $\left(\mathrm{CA}^{* *}\right)$ dressings used within regimens to manage out-patients with Type 1 or 2 diabetes and neuropathic or neuro-ischaemic Wagner Grade 1 or 2 DFU.

Materials and Methods: Patients were randomly assigned to similar protocols including off-loading, AQ or CA primary dressings and secondary foam dressings for 8 weeks or until healing. Assignment was stratified by baseline antibiotic use (Antibiotic) or non-use for the study ulcer, reflecting baseline clinical ulcer-related infection or its risk. Primary efficacy measures were healing outcomes. Safety was measured as adverse events.

Results: AQ and CA groups were comparable at baseline. Both groups improved on all ulcer healing outcomes. The time to healing was 53 days for AQ ulcers and 58 days for CA ulcers. AQ ulcers reduced in depth nearly twice as much as CA ulcers did ( $2.5 \mathrm{~mm}$ versus $1.3 \mathrm{~mm}$; $\mathrm{p}=0.04$ ). AQ subjects also experienced more overall ulcer improvement and less deterioration $(\mathrm{p}=0.06)$, accentuated in the Antibiotic subset $(\mathrm{p}=0.02)$. The safety profiles of both groups were similar.

Conclusion: A regimen including AQ significantly outperformed one using CA on ulcer depth reduction. This study reports the first significant clinical effects of a primary wound dressing containing silver on DFU healing. Product Notations:

*AQ, AQUACEL ${ }^{\circledR}$ Hydrofiber ${ }^{\circledR}$ dressing with $1.2 \%$ ionic silver (AQUACEL and Hydrofiber are registered trademarks of E. R. Squibb \& Sons, L.L.C., Princeton, NJ, USA)

${ }^{* *} \mathrm{CA}$, Algosterile ${ }^{\mathrm{TM}}$ calcium alginate dressing (Algosterile is a trademark of Les Laboratoires Brothier, S.A., ParisNanterre, France); DFU, Diabetic foot ulcers

Supported by ConvaTec, a Bristol Myers Squibb company

1129

The association of resistant staphylococcus aureus and the rate of lower limb amputations in patients with the diabetic foot

V. Fejfarová ${ }^{1}$, A. Jirkovská ${ }^{1}$, J. Skibová ${ }^{1}$, V. Petkov $^{2}$;

${ }^{1}$ Diabetes Center, ${ }^{2}$ Department of Microbiology, Institute for Clinical and Experimental Medicine, Prague, Czech Republic.

Background and Aims: The progression of infection may contribute to lower limb amputation (LLA) in risk patients with the diabetic foot. Microbial resistance complicates further outpatient treatment of diabetic foot infection and may accelerate the progression of infection leading to amputations even if patients could be treated by i.v. antibiotics during hospitalization. The aim of our study was to assess the association of resistant Staphylococcus 
Aureus (S.Aureus) and the rate of LLA in patients with the diabetic foot.

Methods: A total of 191 patients with diabetic foot ulcers and positive swab cultivation, whose were treated in our foot clinic from $12 / 1998$ to $11 / 1999$, were consecutively included into our study. The frequency of amputation was observed during a two-year period. Bacterial findings were established by cultivation of swabs taken from diabetic foot ulcers after local debridement. Microbial sensitivity to antibiotics was tested by sets by sets/using panels/batteries of antibiotics composed composed/developed on the basis of previous analysis of resistance at IKEM and by the recommendations of the National Reference Laboratory on Antibiotics. Microbial sensitivity to different types of antibiotics was determined semi- quantitatively by disc diffusion susceptibility10,(10,Courvalin, 191196; Reese, 1996). The occurrence of S.Aureus resistant to betalactam antibiotics was observed. Osteomyelitis (OM) was diagnosed by X-ray and laboratory markers of infection. Peripheral arterial disease (PAD) was detected by noninvasive methods (Doppler indexes $<0.8$, TcPO $2<30 \mathrm{mmHg}$ ) or angiography. Surgeons indicated LLA, if acute progression of infection or ischemia developed in the study patients.

Results: 50 patients (26\%) underwent LLA (50/191; 44 patients had minor and 6 patients had major LLA) and 141 patients (74\%) had no LLA during the observed period. $16.5 \%$ of all diabetic patients were shown to have resistant S.Aureus. Moreover, a higher occurrence of this pathogen was found in swabs of amputees before LLA compared with patients not undergoing LLA (33\% and $11 \%)$; $\mathrm{p}<0.001$. Based on Stepwise Logistic Regression, resistant S.Aureus (OR 4.34; CI 1.6-12.1), together with other factors such as OM (OR 15.4; CI 6.2-38.0) and PAD (OR $3.21 ; 1.1-9.2)$ were independent important risk factors associated with the rate of LLA. In addition, resistant $\mathrm{S}$. Aureus was significantly associated with LLA especially in patients without $\mathrm{OM}(\mathrm{p}=0.003$; OR 7.1).

We conclude that not only OM and PAD but also the presence of S.Aureus resistant to betalactamsmicro may species could increase the incidence of LLA in patients with the diabetic foot, particularly those without OM.

Supported by MZO 00023001

1130

Change in bacteriological analysis and in susceptibility to antimicrobial agents in diabetic foot infections

M. C. Collina ${ }^{1}$, D. Pitocco ${ }^{1}$, L. Mancini ${ }^{1}$, T. Musella ${ }^{1}$, A. Manto ${ }^{1}$, F. Zaccardi ${ }^{1}$, C. Fabiano ${ }^{1}$, M. Galli ${ }^{2}$,

S. Caputo ${ }^{1}$, G. Ghirlanda ${ }^{1}$;

${ }^{1}$ Internal Medicine, ${ }^{2}$ Orthopedics, Catholic University, Rome, Italy.
Background: Infection is the primary problem requiring hospital admission of the patients with diabetic foot and it is the main reason of amputation. Aim of this study is to perform a bacteriologic analysis on lesions of diabetic outpatients with foot infections in order to characterize the pathogens more frequent, the variability of their prevalence in ten years (from 1995 to 2005) and to test the susceptibility to antimicrobial agents commonly used.

Methods: Were analyzed 100 cultures taken from the following sources: ulcers, abscesses, cutaneous bullae, bone or soft tissue specimens. They were placed in sterile transport containers Portagerm (Bio-Merieux) and promptly delivered to the clinical microbiology laboratory and were processed aerobically and anaerobically

Results: Staphyloccus aureus showed the highest prevalence $(50 \%)$ and this was significantly increased if compared to the prevalence observed ten years before $(20.6 \%)$. Pseudomonas aeruginosa showed the same trend with a prevalence of $23.2 \%$ against $8.7 \%$ at the first observation. The prevalence of streptococci show a not significant decrease $(18.7 \%$ vs $21 \%)$. Furthermore we found a significant increase of polimicrobial infections (56.2 vs $48.6 \%$ ) Regarding the susceptibility to the antimicrobial agents tested teicoplanin, vancomicin, linezolid and cotrimoxazol showed a $100 \%$ of susceptibility against staphyloccus aureus infection but teicoplanin had a lower MIC $(<1,4)$. Ciprofloxacin with a percentage of $80 \%$ seems to be the most active against gram-negative. Imipenem showed the higher appearance of resistence, it was active only in $50 \%$ of infections

Conclusion: This study show that in 10 years there is a change in the prevalence of pathogens and in their susceptibility to antimicrobial agents with a significant increase of polymicrobial infections linked to the appearence of mulit-resistent pathogens as Staphyloccus aureus and Pseudomonas aeruginosa. Furthermore teicoplanin seems to be an anti-microbial agent of first choice in the treatment of Staphyloccus aureus infections.

\section{PS 100}

\section{Glycation and advanced glycation endproducts}

\section{1}

Cellular events and signaling pathways activated by AGE-albumin interaction with the aortic endothelium

L.-D. Popov, M. Simionescu;

Vascular Dysfunction in Diabetes, Institute of Cellular Biology and Pathology "N. Simionescu", Bucharest, Romania. 
Background and Aims: Diabetes is associated with increased formation and accumulation of advanced glycation end products (AGE), a heterogeneous group of compounds resulting from the non-enzymatic reaction between reducing sugars and proteins. Continuously exposed to high concentrations of both glucose and AGEproteins, the diabetic vascular endothelial cells (EC) undergo structural, functional, and biochemical changes that eventually lead to a dysfunctional state. The aim of this study was to investigate (i) the interaction of circulating AGE-albumin (AGE-Alb) with aortic EC in vivo (binding, uptake, and degradation) and (ii) the signaling cascades activated upon AGE-Alb interaction with EC, in vitro.

Materials and Methods: The experimental approaches were: (i) streptozotocin-injected RAP mice perfused in situ with $70 \mu \mathrm{M}$ AGE-Alb adsorbed to $5 \mathrm{~nm}$ colloidal gold (AGE-Alb.Au) followed by electron microscopy (EM); as controls, normal mice were employed; (ii) confluent human aortic EC cultured in DMEM supplemented with $25 \mathrm{mM}$ glucose and exposed to 5,15 and $30 \mu \mathrm{M}$ AGE-alb for 5 to 60 min (controls: cells grown in $5.5 \mathrm{mM}$ glucose and exposure to 5,15 and $30 \mu \mathrm{M}$ albumin). The expression of ERK1/2 (44 and $42 \mathrm{kDa}$ MAPKs) was detected by immunoblotting using antibodies against the tyrosinephosphorylated and total ERK1/2 proteins.

Results: EM studies showed that in vivo, AGE-Alb.Au bound to two EC transport structures: the caveolae and the coated pits. Binding to caveolae was followed by AGEAlb.Au transcytosis, discharge and accumulation in the subendothelium; vascular infusion of anti-RAGE IgG diminished by $\sim 50 \%$ the number of caveolae involved in AGE-Alb.Au uptake, indicating the role of caveolar receptors for AGE (RAGE) in the removal of circulating AGE-Alb. Upon interaction with the coated pits, the ligand was found in coated vesicles and multivesicular bodies, suggesting endocytosis and degradation of AGE-Alb. Although the EC pathways of AGE-Alb transport were identical in diabetic and normal animals, quantitatively, $\sim 20 \%$ more caveolae transcytosed AGE-Alb.Au in diabetic aorta than in control, indicating enhanced permeability in diabetic conditions. In vitro assessment showed that human EC grown in $25 \mathrm{mM}$ glucose and further subjected to $5 \mu \mathrm{M}$ AGE-Alb exhibited a fast (up to $5 \mathrm{~min}$ ) and significant increase ( 4.3 fold) in ERK1/2 phosphorylation (as demonstrated by densitometry); concentrations of 15 and $30 \mu \mathrm{M}$ AGE-Alb slightly augmented ( 1.4 fold) ERK1/2 phosphorylation, as compared to control. A similar sequence of events but at lower amplitude took place at low $(5.5 \mathrm{mM})$ glucose concentration: a rapid stimulation of ERK1/2 phosphorylation ( 2.3 fold) at $5 \mu \mathrm{M}$ AGE-Alb, and a nonsignificant enhancement $(\sim 1.2$ fold $)$ at both 15 and $30 \mu \mathrm{M}$ AGE-Alb. While the expression of total ERK1/ 2 proteins in $25 \mathrm{mM}$ glucose was $\sim 1.6$ fold above the control values (5.5 mM glucose) their concentration was not affected by the exposure to AGE-Alb. No changes in ERK1/2 phosphorylation were found in control samples challenged with 5-30 $\mu \mathrm{M}$ albumin.

Conclusion: The results indicate that in diabetes, AGE-Alb binds to EC-surface AGE-receptors that may be instrumental in the initiation of activation of ERK1/2. They also suggest that ERK1/2 and AGE-proteins contribute to EC activation and to the enhanced proliferative capacity in diabetic vascular endothelium.

Supported by grants from the Romanian Academy and European SSA-SERA project \# 016873

\section{2}

\section{WITHDRAWN}

1133

Influence of insulin resistance, pro- and anti-inflammatory markers, on soluble receptor for advanced glycation end-products (sRAGE)

G. Basta ${ }^{1}$, A. M. Sironi ${ }^{1}$, G. Lazzerini ${ }^{1}$, D. Ciociaro ${ }^{1}$, E. Buzzigoli ${ }^{1}$, S. Del Turco ${ }^{1}$, M. Pettiti ${ }^{1}$, S. Baldi ${ }^{2}$, A. Natali ${ }^{2}$, E. Ferrannini ${ }^{2}$, A. Gastaldelli ${ }^{1}$;

${ }^{1}$ Metabolism Unit, CNR Institute of Clinical Physiology, Pisa, ${ }^{2}$ Dpt of Internal Medicine, University of Pisa, Italy.

Background and Aims: In the development of diabetic micro- and macro-vascular complications, the interaction between advanced glycation end-products (AGEs) or ligands S100A12 with their receptors RAGE is thought to play a major role. RAGE has a circulating secretory receptor form, soluble RAGE (sRAGE), that neutralizing the action of its ligands might exert a protective role. The goal was to investigate if low sRAGE plasma levels were associated with insulin resistance and preferential abdominal fat accumulations, that are strong risk factors for the development of cardiovascular disease (CVD).

Materials and Methods: We studied 158 subjects, 83 with type 2 diabetes (T2DM) and 75 nondiabetic where we measured with ELISA systems sRAGE, NE-(Carboxymethyl)lysine-protein adducts (CML), S100A12, adiponectin, $C$ reactive protein (CRP).

Results: Plasma sRAGE was twice as low in T2DM subjects as in controls (140 [293] versus 735 [482] pg/mL, median [interquartile range], $\mathrm{p}<0.0001)$. Plasma sRAGE levels were inversely related to glycemic control, (as the fasting plasma glucose concentration, $r=-0.66, \mathrm{p}<0.0001$ ), insulin resistance (as HOMA, $\mathrm{r}=-0.39, \mathrm{p}<0.0001$ ), levels of inflammatory markers (CRP, $\mathrm{r}=-0.48, \mathrm{p}<0.0001$ ), and 
directly with adiponectin $(\mathrm{r}=0.25, \mathrm{p}<0.008)$. Low sRAGE levels were also associated with preferential abdominal fat accumulation, as waist-to-hip ratio $(\mathrm{r}=-0.20 \mathrm{p}<0.02)$ and visceral fat volume (VF, measured by MRI in a subgroup of subjects, $\mathrm{n}=84, \mathrm{r}=-0.30, \mathrm{p}<0.006)$, as well as with increased risk for CVD (Framingham score, $r=-0.42$ $\mathrm{p}<0.0001)$. On the other hand, while an inverse correlation was found between sRAGE and S100A12 ( $\mathrm{r}=-0.64$, $\mathrm{p}<0.0001$ ), an important inflammatory molecule strongly connected to RAGE signaling, no association was found with advanced glycation end products such as CML $(\mathrm{r}=0.06, \mathrm{p}=\mathrm{ns})$.

Conclusions: Low values of sRAGE are inversely associated with insulin resistance and pro-inflammatory markers, and directly with anti-inflammatory factors as adiponectin, indicating that this parameter is in first line to be used as a new marker in the predisposition to CVD, particularly in patients with T2DM.

\section{4}

Increased plasma levels of sRAGE in patients with diabetic nephropathy

K. Kankova ${ }^{1}$, M. Kalousova ${ }^{2}$, M. Hertlova ${ }^{3}$, D. Krusova ${ }^{4}$, T. Zima ${ }^{2}$;

${ }^{1}$ Department of Pathophysiology, Masaryk University, Brno,

${ }^{2}$ Institute of Clinical Chemistry and Laboratory Diagnostics, Charles University, Prague, ${ }^{3}$ Dept. of Gastroenterology, Faculty Hospital Brno-Bohunice, Brno, ${ }^{4}$ Dept. of Internal Medicine, St. Anne's Faculty Hospital, Brno, Czech Republic.

Background and Aims: Advanced Glycation End products (AGEs) accumulate in diabetic patients in a greater rate than in age-matched non-diabetic subjects and exert various adverse effects via the Receptor of AGE (RAGE). Soluble RAGE (sRAGE) is a $50 \mathrm{kDa}$ naturally occurring Cterminally truncated form of RAGE that functions as an inhibitor of AGE-mediated signaling events. Decreased levels of supposedly protective sRAGE were ascertained in patients with macrovascular disease and hypertension. Data about the role of sRAGE in diabetic nephropathy are so far lacking. The aim of the study was to analyse the relationship between sRAGE and renal functions in subjects with diabetic nephropathy and, hereby, implicit protective capacity against AGE-mediated toxicity.

Materials and Methods: Study was designed as a crosssectional analysis of a cohort of 255 diabetic patients with variable extent of diabetic nephropathy. Total number of subjects consisted if 36 diabetics with T1DM, 219 with T2DM (approx. 1:1 male-to-female ratio). Mean duration of diabetes was $12.2 \pm 9.4 \mathrm{yrs}$, mean age was $61.3 \pm 14.5 \mathrm{yrs}$.
Among all study subjects, 82 subjects had normoalbuminuria, 50 had persistent microalbuminuria, 112 had persistent proteinuria and 10 subjects had ESRD. Plasma concentration of sRAGE was assessed immunochemically (ELISA) using standard kits (Quantikine, RD Systems). Routine biochemical parameters were measured by certified methods using automated analysers. Relationship between circulating levels of sRAGE and the grade of diabetic nephropathy, renal function parameters and diabetes duration and compensation was analysed by means of nonparametric correlation, difference between groups were tested by Mann-Whitney test.

Results: sRAGE was significantly higher in nephropathy subjects (any degree of DN) than normoalbuminuric patients $(\mathrm{P}=0.0015), 2154.4 \pm 2612 \mathrm{pg} / \mathrm{ml}$ vs. $1622.7 \pm 1981.4 \mathrm{pg} / \mathrm{ml}$, respectively. In patients with nephropathy, sRAGE positively correlated with severity of nephropathy ( $\mathrm{P}=0.0028)$. Further, sRAGE positively correlated with patient's age, duration of diabetes, serum urea, serum creatinine and albumin excretion rate and negatively with glomerular filtration rate (all $\mathrm{P}<0.05$ ). No significant correlation was ascertained with fasting plasma glucose or $\mathrm{HbA}_{1 \mathrm{c}}$.

Conclusion: Contrary to our anticipations, serum sRAGE levels are in fact (i) increased in diabetics with diabetic nephropathy compared to normoalbuminuric subjects and (ii) further rise with decreasing renal function. It remains to be elucidated whether the increase merely reflects decreased renal clearance of sRAGE or up-regulation of sRAGE in order to protect cells against AGE-mediated effects.

Supported by the Grant Agency of the Czech Academy of Sciences and from the Ministry of Education, Youth and Physical Education of the Czech Republic

\section{5}

Effect of AGEs on the expression of fractalkine in cultured human renal mesangial cells

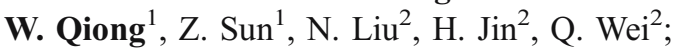

${ }^{1}$ Endocrinology, ${ }^{2}$ Institute of Cardiology, Zhongda Hospital, Southeast University, Nanjing, China.

Background and Aims: Fractalkine (FKN, CX3CL1)is a membrane-bound chemokine which are proinflammatory cytokines due to its ability to activate endothelial cells and promotes strong chemoattractant and adhesion in glomerular diseases. Our previous studies showed that advanced glycosylation end products (AGEs) induced the expression of MCP-1 and RANTES, which belong to CC chemokine family members. The aim of this study was to characterize the effect of AGEs on the expression of fractalkine in cultured human renal mesangial cells (HRMC). 
Materials and Methods: HRMC were exposed to vehicle (control), BSA, AGE-BSA (50 to $400 \mathrm{mg} \cdot \mathrm{L}^{-1}$ ) for $24 \mathrm{~h}$, in turn, HRMC were stimulated with AGE-BSA(200 $\left.\mathrm{mg} \cdot \mathrm{L}^{-1}\right)$ for various lengths of time ( $8 \mathrm{~h}, 16 \mathrm{~h}, 24 \mathrm{~h}, 48 \mathrm{~h}, 72 \mathrm{~h}$ ). Fractalkine mRNA and protein expression in HRMC were analyzed by reverse transcription-polymerase chain reaction (RT-PCR) and Western blotting.

Results: HRMC were incubated with different concentrations of AGE-BSA (50 to $400 \mathrm{mg} \cdot \mathrm{L}^{-1}$ ) for varying period ( 8 to $72 \mathrm{~h}$ ). RT-PCR and Western blotting showed that basal state HRMC expressed a low level fractalkine mRNA and protein. AGE-BSA stimulated HRMC fractalkine mRNA and protein expression in a concentration-dependent manner, The maximal effect of AGE-BSA on HRMC fractalkine mRNA and protein expression was observed at $72 \mathrm{~h}$.

Conclusion: AGEs may induce up-regulation of fractalkine on HRMC. These findings suggest that over-expression of fractalkine may be associated with inflammation in diabetic nephropathy, it might play an important role on progression of diabetic nephropathy.

Supported by National Natural Science Foundation

\section{6}

$\mathbf{N}^{\varepsilon}$-(Carboxymethyl)lysine is increased in plasma and the kidney of spontaneously hypertensive rats and is reduced by losartan and spironolactone, but not by hydralazine

C. G. Schalkwijk ${ }^{1}$, M. Baumann ${ }^{2}$, J. Scheijen ${ }^{1}$,

C. D. A. Stehouwer ${ }^{1}$;

${ }^{1}$ Internal Medicine, University Hospital Maastricht,

${ }^{2}$ Pharmacology, University Maastricht, Maastricht, The Netherlands.

Background and Aims: Pulse pressure is positively associated with risk of incident cardiovascular disease. We recently demonstrated that pulse pressure is strongly and independently associated with plasma levels of the advanced glycation endproduct, $\mathrm{N} \varepsilon$-(Carboxymethyl)lysine (CML) in young type 1 diabetic individuals. Since the kidney plays a major role in the development of blood pressure we studied the accumulation of CML in the kidney during the development of hypertension in spontaneous hypertensive rats (SHR) and compared the effect of the antihypertensive specific angiotensin II receptor blocker losartan, the mineralocorticoid receptor antagonist spironolactone and the nonselective vasodilator hydralazine on the levels of CML in the kidney.

Materials and Methods: We determined plasma levels of CML in aged normotensive rats $(n=8)$ and aged SHR rats $(n=4)$ with tandem mass spectrometry. We analysed renal sections of very young ( 8 weeks of age) normotensive rats
(WKY, n=6) and placebo- $(\mathrm{n}=6)$, losartan- $(\mathrm{L}: 20 \mathrm{mg} / \mathrm{kg} /$ day, $\mathrm{n}=6)$, spironolactone- (S: $1 \mathrm{mg} / \mathrm{kg} / \mathrm{day}, \mathrm{n}=6)$ and hydralazine-treated SHR (H: $15 \mathrm{mg} / \mathrm{kg} /$ day,n=6), which were subcutaneously treated for 4 weeks, for CML accumulation with immunohistochemistry and semiquantitatively analyzed. Mean arterial blood pressure was measured intra-arterially.

Results: SHR $(2.32 \pm 0.69 \mu \mathrm{M})$ showed higher plasma levels of CML as compared to WKY rats $(1.33 \pm 0.43 \mu \mathrm{M}$; $\mathrm{p}<0.05)$. Plasma levels of CML were correlated to pulse pressure in aged WKY rats and SHR (correlation 0.51; Rsquare 0.26). In SHR, all drugs lowered significantly blood pressure (L: $136 \pm 3, \mathrm{~S}: 136 \pm 2, \mathrm{H}: 126 \pm 5$ in comparison to placebo (SHR: $144 \pm 1 \mathrm{mmHg}$ ). In the kidney, CML staining was five-fold increased in proximal tubuli and as a thin endothelial cap in intrarenal arterioles in SHR (3.3 $\pm 0.6 \mathrm{AU})$, in comparison to $\mathrm{WKY}$ rats $(0.7 \pm 0.5 \mathrm{AU}$; $\mathrm{p}<0.01)$. As compared to untreated SHR, both losartan $(1.2 \pm 0.6 ; \mathrm{p}<0.01)$ and spironolactone $(2.0 \pm 0.6 ; \mathrm{p}<0.05)$, but not hydralazine $(3.0 \pm 0.4)$, decreased CML staining in proximal tubuli and intrarenal arterioles in SHR.

Conclusion: The enhanced blood pressure increase in young SHR was associated with an increase of CML in renal parenchym and endothelial cells and may contribute to development and complications of hypertension. Despite similar blood pressure lowering, the effect of losartan and spironolactone on CML reduction indicates renoprotection of angiotensin inhibition beyond blood pressure lowering. Supported by Diabetes Fonds Nederland

1137

Plasma levels of soluble receptor for advanced glycation endproducts (sRAGE) are associated with components of the metabolic syndrome: the CODAM study C. J. H. van der Kallen ${ }^{1}$, M. M. J. van Greevenbroek ${ }^{1}$, E. H. J. Jansen ${ }^{2}$, T. W. A. de Bruin ${ }^{1}$, E. J. H. Feskens ${ }^{3}$, C. D. A. Stehouwer ${ }^{1}$, C. G. Schalkwijk ${ }^{1}$;

${ }^{1}$ Internal Medicine, Maastricht University, ${ }^{2} \mathrm{Lab}$ for Toxicology, Pathology and Genetics, National Institute for Public Health and the Environment, Bilthoven, ${ }^{3}$ Human Nutrition, Wageningen University, The Netherlands.

Background and Aims: The receptor for advanced glycation end products (RAGE) has been implicated in diabetes, inflammation and vascular disease. Soluble (s) RAGE is a C-truncated secretory isoform of the RAGE, that may neutralize the AGEs-mediated damage by acting as a decoy. Recently, sRAGE has been identified as a potentially important factor in the metabolic syndrome and atherosclerosis. Therefore, we studied potential determinants of plasma sRAGE levels, with a focus on components 
of the metabolic syndrome, in individuals without and with type 2 diabetes, the CODAM cohort.

Materials and Methods: Plasma levels of sRAGE were measured with a commercially available Elisa (Quantikine, R\&D systems) in subjects with normal glucose tolerance (NGT, $\mathrm{n}=298$ ), impaired glucose tolerance (IGT, $\mathrm{n}=129$ ) and in type 2 diabetes (DM2, $\mathrm{n}=147)$. To evaluate the relation between the levels of sRAGE with components of the metabolic syndrome, linear regression analyses were performed.

Results: Individuals with IGT and DM2 showed lower plasma levels of sRAGE compared to NGT subjects (NGT: $1408 \pm 599 \mathrm{pg} / \mathrm{ml}$, IGT $1286 \pm 608 \mathrm{pg} / \mathrm{ml}$ and DM2 $1202 \pm$ $598 \mathrm{pg} / \mathrm{ml}$ ) after adjustment for age, gender and BMI (IGT vs NGT $\mathrm{p}<0.05$, DM2 vs NGT $\mathrm{p}<0.01)$. Moreover, gender (standardised (st) beta $0.27, \mathrm{p}<0.001$ ), HDL-cholesterol (st beta $-0.19, \mathrm{p}<0.001$ ), waist circumference (st beta -0.22 , $\mathrm{p}<0.001$ ), diastolic blood pressure (st beta $0.12, \mathrm{p}<0.05$ ) and fasting glucose (st beta $-0.12, \mathrm{p}<0.01$ ) were identified as independent factors associated with plasma sRAGE levels $\left(\mathrm{R}^{2}=0.15, \mathrm{p}<0.001\right)$ in linear regression analysis studying components related to metabolic syndrome (triglycerides, systolic blood pressure and age: not significant). Since waist was one of the strongest predictors of sRAGE, we added the adipokines resistin, adiponectin and leptin to the above described model. This improved the model $\left(\mathrm{R}^{2}=0.215, \mathrm{p}<0.001, \mathrm{p}\right.$ ( $\mathrm{F}$ change $\left.)<0.001\right)$ with significant independent contributions of HDL cholesterol (st beta $-0.21, \mathrm{p}<0.001$ ), waist circumference (st beta -0.23 , $\mathrm{P}<0.001$ ), gender (st beta $0.13, \mathrm{p}<0.05$ ), adiponectin (st beta $0.22, p<0.001$ ) and resistin (st beta $0.09, p<0.05$ ), but not of age, fasting glucose, triglycerides, diastolic and systolic blood pressure, and leptin.

Conclusion: Plasma sRAGE levels are decreased in IGT and DM2 subjects and are strongly related to components of the metabolic syndrome, especially adipocyte function. These data suggest that the sRAGE - RAGE axis is involved in components of the metabolic syndrome and its complications.

Supported by ZonNW 940-35-034 and DFN 98.901

\section{PS 101}

\section{Endothelium, oxidative stress and inflammation}

\section{8}

Diabetes-induced effects on endothelial function and the actions of nitric oxide

A. Soro-Paavonen ${ }^{1}$, W.-Z. Zhang ${ }^{2}$, K. Venardos ${ }^{2}$,

E. Jones ${ }^{3}$, J. Chin-Dusting ${ }^{3}$, M. T. Thomas ${ }^{1}$,

M. T. Coughlan ${ }^{1}$, D. Brasacchio ${ }^{4}$, M. E. Cooper ${ }^{1}$,

D. Kaye ${ }^{2}$, J. M. Forbes ${ }^{1}$;
${ }^{1}$ Diabetic Complications, ${ }^{2}$ Molecular Neurocardiology,

${ }^{3}$ Vascular Pharmacology, ${ }^{4}$ Epigenetics, Baker Heart Research Institute, Melbourne, Australia.

Background and Aims: Endothelial dysfunction with decreased NO (nitric oxide) bioavailability has been well documented in vivo in patients with both Type 1 and Type 2 diabetes. Impaired endothelium-dependent relaxation precedes both micro- and macrovascular complications. The aim of this study was to examine the endothelial function at two different stages (16 and 32 weeks) of diabetes in streptozotozin-induced diabetic rats, and to test whether diabetes induces changes in NO production or in the levels of oxidative stress or circulating advanced glycation endproducts. We also examined whether similar changes in $\mathrm{NO}$ synthase (NOS) activity or NO production occur in vitro in endothelial cells when exposed to either normoglycemic (NG) and hyperglycaemic (HG) conditions.

Materials and Methods: Endothelium-dependent and independent vasorelaxation in the rat aortic rings were determined by constructing concentration-response curves for acetylcholine (Ach) and sodium niroprusside (SNP). Plasma levels of arginine metabolites were determined by high-performance liquid chromatography (HPLC). Carboxymethyl lysine (CML) was determined in plasma, and levels of oxidative stress in aortic tissue. The specific uptake of radiolabeled L-arginine and NO production were measured in bovine aortic endothelial cells (BAECs) after 7-day treatment in $\mathrm{NG}$ or $\mathrm{HG}$ environment.

Results: At 16 weeks of diabetes, the diabetic rats presented significantly delayed vasodilatory response to Ach as compared to the non-diabetic control rats $\left(\right.$ Ach $\mathrm{EC}_{50}$ $-6.95 \pm 0.12$ vs $-7.37 \pm 0.20, P=0.048)$. At 32 weeks, the Ach curve was shifted to the right also in control animals and there were no significant between-group differences. Endothelium-independent vasodilatory responses to SNP were identical between controls and diabetics and showed no age-dependent change. The diabetic rats had significantly increased aortic intima-media thickness (IMT) at 16 weeks as compared to non-diabetic animals $(0.95 \pm$ $0.02 \mu \mathrm{m}$ vs $0.83 \pm 0.01 \mu \mathrm{m}, \mathrm{P}<0.001)$. Aortic superoxide production was increased in diabetic rats at 32 weeks (914 \pm 274 vs $235 \pm 37 \mathrm{U} / 5 \mathrm{mg}$ of tissue, $P=0.034$ in diabetics vs controls, respectively), and plasma CML at 16 weeks of diabetes $(705 \pm 114$ vs $305 \pm 26 \mu \mathrm{mol} / \mathrm{mol}$ lysine, $P=0.018)$. The diabetic animals had significantly decreased plasma levels of NO precursor L-arginine and of NG-hydroxy-Larginine (NOHA), indicating inactive NOS function and decreased NO synthesis. They had increased levels of citrulline and asymmetric NG,NG-dimethyl-L-arginine (ADMA), an endogenous inhibitor of NOS. In vitro, similar basal levels of NO production and L-arginine uptake were observed in the cells in both $\mathrm{HG}$ and NG conditions. 
However, insulin induced a significant increase in NO production in the HG-treated cells, suggesting an increased ATP-availability for the citrulline-to-arginine conversion in the HG environment in vitro.

Conclusion: Our results indicate decreased NO bioavailability in diabetic rats, potentially due to a blockade of the citrulline-arginine cycle. These results are in line with the observed decrease in the endothelium-dependent vasodilatation in these animals.

Supported by the Juvenile Diabetes Research Foundation $(J D R F)$, the National Health and Medical Research Council of Australia, the Academy of Finland, Paulo Foundation, Jalmari, Rauha and Ahokas Foundation

\section{9}

A new mechanism linking IAPP and high blood pressure M. El Assar ${ }^{1}$, S. Vallejo ${ }^{1}$, A. Novials ${ }^{2}$, R. Gomis ${ }^{3}$, L. Rodríguez-Mañas ${ }^{1,4}$;

${ }^{1}$ Unidad de Investigacion, Hospital Universitario de Getafe, Madrid, ${ }^{2}$ Investigación, Fundación Sardá Farriols, Barcelona, ${ }^{3}$ Endocrinology and Diabetes Unit, Hospital Clinic, IDIBAPS, Barcelona, ${ }^{4}$ Servicio de Geriatría, Hospital Universitario de Getafe, Madrid, Spain.

Background and Aims: IAPP has been involved in facilitating the development of High Blood Pressure (HBP), that is a frequent finding in patients with type 2 diabetes. There is very few data about the potential mechanisms by which IAPP could evoke an increase in HPB, being the stimulation of renin-angiotensin system the most known, although some data raise the possibility of the existence of other mechanism(s) in the development of HBP due to IAPP. The aim of this study was to test the interference with endothelium-dependent responses as an added potential mechanism for IAPP induced HBP.

Material and Methods: 15 week-old non-diabetic male Sprague-Dawley rats $(400-450 \mathrm{~g})$ were used. Rats were anaesthetised with $70 \mathrm{mg}$. kg-1 i.p. sodium pentobarbital and killed by bleeding. Aorta and mesentery were removed and placed in Krebs-Henseleit solution (KHS). For isometric tension recording both aortic segments (4-5 mm length) and segments of third branch mesenteric arteries $(2 \mathrm{~mm}$ length; mean internal diameter range between 200 and $400 \mu \mathrm{m}$ ) were mounted in an organ bath and on a small vessel myograph respectively according to a method described previously.

Relaxation to ACh were subsequently assessed in both experimental approaches by adding increasing concentrations of the drug $(10 \mathrm{nmol} / \mathrm{L}$ to $10 \mu \mathrm{mol} / \mathrm{L}$ in aorta; $1 \mathrm{nmol} /$ $\mathrm{L}$ to $10 \mu \mathrm{mol} / \mathrm{L}$ in mesenteric arteries). In other set of experiments the contractile responses to noradrenaline (NA) were evaluated by adding cumulative concentrations to the organ bath $(1 \mathrm{nmol} / \mathrm{L}-10 \mu \mathrm{mol} / \mathrm{L}$ in aorta; $10 \mathrm{nmol} / \mathrm{L}$ $-30 \mu \mathrm{mol} / 1$ in mesenteric arteries). In both set of experiments, in some cases, the curves were performed after incubating some segments for 20 minutes with IAPP ( 5 or $20 \mathrm{pmol} / \mathrm{L}$ ) and/or $100 \mu \mathrm{mol} / \mathrm{l} \mathrm{L} \mathrm{NG-nitro-L-arginine} \mathrm{me-}$ thyl ester (L-NAME).

Results: In both aortic and mesenteric segments the preincubation with 5 and $20 \mathrm{pmol} / 1$ of IAPP did not change the maximal responses to NA. Otherwise the responses induced by $\mathrm{ACh}$ were significantly impaired by the preincubation with IAPP $(20 \mathrm{pmol} / \mathrm{L})$ but not with $5 \mathrm{pmol} / \mathrm{L}$. L-NAME preincubation totally abolished the relaxant responses induced by $\mathrm{ACh}$ in aortic rings, and the presence of L-NAME plus IAPP $(20 \mathrm{pmol} / \mathrm{L})$ did not add any effect to the effect observed when segments were preincubated with L-NAME alone. In contrast to that observed in aorta, in microvessels L-NAME alone did not totally abolish relaxant responses to ACh but impaired them in a significant way, very close to the impairment produced by $20 \mathrm{pmol} / \mathrm{L}$ of IAPP. Otherwise no increased inhibitory effect on vascular relaxation was observed in the presence of L-NAME plus IAPP $(20 \mathrm{pmol} / \mathrm{L})$ in mesenteric microvessels.

Conclusions: We describe a new mechanism to explain the association between IAPP and HBP. Our findings support a direct effect of IAPP on vascular responses in macrovessels (aorta) and microvessels as well. This effect seems to be mediated by the interference of IAPP with the nitric oxide released by the endothelium.

Supported by FIS PI05/1920, FIS PI 04/1955 and RGDM G03/212

\section{0}

The role of oxidative stress in development of vascular insulin resistance in OLETF Rat, a model of type 2 diabetes

S.-M. Son, J. Park, Y. Kang, Y. Kim;

Endocrinology and Metabolism, Pusan National University School of Medicine, Busan, Republic of Korea.

Background and Aims: Reactive oxygen species was shown to induce vascular insulin resistance which is one of major risk factors in the development of vascular complications in diabetes, raising the possible involvement of alteration in insulin receptor substrate (IRS) proteins. This study was conducted to assess whether oxidative stress induced IRS protein degradation and enhanced serine phosphorylation, and to assess their functional importance in OLETF rats, an animal model of type 2 diabetes. 
Materials and Methods: We used aortas of OLETF rats and LETO rats as control to measure IRS-1 protein expression, its serine phosphorylation and downstream metabolic insulin effects were measured.

Results: Superoxide production was markedly increased in aortas of OLETF rats compared to LETO rats. Furthermore, aortas of OLETF rats contained significantly less total glutathione and more $8-\mathrm{OHdG}$ and nitrotyrosine, in comparison to controls. Aortas of OLETF rats exhibited decreased IRS-1 protein (but not IRS-1 mRNA and IRS-2 protein) expression, and increased phosphorylation of IRS1 at Ser 307 compared with LETO rats. Total protein ubiquitination was also increased in aortas of OLETF rats. In contrast, there were no significant differences in expressions of insulin receptor, PI3K, PDK, and Akt/PKB between two groups.

Conclusion: These findings suggest that oxidative stress induces insulin resistance in the vasculature of OLETF rats specifically through increased serine phosphorylation of IRS-1 and its degradation by a proteasome-dependent pathway, providing an alternative mechanism that may explain the association with hyperinsulinemia with diabetic vascular complications

\section{1}

HOMA IR and oxidative stress in non-diabetics

P. J. Kraml ${ }^{1}$, P. Syrovatka ${ }^{1}$, J. Potockova ${ }^{1}$, J. Crkovska ${ }^{2}$, L. Fialova ${ }^{2}$, M. Andel ${ }^{1}$;

${ }^{1}$ 2nd Dept. of Internal Medicine, ${ }^{2}$ Dept. of Medical Biochemistry, Charles University Prague, Czech Republic.

Background and Aims: Oxidative stress has been proposed as on of the key factors in pathogenesis of insulin resistance and type 2 diabetes mellitus. The aim of our study was to determine whether markers of oxidative stress are elevated in relation to higher HOMA IR already in healthy nondiabetics.

Materials and Methods: We examined 67 men (aged 3060 yrs.) with no history of atherosclerotic diseases (coronary heart disease, peripheral vascular disease, cerebrovascular stroke), no treatment for hypertension, heperlipoproteinemia or diabetes and with morning fasting venous plasma glucose below $6.0 \mathrm{mmol} / \mathrm{l}$. The cohort was divided into tertiles of HOMA IR (calculated from fasting glycemia and insulinemia). In subsequent analysis we compared groups from the lowest $(\mathrm{n}=23,47.99 \pm 9.051 \mathrm{yrs}$.) and highest $(\mathrm{n}=23,51.00 \pm 7.472$ yrs.; $\mathrm{P}=0.2220)$ tertiles. All individuals underwent physical and anthropometric examination, sonographic measurement of intima media thickness on common carotid artery (IMT) and endothelium dependent vasodilation on brachial artery (EDV). Morning fasting venous blood was collected for biochemical examination which included oxidized LDL (oxLDL), antibodies to oxLDL (aoxLDL), advanced products of protein oxidation (AOPP), lipid hydroperoxides (HPL), levels of retinol, tocopherol and total plasma antioxidation capacity (TAC). Concentrations of v.Wilebrand factor (vWF) and nitrites/nitrates were used as plasma markers of endothelial dysfunction.

Results: Subjects in the highest tertile of HOMA IR showed significantly higher levels of oxLDL $(49.09 \pm 8.051$ vs $60.39 \pm 7.308 \mathrm{mU} / \mathrm{l} ; \mathrm{P}<0.05)$, AOPP $(78.52 \pm 17.118$ vs $119.67 \pm 32.671 \mu \mathrm{mol} / \mathrm{l} ; \mathrm{P}<0.05)$ but surprisingly also of tocopherol $(16.56 \pm 2.02$ vs $19.54 \pm 1.876 \mathrm{mg} / \mathrm{l} ; \mathrm{P}<0.05)$ although the TAC was not significantly different between the compared groups $(0.12 \pm 0.013$ vs $0.12 \pm 0.011 \mathrm{mmol} / \mathrm{l}$; NS). Comparison of the highest vs lowest tertile groups revealed no significant difference in IMT $(0.57 \pm 0.119$ vs $0.61 \pm 0.144 \mathrm{~mm}$; NS), EDV (3.82 \pm 2.051 vs $2.99 \pm 1.516 \%$; NS) or vWF (121.59 \pm 38.890 vs $141.53 \pm 66.083 \%$; NS).

Conclusion: Higher HOMA IR is accompanied by elevation of certain oxidative stress parameters already in asymptomatic healthy nondiabetic men even before manifestation of endothelial dysfunction.

This study was supported by Internal Grant Agency No. NR/ 8529-3 and by Research Program No. MSM 0021620814

\section{2}

LPS as a mediator of sub-clinical inflammation in obese children and its' association with vascular injury and atherogenesis biomarkers

A. L. Harte ${ }^{1}$, C. M. Kusminski ${ }^{1}$, M. J. Hill ${ }^{1}$, L. Gilardini ${ }^{2}$, A. Girola ${ }^{2}$, N. F. da Silva ${ }^{1}$, L. Alberti ${ }^{2}$, C. Invitti ${ }^{2}$, S. Kumar ${ }^{1}$, P. G. McTernan ${ }^{1}$;

${ }^{1}$ Unit for Diabetes and Metabolism, University of Warwick, Coventry, United Kingdom, ${ }^{2}$ Unit for Metabolic Diseases and Diabetes, Istituto Auxologico Italiano, Milan, Italy.

Background: Obesity and its' later progression to Type 2 Diabetes Mellitus (T2DM) are both known to be associated with sub-clinical inflammation; although the underlying cause for this is unclear. However, studies suggest that bacterial endotoxin (LPS) derived from commensal bacteria in the human gastrointestinal tract may contribute directly to sub-clinical inflammation. Hence, with increasing adiposity the inflammatory response is exacerbated producing inflammatory adipocytokines, such as plasminogen activator inhibitor type-1 (PAI-1), IL-6 and TNF- $\alpha$. This study therefore investigated the pathogenesis of the metabolic syndrome in obese children and its' association with inflammation, with LERC approval. Therefore, for this study, we examined the role of endotoxaemia in childhood 
obesity through the application of multiplex cardiovascular disease (CVD) biomarker immunoassays to investigate the levels of a range of inflammatory and CVD risk markers.

Methods and Materials: Fasted serum was obtained from children with varying degrees of obesity (age \pm SD:13.9 \pm $2.3 \mathrm{yr} ; \mathrm{BMI} \pm \mathrm{SD}: 35.1 \pm 5.2 \mathrm{Kg} / \mathrm{m}^{2} ; \mathrm{n}=60$ ). All children underwent an OGTT and insulin resistance was measured by homeostasis model assessment (HOMA-IR) and insulin secretion by Stumvoll index (ISI). The vascular injury markers for these obese children were measured using a multiplex assay system that analysed interleukin - (IL-) $1 \beta$, 6,8 and 10 , interferon- $\gamma$ (IFN- $\gamma$ ), TNF- $\alpha$, monocyte chemotatic protein-1 (MCP-1), n-terminal brain natriuretic peptide (NT-proBNP), vascular endothelial growth factor (VEGF), plasminogen activator inhibitor 1 (PAI-1), Creactive protein (CRP), fibrinogen, serum amyloid A (SAA), serum amyloid P component (SAP), E-selectin, soluble intercellular adhesion molecule type 1 (sICAM-1), soluble vascular cell adhesion molecule type 1 (sVCAM-1), matrix metalloproteinase -9 (MMP9), myeloperoxidase lipid peroxide modified protein (MPO) and adiponectin.

Results: BMI correlated with several of the inflammatory markers (TNF- $\alpha, \mathrm{r}=0.331 *$; CRP, $\mathrm{r}=0.310^{*}$; fibrinogen, $\mathrm{r}=0.291^{*}$; MPO, $\left.\mathrm{r}=0.348^{* *},{ }^{*} \mathrm{p}<0.05,{ }^{* *} \mathrm{p}<0.01\right)$. However, endotoxin levels demonstrated a significant and positive correlation with the majority of markers for vascular injury and atherogenesis (TNF- $\alpha$, myeloperoxidase; $p<0.05$; PAI1 , matrix metalloproteinase- 9 , monocyte chemotactic protein-1, soluble intercellular adhesion molecule type-1, vascular endothelial growth factor; $p<0.01$ ). These relationships remained significant following adjustment for sex, BMI and HOMA-IR.

Conclusions: In summary, these data highlight that subclinical inflammation in childhood obesity and T2DM may be mediated by circulating endotoxin levels. Furthermore, that children as young as 11 exhibit the same inflammatory profiles as identified in obese adults and may, as a result of long-term sub-clinical inflammation, have increased risk in the pathogenesis of T2DM and CVD at a much earlier age. Supported by Wellcome Trust

\section{3}

Association between markers of subclinical inflammation, oxidative stress, endothelial dysfunction and metabolic parameters in impaired glucose regulation K. Farkas ${ }^{1}$, M. Herold ${ }^{2}$, E. Csöregh ${ }^{2}$, M. Sasvári ${ }^{3}$, A. Somogyi ${ }^{2}$, G. Jermendy ${ }^{1}$;

${ }^{1}$ III. Department of Medicine, Bajcsy-Zsilinszky Hospital, Budapest, ${ }^{2}$ II. Department of Medicine, ${ }^{3}$ Clinical and Experimental Research Institute, Semmelweis University, Budapest, Hungary.
Background and Aims: The aim of our study was to evaluate the associations between markers of subclinical inflammation and endothelial dysfunction - indicative of increased cardiovascular risk-, metabolic parameters and markers of oxidative stress in people with impaired glucose regulation (IGR).

Materials and Methods: The study included 26 subjects with IGR (15 with impaired glucose tolerance [IGT]/11 with impaired fasting glycaemia [IFG], age: IGT:53.06 \pm 3.10, IFG:46., $72 \pm 4.07$ years; body mass index [BMI]: IGT:29.24 \pm 1.17 , IFG:26.20 $\pm 1.12 \mathrm{~kg} / \mathrm{m} 2 ; \mathrm{x} \pm$ SEM) and 15 matched control subjects (C: age:49.60 \pm 3.06 years; BMI:26.27 $\pm 1.01 \mathrm{~kg} / \mathrm{m} 2$ ). Collection of fasting blood sample was followed by a $75 \mathrm{~g}$ oral glucose tolerance test and a second blood sampling 1 hour post load. White blood cell (WBC) count and high sensitivity C-reactive protein (hsCRP, immunoturbidimetry) were measured as markers of subclinical inflammation. Thiobarbituric acid reactive substances (TBARS, spectophotometry) and nitrotyrosine (NT, ELISA) were determined as markers of oxidative stress. Total antioxidant status (TAS, spectrophotometry) was used to assess the activity of the antioxidant defence system. Endothelial function was evaluated by determination of endothelium dependent (flow mediated - FMV) and independent vasodilation of the brachial artery by high resolution ultrasound in the fasting state and one hour post load.

Results: In addition to fasting blood glucose significant difference was found between the 3 groups in waist circumference (C:85.53 \pm 3.37 , IFG:88.82 \pm 3.99 , IGT:99.73 \pm $3.55 \mathrm{~cm} ; \mathrm{p}<0.05)$, waist/hip ratio (C:0.81 \pm 0.03 , IFG:0.87 \pm 0.02 , IGT:0.92 \pm 0.02$)$ and WBC count (C:6.13 \pm 0.23 , IFG:7.03 \pm 0.46 , IGT:7.90 $\pm 0.37 \mathrm{G} / 1)$. There was a trend of increasing hsCRP concentrations through the groups (C:1.31 \pm 0.23 , IFG: $1.97 \pm 0.54$, IGT: $2.65 \pm 0.38 \mathrm{mg} / \mathrm{l}$ ), but the difference did not reach the level of significance. Fasting FMV was in the normal range in all three groups. One hour following the glucose load significant differences could be observed in blood glucose (C:8.38 \pm 0.56 , IFG:10.56 \pm 0.43 , IGT: $12.10 \pm 0 ., 44 \mathrm{mmol} / \mathrm{l})$, TBARS (C:3.83 \pm 0.30 , IFG:5.76 \pm 0.58 , IGT: $6.00 \pm 0.49 \mu \mathrm{mol} / \mathrm{l}$ ), TAS (C:0.99 \pm 0.05, IFG:1.13 \pm 0.06 , IGT:1.22 $\pm 0.05 \mathrm{mmol} / \mathrm{l})$ and NT (C:16.84 \pm 7.89 , IFG:87.17 \pm 21.35 , IGT:168.54.06 \pm $39.55 \mathrm{nmol} / \mathrm{l}$ ) with a significant decrease of FMV only in the IGT group $(13.63 \pm 1.86$ to $5.81 \pm 1.78 \%)$. Significant positive correlation was found between WBC and TBARS $(\mathrm{r}=0.61)$, and NT $(\mathrm{r}=0.53)$ concentrations. hsCRP showed a positive correlation with insulin $(\mathrm{r}=0.43)$ and with 2 hour post load glucose $(r=0.45)$. Fasting TBARS was found to correlate with cholesterol $(\mathrm{r}=0.58)$ and triglyceride levels $(\mathrm{r}=0.47)$. Postload TBARS and glucose $(\mathrm{r}=0.54)$, and postload NT and glucose $(\mathrm{r}=0.47)$ concentrations positively correlated also. In the IGR subgroup $(\mathrm{n}=26) \mathrm{hsCRP}$ inversely 
correlated with HDL cholesterol $(r=-0.44)$ and NT positively correlated with TBARS ( $\mathrm{r}=0.47$ ).

Conclusion: In IGR altered levels of non-classical cardiovascular risk factors and markers of oxidative stress and endothelial dysfunction can be observed that are associated with metabolic parameters. According to the examined parameters subjects with IGT represent a higher risk group.

\section{4}

Urinary orosomucoid excretion is related to inflammation and signs of endothelial dysfunction in patients with type 2 diabetes

M. S. Christiansen ${ }^{1}$, E. Hommel ${ }^{2}$, J. Mølvig ${ }^{3}$,

B. K. Pedersen ${ }^{4}$, E. Magid ${ }^{1}$, B. Feldt-Rasmussen ${ }^{5}$;

${ }^{1}$ Department of Clinical Biochemistry, Amager Hospital, Copenhagen, ${ }^{2}$ Steno, Diabetes Center, Gentofte, Denmark, ${ }^{3}$ Department of Internal Medicine, Amager Hospital, Copenhagen, ${ }^{4}$ Department of Infectious Diseases, Rigshospitalet, Copenhagen, ${ }^{5}$ Department of Nephrology, Rigshospitalet, Copenhagen, Denmark.

Background and Aims: Increased levels of inflammatory proteins and pro-inflammatory cytokines are associated with increased cardiovascular risk. Endothelial dysfunction is related to the development of atherosclerosis and predicts cardiovascular mortality in patients with type 2 diabetes. We have previously shown that increased urinary orosomucoid excretion rate (UOER) is an independent predictor of cardiovascular mortality in patients with type 2 diabetes. The aim of the present study was to evaluate markers of inflammation and endothelial dysfunction in patients with type 2 diabetes and normal or increased UOER.

Materials and Methods: We studied 41 patients with type 2 diabetes (17 with normal UOER and 24 with increased UOER; cut-off value of UOER: $2.04 \mu \mathrm{g} / \mathrm{min}$ ) and 21 healthy subjects. Urinary orosomucoid, S-Orosomucoid and S-C-Reactive protein (CRP) were measured by immunoturbidimetry. P-Interleukin 6 (IL-6), P-Tumor Necrosis Factor-alfa (TNF- $\alpha)$, P-tissue Plasminogen Activator (tPA), P-soluble Intercellular Adhesion Molecule-1 (sICAM) and P-soluble Vascular Cell Adhesion Molecule1 (sVCAM) were measured by ELISA. Endothelial function was also evaluated by measurement of the diameter of the brachial artery by external ultrasound at baseline and after flow-mediated vasodilatation versus post-nitroglycerin (NTG) vasodilatation using sublingual nitroglycerin.

Results: Mean (SD) age was 55 (9.4) years. In the 2 diabetic patient groups mean (SD) HbAlc was $8.4(1.7) \%$ and median (IQR) duration of diabetes was $5.6(3.3-8.1)$ years. Analysis of variance revealed that patients with diabetes had higher concentrations of S-Orosomucoid $(\mathrm{p}<0.001), \quad$ S-CRP $(\mathrm{p}<0.001)$, P-IL-6 $(\mathrm{p}<0.001)$, P-tPA $(p<0.001)$ and P-sICAM $(p<0.003)$ compared with healthy controls. Patients with increased UOER had higher concentrations of S-Orosomucoid $(p<0.001)$, S-CRP $(\mathrm{p}=0.001)$, P-IL-6 $(\mathrm{p}<0.001)$, P-tPA $(\mathrm{p}<0.001)$ and PsICAM $(p<0.002)$ compared with healthy controls and higher P-IL-6 $(\mathrm{p}<0.04)$ than patients with normal UOER. In multiple regression analysis UOER independently predicted increasing values of S-Orosomucoid (OR $(95 \% \mathrm{CI}) 1.52$ (1.23-1.88)), S-CRP (1.66 (1.24-2.22)), P-sICAM (1.38 (1.09-1.76)) and P-tPA (1.75 (1.28-2.39)). The analyses were adjusted for age, sex, systolic blood pressure, body mass index, HbAlc, serum cholesterol and urinary albumin excretion. There were no differences in P-TNF- $\alpha$, PsVCAM, flow-mediated vasodilatation or post-NTG vasodilatation between the three subgroups.

Conclusion: We conclude that patients with increased UOER and type 2 diabetes have a sub-clinical increased level of chronic inflammation and signs of endothelial dysfunction compared with healthy controls. Furthermore UOER is independently related to increasing values of markers of inflammation. Thus the pathophysiological link between increased UOER and cardiovascular mortality may be explained by vascular inflammatory activity and endothelial cell dysfunction in patients with type 2 diabetes.

Supported by Danish Kidney Foundation, Novo Nordisk Foundation, Johan and Lise Boserup Foundation, Bernhard and Marie Klein Foundation for Diabetes Research and Foundation of the Copenhagen Hospital Corporation.

\section{5}

Elevated systemic chemokine concentrations precede the incidence of coronary heart disease and type 2 diabetes: results from the MONICA/KORA Augsburg study, 1984-2002

C. Herder ${ }^{1}$, J. Baumert ${ }^{2}$, B. Thorand ${ }^{2}$, S. Martin ${ }^{1}$, H. Löwel ${ }^{2}$, C. Meisinger ${ }^{2}$, T. Illig $^{2}$, H. Kolb ${ }^{1}$, W. Koenig ${ }^{3}$; ${ }^{1}$ German Diabetes Clinic, Düsseldorf, ${ }^{2}$ Institute of Epidemiology, GSF, Neuherberg, ${ }^{3}$ Department Internal Medicine II - Cardiology, University of Ulm Medical Center, Germany.

Background and Aims: There is evidence from clinical and preclinical studies that chemokines may be involved in leukocyte infiltration into adipose tissue and in the pathogenesis of coronary heart disease (CHD) and type 2 diabetes (T2D). The aim of this study was to test the hypothesis that elevations of systemic levels of the chemokines MCP-1/ CCL2 (monocyte chemoattractant protein-1), IL-8/CXCL8 (interleukin-8) and IP-10/CXCL10 (interferon- $\gamma$-inducible protein-10) precede the incidence of CHD and T2D. 
Materials and Methods: Baseline serum concentrations of MCP-1, IL-8 and IP-10 were measured using a Luminex assay in a case-cohort design based on data from 381 individuals with and 1977 individuals without incident CHD and 526 individuals with and 1695 individuals without incident T2D from the prospective, populationbased MONICA/KORA Augsburg study (1984-2002). The mean follow-up time was 11 years.

Results: Baseline serum levels of MCP-1, IL-8 and IP-10 were higher in $\mathrm{CHD}$ and $\mathrm{T} 2 \mathrm{D}$ cases compared to the respective non-cases ( $\mathrm{P} \leq 0.001$ for all chemokines). After multivariable adjustment for other risk factors, individuals with concentrations of all three chemokines above the median level (compared to those subjects with all concentrations $\leq$ median) had no significantly increased CHD risk (hazard ratio 1.32 (95\% confidence interval 0.88-2.00)), whereas the risk of incident T2D was significantly elevated (HR 1.79 (95\% CI 1.18-2.72)).

Conclusion: Increased serum concentrations of the chemokines MCP-1, IL-8 and IP-10 are associated with incident CHD and incident T2D. This association appears more pronounced for T2D, and our data do not support the hypothesis that these chemokines are also independent risk factors for incident CHD.

Supported by Deutsche Forschungsgemeinschaft (German Research Foundation), European Foundation for the Study of Diabetes

\section{6}

P66shc and oxidative stress in the physiopathology of type 2 diabetes complications

E. Pagnin, E. Iori, A. Gallo, L. A. Calò, A. Avogaro;

Dep. Clinical and Experimental Medicine, University of Padova, Italy.

Background and Aims: Oxidative stress plays an important role in cardiovascular dysfunction and atherogenesis. This is of particular interest in diabetes, a condition characterized by oxidative stress and increased prevalence of cardiovascular disease. The major role played by p66shc in oxidative stressrelated response has been established by the resistance to and reduction of oxidative stress and prolonged lifespan of p66shc knockout mice. Recently we have demonstrated for the first time in type 2 diabetic patients an increase of mononuclear cell p66shc gene expression compared to healthy controls. Our results in patients with type 2 diabetes joined with those described in literature obtained in vitro and in animal models suggest a possible involvement of p66shc in the cellular and molecular mechanisms responsible of the dangerous oxidative stress mediated cardiovascular complications in type 2 diabetes.
In this study we have evaluated in skin fibroblasts of type 2 diabetic patients compared with healthy controls the effect of p66shc gene silencing on the production of reactive oxygen species (ROS).

Materials and Methods: After incubation with insulin (100 nM), ROS production was determined in human fibroblasts obtained from skin biopsies with a fluorescent probe (TEMPO-9-AC). p66shc gene silencing was performed using siRNA methodology.

Results: Insulin induced ROS production was significantly higher in fibroblasts from type 2 diabetic patients than in those from healthy controls $(25,95 \pm 9,97$ vs $21,21 \pm 8,19$ arbitrary unit, a.u., $\mathrm{p}<0,02)$. p66shc silencing didn't change significantly ROS production in healthy controls $(20,30 \pm$ 7,01 vs $21,21 \pm 8,19$ a.u., n.s.), while it induced a significantly decrease of ROS production in type 2 diabetic patients $(18,73 \pm 8,42$ vs $25,95 \pm 9,95$ a.u., $p<0,01)$.

Conclusion: Our results not only confirm the presence of increased oxidative stress in patients with type 2 diabetes, but, also, demonstrate in these patients an essential role played by p66shc in its induction. Since p66shc silencing reduces insulin induced fibroblasts ROS production. This is the first demonstration of a direct involvement of p66shc in insulin induced oxidative stress in type 2 diabetes which could give useful information for a possible therapeutic target in diabetic complications.

1147

Short-term intravenous insulin infusion and endothelial function in poorly controlled type 2 diabetes patients M. Saryusz-Wolska ${ }^{1}$, L. Czupryniak ${ }^{1}$, M. Pawlowski ${ }^{1}$, A. Borkowska ${ }^{1}$, P. Grzelak ${ }^{2}$, L. Stefanczyk ${ }^{2}$, J. Loba ${ }^{1}$; ${ }^{1}$ Diabetology \& Metabolic Diseases Dept, ${ }^{2}$ Radiology Dept, Medical University of Lodz, Lodz, Poland.

Background and Aims: Type 2 diabetes mellitus is associated with endothelial dysfunction, which is particularly enhanced at the stage of poor glycemic metabolic control. The study aimed at assessment of the effect of short-term continuous intravenous insulin infusion (IVII) on endothelial function and insulin resistance-related markers.

Materials and Methods: The study group consisted of 22 type 2 diabetes patients (mean age $51.8 \pm 17.0$ years, BMI $28.5 \pm 2.1 \mathrm{~kg} / \mathrm{m}^{2}$, mean HbAlc $10.6 \pm 1.8 \%$, mean fasting plasma glucose [FPG] $196 \pm 34 \mathrm{mg} / \mathrm{dl}$, mean diabetes duration $8.3 \pm 2.7$ years). IVII consisted of basal infusion rate of mean $1.9 \pm 0.5 \mathrm{IU} / \mathrm{h}$ and three mealtime $90-\mathrm{min}$ boluses (mean 14 \pm 3 IU per bolus). Mean IVII duration was $4 \pm 1.4$ days. Fasting serum soluble vascular cell adhesion molecule (sVCAM), soluble intercellular adhesion molecule (sICAM), sE-selectin, free fatty acids (FFA), adipo- 
nectin, tumor necrosis factor- $\alpha$ (TNF- $\alpha$ ) were measured with ELISA method immediately prior to the beginning and after the completion of IVII.

Results: After IVII mean FPG was $121 \pm 17 \mathrm{mg} / \mathrm{dl}(\mathrm{p}<0.01$ vs baseline). Before and after IVII serum sVCAM concetrations were $695 \pm 127$ and $685 \pm 101 \mathrm{ng} / \mathrm{ml}(\mathrm{p}>0.05)$, sICAM $347 \pm 90$ and $330 \pm 117 \mathrm{ng} / \mathrm{ml}(\mathrm{p}>0.05)$, sE-selectin $75.4 \pm 50.7$ and $64.9 \pm 49.5 \mathrm{ng} / \mathrm{ml}(\mathrm{p}>0.05)$, FFA $0.69 \pm 0.48$ and $0.53 \pm 0.27 \mathrm{mmol} / \mathrm{l}(\mathrm{p}>0.05)$, adiponectin $1877 \pm 2357$ and $1366 \pm 964 \mathrm{ng} / \mathrm{ml}(\mathrm{p}>0.05)$, TNF- $\alpha 31.3 \pm 47.7$ and $11.1 \pm$ $17.1 \mathrm{pg} / \mathrm{ml}(\mathrm{p}>0.05)$, respectively.

Conclusion: Despite marked improvement of glycemic control short-term continuous IVII in type 2 diabetes does not significantly affect endothelial function or insulin resistance-related markers, although apparent tendency towards less impaired endothelial function was noted. Upon our results it may be suggested that achieving improvement of endothelial function in type 2 diabetes patients requires longer duration of hypoglycemic treatment than it is necessary to achieve good glycemic control. However, it may also be speculated that it is solely duration of good glycemic control and not insulin treatment itself which is essential for improving endothelial function.

Supported by Medical University Grant No 502-18-306

\section{PS 102}

\section{Pancreas, liver and metabolic syndrome}

\section{8}

Pancreatic fat content, measured by proton-MRS in type 2 diabetes is associated with $\beta$ cell failure M. E. Tushuizen ${ }^{1}$, M. C. Bunck ${ }^{1}$, P. J. W. Pouwels ${ }^{2}$, J. T. M. van Waesberghe ${ }^{3}$, R. J. Heine ${ }^{1}$, M. Diamant ${ }^{1}$; ${ }^{1}$ Department of Endocrinology/Diabetes Centre, ${ }^{2}$ Department of Physics \& Medical Technology, ${ }^{3}$ Department of Radiology, VU University Medical Centre, Amsterdam, The Netherlands.

Background and Aims: Insulin resistance and central obesity lead to increased lipolysis with high flux of nonesterified fatty acids (NEFA) from adipose tissue and elevated hepatic production of triglyceride (TG)-rich particles. Due to the augmented lipid load in the presence of impaired glucose utilization, non-adipose tissues, including the pancreatic islets, accumulate TG. Excessive TG deposition in pancreatic islets enlarges the intracellular pool of fatty acyl-CoA, thereby providing substrate for nonoxidative metabolic pathways leading to oxidative stress and apoptosis, which, in susceptible subjects ultimately causes $\beta$-cell dysfunction (lipotoxicity). However, in human type 2 diabetes (DM2) pancreatic lipid accumulation in vivo and its association with $\beta$-cell function was never reported. Proton magnetic resonance (MR) spectroscopy may be used to quantify lipid content.

Materials and Methods: We measured pancreatic fat content in the fasting state by localized proton MRspectroscopy in 4 DM2 (only oral blood-glucose-lowering therapy or diet, no TZDs or insulin) and 4 healthy agematched males. Oblique voxels $(10 \times 10 \times 20 \mathrm{~mm})$ were placed in the pancreas, and 8 spectra were acquired and analysed separately. Occasionally, spectra showed dramatic increase of lipid signals, probably due to sudden deep breathing of the subject. These spectra were removed, and only spectra from voxels within the pancreas were subsequently used in the analysis of lipid versus water area. To estimate pancreatic $\beta$-cell function, we performed an oral glucose tolerance test (OGTT) and calculated insulinogenic index adjusted for insulin sensitivity $(\triangle \mathrm{I} 0$ 30/AG0-30/HOMA-IR).

Results: Patients (mean age 60 (SE 4.2) yrs; HbAlc 7.7 $(0.8) \%$ ) versus healthy subjects did not differ in age, BMI, and waist. Mean pancreatic fat content was $42 \%$ in patients (range $9-115 \%$ ) and $12 \%$ in controls (range 3-22\%). $\beta$-cell function was impaired in patients (16.3 (5.4)) compared to controls (61.2 (11.5)). Pancreatic fat content was negatively associated with $\beta$-cell function ( $r=-0.59, P=0.12$ ).

Conclusion: These preliminary results suggest that pancreatic fat content is elevated in subjects with DM2 relative to healthy controls and associated with decreased $\beta$-cell function. This finding may lead to future studies that should elucidate whether antisteatotic therapies targeting pancreatic fat accumulation indeed prevent DM2 in highrisk individuals.

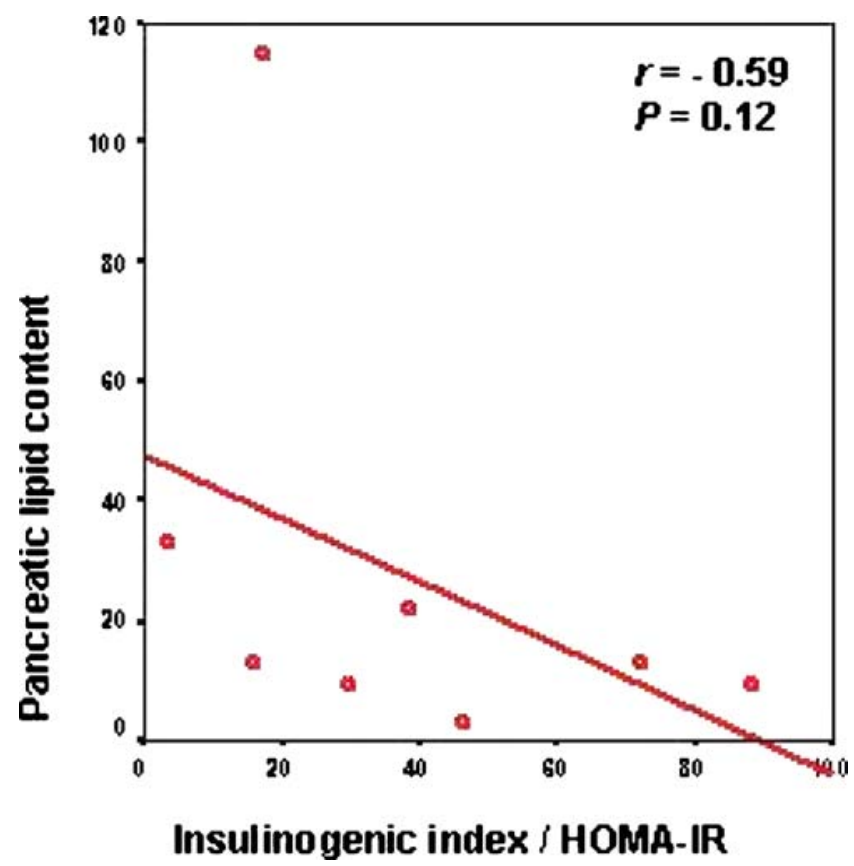




\section{9}

Screening for advanced fibrosis using non-invasive fibrosis biomarkers (FibroTest-FibroSure) in $\mathbf{7 8 9}$ diabetic patients

S. Jacqueminet ${ }^{1}$, R. Morra ${ }^{2}$, M. Munteanu ${ }^{3}$, L. Devers ${ }^{4}$, D. Messous ${ }^{5}$, M. Bernard ${ }^{5}$, S. Jeanne ${ }^{6}$, C. Sachon ${ }^{6}$,

A. Heurtier ${ }^{6}$, D. Bonnefont-Rousselot ${ }^{5}$, A. Mercadier ${ }^{4}$, F. Imbert-Bismut ${ }^{5}$, V. Ratziu ${ }^{7}$, A. Grimaldi ${ }^{6}$, T. Poynard ${ }^{7}$; ${ }^{1}$ Diabetology, Hopital Pitié-SalpÁtrière, Paris, ${ }^{2}$ Biochemistry, ${ }^{3}$ Hepatology, Biopredictive, Paris, ${ }^{4}$ Transfusional Unit, ${ }^{5}$ Biochemistry, ${ }^{6}$ Diabetology, ${ }^{7}$ Hepatology, Hopital PitiéSalpÁtrière, Paris, France.

Background and Aims: Type 2 diabetic patients (DI) vs non-DI have higher risk of non-alcoholic fatty liver disease (NAFLD) and liver cancer death. It is impossible to perform liver biopsy in such number of DI to identify advanced liver fibrosis (few septa F2, many septa F3, cirrhosis F4: F2F3F4). The aim was to identify F2F3F4 using non-invasive fibrosis biomarkers, FibroTest (FT), previously validated in chronic hepatitis $\mathrm{C}, \mathrm{B}$, alcoholic and non-alcoholic steatosis.

Materials and Methods: Consecutive DI seen in a diabetes unit, (HCV HBV HIV neg), were prospectively included, as well as a prospective control group of blood donors (BD). Security algorithms permitted to exclude high-risk (HR) profiles of false negative/positive (HRFNFP)due to extreme values of components of FibroTest (alpha2macroglobin, haptoglobin, apolipoproteinA1, GGT, bilirubin). Analysis used univariate comparison with BD matched for gender, age, alcohol consumption and BMI, and multivariate logistic regression.

Results: From 1,994 subjects pre-included, 217 were excluded for HRFNFP, HCV, HBV, HIV and 1,777 were included: 519 type 2 DI, 237 type 1 DI, 33 other DI and 988 BD. $53 \%$ DI were male, mean age 55 yrs, $67 \%$ DI type 2; $83 \%$ had fasting glucose $\geq 6.1 \mathrm{mmol} / \mathrm{L}, 69 \%$ glycohaemoglobin $\geq 7.5 \%, 40 \%$ cholesterol $\geq 2.0 \mathrm{~g} / \mathrm{L}, 27 \%$ triglycerides $\geq 1.5 \mathrm{~g} / \mathrm{L}, 52 \% \mathrm{BMI} \geq 27,47 \%$ arterial hypertension, $58 \%$ no alcohol consumption, $40 \%<50 \mathrm{~g} / \mathrm{d}$, and $2 \%>50 \mathrm{~g} / \mathrm{d}$. BD were younger $(36 \mathrm{yr})$, had lower BMI $(\mathrm{p}<0.0001)$ and had more alcohol consumption. 239/584 (41\%) DI had more than $30 \%$ steatosis detected by non-invasive biomarkers of steatosis (SteatoTest) versus 11/222 (5\%) BD $(\mathrm{p}<0.0001)$. 41/584 (7\%) DI had NASH (non-alcoholic steatohepatitis) detected by non-invasive biomarkers (NashTest) versus 0/ $222 \mathrm{BD}(\mathrm{p}=0.0006)$.

F2F3F4 were identified by FibroTest in 50/519 (9.1\%) of type $2 \mathrm{DI}$, including $23 \mathrm{~F} 2,15 \mathrm{~F} 3$ and $12 \mathrm{~F} 4$, vs none F2F3F4 among the $988 \mathrm{BD}(\mathrm{P}<0.0001) .9 .1 \%$ type $2 \mathrm{DI}$ had F2F3F4 vs $0.8 \%$ DI type $1(\mathrm{p}<0.0001)$. Independent significant factors associated with $\mathrm{F} 2 \mathrm{~F} 3 \mathrm{~F} 4$ in multivariate regression analysis were type 2 DI $(\mathrm{OR}=5.4 \mathrm{p}=0.03)$, AST $\geq 50 \mathrm{IU} / 1(\mathrm{OR}=9.0 \mathrm{p}=027)$, age $\geq 55 \mathrm{yrs}(\mathrm{OR}=6.6 ; \mathrm{p}=0.009)$ and sex $(\mathrm{OR}=4.9 ; \mathrm{p}=0.002)$.

$71 \%$ F2F3F4 DI had steatosis $>30 \%$ at SteatoTest versus $57 \%$ DI with minimal fibrosis (NS). 22\% F2F3F4 DI had NASH by NashTest versus $9 \%$ DI with minimal fibrosis $(\mathrm{p}=0.01)$.

After exclusion of 170 not-matched type 2 DI, there were still 8.1\% (28/349) F2F3F4 DI versus $0 \%$ BD $(\mathrm{P}<0.0001) .35 / 50$ F2F3F4 DI, had ALT $<50$ IU/L, 18/50 GGT $<50$ IU/L, 16/50 both normal, 21/50 one of them and 13/50 both elevated. Only 2 DI had overt clinical-biological signs of cirrhosis attributable to an ancient heavy alcohol consumption.

Conclusion: The prevalence of advanced fibrosis (F2F3F4), estimated by FibroTest, is very high (9.1\%) in type 2 DI followed in a tertiary center. This study strongly suggests that non-invasive biomarkers could be very useful for the screening of advanced fibrosis in type 2 DI to prevent liver mortality.

\section{0}

Apolipoprotein-AII, insulinoresistance and steatosis in hepatitis $C$ virus-infected patients

J.-M. Petit ${ }^{1}$, A. Minello ${ }^{2}$, L. Duvillard ${ }^{3}$, V. Jooste ${ }^{2}$,

H. Devilliers ${ }^{1}$, V. Texier ${ }^{1}$, S. Baillot-Rudoni ${ }^{1}$, J.-M. Brun ${ }^{1}$, B. Verges ${ }^{1}$, P. Hillon ${ }^{2}$;

${ }^{1}$ Diabetologie, ${ }^{2}$ Hepatologie, ${ }^{3}$ Biochimie, CHU du Bocage, Dijon, France.

Background and Aims: The mechanisms underlying steatosis during hepatitis $\mathrm{C}$ virus $(\mathrm{HCV})$ infection are complex and multifactorial. A new pathophysiological mechanism of viral-induced liver steatosis was described for the core protein of HCV. It has been shown that the $\mathrm{HCV}$-core protein reduces the activity of microsomal triglyceride transfer protein (MTP) and could lead to steatosis in $\mathrm{HCV}$-infected patients. HCV-core protein markedly reduced hepatic microsomal triglyceride transfer protein activity, thereby interfering with VLDL assembly and secretion. Transgenic mice expressing HCV-core protein alone displayed decreased triglyceride secretion, whereas secretion was restored to normal in mice expressing both HCV-core protein and human apolipoprotein-AII (apo A-II). In consequence, it has been suggested that apoAII could be a protective factor against HCV steatosis. One the other hand, it has been demonstrated that increasing plasma concentrations of mouse apoA-II in transgenic mice produced several aspects of the insulin resistance (IR) syndrome including hypertriglyceridemia, obesity, hyperleptinemia and IR. In the same way, some data suggested that the ApoA-II gene is implicated in visceral fat 
accumulation and metabolism of triglyceride-rich lipoproteins. Both visceral obesity and insulin resistance are also implicated in the pathogenesis of HCV-steatosis. The aim of our study was to investigate the role of apo-AII in HCVrelated steatosis.

Materials and Methods: Sixty-five consecutive untreated chronic HCV patients hospitalised for liver biopsy were included for this study to assess the effects of apo-AII, body mass index (BMI), age, insulin sensibility (HOMA), and leptin level on steatosis.

Results: Steatosis was observed in $36(55.3 \%)$ patients. Apo-AII was significantly associated with HOMA $(\mathrm{r}=0.28$; $\mathrm{P}=0.02)$ and with leptin concentrations $(\mathrm{r}=0.45 ; \mathrm{P}=0.0002)$. In univariate analyses, age $(46.6+/-11.5$ vs $37.6+/-12.2$, $\mathrm{p}=0.005)$, BMI $(26.6+/-4.8$ vs $22.8+/-2.8, \mathrm{p}=0.001)$, increased leptin level $(16.6+/-15.4 \mathrm{vs} 11.1+/-11.3 \mu \mathrm{g} / \mathrm{ml}$, $\mathrm{p}=0.05)$, increased HOMA $(2.6+/-1.6$ vs $1.4+/-0.8$, $\mathrm{p}=0.001)$, and increased apo-AII concentration $(0.42+/-$ 0.07 vs $0.36+/-0.10, p=0.01$ ) were associated with steatosis. In multivariate analysis, steatosis was significantly associated with apo-AII concentration, age, gender and BMI whereas steatosis was not associated with leptin, and HOMA.

Conclusion: At the opposite of previous hypothesis, ApoAII is not a protective factor against HCV-steatosis but is significantly associated with the development of liver steatosis. The fact that the plasma levels of apo-AII correlate with HOMA and leptin levels in HCV-infected subjects suggests that apo-AII may contribute to hepatic steatosis progression probably in relationship to visceral obesity, IR or metabolism of triglyceride-rich lipoproteins. These preliminary data need further investigations.

Supported by PHRC 2001

\section{1}

Endothelial function in non-diabetes subjects with nonalcoholic fatty liver disease

E. Szymanska-Garbacz ${ }^{1}$, L. Czupryniak ${ }^{1}$, M. Saryusz-Wolska ${ }^{1}$, M. Jablkowski ${ }^{2}$, J. Bialkowska ${ }^{2}$, M. Pawlowski ${ }^{1}$, A. Borkowska ${ }^{1}$, P. Grzelak ${ }^{3}$, L. Stefanczyk ${ }^{3}$, J. Loba ${ }^{1}$;

${ }^{1}$ Diabetology \& Metabolic Disease Dept, ${ }^{2}$ Infectious Diseases Clinic, ${ }^{3}$ Radiology Dept, Medical University of Lodz, Lodz, Poland.

Background and Aims: Non-alcoholic fatty liver disease (NAFLD) is associated with insulin resistance and glucose intolerance. However, whether NAFLD subjects are at increased risk of vascular injury is unclear. The study aim was to assess endothelial function in non-diabetes subjects with NAFLD.
Materials and Methods: Study group consisted of 30 subjects (mean age $38 \pm 9$ years, body mass index $27.2 \pm$ $3.2 \mathrm{~kg} / \mathrm{m} 2$ ) with mildly elevated (up to 3 times over the upper reference limit) aspartate and alanine aminotransferases (AST and ALT) and 'bright' liver in ultrasound examination, without history of hepatitis viral infections, cardiovascular disease or diabetes. The controls were age-, gender- and body weight matched 20 subjects. All subjects had oral glucose tolerance test (OGTT) performed. Insulin resistance parameters including HOMA index were assessed and plasma endothelial function markers were measured in a fasting state.

Results: AST levels in the study group and controls were $76 \pm 22$ and $23 \pm 9 \mathrm{IU} / 1(\mathrm{p}<0.01)$, ALT $66 \pm 15$ and $21 \pm 9 \mathrm{IU} / 1$ $(\mathrm{p}<0.01)$, respectively. In the study group and control subjects plasma glucose at $0,60,120$ and 180 min OGTT were: $102 \pm 36,171 \pm 53,127 \pm 45,88 \pm 32$ and $82 \pm 24,136 \pm 42,101 \pm$ $22,73 \pm 18 \mathrm{mg} / \mathrm{dl}(\mathrm{p}<0.05)$, and plasma insulin 16.5 \pm 7.6 , $93.0 \pm 56.7,62.7 \pm 43.1,21.5 \pm 17.8$ and $7.1 \pm 3.2,20.3 \pm 7.3$, $15.3 \pm 7.7,10.1 \pm 5.3 \mathrm{mIU} / 1(\mathrm{p}<0.05)$, respectively. HOMA index was $4.7 \pm 3.5$ and $1.4 \pm 0.4$, respectively $(p<0.01)$. Fasting plasma adiponectin in the study group and controls was $501 \pm 209$ and $1450 \pm 423 \mathrm{ng} / \mathrm{ml}(\mathrm{p}<0.01)$, tumor necrosis factor $\alpha$ $18.7 \pm 23.9$ and $9.3 \pm 8.7 \mathrm{pg} / \mathrm{ml}(\mathrm{p}<0.01)$, free fatty acids $0.84 \pm 0.93$ and $0.36 \pm 0.13 \mathrm{mmol} / 1 \quad(\mathrm{p}<0.01)$, soluble vascular cell adhesion molecule (sVCAM) $2316 \pm 1198$ and $1363 \pm 589 \mathrm{ng} / \mathrm{ml}(\mathrm{p}<0.01)$, soluble intercellular adhesion molecule (sICAM) $559 \pm 290$ and $202 \pm 78 \mathrm{ng} / \mathrm{ml}(\mathrm{p}<0.01)$, sE-selectin $71.8 \pm 26.5$ and $27.4 \pm 5.3 \mathrm{ng} / \mathrm{ml} \quad(\mathrm{p}<0.01)$, respectively.

Conclusion: Individuals with NAFLD present with marked insulin resistance and mild glucose intolerance as well as signs of apparent endothelial dysfunction. NAFLD thus might be associated with increased risk of vascular injury; this risk is present before the development of overt diabetes.

Supported by Medical University Grant No 502-18-306

1152

Lipoprotein particle distribution and size, insulin resistance and the metabolic syndrome in Alaska Eskimos. The GOCADAN Study

A. A. Rivellese ${ }^{1}$, L. Patti ${ }^{1}$, G. Annuzzi ${ }^{1}$, G. Riccardi ${ }^{1}$, O. Vaccaro ${ }^{1}$, J. Zhu ${ }^{2}$, D. Kaufman ${ }^{2}$, B. V. Howard ${ }^{2}$;

${ }^{1}$ Dept Clinical \& Experimental Medicine, Federico II University Medical School, Napoli, Italy, ${ }^{2}$ Department of Epidemiology and Statistics, MedStar Research Institute, Hyattsville, MD, United States.

Background and Aims: Insulin resistance is associated with a dyslipidemia characterized by increased VLDL, 
lower HDL and a preponderance of small-dense LDL particles. The former two abnormalities are included in the definition of metabolic syndrome. Within each lipoprotein class there is a particle size and density heterogeneity, whose relationship with insulin resistance and metabolic syndrome has been only partly investigated. Recently, Nuclear Magnetic Resonance (NMR) provides an advanced, rapid and cost-effective method to measure lipoprotein distribution and size in large population samples. Therefore, the aim of this study was to determine how lipoprotein subclass distribution and size are related to insulin resistance and metabolic syndrome in a population based sample of Alaska Eskimos over a wide age range.

Materials and Methods: In 1121 adult (age $\geq 18$ yrs) men and women, VLDL, LDL, HDL particles distribution and size were evaluated by NMR and related to the presence of metabolic syndrome (ATP III criteria) and to the level of insulin resistance (HOMA-IR index).

Results: Subjects with metabolic syndrome $(n=157)$, compared to those without $(\mathrm{n}=964)$, were characterized, independently of gender, age, body mass index, C-reactive protein, current and ex smoking, by: 1) a significant, higher percentage of large/intermediate VLDLs $(\mathrm{p}=0.0001)$ and lower percentages of large/intermediate LDL $(p=0.0001)$ and HDL particles $(\mathrm{p}=0.0001), 2)$ a significant, higher VLDL size $(\mathrm{p}=0.0001)$ and lower sizes of both LDL and HDL (both $\mathrm{p}=0.0001$ ). Dividing the whole population according to tertiles of HOMA-IR, percentage of large/ intermediate VLDL was significantly higher $(\mathrm{p}=0.0008$ and 0.02 , respectively) with increasing HOMA-IR tertile, while percentages of large/intermediate LDL and HDL particles were significantly lower ( $\mathrm{p}=0.02$ and 0.0009 , respectively). VLDL size significantly increased with increasing of HOMA-IR tertile, while LDL and HDL sizes were significantly decreased (all $\mathrm{p}=0.0001$ ).

Conclusion: In this population, both insulin resistance and metabolic syndrome are characterized by a complex spectrum of VLDL, LDL, and HDL distribution abnormalities that are independent of obesity, age and other CVD risk factors.

Supported by U01 HL064244-05

\section{3}

Circulating $\mathrm{CD} 34^{+}$cells, metabolic syndrome and cardiovascular risk

S. Vigili de Kreutzenberg ${ }^{1}$, A. Avogaro ${ }^{1}$, A. Coracina ${ }^{1}$, I. Baesso ${ }^{2}$, C. Agostini' ${ }^{2}$, A. Tiengo ${ }^{1}$, G. Fadini ${ }^{1}$;

${ }^{1}$ Medicina Clinica e Sperimentale, Malattie del Metabolismo, Padova, ${ }^{2}$ Medicina Clinica e Sperimentale, Immunologia ed Ematologia, Padova, Italy.
Background and Aims: Cardiovascular diseases (CVD) are the leading cause of death in western countries and risk assessment has become mandatory in the adult population. Over the past decades, it has become clear that patients with a given combination of risk factors, clustered in the socalled metabolic syndrome (MetS), are more prone to develop CVD. Besides the classical risk factors, novel biomarkers have recently been proposed, among which, circulating progenitor cells (CPCs). CPCs are believed to participate in cardiovascular (CV) homeostasis and their exhaustion has been linked to $\mathrm{CV}$ damage. This work was carried out to assess the relationships between different antigenic profiles of progenitor cells and $\mathrm{CV}$ risk, with special regard to MetS.

Materials and Methods: Two hundred and fourteen subjects (56\% male; age $56.4 \pm 14.9$ years) with different levels of $\mathrm{CV}$ risk participated in the study. CV risk was defined considering: diabetes mellitus, obesity, hypertension, dyslipidemia, smoke, age $>50$ years. MetS components were assessed using ATP-III criteria. 10-year Framingham risk was assessed as well. Flow cytometric analysis was used to quantify six different CPC subtypes $\left(\mathrm{CD} 34^{+}, \mathrm{CD} 133^{+}, \mathrm{CD} 34^{+} \mathrm{CD} 133^{+}, \mathrm{CD} 34^{+} \mathrm{KDR}^{+}\right.$, $\left.\mathrm{CD} 33^{+} \mathrm{KDR}^{+} \mathrm{CD} 34^{+} \mathrm{CD} 133^{+} \mathrm{KDR}^{+}\right)$.

Results: The study sample was representative of an intermediate-high CV risk (mean Framingham risk 18\%). Among the six different progenitor cell subtypes, CD34 $4^{+}$ cells showed the best correlation with all CV parameters. Components of the MetS were all characterised by reduction of $\mathrm{CD}_{4} 4^{+}$cells, and their clustering acted synergically in decreasing $\mathrm{CD} 4^{+}$cell count. Linear correlations between $\mathrm{CD} 34^{+}$cells and age $(\mathrm{r}=-0.40)$, blood glucose $(r=0.34)$, systolic blood pressure $(r=-0.33)$, LDL $(\mathrm{r}=-0.22)$, HDL $(\mathrm{r}=0.23)$, triglycerides $(\mathrm{r}=-0.34)$, and waist circumference $(\mathrm{r}=-0.23)$ were significant after adjustment for $\alpha$-inflation. Significant correlation was also detected between $\mathrm{CD} 34^{+}$cells and 10-year Framingham risk $(\mathrm{r}=-0.40)$, excluding subjects with established CVD, for whom the Framingham algorithm is not applicable. Consistently, a multiple logistic regression showed that $\mathrm{CD} 34^{+}$cell count was an independent predictor of high (>20\%) 10-year risk, suggesting that progenitor cell count may provide further information on cardiovascular risk beyond classical risk factors. Finally, a ROC curve analysis indicated a high performance of $\mathrm{CD}^{+} 4^{+}$cell count in detecting high $\mathrm{CV}$ risk.

Conclusion: These data demonstrate that CD34 identifies CPC linked to $\mathrm{CV}$ risk, showing a close negative correlation between $\mathrm{CD} 34^{+}$cells and CV risk as well as a synergic detrimental effect of clustered metabolic components. CPC count may be used as a surrogate marker of $\mathrm{CV}$ risk whereas extensive antigenic characterisation may not be useful for this purpose. 
1154

Impacts of the metabolic syndrome and Framingham risk score on cardiovascular predictors in patients with type 2 diabetes

G. Koh ${ }^{1}$, D.-M. Lim ${ }^{2}$, K.-Y. Park ${ }^{2}$, D.-H. Lee ${ }^{1}$, H.-J. Chin ${ }^{3}$, J.-M. Lee ${ }^{4}$;

${ }^{1}$ Department of Endocrinology \& Metabolism, Cheju National University Hospital, Jeju, ${ }^{2}$ Department of Endocrinology, Konyang University Hospital, Daejeon, ${ }^{3}$ Department of Internal Medicine, Hankook General Hospital, Jeju, ${ }^{4}$ Department of Endocrinology, Daejeon St. Mary's Hospital, Daejeon, Republic of Korea.

Background and Aims: The metabolic syndrome (MetS) is a constellation of interrelated risk factors to promote the development of atherosclerotic cardiovascular disease (CVD). In type 2 diabetes mellitus (T2DM), however, it has not yet been clarified whether the identification of the MetS improves the prediction of cardiovascular (CV) events. Framingham risk score (FRS) is an established predicting model for CVD. We sought to compare the impact of MetS with FRS on CV predictors in patients with T2DM.

Materials and Methods: One hundred and seven patients with T2DM (50 males and 57 females) were enrolled. Patients with history of CVD, any inflammatory disease and anti-hyperlipidemic medication were excluded. MetS was defined by modified NCEP-ATP III criteria. Highsensitivity C-reactive protein (hSCRP), homocysteine, lipoprotein(a), fibrinogen, uric acid and $\gamma$-glutamy transferase were regarded as CV predictors.

Results: $71.4 \%$ of total patients had the MetS. The levels of all $\mathrm{CV}$ predictors was not different between diabetic patients with and without MetS. Any CV factors were also not significantly different between numbers $(1,2,3,4$ and $5)$ of components of the MetS. However, homocysteine $(r=0.364 ; p<0.001)$, fibrinogen $(r=0.202 ; p<0.05)$ and uric acid $(r=0.298 ; p<0.05)$ levels were positively correlated with FRS. Homocysteine $(8.9 \pm 5.3,9.7 \pm 2.7,11.8 \pm 4.5,12.8 \pm$ 6.1 and $12.5 \pm 3.2 \mu \mathrm{mol} / \mathrm{L} ; \mathrm{p}<0.01)$ and uric acid $(4.1 \pm 2.2$, $4.8 \pm 1.6,4.9 \pm 1.5,6.5 \pm 3.6$ and $5.9 \pm 0.7 \mathrm{mg} / \mathrm{dL} ; \mathrm{p}<0.05)$ levels showed significant differences according to increasing quintiles of FRS.

Conclusion: The MetS is not related to $\mathrm{CV}$ predictors as well as FRS in patients with T2DM. It suggests that categorizing type 2 diabetic subjects as having or not having the MetS does not provide further prediction of CVD.
1155

The relationship of metabolic syndrome and its specific combinations with all cause mortality and coronary heart disease among type 2 diabetic patients

I. D. Protopsaltis, A. Kamaratos, E. Dimou, G. Nikolopoulos, I. Kyriazis, S. Kokkoris, A. Melidonis; Diabetes Center, Tzanio Gen Hospital, Piraeus, Greece.

Background and Aims: Although most studies investigating the relationship of metabolic syndrome (MBS) with all cause mortality or coronary heart disease (CHD) concern general population, there is lack of studies examining this relation among type 2 diabetic patients. The aim was to asses if MBS components or its several combinations have a higher predictive value than MBS alone in the evaluation of CHD and all cause mortality, among type 2 diabetic patients.

Materials and Methods: A total of 600 patients were enrolled in this analysis, from 1989 until 2004 with a mean follow up of ten years. In each patient the presence of MBS (NCEP ATP III), the cumulative number of MBS components, age, sex, blood pressure, HDL, LDL, total cholesterol, $\mathrm{TG}, \mathrm{HbA}_{1 \mathrm{C}}$, smoking status and diabetes duration were determined at baseline. Information regarding death or CHD events was obtained from our diabetic outpatient database and from hospital discharges. Statistical analyses were performed using Kaplan -Meier estimator and Cox proportional Hazard models.

Results: $62.4 \%$ had MBS. Significant predictors for all cause mortality were the presence of MBS (H.R: 1.75), sex (H.R: 1.68), age (H.R: 1.09), and diabetes duration (H.R: 1.02). With respect to CHD incidents, HDL (H.R: 0.97), systolic blood pressure (H.R: 1.012), and total cholesterol (H.R: 1.005) were significant predictors. MBS was not significant predictor in multivariate analysis after adjusting for CHD conventional risk factors. Subjects meeting the triad consisting of diabetes-hypertension- low HDL or the quadruple diabetes- hypertension- low HDL- high TG levels presented the higher probability for developing CHD events. (H.R: 1.79, 1.73 respectively).

Conclusion: The coexistence of MBS in type 2 diabetic patients decreases the survival probability. MS combinations mentioned above seems to confer higher risk regarding CHD prediction than other MS combinations or MS alone. 


\section{6}

Prospective evaluation of International Diabetes Federation 2005 and Adult Treatment Panel III 2001 criteria of the metabolic syndrome among women undergoing coronary angiography

C. H. Saely ${ }^{1,2}$, L. Koch ${ }^{1}$, F. Schmid ${ }^{1}$, T. Marte ${ }^{1,2}$, S. Aczel ${ }^{1,2}$, P. Rein $^{1}$, P. Langer ${ }^{1}$, G. Hoefle ${ }^{2}$, H. Drexel ${ }^{1,2}$; ${ }^{1}$ VIVIT Institute, Feldkirch, Austria, ${ }^{2}$ Department of Internal Medicine, Academic Teaching Hospital Feldkirch, Austria.

Background and Aims: The International Diabetes Federation (IDF) has recently established a worldwide consensus definition of the metabolic syndrome (MetS). The aim of this study was to prospectively evaluate the cardiovascular risk associated with the IDF and Adult Treatment Panel III (ATPIII) MetS definitions in women undergoing coronary angiography.

Materials and Methods: In a prospective study on 241 women undergoing coronary angiography for the evaluation of coronary artery disease we recorded vascular events over 4 years.

Results: From the women enrolled in our study, 34.9\% $(\mathrm{n}=84)$ had the MetS according to the ATP III definition and $47.8 \% \quad(n=115)$ according to the IDF definition. Concordance between the definitions was moderate (Cohen-Kappa coefficient $=0.537 ; \mathrm{p}<0.001$ ). The MetS as defined by the ATP III criteria significantly predicted vascular events (adjusted hazard ratio=2.133 [1.1114.094]; $\mathrm{p}=0.023$ ) but the MetS as defined by IDF criteria did not (adjusted hazard ratio=1.214 [0.635-2.319]; $\mathrm{p}=0.558$ ). Accordingly, event-free survival was significantly lower among women who fulfilled the ATP III but not the IDF criteria than among those who met the IDF but not the ATP III criteria $(\mathrm{p}=0.035)$.

Conclusion: Among women undergoing coronary angiography for the evaluation of CAD, the ATP III and the IDF definitions of the MetS do not identify the same patient populations. The ATP III definition of the MetS confers a significantly higher risk of vascular events than the IDF definition.

\section{PS 103}

\section{Glitazones: mechanisms, effects and costs}

1157

Rosiglitazone inhibits the upregulation of fractalkine induced by AGEs in vivo and in vitro

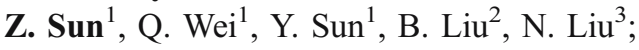

${ }^{1}$ Endocrinology, ${ }^{2}$ Institute of Nephrology, ${ }^{3}$ Institute of Cardiology, Zhongda Hospital, Southeast University, Nanjing, China.

Background and Aims: Our previous studies showed that advanced glycosylation end products (AGEs) induced the expression of CC chemokines such as MCP-1 and RANTES, and played an important role in the progression of diabetic nephropathy (DN). fractalkine (CX3CL1) is a unique membrane-bound $\mathrm{CX} 3 \mathrm{C}$ chemokine that serves as a potent chemoattractant and adhesion of monocytes via its receptor CX3CR1, was observed upregulation by AGEs in cultured human renal mesangial cells (HRMC). This study was to investigate the influence of rosiglitazone on the upexpression of fractalkine by AGEs in HRMC. We also observed the effect of rosiglitazone on the fractalkine expression in rat renal cortex.

Materials and Methods: HRMC were incubated with AGE-bovine serum albumin (AGE-BSA)in presence or absence of rosiglitazone for $24 \mathrm{~h}$. Type 2 diabetic rats were induced by an intraperitoneal injection of low doses of streptozotocin combined with high fat diet, and were randomized to receive rosiglitazone or vehicle treatment. Serum AGE-peptide was measured by flow injection assay. The mRNA and protein expression of fractalkine in HRMC were analyzed by semi-quantitative reverse transcription polymerase chain reaction (RT-PCR) and Western blotting, respectively. The expression of fractalkine in renal cortex of rats was examined with RT-PCR and immunohistochemical staining, respectively. Data were expressed as the mean \pm standard deviation of at least three independent experiments. Comparisons among groups were done by one-way ANOVA.

Results: 1. After incubation of HRMCs with AGE-BSA for 24 hours, both mRNA and protein expression for fractalkine were significantly increased compared to corresponding BSA group. 2. The elevated expressions of fractalkine mRNA and protein were decreased by exposure to rosiglitazone in a concentration dependent manner. 3. The serum AGE-peptide, the level of fractalkine mRNA and protein in renal cortex of diabetic rats was significantly higher than those of normal control. However those of rats treated with rosiglitazone were obviously lower compared to untreated group.

Conclusion: Rosiglitazone can inhibit the upregulation of fractalkine by AGEs in vivo and in vitro. These results suggest rosiglitazone might play a role of reno-protection via supression of AGEs and fractalkine.

Supported by National natural science foundation of China (30070300) 


\section{8}

Pioglitazone acutely influences glucose-sensitive insulin secretion in normal and diabetic human islets

Q. Zhang ${ }^{1}$, F. Zhang ${ }^{1}$, A. Sjöholm ${ }^{2}$;

${ }^{1}$ Research Center, ${ }^{2}$ Internal Medicine, Karolinska Institutet, Stockholm, Sweden.

Background and Aims: Peroxisome proliferator-activated receptor- $\gamma(\operatorname{PPAR} \gamma)$ plays an important role in the regulation of energy homeostasis. Pioglitazone is an orally administered insulin-sensitizing agent that activates PPAR $\gamma$, leading to increased transcription of genes encoding various proteins regulating glucose and lipid metabolism. In contrast to its action on insulin target tissues, little is known about the direct action of the PPAR $\gamma$ agonist on pancreatic $\beta$-cell function. The aim of the study was to investigate acute effects of the PPAR $\gamma$ agonist pioglitazone in vitro on human islets from both non-diabetic and type 2 diabetic subjects.

Materials and Methods: Human islets from adult nondiabetic or type 2 diabetic donors were maintained in tissue culture medium in the presence of $5.5 \mathrm{mM}$ glucose. Pancreatic islets from $o b / o b$ mice were isolated by collagenase digestion. Insulin secretion dynamics from perifused human islets was monitored using micro-columns and factions were collected every two minutes. Insulin secretion from batch-incubated islets was evaluated in 24 well plates during $20 \mathrm{~min}$-incubation. The insulin content in the fractions was measured using human or rat ELISA kits. Cytosolic free $\mathrm{Ca}^{2+}$ concentration $\left(\left[\mathrm{Ca}^{2+}\right]_{\mathrm{i}}\right)$ was measured in Fura-2 pre-loaded single islet cells prepared from human islets, using a time-sharing spectrofluorometer equipped with an inverted epifluorescence microscope.

Results: In $5 \mathrm{mM}$ glucose, pioglitazone caused a slight and transient increase in insulin secretion in non-diabetic, but not diabetic, islets. Continuous presence of the drug suppressed insulin release in non-diabetic islets. In islets from non-diabetic subjects, both high glucose and tolbutamide-stimulated insulin secretion was inhibited by pioglitazone. When islets from either non-diabetic or diabetic subjects were perifused with $5 \mathrm{mM}$ glucose, short-term pretreatment with pioglitazone caused approximately 2-fold increase in insulin secretion but only after drug withdrawal. Pioglitazone pre-treatment of diabetic islets restored their glucose sensitivity. Examination of cytosolic free $\mathrm{Ca}^{2+}$ concentration $\left(\left[\mathrm{Ca}^{2+}\right]_{\mathrm{i}}\right)$ in non-diabetic islets revealed slight $\mathrm{Ca}^{2+}$ transient by pioglitazone at $3 \mathrm{mM}$ glucose. In contrast, the suppressed insulin secretion of the drug at higher glucose concentrations was not associated with changes in $\left[\mathrm{Ca}^{2+}\right]_{\mathrm{i}}$.

Conclusions: Our data indicate bimodal acute effects of pioglitazone directly on human islets and suggest that shortterm pretreatment with pioglitazone primes both healthy and diabetic human islets for enhanced glucose-sensitive insulin secretion. This mechanism might be involved in the $\beta$-cell sparing effects of pioglitazone and other thiazolidinedione PPAR $\gamma$ agonists.

1159

Pioglitazone treatment in patients with type 2 diabetes and a history of myocardial infarction: subgroup analyses from PROactive stratified by gender, age, and duration of diabetes

E. Erdmann ${ }^{1}$, B. Charbonnel ${ }^{2}$, J. Dormandy ${ }^{3}$, M. Massi-Benedetti ${ }^{4}$, A. Skene ${ }^{5}$, R. Wilcox ${ }^{6}$, J. Yates $^{7}$ on behalf of the PROactive investigators,

${ }^{1}$ Kardiologie, Angiologie, Pneumologie, und Internistische Intensivmedizin, Klinik III für Innere Medizin, Koeln, Germany, ${ }^{2}$ Hotel Dieu, Clinique d'Endocrinologie, Nantes, France, ${ }^{3}$ Department of Clinical Vascular Research, St. George's Hospital, London, United Kingdom, ${ }^{4}$ Dipartmento di Medicina Interna, Universita di Perugia, Perugia, Italy, ${ }^{5}$ Isaac Newton Center, Nottingham Clinical Research Limited, Nottingham, United Kingdom, ${ }^{6}$ Department of Cardiovascular Medicine, Queen's Medical Centre, Nottingham, United Kingdom, ${ }^{7}$ Takeda Global Research and Development Center, Lincolnshire, United States.

Background and Aims: Compared with the general population, individuals with type 2 diabetes have an increased incidence of myocardial infarction (MI). PROactive studied the effects of pioglitazone on top of standard therapy on a composite macrovascular endpoint, which included non-fatal MI. This exploratory subanalysis of the larger PROactive study assessed 1,804 male and 641 female patients with a previous MI ( $\geq 6$ months prior to randomisation).

Materials and Methods: PROactive was a double-blind, placebo-controlled study in 5,238 patients with type 2 diabetes and macrovascular disease randomised to pioglitazone or matching placebo in addition to existing glucoselowering and cardiovascular medications, including antihypertensive, antiplatelet, and lipid-modifying drugs. The starting dose of pioglitazone was $15 \mathrm{mg} /$ day, which was titrated to a dose of $45 \mathrm{mg} /$ day, if tolerated. Mean follow-up was 34.5 months. Investigators were encouraged to treat diabetes, dyslipidaemia, and hypertension optimally according to the International Diabetes Federation guidelines. Analyses were performed according to gender, age ( $<65$ years $[\mathrm{n}=1452], \geq 65$ years $[\mathrm{n}=993])$, and duration of diabetes $(<5$ years $[\mathrm{n}=731], \geq 5-<10$ years $[\mathrm{n}=673]$, $\geq 10$ years $[n=1040])$.

Results: The occurrence of a recurrent fatal or non-fatal MI (excluding silent MI) in patients with previous MI was 
significantly lower in those treated with pioglitazone than in those treated with placebo $(5.3 \%$ versus $7.2 \%, \mathrm{HR}=0.72$, $\mathrm{P}=0.045)$. Interaction tests showed that there was no significant impact of gender $(\mathrm{P}=0.53)$, age $<65$ years/ $\geq 65$ years $(\mathrm{P}=0.43)$, or diabetes duration $<5$ years/ $\geq 5-<10$ years $/ \geq 10$ years $(\mathrm{P}=0.46)$ on the hazards ratio for time to fatal/non-fatal MI (excluding silent MI). This suggests that the best estimate for the reduction in risk of recurrent $\mathrm{MI}$ for each subgroup is given by that for the entire previous $\mathrm{MI}$ cohort. Median $\mathrm{HbA}_{1 \mathrm{c}}$ and triglycerides decreased and HDL-C increased to a greater extent in the pioglitazone group than in the placebo group in the total cohort of patients with previous $\mathrm{MI}$ and also in all of the gender, age, and diabetes duration subgroups $(\mathrm{P}<0.0001$ for all comparisons except triglycerides in the $\geq 5-<10$ years duration subgroup, $\mathrm{P}<0.03$ ).

Conclusion: Pioglitazone has been shown to have beneficial effects on hyperglycemia as well as diabetic dyslipidemia and pleiotropic markers associated with macrovascular disease. In this exploratory subset, pioglitazone had a consistently better effect on recurrent MI and metabolic parameters than placebo did, irrespective of gender, age, and duration of diabetes.

\section{0}

Glycaemic and lipid effects of pioglitazone and rosiglitazone in patients naive to oral antidiabetic medications S. Shinall ${ }^{1}$, J. Buse ${ }^{2}$, M. Tan ${ }^{3}$, S. Gbenado ${ }^{3}$,

R. Spanheimer ${ }^{4}$;

${ }^{1}$ Medical \& Scientific Affairs, Takeda Pharmaceuticals North America, Inc., Lincolnshire, United States, ${ }^{2}$ Division of General Internal Medicine and Clinical Epidemiology, University of North Carolina School of Medicine, Chapel Hill, United States, ${ }^{3}$ Lilly Research Laboratories, Eli Lilly and Company, Indianapolis, United States, ${ }^{4}$ Biometrics and Data Management, Takeda Global Research and Development Center, Lincolnshire, United States.

Background and Aims: Therapeutic responses to oral antidiabetic medications (OAM), such as the thiazolidinediones (TZDs) pioglitazone (PIO) and rosiglitazone (ROSI), are affected by factors including baseline A1C values, duration of disease, and history of prior OAM. This subgroup analysis from the GLAI study evaluated the glycemic and lipid effects of PIO and ROSI in patients with type 2 diabetes (T2D) and no prior history of OAM.

Materials and Methods: GLAI was a prospective, randomized, double-blind, multicenter trial comparing glycemic and lipid effects of PIO and ROSI in patients with T2D (WHO criteria) and dyslipidemia (fasting TG levels $\geq 150$ and $<600 \mathrm{mg} / \mathrm{dL}$; fasting LDL-C levels=130 mg/dL). A prespecified subgroup comprised 197 patients previously naïve to OAM (PIO, $n=88$; ROSI, $n=89$ ). Following randomization and a 4-week placebo run-in phase, patients received PIO (30 $\mathrm{mg}$ QD) or ROSI (2 mg BID). At 12 weeks PIO and ROSI were titrated to maximally effective doses of $45 \mathrm{mg}$ QD and $4 \mathrm{mg}$ BID, respectively, and maintained for an additional 12 weeks.

Results: Both treatment groups had similar A1C levels at baseline (Mean+SD, PIO: 7.99 $\pm 0.12 \%$, ROSI: $7.79 \pm$ $0.12 \%$ ). Comparable reductions from baseline in A1C were observed for both treatment arms (PIO: $-1.13 \pm 0.09 \%$, $\mathrm{P}<0.05$; ROSI: $-1.05 \pm 0.09 \%, \mathrm{P}<0.05)$ with no significant between-group difference $(\mathrm{P}=0.491)$. By study's end, 44/88 $(50 \%)$ of PIO and $40 / 89$ (45\%) of ROSI patients reached the ADA target of $\mathrm{A} 1 \mathrm{C}<7.0 \%$. The serum lipoprotein results are shown below.

Week 24 Changes from Baseline

\begin{tabular}{|c|c|c|c|c|c|c|}
\hline & \multicolumn{3}{|c|}{ PIO N=88 } & \multicolumn{3}{|c|}{ ROSI N=89 } \\
\hline & Baseline & $\begin{array}{l}\text { Mean } \\
\text { change } \\
\pm \text { SEM }\end{array}$ & $\begin{array}{l}\% \\
\text { change }\end{array}$ & Baseline & $\begin{array}{l}\text { Mean } \\
\text { change } \\
\pm \text { SEM }\end{array}$ & $\begin{array}{l}\% \\
\text { change }\end{array}$ \\
\hline $\begin{array}{l}\text { TG } \\
(\mathrm{mg} / \mathrm{dL})\end{array}$ & $\begin{array}{l}279.8 \\
\pm 17.2\end{array}$ & $\begin{array}{l}-73.5 \\
\pm 17.8^{*}\end{array}$ & $-16.7 \% *$ & $\begin{array}{l}236.6 \\
\pm 16.9\end{array}$ & $\begin{array}{l}+7.8 \\
\pm 17.8\end{array}$ & $+13.4 \%$ \\
\hline $\begin{array}{l}\text { HDL-C } \\
(\mathrm{mg} / \mathrm{dL})\end{array}$ & $\begin{array}{l}39.06 \\
\pm 1.1\end{array}$ & $\begin{array}{l}+2.1 \\
\pm 0.9\end{array}$ & $+6.6 \%$ & $\begin{array}{c}38.4 \\
\pm 1.0\end{array}$ & $\begin{array}{l}+1.7 \\
\pm 0.9\end{array}$ & $+5.5 \%$ \\
\hline $\begin{array}{l}\text { Non-HDL-C } \\
(\mathrm{mg} / \mathrm{dL})\end{array}$ & $\begin{array}{l}151.35 \\
\pm 3.4\end{array}$ & $\begin{array}{l}-0.4 \\
\pm 3.5^{*}\end{array}$ & $-3.8 \% *$ & $\begin{array}{l}151.2 \\
\pm 3.3\end{array}$ & $\begin{array}{l}30.3 \\
\pm 3.4\end{array}$ & $+12.6 \%$ \\
\hline $\begin{array}{l}\text { LDL-C } \\
(\mathrm{mg} / \mathrm{dL})\end{array}$ & $\begin{array}{l}102.6 \\
\pm 2.4\end{array}$ & $\begin{array}{l}+4.7 \\
\pm 2.4^{*}\end{array}$ & $+8.3 \% *$ & $\begin{array}{l}101.9 \\
\pm 2.4\end{array}$ & $\begin{array}{l}+15.4 \\
\pm 2.4\end{array}$ & $+17.8 \%$ \\
\hline $\begin{array}{l}\text { Total-C } \\
(\mathrm{mg} / \mathrm{dL})\end{array}$ & $\begin{array}{l}191.3 \\
\pm 3.6\end{array}$ & $\begin{array}{l}+1.95 \\
\pm 3.3^{*}\end{array}$ & $+2.2 \% *$ & $\begin{array}{l}190.7 \\
\pm 3.5\end{array}$ & $\begin{array}{l}31.4 \\
\pm 3.3\end{array}$ & $+17.4 \%$ \\
\hline $\begin{array}{l}\text { LDL } \\
\text { particle } \\
\text { conc. } \\
\text { (nm ol/L) }\end{array}$ & $\begin{array}{l}1313.7 \\
\pm 36.7\end{array}$ & $\begin{array}{c}-25.2 \\
\pm 36.9 * *\end{array}$ & ${ }^{+}+3.0 \% * *$ & $\begin{array}{l}1303.9 \\
\pm 36.0\end{array}$ & $\begin{array}{l}105.1 \\
\pm 37.1\end{array}$ & $+11.0 \%$ \\
\hline $\begin{array}{l}\text { LDL - } \\
\text { particle } \\
\text { size }(\mathrm{nm})\end{array}$ & $\begin{array}{l}20.01 \\
\pm .08\end{array}$ & $\begin{array}{l}+0.43 \\
\pm 0.07\end{array}$ & $+2.2 \%$ & $\begin{array}{l}20.04 \\
\pm .08\end{array}$ & $\begin{array}{l}+0.35 \\
\pm 0.07\end{array}$ & $+1.8 \%$ \\
\hline $\begin{array}{l}\text { Apolipo- } \\
\text { protein } \\
\text { B (mg/dL) }\end{array}$ & $\begin{array}{l}1.02 \\
\pm .02\end{array}$ & $\begin{array}{l}-0.02 \\
\pm 0.02 *\end{array}$ & $+1.4 \% *$ & $\begin{array}{l}1.01 \\
\pm .02\end{array}$ & $\begin{array}{l}+0.12 \\
\pm 0.02\end{array}$ & $+12.5 \%$ \\
\hline
\end{tabular}

$* \mathrm{P} \leq 0.005$ or $* * \mathrm{P}<0.05$, between treatment groups

Conclusion: Treatment with either PIO or ROSI yielded comparable $\mathrm{A} 1 \mathrm{C}$ reductions in this subgroup of patients 
naïve to OAM in the GLAI study. After 16 weeks of thiazolidinedione treatment in OAM naïve patients, there was a marked reduction in A1C despite only moderately elevated baseline A1C levels. Approximately half of patients in both treatment groups achieved the ADA target of $\mathrm{A} 1 \mathrm{C}<7.0 \%$ Similar to results from GLAI in both OAM treated and naïve patients, PIO demonstrated significant improvements versus ROSI in several lipid parameters (TG, TC, non-HDL-C, LDL-C, LDL particle concentration, and ApoB) in this OAM naïve subgroup.

\section{1}

Lipid and apolipoprotein levels improve with pioglitazone plus sulfonylurea after treatment conversion from rosiglitazone in subjects with type 2 diabetes and dyslipidemia who continue stable statin therapy

M. Khan, R. Spanheimer, S. Shinall;

Takeda Pharmaceuticals North America, Inc., Lincolnshire, United States.

Background and Aims: A randomized, head-to-head, placebo-controlled study demonstrated that the thiazolidinediones pioglitazone (PIO) and rosiglitazone (ROSI) exerted differential lipid effects in patients with type 2 diabetes and dyslipidemia in the absence of lipid-altering therapy. The PIO-treated patients showed improvements over ROSI-treated patients in triglycerides (TGs), highdensity lipoprotein cholesterol (HDL-C), and LDL particle concentration and size, and smaller increases in low-density lipoprotein cholesterol (LDL-C) and non-HDL-C levels (all results $\mathrm{P} \leq 0.005$ ). The COMPLEMENT study was designed to determine whether the lipid differences observed in the previous study would be similar when subjects were converted from ROSI to PIO therapy while maintaining constant doses of their existing statins and other lipidaltering therapies. The primary outcome measure was fasting TGs; additionally, total cholesterol, LDL-C, HDLC, apolipoprotein (apo) A-I, and apo B were measured. Here we evaluate the lipid-altering effects of PIO when added to an existing sulfonylurea regimen in a subset of patients from a post-hoc analysis of COMPLEMENT.

Materials and Methods: In this multicenter, open-label study, 305 patients with type 2 diabetes and dyslipidemia (TGs $2.26-11.29 \mathrm{mmol} / \mathrm{L}$ ) who had been taking stable doses of ROSI and statin therapy for at least 90 days were converted to PIO at baseline. Of these patients, 130 were on a sulfonylurea-based regimen. All patients were then followed for 17 additional weeks while continuing a constant dose of the existing statin agent (+additional lipid-altering therapies, if already present). PIO was started at $30 \mathrm{mg} / \mathrm{d}$, but could be increased to $45 \mathrm{mg} / \mathrm{d}$ at the investigator's discretion.

Results:

\begin{tabular}{lllll}
\hline & $\begin{array}{l}\text { Baseline } \\
\text { Mean (SD) } \\
(\mathbf{n = 1 3 0 )}\end{array}$ & $\begin{array}{l}\text { Week 17 } \\
\text { Mean } \\
\text { Change (SE) } \\
(\mathbf{n = 1 2 1 )}\end{array}$ & $\begin{array}{l}\text { Week 17 } \\
\text { Mean } \\
\text { \% Change } \\
(\mathbf{n = 1 2 1})\end{array}$ & $\begin{array}{l}\text { P- } \\
\text { value }^{\mathbf{a}}\end{array}$ \\
\hline $\begin{array}{l}\text { HbA1c (\%) } \\
\text { TGs (mmol/L) }\end{array}$ & $3.5(1.13)$ & $0.05(0.070)$ & $\mathrm{NC}$ & $\mathrm{NC}$ \\
$\begin{array}{l}\text { Total Cholesterol } \\
(\mathrm{mmol} / \mathrm{L})\end{array}$ & $5.12(1.877)$ & $-0.71(0.129)^{\dagger}$ & -14.17 & $<0.001$ \\
$\begin{array}{l}\text { LDL-C (mmol/L) } \\
\text { HDL-C }\end{array}$ & $2.65(0.814)$ & $-0.56(0.060)^{\dagger}$ & -10.00 & $<0.001$ \\
$(\mathrm{mmol} / \mathrm{L})$ & $1.08(0.241)$ & $-0.013(0.013)$ & 0.53 & 0.678 \\
$\begin{array}{l}\text { Apo A-I (g/L) } \\
\text { Apo B (g/L) }\end{array}$ & $1.175(0.464)$ & $0.091(0.017)^{\dagger}$ & $\mathrm{NC}$ & $\mathrm{NC}$ \\
& $0.907(0.247)$ & -0.038 & $\mathrm{NC}$ & $\mathrm{NC}$ \\
& & $(0.013)^{\dagger \dagger}$ & & \\
\hline
\end{tabular}

*Baseline $\mathrm{N}=129$, Week $17 \mathrm{~N}=120$

${ }^{\dagger} \mathrm{P}<0.001$

${ }^{\dagger} \mathrm{P}=0.003$

${ }^{\text {a }} \mathrm{P}$ value for $\%$ change

$\mathrm{NC}=$ not calculated

At week 17 following conversion from ROSI to PIO, significant reductions were observed in TGs and total cholesterol: LDL-C decreased and HDL-C increased, but these changes were not statistically significant. Apo A-l levels increased significantly and Apo B levels decreased significantly. In addition, LDL-C particle concentration decreased and LDL-C particle size increased. A1c levels was virtually unchanged after conversion to PIO.

Conclusion: Replacing ROSI with PIO in subjects with type 2 diabetes while maintaining concomitant statin and other lipid-altering therapies resulted in significant improvements in TGs, total cholesterol, minor improvements in LDL-C but no change in HDL-C levels in this subset of patients on a sulfonylurea-based regimen. Both apo A-1 and apo B levels also improved significantly. These combined improvements were independent of glycemic control, which remained stable after treatment conversion.

\section{2}

A one-year, randomized, placebo-controlled, doubleblind trial of rosiglitazone in non alcoholic steatohepatitis: Results of the FLIRT pilot trial

V. Ratziu ${ }^{1}$, S. Jacqueminet ${ }^{2}$, P. Giral ${ }^{3}$, P. Podevin ${ }^{4}$,

L. Serfaty ${ }^{5}$, F. Charlotte $^{6}$, E. Bruckert ${ }^{3}$, C. Bernhardt ${ }^{7}$,

A. Grimaldi ${ }^{2}$, T. Poynard ${ }^{7}$

on behalf of the LIDO Study Group ${ }^{7}$; 
${ }^{1}$ Hepatogastroenterologie, Hospital Pitie Salpetriere, Paris, ${ }^{2}$ Diabetes, ${ }^{3}$ Endocrinology, Hospital Pitie Salpetriere, Paris, ${ }^{4}$ Liver Disease, Hospital Cochin, Paris, ${ }^{5}$ Liver Disease, Hospital Saint Antoine, Paris, France, ${ }^{6}$ Pathology, ${ }^{7}$ Liver Disease, Hospital Pitie Salpetriere, Paris, France.

Background and Aims: Nonalcoholic steatohepatitis (NASH) is a liver disease associated with insulin resistance. Glitazones are promising drugs for NASH but only uncontrolled studies are available thus far. The aim was to test the efficacy and safety of rosiglitazone vs. placebo in patients with NASH.

Materials and Methods: Patients with biopsy-proven NASH were randomized to a one-year treatment with rosiglitazone (RSG, $8 \mathrm{mg} /$ day) or placebo (PLB), followed by a control liver biopsy. End-points were, primary : improvement in steatosis $>30 \%$ (histological response, HR); secondary : normalization of transaminase ALT (biochemical response, BR).

Results: 32 patients were included in the RSG group and 31 in the PLB group. Sex ratio was 1.4, mean age 54 years. 20 Pts had diabetes $(32 \%, 17$ drug-treated, 16 with metformin), 35\% arterial hypertension, mean BMI was $31.1 \mathrm{~kg} / \mathrm{m}^{2}$. RSG significantly improved serum glucose $(-0.93 \mathrm{mmol} / 1 \mathrm{vs}+0.55 \mathrm{mmol} / \mathrm{l}$ in PLB, $\mathrm{p}=0.001)$ and, in non diabetics, median insulin $(-5 \mu \mathrm{UI} / 1$ vs $0 \mu \mathrm{UI} / 1$ in PLB, $\mathrm{p}=0.016)$, HOMA $(-1.18 \%$ vs $0.3 \%$ in PLB, $\mathrm{p}<0.03)$ and QUCKI ( 0.032 vs -0.005 in PLB, $\mathrm{p}<0.01)$. HR occurred in 47\% (15/32) RSG and 16\% (5/31) PLB pts $(\mathrm{p}<0.004)$. BR occurred in $38 \%(12 / 32)$ RSG and $7 \%(2 / 31)$ PLB pts $(p=0.005)$. In RSG treated pts, HR was more frequent in non-diabetics, $(61 \%, 14 / 23)$, than in diabetics $(11 \%, 1 / 9$, $\mathrm{p}<0.01)$ while BR was equivalent $(39 \%, 9 / 23$, vs $33 \%, 3 / 9$, respectively). The antisteatogenic effect of $\mathrm{RSG}$ was correlated with improvement in $\operatorname{ALT}(\mathrm{r}=0.35, \mathrm{p}<0.05)$, QUICKI $(\mathrm{r}=0.66, \mathrm{p}<0.01)$ and HOMA $(\mathrm{r}=-0.55, \mathrm{p}=0.04)$. In the RSG group, 5 pts required dose reduction due to side effects (vs. 1 in the PLB group, $p<0.09$ ) and 1 had to discontinue treatment. Weight gain was $+1.5 \mathrm{~kg}$ in RSG pts and $-1.4 \mathrm{~kg}$ in the PLB pts (p3 $\mathrm{kg}$ was seen in $31 \% \mathrm{RSG}$ $(10 / 32)$ and $13 \%(4 / 31)$ PLB groups $(p<0.08)$. Pedal edema developed in 10 RSG and 8 PLB pts. ALT elevation consistent with hepatotoxicity was not observed in any pt. Conclusion: A one year treatment with RSG, an insulin sensitizing agent, significantly improves liver injury in pts with NASH. Large scale trials are warranted and longer treatment durations should be tested.

Supported by GSK

\section{3}

\section{WITHDRAWN}

1164

\section{WITHDRAWN}

\section{PS 104}

\section{Coronary artery disease and assessment of cardiac function}

\section{5}

\section{Deterioration of adrenergic and cholinergic coronary artery tone and heart function regulation in diabetic dogs}

\section{A. Nescheret;}

Lab. Neurohormonal Regulation of Circulation, Institute of Endocrinology and Metabolism, Kiev, Ukraine.

Background. It is well known that insulin deficiency and hyperinsulinemia in diabetes mellitus evoke neuropathy with disturbances of sympathetic and parasympathetic nervous and hormonal regulation of heart function and vessels tone. However peculiarities and exact mechanisms of heart function and vessels tone regulation disorders induced by diabetes mellitus remain insufficiently studied. Aim. The experimental study was devoted to investigation of peculiarities of adrenergic (Adr) and cholinergic (Ch) coronary artery (CA) tone and heart function regulatory disorders in different stages of diabetes mellitus development.

Materials and Methods. Experiments were carried out on closed-chest dogs under alpha-chloralose anesthesia (50$100 \mathrm{mg} / \mathrm{kg}$, i.v.), catheterization and programmed autoperfusion of left $\mathrm{CA}$, catheterization and continuous drainage of coronary sinus, heart and main vessels catheterization, registration of cardiohaemodynamics, CA resistance and ECG were performed. In metabolically healthy dogs (HD) and alloxane-diabetic dogs (AD, $75 \mathrm{mg} / \mathrm{kg}$ of alloxane, i.v.) Adr and Ch test-reactions of $\mathrm{CA}$ and heart were provoked by bolus intracoronary (i.c.) injection of epinephrine (E, 0.05-5.0 mcg), norepinephrine (NE, 0.05-5.0 mcg) and acetylcholine (Ach, 0.001$1.0 \mathrm{mcg}$ ). Due to different levels of hyperglycemia development in $\mathrm{AD}$ all of them were divided into three groups: HD - with normal level of glycemia (3.8 \pm $0.5 \mathrm{mmol} / \mathrm{l})$; AD1 - hyperglycemia - $7.2 \pm 1.7 \mathrm{mmol} / \mathrm{l}$; AD2 - 9.8+1.7 mmol/1 and AD3-15.6 $\pm 3.4 \mathrm{mmol} / \mathrm{l}$.

Results. In $\mathrm{HD} \mathrm{E}$ or $\mathrm{NE}$ injection into $\mathrm{CA}$ blood flow caused dose-dependent of short duration reflectory $\mathrm{Ch} \mathrm{CA}$ dilation (1 phase) and attenuation of heart contractility then followed by more pronounced and protracted Adr CA resistance decrease and augmentation of heart contractility (2 phase). In AD1 Ch and Adr CA dilation induced by $\mathrm{E}$ or 
$\mathrm{NE}$ injection (5.0 mcg, i.c.) increased $(\mathrm{Ch}:+47.1 \%$ and $+58.3 \%$; Adr: $+66.7 \%$ and $+23.7 \%$, resp.). The same but more pronounced changes of $\mathrm{Ch}$ and $\mathrm{Adr} \mathrm{CA}$ reactions in AD2 were observed (Ch: $+62.5 \%$ and $+100 \%$; Adr: $+74.4 \%$ and $+105.2 \%$, resp.). Both in $\mathrm{AD} 1$ and $\mathrm{AD} 2$ negative reflectory $\mathrm{Ch}$ heart reactions and positive Adr ino- and chronotropic heart reactions augmented. On the contrary, in $\mathrm{AD} 3 \mathrm{Ch}$ and $\mathrm{Adr} \mathrm{CA}$ reactions were diminished ( $\mathrm{Ch}$ and Adr, E: $-29.2 \%$ and $-33.3 \%$; Ch and Adr, NE: $-83.3 \%$ and $-34.2 \%$, resp.). Besides, negative and positive ino- and chronotropic heart reactions were limited or absolutely disappeared. In AD1 CA dilatations induced by Ach (1.0 mcg, i.c.) enhanced $(+23.3 \%)$ whereas in AD2 and AD3 these reactions diminished $(-4.7 \%$ and $-25.6 \%$ resp.).

Conclusion. We suggest that identified peculiarities of $\mathrm{Ch}$ and $\mathrm{Adr} \mathrm{CA}$ and heart function regulatory deteriorations participate in diabetic cardiovascular complications development and must be taken into considerations in practice.

\section{6}

Validation of the French guidelines on the diabetic patients to screen for silent myocardial ischaemia

P. Valensi ${ }^{1}$, J. Pariès ${ }^{1}$, F. Paycha ${ }^{2}$, S. Cattan ${ }^{3}$, J.-R. Attali ${ }^{1}$, E. Cosson ${ }^{1}$;

${ }^{1}$ Endocrinology-Diabetology-Nutrition, Jean Verdier Hospital, Bondy, ${ }^{2}$ Nuclear Medicine, Louis Mourier Hospital, Colombes, ${ }^{3}$ Cardiology, Montfermeil-Le Raincy Inter Communal Hospital, Montfermeil, France.

Background and Aims: French Societies of Diabetology and Cardiology recommended in 2004 to screen for silent myocardial ischemia (SMI) the diabetic patients selected on the following criteria : those with nephropathy or carotid or peripheral artery disease, those with $\geq 2$ other cardiovascular risk factors if type 1 diabetic patients older than $45 \mathrm{yrs}$ or with diabetes duration $>10 \mathrm{yrs}$, and if type 2 diabetic patients older than $60 \mathrm{yrs}$ or with diabetes duration $>$ $15 \mathrm{yrs}$. The aim of this study was to evaluate the impact of these criteria on the prevalence of SMI, the prevalence of coronary stenoses (CS) on angiography and the incidence of cardiac events (CE: cardiac death, myocardial infarction, cardiac failure, coronary revascularization and unstable angina).

Patients and Methods: We retrospectively studied the files of 362 diabetic patients who were screened in 1997-2000 for SMI, defined as an abnormal myocardial scintigraphy, and for CS on angiography (performed in the patients with SMI). Patients were followed up for $41 \pm 24$ months. In this population, 134 patients fulfilled the French screening criteria $(\mathrm{C}+)$ and 228 did not $(\mathrm{C}-)$.
Results: The prevalence of SMI was similar in both groups: 47/134 C+(35.1\%) versus 74/228 C- (32.5\%). The positive predictive value of SMI for identifying patients with CS was higher $(\mathrm{p}<0.02)$ in the $\mathrm{C}+$ group $(24$ out of 39 performed coronary angiographies, 62\%) than in the Cgroup (20/55, 36\%). SMI was predictive of CE (Kaplan Meier, log rank $9.1 ; \mathrm{p}<0.005)$. The incidence of $\mathrm{CE}$ was greater in the $\mathrm{C}+$ group $(11 \%)$ than in the $\mathrm{C}$ - group $(4 \%)$ (Odds Ratio=2.8 [1.2-6.8], $\mathrm{p}<0.02$ ). In the $\mathrm{C}+$ group, 128 were followed-up; a CE occurred in 8 of the $45 \mathrm{SMI}$ +patients, in 6 of them within 3 yrs, whereas a CE occurred in 6 of the 83 SMI- patients, always after 3 yrs.

Conclusions: The present data show that the current French guidelines do identify diabetic patients with high risk of silent $\mathrm{CS}$ and $\mathrm{CE}$, and suggest that patients fulfilling these criteria and with a negative screening should be reassessed 3 years later.

1167

Selecting asymptomatic type 2 diabetic patients or screening for silent myocardial ischaemia: what risk factors should be considered?

A. Sultan ${ }^{1}$, C. Piot $^{2}$, J. Thuan dit Dieudonné ${ }^{1}$, D. Mariano-Goulard ${ }^{3}$, E. Renard ${ }^{4}$, A. Avignon ${ }^{1}$;

${ }^{1}$ Metabolic Disease, ${ }^{2}$ Cardiology, ${ }^{3}$ Nuclear Medicine, ${ }^{4}$ Endocrinology, University Hospital, Montpellier, France.

Background and Aims: Silent myocardial ischemia (SMI) is frequent in type 2 diabetic patients (T2DP) and could contribute to worse outcomes in this population. Recent studies suggest that traditional risk factors (RFs) are not associated with abnormal screening tests, leading the authors to suggest that all T2DP may merit screening stress tests. However, since stress tests in asymptomatic T2DP are mostly performed to identify patients with significant CAD susceptible to benefit from coronary revascularization, we evaluated whether traditional RFs are associated with asymptomatic but angiographycally proven CAD.

Materials and Methods: Seven hundred twenty one asymptomatic T2DP were evaluated for SMI using stress myocardial perfusion imaging; of 222 patients with positive results, 137 underwent coronary angiography, 71 of whom showed significant CAD. Angiography showed no significant lesion in 66 patients. Multivariate logistic regression was used to examine difference in RFs between the latter 2 groups.

Results: Nephropathy (odds ratio 4.0 [95\% CI 1.8-9.5], $\mathrm{P}=0.001)$, HDL-cholesterol $<50 \mathrm{mg} / \mathrm{dl}$ (3.6 [1.5-8.4], $\mathrm{P}=0.004)$, age $>60$ years $(3.2[1.3-7.6], \mathrm{P}=0.009)$, treated dyslipidemia (2.8 [1.5-8.4], $\mathrm{P}=0.004)$ and HbA1c (2.9 $[1.2-7.5], \mathrm{P}=0.025)$ were significant independent predictors 
of asymptomatic CAD in T2DP. A significant association was found between CAD and the number of these factors $(p<0.0001)$. No association was found between CAD and the number of RFs when considering the RFs of current ADA screening guidelines.

Conclusion: Considering that the RFs we identified are all recognized predictors of cardiovascular events in T2DP, we conclude that screening stress tests should be limited to patients who have an association of these RFs, i.e. to patients who have a high risk of cardiovascular events and susceptible to benefit from coronary revascularization.

\section{8}

Increasing prevalence of cardiovascular disease in Mauritius, and a strong association between postprandial hyperinsulinaemia and ECG changes indicating possible ischaemic heart disease

S. Söderberg ${ }^{1}$, J. Shaw ${ }^{2}$, P. Zimmet ${ }^{2}$, R. Reklamaite ${ }^{3}$, A. Tamosiunas ${ }^{3}$, K. Alberti ${ }^{4}$, J. Tuomilehto ${ }^{5}$;

${ }^{1}$ Cardiology, Dept of Public Health and Clinical Medicine, Umeå, Sweden, ${ }^{2}$ International Diabetes Institute, Melbourne, Australia, ${ }^{3}$ Cardiology, Kaunas Institute of Cardiology, Lithuania, ${ }^{4}$ Department of Endocrinology and Metabolic Medicine, Imperial College, London, United Kingdom, ${ }^{5}$ National Public Health Institute, University of Helsinki, Helsinki, Finland.

Background: With increasing westernization, increasing prevalence of obesity is followed by coronary heart disease (CHD) and diabetes. Ethnic differences may be important as people with an Asian background seem to develop obesity-related diseases at lower levels of obesity.

Materials and Methods: Three population-based surveys were performed in Mauritius, in 1987, 1992 and in 1998. Altogether 9688 participated, and more than 50\% were seen more than twice. Life-style related questionnaires, anthropometry, oral glucose tolerance tests, and 12-lead ECGs ( $>35$ years) were included. Blood lipids, glucose, insulin and leptin were analysed. ECG changes were classified as "probable CHD" (mainly anterior Q-waves) and "possible CHD" (mainly STT depression/inversion and LBBB) according to Minnesota code.

Results: Probable changes were more common in men than women, and increased with age, whereas possible changes were more common in women, especially those with an African ancestry.

The incidence of probable ECG changes increased from 1.5 (1987-1992) to 4.4 (1992-1998) per 1000 PY and the incidence of possible ECG changes increased from 24.9 to 38.4 per $1000 \mathrm{PY}$.
In an cross-sectional analysis, approximately $60 \%$ of all subjects with probable ECG changes had abnormal glucose tolerance (impaired fasting glucose, impaired glucose tolerance or diabetes). High levels of postprandial glucose and insulin levels associated with the presence of both types of ECG changes in a multivariate analysis, taking into account traditional risk factors such as age, smoking, hypertension, diabetes, dyslipidemia and obesity. Fasting insulin and glucose levels did not associate with ECG changes once adjusted for traditional risk factors. In addition, only postload insulin levels related to selfreported heart attacks after adjustments.

Conclusion: Postprandial levels of glucose and insulin strongly associated with ECG changes indicating possible or probable CHD in a population of mainly Asian and African ancestry. Over 12 years, the incidence of CHDrelated ECG changes appeared to increase in Mauritius.

1169

Hyperglycaemia and its effect after acute myocardial infarction on cardiovascular outcomes in patients with type 2 diabetes mellitus (HEART2D): Geographic differences in baseline $\mathrm{CV}$ risk factors and treatments I. Aganovic ${ }^{1}$, Z. Milicevic ${ }^{2}$, B. Campaigne ${ }^{2}$, S. Sarwat ${ }^{2}$, D. C. Robbins ${ }^{2}$, E. Gromniak ${ }^{3}$, I. Kowalska ${ }^{4}$, M. Tan ${ }^{2}$;

${ }^{1}$ Internal Medicine, Clinical Centre Zagreb, Croatia, ${ }^{2}$ Eli Lilly and Company, Indianapolis, United States, ${ }^{3}$ Department of Endocrinology, Hypertension and Metabolic Diseases, Pomeranian Medical University, Szczecin, ${ }^{4}$ Department of Endocrinology, Diabetology and Internal Medicine, Medical University of Bialystok, Poland.

Background and Aims: HEART2D study aims to determine whether an insulin regimen targeting postprandial (pp) hyperglycemia reduces incidence of cardiovascular (CV) outcomes in patients with type 2 diabetes mellitus (T2DM) and acute myocardial infarction (AMI) when compared to a regimen that targets fasting/pre-meal blood glucose (BG). Patients are followed up to 5 years after study enrollment. The current analysis evaluates regional differences in baseline CV risk factors (CVRF) and CV treatments.

Materials and Methods: Post AMI (within 21 days of event) T2DM patients were randomized to 1 of 2 treatments; (a) prandial strategy: pre-meal insulin lispro and $\mathrm{NPH}$ at bedtime, if needed or (b) basal strategy: NPH twice-daily or insulin glargine once-daily, followed by twice-daily human premixed insulin, if needed. Treatment targets: prandial strategy, $\mathrm{A} 1 \mathrm{C}<7.0 \%$ and $\mathrm{pp} \mathrm{BG}<7.5 \mathrm{mM}$; basal strategy, $\mathrm{A} 1 \mathrm{C}<7.0 \%$ and fasting/premeal $\mathrm{BG} 4.4-$ $6.7 \mathrm{mM}$. The 5 regions were Eastern Europe $(n=718)$, Middle East $(n=149)$, South Africa $(n=89)$, Western 
Europe/Canada $(\mathrm{n}=84)$ and Western Asia $(\mathrm{n}=70)$. Chisquare and ANOVA were used to test regional differences. Results: One thousand one hundred twenty patients from 105 centres in 17 countries were randomised. The proportion of patients with ST-segment elevated entry MI was variable $(60-83 \% ; \mathrm{p}=.01)$, as it was for left ventricular ejection fraction $(49.5-54.3 \% ; \mathrm{p}=.0003)$ and $\mathrm{QT}$ interval duration $(420-439 \mathrm{~ms} ; \mathrm{p}<0.0001)$. No differences were found for duration of diabetes, albumin/creatinine ratio or A1C, but fasting BG (8.1-8.8 mM; $\mathrm{p}=.01)$ and mean pp BG $(10.6-13.1 \mathrm{mM} ; \mathrm{p}<0.0001)$ differed significantly. Postprandial BG levels were highest in the subpopulation from Western Asia. The following differences in CVRF were found: triglycerides (mean range) 1.6-1.9 mM ( $\mathrm{p}=.07$ ), total cholesterol 3.7-4.7 $\mathrm{mM}(\mathrm{p}<0.0001)$, low-density lipoprotein cholesterol 2.1-2.9 $\mathrm{mM}(\mathrm{p}<0.0001)$, and tobacco use 7-20 yrs $(\mathrm{p}=.03)$. Treatments received for entry AMI also showed variability: primary percutaneous transluminal coronary angiopathy $31-57(\mathrm{p}=0.0005)$, fibrinolysis $9-49 \%$ $(\mathrm{p}<0.0001)$, CABG $1-10 \%(\mathrm{p}<0.0001)$, and GP IIb/IIIa receptor blockers $12-32 \%(\mathrm{p}<0.0001)$. Application of i.v. insulin infusion for AMI was similar across regions: 28 $37 \%(\mathrm{p}=0.4)$.

Conclusions: These regional differences in features of the entry AMI, daily BG profiles, CVRF, and treatment for AMI will be critical to fully understand the results of this trial, and may have implications for calculating sample size and design of future trials on $\mathrm{CV}$ disease and diabetes.

This study was funded by Eli Lilly and Company.

\section{0}

Effects of intensive insulin therapy in non-previously known diabetic subjects with an acute myocardial infarction and hyperglycaemia. The INSUCOR study

I. Conget ${ }^{1}$, B. Benito ${ }^{2}$, X. Bosch ${ }^{2}$, M. Heras ${ }^{2}$, A. Martin ${ }^{1}$, E. Esmatjes ${ }^{1}$;

${ }^{1}$ Endocrinology and Diabetes Unit, ${ }^{2}$ Cardiology Unit, Hospital Clínic i Universitari/IDIBAPS, Barcelona, Spain.

Background and Aim: Hyperglycaemia has been widely associated with a bad prognosis in acute coronary syndrome. The clinical benefit of maintaining glycaemia close to normal levels using insulin in the course of acute myocardial infarction (AMI) has been evaluated mainly in diabetic subjects. Our study aimed to investigate the shortterm effects and the safety of strict glycaemic control using i.v.insulin in subjects with an AMI and hyperglycaemia without known diabetes. Patients and methods: 28 subjects with AMI and a plasma glycaemia (PG) at admission $>$ $140 \mathrm{mg} / \mathrm{dl}$ and after $6 \mathrm{~h}$-fasting $>126 \mathrm{mg} / \mathrm{dl}$ were included in the study. They were randomly assigned to two different treatment groups of 48 hours' duration: Intensive Group (IG, $\mathrm{n}=13$ ), i.v. Intensive insulin therapy to keep PG between $80-110 \mathrm{mg} / \mathrm{dl}$ and Control group (CG, $\mathrm{n}=15)$, s. c. insulin only if $\mathrm{PG}>160 \mathrm{mg} / \mathrm{dl}$. Glucose profile and hs C reactive protein (CRP) were evaluated at admission and after $48 \mathrm{~h}$ of intervention. Clinical and metabolic parameters were analysed during hospitalisation and after 4 weeks. Results: There were not initial differences in clinical characteristics and PG (Age, $62 \pm 12$ yr vs. $67 \pm 10$; Women, 3 vs. 5; PG, $180 \pm 69 \mathrm{mg} / \mathrm{dl}$ vs. $192 \pm 62$, IG and CG, respectively). During the 48 hours of experimental treatments, PG was significantly lower in IG $(104 \pm 8 \mathrm{mg} / \mathrm{dl})$ than in CG $(153 \pm 54 \mathrm{mg} / \mathrm{dl}, \mathrm{p}=0.002)$ without hypoglycaemic episodes in any group. After $48 \mathrm{~h}$, hsCRP was significantly lower in IG $(20.4 \pm 20.0 \mathrm{mg} / \mathrm{l})$ than in CG $(44.3 \pm 28.0 ; \mathrm{p}=0.04)$. This difference disappeared at the moment of discharge. After 4 weeks, glucose tolerance categories (OGTT) were similar in both groups, with only $28.6 \%$ of the subjects being normal in OGTT. $\mathrm{HbA}_{1 \mathrm{c}}$ at admission was significantly higher in those subjects later diagnosed of diabetes.

In summary, the maintaining of near normoglycaemia is feasible and safe using i.v. insulin in subjects without known diabetes with AMI and hyperglycaemia. In addition, it produces attenuation in inflammatory response. Our study also confirms the high prevalence of unknown abnormalities in glucose tolerance in subjects with AMI.

This study was partially supported by a grant from the Spanish Society of Cardiology (Beca Merck Farma $y$ Quimica, October 2004) and from a contribution from GlaxoSmithKline.

\section{1}

Predictors of transition to persistent fibrillation in type 2 diabetic patients with paroxysmal atrial fibrillation

T. T. Akhobadze ${ }^{1}$, R. Kurashvili ${ }^{1}$, L. Dzneladze ${ }^{2}$, M. Dundua ${ }^{1}$, K. Pagava ${ }^{1}$, T. Avazashvili ${ }^{3}$,

T. Khidesheli ${ }^{2}$, N. Nakopia ${ }^{2}$;

${ }^{1}$ Georgian Diabetes Center, Georgian Diabetes Center, Tbilisi, ${ }^{2}$ National Center of Therapy, Tbilisi, ${ }^{3}$ National Center of Therapy, Ministry of Public Health, Tbilisi, Georgia.

Background and Aims: Atrial fibrillation (AF) is often observed in patients (pts) with type 2 diabetes mellitus (T2DM). It has been shown, that P-wave dispersion (Pd) may be used for predicting pts with paroxysmal AF (PAF) from healthy subjects. This study was conducted for identifying PAF predictors of progression to persistent $\mathrm{AF}$ in $\mathrm{T} 2 \mathrm{DM}$ 
Materials and Methods: Participants were 64 pts (37 males/27 females, mean age 59.7+6.3 yrs) with T2DM and PAF in history. In all pts 12-lead surface ECG, 24-hour ECG and blood pressure (BP) monitoring and echocardiography were performed. The maximum P-wave duration (Pmax), minimum P-ware duration (Pmin) and P-wave dispersion $(\mathrm{Pd}=\mathrm{Pmax}-\mathrm{Pmin})$ were calculated on the surface ECG. The rhythm disturbances were studied using Holter ECG. Left ventricular ejection fraction (LVEF), left ventricular mass (LVM) and left atrial diameter were measured by echocardiography. The pts were followed for 12 months. Results: At the end of the study AF became chronic in $34.7 \%$ of pts ( $n=22$, Gr. 1 ). At entry, the pts who developed chronic AF in the future, differed from those who did not $(\mathrm{n}=42$; Gr. 2). Pts age in Gr. 1 was higher than in Gr. 2 $(63.3+5.1$ vs $54.4+6.3$ yrs, $\mathrm{P}=0.000$, respectively). Hypertension was more frequent, and the number of non-dippers was higher in Gr. 1, than in Gr. 2. In Gr. 1-12 (54.5\%) out of 22 pts, and in Gr.2-11(26.2\%) out of 42 pts were nondippers. 24 -hour systolic BP (SBP) was higher in Gr.1 $(143+9$ vs $138+6 \mathrm{mmHg})$; according to Holter ECG data no statistically significant difference in frequency of supraventricular extrasystols between the groups was registered, though episodes of silent myocardial ischemia were more frequent in Gr.1 $(\mathrm{p}<0.01)$. LVM and left atrial diameter were higher in Gr.1, than in Gr.2 $(\mathrm{P}<0.01, \mathrm{P}<0.05$, respectively). LVEF was lower in pts with chronic AF in the future, but not significantly. There was significantly increased $\mathrm{P}$-wave dispersion $(59+7$ vs $48+8 \mathrm{~ms}, \mathrm{P}=0.000$, respectively). Persistent AF in T2DM pts is associated with age, left ventricular mass, lack of nocturnal decline of SBP, silent ischemia episodes, left atrial size, and increased Pwave dispersion.

Conclusion: In T2DM pts risk of chronic atrial fibrillation increases with age, left ventricular mass and left atrial size. Increased P-wave dispersion in T2DM pts with PAF in history is associated with increased risk of PAF transition to persistent atrial fibrillation.

\section{2}

Lower heart rate variability is associated with higher plasma concentration of Il-6 in type 1 diabetis

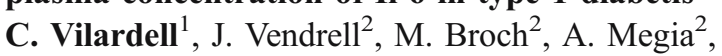
A. Caixàs ${ }^{1}$, O. Giménez-Palop ${ }^{1}$, C. Richart ${ }^{2}$, I. Simón $^{2}$, A. Martínez-Riquelme ${ }^{3}$, D. Mauricio ${ }^{1}$, G. Giménez-Pérez ${ }^{1}$, J. González-Clemente ${ }^{1}$;

${ }^{1}$ Department of Diabetes, Endocrinology and Nutrition, Hospital de Sabadell, (Barcelona), ${ }^{2}$ Diabetes and Research Unit, Hospital Universitari Joan XXIII. Universitat Rovira i Virgili. Institut Pere Virgili., Tarragona, ${ }^{3}$ Associació Catalana de Diabetis, ACD, Barcelona, Spain.
Background and Aims: In type 1 diabetes, cardiovascular autonomic neuropathy (CAN) is associated with cardiovascular risk factors related to insulin resistance, which in turn are associated with low-grade systemic inflammation. As the autonomic nervous system interacts with systemic inflammation, we evaluated CAN to test its possible association with low-grade systemic inflammation.

Materials and Methods: One hundred and twenty subjects diagnosed with type 1 diabetes 14 years before (age 27.4+ $6.6,50 \%$ women), were analysed for: 1) clinical characteristics; 2) plasma levels of soluble fractions of TNF- $\alpha$ receptors 1 and 2, IL-6 and high-sensitivity C-reactive protein; 3) insulin resistance (through an estimation of the glucose disposal rate -eGDR-), and 4) tests for CAN (heart rate variability $-\mathrm{HRV}$ - in response to deep breathing (E/I ratio), HRV in response to the Valsalva manoeuvre and changes in systolic blood pressure responding to standing).

Results: A significant negative correlation was found between $\mathrm{E} / \mathrm{I}$ ratio and plasma concentrations of Il-6 $(r=-0.244, p=0.013)$. This correlation remained significant after adjusting for potential confounders (age, sex, smoking, alcohol intake, HbAlc, waist-to-hip ratio, diastolic blood pressure, triglycerides, HDL-colesterol, retinopathy, nephropathy, peripheral neuropathy and insulin dose $(\mathrm{r}=-0.239, \mathrm{p}=0.031)$. No other significant associations were found between inflammation-related proteins, test for CAN and eGDR.

Conclusion: The current findings suggest a link between low grade inflammation and early alterations of CAN in type 1 diabetes amd may be of importance in the pathogenesis of CAN and/or its clinical implications.

Supported by Fondo de Investigaciones Sanitarias (RG03/ 212, RC03/08) Associació Catalana de Diabetis

1173

Usefulness of pulmonary venous flow in non-invasive assessment of early left ventricular diastolic dysfunction in young non-smokers with type 1 diabetis mellitus

K. Kurnicka, B. Lichodziejewska, A. Lipińska, K. Jankowski, M. Ciurzyński, D. Liszewska-Pfejfer;

Internal Medicine and Cardiology, Medical University of Warsaw, Poland.

Background and Aims: The impairment of left ventricular diastolic function (LVDF) connected with diabetic cardiomyopathy leads to symptomatic heart failure. We studied mitral valve flow (MVF) and pulmonary venous flow (PVF) parameters in young not smoking patients with diabetes mellitus type 1 (DM1) to evaluate LVDF, using an echocardiographic method. 
Materials and Methods: The study group (I) consisted of 72 patients $(30 \mathrm{M}, 42 \mathrm{~F}$, mean age $28.7 \pm 7.0 \mathrm{yrs})$ with DM1, non-smokers, without cardiac disease, with mean DM1 duration $15.4 \pm 7.2$ yrs. Mean HbAlc was $9.6 \pm 2.6 \%$. Thirty healthy non-smokers (15 M, 15F, mean age $29.1 \pm 6.7 \mathrm{yrs})$ served as a control group (II). In MVF: the maximal velocity (Vmax) of early phase - E, Vmax of late phase - A, $\mathrm{E} / \mathrm{A}$ ratio, deceleration time (DT) and isovolumetric relaxation time (IVRT) were measured. In PVF: Vmax of systolic flow (S), Vmax of diastolic flow (D) and S/D ratio were evaluated.

Results: Mean values of BMI, heart rate, systolic and diastolic blood pressure, ejection fraction and LV mass did not differ between both groups. In MVF, the mean value of A was significantly higher $(58.9 \pm 9.5$ vs $53.5 \pm 10.4 \mathrm{~cm} / \mathrm{s}$; $\mathrm{p}=0,02)$, and the $\mathrm{E} / \mathrm{A}$ ratio was significantly lower $(1.35 \pm$ 0.3 vs $1.54 \pm 0.35 ; p=0,01)$ in group I than in group II. Mean value of DT was significantly higher in group I than in II (168.4 \pm 17.7 vs $154.7 \pm 15.9 \mathrm{~ms} ; \mathrm{p}<0,001)$. The MVF results tend to show the impairment of LVDF in young not smoking DM1 patients, despite the fact they remain normal for this age group. In PVF, the mean value of $\mathrm{D}$ was significantly lower $(47.5 \pm 7.6 \mathrm{vs} 55.1 \pm 7.3 \mathrm{~cm} / \mathrm{s} ; \mathrm{p}<0,0001)$, so the $\mathrm{S} / \mathrm{D}$ ratio was significantly higher $(1.13 \pm 0.2$ vs $0.9 \pm$ $0.1 ; p<0,0001)$ in diabetics than in controls. PVF $S / D$ ratio showed systolic dominance $(\mathrm{S} / \mathrm{D}>1)$ typical for impaired LV relaxation and was abnormal for this age group. No correlation between echo-parameters and DM1 duration, HbAlc level, presence of retinopathy, nephropathy or peripheral neuropathy was found.

Conclusion: 1. Echocardiographic assessment of PVF shows an impaired LV relaxation in young, not smoking patients with long term DM1. 2. PVF parameters are especially sensitive markers in detection of early subclinical LV diastolic dysfunction, especially when MVF parameters remain normal.

\section{PS 105}

\section{Predictors of morbidity and mortality}

\section{4}

\section{Abnormal glucose tolerance prior to first myocardial infarction - not as prevalent as expected E. Fhärm ${ }^{1}$, J.-H. Jansson ${ }^{2}$, O. Rolandsson ${ }^{1}$; \\ ${ }^{1}$ Family Medicine, ${ }^{2}$ Internal Medicine, Public Health and Clinical Medicine, Umeå, Sweden.}

Background and Aims: Abnormal glucose tolerance and especially $2 \mathrm{~h}$-glucose is reported to predispose for cardiovascular disease (CVD). In a recently published study from Sweden it was reported that among patients, aged $63.5( \pm 9.4)$ years, who were admitted to hospital for acute myocardial infarction (AMI), a majority (66\%) had an abnormal glucose tolerance i.e. previously unknown diabetes or impaired glucose tolerance (IGT). However, the prevalence of abnormal glucose tolerance in the general population is much lower, so, it has been speculated that AMI as a stressful life event by itself might inflict abnormal glucose tolerance. Our aim was to study the proportion of abnormal glucose tolerance prior to the subjects first AMI. Materials and Methods: A prospective nested case-control study of 508 (M/F 414/94) patients with incident first-ever myocardial infarction (mean age 58.0 7.7 years), and 1751 sex and age matched controls (M/F 1434/317) was performed. Cases and controls had no diagnosed diabetes before participating in a health survey, which included an oral glucose tolerance test (OGTT) prior to the AMI (mean 4.1 \pm 2.7 years). Categorisation of the OGTT was based on the 1999 WHO criteria for diabetes and impaired glucose tolerance. It was thoroughly investigated that none of the cases or controls had AMI prior to the observed event. Myocardial infarction, fatal and non fatal, was diagnosed according to the protocol of the Northern Sweden MONICA data registry.

Results: $5.3 \%(95 \% \mathrm{CI} 3.4-7.2 ; \mathrm{M} / \mathrm{F} 5.6 \% / 4.3 \%)$ of cases and $3.0 \%(95 \% \mathrm{CI} 2.2-3.8 ; \mathrm{M} / \mathrm{F} 3.0 \% / 3.2 \%)$ of controls were found to have a previously unknown diabetes $(\mathrm{p}<0.05)$ whereas $9.4 \%(95 \% \mathrm{CI} \quad 6.9-11.9 ; \mathrm{M} / \mathrm{F} \quad 8.0 \% /$ $16.0 \%)$ of cases and $5.6 \%(95 \% \mathrm{CI} 4.5-6.7$; M/F $5.1 \% /$ $7.9 \%$ ) of the controls had impaired glucose tolerance $(p<0.01)$. There was no significant difference in OGTT beween men and women. Hypertension $(43 \%$ vs. $24 \%$; $\mathrm{p}<0.001)$ and obesity $(22 \%$ vs. $10 \% ; \mathrm{p}<0.001)$ was more frequent among subjects with diabetes/IGT compared to controls. Hypercholesterolemia ( $87 \%$ vs. $84 \%)$ and smoking $(21 \%$ vs $26 \%)$ did not differ significantly.

Conclusion: In this population of AMI patients without previous cardiac events, abnormal glucose tolerance was almost twice as common as in age and sex matched controls. As expected, the subjects with abnormal glucose tolerance also had a higher risk profile with more signs of the metabolic syndrome. However, the proportions of abnormal glucose tolerance in both cases and controls in our study were about one quarter of the earlier published data from Norhammar et al. Our patients were younger and did not include patients with re-infarction which might contribute to the lower proportion of abnormal glucose tolerance in our study. In summary, the noticeable difference in glucometabolic state in AMI patients between our and previous studies calls for further evaluation of the impact of coronary events on patients' metabolic state. Supported by County Council of Västerbotten, Sweden 


\section{5}

Abnormal glucose regulation is common and often
undiagnosed in patients with coronary artery disease
in China
C.-Y. Pan ${ }^{1}$, D.-Y. $\mathrm{Hu}^{2}$, J.-M. Yu ${ }^{2}$;
${ }^{1}$ Department of Endocrinology, Chinese PLA General
Hospital, Beijing, ${ }^{2}$ Centre for Cardiovascular and Pulmo-
nary Blood Vessels, Tongji University, Shanghai, China.

Background and Aims: Coronary artery disease (CAD) is a common cause of death in type 2 diabetes patients, and there is evidence that prediabetes, particularly impaired glucose tolerance (IGT), is also associated with an increased risk of cardiovascular morbidity and mortality. The presence of diabetes or prediabetes at admission for a cardiovascular event has been shown to be associated with poor patient outcome. Studies determining the glucometabolic state of patients with CAD in Europe have prompted this similar investigation in China.

Materials and Methods: The study was conducted in 52 centres in 7 cities in China, and enrolled 3513 patients hospitalised for CAD. Data were collected by case record forms. Glucometabolic state was classified using WHO 1999 criteria; prediabetes includes IGT and impaired fasting glucose. Fasting plasma glucose (FPG) levels were assessed on admission. Standard oral glucose tolerance tests (OGTTs) were performed in patients without known or newly diagnosed (FPG $>7.0 \mathrm{mmol} / \mathrm{L}$ ) type 2 diabetes, before discharge or within 2 months of admission.

Results: The majority of patients were male $(67 \%)$. The mean age was 69 years and the mean body mass index was $24 \mathrm{~kg} / \mathrm{m}^{2}$. Of the patients enrolled, $35 \%$ were admissions for acute $\mathrm{CAD}$ and $65 \%$ were elective admissions for $\mathrm{CAD}$. Acute coronary syndrome was the most common type of CAD diagnosis in both admission groups. At admission, 1153 (33\%) patients had known type 2 diabetes and 97 (3\%) had newly diagnosed diabetes by FPG level. Of the remaining patients, forming the OGTT cohort $(n=2263)$, $27 \%$ had type 2 diabetes and $37 \%$ had prediabetes. The prevalences of type 2 diabetes and prediabetes were similar in the acute ( $28 \%$ and $39 \%$, respectively) and elective ( $26 \%$ and $36 \%$, respectively) admissions groups within the OGTT cohort. When FPG levels (OGTT 0 min) were considered, $10 \%$ of patients had diabetes or prediabetes. In contrast, when postchallenge levels (OGTT $2 \mathrm{~h}$ ) were considered, $64 \%$ of patients had diabetes or prediabetes. Consequently, $87 \%$ of patients with prediabetes and $81 \%$ of patients with diabetes would have remained undiagnosed without the performance of OGTTs. Overall, the proportion of patients with diagnosed diabetes increased from $33 \%$ at the start of the study to $53 \%$ post-OGTT analysis. Glucometabolic state was not associated with the type of CAD diagnosis, but was significantly associated with $\mathrm{HbAlc}$ level, systolic blood pressure, triglyceride level, HDL-cholesterol level and serum creatinine level (all $\mathrm{p} \leq 0.001$ ).

Conclusion: This study adds to the growing evidence that type 2 diabetes and prediabetes are common and often undiagnosed in patients with CAD. The prevalence of abnormal glucose regulation is underestimated by FPG testing alone, and OGTTs should be routinely used to assess the glucometabolic state of patients admitted with CAD. Earlier diagnosis and treatment of diabetes or prediabetes may improve long-term outcomes in this highrisk patient group.

Supported by an unrestricted research grant from Bayer HealthCare Ltd

1176

Diabetes, coronary heart disease and C-reactive protein in high-risk individuals undergoing coronary angiography

G. Babacan ${ }^{1}$, P. Topsever ${ }^{2}$, F. Guzelbulut ${ }^{1}$, M. Eryilmaz ${ }^{1}$, N. Aydin ${ }^{1}$;

${ }^{1} 5$ th Department of Internal Medicine, Haydarpasa Numune Research and Training Hospital, Istanbul, Turkey, ${ }^{2}$ Department of Family Medicine, Kocaeli University, Turkey.

Background and Aims: As a non-specific acute phase reactant, C-reactive protein (CRP) is being discussed as an adjunct predictor for cardiovascular disease which is an inflammatory process. Diabetes (DM) is a condition associated with an increased risk for cardiovascular morbidity and mortality and according to NCEP/ATP 3 guidelines considered a coronary artery disease equivalent. The aim of this study was to evaluate the impact of diabetes on the association of CRP with the presence of coronary heart disease (CHD) in a high-risk population undergoing coronary angiography.

Materials and Methods: One hundred and twenty three consecutive patients (female $\mathrm{n}=50,40.7 \%$, male $\mathrm{n}=73$, $59.3 \%$, mean age $59.40 \pm 10.91$ years) referred to the department of cardiology for diagnostic coronary angiography were enrolled in this cross-sectional, case-control study upon verbal informed consent. Except for sociodemographics like age and gender, tobacco and alcohol consumption, family history of premature CHD, established diagnosis of diabetes mellitus, hypertension, hyperlipidemia and ongoing drug treatments were assessed. Other conditions, likely to confound CRP levels were exclusion criteria. Coronary angiography results were evaluated by two independent cardiologists and patients were dichotomized as cases (stenosis $\geq 50 \%$ of one ore more main coronary artery) and controls (no stenoses). Main outcome 
parameter was serum CRP level. Except for descriptives, Independent-Samples $\mathrm{T}$ Test was used for parametric and Chi-Square Test for non-parametric variables. Odds ratios (OR) were calculated via cross-tabulation with a $95 \%$ confidence interval (CI) and a significance level of 0.05 . In multiple linear regression analysis, interaction models were built with CRP as dependent and CHD and DM as independent variables.

Results: CHD was angiographically detected in 93 cases (75.6\%). Cases were differing from controls with respect to CRP $(0.74 \pm 0.65$ vs. $0.41 \pm 0.25, p<0.001)$ and HDL levels $(57.16 \pm 16.72$ vs. $49.63 \pm 13.22, p=0.013)$ in parametric testing. Older age (females $\geq 55$, males $\geq 45$ years) $(\mathrm{OR}=5.3$, $95 \%$ CI 1.8 to 15.8$)$, male gender $(\mathrm{OR}=4.0,95 \% 1.9$ to 8.3$)$, smoking ( $\mathrm{OR}=1.2,95 \% \mathrm{CI} 1.1$ to 1.5$)$ and hypercholesterolemia $(\mathrm{OR}=3.1,95 \% \mathrm{CI} 1.3$ to 7.7$)$ were associated with CHD in nonparametric testing. Nevertheless, cases were more likely to receive statin therapy $(\mathrm{OR}=1.2,95 \% \mathrm{CI} 1.0$ to 1.5). Conventional risk factors like family history of premature CVD, diabetes and hypertension were not found to be associated with CHD. CRP among cases was $0.27 \mathrm{mg} / \mathrm{dL}$ ( $95 \%$ CI 0.02 to $0.52, p=0.034$ ) higher than in controls after adjustment for age, gender and hypercholesterolemia in multiple regression analysis. DM and CHD significantly interacted in their relationship with CRP $(P$ interaction $=0.078$ ), as shown by a significant positive association of CHD with CRP in non-diabetic individuals (CRP $0.35 \mathrm{mg} / \mathrm{dL}$ higher in $\mathrm{CHD}(+)$ patients, $95 \%$ CI 0.1 to $0.6 ; p=0.012)$ which eroded in the presence of diabetes $(p=0.351)$.

Conclusion: The interpretation of CRP as a screening test for coronary heart disease in diabetic individuals has to be critically reevaluated and investigated further.

Supported by an unrestricted research grant from Bayer HealthCare Ltd

\section{7}

Type 2 diabetes and the coronary angiographic state are mutually independent predictors of future vascular events

P. $\operatorname{Rein}^{1}$, C. H. Saely ${ }^{1,2}$, F. Schmid ${ }^{1}$, L. Koch ${ }^{1}$, T. Marte ${ }^{1,2}$, S. Aczel ${ }^{1,2}$, P. Langer ${ }^{1}$, H. Drexel ${ }^{1,2}$;

${ }^{1}$ VIVIT Institute, ${ }^{2}$ Department of Internal Medicine, Academic Teaching Hospital Feldkirch, Feldkirch, Austria.

Background and Aims: Type 2 diabetes (T2DM) in crosssectional studies is associated with coronary artery disease (CAD) and prospectively confers a strongly increased risk of vascular events. The aim of this study was to investigate to what extent the baseline CAD state accounts for the increased vascular risk of diabetic patients.
Materials and Methods: We enrolled 750 consecutive patients undergoing coronary angiography for the evaluation of CAD. At angiography, CAD was diagnosed in the presence of any irregularities of the vessel wall. Stenoses of at least $50 \%$ were considered significant, and the extent of $\mathrm{CAD}$ was defined as the number of significant stenoses in a patient. Vascular events were recorded over 4 years.

Results: The prevalence of CAD $(87.8 \%$ vs. $80.4 \%$; $\mathrm{p}=$ $0.029)$ and of significant stenoses $(69.5 \%$ vs. $58.4 \%$; $\mathrm{p}=0.010)$ as well as the extent of CAD $(1.7 \pm 1.5$ vs. $1.4 \pm$ $1.5 ; \mathrm{p}=0.014)$ were significantly higher in patients with T2DM $(n=164)$ than in nondiabetic subjects $(n=586)$. Prospectively, T2DM after multivariate adjustment strongly predicted vascular events (adjusted hazard ratio $(\mathrm{HR})=1.66$ [1.15-2.39]; $\mathrm{p}=0.006)$. Also, the presence of CAD $(\mathrm{HR}=2.78$ [1.39-5.57]; $\mathrm{p}=0.004)$, the presence of significant stenoses $(\mathrm{HR}=3.67[2.30-5.85] ; \mathrm{p}<0.001)$ and the extent of CAD (standardized adjusted $\mathrm{HR}=1.62$ [1.411.87]; $\mathrm{p}<0.001$ ) significantly predicted vascular events. These angiographic characteristics still predicted vascular events after additional adjustment for T2DM $(\mathrm{HR}=2.66$ [1.33-5.34]; $\mathrm{p}=0.006,3.57$ [2.24-5.70]; $\mathrm{p}<0.001$, and 1.60 [1.34-1.84]; $\mathrm{p}<0.001$, respectively). Conversely, T2DM remained strongly and significantly predictive of future vascular events after adjustment for the presence and extent of CAD (HR=1.50 [1.04-2.15]; $\mathrm{p}=0.029$ ).

Conclusion: Among angiographied coronary patients, the presence and the extent of CAD are higher in patients with T2DM than in nondiabetic individuals. Prospectively, T2DM and the baseline CAD state are mutually independent predictors of future vascular events.

\section{8}

Mortality of patients with early-onset type 2 diabetes in Japan

Y. Uchigata ${ }^{1}$, M. Okudaira ${ }^{1}$, M. Matsushima ${ }^{2}$, T. Otani ${ }^{1}$, Y. Iwamoto ${ }^{1}$;

${ }^{1}$ Diabetes Center, Tokyo Women's Medical University School of Medicine, Japan, ${ }^{2}$ Department of General Medicine, Jikei University School of Medicine, Tokyo, Japan.

Background and Aims: According to a recent study based on the results of school health checkups involving urinalysis in urban areas in Japan, the number of patients with early-onset type 2 diabetes (E-T2D) has gradually increased, as reported for the number of patients with adultonset T2D. In a survey in which diabetic complications were matched to the duration of disease, we previously reported that nephropathy in those with E-T2D was more advanced than in those with early-onset type 1 diabetes (E- 
T1D). The above results suggest that the prognosis of ET2D is poorer than that of E-T1D, in whom the standardized mortality ratio (SMR) was already reported to be 2.8. The aim of this study is to investigate the mortality and the causes of death in patients with E-T2D.

Patients and Methods: Among 927 patients with E-T2D who consulted our center between January 1, 1980 and December 31, 1990 (age at detection: less than 30 years), the subjects were 642 patients who were treated at our center for 1 year or more. The survival status by January 1 , 2001 was investigated. The end-point was determined using questionnaires mailed to attending physicians or through telephone interviews with patients or their families.

Results: There were no differences of baseline characteristics between the subjects $(n=642,358$ males and 284 females, age at detection and duration at start of follow-up in the center ; $0-9 / 10-19 / 20-29$ ages $=2 / 201 / 439$ and $19 \pm$ $8.8 / 6.7 \pm 8.4 / 7.2 \pm 8.2$, respectively) and the patients treated less than 1 year( $\mathrm{n}=285,173$ males and 112 females, age at detection and duration at start of follow-up in the center ; $0-9 / 10-19 / 20-29$ ages $=0 / 77 / 208$ and $0 / 6.8 \pm 8.6 / 7.4 \pm 8.8$, respectively). The completeness of this study was $84.4 \%$ (542/642). Fifty-one patients had died. The mortality ratio $(/ 1,000$ person-year) was 6.7 and the standardized mortality ratio (SMR) in the subjects was $3.7(95 \% \mathrm{CI} ; 2.2-4.5)$. The cumulative 10-year and 20-year survival rates from the follow-up period after the initial consultation in our center were $95.7 \%$ and $79.5 \%$, respectively. Approximately 33\% of the deceased patients died of major vascular disorders (CVD 17.6\%; IHD 15.7\%), although $67.5 \%$ of the patients had the end-stage renal disease and received dialysis treatment before dying.

Conclusion: The follow-up rate was lower than that in a survey involving patients with E-T1D in our center, though, the frequency of major vascular disorders as the cause of death was more frequent than that in the survey of E-T1D. This was possibly because the age at detection and that during follow-up in the subjects were older than those in the E-T1D in our center and dialysis treatment had effective for surviving but did not overcome the major vascular disorders.

\section{9}

Elevated pregnancy-associated plasma protein A predicts mortality in type 1 diabetic nephropathy

L. Tarnow ${ }^{1}$, M. Christiansen ${ }^{2}$, P. R. Hansen ${ }^{3}$, A. S. Astrup ${ }^{1}$, H.-H. Parving ${ }^{1,4}$;

${ }^{1}$ Clinical Research, Steno Diabetes Center, Gentofte, ${ }^{2}$ Department of Biochemistry, Statens Serum Institute, Copenhagen, ${ }^{3}$ Department of Cardiology, Gentofte Hospital, ${ }^{4}$ Faculty of Health Science, Aarhus University, Aarhus, Denmark.
Background and Aims: Pregnancy-Associated Plasma Protein A (PAPP-A) has been implicated in acute coronary syndromes and carotid and peripheral atherosclerosis. Diabetic nephropathy is characterised by increased cardiovascular morbidity and mortality. This study investigated the prognostic value of circulating PAPP-A levels in a large cohort of type 1 diabetic patients with and without diabetic nephropathy.

Materials and Methods: In a prospective observational follow-up study 198 type 1 diabetic patients with overt

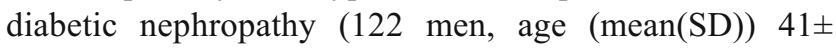
10 years, duration of diabetes $28 \pm 8$ years, GFR $74 \pm$ $33 \mathrm{ml} / \mathrm{min}$ ) and a matched control group of 180 patients with longstanding type 1 diabetes and persistent normoalbuminuria (114 men, age $43 \pm 10$ years, duration of diabetes $27 \pm 9$ years) were followed for $9.3(0.0-9.5)$ years. Plasma PAPP-A concentration was determined by immunoassay at baseline.

Results: In patients with diabetic nephropathy, plasma PAPP-A concentration was elevated $3.6(0.4-51.1) \mathrm{mIU} /$ 1 (median(range)) versus $2.1(0.4-46.6) \mathrm{mIU} / \mathrm{l}$ in normoalbuminuric patients, $\mathrm{p}<0.0001$. Furthermore, proMBP (proform of eosinophil major basic protein) the endogenous inhibitor of PAPP-A was raised in patients with nephropathy versus normoalbuminuria, 2287 (909-9287) and 1612 (780-12970) mIU/l, $\mathrm{p}<0.0001$. For acute coronary syndromes, a PAPP-A threshold of $10 \mathrm{mIU} / 1$ has previously been suggested. Among patients with nephropathy 9 (47\%) patients with plasma PAPP-A above the threshold and 42 (24\%) with values below died from any cause (hazard ratio $2.41(95 \%$ CI $1.17-4.95) \mathrm{p}=0.017)$. This lower mortality was attributable to fewer cardiovascular deaths: $8(42 \%)$ and $30(19 \%)$ above and below the PAPP-A threshold level, respectively (hazard ratio 3.00 (1.37-6.52), $\mathrm{p}=0.006$ ). PAPP-A levels at baseline were elevated in men as compared to women $(4.0(0.4-29.7) \mathrm{mIU} / \mathrm{l}$ versus 3.3 (0.5-51.1) $\mathrm{mIU} / \mathrm{l} \mathrm{p}=0.02$, increased with age and systolic blood pressure, and rose with declining kidney function, $\mathrm{p}<0.01$. The prognostic information of PAPP-A was attenuated after adjustment for confounding factors.

Conclusion: Elevated circulating PAPP-A predicts excess overall and cardiovascular mortality in type 1 diabetic patients with diabetic nephropathy.

\section{0}

Homocysteine levels seem to be the main factor determining familial longevity

A. Ugalde ${ }^{1}$, M. Fabregate ${ }^{1}$, R. Fabregate ${ }^{1}$, O. Sanchez ${ }^{1}$, M. Maties ${ }^{2}$, E. Fernandez ${ }^{1}$, J. Saban-Ruiz ${ }^{1}$; 
${ }^{1}$ Endothelial Pathology Unit, Ramon y Cajal Hospital, Madrid, ${ }^{2}$ Biochemistry Service, Ramon y Cajal Hospital, Madrid, Spain.

Introduction: The increase of mean lifetime has also given rise to an increase in the number of centenarians. Longevity is the result of health-promoting behaviours plus genetic factors that promote disease resistance and long-term survival. If environmental factors did not change from one generation to the next, parent's and children's longevities would be expected to be similar. Some studies do, in fact, show similarities in the age of death in parents and offspring while other investigations confirm that parental survival is an independent predictor of offspring's longevity. Epidemiological studies have enhanced the importance of high-density lipoprotein (HDL) in coronary artery disease (CAD) and longevity. On the other hand, emerging risk factors such as homocysteine (Hcy) have been studied in relation to CAD but not to longevity.

Aims: 1- To evaluate metabolic, haemodynamic and endothelial parameters in a population of high cardiovascular risk which has been divided into different groups based on its family history of longevity. 2- To establish gender-related behavioural differences.

Material and Methods: $\mathrm{N}=227$ patients of high cardiovascular risk (55\% type 2 diabetic), aged 22-95 years $(57,57 \pm 0,94)$. 113/114 male/female ratio. Family history of longevity (FHL): at least one first or second degree relative with a demonstrated survival over 85 years. Anthropometric parameters: waist circumference (centimetres) and waist/ hip ratio.

Biochemical parameters: glucose, HDL, LDL, uric acid (Hitachi autoanalyzer). Hcy (uM/L): IMX Abott.PAI-1 (ng/ $\mathrm{ml}$ ): Biopool-Menarini ELISA kit. Ambulatory Blood Pressure Monitoring (ABPM): the following parameters were evaluated in $24 \mathrm{~h}$, during activity (9:00 to $22: 00)$, rest (22:00 to 6:00) and critical (06:00 to 9:00) periods: average systolic blood pressure (aSBP), average diastolic blood pressure $(\mathrm{aDBP})$, average mean arterial pressure (MAP) [(SBP-DBP)/3+DBP]. Ambulatory pulse pressure (APP). Standard systolic and diastolic blood pressure burden (systolic $>125$ and diastolic $>75 \mathrm{mmHg}$ pressure reading percentages) (SBPB and DBPB). Hemodynamic load $(\mathrm{HL}=$ Heart Rate $\mathrm{x} \mathrm{MAP} / 100)$. SBP variability (SBPV) and DBP variability (DBPV). HR variability (HRV). Statistical analysis: Student's T test. Mann-Whitney's U test. Kolmogorov-Smirnov's test. Chi-squared test. RESULTS: 1 Patients with a family history of longevity (FHL) ( $\mathrm{n}=82,37 / 45$ male/female ratio) presented lower levels of Hcy $(12,25 \pm 0,39$ vs $13,87 \pm 0,83$; $<<0,05)$ compared to those without FHL $(n=145,76 / 69$ male/female ratio). Ages were similar in both groups $(57,97 \pm 1,22$ vs $56,87 \pm 1,46)$. 2 In men, statistically significant differences were found in waist circumference values $(91,62 \pm 1,49$ vs $96,75 \pm 2,16, \mathrm{p}=0,034)$ and Hcy levels $(12,03 \pm 0,58$ vs $15,61 \pm 1,32, p=0,007)$ between both groups. Differences were also found in PAI-1 levels $(45,11 \pm 2,74$ vs $56,88 \pm$ $4,73)$, although at the borderline of statistical significance $(p=0,06)$. No significant differences were found in women.

Conclusions: 1. In the general population, homocysteine levels seem to be the main factor determining familial longevity. 2. In the gender-based study, this discovery is confirmed only in males. 3. Such differences in males were further accompanied by differences in waist circumference and endothelial impact, at the borderline of statistical significance.

\section{1}

N-terminal pro brain natriuretic peptide levels in correlation with renal echo Doppler parameters and microalbuminuria in hypertensive diabetic and nondiabetic patients

M. Szabo ${ }^{1}$, M. German-Sallo ${ }^{2}$, D. Balint-Szentendrey ${ }^{2}$, L. Farkas $^{2}$, G. Suciu ${ }^{1}$;

${ }^{1}$ Diabetes Department, ${ }^{2}$ Cardiological Department, Medical University Hospital Targu Mures, Targu Mures, Romania.

Background and Aims: Elevated $\mathrm{N}$ terminal pro brain natriuretic peptide (NT-proBNP) is related to the presence of cardial dysfunction, but is also a predictor of increased cardiovascular morbidity in microalbuminuric type 2 diabetic patients. Our aim was to study NT-proBNP in hypertensive diabetic or nondiabetic patients, without symptoms of cardial disease, in relation with microalbuminuria, and renal echo-Doppler measurements.

Materials and Methods: Waist circumference, blood pressure, ProBNP, microalbuminuria, creatinine measurements, renal echo Doppler (pulsatility index (PI), resistive index (RI)) was assessed and full echocardiographic examination was performed in 62 hypertensive patients (aged 52,5 \pm 3 years, 38 male). 34 (55\%) of the patients had type 2 diabetes.

Results: Diastolic dysfunction was more frequent in diabetic patients $(89 \%$ versus $68 \%, \mathrm{p}<0,05)$. Pulsatility index and resistive index were higher in patients with type 2 diabetes $(1,11 \pm 0,24$ respective $0,70 \pm 0,1$ versus $1,0 \pm 0,12$ respective $0,65 \pm 0,07, \mathrm{p}<0,05)$. ProBNP correlated also with age $(\mathrm{r}=0,561, \mathrm{p}<0,001)$ and creatinine clearance $(\mathrm{r}=0,412$, $\mathrm{p}<0,05)$. After adjusting for age and creatinine clearance, ProBNP was found significantly higher in diabetic patients ( $177,1 \pm 87$, versus $98,3 \pm 36, \mathrm{p}<0,01)$, but was not significantly correlating with echocardiographic parameters. We could not find any correlation between proBNP and microalbuminuria in our patients, but proBNP was highly 
correlating with renal pulsatility and resistive index $(\mathrm{r}=0,423$, and $0,452, \mathrm{p}<0,01)$.

Conclusion:The present study provides evidence for the correlation of NT-proBNP with the presence of diabetes, and with intrarenal flow deterioration in hypertensive, type 2 diabetic patients, independent of other cardiovascular parameters.

\section{2}

Glomerular filtration rate, cardiovascular endpoints and all-cause mortality in type 2 diabetic patients

A. P. S. Kong ${ }^{1}$, W. Y. So ${ }^{1}$, P. C. Y. Tong ${ }^{1}$, F. C. C. Chow ${ }^{1}$, C. S. Cockram ${ }^{1}$, N. N. Chan ${ }^{2}$, W. B. Chan ${ }^{2}$, R. Ozaki ${ }^{1}$, R. C. W. Ma ${ }^{1}$, J. C. N. Chan ${ }^{1}$;

${ }^{1}$ Medicine and Therapeutics, ${ }^{2}$ Qualigenics Diabetes Centre, The Chinese University of Hong Kong, Shatin, Hong Kong.

Background and Aims: Chronic kidney disease (CKD) predicts cardiovascular disease (CVD) in general population. However, its impact on diabetic populations, especially Chinese, has not been fully studied. We investigated the effects of stages of renal function using estimated glomerular filtrate rate (eGFR) on all-cause mortality and cardiovascular endpoints in a large prospective cohort of Chinese type 2 diabetic patients.

Materials and Methods: Between 1995 and 2000, 4,421 Chinese type 2 diabetic patients without macrovascular disease or end stage renal disease was enrolled. Renal function was assessed by eGFR calculated by the abbreviated Modification of Diet in Renal Disease Study Group (MDRD) formula. Clinical endpoints included all-cause mortality and cardiovascular endpoint (cardiovascular death, new admissions due to angina, myocardial infarction, stroke, revascularization or heart failure).

Results: After a median follow-up of 39.4 months [interquartile range: 20.3 - 55 months], rates of cardiovascular endpoints and all-cause mortality increased from $2.6 \%$ to $24.6 \%(95 \%$ CI [2.0-3.3] to [14.2-35.1], $\mathrm{p}$ for trend $<0.001)$, and $1.3 \%$ to $17.4 \%([0.8-1.8]$ to $[8.2-26.6]$, $\mathrm{p}$ for trend $<0.001$ ) amongst patients with eGFR $\geq 90 \mathrm{ml} /$ $\min / 1.73 \mathrm{~m}^{2}$ to those with eGFR between 15 and $29 \mathrm{ml} /$ $\min / 1.73 \mathrm{~m}^{2}$ respectively. After adjustment for covariates (age, sex, albuminuria, lipid, blood pressure and glycemic control), hazard ratios across different stages of eGFR ( $\geq 90$, $60-89,30-59, \quad 15-29 \mathrm{ml} / \mathrm{min} / 1.73 \mathrm{~m}^{2}$ ) for all-cause mortality were $1.00,1.27,2.34$ and 9.82 ( $\mathrm{p}$ for trend $<0.001$ ), while for cardiovascular endpoints were $1.00,1.04,1.05$ and 3.23 ( $\mathrm{p}$ for trend $<0.001$ ) respectively. Conclusion: Chinese type 2 diabetic patients with reduced eGFR were at high risk of developing cardiovascular endpoints and all-cause mortality, independent of albuminuria and metabolic control.

1183

Reduction of plasma N-terminal pro-brain natriuretic peptide by angiotensin II receptor blockade in type 2 diabetic patients with microalbuminuria

K. Rossing ${ }^{1}$, K. J. Schjoedt ${ }^{1}$, B. R. Jensen ${ }^{1}$, H.-H. Parving ${ }^{1,2}$;

${ }^{1}$ Steno Diabetes Center, Gentofte, ${ }^{2}$ Faculty of Health, Aarhus University, Denmark.

Background and Aims: Raised plasma N-terminal pro-brain natriuretic peptide (NT-proBNP) is independently associated with an increased overall and cardiovascular mortality in nondiabetic and diabetic populations. We investigated the effects of the angiotensin II receptor blocker irbesartan given in the maximally recommended dose (300 $\mathrm{mg}$ daily) and in ultrahigh doses (600 and $900 \mathrm{mg}$ daily) on NT-proBNP in type 2 diabetic patients with microalbuminuria.

Materials and Methods: This double-masked randomized cross-over trial included 52 (41 males) hypertensive Type 2 diabetic patients with microalbuminuria on ongoing antihypertensive medication. At inclusion, previous antihypertensive treatment was discontinued and replaced with bendroflumethiazide $5 \mathrm{mg}$ o.d. for the entire study. Following two months wash-out (baseline), patients were treated randomly with irbesartan 300, 600 and $900 \mathrm{mg}$ o.d., each dose for two months. End-points evaluated at the end of each study period included plasma NT-proBNP (immunoassay, Roche Diagnostics), and as previously reported 24-hours urinary albumin excretion (UAE), 24-hrs ambulatory blood pressure ((ABP)), Takeda-TM2420).

Results: Two months after withdrawal of previous antihypertensive medication, baseline values were: plasma NTproBNP[geometric mean $(95 \% \mathrm{CI})] 32$ (23 to 44$) \mathrm{ng} / \mathrm{ml}$, UAE 93 (72 to 121) $\mu \mathrm{g} / \mathrm{min}$, and ABP [mean (SD)] 140 (10)/77 (7) $\mathrm{mm} \mathrm{Hg.}$

All three doses of irbesartan significantly reduced plasma NT-proBNP, UAE, systolic - and diastolic ABP as compared with baseline.

Reductions in plasma NT-proBNP from baseline were: 20 (4 to 32 ), 25 (3 to 43 ) and 30 (10 to 44$) \%$ with increasing doses of irbesartan (NS between doses). UAE were reduced by 52 (46 to 57 ), 49 (43 to 54 ) and 59 (54 to 63 )\% with increasing doses of irbesartan $(\mathrm{p}<0.01)$. UAE was reduced significantly more by irbesartan $900 \mathrm{mg}$ compared with lower doses with an additional reduction in UAE of 15 (2 to 26)\% by irbesartan $900 \mathrm{mg}$ compared with $300 \mathrm{mg}$ $(p=0.02)$. With increasing doses systolic-ABP was reduced from baseline by 8 (4 to12), 9(5 to13) and 9 (5 to13) 
$\mathrm{mmHg}$, and diastolic-ABP by 6 (4 to7), 7 (6 to 9), and 7 (6 to 9) $\mathrm{mm} \mathrm{Hg}$ (NS between doses).

Conclusion: Plasma NT-proBNP is reduced by irbesartan in type 2 diabetic patients with microalbuminuria. Furthermore there is a tendency towards an increased and dosedependent reduction by ultra-high. doses of irbesartan.

The study was supported by an unrestricted study grant from Sanofi-Aventis, who also provided the study medication. The assay for measurement of NT-proBNP was provided by Roche Diagnostics GmbH, Mannheim, Germany

\section{PS 106}

\section{Diabetic cardiomyopathy}

\section{4}

Critical role of calcium-dependent molecular pathways in type 2 diabetes-associated enhanced activity of the cardiac $\mathrm{Na}+/ \mathrm{H}+$ exchanger

A. Darmellah, D. Baetz, S. Tamareille, C. Rücker-Martin, D. Feuvray;

Hôpital Marie Lannelongue, UMR CNRS 8078, Université Paris-Sud XI, Le Plessis Robinson, France.

Background and Aims: The $\mathrm{Na}^{+}-\mathrm{H}^{+}$exchanger (NHE) is a potential target in terms of attenuation of hypertrophic processes that contribute to heart failure. We investigated sarcolemmal NHE activity and its putative role on left ventricular (LV) abnormalities in a type 2 diabetic animal model.

Materials and Methods: Fluorometric measurements in isolated adult cardiomyocytes demonstrated that LV cardiac hypertrophy in GK (Goto-Kakizaki) rat hearts was associated with enhanced sarcolemmal NHE activity and increased cytosolic $\mathrm{Ca}^{2+}$ levels. Chronic treatment of GK rats with the NHE1 inhibitor cariporide normalized the increase in NHE activity and also significantly reduced LV myocyte hypertrophy. To characterize the molecular pathways driving enhanced NHE activity, we evaluated the role of mitogen-activated protein kinases (MAPKs), protein kinase $\mathrm{C}$ (PKC) isotypes, and $\mathrm{Ca}^{2+} /$ calmodulin-dependent kinase II (CaMKII).

Results: The results demonstrate that the activity of Erk, but not p38 MAPK, was increased markedly, and that $\mathrm{Ca}^{2+}$. dependent PKC and CaMKII are involved. Thus NHE activity, as assessed by the rate of acid efflux carried by the exchanger at an intracellular of 6.85 , was significantly more reduced in GK LV myocytes than in control LV myocytes when each of these kinases was specifically blocked. Only intracellular $\mathrm{Ca}^{2+}$ increase, higher sensitivity of NHE activity to CaMKII as well as to $\mathrm{Ca}^{2+}$-dependent $\mathrm{PKC}$ regressed during reversal of the hypertrophic phenotype by cariporide treatment.

Conclusion: Preventing excessive activation of NHE and subsequent increases in intracellular $\mathrm{Ca}^{2+}$ as well as critical $\mathrm{Ca}^{2+}$-dependent kinases activity may afford protection against LV hypertrophy in type 2 diabetes.

Supported by Fondation de France

\section{5}

Moderate hyperglycaemia affects early MMP activity without effects on late $\mathrm{LV}$ remodeling after myocardial infarction in mice

B. Szepietowska ${ }^{1}$, M. Szelachowska ${ }^{1}$, K. Kaminski ${ }^{2}$,

A. Malkowski ${ }^{3}$, W. Karwowski ${ }^{4}$, M. Kozuch ${ }^{2}$,

A. Leszczynska ${ }^{4}$, K. Sobolewski ${ }^{3}$, W. Musial ${ }^{2}$, M. Gorska ${ }^{1}$, M. Winnicka ${ }^{3}$;

${ }^{1}$ Department of Diabetes, Endocrinology and Internal Medicine, ${ }^{2}$ Department of Cardiology, ${ }^{3}$ Department of Medical Biochemistry, ${ }^{4}$ Department of Clinical and Experimental Pathology, Medical University, Bialystok, Poland.

Background and Aims: Enhanced metalloproteinase (MMP) activity is thought to be one of the major determinants of post-infarction LV remodelling. Former studies revealed MMP activation and development of HF in animals with very high glucose levels. The chronic effects of moderately elevated blood glucose on cardiac muscle are unknown.

The aim of the study was to assess the effects of long-term moderate streptozotocin (STZ) induced diabetes on MMP activity and LV remodelling following experimental MI in mice.

Materials and Methods: Moderate hyperglycaemia (MH) $(200-400 \mathrm{mg} / \mathrm{dL})$ was produced in $4510-12$ week old male C57B16/J mice by 5 injections of STZ ( $45 \mathrm{mg} / \mathrm{kg}$ ); 42 age, sex and strain matched mice served as controls (C). After 8 weeks mice were subjected to left coronary artery ligation (MI) $(n=61)$ or sham operation $(\mathrm{SH})$ $(n=26)$. Early infarct size was measured using Evans blue and TTC, 5 hours after MI. MMP activity was measured 5 days after MI or SH using gelatin zymography. Other mice were sacrificed 8 weeks post MI. Apoptosis assessment (TUNEL), trichrome staining and morphometric measurements of LV were performed.

Results: Staining with Evans blue and TTC revealed a comparable size of infarction in both groups. Five days after MI there was a similarly strong induction of MMP-2 and MMP-9 activity in infarct zone in all animals.

An increased activity of MMP-1 in infarct area only in C MI animals was observed. Increased MMP-9 activity in the 
remote myocardium was observed only in $\mathrm{MH}$ MI and $\mathrm{MH}$ $\mathrm{SH}$ mice, but not in $\mathrm{C}$ animals. MH mice presented similar mortality during 8 weeks as controls. Morphometric parameters, apoptosis and fibrosis in viable myocardium after MI were significantly increased in comparison to $\mathrm{SH}$ animals, but there were no differences between $\mathrm{MH}$ and $\mathrm{C}$ mice, neither in MI nor SH groups.

Conclusion: These data suggest that prolonged moderate hyperglyceamia in mice affects MMP activity early after MI, but unlike in high hyperglycaemia, this effect is probably too modest to affect long term mortality and LV remodelling.

Supported by AMB 3-50679

\section{6}

Right ventricular diastolic function abnormalities in patients with type 1 diabetes mellitus

S. I. Handanis ${ }^{1}$, D. Beldekos ${ }^{1}$, A. Koutsovasilis ${ }^{2}$,

A. Lyras ${ }^{1}$, S. Argyrakis ${ }^{1}$, I. Kyriazis ${ }^{2}$,

A. Melidonis ${ }^{2}$, S. G. Foussas ${ }^{1}$;

${ }^{1}$ Cardiology Dept., ${ }^{2}$ Diabetes Center, Tzanio Gen Hospital, Piraeus, Greece.

Background and Aims: Type I diabetes mellitus (DM) has been associated with left ventricular diastolic dysfunction even before the development of signs and symptoms of heart disease. The aim of the study was the early detection of right diastolic dysfunction in asymptomatic patients with DM type I by the use of tissue Doppler imaging (TDI) indices.

Materials and Methods: We examined 45 patients (24 men, mean age 39,2 $\pm 9,9$ ), with DM type I (Group A) and 31 normal controls (Group B) of comparable age and sex. All the participants underwent a standard 2-D and color Doppler echocardiographic study. Pulsed TDI was performed in apical 4- chamber view.

We measured the LV ejection fraction (EF), transmittal flow velocity $(\mathrm{E}, \mathrm{A}, \mathrm{E} / \mathrm{A})$ and Tran tricuspid flow velocity $\left(\mathrm{E}^{\prime}, \mathrm{A}^{\prime}\right.$, $\mathrm{E}^{\prime} / \mathrm{A}^{\prime}$ ). Additionally TDI measurements of mitral (mitral Em, $\mathrm{Am}, \mathrm{Sm}$ ) and tricuspid annulus velocities (tricuspid Em, Am, $\mathrm{Sm}$ ) were evaluated. A possible correlation was examined for age, diabetes duration and echocardiographic measurements of left and right ventricle with univariate analysis.

The volume of right atrium, RA volume was calculated from the apical 4-chamber view using Simpson's method and consequently indexed to body surface area (RAVI).

Results: All the participants had normal ejection fraction, dimensions and wall thickness of the ventricle. Patients of the group A had impaired right diastolic function compared to the controls of group B.

\begin{tabular}{llll}
\hline & Diabetic patients & Controls & $\mathrm{P}$ \\
\hline EF\% & $68 \pm 5$ & $66 \pm 5$ & $\mathrm{NS}$ \\
$\mathrm{E} / \mathrm{A}$ & $0.95 \pm 0.6$ & $1.8 \pm 5$ & $<0.0001$ \\
E“/A” & $0.94 \pm 0.58$ & $1.77 \pm 0.48$ & $<0.001$ \\
Mitral Sm cm/sec & $6.1 \pm 0.9$ & $6.2 \pm 0.75$ & $\mathrm{NS}$ \\
Mitral Em/Am & $0.9 \pm 0.2$ & $1.6 \pm 0.7$ & $<0.0001$ \\
Tricuspid Sm & $9.8 \pm 1.1$ & $10.1 \pm 1.1$ & $\mathrm{NS}$ \\
Tricuspid Em/Am & $0.89 \pm 0.39$ & $1.38 \pm 0.38$ & $<0.001$ \\
IVCv cm/sec & $8.2 \pm 1.8$ & $9.1 \pm 1.6$ & $\mathrm{NS}$ \\
Right atrium ml/m & $15 \pm 5$ & $14 \pm 3$ & $\mathrm{NS}$ \\
\hline
\end{tabular}

Conclusion: Asymptomatic patients with DM I have early right diastolic dysfunction that can be reliably detected using indices of tissue Doppler echocardiography.

1187

Endothelial dysfunction in patients with diabetes mellitus type 1 and diabetic cardiomyopathy

J. B. Ribchenko, L. Sokolova, O. Voloshina, A. Efimov, N. Tronko;

Diabetology, Endocrinology and Metabolism, Kiev, Ukraine.

Background and Aims: Diabetes influences myocardial and coronary vessels function by coexisting macroangiopathy, microangiopathy, metabolic disturbances and autonomic nervous system neuropathy. All these factors result in diastolic and systolic dysfunction of the heart. Subclinical diastolic dysfunction is served as a marker of a diabetic cardiomyopathy. The diabetic state is also associated with endothelial dysfunction as an initial manifestation of vascular abnormalities in diabetes. The aim of this study was to assess echocardiographic evidence of cardiomyopathy and its association with endothelial function in type 1 normotensive non-proteinuric diabetic patients.

Materials and Methods: Fifty patients with type $1 \mathrm{DM}$ (mean age 30,1 $\pm 1,8$ years) and 20 healthy controls (mean age $29,9 \pm 1,8$ years) were studied. Each patient was underwent echocardiography and assessment of brachial endothelial function. Indexes of left ventricular diastolic filling were measured by Doppler-echocardiography. Endothelial function was investigated using ultrasound assessment of flow-mediated dilatation (FMD). Data are presented as $\mathrm{M} \pm \mathrm{m}$. The results were compared using Student's paired test.

Results: No patient had chest pain or electrocardiographic changes during exercise testing. All patients had a normal ejection fraction $(53,9 \pm 2,3$ vs $56,1 \pm 4,3,(p>0,05)$ in those with and without DM, respectively). A diagnosis of LVDD was made when at least one of the following parameters 
was present in the echocardiographic study: isovolumetric relaxing time $($ IRT) $>100 \mathrm{~ms}$, deceleration time $(\mathrm{DT})>$ $220 \mathrm{~ms}$ or early filling rate peak/late filling rate peak ratio $(\mathrm{E} / \mathrm{A})<1$. According to these criterions $18(36 \%)$ of the 50 diabetic patients had evidence of diastolic dysfunction as assessed by the presence of at least two abnormal variables of mitral inflow velocity. In particular, the ratio of peak early to peak late (atrial) filling velocity was significantly decreased in diabetic compared with control subjects (E/A ratio $0,9 \pm 0,05$ vs $1,2 \pm 0,06, p<0,05)$, the DT was $257,1 \pm$ $28,2 \mathrm{~ms}$ vs $186 \pm 14,6 \mathrm{~ms} \mathrm{p}<0,05$, in those with and without $\mathrm{DM}$, respectively. Also the IRT was increased in diabetic patients compared with control subjects $(130 \pm 14,0 \mathrm{~ms}$ vs $79,3 \pm 6,1 \mathrm{~ms}, \mathrm{p}<0,05)$. The percent change in diameter of the brachial artery in response to reactive hyperemia was significantly impaired in patients with DM compared with control subject, and this impairment was significantly more prominent in patients with diabetic cardiomyopathy than in those without left ventricular diastolic dysfunction. According to our data, the percent change in diameter of the brachial artery in response to reactive hyperemia in patients with diabetic cardiomyopathy $(8,4 \pm 1,5 \%)$ was significantly lower than that in healthy volunteers $(17,6 \pm 1,3 \%),(p<0,05)$, and in patients with DM but without cardiomyopathy $(13,6 \pm$ $0,9 \%),(\mathrm{p}<0,05)$

Conclusion: Our data suggest that 1) more than one-third of young type $1 \mathrm{DM}$ patients with a normal systolic ventricular function have diabetic cardiomyopathy; 2) impairment of endothelial function was significantly more prominent in patients with diabetic cardiomyopathy.

\section{8}

Diabetic cardiomyopathy and endothelial dysfunction associated with microalbuminuria in patients with diabetes mellitus type 1

L. Sokolova, J. Ribchenko, A. Efimov, N. Tronko;

Diabetology, Endocrinology and Metabolism, Kiev, Ukraine.

Background and Aims: Diabetic cardiomyopathy (DMC) is a complication of diabetes mellitus (DM) that is more frequently observed in those patients with microalbuminuria. Left ventricular diastolic dysfunction (LVDD) in patients with diabetes, in absence of another etiology that justifies it, is an early marker of DMC. The diabetic state is also associated with endothelial dysfunction as an initial manifestation of vascular abnormalities in diabetes. On other hand, microalbuminuria is considered an important predictor of cardiovascular events in diabetic patients and as a marker of endothelial dysfunction. The aim of this study was to assess echocardiographic evidence of cardio- myopathy, endothelial dysfunction and their association with microalbuminuria in type 1 diabetic patients without hypertension, coronary artery disease, or autonomic dysfunction

Materials and Methods: Fifty young type $1 \mathrm{DM}$ patients with LVDD, 30 with and 20 without microalbuminuria, and 20 control subjects were studied. Indexes of left ventricular diastolic filling were measured by Doppler-echocardiography. Endothelial function was investigated using ultrasound assessment of flow-mediated dilatation (FMD). Data are presented as $\mathrm{M} \pm \mathrm{m}$. The results were compared using Student's paired test.

Results: A diagnosis of LVDD was made when at least one of the following parameters was present in the echocardiographic study: isovolumetric relaxing time (IRT) $>100 \mathrm{~ms}$, deceleration time (DT) $>220 \mathrm{~ms}$ or early filling rate peak/ late filling rate peak ratio $(\mathrm{E} / \mathrm{A})<1$. According to these criterions LVD function was significantly impaired in both diabetic groups compared with control subjects; moreover, this impairment was significantly greater in microalbuminuric than in normoalbuminuric patients. In particular, the ratio of peak early to peak late (atrial) filling velocity was significantly decreased in diabetic microalbuminuric patients compared with diabetic patients without microalbuminuria and control subjects (E/A ratio $0,7 \pm 0,05$ vs $1,0 \pm$ 0,06 vs $1,3 \pm 0,06, p<0,05$ ), the DT was $257,1 \pm 28,2 \mathrm{~ms}$ vs $196 \pm 14,6 \mathrm{~ms}$ vs $176 \pm 14,6 \mathrm{~ms} \mathrm{p}<0,05$, in those with and without microalbuminuria and control group, respectively. Also the IRT was increased in patients with DM compared with control subjects $(130 \pm 14,0 \mathrm{~ms}$ vs $79,3 \pm 6,1 \mathrm{~ms}$, $\mathrm{p}<0,05$,), but there were no significant differences between diabetic patients with and without microalbuminuria. The percent change in diameter of the brachial artery in response to reactive hyperemia in patients with LVDD and microalbuminuria $(6,4 \pm 1,3 \%)$ was significantly lower than that in control subjects $(12,6 \pm 1,4 \%),(p<0,05)$, and in diabetic patients with LVDD but without microalbuminuria $(8,6 \pm 1,3 \%),(\mathrm{p}<0,05)$

Conclusion: Our data suggest that microalbuminuria is associated with significant changes of cardiac diastolic function and endothelial dysfunction. Microalbuminuria may be an early biochemical marker of these abnormalities. Therefore, microalbuminuric patients should be considered to have a higher risk of cardiovascular complications.

\section{9}

Diabetic retinopathy is associated with left ventricular diastolic dysfunction in young patients with long lasting diabetes mellitus type 1

B. Lichodziejewska, K. Kurnicka, A. Lipińska, K. Jankowski, M. Ciurzyński, D. Liszewska-Pfejfer; 
Internal Medicine and Cardiology, Medical University of Warsaw, Poland.

Background and Aims: Diabetic retinopathy in young patients may coexist with other organs complications, especially subclinical disorders of cardiac function. Echocardiographic doppler evaluation (ECHO) of mitral valve flow (MVF) and pulmonary venous flow (PVF) is a good method for assessment of left ventricular diastolic function (LVDF). The aim of the study was to evaluate the LVDF using ECHO in young patients with long lasting diabetes mellitus type 1 (DM1), with or without diabetic retinopathy. Materials and Methods: 65 patients (pts) with DM1 (33F,

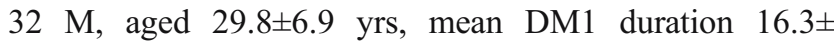
$7.6 \mathrm{yrs}$ ) without evidence of cardiovascular disease were included in the study. Mean HbA1c level was 9.7 $\pm 2.1 \%$. Twenty nine patients $(14 \mathrm{~F}, 15 \mathrm{M})$ with diabetic retinopathy only (non-proliferative or proliferative) completed group I and 36 pts $(19 \mathrm{~F}, 17 \mathrm{M})$ without diabetic retino-, nephro- and peripheral neuropathy formed group II. The control group $\mathrm{C}$ consisted of 30 healthy persons $(15 \mathrm{~F}, 15 \mathrm{M})$. To assess LVDF, the MVF and PVF parameters were evaluated. In MVF, the mean value of the maximal velocity (Vmax) of early phase $\mathrm{E}$ to $\mathrm{Vmax}$ of late phase $\mathrm{A}$ ratio $(\mathrm{E} / \mathrm{A})$ was measured. In PVF the mean value of $\mathrm{Vmax}$ of diastolic flow (D) and Vmax of systolic flow (S) were assessed and the $S / D$ ratio was calculated.

Results:

MVF and PVF results

\begin{tabular}{lllll}
\hline Parameter & $\begin{array}{l}\text { Group } \\
\text { I }\end{array}$ & $\begin{array}{l}\text { Group } \\
\text { II }\end{array}$ & $\begin{array}{l}\text { Group } \\
\text { C }\end{array}$ & $\begin{array}{l}\text { 1. I vs II; } \\
\text { 2. I vs C; } \\
\text { 3. II vs C }\end{array}$ \\
\hline E/A & $1.18 \pm 0.2$ & $1.42 \pm 0.3$ & $1.54 \pm 0.3$ & $\begin{array}{l}\text { 1. } \mathrm{p}=0,003 ; \\
\end{array}$ \\
& & & $\begin{array}{l}\text { 2. } \mathrm{p}<0,0001 ; \\
\text { 3. NS }\end{array}$ \\
S/D & $1.22 \pm 0.2$ & \multirow{2}{*}{$1.06 \pm 0.1$} & $0.9 \pm 0.1$ & $\begin{array}{l}\text { 1. } \mathrm{p}=0,005 ; \\
\text { 2. } \mathrm{p}<0,0001 ; \\
\end{array}$ \\
& & & & $3 . \mathrm{p}<0,0001$ \\
\hline
\end{tabular}

The changes in MVF and PVF parameters (decreased E/A ratio and increased $\mathrm{S} / \mathrm{D}$ ratio) suggest preclinical impairment of LV relaxation in diabetic patients, more pronounced in persons with diabetic retinopathy. There was no correlation between ECHO parameters and DM1 duration and metabolic control of diabetes.

Conclusion: The echocardiographic assessment of MVF and PVF shows that young asymptomatic patients with long lasting DM1 and diabetic retinopathy have more pronounced LV diastolic dysfunction than patients without diabetic complications.
1190

High visceral fat accumulation in subjects with nonischaemic dilated cardiomyopathy

A. M. Sironi, D. De Marchi, D. Neglia, M. Emdin, P. Iozzo, D. Ciociaro, S. Masi, D. Giannessi, M. Lombardi, E. Ferrannini, A. Gastaldelli;

National Research Council - CNR, Clinical Physiology Institute, Pisa, Italy.

Background and Aims: Preferential visceral fat (VF) accumulation, dyslipidemia and alterations in glucose homeostasis are well known risk factors for cardiovascular diseases, but less is known in dilated cardiomyopathy (DCM), especially in the early stages of the disease. Most of the studies were conducted in patients with coronary artery disease (CAD), where the co-existence of other abnormalities confounds the relationship between metabolic variables and left ventricular (LV) function. Thus, the aim of this study was to investigate the relationship between metabolic profile, VF and cardiac function/dimension in subjects with idiopathic DCM.

Materials and Methods: We recruited 30 male non-obese subjects, 16 with previously known non-ischemic DCM (age 56 \pm 3 , BMI 25.9 \pm 0.8 , EF $37 \pm 3 \%$ ) and 14 controls (CT; age $50 \pm 1$, BMI $26.9 \pm 0.4, \mathrm{EF}=66 \pm 2 \%$ ). Exclusion criteria were known diabetes or FPG $>126 \mathrm{mg} / \mathrm{dl}$. All subjects received a $3 \mathrm{~h} 75 \mathrm{~g}$-OGTT for determination of glucose tolerance and $1.5 \mathrm{~T}$ MRI was used to measure VF, subcutaneous fat (SC) and cardiac function (i.e., left ventricular mass, LV-EDMi; left-ventricular end-diastolic volume, LV-EDVi and quantitative ejection fraction, EF). In all subjects BNP was determined as well.

Results: Compared to CT, DCM subjects had larger VF depot ( $122 \pm 16$ vs $\left.86 \pm 5 \mathrm{~cm}^{2}, \mathrm{p}=0.04\right)$ and increased VF/SC ratio $(0.68 \pm 0.08$ vs $0.39 \pm 0.0, p=0.0024)$ despite similar BMI. All subjects had similar FPG $(102 \pm 2 \mathrm{mg} / \mathrm{dl}, \mathrm{p}=\mathrm{ns})$ and fasting plasma insulin (FPI; $13 \pm 1 \mu \mathrm{U} / \mathrm{ml}, \mathrm{p}=\mathrm{ns}$ ) but DCM had increased trygliceride (TG; 124 [78] vs 83 [51] $\mathrm{mg} / \mathrm{dl})$, and TG/HDL (1.6 \pm 0.2 vs $0.9 \pm 0.1)$. After OGTT in CT group 5 subjects were found IGT, but none with type 2 diabetes (T2DM) whereas in DCM group 5 subjects were found IGT and 5 with T2DM. Regarding the cardiac parameters, as expected, DCM had higher values of LVEDVi (127 \pm 9 vs $\left.74 \pm 4 \mathrm{ml} / \mathrm{m}^{2}, \mathrm{p}=0.0002\right)$, LV-EDMi $(105 \pm 6$ vs $\left.73 \pm 4 \mathrm{~g} / \mathrm{m}^{2}, \mathrm{p}<0.0001\right)$ and BNP (26 [88] vs 7 [7] pg/ml). Conclusion: Preferential VF accumulation is present in early stages of DCM and may represent a risk factor for the development of non-ischemic dilated cardiomiopathy. 
1191

Impact of anaemia on the left ventricular structure in type 2 diabetic patients with advanced phase of nephropathy

A. Sato, N. Aki, M. Nagao, T. Babazono, Y. Iwamoto; Diabetes Center, Tokyo Women's Medical University, Japan.

Background and Aims: The excess mortality in patients with diabetic end-stage renal disease (ESRD) is mainly due to cardiovascular events. Left ventricular (LV) hypertrophy is an independent risk factor for myocardial ischaemia, cardiac arrhythmia, sudden death, and heart failure. Chronic anaemia may also lead to adverse cardiovascular consequence. Patients with diabetic ESRD increase rates of LV hypertrophy and anaemia. The aim of this study is to evaluate the impact of anaemia on LV remodeling in type 2 diabetic patients with advanced phase of nephropathy.

Subjects and Method: M-mode echocardiography was performed in consecutive type 2 diabetic patients with nephropathy, whose serum creatinine concentration was $2.0 \mathrm{mg} / \mathrm{dl}$ and more, by one experienced examiner as a base-line (B). During follow-up period, echocardiography was performed 50 type 2 diabetic patients with nephropathy (39 men, age (mean(SD)) 58 (12) years) who did not have overt cardiac diseases (myocardial infarction, valvular failure, hypertrophic cardiomyopathy) at the point of a doubling of the base-line serum creatinine concentration (D). LV remodeling was defined by 4 categories according to LV mass index and relative wall thickness; normal $(\mathrm{N})$, concentric remodeling $(\mathrm{CR})$, concentric hypertrophy $(\mathrm{CH})$, and eccentric hypertrophy $(\mathrm{EH})$. Arterial blood pressure, $\mathrm{HbA} 1 \mathrm{c}$, haemoglobin, and traditional clinical risk factors of CVD were measured at both $\mathrm{B}$ and $\mathrm{D}$ points.

Results: The median duration of follow up was 451(56 1631) days. The change of serum creatinine was from 3.8 (1.1) at B point to $7.3(2.0) \mathrm{mg} / \mathrm{dl}$ at $\mathrm{D}$ point, systolic blood pressure from $151(19)$ to $152(19) \mathrm{mmHg}$, and diastolic blood pressure from $82(10)$ to $76(14) \mathrm{mmHg}$, respectively. In terms of $\mathrm{LV}$ remodeling during $\mathrm{B}$ and $\mathrm{D}$ point, $\mathrm{LV}$ hypertrophy increased as following the progress of diabetic nephropathy, the ratio of $\mathrm{N}(12$ to $12 \%), \mathrm{CR}(12$ to $0 \%), \mathrm{CH}$ (34 to $38 \%$ ), and $\mathrm{EH}$ (42 to $50 \%$ ), respectively. In those whose LV hypertrophy decreased (7 men, 2 women), haemoglobin level significantly increased from $8.8(0.7) \%$ at $\mathrm{B}$ point to $10.0(1.8) \%$ at $\mathrm{D}$ point $(\mathrm{p}=0.002)$, whereas $\mathrm{HbAlc}$, arterial blood pressure, and serum lipid did not change significantly. In those whose LV hypertrophy increased (10 men, 5 women), haemoglobin did not change significantly from $9.5(0.8) \%$ at B point to $8.7(1.5) \%$ at D point (N.S.). There were no differences in age, HbAlc, arterial blood pressure, serum creatinine level, and serum lipid at B point between both groups.

Conclusions: In type 2 diabetic patients with advanced phase of nephropathy, the ratio of LV hypertrophy increases as following the progress of renal insufficiency. Improvement of anaemia during advanced phase of diabetic nephropathy is important for the prevention of LV hypertrophy in type 2 diabetic patients.

1192

Analogue compared to human insulin therapy: effects on metabolism and cardiac function in patients with type 2 diabetes and an intensive conventional insulin therapy (ICD)

H. von Bibra, T. Siegmund, U. Pischa, M. Volozhyna, P.-M. Schumm-Draeger;

Endocrinology, Academic Hospital Bogenhausen, München, Germany.

Background and Aims: Compared to human insulins (HI), analogue insulins (AI) offer the option of optimizing metabolism also in type 2 diabetes (T2D). Especially, fast acting insulin analogues lower postprandial glucose levels more effectively than human regular insulin. However, it is not known whether therapy with AI can also improve the subclinically impaired myocardial function in T2D. This prospective, randomized, open long term study compared the effects of ICD with AI vs. HI on metabolic control and systolic and diastolic myocardial function.

Materials and Methods: Inclusion criteria: T2D with preceding insulin therapy $>1$ year. Goup 1: AI (insulin detemir+insulin aspart) and group 2: HI (NPH-insulin and regular human insulin). Systolic (Vs) and diastolic myocardial function (Ve) were assessed by tissue Doppler before and two hours after a standardized breakfast (48 $\mathrm{g}$ carbohydrates). Both treatment groups were comparable with regard to demographics, cardiac function and metabolic control at baseline. This interim analysis includes data from 60 patients after a 12-month treatment period.

Results: After 12 months, HbA1c did not change in both groups (in the AI group: baseline $6.7 \pm 1.1$, endpoint $6.4 \pm$ $0.9 \%(\mathrm{p}<0.19)$; HI group: baseline $6.5 \pm 0.8$, endpoint $6.5 \pm$ $0.9 \%$. Postprandial serum glucose was reduced $(\mathrm{p}<0.02)$ by $41 \pm 70 \mathrm{mg} / \mathrm{dl}$ in the AI group and by $0 \pm 44 \mathrm{mg} / \mathrm{dl}$ in the HI group ( $p<0.04$ compared to AI). Fasting serum glucose was reduced by $17 \pm 44 \mathrm{mg} / \mathrm{dl}$ in the AI group and by $15 \pm 44 \mathrm{mg} /$ $\mathrm{dl}$ in $\mathrm{HI}$ group. In the AI group, Vs increased significantly from $7.4 \pm 1.0$ to $7.8 \pm 1.1 \mathrm{~cm} / \mathrm{s}(\mathrm{p}<0.03)$ as did Ve $(7.7 \pm 1.1$ to $8.4 \pm 1.6 \mathrm{~cm} / \mathrm{s}, \mathrm{p}<0.03$ ) associated with parellel changes in the postprandial state $(\mathrm{p}<0.02)$. In the HI group, however, Vs (baseline 7.5 \pm 1.1 , endpoint $7.5 \pm 0.9 \mathrm{~cm} / \mathrm{s}$ ) and $\mathrm{Ve}$ 
(baseline $7.6 \pm 1.3$, endpoint $7.9 \pm 1.7 \mathrm{~cm} / \mathrm{s}$ ) did not change in the fasting state and in the postprandial state.

Conclusion: Twelve months ICD-therapy with AI significantly improved postprandial glucose control and cardiac function compared to therapy with HI. The association of lower postprandial glucose levels and improved myocardial function should be taken into consideration when selecting an insulin regimen for people with T2D.

Supported by Novo Nordisk

\section{3}

Optimisation of glycaemic control by insulin pump therapy improves left ventricular contractility in type 1 diabetes

N. H. Andersen, T. K. Hansen, J. S. Christiansen; Department of Internal Mdicine, Aarhus University Hospital, Denmark.

Decreased left ventricular long axis contractility may be the earliest stage in sub-clinical heart failure in type 1 diabetes. The aim of the study was to examine whether optimized glycemic control by insulin pump therapy would improve left ventricular contractility in a group of type 1 diabetic patients.

To assess whether improved blood glucose regulation, by intensified insulin treatment, would influence the left ventricular function, we included 19 consecutive type 1 diabetic patients selected for insulin pump therapy due to poor metabolic control. The main outcome measures were left ventricular strain rate in the long axis plane, examined by tissue doppler echocardiography and changes in Hbalc. Mean follow-up was 45 days (range 35-50). All patients included had an ejection fraction $>0.55 \%$ and fractional shortening $>0.25 \%$ and were free of any cardiac symptoms.

The mean age of the participants was 40 years (range 22 65). Following initiation of insulin pump therapy mean $\mathrm{HbAlc}$ was significantly improved from $8.6 \pm 1.4 \%$ to 7.6 $\pm 1.1 \% \quad(\mathrm{p}<0.01)$. Mean strain rate of 16 myocardial segments was also significantly improved and decreased from $-1.58 \pm 0.30 \mathrm{~s}^{-1}$ to $-1.80 \pm 0.4 \mathrm{~s}^{-1}(\mathrm{p}<0.01$, figure $\mathrm{A})$. The changes in left ventricular function were significantly correlated to the changes in HbAlc (delta-Strain rate vs. delta Hbalc: $r=0.49, \mathrm{p}<0.01$ (figure.B). The changes in left ventricular function were more pronounced in the youngest part of the population (age at baseline vs. delta-Strain rate $\mathrm{r}=-0.47, \mathrm{p}<0.05)$.

In conclusion, optimized glycemic control by insulin pump therapy improves left ventricular long axis contractility, mainly in young patients with type 1 diabetes.
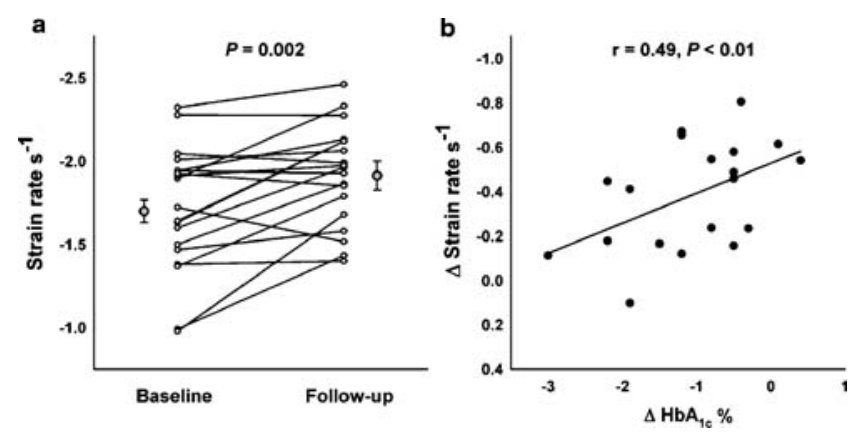

\section{PS 107}

\section{Peripheral endothelial function}

\section{4}

Endothelial barrier protective effect of FTY720 under the hyperglycaemic condition: involvement of focal adhesion kinase, small GTPases and adherens junction proteins

K. Sarai, Y. Shikata, K. Ohmori, N. Watanabe, S. Ohga, M. Sasaki, C. Sato, S. Nishishita, J. Wada, K. Shikata, H. Makino;

Department of Medicine and Clinical Science, Okayama University Graduate School of Medicine, Okayama, Japan.

Background and Aims: Sphingosine 1-phosphate (S1P) is a remarkably effective endothelial cell agonist that induces proliferation, calcium mobilization, adhesion molecule expression, and suppression of apoptosis. Recently, the role of S1P as an endothelial barrier stabilizing mediator has been highlighted. FTY720 is a S1P analog originally developed as a novel immunosuppressant. Phosphorylated form of FTY720 binds to S1P receptors to exert S1P-like biological effects, suggesting endothelial barrier promotion by FTY720. These lines of evidences give raise a question if FTY720 ameliorates diabetic angiopathy, the crucial cause of several diabetic complications, via vascular endothelial barrier enhancement. In the present study, we investigated the role of FTY720 on focal adhesion (FA) and adherens junction (AJ) remodeling associated with actin redistribution under both normoglycemic and hyperglycemic conditions.

Materials and Methods: Human microvascular endothelial cells (HMVECs) were preincubated with hyperglycemic (30 mM) media for $48 \mathrm{~h}$ and then treated with $100 \mathrm{nM}$ FTY720 for $3 \mathrm{~h}$. To monitor effects of hyperglycemic challenge and FTY720 treatment on the distribution of focal adhesion kinase (FAK), $\alpha$-catenin, $\beta$-catenin and VEcadherin, immunfluorescent microscopy was performed. To investigate the possible effect of FTY720 on FA and AJ 
remodeling, $\beta$-catenin was immunoprecipitated and then, amounts of coprecipitated VE-cadherin and FAK was evaluated. Activity of small GTPase Rac and Rho and site-specific FAK tyrosine-phosphorylation were also monitored.

Results: Immunfluorescent microscopy and coprecipitation study revealed the FTY720-induced FAK-associated AJ assembly at cell-cell contacts coincident with the formation of a prominent cortical actin ring. Similar to S1P, sitespecific FAK tyrosine-phosphorylation analysis revealed FTY720-induced FAK $\left[\mathrm{Y}^{576}\right]$ phosphortylation without phosphorylation of FAK $\left[\mathrm{Y}^{397} / \mathrm{Y}^{925}\right]$. Furthermore, FTY720 conditioned the phosphorylation profile of FAK $\left[\mathrm{Y}^{397} / \mathrm{Y}^{576} / \mathrm{Y}^{925}\right]$ in hyperglycemic media to the same pattern observed in normoglycemic media. FTY720 challenge resulted in small GTPase Rac activation under hyperglycemic condition, whereas increased Rho activity in hyperglycemic media was restored to the basal level.

Conclusion: We investigated the role of FTY720 on FA and AJ remodeling in HMVECs under both normoglycemic and hyperglycemic conditions. These results strongly suggest the pivotal role of FTY720 to ameliorate endothelial barrier function disturbed by hyperglycemic challenge, further implying the possibility of FTY720 as a therapeutic agent of diabetic vascular disorder.

This study was supported in part by a Grant-in-Aid for Scientific Research from the Ministry of Education, Science, Culture, Sports, and Technology of Japan.

\section{5}

Protective role for AMPK-activated protein kinase in palmitate-induced HUVEC apoptosis

S.-E. Choi ${ }^{1}$, K.-W. Lee ${ }^{2}$, S.-H. Jung ${ }^{2}$, Y.-K. Kim² ${ }^{2}$,

S.-M. Ahn ${ }^{2}$, Y.-E. Jo ${ }^{2}$, Y.-J. Choi ${ }^{2}$, H.-J. Kim², D.-J. Kim², Y. Kang ${ }^{1}$;

${ }^{1}$ Institutes for Medical Sciences, ${ }^{2}$ Endocrinology and metabolism, Ajou University School of Medicine, Suwon, Republic of Korea.

Background and Aims: Vascular diseases are the principal cause of morbidity and mortality in patients with diabetes. While high plasma free fatty acid levels are associated with atherosclerosis and vascular complication, the mechanism of vascular endothelial apoptosis by palmitate is usually obscured. Therefore, we have studied the role of AMPactivated protein kinase (AMPK) on the death of palmitate induced human umbilical vein endothelial cells (HUVEC).

Materials and Methods: Palmitate induced HUVEC death was measured by DNA ladder, DAPI and MTT assay. To determine the mechanism of HUVEC death by palmitate, we used several inhibitors and AMPK adenovirus
Results: Treatment of palmitate increased HUVEC cell death in a dose and time-dependent fashion. Also palmitate increased the activity of caspase 9, 3 and that of DNA ladder. In investigating the mechanism of palmitate induced cell death, HUVEC were simultaneously incubated with palmitate and several inhibitors, SP600125 (JNK inhibitor), SB203580 (p38 inhibitor), GO6983 (PKC inhibitor), fumonisin B1 (ceramide synthesis inhibitor), NAC (ROS scavenger) and AICAR (AMPK activator). The degree of apoptosis was then measured. The preventive effects of NAC, SP600125 and SB203580 were 30\%, 30\% and $40 \%$ respectively. GO6983 and fumonisin B1 were 3\% and $10 \%$, respectively. AICAR and overexpression of constitutively active AMPK completely blocked palmitate induced HUVEC death.

Conclusion: In this study, palmitate induced HUVEC apoptosis was prevented by AMPK activation. So we think that vascular endothelial apoptosis of palmitate is caused by accumulation of fatty acid through fat synthesis metabolism. This research was supported by the "GRRC" Project of Gyeonggi Provincial Government, Republic of Korea

\section{6}

Acute hyperhomocysteinaemia blunts endothelial dependent and endothelial independent vasodilatation in diabetic patients

K. Makrilakis, I. Xousos, G. Argyrakopoulou,

D. Kyriaki, D. Perrea, N. Bruzidis, M. Bourlakis,

I. Bitsikas, N. L. Katsilambros;

First Department of Propaedeutic Medicine, University Medical School, Athens, Greece.

Background and Aims: Homocysteine (Hcy) is believed to cause vascular damage by interfering with endothelial function, platelet activation, oxidative stress, hypercoagulability and vascular smooth muscle cell proliferation. Diabetic persons are known to have endothelial dysfunction and a very high incidence of atherosclerosis. The aim of this study was to investigate the effect of acute hyperhomocysteinemia on endothelial function of skin microcirculation in diabetic persons, compared to non-diabetic ones. Materials and Methods: We compared 20 type 2 diabetic persons $(59.95 \pm 10.54$ years old, 10 males-10 females, diabetes duration $7.7 \pm 5.1$ years) with 24 non-diabetic ones ( $58.42 \pm 10.93$ years old, 7 males-17 females). Endothelial and non-endothelial dependent vasodilatation of the skin microcirculation was evaluated with the iontophoretic administration of acetylcholine (Ach) and sodium nitroprusside (SNP) respectively, on the forearm, during fasting 
and 4 hours after $0.1 \mathrm{mg} / \mathrm{kg}$ methionine-induced acute hyperhomocysteinemia.

Results: The two groups were comparable regarding age and the other biochemical characteristics, with the exception of body mass index, waist circumference, fasting glucose, HbA1c, HOMA resistance index, fibrinogen and uric acid (higher in diabetics) and HDL-cholesterol (lower in diabetics). Both endothelial-dependent vasodilatation (Ach effect) and endothelial-independent vasodilatation (SNP effect) were significantly lower in the diabetic patients compared to the control group, after induction of acute hyperhomocysteinemia $(\mathrm{p}=0.027$ and $\mathrm{p}=0.08$, respectively). Conclusion: Acute hyperhomocysteinemia causes blunting of endothelial-dependent and endothelial-independent vasodilatation of the skin microcirculation in diabetic persons, compared to non-diabetic ones. This may contribute to the atherogenic potential of Hcy.

\section{7}

Insulin-like growth factor-binding protein-related protein 1 (IGFBP-rP1/MAC25) contributes to endotheliumdependent vasodilation in high-ferritin type-2 diabetes W. Ricart $^{1}$, A. López-Bermejo ${ }^{1}$, J. Khosravi ${ }^{2}$, A. Castro ${ }^{3}$, V. Hwa ${ }^{4}$, K. L. Pratt ${ }^{4}$, R. Casamitjana ${ }^{5}$, R. G. Rosenfeld ${ }^{6}$, J.-M. Fernández-Real ${ }^{1}$;

${ }^{1}$ Endocrinology, Dr. Josep Trueta Hospital, Girona, Spain, ${ }^{2}$ Research \& Development, Diagnostic System Laboratories, Toronto, Canada, ${ }^{3}$ Internal Medicine, Dr. Josep Trueta Hospital, Girona, Spain, ${ }^{4}$ Department of Pediatrics, OHSU, Portland, United States, ${ }^{5}$ Endocrine Laboratory, University Clinical Hospital, Barcelona, Spain, ${ }^{6}$ Pediatrics, Lucile Packard Foundation for Children's Health, Palo Alto, United States.

Background and Aims: Blood letting improves vascular reactivity in high ferritin type-2 diabetic subjects (T2DM). Insulin-like growth factor (IGF)-binding protein-related protein 1 (IGFBP-rP1; also known as mac25 and angiomodulin) is a novel vascular factor involved in oxidative stress whose role in T2DM is largely unknown. We hypothesized that circulating IGFBP-rP1 modulates the vascular changes that follow iron depletion in T2DM.

Materials and Methods: Twenty-six T2DM male patients with high ferritin levels $(>200 \mathrm{ng} / \mathrm{ml})$ randomized to either blood letting (three extractions of $500 \mathrm{ml}$ of blood at twoweek intervals; $n=11)$ or to observation $(n=15)$ were studied. Endothelium-dependent and independent vasodilation were measured in the brachial artery by high-resolution ultrasound at baseline and at 4 months thereafter. The groups were matched for age, BMI, pharmacological treatment, chronic diabetes complication and baseline serum IGFBP-rP1.

Results: Circulating IGFBP-rP1 was significantly associated with endothelium-dependent, but not endotheliumindependent vasodilation at baseline in these subjects (studied as a single group; $\mathrm{r}=0.49, P=0.014$ ). Although blood letting did not have a significant effect on circulating IGFBP-rP1 [mean (SEM) for percentage change at 4 months: $+39 \%$ (13\%) vs. $+25 \%$ (13\%), for intervention and observation groups, respectively; $P=0.44]$, significant correlations were documented between the changes in endothelium-dependent vasodilation at 4 months and both baseline serum IGFBP-rP1 $(\mathrm{r}=-0.48, P=0.016)$ and its change during this time $(\mathrm{r}=0.56, P=0.008)$. Finally, both endothelium-dependent vasodilation and circulating IGFBP-rP1 were significantly correlated with plasma cyclic GMP in these subjects $(\mathrm{r}=0.51, \mathrm{P}=0.04$ and $\mathrm{r}=0.68$, $P=0.003$, respectively).

Conclusion: These results suggest for the first time that IGFBP-rP1 modulates endothelium-dependent vasodilation in T2DM. The contribution of IGFBP-rP1 to the vascular dysfunction of diabetes mellitus merits further research.

A. L-B. is a Research Investigator of the Fund for Scientific Research "Ramon y Cajal" (Ministry of Education and Science, Spain).

\section{8}

Vascular endothelial dysfunction evidenced by decreased flow mediated dilation is an early manifestation in subjects with fasting euglycaemia but impaired 2hour glucose levels

M. J. Theodorakis, P. T. Voidonikola, C. Papamichael, K. S. Stamatelopoulos, A. Protogerou, G. Kollias, P. Katsichti, E. Chryssochoou, N. A. Zakopoulos, M. Alevizaki;

Dept. Clinical Therapeutics, University of Athens, Medical School, Athens, Greece.

Background and Aims: Increased prevalence of hyperglycaemia in the general population is largely manifested as elevated 2 hour post-challenge glucose concentrations. Normal fasting glucose depends on adequate basal insulin secretion and sensitivity, mainly in the liver, in order to control glucose output, whereas impaired 2 hour plasma glucose levels after an OGTT reflect peripheral insulin resistance, particularly in skeletal muscle, the main site for glucose disposal. We investigated the potential effect of postchallenge hyperglycemia on endothelial dysfunction as an early manifestation of vascular disease in subjects with fasting euglycemia. 
Materials and Methods: We carried out an OGTT for 2 hours with two blood samples per subject (baseline and 2 hours post-glucose (75 grams) ingestion), on 38 age- and BMI- matched participants (age $49 \pm 2$ vs $51 \pm 3$ yrs and BMI $28 \pm 1$ vs $29 \pm 1$, respectively), who all had normal fasting plasma glucose levels $(<100 \mathrm{mg} / \mathrm{dl})$. Group A subjects $(\mathrm{N}=24)$ had normal glucose tolerance (2 h-glucose $<140 \mathrm{mg}$ / $\mathrm{dl} ; 91 \pm 5 \mathrm{mg} / \mathrm{dl})$ whereas Group B subjects $(\mathrm{N}=14)$ exhibited impaired $2 \mathrm{~h}$ plasma glucose levels $(165 \pm 7 \mathrm{mg} /$ dl). FMD of the brachial artery was determined by high resolution ultrasonography as a surrogate marker of endothelial function before OGTT was performed.

Results: Plasma insulin, total-, HDL- and LDL cholesterol, triglycerides and the HOMA-IR insulin resistance index did not differ between subject groups. Similarly, glycosylated hemoglobin $\left(\mathrm{HbA}_{1} \mathrm{c}\right)$ was not significantly different between groups ( $4.8 \pm 0.5$ vs $5.0 \pm 0.1 \%$, respectively). However FMD was significantly lower in subjects with impaired $2 \mathrm{~h}$ glucose compared to those with normal glucose tolerance $(2.6 \pm 0.8$ vs $7.9 \pm 0.6 \% ; \mathrm{p}<0.001)$.

Conclusion: In otherwise healthy non-obese individuals with fasting euglycemia, the presence of glucose intolerance after glucose ingestion was associated with diminished FMD compared to the normal glucose tolerant state. This finding could be of particular significance to the early pathophysiology of vascular endothelial dysfunction observed in insulin resistant states such as the metabolic syndrome and demonstrates the importance of optimal glycemic control on the maintenance of normal vascular function.

\section{9}

Rosiglitazone restores endothelial function in Zucker Diabetic Fatty rats

E. Cosson ${ }^{1}$, P. Valensi ${ }^{1}$, A. Behar ${ }^{1}$, F. Cohen-Boulakia ${ }^{1}$, H. Dabiré ${ }^{2}$;

${ }^{1}$ Laboratory of Nutrition, Metabolic Diseases and Cardiovascular Prevention, Paris-Nord University, Bobigny, ${ }^{2}$ U660, ENVA, Maisons Alfort, France.

Background and Aims: Obesity and type 2 diabetes are associated with endothelial dysfunction. The aim of the study was to assess for the first time in Zucker Diabetic Fatty (ZDF) rats, a model of type 2 diabetes, if the insulin sensitizer rosiglitazone may reduce endothelial dysfunction, as well as vascular reactivity to insulin and blood pressure abnormalities. Materials and Methods: Three series of 7-9 rats were studied at 24 weeks of age: Lean (fa/?) controls, ZDF (Gmi$\mathrm{fa} / \mathrm{fa})$ rats treated with rosiglitazone $(7 \mu \mathrm{mol} / \mathrm{kg} /$ day $)$ for 16 weeks (ZDF-ROS) and untreated ZDF rats. Central aortic pressure was measured in anesthetised rats at the end of the treatment. Thoracic aorta was removed and the dose- response curves to insulin $\left(30-10^{5} \mathrm{mIU} / \mathrm{L}\right)$ and acetylcholine (Ach: $\left.10^{-10}-10^{-4} \mathrm{M}\right)$ were studied in vitro after a precontraction with Norepinephrine $\left(10^{-6} \mathrm{M}\right)$, in the presence or absence of N-Nitrow-L-Arginine (L-NNA $3.10^{-5} \mathrm{M}$ ) or indomethacin (IND $3 \cdot 10^{-5} \mathrm{M}$ ).

Results: Body weight was increased in ZDF-ROS rats $(510 \pm$ $47 \mathrm{~g})$ as compared to ZDF rats $(379 \pm 8 \mathrm{~g}, \mathrm{p}<0.01)$ and to controls (429 $\pm 9 \mathrm{~g}, \mathrm{NS})$. In ZDF-ROS rats, systolic blood pressure (SBP: $160 \pm 5 \mathrm{mmHg}$ ) and pulse pressure (PP: $51 \pm$ $4 \mathrm{mmHg}$ ) were increased as compared to untreated ZDF rats (146 $\pm 2 \mathrm{mmHg}, \mathrm{p}<0.05 ; 39 \pm 1 \mathrm{mmHg}, \mathrm{p}<0.01$, respectively) and control rats $(138 \pm 6 \mathrm{mmHg}, \mathrm{p}<0.0001 ; 31 \pm 2 \mathrm{mmHg}$, $\mathrm{p}<0.0001$, respectively). The vasorelaxation response to insulin observed in control rats was inhibited by L-NNA, was absent in untreated ZDF rats and was partially restored in the ZDF-ROS rats. Impaired Ach-induced relaxation in ZDF rats compared to controls was also partially restored in ZDF-ROS rats. L-NNA but not IND blocked Ach-induced relaxation.

Conclusions: Rosiglitazone, despite weight gain, higher SBP and PP that occur here in contrast to human data, restores endothelium-dependent relaxation induced by insulin and Ach in the thoracic aorta of this model of type 2 diabetes.

Supported by GlaxoSmithKline-Ltd

1200

The effect of leucine consumption on endothelial function, plasma glucose and insulin levels in healthy male subjects

G. Argyrakopoulou, A. Kokkinos, N. Tentolouris, D. Perrea, C. Arapostathi, D. Kyriaki, N. Katsilambros; 1st Department of Propaedeutic Medicine, Laiko Hospital, Athens University, Medical School, Greece.

Background: It is known that leucine consumption enhances insulin secretion. Data on the effect of acute hyperglycaemia on endothelial function in healthy subjects are controversial. Recent data showed that hyperinsulinaemia may exert a favourable effect on endothelial function. The combined, however, effect of hyperglycaemia and hyperinsulinamia on endothelial function has not been examined so far. This study examined the potential differential effect of hyperglycaemia and hyperinsulinaemia induced by glucose infusion alone and in combination with leucine consumption on endothelial function in healthy subjects.

Subjects-Methods: Ten healthy, non-obese males (mean age $31.8 \pm 5.5$ years) were studied twice. In one visit, they consumed $250 \mathrm{ml}$ of water (placebo) p.o. at baseline and $30 \mathrm{~min}$ later glucose $35 \%(0.5 \mathrm{~g} / \mathrm{kg}$ of body weight $)$ was infused i.v. (phase A). In the other visit, leucine $25 \mathrm{~g}$ in 
$250 \mathrm{ml}$ water p.o.was consumed at baseline and $30 \mathrm{~min}$ later the same amount of glucose was infused (phase B). Endothelial function was evaluated by measurement of flow-mediated dilatation (FMD) using high resolution ultrasonography at baseline, $40 \mathrm{~min}$ and $90 \mathrm{~min}$ afterwards. Plasma glucose and insulin levels were measured at baseline and every $10 \mathrm{~min}$ after glucose infusion.

Results: At $40 \mathrm{~min}$ of the phase A, FMD decreased significantly-compared with the baseline values- by $73.7 \%$ (median value, $\mathrm{P}=0.006$ ); the respective value in phase $\mathrm{B}$ was $+8.2 \%, P=0.80$ ). At $90 \mathrm{~min}$, there were no significant differences in FMD change after either experiment. Plasma glucose levels increased significantly and to the same degree at all time intervals studied after both experiments. Insulin levels increased much more during phase B as compared to phase A.

Conclusions: In healthy male subjects, acute hyperglycaemia impairs endothelial function. A concomitant, however, increase in plasma insulin levels after combined glucose leucine consumption exerts a protective effect on endothelial function.

\begin{tabular}{|c|c|c|c|c|c|c|c|c|}
\hline & & $\begin{array}{l}0 \\
\min \end{array}$ & $\begin{array}{l}40 \\
\min \end{array}$ & $\begin{array}{l}50 \\
\min \end{array}$ & $\begin{array}{l}60 \\
\min \end{array}$ & $\begin{array}{l}70 \\
\min \end{array}$ & $\begin{array}{l}80 \\
\min \end{array}$ & $\begin{array}{l}90 \\
\min \end{array}$ \\
\hline \multirow{4}{*}{$\begin{array}{l}\text { Glucose } \\
+ \text { placebo } \\
\text { (Phase A) }\end{array}$} & Glucose & 90.0 & 340.9 & 245.1 & 187.5 & 144.2 & 117.4 & 100.7 \\
\hline & (mg/dl) & \pm 7.9 & \pm 40.0 & \pm 25.9 & \pm 42.2 & \pm 49.2 & \pm 42.6 & \pm 31.1 \\
\hline & Insulin & 11.5 & 55.2 & 60.4 & 42.8 & 37.0 & 27.3 & 21.3 \\
\hline & $\begin{array}{l}(\mu \mathrm{IU} / \\
\mathrm{ml})\end{array}$ & \pm 1.7 & \pm 25.7 & \pm 38.1 & \pm 19.7 & \pm 19.0 & \pm 12.5 & \pm 9.1 \\
\hline \multirow{4}{*}{$\begin{array}{l}\text { Glucose } \\
\text { +leucine } \\
\text { (Phase B) }\end{array}$} & Glucose & 89 & 339.6 & 237.7 & 182.6 & 140.4 & 105.9 & 85.1 \\
\hline & $(\mathrm{mg} / \mathrm{dl})$ & \pm 12.4 & \pm 50.2 & \pm 62.0 & \pm 63.2 & \pm 58.6 & \pm 38.7 & \pm 31.4 \\
\hline & Insulin & 13.6 & 142.9 & 166 & 122.8 & 88.5 & 60.0 & 42.2 \\
\hline & $\begin{array}{l}(\mu \mathrm{IU} / \\
\mathrm{ml})\end{array}$ & \pm 7.6 & \pm 92.7 & \pm 79.0 & \pm 49.4 & \pm 35.0 & \pm 24.5 & \pm 19.0 \\
\hline
\end{tabular}

\section{1}

Effects of 17- $\beta$ estradiol treatment on vascular reactivity of ovariectomized and diabetic rats

A. Ceylan Isik ${ }^{1}$, O. Erdogan Tulmac ${ }^{2}$, N. Ari ${ }^{1}$, G. Ozansoy ${ }^{1}$; ${ }^{1}$ Pharmacology, University of Ankara, Faculty of Pharmacy, Ankara, Turkey, ${ }^{2}$ Gynecology, Zekai Tahir Burak Women Health, Education and Research Hospital, Ankara, Turkey.

Background and Aims: Menopause is an independent risk factor for cardiovascular diseases and it's believed that diabetes contributes this situation. Estrogen replacement therapy is associated with reduction in cardiovascular mortality in postmenopausal women. The aim of the present study was to show if there is any effect of $17-\beta$ estradiol (E2) on regulation of responsiveness of aortae from both diabetic (Dia) and non-diabetic ovariectomized (OVX) rats.

Materials and Methods: 4 groups have been used in this study: OVX $(n=8)$, OVX+E2 $(n=8)$, Dia+OVX $(n=8)$ and $\mathrm{Dia}+\mathrm{OVX}+\mathrm{E} 2(\mathrm{n}=10)$. Bilateral ovariectomy was applied to anesthetized animals and diabetes induced by a single i.p. injection of streptozotocin (STZ; $45 \mathrm{mg} / \mathrm{kg}$ ) a week after the operation. E2 treatment has been started on the 3rd day of the operation $(1 \mathrm{mg} / \mathrm{kg}$ day, orally). After 8 weeks blood glucose (BG), total cholesterol $(\mathrm{CH})$ and triglyceride (TG) concentrations have been measured as final values. Blood pressure (BP) levels have been measured by non-invasive tail cuff method weekly. Abdominal aortae have been used for functional studies in organ baths. $\mathrm{KCl}(10-60 \mathrm{mM})$ and phenylephrine (PE; $10^{-8}-10^{-4} \mathrm{M}$ ) were used in cumulative concentrations as contractile agents. In order to show functional endothelium, aortae were pre-contracted with submaximal concentration of PE $\left(10^{-6} \mathrm{M}\right)$ and then acethylcholine (ACh; $10^{-6} \mathrm{M}$ ) was applied to the aortae. Incubations with L-Nitro-L-Methyl Arginine Methyl Ester (L-NAME; $10^{-6} \mathrm{M}$ ), superoxide dismutase (SOD; $10^{-6} \mathrm{M}$ ) and indomethacine (INDO; $10^{-6} \mathrm{M}$ ) were performed to compare the magnitudes of PE-induced submaximal contractions and ACh-induced relaxations $\left(10^{-6} \mathrm{M}\right)$.

Results: Although the BG concentration was not affected by the treatment, $\mathrm{TG}$ and $\mathrm{CH}$ concentrations were significantly normalized $(\mathrm{P}<0.05$ vs. $\mathrm{OVX}+\mathrm{Dia})$. E2 treatment also reduced the BP values in Dia+OVX and OVX groups $(\mathrm{P}<0.05$ vs. $\mathrm{OVX}+\mathrm{E} 2)$. KCl- and PE-induced contractions were found that increased in both Dia and Dia + OVX aortae and reduced by the treatment $(\mathrm{P}<0.05$ vs. $\mathrm{OVX}+\mathrm{E} 2)$. The contractile effect of PE was increased by L-NAME, and reduced by SOD and INDO in all aortic groups. The vasodilator effect of ACh was increased by SOD and INDO, and reduced by L-NAME in all aortic groups.

Conclusion: These results suggests that E2 treatment has some beneficial effect on vascular reactivity in both diabetic and non-diabetic OVX animals and this effect may partly due to the antioxidant property of E2 itself.

\% Changes on PE-induced (10-6 M) Contractions

\begin{tabular}{llll}
\hline & L-NAME & SOD & INDO \\
\hline OVX & $40.18 \pm 0.12^{* \#} \uparrow$ & $46.36 \pm 1.34^{* \#} \downarrow$ & $47.53 \pm 0.46^{*} \downarrow$ \\
OVX+E2 & $64.60 \pm 0.22 \uparrow$ & $21.41 \pm 1.02^{\#} \downarrow$ & $40.37 \pm 0.68^{\ddagger} \downarrow$ \\
OVX+DiA & $41.60 \pm 0.18^{* *} \uparrow$ & $48.00 \pm 0.63^{* \#} \downarrow$ & $45.03 \pm 1.14^{\dagger} \downarrow$ \\
OVX+DiA+E2 & $68.10 \pm 0.10 \uparrow$ & $11.30 \pm 1.32^{*} \downarrow$ & $44.93 \pm 1.11^{\dagger} \downarrow$ \\
\hline
\end{tabular}


\% Changes on ACh-induced (10-6 M) Relaxations

\begin{tabular}{llll}
\hline & L-NAME & SOD & INDO \\
\hline OVX & $77.38 \pm 1.54^{* \#} \downarrow$ & $29.10 \pm 1.32^{* \#} \uparrow$ & $9.57 \pm 0.65^{\dagger \dagger} \uparrow$ \\
OVX+E2 & $88.35 \pm 1.62 \downarrow$ & $22.10 \pm 1.74 \uparrow$ & $11.46 \pm 0.21 \uparrow$ \\
OVX+Dia & $79.33 \pm 1.77^{* \#} \downarrow$ & $32.26 \pm 1.12^{* \#} \uparrow$ & $9.08 \pm 0.16^{\dagger \dagger} \uparrow$ \\
OVX+Dia+E2 & $84.35 \pm 1.83 \downarrow$ & $18.40 \pm 1.22 \uparrow$ & $11.47 \pm 0.37 \uparrow$ \\
\hline
\end{tabular}

$* \mathrm{P}<0.001$ and $\dagger \mathrm{P}<0.01$ vs. $\mathrm{OVX}+\mathrm{E} 2$

\# $\mathrm{P}<0.001$ and $t \mathrm{P}<0.01$ vs. $\mathrm{OVX}+\mathrm{Dia}+\mathrm{E} 2$

1202

The effect of benfotiamine on endothelial function in patients with type 2 diabetes mellitus

D. $\mathrm{Kim}^{1}, \mathrm{H} . \mathrm{Nam}^{2}$;

${ }^{1}$ Dept. of Internal Medicine, Hallym University, Seoul,

${ }^{2}$ Dept. of Internal Medicine, National Medical Center,

Seoul, Republic of Korea.

Background and Aims: Many studies show a strong relationship between hyperglycemia and diabetic microvascular complications, and hyperglycemia seems to have a important role in the pathogenesis of diabetic macrovascular disease. There are a number of potential strategies to pursue to prevent or possibly reverse damage caused by hyperglycemia. Typically, the most effective strategies would be aimed at the vasculature, which appears to be the first organ system to undergo hyperglycemia induced damage. Benfotiamine is a synthetic thiamine derivative that activates the enzyme transketolase to redirect glycolytic metabolites from vascular damaging pathways. Recently in diabetic animals benfotiamine treatment inhibited major pathways implicated in pathogenesis of hyperglycemia induced vascular damage and NF-kB activation. Endothelial dysfunction is earliest sign involved in the pathogenesis of diabetic complications.

Materials and Methods: We evaluated the change of plasma soluble E-selectin, ICAM-1 and VCAM-1 concentration after the administration of benfotiamine in patients with type 2 diabetes. Plasma soluble E-selectin, ICAM-1 and VCAM-1 (a marker of endothelial function) were measured using commercial ELISA kit. Eighteen type 2 diabetes patients (age $57.7 \pm 6.75$ years, BMI $24.6 \pm 1.74 \mathrm{~kg} / \mathrm{m}^{2}$ and DM duration $13.1 \pm 5.64$ years) were studied and benfotiamine $120 \mathrm{mg}$ was administered twice daily for two months. Results: 1. Plama E-selectin level was decreased significantly from $39.3 \pm 3.34 \mathrm{ng} / \mathrm{mL}$ to $34.8 \pm 2.60 \mathrm{ng} / \mathrm{mL}$ after administration of benfotiamine $(\mathrm{P}<0.05)$.

2. Plama ICAM-1 concentration was decreased significantly from $225.4 \pm 18.39 \mathrm{ng} / \mathrm{mL}$ to $205.1 \pm 16.03 \mathrm{ng} / \mathrm{mL}$ after administration of benfotiamine $(\mathrm{P}<0.05)$.
3. Plama VCAM-1 was decreased significantly also from $31.9 \pm 7.81 \mathrm{ng} / \mathrm{mL}$ to $26.6 \pm 5.07 \mathrm{ng} / \mathrm{mL}$ after administration of benfotiamine $(\mathrm{P}<0.05)$.

Conclusion: These results showed that benfotiamine can decrease plasma E-seletin, ICAM-1 and VCAM-1 level and improve endothelial function in patients with type 2 diabetes. So benfotimine might be clinically useful in preventing the development and progression of diabetic vascular complications.

1203

Endothelial progenitor cells are reduced but activated in type 1 diabetic patients with longstanding disease and high $\mathrm{HbA}_{1 \mathrm{c}}$

G. H. Schernthaner ${ }^{1}$, M. Satler ${ }^{1}$, J. Schnabler ${ }^{2}$,

R. Koppensteiner ${ }^{1}$, G. Schernthaner ${ }^{2}$;

${ }^{1}$ Department of Internal Medicine II, Division of Angiology, Medical University of Vienna, ${ }^{2}$ Department of Medicine I, Rudolfstiftung Hospital Vienna, Austria.

Background and Aims: Two recent european epidemiological studies indicate an excess mortality, mainly caused from cardiovascular disease (CVD), in patients with type 1 diabetes (T1DM) despite advances in diabetes care. Since a reduced number of endothelial progenitor cells (EPC) was recently found to predict CVD death and events in nondiabetic patients, our study was aimed to quantify EPC in well-characterised patients with T1DM.

Materials and Methods: 45 patients with T1DM $\left(\mathrm{HbA}_{1} \mathrm{c}\right.$ : $7.8 \pm 1.9 \%$; age: $36 \pm 13$ years; BMI: $24.5 \pm 3.4 \mathrm{~kg} / \mathrm{m}^{2}$ ) were studied in comparison with 30 age- and sex-matched control subjects. The T1DM patients were divided into three groups according to their duration of disease: $<10,10-25$, and $>25$ years. Circulating progenitor cells (CPC, defined as CD34+/CD133+), endothelial progenitor cells (EPC, defined as CD34+/CD133+/CD309+) and activated EPC (actEPC, defined as CD34+/CD133+/CD309+/CD31+) were enumerated by flow cytometry. The EPC/CPC ratio as well as the actEPC/EPC ratio was determined. T1DM patients presenting with diabetic retinopathy were excluded from the study. Results: CPC, EPC and actEPC were significantly reduced in T1DM patients in comparison with control subjects (CPC: $1264 \pm 421$ vs $529 \pm 167, \mathrm{p}<0.001$; EPC: $365 \pm 164$ vs $109 \pm 80, \mathrm{p}<0.001$; actEPC: $150 \pm 59$ vs $62 \pm 48, \mathrm{p}<0.001)$. Interestingly, the EPC/CPC ratio was lower in the T1DM compared with the controls $(21 \pm 13 \%$ to $29 \pm 9 \%, \mathrm{p}<0.001)$, whereas the actEPC/EPC ratio was increased in the T1DM versus controls $(62 \pm 25 \%$ to $42 \pm 8$ to $\mathrm{p}<0.001)$. By univariate regression analysis, age had the strongest association with the decline in CPC $(\mathrm{r}=-0.335, \mathrm{p}=0.003)$, diabetes duration with the decline of EPC $(r=-0.434, p<0.001)$, and 
HbAlc showed the strongest association with the activation of EPC as detected by the adhesion molecule CD31 (HbA1c to actEPC/EPC ratio: $r=0.380, p=0.001$ ).

Conclusion: Type 1 diabetic patients with long duration of disease and high HbA1c have significantly less numbers of CPC and EPC. Remarkably, the rate of activated EPC as determined by expression of CD31 on EPC surface is elevated in these patients. The increase in activated EPC could be a compensatory response to augmented demand of vascular repair in those type 1 diabetic patients, who have longstanding disease, poor glycemic control and are at increased risk for cardiovascular morbidity and mortality.

\section{PS 108}

\section{Arterial stiffness and remodelling}

\section{4}

Metabolic syndrome and intima media thickness - an observation of the RIAD-study

F. Schaper, C. Köhler, M. Hanefeld;

GWT-TUD, Centre of Clinical Studies, Dresden, Germany.

Background and Aims: Several studies have shown that patients with metabolic syndrome have significant more cardiovascular events. Scarce information exist, how far the intima media thickness (IMT) of common carotid arteries as marker of early atherosclerotic changes was influenced by metabolic syndrome (MS).

Materials and Methods: A total of 566 middle-aged subjects participate on the Risk Factors in Impaired Glucose Tolerance for Atherosclerosis and Diabetes (RIAD) were classified as follows: 108 without traits of MS (group 1), 135 with single trait (group 2), 150 with two traits (group 3) and 173 with three or more traits (group 4) of MS. MS was defined by NCEP III ATP criteria. IMT was measured by high resolution ultrasound. Increased IMT was defined as the value over the $3 \mathrm{rd}$ percentile ( $\geq 0,95 \mathrm{~mm}$ ).

Results: No difference in patients with increased IMT was found between group 2 and group 1. Patients with increased IMT were significant more frequent in group 3 and group 4 comparing with group 1 (OR 1,93 vs $1,72, p=0,012$ vs $\mathrm{p}=0,032)$. No difference in patients with increased IMT was found between group 3 and group 4.

Conclusion: Increased IMT was significant more often in patients with MS ( $\geq 3$ traits). These early atherosclerotic changes were not only seen in patients with MS but also in patients with "pre"metabolic syndrome (2 traits).
1205

Postprandial glucose is associated with carotid intima media thickness but postprandial triglycerides are not. The HOORN PRANDIAL STUDY

M. Alssema ${ }^{1}$, R. K. Schindhelm ${ }^{2}$, J. M. Dekker ${ }^{1}$, M. Diamant ${ }^{2}$, G. Nijpels ${ }^{1}$, T. Teerlink ${ }^{3}$, P. G. Scheffer ${ }^{3}$, P. J. Kostense ${ }^{1}$, R. J. Heine ${ }^{1,2}$;

${ }^{1}$ EMGO-institute, ${ }^{2}$ Department of Endocrinology/Diabetes Center, ${ }^{3}$ Department of Clinical Chemistry, VU University Medical Center, Amsterdam, The Netherlands.

Background and Aims: Both postprandial hyperglycemia (ppGL) and postprandial hypertriglyceridemia (ppTG) have been identified as stronger risk markers for atherosclerosis than the respective fasting levels. However, the relative contribution of ppGL and ppTG to atherosclerosis has not been investigated until now. The aim of this study was to compare the associations of ppGL and ppTG with carotid intima media thickness (IMT) following two consecutive high-fat meals or two consecutive high-carbohydrate meals on two separate days.

Materials and Methods: Non-smoking, post-menopausal women, aged 50-65 years were included; 76 women with normal glucose metabolism (NGM, WHO-99 criteria) and 78 women with type 2 diabetes (DM) of whom 37 were using statin medication. Blood samples were taken at both visits and GL and TG were measured before and at $\mathrm{t}=1,2$, 4, 6 and 8 hours following breakfast; lunch was given at $\mathrm{t}=4$. The high-fat breakfast and lunch contained $3.3 \mathrm{MJ}$; $50 \mathrm{~g}$ fat and $56 \mathrm{~g}$ carbohydrates. The high-carbohydrate breakfast and lunch contained $3.2 \mathrm{MJ} ; 4 \mathrm{~g}$ fat and $162 \mathrm{~g}$ carbohydrates. IMT was measured by ultrasound imaging of the right common carotid artery at $10 \mathrm{~mm}$ proximal to the carotid bulb. Linear regression analysis was used to calculate associations with IMT, we tested for interaction with DM and use of statin medication.

Results: Mean IMT was 764 and $780 \mu \mathrm{m}$ for women with NGM and DM, respectively. In the complete study population, age was statistically significantly associated with IMT (standardized regression coefficient $(95 \% \mathrm{CI})$ in $\mu \mathrm{m}$ IMT was $20.2(2.6 ; 37.8))$. In women with NGM, ppGL levels after both the high-fat and the high-carbohydrate meals were positively associated with IMT. Standardized regression coefficients $(95 \% \mathrm{CI})$ in $\mu \mathrm{m}$ IMT were 92.6 $(6.1 ; 179.1)$ for ppGL after the high-fat meals and 98.8 $(-13.7 ; 211.3)$ for ppGL after the high-carbohydrate meals with adjustment for age (Table). 
Associations (standardized beta $(95 \% \mathrm{CI})$ in $\mu \mathrm{m}$ IMT, adjusted for age and use of statins)

\begin{tabular}{lll}
\hline & NGM $(\mathbf{n}=\mathbf{7 6})$ & DM $(\mathbf{n}=\mathbf{7 8})$ \\
\hline Fasting TG & $2.3(-26.0 ; 30.7)$ & $-5.6(-34.1 ; 22.9)$ \\
Fasting GL & $50.4(-45.8 ; 146.6)$ & $\mathbf{3 8 . 6}(\mathbf{6 . 2} ; \mathbf{7 1 . 0}) \#$ \\
Fat meals & & \\
PpTG & $3.9(-23.1 ; 30.9)$ & $-13.4(-40.3 ; 13.6)$ \\
PpGL & $\mathbf{9 2 . 6 ( 6 . 1 ; 1 7 9 . 1 )}$ & $8.6(-18.5 ; 35.6)^{*}$ \\
Carbohydrate meals & & \\
PpTG & $10.0(-16.5 ; 36.4)$ & $-10.1(39.6 ; 19.5)$ \\
PpGL & $98.8(-13.7 ; 211.3)$ & $14.4(-13.1 ; 42.0)^{*}$ \\
\hline
\end{tabular}

\#Women using statins were excluded because of interaction with use of statins $(\mathrm{p}=0.07)$

*Interaction with $\mathrm{DM}(\mathrm{p}<0.15)$

These associations were not explained by BMI, waist, blood pressure, total cholesterol, LDL-c or HDL-c. No association between ppGL and IMT in women with DM was found ( $p$-value for interaction with DM p <0.15). PpTG were not associated with IMT in women with NGM nor in women with DM. In women with DM no interaction was found with use of statins, except for fasting GL ( $p=0.07$ ).

Conclusion: The association between ppGL and IMT was stronger than that of ppTG and IMT. The association between ppGL and IMT in women with NGM suggests that ppGL even in the normal range, is a marker or a risk factor of CVD. The research was financially supported by funding from Novartis and by a grant from the Dutch Diabetes Research Foundation, grant 2001.00.052.

\section{6}

Arterial stiffness is inversely related to adiponectin levels in young, normotensive type 1 diabetic patients

A. Tsiakou, S. Liatis, D. Kyriaki, K. Kyriakopoulos, I. Bitsikas, N. Katsilambros;

1st Internal Medicine Department, Athens University Medical School, Laiko Hospital, Greece.

Background and Aims: Arterial stiffness, a contributing factor to cardiovascular morbidity and mortality, is increased even at the early stages of type 1 diabetes and is associated with the presence of cardiac autonomic neuropathy. Adiponectin is an adipose tissue derived adipokine that has recently attracted much attention as a posible insulinsensitizing and antiatherogenic factor. Plasma adiponectin (ADPN) concentrations are low in individuals with insulin resistance such as those with type 2 diabetes. Only few studies have examined adiponectin levels in patients with type 1 diabetes and have consistently shown that these patients have increased plasma levels of that hormone.
Recently it has been reported that increased adiponectin levels in patients with type 1 diabetes are prospectively associated with a lower risk of coronary artery disease.

Aim: The aim of this study was to investigate the association of arterial stiffness with ADPN levels and autonomic dysfunction in young, normotensive type 1 diabetic patients. Materials and Methods: 43 normotensive type 1 diabetic patients, aged less than 40 years were consecutively selected from the outpatient diabetes clinic of our institution. Subjects on statin therapy as well as those with macrovascular disease or overt nephropathy were excluded. Large artery stiffness was assessed by measurement of carotid-femoral pulse wave velocity (PWV) and plasma ADPN was measured by radio-immuno assay. Autonomic function was estimated on the basis of the battery of classic cardiovascular autonomic function tests (Ewing tests).

Results: Data from 43 subjects were analyzed (mean age $26.8 \pm 5.9$ years, body mass index [BMI] $24.1 \pm 2.9 \mathrm{~kg} / \mathrm{m}^{2}$, $\mathrm{HbA}_{1 \mathrm{C}} 7.5 \pm 1.3 \%, 20$ [45.5\%] men). Mean PWV and ADPN values were $5.5 \pm 0.9 \mathrm{~m} / \mathrm{s}$ and $15.3 \pm 6.8 \mu \mathrm{g} / \mathrm{mL}$, respectively. There was a significant positive correlation of PWV with age $(\mathrm{r}=0.508, \mathrm{p}=0.001)$, duration of diabetes $(r=0.278, p=0.048)$, BMI $(r=0.303, p=0.048)$, waist circumference $(\mathrm{r}=0.448, \mathrm{p}=0.003)$ and systolic blood pressure $(\mathrm{r}=0.375, \mathrm{p}=0.013)$. Nevertheless, PWV showed a reciprocal relationship with inspiration/expiration index $(\mathrm{r}=$ $-0.360, \mathrm{p}=0.018)$ and plasma ADPN $(\mathrm{r}=-0.418, \mathrm{p}=0.01)$. In multivariate stepwise linear regression analysis, PWV was independently associated to plasma ADPN (standardized $\beta$-regression coefficient $=0.480, p=0.001$ ), duration of diabetes $(\beta=0.342, p=0.019)$ and systolic blood pressure $(\beta=0.345, p=0.016)$. The $\mathrm{R}^{2}$ of the statistical model was 0.468 .

Conclusion: Arterial stiffness appears to be inversely related to plasma ADPN concentration in young, normotensive patients with type 1 diabetes without major complications, the latter association being independent of the presence of cardiac autonomic neuropathy. This novel finding suggests that the above adipocytokine might have a protective role against macrovascular disease in the population studied.

1207

High vitamin B6 is associated with lower carotid distensibility and compliance in type 2 diabetes but not in normal or impaired glucose metabolism: the HOORN STUDY

A. M. W. Spijkerman ${ }^{1}$, Y. M. Smulders ${ }^{2}$, J. M. Dekker ${ }^{1}$, T. Teerlink ${ }^{3}$, C. Jakobs ${ }^{3}$, G. Nijpels ${ }^{1}$, R. J. Heine ${ }^{4}$,

L. Bouter ${ }^{1}$, C. D. A. Stehouwer ${ }^{5}$; 
${ }^{1}$ EMGO Institute, ${ }^{2}$ Department of Internal Medicine, ${ }^{3}$ Department of Clinical Chemistry, ${ }^{4}$ Department of Endocrinology, VU University Medical Center, Amsterdam, ${ }^{5}$ Department of Internal Medicine, Academic Hospital Maastricht, Maastricht, The Netherlands.

Background and Aims: High homocysteine and low levels of vitamin B6 have been associated with cardiovascular disease. Our aim was to determine whether homocysteine (Hcy) and vitamin B6 are associated with carotid stiffness in people with and without type 2 diabetes.

Material and Methods: The study was performed among participants of the 2nd follow-up of the Hoorn Study, a population-based study of diabetes and cardiovascular disease. Data on Hcy and vitamin B6 and carotid stiffness was available for 545 people, 237 with normal glucose metabolism (NGM), 112 with impaired glucose metabolism (IGM) and 196 with type 2 diabetes (DM2), 50\% men, mean age 68 years. Distensibility and compliance coefficients (DC and $\mathrm{CC}$ ) of the right common carotid artery were obtained by a single observer with an ultrasound scanner with a $7.5 \mathrm{MHz}$ linear probe (350 series, Pie Medical). Total fasting plasma homocysteine was determined on an Abbott Imx analyser and vitamin B6 with HPLC. Multiple linear regression analysis was used and age, sex and mean arterial pressure were included as standard correction variables.

Results: High Hcy was not associated with carotid stiffness in the various categories of glucose metabolism (see table). In contrast, high vitamin B6 was associated with lower carotid distensibility and compliance coefficients in patients with type 2 diabetes, but not in NGM or IGM. The association of high B6 in type 2 diabetes was independent of Hcy, folate, vitamin $\mathrm{B} 12$, prior $\mathrm{CVD}$, current smoking or renal function.

\begin{tabular}{|c|c|c|c|c|c|c|}
\hline & \multicolumn{3}{|c|}{$\mathrm{CC}$} & \multicolumn{3}{|c|}{$\mathrm{DC}$} \\
\hline & NGM & IGM & DM2 & NGM & IGM & DM2 \\
\hline Hcy 2nd tertile & $\begin{array}{l}0.027 \\
(.412)\end{array}$ & $\begin{array}{c}-0.042 \\
(.518)\end{array}$ & $\begin{array}{c}-0.014 \\
(.630)\end{array}$ & $\begin{array}{c}-0.260 \\
(.642)\end{array}$ & $\begin{array}{c}-0.262 \\
(.793)\end{array}$ & $\begin{array}{l}0.212 \\
(.722)\end{array}$ \\
\hline Hcy 3rd tertile & $\begin{array}{l}0.044 \\
(.186)\end{array}$ & $\begin{array}{c}-0.082 \\
(.210)\end{array}$ & $\begin{array}{c}-0.015 \\
(.066)\end{array}$ & $\begin{array}{l}0.133 \\
(.815)\end{array}$ & $\begin{array}{c}-0.694 \\
(.488)\end{array}$ & $\begin{array}{l}0.907 \\
(.163)\end{array}$ \\
\hline B6 2nd tertile & $\begin{array}{c}-0.058 \\
(.083)\end{array}$ & $\begin{array}{c}-0.046 \\
(.409)\end{array}$ & $\begin{array}{c}-0.032 \\
(.289)\end{array}$ & $\begin{array}{c}-0.537 \\
(.359)\end{array}$ & $\begin{array}{c}-0.746 \\
(.382)\end{array}$ & $\begin{array}{c}-0.411 \\
(.485)\end{array}$ \\
\hline B6 3rd tertile & $\begin{array}{c}-0.056 \\
(.086)\end{array}$ & $\begin{array}{l}0.035 \\
(.554)\end{array}$ & $\begin{array}{c}-0.112 \\
(.000)\end{array}$ & $\begin{array}{c}-0.070 \\
(.902)\end{array}$ & $\begin{array}{c}0.504 \\
(.578)\end{array}$ & $\begin{array}{c}-1.891 \\
\mathbf{( . 0 0 2 )}\end{array}$ \\
\hline
\end{tabular}

Data are linear regression coefficients and (p-values)

Conclusion: In this cohort of elderly people high vitamin B6, but not Hcy, was associated with higher carotid stiffness in patients with type 2 diabetes but not in people with normal or impaired glucose metabolism.

Supported by Netherlands Heart Foundation grant no $2003 B 239$
1208

Predictors of aortic stiffness in elderly type 2 diabetic patients in terms of diabetic nephropathy M. Kameyama $^{1}$, T. Kunito ${ }^{1}$, M. Miyagi ${ }^{1}$, K. Iso ${ }^{1}$, R. Ochiai ${ }^{2}$, K. Kuboki ${ }^{2}$, G. Yoshino ${ }^{2}$;

${ }^{1}$ Division of Diabetes, Department of Internal Medicine (Ohashi), Toho University School of Medicine, Tokyo, ${ }^{2}$ Division of Diabetes, Metabolism and Endocrinology, Department of Internal Medicine (Omori), Toho University School of Medicine, Japan.

Background and Aims: Carotid-femoral pulse wave velocity (PWV) reflects arterial stiffness. Several studies demonstrate that increased PWV is a predictor of future cardiovascular events with diabetes mellitus. However, it is difficult to measure PWV of all diabetic patients because there are many numbers of diabetic patients. The aim of this study was to investigate the predictors of aortic stiffness in elderly type 2 diabetic patients in terms of diabetic nephropathy.

Materials and Methods: We studied cross-sectionally 128 elderly type 2 diabetic outpatients of 65 to 86 years of age $[73.0+/-6.7$ years (mean+/-SD), duration of diabetes $15.9+/$ -7.5 years]. All of the subjects had no evidence of cerebral, cardiac or peripheral vascular complications. PWV was evaluated after 30-minutes bed resting and used as an index of arterial stiffness, and blood pressure (BP) were assessed. At overnight fast, blood sampling were performed: plasma glucose (PG), hemoglobin A1c (HbAlc), serum lipids, creatinine and von Willebrand factor (vWF), a marker of vascular endothelial cell damage. Urinary albumin excretion ratio (AER), and urinary transferrin (Tf) and type IV collagen (Col-IV) were also determined in morning spot urine sample. Creatinine clearance rate $(\mathrm{Ccr})$ was calculated from the Cockcroft-Gault formula. The subjects were categorized in quartiles based on the PWV value. The analysis was carried out on log-transformed AER, Tf and Col-IV values due to the skew distribution of these variables.

Results: The values of PWV in the first, second, third and fourth quartiles were $7.9+/-0.4(n=20), 9.2+/-0.4(n=49)$, $10.5+/-0.3(n=44)$ and $12.1+/-0.4 \mathrm{~m} / \mathrm{sec}(\mathrm{n}=15)$, respectively. Systolic BP in the fourth quartile was significantly higher than that in the first quartile, but diastolic BP was not different. No significant differences were observed in PG, HbAlc and serum lipids among four quartiles. Ccr levels in the third and the fourth quartile of PWV were significantly lower than those in the first quartile. Furthermore, vWF, $\log$ AER and $\log \mathrm{Tf}$ were significantly increased through quartiles of PWV. Moreover, log ColIV levels in the fourth quartile were significantly higher than those in the first quartile. In lineal regression analysis, 
age, duration of diabetes, systolic BP, PG, log AER, log Tf and $\log$ Col-IV in all subjects were positively correlated, and Ccr was inversely correlated with PWV. Stepwise multivariate analysis in all subjects demonstrated that independent predictive values for PWV were $\log$ AER $(\mathrm{F}=10.92, \mathrm{P}=0.001)$, systolic $\mathrm{BP}(\mathrm{F}=7.96, \mathrm{P}=0.006)$, age $(\mathrm{F}=7.58, \mathrm{P}=0.006)$ and $\mathrm{Ccr}(\mathrm{F}=5.10, \mathrm{P}=0.026)$, respectively. Conclusion: These results suggest that AER, systolic BP, age and Ccr are diagnostic predictors of aortic stiffness, and neither urinary Tf nor Col-IV are predictive values of aortic stiffness in elderly type 2 diabetes without macroangiopathy.

\section{9}

Cardiovascular risk factors and intima-media thickness of the common carotid artery in patients with latent autoimmune diabetes of the adult (LADA)

L. Laviola, C. Lattanzio, J. Rinaldi, M. Bellacicco, T. Rizzi, G. Stefanelli, G. Garruti, A. Bellomo Damato, S. Perrini, R. Giorgino, F. Giorgino;

Internal Medicine, Endocrinology and Metabolic Diseases, University of Bari, Italy.

Background and Aims: patients with LADA show different anthropometric and metabolic characteristics as compared to the "common" form of type 2 diabetes (T2DM). The aim of this study was to investigate multiple cardiovascular risk factors and the intima-media thickness of the common carotid artery (CCA-IMT), an early marker of atherosclerosis, in LADA $(n=26, M / F$ 13/13) and T2DM $(n=23, M / F 15 / 8)$ patients.

Materials and Methods: the LADA group included patients who had seropositivity for antibodies to glutamic acid decarboxylase (GAD, RIA method), adult age at diagnosis ( $>25$ yrs old), and at least six months without insulin treatment post-diagnosis.

Results: LADA and T2DM subjects were not different for age $(43.8 \pm 2.9$ yrs for LADA vs. $49.3 \pm 2.2$ yrs for T2DM, $\mathrm{p}=0.14)$, duration of disease $(5.48 \pm 1.0$ yrs for LADA vs. $8.05 \pm 1.80 \mathrm{yrs}$ for T2DM, $\mathrm{p}=0.23$ ), and HbA1c levels $(8.42 \pm \tilde{0} 0.40 \%$ for LADA vs. $8.19 \pm 0.44 \%$ for T2DM, $\mathrm{p}=0.70)$. However, as compared to T2DM subjects, patients with LADA showed reduced fasting C-peptide levels $(1.38 \pm 0.30$ vs. $3.37 \pm 0.39 \mathrm{ng} / \mathrm{ml}, \mathrm{p}=0.0003)$, a trend toward lower BMI $\left(24.42 \pm 0.70 \mathrm{~kg} / \mathrm{m}^{2}\right.$ vs. $25.83 \pm 0.94 \mathrm{~kg} / \mathrm{m}^{2}$, $\mathrm{p}=0.22)$ and waist $(88.2 \pm 2.3 \mathrm{~cm}$ vs. $92.3 \pm 2.2 \mathrm{~cm}, \mathrm{p}=0.17)$ values, and significantly lower arterial blood pressure levels (systolic blood pressure $120.8 \pm 2.8$ vs. $130.5 \pm 2.8 \mathrm{mmHg}$, $\mathrm{p}=0.02$; diastolic blood pressure $76.60 \pm 1.4 \mathrm{mmHg}$ vs.
$81.14 \pm 1.7 \mathrm{mmHg}, \mathrm{p}=0.046)$. The lipid profile also significantly differed in LADA patients since they had lower total cholesterol $(171.7 \pm 6.1 \mathrm{mg} / \mathrm{dL}$ vs. $191.6 \pm 6.7 \mathrm{mg} / \mathrm{dL}$, $\mathrm{p}=0.033)$, LDL cholesterol $(108.8 \pm 5.7 \mathrm{mg} / \mathrm{dL}$ vs. $127.2 \pm$ $6.3 \mathrm{mg} / \mathrm{dL}, \mathrm{p}=0.036)$, and triglyceride $(72.5 \pm 7.5 \mathrm{mg} / \mathrm{dL}$ vs. $129.3 \pm 18 \mathrm{mg} / \mathrm{dL}, \mathrm{p}=0.0061)$ levels, and higher $\mathrm{HDL}$ cholesterol $(48.4 \pm 2.3 \mathrm{mg} / \mathrm{dL}$ vs. $40.5 \pm 2.2 \mathrm{mg} / \mathrm{dL}$, $\mathrm{p}=0.016$ ) levels compared to T2DM subjects. Interestingly, the CCA-IMT, evaluated by high-resolution B-mode ultrasonography, was significantly lower in LADA than in T2DM patients $(0.870 \pm 0.032 \mathrm{~mm}$ vs. $0.955 \pm 0.027 \mathrm{~mm}$, $\mathrm{p}<0.05)$.

Conclusion: therefore, as compared to T2DM individuals, LADA patients show more beneficial anthropometric and metabolic characteristics and lower cardiovascular risk factors and markers that could be associated with lower atherogenesis and frequency of cardiovascular disease in this diabetes subgroup.

1210

ACE inhibitor/angiotensin II receptor blocker ameliorates secular change in brachial-ankle pulse wave velocity in type 2 diabetic patients

N. Tanaka, M. Hosoi, Y. Omura, M. Ueda, T. Yamakita, I. Kawasaki, K. Yoshioka, T. Sato;

Endocrinology and Metabolism, Osaka City General Hospital, Japan.

Background and Aims: Pulse wave velocity (PWV) reflects aortic stifness, being an independent cardiovascular risk predictor in hypertensive and end stage renal disease patients. It is reported that ACE inhibitor/angiotensin II receptor blocker (ACEI/ARB) reduces $\mathrm{PWV}$ in essential hypertensive patients. In type 2 diabetic patients, it is well known that PWV is higher than in normal population, but whether intervention of ACEI/ARB improves PWV is obscure. We examined how much secular change of PWV is influenced by chronic administration of $\mathrm{ACEI} / \mathrm{ARB}$ in type 2 diabetes mellitus patients.

Materials and Methods: We measured brachial-ankle PWV (baPWV) and ankle-brachial index (ABI) by form PWV/ABI (Colin MT Co. Ltd.) in 109 of diabetic inhospital patients or outpatients in our hospital (whose age ranged 48 to 73 years, 59 male and 50 female, HbA1c; $8.3 \pm$ $2.3 \%$ ). We measured baPWV at the beginning and the end of observation period ( $29 \pm 11$ months after), and the change in baPWV values during the observation period was referred as $\triangle \mathrm{PWV}$. All the subjects were divided into ACEI/ARB treated group (group A, n=41) and non-treated 
group (group $\mathrm{C}, \mathrm{n}=68$ ). We investigated clinical profiles and medications such as sulfonylureas, insulin, biguanide, $\alpha$-GI, pioglitazone, statins, fibrates, anti-platelets and $\mathrm{Ca}$ channel antagonists, as well as ACEI/ARB. Those who had low value of ABI $(<0.9)$ were excluded from this study.

Results: Characteristics of the subjects at the beginning and at the end of the observation were as follows; blood pressure (mmHg): $138 \pm 18 / 80 \pm 11,139 \pm 21 / 80 \pm 13$, baPWV $(\mathrm{cm} / \mathrm{s}): 1730 \pm 361,1772 \pm 338$, pulse pressure $(\mathrm{mmHg}): 57 \pm$ $10,59 \pm 11 . \Delta \mathrm{PWV}$ was $47 \pm 286 \mathrm{~cm} / \mathrm{s}$. In group A, profiles were as follows; blood pressure (mmHg): $143 \pm 19 / 84 \pm 13$, $144 \pm 19 / 82 \pm 12$, baPWV (cm/s): $1829 \pm 443,1758 \pm 298$, while in group C, blood pressure (mmHg):134 $\pm 17 / 78 \pm 9$, $135 \pm 22 / 77 \pm 13$, baPWV (cm/s): $1668 \pm 298,1780 \pm 359$. $\triangle \mathrm{PWV}$ was significantly ameliorated in group A (group A/group C: $-53.7 \pm 309 / 110.6 \pm 260, \mathrm{P}=0.0049)$, although values of systolic blood pressure (group A/group C: $0.829 \pm$ 20.7/1.833 \pm 18.8 ) and pulse pressure (group A/group C: $2.355 \pm 10.5 / 1.365 \pm 11.0)$ were not significantly altered during the period. Multiple regression analysis revealed that only administration of $\mathrm{ACEI} / \mathrm{ARB}$ was associated with $\triangle \mathrm{PWV}(\beta=-0.303, \mathrm{P}=0.0046)$ in all those drugs.

Conclusion: In the type 2 diabetic patients, chronic administration of ACEI/ARB ameliorates the secular change in baPWV independent of blood pressure.

\section{1}

The effect of atorvastatin therapy on the elastic properties of arteries in subjects with type 2 diabetes and dyslipidaemia

P. Grigoropoulou ${ }^{1}$, N. Tentolouris ${ }^{1}$, A. Papazafiropoulou ${ }^{1}$, D. Perrea ${ }^{1}$, C. Zoupas ${ }^{2}$, P. Philippidis ${ }^{2}$, N. Katsilambros ${ }^{1}$;

${ }^{1} 1$ st Department of Propaedeutic Medicine, Athens University Medical School, Laiko Hospital, ${ }^{2}$ 2nd Department of Internal Medicine, General Hospital of Athens "G.Gennimatas", Athens, Greece.

Background and Aims: Dyslipidaemia is a major risk factor for cardiovascular disease (CVD) in patients with type 2 diabetes (T2DM), associated with endothelial dysfunction and increased arterial stiffness. Statins are accepted as first-line treatment for such patients, improving endothelial function and exerting a direct antiatherogenic effect in the arterial wall. Pulse wave velocity (PVW) of the aorta, which reflects its elastic properties, has emerged as an important predictor of cardioavascular events. The aim of the study was to assess the effect of atorvastatin on aortic and brachial arterial stiffness by measuring of PWV.

Materials and Methods: A total of 23 patients $(7$ men and 16 women) with T2DM, mean age $61.6 \pm 7.6$ years, duration of diabetes $6.8 \pm 5.6$ years, and dyslipidaemia were examined before, 3, and 6 months after initiation of statin therapy (atorvastatin $10 \mathrm{mg}$ daily). Subjects were eligible if they were 40-75 years of age, had LDL cholesterol (LDL-C) $\geq 100 \mathrm{mg} /$ $\mathrm{dl}$, and if they did not have clinically apparent macrovascular disease. HBA1c, plasma lipids were determined and carotidfemoral PWV (PWVcf) and carotid-radial PWV (PWVcr) were measured by sequential applanation tonometry (SphygmoCor Vx, AtCor Medical, Sydney, Australia).

Results LDL-C values declined significantly at 3 and 6 months by $34 \%$ and $33 \%$, respectively (both $\mathrm{P}$ values $<0.001)$. The same was valid for total triglycerides (decline by $33 \%$ and $20 \%$, respectively, $\mathrm{P}<0.04$ ). No significant changes were found in HBA1c and HDL cholesterol levels. PWVcr values declined by $11 \%$ at 3 months $(\mathrm{P}=0.001)$ and $17 \%$ at 6 months $(\mathrm{P}=0.001)$. The same was valid for PWVcf at 3 and 6 months (decline by $13 \%, \mathrm{P}=0.006$, and by $10 \%, \mathrm{P}=0.004$, respectively).

Conclusion: Statin treatment, except of reducing LDL-C and triglyceride levels in patients with T2DM, improved the elastic properties of the aorta and brachial artery after 6 months therapy.

1212

Atorvastatin improves arterial stiffness in normotensive normolipidaemic persons with type 2 diabetes

K. Pandit, S. Mukhopadhyay, S. Mukhopadhyay,

P. Mukhopadhyay, S. Chowdhury;

Endocrinology, IPGME\&R, Kolkata, India.

Background and Aims: Increased arterial stiffness is an independent predictor of cardiovascular disease and mortality in middle-aged and older adults.

Materials and Methods: Arterial stiffness was measured by pulse wave velocity (PWV) in brachial-ankle segments by automated oscillometry in 71 normotensive and normolipidaemic subjects with type 2 diabetes (40 males and 31 females).

Results: There was no significant correlation between the mean brachial-ankle pulse wave velocity (baPWV) and duration of diabetes, BMI, waist circumference, waist-hip ratio, glycated hemoglobin, LDL and/or HDL cholesterol or spot urine albumin-creatinine ratio at the baseline. However correlation was found between baPWV and systolic blood pressure. 57 patients showing abnormal baPWV were randomized into 2 groups. Patients in group $A(n=29)$ were given $10 \mathrm{mg}$ atorvastatin daily for 6 months. 28 patients in group B, who served as matched controls, were given placebo for the same duration in a double blind manner with both the investigator and the patient being blinded as to the nature of the tablet being administered (atorvastatin or placebo); baPWV was reassessed after 6 months in all patients. 
Conclusion: The atorvastatin group showed statistically significant reduction in baPWV $(1712.03 \pm 349.9 \mathrm{~cm} . / \mathrm{sec}$. vs. $1658.81 \pm 303.26 \mathrm{~cm} . / \mathrm{sec}$., $\mathrm{p}<0.05)$. Although the placebo group also showed some improvement in mean baPWV ( $1692.03 \pm 425.15 \mathrm{~cm} . / \mathrm{sec}$. vs. $1672.78 \pm 425.11$ at baseline, the difference was not statistically significant $(\mathrm{p}>0.1)$.

\section{3}

Aortic vascular smooth muscle cells from insulin-resistant obese Zucker rats show increased proliferative response to serum in comparison to those from lean Zucker rats: involvement of MAPK and VASP activation

I. Russo, P. Del Mese, G. Doronzo, L. Mattiello,

G. Anfossi, M. Trovati;

Diabetes Unit, Department of Clinical and Biological Sciences, University of Turin, Orbassano - Torino, Italy.

Background and Aims: Insulin resistance is a risk factor not only for cardiovascular atherothrombotic events but also for restenosis after percutaneous revascularization. Vascular smooth muscle cell (VSMC) proliferation, which is involved both in atherosclerosis and in post-angioplasty restenosis, is regulated by a complex sequence of intracellular signaling pathways, which involves activation of Mitogen-Activated Protein Kinase (MAPK) and PKCinduced phosphorylation at Ser 157 of Vasodilator-Stimulated Phosphoprotein (VASP). To clarify whether VSMCs show an increased proliferation attitude in the insulinresistant states, we aimed to evaluate whether cultured aortic VSMCs from insulin-resistant obese Zucker fa/fa rats -a classical model of insulin resistance- show differences in serum-induced proliferation, MAPK pathway activation and PKC-dependent pSer157 VASP expression in comparison to VSMC from insulin-sensitive lean Zucker fa/+rats. Materials and Methods: Cultured aortic VSMCs from insulin-resistant obese Zucker fa/fa rats and from insulinsensitive lean Zucker fa/+rats obtained in primary culture in our laboratory were exposed to different concentrations of foetal calf serum (FCS, 1-10\%) for 24-96 hours; at different times, VSMC number, VSMC incorporation of $3 \mathrm{H}$-thimidine, expression of phosphorylated ERK 1 and ERK 2 - steps of the MAPK cascade- and expression of pSer157VASP (Western blot) were measured $(n=4$, results expressed as mean $\pm \mathrm{SEM}$ and referred to $0 \% \mathrm{FCS}$ ).

Results: In response to FCS exposure at different concentrations and times, VSMCs from obese Zucker rats exhibited a significantly greater proliferation in comparison to VSMCs from lean Zucker rats. For instance, a 24-hour VSMC incubation with 5\% FCS induced an increase in VSMC number of $366.6 \pm 26.9 \%$ in obese vs $166.7 \pm 13.3 \%$ in lean Zucker rats $(p=0.0001)$ and an increase in $3 \mathrm{H}$ - thimidine incorporation of $1380.0 \pm 148.54 \%$ in obese vs $878.83 \pm 46.09 \%$ in lean Zucker rats $(p=0.009)$. Incubation (30 min-24 hours) with 1-10\% FCS serum significantly increased expression of phosphorylated ERK-1, ERK-2 and pSer157VASP both in VSMCs from obese $(p=0.03-$ $0.0001)$ and in those from lean $(p=0.05-0.0001)$ Zucker rats: the extent of phosphorylation, however, was greater in obese vs lean Zucker rats, as far as ERK-1 ( $\mathrm{p}=0.003-0$ 0001), ERK-2 $(\mathrm{p}=0.002-0.0001)$, and pSer157 VASP $(p=0.01-0.0001)$ is concerned. The serum-induced phosphorylation of VASP at Ser157 was blunted by the PKCinhibitor staurosporine $(1 \mu \mathrm{mol} / 1)$ both in obese and in lean Zucker rats. In the same cells, no difference was observed in the proliferative response to insulin.

Conclusions: VSMC from insulin-resistant obese $\mathrm{fa} / \mathrm{fa}$ Zucker rats showed -in comparison with VSMC from insulin-sensitive lean fa/+rats- increased FCS-induced DNA synthesis and cell proliferation. These effects correlated with an increased activation of the MAPK pathway and with an increased PKC-induced pSer157VASP phosphorylation. The increased proliferative attitude of VSMC in a classical model of insulin resistance provides a further piece of information on the mechanisms involved in the increased risk of cardiovascular atherothrombotic events and of restensosis after angioplasty in the insulin-resistant states.

Supported by University of Turin

\section{PS 109}

\section{Peripheral arterial disease}

1214

Reduced TIMP3 levels in atherosclerotic plaques from patients affected by type 2 diabetes and impaired glucose tolerance

F. Folli ${ }^{1}$, R. Menghini ${ }^{2}$, E. Martelli ${ }^{3}$, S. Rizza $^{2}$, R. Lauro ${ }^{2}$, M. Federici ${ }^{2}$;

${ }^{1}$ Dept. of Medicine/Division of Diabetes, University of Texas Health Science Center, San Antonio, United States, ${ }^{2}$ Dept. of Internal Medicine, ${ }^{3}$ Dept. of Vascular Surgery, University of Tor Vergata, Rome, Italy.

Background: Tissue Inhibitor of Metalloproteinase 3 (Timp3) is an inhibitor of metalloproteinases (MMPs) and TNF-alpha converting enzyme (TACE) known to be a negative regulator of inflammation, excessive angiogenesis and cell motility. We reported that loss of Timp3 converts the phenotype of Insulin Receptor haploinsufficient mice from normal glucose tolerance to diabetes and vascular inflammation, through up-regulation of TNF-alpha signaling. Furthermore, increased expression of membrane 
TNF-alpha in vascular tissues reduced the extent of atherosclerotic plaque growth in a murine model of atherosclerosis. Increased Tace activity has been recently demonstrated to cause angiotensin-II chronic kidney disease. The aim of this study is to investigate alteration in Timp3 expression in tissues from diabetic and non diabetic patiets. Methods and materials: Here, we explore the levels of Timp3 and TACE by Real Time PCR in: a) skeletal muscle $(\mathrm{n}=70)$ and subcutaneous fat $(\mathrm{n}=106)$ from patients with obesity (BMI $>35)$ obtained through bariatric surgery, and b) carotid atherosclerotic plaques $(n=74)$ from patients with different degrees of glucose tolerance (NGT, $n=41$; IGT, $\mathrm{n}=17 ; \mathrm{DM} 2=16$ all matched for age, sex, BMI and medications other than hypoglycaemic agents).

Results: In patients with severe obesity divided according to glucose tolerance states (NGT, IGT/IFG, DM2) Timp3 and TACE levels were similar in muscle and fat. By contrast, Timp3 levels (but not TACE) were decreased in atherosclerotic plaques from both IGT and DM2 patients compared with NGT $(\mathrm{P}<0.02$ and $\mathrm{p}<0.001$, respectively, 1way ANOVA) (Figure 1). Timp3 levels were inversely correlated to HbA1c $(r=-0.31, p<0.019)$, LDL Cholesterol $(\mathrm{r}=-0.29, \mathrm{p}<0.029)$, Total Cholesterol $(\mathrm{r}=-0.31, \mathrm{p}<0.016)$ and $\mathrm{C}$ reactive Protein $(\mathrm{r}=-0.28, \mathrm{p}<0.030)$. Immunohistochemical analysis confirmed reduced Timp3 levels in plaques from IGT/DM2 patients $(\mathrm{n}=16)$ compared with NGT patients $(\mathrm{N}=16)(\mathrm{p}<0.01, \mathrm{t}$ test $)$.

Conclusions: Our results suggest that reduced Timp3 expression is a feature of macrovascular disease linked to insulin resistance and type 2 diabetes.

\section{5}

Carotid atherosclerosis is influenced by visceral adiposity rather than general obesity in type 2 diabetic patients

D.-J. Chung, D.-H. Cho, M.-Y. Chung;

Department of Endocrinology and Metabolism, Chonnam National University Medical School, Gwangju, Republic of Korea.

Background and Aims: Diabetes mellitus is a major independent risk factor for atherosclerosis. Carotid intimamedia thickness (IMT) is a measure of subclinical atherosclerosis and is predictive of future myocardial infarction and stroke. General obesity as indicated by high body mass index (BMI) has been related to increased risk of clinical events of atherosclerotic diseases. Interestingly, there is some evidence that abdominal obesity, as indicated by high waist-to-hip circumference ratio (WHR) or high waist circumference, is even a stronger predictor of atherosclerotic diseases than general obesity. We studied whether obesity, especially abdominal obesity is associated with carotid atherosclerosis in type 2 diabetic patients.

Materials and Methods: One hundred and seventy-nine Korean type 2 diabetic patients (M: 92, F: 87; mean body mass index (BMI): $23.6 \mathrm{~kg} / \mathrm{m}^{2}$; age range: $34-83$ years) were recruited. All subjects underwent assessment for the degree and distribution of obesity, the presence of coronary artery disease risk factors, and the presence of diabetic complications, and examined the body composition and body-fat percent by measuring bioelectrical impedance using a body fat analyzer. The carotid IMT was assessed using B-mode carotid ultrasound by measuring generally used parameters, including maximum and mean IMT.

Results: Diabetic patients having visceral obesity (waist to hip ratio $>0.9)$ showed greater maximum $(1.17 \pm 0.26$ vs. $0.98 \pm 0.23 \mathrm{~mm} ; \mathrm{p}=0.001)$ and mean $(0.84 \pm 0.17$ vs. $0.70 \pm$ $0.11 \mathrm{~mm} ; \mathrm{p}<0.001)$ IMT of the carotid artery than patients without visceral obesity. But there was no significant difference in maximum and mean carotid IMT between patients without general obesity $\left(\mathrm{BMI}<23 \mathrm{~kg} / \mathrm{m}^{2}\right)$ and patients with general obesity $\left(\mathrm{BMI} \geq 23 \mathrm{~kg} / \mathrm{m}^{2}\right)$. The correlations between visceral obesity and carotid IMT were higher in women $(r=0.35)$ than men $(r=0.28)$. Age, gender, duration of diabetes, blood pressure, total-cholesterol concentration, glycated hemoglobin, cardiovascular risk factors and diabetic microvascular complications were not different according to visceral obesity. On univariate analysis, visceral but not general obesity was the major correlate with maximum and mean carotid IMT in both men and women. On multivariate analysis, carotid IMT was an independent determinant of carotid IMT after adjustment for age, gender and smoking. The association of visceral obesity with carotid IMT appeared stronger in women than in men, which however was not confirmed on multivariate analysis. Conclusion: This study suggested that abdominal obesity rather than general obesity was associated with carotid atherosclerosis in type 2 diabetic patients.

\section{6}

Non-traditional cardiovascular risk factors as contributors to peripheral arterial disease in type 2 diabetes C. Bianchi ${ }^{1}$, G. Penno ${ }^{1}$, G. Daniele ${ }^{1}$, R. Barontini ${ }^{1}$, A. Civitelli ${ }^{1}$, L. Malloggi ${ }^{2}$, A. Piaggesi ${ }^{1}$, S. Del Prato ${ }^{1}$, R. Miccoli';

${ }^{1}$ Department of Endocrinology and Metabolism, Pisa University, ${ }^{2}$ Cisanello Central Laboratory, Pisa University Hospital, Italy.

Background and Aim: Screening for peripheral artery disease (PAD) is not common practice in diabetic patients, so that its prevalence is not yet well documented. We evaluated 
the PAD prevalence in type 2 diabetic patients, its association with traditional and non-traditional risk factors for atherosclerosis and risk of coronary heart disease (CHD).

Patients and Methods: A cross-sectional study was conducted in 1610 Type 2 diabetic patients (age 61,6 \pm 7.0 years; $42 \%$ women and 58\% men). Ankle-brachial pressure index was measured and PAD was defined as $\mathrm{ABPI}<0,9$. $\mathrm{HbA}_{1 \mathrm{c}}$, total-, LDL- and HDL-cholesterol (HDL-C), uric acid, fibrinogen, albumin/creatinine ratio were measured and glomerular filtration rate (GFR) estimated by MDRD. CHD risk was calculated by UKPDS Risk Engine.

Results: Overall PAD prevalence was 18\% (men: 18\%; women: $\left.16 \%, \mathrm{X}^{2}: \mathrm{p}=\mathrm{ns}\right)$, but increased with age $(<40$ years: $2 \%$; $>70$ years: 45,5\%; Chi-Square: $p<0,0001)$ and with diabetes duration ( $<10$ years: $42 \%$; $>10$ years: $58 \%$; ChiSquare: $\mathrm{p}<0,0001)$. The prevalence of traditional cardiovascular risk factors such as smoking (24\% vs. $21 \%)$, hypertension $(72,9 \%$ vs. $66,5 \%)$, obesity (19\% vs. $17 \%)$, LDL-C $>130 \mathrm{mg} / \mathrm{dl}(18,4 \%$ vs. $18,6 \%)$, low HDL-C $(19 \%$ vs. $18 \%$ ) and high triglycerides (19,7\% vs. 17,7\%) was not different between patients with and without PAD. PAD patients had higher levels of fibrinogen $(370 \pm 79 \mathrm{mg} / \mathrm{dl}$ vs. $347 \pm 76 \mathrm{mg} / \mathrm{dl}$; ANOVA: $<<0,0001$ ), higher prevalence of microalbuminuria $(21,3 \%$ vs. $13,7 \%$; Chi-Square: $\mathrm{p}<0,05)$ and lower renal function (GFR 80,7 \pm 24 vs. $89,9 \pm 22 \mathrm{ml} /$ $\min / 1,73 \mathrm{~m}^{2}$; ANOVA: $\left.\mathrm{p}<0,001\right)$ as compared to subjects without PAD. In age- and gender-adjusted analysis, ATPIII metabolic syndrome components and traditional cardiovascular risk factors were not associated with PAD, with the exception of current smoking (OR 1,5; CI: 1,07-2,2; $\mathrm{p}<0,05)$. In contrast, there was a significant correlation between PAD and poor glucose control (OR 1,45; CI: 1,072,$08 ; \mathrm{p}<0,05$ ), high pulse pressure (OR 2; CI: 1,46-2,75; $\mathrm{p}<0,001$ ), reduced GFR (OR 2,16; CI: 1,4-3,3; $<<0,0001$ ), microalbuminuria (OR 1,62; CI: 1,11-2,36; $\mathrm{p}=0,001$ ), fibrinogen (OR 1,78; CI: 1,29-2,47; $\mathrm{p}<0,001$ ) and uric acid (OR 1,5; CI: 1,03-2,19; $<<0,05$ ). Among patients without history of $\mathrm{CV}$ disease, $20 \%$ of subjects with high $\mathrm{CHD}$ risk had $\mathrm{ABPI}<0,9$ while the risk of $\mathrm{PAD}$ in patients with high CHD risk ( $\geq 20 \%$ ) was higher than in those with low risk (OR 2,5; CI: 1,47-4,2; $<<0,001$ ).

Conclusions: The prevalence of PAD, as determined by ABPI, is high in type 2 diabetic patients and is increased by age, diabetes duration, smoking and non-traditional CV risk factors. Patients with PAD also carry greater CHD risk.
1217

Is it worthwhile screening for peripheral arterial disease in subjects with type 2 diabetes mellitus? Results from a nation-wide screening study

J.-M. Gonzalez-Clemente ${ }^{1}$, A. Calle-Pascual ${ }^{2}$, J.-A. Pinies ${ }^{3}$, A. Saavedra ${ }^{4}$, C. Sánchez ${ }^{5}$, D. Bellido ${ }^{6}$, T. Martín ${ }^{7}$, I. Moraga ${ }^{8}$, A. Recasens ${ }^{9}$,

P. De Pablos-Velasco ${ }^{10}$, J. Girbés ${ }^{11}$, J.-A. Rubio ${ }^{12}$, M. Sánchez-Zamorano ${ }^{13}$, D. Mauricio ${ }^{14}$ on behalf of the PADiD Study Group;

${ }^{1}$ Diabetes, Endocrinology and Nutrition, Hospital de Parc Taulí, Sabadell, ${ }^{2}$ Diabetes, Endocrinology and Nutrition, Hospital Clínico San Carlos, Madrid, ${ }^{3}$ Diabetes, Endocrinology and Nutrition, Clínica Pinies, Bilbao, ${ }^{4}$ Diabetes, Endocrinology and Nutrition, Centro de Especialidades Hermanos Sangro, Madrid, ${ }^{5}$ Diabetes, Endocrinology and Nutrition, Hospital Ciudad de Jaén, Jaén, ${ }^{6}$ Diabetes, Endocrinology and Nutrition, Hospital Aquitecto Marcide, El Ferrol, ${ }^{7}$ Diabetes, Endocrinology and Nutrition, Hospital Clínico de Tenerife, Tenerife, ${ }^{8}$ Diabetes, Endocrinology and Nutrition, Hospital de Móstoles, Madrid, ${ }^{9}$ Diabetes, Endocrinology and Nutrition, Hospital General de Granollers, Granollers, ${ }^{10}$ Diabetes, Endocrinology and Nutrition, Hospital Dr. Negrin, Las Palmas de Gran Canaria, ${ }^{11}$ Diabetes, Endocrinology and Nutrition, Hospital Arnau de Vilanova, Valencia, ${ }^{12}$ Diabetes, Endocrinology and Nutrition, Hospital Universitario Principe de Asturias, Madrid, ${ }^{13}$ Medical Department, Bristol Myers Squibb, Madrid, ${ }^{14}$ Diabetes, Endocrinology and Nutrition, Hospital Sant Pau, Barcelona.

Awareness of peripheral arterial disease (PAD) is low among physicians, because the recommended test for its detection (ankle-brachial index -ABI-) is time-consuming and is not usually performed in routine clinical practice. However, PAD is a coronary heart disease (CHD) risk equivalent and a tight control of classical cardiovascular risk factors $(\mathrm{CCRF})$ is recommended in this condition.

Objective: To assess in type 2 diabetes mellitus (T2DM) subjects whether those subjects with PAD have a different control of CCRF in relation to subjects without PAD.

Methods: 31 Spanish Diabetes Departments consecutively screened for PAD (ABI test) 1305 subjects with T2DM (age: 65.4 $\pm 7.7 \mathrm{yr}$, women: $52.2 \%$, diabetes duration: $13.4 \pm 8.9 \mathrm{yr}$ ), recording information on control of CCRF and history of CHD and stroke.

Results: 292 subjects showed PAD $(\mathrm{ABI}<0.9)(23.10 \%$, CI 95\%: 20.78-25.42, after excluding 41 subjects with an ABI > 1.30). According to the presence of other previously known cardiovascular disease (CVD) manifestations (CHD or stroke) and the current diagnosis of PAD, subjects were classified into 4 groups: 1) without previously known CVD and no PAD ( $\mathrm{n}=789) ; 2$ ) without previously known CVD and 
PAD ( $\mathrm{n}=194) ; 3$ ) with previously known CVD and no PAD $(n=182)$, and 4) with previously known CVD and PAD $(n=95)$. The control of the CCRF was (respectively): Nonsmokers: $84.5 \%, 79.3 \%, 93.3 \%, 86.1 \%$ (2 vs 3 p $<0.01$ ); antiplatelet treatment: $36.1 \%, 40.2 \%, 80.2 \%, 79.0 \%$ (2 vs 3 and 2 vs $4 \mathrm{p}<0.01$ ); blood pressure $<130 / 80 \mathrm{mmHg}: 17.9 \%$, $12.9 \%, 20.3 \%, 16.0 \%$ (NS); LDL cholesterol $<100 \mathrm{mg} / \mathrm{dl}$ : $43.8 \%, 35.5 \%, 59.6 \%, 64.3 \%(2$ vs 3 and 2 vs $4 \mathrm{p}<0.02)$; HbAlc $<7.5 \%$ : 50.7\%, 45.5\%, 47.6\%, 52.4\% (NS).

Conclusions: Detection of PAD by the ABI identifies a group of subjects with a poorer control of CCRF than subjects with other clinical manifestations of CVD, thereby increasing the value of ABI in the routine care of subjects with T2DM. This study received an educational grant from BristolMyers-Squibb

\section{8}

\author{
Concordance of ankle-brachial index and toe-brachial \\ index in patients with diabetes \\ V. Martín-Borge, L. Herranz, I. Castro, A. Fernández, \\ L. F. Pallardo; \\ Endocrinología y Nutrición, Hospital La Paz, Madrid, \\ Spain.
}

Background and Aims: To evaluate the influence of peripheral neuropathy, autonomic neuropathy and arterial wall calcification on the concordance of two screening methods, ankle-brachial index (ABI) and toe-brachial index (TBI), used to assess lower limb perfusion in patients with diabetes.

Materials and Methods: We evaluated 221 diabetic patients (73.8\% type $2 ; 66.6 \%$ male) with a mean age of 61.1 \pm 11.9 years and mean duration of diabetes of $16 \pm 11.9$ years. Both brachial systolic pressures, and ankle (posterior tibial and dorsalis pedis) and toe systolic pressures were measured using a Doppler probe (8Mhz). For each patient, the lowest of the four ABI and the lowest of the two TBI obtained were used for the analysis. ABI values $<0.9$ and TBI values $<0.5$ were considered indicators of peripheral artery disease. Peripheral neuropathy was assessed using Boulton's clinical scale, a 10-g monofilament and a $128 \mathrm{~Hz}$ tuning fork. Continuous flow in the waveform doppler display was considered indicator of autonomic neuropathy. An ABI value $\geq 1.30$ was considered indicator of arterial wall calcification. Statistical analysis was performed using SPSS software.

Results: The mean difference between ABI and TBI in our population was $0.33 \pm 0.25$. Patients with arterial wall calcification had a higher mean difference between ABI and TBI than patients without arterial wall calcification $(0.87 \pm 0.39$ vs $0.30 \pm 0.21 ; \mathrm{p}=0.002)$. Mean difference between ABI and TBI was similar for patients with and without autonomic neuropathy $(0.36 \pm 0.26$ vs $0.30 \pm 0.24$; $\mathrm{p}=0.057$ ) and for patients with and without peripheral neuropathy $(0.34 \pm 0.28$ vs $0.31 \pm 0.21 ; p=0.423)$. Among all patients there was a significant linear association $(r=0,506$; $\mathrm{p}=0,000)$ between ABI and TBI. For patients with arterial wall calcification there was no correlation between $\mathrm{ABI}$ and TBI $(r=-0.070 ; p=0,857)$. Autonomic neuropathy and peripheral neuropathy did not modify the relation between ABI and TBI ( $\mathrm{r}=0.562$ and 0.492 respectively).

Conclusion: Our findings indicate that in patients with diabetes TBI is the method of choice to evaluate lower limb perfusion in the presence of overt arterial wall calcification as evidenced by an ABI value $\geq 1.30$. However, in diabetic patients with autonomic neuropathy or peripheral neuropathy there is a good relationship between ABI and TBI.

1219

Clinical significance of toe-brachial pressure index in patients with type 2 diabetes

K. Ikeda, T. Hamaguchi, M. Miuchi, T. Ishikawa, M. Fukamizu, T. Katsuno, H. Konya, J.-I. Miyagawa, M. Namba;

Diabetes and Metabolism, Hyogo Colege of Medicine, Nishinomiya, Japan.

Background: Peripheral obstructive arterial disease (POAD) is a potent predictor of cardiovascular disease (CVD) in diabetic patients. Medical and/or life style intervention in the earlier stage of POAD may also reduce the risk of CVD mortality of the patients. Although the efficacy of ankle brachial pressure index (ABI) for diagnosing of POAD is well demonstrated, its sensitivity is not so high enough for detecting the early stage of POAD, particularly in type 2 diabetes. More sensitive clinical marker for POAD should be established.

Aims: To assess the efficacy of measurement of toebrachial pressure index (TBI) using digital cuff in patients with type 2 diabetes.

Materials and Methods: For 155 patients with type 2 diabetes and without apparent clinical symptoms of the peripheral arterial obstruction, ABI and TBI were screened simultaneously. Also, carotid intima-media thickness (IMT) and pulse wave velocity (PWV) were measured in these patients for evaluating the degree of their atherosclerosis. Comparative analyses of both ABI and TBI in relation to other clinical indication of diabetic complications were performed. Results: Significant positive correlation was found between $\mathrm{ABI}$ and TBI. In that relationship, the cut off value of 0.9 in $\mathrm{ABI}$ for POAD was equivalent to the value of 0.6 in TBI. In 25 cases whose TBI was under $0.6,72 \%$ of the patients had a clinical history of cardiovascular disease, and this rate was much higher than that of the patients whose TBI was 
above 0.6. This group with low TBI had longer duration of diabetes than the group with high TBI. The frequencies of the patients with hypertension and/or hyperlipidemia were also higher in the low TBI group than in the high TBI group. Although $89 \%$ of the patients showed low TBI with low ABI, or high TBI with high ABI, the discrepancy was found in 15 patients whose $\mathrm{ABI}$ was above 0.9 , and their TBI was below 0.6. In these cases with high ABI and low TBI, the average of IMT was $1.42 \mathrm{~mm}$ (normal range $<1.10 \mathrm{~mm}$ ), and their average of PWV was $2,200 \mathrm{~cm} / \mathrm{sec}$ (normal range $<1,600 \mathrm{~cm} / \mathrm{sec}$ ).

Conclusion: In this study, TBI is shown to be as effective marker as ABI for detecting POAD with type 2 diabetes. Furthermore, low TBI may reflect the earlier stage of systemic atherosclerosis in some cases with type 2 diabetes than ABI.

\section{0}

\section{Paradoxical blood flow responses in postmenopausal women with diabetic neuropathy}

N. V. Vorokhobina, T. A. Zelenina, O. F. Malygina,

E. A. Volkova;

Endocrinology, Medical Academy of Postgraduate Education, St. Peterburg, Russian Federation.

Background and Aims: Diabetic neuropathy (DN) is a common cause of morbidity and death among patients with diabetes mellitus (DM). Reduction of nerve blood flow and endoneural hypoxia are attributed to oxidative stress and to alterations in vasoactive agents. Neuropathy alone can be responsible for abnormal blood flow responses in DM. In addition a low blood estrogen level can deteriorate microvascular complications in postmenopausal women with DM. The aim of this investigation was to study a microvascular disorders and effect of estrogen-progestin replacement therapy (EPRT) on microvascular responses in postmenopausal women with DN.

Materials and Methods: We exanimate 55 postmenopausal women with DM (24/31 patients with 1/2 types of DM) and climacteric vasomotor symptoms (mean $[ \pm \mathrm{SD}]$ age $53.2 \pm$ 1.72 years). Written informed consent was obtained from all patients and the institutional review board approved the study. Diagnose of DN was determined by careful neurological examination, electroneurophysiology studies. The wrist and foot digital skin blood flow and microvessels responses to functional tests were assessed by Doppler highfrequency ultrasound examination. We studied the microvessels endothelial function on measuring reactive hyperemia perfusion changes. We investigated the skin vessels response in another extremity to a local cold stimulus. Hemostasis values, glycosylated hemoglobin, serum lipids, plasma levels of total non-enzymatic antioxidants and malondialdehyde were measured. Control group consists from 15 healthy postmenopausal women of the same age. 51 diabetic postmenopausal women received EPRT by estradiol valerate and dienogest during 12 months.

Results: On baseline the paradoxical decreasing of wrist blood flow was obtained in $42 \%$ women with DM type 1 and $52 \%$ women with DM type 2 . All patients with DM type 1 and $71 \%$ with DM type 2 showed the paradoxical increasing of skin wrist blood flow to $87 \%$ as compared whit basal level on a local cooling. However basal foot digital skin blood flow was significantly reduced and there were no vessel responses to the functional testing. Diabetic women had higher baseline levels of malondialdehyde and lower levels of total non-enzymatic antioxidants as compared with control groups. There were higher body-mass index, higher levels of total cholesterol, LDL-cholesterol and fasting triglycerides in postmenopausal women with DM type 2 as compared with patients with DM type 1 and control groups. Paradoxical vasoconstrictor blood flow responses to reactive hyperemia testing was associated with obesity, high levels of total cholesterol, LDL-cholesterol and malondialdehyde. The poorer nerve functional and antioxidant control determined the abnormal increasing of microvascular blood flow to the cold testing. EPRT restored the flow-mediated microvessels dilatation in $77 / 67 \%$ patients and the vessels decreasing on the cold testing in 50/56\% patients. There were decreasing of the total cholesterol, LDL-cholesterol and malondialdehyde due to the EPRT that determined the improvement of nerve function.

Conclusion: The nerve dysfunction in postmenopausal women with DN associated with high total cholesterol and LDL-cholesterol levels, antioxidant control injury and microvessels disorders. EPRT improve quality of life in postmenopausal women with DN by relieved vasomotor climacteric symptoms. In the same time EPRT may have the beneficial influents on peripheral nerve function.

1221

Systemic trail delivery shows anti-atherosclerotic activity in diabetic apolipoprotein E-Null (ApoE) mice R. Candido ${ }^{1}$, P. Secchiero ${ }^{2}$, B. Toffoli ${ }^{3}$, F. Corallini ${ }^{2}$, S. Zacchigna ${ }^{4}$, E. Manca ${ }^{1}$, E. Caroli ${ }^{1}$, P. Da Col ${ }^{1}$, R. Carretta ${ }^{3}$, M. Giacca ${ }^{4}$, G. Zauli ${ }^{5}$, B. Fabris ${ }^{3}$; ${ }^{1}$ Diabetic Centre, A.S.S. 1 Triestina, Trieste, ${ }^{2}$ Department of Morphology and Embryology, University of Ferrara, ${ }^{3}$ Department of Internal Medicine and Neurology, University of Trieste, ${ }^{4}$ Molecular Medicine Laboratory, International Centre for Genetic Engineering and Biotechnology, Trieste, ${ }^{5}$ Department of Normal Human Morphology, University of Trieste, Italy. 
Background and Aims: Diabetes mellitus is associated with a marked increase in atherosclerosis. However, the mechanisms by which diabetes promotes vessel disease have not been fully delineated. Previous in vitro studies have suggested that TNF-related apoptosis inducing ligand (TRAIL) might be involved in the regulation of vascular biology, but its role in vascular physiopathology is still incompletely understood.

Materials and Methods: Both recombinant human TRAIL and an adeno-associated virus (AAV) vector expressing human TRAIL were used to deliver TRAIL in 6-week-old ApoE-null mice, in which diabetes mellitus was induced by injection of streptozotocin ( $50 \mathrm{mg} / \mathrm{kg}$ daily for 5 days) for 3 months. Untreated diabetic and non-diabetic ApoE-null mice were used as controls. Body weight, glycated haemoglobin, atherosclerotic lesion area of the aorta, aortic atherosclerotic plaque collagen content, cellular proliferation and smooth muscle cell and macrophage infiltration were evaluated.

Results: Diabetes in ApoE-null mice was associated with a significant increase in aortic atherosclerotic plaque area (diabetics: $8.8 \pm 1.1 \%$ vs controls: $1.4 \pm 0.9 \%$; $<<0.001$ ) and complexity as assessed by a marked increase in plaque collagen content (diabetics: $34 \pm 9 \%$ vs controls: $17 \pm 8 \%$; $\mathrm{p}<0.001$ ), cellular proliferation (diabetics: $16 \pm 9 \%$ vs controls: $4 \pm 2 \% ; \mathrm{p}<0.001)$ and macrophage- and alfa-smooth muscle actin-positive cell infiltration (diabetics: $13 \pm 7 \%$ vs controls: $7 \pm 4 \%$; $<<0.001)$. Repeated intraperitoneal injections of recombinant human TRAIL and a single intravenous injection of AAV-hTRAIL significantly attenuated the development of atherosclerotic plaques $(4.5 \pm 0.5 \%$ and $5.2 \pm 0.7 \%$ respectively). Moreover, systemic TRAIL administration also markedly decrease overall cellular proliferation $(3.1 \pm 2.6 \%$ and $2.7 \pm 2.3 \%$ respectively) and collagen content $(19 \pm 11 \%$ and $20 \pm 6 \%$ respectively) in the atherosclerotic plaques of ApoE-null animals without significantly affecting the smooth muscle cell content $(21 \pm 9 \%$ and $17 \pm 7 \%$ respectively), resulting in a reduced severity of the atherosclerotic lesions.

Conclusion: Overall, our data indicate that diabetesassociated atherosclerosis is ameliorated by TRAIL administration and indicate that AAV vector offer a novel method of using TRAIL as an innovative drug for the therapy of diabetic vascular diseases.

Treatment of diabetic peripheral arterial disease with neuromodulation FREMS

R. Da Ros, C. Vitale, R. Assaloni, A. Ceriello; Department of Pathology and Medicine, Experimental and Clinical, University of Udine, Italy.
Background: Peripheral arterial disease (PAD) may be asymptomatic, may be associated with intermittent claudication or may be associated with critical limb ischaemia. Patients with critical limb ischemia should be revascularized to minimize the chance of limb loss.

In absence of tissue damage, also with low ankle arterial pressure and low oxymetric values, there are no consistent evidence to support specific treatment. Presence of peripheral neuropathy, with loss of pain sensation, leads diabetic patients with PAD at high risk of limb loss.

FREMS neuromodulation (frequency rhythmic electrical modulation system), utilized for the treatment of diabetic neuropathy, was demonstrate to produce vasoactive products, in particular VEGF. For these evidences we can suppose that FREMS therapy can be useful in the treatment of PAD when oxymetric values are low.

Aim of the study: This prospective study was designed to determine if the use of FREMS neuromodulation in diabetic patients with peripheral limb ischaemia, is effective on oxymetric values and pain free walking distance improvement.

Materials and methods: Fourteen patients with PAD, were evaluated with transcutaneous oxymetry and with a standardized treadmill testing. 18 limbs with oxymetric values (TcpO2) below $50 \mathrm{mmHg}$ were founded. For each patients oxymetric test was repeated after one month to verify values stability. All patients received ten consecutive session of FREMS therapy. After one and three months we performed a transcutaneous oxymetry and patients with claudicatio (n.6) repeated treadmill walking test.

Results: 18 legs were examinated: baseline oxymetric values were $26.9 \pm 14.4 \mathrm{mmHg}($ mean $\pm \mathrm{SD})$. After one month, from the end of FREMS therapy, oxymetric values increased to $38.8 \pm 20.7 \mathrm{mmHg}$, with a $\mathrm{p}$ value of 0.01 compared to the baseline values. Moreover at 3 months values further increased at $48 \pm 10.1, \mathrm{p}<0.01$ compared to baseline values. After FREMS teraphy patients with claudicatio demonstrated improvement in pain free walking distance at one and three months $(\mathrm{p}<0.001)$.

Conclusions: In diabetic patients with PAD, FREMS therapy seems achieve considerable effects on improvement of oxymetric values and pain free walking distance. In absence of indications for revascularization this therapy can achieve a good amelioration without risks for the patients.

1223

Endothelial progenitor cells in subjects with pre-diabetes L. Pucci ${ }^{1}$, G. Penno ${ }^{1}$, D. Lucchesi ${ }^{1}$, M. Barsotti ${ }^{2}$, M. Iorio ${ }^{3}$, R. Di Stefano ${ }^{2}$, A. Balberini ${ }^{2}$, R. Vanacore $^{3}$, R. Miccoli ${ }^{1}$, S. Del Prato ${ }^{1}$;

${ }^{1}$ Endocrinology and Metabolism, ${ }^{2}$ Cardiothorax, ${ }^{3}$ Immunohaematology, Unit II, University of Pisa, Italy. 
Background and Aims: Cumulative cardiovascular risk, and cardiovascular disease status appear to alter number and function of circulating endothelial progenitor cells (EPCs). Furthermore, the level of circulating EPCs seems to predict the occurrence of cardiovascular events. Diabetes has been associated with low levels of circulating EPCs. To investigate whether even subjects with pre-diabetes show altered circulating levels of progenitor cells (CPCs) and endothelial progenitor cells (EPCs).

Materials and Methods: 27 patients with pre-diabetes (8 with impaired fasting glucose, IFG; 14 with impaired glucose tolerance, IGT; and 5 with both IFG and IGT) and 22 age-matched healthy controls were recruited. No subject was on any pharmacological treatment. BMI, waist circumference, OGTT, HbA1c, blood pressure, lipid profile and inflammatory parameters were measured. Metabolic syndrome (MS) was assessed by NCEP/ATPIII criteria. Blood samples were obtained in EDTA tubes in order to assess CPCs (CD34+cells) and EPCs (CD34+KDR+cells) by FACS analysis. Data are expressed as cells number for $\mathrm{ml}$ of blood obtained by ISHAGE protocol and as EPCs/ CPCs ratio.

Results: No differences were observed in EPCs levels and in EPCs/CPCs ratio in subjects with pre-diabetes compared with controls (EPCs: $532 \pm 424$ vs $511 \pm 348 ; / \mathrm{ml}$, p=0.86; EPCs/CPCs ratio: $28.0 \notin \pm 16.1$ vs $22.9 \pm 17.2 \%, \mathrm{p}=0.30$ ). Linear regressions didn not show any significant correlation between EPCs level or EPCs/CPCs ratio and clinical (age, BMI, waist, BP) as well laboratory parameters (fasting or 2$\mathrm{h}$ glucose, HbA1c, glucose area under the OGTT curve, lipids, hs-CRP). Within subjects with pre-diabetes, EPCs $(516 \pm 298$ vs $542 \pm 494 / \mathrm{ml}, \mathrm{p}=0.88)$ and $\mathrm{EPCs} / \mathrm{CPCs}$ ratio $(27.6 \pm 16.4$ vs $28.3 \pm 16.4 \%, p=0.093)$ were superimposable in those with (n. 11, 40\%) and without MS. All controls were free of MS. A positive first-degree family history for type 2 diabetes was present in 29 individuals, 10 controls (45\%) and 19 pre-diabetics (70\%). EPCs (436 \pm 334 vs $651 \pm$ $432 / \mathrm{ml}, \mathrm{p}=0.067)$ and EPCs/CPCs $(21.9 \pm 14.3$ vs $31.1 \pm$ $18.5 \%, \mathrm{p}=0.069$ ) were marginally lower in subjects with a positive family for diabetes. This trend was present both in controls and in pre-diabetics.

Conclusion: These preliminary results suggest that minor impairments of glucose regulation (pre-diabetes) do not affect the levels of circulating EPCs. Furthermore, in prediabetics, levels of EPCs are not modified by the cooccurrence of metabolic syndrome.

\section{PS 110}

\section{Erythrocytes, clotting and thrombosis}

\section{4}

Factor IX activation enzyme on erythrocyte membranes causes hypercoagulability in diabetes

I. Uchimura ${ }^{1}$, M. Ikebukuro ${ }^{1}$, Y. Hirata ${ }^{1}$, M. Kaibara ${ }^{2}$;

${ }^{1}$ Department of Endocrinology and Metabolism, Tokyo Medical \& Dental University, Tokyo, ${ }^{2}$ Computational Biomechanics Unit, Riken, Saitama, Japan.

Background and Aims: Macroangiopathy (MA) such as cerebral infarction, myocardial infarction is a common cause of death in diabetes. These complications are thought due to increased intrinsic coagulation activity. We have discovered new enzyme that activate coagulation factor IX (FIX) on erythrocyte membrane in normal and diabetics. FIX activation enzyme may be a serine protease and the enzyme activity may be identical with that present of $\mathrm{RBC}$ membranes in another study. These data suggests RBC may play important role in developing angiopathy in diabetes with its blood coagulation activity.

Materials and Methods: Blood was obtained from human adult volunteers including normal $(\mathrm{N}: \mathrm{n}=21)$ and diabetic patients (DM n=32). Platelet poor plasma (PPP) was prepared by centrifuging the blood. RBCs were washed in HEPES buffer. The enzyme activity on the surface of erythrocyte was estimated from a comparison of fluorescence intensity with a synthetic substrate (Suc(OMe)-Ala-Ala- Pro-Val-MCA). Time course of fluorescence intensity (FI) was measured using a fluorescence spectrophotometer (Hitachi F-1200). Blood coagulation activity was measured by determination of the onset time of coagulation which was monitored using a damped oscillation rheometer (Kawakamil: Biorheology 32:521, 1995). Hypercoagulable blood showed shortened onset time in logarithmic damping factor (LDF) monitored after adding $\mathrm{CaCl}_{2}$ solution to anticoagulated blood sample. Plasma fibrinogen, plasma thrombin, FXa, FXIIa, FXa, FVIIa, tissue factor(TF) was also measured.

Results: The average of glucose levels and HbAlc levels in diabetic patients were $179.0 \mathrm{mg} / \mathrm{dL}$ and $7.9 \%$, respectively. The onset time of whole blood coagulation is significantly shorter in DM than in N (24.8 min V.S. $35.7 \mathrm{~min})$. And the onset time of PFP with RBC is also shorter in DM than in $\mathrm{N}$ (35.5 min V.S. $46.0 \mathrm{~min}$ ). Fluorescent observation of FIX activation enzyme is higher in DM than in N (13.4 V.S. 8.3). In diabetics with MA, onset time(Ti) was shorter than in diabetics without MA. And Ti became short by $22 \%$ in high PG group. Fluorescent intensity (FI) was higher in diabetics with MA. Plasma TF, thrombin, and fibrinogen levels are higher in DM especially in DM with MA. HbAlc 
levels showed significant positive correlation with FI $(p<0.001)$ and plasma fibrinogen $(p<0.001)$, and showed negative correlation with $\mathrm{Ti}(\mathrm{p}<0.01)$. Plasma TF or thrombin showed no correlation with Ti or FI in diabetics. Plasma FIXa, FXIIa, and FXa showed no difference between $\mathrm{N}$ and $\mathrm{DM}$.

Conclusion: FIX plays an important role in blood coagulation because absence or deficiency of its activity results in hemophilia B. However, epidemiological data showed that the plasma FIXc was associated with several CHD risk factors. We revealed that FIX-activation enzyme was located on the RBC membrane from diabetes. Hyperglycemia in diabetes may be a main cause of hypercoagulability that will increase the incidence of MA such as acute coronary syndrome. We observed that Neutrophil elastase can inactivate FIX and this enzyme activity in diabetes. Measurement of FIX activation enzyme on RBC will be an useful marker for hypercoagulability in diabetes.

\section{5}

Potential reason for diabetic microcirculatory disorders: decreased NO-synthase activity in red blood cells P. Kleinbongard ${ }^{1}$, P. Horn ${ }^{1}$, P. Brouzos ${ }^{1}$, P. Rösen ${ }^{2}$, M. Kelm ${ }^{1}$, T. Lauer ${ }^{1}$;

${ }^{1}$ Medizinische Klinik I, Universitätsklinikum, Aachen, ${ }^{2}$ Institute for Pathobiochemistry and Clinical Biochemistry, Deutsches Diabetes Zentrum, Düsseldorf, Germany.

Background and Aims: Endothelial dysfunction and diminished bioavailability of nitric oxide (NO) are hallmarks for development of vascular complications in diabetes. Recently, we provided evidence for an active endothelial-type NO-synthase in erythrocytes (RBC-NOS) of healthy volunteers which influences RBC deformability and might also be of importance for regulation of microcirculatory blood flow. We hypothesise that diabetes does not only impair bioavailability of NO by endothelium, but also reduces the NO-pool supplied by erythrocytes.

Materials and Methods: RBC-NOS activity and RBC deformability were determined in healthy and type 2 diabetic subjects ( $\mathrm{n}=10$, age-matched). The release of $\mathrm{NO}$ was measured after incubation of erythrocytes with Larginine $(3 \mathrm{mM})$ and inhibition by L-NIO (L-iminoethylornithine hydrchloride, $10 \mu \mathrm{M})$. Changes of RBC-NOS activity were assessed by measurement of nitrite in RBC surrounding plasma by gas phase chemiluminescence. The influence of insulin was tested after incubation of erythrocytes with insulin. Phosphorylation of NOS was determined histochemically. RBC deformability was assessed in relation to $\mathrm{RBC}$ flow rate through a filter system.
Results: RBC NOS activity was significantly decreased in diabetics $(108 \pm 43 \%$ in healthy volunteers to $46 \pm 23 \%$ in diabetics). In parallel RBC-NOS dependent deformability of RBC is significantly reduced $(10 \pm 5 \%$ in diabetics and $15 \pm 8 \%$ in healthy volunteers). In both collectives altered NOS activity and RBC deformability correlate significantly. Insulin significantly increased the release of RBC-NO and activated RBC-NOS by phosphorylation. In erythrocytes of diabetic patients both effect of insulin on RBC-NOS were blunted.

Conclusion: RBC-NOS activity is drastically impaired in type 2 diabetic patients and is resistant to activation by insulin. Both effects may contribute to the reduced deformability of erythrocytes and to the deleterious microcirculatory damages observed in many diabetic patients. This defect may also contribute to the close association of insulin resistance and microvascular defects. Treatment of RBC NOS may therefore be a potential target to prevent an impairment of microcirculatory blood flow and may offer interesting new perspectives in therapy of diabetic patients.

1226

Tissue plasminogen activator (tPA) activity is an early and independent marker of LEAD in type 2 diabetes M. Svensson ${ }^{1}$, D. Sahli ${ }^{1,2}$, G. Dahlquist ${ }^{2}$, K. Boman ${ }^{3}$, J. W. Eriksson ${ }^{1}$;

${ }^{1}$ Dept of Medicine, Umeå University Hospital, ${ }^{2}$ Dept of Pediatrics, Umeå University Hospital, ${ }^{3}$ Dept of Medicine, Skellefteå Hospital, Skellefteå, Sweden.

Background and Aims: Age, hypertension and smoking are well-known risk factors for the development of lower extremity arterial disease (LEAD). In addition, hyperglycemia, dyslipidemia and duration of diabetes are of major importance in diabetic subjects. In this cross-sectional study we addressed the relationship between early, asymptomatic LEAD and "classic" risk factors for LEAD, markers of inflammation, fibrinolysis and adipose-derived factors.

Subjects and Methods: We studied 226 subjects (75 type 1-, 69 with type 2- and 82 non-diabetic, 113 men, 113 women, age 30-70 years) without previously known or suspected LEAD. Peripheral blood pressures were measured at the level of the ankle (ABP) or the great toe (TBP) using a Doppler pen or a pulse oximetry sensor, respectively, for pulse detection. Toe and ankle blood pressure indices (ABI and TBI) were calculated by dividing $\mathrm{ABP}$ and TBP by the systemic, brachial, blood pressure. The subjects were divided into two groups; with normal $(n=183)$ and reduced peripheral blood pressure $(n=43)$, respectively. Reduced blood pressure was defined as at least one absolute blood pressure (ABP or TBP) or index (ABI 
or TBI) below the normal range: ankle $<98 \mathrm{mmHg}$, toe $<79 \mathrm{mmHg}$ or $\mathrm{ABI}<0.9$, $\mathrm{TBI}<0.74$ (cut-offs are means minus 2 SDs for controls in a previous, larger study; Sahli et al, Angiology, 2004). Blood samples were obtained for measurement of lipids, HbAlc, hs-CRP, TNF-alpha, IL-6, adiponectin, leptin, FFA, tPA-activity, tPA-mass, PAI-1 activity and tPA-PAI-1 complex.

Results: In logistic regression analyses, age $(\mathrm{p}<0.05)$, hypertension $(p<0.001)$, diabetes $(p<0.01)$ and high levels of HbA1c $(p<0.001)$, FFA $(p<0.01)$ and also TNF-alpha, IL-6, adiponectin and tPA-activity (all $\mathrm{p}<0.05$ ) were all associated with impaired peripheral circulation (reduced $\mathrm{ABP}, \mathrm{TBP}, \mathrm{ABI}$ or TBI). When adjusting for the "classic" risk factors, i.e. age, hypertension, smoking and HbAlc, only tPA-activity, among inflammatory, adipose-derived or fibrinolytic factors, was an independent risk marker for LEAD $(p<0.05)$. When analyzing each group of subjects, i. e. type 1-, type 2- and non-diabetics, separately, tPAactivity remained an independent risk marker only among subjects with type 2 diabetes $(\mathrm{p}=0.03$ ).

Conclusion: In addition to the "classic" risk factors, tPAactivity may be an independent and early marker for LEAD in type 2 diabetes. Thus, an altered fibrinolytic activity could indicate and possibly contribute to atherosclerosis development in the lower extremities.

1227

Advanced glycation end products stimulate platelet phosphatidylserine externalisation: a role for $5 \mathrm{HT}_{2 \mathrm{~A}}$ receptors and protein kinase $\mathrm{C} \delta$ but not caspase-3

S. M. Marshall ${ }^{1}$, Y. Wang ${ }^{1}$, W. Beck ${ }^{2}$, N. A. Hoenich ${ }^{1}$, M. G. Thompson ${ }^{1}$;

${ }^{1}$ Diabetes Research Group, SCMS, University of Newcastle upon Tyne, United Kingdom, ${ }^{2}$ Gambro Corporate Research, Hechingen, Germany.

Background and Aims: Levels of advanced glycation end products are increased in individuals with diabetes and/or end stage renal disease (ESRD) and may contribute to the greatly increased cardiovascular risk in these patients. Platelets play a crucial role in early events leading to inflammatory responses at sites of vessel wall injury. Externalisation of the membrane phospholipid, phosphatidylserine (PS), via activation of protein kinase $\mathrm{C} \delta$ (PKC $\delta$ ), has been proposed as a key event in aggregation/adhesion of platelets. Alternatively, PS externalisation has also been implicated as a marker for apoptosis when it occurs in a Caspase-3-dependent manner. A specific 'receptor for AGE', RAGE, has been identified, but its presence on platelets has not been demonstrated and it is clear that not all effects of AGE are mediated via RAGE. In particular, a previous study demonstrating additive effects of AGE with ADP showed that they could be blocked via an inhibitor of the $5 \mathrm{HT}_{2 \mathrm{~A}}$ receptor.

The aim of this study was to investigate our hypothesis that AGE elicit platelet PS externalisation via $\mathrm{PKC} \delta$, but independently of Caspase-3, and to identify the AGE receptor(s) involved.

Materials and Methods: Platelet-rich plasma (PRP) was prepared by centrifugation of whole blood from healthy individuals and PS externalisation was quantified by a fluorescence-activated cell-sorter using Annexin V-FITC to determine PS and with CD61-PerCP as a platelet marker. Human serum albumin-AGE (HSA-AGE) was prepared by incubating HSA with $1 \mathrm{M}$ glucose under sterile conditions for six weeks followed by extensive dialysis. PKC $\delta$ activity was blocked by Rottlerin, Z-DEVD-FMK was used to inhibit Caspase-3 and Ritanserin was employed as a specific $5 \mathrm{HT}_{2 \mathrm{~A}}$ receptor antagonist.

Results: $0.5 \mathrm{mg} / \mathrm{ml}$ HSA-AGE elicited a rapid increase in PS externalisation in a sub-population of human platelets. This response was maximal after 10 minutes and still significant after 30 minutes with an optimal effect at $0.5 \mathrm{mg} / \mathrm{ml}$ HSA-AGE $(10263 \pm 171$ vs $2051 \pm 164$ total fluorescent binding sites, $\mathrm{n}=3 ; \mathrm{p}<0.001) .10$ minutes preincubation with the $\mathrm{PKC}$ inhibitor, rottlerin, at a concentration specific for the PKC $\delta$ isoform, $(10 \mu \mathrm{M})$, totally inhibited PS externalisation in response to $0.5 \mathrm{mg} / \mathrm{ml}$ HSA-AGE. Similarly, a 30 minute preincubation with the $5 \mathrm{HT}_{2 \mathrm{~A}}$-specific receptor antagonist, Ritanserin, also prevented PS externalisation in response to $0.5 \mathrm{mg} / \mathrm{ml} \mathrm{HSA-}$ AGE in a dose-dependant manner with complete blockade at $1 \mu \mathrm{M}$. In contrast, 30 minutes preincubation of PRP with an optimal concentration of the Caspase-3 inhibitor, ZDEVD-FMK $(2 \mu \mathrm{M})$, had no effect on HSA-AGE-mediated PS externalisation.

Conclusion: The data demonstrate that HSA-AGE rapidly stimulates platelet PS externalisation via $\mathrm{PKC} \delta$ in a timeand concentration-dependent manner. Moreover, the observation that this response is independent of Caspase-3 suggests that it is linked to platelet aggregation/adhesion rather than apoptosis. In addition, our novel finding that HSA-AGE-mediated PS externalisation is completely inhibited by Ritanserin suggests that this effect is mediated through $5 \mathrm{HT}_{2 \mathrm{~A}}$ receptors rather than RAGE. It may contribute to the increased cardiovascular risk observed in individuals with diabetes and/or ESRD.

Supported by Northern Counties Kidney Research Fund and Hospal Research Fund 


\section{8}

Changes in the level of soluble and thrombocyte surface P-selectin and the number of thrombocyte derived microparticles in diabetes mellitus M. Káplár ${ }^{1}$, Ü. Csongrádi ${ }^{1}$, G. Paragh ${ }^{1}$, M. Papp ${ }^{2}$, I. Földi' ${ }^{2}$, T. Magyar ${ }^{3}$, A. Veszprémi ${ }^{4}$, B. Nagy ${ }^{4}$, J. Kappelmayer" ${ }^{4}$

${ }^{1} 1$ st Department of Medicine, ${ }^{2} 2$ nd Department of Medicine, ${ }^{3}$ Department of Neurology, ${ }^{4}$ Department of Clinical Biochemistry and Molecular Pathology, University of Debrecen, Debrecen, Hungary.

Background and Aims: Thrombocyte hyperfunction is well-known in diabetes mellitus but the causal relationship with diabetic angiopathy is still unanswered. Microparticles are small fragments broken off from the formed elements of the blood and are biologically active, can markedly influence thrombus formation and inflammatory processes. The number of thrombocyte and leukocyte microparticles were both increased in Type 2 diabetes mellitus, and there was a relationship between increased thrombocyte microparticle number and nephropathy. Our aims were to determine the activation level and the number of thrombocyte microparticles and to measure thrombocyte activation in the circulation of diabetic patients and healthy controls. In addition, P-selectin T715P polymorphism was also determined. We investigated the relationship between microparticle number, thrombocyte activation, P-selectin polymorphism and the state of carbohydrate metabolism (HbAlc, existent blood sugar level), carotis intima-media thickness and the occurrence of nephropathy.

Materials and Methods: 100 patients with type II (mean age: $54.89 \pm 7.81$ years, duration of diabetes: $6.89 \pm 5.82$ years), 40 patients with type I diabetes mellitus (mean age: $34.29 \pm 10.08$ years, duration of diabetes: $10.00 \pm 10.17$ years) and 62 healthy controls (mean age: $39.19 \pm 6.12$ years) were included in the study. CD42a indicated the presence of thrombocyte microparticles, while the concomitant occurrence of CD62 (P-selectin) reflected activation. Thrombocyte activation was also monitored by CD62. Measurements were performed by flow cytometry. Carotis intima-media thickness was determined by ultrasound, while the presence of nephropathy was demonstrated by determining urine albumin levels following a 24-hour urine collection.

Results: The activity and number of thrombocyte microparticles were significantly increased in both diabetic groups compared to the controls [thrombocyte microparticle number - type I diabetes: $360 \pm 199 \mathrm{MP} / \mu 1$, type II diabetes: $311 \pm 340 \mathrm{MP} / \mu 1$, control: $122 \pm 68.5 \mathrm{MP} / \mu 1$, type I and type II diabetes vs. control $\mathrm{p}<0,0001$; thrombocyte microparticle activity (mean fluorescence intensity): type I diabetes: $68.2 \pm 48.1$, type II diabetes: $76.5 \pm 102.6$ control:
$18 \pm 8.7$, type I and type II diabetes vs. control $p<0,0001]$. There was a significant positive correlation between thrombocyte microparticle number, P-selectin and soluble P-selectin levels and HbAlc, existent blood sugar level and intima-media thickness. We could not detect any relationship with microalbuminuria.

Conclusion: The level of thrombocyte activation and the number of thrombocyte microparticles are significantly increased in diabetes. Our results suggest that there is a strong correlation between these values and the intima-media thickness, although the causal relationship could not be established. Their role in the pathogenesis of macrovascular complications is likely and their increased level indicate a greater risk for the development of vascular disease. The T715P polymorphism of soluble P-selectin doesn't have any effect on diabetic macrovascular complications.

Supported by Mecenatura

1229

Prognostic value of plasma soluble P-selectin and von Willebrand factor as indices of platelet activation and endothelial dysfunction in 234 patients with hypertension with and without diabetes mellitus

G. I. Varughese ${ }^{1,2}$, J. V. Patel ${ }^{2}$, J. Tomson ${ }^{2}$, G. Y. H. Lip ${ }^{2}$; ${ }^{1}$ Diabetes \& Endocrinology, University Hospital of North Staffordshire, ${ }^{2}$ City Hospital, University Department of Medicine, Birmingham, United Kingdom.

Background and Aims: Abnormal plasma markers of a prothrombotic state have been described in hypertension. We hypothesized that raised plasma levels of soluble Pselectin (sP-sel, an index of platelet activation) and/or von Willebrand factor (vWf, an index of endothelial damage/ dysfunction) might predict vascular events in patients with hypertension with or without diabetes mellitus [DM].

Materials and Methods: We measured sP-sel and vWF levels by ELISA in 234 participants (who did not have prior cardiovascular events) and related these indices to subsequent cardiovascular events over a mean follow up period of 59 months.

Results: Plasma sP-sel levels were a significant predictor of myocardial infarction $(\mathrm{P}=0.03)$, with the greatest risk for those with the highest levels of sP-sel, but were not predictive of cerebrovascular events $(\mathrm{P}=0.53)$, or composite cardiovascular endpoints $(\mathrm{P}=0.06)$. No significant relationships were found between vWF levels and outcome. There was no relationship to the presence or absence of DM at baseline or subsequent development of DM during the follow-up period (Table 1). Conclusion: Among patients with hypertension, raised levels of sP-sel (platelet activation) were predictive of coronary events. vWf (endothelial 
damage/dysfunction) did not predict subsequent cardiovascular events. sP-sel might represent a target for novel therapies or an adjunctive aid to risk stratification in the setting of hypertension. The initial presence of DM or the subsequent development of DM during the follow-up period had no effect on the levels of these prothrombotic markers. Nevertheless, given the high coexistence of hypertension (up to $60 \%$ ) in patients with $\mathrm{DM}$, the results of this study will be applicable to many patients with DM.

Table 1. Cross-sectional data on levels of von Willebrand factor (vWF), soluble P-selectin (sP-sel) and demographics of 234 treated patients with hypertension (with and without cardiovascular (CV) events) after a mean follow-up period of 59 months.

\begin{tabular}{|c|c|c|c|}
\hline \multirow{2}{*}{$\begin{array}{l}\text { Characteristics } \\
\text { at baseline }\end{array}$} & \multicolumn{3}{|c|}{ Patients with hypertension } \\
\hline & $\begin{array}{l}\text { Patients } \\
\text { without } \\
\text { CV Events }\end{array}$ & $\begin{array}{l}\text { Patients } \\
\text { with CV } \\
\text { Events }\end{array}$ & $\begin{array}{l}P \\
\text { value }\end{array}$ \\
\hline Age in years $\pm(S D)$ & $63.4 \pm 8.1$ & $64.2 \pm 6.7$ & 0.55 \\
\hline Number of Patients (\%) & 183 & 51 & - \\
\hline Male gender $(\%)$ & $158(86.3)$ & $44(86.3)$ & 0.99 \\
\hline Smoking history (\%) & $39(21.3)$ & $17(33.3)$ & 0.09 \\
\hline Caucasian ethnicity $(\%)$ & $156(85.2)$ & $42(82.4)$ & 0.93 \\
\hline Body-mass index $\left(\mathrm{kg} / \mathrm{m}^{2}\right) \pm \mathrm{SD}$ & $29.0 \pm 4.2$ & $29.8 \pm 5.0$ & 0.25 \\
\hline Systolic BP $(\mathrm{mmHg}) \pm \mathrm{SD}$ & $148 \pm 15$ & $151 \pm 15$ & 0.13 \\
\hline Diastolic BP $(\mathrm{mmHg}) \pm \mathrm{SD}$ & $84.4 \pm 8.8$ & $83.9 \pm 7.7$ & 0.68 \\
\hline Diabetes at baseline $(\%)$ & $43(23.5)$ & $19(37.3)$ & 0.07 \\
\hline $\begin{array}{l}\text { Diabetes at the end of } \\
\text { follow-up }(\%)\end{array}$ & $61(33.3)$ & $23(45.1)$ & 0.13 \\
\hline $\begin{array}{l}\text { Fasting plasma glucose }(\mathrm{mmol} / \mathrm{l}) \\
\pm \mathrm{SD}\end{array}$ & $6.11 \pm 1.12$ & $6.14 \pm 1.37$ & 0.88 \\
\hline Serum cholesterol $(\mathrm{mmol} / \mathrm{l}) \pm \mathrm{SD}$ & $5.44 \pm 1.17$ & $5.26 \pm 1.14$ & 0.32 \\
\hline $\begin{array}{l}\text { Serum triglycerides } \\
(\mathrm{mmol} / \mathrm{l})[\mathrm{IQR}]\end{array}$ & $\begin{array}{l}1.3[0.9- \\
1.8]\end{array}$ & $\begin{array}{l}1.1[0.8- \\
1.5]\end{array}$ & 0.05 \\
\hline HDL cholesterol $(\mathrm{mmol} / \mathrm{l}) \pm \mathrm{SD}$ & $1.22 \pm 0.35$ & $1.12 \pm 0.25$ & 0.04 \\
\hline $\begin{array}{l}\text { von Willebrand } \\
\text { factor }(\mathrm{IU} / \mathrm{dl}) \pm \mathrm{SD}\end{array}$ & $134 \pm 34$ & $131 \pm 33$ & 0.59 \\
\hline $\begin{array}{l}\text { soluble P-selectin } \\
(\mathrm{ng} / \mathrm{ml})[\mathrm{IQR}]\end{array}$ & $61[50-78]$ & 70 [45-97] & 0.23 \\
\hline
\end{tabular}

1230

Platelet soluble glycoprotein $\mathrm{V}$, a new thrombosis marker, is increased in diabetic patients in relation with poor glycaemic control

L. Kessler ${ }^{1}$, B. Aleil ${ }^{2}$, J. Siméoni ${ }^{1}$, N. Meyer ${ }^{3}$, M.-L. Wiesel ${ }^{2}$, C. Gachet ${ }^{2}$, M. Pinget ${ }^{1}$, J.-P. Cazenave ${ }^{2}$, F. Lanza ${ }^{2}$;
${ }^{1}$ Endocrinologie, Diabète, Maladies Métaboliques, Hôpitaux Universitaires, Strasbourg, ${ }^{2}$ Inserm U311, Etablissement français du sang, Strasbourg, ${ }^{3}$ Santé Publique, Hôpitaux Universitaires, Strasbourg, France.

Background and Aims: Diabetes is a major risk factor for cardiovascular disease. Soluble GPV (sGPV) is a new marker of thrombosis released from the surface of thrombin-exposed platelets. Diabetic patients with poor glycemic control are at increased risk of developing thrombotic cardiovascular complications. Therefore the aim of this study was to measure plasma levels of sGPV in diabetic patients according to glycemic control.

Materials and Methods: Patients and control subjects were prospectively enrolled between January 2002 and November 2004. Plasma levels of sGPV, thrombin-antithrombin complexes (TAT), platelet factor 4 (PF4) and von Willebrand factor (vWf) were determined in 260 diabetic patients (76 with type 1 and 184 with type 2 diabetes mellitus) and in 175 control subjects.

Results: sGPV was significantly increased in diabetic patients as compared to control subjects (median [interquartile range]: 38.7 [29.6-54.0] vs 29.0 [24.1-35.5] ng/ $\mathrm{mL} ; \mathrm{p}<0.0001)$. In the population of diabetic patients, multivariate analysis showed that sGPV levels were strongly associated to glycemic control (hemoglobin Alc: 2.93 [1.50-5.71]; $\mathrm{p}=0.002$; plasma fasting glucose: 2.08 [1.10-3.95]; $\mathrm{p}=0.025)$, duration of the disease (2.33 [1.124.83]; $\mathrm{p}=0.023)$ and platelet count (2.11 [1.25-3.58]; $\mathrm{p}=0.005$ ). In parallel, TAT, PF4 and vWF levels were increased in diabetic patients independently from hemoglobin A1c and plasma glucose.

Conclusion: sGPV is significantly increased in diabetic patients. There is a tight correlation between sGPV and hyperglycemia. sGPV release could be due to thrombin or to circulating platelet metalloproteases present in diabetes. sGPV is a new tool to study the mechanisms linking hyperglycemia and cardiovascular disease.

1231

A new mechanical compression device improves circulation in critical limb ischaemia - a pilot study

R. Karmeli ${ }^{1}$, D. Mannheim ${ }^{1}$, S. Fajer ${ }^{1}$, R. Sachner ${ }^{1}$, S. Raz ${ }^{1}$, O. Galili ${ }^{2}$;

${ }^{1}$ Dept. Vascular Surgery, Carmel Medical Center, Haifa,

${ }^{2}$ Dept. Surgery, Bnei-Zion Medical Center, Haifa, Israel.

Objectives: Critical limb ischemia (CLI) is one of the most challenging problems in vascular medicine; treatment failure will most probably result in limb loss. Patients who suffer from CLI (rest pain, non healing ulcers and 
gangrene) are usually treated by angioplasty or bypass surgery. The relative low patency rate of the above procedures and the high risk patients (diabetic and cardiac morbidity) limits the success and long-term limb salvage. The current study evaluates the effect of a small home-use and portable mechanical calf compression device in patients that do not qualify for vascular treatment.

Methods: Nine (9) patients with CLI were studied prospectively, 6 of them were diabetic. All patients did not meet the criteria for vascular treatment (based on angiography and co-morbidities). Patients used the device 6 hours daily 5 days a week for 6 weeks and were evaluated at baseline, 3 and 6 weeks with a follow-up visit at 12 weeks. Evaluation included: Common Femoral Artery Flow Volume (AFV) using duplex ultrasound, Ankle Brachial Index (ABI), Transcutaneous Oxygen Pressure $\left(\mathrm{TcpO}_{2}\right)$ and Tissue Laser Doppler (TLD). A graded pain scale was used for pain assessment at each visit.

Results: Preliminary data demonstrates that $\mathrm{TcpO}_{2}, \mathrm{AFV}$ and TLD increased significantly in the treated leg and gradually returned to baseline once therapy ended (table). Mean $\mathrm{TcpO}_{2}$ increased up to 10 times the baseline value at 6 weeks following treatment. Resting ABIs did not change during the treatment. The level of pain decreased from 8 to 6.5 following 6 weeks of treatment.

Conclusion: The studied device enhances $\mathrm{TcpO}_{2}, \mathrm{TLD}$ and AFV when used daily. This may be due to improved venous drainage and decreased peripheral resistance, which led to an increased arterial flow and oxygen supply to the tissue. The effect was gradually reversed after suspension of treatment. The results presented here are preliminary in an on-going study and the effect on limb salvage and wound healing is unclear but encouraging. Further studies are needed to determine the optimal role of this mechanical compression device in the treatment of CLI and potentially diabetic foot patients.

Table: Mean ( \pm standard deviation) of $\mathrm{TcpO}_{2}, \mathrm{TLD}$, and $\mathrm{AFV}$ at all time points

\begin{tabular}{|c|c|c|c|c|}
\hline & \multirow{2}{*}{$\begin{array}{l}\text { Baseline } \\
\text { Day } 0\end{array}$} & \multicolumn{2}{|c|}{ Treatment period } & \multirow{2}{*}{$\frac{\text { Follow up }}{3 \text { months }}$} \\
\hline & & 3 weeks & 3 months & \\
\hline $\mathrm{TcpO}_{2}[\mathrm{mmHg}]$ & $12.7 \pm 12.53$ & $33.4 \pm 18.98$ & $36.6 \pm 20.33$ & $25.5 \pm 18.6$ \\
\hline $\begin{array}{l}\text { TLD }[\mathrm{ml} / \mathrm{min} / \\
100 \mathrm{gr} \text { tissue] }\end{array}$ & $1.38 \pm 0.66$ & $1.76 \pm 0.43$ & $1.91 \pm 0.43$ & $1.55 \pm 0.70$ \\
\hline $\mathrm{AFV}[\mathrm{ml} / \mathrm{min}]$ & $118.4 \pm 33.43$ & $107.6 \pm 34.13$ & $115.2 \pm 49.41$ & $85.1 \pm 10.98$ \\
\hline
\end{tabular}

Supported by FlowMedic

\section{PS 111}

\section{Lipid metabolism and lipid-lowering therapy}

\section{2}

High prevalence of low HDL-cholesterol among European type 2 diabetes patients receiving treatment for dyslipidaemia: data from a pan-European survey M. Baccara-Dinet ${ }^{1}$, E. Bruckert ${ }^{2}$, E. Eschwege ${ }^{3}$; ${ }^{1}$ CardioMetabolic Care, Merck Santé, Paris, ${ }^{2}$ Service d'Endocrinologie Métabolique - Hôpital de la Pitié-Salpétrière, Groupe Hospitalier Pitié-SalpÁtrière, Paris, ${ }^{3} \mathrm{U} 780$, INSERM, Paris, France.

Background and Aims: The objective of the study was to determine the prevalence of low HDL-cholesterol according to current National Cholesterol Education Program and International Diabetes Federation criteria $(<1.03 \mathrm{mmol} / \mathrm{L}$ $[<40 \mathrm{mg} / \mathrm{dL}]$ in men and $<1.29 \mathrm{mmol} / \mathrm{L}[<50 \mathrm{mg} / \mathrm{dL}]$ in women) in European type 2 diabetes patients receiving treatment for dyslipidaemia.

Material and methods: The pan-European Survey of HDL-cholesterol measured lipid profiles in 8545 patients followed by a specialist for dyslipidaemia in 11 European countries. Within this population, 3866 patients had a diagnosis of type 2 diabetes mellitus (T2DM).

Results: Compared with non-diabetic subjects, the T2DM population were more likely to be overweight or obese, and were more likely to have a history of hypertension or prior myocardial infarction (MI). Most patients with or without T2DM received treatment with lifestyle modification $(87 \%$ vs. $84 \%$, respectively) and/or pharmacologic lipid-modifying therapy ( $84 \%$ vs. $82 \%)$, usually a statin ( $89 \%$ vs. $91 \%)$. Similar proportions of patients with or without T2DM received fibrates combined with a statin ( $2 \%$ vs. $1 \%)$, fibrate monotherapy (7\% vs. $6 \%$ ) or other treatments (4\% vs. $3 \%$ ). Mean $( \pm \mathrm{SD})$ HDL-cholesterol (mmol/L) was lower $(\mathrm{p}<0.001)$ in $\mathrm{T} 2 \mathrm{DM}(1.22 \pm 0.37)$ than in subjects without T2DM (1.35 \pm 0.44$)$. Mean (SD) HDL-cholesterol was also significantly $(\mathrm{p}<0.001)$ lower in men with T2DM vs. men without T2DM (1.16 $\pm 0.34[\mathrm{n}=2140]$ vs. $1.26 \pm 0.39$ $[\mathrm{n}=2733])$, and in women with T2DM vs. without T2DM $(1.32 \pm 0.38$ [n=1527] vs. $1.53 \pm 0.46[\mathrm{n}=1510])$. Consistent with these observations, low HDL-cholesterol was more common in T2DM vs. no T2DM for all subjects (45\% vs. $30 \%$ ), men (40\% vs. $30 \%$ ) and women (52\% vs. $31 \%$ ).

The T2DM group (men and women combined) also had higher mean triglycerides (TG) $2.32 \pm 2.10$ vs. $1.85 \pm 1.60$ $\mathrm{mmol} / \mathrm{L}$ in the non-T2DM group $(\mathrm{p}<0.001)$, but lower mean LDL-cholesterol (3.02 \pm 1.05 vs. $3.30 \pm 1.14 \mathrm{mmol} / \mathrm{L}$; $\mathrm{p}<0.001)$ and lower mean total cholesterol (5.12 \pm 1.32 vs. $5.38 \pm 1.34 \mathrm{mmol} / \mathrm{L} ; \mathrm{p}<0.001)$. The T2DM group were 
significantly more likely than the no T2DM group (see table) to have high TG, low HDL-cholesterol and high TG, or very low HDL-cholesterol $(<0.9 \mathrm{mmol} / \mathrm{L})$.

Conclusions: Low HDL-cholesterol is common among patients treated for dyslipidaemia in Europe, despite widespread use of statins in this population. Overall, half of women and two-fifths of men with T2DM had low HDLcholesterol, more than half of men and women had high TG, and about one-third of the population had both lipid disorders. Low HDL-cholesterol is an independent risk factor for coronary heart disease, and physicians should be aware of the high risk of low HDL-cholesterol in their statintreated patients, and adjust therapeutic regimens accordingly.

\begin{tabular}{|c|c|c|c|}
\hline & $\begin{array}{l}\text { T2DM } \\
(n=3866)\end{array}$ & $\begin{array}{l}\text { No T2DM } \\
(n=4436)\end{array}$ & $\mathbf{p}$ \\
\hline \multicolumn{4}{|c|}{ Demographics/disease characteristics } \\
\hline Males $(\%)$ & 58 & 64 & $<0.001$ \\
\hline Mean age $(y)$ & $64(10)$ & $61(12)$ & $<0.001$ \\
\hline Mean BMI $\left(\mathrm{kg} / \mathrm{m}^{2}\right)$ & $31(6)$ & $28(4)$ & $<0.001$ \\
\hline $\begin{array}{l}\text { Mean waist circumference } \\
(\mathrm{cm})\end{array}$ & $104(15)$ & $97(14)$ & $<0.001$ \\
\hline Mean SBP/DBP (mmHg) & $\begin{array}{l}141(19) / 81 \\
(10)\end{array}$ & $137(18) / 80(10)$ & $<0.001$ \\
\hline Mean FBG (mmol/L) & $8.3(2.8)$ & $5.6(1.4)$ & $<0.001$ \\
\hline BMI $\geq 25 \mathrm{~kg} / \mathrm{m}^{2}(\%)$ & 85 & 69 & $<0.001^{*}$ \\
\hline $\mathrm{BMI} \geq 30 \mathrm{~kg} / \mathrm{m}^{2}(\%)$ & 48 & 25 & \\
\hline Hypertension (\%) & 82 & 64 & $<0.001$ \\
\hline Prior MI $(\%)$ & 23 & 26 & 0.0015 \\
\hline \multicolumn{4}{|l|}{ Lipids } \\
\hline Low HDL-C (\%) & 45 & 30 & $<0.001$ \\
\hline High TG $(\%)$ & 57 & 42 & $<0.001$ \\
\hline $\begin{array}{l}\text { Low HDL-C and high TG } \\
(\%)\end{array}$ & 32 & 19 & $<0.001$ \\
\hline HDL-C $<0.9$ mmol/L (\%) & 18 & 12 & $<0.001$ \\
\hline
\end{tabular}

*For the overall distribution of BMI. FBG: fasting blood glucose. Figures in parentheses are SD.

Supported by a grant from Merck CardioMetabolic Care.

1233

Endothelial dysfunction in type 2 diabetes mellitus is associated with decreased cholesterol absorption and increased cholesterol synthesis

V. Bláha ${ }^{1}$, A. Šmahelová ${ }^{1}, C$. Andrýs ${ }^{2}$, R. Hyšpler ${ }^{1}$, D. Solichová ${ }^{1}$, A. Tichá ${ }^{1}$, J. Knížek ${ }^{3}$, Z. Zadák ${ }^{1}$;

${ }^{1}$ Department of Metabolic Care and Gerontology, ${ }^{2}$ Department of Immunology, ${ }^{3}$ Department of Medical Biophysics, Medical Faculty Charles University in Hradec Králové, Czech Republic.
Background and Aims: Metabolic abnormalities, associated with Type 2 diabetes, feature a novel factor of low cholesterol absorption and high cholesterol synthesis. We hypothesized an association of endothelial dysfunction and disturbances of cholesterol turnover predisposing to an advanced atherosclerosis in patients with type 2 diabetes.

Materials and Methods: 75 patients, 30 with type 2 diabetes and 30 non-diabetic subjects with a history of cardiovascular disease, and 15 healthy subjects were studied. Plasma sterols, soluble adhesion molecules sCD14, sCD40 Ligand, monocyte chemo-attractant protein-1 (MCP-1), E- and P- selectins were measured with and without atorvastatin therapy.

Results: The patients with Type 2 diabetes mellitus showed significantly higher body weight, body mass index, systolic blood pressure, glycaemia and glycosylated haemoglobin. Quicki insulin sensitivity index was significantly lower. The diabetic patients had significantly higher levels of lathosterol, lathosterol/cholesterol, and lower levels of sitosterol and campesterol and the sterol/cholesterol index. Plasma levels of hsCRP, E-selectin and MCP-1 were significantly higher in patients with diabetes than in healthy subjects. The parameter of MCP-1 significantly correlated with lathosterol $(p=0,0266)$ in the cohort of patients treated with statin. The diabetic patients without statin therapy showed a significant correlation of sE-selectin $(\mathrm{p}=0,0138)$ with the marker of cholesterol absorption - sitosterol and sitosterol/cholesterol ratio; the relationship disappeared with statin treatment. The diabetic patients without statin therapy showed a significant negative correlation of sP-selectin with the marker of cholesterol synthesis - lathosterol $(\mathrm{p}=0,0335)$; the relationship disappeared with statin treatment.

Conclusion: Endothelial dysfunction in Type 2 diabetes mellitus is associated with parameters of decreased cholesterol absorption and increased cholesterol synthesis thus predisposing to adverse cardiovascular outcome of Type 2 diabetes mellitus.

Supported by research project No. MSM0021620820.

1234

Increased cholesterol efflux from cultured fibroblasts to plasma from hypertriglyceridaemic type 2 diabetic patients: role of pre $\beta$-HDL and lipid transfer proteins R. P. F. Dullaart ${ }^{1}$, R. de Vries ${ }^{1}$, A. K. Groen ${ }^{2}$, G. Dallinga-Thie ${ }^{3}$, F. Perton ${ }^{1}$, B. H. R. Wolffenbuttel ${ }^{1}$, A. van $\mathrm{Tol}^{1}$;

${ }^{1}$ Endocrinology, University Medical Center, Groningen, ${ }^{2}$ Experimental Hepatology, Academic Medical Center, Amsterdam, ${ }^{3}$ Vascular Medicine, Erasmus Medical Center, Rotterdam, The Netherlands. 
Background and Aims: Cellular cholesterol efflux represents an early step in the reverse cholesterol transport (RCT) pathway, whereby excess peripheral cholesterol is transported back to the liver. We tested whether, in type 2 diabetes, hypertriglyceridemia is accompanied by increased pre $\beta$-HDL and whether such changes would affect the cellular cholesterol efflux-promoting ability of plasma.

Materials and Methods: In 28 hypertriglyceridemic and 56 normotriglyceridemic type 2 diabetic patients, treated with diet alone or in combination with oral glucose lowering agents, and in 56 normotriglyceridemic control subjects, we determined plasma lipids, HDL lipid composition, plasma pre $\beta$-HDL and pre $\beta$-HDL formation (both expressed in $\%$ of plasma apo A-I), cholesteryl ester transfer protein (CETP) concentration, phosphoplipid transfer protein (PLTP) activity and the ability of plasma to stimulate cholesterol efflux out of cultured human fibroblasts.

Results: HDL cholesterol and HDL phospholipids were lower, whereas HDL triglycerides, plasma pre $\beta$-HDL and pre $\beta$-HDL formation, as well as plasma CETP concentration and PLTP activity were higher in hypertriglyceridemic diabetic patients than in control subjects (all $\mathrm{p}<0.05$ to $\mathrm{p}<0.01)$. Except for lower HDL cholesterol and higher PLTP activity $(\mathrm{p}<0.01)$, these variables were not different between normotriglyceridemic diabetic patients and control subjects. Cellular cholesterol efflux to plasma from hypertriglyceridemic diabetic patients was increased compared to control plasma $(9.0 \pm 0.7 \%$ per $4 \mathrm{~h}$ vs $8.3 \pm 0.9 \%$ per $4 \mathrm{~h}$, $\mathrm{p}<0.01$ ), but did not differ between plasma from normotriglyceridemic diabetic patients $(8.4 \pm 0.8 \%$ per $4 \mathrm{~h})$ and control subjects. Multiple linear regression analysis demonstrated that, in all subjects combined, cellular cholesterol efflux to plasma was positively determined by pre- $\beta$ HDL formation, HDL phospholipids, PLTP activity and HDL triglycerides (multiple $\mathrm{r}=0.49$ ), but not by the diabetic state. Conclusions: Cellular cholesterol efflux to plasma from hypertriglyceridemic diabetic patients is enhanced, probably as a result of increased pre $\beta$-HDL, altered HDL lipids and increased PLTP activity. This early step in RCT is unchanged in normotriglyceridemic diabetic patients.

Supported by Dutch Diabetes Fund

\section{5}

Efficacy and tolerability of rosuvastatin and atorvastatin when force-titrated in patients with the metabolic syndrome as defined by the IDF: results from the ECLIPSE Study

O. Faergeman ${ }^{1}$, F. Sosef ${ }^{2}$, E. Duffield ${ }^{2}$ on behalf of the ECLIPSE investigators,
${ }^{1}$ National Heart and Lung Institute, Imperial College, London, ${ }^{2}$ AstraZeneca, Macclesfield, United Kingdom.

Background and Aims: The metabolic syndrome (MeS) is a complex constellation of disorders that increase the risk of coronary heart disease (CHD). Many patients, particularly those at high risk of CHD, fail to reach their LDL-C goals due to suboptimal treatment. The effect of rosuvastatin (RSV) 10-40 mg and atorvastatin (ATV) 10-80 mg over 24 wks was compared in ECLIPSE, an open-label, randomised, multinational, multi-centre, parallel-group study conducted in 118 centres in Canada and Europe.

Materials and Methods: After a 6-wk dietary lead-in period, 1036 patients with LDL-C $\geq 4.14-<6.47 \mathrm{mmol} / \mathrm{L}$ and CHD, a CHD-risk equivalent or clinical evidence of atherosclerosis were randomised to force-titrated treatment with RSV (10, 20 and $40 \mathrm{mg}$ for 6, 6 and $12 \mathrm{wks})$ or ATV $(10,20,40$ and $80 \mathrm{mg}$ each for $6 \mathrm{wks})$. In this analysis, we report on 2003 European LDL-C goal achievement and percentage change from baseline in lipid levels in patients with or without the MeS (based on the IDF 2005 definition), and the overall ITT (intention-to-treat) study population. Statistical comparisons were performed on the overall ITT population only.

Results: In the overall ITT population ( $n=1015), 66.7 \%$ of patients (RSV, $n=354$; ATV, $n=323$ ) had the MeS at baseline. Significantly more patients treated with RSV than ATV achieved their 2003 European LDL-C goal at all time points $(p<0.001$, Table), and goal achievement for RSV and ATV was similar in patients with or without the MeS (Table). Throughout the study, in the overall ITT population, changes from baseline in LDL-C $(\mathrm{p}<0.001)$, TC $(\mathrm{p}<0.05)$, and HDL$\mathrm{C}(\mathrm{p}<0.01)$ were significantly greater for RSV than ATV. Lipid changes with RSV and ATV in patients with or without the MeS were consistent with those for the overall ITT population (wk 24 data in Table). Analysis of LDL-C goal achievement and changes in lipid levels was also performed for patients with the MeS as defined by NCEP ATP III, and the results were similar to those presented here. Overall, both treatments were well tolerated.

Conclusions: In the ECLIPSE study, RSV 10-40 mg was more effective than ATV $10-80 \mathrm{mg}$ at enabling patients to achieve their 2003 European LDL-C goals and improving the atherogenic lipid profile. The benefits of RSV treatment were consistent in patients with and without the MeS and in the overall ITT population. 


\begin{tabular}{|c|c|c|c|c|c|c|}
\hline & \multicolumn{2}{|c|}{ Overall ITT } & \multicolumn{2}{|c|}{$\begin{array}{l}\text { With } \\
\text { metabolic } \\
\text { syndrome }\end{array}$} & \multicolumn{2}{|c|}{$\begin{array}{l}\text { Without } \\
\text { metabolic } \\
\text { syndrome }\end{array}$} \\
\hline & $\begin{array}{l}\text { RSV } \\
(n=505)\end{array}$ & $\begin{array}{l}\text { ATV } \\
(n=510)\end{array}$ & $\begin{array}{l}\text { RSV } \\
(n=354)\end{array}$ & $\begin{array}{l}\text { ATV } \\
(n=323)\end{array}$ & $\begin{array}{l}\text { RSV } \\
(n=147)\end{array}$ & $\begin{array}{l}\text { ATV } \\
(n=180)\end{array}$ \\
\hline \multicolumn{7}{|c|}{$\%$ patients at LDL-C goal* } \\
\hline $\begin{array}{l}\text { wk } 6 \\
\text { (RSV } 10 \mathrm{mg} \text {, } \\
\text { ATV } 10 \mathrm{mg} \text { ) }\end{array}$ & $52.4^{\dagger}$ & 28.8 & 52.3 & 28.8 & 52.8 & 29.4 \\
\hline $\begin{array}{l}\text { wk } 12 \\
\text { (RSV } 20 \mathrm{mg} \text {, } \\
\text { ATV } 20 \mathrm{mg})\end{array}$ & $71.3^{\dagger}$ & 45.7 & 72.5 & 45.4 & 67.6 & 47.7 \\
\hline $\begin{array}{l}\text { wk } 18 \\
\text { (RSV } 40 \mathrm{mg} \text {, } \\
\text { ATV } 40 \mathrm{mg} \text { ) }\end{array}$ & $80.4^{\dagger}$ & 64.0 & 80.0 & 61.9 & 80.9 & 68.6 \\
\hline $\begin{array}{l}\text { wk } 24 \\
\text { (RSV } 40 \mathrm{mg} \text {, } \\
\text { ATV } 80 \mathrm{mg} \text { ) }\end{array}$ & $82.8^{\dagger}$ & 73.3 & 81.6 & 71.0 & 85.3 & 77.4 \\
\hline \multicolumn{7}{|c|}{$\%$ change from baseline at wk 24} \\
\hline LDL-C & $-57.3^{\dagger}$ & -52.2 & -57.3 & -52.1 & -56.8 & -52.5 \\
\hline $\mathrm{TC}$ & $-41.3^{\ddagger}$ & -39.5 & -41.5 & -39.7 & -40.4 & -39.2 \\
\hline HDL-C & $+8.4^{\dagger}$ & +1.8 & +8.7 & +2.8 & +7.9 & +0.6 \\
\hline TG & -24.6 & $-28.0^{*}$ & -25.7 & -29.5 & -21.4 & -25.3 \\
\hline
\end{tabular}

*2003 European LDL-C goal: $<2.5$ or $3.0 \mathrm{mmol} / \mathrm{L}$, depending on risk category

${ }^{\dagger} \mathrm{p}<0.001$ vs ATV

${ }^{ \pm} \mathrm{p}<0.05$

Supported by AstraZeneca

1236

Lipid-lowering effects of colesevelam hydrochloride $(\mathrm{HCl})$ in patients with type 2 diabetes

S. Abby ${ }^{1}$, M. Kalin ${ }^{2}$, F. Zieve ${ }^{3}$, S. Schwartz ${ }^{4}$, M. Jones ${ }^{1}$; ${ }^{1}$ Sankyo Pharma Inc., Parsippany, United States, ${ }^{2}$ Endocrine Service, Department of Medicine, Memorial SloanKettering Cancer Center, New York, NY, United States, ${ }^{3}$ Diabetes Health Center, Richmond VA Medical Center, Richmond, United States, ${ }^{4}$ Diabetes and Glandular Disease, Diabetes and Glandular Disease Clinic of San Antonio, P.A., San Antonio, United States.

Background and Aims: The importance of lipid control in patients with type 2 diabetes mellitus (T2DM) has been well established.

Materials and Methods: The lipid-lowering effects of colesevelam $\mathrm{HCl}$ in patients with $\mathrm{T} 2 \mathrm{DM}$ were evaluated in a prospective, randomized, double-blind, placebo-controlled, parallel-group pilot study. The trial was comprised of a 4 -week, placebo run-in period followed by a 12-week active treatment period. Eligibility criteria included a diagnosis of $\mathrm{T} 2 \mathrm{DM}, \mathrm{HbA1C} 7.0 \%-10.0 \%$ inclusive at the time of randomization, serum triglycerides $\leq 300 \mathrm{mg} / \mathrm{dL}$, BMI $\leq 40 \mathrm{~kg} / \mathrm{m}^{2}$, and at least 3 months of treatment with a stable dose of a sulfonylurea, metformin or their combination. Eligible subjects were maintained on their existing antidiabetic regimen and were randomized to colesevelam $\mathrm{HCl}(3.75 \mathrm{~g} / \mathrm{d})$ or placebo $(6$ tablets/d). Sixty-five patients (31 receiving colesevelam $\mathrm{HCl}$ and 34 receiving placebo) were randomized.

Results: Colesevelam $\mathrm{HCl}$ treatment resulted in significant reductions compared to placebo in LDL-C $(-11.7 \%$; $P=0.007)$, total-C $(-7.3 \% ; P=0.019)$, apolipoprotein B $(-11.8 \% ; P=0.003)$, and LDL particle concentration $(-209.6 \mathrm{nmol} / \mathrm{L} ; P=0.037)$. Of particular interest is the fact that triglycerides were not significantly increased with colesevelam $\mathrm{HCl}$ compared to placebo (treatment effect $+8 \pm$ $14 \mathrm{mg} / \mathrm{dL} ; P=0.570$ ). No significant treatment differences for other lipid parameters were noted.

Conclusion: These data indicate that colesevelam $\mathrm{HCl}$ is a useful agent for lowering LDL-C, total-C, apolipoprotein B, and LDL particle concentration in patients with T2DM.

\begin{tabular}{|c|c|c|c|c|c|c|c|c|}
\hline & $\begin{array}{l}\text { LDL-C } \\
(\mathrm{mg} / \mathrm{dL})\end{array}$ & & $\begin{array}{l}\text { Total-C } \\
(\mathrm{mg} / \mathrm{dL})\end{array}$ & & $\begin{array}{l}\text { Apo-B } \\
(\mathrm{mg} / \mathrm{dL})\end{array}$ & & $\begin{array}{l}\text { LDL } \\
\text { Particle } \\
\text { Concentr } \\
\text { (nmol/L) }\end{array}$ & ration \\
\hline & Baseline & $\begin{array}{l}\text { Week } \\
12\end{array}$ & Baseline & $\begin{array}{l}\text { Week } \\
12\end{array}$ & Baseline & $\begin{array}{l}\text { Week } \\
12\end{array}$ & Baseline & $\begin{array}{l}\text { Weel } \\
12\end{array}$ \\
\hline $\begin{array}{l}\text { Colesevelam } \\
\text { (n) }\end{array}$ & $\begin{array}{l}123 \\
(25)\end{array}$ & $\begin{array}{l}108 \\
(27)\end{array}$ & $\begin{array}{l}208 \\
(27)\end{array}$ & $\begin{array}{l}197 \\
(17)\end{array}$ & $\begin{array}{l}129 \\
(27)\end{array}$ & $\begin{array}{l}118 \\
(27)\end{array}$ & $\begin{array}{l}1559 \\
(26)\end{array}$ & $\begin{array}{l}1428 \\
(26)\end{array}$ \\
\hline $\begin{array}{l}\text { Placebo } \\
\text { (n) }\end{array}$ & $\begin{array}{l}120 \\
(30)\end{array}$ & $\begin{array}{l}122 \\
(32)\end{array}$ & $\begin{array}{l}196 \\
(32)\end{array}$ & $\begin{array}{l}202 \\
(32)\end{array}$ & $\begin{array}{l}123 \\
(31)\end{array}$ & $\begin{array}{l}129 \\
(31)\end{array}$ & $\begin{array}{l}1524 \\
(29)\end{array}$ & $\begin{array}{l}1603 \\
(29)\end{array}$ \\
\hline
\end{tabular}

Supported by Sankyo Pharma Inc.

1237

Colesevelam $\mathrm{HCl}$ improves glycaemic control in patients with type 2 diabetes (T2DM): a pilot study

M. Jones ${ }^{1}$, S. Schwartz ${ }^{2}$, F. Zieve ${ }^{3}$, M. Kalin ${ }^{4}$;

${ }^{1}$ Sankyo Pharma Inc., Parsippany, ${ }^{2}$ Diabetes \& Glandular Disease, Diabetes \& Glandular Disease Clinic of San Antonio, P.A., San Antonio, ${ }^{3}$ Diabetes Health Center, Richmond VA Medical Center, Richmond, ${ }^{4}$ Endocrine Service, Department of Medicine, Memorial Sloan-Kettering Cancer Center, New York, United States.

Background and Aims: Colesevelam $\mathrm{HCl}$, a bile acid sequestrant, is indicated for lowering LDL-cholesterol, but may also affect blood glucose levels. 
Materials and Methods: The effect of colesevelam $\mathrm{HCl}$ on glycemic control in patients with T2DM, not adequately controlled with their current anti-diabetic regimens, was evaluated in a prospective, randomized, double-blind, placebo-controlled, parallel-group pilot study. The trial consisted of 4-weeks of placebo run-in followed by 12-weeks of active treatment. Eligibility criteria included a T2DM diagnosis, at least 3 months of treatment with a stable dose of a sulfonylurea, metformin or their combination, and A1C $7.0 \%-10.0 \%$ inclusive at randomization. Eligible patients were randomized to colesevelam $\mathrm{HCl}(3.75 \mathrm{~g} / \mathrm{d})$ or placebo (6 tablets/d). Sixtyfive patients were randomized; 59 completed the study. Differences between groups were evaluated by Analysis of Covariance, with baseline values as covariates, and are expressed as least square (LS) means \pm standard error (SE).

Results: Fasting plasma glucose (LS mean \pm SE) was significantly lower in the colesevelam $\mathrm{HCl}$ group compared to the placebo group at weeks $4(-23 \pm 9 \mathrm{mg} / \mathrm{dL}, P=0.016)$ and $8(-18 \pm 7 \mathrm{mg} / \mathrm{dL}, P=0.011)$, and tended to be lower at week $12(-14 \pm 9 \mathrm{mg} / \mathrm{dL}, p=0.118)$. Treatment resulted in $0.5 \%$ reduction in $\mathrm{A} 1 \mathrm{C}$ vs placebo in the total population. Subpopulation analysis of patients with $\mathrm{A} 1 \mathrm{C} \geq 8.0 \%$ at baseline showed that colesevelam $\mathrm{HCl}$ lowered $\mathrm{A} 1 \mathrm{C}$ by $1.0 \%$ compared to placebo $(P=0.002)$.

Conclusion: Thus, colesevelam $\mathrm{HCl}$ not only lowers LDLcholesterol, but may also improve glycemic control in patients with T2DM.

\begin{tabular}{|c|c|c|c|c|}
\hline & Baseline & Week 12 & $\Delta$ & $\begin{array}{l}\text { LS mean } \pm \text { SE } \\
\text { Treatment } \\
\text { Effects }\end{array}$ \\
\hline A1C (\%) & & & & \\
\hline $\begin{array}{l}\text { Colesevelam } \\
\text { HCl }(n=27)\end{array}$ & 7.9 & 7.7 & -0.2 & $\begin{array}{l}-0.5 \pm 0.2 \\
P=0.007\end{array}$ \\
\hline Placebo $(n=32)$ & 8.1 & 8.3 & +0.3 & $\begin{array}{l}-0.5 \pm 0.2 \\
P=0.007\end{array}$ \\
\hline $\begin{array}{l}\text { Fructosamine } \\
(\mu \mathrm{mol} / \mathrm{L})\end{array}$ & & & & \\
\hline $\begin{array}{l}\text { Colesevelam } \\
\mathrm{HCl}(\mathrm{n}=27)\end{array}$ & 299 & 288 & -11 & $\begin{array}{l}-29 \pm 11 \\
P=0.011\end{array}$ \\
\hline Placebo $(n=32)$ & 318 & 330 & +12 & $\begin{array}{l}-29 \pm 11 \\
P=0.011\end{array}$ \\
\hline
\end{tabular}

Supported by Sankyo Pharma Inc.

\section{8}

HDL evaluation approach for reduction in cardiovascular risk: The HEART audit

L. Varadhan, I. Basha, H. Reddy, A. Gopinath, R. Nair,

J. Morrissey, V. Patel;

Diabetes Centre, George Eliot Hospital NHS Trust, Nuneaton, United Kingdom.
Background and Aims: Type 2 diabetes is associated with a 2-4 fold increase in cardiovascular events and major vascular events can be significantly reduced by appropriate management of dyslipidemia. Treatment targeted towards HDL Cholesterol should be started when primary targets for cholesterol are reached. A $1 \%$ reduction in LDL reduces risk of cardiovascular event by $1-1.5 \%$ and an increase in HDL by $0.03 \mathrm{mmol} / \mathrm{L}$ can reduce cardiac event by $2-4 \%$ independent of LDL

The aim of our study was to assess the potential for reducing cardiovascular risk by targeting the secondary lipid target of HDL cholesterol $\geq 1 \mathrm{mmol} / \mathrm{L}$ using Modified release Nicotinic acid, simultaneously assessing its tolerability and impact on glycemic control.

Materials and Methods: 50 patients with type 2 diabetes, with HDL cholesterol less than $1 \mathrm{mmol} / \mathrm{L}$, attending an outpatient clinic in UK, were recruited into the study. Patients on Fenofibrate were excluded. Baseline assessment including BP, $\mathrm{HbA} 1 \mathrm{c}$, lipid profile and UKPDS cardiovascular risk score were calculated at this visit(V1). Eligible patients were then commenced on Modified release Nicotinic acid, starting at $375 \mathrm{mg}$ once daily and gradually increased to $1000 \mathrm{mg}$ once daily over 4 weeks. HDL helpline was available for patients to seek advice during the trial. 34 patients reported for the second visit(V2) in 3 months and were reassessed.

Results: The average age of the patients was 58.2 years, $90 \%$ of patients being caucasian in origin. Average duration of diabetes at presentation was 9.5 years. $82 \%$ of the patients were already on a statin and $23 \%$ of them for hypercholesterolemia. 54\% were on Insulin for glycemic control and $22 \%$ on thiazolidinediones.

$62.5 \%$ of the patients showed improvement in HDL over the trial period (range $0.1-0.4 \mathrm{mmol} / \mathrm{L}$ ). The HDL Cholesterol improved by $12.5 \%$ overall from the first to second visit $(0.8$ vs. $0.9 \mathrm{mmol} / \mathrm{L}, \mathrm{p}<0.05)$. The improvement in HDL was significantly higher at $18.3 \%$ when used as monotherapy. The total cholesterol improved by $15.2 \%$ between the two visits ( 3.8 vs. $3.3 \mathrm{mmol} / \mathrm{L})$ and LDL by $18.8 \%$ (1.9 vs. $1.6 \mathrm{mmol} / \mathrm{L}$ ) whereas triglycerides did not change (2.6 vs. $2.6 \mathrm{mmol} / \mathrm{L}$ ). The diabetes control had not deteriorated after initiation of this therapy (8.28 vs. $8.17 \%)$. The blood pressure remained comparable (135.7/75.8 vs. 134/74.5).

$62.5 \%$ of the patients showed an improvement in the UKPDS risk score, improving from 23.2 (V1) to 16.2 (V2) (range 0.2-15.5; mean improvement 6.5). The risk score improved by $12.7 \%$ overall (23.1 vs. 20.5 ). Considering the patients who showed improvement in HDL, the risk score improved by $19 \%$ ( 27.9 vs. 22.5 ).

8 patients $(16 \%)$ had discontinued therapy during the trial period, 3 due to headache and 2 each due to itching and flushing. 
Conclusion: Nicotinic acid has the potential in improving cardiovascular risk in patients with low HDL cholesterol levels. An increase in HDL could be achieved in more than half of the patients initiated on treatment, which would favourably reflect on the total cardiovascular risk. The drug was well tolerated with neutral effects on HbA1c. Randomized trials with larger numbers may be needed to assess clinical outcomes to this approach.

\section{9}

The safety and efficacy of ezetimibe/simvastatin (E/S) versus atorvastatin (A) in hypercholesterolaemic (HC) patients with type 2 diabetes (T2DM)

T. Mazzone ${ }^{1}$, R. B. Goldberg ${ }^{2}$, J. R. Guyton ${ }^{3}$,

R. S. Weinstock ${ }^{4}$, A. Polis ${ }^{5}$, R. A. Gazzara ${ }^{5}$,

J. E. Tomassini ${ }^{5}$, A. M. Tershakovec ${ }^{5}$;

${ }^{1}$ Department of Medicine, University of Illinois at Chicago,

${ }^{2}$ Department of Medicine, University of Miami School of Medicine, ${ }^{3}$ Department of Medicine, Duke University School of Medicine, Durham, ${ }^{4}$ Department of Medicine, SUNY Upstate Medical University College of Medicine, Syracuse, ${ }^{5}$ Clinical Development, Merck \& Co., Inc., West Point, United States.

Background and Aims: T2DM is considered a coronary heart disease risk equivalent, requiring aggressive lipid management. This study evaluated the safety and lipidmodifying efficacy of E/S vs A at the usual start doses (E/S $10 / 20 \mathrm{mg}$ vs A $10 \mathrm{mg}$ and A $20 \mathrm{mg}$ ) and alternate start doses (E/S 10/40 mg vs A $40 \mathrm{mg}$ ) in HC patients with T2DM.

Material and methods: This was a multicenter, doubleblind, randomized, 6-week, parallel-group study. The primary efficacy endpoint was \% change from baseline in LDL-C, and secondary efficacy endpoints were $\%$ changes from baseline in HDL-C, Total-C, TG, apo B, apo A-I, Non-HDL$\mathrm{C}$, and hs-CRP, and \% attaining LDL-C $<100$ and $<70 \mathrm{mg} / \mathrm{dL}$. Safety was assessed by analyzing adverse experiences (AEs). ANOVA was used for analysis of \% change in lipid and nonlipid measures, logistic regression for \% attaining LDL-C targets, and Fisher's exact test for safety comparisons.

Results: 1229 patients were randomized from 2300 screened. Baseline patient characteristics were comparable across treatment groups. Efficacy results, shown below, were based on 1198 patients $(\mathrm{E} / \mathrm{S}=480 ; \mathrm{A}=718)$ included in the modified intent-to-treat population. The incidences of serious drug-related, clinical, laboratory, or prespecified (GI-, gallbladder-, or hepatitis-related, allergic reaction/rash, ALT, AST, CK) AEs were comparable in the pooled ezetimibe/ simvastatin and pooled atorvastatin treatment groups.

\begin{tabular}{|c|c|c|c|c|c|c|}
\hline \multirow{2}{*}{$\begin{array}{l}\text { Treatment } \\
\text { comparison }\end{array}$} & \multicolumn{2}{|c|}{$\begin{array}{l}\text { EZ/Simva } 10 / 20 \\
\text { vs. Atorva } 10 \mathrm{mg}\end{array}$} & \multicolumn{2}{|c|}{$\begin{array}{l}\text { EZ/Simva } 10 / 20 \\
\text { vs. Atorva } 20 \mathrm{mg}\end{array}$} & \multicolumn{2}{|c|}{$\begin{array}{l}\text { EZ/Simva 10/40 } \\
\text { vs. Atorva } 40 \mathrm{mg}\end{array}$} \\
\hline & $\begin{array}{l}\text { EZ/Simva } \\
20 \mathrm{mg} \\
(\mathrm{N}=238)\end{array}$ & $\begin{array}{l}\text { Atorva } \\
10 \mathrm{mg} \\
(\mathrm{N}=237)\end{array}$ & $\begin{array}{l}\text { EZ/Simva } \\
20 \mathrm{mg} \\
(\mathrm{N}=238)\end{array}$ & $\begin{array}{l}\text { Atorva } \\
20 \mathrm{mg} \\
(\mathrm{N}=240)\end{array}$ & $\begin{array}{l}\text { EZ/Simva } \\
40 \mathrm{mg} \\
(\mathrm{N}=242)\end{array}$ & $\begin{array}{l}\text { Atorva } \\
40 \mathrm{mg} \\
(\mathrm{N}=241)\end{array}$ \\
\hline LDL-C & & & & & & \\
\hline Baseline mean $\dagger$ & 145.0 & 145.2 & 145.0 & 146.6 & 144.0 & 145.9 \\
\hline $\begin{array}{l}\% \text { change from } \\
\text { baselinet }\end{array}$ & -53.6 & -38.3 & -53.6 & -44.6 & -57.6 & -50.9 \\
\hline $\begin{array}{l}\text { Treatment } \\
\text { Difference§\# } \\
(95 \% \text { CI })\end{array}$ & \multicolumn{2}{|c|}{$\begin{array}{l}-15.3 * * * \\
(-17.7,-12.8)\end{array}$} & \multicolumn{2}{|c|}{$\begin{array}{l}-9.0 * * * \\
(-11.5,-6.5)\end{array}$} & \multicolumn{2}{|c|}{$\begin{array}{l}-6.6 * * * \\
(-9.1,-4.2)\end{array}$} \\
\hline \multicolumn{7}{|l|}{ HDL-C } \\
\hline Baseline mean $\dagger$ & 44.5 & 44.8 & 44.5 & 46.5 & 47.4 & 46.0 \\
\hline $\begin{array}{l}\% \text { change from } \\
\text { baselinet }\end{array}$ & 8.0 & 4.3 & 8.0 & 4.5 & 6.3 & 2.3 \\
\hline $\begin{array}{l}\text { Treatment } \\
\text { Difference§\# } \\
(95 \% \mathrm{CI})\end{array}$ & \multicolumn{2}{|l|}{$\begin{array}{l}3.6 * * * \\
(1.5,5.7)\end{array}$} & \multicolumn{2}{|l|}{$\begin{array}{l}3.4 * * \\
(1.3,5.5)\end{array}$} & \multicolumn{2}{|l|}{$\begin{array}{l}4.0 * * * \\
(1.9,6.0)\end{array}$} \\
\hline \multicolumn{7}{|l|}{ TG } \\
\hline Baseline median & 173.3 & 191.0 & 173.3 & 175.0 & 175.3 & 175.5 \\
\hline $\begin{array}{l}\% \text { change from } \\
\text { baselinet† }\end{array}$ & -25.7 & -22.7 & -25.7 & -26.1 & -29.3 & -28.4 \\
\hline $\begin{array}{l}\text { Treatment } \\
\text { Differencett\# } \\
(95 \% \text { CI })\end{array}$ & \multirow{2}{*}{\multicolumn{2}{|c|}{$\begin{array}{l}-4.4^{*} \\
(-7.9,-0.7)\end{array}$}} & \multirow{2}{*}{\multicolumn{2}{|c|}{$\begin{array}{l}-0.5 \\
(-4.2,3.0)\end{array}$}} & \multirow{2}{*}{\multicolumn{2}{|c|}{$\begin{array}{l}-1.1 \\
(-4.7,2.6)\end{array}$}} \\
\hline hs-CRP (mg/L) & & & & & & \\
\hline Baseline median & 2.9 & 2.4 & 2.9 & 2.5 & 2.9 & 2.6 \\
\hline $\begin{array}{l}\% \text { change from } \\
\text { baselinet† }\end{array}$ & -23.3 & -13.7 & -23.3 & -13.8 & -33.3 & -28.6 \\
\hline $\begin{array}{l}\text { Treatment } \\
\text { Differenceft\# } \\
(95 \% \text { CI })\end{array}$ & \multicolumn{2}{|c|}{$\begin{array}{l}-10.2^{*} \\
(-18.5,-1.9)\end{array}$} & \multicolumn{2}{|c|}{$\begin{array}{l}-6.5 \\
(-15.0,1.4)\end{array}$} & \multicolumn{2}{|c|}{$\begin{array}{l}-5.4 \\
(-12.5,1.6)\end{array}$} \\
\hline $\begin{array}{l}\text { Target LDL-C } \\
<70 \mathrm{mg} / \mathrm{dL} \text { n }(\%) \#\end{array}$ & $\begin{array}{l}142 \\
(59.7)^{* * *}\end{array}$ & $\begin{array}{l}51 \\
(21.5)\end{array}$ & $\begin{array}{l}142 \\
(59.7)^{* * *}\end{array}$ & $\begin{array}{l}84 \\
(35.0)\end{array}$ & $\begin{array}{l}180 \\
(74.4)^{* * *}\end{array}$ & $\begin{array}{l}133 \\
(55.2)\end{array}$ \\
\hline $\begin{array}{l}\text { Target LDL-C } \\
<100 \mathrm{mg} / \mathrm{dL} \mathrm{n}(\%) \#\end{array}$ & $\begin{array}{l}215 \\
(90.3)^{* * *}\end{array}$ & $\begin{array}{l}166 \\
(70.0)\end{array}$ & $\begin{array}{l}215 \\
(90.3)^{* *}\end{array}$ & $\begin{array}{l}197 \\
(82.1)\end{array}$ & $\begin{array}{l}226 \\
(93.4)\end{array}$ & $\begin{array}{l}214 \\
(88.8)\end{array}$ \\
\hline
\end{tabular}

${ }^{*} \mathrm{p}<0.05, * * \mathrm{p}<0.01, * * * \mathrm{p}<0.001$ for specified betweentreatment difference.

†Means; †LS Means; §Differences in LS Means; $\dagger \dagger$ Median; ++ Median $(95 \% \mathrm{CI})$

\#Treatment differences are EZ/Simva 10/20 mg minus Atorva $10 \mathrm{mg}$, EZ/Simva 10/20 mg minus Atorva $20 \mathrm{mg}$, and EZ/Simva 10/40 mg minus Atorva $40 \mathrm{mg}$.

Conclusion: Ezetimibe/simvastatin treatment resulted in significantly greater LDL-C-lowering and HDL-C increases at all dose comparisons; generally significantly greater LDL-C goal attainment, particularly at the optional LDL$\mathrm{C}<70 \mathrm{mg} / \mathrm{dL}$ target; and similar reductions in TG and hsCRP compared to atorvastatin usual/alternate start doses. Thus, the dual cholesterol-lowering mechanisms of ezetimibe/simvastatin provided additional lipid-modifying benefits over atorvastatin monotherapy in patients with T2DM. Both treatments were generally well-tolerated.

Supported by Merck/Schering Plough Pharmaceuticals 


\section{0}

Efficacy and tolerability of rosuvastatin $40 \mathrm{mg}$ alone or in combination with ezetimibe $10 \mathrm{mg}$ in patients with type 2 diabetes: results from a subgroup of the EXPLORER Study

C. M. Ballantyne ${ }^{1}$, F. Sosef ${ }^{2}$, E. Duffield ${ }^{2}$

on behalf of the EXPLORER study investigators,

${ }^{1}$ Department of Medicine, Baylor College of Medicine, Houston, United States, ${ }^{2}$ AstraZeneca, Macclesfield, United Kingdom.

Background and Aims: Type 2 diabetes is associated with an increased risk of coronary heart disease (CHD). Lowering LDL-C should be a priority in the control of diabetic dyslipidaemia, yet many patients eligible for statin therapy do not achieve recommended lipid goals in clinical practice. The combination of statins with other lipidlowering agents is an option for patients who require reductions in LDL-C that cannot be achieved with monotherapy. In EXPLORER, the effect of monotherapy with rosuvastatin (RSV) $40 \mathrm{mg}$ and combination therapy with RSV $40 \mathrm{mg}+$ ezetimibe (EZE) $10 \mathrm{mg}$ for 6 weeks was assessed in patients at high risk of CHD.

Materials and Methods: Patients $(n=469)$ with hypercholesterolaemia (LDL-C $\geq 160-<250 \mathrm{mg} / \mathrm{dL}$ for statin naïve patients; $\geq 100-<190 \mathrm{mg} / \mathrm{dL}$ for statin-treated patients) and a history of CHD, clinical evidence of atherosclerosis or a CHD risk equivalent (diabetes mellitus or a 10 -year CHD risk score $>20 \%$ ) were randomised to 6 weeks' treatment with RSV $40 \mathrm{mg}$ alone or in combination with EZE $10 \mathrm{mg}$. The primary endpoint was achievement of the NCEP ATP III LDL-C goal of $<100 \mathrm{mg} /$ $\mathrm{dL}$ at week 6 . Secondary endpoints included achievement of other lipid goals, and changes in lipids and lipoproteins. In this post-hoc analysis, we report on changes in lipid levels and LDL-C goal achievement in patients with or without diabetes from the EXPLORER study, in addition to the overall ITT population. Statistical comparisons were performed on the overall ITT population only.

Results: In the ITT population ( $\mathrm{n}=465), 35.7 \%$ of patients (RSV, n=89; RSV+EZE, $\mathrm{n}=77$ ) had diabetes at baseline. Overall, mean LDL-C levels at baseline were similar in both treatment groups (RSV, $4.93 \mathrm{mmol} / \mathrm{L}[190.8 \mathrm{mg} / \mathrm{dL}$; RSV+EZE, $4.89 \mathrm{mmol} / \mathrm{L}[189.2 \mathrm{mg} / \mathrm{dL}])$. At 6 weeks, LDL-C was reduced to 2.11 and $1.47 \mathrm{mmol} / \mathrm{L}(81.5$ and $56.9 \mathrm{mg} / \mathrm{dL}$ ) in the RSV and RSV+EZE groups, respectively; significantly greater percentage reductions in LDL-C were achieved with RSV+EZE (Table). RSV+EZE was also associated with significantly greater reductions in TC and TG than RSV (Table). HDL-C levels were increased by both RSV and RSV+EZE (Table). Lipid changes in patients with or without diabetes were consistent with those for the overall ITT population (Table). Achievement of LDL-C goals with RSV and RSV+EZE in patients with or without diabetes was consistent with that observed with the overall population (Table). Both treatments were well tolerated.

Conclusions: RSV $40 \mathrm{mg}$ was effective at improving the atherogenic lipid profile and enabled the majority of patients to achieve LDL-C goals. Combination of RSV $40 \mathrm{mg}+\mathrm{EZE} 10 \mathrm{mg}$ produced further improvements in lipid levels and enabled additional patients to reach LDL-C goals. The benefits of RSV monotherapy and combination therapy with RSV+EZE in patients with diabetes were consistent with those observed in high - risk patients without diabetes, and in the population as a whole.

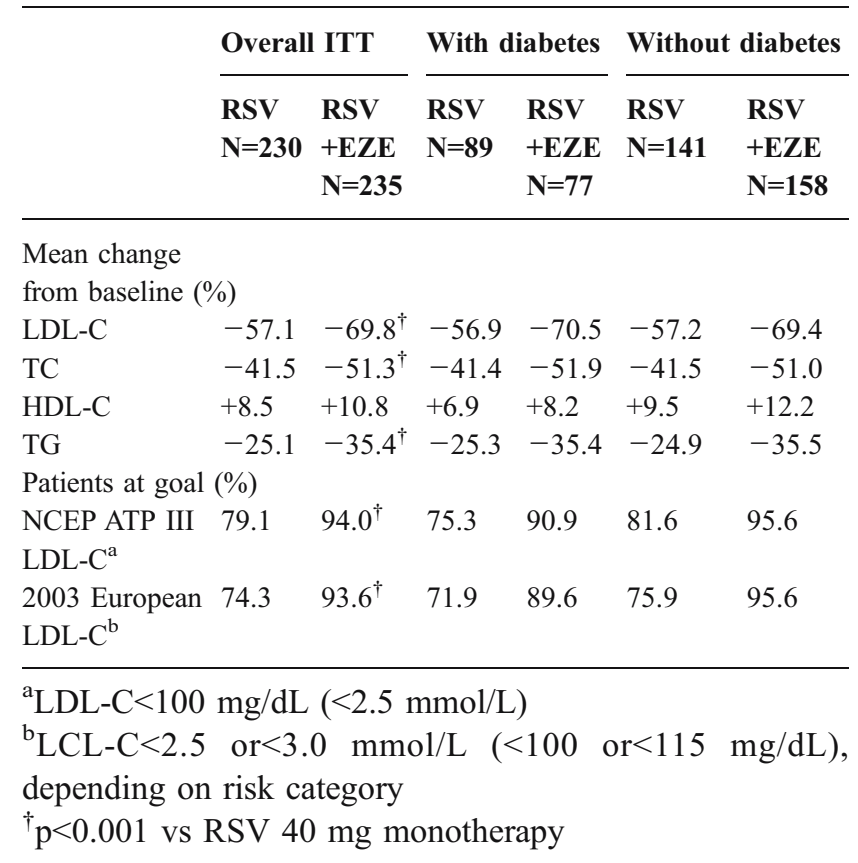

This study was funded by AstraZeneca

\section{PS 112}

\section{Brain, bone and skin in diabetes}

1241

Evidence of a role for acetyl-L-carnitine in diabetesrelated serotonin transmission

T. Kuchmerovska, I. Shymanskyy, G. Donchenko, V. Kopelevich, L. Yanitska, A. Klimenko;

Dept. of Coenzymes, O.V. Palladin Institute of Biochemistry, Kyiv, Ukraine.

Background and Aims: It has been previously shown that diabetes-associated CNS abnormalities are characterized by 
neurotransmission impairments. Several evidences support the concept that a correct mitochondrial function is essential for maintenance of an adequate neurotransmitter secretion. The accumulation of long-chain fatty acids and impaired $\beta$ oxidation due to deficiencies in L-carnitine (LC) and/or its esterified derivatives may have deleterious effects on nerve cells function in diabetes by altering plasma or mitochondrial membrane integrity and perturbing intracellular metabolism and energy production. The study has been designed to establish the possible implications of putative LC deficiency and mitochondrial dysfunction as well as potential benefits from acetyl-L-carnitine (ALC) treatment on serotonin (5-HT) transmission in diabetic brain.

Materials and Methods: All studies were carried out after 5 weeks of STZ-induced diabetes $(60 \mathrm{mg} / \mathrm{kg}$, i.p. $)$ in rats treated for 14 days with or without ALC (150 mg/kg, i.p.). The LC contents were measured in blood serum and brain by radioenzymatic method. The uptake and the release of $\left[2-{ }^{14} \mathrm{C}\right]$ serotonin were determined in purified synaptosomes. The mitochondrial membrane potential $\left({ }_{\Delta} \mathrm{Xm}\right)$ was assayed by spectrofluorimetry using rhodamine123 (Rh123).

Results: Diabetes led to a pronounced decrease in LC levels of serum $(46.4 \pm 3.2 \%)$ and brain $(31.7 \pm 2.7 \%)$ vs control, $p<0.05$. Diabetic mitochondria showed elevation of Rh123 fluorescence $(21.0 \pm 0.19 \%, \mathrm{p}<0.01)$ vs control, indicating decreased ${ }_{\Delta} \mathrm{Xm}$. These changes were associated with the decline in both synaptosomal ATP and respiratory control ratio (ATP/ADP), respectively $2.15 \pm 0.09$ vs $3.19 \pm$ $0.11 \mathrm{nmol} / \mathrm{mg}$ protein and $4.23 \pm 0.21$ vs $6.85 \pm 0.38, \mathrm{p}<0.05$. Compared to control, diabetes caused $40.3 \pm 3.7 \%$ elevation of spontaneous release and $24 \pm 2.9 \%$ decrease in uptake of 5 -HT in synaptic endings, that was accompanied by $24.3 \pm$ $1.8 \%$ downregulation of synaptosomal $\mathrm{Na}^{+}, \mathrm{K}^{+}$-ATPase activity, $\mathrm{p}<0.01$. 5-HT release was 2 -fold more profound in diabetes vs control upon in vitro stimulation by $30 \mathrm{mmol} /$ $1 \mathrm{KCl}$, suggesting augmented diabetes-induced quantal mode of neurotransmitter secretion. ALC treatment normalized serum and brain LC levels and diminished the development of cellular respiration deficits by $81.0 \pm 5.7 \%$ restoration of ${ }_{\Delta} \mathrm{Xm}$ with corresponding, respectively $72.5 \pm$ $4.3 \%$ and $78.3 \pm 5.6 \%$, recovery of ATP content and ATP/ ADP, $p<0.05$. It is most likely that attenuation of mitochondrial dysfunction following ALC administration contributed to significant currently established improvement of $\mathrm{Na} / \mathrm{K}$ pump activity, 5HT uptake and spontaneous release except for the increased synaptosomal response to $\mathrm{KCl}$, which was affected only slightly. It is noteworthy, that $\mathrm{KCl}$-induced 5-HT release is largely independent on ATP synthesis, since this secretogue directly affects plasma membrane potential and ion fluxes. ALC treatment had no lowering effect on blood glucose and brain sorbitol.

Conclusion: The data provide evidence that under energydepleted conditions related to diabetes the decrease in $\mathrm{Na}^{+}$ and $\mathrm{K}^{+}$gradients and subsequent reversal of 5-HT transporters in synaptosomes may be the key mechanism underlying reduced uptake and enhanced spontaneous release of 5HT. ALC modulatory action on serotoninergic system is dependent on energy metabolism, rather than being the consequence of a direct effect on the neurotransmitter secretory machinery.

\section{2}

\section{Lutein effect in the retina and hippocampus of diabetic} mice

M. Muriach ${ }^{1}$, M. Miranda ${ }^{1}$, G. Alexander ${ }^{2}$, R. Blomhoff ${ }^{2}$, J. Barcia ${ }^{1}$, F. Bosch-Morell ${ }^{1}$, J. Romero ${ }^{1}$;

${ }^{1}$ Physiology, Universidad Cardenal Herrera-CEU, Valencia, Spain, ${ }^{2}$ Nutrition, University of Oslo, Norway.

Background and Aims: It has been repeatedly suggested that oxidative stress may play an important role in the pathogenesis of diabetes complications, and it is clear that the elevated glucose levels seen in diabetes and the existence of oxidative stress are inseparable.

The diabetes-induced production of reactive oxygen species (ROS) activates the nuclear factor $\mathrm{kB}$ (NFkB), which activates a variety of target genes which are linked to the development of diabetic complications.

Among these target genes are include the ones involved in inducing programmed cell death, because although in general, this nuclear factor is considered antiapoptotic, it can be proapoptotic depending of the cell type and the stimuli.

Diabetic retinopathy is a frequent complication of diabetes, and in the recent years, evidence is emerging that diabetes affects also the Central Nervous System by impairing the functional and structural integrity of the brain in diabetic patients. One of the mechanisms by which hyperglycemia is known to cause neural degeneration is via the increased oxidative stress that accompanies diabetes.

The aim of this study was to confirm the early biochemical and functional changes in retina and hippocampus of diabetic mice. Oxidative stress markers, NFkB activity and functional tests for retina and hippocampus were studied. We also tested the effects of a treatment with lutein (a natural antioxidant without hypoglycemiant properties),compared to insulin therapy.

Materials and Methods: Male albino mice were induced diabetic by alloxan injection and divided into subgroups: control, control+lutein, diabetic, diabetic+lutein, diabetic + insulin and diabetic + insulin+lutein. Treatments started on day 4 after alloxan injection and animals were sacrificed on day 14. Malondialdehyde (MDA) and glutathione (GSH) concentrations, as well as Glutathione peroxidase (GPx) activity were measured as oxidative stress markers. Functional tests 
for retina and hippocampus were performed: electroretinogram and Morris water maze test (a test for evaluating spatial memory). NFKB activity was measured in transgenic mice containing the NFkB-luciferase reporter gene.

Results: After 15 days of diabetes, lipid peroxidation (MDA) was increased in the retina and hippocampus, while the antioxidants defences (GSH and GPx) decreased. NFkB activity was increased in both tissues. We observed impairment of the electrorretinogram in diabetic mice, but no differences were observed when examined for spatial learning ability. However, a positive correlation was found between latencies in the Morris water maze test and $\mathrm{HbA1c}$ levels. Lutein treatment (even under hyperglycaemic conditions), as well as insulin therapy, prevented all these alterations. Retina appears to be affected earlier than hippocampus by diabetes-induced oxidative stress.

Conclusion: Although a proper glycemic control is desirable to prevent the development of diabetic complications, it is not always achivable. Lutein, a natural antioxidant which is able to modulate NFkB activity, and a scavenger of peroxynitrites, could be an appropriate coadyuvant treatment for the alterations observed in this study. Further studies are needed to elucidate the mechanisms that make the retina more susceptible to diabetes than hippocampus at early stages of these disease, and to determine the role of NFkB on these mechanisms.

This work was supported by projects PI03/1710 from the Fondo de Investigación Sanitaria and PRUCH04/30 from Universidad Cardenal Herrera-CEU

\section{3}

Model for investigating type 2 diabetic patients with suspected sleep apnoea

K. G. Konz ${ }^{1}$, R. Oelze ${ }^{2}$, E. Lensch ${ }^{3}$, M. Berres ${ }^{4}$, I. Plininger ${ }^{1}$, A. Mahnel ${ }^{1}$, C. Jaursch-Hancke ${ }^{1}$, K. Rett ${ }^{1}$; ${ }^{1}$ Endocrinology/Diabetology, ${ }^{2}$ Pneumology, ${ }^{3}$ Neurology, German Clinic for Diagnosis, Wiesbaden, ${ }^{4}$ Medical Biometry, University of Mainz, Germany.

Background and Aims: The prevalence of sleep apnoea has been reported to be increased in type 2-diabetic patients. It seems to be associated with an additional cardiovascular risk. Full polysomnography is time consuming and expensive. An approach based on a logistic regression model and an ambulatory monitoring for cardiorespiratory abnormalities as well as clinical features and signs and symptoms of diabetic complications has been developped to determine which diabetic patient needs full polysomnography.

Materials and Methods: The variables entered in the regression model were derived from 60 type 2 -diabetic patients of both sexes (mean age 60 years, BMI $30.9 \mathrm{~kg} / \mathrm{m}^{2}$, mean diabetes duration 8 years, mean $\mathrm{HbAlc} 8.5 \%$ ) referred to the outpatient clinic for tighter diabetes control. Besides autonomic function tests to assess cardiovascular autonomic neuropathy, motor and sensory nerve conduction velocities of the tibial and sural nerves, as well as an ambulatory monitoring for cardiorespiratory abnormalities recording airflow, respiratory movements, arterial oxygen saturation, heart rate and body position were done.

Results: The prevalence of sleep apnoea was $20 \%$ in the population studied. The logistic regression analysis showed that the age, the motor nerve conduction velocity, and a cardiovascular autonomic neuropathy are predictors of the sleep apnoea. Neither the BMI, nor the duration of the disease, other diabetic complications, or the $\mathrm{HbAlc}$ value are associated with sleep disturbances. A cut off probability of 0.30 gave the prediction model a sensitivity of $75 \%$ and a specificity of $89 \%$ to predict a RDI of $>30$.

Conclusion: The patients age, the motor nerve conduction velocity and a cardiovascular autonomic neuropathy are useful predictors of a sleep apnoea in type 2- diabetes.

\section{4}

Obstructive Sleep Apnoea (OSA) is independently associated with increased prevalence of the metabolic syndrome but not insulin resistance status

A. Gruber ${ }^{1}$, F. Horwood ${ }^{1}$, J. Sithole ${ }^{2}$, N. Ali ${ }^{1}$, I. Idris ${ }^{1}$; ${ }^{1}$ Diabetes and Endocrinology, Sherwood Forest Hospital NHS Trust, Sutton in Ashfield, ${ }^{2}$ Trent RDSU, University of Nottingham, United Kingdom.

Background and Aims: Epidemiological evidence suggests a link between OSA and components of the metabolic syndrome (MS) but whether OSA is associated with the overall syndrome independent of obesity remains unclear. Furthermore, the agreement between MS and insulin resistant state when applied to patients with OSA has not been previously determined.

Materials and Methods: We prospectively recruited 41 subjects with OSA and 38 controls. Anthropometric measurements, lifestyle questionnaires, glucose, lipids, insulin and blood pressure (BP) were measured after an overnight fast. Insulin resistance state was defined as homeostasis model assessment (HOMA) value $>3.99$ and MS were diagnosed according to the International Diabetes Federation (IDF) criteria.

Results: Subjects with OSA were more obese, more insulin resistant, more hyperglycaemic, had higher epworth score (measure of day time somnolence) and systolic blood pressure levels. The prevalence of MS was higher in OSA compared with non-OSA subjects $(74 \%$ vs $24 \%, p<0.001)$. The prevalence of microalbuminuria in both groups was 
negligible. Regression analysis model, adjusting for age, BMI and smoking showed that the prevalence of MS was 5.9 (95\% CI 2.0-17.6) times higher in subjects with OSA. Triglyceride $(\mathrm{p}=0.031)$, glucose $(0.023)$ and Epworth scores (0.003) vales are independently associated with OSA after adjusting for BMI and other covariates whilst insulin resistant status was found not to be significant. Using ROC curve analysis, we found that a waist circumference of $>$ $105 \mathrm{~cm}$ and a neck circumference $>40 \mathrm{~cm}$ would predict MS in patients with OSA at $75 \%$ sensitivity and $65 \%$ specificity. Using $\kappa$ statistics, we found that the agreement between MS and insulin resistant status in this cohort is poor.

Conclusion: Thus, OSA is shown to be associated with the MS independent of obesity. This independent association is driven mainly by the increased triglyceride, glucose and Epworth score values but not insulin resistant status. This observation suggests an alternative pathogenic factor which mediates the increased cardiovascular risk in patients with OSA and MS, other than that due to insulin resistant state. The independent link between Epworth score and MS in patients with OSA implicates the role of daytime sleepiness and chronic hypoxia as a potential mediator.

\section{5}

The components of metabolic syndrome: their impact on the extension of atherosclerosis assessed by the carotid intima-media thickness

A. Marian ${ }^{1}$, I. Marian ${ }^{2}$, N. Hancu'

${ }^{1}$ Diabetes, Nutrition, Metabolic Disease, Clinical Municipal Hospital Cluj-Napoca, Romania, ${ }^{2}$ Cardiology, Medical Clinic No I Cluj-Napoca, Romania, ${ }^{3}$ Diabetes, Nutrition, Metabolic Disease, Diabetes Center and Clinic ClujNapoca, Romania.

Background and Aims: The carotid intima-media thickness (IMT) determined with high resolution ultrasound is an accepted parameter for the assessment of early atherosclerosis.The presence of metabolic syndrome (MetS) represent a risk for the development of both type 2 diabetes and cardiovascular diseases. The atherogenic impact of different components within the MetS is not well established. There is no convergent information on how some MetS component combinations (different numbers and types) can influence preclinical atherosclerosis assessed by carotid IMT. The purpose of this study was the assessment of atherogenic potential of different components of the MetS, using carotid IMT like early atherosclerosis marker. Materials and Methods: We included 131 patients who fulfill the IDF 2005 criteria for the diagnosis of the MetS, men and postmenopausal women, non-smokers, non-diabetic, without a previous treatment for dyslipidaemia.
Carotid IMT was considered to be the maximum value measured bilaterally from multiple incidences. The studied groups do not differ in term of age and gender distribution. Results: IMT increased gradually with the number of MetS components (without counting increased waist circumference, mandatory for the diagnosis) present at the same patient (3 components: $0.96+/-0.18 \mathrm{~mm}$ vs 2 components: $0.91+/$ $-0.15 \mathrm{~mm}, \mathrm{p}=0.04$ and 4 components: $1.05+/-0.17 \mathrm{~mm}$ vs 3 components: $0.96+/-0.18 \mathrm{~mm}, \mathrm{p}=0.04$ ). Low HDLcholesterol levels and the presence of prediabetes showed the strongest impact on the carotid IMT, for the patients with 2 components of MetS. For the patients who had 3 components of the MetS, the low HDL-cholesterol/high triglycerides/hypertension combination was more atherogenic than prediabetes/high triglycerides/hypertension combination $(1.02+/-0.18 \mathrm{~mm}$ vs $0.92+/-0.17 \mathrm{~mm}, \mathrm{p}=0.03)$.

Conclusion: An increase in the number of components of the metabolic syndrome was associated with an increase in the mean carotid intima-media thickness. Among the components of the metabolic syndrome, low HDL-cholesterol and prediabetes showed a stronger impact on carotid atherosclerosis.

1246

Gender risk of non-fatal stroke in type 2 diabetic patients differs depending on the type of treatment M. Khalangot ${ }^{1}$, M. Tronko ${ }^{1}$, V. Kravchenko ${ }^{1}$, A. Vaiserman ${ }^{2}$, V. Gyrianov ${ }^{3}$;

${ }^{1}$ Department of Epidemiology, Institute of Endocrinology and Metabolism, Kiev, ${ }^{2}$ Laboratory of Modelling of Aging Processes, Institute of Gerontology, Kiev, ${ }^{3}$ Department of Informatics, Donetsk Medical University, Ukraine.

Background and Aims: The significant decrease of the gender differences in cardio-vascular risk among type 2 diabetics (T2D) were detected in a number of epidemiological studies. The reason of this phenomenon remains unknown. The different types of treatment are used to treat $\mathrm{T} 2 \mathrm{D}$, including insulin, the excess of which was shown to result in hyperandrogenization of the T2D women (Poretsky L.1991). However, the stratification by type of treatment at an estimation of gender risk in T2D was not carried out till now. The aim of present observational cross-sectional study was the estimation of the genderrelated risk of non-fatal stroke in T2D depending on the type of antidiabetic treatment in Ukraine.

Materials and Methods: In Dec 2005, the data from 11 Ukrainian regions for all living type 2 diabetics already included into the nationwide population-based diabetes register (start of creation Jan 2001) was extracted. Male/ female relative risks (RRs) for non-fatal stroke prevalence were calculated in 3 treatment groups: Diet only (DO) 
7273/15901; oral antidiabetic drugs (OAD) 15109/33913; insulin: 5529/12462 male/female respectively. 95\% confidence intervals $(95 \mathrm{CI})$ were estimated by logarithmic transformation. Chi-square test was used to compare the differences between groups. Male/female odds ratios (OR) were estimated using a logistic regression model after adjustment for age and diabetes duration. The study protocol was approved by the ethics committee of the Institute of endocrinology and metabolism, Kiev.

Results: The higher prevalence of stroke among men was in those patients treated with OAD or DO: RRs=1.38 (1.26$1.51)$ and $1.48(1.25-1.76)$, respectively. The gender differences were absent among those treated with insulin: $R R=0.98$ $(0.87-1.10)$, which is significantly lower $(p<0.001)$ than corresponding OAD or DO RR's levels. Men's stroke OR's were significant $(\mathrm{p}<0.001)$ for $\mathrm{DO}$ and $\mathrm{OAD}$ treated patients: 1.54 (1.29-1.84) and $1.45(1.31-1.59)$ respectively, but not for insulin-treated T2D: $1.06(0.96-1.16), \mathrm{p}=0.22$.

Conclusion: The gender risks of not-fatal stroke in T2D are significantly different depending on the type of treatment. Further research could clarify if it depends upon insulinrelated hyperandrogenisation in T2D women.

This work was funded in the framework of the National Diabetes Mellitus Plan implementation.

\section{7}

Impact of diabetes on type $I$ collagen in relation to availability of vitamins. Role of nicotinamide and nicotinoyl-GABA treatments

I. Shymanskyy, T. Kuchmerovska, L. Bondarenko, T. Pechonova, S. Stepanenko, A. Klimenko;

Dept. of Coenzymes, O.V. Palladin Institute of Biochemistry, Kyiv, Ukraine.

Background and Aims: Diabetes-related dermal changes, impaired wound healing, osteopenia and decreased bone strength may be associated with altered metabolism of type I collagens (I-C). It is assumed that alterations in I-C amino acids as a result of gene polymorphism may strongly affect protein properties and physiological function; however, very limited evidences are present at the moment regarding amino acid (AA) composition of bone and skin I-C and its relevance to abnormal availability of vitamins which are necessary for collagen synthesis in diabetes. We tested whether nicotinamide (NAm) and nicotinoyl-GABA (NGABA) as the drugs with a wide range of metabolic effects can influence I-C formation and its AA composition as well as vitamins availability in diabetes.

Materials and Methods: After 4 weeks of STZ-induced diabetes $(60 \mathrm{mg} / \mathrm{kg}$, i.p.), male Wistar rats were injected for 14 days with or without NAm or N-GABA (200 mg/kg, i.p.).
The contents of I-C after its acid extraction from bones or skins with following step-wise salting out were estimated by the amounts of hydroxyproline (Hyp). The amino acids were assayed by cation exchange chromatography.

Results: Vit. C, PP and E ( $\alpha$-tocopherol) contents were $19.1 \pm$ $6.4,31.0 \pm 1.9,21.8 \pm 2.0$ vs $43.9 \pm 7.1,54.3 \pm 3.8,14.6 \pm 2.4$ in blood serum and $601.3 \pm 72.5,540 \pm 37,49.0 \pm 7.2$ vs $1893 \pm$ $279,795 \pm 52,31.0 \pm 5.1$ in liver, respectively in diabetes vs control ( $\mathrm{nmol} / \mathrm{g}$ tissue, $\mathrm{p}<0.05$ ). Impaired availability of the vitamins may be ascribed to their increased consumption by elevated oxidative stress which we previously identified in various tissues from diabetic rats and may also be due to reduced NADPH-dependent regeneration of vit. C since its level was reversed towards normalisation after NAm and NGABA treatment, $p<0.05$. Restoration of circulatory and liver vit. PP contents and slight decrease in vit. E levels were also seen due to both treatments. I-C depositions in bones and skins were lower in diabetes vs control as it is evident from respectively 5.2- and 2.4-fold decrease in Hyp contents, $\mathrm{p}<0.01$. Diabetes-associated changes in amino acid composition of I-C were largely independent on its localization mainly affecting those AA which are known to be involved in helix formation, cross-linking and charging of the molecules. Besides Hyp lowering, diabetes also significantly reduced collagen Gly, Ala contents in skins and additionally hydroxylysine and Pro in bones, whereas collagen Lys, His, Arg, Glu, Thr, Leu, Phe contents in both organs and Asp, Ser and Tyr in skins were elevated, $p<0.05$. NAm and N-GABA were able to partially restore I-C depositions and normalise AA contents with the efficacy greater in case of N-GABA treatment. The diabetes-induced alterations could be related to abnormal hepatic function as evidenced by 1.7 - and 1.3 -fold increase in the total amount of free amino acids in liver and serum and Fischer index (Leu+ILe+Val/Tyr+Phe) raised in serum (3.58 \pm 0.41 vs $1.73 \pm 0.16$ in control, $\mathrm{p}<0.05)$. Drug treatments exhibited similar beneficial effects on liver and serum AA.

Conclusion: The study confirms the relationship between the diabetes-associated decrease in I-C content, alterations of its amino acid composition and impaired availability of vitamins. The corrective effects of NAm and N-GABA on collagen synthesis and processing, additionally with other possible contributing factors, may be attributed to preservation of vitamin $\mathrm{C}$ and $\mathrm{E}$ functions.

\section{8}

Longitudinal changes in bone mineral density in preand postmenopausal women with type 1 and type 2 diabetes

M. T. Petersen ${ }^{1}$, L. Stoltze ${ }^{1}$, J. O. Christensen ${ }^{2,3}$, O. L. Svendsen ${ }^{2,3}$; 
${ }^{1}$ Internal Medicine, Bispebjerg University Hospital, Copenhagen, ${ }^{2}$ Steno Diabetes Center, Gentofte, ${ }^{3}$ Research Center for Bone Metabolisme, Center for Clinical and Basic Research, Ballerup, Denmark.

Background and Aims: The aim of this longitudinal study was to investigate the spontaneous changes in bone mineral density (BMD) in pre- and postmenopausal women with type 1 (T1D) and type 2 (T2D) diabetes mellitus.

Materials and Methods: 51 pre- and postmenopausal women with type 1 or type 2 diabetes mellitus from the out patient clinic at the Steno Diabetes Center were examined. The population was divided into four groups premenopausal type $1(n=19)$, postmenopausal type 1 $(\mathrm{n}=15)$, premenopausal type $2(\mathrm{n}=7)$, and postmenopausal type $2(n=10))$. At two occasions three years apart BMD in the total body, lumbar spine, femoral neck and nondominant forearm were determined.

Results: In the group of postmenopausal type 1 diabetics a significant decrease in BMD could be detected (total body BMD $-0.87 \%$ p.a). The change in BMD correlated significantly and positively with duration of the menopause suggesting a large fall around the menopause. There were no statistically significant changes in BMD in any of the sites measured in premenopausal $\mathrm{T} 1$ or $\mathrm{T} 2$ diabetics or in postmenopausal T2D.

Conclusion: BMD decreased in postmenopausal type 1 diabetics, but not in premenopausal T1D or T2D. The decrease in postmenopausal T1D were not larger than what could be expected in the normal healthy population.

\section{9}

The effect of diabetes mellitus type 2 on the bone mineral density of males and postmenopausal females D. J. Hadjidakis, A. Mylonakis, I. I. Androulakis, A. E. Raptis, A. Papaefstathiou, S. A. Raptis; 2nd Department of Internal Medicine-Propaedeutic, Research Institute and Diabetes Center, "Attikon" and "Evgenidion" University Hospitals, Athens University, Greece.

Background and Aims: The majority of previous studies have demonstrated a favorable effect of diabetes mellitus type 2 on bone mineral density (BMD). It is still unclear whether the degree of such effect is similar for the two genders. The purpose of the study was the comparison of the degree by which diabetes mellitus type 2 affects the BMD of both genders.

Materials and Methods: One hundred and forty one diabetic males [DM, age $56.9 \pm 5.8$ years (mean value \pm 1 SD), BMI $26.8 \pm 3.1 \mathrm{~kg} / \mathrm{m}^{2}$, disease duration $8.2 \pm 3.4$ years], 137 diabetic postmenopausal females (DW, 57.3 $\pm 4.1,26.7 \pm 3.5$,
$7.4 \pm 3.3$ ), 60 healthy males (HM, age 56.1 \pm 6.7 , BMI 26.8 \pm 3 ) and 301 healthy postmenopausal females (HW, 56.9 \pm 3.3 , 26.2 \pm 3.6$)$ were studied. BMD was measured at the L2-L4 vertebrae and the femoral neck (FN) by DXA. None of the subjects suffered from any other disease or had received any medication with a known effect on bone metabolism. They were all matched for age and BMI. None of the diabetic subjects received insulin. The sex- and age- adjusted BMD values (Z-scores) were used to compare the diabetes' effect between the 2 genders.

Results: Regarding the vertebral BMD values, diabetic persons of both genders presented significantly higher BMD values compared to their respective healthy ones $(\mathrm{p}<0.001)$. On the other hand, although in both genders diabetic patients presented higher FN BMD values compared to healthy ones, only between DW and HW this difference was significant $(\mathrm{p}<0.001)$. Both DM and HM presented significantly higher vertebral Z-score values compared to their female counterparts $(p<0.001)$. Regarding the FN, only HM presented significantly higher Z-score values compared to HW $(p<0.05)$, while DM and DW did not differ significantly. The proportions of osteopenic-osteoporotic subjects (WHO criteria) were significantly higher in the healthy compared to their diabetic counterparts in both genders $(\mathrm{p}<0.01)$. No significant differences were observed in the same proportions between diabetic males and females. In all groups, but HM, a significant positive correlation was observed between either vertebral or FN BMD values and BMI $(r=0.19-0.30, p<0.05)$. No correlations were observed between the BMD values and the disease duration or the HbA1c concentrations in diabetic subjects.

Conclusion: Diabetes mellitus type 2 seems to exert a favorable effect on the bone mineral density of diabetic patients of both genders. This effect seems to be stronger in the trabecular bone of diabetic males and is irrespective of the body weight. Rather the presence than the duration of diabetes seems to correlate to the disease's effect on bone mass.

1250

Detection of free elastin derived peptides among diabetic children

A. Nikolov ${ }^{1}$, G. Nicoloff ${ }^{1}$, C. Petrova ${ }^{2}$, M. Petkova ${ }^{3}$; ${ }^{1}$ Department of Biology \& Pathological Physiology, ${ }^{2}$ Department of Pediatric, ${ }^{3}$ Department of Endocrinology, Medical University, Pleven, Bulgaria.

Background and Aims: Elastin breakdown products are found in the serum of all human subjects. The presence of these elastin-derived peptides (EDP) and the corresponding antibodies in circulation leads to formation of circulating immune complexes (CIC). The aim of this study was to 
determine if serum level of free-EDP (unbound in CIC) correlate with the development of vascular complications in diabetic children.

Materials and Methods: To this end we used a method for detecting immune complexes CIF-ELISA. Isolation of a new glycoprotein complement inhibition factor (CIF) from the parasitic plant Cuscuta europea seed, which appears to bind specifically to complement component $\mathrm{C} 3$ has been provided an unique tool for the measurement of immune complexes by means of ELISA-type techniques (CIFELISA). Free EDP were detected by using a two-step method consisting of: (i) CIF-ELISA to remove immune complexes followed by (ii) an elastin-specific ELISA for detection of EDP.

The levels of free EDP were studied in sera of 81 diabetic children (mean age-13.46 \pm 3.51 years, diabetes duration $5.17 \pm 4.21$ years). Forty-two of the children had vascular complications (group 1) and 39 were without vascular complications (group 2).

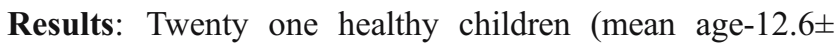
2.47 years) were used as controls. Diabetics showed statistically significant higher levels of free EDP $(68.1 \pm 25$ vs. $51 \pm$ $12.5 \mathrm{ng} / \mathrm{ml} ; \mathrm{p}=0.003$ ) compared to the control group. In group 1, free EDP showed statistically significant higher levels than controls $(78.9 \pm 25.6$ vs. $51 \pm 12.5 \mathrm{ng} / \mathrm{ml} ; \mathrm{p}=0.0001)$. Thirty eight of 81 (47\%) patients were positive for free EDP (30/42$71 \%$ in group 1 and $8 / 39-21 \%$ in group 2). Free EDP levels in all diabetics showed a correlation with insulin dose $(\mathrm{r}=0.23 ; \mathrm{p}=0.041)$, retinopathy $(\mathrm{r}=-0.20 ; \mathrm{p}=0.06)$, and microalbuminuria $(r=0.44 ; \mathrm{p}=0.0001)$. Patients who had vascular pathology showed a correlation of free EDP with microalbuminuria $(\mathrm{r}=041 ; \mathrm{p}=0.01)$, retinopathy $(\mathrm{r}=0.32$; $\mathrm{p}=0.041)$, insulin dose $(\mathrm{r}=0.37 ; \mathrm{p}=0.02), \mathrm{HbAlc}(\mathrm{r}=0.35$; $\mathrm{p}=0.03)$, systolic blood pressure $(\mathrm{r}=0.30 ; \mathrm{p}=0.045)$ and total cholesterol $(\mathrm{r}=0.36 ; \mathrm{p}=0.02)$.

Conclusion: These findings suggest that elevated levels of free EDP are associated with the development of diabetic vascular complications in children. 